


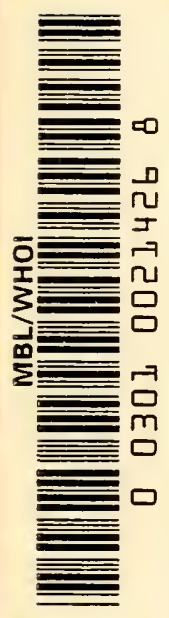




TOPLEY AND WILSON'S PRINCIPLES OF
BACTERIOLOGY AND IMMUNITY 



\title{
TOPLEY AND WILSON'S PRINCIPLES
}

\section{OF BACTERIOLOGY AND IMMUNITY}

\author{
THIRD EDITION
}

REVISED BY

G. S. WILSON, M.D., F.R.C.P., D.P.H., K.H.P. PROFESSOR OF BACTERIOLOGY AS APPLIED TO HYGIENE, UNIVERSITY OF LONDON, LONDON SCHOOL OF HYGIENE AND TROPICAL MEDICINE DIRECTOR OF THE PUBLIC HEALTH LABORATORY SERVICE

\section{AND}

A. A. MILES, M.A., F.R.C.P.

PROFESSOR OF BACTERIOLOGY, UNIVERSITY OF LONDON, UNIVERSITY COLLEGE HOSPITAL MEDICAL SCHOOL

\author{
IN TWO VOLUMES \\ VOLUME I
}

A WILLIAM WOOD BOOK

THE WILLIAMS \& WILKINS COMPANY

BALTIMORE 
Printed in Great Britain by Butler \& 'Tanner Ltd., Frome and London 
To

K. T. AND J. W. 


$$
\text { . }
$$




\section{PREFACE TO THE THIRD EDITION}

APART from the war, this edition has been prepared under the shadow of a double misfortune. Early in 1941, Professor Topley accepted the post of Secretary of the Agricultural Research Council, and thereby took a step that rendered his further participation in this book impossible. To replace him, I was fortunate in enlisting the co-operation of our former colleague, Professor Miles. Together we began the arduous task of revision. Our work, however, had not progressed far before the second blow fell. Quite suddenly in January, 1944, Topley died. The effect of this second misfortume was almost as serious as the first. Although Topley could have made no direct contribution to the text, his criticism and advice would have been constantly available, and he would have helped us to maintain that uniformity of presentation for which he and I had always striven. On Miles, in particular, the burden weighed heavily, since, in taking over those parts of the book for which Topley had previously been responsible, he was deprived of counsel that would doubtless have proved invaluable to him.

There is no call to write a long preface. We have endeavoured not merely to bring the book up to date, but to present the new additions to our knowledge in a manner worthy of their importance. One chapter-that on Soil Microbiology - has been deleted, but two new chapters, on Chemotherapy and on the Bacteriology of Air, have been added. For the sake of convenience we have divided the previous Bacterium chapter into three, giving separate recognition to the genera Shigella and Salmonella. We have also removed the psittacosis-lymphogranuloma group of diseases from the other filtrable virus diseases with which they were associated and awarded them a chapter of their own. Except for these alterations, the form of the book remains unchanged. In the first two editions we tried to ensure that scientific literature from all parts of the world was fairly represented, but in the present edition we have suffered under a handicap imposed by the war, which has seriously curtailed the inflow of journals from many parts of Europe as well as, of course, from Japan. This gap we shall look forward to filling in the future. Partly because of the necessarily one-sided picture we have been obliged to paint, we have thought it wise to present our evidence in greater detail than might otherwise have been necessary, and to be perhaps unduly cautious in drawing our conclusions. The bibliography has been much expanded, so as to cater for the needs of those who use the book more as a work of reference than as a textbook. For the increased length of the new edition we tender our apologies. The war 
has not been conducive to careful leisurely recapitulation, and our plea must be the paradoxical excuse that we have not had time to be more concise.

To those who have assisted us in various ways, we would express our thanks. We are particularly grateful to Dr. N. W. Pirie for his help with some chapters in Part I, to Professor A. Bradford Hill for his advice on Chapter 43, and to Professor S. P. Bedson, Lt.-Col. R. F. Bridges, Dr. A. Q. Wells, Dr. Joan Taylor, Dr. A. W. Stableforth, Dr. R. Lovell, Miss Nancy Hayward, and many of our former helpers for information on particular problems. To Dr. Stuart Mudd and his American colleagues, to Professor A. D. Gardner, Dr. C. F. Robinow, Dr. S. T. Cowan, Dr. N. G. Heatley, Dr. A. Pijper and the publishers of "Endeavour" we are indebted for a number of electron micrographs and photographs ; to Professor J. R. Marrack for Fig. 32 ; to H.M. Stationery Office for permission to reproduce Figures 32, 34, 77, 79, 80 and 81; and to Miss Margaret Rees for the preparation of some new diagrams. We should also like to pay our tribute to the library staffs of the London School of Hygiene and Tropical Medicine, the Bureau of Hygiene, University College Hospital Medical School, and the Radcliffe Science Library, Oxford, for their unfailing courtesy and help in the tracing of numerous references.

G. S. W.

June, 1945. 


\title{
CONTENTS
}

\author{
VOLUME I
}

\section{PART I \\ GENERAL BACTERIOLOGY}

CHAPTER

1. Historical OdtLine

PAGE

2 The Biological Characteristics of Bacteria: Morphology

3. The Biological Characteristics of Bacteria: Metabolism . . . 42

4. The Growth and Death of Bacteria . . . . . . . 80

5. The Resistayce of Bacteria to Physical and Chemical Agents: DisINFECTION . . . . . . . . . . . . $10 \mathrm{I}$

6. Antibactertal Substances used in the Treatment of Infections. . 155

7. The Antigen-Antibody Reactions . . . . . . . . 192

8. The Antigenic Structure of Bacteria . . . . . . . . . 273

9. Bacterial Vartation . . . . . . . . . . . . . 288

10. The Classification of Bacteria . . . . . . . . . 310

11. The Bacteriophage . . . . . . . . . . . 325

\section{PART II}

\section{SYSTEMATIC BACTERIOLOGY}

12. The Methods of Obtaining Pure Cultures, and the Identification of Bacterta . . . . . . . . . . .

13. Description of the Methods used in the Systematic Examination of Bacteria, and a Glossary of the Terms Employed . . . . 364

14. Actinomyces and ACtinobacillus . . . . . . . . . . . 373

15. ERYSipelothrix AND Listerella . . . . . . . . . 395

16. МүсовастеRIUni . . . . . . . . . . . . . . 404

17. Corynebactertum . . . . . . . . . . . . . . . . . 447

18. FUSIFORMIS . . . . . . . . . . . . . . . 477

19. Pfetfferella, and Certain Allied Organisms . . . . . . 486

20. Azotobacter, Rhizobidi, Nitrosomonas, Nitrobacter, Hydrogenomonas,

Methanomonas, Carboxydomonas, and Acetobacter _ . . . 497

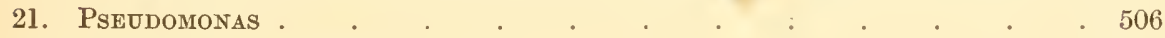

22. Vibrio and SpIRILlum . . . . . . . . . . . . . . 514

23. Neisserta . . . . . . . . . . . . . . . . . 531

24. Streptococcus . . . . . . . . . . . . . . . 559 
OHAPTER

25. Staphylococcus, Micrococcos, Sarcina, Rhodococcus, and Leuconostoc . 607

26. Chromobacterium aNd AChromobacterium . . . . . . 631

27. Proteus and Zopfius . . . . . . . . . . . . 642

28. BACTERIUM . . . . . . . . . . . . . . . 654

29. Shigella. . . . . . . . . . . . . . . . . 685

30. Salmonella . . . . . . . . . . 702

31. Lactobacillus. . . . . . . . . . 750

32. Pasteurella . . . . . . . . . . . . . . . 767

33. HжMOPHLUS . . . . . . . . . . . . 786

34. Brucella . . . . . . . . . . . . 814

35. BACILlUS . . . . . . . . . . . . . 838

36. Clostridiul . . . . . . . . . . . . . 858

37. Miscellaneods Bacteria. . . . . . . . . . . . . 898

38. The Sptrochetes . . . . . . . . . . . . 907

39. RicketTSIA . . . . . . . . . . . . . 928

40. The Pleuropneumonia Group of Organisms . . . . . . . 939

41. The Aninal Viruses: General Properties . . . . . . . . 949

INdex to Volumes I AND II . . . . . . . . i-xliv

\section{VOLUME II}

\section{PAR'T III \\ INFECTION AND RESISTANCE}

42. Types of Immunity. . . . . . . . . . . . . 971

43. The Measurement of Immunity Reactions in the Living Animal. . 980

44. The Mechanisis of Bacterial Infection . . . . . . . 1002

45. The Mechanisis that Hinder or Prevent the Access of Bacteria to the Tissues . . . . . . . . . . . 1019

46. The Mechanisus of Antitoxic Imnunity $\quad$. . . . . . . . 1029

47. The Mechanisms concerned in Specific Antibacterial Immonity . 1034

48. The Aggressive Action of Bacteria . . . . . . 1068

49. The Natural Antibodies: Their Nature, Origin and Behavtour . 1075

50. The Antibody-forimng Apparatus and its Reactions . . . . 1101

51. Anaphylaxis, Hypersenstitineness and Allergy . . . . . . 1136

52. Certain Non-specific Mechanisms in General Imiuntiy . . . 1173

53. Local Iminuntey . . . . . . . . . . . 1180

54. The Influence of Diet, Fatigde, Changes in Temperature and Humidity,

Chemical and Chemotherapeutic Agents and other Factors on General

OR Local IMMUNity . . . . . . . . . . . 1190

55. Immunity in Virus Diseases . . . . . . . . . . . . . . 1225

56. Herd Infection and Herd Imudntty . . . . . . . . . 1245

\section{PART IV}

\section{THE APPLICATION OF BACTERIOLOGY TO MEDICINE AND HYGIENE}

57. Actinomicosis, Actinobacillosis and Related Diseases . . 1269

58. Swine Erysipelas, Erysipeloid, Mouse Septicema, and Infective Mono-

NUCLEOSIS OF RABBITS . . . . . . . . . 1283 
CHAPLER

59. Tuberculosis . . . . . . . . . . . . . . . . . . 1259

60. Leprosy, Rat Leprosy, and Johne's Disease. . . . . . . 1358

61. Diphtheria, and other Diseases due to Corynebacteria . . . 1368

62. Glanders AND Melioidosis . . . . . . . . . . . 1408

63. Cholera . . . . . . . . . . . . . . . . . . . 1418

64. Meningitis . . . . . . . . . . . . . . . . . . . . . . . . . . . . . .

65. GoNorrhes . . . . . . . . . . . . . . . . 1454

66. Scarlet Fever, and other Diseases due to Hæiolytic Streptococci - 1462

67. Pyogenic and Wound Infections . . . . . . . . . . 1490

68. Tine Bacteriology of Rheumatic Infections and of Endocarditis . . 1509

69. ENTERIC Infections . . . . . . . . . . . . . . 1519

70. Bacillary Dysentery . . . . . . . . . . . . . . . 1561

71. Infective Enteritis of Infancy . . . . . . . . . . 1580

72. Bacterial food Poisoning and Botulism . . . . . . . 1592

73. Plague, Pasteurellosis, and Pseudotuberculosis . . . . 1627

74. Acute Respiratory Infections . . . . . . . . . 1653

75. Undulant Fever, Contagious Abortion of Cattle, ani Alifen Infections:

TUlarmita . . . . . . . . . . . 1692

76. Anthrax. . . . . . . . . . . . . . . . . 1730

77. Tetanus . . . . . . . . . . . . . . . . . . . 1746

78. Gas Gangrene: Anaerobic Infections of Animals . . . . 1770

79. Miscellaneous Diseases: Necrobacillosis, Ozena, Rhinoscleroma, Granuloma Texereum, Soft Chancre, Glandular Fever, Infective Hepatitis, Bartonella Infections, and various other Diseases - 1786

80. Relapsing Fever, Avian Spirochetosis, and Vincent's Angina . . 1803

81. Syphilis, Rabit Sypillis, and Yaws . . . . . . . . 1812

82. Weil's Disease and other leptospiral Diseases : Rat-bite Fever . . IS:S

83. Typhus Fever and other Rickettsial Diseases . . . . . 1840

84. Pleuropneumonia, Contagious Agalactia, and Allied Diseases . 1867

85. Filtrable V'irus Diseases. A. Limpliogranuloma-Psittacosis Group 1869

86. Filtrable Virus Diseases-continued. B. Group cilaracterized by Lesions OF THE SKIN . . . . . . . . . . . . . 1884

87. Filtrable Virus Diseases-continued. C. Group characterized by Lesions of the Nervous System . . . . . . . . .

88. Filtrable Virus Diseases-continued. D. Group ciiaracterized bi Catar.

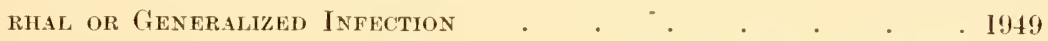

89. Filtrable Viros Diseases-continued. E. Group characterized by Tumour Formation.

90. Tile Normal Flora of the Human Body . . . . . . . 1984

91. Tile Bacteriology of Air . . . . . . . . . . . 2002

92. The Bacteriologr of Water, Shell Fish and Sewage. . . 2012

93. The Bacteriolog of Milk . . . . . . . . . . . 2036 INDEX tO VOLCMES I AND II . . . . . . . . . . . i-xliv 



\title{
PAR'T I
}

\section{GENERAL BAC'TERIOLOGY}

\author{
CHAPTER 1
}

\section{HISTORICAL OUTLINE}

In the study of any branch of science, an acquaintance with the historical development of knowledge is an important element in a clear understanding of our present conceptions. To the student of bacteriology such a basis is essential. It is almost true to say that the clue to the present position of bacteriology is the curious fact that there have been no bacteriologists. From Pastenr onwards, the great majority of investigators have been inore interested in what bacteria do than in what they are, and much more interested in the ways in which they interfere with man's health or pursuits than in the ways in which they function as autonomous living beings. The relations of bacteria to disease, to agriculture, and to various commercial processes, have presented problems which pressed for solution; and, as a result, we have witnessed a reversal of the normal process. We have seen the development of an applied science of bacteriology, or rather its application along many divergent lines, without the provision of any general basis of purely scientific knowledge. The essential interlocking of pure and applied science has, of course, been in evidence here as elsewhere. The necessity for being able to recognize a bacterium, which has been shown to be of importance in some province of human affairs, or of determining the way in which its harmful or beneficial action is brought about, has led to an intensive study of many aspects of bacterial morphology and physiology; but, in general, it may be said that the study of bacteria themselves has been carried out en passant, that amount of knowledge being acquired, or searched for, which would afford adequate data for the solution of some problem in applied bacteriology. Gradually the general structure of our knowledge has been added to, and gaps have been filled. Many of those who have started from some particular application have been led far afield by that desire for knowledge, altogether apart from its technical application, which is the essence of science itself. But this mode of construction has given to the general body of existing bacteriological knowledge a curious patchiness and indefiniteness which are puzzling to the student, and which must be realized and allowed for in any attempt to present the subject as a whole. There can be no question of any future reconstruction $a b$ initio. The history of a science is largely a history of technique, and the foundations of bacteriological technique, which presents many peculiar difficulties, have been well and truly laid by those who have worked in this field since 
the middle of the nineteenth century. The pure bacteriologist of the future will owe a lasting debt to those who have worked on the applied side, and his investigations will necessarily be based upon the knowledge gained by the medical or agricultural bacteriologist. The study of immunology, for instance, has supplied a body of facts, and an armoury of technical methods, which no bacteriologist can neglect, and which will inevitably give to future bacteriological research certain peculiarities of outlook and special methods of attack.

It is customary, in summarizing the history of bacteriology, at least in relation to medicine, to refer to the conception advanced by Fracastorius of Verona (1546), concerning a contagium vivum as the possible cause of infective disease, and to the views advanced by von Plenciz (1762) on the specificity of disease, based on a belief in its microbial origin. A concrete science is, however, seldom advanced to any considerable extent by arguments, however ingenious, which are propounded without appeal to experiment, or to wide and detailed observation ; and the absence of all real progress until the middle of last century is sufficient evidence that the views of Fracastorius, von Plenciz and others have acquired their main significance from knowledge gathered by later generations, rather than from their inherent fertility. The construction and use of the compound microscope was an essential prerequisite to the study of microbial forms, and the reported observation by Kircher (1659) of minute worms in the blood of plague patients forms, perhaps, the earliest attempt at direct microscopical observation in this field. It is, however, more than doubtful whether Kircher could have seen plague bacilli, or indeed any bacterial forms, with the apparatus which he had at his disposal. To van Leeuwenhoek (1683) must be ascribed the credit of placing the science of microbiology on the firm basis of direct observation (Dobell 1932). This Dutch maker of lenses developed an apparatus and technique (Cohen 1937) which enabled him to observe and describe various microbial forms with an accuracy and care which still serve as a model for all workers in this field. He observed, drew, and measured with considerable approximation to truth large numbers of minute living organisms, including bacterial and protozoal forms. It is, perhaps, somewhat surprising that this marked advance was not followed by further rapid progress in our knowledge of bacteria and their activities. Such progress was, however, impossible without further developments in technique. The world of minute living things, opened to morphological study by van Leeuwenhoek, was seen to be peopled by a multitude of dissimilar forms, whose interrelationships it was impossible to determine without preliminary isolation; and, so far as bacteria were concerned, this isolation was not accomplished until the problem of artificial cultivation was solved, almost two hundred years later.

The real development of bacteriology as a subject of scientific study dates from the middle of the nineteenth century, and is the direct outcome of the work of Louis Pasteur (1822-95). Isolated observations of microbial parasites, by Brassi, Pollender, Davaine and others, have priority in particular instances, just as Schultze, Schroeder and Dusch and others initiated technical methods which Pasteur applied to his own researches. But it was Pasteur and his pupils who settled the fundamental questions at issue, and developed a technique which made possible the cultivation and study of bacteria.

Trained as a chemist, Pasteur was led to the study of microscopic organisms by his observations on the phenomena of fermentation. His early studies on the structure of the tartrates, and on molecular asymmetry, had led him to believe 
that the property of optical activity, possesserl by certain organic compounds was characteristic of substances synthesized by living things, as contrasted with substances synthesized in the laboratory. It was known that small amounts of an optically active substance, amyl alcohol, were formed during the fermentation of sugar, especially in association with the lactic fermentation. Since it was impossible to regard the molecule of amyl alcohol as derived from the molecule of sugar by any simple break-down process, he was led to the conclusion that the optically active molecule of the sugar was first broken down to relatively simple substances, which experience had shown to be without optical activity,

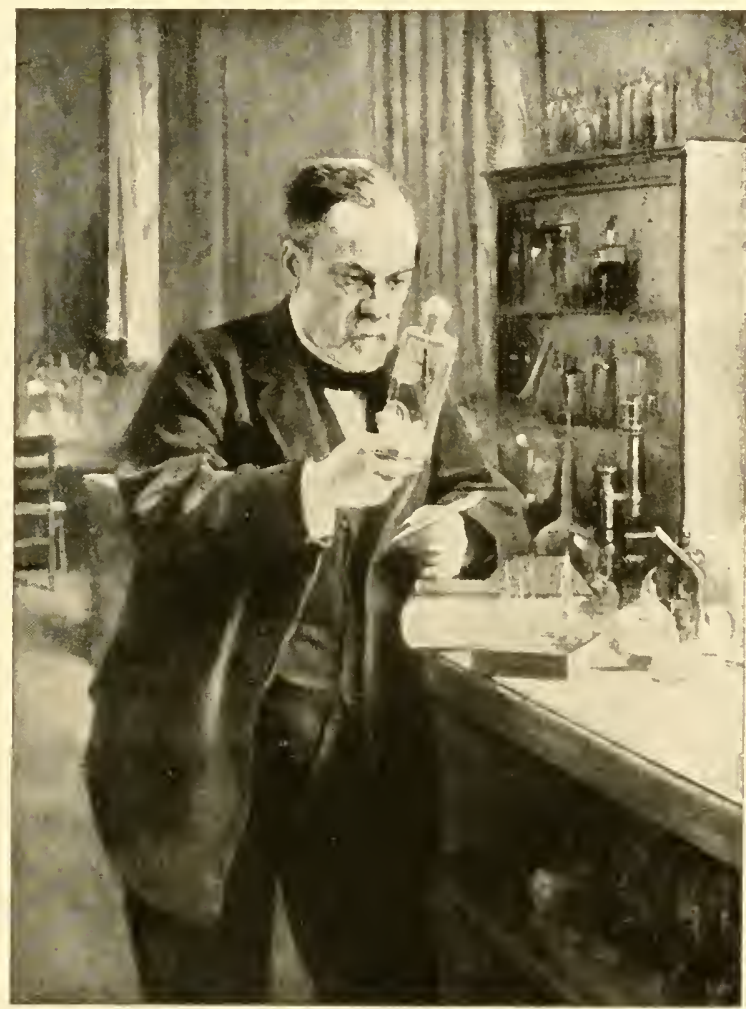

Fig. 1.-Louis Pasteur (1822-1895).

and that from such inactive substances the optically active amyl alcohol was synthesized. For Pasteur this was evidence of the presence and activity of living things, and he therefore started on his study of fermentation with a strong a priori leaning towards the microbial theory of fermentation, and away from the then dominant hypothesis of Liebig. He was prepared to adopt the theories already propounded by Cagniard-Latour in 1836, and by Schwann in 1837, concerning the living nature of the yeast globules, which were always to be found in sugar solutions undergoing alcoholic fermentations, and which bad been described by van Leeuwenhoek in 1680 .

Since, however, it was in the lactic fermentation that the production of amyl 
alcohol had especially been noted, it was this reaction which Pasteur first selected for experimental study, though he had already made numerous observations on material from the vats of the breweries of Lille. He was probably influenced by the fact that the observations of van Helmboltz (1843) had already indicated that the alcoholic fermentation was due to the yeast itself or to some other organized material. Helmholtz had shown that the substance, whatever it might be, which was responsible for initiating alcoholic fermentation, would not pass through membranes that allowed the passage of organic substances in solution but held back particles in suspension. This experiment, successful with alcoholic fermentation, failed with many other ferments and fermentable liquids. Pasteur's mind was naturally addicted to generalization, and his interest lay in the phenomenon of fermentation as a general type of reaction, rather than in one kind of fermentation in particular. It was therefore natural that he should at first neglect the field in which the battle was more evenly balanced between the purely chemical conceptions of Liebig, and the biological theories of Cagniard-Latour, Schwann and Helmholtz, and turn to the field in which Liebig's views had never been successfully attacked. Pasteur's first memoir was published in 1857, and in it he declared the lactic ferment to be a living organism, far smaller than the yeast-cell, but which could be seen under the microscope, could be observed to increase in amount when transferred from one sugar solution to another, and had very decided preferences as regards the character of the medium in which it was allowed to develop; so that, for instance, by altering the acidity of the medium one could inhibit or accelerate its growth and activity. In this memoir Pasteur laid the first foundations of our knowledge of the conditions which must be fulfilled for the cultivation of bacteria.

These studies on fermentation occupied Pasteur almost continuously from 1855 to 1860 , and he returned to them again at intervals during later years. $\mathrm{He}$ was able to show that the fermentation of various organic fluids was always associated with the presence of living cells, and that different types of fermentation were associated with the presence of microscopic organisms which could be differentiated from one another by their morphology and by their cultural requirements. Thus, at this early stage, the idea of specificity entered into bacteriology.

It was impossible for Pasteur to pursue these studies without facing the problem of the origin of these minute living organisms, which he regarded as the essential agents of all fermentations. At this time (1859) there were two opposed schools of thought with regard to the genesis of microbial forms of life. One school, deriving their concepts from the great naturalists of antiquity, believed in the spontaneous generation of living things from dead, and especially from decomposing organic matter. It is of little interest to remember the vague terms in which such conceptions were clothed; but one tendency may be noted, which did not escape the astute mind of Pasteur. The species of animals or plants believed to arise by spontaneous generation were diminishing in number, and the average size of those organisms still included in this category was getting smaller and smaller. In the beginning, the supporters of spontaneous generation were prepared to attribute this mode of origin to relatively large animals. Van Helmont, in the sixteenth century, offered a prescription for making mice. It needed the experiments of Redi (1688) to substitute, for the idea that worms were spontaneously generated in decomposing meat, the truth that these worms were the 
larvæ of flies, and that their appearance could be very simply prevented by protecting the meat with gauze, through which the flies could not pass to deposit their eggs. The discovery by Leeuwenhoek of the world of microbial organisms gave a powerful stimulus to the somewhat decadent theory. Here, at all events, were living things which obeyed no known law of reproduction, and whose existence seemed to lend support to a belief which had long been accepted by eminent authorities, and which had thereby acquired a natural prestige.

From the start of his inquiry, Pasteur leaned towards the opposing school of those who believed that spontaneous generation was a myth, that these microscopic organisms, like other living things, were reproduced in some way from similar pre-existing cells. He had already convinced himself that these organized cells were the active agents of fermentation. Clearly then they could not arise de novo during the changes for which they were themselves responsible, but must have been introduced from without. Their marked specificity, maintained through repeated transferences from one specimen of fermentable fluid to another of the same kind, was strong evidence in favour of their autonomous reproduction. Here again Pasteur had tentatively adopted the correct solution before starting his experimental inquiry, but the main interest of his part in the controversy lies in the consummate skill with which he developed methods which enabled him to give clear demonstrations where others had left doubt and confusion, and which determined the main rules of a technique which has made possible the cultivation and study of bacteria.

Neglecting for the moment the vaguer conceptions of the pre-experimental era, the position in 1859 was as follows. Needham, an Irish priest, had published in 1745 a memoir describing the spontaneous generation of microbial organisms in closed flasks of putrescible fluids, which had been heated to destroy pre-existing life. These views were strongly supported by the celebrated naturalist Buffon in 1749. An Italian abbot, Spallanzani, countered in 1769 with the publication of a series of admirable experiments in which he criticized Needham's results, and showed that, with longer heating, the fluid in such flasks remained clear and sterile. This controversy narrowed into a dispute as to the nature of the principle which survived short periods of heating, but was destroyed by long heating in flasks hermetically sealed. For Spallanzani the principle was a living germ, for Needham it was a "vegetative force," resident in the air, or perhaps in the putrescible fluid. In any case such argument was sterile, and although it was generally admitted that the honours remained with Spallanzani, no final judgment was pronounced.

At this time oxygen was regarded as an element of quite peculiar power and significance, and the experiments of Appert (1810) on the preservation of food-stuffs, by heating and hermetical closure of the containing vessels, followed by a weighty expression of opinion by Gay-Lussac, had led to a general belief that the exclusion of this gas was the essential factor in ensuring the absence of fermentation. Schwann (1837) showed that the air in a flask containing a putrescible fluid, which had been sterilized by boiling, could be renewed by drawing in air which had passed through a glass tube immersed in a bath of fusible alloy kept at high temperature, and by this means he demonstrated that the presence of oxygen alone would not cause the appearance of micro-organisms in the fluid. Unfortunately, in the same memoir, Schwann reported other experiments, in which he introduced heated and unheated air into flasks, containing a sterilized solution 
of sugar in a watery extract of yeast, by inverting the flasks over a mercury bath and admitting the air through the mercury seal. Here his results, as regards the occurrence of fermentation, were altogether uncertain, and his conclusions lost much of their force. Helmholtz (1844) confirmed certain of Schwann's observations. Schultze (1836) had already obtained similar results by admitting to his flasks air which had been drawn through strong potash solutions or through concentrated sulphuric acid. Schroeder and Dusch (1854) showed that the active principle could be removed from the air by drawing it through cotton-wool. This last method was a real advance, since the incoming air had not been subjected to high temperatures, nor to strong chemical reagents. Unfortunately another element of doubt was introduced. Schroeder and Dusch relied, for their preliminary sterilization, on a short period of heating to the boiling-point. They experimented with four kinds of material-water containing meat, malt of beer, milk, and meat without the addition of water. With the first two materials their results were quite uniform: the fluids remained unaltered. With the last two materials fermentation usually occurred. They concluded that there were two kinds of decomposition, associated with the presence of living organisms, the one spontaneous, needing only the presence of oxygen, the other requiring some additional principle, which could be removed from the air by filtration through cotton-wool.

This, then, was the position when Pasteur began his investigations in 1859. In a series of admirable memoirs, starting in 1860 and continuing for more than four years, he wont over the ground already covered, added new and illuminating experiments of his own devising, and terminated the controversy by clear and decisive demonstrations. He showed that the material removed from air by passage through cotton-wool, or through similar filters, contained organized particles which were neither crystals nor starch granules, but which were similar in appearance to the spores of moulds. By introducing these particles into flasks of sterilized organic material, he demonstrated that they were capable of giving rise to the growth of numerous kinds of living organisms. Using other methods, he showed that the air in different situations differed in its content of these germs; that they were numerous in the streets of cities, less numerous in the air of country uplands, rare in the quiet air of closed and uninhabited rooms or cellars, where the dust had deposited and remained undisturbed, and very rare in the pure air of the high Alps, above the level of human habitation. He showed that Schwann's failures were due to his use of mercury, from the surface of which his fluid had acquired the germs, which had settled on it from the air. He showed that the failures of Schroeder and Dusch were due to the inadequate sterilization of their material.

He also showed that certain animal fluids, such as blood or urine, known to be eminently liable to undergo putrefaction, could be collected in such a way as to remain permanently unaltered.

The controversy with Pouchet, Joly and Musset, which continued from 1860 to 1864 , did not lead to the collection of many new facts, except those with regard to the unequal distribution of micro-organisms in the atmosphere; but a later dispute with Bastian, who became a veteran in the dwindling army of the supporters of spontaneous generation, was more fertile, because it caused Pasteur to reconsider some of his ideas, and to elaborate the technical methods which he had partially developed during his re-investigation of the results obtained by Schroeder and 
Dusch. In 1876 Bastian published a communication controverting an early statement by Pasteur that urine, sterilized by boiling, remained free from growth on subsequent incubation. Bastian declared that, if the urine were made alkaline at the start, growth often ensued. Pasteur, on repeating the experiment, was forced to admit the truth of Bastian's statement. A careful retracing of all his steps resulted in the demonstration that fluids with an acid reaction, after sterilization at $100^{\circ} \mathrm{C}$., might remain apparently sterile because certain organisms, which remained alive, were unable to develop, while in an alkaline medium they might grow freely. It was found also that ordinary water frequently contained organisms which were not killed by heating to $100^{\circ} \mathrm{C}$., and that organisms which had become deposited on the surface of glass-ware in the dry state might withstand far higher temperatures. We know now that it is especially for those bacteria which form spores that these conditions hold true. As a result of this controversy Pasteur established the practice of heating fluid material to $120^{\circ} \mathrm{C}$. under pressure for the purpose of sterilization, thus introducing the autoclave into the laboratory, and the practice of sterilizing glass-ware by dry heat at $170^{\circ} \mathrm{C}$. In this connection a very important advance was made by 'Tyndall who, observing that actively growing bacteria are easily destroyed by boiling, and that a certain amount of time is required for bacteria in the resistant, inactive phase to pass into the growing phase in which they are heat-sensitive, introduced the method of sterilization by repeated heatings, with appropriate intervals between them. This method is still known as Tyndallization. It was first described in a letter to Huxley in 1877 (see Bulloch 1930).

While investigating the phenomenon of fermentation, and the problem of spontaneous generation, Pasteur had studied the behaviour of very various kinds of natural organic fluids and solutions, and had succeeded in growing microorganisms on simple synthetic media. As a result he had become assured of the fact that a medium, which is eninently suitable for the growth of one bacterium or mould, may be ill-adapted for the growth of another, and that one of the primary necessities for the successful cultivation of any species of micro-organism is the discovery of a suitable medium for its growth. Quick to grasp the general significance of isolated observations, he pointed out the decisive effect which must be exercised by the selective action of various environmental factors in determining the constitution of any naturally occurring bacterial flora; and he later developed these ideas in connection with the problem of infection.

As the result of these studies Pasteur had collected a mass of data, which enabled him to deal successfully with bacteriological problems that could not previously have been attacked. He had learned the need for the scrupulous sterilization of everything that came into contact with material which was to be submitted to bacteriological examination. He had learned the necessary methods of sterilization, in the steamer, in the autoclave, in the hot-air oven, or by direet flaming, which enabled these conditions to be fulfilled. He had proved the serviceableness of the cotton-wool plug for protecting media in flasks or tubes. He had realized the importance of the constitution of the nutrient material offered to a given bacterium, of the acidity or alkalinity of that medium, and of the oxygen pressure to which it was subjected. Armed with this knowlerge, he proceeded to break new ground.

Pasteur was before all else a scientist, intensely curious, and loving knowledge for its own sake, but he was also a convinced utilitarian, and a Frenchman. He 
desired greatly that his discoveries should benefit mankind in general, France in particular, and, if possible, his neighbours in the first place. Thus we find him investigating with enthusiastic care the troubles of the local vintners or brewers, or vinegar-makers, and many of his memoirs are devoted to the diseases of wines or of beers, and the methods of preventing them. It was in connection with these studies that Pasteur faced a new problem of fundamental importance. $\mathrm{He}$ had shown that ferments were living organisms, that they were specific, that they were reproduced from parent forms and not by spontaneous generation. He was now faced with the problem as to whether one species could change into another, in particular whether mycoderma vini could change into the ordinary yeast of wine. Deceived on this point at first, he resorted as usual to rigorous and repeated experiments, and not only demonstrated that this mutation did not occur, but indicated clearly the conditions which led to its apparent occurrence, and the care which must be exercised before accepting any reported variation of this kind.

Anyone who reads for himself the original memoirs on fermentation and spontaneous generation (see Vallery-Radot, P., 1922-1933) will realize that the possibility of applying this new knowledge to the elucidation of infective disease was already in Pasteur's mind. It nceded only the spur of a request from Dumas to investigate the disease, which was then ruining the silkworm industry in the South of France, to turn his steps permanently towards the study of infective processes. We cannot follow here, even in outline, Pasteur's researches into pébrine, anthrax, chicken cholera, or hydrophobia. Some of them will be referred to in later chapters. We must, however, note certain contributions which Pasteur and his colleagues made to the fundamental data of bacterial infections. It was Pasteur who showed, in the case of anthrax, that a culture of a pathogenic organism could be passed through successive subcultures, in such a way as to dilute, beyond possibility of significant action, any other material introduced with it into the primary culture from the blood or tissues, and still produce the disease when inoculated into a susceptible animal; though it is to Koch that priority must be given as regards many points in the demonstration of the nature and action of the anthrax bacillus. It was Pasteur who introduced into bacteriology the conception of virulence and of attenuation, and who demonstrated the fact that an attenuated bacterial culture will act as a vaccine, that is, will confer immunity against subsequent infection with a virulent strain of the same bacterium. For Pasteur, indeed, a vaccine was synonymous with an attenuated culture, as opposed to a virulent culture on the one hand and to a dead culture on the other. It was Pasteur who, in the case of rabies, showed that it was possible to study the virus of an infective disease by animal passage, when the organisin could not be cultivated, and even to prepare a perfectly efficient vaccine by using suitably treated animal tissue.

Thus, throughout a long scientific life, Pasteur was largely concerned with the practical application of knowledge gained during his studies on fermentation. The correct procedure for preparing good wine, good beer, good vinegar, and the methods of preserving them, the control of pébrine, of anthrax, of chicken cliolera, of hydrophobia, these were the problems which occupied the last thirty years of his life, and the solution of which made his name a household word. But we shall miss the real significance of his work if we fail to realize that his fertile generalizations were of infinitely more importance for the progress of science than were his successful attacks on these isolated problems. 
He had learned how to isolate and cultivate bacteria, and how to study their effect on animals; but with the minutiæ of their morphology or physiology, apart from any significance these might have for the problem in hand, he was not greatly concerned. Duclaux relates that a clever and positive microscopist, who told Pasteur in very cautious language that a certain organism which he had taken for a coceus was in reality a very small bacillus, was much astonished to hear him reply: "If you only knew how little difference that makes to me!"

One further point must be noted. Pasteur and his colleagues had shown how to obtain cultures of micro-organisms, and propagate them indefinitely in the

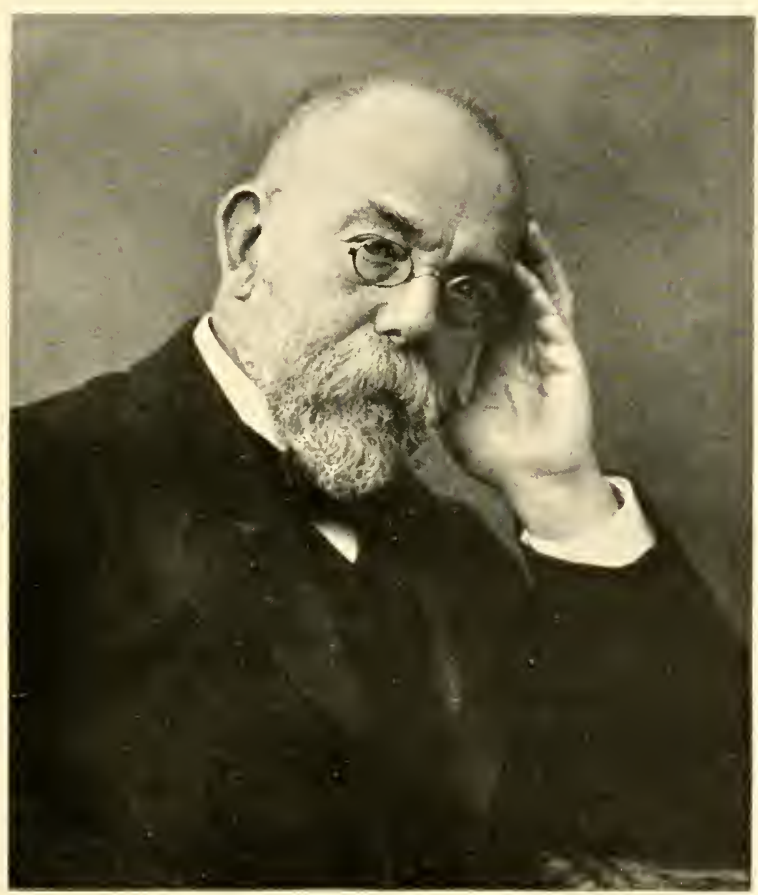

Fia. 2.-Rabert Koch (1843-1910).

laboratory; but the methods which they employed were not well suited to the isolation of pure strains of bacteria from an originally mixed culture, except in those relatively rare cases in which it was possible to employ a highly selective medium. Since all media were employed in the fluid state, the only method of purifying a culture was to make siccessive transfers with very small amounts of material, in the hope that only a few bacteria, all of one kind. would be carried over. Such a technique was very uncertain in its results.

Pasteur, starting as a chemist, founded bacteriology and revolutionized medicine. At about the time when he was propounding his germ theory of disease, a young German physician, some twenty years his junior, was turning from clinical medicine to bacteriology. Robert Koch (1843-1910), at that time a 
practising physician at Wollstein, attacked the problem of anthrax, and produced, as his first contribution to science, a demonstration of the character and mode of growth of the causative bacillus, which opened a new era in bacteriological technique. This memoir he published in 1876. In the following year he published his methods of preparing, fixing, and staining film-preparations of bacteria, using the aniline dyes introduced into histology by Weigert, and described his methods of photographing such preparations. In 1878 he published his memoir on traumatic infective diseases, which remains a classical example of the study of experimental infections in laboratory animals. In 1881 he deseribed his method of preparing cultures on solid media, a technical advance of the first importance, since it made possible the isolation of pure strains of bacteria from single colonies. Solid media prepared from naturally occurring material such as pieces of potato, had previously been used for the isolation of micro-organisms, particularly by mycologists, and the general principles to be observed in the preparation of pure eultures had been clearly enunciated by Brefeld, who had suggested the solidification of a nutrient medium by the addition of gelatin. The media and methods available for the eultivation of fungi were not, however, well suited for bacteria; and it was left for Koch to devise, in the form of his nutrient gelatin, and later, at the suggestion of Frau Hesse, of nutrient agar, a solid, transparent medium, easy to sterilize and handle, and thus admirably adapted for obtaining isolated colonies of bacteria (see Bulloch 1930). In 1882 and 1884 he published his classical papers on the bacillus of tuberculosis. In 1883 he discovered the vibrio of cholera. Already, Koch had enlisted the services of Loeffler and of Gaffky as his assistants. Later came Pfeiffer, Kitasato, Welch and many others, and, with his growing fame, he began to gather round him a group of keen and able young men, who were destined to introduce the methods he devised into the laboratories of many lands. In 1885 he was appointed Professor of Hygiene and Bacteriology in Berlin, and in 1891 he was made Director of the newly-founded Institute for Infective Diseases. His later years were devoted almost entirely to the investigation of bacteriological problems in their relation to the prevention and cure of disease, and many of his contributions to our knowledge will be considered in later chapters. Koch was, above all, an able and carefnl technician. He was greatly aided by the vigour and initiative of the great German chemical and optical firms, and the advances which he made in staining methods, in the use of the microscope for the observation of bacteriological preparations, and in the technique of cultivating bacteria, revolutionized this branch of science.

The fruits of this revolution appeared with surprising rapidity. During the last quarter of the nineteenth century a succession of discoveries was reported, bearing on the relation of bacteria to human and animal disease, which opened a new era in medicine.

In 1874 Hansen described the bacillus of leprosy, and Neisser, in 1879, the gonococcus. In 1880 Pasteur recorded the isolation of the bacillus of fowl cholera, and Eberth observed the bacillus of typhoid fever. In 1881 Ogston published an adequate description of the staphylococcus. In 1882 Koch discovered the tubercle bacillus, and Loeffler and Schütz the bacillus of glanders. In 1883 Koch discovered the cholera vibrio, Febleisen isolated the streptococeus of erysipelas, and Klebs described, but did not isolate, the bacillus of diphtheria. In 1884 Loeffler isolated, and subjected to thorough study, the bacillus which Klebs had briefly described in the previous year, and Gaffky isolated and studied the typhoid bacillus, which 
Eberth had observed four years previously. In 1885 Loeffler discovered the bacillus of swine erysipelas, Kitt the bacillus of hæmorrhagic septicæmia of cattle, and Salmon and Smith the bacillus associated with hog cholera. In the same year Nicolaier observed the tetanus bacillus in soil, inoculation of which produced the disease in animals. In 1886 Fraenkel isolated the pneumococcus, Escherich the colon bacillus, and Loeffler the bacillus of swine plague. In 1887 Weichselbaum discovered the meningococcus, and Bruce the micrococcus of Malta fever. In 1888 Gaertner described the bacillus which bears his name, and Schütz the streptococcus of equine strangles. In 1889 Kitasato cultivated the tetanus bacillus, which had been earlier observed by Nicolaier. In 1892 Pfeiffer isolated the bacillus which he believed to be the cause of influenza, and Welch and Nuttall described the anærobic bacillus now known as $\mathrm{Cl}$. welchii. In 1894 Kitasato and Yersin independently discovered the bacillus of plague. In 1895 Moore isolated the bacillus of fowl typhoid. In 1896 van Ermengem described Cl. botulinum as the cause of a particular variety of food poisoning. In 1897 Bang discovered the bacillus of bovine abortion. In 1898 Shiga isolated the variety of dysentery bacillus which bears his name, and Nocard and Roux described the minute organism of infectious pleuro-pneumonia of cattle.

Thus, by the close of the nineteenth century a great variety of micro-organisms had been identified as occurring in definite association with human or animal disease. In many instances complete demonstration had been afforded that the relation was one of cause and effect. In others, this relation was rendered highly probable. In others, again, there remained good reason for doubting whether the bacterium, whose presence had been demonstrated, played any more important rôle than that of a secondary invader. Beyond dispute, however, the scientific investigation of infective disease had become the province of the bacteriologist.

Another incident had done much to emphasize the importance of bacteria as the cause of disease and death, although it had comparatively little influence on bacteriology itself. Joseph Lister (1827-1912), during his tenure of the Professorship of Surgery at Glasgow, was deeply interested in the post-operative sepsis, which exacted such a terrible toll on the lives of hospital patients. His attention was drawn to Pasteur's work on fermentation, and the analogy between the changes which occur in fermenting organic material and the putrefaction which occurs in wounds suggested to him that in the latter, as in the former, the underlying cause might be the activity of minute living organisms. This led directly to the introduction of his antiseptic technique in surgery, described in 1867, which opened the door to modern surgical methods. Lister's technique has since been replaced by aseptic measures, but this detracts in no way from the merit of his discovery, nor from the debt which we owe to him for fighting the usual battle against the forces of ignorance and prejudice. Nor should it be forgotten that Lister made important contributions to bacteriological technique as such. He devised a method for diluting a bacterial culture and preparing a series of subcultures with so small a volume of the original fluid that many of them remained sterile, the presumption being that those that grew had developed from a single bacterial cell. In this way he isolated, in 1878, a bacterium that caused the souring of milk; and Bulloch (1938) expresses the view that he may perhaps have been the first bacteriologist to obtain a certainly pure culture.

But the revolution inaugurated by Pasteur and extended by Koch spread far beyond the field of medicine. Agriculturists had long been puzzling over the 
problem of soil fertility, without arriving at any very helpful conclusions. One curious phenomenon was the reaccumulation of nitrates in the soil, in spite of their constant removal by the washing action of the rain. It was suspected that these nitrates might be derived in some way from the decomposition of organic material, and in 1877 Schloesing and Müntz, acting on a suggestion made by Pasteur in 1862 , showed by experiment that the formation of nitrates was due to the action of living organisms. Warington, at Rothamsted, confirmed these results in 1878 and 1879 , and showed that two stages were involved, a preliminary conversion of ammonia to nitrites, and a subsequent oxidation of nitrites to nitrates. He believed that these two stages were carried out by different organisms, but failed to isolate or identify them. This problem was solved by Winogradsky in 1890 , who isolated and described both the nitrite- and nitrate-forming organisms. In 1888 Hellriegel and Willfarth described the nitrogen-fixing bacteria which caused the formation of nodules on the roots of leguminous plants. Later Winogradsky described a free-living anaerobic organism which was able to fix atmospheric nitrogen, and Beijerinck, some ten years later, described a large, free-living, nitrogen-fixing aerobic bacterium, which he named Azotobacter, and which has since been extensively studied. The bacteriology of the soil thus became an important part of agricultural science.

In the early years of the bacteriological revolution it had been demonstrated that bacteria attacked plants, as well as animals. In 1878 Burrill described the organism of pear blight, and in 1883 Wakker discovered the bacillus which causes the "yellows" of the hyacinth. This branch of bacteriology has been pursued energetically during the last thirty years, especially by Erwin Smith and his colleagues in America.

The demonstration by Pasteur of the essential nature of fermentation led, as a natural consequence, to the entry of the bacteriologist into the commercial sphere. His help was required in dairy farming, in brewing, in the preservation of foods, and in all those commercial processes in which bacterial activity was desired or feared.

Fig. 3, which sets out in diagrammatic form the time relations of the more important discoveries associated with the work of Pasteur, Koch and Lister, may be of some service in enabling the student to follow the development of our knowledge down to the end of the nineteenth century.

This brief summary will indicate with sufficient clearness to how great an extent the bacteriologist has been occupied with applied problems. He has, by way of description, usually been satisfied if he could determine, for any given bacterium, a number of characters sufficient to differentiate it from the organisms with which he considered that it was most likely to be confused. It is in this way that our knowledge of bacterial characters has slowly grown and it is not surprising that the results should be an arbitrary and rather odd assortment of differential criteria. In such a bacterial group as that comprising the colon and typhoid bacilli, and certain nearly related organisms, it has been demonstrated that fermentation reactions form a reliable method of differentiation, and such reactions have been extensively studied. In another group morphological differences may be more distinctive, or the production of specific toxins may be a well-marked feature in certain species. The soil-bacteriologist employs methods which differ in important respects from those used by other workers. It is the inevitable result that systematic bacteriology has been very generally neglected, and it is only in recent 
years that any real attempt has been made to survey bacterial groups as a whole, and to bring some order out of our chaos.

The first two decades of the present century witnessed no such striking advances in our knowledge of the bacteriology of disease as occurred between 1875 and 1900, and the reason for this slower progress is obvious. The technique developed by Pasteur and Koch had been applied over a very wide field. Those problems which were susceptible of solution by the methods available had, to a great extent,

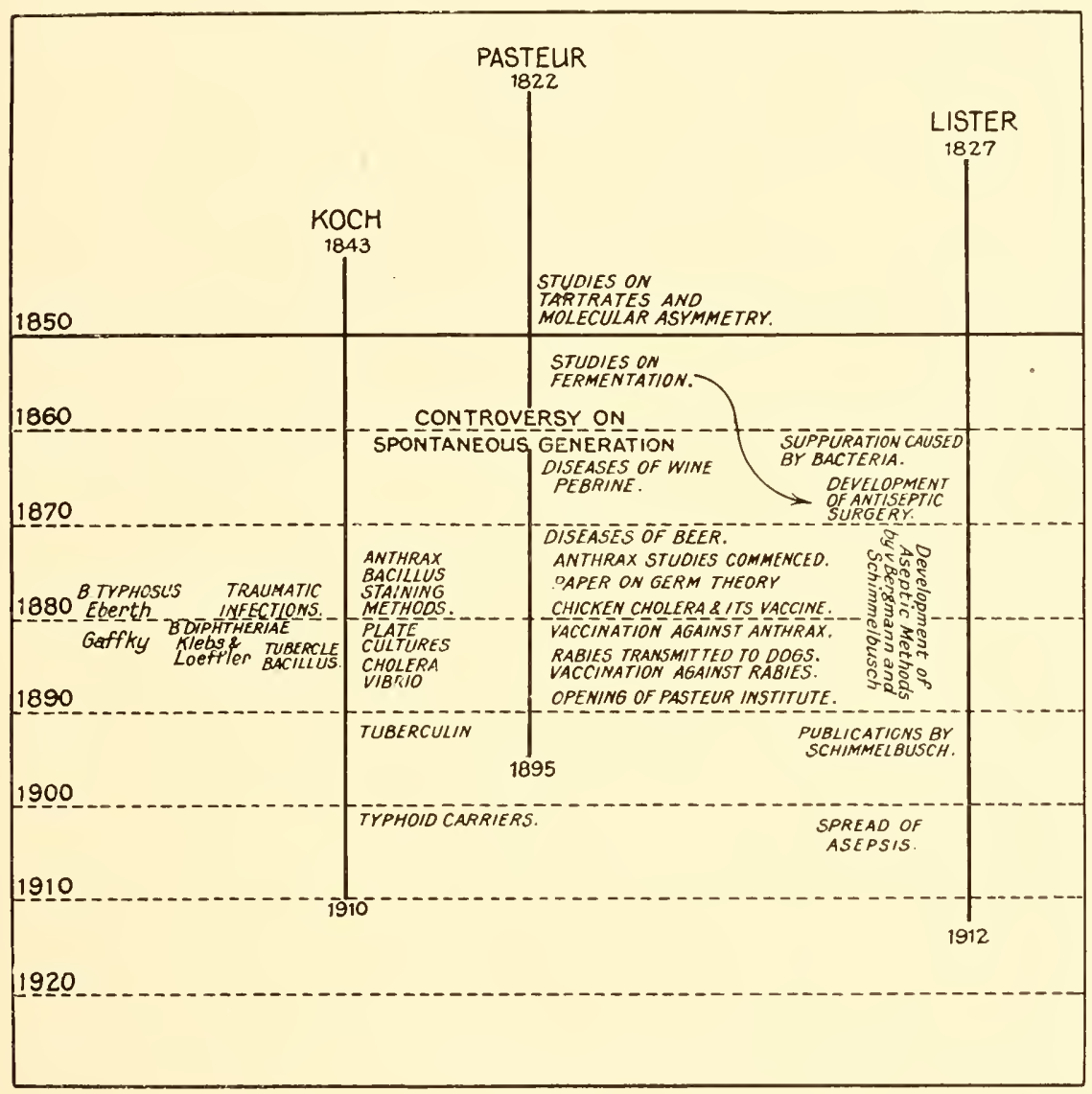

FIG. 3.

been solved, and those which remained unanswered appeared to demand new methods of attack, or at least attack along new lines.

The study of immunity has absorbed the interest and energies of a large number of bacteriologists during the past forty years. This branch of bacteriology derives from Pasteur's studies on chicken cholera, anthrax and rabies, from Metchnikoff's investigations on the cellular reactions in infection, and from the work of Buchner, Nuttall, von Behring, Ehrlich, Bordet and others, who have investigated the reactions between the sera of artificially immunized animals and the bacteria, bacterial products, foreign cells, or foreign proteins with which they have been 
inoculated. The development of our knowledge of these phenomena wiil be discussed in Chapter 7.

The demonstration by Loeffler and Frosch, in 1898, that foot-and-mouth disease was caused by a virus which could pass through a porcelain filter, and was below the limits of direct microscopical observation, opened a new field for investigation. We now know many diseases which can be transmitted by filtered suspensions of material obtained from infected animals, and in which therefore a filter-passing virus is presumably concerned.

There is nothing surprising in the fact that the years from 1900 to 1920 , or thereabouts, were for bacteriology a period of slower development as compared with the riotous growth of the eighteen-eighties. The ground won had to be consolidated. The previous adrance had been a somewhat hasty affair; and many secondary problems had been left for more leisurely solution. Many bacteria were very incompletely described. Many had been described independently by different investigators, so that the same bacterium was masquerading under several different names. Many of the earlier descriptions, especially of the anaerobic bacteria, had been based on impure cultures. Little notice had been taken of resemblances between bacteria, isolated at different times from different sources, unless the practical application of the knowledge gained brought such rescmblances forcibly to the attention of some observer. Little was known about the distribution in nature of bacteria other than those concerned in the causation of disease, or in some commercial or agricultural process, and even here the data were very scanty. Bacterial ecology is, indeed, still almost an unexplored territory. In all these directions the past thirty years have scen a considerable advance. Bacteria have been studied more systematically. Fuller, and more accurate, descriptions have been recorded and errors have been corrected. The labelling of the stock strains of hacteria, scattered throughout the laboratories of the world, has been nore closely scrutinized. Many synonyms have been suppressed, and species that had received several names have been shorn of all but one. The formation of such collections as the National Collection of Type Cultures in this country has provided a much-needed standard of reference.

This re-survey of the bacteriological field on its qualitative side has been associated with a great advance in our quantitative methods. The introduction of bacteriological methods of analysis in the control of water-supplies, milk, and so on, demanded standardized tests yielding numerical answers. Those first employed were in most cases very faulty. There was a failure to realize many of the technical sources of error; and there was a still more general failure to take into account the statistical principles involved in any sampling of this kind. It is, indeed, only within recent years that a satisfactory liaison has been established between the bacteriologist and the statistician; and, even now, it is not as general as it should be. It is not merely a question of the kind of analytical test referred to above. The vast literature of immunity contains record after record which is rendered meaningless by a neglect of the sampling errors involved in working with small groups of animals. Reference to Chapter 43 will provide examples of the ways in which these errors may be avoided.

In the last twenty years or so, there lrave been unmistakal)le signs that bacteriology is on the march again. As always in experimental science it has been a matter of technique. In this case the acceleration has followed the application to bacteriology of the more exact methods of analysis developed by the chemist and the 
physicist. The facts set out in Chapter 3 show clearly the rapid advance that has followed the incursion of the biochemist into the bacteriological domain. Those described in Chapter 6 show the organic chemist and the biochemist initiating a remarkable extension of chemotherapy in relation to bacteria, and the outstanding success of synthetic suphonamide componnds, and of the natural mouldproduct, penicillin; and those described in Chapter 7 show the organic and physical chemist inaugurating a new era in immunology. The remarkable increase within quite recent years of our knowledge of the filtrable viruses (see Chapters 41 and 85-89) has resulted in large part from improved methods of filtration, optical examination and high-speed centrifugation. It is not a rash prophecy that the years ahead of us will be the eighteen-eighties over again.

\section{REFERENCES}

Bulloch, W. (1938) "The History of Bacteriology." Oxford Lniv. Press, London. Cohen, B. (1937) J. Bact., 34, 343.

Dobes., C. (193:) "Antony van Leeuwenhoek and his "Little Animals." John Bale, Sons and Danielsson, London.

Ducladx, E. (1920) "Pasteur, the History of a Mind." Eng. Transl. by E. F. Smith and Florence Herges. Saunders, Philadelphia and London.

Vallery-Radot, P. (192*-33) "Euvres de Pasteur." 6 vols. Masson et Cie, Paris.

Vallery-Radot, R. (1919) "The Life of Pasteur." Eng. Transl. by Mrs. R. L. Deconshire. Constable, London. 


\section{CHAPTER 2}

\section{THE BIOLOGICAL CHARACTERISTICS OF BACTERIA : MORPHOLOGY}

General Considerations.--With the exception of certain observations on the finer structure of the bacterial cell, which will be referred to later, our knowledge of bacterial morphology has been gained from the study of cells which have been cultivated in the laboratory under artificial conditions. The morphology of bacterial cells may be notably affected by the constitution of the medium on which the bacteria are grown, the temperature of incubation, and many other factors. In particular, the cells in a pure culture may show very striking changes with age. It is customary to regard the forms found in young cultures as typical of a given species, and the very different appearances, often met with in old cultures, as due to the occurrence of degenerative changes. How far we are justified in labelling all the morphologically atypical cells that we meet with in old cultures as degeneration or involution forms, is a controversial question which is discussed elsewhere. It must always be remembered, however, that when a description is given of the morphology of any bacterial species, such a description is supposed to apply to the cells found in a young, actively-growing culture, on a medium which is favourable to the growth of that particular species, and incubated at the optimum temperature, unless the contrary is specifically stated. Those who are for any purpose describing the appearances met with in preparations from bacterial cnltures should always recollect that such descriptions have little value unless the exact conditions of cultivation are carefully noted.

Apart from variations associated with age, variations in form, sometimes of a very striking character, may occur in young cultures of a single bacterial species. Different forms of cell may be present in a single culture; or the cells may appear to alter their form in successive subcultures ; or different strains of a single bacterial species may show morphological differences, which persist in successive subcultures carried on over a considerable period of time. A description of the morphology of a given bacterial species should include the characters displayed by the modal form, and the extent to which these characters vary. Variability of form is, in itself, very characteristic of certain bacterial species; while other species show only minor differences in the shape and size of the bacterial cells.

The Size of Bacterial Cells.--Ignoring for the moment the very large bacteria which have been described by a few investigators, and those very minute organisms which will pass through a porcelain filter - the filtrable viruses - it may be said that the dimensions of most cultivable forms are of the order of low multiples or submultiples of $\mu$, i.e. of $1 / 1000$ th of a millimetre. Among the spheroidal forms, the 
parasitic staphylococci and streptococci usually measure between $0.75 \mu$ and $1.25 \mu$ in diameter. Some forms of micrococci or sarcinæ may show an average diameter of 1.5 to $2 \mu$. Among the rod-forms, a relatively large bacillus, such as $B$. anthracis, has a transverse diameter varying between 1 and $1.25 \mu$ and a length varying between 3 and $8 \mu$. A medium-sized bacillus, such as Bact. coli, has a transverse diameter varying between 0.5 and $1 \mu$, and a length of 2 to $3 \mu$. A very small bacillus, such as the influenza bacillus, has an average diameter of 0.2 to $0.4 \mu$, and a length of 0.7 to $1.5 \mu$. Some bacilli, such as $\mathrm{Cl}$. tetani, may combine a small transverse diameter, 0.3 to $0.4 \mu$, with considerable length, 3 to $5 \mu$. All such measurements must, of course, be taken as representing a modal size, corresponding to the modal form, and wide variations may occur. Some species of bacilli, for example, may show occasional filamentous forms, measuring $100 \mu$ or more in length. It remains true that the modal size of a bacterium is one of its distinctive characteristics, and has the advantage that it can be stated in numerical terms. In such forms as the Actinomyces, which are normally filamentous, it is to the transverse diameter alone that we can assign a modal value.

The Shape of Bacterial Cells.-We can recognize three main types of bacterial cell-the coccal or spheroidal, the bacillary or cylindrical, and the spirillar.

The coccal form is distinguished by the fact that any one axis of the bacterial cell is approximately equal to any other. Many forms approximate to true spheres, although it is doubtful whether any living cell is truly spherical. In many cases the spherical form is widely departed from, and the individual cells may be ellipsoidal or show conical distortions, flattenings or indentations, which give the cells, when observed in film preparations, shapes which may be likened to a bean, or a kidney, or a lancet, as the case may be.

The bacillary form is distinguished by the fact that one axis of the cell is markedly longer than either of the others, which are themselves approximately equal. Since it is customary to examine bacteria in film preparations, and to describe them as though they were two-dimensional bodies, it is usual to refer to the long axis and the transverse axis, ignoring any possible departure from the circular form in the cross section of the cylinder. The ratio between the length of these cylindrical cells and their transverse diameter may vary over an enormous range; so that, while some may be almost coccal in appearance, others may be filamentous. Certain modifications of the general cylindrical shape are characteristic of particular bacterial groups. The average ratio of the long axis to the transverse diameter, that is, the thinness or thickness of the cell, is one such character. The ends of the bacillus often show modifications of form which are of differential value. They may be square-cut, rounded, or acutely pointed, or may form definite clubs. Other irregularities in contour, due to the presence within the cell of structures which cause distortion of the cylindrical form, will be considered later.

The spirillar form is characterized by a bending or twisting of the cells, so that they assume curved or spiral shapes. It has become customary to speak of a bacterium which shows a single curve, thus assuming the so-called comma shape, as a vibrio, and of a bacterium which shows a series of curves or twists, thus assuming a corkscrew form, as a spirillum. Vibrios and spirilla are of necessity relatively elongated cells, and they always have rounded or pointed ends.

The study of the finer details of bacterial structure may be carried out in four 
different ways. Wach of them is subject to the limitations imposed by the optical system employed.

Stained or unstained preparations may be examined under the microseope, using light transmitted through a sub-stage condenser of the ordinary type. The limit of resolution, $d$, (that is, the shortest distance by which two particles must be separated in order that they may give distinct images) is determined by the wave-length of the light and the numerical aperture of the objective. It is given by the formula,

$$
\frac{0.5 \lambda}{\text { N.A. }}
$$

where $\lambda=$ wave-length of light used, and N.A. = numerical aperture of objective.

The possible range of $\lambda$ is obviously limited to that part of the spectrum to which the human retina is sensitive, and the numerical aperture is subject to the technical limitations of the optical system employed. In practice, the highest N.A. that can be employed with transmitted light is about $\mathbf{1 \cdot 4}$, and this, when used with monochromatic light with a wave. length of $546 \mathrm{~m} \mu$, corresponding to the green mercury line, gives a resolving power of

$$
\frac{546}{2} \times \frac{1}{1 \cdot 4}=195 m \mu=0.2 \mu \text { (approximately) }
$$

This degree of resolution is obtained only under optimal conditions, and in ordinary practice the limits of resolution are reached with particles that have a diameter of about $0 \cdot 25 \mu$. Merling-Eisenberg (1937) has modified the formula by introducing a factor measuring the intensity of illumination. By suitably reducing this intensity a limit of resolution of $0.08 \mu$ is approached. Under ordinary conditions, however, objects smaller than $0.25 \mu$, though "seen" in the sense of being visible, do not form images that reveal the real size or shape of the particle.

Another method is that known as dark-ground illumination. The bacteria, or particles, are examined unstained, and the light passing through the special sub-stage condenser is directed along a path such that only those rays that are refracted, diffracted, or seattered by the object under examination reach the eye of the observer. Bacteria so examined appear as bright images on a dark background. This method is vastly superior to the former as a means of examining living, unstained organisms, but it is subject to the same limitations in regard to resolution; and, since the highest N.A. at present available for use with illumination of this type is about 1.27 , the smallest particle that can be resolved has a diameter of about $0.35 \mu$.

The third method, which has been developed particularly by Barnard (1919, 1925, 1930), extends the limits of resolution by decreasing the wave-length of the light used. Using ultra-violet light with a wave-length of $257 \mathrm{~m} \mu$ and an optical system of quartz lenses, Barnard has been able to photograph and resolve particles with a diameter of $0.075 \mu$. Beyond this point a limit is again reached, due to the lack of a refracting material that will transmit light of shorter wave-length. The degree of internal structure that may be revealed in certain bacteria by ultra-violet photography, combined with darkground illumination, is illustrated in Fig. 4, for which we are indebted to Mr. Barnard.

The electron microscope devised by Ruska (1934) and by Mlarton (1934) provides the fourth method of examination (see Marton 1941). The fact that an electron beam passing through a maguetic field behaves in a manner closely analogous to a beam of light passing throngh a refracting medium permits the construction of a microscope generally similar to an optical microscope, and its description in the terminology of light optics. The "lenses" are circular electro-magnets whose focus varies as the strength of the applied magnetic field ; and the microscope has a "condenser" coil, an "objective" coil, and a "projective" coil, the last being equivalent to the eyepiece. The "wave. length" is a function of the speed of the electrons, and those used in electron microscopy are equivalent to a wave-length about $1 / 100,000$ th that of visible light. Resolving powers commensurable with this wave-length cannot be attained in practice, since "chromatic" aberration in the magnetic lens cannot be corrected, and "spherical" aberration is over 
a thousand times that of a glass lens. With these restrictions, a theoretical resolution of $1.0 \mathrm{~m} \mu$ is possible, and in practice resolutions of $2.2 \mathrm{~m} / \mu$, equivalent to a magnification of 180,000 , have been attained. For biological purposes magnifications of 10,000 to 50,000 are employed. The different opacities in an object recorded on the photographic plate are due to the scattering of the electron beams, not to the absorption and refraction of light, as in the optical microscope. The intensity of the transmitted beam, for a given electron speed, is roughly proportional to the thickness and density of the material examined. In a manner analogons with staining of optical microscopic objects, certain biological materials may be increased in density (i.e. electron scattering power) by impregnation with salts of heary metals (IIndd and Anderson 1942). 'The value of this method is at present severely limited by the necessity for completely dry specimens for examination, since the microseope works only in a high vacumm. (For a review of the recent achievements of electron microscopy, see Mudd and Anderson 1944.)

The great majority of the studies referred to in this section have, however, been carried out by the direct observation of stained preparations, and have therefore been subject to the narrower optical limitations considered above. As against these limitations must be set the advantage that, in preparations of this kind, we can study the affinity of different cell constituents for certain special stains. In most, if not in all, instances, staiming has been preceded by some form of fixation, so that the possibility of protoplasmic changes due to heat, or other fixing agents, must be borne in mind.

\section{The Nuclear Apparatus.}

One of the most controversial questions in regard to the structure of the bacterial cell is the presence or absence of a nucleus, and its nature if present.

It is impossible to discuss at all fully the many conflicting statements which have been made, and the evidence

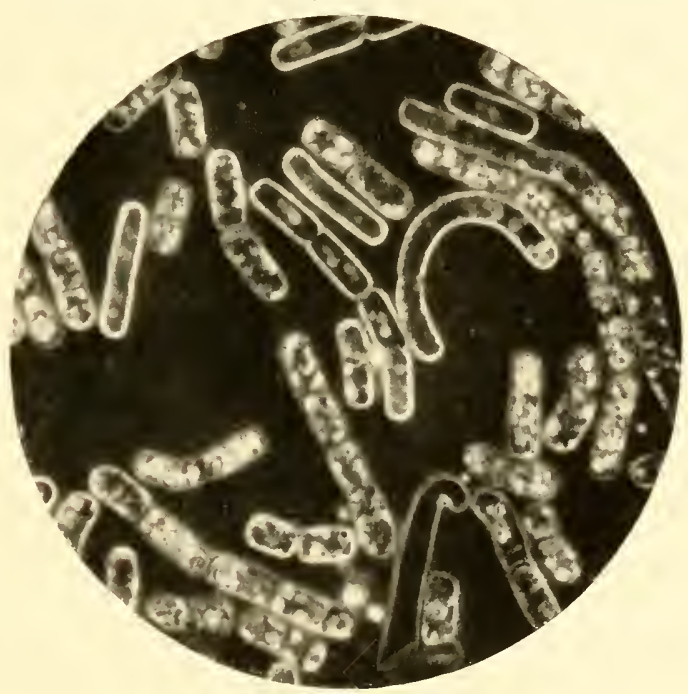

FIG. 4.-B. megatherium.

Unstained, dark-ground illumination, photographed with ultra-violet light $(\times 2,500)$.

on which they have been based. Much of this evidence indeed is of little value since it has been obtained by faulty or inadequate technique. Those who desire fuller information on this subject may be referred to the papers of Dobell (1911), Guilliermond (1907) and Hollande (1931), and to the reviews of Knaysi (1938) and Lewis (1941).

Certain of the earlier workers regarded bacteria as cells possessing no nuclei. This nihilist view perhaps expressed the difficulty of demonstrating bacterial nuclei by methods that depend upon the staining of chromatin. Chromatin, however, is not the essential hereditary material, and its absence does not in any case establish the absence of a nucleus. Horeover, as Lindegren (1935) points out, if the existence of some form of nuclear apparatus is denied, an explanation is required for the constancy of transmission of multiple hereditary characters in bacteria, by a mechanism different from that in most other living forms. The hypothesis of some form of nuclear apparatus has at least the prior claim on our attention. 
Riǔžička (1898, 1903, 1908, 1909) regarded the whole bacterial cell as homologous with the cell-nucleus of more highly developed organisms. There is no substantial evidence, apart from a similarity of staining reactions, for identifying the bacterial cell as nucleus, and, even if there were so, the term nucleus would he a misnomer, for, as Dobell points out, a nucleus must by definition be differentiable from the cytoplasm of the cell.

Meyer (1897, 1899, 1908) has been a prominent exponent of the view that bacteria contain well-defined nuclei and lias brought together the available evidence in his book on the bacterial cell (1912). Many other observers have shared this view (Feinberg 1900, Nakanishi 1901, Ellis 1902-03, 1922, Grimme 1902, Swellengrebel 1906, Amato 1909, Vay 1909, 1910), but it is reasonably certain that the structures observed differed in their nature, so that the observations do not confirm one another. The observations of Menel (1904, 1905, 1909, 1910) and of Rayman and Kruis (1904) strongly suggested the presence of a differentiated nucleus in the bacteria they studied. Their earlier observations have not been confirmed; according to Guilliermond (1907) the structures they describe as nuclei were stages in the formation of transverse septa. Stoughton (1929, 1932) found in a plant pathogen, Bact. malvacearum, a central nucleus-like body differentiable from the cytoplasm by intravitam staining. The constancy of its occurrence, position, and division during cell division suggested a true nucleus. Guilliermond (1933) identified the body as a volutin granule (see below). In one case, the observation by Vejdovský $(1900,1904)$ of a nucleus in an organism he named B. gammari, there is a consensus of opinion that a true nucleus has been demonstrated, but there is good reason to believe that the organism itself is a fungus, not a bacterium.

There remain several possibilities, of which we may mention three : that the nuclear material is diffusely and uniformly seattered through the cytoplasm, that it is disposed in certain chromatin masses in the cytoplasm, and that there is in the cell a separate nuclear apparatus, perhaps consisting of one or more chromosomes, not usually revealed by ordinary staining methods.

The notion of the diffuse nucleus has arisen partly to explain the absence of a demonstrable discrete nuclear body (Zettnow 1918), and partly to account for the occurrence of a uniform coloration of some bacteria when treated by Feulgen's reagent. Feulgen's reagent is used as a test for thymonucleic acid. A diffuse Feulgen reaction of bacteria has been noted by a number of observers (see, for example, Pietschmann and Rippel 1932, Imšenecki 1936). Knnaysi (1938) doubts the specificity of the reaction, and points out that if nucleic acid is demonstrated in this way, it may be merely reserve material in the cell, having no necessary connection with a nuclear apparatus (see also Schaede 1939, Mirsky 1943, Stedman and Stedman 1943). As we have seen, Lindegren (1935) objects to the conception of a diffuse nucleus on genetical grounds; it is necessary to assume the existence of some form of discrete nuclear body, which, by dividing into identical halves, can ensure the genetical constancy of the daughter cells. The view that nuclear material is segmented in certain chromatin masses in the cell has been developed by Schaudinn (1902) in the case of B. biitschlii, by Guilliermond (1907) in the case of several cultivable spore-bearing bacilli, and by Dobell (1911) in the case of bacilli parasitic in reptiles and fishes.

Schaudinn described numerous small chromatin-like granules, which were scattered through the cytoplasm of $B$. bütschlii, a large spore-bearing bacillus. At the start of sporulation, the granules gather into an axially situated spiral. The ends of the spiral inerease in size at the expense of the chromatin granules, and develop into homogeneous masses that stain deeply with nuclear stains. These masses, as they develop into spores, cease to take up the stain, and generally appear as highly refractile unstained bodies. Guilliermond (1907) found a similar pieture in the spore-bearing bacillus studied by him. Cells eight hours old were finely vacuolated, and contained numerous granules of chromatin. At sporulation, however, no axial filament was formed, and a single deeply staining mass appeared at one pole of the cell, gradually increasing in size. Dobell (1911) observed scattered chromatin that was gathered into an axial filament before sporulation 
in certain bacilli, and a permanent axial spiral in others, both of which he regarded as being nuclear in character. In certain large microeocei he observed well-differentiated and homogeneous bodies which took up nuclear stains, divided before cell division occurred, and had the essential characters of nuclei. Reviewing his own results and those of other investigators, Dobell concluded that bacteria are nucleated cells, in which the nuclear apparatus is highly variable in form, appearing sometimes as scattered granules, some. times as a spiral filament, and occasionally as a differentiated nuclens similar to that in cells of higher organisms. Lewis $(1932,1934,1940)$ doubts the reality of nuclear spiral filaments, believing rather that they represent fine strands of cytoplasm compressed by an abundance of volutin granules or fat globules.

In recent years, the evidence for the existence of a discrete nuclear apparatus in bacteria has been accumulating. Hollande (1934) (see also Hollande and Hollande 1932) differentiated three structures in a number of bacilli, which he terms nucleosome, paranucleosome and metanucleosome. The paranucleosome is eosinophilie, and the metanueleosome, which surrounds it, is basophilis. Both are closely associated with the nuclcosome, which is a minute spherical granule staining blue with eosinate of methylene blue. The nucleosome divides by elongation, constriction of the central portion into a thread, and finally separation into two daughter nucleosomes. Later workers confirm Hollande's work to the extent of recognizing bodies, usually referred to as nucleoids, that take part in cell division. Dombrowsky (1936) described two concentrations of protoplasm in Bact. coli, which appeared prior to division; the line of division oecurred between them. In young cells of another coliform bacillus Rosca (1937) found a elromatinic substance developing into a distinct "nucleus" and noted the appearances resembling mitotic figures. Stille (1937) was able to demonstrate Feulgen-reaeting bodies in a large number of bacterial species after they had undergone gentle acid hydrolysis. Young vegetative forms contained two bodies, though cells containing four bodies appeared prior to division. Similar mild hydrolysis reveals nucleoids in Bact. coli and Salm. paratyphi B (Piekarski 1937) and in Proteus vulgaris (Neumann 1941). Piekarski distinguished a primary form with two bodies, and a secondary form, usually found in older cultures, with one only. The bodies were Feulgen positive, and absorbed ultra-violet light of a wave-length known to be strongly adsorbed by thymomeleic acid (Piekarski 1938). Later it was found that all the bacteria studied contained at least one nueleoid (Piekarski 1939, 1940, Piekarski and Ruska $1939 a, b)$. In some cases the nucleoids were demonstrable in electron mierographs. It may be noted here that the electron mieroscoje has so far not revealed very much of the nuelear structure of bacteria (see, for example, von Borries et al. 1938, Lembke and Ruska 1940, Mudd et al. 1942, Knaysi and Mudd 1943). If any nuclear material is present, it must either be masked by other matter more opaque to the electrom beam, or have the same electron scattering power as other cellular constituents.

Robinow $(1942,1944)$ has extended these studies of the nuclear apparatus revealed by preliminary acid treatment of the bacteria. Robinow's results not only confirm and extend those of Stille and Piekarski, but clarify the relation of the Feulgen-positive (chromatinic) bodies to the growth of the bacteria. In old bacteria, the chromatinic bodies are small, and difficult to demonstrate; when the bacteria are transferred to a fresh nutrient medium, the bodies increase in size and stain more deeply, usually laving the form of short, dumbbell shaped rods. Longitudinal divisions of these rods precedes the division of the cell, though several divisions may take place before the evidence of cellular division is evident by ordinary methods of examination. Growing bacilli may therefore contain two or more pairs of chromatinic bodies (Fig. 5). By special staining methods these multinucleate bacilli appear to be banded, indicating the existence of multiple cell units within the single bacillus. In the case of $B$. megatherium the composite nature of the cell can also be demonstrated by a plasmolysing treatment (see p. 28 below). The possibility that single bacillary rods may in fact be multicellular must be borne in mind in the interpretation not only of morphological studies on bacteria, but also of the phenomena of heredity that they display. 
The fact that similar aeid treatment does not destroy the chromatinic structures in other types of cell, and that changes in the bodies are closely correlated with stages in the growth of the cells, makes it highly mnlikely that the dumbbell bodies are artefacts. Robinow concludes that the chromatinic bodies are comparable with the chromosomes of aninal and plant cells. It may be noted that Beebe (1941) found single central Feulgen

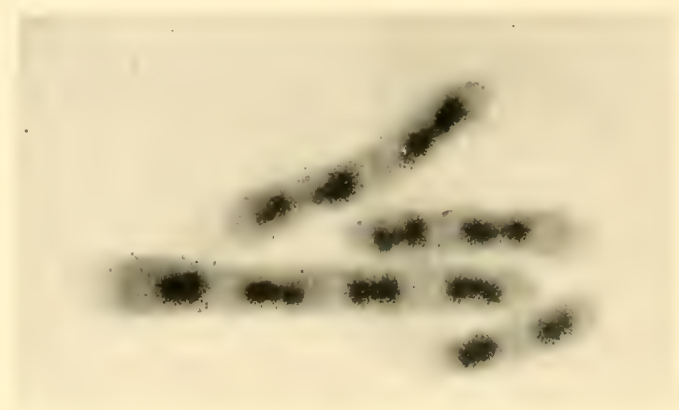

A

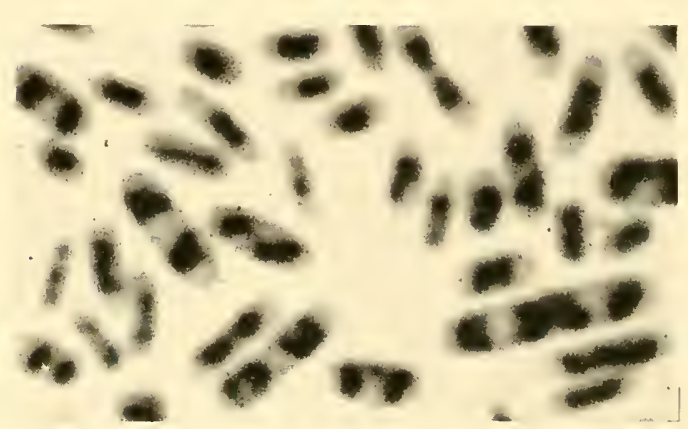

B

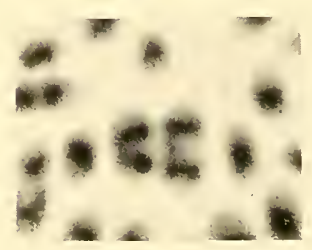

$\mathrm{C}$

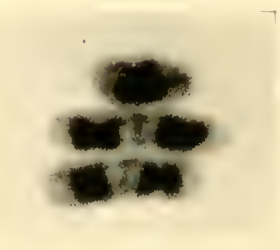

D

Fir. 5.-Chromatinic bodies of bacteria, showing the various shapes taken during growth and division.

A. B. mycoides, B. Bact. coli, C. Proteus vulgaris, D. B. mesentericus. $(\times 4,660)$. (From photographs kindly supplied by Dr. C. F. Robinow.)

positive bodies in a myxoeoceus, which divided with the cell, and which at times developed a dumbhell shape.

The eonception of the nuclear apparatus of baeteria as granules made up of one or more chromosomes has been reviewed by Lindegren (1935). Lindegren himself (1936) deseribed a diplococens whose meleus, visible only at certain stages in division, consisted of a single haploid chromosome with seven ehromomeres. Badian (1933) observed the longiturlinal division of a single rod-like chromosome in B. subtilis, and Chance (I938) 
a mitosis-like effect during the division of a bacillus resembling $B$. mesentericus. Prior to sporulation, the chromosomes of Badian's bacillus underwent division, followed by fusion end-to-end of the resulting two chromosomes, and division of the fused chromosome into four, only one of which was incorporated in the spore. A similar series of events was observed by Allen, Appleby and Wolf (1939) in a spore-bearing bacillus. The normal vegetative cell contained one haploid chromosome. Two kinds of spore were formed, one haploid, the other diploid. In the one case a phenomenon analogous with meiosis occurred before spore formation, in the other after spore germination.

A nucleoprotein granule which persisted in all stages of growth, multiplication, ageing and starvation of the eulture, and which divided before cell division, was found in a staphylococcus by Knaysi (1942). The granule did not correspond to any known cellular reserve material, and was apparently the nuclear apparatus.

Taking the evidence as a whole, it appears that bacteria have a nuclear apparatus which in many respects is analogous to that of the cells of fungi, plants and animals, though the survey of bacteria by modern cytological methods is as yet too limited to permit more than a tentative conclusion that the apparatus seen in a few bacteria will prove characteristic of bacteria in general.

\section{Other Intracellular Granules.}

Nitrogenous Material-Volutin Granules.-In many species of bacteria there are found intracellular granules which possess a strong affinity for nuclear stains, and which are coloured a reddish purple with certain blue or violet stains, especially with polychrome methylene b!ue. These granules were first described by Ernst $(1888,1889,1902)$ and by Babes $(1889,1895)$, and are frequently referred to as the Babes-Ernst granules. They have also been named volutin granules, and from their peculiar staining properties, metachromatic granules. These constituents of the bacterial cell have, from time to time, been credited with almost every conceivable function. There is now, however, very general agreement that they are relatively inert in the cell. Using a cytological term, they are metaplasmatic granules, particles of some substance concerned in cell-metabolism. Their number and size vary according to the medium in which the bacterium is grown, and according to the period of growth. That they form no part of the muclear apparatus is shown by the facts that they can be observed in yeasts and fungi, which possess well-differentiated nuclei, and that they take no active part in cell-division or sporulation. They contain a high proportion of nucleoprotein, and to this they probably owe their affinity for nuclear stains.

The rôle which they play in the chemistry of the bacterial cell is at present unknown; the suggestion of Marx and Woithe (1900), that the presence of these granules is correlated with the virulence of the bacterium, has been completely disproved by the work of several subsequent investigators (Ascoli 1901, Krompecher 1901, Gauss 1902, Ficker 1903, Guilliermond 1906). Gróh (1938) has recently assigned a reproductive rôle to the volutin granules of $C$. diphtherio. His findings still await confirmation.

Carbohydrates.--Glycogen granules and starch granules occur in many species of bacteria, and may be identified by staining with iodine.

Fats and Lipoids.-Fat globules are frequently found in bacterial cells, and may be stained by the usual fat-soluble dyes. Other lipoidal or waxy substances, which may be extracted by the usual solvents, are found in many bacteria, and are particularly abundant in certain species, such as the acid-fast bacilli.

These different forms of granular products of metabolism are by no means equally abundant in different bacterial species. In some, volutin granules tend 
to be numerous and of large size; in others, they are scanty or absent. Some species which seldom contain volutin granules frequently contain fat globules. In others the presence of glycogen or starch is a characteristic feature. The data

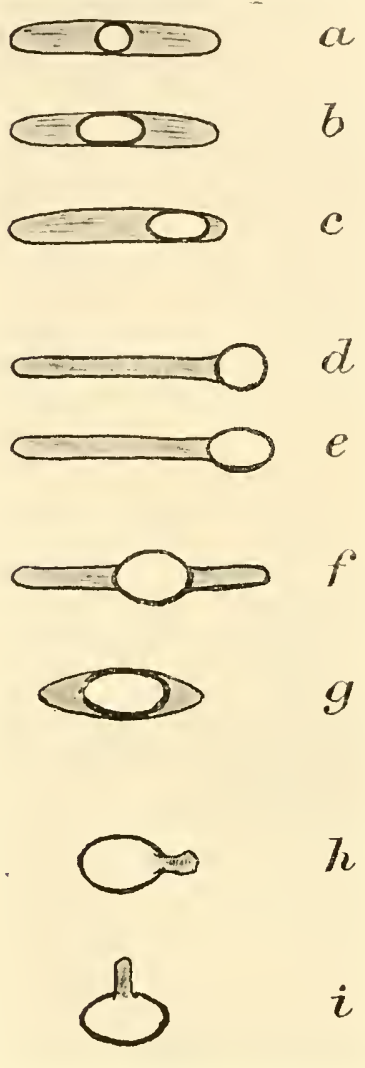

FIG. 6 .

$a-c$. Without distortion of bacterial cell.

a. Spherical equatorial.

b. Oval equatorial.

c. Oval subterminal.

$d-g$. With distortion of bacterial cell.

d. Spherical terminal.

e. Oval terminal.

f. Oval equatorial.

g. Oval equatorial.

$h$ and $i$. Germination of spores.

$h$. Polar germination.

$i$. Equatorial germination. available do not warrant us in saying that any one of these forms of granular material is the peculiar property of certain species or is uniformly absent from others, but their relative abundance or scarcity may be a striking characteristic of a particular bacterial species.

For a good and concise account of these intracellular granules see Zettnow (1918).

Endospores. - The formation of intracellular spores by bacteria was first observed by Cohn (1875), and first studied in detail by Koch (1876) in the case of $B$. anthracis. The fact that the existence of an endospore, and its significance in reproduction, had been clearly demonstrated in the case of one of the first bacteria to be identified as the cause of an important infective disease, led many observers to approach the study of new bacterial species with a bias towards identifying any morphologically differentiated element within the bacterial cell as a spore, or its equivalent. Much of the earlier literature is, for this reason, full of mistaken allusions to spores or sporogenous granules in various bacteria. We now know that the formation of endospores is an important distinguishing characteristic of certain bacterial groups.

The great majority of sporing bacteria which have been adequately studied are monosporous, that is only one spore occurs in any one cell. A few large forms which have been observed by cytologists are di-sporous.

The mode of formation of the spore has been briefly referred to in discussing the nuclear apparatus of the cell. There is general agreement that it is formed by a localized accumulation of chromatin, or of a chromatin-like substance, which at first stains deeply with nuclear stains, but which later becomes surrounded by a spore-wall or membrane, which is impervious to stains in the absence of special preliminary treatment; so that the ripe spore appears as an unstained, highly refractile body, spheroidal or ovoid in shape. As regards the finer details of spore-formation there is less agreement, and it would seem possible that the process differs to some extent in different bacterial species. Many observers have described the appearance, at the commencement of sporulation, of a clear vacuolar area, corresponding in size and shape to the space occupied by the ripe spore, and the subsequent 
accumulation, within this area, of the nuclear material of which the spore is formed. Meyer and his supporters lay great stress on the appearances seen during sporulation, as evidence for the existence of differentiated nuclei. Those who have studied the internal structure of bacterial cells have, for the most part, included the process of spore-formation in their observations, and the papers which have already been referred to contain full discussions of this question.

The situation of the spore within the bacterial cell, which may be terminal, subterminal, or approximately equatorial, gives a distinctive morphology to many bacterial species. In addition, the diameter of the ripe spore is often greater than that of the bacillus which contains it; so that the cell is distorted and, according to the position of the spore, "drum-stick," "barrel," or other irregular forms may be presented (Fig. 6).

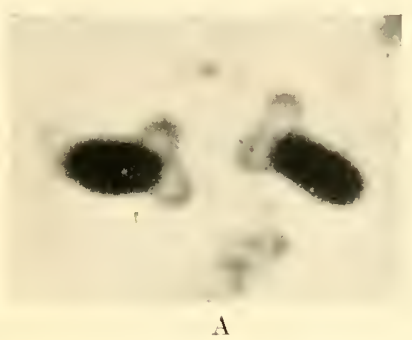

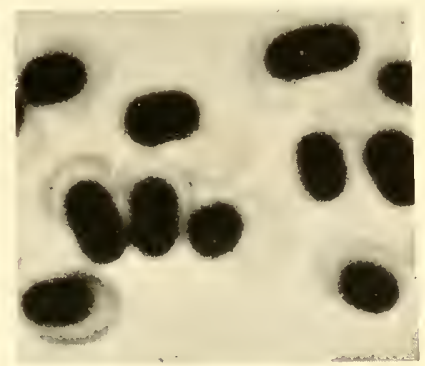

B

Fig. 7.-Germination of spores by equatorial rupture.

A. An unidentified soil bacillus. B. B. mesentericus $(\times 4,660)$.

(From photographs kintly supplied by Dr. C. F. Robinow.)

The germination of a spore, when placed under favourable conditions, may occur by simple enlargement of the spore without obvious rupture or shedding of the spore-envelope, though this is an unusual method. Far more frequently the spore-envelope ruptures, either at one pole or equatorially, and the spore as it develops into a bacillus sheds its enveloping membrane (Figs. 6 and 7).

By careful staining of mildly hydrolysed preparations of spores, Rolinow (1942) was able to distinguish three structures taking part in the germination of spores of $B$. mycoides and other members of the Bacillus group. In the resting spore there was a delicate membrane, containing an apparently structureless rod of cytoplasm and a small, sometimes dumbbell-shaped, chromatinic body attached to, but distinct from, the rod (Fig. 8). With germination, the chromatinic bodies are transformed into ring-shaped bodies, which enter the eytoplasmic rod, and merge with the eytoplasm. At a later stage of germination the chromatinic: bodies reappear and differentiate into the dumblell forms characteristic of the regetative form. Robinow (personal communication) also distinguishes a layer of refractile

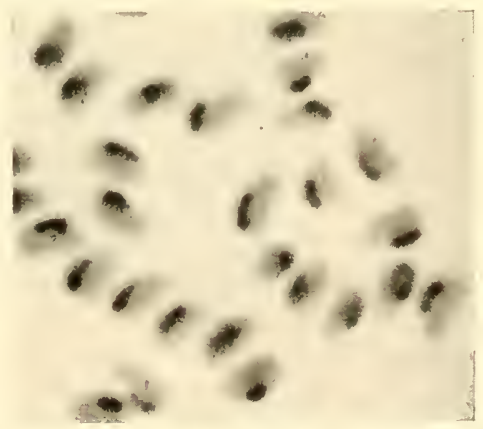

FIG. 8.-Resting spores of $B$. mycoides $(x+6,60)$.

(From a pliotograph kindly supplied by Dr. C. I. Robinow.) 
cytoplasm between the central cytoplasmic core and the spore membrane, and considers that this, rather than the spore membrane itself, is responsible for the refractility commonly seen in unstained preparations of spores.

Most of the available evidence indicates that the spore is simply a resting stage of the bacterial cell, in which it is far more resistant to adverse environmental conditions than it is in the ordinary vegetative form. The spores of bacteria of the Bacillus group have a bound water content of the order of $60-70$ per cent., as compared with 3-21 per cent. in the vegetative cell (Henry and Friedman 1937, Frierman and Henry 1938). By spectrochemical analysis Curran, Brunstetter and Myers (1943) found that the spores contained substantially more Ca, more $\mathrm{Cu}$ and $\mathrm{In}$, but less $\mathrm{P}$ and $\mathrm{K}$ than the vegetative cell. They suggest that heat resistance is associated with, though not necessarily immerliately dependent on, high bound water and ('a content of the spore. The protoplasm of the spore differs materially from that of the vegetable cell; both Howie and Cruickshank (1910) and Lamanna (1940) have demonstrated the antigenic dissimilarity of spores and their parent vegetative forms (see Chapter 8).

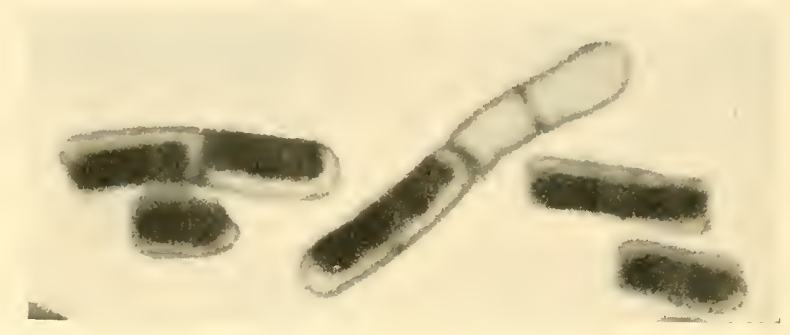

FIG. 9.-The cell wall of $B$. megatherium after plasmolysis, showing transverse septa, both complete and incomplete $(\times 3,500)$.

(From a photograph kindly supplied by Dr. C. F. Robinow.)

The resting spore retains its capacity to germinate for long periods. GrahamSmith (1941), for example, noted the survival for over seventeen years of the dry spores of $B$. anthracis kept in diffuse daylight at room temperature, while Wilson and Shipp (1938) were able to grow sporing bacilli from preserved meats that had been sealed in containers for 114 years.

There is little evidence that spore-formation is associated with any sexual reproductive process. Certain appearances which have been interpreted in this sense by Schaudimn (1902), and Rủžička (1909), have been shown by Dobell (1911) to have no such significance.

However, from their careful studies of the nuclear structure of a spore-bearing bacillus (see above) Allen. Applehy and Wolf (1939) concluded that the spore was not a resting stage, but provided an opportunity for a re-arrangement of nuclear material. In this connection it is noteworthy that Kaplan and Williams (1941), after exploring the cultural conditions which induced spore formation in $\mathrm{Cl}$. sporogenes, concluded that spore-formation was a natural phenomenon in the developmental eycle of the organism. Klieneberger (personal communication) has observed in several species of clostridia a process in which the dumbbell chromatinic bodies present in filamentous forms of the bacteria join into axial structures; these then divide into four chromatinic bodies, one of which matures into the nuclear body of the spore, while the remaining thrce disintegrate. The process suggests a form of autogamy, and that the spore is more than a resting stage in the life history of the bacillus. 
The Cell Wall and the Cytoplasmic Membrane.-There seems to be general agreement that bacterial cells may be differentiated with varying degrees of distinctness, into two zones, which have been variously called cell-membrane and protoplasm, ectoplasm and endoplasm, and even cortex and medulla. Until a

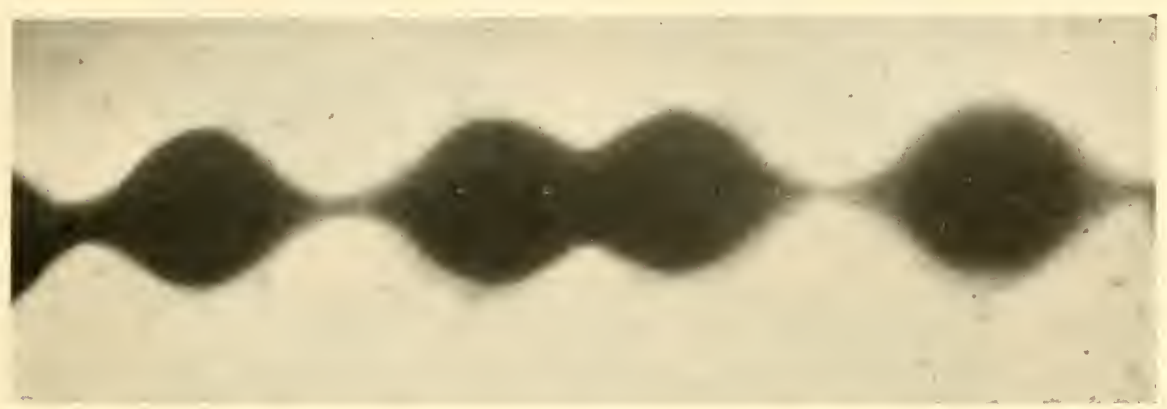

$\mathbf{A}$

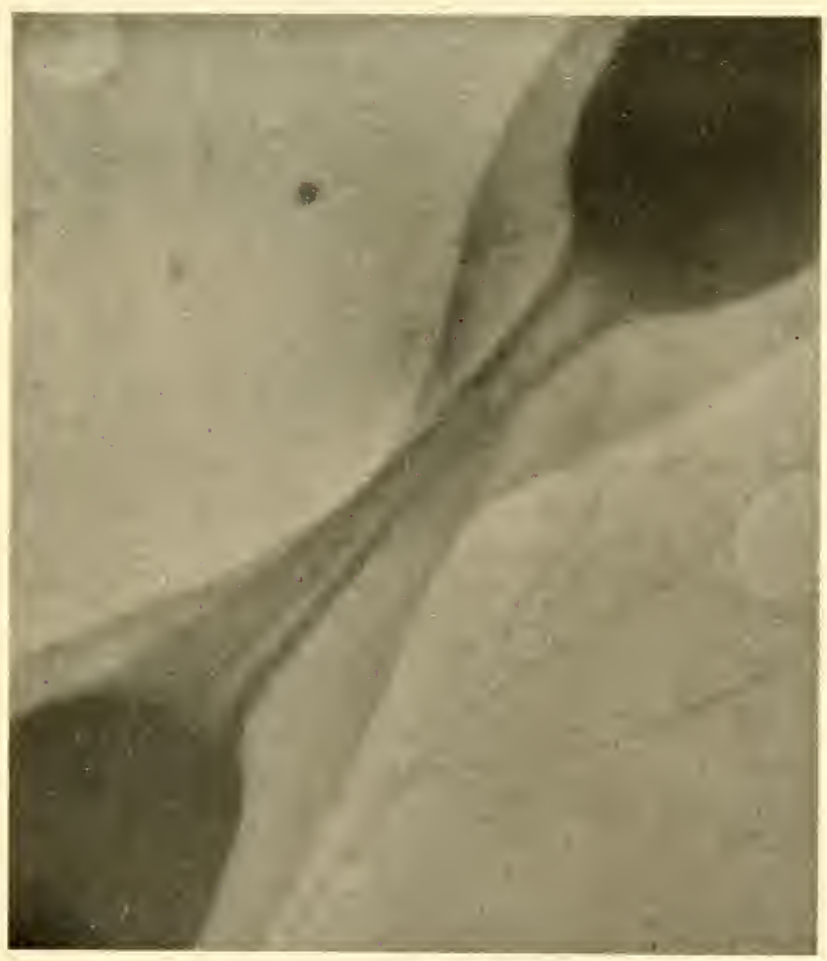

B

Fig. 10.-Electron Mierographs of Bacteria.

A. Str. pyogenes, showing the contimity of the cell wall along the length of the chain $(\times 39,000)$. (Mudd and Lackman, 1941.)

B. B. cereus, at the junction of two cells, joined by an inner thread of cytoplasm and an outer, continuous cell wall $(\times 34,000)$. (Johnson, 1944.)

(From micrographs kindly supplied by Dr. Stuart Mudd and Dr. F. H. Johnson.) 


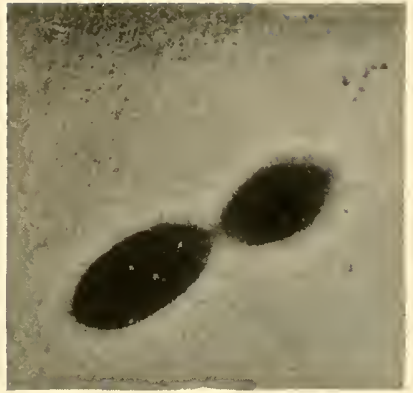

A

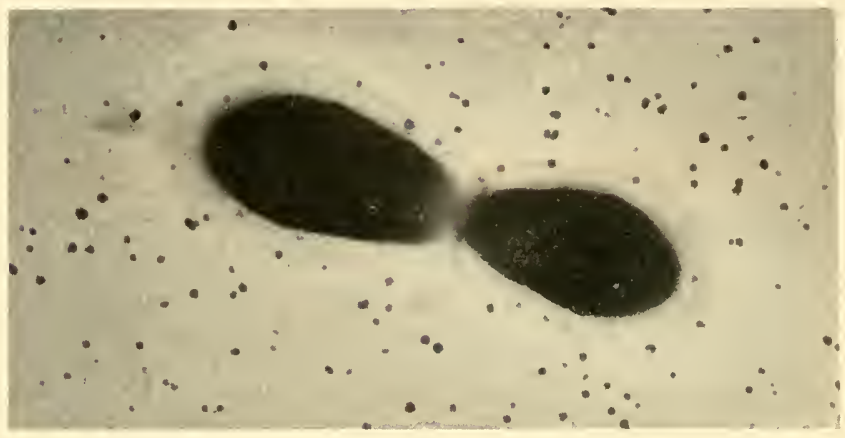

C

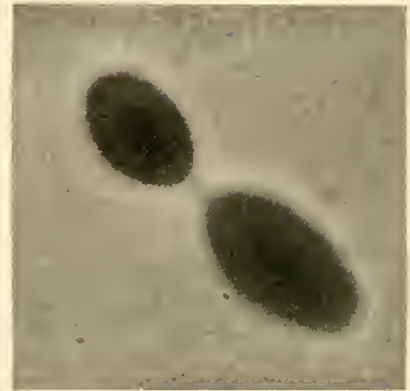

$\mathrm{B}$

Fra. 11.-Electron Micrographs of Pneumococci $(\times 11,000)$.

A and B showing the capsule as a delicate light halo, and continuity of protoplasm

in between the two cocci in A, and of cell wall in B. (Mudd et al., 1943a.)

C showing the capsule as a demarcated halo. (Mudd et al., 1943b.)

(From micrographs kindly supplied by Dr. Stuart Mudd.)

few years ago there was some doubt whether a definite external membrane limited the eetoplasm (Legroux 1925, Legroux and Margrou 1920). The microdissection of bacteria performed by Wámoscher (1930) suggested a definite elastic outer layer, distinct from the imner material of the bacterium.

Fischer (1894) studied the effect of hypertonic salt solutions on bacteria. The cell contents were observed to shrink, leaving what appeared to be a rigid cell wall of the same shape as the bacterial cell before treatment. These plasmolytic experiments have since been repeated by many observers (see, for example, Knaysi 1930), but their significance as evidence for a definite limiting membrane in the bacterial ectoplasm has been obscured by the possible artificial nature of the structures produced. The differenees of opinion (Legroux 1925, Legroux and Margrou 1920) are to a large extent resolved by results of recent demonstrations, hy varions independent techniques, of a definite cell wall.

The changes in the cell wall during the division of bacilli have been studied in detail by Knaysi (1941). Prior to the division of an elongated mother cell into two daughter cells, he observed first a break in the cytoplasmic membrane, and a centripetal movement of the cytoplasm. At the line of demarcation between the cytoplasmic masses of the two dangliter cells, a double intercellular plate was deposited, which formed the two adjacent end walls of the completer daughter cell. In a yeast, the deposition of the two walls preceled the eytoplasmic division. 
According to Knaysi, the frequently reported constriction of the cell wall prior to division is an optical illusion, depending on a constriction of the cytoplasm. The double cell wall is well shown in the electron micrograph of $B$. anthracis made by Mudd and others (1941).

Robinow (1944) also obtained evidence that the transverse divisions of the cytoplasm of dividing bacillary cells are laid down before the bacilli divide in the accepted sense of the term. In some of his preparations (Fig. 9) the transverse walls between daughter cells appear to be growing inwards as annular diaphragms along the plane of the cytoplasmic cell boundary (personal communication).

Electron micrographs show a cell wall that retains its form even after the cells have been fractured, and their contents dispersed, by ultrasonic vibration (Mudd et al. 1941). A rigid cell wall extending along the length of chains of cocci, and connecting adjacent cells in chains of bacilli, was observed by similar means (Figs. 10A, B ; 11B) (Mudd and Lackman 1941; see also Frühbrodt and Ruska 1940, Mudd et al. 1942, Johnson et al. 1943).

The surface of the inner protoplasm takes up stain with greater avidity than the cell wall or the inner protoplasm itself. 'This, according to Knaysi (1938), is the cytoplasmic membrane, made up chiefly of surface active materials, lipoids and lipoproteins, which must be clearly distinguished from the rigid semipermeable cell wall.

Capsules.--Many species of bacteria, such as the pneumococcus, the pneumobacillus and the anthrax bacillus, are characterized by the ability to develop a well-marked capsule, which may be observable in stained or unstained preparations as a clear zone surrounding the bacterial cell or may, after suitable fixing and mordanting, be stained by various methods. The degree to which the capsule is developed is largely determined by the environmental conditions. Thus, the pathogenic capsulated bacteria show their maximal capsule formation when growing in the animal tissues (Babes 1895, Gruber and Futaki 1907, Preisz 1907, Bail 1908, Sauerbeck $1909 a, b$, Eisenberg 1903, 1908, 1909). When such bacteria are grown in artificial culture there is usually a high correlation between the degree of capsulation and the content of the medium in unaltered, or slightly altered, animal protein.

It is possible that the response to a medium containing animal protein is a reaction to an unfavourable environment. For instance, the pnemmobacillus forms capsules in artificial culture when the phase of maximum proliferation has ceased (Hoogerheide 1938). On the other hand, the capsules of a strain of Str. pyogenes developed only during its active growth phase in a serum-enriched medium (Morison 1941).

The capsule is usually regarded as being formed by a thickening and alteration in consistency of the outer layer of the bacterial cell. It was, however, held (Meyer 1912, Zettnow 1918) that the capsular substance was an active secretion. Certain bacteria secrete a mucilaginous material in which large numbers of neighbouring cells are embedded; and some organisms, such as the Type III pneumococcus, form this extracellular mucilaginous material in addition to possessing a well-marked capsule (Fig. 11). It seems, however, more convenient to differentiate between the capsulated and the so-called "mucoid" types of bacteria (see Etinger-Tulczynska 1933), and the fact that capsular material may frequently become separated from the cell can hardly be taken as a proof that it is a secretion in the ordinary sense of that term. But the sharp delimitation of the opaque, 
rigid cell-membrane from the space presumably occupied by the capsule in electron micrographs, and the low density to the electron beam of the capsules themselves (Frühbrodt and Ruska 1940, Mudd, Heinmets and Anderson 1943), together with the known delimitation of the cytoplasmic membrane inside the cell wall, suggest that the hypothesis of a "secreted" capsule is the more likely to be valid. We may note that we have, within recent years, acquired a considerable knowledge of the chemical constitution of bacterial capsules. The capsules of the different types of pneumococei are for instance composed of complex polysaccharides (see p. 279), while the capsule of the anthrax bacillus appears to be composed of a polypeptide material (see p. 281).

Whether the capacity to form a capsule is really confined to those species

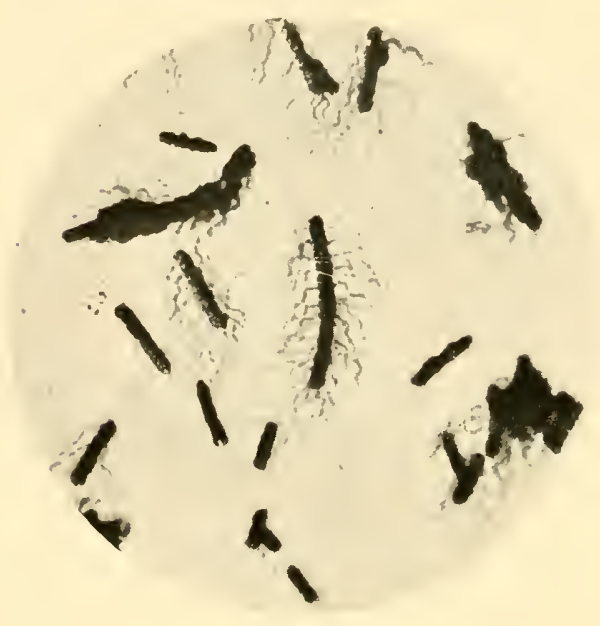

FIG. 12.-Zopfius zenkeri.

Stained to show flagella $(\times 1: 1200)$. that are typically capsulated is very doubtful. Nany observers have described capsule-formation by normally non-capsulated forms under particular environmental conditions ; and mucoid variants of normally nonmucoid species are of relatively common occurrence (see Chapter 9). The absence of a detectable capsule does not exclutle the existence of thin capsules distinct from the cell wall. For example, in a coceobacillus as small as $\mathrm{Br}$. melitensis, the lipopolysaccharide material comprising 10 per cent. of the whole bacillus, if confined exclusively to an external covering, would form a layer less than $20 \mathrm{~m} \mu$ thick (Miles and Pirie 1939).

Flagella.-A large number of bacteria, including a few coccal forms, many bacilli, and most known spirilla and vibrios, are more or less actively motile by means of flagella. These flagella are long, thread-like processes, arranged in various ways on the bacterial cell. They vary greatly in length, but are often longer than the organism to which they are attached. They are extremely slender (Fig. 14A); recent measurements of the flagella of Proteus vulgaris and Salm. paratyphi B under the electron microscope indicate a thickness of 20 to $50 \mathrm{~m} \mu$, at any rate of the electron-opaque part of the structure (Piekarski and Ruska 1939). Flagella were first effectively demonstrated by Loeffler (1890).

According to the observations of Trenkmann (1890), Ellis (1902-03), Fuhrmann (1910) and Meyer (1912), the central portion of the flagellar thread passes through the cell-membrane and is in direct connection with the cytoplasm, and Fuhrmann believes that the proximal extremity is connected with a granule of chromatin, which would then be analogous to the blepharoplast of protozoan flagellates. This conclusion is adversely criticized by Zettnow (1918). Whether the optical methods at present available allow any decision on such points as these seems doubtful.

The arrangement of the flagella on the bacterial cell may take one of four forms. 
There may be a single flagellum at one pole, when the arrangement is called monotrichate. 'There may be a single flagellum at each pole, the amphitrichate condition. There may be a bunch of flagella at one pole, or more rarely at each, an arrangement known as lophotrichate. The flagella may be arranged indiscriminately over the bacterial cell, when they are referred to as peritrichate (see Fig. 12). The cells of any one flagellated species are characterized by one, and one only, of these possible arrangements (see Fig. 13).

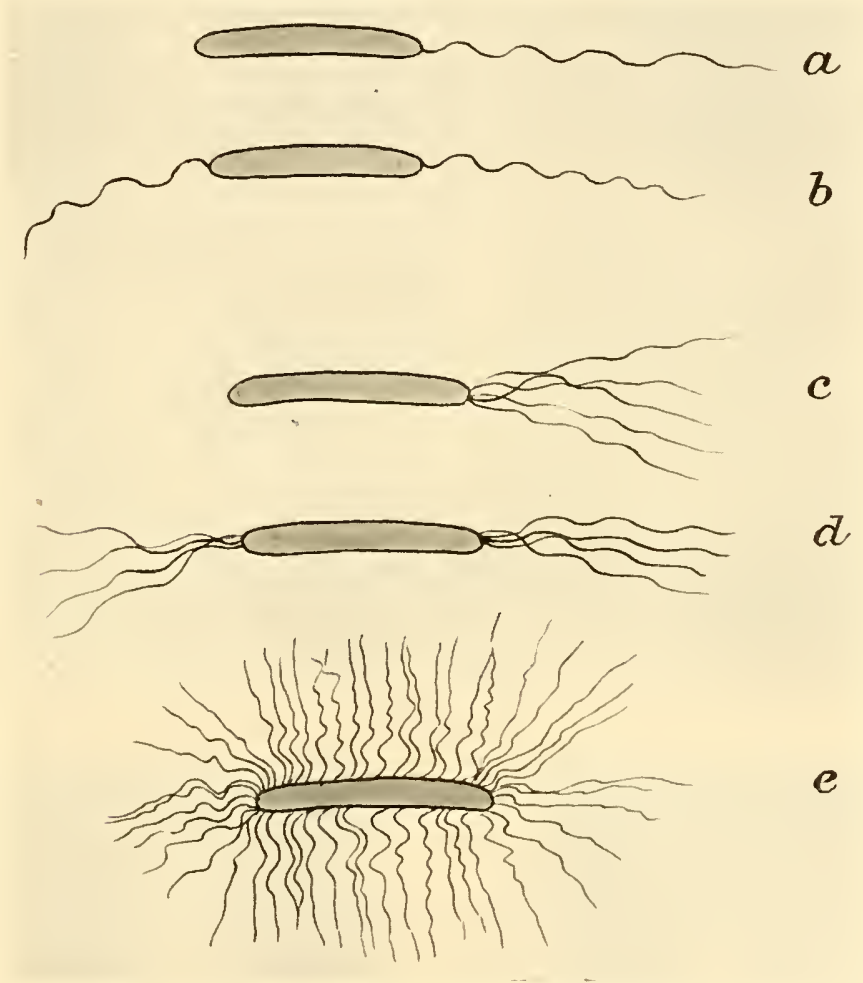

Fig. 13.-Diagram illustrating distribution of flagella.
a. Monotrichate.
b. Amphitrichate.
$c$ and $d$. Lophotrichate.
e. Peritrichate.

Peritrichous flagellation is regarded by Pietschmann (1939) as due to the occurrence of subpolar flagella on a chain of undivided cells. We have ourselves seen this phenomenon in the incompletely divided filamentous forms of monotrichate organisms, but some exceedingly short cells have so many peritrichous flagella that it would be absurd to postulate their multicellular nature merely to avoid the assumption of peritrichous flagellation.

Recently Wei (1936) and Pijper (1938) have described the flagellation of the typhoid bacillus as consisting of two conical spiral structures set up at an angle at the side of the body (Fig. 14c). Pijper found that the two structures were fused into one propelling "tail" organism in fast-moving bacilli ( $\mathrm{B}$ in the figure). He regards the two configurations of flagella as primary and secondary structures. 
In dead bacilli the primary structures disperse into fine threads which he regards as post-mortem artefacts, not as peritrichous flagella which in life form a functional unit. It is, however, legitinate to regard the peritrichous flagellum as the primary structure, for there are analogous cases of functional unity of structures that are

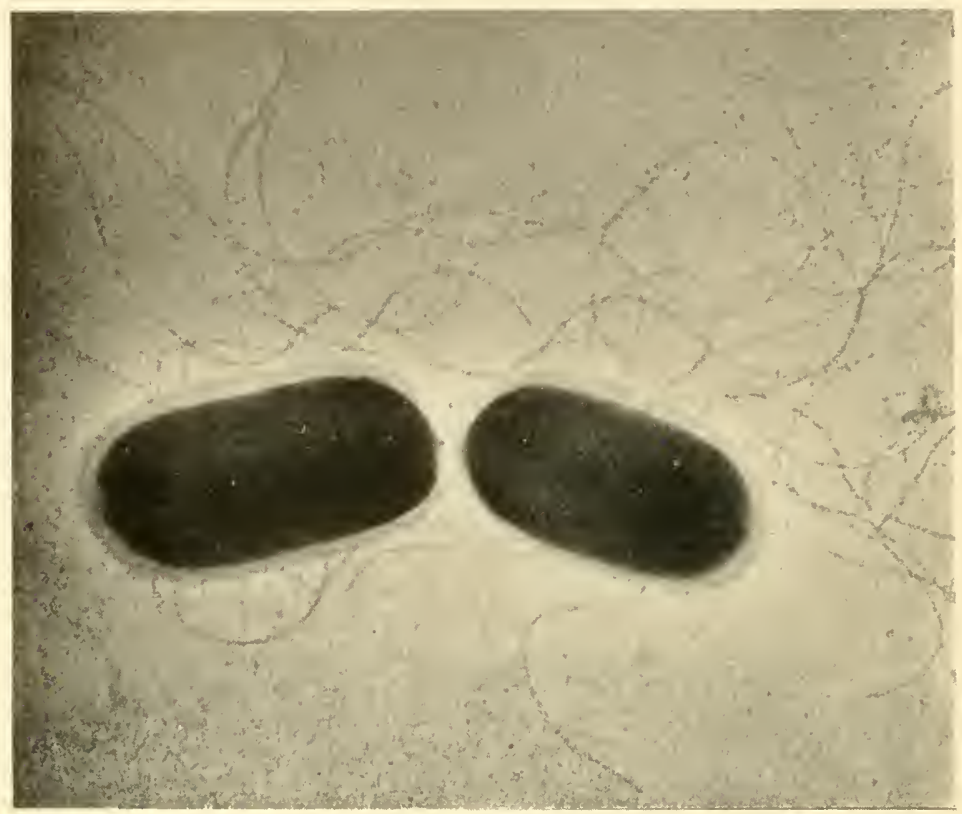

A

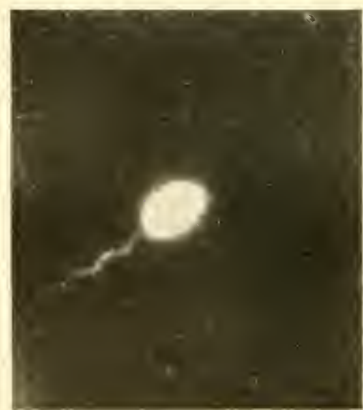

B

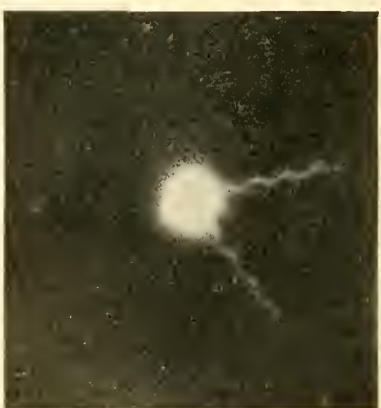

C

Fis, 14.-Bacterial Flagella.

A. Electron micrograph of $C$. tetani, showing protoplasm, cell wall and flagella $(\times 22,000)$. 13. and C. Dark-ground photomicrographs of living Salm. typhi ( $\times 5000)$.

(From photographs kindly supplied by Dr. Stuart Mudd and Dr. A. Pijper.)

made up of morphologically separate flagella. The undulating membrane of certain ciliate infusoria, for instance, consists of a fine row of cilia which can cluring life be separated by mechanical means into single cilia all moving in an uncoordinated mamner (Maier 1903, Chambers and Dawson 1925). 
The nature of flagella, and the modes of generating and transmitting to them the energy required for their propulsive effect, is largely unknown. Under fixed cultural conditions, the speed of a flagellated bacterium is considerable, and to a certain extent characteristic of the strain observed. The highest speeds are recorded for the cholera vibrio, a monotrichate organism, which may travel more than $80 \mu$ per second (see Sanarelli 1919, Ogiuti 1936).

Though flagella are usually confined to vibrios and bacilli, several observers have described motile cocci (Schieblich 1932, Koblmïller 1935, Pownall 1935, Levenson 1938). Under the electron microscope, flagella-like structures have been seen in spirochetes of the genus Treponema (Morton and Anderson 1942).

The results that have been obtained in the detailed study of antigenic structure (see Chapter 8) have shown that the flagellar substance, or at least that portion of it which forms the flagellar surface, is chemically distinct from the substances forming the cell body.

Involution Forms.-In old cultures of many bacteria forms may be found which are quite unlike those seen in young and actively growing cultures. Some of these forms are very typical of the bacterial species in which they occur, as is the case with the so-called involution forms of the plague bacillus, and of some other species of Pasteurella, and the giant forms which occur in cultures of the meningococcus. 'The existence of these and other abnormal forms has long been recognized, and they have been regarded as due to involution or degeneration following the ageing of the culture, and the consequent unfavourable environmental conditions. This view as to their nature has been generally adopted because of the undoubted fact that, under the conditions in which they usually occur, the majority of the organisms are non-viable; while the extensive study of young actively growing cultures has, in general, shown no more striking departure from the normal form than the occurrence of unusually large cells, or occasionally in the rod forms, of very long, almost filamentous cells, due apparently to delay in cell division.

Reproduction.-Although a description of the method of reproduction in bacteria may be regarded as belonging more correctly to a discussion of their physiology than of their morphology, yet the question is so closely bound up with differences in form of the cells, and of the cell-aggregates which result from successive division, that there are many advantages in considering the question in the present chapter.

There is no doubt at all that the method of multiplication of bacteria, under optimal conditions of cultivation in the laboratory, is by simple binary fission, each cell dividing into two daughter cells by constriction, with or without a wellmarked preliminary septation.

In the spheroidal forms division may occur in any diameter. In the ovoid or cylindrical forms division always occurs in the transverse diameter. It is never longitudinal.

In certain gencra of bacteria, sucb as the Actinomyces, the formation of branching filaments is a normal phase of growth and multiplication. In a fow bacillary species, and notably in C.diphtherice and IIyco.tuberculosis, rudimentary branching has frequently been described, and its occurrence in actively growing cells observed under the microscope (Hill 1902). In most bacillary species such rudimentary branching is rare, and is seldom observed in the examination of ordinary stained preparations. Hort $(1917 a, b)$, and Gardner (1925) have, however, noted the occurrence of $Y$ forms in actively growing micro-

P.B. 
scopic cultures of organisms of the coli-typhoid group, and have observed multiplication by elongation and fission from all three points of the $Y$. (See also Wyckoff 1934.) Whether the occurrence of these forms has any special significance is a question that the future must determine.

Spore-formation, which has been described above, is confined to certain species of bacteria. The spore constitutes a special resting and resistant phase of the bacterial cell ; its formation appears to be determined in large part by environmental factors, and there is little ground for regarding it as an integral part of any periodic or essential life-cycle (but see p. 26).

A question still at issue is the occurrence of other forms of reproduction, and in particular of the existence or non-existence of a complex life-cycle, in which sexual processes may or may not play a part. It is not possible to discuss this problem at all fully. It must, however, be noted that there are those who believe that such a life-cycle is a natural characteristic of all bacterial species, and that multiplication by binary fission, which appears to be the only mode of reproduction in young, actively growing cultures, is only one phase of the cycle, which we encourage by the particular artificial conditions under which we choose to study bacterial cells.

Jones $(1913,1914,1920)$ described reproduction by gonidia-like bodies in the case of Azotobacter. These observations were greatly extended by Löhnis and Smith (1916) who described a far more complex life-cycle in this, and in other, species, and applied their results to the reproduction of bacteria in general (Löhnis 1921). Stoughton (1929, 1932) in describing the morphology and cytology of Bact. malcacearum, an organism producing angular leaf-spot in the cotton plant, records the formation, by budding from the bacillary form, of coccus-like bodies that subsequently develop into bacilli, and also the fusion of bacillary cells with the formation of zygospores.

Almquist described various forms of reproductive bodies, mainly minute gonidial structures, in a wide range of bacteria (Almquist 1893-1924, Almquist and Koraen 1918, Koraen 1918). It may be noted that there was a general tendency for these appearances to be observed in old cultures, and sometimes in cultures which had been allowed to undergo a marked degree of drying. However, Allen, Appleby and Wolf (1939) observed the relatively early liberation of intracellular granules from growing vegetative cells of their bacillus. A little later, minute rods were found in the culture, which appeared to have originated in the granule, and which later developed into the modal form of the bacillus. Mcllon (1917-26) has described a variety of forms which he regards as stages in the complex life-cycle of various bacterial species. Enderlein (1925) upholds a similar thesis. Kuhn $(1929,1930)$ has described a remarkable series of morphological forms in common organisms, some of which are interpreted as evidence of a life-cycle, others of whichthe so-called Pettenkofer bodies-are regarded as intracellular parasites. Hadley (1927; see also Hadley, Delves and Klimek 1931) in a detailed review, which should be consulted by those who desire further infurmation on this subject, sets out the available data with regard to the occurrence and possible significance of these various forms, and seeks to relate them to the phenomenon of bacterial dissociation, which we shall discuss in a subsequent chapter. A shorter discussion of the possible modes of bacterial reproduction will be found in papers by Bergstrand (1920, 1921, 1923).

It is, we think, impossible on the evidence at present available to come to any conclusion on the general problem at issue. That a cycle of morphological changes may characterize the life-history of a particular bacterial species under natural conditions there can be no doubt. The detailed studies of Bewley and Hutchinson (1920) and of Thornton and Gangulee (1926) on Rhizobium leguminosarum provide a case in point, though their conclusions have been questioned by Lewis (1938). 
In regard to bacteria in general, there is clearly no justification for ignoring such observations as those recorded above; but the thesis, that the bacterial forms with which we are most familiar in artificial cultures are only stages in some more complex cycle of development, has not in our view been clearly and definitely established. All bacteriologists are familiar with the so-called "involution forms" to which we have already referred; and there is little doubt that many of these forms represent dead or dying cells. It may well be that we have been too ready to. refer to this category every abnormal form encountered; and few would deny that some of the appearances that are met with are strongly suggestive of some more complex form than the simple bacillus. The problem is, however, beset with technical difficulties arising from the small size of the organisms concerned. The crucial test must remain the actual observation of the reproduction of typical bacterial cells from the granules, spheres, or syncytial masses which have been described as stages in the complete life-cycle; and the unequivocal demonstration of this cyclical development demands the isolation of single cells, not only at the commencement of the cycle, but at each successive stage. No harm will be done by maintaining a severely critical attitude at the present stage of the controversy, so long as each new piece of evidence is considered on its merits.

In this connection, the work of Klieneberger and of Dienes may be noted. Klieneberger (1935) describes a minute pleuropneumonia-like organism in symbiosis with Streptobacillus moniliformis, and considers that many of the morphological peculiarities that are commonily regarded as characteristic of the bacillus are, in reality, due to the presence of the symbiont. She suggests that some, at least, of the pleomorphic elements in bacterial cultures that have been described as belonging to a sexual or developmental cycle may have a similar origin. This view has been developed in subsequent publications (see Chapter 40). Dienes (1942), on the other hand, believes that the pleuropneumonia-jike organisms are part of a be cycle not only in the streptobacillus, but in other bacterial species. He has succeeded in inducing their appearance in cultures of Bact. coli, Hamophilus influenza, Flavobacterium, the gonococcus and $F$. funduliformis (see also Klieneberger 1942). Dienes (1943) and Smith (1942, 1943, 1944), have observed in Streptobacillus moniliformis and $F$. funduliformis the formation from the bacterial cell of "large bodies" which may either segment and give rise to bacterial forms, or develop into colonies of pleuropneumonia-like organisms; and state that the large bodies appeared in some cases to arise from the conjugation of two bacterial cells.

We do not propose to enter into the vexed controversy over the existence of filtrable forms of bacteria. The whole crux of the question seems to us to lie in the definition of the term "filtrable," and in the properties of the filter-passing bodies. Bacteria of the same species are known to vary greatly in size under different environmental conditions and at different stages of growth. That certain forms may be small enough to pass through our relatively crude earthenware filters is hardly to be doubted, but whether these forms differ in any of their essential properties from larger members of the same species, and in particular whether they constitute some essential stage in the reproductive cycle of the organism, is still undecided. In our opinion the evidence that has so far been produced does not suggest that they are differentiated in any important respect other than size, and probably this differentiation is of a continuous rather than a discontinuous type.

The Formation of Cell-Aggregates.-When a bacterial cell divides, the two daughter cells may at once part company, or they may remain attached to one another by their cell-membranes. When this incomplete separation persists during many successive cell-divisions, cell-aggregates are produced, the form of which will depend upon the planes in which successive divisions take place, and upon the number of cell-divisions which occur before the cell-aggregate begins to 
separate. The aggregates which are formed in this way are often highly characteristic, and constitute an important factor in the identification of bacterial species. In the spheroidal forms division may occur in any plane, while in the cylindrical forms it always occurs in the transverse diameter. As a result the cocci may, and do, show a greater variety of groupings than the bacillary or spiral forms.

When the successive divisions, in a coccal bacterium, occur in such a way that the daughter cells remain united in pairs for a short period, but these pairs separate before a further division occurs, the organism in question is described as a diplococcus. When several successive divisions occur before separation of the resulting aggregate, and these divisions follow no ordered sequence as regards the planes in which they take place, irregular groups of cocci result, which have been compared to bunches of grapes. Organisms which form this type of cell-aggregate are known as staphylococei, and this term is used as a generic name. When such a series of divisions without separation occurs in planes parallel to one another, the aggregates so formed have the appearance of a chain or chaplet, and organisms which behave in this way are known as streptococci, and are usually classed in a single genus. In some species the cells remain attached while two divisions occur, the second at right angles to the first. The resulting aggregate is a group of four cocci; such an organism is sometimes referred to as a tetracoccus. In some species the typical cell grouping is produced by three divisions, the plane of each being at right angles to the other two. In this way cubes, or packets, each of eight cocci, are produced. The species which form groupings of this type are classed in the genus Sarcina.

Among the cylindrical forms, the only possible departure from the single-cell formation is the adherence of two or more cells in pairs or chains. Such groupings may be referred to as diplobacilli or streptobacilli; but they are not sufficiently constant in a particular bacterial species to be used for purposes of classification. They occur, however, far more often in some species than in others, and their relative frequency may be an important specific character.

Apart from the formation of such united cell-aggregates, the way in which a cell divides influences the grouping of the daughter cells. In certain bacillary forms, such as the diphtheria bacillus, division appears to occur asymmetrically, in the sense that the daughter cells remain attached at one side of the cylinder, after division has procceded across the whole width of the organism from the opposite side. In the early stages of the subsequent growth of the daughter cells, this local attachment seems to act as a hinge, about which the clongating cells swing, so that they come to lie at varying angles to one another, depending on the period which elapses before division becomes complete. The groupings so formed, which have been compared to Chinese letters, or to cuneiform characters, are very characteristic of certain species.

Colony Formation.-When bacteria are grown on solid media, and care is taken to avoid too heavy an inoculation, the individual cells multiply and form isolated colonies. The appearance of these colonies is, in many cases, highly characteristic of the group or species to which a given bactcrium belongs. We know little of the internal structure of bacterial colonies, but that little suggests that the structural differentiation is considerable (Legroux and Margrou 1920, Ravich-Birger and Svinkina 1937), and it is possible that much light may be thrown on bacterial morphology by further study along these lines. 
Two colonial characteristics may be noted here. Certain motile bacilli swarm in continuous films of growth on the surface of solid media, a property which is displayed characteristically by members of the Proteus and Clostridium groups (see Chapters 27 and 36). Other motile bacilli, notably among aerobic and anaerobic sporing bacilli, form rounded colonies in which the constituent bacilli are moving in a clockwise or anticlockwise direction, imparting a rotating motion to the whole colony. As a result of the rotation, the colony wanders over the surface of the solid medium, usually leaving a track in the form of a double line of bacilli, apparently thrown off from the sides of the advancing colony (see Muto 1904, Roberts 1935, Smith and Clark 1938, Russ-Münzer 1938, Shinn 1938, Turner and Eales 1941, Fuller and Norman 1943).

The character of the colonies produced by a given bacterial species is of great importance in identification, and is, for this purpose, best considered in conjunction with other growth-characters, such as the appearances noted in cultures in liquid media, or in the so-called stab-cultures, in which inoculation is made by thrusting a platinum wire axially into a column of solid medium, such as agar or gelatiu. Further description of colony structure is therefore deferred to Chapter 13.

Staining Reactions.--The staining reactions of bacteria, and particularly their response to certain differential stains, might well be regarded as more in the nature of microchemical tests, than methods of demonstrating structure. It would, therefore, be more logical to consider staining reactions in connection with the physiology of the cell than with its morphology, but the way in which a given bacterium reacts to special stains constitutes such an important part of the general picture which we form of it, that it is convenient to deal with this matter in the present chapter.

Reactions to General Bacterial Stains.-Quite apart from the presence of the metachromatic granules, or of other granular material, different bacterial cells may differ in the way in which they take up stains. In some cases the whole cell may be uniformly coloured. In some cylindrical forms the ends of the bacillus may be deeply stained, while the central portion may be almost colourless. This constitutes the so-called polar staining, which is very characteristic of the plague bacillus, and is found in many other bacterial species. Sometimes the cell may stain unevenly throughout its length, so that barred or beaded forms may occur, a feature which is very characteristic of the diphtheria bacillus and many allied organisms, and which occurs to a less marked extent with many acid-fast bacilli. In some cases, in which barred or beaded forms are found, these conditions are associated with the presence of metachromatic granules, but these granules are not the essential cause of such irregularities in staining. It seems clear that, in such forms, there is an unequal distribution throughout the cell of those constituents of the cytoplasm which take up the stain employed, and it has frequently been suggested that they are indicative of plasmolytic changes. It seems doubtful whether plasmolysis can be invoked as a general explanation of this phenomenon, though it is true that there is a general tendency for the frequency of grauular and barred forms to increase with the age of the culture, and to be notably scanty in very young cultures. They are, however, so plentiful in young and actively growing cultures of certain species that it seems impossible to regard them as resulting entirely from degenerative changes.

The Differential Staining of Bacterial Species.-In the application of numerous stains, mordants, and differential decolorizing reagents to the study of bacteria, 
the empirical discovery has been made that certain staining reactions are highly characteristic of certain groups of bacteria. Two such staining reactions are especially important in this respect.

The Gram Stain.-Gram (1884) descriked a staining method which has been of tle greatest service in differentiating bacterial groups, and which has been extensively studied, and frequently modified, by subsequent workers. This reaction depends on the fact that, when certain bacteria are stained with certain aniline dyes, such as gentian-violet, methylviolet and others, and are subsequently treated with a solution of iodine in potassium iodide, a mordanting action occurs which prevents the subsequent decolorization of the bacteria on treatment with alcohol. Other bacteria, after similar treatment, are readily decolorized. This difference between the retention of the stain by Gram-positive bacteria and its loss by Gram-negative forms is correlated with certain other characters. Thus (Kruse 1910) Gram-negative organisms are more susceptible to solution in alkalies, or to digestion by enzymes, than are Gram-positive organisms; they are also more susceptible to lysis by an immune serum in the presence of complement. According to Stearn and Stearn (1930), they are more resistant to the lethal action of oxidizing, and perhaps of reducing, agents. Brudny (1908) and Eisenberg (1909) would ascribe the difference between Gram-positiveness and Gram-negativeness to a difference in the permeability of the cell-membrane; while Sander (1935), who has been able to render Gram-positive bacteria Gram-negative by treatment with a variety of reagents and to demonstrate that this change is reversible, regards the determining factor as the state of dispersion of the bacterial protoplasm. Some observers, on the other hand, and notably Deussen (1918), incline towards a more purely chemical theory, and Stearn and Stearn (1928) regard differences in intracellular pH as the determining factor.

Recently Henry and Stacey (1943), by extraction with bile salt, succeeded in removing from Gram-positive organisms a substance they identify as magnesium ribonucleate, and in thereby rendering the organisms Gram-negative. If the organisms are protected from oxidation, the ribonucleate will recombine and the Gram-staining property will be restored. Gram-negative organisms do not yield the salt and do not combine with it to become Gram-positive. The Gram-positive material appears to be a high molecular conıplex of a reduced basic protein and magnesium ribonucleate. It was noted too that removal of the ribonucleate facilitated observation of stained nuclear bodies, a fact which supports Knaysi's contention that the Gram-staining material is concentrated at the cytoplasmic membrane. The difference between Gram-positive and Gram-negative organisms is particularly evident in their susceptibility to various groups of antibacterial agents, both inorganic and organic (see Chapter 6), and it is probable that investigation of these biochemical differences will lead to a more precise elncidation of a staining reaction first introduced on an entirely empirical basis.

In employing Gram's method it must always be remembered that the differentiation is not absolutely sharp and specific as regards a given bacterium at all stages of its growth, or as regards all bacterial species. Those organisms which are completely Gram-negative never retain the stain, but those organisms which are Gram-positive frequently fail to retain the stain when preparations are made from old cultures. This is indeed easy to understand, since such cultures consist largely of dead, dying or degenerate cells, whose physical and chemical properties must be greatly altered. Certain bacterial species show reactions to this method of staining which are of an intermediate type, with the result that they are extremely sensitive to small changes in technique, sometimes appearing to be Gram-positive and at others Gram-negative. Due allowance must be made for all these points in determining the reaction of any given species.

Acid-fastness.-The fact that certain bacteria, after being stained with warm solutions of fuchsin, resist the decolorizing action of strong mineral acids was observed by Ehrlich (1882) and confirmed by Ziegl $(1882,1883)$ who modified the technique of staining. This reaction is highly characteristic of the tubercle bacillus, and of an allied group of organisms 
which are collectively known as the acid-fast bacilli. It is of interest to note that these bacilli are peculiarly resistant to the action of such solvents as strong solutions of alkalies, or mixtures of alkalies and sodium hypochlorite (antiformin), as also to the action of digestive ferments. In this connection it may be noted that acid-fast bacilli are also Gram-positive, though the majority of Gram-positive organisms are not acid-fast. The substance which confers this property of acid-fastness on the tubercle bacillus and allied forms has been studied by Klebs (I896), Koch (1897), Bulloch and Macleod (1904) and Tamura (1913). It was at first regarded as an unsaturated fat, but is now generally believed to be lipoidal or waxy in nature. Bulloch and Macleod state that it has the chemical properties of an alcohol, and Tamura isolated an alcohol which had the property of acidfastness, and which he named "mykol." (For further details see Chapter 16.)

\section{REFERENCES}

Allen, L. A., Applebi, J. C. and Wolf, J. (1939) Zbl. Bali., IIte Abt., 100, 3.

Almquist, A. and Koraen, G. (1918) Z. Hyg. InfektKr., 85, 347.

ArmQuist, E. (1893) Z. Hyg. InfehtKr., 15, 283; (1904) Zbl. Bakt., 3\%, 18 ; (1905) Z. IIyg. Infekthr., 52, 179; (1907) Zbl. Bakt., 45, 49I ; (1911) Ibid., 60, 167 ; (1917) Z. IIyg. InfektKr., 83, 1 ; (1922) J. infect. Dis., 31, 483; (1924a) Ibid., 35, 341 ; (1924b) Z. Hyg. InfektKr., 101, 15 .

Avato, A. (1909) Zbl. Bakl., 48, 385.

Ascoli. (190I) Dtsch. med. Wschr., 20, 313.

BABES, V. (1889) Z. Hyg. InfektKr., 5, 173; (1895) Ibid., 20, 412.

Badian, J. (1933) Arch. Mikrobiol., 4, 409.

BAIL, O. (1908) Zbl. Bakt., 46, 488.

BARNARD, J. E. (1919) J. R. micr. Soc., p. I ; (1925) Lancet, ii. 117 ; (1930) "A System of Bacteriology," Med. Res. Council London, 1, 115.

BEEBE, J. II. (1941) J. Bact., 42, 193.

Bergstrand, H. (1920) J. infect. Dis., 2\%, I ; (1921) Johns Hopk. Hosp. Bull., 32, 234; (1923) J. Bact., 8, 365.

Bewley, W. F. and Hutchinson, M. B. (I920) J.agric. Sci., 10, 144.

Borries, B. von, Ruska, E. and Ruska, H. (1938) Klin. Wschr., 17, 921.

BRUdNy, V. (1908) Zbl. Bakt., IIte Abt., 21, 62.

Bulloch, W. and Macleod, J. J. R. (1904) J.Hyg., Camb., 4, 1.

Chambers, R. and Dawsox, J. A. (I925) Biol. Bull., 48, 240.

Chance, H. L. (1938) J. Bact., 35, 347.

Conn, F. (1875) Beitr. Biol. Pflanz., 1, 2.

Currain, H. li., Brunstetter, B. C. and Mrers, A. T. (1943) I. Bact., 45, 485.

Deusses, E. (19I8) Z. Hyg. InfektKr., 85, 235.

Dienes, L. (1942) J. Bact., 44, 37 ; (1943) Proc. Soc. exp. Biol., N.Y., 53, 84.

Dienes, L. and Surth, W. E. (1942) Proc. Soc. exp. Biol., N.Y., 51, 297; (I!43) Ibid.. 53, $195 ;(1944)$ J. Bact., 48, 125.

DoBell, C. (1911) Quart. J. micr. Sci., 56, 395.

Dombrowsky, K. H. (1936) Zbl. Bakt., 13\%, 160.

Ehrlich, P. (I882) Disch. med. H schr., 8, 269.

Eisenberf, P. (1903) Zbl. Bakt., 34, 739; (I908) Ibid., 45, 44, 134, 638; (1909) Ibid., $49,465$.

Ellis, D. (1902-03) Zbl. Bakt., 33, 1, 81, 161 ; (1922) Brit. med. J., ii. 731 .

Enderlein. (I925) "Bakterien-Cyclogenie, etc." Berlin.

Ernst, P. (1888) Z. Hyg. InfektKr., 4, 25 ; (I889) Ibid., 5, 428 ; (1902) Zbl. Bakt., IIte Abt., $8,1,34,65,97$.

Etinger-Tulczinska, R. (1933) Z. Hyg. Infektir., 114, 769.

Feinberg. (1900) Zbl. Batit., 27, 417.

FickER, M. (1903) Arch. Hyg., 46, 171.

Fischer, A. (I894) "Untersuchungen über Bakterien." Berlin.

Friedmax, C. A. and Hexry, B. S. (I938) J. Bact., 36, 99.

FRÜHBRoDT, E. and Reska, H. (1940) Arch. Jikrobiol., 11, 137.

Fumbuan, F. (I910) Zbl. Bakt., IIte Abt., 25, 129.

Fuller, W. H. and Norman, A. G. (1943) J. Bact., 46. 273.

Gardner, A. D. (1925) J. Path. Bact., 2\%, 189.

Gadss, C. J. (1902) Zbl. Bakt., 31, 92.

Grallah-Surtil, G. S. (1941) J. Hyg., Camb; 41, 196.

GraM, C. (I884) Fortschr. II cd., 2, 185.

Grimue, A. (1902) Zbl. Bakt., 32, 1, 81, I61, 241, 321. 
GRoн, E. (1938) Zbl. Bakt., 141, 220.

Gruber, M. and FutaKi, K. (1907) Münch. med. Wrshr., 54, 249.

Gulllerwond, A. (1906) Bull. Inst. Pasteur, 4, 145; (1907) Ibid., 5, 273, 321 ; (1933) C. R. Soc. Biol., 113, 1095 .

HaDLEY, P. (1927) J. infcet. Dis., 40, 1.

Hadley, P., Delves, E. and Klinek, G. (1931) J. infect. Dis., 48, 1.

Henry, B. S. and Friedmax, C. A. (1937) J. Buct., 33, 323.

Hexry, H. and STACEY, M. (1943) Nature, Lond.. 151, 671.

Hill, H. IV. (1902) J. med. Res., \%, 115, 202.

Hollanie, A. C. (1934) Arch. Protistenk., 83, 465.

Hollande, A. C. and Hollaxde, G. (1932) Arch. Zool. exp. gén., 72, No. 6.

Hoogerieide, J. C. (1939) J. Bact., 38, 367.

Hort, E. C. (1917a) Brit. med. J., i. 571; (1917b) Ibid., ii. 377.

Howie, J. W. and Cruicksfink, J. (1940) J. Path. Buct., 50, 235.

Mišenecki. A. (1936) Zbl. Baht., IIte Abt., 94, 330.

Johnson, F. H. (1944) J. Bact., 4\%, 551 .

Johison, F. H.. Kworyin, N. and WARrex, G. (1943) J. Bact., 46, 167.

Jonzs, D. H. (1913) Zbl. Bakt., IIte Abt., 38, 14; (1914) Ibid., 40, 170; (1920) J. Bact., 5,325 .

Kiflan, I. and Williams, J. W. (1941) J. Bact., 42, 265.

KLEBS, E. (1896) Zbl. Bakt., 20, 188.

Klieneberger, E. (1935) J. Path. Bact., 40, 93 ; (1942) J. Hyq., Camb., 42, 485.

Kxaysi, G. (1930) J. Bact., 19, 113 ; (1938) Botan.Rev., 4, 83; (1941) J. Bact., 41, 141; (1942) Ibid., 43, 365 .

KNAYsi, G. and IludD, S. (1943) J. Bact. 45, 349.

Kосн, R. (1876) Cohns Beitr. Biol. Pflanz., 2, 277; (1897) Dtsch. mcd. Irschr., 23, 209.

KioblyïLler, L. O. (1935) Zbl. Baht., 133, 310.

Koraen, G. (1918) Z. Hyg. InfehtKr., 85, 359.

Krompecher, E. (1901) Zbl. Baht., 30, 385.

Kruse, W. (1910) Hünch. med. W schr., 5\%, 685.

КонN, P. (1929) Med. Klin., 25, 1351 ; (1930) Ibid., 26, 739.

Lamaña, C. (1940) J. infect. Dis., 6\%, 193, 205.

Legroux, R. (1925) Ann. Inst. Pasteur, 39, 382.

Legroux, R. and Margrou, J. (1920) Ann. Inst. Pasteur, 34, 417.

Lenibe, A. and ReskA, H. (1940) Klin. H'schr., 19, 217.

Levensox, S. (1938) Ann. Inst. Pasteur, 60, 99.

LEwIS, I. M. (1932) J. Bact., 24, 381 ; (1931) Ibid., 28, 133 ; (1938) Ibid., 35, 573 ; (1940) Ibicl., 40, 271 ; (1941) Bact. Rev., 5, 181.

Lindegrex, C. C. (1935) Zbl. Bakt., Ilte Abt., 92, 40 ; (1936) Ibid., 93, 389.

LOEFFLER, F. (1890) Zbl. Bakt., \%, 625.

LöHvis, F. (1921) Mem. nat. Acad. Sci., 16, 5.

Löнуis, F. and Surth, N. R. (1916) J. agric. Res., 6, 675.

MaIer, H. N. (1903) Arch. Protistenk., 2, 73.

Marton, L. (1934) Bull. Acad. Belg., Cl. Sci.. 20, 439 ; (1941) J. Bact., 41, 397.

Marx, H. and Woithe, F. (1900) Zbl. Balit., 28, 1.

Mellon, R. R. (1917) J. Bact., 2, 81, 269; (1920) J. med. Res., 42, 61 ; (1921) Ibid., 42, 111 ; (1925a) J. Bact., 10, 481; (1925b) Ibid., 10, 579 ; (1926) Ibid., 12, 409.

IIence, E. (1904) Zul. Balut., IIte Abt., 12, 559; (1905) Ibid., 15, 544; (1909) Arch. Protistenk., 16, 62; (1910) Ibid., 19, 127.

Merlixg-Eisexierg, K. B. (1937) J. Quekett Hicr. Cl., 1, 311, 324.

MEYER, A. (1897) Flora, 84, 185; (1899) Ibid., 86, 428; (1908) Ibid., 98, 335; (1912) "Die Zelle der Bakterien." Jena.

Miles, A. A. and Pirie, N. IV. (1939) Brit. J. exp. Path., 20, 278.

MIrsiry, A. E. (1943) Advances in. Enzymology, 3, 1.

Morison, J. E. (1941) J. Path. Bact., 53, 1.

Mortox, H. E. and Axdersox, T. F. (1942) Amer. J. Syph., 26, 565.

MUdD, S. AND Axdersox, T. F. (1942) J.exp. Med., 76, 103; (1941) J. Amer. med. $A s s ., 126,561$.

Mudo, S., Heinuets, F. and Avdersox, T. F. (1943a) J. Bact., 46, 205; (1943b) J.exp. Med., $78,327$.

MUdD, S. and Lackmax, D. B. (1941) J. Bact., 41, 415.

Mudd, S., Polevitzky, K. and Andersox, T. F. (1942) Arch. Path., 34, 199.

Mud, s., Polevitzky, K.. Axdersox, T. F. and ChanBers, 1. A. (1941) J. Bact., 42, 251,

Hото, T. (1904) Zbl. Balt., 3\%, 321.

Nakanishi, K. (1901) Zbl. Bakt., 30, 97, 145, 193, 225.

Nevmans, F. (1911) Zbl. Balit., IIte Abt,, 103, 385.

OGiuti, K. (1936) Jap. J. exp. Hed., 14, 19. 
Piekarski, G. (1937) Arch. Mikrobiol., 8, 428; (1938) Zbl. Bakt., 142, 69; (1939) Ibid., 144, 140; (1940) Arch. Vikrobiol., 11, 406.

Piekarski, G. and Ruska, H. (1939a) Arch. Wikrobiol., 10, 302; (1939b) Klin. Wschr., $18,383$.

Pietschunax, K. (1939) Arch. Hikrobiol., 10, 133.

Pietschmaxn, K. and Rippel, A. (1932) Arch. Wikrobiol., 3, 422.

Pijper, A. (1938) J. Path. Bact., 4\%, 1.

Pownall. M. (1935) Brit. J.exp. Path., 16, 155.

Preisz, H. (1907) Zbl. Baht., 44, 209.

Ravich-Birger, H. D. and Siriskina, A. A. (1937) G. Batt. Immunol.. 18, 170.

RaYMaN, B. and KRUIS, K. (1901) (see Guilliermond, 1907).

Roberts, J. L. (1935) J. Bact., 29, 229.

Robinow, C. F. (1942) Proc. Roy. Soc., B., 130, 299; (1944) J. Hyg., Cumb., 43, 413.

Rosca, T. (1937) Areh. roum. Palh. exp. Ticrobiol., 10, 267.

Ruski, E. (1931) Z. Physik., 87, 580.

Russ-llïxzer, A. (1938) Zbl. Bakt., 142, 175.

RŮžıčKa, V. (1895) Zbl. Bakt., 23, 305; (1903) Arch. IIyg., 46, 337; (1908) Ibid., 64, 219; (1909) Zbl. Bakt., IIte Abt., 23, 259.

Saxarelli, G. (1919) Ann. Inst. Pusteur, 33, 569.

SaNder, F. (1935) Zll. Bakt., 133, 385.

Saukrbeck, E. (1909a) Zul. Bakt., 50, 2s9; (1909b) Z. IIyg. Infekthr., 63, 313.

Schaede, R. (1939) Arch. Mikrobiol., 10, 473.

Schaudinx, F. (1902) Arch. Protistenk., 1, 306.

Schieblich, M. (1932) Zbl. Balt., 124, 269.

ShInN, L. E. (1938) J. Bact., 36, 419.

Syith, N. l?. and Clark, F, 1\%. (1938) J. Bact., 35, 59.

Stears, A. E. and Stears, E. W. (1928) Univ. Mo. Stud., 3, No. 2, 1.

Stears, E. W'. and Stearn, A. E. (1930) J. infect. Dis., 46, 500.

Stediax. E. and Stedmax, E. (1943) Nuture, Lond., 152, 267.

STILLE, B. (1937) Arch. Mikrobiol., 8, 125.

Stodgiton, P. H. (1929) Piroc. roy. Soc., B, 105, 469 ; (1932) Ibid., 111, 46.

Swellexgrebel, N. H. (1906) Zbl. Bakt., IIte Abt., 16, 617, 673.

TAMURA, S. (1913) Z. physiol. Chem., 8\%, 85.

Thorston, H. G. and Gaxaulee, N. (1926) Proc. roy. Soc., B, 99, 427.

Trenkians. (1890) Zul. Balt., 8, 385.

Turner, A. W. and Eales. C. E. (1941) Aust. J. exp. Biol. med. Sci., 19, 167.

VAY, F. (1909) Zbl. Balt., 52, 305; (1910) Ibid., 55, 193.

Yejdovskŕ, F. (1900) Zbl. Balt., IIte Abt., 6, 577; (1904) Ibid., 11, 481.

WÁHoscher, L. (1930) Z. IIyg. InfektKr., 111, 422.

WEI, H. (1936) Chin. med. J., Suppl. I. 135.

Wilsox, G. S. and Shipp, H. L. (1938) Chem. Industr., ry, 834.

Wyскоғғ, R. W. G. (1934) J. exp. Med., 59, 381.

Zetrnow. (1918) Z. IIyg. Infelthr., 85, 17.

ZienL, F. (1882) Dtsch. med. Wschr., 8, 451 ; (1883) Ibid., 9, 247. 


\section{CHAPTER 3}

\section{THE BIOLOGICAL CHARACTERISTICS OF BACTERIA METABOLISM}

\section{The Chemical Constitution of Bacterial Cells.}

It would seem that bacterial cells are formed on the same general chemical pattern as the cells of other living organisms, with certain characteristics that ally them closely to the fungi. The determination of the exact chemical composition of any given bacterial species or strain is rendered peculiarly difficult by variations induced by differences in the nutrient media in which they are grown. (See Cramer 1891-97, Nicolle and Alilaire 1909, Dawson 1919, Fulmer et al. 1921, Hunter 1923, Buchanan and Fulmer 1928-30, Eckstein and Soule 1931.) Apart from the variability induced by environmental factors, any bacterial species or strain may give rise to variants that differ sharply from the parental type in certain of their metabolic activities. When due allowance is made for these disturbing factors, the technical problem remains a difficult one. The collection of an adequate mass of bacterial cells for detailed chemical analysis makes large demands on time and apparatus; and the use of the chemically complex media that are necessary to secure abundant growth of certain bacterial species greatly increases the difficulty of interpreting the analytical results, particularly in regard to any constituents that are present in small amount. It is not therefore surprising that our knowledge is as yet fragmentary.

The difference in chemical constitution between different bacterial genera or species are, as would be expected, wider than between different strains or variants belonging to a single species. In this section we may confine our attention in the main to those chemical constituents that are shared by bacterial cells in general, noting in passing certain divergencies that serve to illustrate the kind of differences in chemical structure that have been observed.

The Water Content of the Bacterial Cell.-In common with all living cells bacteria contain a high proportion of water. Estimations of the water content of different bacteria carried out by different observers have varied widely and figures as high as 90 per cent. have sometimes been recorded; but those given by Nicolle and Alilaire (1909) range, with few exceptions, from 73 per cent. (Bact. coli) to 80 per cent. (Proteus vulgaris).

The Ash Content of Bacteria.-The figures recorded for the ash content of bacteria vary very widely. Buchanan and Fulmer (1928-30) quote figures ranging from $2 \cdot 0$ to 13.94 per cent. of dry weight, omitting one widely discrepant figure. It seems probable that the ash content is particularly liable to be affected by the medium on which the bacterium is grown. Thus Fulmer and his colleagues (1921) record a reduction in the ash content of a yeast from 6.3 per cent. to 3.0 per cent. as the result of growing it in a medium free from magnesium and calcium salts. 
There is general agreement that a high proportion of the total ash consists of phosphoric acid, 10-45 per cent. or more reckoned as $\mathrm{P}_{2} \mathrm{O}_{5}$ among most bacterial species, 40-70 per cent. or more among the acid-fast forms (see Tamura 1913b, Buchanan and Fulmer 1928-30). Among the mineral constituents that have been identified are $\mathrm{Ca}, \mathrm{Mg}, \mathrm{Fe}, \mathrm{Na}, \mathrm{K}$ and the $\mathrm{Cl}$ and $\mathrm{SO}_{4}$ ions.

The Protein and other Nitrogenous Constituents of Bacteria.-The recorded figures for total nitrogen are widely discrepant. Buchanan and Fulmer quote figures varying betwcen 1.8 and 15 per cent. of dry bacterial substance; and the percentages recorded by different observers for the same bacterial species show little agreement. The usual range would appear to be from 8 to 15 per cent. Nicolle and Alilaire's figures vary only between 8.28 and 10.79 per cent., but recent determinations carried out by Linton, Mitra and Shrivastava (1934) on the cholera vibrio give values of $12 \cdot 17-15 \cdot 57$ per cent.

In regard to the nature and amount of the coagulable proteins of bacterial cells our knowledge is curiously scanty. Boivin and Mesrobeanu (1934), who record a total N figure of 13.70 per cent. dry weight for Bact. coli, find that only 0.65 per cent. of this nitrogen, reckoned on the same basis, is soluble in trichloracetic acid, the remaining 13.05 per cent being precipitated. Their figures for other organisms vary over a considerable range, but in every case the nitrogen precipitated by trichloracetic acid forms more than 80 per cent. of the total. It should be noted that acids will precipitate most forms of macromolecular nitrogen from solutions containing precipitable proteins. The separation of proteins from such mixtures has seldom been attempted, but Linton, Mitra and Shrivastava (1934) record in the case of $\mathrm{I}$. cholerce that the coagulable protein is almost all in the form of globulin, i.e. is precipitated by half-saturation with ammonium sulphate. As regards the non-coagulable nitrogen, Boivin and Mesrobeanu calculate that about a quarter is present as polypeptides or amino-acids, and about another quarter as ammonium compounds. The percentages falling in these categories vary over a considerable range.

We have more information in regard to the units of which bacterial protein is built up; and these seem, in general, to be the same as those that constitute proteins of other living cells. Numerous observers have carried out estimations by the methods of van Slyke, and the results show fair general agreement, though indicating significant differences in the proteins of different species. Thus, to take a few illustrative examples, in acidfast bacilli, Tamura (1913b) has reported that 63.62-66.74 per cent. of the total nitrogen is present as mono-amino-acid-nitrogen, 13.71-15.2I per cent. as basic amino-acid-nitrogen, while Johnson and Coghill (1925) record 47.39-52.10 per cent. and $11 \cdot 35-14 \cdot 43$ per cent. for the tubercle bacillus. For the diphtheria bacillus Tamura (1914) gives corresponding figures of 54.62 per cent. and 16.89 per cent., Hirsch (1931) gives 44.80-47.41 per cent. and 16.67-17.67 per cent. For Bact. coli Eckstein and Soule (193I) give 42.90-45.71 per cent. and 16.45-19.82 per cent., and for $V$. cholerce, Linton, Mitra and Shrivastava (1934) give 54.84-57.11 per cent. and 24.08-26.03 per cent. These figures, it may be noted, refer to the total nitrogen present in the mono-amino and basic amino-acids, not to the nitrogen present in the amino form. Among the amino-acids that have been identified in bacterial proteins, arginine, histidine, lysine and tyrosine appear to be almost always present ; leucine and tryptophan have both been frequently demonstrated. The figures for cystine vary ; some observers have failed to demonstrate its presence, others have found it in relatively small amounts. There seems little doubt that the relative proportions of different amino-acids in different bacterial proteins vary significantly. Thus Tamura $(1913 a)$ records the presence of relatively large amounts of $l$-phenylalanine in the proteins of an acid-fast bacillus; while (1914) he failed to identify this acid in the proteins of the diphtheria bacillus, which contained an unusually large amount of tyrosine. The capsule of the anthrax bacillus contains a large amount of a polypeptide which on hydro. lysis yields $d(-)$-glutamic acid in a state of almost clremical purity (Ivánovies and Bruckner 
$1937 a, b)$. The presence of various amino-acids in detectable amounts, and their quantitative relationship, might be largely influenced by the media employed for growth. The cultural conditions, for example, which lear to a diminution in the capsule formation of the anthrax bacillus would markedly diminish its content of $d(-)$-glutamic acid, and with certain strains, growth in an atmosphere containing 20 per cent. $\mathrm{CO}_{2}$ markedly increases capsule formation (Iránovics 1937). But Tamura (1913b) records closely similar figures for the mono-amino-nitrogen and basic amino-nitrogen in cultures of an acid-fast bacillus grown in nutrient broth and on a synthetic medium. In any case the findings recorded above give a very incomplete picture. Detailed and comprehensive analyses have, for the most part, still to be made.

There is one further point in connection with the nitrogenous constituents of bacterial cells, on which all observers are agreed-their high content in nucleo-proteins. (See Nishimura 1893, Galeotti 1898, Aronson 1900, Stoklasa 1908, Tamura 1913a, Schaffer ot al. 1922, Buchanan and Fulmer 1928-30, Boivin and Mesrobeanu 1934, Sevag, Smolens and Lackman 1940, Stokinger, Ackerman and Carpenter 1944.) The presence of nucleins had been suspected by many of the earlier bacteriologists because of the affinity of bacterial cells for nuclear stains; and the work of the observers quoted above, and of others, has confirmed this conclusion by direct chemical analysis. Such substances as guanine. xanthine, hypoxanthine and adenine have frequently been demonstrated, and the high content of phosphorus has been noted above. Boivin and Mesrobeanu (1934) and Mesroheanu (1936) report that the purine nitrogen of undried cells varied from 0.18 to 0.29 per cent. among six species examined, being about 10 per cent. of the total bacterial nitrogen. Of this purine nitrogen 73-94 per cent. was in the form of nucleic acid, the rest being made up of nucleotides, nucleosides and free purine bases.

Both ribonucleic acid (the "yeast" type nucleic acid) and desoxyribonucleic acid (the "thymus" type of nucleic acid) have been isolated from bacteria. Coghill (193I) isolated ribonucleic acid from Myco. phlei, Heidelberger and Kendall (1931) from streptococei, and Thompson and Dubos (1938) from pneumococci, of which it constituted 2-5 per cent. of the dry weight. Nucleic acid of the desoxyribose type occurs in Bact. coli (Schaffer et al. 1922) and Myco. tuberculosis (Johnson and Brown 1922). Sevag, Smolens and Lackman (1940) found 20 per cent. of the dry weight of Str. pyogenes to be nucleic acid, and identified 10-30 per cent. of it as of the desoxyribose type (see also p. 306). The gonococcus also contains about 20 per cent. of nucleic acid (Stokinger et al. 1944).

Carbohydrate Constituents of Bacterial Cells.-Buchanan and Fulmer quote total carbon figures ranging from a little below to a little above 50 per cent. dry weight for various bacterial species, with one widely discrepant finding. The significance of this figure in relation to carbohydrate content is dubious. The actual carbohydrate content is probably a great deal lower, and the content of polysaccharides is certainly lower. In their analysis of the gonococcus, Stokinger, Ackerman and Carpenter (1914) found 5-9 per cent. of the dry weight was carbohydrate. We have ourselves observed crude polysaccharide yields of between 5 and 20 per cent. from over thirty different strains of Proteus vulgaris.

There is very little evidence that bacteria share with the majority of vegetable cells the capacity of forming a cellulose envelope, or that cellulose enters in any way into their composition (see Buchanan and Fulmer 1928-30.) They do, however, form a variety of polysaccharide gums; and the studies of recent years have shown quite clearly that complex polysaccharides are of common occurrence in the surface layers of bacterial cells, and in their capsules when these are present. These polysaccharides play an important, often a dominant, part in determining antigenic specificity (see Chapter 8).

We have noted in the preceding chapter that certain bacteria may contain granules that have been varionsly identified, on the basis of staining reactions, 
as starch or glycogen, but of the significance of these intracellular granules we know little, and their exact nature is still in dispute.

Bacterial Fats, Lipins and Waxes.-Buchanan and Fulmer quote figures ranging from $1.56-40.8$ per cent. dry weight for the ether-extractable substances in various species of bacteria. Nicolle and Alilaire record percentages of $6 \cdot 31-15 \cdot 77$ for acetone-extractable substances, but the range of species covered is not the same. Lipin values of less than 10 per cent. have been recorded for certain members of the Salmonella group (Williams, Bloor and Sandholzer 1939), of 5-6 per cent., for Brucella (Stahl and Hamann 1941) and of 10-14 per cent. for the gonococcus (Stokinger et al. 1944). There is no doubt that the production of particular lipins or waxes characterizes particular bacterial species. We have, for instance, noted in the previous chapter the production by the tubercle bacillus of a wax on which its acid-fastness depends (see also Chapter 16). We know so little of the part played by fats, lipins and waxes in the economy of the bacterial cell that, for the moment, it will suffice to note their presence. For such data as are available in regard to their chemical constitution reference may be made to Buchanan and Fulmer (1928-30).

\section{The Study of Bacterial Metabolism.}

In the study of bacterial metabolism, whether we are concerned with sul)stances utilized by bacteria as sources of food or energy, with the products of metabolism, or with the mechanisms by which dissimilation and assimilation of foodstuffs is achieved, the most striking feature is the extreme diversity of bacterial activity. It is possible to give a generalized description of metabolism that will apply with minor modifications to the nutrition of all the higher animals, but in bacteria, the diverse metabolic activities appear to have been evolved by adaptation to the widest variety of environmental conditions; so that one species or another can take advantage of almost any thermodynamically suitable type of foodstuff and any moist environment within a temperature range rather wider than that suitable for most other groups of organisms. Certain groups of bacteria, however, share a sufficient similarity of metabolic activities as to make them suitable for discussion in an introduction to the subject of bacterial metabolism. Among these are the bacteria that primarily interest the medical bacteriologist, and we shall endeavour to select these for illustrative examples. The studies of the kind that conceru us have, for the most part, been carried out by biochemists, and mark the rapid development of microbiological chemistry as a distinctive branch of biological science. Although work in this field was initiated by Pasteur, and developed extensively by him within the limits of the chemical knowledge and technique of his time, it has been largely neglected by his bacteriological successors. These have, for the most part, been content to use the bacterial fermentation reactions as diagnostic tools, without inquiring in any systematic way into the actual chemical changes concerned. The common practice has been to map out the fermentative abilities of different bacterial species, using an arbitrarily selected series of carbohydrate and other substrates which experience has shown to possess differential value, and noting the production of acid by a colour change in a suitable indicator, or the production of gas by observing its collection within a small inverted tube contained in the culture medium. The study of the changes produced in nitrogenous substrates has been even more limited and arbitrary the production of indole from a tryptophan-containing substrate, the production of $\mathrm{H}_{2} \mathrm{~S}$ from sulphurcontaining amino-acids, and so oll. This neglect bas been natural enough. The 
medical bacteriologist, at least, has been mainly interested in other bacterial activities; but the time has quite clearly arrived when the chemical aspects of bacteriology and immunity must be mastered by all serious students of these branches of biology.

The metabolism of bacteria, as of other living cells, is dependent on, and regulated by, a complex system of enzymes and catalysts, whose activity is conditioned by a variety of factors, such as temperature of incubation, the $\mathrm{pH}$ of the medium, the presence or absence of molecular oxygen, and the presence or absence of a particular food-stuff. In order that any given bacterium may grow and multiply, these various conditioning factors must fall within a range limited by the requirements of the enzyme systems that are available. Many bacteria possess more than one enzyme system for dealing with a given type of substrate, and a change of enviromment is accompanied by a change in the predominating enzyme system.

The prime needs of a bacterium are substances that it can assimilate and synthesize into protoplasm; and energy necessary for these syntheses, and for movement (if it is motile), reproduction and the maintenance of structure. Both are obtained by the decomposition of suitable substrates. No sharp dividing line can be drawn between substrates that act mainly as sources of energy and those which are needed for synthesis of bacterial proteins, carbohydrates, fats, enzymes, etc. But it is convenient to distinguish the two types of activity, and discuss the first under bacterial respiration and fermentation, and the second under bacterial nutrition. Bacterial nutrition we shall leave till a later section, but before examining the nature of respiration and fermentation in detail, we shall briefly discuss the general implications of the energy-producing mechanisms in living cells.

The Respiration of Bacteria.-The term respiration, with its comnotations for the student of mammalian physiology of the mechanical intake of oxygen for the purpose of oxidizing food substances, is apt to confuse the student of bacteriology unless he realizes that more than the direct utilization of atmospheric oxygen is concerned. Respiration covers all those metabolic mechanisms that are employed in providing energy, as opposed to those that are concerned in synthesis. It happens that many energy-yielding reactions, that is, exothermic reactious, are oxidations in the simple sense of the word and take place in the cell when molecular oxygen is supplied. But this concept of oxidation as the union of oxygen with a given substance is as limited in its applications to biology as it is to inorganic chemistry. In neither does the process of oxidation necessarily iuvolve the transfer of oxygen.

The simple reaction

$$
\begin{aligned}
& \text { is as much an oxidation as the more obvious } \\
& \qquad 2 \mathrm{FeO}+\mathrm{O} \rightarrow \mathrm{Fe}_{2} \mathrm{O}_{3} .
\end{aligned}
$$

$$
\mathrm{FeCl}_{2}+\mathrm{Cl} \rightarrow \mathrm{FeCl}_{3}
$$

The common feature of these two oxidations, and indeed of all oxidations, is ehange in the electronic state of the substances concerned. If the oxidation of the ferrous to the ferrie chloride takes place in solution, in which the participants in the reactions are ionized, the whole process may be written

$$
\mathrm{Fe}^{++}+2 \mathrm{Cl}^{-}+\mathrm{Cl} \rightarrow \mathrm{Fe}^{+++}+3 \mathrm{Cl}^{-} \text {. }
$$

The ferrous salt is oxidized, and the chlorine atom reduced. The oxidation of the iron may be written

$$
\mathrm{Fe}^{++} \rightarrow \mathrm{Fe}^{+++}+\text {one free electron }
$$

and the reduction of the chlorine by

$$
\mathrm{Cl}+\text { one free electron } \rightarrow \mathrm{Cl}^{-} \text {. }
$$


The essential factor in any oxidation then is the removal of electrons; in any reduction, their addition. Regarded in this light, bacterial respiration covers those processes of electron transfer in the mixture of substrate and bacteria which yield free energy. And just as in simple inorganic reactions the oxidation of one substance implies the reduction of another, so in a study of bacterial metabolism we must consider not only the substrate to be oxidized by the bacterium, but the substances, either in the bacterium or in its environment, which must be reduced in order that the biological oxidations may take place.

Besides the fundamental conception of oxidation as electron transfer, we are concerned in metabolic chemistry with the substances to and from which the electrons are transferred. Thus biological oxidations may be expressed simply in terms of the transfer of either hydrogen or oxygen; but in using this interpretation it must be remembered that it is a particularization of the gencral hypothesis of electron transfer. The addition of oxygen to a molecule, or the removal of hydrogen from it, entails a decrease in clectrons; both are oxidations. Similarly the removal of oxygen from, and the addition of hydrogen to, a molecule are equally reductions. In the transfer of either oxygen or hydrogen, at least two molecules are concerned. One, to supply the oxygen or hydrogen, is called the donator ; the other, to which they go, is ealled the acceptor.

In donating oxygen a molecule is reduced, in accepting oxygen it is oxidized. In donating hydrogen a molecule is oxidized, in accepting hydrogen it is reduced. In both cases the reaction is catalysed, and the catalyst is regarded as " activating " either oxygen or hydrogen.

The theory advanced by Wieland (1913, 1921, 1922) regards hydrogen activation, and consequent hydrogen transport, as the essential mechanism of cellular oxidations. The removal of hydrogen from a molceule may be preceded by the addition of a molecule of water, in which case oxygen is in fact added; or there may be no preliminary addition of water, in which case the molecule is oxidized by the simple loss of hydrogen. In both cases a suitable hydrogen acceptor must be provided. In general terms these reactions may be expressed as follows:

(a) $\mathrm{X}+\mathrm{H} \cdot \mathrm{OH}+\mathrm{A}=\mathrm{XH} \cdot \mathrm{OH}+\mathrm{A}=\mathrm{XO}+\mathrm{AH}_{2}$.

(b) $\mathrm{XH}+\mathrm{A}=\mathrm{X}+\mathrm{AH}$.

In (a) $\mathrm{X}$ represents the substrate to be oxiclized and $\mathrm{A}$ the hydrogen acceptor. Watcr is first added to $\mathrm{X}$ and the hydrogen of the compound $\mathrm{XH} \cdot \mathrm{OH}$ is then activated and passed on to A, leaving $\mathbf{X}$ oxidized. In (b) the compound $\mathrm{XH}$ is the substrate to be oxidized. Oxidation oceurs by the activation of the hydrogen and its transference to $A$.

An example of the first type of reaction is afforded by the oxidation of an aldehyde to an acid with previous hydration,

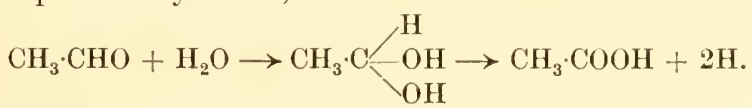

An example of the second type of reaction is afforded by the oxidation of an alcohol to an aldehyde by removal of hydrogen.

$$
\mathrm{CH}_{3} \cdot \mathrm{CH}_{2} \cdot \mathrm{OH} \longrightarrow \mathrm{CH}_{3} \cdot \mathrm{CHO}+2 \mathrm{H} \text {. }
$$

The hydrogen in such reactions is seldom liberated in the gaseous state. In almost all the reactions with which we are here concerned, a hydrogen acceptor must be provided. The enzyme that activates the hydrogen in the substrate to be oxidized, and so brings about its transport, is known as a dehydrogenase.

The Mechanism of Hydrogen Transport. -The phenomena of hydrogen transport in bacteria may be demonstrated by a technique developed by Quastel and his colleagues, which consists in observing the behaviour of washed hacterial cells suspended in an appropriate buffer solution containing the necessary inorganic ions, when incubated in an evacuated Thunberg tube in the presence of a substrate and an indicator dye such as methylene blue. The reactions are usually completed within 30 minutes or less, so that although a small degree of bacterial multiplication 
may take place during the experiment, the enzymic activities displayed are not necessarily those of proliferating cells, nor even of living cells (Cook and Stephenson 1928, Sandiford and Wooldridge 1931). In the Thunberg tube, Bacterium coli, for example, may be shown to possess an enzyme that is capable of oxidizing succinic acid to fumaric acid in the presence of methylene blue (Quastel and Whetham 1924, Quastel, Stephenson and Whetham 1925). A reversible equilibrium is set up.

Succinic acid and methylene blue $\longleftrightarrow$ fumaric acid and leuco-methylene blue.

The enzyme responsible, succinic dehydrogenase, activates the hydrogen. The methylene blue acts as a hydrogen acceptor, and in doing so is reduced to the colourless compound leuco-methylene blue. It will be noted that the methylene blue does not require activation to become a hydrogen acceptor. In the dehydrogenase systems occurring in nature, it is probable that most of the hydrogen acceptors are activated by enzymes. The natural hydrogen acceptors may be either intermediate products of carbohydrate or protein dissimilation, or molecular oxygen itself. In the first case we have a biological oxidation in the absence of air, which we call an anaerobic oxidation, and in the second case an aerobic oxidation, in which oxygen is acting as the hydrogen acceptor. Dehydrogenases may catalyse the transfer of hydrogen from the donator directly to oxygen as the acceptor, but this mechanism appears to be relatively rare.

The transport of oxygen in the cell is usually brought about by a complex system of oxygen carriers, and the oxygen is activated by enzymes which receive the general name of oxidases. Warburg $(1925 a, b)$ pictures the molecular oxygen uniting in the cell with some complex organic substance containing iron in the reduced, or ferrous state, and converting it into ferric iron. In the presence of an oxidizable organic molecule and a suitable oxidase oxygen is transferred and the iron returns to the ferrous condition. A natural carrier of oxygen, cytochrome, has been demonstrated in the cells of animals, yeasts and bacteria by Keilin (1925, 1926, 1928-29, 1930), and Keilin and Hartree $(1938 a, b, c)$. Cytochrome is a respiratory pigment, made up of a number of related hæmatin compounds, which plays an important part in cell respiration. Keilin's studies supply the link between the Wieland hypothesis of hydrogen transport and the Warburg hypothesis of oxidation by iron-containing compounds.

Keilin's view of cellular oxidations may be briefly summarized as follows: Organisms whose respiration demands molecular oxygen contain a widely distributed respiratory pigment cytochrome composed of three main hæmatin compounds, the components $a, b$ and $c$. The pigment also contains an unbound hæmatin compound similar to the protohæmatin of hæmoglobin, and a hæmochromogen precursor of cytochrome. Of these, the $b$ component of cytochrome, the protohæmatin and the hæmochromogen precursor are autoxidizable. The $a$ and $c$ components are oxidized in the presence of a thermostable enzyme cytochrome oxidase; all factors that destroy or inhibit this oxidase diminish the oxygen uptake of the cell. Some bacterial cells, e.g. B. subtitis, have the full complement of cytochrome components, and a fourth component $a_{3}$. Bact. coli, on the other hand, has no $a, a_{3}, c$ or cyto-oxidase, but only $b$, and another component $a_{2}$, which may possibly act as an oxidase to $b$ (Keilin and Harpley 1941). Cytochrome therefore acts as a carrier between two activating mechanisms of the cell, the cytochrome oxidase activating molecular oxygen, and the dehydrogenase activating the hydrogen of various organic substrates, metabolic intermediaries and coenzymes. The autoxidizable hæmatin compound $b$, the unbound hæmatin, and 
the hæmochromogen precursor to cytochrome may act as carriers between hydrogen donators and molecular oxygen. They may also act as direct catalysts, promoting the oxidation of substrates that are not activated by specific dehydrogenases. The various hæmatin compounds of the cell are also responsible for the catalase and peroxidase reactions that occur in the presence of $\mathrm{H}_{2} \mathrm{O}_{2}$. The $\mathrm{H}_{2} \mathrm{O}_{2}$, which is an end-product in a number of bacterial oxidations, is reduced in the presence of catalase. Catalase production is probably limited to bacterial species capable of aerobic respiration (Kluyver 1924). Callow (1923, 1924) found oxygen utilization minimal in bacteria producing no catalase (see section on Aerobiosis and Anaerobiosis, p. 70).

Substituting cytochrome for methylene blue in the succinic dehydrogenase system mentioned above, we may represent the oxidation of succinic acid in two stages, the first catalysed by the dehydrogenase, the second by cytochrome oxidase.

Succinic acid and cytochrome $\rightarrow$ reduced cytochrome and fumaric acid.

Reduced cytochrome $+\mathrm{O}_{2} \rightarrow$ cytochrome + water.

This reaction has not been directly demonstrated in bacterial cells, nor can many of the dehydrogenase systems directly reduce oxidized cytochrome. But it appears that the dehydrogenase systems capable of reacting directly with reduced cytochrome may act as intermediate links between cytochrome and other dehydrogenase systems, by which they are themselves reduced.

Many of the dehydrogenase systems, in addition to hydrogen donator, hydrogen acceptor, dehydrogenase, water, inorganic ions and the correct $\mathrm{pH}$, require the presence of co-enzymes. A co-enzyme may be defined as a thermostable substance necessary in addition to enzyme and substrate to initiate a reaction. Co-enzymes are usually organic in nature, and their molecules are small enough to pass through semi-permeable membranes that hold back the larger enzyme molecules. Hence existence of a co-enzyme is usually demonstrated by the inactivation of a natural enzyme preparation after it has been dialysed, and its reactivation by addition of the dialysate. Co-enzyme I, which has been identified as pyridine nucleotide diphosphate, takes part in many enzyme reactions. Co-enzyme I and its dehydrogenase constitute one of the dehydrogenase systems capable of direct reaction with cytochrome. Its relation to the cytochrome system may be represented (Dewan and Green 1938) as follows :

A $\mathrm{H}_{2}+$ Co-enzyme $\mathrm{I} \stackrel{\text { dehydrogenase for } \mathrm{A}}{\longrightarrow} \mathrm{A}+\mathrm{H}_{2}$-co-enzyme $\mathrm{I}$

$\mathrm{H}_{2}$-co-enzyme $\mathrm{I}+$ oxidized cytochrome dehydrogenase for co-enzyme I co-enzyme $\mathrm{I}+\mathrm{H}_{2}$ cytochrome

The following diagram of the general oxidative mechanisms as they involve cytochrome itself have been taken, with slight modification, from that given by Keilin (1928-29).

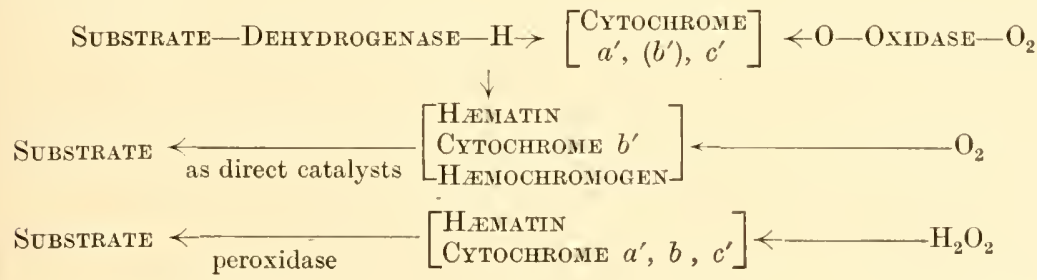


These are not the only mechanisms affecting the transfer of hydrogen to molecular oxygen. Many animal, yeast and bacterial cells, for example, contain flavoprotein (Schuitz and Theorell 1938). Flavoprotein, the "yellow enzyme" of Warburg and Christian (1933), is riboflavin phosphate combined with a protein group; it is spontaneously oxidizable by molecular oxygen, but acts also as a hydrogen carrier between dehydrogenase systems and the cytochrome system (Ogston and Green 1935), and anaerobically between two dehydrogenase systems (Dewan and Green 1938). It is reduced by co-enzyme I and may be related to the dehydrogenase of co-enzyme I mentioned above, which Straub, Corran and Green (1939) have shown to be a flavoprotein.

The dehydrogenase systems are reversible (Qnastel and Whetham 1924, Green and Stickland 1934). If the reaction of succinic acid and methylene blue is allowed to proceed in the presence of Bact. coli dehydrogenase until the dye is reduced, and an excess of fumaric acid added, the lenco-methylene blue gradually becomes reoxidized. The reversal is due to the action of the enzyme, for the destruction of the enzyme by heat or the addition of an antiseptic completely inhibits the reoxidization of the methylene blue. Since these reactions are reversible, it is obvious that they will not take place in a given direction unless the hydrogen donators and acceptors are in a suitable physical state for the removal of electrons from the substance to be oxidized.

Biological Redox Systems. - In a given reversible oxidation-reduction system the state of oxidation may be measured by the tendency of the system to give up electrons, and the state of reduction by the tendency to take up electrons.

If a platinum electrode is immersed in a fluid containing such a system, an electrical "half-eell" is produced, and a potential difference, depending on the availability of eleetrons in the system, is set up at the electrode. When the half-cell is put into a circuit with the normal hydrogen electrode as a standard half-cell, the electromotive force that develops will be a measure of the electrode potential in the system compared with that of the standard half-cell. This force, measured in volts, is designated the Eh of the system. A marked tendency to reduction results in a flow of electrons from the standard half-cell; i.e. the current flows from the system to the half-eell and the Eh is positive. A system with a marked tendency to oxidation, on the other hand, sinee electrons flow to the standard cell half-cell, is indicated by a negative Eh.

The conception of Eh, the oxidation-reduction (or redox) potential (see Clark ct al. 1928, McLeod 1930, Hewitt 1936) as a measure of reducing intensity may be applled to the reversible enzyme systems of the dehydrogenase type. Oxidation, for example, will not proceed in a given system except in the presence of a system with a higher El. Thus it will be seen that the absolute Eh value is of less importance than its value compared with other systems, for a system of a high reducing intensity, characterized by a low Eh, will be further reduced by a system with an even lower Eh. For the general characterization of each system, the Eh is determined in standard eonditions, namely when equivalent amounts of the reduced and oxidized forms are present in equilibrium at $\mathrm{pH} 7.5$ and a temperature of $30^{\circ} \mathrm{C}$. This characteristic $\mathrm{Eh}$ is designated $\mathrm{E}_{0}{ }^{\prime}$, and in general it may be said that, in the proper circumstances, a system of a given $\mathrm{E}_{0}{ }^{\prime}$ will in the oxidized state be reduced by a system of lower $\mathrm{E}_{0}{ }^{\prime}$ and in the reduced state will be oxidized by a system of higher $\mathrm{E}_{0}{ }^{\prime}$. For example, the $\mathrm{E}_{0}{ }^{\prime}$ of the succinic-dehydrogenase system is about 0.00 volt, and that of cytochrome-cytochrome oxidase about +0.12 volt. On electronic grounds it is to be expeeted that the oxidation of the succinic acid to fumaric will occur in the presence of oxidized cytochrome. 


\section{The Chemical Changes produced by Bacteria in various Substrates}

On the view outlined above, the oxidation of a complex substance by bacteria may be pictured as the transfer of hydrogen from one substance to another, each step being brought about by one of a graduated series of oxidation-reduction systems with appropriate enzymes, carriers and co-enzymes, the whole resulting in an even flow of energy for cell maintenance and syntheses. We shall now examine in more detail the action of bacteria in the different types of foodstuffs.

The Action of Bacteria on Carbohydrates and Allied Substances.-The majority of the bacteria with which we are concerned in this book are able to attack the hexose sugars. Occasionally the end result is complete oxidation to water and carbon dioxide. This is, however, an unusual type of reaction, limited to a few species and demanding a copious supply of oxygen. Varions reactions leading to less complete oxidation are more common; and one of the most characteristic types of bacterial fermentation is that in which molecular oxygen plays no direct part, the reaction consisting essentially in the splitting of a complex molecule, usually by hydrolysis, with a rearrangement of the oxygen atoms, so that one portion of the hydrolysed molecule is oxidized while the other is reduced. An example of this general type of reaction is afforded by the studies of Harden (1901, 1905), and of Harden and Walpole (1906) on the fermentation of dextrose by Bact. coli. The main products of fermentation are lactic acid, acetic acil, ethyl alcohol, carbon dioxide and hydrogen, and the reaction appears to be approximately represented by the formula,

$2 \mathrm{C}_{6} \mathrm{H}_{12} \mathrm{O}_{6}+\mathrm{H}_{2} \mathrm{O}=2 \mathrm{CH}_{3} \cdot \mathrm{CHOH} \cdot \mathrm{COOH}+\mathrm{CH}_{3} \cdot \mathrm{COOH}+\mathrm{C}_{2} \mathrm{H}_{5} \cdot \mathrm{OH}+2 \mathrm{CO}_{2}+2 \mathrm{H}_{2}$ although small amounts of other substances, such as succinic acid, are produced.

In some instances, as in the fermentation of dextrose by the typhoid bacillus, no free gas is evolved. It was suggested by Harden that in this case that part of the reaction which, with such an organism as Bact. coli, leads to the evolution of equal parts of $\mathrm{CO}_{2}$ and $\mathrm{H}_{2}$, stops short at the formation of formic acid, $\mathrm{H} \cdot \mathrm{COOH}$ (see also Pakes and Jollyman 1901). This suggestion was strengthened by the observation of Sera (1910), while Grey (1913-14) has shown that formic acid can be identified as an intermediate product in the fermentation of dextrose by Bact. coli.

This type of reaction is, however, by no means the only one that occurs during the cleavage of carbohydrates and allied substances by bacteria.

The existence of wide variations is well illustrated by the results recorded by Birkenshaw, Charles and Clutterbuck (1931). Using the carbon-balance-sheet method employed by Raistrick and his colleagues (1931) in their extensive studies of the metabolism of moulds, they determined the relative proportions of different metabolic products formed by twenty different bacterial species growing in a synthetic medinm containing glucose. In the case of Bact. coli and of certain nearly related organisms, up to 30.4 per cent. of the carbon of the glucose was recovered after fermentation in the form of lactic acid, $5 \cdot 0-14 \cdot 4$ per cent. was recovered in the form of volatile acids, while the amount present as butylene glycol $\left(\mathrm{CH}_{3} \cdot \mathrm{CHOH} \cdot \mathrm{CHOH} \cdot \mathrm{CH}_{3}\right)$ was negligible. On the other hand, a coliform organism of a different type, Buct. asiaticum mobile, yielded 26.8-31.0 per cent. of the carbon as butylene glycol, but none as lactic acid. Two anaerobes $(\mathrm{Cl}$. saccharobutyricum and $C l$. pasteurianum) yielded a large proportion of carbon 
as volatile acids (3t.8 per cent. and $37 \cdot 8$ per cent.) but formed little if any lactic acid or butylene glycol.

Many hypotheses have beeu put forward to explain the precise mechanism of the dissimilation of the relatively simple carbohydrates. It is impracticable to deal with them fully in this chapter, and the student is referred to the monograph of Stephenson (1939) and the review of Werkman (1939) for a detailed consideration of the subject. As an illustration of the complexity of the mechanisms so far elucidated and the multiplicity of enzymes and carriers that may take part we may cite the Embden-Meyerhof-Parnas scheme for the anaerobic dissimilation of glucose by animal tissues and yeast cells.

According to this scheme the hexose first reacts with adenosine triphosphate in the presence of a phosphorylase to produce a hexose diphosphate and adenosine monophosphate. The hexose diphosphate then breaks down into two molecules of triosephosphate under the influence of an aldolase. The triosephosphate combines with acetaldehyde in the presence of a mutase and co-enzyme I to form one end-product of the breakdown, ethyl alcohol, and 3-phosphoglyceric acid. An enolase changes the 3-phosphoglyceric acid into phosphopyruvate by the removal of a molecule of water, and the phosphopyruvate is dephosphorylated in the presence of the adenosine monophosphate (left from the phosphorylation of the hexose) to form pyruvic acid and adenosine triphosphate. The adenosine monophosphate (adenylic acid) thus acts as a co-enzyme in the transfer of phosphate from phosphopyruric acid to glucose. The pyruvic acid, according to the enzymic condition of the cells concerned, may react with more triosephosphate to yield lactic acid and phosphoglycerate, or be acted upon by a carboxylase in the presence of co-carboxylase to give carbon dioxide and acetaldehyde. The carbon dioxide is another end-product, and the acetaldehyde is available for the reaction with triosephosphate to form alcohol.

The co-enzyme I acts as a hydrogen carrier in the third system, the full reaction being

Acetaldehyde and reduced co-enzyme $\rightarrow$ ethyl alcohol + oxidized co-enzyme.

Triosephosphate + oxidized co-enzyme $\rightarrow$ phosphoglyceric acid + reduced co-enzyme.

The cycle, which is one of many possible cycles, is most easily demonstrated in animal tissues, and in extracts of yeast cells. For technical reasons active extracts of bacterial cells are difficult to prepare, but evidence is accumulating that the Embden-MeyerhofParnas scheme is applicable to anaerobic glycolysis of some bacteria. The evidence is of four kinds.

Firstly, the presence of the enzymes concerned may be inferred by comparing the action of relatively specific enzyme poisons on the dissimilation by the cells under study, with its action on defined enzyme systems.

Secondly, the presence of the various oxidative, hydrolytic and phosphorylating systems may be demonstrated by adding the hypothetical intermediaries to a suspension of the cells, and measuring its power to deal with them; but, as Stephenson (1939) points out, though this demonstrates that a particular metabolic path may be followed in experimental dissimilation, it is not necessarily followed in natural fermentation.

Thirdly, the unknown dialysable carriers may be removed from the enzyme preparation, and the need for them demonstrated by adding known carriers from other sources.

And lastly, the intcrmediate products postulated may be isolated from the fermentation system, particularly after the dissimilation has been interrupted by various enzyme poisons.

Virtanen (1924, 1925) and Virtanen and Karström (1931) demonstrated that phosphorylated hexoses occur in bacterial metabolism. A key intermediate, phosphoglyceric acid, has been isolated from a large number of bacteria by Werkman and his colleagues 
(see Werkman 1939) and from Bact. coli by Endo (1938) ; moreover, it is formed during the breakdown of hexose diphosphate.

The difficulty of obtaining active cell-free extracts of bacteria similar to those of the much more extensively studied yeasts has been overcome by disintegrating bacteria by ultra-sonic vibrations (see Chapter 5) or in special crushing mills (Booth and Green 1938). By the latter means, for example, Still (1940) has demonstrated that cell-free preparations of Bact. coli will catalyse the oxidation of triosephosphate (3-phosphoglyceraldehyde) to 3-phosphoglyceric acid.

It must be emphasized that this is one example only of the possible ways of anacrobic glycolysis. Indeed, it is probable that two or more processes of glycolysis, only one of them involving phosphorylation, may oceur at the same time in a bacterial culture (see Tasman and Brandwijk 1938). The applicability of the Embden-Meyerhof-Parnas scheme to bacteria is by no means fully established, but it serves to illustrate not only the complexity of the mechanisms, but their dependence, in certain cases at any rate, on a number of enzymes and carriers, which themselves may be intermediaries between stages on other metabolic paths.

Among the intermediate products of the carboliydrate metabolism of bacteria, pyruvic acid holds an important place, whether the metabolic path actually followed corresponds to the Embden-Heyerhof-Parnas scheme or to one of the alternative schemes that have been proposed. It is a source of acetic, succinic, butyric and fumaric acids, of ethyl and iso-propyl alcohol, acetone, glycerol, acetylmethylcarbinol, butylene glycol, carbon dioxide and hydrogen. Werkman (1939) lists the following examples of anaerohic dissimilation of pyruvic acid produced by bacteria and yeasts.

1. Decarboxylation, giving acetaldehyde and $\mathrm{CO}_{2}$ (Sarcina ventriculi, Smit 1930).

$$
\mathrm{CH}_{3} \cdot \mathrm{CO} \cdot \mathrm{COOH} \rightarrow \mathrm{CH}_{3} \mathrm{CHO}+\mathrm{CO}_{2} \text {. }
$$

2. Hydrolysis, giving acetic and formic acids (Bacteriacexe, Tikka 1937).

$$
\mathrm{CH}_{3} \cdot \mathrm{CO} \cdot \mathrm{COOH}+\mathrm{H} \cdot \mathrm{OH} \rightarrow \mathrm{CH}_{3} \mathrm{COOH}+\mathrm{HCOOH} \text {. }
$$

3. Dismutation of the acetaldelyde produced by decarboxylation, giving acctic acid and alcohol (? many acetic acid bacteria.)

$$
2 \mathrm{CH}_{3} \mathrm{CHO} \rightarrow \mathrm{CH}_{3} \mathrm{COOH} ; \mathrm{C}_{2} \mathrm{H}_{5} \mathrm{OH} \text {. }
$$

4. Condensation of the acetaldehyde to produce acetylmethylcarbinol, and reduction, giving butylene glycol (Bact. aerogenes, Neuberg and Reinfurth 1923-24).

$$
\begin{gathered}
2 \mathrm{CH}_{3} \mathrm{CHO} \longrightarrow \mathrm{CH}_{3} \cdot \mathrm{CO} \cdot \mathrm{CHOH} \cdot \mathrm{CH}_{3} \cdot \\
\mathrm{CH}_{3} \cdot \mathrm{CO} \cdot \mathrm{CHOH} \cdot \mathrm{CH}_{3}+2 \mathrm{H} \longrightarrow \mathrm{CH}_{3} \cdot \mathrm{CHOH} \cdot \mathrm{CHOH} \cdot \mathrm{CH}_{3} \cdot
\end{gathered}
$$

5. Dismutation and reduction, giving acetic and lactic acids, and $\mathrm{CO}_{2}$ (lactic acid bacteria, Nelson and Werkman 1936, Staph. aureus, Krebs, 1937).

$$
\begin{gathered}
\mathrm{CH}_{3} \mathrm{CO} \cdot \mathrm{COOH}+\mathrm{HOH} \rightarrow \mathrm{CH}_{3} \mathrm{COOH}+2 \mathrm{H}+\mathrm{CO}_{2} \cdot \\
\quad \mathrm{CH}_{3} \cdot \mathrm{CO} \cdot \mathrm{COOH}+2 \mathrm{H} \rightarrow \mathrm{CH}_{3} \cdot \mathrm{CHOH} \cdot \mathrm{COOH} .
\end{gathered}
$$

The metabolism of pyruric acid is closely connected with thiamin (vitamin $B_{1}$ ). The co-enzyme necessary for reaction 1, a cocarboxylase, has been identified as the diphosphate of thiamin and is necessary for the breakdown of pyruvic acid by Bact. acidificans longis. simum (Lipmann 1937), Staph. aureus (Hills 1938), propionic acid bacteria (Silverman and Werkman 1939), and Str. hocmolyticus and gonococci (Barron and Lyman 1939).

As noted above (p. 45), for the purposes of classification of bacteria the power of a species to break down a given carbohydrate is usually measured by the production of acid or of acid and gas. The gases produced in macroscopic quantities from small volumes of fluid medium are usually $\mathrm{CO}_{2}$ and $\mathrm{H}_{2}$. We have noted a number of ways in which hexose sugars may be supposed to give rise to various acids and $\mathrm{CO}_{2}$. Pakes and Jollyman (1901) tested certain members of the Bacterium group and showed that all those 
producing gas from dextrose also did so from formate. The production of molecular hydrogen from formic acid was shown by Stephenson and Stickland (1932) to be due to hydrogenlyase, which catalyses the reaction

$$
\mathrm{H} \cdot \mathrm{COOH} \rightarrow \mathrm{H}_{2}+\mathrm{CO}_{2} \text {, }
$$

a reaction that Woods (1936) showed to be reversible. Strains of Bact. coli that produce no gas have no formic hydrogenlyase (Ordal and Halvorsen 1939). Formate is not necessarily the intermediary in hydrogen production, for though. $\mathrm{Cl}$. tetanomorphum produces hydrogen from dextrose and pyruvate, it will not do so from formate (Wroods and Clifton 1937). As we shall see in the section on protein metabolism, the hydrogen may also be produced by the hydrolytic deamination of amino-acids.

So far we have considered examples of anaerobic breakdown of carbohydrates. These substances are incompletely oxidized and the partly oxidized substrates often act as hydrogen acceptors promoting the further oxidation of substances occurring in another chain of metabolic events. The utilization of molecular oxygen results in a more complete oxidation and the liberation for cell synthesis of more free energy than can be obtained by anaerobic glycolysis.

Many bacteria are unable to utilize certain carbohydrate substances unless oxygen, either molecular or combined, is present. Thus Bact. coli, supplied with organic acids such as lactic, fumaric, succinic or pyruvic as sole sources of carbon, will not grow except in the presence of air (Stephenson and Whetham 1924). With the addition of nitrate, anaerobic growth takes place, oxidation of the organic acid being achieved at the expense of the nitrate, which is reduced to nitrite. The activation of the nitrate to become, in terms of Wieland's hypothesis, a hydrogen acceptor, is due to a specific enzyme which Bact. coli happens to possess in common with other bacteria capable of both aerobic and anaerobic dissimilation.

The participation of oxygen as a hydrogen acceptor in glycolysis does not usually take place without the intervention either of carriers, or catalysts, or both. The cytochrome-cytochrome oxidase systems, involving hæmin compounds, appear to be the most important in this respect, though certain bacteria contain respiratory pigments which enable them to utilize oxygen after the cytochrome systems have been poisoned by cyanide. As an example, we may cite the pigment pyocyanin found in Pseudomonas pyocyanea, which Friedheim and Michaelis (1931) showed to be autoxidizable. Green, Stickland and Tarr (1934) demonstrated its ability to act as a hydrogen carrier between pairs of dehydrogenase systems sturlied in the test-tube. Added to suspensions of pigment-free strains of Pseudomonas pyocyanea pyocyanin strongly stimulates the oxygen uptake (Friedheim 1931). The stimulation may in part be due to the addition of an effective carrier between dehydrogenase and cytochrome systems, but in certain circumstances (Friedheim 1934) the pyocyanin carries hydrogen directly to molecular oxygen. The violet pigment of Chromobacterium violaceum acts in an analogous manner. Chr. violaceum frequently gives rise to non-pigmented variants, which, suspended in a buffer solution, take up oxygen at a moderate rate. A solution of the pigment of Chr. violaceum takes up no oxygen, but the addition of the dissolved pigment to the suspension results in a two- to three-fold increase in oxygen uptake (Friedheim 1932).

Many organisms contain enzyme systems for both aerobic and anaerobic glycolysis; the predominance of one system or the other in the cell at a given moment depends on the presence or absence of molecular oxygen in the environment. The mere presence of oxygen, however, does not necessarily impose aerobic 
glycolysis on the organism. Stephenson and Whetham (1924) compared the aerobic and anaerobic breakdown of dextrose by Bact. coli in a free supply of air. The breakdown in the early stages of growth was almost anaerobic; oxygen uptake was minimal, and lactic acid and similar incompletely oxidized substances were produced. During the later stages of growth, when the release of free energy from these incomplete oxidations was diminishing, atmospheric oxygen was freely utilized, and $\mathrm{CO}_{2}$ given off.

A large number of carbohydrate substances are known to be attacked by bacteria : they range from 3-carbon compounds, through pentoses, hexoses and disaccharides to polysaccharides like cellulose, starch and dextrin. Many of the bacterial species that attack hexose sugars also ferment the related hexahydric alcohols, mannitol, sorbitol and dulcitol, which are apparently broken down in an analogous manner. By analogy with yeast metabolism the breakdown of the more complex disaccharides and polysaccharides is presumably achieved by a preliminary hydrolysis to simple sugars. Saccharose is first hydrolysed to a mixture of dextrose and fructose ; lactose to dextrose and galactose, and maltose to dextrose; the enzymes concerned are, respectively, invertase, lactase and maltase. When hydrolysis has reached this stage, the utilization of the hexose sugars proceeds along the lines indicated above. Direct evidence that this is the normal mode of cleavage of disaccharides in bacteria is scanty (see Fleming and Neill 1927). Moreover, some yeasts ferment certain complex sugars at a greater rate than they ferment dextrose, a fact difficult to reconcile with the hypothesis of a preliminary breakdown to the simple sugar (see Sobotka and Holzman 1934, Nord and Engel 1938, O'Connor 1940, Leibowitz and Hestrin 1942). Wright (1936, 1937), finding that certain milk streptococci ferment lactose and saccharose more rapidly than they ferment the constituent monosaccharides, suggested that in bacteria also the disaccharides may be attacked directly, without previous hydrolysis to monosaccharides. The production of acids from saccharose by a number of bacterial species, including Bact. coli, was inhibited by low concentrations of iodoacetic acid, and yet no reducing sugar could be found in the test cultures. The concentration of iodoacetic acid was nevertheless insufficient to inhibit the lydrolysis of saccharose by yeast invertase.

Little is known of the processes of carbohydrate synthesis in bacteria. The best-established synthesis is that of 4-carbon carboxylic acids from 3-carbon compounds and $\mathrm{CO}_{2}$ (see Werkman and Wood 1942, van Niel et al. 1942, Evans et al. 1943). Among the more complex compounds, we may note the formation of the polyglucoside dextran, and the polyfructoside levan, from disaccharides, by the action of cell-free extracts. These polysaccharides are contained in the capsular material of several bacteria. Hehre and Sugg (1942) produced a serologically specific (p. 279) dextran by the action on sucrose of a cell-free enzyme preparation from Leuconostoc mesenteroides. Hestrin and Avineri-Shapiro $(1943,1944)$ studied the formation of levan from sucrose and raffinose with enzymes extracted from a strain of Bact. aerogenes. They suggested that the energy requisite for the synthesis of the polysaccharide might be produced by the breakdown of part of the substrate into an aldose.

The Action of Bacteria on Proteins and other Nitrogenous Substances.-The breakdown of proteins that occurs under natural conditions has long been known to depend on the action of bacteria. This proteolytic activity is, however, confined to particular species, many of which are anaerobic; it is at least doubtful whether even the proteolytic species are able to utilize complex proteins for growth in the absence of other sources of nitrogen.

Bainbridge (1911) found that many bacteria, including Proteus vulgaris, Bact. 
coli and staphylococci, were unable to grow in solutions of pure egg albumin, or of serum proteins, but that Proteus vulgaris was able to break down such complex proteins provided that a sufficient supply of nitrogen in an assimilable form was added to the medium. Similar results have been recorded by Rettger and his colleagues (Sperry and Rettger 1915, Rettger, Berman and Sturges 1916, Berman and Rettger 1918). Of several gelatin-liquefying bacteria studied by Berman and Rettger, none conld utilize egg albumin in the absence of other sources of nitrogen. Only three species, B. subtilis, Chr. prodigiosum and Proteus vulgaris, could break down peptone. With proteoses purified by precipitation, B. subtitis and Chr. prodigiosum cansed complete breakdown. Only B. subtilis, Chr. prodigiosum and Proteus vulgaris could attack casein. B. subtilis and Chr. prodigiosum conld not only liquefy gelatin, but could use it as a source of nitrogen; but such organisms as Staph. aureus or Bact. cloacx, although they caused rapid liquefaction of the gelatin when provided with another source of nitrogen for growth, showed no ability to ulitize the liquefied substance.

The initial stage of liquefaction of protein gels like coagulated serum or gelatin is brought about by protemases that, by opening the peptide linkage, reduce the protein to polypeptides and dipeptides. The bacterial proteinases are readily separated from culture by filtration, and apparently act extracellularly. The breakdown into constituent amino-acids is effected by polypeptidases and dipeptidases. The peptidases have been studied in yeasts, but, being apparently for the most part intracellular, they have as yet received no extensive study in bacteria, for, as noted on p. 53, adequate methods of extraction of intracellular enzymes have not long been available.

Many of the anaerobes are conspicuous for their ability to break down complex proteins, and the method of cleavage has been studied by several workers (Wolf and Harris 1918, Harris 1919, Wolf $1919 a, b$; see also Weil and Kocholaty 1937, Kocholaty, Weil and Smith 1938, van Heyniugen 1940). From a study of the proteinases of Chr. prodigiosum, Ps. pyocyanea and Ps. fuorescens liquefaciens, Maschmann (1937) concluded that enzymes of the different bacteria were identical, but were not of the same type as the animal proteinase trypsin or the plant proteinase papain.

The hydrolytic degradation of protein is apparently due to the action of specialized enzymes, which are produced in adequate amount when the bacteria concerned are supplied with immediately assimilable food material, including nitrogen ; during their consequent growth they are able to produce sufficient enzyme to initiate protein cleavage and thereby increase the available nitrogen.

Bacteria utilizc amino-acids in a variety of ways, similar in extent and complexity to those that have been described for the utilization of carbohydrates. As in our discussion of those substances, we can do no more than note some of the biochemical processes concerned; for greater detail the student is referred to the monograph of Stephenson (1939) and the review of Gale (1940). The amino-acids may be deaminated to yield the corresponding hydroxy acid, by desaturation at the $\alpha-\beta$ linkage to give the unsaturated acid, by reduction to give the saturated acid, by reduction and decarboxylation to give the hydrocarbon; or by oxidation. Decarboxylation results in the corresponding amine. Ncither decarboxylation nor deamination are processes yielding much energy, and in bacteria with alternative energy-yielding mechanisms, like the streptococci (see Ehrismann and Dramburg 1937), the reactions are not important in the bacterial economy. Where the amino-acid is one of the chief sources of food, oxidation to compounds with fewer carbon atoms provides the requisite energy for synthesis, etc. The formation of 
indole from tryptophan, which is widely used as a qualitative biochemical test in the identification of bacterial species, provides a good example of the breakdown of an aminoacid. Thick washed suspensions of Bact. coli in phosphate buffer will convert this aminoacid ( $\beta$-indole $\alpha$-amino propionic acid) to indole in the presence of oxygen. The oxygen taken up corresponds to the complete oxidation of the side chain to carbon dioxide and water (Woods 1935). In the absence of air, the compound is only deaminated, with the formation of $\alpha$-indole propionic acid. The meehanism of the oxidative attack is obscure and many complex series of steps have been proposed for this aerobie dissimilation, bnt the recent work of Fildes (1938), and Baker and Happold (1940), suggest that one enzyme, a tryptophanase, is responsible for the action, breaking the link between the indole ring and the $\alpha$-carbon atom of the side chain. The oxidation of the side chain to ammonia, $\mathrm{CO}_{2}$ and water follows after the liberation of the indole. A similar mechanism, but one involving a dismption and resynthesis of the indole nueleus, is proposed by Krebs, Hafez and Eggleston (1942).

The observation that a free supply of a fermentable carbohydrate will " spare " amino-acids, peptides or proteins in a medium was established by several workers (see Hirschler 1886, Smith 1897, Peckham 1897, Glem 1911, Kendall, Day and Walker 1914, Kendall and Walker 1915, Jones 1916). The hypothesis of proteinsparing formulated on the analogy with mammalian physiology was deduced from the fact that ammonia production in the medium was largely inhibited by the carbohylrate. But, as Stephenson (1939) points out, the analogy is imperfect, for, in the mammalian ease, the sparing is judged by a diminished excretion of urea, an end-product of metabolism, whereas ammonia may be a source of energy for a bacterium. Indeed, Raistrick (1919) and Raistrick and Clark (1921) lave shown that in a medium containing known amino-acids, their decomposition is increased, not lessened, by the addition of the fermentable substance glycerol. The ammonia produced in these cireumstances is undetectable, sinee it is utilizerl by the bacteria for synthesis. It may be noted also that Berman and Rettger (1918) were unable to demonstrate any protein-sparing as the result of the addition of carbohydrates, except in those instances in which the rapid fall in $\mathrm{pH}$ resulting from carbohydrate breakdown caused an inhibition of further bacterial growth; and that Heap and Cadness (1921) have shown that the presence of glucose greatly increases the rate of $\mathrm{H}_{2} \mathrm{~S}$ production from peptone by an organism that forms this gas during protein-cleavage. In regard to the eleavage of more complex protein molecules de Bord (1923) has shown that the addition of a fermentable carbohydrate to a protein-containing medium causes an increase in the concentration of amino-nitrogen induced by bacterial growth. These findings are in accord with later observations by Kendall (1922), which indicate that the effect of added carbohydrate is to lessen the utilization of proteins as a source of energy, not as material for synthesis.

A number of anaerobie bacteria belonging to the Clostridium group are ineapable of gross utilization of carbohydrates (see Chapter 36), and are dependent upon amino-acids as energy sources. Although bacteria have been described which find adequate energy sources in the employment of chemical mechanisms that, from the formal thermodynamic point of view are comparatively unrewarding, the vigorous growth of the clostridia in media containing protein or protein digests has stimulated the search for other anaerobic energy-yielding mechanisms. Stickland $(1934,1935)$ demonstrated a number of amino-acid dehydrogenase systems in $\mathrm{Cl}$. sporogenes. Certain amino-acids (alanine, valine and leucine) were hydrogen donators, and others (glycine, proline and hydroxyproline) hydrogen acceptors. 
He noted that it would be difficult to picture any reaction by which a single aminoacid could break down anaerobically to yield energy, and that the most probable reaction would involve two amino-acids, hydrogen being transported from one to the other by the action of the bacterial enzymes. The general type of such a reaction might be represented as

$$
\begin{aligned}
& \mathrm{R} \cdot \mathrm{CHNH}_{2} \mathrm{COOH}+\mathrm{R}^{\prime} \cdot \mathrm{CHNH}_{2} \cdot \mathrm{COOH}+\mathrm{H}_{2} \mathrm{O} \\
& \rightarrow \mathrm{R} \cdot \mathrm{CO} \mathrm{COOH}+\mathrm{R}^{\prime} \cdot \mathrm{CH}_{2} \mathrm{COOH}+2 \mathrm{NH}_{3} \text {. }
\end{aligned}
$$

Stickland demonstrated paired oxido-reductive deaminations of this kind between alanine and proline, cysteine and arginine. Woods (1936) suggests that, since these coupled reactions occur at a rate similar to those of aerobic oxidations, they may constitute an important part of the respiratory mechanism of the cell. The same type of activity has been observed in $\mathrm{Cl}$. botulinum, but not in $\mathrm{Cl}$. tetani, which attacks amino-acids singly (Clifton 1940, 1942). Another energy-yielding decomposition was described by Woods and Clifton (1937, 1938), an anaerobic oxidative deamination of amino-acids by $\mathrm{Cl}$. tetanomorphum, in which no hydrogen acceptor is required, since molecular hydrogen is produced as one of the endproducts. Hoogerheide and Kocholaty (1938) confirmed the evidence for coupled Stickland reactions in $\mathrm{Cl}$. sporogenes, and found in addition that gaseous hydrogen can be utilized by the organism, certain amino-acids acting as hydrogen acceptors (see also Woods and Trim 1942, Guggenheim 1944).

As with carbohydrates, our knowledge of protein metabolism is confined mainly to relatively simple systems involving decompositions, and to defining the species of bacterium, and the conditions in which these decompositions take place. Of protein synthesis we know very little, except that it may be catalysed by enzymes that our methods of study have hitherto revealed as concerned only in decomposition. The enzyme aspartase in Bact. coli, for example, which deaminates aspartic acid to give fumaric acid and ammonia, will in suitable conditions catalyse the formation of aspartic acid from ammonia and fumaric acid (Cook and Woolf 1928).

The Action of Bacteria on Fats.-It has long been known that many bacteria are able to decompose fats (see von Sommaruga 1894, Rubner 1900, Eijkman 1901, Carrière 1901, Schreiber 1902, Orla-Jensen 1902, Huss 1908, Söhngen 1911, Wells and Corper 1912, Kendall et al. 1914, Avery and Cullen 1920, Stevens and West 1922, Michaelis and Nakahara 1923, Neill and Fleming 1927, van der Walle 1927, Collins and Hammer 1934). The bacterial lipase induces a simple hydrolysis into glycerol and fatty acid (Trussell and Weed 1937), and, under suitable conditions, the glycerol is further decomposed and the fatty acid oxidized (see Harden 1930).

Lipolytic activity is displayed by many parasitic and pathogenic species, such as Bact. coli, Staph. aureus, streptococci, the pneumococcus and the tubercle bacillus, as well as by saprophytic organisms. But, here as elsewhere, there seem to be wide differences in activity between different bacteria. Many of the parasitic forms are feebly lipolytic, while certain saprophytic species, such as the bacterium isolated by Huss (1908) from milk, are extremely active.

The actively lipolytic bacteria are of considerable inclustrial importance, since they cause rancidity in butter and other fat-containing foods. It is possible, also, that such species play some part in sewage-purification.

It may be noted, in comnection with the fat metabolism of bacteria, that one 
species, at least, can form fat from carbohydrate (Stephenson and Whetham 1922, 1923).

\section{The Nature of Enzymes and Site of Enzyme Action.}

The elucidation and the nature of bacterial enzymes, their mode of action, and even the identity of certain enzymes, which in our present state of knowledge are often little more than names attached to something catalysing a recognizable reaction, must await the isolation of enzymes in a pure state. For the most part enzymes appear to be proteins or closely associated with proteins. They differ in their susceptibility to changes of hydrogen-ion concentration, salt concentration, temperature, and to exposure to various chemicals. For example, Quastel and Wooldridge $(1927 a, b)$ found that in general the dehydrogenases of Bact. coli behaved in the same way when the bacterial suspensions were subjected to increasing temperature, $\mathrm{pH}$, exposure to nitrite, benzene, toluene, phenol, ether, chloroform and propyl alcohol. The first affected were those acting on alanine, glycerol, glycol, the sugars and glutamic acid; next, those acting on lactic, succinic and fumaric acid; and finally the formic and acetic dehydrogenases. With strong solutions of $\mathrm{KCN}$ and to a certain extent with $\mathrm{H}_{2} \mathrm{O}_{2}$, the picture was reversed, the formic and acetic dehydrogenating systems being the least resistant. Extending these studies to non-toxic inhibitors, the authors (1928) found that the lactic dehydrogenase of Bact. coli was specifically inhibited by compounds having in common the groups - $\mathrm{CO} \cdot \mathrm{COOH}$ or $-\mathrm{CHOH} \cdot \mathrm{COOH}$, and the succinic dehydrogenase by compounds with the groups $\equiv \mathrm{C} \cdot \mathrm{CHOH} \cdot \mathrm{COOH}$ or $\equiv \mathrm{C} \cdot \mathrm{CH}_{2} \mathrm{COOH}$ in common. On these groups presumably depends the specific adsorption of the compounds at that part of the enzymic surface responsible for the activation of the lactic or the succinic acid, with a consequent inhibition of dehydrogenase activity.

The multiplicity of enzymic activities exhibited by a simple species-Bact. coli, for instance, is capable of activating over fifty types of dehydrogenations, and both micrococci and streptococci exhibit a wide variety of dehydrogenase activity - inspired the doubt as to whether each enzyme would prove to be a separate chemical entity, on the grounds that the single small bacterial cells would not be large enough to contain them (Grey 1924). The problem of accommodating a large variety of enzymes in a bacterium is not, however, as difficult as first appears. To some extent the production of an enzyme in quantity is conditioned by the presence of a substrate in the bacterial environment (see Chapter 9); so that in the absence of the substrate it is necessary to postulate the presence only of a few molecules of a given enzyme in order that the cell may exhibit the enzyme activity when the substrate is added. The volume of an enzyme molecule, if we take pepsin, with a molecular weight of about 37,000 , as a model, will be of the order of 20 to $30 \mathrm{~m} \mu^{3}$. The volume of an average cell of Bact. coli is about $700 \times 10^{6} \mathrm{~m} \mu^{3}$. Even if we assume a major part of this volume is occupied by non-enzymic material, there is ample room for several thousand enzyme molecules.

In some cases, however, the multiplicity of enzymes may be more apparent than real. Bernheim, Bernheim and Webster (1935) observed that suspensions of Proteus vulgaris.were able to oxidize practically all the known "natural" (l-form) amino-acids. Stumpf and Green (1944) found that this activity was due to a number of enzymes, all but one of which were relatively unstable, and disappeared with ageing of the suspension. The remaining stable enzyme, which they also found in Bact. aerogenes and $P s$. pyocyanea, had nevertheless a wide 
range of action, and catalysed the oxidative deamination of no less than eleven amino-acids.

Quastel (1926) and Quastel and Wooldridge $(1927 a, b)$ suggest that the activation of substrate molecules after their specific adsorption to the enzyme surface is due to their polarization by electrical fields which characterize the "active centres" of cellular and intracellular structures. The active centres are conceived as being developed as the result of molecular strain or distortion of certain groups or molecules brought about by the intermolecular or intramolecular forces that determine the formation of large colloidal aggregates.

As an alternative to the process of absorption and activation, Woolf (1931) suggests that activation results from the distortion of the substrate molecule which occurs when enzyme donators and acceptors combine at the enzymic surface. Direct evidence of such a combination was produced by Stern (1936), who by optical means was able to demonstrate the formation of an intermediate compound, not merely an absorption complex, between substrate and enzyme, in the decomposition of monoethyl hydrogen peroxide by animal liver catalase.

Besides the configuration of the active groups on the surface of the enzymic particle, their activity may also be determined by the spatial relationship of one group with another. The oxygen uptake resulting from the action upon lactic acid of the lactic dehydrogenase and the cytochrome-cytochrome oxidase system of Bact. col $i$ is not increased if cytochrome $c$ and cytochrome oxidase from heart muscle are added to the mixture (Keilin and Harpley 1941), suggesting that the components of the bacterial dehydrogenase system are intimately bound to the protein of a single colloid particle, together with the native cytochrome system, to form a single oxidizing system whose efficiency depends on the mutual accessibility of the components. Succinic dehydrogenase and cytochrome oxidase (Potter 1941) and co-cnzyme I and cytochrome $c$ reductase (Lockhart and Potter 1941) have been found in similar association on particles produced by cell-disintegration. In other words, the efficiency and, it might be added, the specificity, of an enzyme system depends not only on the integrity of the components, but on their spatial distribution in the cell containing them. The various cytochrome compounds, indeed, afford an excellent example of the part the so-called "protein-carrier" plays in determining the nature of an enzyme. Recent work has shown that a common feature of these compounds is an iron atom, held by some of its co-ordination valencies to the pyrrol nuclei, forming a tetrapyrrol compound, while others attach this tetrapyrrol compound to the protein. The activity of the resulting compound, i.e. whether it behaves as a cytochrome, cytochrome oxidase, etc., is determined by the nature of the protein. It follows that the distribution of these respiratory pigments, for example, among the bacteria, will be conditioned more by the nature of the proteins available for conjugation, than by the distribution of the pyrrolic iron compounds (Keilin 1943).

Certain observations by Penrose and Quastel (1930) are of interest from this point of view. There is an organism (Micrococcus lysodeikticus) that is peculiarly susceptible to lysis by an active substance (lysozyme), which is contained in various tissues and secretions (see p. 1020). A suspension of this organism activates lactic acid, glucose, lærulose and glutamic acid, among other substances, as hydrogen donators. After dissolution by lysozyme the bacteria are found to have lost completely their power to activate the hexose sugars and glutamic acid, while retaining some 30 per cent. of their activity against lactic acid. 


\section{The Nutritional Requirements of Bacteria}

Some bacterial species can live and multiply when provided only with very simple food materials; others require far more complex substances. Orla-Jensen (1909) distinguished three main bacterial groups on the basis of their food requirements. The groups are:

(1) Bacteria, obtaining both their carbon and nitrogen from inorganic sources, such as $\mathrm{CO}_{2}, \mathrm{CH}_{4}, \mathrm{NH}_{3}$ or atmospheric nitrogen itself.

(2) Bacteria obtaining nitrogen from inorganic sources but requiring organic substances as a source of carbon.

(3) Bacteria demanding organic substances as sources for both carbon and nitrogen.

The first group consists of autotrophic, the second and third groups of heterotrophic bacteria. The limitations of this grouping have been discussed at some length by Knight (1936), who points out that the difference between autotrophs and heterotrophs is quantitative, the autotrophs using the far more costly means of obtaining their energy; but that the quantitative difference is so large that it amounts to a qualitative difference. This we express in terms of the characteristic methods whereby energy is obtained for carbon assinilation; namely, in the case of autotrophs by the use of radiant energy or the oxidation of inorganic compounds, in the case of heterotrophs by use of carbon compounds already partly synthesized on the paths leading to protoplasm. Knight's monograph, which has been drawn on freely in this and the following sections, should be consulted for details of the argument. Like those who have previously attempted a classification of bacteria upon nutritional grounds, he arranges bacterial species in classes corresponding to increasing complexity of nutritional requirements, but maintains that these are to be regarded only as stages, which merge into one another; there are, for example, heterotrophic bacteria that with training can adapt themselves to an autotrophic existence. We shall proceed to discuss the different nutritional types under the headings of Knight's four stages.

Stage 1.-Carbon is assimilated as $\mathrm{CO}_{2}$, and $\mathrm{N}_{2}$ from inorganic sources, especially ammonia. The energy required for this assimilation (the reduction of the $\mathrm{CO}_{2}$ and the synthesis of protoplasm) is derived from the oxidation of simple inorganic compounds, or from the use of rediant and chemical energy.

The classical studies of autotrophs in Stage 1 have been made upon soil organisms, especially those concerned in the nitrogen cycle. The nitrosifying bacteria of the soil obtain energy by the oxidation of ammonia to nitrites, the nitrifying bacteria by the oxidation of nitrites to nitrates. The relation between the ammonia or nitrite oxidized, and the $\mathrm{CO}_{2}$ assimilated, is a quantitative one (Winogradsky $1890 a, b, c)$. Among the substances oxidized by autotrophs are molecular hydrogen (Kaserer 1905, 1906, Niklewshi 1908) and sulphur and sulphur componnds (Winogradsky 1889). A special class of bacteria obtains energy by a photosynthetic mechanism depending on the action of light on a cell pigment, and in this respect forms a link with the blue-green algæ and the chlorophyll-containing higher plants. These photosynthesizing species include the purple and green sulphur bacteria (see van Niel 1931, 1933, 1944, Muller 1933, Roslofson 1934, 1935, and Gaffron $1934,1935,1944)$. The purple sulphur bacteria contain a red and a green pigment, the green alone beiug concerned in photosynthesis; $\mathrm{H}_{2} \mathrm{~S}$ is oxidized to sulphur, 
and the sulphur to sulphate. The green sulphur bacteria also utilize $\mathrm{H}_{2} \mathrm{~S}$, oxidizing it to sulphur.

Stage 2.--Energy and carbon compounds for assimilation are derived by utilization of carbon compounds more reduced than $\mathrm{CO}_{2}$, which is not assimilated; by the assimilation of nitrogen from simple sources $\left(\mathrm{N}_{2}, \mathrm{NH}_{3}, \mathrm{NO}_{3}\right)$ the organisms can synthesize their protoplasm.

In this group are found a few species utilizing carbon monoxide, methane and other hydrocarbons. Of particular interest is $B$. oligocarbophilus, which, though capable of obtaining its energy by the oxidation of $\mathrm{CO}$, can also utilize simple organic compounds like formic, acetic and butyric acids (Lantzsch 1922), constituting a transitional type between Stage 2 and Stage 3.

Stage 3.-Energy and carbon compounds for assimilation are derived by utilization of carbon compounds more reduced than $\mathrm{CO}_{2}$; amino-acids are required for nitrogen assimilation, some as specific components for the synthesis of protoplasm; ammonia cannot be used as a nitrogen source.

Stage 4.-Energy and carbon compounds for assimilation are derived by utilization of carbon compounds more reduced than $\mathrm{CO}_{2}$. An array of amino-acids are needed for nitrogen assimilation, as specific components for synthesis of protoplasm. Accessory growth-promoting substances are also required, some organisms requiring more than one.

Knight regards the nutritional series obtained by arranging bacteria in order of increasing complexity of nutritional requirements as a possible model for the evolutionary process that has produced the bacterial species at present in existence.

The interpretation of species differences along evolutionary lines is of necessity speculative, and particularly so among the bacteria.

The autotroph capable of obtaining its energy from simple carbon and nitrogen compounds provides us with a possible model for the forms of life that first appeared in an enviromment presumably consisting of little but what we now regard as inorganic compounds. But it does not by any means follow that the original bacteria had in fact a complex metabolism of this kind, capable of building complex proteins, carbohydrates, fats and vitamins from the simplest materials. On this basis, we might suppose that heterotrophs evolved from autotrophs by adaptation to environments richer in orgamic matter, whereby they were able to dispense with enzymes concerned in the synthesis of less complex compounds. With increasing parasitism, the organisms would increasingly lose synthetic power, until a stage was reached when the parasite was, perhaps, dependent on another living organism not only for the supply of already synthesized complex food materials, but for the maintenance of a strictly regulated environment to utilize the food. Thus, we should progress from the bacteria in Stage 4 to stricter parasites like $M y c o$. leprox, and finally to the rickettsiae and the viruses, which need the specific environment of the cytoplasm or nucleus of certain cells for their development, and have few or no demonstrable metabolic processes.

The evolutionary process may, in fact, have gone the other way, the highly complex autotroph being the final stage of a process which started in a virus-like organism, proceeding by the gradual acquisition of new assimilative and synthetic powers.

The study of natural and induced bacterial variation (Chapter 9) gives us no valid indication of trends in nutritional evolution. Variation proceeds in both directions; fastidious strains of limited synthetic powers may be trained to utilize 
simpler compounds as foods; and under other conditions bacterial strains may lose synthetic powers and become more heterotrophic. It is impossible to say of a given bacterium whether it has descended from an autotrophic or a heterotrophic ancestor.

Among groups of bacteria that are closely related on other grounds, there are grades of nutritional relationships that may, perhaps justifiably, be regarded as stages in evolution. But with bacteria as a whole, we must beware of regarding the nutritional series exclusively as the trunk of an evolutionary tree, when it is perhaps more likely that each nutritional group represents the end results of evolutionary branches whose common origin is now lost to us.

Moreover, as van Niel (1943) suggests in a review of the biochemistry of the autotrophs, the distinction between heterotrophs and autotrophs is not only difficult, but sometimes illusory. As an example he quotes two bacteria utilizing molecular hydrogen, which, in mixed culture, had the nutritional requirements of an autotroph, but which, in fact, were both heterotrophs requiring thiamin. One bacterium supplied the other with pyrimidine for the synthesis of the vitamin, and was in its turn rewarded by the supply of thiazole for the same purpose. It is possible that the present-day autotrophs are ultimately as dependent on heterotrophs as heterotrophs are on autotrophs, though the dependence in most cases is not as immediate and apparent as in this obvious example of interdependence.

Essential Nutrients and Growth-stimulating Substances.-The bacteria with which we are concerned in this book are mainly heterotrophs in Stages 3 and 4. The elucidation of the growth requirements of many pathogenic species of bacteria is still incomplete, but in recent years the nutrition of a number has been studied in detail. The media usually employed for the cultivation of bacteria have been derived empirically, and for the most part contain complex organic matter of animal origin, like peptones, meat extracts, serum or egg albumin. With increasing biochemical knowledge of the constitution of organic materials, it has been possible to simplify many of these media to the point where all the constituents are chemically defined, and in certain cases to build up the media with ingredients that have been synthesized in the laboratory. Both "synthetic" and "defined" ingredients should whenever possible be tested, for both may contain chemically undetectable traces of organic substances which may be accessory growth-factors; but since it is unlikely that both preparations contain the same impurities the probability that the observed result is due to a contaminant is thus reduced.

With a battery of amino-acids, carbohydrates, and vitamins, a simple basal solution of inorganic salts may be enriched until the mixture supports the growth of bacteria. Alternatively, solutions of peptone, or watery extracts of yeast, may be fractionated, and the fractions found necessary for growth chemically identified. The similarity of metabolic processes in all living cells, whether fungal, bacterial, plant or animal, often makes possible a short cut in the identification of nutrients in culture media, especially with vitamin-like substances. A particular fraction, before the active principle is identified, may exhibit the properties of a known animal or yeast vitamin, and tests with the known vitamin rapidly elucidate the composition of the medium under analysis. But even though the minimum growth-requirements of a bacterium have been established by these methods, we have by no means necessarily defined the essential nutrients of the organisms. The inorganic requirements may apparently be satisfied by the ions potassium, sodium, calcium, magnesium, iron, phosphate, sulphate, carbonate and chlorides, 
but an undetectable though essential trace of metal may also be present (cf. the necessity for molybdenum in the fixation of nitrogen by Azotobacter; Burk 1934).

A particular ingredient may play no essential part in the metabolism of the bacterium, but promote growth by neutralizing a toxic substance in the medium, or by changing the physical state of the medium (see, for example, O'Meara 1937) to one more suitable to essential metabolic processes. Thus the addition of charcoal to a medium is said to improve its growth-promoting properties for gonococci and meningococci (Glass and Kennett 1939) and for tubercle bacilli (Nassau 1942). According to Glass and Kennett, the charcoal acts as an adsorbent of inhibitory substances in the medium, perhaps toxic metabolic products, or by catalysing certain oxidations. Substances even more inert than charcoal are beneficial to growth. For example, Bact. coli will grow better in distilled water containing $\mathrm{CO}_{2}$ and ammonia if talc is added, the talc acting presumably by reason of a surface action that renders the gases more available as nutrients (Bigger and Nelson 1943); and low concentrations of agar, by reducing the rate of diffusion of oxygen from the air, stabilize redox-potentials obtaining in fluid media at levels suitable for the growth of anaerobic bacteria (Reed and Orr 1943; see also Gould 1944). Zobell (1943) in a study of the growth of-bacteria found in sea-water, concluded that solid surfaces promoted growth by concentrating nutrients through adsorption, by providing a resting-place for bacteria, and by retarding the diffusion of exo-enzymes and hydrolysates away from the cell, thereby enhancing the assimilation of substances hydrolysed outside the cell. Certain proof of the participation of a given "essential nutrient" in bacterial metabolism is difficult to obtain, though the marking of food substances with radioactive elements (see $\mathrm{CO}_{2}$ requirements, below) may increase the certainty. Assuming that a basal medium has been established, of which separate ingredients are free of impurities, we are still faced with various difficulties. Gladstone (1939), for example, grew a strain of the anthrax bacillus in a defined medium containing a number of amino-acids. The medium ceased to support growth if either valine or leucine was removed, and supported growth feebly if isolencine, glycine or cystine was absent. Glycine and cystine were subsequently proved to be synthesized by the organism, but the three remaining acids were apparently essential for growth. However, the medium could be made to support growth by the removal of all three amino-acids; singly, each had a toxic effect; that of valine was counteracted by leucine, vice versa, and that of isoleucine by valine and lencine together. None of them was an essential mutrient. (In this connection see also McLeod and Wyon 1921, Wyon and McLeod 1923, Ruggierini 1933.) Again, the mutrients may be tested in too complex a form, and a precursor of the substance prove equally effective. Thus Staph. aureus requires thiamin (vitamin $B_{1}$, Knight 1937), but it can synthesize thiamin if the two constituents, thiazole and pyrimidine, are substituted in the medium (Knight and McIlwain 1938).

Considerable difficulty arises when we attempt to define the meaning of the term "essential nutrients". We may define them as essential for growth of a bacterium, no matter how that growth may be achieved. In that case we must to some extent ignore the changes that the experimental procedure may induce in the bacterium, for its properties in the final medium may be markedly different from those in the starting medium. We may illustrate this by the nutrition of the typhoid bacillus. Braun and Cahn-Bronner (1921, 1922) grew Salm. typhi, Salm. paratyphi $B$, and Salm. enteritidis in media containing mineral salts, various 
three- and four-carbon organic acids, and ammonium salts as a source of nitrogen. One strain of Salm. typhi failed to grow on any of the media, nor would it grow when glucose was substituted for the organic acids as a carbon-source; but it grew when provided with tryptophan. The variation in the tryptophan requirements of different strains of Salm. typhi was studied by Fildes and his colleagues (Fildes, Gladstone and Knight 1933, Fildes and Knight 1933). The organism grew in a basal medium containing sodium citrate, magnesium sulphate, phosphate buffer and glucose, together with fourteen amino-acids : alanine, glycine, valine, glutamic acid, asparagine, tyrosine, phenylalanine, proline, histidine, arginine, leucine, lysine, cystine and tryptophan. Any one of the first ten could be omitted without affecting growth; the omission of leucine or cystine caused delay in growth, and in the absence of tryptophan there was no growth. The concentration of tryptophan necessary for growth in the presence of other sources of nitrogen was as little as 0.00064 per cent. Next, two strains of Salm. typhi were trained to grow in the absence of tryptophan, with ammonium chloride as the sole source of nitrogen; they had evidently adapted themselves to synthesize the amino-acid, for it was demonstrable in the bacterial cell by the glyoxylic reaction. It would seem therefore that tryptophan is an essential constituent of the typhoid bacillus; that most strains when first removed from media that contain it are unable to synthesize it; but that they can be trained to do so by gradually accustoming them to a medium from which it is absent. Several other species were examined by the same method (see also Fildes 1935, Fildes and Richardson 1935), and from these observations Fildes and his colleagues concluded that tryptophan was essential in the metabolic processes of the bacteria studied (an " essential metabolite"). They divided bacteria into three groups: (1) Those that can synthesize tryptophan; such as autotrophic bacteria, and, among the potentially pathogenic organisms examined, Bact. coli, Ps. pyocyanea and Salm. typhi-murium: (2) Those that are unable to synthesize tryptophan for themselves, but car be trained to do so; e.g., certain strains of Salm. typhi, C. diphtherice, Staph. aureus and probably Myco.tuberculosis: (3) Those that cannot synthesize tryptophan, yet for which it is an essential nutrient; to this group belong $\mathrm{Cl}$. tetani, $\mathrm{Cl}$. sporogenes, $\mathrm{Cl}$. botulinum and $\mathrm{B}$. anthracis. As a result of analyses of cultures of Salm. typhi, Burrows $(1939 a, b)$ concludes that in no circumstances is tryptophan an essential nutrient for the organism, but that for those strains whose synthetic powers are small, tryptophan acts as a stimulant of growth.

The distinction between a stimulant and an essential nutrient is important, for if we are to define bacteria studied in this manner by their synthetic abilities, either manifest or latent, we may regard organisms as devoid of a particular synthetic ability, which in fact may have it but in a degree insufficient to allow growth to take place; and later researches may demonstrate this ability, either by successful training of the organism, or by developing more sensitive methods of demonstrating small amounts of the metabolite in question.

Again, the medium containing minimum requirements for growth may not permit the development of the full characteristics of the parent strain. Gladstone (1937) succeeded in training certain strains of Staph. aureus to utilize ammonia as a sole source of nitrogen. Neither coagulase nor $\alpha$-hæmolysin (see Chapter 25) was produced in this medium ; for the production of optimum quantities of hæmolysin, arginine, glycine, proline, phenylalanine and valine were necessary. The trained Staph. aureus exhibited all its initial metabolic properties when transferred to ordinary rich laboratory media. 
A nutrient may therefore be defined as a substance that is essential either for minimal growth, perhaps under restrieted conditions, of an organism trained to be as unexacting as possible, or for optimal growth. The studies of Mueller (1940), for example, on the nutritional requirements of the diphtheria bacillus, have been conducted on the basis of optimal growth, which he defines as the best attainable upon empirically devised media. In the first case, a medium is sought that will promote the growth of at least one of the cells originally inoculated, and the production of a trained culture is most probably due to selection of eells with the greatest synthetic powers. In the second ease, not only the reproduction, but the ready and profuse growth, of all the viable cells of the inoculum is aimed at. The substances required for this purpose, over and above the minimal requirements, are generally called stimulants of growth, though Lwoff (1938) in his review of growth factors for micro-organisms distinguishes stimulants from "starting factors," which are substances essential for the growth of an organism at the commencement of its training, but which are dispensed with by the trained organism. In some cases, however, the addition of a starting factor to the minimal medium enhances growth, and the distinction breaks down. A true stimulant presumably aets by supplying in abundance an essential metabolite that the bacterium itself synthesizes only at a relatively slow rate.

With these reservations in mind, we may note that for many heterotrophic bacteria in Stages 3 and 4, the essential nutrients, carbohydrate, nitrogenous, and growth factors (vitamins), have been defined. Among bacteria pathogenic for man we have already eited Staph. aureus and C. diphtherice, and to these we may add Str. homolyticus (see Mellwain 1940); Group D streptococei (Woolley and Hutchings 1940); Str. salivarius (Smiley, Niven and Sherman 1943); Str. lactis (Niven 1944), and Str. focalis (Niven and Sherman 1944); Myco. tuberculosis (see, for example, Lockemann 1942); N. meningitidis (Frantz 1942); N. gonorrhcece (Gould, Kane and Nueller 1944); Br. abortus, melitensis and suis (Koser, Breslove and Dorfman 1941, Koser and Wright 1942, McCullough and Dick 1942); and certain members of the Salmonella group (Johnson and Rettger 1943). The requirements of different strains of organisms belonging to the same species may vary widely, some having greater, some lesser synthetic powers than the strain for which the essential nutrients were first defined. There is a large number of heterotrophic bacterial species for which the essential nutrients are as yet only partly defined; for the most part the carbohydrate and amino-acid requirements are readily established. For example, the main body of essential nutrients is defined for certain clostridia (Elberg and Meyer 1939); pneumococeus Type III (Badger 1944); Br.tularensis (Tamura and Gibby 1943); certain pasteurellæ (Berkman 1942); and Cl. tetani (Mueller and Miller 1942, Feeney, Mueller and Miller 1943, Pickett, 1943). These organisms are presumably in Stage 4, since the remaining unidentified factors are usually active in low concentrations and are probably accessory growth factors.

\section{The Vitamin Requirements of Bacteria.}

In addition to foodstuffs needed for energy and synthesis, many of the heterotrophic bacteria require vitamins. A vitamin or accessory growth factor is an essential nutrient active in concentrations so small that it is unlikely to act as an important source of either carbon, nitrogen or energy. Such substances range from inorganic ions to complex organic substances like thiamin or riboflavine, and the majority so far described appear to be in some way connected with the function or the structure of enzyme-co-enzyme systems of the organism. It is customary to limit the term vitamin to organic compounds, but it should be noted that, on the ground of their aetivity in low concentrations, many inorganic substances have equal claim to the title. In certain conditions, for example, the 
toxin production by $C$. diphtherice is maximal with an optimal concentration of iron as low as $2.8 \times 10^{-6}$ M (Pappenheimer and Johnson 1936), and maximal toxin production by $\mathrm{Cl}$. tetani is similarly conditioned (Mueller et al. 1943).

It is impossible to review the relevant literature of the organic accessory growth factors here; summaries of recent work will be found in the reviews of Lwoff (1938) and Koser and Saunders (1938). In recent years, the identification and purification of many bacterial growth factors makes it possible to assign to them the rôle of vitamin. As noted above, tryptophan is essential for Salm. typhi, whose needs are satisfied by a concentration of $0.0006 t$ per cent., if an alternative source of nitrogen is supplied for the synthesis of protoplasm. Among other amino-acids we may note that cysteine is an essential accessory growth factor for Staph. aureus, being active in concentrations of less than $10^{-6} \mathrm{M}$, though less complex S-compounds can replace it in a defined medium (Fildes and Richardson 1937), and glutathione is similarly essential for N. gonorrhoee (Gould 1914); $\beta$-alanine is a growth factor for $C$. diphtherice. (Mueller and Cohen 1937), though the organism is able to produce it from $l$-carnosine (Mueller 1938). In $C$. diphtherice $\beta$-alanine may be the precursor of pantothenic acid; but Evans, Handley and Happold (1939) fomnd certain gravis strains of $C$. diphtherice which needed both $\beta$-alanine and pantothenic acid. Pantothenic acid is apparently essential for certain streptococei (McIlwain 1939, 1940, Woolley and Hutchings 1940, Schuman and Farrell 1941), lactic-acid bacteria (Snell, Strong and Peterson 1939), Brucella abortus (Koser, Breslove and Dorfman 1941), Pr. morgani (Dorfman, Berkman and Koser 1942) and Sh.flexneri (Weil and Black 1944). Glutamine, which can be synthesizer by the cocci from glutamic acid, is required by Str. homolyticus (Fildes and Gladstone 1939, McIlwain, Fildes, Gladstone and Knight 1939); it accelerates the growth of a large number of organisms. Of the recognized animal vitamins, thiamin was first recognized as a growth stimulant for bacteria by Tatum, Wood and Peterson (1936) working with the propionic acid bacteria. Staph. aureus, which also needs thiamin as an essential metabolite, can synthesize it from pyrimidine and thiazole (Knight 1937, Knight and IIcIlwain 1938). Thiamin, the phosphate of which acts as a co-enzyme in carboxylase systems, probably plays a fundamental metabolic rôle in all micro-organisms (see, for example, Quastel and Webley 1941, 1942), those for which it is not an essential nutrient being able to synthesize it. Some dysentery bacilli, for example, apparently synthesize thiamin in a defined medium containing nicotinic acid (Dorfman, Koser, Reames, Swingle and Saunders 1939).

The rôle of nicotinic acid has been studied extensively. It is an essential nutrient of Staphylococcus (Knight 1937, Holiday 1937); C. diphtherice (Mueller 1937), some dysentery bacilli (Koser, Dorfman and Saunders 19t0) ; Proteus (Fildes 1938, Pelczar and Porter 1940); Brucella (Koser, Breslove and Dorfman 1941); and Hom. pertussis (Hornibrook 1940) ; though, as with other essential nutrients, not all strains of a given species require it. The significance of nicotinic acid lies in its relationship with co-enzyme I and co-enzyme II, which are respectively diphosphopyridine nucleotide and triphosphopyridine nucleotide. It is supposed that nicotinic acid, or nicotinamide, is synthesized into co-enzyme I by organisms that cannot themselves synthesize the pyridine ring. As we have seen, co-enzyme I is essential in certain dehydrogenase systems.

Hcom. influenzce and Hcom. para-influenzo have even more restricted synthetic powers. Hom. para-influenzo requires co-enzyme I already synthesized for many 
of its dehydrogenations (Lwoff and Lwoff $1937 a, b$ ). According to Schlenk and Gingrich (1942) the co-enzyme can be replaced by the nicotinamide riboside, while a mixture of nicotinamide, $d$-ribose and adenylic acid is inactive. The utilization of the riboside may thus be considered the limit of adaptability of this organism (Gingrich and Schlenk 1944) in the direction of the synthesis of co-enzyme I. Many bacteria are capable of synthesizing co-enzyme I from simpler substances and can therefore stimulate the growth of the influenza and para-influenza bacilli by reason of their co-enzyme content. For instance, certain lactic-acid bacteria can utilize the purine and pyrimidine bases (Stokstod 1941), and Cl. tetani the purine base alone. The conception of nicotinic acid simply as a building block for co-enzyme I in all bacteria has recently been questioned by Saunders, Dorfman and Koser (1941). In a deficient medium, the stimulating effect of co-enzyme I on dysentery bacilli was less than that of the nicotinamide supposed to serve for its synthesis. Co-enzyme I split by hydrolysis, on the other hand, was as active as nicotinamide. It is possible that in this case the nicotinamide may be utilized to form a respiratory hæmochromogen with hæmin. The close relation of nicotinic acid with glycolysis is illustrated by the action of Salm. paratyphi $A$ and Sh. shigce on fermentable carbohydrates. According to Kligler and Grossowicz (1940, 1941), nicotinic acid is not a growth-promoting substance for these organisms. In its absence there is a slow and partial aerobic glycolysis, and in consequence only a slow protein dissimilation. In its presence good growth occurs and the carbohydrate is rapidly oxidized with the production of $\mathrm{CO}_{2}$. In Staph. aureus, nicotinic acid is essential for the oxidation of glucose, which under aerobic conditions is converted into pyruvic and lactic acids. The addition of thiamin as well permits the oxidation of the pyruvate; glycolysis as a whole is more active, and the end products are acetic and lactic and carbonic acids. In the absence of nicotinic acid in a synthetic medium, glucose has an inhibitory effect on growth, perhaps by interfering with the oxidation of the essential amino-acids present (Kligler, Grossowicz and Bergner 1943a, b).

Just as the influenza bacillus is incapable of synthesizing the co-enzyme needed for its dehydrogenase systems, in the same way it requires a supply of hæmin ready made for incorporation in the enzyme systems concerned with oxygen transport. It has long been known that Ham. influenzœ requires hæmatin (the $\mathrm{X}$ factor, see Chapter 33 ) and that, grown anaerobically, the organism can dispense with it. Lwoff and Lwoff $(1937 c)$ concluded from respiratory studies that the hæmatin was needed for the synthesis of cytochrome, cytochrome oxidase, catalase and peroxidases, i.e. it was essential to the completion of the chain of mechanisms transferring hydrogen to atmospheric oxygen.

Among other accessory growth factors of known composition needed by bacteria we may briefly mention riboflavin for lactobacilli (Snell and Peterson 1940), and streptococci (McIlwain 1940, Woolley and Hutchings 1940, and Schuman and Farrell 1941); lactoflavin for propionobacteria and streptococei (Orla-Jensen, Otte and Snog-Kjaer 1936); and pimelic acid for $C$. diphtherice (Mueller 1937); $p$-aminobenzoic acid (see p. 162); pyridoxin for certain streptococei (Pappenheimer and Hottle 1940, Schuman and Farrell 1941); and oleic acid for C. diphtheriae (Cohen, Snyder and Mueller 1941) and Erysipelothrix (Hutner 1942).

It will be scen that the majority of bacterial vitamins are also vitamins for other organisms, including the higher maminals. Pimelic acid is of particular interest, since it has not been recognized as a vitamin in other types of organisms, nor is it a compound that seems likely to play the part of a co-enzyme in bacterial metabolism. Its significance 
as a growth factor, however, has become a little clearer with the identification of biotin (du Vigneaud, Hofmann and Melville 1942). Wildiers (1901) described a growth-promoting fraction in yeast extract, to which he gave the name "bios." Many of the individual growth-promoting substances in bios have been identified, and include $i$-inositol, $l$-leucine, $\beta$-alanine, thiamin and pantothenic acid (see lioser and Sannders 1938) and pyridoxin (Schultz et al. 1939, Eakin and Williams 1939). There remained biotin (Kögl and Tönnis 1936), a remarkable growth factor active in concentrations of $10^{-10}$ or less. Biotin proves to be a keto-imidazolido-thiophane-valeric acid, and it is possible that pimelic acid may contribute the fatty-acid chain in the bacterial synthesis of biotin by $C$. diphtherice. In support of this, du Vigneaud, Dittmer, Hague and Long (1942) found that pimelic acid and biotin behaved alike in the growth stimulation of $C$. diphtherice. Working with the mould Aspergillus niger, Eakin and Eakin (1942) showed that not only was pimelic acid a growth stimulant, but its addition to the medium inereased the biotin content of the culture about twenty-fold.

Biotin itself has been found essential for a number of bacteria, including Str. pyogenes (Hottle, Lampen and Pappenheimer 1941), Staph. aureus (Porter and Pelczar 1941), and Br. abortus (Koser, Breslove and Dorfman 1941).

Biotin preparations are apparently essential for $\mathrm{Cl}$. acetobutylicum (Oxford, Lampen and Peterson 1940), Brucella (Koser, Breslove and Dorfman 1941), Str. pyogenes (Hottle, Lampen and Pappenheimer 1941), and Staph. aureus (Porter and Pelczar 1941). There are also many factors as yet not fully identified, such as an apparently lipoid stimulating factor for the tubercle bacillus (Boissevain and Schultz 1938). Another vitamin-like substance as yet unidentified that appears to have a wide distribution in bacteria, first isolated by Knight and Fildes (1933) from a yeast gum, was necessary for $\mathrm{Cl}$. sporogenes and $\mathrm{Cl}$. botulinum (Fildes 1935). It is probably synthesized by a number of organisms, including Salm.typhi-murium and the tubercle bacillus.

The identity of many bacterial and animal vitamins, and the discovery of large numbers of bacterial species for which the various vitamins are essential nutrients, enables us to use bacteria in the assay of these substances for therapeutic and other purposes. The vitamin for assay is added in varying concentrations to cultures of the bacterium for which it is an essential nutrient, in a medium lacking the vitamin, and the growth-promoting effect estimated. Clearly the assay will be accurate only with bacteria whose growth requirements are defined in every respect. Even so, errors will arise, for minute traces of contaminating vitamius may be present in the materials used for the basal medium, and the preparation of vitamin to be assayed may contain substances which are not essential nutrients, but nevertheless have a stimulating action on growth.

(For a discussion of the B-vitamin content of media in common bacteriological use, see Stokes, Gunness and Foster 1944).

\section{The Gaseous Requirements of Bacteria.}

Examples of autotrophic bacteria utilizing hydrogen and $\mathrm{CO}_{2}$ have been given in previous sections, but $\mathrm{CO}_{2}$ has been considered only as a main source of carbon. $\mathrm{CO}_{2}$ may also act as a subsidiary source of carbon, or as an accessory growth factor. Reference to Chapter 34 will afford a striking example of a heterotroph, which on first isolation from the tissues fails to grow in air unless 5 to 10 per cent. of $\mathrm{CO}_{2}$ is added to the atmosphere. Other species flourish only in a similar excess, while others still require $\mathrm{CO}_{2}$, but in smaller concentrations.

Numerous observations of the $\mathrm{CO}_{2}$ effect suggest that this gas is necessary for the growth of many bacterial species, including anaerobes (see, for instance, Chapin 1918, Cohen and Fleming 1918, Rockwell and McKhann 1921, Rockwell 1921, 1923, 1924, Rockwell and Highbergher 1926, 1927, Valley and Rettger 1927, Valley 1928, NcLeod, Coates, 
Happold, Priestley and Wheatley 1934, Aitkin, Barling and Miles 1936, Nye and Lamb 1936, Khairat 1939, Fleming 1941, Kempner and Schlayer 1942).

In some cases the gas stimulates, not growth, but the appearance of some particular metabolite. On artificial media, for example, Staph. aureus (Chap. 25) produces significant amounts of its characteristic toxin in air containing 20 per cent. of $\mathrm{CO}_{2}$, and the anthrax bacillus its capsular substance (Ivánovics 1937) in the presence of an excess of $\mathrm{CO}_{2}$.

The use of defined media has made the study of $\mathrm{CO}_{2}$ requirements more exact. Walker (1932) found that Bact. coli failed to grow in a liquid synthetic medium when incubated in a current of $\mathrm{CO}_{2}$-free air. Walker concluded that $\mathrm{CO}_{2}$ was necessary for growth, and that the lag phase (sce Chapter 4 ) represented the time taken by the organism to produce in its immediate vicinity a growth-promoting concentration of $\mathrm{CO}_{2}$. Gladstone, Fildes and Richardson (1935) demonstrated a similar inhibition for Salm. typhi, Ps. pyocyanea, B. subtilis, B. anthracis, $C$. diphtherice, $C l$. sporogenes and $C l$. welchii by the passage of $\mathrm{C}^{\prime} \mathrm{O}_{2}$-free gas through culture media.

The nature of the stimulating action of $\mathrm{CO}_{2}$ is not clear. Carbon dioxide is utilized, even by heterotrophic bacteria. Wood and Werkman (I936, 1938, 1940) and Krebs and Eggleston (1941) demonstrated its fixation by propionobacterịa and its probable rôle in the synthesis of a four-carbon chain from pyruvic acid. Wood and his colleagues (1940), by using $\mathrm{CO}_{2}$ made from the radioactive carbon isotope $\mathrm{C}_{13}$, was able to trace the isotope as far as succinic acid. Bact. coli and some clostridia utilize $\mathrm{CO}_{2}$ (Ruben and Kamen 1940). Hes (1938), on the other hand, suggests that the $\mathrm{CO}_{2}$ acts as a catalyser in several oxido-reduction reactions. The possible interrelationship of $\mathrm{CO}_{2}$ with enzyme systems is evident from the work of Pappenheimer and Hottle (1940), who found that adenylic acid was a growth stimulant for Str. pyogenes if the $\mathrm{CO}_{2}$ concentration was as low as 0.25 per cent., but was dispensable if the concentration was raised to 2.5 per cent. Similarly, McCullough and Dick (1942) found that the incapacity of forty-one strains of Br. abortus to grow in the absence of a high concentration of $\mathrm{CO}_{2}$ in the atmosphere (see Chapter 34) was associated with incapacity to grow in a certain medium containing aminoacids and four vitamins. After the strains had been trained to grow in air, thirty of them grew in the basal medium.

\section{Aerobiosis and Anaerobiosis.}

It is customary to divide bacteria into three categories in regard to their behaviour towards molecular oxygen: (1) the obligatory anaerobes-or anaerobes withont the qualifying adjective-which will grow only when oxygen is rigorously excluded (2) the facultative anaerobes, which will grow both aerobically and anaerobically, i.e. in the presence or absence of oxygen, and (3) the obligatory aerobes, which will grow only when supplied with molecular oxygen.

Most of the organisms in Class (2)-to which incidentally the great majority of the bacteria with which we are concerned belong-are able to grow over a very wide range of oxygen pressures, but some species prefer a relatively restricted range, lying well below that of ordinary atmospheric conditions.

It shonld be noted that in the fluid cultures commonly employed in bacteriology, the degree of oxygenation in cultures of aerobic bacteria is by no means necessarily optimal. The oxygen content in the depths of a broth culture may be very low (Rahn and Richardson 1941). In many cases, forced aeration of the culture greatly increases growth (see Wilson 1930, Rahn and Richardson 1942), though the effect may in some cases be due as much to removal of $\mathrm{CO}_{2}$ as to the increased oxygen supply.

In a large number of aerobic and facultatively anaerobic bacteria, the capacity 
to grow in the presence of uxygen, and to utilize it, depends on the possession of a cytochrome-cytochrome oxidase system. The molecular oxygen may, however, be ntilized by other respiratory mechanisms, for some facultative anaerobes have no cytochrome. When the organisms are deprived of oxygen, the predominant metabolic mechanisms change, with a consequent change in the nutritional requirements of the organism. It was Pasteur's observations on the different metabolic products associated with the aerobic and anaerobic growth of certain bacterial species that led him to place so great an emphasis on the absence of oxygen as a determinant factor in bacterial fermentation. We have already noted some of the changes in nutritional requirements consequent on anaerobic growth. The change may concern energy sources or essential nutrients, or accessory growth factors. Anaerobically, $H$. influenze can dispense with hæmatin, since it has no need of hæmochromogen catalysts for oxygen transfer. The pyrimidine base uracil is required as an essential nutrient in addition to thiamin and nicotinic acid when Staph. aureus is grown anaerobically.

It is clear that the respiratory enzyme systems of bacteria may be adapted to aerobic or anaerobic oxidations. It is much less clear, however, why the strict anaerobes should be incapable of growth in the presence of oxygen. The explanation of this inhibitory action of oxygen so far advanced has been made in terms of oxidation-reduction potentials in the medium, or the production by the anaerobes of toxic oxidation products when exposed to air.

The electrical measurement of reducing intensity developed in a culture of bacteria gives us a positive or negative value for the Eh, in volts. The greater the reducing intensity, the more negative this ralue. The Eh level in a culture is dependent upon a large number of factors. Alterations of $\mathrm{pH}$, for example, will alter the concentration of available electrons, and so alter the Eh. Moreover, a particular Eh value cannot be ascribed to a particular oxidation-reduction system in the culture, but may be the resultant of several systems, each of which influences the observed level according to its characteristic $\mathbf{E}^{\prime}$, its relative predominance in the culture, its spced of action, and the "poising" effect it has upon the culture as a whole. But though it has proved impossible to sort out all these contributory factors, or to make any valid inferences about Eh levels inside or at the surface of bacterial cells themselves, observations upen the Eh changes in culture throw some light upon the conditions governing the growth of aerobic and anaerobic bacteria. For instance, the aerobic growth of Str. pyogenes in a medium having an initial Eh of +0.3 volt is accompanied by a gradual fall in potential to ahout $0.15-0.2$ volt. The spores of an anaerobe like $\mathrm{Cl}$. tetan $i$ will not grow in a similar medium unless the starting Eh is as low as +0.11 volt (Knight and Fildes 1930), though once established, the organism itself reduces the Eh to still lower levels. The limiting potentials that will allow the growth of certain bacterial species have been determined in a number of cases (see McLeod 1930, Hewitt 1936, Gillespie and Rettger 1938a, b, Gillespie and Porter 1938, Stephenson 1939). It appears that, in general, anaerobic organisms, unlike the aerobes, are unable to reduce ordinary aerobic media to the Eh level at which they can germinate; though once growth is established, they can maintain it at a low level; the medium must be partially reduced for the culture of anaerobes. In bacteriological practice, the reduction is usually achieved by the exclusion of molecular oxygen from the culture. The reduction of oxygen pressure by this means is not, however, a necessary feature of anaerobic eulture (see, for example, Kligler and Guggenheim 1938); anaerobiosis may be achieved, even though molecular oxygen has access to the medium, by the addition of reducing substances like thioglycollic acid (Quastcl and Stephenson 1926), reduced iron (Hastings and McCoy 1932), granules of cooked meat (Lepper and Martin 1929, 1930a, $b$ ), or by the concomitant growth of an aerobic organism capable of bringing the Eh down to the required level (see also Reed and Orr 1943). It may be that oxygen per se is inhibitory, 
but that the presence of reducing substances in the medium prevents its direct access to the organisms; or the inhibiting effect may be due to the formation of oxidation products through the mediation of catalysts which are inactivated in cultures at Eh levels permitting the growth of obligate anaerobes. The change over from aerobic to anaerobic growth may in fact be brought about by artificial inactivation of enzyme systems. BrolKahn and Mirsky (1938), by cyanide-inhibition of enzymes capable of reacting with molecular oxygen, induced Bact. coli to break down glucose anaerobically in the presence of air, and it is obvious that similar inhibitions may take place as a result of changes in metabolic processes that are reflected in the lower Eh levels of anaerobic cultures. The nature of the hypothetical inhibitory oxidation products is still in doubt. It has long been known that certain bacteria are sensitive to hydrogen peroxide (Traugott 1893, Freer and Novy 1902). This sensitivity is associated with absence of catalase production. The pneumococcus, for instance, produces $\mathrm{H}_{2} \mathrm{O}_{2}$, to which it is sensitive, and no catalase (McLeod and Gordon 1922, Avery and Morgan 1924, Avery and Neill 1924a, b, $c$, Neill and Avery 1925). Other organisms, like $S h$. shigo, are moderately sensitive, but produce ncither $\mathrm{H}_{2} \mathrm{O}_{2}$ nor catalase. Most bacterial species produce catalase more or less actively (see Gottstein 1893, Löwenstein 1903, Rywosch and Rywosch 1907). The fact that anaerobes produce no catalase led McLeod and Gordon $(1925 a, b)$ to suggest that the sensitivity of obligate anaerobes to oxygen is due to their readiness to form in its presence inhibitory concentrations of $\mathrm{H}_{2} \mathrm{O}_{2}$. The acceptance of this hypothesis depends on the demonstration of oxygen utilization and $\mathrm{H}_{2} \mathrm{O}_{2}$ production by the anaerobes in the presence of oxygen. McLeod and Gordon grew the anaerobes in blood media, and inferred the production of $\mathrm{H}_{2} \mathrm{O}_{2}$ from certain colour changes in the medium. Similar changes, however, can be produced by reducing systems in the absence of air (Anderson and Hart 1934) and do not necessarily indicate $\mathrm{H}_{2} \mathrm{O}_{2}$. Moreover, Cook and Stephenson (1928) were unable to demonstrate oxygen utilization by $\mathrm{Cl}$. sporogenes in conditions that would have detected the oxygen uptake equivalent to the formation of $1: 50,000 \mathrm{H}_{2} \mathrm{O}_{2}$ in their suspensions.

\section{Other Factors Influencing the Growth of Bacteria.}

Hydrogen Ion Concentration.-For any giren species of bacterium there is an optimal, and relatively narrow range of $\mathrm{pH}$ allowing vigorous growth, and a wider range extending on each side of the optimum over which growth occurs less vigorously. For most of the bacteria with which we are concerned the optimal $\mathrm{pH}$ lics a little to the alkaline side of neutrality $(\mathrm{pH} 7 \cdot 2-7 \cdot 6)$. The range of $\mathrm{pH}$ over which growth is possible has not been accurately determined for many bacterial species, but for most pathogenic species it would appear to extend over some such range as $\mathrm{pH} 5.0$ to $\mathrm{pH} 8.0$ (see Chapter 9).

There are, however, species that show very distinctive variations from this modal range of sensitivity. Azotobacter, for instance, is very sensitive to acids, and will not grow in pure culture at a $\mathrm{pH}$ lower than 6.5 (Fred and Davenport 1918). The aciduric bacilli of the genus Lactobacillus are, on the other hand, highly resistant to acids, and will apparently grow to some extent at $\mathrm{pH} 4.0$ or even less; though the power to grow has not, in this instance, been decisively distinguished from the power to resist the lethal action of the acid (McIntosh et al. 1922, 1924). The cholera vibrio is very tolerant of alkali, relatively sensitive to acid. Its optimum for growth is $\mathrm{pH} 7 \cdot 6-8 \cdot 0$ and its limits for growth about $\mathrm{pH} 6 \cdot 4-9 \cdot 6$. The enterococcus affords a good example of an organism with a wide growth-range, extending from $\mathrm{pH} 4.8$ to $\mathrm{pH} 11.0$ (Downie and Cruickshank 1928, Davis and Thiel 1939).

Temperature.-We need only note in this section that, for each species of bacterium, there is an optimal temperature for growth and a range of temperature over which growth is possible. For most pathogenic bacteria the optimal temperature for growth is in the neighbourhood of $37^{\circ} \mathrm{C}$., and the range of temperature over which growth occurs is approximately $15^{\circ}-40^{\circ} \mathrm{C}$. Here as elsewhere, however, there are wide variations, especially when we include in our survey non-pathogens as well as pathogens. In their reactions to changes in temperature, bacteria as a whole display in a striking fashion their capacity for adaptation to a wide range of environmental conditions. 
To take a few illustrative examples among pathogenic and potentially pathogenic species, Bact. coli grows best at $37^{\circ} \mathrm{C}$., but will grow at any temperature within the range of $15^{\circ}-45^{\circ} \mathrm{C}$. Among the Gram-negative cocci there are characteristic differences in reaction to temperature; thus $N$. gonorrhece, N. meningitidis and $N$. catarrhalis all show optima at about $37^{\circ} \mathrm{C}$.; but the range of growth of $N$. catarrhalis extends from approximately $18^{\circ}$ to $42^{\circ} \mathrm{C}$., while $N$. gonorrhoce and $N$. meningitidis show a very restricted range of about $30^{\circ}$ to $38^{\circ} \mathrm{C}$. The different types of tubercle bacilli show temperature optima in conformity with the body-temperatures of the host-species that they infect; thus the human and bovine types of tubercle bacillus grow best at $37^{\circ} \mathrm{C}$. and fail to grow below $30^{\circ} \mathrm{C}$.; the avian type grows best at $40^{\circ} \mathrm{C}$, and again fails to grow below $30^{\circ} \mathrm{C}$, while the cold-blooded type grows freely at $22^{\circ} \mathrm{C}$.

As examples of non-pathogenic species that depart from the temperature optima giren above we may note (see Buchanan and Fulmer 1928-30) that bacteria have been isolated from fish, brine and similar sources that grow well at $0^{\circ} \mathrm{C}$.; while from a variety of natural sources (soil, excreta, silos and especially hot springs) thermophilic species have been isolated that have optima at $55^{\circ} \mathrm{C}$. or over, and are able to multiply at a temperature of $75^{\circ} \mathrm{C}$. These thermophiles are of considerable economic importance, since they are a source of difficulty when it is desired to sterilize any material at a relatively low temperature (see Chapter 5).

There appears to be a definite relationship between the thermolability of enzyme systems in a given organism, and the maximum temperature at which growth occurs. In a study of eighteen species, including thermophilic organisms, Edwards and Rettger (1937) demonstrated close agreement between the minimum temperatures at which the three thermolabile respiratory enzymes - catalase, succinic dehydrogenase, and cytochrome oxidase-were destroyed and the maximum growth temperatures. The temperature at which the relatively thermostable peroxidase was destroyed was not related to growth temperatures in this way.

The relationship also appears to hold for spores, for Lamanna (1942), working with seventy-two strains of the spore-bearing bacillus species, found an association between the maximum temperature at which growth took place and the length of time the spore resisted heating at $95^{\circ} \mathrm{C}$.

In this connection, we may note the effects of heat on bacteria, prior to their growth in a given mcdium. If the death of a bacterium depends on the inactivation of certain enzymic systems, or the destruction of some essential nutrient, we might expect that sub-lethal degrees of heating would destroy some but not all of the essential metabolic functions of the bacterium. For instance, yeast cells, increasingly exposed to heat, first lose their ability to reproduce, and then their fermentative ability (Rahn and Barnes 1932). The count of viable bacteria from cultures subjected to sub-lethal doses of heat or ultra-violet light may be greatly increased if the test media are enriched with blood or extra carbohydrates (Fay 1934, Curran and Evans 1937, Nelson 1943). Davis (I940) records a phenomenon he refers to as "pseudo-death" among spores of $\mathrm{Cl}$. welchii. Only a small number of originally viable spores resisted a certain exposure to heat sufficiently to produce colonies when subsequently seeded on to a standard agar medium. In some cases the area of agar seeded with heated spore suspension was free of colonies after 5 days' incubation. When a fresh culture of $\mathrm{Cl}$. welchii was grown on the agar adjacent to the original inoculum, a number of the heated spores germinated. Presumably these "pseudo-dead" spores were without some heat-labile substance essential for germination, which was supplied by the growing $\mathrm{Cl}$. welchii.

\section{Other Biochemical and Physiological Activities of Bacteria.}

We have endeavoured to present a broad picture of bacterial metabolism, particularly as it affects the heterotrophic organisms that constitute the greater part of the bacteria pathogenic for man and the higher animals. Many other activities of bacteria-the production of pigments and of various toxic substances, 
their action on blood cells and blood pigments, their particular growth requirements - are specialized activities, so characteristic of particular bacterial species that it will be convenient to consider then in later chapters. Mention may be made here of the work of Reid (1937) and Kharasch, Conway and Bloom (1936) on the effect of food and inbibitory substances on the formation of pigment by growing cultures of chromogenic bacteria, and by Baker (1938) on the influence of light on pigment production by a species of non-pathogenic Mycobacterium. In Chapter 9 we shall consider the physiological changes that constitute one aspect of bacterial variation, and in Chapter 6 the relation of chemotherapy to the metaholic processes of bacteria.

\section{REFERENCES}

Aitken, R. S., Barling, B. and Miles, A. A. (1936) Lancet, ii, 780.

Andersox, A. B. and Hart, P. d'A. (I934) J. Path. Bact., 39, 465.

Aronson, H. (1900) Arch. Kinderheilk., 30, 23.

Avery, O. T. and Cullen, G. E. (I920) J. exp. Med., 32, 571.

Avery, O. T. and Morgan, H. J. (I924) J. exp. Med., 39, 275, 289.

Avery, O. T. and Neill, J. M. (1924a) J. exp. Med., 39, 347; (1924b) Ibid., 39, 357 ; (1924c) Ibid., 39, 543.

BADGER, E. (1944) J. Buct., 47, 509.

Batnbridge, F. A. (1911) J. Hyg., Camb., 11, 341.

BAKER, J. A. (1938) J. Bact., 35, 625.

BAKER, J. Wr. and HAPPOLD, F. C. (1940) Biochem. J., 34, 657.

Barrox, E. S. G. and Lyman, C. M. (1939) J. biol. Chem., 12\%, 143.

Berman, N. and Retteer, L. F. (1918) J. Bact., 3, 367, 389.

Berkman, S. (1942) J. infect. Dis., 71, 20I.

Bernheim, F., Bervheim, M. L. C. and Webster, M. D. (1935) J. biol. Chem., 110, 165. Bigger, J. W. and Nelson, J. H. (1943) J. Path. Bact., 55, 321.

Birkenshaw, J. H., Charles, J. H. V., and Clutterbeck, P. W. (1931) Biochem. J., 25,1522 .

Boissevain, C. H. and Schultz, H. W. (1938) Amer. Rev. Tuberc., 38, 624.

Borvin, A. and Mesrobeanu, L. (I934) Arch. roum. Path. exp. Microbiol., 7, 95.

Bоoth, V. H. and Grees, D. E. (1938) Biochem. J., 32, 855.

Bord, G. DE. (1923) J. Bact., 8, 1.

BraUn, H. (1931) Zbl. Bakt., 122, 5.

Braun, H. and Cahn-Bronner, C. E. (1921) Zbl. Balt., 86, 1, 196, 380 ; (1922) Biochem. Z., $131,226,272$.

Braun, H. and Goldschmidt, R. (1924) Biochem. Z., 146, 573 ; (1927) Zbl. Bakt., 101, 283, 330; (1928a) Ibid., 10\%, 329; (1928b) Ibid., 109, 353.

Braun, H., HofMeier, K., and Mündel, F. (1929) Zbl. Bakt., 113, 530.

Braun, H. and Kondo, S. (1924) Klin. Wschr., 3, 10.

Braun, H. and Lisch, H. (1928) Zbl. Bakt., 10\%, 35.

Braun, H. and MÜNDEL, F. (1927) Z Zbl. Bakt., 103, I82 ; (1929) Ibid., 112, 347.

Bradn, H., Stamatelakis, A., and Kondo, S. (1924) Biochem. Z., 145, 381.

Braun, H. and VÁSÁrhelyi, J. von. (1933) Zbl. Bakt., 12\%, 105.

Braun, H. and Wördehoff, P. (1933) Zbl. Bakt., 128, 50.

Broli-KAHN, R. H. and Mirsky, I. A. (1938) J. Bact., 35, 455.

Broos, J. C. (1929) Brit. J. exp. Path., 10, 71.

BuGHanan, R. E. and FUlmer, E. I. (1928-30) "Physiology and Biochemistry of Bacteria." (3 vols.) New York and London.

BURK, D. (1934) Ergebn. Enzymforsch, 3, 23.

Burrows, W. (1933) J. infect. Dis., 52, 126; (1939a) Ibid., 64, 145; (1939b) Ibid., 65, 134. Callow, A. B. (1923) J. Path. Bact., 26, 320 ; (1924) Biochem. J., 18, 507.

Callow, A. B. and Robinson, M. E. (1925) Biochem. J., 19, 19.

Carrière, G. (1901) C. R. Soc. Biol., 53, 320.

Cirapin, C. W. (1918) J. infect. Dis., 23, 342.

CLARK, W. M. (1928) "The Determination of Hydrogen Ions." Baltimore and London.

Clark, W. M., et al. (1928) Bull. U.S. hyg. Lab., No. 151.

Cliftos, C. L. (1940) J. Bact., 39, 485; (1942) Ibid., 44, 179.

Coghill, R. D. (1931) J. biol. Chem., 90, 57. 
Cohen, M. B. and Fleming, J. S. (1918) J. infect. Dis,, 23, 337.

Cohex, S., Siyder, J. C. and Mueller, J. H. (1941) J. Bact., 41, 581.

Colijns, M. A. and Hammer, B. W. (1934) J. Bact., 27, 473, 487.

Соок, P. P. (1931) Zbl. Bakt., 122, 329.

Cook, R. P. and Stephexson, M. (1928) Biochem. J., 22, 1368.

Cook, R. P. and Woolf, B. (1928) Biochem. J., 22, 474.

Cramer, E. (1891) Arch. Hyg., 13, 71 ; (1893) Ibid., 16, 151 ; (1894) Ibid., 20, 197 ; (1895) Ibid., 22, 167 ; (1897) Ibid., 28, 1.

Currax, H. R. and Evans, F. R. (1937) J. Bact., 34, 179.

DAvis, H. (1940) Quart. J. Pharm., 13, 14.

D)avis, J. G. and Thiel, C. C. (1939) J. Dairy Res., 10, 455.

Dawsox, A. I. (1919) J. Bact., 4, 133.

Dewax, J. G. and Green, D. E. (1938) Biochem. J., 32, 626.

Dorflax, A., Berkuax, S. and Koser, S. A. (1942) J. biol. Chem., 144, 393.

Dorfuan, A., Koser, S. A., Reames, H. R., Swingle, K. F. and Saunders, F. J. infect. Dis., 65. 163.

Downie, A. W. and Cruickshank, J. (1928) Brit. J. exp. Path., 9, 171.

Eakin, R. E. and EAKIN, E. A. (1942) Science, 96, 187.

Eakin, R. E. and Trilliams, R. J. (1939) J. Amer. chem. Soc., 61, 1932.

Eckstein, H. C. and Soule, M. H. (1931) J. biol. Chem., 91, 395.

EDwards, O. F. and RetTger, L. F. (1937) J. Bact., 34, 489.

Emrisulans, O. (1937) Z. Hyg. Infekthr.. 119, 572.

Emrismaxi, O, and DrambURG, K. (1937) Ibid., 119, 623.

Eijkman, C. (1901) Zbl. Balt., 29, 841.

Elbera, S. S. and IIEYer. K. F. (1939) J. Bact., 3\%, 429.

Elspex, S. B. (1938) Biochem. J., 32, 187.

Evdo, S. (1938) Biochem. Z.. 296, 56.

Evaxs, E. A., Vexpeslaxd, B. and Slotin, L. (1943) J. biol. Chem., 147, 771.

Evaxs, W. C., Haxdeey, W. R. C. and Happold, F. C. (1939) Brit. J.exp. Path., 20, 396 ; (1941) Biochem. J., 35, 207.

FAY, A. C. (1934) J. agric. Res., 48, 453.

Feeney, R. E., Mueller, J. H. and Miller, P. A. (1943) J. Bact., 46, 559.

Fildes, P. (1935) Brit. J. exp. Path., 16, 309; (1938) Biochem. J., 32, 1600

Fildes, P. and Gladstone, G. P. (1939) Brit. J. exp. Path., 20, 334.

Fildes, P., Gladstone, G. P., and Knight, B. C. J. G. (1933) Brit. J. exp. Path., 14, 189.

Fildes, P. and Knight, B. C. J. G. (1933) Brit. J. exp. Path., 14, 343.

Fildes, P. and Richardson, G. 11. (1935) Brit. J.exp. Path., 16, 326 ; (1937) Ibid., 18, 292.

Fischer, H. and Hasenkamp, J. (1935) Liebigs Ann., 515, 148.

Flemixg, A. (1941) Lancet, i, 110.

Flemisg, W. L. and Neili, J. M. (1927) J. exp, Med., 45, 169, 947.

Frantz, I. D. (1942) J. Bact., 43, 757.

Fred, E. B. and DAVenport, A. (1918) J. agric. Res., 14, 317.

FreI, W. L. (1934) "Atmungsfarbstoffe bei pflanzlichen Mikro-organismen." Festschrift Zangger, 1, 805.

Freer and Novy. (1902) Amer. chem. J., 2\%, 161.

Friedheim, E. A. H. (1931) J.exp. Ned., 54, 207; (1932) C. R. Soc. Biol., 110, 353 ; (1934) Biochem J., 28, 173.

Friedheim. E. and Michaelis, L. (1931) J. biol. Chem., 91, 355.

Fulmer, E, I., Nelson, V. E., and Sherwood, F. F. (1921) J. Amer. chem. Soc., 43, 191.

Gaffron,. H. (1933) Biochem. Z., 260, 1; (1934) Ibid., 269, 447; (1935) Ibid., 275, 301 ; (1935) $2 \% 9,1$.

Gale, E. F. (1940) Bact. Rev., 4, 135.

Galeotti, G. (1898) Z. physial. Chem., 25, 48.

Gillespie, R. IT. H. and Porter, J. R. (1938) J. Bact. 36, 633.

Gillespie, R. IV. H. and Rettger, L. F. (1938a) J. Bact., 36, 605; (1938b) Ibid.. 36, 621. Gingrich, W. and Schlexk, F. (1944) J. Bact., 4\%, 353.

Gladstone, G. P. (1937) Brit. J.exp. Path., 18, 322 ; (1938) Ibid.. 19, 208; (1939) Ibid.. $20,189$.

Gladstone, G. P., Fildes, P., and Richardson, G. M. (1935) Brit. J. exp. Path., 16, 335. Glass, V. and Kennett, S. J. (1939) J. Path. Bact., 49, 125.

Glenv, T. H. (1911) Zbl. Bakt., 58, 481.

Gotwstein, A. (1893) Virchows Arch. 133, 295.

Gould, R. G. (1944) J. biol. Chem., 153, 143.

Gorld, R. G., Kane, L. II. and Niteller, J. H. (1941) J. Buct., 4\%, 287.

Grees, D. E. and Stichland, I. H., (1934) Biochem. J., 28, 898.

Green, D. E., Stickland, L. H., and Tarr, H. L. A. (1934) Biochem. J., 28, 1812 ,

Grey, E. C. (1913-14) P'roc. roy. Soc. B. 461 ; (I924) Ibid., 96, 156.

Guggenheim, K. (1944) J. Bact., 4\%, 313. 
HARDEN, A. (1901) J. chem. Soc., 79, 610 ; (1905) J. Hyg., Camb., 5, 488; (1930) "The Metabolism of Bacteria." "System of Bacteriology," 1, 208. Med. Res. Coun. London.

Harden, A. and Walpole, G. S. (1906) Proc. roy. Soc., B, 77, 399.

Harris, J. E. G. (1919) J. Path. Bact, 23, 30.

Hastings, E. G. and MICCoy, E. (1932) J. Bact., 23, 54.

Heap, H. and Cadness, B. (1924) J. Hyg., Camb., 23, 77.

Hehre, E. J. and Sugg, J. Y. (1942) J. exp. Med., "75, 339.

Heidelberger, M. and Kexdall, F. E. (1931) J.exp. Med., 54, 515.

HES, J. W. (1938) Nature, Lond., 141, 647.

Hestrin, S. and Aviveri-Shapiro, S. (1943) Nature, Lond., 152, 49 ; (1944) Biochem. J., $38,2$.

HewitT, L. F. (1936) "Oxidation-Reduction Potentials in Bacteriology and Biochemistry." 4th Ed. L.C.C., London.

Heynixaen, W. E. van (1940) Biochem. J., 34, 1540.

Hills, G. II. (1938) Biochem. J., 32, 383.

Hinscri, J. (1931) Z. Hyg. InfektKr., 112, 660.

Hirsch, J. and Müller, A. W. (1933) Z. Hyg. InfektKr., 115, 443.

Hirschler. (1886) Z. physiol. Chem., 10, 306.

Holiday, E. R. (1937) Biochem. J., 31, 1299.

Hoogerheide, J. C. and Kocholaty, W. (1938) Biochem. J., 32, 949.

Hopkivs, F. G. (1921) Biochem. J., 15, 286 ; (1923)Lancet, i. 1251 ; (1929) J. biol. Chem., 84, 269.

Horvibiook, J. W. (1940) Proc. Soc. exp. Biol. N.Y., 45, 598.

Hosoya, S. and Kuroya, M. (1923) Sci. Rep. Inst. infect. Dis. Tokyo. Univ., 2, 233, 265. Hottle, G. A., Lampen, J. O. and Pappenhenmer, A. M. (1941) J. biol. Chem., 137, 457.

Hughes, T. P. (1932) J. Bact., 23, 437.

Hunter, O. W. (1923) J. agric. Res., 24, 263.

Huss, H. (1908) Zbl. Bakt., IIte Abt., 20, 474.

HUTNER, S. H. (1942) J. Bact., 43, 629.

IvÁxovics, G. (1937) Zbl. Bakt., 138, 449

Ivánovics, G. and BruCKNeR, V. (1937a)Z.ImmunForsch., 90, 304; (1937b) Ibid., 91, 175.

Johnson, E. A. and Rettaer, L. F. (1943) J. Bact., 45, 127.

Johnson, T. B. and Brows, E. B. (1922) J. biol. Chem., 54, 721, 731.

Johnson, T. B. and CoonnL, R. D. (1925) J. biol. Chem., 63, 225.

JoNes, H. M. (1916) J. infect. Dis., 19, 33.

KASERER, H. (1905) Zbl. Bakt., IIte Abt., 15, 573 ; (1906) Ibid., IIte Abt., 16, 681.

KeILIN, D. (1925) Proc. roy. Soc., B, 98, 312; (1926) Ibid., 100, 129 ; (1928-29) Ibid., 104, 206 ; (1930) Ibid., 106, 418; (1943) Proc. biochem. Soc., Biochem. J., 3\%, xxi.

KeILIN, D. and HARPLEY, C. H. (1941) Biochem. J., 35, 688.

Keilin, D. and Hartree, E. F. (1938a) Proc. roy. Soc. B., 124, 397 ; (1938b) Ibid., 125, $171 ;$ (1938c) Nature, Lond., 141, 870.

KeMPNER, W. and SchlaYer, C. (1942) J. Bact., 43, 387.

Kendall, A. I. (1922) J. infect. Dis., 30, 211.

Kendall, A. I., DaY, A. A., and Walker, A. W. (1914) J. Amer. chem. Soc., 36, 1962.

Kendall, A. I. and Walker, A. W. (1915) J. infect. Dis., 17, 142.

Kinairat, O. (1940) J. Path. Bact., 50, 491, 497.

Kharasch, iI. S., Conway, E. A. and Bloon, W. (1936) J. Bact, 32, 533.

Kligler, I. J. and Grossowicz, N. (1940) Nature, Lond., 146, 652 ; (1941) J. Bact., 42, 173.

Ḱligler, I. J., Grossowicz, N. and Beroner, S. (1943a) J. Bact., 46, 399 ; (1943b) Proc. Soc. exp. Biol. N.Y. 52, 332 .

Ḱligler, I. J. and Gugoeniteim, K. (1938) J. Bact., 35, 141.

Kluyver, A. J. (1924) Z. physiol. Chem., 138, 100.

Knight, B. C. J. G. (1935) Brit. J. exp. Path., 16, 315 ; (1936) Spec. Rep. Ser. med. Res. Coun. Lond., No. 210: (1937) Biochem. J., 31, 731, 966.

Knieht, B. C. J. G. and Fildes, P. (1930) Biochem. J., 24, 1496 ; (1933) Brit. J. exp. Path., 14,112 .

Knight, B. C. J. G. and McIlwaix, H. (1938) Biochem. J., 32, 1241.

KNORR, M. (1925) Ergebn. Hyg. Bakt., 7, 641.

Kiocholaty, W., Weil, L. and Sirth, L. (1938) Biochem. J., 32, 1685.

Köol, F. and Haagen Sмit, A. J. (1931) Proc. Acad. Sci. Amst., 34, 1411.

Kö̈G, F. and Tönnis, B. (1936) Z. physiol. Chem., 242, 43.

Koser, S. A., Breslove, B. B. and Dorfman, A. (1941) J. infect. Dis., 69, 114.

Koser, S. A., Dorfman, A. and Saunders, F. (1940) Proc. Soc. exp. Biol., N.Y., 43, 391.

Koser, S. A. and Retteer, L. F. (1919) J. infect. Dis., 24, 301.

Koser, S. A. and SAUNders, F. (1938) Bact. Rev., 2, 99.

Koser, S. A. and Wright, II. H. (1942) J. infect. Dis., '71, 86.

KREBS, H. A. (1937) Biochem. J., 31,661.

Krebs, H. A. and Egaleston, L. V. (1941) Biochem. J., 35, 676. 
Krebs, H. A., Hafez, M. M. and Egglestox, L. V. (1942) Biochcm. J., 36, 306.

LAMAXXA, C. (1942) J. Bact., 44, 29.

Lantzsch, K. (1922) Zbl. Bakt., IIte Abt., 5\%, 309.

Lepper, E. and Martin, C. J. (1929) Brit. J.exp. Path., 10, 327 ; (1930a) Ibid., 11, 137; (19305) Ibid., 11, 140.

Leibowitz, J. and Hestrin, S. (1942) Biochem. J., 36, 772.

Linton, R. W., Mitra, B. N., and Shrivastava, D. L. (1934) Indian J.med. Res., 21, 635.

Lipian, F. (1937) Enzymologia, 4, 65.

Lockemann, G. (1942) Z. Hyg. Infelthr., 124, 373.

Lockhart, E. E. and Potter V. R. (1941) J. biol. Chem., 13\%, 1.

Löwenstein. (1903) Wien. klin. Wschr., 16, 1393.

LwoFf, A. (1938) Ann. Inst. Pasteur, 61, 580.

Lwoff, A. and Lwoff, II. (1937a) Proc. roy. Soc., B., 122, 352 ; (1937b) Ibid., 122, 360; (1937c) Ann. Inst. Paiteur, 59, 129.

IICUllough, N. B. and Dick, L. A. (1942) J. infect. Dis., 71, 193, 198.

McIlwaix, H. (1939) Brit. J. exp. Path., 20, 330; (1940) Ibid., 21, 25.

McIlwat, H., Fildes, P., Gladstone, G. P. and Knight, B. C. J. G. (1939) Biochem. J., $33,223$.

McIntosh, J., James, W. W., and Lazarus-Barlow, P. (1922) Brit. J. exp. Path., 3, 138; (1924) Ibid., 5, 175.

McLeod, J. W. (1930) System of Bacteriology, Med. Res. Coun., London, 1, 263.

Mcleon, J. W., Coates, J. C., Happold, F. C., Priestley, D. P. and Wireatley, B. (1934) J. Path. Bact., 39, 221.

McLeod, J. W. and Gordox, J. (1922) Biochem. J., 16, 499 ; (1923a) J. Path. Bact., 26, 326 ; (1923b) Ibid., 26, 332 ; (1924) Biochem.J., 18, 937 ; (1925a) J. Path. Bact., 28, 147 ; (1925b) Ibid., 28, 155.

McLeod, J. W. and Wros, G. A. (1921) J. Path. Bact., 24, 205.

Maschuan, E. (1937) Biochem. Z., 294, 1.

Mesrobeanu, L. (1936) Arch, roum. Path. exp. Microbiol., 9, 121.

MEYER, K. (1934) Zbl. Bakt., 131, 289, 291.

Michaelis, L. and Nakahara, Y. (1923) Z. Immun Forsch., 36, 449.

Muller, F. M. (1933) Arch. Mikrobiol., 4, 131.

Mueller, J. H. (1937) J. Bact., 34, 163,429; (1938) J. biol. Chem., 123, 421; (1940) Bact. Rev., 4, 97.

Mueller, J. H. and Cohen, S. (1937) J. Bact., 34, 381.

Mueller, J. H. and Miller, P. A. (1942) J. Bact., 43, 763.

Mueller, J. H., Schoenbach, E. B., Jezukawicz, J. J. and Miller, I'. A. (1943) J. clin. Invest., 22, 315 .

Nassau, E. (1942) J. Path. Bact., 54, 443.

Neill, J. M. and Avery, O. T. (1925) J. exp. Med., 41, 285.

NeIl, J. M. and Flemiso, IV. L. (1927) J. exp. Med., 45, 937.

Nelson, F. E. (1943) J. Bact., 45, 395.

Nelson, M. E. and IVerkiax, C. H. (1936) Iowa St. Coll. J. Sci., 10, 141.

Neuberg, C. and Renvfurth, E. (1923-24) Biochem. Z., 143, 553.

Nicolle, M. and Alilaire, E. (1909) Ann. Inst. Pasteur, 23, 547.

Niel, C. B. vaN. (1931) Arch. Mikrobiol., 3, 1 ; (1933) Yearb. Carneg. Instn, No. 32, 184; (1943) Physiol Rev., 23, 338 ; (1944) Bact. Rev., 8, 1.

Niel, C. B. van, Ruben, S., Carson, S. F., Kamex, M. D. and Foster, J. W. (1942) Proc. nat. Acad. Sci., Wash., 28, 8.

Niklewski, B. (1908) Zbl. Bakt., IIte Abt., 20, 469.

Nismiura, T. (1893) Arch. Hyg., 18, 318.

Niven, C. F. (1944) J. Bact., 47, 343.

Niven, C. F. and Shermax, J. M. (1944) J. Bact., 4\%, 335.

NoAcK, K. and SchNeIder, E. (1933) Naturwiss., 21, 835.

Nord, F. F. and ExgeL, W. (1938) Biochem. Z., 296, 153.

Nye, R. N. and Lamb, II. E. (1936) J. Amer. med. Ass., 106, 1075.

O'Connor, R. (1940) Biochem. J., 34, 1008.

OGston, F. J. and Green, D. E. (1935) Biochem. J., 29, 1983.

O'Meara, R. A. Q. (1937) J. Path. Bact., 45, 541.

Oppenheimer, C. (1926) "Die Fermente und ihre Wirkung." 5th Edit., Leipzig.

Ordal, E. J. and Halvorson, H. O. (1939) J. Bact., 38, 199.

Orla.Jensen. (1902) Zbl. Bakt., IIte. Abt., 8, 11 ; (1909) Ibid., 22, 305.

Orla-Jensen, S., Otte, N. C. and SNog-KJaer, A. (1936) Zbl. Balit., IIte Abt., 94, 434, $447,452$.

Oxford, A. E., Lampen, J. O. and Peterson, W. H. (1940) Biochem. J., 34, 1588.

Pakes, W. C. C. and Jollyman, W. H. (1901) J. chem. Soc., 79, 322.

Pappenheimer, A. M. (1935) Biochem. J., 29, 2057. 
Pappeninimer, A. M. and Hottle, G. A. (1940) Proc, Soc. exp. Biol. N.Y., 44, 645. Pappenhemer, A. II. and Jomssox, S. J. (1936) Brit. J. exp. Path., 17, 335.

Peckham, A. W. (1897) J. exp. Med., 2, 549.

Pelczar, M. J. and Porter, J. R. (1940) J. Bacl., 39, 429.

Penrose, M. and Quastel, J. H. (1930) Proc. roy. Soc., B, 10\%, 168.

Peskett, G. L. (1933) Biol. Rev., 8, 1.

Pickett, H. J. (1943) J. biol. Chem., 151, 203.

Porter, J. R. and Pelczar, M. J. (1941) J. Bacl., 41, 173.

Potter, I. R. (1941) J. biol. Chem., 141, 775.

Quastel, J. H. (1925) Biochem. J., 19, 641 ; (1926) Ibid., 20, 166.

Quastel, J. H. and Stephenson, M. (1926) Biochem. J., 20, 1125.

Quastel, J. H., Stephenson, M., and WhethaM, M. S. (1925) Biochem. J., 19, 304.

Quastel, J. H. and Webley, D. II. (1941) Biochem. J., 35, 192 ; (1942) Ibid., 36, 8.

Quastel, J. H. and Whethà, M. S. (1921) Biochem. J., 18, 519 ; (1925a) Ibid., 19, 520; (1925b) Ibid., 19, 645.

Quastel, J. H. and Wooldridge, W. R. (1925) Biochem. J., 19, 652 ; (1927a) Ibid., 21, 148; (1927b) Ibid., 21, 1224; (1928) Ibid., 22, 689.

Rahn, O. and Barves, M. N. (1933) J. gen. Physiol., 16, 579.

Rahn, O. and Richardson, G. L. (1941) J. Bact., 41, 225; (1942) Ibid., 44, 321.

Raistriok, H. (1919) Biochem. J., 13, 44.

Raistrick, H. and Clark, A. B. (1921) Biochem. J., 15, 76.

Raistrick, H., et al. (1931) Philos. Trans. B, 220, 1.

ReEd, G. B. and ORR, J. H. (1943) J. Bact., 45, 309.

ReID, R. D). (1937) Zbl. Bakt., IIte Abt., 95, 379.

Rettger, L. F., Berman, N., and Sturges, W. S. (1916) J. Bact., 1, 15.

Rockwell, G. E. (1921) J. infect. Dis., 28, 352; (1923) Ibid., 32, 98 ; (1924) Ibid., 35, 381. Rockweli, G. E. and Hrahberoer, J. H. (1926) J. infect. Dis., 38, 92 ; (1927) Ibid., 40, 438.

Rockwell, G. E. and McKhann, C. F. (1921) J. infect. Dis., 28, 249.

Roslofson, P. A. (1934) Proc. konink. Akad. Wetensch., Amsterdam, 37, 660 ;

"On the Photosynthesis of the Thiorhodaceae." (Thesis.) Utrecht.

Ruben, S. and Kínien, 11. D. (1940) Proc. nat. Acad. Sci., Wash., 26, 418.

RubNer, M. (1900) Arch. Hyg., 38, 67.

Rugaierixi, G. (1933) Biochim. Terup. sper., 20, 280.

Rywosch, D. and Rywosch, M. (1907) Zbl. Bakt., 44, 295.

SANDiford, B. R. and Wooldridae, W. R. (1931) Biochem. J., 25, 2172.

SaUnders, F., Dorfman, A. and Koser, S. A. (1941) J. biol. Chem., 138, 69.

Scimaffer, A. J., Folkoff, C. and Bayne-Jones, S. (1922) Johns Hopk. Hosp. Bull., 33,151 .

Schlexk, F. and Gixarich, W. (1942) J. biol. Chem., 143, 295.

Schnerder, E. (1931) Z. physiol. Chem., 226, 221.

SCHREIBER, K. (1902) Arch. Hyg., 41, 328.

Schultz, A. S., Atrin, L. and Frex, C. N. (1939) J. Amer. Chem. Soc., 61, 1931.

SchumaN, R. L. and FARrell, I. A. (1941) J. infect. Dis., 69, 81.

今̈снӥтz, F. and 'Tinerell, H. (1938) Biochem. Z., 295, 246.

SerA, Y. (1910) Z. Hyg. InfektKr., 66, 141, 162.

SERqENT, A. L. (1928) "Les facteurs de croissances des microbes sur milieux artificiels." Paris.

Sevac, II. G., Sirotens, J. and Lackuan, D. B. (1940) J. biol. Chem., 134, 523.

SilvermaN, M. and Werkian, C. H. (1939) Enzymologia, 5, 385.

Sulley, K. L.. Nivex, C. F. and Sierian, J. M. (1943) J. Bact., 45, 445.

Sмiтн, 'T. (1\$97) J. exp. Med., 2, 513.

Sneli, E. E. and Peterson, W. H. (1940) J. Bact., 39, 273.

Snell, L. E., Strong, W. M. and Peterson, W. H. (1939) J. Bact., 38, 293.

SовотKа, H. and Holzmas, M. (1934) Biockem. J., 28, 734.

Söhngen, N. L. (1911) Jber. Fortschr. Chem., 41, 788, 789.

Sommaruga, E. von. (1894) Z. Hyg. Infekthr., 18, 441.

Sörensen, S. P. L. (1909) Biochem. Z., 21, 131.

Sperry, J. A. and RetTger, L. F. (1915) J. biol. Chem., 20, 445.

S'rahl, W. H. and Hamann, E. E. (1941) Mich. agr. Exp. Sta. Bull.. No. 177, p. 17.

S'Tephessox, M. (1928) Biochem. J., 22, 605; (1939) "Bacterial Metabolism "2nd. ed., Longmans, Green \& Co., London.

Stephenson, M. and Stickland, L. H. (1931) Biochem. J., 25, 205, 215 ; (1932) Ibid., 26, 712 .

Stephenson, M. and Whethan, M. S. (1922) Proc. roy. Soc., B, 93, 262 ; (1923) Ibid., 95, 200 ; (1924) Biochem. J., 18, 498.

Stern, K. G. (1936) J. biol. Chem., 114, 473.

Stevens, F. A. and West, R. $(1922) \mathrm{J}$. exp. Med., 35, 823. 
Sticklan1), L. H. (1929) Biochem. J., 23, 1187 ; (1931) Ibid., 25, 1543; (1934) Ibid., 28, $1746 ;$ (1935) Ilid., 29, 285.

StilL, J. L. (1940) Biochem. J., 34, 1374.

s'tokes, J. 1., Gunness, I. and Foster, J. W. (1944) J. Buct., 4\%, 293.

Stokinger, H. E., Ackerman, H. and Carpenter, C. I. (1944) J. Bact., 4\%, 129.

Stoklasa, J. (1908) Zbl. Bakt., IIte Abt., 21, 620.

sтokstad, E. L. R. (1941) J. biol. Chem., 139, 475.

Straub, F. B., Corran, H. S. and Green, 1). E. (1939) Nature, Lond., 143, 119.

S'Tumpe, I'. K. and Greex, D. E. (1944) J.biol. Chem., 153, 387.

TAmora, J. 'T. and GibBY, I. W. (1943) J. Biect., 45, 361.

Tamura, S. (1913a) Z. physiol. Chem., 87, 85 ; (1913b) Ibid., 88, 190 ; (1914) Ibid., 89, 289.

Tarr, H. L. A. (1933) Biochem. J., 2\%, 136.

Tasman, A. and Brandwizk, A. C. (1938) J. infect. Dis., 63, 10.

Tatum, E. L., WVood, H. G. and Peterson, IV. H. (1936) Biochem. J., 30, 1 s!s.

Thompson, R. H. S. and Dubos, R. J. (1938) J. biol. Chem., 125, 65.

Tikia, J. (1935) Biochem. Z., 2\%9, 264.

TraugotT, R. (1893) Z. Hyg. InfektKr., 14, 427.

Trussell, R. E. and Weed, L. A. (1937) J. Bact., 33, 381.

Valley, G. (1928) Quart. Rev. Biol., 3, 209.

VALLey, G. and RetTger, L. F. (1927) J. Bact., 14, 101.

Vigneaud, V. ud, Ditther, K.. Hague, L. and Long, J). (1942) Science, 96, 186.

Vigneaud, I. du, Hofmans, K. and Melville, D. B. (1942) J. Amer. chem. Soc. 64, 188.

Virtanen, A. I. (1924) Z. physiol. Chem., 138, 136; (1925) Ibid., 143, 71.

Virtanen, A. 1. and Karström, H. (1931) Actu chem. fenn., B., 7, 17.

Walker, H. H. (1932) Science, 76, 602.

WALLE, N. VAN DER. (1927) Zul. Bakt., IIte Abt., \%0, 369.

Warburg, O. (1925a) Ber. dtsch. chem. Ges., 58, 1001; (1925) Sciencc, 61, 575.

W

Weil, A. J. and Black, J. (1944) Proc. Soc. cxp. Biol. N.Y., 55, 24.

Weil, L. and Kucholaty, W. (1937) Biochem. J., 31, 1255.

Wells, H. G. and Corper, H. J. (1912) J. infect. Dis., 11, 388.

Werkian, C. H. (1939) Buct. Rev., 3, 187.

Werkman, C. H. and Wood, 11. G. (1942) Adernces Enzymology, 2, 135.

Wiela do. (1913) Ber. dtsch. chem. Ges., 46, 3327 ; (1921) Ibid., 54, 2353; (1922) Ergebn. Physiol., 20, 477.

Wildiers, E. (1901) La C'ellule, 18, 311.

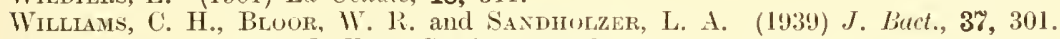

Wilson, G. S. (1930) J. Ityg., Camb., 30, 433.

Winogradsis, s. (1s89) Ann. Inst. Pasteur, 3, 49; (1590a) Ibid., 4, 213 ; (1s90b) Ibid., 4, 257 ; (1890c) Ibid., 4, 760.

Wolf, C. G. L. (1919a) J. Path. Bact., 22, 270; (1919b) Ibid., 22, 289.

Wolf, C. G. L. and Harris, J. E. G. (1918) J. Path. Bact., 22, 1.

Wood, H. G. and Werkias, C. H. (1936) Biochem.J., 30, 48 ; (1938) 1bid., 32, 1262; (1940) Ibid., 34, 7, 129.

Wood, H. G., Merkian, C. H., HemixgWaY, A. and Nier, A. O. (1940) J. biol. Chem,, $135,759$.

Woods, D. D. (1935) Biochem. J., 29, 640, 649; (1936) Ibid., 30, 1934.

Woods, D. D. and Clifton, C. E. (1937) Biochcm. J.. 31, 1774; (1938) Ibid., $32,345$.

Woons, D. D. and Trim, A. R. (194:) Biochem. J., 36, 501.

Woolf, B. (1931) Biochem. J., 25, 342 .

Woolley, D. W. and Hutchisgs, B. L. (1940) J. Butt., 39, 287.

Wrigit, H. D. (1936) J. Path. Bact., 43, 487; (1937) Ibid., 45, 117.

Wyon, G. A. and McLeod, J. W. (1923) J. Hyg., Camb., 21, 376.

Yudkis, J. (1932) Biochem. J., 26, 1859; (1933) Ibid., 27, 1849; (1934) Ibid., 28, 1463.

ZoBell, C. L. (1943) J. Bact., 46, 39. 


\section{CHAPTER 4}

\section{THE GROWTH AND DEATH OF BAC'TERIA}

LEsT the title of this chapter should prove misleading, it may be pointed out that we are not here concerned with the broader aspects of growth, such as the temperature and food requirements of bacteria, or their metabolism and respiration. These have already been briefly dealt with in Chapter 3 . In the present chapter we restriet ourselves to what we may describe as the dynamic aspeets of growth-a study that deals essentially with the rate of change iu a bacterial population.

\section{Technique of Counting Bacteria}

Bacteria may be counted in such a way as to obtain an estimate either of the total number of organisms alive and dead, or of the number of living organisms only. The first we shall refer to as the Total Count, the second as the Viable Count. Each method is suited to various purposes, and the choice of which to employ must depend on the type of information desired. For many purposes, such as calculation of the generation time of bacteria, and the quantitative study of bacterial metabolism, both methods should be used in conjunction.

The general principles underlying the counting of bacteria may be briefly mentioned. [For further details the reader is referred to textbooks on practieal bacteriology, and for a critical review of the different methods that may be employed, with the main sourees of error involved, to articles by Wilson (1922) and Wilson et al. (1935).]

\section{(1) Total Count.}

(a) Direct Counting under the Microscope of a Stained Preparation on a Slide.-First described by Eberle (1896), this method has been used fairly cxtensively, and forms the basis of the Breed (1911) method for counting bacteria in milk. A drop of known volume is spread over a known area on a slide, dried, fixed, stained, and examined under a microscope. The organisms in a given number of fields are counted, and knowing the area of a given field with a particular combination of objective, tube length and ocular - this can be obtained by means of shide and eyepiece micrometers-it is possible to calculate the total number of organisms present in the original suspension. This method, though valuable for certain purposes, is open to a number of technical objections, one of the most important of which is that not all organisms-particularly when dead-stain sufficiently deeply to be visible under ordinary illuminating conditions. The method, therefore, affords an estimate of the number of stainable bacteria, not necessarily of the total bacteria.

(b) Wright's Method (1902).-A known volume of the bacterial suspension is mixed with a known volume of normal human blood. A smear preparation is made on a slide, dried, fixed, stained, examined under the microscope, and the number of bacteria and red cells in a given number of fields is counted. Since, in the blood of the normal adult male there are about 5.5 million red cells per c.mm., and since the numerical relationship of 
the bacteria to the red cells is known, it is easy to estimate the number of bacteria in the suspension. Owing mainly to the impossibility of obtaining a perfectly homogeneous distribution of the red cells and bacteria on the slide, this method is subject to a very considerable experimental error.

(c) Microscopical Examination of the Organisms in a Counting Chamber.-Though an ordinary hæmatocytometer may be used and the bacterial suspension stained with a suitable dye, it is much better to employ a Helber chamber (1904). The Helber slide itself should be $2 \mathrm{~mm}$. thick, the depth of the chamber $0.02 \mathrm{~mm}$., and the area of each small square $0.0025 \mathrm{sq} . \mathrm{mm}$.; it should be provided with a cover slip 1-2 mm. thick. The organisms are examined unstained, under dark-ground illumination, a special condenser being provided for this purpose. A $\frac{2}{3}$ inch objective and a $\times 25$ compensating ocular afford a suitable combination for counting most organisms. This method is undoubtedly the most accurate we possess. Provided a number of technical points are attended to, there is no reason why counts should vary by more than \pm 10 per cent. from the real value.

(d) The Opacity Method.-This is essentially an indirect method of bacterial enumeration. The opacity of the suspension to be estimated is compared. either by the naked eye or by means of a nephelometer (see Liese 1926, Strausz 1930, Nilatz and Rottier 1936), photometer (Mestre 1935), or photo-electric cell (Pulvertaft and Lemon 1933, Alper and Sterne 1933, Longsworth 1936), with a control suspension of standard opacity, the number of organisms in which has been counted by one of the direct methods just described. Though very rapid and of great value for many purposes, this method suffers from the disadvantage that the opacity to which bacteria give rise appears to be determined not only by their numbers, but also by their size and their optical density. Since different strains of the same species, and even organisms of the same strain at different periods of growth (Wilson 1926), vary in size, the opacity method cannot do more than afford an approximate estimate of the numbers of bacteria in a given suspension. According to Liese (1926), who worked with spherical organisms, the opacity is determined by the surface area of the organisms $\left(4 \pi r^{2}\right)$ and therefore varies with the square of the radius. Thus a suspension containing 500 million cocci per $\mathrm{ml}$. with an average diameter of $2 \mu$ would be four times as dense as that of a suspension containing the same number of cocci with an average diameter of $1 \mu$. According to Strausz (1930), however, it is doubtful whether the relationship is quite so simple as this. Considering that some of the light must pass through the organisms themselves, it is difficult to a void the conclusion that volume and density probably play a part in determining the opacity of a bacterial suspension. Though unsatisfactory for the exact enumeration of bacteria, the opacity method is very useful, on account of its rapidity, for approximate estimations, and is peculiarly well adapted for the standardization of vaccines, in which the important factor is not the number of individual organisms, but the total amount of bacterial protoplasm per ml. (For very useful information on the micrometric measurement of bacteria, and for tables giving the relationship of volume to surface area, see Skar 1934.)

(e) The Centrifugal Method.-This is again an indirect method. It consists briefly in centrifuging the suspension in a capillary tube, measuring the height of the column of deposited bacteria, and estimating their number by calculations based on their average diameter or their specific gravity (see Schmidt 1926, Schmidt and Fischer 1930). As an approximate method of ascertaining the bacterial content of a suspension, particularly when the organisms are clumped, this method is often useful, but the doubtful validity of some of the assumptions made in the calculations, and the various factors that may interfere with the sedimentation of the bacteria under a given centrifugal force, are such as to render the final result subject to a considerable error.

(2) Viable Count.

(a) The Dilution Method.-This method, which is based on Lister's original method of dilution to extinction, consists in diluting the suspension to a point beyond which unit quantities are sterile. In practice several consecutive dilutions are made, and from each 
of these a number of tubes of medium, usually liquid, are inoculated with suitable unit quantities. 'The tubes are incubated and from the number in which growth oecurs, the probable number of bacteria in the original suspension may be calculated from formulæ, such as those worked out by HeCrady (1915), Stein (1917), Greenwood and Yule (1917), Halvorson and Ziegler (1933a, $b$ ), and Gordon and ZoBell (1938). (For useful tables see MeCrady 1918, Hoskins 1934.) This method, or one of its numerous modifications, is frequently adopted when an approximate estimate of the numbers of living bacteria in a suspension is required. It has the great advantage of being applieable to organisms that cannot be counted by the ordinary plating method. It is used, for example, in the determination of coliform organisms in water, because no satisfactory plating medium has yet been devised for differentiating eoliform bacilli in mixed culture from other baeteria, though several suitable liquid media are available. It is frequently used in filtrable virus work; for this purpose quantities of the dilutions are inoculated into a susceptible animal, generally by the intradermal route, and the occurrence of a specific skin reaction is regarded as evidence of the presence of the organism in the corresponding dilution. As Halvorson and Ziegler $(1933 a, b)$ have pointed out, the method is subjeet to a very large experimental error, depending mainly on the numbers of tubes seeded from the different dilutions. Even with 40 tubes to eaeh dilution the count is liable to vary between about 38 per cent. below and 47 per eent. above the true count, while with only 5 tubes to each dilution the corresponding figures are -70 and +260 per cent. The method is not therefore suitable for exact bacterial enumeration.

(b) The Plating Method.-This is generally performed by a modification of Koch's original plating method. It consists essentially in preliminary dilution, if necessary, of the suspension, the plating out of unit quantities of suitable dilutions into a suitable solid medium, and the counting of the number of colonies that develop after incubation. The average number of colonies per plate multiplied by the reciprocal of the dilution affords an estimate of the number of living organisms in the original suspension. Instead of plates, roll-tubes may be used. Provided that the bacteria are homogeneously distributed in the suspension, that not more than one species of organism is present, and that attention is paid to a large number of technical points, accurate counts may be obtained by this method. Departure from these provisos may often entail, however, experimental errors of considerable magnitude. (For sources and measurement of errors see Wilson et al. 1935, Jennison and Wadsworth 1940.)

Miles and Nisra (1938) have described a method of plate counting in which the bacterial suspension, instead of being mixed with the melted agar, is deposited in the form of drops on the surface of the solid medium and the count estimated from the number of colonies that develop.

\section{The Growth Curve}

If a given bacterium is seeded into a liquid medium of suitable composition; and incubated at a suitable temperature, it will be found that its growth will follow a definite course. This course is most conveniently represented in graphical form (Fig. 15), the logarithms of the numbers of bacteria along the ordinates being plotted against the time in hours along the abscissæ.

The growth curve may be arbitrarily divided into four phases: (1) The lag phase, $a$ to $b$, lasting for a few hours, during which multiplication is slow. In the early part of this phase there may be no apparent growth; in fact many of the organisms may die, so that there is an actual diminution in their numbers. Within a short time, however, growth becomes apparent, and gradually increases in pace till the beginning of the next phase. (2) The logarithmic phase, $b$ to $c$, in which regular division of the organisms occurs at maximum speed. Since their increase is in geometric progression, it follows that when the logarithms of their numbers are plotted against the time in hours they fall on an ascending straight line. (3) The 
stationary phase, $c$ to $d$, during which the organisms cease to multiply at maximal rate, so that their increase in number becomes less and less, till ultimately it ceases; the number present in a unit volume remains approximately constant for an appreciable length of time. During this phase the number of freshly formed bacteria roughly counterbalances the number of those that are dying. (4) The phase of decline, $d$ to $e$, during which the organisms gradually diminish in number, till finally the eulture becomes sterile.

So far we have been considering the viable bacteria only. If, however, a count is performed of the total number of organisms alive and dead in the culture, a different eurve is obtained. It will be seen that this eurve runs more or less parallel to the curve of the viable bacilli till the period of decline sets in, after which the two diverge, the total curve remaining practically stationary, or rising very slightly. It will be noticed, moreover, that the total curve is throughout

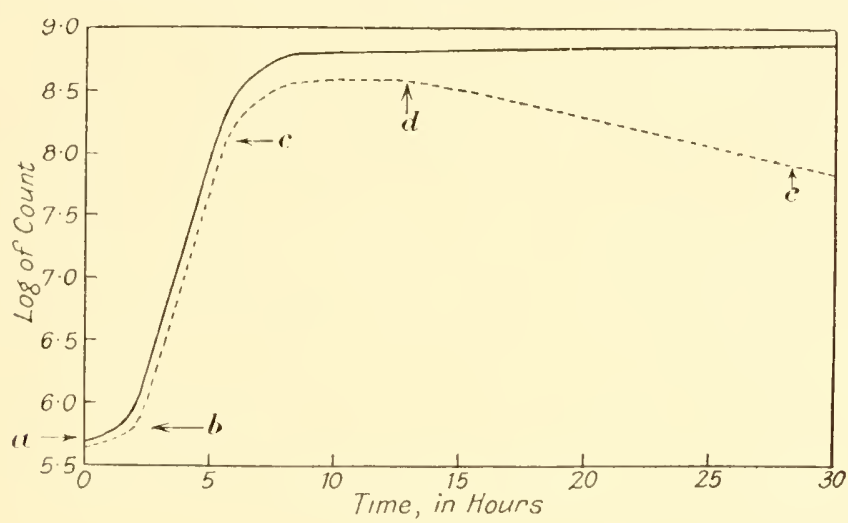

FIG. 15.

Continuous line $=$ Total number of bacteria alive or dead. Interrupted line $=$ Number of living or viable bacteria.

somewhat higher than the viable curve. The probable explanation of this will be given later.

We must now consider each of the phases in detail.

\section{The Lag Phase}

It is important at the outset of any discussion on growth to define our terms. Failure to do this, has been responsible for much confusion in the past. Growth may oceur either in size or in numbers. The individual cell may get progressively larger, or it may divide and give rise to two daughter cells. Growth in size may be referred to as cell enlargement, growth in numbers as cell multiplication.

The observations of early workers in this field (Mïller 1895, Rahn 1906, LaneClaypon 1909, Coplans 1910, Penfold 191t, Ledingham and Penfold 1914, Chesney 1916, Slator 1917, Buchanan 1918) were made almost exclusively on cell multiplication. They found that the lag phase, during which little or no multiplication occurs, was affected by a number of factors, such as the size and age of the inoculum, the frequency of transplantation of the parent culture, the nature of the organism, the composition of the medium, and the temperature at which the culture is incubated. Generally speaking, the lag phase tends $(a)$ to be long when a small 
inoculum is used, when the inoculum is taken from an old culture, when the medium is unsuitable, and when the culture is incubated at a temperature unfavourable to maximal growth, and $(b)$ to be short when the opposite conditions obtain. The most striking factor of all, however, is the stage of growth of the parent culture from which the inoculum is taken. Organisms from a culture in the lag, the stationary, or the decline phase of growth fail to multiply for some time when introduced into a fresh medium, whereas organisms from a culture in the logarithmic phase show no lag, but continue to multiply at the same rate as in the parent culture (Miiller 1895). During the logarithmic phase the organisms are known to be multiplying at maximal rapidity, and since this phase lasts for not more than a few hours, it follows that the organisms must all be comparatively young. By the term "young" we mean an organism that has been generated within a comparatively recent time, from a few minutes to about an hour. The suggestion, therefore, is that young organisms inoculated into a fresh medium multiply without lag, and that old organisms exhibit a lag phase before beginning to grow.

Numerous explanations were put forward to explain the phenomena of lag. It was suggested by Rahn (1906) that before multiplication could occur some essential substance or "bios" had to be excreted by the organisins into the medium. Penfold (1914) modified this view by supposing that certain bodies necessary for the synthesis of protoplasm had to accumulate within the bacteria themselves before multiplication became possible. Chesney (1916) regarded the phenomenon of lag as an expression of injury which the bacterial cell had sustained in its previous environment. Ledingham and Penfold (1914) postulated an inherent difference in the multiplication rate of the individual cells in the inoculum, and suggested that during the lag phase a selection of the more rapidly multiplying cells was occurring, the result of which did not become manifest till the beginning of the logarithmic stage. Experimental evidence does not support this view (Kelly and Rahn 1932, Topley and Wilson 1936).

These explanations need not be discussed further, because more recent work has brought to light one supremely important fact of which the earlier observers were ignorant, and which goes a long way towards explaining the difference in behaviour of young and old organisms. Briefly stated, the lag phase is not a phase of rest, as had been previously supposed, but a phase of intense growth activity during which cell enlargement but little or no cell division occurs. The evidence for this statement must now be considered in some detail.

\section{Evidence of Growth without Multiplication during the Lag Phase.}

(1) Increase in Size.-If a small number of organisms from a 24-hours' culture of Bact. coli, for example, are seeded into an agar medium, which is then spread between a slide and a cover glass and observed on a warm stage by dark-ground illumination, the first change observed is not division, but a gradual and progressive enlargement affecting a certain proportion of the organisms. This enlargement becomes visible shortly after the preparation has been put up and continues till the organisms reach a certain size, when they finally divide. The rate at which the enlargement occurs varies with the different organisms in the preparation. The time elapsing between the incubation of such a preparation and the first division varies from about $1 \frac{1}{2}$ to 3 hours; but after the primary division has occurred, subsequent generations may be produced at the rate of about one in every 30 to 60 minutes. Examination of such a preparation at the end of about five hours will reveal a small proportion of cells that 
have apparently remained unaltered, and a large proportion that have divided; amongst this latter group considerable variation will be found in the number of daughter cells produced, these varying in different micro-colonies from 2 to about 16 or even more. Measurements of the size of these daughter cells show that they are considerably larger than the original cells used for seeding the preparation. Clark and Ruehl (1919) were the first to draw serious attention to increase in size of the bacterial cell during growth. Using a micrometer, they measured the size of seventy strains of organisms belonging to thirty-seven different species at intervals during their growth. With the exception of members of the diphtheria group, they found that the young organisms in cultures from 4 to 9 hours old were much larger than those in cultures 24 hours old. Henrici (1926, 1928), who largely confirmed their work, found in a culture of $B$. megatherium, that the average length of the original cells was $3.4 \mu$, but that during the phase of maximum multiplication the average length of the constituent organisms was about $15 \mu$. After this phase was over, the average length of the organisms decreased till in 10 hours it approximated to that of the organisms used for the original seeding. Similar observations on Salm.typhi-murium showed that the average size of the cells in a 4-hours' agar culture was $2.35 \times 0.79 \mu$, whereas in the same culture after 26 hours it was only $1.13 \times 0.49 \mu$ (Wilson 1926). Estimations made from these measurements showed that the cells from the young culture were nearly six times the volume of those from the older culture. By special staining methods, appearances indicative of nuclear division may be demonstrated in some bacteria before true cell division occurs (see Chapter 2).

(2) Increase in Respiratory Activity.-Observations by various workers (for references see Walker et al. 1934, Winslow and Walker 1939) on the biochemical activity of the cells during the lag phase have shown that this phase is by no means a period of rest. Respiratory and metabolic processes, such as an increased oxygen uptake, a fall in oxidation-reduction potential, and a rise in the output of heat, of $\mathrm{NH}_{3}$, and of $\mathrm{CO}_{2}$, become detectable soon after the organisms have been inoculated into the medium. The rate at which these changes proceed increases till it reaches its culminating point during the first hour of the logarithmic phase of growth. These observations render it clear that, though the cells are not dividing, they are metabolizing very actively during the so-called lag period.

(3) Increase in Susceptibitity to Disinfectants.-Further evidence of change in the bacterial cells during the lag phase is afforded by a study of their resistance to disinfecting agencies. Schultz and Ritz (1910) showed that in young cultures of Bact. coli, 3 to 6 hours old, the organisms were heat-sensitive, whereas in older cultures, 8 to 24 hours, they were nearly all resistant, when tested at $53^{\circ} \mathrm{C}$. for 25 minutes. Similarly Reichenbach (1911), working with Salm. paratyph $B$, found that the younger the cultures were, the higher was the proportion of heat-sensitive organisms. Sherman and Albus (1923) found that in a culture of Bact. coli the freshly formed bacteria were more susceptible to the action of inimical agents such as heat, cold, and disinfectants, than were the older bacteria. Taking advantage of these differences, they attempted (Sherman and Albus 1924) to study the relative numbers of the two kinds of bacteria present during the lag phase of growth. A 1 per cent. peptone water medium was inoculated from a culture of Buct. coli which had been grown in the same medium at room temperature for a week; the inoculum consisted therefore of old bacteria. The culture was incubated at $37^{\circ} \mathrm{C}$., and plate counts were made at intervals. At the 
same time $1 \mathrm{ml}$. portions of the culture were transferred to $100 \mathrm{ml}$. of 5 per cent. sodium chloride solution; the mixture was allowed to stand for 1 hour at $20^{\circ} \mathrm{C}$., after which a count was performed on it, the numbers being calculated in terms of the $1 \mathrm{ml}$. of added broth culture. The results are given in Table 1.

TABLE 1

\begin{tabular}{|c|c|c|c|c|c|}
\hline \multicolumn{3}{|c|}{$\begin{array}{l}\text { Time after } \\
\text { Inoculation. }\end{array}$} & $\begin{array}{l}\text { Original } \\
\text { Culture. }\end{array}$ & $\begin{array}{l}\text { After } 1 \text { hour in } \\
5 \text { per cent. } \mathrm{NaCl} \text {. }\end{array}$ & $\begin{array}{l}\text { Mortality, } \\
\text { per cent. }\end{array}$ \\
\hline $\begin{array}{l}0 \text { hours } \\
1 \text { hour. } \\
1 \frac{1}{2} \text { hours } \\
2 \text { hours } \\
2 \frac{1}{2} \text { hours }\end{array}$ & 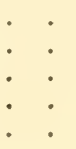 & $\dot{\cdot} \cdot$ & $\begin{array}{r}96,000 \\
80,500 \\
90,500 \\
143,000 \\
255,000\end{array}$ & $\begin{array}{l}82,500 \\
60,500 \\
41,000 \\
33,000 \\
16,500\end{array}$ & $\begin{array}{l}14 \cdot 06 \\
24 \cdot 82 \\
54 \cdot 70 \\
76 \cdot 93 \\
93 \cdot 54\end{array}$ \\
\hline
\end{tabular}

This table shows that the young bacilli, formed after the culture has entered on the logarithmic phase of growth, are very much more susceptible to the action of 5 per cent. $\mathrm{NaCl}$ solution than are the old bacilli inoculated at the start-a mortality of 93.54 per cent. as against 14.06 per cent. In addition it will be noticed that during the lag phase, lasting from 0 to $1 \frac{1}{2}$ hours, though there is no increase in the number of bacteria, there is a progressive increase in their susceptibility to salt solution. Sherman and Albus interpret this as indicating that, during the lag phase, the old bacteria which have been inoculated are undergoing a process of rejuvenescence, which fits them for reproduction. Numerous instances are quoted from other fields of biology, such as the rejuvenescence of Paramocium, the growth curves of colonies of fruit flies, etc., that may be regarded as exemplifying the same biological law of growth. Prior to active growth, there is a latent phase of preparation, during which the old cells are being rejuvenated, and fitted for reproduction.

Sherman and Naylor (1942) made the interesting observation that cells of Bact. coli taken during the logarithmic phase, cooled gradually to $1^{\circ} \mathrm{C}$. and stored at this temperature. retained the characteristics of young bacteria-namely, ability to multiply without lag when transferred to fresh medium at $37^{\circ} \mathrm{C}$. and greater susceptibility to inimical agents (in this ease cold shock) - for as long as 36 days. Cells of Str. lactis, on the other hand, rapidly acquired at $1^{\circ} \mathrm{C}$. the characters of senescence. What this difference was due to, it is impossible to say; but the observations of Sherman and Naylor may be taken in conjunction with those of Anderson and Meanwell (1936), who found that a thermoduric milk streptococcus (i.e. one capable of resisting heat at $60^{\circ} \mathrm{C}$. for 30 minutes) with which they were working became less rather than more susceptible to heat during the lag phase. Generalizations, therefore, based on the behaviour of coliform bacilli alone, must be accepted with reserve.

(4) Diminution in the Electrophoretic Charge.-Working with Bact. coli, Moyer (1936) found that when the organisms were introduced into a fresh medium their rate of migration in a cataphoretic cell decreased during the first two hours, remained low during the third and fourth hours, and then rose towards the end of the logarithmic phase. He concluded that these changes were probably due to an alteration in the electro-chemical state of the bacterial surface during the growth cycle.

(5) Decreased Susceptibitity to Non-sperific Agglutination.- Though fow observations have so far been made, the results recorded by Macgregor (1910) on agglutina- 
tion of meningococci by normal serum, Gillespie (1914) on the salt agglutination of pneumococci, and Sherman and Albus (1923) on the acid agglutination of Bact. coli, all suggest that young cells are less susceptible than old to non-specific agglutination. This presumably is due to an alteration in the electric charge or to some other change on the surface of the cells.

Summarizing the data yielded by these observations, we see that during the lag phase of growth there is a progressive enlargement of the cells before division occurs, associated with a rapid increase in their metabolic activity and their susceptibility to heat and disinfectants. Before a cell can divide, it must increase in size. The so-called lag phase of growth seems to be occupied mainly by this process of cell enlargement. $U_{p}$ to this point all recent workers are in agreement. On two subjects, however, there is some discrepancy of opinion. Firstly, do all cells, when inoculated into a fresh melium, begin to grow at once? In other words, is there no such thing as a true lag phase? It is a little difficult to answer this question, but the available evidence suggests fairly strongly that under certain conditions cells introduced into a fresh medium take some time to adjust themselves to the new conditions before they start to grow, even in size. The duration of this "phase of adjustment" varies with the conditions. While it lasts, it constitutes a true lag phase. If the organisms in the inoculum are vigorous and unharmed and the medium into which they are introduced is favourable, cell enlargement may oceur at once, and no true lag phase will be seen. If, on the other hand, they are suffering from the toxic effects of their previous enviromment, or the medinm into which they are inoculated has an unfavourable $\mathrm{pH}$ or salt content, then some time may elapse before cell enlargement begins.

Secondly, are we justified in referring to the orderly change that occurs in the enlargement and division of a bacterial cell as a life-cycle? Apart from mere difference of opinion on the advisability of using such a term, the answer must depend on whether there is a real differentiation of morphology and function at different stages of growth. The observations of Henrici (1928), in particular, leave no doubt about the differences in size, structure, and staining affinity of bacterial cells at different stages of growth, but the evidence in regard to their metaholic activity is a little conflicting. Hershey (1939), for instance, maintains that the metabolic activity is the same for cells at all stages of growth, provided that it is expressed in terms of amount of bacterial protoplasm rather than in terms of individual cells. It is true that in absolute values a cell in the late lag or early logarithmic phase uses more oxygen and produces more $\mathrm{CO}_{2}$ than a ceil in the stationary or decline phase, but this, according to Hershey, is merely due to a difference in size of the cells. The respiration of any unit amount of protoplasm is the same with cells at all stages of growth. Huntington and Winslow (1937) and Winslow and Walker (1939), however, do not agree with this view. They found that the rate of $\mathrm{CO}_{2}$ production per cubic micron of bacterial substance was much higher during the lag and early logarithmic phases than during the stationary phase. They believe, therefore, that young protoplasm is characterized by a greater rate of metaholism, and that the use of such a term as life-cycle for bacteria is justifiable. Further evidence is required to settle this important difference of fact.

If we accept the view of Winslow and Walker, we shall summarize as follows:

On the ground $(a)$ that newly generated bacilli in the logarithmic phase, when transferred to a fresh medium, start multiplying without exhibiting any lag phase ; (b) that bacilli from cultures in all other stages show a lag phase before they start 
dividing; and (c) that during this lag phase the cells are undergoing a rapid metamorphosis, resembling that perhaps described by Child (1915) in the rejuvenescence of infusoria, flat worms, and marine algæ, which renders them similar to the young actively dividing bacilli of the logarithmic phase : it may be couclucled that the lag phase is essentially a period in which the protoplasm of the old, but still viable, bacteria in the inoculum is acquiring the characteristics of young protoplasm. The lag phase appears to be a phase of rejuvenescence.

\section{Logarithmic Phase}

The logarithmic phase is that period during which regular and maximum multiplication is occurring. Increase in the number of organisms is by geometrical progression, so that, if the logarithms of their numbers are plotted against time, they will fall along an ascending straight line. If counts are made at intervals, it is easy to calculate the number of generations during the phase, and the length of each generation.

Thus if $a=$ number of organisms at the beginning of a given time

- and $b=$ " ", , , end , , , ,

then at the end of the first generation,

$$
b=a \times 2
$$

at the end of the second generation,

$$
b=a \times 2 \times 2
$$

at the end of $n$ generations,

$$
b=a \times 2^{n}
$$

To find the value of $n$, i.e. the number of generations, we may write

or

$$
\begin{aligned}
\log b & =\log a+n \log 2 \\
n & =\frac{\log b-\log a}{\log 2} .
\end{aligned}
$$

Further, if there have been $n$ generations in time $\mathrm{T}$, the generation time $\mathrm{G}$ can be calculated from the formula

$$
\mathrm{G}=\frac{\mathrm{T}}{n}
$$

It has generally been held that during the logarithmic phase all the bacteria are alive and all are actively dividing. Thus, as represented in formula (1), 2 bacteria give rise to 4,4 to 8,8 to 16 , and so on. If this assumption were correct, then all the organisms present during this phase should be viable, and the total count would be identical with the viable count.

This may be true when, as Kelly and Rahn (1932)' have found, organisms are growing under optimal conditions and are followed for a few generations only. Observations, however, on ordinary broth cultures have shown that the total number of organisms generally exceeds the number of viable organisms, even during the logarithmic phase (Wilson 1922, 1926, Régnier, David, and Kaplan 1932, Buice 1933-34, Jordan and Jacobs 1944).

The most probable explanation of this fact is that during the period of maximum growth some of the organisms that are generated fail to survive. If, for example, 80 per cent. of the organisms produced during a given generation continued to live and divide, while 20 per cent. died, then at the end of the logarithmic phase the total number of organisms alive and dead would exceed the number of living. 
The increase in the living organisms would still occur by geometrical progression, and the resultant curve of plotting the logarithms against time would still fall on a straight line; the only difference would be that instead of the number of organisms being doubled in each generation, their factor of increase, or generation index, would be $1 \cdot 6$.

Thus in Table 2 it is supposed that only 50 per cent. of the organisms inoculated are viable. The ratio of viable to total organisms, i.e $\frac{V}{T}$, increases with each generation. After about ten generations, it rapidly approaches the value $p-1$, where $p=$ the generation index. The value of $p$ can therefore be obtained with fair accuracy towards the end of the logarithmic phase by simply calculating the viable : total ratio and adding 1 . In the example quoted it is approximately $1 \cdot 6$.

TABLE 2

Calculations of Viable and Total Organisus with a 20 per cent. Death-rate per Generation

\begin{tabular}{|c|c|c|c|c|c|}
\hline & & $\begin{array}{l}\text { No, of Viable } \\
\text { Org. per ml. }\end{array}$ & No. of Dead Org. per ml. & $\begin{array}{l}\text { Total No. of } \\
\text { Org. per ml. }\end{array}$ & $\begin{array}{c}\text { Ratio Viable : } \\
\text { Total. }\end{array}$ \\
\hline At start & . & 1,000 & 1,000 & 2,000 & 0.500 \\
\hline At end of lst Gen. & . & 1,600 & $1,400(1,000+$ & 3,000 & 0.533 \\
\hline At end of 2nd Gen. & . & 2,560 & $2,040(1,400+640)$ & 4,600 & 0.557 \\
\hline At end of 3rd Gen. & . & 4,096 & $3,064(2,040+1,024)$ & 7,160 & 0.572 \\
\hline At end of 4 th Gen. & - & 6,554 & $4,702(3,064+1,638)$ & 11,256 & 0.582 \\
\hline
\end{tabular}

If the viable and total counts are known both at the start and at the end of $x$ generations, then $p$ can be ascertained at any time during the logarithmic phase, since

$$
\frac{\mathrm{V}_{x}-\mathrm{V}_{0}}{\mathrm{~T}_{x}-\mathrm{T}_{0}}=p-1
$$

or

$$
p=\frac{\mathrm{V}_{x}-\mathrm{V}_{0}}{\mathrm{~T}_{x}-\mathrm{T}_{0}}+1
$$

where $\mathrm{V}_{0}$ and $\mathrm{T}_{0}=$ number of viable and total organisms respectively at the start, and $\mathrm{V}_{x}$ and $\mathrm{T}_{x}=$ number of viable and total organisms respectively at the end of $x$ generations.

Experiments on the growth of Salm. typhi-murium in broth have shown that the average ratio of viable to total organisms at the end of the logarithmic phase is about $0 \cdot 8$. This corresponds to a death-rate of about 10 per cent. per generation, and to a generation index of $1 \cdot 8$. Hence in calculating the generation time, formula (1) has to be changed to

$$
n=\frac{\log b-\log a}{\log 1 \cdot 8}
$$

Employing this formula, the number of generations is greater than by the old formula, and the generation time necessarily shorter.

It must be understood that the factor of increase to be used in the formula may vary with each experiment, and can only be ascertained by the performance of a total and a viable count on each culture.

1 For this formula we are very much indebted to Dr. H. G. W. Hoare. 
TABLE 3

GRowTH of Salm. typhi-murium IN BROTH (Wilson 1922)

\begin{tabular}{|c|c|c|c|c|c|c|}
\hline $\begin{array}{l}\text { Time in } \\
\text { Minutes. }\end{array}$ & $\begin{array}{l}\text { Viable Count } \\
\text { per ml. }\end{array}$ & $\begin{array}{l}\text { Iogarithms } \\
\text { of Viable } \\
\text { Count. }\end{array}$ & $\begin{array}{l}N_{1}-N_{0} \\
N_{2}=N_{1} \\
\text { ete. }\end{array}$ & $\begin{array}{l}t_{1}-t_{0} \\
t_{2}=t_{1} \\
\text { etc. }\end{array}$ & $\begin{array}{c}N_{1}-N_{0} \div t_{1}-t_{0} \\
N_{2}-N_{1} \div t_{2}-t_{1}, \\
\text { etc. }\end{array}$ & $\begin{array}{l}\text { Mid-time } \\
\text { Interval. }\end{array}$ \\
\hline 0 & Not counted & - & - & - & - & - \\
\hline 40 & 828,800 & $5 \cdot 9184$ & - & - & - & - \\
\hline 80 & $1,539,000$ & $6 \cdot 1872$ & 710,200 & 40 & 17,755 & 60 \\
\hline 120 & - $4,363,000$ & $6 \cdot 6398$ & $2,824,000$ & 40 & 70,600 & 100 \\
\hline 160 & $12,150,000$ & $7 \cdot 0845$ & $7,787,000$ & 40 & 194,675 & 140 \\
\hline 200 & $32,490,000$ & $7 \cdot 5117$ & $20,340,000$ & 40 & 508,500 & 180 \\
\hline 240 & $81,640,000$ & $7 \cdot 9119$ & $49,150,000$ & 40 & $1,228,750$ & 220 \\
\hline 280 & $155,900,000$ & $8 \cdot 1928$ & $74,260,000$ & 40 & $1,856,500$ & 260 \\
\hline 320 & $271,700,000$ & $8 \cdot 4341$ & $115,800,000$ & 40 & $2,895,000$ & 300 \\
\hline 370 & $334,300,000$ & $8 \cdot 5241$ & $62,600,000$ & 50 & $1,252,000$ & 345 \\
\hline 440 & $351,400,000$ & $8 \cdot 5458$ & $17,100,000$ & 70 & 244,286 & 405 \\
\hline
\end{tabular}

Instead of plotting the logarithms of the bacterial numbers against time, Lemon (1933) has pointed out that interesting information may be obtained by estimating the actua] rate of growth in unit intervals of time.

If for each period of time $\mathrm{T}$ the count is $\mathrm{N}_{0}, \mathrm{~N}_{1}, \mathrm{~N}_{2} \ldots$ etc. at times $t_{0}, t_{1}, t_{2} \ldots$, the following formula may be used:

$$
\frac{\left(\mathrm{N}_{1}-\mathrm{N}_{0}\right)}{\left(t_{1}-t_{0}\right)_{\frac{1}{2}\left(\mathrm{~T}_{1}\right)}} \text { and } \frac{\left(\mathrm{N}_{2}-\mathrm{N}_{1}\right)}{\left(t_{2}-t_{1}\right)_{\frac{1}{2}\left(\mathrm{~T}_{3}-\mathrm{T}_{2}\right)+\mathrm{T}_{1}}} \text { and } \frac{\left(\mathrm{N}_{3}-\mathrm{N}_{2}\right)}{\left(t_{3}-t_{2}\right)_{\frac{1}{2}\left(\mathrm{~T}_{2}-\mathrm{T}_{2}\right)+\mathrm{T}_{2}}} \text {, etc. }
$$

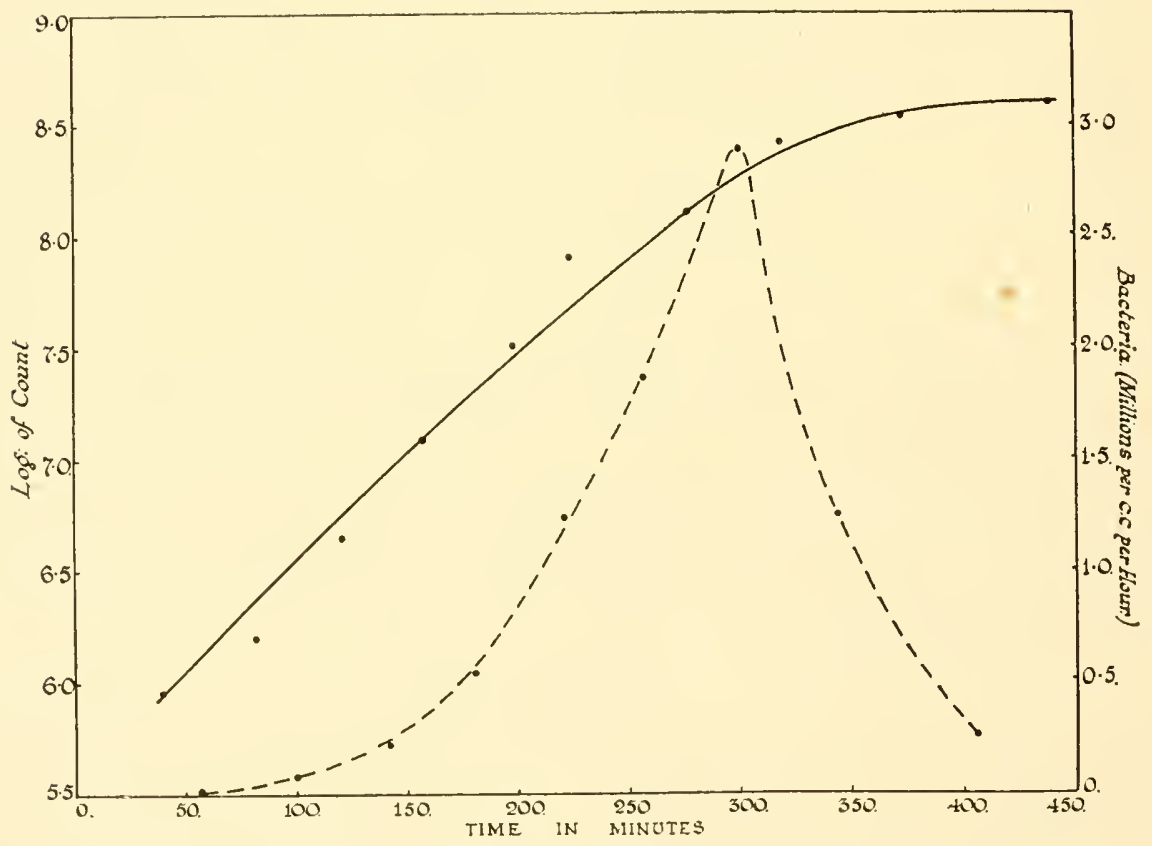

Fig. 16.-Growth of Sulm. typhi-murium in Broth, PLOtTed aCCording TO LEMON's FORMULA.

Continuous line $=$ Numbers plotted logarithmically against time.

Interrupted line $=$ Rate of arithmetic increase plotted against time. 
The results are plotted on a graph whose abscissæ represent $\mathbf{T}$ and whose ordinates indicate the rate of propagation. The numerator and the first term of the denominator in the formula represent the arithmetic increase in the number of organisms in unit time. The second term or "suffix" of the denominator is not part of the divisor but indicates the mid-point of the period during which the actual increase has occurred. The use of the formula is exemplified in Table 3 and Fig. 16.

It will be observed that, while the curve of the logarithms indicates that a steady rate of multiplication is occurring during the $1-5$ hour period, the curve given by Lemon's

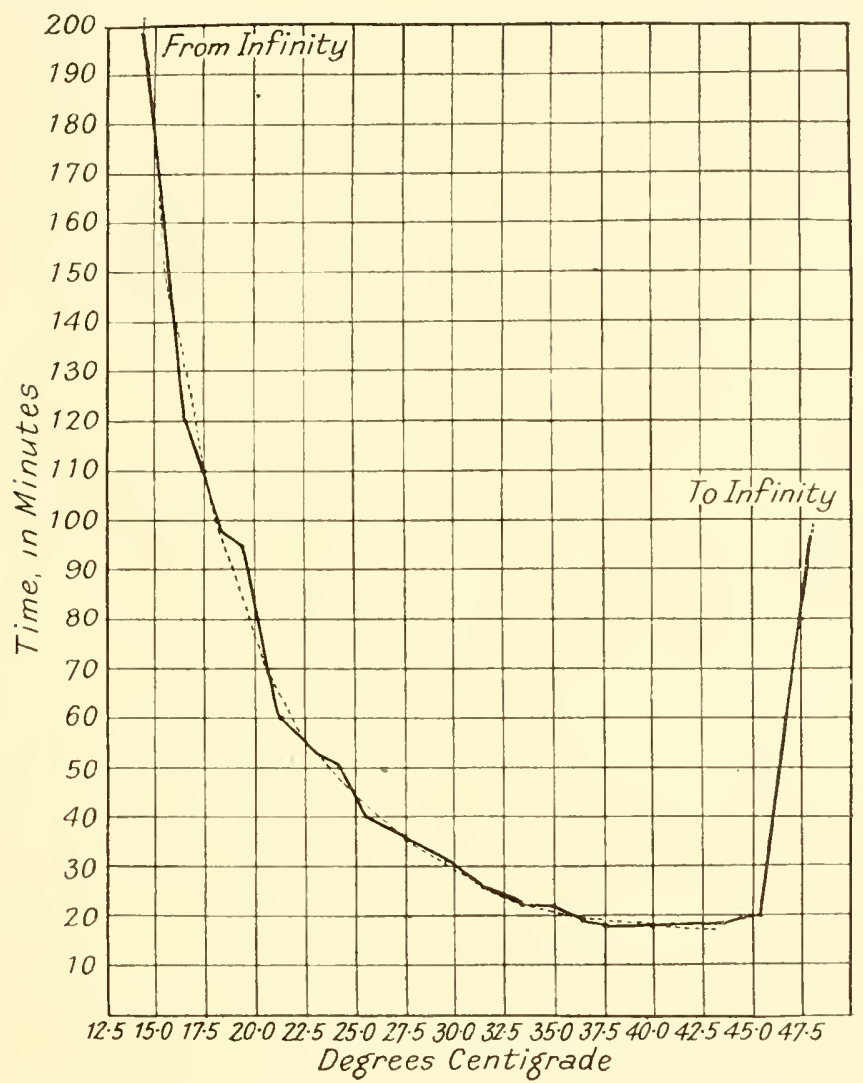

Fig. 17.-The Growth Rate of Bact. coli at Different Temperatures.

Continuous line $=$ Curve plotted from actual observations.

Interrupted line $=$ Smoothed curve.

(After Barber.)

formula shows that the actual rate of arithmetic increase rises rapidly to reach a peak at 5 hours, after which it falls steeply. The reason for the rapid rise is, not that the organisms are dividing more rapidly at the end than at the beginning of the logarithmic phase, but that, owing to the fact that the actual numbers of bacteria are constantly increasing throughout this phase, the progeny of any given generation expressed arithmetically must be greater than that of any previous generation. Lemon's method of calculating the growth rate is likely to be of advantage in physiological and biochemical problems when it is desired to study the rate of change in a chemical substrate in relation to the absolute numbers of organisms produced. 

factors.

The generation time during the logarithmic phase is influenced by several

(1) Temperature of Incubation of Culture.-Working with Bact. coli, Chick (1912) found that between $20^{\circ}$ and $40^{\circ} \mathrm{C}$., each rise of $1^{\circ} \mathrm{C}$. increased the rate of growth 1.072 times; the temperature coefficient for every rise of $10^{\circ} \mathrm{C}$. was $1.072^{10}$, i.e. 2.01. This agrees closely with Lane-Claypon's (1909) figure of $2 \cdot 2$. Barber (1908), who made an exhaustive study of the rate of growth of Bact. coli, constructed the curve shown in Fig. 17. In this it will be seen that at a temperature of $15^{\circ} \mathrm{C}$. the generation time is $180^{\prime}$, at $25^{\circ} \mathrm{C} .44^{\prime}$, at $35^{\circ} \mathrm{C} .22^{\prime}$ and at $40^{\circ} \mathrm{C} .17^{\prime}$. The curve corresponds fairly closely to the quarter of an ellipse. Further, as the increase is geometrical, by plotting the logarithms of the generation times, we shall find that the points fall on a descending straight line. The maximum rate of growth of Bact. coli occurs at about $37^{\circ} \mathrm{C}$., but between $37^{\circ}$ and $46^{\circ} \mathrm{C}$. there

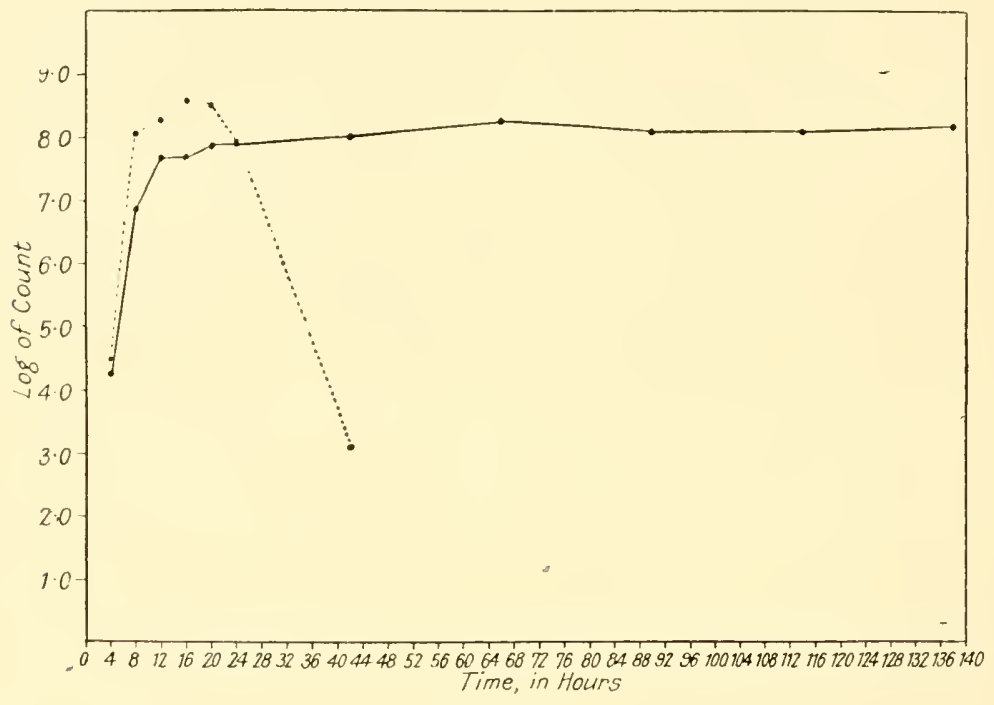

Fig. 18.

A. Continuous line $=$ Viable count of Salm. typhi-murium in peptone water.

B. Interrupted line $=$ Viable count of Salm.typhi-murium in peptone water +2 per cent. glucose. (After Heap and Cadness.)

is little change in the generation time. For Salm. typhi, Müller (1895) found the maximum rate of growth to be between $37^{\circ}$ and $40.4^{\circ} \mathrm{C}$. ; at a temperature of $44 \cdot 5^{\circ} \mathrm{C}$. the bacilli died rapidly.

(2) Nature of Medium.-Penfold and Norris (1912) found that by increasing the concentration of peptone in a peptone water medium from 0.125 per cent. to 1 per cent., the generation time of Salm. typhi was reduced from almost infinity to $40^{\prime}$. They likewise found that the addition of glucose to the medium further reduced the generation time. Other workers have also commented on the beneficial effect of glucose ; thus Heap and Cadness (1924) found that the addition of 2 per cent. glucose to a 3 per cent. peptoue water medium greatly increased the growth of Salm. typhi-murium. (Fig. 18).

(3) Nature of Organism. - Some organisms appear to grow more rapidly than others. Mason (1935) has compiled from the literature the generation times of 
various species of bacteria growing under optimal conditions. Among the most rapidly growing organisms are the members of the coli-aerogenes group, with a generation time of less than 20 minutes. Some of the large spore-bearing bacilli grow at about the same rate, while the thermophilic species may grow even faster. Organisms of the Salmonella, Proteus, and probably Vibrio, groups have a generation time of 20-30 minutes, Staplyylococcus and Streptococcus of 25-35 minutes, Pseudomonas of 30-40 minutes, Corynebacterium of 35-40 minutes, Clostridium of 35-50 minutes, Lactobacillus of 40-80 minutes, Rhizobium of 100 minutes, Azolobacter of 30-240 minutes, and Phytomonas of 75-165 minutes. Mycobacterium multiplies very slowly; the slowest of all appear to be Nitrobacter and Nitrosococcus.

Within certain limits definable for each organism, the duration of the logarithmic phase varies-

(1) inversely with the temperature. For example, Lane-Claypon (1909), and Jennison (1935), working with Bact. coli, made the following observations on the duration of the logarithmic phase at different temperatures:

$$
\begin{array}{r}
6-7 \frac{1}{2} \text { hours at } 37^{\circ} \mathrm{C} \text {. } \\
8-8 \frac{1}{2} \text { hours at } 30^{\circ} \mathrm{C} \text {. } \\
11-15 \text { hours at } 25^{\circ} \mathrm{C} \text {. } \\
14-24 \text { hours at } 20^{\circ} \mathrm{C} \text {. }
\end{array}
$$

(2) inversely with the size of the inoculum.

(3) directly with the quantity of culture medium.

If a flask of $50 \mathrm{ml}$. of broth is seeded with one million living Bact. coli, then the logarithmic phase at $37^{\circ} \mathrm{C}$. will last, generally, between 3 and 4 hours.

At the beginning of this section the logarithmic phase was defined as that period during which regular and maximal multiplication was occurring. Recent work, however, has cast some doubt on the accuracy of this definition. Rogers and Greenbank (1930) and Hirsch (1933), for example, have obtaiued evidence that the rate of growth during this phase is intermittent. Whether measured by the increase in numbers ( $V$. melchnikovi) or by the oxygen consumption (mouse typhoid bacillus), Hirsch found that. 3 or 4 peaks of growth occurred every hour. It is conceivable that these results may have been partly influenced by the periodic shaking of the culture, since the effect of aeration in stimulating growth is very marked. But this criticism does not apply to the results of Rogers and Greenbank, who inoculated their organisms (Bact. coli and Str. lactis) at one end of a tube 15 metres long and measured the rate of advancing growth by observing the decolorization of a dye in the medium. The rate of advance was intermittent, and the intermissions showed a striking degree of periodicity. When plotted against time, the curves depicting the rate of growth showed a moderate peak every 4-5 hours with a much higher peak every 15-20 hours throughout the 8 days required for the organisms to reach the distal end of the tube. It will be noted that the technique used by Rogers and Greenbank was very different from that of Hirsch, and it is by no means certain that the two sets of observers were dealing with the same phenomenon.

Further evidence that growth is not uniform throughout the logarithmic phase is suggested by the observations of Walker and his colleagues (1934) on the rate of $\mathrm{CO}_{2}$ production by $\mathrm{Bact}$. coli in a peptone water medium. It was found that the maximum output of $\mathrm{CO}_{2}$ per unit volume of bacterial substance occurred during the third hour of growth, i.e. the first hour or so of the logarithmic phase, 
after which a progressive fall occurred. It must be pointed out, however, that this might equally well be explained by assuming that the type of metabolism had changed during a period of constant growth.

Care must, of course, be exercised in drawing any conclusions on the rate of multiplication from the rate of growth, since it has already been pointed out that, owing to variations in cell size, increase in bacterial numbers may give a very fallacious idea of the true rate of growth, and the converse is equally true. Further observations at frequent intervals on the numerical increase occurring during the logarithmic phase, made with due attention to multiple small factors that may influence the result, are required before concluding that the rate of cell-division during this phase is discontinuous.

\section{Stationary Phase}

After multiplying at a maximum rate for a variable length of time during the logarithmic phase, the organisms become less active, and divide less frequently, till finally their numbers remain practically constant. What is responsible for this decrease in the reproduction rate? The natural suggestion is that it results from an exhaustion of the food supply. Against this view, however, is the fact that if a culture that has reached the decline phase is sterilized by boiling, and then reinoculated with the same organism, growth occurs in the usual way, though the actual numbers attained may not be so great as in the primary culture (GrahamSmith 1921). The same result is obtained if the culture is sterilized by filtration instead of by boiling, provided that the organism has not produced some volatile substance which would be removed during boiling, as in the special case of the pneumococcus. Penfold (1914) found that if a 24-hours' culture of Bact. coli was centrifuged, and the supernatant fluid was incubated at $37^{\circ} \mathrm{C}$., fresh growth took place. It is probable, therefore, that some other factor than exhaustion of the food supply is responsible for the cessation of maximal growth.

We have already mentioned Chesney's hypothesis, that toxic substances are produced during the phase of multiplication, and that these so injure the bacteria as to delay their subsequent division when introduced into a fresh medium. Although this explanation may hold good for the pneumococcus, which produces $\mathrm{H}_{2} \mathrm{O}_{2}$ in considerable amount, it does not seem to be of more general applicability. We may similarly regard as special cases those instances in which the reaction of the medium is rendered acid during growth owing to the inclusion of a fermentable carbohydrate. In such cases neutralization of the acid by the addition of alkali often suffices to enable growth to occur (Kojima 1923).

Working with the fruit-fly Drosophila melanogaster Pearl and Parker (1922) found that the effective reproduction rate decreased as the population density became greater.

Following on these observations Bail (1929) carried out a number of experiments on different bacteria, from the results of which he concluded that in any fluid culture there was a limiting population density that could not be exceeded. This he referred to as the $\mathrm{I}$-concentration. A few of his findings may be briefly recorded.

(1) Any given species of bacterium reaches in a fluid medium a particular and constant M-concentration of living organisms, the value of which differs with different species. 
(2) With a large inoculum the M-concentration is reached rapidly, with a small inoculum more, slowly.

(3) Living bacteria introduced into fresh broth in M-concentration are unable to multiply.

(4) Living bacteria introduced into fresh broth in a concentration greater than M die off until the M-concentration is reached.

(5) If a culture that has reached its M-concentration is centrifuged and then re-incubated, fresh growth will occur in the clear supernatant fluid till the 11 -concentration is reached. If, however, after centrifuging, the deposit is shaken up so that the organisms are distributed once more throughout the medium, no growth will occur. This seems to show that renewed growth is dependent not on the total number of bacteria in a given volume of medium, but on their mode of distribution within the medium.

(6) If a culture that has reached its M-concentration is heated to $55^{\circ} \mathrm{C}$. for a time sufficient to destroy the majority but not all of the living bacteria, and is then re-incubated, fresh growth will occur till the M-concentration is again reached. This seems to indicate that heat-killed bacteria do not appreciably interfere with the biological space available.

(7) Ordinary meat broth can be diluted 25 times or more without affecting the level of the M-concentration, slowing that it is not due to exhaustion of the medium that growth ceases. In the diluted broth the turbidity, i.e. the total count, is much less, but the number of living bacteria, i.e. the M-concentration, is the same as in the undiluted broth.

(8) The addition of an enriching substance, such as glucose, to the broth increases the total number of bacteria produced, but the M-coneentration of viable organisms remains unchanged.

(9) Growth does not cease with the attainment of the M-concentration; fresh organisms continue to be produced, but an equivalent number die off so that the M-value remains constant.

Many of these findings were confirmed by Fukuda (1929). This worker, however, pointed out that some of them, particularly Nos. 5, 7 and 8, held true only with certain organisms. He found moreover that, if broth cultures of Ps. pyocyanea were sterilized by heat and re-inoculated with fresh organisms, growth occurred till the original M-concentration was reached. This experiment could be repeated two or three times on the same culture, though with Salm. gallinarum only one quarter of the M-concentration was reached after the first heating.

von Wikullil (1932) brought further evidence in support of Bail's hypothesis. He found that, if two organisms $A$ and $B$, each having the same $\mathrm{H}$-concentration of 1,600 million per ml., were inoculated simultaneously in the same numbers into a tube of broth, the final M-concentration of the mixed culture was still only 1,600 million per ml., organism A constituting 800 million and organism B 800 million. The total physical space available had now to be shared between the two organisms. If two organisms, $\mathrm{A}$ and $\mathrm{C}$, having respective $\mathrm{H}$-concentrations of 1,600 million and 300 million per ml., were inoculated simultaneously into the same tube of broth the final M-concentration was again 1,600 million per $\mathrm{ml}$, but this time the whole viable population consisted of organism A. The apparent explanation was that $\mathbf{A}$ grew more rapidly than $\mathrm{C}$, and so monopolized the available space.

Bail and his followers were more interested in making observations than in 
explaining them. The problem still remains why it is that bacteria cease dividing at maximal speed when a certain population density is reached.

A suggestion which we put forward in 1936, and which has reeeived some support from the observations of Hershey and Bronfenbrenner (1937) and of Rahn and Richardson (1942), is that the progressive retardation in growth may often be due to a deficiency of oxygen in the culture. There are certain observations that seem to support this view. We have already seen that the addition of 2 per cent. glucose to a broth medium greatly increases the growth of Salm.typhi-murium. The mechanism by which this increase is brought about is unknown, but it is not unreasonable to suppose that the glucose provides a source of energy supply, which the organisms are able to utilize in the absence of readily available oxygen. In support of this we have found that the total number of organisms per ml. in a given culture can be regulated by altering the available amount of oxygen. Thus, in a casein broth culture of Salm. typhi-murium incubated anaerobieally, the total count after 24 hours is about 500 million organisms; in a culture incubated aerobically it is about 2,000 million organisms; and in a culture through which pure oxygen is bubbled continuously, it is about 8,000 million organisms (IVilson, 1930). There seems no doubt that a liberal supply of oxygen enables growth to continue for some time after it has ceased in a culture incubated anaerobically or under ordinary aerobic conditions. The suggestion is that, in eultures incubated aerobically, growth continues until the increasing density of the organisms renders it impossible for each individual organism to obtain sufficient oxygen to meet its requirements. These findings apply, of course, only to aerobic and facultatively anaerobic bacteria.

This explanation, it will be observed, does not fit all of Bail's faets, though it explains many of them. The main discrepaney is in the effect on the II-concentration of adding certain substrates. Bail found that the addition of glucose, for example, led to an increase in the total number of bacteria, while leaving the M-concentration of viable bacteria unaltered. Our experience, both with glncose and with increased aeration, entirely fails to support this finding. The more favourable the conditions for growth are, the higher is the viable population that the medium can support. It is true that in a medium containing a fermentable sugar the viable population may fall rapidly after having reached a height considerably greater than in the same medium without sugar (see Fig. 18), but this appears to be due to the disinfectant action of the acid produced, and not to any specific effect of bacterial density. That this is so is shown by the effeet of continuous oxygen passage in the experiments quoted above, in enabling a broth medium to support four times its usual M-population.

Broom (1929) and Gildemeister and Neustat (1935) have brought evidence to show that the amount of growth of many organisms appears to depend to a considerable extent on the presence in the medium of casily assimilable carbon compounds, such as glucose. The addition of these to a culture in which growth has ceased may rapidly enable a fresh crop of organisms to be produced. The apparent ability of some organisms to inhibit the growth of others in mixed cultures seems to be due not only to their more rapid growth, but also to their more active fermentative power, which enables them to break down certain compounds more readily and so deprive the weaker organisms of their requisite nutritive materials.

The fact that mere aeration of a culture may enable fresh bacterial multiplication to occur suggests that there are at least two factors leading to cessation of growth. One is the exhaustion of easily assimilable food-stuffs; the other is 
oxygen starvation. It seems not improbable that, once the simpler food-stuffs have been broken down, the attack on the more complex compounds can be successful only in the presence of abundant oxygen.

It must not be thought that during the stationary phase all growth has ceased. In most cases multiplication continues for some time after the cessation of the logarithmic phase, but the rate of division progressively diminishes. The time taken to reach the maximum number varies. The temperature of incubation is important; thus, Graham-Smith (1921), working with Staphylococcus aureus, found that the maximum number of viable organisms was reached on the 2 nd day at $37^{\circ} \mathrm{C}$, on the 5 th day at $27^{\circ} \mathrm{C}$, and on the 8 th day at $17^{\circ} \mathrm{C}$. Much depends, too, on the nature of the medium.

Not only are fresh organisms being formed during the stationary phase, but large numbers are dying. Once the maximum number of organisms has been reached, and before the phase of decline, the multiplication rate just suffices to balance the death rate, so that the number of living bacteria remains constant.

\section{Phase of Decline}

After lasting for a variable time-from about an hour to several days-the stationary phase passes gradually into the phase of decline. The numbers decrease slowly over a period of days, weeks, or months, till all the organisms are dead. With some organisms, such as the pneumococcus, this phase is short, and the culture may be sterile in 2 to 3 days; with other organisms, such as Bact. coli, it may last for months. It is possible that growth is not entirely in abeyance during this stage, for if counts are performed at daily intervals, spasmodic rises are some-

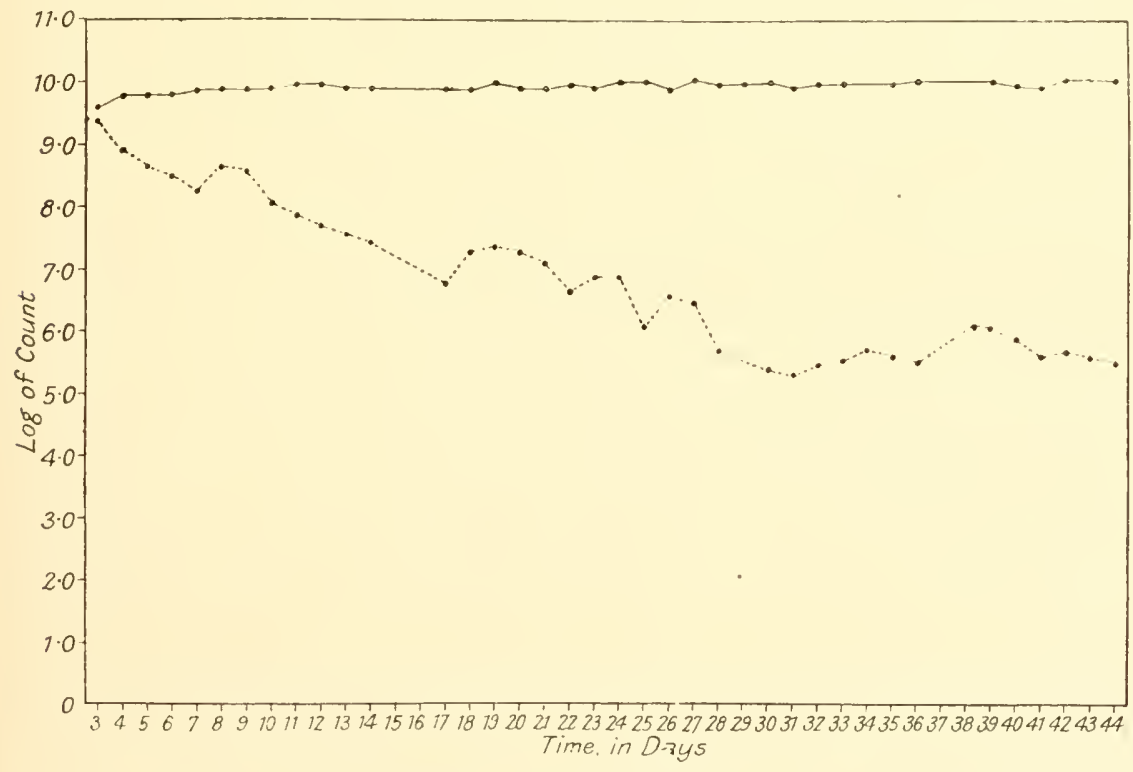

Frg. 19.-Growth of Salm. typhi-murium in Broth, showing the prolongev Decline Phase.

Continuous line $=$ Total number of bacteria alive and dead.

Interrupted line $=$ Number of living, or viable bacteria.

P.B. 
times noticed, indicative of the production of fresh organisms. Moreover, there is a gradual rise in the total count, pointing to the same conclusion (Fig. 19).

This is the curve obtained for a culture of a coliform or similar organism in a nutrient broth medium. In a medium containing a fermentable sugar, such as glucose broth, the curve is very different. Instead of descending slowly and irregularly, it passes rapidly down in an oblique straight line, till it reaches the abscissa (Fig. 18).

This difference is almost certainly due to the disinfectant action of the acid produced in the culture. The death of the organisms in Curve B is similar to the velocity of a unimolecular reaction, and can be represented by the formula

$$
\mathrm{K}=\frac{1}{t} \log \frac{n_{1}}{n_{2}}
$$

where $\mathrm{K}$ is the velocity constant, $t$ the interval of time between successive observations, $n_{1}$ the number of living bacteria present at the beginning, and $n_{2}$ the number present at the end of time $t$. This point will be more fully discussed in Chapter 5. For further information on the growth phases of bacteria, the reader is referred to a detailed consideration of this subject by Buchanan and Fulmer (1928).

\section{Dormancy of Bacteria.}

G. S. Burke (1923) inoculated a series of tubes of glucose peptic digest agar and of glucose peptic digest broth with $\mathrm{Cl}$. botulinum, seeding one spore into each tube. She sealed the tubes to prevent desiccation, incubated them at $37^{\circ} \mathrm{C}$, and noted each day in how many tubes growth had occurred. In the agar medium the majority developed in 10 days, but occasional spores continued to germinate up till the 92nd day. In the broth the majority developed in 14 days, but one or two germinated daily until the 33rd day, and thereafter at intervals till the 144th day.

V. Burke, Sprague and Barnes (1925) obtained similar results with B. subtilis, B. megatherium, and Bact. coli, thus showing that the phenomenon is not confined to anaerobic bacteria or the germination of spores. It is clear from these experiments that bacteria may lie dormant for long periods without multiplying. Superficially, this resembles the phenomenon of lag, but it is possible that dormancy and lag are dependent on different factors. G. S. Burke (1923) draws attention to the similarity in behaviour of dormant spores and the seeds of certain of the higher plants. She suggests that in each instance the cause of the dormancy lies in the cell itself, and is connected with the degree of permeability of the wall.

It is this dormancy which appears to be responsible for the occasional failure of the intermittent process of sterilization. Probably owing to this, too, is the fact that a culture may be contaminated with an organism without signs of the contamination becoming evident till after three or four subcultures have been made.

Mitogenetic Rays.-The extensive work of Gurwitsch and his followers (for references see Bateman 1935) has suggested that certain plant and animal tissues under suitable conditions may emit so-called mitogenetic rays, which are able to stimulate growth in tissue cells placed in a position favourable for their absorption. According to some workers, the radiation is of the short ultra-violet type, but attempts to confirm this by physical methods have not so far been successful. The rays, if they exist at all, have therefore to be detected by biological means. 
So far as bacteria are concerned, the growth-stimulating effects that have been reported have usually been well within the limits of the experimental error of the technique. While not denying the existence of this effect, we feel bound to adopt a strictly sceptical attitude until quantitative results that will stand the usual tests of statistical significance are forthcoming.

\section{REFERENCES}

Alper, T. and Sterne, M. (1933) J. Hyg., Camb., 33, 497.

Anderson, E. B. and Meaniwell, L. J. (1936) J. Dairy Res., \%, 18:.

BATL, O. (1929) Z. ImmunForsch., 60, 1.

BARBER, M. A. (1908) J. infect. Dis., 5, 379.

Bateman, J. B. (1935) Biol. Rev., 10, 42.

Breed, R. S. (1911) Zbl. Bakt., IIte Abt., 30, 337.

Broom, J. C. (1929) Brit. J. exp. Path., 10, 71.

Buchanan, R. E. (1918) J. infect. Dis., 23, 109.

Buchanan, R. E. and Fulmer, E. I. (1928) "Physiology and Biochemistry of Bacteria." London.

Buice, IV. A. (1933-34) Zul. Ba!t., IIte Abt., 89, 387.

Burke, G. S. (1923) J. infect. Dis., 33, 274.

Burke, V., Sprague, A. and Barnes, L. Y. (1925) J. infect. Dis., 36, 555.

Chesney, A. M. (1916) J. exp. Hed., 24, 387.

Chick, H. (1912) J. Hyg., Camb., 12, 414.

CHLd, C. М. (1915) "Individuality in Organisms." Chicago.

Clark, P. F. and Puenl, W. H. (1919) J. Bact., 4, 615.

Coplans, M . (1910) J. Path. Bact., 14, 1.

Eberle, R. (1896) Zbl. Bakt., 19, 2.

Fuguda, T. (1929) Z. ImmunForsch., 60, 88.

Gildemeister, E. and Neustat, M. (1935) Zbl. Bakt., 133, 101.

Gillespie, L. J. (1914) J. exp. Hed., 19, $2 \mathrm{~s}$.

Gordon, R. D. and ZoBerl, C. E. (1938) Zul. Bukt., IIte Abt., 99, 318.

Graham-Smith, G. S. (1921) J. Hyg., Camb., 19, 133.

Greenwood, M. and Yule, G. U. (1917) J. Iyg., Camb., 16, 36.

Halvorson, H. O. and Ziegler, N.R. (1933 a) J.Bact., 25, 101 ; (1933b) Ibid., 26, 331, 559.

HeaP, H. and CAdness, B. H. E. (1924) J. Ifyg., Camb., 23, 77.

Helber, E. (1904) Dtsch. Arch. klin. Hed., 81, 317.

Henrici, A .T. (1926) J. infect. Dis., 38, 54; (1928) "Morphologic Variation and the Rate of Growth of Bacteria." London.

Hershey, A. D. (1939) J. Bact., 3\%, 285.

Hershey, A. D. and Bronfenbrenner, J. (1937) Proc. Soc. exp. Biol., N.Y., 36, 556.

Hinsch, J. (1933) Klin. U'schr., 12, 191.

Hoskins, J. K. (1934) Publ. Hlth. Rep., Wash., 49, 393.

Huntington, E. and Winslow, C.-E. A. (1937) J. Bact., 33, 123.

Jenvisov, M. W. (1935) J. Bact., 30, 603.

Jennison, M. W. and Wadsworth, G. P. (1940) J. Bact., 39, 389.

Jordan, R. C. and JACoBs, S. E. (1944) J. Bact., 48, 579.

KELly, C. D. and RAHN, O. (1932) J. Bact., 23, 147.

Koлгмa, S. (1923) Sci. Rep. Inst. infect. Dis. Tokyo Univ., 2, 305.

Lane-Claypon, J. E. (1909) J.Hyg., Camb., 9, 239.

Ledingham, J. C. G. and Penfold, W. J. (1914) J. Hyg., Camb., 14, 242

LeMon, C. G. (1933) J. Hyg., Camb., 33, 495.

LiESE, W. (1926) Z. Hyg. Infeltkr., 105, 483.

Longsworth, L. G. (1936) J. Bact., 32, 307.

MLason, M. M. (1935) J. Bact., 29, 103.

McCrady, M. H. (1915) J. infect. Dis., 1\%, 183; (1918) Canad. publ. IIlth. J., 9, 201.

MacGregor, A. S. M. (1910) J. Puth. Bact, 14, 503.

Mestre, H. (1935) J. Bact., 30, 335.

Mmatz, J. M. IV. and Rottier, P. B. (1936) Zbl. Bakt., IIte Abt., 94, 227.

Miles, A. A. and Misra, S. S. (1938) J. Hyy., Camb., 38, 732.

MOYER, L. S. (1936) J. Bact., 32, 433.

MÜller, M. (1895) Z. Hyg. InfektKr., 20, 245.

Pearl, R. and Parker, S. L. (1922) Proc. nat. Acad. Sci. Wash., 8, 212 ; quoted from Maclagan, D. S. (1932) Proc. roy. Soc., B, 111, 437.

Penfold, W. J. (1914) J. Hyg., Camb., 14, 215.

Penfold, W. J. and Norris, D. (1912) J. Hyg., Camb., 12, 527. 
Pulvertaft, R. J. V. and Lemon, C. G. (1933) J. Hyg., Camb., 33, 245.

RAHN, O. (1906) Zbl. Bakt., IIte Abt., 16, 417.

RahN, O. and Ricinardsox, G. L. (1942) J. Bact., 44, 321.

Réonier, J., David, R., and Kaplan, A. (1932) C. R. Acad. Sci., 194, 323.

Reichenbach, H. (1911) Z. Hyg. InfektKr., 69, 171.

Rogers, L. A. and Greenbank, G. R. (1930) J. Bact., 19, 181.

Sснмidт, H. (1926) Z. Hyg. InfektKr., 106, 314.

Schmidt, H. and Fischer, E. (1930) Z. Hyq. InfektKr., 111, 542.

Schultz, J. H. and Ritz, H. (1910) Zbl. Bakt., 54, 283.

Shermax, J. M. and Albus, W. R. (1923) J. Bact., 8, I27 ; (1924) J. Bact., 9, 303.

Sherman, J. M. and NAYlor, H. B. (1942) J. Bact., 43, 749.

SKAR, O. (1934) Z. InfektKr. Haustiere, 46, 110.

Slator, A. (1917) J. Hyg., Camb., 16, 100.

Stein. (1917) Engng. News. Rec., 78, No. 8, 391.

Strausz, W. (1930) Zbl. Bakt., 115, 228.

Topley, W. W. C. and Wilson, G. S. (1936) "The Principles of Bacteriology and Immunity," 2nd ed., p. 70. Ed. Arnold \& Co., London.

Walker, H. H., Winslow, C.-E. A., Huntington, E., and Mooney, M. G. (1934) J. Bact., $27,303$.

WikdLLIL, L. v. (1932) Zbl. Bakt., 126, 488.

Wilson, G. S. (1922) J. Bact., 7, 405; (1926) J. Hyg., Camb., 25, 150 ; (1930) Ibid., 30, 433 .

Wilson, G. S., Twiog, R. S., Wrioht, R. C., Hendry, C. B., Cowell, M. P., and Maier, I (1935) Spec. Rep. Ser. med. Res. Coun., Lond., No. 206.

Winslow, C.-E. A. and Walker, H. H. (1939) Bact. Rev., 3, 147.

Wrioht, A. E. (1902) Lancet, ii. 11. 


\section{CHAPTER 5}

\section{'THE RESISTANCE OF BACTERIA TO PHYSICAL AND CHEMICAL AGENTS : DISINFECTION}

\section{INTRODUCTORY}

THE early investigations of the problem of disinfection, which may be said to have commenced with Pringle's observations in 1750 , were largely concerned with a study of the efficacy of various substances in hindering putrefaction. A century and a quarter later Bucholtz (1875), using as his medium an infusion of tobacco leaves, conducted a series of investigations on disinfectants ; and Baxter (1875), working with vaccine lymph and glanders nodules, showed the influence of organic matter in diminishing the activity of disinfectants.

The next advance, illustrating the importance of technique, was made by Koch in 1881, when he introduced an exact method of comparing the germicidal power of different substances. In place of fluids swarming with different organisms of varying resistance, he tested the action of disinfectants on pure cultures of bacteria of approximately equal resistancc. By drying anthrax spores on silk threads of the same length, immersing them in a solution of the substance to be tested, and subsequently transferring them to a nutrient medium in order to ascertain if the bacteria were still alive, he collected a considerable quantity of information on the relative activity of different disinfectants. His work was criticized and his methods improved by Geppert $(1889,1891 a, b)$. In 1897 Krönig and Paul published their classical paper, describing a new method for the quantitative study of disinfection, and demonstrating that in a culture submitted to the influence of a germicidal agent bacteria die, not simultaneously, but in an orderly sequence. To Madsen and Nyman (1907) and to Chick (1908, 1910, 1912) must be ascribed the merit of analysing the various factors upon which disinfection depends, and of showing that the law underlying the death of bacteria is similar to that underlying a simple unimolecular chemical reaction.

Starting from empirical obscrvations on the preservation of dead human bodies, the study of disinfection has progressed through the qualitative stage to the quantitative stage, and has now reached a point when the ultimate solution of the problem lies in the domain of the physical chemist.

The subject of disinfection is large, and can be treated from different aspects. In the present chapter we shall make no attempt to deal with it exhaustively; on the contrary, we shall purposely neglect a considerable part of the subject dealing with the use of germicides in practice, as we consider this to fall within the province of the hygienist. Our main endeavour is to discover as far as possible the underlying principles of disinfection, to discuss the laws governing the killing of bacteria, and to point out the importance of a thorough knowledge of these 
laws and principles to any one who, whether engaged in medicine, hygiene, dairyfarming, food-preservation, or agriculture, is confronted with the problem of controlling bacterial activity.

\section{Physical Agencies}

Light.-Downes and Blunt in 1877 (1877, 1878) showed that exposure of a putrescible fluid to sunlight was sufficient to sterilize it. They observed that this effect was produced only in the presence of air, and were therefore led to regard the germicidal property of light as depending on oxidation. Duclaux (1887) in 1886 showed that in sunlight vegetative bacilli were killed more rapidly than spores. The following year Roux (1887) exposed anthrax spores in a nutrient medium to the sun. Some were contained in glass tubes with plenty of air above the level of the liquid, others in glass tubes containing no free air; the former were destroyed in 29 hours, the latter survived longer than 83 hours. This was a confirmation of the work of Downes and Blunt, but Roux went further; he found that if nutrient broth was exposed to the sun in a layer $5 \mathrm{~mm}$. deep for 3 or 4 hours, it became changed in such a way as no longer to permit of the germination of anthrax spores, though still remaining suitable for the growth of the vegetative bacilli. This antiseptic property was lost after the broth had been allowed to stand for a time in the dark. Broth exposed to the sun in a sealed glass tube containing no free oxygen was unaffected. It was clear, therefore, that not only was sunlight in the presence of air able to destroy anthrax spores, but that it was able to produce an alteration in a nutrient medium-an alteration which was of a transient nature, suggestive of the activity of some volatile or unstable compound. This action of sunlight was reinvestigated by Burnet (1925); working with staphylococci, he found that they would not grow on agar plates that had been exposed to the sunlight, thongh growing quite satisfactorily on control plates that had been kept in the dark. He was able to show that the reason for this is that under the influence of sunlight hydrogen peroxide is produced, and that this substance is so powerful that its inhibitory effect is noticeable even in a dilution of $1-40,000$ (see Chapter 3 ).

To return to the action of light on the bacteria themselves: Ward in 1892 exposed gelatin and agar plates seeded with anthrax spores to the autumn sunlight for 6 hours, each plate being shaded in such a way that only part of it received the direct rays of the sun. After incubation, a growth of anthrax bacilli was found to have occurred in the protected but not in the exposed portion. The inhibitory action of the sun was not due to the heat rays, because the temperature of the plates at no time rose above $18^{\circ} \mathrm{C}$.; nor was it due to desiccation or other alteration of the medium, since exposed plates seeded with fresh spores proved quite suitable for growth. Ward therefore concluded that the germicidal effect of the sun was due to its actinic rays. Further work showed that if a spectrum was thrown across an agar plate, the inhibitory effect of the light was stronger at the blue than at the red end. This was the first demonstration of the selective action of violet light. Some years later Barnard and Morgan (1903), working with the arc spectrum of carbon and of various metals, made more extensive observations, which led them to conclude that the bactericidal action of light was almost entirely due to those radiations in the ultra-violet region which are included between the wave lengths 3287 and $2265 \AA$.U., that is, the light between the visible 


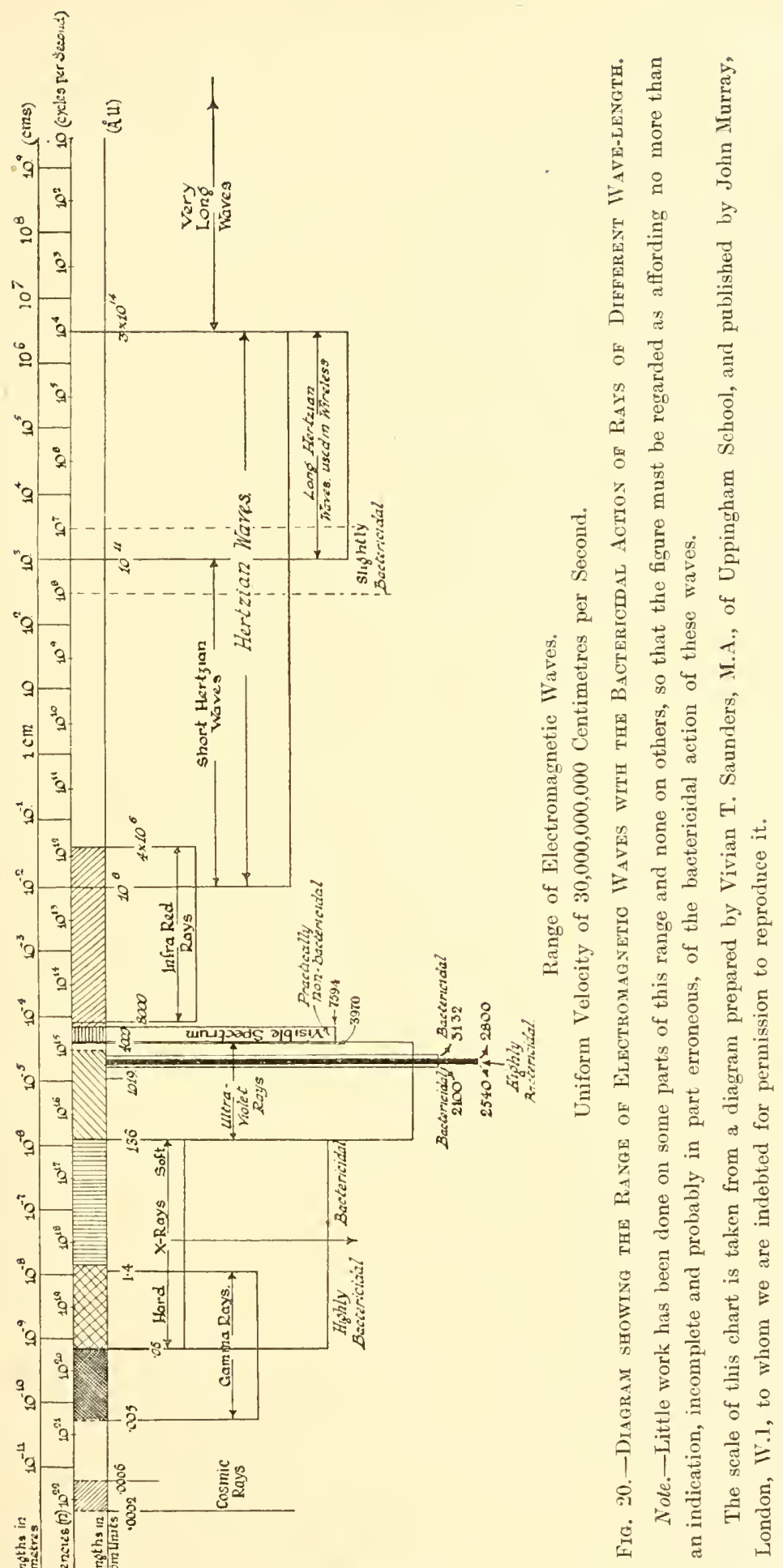


violet and the extreme ultra-violet. No other portion of the spectrum had any effect whatever. Their work has been confirmed by Browning and Russ (1917), who used a quartz spectrometer illuminated by an arc of pure tungsten, which is very rich in ultra-violet rays. The spectrum was thrown across a gelatin or agar plate seeded with staphylococci, the plate incubated after irradiation, and then used as an ordinary photographic negative for producing positive contact prints. An exposure of 6 minutes was sufficient to destroy the organisms in that region illuminated by rays of 2380 to 2940 Angström units. (An Angström unit is equal to $1 \times 10^{-7} \mathrm{~mm}$., i.e. $1 / 10,000,000$ th $\mathrm{mm}$. One $\mathrm{m} \mu$ is equal to $1 \times 10^{-6} \mathrm{~mm}$., i.e. $1 / 1,000,000$ th mm., or $1 / 1,000$ th $\mu$, or 10 Angström units.) There was a sharp line of demarcation at $2960 \AA . U$.; rays of a longer wave length than this were almost devoid of germicidal action. It was found that the rays between 2960 and $2100 \AA . U$. were highly germicidal, but that the most active were those between 2800 and $2540 \AA$.U. As the limit of retinal sensibility is reached when the wave length falls to $3970 \AA$.U., it follows that the most actively germicidal portion of the spectrum is in that part which is invisible to the human eye (Fig. 20). More recent work by Gates (1930), Ehrismann and Noethling (1932), Buchholz and v. Jeney (1935), Prudhomme (1937), and Rouyer and Servigue (1938), has shown that two of the most active wave lengths are 2650 and $2530 \AA$.U. Different organisms appear to vary in their susceptibility to different wave lengths.

Ultra-violet light is lethal, not only to bacteria, but to other unicellular organisms such as amœbæ (Barr 1923), and to the tissue cells of animals and plants. It is, in fact, a protoplasmic poison. Though the short rays are undoubtedly the most active, the other rays of sunlight are not completely devoid of germicidal power; they require, however, very much longer to produce their lethal effect (Thiele and Wolf 1907). Laroquette (1918) found that blue was more active than yellow, and yellow than red; green light was the poorest of all. The time necessary for destruction of micro-organisms by ultra-violet light depends on the intensity of the light, the distance of the source of illumination, and the nature of the medium in which the organisms are exposed. In general, the Bunsen-Roscoe law holds true ; that is, within given limits the product of the intensity of irradiation and the length of exposure is constant. The temperature of the organisms at the time of exposure, provided that it is within the normal limits of viability, seems to have no effect on the action of ultra-violet light (Rentschler et al. 1941).

The germicidal effect of sunlight under natural conditions varies greatly. Its action is complex, due partly to the actinic and partly to the calorific rays, which act by dehydration. Apart from its action on the organisms themselves, it has an action on the medium in which they are growing. In southern lands, the combined effect of the ultra-violet rays and the heat rays render sunlight highly efficient as a germicidal agent; thus Semple and Greig (see Hewlett 1909) in India found that Salm. typhi exposed to the sun on pieces of white drill cloth were killed in 2 hours; controls kept in the dark were still alive after 6 days. In the civilized smoke-covered towns of the north, the ultra-violet rays are often very weak, being largely filtered off by the impurities in the atmosphere, thus depriving sunlight of most of its activity. That light under these conditions is not entirely without effect, however, has been shown by Garrod (1914), who found that in hospital wards hæmolytic streptococci could be readily demonstrated in dust from the darker portions of the ward, but not from dust on window sills and other parts exposed to diffuse daylight. 
Ultra-violet light generated by a Cooper Hewitt Mercury vapour lamp has been used for the sterilization of drinking water. Foulds (1911) found that it was quite easy to ensure a 99 per cent. reduction in the bacterial count, including all Bact. coli, by this method. For further information, see Thresh and Beale (1910).

Mode of Action of Ultra-violet Light.- Ultra-violet light is peculiar in that its penetrating power is very low. Even a thin layer of glass, such as a cover slip, is able to filter off a large proportion of the rays. The same power is possessed by proteins. It follows therefore that its action must be chiefly on the surface of the body which it irradiates. Wesbrook (1896), by an ingenious series of experiments, showed that when tetanus or cholera cultures were irradiated by the sun in the presence of air, a consumption of oxygen took place. D'Arcy and Hardy (1894) came to the conclusion that under the influence of ultra-violet light, some oxidizing substance is produced-possibly ozone-which is responsible for its bactericidal effect. This is very doubtful. There seems to be no question that the destructive action of ultra-violet light is manifest in the absence of atmospheric oxygen (Thiele and Wolf 1906, 1907, Blum 1932, Buchholz and v. Jeney 1935), so that ozone can hardly be responsible. Nor does it seem likely that hydrogen peroxide is generated in sufficient quantities to prove lethal, since Ehrismann (1930) found that, to produce a destruction of bacteria similar to that calsed by ultra-violet light, a concentration of 30 per cent. $\mathrm{H}_{2} \mathrm{O}_{2}$ was required.

There is evidence that the ultra-violet rays act by inducing some change in the protein molecule.

Thus they are destructive, not only of organized cells, but of cellular products, such as tetanus toxin (Kitasato 1891, Fermi and Pernossi 1894, Wesbrook 1896), and serum complement (Sellards 1918, Brooks 1920). Dreyer and Hanssen (1907), working with solutions of various albumins and globulins, exposed in very thin layers, found that the rays caused a true coagulation of the proteins, which were no longer soluble in weak acids or alkalies. Corroboration of this view is found in the experiments of Tchahotine (1921). Working with the eggs of sea urchins, which are rich in lecithin, he found that if they were stained with neutral red and then irradiated with ultra-violet light, the colour changed after a time to yellow, indicating the presence of alkali within the cell. Further work seemed to show that the action of the rays was on the superficial membrane of the cell, which was rendered more permeable to the $\mathrm{OH}$-ions of the medium; these, on penetrating the cell, were responsible for the change of the neutral red to yellow. In support of this, it was found that if the eggs were irradiated in a neutral solution, no change in the colour of the dye took place. From these and further experiments he comes to the conclusion that the rays act primarily on the superficial layer of the cell, coagulating its colloids, and rendering it mare permeable to the ions in the surrounding medium. Ehrismann (1930) exposed saline suspensions of various bacteria to ultra-violet irradiation for 6 hours, and observed that a decrease in the opacity occurred, accompanied by a fall in the total count. No change in $\mathrm{pH}$, however, was noticed. Agencies such as increased acidity, higher temperatures, formol, and mercuric chloride, that tended to hasten the coagulation of protein, partly or completely inhibited the clearing effect of the rays. Analysis showed that the total nitrogen in the suspending fluid was increased, though the amino-nitrogen figure remained unaltered.

The conclusion appears to be that the rays produce a colloidal change in the protoplasm leading to the solution of certain of its constituents. Whether true autolysis occurs in addition is still doubtful. 
Buchholz and v. Jeney (1935) suggest that the reaction may possibly be of photo-chemical nature. These workers point out that the highly lethal waves 2650 and $2530 \AA$.U. correspond respectively to energy values of $4 \cdot 6$ and $4 \cdot 8$ volts, and conclude that this amount of energy is required to displace sufficient electrons from the bacterial protoplasm to give rise to irreversible photo-chemical alterations and thus bring about the death of the cell. Wyckoff (1932), who has studied this aspect of the problem in the light of the quantum theory, finds that about 4 million quanta of energy are required to kill a single coliform bacillus, showing that death is not due, as it appears to be with the cathode rays, to a single quantum absorption, but to some more generalized effect on the bacterial protoplasm. According to Lea and Haines (1940), about 100 times more energy is required to kill a bacterium when administered as ultra-violet light than as $\mathrm{X}$-rays, suggesting that the quantum yields of ultra-violet light are very small. They conclude with Wyckoff that the euergy of a single ultra-violet light quantum is not usually sufficient to cause ionization. A similar conclusion has also been reached by Rentschler, Nagy and Mouromseff (1941).

The mode of action of ultra-violet light is still obscure, but the work of Gates (1930) and Ehrismann and Noethling (1932) renders it probable that it depends on the alteration of certain molecular groupings in the cell having high specific absorption spectra for these rays. The exact nature of the effect produced must await further observation.

Photodynamic Sensitization.-We have seen that the visible rays of the spectrum have only a weak germicidal action on bacteria. It has been found, however, by Raab (1900) and by v. Tappeiner (1900) that certain fluorescent dyes are able to sensitize infusoria to the action of these rays, so that they become almost as lethal as the ultra-violet rays. Thus it was shown that paramœcium suspended in a solution of acridin or eosin was killed very much more rapidly when exposed to diffuse sunlight, which was itself harmless, than when kept in the dark. Examining this phenomenon more closely, v. Tappeiner projected a spectrum across a table, and placed a culture of paramœcium, suspended in 1-800 solution of eosin, in the red, green, violet, and ultra-violet parts. The culture exposed to the green rays was killed in 2 to 4 hours, whereas the cultures exposed to the other rays appeared to be unharmed. This is of double interest; firstly, because the green rays by themselves are the least active in germicidal power; and, secondly, because it is in green light that eosin fluoresces most strongly. Similarly with acridin, death was most rapid when the suspension was exposed to the violet rays, these being the rays which cause acridin to fluoresce. For the sensitization to occur it was essential for the paramœcium to be in close contact with the fluorescing particles. When the cultures were exposed to light that was simply filtered through eosin, they remained unharmed; the eosin had to be dissolved in the actual culture before its sensitizing action became apparent.

Later, v. Tappeiner and Jodlbauer (1904) and Jodlbauer and v. Tappeiner (1904) demonstrated that the photodynamic action of dyes is manifest not only in relation to infusoria, but in relation to bacteria, toxins, and to a less extent antitoxins, while more recently the bacteriophage (Clifton 1931) and filtrable viruses (Perdrau and Todd 1933b) have been found susceptible.

Burge and Neill (1915), exposing various micro-organisms to ultra-violet light, found that the non-fluorescent were killed more rapidly than the fluorescent bacteria; their supposition is that the latter protect themselves from the coagulating 
effect of ultra-violet light by converting the short wave lengths into longer waves, and thus disposing of the energy of the absorbed short waves that would otherwise be spent in coagulating them. The non-fluorescent bacteria are unable to do this, and hence succumb. In this connection, it is interesting to note that fluorescent bacteria may themselves exercise a sensitizing action on infusoria, thus taking the place of complex dyes. Jodlbauer and v. Tappeiner (1904) found that paramœcium, suspended in a killed broth culture of $P s$. pyocyanea, was killed in an hour if exposed to diffuse daylight, while surviving for 24 hours in the dark. Gramnegative organisms appear to be more resistant than Gram-positive ones, especially to the longer wave lengths (see T'ung 1935, Dreyer and CampbellRenton 1936).

Later work by Schmidt and Norman (1920) suggests that the photodynamic effect of certain dyes is not dependent simply on their power of fluorescence.

They showed that red blood cells mixed with eosin and exposed to sunlight were hæmolysed, even if the rays that cause eosin to fluoresce were filtered off before reaching the solution. Again, in a mixture of red cells, eosin, and a protective substance, such as tyrosine, exposed to sunlight, there was no hæmolysis even though the solution was fluorescing strongly. Chifton (1931) found that a staphylococcal bacteriophage suspended in $0 \cdot 01-0 \cdot 1$ per cent. methylene blue solution was inactivated by exposure to sunlight for 5 minutes or more. The reaction did not occur in vacuo, or in the presence of an active reducing agent such as cysteine hydrochloride $(0.01$ per cent.). Perdrau and Todd (1933a), besides confirming these observations and finding that the optimal concentration of dye was about $1-100,000$, showed that the interposition of a green screen prevented the reaction, while a red screen did not.

The mode of action of dyes, in causing sensitization to light that is not itself markedly germicidal, is not very clear, but it would appear that as the dye must be adsorbed on to the surface of the cell, and as oxygen is necessary for the effect to take place, the process is probably due to an activation of oxygen, or to an oxidation product of the dye (Bayliss 1924). (For a review of the whole subject see Blum 1932.)

Electricity.-(1) Direct Currents. Little work has been done. Prochownick and Spaeth (1890) passed a galvanic current through simple saline suspensions of $B$. anthracis, $B$. subtilis, and staphylococci without nuch effect; after 2 hours B. subtilis had lost its motility, but was quite capable of growth. In another experiment the electrodes were coated with agar, seeded with organisms, immersed in saline, and a current passed through. No effect was noticeable at the cathode, but around the anode the organisms were killed. Thus a 60 milliamp. current destroyed Staphylococcus aureus in 15 minutes, and a 230 M.A. current destroyed $B$. anthracis in 30 minutes. They concluded that the effect was due not to the electricity per se, but to the nascent chlorine which was evolved at the anode from the electrolytically dissociated saline. Similar results were obtained in the same year by Apostoli and Laquerrière (1890). They employed a constant galvanic current, which was passed through a broth culture of $B$. anthracis into which the electrodes, situated a short distance apart, had been inserted. A current of 300 M.A. was fatal in 5 minutes; one of 200-250 M.A. failed to sterilize the culture in this time. They found that the action of the constant galvanic current was in direct relation to the intensity of the current, measured in milliampères ; that it depended far more on the intensity of the current than on the time for which it acted; and that the lethal effect was confined to the positive pole. They excluded 
the effect of heat, and were able to show that the sterilizing action of the constant current was due to the liberation of acids and of nascent oxygen at the anode.

(2)Low-Frequency Currents. Beattie and Lewis (1920) were able to kill over 99.9 per cent. of organisms in milk by exposure for 4 minutes to an electric current, with a terminal voltage of about 4,000, and an amperage of about 2. Most of the sterilizing action appeared to be due to heat, as the temperature rose to between 60 and $64^{\circ} \mathrm{C}$., but the authors considered that this alone was insufficient to account entirely for the effect.

(3) High-Frequency Currents. Apart from the early experiments of D'Arsonval and his colleagues in 1893 to 1896 (for references see Fabian and Graham 1933), little work has been carried out till recently on the action of high-frequency currents. During the past few years, however, a number of workers have made observations on the effect of these currents on bacteria, bacteriophage, toxins, and antibodies (Szymanowski and Hicks 1932, Hicks and Szymanowski 1932, Lentze 1932, Fabian and Graham 1933, Hasché and Leunig 1935, Gale and Miller 1935). The results are not easy to summarize, since the conditions of exposure used by different workers were often very different. In Fabian and Graham's experiments a gradual destruction of Bact. coli was brought about by exposure to a high-frequency displacement current of 10 megacycles per second and an intensity of 0.8 amps, but even after 8 hours the suspension was not sterile. The higher frequencies used by most of the other workers appeared to be less harmful. Whether the current acts mainly by generation of heat in the medium, or by setting up intense electronic and ionic linear agitation within the cells, is doubtful. The observations of Bessemans and van Meirhaeghe (1937), Ozzano and Re (1937), and Hasché and Loch (1937), all suggest that high-frequency currents have little effect on bacteria apart from the heat generated. [1 megacycle $=1,000$ kilocycles $=1,000,000$ alternating cycles. Since $\mathrm{V}=n \lambda$, when $\mathrm{V}=$ velocity of travel (186,000 miles per second), $n=$ frequency, or number of vibrations of the wave per second, and $\lambda=$ the wave length, it can be calculated that a frequency of 10 megacycles corresponds approximately to a wave length of 30 metres.] Short-wave therapy, using radiations of 3 to 30 metres in wave length, is now on trial in clinical medicine for the treatment of certain inflammatory processes.

Cathode Rays.-Wyckoff and Rivers (1930), working with Bact. coli, Salm. typhi-murium, and Staph. aureus, bombarded single bacteria on the surface of an agar plate with a known number of cathode rays. The proportion of surviving organisms was estimated from colony counts made after incubation of the plates. The cathode rays were generated in a Coolidge type electron tube working at a voltage of approximately 155 kilovolt. Destruction of the organisms occurred in the usual semi-logarithmic fashion (see p. 137). After 20 seconds $83 \cdot 1-93 \cdot 9$ per cent. of the organisms were dead. Quantitative analysis rendered it evident that the absorption of a single electron was generally sufficient to cause death.

The action of cathode rays seems to depend on the release of large numbers of ions consequent on the absorption of an electron. A single 150-kilovolt electron will liberate about $10^{4}$ ions within less than 0.001 c.mm. The effect of such an ionic shower on organisms as small as those mentioned seems to be almost invariably lethal, though with yeast cells injury, and not death, may result (Wyckoff and Luyet 1931).

Röntgen Rays.-According to Rieder (1902) the cholera vibrio, when exposed on an agar plate at a distance of 10-12 cm. from the anti-cathode, is killed by 
Röntgen rays in 20 to 30 minutes. Feistmantel (1902) found that irradiation for 50 minutes of Actinomyces farcinica, exposed $10 \mathrm{~cm}$. away from the anti-cathode, had apparently no effect. Wyckoff $(1930 a, b)$ exposed Bact. coli and Salm. typhi-murium on the surface of agar plates to soft $\mathrm{X}$-rays, obtained either from a tungsten tube operated at low voltage, or by the characteristic $\mathrm{K}$-radiation of copper. Destruction occurred semi-logarithmically, but less rapidly than with cathode rays (see above). Thus after 20 seconds' exposure to filtered copper radiation only 19.6-33.3 per cent. of organisms were dead. Analysis showed that only about one in twenty of the absorbed quanta of these radiations proved lethal.

According to Wyckoff, the X-rays incident upon a cell either pass through without altering it, or else give up one or more quanta whose energy content is connected with the wave-length $\lambda$ of the rays through the relation

$$
\mathrm{E}=h v=h \frac{c}{\lambda}
$$

where $h$ is Planck's constant, $v$ is the frequency of the rays, and $c$ is the velocity of light.

As the result of such an absorption a high-velocity electron is liberated. This electron gives rise to a chain of ions in the matter through which it passes and to $\mathrm{X}$-rays which, in their turn, liberate more ions of less and less energy. The changes caused by $\mathrm{X}$-rays in protoplasm are naturally identified with the physicochemical changes induced by this ionic shower. The fact that only one in twenty of the absorbed quanta proves fatal suggests that the vital elements capable of being destroyed by a direct quantum hit occupy only about one-twentieth of the cell volume. The harder the $\mathrm{X}$-rays are, the nearer do they approach in their killing effect to the cathode rays. More recent work with Bact. coli by Pugsley, Oddie, and Eddie (1935) yielded results which scemed to show that, provided a correction factor was introduced for lack of uniformity of the $\mathrm{X}$-ray beam, the organisms died in an exponential manner. Discussing these results, the authors came to the conclusion that the one-quantum-hit-to-kill explanation, first put forward by Crowther (1926), appeared to account most satisfactorily for the type of curve obtained.

This explanation is supported by the observations of Lea, Haines and Bretscher (1941). Comparing the bactericidal action of $\mathrm{X}$-rays, neutrons, and radioactive radiations, these workers found that the effect of a given dose was independent of the temperature and $o$ the rate at which the radiation was applied, and that the mean lethal dose was correlated with the ionization density of the radiation, being greatest for those radiations which produced their ionizations closest together. These findings are explained on the assumption that each organism contains a number of sensitive "targets," and that the destruction of any one target is sufficient to impair the viability of the organism. Bact. coli is estimated to contain about 1,000 targets, each of a diameter of $8.6 \mathrm{~m} \mu$, equivalent in size to a molecule having a molecular weight of $2 \times 10^{5}$.

Sub-lethal doses of $\mathrm{X}$-rays may bring about changes in the morphology and growth characteristics of bacteria (see Levin and Lominski 1935, Forfota and Hámori 1937). It may be noted that bacteriophages and filtrable viruses have been shown to be susceptible to soft X-rays (Wright and Kersten 1937, Moore and Kersten 1937).

Radium.-Bruynoghe and Dubois (1925) found that exposure of Leptospira icterohomorrhagice for 26 hours to $8 \mathrm{mgm}$. of radium, enclosed in a platinum cell $\frac{1}{5} \mathrm{~mm}$. in thickness, rendered the organism incapable of growing in vitro or of giving rise 
to disease in the guinea-pig, but did not interfere with its motility. Bruynoghe and Le Fèvre de Arric (1925) stated that they were able to deprive the viruses of rabies and of herpes of their virulence for rabbits by exposure in fairly high dilution to radon, in a dose of 5 millicuries for 48 hours. Danysz (1906) failed to produce any attenuation of the rabic virus by exposure for 20 hours to the $\beta$-and $\gamma$-emanations from $20 \mathrm{mgm}$. of radium bromide. Bisceglie (1926) claims to have lowered the virulence for guinea-pigs of a human strain of tubercle bacillus by exposure of three successive generations to $5 \mathrm{mgm}$. of radium bromide, the exposure being maintained for 5 days. Morphologically the bacilli of the third generation had lost their acid-fast properties to a considerable degree ; thread forms, occasionally showing branching, were numerous, and large numbers of Gram-positive Much granules were visible. According to Suess (1908), exposure of tubercle bacilli to highly active radium emanations for 2 days had apparently no effect on their morphology, growth, or pathogenicity. von Schroetter (1927) finds that bacilli and cocco-bacilli exposed to radon, in a dose varying for different organisms from 0.5 to 40.0 millicuries of an intensity of 5-250 microcuries, tend to elongate and become filamentous ; cocci, on the other hand, swell, increasing more or less equally in size in all diameters. Spirochætes do not change their size; they are eventually killed by the rays, but they remain motile for' a considerable time. Spencer $(1934,1935)$ implanted radium needles in tubes of broth inoculated with Salm. typhi, Proteus X19, or Str. pyogenes and incubated at $37^{\circ} \mathrm{C}$. There was at first a slight retardation of growth, but after 24 hours the growth was similar to that in control tubes. After 8-10 daily transfers the irradiated organisms sometimes grew more luxuriantly and tended to develop filamentous forms or, with streptococci, to grow in long chains. On the other hand irradiation at $0^{\circ} \mathrm{C}$. proved fatal within a few days. Lea, Haines, and Coulson (1936) have recently studied the effect of $\alpha$ and $\beta$-rays on Bact. coli, Staph. aureus, and $B$. mesentericus exposed in very thin gelatin films. Death of the organisms occurred exponentially. The rate of disinfection was found to be independent of the temperature, and proportional to the intensity of the radiation. All three organisms were equally sensitive to $\alpha$-rays, but towards $\beta$-rays $B$. mesentericus differed from the other two organisms. The authors conclude that the action of the radiation can be explained best on the "target" hypothesis. In a later paper Lea, Haines and Coulson (1937) record that the death-rate of Bact. coli and B. mesentericus exposed to $\gamma$-radiation was of the exponential type, and that the mean lethal ionization doses were approximately the same as those previously observed for $\beta$-rays.

Sonic and Supersonic Waves.-Starting with the experiments of Wood and Loomis in 1927, several observations have been made of recent years on the destruction of organized cells by high-frequency sound waves (for references up to 1932 see Chambers and Gaines 1932). Sonic waves, i.e. waves of audible frequency, of about 8,900 cycles per second, produced by a nickel tube vibrating in a strong electromagnetic field in resonance with a 2,000 -volt oscillating power circuit, are said to be able to bring about a considerable destruction of coliform and certain other bacteria exposed to them for sufficient lengths of time.

The method has been used by Chambers and Flosdorf (1936) for the liberation of antigenic constituents from Salm. typhi and hæmolytic streptococci. Death was found to occur logarithmically, the slope of the survival curve being a function of the sound intensity, i.e. of the amplitude when the frequency was constant. The suspensions were not com- 
pletely sterilc at the end of an hour, but filtrates showed the presence of the typhoid Vi antigen and the streptococcal labile antigen respectively. Rivers, Smadel and Chambers (1937) were successful in bringing about a considerable degree of destruction of vaceinia virus in the form of washed elcmentary bodies, but not in ordinary tissue suspensions. The presence of protein in the suspension interferes with the effect of the waves; this may explain why attempts to destroy bacteria in milk, bacteriophage in cultures, and viruses in tissue suspensions have often proved a failure (Beckwith and Weaver 1936, Scherp and Chambers 1936).

Supersonic waves, i.e. waves above audible frequency, of 200,000 to $1,500,000$ cycles per second, produced by connecting a piezo-electric crystal with a highfrequeney oscillator, are also credited with bactericidal power. The observations of Beckwith and Olson (1932), Yen and Liu (1934), and Takahashi and Christensen (1934) suggest that a considerable destruction of bacteria, and even of filtrable viruses, nay be brought about by exposure to these waves for an hour or so. Païe and his colleagues $(1935 a, b)$, however, found that ultrasonic waves of a frequency of 280,000 cycles per second had no destructive action in 2 hours on certain toxins, a coli bacteriophage, the herpes virus, or a number of different micro-organisms, while completely sterilizing a culture of Paramœcium in 5 minutes.

Too little work has yet leen carried out to justify a critical discussion of the results. It is generally believed that the action of the waves, which are of course molecular and not electro-magnetic, is due to the disruption of the cell as a result of the violent agitation set up in its contents. According to Liu and Yen (1934) no effect is produced on cells exposed in vacuo, suggesting that cavitation of dissolved gases plays an important part in the disruption of the bacteria. Probably a relationship exists between the wave length and the size of the organism or molecule exposed. Against the simple disruption explanation may be set the observations of Rivers, Smadel and Chambers (1937) on the effect of sonic waves on washed raccine virus. Though the infective titre of the suspension fell from $10^{-8}$ to less than $10^{-2}$, no microscopical evidence of disintegration of the elementary bodies conld be obtained, nor was there any increase in the opacity of the suspension. The authors, therefore, suggest that the destructive effect may be due to the formation of some oxidizing substance from the water.

Desiccation.--If dried on silk threads or glass slips, the proportion of organisms surviving for any given length of time varies with a great number of factors, such as the species of bacterium, the initial numbers present, the nature of the suspending medium, the rapidity of drying, and the temperature and gaseous nature of the environment (see Ficker 1898). Anthrax spores clried on silk threads may survive for over 20 years, while many of the pathogenic non-sporing bacteria die in a few hours. Paul, Birstein, and Reusz (1910a), working with staphylococci dried on garnets, found that the velocity of disinfection was equal to the square root of the oxygen concentration. The lower the temperature at which the organisms were kept after being dried in this way, the smaller was the proportion that succumbed (Paul 1909).

More recent work (see Otten 1930, 1932, Elscr, Thomas, and Steffen 1935, Flosdorf and Mudd 1935) has shown that even non-sporing pathogenic organisms are able to survive drying indefinitely, provided that desiccation is complete and that the dried organisms are maintained in a high vacuum $(0.01 \mathrm{~mm}$. $\mathrm{Hg}$ or less). Even such sensitive organisms as the meningococcus and the gonococcus remain 
alive and virulent for years under these conditions. The dried organisms are resistant to quite high temperatures. Typhoid bacilli, for example, dried and sealed in vacuo, are said to survive exposure to a temperature of $115^{\circ} \mathrm{C}$. for over 30 minutes. Serum and complement can also be preserved satisfactorily by drying from the frozen state (see Hartley 1936, Flosdorf and Iudd 1938).

The method is now being used extensively for the preservation of stock cultures. In practice $0.5-1.0 \mathrm{ml}$. quantities of a thick suspension of the organisms in broth are distributed into suitable tubes. Drying is carried out as rapidly as possible in vacuo in a desiccator over phosphorus pentoxide. The tubes are then evacuated individually with an efficient pump, and sealed off in the flame. To recover the organisms, an optimal medium is desirable for primary cultivation.

Cold.-Very much less attention has been paid to the effect of cold on bacteria than to the effect of heat. This may undoubtedly be attributed to the fact that, although cold is an excellent means of preventing putrefaction, it has very little germicidal action. Macfadyen (1900) exposed cultures of Bact. coli, Salm. typhi, B. anthracis, V. cholero, Proteus vulgaris, and Staphylococcus aureus for 20 hours to liquid air at a temperature of $-182^{\circ}$ to $-190^{\circ} \mathrm{C}$. After exposure the organisms grew well on subculture, and manifested their usual biochemical activities. He noticed that photogenic bacteria, when frozen, became non-luminous, but, when re-thawed, their luminosity returned with unimpaired vigour. In another experiment he exposed the same organisms in broth suspensions enclosed in fine quill tubing for 7 days to liquid air; subsequently no structural alteration could be detected in the bacteria, and all grew well on subculture. Macfadyen and Rowland (1900) found that the same organisms in sealed glass tubes withstood immersion for 10 hours in liquid hydrogen at a temperature of $-252^{\circ} \mathrm{C}$. ; microscopically and culturally the bacteria appeared to be unaltered. Paul and Prall (1907) exposed staphylococci, which had been dried on garnets, to liquid air, and found that under these conditions they retained thcir viability for several months, and showed no appreciable alteration in their resistance to disinfectant agencies.

Haines (1938) found that rapid freezing with solid carbon dioxide at $-70^{\circ} \mathrm{C}$. killed a high proportion of some organisms, but had little effect on others. If the frozen organisms were subsequently stored at $-20^{\circ} \mathrm{C}$., they died off very slowly, but if they were stored at $-1^{\circ}$ or $-2^{\circ} \mathrm{C}$. they died rapidly. Evidence was brought to suggest that at the latter temperatures denaturation and subsequent flocculation of the bacterial protein occurred similar to the changes that have been observed in muscle. In practice, a temperature of $-70^{\circ} \mathrm{C}$. is very useful for the preservation of many bacteria and filtrable viruses.

Dry Heat.-We have seen that disinfection by drying is influenced by numerous small factors; in disinfection by heat, though numerous small factors may play a part, the one factor, heat, is so important that it overshadows them. We can therefore be more precise in our figures regarding this method of disinfection. Koch and Wolffhügel (1881) were the first to make exact measurcments of the effect of heat on micro-organisms. They found that vegetative bacteria were killed by a temperature of just over $100^{\circ} \mathrm{C}$. in $1 \frac{1}{2}$ hours; many, of course, succumbed well within this interval, but this was the time necessary for complete sterilization. Spores, on the other hand, were much more resistant, requiring a temperature of $140^{\circ} \mathrm{C}$. for 3 hours for destruction. On what this superior power of resistance of spores depends is not known. Probably it is related to their 
lower water content, since there is evidence to show that desiccation can raise the time-temperature limit necessary to cause coagulation of proteins (see Hewlett 1909, Cameron 1930). The resistance of both vegetative bacteria and of spores varies considerably with the different species, some being killed much more rapidly than others. The spores of moulds are intermediate in resistance between the vegetative and sporing bacteria ; they require a temperature of $110-115^{\circ} \mathrm{C}$. for $1 \frac{1}{2}$ hours for their destruction.

As with desiccation, the higher the temperature, the shorter is the survival time. Thus, if the temperature is raised from $140^{\circ}$ to $160^{\circ} \mathrm{C}$. spores are killed in 1 to $1 \frac{1}{2}$ hours. At $400^{\circ} \mathrm{C}$. they are killed in 20-30 seconds (Oag 1940).

Koch did not regard dry heat as an efficient method of disinfection. Though satisfactory when dealing with naked bacteria, it is quite ineffectual within the times usually employed when the bacteria are protected by textile or other relatively non-conducting material; this is due to the low power of penetration of hot air. Thus, when a bundle of tow measuring $55 \times 50 \mathrm{~cm}$. was exposed to a temperature of $140-150^{\circ} \mathrm{C}$, the interior after 3 hours had only reached the temperature of $74.5^{\circ} \mathrm{C}$. - a temperature quite inadequate to kill the spores enclosed in the bundle. Noreover a temperature of $140^{\circ} \mathrm{C}$. is sufficient in a short time to ruin most cloth fabrics.

Flaming is a useful method of surface disinfection for non-inflammable substances; its efficacy appears to depend on the temperature to which the exposed surface is raised (Mayser 1925).

Moist Heat.-Koch (Koch et al. 1881), in conjunction with Gaffky and Loeffler, was the first to make a quantitative study of the germicidal action of moist heat. He found that the temperature required for sterilization of spores was much lower than with dry heat. Thus anthrax spores were killed in 10 minutes at $95^{\circ} \mathrm{C}$., and spores present in garden earth in less than 10 minutes at $105^{\circ} \mathrm{C}$. He also showed that steam under pressure is more efficient than steam at atmospheric pressure. For the disinfection of clothes, too, he found moist heat to be preferable to dry heat, as it has a greater penetrating power. Thus after 4 hours' dry heat at $140-150^{\circ} \mathrm{C}$. the temperature inside a roll of flannel was only $83^{\circ} \mathrm{C}$., and the contained spores germinated freely, whereas after $1 \frac{1}{2}$ hours of moist heat at $120^{\circ} \mathrm{C}$., the temperature inside was $117^{\circ} \mathrm{C}$, and all the spores were dead. Koch was greatly impressed by the value of boiling water; from numerous experiments he concluded that even spores seldom survive its action for more than a few minutes. We now know that Koch rather over-estimated its efficacy, for there are certain bacteria the spores of which will resist the action of boiling water for hours. This is especially marked with the thermophilic bacteria; thus, Bigelow and Esty (1920) exposed the spores of thermophilic organisms, suspended in a mutrient medium of $\mathrm{pH} 6 \cdot 1$ in sealed glass tubes, to various temperatures in oil baths, with the following results:

Temperature.
$100^{\circ} \mathrm{C}$.
$110^{\circ} \mathrm{C}$.
$120^{\circ} \mathrm{C}$.
$130^{\circ} \mathrm{C}$.
$140^{\circ} \mathrm{C}$.

Killed In 1,320 minutes. 225 23, $3 \cdot 5$ $1 \cdot 0$

Thus at a temperature of $100^{\circ} \mathrm{C}$. they remained viable for nearly a day. 
Mündel (1937) has found that the addition of 2 per cent. washing soda $\left(\mathrm{Na}_{2} \mathrm{CO}_{3}\right)$ greatly inereases the disinfecting power of boiling water, as well as reducing the tendency of metal instruments to rust. Thus, spores in a 0-35 per cent. suspension of earth in water resisted boiling for about 10 hours, but were killed in a 2 per cent. solution of soda at $98^{\circ} \mathrm{C}$. in 10 to 30 minutes.

It is on account of the resistance of spores to boiling water that the autoclave has largely displaced the steamer in laboratory practice. Steam is still employed at atmospheric pressure for the sterilization of certain media, the physical or chemical composition of which would be altered by steam under pressure, but where this is necessary we take advantage of Tyndall's observation, and submit the medium to steaming for 30 minutes on 3 successive days; any sporing organisms that have not been killed on the first day germinate, and thus become susceptible to exposure on the second day. It must be realized that tyndallization can be successful only if the nature of the medium and the conditions to which it is subjected between successive heatings are such as to enable all the spores to germinate. It is quite inapplicable, for instance, to the sterilization of bacterial suspensions in a non-nutrient fluid. Similarly, it is unsuitable for the destruction of anaerobic spore-bearing bacteria the spores of which are unable to germinate aerobically, or of thermophilic spore-bearing organisms which fail to grow at a temperature below $50-55^{\circ} \mathrm{C}$.

Steam under pressure, on the other hand, is so effective that a single sterilization usually suffices. In the autoclave the steam, while being submitted to pressure, still remains saturated with moisture. This is most important. Steam which is superheated behaves like a gas, and condenses very slowly on objects cooler than itself. Steam that remains saturated with moisture is much more effective, as it rapidly condenses on objects cooler than itself, and, by giving up its latent heat, quickly raises them to its own temperature. All air must be expelled from the autoclave before the pressure is allowed to rise ; otherwise the temperature developed by a given pressure of steam will be less than that reached by saturated steam (see Rishworth 1938, Spooner and Turnbull 1912). Though there are a few exceptions, it is safe to say that saturated steam under a pressure of $15 \mathrm{lbs}$. per square inch, i.e. with a temperature of about $120^{\circ} \mathrm{C}$, is sufficient to sterilize any medium in 30 minutes. This is therefore the exposure to which the usual media are submitted.

The higher the temperature, provided the steam remains saturated, the more rapid is the sterilization. This is clear from the results of Bigelow and Esty. But there are certain factors other than temperature that affect the time necessary for sterilization by steam. One of the most important is the $\mathrm{H}$-ion concentration of the medium.

It will be remembered that Pasteur in his experiments on spontaneous generation (see Chapter 1) found that boiling was more lethal in an acid than in an alkaline medium. This lias since bcen confirmed repeatedly. Bigelow and Esty (1920), for example, working with the spores of thermophilic organisms, found that when suspended in an acid medium of $\mathrm{pH} 4 \cdot 6$ they were destroyed by a temperature of $120^{\circ} \mathrm{C}$. in 2 minutes, whereas in a less acid medium of $\mathrm{pH} 6.1$ it required 9 minutes to destroy them. Chick (1910) likewise found that minute quantities of acid or alkali, too small of themselves to produce any germicidal action, had a very marked influence on the power of disinfection by hot water. With these substances the rate of disinfection was increased, but much more with the acid than with the alkali. Thus, working with Salm. typhi suspended in distilled water, she 
found that the addition of sufficient alkali to render the solution $\mathrm{N} / 7,000$ alkaline increased the mean rate of disinfection at $54^{\circ} \mathrm{C}$. about 1.5 to 2 -fold; a similar addition of acid increased it 5 to 7 -fold. Further addition of alkali influenced the rate of disinfection but little, whereas further addition of acid rendered it too rapid for study.

Other factors are the age of the culture and the nature of the suspending medium. Young organisms are generally more susceptible to the action of heat and of chemical disinfectants than old, while the presence of protein in the suspension, or of sugar in considerable concentration (Fay 1934), tends to protect the organisins to some extent. (For general information on sterilization by stean, see Underwood 1934, Konrich 1938, and on the sterilization and testing of dressings, in particular, see Hayes 1937, Savage 1940, Chisholm 1941, Report 1942.)

Thermal Death Point of Bacteria.-The mode of action of heat on bacteria appears to be one of protein coagulation. Chick and Martin (1910) showed that heat coagulation of proteins is an orderly process, the rate of which varies with the alteration of temperature, reaction of the medium, and other conditions. The actual process of coagulation consists of two stages : in the first, known as denaturation, the water reacts with the protein; in the second, known as agglutination, the altered protein separates out in a particulate form. In the case of hæmoglobin the coagulation occurs logarithmically, the rate at any moment being proportional to the concentration of uncoagulated protein. Very much the same law appears to be applicable to bacteria. The higher the temperature to which they are submitted, the more rapidly is their cellular protein coagulated. Between different organisms there are considerable variations; thus some vegetative bacteria, such as the gonococcus, are destroyed by heat at $47^{\circ} \mathrm{C}$. in a few minutes; others, such as the enterococcus, withstand a temperature of $60^{\circ} \mathrm{C}$. for nearly an hour. It must not, however, be supposed that these temperatures are to be regarded as specific thermal death points, irrespective of the time of exposure. Chick (1910) bas shown that the death of bacteria under the influence of heat is due to a protein coagulation; that this phenomenon occurs not at one definite point on the temperature scale, but over a considerable range of temperature; and that therefore the death of bacteria within a given range is mainly a function of time. To take for example Salm. typhi: the thermal death point of this organism is usually given as $55^{\circ} \mathrm{C}$. In experiments carried out between $49^{\circ}$ and $59^{\circ} \mathrm{C}$. the temperature coefficient, i.e. the rise in the velocity of disinfection, was found to be 1.635 for $1^{\circ} \mathrm{C}$. Given a value for $k$ (see p. 137) of 0.111 at $49^{\circ} \mathrm{C}$., it can be calculated that a suspension containing 100,000 bacilli per $\mathrm{ml}$. would be sterilized in about 2 hours at $47^{\circ} \mathrm{C}$, in 48 minutes at $49^{\circ} \mathrm{C}$, in 18 minutes at $51^{\circ} \mathrm{C}$, in 7 minutes at $53^{\circ} \mathrm{C}$, in $2 \frac{1}{2}$ minutes at $55^{\circ} \mathrm{C}$, and in 21 seconds at $59^{\circ} \mathrm{C}$. If therefore a suspension was gradually heated, death might apparently take place suddenly at $55^{\circ} \mathrm{C}$. But it is clear that this cannot be regarded as a point possessed of any special significance; it is merely a point near the upper end of a series of temperatures, each of which in itself can legitimately be regarded as a thermal death point. It follows that for purposes of comparison of the heat susceptibility of organisms of different species, it is essential to use suspensions of equal numbers of bacteria, and to ascertain at what temperature complete sterilization is produced within a given time. Even with these precautions, as we shall see later, there is a certain inaccuracy, due to the apparent variation in susceptibility of organisms of the same species in the same suspension, resulting in the survival of some long after the majority have been killed. 
Effect of Heat on Subsequent Multiplication.-In his studies on disinfection, Koch noticed that spores which had been heated but not quite killed required longer to germinate than unheated spores. Similar observations have been recorded by numerous workers both with spores and with vegetative bacteria. The conclusion usually drawn is that during the process of heating, the organisms are damaged in some way, so that their ability to multiply when subsequently transferred to suitable conditions is interfered with. Certain figures of Eijkman (1908) lend support to this view; he heated a suspension of Bact. coli in saline at $52^{\circ} \mathrm{C}$. and after varying intervals he made duplicate plates to ascertain the number of organisms remaining alive. One set of plates was counted after 3 days' incubation, and the other set after 15 days' incubation. The results are shown in Table 4.

TABLE 4

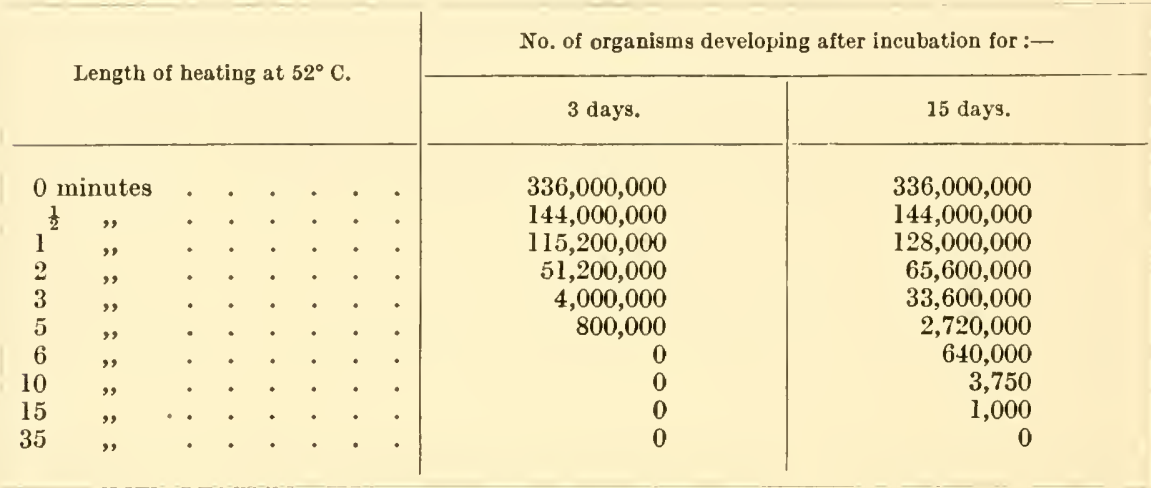

It will be noticed that during the first 30 seconds the heat, though 'killing over 50 per cent. of the organisms, does not interfere with the reproduction of the remainder. Subsequently, the longer the organisms are exposed, the greater is the difference between the results of the two series of plates. This suggests that a certain proportion of the remaining viable organisms are injured, and that the longer they are subjected to heat, the greater is the interference with their reproductive power.

Similar results have been obtained by Allen (1923), who, working with milk, found that the generation time of non-sporing organisms which had been pasteurized was longer than that of the untreated organisms, indicating an attenuation of the pasteurized organisms.

A different interpretation has, however, been proposed. Eckelmann (1917) suggests that the reason why a certain proportion of heated organisms require a long time to germinate is not because they are suffering from the effects of heat, but because they are provided with a more resistant cell membrane, which, while allowing them to withstand temperatures that prove lethal to their fellows, interferes with their rapid reproduction. According to her, heat would act as a selective agency, killing off all the bacteria with thin cell membranes and a power of rapid reproduction, and leaving intact the bacteria with thick, relatively impermeable cell membranes and a restricted power of reproduction. Burke (1923) adheres to the same view. She found that the spores of $\mathrm{Cl}$. botulinum frequently took several days to germinate, even when placed under optimum conditions. When the spores were heated, the germination period was increased, generally 
in proportion to the length of exposure; she found that they might lie dormant for as long as 426 days. Her conclusions are that the reason why the spores resist heat is because they are characterized by the possession of an impermeable membrane, which is also the cause of their delayed germination. The thicker the membrane, the more resistant is it to heat, and the longer does the organism take to develop.

The evidence in favour of the second view does not appear to us to be as convincing as that in favour of the first. Eijkman's figures, given in Table 4, are very striking, and, assuming their general validity, it is difficult to avoid concluding that the effect of heat is to increase the lag period of such organisms as remain viable. That the escape of the few is dependent on the possession of a relatively impermeable cell membrane is quite possible, but it fails to explain why in Eijkman's experiments before heating all organisms developed in 3 days, whereas after heating some failed to develop for 15 days.

Though sub-lethal heat may delay germination of spores, the opposite effect has been recorded by some workers. Christian (1931a,b), for example, working with an aerobic spore-bearing baeillus isolated from tainted milk, found that germination appeared to be stimulated by heating the spores to $100^{\circ} \mathrm{C}$. for 30 minutes at the time of inoculation. Evans and Curran (1943) also observed that heating spores of some species of aerobic spore-bearing baeilli for 10 minutes at $85^{\circ} \mathrm{C}$. often stimulated germination. In general, mild heating followed by 3 hours' incubation led to about the same degree of germination as 24 hours' ineubation without pre-heating.

\section{Chemical Agencies}

Distilled Water.-The evidence concerning the action of distilled water on the viability of bacteria is most conflicting. Spores are undoubtedly able to survive for a long time; thus Koch found that spores of the anthrax bacillus remained alive for more than 90 days. But with vegetative organisms it is otherwise. Some workers have found that they will survive for weeks, others that they are destroyed in a few hours. Such confusion can be explained only by differences in technique. One such difference of primary importance is the nature of the vessel from which the water is distilled. When a metallic still is used, traces of the metal are carried over into the distillate and undoubtedly exercise a deleterious effect on the bacteria. Ficker (1898) found, for instance, that water containing copper sulphate in a dilution of $1 / 50,000,000$ was sufficient to kill cholera vibrios in 1 hour, while Hoder (1932) found that distilled water containing 1 part of copper in 10 million sterilized a suspension of Ps. pyocyanea in 2 hours.

But even those who state that they used glass-distilled water have obtained varied results. One reason for this discrepancy may lie in the number of bacteria inoculated. In this connection some striking figures are reported by Ficker (1898). In one experiment he seeded pure glass-distilled water with $60,000,000$ cholera vibrios per $\mathrm{ml}$, and found that they remained viable for several months. In another experiment, in which he reduced his inoculum to 10,000 per ml., the organisms were nearly all dead in 2 hours. He explains such a difference on the assumption that the inoculation of large numbers of organisms into distilled water converts this into a dilute nutrient medium, no longer possessing the essential purity of the initial menstruum. The $\mathrm{H}$-ion concentration of the water may also be an important factor. Thus Winslow and Falk (1923) give the following figures, compiled from no fewer than seventy-nine tests: 
Viabiltty of Bact. coli in Distilled Water after 9 Hodrs at $37^{\circ} \mathrm{C} . *$

\begin{tabular}{c|c|c|c|c|c|c|}
$\mathrm{pH}$ & 4.0 & 5.0 & 6.0 & 7.0 & 7.5 & 8.0 \\
\hline Percentage of organisms surviving & 1 & 82 & $106 \dagger$ & 54 & 35 & 12 \\
\hline
\end{tabular}

* $\mathrm{pH}$ was adjusted by minute additions of acid or alkali.

$\dagger$ This figure suggests that a slight increase in the numbers of surviving organisms may have occurred.

The maximum viability occurs at $\mathrm{pH} 6 \cdot 0$. Cohen (1922) has likewise shown that when the $\mathrm{pH}$ of water is stabilized by the addition of buffer salts, the results are much more regular.

Other factors that may influence the action of distilled water on bacterial viability are traces of alkali absorbed from the glass, the amount of $\mathrm{CO}_{2}$ absorbed from the air, the quantity of dissolved oxygen, and the temperature at which the suspension is maintained. Whipple and Mayer (1906), studying the length of life of Salm. typhi in sterile tap water, showed that it remained viable for nearly 2 months when the water was exposed to the air, but died out in 4 days when the water was kept under anaerobic conditions. Houston (1914), likewise working with Salm. typhi, found that, when suspended in water kept at $0^{\circ} \mathrm{C}$., it lived for 8 weeks, at $18^{\circ} \mathrm{C}$. for 3 weeks, and at $37^{\circ} \mathrm{C}$. for only 1 week.

Summing up, we may say that the length of life of vegetative bacteria in distilled water is influenced by a large number of factors. When these factors are all favourable, the organisms may remain viable for considerable periods; when unfavourable, they may die out in a very short time; further, the effect varies greatly with different species of organisms. There is no evidence that distilled water acts by causing disruption of bacteria, as it does of many unicellular organisms; bacteria are too resistant to changes in osmotic pressure for this to be probable.

Acids.-Krönig and Paul (1897) were the first to show that the disinfectant action of acids in general is proportional to their degree of electrolytic dissociation, i.e. to the H-ion concentration of their solutions. Some figures of Winslow and Lochridge (1906) wili make this clear. Comparing the strengths of $\mathrm{HCl}$ and $\mathrm{H}_{2} \mathrm{SO}_{4}$ necessary to produce a 99 per cent. and a 100 per cent. reduction in the numbers of Bact. coli in 40 minutes, they observed that the disinfectant action of these two acids was in proportion to their degree of dissociation. Their results are given in Table 5 .

\section{TABLE 5}

Showing Percentage Reduction of Bact. coli in 40 Minutes bx Acids of Different Strengths.

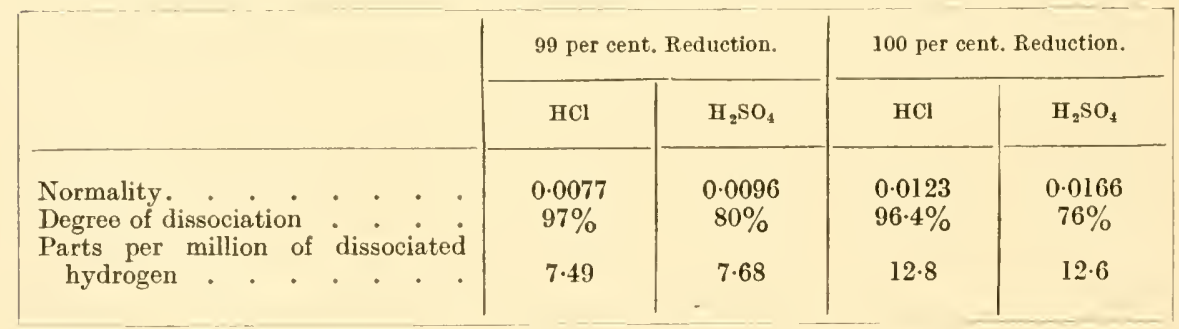


It will be noticed that to cause a 99 per cent. reduction, the strength of $\mathrm{HCl}$ required was 0.0077 normal, whereas that of $\mathrm{H}_{2} \mathrm{SO}_{4}$ was rather greater, 0.0096 normal. But as the degree of dissociation was greater with $\mathrm{HCl}$ than with $\mathrm{H}_{2} \mathrm{SO}_{4}$, the final concentration of $\mathrm{H}$-ions in the two solutions was practically identical.

From this experiment we may, therefore, conclude that the disinfectant action of mineral acids in high dilution is a function of their degree of dissociation, and hence of their resulting $\mathrm{H}$-ion concentration. Incidentally, we may notice that a considerably higher concentration of acid is necessary to sterilize a bacterial suspension completely than to reduce its numbers by 99 per cent. This point will be dealt with under the section dealing with the physical factors concerned in disinfection.

The effect of the H-ion concentration of the medium on bacteria suspended in it is rather complex. There is, first of all, an optimum concentration for growth ; for Bact. coli this is about $\mathrm{pH} 7 \cdot 6$. There is, secondly, an optimum concentration for survival ; for Bact. coli this is about $\mathrm{pH} 6 \cdot 0$. Thirdly, there is a point at which the acid-tolerance of the organism fails ; this for Bact. coli is abont $\mathrm{pH} 4 \cdot 6$. During growth in a medium containing a fermentable carbohydrate, Bact. coli produces acid, which raises the $\mathrm{H}$-ion concentration of the medium to about $\mathrm{pH} 5 \cdot 0$. This degree of acidity can be well tolerated, but if the acidity is increased beyond this point, instead of continuing to grow, the organisms cease multiplying and rapidly die. And lastly, there is evidence that the $\mathrm{H}$-ion concentration most suitable for certain fermentative processes is different from the optimum $\mathrm{pH}$ for growth (Cohen and Clark 1919, Gale 1940).

Here, however, we are dealing with the acid-tolerance of micro-organisms, and this limit varies with different species. In Winslow and Lochridge's experiments, already referred to, the parts per million of dissociated hydrogen necessary to sterilize a suspension of Bact. coli in 40 minutes were 12.80 ; to sterilize a suspension of Salm. typhi only 4.85 were required.

Apart, however, from the action of their free $\mathrm{H}$-ions, certain acids have another disinfectant action on bacteria, which appears to be dependent on the nature of the molecule. To produce a 99 per cent. reduction in the number of Bact. coli in 40 minutes, Winslow and Lochridge (1906) found that a $0.0812 \mathrm{~N}$ solution of acetic acid, or an $0.0097 \mathrm{~N}$ solution of benzoic acid was required. The degree of dissociation of each acid at its respective concentration is only about 1 per cent., so that the amount of dissociated hydrogen in the acetic acid was 1.2 parts per million, and in the benzoic acid 0.1 parts per million. It will be remembered, however, that when $\mathrm{HCl}$ was used, $7 \cdot 49$ parts per million were necessary. From this it is evident that the toxic action of acetic and of benzoic acid depends on some other factor than their $\mathrm{H}$-ion concentration. This other factor must be either the anion or the undissociated molecule. There is some evidence that the bactericidal activity of the monobasic series of organic acids increases with increase in molecular weight and decrease in surface tension, while with the dibasic organic acids the reverse holds true (Reid 1932). Halogenation of the fatty acids is said to increase their germicidal power (Tetsumoto 1937). 'The subject, however, is complex, and 110 general statement can yet be made; it will be discussed further in the section dealing with salt action.

Summary.-(1) The disinfectant action of mineral acids is proportional, not to their normal strength, but to the number of free $\mathrm{H}$-ions per unit volume.

(2) The organic acids are only slightly dissociated, so that their H-ion con- 
centration is relatively low. As, however, they have a markedly germicidal effect, it must be concluded that this is a property of the whole molecule or of the anion and is specific for each acid; acetic acid has, for example, only 10-20 per cent. of the toxicity of benzoic acid.

(3) Certain other acids, such as fluoric acid and nitric acid, have a specific action, which is probably due to the anion.

Alkalies.-By similar experiments to those described in the section on acids, Krönig and Paul (1897) showed that the disinfectant action of alkalies was dependent on their degree of dissociation, and hence on their concentration of $\mathrm{OH}$-ions.

Thus, of the bases $\mathrm{KOH}, \mathrm{NaOH}, \mathrm{LiOH}$, and $\mathrm{NH}_{4} \mathrm{OH}, \mathrm{KOH}$ shows the highest degree of dissociation, and is hence the most actively germicidal; $\mathrm{NH}_{4} \mathrm{OH}$ is dissociated the least and is the least actively germicidal (Table 6).

TABLE 6. (Krönig and Paul 1897.)

Disinfection of Anthrax Spores by Alkalies. Initial number of spores was about 6,800

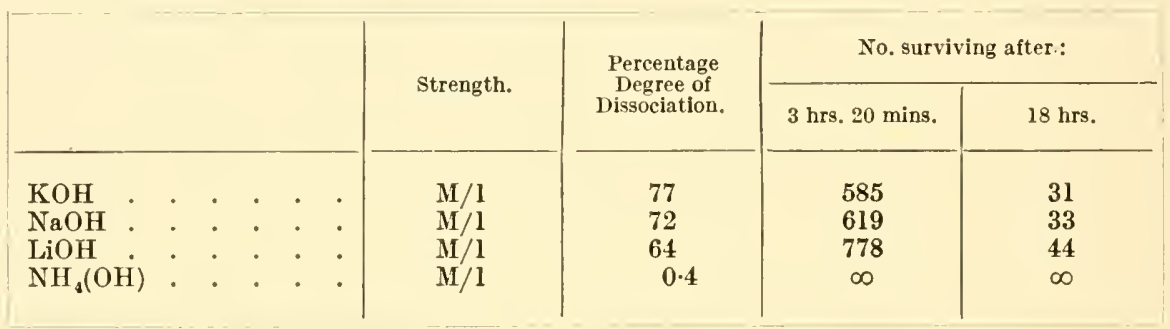

When we turn to other bases, we find exceptions. Thus, $\mathrm{Ba}(\mathrm{OH})_{2}$ is less dissociated than $\mathrm{KOH}$, but is very much more toxic; similarly with the hydroxides of the other alkaline earths. The reason for this, as we shall see in the section on salts, is that the metallic ion is frequently highly toxic, and assists the hydroxylion in its germicidal activities.

Summarizing, we may say that unless a toxic metallic ion is present, the disinfectant action of an alkali is proportional to its degree of dissociation, and hence to its concentration of hydroxyl-ions.

Just as bacteria possess a limit of acid-tolerance, so they possess a limit of alkali-tolerance. Cohen (1922) found that for Salm. typhi this was about pH 8.7. It is of interest to note that $\mathrm{H}$-ions appear to be more toxic than $\mathrm{OH}$-ions in similar concentration.

Salt Action.--Though this chapter primarily concerns the bactericidal action of various physical and chemical agencies, it is convenient to introducc here the subject of salt action in general.

We have seen that distilled water cannot be considered a satisfactory menstruum for bacteria. Many of the vegetative organisms die rapidly in it, and few survive for long. Ficker (1898) was the first to make direct observations on the action of physiological saline on bacteria. His results showed that instead of being harmless, it was actively bactericidal. Subsequent workers have confirmed his observations, and have demonstrated that the bactericidal effect is due to the toxicity of the sodium ion.

Delépine and Greenwood (1914), working with a number of heavy metals- 
copper, silver, zinc, cadmium, mercury-found that, though in certain concentrations they had a strong inhibitory action on bacterial growth, in lower concentrations they had the reverse effect, actually stimulating growth. Winslow and Hotchkiss (1922) found that the same held true for some of the lighter metals (Table 7).

\section{TABLE 7}

Effect of different Concentrations of Salts on Growth of Bacl. coli in 1 per cent. Peptone Water.

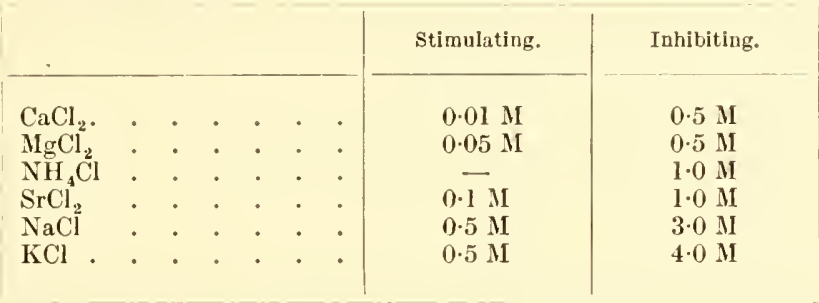

A further point of interest was brought out by Sherman and Holm (1922), who showed not only that $\mathrm{NaCl}$ stimulated growth in a concentration of 0.1 to $0.3 \mathrm{M}$, but that it widened the range of $\mathrm{H}$-ion concentration within which Bact. coli would grow. Taking just visible turbidity of the culture as the sign of growth, they obtained results set out in Table 8 .

TABLE 8.

Growti of Bact. coli in Miedia of Different pH, in the Presence and Absence of NaCl.

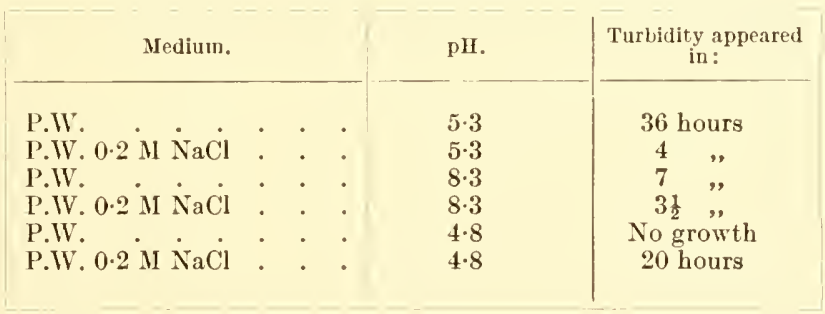

Thus the addition of $0 \cdot 2 \mathrm{M} \mathrm{NaCl}$ to a 1 per cont. solution of peptone in distilled water increased the rate of growth at unfavourable $\mathrm{pH}$ concentrations, and actually enabled the organisms to grow at $\mathrm{pH} 4.8$ - a concentration at which in plain peptone water they refused to grow at all.

It must be pointed out that a salt which is bactericidal in an aqueous solution may exert a stimulating effect when added in the same concentration to a nutrient medium.

Having referred to the favourable action of many weak solutions of salts on the growth and on the survival of bacteria, we must now pass on to consider their toxic action. This is the action which appealed particularly to the early workers. Koch in 1881 drew attention to the toxic action of salts, especially to the salts of the heavy metals, such as mercury and silver. There is no doubt that Koch overestimated the germicidal effect of these substances. Geppert (1889) pointed out that if the excess mercury or silver was removed at the end of the test by bubbling $\mathrm{H}_{2} \mathrm{~S}$ through the suspension, considerably higher concentrations of the 
heavy metal were required to destroy the organisms than had been found by Koch. Geppert showed that minute concentrations of heavy metals were sufficient to inhibit growth, but were unable to kill the organisms. Observations by Fildes (1940) have shown that mercury is even less bactericidal than it was thought to be by Geppert. Fildes has brought some evidence to suggest that mercury acts by combining with the - SH groups of the bacterial cell, which are essential for metabolism. If the mercury is neutralized by the addition of - $\mathrm{SH}$ compounds, like glutathione, cysteine, or thioglycollate, bacteria are able to grow after treatment with strong mercury solutions which previous workers have regarded as being actively germicidal. It seems possible, therefore, that mercury, and perhaps other of the heavy metals, act by interfering with essential metabolites of the cell. In virtue of this property, its bacteriostatic power is high, but its bactericidal effect is comparatively low. In estimating the bactericidal effect in practice, it is necessary to remove the excess of mercury from the suspension at the end of the test period by treatment with $\mathrm{H}_{2} \mathrm{~S}$ or ammonium sulphide, and then to cultivate the organisms in a liquid medium containing 1 per cent. thioglycollate in order to provide an adequate concentration of - $\mathrm{SH}$ groups. Records of the germicidal effect of mercury solutions not based on the use of this method must be regarded as unreliable. The same criticism applies to the organic salts of mercury, such as phenyl mercuric nitrate and several proprietary preparations. If thioglycollate is added to the broth used for subculture, none of these substances is able to destroy Staph. aureus or Buct. coli in a 1/1,000 dilution in 10 minutes at room temperature (Hoyt, Fisk and Burde 1942).

Krönig and Paul (1897), and later Paul and Prall (1907), made the very important discovery that the toxicity of solutions of $\mathrm{HgCl}_{2}$ depends not on the molecular concentration of the salt but on the concentration of free $\mathrm{Hg}$-ions in the solution. Thus the halogen salts of mercury were found to be active in proportion to their degree of electrolytic dissociation,

$$
\mathrm{HgCl}_{2}>\mathrm{HgBr}_{2}>\mathrm{HgI}_{2} .
$$

Solutions of salts in which the mercury was combined with a complex anion, and in which the degree of dissociation was poor, such as mercury acetate or cyanide, were found to be much weaker in germicidal power. The behaviour of the salts of the heavy metals is therefore analogous to that of the mineral acids, the toxicity being in proportion to the concentration of free metallic ions and of free $\mathrm{H}$-ions respectively.

The mode of action of heavy metals themselves, as apart from their salts, is not clear. Their toxicity may be demonstrated either by adding them to distilled water, or by placing them, in the form of a bar or coin, on the surface of an inoculated agar plate. Kling (1932) believes that the pure metal goes into actual solution. On the other hand the experiments of Hofmann (1929) and Pilod and Codvelle (1932), both of whom found that oxygen was necessary for the manifestation of toxicity, suggest that an oxide of the metal is formed which then undergoes ionization. Pure metals, of course, cannot ionize, and their failure, whether in aqueous or colloidal solution, to prove toxic under anaerobic conditions, points strongly to the necessity of preliminary salt formation followed by their ionic dissociation.

A vast amount of work has been done on the effect of different salts on bacteria. As the salts of mineral acids are electrolytically dissociated, it is clear that their action may be due either to the undissociated molecule, to the anion, to the cation, or to all three in combination. To assess the importance of each of these factors, 
comparative tests have been made with salts of one metal combined with different anions, and of one anion combined with different metals. These tests have been conducted not only on various bacteria, but on protozoa, and on the eggs of certain fish. On the whole the results have been reasonably concordant, as may be seen from Table 9, in which the cations are arranged in order of ascending toxicity. It must be understood that strict comparison of the action of different salts can be undertaken only in media of the same $\mathrm{H}$-ion concentration.

TABLE 9 (modified from Falk 1923).

Cations in Series of Increasing Toxictty.

\begin{tabular}{|c|c|c|c|}
\hline $\begin{array}{c}\text { Eisenberg (1919). } \\
\text { Bacteria. }\end{array}$ & $\begin{array}{l}\text { Winslow and } \\
\text { Hotchkiss (19922). } \\
\text { Betet. coli. }\end{array}$ & $\begin{array}{l}\text { Woodruff and } \\
\text { Bunzel (1909). } \\
\text { Paramecium. }\end{array}$ & $\begin{array}{c}\text { Mathews }(1904 a, b) \text {. } \\
\text { F'undulus Eggs. }\end{array}$ \\
\hline $\begin{array}{l}\mathrm{Na} \\
\mathrm{K} \\
\mathrm{NH}_{4} \\
\mathrm{Li} \\
\mathrm{Mg} \\
\mathrm{Sr} \\
\mathrm{Ca} \\
\mathrm{Ba} \\
\mathrm{Hn} \\
\mathrm{Ce} \\
\mathrm{Th} \\
\mathrm{Fe}^{\cdot} \\
\mathrm{Yt} \\
\mathrm{Cr} \\
\mathrm{U} \\
\mathrm{Zn} \\
\mathrm{Fe} \cdot . \\
\mathrm{Ti} \\
\mathrm{Be} \\
\mathrm{Al} \\
\mathrm{Ne} \\
\mathrm{Pb} \\
\mathrm{Cu} \\
\mathrm{Tl} \\
\mathrm{Zr} \\
\mathrm{Ni} \\
\mathrm{Cd} \\
\mathrm{Co} \\
\mathrm{Au} \\
\mathrm{Pt} \\
\mathrm{Hg} \\
\mathrm{Ag}\end{array}$ & $\begin{array}{l}\mathrm{K} \\
\mathrm{Na} \\
\mathrm{NH}_{4} \\
\mathrm{Li} \\
\mathrm{Sr} \\
\mathrm{Mg} \\
\mathrm{Ca} \\
\mathrm{Ba} \\
\mathrm{Mn} \\
\mathrm{Ti} \cdot \cdots \\
\mathrm{Sn} \\
\mathrm{Ni} \\
\mathrm{Ti} \cdot \\
\mathrm{Zn} \\
\mathrm{Cu} \\
\mathrm{Fe} \cdot \\
\mathrm{Fe} \cdots \\
\mathrm{Co} \\
\mathrm{Pb} \\
\mathrm{Al} \\
\mathrm{Ce} \\
\mathrm{Cd} \\
\mathrm{Hg}\end{array}$ & $\begin{array}{l}\mathrm{K} \\
\mathrm{Ca} \\
\mathrm{Zn} \\
\mathrm{Sr} \\
\mathrm{Mg} \\
\mathrm{MIn} \\
\mathrm{Co} \\
\mathrm{Ni} \\
\mathrm{Cd} \\
\mathrm{Cu} \\
\mathrm{Ag} \\
\mathrm{Pb} \\
\mathrm{Fe} \\
\mathrm{Hg}\end{array}$ & $\begin{array}{l}\mathrm{Sr} \\
\mathrm{Hg} \\
\mathrm{Ba} \\
\mathrm{K} \\
\mathrm{NH}_{4} \\
\mathrm{Al} \\
\mathrm{Ca} \\
\mathrm{Na} \\
\mathrm{Nn} \\
\mathrm{Li} \\
\mathrm{Fe} \cdot \\
\mathrm{Ni} \\
\mathrm{Co} \\
\mathrm{Zn} \\
\mathrm{Au} \\
\mathrm{Cd} \\
\mathrm{Cu} \\
\mathrm{Fe} \cdots\end{array}$ \\
\hline
\end{tabular}

From this table it will be seen that on the whole those metals of low atomic weight are less toxic than those of high atomic weight, though there are many exceptions.

To give some idea of the actual strengths necessary to cause inhibition of growth of Bact. coli, some results of Hotchkiss (1923) are given in Table 10. She divides her salts into two groups, the more toxic ones comprising those of the heavy metals, and the less toxic, comprising those of the alkali metals and of the alkaline earth metals. The salts of Group I give neutral solutions; those of Group II, owing to hydrolysis, yield solutions with an acid reaction. 
TABLE 10

Salt Concentrations that limit Growth of Bact. coli in l per cent. Peptone Water. Incubation period, 3 days. Molar concentration.

GROUP I.

GROUP II.

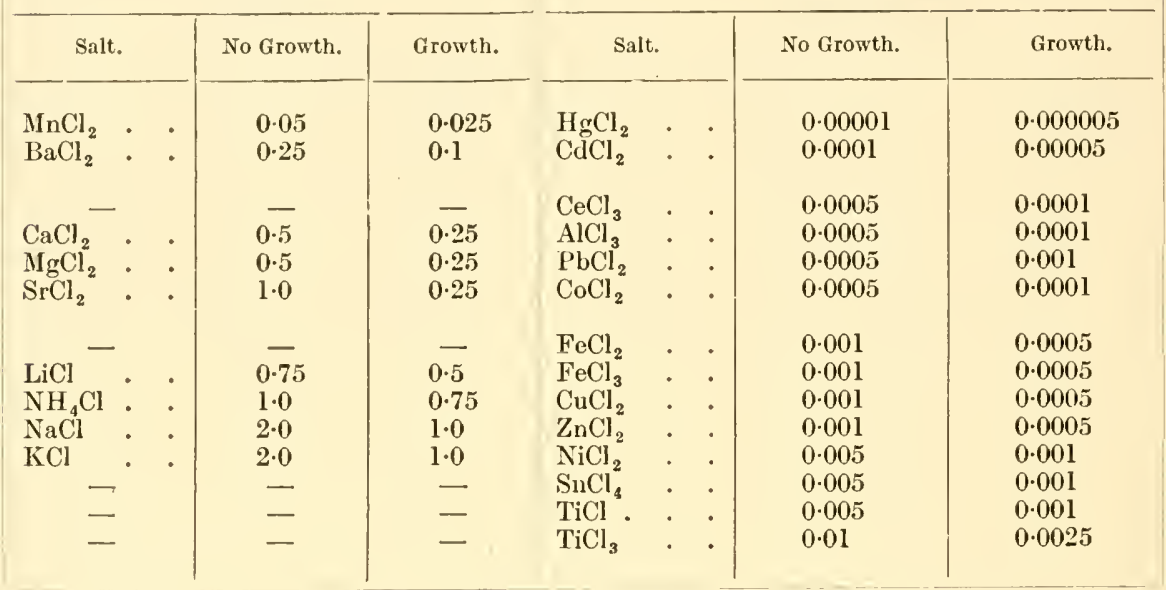

A further point may be noted from this table, namely, that the bivaient cations tend to be more toxic than the monovalent cations.

Very much less work has been done on the effect of anions on the growth of bacteria. Falk (1923) points out that the anions play an essentially different part in metabolism from the cations. The former are intimately related to the nutritive metabolism-particularly the anions that contain carbon, sulphur, nitrogen or oxygen-whereas the latter are concerned with the regulative metabolism of the organism. Nevertheless, the anious in certain concentrations do undoubtedly possess a toxic action on bacteria. Some figures of Holm and Sherman's (1921) will exemplify this point. They grew Bact. coli in 1 per cent. peptone water, to which were added various sodium salts, the $\mathrm{H}$-ion concentration being kept practically constant, and compared the rate of growth in the different tubes (Table 11).

TABLE 11

1 per cent. peptone

\begin{tabular}{|c|c|}
\hline ", & , \\
\hline , & , , \\
\hline ," & , \\
\hline , & ,. \\
\hline , & , , \\
\hline ," & , \\
\hline ," & , \\
\hline , & " \\
\hline :, & , \\
\hline ," & , \\
\hline
\end{tabular}

$0.2 \mathrm{M} \mathrm{NaCl} \cdot \cdot \cdot$

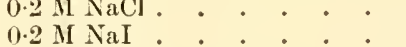

$0.2 \mathrm{M} \mathrm{NaNO}_{3}$.

$0.2 \mathrm{M} \mathrm{Na}_{2} \mathrm{SO}_{4}$.

$0.2 \mathrm{M}$ (mixture of $\mathrm{NaII}_{2} \mathrm{PO}_{4}$ and

$\mathrm{Na}_{2} \mathrm{HPO}_{4}$ ).

$0.2 \mathrm{NI} \mathrm{Na}$ lactate

0.2 II Na oxalate

$0.2 \mathrm{M} \mathrm{Na}$ acetate

$0 \cdot 2$ M Na citrate

$0.2 \mathrm{M} \mathrm{NaF}$.

\section{TABLE 11}


From this it will be seen that the $\mathrm{Cl}$-ion was the least, and the $\mathrm{F}$-ion the most toxic.

One of the most extensive studies is that of Eisenberg (1919), who arranges the anions in order of toxicity thus: $\mathrm{SO}_{4}<\mathrm{S}_{2} \mathrm{O}_{3}<$ Tartrate $<\mathrm{H}_{2} \mathrm{PO}_{2}<\mathrm{MoO}_{4}<$ $\mathrm{Cl}<\mathrm{Br} ; \mathrm{NO}_{3}<\mathrm{SO}_{3}<\mathrm{Fe}(\mathrm{CN})_{6}{ }^{\prime \prime} "<$ Acetate $<\mathrm{ClO}_{3}<$ Citrate $<\mathrm{HPO}_{3}<$ Oxalate $<$ Formate $<\mathrm{CNS}<\mathrm{ClO}_{4}<\mathrm{BrO}_{3}<\mathrm{I}<\mathrm{H}_{2} \mathrm{PO}_{4}<$ Benzoate $<$ Nitroprusside $<\mathrm{HAsO}_{4}<\mathrm{CrO}_{4}<\mathrm{P}_{2} \mathrm{O}_{7}<\mathrm{NO}_{2}<\mathrm{F}<\mathrm{BF}_{4}<\mathrm{HF}_{2}<\mathrm{BO}_{3}<\mathrm{B}_{4} \mathrm{O}_{7}<$ $\mathrm{Fe}(\mathrm{CN})_{6}{ }^{\prime \prime} '<$ Salicylate $<\mathrm{HSeO}_{3}<\mathrm{IO}_{3}<\mathrm{S}_{2} \mathrm{O}_{8}<\mathrm{S}_{2} \mathrm{O}_{7}<\mathrm{TeO}_{4}<\mathrm{SbS}_{4}<\mathrm{OsO}_{4}<$ $\mathrm{IO}_{4}<\mathrm{Cr}_{2} \mathrm{O}_{7}<\mathrm{TeO}_{3}$.

The action of salts depends to a large extent on the medium in which they are dissolved; thus they are more active when dissolved in distilled water than when dissolved in a medium containing protein. This is an observation that has been made frequently (Behring 1890, Krönig and Paul 1897, Chick and Martin 1908). Probably it is due to the fact that many cations combine with proteins to form an insoluble albuminate; hence the concentration of free ions in the medium is diminished.

Another important observation is that different bacteria vary in susceptibility to the same salt. v. Eisler (1909) found that B. subtilis was killed by N/10 LiCl. whereas the El Tor vibrio was unharmed by N/5 LiCl. Eisenberg (1919) found that $B$. anthracis possesses more than the average resistance to fluorides, iodates and oxalates; $C$. diphtherioe to tellurates, tellurites, $\mathrm{Ni}$ and $\mathrm{Cu} ; \mathrm{Salm}$. typhi to $\mathrm{Sr}$ salts; the pneumococcus to ferricyanides and tellurites; and $V$. cholera to chlorates and perchlorates. Certain organisms may be grouped together as having a similar susceptibility to the action of salts ; thus, Staphylococcus pyogenes and Staphylococcus candicans; $C$. diphtherioe and the diphtheroid bacilli; Salm. typhi and Bact. coli; Chromo. prodigiosum and Chromo. kielense are grouped in pairs, each member of the pair exhibiting a similar susceptibility to different salts.

As well as this relationship, however, there is a difference in resistance exhibited between members of the Gram-positive and the Gram-negative group of organisms. Eisenberg found that many salts are more toxic to the Gram-positive than to the Gram-negative bacteria. This holds not merely for particular salts, but for their constituent anions and cations. On the other hand, some salts, such as potassium tellurite (Fleming 1932, Fleming and Young 1940) and sodium azide (Snyder and Lichstein 1940, Mallmann et al. 1941), are more toxic to Gram-negative than to Gram-positive bacteria. This property is now made use of in the preparation of selective media.

The difference in susceptibility to sodium chloride has been suggested by Schoop (1935) as a criterion for bacterial classification. He divides bacteria into three classes: (1) those that grow in ordinary media, but not in media containing 10 per cent. $\mathrm{NaCl}$-non-halophiles; (2) those that grow in both media-facultative halophiles; (3) those that grow only in media containing 10 per cent. $\mathrm{NaCl}$-obligatory halophiles. The last group of organisms are found mainly in sea water, and in sand and mud adjacent to the sea.

Antagonistic Effect of Salts.-Hitherto we have bcen considering the effect on bacteria of solutions containing one salt; we must now examine the effect of solutions containing more than one salt.

Flexner (1907) found that an 0.85 per cent. solution of $\mathrm{NaCl}$ caused rapid disintegration of the meningococcus; but that when a calcium salt was added to the solution, this disintegration no longer occurred. The conclusion he drew was that $\mathrm{NaCl}$ by itself is toxic to the meningococcus, but that its toxic action 
can be neutralized by a salt of calcium. Students of physiology will recall the similar observations made by Ringer on heart muscle in 1880. Shearer (1919) found that living bacteria offered a considerable resistance to the passage of an electric current, depending apparently on the relative impermeability of the cell membrane. Using, therefore, electrical conductivity as his criterion of viability, he obtained evidence suggesting that a 0.85 per cent. solution of $\mathrm{NaCl}$ was toxic to the meningococcus, but that this toxic action could be neutralized by the addition of a trace of $\mathrm{CaCl}_{2}$ or other bivalent salt. On the other hand, it appeared doubtful whether the toxic action of a bivalent could be neutralized by the addition of a monovalent salt.

Similar results have been obtained by other workers. v. Eisler (1909) found that the inhibitory action of $\mathrm{LiCl}$ on $B$. subtilis could be counteracted by the addition of a divalent, but not of a monovalent salt. Thus, N/10 LiCl was counteracted by $\mathrm{N} / 20 \mathrm{CaCl}_{2}$, by $\mathrm{N} / 200 \mathrm{BaCl}_{2}$, or by $\mathrm{N} / 200 \mathrm{MgSO}_{4}$. Further, he showed that the inhibitory effect of a divalent salt could be counteracted by either a mono- or a divalent salt. Thus, $\mathrm{N} / 750 \mathrm{MnSO}_{4}$ was counteracted by $\mathrm{N} / 200 \mathrm{Ca}\left(\mathrm{NO}_{3}\right)_{2}$ and by $\mathrm{N} / 100 \mathrm{KCl}$. This latter conclusion differs from Shearer's.

It must not be thought that the mere addition of a divalent to a monovalent salt will render the solution favourable; the two salts must be present in definite proportions. If not, instead of being harmless to the organism, the solution may be actively toxic. Thus Winslow and Falk (1923) found that 0.145 M solution of $\mathrm{CaCl}_{2}$ mixed with a solution of $\mathrm{NaCl}$ of two or three times this strength was highly toxic to Bact. coli. As the proportion of $\mathrm{NaCl}$ was increased to four times the strength of the $\mathrm{CaCl}_{2}$ solution, the toxicity of the solution diminished very markedly. That is, a solution of $0.145 \mathrm{M} \mathrm{CaCl}_{2}+0.290 \mathrm{M} \mathrm{NaCl}$ was toxic; a solution of $0.145 \mathrm{M} \mathrm{CaCl}{ }_{2}+0.680 \mathrm{M} \mathrm{NaCl}$ was non-toxic. A further increase of $\mathrm{NaCl}$ rendered the solution again toxic.

This antagonistic effect of salts brings us to the conception of a balanced solution. A balanced solution is one in which the proportion of the different salts is so ordered that their individually toxic effects are neutralized. In such a solution, bacteria are able to survive very much longer than in a solution of any one of the constituent salts. Ringer's solution is of this type, and has the following composition :

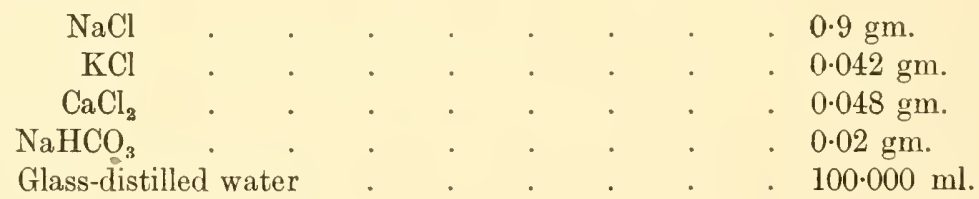

Winslow and Dolloff (1928) have, however, drawn attention to a possible fallacy in the interpretation of the antagonistic effect of salts. According to them, all cations appear to stimulate growth in a certain low concentration and to inhibit it in a certain higher concentration. So far as viability is concerned, therefore, they would postulate an optimum ionic concentration for each organism, depending probably upon an alteration in the permeability of the cell wall. They would explain the apparently antagonistic effects of monovalent and divalent salts as being due not to a qualitative antagonism between the two cations, but to the production in the suspension of a more favourable ionic concentration for the survival of the bacteria. In support of this they quote experiments in which the toxic action of a given salt in dilute solution has been annulled by increasing the con- 
centration of the same salt. How far this explanation is of general applicability, it is as yet impossible to say.

The interaction of various salts is of considerable importance in disinfection. The germicidal action of any one salt may be increased or diminished by the addition of any other. For example, the addition of $\mathrm{NaCl}$ is said to lower the toxicity of $\mathrm{HgCl}_{2}$, but to increase the toxicity of mercuric nitrate, sulphate, or acetais. In estimating, therefore, the toxicity of a salt, the saline content of the solution in which it is acting must be defined. Norton and Hsu (1916) showed that salts were able to modify the germicidal power of acids. When ammonium formate was added to formic acid, the $\mathrm{H}$-ion concentration of the solution decreased as a result of an increase in the concentration of undissociated acid molecules, and its disinfectant power was lowered; when sodium nitrate and sodium chloride were added in very small quantities to formic acid, the degree of dissociation of the acid was hardly affected, but its disinfectant power was considerably increased. They conclude therefore that the addition to an acid of a salt containing an anion common to this acid diminishes its disinfectant power; the addition of a salt which does not have any appreciable effect on the dissociation of the acid greatly increases its disinfectant power.

Not only do salts assist or antagonize the action of each other; they have a similar effect on disinfectants of quite different chemical constitution. Scheurlen (1895) showed, for instance, that the addition of sodium chloride in a concentration of 24 per cent. to a solution of phenol increased its disinfectant power. Beckman (1896) confirmed this, and found that with staphylococci the addition of even 1 per cent. $\mathrm{NaCl}$ to 1 per cent. phenol apparently increased its activity. With anthrax spores, the addition of 6 per cent. $\mathrm{NaCl}$ to 1 per cent. phenol had no effect; 12 per cent. $\mathrm{NaCl}$ increased its activity slightly, and 24 per cent. $\mathrm{NaCl}$ increased its activity very greatly. Thus 1 per cent. phenol alone failed to kill a suspension of 24,800,000 spores in 8 days ; 1 per cent. phenol +24 per cent. $\mathrm{NaCl}$ killed them completely in between 5 and 24 hours. Römer (1898) confirmed the work of Beckman, showing that the greater the amount of salt added, the greater was the increase in disinfectant power. As a rule, the more toxic a salt is in itself, the more does it supplement the action of the disinfectant (Eisenberg and Okolska 1913).

Mode of Action of Salts.-In endeavouring to explain the action of salts on bacteria, we must remember that we are dealing with a complex problem of which there is no simple solution. Many factors are concerned, and the most we can do here is to discuss the most important in turn.

(1) The Osmotic Effect.-It is doubtful whether salts, except in bigh concentrations, exert any influence on bacteria by virtue of their osmotic pressure. Bacteria differ in this way from practically all other living cells. Thus Fischer (1900) observed that $B$. subtilis grew well in an infusion containing 9 per cent. $\mathrm{NaCl}, 11$ per cent. $\mathrm{KCl}$, or 10 per cent. $\mathrm{KNO}_{3}$; and Knaysi (1930b) found that to demonstrate plasmolysis in this organism a 25 per cent. solution of $\mathrm{NaCl}$ was required. Though salts have little direct osmotic action on bacteria, they may exert an indirect action by causing a dehydration of the proteins on which the organisms are growing. It is this dehydrating action of salts which is relied on in many processes of food preservation.

(2) Oxidation.- Salts and certain allied bodies that contain a high proportion of oxygen, or that are able to liberate oxygen from other compounds, have long 
been known to be highly germicidal. Krönig and Paul (1897) compared the disinfectant activity of certain oxidizing agents with their oxidative capacity, as measured by the method of electrical oxidation chains. According to this method, oxidizing agents are arranged in order of decreasing oxidizing capacity thus: $\mathrm{HNO}_{3}$, dichromic acid, chloric acid, $\mathrm{Cl}_{2}, \mathrm{H}_{2} \mathrm{~S}_{2} \mathrm{O}_{8}$, and permanganic acid. This order was, with the cxception of chlorine, the same as that of the germicidal action of these substances. Chlorine, bromine and iodine were found to be germicidal in inverse order to their atomic weight. 'Their action appears to depend on the liberation of nascent oxygen. Ozone is another powerful oxidizing agent; likewise $\mathrm{H}_{2} \mathrm{O}_{2}$, a 3 per cent. solution of which kills anthrax spores in an hour. One of the most commonly used of this group of chemical substances is $\mathrm{K}_{2} \mathrm{Mn}_{\mathbf{2}} \mathrm{O}_{8}$; like $\left(\mathrm{NH}_{4}\right)_{2} \mathrm{~S}_{2} \mathrm{O}_{8}$ its action is increased by the presence of $\mathrm{HCl}$. Krönig and $\mathrm{Paul}$ prepared a mixture containing 1 per cent. $\mathrm{K}_{2} \mathrm{Mn}_{2} \mathrm{O}_{8}$ and $1 \cdot \vec{i}$ per cent. $\mathrm{HCl}$ dissolved in water, and found that it would kill anthrax spores in 30 seconds. A similar mixture containing $3 \cdot 7$ per cent. $\left(\mathrm{NH}_{4}\right)_{2} \mathrm{~S}_{2} \mathrm{O}_{8}$, and $1 \cdot 1$ per cent. $\mathrm{HCl}$ was found by Andrewes and Orton (1904) to exercise an effect very nearly as powerful. Both these are extremely potent, but even more potent is $\mathrm{HOCl}$, which in a concentration of 0.01 per cent. kills anthrax spores in 30 seconds. So far as activity is concerned, this is one of the most powerful germicides we know. Bleaching powder acts by virtue of its ability, when acted upon by weak acids such as $\mathrm{H}_{2} \mathrm{CO}_{3}$, to yield nascent oxygen, which then combines to form $\mathrm{HOCl}$. $\mathrm{HOCl}$ combines with organic substances containing the $=\mathrm{NH}$ group, to form chloramines.

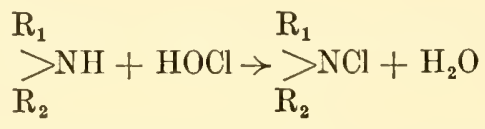

It is found that all bodies containing the $\mathrm{NCl}$ group are strongly antiseptic (Dakin 1915).

(3) Reduction.-Certain salts, such as the sulphites and the ferrous compounds, appear to act by virtue of their reducing power. Apart from such salts there are other substances that act mainly as reducing agents-sulphurous acid and formaldehyde. A 5 per cent. solution of formaldehyde, i.e. a 1-8 dilution of the commercial formalin, kills anthrax spores in between 1 and 2 hours.

(4) Molecular Action.--In a previous section we saw that certain acids, such as acetic and benzoic, and in fact most of the organic acids, act not by virtue of their H-ion concentration but by virtue of the undissociated molecule. Benzoic acid is dissociated very slightly, and its strong disinfectant power must therefore be attributed to the benzoate anion or to the undissociated molecule. Probably the same explanation will account for the action of the salts of the organic acids. The combination of mercury salts with the - $\mathrm{SH}$ compounds of bacteria described by Fildes (sec p. 122) is regarded by Albert (1942) as an example of molecular action.

(5) Ionic Action.-It is clear that salts which are frecly dissociated in solution owe their germicidal power to the action of the ions into which they are dissociated. The way in which these ions act is a matter for speculation. Bayliss (1924) points out that there are three ways in which electrolytes may exert their influence on living matter. (a) They may produce effects through the electrical charges that they bear; this is specially marked with ions with valencies above 
one, and bears no relation to the chemical nature of the ions. Thus the effect of $\mathrm{Ca} \cdot$ cannot be distinguished from $\mathrm{Ba} \cdot$. These effects, especially in the case of the multivalent ions, are manifest even in very dilute solutions. (b) They may affect the nature of the solvent in which they are dissolved-the so-called lyotropic effect. This action has been studied by several workers, prominent among whom are Hofmeister (1888, 1889) and Freundlich (1903). Hofmeister, working with a number of neutral salts, found that these could be arranged in a definite order relative to their action on the coagulation of colloids, and on other physical properties of proteins. Freundlich, studying the effect of electrolytes on the compressibility, surface tension, solubility, viscosity, and other properties of proteins, was likewise able to arrange them in a definite order. He concluded that the main effect was exerted not on the proteins directly, but upon the solvent; modifications in the solvent thus affected the proteins. For this reason he spoke of the salt effects as "lyotropic" effects; his series of salts hence bears the name "lyotropic series" in distinction to the "Hofmeister series." Both series, however, are similar in many respects. Holm and Sherman (1921) and numerous other workers have found a general concordance between the ionic series of Hofmeister or of Freundlich and the stimulative or toxic action of the salts of these series. (c) They may operate through a specific influence, which is more intimately connected with the chemical properties of the ions. Thus sodium and potassium, though having the same electrical charge, and exercising very similar lyotropic effects, are yet totally different in their action on heart muscle. As this action is shown by solutions so dilute that undissociated molecules are nearly absent, we know that this difference must be attributed to some specific or chemical property of the ions.

Other explanations of ionic action have been put forward, some of them modifications of the ones already given. Amongst these may be mentioned Loeb's $(1899,1900)$ hypothesis of ion-protein combination, Mathews' $(1904 a, b$, 1905, 1906) conception of ionic potential, and Zwaardemaker's (1918, 1919-20) radioactivity hypothesis. For these and for further information on this subject the reader is referred to an admirable summary by Falk (1923) - a summary which has been freely drawn on in this section.

There is one further point. We have treated the action of anions and of cations separately; whether both are sometimes required simultancously for the production of the bactericidal effect is not at all clear. It seems probable that in a salt such as $\mathrm{HgCl}_{2}$, in which a toxic cation is united to a weakly toxic anion, almost the entire action of the salt must be referred to the $\mathrm{Hg}$-ion; in a salt such as $\mathrm{K}_{2} \mathrm{Mn}_{2} \mathrm{O}_{8}$, on the other hand, in which a weakly toxic cation is united to a powerful anion, the action must be referred to the permanganate ion.

Summary of Salt Action.

(1) There is a certain concentration for nearly all salts which stimulates bacterial growth; this concentration is generally very low.

(2) There is, for nearly all salts, a limit beyond which the stimulating action passes over into a toxic action; on the whole, the higher the concentration, the more evident does the toxic action become.

(3) The toxic effect of univalent salts can be neutralized by the addition in suitable proportions of a divalent salt. In most instances, too, it is possible for a univalent salt to neutralize the toxic action of a bivalent salt. This action is known as the antagonistic action of salts.

P.B. 
(4) Electrolytes with bivalent cations are generally more toxic than those with univalent cations. Thus $\mathrm{Ba} \cdot$ is more powerful than $\mathrm{Na}$.

(5) On the whole, the salts of the heavier metals are more toxic than those of the lighter metals; thus $\mathrm{HgCl}_{2}$ is more toxic than $\mathrm{CaCl}_{2}$. But there is no strict quantitative relation between the atomic weight of a metal and its toxicity.

(6) On the other hand there is a fairly close relationship between the lyotropic and the toxic effects of a salt.

(7) The toxic action of salts is less marked in protein solutions than in distilled water. Thus the activity of $\mathrm{HgCl}_{2}$ is decreased markedly in the presence of blood serum.

(8) The more favourable the nutrient qualities of the medium in which the bacteria are suspended, the less manifest is the toxic effect of salts, and of germicidal agents in general, upon them.

(9) Different organisms vary in their susceptibility to the disinfectant action of the same salt. Closely allied organisms respond in much the same way to the same salts.

(10) There is evidence that the Gram-positive organisms, with a few exceptions, are more susceptible to the disinfectant action of salts than the Gram-negative organisms.

(11) The addition of a salt to a solution of a germicide-whether itself a salt or not-may increase or decrease the action of the latter. This action may be due partly to the effect on the electrolytic dissociation of the germicide; partly, in a colloidal solution, to an effect on the dispersion coefficient of the disinfectant; and partly perhaps to the disinfectant action of the salt itself.

(12) There is little evidence that salts, except in high concentrations, owe their germicidal action to the osmotic pressure that they exert, since bacteria are strongly resistant to variations of osmotic pressure; but they may act by dehydrating the proteins of the medium in which they are suspended.

(13) The action of salts is complex. It may be referred to an oxidation effect, a reduction effect, a molecular effect, or an ionic effect. Other effects, namely, the sensitization of organisms to $\mathrm{CO}_{2}$, and their interference with proteolytic enzymes, have not been considered in this chapter; for details of these the reader is referred to an article by Rockwell and Ebertz (1924).

Soaps and Synthetic Detergents.- Several workers have studied the germicidal effect of soaps, with results that have been at times contradictory. Many of the discrepancies can be ascribed to the use of different test organisms, since certain soaps are highly bactericidal to some organisms and comparatively inert to others. For example, pneumococci are very sensitive to the soaps of the unsaturated fatty acids-oleic, linoleic, linolenic-but much less so to soaps of the saturated fatty acids-stearic, palmitic, myristic, lauric. According to Lamar (1911) virulent pneumococci are killed by a 0.5 per cent. solution of sodium oleate in 15-30 minutes. Bayliss (1936) found that to kill pneumococci in 10 minutes a 0.1 per cent. solution of sodium palmitate was required, but only a 0.004 per cent. solution of sodium oleate. Lamar (1911) also noticed that sodium oleate, even in high dilution such as $1: 20,000$, greatly accelerated the autolysis of pnemmococci, and favoured their lysis by normal or immune serum. Hæmolytic streptococci, meningococci, gonococci, and diphtheria bacilli resemble pneumococci in their greater sensitivity to soaps of the unsaturated fatty acids. On the contrary, the Gram-negative bacilli of the coli-typhoid group are fairly susceptible to soaps of the saturated fatty 
acids, but are resistant to soaps of the unsaturated fatty-acid series (Reichenbach 1908, Walker 1924, 1925, 1926, Belin and Ripert 1937). Staphylococci are resistant to all the common soaps in neutral and alkaline solution (Walker 1924, Bayliss 1936), but according to Eggerth (1926) they are susceptible at $\mathrm{pH} 5$. The most generally useful soap in practice is sodium lanrate, since it acts on pneumococci, streptococci, and typhoid bacilli, though not on staphylococci (Walker 1924). There is a suggestion that the germicidal activity of soaps increases with increase in molecular weight in the saturated fatty-acid series, but decreases in the unsaturated fatty-acid series (Walker 1924, Bayliss 1936). Of commercial soaps, Nichols (1920) found yellow or brown bar soap, such as is used in washing dishes, to be effective in a 1:200 concentration in killing pneumococci and streptococci (see also Colebrook and Maxted 1933). Soap prepared from coconut oil, such as salt-water soap, is more germicidal than any other soap to the typhoid bacillus (Hamilton 1917, Walker 1925, 1926). If a stiff lather is made on the hands, even Bact. coli is killed within a minute. The germicidal effect of soaps is increased by rise in temperature (Walker 1924).

The mode of action of soaps in destroying bacteria is far from clear. It is certainly not due entirely to free alkali, since this may be present in much too small an amount to have any deleterious effect at all.

Reichenbach (1908), however, thought that alkali might play a part in some soaps. He observed, for example, that with salts of the higher fatty acids the germicidal effect decreased much more slowly on progressive dilution than with salts of the lower fatty acids, and explained this by the greater hydrolysis of the former group with the consequent liberation of free alkali. Eggerth (1926) found that, generally speaking, soaps of the lower nembers of the fatty-acid series were more active in acid solution, the higher members in alkaline; the point of transition varied with the test organism. He explained this result in terms of the effect of the $\mathrm{pH}$ on the dissociation resiclue and on the solubility of the soap. Lamar (1911) is of the opinion that the soap acts on the lipoiclal moiety of the eells, rendering them more permeable to germicidal substances in the solution. This would explain the adjuvant effect of soap on bacterial lysis by serum, or by substances such as aromatic oils, which are often added to commercial soap, and would presuppose a germicidal effect of the soap itself.

Summarizing, we may say that soaps show a strongly selective action towards bacteria, most of the pathogenic respiratory organisms being killed more readily by soaps of the unsaturated fatty acids, and most of the pathogenic intestinal organisms more readily by soaps of the saturated fatty acids. In practice, thorough washing of the hands in a stiff lather with a minimum amount of hot water, preferably using yellow bar household soap, can be relied upon to kill a high proportion of pathogenic organisms on the hands, with the exception of Staphylococcus aureus.

It is possible, as Noguchi (1907) suggests, that soaps in blood and lymph are responsible for some part of the natural defence mechanism of the body, since, in his experience, mixtures of soap and inactivated serum resembled complement in many respects. Burtenshaw (1942) likewise suggests that soaps and long-chain fatty acids are mainly concerned in the auto-disinfecting action of the skin. (For a study of soap derivatives, see Eggerth $1929 a, b, 1931$, and for a review of "germicidal" soaps, see Morton and Klauder 1944).

During recent years a large group of synthetic detergents have been used in industry. Some of these substances are highly bactericidal and are finding a place in surgery for the cleansing of skin and other surfaces. They are classified 
into cationic and anionic detergents, according to whether the location of the longchain hydrophobic group is in the cationic or anionic portion of the molecule. Cetyltrimethylammonium bromide, for example, is a cationic detergent, sodium cetyl sulphate an anionic detergent. Generally speaking, these substances are good wetting and cleaning agents, are relatively non-irritant to raw surfaces and destroy vegetative bacteria in dilutions varying from $1: 100$ to $1: 16,000$ or so. Some are toxic to leucocytes and others precipitate proteins. Their bactericidal action is often greatly diminished by the presence of organic matter and of phospholipins; their penetrating power is usually low; and some organisms, like Ps. pyocyanea, may prove very resistant to them. On the whole, the cationic group appears to be more germicidal than the anionic group, and Gram-positive are more affected than Gram-negative bacteria (see Miller and Baker 1940, Baker et al. 1941a, b, Barnes 1942, Hoyt et al. 1942, Williams et al. 1943, Iland 1944).

Alcohols and Ethers.-Epstein (1897) found that absolute ethyl alcohol was not a germicide, but that when diluted it became germicidal. Minervini (1898) confirmed this, and showed in addition that alcohol had little or no action on spores. For the destruction of vegetative bacteria the optimal strength depends on the degree of moisture present. A final concentration of 50-70 per cent. appears to be most effective. Thus, an equal amount of absolute alcohol should be added to an aqueous suspension of bacteria, whereas for dry bacteria a solution of alcohol already diluted to 50-60 per cent. should be used. For the disinfection of moist hands 80-96 per cent. alcohol is recommended; for the disinfection of dry hands 70-80 per cent. alcohol is better. Dry vegetative bacteria are destroyed less rapidly than moist-presumably because the penetration of alcohol takes longer (Table 12).

\section{TABLE 12}

The taken by Different Strengths of Ethyl Alcohol to destroy Dry and Moist Staphylococci.

(After Russ 1904.)

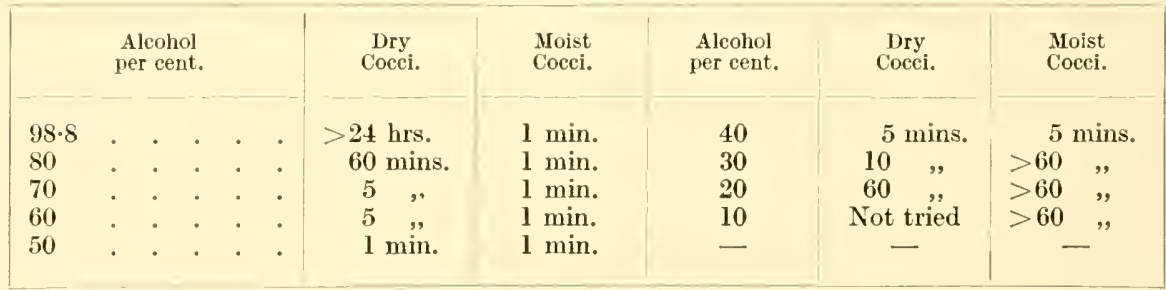

Note.-The dry staphylococci were dried on silk threads; the moist staphylococci were in aqueous suspension.

The presence of protein increases the disinfection time of alcohol, but not to any considerable extent. The addition of a dilute mineral acid or alkali greatly increases its activity, enabling alcohol to kill spores. Thus Coulthard and Sykes (1936) found that a solution of 70 per cent. alcohol containing 1 per cent. sulphuric acid destroyed spores of $B$. subtilis in less than 24 hours, and a solution of 70 per cent. alcohol containing 1 per cent. sodium hydroxide in 24-48 hours. Alcohol lowers the germicidal effect of some substances, like the heavy metal salts, phenol and formaldehyde, that are dissolved in it (Koch 1881, Krönig and Paul 1897), but is said to raise the germicidal effect of others, such as iodine. In fact, a strong 
tincture of iodine- -4.5 per cent. iodine in 70 per cent. alcohol, together with 2 per cent. potassium iodide to stabilize the iodine-is one of the best skin disinfectants known. Though the bactericidal activity of alcohol is negligible below $10-20$ per cent., it may prove bacteriostatic to many organisms in concentrations as low as 1 per cent. (Wirgin 1902). As an antiseptic for the preservation of vaccines, 25 per cent. alcohol has been found to be rather more potent than 0.5 per cent. phenol (see Cruickshank et al. 1942). Commercial alcohol as a rule contains spores, so that for surgical or biological use it should be filtered through a Berkefeld or similar candle (not a Seitz, which is effective only in the presence of water) or distilled.

Ritchie (1899) showed that the germicidal action of different alcohols increased with their molecular weight, ethyl alcohol being more potent than methyl, propyl than ethyl, and butyl than propyl alcohol. This has been confirmed by subsequent workers (Wirgin 1904, Tilley and Schaffer 1926, Tilley 1939, Lockemann, Bär and Totzeck 1941). For disinfection of the skin, 80 per cent. propyl alcohol is particularly useful ; it is more bactericidal than ethyl alcohol, it is a better fat solvent, and it is not so volatile. (For a detailed review of the disinfectant action of alcohol, see Sobernheim 1943, and for its value as a hand disinfectant, see AhIfeld and Vahle 1896, Neufeld and Schiemann 1943).

The ethers are pussessed of some degree of germicidal activity. Cultures of non-sporing bacteria incubated in an atmosphere saturated with the vapour of diethyl ether- $\mathrm{C}_{2} \mathrm{H}_{5} \mathrm{OC}_{2} \mathrm{H}_{5}$-exhibited no growth; subcultures showed that the organisms had been killed in a period varying from about 1 to 48 hours (Topley 1915). Direct immersion of Bact. coli in 50 per cent. ether proved fatal in about 3 minutes at room temperature. On the other hand, exposure of $\mathrm{Cl}$. septicum to pure ether failed to destroy the spores in 24 hours. According to Krönig and Paul (1897) ethereal solutions of disinfectants are almost without effect on anthrax spores.

Phenols and Cresols.- Under this heading we shall consider the action of those bodies that are obtained from the destructive distillation of coal, and that pass over between the temperatures of $170^{\circ}$ and $270^{\circ} \mathrm{C}$. Phenol itself in certain proportions is able to pass into solution in water, but most of the bodies in this group do not do so ; when mixed with water they form emulsions of varying degrees of fineness. Their mode of action is therefore different from the action of the germicides which we have so far considered. The phenols and cresols have a fairly high germicidal activity when employed in solutions above a given concentration; but it requires quite a low degree of dilution to deprive them entirely of this activity. In this respect they differ markedly from the saline disinfectants (see p. 143).

It has been supposed that phenol acts by its formation in contact with proteins of an insoluble albuminate and of other chemical compounds. Reichel (1909), however, who studied the dispersion phases of phenol between oil and water, brought evidence to suggest that the action is not so much chemical as physical, the phenol being capable of passing into solution in such substances as coagulated albumin, certain lipins, and the cytoplasm of bacteria. He suggests therefore, that its disinfectant action results from its penetration into the bacterial cell in the form of a colloidal solution.

The emulsified disinfectants, such as the cresols, probably act in much the same way as phenol, but their germicidal activity is usually somewhat higher. By virtue of their emulsoid state, their particles are adsorbed on to the surface of 
suspended matter, and hence their concentration is increased in the immediate neighbourhood of the bacteria. This action is interfered with by the presence of other suspended organic matter, which serves to adsorb the germicide, and thus lower its effective concentration around the bacteria. Emulsoids of the cresol group are generally most active when freshly made up in solution; after a day or two, probably because of an alteration in their colloidal state, their activity diminishes. Some of the cresols can be employed in true solution, but their solubility in water is very low. Para-chlor-meta-cresol, for example, has a solubility of about $1: 300$; towards naked bacteria it is approximately ten times as active as phenol (see Withell 1942a).

According to Klarmann, Shternov, and Gates $(1934 a, b)$, the germicidal activity of phenol derivatives is increased by halogen substitution, and is still further intensified by the introduction of aliphatic or aromatic groups into the nucleus of these compounds. Their general formulæ are<smiles>OCCCCl</smiles>

para-Chlorophenol Derivative.

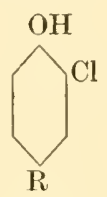

ortho-Chlorophenol Derivative.

where $\mathrm{R}$ is an aliphatic or aromatic group. Some of the compounds tested by these workers, such as para-chlorophenol or ortho-chlorophenol derivatives with $n$-butyl to $n$-octyl substituents, proved highly destructive to bacteria, while being comparatively non-toxic to mice on subcutaneous injection.

The chloroxylenols, many of which form a clear solution in water, have come into prominence of late years, mainly for skin disinfection. They are comparatively non-icritant, but their bactericidal power, on the whole, is considerably less than that of phenol; unless employed in 30, 50, or even 100 per cent. concentration, they cannot be relied upon to destroy staphylococci on the skin (see Colebrook 1941). (For useful information on the use of tar derivatives in practice, see Report 1942,1944 .)

Dyes.-Though a few desultory observations had bcen made at various times on the effects of aniline dyes on bacteria, Churchman (1912) was the first to investigate them thoroughly. Working with gentian violet, he found that if 5 drops of a saturated aqueous solution of this dye were added to broth cultures of different organisms, the mixtures allowed to remain for an hour, and transplants then made on to agar, the Gram-negative organisms grew satisfactorily, but the Gram-positive organisms failed to develop. A similar selective property could be demonstrated by seeding the fresh unstained organisms on to plates, one-half of which contained plain nutrient agar, and the other half nutrient agar containing a dilution of about 1-100,000 gentian violet. A large number of different bacteria were tested to ascertain if there was a perfect correlation between Gram-positiveness and inability to grow in media containing gentian violet. This was found not to be the case ; about 90 per cent. of the Gram-positive organisms were killed by gentian violet and failed to grow on media containing it, but the remaining 10 per cent., comprising the acid-fast group in particular, were not affected. Similarly, though about 90 per cent. of the Gram-negative organisms were resistant, the remaining 10 per cent. were susceptible. 
The difference between the Gram-positive and the Gram-negative organisms is merely one of degree. There is, moreover, a considerable variation in the susceptibility of different species of Gram-positive bacteria. Garrod (1933a) has shown, for example, that staphylococci are much less resistant to the violet dyescrystal violet, methyl violet, Hofmann violet, gentian violet, Dahlia - than streptococci. The presence of $1 / 1,000,000$ gentian violet in nutrient broth or in 5 per cent. serum broth is sufficient to inhibit the growth of staphylococci, while streptococci can grow in the presence of $1 / 250,000$, and sometimes even stronger concentrations of this dye.

Churchman (1923a) stated that, just as gentian violet had a bacteriostatic effect on most Gram-positive organisms, so acid fuchsin had a similar effect on Gram-negative organisms. Garrod (1933b) has recently examined this statement, and concluded that it is untrue. He finds that aniline dyes generally, whether of the basic or acid type, destroy Gram-positive more readily than Gram-negative bacteria. On the other hand, Churchman's results gain some support from the work of Stearn and Stearn $(1926,1928)$. From a study of the reactions of different bacteria to different stains, these workers conclude that Gram-positive bacteria have a lower isoelectric point than Gram-negative bacteria. Hence Gram-positive bacteria combine more actively with basic, and Gram-negative with acid dyes. The subject clearly needs further investigation.

The aniline dyes have, on account of their marked germicidal effect on bacteria, been used for the treatment of wounds. Browning and his colleagues (1917) recommended flavine-diamino-methyl-acridinium chloride. Though they found that brilliant green sulphate, malachite green, crystal violet, and flavine strongly inhibited the growth of staphylococci and Bact. coli, flavine was the only one that was more active in the presence of serum (Table 13). Churchman (1923b) used a mixture of gentian violet and acriflavine.

TABLE 13 (modified from Browning et al. 1917).

Showing Concentrations of Different Substances necessary to inhibit the Growth OF Staphylococcus aureus AND Bact. coli.

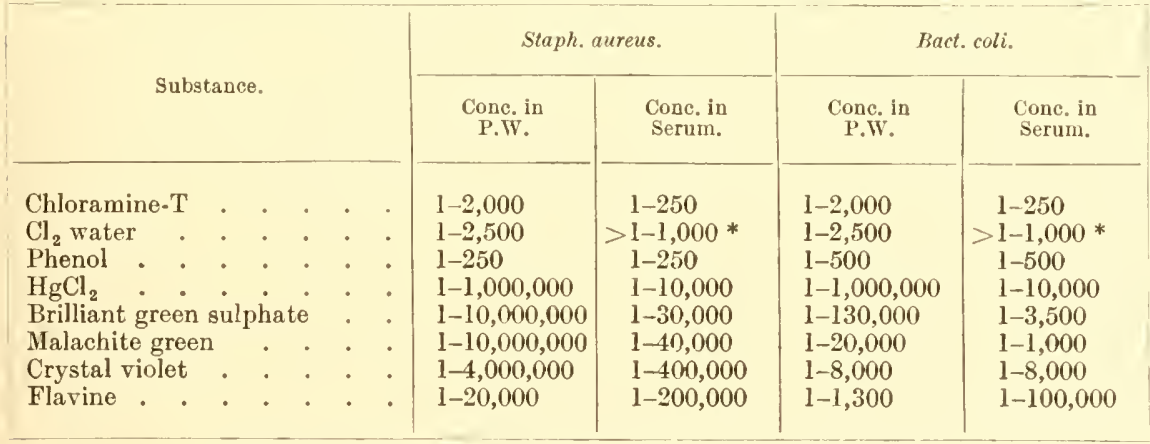

* These concentrations were insufficient to prevent growth.

Table 13 is of interest in showing not only that flavine is much stronger in its inhibiting action in ox serum than in peptone water, but that $\mathrm{HgCl}_{2}$ and the chlorine group of germicides are markedly diminished in activity in the presence of serum, whereas phenol remains unaffected. This diminution of activity in the presence of organic matter will be referred to later. 
Hitherto, the dye treatment of wounds has not fulfilled the expectations of its advocates, probably because the dyes can seldom be present continuously in sufficient concentration in every part of the wound to inhibit bacterial growth completely. As Browning (1933) points out, the destruction of organisms in the centre of masses of necrotic tissue or blood clot is probably beyond the power of any disinfectant. Moreover, many of the dyes inhibit leucocytic activity and cause damage to the tissues (see Fleming 1940, Russell and Falconer 1941, 1943, Manifold 1941, Rubbo et al. 1942, Russell and Beck 1944). They may, however, have some value in the temporary prevention or control of infection (see McIntosh and Selbie 1942, Browning 1943).

Essential Oils.- Chamberland (1887) tested the disinfectant action of a large number of essential oils, by exposing anthrax spores and anthrax bacilli to their vapours in closed tubes. After 4 days' exposure at $37^{\circ} \mathrm{C}$. only one oil was successful in killing the spores-namely, oil of Ceylon cinnamon. Anthrax bacilli, contained in blood, were killed by oil of vespetro in 18 hours at $37^{\circ} \mathrm{C}$., in 40 hours by oil of angelica, and in 65 hours by oil of Ceylon cinnamon. Other oils the vapours of which were germicidal, though less actively so, were oil of geranium and oil of marjoram.

He then tested the effect of the oils in a solution of alcohol and saponin. By this method he found the most active in killing anthrax bacilli were oils of marjoram, cinnamon, sandal-wood, clove, juniper, and Artemesia annua. He draws attention to the fact that cinnamon and marjoram oils are strongly active both in the gaseous and in the liquid state. Similar observations were made by Cadéac and Meunier (1889). They worked with Salm. typhi and Pf. mallei, which were allowed to remain in contact with the pure oil for a given time, and then seeded on to agar. Table 14 shows some of their results.

TABLE 14

Tine necessary to kill Salm. typhi.

Ceylon cinnamon oil

Clove oil. . . . . . . .

IVild thyme oil

Oil of geranium.
12 minutes

25

35

50

Many other oils did not kill for 24 to 48 hours, some not for 4 to 10 days, and some not even in 10 days. Garlic vapour has quite a strong bacteriostatic, and even a moderately germicidal, effect (see Böcker 1938).

It will be seen that certain of the essential oils, if applied pure, are fairly active germicides. The majority, however, are more valued for their antiseptic than for their disinfectant action. For t'is purpose they were used extensively by the ancient Egyptians in the process of embalming, with results which can be seen at the present day (see Risler 1936).

Vegetable oils that have no germicidal action themselves deprive other germicides, which are dissolved in them, of most of their activity; in this respect they resemble alcohol. Koch, for example, found that phenol dissolved in vegetable oils, such as olive or cotton-seed oil, was only slightly active. McNaster (1919) has since confirmed this, but has pointed out that mineral oils do not have this effect. Phenol dissolved in paraffin oil, for example, is nearly as active as when dissolved in water. 
With regard to animal oils, Harris, Bunker, and Milas (1932) find that some, such as seal oil and tuna oil, give off vapours which are germicidal, while others, such as cod-liver oil and sardine oil, become germicidal only after exposure to sunlight or ultra-violet light. It is possible that $\mathrm{H}_{2} \mathrm{O}_{2}$ is given off by the animal oils, and that its rate of evolution is accelerated by irradiation.

Sulphonamides and Mould Products.- These two groups of substances, whose chief interest lies in their ability to control infection in the body, will be more couveniently discussed in Chapter 6.

\section{The Dynamics of Disinfection}

Reaction Velocity.-The figures obtained by Krönig and Paul (1897) in their work on the disinfection of anthrax spores by $\mathrm{HgCl}_{2}$ were submitted by Madsen and Nyman (1907) to a mathematical analysis, with the result that the reaction velocity of disinfection was found to be similar to that obtaining in a unimolecular reaction. Madsen and Nyman themselves made fresh experiments, using the garnet method, and were able to confirm the findings of Krönig and Paul. In the following year Chick (1908), working independently, reached the same conclusions with regard to the analogy between disinfection and a unimolecular reaction (Fig. 21).

In a unimolecular reaction only one of the reacting substances need be regarded as undergoing change, the rate of change being proportional to the concentration of this substance. Examples in chemistry are the inversion of cane sugar by acids, the decomposition of $\mathrm{AsH}_{3}$ into $\mathrm{As}$ and $\mathrm{H}_{2}$, and the disintegration of radio-active substances. When only one of the reacting substances is undergoing change, the velocity of this change according to the Law of Mass Action will depend upon the concentration of this substance at any given moment, the temperature and other conditions remaining constant. This statement may be expressed by the relation

$$
\mathrm{V}=\mathrm{C} . k
$$

in which $\mathrm{V}$ represents the velocity of the reaction, $\mathrm{C}$ the concentration of the

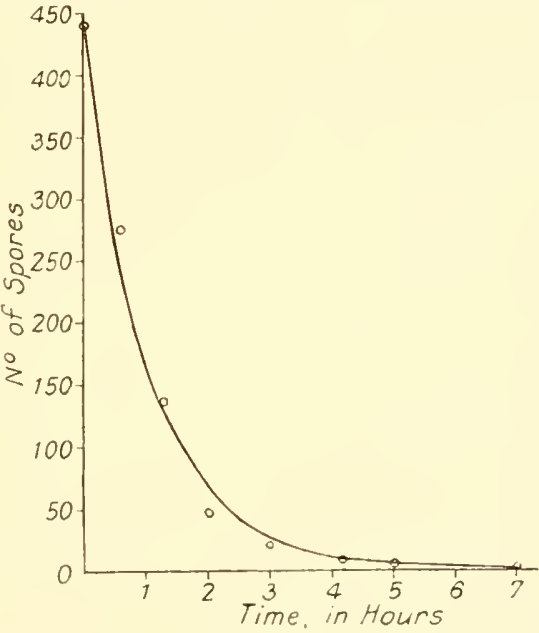

FIG. 21.

Disinfection of anthrax spores with 5 por cent. phenol at $33.3^{\circ} \mathrm{C}$. The curve is drawn through a series of calculated points; the circles represent the experimental observations.

(After Chick.) substance, and $k$ a constant depending on the nature of the substance. The velocity may be expressed by $\frac{d x}{d t}$, in which $x$ represents the amount of substance changed in time $t$; if the original amount of substance is designated by $a$, then $a-x$ will represent the amount remaining after time $t$. The equation may now be written :

$$
\frac{d x}{d t}=k(a-x)
$$




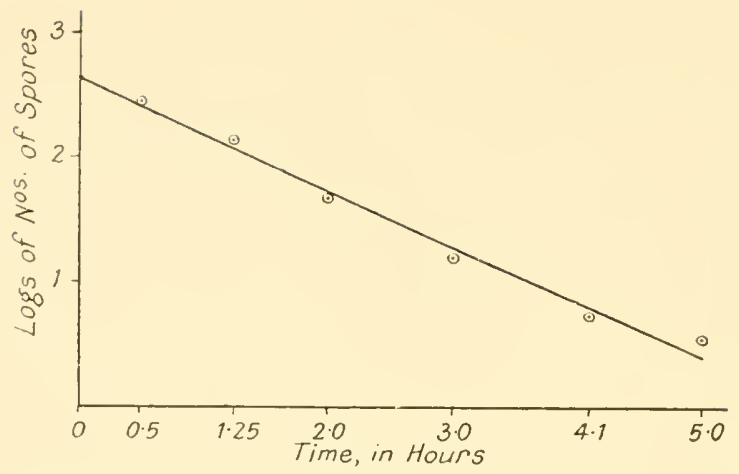

Fia. 22.

Disinfection of anthrax spores with 5 per cent. phenol at $33 \cdot 3^{\circ} \mathrm{C}$. The curve is drawn through a series of calculated points; the circles represent the experimental observations. This curve is constructed from the same observations as those used for Fig. 21, but the numbers of organisms are expressed logarithmically.

(After Chick.)

which, on integration, becomes :

$$
k=\frac{1}{t} \log \frac{a}{a-x}
$$

This is the equation representing the velocity of a unimolecular reaction and is often spoken of as the logarithmic law. If $\log (a-x)$ be plotted against time in this equation, the resulting graph will be a straight line (Fig. 22).

We may adapt the unimolecular reaction definition to the process of disinfection by saying that at any moment the reaction velocity is proportional to the number of surviving bacteria per unit volume. For example, let us suppose that there are 100,000 organisms being submitted to disinfection, and that the rate is one at which 90 per cent. of the organisms are killed in each minute. Then :

Time.

After 0 minutes $\begin{array}{ll}, & \text { I } \\ , & 2 \\ , & 3 \\ , & 4\end{array}$
Nos. Surviving.

100,000
$1 / 10 \times 100,000$ or 10,000
$1 / 10 \times 10,000$ or $1,000$.
$1 / 10 \times 1,000$ or 100
$1 / 10 \times 100 \quad$ or 10

Supposing that $B$ represents the initial number of living organisms, and $b$ the final number, then the reaction velocity may be expressed by the equation :

$$
k=\frac{1}{t} \log \frac{B}{b}
$$

Chick, using the drop method, made experiments on the disinfection of anthrax spores by 5 per cent. phenol. Her results are given in Table 15 and Figs. 21 and 22 .

\section{TABLE 15}

Anthrax Spores. 5 Per Cent. Phenol. $33 \cdot 3^{\circ} \mathrm{C}$.

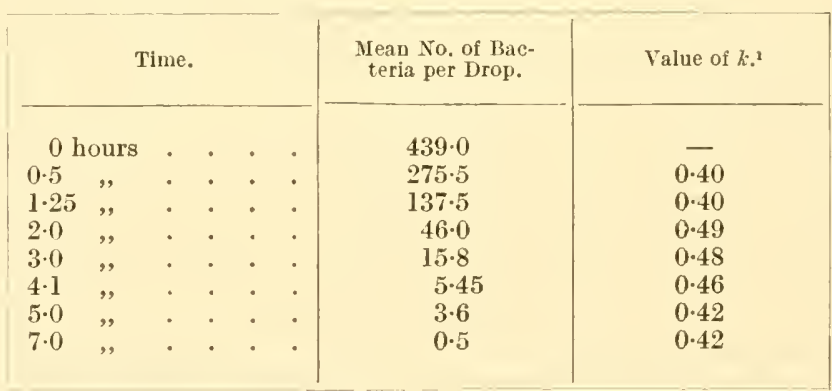

1 Values of $k$ are calculated to base 10 and not to base $e$, and in hours, not in minutes as in Table 18. 
It will be seen from Table 15 that $k$ has a mean value of 0.44 ; from Fig. 21 that the velocity of the reaction becomes slower and slower, till it is almost negligible (in theory the reaction never reaches completion), and from Fig. 22 that the logarithms of the numbers of surviving organisms plotted against time in hours fall along a descending straight line.

In the case of vegetative bacteria, she (Chick 1908, 1910) found that though the disinfection of some organisms such as Salm. typhi and Bact. coli conformed to the unimolecular reaction formula, with others there was a slight departure from it. Thus with Staphylococcus aureus exposed to 0.6 per cent. phenol at $20^{\circ} \mathrm{C}$., there was invariably a lag period, lasting about 4 minutes before the rate became strictly proportional to the number of bacteria (Fig. 23). Paratyphoid bacilli behaved in the opposite way. Instead of there

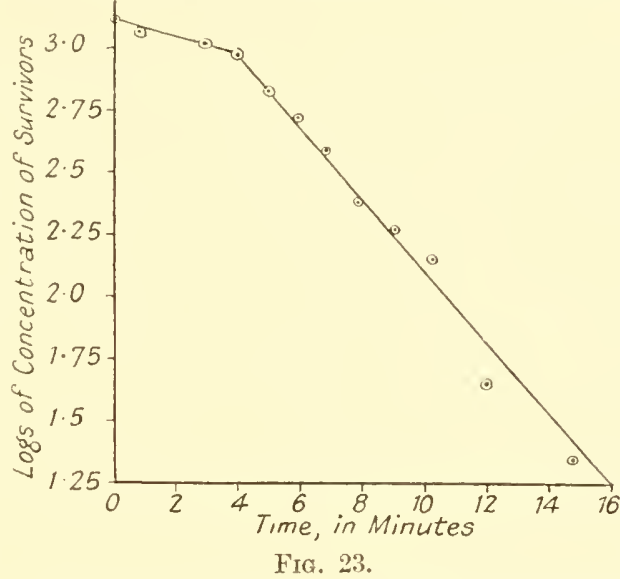

Disinfection of Staph. aureus with $0 \cdot 6$ per cent. phenol at $20^{\circ} \mathrm{C}$. The numbers of organisms are expressed logarithmically.

(After Chick.)

being a lag phase, there was a preliminary rush during which the rate of disinfection proceeded faster than it should have done according to the equation

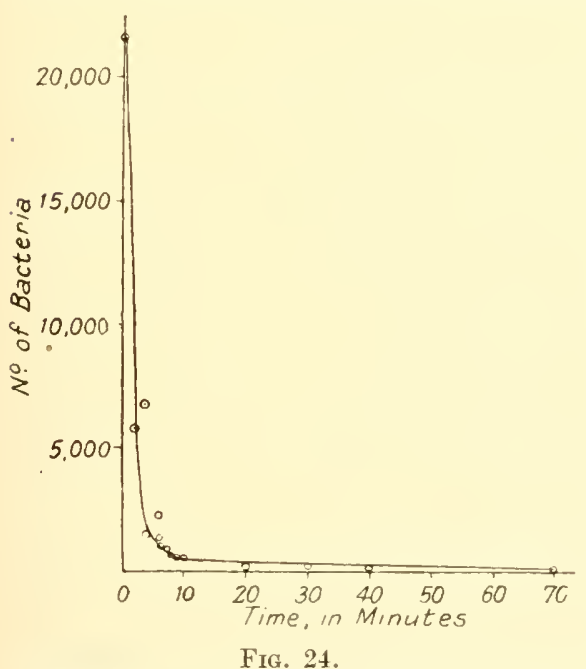

Disinfection of a 24-hours' culture of paratyphoid bacilli with $0 \cdot 6$ per cent. phenol at $20^{\circ} \mathrm{C}$. (After Chick.)
(Fig. 24).

Chick confirmed her work on disinfection by phenol by showing (1) that the death of Bact. coli under the influence of a bactericidal serum conforms to the unimolecular reaction law (Chick 1912); and (2) that the same law holds in the process of disinfection by hot water (Chick 1910); she pointed out the close parallel that exists between disinfection by hot water and the heat coagulation of proteins. Paul, Birstein and Reusz (1910a) found that the killing of Staphylococcus aureus by drying proceeds in accordance with the law of a unimolecular reaction. The results obtained by Clark and Gage (1903) in the study of disinfection by sunlight may also be interpreted in the same way. Robertson (1914) has adduced mathematical evidence in favour of this view.

It does not, of course, follow from such results as these that a unimolecular reaction, in the chemical sense, is actually taking place; the recorded observations 
merely show that a logarithmic curve describes the death of bacteria under the action of a disinfectant, just as it describes a chemical reaction whose rate is governed by the concentration at any moment of one of the reacting substances. Two alternative explanations have been offered to account for the form of the curves which Chick has described. The first of these suggests that the varying resistance of the bacteria in any given suspension can be described in the form of a frequency curve, as is almost certainly the case, and that the survival curves described by Chick are simply an expression of this difference in resistance. The obvious objection to this hypothesis, as Chick has pointed out, is that the form of the frequency curve describing the distribution of resistance must be supposed to be of the extreme skew form, if it is to account for the experimental results. Withell $(1942 a)$, however, has shown that the distribution of bacterial resistance is normal if the survival times are plotted on a logarithmic instead of an arithmetic scale. Such a logarithmic distribution of a characteristic has been noted in pharmacological and zoological work (see Gaddum 1933, Hemmingsen, 1934), and its occurrence in bacteria need not therefore occasion surprise.

An alternative explanation (Chick 1910) is that the death or survival of any given bacterium during any interval of time is determined by a multitude of small and independent causes-by "chance" in the statistical sensethe presence of the disinfectant weighting the chance of survival against each bacterium to a constant degree, for any given concentration of the disinfectant, and with other controllable conditions held constant. If the chance of each bacterium dying during any unit of time is $x$, and remains $x$ over the whole period of the experiment, then the death rate will be the same during each unit of time; the survivors at the end of any one time interval will suffer the same proportionate decrease in their numbers during the time interval which follows, and a logarithmic curve of decrease will result. This explanation does not, of course, mean that variations in resistance of individual bacteria play no part in disinfection. With vegetative bacteria, the rate of death is often represented by a sigmoid curve rather than by a straight line, suggesting that differences in resistance dependent on the age of the individual organisms are responsible for the deviation. The real question is whether, in the disinfection of spores that do not differ materially in age, the exponential type of curve is due to chance in the statistical sense, or to a frequency distribution of resistance of the logarithmic type. As Irwin (1942) points out, it would require very accurate data to distinguish between the two. (For a further discussion of this subject see Eijkman 1908, Hewlett 1909, Reichel 1909, Reichenbach 1911, Loeb and Northrop 1917, Brooks 1919, Cohen 1922, Knaysi 1930a, Knaysi and Gordon 1930, Bancroft and Richter 1931, Jordan and Jacobs, 1944.)

Concentration of Disinfectant.-Chick (1908) found that the relationship between the concentration of a disinfectant and the time taken for disinfection is not a simple but an exponential one, the exponent of the concentration being a factor varying with each disinfectant. That is to say, doubling the concentration of phenol does not halve the time necessary for the completion of the reaction as might be expected, but diminishes it to a far greater extent. Watson (1908), working on Chick's figures, found that the relation could be expressed by the formula

$$
\mathrm{C}^{n} t=\text { a constant }
$$

where $\mathrm{C}$ is the concentration, $n$ a constant varying with each disinfectant, and $t$ 
the time necessary for disinfection. This equation represents the relation when one molecule of one substance reacts with an excess of molecules of a sccond. For purposes of calculation it may be written

$$
n \log \mathrm{C}+\log t=\mathrm{a} \text { constant, }
$$

that is, the relation between $\log \mathrm{C}$ and $\log t$ is a linear one.

An example will make this clear (Table 16).

TABLE 16

Disinfection of Paratyphord Bacilli by Phenol at $20^{\circ} \mathrm{C}$.

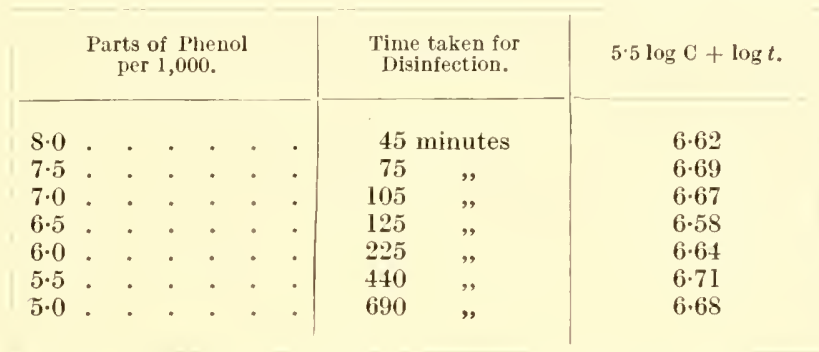

In this table the value of $n$ is taken as 5.5 ; the method of calculating this we shall consider presently. It will be seen that the values of the coustant are closely similar. If the logarithms of the concentrations are plotted against the logarithms of time, the resulting curve is found to be linear (Fig. 25).

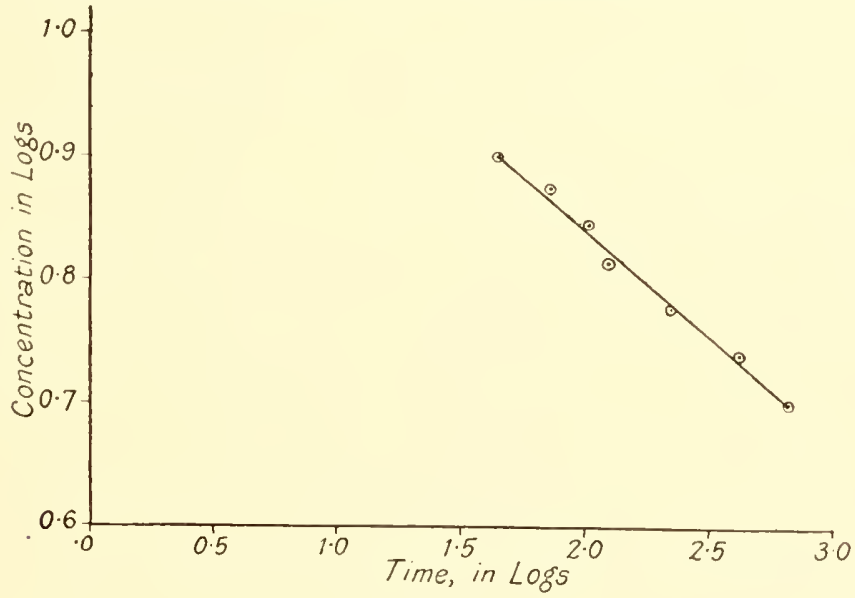

FIG. 25.

Disinfection of paratyphoid bacilli with varying concentrations of phenol. Both the numbers of the bacteria and time are expressed logarithmically.

(After Watson, from observations by Chick.)

For dealing with the salts of the heavy metals, a slight modification of the formula is required, due to the fact that these salts are dissociated in solution, and their action depends not on their molecular but on their ionic concentration. If the concentration of $\mathrm{Hg}$-ions is substituted for concentration of $\mathrm{HgCl}_{2}$, for example, then the formula holds good (see Table 17). 
Paul, Birstein and Reusz (1910b) found that the value of the velocity constant $k$ for aqueous solutions of $\mathrm{HCl}$ was approximately proportional to the square root of its concentration.

TABLE 17

Disinfection of Paratyphoid Bacilli by $\mathrm{HgCl}_{2}$ at $20^{\circ} \mathrm{C}$.

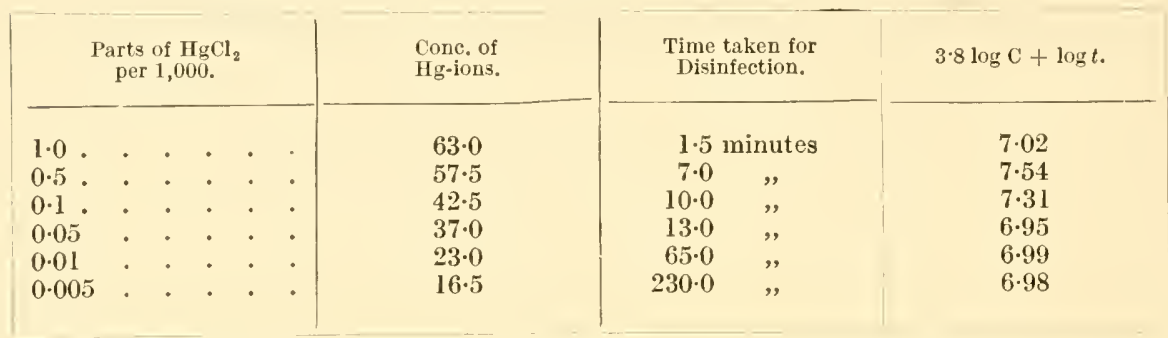

Calculation of Exponent $\boldsymbol{n}$.- The exponent $n$ may be regarded as a concentration coefficient varying with each disinfectant. To calculate its value, we use the formula

$$
{ }^{1} \mathrm{KC}^{n} t=\log \frac{\mathrm{B}}{b}
$$

$k_{1}$ is determined for concentration $\mathrm{C}_{1}$, and $k_{2}$ for concentration $\mathrm{C}_{2}$ in a given experiment. Then

$$
n=\log \frac{k_{2}}{k_{1}} \div \log \frac{\mathrm{C}_{2}}{\mathrm{C}_{1}}
$$

Taking the figures in Table 16 ,

$$
\text { let } 7.0 \text { parts of phenol per } 1000=\mathrm{C}_{2} \text { and } t=105^{\prime}
$$

let 5.0 parts of phenol per $1000=\mathrm{C}_{1}$ and $t=690^{\prime}$

$$
k_{2}=\frac{1}{t} \cdot \log \frac{\mathrm{B}}{\mathrm{b}}
$$

$\mathrm{B}$ in this experiment was $30,000,000 ; b$ can be taken as 1 . Then :

$$
\begin{aligned}
k_{2} & =\frac{1}{105} \cdot \log 30,000,000 \\
& =0 \cdot 0712 \\
k_{1} & =\frac{1}{690} \cdot \log 30,000,000 \\
& =0 \cdot 0108 \\
n & =\log \frac{k_{2}}{k_{1}} \div \log \frac{\mathrm{C}_{2}}{\mathrm{C}_{1}} \\
& =\log \frac{0 \cdot 0712}{0 \cdot 0108} \div \log \frac{7}{5} \\
& =\log 6 \cdot 5 \div \log 1 \cdot 4 \\
& =5 \cdot 5
\end{aligned}
$$

For $\mathrm{HgCl}_{2}$, when the concentration of $\mathrm{Hg}$-ions only was considered, the value of $n$ was found to be 3.8; for the Ag-ions of $\mathrm{AgNO}_{3}, 0.86$. If the molecular concentration of $\mathrm{HgCl}_{2}$ is considered, then $n$ is equal to about 1 .

The value of $n$ for any given disinfectant is very important, because it gives us

$1 \mathrm{~K}$ is the true velocity constant of the disinfectant, being independent of the concentration, and thus differing from $k$, which is constant only at a given concentration. 
information that is not conveyed by the simple reaction velocity. For phenol let us take $n=6$, and for $\mathrm{HgCl}_{2} n=1$. Then a doubling of the concentration of $\mathrm{HgCl}_{2}$, i.e. $\mathrm{C}^{n}$ or $2^{1}$, will halve the time taken for completion of the reaction; doubling the concentration of phenol, i.e. $\mathrm{C}^{n}$ or $2^{6}$, will diminish it 64 times. Conversely, halving the concentration of $\mathrm{HgCl}_{2}$ doubles the time of the reaction; halving the concentration of phenol increases it 64 times.

A substance with a high value of $n$ is actively germicidal above a given concentration; it requires, however, but a low degree of dilution to abolish its germicidal activity entirely. In contrast, a substance with a low value of $n$, while being actively germicidal in solutions above a given concentration, exercises an inhibiting effect on the growth of bacteria even when employed in high dilution.

One further point may be dealt with here, namely the question of whether the numbers of bacteria present in a suspension affect the reaction velocity. Working with $\mathrm{HgCl}_{2}$ and anthrax spores, Madsen and Nyman (1907) found the numbers of spores to be of no importance; a suspension containing 124,800 was sterilized as rapidly as one containing only 7,750. Eisenberg and Okolska (1913), however, divided the disinfectants into 3 classes: in the first, comprising alcohol, phenol, and formaldehyde, the numbers of bacteria had but little effect, i.e. a concentration of disinfectant that would destroy a given number of bacteria would also destroy 100 times that number. In a second group, comprising acetone, $\mathrm{HgCl}_{2}$ and $\mathrm{K}_{2} \mathrm{In}_{2} \mathrm{O}_{8}$, the numbers of bacteria proved to be of importance; thus a concentration that destroyed a given number failed to destroy 10 times that number. A third group comprising $\mathrm{HCl}, \mathrm{H}_{2} \mathrm{SO}_{4}$, oxalic acid, $\mathrm{KOH}$, and other bodies, occupied an intermediate position between the first two classes.

Temperature Coefficient.-As the temperature increases in arithmetical progression, the velocity of the reaction increases in geometrical progression, or mathematically expressed

$$
\frac{k^{\prime}}{k}=\theta^{\left(\mathrm{T}^{\prime}-\mathrm{T}\right)}
$$

in which $k^{\prime}$ and $k$ are the velocity constants of the reaction at temperatures ' $\mathrm{T}^{\prime}$ and $\mathrm{T}$ respectively, and $\theta$ is the temperature coefficient.

In the disinfection of paratyphoid bacilli by 0.6 per cent. phenol at $20^{\circ}$ and at $30^{\circ} \mathrm{C}$., Chick (1908) obtained the following figures (Table 18):

TABLE 18

Paratyphoid Bacilli. Phenol 6 per 1,000 .

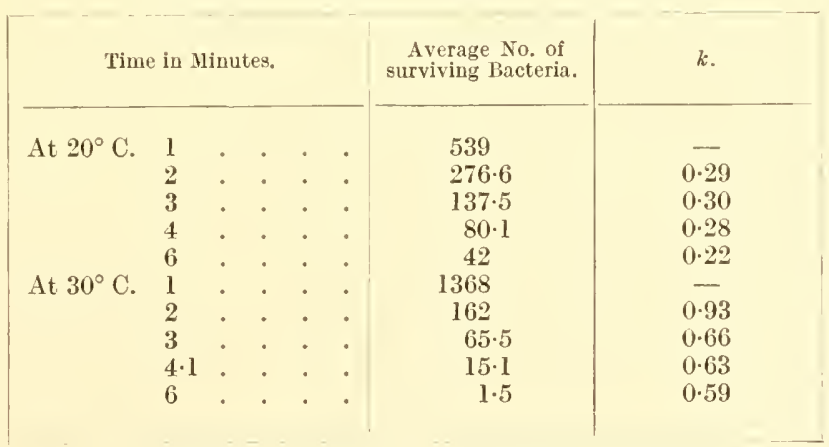

The mean value of $k$ at $20^{\circ} \mathrm{C} .=0 \cdot 27$.

The mean value of $k^{\prime}$ at $30^{\circ} \mathrm{C} .=0 \cdot 7$. 
To find the value of $\theta$

$$
\begin{aligned}
\frac{k^{\prime}}{k} & =\theta^{\left(\mathrm{T}^{\prime}-\mathrm{T}\right)} \\
\theta^{10} & =\frac{0 \cdot 7}{0 \cdot 27} \\
& =2 \cdot 592 \\
\theta & =2 \cdot 592^{0.1} \\
& =1 \cdot 1 \\
\theta & =1 \cdot 1 \text { for } 1^{\circ} \mathrm{C} . \\
& =1 \cdot 11^{10} \text { or } 2 \cdot 6 \text { for } 10^{\circ} \mathrm{C} .
\end{aligned}
$$

A similar experiment is shown diagrammatically in Fig. 26.

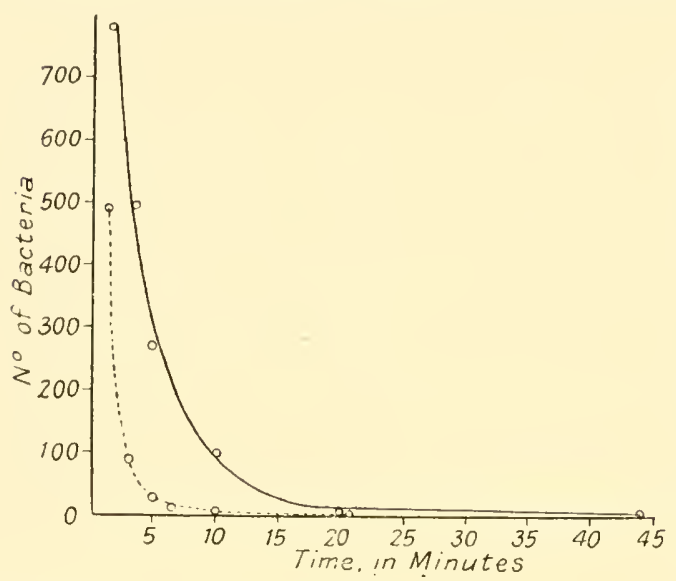

FIG. 26.

Disinfection of a 24-hours' culture of paratyphoid bacilli with 0.6 per cent. phenol at different temperatures.

Continuous curve $=11^{\circ} \mathrm{C}$.

Interrupted curve $=21^{\circ} \mathrm{C}$. (After Chick.)

Phenol 6 per 1,000 .

$11^{\circ} \mathrm{C}$.

$21^{\circ} \mathrm{C}$.

Most observers have obtained higher values of $\theta$ for phenolgenerally about 7 or 8 for each rise in temperature of $10^{\circ} \mathrm{C}$. For $\mathrm{HgCl}_{2}$ the temperature coefficient is lower, geuerally 2 to 4 for $10^{\circ} \mathrm{C}$.

An alternative method of estimating the value of $\theta$ is to start with a suspension of organisms the number of which need not be estimated, and determine how long it takes for complete sterilization at two or more different temperatures. Since the time taken for the completion of a reaction may be considered as inversely proportional to the velocity, there is no need to estimate the value of $k$. Thus :

Paratyphoid bacilli.

Time elapsing in minutes.

Then $\theta=\frac{2 \cdot 2}{1 \cdot 0}=2 \cdot 2$ for $10^{\circ} \mathrm{C}$, or $2 \cdot 2^{0 \cdot 1}=1.08$ for $1^{\circ} \mathrm{C}$.

In disinfection by hot water a very much higher value is obtained for $\theta$. Thus Chick (1910), working with Salm. typhi at temperatures of $49^{\circ} \mathrm{C}$. and $54 \cdot 1^{\circ} \mathrm{C}$, found that the velocity constant of the reaction was increased 13.1 times for the $5^{\circ} \mathrm{C}$. rise in temperature, i.e. $\theta=1.67$ for $1^{\circ} \mathrm{C}$, , or about 170 for $10^{\circ} \mathrm{C}$.

The consistent effect of rise of temperature on the velocity of disinfection points to a close analogy with an ordinary chemical reaction. Arrhenius elaborated a formula, which has been found to be applicable to many chemical re- 
actions, and it is interesting therefore to ascertain whether it applies also to the reaction of disinfection. The formula is:

$$
\mathrm{A}=\frac{\mathrm{T}_{0} \cdot \mathrm{T}_{n}}{\mathrm{~T}_{0}-\mathrm{T}_{n}} \cdot \log \frac{\mathrm{K}_{0}}{\mathrm{~K}_{n}}
$$

where $K_{0}$ and $K_{n}$ are the velocity constants of the reaction in question corresponding to the absolute temperatures $\mathrm{T}_{0}$ and $\mathrm{T}_{n}$ respectively, and $\mathrm{A}$ is a constant. As the time taken for the completion of a reaction may be considered as inversely proportional to the velocity of the reaction, this equation may be re-written thus:

$$
\mathrm{A}=\frac{\mathrm{T}_{0} \cdot \mathrm{T}}{\mathrm{T}_{0}-\mathrm{T}_{n}} \cdot \log \frac{t_{n}}{t_{0}}
$$

where $t_{n}$ and $t_{0}$ are the times taken to complete the reaction at absolute temperatures $\mathrm{T}_{n}$ and $\mathrm{T}_{0}$ respectively.

This formula was found by Madsen and Nyman to apply to the killing of anthrax spores by $\mathrm{HgCl}_{2}$. Chick showed that it was likewise applicable to the disinfeetion of paratyphoil bacilli by $\mathrm{HgCl}_{2}, \mathrm{AgNO}_{3}$ and phenol (Table 19).

TABLE 19

$\mathrm{HgCl}_{2}$ 1/10,000. Paratyphoid Bacilli. (MIodified from Chick 1908.)

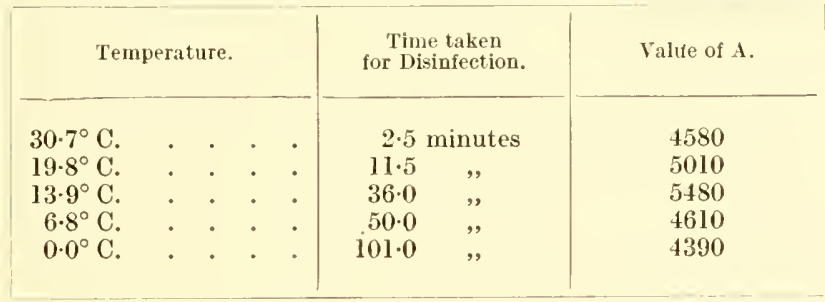

For $\mathrm{AgNO}_{3}$ the value of A was 5450, and for phenol 8430.

Whether rise of temperature affects the velocity of disinfection in a simple logarithmie manner or in aceordance with the law of Arrhenius, it is impossible to say, for within a small range of temperature the two become almost identical (Chick 1910). Bělehrádek (1926) brings evidence to suggest that Arrhenius' formula is not generally applicable to the influence of temperature on biological proeesses.

It must be pointed out that when an organism is suspended in a favourable medium, the effect of temperature on inhibition of growth may be the exact opposite of that on disinfection. Thus Behring found that the growth of anthrax bacilli at room temperature was inhibited in the presence of $1 / 400,000 \mathrm{HgCl}_{2}$; at $37^{\circ} \mathrm{C}$. a concentration of $1 / 100,000$ was necessary to inhibit growth. It appears that the rise of temperature favours the growth of the bacilli more than it does the action of the germicide. Similar results were obtained by Chick (1908) with paratyphoid bacilli and phenol.

Standardization of Disinfectants.-In the practical applieation of disinfection, it is desirable to have some measure of the relative germicidal aetivity of different disinfectants. The first method devised for this purpose was Koch's (1881) thread method. Suitable organisms, such as anthrax spores, were dried on silk threads, submitted to the action of the disinfeetant, and subsequently washed, and trans. 
ferred to a solid medium in order to ascertain whether all the spores had been killed. This method is open to certain fallacies to which attention was first drawn by Geppert $(1889,1891 a, b)$. Geppert found that if, after a short time in mercuric chloride solution, the spores were transferred to a culture medium, they failed to grow, but that if they were inoculated into a guinea-pig, they gave rise to anthrax. The reason for this appeared to be that sufficient $\mathrm{HgCl}_{2}$ was carried over by the thread into the broth to prevent germination of the spores occurring; in the tissues of the guinea-pig, however, this quantity was neutralized, and the spores that were still alive were able to develop. The truth of this explanation was shown by the fact that cultivation of the spores proved successful, provided the threads were treated with ammonium sulphide to neutralize the mercury before being inoculated into the broth. The thread method, therefore, yielded higher values than the disinfectant actually possessed. Geppert's work was of considerable importance, particularly in relation to disinfection by salts of the heavy metals. These substances have a low concentration exponent, and therefore act as antiseptics even in high dilution. Since it is impossible by this method to remove all traces of the disinfectant from the interstices of the thread, Krönig and Paul (1897) replaced the threads by garnets; they introduced a further improvement, which rendered the test quantitative, by plating out the washings from the garnets, and counting the number of colonies that developed. The two tests that are chiefly used at the present time, either in their original or in a modified form, are the Rideal-Walker and the Chick-Martin methods.

The Rideal-Walker Drop Method (Rideal and Walker 1903).- In this method similar quantities of organisms are submitted to the action of varying concentrations of phenol and of the germicide to be tested. Subcultures are made into broth every $2 \frac{1}{2}$ minutes up to 15 minutes and the tubes incubated at $37^{\circ} \mathrm{C}$. for 3 days. That dilution of disinfectant $\mathrm{X}$ which sterilizes the suspension in a given time is divided by that dilution of phenol which sterilizes the suspension in the same time, and a phenol coefficient obtained. Thus:

TABLE 20

Salm. typhi, 24 hodrs' Broth Colture at $37^{\circ} \mathrm{C}$. Temperature at which Test was CONDUCTED $60^{\circ} \mathrm{F}$.

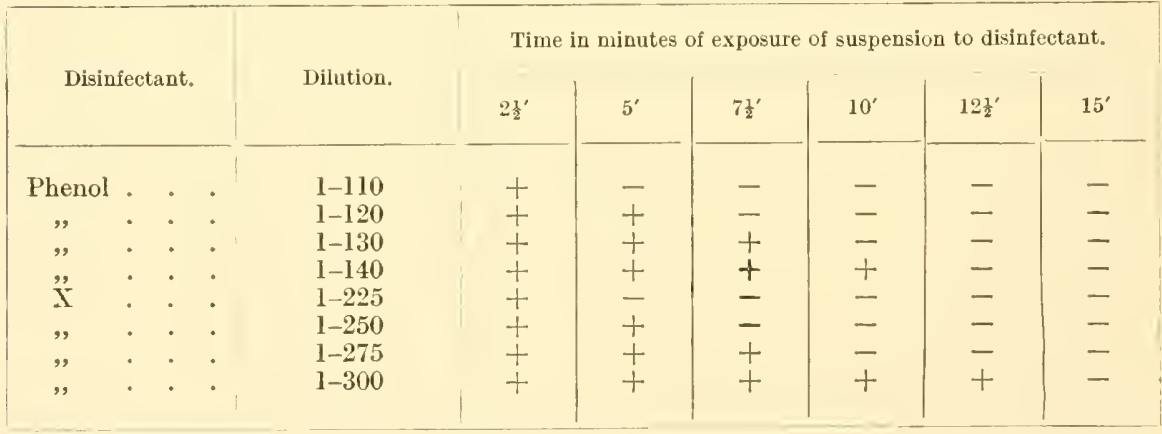

$+=$ growth.

$-=$ no growth.

The phenol coefficient of $\mathrm{X}$ is therefore $\frac{275}{130}=2 \cdot 1$.

(For a full discussion of the Rideal-Walker method see Lancet, 1909, ii, 1516.) 
The Chick-Martin Test.-In the Rideal-Walker method the disinfectant acts in pure solution. But, in practice, disinfectants have usually to act in solutions containing organic matter. As the presence of organic matter seriously lessens the activity of most disinfectants, Chick and Martin (1908) suggested that the disinfectant should be tested on the organisms not in distilled water, but in water containing a suspension of 3 per cent. dried human fæces. Further, instead of allowing the time to vary, as in the Rideal-Walker method, they fix a time limit of 30 minutes for the action of the disinfectant, making subcultures at the end of this time. The phenol coefficient of disinfectants, especially those of the emulsified disinfectants, is distinctly lower by this method. Thus whereas the activity of phenol was reduced about 10 per cent. in the presence of 3 per cent. fæees, that of the commercial cresols was reduced $30-50$ per cent.

There are certain general principles governing the estimation of disinfectant power that must be rigidly adhered to. Great care must be taken to use media that are alike in composition and in $\mathrm{H}$-ion concentration. The organisms used for the test must always be grown and subcultured under exactly the same conditions. Disinfectants such as $\mathrm{HgCl}_{2}$, which, as we have already seen, are antiseptic even in extremely high dilution, must be neutralized, preferably with a saturated solution of $\mathrm{H}_{2} \mathrm{~S}$, at the termination of their action, and thioglycollate must be added to the medium in which subcultures are made to provide the necessary - SH groups. With phenol, clilution is unnecessary, as the inoculation of a loopful of suspension into $5 \mathrm{ml}$. of broth dilutes the phenol sufficiently to destroy its antiseptic action. In comparing salts, molar concentrations, either in their multiples or submultiples, should be used. We have seen that the great majority of organisms are killed long before the culture is completely sterilized; there are always a few organisms that resist disinfection for a considerable period after the remainder are destroyed. Therefore any method that takes sterilization as its end-point is bound to be fallacious. This is one of the greatest objections to all the current methods. Similarly any method in which the time is fixed is unsatisfactory.

Even when the best available standard methods are employed, and the temperature is kept constant, there still remain very important theoretical objections. The present methods depend on the fixation of all but one variable, and the comparison of different disinfectants on this variable. Thus, under standard conditions we learn the concentrations necessary to kill a given organism in a given length of time. It is obvious that the results we obtain must vary with the conditions we lay down. Suppose the time taken be 2.5 minutes, as it sometimes is in the Rideal-Walker method, then for the sterilization of Salm. paratyphi the phenol coefficient of $\mathrm{HgCl}_{2}$ would work out at just over 2 ; but if the time taken is 30 minutes, as it is in the Chick-Martin test, the phenol coefficient works out at 550 (Chick 1908). Again, an increase in the concentration of phenol increases the rate of disinfection far more than a similar increase in the concentration of $\mathrm{HgCl}_{2}$; doubling the concentration of the former shortens the reaction 64 times, of the latter only twice. The effect of temperature also is important, for a rise in temperature increases the rate of action with some disinfectants, such as phenol and the emulsified disinfectants, far more than with others, such as the salts of the heavy metals.

The Rideal-Walker method has been severely and justly criticized. It tells us nothing about the effect of change in concentration or in temperature; it measures only the reaction velocity at a given concentration and during a particular interval 
of time. Nor does it give us any information about the toxicity of the disinfectant to the tissues, its anti-leucocytic power, its activity in the presence of protein or lipins, or its selective action on different bacteria. Any attempt to express by a single index a number of dissociated properties is clearly illogical. The phenol coefficient, as given by the Rideal-Walker method, is at best a grossly over-simplified answer to a very difficult problem, and at worst little short of bacteriological prostitution. To scientific workers, and to those interested in human and animal therapy, the result is meaningless. Were it not for the big financial interests concerned in the commercial production of disinfectants, the test would doubtless die of disuse.

What is the remedy? Several have been proposed, but wone is altogether satisfactory. Phelps (1911) suggests that a method of standardization should comprise the determination of $k, n$, and $\theta$ for each disinfectant. By the formula

$$
\mathrm{KC}^{n} t=\log \frac{\mathrm{B}}{\mathrm{b}}
$$

we can calculate the reaction velocity at any concentration, and by the formula

$$
\mathrm{K}_{\mathrm{T}_{1}}=\mathrm{K}_{20^{\circ}} \times \theta^{\left(\mathrm{T}-20^{\circ}\right)}
$$

where $K_{\mathrm{T}_{2}}$ represents the velocity to be calculated at the temperature desired and $\mathrm{K}_{20^{\circ}}$ the velocity actually determined at a temperature of $20^{\circ} \mathrm{C}$. (or any other convenient temperature), we can determine the value of the temperature coefficient $\theta$.

One objection to this method is that the reaction velocity often varies during the progress of disinfection. To meet this difficulty, Hobbs and Wilson (1942) suggested that the value of $k$ should be taken in the middle stage of the reaction. Withell $(1942 a, b)$ has improved on this by proposing that the time taken to destroy 50 per cent. of the organisms - L $t 50$ - should be selected as a comparative measure of bactericidal efficiency, and has pointed out that this value can be most easily determined by the use of probit logarithm of time graphs (see Bliss 1938, 1941). Withell (1942b) has also drawn attention to the danger of attempting to compare two bactericidal agents that yield different types of time-survivor curve. Many of these are at variance with that yielded by phenol, and a phenol coefficient is therefore inapplicable.

If Phelps' method is adopted, then it is advisable to test the disinfectant against two or three different organisms, as suggested by Eisenberg (1919), since it is known that some germicidal agents have a strongly selective action on certain bacteria. The information derived from the use of this method is considerably greater and more valuable than that supplied by the Rideal-Walker coefficient. Nevertheless, it still leaves us ignorant of a number of important properties of the disinfectant, particularly its toxicity to body tissues and its activity in the presence of cellular material. For this reason, the behaviour of the disinfectant in tissue suspensions or cultures can be studied (see Bronfenbrenner et al. 1939, Salle et al. 1939, Welch and Brewer 1942).

In conclusion, we may say that work of recent years, particularly on the sulphonamides, has shown how inadequate any laboratory test of germicidla activity is to indicate the behaviour of a given substance in the animal body. Attention, in the future, is therefore likely to be devoted far more than in the past to a study of disinfectant agents in the presence of living tissues. 
Practical Application of Germicides.-We shall not give more than a brief account of the use of germicides in practice, and shall confine ourselves to general remarks, illustrating the application of the principles that we have already considered.

Gaseous Disinfectants.-The gaseous disinfectants most commonly employed are sulphur dioxide, chlorine, and formaldehyde. The first two are active only in a moist atmosphere. Thus sulphur dioxide combines with water to form sulphurous acid, and chlorine to form hypochlorous acid. To be successful in the destruction of vegetative bacteria, sulphur dioxide should be present in a concentration of 2-3 per cent., chlorine of 1 per cent., and formaldehyde 1-2 per cent. of the atmosphere. Ozone is sometimes used for the sterilization of water and for meat preservation. Heise (1917) found that concentrations by volume of about 1/1000 destroyed 95 per cent. of coliform organisms on the surface of an agar plate in 1 minute, concentrations of about $1 / 270,000$ in 1 hour, and concentrations of about $1 / 720,000$ in 3-4 hours. The gas has little penetrating power, and is of value only for the destruction of organisms unprotected by colloidal or other material (see Elford and van den Ende 1942). The use of aerosols and vapourized disinfectants for sterilization of the air - as apart from fumigation-is considered in Chapter 91.

Liquid Disinfectants.-In actual practice it is inevitable that disinfectants should be employed more or less empirically; it is impossible, from knowledge gained in the laboratory, to predict exactly the length of time requisite for the complete sterilization of any material. Realizing this, we err on the safe side, and arrange our conditions so as to obtain sterilization in a time much shorter than that which is actually allowed. To do this, however, it is necessary to take into consideration the principles that we have already considered, so far as they are known, and pay particular attention to such variables as the nature of the organism, the material in which it is contained, the $\mathrm{H}$-ion concentration, the salt content, and the temperature at which the reaction is to proceed. Having considered these, the disinfectant to be chosen, the concentration in which it shall be allowed to act, and the time for which its action shall continue, may be determined. For general purposes, we may lay down a few simple rules.

(1) Spores are more resistant than vegetative bacteria.

(2) Bacteria possessing a high content of lipins, such as the acid-fast bacilli. are very resistant to liquid disinfectants. Tubercle bacilli in sputum may withstand 5 per cent. phenol for 24 hours, but they are killed by boiling in 1 minute.

(3) For the destruction of spores and acid-fast bacilli heat is preferable to chemical disinfectants.

(4) Bacteria suspended in a protein medium are more resistant than those in a non-protein medium.

(5) If the protein medium is also a good nutrient medium, the organisms are even more resistant.

(6) Disinfection by nearly all germicides proceeds more quickly in an acid than in an alkaline medium. There is evidence that a given concentration of $\mathrm{H}$-ions is more bactericidal than of $\mathrm{OH}$-ions.

(7) The effect of salts in the medium depends on their nature and on their concentration. In general, salts increase the action of phenol and of the emulsified disinfectants, but diminish that of $\mathrm{HgCl}_{2}$. 
(8) The higher the temperature at which a disinfectant is allowed to act, the more rapid is the process of sterilization.

(9) Some germicides dissolved in alcohol or in vegetable oils are deprived of the greater part of their power, but an alcoholic solution of iodine is a potent skin disinfectant.

(10) Doubling the concentration of $\mathrm{HgCl}_{2}$ halves the time taken for sterilization; doubling the concentration of phenol diminishes it about 64 times.

(11) For use in a protein medium, the acid disinfectants, such as hypochlorous acid, bleaching powder, and the disinfectants that can be employed combined with acids, are most effective. The alkalies are also reliable.

(12) In the presence of organic matter, whether in solution or in suspension, the activity of certain disinfectants is markedly lowered, especially with the emulsified disinfectants, with oxidizing agents, and with the salts of the heavy metals. Phenol is much less affected, and likewise certain dyes, such as flavine.

(13) Salts of the heavy metals-mercury, silver, and copper-are mainly of value for their bacteriostatic effect; their ability to kill bacteria has been grossly overestimated in the past, and is in reality comparatively low.

(14) For the disinfection of varnished or greasy surfaces, an emulsified disinfectant or a bactericidal wetting agent is to be recommended; aqueous solutions are of little value.

(15) For the disinfection of metal instruments, all substances that act on the metal, causing rust or other change, must be avoided.

(16) For references to disinfection of the hands and skin, the following papers may be consulted: Ahlfeld and Vahle (1896), Colebrook (1930, 1941), Colebrook and Maxted (1933), Neufeld and Schiemann (1943).

(17) For instructions in the use of liquid disinfectants in hospitals, see Report (1944).

Solid Disinfectants.-These are generally made up in the form of powders, with a basis of lime, silicious matter, or vegetable fibre. Phenol is the commonest disinfectant incorporated. To destroy bacteria they must pass into solution; ; in the dry state they act merely as deodorants.

\section{REFERENCES}

Ahlesed, F. and VAHLe, F. (1896) Dtsch. med. W'schr., 22, 81.

Albert, A. (1942) Lancet, ii, 633.

AlleN, P. W. (1923) J. Bact., 8, 555.

ANDREWES, F. W. and ORTON, K. J. P. (1904) Zbl. Bakt., 35, 645, 811.

Apostoli, G. and Laquerrière, A. (1890) C. R. Acad. Sci., 105, 918.

D'Arcy, R. F. and Hardy, W. B. (1894) J. Physiol., 1\%, 390.

Baker, Z., Harrison, R. W. and Miller, B. F. (I94la)J.exp. Med., 73, 249 ; (1941b) Ibid., $74,611,621$.

Bancroft, W. D. and Richter, G. H. (1931) J. phys. Chem., 35, 511.

Barnard, J. E. and Morgan, H. de R. (1903) Proc. roy. Soc., r', 126.

BARNES, J. II. (1942) Lancet, i, 531.

BARR, C. E. (1923) J. med. Res., 44, 79.

Baxter. (1875) Rep. loc. Govt. Bd. publ. Hlth., New Ser., No. 5, appendix p. 216.

BAYLISS, M. (1936) J. Bact., 31, 489.

BAYliss, W. II. (1921) "Principles of General Physiology," 4th ed. London.

Beattie, J. M. and Lewis, F. C. (1920) Spec. Rep. med. Res. Coun. Lond., Ser., No. 49.

BeckMan, J. W. (1896) Zbl. Bakt., 20, 577.

Beckwith, T. D. and Olson, A. R. (1932) Proc. Soc. exp. Biol., N.Y., 29, 362.

Beckwith, T. D. and Weaver, C. E. (1936) J. Bact., 32, 361.

BeHriNg. (1890) Z. Hyg., InfektKr., 9, 395. 
BĚlehrádeK, J. (1926) Nature, 118, 117.

Belin, M. and Ripert, J. (1937) C. R. Soc. Biol., 124, 612.

Bessemans, A. and Meirha eghe, A. van. (1937) Bull. Acad. Méd., 118, 263.

Bigelow, IV. D. and Esty, J. R. (1920) J. infect. Dis., 2\%, 602.

Bisceglie, $V$. (1926) Z. ImmunForsch., 49, 272.

BLiss, C. I. (1938) Quart. J. Pharm., 11, 192; (1941) J. Amer. pharm. Ass., 29, 465.

Budм, H. F. (1932) Physiol. Rev., 12, 23.

BöскеR, O. E. (193s) Z. Hyg. Infelthr., 121, 166.

Bronfenbrenner, J., Hershey, A. D., and Doubly, J. (1939) J. Bact., 37, 583.

Brooks, S. C. (1919) J. gen. Physiol., 1, 61 ; (1920) J. med. Res., 41, 411.

Browning, C. H. (1933) Brit. dent. J., 54, 389; (1943) Brit. med. J., i, 311.

Browning, C. H. and Russ, S. (1917) Proc. roy. Soc., B, 90, 33.

Browning, C. H., Kennaway, E. L., Gulbransen, R., and Thornton, L. H. D. (1917) Brit. med. J., i, 73.

Bruysoghe, R. and Dubois, A. (1925) C. R. Soc. Biol., 93, 819.

Bruy noghe, R. and Le Fèvre de Arric, M. (1925) C. R. Soc. Biol., 93, 852.

Buchholz, J. and Jeney, A. v. (1935) Zbl. Baht., 133, 299.

Bucholtz, L. (1875) Arch. exp. Path. Pharmak., 4, 1.

Burge, IV. E. and Neill, A. J. (1915) Amer. J. Physiol., 38, 399.

Burke, G. S. (1923) J. infect. Dis., 33, 274.

Burnet, F. M. (1925) Aust. J. exp. Biol. med. Sci., 2, 65.

Burtenshaw, J. I. L. (1942) J. Hyg. Camb., 42, 184.

Cadéac and Meunier, A. (1889) Ann. Inst. Pasteur., 3, 317.

Camerox, A. T. (1930) Trans. roy. Soc. Can., 3rd Series, 24, Section V, 53.

Chamberland, M. (1887) Ann. Inst. Pasteur, 1, 153.

Chambers, L. A. and Flosdorf, E. W. (1936) Proc. Soc. exp. Biol., N.I., 34, 631.

Chambers, L. A. and Gaines, N. (1932) J. cell. comp. Physiol., 1, 451.

Снiск, H. (1908) J. Hyg., Camb., 8, 92; (1910) Ibid., 10, 237 ; (1912) Ibid., 12, 414.

Chiok, H. and Martin, C. J. (1908) J. Myg., Camb., 8, 698; (1910) J. Physiol., 40, 404.

Chisholi, J. (1941) Brit. med. J., i, 135.

Christian, M. I. $(1931 a)$ J. Dairy Res., 3, 113 ; (1931b) Nature, Lond., 12\%, 558.

Churchia , J. IV. (1912) J. exp. Med., 16, 221 ; (1923a) Ibid., 3\%, 1 ; (19236) Ibid., 38, 1.

Clark and Gage. (1903) 34th Rep. State Bd. Hlth., Mass.

Clifton, C. E. (1931) Proc. Soc. exp. Biol., N.Y., 28, 745.

Cohen, B. (1922) J. Bact., 1, 183.

Cohen, B. and Clark, W. M. (1919) J. Bact., 4, 409.

Colebrook, L. (1930) Interim Rep. depart. Comm. Maternal Mortality and Morbidity, Appendix D, Min. Hlth., Lond.; (1911) Bull. War Med., 2, 73.

Colebrook, L. and Maxted, W. R. (1933) J. Obstet. Gynoec., 40, 966.

Codlthard, C. E. and Srkes, G. (1936) Pharmaceut. J., 83, 79.

Crowther, J. A. (1926) Nature, 118, 86.

Cruickshank, J. C., Hobbs, B. C., McFarlax, A. M. and Maier, I. (1942) Brit. meil. J., ii, 182.

Dakin, H. D. (1915) Brit. med. J., ii, 318.

Danysz, J. (1906) Ann. Inst. Pasteur, 20, 206.

Delépine A. S. and Greenwood, A. (1914) J. R. sanit. Inst., 35, 317.

Downes, A. and Blunt, T. P. (1877) Proc. roy. Soc., 26, 485; (1878) Ibid., 28, 199.

Dreyer, G. and Campbeld-Rentos, M. L. (1936) Proc. roy. Soc., B., 120, 147.

Dreyer and Hanssen. (1907) C. R. Acad. Sci., 145, 234.

Duclaux, E. (1887) Ann. Inst. Pasteur., 1, 88.

Echelian, E. (1917) Zbl. Bakt., IIte Abt., 48, 140.

Eggerth, A. H. (1926) J. gen. Physiol., 10, 147; (1929a) J.exp. Med., 49, 53; (1929b) Ibid., 50, 299; (1931) Ibid., 53, 27.

Ehrismane, O. (1930) Z. Hyg. InfehtKr., 111, 618.

Ehrisman, O. and Noethling, IV. (1932) Z. Hyg. InfektKr., 113, 597.

EijkMan, C. (1908) Biochem. Z., 11, 12.

Eisenberg, P. (1919) Zbl. Bakt., 82, 69.

Eisenberg, P. and Okolska, M. (1913) Zbl. Bakt., 69, 312.

Eisler, M. v. (1909) Zbl. Bakt., 51, 546.

Elford, W. J. and Ende, J., van DEN. (1942) J. Hyg., Camb., 42, 240.

Elser, W. J., Thomas, P. A., and Steffen, G. I. (1935) J. Immunol., 28, 433.

Epstein, F. (1897) Z. Hyg. InfehtKr., 24, 1.

Evans, F. R. and Curran, H. R. (1943) J. Bact., 46, 513.

Fabian, F. W. and Grahai, H. T. (1933) J. infect. Dis., 53, 76.

FaLK, I. S. (1923) Abst. Bact., 7, 33, 87, 133.

FAY, A. C. (1934) J. agric. Res., 48, 453.

Feistmantel, C. (1902) Zbl. Bakt., 31, 433.

Ferir, C. and Pernossi, L. (1894) Z. Hyg. InfektKr., 16, 385. 
Ficker, M. (1898) Z. Hyg. InfektKr., 29, 1.

Fildes, P. (1940) Brit. J. exp. Path., 21, 67.

Fischer. (1900) "The Structure and Functions of the Bacteria." Trans. by A. C. Jones. Oxford.

Flemino, A. (1932) J. Path. Bact., 35, 831.

Fleming, A. (1940) Proc. R. Soc. Med., 33, 487.

Flemisg, A. and Young, II. Y. (1940) J. Path. Bact., 51, 29.

Flexner, S. (1907) J. exp. Med., 9, 105.

Flosdorf, E. IT. and ModD, S. (1935) J. Immunol., 29, 389; (1938) Ibid. 34, 469.

Forfota, E. and Hámori, A. (1937) Zbl. Bakt., 139, 163.

Foulds, M. (1911) J. R. Army. med. Cps., 16, 167.

Freundeich, H. (1903) Z. phys. Chem., 44, 129.

Gaddum, J. H. (1933) Spec. Rep. Ser. med. Res. Coun., Lond., No. 183.

Gale, C. K. and Miller, D. (1935) J. lab. clin. Med., 21, 31.

Gale, E. F. (1940) Bact. Rev., 4, 135.

Garrod, L. P. (1933a) St. Bart's Hosp. med. Rep., 66, 203 ; (1933b) Brit. J. exp. Path., 14, 182; (1944) Brit. med. J., i, 245.

Gates, F. L. (1930) J. gen. Physiol., 14, 31.

Geppert, J. (1889) Berl. klin. Wschr., 26, 789, 819; (1891a) Dtsch. med. Wschr., 1\%, 797, 825,855 ; (1891b) Ibid., 17, 1065.

Haines, R. B. (1938) Proc. roy. Soc., B., 124, 451.

Haniltos, H. C. (1917) Amer. J. publ. Illth., 7, 282.

Harris, R. S., Bunker, J. W. M., and Milas, N. A. (1932) J. Bact., 23, 429.

Hartley, P. (1936) Quart. Bull. Hlth. Org., L.o. N., 5, 735.

Hasché, E. and Leunio, H. (1935) Dtsch. med. W'schr., 61, 1193.

Hasché, E. and Loch, P. (1937) Z. Hyg. InfektKr., 120, 209.

HaYes, S. N. (1937) Brit. med. J., i, 911.

HeIse, R. (1917) Arb. ReichsgesundhAmt., 50, 204, 418.

Hemingsex, A. MI. (1934) Vidensk. Medd. Dansk naturhist. Foren. Kobenhav., 98, 125.

Hewlett, R. T. (1909) Lancet, i. 741, 815, 889.

Hicks, R. A. and Szymanowski, W. T. (1932) J. infect. Dis., 50, 466.

Hoвbs, B. C. and Wilson, G. S. (1942) J. Hyg., Camb., 42, 436.

HODER, F. (1932) Z. ImmunForsch., 74, 455.

Hofmann, P. (1929) Zbl. Bakt., 114, 216.

Hofmeister, F. (1888) Arch. exp. Path. Pharmak., 24, 247 ; (1889) Ibid., 25, 1.

Holi, G. E. and Sherman, J. M. (1921) J. Bact., 6, 511.

Нотснкіss, M. (1923) J. Bact., 8, 141.

Houston, A. C. (1914) 10th Res. Rep. metrop. Wat. Bd.

Hoyt, A., Fisk, R. T. and Burde, G. (1942) Surgery, 12, 786.

Ilasd, C. N. (1944) Lancet, i, 49.

IRwIN, J. O. (1942) J. Hyg., Camb., 42, 328.

Jodlbader, A. and TAPpeiner, H. v. (1904) Münch. med. Wschr., 51, 1096.

Jordax, R. C. and JACOBS, S. E. (1944) J. Hyg., Camb., 43, 275.

Kitasato, S. (1891) Z. Hyg. InfektKr., 10, 267.

Klarmans, E., Shternov, V. A., and Gates, L. W. (1934a) J. Lab. clin. Med., 19, 835; (1934b) Ibid., 20, 40.

Klino, A. (1932) C. R. Acad. Sci., 194, 1402.

KNAYSI, G. $(1930 a) \mathrm{J}$. infect. Dis., 4\%, 293, 322, 328; (1930b) J. Bact., 19, 113.

KNaYsi, G. and Gordox, M. (1930) J. infect. Dis., 47, 303, 318.

Kосп, R. (1881) Mitt. ReichsgesundhAmt., 1, 234.

KосH, R. and WolffHügel, G. (1881) Mitt. ReichsgesundhAmt., 1, 301.

Kосн, R., GaffKY and Loeffler. (1881) Mitt. ReichsgesundhAmt., 1, 322.

Kosrich, F. (1938) "Die bakterielle Keimtötung durch Wärme." Ferd. Enke Verlag, Stuttgart.

KRönio, B. and PAUL, T. (1897) Z. Hyg. InfektKr., 25, 1.

LaMiar, R. V. (1911) J.exp. Med., 13, 1, 380.

LaRoQUetTe, M. DE. (1918) Ann. Inst. Pasteur., 32, 170.

Lea, D. E. and Halves, R. B. (1940) J. Hyg., Camb., 40, 162.

Lea, D. E., Haises, R. B. and Bretscher, E. (1941) J. Hyg., Camb., 41, 1.

Lea, D. E., Hatnes, R. B. and Coulson, C. A. (1936) Proc. roy. Soc., B., 120, 47 ; (1937) Ibid., 123, 1.

Lentze, F.-A. (1932) Zbl. Bakt., 126, 508.

Levis, B. S. and Lominsizi, I. (1935) C. R. Acad. Sci., 200, 863.

Lid, S. C. and Yen, A. C. H. (1934) Proc. Soc. exp. Biol., N.Y., 32, 485.

Lockemaxx, G., Bär, F., and Totzeck, IV. (1941) Zbl. Bukt., 14\%, 1.

Loeb, J. (1899) Pflüg. Arch. ges. Physiol., 75, 303; (1900) Amer. J. Physiol., 3, 327.

Loeb, J. and Northrop, J. H. (1917) J. biol. Chem., 32, 103.

Macfadyex, A. (1900) Proc. roy. Soc., B., 66,180, 339. 
Macfadyen, A. and Rowland, S. D. (1900) Proc. roy. Soc., B, 66, 488.

MeIntosh, J. and Selbie, F. R. (1942) Lancet, ii, 750.

McMaster, P. D. (1919) J. infect. Dis., 24, 378.

Madsen, T. and NyMan, M. (1907) Z. Hyg. Infekthr., 5\%, 388.

Maldmaxx, W. L., Botwright, W. E. and Churchild, E. S. (1941) J. infect. Dis., 69, 215. M ANIfold, M. C. (1941) Brit. J. exp. Path., 22, 111.

M lathews, A. P. (1904a) Amer. J. Physiol., 10, 290 ; (1904b) Ibid., 11, 455 ; (1905) Ibid., 12. $419 ;(1906) \mathrm{J}$. infect. Dis., 3, 572.

NAYSER, H. (1925) Zbl. Bakt., 94, 238.

MILLER, B. F. and BAKER, Z. (1940) Science, 91, 624.

Minervini, R. (1898) Z. Hyg. InfektKr., 29, 117.

lloore, H. N. and Kerstex, H. (1937) J. Bact., 33, 615.

Mortov, H. E. and Kratder. J. V. (1944) J.Amer. med. Ass., 124, 119.5.

MÜNDEL, O. (1937) Z. IIyg.. InfelitKr.. 120, 267.

Nedfeid, F. and Schiemans, O. (1943) Z. Hyg. InfektKr., 124, 751.

Nichols, H. J. (1920) J. Lab. clin. Med., 5, 502.

Nogгсні, H. (1907) Biochem. Z., 6, 327.

Norton, J. F. and Hso, P. H. (1916) Z. Hyg. InfektKr., 18, 180.

OAG, R. K. (1940) J. Path. Bact., 51, 137.

Otten, L. (1930) Zbl. Bakt., 116, 199 ; (1932) Trans. Far East. Ass. trop. Med. 8th Congr. Bangkok, 1930 , p. 89.

Ozzaxo, T. and Re, C. (1937) G. Batt. Immun., 19, 535.

Païc, M., Dedtsch, V., and Borcila, I. (1935a) C. R. Soc. Biol., 119, 1063.

Païc, M., Haber, P., Voet, J., and Fliasz, A. (1935b) C. R. Soc. Biol., 119, 1061.

Paul, T. (1909) Biochem. Z., 18, 1.

Paul, T. and Prall, F. (1907) Arb. ReichsgesundhAmt., 26, 73.

Paul, T., Birstein, G., and Reusz, A. (1910a) Biochem.Z., 25, 367; (1910b) Ibid., $29,202$. Perdrad, J. R., and Todd. C. (1933a) Proc. roy. Soc., B, 112, 277 ; (1933b) Ibid., $112,288$.

Phelps, E. B. (1911) J. infect. Dis., 8, 27.

Pilod and Codvelle. (1932) C. R. Acad. Sci., 194, 497.

Pringle. (1750) Philos. Trans., 46, 480, 525.

Prochownick, L. and Spaeth, F. (1890) Dtsch. med. Wschr., 16, 564.

Prudhomine, R. O. (1937) C. R. Soc. Biol., 126, 289.

Pugsley, A. T., Oddie, T. H., and Eddy, C. E. (1935) Proc. roy. Soc., B, 118, 276.

RAAB, O. (1900) Z. Biol., 39, 524.

Reichel, H. (1909) Biochem. Z., 22, 149.

REICHENBACH, H. (1908) Z. Hyg. InfektKr., 59, 296 ; (1911) Ibid., 69, 171.

REID, J. D. (1932) Amer. J. Hyg., 16, 540.

Rentschler, H. C.. Nagy, R. and Modronseff, G. (1941) J. Bact., 41, 745.

Report. (1942) "The Prevention of "Hospital Infection" of Wounds." Med. Res. Coun., Lond., JVar Memo., No. 6.

Report. (1944) "The Control of Cross Infection in Hospitals." Med. Res. Coun., Lond., War Memo., No. 11.

Rideal, S. and WALker, J. T. A. (1903) J. R. sanit, Inst., 24, 424.

RIEDER, H. (1902) Münch. med. Wschr., 49, 402.

Rishworth, H. R. (1938) Brit. med. J., ii, 574.

Risler, J. (1936) C. R. Acad. Sci., 203, 517.

Ritchie, J. (1899) Trans, path. Soc. Lond., 50, 256.

Pivers, T. M., Sirader, J. E. and Chambers, L. A. (1937) J. exp. Med., 65, 677.

Robertson, T. B. (1914) J. Hyg., Camb., 14, 143.

Rockwell, G. E. and Ebertz, E. G. (1924) J. infect. Dis., 35, 573.

RöMer, C. (1898) Mïnch. med. Wschr., 45, 298.

Roux, E. (1887) Ann. Inst. Pasteur, 1, 445.

Rouyer, MI. and Servigne, M. (1938) Ann. Inst. Pasteur, 61, 565.

Rubbo, S. D., Albert, A. and Maxwell, M. (1942) Brit. J. exp. Path., 23, 69.

Ross, V. (1904) Zbl. Bakt., 3\%, 115, 280.

Russele, D. S. and Beck, D. J. K. (1944) Brit. med. J., i, 112.

Resselt, D. S. and Falconer, II. A. (1941) Brit. J. Surg., 28, 472 ; (1943) Lancet, i, 580.

Salle, A. J., McOnie, W. A., Shechmeister, I. L. and Foord, D. C. (1939) J. Bact., 3\%, 639.

Savage, R. M. (1940) Quart. J. Pharm., 13, 237.

Scherp, H. W. and Chambers, L. A. (1936) Proc. Soc. exp. Biol., N. Y., 35, 495.

Scheurlex, E. (1895) "Die Bedeutung des Molecularzustandes der wassergelösten Desinfectionsmittel für ihren Wirkungswerth." Strassburg.

Schmdt, C. L. A. and Norman, G. F. (1920) J. infect. Dis., 2\%, 40.

Schоор, G. (1935) Zbl. Bakt., 134, 14.

Schroetter, H. v. (1927) Zbl. Bakt., 104, Beiheft. p. 205.

Sellards, A. W. (1918) J. med. Res., 38, 293. 
Shearer, C. (1919) J. Hyg., Camb., 18, 337.

Sherman, J. M. and Holm, G. E. (1922) J. Bact., \%, 465.

SNyder, M. L. and Lichstein, H. C. (1940) J. infect. Dis., 6\%, 113.

Sobernherm, G. (1943) Schweiz. med. W schr., 73, 1280, 1304, 1333.

Spencer, R. R. (1934) Publ. Hlth Rep., Wash., 49, 183; (1935) Ibid., 50, 1642.

Spooner, E. T. C. and Turnbull, L. H. (1942) Bull. War Med., 2, 345.

Stearn, E. W. and Stearn, A. E. (1926) J. Bact., 11, 345; (1928) Univ. MIo. Stud., 3, No. 2,1 .

Suess, E. (1908) Z. Tuberk., 12, 480.

Szymanowski, W. T. and Hicks, R. A. (1932) J. infect. Dis., 50, 1.

Takafashi, W. N. and Christensen, R. J. (1934) Science, 79, 415.

TAPPEINeR, H. v. (1900) Münch. med. Wschr., 4\%, 5.

TAPPEINer, H. v. and JodlbaUer, A. (1901) Münch. med. Wschr., 51, 737.

Tснанотіne, S. (1921) Ann. Inst. Pasteur, 35, 321.

Tetsumoto, S. (1937) Jap. J. exp. Med., 15, 1.

Thiele, H. and Wolf, K. (1906) Arch. Hyg., 5\%, 29 ; (1907) Ibid., 60, 29.

Thresh, J. C. and Beale, J. F. (1910) Lancet, ii. 1849.

'Tilley, F. W. (1939) J. Bact., 38, 499.

Tilley, F. W. and Schaffer, J. MI. (1926) J. Bact., 12, 303.

Topley, W. W. C. (1915) Brit. med. J., i, 237.

T'Una, T. (1935) Proc. Soc, exp. Biol., N.Y., 33, 328.

UNDERWood, W. B. (1934) "Textbook of Sterilisation." Amer. Sterilizer Co., Eerie, Pa.

WALKER, J. E. (1924) J. infect. Dis., 35, 557; (1925) Ibid., 3\%, 181 ; (1926) Ibid., 38, 127.

WARD, H. M. (1892) Proc. roy. Soc., 52, 393.

Watsox, H. E. (1908) J. IIyg., Camb., 8, 536.

Welch, H. and Brewer, C. M. (1912) Amer. J. publ. Hlth., 32, 261.

WesBrook, F. F. (1896) J. Path. Bact., 3, 70.

Whipple, G. C. and MAYer, A. (1906) J. infect. Dis., Supp. 2, p. 76.

Williams, R.. Clayton-Cooper, B., Duncan, J. M. and Miles, E. M. (1943) Lancet, i, 522.

Winslow, C.-E. A. and Dolloff, A. F. (1928) J. Bact., 15, 67.

Winslow, C.-E. A. and FALK, I. S. (1923) J. Bact., 8, 237.

Winslow, C.-E. A. and Нотchisiss, M. (1922) Proc. Soc. exp. Biol., N.Y., 19, 314.

Winslow, C.-E. A. and Lochridge, E. E. (1906) J. infect. Dis., 3, 547.

Wirgin, G. (1902) Z. Hyg. InfektKr., 40, 307; (1904) Ibid., 46, 149.

Withell, E. R. (1942a) J. Hyg., Camb., 42, 124; (1942b) Ibid., 42, 339.

Woodruff, L. L. and Bunzec, H. H. (1909) Amer. J. Physiol., 25, 190.

Wright, E. V. and Kersten, H. (1937) J. Bact., 34, 639.

Wyскоғғ, R. W. G. (1930a) J. exp. Med., 52, 435; (1930b) Ibid., 52, 769 ; (1932) J. gen. Physiol., 15, 351.

WyсKOfF, R. W. G. and LUYет, B. J. (1931) Radiology, 17, 1171.

Wyckoff, R. IV. G. and Rivfre, T. M. (1930) J. exp. Med., 51, 921.

Yen, A. C. H. and Liu, S. C. (1931) Proc. Soc, exp. Biol., N.Y., 31, 1250.

Zwandenaker, H. (1918) Amer. J. Physiol., 45, 147; (1919-20) J. Physiol., 53, 273. 


\section{CHAPTER 6}

\section{ANTIBACTERIAL SUBSTANCES USED IN THE TREATMENT OF INEECTIONS}

Since the discovery of the disinfectant action of substances on micro-organisms many attempts have been made to use antimicrobial substances for treating infections of the living animal. Most of these attempts were abortive, for a great number of disinfectants belong to the class of general protoplasmic poisons, and are as likely to kill the host's tissues as they are to kill the parasites infecting the tissues. Only antimicrobial substances with a selective action on the parasite would be suitable; and the ideal therapeutic agent would be entirely selective, having no action whatsoever on the host's tissues. This ideal has seldom been approached, and we have for the most part to be content with substances that are antimicrobial in concentrations that do not seriously damage the host. There are two types of antibacterial substances to consider, namely, synthetic drugsthe chemotherapeutic agents, etc.--and natural substances, like quinine or penicillin, which are elaborated by plants, moulds and bacteria-the so-called antibiotic agents.

Chemotherapy was first consistently successful with protozoal and spirochætal infections, as a result of the great pioneer work of Ehrlich, which started in 1905 with a systematic search for a cure for syphilis. The research culminated in the establishment of arsphenamine, an organic compound of arsenic, as a cure both for syphilis and for a number of other spirochætal diseases. The organical arsenicals, and synthetic dyes like trypan red and trypan blue, were also useful in the treatment of trypanosomiasis. It is beyond the scope of this book to treat of the chemotherapy of these and other protozoal infections; the student is referred to the standard work of Kolmer (1926) for details. There are, however, a number of noteworthy points to bear in mind in approaching the chemotherapy of bacterial infections.

Ehrlich devised a measure of chemotherapeutic efficiency, the therapeutic index, which in essence is the ratio of the dose tolerated by the host to the dose that cures the infection. Clearly the measure can give us no more than an indication of the magnitude of the differences in susceptibility to the drug of the host and the parasite. The index would be precise only in an animal whose tissues were uniformly infected by the parasite, receiving a single systemic injection of the drug. Variation in susceptibility of different tissues, uneven distribution of parasites in certain organs or in closed lesions of the host, and repeated doses of the drug in sub-toxic concentration, may alter the circumstances so as to make the ratio inapplicable. Nevertheless, for the purposes of investigation, as far as possible using standardized experimental animals with an infection that conforms 
to a fairly constant pattern, the ratio is a valuable index of therapeutic efficiency. For example, using rats suffering from an experimental trypanosomiasis, the ratio of the minimal lethal dose to the minimal curing dose is unity for arsenic acid and 4 for atoxyl. Arsenic acid is clearly useless, and atoxyl not very satisfactory. Under the same conditions, arsphenamine has a ratio of 37 .

Although a great deal of precise chemical investigation accompanied the search for chemotherapeutic agents, we have to-day little knowledge that will enable us to predict what type of substance is likely to prove effective against a given parasite. The successful discoveries have been largely the result of trial and error. Widely different substances prove to be similar in their efficacy on one type of infection, though once a type of substance has proved efficacions it is usually possible to relate its molecular structure to its chemotherapeutic activity. For example, trivalent arsenic attached to a benzene ring is most effective against trypanosomes when there is an amino group in the para position, and against spirochretes when there is a hydroxyl group in the para position and an amino group in the ortho position. Thus, arsphenamine has the formula

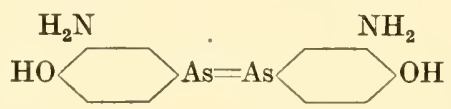

Another general point of importance is that the drug does not necessarily act in the form in which it is administered. Atoxyl, $\mathrm{H}_{2} \mathrm{~N} \longrightarrow \mathrm{AsO}_{3} \mathrm{HNa}$, for example, is a pentavalent arsenical, but is reduced to the active trivalent form in the body.

It is supposed that chemotherapeutic drugs act either by weakening the parasite so that it is easily eliminated by the defence mechanisms of the tissues, or by killing the parasite outright. Experience with experimental trypanosomiasis shows that, if small doses of the drug are given, the "weakening" process may not only be ineffective, but that subsequent generations of the parasite may develop immunity to the drug and become "drug-fast."

\section{CHEMOTHERAPEUTIC AGENTS ACTIVE AGAINST BACTERIA}

The development of chemotherapy in spirochrtal and protozoal infections in the early years of the century had no parallel in bacterial infections. In 1911 Morgenroth and Levy (1911) were able in a certain proportion of cases to protect mice against pneumococcal infection by ethyl dihydrocupreine, but apart from this there is little to note until 1935, when Domagk $(1935 a, b)$ reported that the compound sulphonamido-crysoïdin (Prontosil) would cure streptococcal infection in mice. The curing dose was between one-tenth and one-fiftieth of the tolerated dose for the acute infection that followed the intraperitoneal injection of ten lethal doses of Str. pyogenes, and between one-hundredth and one five-hundredth for a less acute infection. The interval between infection and the start of successful therapy was in some cases as long as three days. Domagk's observations were quickly confirmed by French and British workers (see Levaditi and Vaisman 1935a, b, Nitti and Bovet 1935, Buttle 1935, Colebrook and Kenny 1936). Sulphonamido-crysoïdin, however, was active only in the animal body, and had no effect on streptococci in vitro. Tréfouël and his colleagues (1935) supposed that the in vivo action was due to a breakdown product of the drug, and showed that the 
sulphonamide half of the molecule was active both in the test-tube and the infected mouse (see also Goisseidet et al. 1936, Buttle, Gray and Stephenson 1936). That sulphonamido-crysoïdin was broken down to yield the active sulphonamide in the body was demonstrated by Fuller (1937), and para-aminobenzene sulphonamide was thereby established as the active principle of prontosil.

\section{The Sulphonamide Series.}

Since the establishment of $p$-aminobenzene sulphonamide as the active portion of the prontosil molecule, a large number of active sulphonamide compounds have been synthesized in the search for compounds with increased antibacterial power or range, or for compounds improved in respect of the animal under treatment, such as increased solubility, decreased toxicity, or altered rates of absorption and excretion. They may be conveniently divided into two classes, according to their relation to $p$-aminobenzene sulphonamide, which we may take as the starting substance of the series. In one class, substitution has taken place in the anino group; in the other class, in the amide group. Examples of these classes are depicted in Fig. 27. In each example both the formal chemical name, and the convenient chemical abbreviation of the formal name, are given; the names in brackets are proprietary names, and are inserted either for historical reasons, or because there is no convenient chemical abbreviation (see Ardley 1941). Para-aminobenzene sulphonamide, being the amide of sulphanilic acid, is universally referred to as sulphanilamide.

Nearly all compounds of the first class are inactive per se and must be broken down in the body to yield an active para-amino compound. Sulphonamidocrysoidin is the only example given, though there are many others whose structure is therapeutically justified. The compounds of the second class are more interesting from the bacteriological point of view, because great improvements in the antibacterial action of sulphanilamide have been achieved by substitution in the amide group, the most successful substituents being heterocyclic ring compounds.

Sulphanilamide was chiefly successful against streptococci and pneumococci, and some of the more complex compounds are selective in that they are particularly effective against a certain species. In general, however, the more powerfully antibacterial the sulphonamide the wider its range of action, and it is now clear that most species of medically important bacteria are in some degree susceptible to one or other of the sulphonamide drugs.

The bacterial species include the coli-typhoid-dysentery group (Buttle et al. 1937; Helmholz 1937, Kenny et al. 1937, Cokkinis 1938, Libby and Joyner 1940, Rammelkamp and Jewell 1940, Cooper and Keller 1943); the Brucella group (Nitti, Bovet and Depierre 1937, Francis 1938, Wise 1942); the Corynebacterium group (Murray 1940, Ouyang 1941); Cl. welchii (Long and Bliss 1937a); the Hamophilus group (Long and Bliss 1937b, Pittman 1942) ; meningococei (Long and Bhiss 1937b, Neter 1938a, b) and gonococci (Buttle 1937, Wengatz et al. 1938, Levaditi and Vaisman 1938, Felke 1938); the Mycobacterium group (Rist 1939, Rist et al. 1939, Fitzgerald and Feinstone 1943); Proteus vulgaris and Ps. pyocyanea (Helmholz 1937); Staph. aureus (Domagk 1937, Colebrook and Purdie 1937); shigellæ and V. choleroe (Marshall et. al. 19t0) and B. anthracis (Cruickshank 1937).

The substituted sulphonamides exemplified in Fig. 27 are sulphapyridine (Whitby 1938), sulphathiazole (Fosbinder 1939, McKee et al. 1939), sulphadiazine (Roblin et al. 1940, Feinstone et al. 1940), and sulphaguanidine (Marshall et al. 1940, 1941). Sulphapyridine was the first of the substituted heterocylic compounds 
to be proved active, and was successful in the treatment of pneumococcal, meningococcal and gonococcal infections. Sulphathiazole and sulphadiazine were improvements particularly in respect of Staph. aureus infection, and as an example of the selectivity that a drug may display it is interesting to note that sulphadiazine
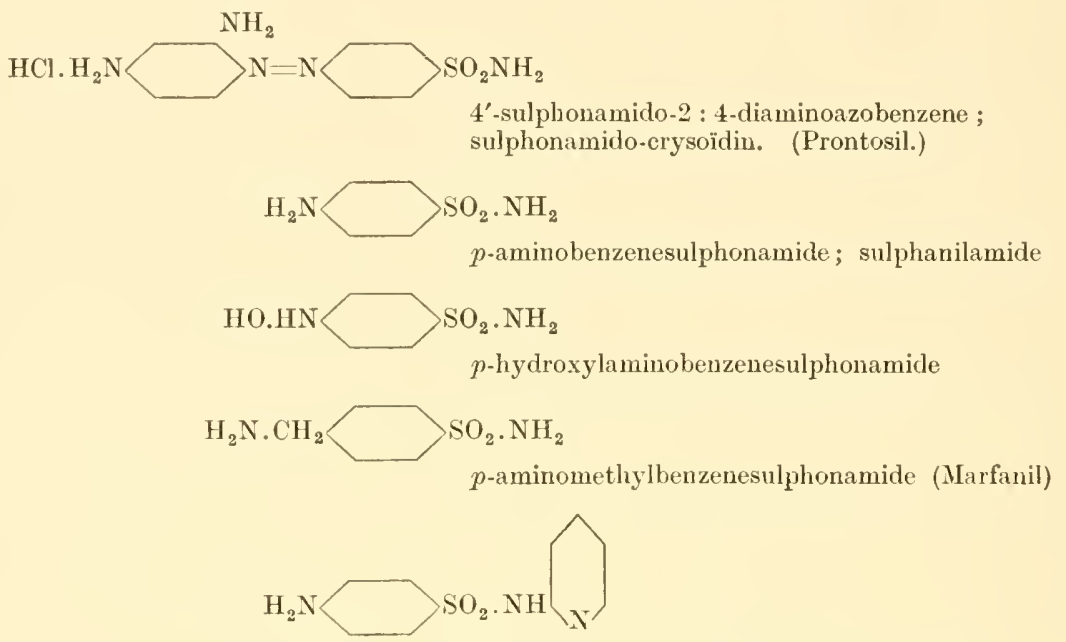

2 -( $p$-aminobenzenesulphonamido)-pyridine; sulphapyridine

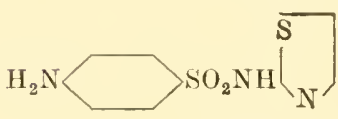

$2-(p$-aminobenzenesulphonamido)-thiazole; sulphathiazole

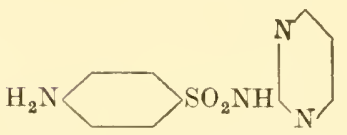

2-( $p$-aminobenzenesulphonamido)-pyrimidine ; sulphadiazine<smiles>NC(N)=[N+]=C1CCNCC1</smiles>

sulphanilylguanidine; sulphaguanidine

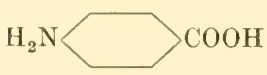

$p$-aminobenzoic acid

FIG. 27.

proved to be effective against Friedländer's bacillus, which had hitherto proved insusceptible to sulphonamides.

Sulphaguanidine is included as an example of a modification designed to be antibacterial, soluble and yet absorbed with difficulty from the alimentary tract; suitable, that is, for the treatment of infections of the intestinal tract. 


\section{Definition of Some Terms.}

The terminology of antibacterial effects produced by all kinds of compounds is confused. The failure of an inoculum of bacteria to grow after exposure to a given compound is referred to sometimes as a bacteriostatic effect, sometimes as a bactericidal effect. The agent producing this effect may be called variously an antiseptic, a bacteriostatic, a bacterial antagonizer, an inhibitor or a suppressor of growth, and so forth. Agents which protect the bacterium from the antibacterial action may be referred to as a deviating substance, an antagonizer, a reversing substance, or an inhibiting substance.

To speak formally, the term bacteriostasis should be applied only to those cases in which there is no change in the count of viable bacteria during exposure to a bacteriostatic substance. But a bacterium whose reproduction is held up by some agent may die of starvation, or what we loosely call senescence, so that in a bacterial population exposed to a bacteriostatic agent, the number of viable organisms will gradually decrease. This being so, the distinction between a bacteriostatic and a bactericidal effect rests to a large extent on the rapidity of bacterial death; the slower the death rate, and the more it deviates from the rapidly initiated unimolecular reaction characteristic of antiseptic action, the more bacteriostatic and less bactericidal is the effect. In many cases the distinction may be clearly drawn, even between the effect of a high and a low concentration of one substance; in other cases, the distinction is difficult to make and, when made, may be invalid.

With regard to the terms for antibacterial agents and substances that protect bacteria from them, we propose to adopt generally the names suggested by Henry (1943) for these substances in the sulphonamide group. Substances that diminish the growth of bacteria will be referred to throughout the chapter as inhibitors, and substances that reverse the effect of these inhibitors as antagonizers.

\section{The In Vitro action of Sulphonamide drugs on Bacteria.}

We are in this chapter concerned with the direct antibacterial action of the sulphonamides. Their action in the body is discussed in Chapter 54. It is necessary to anticipate the discussion only in one respect, namely to adopt the generally accepted hypothesis that their in vivo therapentic effects are referable entirely to the damage they inflict on the infecting bacteria. The damage is qualitatively the same as that which occurs in vitro. It often appears to be quantitatively different; but this is to be expected, since the disappearance of living bacteria from the tissues is conditioned firstly by the inhibitory action of the drug, and secondly by the natural defence mechanisms that come into play against the already damaged bacteria. The antibacterial effect of a sulphonamide in vitro can be demonstrated quite simply. If small measured inocula of a sulphonamidesusceptible streptococcus are added to a nutrient fluid containing the sulphonamide drug, the increase in population with incubation of the culture is very small compared with that of a control culture containing no drug.

For example, at $\mathrm{pH} 7.6 \mathrm{in}$ horse digest broth, a concentration of 1 part in 40,000 inhibited the growth of an inoculum of Str. pyogenes. Concentrations above 1 in 100 were bactericidal in that the inoculum itself was killed (Oag 1939). Low concentrations are apparently bactericidal with prolonged action, but the effect appears to be due more to a starvation of the organisms than to a direct killing. Thus Neter (1941) found that hæmolytic streptococei died as quickly in a non-nutrient medium as they did in a nutrient medium containing 1 per cent. sulphanilamide. 
The bacteriostatic action varics directly as the concentration of the drug, and inversely as the concentration of the bacteria. This latter relation cannot be simply explained in terms of the amount of sulphonamide available per bacterium, for in any given test only a small fraction of the drug is taken up by the bacteria (McIntosh and Whitby 1939, Kohn and Harris 1941, Rose and Fox 1942, Pike and Acton 1942). With organisms that grow optimally at $37^{\circ} \mathrm{C}$. a rise in temperature above this level greatly increases the sulphonamide activity. White (1939) found that only $1 / 100$ th of the amount active at $37^{\circ} \mathrm{C}$. was required at $39^{\circ} \mathrm{C}$. The degree of bacteriostatic action is greatly influenced by the medium used for the test. In general, the richer the medium, the less effective the drug. From the point of view of precision work in bacterial metabolism the variability of common ingredients of routine media, like peptone, tissue extracts and blood fluids, is enormous. Nevertheless, in carefully prepared routine media, comparative tests of sulphonamide inhibition are valid, though absolute measures are unreliable, owing to the variable amounts of substances that antagonize the action of the drug (see, for example, MacLeod 1940, Lewis and Snyder 1940, MacLeod and Mirick 1942). Antagonizers are present in peptone (Lockwood 1938, Weld and Mitchell 1939), in serum, and in extracts and hydrolysates of animal tissue (Landy and Dicken 1942, Lewis 1942). Reliable results are possible only in defined media where the antagonistic effect of ach ingredient may be determined with certainty. It is generally believed that in low concentration the sulphonamide drugs exert only a bacteriostatic effect, but the careful observations of Wolff and Julius (1939) suggest that they are lethal to actively dividing organisms, and that at other stages of the culture cycle bacterial growth is merely inhibited. The analogy to penicillin is very striking (see p. 183).

\section{The In Vitro Resistance of Bacteria to Sulphonamides.}

The resistance of bacteria to a sulphonamide is measured in terms of the minimum concentration of the drug that prevents the growth in a defined medium of a standard inoculum of the strain under test. Without careful standardization of the medium and conditions of test (see, for example, Strauss et al. 1941) these measures serve only as rough indications. Comparative tests, however, show that, although bacteria as a whole are generally susceptible to the action of one or other of the sulphonamides, there are wide variations in susceptibitity, both from one species to another, and from strain to strain within a species. Thus, although the species Str. pyogenes is relatively susceptible to sulphanilamide, and Staph. aureus relatively resistant, yet in an examination of a large number of strains of both species, there would be a wide overlap of susceptible strains of Staph. aureus and resistant strains of Str. pyogenes. Strain-variations in natural resistance have been noted by a number of observers (see, for example, Green 1940, Green and Bielschowsky 1942, Poston and Orgain 1942, Felsenfeld 1943) and must be taken into account in the therapy of infections by sulphonamides, since clearly the assumption of a general level of susceptibility for a given species may have serious consequences if applied consistently to the treatment of all infections by that species.

Of even greater importance is the demonstration that resistance to sulphonamides can be acquired in the test-tube. Bacteria may be trained by serial subculture in media containing non-bacteriostatic concentrations of the sulphonamide (Green 1940, MacLeod 1940, Strauss et al. 1941, Schmidt et al. 1942, Kirby and Rantz 1943, Kirby 1943, Lankford et al. 1943, Harris and Kohn 1943, McIntosh 
and Selbie 1943b). The degree of resistance acquired appears to depend mainly upon the strain, but partly upon the culture medium in which the training takes place. The concentration of drug required to induce resistance is proportional to the bacteriostatic potency of the drug used, and the resistance, once acquired, is sufficiently established as a character of the strain to withstand serial subculture in media free from the sulphonamide.

Resistance can also be induced in vivo (MacLean et al. 1939, Schmidt and Hilles 1940, Frisch and Price 1941, Vivino and Spink 1942). For example, Schmidt, Sesler and Dettweiler (1942) infected sulphapyridine-treated mice with virulent pneumococci, pooled the cultures from the heart blood of the mice which died, and with the pooled cultures infected another group of sulphapyridine-treated mice. Two to three repetitions of this process caused an appreciable increase of in vitro resistance, and three to nine repetitions produced a maximal degree of resistance. The acquired resistance was sufficiently established to withstand 215 passages through normal mice. The effect of the drug does not appear to be one of selective breeding out of naturally resistant variants, but a direct action on all the metabohing cells in the culture.

The practical implication of in vivo induction of resistance, namely the possibility that inadequate doses of sulphonamide given to an infected animal might enable the infecting organism to develop a resistance even to adequate therapy by the same drug, is discussed more fully in Part IV.

The nature of the resistance is not clear. It appears to be specific for the sulphonamides, because an increase in resistance to one member of the series is accompanied by an increase in resistance to the other members. This sulphonamide cross-resistance is not quantitatively complete, for high concentrations of the more potent sulphonamides will inhibit bacteria that are fully resistant to less potent sulphonamides (see Kirby and Rantz 1943). Nevertheless, its qualitative specificity is sufficiently striking to suggest that in all cases the acquisition of resistance depends on a metabolic response to a common feature of the sulphonamides, namely the para-aminobenzene part of the molecule. Sulphonamideresistance is not associated with resistance to antibacterial agents such as propamidine, the acridines (McIntosh and Selbie 1943b), penicillin (Powell and Jamieson 1942b, McKee and Rake 1942, McKee, Hamre and Rake 1943, Tillett et al. 1943, Schmidt and Sesler 1943), and certain sulphones (Evans et al. 1944).

\section{Sulphonamide Antagonists. para-Aminobenzoic Acid.}

Stamp (1939) was the first to demonstrate sulphonamide antagonizers in bacteria. From broth cultures of a Group A and a Group C streptococcus he isolated a fraction antagonizing both sulphanilamide and sulphapyridine. The substance was heat-stable, of low molecular weight, and appeared to contain amino-acids; its action was specific for the sulphonamides, but independent of the nature of the organisms inhibited by the sulphonamide. Stamp suggested that the antagonizer was either a metabolite or part of an enzyme system essential for the growth of the organism. Green (1940) found a similar antagonist in $\mathrm{Br}$. abortus, and in various animal and vegetable materials, and postulated that resistance of bacteria in presence of sulphonamide depended on their capacity for an initial proliferation rapid enough to produce a high intracellular concentration of antagonizer (see also Pike and Foster 1944).

The indications that an antagonizing fraction isolated from yeast contained P.B. 
an aromatic amino compound led Woods (1940) to test the antagonizing action of $p$-aminobenzoic acid. It proved to be a powerful antagonizer; in a defined basal medium containing streptococci, one molecule of the acid antagonized the bacteriostatic action of 26,000 molecules of sulphanilamide. The probable presence of $p$-aminobenzoic acid in yeast was later established by Rubbo and Gillespie (1940), Blanchard (1941) and McIlwain (1942b). (See also Ratner et al. 1944.)

Subsequent observers confirmed Woods' findings (see Green and Bielschowsky 1942, Rubbo and Gillespie 1942, Wood 1942) and showed that the ratio of molar bacteriostatic concentrations of sulphanilamide to the antagonizing concentration of $p$-aminobenzoic acid varied between 26,000:1 and 1000:1. The acid antagonizes all the sulphonamides, and Wood (1942) observed that the greater the bacteriostatic activity of the sulphonamide, the smaller is the ratio, i.e. the greater its ability, molecule per molecule, to counteract the antagonizing effect of $p$-aminobenzoic acid.

\section{The Woods-Fildes Hypothesis.}

The structural similarity of sulphanilamide and $p$-aminobenzoic acid led Woods (1940) and Fildes (1940a) to propound an hypothesis of sulphonamide action that has proved exceedingly fruitful in the investigation of antibacterial substances. The Woods-Fildes hypothesis made precise the previously held notion that sulphonamides in some way interfëred with an essential metabolic function of the bacterium. Woods and Fildes contend that $p$-aminobenzoic acid is an essential metabolite for susceptible bacteria, whose growth is inhibited when sulphonamides, by reason of their structural similarity to the natural metabolite, block the enzyme system concerned with this particular part of the essential metabolism of the organism. Inhibitor and antagonist compete for the enzyme system, and bacteriostasis results when the inhibitor is successful in the competition.

The degree of competition in a given organism may be expressed by the ratio $\mathrm{C}_{1} / \mathrm{C}_{\mathrm{H}}, \mathrm{C}_{1}$ being the molar concentration of inhibitor that is just bacteriostatic in the presence of a molar concentration $\mathrm{C}_{M}$ of the corresponding metabolite (McIlwain 1942b). Thus the ratio, for which McIlwain proposed the name " antibacterial index," was found by Woods to be 26,000 for Str. pyogenes, sulphanilamide and $p$-aminobenzoic acid. Among other things, it can be used to predict the chemotherapeutic efficiency of a drug, if the concentration of the metabolite (i.e. the antagonist) in the tissues of the animal is known (see below, p. 164).

The facts of $p$-aminobenzoic acid antagonism to the sulphonamides have been amply confirmed both in vitro and in the living animal. The Woods-Fildes hypothesis of its action is widely accepted. However, it is not consistent with all the phenomena of sulphonamide action, and for this reason we shall examine both the corroborative evidence and the inconsistencies in some detail.

\section{para-aminobenzoic Acid as Essential Metabolite.}

In two species of bacteria there is no doubt that $p$-aminobenzoic acid is an essential metabolite, for it is demonstrably an essential nutrient. Both $\mathrm{Cl}$. acetobutylicum (Rubbo et al. 1941, Rubbo and Gillespie 1942, Lampen and Peterson 1941) and Acetobacter suboxydans (Lampen et al. 1942) require the acid for growth, and both are susceptible to an inhibiting action of sulphonamides that is reversed by excess of the acid (see also Kuhn and Schwartz 1941, Wiedling 1941, Isbell 1942, Landy and Dicken 1942). In one other species, a soil bacillus isolated by Mirick (1943), an enzyme system capable of oxidizing $p$-aminobenzoic acid has been demonstrated; the growth of the organism was inhibited by sulphapyridine. 
In other species susceptible to the action of sulphonamides, the evidence for $p$-aminobenzoic acid as an essential metabolite is indirect. 'The acid appears to be present in yeast : and it can be recognized in extracts of bacteria either chemically or biologically, utilizing either bacteria for which it is an essential nutrient, or bacteria like Miriek's, which oxidize it. But in both cases the specificity of the test is open to question. Landy and his colleagues (1943), using Acetobacter suboxydans for microbiological assay and checking their results by chemical methods, found $0.042 \mu \mathrm{g}$ per ml. in the culture fluid of staph. aureus. They found also that strains which had been made resistant to sulphathiazole by training in sulphathiazole-broth contained 70 times as much $p$-aminobenzoic acid as the normal strains. These workers correlate this resistance with the power to synthesize an excess of $p$-aminobenzoic acid (see also Spink et al. 1944, Housewright and Koser 1944). Sulphonamide-resistant strains of Bact. coli, Sh. shigce and V. cholerce, on the other hand, showed no associated increase in content of $p$-aminobenzoic acid.

Stokinger and his colleagues (1942) found that sulphonamide-resistant gonococei contained more $p$-aminobenzoic acid than susceptible strains, but since azochloramide, which inhibits $p$-aminobenzoic acid, had no effect on resistance, they concluded that increased resistance was not a function of increased synthesis of the acid.

\section{Analogous Systems with Other Essential Metabolites.}

The most impressive evidence for the Woods-Fildes hypothesis, apart from the observations upon which it was originally founded, lies in the success of its extension to other systems. Fildes (1941) applied it indirectly to the metabolism of a strain of Salm. typhi which needed tryptophan as an essential nutrient. Indole could replace the tryptophan in basal media, and the essential tryptophan was syuthesized from it. It was found that indoleacrylic acid inhibited growth of the organism in indole-containing, but not in tryptophan-containing media. Tryptophan antagonized the inhibition, but there was no constant ratio between antagonizing and inhibiting concentrations of the two substances. Fildes supposed that the indoleacrylic acid competed for the services of an enzyme with a product intermediate between indole and the tryptophan. Indoleacrylic acid had the same action on the strain after it had been trained to grow in a medium containing ammonia as the sole source of nitrogen, suggesting that the hypothetical intermediate substance was still an essential metabolite whose activity could be specifically inhibited.

More direct tests of the hypothesis were made by McIlwain $(1940,1941 a$, $1942 a, b)$.

Para-aminobenzoic acid is of the form R.COOH, and sulphanilamide, of the form $\mathrm{R} \cdot \mathrm{SO}_{2} \mathrm{NH}_{2}$, is an analogue. NcIlwain prepared analogues of various bacterial nutrients, either of the form $\mathrm{R} \cdot \mathrm{SO}_{2} \mathrm{NH}_{2}$ or $\mathrm{R} \cdot \mathrm{SO}_{3} \mathrm{H}$, and showed that they inhibited the growth of bacteria for which the nutrient was essential. Thus nicotinic acid $\mathrm{N}$

$\mathrm{COOH}$ is an essential nutrient for Staph. aureus and Proteus vulgaris, and pyridine sulphonamide $\mathrm{N}$

$\mathrm{SO}_{2} \mathrm{NH}_{2}$ is an inhibitor of their growth in a basal medium containing a nicotinic acid. The same relationship held between aminocarboxylic acids like glycine, alanine and valine, which werc known to be concerned in the growth of the test organisms, often as essential nutrients, and their sulphonic acid analogues. Again, in confirmation of the work of Snell (194I), pantothenic acid (see p. 67)

\section{$\mathrm{HO} \cdot \mathrm{CH}_{2} \mathrm{C}\left(\mathrm{CH}_{3}\right)_{2} \cdot \mathrm{CH}(\mathrm{OH}) \cdot \mathrm{CO} \cdot \mathrm{NH} \cdot \mathrm{CH}_{2} \cdot \mathrm{CH}_{2} \cdot \mathrm{COOH}$}


and pantoyltaurine, in whieh the $-\mathrm{COOH}$ group is replaced by $-\mathrm{SO}_{3} \mathrm{H}$, were found to be similarly related (see also Barnett and Robinson 1942). Recently McIlwain (I943a) has further elucidated the action of pantoyltaurine by comparing the metabolism of resistant and susceptible strains of $C$. diphtherice and Str. pyogenes. Pantothenic acid is an essential nutrient for susceptible strains of both species. Resistance could be induced in $C$. diphtherice by serial subculture either in media containing sub-inhibiting amounts of pantoyltaurine, or in media lacking pantothenate; and all resistant strains were shown to have developed the power to use $\beta$-alanine, which they presumably synthesized into pantothenic acid. Resistant Str. pyogenes had developed no ability to synthesize pantothenate. They were, however, susceptible to salicylate and pantoyltaurine, suggesting that resistance to pantoyltaurine alone is due to the possession of an alternative metabolic proeess, which in turn is susceptible to salicylate (see also Ivánovics 1942). McIlwain and Hughes (1944) have also shown that pantothenate is metabolized by Str. pyogenes during glycolysis, but that neither absence of pantothenate nor its antagonization by pantoyltaurine affects the glycolysis. On the other hand, either the absence of pantothenate, or the addition of pantoyltaurine, or the inhibition of glyeolysis, will inhibit growth. These facts are most conveniently explained by assuming that both glycolysis and pantothenate are necessary for the formation of an essential growth-metabolite in the cell.

Pantoyltaurine is of particular interest since McIlwain (1942a) was able to collect data from which he predicted its in vivo action. The antibacterial index for Str. pyogenes was as low as 500 ; the molar concentration of pantothenic acid likely to be found in animal tissues lay between $10^{-6}$ and $10^{-5}$; and the molar concentration of pantoyltaurine required to inhibit the streptococci in the presence of this amount of pantothenic acid was well below the maximum tolerated concentration. The prediction that pantoyltaurine would be chemotherapeutic in vivo was amply confirmed by McIlwain and Hawking (1943). Rats were protected against 10,000 lethal doses of Str. pyogenes, and the protective effect was abolished by artificially raising the pantothenate concentration of the blood. The drug was iueffective in mice, in whose tissues the natural pantothenate content is higher.

Woolley and White (1943) have also demonstrated a resistance to an antibacterial substance of the Woods-Fildes type, which, like that of Str. pyogenes to pantoyltaurine, does not depend on an ability to synthesize increased amounts of the essential metabolite. Pyrithiamine, the pyridine analogue of thiamin, inhibited the growth of yeast and bacteria in direct proportion to their natural thiamin requirements. But the resistant strains produced neither thiamin nor any other pyrithiamine antagonist in detectable amounts. Failure to demonstrate increased synthesis of the essential metabolite by drug-resistant organisms does not, however, invalidate the Woods-Fildes hypothesis. The resistant organism may develop a different unrelated path along which to carry its essential metabolic processes.

Further examples of antagonism are described in connection with other antibacterial agents. We may note here, for example, that polyamines like triethylenetetramine and tetraethylenepentamine antagonize the bacteriostatic effect of mepacrme on Bact. coli (Silverman and Evans 1943) and of propamidine on L. casei and Str. lactis (Snell 1944). IIcIlwain (1941b) has applied the Woods-Fildes hypothesis to the antagonism of nucleic aeid and related substances to acriflavine. As in the sulphonamides, there was a constant ratio between inhibitor and antagonist. Amino-acid concentrates, especially in the presence of artificial hydrogen carriers like methylene blue, were also antagonistic, but with increasing concentrations of inhibitor, increasing concentrations of antagonists became ineffective. Mcllwain concludes that the acriflavine competes with nucleic acid 
and like substances for an essential enzyme system, and that the amino-acid concentrates are effective because they are substrates or products of the essential enzyme, which to some extent can be replaced by artificial hydrogen carriers.

\section{Other Sulphonamide Antagonists.}

There is a large group of substances capable of antagonizing sulphonamides. For example, antagonizers are found in necrotic tissue and abscesses (Lockwood et al. 1938), in pus (MacLeod 1940), and, as we have already noted, in the complex organic ingredients of routine culture media (see Strauss and Finland 1941). In some of these the active principle may be $p$-aminobenzoic acid, though it is unproven. Among other substances structurally unrelated to $p$-aminobenzoic acid that are reported as antagonists are methionine (Kohn and Harris 1941), co-enzyme I (West and Coburn 1940), nicotinic acid and nicotinamide (Dorfman et al. 1940), urethane (Johnson 1942), purine bases (Martin and Fisher 1942, Snell and Mitchell 1942) and certain amino-acids.

Kohn and Harris postulated that a methionine phase of metabolism was secondary to a phase for which $p$-aminobenzoic acid was a catalyst, and that when the latter was blocked by sulphanilamicle, added methionine enabled the essential metabolism to carry on. With regard to co-enzyme I and nicotinamide, Strauss, Dingle and Finland (194I) conld not confirm West and Coburn's observation that co-enzyme I antagonized the inhibition of staphylococei by sulphapyridine, but found a partial antagonism of sulphaguanidine by a combination of uracil, pyruvate and adenylic acid. Koser and his colleagues (Dorfman and Koser 1942, Berkman and Koser 1943) have defincd some of the conditions in which co-enzyme I and nicotinanide are active. Using a strain of Sh. sonnei in a basal medium they found that these substances antagonized the inhibition of its respiratory activity by sulphapyridine and sulphathiazole, but not inhibition by sulphanilamide, sulphadiazine, sulphaguanidine or sulphacetamide. Para-aminohenzoic acid was antagonistic to all the sulphonamides tested, though its effect on sulphapyridine and sulphathiazole was less than that of nicotinamicle. They concluded that the radicle attached to the sulphonamide part of the molecule could also affect the enzyme systems of the cell, and that in this case the structural similarity of the pyridine or thiazole rings in sulphapyridine and sulphathiazole with nicotinic acid was responsible for inhibition of respiratory activity dependent upon nicotinamide (pyridine-carboxamide) and co-enzyme I (diphospho-pyridine nucleotide) (see also v. Euler 1943, Tę̧ly et al. 1943). Antagonism by amino-acids is illustrated by a recent report of Sevag and Green (1944) who reversed the sulphonamide inhibition of Staph. aureus in a medium containing glucose and certain anino-acids by the addition of tryptophan.

\section{Alternative Hypotheses of Sulphonamide Action.}

The success of the Woods-Fildes hypothesis in predicting the inhibitory activity of analogues of growth factors does not necessarily confirm the truth of the hypothesis in relation to sulphonamide-action, though it provides a strong incentive to give the hypothesis priority of place. The student is referred to the review of Henry (1913) for a full discussion of the objections to the hypothesis. We shall do no more than summarize the main points.

Neither the competitive relationship between sulphonamides and $p$-aminobenzoic acid, nor their structural similarity, necessarily signifies a competition for an enzyme system utilizing $p$-aminobenzoic acid. In the first place $p$-aminobenzoic acid antagonizes sulphonamide inhibition of systems in which it can play no essential part; e.g. the carboxylase system of Stuph. aureus (Sevag et al. 1943), the digestion of starch by diastase, and the adsorption of methylene blue to charcoal (Eyster 1943). Moreover, the rates of inhibition and antagonization may differ 
markedly, a fact difficult to reconcile with the hypothesis of direct competition between the two (Hirsch 1944). (See p. 165, and Johnson et al. 1944, for competition of relatively nnrelated structures.) In the second place, sulphanilamide may be antagonized, as we have seen, by substances that have no structural relation to it.

The hypothesis postulates that $p$-aminobenzoic acid is an essential metabolite in a wide variety of plants and animals (see Fildes 1940 1,1941 ). That it is a growth factor for certain bacteria and animals is no more than suggestive; at present its wide distribution, and a similar distribution of enzyme systems that utilize it, remain unproven. Two other of Henry's points, the limited value of the evidence of $(a)$ increased $p$-aminobenzoic acid production by sulphonamideresistant strains, and $(b)$ analogous systems of growth factors and inhibitors, we have already dealt with. There remains the most cogent objection, that animal and bacterial respiratory systems are inhibited by sulphanilamide. In two systems, the sea urchin's egg (see Henry 1943) and perhaps Br. tularensis (Tamura 1944), the inhibition is not reversed by $p$-aminobenzoic acid. Sulphonamides appear to have a direct action on the respiratory enzymes of bacteria, both aerobic and anaerobic (Sevag and Shelburne 1942, Dorfman and Koser 1942, Berkman and Koser 1943); on bacterial dehydrogenases (Macleod 1939, Fox 1942), and on cocarboxylase (Sevag, Shelburne and Mudd 1942, Sevag et al. 1943). Sevag and his colleagues conclude that sulphonamides inhibit oxidative enzymes (see also Sevag and Green 1944) and, therefore, the growth of the bacteria. Henry groups the sulphonamides with "indifferent" cell inhibitors like narcotics, inhibiting a specific fraction of the total oxidative reactions of the cell upon which cell division depends. Like the narcotics, the sulphonamides stimulate in low, and inhibit in higher, concentrations (Finklestone-Sayliss et al. 1937, Green and Bielschowsky 1942, Lamanna and Shapiro 1943). Like narcotics, they act upon a wide variety of tissues. For example, they inhibit the growth of tissue cultures of tomato plants (Bonner 1942) and wheat and oat seedlings (Brian 1944, Jones 1944); and they inhibit the reproductive division of flagellates (Lwoff et al. 1941). The specificity of $p$-aminobenzoic acid as an antagonizer does not imply that this substance, or any other antagonizer, necessarily acts by specific interference. The antagonizer may act as a non-specific growth stimulant (see, for example, Rantz and Kirby 1944a), though it is pertinent to note that Lynch and Lockwood (1941) distinguished clearly between the antagonistic action of peptone in a human serum medium, which was due to growth stimulation, and that of $p$-aminobenzoic acid, which was not. Alternatively, antagonizers may combine directly with the inhibitor, forming an inactive complex. 'This is unlikely to be the case with $p$-aminobenzoic acid and a sulphonamide, for they do not react in the absence of bacteria. The antagonism of mercapto compounds to the disinfectant action of $\mathrm{HgCl}_{2}$ (Fildes 1940b) may be of this nature. Again, cationic antiseptics of the long-chain fatty-acid type, which presumably act by disorganizing the lipoid membrane of the bacterial cell, are antagonized by the addition of phospholipins (Baker, Harrison and Miller 1941). Finally, antagonizers may shield the susceptible enzyme system from the inhibition, without blocking the enzyme action. Here again cationic antiseptics provide a model for the hypothesis. (See also Penicillic Acid, p. 178). Valko and DuBois (1944) reversed the antibacterial action of a highly toxic cation, $\mathrm{N}$-dodecyl dimethyl ammonium chloride, displacing it by the addition of a relatively non-toxic cation like $\mathrm{N}$-hexadecyl dimethyl ammonium 
chloride. Here we have a similarity of structure between inhibitor and antagonizer, but the antagonizer is not on the face of it likely to be an essential metabolite for the bacteria concerned. It is, however, doubtful whether this mechanism will serve as a model for all inhibitor-antagonist relationships, for Mcllwain (1944) has recently shown, both with sulphonamide- $p$-aminobenzoic acid and with pantoyltaurine-pantothenic acid systems, that there is no gross displacement of antagonizer by inhibitor in the resting bacterium. Inhibition and antagonism take place only in actively growing cells.

There are at present insufficient data for judging the validity of these conflicting hypotheses, and fresh data may demand still different ones. It is clear that the Woods-Fildes hypothesis, though it holds for certain classes of cellinhibitors, is not applicable to other classes. Further knowledge of the hypothetical $p$-aminobenzoic acid metabolism, and its relation to other metabolic functions inhibited by sulphonamides, may permit a modification of the hypothesis to include discrepant facts. Meanwhile, its neatness and the brilliance of its applications must not be allowed to justify the assumption of its universality.

\section{The Relation of Chemical Structure and Antibacterial Activity in the Sulphonamides.}

The activity of the sulphonamides depends on the integrity of the $\mathrm{H}_{2} \mathrm{~N} \longrightarrow \mathrm{S}$ radicle. Replacement of the para-amino group produces inactive compounds, except in the case of the para-nitro (see King and Henschel 1941) and parahydroxylamine groups.

The compound known as Marfanil (Fig. 27) is an exception to this generalization; it is active, even though an amino-methyl group is substituted for the amino group. Its activity, however, is of a different kind from that of the sulphonamide series, since it is not antagonized by $p$-aminobenzoic aeid, or by substances present in blood and pus; nor is there any cross-resistance between Marfanil- and sulphonamide-resistant strains, (see below) (Miller et al. 1940, Klarer 1941, Domagk 1943).

The activity of hydroxylaminobenzene sulphonamide led Mayer (1937) to postulate that sulphanilamide acted by reason of its oxidation to this componnd. Mellon (1940) assumed that the hydroxylamino compound, by inhibiting catalase, produced a lethal accumulation of $\mathrm{H}_{2} \mathrm{O}_{2}$ in the bacteria. Burton and his colleagues (1940) measured the in vitro activity of both the nitro- and hydroxylamino-forms, and concluded that the "active" substance in sulphanilamide action, at least on aerobic bacteria, was the hydroxylamino-form or an oxidation product intermediate between it and the nitro-form. Green and Bielschowsky (1942) objected to this interpretation on the grounds that the hydroxylamino-form was not antagonized by $p$-aminobenzoic acid, an objection which is valid only if it is assumed that the "active" substance must be formed prior to its absorption by the enzymes for which $p$-aminobenzoic acid competes. Nevertheless, competition between the two is demonstrable (McLeod et al. 1914), and Mellon's interpretation of the significance of the hydroxylamino group remains a possible one.

The hypothesis of action by hydroxylamino compounds is in essence an extension of a more general hypothesis of activity as a function of redox-potential developed by the active substance. The latter hypothesis (see Dubos 1929, below) was elaborated by von Jancsó and von Janesó (1936) to cover chemotherapcutic interference in general, and relates the activity to the power to poise the $\mathbf{E h}$ of the cell and its environment at a level unfavourable for cell growth or development. With sulphonamide, it is supposed that the high lethal oxidizing intensity is aehieved by the $\mathrm{H}_{2} \mathrm{O}_{2}$. 
The necessity for the para position holds also for the antagonistic effect of $p$-aminobenzoic acid. As Rubbo and Gillespie (1942) showed, $o$ - and $m$-aminobenzoic acid are poor antagonizers, and very poor essential nutrients for $\mathrm{Cl}$. acetobutylicum, as compared with the para form.

The $\mathrm{S}$ of the radicle can be replaced by arsenic, carbon, phosphorus, selenium and tellurium, though not all the compounds so formed are antibacterial, and not all the antibacterial compounds are antagonized by $p$-aminobenzoic acid. (Rosenthal et al. 1939, Green and Bielschowsky 1942, Hirsch 1942). The substance 4: 4'-diaminobenzil, $\left(\mathrm{H}_{2} \mathrm{~N} \cdot \mathrm{C}_{6} \mathrm{H}_{4} \cdot \mathrm{CO} \cdot \mathrm{CO} \cdot \mathrm{C}_{6} \mathrm{H}_{4} \cdot \mathrm{NH}_{2}\right)$ is sulphur-free, and yet behaves as an active sulphonamide, being antagonized by $p$-aminobenzoic acid (Kuhn, Möller and Wendt 1943).

As will be seen from Fig. 27, most of the sulphonamides in general use are derived from sulphonilamide by the introduction of substituents - notably heteroeyclic radicals into the amide gromp. Methylation of the pyrimidine ring of sulphadiazine in the 4 - and $4: 6$ - position yields sulphamerazine and sulphamezathine respeetively, which have a similarly high degree of activity. The compound $\mathrm{N}_{1}$-3,4-dimethyl benzoyl sulphanilamide

$$
\mathrm{H}_{2} \mathrm{~N} \cdot \mathrm{C}_{6} \mathrm{H}_{4} \cdot \mathrm{SO}_{2} \mathrm{NH} \cdot \mathrm{CO} \cdot \mathrm{C}_{6} \mathrm{H}_{3}\left(\mathrm{CH}_{3}\right)_{2}
$$

is also active, but alteration of the position of the methyl groups in the benzoyl residue completely destroys the activity (Läuger and Martin 1943).

The bacteriostatic potency of the sulphonamides varies directly with their ability to counteract the antagonistic effect of $p$-aminobenzoic acid, i.e. the stronger the drug, the lower its antibacterial index. At about $\mathrm{pH} \mathrm{7,} \mathrm{that} \mathrm{of} \mathrm{sulphanilamide}$ lies between $26,000: 1$ and $1000: 1$. At this $\mathrm{pH}$, however, $p$-aminobenzoic acid is almost fully ionized, sulphanilamide very little, and the ratio in terms of anions may be near unity (Fox and Rose 1942). The acid dissociation constants (Ka) of the drugs increase with activity, that of sulphanilamide being $2.2 \times 10^{-11}$, sulphapyridine $5 \cdot 1 \times 10^{-9}$, and sulphathiazole $6 \cdot 2 \times 10^{-8}$, the last approaching most closely to $p$-aminobenzoic acid, whose $\mathrm{Ka}$ is $1.2 \times 10^{-5}$ (Schmelkes et al. 1942). Albert and Goldacre (1942), on the other hand, suggest that the activity of sulphanilamide and $p$-aminobenzoic acid may be a function of their feeble basicity ; both have a $\mathrm{Kb}$ of about $10^{-11}$. The association between concentration of anions and activity is not, however, constant. Maximum activity may be displayed at a $\mathrm{pH}$ at which the solution must contain a mixture of dissociated and undissociated forms (Cowles 1942, Brueckner 1943). It is suggested that both forms are necessary for activity, the non-ionized form alone being capable of penetrating the cell, but the ionized form being an active inhibitor. A similar explanation may apply to Bell and Roblin's (1942) data, which showed that the relation of activity with a high $\mathrm{Ka}$ value held good only for a given range of compounds. Above a certain point, activity fell off with increasing Ka. Bell and Roblin, however, postulated that activity depended on the electronegativity of the $\mathrm{SO}_{2}$ group, as well as on ionizing capacity. Compounds with the highest $\mathrm{Ka}$ are those with substituents on the amide nitrogen possessing a high electron-attracting power and, as a consequence, increasing activity due to increasing acid strength may be counteracted by decrease in the electronegativity of the $\mathrm{SO}_{2}$ group. Kumler and Daniels (1943) discuss in some detail the association of activity with the polarity of the molecule, and suggest that it is immaterial whether the sulphona- 
mide acts as an anion, cation or neutral molecule, so long as the structure of the compound permits a separation of charge, with the formation of a quininoid structure by movement of bonds after ionization of the amino gromp. The essence of the polarity in this comnection is the electropositivity of the $\mathrm{NH}_{2}$ group.

The general form of the sulphonamides is $\mathrm{H}_{2} \mathrm{~N} \cdot \mathrm{C}_{6} \mathrm{H}_{4} \cdot \mathrm{SO}_{2} \cdot \mathrm{NH} \cdot \mathrm{R}$. If the substituent $\mathrm{P}$ (e.g., a thiazole or a pyridine ring componnd) is an electron acceptor, the negative charge on the $\mathrm{SO}_{2}$ increases, and the positive eharge on the $\mathrm{NH}_{2}$ becomes greater. Substitutions in other parts of the p-aminobenzene sulphonamide molecule that interfere with the induction of this positive charge will produce inactive compounds (see also Jensen and Schmith 1942).

The conception of activity in terms of the highly polar amino group is supported by the work of Bradbury and Jordan (1942), who found that sulphanilanide, sulphathiazole, sulphapyridime and $p$-aminobenzoic acid all modified the electrophoretic mobility of Bact. coli in the same way. Inactive benzene ring compounds and non-resonating isomers of the active compounds did not produce the characteristic change in mobility. As the result of testing a large number of derivatives and analogues of $p$-aminobenzoic acid. Johnson. Green and Pauli (1944) confirmed the importance of the amino group in the para position and concluded that inhibitory activity may be determined primarily by the chemical reactivity of the functional group rather than by structural similarity of the substance to $p$-aminobenzoic acid.

In conclusion, it should be noted that though sulphonamides like sulphathiazole and sulphadiazine approach the maximum attainable activity in vitro as judged by their physicochemical characters, they are not necessarily the lest attainable for chemotherapy, which must take into consideration absorption, excretion and toxicity in the host, as well as susceptibility to antagonism by products of the host's metabolism.

\section{The Relation of Chemical and Antitacterial Activity in other Compounds.}

It will be clear from the foregoing that though within a group of chemically related compounds activity and chemical structure are closely comnected, no ready generalizations in this respect about antibacterial substances are possible in the present state of our knowledge. There are, however, certain noteworthy features which widely different types of compounds, whether antiseptic or chemotherapeutic, have in common. It is beyond the scope of this book to deal with them in detail. The student is referred to the paper by Albert (1942), upon which we have drawn in the following paragraphs.

We are not for the moment concerned with antiseptics which act by an immediate and extensive disruption of the economy of the bacterial cell, such as we see in the coagulative antiseptics. Apart from these, antibacterial substances range from the frank antiseptics that are general protoplasmic poisons, to the highly selective chemotherapeutic agents, with all grades of selectivity of action in between.

The life of the cell depends on the smooth working together of many systems and any agent that interferes with this will be an antiseptic. Since all the enzymes about which there is chemical information have proved to be proteins, it is not surprising that agents affecting all proteins are normally antiseptics. Oxidizing agents, halogens and formaldehyde fall into this category. Their mode of action on a protein is seldom understood, but this is a general problem of protein chemistry rather than specifically the conceru of bacteriology. Agents that upset the relations of lipins and proteins to one another will kill the cell, for it is on these relations 
that the structure of the interfaces generally depends. The lipoid solvents (chloroform, toluene, etc.) fall into this category as do the phenols, cresols and soaps, for, although these can act as protein coagulants, they are generally antiseptic at higher dilutions than are necessary for coagulant action. Of greater interest are the selective agents that are not classifiable in these general terms.

Crystal violet, a triphenylmethane dye used for the surface treatment of wound infections, is bacteriostatic in low concentrations-an effect, according to a number of observers, which is due to the poising of the Eh of the bacterial environment at a level too high for cell development (Dubos 1929, Ingraham 1933, Fildes 1940b, Hoffman and Rahn 1944 ; but see Stearn 1930, for a contrary view). In a like manner, quinones are antibacterial and redox-active. There is, however, little evidence, either in synthetic quinones, or in natural quinones like citrinin and penicillic acid (see below), that they owe their activity to interference with optimal Eh values for bacterial growth, though Page and Robinson (1943) found that the $\mathrm{E}_{0}{ }_{0}$ values of a series of quinones which were markedly active against Staph. aureus clustered round a potential of about +0.03 volt.

Antibacterial activity in many compounds may be interpreted in terms of their capacity to neutralize acidic or basic groups in the bacterial cell forming feebly ionized complexes (see Stearn and Stearn 1924). The anionic antiseptics, like soaps and the "acid" dyes, combine with basic groups. "The kationic antiseptics, which include basic dyes like brilliant green or crystal violet, the aminoacridine antiseptics, and the higher aliphatic amines like cetyltrimethylammoniumbromide combine with acidic groups, and since acidic groups preponderate in most bacteria, particularly in Gram-positive organisms, it is to be expected that the kationic antiseptics will be more active than anionic antiseptics, and that Gram-positive organisms will be more readily killed than Gram-negative; and this is indeed the case.

Another aspect of the difference in susceptibility of Gram-positive and Gramnegative bacteria was pointed out by Miller and his colleagues (1942). Proceeding from the fact that gramicidin, and the anionic detergents, acting only on Grampositive bacteria, were antagonized by certain phospholipins, they suggested that Gram-negative bacteria owed their insusceptibility to the presence of such phospholipins. In support of this hypothesis, they record experiments in which Bact. coli was made susceptible to tyrothricin (a mixture of gramicidin and tyrocidin) by the addition of protamine sulphate, a precipitant of phospholipins. Thus a slightly inhibitory concentration of the protamine, together with a non-inhibitory concentration of tyrothricin, completely inhibited the respiration of Bact. coli.

In the mono-aminoacridine series of compounds, antibacterial activity increases with basic strength up to a certain point, but compounds with a basic strength greater than this are no stronger as antiseptics (Albert, Rubbo and Goldacre 1941). It is probable that the compounds of extreme basicity are too active to form non-ionized neutralization compounds in the bacteria. We have already noted Albert and Goldacre's view (1942) that basicity and activity of the sulphonamides are associated, and it is of interest that in both series of drugs those whose structure permits resonance and induction of polarity tend to be the most active.

In addition to basicity, surface activity may be a feature of a compound contributing to the bactericidal power of a kationic antiseptic. Albert suggests that the distinction between the acridines and the kationic detergent antiseptics, the one group mainly bacteriostatic, the other bactericidal, may possibly lie in 
the power of the latter to lower surface tension, and thus to concentrate on or in the bacterial cell. Fuller (1942) reached the same conclusion from a study of 40 long-chain aliphatic compounds - amines, amidines, guanidines and quaternary ammonium compounds. Gram-positive organisms were more susceptible than Gram-negative organisms; though the strongly basic guanidines and quaternary compounds had a relatively greater effect on Gram-negative bacteria, the less strongly basic amines on Gram-positive bacteria. Basicity, however, was a less important factor than chain length (and presumably, therefore, surface activity), for bacteriostatic activity increased with the length of the carbon chain to a maximum and, with some of the series, declined with a further increase in chain length.

\section{Other Compounds used in Antibacterial Therapy}

\section{Arsenic Compounds.}

The organic arsenicals have been tested in the treatment of experimental and natural bacterial infeetion. For instance, Bierbaum (1912) demonstraterl some protection of mice infected with Ery. rhusiopathic by treatment with arsphenamine and neoarsphena. mine. Kolle, Leupold, Schlossberger and Hundeshagen (1921) studied the same infection, and extended the range of compounds tested to anino-arsenobenzol compounds. Compounds which were active in vivo had little in vitro activity, and they conchded that the active form was produced in the animal body. Christison (1934) also demonstrated a curative effect in this disease of certain arsenopyridine compounds, notably 2-pyridine3-amino-5-arsenic acid. Allison (1918) found that arsphenamine had a protective effect in rabbits against Str. pyogenes infeetions.

Arsphenamine and neoarsphenamine are bactericidal in vitro, in watery solution, in blood, serum, and in the presence of leucocytes (Schiemanu 1915, Douglas and Colebrook 1916, Osgood 1943). Susceptible bacteria inchude B. anthracis, Pf. mallei, Ery. rhusiopathia, Staph. aureus, Str. pyogenes, C. diphtherice, and Ham. influenza. The compounds most active in vitro appear to be those with an arsenoxide group. Peters (1943) and Albert, Falk and Rubbo (1944) suggest that the arsenoxides interfere with thiol groups in the essential enzyme systems of the bacteria, and in this respect resemble the mercurial antiseptics, whose action was first interpreted by Fildes $(19406)$ along the same lines. Neoarsphenamine has been used in the treatment of human infections, notably puerperal fever (Colebrook 1928); anthrax (sce, for example, Lucchesi and Gildersleeve 1941) and Str. viridans endocarditis (Osgood 1942, 1944). The low therapeutic index that obtains for these drugs when used against bacterial infections precludes their unrestricted use in human infections; Osgood maintains that their use is justified in infections with a mortality greater than five per cent., if safer drugs prove ineffective.

\section{Sulphones.}

Diaminodiphenyl sulphone $\left(\mathrm{H}_{2} \mathrm{~N} \cdot \mathrm{C}_{6} \mathrm{H}_{4} \cdot \mathrm{SO}_{2} \cdot \mathrm{C}_{6} \mathrm{H}_{4} \cdot \mathrm{NH}_{2}\right)$ and its acetyl derivative are more active than sulphanilamide against streptococci, both in vitro and in vivo, and though they are more toxic, their high activity ensures a higher chemotherapentic index than sulphanilamide (Buttle et al. 1937, Baner and Rosenthal 1938). An N-phosphoryl derivative of diaminodiphenyl sulphone, which was less toxic in mice, proved to be active against streptococcal and pneumococcal infections (Smith, Rosenthal and Jackson 1942).

"Promin," which is diaminodiphenyl sulphone $\mathrm{N}, \mathrm{N}$ ' diglncose sulphonate, has been used with some success in the treatment of tuberculous infections of guinea-pigs. The life of the animals is prolonged, the character of the tuberculous lesion is changed, and in some cases regression of the lesion occurs (Feldman. Iann and Hinshaw 1942 $a, b$, Barach, Molomut and Soroka 1942, Felhman and Hinshaw 1943). Its action in human tuberculosis is less striking, partly owing to its relatively low therapeutic index (Hinshaw, Pfuetze and Feldman 1943, Tytler and Lapp 1942. Heaf et al. 1943 ; see also Raiziss 1943, Callomon 1943). 
Two sulphones, $p$-methylsulphonylbenzamidine $\left(\mathrm{CH}_{3} \cdot \mathrm{SO}_{2} \cdot \mathrm{C}_{6} \mathrm{H}_{4} \cdot \mathrm{C}(\mathrm{NH}) \cdot \mathrm{NH}_{2}\right)$ and $p$. methylsulphouylbenzylamine $\left(\mathrm{CH}_{3} \cdot \mathrm{SO}_{2} \cdot \mathrm{C}_{6} \mathrm{H}_{4} \cdot \mathrm{CH}_{2} \cdot \mathrm{NH}_{2}\right)$, have a marked in vitro action against clostridia and Str. pyogenes. The former, designated $V$ 187, has proved highly effective in the treatment of experimental gas gangrene of the guinea-pig. It is not antagonized to any marked extent by body fluids. The latter (V 335) is also effective against gas gangrene in the guinea-pig, but is more readily antagonized by the body fluids. Neither substance is antagonized by $p$-aminobenzoic acid; and both act equally well on normal and sulphonamide-resistant bacteria (Evans et al. 1944).

Propamidine. Propamidine, $4: 4^{\prime}$-diamidino-diphenoxy-propane, first proved an effec-

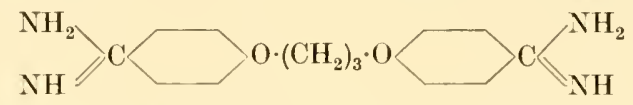

tive therapeutic agent for animal trypanosomiasis (Lourie and Yorke 1939) with a therapeutic index of abont 30. It is also bacteriostatic in low eoncentrations, Str. pyogenes, for example, being inhibited in dilutions of $1: 2 \times 10^{6}$, and non-toxic for leneocytes in dilutions of 1:1,000; it has been used successfully in the local treatment of infections of open wounds and burns (Thrower and Valentine 1943) and of conjunctivitis due to the Morax-Axenfeld bacillus (Valentine and Edwards, 1944).

It is noteworthy that propamidine bears a structural resemblance to stilbene, and that a number of stilbene derivatives have a high bactericidal activity. For example, deoxy diethyl stilbœstrol $\left(\mathrm{HO}_{6} \cdot \mathrm{C}_{6} \mathrm{H}_{4} \cdot \mathrm{C}\left(\mathrm{C}_{2} \mathrm{H}_{5}\right): \mathrm{C}\left(\mathrm{C}_{2} \mathrm{H}_{5}\right) \cdot \mathrm{C}_{6} \mathrm{H}_{5}\right)$ inhibits Str. pyogenes in a dilution of $1: 2 \times 10^{6}$ (Brownlee, Copp, Duffin and Tonkin 1943; see also Faulkner 1943).

\section{Amino-acridine Compounds (Flavines).}

The claim of the amino-acridine compounds to chemotherapentic rank is at present not clear. Their toxicity is such that they are suitable only for application to the surface of infected tissues. The degree of tissue intoxication that justifies submitting infected tissues to the undoubted in vivo antibacterial action of the drugs is in dispute. MeIntosh and Selbie (1942, 1943a) employed proflavine successfully in conjunction with the sulphona. mides in the treatment of experimental gas gangrene. For a full discussion of these drugs from the chemotherapeutic point of view, the reader is referred to the review of Browning (1943).

\section{NATURAL ANTIBACTERIAL SUBSTANCES FROM MICRO-ORGANISMS}

Since the early days of bacteriology and mycology, the inhibition of growth of one micro-organism by the growth of another has been a familiar phenomenon, and its possible significance in the therapy of infectious diseases commented upon. The application of these natural bactericidal or bacteriostatic substances to the treatment of infections was usually limited by the low therapentic index of the materials available; the doses tolerated by the animal were ineffective against the infecting organism. In recent years, however, improvements in methods of isolation, and a clearer understanding of the antibacterial effects, have led to the description of a large number of antibacterial substances from bacteria and moulds, some of which are therapeutically active in experimental infection, and one at least, penicillin, which is highly effective in the treatment of infective disease in the human subject.

The reader is referred to the comprehensive reviews of Porter and Carter (1938) and of Waksman (1941) for a discussion of antagonisms between micro-organisms and of the earlier work on natural antibacterial substances, which is beyond the 
scope of this book. We shall confine the discussion to a few illustrative examples, and note some of the more recently described substances, with especial emphasis on those that have proved their therapeutic value.

\section{Antibacterial Substances from Bacteria.}

Ps. pyocyanea was among the first bacteria to be studied from the point of view of its elaboration of antibacterial substances. Cultures of the organism yield a number of them :- pyocyanase, first prepared by Emmerich and Löw (1899), and Emmerich, Löw and Korschun (1902), depends upon its content of fatty acids of high molecular weight for its activity (Birch-Hirschfeld 1934, Hettche 1934) and affects a large variety of Gram-positive and Gram-negative bacilli including pathogenic cocci, Salmonella bacilli, the diphtheria bacillus (Wagner 1929, Kramer 1935) and Br. abortus (Kocholaty 1942); pyocyanin, a respiratory pigment (see Ehrismamn 1934, and Chapter 3) ; $\alpha$-hydroxyphenazine and a colourless compound having a strongly lytic action on $V$. cholere (Schoental 1941). Iodinin, an antibacterial substance from Chromo. iodimum is a N-oxide of a hydrophenazine, and is apparently specifically antagonized by uaphthoquinone (Mcllwain $19436)$.

Bacteriolytic enzymes of bacterial origin were described by Malfitano (1900, 1903). Nicolle (1907) isolated from B. subtitis a lytic agent active against saline suspensions of staphylococci, pneumococci, the anthrax, plague and glanders bacilli, and salmonellie (see also Jobling and Petersen 1914, Jobling, Petersen and Eggstein 1915, Sartorius 1924, Much 1925).

The imhibitory actions of certain bacteria were not obvionsly due to separable enzymes. Thus, certain strains of Bact. coli, which inhibited the in vitro and in vivo growth of other bacteria, would act only if whole, living cells were present. In culture, they overgrew and eliminated Salmonella and dysentery bacilli, streptococci, staphylococci, B. anthracis and C. diphtherice (Nissle 1930, 1932, 1933, Gundel and Kliewe 1932, Koch and Krämer 1932, Besta and Kuhn 1934, Makowsky 1936, Reploh 1937, Maner 1939). The in vivo inhibition is not marked. For example, Gundel and Kliewe infected mice subcutaneously with mixtures of Bact. coli and a lethal dose of $B$. anthracis. A number of the mice survived, and the survival time of those that died was increased, as compared with mice receiving the lethal dose alone. Other observers have reported the inhibition of $C$. diphtherice by various streptococci. Inhibition by the live cultures was observed in the subcutaneous tissue of the guinea-pig (Besta and Kuhn 1934, Weigmann and Hölzl 1940, Hölzl 1941), and Dulisconet (1939) reported on the successful treatment of throat carriers of $C$. diphtheria by killed cultures of an inhibitory organism.

Two of the most interesting developments in the isolation of antibacterial substances we owe to the work of Dubos. The first was directed to a specific end, the isolation of a bacterium whose enzymes would destroy the polysaccharide substance in the capsules of Type III pneumococcus. This was accomplished by testing large numbers of bacterial strains, mostly spore-bearing aerobic bacilli, in a medium containing the polysaccharide as the sole source of carbon. A bacillus which grew in this medium was trained to produce large amounts of a polysaccharidesplitting enzyme, which in a purified form was subsequently used with success in the treatment of experimental Type III pneunococcus infection of the rabbit (see Chapter 74). The enzyme was specific, and affected only Type III pneumococci. Dubos extended this search to bacilli with a wide range of antibacterial action by serial subculture of samples of soil in a medium containing large quantities 
of dead cocci. The medium favoured the growth of the soil bacilli most capable of utilizing the coccal protoplasm as a source of food, and, by selection, certain spore-bearing bacilli were finally obtained from which antibacterial substances could be isolated (Dubos 1939) (see also Stokes and Woodward 1942). Similar bacilli were isolated from manure, sewage and chcese. Working mainly with one of them, B. brevis, Dubos and Hotchkiss (1941) obtained a mixture of bactericidal substances, tyrothricin, from which they separated two erystalline substances, gramicidin and tyrocidin.

Tyrocidin had the following properties. It was a polypeptide with a free basic $\mathrm{NH}_{2}$ group, a weakly acidic or phenolic group, and contained tryptophan, tyrosine and dicarboxylic amino-acids; about one-fifth of the amino-acid produced on hydrolysis was composed of $d$-amino-acids. The molecular weight was in the region of 2,500 . It was bacterieidal in vitro for Gram-positive and Gram-negative organisms, but was also markedly toxic for animal tissues and leucocytes, losing much of its antibacterial activity when in contact with them. It appeared to act as a general protoplasmic poison. (See also Downs 1943.)

Gramicidin was also a polypeptide, but with no free acid or basic groups, about half the amino-aeids being of the $d$-form. The molecular weight was about 1,400. Gramicidin killed only Gram-positive organisms in vitro. It protected mice against infections by pneumocoeci, streptococei and staphylococci; one $\mu \mathrm{g}$. of the substance, for example, was effective when injected intraperitoneally along with 10,000 lethal doses of pnemmococci. Though highly toxic when given by the parenteral route, the curative dose was well below the dose tolerated by the animal.

The high content of the "unnatural" $d$-amino-acids in both substances suggested that their peenliar properties might in part be dependent on these bodies. Tyrocidin on the whole behaved like an antiseptic; gramicidin acted in a more specific manner, like the sulphonamides (Hotchkiss and Dubos 1940, Dubos and Hotchkiss 1941, Hotchkiss 1941, Lipmann, Hotchkiss and Dubos 1941, Dubos, Hotchkiss and Coburn 1942). (See also Tishler et al. 1941.) It is noteworthy that Fox, Fling and Bollenback (1944) found that $d$-leucine inhibited the growth of $L$. arabinosus, an organism for which $l$-leucine is an essential nutrient, and suggested that the $d$-isomer might act by interfering with the uptake of the $l$-isomer (cf. the antagonism of $p$-aminobenzoic acid and sulphanilamide.)

As the result of a study of the products of acid-hydrolysis, Gordon, Mlartin and Synge (1943) conclude that the structural unit of gramicichin is a cyclopeptide made up of 24 amino-acid residues:-6 $d$-leucine, $6 l$-tryptophan, $5 d l$-valine, $3 l$-alanine, 2 glycine and 2 unknown hydroxyamino compounds. Tyrocidin they found to contain eight different amino-acids, of which phenylalanine was in the $d$-form, the rest being mainly in the $l$-form. (See also Christensen 1943, 1944 and Synge 1944.)

Gramicidin is hæmolytic, and lowers surface tension, though destruction of its antıbacterial activity by heat does not alter the surface activity (Heilman and Herrell $1941 a, b$ ). Tyrocidin lyses both red cells and leucocytes, and both substances are toxic when injected into animal tissues. Administered by mouth to dogs and mice, neither is toxic and neither has any effect on infections (Rammelkamp and Weinstein 1942, Robinson and Molitor 1942). For successful therapy, gramicidin and tyrocidin must be brought into immediate contact with the infecting bacteria. Thus oral, subcutaneous or intravenous administration has no effect on intraperitoneally infected mice, and mice infected systemically are not protected by intraperitoneal administration (Robimson and Graessle 1942). Serial subculture in low, but increasing concentration of gramicidin induced resistance in a susceptible strain of Staph. aureus, and the development of resistance was accompanied by the production of colony variants (Phillips and Barnes 1942). The antibacterial activity of gramicidin is destroyed by gentle hydrolysis in dilute alkali, insufficient to destroy its polypeptide structure. 
Another type of gramicidin. Gramicidin S, has been derived from an unidentified spore-bearing bacillus by Gause and Brazhnikova (1944). It is reported to be more active than tyrotbricin against streptococei and pneumococei ; and, when locally applied, to be curative in suppurative infections of man, especially those due to Staph. aureus.

\section{Antibacterial Substances from Moulds.}

Among the moulds the Actinomycetes, the Penicillia and the Aspergilli have yielded antibacterial agents of various kinds. Wilkins and Harris $(1942,1943 a)$, in an examination of 200 mould cultures for inhibitors of Staph. aureus, Bact. coli and Ps. pyocyanea, record the greatest number of successes in the Penicillia and Aspergilli, few in Phycomycetes and Ascomycetes. Inhibitors of the staphylococcus were the most common, those of the Gram-negative bacilli less common. Atkinson (1943a) found a number of activestrains among Penicillia, and few among Aspergilli, Mucor and "pink moulds." The same substance may be produced by several distinct species of mould (see, for example, White 1943, McKee and MacPhillamy 1943, Florey et al. 1944, Waksman 1944). One mould may produce several kinds of antibacterial substance (see, for example, Waksman and Bugie 1943, Waksman and Geiger 1944), and not all members of a species necessarily produce the same substance, or produce it at all.

One of the first mould-products to be applied to the treatment of infections was an extract of a Streptothrix-like white mould, from which Gratia and Dath $(1924,1925,1926)$ were able, by growth on dead Staph. aureus, to produce a powerful lytic agent for living Staph. aureus and other bacteria. They also noted lytic agents for intestinal coliform bacilli in another white mould, and for $B$. anthracis from an undetermined strain of Penicillium (see also Lieske 1921). The first agent, which they termed a mycolysate, was employed, together with a staphylococcal bacteriophage, in the successful treatment of Staph. aureus carbuncles in man (Gratia and Dath 1930).

In 1928 Fleming (1929) observed the suppression of growth of Staph. aureus round a contaminating colony of a Penicillium that had grown on the agar plate. The inhibitory substance, to which he gave the name penicillin, could be extracted from cultures of the Penicillium, and proved active against Staph. aureus, Str. pyogenes, the gonococcus, the meningococcus, and certain strains of Str. viridans. The enterococcus, and Gram-negative organisms including Bact. friedländeri, H. influenzo, Sh. flexneri and Past. pseudotuberculosis, were relatively insusceptible. The crude filtrates of penicillin were non-toxic to leucocytes in antibacterial concentrations.

The combination of high antibacterial activity with low toxicity for leucocytes led Fleming to suggest and test its application to local infection in man. The amount of penicillin in culture filtrates of the mould was, however, low, and owing to its instability (Fleming 1932, Clutterbuck et al. 1932) attempts at that time to concentrate it proved abortive. Not till Florey and his colleagues (see p. 179) overcame the difficulties in its preparation and demonstrated its remarkable therapeutic powers was its potential value in human medicine fully realized and an active search begun for other similar products.

Not all of the substances listed below have the qualities necessary for a successful therapeutic agent. The curing dose is often so close to the tolerated dose that therapy would be successful only under carefully controlled conditions, though it is possible that some of the less well-defined mould products are mixtures of an antibacterial agent and toxic materials which may later prove to be separable. 
The substances are nevertheless of great interest apart from their possible use in therapy. Those with a selective action on certain bacterial species are useful for ineorporation into culture media for suppressing the growth of unwanted baeteria (see Chapter 12); the properties of some of them afford an explanation of microbial antagonisms observed in nature; and, where their constitution is known, many of the substanees are valuable in relating antibacterial activity to molecular strueture, or as reagents for exploring the metabolism of the organisins upon which they act. It is noteworthy that most of the substanees with a high therapeutic index aet predominantly or exclusively upon Gram-positive bacteria, with the exception of streptothricin. Those acting on both Gram-positive and Gram-negative bacteria appear, like the antiseptics, to be general protoplasmic poisons. None is predominantly or exclusively active against Gram-negative bacteria.

Numerical measurements of antibaeterial powers, and of toxicity to animal tissues afford only a erude means of comparing the various substances. Apart from the varying eriteria of a given type of activity, the measures are dependent on the degree of purity of often undefined substances, the strains of bacteria tested, the type of culture medium, and the animal or type of animal tissue employed. It may, however, be said that most of the substances are bacteriostatic in concentrations lower than 1: 5,000, and many are baetericidal in higher concentrations.

For a general eomparison of a number of these substances, with a number of antiseptics, see Waksinan and Woodruff (1942).

Actinomycetin. The bacteriolytic substance produced by sporulating cultures of a Streptothrix-like monld (Actinomyces albus) has already been mentioned (Gratia and Dath 1924). Welsch (1936, 1937, 1938, 1939, 1940, Welsch and Elford 1937) has studied the lysin, to which he gave the name actinomycetin, in some detail. It appeared to be a proteolytic enzyme, and was inactivated by heating to $54^{\circ}-69^{\circ} \mathrm{C}$., by ultra-violet radiation, and by strong acids. It induced bacteriolysis over a wide range of $\mathrm{pH}$, with an optimum at $\mathrm{pH} 7 \cdot 5$ to $8 \cdot 5$. Lysis appears to depend on at least two factors, a lethal substance which kills the bacteria, and a bacteriolytic enzyme eitber from the Actinomyces, or from the bacteria themselves (see also Welseh 1942).

Actinomyein A and B. These substances were isolated from cultures of Actinomyces antibioticus, a soil organism, by Waksman and Woodruff $(1940 a, b, 1941)$. Staphylococci and streptococci are inhibited in dilutions of over $1: 2.5 \times 10^{6}$, and Salmonella bacilli in $1: 25,000$.

Actinomycin A is a water-soluble, red pigment, with a molecular weight of about 800 , and appears to be a polycyclic nitrogen compound, having a reversible redox system of the quinone type (Waksman and Tishler 1942). It is both bactericidal and bacteriostatic, inhibiting Gram-positive organisms in a dilution of $1: 10^{8}$, and Gram-negative organisms at dilutions of $1: 5,000$ to $1: 10^{5}$. It is active against aerobic and anaerobic bacteria, and against certain fungi. Actinomycin $\mathrm{A}$ is highly toxic to animals. Doses, either oral or parenteral, of $1 \mathrm{mgm}$. per kilogram body weight are lethal for mice, rats and rabbits, the animals dying in 15-20 hours with respiratory failure. Post mortem there are gross pathological changes in liver, kidney and spleen. Sublethal doses have no protective effect in mice infected with Str. pyogenes or Type I pneumococci (Robinson and Waksman 1942). Actinomycin A inhibits fibrinolysis by Str. pyogenes, the production of coagulase by Staph. aureus, but does not affect either tetanus or diphtheria toxin (Neter 1942, 1943) or staphylococcal toxin (Blair and Hallman 1943).

Actinomycin B is a water-insoluble substance. It is markedly bactericidal, but not bacteriostatic, and is highly toxic to animals. 
Aspergillic Acid. This acid has been isolated in crystalline form from Aspergillus fluvus, and has a provisional formula $\mathrm{C}_{12} \mathrm{H}_{20} \mathrm{~N}_{2} \mathrm{O}_{2}$. It is bacteriostatic and bactericidal for a wide range of organisms, including both Gram-positive and Gram-negative species. It is toxic for mice, and no therapentic effect conld be obtained in mice infected with pneumococei or Str. pyogenes (White 1940, White and Hill 1943). Gram-positive bacteria are more susceptible than Gram-negative bacteria. Thus Jones and his colleagues (1943) inhibited $30 \times 10^{6}$ pneumococei with $4 \mu \mathrm{g}$ of the acid, $5,000 \mathrm{Cl}$. welchii with $40 \mu \mathrm{g}$, and only 500 Bact. coli with $30 \mu \mathrm{g}$.

Citrinin. Citrinin is a chemically defined, yellow substance, readily obtainable in large quantities from Penicillium citrinum (Hetherington and Raistrick 1931, Coyne, Raistrick and Robinson 1931). It is inhibitory for Gram-positive bacteria and, to a lesser extent, for Gram-negative bacteria (Oxford 1942a).

Clavacin. (Claviformin, clavatin, patulin). Clavacin is a water-soluble, acidie substance from Aspergillus clavatus. It is bactericidal, moderately bacteriostatic in low concentrations ( $1: 50,000$ to $1: 500,000)$, and is active against Gram-positive and Gramnegative bacteria. It is toxic to animals (Waksman, Horning and Spencer 1943). The same substance was isolated from Penicillium claviforme as claviformin (Chain, Florey and Jennings 1942); and from Penicillium patulum as patulin by Raistrick and his colleagues (1943), who identified it as anhydro-3-hydroxymethylene-tetrahydro- $\gamma$-pyrone2 -carboxylic acid. They reported that its application to the nasopharyngeal mucosa of man was beneficial in the treatment of the common cold. Stuart-Harris, Francis and Stansfeld (1943) and Stansfeld, Francis and Stuart-Harris (1944) could not eonfirm this, nor conld a specially appointed Committee of the Medical Research Council (Report 1944). A similar substance was oltained from Aspergillus clacatus (Bergel et al. 1943, Hooper et al. 1944), and from Aspergillns giganteus (Florey et al. 1944). Chain, Florey and Jennings (1944) identified elaviformin with patulin, and found it to be more toxic to leueocytes and tissues than to bacteria. Katzman and his colleagues (1944) eonfirm the identity of clavacin and claviformin, while Waksman (1944) claims that fumigacin and helvolic acid (sce below) are also identical with clavacin.

Flavacin. A chemically undefined, organic acid from Aspergillus farus, which is bacteriostatic for Gram-positive bacteria, but has little effect on Gram-negative bacteria. In purified preparations it is moderately toxic to mice; and fom limited experimental tests appears to protect mice infected with Type I pneumococei (Bush and Goth 1943).

Fumigacin. Fumigacin is a chemically undefined substance from Aspergillus fumigatus, soluble in alcohol, sparingly soluble in water. It is active mainly against Grampositive bacteria, and is bacteriostatic in low, bactericidal in high concentrations; it is fairly toxic to animals (Waksman, Horning and spencer 1943).

Fumigatin. Fumigatin, first isolated from Aspergillus fumigutus by Anslow and Raistrick (1938), has since been synthesized (Baker and Raistrick 1941). It is a quinone with the molecular structure 3 -hydroxy-4-methoxy-2:5-toluquinone. It is strongly antibacterial, particularly against $V$. cholerce, B. anthracis and Staph. aureus (Oxford and Raistrick 1942 ; see Spinulosin below).

Gigantic Acid. An antibacterial substance of the penicillin type from Aspergillus giganteus (Philpot 1943).

Gliotoxin. A highly toxic, erystalline, sulphur-containing antifungal and antibacterial substance, with a provisional formula $\mathrm{C}_{14} \mathrm{H}_{16} \mathrm{~N}_{2} \mathrm{~S}_{2} \mathrm{O}_{4}$, found in Gliocladium fimbriatum and certain other fungi (Weindling and Emerson 1936, Weindling 1937, 1941, Waksman and Woodruff 1942).

Helvolic Acid. A crystalline substance, with a provisional formula $\mathrm{C}_{32} \mathrm{H}_{44} \mathrm{O}_{8}$. from a variety of Aspergillus fumigatus. It has little action on Gram-negative bacteria, but inhibits Gram-positive cocci and clostridia in high dilution. Bacteria become resistant by serial subculture in media containing low concentrations of the acid. Its activity is not markedly inhibited by blood or serum, and bacteriostatic concentrations are attained in the animal body without untoward toxic effects. 
In experimental streptococcal and staphylococeal infections, its chief effect was to prolong survival time, but not to prevent death (Chain, Florey, Jennings and Williams 1943).

Penatin. (Notatin). Penatin, as well as penicillin, is produced in cultures of P. notatum. An acid medium favours the production of penatin, which can be detected by using Bact. coli (which is insusceptible to penicillin) as the indicator organism in titrations of bacteriostatic power. Penatin is a relatively stable, water-soluble substance, active against Gram-positive and Gram-negative bacteria in dilutions of $1: 12.5 \times 10^{6}$ to $1: 250 \times 10^{6}$. Serum does not interfere with its action. It is active only in the presence of glucose, which it decomposes with the production of $\mathrm{H}_{2} \mathrm{O}_{2}$. In animals, it is moderately toxic, with local reactions at the site of injection (Kocholaty 1942, 1943a,b). The same substance was described by Coulthard and his colleagues (1942) under the name of notatin. It is a glucose-aerodehydrogenase, with the properties of a flavoprotein. The presence of oxygen and glucose, and the absence of a catalase, are necessary for its antibacterial activity, which is mainly due to the $\mathrm{H}_{2} \mathrm{O}_{2}$ produced in these circumstances (Birkinshaw and Raistrick 1943). A similar substance, Penicillin B, was extracted from cultures of $P$. notatum by Roberts and his colleagues (1943). Like notatin, it was a flavoprotein, producing $\mathrm{H}_{2} \mathrm{O}_{2}$ from glucose, but unlike notatin, the glucose could be replaced by xylose, maunose or galactose (van Bruggen et al. 1943). It is noteworthy that a milk flavoprotein has been described, whose antibacterial action also depends on its power to produce $\mathrm{H}_{2} \mathrm{O}_{2}$ from the medium containing the bacteria (Green and Pauli 1943).

Penicidin. Penicidin is a relatively stable, diffusible substance, found in a number of penicillia, bactericidal for Salm. typhi at 1: 100,000. Among the penicillia so far tested, there appears to be a negative association between the capacity to produce penicidin and penicillin-like substances. Penicidin activity, unlike that of penatin, is not suppressed under anaerobic conditions, but it is suppressed by - $\mathrm{SH}$ compounds (Atkinson 1942, 1943b, Atkinson and Stanley 1943).

Penicillic Acid. A chemically defined, water-soluble, aliphatic keto acid from Penicitlium cyclopium. In the animal body it has about the same toxicity as phenol, but has about 100 times as good an antibacterial action on Staph. aureus and Bact. coli, and it may therefore prove to be chemotherapeutically useful (Oxford, Raistrick and Smith 1942, Oxford 1942a). Penicillic acid, and certain bacteriostatic quinones (see spinulosin, below) are antagonized by peptone and certain amino-acids, as the result of combination between the antibacterial agent and the antagonizer (Oxford 1942c).

Penicillin. A water-soluble substance from $P$. notatum, active mainly against Grampositive bacteria (see below).

Proactinomycin. A chemically undefined, relatively stable substance, from a Proactinomyces. Bacteriostatic titres range from $1: 1.5 \times 10^{6}$ for pneumococci, $1: 5 \times 10^{5}$ for Staph. aureus and Str. pyogenes, to $1: 2 \times 10^{3}$ for Gram-negative intestinal bacilli. It is more toxic than penicillin to lencocytes (Gardner and Chain 1942).

Spinulosin. A substance isolated from Penicillium spinulosum by Birkinshaw and Raistrick (1931). It is a toluquinone, like fumigatin, differing from it only in possessing an additional hydroxy group in the 6 position. It has the molecular structure $3: 6$-dihydroxy-4-methoxy-2:5-toluquinone. It is weakly antibacterial, and is chiefly of interest in comparison with fumigatin, since the additional hydroxy group is associated with a marked decrease in antibacterial activity (Oxford and Raistrick 1942). A study of a number of toluquinones and benzoquinones revealed that the introduction of the methoxy group $\mathrm{OCH}_{3}$ into the quinone often resulted in an inerease, or of the hydroxy group, in a decrease in activity. The substitution of the methoxy by hydroxy in an active compound also decreased its activity (Oxford 1942b).

Streptomycin. A chemically undefined water-soluble substance from an umidentified species of Actinomyces, apparently related to streptothricin. Like streptothricin, it acts on Gram-negative and Gram-positive bacteria (Schatz, Bugie and Waksman 1944). 
Streptothricin. A chemically undefined substance from Actinomyces lavendulce. It is an organic base, active in low concentrations against Gram-negative and to a lesser extent Gram-positive bacteria (Waksman and Woodruff 1942, Kocholaty 1942, Waksman 1943). Doses sufficient to kill $\mathrm{Br}$. abortus infecting a developing chick embryo were not toxic (Metzger et al. 1942).

Miscellaneous Mould Products. Glister (1941) reports a substance from Aspergillus flavus with a bacteriostatic titre of $1: 200,000$ for Gram-negative bacteria. A citrinin-like substance has been obtained from an Aspergillus of the candidus type (Timonin 1942). IIcKee and MacPhillamy (1943) and White (1943) report penicillin-like substances from Aspergillus flavus and Aspergillus flavipes, and recently Florey and his colleagues (1944) have obtained penicillin-like substances from five species of penicillia, namely, P. fluorescens, rubens, avellanum, baculatum, and turbatum.

\section{Penicillin.}

We have already discussed the circumstances that led to Fleming's discovery of this remarkable substance, and his adumbration of its significance in the therapy of infection. Its production in bulk, its concentration in a stable, highly active form, the methods of its assay, and the definition of its powers and limitations in the therapy of animal and human infection, we owe to the work of Florey and his colleagnes (Chain et al. 1940, Abraham et al. 1941). In their hands penicillin proved to be outstanding among antibacterial agents.

It is active in low concentrations, the growth of Strph. aureus being inhibited by 1 part in 50 millions of a highly purified preparation. It has little toxicity for animal tissue. Mice weighing $25 \mathrm{gm}$. are unaffected by injection of $20 \mathrm{mgm}$. of the purified preparation. It is active, as Fleming showed, against a wide range of Gram-positive bacteria, and its in vivo activity parallels its in vitro activity. But for the fact that penicillin is rapidly eliminated from the animal body, which necessitates frequent administration to maintain therapeutic concentrations in the tissues, the chemotherapeutic index (the ratio of tolerated dose to curing dose) would be enormous. As it is, in vitro ratios of toxic and bacteriostatic concentrations are very large. For example, human leucocytes will tolerate 250,000 times the concentration of penicillin needed for the total inhibition of Staph. aureus. Florey and his colleagues described penicillin as a weak acid of low molecular weight, forming stable salts with alkaline earth metals, soluble and stable in organic solvents and in water between $\mathrm{pH} 5$ and 7 . They concentrated it from filtrates of cultures of Penicillium notatum by acidification and extraction with chloroform and amyl acetate, and re-extracted the penicillin into water by adjusting the $\mathrm{pH}$ to neutrality. The yield of penicillin is relatively small. For example, 100 litres of culture filtrate yielded $1 \mathrm{gm}$. of a material inhibiting Staph. aureus in a dilution of 1 in $10^{6}$ (Abraham et al. 1941). Crude preparations, containing about 1 per cent. of penicillin, inhibited Gram-positive organisms in low concentrations and were only slightly toxic to animals and man. The chief toxic effect was the induction of rigors in man, and was due to an impurity which was later removed by more elaborate methods of preparation.

Penicillin is destroyed by dilute acid and alkali, by heavy metals, by primary amines, primary alcohols, ketonic reagents and oxidising agents. It is most conveniently handled as a salt of sodium, calcium or barium.

Recent improvements in the production of penicillin have included methods of counteracting the accumulation of acid in the growing cultures, for acidity depresses the penicillin yield. This may be achieved by the use of buffers (Challinor and McNaughtan 1943), 
or by the addition to the medium of traces of zinc, which, by catalysing the complete oxidation of the dextrose, prevents the accmmulation of gluconic acid, a product of incomplete oxidation of the dextrose (Foster, Woodruff and MeDaniel 1943). P. notatum throws variants during its growth in stock cultures, and it is essential to maintain a master culture of high penicillin-producing capacity. In fluid cultures, $P$. notatum grows as a felt on the surface of the medium, with consequent limitations in the exposure of the growing mould to air and to the nutrients in the fluid. Clifton (1943) introduced a method resembling that used in the commercial process for the rapid production of vinegar, in which the culture is kept continuously trickling over wood shavings packed in an aerated columnar container. Improved methods of fractionation, including eloromatographic methods, have also improved the vield from crude culture filtrates (Abraham, Chain and Holiday 1942).

Bacteriological Aspects. Fleming's original observations on the range of in vitro activity of penicillin have been amply confirmed. Staph. aureus, Str. pyogenes, $N$. meningitidis and $N$. gonorrhoeae, the gas-gangrene clostridia and Actino. bovis are among the most susceptible; pneumococci and Str. viridans a little less so. If we take the amount of penicillin required to inhibit a certain number of susceptible Staph. aureus as mity, the inhibiting dose for these other bacteria lies between 0.25 and 4. C. diphtheria is slightly less susceptible, the dose being from 10-30; for Str. focalis, Salm. enteritidis and Br. abortus and melitensis, it is from 30-100 ; for Proteus vulgaris, Sh. shiga, Past. pestis, 150-500 ; for Bact. coli, aerogenes and friedländeri, 1,000-16,000; and for Myco. tuberculosis, Ps. pyocyanea, L. icterohamorrhagia, V. cholerce, Hamophilus, yeasts and moulds, the imhibiting dose is of this order, or higher (Bornstein 1940, Abraham et al. 1941, Florey and Jennings 1942, Hobby, Meyer and Chaffee 1942a, McKee and Rake 1942 ", McKee, Hamre and Rake 1943, McKee, Rake and Menzel 1944, Helmholz and Sung 1944). Ery. monocytogenes (Listerella) resists doses of 40 (Foley, Epstein and Lee 1944). These figures are for the most part determined for a few strains only, and as might be expected, the inhibiting dose varies from strain to strain in a species. Thus, it may vary up to 25 -fold for pneumococci, and up to 100 -fold for Staph. aureus.

Bacteria may be trained to grow in gradually increasing concentrations of penicillin. By this method the resistance of Staph. aureus may be increased up to a 1,000-fold (Abraham et al. 1941). Rammelkamp and Maxon (1942) reported a 64-fold increase in strains habituated to penicillin in vitro, and up to a 100 -fold increase in strains from four of fourteen patients treated with penicillin (sec also McKice and Honck 1943). Penicillinresistant pneumococei have been induced in vivo by passage through penicillin-treated mice. The resulting strains were resistant in vitro, and remained so after 30 passages through normal mice (Schmidt and Sesler 1943). By similar means, Rake and his colleagues (Rake et al. 1944) induced resistance in Staph. aureus and pneumococci. It should be noted that resistance induced $i$ vivo is not necessarily due to an increased insusceptibility to penicillin, for Rake and his colleagues found that one strain of Staph. aureus, when passed through penicillin-treated mice, gained in mouse-virulence, but its in vitro resistance was unchanged; the strain had clearly adapted itself to the antibacterial action of the mouse tissues, but not to the antibacterial action of penicillin.

Solutions of penicillin exposed to air rapidly lose their potency, owing to contamination by bacteria, micrococei, and bacilli of various kinds that elaborate enzymic substances destroying the penieillin. Abraham and Chain (1940) described a penicillinase in Bact. coli, and a similar substance has been described in paracolon bacilli (Harper 1943), in certain naturally insensitive strains of Staph. aureus (Kirby 1944), and in B. subtilis (Ungar 1944, Duthie 1944). These enzymic substances are found in filtrates, cultures and extracts of the bacteria. They are relatively stable. Their relation to the structure of penicillin and of penicillin-like substances is not yet known. At present, their interest 
is three-fold. In the first place, penicillin must be guarded against their action, during its production and use, by the careful exclusion of all eontaminating bacteria. In the second place, they may be cmployed for the destruction of penicillin in mixtures of penicillin and bacteria, as, for example, in the cultivation of material from penicillin-treated tissues. Finally, their immediate interest lies in the relation of penicillinase production to penicillin resistance. Though only a few observations have been made, it appears that most naturally resistant bacteria of medical importance are not penicillinase-producers; and as regards induced resistance, Rake and his colleagnes (1944) conld find no evidence of penicillinase in Staph. aureus habituated to growth in penicillin broth.

The acquisition of penicillin resistance is not accompanied by increased resistance to certain other antibacterial agents, and vice versa. This holds for sulphonamides (see above) acridines (McIntosh and Selbie 1943b) and helvolic aeid (Chain, Florey, Jennings and Williams 1943). Schnitzer and his colleagues (1943), however, found that Strph. albus strains grown on medium containing $\mathrm{BaCl}_{2}$ had increased in penicillin-resistance to the same extent as penicillin-trained strains.

Preparations of actinomycin A (Waksman and Tishler 1942), notatin (Coulthard et al. 1942, Kocholaty 1943b), and gramicidin (McKee, Rake and Ienzel 1944) are, like penicillin, inhibitory in dilutions greater than $1 / 10^{8}$, but all of them are considerably more toxic than penicillin to animal tissues. None of the other antibacterial agents, including the sulphonamides, has an in vitro activity of this order.

Apart from penicillinase and destructive chemical agents, there are no reports of any substances that antagonize penicillin to any conspicuous extent. It retains its in vitro activity in the presenee of peptone, blood, serum, pus and tissue autolysates (Abraham et al. 1941, Hoblyy, Heyer and Chaffee 1942a, Dawson et al. 1943), though according to Bigger (194t $\iota$ ), some destruction occurs in blood (see also Humphrey 1944).

Modes of Assay. In the absence of chemieally defined penicillin, any measure of penicillin must be arbitrary. The measure in general use, the Oxford unit, is arbitrarily based on the activity of a certain buffered preparation of the substance.

The usual method of assay, which has proved sufficiently accurate for both research and therapeutic purposes, consists in placing measured volumes of a series of dilutions of penicillin into cups formed by open, upright glass cylinders, the lower rims of which are embedded and sealed in the surface of an agar plate inoculated with a standard strain of Staph. aureus. The penicillin diffuses through the agar forming the floor of the cup, and spreads outwards from under the lower edges of the eylinders. Incubation of the agar plate reveals clear circular areas where growth of the Staph. atreus has been inhibited round and under the cup. Within certain limits, the diameter of the zone of inhibition is independent of the number of organisms inoculated; and under standardized conditions the diameter of the inhibition zone produced by high dilutions of penicillin is proportional to the concentration of penicillin (Fig. 28). By this means, preparations of unknown strength may be titrated against a standard preparation. Modifications of the method include cutting circular discs out of the agar plate, and sealing the bottom of the cup so formed with a little molten agar (Fleming 1942); and the use of a sensitive strain of spore-bearing bacillus, which can be preserved more readily than Staph.aureus as a standard dried culture (ef. the cup-plate method of Pose and Miller 1939; see also Wilkins and Harris 1943b, Foster and Woodruff 1944, Heatley 1944).

Potency may also be measured in fluid cultures or in body fluids containing penicillin by noting the dilution that inhibits growth of the test bacterium. The inhibition may be judged by inspection (see Fleming 1942, 1943, Florey and Florey 1943); nephelometric measurements of growth-turbidity (Foster and Woodruff 1943, Foster and Wilker 1943); 


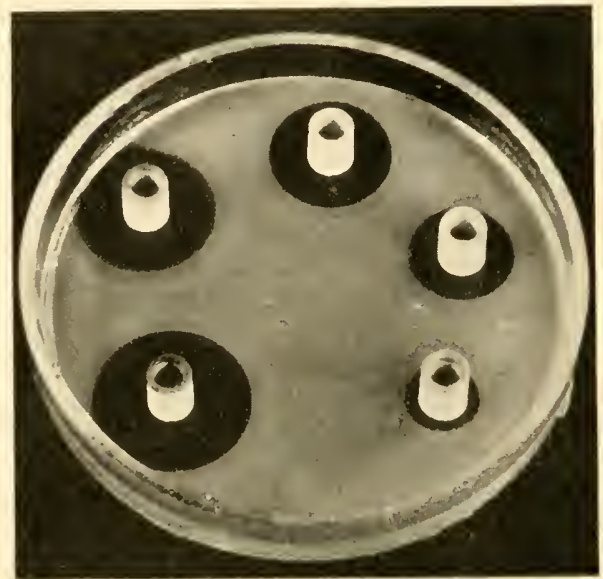

Fic. 28. - The assay of penicillin by the plate methorl.

With falling concentrations of penicillin in the cups, the zone of inhibition of Strph, aureus is progressively less.

(From a photograph kindly supplied by lur. x. G. Heatley.)

or by the absence of some characteristic growth-product of the test organism, for example, the absence of hæmolysin of Str. pyogenes detectable in slide-cells (Wright, Colebrook and Storer 1923) or in culture media (Rake and Jones 1943, Wilson 1943) containing red blood cells; or the absence of acid production in a medium containing an indicator and a carbohydrate fermented by the test bacterimi (Fleming 1942). Strains of species other than Staph. aureus are suitable for assay. Rammelkamp (1942), for example, employed a highly sensitive strain of Str. pyogenes for the measurement of low concentrations of penicillin in small quantities of fluid. (See also Lochhead and Timonin 1943.)

Laboratory Use of Penicillin. The selective action of penicillin in vitro has been fruitfully employed in devising selective media for isolating resistant organisms from mixtures. Following Fleming's original observations on this aspect of penicillin (Fleming 1929) we may note its use for isolation of $H$. influenze and other hæmophilic bacteria (Fleming and Naclean 1930, Fleming 1932, Schoenbach and Seidman 1942): H. pertussis (Naclean 1937, Buxbaum and Fiegoli 1943, Cruickshank 1944) and the acne bacillus (Craddock 1942).

Mode of Action and Constitution. Little is known of the mode of action of penicillin on bacteria. It is bacteriostatic in low concentrations, and appears to be bactericidal in high concentrations. In low concentrations its action is relatively slow (Fleming 1929, Heilman and Herrell 1942). In completely inhibitory concentrations, bacterial respiration may continue for some hours, and some bacteria survive for 24 hours or longer (Abraham et al. 19+1). The rate of killing depends to some extent on the bacterium employed, and for any one strain is relatively constant until some 99 per cent. of the bacteria are dead, after which the numbers of survivors may remain constant, or even increase slightly. Antibacterial activity is marked at $37^{\circ} \mathrm{C}$, slight at $18^{\circ} \mathrm{C}$., absent at $4^{\circ} \mathrm{C}$. The rate increases with diminishing inoculum, and with increasing concentrations of penicillin, though only to a certain point, beyond which the rate does not increase. Within the limits of experimental error, no utilization of penicillin is detectable (Hobby, Meyer and Chaffee 19ł2b). Though a bacterial strain may be trained to 
grow in the presence of penicillin, the immediate survivors of short exposures to peuicillin have not gained in penicillin-resistance. Working with Staph. aureus, Foster and Wilker (1943) could find no change in susceptibility of survivors taken at all stages during growth-inhibition, and concluded that inhibition was due to a prolongation of the average generation time of the culture. With $B$. adhorens, aeration of the culture, which presumably increases the rate of growth of the bacillus, increased the rate of killing.

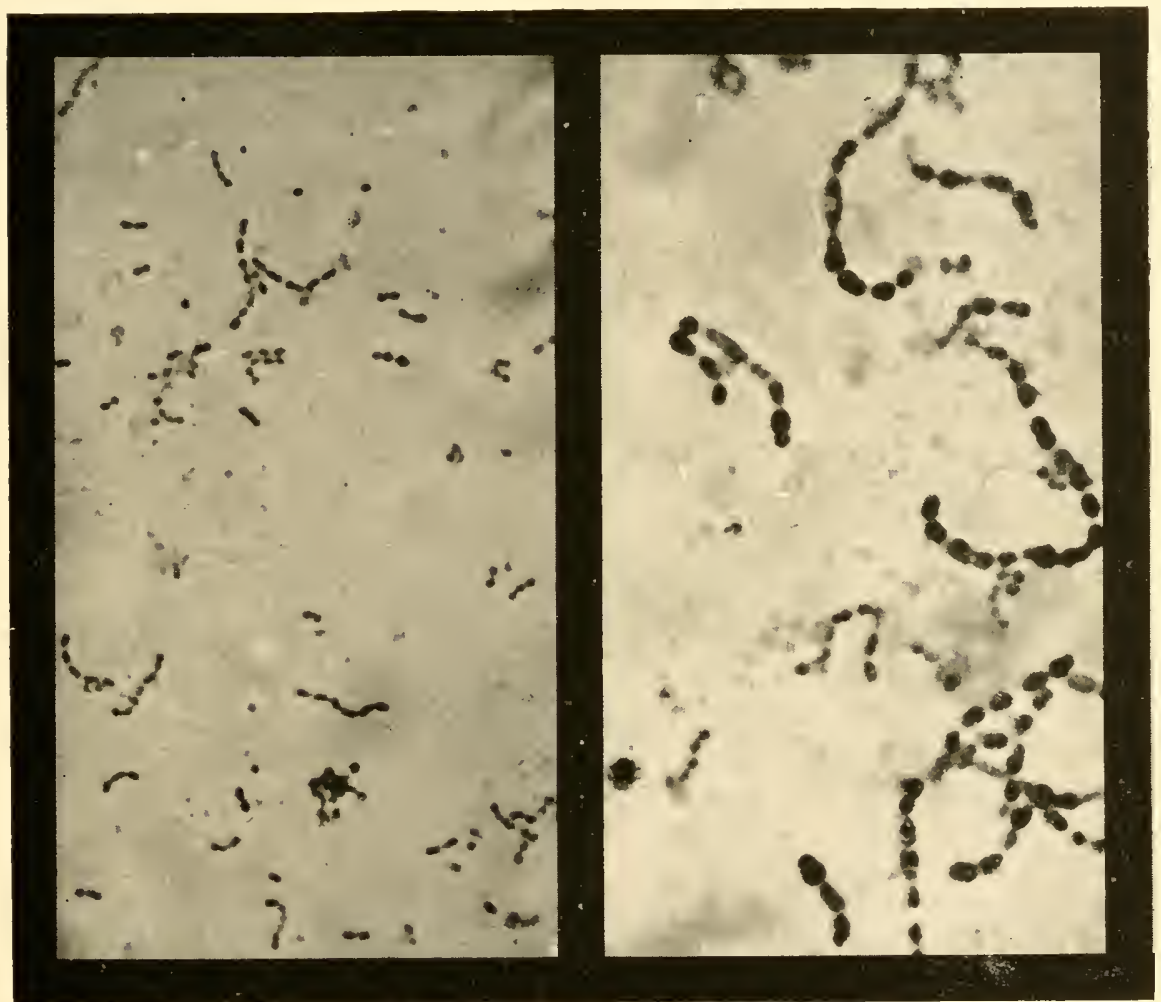

A

B

Fig. 29. - Str. pyogenes grown in media containing: A, no penicillin, and B, sub. bacteriostatic concentrations of penicillin $(\times 1000)$.

(From photographs kindly supplied by Prof. A. D. Gardner.)

Gardner (1940) studied the changes induced in bacteria by growth in a concentration of penicillin 8 to 30 times lower than that causing inhibition. Spherical enlargement and imperfect fission were observed in staphylococci and streptococci, (Fig. 29) and Gram-positive bacilli; and swelling and sometimes bursting in Gram-negative bacilli like Bact. coli and Salmonella bacilli. No morphological changes were seen in penicillin-susceptible meningococci. Gardner concluded that penicillin, like the sulphonamides, caused a failure of fission which led to cellular enlargement and, in some cases, to autolysis. Macroscopically, visible lysis occurs with some cultures of Staph. aureus (Fleming 1929, Smith and Hay 1942, Rantz and Kirby 1944b), and is probably a feature of strains possessing a powerful auto- 
lytic enzyme that is released from penicillin-inhibited cells. Smith and Hay observed that swelling and lysis were associated with active growth, since fully grown suspensions showed neither effect. Rantz and Kirby suggest that staphylococci must divide if penicillin is to be effective, a suggestion also made by Rammelkamp and Keefer (1943b) to explain the prolonged survival of a few bacteria in cultures exposed to inhibitory concentrations of penicillin. Bigger (1941b) concluded from a study of the death rate of large inocula of Staph. aureus in penicillin broth that growing organisms were killed even by low concentrations of the drug. A few cocci, however, survived prolonged exposure to penicillin, but on removal to penicillin-free broth grew freely, and in this state proved to be fully susceptible to the lethal action of penicillin. Bigger suggests that penicillin is bactericidal only for growing organisms; on organisms in the lag phase of growth, it may bave a purely bacteriostatic action, which, by preventing growth, renders the organisms insusceptible to the lethal effect of the drug.

In the absence of more extensive data, it is umprofitable to speculate on the mode of action of penicillin in vitro. If it is generally confirmed that only dividing bacteria are susceptible, it is possible that penicillin may act directly on an essential part of the mechanism of fission; but it is equally possible that it interferes with the metabolism of a substance essential for fission, but one of which the bacterium possesses a reserve, the reserve not being used up until the bacterium has multiplied for a few generations.

Little work has been made public on the constitution of penicillin. The earlier reports (Abraham, Chain and Holiday 1942, Catch et al. 1942, Neyer et al. 1942) deal with the analysis of impure preparations. More recently, two breakdown products of highly active preparations have been described; penicillamine, an amino-acid accomting for over half the total nitrogen of penicillin (Abraham et al. 1943) and penillic acid, produced on acid-inactivation of penicillin (Duffin and Smith 1943). Meyer, Hobby and Dawson (1943) have prepared methyl, ethyl and N-butyl esters of penicillin in the hope of obtaining products more stable and less rapidly excreted than penicillin. The esters were less active than penicillin in vitro, but were active in experimental streptococcal infection in mice both by the subcntaneous and oral routes. They were more toxic than penicillin, and presumably owed their activity to hydrolysis in the animal body, with liberation of penicillin.

Action in the Animal Body. The toxicity of penicillin in therapeutic concentrations appears to be due to impurities, since it varies inversely as the number of arbitrary units per milligram of preparation (Robinson 1913, Hamre et al. 1943) (see also Hobby et al. 1942e, Herrell and Heilman 1943). The mode of administration to man and animals, and the absorption, distribution and excretion have been studied in some detail. Penicilin is ineffective by mouth and per rectum; it is inactivated by the acid of the gastric juice, and by the bacteria of the rectal contents. Given parenterally, it is rapidly excreted and a variahle amount, sometimes up to 75 per cent., is found in the urine. It is not clear whether the remainder is destroyed in the body. Penicillin does not pass easily between the blood stream and serous cavities, and for treating infections of the meninges, pleural cavity, peritoneum and joints, it must be introduced directly (Fleming 1913, Florey and Florey 1943, Pilcher and Meacham 1943).

Like that of sulphonamides, the therapeutic action of penicillin is apparently purely antibacterial, serving to increase the efficacy of the natural defence mechan- 
isms of the body by inhibiting the growth of the infecting organisms. In general, except when the lesions produced by the infecting organisms are inaccessible to the penicillin introduced, the in vivo potency of penicillin parallels the in vitro potency. The first experimental infections successfully treated with penicillin were of mice infected intraperitoncally with Slr. pyogenes and Staph. aureus, and intramuscularly with $\mathrm{Cl}$. septicum (Chain et al. 1940). It was later shown to be effective in mice infected with virulent pneumococei (Hobby et al. 1942c); with N. meningitidis (Dawson et al. 1943); with Cl. welchii (MeIntosh and Selbie 1943a, McKec, Hamre and Rake 1943, Hac and Hubert 1943, 1944); and with Staph. aureus (Powell and Jamieson 1942b). Robinson (1943) was unable to demonstrate any action on mice infected with Myco. tuberculosis, Trypanosoma equiperdum or influenza virus.

Little information is yet available on the effect of penicillin on virus infections. The evidence so far obtained suggests that most viruses are insusceptible, but that two viruses belonging to the lymphogranuloma group (see Chapter 85), namely mouse pneumonia virus and the lymphogranuloma virus itself, constitute possible exceptions to this rule.

\section{REFERENCES}

Abrahali, E. P., Baker, W., Chah, E., and Robinsox, R. (1943) Nature, Lond., 151, 107.

Abraham, E. P'. and Cinaf, E. (1940) Nature, Lond., 146, 837.

Abrahai, E. P., Chain, E., and Holiday, E. R. (1942) Brit. J. exp. Path., 23, 103.

Abrahai, E. P., Gardener, A. D., Chain, E., Heatley, N. G., Fletcher, C. M., Jennings, M. A., and Florey, H. W. (1941) Lancet, ii, 177, 191.

Albert, A. (1942) Lancet, ii, 633.

Albert, A., FalK J. E., and Rubbo, S. D. (1944) Nature, Lond., 153, 712.

Albert, A. and Goldacre, R. (1942) Nature, Lond., 149, 245.

Albert, A., Rubbo, S. D., and Goldacre, R. (1941) Trature, Iond., 147, 332.

Allison, C. S. (1918) J. med. Res., 38, 55.

Anslow, W. K. and Raistrick, H. (1938) Biochem. J., 32, 6s7.

ArdeEY, D. G. (1941) Lancet, ii, 625.

Atkinson, N. (1942) Aust. J.exp. Biol. med. Sci., 20, 287; (1943a) Ibid., 21, 15, 127; (1943b) Mcd.J. Aust., 1, 359.

Atkinson, N. and Stanley, N. F. (1943) Alst. J. exp. Biol. med. Sci., 21, 249, 255.

Baker, W. and RaIstrfek, H. (1941) J. chem. Soc, ii, 670 .

Baker, Z., Harrison, R. W., and Miller, B. F. (19.1) J. cxp. Med., 74, 621.

Barach, A. L., Molonit, N., and Soroka, I. (194:) Amer. Rev. Tubcrc., 46, 268.

BarnetT, J. W. and Robinson, F. A. (1942) Biochem. J., 36, 364.

Bader, H. and Rosenthal, S. M. (1938) Pub. Illh Rep., Wash., 53, .40

Bell, P. H. and Roblin, R. O. (1942) J. Amer. chem. Soc., 64, 2905

Bergel, F. el al. (1943) Nature, Lond., 152, 750.

Berkman, S. and Koser, S. A. (1943) J. infect. Dis., 73, $57^{\circ}$

Besta, B. and livin, H. (1931) Z. IIyg. InfehtKr., 116, 520.

Bierbaum, K. (1912) Dtsch. med. Wschr., 38, 2012.

BrgGer, J. W. (1944a) Lancel, ii, 400 ; (1944b) Ibid., ii, 197.

Birch-Hirschfeld, L. (1934) Z. Hyg. InfektKr., 116, 304.

Bmkinshaw, J. H. and Raistrick, H. (1931) Philos. Trans. B., 220, 245; (1943) J.biol. Chem., 148, 459.

Blair, J. E. and Halluan, F. A. (1943) J. infect. Dis., 72, 246.

Blanchard, K. C. (1941) J. biol. Chem., 140, 919.

Bonner, J. (1942) Proc. U.S. nat. Acad. Sci., Wash., 28, 321.

Bornstein, S. (1940) J. Bact., 39, 383.

Bradbury, F. R. and Jordan, J). O. (1942) Biochem. J., 36, 287.

Brian, P. W. (1944) Nature, Lond., 153, s3.

Browning, C. H. (1943) Brit. med. J., i, 341

Brownlee, G., Copp, F. C., Duffin, W. M., and Tonkin, I. II. (1943) Biochem. J., 37, 572.

Brueckner, A. H. (1943) Yale J. Biol. Med., 15, 813.

Bruggen, J. T. vaN et al. (1943) J. biol. Chem., 148, 365 
Burton, H., McLeod, J. IV., Mcleod, T. S., and Mayr-Harting, A. (1940) Brit. J.exp. Path., 21, 288.

Bush, M. T. and Goth, A. (1943) J. Pharmacol., 78, 164.

Buttle, G. A. H. (1935) Lancet., ii, S40; (1937) Proc. roy. Soc. Med., 31, 154.

Buttle, G. A. H., Gray, W. H., and Stephenson, D. (1936) Lancet, i, 1286.

Buttle, G. A. H., Parish, H. J., McLeod, M., and Stephenson, D. (1937) Lancet, i, 681 .

Buttle, G. A. H., Stephenson, D., Smith, S., Dewing, 'T., and Foster, G. E. (1937) Lancet, i, I33I.

Buxbaum, L. and Fiegoli, N. F. (1943) J. Bact., 46, 543.

Callowon, F. F. T. (1943) Amer. Rev. Tuberc., 4\%, 97.

Сатсн, J. R., Cook, A. H., and Herlbron, I. M. (1942) Nature, Lond., 150, 633.

Chann, E., Florey, H. IV., Gardner, A. D., Heatley, N. G., Jennings, M. A., OrREwine, J., and Sanders, A. G. (1940) Lancet, ii, 226.

Chain, E., Florey, H. W., and Jennings, M. A. (1942) Brit. J. exp. Path., 23, 202 ; (1944) Lancet, i, 112.

Chain, E., Florey, H. IV., Jennings, M. A., and Williams, T. I. (1943) Brit. J.exp. Path., 24, 108.

Challinor, S. W. and MacNaughtan, J. (1943) J. Puth. Buct., 55, 441.

Christensen, H. M., Edwards, R. R., and Piersia, H. D. (1941) J. biol. Chem.. $141,187$.

Christensen, H. M. (1943) J. biol., Chem., 151, 319; (1944) Ibid., 154, 427.

Christison, M. H. (1931) Zbbl. Balt., 131, 193.

Clifton, C. E. (1943) Science, 98, 69.

Clutterbuck, P. W., Lovell, R., and Raistrick, H. (1932) Biochem. J., 26, 1907.

Cokkivis, A. J. (1938) Brit. med. J., i, 1151.

Colmbrook, L. (1928) Spec. Rep. Ser. med. Res. Coun., Lond., No. 119.

Colebrook, L. and KenNy, M. (1936) Lancet, i 1279.

Colebrook, L. and Purdie, A. IV. (1937) Lancet, ii. 1237, 1291.

Cooper, M. L. and Keller, H. M. (1943) J. Pediat., 22, 418.

CoulthaRd, C. E. et al. (1942) Nature, Lond., 150, 634.

Cowles, P. B. (1942) Yale J. Biol. Med., 14, 599.

Coyne, F. P., Paistrick, H., and Robinson, R. (1931) Philos. Trans B., 220, 297.

CradDock, S. (1942) Lancet, i, 558.

Cruickshank, J. C. (1939) Lancet, ii, 681.

Cruickshank, R. (1944) Lancet, i, 176.

Dawson, M. H., Новby, G. L., Meyer, K., and Cifaffee, E. (1943) Ann. interi. Med., $19,707$.

DoMAGK, G. (1935a) Dtsch. med. Wschr., 61, 250; (1935b) Ibid., 61, 829; (1937) Klin. Wschr., 16, 1412; (1943) Dtsch. med. Wschr., 69, 379.

Dorfuna, A. and Koser, S. A. (1942) J. infect. Dis., Y1,

Dorfman, A., Rice, L., Koser, S. A., and Saunders, F. (1940) Proc. Soc. exp. Biol., N.Y., 45, 750 .

Dodglas, S. R. and Colebrook, L. (1916) Lancet, i, 181.

Downs, C. M. (1943) J. Bact., 45, 137.

Dubos, R. (1929) J. exp. Med., 49, 575; (1939) Ibid., \%0, 1, 11.

Dubos, R. J. and Нотснкiss, R. D. (1941) J. ехр. med., 73, 629.

Dubos, R. J., Нотснкiss, R. D., and Coвurn, A. F. (1942) J. biol. Chem., $146,421$.

Duffin, W. M. and SiIth, S. (1943) Nature, Lond., 151, 251.

Duliscoent (1939) Arch. Méd. Pharm. nav., 129, 410.

Duthie, E. S. (1944) Brit. .J. exp. Path., 25, 96.

Ehrisiann, O. (1934) Z. IIyg. Infekthr., 116, 209.

Emuerich, R. and Löw, O. (1899) Z. Hyg. InfektKr., 31, 1.

Emmerich, R., Löw, O., and Korschú, A. (1902) Zbl. Bakt., 31, 1.

Euler, H. vox. (1943) Ber. dtsch. chem. Ges., 75, 1876.

Evans, D. G., Frller, A. 'T., and Walker, J. (1944) Lancet, ii, 523.

Eyster, H. C. (1943) J. cellular comp. Physiol., 21, 191.

FaUlkner, G. H. (1943) Lancet, ii, 38.

Feinstone, W. H., William, R. D., Wolff, R. T., Huntingdon, E., and Crossley, M. L. (1940) Johns Hopk. Hosp. Bull., 6\%, 427.

Feldman, W. H. and Hinshaw, H. C. (1943) Amer. Rev. Tuberc., 48, 256.

Feldian, W. H., Mann, F. C., and Hinshaw, H. C. (1942a) Amer. J. Path, 18, 750; (1942b) Amer. Rev. Tuberc., 46, 187.

FELKE, H. (1938) Klin. Hschr., 17, 13.

Felsenfeld, O. (1943) J. Bact., 45, 25.

Fudes, P. (1940a) Lancet, i, 955; (1940b) Brit. J. exp. Path., 21, 67 ; (1941) Ibid., $22,293$.

Finklestone-Sayliss, H., Paine, C. G., and Patrick, L. B. (1937) Lancet, ii, 792.

Fitzenald, R. J. and Feinstone, W. H. (1943) Proc. Soc. exp. Biol., N.Y., 52, 27. 
Fleming, A. (1929) Brit. J. exp. Path., 10, 2.26; (1932) J. Path. Bact., 35, 831 ; (1942) Lancet, i, 732 ; (1943) Ibid., ii, 434.

Fleming, A. and Maclean, I. II. (1930) Brit. J.exp. Path., 11, 127.

Florey, H. W., Heatley, N. G., Jennings, M. A., and WilliaMs, T. I. (1944) Nature, Lond., 154, 269.

Florey, H. W. and Jennings, M. A. (1942) Brit. J. exp. Path.. 23, 120.

Florey, H. W., Jennings, M. A., and Pillpot, F. J. (194t) Nature, Lond., 153, 139.

Florey, M. E. and Florey, H. W. (1943) Lancet, i, 397.

Foler, E. J., Esstein, J. A., and Lee, S. W. (1944) J. Buct., 4\%, 110.

Fosbinder, R. J. and Walter, I. A. (1939) J. Amer. chem. Soc., 61, 2032.

Foster, J. W. and Wilker, 13. I. (1943) J. Bact., 46, 377.

Foster, J. W. and Woodruff, H. B. (1943) J. Bact., 46, 187 ; (1944) Ibid, 4\%, 43.

Foster, J. W., Woodruff, H. B., and HcDaniel, L. E. (1943) J. Bact., 46, 421.

Fox, C. L. (1942) J. Bact., 43, 68.

Fox, C. L. and Rose, H. M. (1942) Proc. Soc. exp. Biol. N.Y., 50, 142.

Fox, S. W., Fling, M., and Bollenbark, G. N. (1944) J. biol. Chrm., 155, 465.

Francis, A. E. (1938) Lancet, i, 496.

Frisch, A. W. and Price, A. E. (1941) Ann. intern. Wed.. 15, 987.

Fuller, A. T. (1937) Lancet, i, 194; (1942) Biochem. ... 36, .74.

Gardener, A. D). (1940) Nature, lomel., 146, s37.

Gardener, A. D. and Chain, E. (194:) Brit. J. exp. Path., 23, 1:23.

Galse, G. F. and Brazhnikova, 11. G. (1944) Nature, Loml., 154, 71).3.

Glister, G. A. (1941) Nature, Lond., 148, 470.

Goisseidet, P., Despois, R., Cialliot, P., and Mayer, R. (1936) C.R. Soc. Biol., 121, 108:.

Gordon, A. H., Martin, A. J. P., and Synge, R. L. M. (1943) Biochem. J., 3\%, 86, 313.

Gratia, A. (1930) C. R. Soc. Biol., 104, 1058.

Gratia, A. and Dath, S. (1924) C. R. Soc. Biol., 91, 1442; (1925) Ibid., 92, 461, 1125; (1926) Ibid., 94, 1267.

Green, D. E. and Pauli, R. (1943) Proc. Soc.exp. Biol., N.Y., 54, I48.

Green, H. N. (1940) Brit. I. exp. Path., 21, 38.

Green, H. N. and Brelscimowsir, F. (194:) Brit. J. exp. Path., 23, 1, 13.

Gundel, M. and KLiewe. H. (1932) Zbl. Baht., 124, 519.

Hac, L. R. and Hubert, A. C. (1943) Proc. Soc.exp. Biol., N.Y., 53, 111 ; (1944) J. infect. Dis., 74, 164.

Hamre, D. M., Rake, G., Mcliee, C. M., and MacPhillay, H. B. (1943) Amer. J.mer. Sci., 206, 64:.

HARPER, G. J. (1943) Lancet, ii, 569.

Harris, J. S. and KoHs, H. I. (194\%) J. l'harmacol., 73, 353; (1943) J. Immunol., 46, 189.

Heaf, F. R. G., Hurford, J. Y., Eiser, A., and Franklix, L. M. (1943) Lancet, i, 702.

Heatley, N. G. (1941) Biochem. J., 38, 61.

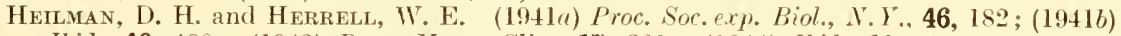
Ibid., 46, 480; (1942) Proc. Mayo. Clin., 1\%, 321 ; (1944) Ibirl.. 19, 57.

Hecmolz, H. F. (1937) Proc. Mayo Clin., 12, 244.

Helmholz, H. F. and Sung, C. (1944) Proc. Mayo Clin., 19, 376.

Henry, R. J. (1943) Bact. Rev.. \%, 175.

Herrell, IT. E. and Heilian, D. (1943) Amer. J. med. Sci., 205, 157.

Hetherincton, A. C. and Raistrick, H. (1931) Philos. Trans. B., 220, 269.

HеттсHе, H. O. (1934) Z. Imm*unForsch., 83, 499.

Hinshaw, H. C., PFuetze, K., and Feldmax, IV. H. (1943) Amer. Rev. Tuberc., 47, 26.

Hinsch, J. (1942) Science, 96, 139 ; (1944) J. Immunol., 48, 199.

Hobiy, G. L., Neyer, K., and Chaffee, E. (1942a) Proc. Soc. exp. Biol., N.Y., 50, 277 ; (1942b) Ibid., 50, 281; (1942c) Ibid., 50, 285.

Hofmiann, C. E. and RahN, O. (1944) J. Bact., 47, 177.

Holiday, E. R. (1942) Brit. J. exp. Path., 23, 115.

HölzL, H. (1941) Z. IIyg. InfektKr., 123, 500.

Hooper, I. R. et al. (1944) Science, 99, 16.

Hотснкiss, R. D. (1941) J. biol. C'hem., 141, 171; (1943) J. Bact., 45, 64.

Нотснкіss, R. D. and Debos, R. J. (1940) Ibil., 132, 791, 793 ; 136, 803; (1941) Ibid., 141, 155.

Housewright, R. D. and Koser, S. A. (I944) J. infect. Dis., 75, I13.

Humphrey, J. H. (1944) Nature, Lond., 154, 765.

INGRAHAM, II. A. (1933) J. Bact., 26, 573.

ISBELL, H. (1942) J. biol. Chem., 144, 567.

Ivánovics, G. (1942) Z. ImmunForsch., 102, 238. 
JAncsó, N. von and JAxesó, H. vos. (1936) Z. ImmunForsch., 88, 275.

JeNsen, K. A. and Schmiт, K. (1942) Z. ImmunForsch., 102, 261.

Jobling, J. W. and Petersen, W. (1914) J. exp. Med., 19, 251, 459; 20, 37, 321, 452.

Jobling, J. W., Petersen, W., and Eggsteix, A. A. (1915) Ibid., 21, 239 ; 22, 129, 141 , $401,568,590,597$.

Joinnson, F. H. (1942) Science, 95, 104.

Johnson, O. H., Green, D. E., and Pauli, R. (1944) J. biol. Chem., 153, 37.

Jones, H., Rake, G., and HanRe, D. M. (1943) J. Bact., 45, 461.

Jones, R. F. (1941) Nature, Lond., 153, 379.

Katzian, P. A. et. al. (1944) J. biol. Chem., 154, 475.

Kensy, M., Johnston, F. D., and Haebler, T. vos. (1937) Lancet, ii, 119.

King, J. 'T. and Henschel, A. F. (1941) Proc. Soc. exp. Biol., N.Y. 4\%, 400.

Kirby W. I. M. (1943) Proc. Soc. exp. Biol., N.Y., 52, 175; (1944) Science, 99, 452. Kirby, W. M. M. and Raxtz, L. A. (1943) J. exp. Med., 7\%, 29.

KLARER, J. (1941) Klin. Wschr., 20, 1250.

KосH, F. E. and KRä山ER, E. (1932) Zu. Baht., 123, 308.

Kocholaty, W. (1942) J. Bact., 44, 469; (1943a) Science, 9\%, 1st; ; (1943b) Arch. Biochem., 2, 73.

Kohy, H. I. and Harris, J. S. (1941) J. Pharmacol., 73, 343.

Kolle, W., Leupold, H., Schlossberger, H., and Hundeshagex, K. (1921) Arb. Inst. exp. Therap. Frankfurt, 14, 43.

Kolmer, J. A. (1926) "Principles and Practice of Chemotherapy." I'hiladelphia.

KRAMER, H. (1935) Z. ImmunForsch, 84, 505.

Kunn, R., Möller, E. F., and Wenot, G. (1943) Ber. chem. Ges., Frunkfurt, \%6, 40.5.

KonN, R. and Schwarz, K. (1941) Ber. chem. Ges., Frankfurt, 74, 1617

Kumler, IV. D. and Daniels, T. C. (1943) J. Amer. chem. Soc, 65, 2190.

Lamanna, C. and Silapiro, I. M. (1943) J. Bact., 45, 385.

Lamper, J. O. and Peterson, W. H. (1941) J. Amer. chem. Soc., 63, 2283.

Lampen, J. O., Underkofler, L. A. and Peterson, W. H. (1942) J. biol. Chem., 146, 277.

Landy, M. and Dicken, D. M. (1942) J. biol. Chem., 146, 109.

Landy, M., Jarkum, N. IT., Oswald, E. J., and Streightoff, F. (1943) Science, $9 \%, 265$.

LANKFord, C. E., Scott, V. and Cooke, IV. R. (1943) J. Bact., 45, 201.

LäUger, P. and AIartin, H. (1943) Schweiz. med. Wschr., 73, 399.

Levaditi, C. and Vaismax, A. $(1935 a)$ C. R. Acad. Sci., 200, 1694; (1935b) C. R. Soc. Biol., 119, 946 ; (1938) Ibid., 12\%, 1428.

Lewis, J. C. (1942) J. biol. Chem., 146, 441.

Lewis, K. H. and SNyDER, J. E. (1940) J. Bact., 39, 28.

Libiy, R. L. and Joyner, A. L. (1940) J. infect. Dis., 6\%, 67.

Lieske, R. (1921) "Morphologie und Biologie der Strahlenpilze." Leipzig.

Lipmann, F., Hotchiss, R. D., and Dubos, R. J (1941) J. biol. Chem., 141, 163.

Lochread, A. G. and Timonin, M. (1943) Canad. J. publ. Hlth., 34, 236.

LOCKWOOD, J. S. (1938) J. Immunol., 35, 155.

Lockwood, J. S., Coвurn, A. F., and Stokinger, H. E. (193s) J. Amer. med. Ass., 111, 2259.

Long, P. H. and Bliss, E. A. (1937a) Canad. med. Ass. J., 3\%, 457; (1937b) J. Amer. med Ass., 108, 32.

Lourie, E. M. and Yorke, IV. (1939) Ann. trop. Med. Parasit., 33, 289, 305.

Lucchesi, P. F. and Gildersleeve, N. (1941) J. Amer. med. Ass., 116, 1506.

Lwoff, A., Nitti, F., T'RéfouëL, J., and Hamox, V. (1941) Ann. Inst. Pasteur, 6\%, 9. LYNCH, H. M. and Lockwood, J. S. (1941) J. Immunol., 42, 435.

IcIlwain, H. (1940) Brit. J.exp. Path., 21, 136; (194la) Ibid., 22, 148 ; (1941b) Biochem. J., 35, 1311 ; (1942a) Brit. J.exp. Path., 23, 95; (1942b) Itid., 23, 265 ; (1942c) Biochem. J., 36, 417 ; (1943a) Brit. J. exp. Path., 24, 203, 212; (1943b) Biochem. J., 37, 265; (1944) Proc. biochem. Soc., Biochem. J., 38, viii.

McIlwain, H. and HawkiNG, F. (1943) Lancet, i, 449.

McIlwain, H. and Hugires, D. E. (1944) Biochem. J., 38, 187.

McIntosh, J. and Selbie, F. R. (1942) Lancet, ii, 750; (1943a) Ibid., ii, 2.24; (1943b) Brit. J. exp. Path., 24, 246.

McInтosн, J. and W'нiтвY, I. E. H. (1939) Lancet, i, 431.

McKee, C. M., Hame, D. M. and Rake, G. (1943) Proc. Soc. exp. Biol., N. Y., 54, 211.

McKee, C. M. and Houck, C. L. (1943) Proc. Soc. exp. Biol., N.Y., 53, 33.

McKee, (. M. and MacPhillamy, H. B. (1943) Proc. Soc.exp. Biol., N. Y., 53, 247.

Mckee, C. M. and Rake, G. (1942a) J. Bact., 43, 645; $(1942 b)$ P'roc. Soc. exp. Biol., N. Y., $51,275$.

Mchee, C. M., Rake, G., Greep, R. O., and Dyke H. B. vax. (1939) Proc. Soc. exp. Biol. N.Y., 42,417 . 
Mickee, C. M., Rake, G., and Menzel, A. E. O. (1944) J. Immunol, 48, 259.

Mlaclean, I. H. (1937) J. Path. Bact., 45, 472.

Nlaclean, I. H., Rogers, K. B., and Flemino, A. (1939) Lancet, i, 562.

MacLeod, C. M. (1939) Proc Soc. exp. Biol., N.Y., 41, 215; (1940) J.exp. Med., 72, 217.

MacLeOd, C. II. and DAdDi, G. (I939) Proc. Soc. exp. Biol., N.Y., 41, 69.

MacLeOD, C. M. and Mirick, G. S. (1942) J. Bact., 44, 277.

McLeod, J. W., Mayr-Harting, A., and Walker, N. (1944) Brit. J. exp. Path., 25, 27.

MakowskY, L. (1936) Zbl. Bakt., 138, 194.

Malfitano, G. (1900) C. R. Acad. Sci., 131, 295 ; (1903) C. R. Soc. Biol., 55, 841.

MANER, R. (1939) Z. Hyg. InfektKr., 122, 249.

Marshall, E. K., Bratton, A. C., Evwards, L. B., and Walker, E. (1941) Johns Hopk. Hosp. Bull., 68, 94.

Marshall, E. K., Bratton, A. C., White, H. J., and Litchfield, J. T. (1940) Johns Hopk. Hosp. Bull., 6\%, 163.

Martin, G. J. and Fisher, C. V. (1942) J. biol. Chem., 144, 289.

MAYER, R. L. (1937) Bull. Acad. Mléd., 11\%, 727.

Mellon, R. R. (1940) Amer. J. med. Sci., 199, 749.

Mletzger, H. J. Waksman, S. A., and Pugit, L. H. (1942) Proc. Soc.exp. Biol., N.Y., 51, 251.

Meyer, K., Chaffee, E., Нobby, G. L., Dawson, M. H., Schwenk, E., and Fleischer, G. (1942) Science, 96, 20.

Meyer, K., Hobby, G. L., and Dawson, M. H. (1943) Proc. Soc. exp. Biol., N.Y., 53, 100.

Miller, B. F., Abrams, R., Dorfian, A., and Klein, M. (1942) Science, 96, 428.

Miller, E., Sprague, J. M., Kissinger, L. W., and McBurney, L. F. (1940) J. Amer. chem. Soc., 62, 2099.

Virick, G. S. (1943) J. exp. Med., 78, 255.

Mloragues, V., Pinkerton, H., and Greiff, D. (1944) J. exp. Med., 79, 431.

Morgentoth, J. and LevY, R. (1911) Berl. klin. Wschr., 48, 1560, 1979 .

МUсн, Н. (1925) Mïnch. mal. Wschr., \%2, 374.

Mlurray, J. F. (1940) S. Afr. med. J., 5, 110.

Neter, E. (1938a) Proc. Soc. exp. Biol., N.Y., 38, 37 ; (1938b) Ibid., 39, 84 ; (1941) J. infect, Dis., 68, 278; (1942a) Proc. Soc. exp. Biol.. N.Y., 49, 163; (19426) science, 96, 209.

Nicolle, M. (1907) Amn. Inst. Pusteur, 21, 613.

Nisz.Le, A. (1930) Arch. IIyg., 103, 124; (1932) Klin. W'schr., 11, 1456 ; (1933) Münch. med. Wschr., 80, 760.

NitTi, F. and Bovet, D. (1935) C. R. Soc. Biol., 119, I277.

Nitti, F., Bovet, D. and Depifre, F. (1937) C. R. Soc. Biol., 124, 16.

OAG, R. K. (1939) Edinb. med. J., 46, 542.

OsGood, E. E. (1942) Arch. intern. IIed., 69, 746 ; (1943) J. Lab. clin. Hed., 28, 953 ; (1944) Canad. med. Ass. J., 50, 1.

Ouyaxg, G. (1941) Proc. Soc. exp. Biol., N.Y. 48, 41.

Oxford, A. E. (1942a) Chem. Ind., 20, 4s ; (1942b) Ibid., 20, 189 ; (1942c) Biochem. J., 36,438 .

Oxford, A. E. and Raistrick, H. (1942) Chem. Ind., 20, I28.

Oxford, A. E., Raistrick, H., and simth, G. (I942) Chem. Ind., 20, 22.

Page, J. E. and Robinson, F. A. (1943) Brit. J. exp. P'ath., 24, 89.

Peters, L. (1943) J. Phurmocol., 79, 32.

Phillips, R. L. and Barnes, L. H. (1942) J. Franklin Inst., 233, 396.

Philpot, F. J. (1943) Nature, Lond., 152, 725.

Pike, R. M. and Acton, E. V. (1942) Proc. Soc. exp. Biol. N.Y., 49, 445.

Pike, R. II, and Foster, A. Z, (1944) J. Bact., 47, 97.

Pilcher, C. and Meacham, IV. F. (1943) J. Amer. med. Ass., 123, 330.

Pittman, 11. (1942) Pub. Hlth. Rep., Wash., 5\%, 1899.

Porter, C. L. and Carter, J. C. (1938) Bot. Rev., 4, 165.

Poston, M. A. and Orgain, E. S. (1942) Amer. J. med. Sci., 203, 577.

Powell, H. M. and Jamieson, W. A. (1942a) Proc. Soc. exp. Biol., N.Y., 49, 387 ; (1942b) J. Indiana St. med. Ass., 35, 361.

Raistrick, H., Birkinshaw, J. H., Michael, S. E., Bracken, A., Gye, W. E., Hopkins, IV. A., and Greenwood, M. (1943) Lancet, ii, 625.

RaIziss, G. W. (1943) Science, 98, 350 .

RAke, G. and Jones, H. (1943) Proc. Soc. exp. Biol., N.Y., 54, 189.

Rake, G., McKee, C. M., Hamre, D. M., and Houck, C. L. (1944) J. Immunol., 48, 271.

Rammelkamp, C. H. (1942) Proc. Soc. exp. Biol., N.Y., 51, 95.

Ranimelkamp, C. H. and Jewell, M. L. (1940) Proc. Soc. exp. Biol., N.Y., 45, 169.

Ramielkamp, C. H. and Keefer, C. S. (1943a) J. clin. Invest., 22, 425 ; (1943b) Ibid., $22,649$.

Rammelkamp, C. H. and Maxon, T. (1942) Proc. Soc. exp. Biol., N.Y., 51, 386. 
Rammelkamp, C. H. and Weinstein, I. (1942) J. infect. Dis., 71, 166.

Rantz, L. A. and Kirbi, IV. M. M. (1944ı) J. Immunol., 48, 29 ; (1944b) Ibid.. 48, 335. Ratner, S., Blancifard, 11., Coivrn, A. F., and Greex, D. E. (1944) J. biol. Chem., 155, 689 .

RePLOH, H. (1937) Z. ImmunForsch., 90, 29.

Report of Patulin Trials Committee. (1944) Lancet, ii, 373.

Rist, N. (1939) C. R. Soc. Biol., 130, 972.

Rist, N. Bцосн, F. and Hamon, V. (1939) Ibid., 130, 976.

Roberts, E. C. et al. (1943) J. biol. Chem., 147, 47.

Robinson, H. J. (1943) J. Pharmacol. $7 \%, 70$.

Robinson, H. J. and Graessle, O. E. (1942) J. Pharmacol., \%6, 316.

Robinson, H. J. and Holitor, H. (1942) J. Pharmacol., 74, 75.

Robinson, H. J. and Waksman, S. A. (1942) J. Pharmacol., 74, 25.

Roblin, R. O., Williais, J. H., Winnek, P. S., and English, J. P. (I940) J. Atner. chem. Soc., 62, 2002.

Rose, H. M. and Fox, C. L. (1942) Science, 95, 412.

Rose, S. B. and Miller, R. E. (1939) J. Bact., 38, 525.

Rosenthal, S. M., Bader, H., and Elvove, E. (1939) Publ. Hlth. Rep., Wash., 54, 1317. Rubbo, S. D. and Grllespie, J. M. (1940) Nature, Lond., 146, 838 ; (1942) Lancet, i, 36.

Rubbo, S. D., Maxwell, M., Fairbridge, R. A., and Gillespie, J. M. (1941) Aust. J. exp. Biol. med. Sci., 19, 185.

SARTORIUS, F. (1924) Zbl. Bakt., 93, 162.

Schatz, A., Bugie, E., and Waksman, S. A. (1944) Proc. Soc. exp. Biol., N.Y., 55, 66.

SCHimiran, O. (1915) Z. ImmunForsch., 24, 167.

Schmelkes, F. C., Wyss, O., Marks, H. C., Judwig, B. J., and Strandskov, F. B. (1942) Proc. Soc. exp. Biol., N.Y., 50, 145.

Schmid, L. H. and Hilles, C. (1940) Proc. Soc. exp. Biol., N.Y., 43, 228.

Schumt, L. H. and SEsler, C. L. (1943) Proc. Soc. exp. Biol., N.Y., 52, 353.

Schmidt, L. H., Sesler, C. L., and DetTwemler, H. A. (1942) J. Pharmacol. exp. Therap., 74, 175 .

Scinitzer, R. J., Camagni, L. J., and Buck, II. (1943) Proc. Soc. exp. Biol., N.Y., 53, 75. Schoenbach, E. B. and Seidman, L. R. (1942) Proc. Soc. exp. Biol., N.Y., 49, 108.

Schoental, R. (1941) Brit. J. exp Path., 22, 137.

Sevag, M. G. and Green, M. H. (1944) Amer. J. med. Sci., 20\%, 686.

Sevag, M. G., Henry, J., and Richardson, R. (1943) Amer. J. med. Sci., 205, 877.

SevaG, M. G. and Shelburne, M. (1942) J. Bact., 43, $41 \mathrm{I}, 421,447$.

Sevag, M. G., Shelburne, M., and Mudo, S. (1942) J. gen. Physiol., 25, 805.

Silverman, M. and Evans, E. A. (1943) J. biol. Chem., 150, 265.

Sмiтн, L. D. and HAY, T. (1942) J. Franklin Inst., 233, 598.

Suith, M. I., Rosenthal, S. M., and Jackson, E. L. (1942) Pub. Hlth. Rep., Wush., 5\%, 1534 .

SNELl, E. E. (1941) J. biol. Chcm., 141, 121 ; (I944) Ibid., 152, 475.

Snell, E. E. and Mitchell, H. K. (1942) Arch. Biochcm., 1, 93.

Spink, W. W., Wright, L. D., Vivino, J. J., and Skegas, H. R. (1944) J. exp. Med., 79, 331.

Stamp, T. C. (1939) Lancet, ii, 10.

Stansfeld, J. M., Francis, A. E. and Stuart-Harris, C. H. (1944) Lancet, ii, 370.

Stearn, A. E. (1930) J. Bact., 19, 133.

Stearn, A. E. and Stearn, E. W. (1924) J. Bact., 9, 49 I.

Stokes, J. L. and Woodward, C. R. (1942) J. Bact., 43, 253.

Stokinger, H. E., Charles, P. C., and Carpenter, C. M. (1942) J. Bact., 44, 261.

Strauss, E., Dingle, J. H., and Finland, M. (1941) J. Immunol., 42, 313.

Strauss, E. and Finland, M. (1941) Proc. Soc. exp. Biol., N.Y., 47, 428.

Stuart-Harris, C. H., Francis, A. E., and Stansfeld, J. M. (1943) Lancet, ii, 684.

Synat, R. L. M. (1944) Biochem. J., 38, 285.

TAMURA, J. T. (1944) J. Bact., 47, 529.

Teply, L. J., Axelrod, A. E., and Elvenjem, C. A. (1913) J. Pharmacol., 7y, 207.

Thrower, W. R. and Valentine, F. C. O. (1943) Lanct, i, 133.

Tillett, W. S., Cambier, M. J., and Harris, W. H. (1943) J. clin. Invest., 22, 249.

Timonin, M. I. (1942) Science, 96, 494.

Tishler, M., Stokes, J. L., Trenner, N. R. and Conn, J. B. (1941) J. biol. Chem., $141,197$.

Tréfou ËL, J., TréfouëL, J., Niți, H., and Bovet, D. (1935) C. R. Soc. Biol., 120, 756.

Tytler, W. H. and LAPP, A. D. (1942) Brit. med. J., ii, 748.

Ungar, J. (1944) Nature, Lond., 154, 236.

VAlentine, F. C. O. and Edwards, A. M. (1944) Lancet, i, 753.

VALKO, E. I. and DUBoIs, A. S. (1944) J. Bact., 4\%, 15.

Vivino, J. J. and Spink, W. W. (1942) Proc. Soc. exp. Biol., N.Y., 50, 336. 
WAGNER, W. (1929) Z. ImmunForsch., 63, 483.

WAKSMAN, S. A. (1941) Bact. Rev., 5, 231 ; (1943) J. Bact., 46, 299 ; (1944) Science, 99, 220.

Waksman, S. A. and Bucie, E. (1943) Proc. nat. Acad. Sci., 29, 282.

WaKsman, S. A. and Geiger, W. B. (1944) J. Bact., 4\%, 391.

Waksman, S. A., Horning, E. S., and Spencer, E. L. (1943) J. Bact., 45, 233.

Waksman, S. A. and Tishler, M. (1942) J. biol. Chem., 142, 519.

Waksman, S. A. and Woodruff, H. B. (1940a) J. Bact., 40, 581 ; (1940b) Proc. Soc. exp. Biol. N.Y., 45, 609 ; (1941) J. Bact., 42, 231 ; (1942) Ibid., 44, 373.

Weiguan , F. and HöLzL, H. (1940) Z. Hyg. InfektKr., 122, 673.

Weindlina, R. (1937) Phytopathology, 2\%, 1175; (1941) Ibiil., 31, 991.

Weindling, R. and Emerson, O. H. (1936) Ibid., 26, 1068.

WELD, J. T. and HitChell, L. C. (1939) J. Bact., 38, 335.

Welsch, M. (1936) C. R. Soc. Biol., 123, 1013 ; (1937) Ibid., 124, 1240 ; 126, 241, 1254 ; (1938) Ibid., 12\%, 347; 128, 795, 1172, 1175; (1939) Ibid., 130, 104, 797, 800; 131, 1296 ; (1941) J. Bact., 42, 801 ; (1942) Ibid., 44, 571.

Welsch, M. and Elford, W. J. (1937) C. R. Soc. Biol., 125, 1053.

Wengatz, H. F., Boak, R. A., and Carpenter, C. M. (1938) J. Bact., 35, 36.

West, R. and Coburn, A. F. (1940) J. exp. Med., \%2, 91.

Wнiтвх, L. E. H. (1938) Lancet, i, 1210.

White, E. C. (1940) Science, 92, 127; (1943) Proc. Soc. exp. Biol., N.Y., 54, 258.

White, E. C. and Hill, J. H. (1943) J. Bact., 45, 433.

WHiте, H. J. (1939) J. Bact., 38, 549.

WiedLing, S. (1941) Science, 94, 389.

Winkins, W. H. and Harris, G. C. M. (1942) Brit. J. exp. Path., 23, 166; (1943a) Ibid., 24, 141; (1943b) Ann. appl. Biol., 30, 226.

Wilson, U. (1943) Nature, Lond., 152, 475.

Wise, B. (1942) J. Pharmacol., 76, 156.

Wolff, L. K. and Julios, H. W. (1939) Ann. Inst. Pasteur, 62, 616.

Woods, D. D. (1940) Brit. J. exp. Path., 21, 74.

Wood, W. B. (1942) J. exp. Med., 75, 369.

Woolley, D. W. and White, A. G. C. (1943) J. exp. Med., "78, 489.

Wright, A. E., Colebrook, L., and Storer, E. J. (1923) Lancet, i, 365, 417, 473. 


\section{CHAPTER 7}

\section{THE ANTIGEN-ANTIBODY REACTIONS}

TнE problems of infection and resistance--the nature of the mechanisms which determine the course of events when a potentially pathogenic parasite gains access to an animal host-will be discussed in later chapters.

Very early in the development of this field of research investigators began to study the reactions which occur when blood, blood serum, or other body fluids are allowed to react in the test-tube with bacteria, or with bacterial products. Almost from the first, the study of these reactions was pursued by many workers without particular reference to the rôle, if any, which they played in the combat between parasite and host; and it soon became clear that they provided a new technique, which could be applied to a variety of biological problems quite apart from the study of disease.

The development of our knowledge of the various reactions which may occur when the blood or serum of a given animal is mixed with various bacteria, bacterial products, foreign cells, or foreign proteins, may be briefly summarized as follows.

In 1888 Nuttall demonstrated that the defibrinated blood of certain animals had the power of killing certain bacteria. Buchner $(1889 a, b, c)$ showed that this bactericidal power was possessed by the cell-free serum, and that it was lost when the serum was heated to $55^{\circ} \mathrm{C}$. for 1 hour.

In 1890 von Behring and Kitasato showed that the serum of animals which had received repeated injections of non-lethal doses of tetanus toxin, or of diphtheria toxin, had acquired the property of specifically neutralizing these toxins, and thus preventing their poisonous effect.

Between 1893 and 1895 Pfeiffer (see also Pfeiffer and Issaeff 1894) recorded the occurrence of bacteriolysis, or granular degeneration followed by partial dissolution, in cholera vibrios and some other bacteria, when these were introduced into the peritoneal cavity of guinea-pigs which had previously received inoculations of killed cultures of the particular bacteria in question. They showed, also, that the substances which determined this bacteriolysis were present in the blood serum, and in other body fluids.

In 1895 Bordet published his classical paper on the properties of the sera of immunized animals. We may note here that, although the idea of increased resistance to infection was implicit in the early conception of the process of immunization, this term soon came to possess a wider meaning. Any animal, into whose tissues has been introduced any antigenic foreign substance, dead or living, and whose serum has, in consequence, gained the property of reacting in some way with that particular substance, is spoken of as having been immunized against it; and such a serum is referred to as an immune serum or an antiserum. Bordet, extending the observations of Buchner, showed that two different substances are 
involved in the phenomenon of bacteriolysis. One of these is present in any normal serum, is inactivated by heating to $55^{\circ} \mathrm{C}$. for 1 hour, and is not increased in amount as the result of immunization. The other, if present in a normal serum, is seldom found in any considerable amount. It appears, or increases greatly in amount, in response to immunization with a particular bacterium, and is not inactivated by heating at $55^{\circ} \mathrm{C}$. He noted in the same paper that, when cholera vibrios are subjected to the action of an immune serum, they often aggregate into clumps before lysis occurs, a phenomenon which had, indeed, been casually recorded by previous observers.

In 1896 Gruber and Durham published the first detailed studies of this aggregation, or agglutination, of bacteria.

In 1897 Kraus made the observation that the addition of filtrates of cultures of the plague bacillus, or of the cholera vibrio, to the serum of an immunized animal led to the formation of a precipitate, and that this reaction, like the reactions of bacteriolysis and agglutination, was specific; that is, the filtrate of a cholera culture reacted with its own, or homologous antiserum, but not with the plague antiserum, and vice versa.

In 1898 Bordet described the appearance of lytic antibodies in the serum of animals which had been injected with the blood of an animal of some other species, and showed that the lysis of the red corpuscles, like the lysis of bacteria, depended upon the interaction of two distinct substances, the thermolabile, ferment-like body, present in normal serum and a specific thermostable substance, developed as the result of immunization, which he referred to as the sensitizing substance. The thermolabile component, which Buchner named alexine, is now generally known as complement. Bordet's paper had far-reaching results, for it substituted the simple technical procedure of observing the lysis of red cells in the test-tube for the more difficult process of determining the death of bacteria. The natural result was that many investigators in this field turned their attention to the phenomenon of hrmolysis, often with the tacit assumption that the data obtained could be transferred by analogy to the interaction of any serum with any bacterium, an assumption which now appears to le largely nuwarranted.

In 1901 Bordet and Gengou showed that the absorption of complement by sensitized bacteria could be demonstrated without relying on the observation of lysis, or other change, in the bacteria themselves. Using many species of bacteria, they found that, if a suspension of a given organism were mixed with the corresponding immune serum in the presence of complement, the removal of complement from the fluid could be demonstrated by the addition of sensitized red cells, which failed to undergo hæmolysis. In the following year Gengou (1902) showed that the presence of organized material was unnecessary, and that the interaction of soluble proteins with antisera prepared against them was associated with a similar absorption of complement.

Denys and Leclef, in the course of experiments on antistreptococcal immunity, which were recorded in 1895, showed that the phagocytosis of bacteria by leucocytes was promoted by an action of the blood serum exerted on these bacteria, and Mennes (1897) confirmed and extended their observations. For some cause, which is difficult to understand in view of the inherent importance of these observations, little attention was paid to them by other workers during the years which immediately followed. In 1902 Leishman again, and independently, demonstrated the effect of serum in stimulating phagocytosis, but did not analyse the mechanism 
involved. In 1903 Wright and Douglas, in an extensive series of experiments, demonstrated that this action of normal serum was due to a thermolabile substance which acted directly upon the bacteria and not upon the leucocytes. To this substance they gave the name of opsonin. Neufeld and Rimpau (1904, 1905) demonstrated the presence of thermostable substances in the blood serum of animals immunized against streptococci and pneumococci, which acted specifically on these bacteria in such a way as to increase the degree to which they were ingested by phagocytic cells. 'T'o these substances they gave the name of bacteriotropins.

Thus, in the earliest years of the present century, we had at our disposal a considerable body of facts with regard to the action of the sera of normal and of immunized animals on bacteria, foreign cells and foreign proteins. Little indeed has been added, during the intervening years, to our knowledge of what may happen when a normal or immune serum is mixed in vitro with the material against which it is active. Investigators during this period have been mainly engaged in trying to discover how these reactions are brought about. On this aspect of the problem a mass of information has been collected; and, although the correlation of the ascertained facts has been a difficult matter, and our understanding of the underlying mechanism of the serum reactions is still far from complete, we seem during the last two decades to have made appreciable progress towards an orderly arrangement of evidence and a generalization of theory, which has resulted in a clearer conception of the processes involved.

Terminology.- With the gradual development of our knowledge of the serum reactions new names have been invented to describe the phenomena observed and to designate the substances which are assumed to be the essential reagents. As in other terminologies which have grown naturally and have never yet been systematized, the terms employed are often ill-defined, and there is much overlapping. There has indeed been a riotous creation of hypothetical entities in the discussion of the available data, and this redundant growth is responsible for many of the obstacles encountered by the student in mastering the facts of a subject, which in its main outlines is not intrinsically difficult. It is, however, quite impossible to dispense with the use of some kind of scientific shorthand, and it is therefore necessary to gain an adequate acquaintance with the terms in common use, and especially to realize their limitations.

It is usual to refer to the substances which make their appearance in the blood serum, in response to the inoculation of foreign substances of various kinds, and which react with these substances, in vitro or in vivo, in some observable way, as antibodies. The foreign substances which, on inoculation into the tissues, stimulate the formation of these antibodies, are referred to as antigens. These two terms may be defined as follows.

An ANTIGEN is any substance which, when introduced parenterally into the animal tissues, stimulates the production of an antibody, and which, when mixed with that antibody, reacts specifically with it in some observable way.

An ANTIBODY is any substance which makes its appearance in the blood serum or body fluids of an animal, in response to the stimulus provided by the introduction of an antigen into the tissues, and reacts specifically with that antigen in some observable way.

The introduction of the antigen into the tissues is usually made by the parenteral route, since the antigen must reach the tissues in an unaltered state. Antigens 
administered via the alimentary canal are either not absorbed, or are partly or wholly disintegrated by the digestive fluids.

It will be noted that an antigen, as defined, has two essential attributes. It must react with the specific antibody, i.e. the antibody produced in response to its entrance into the tissues; and it must have the power of stimulating the production of that antibody. Examples will later be noted of substances which react specifically with a given antibody, without possessing the power of stimulating its formation. Such substances are not true and complete antigens, though they are closely related to this class by the property of specific reaction.

The term antibody, as defined, has also two attributes, that of specific reaction with the antigen, and that of being produced in response to the stimulus provided by the access of that antigen to the tissues. In practice it is not customary to insist on the demonstration of the second attribute. When we prepare an antiserum by inoculation, or find antibodies in the serum of a man or animal suffering from a particular bacterial infection, the condition of specific stimulation may be assumed to have been fulfilled. But there are numerous cases in which we find, in the blood of normal and untreated animals, substances which react with various foreign cells or bacteria in ways which are essentially similar to those observed when we mix an antigen with the specific antibody which has been produced by artificial immunization. It is usual to refer to these substances as " natural " or "normal" antibodies. They may, in many cases, have arisen as the result of natural infection; in others they are probably an expression of the chemical interrelationships between living organisms, which have arisen during the long course of evolution (see Chapter 50).

Having decided upon a generic term for each of the two essential reagents, it became necessary to name in some way the particular substances which were assumed to be involved in the different reactions which were observed. Little being known of their nature, they were named according to what they did, instead of according to what they were. The names given described the reaction observed when they were mixed with the corresponding antigens, the suffix -in being added to the descriptive term. Thus, an antibody which caused lysis was called a lysin, a harmolysin if it acted upon red blood corpuscles, a bacteriolysin if it acted upon bacteria. An antibody which gave rise to agglutination was called an agglutinin, an antibody which caused precipitation a precipitin, and so on.

The actual material, organized or unorganized, with which these antibodies reacted, was in general provided with some well-recognized name; so that one could speak of bacteria, or of red blood corpuscles, or of foreign proteins, as antigens. But it was clearly realized that many of these antigenic materials were highly complex, and that the antigenic property was almost certainly confined to some particular part of the material in question. It thus became customary to use a term, derived from the name of the antibody, to denote the essential part of the antigenic material which functioned as a stimulus to antibody production and reacted specifically with the antibody so formed. This was accomplished by adding the suffix -ogen to the name of the corresponding antibody.

Thus bacteria, when injected into the tissues, act as antigens and stimulate the production of agglutinins; but the particular part of the bacterial substance which provides the specific stimulus is spoken of as an agglutinogen and one bacterium may possess several different agglutinogens.

Although the terms agglutinogen, precipitinogen, etc., were in common use until 
recent years they are now rarely employed. It is usually better, for reasons that are discussed in later sections of this chapter, to rely on the term antigen, allowing the context to make clear which particular antigen or antigenic component is indicated.

These general rules of nomenclature have certain exceptions. When the antigen itself possesses some well-marked biological activity, which is neutralized by the antibody, the antibody is named by adding the prefix anti- to the name of the antigen. Thus, the antibody which neutralizes a toxin is called an ant $i$ toxin, the antibody which neutralizes snake-venom is called antivenin, and so on. In many cases descriptive terms have been retained for the designation of antibodies, instead of coining new names, and this practice has much to recommend it in the present state of our ignorance.

It will be observed that the thermolabile substance, which is present in normal serum and which appears to be the active agent in bringing about the lysis of sensitized cells and the death of sensitized bacteria, demands a separate name. This substance is not an antibody, since it is not increased in amount as the result of immunization, and it must for the moment be regarded as in a class by itself. Its original name of alexine has become very generally replaced by the name complement, which was introduced by Ehrlich. It may be defined as follows.

COMPLEMENT (or ALEXINE) is a thermolabile substance, or complex of substances, present in varying concentrations in the blood serum of most normal animals, which has the property of bringing about the lysis of certain cells and bacteria, in conjunction with certain antibodies which render the cells or bacteria sensitive to its action.

This definition is incomplete, for it begs the question as to whether complement, acting by itself, produces any significant reaction whatever. It describes, however, with sufficient accuracy, the part which complement plays in the particular reactions with which we are concerned.

\section{Theories of the Mechanism of the Serum Reactions.}

Before entering on a detailed discussion of the various serum reactions, it is well to have a general idea of the theories which have been propounded to account for the phenomena observed.

One theory, which has played a prominent part in immunological studies, was propounded by Ehrlich $(1898,1900)$. It has been developed and modified with extreme ingenuity by himself and by his colleagues to meet the demands made upon it by the continuous accumulation of new and often disconcerting facts, which had to be fitted into a structure growing more and more complex, and obviously becoming a little strained, as one new hypothesis after another was added to the central conception.

According to this "side-chain" theory we should regard the cell as being built up of highly complex chemical aggregates, with attached groupings, or side chains, the normal function of which is to anchor nutrient substances to the cell, and in some cases to act upon and modify them, as a preliminary to their incorporation into the essential cell substance. These side-chains, or receptors, thus form the point of contact between the cell substance and any other materials which gain access to the fluids in which the cell is bathed. It is only by gaining attachment to these receptors that substances of the class to which antigens belong can exert any action on the cell, and so stimulate it to activity. In Ehrlich's view the antigens, whatever their nature, attach themselves to these cell-receptors. Since the antigens are in all cases foreign substances, which have no part in the 
normal economy of the cell, the receptors in question are diverted from their normal function. Stimulated by this derangement of its normal mechanism, the cell produces new receptors, of the same type as those thrown out of action; and, acting in accordance with a physiological habit which may frequently be observed in living things, the process is carried to excess. The superfluous receptors are shed by the cell into the surrounding fluids, and it is these shed receptors which

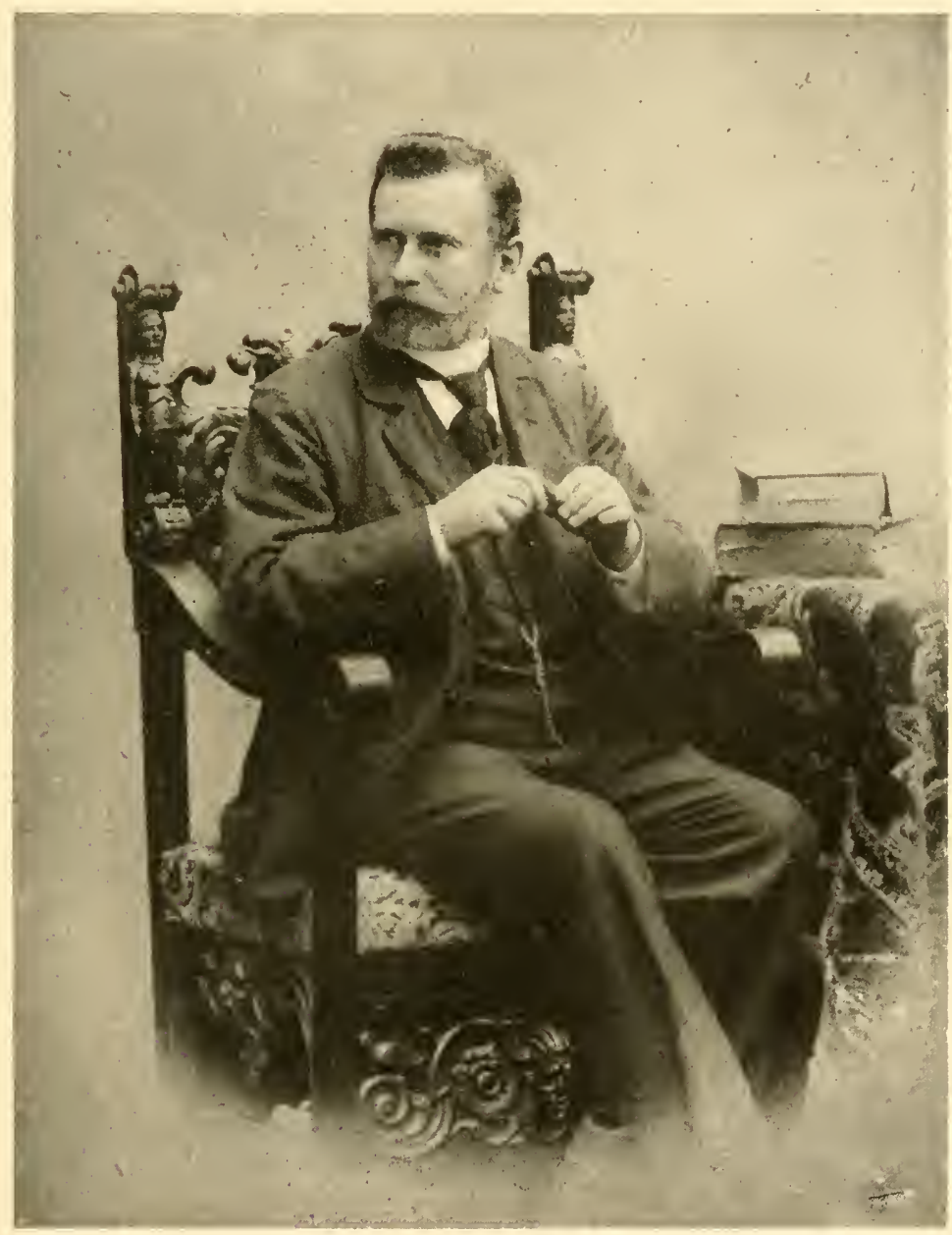

Fig. 30.-Paul Ehrlich (1854-1915).

constitute the specific antibodies. The essential feature of these antibodies is that they unite with the corresponding antigens. Ehrlich therefore endowed each of his receptor's with a special chemical grouping, the haptophore group, which entered into chemical union with a corresponding group of the antigen. If neutralization alone resulted from the union, the requirements were met at this stage. If, however, the antigen became altered in some other recognizable way, as in agglutination 
or precipitation, Ehrlich postulated the existence of another grouping in the receptor, which determined the particular change in the condition of the antigen after the antibody was anchored by its haptophore group. This second, active, group was named by Ehrlich the ergophore group. In certain cases, which we discuss more fully below, it became necessary to postulate the existence of receptors which, while themselves inactive, served to unite an antigen to a second active substance, the complement or alexine to which we have referred above. To meet

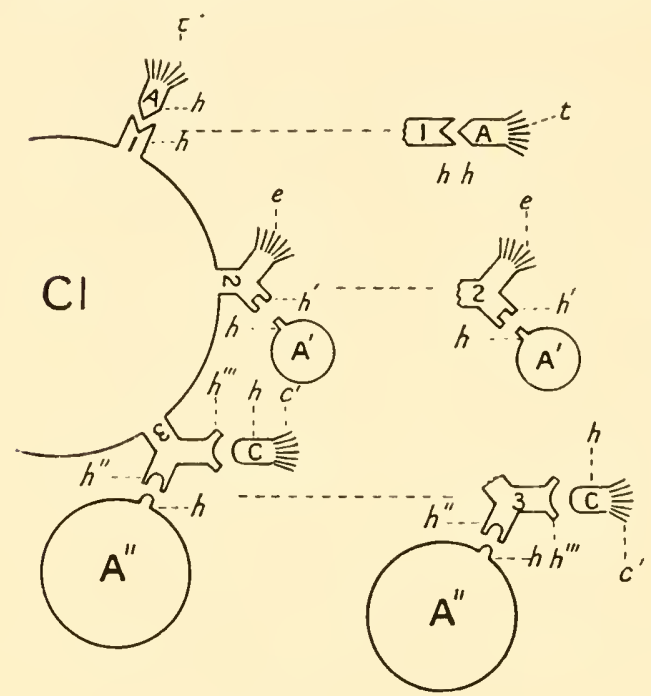

Fig. 31.

Cl. Cell.

1. Receptor of lst order with its haptophore group $h$.

2. Receptor of 2 nd order with its haptophore group $h^{\prime}$ and its ergophore group $e$.

3. Receptor of 3rd order with its two haptophore groups, $h^{\prime \prime}$ the eytophilic group, and $h^{\prime \prime \prime}$ the complementophilic group.

A, A', A". Various antigens, each with its haptophore group $h$; A has in addition an ergophore, or toxophore group $t$.

c. Complement, with its haptophore group $h$, and its ergophore group $c^{\prime}$. this case Ehrlich postulated the existence of receptors with two haptophore groups, one of which became attached to the antigen to be acted upon, and one to the complement which was the acting substance. Both these groups were to be regarded as strictly specific in their chemical affinities. The one which combined with the cell or other antigen to be acted upon was known as the cytophilic group, the one which combined with the complement was referred to as the complementophitic group. Ehrlich named this type of receptor an amboceptor, because both groups were supposed to be of the haptophore type. He also referred these three types of receptors to three orders, the first having a single haptophore group, the second one haptophore and one ergophore group, and the third two haptophore groups.

Fig. 31 gives a diagrammatic representation of Ehrlich's general conception.

Ehrlich regarded these receptors as definite chemical entities which entered into firm union with antigens, or with complement, by linkage of the corresponding haptophore groups. When it was found that such antigens as the toxins could be so treated as to lose their toxicity without losing their power of combining with antitoxin, he assumed that the toxophore group had been altered or destroyed, while the haptophore group remained intact. Similarly he postulated the existence of a modified complement, in which an intact haptophore group was associated with an ergophore group which had lost its functioning power. It will be realized that such a theory lent itself readily to schematic representation of the various reacting substances, and their assumed modifications, and that there was a natural tendency to elaborate the assumed structure, and vary the functional activity of the various groups, to account for new phenomena not readily explicable by the unmodified hypothesis. This is in fact what happened. 
As an instance we may cite the quantitative results obtained in the neutralization of toxin by antitoxin (see pp. 238-240). These could not be reconciled with the sidechain theory in its simple, unmodified form. In order to retain the conception of firm union between antigen and antibody in constant proportions, Ehrlich found it necessary to postulate the existence of a large number of different toxin components with varying degrees of affinity for antitoxin. Similarly, some of the results obtained in his studies on hæmolysins made it necessary to assume the intervention of receptors of considerable structural complexity. The consequent coining of a host of new terms, designating components the actual existence of which was extremely doubtful, served to confuse the problem rather than to clarify it. In spite of these failings, Ehrlich's theory has the outstanding merit that it has kept firm hold on chemical specificity as the essential feature of the antigen-antibody reactions. If we compare his picture of the activities of the living cell with the hypothesis of cellular metabolism referred to on p. 60 we shall note obvious points of similarity; though the studies of recent years have largely modified the cruder and more hazy concepts of cellular processes on which the side-chain theory had of necessity to be built. The strains to which this theory was subjected arose, in the main, from the effort to retain the conception of firm chemical union in constant proportions. We shall sce later the direction in which an escape from this dilemma may be found. Neanwhile we may turn to other theories that have had an important influence on the development of our present views.

An alternative theory, proponnded by Bordet $(1899,1903)$, denies the applicability of the laws of ordinary chemical union to reactions between antigens and antibodies, and regards them as belonging to the class of colloidal reactions, in which the determining conditions are physical rather than chemical. On this view the laws which describe the phenomena associated with adsorption at surfaces and interfaces should be found to hold true, when antigens interact with antibodies. The supporters of this hypothesis have been energetic opponents of the view put forward by Ehrlich and his school, and the output of immunological literature during the first twenty years of the century was largely concerned with this controversy. Bordet's theory makes no attempt to present a complete picture of the mechanism underlying the serum reactions. He himself insisted that his conclusions are so little removed from the facts observed, that they scarcely merit the name of hypotheses. He quite frankly leaves many observed phenomena unexplained, contenting himself with the claim that all the available data are consistent with the limited views he has expressed, while pointing out inconsistencies between these data and the more detailed conceptions of the supporters of the side-chain theory. The essential simplification introduced by Bordet's hypothesis was that, in bringing antigen-antibody reactions into the category of colloidal phenomena, it abandoned the necessity for assuming combination in constant proportions. One colloid may combine with another in proportions that vary over a wide range. This advantage was gained at the initial expense of leaving specificity out of account; but more recent studies of the factors that determine adsorption at surfaces and interfaces (see pp. 263-265) have gone far towards filling this gap.

Arrhenius (1904, 1915), alone and in co-operation with Madsen (1902, 1904), has attempted to retain the advantages of the chemical basis of Ehrlich's theory, while avoiding the necessity of making a fresh assumption to meet each new fact observed. He has suggested that the reaction of an antigen with its antibody is 
not analogous to that between a strong acid and a strong base, but is a reversible action of the type which occurs between weak acids and weak bases, the equilibrium attained in any particular case being determined by the concentration of the reacting substances, in accordance with the law of mass action.

Our present concept of the mechanism of the antigen-antibody reactions is derived, in varying degree, from each of these three theories. Its essential core is provided by Ehrlich's elassical hypothesis, simplified and made more precise by the pioneer chemical studies of Landsteiner and his colleagues (see pp. 252-258). It follows Bordet in postulating the union of antigen with antibody in varying proportions. It adopts the assumption of Arrhenius and Madsen that the antigenantibody compound is dissociable, at least in some cases and under certain conditions.

Before discussing how this synthesis of conflicting theories has been brought about, it will be convenient to give a brief description of each of the antigen-antibody reactions in turn.

\section{The Precipitin Reaction.}

Our knowledge of the mechanism of this reaction has, in the main, been ohtainerl during recent years ; but it forms so convenient a starting-point for a general description of the in vitro reactions between antigens and antibodies that we may with advantage ignore historical succession. In this reaction the antigen, as well as the antibody, is initially in a state of colloidal solution. We can therefore work with chemically purified antigens, such as crystalline egg albumin, or one of the bacterial polysaccharides that will be deseribed in future chapters. In this way we can eliminate the complexities that are introduced by the presence in the reacting system of several different antigenic components. We can also, as Landsteiner and his colleagues have shown (see pp. 252-255), prepare synthetic antigens, the specificity of which is determined by known ehemical groupings, and study their flocculation by antisera prepared against them. Moreover, in the precipitin reaction our data in regard to the quantitative relationships in our reacting system have attained their greatest precision.

Specific, serological precipitation occurs when any antigen in solution is allowed to react with its corresponding antiserum in the presence of electrolytes, provided that the concentration of each of these reagents, and the experimental conditions, such as temperature of incubation and time of incubation, are suitably arranged.

The rate of formation of the antigen-antibody compound, and of its floceulation, varies according to the conditions of the experiment. It is hastened by any procedure that increases the frequency of impact between the molecules, or particles, of antigen and antibody, or between the first-formed particles of the antigenantibody complex (see Eagle 1932). Such procedures include any decrease in the total volume of fluid in the reacting system, with a consequent decrease in the space between the reacting particles, and any factor that increases their rate of movement, such as shaking or convection currents in a tube partially immersed in a waterbath. It is also hastened by increasing the concentration of electrolytes up to an optimal point, beyond which a further increase may cause a retardation. An increase in temperature up to an optimum also increases the speed of floceulation, and it seems doubtful whether this effect is wholly due to an increased frequency of impact. With some reacting systems the higher water-bath temperatures (37-55 (C) may be found unsuitable, since the antigen-antibody compound may be partly soluble in this range. 


\section{The Zone Phenomenon. The Optimal Antigen-Antibody Ratios.}

We may specify the precipitin content of an antiserum by determining the smallest amount that will give a visible precipitate with a standard amount of antigen; and the antigen content of a solution may be similarly specified by the smallest amount precipitating with a standard amount of antiserum. Both these end-point methods, however, have a limited application, because there is no simple relationship between the degree and speed of precipitation, and the relative concentration of the two reactants; the end-points may vary widely with small variations in the conditions of the titration.

If we examine a series of tubes containing a unit volume of an antibody solution, to which unit volumes of graded concentrations of antigen have been added, we shall see an end-point with one of the diluter solutions of antigen. As the antigen concentration increases, the precipitate appears sooner and in greater amount, but beyond a certain point the speed of precipitation and the amount of precipitate falls off with increasing antigen concentration, and a point is finally reached at which there is no precipitation at all.

The characters of this zone of marked precipitation were first investigated in detail by Dean and Webb (1926) using rabbit antisera prepared against horse serum protein antigens. Table 22 illnstrates their results. It shows the degrees of reaction 32 minutes after mixing increasing dilutions of antigen with a constant 1 : 20 dilution of a particular rabbit antiserum.

TABLE $2: 2$

Showing the State of Reacting Mixtures 32 Minutes after mixing a 1 : 20 Dilution of Antiserul with increasinf Dilutions of Antigen (Horse Serum)

\begin{tabular}{|cl|}
\hline bilution of Antigen. & Appearance of Mixture. \\
$1: 10$ & \\
$1: 20$ & Clear. \\
$1: 40$ & Clear. \\
$1: 80$ & Trace of opalescence. \\
$1: 160$ & Opalescence. \\
$1: 320$ & Opalescence. \\
$1: 640$ & Turbid : small particles. \\
$1: 1,280$ & Turbid. \\
$1: 2,560$ & Opalescence. \\
$1: 5,120$ & Trace of opalescence. \\
$1: 10,240$ & Trace of opalescence. \\
$1: 20,480$ & Trace of opalescence. \\
\hline
\end{tabular}

Flocculation is most advanced in the tube containing equal volumes of $1: 20$ antiserum and 1:320 of antigen. In terms of the original rabbit antiserum and the solution of horse serum proteins, the ratio of antigen to antibody in this tube is $1: 16$. Mixtures in which this ratio is departed from, in the direction either of excess or deficiency of antigen, flocculate more slowly or not at all.

Dean and Webb called the antigen-antibody ratio that gave most rapid flocculation, the optimal proportion or the optimal ratio. For a given antiserum and antigen, they fomd that the ratio was constant for all dilutions of antiserum. In the example quoted, repetition of the titration with a $1: 40$ dilution of antisermm gave the most rapid floceulation with a $1: 640$ dilution of antigen, and a $1: 10$ dilution was optimal with a $1: 160$ dilution of antigen. 
These observations have since been confirmed by many other workers, and with a number of different antigen-antibody systems. In these systems, the optimal ratio as determined by Dean and Webb's method is a constant, and the methor affords a reliable method of determining $(a)$ the antibody content of two or more solutions in terms of a fixed amount of antigen, or $(b)$ the antigen content of two or more solutions of antigen in terms of a fixed amount of antibody.

A few examples may make these points clear.

Dean and Webb titrated 33 samples of rabbit-v-horse antisera, and found the optimal ratio varied from $1: 177$ to $1: 14$. Taking the two sera exhibiting these extreme ratios, we see that one part by volume of antigen (horse serum) gave optimal flocculation with 177 and with 14 parts by volume of the two antisera. Since one part by volume of antigen requires a constant amount of antibody for optimal flocculation, this amount must be contained in the one in 177 volumes, and in the other in 14 volumes; and the second serum contains $177 / 14=12 \cdot 6$ times the concentration of antibody in the first.

Taylor, Adair and Adair (1932) have used this method to determine the amount of a given antigen in a naturally-occurring mixture. Thus, they have estimated the percentage of egg albumin in egg white, and the amount of globulin in horse serum, using in each case chemically purified antigens for their titrations. The results obtained were in close agreement with previous determinations, carried out by the usual methods of chemical analysis, as shown by the following comparative figures:

\begin{tabular}{|c|c|c|c|c|c|c|c|c|}
\hline \multicolumn{8}{|c|}{ Percentage of crystalline egg albumin in egg white. } & P'er cent. \\
\hline Hopkins (1900) & . & $\therefore$ & • & . & • & . & . & $6 \cdot 0$ \\
\hline IVu and Ling (1927). & . & : & . & . & • & . & . & $8 \cdot 5$ \\
\hline Optimal ratio method & . & . & . & . & • & . & . & $7 \cdot 29$ \\
\hline \multicolumn{8}{|c|}{ Percentage of globulin in horse serum. } & Per cent. \\
\hline Hammarsten (1878). & & . & . & . & . & . & . & . 4.57 \\
\hline Gibson and Banzhaf (1 & & . & . & . & . & . & . & $4 \cdot 07$ \\
\hline Optimal ratio method & . & . & . & . & . & . & . & $4 \cdot 46$ \\
\hline
\end{tabular}

The validity of the calculations depends among other things on the assumption that there is one type of antibody reacting with a single antigen in solution. With solutions containing two antibodies and two antigens the titration series may yield two distinct zones, or a single zone that is narrow or broad according to the degree of coincidence of two optimally reacting mixtures in the test-tubes. (See, for example Dean, Taylor and Adair 1935, Goldsworthy and Rudd 1935, Pochon 1936).

It will be noted that Dean and Webb, in determining the optimal ratio for flocculation, kept the amount of antiserum constant, and varied the amount of antigen. The ratio determined by this method may conveniently be referred to as the constant-antibody optimal ratio, or constant-antibody O.R.

Prior to these studies, Ramon (1922) had shown that diphtheria antitoxin could be titrated by mixing falling amounts of antitoxic serum with constant amounts of toxin, and noting which tube in such a series first showed flocculation. The fact that this technique was introduced as a practical method of standardizing an important therapentic reagent and that controversy centred round its relation to the current in vivo methods of standardization probably delayed the recognition of its wider theoretical significance. We may conveniently refer to the optimal antigen-antibody ratio, as determined by Ramon's technique, as the constantantigen optimal ratio, or constant-antigen O.R. 
What is the relation between the constant-antibody O.R. and the constantantigen O.R.? The obvious expectation would be to find them identical ; but in fact they may be very different.

Duncan (1932a), working with a crude polysaccharide from a yeast-like fungus and the corresponding antiserum, records a constant-antibody $O . R$. of $1: 8$ and a constant-antigen O.R. of $1: 64$, the latter corresponding to a mixture containing a concentration of antibody eight times greater than the former. Taylor (1933), working with crystalline egg albumin as an antigen, and a considerable number of different antisera prepared by the injection of rabbits, found that the antibody concentration at the constant-antigen O.R. was approximately $1 \cdot 65$ times the concentration at the constant-antibody O.R. Noreover, ten antisera, tested by both methods, gave figures that did not fluctuate widely about this mean value. It would appear that, for any given system, the constant-antibody O.R., the constantantigen O.R., and hence the ratio of one to the other, are constant values, thongh the constant-antibody O.R. may differ widely from the constant-antigen O.R. In different systems, however, the relation of one optimal ratio to the other may vary over a considerable range.

To make the relation between the two optimal ratios quite clear, we may consider an actual titration, in which the times of first flocculation are recorded for a series of mixtures of antigen (horse serum) and antiborly (rabbit-v.-horse antiserum). The figures, for which we are indebted to our colleague Dr. J. C. Cruickshank, are set out in Table 23. Dilutions of antigen are given in the top row, dilutions of antiserum in the first column. Times are recorded in minutes. Each row corresponds to a constant-antibody titration, each column to a constant-antigen titration. The shortest time in each row which corresponds to the constant-antibody O.R. is underlined with a single line. The shortest time recorded in any column corresponding to the constant-antigen O.R. is doubly underlined. Where the shortest time in any column is the first recorded figure it is not underlined The blank spaces in the table correspond to tubes in which no visible flocculation occurred during the period of observation. It will be noted that they fall in the zone of gross antigen excess.

TABLE 23

Showing the Floceulation Time in Minutes of Different Mixtures of an Antigen (HoRse SERuM) With its Homologous Antibody

\begin{tabular}{|c|c|c|c|c|c|c|c|c|c|c|c|c|c|c|}
\hline \multirow[b]{2}{*}{$\begin{array}{c}\text { Antibody } \\
\text { Dilu- } \\
\text { tions. }\end{array}$} & \multicolumn{14}{|c|}{ Antigen Dilutions. } \\
\hline & \begin{tabular}{l}
8 \\
\hdashline \\
-7
\end{tabular} & $\begin{array}{l}10 \\
\because \because \\
-1\end{array}$ & $\begin{array}{l}\stackrel{8}{ } \\
\dddot{-1} \\
-1\end{array}$ & \begin{tabular}{l}
$\stackrel{9}{2}$ \\
\hdashline \\
\hdashline
\end{tabular} & $\begin{array}{c}8 \\
: ! \\
-1\end{array}$ & $\begin{array}{l}\stackrel{8}{\circ} \\
\because \\
-\end{array}$ & \begin{tabular}{l}
$\mathscr{8}$ \\
$\dddot{\sim}$ \\
\hdashline
\end{tabular} & \begin{tabular}{l}
8 \\
8 \\
\hdashline-1
\end{tabular} & \begin{tabular}{l}
8 \\
\hdashline \\
\hdashline \\
$\ddot{\infty}$
\end{tabular} & $\stackrel{8}{\&}$ & $\begin{array}{l}\mathscr{B} \\
- \\
- \\
-\end{array}$ & \begin{tabular}{c}
$\stackrel{8}{7}$ \\
\hdashline$i$ \\
\hdashline
\end{tabular} & $\begin{array}{l}8 \\
81 \\
8 \\
\because \\
\because\end{array}$ & $\begin{array}{l}\stackrel{\Xi}{\vec{b}} \\
\ddot{+} \\
\ddot{-}\end{array}$ \\
\hline $1: 2 \cdot 5$ & $2 \frac{3}{4}$ & $2 \frac{1}{2}$ & $2 \frac{1}{4}$ & $2 \frac{1}{4}$ & $2 \frac{1}{2}$ & $2 \frac{3}{4}$ & $3 \frac{1}{4}$ & 5 & 6 & $10 \frac{1}{2}$ & 17 & 29 & 60 & 110 \\
\hline $1: 5$ & 4 & $3_{4}^{3}$ & $3 \frac{1}{2}$ & 3 & 23 & $3 \frac{1}{4}$ & $3_{4}^{3}$ & $5 \frac{1}{2}$ & 7 & 10 & 14 & 25 & 40 & 50 \\
\hline $1: 7 \cdot 5$ & 8 & $6 \frac{1}{2}$ & 6 & $5 \frac{1}{2}$ & $4 \frac{3}{4}$ & $4 \frac{1}{2}$ & $5 \frac{1}{4}$ & 6 & $7 \frac{1}{2}$ & $10 \frac{1}{2}$ & 12 & 20 & 33 & 45 \\
\hline $1: 10$ & $14 \frac{1}{2}$ & 13 & 12 & $9 \frac{1}{2}$ & 8 & $6 \frac{1}{4}$ & 6 & $6 \frac{1}{2}$ & $8 \frac{1}{2}$ & 11 & 13 & 16 & 28 & 40 \\
\hline $1: 15$ & 53 & 27 & $23 \frac{1}{2}$ & 17 & 15 & $11 \frac{1}{2}$ & 8 & $7 \frac{1}{2}$ & 10 & 13 & $14 \frac{1}{2}$ & $18 \frac{1}{2}$ & 25 & 38 \\
\hline $1: 20$ & 134 & 60 & 39 & 34 & 30 & 25 & 19 & $15 \frac{1}{2}$ & $13 \frac{1}{2}$ & 16 & $18 \frac{1}{2}$ & $2: 2$ & 28 & 35 \\
\hline $1: 30$ & - & - & 102 & 62 & 55 & 50 & 35 & 28 & 22 & 20 & 23 & 25 & 30 & 41 \\
\hline $1: 40$ & - & - & - & 120 & 83 & $7 \ddot{2}$ & 6.5 & (ii) & 50 & 40 & 38 & 42 & 45 & 55 \\
\hline $1: 60$ & - & - & - & - & - & 125 & 100 & 93 & 85 & 68 & $5:$ & 51 & 6.5 & 80 \\
\hline
\end{tabular}


Considering first the rows in the table, we see that the constant-antibody O.R. is $1 / 5: 1 / 200,1 / 7 \cdot 5: 1 / 300$, etc.; that is, $1: 40$. Considering columns, the constant-antigen $0 . R$. is $1 / 5: 1 / 1,200,1 / 7 \cdot 5: 1 / 1,600$ etc. ; that is $1: 240$. In this instance, therefore, the constant-antigen optimal mixture contains six times as much antibody as the constant-antibody optimal mixture. The two optima are on the face of it quite arbitrary, depending on which reagent we vary in comparing reactions taking place in a constant volume. For a 1/30 dilution of antibody, 1/1,200 antigen precipitates faster than any other dilution; but this particular dilution of antigen can be made to precipitate even more quickly by increasing the concentration of antibody to $1 / 5$, when precipitation occurs in 10 , as compared with 20 minutes.

\section{The Two Optimal Ratios and Chemical Equivalence.}

If the precipitates are removed by centrifugation the supermatant fluid remaining may be tested, by the addition of more antibody or more antigen, for the presence of residual antigen or antibody. Dean and Webb (1926) found that at constant-antibody optimum there was neither antigen nor antibody in the supernatant fluid and confirmatory results have been recorded by Taylor $(1931,1933)$, Smith (1932) and Duncan (1932a). Duncan also found that at the constantantigen $\mathrm{O} . \mathrm{R}$. of the system with which he was working the supernatant fluid contained a gross excess of antibody. It seems that in many systems the amounts of antibody and antigens in an optimally reacting mixture, as defined by the constant-antibody titration, are equivalent. For this reason, the constant-antibody optimal ratio is obviously important in determining the nature of the combination between antigen and antibody. However, the Ramon method of titrating antitoxin gives a constant-antigen O.R. and the close agreement between in vitro and in vivo tests indicates that the ratio corresponds to a mixture in which toxin is first neutralized by antitoxin; that is, an equivalent mixture. The fact is, however, not irreconcilable with the generally observed equivalence at the constantantibody O.R., for the two ratios in diphtheria toxin-antitoxin titrations happen to differ very little from one another (see Miles 1933, Boyd 1941, Boyd and Purnell 1944).

The second problem that falls for discussion at this stage is that raised by Bordet's hypothesis. Do antigen and antibody combine in constant proportions, or may they combine in varying proportions, according to their relative concentrations in the reacting mixture? The precipitin reaction affords particularly favourable opportunities for attacking this important problem.

Confining ourselves to the constant-antibody titration, we may distinguish three zones - the central equivalence zone, the zone of antigen excess and the zone of antigen deficiency, which is usually referred to as antibody excess, though this term should properly be used for the constant-antigen titration series. The zone of antigen excess may be further divided into two-a region where a marked excess of antigen totally inhibits precipitation, and a region of moderate excess, where some precipitation occurs. The equivalence zone may be narrow and confined to mixtures in the optimal ratio, or the range of complete precipitation of both reagents may be so broad that the antigen concentration at the antigen excess end of the equivalence zone may be eight times, and is often two or three times, that at the antibody excess end of the zone.

These are the kind of results obtained with rabbit and horse antisera. With other animals, notably the guinea-pig, it is sometimes impossible to obtain any zone of complete 
neutralization of antigen by antibody, a failure perhaps due to a ready dissoeiability of these particular antigen-antibody complexes (Yomans and Colwell 1940, Colwell and Youmans 1941). The nature of the antigen may also affeet the equivalenee point to a marked degree. Thus, Youmans and Colwell (1943), working with rabbit and guinea-pig antisera to whelk hæmocyanins, report that, in eontrast to other antigens, the constantantibody optimal ratio was well in the region of antibody excess, and bore no relation to the antibody content of the sera.

Such a wide range of equivalent combining proportions argues either heterogeneity of the reagents, or multiple combining proportions of homogeneous antibody particles with homogeneous antigen particles. In many of the systems studied, it is probable that the antibody and antigen particles concerned vary either in kind or in combining capacity, but in others the evidence for homogeneity of the reagents is sufficient to permit deductions about the combining proportions of antigen and antibody.

\section{Analysis of Antigen-Antibody Complexes.}

Antibody is a globulin (see pp. 242-246) and the chemical analysis of antigenantibody precipitates yields little information about combining proportions where the antigen is itself a protein of similar gross composition. Heidelberger and Kendall (1929) took advantage of the nitrogen-free purified polysaccharide, obtained from a Type III pneumococcus, to make a quantitative study of specific precipitates. The polysaccharide precipitates with a Type III pneumococcus antiserum, and micro-Kjeldahl estimations of the nitrogen in the precipitates, give a measure of the antibody in combination. In the zone of antibody excess, and in the equivalence zones where no antigen is detectable in the supernatant fluid, the precipitate contains all the antigen. They found antigen-antibody ratios ranging from $1: 125$ with the lowest concentration of antigen used to $1: 69$ at the beginning of the antigen excess zone, and attributed the varying proportions of antigen and antibody to the presence in the precipitates of mixtures of three different compounds, each formed by union of antigen and antibody in constant proportions. That is, symbolizing antibody and antigen by $\mathrm{A}$ and $\mathrm{G}$ respectively, they postulated a series of compounds $A G_{1}, A_{2}, A G_{3}$, etc.

Working with an azo-protein antigen (see pp. 253, 254) whose concentration in the precipitate could be determined eolorimetrieally, Heidelberger, Sia and Kendall (1930) found the antigen-antibody ratios in precipitates from mixtures in antibody excess, equivalence zone and antigen excess were $1: 14 \cdot 1,1: 6 \cdot 7$ and $1: 3$. With hæmoglobin as antigen, Breinl and Haurowitz (1930) found the precipitate might contain from 6 per cent. to 24 per cent. of antigen according to the proportions present in the reacting mixture.

With azo-proteins and iodo-proteins as antigens, Marrack and Smith (1931b) found that the proportions of antigens in the precipitate at the limit of antigen excess was about $I \cdot 5$ times as great as at the equivalence point. It is clear that though antigen and antibody unite to give optimal floceulation only when mixed in one proportion, yet they ean unite in a series of varying proportions. Marrack and Smith $(1931 a, b)$ and Marrack (1934, 1938) would represent the componnds formed by the symbols $\mathrm{A}_{m} \mathrm{G}_{n}$, where $\mathrm{A}$ and $\mathrm{G}$ represent antibody and antigen respectively, and $m$ and $n$ may take any integral values within a certain range.

Heidelberger and Kendall $(1935 a, b, c)$ adopted the conception of union in varying proportions from a quantitative analysis of other precipitin systems. Their results are illustrated in Fig. 32, which shows the relation between the amount of antigen added to a constant amount of antibody in a constant reacting volume, 
to the amount and composition of the precipitate. The antibody excess zone is to the left, the equivalence zone or point is indicated by the arrows, and the antigen excess zone is to the right. As the a mount of antigen added is increased, the amount of antibody in the precipitate rises to a maximum. The maximum is not, as might be expected, attained in the equivalence zone, but increases in the antigen

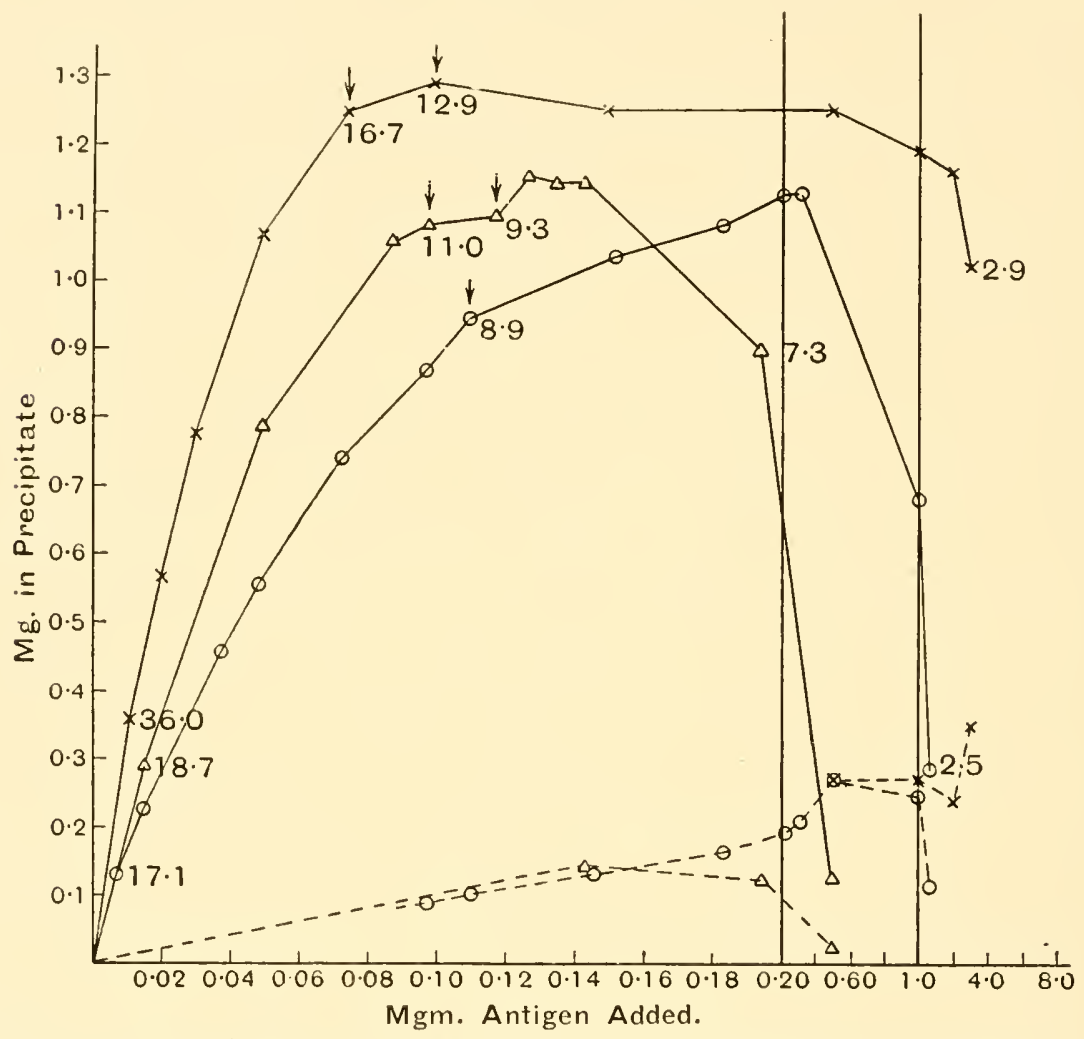

FIG. 32.-Amounts of antiboly nitrogen and antigen in the preeipitate formed from $1 \mathrm{ml}$. of antibody solution, on the addition of the amonnts of antigen shown as abscissa.

Continuons lines antibody nitrogen; broken lines antigen; Type III pneumocoecns polysaccharide (SSS III) in $\mathrm{mgm}$. of polysaceharide.
$x$ SSS III and homologous antibody.
$\odot$ Azo-protein and homologous antibody.
$\triangle$ Egg albumin and homologous antibody.

The arrows show the points at which neither antigen nor antibody was detectable in the supernatant fluids. The figures against points on the curves show the ratio of antibody to antigen in the precipitate at these points.

The scale of the abseissæ is changed twice in order to bring the whole curve into the figure.

From the figures of Heidelberger and Kendall (1935). Office.)

(Reproduced from Marrack (1938) by kind permission of the Author and H.M. Stationary

excess zone. According to tests of the supernatant fluid, all the available antibody was removed from mixtures in the equivalence zone, so that the antibody represented by the extra weight of precipitate in the antigen excess zone must be incapable of forming a precipitate by itself, though capable of entering into combination with antigen and precipitating antibody. The existence of such antibody 
in anti-egrg albumin sera has recently been demonstrated by Heidelberger, Treffers and llayer (1910).

With greater antigen excess, both the amount of antigen and the amount of antibody precipitated fall off, until finally no precipitate forms. We might expect the increase in antibody precipitated, with increase of antigen in the antibody excess zone; the striking feature of the curves is the inhibition of precipitation, partial and total, in the region of antigen excess. This feature is discussed below. We must digress for a moment to consider factors, other than the presence of antigen and antibody in optimal proportions, that determine the flocculation of the antigen-antibody compound.

\section{The Factors that Determine the Flocculation of the Antigen-Antibody Compound.}

It has already been noted that, in the precipitin reaction, both antigen and antibody are in a state of colloidal solution. Both antigen and antibody are hydrophile colloids. That is, they carry at their surfaces groups with a considerable attraction for water, so that each particle is surrounded by a more or less loosely attached "atmosphere" of water, which maintains it in watery solution. If some change occurs that makes these hydrophile groupings inaccessible to water, or decreases their affinity for water, the antigen, or antibody, or both may become hydrophobe colloids, and precipitate from solution. The many features that antigen-antibody precipitation has in common with the non-specific precipitation of colloids suggest that in the former we are dealing with a change from the hydrophile to the hydrophobe state.

We have noted above that electrolytes play an essential part in the precipitin reaction. One effect that they produce is a lowering of the electric charge on the molecules of the antibody or of the antigen or of the antigen-antibody complex. The action of electrolytes has, however, been studied in much more detail in relation to agglutination; and will be more conveniently discussed when dealing with that reaction (see pp. 211, 213).

In most of the precipitating reactions that have been studied, by far the greater part of the precipitate is derived from the antibody (see above). There has, therefore, been a natural tendency to regard the flocculation of the antigen-antibody compound as due to some change in the antibody protein. It has been usual to picture the particles of antigen as becoming covered with antiboly globulin, this globulin then undergoing a change-perhaps becoming denatured-that alters its condition from hydrophile to hydrophobe (see Dean 1917, Eagle 1930, 1932, Boyd and Hooker 1934, Marrack 1938).

Antibody protein, and some protein antigens, contain lipoid substances, extractable by alcohol, ether and other fat solvents. The extraction of lipins in such a way as to avoid denaturing the proteins has a marked effect on antibody proteins.

Hartley (1925) found that ether extraction of rabbit antibody to horse sernm proteins greatly reduced its eapacity to form speeifie precipitates. It seemed that thorough extraction with ether prevented the antibody protein changing from the hydrophile to the hydrozhobe state as the result of antigen-antibody union. That union took place was shown by the action of ether-extracted antitoxic serum, which, though unable to precipitate with diphtheria toxin, nevertheless neutralized it. Horsfall and Goodner $(1935,1936 a, b)$ eonfirmed and extended these observations. They showed that though extraeted horse and rabbit antipneumococcal sera retained their protective power, their agglutinating and precipitating powers were reduced or lost. Small amounts of extracted lipin restored the flocculating powers to the sera. Cephalin restored the activity of 
extracted rabbit, rat, guinea-pig and sheep antisera, and lecithin that of extracted horse, man, mouse, eat, dog and goat antisera. Although it is possible that a small amount of lipin is an essential constituent of antibody globulin (Chow and Goebel 1935), the large amounts often found in specific precipitates are adsorbed non-specifically during precipitation, and are essential neither to the eomplete antibody molecule nor to precipitation.

Native antibody may be a lipo-globulin complex containing this small amount of lipin (see Chow and Goebel 1935). Heidelberger (1939) suggests that the action of lipin is essentially mechanical, providing nuclei round which specific precipitation is initiated.

\section{The Inhibiting Effect on Precipitation of Antigen or Antibody Excess.}

We may now return to the inhibitory effect exerted by antigen or antibody excess, and to the observed difference between the constant-antibody $0 . R$. and the constant-antigen O.R.

Boyd and Hooker $(1934,1938)$ pointed out that the ratio of antibody to antigen in optimal precipitates varied inversely as the molecular weight of the antigen. For example with the Type III pneumococcal polysaccharide, with an estimated molecular weight of 4,000 , the mean ratio was 60 ; with serum albumin of molecular weight 70,200 , the mean ratio was $7 \cdot 4$, and with a molluse hæmocyanin of molecular weight $3,000,000$, the mean ratio was 1.53 . The relation holds with sufficient accuracy over a whole series of precipitin systems. Since the molecular weight of the rabbit and horse antibodies used in the majority of the systems was in the region of 150,000 , it follows from the size of the ratio that the antigen molecules concerned must each possess a number of similar reactive groups capable of combining with antibody. At the optinum, the amount of antibody taken up is consistent with the hypothesis that it forms a layer of contiguous units of antibody protein (see also Kleczkowski 1941a).

We may call the reactive groupings on antigen and antibody "valencies," though, unlike the valency of orthodox chemistry, they are not simple atomic valencies, but combining complexes. On this basis, antigens are multivalent, a fact which may also be deduced from the reaction of antigens with artificially introduced reactive groups. (See p. 262).

There is more doult about the valency of antibody. Analysis of precipitates formed in the presence of a great excess of antigen should help to decide this point by telling us the maximum number of antigen molecules that combine with a single molecule of antibody (see Hooker and Boyd 1942). Unfortunately, for technical reasons, the composition of precipitates in this region is difficult to determine with accuracy, but the available figures indicate that nsually one, and, in rare cases, two, antibody molecules combine with one of antigen. On this hasis, therefore, antibody is usually monovalent, or at most divalent.

\section{The "Two-Stage" and the "Lattice" Hypotheses.}

If we accept the hypothesis of the monovalent antiborly, we are committer to an explanation of the phenomena of precipitation along the lines of Bordet, namely that there is first a stage of specific combination, followed by a second stage of non-specific flocculation depending on the presence of electrolytes and serum lipins. The union of antibody globulin with antigen renders it hydrophobe, and consequently salt-sensitive. The antigen-antibody complexes are forced out of solution, and having more attraction for one another than each separate particle has for water, they cohere and eventually form a visible precipitate. This is the "two-stage" hypothesis.

On the other hand, there are phenomena in precipitation and agglutination 
that are more readily explained by the assumption of multivalent antibody as well as of multivalent antigen. According to this view, first outlined hy Marrack (1934), the specific precipitate consists of antigen particles linked to one another by antibody to form a continuous mass in which antigen and antibody alternate (Fig. 33C). The initial combination and subsequent precipitation as a "lattice" of antigen and antibody, result from binding of the particles at their specific combining sites. This is the "lattice" hypothesis, and it will be seen that its validity depends on the demonstration of multivalent antibody.

Let us see how far the two hypotheses fit the observed facts. The constant-antibody optimum is defined in terms of the velocity of precipitation, which will be determined by differences in concentration, composition and flocculability of the antigen-antibody compound (see Viles 1933).

Given a constant amount of antibody, the speed of flocculation will increase with increasing concentration of antigen, since, in the standard volume used in the titration, there is a greater concentration of antigen particles to act as centres of aggregation, and the speed inrreases until the equivalence zon is reached. The falling off in the speed of precipitation that occurs if the amount of antigen added is greater than the optimal amount is attributed to a reduced precipitability of the compound formed in the presence of excess antigen.

According to Bordet's two-stage hypothesis, the optimal mixture contains enough antibody to sensitize fully a naximum number of antigen particles; in the antigenexcess zone, the available antibody for each antigen particle is insuf-

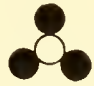

(A)

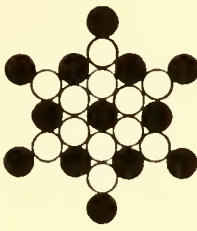

$\left(A_{1}\right)$

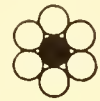

(B)

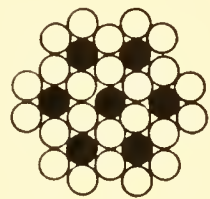

$\left(B_{1}\right)$

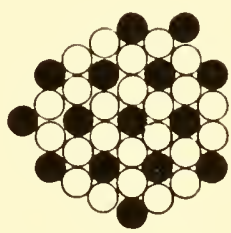

(C)

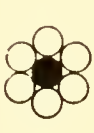

(D)

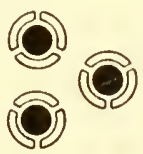

(E)

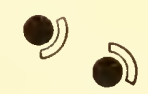

(F)

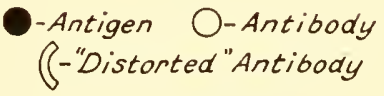

Fig. 33. - Fichematic representation of various antigen-antibody componnds, according to the lattice (A to (C) and the two-stage (I) to F) hypotheses. ficient to sensitize it fully, and with a great excess of antigen, there is no sensitization at all. We shall consider the nature of this sensitization in detail in the section on agglutination. Briefly, the hydrophole nature of the antigen-antibody complex is thought to be due to the distortion of the antibody molecule as a result of its combination with the antigenic surface, with a consequent partial denaturation, and the simultaneous occlusion of hydrophile groups on the surface of the antigen. In the region of antigen excess, then, though there may be maximum distortion of the little antibody that combines with the antigenic particle, sufficient 
hydrophile groups remain to slow, or totally to inhibit, precipitation. The compounds are schematically represented in Fig. 33, where D, E and F represent an antigen molecule after combination in the zones of antibody excess, equivalence and antigen excess.

The compounds formed according to Marrack's lattice hypothesis are also illustrated in Fig. $33 \mathrm{~A}-\mathrm{C}$, which represent two-dimensionally the reaction of a hypothetical hexavalent antigen and trivalent antibody. In gross antigen excess, all three antibody valencies are occupied by antigen; the solution consists of quadrimolecular complexes which cannot combine with one another to form larger complexes because only antigen valencies remain unsatisfied. In a lesser antigen excess, complexes as large as $A_{1}$ are formed; but these cannot aggregate more fully since again only antigen valencies are unsatisfied. $B$ and $B_{1}$ illustrate similarly two complexes formed in antibody excess, and $\mathrm{C}$ represents an early stage in lattice formation at the equivalence point; there are free antigen and antibody valencies for the extension of the lattice indefinitely.

On this view the hydrophobe character of the larger aggregates is ascribed not only to a loss of attraction for water, but also to a specific attraction between the particles or molecules of which the aggregates are composed. This conception, as Marrack points out, is entirely compatible with the dependence of flocculation on the presence of salt in moderate concentration. The action of the electrolyte, in reducing the negative charge on the molecules, or particles, will assist aggregation, whether this be due to a loss of attraction for water or to mutual attraction hetween one molecule, or particle, and another.

Similar arguments apply to the constant-antigen titration. As antibody concentration increases, the antigenic particles are more and more rapidly and fully sensitized, and the speed of flocculation increases. In antibody excess, the complexes formed are more soluble than those at the optimum. The marked inhibitory effect of excess antigen, however, in the constant-antibody titration is not usually paralleled in the constant-antigen titration by inhibition in antibody excess. As we have seen (p. 203), the addition of antibody in excess of constantantigen equivalence produces even more rapid flocculation, so that the constantantigen optimal ratio of antigen to antibody is higher than the equivalent ratio (see also Duncan 1937). Marrack (1934) suggested that if antibody but not antigen were denatured during the combination of the two, compounds like A (Fig. 33) would be soluble, since the antibody is protected by the fully hydrophile antigen, whereas compounds like $B$ would be hydrophobe, since partial denaturation of the antibody at the surface would take place. Moreover, since it appears that antigen has a greater valency than antibody, in antibody excess there will be a greater packing of antibody round the antigen than of antigen round antibody in antigen excess. We have already noticed that in constant-antigen titrations of systems like diphtheria toxin and horse antitoxin antibody excess has a sharply inhibiting effect. Thus precipitation may be completely inhibited by doubling the concentration of antibody that gives optimal precipitation (Healey and Pinfield 1935, Pappenheimer and Robinson 1937). The work of Pappenheimer (1940) suggests that the narrow flocculating zone, sharp inhibition by antibody excess, and the easily elicited Danysz phenomenon of horse antitoxic sera are dependent on the nature of the antibody. Horse antisera to diphtheria toxin and ovalbumin exhibited all three properties, whereas the corresponding rabbit antisera to the two antigens did not. Boyd (1941) came to a similar conclusion from a study 
of the floceulation rates of 20 precipitating antisera. Horse antisera, and some rabbit antisera, gave the two optimal ratios, sometimes very close together. Other antisera, mainly rabbit, gave only a constant-antibody O.R. ; the constant-antigen O.R. was indefinite or absent, the speed of floceulation being approximately constant for all antibody-excess mixtures. Horse antibody is more soluble than rabbit antibody, and the failure of horse-antibody compounds to precipitate in antibody excess may be due to this greater solubility (see Brown 1935).

\section{The Agglutination Reaction.}

The original description of the agglutination reaction by Gruber and Durham (1896) was concerned with the flocculation of bacteria ; but any foreign cells-yeasts and other fungi, red blood corpuscles coming from another species, etc.-will stimulate the formation of agglutinins when injected into the tissues, and will be specifically flocculated in the test-tube under the influence of the antiserum so produced.

The reacting system differs from that concerned in precipitation, in that the antigen is not in colloidal solution but forms part of the structure of an organized cell, usually part of its surface. It is the behaviour of the sensitized cells that we observe, not that of the isolated antigen-antibody compound. As a result, our system frequently becomes highly complex. It is not often that the surface of a bacterial cell is characterized by a single antigenic component. In many cases several different antigens are concerned; and the injection of the bacterial cells into a rabbit, or other animal, will induce the formation of a corresponding number of different antibodies. The problem of the antigenic structure of bacteria is, however, so important that it will be more convenient to deal with it in another chapter. For the moment we may confine our attention to the agglutination reaction as such, merely noting that the possible intervention of a multiplicity of antigens and antibodies is one of the factors that has to be allowed for when trying to interpret any experimental findings.

When observing agglutination we can, if we choose, watch the formation of bacterial aggregates under a microscope. This method was once commonly employed in diagnostic agglutination tests and still has its uses. But in quantitative titrations the macroscopic method, in which bacterial flocculation is observed with the naked eye, or with the aid of a hand lens, is greatly to be preferred.

The reactions that determine the agglutination of bacteria are essentially similar to those that determine the formation of a precipitate when a soluble antigen reacts with its homologous antibody. It was, indee $\hat{i}$, in conneetion with agglutination that the essential rôle of electrolytes was first clearly demonstrated (Bordet 1899). If bacteria are allowed to react with an agglutinating antiserum in a salt-free medium there is usually no aggregation of the bacterial cells. That the antibody under these conditions unites with the antigen may, however, be shown by demonstrating its absence from the supernatant fluid after centrifugation of a mixture in which bacteria are present in excess, or by adding an electrolyte to the resuspended cells, when agglutination occurs (see also Joos 1901, 1902, Bechhold 1904, Porges 1906, Porges and Prantschoff 1906). The concentration of electrolytes is not without effect on the amount of antibody absorbed by bacteria or other cells. In the analogous case of the binding of hæmolysin by red cells, or by red-cell stroma, there is evidence that the amount of antibody bound at or about neutrality ( $\mathrm{pH} 7$ ) is greatly reduced in the absence of electrolytes (Coulter 1920-21, von Euler and Brunius 1931). Changes in $\mathrm{pH}$ also affect the absorption of antibody by antigen, particularly in the absence of salts (Coulter 1920-21, von Euler and Brunius 1931, de Kruif and Northrop 1922-23). Many of these effects probably result from a change in the dissociation constant 
of the antigen-antibody compound; but it is possible that some of them, for instance the lessened absorption of agglutinins by typhoid bacilli at a $\mathrm{pH}$ below 4.0 observed by de Kruif and Northrop, may be due to the destruction or inactivation of the bacterial antigen concerned (sec Duncan 1935).

We are, in the study of such reactions as these, dealing with systems of great physical and chemical complexity, about which we know relatively little. We should therefore be very cautious in ascribing any observed phenomenon to the influence of a particular physical or chemical factor until other possibilities have been satisfactorily excluded.

Before considering the effect of electrolytes, and other factors, on the actual flocculation of bacteria, it will be convenient to note certain quantitative data in regard to the absorption of agglutinins by bacterial cells, when these ancillary factors are kept constant. Here, as elsewhere, the observed relationships are inexplicable on the hypothesis of firm union in simple multiple proportions. For instance, Eisenberg and Volk (1902) showed that, when a constant amount of bacterial suspension was allowed to react with varying concentrations of an agglutinating serum, proportionately more agglutinin was absorbed from the more dilute serum, though absolutely more was absorbed from the more concentrated serum. When a constant dilution of antiserum was absorbed with varying amounts of bacteria, the amount of agglutinin bound did not bear a linear relation to the absorbing dose of bacterial cells. With increasing doses of bacteria, the amount of agglutinin removed became proportionately less. Such a relationship is analogous to that observed in any adsorption process. Craw (1905) noted, in studying the absorption of agglutinin by bacteria, a phenomenon analogous to that described by Danysz in the toxin-antitoxin reaction (see p. 216).

\section{The Zone Phenomenon in Agglutination.}

The system with which we are dealing in the agglutination reaction differs sharply from that concerned in precipitation in that the floccules consist mainly of the antigencarrying material, the concentration of which in the initial mixtures can be judged from measures of opacity, or from actual counts, of the bacterial suspension. For this reason quantitative measures of the agglutination reaction are most conveniently performed by the constant-antigen method, varying the concentration of antisera. The constantantiborly technique is possible, but has the disadvantage that the initial heavy opacity of concentrated antigen suspensions may mask moderate degrees of flocculation. Titration of bacterium-agglutinin systems by both techniques yields optimally flocculating zones, though they are less well defined than precipitin zones. The failure of some bacterial suspensions to agglutinate in the higher concentrations of the dilution series of homologous antiserum, the "pro-zone," has been noted by many workers. In other systems, the inhibition of agglutination is not absolute but can be observed, particularly with light bacterial suspensions (Heuer 1922, da Costa Cruz 1929).

Duncan (1932b) and Miles (1933) studied the constant-antigen optimum and its relation to the constant-antibody optimum. As in precipitin systems, the antibody content of the constant-antibody optimal mixture was greater than that in the constant-antigen optimal mixture. In one system, Duncan found the difference to be six-fold. Equivalence zones cannot be fully defincd, since removing a precipitate by centrifugation in the zones of antigen-excess also brings down unagglutinated antigen, but the equivalence zone appears to cover a wide region of the constant-antigen series, including the optimal mixture.

Shibley (1929) observed the pro-zone phenomenon in antisera that had been exposed to moderate heat, and attributed it to a modification of antibody, whereby it was preferentially absorbed by the bacteria, but had lost its power to sensitize. The probable 
nature of the modification is made elear by the work of Bawden and Kleezkowski (1942b) who showed that, under the influence of heat, a combination hetween the albumins and the antibody globulin of the antiserum occurred. The resulting complex combined with antigen without precipitation and strongly inhibited the reaction between unmodified antibody and the homologous plant-virus antigen. The pro-zones of ummodified antisera are not necessarily due to preferentially absorbed non-agghutinating antibodies, for Miles (1939) sensitized Brucella suspensions in the absence of salt, with both optimal and $16 \times$ optimal amounts of a strongly pro-zoning antiserum, and, after removal of nnabsorber antibody, found that on the addition of saline the rate of flocculation of both suspensions was identical.

As in precipitin titration, multiple zones of maximal flocculation oceur in eertain systems. Miles (1939) demonstrated in a Brucella system two such zones, which appearerl to be due to the reactions of separate smooth and rough antigens on the bacterial surface with their respective antibodies.

The optimal proportions method is not, however, commonly employed in determining the antibody content of an agglutinating serum. In this case, the optimal ratios of any two sera bear the same relation to one another as do the end-points observed when increasing dilutions of the sera are titrated against a constant amount of bacterial suspension; and the end-point is more easily determined.

In performing such titrations the experimental conditions, time and temperature of incubation and so on, must be so arranged that the true end-point is attained. If great accuracy is required, a definite degree of agglutination must be selected as marking this end-point, and this is usually taken to be the least degree of floceulation that can be easily observed by transmitted light against a dark background, when viewed by the naked eye, or with the aid of a hand lens. When the amount of antiserum in successive tubes is halved, as is commonly the ease, it will often happen that no tube shows the standard degree of agglutination. When this is so, a good approximation can sometimes be obtained by using an appropriate interpolation table based on a sufficient series of preliminary experiments (Dreyer and Inman 1917).

\section{The Effect of Salts on the Combination of Antigen and Antibody.}

Turning now to the mechanism concerned in the actual flocculation of bacteria, we may consider first the rôle of electrolytes in this phase of the reaction. The isoelectric point of many bacteria is in the neighbourhood of $3 \cdot 0$; at this $\mathrm{pH}$ the bacterium is not an uncharged particle, but rather a particle with its positive and negative charges balanced. When suspended in neutral solutions of low salt content, the ionization of those groups responsible for the positive charge is reduced, leaving an excess negative charge. Under these conditions, therefore, the bacteria move towards the anode in an electrophoretic cell (see Putter 1921, Northrop 1922, 1928, Winslow et al. 1923 and many subsequent observers). If the $\mathrm{pH}$ is lowered below the isoelectric point, the ionization of the groups responsible for the negative charge is reduced and the bacteria drift towards the kathode.

Agglutination depends in part upon the electrostatic attraction between opposite charges on different bacteria, but at $\mu \mathrm{H}$ values where all the bacteria carry an excess of one charge, this is impeded by the electrostatic repulsion that like charges have for one another. Many observations have been maile on the flocculating effect of salts and acids on bacteria, altogether apart from the action of agglutinating antibodies (Bechhold 1904, Michaelis 1911, Beniasch 1911). The range of 
pH over which acid agglutination oceurs may vary considerably from one bacterial species to another, and has sometimes been employed as a differential criterion, but its usefulness in this respect would seem to be very limited (see Sgalitzer 1914, Buchanan 1919).

The relation of salt agglutination to agglutination by speeific antisera has been studied in some detail by Northrop and de Kruif $(1922 a, b)$. Working with Salm. typhi, they showed that, with those kations that caused a great reduction in surface potential in low concentrations, floceulation of either sensitized or unsensitized bacteria tends to occur when the surface potential is reduced below about 15 mvt. With kations that reduce the surface potential only in higher concentrations unsensitized bacteria are not agglutinated, even when the surface potential is rednced to zero, while sensitized bacteria flocculate when the potential falls below 15 mvt. The effects produced on sensitized and unsensitized Salm. typhi by the trivalent kation $\mathrm{Al} \cdots$, the bivalent kation $\mathrm{Ba} \cdot$, and the monovalent kation $\mathrm{Na}$, are shown in Fig. 34 (Northrop and de Kruif 1922a).

In an attempt to determine why unsensitized bacteria fail to agglutinate at relatively high salt concentrations, even when their surface potential is reduced to zero, Northrop and de Kruif (1922a) studied the effect of salt concentration on the "cohesive force" of bacterial cells, by measuring the forces required to separate two cover-slips coated with bacteria. They found that the mutual attraction of unsensitized bacterial cells was reduced with increasing salt concentration, whereas this effect was not produced when the bacteria had previously been covered with serum protein. It would seem then that one of the ways in which serum protein, or a specific antiborly, exerts its effect is by so altering the bacterial surface that it reacts to a reduction of surface potential in relatively high salt concentrations in the same way that unsensitized bacteria react to an equal reduction of potcntial in very low salt concentrations.

Before leaving this question of the influence of electrolytes on agglutination, or on other antigen-antibody reactions, we may note briefly the effect of salt concentrations higher than those that we have considered in the previons paragraphs. High concentrations of sodium chloride ( $2 \mathrm{~N}$ or above) inhibit both precipitation and agglutination. These reactions may be delayed in concentrations of $0 \cdot 2-0 \cdot 5 \mathrm{~N}$ sodium chloride. (See Streng 1909, Friedberger and Goldschmidt 1910, Landsteiner and Welecki 1911, Eagle 1932.) Schmidt (1930) noted that the reaction was delayed in high concentrations of various salts, and that the order of activity of the anions was approximately that of the Hofmeister series,

namely $\left(\mathrm{ClO}_{4}{ }^{\prime}>\mathrm{SCN}^{\prime}>\mathrm{ClO}_{3}{ }^{\prime}>\mathrm{NO}_{3}{ }^{\prime}>\mathrm{Br}^{\prime}>\mathrm{IO}_{3}{ }^{\prime}>\mathrm{SO}_{4}{ }^{\prime \prime} / 2>\mathrm{Cl}^{\prime}>\mathrm{NO}_{2}{ }^{\prime}>\mathrm{F}^{\prime}\right)$

and Marrack and Smith (1930) found that toxin-antitoxin floccules were dispersed by strong salt solutions, the order of activity being $\mathrm{I}^{\prime}>\mathrm{SCN}^{\prime}>\mathrm{Br}^{\prime}>\mathrm{NO}_{3}{ }^{\prime}$. (See also p. 230.) It is probable that the effect is due to a direct action of the salt on the serum proteins.

The effect of specific antibody itself on the surface charge of bacteria, at the $\mathrm{pH}(7-8)$ and the salt concentration $(0.9$ per cent. $=0.15 \mathrm{~N} \mathrm{NaCl})$ at which agglutination reactions are usually carried out, may be negligible (Shibley 1926, Marrack 1934); though some workers (see Brown and Broom 1929) have ascribed great importance to this effect.

There seems little doubt that the essential effect of sensitization is not its direct action on the surface charge, but the fact that sensitized bacteria react as hydrophobe colloids, even in moderately high salt concentrations, while unsensitized bacteria do not. These hydrophobe particles remain dispersed only when their surface potential is maintained at a level greater than about 13-15 mvt. When the charge is reduced below this level by the action of electrolytes the bacteria floceulate. It may be noted (Streng 1909, Northrop and de Krnif 19226) that when the amount of antibody combined with the bacteria is very small, agglutination may occur only over a very narrow range of salt concentration. 
Later studies on the effect of salt have included measures not only of sensitivity of the antigen-antibody complex to flocenlation by salt, but the degree of combination. It is obvions that both are affected. Duncan (1934), working with agglutinin systems, showed that with flagellar agglutinins the amonnt of antibody combining was increased by increasing the salt concentration from $0.001 \mathrm{~N}$ to $1.2 \mathrm{~N}$; but similar increases reduced the combination of somatic agglutinins. The salt concentration, by determining the amount of antibody combining with bacteria, influences the position of the optimum in both titrations (see also Platt 1938). Similar results were obtained by Heidelberger. Kendall and Teorell

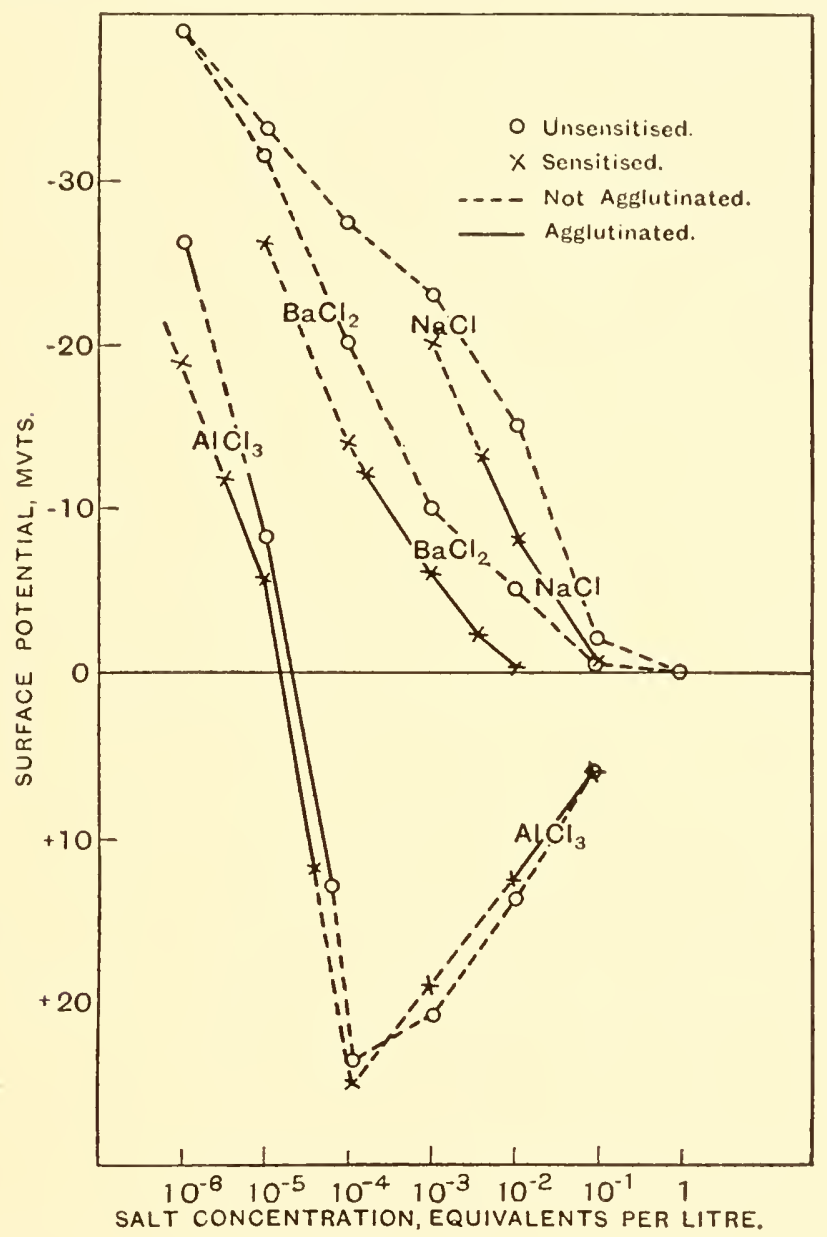

Fig. 34 .

(1936) and Heidelberger and Kendall (1936) working with 'Type III pneumococcal polysaccharide precipitin systems. Proving first that the reduction of precipitation in high salt concentration was due to reduced combination of antigen and antibody, and not to the increased solubility of the antigen-antibody complex, they were able to separate a fully reactive antibody, by dissociation of optimal precipitates in strong salt solutions. Their results were confirmed in other systems by Marrack and Höllering (1938). The relationship also held with bacteria (Heidelberger, Grabar and Treffers 1938). 


\section{The Reversibility of the Union between Antigen and Antibody.}

The formation and precipitation of insoluble antigen-antibody conplexes imply a certain degree of irreversibility, a degree which is illustrated by the Danysz phenomenon referred to above. Danysz (1902) showed that if a constant amount of diphtheria toxin is added to a constant amount of antitoxin, the toxicity of the mixture varies according to the way in which the addition is made. If, for instance, to a given amount of antitoxin, the equivalent amount of toxin is added all at once, the mixture is non-toxic. If the same amount of toxin is added in two or more fractions, with an interval of 15 minutes or more between each addition, the mixture will be highly toxic. The fraction of toxin added earlier unites with more than its equivalent amount of antibody, so that there is insufficient antibody to neutralize the later fractions, and there is, during the period of the experiment, no detectable redistribution of antibody among the toxin molecnles. The solubility of the compounds formed in antigen or antibody excess, however, suggests that if the union was reversible, a precipitate formed from equivalent amounts should dissolve in excess of antigen or antibody. The evidence on this point is conflicting.

Little solution in these circumstances could he detected in precipitates of the following antigens with their respective antisera: Types 1 and Il pneumococeal polysaccharides (Sobotka and Friedlander 1928); hæmoglobin (Breinl and Haurowitz 1930). On the other hand, the following specifie precipitates are soluble: diphtheria toxin in antibody and antigen excess (Healey and Pinfield 1935); Type III polysaccharide (Heidelberger and Kendall 1935a); hæmocyanin (Hooker and Boyd 1936), and ovalbumin (Boyd 1940).

Boyd found that the ovalbumin precipitates were as readily soluble after 10 months' storage in the cold. In other cases the antigen-antibody union appears to become less reversible with time. For instance, both Enders and Shaffer (1937) and Morris (1940) found that dilute mixtures of living Type I pneumococei and protective antibody were at first infective, but became less so if the mixtures were allowed to stand before injection into mice. It should be noted that the fact of decreased dissociability with age is not necessarily due to increasing firmness of combination; an irreversible denaturation of the antibody protein in the compound may take place (see also Chapter 11, p. 342).

The solubility of antigen-antibody complexes in high salt concentrations and, in some cases, in antigen or antibody excess, suggests that dissociation of the complex takes place. The establishment of this fact is important in deciding which of the better-known chemical and physical processes best describes the phenomena of precipitation and agglutination. With Bordet, we may interpret these reactions as adsorption phenomena, or with Arrhenius and Madsen, as obeying the mass law. In the equations both for the mass law and the adsorption isotherm of Freundlich (see below) the amount of absorption complex, or of compound, present in the system at equilibrium devends on the concentration of one or more of the reactants. However, in most precipitin systems studied (see p. 219) the composition and amount of the antibody-antigen complex is dependent, not on the final, but on the initial concentration of the reactants, a fact that implies a relatively high degree of irreversibility of the antigen-antibody union. The union takes place probably within a few seconds, in systens that precipitate visibly in a few minutes (see Burnet 1931, Boyd and Hooker 1938, Follensby and Hooker 1939, Heilelberger, Treffers and Mayer 1940, and Boyd, Comn, Gregg, Kistiakowsky and Roberts 1941). In this case the laws will apply only in the earlier stages of the reaction and their application to data obtained mainly from 
a consideration of the later stages will necessitate assumptions whose validity may not be independently demonstrable.

Before discussing the evidence for the two views of the reaction, we must first understand how far the two mechanisms--adsorption and chemical combination-can be distinguished.

In the formation of chemical compounds of the type involving intramolecular rearrangement of atoms, whether these compounds are freely dissociable or not, the forces that hold the constituent atoms of the compound together are, according to the electronic theory of valency, derived from a change in the distribution of the electrons (see Marrack 1938). In some cases one atom may simply give up one electron to another. Two oppositely charged ions are produced. In this case, no true compound is formed; for in solution, the ions exist independently; and in the crystal, although the ions occur in a ratio corresponding to the conception of a compound in definite proportion, there is no continuous pairing of oppositely charged ions to form molecules of the compound, but an interpenetrating lattice of the different kinds of ions, in which each ion is in an electrostatic relation with all its immediate neighbours. In other cases electrons are shared between the atoms concerned. The shared electrons may be contributed by one of the atoms concerned (co-valent hond) or by both atoms (co-ordinated bond). Combination of this kind must, of necessity, obey the law of simple multiple proportions.

There is no clear distinction between the formation of componnds by co-valent or co-ordinate bonds, and the formation of absorption complexes. In the latter, two molecules are held together by the attraction of the nuclei of certain atoms in one molecule for the electrons of atoms in the other, and vice versa, according to the distribution of electrically active polar groups on the molecular surfaces. With small molecules the distinction can be made because it is technically possible to separate and identify the constitient molecules, and to determine whether or not they were united in constant proportions. Large molecules are more difficult to separate and identify, because absolute differences, such as the presence or absence of a methyl group, are proportionately small; and by the same token, the law of simple multiple proportions is meaningless as soon as the molecular weights of the reacting substances are so large that the removal or insertion of an atom is no longer detectable by available analytical methods.

We may assume, if we wish, that adsorption depends primarily on intermolecular forces as opposed to intramolecular forces, and that combination depending on the intermolecular attractions will take place in varying proportions; that is, combination will not be subject to the law of simple multiple proportions. Nevertheless, the existence of compounds showing varying proportions of the two constituents will not be an absolute criterion for the differentiation of adsorption compounds from those that are formed by the intramolecular union of atoms. If a molecule is very large, and contains numerous active groups, it may be very difficult to obtain a satisfactory differentiation on this basis. The nitro-compounds of cellulose, for instance, have a composition that varies over a wide range. Moreover, a compound that is formed as the result of intermolecular forces may undergo secondary changes in which intramolecular forces are involved. We may, however, take it as a safe guiding rule that if the combination of two substances is shown to be subject to the law of simple multiple proportions, it is an indication that intramolecular forces are involved, while an apparent failure to obey this law suggests, though it does not prove, that we are dealing with an adsorption compound. 
When we come to the results obtained in partial neutralization, or partial adsorption experiments, we find that they are not compatible with the concept of firm union in constant proportions; but that they afford no secure basis for distinguishing between an adsorption reaction and a combination due to intramolecular forces that is subject to the law of mass action.

The law which describes the relation between the amount of a given substance adsorbed from a solution, and the concentration of that substance remaining in solution when equilibrium has been reached, has been formulated by Freundlich (see Freundich 1906, 1922, Freundlich and Neumann 1909), and expressed in the form of the following equation :

$$
\frac{x}{m}=a \mathrm{C}^{n}
$$

where $x$ is the amount of the substance adsorbed by the surface $m, C$ is the final concentration of the substance in the fluid, $a$ is a constant depending on the units of measurement, and $n$ is a constant less than unity.

If we let $x$ represent the adsorption on unit surface, the equation will become:

$$
x=a \mathrm{C}^{n}
$$

or

$$
\log x=\log a+n \log \mathrm{C} .
$$

The curves of the two equations are shown in Figs. 35 and 36.

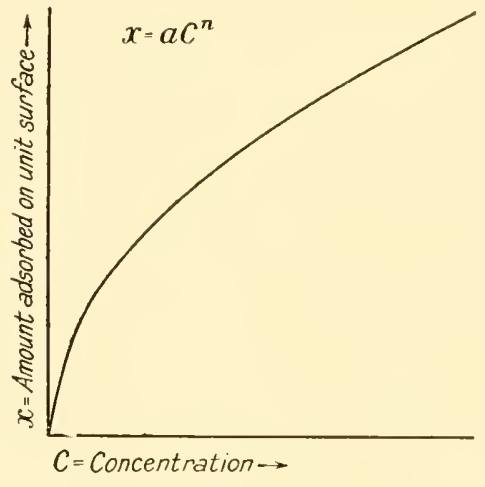

Fig. 35.

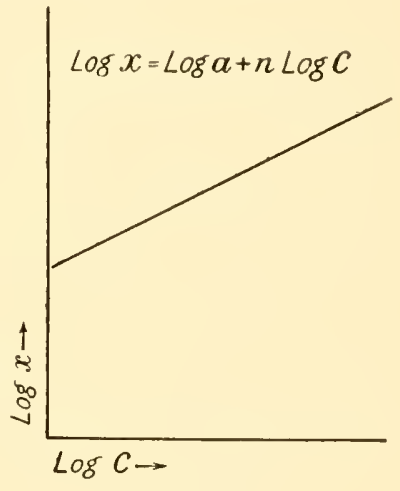

Fig. 36 .

It is clear that this formula expresses the fact that the amount of substance adsorbed by unit surface increases with increasing concentration, not in direct proportion to this increase, but to some root value of it. If, for instance, the value of $n$ were $0 \cdot 5$, the amount adsorbed by unit surface would increase as the square-root of the concentration. It follows that proportionately more of a dissolved substance will be adsorber from a weak solution than from a strong one.

The figures recorded in many antigen-antibody reactions, and particularly in the neutralization of toxin by antitoxin, fit a curve of this type very closely over a considerable part of their range, but the calculated and observed values usually differ significantly with a very great excess of either reagent (see, for instance, von Krogh 1911). This discrepancy is not surprising (see Marrack 1938). The Freundlich isotherm, in its classical form, does not approach to a maximum at high concentration of one reagent, but rises continuously, though at a decreasing rate. This seems very unlikely to describe the course of any antigen-antibody reaction. The adsorbing reagent would be expected to become fully saturated 
at some point. Modified equations have in fact been evolved that allow for such a saturation limit.

With this proviso, the demonstration that there is a close correspondence over most of the range between observed values and values calculated on the basis of Freundlich's isotherm, clearly suggests the possibility that antigen-antibody reaetions are examples of adsorption phenomena. But an equation derived from the law of mass aetion gives a eurve that has a close resemblanee to the Freundlich isotherm over a great part of its range. It differs from it in rising rather less steeply at low coneentrations of the reagent undergoing adsorption, and asymptoting towards a maximum at high concentrations. Arrhenius and Madsen (see Arrhenius 1915) give several examples in which there is a elear correspondence between the observed values and those calculated on the mass-action hypothesis over a considerable range of antigen-antibody reactions, though here again there are usually diserepaneies at high concentrations of one or other reagent. Biltz (1910) found that the curve of neutralization of tetanolysin by an antitetanic serum would fit the Freundlich isotherm, but would also fit a eurve based on the mass-action equation. It does not in fact seem possible, on the basis of the recorded data, to decide between adsorption and mass action by invoking the form of the nentralization curves obtained.

The agreement of experimental data with a theoretical equation does not guarantee its validity; whether the equation is derived from the mass law or Freundlich's isotherm. The adsorption isotherm can be used to describe a number of different statistical phenomena such as association between death rates and overerowding (Brownlee 1925). It describes, in fact, the statistical behaviour of many complex systems of interrelated events, and the agreement of data with it should probably be regarded as evidence of a complex reaction, and not as an indication of the nature of the faetors producing the complexity.

The mass law has been applied by Heidelberger and his colleagues to the extensive data they obtained from an analysis of various specific precipitin reactions; Type III pneumococcal polysaceharide, azoprotein-dye, egg albumin (Heidelberger and Kendall 1935a, b, $c, 1937$ ); thyroglobins (Stokinger and Heidelberger 1937), and serum albumin (Kabat and Heidelberger 1937).

The hypothesis assumes that antibody may be considered as homogeneous, that antigens and antibody are multivalent with respect to one another, and that before precipitation, there must oecur a competing set of bimolecular reactions, whose nature depends on the relative eoneentrations of antigen and antibody. If antigen and antibody combine in equimolecular proportions an equation applicable to all cases in which there is excess of antibody is

$$
y=2 x-x^{2} / \mathrm{A},
$$

where $y$ is the number of molecules of antibody precipitated when $x$ moleeules of antigen are added, and $A$ is the number of antibody moleeules precipitated at the equivalence point of a constant-antibody titration. There are a number of objections to this derivation. Among them is the fact that there are not always equimolecular amounts of antigen and antibody at the equivalenee point (see Marrack 1938, Heidelberger 1939). Without assuming equimolecular combination at equivalence, the following equation deriver from experimental data is found applicable to many systems:

$$
y=2 \mathrm{R} x-\mathrm{R}^{2} x^{2} / \mathrm{A},
$$

where $x, y$ and $\mathrm{A}$ are expressed in milligrams, and $\mathrm{R}$ is the ratio of antigen to antibody at the equivalence point. This, and other similar equations, are empirieally suecessful 
in characterizing antisera in terms of standard antigenic preparations and vice versa. Antibody content may be expressed in terms of maximally precipitated nitrogen, and degrees of antigenic similarity as the proportion of homologous antibody precipitable by different antigenic preparations. Heterologous systems are not so readily characterized by this method. For instance, Pennell and Huddleson (1938) found a good fit with the precipitation of Brucella abortus and melitensis endo-antigens, by homologons antisera, but the fit was poor with the cross-reacting systems, abortus antigen and melitensis antiserum. This may be due to peculiarities of the antigenic relationship between these two organisms (see Chapter 34). A more important objection is that the relation does not hold for the region of antigen excess (Malkiel and Boyd 1937); though Heidelberger (1939) points out that this region is characterized by the co-existence of soluble and insoluble antigen-antibody complexes in equilibrium, and his original assumptions do not necessarily apply.

Hershey (1941 $a, b, 1942,1943 a)$ has expressed the main features of specific precipitation in a deseriptive theory that differs from those of Heidelberger and Kendall in a number of respects. The mass law is not invoked. Hershey adopts the notion of competing bimolecular reactions, but unlike Heidelberger and Kendall assumes that the initial antigen-antibody combination is reversible. The compounds may be characterized in terms of valency of antigen and of antibody, and a dissociation constant $k$. Of special interest is his restricted theory, in which the lattice hypothesis is assumed. That is, both antigen and antibody are multivalent, and not only the initial combination, but the later aggregation is determined by antigen and antiborly valencies. The original papers must be consulted for the details of the theory. It may be noted that, by assuming that aggregates as well as initial compounds have a determined lattice strueture, the assumption of irreversibility of reaction is not only unnecessary but is irreconcilable with the lattice hypothesis $(1943 \alpha)$. The agreement of the theory with available data is as good as that of Heidelberger and Kendall's, and the theory is applicable over a wide range of the precipitin reaction. 'The consequences of the restricted theory are compatible with a large number of observed facts including insolubility of precipitates, the different effects of antigen and antibody excess, and the varying relation of optima with various points of reference in the equivalence zone. Taken in conjunction with the experimental data, the success of the restricted theory at least provides an additional reason for giving the lattice hypothesis the first place among the working hypotheses of serology. (See also Ghosh 1935, Kendall 1942, and Pauling, Pressman, Campbell and Ikeda 1942.)

\section{Tests of the Lattice Hypothesis.}

The description of the precipitin reaction in terms of Heidelberger and Keudall's mass-law equations corresponds in essentials with the lattice hypothesis of Marrack. Both depend on the assumption that antibodies are multivalent. The main objections advanced against the conception of multivalent antibody are firstly the difficulty of conceiving the mode of formation of multivalent antibodies in the animal, and secondly that the assumption of multivalence is unnecessary to deseribe the phenomena of precipitation. The first point is dealt with more fully in a later section (p. 251). Hooker and Boyd (1937) object to the assmmption of multivalence on the grounds that inhibition by antibody excess is rare; that non-specific adjuvants like electrolytes and lipins are necessary for precipitation; that the surface properties of bacteria sensitized by antibody are those of a hydrophobic, salt-sensitive, denatured globulin; and that in the presence of excess of antibody the rate of flocculation of an antigen is similar to a simple colloid undergoing non-specific flocculation. The same authors (1942) point ont that analysis of precipitates formed in the zone of antigen excess, where antiborly is not likely to have all its valencies satisfied, seldom reveals a molecular ratio of antigen to antibody of more than unity. 
Eagle (1938) subjected diphtheria antitoxin and antipneumococal serum to progressively intensive treatment with formaldehyde. At one stage the antibodies lost their precipitating power, but were still protective, a separation of activities that suggests a non-specific element in aggregation. Kleczkowski (1941b) and Bawden and Kleczkowski (1942b) made complexes of antilody globulin with albumin, in which the antibody retained its specific combining power, but was incapable of in vitro flocculation. Phenomena of this kind do not necessarily invalidate the lattice hypothesis, but they give an indication of the degree to which non-specific factors may be operative in aggregation.

Topley, Wilson and Duncan (1935) devised a test of the multivalence of antibody, using mixed agglutination systems. Two antigens are chosen, of approximately equal particle size, and in similar concentration. The amount of antibody which in each case will flocculate the antigen in a given time is determined, and the reaction of the two antigens and antibodies allowed to take place in a mixture. If the lattice hypothesis holds, the aggregates that form will consist of one antigen or the other, since they are held together by specific antibody. If flocculation is non-specific, the aggregates will be mixed. Mloreover, in the first case, the speed of flocculation of each antigen will be independent of the presence of the other; but if antibody merely sensitizes the antigens to the action of electrolyte, then the concentration of flocculable material in the mixtures will be doubled, and the speed of the mixed reaction much greater than that of either reaction separately. Topley, Wilson and Duncan found that pneumococci, and the microscopically distinguishable coliform bacilli, formed separate aggregates. With mixtures of two distinguishable types of red blood cell, Abramson (1935) and Hooker and Boyd (1937) found mixed aggregates. Wiener and Herman (1939), on the other hand, found separate aggregates with mixtures containing red cells and a precipitating antigen, red cells and pneumococci, and two types of red cells. Mixed aggregates were formed with the latter when antibody was in excess. Non-specific flocculants also gave mixed aggregates. With precipitating systems, Hooker and Boyd (1937) found an increased speed of flocculation in mixtures, indicating non-specific aggregation. Duncan (1938) confirmed this but, with agglutinating systems, found no increase in speed of agglutination. He suggested that both mechanisms were operative, but that one or the other might predominate according to the system and the circumstances of its reaction. Heidelberger and Kendall (1937) saturated pneumococei with horse antibody. The addition of more pneumococci to the over-sensitized cells resulted in prompt agglutination of all the pneumococci, a result strongly suggesting that, in addition to the valencies employed in bincing antibody to the original pneumococci, there were on the bound antibody free valencies available for combination with the added cocci. Boyd and Hooker (1938) suggest that in mixed systems specific combination may operate in the second stage, and a lattice may form in antigen excess ; in antibody excess, the "two-stage" reaction appears to take place, for they fornd that red cells saturated with a very marked excess of antibody were still susceptible to the flocculating action of salts. Hershey's (1943b) recent experiments, made on somewhat similar lines with bacteriophage, also support the lattice hypothesis. A living Bact. coli hacteriophage adhered firmly to a precipitate consisting of dead coli bacteriophage and homologous antibody. Other specific aggregating systems, with e.g. staphylococcal phage, serum proteins, pneumococcal polysaccharide, absorbed only a small amount of the phage. $\mathrm{He}$ obtained no evidence that the particles of antigen were rendered non-specifically " sticky" by their coating of antibody, a condition to be expected if aggregation was non-specific.

Another experimental approach to the problem of antibody valency is possible. No safe assumption can be made of the precise valency of natural antigens. But, as we shall see later (p. 258), certain fractions of natural antigens will react with antibody, provided they possess the chemical groupings that characterize the full antigen. These reacting fractions are called haptens, and in some cases can be prepared synthetically. The reaction of monovalent and divalent synthetic haptens with antibody should provide a clue to the valency of antibody. Mono- 
valent haptens do not precipitate with homologous antibody, though they can be shown to combine with it. It is argued (Marrack 1938, Pauling 1940) that if antibody is divalent, divalent haptens should aggregate with it in long chains; with tri- or multi-valent antibody, divalent haptens could form a lattice and precipitate.

Precipitation of divalent haptens was observed by Landsteiner and van der Scheer (1932b) and by Pauling, Campbell and Pressman (1941); and Pauling, Pressman and Ikeda (1942) showed that the hapten-antibody ratio was constant throughout the titration range of a divalent hapten with homologous antiserum. The observed molar ratio was 0.75 , as against the ratio of unity to be expected if both the antigen and the antibody were bivalent. Hooker and Boyd (194I $b$ ) and Boyd (1942) obtained no evidence of precipitation or of the formation of long chains of alternating antigen and antibody. Landsteiner and van der Scheer's haptens may have polymerized in solution and so formed multivalent hapten particles; though there is no positive evidence that the same criticism is applicable to the results of Pauling and his co-workers. Boyd (1942), from a study of specifically precipitable trivalent arsonic haptens, concluded that their precipitability depended, not on their power to form a lattice, but on the closeness of their combining groups to one another. When the arsonic combining groups were attached to a compound so that they were separated by a relatively large molecule, the hapten was not precipitable by antibody. Boyd suggests that after the arsonic groups of this latter type of hapten have united with antibody, the number of hydrophile groups remaining free is sufficient to keep the compound in solution. When these groups were rendered less hydrophilic by acetylation, the haptens became precipitable. Precipitation on this basis is due to the lowering of solubility by mutual neutralization of polar groups in antiborly and napten, and by crowding of antibody molecules together so that other polar groups are occluded. This "oeclusion" hypothesis is an extension to haptens of the "two-stage" theory as applied to the reaction of full antigens in antibody excess. The serological reactions of haptens, however, are not strietly parallel to those of full antigens, and this lack of parallelism may invalidate any conclusions that we may draw about the antigenantibody reactions. For example, Woolf (1941), working with antigens synthesized by conjugating protein to hapten, observed that the antigen-antibody ratios were dependent, as in other systems, on the amounts, and not on the concentrations of the reactants. Iuhibition of the reaction by hapten, on the other hand, depended on its concentration. a fact which suggested that hapten-antibody compounds were far more dissoeiable than antigen-antibody compounds. Moreover, Hershey (1942) points out that experiments with haptens are unlikely to indicate anything about the valency of antibody, since the discrepaney in size between the reactive groups of the haptens and a reactive pateh (i.e. unit "valency") on the antibody is such that a modified lattice will form in the early stage of preeipitation, whether antibody is monovalent or not. In a later paper (19i4) he shows on theoretieal grounds that the combination of antibody with divalent hapten would not result in specific precipitation, since it is highly unlikely that sufficiently long chains of alternating antigen and antibody molecules could form; precipitation in such circumstances would imply either a non-specific effect, or irreversible antigen-antibody linkages; or, as already noted, either an increase in the valency of hapten by its polymerization, or an effective multivalence of unit reactive patches on the antibody molecule.

It is impossible at present to decide between the lattice and the two-stage hypotheses, at any rate as mutually exclusive hypotheses. The proponents of both are united in assuming that the initial combination is determined by specific chemical factors, and that antigens are multivalent. It should be noted that the demonstration of the multivalence of antibody will not alone prove that a specific lattice is formed; it will be necessary also to show that the antigen-antibody linkages are specific, and that the bonds between the molecules are reversible 
(Hershey 1944). The resolution of the controversy either by the establishment of one or the other hypothesis, or their merging into a more general hypothesis, must await further research.

\section{The Microscopic Appearance of Specific Aggregates.}

There are few observations of specific aggregates, either during or after their formation. which yield any information about the nature of the antigen-antilody union. Pijper $(1938,194] a, b)$ records an interesting study of the agglutination of Salm. typhi made by dark-ground cinematography. He eonfirms the well-established faet that flagellar antibody first immobilizes the organisms by a direet aetion on the flagella, after which aggregates are formed by the adhesion to one another of the motionless flagella of bacteria fortuitously brought into contaet by Brownian movement or convection currents (Fig. 37). Agghutination by antibody to the somatie antigens results in the formation of closely paeked clumps of bacteria, whieh retain their motility since the flagella are not immobilized. The einematograph record shows the motile, sensitized baeteria swimming in all directions aeross the field along relatively straight lines; and many of them are seen swimming directly towards already formed aggregates, a phenomenon that Pijper attributes to

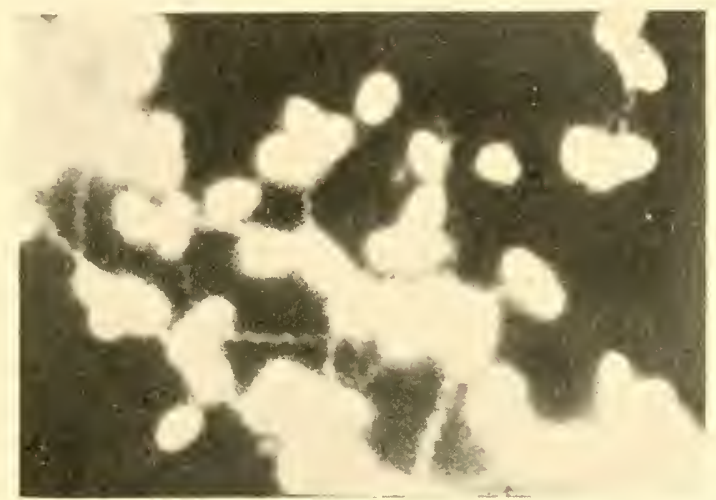

FIG. 37.-Dark-ground photomicrograph of Sulm. typhi aggregater by flitgtllar antiborlies. (From a photograph kindly supplied l,y 1)r. A. Pijper.)

attraetion exerted by the aggregatc. It is difficult to imagine that the mion of antigen and antibody could generate a field of force capable of acting at distanees of several microns, and it appears to us that the apparent attraction ean be explained by postulating a straight-line course for the motile sensitized bacilli over short distances. If an aggregate is in the line of travel, a head-on collision with the aggregate, and arlhesion to it, will give the impression of attraction.

The bacterial aggregates formed during agglutination varied with the antibody used. Sensitization by flagellar antibody gives the fortuitous pattern already noted ; by somatic $\mathrm{O}$ antibody, an end-to-end arrangement (Fig. 38); and by Vi antibody (see Chapter 8), the characteristic paeking depieted in Fig. 39. The factors that determine these patterns are matters for speeulation. It may be noted in this connection that Nliles and Pirie (1939) observed specific precipitates of antibody and native antigen from $\mathrm{Br}$. melitensis which appeared to be built up of bacterial fragments adhering to each other end-to-end.

During the action of flagellar antibodies, Pijper observed the deposition of granules on the flagella. Mudd and Anderson (1941) found a patehy distribution of thickenings on the flagella of specifieally sensitized Salm. typhi. Neasured in electron micrographs, the increase in thickness varied from 1 to $17 m \mu$. Similar thickenings oecurred on the cell wall. The dimensions of the thiekenings were of an order of size compatible with those of a unimolecular layer of rabbit antibody. 
The sensitized patches appeared to be sticky. not only for each other, but for nonspecific particles. No estimate of the degrees of stickiness is possible from an electron

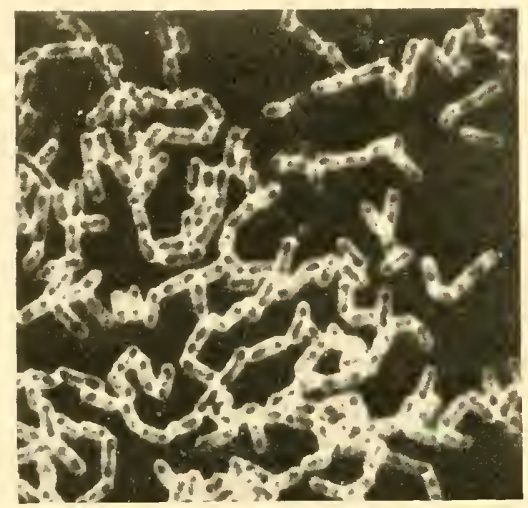

Fig. 35.-Dark-ground photomicrograph of Salm. typhi aggregated by somatic antibodies.

(From a photograph kindly supplied by Dr. A. Pijper.) micrograph. but if we assume that the non-specific and specific stickiness were of the same order, the observation provides evidence in favour of the two-stage rather than the lattice hypothesis.

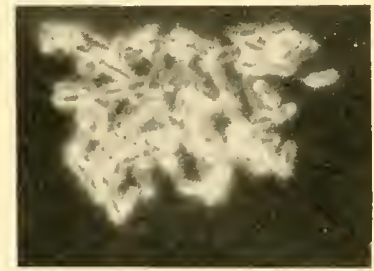

F1G. 39.-Dark-ground photomicrograph of salm. typhi aggregated by $\mathrm{Vi}$ antibodies.

(From a photograph kindly supplied by Dr. A. Pijper.)

In a mixture of a tobacco mosaic virus, having a particle width of $15 \mathrm{m \mu}$, and its homologous antibody, Anderson ant Stanley (1941) demonstrated that, in antibody excess, the increase in width of the virus particles was compatible with the hypothesis that highly elongated rabbit antibody molecules, about $4 \times 27 \mathrm{~m} \mu$, were attached radially to the surface of the antigen. The authors point out that electron micrographs of the aggregates suggest a rough lattice formation. Such a resemblance is irelevant in a consideration of Marrack's hypothesis, in which "lattice" is used more in a crystallographic, than in a secular, sense of the term.

\section{The Lysis of Red Blood Cells.}

Although the original observations on the phenomenon of lysis were made on bacteria, Bordet's demonstration that a similar reaction could be obtained with red blood corpuscles, and the numerous and detailed investigations of Ehrlich and his school into the interactions between red cells and their corresponding antisera, turned the attention of the great majority of workers from bacteriolysis to hæmolysis; and the greater part of our knowledge of the mechanism of lytic reactions in general is based on the data obtained in studying the lysis of red cells. For this reason we shall discuss hæmolysis before considering the lysis of bacteria.

It is not possible, in the space at our disposal, to give any account of the historical development of our knowledge of this subject. Reference must be made to textbooks dealing particularly with immunological reactions, or to the collected papers of Bordet, and of Ehrlich, who have been the protagonists in this particular controversy. The main facts, which are not in dispute, are as follows:

The phenomenon of hæmolysis consists in a laking of the red blood corpuscles, that is, in a setting-free of their contained hæmoglobin, and not in a true solution. The cell stromata remain undissolved, though altered in size and shape, and probably in other physical and chemical characteristics.

As has already been noted, it was shown by Bordet (1898) that hæmolytic sera, like the bactericidal sera studied by Buchner, are inactivated by heating for 30 
minutes at $56^{\circ} \mathrm{C}$. He also showed that this inactivation concerned not the hæmolytic antibody itself but a second non-specific thermolabile factor, which caused the lysis of the red cells when these had been sensitized by the specific hæmolysin. This non-specific, thermolabile factor, which is present in all normal, fresh, unheated sera, was named alexine by Buchner. It is now generally known by the name complement employed by Ehrlich.

The fundamental reactions that demonstrate the nature of the lytic reaction may be briefly summarized as follows: The defibrinated or citrated blood of a suitable animal, such as the sheep, is centrifuged, and the deposited red cells are separated and washed several times in saline to free them from the last traces of serum. The washed cells are then made into a 5 per cent. suspension in saline. The serum from some convenient animal-usually a rabbit-that has received repeated injections of washed sheep corpuscles and has in consequence produced a specific hæmolysin to high titre, is heated at $56^{\circ} \mathrm{C}$. for 30 minutes to inactivate the normal complement. The fresh, unheated serum of some other animal, usually the guinea-pig, is used as a source of complement. When these reagents are mixed in various combinations, and the mixtures incubated at $37^{\circ} \mathrm{C}$, the following results are obtained :

(1) Red cells + Hæmolysin $\rightarrow \quad$ No hæmolysis.

(2) Red cells + Complement $\rightarrow \quad$ No hæmolysis.

(3) Red cells + Hæmolysin + Complement $\rightarrow$ Complete hæmolysis.

If mixtures (1) and (2) are centrifuged, and the deposit and supernatant fluid examined separately for the presence of hæmolysin and complement, the following results will be noted, provided that the proportions of the reagents in the original mixtures have been suitably adjusted.

(4) Deposit from (1) + Complement $\rightarrow$ Complete hæmolysis.

(5) Supernatant from (1) + Red cells + Complement $\rightarrow$ No hæmolysis.

(6) Deposit from (2) + Hæmolysin $\rightarrow$ No hæmolysis.

(7) Supernatant from (2) + Red cells + Hæmolysin $\rightarrow$ Complete hæmolysis.

Reaction (4) shows that hæmolysin has combined with the red cells in (1) and sensitized them to the lytic action of complement. Reaction (5) confirms this by demonstrating the absence of hæmolysin from the supernatant fluid. Reaction (6) shows that complement has not combined directly with the red cells in (2). Reaction (7) confirms this by demonstrating the presence of complement in the supernatant fluid.

The controversy that arose between Bordet and his co-workers on the one hand, and Ehrlich and his school on the other, concerned the mode of union between the complement and the sensitized red cells. As already indicated, Ehrlich's conception of the hæmolytic antibody was that of a special type of side-chain, which he referred to as a receptor of the third order, or an amboceptor. He endowed this hypothetical receptor with two haptophore, or combining groups, one of which united with the red cell, and was named by him the cytophilic group, while the other united with the complement, and was named the complementophilic group. Ehrlich's conception of the structure and mode of action of an amboceptor may be represented as in Fig. 40A. In the diagram, R.C. represents the red cell, and $\mathrm{R}$ one of its receptors; $\mathrm{A}$ the amboceptor, or hæmolysin, attached to the receptor of the red cell by its cytophilic haptophore group $C y$, and to the complement by its complementophilic haptophore group $\mathrm{Cm}$; C repre-

P.B. 
A

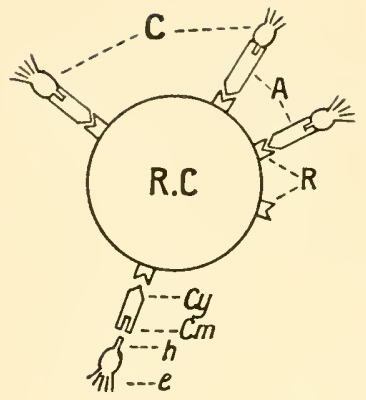

FIG. 40.
B

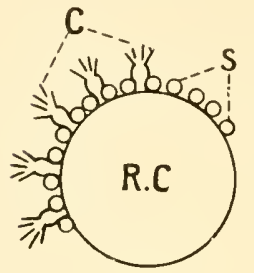

sents the complement, with its haptophore group $h$, by which it is attached to the complementophilic group of the amboceptor, and $e$ its ergophore group, in virtue of which it brings about lysis of the red cell, when united to it by the hæmolytic amboceptor.

Bordet's conception of the action of the hæmolytic antibody, which he refers to as the "substance sensibilisatrice," or sensitizer, is essentially different. $\mathrm{He}$

denies that the sensitizer acts as a link between the red cell and the complement, or that the complement ever unites with the sensitizer as such. He regards the combining affinities of the hæmolysin as being directed entirely upon the red cell. The complement, in his view, unites not with the hæmolysin, but with the complex, red cell + hæmolysin, or, to put it iu another way, with the red cell as altered by union with the hæmolysin. Bordet's conception may be represented diagrammatically in Fig. 40B. R.C. represents the red cell; S the sensitizer or hæmolysin, which has united with the red cell, and altered the physical conditions at the sur. face; and $\mathrm{C}$ the complement, uniting with the red cell which has been so altered.

It will be seen that the controversy can be narrowed down to the question: what evidence is there for the existence of a complementophilic group of the hæmolysin? We have not space to present all the experimental data which have been advanced by each side in turn, nor the arguments which have been based upon them. It may, we think, be safely asserted that Ehrlich and his supporters have, in this respect, failed to substantiate their position, and that the weight of evidence strongly upholds Bordet's view.

The quantitative aspects of the union between red cells and hæmolysin have been studied by many observers, and the results recorded have been of the same general kind as those observed with mixtures of soluble antigens and the corresponding antibodies, or with bacteria and their homologous agglutinins. They are not compatible with the view that red cells and hæmolysin unite in constant proportions, in a firm chemical union. They are compatible with the view that the union obeys the laws of an adsorption process-for instance, Bordet was able to demonstrate a reaction analogous to the Danysz phenomenon. They show (see Muir 1909) that the red-cell-hæmolysin compound can be dissociated under certain conditions, though the dissociation is not of the type that occurs on simple dilution of a dissociable chemical compound. There is, indeed, no reason to suppose that the laws that determine the union of a hæmolysin with the specific antigenic components of a red cell differ in any way from those that determine the union of precipitins or agglutinins with their corresponding antigens.

Not all the antigenic groups on the surface of the red cell appear to take part in the sensitization of the cell to the action of complement. Heidelberger, Weil and Treffers (194I), calculate that, in a red cell suspension sensitized by one minimal hæmolytic dose of hremolysin (see below), at most only 14 per cent. of each cell surface is occupied by antibody. A figure of the same order, 6 per cent., was estimated by Haurowitz and Yenson (1943).

The effect of electrolytes and hydrogen-ion concentration on the union of hrmolysin with red cells has been referred to on p. 211 , in relation to the union of agglutinins with 
bacteria. It may be noted (see, for instance, Markl 1902, Topley 1915) that electrolytes in high concentration have an inhibitory effect on the union of complement with sensitized red cells, and on the resulting hæmolysis.

The effect of temperature on hæmolysis differs somewhat from its effect on precipitation and agglutination, because we have an additional reagent to consider - the complementand this reagent is thermolabile. Union between red cells and hæmolysin occurs readily at $0^{\circ} \mathrm{C}$ (see, for example, Ueno 1938). At this temperature complement also unites with sensitized red cells, though very much more slowly, but lysis fails to oceur over any ordinary period of observation, umless excess of hemolysin and complement is present. At room temperature lysis occurs, but so slowly that this temperature is unsuitable for experimental purposes. The optimal temperature for hemolysis is in the neighbourhood of $37^{\circ} \mathrm{C}$. At temperatures much above this the complement is inactivated, the rapidity of inactivation increasing as the temperature is raised.

The problem of the titration of a hrmolytic serum differs from that of titrating precipitins or agglutinins in that we are faced with three dependent variables instead of two. Our essential reagents are red cells, hæmolysin and complement. Of these reagents, it is natural to keep our red cells constant, regarding as our end-point the lysis of a specified quantity of red cells, in a specified time, under specified conditions. It is a common practice to use a 5 per cent. suspension of red cells in saline, and to employ some convenient volume of this suspension, such as $0.5 \mathrm{ml}$., in our tests. We have two reagents left, hæmolysin and complement. The natural, and usual, plan is to vary the amount of the reagent that we want to measurethe hæmolysin-while keeping the complement constant. But here we meet a difficulty. We can measure our reagents only in terms of their activity, and they are dependent variables; the more complement we add, up to a point, the less hæmolysin we need, and vice versa. We get out of our difficulty by making use of the kind of relation that exists between our variables. However much complement we add, we need a certain amount of hæmolysin. However much hæmolysin we add, we need a certain amount of complement. Noreover, the limit at which further additions of one reagent make no appreciable difference to the required amount of the other is not a high multiple of the minimal dose. So we define our units of measurement as follows:

The Minimal Hamolytic Dose (M.H.D.) of hamolysin is the smallest amount that will cause complete lysis of an arbitrarily selected amount of red cells, in the presence of excess of complement, in 1 hour at $37^{\circ} \mathrm{C}$.

The Minimal Hamolytic Dose (M.H.D.) of complement is the smallest amount that will cause complete lysis of an arbitrarily selected amount of red cells, in the presence of excess of hamolysin, in 1 hour at $37^{\circ} \mathrm{C}$.

In an actual titration we proceed as follows: We first titrate our complement, which must be used fresh, adding rarying dilutions of our complement-containing serum to mixtures of red cells and a hæmolytic serum of known titre. A hæmolytic serum, it may be noted, remains stable over a considerable period of time. In this titration we use excess of hæmolysin say 5 or 6 M.H.D. We note the last tube that shows complete hæmolysis, and this gives us the H.H.D. of our complement. We now dilute our complement so that the volume we intend to use, commonly $0.5 \mathrm{ml}$., contains 3-5 M.H.D., and to a series of tubes we add $0.5 \mathrm{ml}$. of our red cell suspension, $0.5 \mathrm{ml}$. of our diluted complement, and $0.5 \mathrm{ml}$. of the hremolysin under test, the dilution of the latter increasing from tube to tube. After 1 hour at $37^{\circ} \mathrm{C}$. we note the last tube that shows complete hæmolysis, and this gives us the M.H.D. of our hæmolysin. 


\section{The Lysis of Bacteria.}

Although we talk of bacteriolysins, antibodies that lyse bacteria, and bactericidins, antibodies that kill them, the two effects are not differentiated in practice, and though we often talk of the bacteriolytic titre of a serum it is the bactericidal effect that we actually measure. It is true that in many instances, as in Pfeiffer's classical experiments on the lysis of the cholera vibrio, gross degenerative changes in the bacteria have been observed, and there is no doubt that those bacteria that are susceptible to the lethal action of complement in the presence of a specific antibody undergo a change in structure that is analogous to the lysis of red blood corpuscles. The change is not, however, of the same dramatic kind; and the naked-eye observation of the changes in a turbid bacterial suspension is not a practical method of observing bacteriolysis. The principles involved in the reaction do not differ from those concerned in hæmolysis, and the method employed in titration is essentially similar.

A very light bacterial suspension is employed, and the serum under test, after its natural complement has been inactivated by heat, is mixed in increasing dilution with a constant amount of the bacterial suspension and a constant amount of complement. The surviving bacteria in the mixtures are counted after varying intervals by some suitable cultural method, and the highest dilution of serum that produces a significant killing effect is noted. The method contains many possible sources of technical error. Gengou (1899), for instance, drew attention to the fact that bacteria are agglutinated as well as lysed by a specific antiserum, and that this effect may greatly reduce the number of colonies in a plate count, since a clump of bacteria will produce a single colony. When the original mixtures are incubated for long periods before plating, this effect will be counterbalanced by the multiplication of those bacteria that have been agglutinated but not killed; but this possible source of error must be considered in estimating the significance of any reduction of the viable count over a short period, and a control mixture containing the bacteriolytic serum without complement must always be included in the test.

It was noted by Neisser and Wechsberg (1901) that a marked pro-zone often occurred in tests of this kind. A particular dilution of serum might exert no bactericidal action, while a much higher dilution resulted in a complete killing of the bacteria. This phenomenon was seized on by the Ehrlich school as an example of the union of complement with free amboceptor, the hypothesis being that complement united indifferently with all the amboceptor present and that, when this was present in excess, chance would favour the union of all the complement, or the greater part of it, with the amboceptor that was not attached to bacteria. We know, however, from our experience with precipitation and agglutination, that a similar inhibitory effect is produced by a great excess of antibody in reactions in which complement plays no part; and it is clear that the use, in bactericidal tests, of very thin bacterial suspensions will favour the frequent occurrence of zones of gross antibody excess. The Neisser-Wechsberg phenomenon cannot, therefore, be regarded as affording any support to the view that complement combines directly with antibody.

A point of considerable importance in relation to the bactericidal, or bacteriolytic, reaction is that its occurrence depends in large part on the nature of the bactcrial cell. Certain bacteria, such as the cholera vibrio, the typhoid bacillus, and most Gram-negative bacilli, are readily killed and lysed when acted on by complement after sensitization by a specific antibody. Other bacteria, such as the Grampositive cocci, are insusceptible to the direct killing action of antibody and com- 
plement. And it should be noted that this insusceptibility is not due to any failure of the sensitized cell to combine with complement (see Buxton 1905a, $b, c$, Muir and Browning 1909).

\section{The Nature of Complement.}

We have seen that complement is a non-specific substance present in all normal sera, and not increased in amount as the result of immunization. It does not follow that complement is a single substance, or a single system. Different kinds of antibodies, for instance the antibodies acting on different species of red cells, might require different kinds of complement, all, or many of which, might be present in any specimen of normal serum. This question may be analysed into at least three components. (1) As regards one kind of lysis, for instance that of red cells, is the complement present in a given specimen of serum a single entity, or are there separate complements corresponding to the different hæmolytic antibodies? (2) If only one kind of complement is concerned in hæmolysis, is it the same, or a different complement, which brings about the lysis of bacteria, or of other organized cells? (3) If the complement in a given specimen of serum is one and the same, irrespective of the kind of cells which are lysed, or of the particular antibodies which are sensitizing them, is the complement in different sera, and particularly in sera from different animal species, always the same?

This problem afforded one of the most closely debated points in the long controversy between Ehrlich and Bordet, and the question has been investigated by many other workers. Space does not allow us to reproduce the arguments employed, nor the experimental evidence on which these arguments were based. For this reference is best made to the original papers, which contain interesting examples of the complexity of the hypothetical receptor-apparatus employed by Ehrlich to describe his experimental results. The provisional answers to these questions which are, in our view, afforded by the available evidence, are as follows :

There is no evidence that more than one kind of complement is concerned in hæmolysis.

The evidence strongly suggests that the complement, in a given serum, which causes lysis of one type of cell, for instance the red cell, is identical with that which causes lysis of another type of cell, for instance a bacterium.

It is clear that sera derived from different animal species show qualitative differences in their complementary activities; and the available evidence indicates that the differences are due to both qualitative and quantitative variations in the components of the complement concerned.

Accepting the view that there is little, if any, evidence in favour of the existence of a multiplicity of complements in the sense employed above, it remains to inquire whether complement is a single chemical substance, or is a name for a property of normal serum that is dependent on a number of different factors. Here the answer is not in doubt. The complementary action of fresh, unheated serum depends on the interaction of a number of separate components, of which there appear to be four.

Complement becomes inactive if the euglobulins are precipitated by removal of electrolytes. The remaining fluid is inactive. If the precipitate is removed, and dissolved in saline, it also is inactive, but a mixture of the two fluids will cause hæmolysis of sensitized red cells (Ferrata 1907, Brand 1907, Liefmann 1909, Skwirsky 1910, Amako 1911, Gengou 1911.) The "globulin" fraction is known as " mid-piece," and the soluble albumin fraction as "end-piece," because 
mid-piece unites directly with sensitized cells whereas end-piece unites with such cells only in the presence of mid-piece. In various complement-fixation reactions, mid-piece is mainly absorbed, the greater part of the end-piece being left in solution. Both mid-piece and end-piece are inactivated by heat.

It has long been known that a serum loses its complementary power after absorption by yeast. Such a serum is rendered active again by adding serum in which mid-piece and end-piece have been destroyed by heating to $56^{\circ} \mathrm{C}$. (Coca 1914). This third heat-stable component is present in both mid-piece and end-piece, as prepared by Liefmann's method of mid-piece precipitation by $\mathrm{CO}_{2}$ (Whitehead, Gordon and Wormall 1925).

Gordon, Whitehead and Wormall $(1926 a, b)$ demonstrated a fourth component of complement which is heat-stable, but not absorbed by yeast. It is specifically inactivated by treating the serum with ammonia. Though this component is necessary for the hæmolytic action of complement, it is not necessary for the complementary effect of normal serum in the opsonic reaction (see p. 236). The existence of the fourth component was confirmed by Deissler (1932) and many subsequent workers.

The demonstration of components by inactivation with specific chemicals must be viewed with caution, for activity is associated with proteins, and agents which denature the proteins will also destroy complement. The postulation of a benzene-inactivated component by Tokano (1936), a dialysable component by Chow and Zia (1938) and of the necessity for ionized calcium (Ottolenghi and Mori 1905) were probably based on observations of denaturation (sec Jones and Ecker 1940, Pillemer and Ecker 194la, Ecker and Pillemer 1942).

Complement is most active at a $\mathrm{pH}$ of about 7.3 in the presence of physiological saline. Its activity is progressively reduced by increases and decreases of $\mathrm{pH}$ and salt concentration. Its inactivation by various salts was studied by Gordon and Thompson $(1933 a, b)$, who found that the activity of various ions fell into the general order of the Hofmeister series $\left(\mathrm{SCN}^{\prime}>\mathrm{I}^{\prime}>\mathrm{Br}^{\prime}=\mathrm{NO}_{3}{ }^{\prime}>\mathrm{SO}_{4}{ }^{\prime \prime} / 2>\mathrm{Cl}^{\prime}\right)$. Some of the salts produced a reversible, others an irreversible inactivation. It loses activity in a few days at $0^{\circ} \mathrm{C}$. and in a few minutes at $56^{\circ} \mathrm{C}$. According to Wehmeyer (1941) the inactivation of complement at any given temperature between $4^{\circ} \mathrm{C}$. and $55^{\circ} \mathrm{C}$. proceeds like one unimolecular reaction between $0^{\circ} \mathrm{C}$. and $37^{\circ} \mathrm{C}$., and another between $50^{\circ} \mathrm{C}$ and $56^{\circ} \mathrm{C}$. At the lower temperatures only mid-piece is labile, at the higher, both mid-piece and end-piece are labile, end-piece predominantly so; in the $30^{\circ}-50^{\circ} \mathrm{C}$. range, the rate depends on the velocities of two unimolecular reactions.

Dry complement that has been kept for months is still active when reconstituted (Craigie 1931). Filtration through a porcelain candle (Strong and Culbertson 1934) inactivates complement mainly by retention of mid-piece and end-piece.

Many attempts have been made to isolate the components of complement (see Ecker and Pillemer 1942). The association of mid-piece activity and endpiece activity with "globulin" and "albumin" fractions of serum is to a large extent illusory, for, as Pillemer and Ecker (1941b) have shown, the electrophoretic patterns of both fractions show four distinct proteins, two of those in "globulin" fraction differing from any observed when normal guinea-pig serum is analysed by this method. Inactivation of the third and fourth components has little effect on these patterns These authors propose the symbols $C^{\prime} 1, C^{\prime} 2, C^{\prime} 3$ and $C^{\prime} 4$ for the active principles in mid-piece, end-piece, third and fourth component respectively. By carefully controlled methods, Pillemer, Ecker, Oncley and Cohn (1941) have isolated three fractions with high $C^{\prime} 1, C^{\prime} 2$ and $C^{\prime} 4$ activity from 
guinea-pig serum. $C^{\prime} 1$ is a euglobulin containing $2 \cdot 7$ per cent. carbohydrate. A muco-euglobulin fraction of carbohydrate content 10.3 per cent. contains $C / 2$ and $C^{\prime} 4$. The $C^{\prime} 2$ is heat-labile and the $C^{\prime} 4$ relatively heat-stable. According to Pillemer, Seifter and Ecker (1941) $\mathrm{C}^{\prime} 4$ is a carbohydrate, whose carbonyl groups are specifically attacked during inactivation by ammonia and other compounds, and which is normally carried by $\mathrm{C}^{\prime} 2$, the two forming a globulin-carbohydrate complex. $C^{\prime} 1$ accounts for 0.6 per cent., and $C^{\prime} 2$ and $C^{\prime} 4$ for 0.17 per cent. of the total serum protein.

More recently Ecker and his colleagues have made a similar analysis of human complement. Four components are present with properties analogous to the four in guinea-pig sera. $C^{\prime} 1$ has been isolated, and closely resembles guinea-pig $C^{\prime \prime} 1$; both have similar electrophoretic mobilities and sedimentation constants. The carbohydrate content of human $\mathrm{C}^{\prime} 1$ was slightly higher, $3 \cdot 2-3 \cdot 7$ per cent., and so was the apparent isolectric point, $\mathrm{pH} 6 \cdot 0-6 \cdot 4$ as against $\mathrm{pH} 5 \cdot 2-5 \cdot 4$. In human serum, C'1 constituted about $0 \cdot 6-0 \cdot 8$ per cent. of the total serum protein. Of all four components in the two species, only $\mathrm{C}^{\prime} 3$ was completely interchangeable in hæmolytic tests. Human $\mathrm{C}^{\prime} 1$ and $\mathrm{C}^{\prime} 4$ could replace the guinea-pig $\mathrm{C}^{\prime} 1$ and $\mathrm{C}^{\prime} 4$, and guinea-pig $C^{\prime} 2$ could replace human $C^{\prime} 2$, but the corresponding converse replacements were either less effective or ineffective (Ecker, Pillemer and Seifter 1943, Seifter, Pillemer and Ecker 1943, Pillemer, Seifter, San Clemente and Ecker 1943).

\section{Complement and Vitamins.}

The relation of complement to vitamin $\mathrm{C}$ has received much attention in recent years. The reduced complement titres in guinea-pig sera during the winter months (Tokunaga 1928, Marsh 1936, Horgan 1936) suggests a dietary deficiency assoeiated with a lack of green food. In the guinea-pig Harde and Thomson (1935) rlemonstrated an association between vitamin $\mathrm{C}$ intake and complement titres; Ecker, Pillemer, Wertheimer and Grodis (1938) and Chu and Chow (1938) a correlation betwcen complement and serum vitamin $\mathrm{C}$ levels. The connection between the two substances may lie in the capacity of aseorbic acid to take part in redox mechanisms in the blood (Eeker, Pillemer, Martiensen and Wertheimer 1938). Ascorbie acid reactivates both complement deprived of its C'3 and complement inactivated by oxidation, and increases the titre of complement from scorbutic guinea-pigs. Hexoxidase, which oxidizes aseorbic acid. will rednce complement activity in vitro, apparently by acting on ('/3.

On the other hand, Zilo (1915) and Koch and Smith (1924) found high complement titres in scorbutic animals, and. like Heinicke (1934) and Kapmick and Cope (1940), were unable to demonstrate any relation between complement and ascorbic acid levels (see also Crandon, Lund and Dill 1940, Feller et al. 1942).

These results have been confirmed (Spink et al. 1942. Agnew et al. 1942): in human subjects with low serum ascorbic acid the injection of ascorbic acid did not raise the complement titre; nor was oxidation of ascorbic acid with $\mathrm{CuCl}_{2}$ accompanied by a reduction of the complement titre. In guinea-pigs, variations in serum ascorbic acid induced by diet were not reflected in complement titres. Similarly Kodicek and Traul, (1943) conld not alter complement levels in any significant manner by variations of ascorbic acid intake in guinea-pigs; they suggested that since there was a wide nomal variation in eomplement titres, previously observed associated rariation in the levels of the two substances may have been fortuitous. Noreorer, the in vitro bactericidal power of human blood was not altered by an inerease or decrease of ascorbic acid (Spink, Agnew, Mickelsen and Dahl 1942). These authors, however, noted differences between the effieacy of synthetic ascorbic acid and native vitamin $\mathrm{C}$, and the diserepaneies may perhaps depend on the action of substances associated with native vitamin C. The vitamin is certainly 
among the reducing agents that reactivate oxidized complement, and there appears to be a variable relation between the two, whose nature is as yet obscure.

The hypothetical dietary deficiency in complement-poor guinea-pigs associated with lack of green food may conceivably be a vitamin K deficiency, for Büsing and Zuzack (1943) report a marked correlation between the complement titre and vitamin $\mathrm{K}$ intake in the young chick.

\section{Complement in Other Animals.}

The greater part of the work on complement has been concerned with guinea-pig scrum. The quantitative and qualitative variations in complement among different species of animal have received little study. Shrigley and Irwin (1937) found no association between hrmolytic and bactericidal activity in guinea-pig, sheep or rabbit sera, but noted a wide species variation of both. Eeker, Pillemer and Kuehn (1942) fractionated the sera of man, dog, eat, guinea-pig, monkey, rabbit and sheep, and found wide species differences in the effect on opsonic powers of measures designed to inactivate or remove each of the four components. No generalization about complement titres in mammals is possible without specifying the components present, and the method of measuring the complement-e.g. bactericidal or hrmolytic-and it must be remembered that in all cases the titre is determined by the amount of the least plentiful component. In human sera, for example, it is $C^{\prime} 2$ (Hegedüs and Greiner 1938, Heidelberger and Mayer 1942) and to some extent C'3 (Dozois, Seifter and Ecker 1944). Nouse serum, which has no complementary activity, lacks C'2 (Brown 1943).

\section{Complement Fixation.}

We have noted that the absorption of complement is a general property of bacterial cells that have been sensitized by a specific antibody; though some bacteria undergo lysis as a result of this absorption, while others do not. That complement fixation, apart from the observation of any resulting change in the bacterial cells, could be used as a general method for the detection and titration of specific antibodies was first demonstrated by Bordet and Gengou (1901). In these experiments a suspension of a given bacterium was allowed to react with a specific antiserum in the presence of complement. After time had been allowed for the reaction to take place, red cells and a suitable dilution of hæmolysin were added, and the mixture was incubated again for 1 hour at $37^{\circ} \mathrm{C}$. It was found that in these circumstances no lysis tock place, showing that no free complement was present, and it was reasonably inferred that it had been absorbed by the bacteriumsensitizer complex.

The reaction may be summarized as follows:

(a) Bacteria + Sensitizer + Complement $=$ Fixation.

(b) $(a)+$ Red Cells + Hæmolysin $=$ No hæmolysis.

It will be noted that $(b)$ is simply an indicator reaction. It has no connection with the fixation of complement by the sensitized bacteria. That reaction has already occurred in $(a)$.

Using this technique Bordet and Gengou demonstrated sensitizers for Past. pestis, B. anthracis, Salm. typhi, the bacillus of swine plague, and Proteus vulgaris in the corresponding antisera.

The following year Gengon showed that the same phenomenon occurs when soluble proteins are allowed to react with their specific antisera in the presence of complement. He demonstrated specific complement fixation, using as antigens cow's milk, egg-white, horse fibrinogen, and heated dog serum, and as antibody in each case the serum of a rabbit which had been immunized against the corresponding protein. 
It thus became clear that complement fixation was a general reaction, liable to occur when any antigen was allowed to react with its specific antibody in the presence of complement.

The strdy of the relation between complement fixation and precipitation has yielded results of great theoretical importance. Gay (1905) showed that the precipitate formed by the interaction of an antiserum with the corresponding antigen frequently had the power of absorbing complement. This suggested that precipitation and complement fixation might be two aspects of a single reaction. Muir and Martin (1906b) found that, although there was a close correlation between precipitation and complement fixation, the correlation was not absolute. They showed that complement fixation might occur in the absence of precipitation, and that, in the presence of a constant amount of antiserum, there was a particular amount of antigen that gave maximal complement fixation, while amounts much greater, or much less, than this might fix little or no complement.

Dean (1912) elucidated the cause of thesc earlier discrepancies. In constant-antibody titration series he found that mixtures giving maximal complement fixation contained less antigen than mixtures giving maximal precipitation; in the one antibody was in excess, in the other antigen was in excess. He showed also that complement was fixed in the carly stages of precipitation, before visible flocculi had formed, and concluded that fixation was maximal in slowly aggregating mixtures of antigen and antibody, where the relative persistence of small aggregates ensured a large total absorbing surface. The two maxima were not due to the reactions of different antibodies but were different secondary results of a single antigen-antibody reaction. Goldsworthy (1928) confirmed these results, but showed that the relation of the maximal complement-fixing and precipitating mixtures described by Dean were determined by the particular system he used. With slowly reacting antisera, the maximal fixing mixture coincided with the optimal ratio ; with rapidly reacting antisera, it was in the region of antibody excess. The determiming factor appeared to be the exposure to complement of a maximal absorbing surface of antigen-antibody particles for the maximum period. Later studies show that there is no constant relation between the point of maximal fixation and the established reference points of constant-antibody titrations. With an ovalbumin system, the point coincided with the equivalence ratio (Maltaner and Maltancr 1940); with pneumococcus carbohydrate, Goodner and Horsfall (1936) found it equal to the ratio for maximal precipitation in the antigen excess region. Rice and Sickles (1942) and Rice (1943) in a more extensive study of the pneumococcal carbolydrate system record a close relation between the ratio for optimal fixation and the antibody content of the serum, and note that the ratio uswally falls in the region of antibody excess. Apparently the size of the antigenic particle employed appears to affect the ratio. Both Spooner and Bawden (I935) and Platt (1936), working respectively with tobacco mosaic virus and pneumococei, found the ratio in the antigen excess region. They also noted that the ratio was higher with low than with high serum concentrations. According to Platt the result is due to the fact that with a particulate antigen the complement-absorbing surface is determined by the concentration of antigen, and a large number of cocci sub-optimally sensitized for agglutination would absorb more complement than fewer fully sensitized cocci.

\section{The Rôle of the Components in Fixation.}

During the fixation of complement very little protein is removed from guineapig serum. Haurowitz (1940) found little was added to the weight of specific precipitates formed in its presence. Heidelberger (1941) estimated that $0 \cdot 25-0 \cdot 40$ mgm. per ml. of complement protein, mainly C'1, was added to specific precipitates. Similar proportional weights are added when hæmolytic antibody combines with washed red-cell stromata (Heidelberger and Treffers 19.1). In the sheep redcell hæmolytic system, from 2 to 7 molecules of C'1 were fixed for every molecule 
of hæmolytic antibody, according to the initial concentrations of the various reagents (Heidelberger, Weil and Treffers 1941). The regularity of the molecular ratio in a given system, and the fact that $\mathrm{C}^{\prime} 1$ is in excess of hæmolysin, provides quantitative confirmation of the view that the action of complement is not enzymic. However, Haurowitz and Yenson (1943), in confirming the quantitative findings of Heidelberger and his colleagues, estimated that, while the complement required for hæmolysis, if spread in a unimolecular film on the surface of the red cell, would cover 6 per cent. of it, the amount of saponin or oleic acid required for non-specific lysis of a red cell was over 1,000 times the amount required for a complete unimolecular film. In their view, the high activity of a small amount of complement suggested an enzymic nature. It should be noted that heat inactivation of complement does not destroy all its combining powers (Heidelberger 1941, Pillemer, Chu, Seifter and Ecker 1942).

By testing the complementary activity of all possible recombinations of the varions fractions and mixtures of fractions of guinea-pig complement, Pillemer, Seifter and Eeker (1942) have shown that $C^{\prime} 1$ is not the only combining component of eomplement. C'4 and $\mathrm{C}^{\prime} 2$ are also fixed, but contribute little in the absence of $\mathrm{C}^{\prime} 1 . \mathrm{C}^{\prime} 1$ and $\mathrm{C}^{\prime} 2$ are fixed in the absence of $\mathrm{C}^{\prime} 3$ and $\mathrm{C}^{\prime} 4$ (see also Ueno 1938). Little $\mathrm{C}^{\prime} 3$ is fixed, but this acts in a manner similar to a catalyst to produce the complementary effect (e.g. hremolysis) on systems with which $\mathrm{C}^{\prime} 1, \mathrm{C}^{\prime} 2$ and $\mathrm{C}^{\prime} 4$ have combined.

With hnman complement, they found the fixation of $\mathrm{C}^{\prime} \mathbf{2}$ and $\mathrm{C}^{\prime} 3$ varied with the nature of the bacterium employed as antigen. In the absence of $\mathrm{C}^{\prime} \mathrm{I}$ or $\mathrm{C}^{\prime} 4$, very little fixation occurred. In the absence of $\mathrm{C}^{\prime} 2, \mathrm{C}^{\prime} 4$ was fixed in large amounts, and in the absence of $\mathrm{C}^{\prime} 3$, both $\mathrm{C}^{\prime} 2$ and $\mathrm{C}^{\prime} 4$ were fixed. The influence of the different components upon fixation of others was, as in the guinea-pig, curiously arbitrary. As with guinea-pig complement, all four components were necessary for the two specific complement effeets, lysis of sensitized red cells, and death of sensitized bacteria. Neither purified $C^{\prime} I$ and $\mathrm{C}^{\prime} 2$, nor fractions containing $\mathrm{C}^{\prime} 3$ or $\mathrm{C}^{\prime} 4$, were active alone, though, if fixed to the cell, they were active on the subsequent addition of the remaining components. Bactericidal and hemolytic power of single components and of various mixtures ran exactly in parallel, indicating a close similarity of action on the respective cell surfaces (Dozois, Seifter and Eeker 1943, 1944; Seifter, Dozois and Eeker 1944).

The nature of the fixation is still in doubt. Heidelherger, Weil and Treffers (1941) suggest that complement-combining components differ from normal globulin in being able to form loose, dissociable mions with antibody molecules, and that these unions are stabilized when the components are surrounded by antibody molecules in specific antigen-antibody aggregates. In cases where excess of the component combines in presence of minimal amounts of antibody, a similar loose combination with antigen is postulated.

Complement is inhibited by varions anticoagulants, but they appear to act by reason of their acidie or basic nature or of their large molecular size, and their aetion does not indicate an immediate connection between coagulation and complement (Wadsworth, Maltaner and Maltaner 1937, Eeker and Pillemer 1941). For example, C'2 and C'4 are unstable in acid solutions, which at the same time depress the ionization of ealcium necessary for coagulation (Pillemer and Eeker 194l $\ell$ ); again. the action of lipoid solvents in reducing complementary activity may be attributed to their independent action on hydrated complement, for the dehydrated complement may be extracted by alcohol and ether without loss of activity on reconstitution with water (Ecker, Pillemer and Grabill 1938). The tanning agent, sodium hexametaphosphate, inactivates complement aplarently by an alteration of the groups on which the activity depends, as a result of combination with the basic groups of the proteins concerned (Gordon and Atkin 1941). 


\section{Opsonins and Bacteriotropins.}

Wright and Douglas $(1903,1904)$ named and described the opsonins-thermolabile, relatively non-specific substances, occurring in normal serum, acting on a variety of bacteria and rendering them liable to phagocytosis by leucocytes. Neufeld and Rimpau $(1904,1905)$ named and described the bacteriotropins-thermostable antibodies occurring in the serum of immunized animals, acting specifically on the bacteria against which the animals had been immunized, and rendering them liable to phagocytosis.

The mode of action of the bacteriotropins is clearly analogons to that of the precipitins, agglutinins and lysins. Like these antibodies they are relatively thermostable. Like them they unite specifically with an antigen carried by the bacterial cell. We can safely assume that the anchoring of the antibody globulin to the antigen alters the condition at the cell surface in such a way as to make it easier for the leucocytes to engulf the bacteria, just as it makes the bacteria saltsensitive, and, in certain cases, renders the cell membrane more permeable. It would appear that one important factor is a lowering of the negative charge, and hence of the difference in electrical potential between the bacteria and the surrounding fluid.

Falk and Matsuda (1926) found that alterations in the charge carried by pneumococei induced by the addition of lanthanum nitrate or sodimm oleate had a striking effect on the phagocytosis of these organisms, and Broom and Brown (1930) were able to decrease the phagocytosis of staphylococci, previonsly sensitized with serum, by preventing the usual rednction in surface charge by the addition of potassium ferrocyanide. Mudd and others (1929) studied the changes induced by specific antisera in four strains of acid-fast bacilli. They found that sensitization $(a)$ increased the cohesiveness of the bacilli ; $(b)$ decreased the electric charge, as evidenced by a decrease in velocity of cataphoresis; (c) decreased the "wettability" of the bacilli by oil, as evidenced by their distribution at an interface between tricaprin and normal saline, and $(d)$ inereaser their susceptibility to phagocytosis.

Bacteria may be opsonized by non-specific substances, taming agents like gallotannie acid (Reiner and Fischer 1929, Reiner and Kopp 1929), tannie acid (Frend 1929) and ahm, ehrome and ferric salts (Neufeld and Etinger-Tulcyznska 1929). Gordon and Thompson (1936) and Gordon and Atkin (1938) made a systematic study of the artificial opsonization of Salm. typhi and Staph. aureus. Metallie tanning agents appeared to act by combination with carboxyl groups, the vegetable tamins with the basie groups of the bacterial proteins. The two organisms differed in their susceptibility to certain agents; Staph. aureus, perhaps by virtue of having on its surface more acidie groups than Salm. typhi, was more susceptihle to protamine, a highly basic protein. These authors noted that while potassium oxalate and distilled water reversed opsonization, opsonization by specific serum was more diffient to reverse.

There remains the problem of the relation of the normal opsonins of Wright and Donglas to the bacteriotropins of Neufeld and Rimpau. In their thermolability the opsonins resemble complement; and their identity with this serum constituent seemed at first to be rendered probable by the observation of Muir and Martin (1906a) that such complexes as red cells and hæmolysin, a protein antigen and its corresponding antibody, or bacteria and a specific antibacterial serum, all removed the opsonin from a normal serum, at the same time as they removed the complement. The observation of Neufeld and Hüne (1907), that ahsorbing a normal serum with yeast had a similar double effect, seemed to point in the same direction. But there were difficulties in this simple conception. T'he normal serum opsonins 
do not show the same strict specificity as the bacteriotropins-normal unheated serum promotes the phagocytosis of a wide variety of antigenically unrelated bacteria-but from the first there was evidence that suggested the presence of specific factors of one kind or another. Thus, it has been the general experience that the serum of any one person varies in its opsonic effect on different bacteria; that the sera of different individuals may show striking differences when tested against the same bacterium; and that any one person may show a variation in the opsonizing power of his serum for a particular bacterium, especially as the result of infection with that organism, or of artificial immunization. Variations of this kind have been studied extensively by Wright and his colleagues (see Wright 1909). Moreover, evidence pointing in the same direction was obtained by in vitro experiments. Bulloch and Western (1906) succeeded in removing the opsonic power of normal serum for particular bacteria by selective absorption. Other workers were unable to confirm these results, but the careful studies of Hektoen (1908) on the normal opsonins acting on the red blood corpuscles of different animal species afforded strong support for the correctness of Bulloch and Western's contention.

The explanation of these anomalous findings would appear to lie in the fact that the opsonic effect of normal serum resembles its hæmolytic and bacteriolytic effects in being dependent on both antibody and complement. Chapin and Cowie (1907) showed that normal serum may have its opsonic power for a staphylococcus removed by absorption with that organism in the cold, but that such absorbed serum may still have the power of reactivating normal serum that has been inactivated by heat. The cocei that have been used for absorption, when washed and resuspended in saline, show little if any increased susceptibility to phagocytosis in the absence of unheated serum. Later (Cowie and Chapin 1907) they showed that normal serum loses almost all its opsonic power when diluted fifteen times with saline. If, however, such diluted serum is added to another sample of serum that has been inactivated by heating at $55^{\circ}-60^{\circ} \mathrm{C}$. for 10 minutes the mixture is almost as active in promoting phagocytosis as was the original unheated, undiluted serum. It would appear that normal serum contains specific sensitizing antibodies, present in amounts so small that they are ineffective in a dilution of $1: 15$ or more. Even in undiluted serum these antibodies are unable, by themselves, to alter the bacterial surface sufficiently to promote phagocytosis; but when complement is adsorbed by the incompletely sensitized bacteria the necessary change in surface conditions is produced. It would seem, also, that complement is not without effect on the action of the bacteriotropins; for G. Dean (1907) has shown that the action of a heated antiserum is increased by the addition of a little unheated normal serum. Sleeswijk (1908) has confirmed Dean's results, and concludes that sensitization, as a preliminary to phagocytosis, is primarily dependent on a specific antibody. When this antibody is present in adequate concentration it can produce its effect in the absence of complement, though added complement may enhance it. When the antibody is present in very small amount complement is necessary to induce adequate sensitization. (See also Ward and Enders 1933.)

It is of interest to note that the action of complement in opsonization appears to differ in some way from its aetion in hæmolysis ; since, as Gordon, Whitehead and Wormall (1926b) have shown, the addition of ammonia renders complement inactive as a hrmolytic agent, but removes none of its opsonie activity.

Gordon and Thompson (1935) inactivated complement by ammonia, Congo red, acid, 
alkali and hypotonic solutions and certain sodium and potassium salts. In each case, by adjusting the conditions of treatment it was possible to destroy the hremolytic activity without loss of opsonic activity. They concluded that the opsonic system differs markedly from the complement system. Haltaner (1935) suggested that ammonia-treated serum opsonized because the fourth component was supplied by the leucocytes employed in the opsonic mixture. Gordon (1937) confirmed Ialtaner's observations, but maintained that the opsonin was not closely related to complement, since specifically sensitized red cells that have absorbed from ammonia-treated serum all the components of complement excepting fourth component are not lysed in the presence of leucocytes, a source of fourth component. It is possible, however, on the basis of these experiments to conclnde that all but the fourth component is concerned in opsonization, especially since Pillemer, Seifter and Ecker's demonstration (1942) that no hæmolysis takes place unless $\mathrm{C}^{\prime} 4$ is fixed previously, or simultaneously with $\mathrm{C}^{\prime} 1$. On the other hand, the experiments of Ecker, Pillemer and Kuehn (1942) clearly demonstrate the lack of any constant association, in the sera of a number of mammals, of opsonization with one or other of the four recognized components of complement.

Ecker, Weisberger and Pillemer (1942) and Ecker, Pillemer and Kuehn (1942) measured the opsonic action of large numbers of sera from varions mammals on staphylococci, both alone and in conjunction with staphylococcal antibody (bacteriotropin). In general, mixtures of heat-labile opsonin and specific bacteriotropin had less opsonic activity than cither acting separately. There was no enhancement of opsonic activity except with weak concentrations of opsonin and antibody. Though there was no relation between opsonic power and hrmolytic complement titre in any of the species studied, yet the processes inactivating complement also reduced opsonic power. All the normal opsonins were heat-labile; those of the guinea-pig, monkey and sheep were inactivated by ammonia ; those of the monkey and sheep by absorption with yeast. The opsonic powers were variously distributed between mid-piece and end-piece fractions, mid-piece predominating markedly in man and guinea-pig, and moderately in monkey and cat, little in shcep and rabbit. In the dog, neither fraction was opsome, but a mixture of the two was.

The observation of Gordon and Thompson (1937) that protamine could opsonize bacteria led Gordon and Atkin (1939) to test globin. Globin oceurring naturally in the body as hæmoglobin is a distinctly basic protein, forms salts with acid proteins like casein, and proved to be a good artificial opsonin. This observation not only offers a possible analogy for a natural opsonin, but clearly shows that body proteins other than those known to be components of complement can act as opsonins.

The quantitative measurement of opsonic or bacteriotropic action is a technical problem of great difficulty. The system is a very complex one, including living phagocytic cells the uniform distribution of which is exceedingly difficult to ensure. It is impossible, even when the tubes are rotated by one of the various mechanisms that have been devised, to obtain results of the same order of accuracy as in the precipitin, agglutination or hæmolytic reactions (see, for example, Hanks 1940).

In the method employed by Wright and his colleagues, serum, leucocytes and bacterial suspensions are mixed in capillary tubes and incubated at $37^{\circ} \mathrm{C}$. for 15-30 minutes. Their contents are then expelled on to slides. Films are prepared and stained, and the bacteria contained in the first 50-100 leucocytes encountered are enumerated. The relative opsonic effect of two sera is expressed as the ratio between the numbers of bacteria taken up, under their influence, by the same number of leucocytes. As might be supposed, the experimental error is a high one (see Greenwood 1913). An alternative method, suggested by Klein (1907), is to fix on some particular degree of phagocytosis as an arbitrary end-point, and to dilute the serum under test until this end-point is reached; but it seems probable that the experimental error will still be large. 
The test has recently been revived by Huddleson and his colleagues (1933) as the opsono-cytophagic reaction and used as a measure of the immune response in brucella infections and pertussis. No attempt is made to measure a precise ratio of control and test opsonic effects, but bloods are arbitrarily graded according to the number of bacteria ingested by a standard number of leueocytes.

\section{The Toxin-Antitoxin Reaction.}

This reaction hardly falls into the same category as those we have been considering, since the titrations are carried out in vivo. There is, however, no doubt at all that the neutralization of toxin by antitoxin depends on the same factors, and is governed by the same laws, as any other antigen-antibody reaction; and we have seen that, when toxin and antitoxin are mixed in the test-tube, specific precipitation occurs. It is convenient to discuss in this chapter some of the peculiarities recorded in the in vivo tests, since many of these were noted during the pioneer studies of Ehrlich (1897) on the standardization of diphtheria antitoxin, and so formed the basis of the controversy in regard to antigen-antibody reactions in general.

The starting-point of any method of standardization is the definition of units of measurement. When these units have to be defined in terms of some in vivo reaction, the resulting measurements are liable to errors of a kind different from those involved in in vitro titrations. These errors and the ways in which they can be avoided or allowed for are discussed in Chapter 43. For the moment we are concerned only with the general nature of the quantitative results that have been recorded.

The first reagent to which a unit was assigned was the toxin. This unit, the Minimal Lethal Dose, may be defined as follows:

The Minimal Lethal Dose (M.L.D.) of diphtheria toxin is the least amount that will, on the average, kill a guinea-pig of $250 \mathrm{gm}$. weight within 96 hours after subcutaneous inoculation.

Ehrlich (1897) defined the unit of antitoxin in terms of the M.L.D. as the smallest amount of antitoxin that will neutralize 100 M.L.D. of toxin. This left the M.L.D. as the fundamental unit; but it was soon discarded. Toxin, on storage or on treatment with a variety of physical or chemical reagents, has a tendency to lose its toxicity while retaining its combining power for antitoxin. It is converted into toxoid. Under these conditions the definition of a unit of antitoxin, A.U., in terms of the number of II.L.D. of toxin that it will neutralize clearly becomes impossible, since no standard stable toxin can be preserved. An antitoxic serum, when dried in vacuo and stored at $0^{\circ} \mathrm{C}$., remains stable over long periods of time, and hence provides an excellent standard of reference. Ehrlich's original antitoxin has been adopted as an international standard, and the correct definition of a unit of diphtheria antitoxin is as follows:

One unit of Diphtheria Antitoxin (1 A.U.) is contained in that amount of antitoxic serum that has the same total combining capacity, for toxin and toxoid together, as one unit of Ehrlich's original antitoxin.

The fact that one unit of Ehrlich's original antitoxin happened to neutralize 100 M.L.D. of the particular toxic filtrate with which he was working has now only a historical interest. The units of other antitoxins are defined in a similar way, some particular specimen of the antitoxic serum in question being selected as an arbitrary standard, against which all subsequent samples are measured.

The actual procedure consists in first determining the quantity of a given toxic 
filtrate that is neutralized, or nearly neutralized, by one unit of the standard antitoxin, and then determining the amount of the antitoxic serum under test that will neutralize, or nearly neutralize, this amount of toxic filtrate. This amount of the antitoxic serum will contain 1 A.U. Since the two tests are performed within a few days of one another there will be no significant change of toxin to toxoid during the interval, and the proportions of the two reagents in the toxic filtrates will remain constant.

This method of titration led to the definition of two other doses of toxin"toxin" here, as in the case of the M.L.D., referring in practice to a toxic filtrate, containing both toxin and toxoid.

The Limes $N u l\left(L_{0}\right)$ dose of diphtheria toxin is the largest amount of toxin that, when mixed with one unit of antitoxin and injected subcutaneously into a guinea-pig of $250 \mathrm{gm}$. weight, will, on the average, give rise to no observed reaction.

Actually, the $\mathrm{L}_{0}$ dose is usually recorded as the dose that, when tested in this way, gives rise to a minimal local cedema.

The Limes Tod $\left(L_{+}\right)$dose of diphtheria toxin is the smailest amount of toxin that, when mixed with one unit of antitoxin and injected subcutaneously into a guineapig of $250 \mathrm{gm}$. weight, will, on the average, kill that guinea-pig within ninety-six hours.

Other doses of toxin, determined by other methods of testing, have been defined in terms of their combining power for antitoxin, and are now commonly employed for standardization purposes (see Chapter 61), but the relation between the $\mathrm{L}_{0}$ and $\mathrm{L}_{+}$doses is the matter that concerns us here.

If toxin combined with antitoxin in constant proportions giving firm chemical union it would be expected that

$$
\mathrm{L}_{+} \text {toxin }-\mathrm{L}_{0} \text { toxin }=1 \text { M.L.D. }
$$

In fact it does not. The difference between the $\mathrm{L}_{+}$and the $\mathrm{L}_{0}$ dose has been found, with different toxic filtrates, to vary from 10 M.L.D. to 100 M.L.D. or more.

Ehrlich attempted to account for this phenomenon, to which his name has often been attached, by postulating the existence, in toxic filtrates, of a special modification of toxoid, epitoxoid, having less affinity than toxin, or unmodified toxoid, for antitoxin. Over the range between $\mathrm{L}_{0}$ and $\mathrm{L}_{+}$doses he assumed that the additional toxin added merely displaced epitoxoid, and that only when all epitoxoid had been displaced from its union with antitoxin did the added toxin remain free to exert its lethal effect. Whether toxoid differs from toxin in its affinity for antitoxin, or whether varieties of toxoid exist that differ from one another in this respect, we do not know with any certainty. Since we have many reasons other than the observed difference between the $\mathrm{L}_{0}$ and the $\mathrm{L}_{+}$doses of toxic filtrates for discarding the hypothesis of chemical union in constant proportions between antigen and antibody, we are not faced with Ehrlich's dilemma, and have no need to postulate the existence of epitoxoid, or of any of the other special varieties of toxin and toxoid that he evolved to explain the results observed in his later studies on partial neutralization.

These studies, and those of many subsequent observers, made it clear that, when varying amounts of antitoxin are added to a constant amount of toxin, the curve of neutralization is not linear, as it would be if we were studying the neutralization of a strong acid by a strong base. (See Arrhenius and Madsen 1902, 1904, Arrhenius 1915, von Krogh 1911, Glenny et al. 1925.) The observed departure from linearity is not peculiar to diphtheria toxin and antitoxin, but is characteristic of toxin-antitoxin reactions in general (see, for instance, Burnet 1931); and, as we 
have seen, is equally well exemplified by the results obtained when studying the absorption of any antibody by any antigen.

We have already discussed the Danysz phenomenon (p. 216). It was first observed in mixtures of diphtheria toxin and antitoxin, but a similar phenomenon has been shown to occur in other antigen-antibody reactions, and analogies have been drawn with chemical reactions of other kinds. Noriyama (1937), for instance, noted that the precipitating effect of tannic acid on proteins varies according to the way in which the reagents are mixed. He was able to elicit Danysz effects in urease-anti-urease and ricin-antiricin systems when tannin replaced the specific antibody in the systems. He attributed the effects to the capacity of the complex proteins concerned to form several quantitatively different compounds according to the proportions present at first mixing-compounds which, being incompletely reversible, do not contribute to a redistribution of two reagents when more of one reagent is added. Pappenheimer (1942), in a recent review, points out that both the discrepancy between $\mathrm{L}_{0}$ and $\mathrm{L}_{+}$doses of diphtheria toxin and the Danysz effect can be adequately explained on the assumption that toxin ( $\mathrm{T}$ ) and antitoxin (A) form compounds varying in constitution from $\mathrm{T}_{2} \mathrm{~A}$ to $\mathrm{TA}_{8}$ (see Pappenheimer, Lundgren and Williams 1940). In the neutral mixture, the average composition of the complexes may be $\mathrm{TA}_{2}$. The addition of an excess of $\mathrm{T}$ would result in complexes TA and $\mathrm{T}_{2} \mathrm{~A}$, and only with toxin in marked excess would enough be left free to kill the guinea-pig. Similarly in the Danysz effect; the first fraction of toxin would form slowly reversible complexes, like, for example, TA and its polymers, leaving insufficient antitoxin for union with the remainder of the toxin added later.

The state of toxin and antitoxin in over-neutralized, neutral and underneutralized mixtures of the two has been studied by Eagle (1937), who precipitated toxin-horse-antitoxin mixtures with an antiserum to horse globulin. Assuming that free toxin was not carried down in the precipitate, Eagle was able to show that antitoxin unites with excess of toxin to form a toxic complex, and toxin with an excess of antitoxin to form a complex with antitoxic properties. Only in one proportion was a precisely neutral complex found. Here again we have recorded analogous findings in the reactions of other antigens with other antibodies. This phenomenon is more readily explicable on the adsorption hypothesis than on the theories advanced by Ehrlich, or by Arrhenius and Madsen.

\section{Other Manifestations of the Antigen-Antibody Reaction.}

The addition of antigens to antibody even in optimal proportions is not always followed by a visible precipitation. Either of the two reagents may be too weakly reactive or too dilute. The classical method of detecting the union of antigen and antibody in these circumstances is by the complement-fixation reaction (see p. 232) ; but the reaction may be made manifest by other less elaborate means. The high sensitivity of the varions flocculation tests for syphilis (see Chapter 81) is due to the addition of agents, such as lipins, which shift the precipitating system more to the hydrophobe state. Again, dyes may act both as sensitizers and as indicators in precipitin reactions. For instance Dean (1937), in a study of non-specific precipitation of proteins by the electropositive dye isamine blue, found that appropriate dilutions of the dye added to constant-antibody titration series markedly increased the sensitivity of the titration; Berger (1943) made use of Janus green and Victoria blue for the same purpose in the flocculation test for syphilis. Cannon and Marshall (1940), taking advantage of the fact that the amount of antibody required for the surface sensitization of large particles is smaller than that required for the surface sensitization of the same bulk of material in a more finely divided 
state (see Zinsser 1930), devised a method of detecting small non-precipitating amounts of antibody by titrating them against suspensions of eollodion particles coated with the appropriate antigen (see also Lowell 1943).

The fine turbidity which preeedes flocculation in strongly reacting antigen-antibody systems, and whieh may be the end-result in weakly reacting systems, can be measured by photoelectric and other types of nephelometer, and the measures made the basis of titrations of antigen or antibody (see, for example, Libby 1938, Pope and Healey 1938, Bukantz, Cooper ånd Bullowa 1941). Advantage may also be taken of plysicoehemieal properties of antigen-antibody eompounds other than precipitability in the presence of eleetrolyte. Thus, du Noüy and Hamon $(1935,1936)$ found a well-marked maximum of viscosity to oecur in mixtures containing optimal proportions of diphtheria toxin and antitoxin.

After initial sensitization, which is relatively rapid, the agglutination of bacteria depends on their rate of collision under the influence of Brownian movement and convection currents in the suspending fluid. If the cells are brought together rapidly by centrifugation, agglutination may be rapidly detected by the resistance to resuspension of the centrifuged mass, as compared with that of a control preparation of bacteria without antibody.

An interesting consequence of an antigen-antibody reaction is the swelling of pneumoeoccal eapsules in the presenee of specific anti-capsular rabbit serum, first deseribed by Neufeld 1902, Neufeld and Etinger-Tulezynska 1931 (see Chapter 24). The swelling reaction can be elicited in both living capsulated pneumococci, and in desiceated organisms (Brown 1938). The swelling may be reversed if the antibody is destroyed by beat (EtingerTulezynska 1933) or by digestion of serum proteins with papain (Kalmanson and Bronfenbrenner 1942). It is reversed if the antibody is removed by repeated washing, or by adding an excess of specific polysaccharide to a suspension of washed swollen eells (Kempf and Nungester 1942).

Aceording to Hershey (1940), the capsule does not appear to present a smooth surface to the action of antibody, for the amount absorbed is greater than such a surfaee could accommodate as a elosely packed unimoleeular layer; it is possible that the capsular polysaceharide is in the form of a loose fibrous gel, whose surface and interstiees provide ample surface for the adsorption of large amounts of antibody. The electron-mierographie studies of Mudd and his eolleagues support this view. As we have already noted, homologous antibody is deposited on Salm. typhi in an electron-opaque layer about as thick as a mimoleeular film of globulin (Mudd and Anderson 1941). Under the intluence of rabbit antibody, the pneumococeal capsule, which is relatively transparent to the electron beam and appears to be a low density gel, beeomes opaque, and increases in thiekness. The inerease in thickness is more than 25 times that of a unimolecular layer of antibody, even assuming that the globulin is paeked with its maximum length perpendicular to the surfaee of the capsule (Mudd, Heimmets and Anderson 1943). There is elearly some adsorption of non-specific material, for the opaeity of swollen capsule is increased in the presence of non-speeific serum proteins. It seems probable that the swelling is not purely an antigen-antibody effect. Jolnson and Dennison (1944) measured the volume increase during swelling by two independent methods; it varied from 2.9 to $9.5 \mu^{3}$ by one method, and from $7 \cdot 4$ to $10 \cdot 1 \mu^{3}$ by the other. They concluded that the volume increase could not be aecounted for solely by the molecular volume of antibody absorbed, but might be due to a hydration of the capsule following the antigenantibody reaetion.

We may refer to the "adhesion phenomenon," in whieh spirochaetes specifically sensitized by antibody adhere readily to baeterial cells added to the reacting mixture: Advantage is taken of this behaviour in the serological study of spirochaetes (p. 912). 


\section{THE QUALITATIVE ASPECTS OF THE ANTIGEN-ANTIBODY REACTIONS-IMMUNOLOGICAL SPECIFICITY}

This problem has been attacked mainly from the side of the antigens, but it will facilitate our discussion of the recorded data to deal first with the probable structure of the serum antibodies with which they react.

\section{The Nature and Properties of the Serum Antibodies.}

We have already made frequent references to the view that the serum antibodies are specialized globulins. The properties of normal serum globulins have been established by a variety of methods. The fractionation of serum by precipitants like ammonium sulphate yields a mixture of proteins differing from one another physically, and chemically in lipin and carbohydrate content (see Tiselius $1937 a$, Stenhagen 1938, Hewitt 1938). It is difficult to decide how far these fractions are the results of the chemical handling the native protein receives in its preparation. The measurement of the rate of migration of serum proteins in an electrical field (Tiselius $1937 a$ ) provides convineing evidence that native serum contains several well-defined components. After a given period of exposure in the Tiselius electrophoretic apparatus, the proteins of native serum separate into a number of zones, each containing particles having approximately the same electrophoretic mobility. In normal horse and rabbit sera, the albumin forms a single well-defined zone, and there are three components of globulin, $\alpha, \beta$ and $\gamma$, the $\alpha$ being the most, and the $\gamma$ the least mobile.

The current hypotheses of protein structure are none of them sufficiently developed to warrant description in a book of this kind. The student may consult the reviews of Pauling and Niemann (1939), Svedberg (1939) and Synge (1943) for details of the controversies regarding the precise architecture of the proteins. It is sufficient for our purpose to note those features of protein chemistry that are relevant to the understanding of serology in its present state. In the first place, it is generally accepted that the proteins are built up of polypeptide chains of amino-acids, and that the pattern of recurrences, whether regular or irregular, of the various amino-acids in the chains will vary from protein to protein according to the relative proportion of constituent amino-acids. A secondary structure is imposed on the protein molecule by the folding of the chains so that several of them are held together by hydrogen-bonding and other interatomic electrical forces. In the so-called fibrous proteins, the arrangement of chains, as revealed by X-ray analysis, is markedly regular (see Astbury 1943); keratin and silk fibroin are examples of this type of protein. The globulins belong to the class of nonfibrous proteins, and here it is supposed that polypeptide chains are primarily folded into a lamina that has approximately the thickness of the polypeptide chain, and that these laminæ are built up into a more or less globular protein molecule, being held together by interatomic forces similar to those that hold the chains in laminæ. Whether the laminar structure exists or not, it is a fact that certain proteins can be spread on an air-water interface into films whose thickness is much less than the known diameter of the protein molecule; some proteins are denatured by this process, but others, including serum proteins, will re-form into "globular" molecules after spreading.

That is to say, although protein molecules compared with smaller molecules 
are relatively rigid structures, by reason of the large number of interatomic forces which, acting together, stabilize the geometric form of the molecule, they are also capable of some distortion, which may or may not be reversible. In much of the earlier work on protein molecules, the assumption was made that protein molecules behaved as spheres, i.e. were isodimensional. It is now evident that some molecules, including the glolulins, are markedly anisodimensional, behaving as though they were rod-shaped, or at any rate larger in one dimension than in any other. The term "globular," applied to the non-fibrous proteins, does not imply that they necessarily behave as spherical or nearly spherical units. For example, in the serum reactions, the shape of the molecule, and the degree to which it may be changed, will affect the range of permissible speculation about the structure of the antigen-antibody complexes.

The molecular weight of proteins may be determined from their sedimentation rate in the intense gravitational fields of high-speed ("ultra "-) centrifuges. Those of non-fibrous proteins range from 17,500 for lactalbumin, to over 6 millions for molluse hæmocyanins. The molecular weight of the normal serum globulins and the majority of antibody globulins in the horse and rabbit are of the order of 150,000 (see Svedberg 1937, Heidelberger and Pedersen 1937). Neurath (1939) estimated that the physicochemical properties of rabbit antibody globulin were consistent with those of an ellipsoid, $27 \mathrm{m \mu}$ in length, and a maximum width of $4 m \mu$. Svedberg has pointed out that the molecular weights of the non-fibrous proteins cluster round certain values which are whole-number multiples of the lowest weight recorded, 17,500, and suggests that they are built up from units having a molecular weight of approximately 35,000. This relationship of the molecular weights of proteins, whether animal or vegetable in origin, presumably reflects a similarity of the enzyme systems that take part in protein-synthesis. On this view, the rabbit antibody globulin molecule would correspond to four Svedberg "units." As we shall see, this possibility has been raised in connection with the valency of antibody by Hooker and Boyd, who argue that if the four units each carried an antibody receptor, and were arranged linearly, the whole molecule would be multivalent, and markedly anisodimensional (p. 251).

\section{The Purification of Antibody.}

Antibodies are precipitated from serum by agents that bring down the serum globulin. Precipitation of antisera with sodium or ammonium sulphate, with alcohol at various temperatures, or by dialysis, brings down the greater part of the contained antibody with the globulin fractions. (See Banzhaf and Gibson 1907, Gibson and Collins 1907, Ledingham 1907, Mellanby 1908, Hartley 1914, Felton 1926, 1928, 1932, Maitland and Burbury 1927, Barr, Glemny and Pope 1931, Barr and Glenny 1931, Laidlaw and Dunkin 1931, Barr 1932, and many others.)

A considerable degree of purification of antibody solutions is possible where the antibody is confined to one particular fraction. By fractionation Felton (1926, 1932) purified the antibody to the Type I pnemmococeus, and Laidlaw and Dunkin (1931) the antibody to distemper virus.

Specific precipitation of serum globulins by antisera prepared in an animal of another species also brings down the antibodies (Landsteiner and Prášek 1911, Eisler 1920, Smith and Marrack 1930). Moreover, the inactivation of antibodies by heat corresponds in general with the denaturation of proteins by heat (see Streng 1909, Madsen and Streng 1910, Marrack 1938). Many of the earlier studies of 
this phenomenon are complicated by the fact that the heat inactivation was carried out in the presence of other serum proteins, and, as the work of Kleczkowski (1941b), van der Scheer, Wyckoff and Clarke (1941a), Bawden and Kleczkowski (1942b) Jennings and Smith (1942), and Krejci, Jennings and Smith (1942) shows, heating mixed solutions of antibody globulin and proteins like albumin or casein in the presence of salts (Kleczkowski 1943) produces globulin-albumin and globulincasein complexes which display no direct antibody activity, but which cannot be considered as denatured in the ordinary sense of the word.

The study of the antigen-antibody complex offers a more direct approach to the nature of antibody, since protein united with antigen has presumably been selected from the antiserum by reason of its specific combining powers. A certain amount of non-specific material may be adsorbed by the complex-such adsorption occurs in complement fixation-but the amounts are relatively too small to invalidate the assumption that the bulk is in fact antibody. For instance, Heidelberger and Landsteiner (1923), Marrack and Smith (1931b) and Haurowitz and Breinl (1933) have shown that non-specific coloured proteins are not carried down in the precipitate when an antigen reacts with its specific antibody; and Dean, Taylor and Adair (1935), using the optimal proportions technique and examining a sample of antiserum containing antibodies to purified egg albumin and purified horse-serum albumin, found that either antibody could be specifically precipitated by the corresponding antigen without affecting the titre of the other. It has been established that antigen-antibody complexes contain considerable quantities of protein from the antiserum, and that this protein has the general characters of serum globulin. Thus the precipitate formed when $2.5 \mathrm{mgm}$. of Type II pneumococcal polysaccharide, which contains no nitrogen and is therefore particularly suitable for work of this kind, reacts with its homologous antiserum, has been found to contain $37 \mathrm{mgm}$. of serum protein (Felton and Bailey 1926); and the antibody titre of a given antiserum nua be measured with considerable accuracy by determining the amount of protein that is removed by a soluble or particulate antigen (Heidelberger and Kendall 1929, Heidelberger et al. 1930, Heidelberger and Kabat 193t).

That the protein so bound has the general properties of globulin is attested by such observations as those of Marrack and Smith (1930, 1931a), who found that the diphtheria toxin-antitoxin compound showed the same ultra-violet absorption spectrum as serum globulin, or those of Breinl and Haurowitz (1930), who found that a precipitate, containing about 10 per cent. of hæmoglobin and 90 per cent. of material derived from a homologous antiserum, showed the same proportions of tyrosine, cystine, histidine and arginine as did serum globulin when examined by the same technique.

Antigen-antibody complexes act as antigens, and induce in animals "antiantibodies" that are specific for antibody in that they combine with the antigenantibody complex, but not with antigen alone. The antisera will also react with normal serum globulins (Audo 1937; see also Marrack and Duff 1938). Another noteworthy feature of such antisera, to which we shall refer later, is their capacity to react with specific precipitates of other antigens, provided that the antibody in these precipitates was produced in the same species of animal (Treffers and Heidelberger 1941). However, the properties of antibody protein as revealed in the antigen-antibody complex are open to the objection that the antibody may have been altered by its union with antigen. 
Many attempts have been made to dissociate antibodies from an antigenantibody complex in a protein-free condition. The dissociation of the antigenantibody compound may be accomplished in a variety of ways, and certain of the antibody solutions so obtained have contained very little protein (see Landsteiner and Jagie 1903, Muir 1903, Bail and Tsuda 1909, Spät 1910, Kosaki 1918, Huntoon and Etris 1921, Huntoon et al. 1921, Loeke and Hirsch 1925), but in none of these instanees has it been satisfactorily demonstrated that the amount of protein present was insuffieient to aceount for the autibody aetion observed (see Eagle 1930). Similar attempts have been made by adsorption on to various reagents other than the specific antigen, followed by elution with various finids at different pH levels. Some of these attempts have been elaimed as suceessful (see Frankel and Olitzki 1930, Olitzki and Frankel 1931, Frankel 1932, Olitzki 1932), but these claims have not been confirmed by subsequent workers (see Marrack 1934, Rosenheim 1935).

Northrop (1941) and Rothen (1941) prepared purified diphtheria antitoxin from toxin-antitoxin precipitates by digestion with trypsin. The purified antitoxin had a molecular weight in the region of 90,000 . By fractional precipitation Northrop isolated a crystalline antitoxic protein which appeared to be antigenieally distinct from normal horse proteins.

Later studies of the effect of elect=olytes on specific aggregation (see p. 215) led to the preparation of dissoeiated antibody solutions of a high degree of purity (Heidelberger and Kendall 1936, Heidelberger, Grabar and Treffers 1938). Heidelberger and his associates were able to purify both rabbit and horse antisera by salt dissociation of agglutinated pneumoeocci, Types I, II and III. Dissoeiated and native antibody behaved alike in serological reactions. Dissociated antibody had the properties of a globulin. The moleeular weight of dissociated rabbit antibody, caleulated from its sedimentation rate in the ultracentrifuge, was, like that of antibody in native antiserum and of globulin in normal serum, in the region of 150,000 to 196,000 .

\section{Varieties of Antibody Globulin.}

Horse antibody to the Type III pneumocoecus has a molecular weight over 4 times that of the corresponding rabbit antibody. A similar component was found in other preparations of horse antisera, but not in normal horse serum (Heidelberger and Pedersen 1937). Later, Kabat (1939) showed that these heavier antipneumococcal globulins, of moleeular weight 910,000 to 930,000 , were formed also in the eow and pig.

These antibodies of high molecular weight differ in certain respects. For instance, "heavy" antiborly to pnemmoeoceal polysaccharide will not fix guinea-pig or human eomplement in the presence of homologous antibody, whereas ox antibody of similar weight does so (Heidelberger and Treffers 1941). Heavy antibodies do not appear to be quite so firmly constituted as antibodies of a lower molecular weigit, for they are disaggregated by relatively mild treatment with barium hydroxide without mueh reduction of precipitating power. Not all horse antibody has this high molecular weight; for example, diphtheria, scarlet fever, tetanus and $\mathrm{Cl}$. welchii antitoxins from the horse, when diluted, contain no abnormally heavy protein molecules (Fell, Stern and Coghill 1940).

The significance of the large antibody molecules is not elear, but it is evident that response to immunization on the part of eertain animals is more than the production of normal globulins modified only in configuration; a new type of globulin may in fact be formed. 
The serum globulins as a whole behave in the ultracentrifuge as though they were homogeneous, but they are separable, as we have already seen, into fractions of differing solubilities. Antibodies are distributed variously among the fractions (see, for example, Adair and Taylor 1936). The distribution depends on the animal immunized, the nature of the antigen, and the mode of fractionation.

It is still questionable whether the euglobulins and pseudoglobulins are artefacts produced during fractionation of serum. Rabbit antisera to horse and human serum proteins react to some extent separately with euglobulins and pseudoglobulins, suggesting that the two exist as such in the native serum (Harris and Eagle 1935).

Marrack and Duff (1938) were unable to demonstrate an absolute serological distinction between euglobulin and pseudoglobulin, which neither singly nor in mixture had the full serological reactivity of native globulin. Treffers and Heidelberger (194la) also found only a partial serological distinction between the water-soluble and water-insoluble globulins.

The increase in serum globulin during immunization has been noted by several observers (see Marrack 1938), thongh not all of it is attributable to the formation of antibodies (Liu, Chow and Lee 1937, Boyd and Bernard 1937). In Boyd and Bernard's experiments with a variety of antigens, the antibody never accounted for more than 35 per cent. of the increase, though, as the authors point out, some of the serologically inert proteins may have been antibodies of a reactivity too weak to be detected by their methods.

Ando and his colleagues (Ando, Kee and Komiyama 1937, Ando, Kee and Manako 1937, Ando, Manako, Kee and Takeda 1937, Ando, Manako and Takeda 1938, Ando, Takeda and Hamano 1938), by immunizing rabbits with antigen-antibody aggregates, distinguished two types of globulin, A and B in borse sera, A predominating in the watersoluble, $\mathrm{B}$ in the water-insoluble fractions. Both normal sera and antisera contained $A$ and $B$ globulins. Immunization with diphtheria toxin, or Shiga's dysentery bacilli, increased the A globulins ; B globulins were increased during immunization with pneumococci, Salm. typhi and other bacterial antigens.

The globulin components revealed by Tiselius's electrophoretic apparatus undoubtedly exist as such in native serum. Protein particles of similar molecular weight may prove to be heterogeneous by electrophoresis, and Tiselius (1937b) showed that antibody preparations of Heidelberger and Pedersen (1937), which resembled normal globulins in the ultracentrifuge, differed strikingly from the normal in their rate of migration in an electrical field. By comparing antibody to egg albumin and to pneumococci, before and after absorption with the homologous antigens, Tiselins and Kabat (1939) demonstrated that, in the rabbit and the monkey, antibody was electrophoretically identical with the $\gamma$ component of normal globulin: horse antibody migrated as a new component, midway in mobility between the $\beta$ and the $\gamma$ components. In the hands of Moore, van der Scheer and Wyckoff (1940) and van der Scheer, Lagsdin and Wyckoff (1941) the high molecular weight antibody in horse antipneumococcal sera migrated with the normal $\gamma$ globulin. Van der Scheer, Wyckoff and Clark $(1940,1941 b)$ described in some antisera an increase in $\gamma$ globulin, in others a new component $T$, both associated with an increase in antibody. The $\mathrm{T}$ component was midway in mobility between the $\beta$ and $\gamma$ components (see also Rothen 1941). It appears more readily in antitoxic, as distinct from antibacterial sera, though not regularly so. Kekwick and Record (1941) fonnd two antibody fractions in diphtheria antitoxin from the horse, one associated with the $\beta$, the other with the $\gamma$ component. With optimal amounts of toxin, the $\beta$ fraction floceulated slowly and formed a compound with the composition $\left(\mathrm{TA}_{2}\right)_{n}$. The $\gamma$ fraction flocenlated 20 times as rapidly, and formed compounds of the type $\left(\mathrm{TA}_{4}\right)_{n}$. The $\gamma$ fraction, which is precipitated with the englobulin during salt fractionation, predominated in carly bleedings from immunized horses; in the later bleedings, the $\beta$ fraction constituted the main antitoxin content (Kckwick, Knight, MacFarlane and Record 1941).

We may note one more variety of antibody, recently described by Race (1944). The 
erythrocytes of certain human subjects contain an antigen D, which is one of the natural Rh antigens (Chapter 49). An antibody to D occurs naturally in the sera of other human subjects, and, like other natural antibodies to the blood group antigens, it agglntinates erythrocytes containing the corresponding antigen. In certain subjects, however, Race found "incomplete" antibodies to D, which combined specifically with D-containing erythrocytes, but failed to agglutinate them. The properties of this naturally occurring "incomplete" antibody in many respects resemble the reacting but non-flocculating albumin-antibody-globulin complexes made artificially by Bawden and Kleczkowski (1942) (see pp. 213, 244).

The Modification of Antibody by Physical and Chemical Means.-Although there is a general parallelism between gross alterations of the globulin nature of antibodies, and their activity with respect to antigens, yet the protein can in many cases be altered considerably without destroying its efficacy as an antibody.

If, for instance, Type II pnenmococcal antibody is "unfolded" in a mono-layer on an air-water interface, it loses its capacity to combine with the specific carbohydrate of the Type II pnenmococcus (Danielli, Danielli and Narrack 1938).

A similar unfolding of normal proteins appears to take place in urea solutions (see Bernheim et al. 1942), possibly by the weakening of the hydrogen and other bonds responsible for maintaining the association of adjacent polypeptide chains. In urea solutions at $\mathrm{pH} 7$.8 diphtheria antitoxin slowly loses its neutralizing power for toxin, and more rapidly at a higher or a lower $\mathrm{pH}$ (Wright 1944). These results suggest that the antibody activity is closely associated with integrity of the general molecular structme and that destruction of the antibody configuration is associated with ionization of basic or acidic groups.

Chemical modification by moderate treatment with formaldehyde, which reacts irreversibly with the protein at the surface of the moleeule, has little effect on antibody activity (see Mudd and Joffe 1933, Ivanoff 1936, and Eagle 1938). As Eagle showed, stronger treatment with formaldehyde, or with a reactive diazo compound, removed first the precipitating and then the protective power of antipneumococcal sera and diphtheria antitoxins, a fact we have already noted in discussion of the lattice hypothesis. Again, the amino groups of an antibody globulin molecule may be treated with ketene gas until one-third of them are acetylated, without marked loss of the power to combine with antigen (Goldie and Sandor 1937). The chemical groups on the surface of globulin moleenles responsible for the antigenic power of the protein do not appear to form part of the specific combining groups of the antibody. The destruction of activity of horse antipneumoeoccal sera by photodynamic oxidation in the presence of methylene blue only reduces the power of the proteins to precipitate with anti-horse serum (Ross 1938). The independence in the antibody molecule of the antibody-combining group and the groups that determine antigenic specificity has been demonstrated serologically by several workers. Smith and Marrack (1930), and later Eagle (1936), demonstrated that diphtheria antitoxin from the horse, which has combined with a rabbit anti-horse precipitin, would react with toxin. Eagle in addition showed that antitoxin combined with toxin could still react with the rabbit antibody. Treffers and Heidelberger $(1941 a, b)$ immunized animals with specific precipitates formed by the combination of various antigens with their homologous antibodies, both horse and rabbit. The reaction of the resulting antiantibodies was conditioned solely by the species of animal from which the antibody was obtained; any difference between various antibodies that might be expected from the fact that they each possessed different combining groups for their homologous antigens, was not demonstrable by serological methods.

The Enzymic Digestion of Antibodies.-Hydrolysis by enzymes ultimately destroys the antibody activity of serum globulin (see Marrack 1938). Partial hydrolysis under controlled conditions is selective in its action on the varions serum proteins. For instance, Pope $(1939 a, b)$, investigating the Parfentjev patent for the refinement of antitoxin, con- 
cluded that by heat treatment in an acid solution, diphtheria antitoxin globulin was partially denatured, and could be refined by proteolytic enzyme hydrolysis, leaving an undenatured portion which carried most of the antitoxic activity. Similarly, Petermann (1942) demonstrated that diphtheria antitoxin was split into approximate halves of different solubilities by papain digestion, one half carrying the antitoxic activity, the other leeing inert. If digestion was prolonged so that the halves split into quarters, no antitoxic activity remained. The process appeared to be one of enzyme cleavage, and not a denaturation. The antibody loses most of its original antigenic activity as a result of its "proteolytic refincment" (Weil, Parfentjer and Bowman 1938). The change is reflected in the distribution of electrophoretic components. Refined antisera show a diminution or absence of albumin, disappearanec of the $\mathrm{T}$ component in diphtheria antitoxin and its replacement by a new $\gamma$ component (van der Scheer, Wyckoff and Clarke $1941 c$ ), or a relative increase in the $\beta$ and $\gamma$ components (Fell, Stern and Coghill 1940).

From this fact, and the varying distribution of antibody among the components, it is elear that the characterization of an antibody globulin by its electrophoretic mobility has no necessary connection with the fact that it carries immunologically active groups.

An interesting example of partial digestion of antibody is reeorded by Kalmanson and Bronfenbremner (1943). A bacteriophage just neutralized by homologous rabbit antibody was restored to activity by papain digestion of the globulin on its surface. The digestion of over-neutralized bacteriophage, on which there were many more antibody molecules, did not reverse the neutralization. Some part of the antibody apparently remained on the phage particles after digestion, for just-neutralized phage after digestion would sensitize guinea-pigs to rabbit globulin ; and over-neutralized phage after digestion was found to be incapable of stimulating phage antibody on injection into a rabbit, presumably becanse the antibody remnants formed an obscuring layer on its surface.

Rosenheim (1937) has drawn attention to an increase in the resistance of antibodies to pepsin and trypsin hydrolysis that takes place as immunization proceeds. She found that " $\mathrm{H}$ " bacterial agglutinins (see p. 276) from late bleedings of an immunized rabbit were resistant to a degree of digestion that destroyed 80 per cent. or more of agglutinins from the first bleeding. There did not, however, appear to be a corresponding increase in the resistance of the serum proteins to digestion. " $O$ " antibodies did not become resistant in this way. Both Pappenheimer and Robinson (1937) and Pope (1939a) noted that the resistance of diphtheria antitoxin to proteolytic refinement varied with the stage and the method of immunization of the horse.

\section{Unity and Diversity of Antibodies.}

Immunization with a single antigen yiclds an antiserum which can display a variety of specific phenomena; for instance, precipitation, neutralization of toxin, protection of an infected animal. In the interpretation of antibody reactions, there are two questions about the variety of antibodies that need an answer. In the first place, are all these manifestations due to a single kind of antibody, or does a precipitin, for example, differ fundamentally from an antitoxin, when the toxin serves as antigen in both reactions? In the second place, in a solution that displays a given kind of serological activity, are all the molecules of antibody similar? In other words, in a monospecific antibody solution, are there antibodies with different functions, and are those of the same function homogeneous?

The view that precipitins, opsonins, antitoxins, etc., differed in kind, held the field for many years; but the unitarian hypothesis that these were due to the same kind of antibody in different circumstances has now gained almost universal acceptance. This has been due mainly to the work of Dean (1917) in this country, to that of Zinsser (1921) in America, and to Nicolle and Césari (1922) in France ; though a similar view had been adumbrated by Bail and Hoke (1908). We have seen how Dean established the essential identity of precipitation and complement 
fixation. We have noted that precipitation is a general form of reaction between all antigens and all antibodies, including toxin and antitoxin. And we have seen that the union of antigen and antibody at the surface of a bacterial cell sensitizes it alike to the floeculating action of electrolytes, to the lytic action of complement, and to the phagocytic action of leucocytes.

There are too, in records of the assay of the protective power of antisera, many instances of complete parallelism between the protective power and some in vitro agglutination, antihæmolytic or antitoxic reaction. For instance, the typespecific precipitin content of antimeningococcal sera was found to parallel their protective power in mice (Pittman 1943).

The detailed evidence has been reviewed by Marrack (1938). It remains to note that Delves (1937) found that precipitating antibodies to pure human albumin and pseudoglobulin would agglntinate and opsonize collodion partieles coated with homologous antigen; that Gerlough, Palmer and Blumenthal (1941) were able to establish the identity of precipitins, agglutinins and protective antibodies in antisera to six different types of pneumococeus; and finally, by their method of dissociation of agglutinated bacteria, Heidelberger and Kabat (1936) and Alexander and Heidelberger (1940) were able to demonstrate directly the identity of the agglutinin and preeipitin to Type I pneumococeus and to Hamophilus influenze. Type b.

This conception of the serum reactions does not, of course, in any way modify our belief in a multiplicity of antibodies corresponding to a multiplicity of antigens. A red cell, a bacillus, or a erude protein solution such as horse serum, contain many antigens and give rise to many antibodies. The unitarian hypothesis, as Zinsser (1921) has emphasized, implies simply that the injection into the tissues of a chemically pure antigen will lead to the formation of one antibody capable of producing all the various manifestations of antigen-antibody union.

When we discuss, in the next chapter, the antigenic structure of bacteria, we shall see that there is a sense in which it would be correct to differentiate one antibody from another in terms of function-to say, for instance, that a particular antibody is an agglutinin but not a lysin. This difference in functional activity, however, is determined not by a difference in the nature of the antibody but by a difference in the structural position of the antigen to which it is attached.

The Homogeneity of Monospecific Antibodies.-It is often assumed that the behaviour of antibody in solution, provided that it has been formed in response to a single antigen, is the sum of the effects of a homogeneous collection of antibody globulin molecules. We have already discussed a number of phenomena that indicate a heterogeneity of the antibody in a given seram. Thus, in monospecific sera there is an antibody that precipitates with antigen only in the presence of fully reacting antibody (Marrack and Smith 1931b, Heidelberger and Kendall 1935a, b, $c$, Heidelberger, Treffers and Mayer 1940). Heidelberger postulater univalence for this low-grade antibody, and multivalence for the more "avidly" " reacting antibody. Landsteiner and van der Seheer (1940) found in an anti-hen ovalbumin serum two kinds of antibody both precipitating with hen ovalbumin; turkey albumin precipitated readily with one, and failed to precipitate with the second, though there was evidence of combination. Other evidence of heterogeneity of antibody is provided by the change in quality of antibody during the course of immunization. The broadening of reactivity and the increase in avidity of antiserum with prolonged immunization has been observed many times. Hooker 
and Boyd (1941a) suggest that they may be due to $(a)$ the formation of antibodies to minor antigenic determinants, $(b)$ the increase in the number of reactive groupings on the antibody, or $(c)$ the wider affinity of the combining groups, due to the formation of a larger and more complex reactive patch on the antibody surface. The broadening may be accompanied by a change in the nature of the globulins. Raffel, Pait and Terry (1940) found the earlier, less avid antibody to be associated with the water-insoluble globulins, the later, avid antibody with water-soluble globulins. The evidence of heterogeneity found among fractions of antiserum made by precipitation with salts is less convincing, because it is difficult to exclude artefacts, but the differences, noted above, in reactivity displayed by electrophoretic fractions of antisera cannot be lightly dismissed.

Goodner and Horsfall (1937) obtained other evidence of heterogeneity, in antipneumococeal sera. In several rabbit antisera, the ratio of precipitin content to protective power was constant. but in horse antisera it was inconstant. Moreover, in individual sera of both species the protective power of antibody left after some of it had been precipitated by antigen varied with the amount of antigen added, though the rariation was much greater in the horse. When horse antiserum was fractionated, the pseudoglobulin was found to contain antibody with a protective power only one-seventh that in the euglobulin fraction, though the pseudoglobulin antibody was far more reactive as precipitin. In an electrophoretic fractionation of diphtheria antitoxin, the antibody $\gamma$ component combined with twice as much nitrogen per flocculating unit as that from the $\beta$ component. Goodner, Horsfall and Baner (1938) also demonstrated heterogeneity in the size of antibody particles in both rabbit and horse antisera. For example, in horse antisera very large particles, corresponding to a pore size of $175 \mathrm{m \mu}$ in a collodion membrane filter, were found in concentrated serum, and of $83 m \mu$ in native serum. Dihution in saline reduced the particle size in both types of serum.

The antibodies formed in response to the injection of what is apparently a single antigen may also display varying kinds of serological specificity. Thus, Landsteiner and van der Scheer (1939), working with proteins modified by coupling to pentapeptides (made up of various combinations of glycine and leucine), showed that all the antibodies produced in response to any one peptide-protein were absorbed by the same peptide coupled to an antigenieally unrelated vehicle, namely erythrocyte stromata. Nevertheless, varying amounts of the total antibody could be removed from the sera prepared against one peptide. by absorption with stromata to which one of the other peptides had been eompled. Absorption occurred even when the absorbing peptide could not conceivably have been a breakdown product of the antibody-producing peptide.

It is clear that in horse sera, at any rate, apparently monospecific antibodies display heterogeneity both with regard to their reactivity as antibodies, and to their other physicochemical properties. Monospecific rabbit antibodies appear to be less heterogeneous, at least functionally. Indeed, the degrce of homogeneity may be surprisingly high. Thus, Hershey, Kalmanson and Bronfenbrenner (1943) record an analysis of a number of rabbit anti-phage sera, with a 25 -fold range of combining power, in which there was full correlation between three varying qualities, specific rate of phage neutralization, neutralizing capacity, and precipitating capacity; and no tests revealed any heterogeneity of antibody in any one serum.

\section{The Valency of Antibody.}

It is obvious that the conception of monospecific antibody molecules of different valencies will help to explain some of the observed heterogeneity of antibody in the natural state. As we have seen, the assumption of multiralent antibody is 
an essential part of the lattice hypothesis, and on p. 220 we have indicated a number of objections to the lattice hypothesis which imply the rejection of a multivalent antibody. Nevertheless, variations in "avidity" and precipitating power of antibody obtained during a prolonged course of immunization are conveniently explained in terms of variations in valency. But, as we should expect from our ignorance of globulin metabolism in the animal body, there are no impressive data to support the speculation. The most cogent objection to the theory of multivalence (see Hooker and Boyd 1942) lies in the fact that immunization with a known mixture of distinct antigens does not result in polyspecific antibody (see, for example, Hektoen and Boor 1931, Dean, Taylor and Adair 1935). It is argued that, if during the synthesis of antibody in the animal there was in the growing antibody globulin molecule more than one region that could be influenced by antigen, there is no good reason why two or more regions on one molecule should not be influenced by separate antigens, and thus give rise at least to a dispecific antibody.

The conception of dispecific antibody offers a simple explanation of the results of antibody response to certain natural complex antigens (Meyer I936, Miles I939), but since the antigenic constituents of the mixtures were not fully defined, the success of the hypothesis is not good evidence of its validity. The difficulty may in part be resolved by assuming that monoralent Svedberg units, of molecular weight 35,000 , are in fact formed in the body, and that these are later united into larger molecules. which are multivalent. But here again, unless we postulate that the units are always built on to one another in conjunction with a single antigen particle, there is no good reason for assuming that Svedberg units of diverse specificity should not be joined to form a polyspecific antibody.

Molecules of antigen having on the surface two antigenic determinants may give rise to apparently dispecific antibodies. For example, Heidelberger and Kendall (1934), immunizing rabbits with oralbumin modified by an azo-dye, produced antibodies to ovalbumin alone and to azo-protein alone, and also antibody that reacted equally well with azo-protein or ovalbumin (see also Singer 1942). Landsteiner and van der Sicheer (1938), on the other land, conld find no evidence of a double specificity even in antibodies prepared against a single azo-antigen having two haptenic groups. They prepared antigens with aminosuccinanilic acid (S). with aminophenylarsenic acid (A) and with a compound of the.two (SA). Antisera to SA preeipitated with $S A, A$ and $S$, but sera absorbed with A precipitated only with $S$, and sera absorbed with $S$, only with $A$. The various antisera contained one or two kinds of specific antibody, but never dispecific antibodies. Haurowitz and Schwerin (1943) obtained analogons results by immunizing rabbits with a globulin antigen containing both $p$-azo-phenylarsonic (A) and $m$-phenylsulphonic groups (S) or with a globulin containing both $\mathrm{A}$ groups and di-iodotyrosine ( $\mathrm{T}$ ) groups. The resulting antisera contained antibodies against $\mathrm{A}$ and $\mathrm{S}$, and against $\mathrm{A}$ and $\mathrm{T}$ respectively, but there was no evidence of dispecific antibodies against $(A+S)$ or $(A+T)$.

With regard to the apparent variations in combining power displayed by monospecific antibody, Hershey (1941b) points out that it is not necessary to invoke differences in valency to explain the properties of "low grade" or of highly avid antibody. The "affinity" of a unit reactive patch on the antigen molecule for unit reactive patch on the antibody may be described in terms of a dissociation constant $k$, and Hershey has ingeniously subsumed most of the observed individual variations of antibody under variations in $k$. For example, a small $k$ explains large maximal antigen-antibody ratios, high avidity, and broadened reactivity in sera taken late in immunization. Heidelberger's univalent antibody, and nonprecipitable antibody left after absorption of precipitable antibody, would have a large $k$. Variations in $k$ would be independent of the nature of the immune 
response, and of valency ; and variations in $k$ would explain the effect of salts, lipins and other non-specific substances on the progress of immune reactions.

The analysis of specific precipitates in the region of antigen excess should provide the best direct evidence for or against the multivalence of antibody. In their review Hooker and Boyd (1942) point out that in only two systems have the recorded estimates of the molecular ratio of antigen to antibody been more than unity, namely, with diphtheria toxin (Pappenheimer, Lundgren and Williams 1940), and with Heidelberger and Kendall's (1934) azo-dye ovalbumin antigenand in both cases the value of 2 is in doubt. We have already noted that the value of 2 accords well with Hershey's descriptive theory of the lattice hypothesis. It is clear that precise analyses of a large number of antigen-antibody compounds of this kind are required to settle this point.

We may summarize the position with regard to the unity or diversity of antibodies as follows.

The antibodies in a monospecific antiserum prepared against a single antigen may vary in physicochemical properties, such as particle size, electrophoretic mobility, readiness to dissociate from union with antigen, and perhaps in valency; in "combining power" and "avidity" ; in resistance to destructive agents ; and in capacity to form complexes with antigens that are susceptible to other substances in the reacting system, such as electrolyte, complement, or a phagocyte. In no case, however, is there good evidence of association between a given set of physicochemical properties, and a particular type of serological reactivity. The heterogeneity in a collection of antibody particles present in a certain preparation may affect the different serological reactions in different degrees, but there is no reason to suppose that opsonins, precipitins, antitoxins and so forth differ in kind, either constantly or fundamentally. In this sense then, we may accept the hypothesis of the unity of antibodies.

\section{The Nature and Properties of Antigens}

The two outstanding characters of antigens, their power to stimulate antibody production and their specificity, may be to some cxtent explained in terms of their chemical constitution. The basis of specificity is the more clearly understood, and it will be convenient to discuss it first.

\section{The Basis of Specificity.}

Specificity is not absolute. There is no one natural antigen of which it would be safe to predict that it would not react with antibody prepared against another antigen. The variety of syntheses of which living tissues are capable, though wide, is limited, and many tissue constituents in one organism are similar to those in another organism. Where these constituents are antigenic, we often find the similarity reflected in the serological reactions of the antigens with homologous and heterologous antibodies.

Thus the lens proteins of mammals are antigenically similar, though they differ markedly from other proteins found in the animal body. This type of specificity is referred to as "organ" specificity, and has been noted in antigens from brain, testes and placenta, and in mammalian proteins like keratin, fibrinogen and thyroglobulin (see Landsteiner 1936).

On the other hand, antigens are often "species" specific. We can differentiate by serological tests between the egg albumin of the duck and of the hen (Dakin and Dale 1919), between the hæmoglobins of different animal species (Higashi 
1922, Landsteiner and Heidelberger 1923, Hektoen and Schulhof 1923), and between different vegetable proteins (Wells and Osborne 1911, Wells 1915, Jones and Gersdorff 1923, Lewis and Wells 1925, Wells et al. 1927).

As the classical researches of Nuttall (1904) indicated in the first place, this degree of specificity may be closely correlated with biological elassifications, so that we might expect cross-reactions between the serum proteins of man and monkey, but none between those of man and ox. But, though this holds to a large extent, later studies have revealed relationships between the antigens of organisms as widely separated as man and Shiga's dysentery bacillus (see Chapter 8). A serological relationship, therefore, between two organisms can strengthen a relationship already established on other biological grounds, but it cannot by itself be considered as good evidence of anything more than a similarity of perhaps a small part of the metabolism of the two organisms.

Analysis in many cases reveals chemical differences between native antigens, but the complexity of the antigenic particles concerned is such that analyses are necessarily crude. Far more significant information has been gained by altering the chemical of protein antigens along certain limited and well-defined lines, and noting the resulting changes in their immunological reactions.

Obermayer and Pick (1906) (see also Pick 1912) showed that the nitration or halogenation of proteins - that is, the introduction of the nitro-group or of a halogen element such as iodine-profoundly altered the antigenic reactions of the treated protein. Serum proteins so treated lost their species specificity, but they gained a new specificity, shared by normally unrelated serum proteins that had been chemically altered by the same procedure. Thus an antiserum prepared against the nitrated serum of a particular amimal species failed to react with the unaltered serum of that species, but reacted with a wide range of nitrated sera from other animals. Since it was known that the nitro-group and the halogen elements entered into the benzene ring of certain of the amino-acids which build up the complex protein molecule, Obermayer and Pick were led to attach particular importance to these chemical groupings as factors determining immunological specificity (sce also Wormall 1930, Johnson and Wormall 1932, Snapper and Grunbaum 1936, Shahrokh 1943).

Landsteiner and his colleagues, however, have shown that the salt-forming groups of the amino-acids (the carboxyl-, hydroxyl- and amino-groupings) play an equally important part. By esterification, methylation and acetylation, they have succeeded in altering the immunological specificity of proteins (see Landsteiner and Prášek 1914, Landsteiner and Lampl 1917a, Landsteiner 1917).

This relatively simple modification of active groups on the surface of the antigen was largely extended by Landsteiner and his colleagues (see Landsteiner 1930, 1933, 1936), who utilized the diazo reaction to introduce larger modifying groups into the protein molecule. The substances for introduction contain an $-\mathrm{NH}_{2}$ group, usually attached to a ring structure in the molecule. The $-\mathrm{NH}_{2}$ group is converted to $-\mathrm{N}: \mathrm{N} \cdot \mathrm{Cl}$ in presence of $\mathrm{NaNO}_{2}$ and $\mathrm{HCl}$, and the resulting diazo compound mixed with the protein. The diazo compound reacts with the phemolic groups of tyrosine (see Fig. 41), with the imidazole ring of histidine, and probably with the indole group of tryptophan, the - NH group of proline and hydroxyproline and all the aliphatic $-\mathrm{NH}_{2}$ groups on the protein surface (Eagle and Vickers 1936). By varying the conditions of the reaction it is possible to control the amount of modifying substance introduced into the protein. Fig. 41 illustrates the coupling of atoxyl ( $p$-amino benzene arsenic acid) to a protein, on the assumption that it unites with the phenolic group of the tyrosine residues in the protein molecule. 
The results obtained with azo-proteins were criticized by Clutton, Harington and Mead (1937) on the ground that the diazo link is highly artificial and is never found in natural substances. They devised means of coupling carbohydrate to tyrosine, by an $\mathrm{O}$-glucosido linkage, and attaching the compound to the $\mathrm{NH}_{2}$ groups of protein. Subsequent work (Clutton, Harington and Yuill 1938), however, showed that the nature of the linkage to the protein had no observable effect on the modification of antigenic specificity induced by the glucosido-tyrosyl groups themselves. Another objection to the diazo method is the drastic chemical treatment to which the proteins are subjected during diazotization. Hopkins and Wormall $(1933 a, b)$ were able to modify the specificity of proteins by the much gentler action of phenyl iso-cyanate in alkaline solution. The iso-cyanate reacts with free $-\mathrm{NH}_{2}$ groups to form a substituted urea. The results of chemical

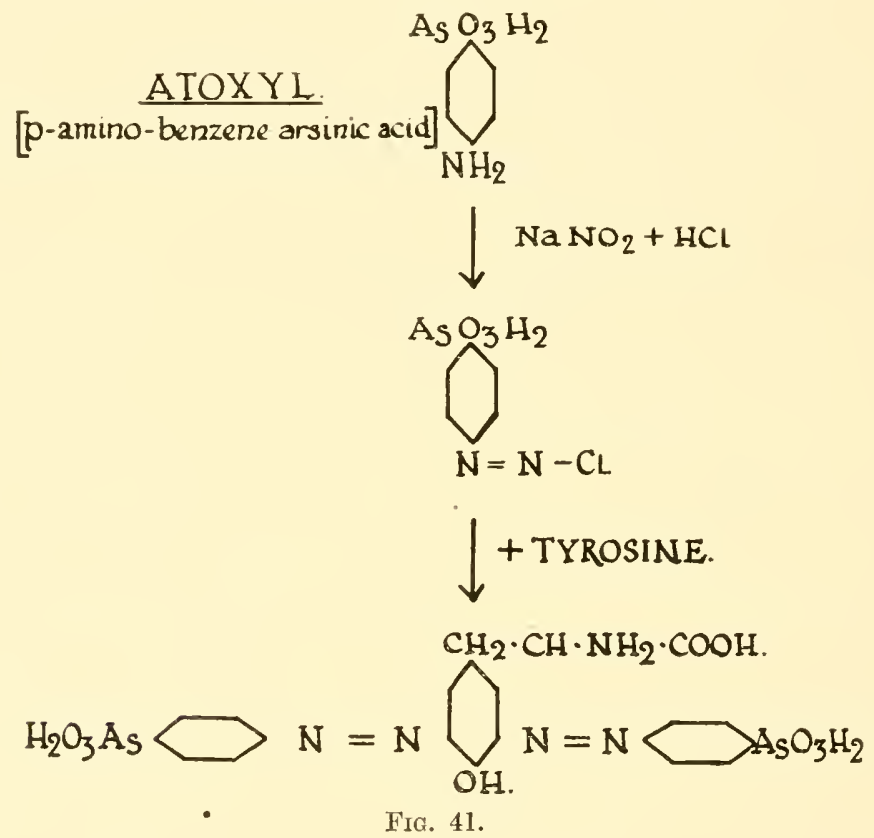

modification by all these methods are essentially similar, and we may take those obtained by the extensively employed azo-proteins as valid.

In general the protein to which such a grouping has been attached will retain its species specificity, though usually with some loss of potency. Thus an antigen prepared by coupling atoxyl to horse globulin will react with an antiserum prepared against any atoxyl-azo-protein, in virtue of the atoxyl grouping; and with an antiserum prepared against horse globulin or against an azo-protein prepared from horse globulin, in virtue of the specific horse-globulin groupings. This difficulty is overcome by coupling any grouping that it is desired to study to two immunologically unrelated proteins. One of these is used as the antigen for the preparation of the antiserum, the other is used for the in vitro tests. Thus, atoxyl may be coupled with horse globulin, and the atoxyl-azo-protein so prepared may be used for the immunization of a rabbit. The serum so prepared will react with this antigen 
in the test-tube in virtue of antibody groupings that unite specifically with unaltered horse globulin. But if atoxyl is also coupled to chicken globulin, and the atoxylazo-protein so prepared is used in the in vitro tests, the precipitation that occurs will depend solely on antibody groupings acting specifically on atoxyl, since horse globulin and chicken globulin show no antigenic relationship.

Using these methods, it has been possible to prepare antisera that give specific precipitation with synthetic antigens in which the active groupings are provided by such substances as metanilic acid, atoxyl, lævo-, dextroand meso-tartaric acid, glucosides, galactosides, and so on (Landsteiner and Lampl 1917b, Landsteiner 1919, 1930, Landsteiner and van der Scheer 1928, 1929, 1931, 1932a, b, 1934a, b, Avery and Goebel 1929, Avery, Goebel and Babers, 1932, Goebel, Avery and Babers 1931), dipeptides and pentapeptides (Landsteiner and ran der Scheer 1932a, 1939), pyrazolon com-
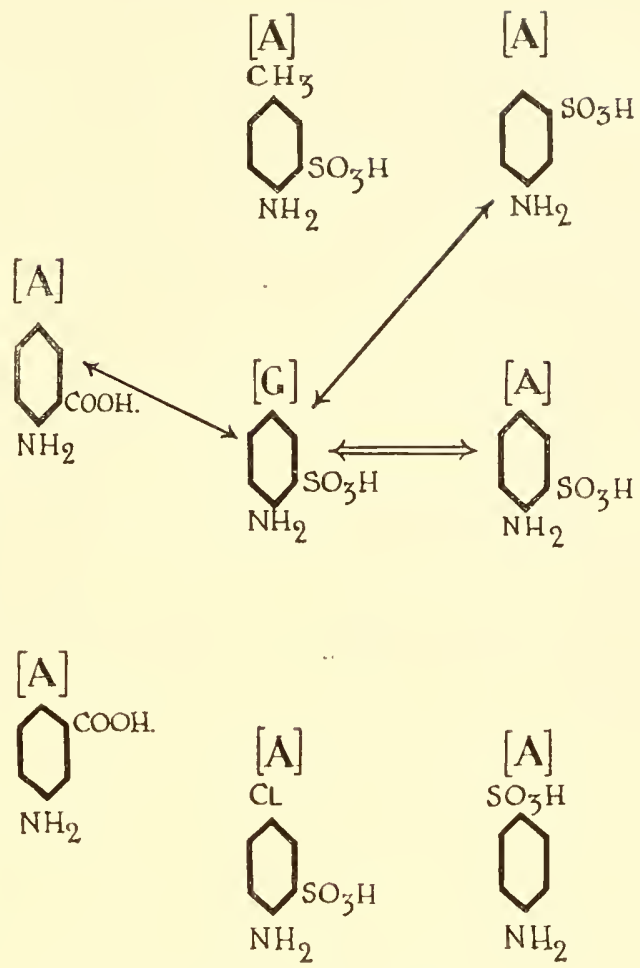

Fia. 42.-Precipitation. pounds (Erlenmeyer and Berger 1934, Harte 1938), pyridine (Landsteiner and Pirie 1937), strychnine (Hooker and Boyd 1940), thyroxin (Clutton, Harington and Yuill 1938), aspirin (Butler, Harington and Yuill 1940, and histamine (Fell et al. 1943).

These results clearly demonstrate an immunological specificity dependent upon chemical groups of known structure. In the last three examples the specific action of the antibodies concerned could be shown by a marked reduction of pharmacological activity of the thyroxin, aspirin and histamine in animals treated with antisera prepared against thyroxyl protein, aspiryl protein and histamine protein respectively (sce also Singer, p. 259).

The comparison of antigens modified by optically active isomers, as, for instance, with dextro-, meso- and lævo-tartaric antigens, or with glucoside and galactoside antigens (see also Woolf, Marrack and Downie 1936), in which the isomers differ only in the arrangement round a single carbon atom, shows clearly that, as might be expected, stereo-chemical differences in structure are important determinants of immunological specificity.

Specificity depends in part on the nature of any grouping in a complex organic molecule, and in part on the position in the molecule which the grouping occupies. Fig. 42 gives an example-the cross precipitations observed with an antigen prepared from o-amino-benzene-sulphonic acid (see Landsteiner 1919, Marrack 1934).

In the centre of the figure, labelled [G], is represented the active grouping of the azo-protein used as an antigen in the precipitation tests. Around it, labelled [A], are placed antibodies prepared against antigens synthesized by coupling the groupings shown to some unrelated protein. The double-headed arrows in- 
dicate the instances in which precipitation occurs. The reaction is strongest with the homologous antigen, containing the $\bigcup_{-}-\mathrm{SO}_{3} \mathrm{H}$ group; but precipitation occurs when the $-\mathrm{SO}_{3} \mathrm{H}$ group is shifted to the meta position $\left(\bigcirc-\mathrm{SO}_{3} \mathrm{H}\right)$, or when it is replaced, in the ortho position, by $-\mathrm{COOH}\left(\bigcirc_{-\mathrm{COOH}}\right)$. It does not occur when the $-\mathrm{SO}_{3} \mathrm{H}$ group is shifted to the para position $\left(0_{1}^{1}\right)$, or when the $-\mathrm{SO}_{3} \mathrm{H}$ group in the ortho position is replaced by a $-\mathrm{COOH}$ group in the meta position $\left(\bigcirc^{-\mathrm{COOH}}\right)$. The reaction is also abolished by introducing a methyl or chlorine group in the para position, even though the $-\mathrm{SO}_{3} \mathrm{H}$ group is retained in the ortho position.

Another example, illustrating the importance of spatial configuration and the immunological equivalence of different chemical groups so long as this spatial configuration is maintained, is afforded by the studies of Erlenmeyer and Berger (1932). They found that azo-proteins prepared from the compounds $\mathrm{NH}_{2} \square-\mathrm{O} \square \square$, $\mathrm{NH}_{2} \bigcirc-\mathrm{NH}-\square$ and $\mathrm{NH}_{2} \bigcirc \bigcirc-\mathrm{CH}_{2}-\square$ behaved similarly in precipitation reactions, though $\mathrm{NH}_{2} \square-\mathrm{C}-\square$ reacted differently.

0

The studies of Landsteiner and van der Scheer $(1932 a, 1934 b)$ on peptides also illustrate the effect of relative position on active groups in a complex molecule.

They prepared synthetic azo-proteins from the dipeptides glycyl-glycine, leucyl-lencine, glycyl-leucine and leucyl-glycine. It was found that the terminal amino-acid earrying the carboxylic group had the greater influence on immunological specificity. Thus a glycylleucine antiserum reacted best with the corresponding antigen, less effectively with a leucyl-leucine antigen and much less effectively with a glycyl-glycine or leucyl-glycine antigen. The nature of the pemultimate amino-acid also influenced the reaction, and in later studies (1939) with pentapeptides made from various combinations of glycine and leucine, it was found that the arrangement of all five members of the polypeptide chain affected spccificity.

The influence of the whole substituted group, apart from that of active acidic or other sub-groups, is well shown in the studies of Erlenmeyer and Berger (1932), Mntsaars and Grégoire (1936), and Jacobs (1937), with compounds containing more than one benzene ring. Here the nature of the whole compound was prominent in determining specificity.

To obtain precipitation with these synthetic antigens and the corresponding antisera it is in most cases necessary to employ the complete antigen, i.e. the active group coupled to a suitable protein.

The specificity of an antiserum, however, can be tested by adding to it the active substance, alone or coupled to a substance simpler than protein. Combination of the group with the antibody is then tested by adding the complete antigen. If precipitation is inhibited, we may conclude that the simpler fragment of antigen has combined with the antibody, forming a soluble compound and blocking its combining groups. 
When this procedure is adopted, it is found that relatively simple substances may function as incomplete, or partial antigens, in the sense that they combine specifically with corresponding antibodies. Thus, diazotized atoxyl coupled with tyrosine, instead of with an intact protein molecule, does not form a precipitate when mixed with the corresponding antiserum, but it inhibits the precipitation that would normally occur when this antiserum is mixed with an atoxyl-azoprotein. A similar inhibition may be demonstrated with atoxyl itself in higher concentrations, or even with arsenic acid when this is added in sufficient amount (Laudsteiner 1920).

When the inhibition reaction is used to determine the immunological behaviour of non-identical but structurally related organic compounds, it is found that the specificity is less strict than that displayed in cross-precipitation tests. Fig. 43 illustrates the findings in relation to $o$-amino-benzoic acid (see Landsteiner and van der Scheer 1931, Marrack 1938). The antibody prepared against an azo-proteiu containing this grouping, indicated at the top of the figure, gives specific precipitation with another azo-protein, containing the same active grouping but a different protein-component, indicated at the bottom of the figure. Inhibition by various other groupings is indicated by the horizontal broken arrows. The reaction is inhibited not only by benzoic acid, or benzoic acid substituted either in the ortho or meta position by the methyl group, but by compounds showing considerable structural differences, such as thiophene carboxylic acid and naphthoic acid.

The specific combination of certain azo-dyes with the correspouding antibodies, without the formation of a precipitate, has also been demonstrated by Marrack and Smith (1932), using an ingenious colorimetric technique.

We have already noted
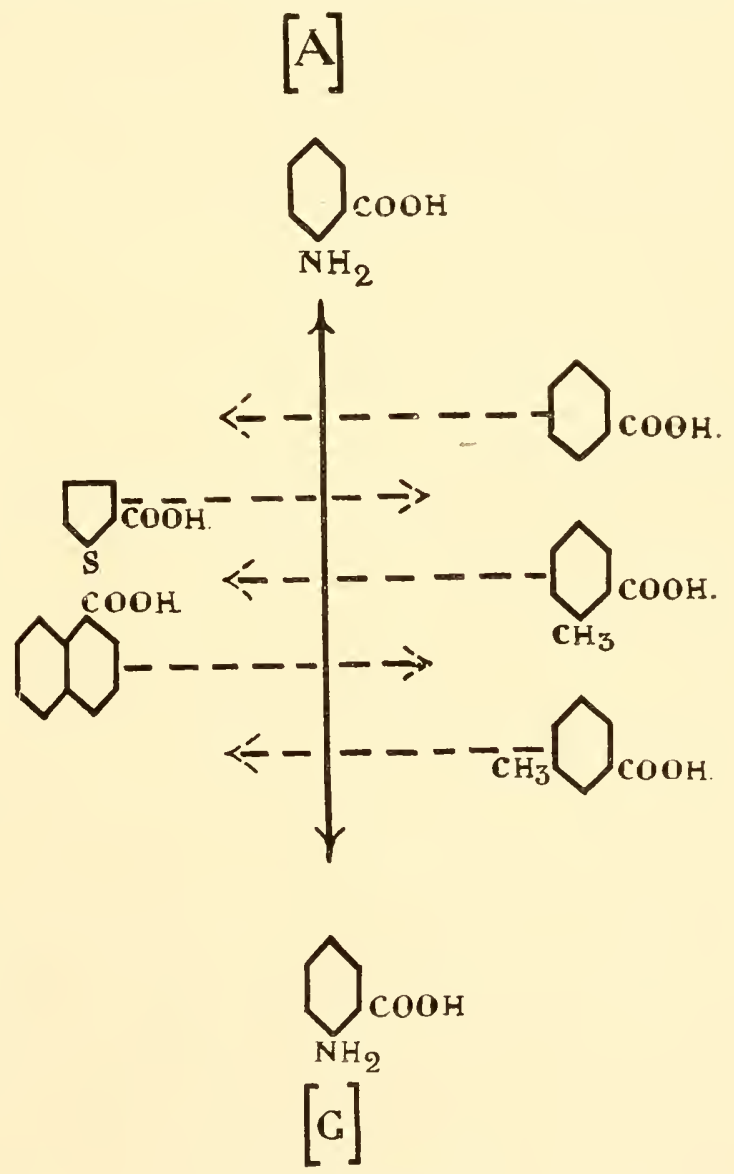

Fig. 43.-Inhibition.

that the partial antigens that inhibit precipitation do not generally themselves yield a precipitate in vitro. Neither will they stimulate the production of precipitins in vivo. In the definition of an antigen that was given at the beginning P.B. 
of this chapter the capacity to act as a stimulant of antibody production was included among its essential properties - it is, indeed, the property from which its name is derived. The difference in behaviour between substances of vảrying complexity, all bearing the same active specific grouping, led Landsteiner to formulate the conception of the hapten, or partial antigen, which, while bearing the specific grouping and therefore combining with the homologous antibody, lacks certain of the properties of the complete antigen.

In subsequent chapters frequent reference will be made to various polysaccharide components derived from different types and species of bacteria. Many of these polysaccharides, from the immunological point of view, occupy a position intermediate between Landsteiner's simpler haptens and complete antigens. They react specifically with the homologous antisera in vitro, giving precipitation; but they do not induce antibody formation in vivo, or, if they do so at all, the stimulus they provide is of an altogether different order from that provided by the complete antigen as it occurs in the bacterial cell. It seems likely that the range of activity of a partial antigen is determined in the main by its molecular size. Landsteiner and van der Scheer (19326) coupled succinic, adipic and suberic acids, through their aniline compounds, to proteins, and prepared antisera against the complete antigens so produced. Partial antigens were prepared by coupling the same compounds to resorcinol. These partial antigens gave precipitation with the corresponding antisera, and it was noted that the partial antigen derived from suberic acid gave particularly strong precipitin reactions, probably on account of its long aliphatic side chains.

It is convenient to classify the antigenic complexes we have described under the following headings :

(a) Simple haptens, possessing immunological specificity, combining with the homologous antibody and so preventing precipitation, but neither forming a precipitate in vitro, nor stimulating antibody production in vivo.

(b) Complex haptens, possessing immunological specificity, combining with the homologous antibody and forming a precipitate, but not stimulating antibody production in vivo.

(c) Complete antigens, possessing immunological specificity, combining with the homologous antibody and forming a precipitate, and stimulating antibody production in vivo.

It must not, however, be supposed that there is any sharp natural demarcation between our named classes. It seems likely that the characters that determine full antigenic potency are high molecular weight, associated with a large molecular surface and a multiplicity of active specific groupings, and the relative activity of these groupings themselves. If we were in a position to give a detailed description of the chemical structure and immunological properties of a very large number of antigenic substances, ranging from the simplest to the most complex, we should probably find that they formed a continuous rather than a discontinuous series, the falling-off in immunological activity as we passed from large and complex to small and simple molecules being marked by the gradual loss first of one immunological property, then of another, until we were left only with the ability to combine specifically with the antibody under optimal conditions of concentration and other relevant factors. 


\section{The Basis of Antigenicity.}

The distinction between haptens and antigeus brings us to the second of our inquiries, the basis of antigenicity. It seems likely that the characters determining full antigenic potency of a substance are high molecular weight, and the presence on the molecular surface of groupings that are distinctive either by reason of their molecular shape, or by reason of high electrochemical activity of certain sub-groups within the group. There are no rigid criteria by which we may judge whether a substance is likely to be antigenic or not. There are only a number of features associated with antigenicity.

It is sometimes stated that substances are antigenic because they are "foreign" to a given species of animal. This criterion is not very helpful. If we define "foreign" as "harmful" to the animal, we shall cover antigens like snake venom or diphtheria toxin, but not non-toxic antigens like ovalbumin. If we define it as "derived from a different species of organism," we shall expect, for example, insulin from the ox to be antigenic in the rabbit, which is not the ease. If, quite unjustifiably, we define it as " not normally present in the tissues of the animal," we assume either that we have a complete specification of the normal constituents of these tissues, or that we can always recognize an abnormality; and we shall find in a number of cases that our only criterion of abnormality of a substance is the response of the tissues to it as an antigen. That is, the term "foreign" can be correctly used to describe all antigens only when it is synonymous with the term "antigenic."

We have already noted that antibody response may be conditioned by various distinguishable groupings on the surface of the antigenic particle, and we may reasonably expect that antigenicity itself will be determined by certain combinations of chemical groups. These groups, and combinations of them, are called antigenic "determinants," but in seeking to relate antigenicity to the determinants of an antigen we must not be misled by a too restricted notion of a determinant. To call atoxyl a determinant in atoxyl-azo-protein does not necessarily imply an additive effect when atoxyl is linked to the protein. The true determinant is atoxyl in certain positions on the surface of the protein. The atoxyl covers some native groups, or renders them in some way inaccessible to antibody; and the reactivity of other surface groups may be altered by the presence of the new group. Moreover, the determinant effect that we attribute to atoxyl may depend on its electrochemical and spatial relations with the rest of the surface, whether antigenic or not.

We may illustrate this distinction by reference to the determinant effect of organic arsenic compounds. Singer (1942) prepared antisera to arsenic-ox globulin, and used it to protect mice against lethal doses of the synthetic antigen. Successive adsorption of the sera with arsenic-rabbit globulin and ox-globulin did not remove all protective power; clearly, antibodies to the protein-determinant complex were left in the serum.

The sphere of influence of the determinant will clearly depend on the chemical structure of the antigen. A protein antigen like horse globulin could not be spread in a unimolecular film without losing reactivity with antibody (Danielli, Danielli and Marrack 1938). Here the relationship of amino-acids in the complete protein molecule was a determinant of specificity. On the other hand, the films of nucleoprotein and other fractions of streptococci spread in thin expanded films on the surface of slides still retained a conspicuous degree of specific reactivity (Bateman, Calkins and Chambers 1941, Chambers, Bateman and Calkins 1941). 
Similarly with the fractionation of a native antigen; the loss of specificity that follows the removal of a recognizable chemical compound does not necessarily mean that the compound as such is the determinant; and even if the specificity is restored when the compound is reintroduced the essential determination of specificity lies in the restoration of the relations between the compound and the remainder of the molecule.

Full proteins are usually antigenic, though among the larger mammals the antibody response to protein antigens of other mammalian species is partly conditioned by the function as well as the structure of the protein. As Harington (1940) has pointed out, the metabolic proteins, like serum globulin, are fully antigenic ; the storage proteins, like lens proteins and casein, less so ; and hormonal proteins, like insulin and thyroglobulin, apparently shared by a large number of animal species, have little or no antigenic power.

The early successes in the study of protein antigens, the early failures to demonstrate antigenic response to the injection of carbohydrates and fats, and the fact that most of the known antigens contained at least a substantial amount of protein, led to the supposition that all antigenicity was conferred by protein, and that in the alleged examples of non-protein antigens, the substances were contaminated with chemically undetectable, but immunologically active, traces of protein. It is in many cases difficult to exclude the possibility of antigenic contaminants in a purified preparation. The scanty work, for example (sce Marrack 1938), on the antigenicity of lipins, suffers from this defect. There is another way in which a substance may be erroneously called antigenic. It may combine with the native proteins of the animal into which it is introduced, and convert them into "foreign" proteins that stimulate antibody formation. We shall discuss examples of this instructive phenomenon in connection with hypersensitivity (Chapter 51). But carbohydrate substances have been prepared whose protcin content is so low that the possibility of an effective antigenic stimulus may be neglected, but which act as full antigens, and the unique efficacy of proteins in conferring full antigenicity can no longer be upheld.

Among the proteins, gelatin is not antigenic. It lacks tyrosine and tryptophan, and has only a little phenylanine, a fact which suggests that the aromatic radicles are necessary for antigenicity (see Wells 1925). Hopkins and Wormall (1933b) failed to make gelatin antigenie by introducing aromatic radicles. Hooker and Boyd (1933), however, demonstrated that antibodies were formed to a diazo arsanilie acid gelatin complex, and Clutton, Harington and Yuill (1938) had a similar success with glucosido-tyrosyl gelatin. The absence of an aromatic radicle is not, however, the sole reason for non-antigenicity. Insulin, which is a full protein, containing aromatic radicles, is in no sense a full antigen. Various workers have reported anaphylactic sensitization with insulin preparations (Barral and Roux 1931, Lewis 1937, Bernstein, Kirsner and Turner 1938), and Wasserman, BrohKahn and Mirsky (1940) record the production of complement-fixing antibodies in the rabbit. Insulin nevertheless does not stimulate frankly precipitating antibodies, but the introduction of a glucosido-tyrosyl residue converted insulin into a full antigen (Clutton, Harington and $Y$ uill 1938). That tyrosine and other aromatic radicles are not all-important is also shown by the masking of original specificity that occurs when determinants are linked to amino-acid residues containing no aromatic rings. From the alterations induced in serum pseudoglobulin by different degrees of substitution of azo-atoxyl, Haurowitz, Sarafian and Schwerin (1941) concluded that specificity depended on a determinant arrangement of tyrosine, free amino and perhaps other groups on the molecular surface. In native lens proteins, the sulphydryl groups are apparently important determinants (Ecker and Pillemer 1940). 
A single protein molecule may possess more than one set of determinants, in the sense that on injection into an animal two distinct types of specific antibody are produced (Hooker and Boyd 1936). Haurowitz (1937) also was able to demonstrate distinct antibodies to globulin, arsanil-ązo-globulin and to the arsanilic group alone, in rabbits immunized with an apparently homogeneous preparation of arsanil-azo-globulin.

Further, Haurowitz, Tunca and Schwerin (1943) have shown that non-antigenicity may in fact be due to failure of retention on the part of the animal. One hour after injection of arsanil-azo-gelatin, rabbit liver contained $4 \cdot 4$ per cent. of the total dose, as compared with 34 per cent. arsanil-azo-globulin, while the corresponding figures in urine were 60 per cent. and $9 \cdot 2$ per cent. Their observation obviously indicates a reconsideration of many examples of non-antigenicity that have been attributed to the absence from the antigenic molecule of substances supposed to render it capable of stimulating antibody-production. A failure of adsorption on to certain cells, or of combination with body substances, may also underlie the varying response of different species of animals to the Type I pneumococcal polysaccharide. It is antigenic, or at least capable of stimulating a specific immunity, in man, horse, cat, dog and mouse, but not in the sheep, rabbit, guineapig or rat (see Horsfall and Goodner 1936, Downie 1937).

The Antigenicity of Denatured and Degraded Proteins. - Denaturation by heat or by prolonged exposure to alkali, which lead to racemization, changes the antigenic behaviour of proteins (see Hartley 1931, Wells 1929, Marrack 1938). Of particular interest is the effect of reversible denaturation. When horse or ox serum albumin was denatured by strong urea solution, and regenerated by dialysis, the protein was found to be less antigenic, but apparently had lost none of its specificity (Erickson and Neurath 1943, Martin, Erick. son, Putnam and Neurath 1943). The loss of antigenicity was attributed to breakdown of internal structure of the molecule, the retention of specificity to the preservation of a characteristic arrangement of amino-acid residues in the polypeptide chains.

These authors also noted an association between antigenicity of various protein prepara. tions and their carbohydrate content. Crystalbumin, which was the most feebly antigenic of the preparations studied, contains no carbohydrate. Lack of carbohydrate cannot be the sole reason for low antigenicity, for the regenerated, feebly antigenic albumins had lost none of their original carbohydrate; though it is possible that the mode of linkage may have been drastically altered. The earbohydrate in some proteins may be loosely attached, but in others, like serum and egg-white protein, it forms an integral part of the molecule, and is not separable until polypeptide chains are broken. Some influence on antigenicity is to be expected. Indeed, the accumulating evidence that a number of native antigens consist of complexes of polypeptides, lipins and carbohydrates suggests that the antigenicity of a protein may depend on the presence of its non-protein components. The striking example of the dependence of antigenicity upon a complex of protein and non-protein sulsstances is provided by Morgan and Partridge's (1941) analysis of a bacterial antigen in Shigella shigce. This was separable into a feebly antigenic poly. peptide and a non-antigenic polysaccharide; recombination of the two restored the full antigenic properties. On the other hand, there is no evidence that the carbohydrates associated with some of the fractions separable from serum albumins and globulins (Coghill and Creighton 1938, Rimington and van den Ende 1940) and from egg albumin and ovomucoid (Ferry and Levy 1934, Sevag and Seastone 1934, Neuberger and Yuill 1940) have any effect on antigenic specificity. The extent to which these carbohydrates are integral parts of native protein, however, is not yet clear.

The immunological reactivity of proteins decreases with progressive hydrolysis. The stage at which the hydrolysed protein ceases to react serologically varies with the protein 
(see Stull and Hampton 1941). Landsteiner (1942) found that polypeptide chains of 8-12 amino-acids from hydrolysed silk fibroin were haptens in that they inhibited the reaction between fibroin and specific antibody, and concluded that the determinant units of the protein were of this size.

A number of mechanisms can be used by an animal to remove or neutralize a substance that has been introduced into it; such as excretion, if the substance is small enough to get through the kidney; hydrolysis, if suitable enzymes are present; absorption by phagocytes or by the cells of the reticulo-endothelial system (see Chapter 50); or, in the case of substances like insulin, whatever mechanisms normally remove indiffusible hormones, etc., after they have produced their physiological effect. In these circumstances a substance will be antigenic only if it is too big to be excreted, too stable to be hydrolysed and so unusual that it is more readily handled by the mechanism resulting in antibody-formation than by the more direct physiological modes of destruction and elimination.

In summary, then, it appears that to be an antigen a substance must have certain minimum chemical properties, but it must also be so constructed that it cannot be handled by one of the readily available mechanisms of elimination, and thus removed before it has time to exert an antigenic stimulus.

\section{The Effective Number of Determinants, and the Valency of Antigen.}

The valency of native antigens may be estimated from analysis of specific precipitates in the region of antibody excess. This figure gives only the minimal number of combining groups on the antigen molecule, since spatial considerations may not permit their full saturation with antibody in the precipitate. The values range from 5 for crystalline ovalbumin (molecular weight about 42,000) (Heidelberger and Kendall 1935c, Heidelberger 1938) to 231 for the hæmocyanin (molecular weight $5-6 \times 10^{6}$ ) of the crab Viviparus (Malkiel and Boyd 1937).

The number of valencies is correlated with the surface area of the antigenic protein molecules (Hooker and Boyd 1942), and it appears that the maximal size of a determinant, supposing it to be protein in nature, is of the order of 30 aminoacids.

By coupling arsanilic acid in varying proportions to casein, and testing the compounds against antisera prepared against another arsanil-azo-protein, Hooker and Boyd (1932) calculated that an average of at least 13 introduced groups per molecule of casein was necessary for precipitation. Haurowitz, Krans and Marx (1936) similarly estimated 10-20 groups for arsanil-azo-globulin. The assumption is made that the protein particles are of full molecular weight. During the preparation of azo-proteins, however, the average particle size may decrease (Hooker, personal communication) and the minimal number of reacting groups may in fact be lower than the calculated 10-13.

\section{Unrecognized Antigens.}

Our definition of an antibody implies that it should react in a detectable manner with the corresponding antigen, and we consequently infer antigenicity when a substance reacts specifically in a detectable manner with the "antiserum " prepared against it. It is nevertheless possible for a substance to be antigenic in the formal sense of stimulating antibody production, and yet be non-reacting. As we saw in the preceding section, 10-20 arsanilic acid determinants were necessary for the precipitation of arsanil-azo-protein by its antibody. Yet the introduction of an average of one arsanilic acid group per molecule of protein gives 
a preparation that stimulates the production of antibodies to the azo compound (Haurowitz 1937).

The important observation of Bawden and Kleczkowski (1941, 1942a) that, when antigens like bushy stunt virus or human serum globulin are heated in the presence of a serum albumin, complexes are formed which, though non-precipitating, are still antigens, demands a similar modification of the conception of antigenicity. The antiserum to the albumin-globulin complex precipitated with globulin alone; the precipitation was inhibited by the complex, which in this respect behaved like a hapten. There is no good reason to suppose that antigenic, but non-reacting substances do not occur in nature. The examples from experimental serology suggest that they may be discovered in the first case by reaction between the "antiserum" and a heterologous antigen that happens to possess a large number of the determinant groups characterizing the "antigen," and in the second case, by reactions between "antiserum" and degradation products of the apparently non-antigenic substance, though it would be hard to predict the kind of degradation processes likely to yield positive results. Bawden and Pirie (1944) have provided a natural example of the first kind. Extracts of the leaves of tomatoes infected with the bushy stunt virus contain a substance which, on injection into rabbits, yields an antiserum that does not precipitate with the substance, but precipitates with bushy stunt virus obtained from sap. In this case, the nonprecipitating antigenic complex consisted of virus and a chromoprotein; on separation, the former precipitated with the antibody, and the latter had an inhibiting effect on this precipitation.

\section{The Nature of the Antigen-antibody Union.}

The large body of evidence makes it quite clear that the antibody receptor is adapted with a high degree of precision to the surface of native antigens, and that the adaptation is changed by imposing additional determinants on the antigenic surface. The nature of the adaptation is not clear. The surface of a large molecule, which may as a whole be electrically neutral, is characterized by positively or negatively charged atomic groupings, which create localizerl electric fields in the immediate neighbourhood of the molecule, operative over a very short distance.

When two molecules approach each other closely, these fields, if of opposite sign, will cause an attraction. In large molecules-and it will be remembered that it is with reactions between large molecules that we are mainly concernedthe electric fields set up may be very numerous, and they will have a quite definite spatial arrangement. Taken in conjunction with the shortuess of the distance over which these intermolecular forces are operative, this complexity and constancy of pattern provide just the conditions required for specificity. Two molecules possessing at their surface electric fields so arranged that, when they come into close contact, there will be multiple points of attraction between them, will tend to adhere to one another. Two molecules possessing the same number of electric fields, but with these so arranged that close contact cannot be made at many points simultaneously, will show no tendency to adhere. The better the fit, in this specialized sense, the greater will be the total force of attraction.

One of the best available examples of the action of these intermolecular forces is the selective formation of mixed crystals. The molecules involved must conform to the required pattern in regard to their dimensions and the relative position of their active groupings, or atoms. As a result most crystals consist of one kind of molecule alone; but molecules of different kinds may be built into a single crystal, provided 
that the structural differences between them do not exceed certain limits. Thus, for example, benzene

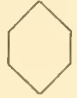
and thiophenc<smiles>C1CCSC1</smiles>
form mixed crystals, as do

azo-benzene
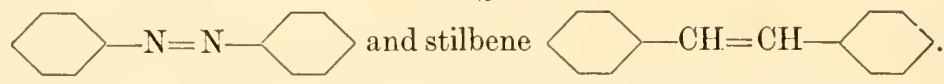

The formation of mixed crystals might, perhaps, be regarded as analogous to the formation of a precipitable antigen-antibody compound, particularly if we adopt Marrack's lattice hypothesis. It is of interest to note that the phenomenon of crystallization also presents an analogy to the inhibition of precipitation by haptens. Many crystals will specifically adsorb on to their surfaces the molecules of another substance, with which they will not form mixed crystals. Such adsorption inhibits the growth of the erystal by the addition of further molecules of the substance of which the crystal is composed. The conditions, in regard to molecular size and position of active groupings, that determine the specificity of adsorption on the surface of a crystal, are less strict than those that determine availability for building into a complete crystal lattice. This would be expected, since only one aspect of the adsorbed molecule need conform to the distribution of active groupings on the surface of the molecule. Relatively small differences may, however, be sufficient to determine the occurrence or non-occurrence of adsorption. Thus (France 1930) the dye

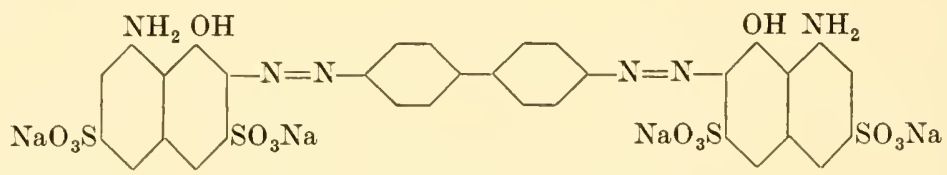

is adsorbed on the cube faces of potash alum, while the dye

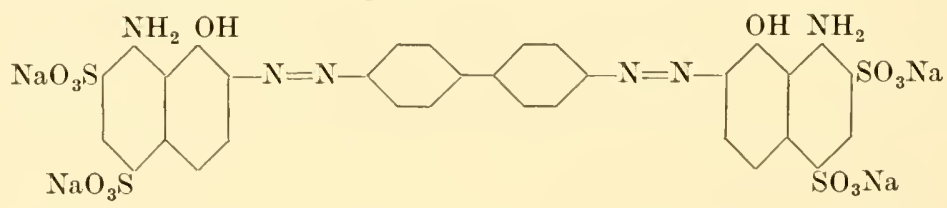

is not.

These analogies cannot be pressed far, for many of the substances which are known to act as antigenic determinants could not find, in any of the geometrically possible rearrangements of the polypeptide chains of an antibody globulin, configurations that are specific in the sense that a surface of a crystal fits molecules of its own kind. Moreover, Pauling, Campbell and Pressman (1943) point out that the attractions between permanent electric charges and dipole moments on the molecule are small in water, and have probably little effect in antigenantibody reactions; the attractions are more likely to be due to van der Waals' forces, and to hydrogen-bonding. Van der Waals' forces are due to momentarily induced charges between molecules having no permanent dipole moments. Considered singly, these forces are small, and would be strong enough to account for attraction of antigen and antibody only if large areas of each molecule were approximated sufficiently to give a summation effect of hundreds of van der Waals' attractions. Hydrogen-bonding occurs when the hydrogen attached to a strongly electronegative atom is attracted to the unshared electron pair of another electronegative atom (co-ordinate covalency). Since all these forces are non-specific, 
specificity must depend on the existence of an area on the surface of the antibody molecule that is in structure complementary to an area on the antigen molecule, enabling large surfaces to approach close enough for the forces to operate over a high proportion of the area of contact. Van der Waals' forces, for example, decrease as the 7 th power of the distance and are active only at distances of 0.1 to $0.3 \mathrm{~m} \mu$.

The complementary nature of the two surfaces must include adaptation on the part of the antibody to different determinants, their spatial relationships, and the relative accessibility of the various groups. Cross-reactions between heterologous antigens and antibodies, when there is good reason to believe they are due to a monospecific antibody, and not to other types of antibody in the antiserum, would result from the juxtaposition of less complementary areas, so that the attractions are feebler, the dissociation of the antigen-antibody link greater, and, as has been observed, the inhibition with haptens more effective, than in homologous reactions (Landsteiner and van der Scheer 1936).

Since it is likely that added determinants protrude from the surface of "synthetic" antigens, Hooker and Boyd (1942) suggest that the corresponding surface of the antibody is a depression. Indeed it may be that a spatial protrusion is a necessary condition for determinant action. This speculation is supported by the failure of all attempts to produce specific anti-antibodies, for if the antibody receptors were depressions on the surface of the globulin molecule they would on this basis have no determinant action. The anti-antibodies, as we have seen, react equally well with normal globulins from the same animal, and, when they react with the antibodies, do not interfere with the original antibody receptors.

\section{REFERENCES}

Abramson, H. A. (1935) Nature, Lond., 135, 995.

Adair, M. E. and TAYlor, G. L. (1936) J. IIyg., Camb., 36, 564.

AgNew, S., Sprnk, W. W. and IICKelsen, O. (194:2) J. Immunol., 44, 297.

Alexander, H. E. and Heipelberger, M. (1940) J.exp. Hed., 71, 1.

Aмако, T. (1911) Z. ImmunForsch., 8, 168.

Anderson, T. F. and stanlex, W. M. (1941) J. biol. Chem., 139, 339.

ANDO, K. (1937) J. Immunol., 33, 41 .

AxDo, K., KEe, R., and Komirana, T. (1937) I. Immunol., 32, 181.

Axpo, K., Kee, R., and MAnako, K. (1937) J. Immunol., 32, 83.

Axdo, K., Maxako, K., Kee, R., and TAkEDA, S. (1937) J. Immunol., 33, 27.

Axdo, K., Maxako, K., and Takeda, S. (1933) J. Immunol., 34, 295.

Axdo, K., Takeda, S., and Haraxo, II. (1938) J. Immunol., 34, 303.

Arrhexius, S. (1904) Arb. ReichsgesundhAmt., 20, 559; (1915) "Quantitative Laws in Biological Chemistry." London.

Arrhexius, S. and Madsen, T. (1902) Festskrift Staatens Serum Inst., No. 3 ; (1904) Zbl. Bakt., 36, 612 ; (1904) Ibid., 3\%, 1.

Astbury, W. T. (1943) Advances in Enzymology, 3, 63.

Avery, O. T. and Goeber, W. F. (1929) J. exp. Med., 50, 533.

Avery, O. T., Goebel, IV. F., and Babers, F. H. (1932) J.exp. Med., 55, 769.

BAIL, O. and Hoke, E. (1908) Arch. Hyg., 64, 313.

BAIL, O. and TsUdA, K. (1909) Z. ImmunForsch., 1, 546.

BaxzhaF, E. J. and Gibsos, R. B. (1907) J. biol. Chem., 3, 253.

BARr, M. (1932) J. Path. Bact., 35, 913.

Barr, M. and Glensy, A. T. (1931) J. Path. Bact, 34, 539.

Barr, M., Glenvy, A. T., and Pope, C. G. (1931) Brit. J. exp. Path., 12, 217.

Barral, P. and Roux, J. (1931) C. R. Soc. Biol., 106, 292.

Batemax, J. B., Calkixs, H. E. and Chambers, I. A. (1941) J. Immunol., 41, 321.

Bawdex, F. C. and Kleczkowski, A. (1941) Brit. J.exp. Path., 22, 20s: (194:a) Ibid., 23, 169 ; $(19426)$ Ibid., 23, 175.

Bawdex, F. C. and Pirie, N. W. (19t4) Brit. J. exp. Path., 25, 68. 
Bechinold, H. (1904) Z. phys. Chem., 48, 385.

Behring, von and Kitasato. (1890) Dtsch. med. W'schr., 16, 1113.

Benlasch, M. (1911) Z. ImmunForsch., 12, 268.

Berger, F. II. (1943) Brit. J. exp. I'ath., 24, 252.

Bernheim, F., Neurath, H., and Erikson, J. O. (1942) J. biol. Chem., 144, 259.

Bernstein, C., Kirsner, J. B., and Turner, W. J. (1938) J. Lab. clin. Med., 23, 938. Biltz, IV. (1910) Biochem. Z., 23, 27.

Bordet, J. (1895) Ann. Inst. Pasteur, 9, 462 ; (1898) Ibid., 12, 688 ; (1899) Ibid., 13, 225; (1903) Ibid., 1\%, 161.

Bordet, J. and Gengou, O. (1901) Ann. Inst. Pasteur, 15, 290.

Boyd, W. C. (1940) J. Immunol., 38, 143; (1941) J. exp. IIed., 74, 369 ; (1942) Ibirl., $75,407$.

Boỹ, W. C. and Bernard, H. (1937) J. Immunol., 33, 111.

Boyd, W. C., Conn, J. B., GregG, D. C., Kistiakowsky, G. B., and Roberts, R. M. (1941) J. biol. Chem., 139, 787.

Boxd, W. C. and Hooker, S. B. (1934) J. gen. Physiol., 17, 341 ; (1938) Proc. Soc. exp. Biol., N.Y., 39, 491.

Boyd, W. C. and Purnelt, M. A. (1944) J. exp. Med., 80, 289.

BRAND, E. (1907) Berl. klin. W schr., 44, 1075.

Breinl, F, and Haurowitz, F. (1930) Z. physiol. Chem., 192, 45.

Broov, J. C. and Brows, H. C. (1930) Brit. J. exp. Path., 11, 305.

Brown, A. M. (1935) Brit. J. exp. Path., 16, 554.

Brown, G. C. (1943) J. Immunol., 46, 319.

Brown, H. C. and Broou, J. L. (1929) Brit. J. exp. Path., 10, 387.

Brown, H. R. (1938) J. Lab. clin. Med., 23, 1277.

Brownlee, J. (1925) J. IIyg., Camb., 23, 437.

BUChaNAN, R. E. (1919) J. Bact., 4, 73.

Buchner, H. (1859a) Zbl. Bakt., 5, 817; (1889b) Ibid., 6, 1; (1889c) Ibid., 6, 561.

Bukantz, S. C., Cooper, A., and Bullowa, J. G. I. (1941) J. Bact., 42, 15.

Bulloch, W. B. and Western, G. T. (1906) Proc. roy. Soc., B, 7\%, 531.

Burnet, F. M. (1931) J. Path. Bact., 34, 471.

Büsing, K. H. and Zuzack, H. (1943) Z. ImmunForsch., 102, 401.

Butler, G. C., Harington, C. R., and Yulli, M. E. (1940) Biochem. J., 34, 838.

Buxtow, B. H. (1905a) J.med. Res., 13, 305; (1905b) Ibid., 13, 431 ; (1905c) Ibid., 13, 461.

Cannon, P. R. and Marshali, C. E. (1940) J. Immunol., 38, 365.

Chanbers, L. A., Bateman, J. B., and Calkins, H. E. (1941) J. Immunol., 40, 483.

Chapin, W. S. and Cowie, D. M. (1907) J. med. Res., 17, 213.

Chow, B. F. and Goeber, W. F. (1935) J. exp. Med., 62, 179.

Chow, B. F. and ZıA, S. H. (1938) Proc. Soc. exp. Biol. N.Y., 38, 695.

Chu, F. and Chоw, B. F. (1938) Proc. Soc. exp. Biol. N.Y., 38, 679.

Clutton, R. F., Harington C. R., and Mead, T. H. (1937) Biochem. J., 31, 764.

Clutton, R. F., Harington, C. R., and Yuill, M. E. (1938) Biochem. J., 32, 1111 , 1119.

CocA, A. F. (1914) Z. ImmunForsch., 21, 604.

Coghill, R. D. and Creighton, M. (1938) J. Immunol., 35, 477.

Colwell, C. A. and Youmans, G. P. (1941) J. infect. Dis., 68, 226 ; J. Immunol., $42,79$. Costa Cruz, J. DA. (1929) C. R. Soc. Biol., 100, 932.

Coulter, C. B. (1920-21) J. gen. Physiol., 3, 513.

Cowie, D. M. and Chapix, W. S. (1907) J. med. Res., 17, 57, 95.

Craigie, J. (1931) Brit. J. exp. Path., 12, 75.

Crandon, J. H., Iunnd, C. C., and Dill, D. B. (1940) Tew Eng. med. J., 223, 353.

Craw, J. A. (1905) J. Hyg., Camb., 5, 113.

DAKIN, H. D. and DALE, H. H. (19L9) Biochem. J., 13, 248.

Dale, H. H. and Hartley, P. (1916) Biochem. J., 10, 110.

Danielit, J. F., Danielli, M., and Marrack, J. R. (1938) Brit. J. exp. Path., $19,393$.

Danysz, J. (1902) Ann. Inst. Pasteur, 16, 331.

Deav, G. (1907) Proc. roy. Soc., B, 79, 399.

Deax, H. R. (I912) Z. ImmunForsch., 13, 84; (1917) Lancet, i. 45; (1937) J. Path. Bact., 45, 745.

Dean, H. R., Taylor, G. L., and Adair, M. E. (1935) J. IIyg., Camb., 35, 69.

DeAs, H. R. and WebB, R. A. (1926) J. Path. Bact., 29, 473.

DeISSLER, K. (1932) Z. ImmunForsch., 73, 365.

Delves, E. (1937) J. infect. Dis., 60, 55 .

Denys, J. and Leclef, .J. (1895) La Cellule, 11, 177.

Downie, A. W. (1937) J. Path. Bact., 45, 149.

Dozois, T. H., Seifter, S., and Ecker, E. E. (1943) J. Immunol., 47, 215 ; (1944) Ibid., 49,31 . 
DREYER, G. and InMAN, A. C. (1917) Lancet, i. 365 .

Duncan, J. T. (1932a) Brit. J. exp. Path., 13, 489; (1932b) Ibid., 13, 498 ; (1934) Ibid., 15, 23; (1935) Ibid., 16, 405; (1937) Ibid., 18, 108; (1938) Ibid., 19, 328.

EAGLE, H. (1930) J. Immunol., 18, 393 ; (1932) Ibid., 23, 153; (1936) Ibid., 30, 339 ; (1937) Ibid., 32, 119; (193s) J. exp. Ued., 6\%, 495.

EAGLe, H. and ViChers, P. (1936) J. biol. Chem., 114, 193.

Ecker, E. E. and Pillemer, L. (1940) J. exp. Led., 71, 585 ; (1941) J. Immunol., 40, 73 ; (1942) Ann. N.Y. Acad. Sci., 43, 63.

Ecker, E. E., Pillemer, L., and Grabil, F. J. (1938) Proc. Soc. exp. Biol., M.Y., $38,318$.

Ecker, E. E., Pillemer, L., and Kuefhn, A. O. (1942) J. Immunol., 43, 245.

Echer, E. E., Pillemer, L., Martiensen, E. W., and Wertheimer, D. (1939) J. biol. Chem., 123, 351 .

Ecker, E. E., Pillemer, L., and Seifter, S. (1943) J. Immunol., 4\%, 181.

Ecker, E. E., l'iliemer, L., Werthenner, D., and Gradis, H. (1938) J. Immunol., $34,19$.

Ecker, E. E., Weisberger, A. S., and Pillemer, L. (1942) J. Immunol., 43, 227.

Eнrцich, P. (1897) Klin.Jb., 6, 299; (1898) Dtsch. med. Wschr., 24, 597; (1900) Ploc. roy. Soc., $B, 66,424$.

EiseNBerg, P. and VOLK, R. (1902) Z. Hyg. InfehtKr., 40, 155.

Eisler, M. (1920) Zbl. Bakt., 84, 46.

ENDERS, J. F. and SHAFFER, II. F. (1937) J. Imminol., 32, 379.

Erickson, J. O. and Neuratir, H. (1943) J. exp. Mcd., 78, 1.

Erlenveyer, H. and Berger, E. (1932) Biochem. Z., 252, 22: ; (1934) Arch. exp. Prath. Pharmak., 17\%, 116.

Etinger-TulczYNSKa, R. (1933) Z. IIyg. InfektKr., 114, 769.

Euler, H. vos, and Bruxies, E. (1931) Z. ImmunForsch., 72, 65.

Falk, I. S. and Matsuda, T. (1926) Proc. Soc. exp. Biol., N.Y., 23, 781.

Fell, N., Rodney, G., and Marshall, D. E. (1943) J. Immunol., 4\%, 237.

Fell, N., Sters, K. G., and Coghill, R. D. (1940) J. Immunol., 39, 223.

Feller, A. E., Roberts, L. B., Ralli, E. P., and Francis, T. (1942) J. clin. Invest., 21, 121.

Felton, L. D. (1926) Bull. Johns Hopk. Hosp., 38, 33 ; (1928) J. infect. Dis., 43, 543; (1932) J. Immunol., 22, 453.

Felton, L. D. and Balley, G. H. (1926) J. infect. Dis., 38, 131.

Frirrata, A. (1907) Berl. Klin. Wrehr., 44, 366.

Ferry, R. M. and Levy, A. H. (1934) J. biol. Chem., 105, xxrii.

Follexsby, E. M. and Hooker, s.. B. (1939) J. Immunol., 3\%, 367.

France, W. G. (1930) Coll. Symp. Honogr., \%, 59. (See Marrack 1934.)

Frankel, II. (1932) Proc. roy. Soc., B, 111, 165.

Frankel, M. and Olitzki, L. (1930) Nature, Lond., 126, 723.

Freund, J. (19:9) Proc. Soc. exp. Biol., N.Y., 26, 876.

Freundlich, H. (1906) Z. phys. Chem., 5\%, 385 ; (1922) "Kapillarchemie." Leipzig.

Freundeich, H. and Neuniax, W. (1909) Z. phys. Chem., 6\%, 538.

Friedberger, E. and Golnschmidt, E. (1910) Z. ImmunForsch., 6, 299.

GaY, F. P. (1905) Zbl. Bakt., 29, 603.

GexGou, O. (1899) Ann. Inst. Pasteur, 13, 642 ; (1902) Ibid., 16, 734 ; (191 I) Z. Immun. Forsch., 11, 143.

Gerlough, T. D., Palmer, J. W., and Blumenthal, R. R. (1941) J. Immmol., 40, 53.

Ghosh, B. N. (1935) Indian J. med. Res., 23, 285.

Gibsox, R. B. and Banzhaf, E. J. (1910) J. exp. Med., 12, 411.

Gibsox, R. B. and Collins, K. R. (1907) J. biol. Chem., 3, 233 .

Glenny, A. T., Pope, C. G., and Waddington, H. (1925) J. Path. Bact., 28, 279.

Goebel, W. F., Avery, O. T., and Babers, F. H. (1934) J. exp. Med., 60, 599.

Goldie, H. and SAxpor, G. (1937) C. R. Soc. Biol., 126, 291.

Goldsworthy, N. E. (1928) J. Path. Bact., 31, 220.

Goldsworthy, N. E. and Rudd, G. V. (1935) J. Path. Bact., 40, 169.

Goodner, K. and Horsfall, F. L. (1936) J. cxp. Med., 64, 201; (1937) Ibid., 66, 413, $425,437$.

Goodner, K., Horsfall, F. L., and BaUer, J. H. (1938) J. Immunol., 35, 439, 451.

Gordox, J. (1937) J. Immunol., 32, 375.

Gordox, J. and Atkin, IV. R. (1938) Brit. J. exp. Path., 19, 204; (1939) J. Path. Bact., 48, 477 ; (1941) Brit. J. exp. Path., 22, 226.

Gordon, J. and Thompsox, F. C. (1933a) Brit. J. exp. Path., 14, 33; (1933b) Ibid., 14, 277 ; (1935) Ibid., 16, 101 ; (1936) Ibid., 17, 159; (1937) Ibid., 18, 390.

Gordon, J., Whitehead, H. R., and WormaLe, A. (1926a) Biochem. J., 20, 1028 ; (1926b) Ibid., 20, 1036. 
Greenwood, M. (1913) Lancet, i. 158.

Gruber, M. and DurнaM, H. E. (1896) Mïnch. med. Wschr., 43, 285.

Hammarsten, O. (1878) Arch. ges. Physiol., 17, 413.

Hanks, J. H. (1940) J. Immunol., 38, 159.

Harde, E. and Tuomson, A. E. (1935) C. R. Acud. Sici., 200, 1425.

Harington, C. R. (1940) J. Chem. Soc., i, 119.

Harris, T. and Eagle, H. (1935) J. gen. Physiol., 19, 383.

Harte, R. A. (1938) J. Immmnol., 34, 433.

Hartcey, P. (1914) Mem. Dept Agric. India, 4, 178; (1925) Brit. J. exp. Path., 6, 180; (1931) Med. Res. Coun. Lond. "System of Bacteriology," 6, 22t.

Haurowitz, F. (1937) Klin. II'schr., 16, 257; (1940) 3rd int. Congr. Microbiol., s19.

Haurowitz, F. and Breinl, F. (1933) IIoppe-Seyl. Z., 214, 111.

Hacrowitz, F., Kraus, F., and llarx, F. (1936) Z. physiol. Chem., 245, 23.

Haurowitz, F., Sarafian, K., and Schwerin, P. (194I) J. Immunol., 40, 391.

Hadrowitz, F. and Scinerin, P. (1943) J. Immunol., 4\%, 111.

Hatrowitz, F., Tunca, M. and Schweris, I'. (1943) Biochem. J., 37, 249.

Haurowitz, F. and Yenson, M. M. (1943) J. Immunol., 47, 309.

Healey, H. and Pineield, S. (1935) Brit. J.exp. Path., 16, 535.

Hegedüs, A. and Greiner, H. (1938) Z. ImmunForsch., 92, 1.

Heidelberger, M. (1938) J. Amer. chem. Soc., 60, 242; (1939) Bact. Rev., 3, 49; (1941) J. exp. Mèl., 73, 681.

Heidelberger, M., Grabar, P., and Treffers, H. P. (1938) J. exp. Med., 68, 913.

Heidelberger, M. and Kabat, E. A. (1934) J. exp. Med., 60, 643; (1936) Ibid., 63. 737.

Heidelberger, M. and Kendall, F. E. (1929) J.exp. Med., 50, 809 ; (1934) Ibid., 59, 519; (1935a) Ibid., 61, 559, 563; (1935b) Ibid., 62, 467; (1935c) Ibid., 62, 697 ; (1936) Ibid., 64, $161 ;(1937)$ Ibid., 65, 647.

Heidelberger, M., Kendall, F. E., and Teorell, T. (1936) J.exp. Med., 63, 819.

Heidelberger, M. and Landsteiner, K. (1923) J. exp. Med., 38, 561.

Heidelberger, M. and MAYer, M. (1942) J.exp. Med., \%5, 285.

Heidelberger, M. and Pedersen, K. O. (1937) J. exp. Med., 65, 393.

Heidelberger, M., Sia, R. H. P., and Kendall, F. E. (1930) J. exp. Med., 52, 477.

Heidelberger, M. and Treffers, H. P. (1941) J. gen. Pliysiol., 25, 523.

Heidelberger, M., Treffers, H. P., and Mayer, M. (1940) J.exp. Med., 71, 271.

Heidelberger, M., Weil, A. J., and Treffers, H. P. (1941) J. exp. Med., 73, 695.

Heinicke, A. (1934) Z. ImmunForsch., 83, 245.

Hertoen, I. (1908) J. infect. Dis., 5, 249.

Heктоen, L. and Boor, A. K. (1931) J. infect. Dis., 48, 588.

Heztoen, L. and Schulhof, K. (1923) J. infect. Dis., 5, 249.

Hershey. A. D. (1940) J. Immunol., 39, 383; (194la) Ibid., 42, 45.), 485 ; (1941b) Ibid., 42, 515; (1942) Ibid., 45, 39; (1943a) Ibid., 46, 249; (1943b) Ibid., 47, 77; (1944) Ibid., 48, 381 .

Hershey, A. D., Kalianson, G., and Broneenbrenner, J. (1943) J. Immund., 46, 267, 281.

HeUer, G. (1922) Z. Hyg. Infehthr., 95, 100.

Hewite, L. F. (1935) Biochem. J., 32, 26, 1554.

Higasil, S. (1922) J. Biochem., Tokyo, 2, 315.

Hooker, S. B. and BuYd, W. C. (193:) J.Immunol., 23, 465: (1933) Ibid.. 24, 141 ; (1936) Ibid., 30, 33, 41 ; (1937) Ibid., 33, 337; (1940) Ibid., 38, 479; (194I) Proc. Sor. exp. Biol., N.Y., 47, 187; (1941b) J. Immunol., 42, 419; (1942) Ibid., 45, 127.

Hopkins, F. G. (1900) J. Physiol., 25, 306.

Hopkins, S. J. and Worinal, A. (1933a) Biochem. J., 27, 740; (1933b) Ibid., 27, 1706. Horgan, E. S. (1936) Nature, Lond., 13\%, 872.

Horsfall, F. L. and Goonner, K. (1935) J. exp. Med., 62, 485; (1936a) J. Immunol.. 31, $135 ;(1936 b)$ J. exp. Hed., 64, 583.

Huddeson, I. F., Johnson, H. WT. and Hamann, E. E. (1933) Amer. J. publ. Illik., 23, 917.

Huntoon, F. M. and Etris, S. (1921) J. Immunol., 6, 123.

Huntoon, F. M., Masucci, R., and Hannum, E. (1921) J. Immunol., 6, 185.

Hyde, R. R. (1923) J. Immunol., 8, 267.

IWANOFF, K. (1936) Z. Infelitir., 118, 197.

J ACOBs, J. (1937) J. gen. Physiol., 20, 353.

Jennings, R. K. and Sirth, L. D. (1942) J. Immunol., 45, 105.

Johnson, F. H. and Dennison, W. L. (1944) J. Immunj., 48, 317.

Johnson, L. R. and Tormall, A. (1932) Biochem. J., 26, 1202.

Jones, C. B. and Echer, E. E. (1940) Proc. Soc, exp. Biol, N.Y., 44, 2 (i.4. 
Jones, D. B. and GersdorfF, C. E. F. (1923) J. biol. Chem., 56, 79.

Joos, A. (1901) Z. Hyg. InfektKr., 36, 422; (1902) Ibid., 40, 203.

Кават, E. А. (1939) J. exp. Med., 69, 103.

Kabat, E. A. and Heidelberger, M. (1937) J. exp. Med., 66, 229.

KAPNICK, I. and Cope, O. (1940) Endocrinology, 2\%, 543.

Kilmansoy, G. M. and Bronfenbrenner, J. (194:2) Science, 96, 2I ; (1943) J. Immunol. $47,387$.

Kekwick, R. A., Macfarlane, M. G., KNight, B. C. J. G., and Record, B. R. (1941) Lancet, i, 571.

Kekwick, R. A. and Pecond, B. R. (1941) Brit. J. exp. Path., 22, 29.

Keupf, A. H. and Nungester, W. J. (1942) J. infect. Dis., $71,50$.

Kendall, F. E. (1942) Ann. N.Y. Acad. Sci. 43, 85.

Kleczkowski, A. (1941a) Brit. J. exp. Path., 22, 44; (1941b) Ibid., 22, 18s, 19.2 ; (1943 Biochem. J., $3 \%, 30$.

KLess, H. (1907) Johns Hopk. Hosp. Bull., 18, 245.

Kocr, M. L. and Sнrт, A. H. (1924) Proc. Soc. exp. Biol., N.Y., 21, 366.

Konicek, E. and Traub, B. (1943) Biochem. J., 37 456.

Kosaki, M. (1918) J. Immunol., 3, 109.

Kraus, R. (1897) Wien. klin. Wschr., 10, 736.

Krejci, L. E., Jennings, R. K., and Sirth, L. D. (1942) J. Immunol., 45, 111.

KROGH, I. voN. (1911) Z. Hyg. InfektKr., 68, 251.

Kruif, P. H. de and Northrop, J. H. (1922-23) J. gen. Physiol., 5, 127.

LaIdlaw, P. P. and Dunkin, G. W. (1931) J. comp. Path., 44, 1.

LANDSTEIner, K. (1917) Z. ImmunForsch., 26, 122; (1919) Biochem. Z., 93, 108 ; (1920) Ibid., 104, 280; (1930) Naturwissenschaft., 18, No. 29, 653; (1933) "Die Spezifizität der serologischen Reaktionen." Julius Springer, Berlin; (1936) "The Specificity of Serological Reactions." Baltimore; (1942) J. exp. Ucd., 75, 269.

Landsteiner, K. and Heidelberger, M. (1923) J. gen. Physiol., 6, 31.

LANDSTEINER, K. and JAGIC, N. (1903) Münch. med. Wschr., 50, 761.

Landsteiner, K. and Lampe, H. (1917a) Z. ImmunForsch., 26, 133, 258; (1917b) Biochem. Z., 86,343 .

Landsteiner, K. and Pirie, N. W. (1937) J. Immunol., 33, 265.

Landsteiner, K. and Prášek, E. (1911) Z. ImmunForsch., 10, 68 ; (1914) Ibid., 20, 211.

JANDSTEINER, K. and Sciner, J. vaN DER. (1928) J.cxp. Hcd., 48,315; (1929) Ibid., 50, 407 ; (1931) Ibid., 54, 295 ; (1932a) Ibid., 55, 781 ; (1932b) Ibid., 56, 399 ; (1934a) Ibid., 59, 751; (1934b) Ibid., 59, 769; (1936) Ibid., 63, 325; (1938) Ibid., 67, 709; (1939) Ibid., 69, 705; (1940) Ibid., 71, 445.

LANDSTEINER, K. and WeLECKI, S. (1911) Z. ImmunForsch., 8, 395.

LedinghaM, J. C. G. (1907) J. Hyg., Camb., \%, 65.

LeIshman, W. B. (1902) Brit. med. J., i. 73.

Lewis, J. H. (1937) J. Amer. med. Ass., 108, 1336.

Lewis, J. H. and Wells, H. G. (1925) J. biol. Chem., 66, 37.

Liвву, R. L. (1938) J. Immunol., 34, 269; 35, 289.

Liefaidn, H. (1909) Münch. med. Wschr., 56, 2097.

Liv, S-C., Chow, B. F., and Lee, K-H. (1937) Chin. .J. Physiol., 11, 201.

Locke, A. and Hirsch, E. F. (1925) J. infect. Lis., 3\%, 419.

Low ELL, F. C. (1943) J. Immunol., 46, 177.

Madsen, T. and Streng, O. (1910) Z. phys. Chem., 70, 263.

Maitland, H. B. and Burbury, Y. M. (1927) J. comp. Path., 40, 93.

Malkiel, S. and Buyd, W. C. (1937).J. exp. Med., 66, 383.

Maltaner, E. (1935) Proc. Soc. exp. Biol., N.Y., 32, 1555.

Maltaner, E. and Maltaner, E. (1940) 3rd int. Congr. Microbiol., 781.

MaRkL. (1902) Z. Hyg. InfektKr., 39, 86.

Marrack, J. R. (1934) Spec. Rep. Ser. med. Res. Coun. Lond., No. 194; (I93s) Ibid., No. 230 .

Marrack, J. R. and Duff, D. A. (1938) Brit. J. exp. Path., 19, 171.

Marrack, J. and Höllering, H. F. (1938) Brit. J. exp. Path., 19, 424.

Marrack, J. and Sirti, F. C. (1930) Proc, roy. Soc., B, 106, I ; (1931a) Brit. J. exp. Path., 12, 30; (1931b) Ibid., 12, 182; (1932) Ibid., 13, 394.

Marsh, F. (1936) Nature, Lond., 13\%, 618.

Martin, D. S., Erickson, J. O., Putnai, F. W., and Neuratir, H. (1943) J.gen. Physiol., $26,533$.

Mellaxby, J. (1908) Proc. roy. Soc, $B, 80,399$.

Mennes, F. (1897) Z. Hyg. InfektKr., 25, 413.

Meyer, K. (1936) Ann. Inst. Pasteur, 56, 684.

Mich Aelis, L. (1911) Dtsch. med. Wschr., 37, 969.

Miles, A. A. (1933) Brit. J. exp. Path., 14, 43 ; (1939) Ibid., 20, 63. 
Miles, A. A. and Pirie, N. W. (1939) Brit. J. exp. Path., 20, 109.

MoOre, D. H., VAN DeR Scheer, J., and WYCkofF, R. WV. G. (1940) J. Immunol., 38, 221.

Moriyama, H. (1937) J. Shanghai Sci. Inst., Seet. IV, 2, 279.

Morgan, W. T. J. and Partridae, S. M. (1941) Biochem. J., 35, 1140.

Morris, M. C. $(1940) \mathrm{J}$. Immunol., 39, 369 .

Mudd, S. and Anderson, T. F. (1941) J. Immunol., 42, 251.

Mudd, s., Heinuets, F., and Anderson, T. F. (1943) J.exp. Med., 78, 327.

MUdd, S. and Joffe, E. IT. (1933) J. gen. Physiol., 16, 947.

Mudd, S., Lucké, B., McCutcheos, M., and Strumi, M. (1929) J. exp. Med., 49, 779.

Muir, R. (1903) Lancet, ii. 100, 446 ; (1909) "Studies on Immunity." London.

Muir, R. and Browning, C. H. (1909) J. Path. Bact., 13, 76.

Muir, R. and Martin, W. B. M. (1906a) Brit.med.J., ii, 1783; (1906b) J. IIyg., Camb., 6, 265.

Mutsaars, W. and Grégoire, P. E. (1936) C. R. Soc. Biol., 123, 144.

Neisser, M. and Wechsberg, F. (1901) Münch. med. Wschr., 48, 697.

Nevberger, A. and Yulle, M. E. (1940) Biochem. J., 34, 109.

NeUfeld, F. (1902) Z. I1 yg. Infelikr., 40, 54.

Neufeld, F. and Etinger-Tulczinska, R. (1929) Zbl. Bakt., 114, 252 ; (1931) Z. Hyg. InfektKr., 112, 492 .

Neufeld, F. and Hüne. (1907) Arb. ReichsgesundhAmt., 25, 164.

Neufeld, F. and Rimpad, R. (1904) Dtsch. med. Wschr., 11, 1458; (1905) Z. Hyg. InfektKr., $51,283$.

Neuratir, H., (1939) J. Amer. chem. Soc., 61, 1841.

Neurath, H.. and Saum, A. II. (1939) J. biol. Chem., 128, 347.

Nicolle, M. and Césari, E. (1922) Ann. Inst. Pasteur, 36, 463.

Northrop, J. H. (1922) J. gen. Physiol., 4, 629; (1928) "The Newer Knowledge of Bacteriology and Immunology." Jordan \& Falk, Chicago, p. 782; (1941) J.gen. Physiol., 25, 465.

Northrop, J. H. and Kruif, P. H. DE. (1922a) J. gen. Physiol., 4, 639 ; (1922b) Ibid., 4, 655.

Nö̈r, P. L. in and Hamon, V. (1935) C. R. Acad. Sci., 200, 1250; (1936) Ann. Inst. Pasteur, 56, 359.

Nuttale, G. (1888) Z. Hyg. InfektKr., 4, 353.

Nuttall, G. H. F. (1904) "Blood Immunity and Blood Relationships." Cambridge.

Obermayer, F. and Pick, E. P. (1906) Wien. klin. Wschr., 19, 327.

OLITzKI, L. (1932) Z. ImmunForsch., 76, 296.

Olitzki, L. and Frankel, M. (1931) Proc. Soc. exp. Biol., N.Y., 28, 492.

Ottolenghi, D. and Mori, N. (1905) Zbl. Bakt., 38, 338, 468.

Pappenheimer, A. H. (1940) J. exp. Med., 71, 263; (1942) J. Bact., 43, 273.

Pappenheimer, A. M., Lundgrex, H. P., and Williams, J. W. (1940) J. exp. Med., $\mathbf{7 1 ,}$ 247.

Pappenineimer, A. M. and Robinson, E. S. (1937) J. Immunol., 32, 291.

Padling, L. (1940) J. Amer. chem. Soc., 62, 2643.

Patling, L., Campbell, D. H., and Pressian, D. (1941) Proc. nat. Acud. Sci. Wash., 2\%, 125; (1943) Physiol. Rier., 23, 203.

Palling, L. and Niemann, C. (1939) J. Amer. chem. Soc., 61, 1860.

Pauling, L., Pressian, D., Campbell, D. H., and Ifeda, C. (1942) J. Amer. chem. Soc., $64,3003$.

Pauling, L., Pressian, D., and Ineda, C. (1942) J. Amer. chem. Soc., 64, 3010.

P'enneli, R. B. and Huddleson, I. F. (1938) J. exp. Med., 68, 73, 83.

Pétermann, M. L. (1942) J. biol. Chem., 144, 607.

P'Feiffer, R. (1893) Z. IIyg. InfektKr., 11, 393; (1894a) Ibid., 16, 268; (1894b) Ibid., 18, 1 ; (1895) Ibid., 19, 75.

Pfeiffer, R. and IssaefF. (1894) Z. Hyg. Infektkr., 1\%, 355.

Pick, E. P. (1912) Kolle and Wassermanns "Hdb. d. path. Mikroorg" ; IIte Aufl., 1, 685 PiJper, A. (1938) J. Path. Buct., 4\%, 1 ; (1941a) Ibid., 53, 431 ; (1941b) J. Bact., 42, 395.

P'illemer, L., Chu, F., Seifter, S., and EcKer, E. E. (1942) J. Immunol., 45, 51.

Pillemer, L. and Ecker, E. E. $(1941 a)$ J. Immunol., 40. 101 ; (1941 $b)$ Science, 94, 437.

Pillemer, L., Ecker, E. E., Oxrley, J. L., and ('ohn, E. J. (1941) J. exp. Med., 74, 297.

Pillemer, L., Seifter, J., and Ecker, E. E. (1941) J. Immunol., 40, 89 ; (1942) J. exp. Med., 75, 421.

Pillemer, L., Seifter, S., San Clemente, C. L., and Ecker, E. E. (1943) J. Immunol., $4 \%, 205$.

Pittian, M. (1943) Publ. Hlth. Rep.. Wash., 58, 139.

Platt, A. E. (1936) Aust. J. exp. Biol. med. Sci., 14, 101 ; (1938) Ibid., 16, 275.

Pochon, J. (1936) C. R. Soc. Biol., 121, 387.

Pore, C. G. (1939a) Brit. J. exp. P'uth., 20, 132; (1939b) Ibid., 20, 201. 
Pope, C. G. and Healey, M. (1935) Brit. J. exp. Path., 19, 397.

Porges, O. (1906) Zbl. Bakt., 40, 133.

Porges, O. and Prantschoff, A. (1906) Zbl. Bakt., 41, 466, 546, 658.

Putter, E. (1921) Z. ImmunForsch., 32, 538.

RACE, R. R. (1944) Nature, Lond., 153, 771.

Raffel, S., Pait, C. F., and Terry, M. C. (1940) J. Immunol., 39, 317.

Ramon, G. (1922) C. R. Soc. Biol., 86, 661, 711, 813.

Reiner, 1. and Fischer, O. (1929) Z. ImmunForsch., 61, 317.

ReINer, L. and KopP, H. (1929) Z. ImmunForsch., 61, 397.

Rice, C. E. (1943) J. Immunol., 46, 427.

Rice, C. E. and Sickles, G. R. (1942) J. Immunol., 43, 319.

Rinington, C. and Ende, M. van Des, (1940) Biochem. J., 34, 941.

Rosenhem, A. H. (1935) J. Path. Bact., 40, 75; (1937) Biochem. J., 31, 54.

Ross, V. (1938) J. Immunol., 35, 351, 371.

Pothen, A. (1941) J. gen. Phys., 25, 487.

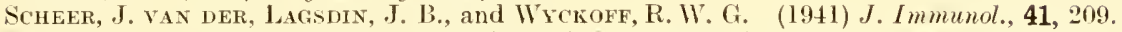
SCHEER, J. YAN DER, WYCKOFF, R. IV.G., and Clarke, F. H. (1940) J.Immumol., 39, 65; (19+1a) Ibid., 40, 39 ; (1941b) Ibid., 40, 173; (194lc) Ibid., 41; 349.

Sichumpt, S. (1930) C. R. Soc. Biol., 103, 101

Seifter, S., Dozois, T. F., and Ecker, E. E. (1941) J. Immunol., 49, 45.

Neifter, S., Pillemer, J., and Ecker, E. E. (1943) J. Immunol., 4\%, 195.

Sievag, M. G. and Seastone, V. (1934) Z. ImmunForsch. 83, 464.

Sgalitzer, II. (1914) Z. Hyg. InfektKr., 76, 209.

Simahrokh, K. B. (1943) J. biol. Chem., 151, 659.

Sinblex, G. S. (1926) J. exp. Med., 44,667; (1929) Ibid., 50, 825.

SHrigleY, E. II. and IrWin, M. R. (1937) J. Immunol., 32, 251.

Singer, E. (1942) Aust. J. exp. Biol. med. šci., 20, 209.

Skwirsky, P. (1910) Z. ImmunForsch., 5, 538.

Sleeswijk, J. G. (1908) Zbl. Bakt., 46, 513.

Sirth, F. C. and Marrack, J. (1930) Brit. J. exp. Path., 11, 494.

Siith, WV. (1932) J. Path. Bact., 35, 509.

Sinapper, I. and Grunbaru, A. (1936) Brit. J. exp. Path., 1\%, 361.

SobotkA, H. and Friedlander, M. (19:3) J.exp. Med., 47, 57.

Spät, W. (1910) Z. ImmunForsch., 7, 712.

SPink, W. W., AgNew, S., and Mickelsex, O. (1942) J. Immunol., 44, 289, 297.

SPink, W. IV., AGNew, S., Mickelsen, O., and DAHL, L. II. (194:) J. Immunol., 44, 303.

Spooner, E. T. C. and BAwden, F. C. (1935) Brit. J. exp. Path., 16, 2I8.

Stenhagen, E. (1938) Biochem. J., 32, 714.

Stokinger, H. E. and Heidelberger, .I. (1937) J. exp. Hcd., 66, 251.

Streng, O. (1909) Z. Hyg. InfektKr., 62, 281.

Strong, P. S. and Culbertson, J. T. (1934) J. Hyg., Camb., 34, 522.

STtul, A. and Hampton, S. F. (1941) J. Immunol., 41, 143.

SYedberg, T. (1937) Nature, Lond., 139, 1051 ; (1939) Proc. roy. Soc. B., 12\%, 1.

SYNGE, R. L. M. (1943) Chem. Rev., 32, 135.

TAKANo, Y. (1936) Z. ImmunForsch., 8\%, 29.

Taylor, G. L. (1931) J. Hyg., Camb., 31, 56 ; (1933) Ibid., 33, 12.

Taylor, G. L., Adair, G. S., and AdAir, II. E. (1932) J. Hyg., Camb., 32, 340.

'Tiselius, A. (1937a) Biochem. J., 31, 1464; (1937b) J. exp. Med., 65, 641.

Tiselius, A. and Kават, E. A. (1939) $J$.exp. Med., 69, 119.

Tokunaga, H. (19:8) Zbl. Bakt., 10\%, 283.

Toplex, W. W. C. (1915) Proc, roy. Soc., B, 88, 396.

Topley, W. W. C., Wilson, J., and Duncan, J. T. (1935) Brit. J. exp. Path., 16, 116.

Treffers, H. P. and Heidelberger, M. (1941a) J.exp. Med., 73, 125 ; (1941b) Ibid., '73, 293.

Ueno, S. (1938) Jap. J. med. Sci. VII, Soc. Mcd. Hyg., 2, 201, 225.

Wadsorth, A., Maltaner, F., and Maltaner, E. (1937) J. Immunol., 33, 297.

WARD, H. K. and Enders, J. F. (1933) J. exp. Ied., 5\%, 527.

Wasserman, P., Broir-Kahn, R. H. and Mrrsky, I. A. (1940) J. Immunol., 38, 213.

WeHMEYER, P. (1941) Z. ImmunForsch., 100, 179.

Weil, A. J., Parfentjev, 1. A., and Bowman, K. L. (1918) J. Immunol., 35, 399.

Wells, H. G. (1915) J. infcct. Dis., 16, 259 ; $(1925)$ "The Chemical Aspects of Immunity." New Tork; (1929) "The Chemical Aspects of Immunity," 2nd Edit. New York.

Wells, H. G., Lewis, J. H., and Jones, D. B. (1927) J. infect. Dis., 40, 326.

Wells, H. G. and Osborne, T. B. (1911) J. infect. Dis., 8, 66.

Whitehead, H. R., Gordox, J., and Worvall, A. (1925) Biochem. J., 19, 618.

WIENER, A. S. and HERMAN, II. (1939) J. Immunol., 36, 25.

Winslow, C.-E. A., Falk, I. S., and Caulfield, M. F. (1923) J. gen. Physiol., 6, 177. 
Woolf, B. (1941) Proc. roy. Soc., B., 130, 60.

Woolf, B., Marrack, J. R., and Downie, A. W. (1936) Chem. Ind., 14, 156.

Wormale, A. (1930) J. exp. Med., 51, 295.

Wright, A. E. (1909) "Studies in Immunization." London.

Wright, A. E. and Dovglas, S. R. (1903) Proc. roy. Soc., B, 72, 361 ; (1901) Ibid., 73, $1: 6$

Wright, G. G. (1944) J. exp. Med., 79, 455.

WU, H. and Ling, S. M. (1927) Chin. J.P Physiol., 1, 431.

Youmans, G. P. and Colwell, C. A. (1940) J. infect. Dis., 66, 235; 6\%, 48; (1943) J. Immunol., 46, 217.

ZıLo, S. (1919) Biochem. J., 13, 172.

Zinsser, H. (1921) J. Immunol., 6, 289; (1930) Ibid., 18, 483 


\section{CHAPTER 8}

\section{THE ANTIGENIC STRUCTURE OF BACTERIA}

A Complete description of the antigenic structure of a bacterial cell would include (a) the number and kind of different antigens present, $(b)$ their relative proportions, and $(c)$ their position in the cell or cell appendages.

Though in no instance has a complete description of this kind been achieved, yet for certain bacterial species we can construct working models of the antigenic structure, which, though crude, have at least enabled us to solve a number of immunological puzzles; and it seems certain that further advance along the same lines will enable us to solve many more.

We may start our discussion by considering the methods that are available for studies of this kind.

\section{Antigenic Analysis by Selective Qualitative Absorption of Antibodies.}

The fact that a single bacterial cell contains many antigens was established during the earliest studies on the agglutination reaction. The original observations of Gruber and Durham (1896) showed that the specificity of this reaction was not peculiar to each species of bacterium. For instance, while the colon bacillus was sharply differentiated from the typhoid bacillus and both from certain vibrios, these vibrios were not so clearly differentiated from one another. Durham (1901) as the result of a more detailed study of these group agglutinations enunciated quite clearly the hypothesis of a multiplicity - as he called it, a mosaic-of antigens within a single bacterial cell. Employing small letters to denote the antigenic bacterial components, and capitals to denote the corresponding agglutinins in the antisera, he suggested that the actual agglutinins and agglutinogens involved might be represented as follows:

\begin{tabular}{lll} 
& \multicolumn{3}{c}{ Agglutinogens. } & \multicolumn{2}{c}{ Aggiutinins. } \\
Bacterium 1. $a, b, c, d, e$. & Serum 1. A, B, C, D, E. \\
Bacterium 2. $c, d, e, f, g, h$. & Serum 2. C, D, E, F, G, H. \\
Bacterium 3. $e, f, g, h, j, k$. & Serum 3. E, F, G, H, J, K.
\end{tabular}

The serum prepared against Bacterium 1 would contain the agglutinins A, B, C, D, E, and in virtue of the presence of each and all of these it would agglutinate the corresponding bacterium. It would also agglutinate Bacterium 2 in virtue of agglutinins $\mathrm{C}, \mathrm{D}$, and $\mathrm{E}$, and Bacterium 3 in virtue of agglutinin $\mathrm{E}$.

The method that has been employed in analysing the antigenic structure of a particular bacterial strain - the method of agglutinin absorption-was introduced by Castellani in 1902; though a precisely similar method had been previously employed by Ehrlich and his co-workers in demonstrating the multiplicity of 
hæmolytic antibodies. When an agglutinating serum is exposed to an excess of bacterial cells that contain some or all of the corresponding agglutinogens the agglutinins that find their connterparts in the antigenic structure of the bacteria are bound, or absorbed, and thus removed from the fluid in which the bacteria are suspended. If the bacteria and the antibodies adsorbed to them are separated by centrifugation, the supernatant fluid can be tested as regards its remaining agglutinins. For instance, taking the illustrative example given above, if Serum 1 were absorbed with an excess of Bacterium 1 all the agglutinins A, B, C, D, and E would be removed and the supernatant fluid would have no agglutinating action. If it were absorbed with Bacterium 2, C, D and $\mathrm{E}$ would be removed, but $\mathrm{A}$ and $\mathrm{B}$ would remain. The supernatant fluid would not agglutinate Bacterium 2 the absorbing strain-nor Bacterium 3 (since that bacterium, does not contain agglutinogens $a$ or $b$ ); but it would still agglutinate Bacterium 1 in virtue of the remaining agglutinins $\mathrm{A}$ and $\mathrm{B}$. If it were absorbed with Bacterium 3, the single common agglutinin $\mathrm{E}$ would be removed. The supernatant fluid would (as always) fail to agglutinate the absorbing strain ; but it would still agglutinate Bacterium 1 in virtue of agglutinins $\mathrm{A}, \mathrm{B}, \mathrm{C}$, and $\mathrm{D}$, and Bacterium 2 in virtue of agglutinins $\mathrm{C}$ and $\mathrm{D}$.

A note may be interpolated here with regard to two terms that are sometimes loosely applied. The antiserum that is produced by the inoculation into a suitable animal of a particular bacterium is frequently referred to as a homologous serum. A serum that agglutinates the same bacterium but has been produced by the inoculation of some other bacterium, differing in one or more of its antigenic components, is termed a heterologous serum. Used in this sense the terms are useful and logical, and may be applied either to an antiserum in relation to a bacterium, or to two antisera or two bacteria in relation to one another, implying in either case complete correspondence between active combining groups. Thus, a serum that contains the active antibody groupings $\mathrm{A}, \mathrm{B}, \mathrm{C}, \mathrm{D}$ is homologous with a bacterium containing the active antigenic groupings $a, b, c, d$; two bacteria each containing the active antigenic groupings $d, e, f, g$ are homologous with one another, and so on. But an antiserum containing the active groupings A, B, C, D is not homologous, either with a bacterium containing the active groupings $a, b, c, e$, or $a, b, c, d, e$, or $a, b, c$. The terms cannot, logically, be applied to single antigens or single antibodies. They describe the relation between a group of different antibodies in a serum and a group of antigens attached to a bacterial cell, or between the group of antigens attached to one bacterial cell and the group attached to another. We are also, it should be noted, using the terms qualitatively, not quantitatively.

Suppose that we have an unknown bacterium $x$, which we suspect to be antigenically homologous with a known bacterium $y$, and suppose that we have available an anti-y serum. We absorb the anti- $y$ serum with $x$ and then test it against $y$. If it now fails to agglutinate $y$ we may assume that all the active antigenic groupings in $y$ are also present in $x$. But we have not excluded the possibility that $x$ has additional active antigenic groupings, not present in $y$. So we prepare an anti- $x$ serum, and then absorb it with $y$. If, after absorption, it fails to agglutinate $x$, we assume that all the active antigenic groupings present in $x$ are also present in $y$. That is, $x$ and $y$ are antigenically homologous. This double cross-absorption method is often spoken of as the "mirror test."

The results of agglutination and adsorption tests with related strains of bacteria and their respective antisera, when interpreted in the light of these principles, have in many cases provided detailed pictures of the antigenic make-up of certain bacteria. These pictures are for the most part qualitative, though it is usually possible to guess roughly which of the antigens predominate. The validity of these qualitative interpretations depends on the assumption that the separate antigenic components react only with 
corresponding antibodies in the serum, and that the various antibodics in the serum reflect the various antigens in the bacterium.

We have seen on p. 251 that certain complex antigens appear to possess more than one kind of determinant, and stimulate the production of an antibody with two or more specificities (see Burnet 1934, Heidelberger and Kendall 1934, Morgan 1936a, Meyer 1938, 1939, Miles 1939). If then we postulate a bacterium $x$ with an antigenic complex $a b$, and a second bacterium $y$ with an antigen $a$, the results of absorption tests would indicate that $x$ and $y$ were antigenically identical, since $a b$ would remove all $\mathrm{A}$ agglutinins from the anti- $y$ serum and $a$ would remove the AB agglutinins from the anti- $x$ serum. Indeed, the only way we should ever discover the existence of the complex $a b$ would be in comparison with two other bacterial species, which happen respectively to possess $a$ only and $b$ only. If, on the other hand, we postulate that the response to bacterium $x$ (with the complex $a b$ ) consists of separate antibodies A and B, then absorption tests would lead us to postulate that $x$ and $y$ had an antigen in common, and that $x$ had in addition a distinct antigen peculiar to itself. The extraction and separation of the various antigens provides a way out of this impasse. The different antibodies may then be removed from the bacterial antisera in specific precipitates, and the precise specificity of the remaining antibodies for the other antigens determined.

\section{Quantitative Techniques in Antigenic Analysis.}

The methods of antigenic analysis discussed above yield qualitative answers. Estimation of the relative amounts of the various antigens is possible only if certain assumptions are made. These are, that there is a quantitative correspondence between the different kinds of antibodies in a serum and the different kinds of antigens present in the homologous bacterium; that all the antigens have the same antigenic capacity, weight for weight, and that each of the antigens reacts with its corresponding antibody with the same degree of intensity. None of these assumptions is warranted, and they are indeed seldom made explicitly, though they are sometimes implicit in the inferences drawn from analytical data. It will be clear from Chapter 7 that the last two assumptions are unjustified. The first requires a little amplification.

If a bacterium $y$ removes half the antibody from anti- $x$ serum, the fact that a large amount of antibody peculiar to $x$ remains is sometimes interpreted as indicating a large amount of corresponding antigen in $x$. But, as Miles (1939) pointed out, the antibody response to many repeated doses of a small amount of antigen may be as great as that to many doses of a large amount, so that after prolonged immunization with a bacterium containing a major and a minor antigen, the corresponding antibodies may be in equal concentration in the serum. Removal of the antibody to the major antigen would in this case leave a large amount of antibody representing what was in fact a minor antigen.

Nevertheless, though quantitative correspondence of antibody and antigens is unlikely ever to occur, it is clearly important to know how much of the varions antigens and antibodies are contained in the two reagents, a bacterial suspension and an antiserum, at our disposal. A knowledge of the antibody concentrations is particularly useful. For example, the quantitative relationship in antigen-antibody reactions described in Chapter 7 permits the measurement of the concentration of one antigen in a mixture of antigens derived from a bacterinm.

Heidelberger and his colleagues (see Heidelberger and Kabat 1934, 1936, 1937, 1938, Alexander and Heidelberger 1940, Henriksen and Heidelberger 1941) have measured specific agglutinin content in terms of maximum antibody nitrogen adsorbed by known doses of bacterial suspensions. The data so obtained permit 
comparison of bacterial strains in terms of the proportion of total antibody they will absorb from a specific antiserum. Duncan $(1932 a, b)$ and Miles (1933, 1939) attacked the problem by means of the optimal proportions technique. The equivalence point between a bacterial suspension and an antiserum may be the resultant of the reaction of a number of different antigens and their corresponding antibodies which may to some extent be distinguished by appropriate variations of the experimental conditions.

This leads us to the problem of differentiating two types of bacteria, both of which contain the same antigenic components, but in different proportions. Using the qualitative differential absorption technique, with maximal absorbing doses of bacteria, the two types would appear identical, since each would absorb all the agglutinins from both the homologous and heterologous serum, using those terms in this case to include quantitative as well as qualitative relations. By careful adjustment of the absorbing dose it is, however, sometimes possible to demonstrate a quantitative difference of this kind. An example, dealing with the differentiation of Br.melitensis from Br.abortus, will be found on p. 824.

\section{The Study of Bacterial Variation as a Guide to Antigenic Structure.}

Bactcrial variation, as a general phenomenon, is dealt with in the succeeding chapter; but the detailed study of the antigenic differences displayed by bacterial variants has played so large a part in the formation of our present views in regard to antigenic structure that we must anticipate a little and discuss in the present scetion certain examples that will help to illustrate the kind of antigenic ehanges that oceur.

Smith and Reagh (1903) isolated a non-motile variant of the hog cholera bacillus, and compared its agglutination reactions with those of the normal, motile, flagellated type of this organism. They found that a serum prepared against the motile strain agglutinated both the motile and the non-motile strains; but the titre was much higher for the motile than for the non-motile, and the clumps formed by the motile strain were fluffy and formed rapidly, while the clumps formed by the non-motile strain were tight, small and granular, and formed far more slowly. A serum prepared against the non-motile strain agglutinated the motile and non-motile strains to the same degree, giving in each case the slow, granular type of agglutination. The serum prepared against the motile strain, when absorbed with the nonmotile strain, lost its power of agglutinating the non-motile bacilli, but retained its power of agglutinating the motile strain. The serum prepared against the nonmotile strain lost its agglutinating power for both. strains when absorbed with the motile strain. Smith and Reagh concluded that the normal, motile hog cholera bacillus has two kinds of antigens, one contained in the flagella, the other in the cell body. The non-motile type has lost the flagellar antigens and retains the body antigens only. Beyer and Reagh (1904) extended these observations and showed that the flagellar antigens were so altered by heating to $70^{\circ} \mathrm{C}$. for 15 minutes that the heated bacilli no longer gave the flagellar type of agglutination, but reacted like the non-motile strain. The somatic, or body, antigens were not affeeted by this treatment and still gave the characteristic slow, granular agglutination (see also Orcutt 1924, Craigie 1931).

These observations of Smith and Reagh and of Beyer and Reagh demonstrated all the essential points of difference between the flagellar and somatic types of agglutination, but they passed almost unnoticed until similar findings were recorded by Weil and Felix (1917) in connection with their work on the diagnostic significance of the agglutination of a particular strain of proteus bacillus by the blood of a patient 
suffering from typhus fever. The proteus bacillus, in its normal, flagellated form, grows on the surface of nutrient agar as a thin sprearing film which resembles the mist produced by breathing on glass, and was named the Hauch form-the "breath " form, by Weil and Felix. A non-flagellated variant grew in isolated colonies with no thin, spreading growth between them; this was the Ohne Hauch form-the form without an exhalation. As is the way in laboratory shorthand these soon became the " $\mathrm{H}$ " and " $\mathrm{O}$ " forms; and so the thermolabile, flagellar antigens are now the $\mathrm{H}$ antigens, and the thermostable, somatic antigens are the $\mathrm{O}$ antigens.

Arkwright (1920, 1921, 1924), working with bacteria of the typhoid-paratyphoiddysentery group, described a type of variation that has proved particularly instructive from this point of view. He noted that a particular type of variant was of relatively common occurrence among these bacilli ; and this variant differed from the parent form in several characteristic ways. Thus, the normal parent form gave smooth colonies on a solid medium and a diffuse growth in broth, and was not auto-agglutinable in normal saline ( 0.85 per cent.). The variant form gave rough or granular colonies on solid media and a granular growth in broth, and was autoagglutinable in normal saline, though a stable suspension could usually be prepared in distilled water, or in a saline solution with a greatly decreased salt-content $(02-0 \cdot 4$ per cent.). These differences in colony form, growth in broth, and saltscnsitiveness were associated with a profound change in antigenic structure. The normal parent form reacted specifically as regards its agglutinability by immune sera; the variant was agglutinated not only by its own antiscrum but by antisera prepared against many other bacteria in the rough state, some of which were only distantly related to the parent form according to the ordinarily accepted bacteriological criteria. By the usual transition-through shorthand to symbols-the normal form, giving smooth colonies, became the Smooth (or S) form; the variant, giving rough colonies, became the Rough (or R) form. And here again we have become entangled in the web of our words, for we have never defined exactly the criteria of roughness and smoothness. The implicit meaning is certainly not the meaning we want; since, as we shall see, the correlation between colonial form and antigenic structure is by no means constant. If we give Smooth and Rough their obvious or commonsense meaning, then we shall want new terms for the underlying changes that really interest us. If we employ the legitimate licence of scientific terminology, and say that by Smooth we mean one sort of antigenic structure and by Rough another, then we must at least define quite clearly what we mean. Up to the present we have evaded our difficulties, and so we find that the rough type of one species is recorded as having the more important characters of the smooth type of another species, as in the case of the anthrax bacillus (see Preisz 1904, Eisenberg 1912, Bordet and Renaux 1930, Tomcsik and Szongott 1932, 1933); or it becomes necessary to invent new descriptive terms, such as matt and glossy, as in the case of the hæmolytic streptococcus (see Todd 1928a, $b$, Todd and Lancefield 1928). Probably the most convenient convention is to use the symbols $\mathrm{S}$ and $\mathrm{R}$ to distinguish the antigenic variation, and retain, as far as is necessary, the words Smooth and Rough as descriptions of colonial variants. There will thus be no contradiction in asserting that the $\mathrm{S}$ form of $B$. anthracis grows as a rough colony.

In most of the cases with which we are familiar the change from $\mathrm{S}$ form to $R$ form (the $S \rightarrow R$ variation) is associated with a loss of virulence-an association of obvious importance to the immunologist-as well as with a particular kind 
of change in antigenic structure. We have very good reasons for believing that the change in antigenic structure determines the loss of virulence, and it certainly determines the change in antigenic value of the variant strain regarded as an immunizing agent. We may, then, for our immediate purpose and to avoid confusion, explicitly limit ourselves to this antigenic variation and its consequences, noting that if we define the $S \rightarrow R$ variation in this way we are acting in defiance of general usage.

In terms of antigenic structure we may say that this variation consists in the loss of the heat-stable somatic antigen that characterizes the surface of the normal virulent bacterial cell. This loss may be associated with an uncovering of some other somatic antigen, which then dominates the antigenic behaviour of the strain.

Variation of this type has now been described in a wide range of different bacterial species-in the Pasteurella group (de Kruif 1921, Webster 1925), in pneumococei (Griffith 1923, Reimann 1925, 1927), in staphylococci (Bigger, Boland and O'Meara 1927), in streptococci (Todd 1928 $\iota$ ) and in a host of other organisms. We are probably justified in regarding it as a type of variation to which pathogenic bacteria are inherently liable.

Sometimes (see White 1932, 1933, Henderson 1939) this variation may proceed still further, and another antigenic constituent may be lost. The antigenic behaviour of the organism may then be dominated by a component that, in the normal state, was altogether latent. It may be noted $(a)$ that the $\mathrm{S} \rightarrow \mathrm{R}$ variation is quite independent of the $\mathrm{H} \rightarrow \mathrm{O}$ variation (rough variants are often flagellated), and $(b)$ that loss variations of the $\mathrm{S} \rightarrow \mathrm{R}$ or more deeply seated types are often irreversible.

There is another kind of antigenic variation to which reference must be made before we pass to the next section of our discussion.

Andrewes $(1922,1925)$ described a curious phasic variation in the $H$ antigens of certain flagellated species. Taking two bacteria, $x$ and $y$, that showed the flagellar type of agglutination when tested either against an anti- $x$ or against an anti- $y$ serum, and therefore possessed at least one $\mathrm{H}$ antigen in common, he absorbed the anti- $x$ serum with bacillus $y$ and thus obtained a serum that agglutinated $x$ but not $y$. He then took a broth culture of $x$ and plated it on a solid medium, thus obtaining separate colonies. Subculturing from several of these, he obtained different cultures, each representing a single bacillus in the original broth culture. When he tested these different cultures against the anti- $x$ serum, rendered specific by absorption, and against the anti- $y$ serum, containing the common, or "group " antibody, he found that his subcultures fell sharply into two classes. Those of one class were agglutinated to titre by the specific anti- $x$ serum, but not at all by the anti- $y$ serum. Those of the other were agglutinated to titre by the anti- $y$ serum, but not at all by the specific anti- $x$ serum. The only possible conclusion would seem to be that the original culture of $x$ showed cross-agglutination with the anti- $y$ serum not because each bacillus in the culture possessed two antigenic components, say $a$ and $b$, in their flagella, $a$ being specific for $x$ and $b$ being shared by $y$, but because some of the bacilli possessed $a$ alone, and others $b$ alone. We need not, for the moment, worry as to whether the $a$ bacilli do or do not possess a trace of $b$ and vice versa. Similarly Andrewes was able to show that the $y$ culture, which agglutinated with anti- $x$ and anti- $y$ sera, contained some bacilli possessing the common flagellar antigen $b$ and others containing an antigen $c$ specific for $y$. The bacilli that possessed the group antigen only were referred to as being in the group 
phase; those that possessed the specific antigen only as being in the specific phase. If repeated plate cultures were prepared from a strain that was in the specific phase, bacilli in the group phase turned up sooner or later; similarly a strain in the group phase gave rise to sub-strains in the specific phase. The variations might occur in either direction. Species or types of bacteria that show this curious variation in their flagellar antigens are termed diphasic. For completeness, we should note the association of one other type of bacterial change, not strictly a variation, with change of antigenic structure; namely, the transformation of a vegetative form into a spore. Not only is there a transformation of antigenic structure (Howie and Cruickshank 1940), but the characterization of the spore antigens reveals relationships between bacteria that are not evident on the basis of the vegetative forms of the antigens (Lamanna 1940). We do not know of any example of true diphasic variation affecting the somatic antigens.

In diphasic variation, the antigenic constitution of the flagella does not always alternate between a "specific" and a "group" phase. In some of the diphasic Salmonella types the alternate phase possesses antigens that characterize the "specific" phases of other Salmonella types (Kauffmann 1936, Kauffmann and Tesdal 1937, Kauffmann 1939). Kauffmann used the notation $\alpha \rightarrow \beta$ variation to distinguish it from the commoner type of diphasic variation. The $\beta$ phases have nothing in common with flagellar " group " phases, but they are relatively restricted in range, all being characterized by two antigens, and most of them possessing one of three subsidiary antigens (see Chapter 30). It may be noted that the $H$ and $O$ antigens vary independently. Thus, Boivin and Mesrobeanu (1937) induced the following six variants in Salm. typhi-murium-smooth $\mathrm{O}+$ specific $\mathrm{H}$, smooth $\mathrm{O}+$ group $\mathrm{H}$, smooth $\mathrm{O}$; and rough $\mathrm{O}+$ specific $\mathrm{H}$, rough $\mathrm{O}+\operatorname{group} \mathrm{H}$, rough O. (See also Boivin, Izard and Sarciron 1939.)

\section{The Application of Chemical Methods in the Analysis of Antigenic Structure.}

A few examples will suffice us here, since the chemical constitution of the antigenic components that characterize the various species of bacteria is described more fully, where it is known, in the chapters devoted to systematic bacteriology.

Zinsser and Parker (1923) isolated substances from pneumococci, staphylococei, the influenza bacillus, the typhoid bacillus and the tubercle bacillus, which gave none of the ordinary protein reactions, except a very weak xantho-protein reaction, but gave specifio precipitation and complement fixation with the corresponding antisera prepared by the injection of whole organisms. These non-protein antigens failed to stimulate antibody production on injection.

In the same year Heidelberger and Avery published the first of a series of papers dealing with a chemical study of the antigenic constituents of the pneumococcus (Heidelberger and Avery 1923, 1924, Avery and Heidelberger 1923, 1925, Avery, Heidelberger and Goebel 1925, Avery and Morgan 1925, Avery and Neill 1925). From the three classical types of pneumococci, in their normal smooth form, partial antigens were separated, which were found to have the chemical structure of complex polysaccharides, failed to stimulate antibody production in vivo, but gave specific precipitation at extraordinarily high dilutions when mixed with antisera prepared by the inoculation of rabbits with the corresponding strains of pneumococci.

In rough variants, which are non-capsulated, these polysaccharide antigens are absent; but there remains a protein antigenic component which is common to the rough variants of all types, and is also present in the smooth capsulated forms, though in these their presence is masked by the type-specific capsular polysaccharide.

The history of the pneumococcal polysaccharide illustrates one of the drawbacks of 
chemical fractionation, namely that the treatment used may partly but fundamentally alter the substance we are looking for. The pneumococcal polysaccharide as originally isolated was not antigenic. By less chemically active fractionation, an acetylated form of the substance is produced, which is immunologically far more significant (Avery and Goebel 1933).

Lancefield (1928) separated antigenic components from hæmolytic streptococci by chenical methods. She found that the component that confers type-specificity is acidsoluble and contains some 14 per cent. of protein nitrogen. In its purified state it is hapten-like, in that it gives specific precipitation in the test-tube but fails to stinulate antibody-production in vivo. In addition to this type-specific antigen there is a polysaccharide component that is shared by many types of hæmolytic streptococci, but differentiates the species into large sub-groups (Lancefield 1933); and there is a nucleoprotein antigen that is shared by a wide variety of streptococci, hæmolytic and nonhæmolytic. Studies by Todd and Lancefield (1928) (see also Lancefield and Todd 1928) have shown that the change from the virulent matt to the avirulent glossy form is associated with the loss of the type-specific component, the polysaccharide component being retained.

The capsulated bacillus of Friedländer has given results entirely analogous to those obtained with the pneumococcus (Heidelberger, Goebel and Avery 1925, Julianelle 1926). The species may be divided into a number of sharply demarcated serological types. This type-specificity is conferred by a polysaccharide hapten present in the capsule. The body of the bacillus contains a nucleo-protein antigen that is shared by all types.

Polysaccharide haptens have also been isolated from the tubercle bacillus (Laidlaw and Dudley 1925, Enders 1929), from Bact. lactis aerogenes (Tomcsik and Kurotchkin 1928), from organisms of the Salmonella group (Furth and Landsteiner 1928, 1929), from cholera vibrios (Landsteiner and Levine 1927, Jermoljewa and Bujanowskaja 1930), from Shiga's dysentery bacillus (Meyer 1930, Morgan 1931), from the anthrax bacillus (Tomesik 1930, Schockaert 1928, Tomcsik and Szongott 1932) and from yeasts (Mueller and Tomesik 1924, Stone and Garrod 1931, Duncan 1932a).

Recently the isolation of antigens more nearly in the native form has been facilitated by gentler and in some cases more specific methods of fractionation. Among these we may mention trichloracetic acid (Boivin and Mcsrobeanu 1933) and diethylene glycol (Morgan 1937) for the extraction of the main antigenic complexes from salmonella and dysentery bacilli (see Chapters 29 and 30); Raistrick and Topley's (1934) method for the same type of substance by tryptic digestion of acetone-extracted bacteria; and Fuller's (1938) formamide method of extracting group-specific components from streptococci. Sonic vibrations apparently liberate antigens from bacteria in a more native form. Mudd and his colleagues (1937) succeeded by this means in isolating from Str. pyogenes a labile antigenic substance built up of smaller, serologically specific fractions extracted by Lance. field. By similar methods, Sevag and his colleagues (1941) separated from the disintegrated streptococcal cells large numbers of antigenic "macromolecular" particles containing lipins, nucleic acid, protein, carbohydrate and a pigment.

Julianelle and Wieghard (1934), and Thompson and Khorazo (1937), isolated carbohydrate substances responsible for the type-specificity of Staph. aureus. By milder procedures Verwey (1940) isolated an additional, type-specific protein antigen. According to Menzel and Rake (1942) the type-specific substance of the Type II meningococcus owes its serological activity to a carbohydrate-polypeptide complex differing from the type-specific polysaccharide and type-specific proteins described for other species of cocci. (For the characterization of protein antigens in the gonococcus, see Stokinger et al. 1944; and of various antigenic fractions in C. diphtheria, see Wong and 'T"ung 1939, 1940, Wong 1940. and Hoyle 1942.)

The case of the anthrax bacillus presents points of interest. The normal virulent form of this bacillus is capsulated and gives a rough colony on agar ; the avirulent variant is noncapsulated and gives a smooti colony on agar (Preisz 1904, Eisenberg 1912). This, then, is one of the cases in which the normal relation between smoothness and virulence is reversed. 
It was natural to suppose that the polysaccharide component isolated from $B$. anthracis was the capsular material; but Tomesik and Szongott $(1932,1933)$ report that this is not the case. They state that the polysaccharide is a somatic component common to the virulent, capsulated rough-colony-forming type, and to the avirulent, non-capsulated, smooth-colony-forming type. The capsular substance is a protein-like substance. Ivánovics and his colleagues identified the capsular substance as a polypeptide which on hydrolysis yielded $d(-)$-glutamic acid almost in the amount to be expected if the starting substance were built up of glutamic acid only. This antigenically distinet capsular polypeptide was also found in $B$. mesentericus and certain other members of the Bacillus group (Ivánovics and Erdös 1937, Ivánovies and Bruckner 1937a, b, 1938).

There is another way in which clemical methods may be applied in the investigation of antigenic structure. Bacteria may be treated with various chemical solvents, or other reagents, with or without heat, and the effect of this treatment on their immunological behaviour may be studied (see White 1927, 1928, 1929, 1932, 1933).

As an example of an indirect method of chemical analysis, we may cite the comparison of the serological reactions of a natural antigen with that of a "synthetic " antigen whose hapten is of a known chemical nature. Thus Goebel (1936) confirmed the importance of glucuronic acid in the molecular structure of the capsular polysaccharide of Types II. III and VIII pneumococci, by demonstrating the cross-reactions between the polysaccharides and antisera to glucuronic acid azo-proteins. The method is not entirely specific since a group of non-identical haptens having certain chemical similarities may cross-react with one another. Thus the precipitation of antibody to galactoside-azoprotein by the polysaccharide of pneumococcus Type I confirms the importance of the galacturonic acid identified in the polysaccharide molecule; but antisera to azo-protein antigens containing a benzene carboxylic radicle (which has never been identified in Type I pneumococci) also precipitate with the polysaccharide (Goebel and Hotchkiss 1937). The cross-reaction in this case appeared to depend on the reactivity of the antisera with acidic groups, irrespective of the hapten radicle that bore them (see also Goebel 1940).

The first essential for the characterization of a bacterial product is the certainty that it has been synthesized by the bacterium and not derived from the culture media from which it was obtained. It will be obvious that blood and other animal proteins used in culture and adsorbed to the bacterium, may induce the formation of antibodies that are later confused with those induced by the bacteria (see, for example, Bliss 1938).

Bailey and Raffel (1941) record that even infusion broths will substantially modify the antigenicity of bacteria grown in them. In association with bacteria, non-antigenic substances like agar may be antigenic (Sordelli and Nayer 1931, Morgan 1936b, Sickles and Rice 1938). The mechanism of this association is unknown, but Partridge and Morgan (1942) have provided a model for it by making artificial antigenic complexes of agar and the conjugated protein component of the endotoxin of Sh. shigce.

The development of culture media whose components are all known and all of relatively low molecular weight will eliminate this source of error. (See, for example, Freeman et al. 1940.)

It should be emphasized that only in a few cases has the chemical constitution of antigens been fully determined. The antigenic substances isolated from bacteria are usually either large molecules, or large associations of molccules. The interpretation of their biological activity in terms of chemical structure must always be tentative in the absence of satisfactory evidence that the product is pure. Unfortunately the biologist's and the chemist's conception of purity are not always coincident, and each tends to use criteria borrowed from the other, without full appreciation of their limitations. For instance, the fact that a substance has 
undergone several successive precipitations by the same reagent, or successive crystallizations from the same solvent, is no guarantee of its purity, for loosely bound heterogeneous complexes will exhibit constant precipitability, and mixed and contaminated crystals are common in biochemistry. For further details on this important point, reference should be made to Pirie's (1940) review of the subject.

\section{The Sharing of Antigenic Components between Unrelated Bacteria, or between Bacteria and Other Cells.}

In the course of the numerous studies that have been made on antigenic structure, instances have come to light of the sharing of a particular antigenic component by bacteria that show no systematic relation to one another, or by bacteria on the one hand and plant and animal tissues on the other. It is possible in some cases that the reported immunological cross-reactions may have been due to the presence of contaminating antigens discussed in the previons section. It will be noted that the various types of pneumococci figure largely in the examples given below. This predominance is not necessarily a peculiarity of the species; more probably it is a reflection of the extensive American work on the pneumococcal antigens.

Thus, pneumococeus 'Type I and certain coliform bacilli are serologically related (Barnes and Wight 1935). The capsular polysaccharide of pnemococcus Type II is closely similar to that of the Type B pneumobacillus (Avery et al. 1925, Julianelle 1926); to polysaccharides isolated from a species of yeast (Sugg, Richardson and Neill 1929) and to a species of Leuconostoc (Sugg and Hehre 1942); and cross-reactions occur between this type and Past. lepiseptica (Dingle 1934).

Antisera to Types II and III pneumococci react with a polysaccharide obtained by partial hydrolysis of gum arabic (Heidelberger, Avery and Goebel 1929), and of gum acacia, cherry and other vegetable gums (Marrack and Carpenter 1938). Miller and Boor (1934) report cross-reactions between Type III pneumococcus on the one hand, and meningococeus and gonococeus on the other, and Kauffmann and Langvad-Nielsen (see Morch 1942) between Type XXXV and a Salmonella species. There are similar relationships between Type VI pneumococcus and H. influenze Type $a$ (Chapman and Oshorne 1942, Neter 1943, Zepp and Hodes 1943).

The polysaccharide of pneumococcus Type XIV is related to that which characterizes the antigen of the human Group A red blood cell (Gocbel et al. 1939), and both are related to the somatic polysaccharide of the anthrax bacillus (Ivànovics 1940).

Many other examples could be cited of relationship between markedly differing species, particularly within the group of the Gram-negative intestinal bacilli. Perhaps one of the most striking antigenic relationships is that between certain species of Proteus vulgaris and certain species of Rickettsia (see Chapter 39), where the antigenic varieties in one group are to some extent paralleled by antigenic varieties in the other.

An equally curions example of this sharing of antigenic components is afforded by the presence in a wide variety of bacteria of Forssman's heterophile antigen (see p. 1089), which is a constituent of the red blood corpuscles and tissue cells of certain animal species (see Rothacker 1913, Iijima 1923, Schmidt 1925, Meyer 1926, 1930, 1931, Meyer and Morgan 1935, Powell 1926, Yasui 1929, Combiesco et al. 1930, Eisler 1931a, b, Eisler and Howard 1931, 1932, Bailey and Shorb 1931, Buchbinder 1935, Morgan 1937, Goebel and Adams 1943, Goebel et al. 1943).

There is, of course, nothing very bizarre in the occurrence of identical, or closely similar, antigenic groupings in living cells that have no close systematic relationship. The specificity that antigen-antibody reactions detect is, as we have seen, a chemical one. The fact that it is also biological, in the systematic sense, depends on the 
way in which the chemical substances, or the complexes of enzyme systems that synthesize them, are distributed in nature. It is very probable that, as our search extends, we shall come on one instance after another in which antigenically similar substances are found in entirely dissimilar biological situations.

The Localization of Antigenic Components in the Bacterial Cell.---The observations recorded above, and many others that will be referred to in subsequent chapters (see particularly the antigenic structure of the typhoid-paratyphoid group, Chapter 30 ). not only tell us that there are many different antigens in bacterial cells, and that these antigens have different chemical constitutions; they give us some indication of whereabouts in the bacterial cell these different antigens are placed. They indicate also that this antigenic anatomy is a factor of primary importance in determining that cell's immunological behaviour. Our conception with regard to the way in which such components are actually arranged can be set out most easily in diagrammatic form. But it must be emphasized that our diagram is not a picture. By placing an antigen at the cell surface we are implying that it behaves as though it were there. By placing it beneath the surface we mean that it seems, in the normal form of the organism, to be overshadowed by some other bacterial component. How the components are really arranged we do not know, except that we can certainly allocate some to flagella or capsules, and are almost certainly right in supposing that changes in antigenic behaviour are associated with changes at the cell surface. It is quite likely that antigenic variation is associated with a change in the amount of a particular antigenic component, as well as a change in its situation. A component, for instance, that has been unmasked by the loss of another component may be produced in greater amount when it assumes a dominant position on the active surface of the cell. Perhaps in some cases it is only represented in the normal cell in a rudimentary form.

Bearing these caveats in mind we may consider the diagrammatic arrangements of antigens set out in Fig. 44.

At $\mathrm{A}$ is represented a portion of a flagellated bacillus in its normal, virulent, smooth form, with one antigen at the surface of its flagella, a second and a third antigen at the cell surface, a fourth antigen situated more deeply and masked by antigens two and three, a fifth antigen situated more deeply still, and a sixth antigen situated centrally in the cell body.

At $\mathrm{B}$ is a portion of a bacillus having the same antigens, but without flagella. We might regard it as an $O$ variant derived from $A$. Alternatively we might alter the nature of the antigens, maintaining their arrangement, and regard it as the normal, smooth form of any non-flagellated organism.

At $\mathrm{C}$ is a rough variant from $\mathrm{A}$. It has kept its flagella and its normal flagellar antigen; but it has lost the antigenic components that determined the nature of the cell surface in the normal, smooth form. A deeper antigen has been unmasked. It will be noted that a few of the antigenic components that originally lay more deeply still are represented as having now found a place at the cell surface.

In some cases (see White 1933) antigenic variation by loss may proceed still further. The characteristic rough somatic antigen may disappear, and the bacterial surface may be dominated by components that lay very deeply in the normal, smooth form.

At $\mathrm{D}$ is an organism, such as the pneumococcus, having a capsule that consists mainly of one kind of antigen (or hapten). Beneath it, in the cell body, is another antigen which is entirely covered by the capsule. 

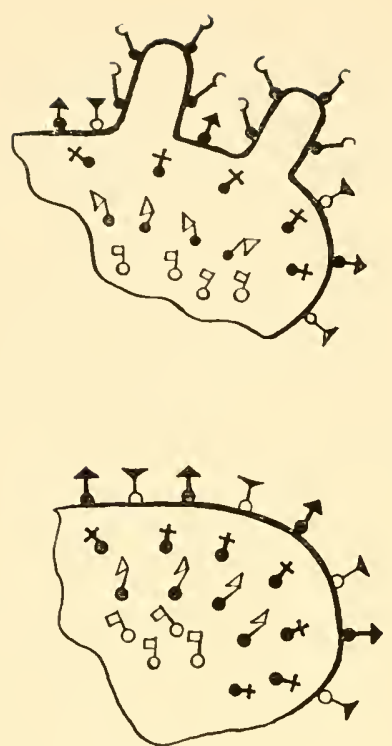

B.
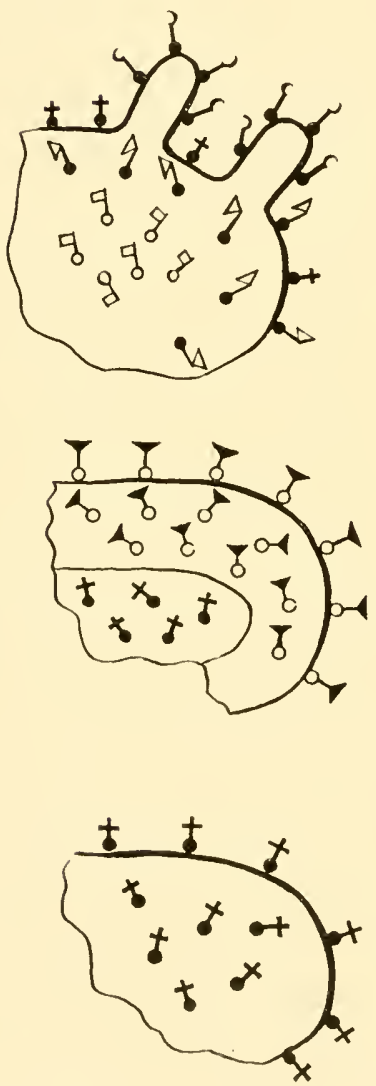

f. Flagellar Antigen.

J. -Predominant Surface Antigen.

A.. $t \quad$ More deeply situated Antigen.

9. -Still more deeply situated Antigen.

q -Centrally situated Antigen.

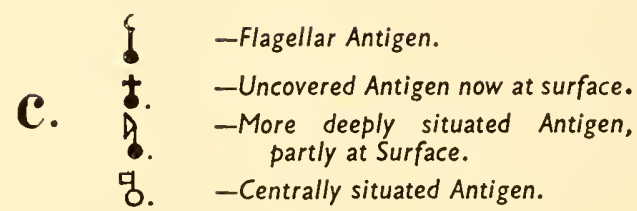

T. - Capsular Antigen.

t. -Antigen of Cell Body.

E. $\begin{gathered}\text { Antigen of Cell Body now at Sur- } \\ \text { face. }\end{gathered}$

Frg. 44. 
At $\mathrm{E}$ is a rough variant from $\mathrm{D}$. The capsule has gone, and with it the characteristic capsular antigen. The character of the cell surface is now determined by the antigen that before was hidden.

All these diagrams, and particularly D and E, are much too simple to be true. There are probably a very large number of antigens, or antigenic groupings, in any bacterial cell. But it is true that the character of the cell surface appears in most cases to be dominated by a few characteristic antigenic components, and that this surface may be profoundly altered by the replacement of these components by a few others. It is probable that the $S \rightarrow R$ variation, at least as it manifests itself in a culture of bacteria, is not a sudden, all-or-none mutation, but a more gradual or step-like process, with intermediate forms in which the normal surface antigens are still present, though in diminished amount (see, for instance, Wilson 1930).

\section{Antigenic Stracture in Relation to the Different Antigen-Antibody Reactions.}

When antibodies unite with antigens that form part of a bacterial cell, the effect on the cell will vary according to the position that the antigen oceupies.

If the antigen is situated on the surface of the flagella the antigen-antibody compound will be formed in this situation, the usual change in the direction of salt-sensitiveness will oceur, and, in the presence of electrolytes, the bacilli will flocculate in the large, loose clumps characteristic of flagellar agglutination.

Antigen-antibody union in this situation does not, however, render the bacterium sensitive to lysis by complement, and it is relatively ineffective in promoting phagocytosis (see Felix 1924, Braun and Nodake 1924, Felix and Robertson 1928). For sensitization of this type to occur antigen-antibody union must take place at the bacterial surface.

It would seem, also, that the aggregates formed in flagellar agglutination are not of the kind that readily fix complement; though there is some uncertainty on this point (see Hofmeier 1927, Springut 1927). It seems possible that the absence, or paucity, of complement fixation may be due simply to the physical character of the aggregates and the rapidity with which they are formed.

The more deeply seated antigens will play no part in the serological reactions of the intact cell in its normal smooth form. They are inaccessible to the antibody. In suspensions that have been allowed to autolyse, or that have been broken up in some other way, these antigens may be liberated. They will then unite with the corresponding antibody to form a precipitate, and if complement is present and the conditions favourable this complement will be fixed.

To revert for a moment to the problem of the unity or diversity of antibodies, it is logical to say that an antibody acting on a flagellar antigen is an agglutinin, but not a lysin; that an antibody acting on a surface antigen is an agghtinin and a lysin, and a complement-fixing antibody, and an opsonin; and that an antibody acting on an antigen that is normally situated below the bacterial surface is, when tested against bacterial extracts or autolysates, a precipitin and a complement-fixing antibody, but is not, so far as the normal cell is concerned, an agglutinin, or a lysin or a complement-fixing antibody or an opsonin. But none of these differences in reaction are determined by differences in the nature of the antibody, apart from its combining group; they depend on differences in the situation of the antigen with which it unites. 


\section{REFERENCES}

Alexander, H. E. and Heidelberger, M. (1940) J.exp. Med., Y1, 1.

Andrewes, F. W. (1922) J. Path. Bact., 25, 505; (1925) Ibid., 28, 345.

Arkwright, J. (1920) J. Path. Bact., 33, 358; (1921) Ibid., 34, 36 ; (1924) Brit. J. exp. Path., 5, 23.

AverY, O. 'T. and Goebel, W. F. (1933) J.exp. Med., 58, 731.

Avery, O. T. and Heidelberger, M. (1923) J.exp. Med., 38, 81 ; (1925) Ibid., $42,367$.

Avery, O. T., Heidelberger, H., and Goeber, IV. F. (1925) J. exp. Med., 42, 709.

Avery, O. T. and Morgan, H. J. (1925) J. exp. Med., 42, 347.

Avery, O. T. and Neilu, J. M. (1925) J. exp. Med., 42, 355.

Bailey, G. H. and Raffel, S. (1941) Amer. J. IIyg., 33, 86.

Batley, G. H. and Shorb, M. S. (1931) Amer. J. Hyg., 13, 831.

Barnes, L. A. and Wight, E. C. (1935) J. exp. Med., 62, 281.

Beyer, H. G. and Reagh, A. L. (1904) J. med. Res., 12, 313.

Bigger, J. W., Boland, C. R., and O'Meara, R. (1927) J. Path. Bact., 30, 261.

Bliss, E. A. (1938) J. Immunol., 34, 337.

Boivin, A., Izard, Y., and Sarciroy, R. (1939) C. R. Soc. Biol., 131, 867, 870.

Botvin, A. and Mesrobeane, L. (1933) C. R. Soc. Biol., 112, 76 ; (1937) Rev. Immunol., Paris, 3, 319.

Bordet, J. and Renaux, E. (1930) Ann. Inst. Pasteur, 45, 1. .

Braun, H. and Nodake, R. (1924) Zbl. Bakt., 92, 429.

Buchinder, L. (1935) Arch. Path., 19, $8 \pm 1$.

Burnet, F. M. (1934) Brit. J. exp. Puth., 15, 354.

Castellani, A. (1902) Z. Hyg. InfektKr., 40, 1.

Chapman, O. D. and Osborne, W. (1942) J. Bact., 44, 620.

Combiesco, D., Statmatesco, S., Nestoresco, N., and Adal, C. (1930) Arch. roum. Path. exp. Microbiol., 3, 241.

Craigie, J. (1931) J. Immunol., 21, 417.

Dingle, J. H. (1934) Amer. J. Hyg., 20, 148.

Duncan, J. T. (1932a) Brit. J. exp. Path., 13, 489; (1932b) Ibid., 13, 498.

Dorhai, H. E. (1901) J. exp. Med., 5, 353.

Eisen BerG, P. (1912) Zbl. Bakt., 63, 305.

Eisler, M. (1931) Z. ImmunForsch., \%0, 48; (1931) Ibid., 73, 37.

Eisler, I. and Howard, A. F. (1931) Z. ImmunForsch., 71, 473; (1932) Ibid., 76, 461. ENders, J. F. (1929) J. exp. Med., 50, 777.

FELIX, A. (1924) J. Immunol., 9, 115.

Felix, A. and Robertson, M. (1928) Brit. J. exp. Path., 9, 6.

Fremian, G. G., Challinor, S. W., and Wilson, J. (1940) Biochem. J., 34, 307.

Fuller, A. T. (1938) Brit. J. exp. Path., 19, 130.

Furth, J. and Landsteiner, K. (1928) J. exp. Med., 47, 171 ; (1929) Ibid., 49, 727.

GoebeL, W. F. (1936) J. exp. Ned., 64, 29; (1940) Ibid., 72, 33.

Goebel, W. F. and ADais, M. H. (1943) J.exp. Med., ry, 435.

Goebel, IV. F., Beeson, P. B., and Hodgland, C. L. (1939) J. biol. Chem., 129, 455.

Goebel, W. F. and Hotchisiss, R. D. (1937) J. exp. Med., 66, 191.

Goebel, W. F., Shedlovsky, T., Lavin, G. I., and Adaus, M. H. (1943) J. biol. Chem., $148,1$.

Griffith, F. (1923) Rep.publ. Hlth. med. Subj., Lond. No. 18.

Gruber, M. and DurhaM, H. E. (1896) Münch. med. Wschr., 43, 285.

Heidelberger, M. and Avery, O. T. (1923) J.exp. Med., 38, 73; (1924) Ibid., 40, 301. Heidelberger, M., Avery, O. T., and Goebel, W. F. (1929) J. exp. Med., $49,817$.

Heidelberger, M., Goebel, IV. F., and Avery, O. T. (1925) J. exp. Med., 42, 701.

Heidelberger, H. and Ka bat, E. A. (1934) J.exp. Med., 60,643; (1936) Ibid., 63, 737 ; (1937) Ibid., 65, 8s5; (1938) Ibid., 6\%, 545.

Heidelberger, M. and Kendall, F. E. (1934) J.exp. Mcd., 59, 519.

Henderson, D. W. (1939) Brit. J. exp. I'ath., 20, 11.

Henriksen, S. D. and Heidelberger, M. (1941) J. exp. Med., 14, 105.

HoFMeIER, K. (1927) Z. ImmunForsch., 50, 71.

Howie, J. W. and Cruickshank, J. (1940) J. Path. Bact., 50, 235.

Hoxle, L. (1942) J. IIyg., Camb., 42, 416.

Iіліма, T. (1923) J. Path. Bact., 26, 519.

Ivánovics, G. (1940) Z. ImmunForsch., 98, 373.

Ivánovics, G. and BruckNer, V. (1937a) Z. ImmunForsch., 90, 304; (1937b) Ibid., 91, 175 ; (1938) Ibid., 93, 119.

Iváxovics, G. and Erdös, L. (1937) Z. ImmunForsch., 90, 5.

JeRMolJEWA, Z. W. and BUJANowskaJA, I. S. (1930) Z. Immun Forsch., 68, 346.

JulianelleE, L. A. (1926) J. exp. Med., 44, 113, 683, 735. 
Julianelle, L. A. and Wieghard, C. W. (1934) Proc. Soc. exp. Biol. N.Y., 31, 947.

KaUfFMan, F. (1936) Z. IIyg. InfehtKr., 118,540; 119, 103; (1939) Acta path. microbiol. scand., 16, 278.

KaUffian, F, and Tesdal, M. (1937) Z. IIyg. InfektKr., 120, 168.

KRUIF, P. H. DE. (1921) J. exp. Med., 33, 773.

Laidlaw, P. P. and Dodlex, H. W. (1925) Brit. J. exp. Path., 6, 197.

LamanNa, C. (1940) J. infcct. Lis., 6\%, 193, 205.

LANCEField, R. C. (1928) J. exp. Med., 47, 91, 469, 857; (1933) Ibid., 5\%, 571.

Lancefield, R. C. and Todd, E. IV. (1928) J.exp. Med., 48, 769.

Landsteiner, K. and Levine, P. (1927) J. exp. Med., 46, 213.

Marrack, J. and Carpenter, B. R. (1938) Brit. J.exp. Path., 19, 53.

Menzel, A. E. O. and RAkE, G. (1942) J. exp. Hed., $75,437$.

MEYER, K. (1926) Z. ImmunForsch., 45, 97; (1930) Ibid., 68, 98 ; (1931) Ibid., 71, 331 ; (1935) C. R. Soc. Biol., 129, 485; (1939) Ann. Inst. I'asteur, 62, 282.

Meyer, K. and Moraan, W. T. J. (1935) Brit. J. exp. Path., 16, 476.

IInes, A. A. (1933) Brit. J. exp. Path., 14, 43; (1939) Brit. J. exp. Path., 20, 63.

Miller, C. P. and Boor, A. K. (I934) J. exp. Med., 59, 75.

Morch, E. (1942) J. Immunol., 43, 177.

Morgan, W. T. J. (1931) Brit. J. exp. Path., 12, 62; (1936a) Rep. int. Congr. Microbiol., 423; (1936b) Biochem. J., 30, 909; (1937) Ibid., 31, 2003.

IIudd, S., Czarnetzky, E. J., Ретtit, H., and Lackman, D. (1937) Publ. Hlth. Rep., Wash., $52,434$.

Mueller, J. H. and Tomasik, J. (1924) J. exp. Med., 40, 343.

Neter, E. (1943) J. Immunol., 46, 239.

Orcutt, M. L. (1924) J. exp. Med., 40, 43, 627.

Partridge, S. M. and Morgan, W. T. J. (1942) Brit. J. exp. P'ath., 23, 84.

Pirie, N. W. (1940) Biol. Rev., Camb., 15, 377.

Powell, H. M. (1926) J. Immunol., 12, 1.

Preisz, H. (1904) Zbl. Bakt., 35, 280, 416, 537, 657.

Raistrick, H. and Topley, W. W. C. (1934) Brit. J. exp. Path., 15, 113.

Remmane, H. A. (1925) J. exp. Med., 41, 587 ; (1927) Ibid., 45, 1, 807.

ROTHAOKER, A. (1913) Z. ImmunForsch., 16, 491.

ScHiIDT, H. (1925) Z. Immun Forsch., 43, 422.

Schockaert, J. (1928) C. R. Soc. Biol., 99, 1242.

Sevag, I. G., Smolexs, J. and Stern, K. G. (1941) J. biol. Chem., 139, 925.

Sickles, G. I. and Rice, C. E. (1938) J. Immunol., 34, 235.

Simth, T. and Reach, A. L. (1903) J. med. Res., 10, 89.

Sordelli, A. and Mayer, E. (1931) C. R. Soc., Biol., 10\%, 736.

Sprivgut, E. (1927) Z. ImmunForsch., 52, 25.

Stokinger, H. E., Ackerian, H., and Carpenter, C. I. (1944) J. Bact., $47,141$.

Stone, K. and Garrod, L. P. (1931) J. Path. Bact., 34, 429.

SugG, J. Y. and Hehre, E. J. (1942) J. Immunol., 43, 119.

Suga, J. Y., Richardson, L. V., and Neill, J. M. (1929) J.exp. Med., 50, 579.

'Thompson, R. and Khorazo, D. (1937) J. Bact., 34, 69.

Topd, E. W. (1928a) Brit. J. exp. Path., 9, 1; (1928b) J.exp. Med., 48, 493.

Todd, E. W. and Lanoesield, R. C. (1928) J.exp. Med., 48, 751.

Tomcsik, J. (1930) Z. Hyg. Infekthr., 111, 119.

Tomcsik, J. and Kurotohis, T. J. (1928) J. exp. Med., 47, 379.

Toucsik, J. and Szongotт, H. (1932) Z. ImmunForsch., 76, 214; (1933) Ibid., 78, 86.

VERWEY, W. F. (1940) J. exp. Hed., \%1, 635.

WeBster, L. T. (1925) J. exp. Med., 41, 571.

WeIL, E. and FerIx, A. (1917) Wien. klin. Wschr., 30, 1509.

Whiтe, P. B. (1927) J. Path. Bact., 30, 113; (1928) Ibid., 31, 423 ; (1929) Ibid., $32,85$. (1932) Ibid., 35, 77 ; (1933) Ibid., 36, 65.

Wilson, G. S. (1930) J. Hyg., Camb., 30, 40.

Wong, S. C. (1940) Proc. Soc. exp. Biol., N.Y., 45, 850.

Wong, S. C. and T'ung, T. (1939) Proc. Soc.exp. Biol., N.Y., 42, s24; (1940) Ibid., 43, 749.

YAsUi, K. (1929) Z. ImmunForsch., 63, 440.

Zepp, H. D., and Hodes, H. L. (1943) Proc. Soc. exp. Biol., N.Y., 52, 315.

Zinsser, H. and Parker, J. T. (1923) J. exp. Med., 37, 275. 


\section{CHAPTER 9}

\section{BACTERIAL VARIATION}

In the earlier bacteriological writings, from the days of Pasteur and Koch onwards, there will be found seattered references to certain bacterial strains that have deviated, in one way or another, from the modal form of the particular species concerned. In the earliest days of all, when the doctrine of spontaneous generation was dying, but not yet dead, it was, indeed, the fixity rather than the variability of bacterial species that was in dispute. It was not, however, until the beginning of the present century that any serious attempt was made to study bacterial variation as a problem suigeneris, or to apply to bacteria the concepts that had proved so fruitful in the study of the higher plants and animals. During the first decade or so of the present century these attempts were sporadic ; but during the last twentyfive years an immense impetus has been given to this line of inquiry by a series of converging studies, and the relevant literature has expanded from a trickle to a flood.

The significance of many of the observations that have been recorded is at the moment exceedingly difficult to assess. Some of them serve to illustrate the wide range of variation that may occur within a single bacterial species, but tell us little or nothing in regard to the relative frequency of the different variants described, or the factors on which the variation depends. Others are concerned, at least in part, with problems that have been described in Chapter 2-the existence of a complex bacterial life-cycle, of filtrable forms of bacteria, of some form of sexual reproduction and so on. In the present chapter we shall discuss the general problem of bacterial variation, as illustrated by a series of observations the selection of which must of necessity be to some extent arbitrary. A more detailed account of certain variants will be found in the systematic deseriptions of the different genera and species given in later chapters.

\section{Terminology.}

The application to bacteria of terms that have been coined to express changes in form or function occurring in higher plants or animals is not without its dangers ; and it is possible that there is little real justification for the use of such a term as mutation, in connection with the variations which bacteria may undergo. Some biologists would attach two implications to the use of this term: the suddenness of the change - the variation per saltum-and the permanency of the change, once it has occurred. Dobell (1913) regards as a mutation any permanent change, which is transmitted to subsequent generations of bacteria, without any implica. tion in regard to the suddenness or gradualness of the change, or the manner of its acquisition. We must, at all events, remember that most of our conceptions with regard to variation and heredity have been built up on data derived from observations and experiments on living things which pass through a sexual cycle; 
and, so long as we regard bacteria as asexual organisms multiplying by simple binary fission, we must avoid the tendency to misapply concepts which have their essential basis in the segregation, and conjugation, of a special system of reproductive cells. Some of the concepts are applicable; as we noted in Chapter 2, the persistence of specific characters through a large number of generations of a bacterium implies an hereditary mechanism of some kind, and one that behaves as a unit within the bacterial cell. For the purposes of bacterial genetics, it is irrelevant whether a nuclear apparatus has been demonstrated morphologically or not. A logical deduction from the mathematical data of genetical experiments with other species leads to a chromosome and gene mechanism. We have as yet insufficient data on bacterial heredity to justify the assumption that here also there is a similar mechanism, but in the meantime, it is convenient, and indeed sensible, to use those concepts of general genetics which are applicable to bacteria.

We may postulate a haploid or a polyploid nucleus; scanty morphological studies suggest that haploid and perhaps diploid nuclei are likely to be commonest in bacteria. The mutations observed in bacteria are characterized by absence of the intermediate forms, and a moderate readiness to reversion. If these are due to genic changes in the nucleus, the most likely of all known types of genic change is transgenation (Lindegren 1935). It should be noted, however, that single-cell cultures, which are essential for the proper study of bacterial genetics, do not necessarily represent a single cell in the genetical sense, for many single cells appear to contain more than one nuclear unit. In this respect, at least, a technique for the morphological demonstration of a nucleus is a necessary foundation for the formulation of a "mathematical" nucleus referred to above.

In one sense, bacteria offer a fruitful field for the study of mutations. Observable mutations occur with great rarity among more complex multicellular organisms. If bacterial mutations are of the same order of rarity, the bacteriologist will have ample opportunities for observing them, since the colony that grows from a single cell after a day's incubation on an artificial medium may contain $10^{7}-10^{9}$ individual viable cells, representing the end result of an even greater number of divisions during which mutation could have occurred.

The term "bacterial dissociation" is frequently employed to denote a particular type of bacterial variation (see Hadley 1927, 1937, and Morton 1940, for detailed reviews). This term, in its generally accepted sense, denotes the appearance, in a bacterial culture, of forms which differ sharply, in one or more characters, from the "normal" forms of the parent strain; that is, the strain may be said to have undergone dissociation into two types, differentiated from each other in colony-form, in antigenic structure, or in some other way. The variation must be discontinuous in type, even though there is some overlapping; and the dissociated, or variant form, must be sufficiently stable to maintain its new characters over several generations, whether or not it eventually reverts, wholly or in part, to the normal form from which it was derived.

Those who uphold the view that bacteria pass through a complex life-cycle have naturally sought to relate the phenomenon of dissociation to the phases of this cyclical development (Hadley 1927); but the term may be employed in a purely descriptive sense, without any reference to the possible existence of modes of reproduction other than simple binary fission. It is, however, doubtful whether "dissociation" has any advantage over "variation" as a descriptive term ; and, since it is desirable, at the present stage, to avoid any implication in regard to

P.B. 
the underlying mechanisms involved, it seems wiser to adhere to the older name, which serves our purpose well because of its very vagueness.

\section{Correlated Variations.}

An observed variation in any given bacterial character clearly gains in significance if it is found to be uniformly or frequently associated with a change in some other character, or characters. It is a fairly safe assumption that correlated variations of this kind indicate some relatively major change in genetic make-up. Whether the correlated character changes are different expressions of a single character factor, or are due to changes in two or more genetic factors that are themselves associated as a result of the reproductive mechanisms of the cell, we cannot tell; but in a few instances, as in the smooth $\rightarrow$ rough variations to which we have already referred in Chapter 8 , and which we shall shortly describe in more detail, we can relate many of the associated character changes to a loss of the ability to synthesize and store a particular chemical component of the bacterial cell.

\section{Impressed Variations.}

By an impressed variation is meant a variation that occurs in response to a particular environmental stimulus; so that, by applying the stimulus, we can induce the variation at will. It should be noted that, if this term is to be applied in its strict sense, it is the genetic variation that must be impressed, not merely the character change by which this variation is recognized. We know, for instance, certain genetic variations in insects that lead to the appearance of well-defined character changes under particular environmental conditions. In the absence of these conditions the insects appear to conform to the normal type, though the genetic differences, which are themselves quite independent of these envirommental factors, persist all the time. The criteria that justify the conclusion that a particular variation has been induced by a particular environmental stimulus are clearly that the application of the stimulus should regularly be followed by the appearance of the variation in question, and that the variant form should persist, over many generations at least, after the stimulus has been withdrawn. The appearance of a variant form in response to a given stimulus, followed by immediate reversion to the normal form when the stimulus is no longer applied, should be regarded as a temporary adaptation to a changed environment, rather than as a variation in the sense in which that term is used here. It is often extremely difficult, in the light of our present knowledge, to determine in which category a given change in bacterial form or function should be placed.

It will be convenient to discuss the variability of each of the more important bacterial characters in turn, indicating, where possible, whether the variation in question is correlated with others, and whether it occurs naturally or in response to any known change in environment.

\section{Variations in Morphology.}

There are innumerable accounts in the literature of changes in shape, size or structure of bacterial cells. Some of these, such as the involution forms that appear in cultures of the plague bacillus when that organism is grown on agar with a high salt-content, are clearly a direct response to an environmental stimulus, and are not inherited. In other instances it is very difficult to tell whether or not true variation has occurred. From among the many examples available, we may select the following as illustrating the kinds of variation that have significance from our present point of view. 
Barber (1907), starting with a single strain of Bact. coli, selecterl and subeultured, by means of a micromanipulator, individual bacterial cells that had grown to an unusual length. In this way he was able to isolate three strains that grew in the form of long rods throughout many sucessive generations, showing no tendency to revert to the modal short bacillary form of the original parent strain. This would appear to afford an instance of the perpetuation of a natural heritable variation by simple selection.

We have already, in Chapter 8 , noted the occurrence of the $\mathrm{H} \rightarrow \mathrm{O}$ variation - the loss by a flagellated organism of the capacity to produce flagella, associated of course with a loss of motility. When this variation occurs naturally, the variant O form usually shows no tendency towards reversion. The importance of this change from the point of view of antigenic analysis led to a search for methods by which it could be induced at will. It has been found that growth on agar containing $0 \cdot 1$ per cent. phenol largely, or completely, suppresses the formation of flagella (Braun 1918); but the non-flagellated cells so obtained give rise to the normal flagellated form when subcultured on ordinary media; so that we are here dealing not with an impressed variation but with a temporary adaptation to environment.

Another striking morphological variation is the occurrence of asporogenous variants of such spore-bearing bacilli as B. anthracis (Preisz 1904, Eisenberg 1912). Many of these naturally-occurring asporogenous variants have shown no tendency to revert to the normal spore-bearing form. Pasteur $(1881 a, b)$ found that the growth of $B$. anthracis at $42.5^{\circ} \mathrm{C}$. for about a month resulted in a great decrease in the frequency of spore formation, as well as in a decrease in virulence; and Roux (1890) obtained asporogenous strains of this organism by growing it in the presence of low concentrations of antiseptics. Whether the asporogenous strains obtained by these methods were examples of an impressed variation or of a temporary adaptation to environment is difficult to determine. Iater experiments by Bordet and Reuaux (1930), however, strongly suggest the occurrence of an impressed variation of the genetic type. They found that certain strains of $B$. anthracis, yielding the normal, flat, filamentous colony when grown on solid media, gave rise on prolonged incubation to a characteristic type of papillary daughter colony consisting of asporogenous bacilli. By repeated subculture from these daughter colonies a completely asporogenous strain of $B$. anthracis could be isolated. When the medium on which these strains of $B$. anthracis were grown was deprived of its calcium by treatment with oxalate, spore formation was stimulated, and the papillary, asporogenous daughter colonies did not appear. When the calcium content of the medium was increased, by the addition of a little calcium chloride, the frequency of spore formation decreased and the papillary, asporogenous daughter colonies became very numerous. Repeated subcultures from these daughter colonies again produced a completely asporogenous strain (see also Bordet, P. 1930).

There would seem to be an interesting difference between the asporogenous strains of $B$. anthracis obtained by the method of Pasteur, and those obtained by the method of Bordet and Renaux. Although growth at $42.5^{\circ} \mathrm{C}$. for several days leads to a decrease in the average virulence of a culture and to the appearance of various abnormal bacillary forms, many of which are non-spore-bearing, Preisz (1911) was unable to find any correlation between the capacity to form spores and virulence as tested by animal inoculation. Spore-bearing strains might be virulent 
or avirulent; so might asporogenous strains. In Bordet and Renaux's experiments the actively sporogenous strains obtained by cultivation on oxalated media were highly virulent, while the asporogenous strains obtained by subculturing from the daughter colonies on media with a high calcium content were completely avirulent. It would seem, then, that loss of ability to form spores is sometimes, but not always, associated with loss of virulence. The most reasonable hypothesis would seem to be, either that asporogenous strains may be produced by different genetic mechanisms, or that the presence in a medium of excess of calcium stimulates some genetic change in addition to that on which the loss of spore-bearing capacity depends. We should not, it may be noted, on the basis of these divergent results, be justified in regarding this loss and the loss of virulence as correlated variations in the usually accepted sense. The use of precisely defined media will in all probability throw light on a number of morphological variations, though there are at present only a few isolated observations on this point. For example, in an amino-acid glucose salt medium, and sub-optimal amounts of an unidentified growth factor present in liver extract, gonococcal strains produced large, distorted, swollen, "vacuolated," and dumb-bell forms; in the presence of ample growth factor, the cells grew in characteristic diplococcal forms (Lankford, Scott, Cox and Cooke 1943). Badger (1944) records an interesting variation in a Type III pneumococcus for which choline was an essential nutrient. The choline could be replaced by ethanolamine, and in the presence of the latter, the pneumococcus grew in characteristic long chains. Again, Pappenheimer and Shaskan (1944) found that $C l$. welchii grew as regular rods in a medium containing enough iron to ensure maximum growth, toxin production and breakdown of carbohydrate. With a reduction of iron content only, there was a depression of these activities, and the clostridia grew as curved, elongated and entirely atypical bacilli.

There are many other ways in which bacterial variants may depart from the normal morphological type of the species to which they belong. A capsulated organism, such as the pneumococcus, may, for instance, give rise to a non-capsulated variant. This particular variation is, however, associated with other important changes in behaviour, and it will be more convenient to consider it in the section dealing with antigenic variation.

\section{Variations in Biochemical Reactions.}

Since the earliest days of bacteriology, differences in fermentation reactions have been extensively utilized in differentiating bacterial species or types that belong to the same genus or group, as judged by morphological or other criteria. To be of use from this point of view the fermentation reactions of any given species, or type, must, of course, be constant. This has been found to be the case, to the extent that it is usually possible to select empirically a number of substrates that a given organism, in its normal form, will consistently alter or leave unaltered. It has, however, been found that for any given bacterial species there are other substrates that are sometimes fermented, sometimes not; from the systematic point of view we should say that those particular substrates, in relation to that particular organism, had little differential value. Similarly, some bacterial species vary more than others in their fermentative abilities ; and the differential value of fermentation tests therefore varies from one bacterial group to another.

In trying to assess the significance of the numberless recorded instances of variants that differ in their fermentation reactions from the parent strain from which they were derived, we must keep these facts constantly in mind. We may 
regard any given bacterial species as equipped with an armoury of enzymic mechanisms of the kind discussed in Chapter 3. Some of these may perhaps readily be lost by disuse, and as readily regained if called into activity by appropriate stimuli. Others may, perhaps, be easily adapted to deal with some substrate that does not differ too greatly from that which the enzyme naturally attacks. Others, again, will depend on more constant and fundamental cell mechanisms; these will be lost, regained, or altered only as the result of some deep-seated variation in cell structure.

It is indeed impossible to draw any hard and fast line between those changes in fermentative ability that arise as temporary adaptations to environment, and those that may be regarded as variations of a more permanent kind, though certain instances can be assigned with some confidence to one category or the other.

Adaptive and Constitutive Enzymes. - The enzyme response of a bacterium to a substrate may fall into one of two classes. The enzyme may be produced only in the presence of the substrate or it may be produced whether the substrate is there or not. Thus, Wortmann (1882) described a bacterium that produced amylase in a starch medium, but none in a starch-free medium, and contrasted it with a yeast, which produced invertase whether sucrose was present or not. For these two classes Karström $(1930,1937)$ proposed the names "adaptive" and "constitutive" enzymes. For example, the production of molecular hydrogen from glucose or formic acid by Bact. coli was found to be due to two "adaptive" enzymes, one a glucose hydrogenlyase, the other a formic hydrogenlyase (Stephenson and Stickland 1932, 1933, Yudkin 1932). Not only were the enzymes adaptive, but they were apparently formed in resting, as well as in reproducing cells. Some protoplasmic growth, however, appeared to be necessary, since in the absence of nutrient broth no enzymes were formed (see also Stephenson and Yudkin 1936). The adaptation disappeared in the absence of the stimulating substrate, and was therefore not heritable.

Enzyme formation may also be conditioned by the presence of materials which are unrelated to the substrate, presumably because they are needed for enzyme synthesis (see Jacoby 1916, 1917, 1918, Passmore and Yudkin 1937). Quastel (1937) developed this concept by postulating that enzymes themselves were metabolites whose rates of formation and destruction follow the same physicochemical laws as those controlling the metabolism of other metabolites in the cells; the presence of substrate, therefore, might or might not be the particular condition necessary for the production of an enzyme. In his view, the adaptive and constitutive enzymes represent the limits of variability of cellular enzymes, the constitutive having the least, the adaptive the greatest range under different environmental conditions.

Euler and Cramér (1913) were able to stimulate the production of invertase in a yeast by the addition of fructose or glucose, and also by mannose. It appears, therefore, that not only substrates, but the products of hydrolysis of substrates and chemicals related to the substrate act as stimulants of adaptive enzymes. According to Yudkin (1938) the Mass Law serves to reconcile many of these phenomena of enzyme adaptation. If it is assumed that the enzyme and its precursor are in a state of equilibrium in the cell, with the precursor predominating and the enzyme in undetectable amounts, a substrate, its hydrolytic products, or a related substance will, by combining with the enzyme, shift the equilibrium so that more enzyme is formed from the precursor. The mere presence of the substrate is 
insufficient; it must be utilized by the metabolizing cell. Thus, the production of the enzyme hydrolysing the specific polysaccharide of Type III pneumococcus by Dubos and Avery's (1931) bacillus was retarded when nutrients more readily assimilable than the polysaccharide were added to the culture (see also Dubos 1940).

It should be noted that the appearance of an enzyme does not necessarily imply its increased production; the effect may be due to activation of an enzyme already existing in considerable quantity. It may also be due to the removal of an inhibitor, rather than the addition of a substrate. For instance, the tryptophanase system of Bact. coli is adaptive, but when tryptophan is present, glucose and phenylalanine will inhibit the production of the enzyme system by resting bacteria (Evans, Handley and Happold 1940). Dawson and Happold (1943) suggest that the phenylalanine may act by competing with tryptophan for a labile component common to two enzyme systems, one the tryptophanase, the other concerned with carbohydrate storage in the cell.

Kocholaty and his colleagues (Kocholaty and Hoogerheide 1938, Kocholaty and Weil 1938) report enzyme adaptations to variations in $\mathrm{pH}$. They produced a shift in the $\mathrm{pH}$ optima of, for example, the alanine and pyruvic dehydrogenases of $\mathrm{Cl}$. sporogenes by varying the $\mathrm{pH}$ of the culture media; and found that $\mathrm{Cl}$. histolyticum grown in a casein medium produced proteinases with an optimum activity at $\mathrm{pH} 7 \cdot 0$, but when grown in a casein-glucose medium at a lower $\mathrm{pH}$, produced proteinases with an optimum at $\mathrm{pH}$ 6-0. Cells from glucose-casein cnltures, when transplanted to the plain casein medium within 20 hours, had produced the proteinase with an optimum at $\mathrm{pH} 7.0$. They were also able to train $\mathrm{Cl}$. histolyticum to attack casein or gelatin alone, though normally it attacks both equally well. This specificity was very labile, however, for when aminoacids not present in the homologous protein were adder to the medium the resulting enzymes attacked both proteins. To explain these phenomena, they sought to combine Yudkin's mass action theory with Quastel's theory of enzymes as metabolites by assuming that relatively few colloidal carriers are available for a number of enzymes, and that under different conditions these combine with different active groupings to form enzymes of different activity or specificity. On this basis Yudkin's precursor may be a colloidal carrier in equilibrium with varions prosthetic gronps of enzymes. On the other hand, this type of $\mathrm{pH}$ adaptation may depend on a multiplieity of enzymes. van Heyningen (1940), for example, deseribed two proteinases in Cl. histolyticum, one activated by cysteine, appearing in the first 12 hours of growth, one appearing later which was inhibited by cysteine.

In other organisms, a close relation is demonstrable between the pH optima of enzymes and the cultural conditions in which the enzymes come into play. Thus, Bact. coli, Str. facalis and certain clostridia produce amines from aminoacids by specific decarboxylases that act only between the limits $\mathrm{pH} 2.5$ and 5.5 . These decarboxylases are formed only when the organisms are grown in acid media (Gale 1940, 1941). Again, at a pH in the vicinity of $5 \cdot 0$, Bact. aerogenes decomposes pyruvic acid with the formation of acetylmethylcarbinol; at a higher pH, this activity is entirely suppressed, and decomposition proceeds by breakdown into acetic and formic acids (Silverman and Werkman 1941). Extending these investigations, Gale and Epps (1942) showed that Bact. coli could grow in a casein digest at any $\mathrm{pH}$ between 4.5 and 9.0 and that, though with changing growth $\mathrm{pH}$, the enzymic constitution of the bacteria changed, there was no evidence of any shift in the pH optima of individual enzymes. The enzymes concerned fell into two groups. The formation of those in the first group increased as the growth $\mathrm{pH}$ deviated from the $\mathrm{pH}$ at which their action was maximum, so that in each cell the drop in activity due to the $\mathrm{pH}$ change was compensated by the 
increased production of enzyme. Catalase, urease and formic dehydrogenase were included in these enzymes, one of whose essential functions appeared to be removal of inhibitory products of metabolism; these were maintained at an efficient level over a large $\mathrm{pH}$ range. The enzymes of the second group displayed only a small degree of compensatory formation when the growth $\mathrm{pH}$ shifted from the $\mathrm{pH}$ of their optimal activity. They behaved like adaptive enzymes, but adaptive in respect of $\mathrm{pH}$ rather than of substrates. Nevertheless, they contributed to the stability of cellular equilibrium. Thus, in an acid medium, amino-acid decarboxylases, and consequently amines, were produced; on the other hand, amino-acid deaminases, and consequently hydroxy acids, were produced in an alkaline medium.

Adaptation occurs much more readily in physiologically young than in older cells (see, for example, Hegarty 1939), and we have already seen that adaptation takes place in resting bacteria. The independence of reproduction and some forms of adaptation is confirmed by the interesting study of Doudoroff (1940) on the adaptation of Bact. coli to sodium chloride.

When the cultural conditions, such as air supply, concentration of nutrient, and $\mathrm{pH}$, were standardized, a fairly constant fraction of a fresh-water culture of Bact. coli was able to reproduce when inoeulated into a medium of definite $\mathrm{NaCl}$ concentration. The acelimatization to salt was independent of reproduction, since bacteria in the stationary phase were far more readily acclimatized than those in the phase of logarithmic growth or the phase of decline. Only acclimatized eells were capable of reproduetion in saline media, but if after acelimatization the non-dividing cells were returned to salt-free media, they rapidly lost the capacity to grow in saline media. Once acelimatized, the cells could be propagated in saline media withont further acelimatization, though the division rate was considerably lower than that in salt-free eultures.

Optimal acchimatization to growth, even in high salt eoneentration, was achiever by preliminary exposure to a single intermediate salt concentration. In other words, the adaptive response of the bacteria consisted firstly of a reversible acclimatization, independent of reproduction, and secondly of a selection of the cells with the widest range of potentialities for growth in various concentrations of saline.

The distinetion between adaptive and constitutive enzymes is perhaps more a quantitative than a qualitative one. The production of constitutive enzymes varies according to the conditions of culture, and sometimes according to the presence or absence of the substrate. Moreover, adaptive enzymes are constitutive in the sense that they are a constant feature of the bacterial cell. As far as we are aware, there is no recorded instance of a bacterial strain losing the capacity to make a given adaptive enzyme response by contimuous subculture in media which preserve the general characters of the strain. In other words, though by definition the adaptation is not heritable, the specific adaptability certainly is. We do not know whether the potentiality for adaptation is maintained as a precursor or as the enzyme itself, but in some cases it is possible that the enzyme is present as such when no substrate is present, though in undetectable amounts.

Variation and Mutation in Enzyme Systems.-At the other end of the scale of variation, we have those changes which are presumably due to the selection, over a large number of bacterial generations, of relatively rare mutations. Changes in enviromment of the kind that induce an adaptive response in bacteria may also serve to make mutations manifest. In a case of this kind, the distinction between the two effects would lie mainly in the persistence of the mutation effect when 
the original environment was restored. We must nevertheless guard against a too literal interpretation of this difference, for as we have pointed out above, bacterial generation under experimental conditions is so rapid as to compensate for a very low mutation rate (the ratio of the number of mutations to the total number of cell divisions). It follows that if mutations in the reverse direction were as frequent as those in the direction of an observed variation, variation and reversion might be accomplished with such speed as to suggest a temporary adaptation.

Mntations are not equally rare in all bacterial strains. Indeed, some produce variants so constantly and in such relatively large numbers that they are often designated as "unstable" strains. Deskowitz (1937) concluded from a study of unstable colony variants of Salm. typhi-murium that unstable strains differed from stable only in having a mutation-rate of the order of 1 per cent. or more. The existence of these "unstable" strains raises a practical point of some importance in distinguishing adaptation from true mutation.

We have seen in Chapter 3 that the typhoid bacillus, though normally dependent for its growth on the presence of tryptophan, can be trained to synthesize this essential metabolite from ammonium salts; and in Chapter 6 there are numerous examples of habituation of bacteria to growth in otherwise inhibitory concentrations of various antibacterial agents. These changes, in most cases permanent, are excellent examples of impressed variation in biochemical constitution. A few examples from recent work on the vitamin metabolism of bacteria and yeasts will serve to show the effects of such training, and the degree of success which attends it.

Wood, Anderson and Werkman (1938) trained a strain of propionic acid bacteria to dispense with thiamin, and later showed (Silverman and Werkman 1939) that the trained cultures were synthesizing a substance that had the biological qualities of thiamin. Leonian and Lilly $(1942,1943)$ induced several strains of the yeast Saccharomyces cerevisice to grow without one or more of certain essential vitamins, including thiamin, pyridoxin, inositol, pantothenic acid, and in some cases biotin. At least two of the variants grew without all five vitamins, and in some cases the growth in the absence of one vitamin induced the power to dispense with other vitamins as well. Some variants reverted easily, but many of them reverted only after six months' subcultivation on a medium containing all the vitamins.

Koser and Wright (1943) trained four strains of dysentery bacilli to dispense with nicotinamide. The variants could be obtained either by serial subculture in a glucose amino-acid medium containing diminishing quantities of nicotinamide; or by incubation of a large inoculum in a nicotinamide-free medium. Under the latter conditions the proportion of variants to the total of viable cells increased during incubation. The variants resembled the parent strains both antigenically and in their gross fermentation reactions, but never developed as luxuriantly as in media containing optimal amounts of the vitamin. Nevertheless, they had acquired the power to synthesize a substance physiologically equivalent to nicotinamide, for filtrates of their cultures in vitamin-free media supported the growth of strains of bacteria known to require nicotinamide.

Whereas the typhoid bacillus appears to acquire the ability to synthesize tryptophan with relative ease, its normal inability to attack lactose seems to depend on a more fundamental peculiarity of cell organization, since it is extremely difficult to produce lactose-fermenting strains of this organism, even by prolonged "training" in media containing this substrate as the main available source of carbon. Twort (1907) has succeeded in producing one such strain; and we may perhaps regard the extreme rarity with which this change has been induced as 
evidence that it depends on a major variation in cell organization, though it must be noted that Penfold $(1910 a)$ found a lactose-fermenting strain of this organism to be very unstable, with a marked tendency to revert to the non-lactose-fermenting form.

In this connection we may refer to an interesting type of variability in enzymic activity which was first demonstrated by Massini in 1907. This observer described a coliform bacillus whieh, on first isolation, failed to ferment lactose, and hence gave rise to colourless colonies on an agar medium, containing lactose and an indicator that gave a red colour in the presence of acid. From the third day of incubation onwards, small papillæ began to appear on these colonies, and took on a red tint, indieating that the bacilli composing them were breaking down the lactose with the formation of acid. Subcultures from these papillæ gave non-papillated red colonies, showing that the power to ferment lactose had been transmitted to the descendants of the bacilli which had originally formed the red papillæ; and repeated subcultures showed that this power was not subsequently lost. Subcultures from the colourless parts of the original colonies, however, gave rise to colourless colonies on which red papillø appeared after about 3 days, just as in the case of the original culture; and repeated subcultures from the colourless portions of the colonies of successive generations gave similar results. Thus, the non-lactose-fermenting form of this organism showed a constant tendeney, when grown on a lactose-containing medium, to give off lactose-fermenting variants in which the new character appeared to be permanent. To this organism Massini gave the name of Bacterium coli mutabile. Such a bacterial strain may, as Dobell has pointed out, be likened to the ever-sporting races of plants which have frequently been described. Massini's observations have been confirmed, in all essentials, by many subsequent workers (Burk 1908, Benecke 1909, Burri 1910, Kowalenko 1910, Baerthlein 1912 $(1, b)$; while Benecke, and Kowalenko, added greatly to the significance of their results by starting with a culture obtained from a single bacterial cell, thus eliminating the possibility that the phenomena resulted from an original admixture of strains. In a careful quantitative study, Lewis (1931) showed that the mutation rate in this organism was such that when it was grown in a lactosefree culture medium, one in every 100,000 viable cells produced a lactose-fermenting colony. Lewis also observed that the proportion of variants was relatively constant. A relatively constant proportion of variant and normal cells has been recorded by other observers as characterizing certain mutating strains (see, for example, Solotorovsky and Buchbinder 1941).

Experiments along similar lines, employing other species belonging to the same bacterial group, have been carried out by Penfold (1910a and $b, 1911 a, b$ and $c$, 1912) and Mïller (1908, 1911). The observations recorded by these observers have made it clear that the behaviour of Massini's Bact. coli mutabile is by no means a bacteriological curiosity, but that, given a substrate appropriate to the particular species under investigation, many members of the coli-typhoid-dysentery group will adapt themselves to a particular nutrient material which they do not immediately attack by giving rise to variants endowed with the power of breaking down this particular substrate.

Thus, Salm. typhi usually fails to ferment dulcitol; but when grown on a medium containing that alcohol, it gives colonjes which develop dulcitol-fermenting papillæ (Penfold 1910a, $b, 1911$ ) ; the same organism behaves similarly towards rhamnose (Müller 1911); while Salm. paratyphi $B$ behaves similarly towards 
raffinose. From Muiller's account it would appear that the rhamnose-fermenting variants of Salm. typhi, and the raffinose-fermenting variants of Salm. paratyphi B, are non-reverting modifications of the parent strain. Apparently, also, subcultures from the non-fermenting portions of the colonies, in these two organisms, showed the same tendency to throw off fermenting variants in the form of papillæ, as was observed by Massini in the case of Bact. coli mutabile. Penfold's observations do not, however, confirm the absence of a tendency towards reversion. In connection with the appearance of dulcitol-fermenting forms of Salm. typhi, in particular, he finds that subcultures from the dulcitol-fermenting papillæ, or from fermenting cultures in dulcitol peptone water, show a marked tendency to revert to the nonfermenting parent form during the earlier generations. If the selective process is continued through a long series of successive generations the tendency to reversion becomes less and less; though it appears doubtful whether absolute permanency is ever attained. Penfold concludes from his results that the more rapidly a particular species acquires the ability to ferment a particular substrate, the less tendency is there for subsequent reversion; while the longer and more rigorons is the training required to bring about the appearance of fermenting variants, the longer must that training be afterwards continued to make a lasting impression on that particular strain.

Certain experiments carried ont by Penfold $(1911 b, c)$ and by Revis (1911, 1912) have brought to light a different type of impressed variation in bacteria. Penfold found that, by growing certain strains of Bact. coli on an agar medium containing sodium monochloroacetate, he was able to isolate strains which retained the power of producing acid in all the usual carbohydrate media, but which had lost the power of producing gas in many of them. Moreover, he found that while the power to produce gas from sugars, or from substances giving rise to sugars on hydrolysis, was usually suppressed, the power of forming gas from alcohols, such as mannitol or dulcitol, was usually unaffected, or but slightly diminished. The only exception noted was in the case of rhamnose, a methyl pentose, which was fermented with gas formation by the variant strains.

Goodman (1908) obtained variants showing differences in fermentative ability by an essentially different technique. Starting with a particular strain of $C$. diphtheriox, which produced a certain degree of acidity in dextrose broth, he inoculated 15 tubes of this medium from a single colony, and determined the degree of acidity attained after a few days. From the tube showing the highest acidity he inoculated 15 tubes of the same medium and a similar number from the tube showing the lowest acidity. This process he repeated through 36 successive subcultures. At the end of this series the high-acid strain produced a titratable acidity more than twice as great as the parent strain, while the low-acid strain produced no acidity at all. As would be expected this strain had also lost its power to produce acid from maltose, but it is of interest to note that its power to produce acid from dextrin was almost unaffected. These observations would appear to afford an example of the separation of a bacterial strain into a fermenting and a non-fermenting variant, by a simple process of selection without any modification of the environmental conclitions ; for thongh it is true that the high-acid strain was in fact subjected to a high concentration of hydrogen-ions during its successive subcultures, while the low-acid strain was not, it is difficult to understand how the latter condition could lead to the production of a variant which had lost its power to ferment dextrose and maltose, while retaining its power to ferment dextrin. 
Variations in Pigment Production.-It has long been recognized that different strains of a particular bacterial speeies, which gives rise to a coloured growth on the ordinary laboratory media, may vary widely in their power of pigment produc. tion; and that any particular strain may lose this power as the result of repeated subeulture, and may regain it for no apparent reason at some later period. In many cases it has been demonstrated that particular environmental conditions are favourable or unfavourable to pigment production, but there are many cases on record in which we cannot reasonably attribute the loss of piginentation to such external influences.

An interesting series of observations have been recorded by Rettger and Sherrick (1911), who studied a strain of the rerl Chromobacterium prodigiosum, which had partially lost its pigment-producing capacity. By suceessive subcultures from growths on solid media, using in one series the most pigmented part of the growth and in another that part which showed least pigmentation, they were able to separate a strain which produced an intensely coloured growth, and a strain which gave almost colourless colonies. The segregation of these two types oceurred early in the series of subeultures, and there appeared to be some tendency for the highly pigmented variant to revert to the slightly pigmented type, though the property of intense pigmentation was successfully maintained by selection through a long series of subeultures. There was, however, no apparent tendency for the nonpigmented variant to aequire the property of pigment production. Within recent years there have been numerous records of colourless variants of species that are normally pigmented. In sone eases, at least, these variants differ sharply from the parent strain in the form of colony produced, as well as in the absence of pigment, and in such instances the variation appears to have much in common with the type discussed in the succeeding section.

\section{Antigenic Variations and the Changes in Colonial and other Characters Associated with Them : the Smooth-Rough Variation.}

The Smooth $\rightarrow$ Rough $(\mathrm{S} \rightarrow \mathrm{R})$ type of variation has already been referred to in our diseussion of antigenic structure (pp. 277, 278), but it is of such fundamental importance in the general problem with which we are here concerned that it is necessary to consider it in considerably greater detail.

Arkwright (1920, 1921, 1924) deseribed variants of bacteria belonging to the coli-typhoid-dysentery group which were characterized by the formation of rough or granular colonies on solid media (see Fig. 45), by giving granular growths in broth or peptone water, and in many cases by undergoing spontaneous agglutination in the presence of 0.85 per cent. sodium chloride.

These properties - colonial roughness, granular growth in fluid media, ancl instability in saline-are associated, in some species at least, with recognizable changes in morphology (Wilson 1930) and

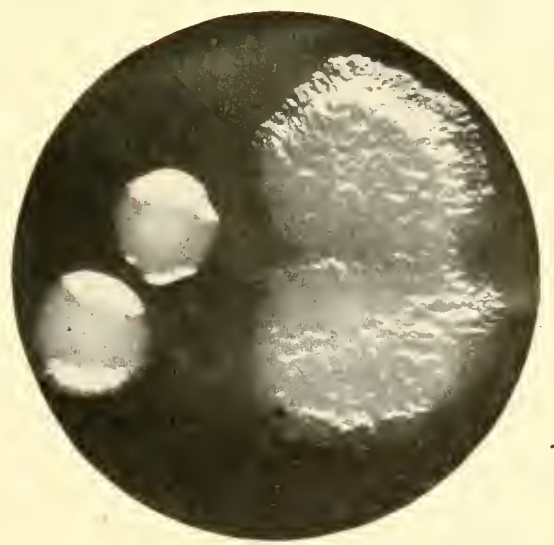

Fig. 45.-Salm. typhi.

Smooth and rough colonies, 24-hours' growth on agar $(\times 8)$. 
with an alteration in the method of cell division (Nutt 1927). If normal smooth strains of Salm. typhi-murium are grown in a thin layer of agar between a slide and a cover-slip and watched under the microscope, it will be noted that each cell division is soon followed by separation of the daughter cells, which slip past each other and come to lie side by side. In rough variants derived from these smooth strains, the daughter cells tend to adhere, end to end, for some time after division has occurred. Short chains are formed and angular bends develop at the junctions between adjacent cells. The tendency of rough bacilli to agglutinate spontaneously in normal saline appears to be due in many cases to the presence, at the bacterial surface, of some lipoidal substance, since extraction with alcohol at a temperature of $50-60^{\circ} \mathrm{C}$. removes the salt-sensitiveness of many rough strains (White 1927). It seems probable that this alcohol-soluble constituent is present in the normal smooth form as well as in the rough variant, but in the former it does not determine the character of the cell surface.

In the organisms referred to above, and in many others, the $\mathrm{S} \rightarrow \mathrm{R}$ variation is associated with the loss of the polysaccharide antigen that characterizes the surface of the normal smooth form; and, though the rough variant has a polysaccharide constituent of its own, there seems little doubt that the change from smoothness to roughness is associated with a relative increase of lipoid as compared with polysaccharide components at the cell surface, and a consequent change in colloidal behaviour from hydrophile to hydrophobe. The hydrophobe qualities of a suspension of rough variants is reflected by increased agglutinability by salt and other non-specific agents, like the dye trypaflavine. There is not, however, any dependable comnection between this degree of agglutimability and roughness. For example, trypaflavine agglutinates not only rough variants of bacteria in the coli-typhoid group, but also Vi strains of Salm. typhi, and flagellated salmonella bacilli in the group phase (Sertic and Boulgakov 1936, 1937, Hirsch 1937). There would also appear to be a laying-bare of protein constituents; since rough variants of salmonella bacilli, and certain other organisms, usually give a positive Millon reaction, while normal smooth strains do not (see White 1929b).

In the particular case of the pneumococcus the change from smoothness to roughness is associated with the loss of the characteristic capsule, and with it of the specific capsular polysaccharide that determines type-specificity. Among the antigenic components that are left are a nucleo-protein antigen and a minor polysaccharide component that is common to all pneumococcal types (see Avery and Heidelberger 1923, Griffith 1923, Reimann 1925, Tillett, Goebel and Avery 1930).

A similarly associated loss of capsule and virulence has been observed in Pasteurella septica (Priestley 1936) and H. influenzae (Chandler, Fothergill and Dingle 1937), which, like the pneumococcus, had at the same time lost their capacity to produce specific soluble substances. In bacilli with no morphologically distinguishable capsule, there is nerertheless a demonstrable loss of antigen characterizing the virulent form. Thus, Boivin and Nesrobeanu (1936) could extract 8-9 per cent. of $O$ antigen from smooth Salm. typhimurium but none from rough forms; and Miles and Pirie (1939) found a progressive diminution in the yield of main antigen from $\mathrm{Br}$. melitensis as cultures increased in agglutinability by heat, salt, and a specific antiserum prepared against rough strains.

It must not be supposed, however, that the mere power to synthesize large amounts of polysaccharide substances, whether they appear as a capsule with a defined edge, as a mucoid extra-cellular substance, or as increased cellular content, necessarily characterizes the virulent smooth form. For example, Boivin, Mesrobeanu, Magheru and Magheru (1936) found a high polysaccharide content in all of five smooth strains of Bact. coli, but 
it was also high in five of eight intermediate strains, and in one of seven rough strains. Among the coli-typhoid group, indeed, the production of large ainounts of polysaccharide mueoid substance is sometimes associated with no change, or with a fall, in virulence. Mucoid variation is often favoured by growth at $25^{\circ} \mathrm{C}$. instead of $37^{\circ} \mathrm{C}$. (Birch-Hirschfeld 1935, Morgan and Beckwith 1939, vor dem Esche 1940b). Vor dem Esche (1940a) noted the appearance of mucoid variants in the stool of a woman who had been treated by inoculations with an autogenous vaceine during convalescence from paratyphoid infection.

In many of these instances the change from smoothness to ronghness is also associated with a complete or partial loss of virulence, a factor which is clearly of the first importance from the point of view of the medical bacteriologist.

Again (see Chapter 11) the $\mathrm{S} \rightarrow \mathrm{R}$ variation is usually associated with a change in sensitivity to the lytic action of various strains of bacteriophages, the filtrable viruses that propagate on, and at the expense of the bacterial cells.

Changes that are exactly similar have been described in Pasteurella (de Kruif 1921, Webster 1925), many other bacilli of the coli-typhoid group (White 1926), Staph. aureus (Bigger et al. 1927), and in a large number of other species (see, for instance, Hadley 1927). We may, indeed, regard it as a variation to which most if not all bacteria are subject.

The $\mathrm{S} \rightarrow \mathrm{R}$ variation affords an excellent example of a correlated variation. If we accepted the change in colony form as the essential criterion of the change from roughness to smoothness, we should note that the rough variant differed from the smooth form in the following characters:

(1) Roughness or granularity of colonies.

(2) Instability in saline.

(3) Loss of the antigenic component characterizing the surface of the bacterial cell in the normal smooth form, whether this component is normally present in the form of a definite capsule or not.

(4) Loss of virulence, partial or complete.

(5) Altered sensitivity to various bacteriophages.

In accepting such a list of correlated characters we are, however, met with the difficulty that the variations observed in certain bacterial species fail to fall into line.

For instance, the normal, virulent, capsulated form of the anthrax bacillus gives a flat, uneven, filamentous colony, while the avirulent, non-capsulated variant is raised, circular and smooth (Preisz 1904, 1911, Eisenberg 1912). 'T'he hæmolytic streptococci appear also to form au exception to the general rule. The colony given by the normal virulent form is finely granular, not smooth, and the avirulent variant, which has lost its type-specific antigen, is smoother, not rougher than the normal form. For this reason Todd (1928), who first gave a detailed description of these variants, used the term " matt" to describe the normal colony form, and "glossy" to describe that of the avirulent variant.

According to Dawson, Hobby and Olmstead (1938) the range of variation possible between highly virulent and non-virulent forms of streptococei is much wider than that indicated by Todd's matt $\longrightarrow$ glossy. They described in a number of hæmolytic streptococei four types of variants - a capsulated II variant, forming large, watery, mucoid colonies, an MS variant, forming colonies corresponding to Todd's " matt" variants, a non-capsulated S variant, corresponding to Todd's "glossy," and an R variant. With progressive loss of characters associated with virulence, the variation proceeds as follows :$\mathrm{M} \rightarrow \mathrm{MS} \rightarrow \mathrm{S} \rightarrow \mathrm{SR} \rightarrow \mathrm{RS} \rightarrow \mathrm{R}$, where SR and RS represent variants intermediate between $S$ and $R$ forms (see also Morison 1940, Seastone 1943). A similar series of variants 
occurs in other speeies. It has been deseribed, for exanple, in $\boldsymbol{H}$. tetragenus (Reimann 1937a) and in Hem. influenze (Chandler, l'othergill and Dingle 1939). In the influenza bacillus, the $\mathrm{H} \longrightarrow \mathrm{S} \rightarrow \mathrm{R}$ variation oceurred spontaneously ; the reversion $\mathrm{R} \rightarrow \mathrm{S}$, but not $\mathrm{S} \rightarrow \mathrm{M}$, conld be induced by serial passage through mice. There is also another colonial variant, found in a number of bacterial species, which does not fall into line with the $\mathrm{S} \rightarrow \mathrm{R}$, or the extended $\mathrm{Nl} \rightarrow \mathrm{S} \rightarrow \mathrm{R}$ scheme of variation (see Mlorton 1940). It is the D (dwarf eolony) variant described by Hadley $(1927,1937)$, and must be distinguished from his G (gonidial) variants, which give minute colonies that in some cases are said to contain filtrable elements capable of growing into the normal, modal forms of the parent bacteria. The D variant is characterized mainly by the small size of the colony, in comparison with the $S$ variant. The variations $S \rightarrow D$ and $D \rightarrow S$ have been observed in a number of species. In many cases, colony size is the only conspicuous feature distinguishing $\mathrm{D}$ from $\mathrm{S}$ variants. Thus, the D and $\mathrm{S}$ variants of a strain of Salm. typhi (Morris, Sellers and Brown 1941) and of a Group C streptococcus (Morton and Sommer 1944) differed little in virulence, or in biochemical and serological reactions.

Nevertheless, in general, there is in each of the species we have described a variation of the same essential character as those listed above-loss of normal, type-specific, surface antigen associated with loss of virulence. There are, however, variations within the $\mathrm{S}$ form, both natural, and induced by exposure to antisera or bacteriophage, which have no obvious connection with $\mathrm{S} \rightarrow \mathrm{R}$ variation by loss. For example, Takita (1937) records a change from "specific" antigens to "group" antigens in the Sh. flexneri group, analogous to the diphasic flagellar variation in the salmonellae; and Kauffman (1941) records a variation by loss of one of the sub-types of XII somatic antigen in certain strains of Salmonella.

There are other reasons also for rejecting the hypothesis of a necessary connection between virulence and the cultural and antigenic characters of smoothness. A loss of, or a considerable change in, virulence may occur apart from the loss of the specific antigenic component characterizing the normal smooth form.

Wilson (1928) observed variants of Salm. typhi-murium which were both smooth and avirulent, and which must be regarded as true variants, as judged by their failure to revert easily to the virulent parent type. Boivin (1939) found that two strains of Salm. typhi-murium, one with a mouse M.L.D. of over 10,000 bacilli, the other with an M.L.D. of under 200 , both yielded approximately the same amount of "smooth" lipopolysaccharicle endotoxin, and that the two endotoxins were equally toxic and had equal immunizing power. Thus virulence may vary independently of the $\mathrm{O}$ antigen. Again, Shaffer, Enders and $\mathrm{Wu}$ (1936) described two strains of Type III pneumocoeens, which were both fully capsulated, and antigenically identical. One was avirulent for rabbits, and had a greater tendency than the other, a virulent strain, to lose its capsule. The rough strains of both could be induced to revert to the smooth forms by growth in the presence of smooth killed cultures (see p. 305 below), and, whether the killed culture was of the original virulent or avirulent strain, both reverted to their original high or low virulence. The virulence, in fact, was not an expression of the degree of "smoothness" of the two strains, but dependent on some stable difference in physiological behaviour. Large changes in virulence can in fact be induced in a strain without altering its smooth characters. Thus, Hadley and Wetzel (1943), starting with a rough variant of an alpha-hæmolytic streptococcus, raised its virulence by serial passage through mice. The total increase in virulence during the transformation from rough to smooth was 140-fold; subsequent passage of the smooth form through mice raised its virulence 5,000-fold. It may be noted also that Bhatnagar (1940) and Jawetz and Meyer (1944) could detect no difference in the antigenic surface of virulent and avirulent strains of Past. pestis, though, as the latter authors suggest, in this case virulence may have been associated with an antigenic com. ponent that did not appear on the surface of the bacillus. 
The exact nature of the change in colony form, though it happened to give the conventional name to this particular kind of variation, is clearly of quite secondary importance. We may note as a point of interest that the avirulent, non-specific variants of B. anthracis and Str. pyogenes, which happen to give smoother colonies than the normal, virulent forms, have protein instead of polysaccharide components as their dominant surface antigens when in the normal virulent state.

We can, if we wish, use some term other than Smooth $\rightarrow$ Rough to denote these particular variations, but it seems undesirable to do so, since they belong, in all essentials, to the same category. Alternatively we could abandon "smooth" and "rough" altogether as descriptive terms, and select some new name to describe the loss of the normal surface antigen that is the essential factor concerned. It seems simpler, as we have suggested in Chapter 8 , to use the initial letters of the terms "smooth" and "rough" to designate the variation of which the first examples observed happened to be associated with smooth and rough colony formation, but to dissociate " $\mathrm{S}$ " and " $\mathrm{R}$ " from a clesignation of colony form. We should then define the P. variant as differing from the normal $\mathrm{S}$ form in the following ways :

(1) Loss of the antigenic component characterizing the surface of the bacterial cell in the normal smooth form, whether this component is normally present in the form of a bacterial capsule or not.

(2) Loss of virulence, partial or complete.

(3) Altered sensitivity to various bacteriophages.

(4) A change in colony form, usually, but not always, in the direction of increased granularity or roughness.

(5) A change in the hydrophobe or hydrophile properties of the cell, usually but not always in the direction of a decreased affinity for water, and a consequently increased sensitivity to the flocculating action of electrolytes.

It must not be supposed that the $S \rightarrow R$ variation represents the limit of the loss of particular antigenic components that bacterial variants may display. An excellent example of this progressive variation by loss is provided by the detailed studies which White has carried out on members of the typhoid-paratyphoid group of bacteria (see White $1926,1927,1928,1929 a, b, 1931 a, b, 1932,1933)$. The polysaccharide components that characterize the surface of the bacterial cell in the normal smooth form are shared by certain types, which are further differentiated from one another by the antigenic components contained in the flagella (see Table 47, p. 713). With these flagellar antigens we are not here concerned. When the normal smooth polysaccharide antigen is lost, the surface of the cell is dominated by antigenic components that are shared by all members of the typhoid-paratyphoid group, and by some related bacteria. These include a polysaccharide component that differs from that characterizing the normal smooth form, and another antigen, or pair of antigens, that are apparently protein in nature and have been named by White $\rho_{1}$ and $\rho_{2}$. As the result of further variation the rough or $\mathrm{R}$ form may lose its particular polysaccharide component and then give rise to a form, the antigenic behaviour of which is determined entirely by the components $\rho_{\mathbf{I}}$ and $\rho_{2}$. Situated still more deeply in the bacterial cell is another antigen, which White has named $\mathrm{T}$; but it seems doubtful whether this component is ever exposed at the cell surface as the result of lossvariation.

It may be noted that the $\mathrm{S} \rightarrow \mathrm{R}$ variation, and still more the progressive loss-variations referred to in the preceding paragraph, are relatively irreversible. 
The $\mathrm{S} \rightarrow \mathrm{R}$ variation occurs frequently under ordinary laboratory conditions of cultivation, and may be readily induced by the methods that we shall shortly describe. The reverse change $(R \rightarrow S)$ seldom, if ever, occurs under the ordinary conditions of cultivation and it is very difficult, though not impossible, to induce it by any specific stimulus, when the original $\mathrm{S} \rightarrow \mathrm{R}$ change has been complete. The evidence indicates that this $\mathrm{S} \rightarrow \mathrm{R}$ variation, at least as a quality of a culture as distinct from that of a component cell, is not a sudden "all-or-none" process, but a gradual or step-like change, so that intermediate SR forms appear between the typical $\mathrm{S}$ and the fully degraded $\mathrm{R}$. In these partially degraded SR variants reversion to the normal $\mathrm{S}$ form may be more easily induced.

Prolonged growth of a normal smooth strain in any of the ordinary fluid media of the laboratory, followed by plating on ordinary agar, will usually result in the appearance of a proportion of rough, or partially rough, colonies. A bacteriophage that causes lysis of the normal smooth strain provides another, and very potent, method by which this change can be induced (see Chapter 11). The contamination of a bacterial culture with a bacteriophage is not, however, an entirely desirable procedure for this particular purpose; and the best method available is that introduced by Griffith (1923), who showed in the particular case of the pneumococcus, that rough variants could readily be produced by growing the normal smooth form in the presence of an antiserum acting on the type-specific capsular polysaccharide. This method, the efficacy of which has been repeatedly confirmed by other workers, appears to be of quite general applicability.

The induction appears to act partly by selection. In a broth culture bacilli with a great deal of the antigen in question will be flocculated in a deposit by the antibody, and variants with none or less of it will tend to remain in suspension, so that a sample from the upper part of the fluid will contain a relatively high proportion of the variants. A method that can be used with motile flagellated bacteria is growth in a semi-solid agar medium containing flagellar artibodies; those bacteria that by reason of their motility spread from the original inoculum through the semi-solid agar will tend to be those with little of the homologous flagellar antigen. By this second method Gnosspelius (1939) induced flagellated variants in diphasic salmonella bacilli possessing antigens different from the original characteristic "type" or "group" antigens. The variation was irreversible, and though the new antigenic types retained their capacity for diphasic variation, it was found that only the phase which had undergone impressed variation had altered. Thus, the "new" group phase alternated with the "original " type phase, and a " new" type phase alternated with the "original" group phase. Eriksson and Malmström (1939) induced a similar variation in Salm. newport, and found that the "new "flagellar variant had acquired an antigen of the $\beta$ type; i.e., an $\alpha \rightarrow \beta$ variation (see p. 716) had been induced. These organisms were originally diphasic. Starting with the monophasic Salm. paratyphi A, Bruner and Edwards (1941) induced four variants, one in the original specific phase, one corresponding to the group phase of diphasic salmonellæ, and two new phases with antigenic components unlike any described for the salmonellæ.

It is clear that induced variations, which in this case revealed not only a potentiality for diphasic variation, but also two hitherto undiscovered antigens, offers a means of exploring hidden antigenic relationships within groups of bacteria.

As an example of a phage-induced variation, we may cite the production from typhoid bacilli with $\mathrm{Vi}, \mathrm{O}$ and $\mathrm{R}$ antigens, of variants which have either $\mathrm{Vi}$ and no $\mathrm{O}$ antigen, or $\mathrm{O}$ antigen without $\mathrm{Vi}$ (see, e.g., Kauffmann 1936, Craigie and Brandon 1936).

We are then, in the change from $\mathrm{S}$ to $\mathrm{R}$, dealing with a striking example of a variation, of a very definite type, that can be induced at will by certain specific 
stimuli. It seems probable that bacteria afford particularly favourable material for this field of biological study.

Although the colonial changes associated with the $S \rightarrow R$ variation have been described in particular detail, it must not be supposed that they constitute the only variations in colony form to which bacteria are subject. Such is far from the case. We have already noted D variants, and, in the coli-typhoid group of bacilli, the occurrence of mucoid variants that clearly do not conform with the $\mathrm{II}$ forms in the $\mathrm{M} \rightarrow \mathrm{S} \rightarrow \mathrm{R}$ series observed in pneumococci, streptococci and the influenza bacillus. This type of variation is not infrequently stimulated when a non-mucoid bacterium is submitted to the action of a bacteriophage to which it is sensitive. It seems likely that most variations associated with a change in colony form will be found to be associated also with a change in the antigenic components at the bacterial surface, but not necessarily with that particular change on which the $\mathrm{S} \rightarrow \mathrm{R}$ variation depends.

\section{The Transmutation of Antigenic Types.}

The loss of a specific antigenic component in the $\mathrm{S} \rightarrow \mathrm{R}$ variation and its reappearance when, as oceasionally happens, the rough variant again gives rise to the normal smooth form, naturally raises the question as to whether it is possible for a rough strain to acquire the power of synthesizing, not the specific antigen that characterized the smooth strain from which it was derived, but some different antigen that is characteristic of another serological type belonging to the same species. Is it possible, for instance, to transmute a smooth Type I pneumococcus, via the non-capsulated rough variant, into a smooth Type II or Type III pneumococcus? The problem is so important, in its biological interest and implications, that the evidence must be considered in some detail.

The pioneer experiments in this field were those of Griffith (1928). He injected mice subcutaneously with living cultures of rough avirulent pneumococci, mixed with large amounts of heat-killed smooth pneumococci belonging to the same or another type. From the animals so inoculated smooth virulent pneumococci were frequently recovered; not only was a rough strain induced to revert to the smooth type from which it was derived, but a rough variant from a Type II strain was changed to a smooth Type I strain, a rough variant from a Type I strain to a smooth Type II strain, rough variants of Type I or Type II strains to a smooth Type III strain, and so on.

These results were confirmed by Neufeld and Levinthal (1928), by Reimann (1929) and by Dawson $(1930 a, b)$. The study of this phenomenon was considerably advanced by the experiments of Dawson and Sia (1931), who were able to bring about a similar change in vitro by growing rough variants in a medium containing a heavy suspension of heat-killed smooth pneumococci of the type it was desired to produce. The addition of an anti-rough serum greatly assisted the transmutation, but was not an essential factor. In further experiments (Sia and Dawson 1931) it was found that the transmutation could not be induced by growing rough pneumococei in the presence of purified pneumococcal polysaccharide, and that heat-killed smooth pneumococci obtained from old autolysed cultures, or from suspensions that had been subjected to repeated freezing and thawing, were unsuitable for this purpose. These results clearly demonstrated that the presence of the polysaccharide antigen belonging to a given type could not induce a rough variant to manufacture that particular antigenic component, and pass on the capacity 
to synthesize it to subsequent generations, and they suggested that some enzyme, readily liberated from the pneumococcal cells, destroys some substance that is an essential stimulant of this change. Alloway (1932) was able to change rough variants of Type II pneumococci to smooth Type III or smooth Type I, by growing the former on Berkefeld filtrates of extracts derived from the latter, together with normal pig serum, which contains anti-R agglutinins. In further experiments (Alloway 1933) he substituted, for the Berkefeld filtrate, a preparation obtained by dissolving pneumococci of the required type in a solution of sodium desoxycholate, and precipitating the extract so obtained with alcohol. When rough pneumococci were grown in serum broth to which a saline extract of such a precipitate was added they gave rise to smooth strains of the type from which the extract was derived.

Recently, Avery, MacLeod and McCarty (1944), in defining both the conditions for effecting the transformation and in making a presumptive identification of the substance inducing the transformation, have made a noteworthy advance in the subject of bacterial variation. To effect the transformation, a reactive variant must be selected from an irreversibly rough (R) strain of Type II pneumococcus. It is grown in broth free from as yet unidentified inhibitors, containing $R$ antiserum free from enzymes that destroy the transforming principle, and the transforming substance itself. This substance, isolated from a Type III pneumococcus, was active in a dilution of $1: 6 \times 10^{11}$, appeared to be homogeneous, with a particle weight of 500,000, and had the gross chemical constitution, the exclusive susceptibility to a specific depolymerase, and certain physicochemical properties, of a desoxyribonucleic acid.

It appears that this particular type of nucleic acid interacts with the $\mathrm{R}$ cell to give rise to a series of enzyme reactions culminating in the synthesis of Type III capsular polysaccharide. Once the transformation is established, it is permanent, and the transformed cell continues to produce both polysaccharide and the specific desoxyribonucleate. We have in this phenomenon an outstanding example of an impressed genetic variation; i.e., a type-specific, heritable mutation induced by a specific chemical agent. The mechanism of its action is unknown, but we may provisionally ascribe it to a direct effect on a bacterial gene.

\section{Variations in Virulence or Toxigenicity.}

Variations in the characters on which bacteria depend for the production of disease in man and animals are clearly of particular importance to the medical bacteriologist. It would, however, be altogether impossible in the course of a general survey to give illustrative instances of the innumerable types of variation that are associated with some change in virulence, or in toxigenicity. We may, however, note a few general principles, leaving particular instances to be dealt with in the systematic description of the different bacterial species, or in the chapters devoted to the diseases to which they give rise.

Taking virulence to mean the capacity for tissue invasion, and toxigenicity to mean the power to produce a soluble toxin (see Chapter 44) we may note that these two characters depend on different factors, so that, where both are present in the same bacterial species, they may vary independently. A hæmolytic streptococcus, for instance, may lose its power of invading the tissues of a particular animal host without necessarily losing its power to produce a filtrable hæmolysin.

We have seen that $R \rightarrow S$ variation may be impressed on relatively rough strains of infective bacteria by passage through a susceptible animal. The varia- 
tion is nsually considered to be the result of selection of a few virulent organisns, which are either present in, or develop from, the injected culture.

Zelle (1942), working with a strain of Salm. typhi-murium, has recently provided evidence of diseontinuous variations of $S$ and $R$ forms, and their selection by the environment in the infected host. For example, an unstable S variant was shown, by a micro. technique of separating cells as they divided in vitro, to throw stable $\mathbf{R}$ mutants. In one experiment, the division of an $\mathrm{S}$ cell, the unstable variant, into an $\mathrm{S}$ and an $\mathrm{R}$ danghter cell, was observed directly. The $\mathbf{R}$ mutation was observed twice in 296 divisions, a relatively high mutation rate which conforms to the hypothesis already noted, that instability is a manifestation of high mutation rates of the order of 1 per cent. Hixtures of small numbers of stable virulent variants with large numbers of less virulent stable variants were injected into mice, the organs of which after death yielded a culture with an increased proportion of the virulent variants. In other tests, virulent and avirulent variants were injected into inbred strains of susceptible and highly resistant mice. It was hoped to demonstrate a differential effect of resistant and susceptible host environments on the variants, such as a reduction in virulence of highly virulent strains propagated in the presumably less selective tissues of the susceptible mice. The only change observed, however, was an enhancement of virulence of certain variants, and this oceurred both in susceptible and in resistant mice, showing that whatever the general susceptibility of the mice, any- less virulent mutant, thrown by an injected strain of given virulence, was always more readily destroyed than the bacteria from which it arose.

Non-toxigenic strains of $C$. diphtheria, $C l$. tetani and other normally toxigenic species have been frequently described. There is no reason to suppose that this loss of toxigenicity is in any way related to the $S \rightarrow R$ variations, since there is no evidence that the production of a filtrable toxin is affected by the presence or absence of the smooth somatic antigen.

\section{REFERENCES}

Alloway, J. I. (1932) J. exp. Med., 55, 91; (1933) Ibid., 5\%, 265.

Arkwright, J. A. (1920) J. Path. Bact., 23, 358; (1921) Ibid., 24, 36; (1924) Brit. J. exp. Path., 5, 23.

Avery, O. T. and Heidelberger, M. (1923) J.exp. Med., 38, 81.

Avery, O. T., Macleon, C. M., and McCarty, M. (1944) J. exp. Med., 79, 137.

BADGER, E. (1944) J. Bract., 4\%, 509.

Baerthlein. (1912a) Zbl. Bakt., 66, 21 ; (1912b) Ibid., Ref., 54, 178.

Barber. (1907) Kansas Univ. Sci. Bull., 4, No. 3, 1.

BENECKE, W. (1909) Z. indukt. Abstamm.-u. VererbLehre, 2, 215.

Bhatnagar, S. S. (1940) Indien J. med. Res., 28, 1, 17.

Bigger, J. W., Boland, C. R., and O'Meara, R. A. Q. (1927) J. Path. Bact., 30, 261.

Birch-Hirscifeld, L. (1935) Z. Hyg. InfektKr., 11\%, 626.

Bolvin, A. (1939) C. R. Soc. Biol., 132, 370.

Borvin, A. and Ilesrobeanu, L. (1936) C. R. Acad. Sci., 203, 1402.

Boivin, A., Mesrobeanu, L., Magheru, G., and Magheru, A. (1936) C. R. Soc. Biol., 121. 169.

Bordet, J. and Renaux, E. (1930) Ann. Inst. Pasteur, 45, 1.

Bordet, P. (1930) Ann. Inst. Pasteur, 45, 26.

Braun, H. (1918) Berl. klin. Wschr., 55, 637.

Bruner, D. II. and Edwards, P. R. (1941) J. Bact., 42, 467.

Burk, -A. (1908) Arch. Hyg., 65, 325.

Burri, R. (1910) Zbl. Bakt., IIte Abt., 28, 321.

Chandeler, C. A., Fotmergill, L. D., and Dingle, J. H. (1937) J. exp. Med., 66, 789 (1939) J. Bact., 3\%, 415.

Craigie, J. and Brandon, K. F. (1936) J. Path. Bact., 43, 233, 249.

Dawson, J. and Happold, F. C. (1943) Biochem. J., 3\%, 389.

Dawson, M. H. (1930a) J. exp. Med., 51, 99 ; (1930L) Ibid., 51, 123.

Dawson, M. H., Новву, G. L., and Ormstead, M. (1938) J. infect. Dis., 62, 138. 
Dawson, M. H. and Sia, P. H. P. (1931) J. exp. Med., 54, 681.

Deskowitz, M. W. (1937) J. Bact., 33, 349.

DoBell, C. (1913) J. Genetics, 2, 325.

DoudorofF, H. (1940) J. gen. Physiol., 23, 585.

Dubos, R. (1940) Bact. Rev., 4, 1.

Dubos, R. and Avery, O. T. (1931) J.exp. Med., 54, 51.

Eisenberg, P. (1912) Zbl. Bakt., 63, 305.

Eriksson, E. and Maliström, F. (1939) Z. Hyg. InfektKr., 121, 629.

Esche, P. vor DEM. (1940a) Z. ImmunForsch., 98, 75; (1940b) Itid., 98, 333.

Euler, H. and Cramêr, H. (1913) Hoppe-Seyl. Z., 88, 430.

Evans, W. C., Handley, W. R. C., and Happold, F. C. (1940) Biochem. J., 35, 207.

GALE, E. F. (1940) Biochem. J., 34, 392, S46, S53; (1941) Ibid., 35, 66.

GALE, E. F. and EPPS, H. M. R. (1942) Biochem. J., 36, 600.

Gnosspelius, A. (1939) Z. Ilyg. Infekthr., 121, 529.

Goodian, H. M. (1908) J. infect. Dis., 5, 421.

Griffith, F. (1923) Min. Hlth., Rep. publ.Hlth. med. Subj., 18 ; (1928) J.Hyg., Camb., 27, 113.

Hadley, P. (1927) J. infect. Dis., 40, 1; (1937) Ibid., 60, 129.

Hadley, 1'. and Wetzel, V. (1943) J. Bact., 45, 529.

Hegarty, C. P. (1939) J. Bact., 3\%, 145.

Heyningen, IV. E. vaN. (1940) Biochem. J., 34, 1540

Hirsch, W. (1937) J. Path. Bact., 44, 349.

JАCOBY, M. (1916) Biochem. Z., 7\%, 124, 402, 405; (1917) Ibid., 79, $35 ; 80,357 ; 81$, $332 ; 83,74 ; 84,358 ;$ (1918) Ibid., 86, 329; 88, 35.

JAWETZ, E. and MEYER, K. F. (1943) J. infect. Dis., 73, 124.

Karström, H. von. (1930) see StePHenson and Stickland (1932) ; (1937) Ergebn. Enzymforsch., $\%, 350$.

Kaufflann, F. (1936) Z. Hyg. InfehtKr., 119, 103; (1941) J. Bact., 41, 127.

Kocholaty, W. and Hoogerheide, J. C. (1938) Biochem. J., 32, 437.

Косноlaty, IV. and Weil, L. (1938) Biochem. J., 32, 1696.

Koser, S. A. and Wright, M. H. (1943) J. Bact., 46, 239.

Kowalenko, A. (1910) Z. Hyg. InfektKr., 66, 277.

KRUIF, P. DE. (1921) J. exp. Med., 33, 773.

Lankford, C. E., Scott, V., Cox, M. F., and Cooke, W. R. (1943) J. Bact., 45, 321. Leonian, L. H. and Lilly, V. G. (1942) Science, 95, 658; (1943) J. Bact., 45, 329.

LEwIS, I. M. (1934) J. Bact., 28, 619.

Lindegren, C. C. (1935) Zbl. Bakt., II te Abt., 92, 40; 93, 113.

Massini, R. (1907) Arch. Hyg., 61, 250.

Miles, A A. and Pirie, N. W. (1939) Brit. J. exp. Path., 20, 83.

Morgan, H. R. and Beckwith, T. D. (1939) J. infect. Dis., 65, 113.

Morison, J. E. (1940) J. Path. Bact., 51, 401.

Morris, J. F., Sellers, T. F., and Brown, A. W. (1914) J. infect. Dis., 68, 117.

Mortox, H. E. (1940) Bact. Rev., 4, 177.

Mortoy, H. E. and Sommer, H. E. (1944) J. Immunol., 48, 123.

Müller, P. (1908) Zbl. Bakt. Ref., 42, Beitr., 57 ; (1911) Zbl. Bakt., 58, 97.

Neufeld, F. and Levinthal, W. (1928) Z. ImmunForsch., 55, 324.

Nutт, M. M. (1927) J. Hyg., Camb., 26, 44.

Pappenheimer, A. M. and Shaskan, E. (1944) J. biol. Chem., 155, 265.

Passmure, R. and Yudkin, J. (1937) Biochem.J., 31, 318.

Pasteur, L. (1881a) C. R. Acad. Sci., 92, 429; (1881b) Ibid., 92, 666.

Penfold, W. J. (1910a) Brit. med. J., ii. 1672; (1910b) J. Path. Bact., 14, $406 ;(1911 a)$ J.Hyg., Camb., 11, 30 ; (1911b) Proc. roy. Soc. Med., 4 (Path. Sec.), 97 ; (1911c) J. IIyg., Camb., 11, 487 ; (1912) Ibid., 12, 195.

Preisz, H. (1904)'ZZbl. Bakt., 35, 280, 416, 537, 657; (1911) Ibid., 58, 510.

Priestley, F. W. (1936) J. comp. Path., 49, 348; Brit. J. exp. Path., 17, 374.

Quastel, J. H. (1937) Enzymologia, 2, 37.

Reimann, H. A. (1925) J. exp. Med., 41, 587; (1929) Ibid., 49, 237; (1937a) J. Bact., 33, 499 ; (1937b) Ibid., 33, 513.

RetTger, L. J., and Sherrick, J. I. (1911) J. med. Res., 24, 265.

Revis, C. (1911) Zbl. Bakt., IIte Abt., 31, 1 ; (1912) Proc. roy. Soc., B, 85, 192.

Roux, E. (1890) Ann. Inst. Pasteur, 4, 25.

Seastone, C. V. (1943) J. exp. Med., ryy, 21.

Sertic, V. and Boulgakov, N. A. (1936) C. R. Soc. Biol., 123, 951; (1937) Ibid., 124, 217.

Shaffer, M. F., Enders, J. F., and IVU, C.-J. (1936) J.exp. Med., 64, 281.

Sia, R. H. P. and Dawson, M. H. (1931) J. exp. Med., 54, 701.

Silverhan, M. and Werkman, C. H. (1939) J. Buct., 38, 25 ; (1941) J. biol. Chem., $138,35$.

Solotorovsky, M. and Buchbinder, I. (1941) J. Immunol., 40, 243. 
Stephenson, 11. and Stickland, L. H. (1932) Biochem. J., 26, 712; (1933) Ibid., 27 1528.

Stephenson, M. and Yudhin, J. (1936) Biochem. J., 30, 506.

ТАКітA, J. (1937) J. Hyg., Camb., 3\%, 271.

Tillett, W. S., Goebel, W. F., and Avery, O. T. (1930) J. exp. Med., 52, 895.

Tond, E. W. (1928) Brit. J.exp. Path., 9, 1.

Twort, F. IV. (1907) Proc, roy. Soc., B, 79, 329.

Webster, L. T. (1925) J. exp. Hed., 41, 571.

Wнiте, P. B. (1926) Spec. Rep. Ser. med. Res. Coun., Lond., No. 103 ; (1927) J. Path. Bact.

30,113; (1928) Ibid., 31, 423; (1929a) Ibid., 32, 85 ; (1929b) Med. Res. Coun. "System of Bacteriology," 4, 86; (1931a) J. Path. Bact., 34, 23; (1931b) Ibid., 34, 325; (1932), Ibid., 35, 77; (1933) Ibid., 36, 65.

Wilson, G. S. (1928) J. Hyg., Camb., 28, 295; (1930) Ibid., 30, 40.

Wortuans, .T. (1882) Hoppe-Sfyl. Z., 6, 287.

Woon, H. G., Andersen, A. A., and Werkman, C. H. (1938) J. Buct., 36, 201.

Tudkin, .J. (1932) Biochem. J., 26, 1859: (1938) Biol. Ree., 13, 93.

ZELLE, M. R. (1942) J. infect. Dis., 71, 131. 


\section{CHAP'TER 10}

\section{'THE CLASSIFICATION OF BACTERIA}

As has been indicated in preceding chapters, it is the behaviour rather than the nature of bacteria which has interested the bacteriologist. It is not surprising, therefore, to find that the field of systematic bacteriology has been very largely neglected. The study of bacteria has indeed never passed through that phase of detailed and accurate description, which has formed so important a part of the foundations of botany and zoology.

This neglect is not entirely attributable to lack of interest. In dealing with the morphology of bacteria, we have pointed out the difficulties which are inherent in any study of bacterial structure. As a result of these difficulties, the bacteriologist has come to rely very largely on physiological characters in the differentiation of bacterial groups, and the study of the antigen-antibody reactions has led to the elaboration of a technique which is peculiar to this field of biology. In addition to these methods of studying bacteria in artificial culture, the medical bacteriologist, who is primarily interested in the rôle of micro-organisms in disease, has naturally developed the habit of testing the pathogenicity of the strains he has isolated by the experimental infection of laboratory animals.

Employing a combination of these methods, the bacteriologist has learned by experience to identify a large number of well-differentiated and stable bacterial types; and to these he has given names. The criteria that have determined the classification and nomenclature of bacteria are not, therefore, such as would be accepted by the systematist in any other branch of biology; and the bacteriologist himself has not in general troubled overmuch as to the validity of a system which has developed rather as the result of luck than of cunning.

The inconvenience of a total absence of classification, reflected in a chaotic nomenclature, has, however, been so great, that various attempts have been made to introduce some sort of order into the bacteriological household. We cannot here enter into any historical description of the various systems which have been propounded; except to note that a comparison of those suggested by Zopf (1885), Migula (1894), Kruse (1896), Lehmann and Neumann (1896) and Orla-Jensen (1909) will reveal how widely the lines of cleavage may differ, when a large biological group is viewed from different angles. Those who desire more detailed information on this aspect of the question are referred to the two reports of the Committee of the Society of American Bacteriologists on characterization and classification of bacterial types $(1917,1920)$; the monograph by Buchanan (1925) ; the manual by Bergey and his colleagues (1939) ; and to a paper by Buchanan and others (1928), which sets out in diagrammatic form the classifications suggested by Migula, Orla-Jensen, Buchanan, Castellani and Chalmers (1920), Lehmann 
and Neumann, Bergey and his colleagues, and the earlier Committee of the Society of American Bacteriologists which reported in 1917 and 1920.

As a result of the activities of the American Society, the whole question of bacteriological classification and nomenclature has been reopened during recent years. It cannot be said that the system propounded by the American Committee (1920) has met with the entire approval of bacteriologists in general ; while systematists in other biological sciences would probably question the validity of the whole basis upon which the classification is founded. There does not, however, appear to be any compelling reason for the bacteriologist to abandon, for purposes of classification, the criteria on which he has come to rely for purposes of identification; and few of us would be willing to admit that our systematic grouping must have a purely morphological basis, simply because structural differences have been found to afford adequate classificatory criteria in the case of more highly differentiated plants and animals. While admitting that morphological differences must be given their full weight, and accepting them as the natural basis for our primary subdivisions, we might argue that our differential criteria, depending as they do on differences in chemical structure rather than on the gross architecture of the cell, come nearer to the heart of the matter than do those adopted by botanists or zoologists.

We may note that our assessment of the significance of any particular differential criterion rests largely on a statistical basis. Our first concern is to determine the variability of a given character within a particular bacterial strain. If it is constant, it may be of value for purposes of classification. If it varies, but in such a way that the variation is itself characteristic, it may still have classificatory value. If it varies in an entirely random and unpredictable fashion, it cannot be used for purposes of identification or classification. Once it has been shown that a given character is of service in identifying a particular strain, we can examine the distribution of this character among a sample of strains which possess other characters in common. In this way we gradually obtain a picture of the frequency distribution of many different characters among large samples of strains. The significance we attach to any particular character then depends in the main on its association with other characters. If we find that a particular group of strains resemble each other in several different characters, and differ in these same characters from ali other groups, we feel justified in regarding the group as biologically valid and in attaching an added significance to each of the associated characters, as a differential criterion within the larger group of which our homogeneous group forms a part. If, on the other hand, one particular character varies independently of all other characters, within a group which has many other characters in common, we shall not in general attach the same significance to it, from the point of view of classification. In assessing characters in this way, we shall not of course accord all characters equal rank, a priori, and limit our consideration entirely to their frequency distribution and degree of association. Some characters will be given more weight than others, and our arrangement of characters in descending order of importance will depend entirely on the function we envisage for our classification of bacteria. The classification may be predominantly utilitarian. For example, as medical bacteriologists, we might concentrate on the outstanding features of the medically important bacteria, and ignore all other bacterial species, excepting those which are sufficiently like the medically important species to cause trouble in identification. This is, in fact, the working procedure 
in applied medical bacteriology, but it is not a sound basis for a classification of bacteria in general. A classification intended to accommodate the species familiar to animal, plant, industrial, biochemical and "pure" bacteriologists should depend mainly upon characters whose selection is securely based on agreed general principles. Its design should be consistent in that, should a species be discovered with a hitherto unknown combination of differential characters, it could be accommodated in the system without dislocating it. Clearly, any working classification will be a compromise between the utilitarian and the "logical " classification based on a priori conceptions of the relations of bacterial species.

The most promising a priori conception upon which to base a classification, and one which has proved fruitful in many other branches of biology, is the conreption of species in a phylogenetic series. We have already discussed in Chapter 3 the relation of nutritional requirements to the possible evolution of bacterial species, and some of the dangers of a too-ready acceptance of the phylogenetic hypothesis. Our chief objection to the phylogenetic conception of the nutritional series was the impossibility of deciding which end of the series-organisms with the most complex or organisms with the least complex nutritional requirements-represented the starting point. With morphological characters there is perhaps less difficulty, for the spherical shape being the simplest and the most economical shape that could be taken by a unicellular organism (see Thompson 1942) may with some justification be taken as the primitive type. From this primitive coccus we can assume developments in the direction of aggregates of cocci, of bacteria, and of thread and mycelial forms, and in some members of each group postulate the acquisition of flagella, capsules, and other of the more striking morphological features of bacteria.

In their valuable review of bacterial classification, Kluyver and van Niel (1936) in fact make this assumption the starting point of their proposed natural system of classification. Each of the main morphological groups springing from the cocci is subdivided according firstly to the main sources of energy of the bacteria, and secondly according to the most favoured substrates and their modes of dissimilation. Thus, there are the photosynthesizing autotrophs and heterotrophs, and the chemosynthesizing autotrophs and heterotrophs, four groups that are further divided according to their most favoured modes of dissimilation.

In choosing these modes, Kluyver and van Niel point out that the type of attack is more important than the range of attack. Thus, the difference between an organism which splits glucose into lactic acid, and one which splits it into butyric and acetic acid, $\mathrm{CO}_{2}$ and hydrogen, is more fundamental than the difference between two organisms of the first type, one of which attacks maltose.

The principles have been further developed by Stanier and van Niel (1941), who propose the term Monera to cover all micro-organisms without true nuclei, plastids and sexual reproduction. Fig. 46 summarizes the proposed arrangement of llonera. The reader is referred to the original paper for details of their system, which may be compared with that set out on p. 319.

In our preoccupation with bacteria, parasitic on the larger animals, we are, however, concerned with distinctions that as yet do not fall into the province of the morphologist and biochemist, and while agreeing with the criteria governing the subdivisions that can be achieved in this province, we shall not find them particularly helpful with characters like pathogenicity or antigenic structure. Both are expressions of certain biochemical features in the organism, and may 
ultimately fall into line with better studied features of bacterial economy, but until then we have no a priori grounds for evaluating the biological importance in elassification of antigenic structure, or, say, the power of producing a characteristic toxin.

A. Organisms photosynthetic with the evolution of oxygen and possessing the typical green plant chlorophylls, phycocyanin and sometimes phycoerythrin, and colourless, non-photosynthetic counterpart, clearly recognizable as such.

Division I. Myxophyta.

B. Organisms not so characterized.

1. Unicellular or mycelial organisms with rigid cell walls. Motility, when present, by means of flagella. Entospores, eysts, or conirlia may be formed.

Class 1. Eubacteriæ.

(a) Organisms photosyuthetic, but not producing oxygen.

Order 1. Rhodobacteriales.

(b) Non-photosynthetic organisms.

1. Unicellular.

Order 2. Eubacteriales.

2. Mycelial organisms.

Order 3. Actinomycetales.

II. Unicellular rod-shaped organisms, without rigid cell walls. Always crecping motility. Hicrocysts and fruiting bodies may be formed.

Class 2. Myxobacteriæ.

One order. Myxobacteriales.

III. Unicellular, spiral organisms withont rigid cell walls. Motility by means of an elastic axial filament or modified fibrillar membrane.

Class 3. Spirochretæ.

One order. Spirochætales.

IV. Organisms not falling into the previous classes.

FIG. 46.

Our main trouble is that we have no rules, and the few conventions which take their place are honoured as much in the breach as in the observance. It seems quite clear that nothing but some form of international agreement with regard to classification and nomenclature will put an end to the existing state of chaos. Whether it will be possible to adopt, in their entirety, the rules of botanical nomenclature, is a problem which only the future can decide. There are obvious advantages in adopting the Linnæan binomial nomenclature, which has served the purposes of zoologists and botanists in general; but it is doubtful whether the bacteriologist will not be forced to make frequent use of additional terms, designating races, varieties, or types. The frequent use of trinomial or quadrinomial names is, however, a cumbersome procedure ; and it may be found necessary to regularize the use of letters or numbers, which is a current convention in bacteriological terminology. The Linnæan admonition, "varietates levissimas non curat botanicus," may serve the turn of the systematic botanist, and the bacteriologist would probably be well advised to bow to it in naming those groups 
which he intends to regard as genera or species; but he cannot ignore small differences, and he needs a vocabulary which will allow him to talk or write about the bacterial types which interest him.

This leads to the consideration of another difficulty, which has grown acute during recent years, and will clearly increase rather than diminish in the absence of some agreed international ruling. There is no agreement at all as to the end from which a bacteriological classification should start. Are we to begin by an intensive study of one or another relatively small group, seeking to differentiate within it all the identifiable and stable types, and giving names to these? Or are we first to differentiate the larger groups, and only when these have been adequately demarcated seek to divide them into their constituent species, varieties or types ? As a method of mapping out the ground either approach will serve; but they lead, unfortunately, to quite incompatible nomenclatures. The method of the intensive study of a small, or relatively small, bacterial group has been adopted by several groups of workers within recent years, and has resulted in such conspicuously successful systematic descriptions as those of the Salmonella group (see Chapter $30)$, or of the hæmolytic streptococci and the pneumococci (see Chapter 25). In each of these instances the final differentiation has depended, entirely or almost entirely, on an analysis of antigenic structure. The workers who have been engaged in the study of the Salmonella group have, however, adopted, at the extreme end of the scale, differentiable types listed as Salmonella typhi, Salmonella dublin, Salmonella eastbourne, and so on. Those who have studied the pneumococci and hæmolytic streptococci have, we think more wisely, labelled their recognizable types with numbers, or letters, or letters and numbers combined; though, except in the case of the pneumococci, there is as yet no general agreement as to the lettering or the numbering.

We do not, ourselves, think that the agreed definition of species and genera, which has still to be achieved, should be prejudged by the results obtained by antigenic analysis, particularly in the light of recent work on the sharing of antigens among species generally regarded as distinct (see Chapter 8). In the coli-typhoiddysentery group, however, there are major distinctions, both antigenic and otherwise, which serve to divide the salmonella and the dysentery bacilli from the colon group of organisms in the genus Bacterium, and in the light of the growing number, we have accordingly assigned the enteric and food-poisoning bacilli to the genus Salmonella and the dysentery to the genus Shigella.

While remaining convinced that, in naming genera and species, weight should be given to other criteria in addition to antigenic relationships, we should wish to record our entire agreement with those who, like White (1937), hold that antigenic analysis affords the best available method of differentiating the ultimate types or varieties into which bacteria are divided, that the antigenic similarities and differences provide a most valuable clue to the natural relationships of these types and the lines along which they have probably been evolved, and that each type or variety so differentiated should be given a distinctive label. We may perhaps add that this distinctive labelling is of particular importance to the medical bacteriologist, since it is the antigenic make-up of a bacterium that determines all its immunological reactions, in the body as well as in the test-tube. We must, however, try to be consistent. We ought not to use letters and numbers for one set of labels, specific names for another, though current bacteriological usage compels us to do both. 
Apart from the increasing importance of antigenic analysis in the classification of bacteria, it may be noted that there is a growing tendency to enlarge the range of criteria employed in the differentiation of types and species, and to rely less exclusively on the somewhat crude series of fermentation reactions that played so large a part in earlier systematic studies. The reaction of an organism to variations in the partial pressure of carbon dioxide, its resistance to various dyes, its tolerance of a high concentration of hydrogen-ions, all these and many other criteria are being increasingly employed in defining bacterial groups, and in tracing the relation of one group to another.

There is one criterion commonly employed whose use, we believe, should be discontinued, from both the formal and the utilitarian standpoint, namely, the ecological. The relationship of an organism in its natural state to other forms of life in its environment is conditioner by many other factors besides those inherent in its own protoplasm; and in the absence of knowledge about those factors, we cannot say what features of the habitat of an organism are necessarily connected with it. Consequently, habitat is on a priori grounds likely to be misleading as a differential character. It will be even more misleading on utilitarian grounds, since we classify bacteria in order to make precise bacteriological explorations of our enviromment. If, then, the definition of a species includes habitat in a given type of environment, we may delay its recognition in another equally important environment. It is, for example, only recently that the probable identity of Phytomonas polycolor, a tobacco-plant pathogen, and Ps. pyocyanea, an organism infecting wounds in war, has been recognized (Elrod and Braun 1941).

One essential character of any systematic nomenclature is stability; and those who have to read or write about bacteria at the present time are in a singularly unhappy position in this respect. When the same organism is masquerading as Bacillus typhosus, Bacterium typhosum, Salmonella typhi or Eberthella typhi, while another answers with equal readiness to the names of Micrococcus melitensis, Bacillus melitensis, Brucellı melitensis or Alkaligenes melitensis, all printed in italics with a capital letter to the generic name, the student, or even the more practised reader of bacteriological literature, may be excused some degree of confusion.

It would, perhaps, be simplest to await some agreed solution of our difficulties, and use the moribund nomenclature which was current before the first world war, till some better system with authoritative support is offered in its stead. There are, however, real disadvantages in such a course. It is desirable, especially from the student's point of view, that a name should be as informative as possible. The scientific name of a living organism should tell us as much as possible about that organism itself, and about its relation to other organisms with different names. The latter problem is the particular concern of the systematist; and it may be many years before we know enough about the relationship of bacteria to evolve a system of classification in which those relationships can be adequately expressed. It is, however, possible to allot names to bacterial groups, which will give us a considerable amount of information with regard to the species, races, or types, of which they are constituted. In this respect the conventional bacteriological nomenclature of the past fifty years has been a conspicuous failure. Nothing could be less informative than the name Bacilius, when that name is applied to any rod-shaped bacterium; and the student, who has memorized the 
names $B$. typhosus, B. pestis, B. anthracis, and B. tuberculosis, has obtained very poor value for his effort. If, adopting a more rational nomenclature, he memorizes the names Salmonella typhi, Pasteurella pestis, Bacillus anthracis, and Mycobacterium tuberculosis, he will, when he has studied the groups concerned, have a very useful picture of each of these organisms as typifying a separate genus; and the fact that some other organism is called Salmonella enteritidis, or Pasteurella aviseptica, or Bacillus subtilis, or Mycobacterium phlei, will convey to him some knowledge of its salient characteristics.

We are, ourselves, convinced that the correct approach to bacteriology, irrespective of the particular field in which the student intends ultimately to work, is to gain some knowledge of bacteria as living things; and such knowledge can most easily be obtained by grouping like forms together for the purposes of study, comparing them with other groups, and noting the differences and resemblances. For these groups we need names, even if they must, for the moment, be regarded as provisional.

This requirement can be fulfilled by adopting one of the several systems of classification and nomenclature that have been advocated within recent years. This is quite definitely a policy adopted faute de mieux. None of these systems has received any official or international sanction. As Buchanan, Breed and Rettger (1928) point out, neither of the systems drawn up by committees appointed by the Society of American Bacteriologists has been officially approved by that Society. The selection of one of the existing systems therefore remains a matter of personal choice; and, whichever system is selected, there is no reason to suppose that it, or the nomenclature based upon it, will receive international sanction without modification.

The terms "genus" and "species," as applied to bacteria, seem to us to defy definition, except as designations for two convenient groupings, of which the genus is the larger including group, and the species the smaller included group. For this reason, and because of the absence of any form of international agreement, we doubt the usefulness, at the present time, of naming orders, families, sub-families, and tribes. Nor do we feel that the time is ripe for the creation of large numbers of genera, or for the erection of an inelastic system into which all known varieties of bacteria are to be forced, each with its appropriate label.

It appears to us that the classification advocated in the final report of the first American Committee (1920) offers a carefully constructed scheme on which a useful nomenclature can be based, and that it has been designed on general lines which most bacteriologists would regard as sound; moreover, the Committee themselves make no claim to finality, and are at pains to indicate the tentative nature of some of the groupings they suggest.

We have therefore adopted the system of nomenclature set out in the final report of the first American Committee (Winslow et al. 1920), with a few minor modifications. We have merged the genus Diplococcus in the genus Streptococcus, since it appears to us that the pneumococcus should be included in the latter group. We have adopted the genus Brucella, which has already received unofficial recognition from many bacteriologists; since the group which contains the bacillus of Malta fever, and the bacillus of bovine abortion, appears to have as good a title to generic rank as the group which contains the plague bacillus and the bacilli causing hæmorrhagic septicæmia in animals, though we frankly admit 
that the content of the Brucella group is extremely difficult to define. As mentioned already, we have separated the genera Salmonella and Shigella from the more inclusive genus Bacterium. We have combined the genera Erythrobacillus and Chromobacterium under the latter name, since it does not appear to us that the differences in morphology, physiological reactions or habitat between Erythro. prodigiosus and such species as $\mathrm{Chr}$. indicum ruber merit separate generic rank. The final classification of these small saprophytic, chromogenic bacilli must await a more detailed study of the group; and it seems doubtful whether pigment formation should be accepted as a generic character. We have excluded the genus Erwinia, since the differentiation of coliform organisms of plant origin from those met with in animal tissues seems to rest on no satisfactory basis.

We append to this chapter a diagrammatic representation (Fig. 47) of the classification given in the final report of the first American Committee (see Buchanan et al. 1928) with the modifications referred to above, noting that we are concerned with the list of genera, rather than with the grouping of these genera into tribes, families or orders.

We also include in this chapter a summarized description of the characters of each genus, taken from the final report of the first American Committee (Winslow et al. 1920), and emended in some cases in the light of more recent studies of the various groups.

In the remainder of this book we shall employ these generic names, when referring to any species which appears to be clearly assignable to one of the listed genera. In general, bacteria which can be so assigned are already provided with a specific name, which is not in dispute. Such binomial names will be printed in italic, the generic name being given a capital letter, and used in an abbreviated form.

Other well-recognized designations for various bacteria will, of course, be freely used, with the recognition that we are using the common name for a particular organism, instead of its scientific name-a practice universally followed in biological science. Such common names will be printed in ordinary type, and without a capital initial letter. For instance, the scientific name of the organism which causes tuberculosis will be written as : Mycobacterium tuberculosis, or more shortly as Myco. tuberculosis, but it will be generally referred to as the tubercle bacillus; similarly with Corynebacterium diphtheria, $C$. diphtheria, or the diphtheria bacillus; Pasteurella pestis, Past. pestis and the plague bacillus; Brucella abortus, Br. abortus or the bacillus of bovine abortion; Neisseria gonorrhoce, $N$. gonorrhoee or the gonococcus: Streptococcus pneumonice, Str. pneumonice or the pneumococcus.

It is a common practice to refer to members of the Streptococcus and Staphylococcus groups as streptococei and staphylococci respectively. These terms are colloquial expressions, and we see no reason why a similar use of other generic names in the plural, or of a limited adjectival use of the generic name in the singular, should not be sanctioned. Thus, "the rickettsiæ" refers to the members of the genus Rickettsia, "the brucellæ" to the members of the genus Brucella, "the pasteurellæ " to the members of the genus Pasteurella, and so forth. A salmonella antigen means an antigen met with in one or more members of the Salmonella genus, a proteus type of growth means a growth characteristic of members of the Proteus genus, and so on; but when the genus is specifically referred to, the adjective should preferably be printed with a capital and in italic, as, for instance, " members of the Salmonella group." The procedure is convenient, the terms 
employed in most cases euphonious, and the meaning with but two exceptions is unambiguous.

The two exceptions are the terms "bacillus" and "bacterium." The term bacillus means any rod-shaped organism, and the term bacterium means any organism whatever in the general class of Schizomycetes. Hence, neither of them is available for designating members of the two well-defined genera, Bacillus and Bacterium. In these cases we have retained the customary circumlocutions, "members of the Bacillus group" and " members of the Bacterium group."

There remain a considerable number of bacteria, which cannot yet be accorded a scientific name: in some cases because the available descriptions are not sufficiently detailed to allow us to determine their systematic relationships; in others, because the characters, as described, do not seem to warrant the inclusion of the organism in any of the recognized genera. No useful purpose would be served by suggesting new generic names, which would have no validity. As we have emphasized above, any system of nomenclature employed at the present time must be a temporary expedient, pending some form of international agreement, and it appears to us that our aim should be to use those names which seem most likely to be retained when such agreement is reached. When dealing with those organisms which, at present, defy classification, we have therefore frankly abandoned the use of a scientific name, with its conventional italic and capital letter, and have employed the most convenient designation available. It is unfortunate that many of these organisms have been given the generic name of Bacillus. As this name is reserved for the spore-bearing aerobes, it cannot be used, in the conventional form, for bacteria which do not in fact belong to that genus. We have, in general, adopted the expedient of referring to Bacillus $x$ as " the $\mathrm{x}$ bacillus." In describing bacteria whose title to specific rank appears to us doubtful, though their generic position is not in doubt, we have in some cases employed a similar convention.

We would here add a protest against the habit, which is unfortunately frequent among medical bacteriologists, of coining new names for bacterial strains which they have isolated, without appending an adequate description of the organism or determining whether the organism in question corresponds with one that has already been described. This laxity has in the past led to much confusion; and it would be greatly to the advantage of bacteriology in general if editorial authority could be exerted to prevent the publication in medical or scientific journals of descriptions of newly-named bacterial species, where these requirements are not properly fulfilled. The existence in this country, and in America, of adequate collections of type cultures provides the material for such comparisons as may be necessary.

Fig. 47 provides a summary, in chart form, of the American classification, with the modifications referred to above.

The following are the genera included in this classification, given in the order in which they are listed in the chart:

Actinobacillus.-Gram-negative, non-acid-fast rods, sometimes occurring in long chains or in unjointed filaments. In lesions in the animal body no mycelium is formed, but at the periphery finger-shaped cells or clubs may be visible.

Type species. Actinobacillus lignieresi.

Leptotrichia.-Thick, long, straight or curved threads, unbranched, frequently clubbed at one end and tapering to the other. Gram-positive when young. Threads fragment 
into short, thick rods. Anaerobic or facultative. Non-motile. Filaments sometimes granular. No aerial hyphæ or conidia. Parasites or facultative parasites.

Type species. Leptotrichia buccalis.

\section{SCHIZOMYCETES.}

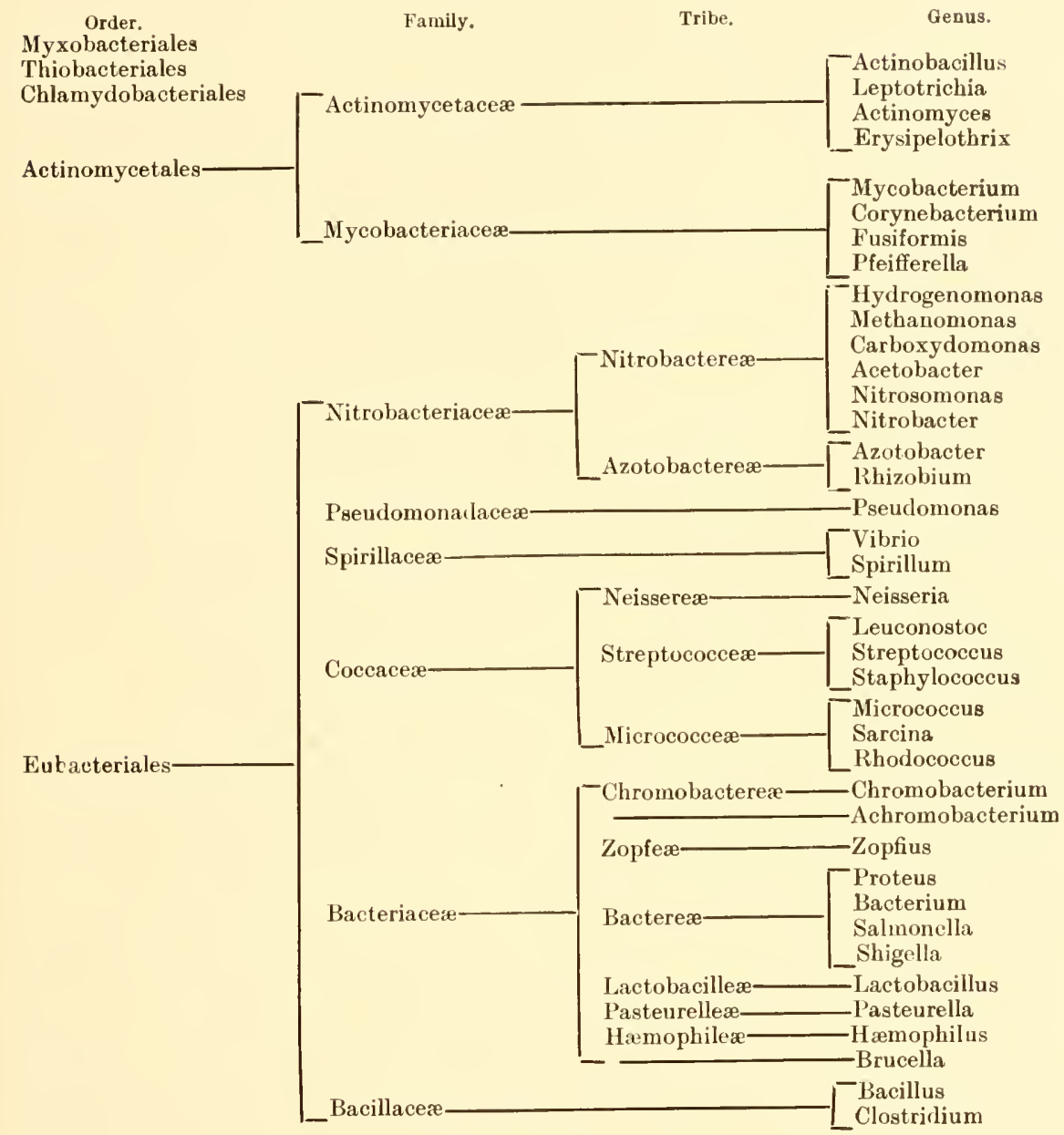

Fig. 47.

Actinomyces.-Organisms growing in the form of a much-branched mycelium, which raay break up into segments or produce "spores." Aerial mycelium often formed under suitable conditions. Mainly aerobic, but may be microaerophilic or even anaerobic. Usually saprophytic, but some species are parasitic on plants or animals, and may give rise to disease. In animal body organisms are frequently arranged in colonies composed of radiating threads with clubbed ends. Non-motile. Some species are acid-fast.

Type species. Actinomyces bovis Harz.

Erysipelothrix.-Rod-shaped organisms with a tendency to the formation of long filaments, which may show branching. The filaments may also thicken and show 
characteristic granules. No spores. Motility slight or absent. Gram-positive. Slight fermentative activities. Microaerophilic. Usually parasitic.

Type species. Erysipelothrix rhusiopathice.

Mycobacterium.--Slender rods which are stained with difficulty but which, when once stained, are acid-fast. Cclls are sometimes swollen, clavate, cuneate, or even branched. Non-motile. Gram-positive. No endospores. Growth on media slow. Aerobic. Several species are pathogenic to animals.

Type species. Mycobacterium tuberculosis.

Corynebacterium.-Gram-positive rod-like forms, arranged usually in a palisade. Not acid-fast. Often with club-shaped swellings at the poles, generally with irregularly staining segments or granules. Non-motile, non-sporing. Growing aerobically or under microaerophilic conditions, but often capable of anaerobic cultivation. Never forming gas in carbohydrate media, in which they may or may not produce acidity. They may or may not liquefy gelatin or serum. Some species produce a powerful exotoxin.

Type species. Corynebacterium diphtherice.

Fusiformis.-Obligate parasites. Anaerobic or microaerophilic. Cells frequently elongate and fusiform, staining somewhat unevenly. Filaments sometimes formed. Non-branching; sometimes highly pleomorphic. Non-motile. No spores. Reaction to Gram's stain variable, but mainly Gram-negative. Growth in laboratory media feeble.

Type species. Fusiformis termitidis.

Pfeifferella.-Small, slender, usually non-motile, Gram-negative rods, often staining irregularly, and sometimes forming threads or showing a tendency towards branching. Growth on all media is rather slow; gelatin may be slowly liquefied; fermentation of carbohydrates is very weak; characteristic brown honey-like growth on potato.

Type species. Pfeifferella mallei.

Hydrogenomonas.- Monotrichate short rods capable of growing in the absence of organic matter, and securing growth energy by the oxidation of hydrogen (forming water).

Type species. Hydrogenomonas pantotropha.

Methanomonas.--Monotrichate short rods capable of growing in the absence of organic matter and securing growth energy by the oxidation of methane (forming carbon dioxide and water).

Type species. Methanomonas methanica.

Carboxydomonas.-Rod-shaped cells capable of securing growth energy by the oxidation of carbon monoxide (forming carbon dioxide).

Type species. Carboxydomonas oligocarbophila.

Acetobacter.-Cells rod-shaped, frequently in chains, non-motile. Cells grow usually on the surface of alcoholic solutions as obligate aerobes, securing growth energy by the oxidation of alcohol to acetic acid. Also capable of utilizing certain other carbonaceous compounds, as sugar and acetic acid. Elongated, filamentous, club-shaped, swollen and even branched cells may occur as involution forms.

Type species: Acetobacter aceti.

Nitrosomonas.-Cells rod-shaped or spherical; motile or non-motile; motile forms possess polar flagella. Capable of securing growth energy by the oxidation of ammonia to nitrites. Growth on media containing organic substances scanty or absent.

Type species. Nitrosomonas europaa.

Nitrobacter.-Cells rod-shaped, non-motile, not growing readily on organic media or in the presence of ammonia. Cells capable of securing growth energy by the oxidation of nitrites to nitrates.

Type species. Nitrobacter winogradskyi. 
Azotobacter.- Relatively large rods, or even cocci, sometimes almost yeast-like in appearance, dependent primarily for growth energy upon the oxidation of carbohydrates. Motile or non-motile; motile forms possess a tuft of polar flagella. Obligate aerobes; usually growing in a film upon the surface of the culture medium. Capable of fixing atmospheric nitrogen when grown in solutions containing carbohydrates and defieient in combined nitrogen.

Type species. Azotobacter chroococcum.

Rhizobium.-Minute rods, motile when young. Specialized forms abundant and charaeteristic when grown under suitable conditions. Obligate aerobes, capable of fixing atmospheric nitrogen when grown in the presence of carbohydrates and in the absence of compounds of nitrogen. Produce nodules upon the roots of leguminous plants.

Type species. Rhizobium leguminosarum.

Pseudomonas.--Rod-shaped organisms, usually motile, by means of polar flagella. Generally Gram-negative. Non-sporing. Aerobic; some species are facultative anaerobes. Frequently produce a water-soluble pigment, which is yellow, green, blue, purple, or brown in colour, and which diffuses through the medium. Some species form a nondiffusible yellow pigment, and some species are photogenic. Fermentation of carboliydrates as a rule not active. Frequently gelatin-liquefiers, and active ammonifiers. Common in soil and water. Many yellow species are plant parasites.

Type species. Pseudomonas pyocyanea.

(On grounds of priority the American Committee recommend that this organism should be ealled $P$ s. aeruginosa.)

Vibrio.-Short, curved, rigid rods, arranged singly or united into S-forms or spirals. Motile by a single polar flagellum, which is usually relatively short. (Some species may have two or three polar flagella.) Non-sporing. Usually Gram-negative. Aerobic and facultatively anaerobic. Many species liquefy gelatin and are active ammonifiers. Commonly found in water. Most species are saprophytic; a few are pathogenic to man.

Type species. Vibrio cholerce.

Spirillum.-Rigid rods of spiral form, varying considerably in the number, length and breadth of the spirals. Usually motile by means of a tuft of polar flagella (5 to 20 ). The flagella occur at one or both poles; their number varies greatly, and is difficult to determine, since in stained preparations several are often united into a common strand. Generally Gram-positive. Some species form a reddish-yellow or greenish-yellow pigment. Found in water or putrid infusions.

Type species. Spirillum undula.

Neisseria.-Gram-negative cocci, usually arranged in pairs. Strict parasites, often growing poorly on ordinary media, but growing well on serum media. Frequently pathogenic.

Type species. Neisseria gonorrhoce.

Leuconostoc.-Spherical or ovoid cells, arranged in pairs and chains; the cocci are surrounded by a gelatinous envelope, which unites them into zooglœal masses. Usually Gram-positive, but decolorize easily. Saprophytes, usually growing in cane-sugar solutions.

Type species. Leuconostoc mesenteroides.

Streptococcus.-Spherical or ovoid cells, arranged in short or long chains, or in pairs. Non-sporing, usually non-motile. Most species Gram-positive. Some species form capsules. Growth tends to be relatively slight on artificial media, and some species grow poorly in the absence of added native protein. Several species produec characteristic changes in media containing blood. Various carbohydrates are fermented with the production of acid. Most species fail to liquefy gelatin. Most species are aerobic and facultatively anaerobic; some are anaerobic. Many species are normally parasitic on man or animals. Some species are highly pathogenic, and some produce soluble toxins.

Type species. Streptococcus pyogenes.

P.B. 
Staphylococcus.- Spherical or ovoid, non-motile, Gram-positive cells, arranged in grape-like clusters on solid media, and in pairs, small groups, or short chains in liquid media. On agar the growth is of a golden, white, or yellow colour. Great variation in biochemical activities, hæmolytic power, and pathogenicity. Actual or potential parasites.

Type species. Staphylococcus aureus.

Micrococcus.-Spherical or ovoid cells, non-motile, arranged in pairs, tetrads, or groups, but not in grape-like clusters or chains. Generally Gram-positive. Grow freely on ordinary media. Sometimes produce a yellowish pigment. Gelatin liquefaction is not constant, and is usually slow. Fermentative activities weak. Usually non-pathogenic to man or animals.

Type species. Microcorrus luteus.

Sarcina.-Has same characters as Micrococcus, except that cell division occurs, under favourable conditions, in three planes, so that cubical packets are formed.

Type species. Sarcina ventriculi.

Rhodococcus.--Spherical or ovoid cells occurring in groups or regular packets. Usually Gram-positive, but are easily decolorized. Growth on agar abundant with formation of red pigment. Weak fermentative powers. Gelatin rarely liquefied. Nitrates generally reduced. Saprophytes.

Type species. Rhodococcus rhodochrous.

Chromobacterium.--Small, non-sporing, acrobic rods, usually motile and usually Gramnegative, producing a yellow, red, or violet pigment, which is generally insoluble in water. Saprophytic; commonly found in water or soil.

Type species. Chromobacterium violaceum.

Achromobacterium.-Motile or non-motile, Gram-negative rods, usually small to medium in size, forming no pigment on agar, and varying in their fermentative ability. Optimum temperature for growth about $25^{\circ} \mathrm{C}$., but often good growth at $37^{\circ} \mathrm{C}$. Saprophytic; commonly found in water, soil, and milk.

Zopfius.-Long rods, occurring in evenly curved chains. Gram-positive. Motile. Spider-web growth on solid media. Facultative anaerolses. Carbohydrates and gelatin not attacked; hydrogen sulphide not formed.

Type species. Zopfius zopfi.

Proteus.-Highly pleomorphic rods, filaments and curved cells being common in young cultures. Gram-negative. Actively motile. Characteristic spreading growth on moist media. Often liquefy gelatin, and often Iroduce vigorous decomposition of proteins. Ferment glucose and usually sucrose, but not mannitol or lactose, with production of acid and gas.

Type species. Proteus vulgaris.

Bacterium.-Gram-negative, non-sporing rods : often motile, with peritrichate flagella : some species capsulated. Easily cultivable on ordinary laboratory media. Aerobic and facultatively anaerobic. All species ferment dextrose with the formation of acid, or acid and gas. Many species are active fermenters of a wide range of carbohydrates and allied substrates. Typically intestinal parasites of man and animals, although some species may occur in other parts of the body, on plants, or in the soil. Many species are pathogenic.

Type species. Bacterium coli.

Shigella. - Gram-negative, non-motile rods, $2-3 \mu$ long by $0 \cdot 5-0 \cdot 7 \mu$ broad. Noncapsulated; non-sporing. Ferment a variable number of carbohydrates with the production of acid. Lactose is not attacked except by some species, and then not for two days or more. Reduce nitrates to nitrites, form ammonia but not hydrogen sulphide, 
are Voges-Proskauer negative, and fail to grow in Koser's citrate. Ficultative anacrobes. Some species are antigenieally related. One species produces a toxin. Most species are pathogenic to man, giving rise to dysentery or sometimes acute gastro-enteritis. Found, as a rule, in the intestinal tract of human dysentery patients and contacts.

Type species. Shigella shiga.

Salmonella.-Gram-negative, non-sporing rods, usually $1-3 \mu$ long by 0.5-0.7 $\mu$ broar. Primarily intestinal parasites, widely distributed in man. mammals and birds. With few exceptions all species are motile, by perit richate flagella. Easily cultivable on ordinary media. Aerobic and facultatively anaerobic. Apart from a few species that form acid only, acid and gas are produced from glucose, mannitol, dulcitol, and sorbitol. Lactose, sucrose, and adonitol, and, except rarely, saliein are not fermented. Indole and acetylmethylearbinol are not formed. Gelatin is seldom liquefied. $\mathrm{H}_{2} \mathrm{~S}$ production is usual. The species are closely related to each other by somatic and Hagellar antigens; most species are diphasie. Pathogenic for man, animals, birds, or all three, giving rise to foodpoisoning, enteritis, or typhoid-like infections.

Lactobacillus.-Rods, often long and slender. Gram-positive; non-motile; without endospores. Usually produce acid from earbohydrates, as a rule lactic. Some species grow best at $40^{\circ}$ to $44^{\circ} \mathrm{C}$, and some species are microaerophilic. Surface growth on media poor.

Type species. Lactobacillus caucasicus.

Pasteurella.-Small, Gram-negative, oroid bacilli, showing bipolar staining. Aerobic and facultatively anaerobic. Powers of earbohydrate fermentation relatively slight; no gas produced. Gelatin not liquefied. Parasites in man and animals, producing characteristic infections.

Type species. Pasteurella aviseptica.

Hæmophilus.-Minute rods, sometimes almost coccal, sometimes thread-like; may be highly pleomorphic. Non-motile; non-sporing; Gram-negative; not acid-fast. Dependent for their growth on the presence of some factor, which is supplied by blood pigments, and by certain plant tissues. Some species require for their growth a second factor, which is present in blood, in most plant tissues, in yeast, or in the cells of other bacterial species. All known species appear to be obligatory parasites: some are patho. genic.

Type species. Hamophilus influenza.

Brucella.-Small, non-sporing, Gram-negative eoceo-bacilli. Non-motile. Grow rather poorly on ordinary media or may require special media. Aerobic; no growth under strict anacrobic conditions. Growth often improved by $\mathrm{CO}_{2}$. Little or no fermentative action on carbohydrates. Usually tend to produce alkali in litmus milk, and a brown pigmentation on potato. Strict parasites, occurring in man and animals, and produeing characteristic infections.

Type species. Brucella melitensis.

Bacillus.-Aerobic, spore-bearing rods, usually Gram-positive. Often oceur in long threads, and form rhizoid colonies. Form of rod not greatly ehanged at spornlation. Liquefy gelatin. Mostly saprophytes.

Type species. Bacillus subtilis.

Clostridium.-Anaerobic or microaerophilic rods, producing endospores, which are usually wider than the vegetative organisms in which they arise-so-called clostridium forms. Generally Gram-positive. In young cultures often decompose protein media through the agency of enzymes, and often ferment carbohydrates. Many species are pathogenic.

Type species. Clostridium butyricum. 


\section{REFERENCES}

Bergey, D. H., et al. (193!) "Manual of Determinative Bacteriology." 5th Edit. Baillière, Tindall and Cox, London.

Buchanas, R. E. (1925) "General Systematic Bacteriology." Baltimore.

Buchanan, R. E., Breed, R. S., and Rettger, L. F. (1928) J. Bact., 16, 387.

Castellani, A. and Chalmers, A. J. (1920) Ann. Inst. Pasteur, 34, 600.

Ellrod, R. P. and Braun, A. C. (1941) Science, 94, 520.

Kluvier, A. J. and Niel, C. B. VAs. (1936) Zbl. Bal.t., IIte Abt., 94, 369.

Kruse, W. (1896) "Die Mikroorganismen." Flügge, Leipzig.

Lehmane, and Necmann. (1896) (See 1920) "Atlas u. Grundriss der Bakt. u. Lehrb., ete." 6th Edit. Munich.

Migula, W. (1894) (See 1900) "System der Bakterien," Bd. II, Jena.

Orla-Jensen, S. (1909) Zbl. Bakt., IIte Abt., 22, 305.

Reports. Comm. Soc. Amer. Bacteriol. Winslow, C.-E. A., Broadhurst, J., Buchanax, R. E., Krumwiede, C., Rogers, L. A., and Sinth, G. H. (1917) J. Bact., 2, 505 ; (1920) Ibid., 5, 191 .

Stanier, R. Y. and Niel, C. B. vas. (1941) J. Bact., 42, 437.

Thompson, D'A. W. (1942) "On Growth and Form," 2nd. ed., Camb. Univ. Press.

White, P. B. (1937) Zbl. Balit., IIte Abt., 96, 145.

Zopf, W. (1885) "Die Spaltpilze." 3. Aufl. Breslau. 


\section{CHAPTER 11}

\section{THE BAC'TERIOPHAGE}

Is 1915, Twort described a curious degenerative change that he had observed in cultures of a staphylococcus derived from calf-lymph. He was able to transmit this change from one culture of the susceptible organism to another, by placing on the surface of an inoculated agar slope a drop of a highly diluted filtrate from a suspension of an earlier growth that had undergone the degenerative change. In 1917 d'Herelle recorded his first series of observations on the lytic properties of filtrates of mixed cultures obtained from the frees of patients suffering from bacillary dysentery. In these preliminary studies, he was able to demonstrate the occurrence of rapid and generalized lysis in a growing broth culture of a dysentery bacillus to which some of the filtrate from the original mixed culture had been added, and the transmission of the lytic agent in a prolonged series of cultures of the susceptible bacterium, by the addition to each new culture of a filtrate obtained from the preceding one after lysis had occurred.

There can be no doubt, though d'Herelle has strenuously opposed this view, that these two descriptions afford different examples of the same essential process. With our present knowledge, we are, indeed, able to identify earlier records of curious happenings and appearances in bacterial cultures as instances of this lytic change; but in none of these cases was the nature of the process studied in any detail, nor was its transmission by bacteria-free filtrates demonstrated. 'Twort's original paper, on the other hand, contains a complete demonstration of all the essential features of this important and significant reaction; and it has lence come to be generally known as the "Twort-d'Herelle phenomenon."

D'Herelle's observations, which he has recorded in numerous papers from 1917 onwards and collected in three monographs (d'Herelle 1921, 1926, 1930), have, however, been far more detailed and extensive, and have played a major part in the development of our present conceptions. The name that he applied to the lytic agent, Bacteriophage, has come into general use, familiarly shortened to the diminutive phage; and the view, consistently maintained by him, that this agent is a filtrable virus, parasitic on bacterial cells, has won increasing support, particularly within recent years.

An extensive literature has grown around this subject; but we can here do no more than summarize certain of the more important observations, and the conclusions that have been drawn from them. The results olstained in recent studies have, indeed, deprived many of the earlier records of all save historical interest. 


\section{The General Characters of Bacteriophage Lysis.}

The observations of d'Herelle, of Twort, and of other early workers established the following facts in regard to the behaviour of the lytic agent.

(1) It will pass through filters that hold back all bacteria.

(2) It acts upon susceptible bacteria in such a way as to bring about their lysis during the phase of active bacterial growth. This lytic action

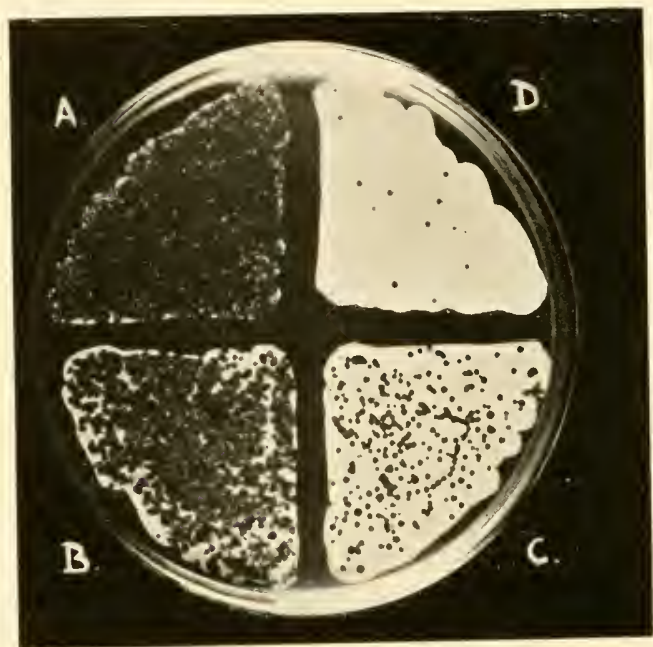

Fig. 48.

Effect of increasing dilutions of a phage on bacterial growth on an agar plate. may be demonstrated in several different ways.

(a) A phage-containing filtrate may be added to a broth, or peptone-water, culture of a susceptible bacterium, the addition bcing made either at the time the broth is inoculated, or during the early stages of bacterial growth. The addition of an active filtrate will result, after a variable period, in a relatively sudden cleariug of the turbid, growing culture. This clearing may be partial, or apparently complete; but, even in the latter case, prolonged incubation usually results in renewed bacterial growth.

(b) The surface of an agar plate may be thickly inoculated with a susceptible bacterium, so as to give a confluent growth, and a few drops of a phage-containing filtrate may be spread over this inoculated surface before the plate is incubated. If the filtrate is rery active, and has not been diluted, no growth may develop over the area on which it has been spread, or thero may be a few "resistant" colonies (Fig. 48A). With a moderate dilution of the filtrate there will result irregular, confluent areas of clearing (Fig. 48B). With a still greater dilntion there will be a number of well-separated clear areas, each of them circular, or roughly circular, in outline (Fig. 48c). These are the tâches vierges, plaques or plages, of the French authors, the Löcher of the German. As the phage filtrate is still further diluted, these plaques become fewer and fewer (Fig. 48n), a linear relation existing between the degree of dilution and the number of plaques, at least over a considerable range.

Phage action may also be demonstrated on

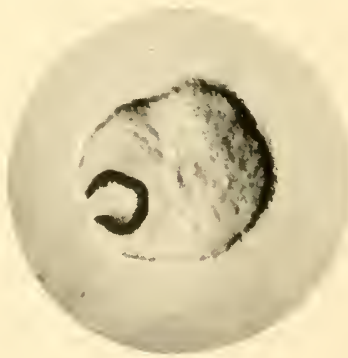

Fig. 49.

Colony of Bact. coli on agar, showing a "bitten" seg. ment at the periphery, due to the action of a bacterio. phage $(\times 8)$.

a solid medium by the appearance of "litten," or " nibbled" colonies, of the type shown in Fig. 19, or, in certain rather cxceptional instances, ing 
the occurrence of a vitreous, or granular, degeneration in colonies that have already attained a relatively advanced stage of growth.

(3) The lytic agent may be propagated indefinitely in association with growing cultures of the bacteria on which it acts. A few drops of a filtrate from the first culture in which lysis has occurred may be added to a second young, growing culture of the bacterium; this may be filtered after lysis and the filtrate added to a third freshly inoculated culture, and so on in series. A phage filtrate may be active in very high dilution $\left(1 \times 10^{-8}\right.$ or higher), and in successive passages, carried out as above, the original titre is often increased during the earlier transfers, and is then maintained. It is clear, therefore, that the lytic agent, whatever it may be, is actively reproduced during the lytic process. There are few instances of its reproduction in the absence of bacterial cells, or in the presence of dead bacterial cells (see Krueger and Baldwin 1937). Under certain conditions, however, it can be produced in the presence of bacterial cells that, though living, are not growing or dividing. There has been some controversy on this point (see Otto and Munter 1923, Twort 1925, 1926, Gratia and Rhodes 1926, Gohs and Jacobsohn 1927, Bronfenbrenner and Muckenfuss 1927), but recently Krueger and his colleagues have defined conditions in which phage appears to be produced without growth of the bacterial cell (Scribner and Krueger 1937, Krueger and Fong 1937, Northrop 1939).

(4) Any given lytic agent will be found to be most active against one bacterial species or type, or against a few species or types that are known to be related to one another. Against unrelated species, or types, there is usually no action. The phage is, then, a highly specific agent.

(5) Phage lysis has been observed in a large number of bacterialspecies. It has also been reported in yeasts and actinomyces (Wiebols and Wieringa 1936).

(6) A secondary result of phage action is the appearance, in the bacterial culture that is undergoing lysis, of variants that are resistant to the action of the particular phage concerned. These variants are usually susceptible to the action of other phages.

(7) A large number of bacteria are known to carry a lytic agent to which they are themselves resistant. These phage-resistant carrier strains are called lysogenic. Lysogenic strains are indistinguishable from others except for the fact that they release an agent which is actively lytic for phage-sensitive bacteria. A lysogenic strain, and the bacteria sensitive to the lytic agent it carries, usually belong to the same species or group of micro-organisms.

(8) One of the commonest natural habitats of the phage is the intestinal tract of man and animals, and filtrates active against one or more bacteria can almost always be obtained from any specimen of fæces, or from material that has been subjected to fæcal pollution.

\section{The Nature of the Bacteriophage.}

As we have seen, d'Herelle has consistently maintained the view that the phage is a filtrable virus, parasitic on bacteria. This hypothesis has always been in accord with many of the most striking features of phage behaviour (see Flu 1923, Reichert 1924, Schuurman 1925). There can be little doubt that it would have gained early and general acceptance but for the fact that certain recorded observations seemed almost irreconcilable with it. 
Because of these difficulties in accepting the virus hypothesis, various alterna . tive theories have been propounded.

Kabéshima (1920) suggested that the phage was a catalyst, activating a pro-ferment present in the bacteria themselves. On this view the liberation of the ferment in an active form would, of course, have to be regarded as an essential consequence of the lysis of the bacterial cells, the process, when once set going, being self-reproducing.

Bordet (see Bordet and Ciuca 1920, Bordet 1923, 1925) sought to reconcile the con ception of an inanimate phage with its reprcduction in an unlimited series in a rather different way. He suggested that the phenomenon was a true antolysis, the active agent being produced exclusively from the bacteria themselves. The origin of the autolysis he traced to a disturbance of the normal equilibrium between the assimilative and metabolic activities of the bacterial cell, adding the supposition that the substances set free during the autolysis of the cells initially affected were able to act in some way upon susceptible but hitherto unaffected cells, and to initiate in them the same series of antolytic changes. Once started, the process would thus be transmissible in series, provided that susceptible bacteria were present, and that these bacteria were metabolically active.

Northrop (1939) emphasized the greater likeness of many of the characteristics of phage production and action to the production and action of enzymes, and in support of his thesis demonstrated the similarity in the production of an extracellular gelatinase and of phage from a lysogenic strain of $B$. megatherium.

Another hypothesis, advanced by Hadley (1927, 1928), and since put forward in a rather different form by Wollman $(1925,1927,1928,1929,1934 a, b, 1935)$ [see also Wollman and Wollman, 1932, and Lwoff, 1936], assumes the phenomenon to be purcly bacterial in origin, but relates it to the genetic, not to the metabolic activities of the bacterial cell. The phage, on this view, would be regarded as analogous to some genc-earrying constitnent of the bacterial cell, or some filtrable phase in a complex life-cycle. It must, of course, be assumed that the addition of this cellular component, or bacterial phase, to a young culture of susceptible organisms, so alters their genetic behaviour that they undergo lysis during the process of multiplication, and, in so doing, reproduce the active agent in large amount. As further evidence for their view, Wollman and Wollman (1936, 1938) maintain that each living cell of a phage-carrying strain of $B$. megatherium liberates one, and only one particle of phage; if the phage were an externally infecting virus, it is argned, the number present in a carrier cell should vary from cell to cell. Gratia (1936) and Flu (1938a), working with the same strain of bacterium, have both been unable to elicit the one-to-one ratio deseribed by the Wollmans; both find the number of particles liberated can exceed the number of baeterial cells (see also Lewis and Worley 1936).

There are, we think, adequate reasons for accepting the virus hypothesis, at least as the most probable explanation of all the recorded facts. In setting ont the evidence that seems to us to justify this conclusion, it will be convenient to discuss, seriatim, certain aspects of the nature and behaviour of the phage; but before doing so one point may be made clear, since it will be involved in our consideration of each other question in turn.

D'Herelle, for reasons that are not easy to appreciate, has upheld the view that the phage is a single living organism, Protobios bacteriophagum, which may adapt itself to live at the expense of a wide variety of different bacteria. The very extensive evidence that is now available is quite incompatible with this view. There is not one phage, but an enormous number of different phages; and in order to obtain constant and reproducible results it is as necessary to work with pure strains of phage as with pure cultures of bacteria. Neglect of this precantion has rendered many recorded studies of very doubtful value.

Pure strains of phage may be obtained in two ways. Since a given strain of 
bacterium will in many cases be susceptible to only one of the phages contained in a crude mixed filtrate, repeated transfer in growing cultures of this bacterium will of ten eliminate all phages except the one to which it is sensitive. This method is not, however, always reliable. A crude filtrate will often be found to contain more than one phage acting on the test bacterium selected. In such a case each of these phages may be propagated in successive transfers. It often happens that one or more are reproduced more rapidly than the others, and so have a better chance of transfer; but such phage cultures may remain mixed throughout a number of generations. An alternative, and better, method is to pick from isolated plaques on agar plates, just as we pick isolated colonies in attempting to purify a mixed bacterial culture. By a combination of these two methods it is usually possible to obtain phage strains of undoubted purity; but the procedure may be laborious, and contamination is very liable to occur. For these reasons strict attention to details of technique is required in any work of this kind (see Asheshov et al. 1933a, Rakieten and Rakieten 1937).

The ways in which various strains of phage can be differentiated from one another will be considered in later sections of this chapter. At the moment we are concerned only with the point that such strains exist, and are identifiable.

\section{The Physical State of the Phage. Is it Particulate or in Solution?}

Given a bacteria-free filtrate containing active phage, we may determine whether it behaves as a homogeneous solution or whether the activity is distributed in a discontinuous fashion throughout the solution. The answer will depend entirely on the sensitivity of the method of examination. The question, therefore, "Is phage particulate?," must be understood in the restricted sense, "Is the lytic activity in the solution continuous or discontinuous?" Should it prove to be continuous, the fact is not evidence that phage is not particulate in the general sense of the term, hut merely that the technique was too insensitive to detect the separate units in action.

As d'Herelle showed, isolated plaques form on agar cultures over which a high dilntion of phage filtrate has been spread. As further evidence that the phage activity was discontimuous, d'Herelle noted that when a very high dilution of phage filtrate was distributed in equal volumes among a large number of tubes, each containing a young growing culture of a sensitive bacterium, lysis occurred in some tubes but not in others, indicating that the active agent was not uniformly dispersed in solution. This observation has been confirmed by a number of sub. sequent observers (Bronfenbrenner and Korb 1925b, McKinley and Holden 1926. Bronfenbrenner 1927); and both Feemster and Wells (1933) and Luria (1940) have shown that the proportion of tubes showing lysis among large numbers inoculated with varying amounts of plage filtrate is in accord with statistical expectations based on the assumption that the agent is dispersed in lytic particles.

A possible escape from the obvious implication of these observations was provided by the assumption that only a few of the bacteria in any culture were susceptible to phage action. This assumption had little inherent probability and could hardly account for the test-tube experiments referred to above, but it was accepted by some observers, mainly on aceount of other observations that secmed to tell against a grossly particulate nature. Thus Bordet (1925) stated that it was impossible to concentrate a phage filtrate by centrifugation at high speeds. We now know that this can readily be done if the speed is adequate. Olsen and Yasaki (1923) stated that the pluage was volatile. This would have finally disposed of the view that it was particulate, or a living agent of any nature; 
but the results recorded were undoubtedly due to technical errors (Spät 1924, Borchardt 1924, Gildemeister and Herzberg 1924b, Meissner 1924b, Gercke 1925, Bronfenbrenner and Korb 1925a).

The size of the lytic particle in a number of phage preparations has been determined and it has been found that different strains of phage are each characterized by a certain relatively narrow range of particle sizes.

The size has been estimated in various ways. Filtration through membranes of approximately known pore-sizes indicated diameters of the order of 20-50m (Stassano and de Beaufort 1925, Bechhold and Villa 1926, Zinsser and Tang 1927). Elford and Andrewes (1932) used carefully graded collodion membranes, and showed that different pure-strain phages had each a different particle size and that size was independent of the particular strain of bacterium upon which they were being propagated. One phage, for instance, had a mean diameter of 8-12 $m \mu$. The diameters of a group of phages lay between 50 and $75 \mathrm{m \mu}$, while other strains gave intermediate values. Yaoi and Sato (1935) recorded similar results. Schlesinger (1932-33a, 1933), Elford (1936), McIntosh and Selbie (1937) and Païc, Krassnoff, Haber, Reinié and Voet (1938) estimated the diameter of phage-particles by measuring their rate of sedimentation in a high-speed centrifuge. The results were consistent with those observed by filtration and confirmed the existence of a wide range of particle-size. Wollman and Lacassagne (1940) estimated a similar range of sizes, from the results of inactivation of phages by $\mathrm{X}$-radiation (see also Luria and Exner 1941).

Reference to Chapters 2 and 41 will show that the larger phages are big enough to be demonstrated by modern microscopical methods. Merling-Eisenberg (1938) and Eisenberg-Merling (1941) obtained photomicrographic images in visible light of staphylococcus and Bact. coli phages, which on photometric measurement yielded estimates of size similar to those obtained by ultrafiltration and ultracentrifugation studies. Barnard, using the technique of photography by monochromatic ultra-violet light, has obtained photographs of a phage of this type, which show it to be composed of uniformly sized particles with a diameter of about $50 \mathrm{~m} \mu$ (see Burnet 1933e). These large phages produce in lysed broth cultures a turbidity that can be detected by its Tyndall effect, and the intensity of this effect has been found to provide an accurate measure of the concentration of the phage in the filtrate (Schlesinger 1932-33b, Schuurman and Schuurman-ten Bokkel Huininck 1936).

Electron micrographs reveal not only the size of phage particles, but also a structure. Ruska (1941) noted "sperm-shaped" particles in his photographs, and more recently Luria, Delbrïck and Anderson (1943) have shown that three of four Bact. coli phages they studied had an opaque "head" consisting of a pattern of granules, about $80 \mathrm{m \mu}$ in diameter, and a less opaque "tail," about $120 \mathrm{m \mu}$ long (Figs. 50, 51, 52).

Our conception of the lytic particle, of a size that closely characterizes the various types of phage, as the ultimate unit of phage, does not accord with recent descriptions of certain physical and serological properties of Bact. coli phage. By diffusion experiments with dilute solutions Kalmanson and Bronfenbremer (1939) deduced a particle weight of 1,500,000 for a coli phage. The particle weight of the corresponding lytic unit was 27 times as great. Again, the maximum amount of antibody that combined with a lytic unit of phage was found to be far more than could be accommodated on the surface of a simple lytic unit. By postulating sub-units of $1,500,000$ particle weight, the quantitative serological data fall into line with those from reactions of other antigen-antibody systems (Hershey, Kalmanson and Bronfenbremner 1943). The lytic unit appears to be made up of about 30 independent diffusing units, bound by 25 per cent. of carrier 


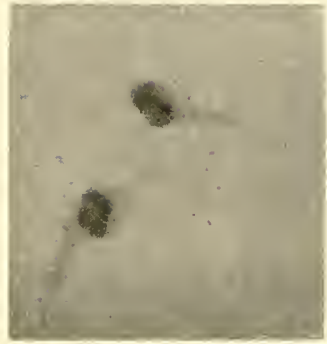

$a$

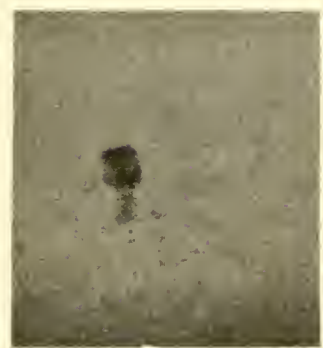

$c$

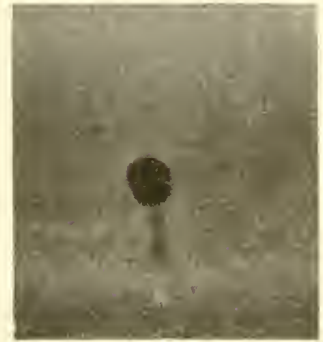

$b$

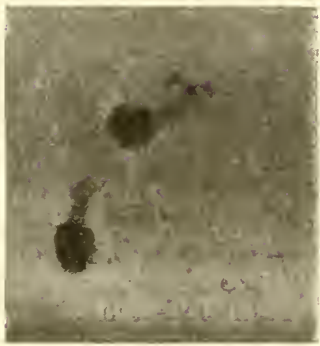

$d$

FIG. 50.-Electron micrographs of a Bact coli. phage. $(\times 53,000)$. (From a photograph kindly supwlied by Dr. S. E. Luria)

protein into an aggregate that in concentrated solutions behaves in lysis, in the photographic field, in the ultracentrifuge and in the ultrafilter as a unit particle. The titration of lytic activity by the plaque method thus reveals ouly one in thirty of the sub-units. The lytic unit readily dissociates into diffusing particles, also containing carrier protein, which in turn are made up of ultimate particles having a particle weight of 50,000. If these facts are confirmed, then the chemical descriptions of phage as a nucleo-protein (see Schlesinger 1934, Northrop 1938) may prove to be descriptions of carrier protein only. The identity of the protein isolated from phage preparations with phage itself has already been questioned on technical grounds (see Moriyama and Ohashi 1937, Flu 1938b).

The justifiable inferences from all these observations take us far beyond the conclusion that the phage is particulate. They accord well with the view that phages are filtrable viruses. They accord badly with any other hypothesis that has yet been propounded. A particulate lytic agent might conceivably consist of particles of bacterial protoplasm on to which some active principle had been adsorbed. If so, the adsorbing material, and the nature of the complex formed, must be specific for each phage in a high degree, or the particles resulting from disruption of a bacterium by one type of phage would be of varying sizes, and would be unlikely to differ consistently in size from those produced by the action of another phage.

\section{Other Characters of the Phages.}

Most phages, within the $\mathrm{pH}$ range over which they remain active, appear to carry a negative electric charge, in this way resembling bacteria and filtrable viruses (Todd 1927, Krueger et al. 1929, Burnet and NcKie 1930a, Natarajan and Hyde $1930)$. 
The fact that phages will grow only in the presence of multiplying or living bacteria renders the study of their metabolic activities exceedingly difficult. Apart from the work of Schïler (see Editorial 1935), which suggests that phosphatase is the only hydrolytic enzyme possessed by the bacteriophage, such studies as have been recorded have, in fact, yielded negative or ambiguous results (Bronfenbrenner 1924-25, 1926, Gózony and Surányi 1925, Kauffmann 1925-26, Bronfenbrenner and Reichert 1926-27, Schwartzman 1926-27, Bachmann and Wohlfeil 1927).

The resistance of various phages to heat and to other physical or chemical agents has been studied in some detail. Certain early statements ascribed to lytic filtrates a degree of resistance to heat, and to such chemical agents as chloroform, toluene, alcohol, acetone, etc., that seemed to place them in a category apart from other living things, except the spore-bearing bacteria (see Kabéshima 1920). These statements played a not-inconsiderable part in the controversy as to whether the phage was a living or non-living agent; but they were not in accord with all the records existing at the time-Twort (1915), for instance, had stated that his lytic agent was inactivated by heating at $60^{\circ} \mathrm{C}$. for 1 hour-and they have since been shown to have no general validity. All the evidence suggests that different strains of phage, like different species of bacteria, differ from one another in their resistance to heat, to drying and freezing (see Knorr and Ruf 1935, Campbell-Renton 1941), to shaking (Campbell-Renton 1942) and to various other physical and chemical reagents, and that the range of sensitivity, taken as a whole, is much the same in the two cases.

A few observations of general interest in regard to this particular problem may be briefly noted. Baker and Nanavutty (1929) found that the time relations of the inactivation of a Shiga phage by ultra-violet light were closely similar to the killing of Bact. coli by the same agent. The time required to inactivate the Shiga phage, or a staphylococcal phage, did not differ greatly from that required to kill Bact. coli; but the time required to inactivate trypsin was 20-30 times as long, and for the inactivation of diastase about 120 times as long. In this respect, therefore, the phages studied behaved as living things, not as Cerments (see also Gates 1934). Wright and Kersten (1937) and Campbell-Renton (1937) noted a wide variation in the sensitivity of different phages to ultra-violet light.

Schultz and Krueger (1928) recorded the inactivation of a staphylococcus phage by methylene blue. Clifton (1931) found that this inactivation occurred when the mixture was exposed to sunlight, but not in the dark, and concluded that it was due to the oxidation of the phage by the photodynamically activated dye. Perdran and Todd (1933), using more exact methods, record similar results. Different phages have been found to vary widely in their sensitiveness to this photodynamic action, thongh none is entirely resistant (Burnet 1933b, 1934). Staphylococcus phage may also be inactivated by safranine; the inactivation is partly photodynamic (Krneger and Baldwin 1935).

Phage is inactivated by aldehydes and the inactivation reversed by the addition of substances with free amino or imino groups, which compete with the phage for the aldehycle (Kendall and Colwell 1938, Kligler and Oleinik 1943). The activity of the phage appears to depend in part upon certain of the free $-\mathrm{NH}_{2}$ or $-\mathrm{NH}$ groups on its surface. Levin and Lominski (1936) record reversible inactivation of phage by lecithin, which appears to prevent access of phage to sensitive bacteria. Williams, Sandholzer and Berry (1940) record the inactivation of a phage by cholesterol and cephalin, by bacterial and certain non-bacterial phospholipins, but not by lecithin, sphingomyelin or plasma phospholipins.

A well-marked antagonistic action on the viability of phages, of $\mathrm{Ca}, \mathrm{Ng}$. Ba and $\mathrm{Sr}$ ions on the one hand, and of $\mathrm{Na}$ and $\mathrm{K}$ ions on the other, can be demonstrated (Burnet and MeKie 1930, , Sertic $1937 a$, Gratia 1940), suggesting that these ions are acting on a protein constituent at the surface of a living cell. Diminution in phage counts are also 
observed after treatment of the phage at $50^{\circ} \mathrm{C}$. with varions anions (Ohashi 1938). The inhibition of lysis, by which inactivation is measured, may be due to an action, not on the phage particle itself, but on the sensitive bacterium. For instanee, Gest (1943) has shown that with high concentrations of univalent cations, like $\mathrm{Na}$, and moderate concentrations ( 0.1 molar) of $\mathrm{Mg}$ ions, plate lysis may be completely masked by overgrowth of resistant bacteria, which are apparently produced in large numbers in the presence of the cations. Some of the larger phages are rapidly inactivated by a strong urea solution, a reagent that acts similarly on many bacteria and viruses (Burnet 1933b, 1934).

It has been shown by several workers that some, but not all, phages are unable to produce lysis in bacterial cultures growing in a medium from which the calcium ions have been removed by previous treatment with citrate (Stassano and de Beaufort 1925. Bordet 1926, Asheshov 1926, Burnet 1933b). This fact allows a useful differentiation of phages into "citrate-sensitive" and "citrate-resistant" strains; but it should be noted that an initially citrate-sensitive phage may often be trained to produce lysis in a citratecontaining medium.

\section{The Mechanism of Phage Lysis.}

In considering the mechanism of phage lysis we may first deal with the phenomenon in a general sense, leaving the problem of specificity for later consideration.

D'Herelle's conception of the lytic process, based of course on the view that the phage is a parasitic virus, is simple and straightforward. He believes that a phage particle enters a growing bacterial cell, multiplies within it, and causes its more or less explosive disintegration when the limit of distension has been reached. This limit appears to vary over a considerable range ; but disruption usually occurs when the number of particles in the cell have reached some figure between 6 and 60 . In support of this view, d'Herelle states that the addition of very small amounts of phage to a growing culture is followed by a step-like increase in phage titre during the earlier stages of growth, successive sudden increases occurring at intervals of 20 to 30 minutes. After a few such jumps in titre the phage concentration rises logarithmically until lysis occurs. This change from a discontinuous to a continuous rise would, of course, be expected; since the successive increases would soon get out of step, and their combined effect would give a steady rise in titre.

Burnet $(1929 c)$ has recorded observations that are in entire accord with d'Herelle's view. To a number of small tubes, each containing a young, actively growing culture of a sensitive bacterium, he added a phage filtrate so highly diluted that, on the average, each tube received a single particle. At short intervals thereafter the whole contents of one of the tubes was spreact on the surface of an agar plate, and the resulting plaques were counted. The results showed that there was no detectable increase during the first 20 minutes or so. After this time there was a sharp and sudden rise. Thus, a series of tubes plated at one-minute intervals, over the appropriate time range, gave $1,0,1,2,0,1,80$, $0,1,120,1,230,0$ and 100 plaques. The absence of values intermediate between $I$ and 80 , makes it clear that the free phage in the culture was not increasing by twofold steps, as would occur during the early generations of a single bacterium multiplying by binary fission; and the only obvious explanation of such findings is that the phage particles are dividing in, or on, a bacterium, and are suddenly liberated into the surrounding fluid when that bacterium disrupts. These observations have been fully confirmed by Ellis and Delbrück (1939) and Hershey and Bronfenbrenner (1943).

The process of phage lysis has also been studied quantitatively by observations carried out on a lysing culture as a whole (see, for instance, Lepper 1923). Krueger and Northrop (1931) determined the rates of growth of staphylococei and staphylococcal phage in a lytic mixture. After a short lag period, the concentration of bacteria and phage both increased steadily up to the moment of visible lysis. The rate of increase of phage was consistently greater than that of the bacteria. Clifton and Horrow (1936) demonstrated 
a similar relationship in a lytic mixture of Puct. coli and a coli phage. In both cases, massive lysis was initiated when the number of phage particles per bacterial eell reached a certain value. In the Buct. coli system it was 1,600. The steady rates of phage production, as Burnet (1934) points out, are not incompatible with the coneeption of liberation of phage in bursts from infected bacteria, but may well represent the statistical average of large numbers of sudden bursts occurring at different times. Krueger and Fong (1937) later showed that the relationship they had observed between baeterial count and phage count was peculiar to their conditions of measurement, which happened to have been near the optimum both for phage production and bacterial growth; and, as we have already noted, they were apparently able by altering these conditions to demonstrate increase of phage in the absence of bacterial multiplication. This important observation, that phage is liberated in large amounts from infected bacteria in the absence of bacterial multiplication, has been repeated by Spizizen (1943a), but in view of the large number of previous negative reports, it may be wise to await its further confirmation.

We may distinguish three stages in the production of phage, namely, adsorption of phage on the bacteria (Fig. 51), a period of constant phage count in which phage grows in or on the bacteria, and release of phage when the phage count rises (Fig. 52).

Adsorption. - The rate of adsorption depends on the concentration of both phage and bacteria. The adsorption capacity of a bacterial species for a phage to which it is sensitive varies with the strain (see for instance, Hershey and Bronfenbrenner 1943), and the physiological state of the baeterial cell. Delbrück (1940) found that an actively growing culture of Bact. coli adsorbed 200 particles per cell, while the cells of starved culture of the same strain adsorbed only 20 particles per cell. When a phage filtrate is adsorbed by excess of bacteria a certain amount of residual phage is left, which was regarded by Krueger (1931) as evidence that adsorption was reversible. An alternative view (Schlesinger 1932-33a, $b$, and Delbrück 1942) regards the residual phage as having lessened affinity for the bacterium.

Constant Period.-The constant period varies in the same wây that division of bacteria varies with temperature (Ellis and Delbrück 1939).

Release of Phage.-At the end of the constant period, the release of phage occurs steadily for a certain period, during which all the infected bacteria one after the other are lysed, and then ceases, a step being followed by a halt in the curve of phage production. The released phage is adsorbed to other bacteria, and in due time a second "step release" oecurs, and so on, each succeeding step being less clearly marked as the cycles of adsorption, growth and release in each infected bacterium overlap one another in time. Within certain limits, the constant period and the size of the step release are independent of the number of phage particles adsorbed, and it appears that if more than one particle is adsorbed, only one of them actively induces the production of more phage. Lysis of the growing culture as a whole occurs when the phage concentration reaches a threshold limit for the number of bacteria present. In his studies of Bact. coli phage systems Delbrück (1940), demonstrated in bacteria, at a given stage of growth, a close similarity of the adsorption capacity of the bacterium, the threshold limit for lysis, and the yield of phage at the end of the constant period, and suggested that each bacterium might produce as many phage particles as it had receptors to which adsorption could take place. Using a similar system, Hershey and Bronfenbrenner (1943) did not observe any simple relation between phage yield and adsorptive capacity.

It may be noted that phage particles, when present in adequate concentration, are able to kill bacteria without producing lysis. Andrewes and Elford (1932) have demonstrated this direct killing aetion by studies with a citrate-sensitive coli-phage. Mixtures of this phage with a sensitive Bact. coli, when plated on citrate agar gave normal growth, without lysis, so long as the phage concentration was below a certain limit. But when this limit was exceeded 95-99 per cent. of the Bact. coli were killed, in the sense that they failed to develop on the citrate agar, although there was, of course, still no lysis. 


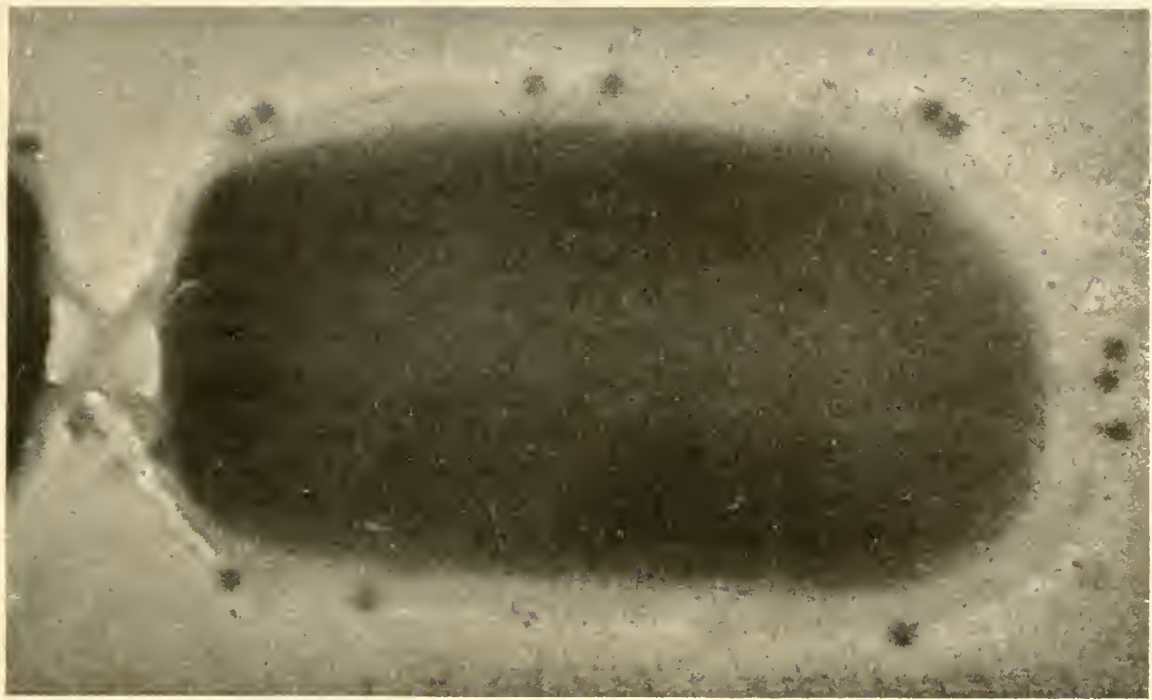

FIG. 51.

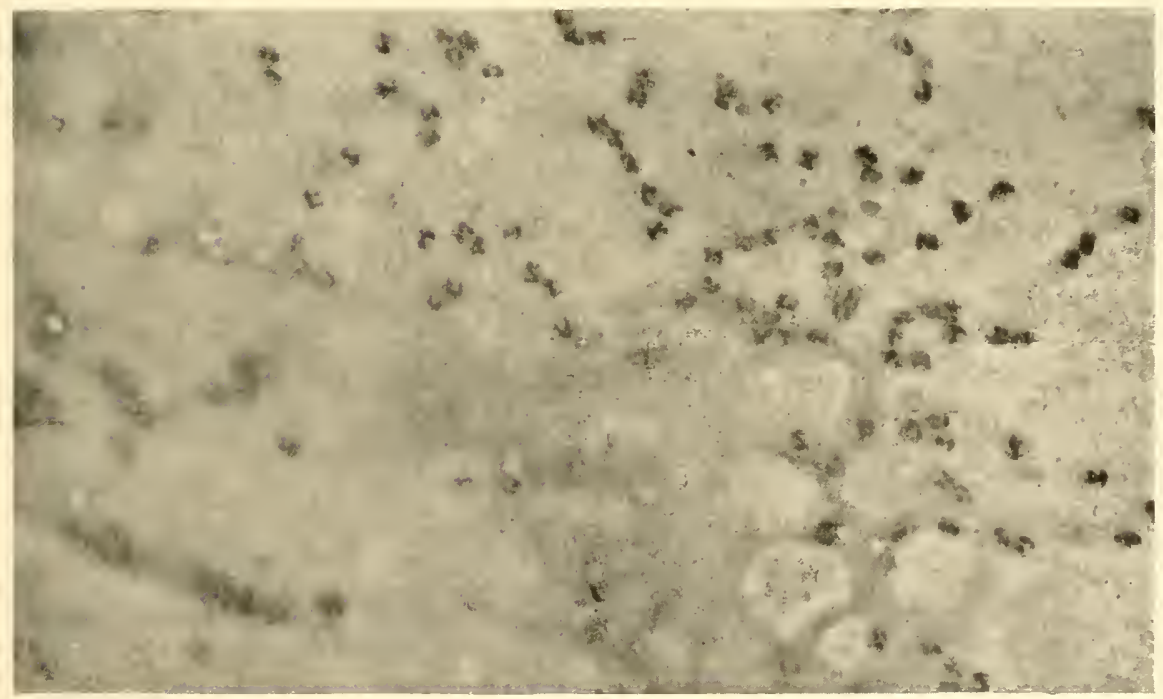

FIG. 52.

FIGS. 51 and 52.-Electron micrographs of phage action.

F1G. 51. Adsorption of phage to the surface of Bact coli. ( $\times 54,000)$.

FIG. 52. The release of phage from a lysed Bact. coli. The outline of the cell wall, and the bacterial debris are seen at the left of the picture. $(\times 21,000)$.

(From micrographs kindly supplied by Dr. S. E. Luria.)

It should also be noted that, although the multiplication of phage particles and the characteristic lysis associated with it demand the presence of living bactcria, the phage is readily absorbed by dead bacterial cells (sec Krucger 1931). As we shall see in a later section, this absorption is specific. 
Apart from these quantitative data, it is, of course, possible to observe the occurrence of phage lysis under the microscope, and this has in fact been done (see d'Herelle 1921, 1926, Twort 1922, Wollman 1925, Burnet 1925, v. Preisz 1925, Manninger 1926, da Costa Cruz 1926, Bronfenbrenner et al. 1927, Bronfenbrenner 1928, Bayne-Jones and Sandholzer 1933). Not all of these observations have been made under the best modern conditions of microscopy or photomicrography, so that their value, particularly in regard to matters of detail, varies considerably. They differ, also, in certain particulars of the actual happenings that are described, and there can be little doubt that phage lysis may, in fact, occur in several different ways, according to the nature of the phage and bacterium involved.

Swelling of the bacterial cells is usually seen in the early stages of the process, and is sometimes accompanied by the development of granules. The disruption of the swollen bacterial cell is usually very sudden. The cell may appear to explode, giving place to a cloud of granular debris, or it may lose its outline and disappear, without the liberation of the granular cloud.

Merling-Eisenberg (1938) (see also Eisenberg-Merling 1941) was able to make direct observations on the disruption of invaded bacteria; he found that 150-200 phage particles were liberated from cells of Bact. coli and 2-4 from cells of Staph. aureus. Delbrück (1940) distinguishes two forms of lysis, lysis from without and lysis from within. Lysis from without occurs almost instantly when plage is adsorbed at the threshold limit. The cells swell into spherical bodies. Iysis from within occurs in Bact. coli after the adsorption of one or a few particles, but not until the phage has nultiplied to the threshold limit. The cells appear to fade more or less quickly. Electron micrographic studies of Bact. coli undergoing phage lysis (Luria, Delbrück and Anderson 1943) gave precise pictures of the adsorption of phage, lysis of the cell, and liberation of phage particles from the interior of the cell, which were mumerically in accord with the results obtained by concurrent phage counts of the infected cultures studied. They also revealed that in multiple infection of a cell, the adsorbed particles did not seem to enter the cell, but remained attached to the cell wall.

The mechanism of the aetual disintegration of lysed bacteria is not understood. In some cases it is doubtful whether such disintegration occurs, at any rate at the moment when the turbidity of the eulture disappears. With some phages, the absence of microscopically apparent disintegration at this moment suggests that the main change is an approximation of the optical eharacter of the bacteria to that of the suspending medium.

The liberation by phage of a lytic enzyme at the time of lysis was postulated by d'Herelle (1926). Sertic (1929, 1937b; Sertic and Boulgakov 1939) observed a halo round each plaque formed by certain coli phages in an opaque growth of Bact. coli. The halo was due to the induction of variants of the Bact. coli used, by a "lysin" which would pass an ultrafilter; the variants grew as a transparent film. The addition of the plage-free lysin to a mixture of Bact. coli and a phage to whieh it was insensitive resulted in lysis. The lysin apparently destroyed certain antigenic constituents of the insensitive Bact. coli, thereby creating a variant of different sensitivity (see also Schuurman 1936). Evans (1940) was able to enhance the activity of streptococcal phages on resistant strains by adding a small quantity of a susceptible strain to the mixture; she explained the reaction in terms of "nascent" pluge. It is probable that she was dealing with a lysin similar to that of Sertic. Campbell-Renton (1937) observed a related phenomenon when phage filtrates inactivated by ultra-violet light were tested on sensitive organisms: there was a diffuse lysis of the bacteria without the formation of plaques. In both these cases the lytic agent appeared to be derived from the bacteriophage. This view was also supported by Gratia (1937). On the other hand, Bronfenbremer and Muckenfuss (1927) obtained a lytic enzyme from both normal and phage-lysed cultures of staphylococci. Wollman $(1934 a)$ upheld the bacterial origin of lytic enzymes. Phage action can be enhaneed 
by lytic enzymes other than those derived from bacteria or phage. Lysozyme, for instauce, will enliance the action of cholera phage (White 1937).

Pirie (1939) observed an increase in reducing sugars and a decrease in precipitable carbohydrate during lysis by coli phage. No enzyme was detected in the phage itself, but the coli bacillus yielded an enzyme that attacked the bacterial cellular polysaccharides. Though no direct connection exists between this phenomenon and lysis by the phage, the association of lysis with the enzymic destruction of those cellular elements known to adsorb phage is at least suggestive. In a later paper (1940) Pirie records the release of adsorbed phage from $B$. megatherium by treatment with lysozyme, and the destruction of the bacterium's capacity to absorb phage by preliminary incubation with lysozyme. The lysozyme hydrolysed a earbohydrate present in $B$. megatherium, and, as Pirie suggests, it is possible that carbohydrate is one that determines the specific absorption of the phage.

\section{Phage Production and Phage Precursor.}

The rapid production of phage from bacteria following the adsorption of one or a few phage particles has suggested to some workers the possibility that the substances of bacterial origin from which the phage is derived exist as a precursor requiring only a few comparatively small transformations to convert it into phage. The analogy of the activation of a pepsinogen by a pepsin has been cited in this connection. It breaks down, however, in one important respect, for with the activation of enzyme precursors by similar enzymes, it is the nature of the precursor which determines the enzyme produced. Thus chicken pepsin converts swine pepsinogen into swine pepsin, and swine pepsin converts chicken pepsinogen into chicken pepsin (Herriott, Bartz and Northrop 1938). A bacterium, on the other hand, may be attacked by several distinct phages, and each phage reproduces itself. If a single phage precursor is postulated, it must then be sufficiently primitive in form to yield a variety of phages when suitably activated. Krneger and Baldwin (1937) made a cell-free extract of staphylococci in the presence of which the titre of a phage filtrate doubled in 2 hours. In staphylococci, this phage precursor was more easily destroyed than either the cells which produced it, or the phage into which it was converted. Apparently protein in nature, its activity was reduced by antistaphylococcal serum, by heating to $45^{\circ} \mathrm{C}$. for 20 minutes, by iodoacetic acid, and by light in the presence of methylene blue. It was not liberated from cells disintegrated by sonic vibrations (see Krueger, Scribner and Mecracken 1940, Krueger, Brown and Seribner 1941).

Spizizen (1943b) has opened up a new field in the study of phage precursors by applying the methods elaborated by workers on bacterial nutrition to the study of phage production by Bact. coli in phosphate buffer. Several amino-acids, certain phosphorylated compounds (including nucleic acid and co-enzyme I), t-carbon dicarboxylic acids, and ferric, ferrous, magnesium and manganese ions, stimulated phage production. Glycine and glycine anbydride were particularly effective in this respect, inducing a marked increase in phage in the absence of bacterial multiplication. The production of phage was even greater if the organisms were exposed to the glycine anhydride for several hours before the addition of the infecting dose of phage, suggesting that, in the interval, the cells either har adapted themselves to a more rapid glycine metabolism, or had built up reserves of a phage precursor. It appears that certain fundamental metabolic processes in the cell are required for phage multiplication but not necessarily all the processes that lead to cell multiplication. The specificity of some of these processes is illustrated by the fact that the action of glycine was specifically inhibited by the 
addition of its sulphone derivatives, amino-methane sulphonic acid, and that the inhibiting action displayed by sulphanilamide was antagonized by $p$-aminobenzoic acid (see Chapter 6).

\section{The Factors that Determine Phage Specificity.}

It has been well established, from the earliest days of bacteriophage study, that any given strain of phage has a relatively limited range of activity. A coliphage, for instance, will not act on a staphylococcus, or on a diphtheria bacillus. It was soon found, however, that phage specificity, while varying widely in range with different strains of phage, might be much narrower than this. A coli-phage would act on some strains of Bact. coli, but not on others, and so on. The obvious assumption was that different strains of the same bacterial species differed in " phage sensitivity," but no attempt was made by the earlier workers to determine on what factors this sensitivity depended. A very significant advance in our knowledge of this problem has been made by the studies of Burnet and his colleagues.

In a study of the activity of different strains of phage on the species and types included within the Salmonella group of bacteria, it was found (Burnet 1927) that there was a close relation between the sensitiveness of different bacterial species, or types, to a particular phage, and the distribution of particular surface somatic antigens. Thus, a particular phage was active against Salm. typhi, Salm. enteritidis and Salm. pullorum, all of which possess the somatic antigen IX, but was inactive against Salm. paratyphi A and Salm. cholera-suis, which do not possess this antigen. If, however, the normal smooth strains of these species were replaced by their rough variants, in which the smooth somatic antigens are lost, their place being taken by a common rough somatic antigen, then a phage that attacked one rough variant would attack all the others. Some phages were found to attack only the normal smooth forms, their range of activity then being determined by the distribution of the various smooth somatic antigens. Other phages attacked only rough forms. A few phages were able to attack both rough and smooth forms (Burnet $1929 a)$.

Further study revealed similar correspondence between antigenic structure and sensitivity to various phages. Schmiclt (1931) noted that different species of salmonella bacilli eould be differentiated by their sensitiveness to different selected phages. In some cases the correspondence is exact, in others less so. Lately it has proved possible to extend the range of phages specific for different antigenic varieties of certain bacterial species by adapting phages to antigemic types for which at first they show little affinity. For example, following the discovery that the action of certain phages on Salm. typhi depended on the presence of the Vi antigen of Felix and Pitt (1934) (see Sertic and Boulgakov 1936a, Scholtens 1936, 1937, Craigie and Brandon 1936a), Craigie and Yen (1938) were able to develop phages with selective action against 11 different varieties of Salm. typhi. In a similar manner Felix and Callow (1943) were able to adapt naturally occurring phages active against $\mathrm{Vi}$ forms of Salm. paratyphi $B$, and so extend the range of phages selective for different varieties of the bacilli. It will be obvious that once the correspondence hetween a bacterial and phage type is established, bacterial strains may be identified and assigned to a given phage type by tests with known strains of phage.

In many cases, a group of bacteria, homogeneous by serological tests, proves on phage testing to consist of a number of stable sub-types. The recognition of these sub-types, as Craigie and Yen first demonstrated, adds greatly to the precision of bacteriological surveys for epidemiological purposes. (See also Helmer, Kerr, Dolman and Ranta 1940, Lazarus 1940, 1941, Dolman, Kerr and Helmer 1941, Desranleau 1942, Hutchinson 1943, Felix 1943.)

There are a number of bacterial species in which the relationship of antigenic structure 
and phage sensitiveness has been recorded; in the Flexner group of dysentery baeilli (Burnet and HcKie 1930b, Clauberg and Marcuse 1932, Radojěič 1936, Miller 1937, Wheeler and Burgdorf 1941); among rough and smooth dysentery strains (Denys 1932) ; among variants of a Bact. coli strain (Sertic and Boulgakov 1937); among rough and smooth strains of $V$. cholerce (Asheshov et al. 1930, 1933b); in Salm. paratyphi $C$ (cholerce-suis) (Levine and Frisch 1936, Frisch and Levine 1936); in Group A streptococci (Evans 1936, 1940, 1942, Evans and Verder 1938, Evans and Sockrider 1942); among certain serological types of $C$. diphtherice (Keogh, Simmons and Anderson 1938); in Staph. aureus (Burnet and McKie 1929, Williams and Timmins 1938, Fisk 1942a, b); and in mucoid strains of Bact. coli, Bact. aerogenes and Bact. friedlünderi (Rakieten, Eggerth and Rakieten $1940)$.

It should be noted that these varieties of antigens and combinations of antigens associated with the specificity of phages are somatic antigens. Sertic and Boulgakov (1936b). however, record a phage which acted only on flagellated forms of Salm. typhi.

The intimate relationship between phage sensitivity and the nature of the surface bacterial antigens has been confirmed by observing the effects on phage lysis of different bacterial extracts. Levine and Frisch (1934) tested the fractions obtained by alcoholic precipitation of saline extracts prepared from bacteria of the Salmonella and Shigella groups, and found that they specifically inhibited the lysis of the homologous organisms by phage. Gough and Burnet (1934), working with more highly purified fractions, record similar results. The fractions obtained in this way were known to consist mainly of the specific polysaccharide somatic antigens. Still further confirmation has been obtained by the demonstration by Levine and his colleagues (Levine, Frisch and Cohen 1934, Levine and Frisch 1935), that heat-killed bacteria of the Salmonella group absorb phages specifically, in general accordance with their antigenic structure as revealed by agglutination teste.

Working with staphylococcal phage, Rakieten, Rakieten and Doff (1936) demonstrated that antolysates of sensitive staphylococci inhibited phage action, but those of resistant cocci did not : the same specificity was displayed by heat-killed staphylococei. Freeman (1937) found that crude preparations of the specific polysaccharide of a staph. aureus strain inactivated a phage to which it was sensitive (see also Burnet and Freeman 1937). The rates of inactivation of a phage hy eulture filtrates are not compatible with the view that total inactivation follows simply from combinations of phage and inhibitory substances (Ellis and Spizizen 1941); there appear to be two eompeting processes, one producing inactivated, the other partly inactivated phage, and full inactivation is achieved only by circumstances favouring one process at the expense of the other (see below, PhageAntiplrage Reaction).

There can, then, be no doubt at all that the antigenic structure of the bacterial surface is one of the main factors in determining the accessibility of the bacterial cell to a given strain of phage; and we must suppose the phage particles to be so constituted that those of one strain can find attachment, or entry, only at an area of bacterial surface that is characterized by a particular antigenic structure, while those of another strain have wider potentialities, and are able to attack a bacterium through more than one type of antigenic surface.

It should, however, be emphasized that this is not the only factor that determines phage sensitivity, or, at least, that there is not complete parallelism between sensitivity and antigenic structure as revealed by the available serological tests. Burnet (1929b), for instance, notes that a phage-resistant strain, appearing as the result of the lytic action of a particular phage on a particular sensitive bacterium, may show exactly the same antigenic structure, as revealed by serological tests, as the sensitive strain from which it was derived. He notes further that the resistant strain not only shows no lysis, but fails to adsorb the phage; so that some change 
in surface structure would appear to be concerned, though it is not detectable by the ordinary serological methods.

\section{The Variations Induced in Bacteria in Response to Phage Lysis.}

In an earlier section of this chapter, it was noted that a secondary bacterial growth usually appears in a culture that has undergone phage lysis. If this growth is further examined, it will be found to be composed of bacteria that are resistant to the strain of phage that caused the lysis, though they will usually be sensitive to other strains of phage.

Sometimes these resistant strains show a well-marked antigenic difference from the original sensitive strain; for instance, the lysis of a smooth strain of a dysentery bacillus by an anti-smooth phage is frequently followed by the appearance of characteristically rough resistant variants. But, as we have seen, resistant strains are not always of a recognizably different antigenic type from the original sensitive strain; and, in the great majority of cases, we have at present no evidence as to whether the change in phage sensitivity has, or has not, been accompanied by a change in antigenic structure. What we do know is that submission to the action of a phage is one of the most potent methods of inducing bacterial variation (see Arkwright 1924, Hadley 1927, 1928), that the variants so produced may be of many different kinds, that they share the character of resistance to the phage that induced the variation, and that some at least of them differ antigenically from the parent strain.

The kind of variation induced is not necessarily similar to variations which, like those noted in Chapter 9, occur in other circumstances. These latter variations may be indeed inhibited by the presence of phage. For instance, Lewis and Worley (1936) noted that spontaneous dissociation of $B$. megatherium occurred much less promptly in the presence of phage than in its absence.

It is clear that the existence of phage-resistant strains of bacteria, and the possibility of producing them at will, afford a method of studying in greater detail the phages acting on single bacterial species.

Bail (1923) was the first to stress the importance of cross-resistance tests between different phages and different bacterial strains as a method of phage differentiation ; and many others have since employed this method in the study of phages acting on particular groups or species of bacteria, such as the Salmonella group (Burnet 1929b), the shigella group (Burnet and MeKie 1930b, Morison 1932), or the cholera vibrios (Asheshov et al. 1930, 1933b, Morison 1932). Many of these studies, however, were concerned with differences in phage sensitivity between different bacterial species or types, as well as between different variants derived by phage action from a single bacterial strain. It is with differences of the latter type, and their application as a method of separating and identifying different phages that are active against a single bacterial species or type, that we are here concerned. This method has been very extensively developed by Asheshov and his colleagues, and we may take an illustrative example of an experiment performed according to their technique (Asheshor et al. 1933b).

Suppose that we have three strains of phage, all acting on the same strain of a given bacterium, but which we suspect, for one reason or another, to belong to different types. We allow each of the three phages, which we may label I, II and III, and a mixture of them (I-II-III), to act on four separate cultures of our sensitive bacterium growing in a fluid medium. We allow lysis to occur, and a secondary growth of resistant bacteria to follow it. We take a large agar plate, and mark it off into 16 squares $(4 \times 4)$. Over each square in the first column of 4 squares we make a thick seeding from the secondary growth from our Type I phage, over each square in the second column we seed the secondary 
growth from our Type II phage, over the squares in the third column the secondary growth from our Type III phage, and over the squares in the fourth and last column the secondary growth from the culture containing all three phages. Then we take a filtrate containing Type I phage, and spread a small drop of it over a circular area in the middle of the top square of each column (i.e. of each square of the first row). In each square of the second row we make a similar inoculation of Type II phage, in each square of the third row an inoculation of Type III phage, and in each square of the fourth and last row, an inoculation of phages I, II and III.

If the phages actually belong to different types, as judged by this test, the results will be as shown in Fig. 53, in which the dark circles indicate the occurrence of confluent lysis over the circular area inoculated with the phage.

It will be noted that Phage I acts on the secondary growth from Phages II and III, but not on the secondary growth from I'hage I, and so on. None of the three phages acts on the secondary growth from the culture submitted to the action of all three phages. The mixture of three phages acts on the secondary growth from each of the three separate phages.

If we desire to test another phage, to see whether or not it is identical with any of our three Types I, II, or III, we include it in such a series of tests, adding the additional row and column to our squares. If it is identical with any of our three types it will behave as that type does. If it is a new type (say Type IV) then it will lyse the secondary growths from Types I, II and III, and its own secondary growth will be lysed by each of these types.

This leads us to a consideration of other ways in which phages may be differentiated from one another. One of these is the method of antigenic analysis, which has been so extensively employed in the identification and classification of bacteria.

\section{Phages as Antigens. The Phage-Antiphage Reaction.}

It was shown by Bordet and Cinca (1921) that the phage is antigenic, and that an antiphage serum has the power of neutralizing the phage and so preventing its Iytic action on bacteria. Numerous workers have since studied this phenomenon, and it has been clearly shown $(a)$ that the inhibition of phage lysis is due to antibodies acting on the phage itself, and not to the antibacterial antibodies that are usually present in an autiphage serum, $(b)$ that the antibodies are specific for particular types of phage, phages thus showing an antigenic specificity of exactly the same kind as that displayed by bacteria, but $(c)$ that the antibodies have no action on the bacteria from which the phage arose; that is, phage and bacterial host are serologically distinct. Many of the papers already referred to contain 
references to the antigenic behaviour of the phages studied, as well as to their other properties.

The studies of Burnet and his colleagues clearly show the close analogy of the serological reactions of phage with those of bacteria. Bacteria which have been allowed to adsorb large amounts of a particular phage, will absorb the antibody from a homologous speeifie antiphage serum, from which all antibodies aeting on the bacteria themselves have been removed. The treated bacteria are agglutinated by the antiphage serum (Burnet 1933c).

Filtrates from phage preparations contain soluble substances that inhibit the reaction of the phage with its antibody and will to some extent reverse a phageantibody union that has already taken place (Burnet 1933d, Burnet et al. 1937). The inhibitory substance here is analogous with the speeifie soluble substance that characterizes many speeies and types of bacteria. Suspensions of largeparticle phages free from bacteria and bacterial debris are agglutinable by antiphage sera (Burnet 1933c, Merrill 1936), and the agglutinating power of the serum for various related phages runs parallel with its power of phage-neutralization.

The quantitative relations between a phage and its homologous neutralizing scrum were first studied in detail by Andrewes and Elford $(1933 a, b)$, who found that, over a considerable range of phage dilutions. a given amount of antiserum neutralized a constant percentage of the phage present, irrespective of the number of phage particles exposed to its aetion (see also Clifton, Mueller and Rogers 1935). This certainly indicates that the particles are inactivated individually and not by aggregation; for if aggregation was effective in reducing the phage-count in the serum-phage mixture, we should expect a greater percentage drop in the count as the eoneentration of phage, and therefore the likelihood of collisions of sensitized phage partieles, increased. The results suggest also that, as we have seen in other antigen-antibody systems (Chapter 7 ), the phage or the antibody, or both, may be heterogeneous, and that in any phage preparation a certain percentage of the particles either unite feebly with antibody, or are in some way insusceptible to inactivation after union (see also Kalmanson and Bronfenbrenner 1942). The validity of these interpretations is questioned by Hershey and his colleagues (1943, 1944) on the grounds that there is little direct, independent evidence of heterogeneity of either antibody or phage. They have observed two types of antibody effect in a coli phage. Thus, with a given amount of homologous antiserum, the phage was sensitized, and its infectivity thereby increased; with double this amount the phage was neutralized and became non-infective. The variations in activity displayed by phage-antiserum mixtures may therefore be interpreted in terms of a homogeneous phage, varying proportions of which are unaltered, increased in infectivity, or decreased in infectivity, according to the degree of union with an essentially homogeneous antibody.

Though there are obvious parallels between inactivation of phage by adsorption to bacterial extracts, and by antiphage sera, it does not appear that the two inactivators unite with the same part of the phage particle (Burnet and Freeman 1937, Burnet et al. 1937), for antigenically similar phages lyse antigenically unrelated bacteria, and phages for antigenically similar bacteria may themselves be antigenically unrelated. Moreover, by adaptation, the affinity of a phage for a bacterium may be profoundly modified without any alteration in antigenic quality. Nevertheless, the active sites of umion of antibody and bacterial inactivator appear to be close together on the phage particle, for union with antibody blocks union with bacterial inactivator, though in some cases the phage is only partly nentralized by the antibody, prodneing lysis on the prepared plate after a delay which may represent the time required for a freeing or a redistribution of the antibody on the phage particle, making the bacterial receptor again accessible. The variation observed in the degree of neutralization and in the rate of the phage-antibody reaction with temperature (Hershey 1941) suggests that the surface of the phage particle 
varies in reactivity, so that not all eollisions of antibody and phage in the nentralization mixtures are fruitful (Kalmanson, Hershey and Bronfenbrenner 1942). Even with fully neutralized phage, there is no evidence that union of antibody with phage alters it permanently, even after prolonged contact (Kalmanson, Hershey and Bronfenbrenner 1942). After 56 days' contact with antibody at $37^{\circ} \mathrm{C}$., Kalmanson and Bronfenbrenner (1943) were able to reactivate a just-neutralized phage by digesting the inactive phage with papain. The digestion apparently removed only a portion of the combined antibody molecules, but in doing so made the bacterial receptors fully accessible.

The relations of phage-inactivation by antisera and by bacterial antigens are summarized in Fig. 54, which is modified from a scheme devised by Delbrück (1942).

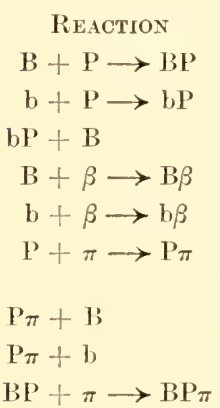

\section{RESULT}

Adsorption of phage to bacteria; lysis.

Adsorption of phage to free bacterial antigen; inactivation of phage. No lysis, or delayed lysis, according to degree of phage-inactivation. Antigen-antibody union; agglutination of bacteria. Antigen-antiboly union; precipitation of bacterial antigen.

Antigen-antibody union; inactivation of phage; (agglutination of large. particle phages).

No lysis, or delayed lysis, according to degrce of phage-inactivation.

No adsorption of phage to free bacterial antigen.

Antigen-antibody union; agglutination of phage-coated bacteria by antiphage serum.

FIG. 54. The reactions of phage-inactivation by bacterial antigens and specific

$\mathbf{B}=$ bacterium. antiphage sera.

$\mathrm{b}=$ antigenic component of bacterium associated with specific adsorption of phage.

$\beta=$ antibody to $\mathrm{b}$.

$\mathrm{P}=$ phage with affinity for $\mathbf{B}$.

$\pi=$ antibody to $\mathrm{P}$.

\section{Other Methods of Phage Differentiation, and the Identification and Classification of Phages.}

The phage plaques that have so frequently been referred to in preceding sections have characters that are of great value in the differentiation of one phage from another. For any one phage, acting on any one sensitive bacterium, the plaques formed on an agar plate are usually closely similar, both in size and in form. But different phages acting on the same bacterium may give plaques that differ sharply and characteristically from one another.

To take first the character of plaque size, the existence of large-plaque-forming phages and small-plaque-forming phages has long been recognized, and used as a basis for differentiation (see Bail 1923). Elford and Andrewes (1932) were able to show that there is an inverse relation between plaque size and phage size. The small phages give big plaques, and vice versa. It is an obvious assumption that the small phages can diffuse more readily through the surface bacterial growth, and so extend over a larger radius. The probability of this assumption is increased by the demonstration by Andrewes and Elford (1933b) that a large-plaque-forming phage, incompletely neutralized by an antiphage serum and probably aggregated into small clumps, gives small instead of large plaques.

But size is not the only character that differentiates one pliage plaque from another; there are often marked differences in the edge of the circular clearings. 
These may be entire or eroded, sharp or bevelled. They may, or may not, be surrounded by a halo in which the bacterial growth is altered in appearance, though not completely lysed. Phage plaques, indeed, present variations in morphology as distinctive as those shown by bacterial colonies, and have the same classificatory

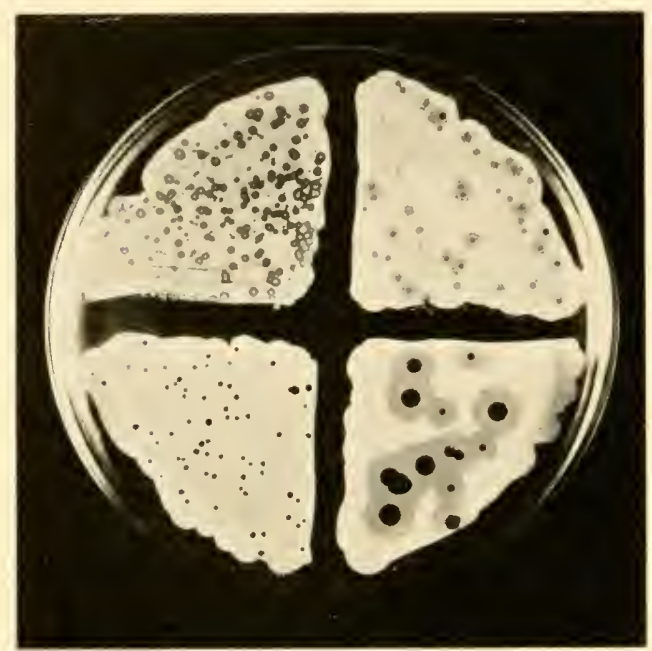

FIG. 55.

Different types of plaque produced by different phages acting on the same bacterial culture. value. Asheshov (1924) (see also Asheshov el al. 1933b) has paid particular attention to these differences in plaque morphology, and Fig. 55 illustrates the differences, in size and form, that may be observed among the plaques produced by different phages acting on the same strain of a sensitive bacterium.

If, then, we seek to classify any particular group of phage strains, we can make use of the following series of tests.

(A) The determination of the species of bacteria against which each phage is active. This will enable us to differentiate broad groups - coli-phages, dysentery phages, salmonella phages, staphylococcal phages, cholera phages, and so on.

(B) By more detailed tests of cross-resistance we can subdivide these broad groups, each into a number of different types - so many different types of dysentery phage, so many of cholera phage, and so on.

(C) We can apply to the same nroblem of the subdivision of our broad groups the method of antigenic analysis, using specific antiphage sera.

(D) We can study the size and morphology of the plaques formed, and divide our original groups of phages into large-plaque-forming strains, small-plaque-forming strains, and so on.

(E) We can also apply certain other biological tests, such as relative resistance to heat, or to the photodyuamic action of methylene blue, or to the presence of citrate in a medium.

When, in fact, we apply several of these tests, our confidence in each of them is increased by funding that the results they give are highly correlated. The classification derived from detailed resistance tests corresponds closely with that derived from neutralization tests with antiphage sera. The phages producing a particular type of plaque, when acting on a particular sensitive bacterium, are usually found to fall into the same antigenic group. An admirable series of studies in which this correlation is hrought out very clearly have been recorded by Burnet $(1933 a, b)$; and other evidence, all pointing in the same direction, will be found in the studies of Asheshov and his colleagues (1930, 1933b), in papers by Sertic and Boulgakov $(1935 a, b)$, and in many of the other records referred to above. The correspondence is not, of course, absolute. Just as some groups of bacterial strains would be divided into the same species or types, whether classified on the basis of 
fermentation tests or antigenic structure, while other groups that are identical in regard to their fermentation reactions may be divided into different types on the basis of serological tests, so one particular method of analysis may serve to separate strains of phage that would be grouped together when tested by another. But correlation of characters is as frequent among the phages as it is among the bacteria, and leaves no reasonable doubt that, by making full use of the methods now at our disposal, we can separate and identify phage types that have just as much claim to be regarded as biological entities as have the various species or types of bacteria.

\section{Variation and Adaptation in Phages.}

The literature contains very numerous records of phage variation and phage adaptation. D'Herelle, as has been noted, regards the phage as a single virus, that may become adapted to attack a wide variety of bacterial species. Many of the earlier accounts of adaptation must be discounted, because the technique adopted did not ensure the purity of the original filtrates. There are, on the other hand, numerous observations which show quite clearly that adaptation occurs, though its range is probably more limited than was at one time supposed. Here, again, the evidence suggests that the phage behaves in the same way as other known micro-organisms.

We may note, for example, that d'Herelle and Rakieten (1935) adapted a staphylococcus bacteriophage, initially sensitive to neutralization by antiphage serum, to lyse cocci in the presence of high serum concentrations. Burnet and Lush (1936) observed a mutant from a Staph. albus phage. The two varieties were similar, both serologically and by cross-tests on resistant survivors (see preceding section), though one had less lytic power than the other. But in one case the resistant strain was lysogenic and in the other, not; and infection of a culture with the weak variant protected it against lysis by the strong variant.

\section{The Ecology of Phages.}

The problem of phage ecology raises points of the greatest biological interest and importance. We have already noted that phages acting on one or other of the normal or pathogenic intestinal bacteria can almost always be isolated from fæces, from sewage, or from polluted water supplies (see, for instance, Sonnenschein 1927, Stewart and Ghosal 1931, Gildemeister and Watanabe 1931, Schlossmann 1932, von Vagedes and Gildemeister 1934, De and Paul 1940, de Assumpção and Leite e Silva 1942, Guelin 1943). This clearly suggests that wherever particular species of bacteria occur in nature, there also are likely to be found phages to which these bacteria are sensitive. But that is merely the first, and least interesting step in the problem.

Just as it was tacitly assumed, in the earliest days of medical bacteriology, that a pathogenic bacterium, when it became parasitic on its natural host would always, or almost always, cause the specific disease of which it was the causal agent, so it was assumed in the early days of bacteriophage studies that a phage, if it became attached to, and multiplied in association with, a particular bacterium would cause phage lysis. The conception of phage-carriers among bacteria, or of a normal phage flora living symbiotically with certain bacterial species, came relatively late.

The demonstration that a phage, active against a particular species of bacterium, could sometimes be isolated from an old laboratory culture of that organism by repeated filtration, and the addition of each successive filtrate to a fresh broth culture of the bacterium 
in question (Gildemeister and Herzberg 1923, Kuttner 1923, Kline 1927, Hadley 1928, Fukuda 1928, Klieneberger 1932) was at first regarded as a powerful argument against the virus theory, and in favour of the view that the phage was a product, enzymic or other, of the bacteria themselves. It was indeed the failure of many workers to confirm these findings (Ogata 1924, Reichert 1924, Meissner 1924a, Arkwright 1924, Sonnenschein 1927), rather than any realization that they were quite compatible with the virus theory, that deprived them of much of their force. It is quite probable (see Burnet 1934) that some, at least, of the positive findings were due to technical errors. But in the light of our present knowledge it would be by no means surprising if many of them were correct.

Our present conception of the real nature of phage-bacterium parasitism has been determined largely by the proved existence of what are known as "lysogenic strains" of bacteria.

The classical examples of these lysogenic strains are the Bact. coli strain, which was first described by Lisbonne and Carrère (1922) and later studied by Bordet and Renaux (1928), and another Bact. coli strain which was studied by Gildemeister and Herzberg (1924a). Both these strains, although they themselves show no evidence of phage lysis, regularly yield filtrates which lyse Sh. shiga, i.e. they are permanent carriers of a phage to which they are themselves resistant, but to which Sh. shigce is sensitive.

Such strains were, for a time, regarded as bacteriological anomalies, but such a view is no longer tenable. Here, again, our present concepts have been largely influenced by the studies of Burnet (1932, 1934).

In a careful study of 34 strains of Salm. enteritidis he found that 27 were lysogenic, in the sense that they yielded a phage to which they were themselves resistant, but which produced transmissible lysis in other, specially susceptible bacterial strains. From these 27 lysogenic strains three different types of phage were obtained, A, B, and D; fourteen strains yielded phage $B$, seven phage $D$, two $A$ and $B$, and one A and D. Phage A was also frequently isolated from lysogenic strains of Salm. paratyphi A, Salm. paratyphi $B$ and Salm.typhi-murium, while phages B and D, and another phage $\mathrm{N}$ were sometimes present in strains belonging to these species. Such results as these, as Burnet points out, are incompatible with the view that the lytic agent in these lysogenic strains is some com. ponent of the genetic apparatus of the bacterial cell.

In the case of Salm. paratyphi C, on the other hand, all strains examined, whether they had been isolated in Russia, South America, or the East Indies, were found to be carrying a single antigenic type of phage. A similar state of affairs may, as Burnet points out, exist in the case of $C$. diphtherice, since Smith and Jordan (193I) found that every strain examined was lysogenic when tested against a single, sensitive indicator strain of the same species. The occasional occurrence of "nibbled" colonies that have been noted by many workers in certain bacterial cultures represent, in Burnet's view, lysogenic strains in which the resistance of the carrying bacterium is slightly unstable.

We must, then, if we are to accept the virus hypothesis, to which all other evidence clearly points, also accept the view that symbiosis between phage and bacterium is an exceedingly common event; so common that it wonld at the moment be unwise to assert that any bacterial strain was certainly not carrying phage.

What place this symbiotic process will eventually take in our conceptions of bacterial structure, bacterial variation, antigenic behaviour, and other similar problems we cannot yet proplesy. It may be a relatively minor one; but it may not.

(For some of the methods of unmasking lysogenic strains, see Flu 1938b, Lominsti 1938, Fisk 1942a). 
We may note here one of the few instances of a geographical distribution of a phage. White (1937), examining a number of strains of $V$. cholerce, found a certain phage in all strains isolated in India, but in none of several strains of Chinese or Japanese origin.

In the carrier state represented by a lysogenic strain (see Burnet 1934) it is clear that the multiplication of phage and bacterium must be so co-ordinated that, when a bacterium divides, each daughter cell receives its quota of phage. It has in fact been shown (den Dooren de Jong 1931, Cowles 1931) that when a lysogenic strain is a spore-bearer, the phage is present in the spore and in the new generations that arise from it. It is also noteworthy that in such strains the phage appears to share the heat resistance of the host, being relatively resistant in the spore, and susceptible when in the vegetative cell. Wollman and Wollman (1939) regard this fact as strong evidence for the bacterial origin of phage.

One other aspect of the ecology of phages must be mentioned, namely, the animal origin of phage. Certain workers concluded that the passage of various bacteria through the intestinal tract of warm-blooded animals results in the appearance of phages not previonsly present either in the animal's intestine or in the cultures administered. Neither Naito (1936), who worked with hens reared from the egg on sterile diets, nor Glaser (1938), who used aseptically reared flies, conld find support for this notion. The recorded cases appear to have been due to insufficiently sensitive methods for detecting phage. Ohashi (1939), for instance, obtained phage more easily from the viscera than from the froces or intestinal mucosa of mice.

The possible relation of phages to bacterial infections in man and animals is considered in Chapter 54.

\section{REFERENCES}

Andrewes, C. H. and Elford, W. J. (1932) Brit. J. exp. Path., 13, 13; (1933a) Ibid., 14, 367; (1933b) Ibid., 14, 376.

Arkwright, J. A. (1924) Brit. J. exp. Path., 5, 23.

Asheshov, I. N. (1924) J. infeet. Dis., 34, 536; (1926) C. R. Soc. Biol., 94, 687.

Asheshov, I. N., Asheshov, I., Khan, S., and Lahiri, M. N. (1930) Indian J. med. Res., 17, 971 ; (1933a) Ibid., 20, 1101.

Asheshov, I. N., Asheshov, I., Khan, S., Lahiri, M. N., and ChatterJi, S. K. (1933b) Indian J. med. Res., 20, 1127.

Assumpção, L. de and Leite e Silva. (1942) Arq. Hig. Saude públ., 14, 99.

Bachman, W. and Wohlfeil, T. (1927) Zbl. Bakt., 104, 256.

BAIL, O. (1923) Z. ImmunForseh., 38, 57.

Baker, S. L. and Nanavetty, S. H. (1929) Brit. J. exp. Path., 10, 45.

Bayne-Jones, S. and Sandholzer, L. A. (1933) J. exp. Med., 5\%, 279.

BeChHold, H. and Villa, L. (1926) Z. IIyg. InfehtKr., 105, 601.

BorCHARDT, W. (1924) Klin. W'schr., 3, 278.

Bordet, J. (1923) Brit. med. J., i. 175 ; (1925) Ann. Inst. Pasteur, 39, 717 ; (1926) C. R. Soc. Biol., 94, 403.

Bordet, J. and Cluca, M. (1920) C. R. Soc. Biol., 83, 1296 ; (1921) Ibid., 84, 280.

Bordet, J. and Renaux, E. (1928) Ann. Inst. Pasteur, 42, 1283.

Bronfenbrenner, J. (1924-25) Proc. Soc. exp. Biol., N.Y., 22, 81 ; (1926) Science, 63, 51 ; (1927) J.exp. Med., 45,373 ; (1928) "Filterable Viruses" (Rivers) Baillière, Tindall and Cox, London, p. 373.

Bronfenbrenner, J. J. and Korb, C. (1925a) J.exp. Med., 41, 73 ; (1925b) Ibid., 42, 483.

Bronfenbrenner, J. and Muckenfuss, R. S. (1927) J. exp. Med., 45, 887.

Bronfenbrenner, J., Muchenfuss, R. S., and Hetler, D. M. (1927) Amer. J. Path.. 3,562 .

Bronfenbrenner, J. J. and Reichert, P. (1926-7) Proc. Soc. exp. Biol., N.Y., 24, 176. 
Burnet, F. M. (1925) J. Path. Bact., 28, 407 ; (1927) Brit. J. cxp. Path., 8, 121 ; (1929a) J. Path. Bact., 32, 15 ; (1929b) Ibid., 32, 349 ; (1929c) Brit. J. exp. Path., 10, 109 ; (1932) J. Path. Bact., 35, 851 ; (1933a) Ibid., 36, 307 : (1933b) Ibid., 3\%, 179 ; (1933c) Brit. J. exp. Path., 14, 93 ; (1933d) Ibid., 14, 100; (1933e) Ibid., 14, 302; (1934) Biol. Rev., 9, 332 .

Burnet, F. M. and Freeman, M. (1937) A ust. J. exp. Biol. med. Sci., 15, 49.

Bunnet, F. M., Keogh, E. V., and Lush, D. (1937) Aust. J. exp. Biol. med. Sci.. 15, 227. Burnet, F. M. and Lush, D. (1936) Aust. J. exp. Biol. med. Sci., 14, 27.

Burnet, F. M. and McKie, M. (1929) Aust. J. exp. Biol. med Sci., 6, 21 ; (1930a) Ibid., 7, 183, 199; (1930b) J. Path. Bact., 33, 637 ; (1933) Ibid., 36, 299.

Campbell-Renton, M. L. (1937) J. Path. Bact., 45, 237 ; (1941) Ibid., 53, 371 ; (1942) Ibid., 54, 235.

Clauberg, K. W. and Marcuse, K. (1932) Zbl. Bakt., 124, 29.

Clifton, C. E. (1931) Proc. Soc. exp. Biol., N.Y., 28, 745.

Clifton, C. E. and Morrow, G. (1936) J. Bact., 31, 441.

Cliftox, C. E., Mueller, E., and Rogers, W. (1935) J. Immunol., $29,377$.

Costa Cruz, J. Da. (1926) C. R. Soc. Biol., 95, 1501.

Cowles, P. B. (1931) J. Bact., 22, 119.

Craigie, J. and Brandon, K. F. (1936a) Canad. publ. Hlth. J., 27, 165; (1936b) J. Path. Bact., 43, 233.

Craigie, J. and Yex, C. H. (1938) Canad. publ. Hlth. J., 29, 448, 484.

DE, S. P. and PauL, S. (1940) Calcutta med., J., 3\%, 499.

Delbrück, 11. (1940) J.gen. Physiol., 23, 631, 643; (1942) Advanccs Euzymology, 2, 1. Denys, P. (1932) Ann. Inst. Pasteur, 48, 349.

Jesranleat, J. H. (1942) Canad. publ. IIlth. J., 33, 122.

Dolman, C. E., Kerr, D. E., and Helmer, D. E. (1941) Canal. publ. IIlth. J., $32,113$. Dooren de Jong, L. E. Den. (1931) Zbl. Bakt., 120, 1.

Editorial (1935) Lancet, i. 818.

Eisenberg-Merling, K. B. (1941) J. Path. Bact., 53, 385.

Elford, W. J. (1936) Brit. J. exp. Path., 17, 399.

Elford, W. J. and Andrewes, C. H. (1932) Brit. J. exp. Path., 13, 446.

Ellis, E. L. and Decbrück, M. (1939) J. gen. Physiol., 22, 365.

Ellis, E. L. and Spizizen, J. (1941) J. gen. Physiol., 24, 437.

Evans, A. C. (1936) J. Bact., 31, 423; (1940) Ibid., 39, 597 ; (1942) Ibid., 44, 207.

Evans, A. C. and Sockrider, E. M. (1942) J. Bact., 44, 211.

Evans, A. C. and Verder, E. (1938) J. Bact., 36, 133.

Feenster, R. F. and Wells, W. F. (1933) J.exp. Med., 58, 385.

Felix, A. (1943) Brit. med. J., i, 435.

Felix, A. and Callow, B. R. (1943) Brit. med. J., ii, 127.

Felix, A. and Pitt, R. M. (1934) J. Path. Bact., 38, 409.

Fisk, R. T. (1942a) J. infect. Dis.; 71, 153; (1942b) Ibid., 71, 161.

Fud, P. C. (1923) Zbl. Bakt., 90, 362; (193sa) Ann. Inst. Pasteur, 60, 610; (1938b) Acta leidensia (Schol. med. trop.), 12-13, 103, 113, 118.

Freeman, M. (1937) Aust. J exp. Biol. med. Sci., 15, 221.

Frisch, A. W. and Lerine, P. (1936) J. Immunol., 30, 89.

FUKUdA, Y. (1928) Z. ImmunForsch., 54, 369.

Gates, F. L. (1934) J. exp. Med., 60, 179.

Gerске, А. (1925) Zbl. Bakt., 94, 387.

Gest, H. (1943) J. infect. Dis., 73, 158.

Gildemeister, E. and HerzberG, K. (1923) Zbl. Bakt., 91, 12 ; (1924a) Ibid., 93, 402; (1924b) Klin. Wschr., 3, 186.

Gildemeister, E. and Wataxabe, H. (1931) Zbl. Bakt., 122, 556.

Glaser, R. II. (1938) Amer. J. Hyg., 27, 311.

GoHs, W. and JACOBsohn, I. (1927) Z. ImmunForsch., 53, 12.

Gough, G. A. C. and Burnet, F. M. (1934) J. Path. Bact., 38, 301.

Gózony, L. and Surányi, L. (1925) Zbl. Bakt., 95, 353.

Gratia, A. (1936) Ann. Inst. Pasteur, 56, 307; (1937) C. R. Soc. Biol., 126, 418 ; (1940) Ibid., 133, 445, 702 .

Gratia, A. and Rhodes, B. (1926) Lancet, i. 204.

Guelis, A. (1943) Ann. Iust. Pasteur, 69, 219.

HadLey, P. (1927) J. infect. Dis., 40, 1 ; (1928) Ibid., 42, 263.

Helmer, D. E., Kerr, D. E., Doluan, C. E., and Ranta, L. E. (1940) Canad. publ. Hlth. J., $31,433$.

D'Herelle, F. (1917) C. R. Acad. Sci., 165, 373; (1921) "Le Bactériophage: son rôle dans l'immunité." Masson, Paris. Eng. transl., Baltimore and London, 1922. (1926) "Le Bactériophage et son Comportement." Masson, Paris. Eng. transl., Baltimore and London. (1930) "Bacteriophage and its clinical applications." Thomas, Springfield, Illinois. 
D'Herelle, F. and Rakieten, M. L. (1935) J. Immunol., 28, 413.

Herriott, R. M., Bartz, Q. R., and Northrop, J. H. (1938) J. gen. Physiol., 21, 575. Hershey, A. D. (1941) J. Immunol., 41, 299.

Hersiey, A. D. and Bronfenbrenner, J. (1943) J. Bact., 45, 211.

Hershey, A. D., Kalmanson, G., and Bronfenbrenner, J. (1943) J. Immunol., 46, 267, 281 ; (1944) Ibid., 48, 221.

Hutchinson, J. R. (1943) Brit. med. J., ii, 130.

Kabéshima, T. (1920) C. R. Soc. Biol., 83, 219.

Kilmanson, G. and Bronfenbrenner, J. (1939) J. gen. Physiol., 23, 203.

Kalmanson, G. M. and Bronfenbrenner, J. (1942) J. Immumol, 45, 13 ; (1943) Ibid., $4 \%, 387$.

Kalianson, G. M., Hersiey, A. D., and Bronfenbrenner, J. (1942) J. Immunol., 45, 1.

KaUfemann, F. (1925-26) Z. Hyg. InfektKr., 105, 594.

Kendall, A. I. and Colwell, C. A. (1938) J. infect. Dis., 63, 81.

Keogh, E. V., Simmons, R. T., and Anderson, G. (1938) J. Path. Bact., 46, 565.

Klieneberger, E. (1932) Zbl. Bakt., 123, 318.

Kligler, I. J. and OleiNik, E. (1943) J. Immunol., 47, 325.

Kline, G. M. (1927) J. Lab. clin. Med., 12, 1074.

KNorr, M. and Rur, H. (1935) Zbl. Bakt., 133, 289.

Krueger, A. P. (1931) J. gen. Physiol., 14, 493.

Krueger, A. P. and Baldwin, D. M. (1935) J. infect. Dis., 5\%, 207 ; (1937) Proc. Soc. exp. Biol.. N.Y., 3\%, 393 .

Kirueger, A. P., Brown, B. B., and Scribner, E. J. (1941) J. gen. Physiol., 24, 691.

Krueger, A. P. and Fong, J. (1937) J. gen. Physiol., 21, 137.

Krueger, A. P. and Northrop, J. H. (1931) J. gen. Physiol., 14, 233.

Krueger, A. P., Ritter, R. C., and Shith, S. P. (1929) J. exp. Med., 50, 739.

Krueger, A. P., Scribner, E. J., and Mecracken, T. (1940) J. gen. Physiol., 23, 705.

KutTNer, A. G. (1923) J. Bact., \%, 49.

LAzARUS, A. S. (1940) Amer. J. publ. Ilth., 30, 1177 ; (1941) Ibill., 31, 60.

LePper, E. (1923) Brit. J. exp. Path., 4, 204.

Levin, B. S. and Lominski, I. (1936) C. R. Soc. Biol., 122, 1286.

lievine, P. and Frisch, A. W. (1934) J.exp. Med., 59, 213; (1935) J. infect. Dis., 5\%, 104; (1936) J. Immunol., 30, 63.

Levine, P., Frisch, A. W., and Cohen, E. V. (1934) J. Immunol., 26, 321.

LEwis, I. M. and Worley, G. (1936) J. Bact., 32, 195.

Lisbonne, M. and Carrère, L. (1922) C. R. Soc. Biol., 86, 569.

Lominski, I. (1938) C. R. Soc. Biol., 129, 264.

Luria, S. (1940) Ann. Inst. Pasteur, 64, 415.

Lurla, S. E., Delbrück, M. and Anderson, T. F. (1943) J. Bact., 46, 57.

Luria, S. E. and Exner, F. M. (1941) Proc. nat. Acal. Sci. H'ash., 27, 370.

Lwoff, A. (1936) Ann. Inst. Pasteur, 56, 165.

McIntose, J. and Selbie, F. R. (1937) Brit. J. exp. l'ath., 18, 162.

McKinley, E. B. and Holden, M. (1926) J. infect. Dis., 39, 451.

Manninger, R. (1926) Zbl. Bakt., 99, 203.

Meissner, G. (1924a) Zbl. Bakt., 91, 149; (1924b) Ibid., 92, 424.

Merling-Eisen bero, K. B. (1938) Brit. J. exp. Path., 19, 33s.

MerRILL, M. H. (1936) J. Immunol., 30, 169.

Miller, A. A. (1937) Ann. Inst. Pasteur, 58, 709.

Morison, J. (1932) "Bacteriophage in the treatment and prevention of cholera." Lewis \& Co., London.

Moriyama, H. and Ohashi, S. (1937) J. Shanghai Sci. Inst., Sect. IV, 3, 155, 161.

NaITo, R. (1936) Zbl. Bakt., 138, 34.

Natarajan, C. V. and Hyde, R. R. (1930) Amer. J. Hyg., 11, 652.

Northrop, J. H. (1938) J. gen. Physiol., 21, 335 ; (1939) Ibid., 23, 59.

Ogata, N. (1924) Zbl. Bakt., 93, 329.

Ohashi, S. (1938) J. Shanghai Sci. Inst., Sect. IV, 3, 279; (1939) Ibid., 5, 1.

OLsEN, O. and Y ASAKI, Y. (1923) Klin. Wschr., 2, 1879.

OtTo, R. and Munter, H. (1923) Z. Hyg. InfektKr., 100, 402.

Païc, M., Krassnoff, D., Haber, P., Reinié, L., and Toet, J. (1938) Ann. Inst. Pasteur, $60,227$.

Perdrad, J. R. and Todd, C. (1933) Proc. roy. Soc., B, 112, 277.

Pirie, A. (1939) Brit. J. exp. Path., 20, 99 ; (1940) Ibid., 21, 125.

Preisz, H. von. (1925) "Die Bakteriophagie, etc." Fischer, Jena.

RADOJC̆ıč, M. I. (1936) Zbl. Bakt., 136, 326.

Rakieten, M. L., Eggerth, A. H., and Rakieten, T. L. (1940) J. Bact., 40, 529.

Rakieten, M. L. and Rakieten, T. L. (1937) Yale J. Biol. Med., 10, 191.

Rakieten, M. L. Rakieten, T. L., and Doff, S. (1936) J. Bact., 32, 505. 
REICHERT, F. (1924) Zbl. Bakt., 91, 235.

Reska, H. (1941) Naturwiss., 29, 367.

Schlesinger, M. (1932-33a) Z. IIyg. InfektKr., 114, 161 ; (1932-33b) Ibid., 114, 746; (1933) Biochem. Z., 264, 6; (1934) Bioehem. Z., 273, 306.

Schlossmann, K. (1932) Z. Hyg. InfektKr., 114, 65.

Sснмidт, A. (1931) Zbl. Bakt., 123, 202, 207.

Scholtens, R. T. (1936) J. Hyg., Camb., 36, 452 ; (1937) Ibid., 3\%, 315.

Schultz, E. W. and Krueger, A. P. (1928) Proc. Soc. exp. Biol., N.Y., 26, 97.

Schudrman, C. J. (1925) Zbl. Bakt., 95, 97; (1936) Ibid., 13\%, 438.

Schudrman, C. J. and Schudrman-ten Bokkel Huinink, A. Ml. (1936) Zbl. Bakt., 136, 264.

Schwartzman, G. (1926-27) Zbl. Bakt., 101, 62.

Scribner, E. S. and Krueger, A. P. (1937) J. gen. Physiol., 21, 1.

Sertic, V. (1929) C. R. Soc. Biol., 100, 477; (1937a) Ibid., 124, 14, 98; (1937b) Ibid., $126,1074$.

Sertic, V. and Boulgakov, N. (1935a) C. R. Soc. Biol., 119, 983 ; (1935b) Ibid., 119, 985.

Sertic, V. and Boulgakov, N. A. (1936a) C. R. Soc. Biol., 122, 35 ; (1936b) Ibid., 123, 887 ; (1937) Ibid., 126, 734; (1939) Ibid., 132, 444.

Smith, G. F. and Jordan, E. O. (1931) J. Bact., 21, 75.

Sonnenschein, C. (1927) G. Batt. Immun., 2, 32.

Spät, W. (1924) Med. Klin., 20, 184.

SPIZIZEN, J. (1943a) J. infect. Dis., 73, 212 ; (1943b) Ibid., 73, 222.

Stassano, H. and de Beaufort, A.-C. (1925) C. R. Soc. Biol., 93, 1378.

Stewart, A. D. and Ghosal, S. C. (1931) Indian J. med. Res., 19, 137.

Topd, C. (1927) Brit. J. exp. Path., 8, 369.

Twort, F. W. (1915) Lancet, ii. 1241 ; (1922) Brit. med.J., ii. 293 ; (1925) Lancet, ii. 642 ; (1926) Ibid., i. 416.

Vagedes, K. von and Gildemeister, E. (1934) Zbl. Bakt., 131, 414.

Wheeler, K. M. and Burgdorf, A. L. (1941) Amer. J. publ. IIlth., 31, 325.

White, P. B. (1937) J. Path. Bact., 44, 276.

Wiebols, G. L. and Wieringa, K. T. (1936) "Fonds Landbouw Export Bureau, 1916-18. No. 16."

Williams, C. H., Sandholzer, L. A., and Berrý, G. P. (1940) J. Bact., 40, 517.

Williams, S. and Timmins, C. (1938) Med. J. Aust. ii, 687.

Wollman, E. (1925) Ann. Inst. Pasteur, 39, 789 ; (1927) Ibid., 41, 883 ; (1928) Bull. Inst. Pasteur, 26, 1 ; (1929) Ann. Inst. Pasteur, 43, 359; (1934a) C. R. Soc. Biol., 115, 1616. (1934b) Bull. Inst. Pasteur, 32, 945 ; (1935) Lancet, ii. 1312.

Wollman, E. and Lacassagne, A. (1940) Ann. Inst. Pasteur, 64, 5.

Wollman, E. and Wollman, E. (1932) Ann. Inst. Pasteur, 49, 41 ; (1936) Ibid. 56, 137 ; (1938) Ibid., 60, 13; (1939) C. R. Soc. Biol., 131, 442.

Wright, E. V. and Kersten, H. (1937) J. Bact., 34, 639.

YAOI, H. and SATO, K. (1935) Jap. J. exp. Med., 13, 565.

Zinsser, H. and TANG, F. F. (1927) J. exp. Med., 46, 357. 


\title{
PART II \\ SYSTEMATIC BACTERIOLOGY
}

\author{
CHAP'TER 12
}

\section{THE METHODS OF OBTAINING PURE CULTURES, AND THE IDENTIFICATION OF BACTERIA}

\section{Methods of Obtaining Pure Cultures of Bacteria}

ONE of the first essentials in the study of bacteriology is the preparation and maintenance of pure cultures of bacteria. Neglect of this leads inevitably to confusion. During the sixties and seventies of last century, micro-organisms were perforce cultivated in liquid media ; and as the preparation of pure cultures in such media is often difficult and sometimes impossible relatively little progress in the identification of particular species was made in these years. It was the introduction of solid media by Robert Koch in 1881 that rendered possible the easy separation of different organisms from one another in a mixed culture, and provided a means for distinguishing macroscopically between different species of bacteria. Koch found that on a suitable solid medium, such as potato or gelatin, most organisms formed characteristic colonies by which they could be readily identified; by inoculation of separate colonies into tubes of a liquid medium, pure cultures could be obtained. These could again be streaked on to a solid medium, and if the resulting colonies were all of the same appearance, it might be concluded that the liquid culture probably contained only one species of bacterium. This method-Koch's plating method-affords the simplest and most rapid means of separating one organism from another; we shall later describe it more fully.

We have now at our command numerous methods of purifying cultures. Some are of limited utility, or are suited solely to certain organisms; others are of wider applicability. Without discussing the technical details we shall give a brief description of the principles underlying the more important of these methods.

A. Dilution Method.-In point of time, this was the first method introduced for obtaining pure cultures of a bacterium, a method which we owe to Lister (1878). The mixed culture is diluted with sterile tap water, or other suitable fluid, till there is only about one organism in every two drops of the mixture. A series of tubes containing broth is then seeded, each with one drop of the diluted culture. If the dilution has been correctly gauged, there should be a growth in approximately 
every alternate tube. This method affords one no certainty that the cultures obtained are pure; further study must be undertaken to ascertain this. The objections to the method are that too much guess-work is involved in judging the correct dilution, and that several tubes of medium are inevitably wasted. It is useful, however, in a modified form in conjunction with the plating method. That is to say, when the culture is thick, it is wise to dilute it considerably before plating out; in this way there is more likelihood of obtaining single colonies from single organisms.

B. Koch's Plating Method.-Originally the solid medium used was spread out in the melted state on microscopic slides and allowed to set; these were then streaked with a needle dipped in the culture, and incubated in a moist bell-jar. Later, large glass plates were used; these had to be specially levelled by means of adjustable screw supports, and covered with a bell-jar. The method now employed is to pour the melted medium into Petri dishes; each of these is provided with a cover, which protects the medium from contamination. The plating method may be employed in one of two ways. Either the culture material may be streaked on the surface of the solid medium, or it may be mixed with the medium in the melted state, poured out into Petri dishes, and allowed to set. The former method results in a surface growth, the latter in a growth throughout the whole thickness of the medium. As a rule the former method is the more useful. In streaking the surface of the medium, a drop of the fluid culture may be placed in the centre of the dish, and spread out in all directions by means of a sterile glass or metal rod bent at a right angle. This results in an even distribution of organisms over the plate. If, however, single colonies are particularly desired-and this is usualit is best to make a series of streaks across the plate with a platinum loop dipped in the culture; the streaks should be about $10 \mathrm{~mm}$. apart, and may be crossed at right angles; the platinum loop should not be re-charged with culture during the process. If preliminary dilution has not been performed, it is often advisable to continue the streaking over a second or even a third plate without re-charging the loop. On the first plate the growth may be entirely confluent; but on the second and third, single colonies will generally be obtained. These single colonies can then be examined with a hand lens, and picked off with a platinum needle into broth. Except with certain organisms, single colonies obtained in this way, especially if the culture has been previously diluted, are generally derived from single organisms, and are hence pure. The purity of colonies picked from overcrowded plates is less certain. In using the surface streak method it is important that the plates should be fairly dry ; if there is a film of moisture on the medium resulting partly from condensationand partly from expression-organisms, particularly if motile, are apt to form a confluent growth over the whole surface. This point must be borne in mind, especially when attempting to isolate anaerobic bacteria. These organisms, instead of growing up from the medium, frequently spread in a thin layer over it; the edges of the colonies are difficult to define, and if there is a film of moisture over the medium, they coalesce, thus rendering their isolation impossible. The pour-plate method is, in general, of less value ; the deep colonies are not usually as characteristic as the surface colonies, and to pick them off involves stabbing the medium with a platinum wire - a process that takes longer than the simple one of surface picking. In carrying out this method of plating, a tube containing $15 \mathrm{ml}$. of the solid medium is heated in water till the medium is melted; for gelatin a temperature of $30^{\circ} \mathrm{C}$. will suffice; for agar the water must be boiled. 
The gelatin tube can be inoculated directly with a drop of the diluted culture; the agar tube should be cooled to $45^{\circ} \mathrm{C}$. before inoculation. The culture is then thoroughly mixed by rotation of the tube, and the mixture is poured out into a Petri dish and allowed to set. Gelatin sets at about $25^{\circ} \mathrm{C}$, agar at $38^{\circ} \mathrm{C}$. In warm weather the gelatin plates should be set on ice.

C. Shake-tube Method.-This is used chiefly for anaerobes. A test-tube containing about $15 \mathrm{ml}$. of solid medium, generally made up with a reducing agent such as glucose, is heated till the medium is melted; a drop of culture is delivered into the medium, which has been cooled to a suitable temperature, and thoroughly mixed by gentle shaking and rotation. The medium is then allowed to set in the tube in a vertical position. Incubation is carried out aerobically or anaerobically. The great value of this method is that it affords a simple means of grading the oxygen pressure in the medium; on the surface the pressure is atmospheric; at the bottom of the tube, particularly if a reducing agent has been added, the conditions are completely anaerobic. Thus the aerobic bacteria grow at or just below the surface; the anaerobic bacteria grow near the bottom; and the facultative aerobes and anaerobes are distributed throughout the medium. The single colonies, which appear, are often fairly characteristic. To pick them off, the test-tube is cut round with a diamond at the middle of the column of medium, the two halves of the tube drawn apart, and the medium allowed to fall gently into a sterile Petri dish. A stout platinum wire is then used to fish the colonies; it is stabbed into the medium over the particular colony desired, taking care that no other colony is touched on the way; and as soon as it has come into contact with the colony, it is withdrawn and inoculated into broth. Sometimes it is advisable to cut the medium into pieces with a sterile scalpel before attempting to pick off the colonies. This method was used at one time for purifying anaerobes; but now that the technique of obtaining anaerobiosis has improved, it has been largely replaced by the more reliable surface plating method. In order to avoid breaking a test-tube every time a colony has to be picked off, it is better to use a Veillon tube instead of the ordinary test-tube. This consists of a piece of glass-tubing, about $1 \mathrm{~cm}$. in diameter, and 8 or 10 inches long. One end is fitted

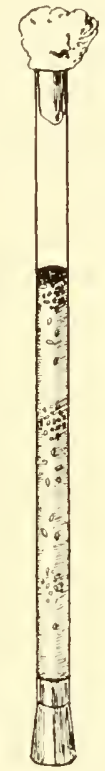

Fig. 56.VeILLON TUBE. with a rubber cork, the other with a cotton-wool plug. The medium is poured into the tube till it reaches about half or two-thirds of the way up. Inoculation is performed in the usual way. When it is desired to pick off a colony, the rubber cork and the woollen plug are removed, and the whole column of medium expelled by a stout glass rod into a Petri dish. The tubes and corks can be used over and over again.

D. Motility.-Various methods have been devised for making use of motility to separate motile from non-motile organisms. Rovida's (1925) tube, which is a modification of that invented by Carnot and Garnier (1902), may be used for this purpose. It consists of a large test-tube to the bottom of which is fused a glass tube of $7 \mathrm{~mm}$. internal diameter. This inner tube has a constriction near the lower end, below which are three small holes to afford a communication between the inside of the inner and the outer tubes. Above the constriction there is a 
plug of glass wool, which supports a layer of sand. Broth is poured into both tubes till it reaches the same level. The mixed culture is seeded into the broth of the outer tube, and the apparatus is put in the incubator. The organisms that are motile will pass through the holes into the inner tube, grow up through the wool and sand, and produce a turbidity in the broth of the inner tube ; from this they may be recovered in pure culture. The non-motile organisms remain confined to the broth of the outer tube. An alternative method described by Craigie (1931) is now extensively used for the separation of motile from nonmotile organisms of the Salmonella group. It consists of an ordinary test-tube, $6 \times \frac{5}{8}$ in., containing a piece of glass tubing 4 in. long, having the bottom cut off obliquely. About 6-8 ml. of melted semi-solid ( 0.25 per cent.) nutrient agar are poured into the outer tube. After autoclaving, the agar is cooled to $45^{\circ} \mathrm{C}$., and the inner tube, which projects above the level of the agar, is inoculated with the organism under test. If motile organisms are present, or appear as the result of incubation, they grow down the inner tube and up the outer tube, from the top of which they may be subcultured in a day or two's time (see also Tulloch 1939).

E. Optimum Temperature.-It is sometimes possible to make use of the optimum temperature of growth of an organism when it is desired to obtain it in pure culture, as for instance when one wants it to multiply freely in a mixed culture. The thermophilic bacteria may be separated from other organisms by incubating the medium at about $60^{\circ} \mathrm{C}$.; none of the ordinary bacteria will grow at this temperature, so a pure growth of the thermophilic organisms is obtained. Again, certain bacteria will not grow at $22^{\circ} \mathrm{C}$., whereas others will. If a mixture of the two is incubated at $22^{\circ} \mathrm{C}$., only one type will develop; this may then be picked off pure. In this way $N$. catarrhalis may be separated from the meningococcus. In water analysis, when it is desired to know the numbers of potentially pathogenic bacteria in a given sample, the cultures are incubated at $37^{\circ} \mathrm{C}$.; many of the saprophytic forms fail to grow at this temperature, and the resulting growth consists largely of potential parasites.

F. Aerobic and Anaerobic Incubation.-This is a simple method of separating aerobes from anaerobes. Incubated aerobically, the strict anaerobes will not grow; incubated anaerobically, the strict aerobes will not grow. And as most facultative anaerobes seldom grow as well under anaerobic as under aerobic conditions, anaerobic incubation favours the strict anaerobes more than the facultative ones.

G. Heating.-Heating a mixed culture at $80^{\circ} \mathrm{C}$. for 10 minutes will destroy all the vegetative non-sporing bacteria, while leaving the spores unaffected. This method is largely used in the preliminary purification of the anaerobes. In the body, most of the organisms with which the anaerobes are likely to be contaminated are non-sporing cocci and bacilli ; all of these are destroyed at $80^{\circ} \mathrm{C}$., and consequently the anaerobic organisms alone develop.

H. Selective Bactericidal Substances.-Certain substances with a germicidal action are useful in destroying susceptible organisms, while leaving the more resistant unaffected. One of the best examples of this method is the isolation of the tubercle bacillus from sputum by the use of antiformin. Tubercle bacilli are very resistant to chemical disinfectants, even though they are easily killed by heat. If the sputum, which generally contains numerous other organisms, is treated with 15 per cent. antiformin (equal parts of 15 per cent. $\mathrm{NaOH}$ and Liq. sodæ chlorinatæ 
B.P.), for a time varying from 5 to 60 minutes according to the thickness of the sputum, and inoculations are then made on to egg medium, the tubercle bacilli will develop in pure culture; without the antiformin they would be overgrown in 24 hours or less. Fifteen per cent. sulphuric acid is often used for the same purpose. Subcultures should be made at intervals of from 5 to 20 minutes.

I. Agglutinating Serum.--If it is suspected that relatively few organisms of a particular species are present in a bacterial culture or suspension, so that direct plating is unlikely to prove successful, it is sometimes possible to concentrate them by adding a specific high-titre agglutinating serum, incubating for 2 hours, and centrifuging. The organisms, which are clumped together, are easily thrown down, and are present almost exclusively in the deposit. Plates may then be streaked from this directly. This method is sometimes of value in isolating the typhoid bacillus from water. The same principle underlies the method of separating the phases of motile diphasic organisms of the Salmonella group. If, for example, a Craigie tube (see p. 354), containing semi-solid agar to which a small quantity of agglutinating serum active against Phase 1 has been added, is inoculated with the strain under test, the Phase 1 organisms will be agghutinated and rendered non-motile, whereas the Phase 2 organisms will grow down the inner and up the outer tube, and be recoverable from the surface of the agar (see Tulloch 1939).

J. Filtration.-This method may be used to separate the filtrable viruses from the ordinary bacteria, or if a fairly coarse candle is used, such as a Berkefeld $\mathrm{N}$ or $\mathrm{V}$, it may be used to separate very small bacteria like Bact. pneumosintes from larger organisms. Many spirochætes will also pass through Berkefeld filters; this property is made use of in separating them from the bacteria with which they are often contaminated.

K. Selective and Enrichment Media.-For the isolation of special organisms from others that are likely to overgrow it in culture, it is common to add to the medium substances having either a stimulating effect on the organism it is desired to cultivate or an inhibitory effect on those it is desired to suppress. If the substance is added to a liquid medium the result is an absolute increase in the numbers of the special organism, which becomes at the same time relatively more numerous to others than in the original material used for the inoculum. Such a liquid medium is known as an enrichment medium. If, on the other hand, the substance is added to a solid medium it acts selectively, enabling a greater proportion of the special organisms to form colonies than would otherwise have been possible. Such a solid medium is known as a selective medium. It is common to use the two types of media in conjunction, inoculating the material first into a liquid enrichment medium so as to obtain a relative and absolute increase in the numbers of the special organism, and then plating the culture on to a solid selective medium so as to favour the development of colonies of the special organism at the expense of others. It is important to realize that colonies on a selective medium are not necessarily pure. At the base of the colony other organisms may be present which, though unable to develop on the selective medium itself, will nevertheless grow rapidly when transferred to a non-selective medium. Thus, fermentation results may be misleading if the sugar tubes are inoculated directly from colonies on a selective medium. It is therefore wise to re-plate colonies from a selective medium on to a plain medium to ensure their purity before testing the biochenical, antigenic or other properties of the organism under study. 
Blood, serum, and ascitic or hydrocele fluid are substances that are frequently used to stimulate the growth of certain organisms; glucose and other sugars, extracts of vegetable and animal tissues, and certain salts such as potassium nitrate, are likewise used for the same purpose. On the other hand certain aniline dyes, phenol, telluric acid, bile salts, and numerous other substances are used for inhibiting the growth of various organisms. Gentian violet, in a concentration of about 1-10,000, suppresses the growth of most Gram-positive organisms, while allowing most Gram-negative organisms to develop. Used at 1-500,000 it is of value in separating streptococci from staphylococci, since the latter organisms are inhibited by this concentration. Brilliant green is often used for preventing the growth of lactose-fermenting organisms in cultures from the stools. It is added to the frcal suspension in broth in a concentration of about 1-150,000; the culture is incubated, and plated out on a suitable medium after 18 to 24 hours. Many Gram-negative bacteria are inhibited by potassium tellurite in a concentration of 1-80,000 or more, while penicillin exercises an inhibitory action mainly on Gram-positive bacteria (Fleming 1932).

L. Indicator Media.-These are media that contain an indicator which changes colour when a certain organism or group of organisms develops. Thus, if it is known that the organism which it is desired to cultivate produces $\mathrm{H}_{2} \mathrm{~S}$, lead acetate may be added to the medium; the colonies of the organism are coloured brown, owing to the production of lead sulphide, and can be readily picked off for identification. The diphtheria bacillus reduces sodium tellurite, whereas many of the organisms likely to be associated with it in a throat swab do not. When this substance is added to the medium, the colonies of the diphtheria and of the diphtheroid bacilli are coloured black, whereas those of the streptococci and numerous other organisms are colourless. Litmus and neutral red are two dyes that are frequently used to indicate the production of acid from some carbohydrate incorporated in the medium. Colonies of organisms that ferment the sugar are coloured red owing to the production of acid, whereas those that do not do so take on the alkaline colour of the dye-blue and yellow respectively. Blood is a very useful indicator. Some organisms produce no alteration in it, others form from it a green pigment, while others lyse it completely. It is usually added to agar in a concentration of 5 per cent. The colonies of the first class leave the medium unchanged; those of the second class are surrounded by a greenish ring; those of the third class by a perfectly clear transparent ring. It is used particularly in the differentiation of the streptococci.

Selective and indicator media are frequently combined. Thus in MacConkey's medium, bile salts are added to inhibit the growth of organisms other than those capable of multiplying freely in the intestine, and lactose and neutral red are added to distinguish the lactose-fermenting coliform organisms from the non-lactosefermenting group.

M. Pathogenicity Methods.-The introduction of the pathogenicity method of separating organisms from one another we owe to Koch (1880). By this means he succeeded in separating streptococci from Erysipelothix muriseptica. When the mixed culture was injected into the ear of a house mouse, Ery. muriseptica proliferated, invaded the blood stream, and could be obtained in pure culture from the heart's blood after death; the streptococcus proliferated locally but did not invade the blood stream. As it was mixed in the local lesion with Ery. muriseptica it could not be obtained in pure culture. But Koch found that if the mixed culture 
was injected into a field mouse, the streptococci proliferated, invaded the bloodstream, and caused death, while Ery. muriseptica did not grow at all ; the streptococcus was therefore obtained in pure culture from the blood. This principle is of wide application. It is used particularly to isolate organisms that are pathogenic to a certain laboratory animal from other closely similar organisms that are not pathogenic. Thus $B$. anthracis can easily be separated from $B$. subtilis or $B$. megatherium by the injection of a mouse or a guinea-pig. It is also used to isolate pathogenic organisms which are not easy to grow in culture, or which are readily over-grown by contaminating organisms. As examples, we may quote the tubercle bacillus in pus, or the pneumocoecus in sputum. The contaminating organisms are rapidly killed in the animal body, whereas the pathogenic organism multiplies and can be recovered in pure culture from the tissues.

N. Single Cell Methods. - The aim of these methods is to obtain a culture of a given organism from a single bacterial cell. If this can be carried out successfully, then the resultant culture must obviously be pure. If the technique for singlecell isolation was simple and flawless, this would be the ideal method for the purification of cultures; in fact, however, several of the methods advocated for this purpose suffer from optical or other defects, which seriously detract from their value. In Barber's (1908) method the culture is diluted, and a series of tiny droplets prepared. These are placed on the under surface of a cover-slip forming the roof of a special chamber, and examined under the microscope. When a drop is found containing only one organism, it is picked off with a special capillary pipette and transferred to a fluid medium. This method has been widely used, but it suffers from the defect that in viewing a spherical droplet the optical conditions are such as to render accurate observation of particles at the water-air interface very difficult or impossible. Hence there is no absolute certainty that a single cell has been picked. A method devised by Topley, Barnard and Wilson (1921) eliminates these particular optical defects. A loopful of a young gelatin culture at $37^{\circ} \mathrm{C}$. is placed on a slide, and covered with a quartz cover-glass. Under darkground illumination a single organism is picked out which is well removed from any other organisms, and is covered with a minute droplet of mercury. The preparation is exposed for a short time to ultra-violet irradiation with the object of destroying all organisms except the single one that has been protected by the mercury droplet. After incubation overnight the preparation is again examined, and, if successful, a colony will be observed at the site previously occupied by the protected organism. This can then be transferred to a liquid medium. Adequate controls are necessary to prove that the irradiation was sufficient to kill all nonprotected organisms. Several other methods have been described.

\section{The Identification of Micro-organisms}

Having once obtained a pure culture of a particular organism it is necessary to establish its identity by an appropriate series of tests. This may require a few weeks, or it may take several months to complete. Many of the reactions may have to be tested three or four times to make sure of their consistency. It is often desirable to prepare photographs recording the morphology and colonial appearances 
on the most important media ; these will be found of great value for future comparison. For studying the properties of an organism the following scheme is suggested.

A. Morphology.--Under this heading we include the shape and size of the organism, its arrangement, motility, the number and distribution of flagella, the shape and situation of spores, and capsule formation. It is impossible to study all these properties on a single medium ; motility for example should be looked for in a young rapidly growing broth culture, preferably not more than 6 to 8 hours old ; flagella are sought for on a young agar culture; spores in a culture that has been growing for some days; capsules in a pathological exudate, and so on. The shape and size of the organisms are subject to considerable variation, and it is important to gain some idea of the extent of this variation. With a few exceptions, such as the corynebacteria, most organisms are larger in a young than in an old culture (Henrici 1926). Neasurements, for example, of Salm. typhi-murium in a 4hours' culture on agar showed that the average size was $2.35 \mu \times 0.79 \mu$; in the same culture after 26 hours the size was only $1.13 \mu \times 0.49 \mu$. In volume the organisms from the young culture were over five times that from the old. On further incubation the average size decreased still more (Wilson 1926). When taking measurements of a given strain it is therefore important to record the age of the culture from which they were taken. Even in one and the same preparation the individual organisms may vary considerably in size and shape ; this may be so marked as to justify the term "pleomorphic." Thus coccoid, bacillary, and filamentous forms may all be present together; or besides the usual rods there may be club forms, navicular forms, granular forms, large bloated forms, shadow forms, and so on. Moreover, the appearance of the organisms is often considerably influenced by the type of medium on which they are grown. Chain formation, for example, is more evident in liquid media than on solid. The typical morphological appearance of the diphtheria bacillus is seen best on Loeffler's serum ; on agar the organisms tend to be more solid and less granular. The nature of the medium often influences the production of spores and of capsules. Some organisms, such as $B$. anthracis, form spores readily in artificial culture, but never do so in the animal body. On the other hand, capsules are quite frequently found in the body, but less often in artificial culture. The arrangement of the organisms should be carefully studied ; if they are cocci, they may be arranged singly, in pairs, tetrads, packets, clusters, or chains ; if bacilli, they may be arranged singly, in pairs endto-end, in bundles, chains, clusters, or in Chinese-letter forms in which the individual bacilli lie more or less at right angles to each other : if vibrios, they may be arranged singly, in S-forms, semicircles, in wavy chains composed of S-forms strung end-to-end, or they may present the fish-in-stream appearance. Though most organisms show two or three types of arrangement, it is usual for one of these to be predominant; this comes to be regarded as the typical arrangement. It cannot be emphasized too strongly that the morphology of bacteria is subject to variation, depending on the age of the culture, the nature of the medium, the particular strain used, the temperature of incubation, and a number of other factors; the extent of this variation can be learnt only by experience.

B. Staining Reactions.-The morphology of bacteria may be studied in a hanging-drop preparation, by dark-ground illumination, or in stained films. By each of these methods different information may be gained. Staining methods, in addition to revealing the morphology of the organism, may render evident 
differences in the chemical constitution of different organisms, or of different parts of the same organism. For studying the morphology, it is advisable to use a weak stain ; otherwise so much dye may be absorbed as to alter the appearance of the organism. Gram's stain is of great value, in that it serves to divide all bacteria into one or other of two classes - the Gram-positive and the Gram-negative. The Ziehl-Neelsen method of staining is likewise of value, since it serves to distinguish the acid-fast from the non-acid-fast bacilli. Numerous other stains are used for special purposes, such as the demonstration of flagella, capsules, spores, and metachromatic granules.

By a study of the morphology and the staining reactions, it is generally possible to identify the group to which a given organism belongs. In certain instances, when the origin of the organism is known, it is possible to make a presumptive diagnosis of its actual identity; though it should be clearly understood that, in medical bacteriology, a provisional identification of this kind is valid only if in consonance with a clinical diagnosis established on other grounds. Thus acid-fast bacili in the cerebro-spinal fluid of a patient with clinical symptoms of meningitis may provisionally be identified as tubercle bacilli; Gram-negative diplococci in the pus of an infant with ophthalmia neonatorum are probably gonococci ; and Gram-negative bipolar-staining ovoid bacilli in the gland-juice from a patient with an inguinal bubo, in an area where plague is prevalent, may provisionally be regarded as plague bacilli. As a rule, however, it is impossible to identify an organism by morphology and staining alone.

C. Cultural Reactions.--Under this heading must be included a study of the surface, and often of the deep, colonies formed on solid media, and of the type of growth in fluid media. Nutrient agar is the usual medium on which colony formation is studied, but if the organism fails to grow on agar, then some other medium must be chosen. The colonies are best examined after 24 hours' incubation at $37^{\circ} \mathrm{C}$, and again at intervals for a week. In describing them, particular attention should be paid to their shape, size, elevation, structure, colour, transparency, surface, edge, consistency, and emulsifiability ; differentiation into central and peripheral areas should also be noted. The type of growth following a streak inoculation on an agar slope should be studied, attention being paid particularly to the profuseness of growth, to the elevation, colour, surface, and edge, and to any change in the medium itself. The type of growth in a gelatin stab culture should also be studied, and notes made of the degree and extent of the growth, the presence of a surface growth, the presence or absence of liquefaction, and if liquefaction occurs, of the particular type which it assumes (see Chapter 13). In any systematic examination, the growth should be studied on certain special media such as Loeffler's serum, glycerine potato, and coagulated egg.

The cultural reactions of the different groups of bacteria are fairly distinctive, and even within a given group there may be differences between the members. Some organisms moreover have a characteristic form of growth, which enables them to be distinguished from morphologically similar organisms. As a rule, however, a study of the cultural reactions merely indicates the group to which a given organism belongs; it does not distinguish between the different members. It serves to confirm the conclusions reached from the examination of the morphology and staining reactions.

D. Resistance--Organisms vary considerably in their resistance to inimical agencies. Roughly speaking, three classes may be distinguished : 
(1) The bacteria that are susceptible to low degrees of heat, and low concentrations of chemical disinfectants; this class includes the non-sporing bacteria and the vegetative forms of the spore-bearing bacteria. They are destroyed by moist heat at $60^{\circ} \mathrm{C}$. in half an hour and by 1 per cent. phenol within an hour.

(2) The bacteria that are susceptible to low degrees of heat, but are resistant to low concentrations of disinfectants; this class includes the acid-fast bacteria, which are killed at $60^{\circ} \mathrm{C}$. in half an hour, but resist destruction by chemical agents in the cold often for several hours.

(3) The bacteria that are resistant both to low degrees of heat and low concentrations of disinfectants; this class includes the sporing forms of the spore-bearing bacteria. To kill them with certainty, steam under pressure at a temperature of $120^{\circ} \mathrm{C}$. for half an hour should be employed, or high concentrations of disinfectants, for example, 5 per cent. phenol, maintained for several hours.

A study of the resistance of a given bacterium will, as a rule, merely serve to confirm the conclusions already reached by the three previous methods of examination, but occasionally it is in itself of some diagnostic importance. Thus certain of the non-sporing vegetative bacteria, for example the enterococcus, are not destroyed at $60^{\circ} \mathrm{C}$. in half an hour; they require a temperature of $65^{\circ} \mathrm{C}$. ; this abnormal heat resistance is of value in differentiating this species of streptococcus from other species, which are readily killed at the lower temperature. Whenever an organism is suspected of forming spores, the heat resistance must be tested, and not till the suspected spores have definitely been found to be resistant to heat should the conclusion be reached that they really are spores. Many forms have been interpreted in the past as being true spores, which on subsequent examination have been found to be devoid of the characteristic property of heat resistance.

E. Metabolism.- Under this heading is included a study of the oxygen pressure required for growth, the optimum temperature for growth, pigment formation, hæmolysin production, and the effect on growth of adding different substances to the medium. It is usual to divide bacteria into 3 classes according to their oxygen requirements: (1) Strict aerobes: these organisms will grow only in the presence of free oxygen. (2) Strict anaerobes: these will grow only in the absence of free oxygen. It must be noted, however, that growth will occur in the presence of molecular oxygen, provided the medium contains a reducing system capable of bringing about a sufficiently low $\mathrm{O}-\mathrm{R}$ potential. (See Chapters 3 and 36.) (3) Facultative anaerobes: these grow best under aerobic conditions, but are able to grow under anaerobic conditions. To these may be added a fourth class, the microaerophiles, comprising those organisms that grow best under a pressure of oxygen lower than that of the atmosphere. According to their temperature requirements bacteria may be divided into (1) the mesophilic, which have an optimum temperature between $20^{\circ} \mathrm{C}$. and $40^{\circ} \mathrm{C}$, and (2) the thermophilic, which have an optimum temperature between $60^{\circ} \mathrm{C}$. and $70^{\circ} \mathrm{C}$. In medical bacteriology, the important distinction lies between those organisms that will grow at room temperature as well as at $37^{\circ} \mathrm{C}$, and those that will grow at $37^{\circ} \mathrm{C}$. but not at room temperature. The latter class includes many of the highly parasitic organisms. The power to hæmolyse may be studied by growing the organism on blood agar plates, or by mixing varying dilutions of a broth culture with a suspension of washed red cells. This property is of considerable import- 
ance, and is employed as a primary criterion for differentiating between the members of the streptococcal group. The effect on growth of adding blood, serum, glucose, nitrates, and bile salts to the medium is important, since it is often of differential value. The formation of pigment should be studied on various media and at different temperatures; as a rule it will be found that pigment is best formed on the surface of a solid medium at a temperature of $25-30^{\circ} \mathrm{C}$. In liquid media, or in the depth of solid media, and at temperatures above $35^{\circ} \mathrm{C}$., pigment is formed less abundantly ; under strict anaerobic conditions it is formed only by exceptional organisms, such as $F$. melaninogenicus.

A study of the salient metabolic functions of an organism as a rule adds considerably to the information derived from the previous methods of examination. Oxygen and temperature requirements, and pigment formation, especially are of great classificatory value, being frequently used for the differentiation of species.

F. Fermentation Reactions and Other Biochemical Properties.-Under this heading we include a study of the fermentative action on certain carbohydrates and alcohols (colloquially spoken of as "sugars"); of the proteolytic powers, especially the digestion of gelatin, egg, and serum; of the fat-splitting powers; of the power to reduce certain dyes such as methylene blue and litmus, or certain salts such as nitrate and tellurite; of the production of catalase; the production of indole from peptone; the formation of $\mathrm{NH}_{3}$ and $\mathrm{H}_{2} \mathrm{~S}$; the final hydrogen-ion concentration in glucose broth; and the power to utilize certain salts such as tartrates and citrates.

As a rule the fermentation of sugars is observed qualitatively, the formation of acid being rendered evident by the inclusion in the medium of an indicator, and the liberation of gas by an inverted Durham tube, or by a special fermentation tube. For the testing of the other biochemical properties, certain fairly stereotyped methods have been evolved, which it is unnecessary to describe here.

In bacterial differentiation the biochemical reactions are often of the greatest importance; in many groups of organisms the classification is made on the basis of sugar fermentation, of proteolytic power, or of both tests taken together. The sugar tests especially afford a means of bringing nut the finer distinctions between closely allied organisms. The oxidation of tartrates and citrates is employed in the differentiation of the coliform group of bacteria.

G. Antigenic Structure--For identifying bacteria, the serological reactions most frequently employed are agglutination and complement fixation. Provided that adequate controls are used, these reactions-particularly agglutinationafford the most rapid and reliable method of identifying a given bacterium. For the identification to be complete the organism should be agglutinated to titre by a serum prepared against the organism which it is supposed to resemble, and should absorb all the agglutinins from that serum; moreover the type organism should be agglutinated to titre by a serum prepared against the unknown organism, and should likewise remove all agglutinins from it. That is to say, there should be complete cross-agglutination and cross-absorption between the two sera and the two organisms. In certain groups of bacteria, the serological method is found to be much the quickest and most satisfactory way of distinguishing between the different members, and it is therefore extensively used for rapid identification. In other groups the agglutination method is not of much help, either because there are numerous varieties within the species, or because the organisms are autoagglutinable, or for some other reason. Apart from affording a rapid means of 
identification of certain bacteria, the serological method is as a rule the most delicate method available for bringing out the finer distinctions between closely allied organisms. In this respect it is more valuable even than the biochemical tests. It is often the only method available for differentiating between the sub-species or varieties of a given species of organism. Of recent years increasing attention has been paid to the precipitin test, which is particularly useful when homogeneous suspensions of bacteria cannot be obtained for agglutination, or when, as with the haemolytic streptococci, a particular antigen can be extracted from the organisms and recoguized quickly by a simple precipitin technique.

H. Pathogenicity. - The pathogenicity of bacteria is usually tested on laboratory animals, especially the guinea-pig, rabbit, rat, and mouse. It may be advisable to introduce the organism directly into the tissues by inoculation subcutaneously, intramuscularly, intraperitoneally, or intravenously; or it may be given by the mouth, or in the form of a spray, which the animal is made to inhale. Pathogenicity tests are open to numerous errors, but provided these are adequately guarded against, they often afford very important information. This is limited, however, to certain groups of organisms. In the study of the purely saprophytic bacteria and of certain bacteria that are harmless to laboratory animals, the pathogenicity test is of no value except to establish the absence of virulence. It is used chiefly in distinguishing virulent from avirulent members of the same genus or species. But it is also used to distinguish between closely allied organisms, both of which are virulent to the same animal, but which produce in it lesions of varying extent or localization; or which differ in their virulence to different species of animal.

For the complete identification of a pathogenic species, it may be necessary to determine whether or not it forms a soluble exotoxin ; that is to say, whether sterile filtrates of cultures grown for a suitable time in suitable fluid media produce death with characteristic lesions. In species which produce such exotoxins, neutralization with a specific antitoxin may play an important part in identification. In the description of any newly isolated pathogenic species a record of the toxicity or non-toxicity of filtrates should always be included.

It will be realized that the complete identification of an unknown organism is often a lengthy proceeding. As a rule it is easy to refer it to its proper genus; this can be done by simple examination of the morphological and staining reactions, aided at times by the cultural reactions. But its more exact denomination requires the use of the most delicate tests at our disposal, namely the biochemical, serological, and pathogenicity tests. It is advisable never to place too much weight on any one test; errors of technique or of interpretation are always liable to occur. If a large series of tests is carried out, and the organism is studied by several different methods, then the chances of being misled are very greatly reduced.

The complete identification of a given organism with any known type is not, of course, always possible; within the type species there are often varieties differing in minor respects from the type organism. It is very important to realize this; in any large collection of organisms of apparently the same species, there will almost invariably be found a number that differ from the rest in one or more of their properties; sometimes these differences are so numerous, or a single one of them may be so important, that it is necessary to revise one's classification.

If the characters of the organism differ from those of any described species, 
and if it is desired to preserve or to publish a description of the new species or type, it is essential to inake a full and careful record of all its characters and reactions, so that they will be available for comparison with organisms isolated at a future date, or with organisms isolated by other observers. Such a record should always contain careful comparisons with those species or types which most nearly resemble the newly isolated organism.

\section{REFERENCES}

BARBER, M. A. (1908) J. infect. Dis., 5, 379.

Carnot, P. and Garnier, M. (1902) C. R. Sor. Biol., 54, 748.

Craigie, J. (1931) J. Immunol., 21, 417.

Fleming, A. (1932) J. Path. Buct., 35, 831.

Henrici, A. T. (1926) J. infect. Dis., $38,54$.

KоCH, R. (1880) "Investigations into the Etiology of the Traumatir Infective 1)iseases." New Sydenham Soc., Lond.; (1881) Mitt. ReichsgesundhAmt., 1, 1.

Lister, J. (1878) Quart. J. micr. Sci., 18, 177.

RovidA, G. (1925) Sperimentale, 79, 1053.

Topley, W. W. C., Barnard, J. E., and Wilson, G. S. (1921) J. Hyg., Camb., 20, 221.

Tulloch, W. J. (1939) J. Hyg., Camb., 39, 324.

Wilson, G. S. (1926) J. Hyg., Camb., 25, 150. 
CHAPTER 13

\section{DESCRIPTION OF THE METHODS USED IN THE SYSTEMATIC EXAMINATION OF BACTERIA, AND A GLOSSARY OF THE TERMS EMPLOYED}

WE have already dealt in Chapter 12 with the methods of isolating pure cultures of bacteria, and with the various criteria that are employed in their identification. In the present chapter we describe a routine which may be used in examining the various morphological, cultural, and biochemical properties of bacteria, and define the terms which we shall employ in the description of these properties.

Morphological Appearance of Bacteria.-The chief points to be noted are the following :

Shape.-Spheres, short rods, long rods, filaments, commas, or spirals.

Axis.-Straight or curved.

Size.-Length and breadth.

Sides.-Parallel, bulging, concave, or irregular.

Ends.-Rounded, truncate, concave, or pointed.

Arrangement.-Singly, in pairs, in chains, in fours, in groups, in grape-like clusters, in cubical packets, in bundles, or in Chinese letters.

Irregular Forms.-Variations in shape and size; club, filamentous, branched, navicular, citron, fusiform, giant swollen forms and shadow forms.

Motility.-Motile or non-motile.

Flagella.-Monotrichate, amphitrichate, lophotrichate, peritrichate (Fig. 13, p. 31). Endospores.-Spherical, oval, or ellipsoidal ; equatorial, subterminal, or terminal ; single or multiple; causing bulging of bacillus or not (Fig. 6, p. 24).

Capsules.-Present or absent.

Staining.-Even, irregular, unipolar, bipolar, beaded, barred; and variations in depth between different organisms. Presence of metachromatic granules; reaction to Gram and to Ziehl-Neelsen stains.

\section{Surface Colonies on Solid Media.}

Shape.-Circular, irregular, radiate, rhizoid.

Size.-In millimetres.

Elevation.-Effuse, raised, low convex, convex or dome-shaped, umbonate, umbilicate; with or without bevelled margin.

Structure--Amorphous ; fine, medium, or coarsely granular ; filamentous, curled. Surface.-Smooth ; contoured, beaten-copper ; rough ; fine, medium, or coarsely granular; ringed; papillate; dull or glistening.

Edge.-Entire, undulate, lobate, crenated, erose, fimbriate, curled, effuse. 
Colour.-Colour by reflected and transmitted light; fluorescent, iridescent, opalescent, self-luminous.

Opacity.-Transparent, translucent, or opaque.

Consistency.-Butyrous, viscid, friable, membranous.

Emulsifiability.-Easy or difficult ; forms homogeneous, granular, or membranous suspension when rubbed up in a drop of water with a platinum loop.

Differentiation.-Differentiated into a central and a peripheral portion (Fig. 57).

\section{Growth on Stroke Culture.}

Degree.-None, scanty, moderate, abundant, profuse ; discrete, or confluent.

Form.-Filiform, spreading, rhizoid.

Elevation.-Effuse or raised.

Surface.-Smooth; contoured ; beaten-copper ; rough ; finely, moderately, or coarsely granular; papillate; heaped-up; dry or moist.

Edge.-Entire, undulate, lobate, crenated, erose, fimbriate ; curled, effuse.

Colour, Opacity, Consistency, and Emulsifiability.-As for colonies.

Odour.-Absent, decided, resembling-_.

Medium.-Coloured; digested; crystal formation (Fig. 57).

\section{Growth in Stab Culture.}

Degree.-As for stroke culture. Also position of optimal growth.

Form.--Filiform, beaded, with or without branching.

Extent.-Depth in tube to which growth occurs.

Surface.-Surface growth present or absent; if present, diameter, surface, and edge.

Colour and Opacity.-As for stroke culture.

Liquefaction.-Present or absent; if present, crateriform, napiform, infundibuliform, saccate, or stratiform.

Medium.-As for stroke culture (Fig. 57).

\section{Growth in Shake Culture.}

Position.-Uniform growth throughout tube; or position of optimal growth.

Surface.-Surface growth present or absent.

Colonies.-Size, shape, colour, opacity, outgrowths from periphery, if any.

Gas.-Present or absent; medium disrupted.

Medium.-Coloured, digested, or rendered turbid.

\section{Growth in Fluid Medium.}

Degree.-None, scanty, moderate, abundant, or profuse.

Turbidity.-Present or absent; if present, slight, moderate, or dense ; uniform, granular, or flocculent.

Deposit.-Present or absent ; if present, slight, moderate, or abundant; powdery, granular, flocculent, membranous, or viscid; disintegrating completely or incompletely on shaking.

Surface Growth.-Present or absent ; if present, ring growth around wall of tube ; or surface pellicle, which is thin or thick, with a smooth, granular, or rough surface, and which disintegrates completely or incompletely on shaking.

Odour.-Absent, decided, resembling-. 


\section{Growth in Blood Agar.}

Colonies.-Description of surface colonies.

Hamolysis.-Present or absent; if present, of $\alpha$ - or $\beta$-type.
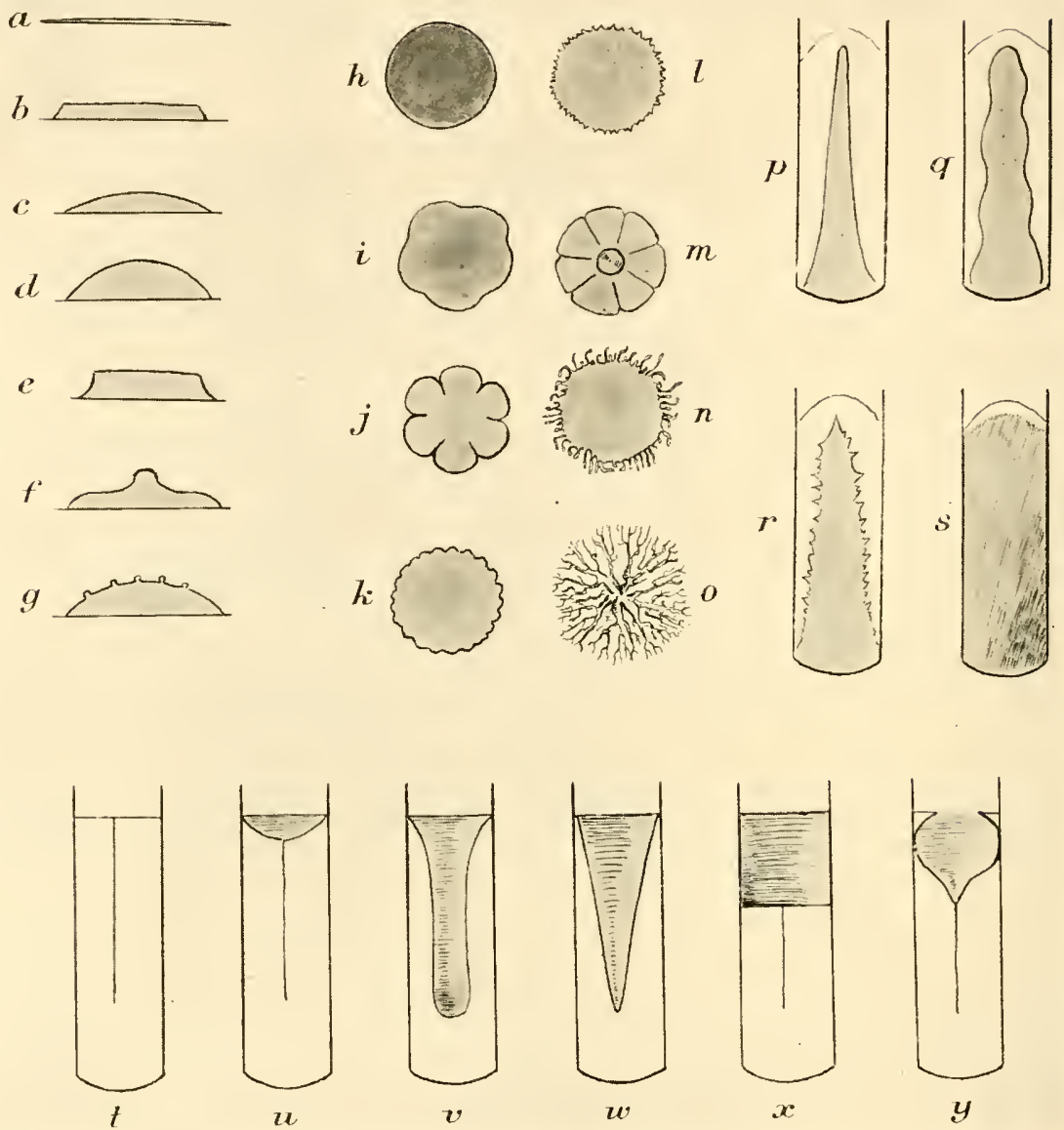

FIG. 57.

$a-g$. Elevation of colonies. $a$. Flat or effuse. b. Raised. $c$. Low convex. $d$. Convex or dome-shaped. e. Raised with concave bevelled edge. $f$. Umbonate. $g$. Convex with papillate surface. $h \rightarrow 0$. Edge of colonies. $h$. Entire. $i$. Undulate. $j$. Lobate. $k$. Crenated. $l$. Erose or dentate. $m$. Radially striated periphery with lobate edge. $n$. Fimbriate. o. Rhizoid or arborescent. $p-s$. Growth on agar stroke culture. p. Filiform. $q$. Slightly spreading with undulate edge. $r$. Slightly spreading with erose edge. $s$. Spreading. $t-y$. Growth in gelatin stab culture. $t$. Filiform growth without liquefaction. $u$. Crateriform liquefaction. $v$. Saccate liquefaction. $w$. Infundibuliform liquefaction. $x$. Stratiform liquefaction. $y$. Napiform liquefaction.

Resistance.-Tested usually by placing a 24 -hours' broth culture, containing $5 \mathrm{ml}$. of medium in a $\frac{5}{8}$-inch test tube, in a water-bath at such temperatures as $55^{\circ} \mathrm{C}$. for 1 hour, $60^{\circ} \mathrm{C}$. for half an hour, and $80^{\circ} \mathrm{C}$. for ten minutes, and sub- 
culturing into a favourable medium. This is, of course, merely a rough differential test. For accurate purposes, the column of fluid culture should be enclosed in a capillary tube thin enough to ensure rapid heating of the organisms to the temperature of the water in the bath.

\section{Metabolic Properties.}

Oxygen Pressure required for Growth.-Aerobic, facultatively anaerobic, obligatory anaerobic, microaerophilic.

Increased Carbon Dioxide pressure required for Growth.-The growth of many organisms is favoured by a partial pressure of $\mathrm{CO}_{2}$ higher than that -0.03 per cent.-in the atmosphere, and some entirely fail to grow in the absence of a raised pressure.

Effect of Temperature on Growth.-Limits between which growth occurs ; optimal temperature for growth.

Pigment Formation.-Tested usually on an agar slope incubated at $22^{\circ} \mathrm{C}$, , or left at room temperature in the light after preliminary incubation at $37^{\circ} \mathrm{C}$.

Effect of Modifying the Constitution of the Medium.-Effect on growth of adding to the medium blood, serum, ascitic fluid, glucose, glycerine, potassium nitrate, bile salts, or other substances.

\section{Biochemical Reactions.}

Fermentation of Sugars.-Tested in 1 per cent. peptone water containing 1 per cent. of the sugar and Andrade's indicator. A Durham's tube is included. For certain groups of organisms, which do not grow well in this medium, 5 per cent. of serum is added. Horse serum may be used as a rule, but in testing the fermentation of maltose it is better replaced by human or rabbit serum, since it contains an enzyme, maltase, which may lead to a false reaction (Hendry 1938). Acid, or acid and gas production, is noted.

Litmus Milk.-No change, acid or alkali; clot; clot disrupted by gas; peptonization; saponification. The term "clot" is unfortunately used for both an acid clot and a rennet clot. An acid clot results from the precipitation of the caseinogen; it is soft, gelatinous, does not retract, and can be completely dissolved in alkali. A rennet clot is due to the coagulation of the caseinogen under the influence of bacterial enzymes. A few hours after its formation it retracts with the expression of a clear greyish-coloured fluid called whey; the clot itself is firm and cannot be dissolved by alkali. Calcium caseinogenate is soluble in water. When acid is produced from the lactose of the milk, the calcium combines with it, and the caseinogen, which is insoluble, is precipitated. This is the mechanism of formation of the acid clot. In coagulation by rennet the soluble calcium caseinogenate is converted into insoluble calcium caseinate, which forms the curd.

Indole.-Tested in 1 per cent. peptone water after 5 days' growth, using Böhme's reagents. One $\mathrm{ml}$. of ether is added to the culture, which is shaken thoroughly, and then allowed to stand till the ether collects on the surface. $1 \mathrm{ml}$. Solution $\mathrm{A}$ is run down the side of the tube; if no colour appears within a minute, $1 \mathrm{ml}$. of Solution $B$ is added. A positive reaction is characterized by a colour varying from a faint pink to a deep magenta. According to Happold and Hoyle (1934) xylene is better than ether. 


\section{Solution A:}

parı-dimethylaminobenzaldehyde $4 \mathrm{gm}$.

96 per cent. Alcohol . . 380 ,

Concentrated $\mathrm{HCl}$. . 80 "

\section{Solution B :}

Saturated watery solution of potassium persulphate.

An alternative method, depending on the volatility of indole at $37^{\circ} \mathrm{C}$., is recommended by Holman and Gouzales (1923). It consists in placing a strip of filter paper, soaked in a saturated watery solution of oxalic acid and subsequently dried. between the cotton-wool plug and the tube. The paper should be carefully folded so as to present the maximum surface to the volatilizing indole, which turns it a pink colour.

Some organisms form indole, but break it down more rapidly than they produce it, and hence may give a false negative reaction (Reed 1942).

Methyl-Red Test (M.R.).- Tested by adding 5 drops of an 0.04 per cent. solution of methyl red to a culture in glucose phosphate medium (peptone $0.5 \mathrm{gm}$., $\mathrm{K}_{2} \mathrm{HPO}_{4} 0.5 \mathrm{gm}$., glucose $0.5 \mathrm{gm}$., water $100 \mathrm{ml}$., $\mathrm{pH} 7.5$ ). Culture grown for 5 days at $30^{\circ} \mathrm{C}$ or 3 days at $37^{\circ} \mathrm{C}$.

Red colour = positive.

Yellow colour $=$ negative.

Voges-Proskauer Test (V.P.).-Tested by adding $1 \mathrm{ml}$. of a 10 per cent. solution of $\mathrm{KOH}$ to a glucose phosphate culture grown for 5 days at $30^{\circ} \mathrm{C}$. or 2 days at $37^{\circ} \mathrm{C}$. The colour develops slowly, and the test should be read after 18 to 24 hours.

Pink fluorescence $=$ posiúıe.

No coloration = negative.

A higher proportion of positive reactions is obtained by the use of O'Meara's (1931) modification. A knife point of creatine is added to the culture, followed by $5 \mathrm{ml}$. of 40 per cent. sodium hydroxide. The tube is shaken thoroughly for 2 to 5 minutes. A positive reaction is characterized by the appearance of a pink colour within about 2 minutes, unaccompanied by fluorescence; the development of the colour may, however, be delayed for an hour or longer.

An even more sensitive test for acetylmethylcarbinol is that described by Barritt (1936). It consists in adding $0 \cdot 6 \mathrm{ml}$. of a 5 per cent. alcoholic solution of $\alpha$-naphthol and $0.2 \mathrm{ml}$. of 40 per cent. $\mathrm{KOH}$ solution to $1 \mathrm{ml}$. of culture. In a positive reaction a pink colour appears in 2-5 miuutes, deepening to magenta or crimson in half an hour. In a negative reaction the mixture remains colourless for an hour or so, when it may become copper-coloured owing to the action of $\mathrm{KOH}$ on the $\alpha$-naphthol. Traces of pink coloration are best neglected.

Nitrate Reduction.-Tested on a broth culture containing $0 \cdot 1$ per cent. $\mathrm{KNO}_{3}$, grown for 5 days at $37^{\circ} \mathrm{C}$., by the Griess-Ilosva method.

\section{Solution A :}

$$
\begin{aligned}
& \alpha \text {-naphthylamine . . . } 1 \mathrm{gm} \text {. } \\
& \text { Water. . . } 22 \mathrm{ml} \text {. }
\end{aligned}
$$

Dissolve, filter, and then add $180 \mathrm{ml}$. of dilute acetic acid (sp. gr. 1.04). 
Solution B :

Sulphanilic acid . . $0.5 \mathrm{gm}$.

Dilute acetic acid . . $150 \mathrm{ml}$.

Add $1 \mathrm{ml}$. of Solution A, followed by $1 \mathrm{ml}$. of Solution B.

Pink, red, or maroon colour $=$ positive.

No coloration = negative.

A negative reaction may sometimes be due to the reduction of the nitrite to gaseous nitrogen almost as rapidly as it is formed, or to the prorluction of hydroxylamine. The first possibility may be examined by growth in a gas fermentation tube, or by chemical estimation of the nitrate, the second by testing for nitrite in the way just described, after preliminary oxidation of the hydroxylamine with iodine (see Lindsey and Rhines 1932, Com 1936, Reed 1942). A control tube should always be tested.

Ammonia.-Tested on a peptone water culture, grown for 5 days at $37^{\circ} \mathrm{C}$, by adding Nessler's reagent.

Brown colour = positive.

Faint yellow colour $=$ negative.

Hydrogen Sulphide.-Tested on lead acetate medium (heart extract broth containing 4 per cent. peptone and 2.5 per cent. agar. Sterilize, and add an equal quantity of a sterile $0 \cdot 1$ per cent. solution of basic lead acetate.)

Brown or black coloration $=$ positive.

No coloration = negative.

The lead acetate may be replaced by 0.05 per cent. ferric ammonium citrate or 0.03 per cent. ferrous acetate (Zobell and Feltham 1934). A higher proportion of positive reactions is obtained with some organisms by incubating at $30^{\circ} \mathrm{C}$. instead of $37^{\circ} \mathrm{C}$. (Tittsler 1931). The most delicate method is to grow the organisms in a slope tube of liver extract agar, and to include between the cotton-wool plug and the tube a slip of filter paper soaked in 10 per cent. lead acetate solution and subsequently dried. The amount of browning or blackening of the paper is measured in millimetres. A fresh slip may be inserted daily.

Methylene Blue Reduction.-Tested on a 24 -hours' broth culture at $37^{\circ} \mathrm{C}$. Add

1 drop of 1 per cent. aqueous methylene blue, and incubate at $37^{\circ} \mathrm{C}$.

Complete decolorization $=$ strong positive.

Green coloration = weak positive.

No decolorization = negative.

Catalase.-Tested on a 24-hours' agar slope culture at $37^{\circ} \mathrm{C}$. One ml. of $\mathrm{H}_{2} \mathrm{O}_{2}$ (10 vols.) is poured over the growth, and the tube is set in an inclined position.

Gas bubbles produced $=$ positive.

No gas produced = negative.

For an account of the methods of examining the antigenic structure and the pathogenicity of bacteria, reference must be made to the chapters dealing with the particular organism under consideration.

\section{Glossary of Descriptive Terms}

Aerobic: growing in the presence of free oxygen; strictly aerobic, growing only in the presence of free oxygen. 
Amorphous (colonies): without visible differentiation in structure. Amphitrichate: having a single flagellum at each pole.

Anaerobic: growing in the absence of free oxygen; strictly anaerobic, growing only in the absence of free oxygen; facultatively anaerobic, growing both in the presence of and in the absence of oxygen. It must be noted, however, that growth of even strict anaerobes will occur in the presence of molecular oxygen, provided the medium contains a reducing system capable of bringing about a sufficiently low $\mathrm{O}-\mathrm{R}$ potential (see Chapters 3 and 36 ).

Beaded (stained bacteria) : deeply staining granules arranged at regular intervals along the course of the rod. (In stab or stroke culture) : disjointed or semi confluent colonies along the line of inoculation.

Beaten-copper : multiple small crateriform depressions on the surface of a growth, resembling beaten copper.

Bipolar: at both ends or poles of the bacterial cell.

Butyrous : growth of butter-like consistency.

Chains: four or more bacterial cells attached end-to-end.

Chromogenesis: the production of colour.

Citron: shaped like a lemon, having a small knob at each end.

Clavate: club-shaped.

Coagulation: formation of a firm clot in milk with the subsequent separation of the casein from the whey.

Contoured : an irregular, smoothly undulating surface.

Convex: the segment of a sphere of short radius; Low convex, the segment of a sphere of long radius.

Crateriform: a sancer-shaped liquefaction of the medium.

Crenated: small, shallow indentations of the edge, which has a scalloped appearance.

Cuneate: wedge-shaped.

Curled: composed of parallel chains in wavy strands, as in anthrax colonies.

Effuse: growth thin, hardly raised at all from the medium.

Endospores: thick-walled spores formed within the bacterial cell.

Entire: with an even margin.

Equatorial: situated about equidistant from each end.

Erose: border showing fine, pointed, tooth-like projections.

Filaments : applied to morphology of bacteria, refers to thread-like forms, generally unsegmented; if segmented, to be distinguished from chains (q.v.) by the absence of constrictions between the segments.

Filamentous : growth composed of long, often interwoven threads.

Filiform: in stroke or stab cultures, a uniform growth confined to the line of inoculation.

Fimbriate: fine, sometimes recurved, processes projecting from the edge of the colony or growth.

Flocculent : containing small adherent masses of bacteria of various shapes floating in the culture fluid, or deposited at the bottom.

Fluorescent: having one colour by transmitted light and another by reflected light.

Friable: growth dry and brittle, when touched with a platinum needle.

Granular: composed of granules; fine, medium or coarse. 
Hamolysis : on blood agar plate. $a$-hæmolysis : colonies surrounded by a greenish ring. $\beta$-hæmolysis: colonies surrounded by an area of clearing, which is transparent (see Chapter 24).

Heaped-up: irregular, coarse processes projecting considerably above the level of the rest of the growth.

Infundibuliform: in form of a funnel or inverted cone.

Iridescent: exhibiting changing rainbow colours in reflected light.

Lenticular: surface colony, which is convex and translucent, and which acts like a plano-convex lens, giving an inverted image of an object viewed through it. Deep colony, which is shaped like a lentil.

Lobate: having the margin deeply undulate, producing lobes (see Undulate).

Lophotrichate: having a tuft of flagella at one or both poles.

Luminous: glowing in the dark, phosphorescent.

Maximum Temperature: temperature above which growth does not take place.

Membranous: growth thin, coherent, like a membrane.

Microcerophilic: growing best under a lowered oxygen pressure.

Minimum Temperature: temperature below which growth does not take place.

Mirror-like: having a smooth glistening surface, in which reflections of surrounding objects, e.g. window bars, can be seen.

Monotrichate: having a single flagellum at one pole.

Napiform: liquefaction in form of a turnip.

Navicular: shaped like a boat.

Opalescent: coarsely iridescent, like an opal.

Opaque: objects, e.g. window bars, cannot be seen through growth.

Optimum Temperature: temperature at which growth is most rapid.

Papillate: growth beset with small nipple-like processes.

Pellicle: bacterial growth forming either a continuous or an interrupted sheet over the culture fluid.

Peptonization: rendering curdled milk soluble by the action of peptonizing enzymes.

Peritrichate: having flagella disposed around the organism.

Punctiform: very small, but visible to naked eye; under $1 \mathrm{~mm}$. in diameter.

Radiate: showing fissures or ridges arranged in a radial manner.

Raised: growth thick, with a comparatively flat surface, and with abrupt or terraced edges.

Rhizoid: growth of an irregular branched or root-like character, as in $B$. mycoides.

Ring: growth at the upper margin of a liquid culture, adhering to the glass.

Ringed: having one or more circular depressions or elevations on the surface, sometimes giving a draughtsman-like appearance.

Rough: general term for an irregular surface, the irregularity being of a coarsely granular type, or resembling morocco-leather or a relief map.

Saccate: liquefaction in form of an elongated sac, tubular, cylindrical.

Spreading: growth extending much beyond the line of inoculation, i.e. several millimetres or more; sometimes over an entire tube or plate.

Stratiform: liquefying to the walls of the tube at the top and then proceeding downwards horizontally.

Subterminal: situated towards the end.

Terminal: situated at the extreme end. 
Translucent: objects, e.g. window bars, are visible through growth, but growth is not water-clear.

Transparent: growth is water-clear.

Truncate: ends abrupt, square.

Turbid: cloudy; may be a uniform, flocculent, or granular turbidity.

Umbonate: having a button-like, raised centre.

Undulate: border wavy, with shallow sinuses.

Unipolar: at one end only of the bacterial cell.

Viscid: sticky, semi-fluid; on withdrawal of the needle, the growth follows it in the form of a thread; sediment on shaking rises as a coherent swirl.

\section{REFERENCES}

Barritt, M. H. (1936) J. Path. Bact., 42, 441.

Coxw, H. J. (1936) J. Bact., 31, 225.

Happold, F. C. and Hoyle, L. (1934) Biochem. J., 28, 1171.

Hendry, C. B. (1938) J. P'ath. Bact., 46, 383.

Holman, W. H. and Gonzales, F. L. (1923) J. Bact., 8, 577.

Lindsey, G. A. and Rhines, C. 1l. (1932) J. Bact., 24, 489.

O'Meara, R. A. Q. (1931) J. Path. Bact., 34, 401.

REED, R. W. (1942) J. Bact., 44, 425.

Tittsler, R. P. (1931) J. Bact., 21, 111.

Zobell, C. E. and Feltham, C. B. (1934) J. Bact., 28, 169. 


\title{
CHAPTER 14
}

\section{ACTINOMYCES AND ACTINOBACILLUS}

\author{
ACTINOMYCES
}

\section{Definition. Actinomyces, Harz 1877.}

Organisms growing in the form of a much-branched mycelium, which may break up into segments or produce "spores." Aerial mycelium often formed under suitable conditions. Mainly aerobic, but may be microaerophilic or anaerobic. Usually saprophytic, but some species are parasitic on plants or animals, and may give rise to disease. In animal body organisms are frequently arranged in colonies composed of radiating threads with clubbed ends. Non-motile. Some species are acid-fast. The type species is Actinomyces bovis Harz.

THE term Actinomyces bovis was originally given by Harz to a mould-like organism which was found by Bollinger (1877) in the lesions of cattle suffering from a peculiar disease of the tongue and jaw, now known as Actinomycosis. This organism was first cultivated by Wolff and Israel in 1891 under anaerobic conditions. An aerobic organism, which is occasionally present in actinomycotic lesions, but which is probably not rtiologically related to the disease, was isolated in the same year by Bostroem (1891). In order to avoid confusion with the anaerobic pathogenic type, we have suggested that Bostroem's organism should be called Actinomyces graminis. Since then a number of similar organisms have been isolated from a variety of diseases in man and animals, and from such situations as soil, grains, and grasses.

These organisms appear morphologically as jointed or unjointed filaments, which frequently show true branching. In culture, rod forms are not uncommon. In the animal body, many of the pathogenic species are characterized by the formation of granules of varying size, which are found to consist of a filamentous mycelium surrounded by radiating clubs - a picture which is responsible for the term "ray-fungus." (Botanically the term "ray" refers to the marginal portion of a composite flower, consisting of ligulate florets arranged radially.) In their staining reactions some species are acid-fast, though the majority are non-acid-fast. It is evident that these organisms bear some resemblance to those of Mycobacterium, and in the classification furnished by the American Committee of Bacteriologists in 1917 (Report 1917), Actinomyces and Mycobacterium were included in a single family, known as the Mycobacteriacece. In the 1920 report (Report 1920), however, it was decided to create a separate family of Actinomycetacece, which should contain the genera Actinobacillus, Leptothrix, Actinomyces, and Erysipelothrix. For descriptive purposes it is convenient to consider these genera separately, and in the present chapter we shall confine ourselves to a description of Actinomyces and Actinobacillus. 
Other terms such as Streptothrix or Nocardia have been applied to organisms of the Actinomyces group. Since the term Streptothrix was given by Corda in 1839 to a genus of fungi belonging to the Hyphomycetes, quite different from the group that we are considering, this name is obviously inapplicable. The term Nocardia was coined later than Actinomyces, and is likewise inapplicable.

Habitat.-Many members of this group lead a saprophytic existence on grains and grasses, and in water. As these substances are widely used as foods, it is not unnatural that by their means Actinomyces often gains access to the alimentary and respiratory tracts of man and other animals. They have been isolated chiefly from man and cattle, and also from pigs, chickens, rabbits, dogs, elephants, lizards, and oysters (Foulerton 1910). Most members live in the soil, where they play an important part in the biological processes that are occurring there.
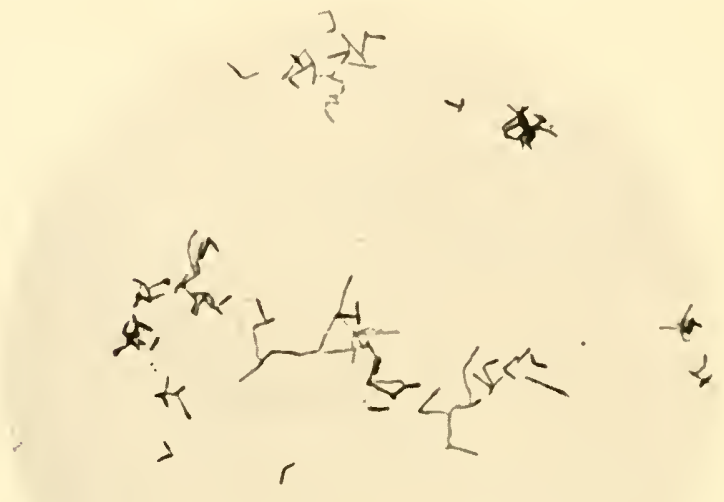

Fig. 58.-Actinomyces bovis $\times 1000$.

From an agar slope culture, 14 days, $37^{\circ} \mathrm{C}$. anaerobically.

There is at least one species that appears to be a strict parasite in man and animals. Numerous members are pathogenic for plants, causing such diseases as potato scab.

Morphology.--On culture media the morphology is variable. In ordinary film preparations the a n a e robic Wolff-Israel type occurs chiefly as rods, $3-4 \mu$ long by $0.6 \mu$ broad, which from their arrangement, their clubbed ends, and their irregular staining bear a resemblance to certain members of the corynebacteria ; careful search, however, will generally reveal a few definite filaments, some of which may show true branching (Fig. 58). The aerobic type in young cultures occurs chiefly as long unsegmented straight or wavy filaments, which show simple or dichotomous branching (Fig. 59), and which not infrequently grow upwards from the surface as aerial hyphæ. Later these filaments undergo segmentation, and break up into rod forms of varying length and oval coccoid bodies, which are usually referred to as spores. In some strains of the aerobic type segmentation is visible within 24 hours; in others it may not occur for 3 weeks or more. On solid media the filaments are arranged in loose groups or in a tangled mycelium, but in broth definite colonies occur consisting of a densely matted central core of filaments and a peripheral zone in which the filaments are more loosely disposed (Fig. 63). These colonies in liquid media are common to both the aerobic and the anaerobic types. The rods and filaments may stain evenly, but as a rule granular staining is evident. After growth for some time in liquid media both the aerobic and the anaerobic types may show involution 
forms, consisting mainly of spherical or club-shaped swellings on the ends of the filaments.

If, instead of making film preparations, the growth of the organisms is followed by Orskov's (1923) agar-block technique, it will be seen that in some species the aerial mycelium gives rise, without preliminary segmentation, to circular or oval "spores." There is evidence that these "spores" are rather more resistant to inimical agencies generally than the plain mycelium. They may, for example, resist moist heat at $65^{\circ} \mathrm{C}$. for as long as 3 hours. The segmented mycelium, observed in many species, is no more resistant, however, than the unicellular mycelium.

All members are non-motile and all, with a few possible exceptions, are Grampositive. The anaerobic types are miformly non-acid-fast. The aerobic types may be differentiated into :

(1) acid-fast; these resist decolorization with 1 per cent. sulphuric acid for 5 minutes, but are usually decolorized by the application of 25 per cent. $\mathrm{H}_{2} \mathrm{SO}_{4}$ for a similar length of time; there is, however, a marked variation in the acid-fastness of different species. (2) Non-acid-fast.

In the animal body the morphology is often different from that on culture media. The anaerobic Wolff-Israel type grows in the form of definite colonies, which appear in the pus or in sections of the tissues as granules or "Drusen." When crushed and ex-
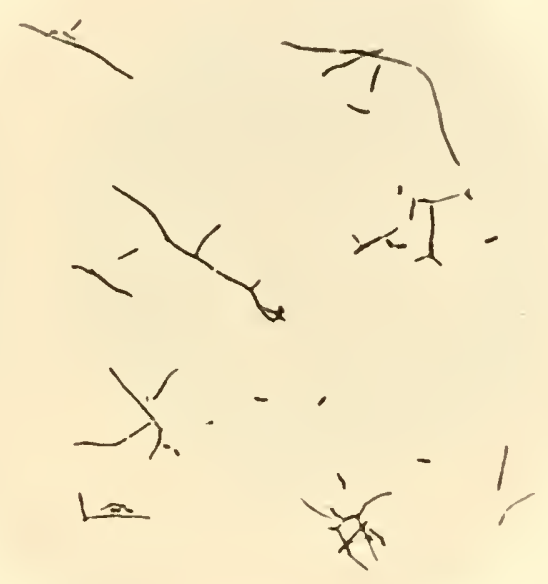

FIG. 59.-Actinomyces graminis $\times 1000$. From a broth culture, 24 hours, $37^{\circ} \mathrm{C}$. aerobically.

amined microscopically these granules are seen to consist of a central filamentous Gram-positive mycelium surrounded by a peripheral zone of large, Gram-negative clubs. In old colonies the mycelium is replaced by a mass of short Gram-positive rods and coccoid bodies, which appear to have resulted from the disintegration of the filaments. The clubs vary in size, but may be as long as $10 \mu$ and as broad as $5 \mu$. Their mode of origin has given rise to much discussion. On the whole it seems probable that there are two entirely different types of club, one observed in artificial culture and derived from the organism itself, the other observed in the animal body and derived from the host. The first type, or "culture club" as Ørskov (1923) calls it, represents the swollen end of the mycelial filament. The second type or "tissue club," as we may call it, appears to be due to the deposition around the end of the filament of some material, probably rich in lipoid, by the tissues of the host. Tissue clubs are observed, not only in ray-fungus infections, but also in lesions caused by other 
organisms such as tubercle bacilli and staphylococci. Even dead tubercle bacilli are said to stimulate their production.

In sections of tissues the filaments may be differentiated from the clubs by a modified Ziehl-Neelsen stain. If a seetion is stained with carbol-fuchsin, decolorized for 20 to 30 seconds with 1 per cent. $\mathrm{H}_{2} \mathrm{SO}_{4}$, and counterstained with methylene blue, the clubs appear red and the filaments blue.

The aerobic types, when growing in the animal body, generally form a tangled mycelium without evidence of ray or of elub formation; but exceptions do occur, as with Actinomyces madurce, which forms definite granules similar to those of the Wolff-Israel type.

The most striking feature of the Actinomyces is their pleomorphism. All forms may be seen-filaments, rods, cocci, and even spirilla. In the anaerobic type rod forms predominate in eulture, in Actinomyces madurce filaments. But in most of the aerobic types all forms are seen, coexisting in a single culture. For a detailed

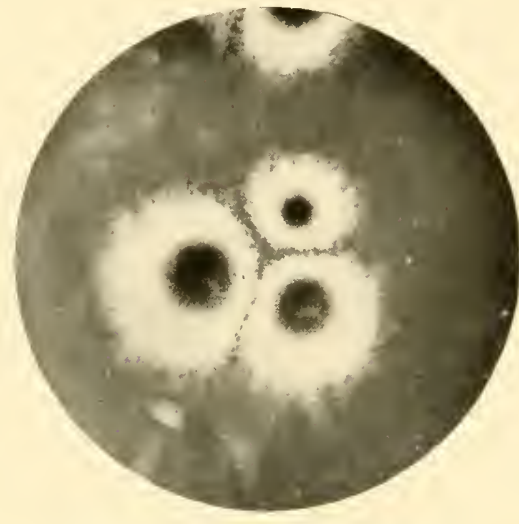

FIG. 60.-Actinomyces graminis.

Colonies on agar plate, 7 days, $37^{\circ} \mathrm{C}$. aerobically $: \times 8$.

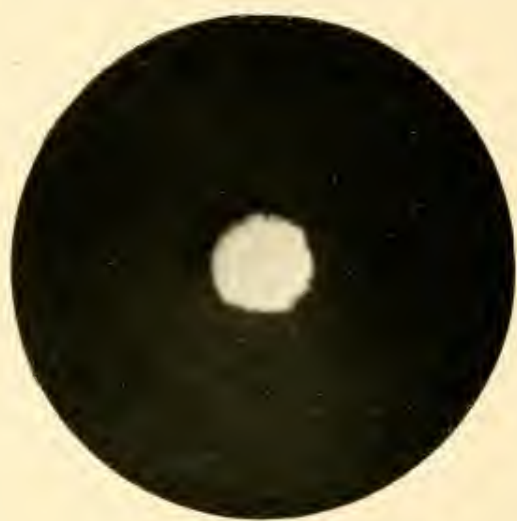

FIG. 61.-Actinomyces bovis.

Colony on agar plate, 14 days, $37^{\circ} \mathrm{C}$. anaerobically $: \times \dot{8}$.

description of their morphology, the reader is referred to a monograph by Lieske (1921).

Cultural Reactions.-In general, growth on artificial media is readily obtained. The usual media suffice, but the addition of glucose or glycerol is beneficial. The aerobic types, with a few exceptions, multiply rapidly, so that in 24 hours definite evidence of growth is visible on agar. The anaerobie types, on the other hand, grow more slowly, taking 3 or 4 days to form macroscopic colonies. Great diversity of cultural appearance is noticeable, particularly in the aerobie species. The deseriptions that follow refer only to some of the eommoner types.

On an agar plate the aerobic types form round, low convex, opaque, finely granular colonies, which later undergo differentiation into a raised, knob-like, sometimes radially striated eentre and an effuse, ground-glass-like periphery. The surface is finely granular and often has a "ehalk powder" covering due to the formation of aerial spores; the edge is rhizoid, indented, or feathery (Fig. 60). Iost strains form pigment-yellowish, pink, or orange in colour-which becomes apparent after a few days' incubation, and which may show progressive alterations in tint. This is espeeially noticeable in eultures that have been incubated at $37^{\circ} \mathrm{C}$., 
and subsequently left in the dark at room temperature. under suitable conditions, aerial hyphæ may develop, giving rise to a characteristic bloom on the surface of the colony - the chalk powder appearance just described.

The anaerobic Wolff-Israel type forms smaller colonies, not apparent for 3 or 4 days; they are more compact, greyish or porcelain white in colour, and have a nodular surface (Fig. 61).

On glycerol or glucose agar the aerobic types give a luxuriant, confluent, heaped-up, worm-cast, pigmented growth, adherent to the medium, of tough consistency, and difficult to emulsify (Fig. 62). The anaerobic type grows in the form of discrete colonies, which are only slightly adherent to the medium, and are much easier to emulsify.

In a glucose agar shake culture the aerobic types give a thick pigmented growth confined entirely or almost entirely to the surface. The anaerobic type gives a characteristic band-like growth situated about 0.5 to $1 \mathrm{~cm}$. below the surface, with a few larger discrete colonies scattered throughout the medium below. No growth at all occurs in the upper few millimetres.

In broth the aerobic types often form a thick, dry, dull, scaly or nodular, pigmented surface pellicle, which may extend for some distance up the sides of the tube. A ropy or membranous, sometimes pigmented sediment

After a variable time

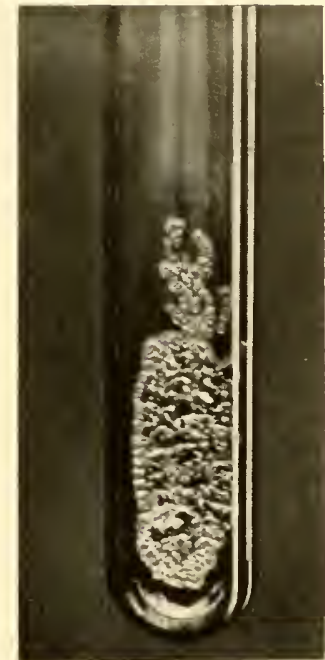

Fig. 62.-

Actinomyces graminis.

Culture, 14 days, $37^{\circ} \mathrm{C}$. glycerine agar slope, aerobically.

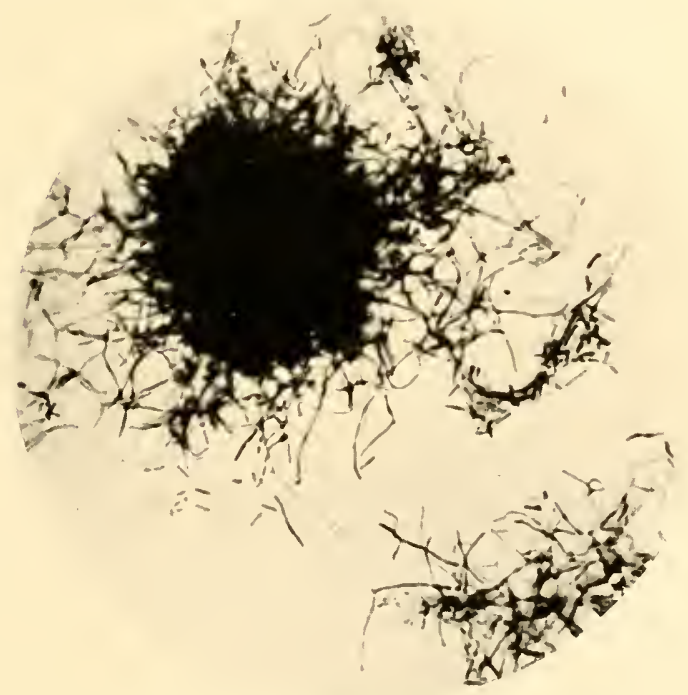

FIG. 63.-Actinom yces graminis $\times 1000$.

A small granule composed of radiating filaments; from a broth culture, 24 hours, $37^{\circ} \mathrm{C}$. aerobically.

be ascertained from the descriptions of the individual species.

forms, augmented by frequent deposits from the surface membrane. The broth remains clear, or at most shows a finely granular turbidity. Aerial hyphre may sprout from the surface pellicle. Sometimes growth commences at the bottom, and characteristic fluff-balls, resembling the head of a seeding dandelion, develop. The anaerobic type grows in the form of compact whitish granules with a nodular surface deposited at the bottom of the tube; there is no turbidity and no surface growth. Nitrate broth is more favourable for growth than ordinary broth.

The cultural characteristics on other media can 
Resistance.-The members of this group show no special resistance to heat or disinfectants. Most are killed in 15 minutes when exposed to moist heat at $60^{\circ} \mathrm{C}$. Some attempts have been made to find whether filaments containing so-called spores are specially resistant. Vincent (1894), working with Actinomyces madura, stated that the spores were killed at $85^{\circ} \mathrm{C}$. in 3 minutes, whereas the non-sporing forms were killed at $60^{\circ} \mathrm{C}$. in 3 to 5 minutes. Orskov (1923) has likewise found that the "spores" are more resistant than the plain mycelium; they may survive exposure to moist heat for 3 hours at $65^{\circ} \mathrm{C}$. Goyal (1936), however, was unable to show that the "spores" were any more resistant than the filaments; both were killed by a time-temperature combination varying with different strains from 30 minutes at $60^{\circ} \mathrm{C}$. to more than one hour at $72^{\circ} \mathrm{C}$. There is, of course, a great difference between the resistance of the Actinomyces " spores" and that of true bacterial spores.

Cultures of the aerobic type, if kept at room temperature, remain viable for months; cultures of the anaerobic type usually die out in about 6 to 8 weeks. If kept in the incubator they die very much more quickly.

Growth Requirements.-There is a fairly sharp division between the aerobic and the anaerobic types. The aerobic types are unable to grow under strictly anaerobic conditions, while the anaerobic types are unable to grow, at any rate on solid media and when first isolated, in the presence of air. In liquid media, especially in nitrate broth, and in the depths of solid media, such as glucose agar, the anaerobic types may flourish when kept under aerobic conditions, showing that they are not strict anaerobes, but rather organisms having a preference for a low oxygen pressure-micro-aerophiles. Intermediate types are met with (Bruns 1899, Lignières and Spitz 1903), usually having a preference for anaerobic conditions (Naeslund 1925). The development of both aerobic and anaerobic types is favoured by the addition of 10 per cent. $\mathrm{CO}_{2}$ to the atmosphere. The range of temperature over which the aerobic species are able to grow is very wide. Many of the water and soil strains multiply even at $3-6^{\circ} \mathrm{C}$., while nearly all strains grow between $6^{\circ}$ and $30^{\circ} \mathrm{C}$. About $30^{\circ} \mathrm{C}$. some strains fail to grow, but the majority have their optimum temperature round about $37^{\circ} \mathrm{C}$. Thermophilic species are encountered with an optimum temperature between $40^{\circ}$ and $70^{\circ} \mathrm{C}$. The anaerobic strains are more fastidious in their requirements, and fail to grow if the temperature varies more than a few degrees from the optimum of $37^{\circ} \mathrm{C}$. (Lieske 1921). Growth is improved as a rule by the addition of glucose or glycerol, sometimes by blood or serum.

Biochemical Characteristics. - The anaerobic type produces acid, but no gas, in glucose, maltose, lactose, and sucrose; strains of human origin (see p. 379) ferment also mannitol and salicin (Erikson 1940). Fermentation is often very slow. The aerobic types have, as a rule, no action on these substances. Many of the aerobic types turn litmus milk slightly alkalime and peptonize it slowly; the anaerobic type turns it acid (Negroni and Bonfiglioli 1937-38, Slack 1942). A few members are proteolytic, digesting gelatin and serum, but the majority have no proteolytic power. The anaerobic type cannot grow in the presence of bile salts; some of the aerobic types are able to do so.

None of the strains that we have tested was hæmolytic, but Waksman (1918) has noted hæmolysis in certain strains, and has correlated this property with the power to digest proteins. 
Pigment formation is characteristic of many of the aerobic species, the usual colour being some shade of pink or brown.

Antigenic Structure-Colebrook (1921), working with three strains of the Wolff-Israel type, found that their agglutination reactions differed. Aokı $(1936 ı, b)$, using agglutination and complement-fixation reactions, found that 6 anaerobic strains all fell into one group, but that 19 aerobic strains fell into eight different groups. The careful observations of Erikson (1940) have shown that by agglutination, aided when necessary by absorption of agglutinins, the anaerobic species can be divided into (1) a group containing strains of human origin, which are antigenically homogeneous though differing in their degree of agglutinability, and (2) a group containing strains of bovine origin, likewise homogeneous, but with much less power of giving rise to antibodies in rabbits. It must be noted, however, that all of Erikson's hovine strains were of Australian origin; whether European strains behave similarly is not known.

Pathogenicity.-The anaerobic Wolff-Israel type appears to be responsible for actinomycosis in man and cattle. The aerobic types are mostly saprophytic, but occasionally they are able to give rise to chronic granulomatous lesions in man and other animals.

For laboratory animals both types have a low pathogenicity. Inoculated in a large dose subcutaneously into rabbits they give rise to a circumscribed abscess, which may persist for weeks or months; in the pus Drusen may often be found (Hassegawa et al. 1938). Intraperitoneal injection of the Wolff-Israel type into guinea-pigs and rabbits may give rise to small nodules containing typical granular pus, but the lesions are neither extensive nor fatal. By the repeated inoculation of massive doses intravenously Slack (1942) was able to kill rabbits in 6-10 weeks, and to demonstrate post mortem the presence of macroscopic or microscopic abscesses and focal necroses in the lungs and liver, sometimes containing granules with hyalinized clubs. The acid-fast members of the aerobic group, such as Actinomyces asteroides, are more pathogenic. Injection of this organism by the subcutaneous, intravenous, or intraperitoneal route leads to a progressive fatal infection of guinea-pigs and rabbits in 5 days to 4 weeks. Post mortem, small tubercles are found scattered throughout the organs, being especially numerous in the lumgs, liver, and spleen (Eppinger 1891, MacCallum 1902). Microscopically these nodules contain tangled filaments, sometimes arranged in the typical ray form.

Variation in Actinomyces bovis.-Not all the anaerobic strains isolated from actinomycotic lesions conform to the description that we have given. Lentze (1938) pointed out that in certain cases an anaerobic bacillus of more diphtheroid appearance might be found, differing in its morphological, cultural, biochemical and antigenic characters from the classical Wolff-Israel type. He designated the new form as the S and the old as the R form. Erikson (1940), however, rightly objects to this terminology, and since the majority of the new strains are of animal origin, refers to Lentze's $\mathrm{S}$ and $\mathrm{R}$ forms by the terms bovine and human respectively. Whether these different forms are stable varieties, analogous to the bovine and human types of the tubercle bacillus, must await further observation.

The chief differential characters of strains of bovine origin are as follows :The mycelium undergoes fragmentation very rapidly so that rod forms are predominant; extensive ramification is rare; growth is scanty; the colonies are smoother, softer, have an entire edge, and are not adherent to the medium ; aerial hyphæ are not formed, as they may occasionally be by the classical form (Erikson 
1940); in liquid media there is sometimes a slight turbidity, and a wispy or light flocculent deposit; mannitol and salicin are seldom fermented; and antigenically they constitute a separate group.

\section{Classification}

Lieske (1921), who made an extensive study of the Actinomyces from the point of view of a botanist, found so much variation in the behaviour of individual strains that he was unable to arrive at any satisfactory classification. Subsequent workers, however, have felt that Lieske overestimated the difficulties of this task. Attempts at classification have been made along both morphological and physiological lines. The most prominent exponent of the former group during recent years is Orskov (1923), who devised the following scheme:

Group I. "Spores" give rise to a unicellular branching mycelium. This affords the substratum for an aerial mycelium, which consists of rather thickerbranched filaments. "Spores" are formed from the aerial hyphæ without any previous segmentation of the cytoplasm. "Spore" formation commences at the tip of the thread and proceeds towards the base. "Spores" are more resistant to heat than the plain mycelia. Minor points are that the primary mycelium is of cartilaginous consistency, and often sends roots into the agar. The aerial mycelium may arise centrally or peripherally, and may not appear for a long time. Condensation of the cytoplasm occurs at regular intervals. Whole appearance of organism is fairly uniform. Gelatin is often liquefied. This group seems to include most of the non-acid-fast aerobic species.

Group IIA. Both the primary and the aerial mycelium are of the same diameter, and both undergo early segmentation into irregular fragments. The aerial mycelium arises very early in the culture. It starts at the centre of the primary mycelium and spreads concentrically towards the periphery. No "spore "formation occurs, and the segmented mycelial fragments are no more resistant to heat than the unicellular mycelium. The whole appearance is very pleomorphic; coccoid, bacillary, filamentous, clubbed, and irregular-shaped forms are common. The growth is generally soft in consistency, and does not adhere to the medium. A reddish insoluble pigment is frequently formed. Gelatin is not usually liquefied. This group includes many of the aerobic acid-fast species, such as Actinomyces asteroides and Actinomyces farcinicus.

Group IIB. Differs from Group IIA in forming no aerial mycelium. [Erikson (1935), however, denies this, and would do away with the distinction between the $A$ and B groups.] Besides segmentation, the so-called angular division is common in this sub-group, and is responsible for the diphtheroid appearance of these organisms in film preparations. Group IIB comprises mainly the anaerobic species, of which the most important is the pathogenic organism described by Wolff and Israel.

Group III. The characteristic feature of this group is the formation of oval "spores" at the extreme tips of the mycelial branches. No aerial mycelium is produced. Only one species has so far been recognized-Actinomyces chalcece.

Naeslund (1925) put forward a classification based primarily on physiological characteristics. We have modified it very slightly, and present it in the following form, paying attention mainly to the organisms that are parasites or potential parasites of animals. 
Actinomyces.

A. Predominantly anaerobic types. Actinomyces bovis Harz.

B. Predominantly aerobic types.

(1) Non-acid-fast. Actinomyces graminis Bostroem.

Actinomyces capro.

Actinomyces madurce.

Actinomyces somaliensis.

(2) Acid-fast. Actinomyces farcinicus.

Actinomyces asteroides.

Actinomyces gypsoides.

C. Facultative aerobic types. Actinomyces muris.

The creation of a special subdivision for the facultative aerobic types is dictated, partly by convenience, and partly by the differences of the main species Actinomyces muris from the anaerol,ic Wolff-Israel type.

There seems little doubt that the organism Streptobacillus moniliformis, to which so much attention has been called in recent years by Levaditi, Nicolau, and Poincloux (1925), Parker and Hudson (1926), Levaditi, Selbie and Schoen (1932), Strangeways (1933), and Mackie, van Rooyen, and Gilroy (1933), is the same as the organism isolated by Schottmüller (1914), Blake (1916), and Tileston (1916), from one type of rat-bite fever in human beings, and called Streptothrix muris ratti. Since the original name for this organism claims priority over Streptobacillus moniliformis, we propose to adopt it. Modification, however, is necessary, partly to suit the binomial nomenclature, and partly because the term Streptothrix is not valid. In its place we suggest the name Actinomyces muris.

The inclusion, however, of this organism in the Actinomyces group is admittedly tentative, and may well have to be revised in the light of future work. The observations of Klieneberger, of Dienes, of Heilman and others (see pp. 939, 945) have revealed the existence of a group of small pleomorphic bodies resembling in many ways organisms of the pleuropneumonia group. The first of these bodies was isolated from a culture of Streptobacillus moniliformis. Whether it was a variant form of this organism or a symbiont remains doubtful, but since the other pleuropneumonia-like bodies that have been described do not appear to have been associated with Streptobacillus moniliformis, it seems premature to transfer this organism to the pleuropmeumonia group and eall it, as Heilman (1941) suggests, Asterococcus muris. We propose, therefore, to include it temporarily at least in the Actinomyces genus, but to describe the pleuropneumonia-like bodies associated with it in the chapter on the pleuropneumonia organism and associated forms. Van Rooyen (1936) would exclude Streptobacillus moniliformis from the Actinomyces group, because he was unable to demonstrate the presence of true branching in this organism; but his observations are contrary to those of most other observers, and the reasons he advances for assigning it to the Hamophilus group are not convineing. There is some ground for believing (see Dienes and Edsall 1937) that the organism isolated by Theobald Smith from the pneumonic lungs of calves, and called by him $B$. actinoides, is closely related to, if not identical with, Streptobacillus moniliformis. Since there is still some doubt about this, we shall for the moment describe it in the Actinobacillus group (see p. 389) to which its normal morphological appearance would naturally assign it.

Erikson (1935), who has recently studied a number of new parasitic species of Actinomyces, has suggested a scheme of classification containing both morphological and physiological characteristics. She accepts Orskov's grouping, but does away with the distinction between his Groups IIA and IIB. She objects to the use of oxygen requirements as a basis of classification on the ground that the distinction 
between the aerobic and anaerobic species is not sufficiently sharp. Instead, she places reliance on pigment formation, and proteolytic action.

Waksman and Henrici (1943) suggest a classification based primarily on the fragmentation or not of the mycelium. Organisms in which the mycelium breaks up into bacillary or coccoid elements they would place in the family Actinomycetacex; this would comprise two genera-Actinomyces for the anaerobic and Nocardia for the aerobic species. Organisms in which the mycelium does not fragment they would place in a new family Streptomycetacea ; this would comprise two genera-Streptomyces for those species in which multiplication occurs by conidia in chains from aerial hyphæ, and Micromonospora for those species in which multiplication occurs by single terminal spores or short sporophores. It will be realized that agreement on the classification of members of the Actinomyces group is still far from being reached.

A detailed description of some of the more important members is appended, followed by notes on others that are of less importance. These descriptions are based in part on our own observations of relatively few strains. For a differential table see p. 392 (Table 24), and for a general description of different types see Lieske (1921), Naeslund (1925), Setti (1929), and Rosebury (1944).

\section{Actinomyces bovis Harz}

Isolation.-Described by Bollinger in 1877, named Actinomyces bovis by Harz in 1877, and first isolated by Wolff and Israel in 1891.

Habitat. - Strict parasite found in lesions of actinomycosis in man and cattle. Frequent in human month and in salivary calculi.

Morphology.-Glycerol agar, 7 days at $37^{\circ} \mathrm{C}$. Long and short rods predominate; long continuous or segmented threads with a straight or curved axis, showing simple or dichotomous branching; S-shaped or spiral organisms; coccoid forms. The rods resemble, and are arranged like, certain members of the corynebacteria; sides parallel or irregular; ends rounded, clubbed or tapered; axis straight or curved; great variation in appearance; irregular staining is usual ; granular and beaded forms are not uncommon. Non-motile. Non-sporing. Gram-positive. Non-acid-fast.

Agar Plate. -7 days at $37^{\circ} \mathrm{C}$. anaerobically. Poor growth of round, $0 \cdot 5-1 \cdot 0 \mathrm{~mm}$. in diameter, convex, opaque, amorphous colonies with smooth dull surface and entire edge ; greyish-white by transmitted, porcelain white by reflected light; butyrous or friable consistency; emulsifiability not difficult as a rule. 21 days, rather larger, 1-1.5 mm. in diameter, umbonate, with slightly irregular nodular surface and lobate edge; differentiated into a glistening raised centre and a dull shelving periphery resembling a rosette. Colonies may grow into medium.

Agar Slope. -7 days at $37^{\circ}$ C. anaerobically. Moderate growth of discrete colonies similar to those described. Numerous greyish-white floccular masses of coarsely granular structure in water of condensation; they are irregular in shape, have an irregular edge, and are opaque. In the condensation water there is also a finely granular turbidity.

Gelatin Stab.-No growth at $23^{\circ} \mathrm{C}$. After 12 days at $37^{\circ} \mathrm{C}$, the culture shows, when cooled, a band of growth $4 \mathrm{~mm}$. deep with its upper margin $1 \mathrm{~mm}$. below the surface. Growth consists of very fine greyish-white interlacing filaments, looking like cotton-wool. No liquefaction.

Broth.-5 days at $37^{\circ} \mathrm{C}$. anaerobically. Poor to moderate growth; deposit of compact, white, mulberry-like granules with nodular surface, often adherent to each other; not disintegrated on shaking. No turbidity; no surface growth; no odour. 
Glucose Agar Shake. -5 days at $37^{\circ} \mathrm{C}$. No growth for $1 \mathrm{~cm}$. below surface. Then comes a turbid band, about $0.8 \mathrm{~mm}$. deep, consisting of large numbers of tiny colonies. Throughout the rest of the medium are scattered discrete, irregularly round, opaque. greyish-white colonies, about $0 \cdot 1-1 \cdot 0 \mathrm{~mm}$. in diameter, with smooth or slightly knobby surface.

Loeffler's Serum. -7 days at $37^{\circ} \mathrm{C}$. anaerobically. Moderate, partly confluent, raised, shiny growth of low convex, rounded colonies about $0.5 \mathrm{~mm}$. in diameter. No liquefaction.

Glycerol Egg.-14 days at $37^{\circ}$ C. anaerobically. Poor, slightly raised, confluent growth with finely granular surface due to imperfect fusion of colonies. No liquefaction. MacConkey.-No growth in either solid or liquid medium.

Potato.-14 days at $37^{\circ} \mathrm{C}$. anaerobically. Very poor growth of discrete, round, $1 \mathrm{~mm}$. in diameter, whitish low convex colonies with smooth glistening surface and entire edge.

Resistance.-At $37^{\circ} \mathrm{C}$. cultures live for about 1 to 4 weeks, sometimes longer. Dried on glass and kept in the dark, organisms may live for 7 weeks or more. Killed by moist heat at $60^{\circ} \mathrm{C}$. in 15 minutes.

Metabolism.-Anaerobe of the microaerophilic type. Will not grow on surface culture exposed to the air. Optimum temperature for growth $37^{\circ} \mathrm{C}$.; growth below $30^{\circ} \mathrm{C}$. is either very slight or absent. Optimum $\mathrm{pH} 7 \cdot 3-7 \cdot 6$. No hæmolysis of horse red cells. No pigment formation. Growth is improved by nitrates, glycerol, blood, an increased partial pressure of $\mathrm{CO}_{2}$, and sometimes by glucose.

Biochemical.-Acid, no gas, in glucose, maltose, mannitol, lactose, sucrose, and salicin within 21 days under anaerobic conditions. L.M. acid. Indole - ; M.R. - ; V.P. - ; Nitrate reduction ++ ; $\mathrm{H}_{2} \mathrm{~S}-; \mathrm{NH}_{3}+$; M.B. reduction - ; Catalase -

Antigenic structure.-Two groups distinguishable by agglutination, one containing strains of human, the other of bovine origin.

Pathogenicity.-Responsible for actinomycosis in man and cattle. Very slight pathogenicity for laboratory animals. Intraperitoneal inoculation of a broth culture into a rabbit or guinea-pig may be followed by appearance of snall nodules, chiefly in the great omentum, containing the typical clubbed colonies of Actinomyces. The animals live indefinitely.

\section{Actinomyces maduræ}

Isolation.-Isolated from pale variety of Madura foot by Vincent in 1894. Called by him Streptothrix madure.

Habitat.-Found in pale variety of Madura foot. Saprophytic existence probable, but not demonstrated.

Morphology.-Glycerol agar, 14 days at $37^{\circ} \mathrm{C}$. Long, non-segniented filaments, $0 \cdot 4-0 \cdot 6 \mu$ thick, showing true and false branching; sides parallel, ends often tapering. Arranged in a mycelium; sometimes aggregated into dense masses. Stain evenly. Later fragmentation may occur with production of ovoid bodies. Non-motile. Gram-positive. Non-acid-fast.

Agar Plate. -5 days at $37^{\circ} \mathrm{C}$. Small, round, convex colonies about $0.5 \mathrm{~mm}$. in diameter. 14 days, larger, 1-3 mm. in diameter, greyish-yellow, opaque, irregularly heapedup, nodular, umbonate colonies, resembling worm casts, which have a smooth glistening surface. Very adherent to medium; consistency horny; very difficult to emulsify. Whole colony looks like a rosette.

Agar Slope. -7 days at $37^{\circ} \mathrm{C}$. Poor growth of discrete, dull, greyish-white opaque irregularly heaped-up colonies with nodular surface.

Gelatin Stab.-14 days at $20^{\circ} \mathrm{C}$. Moderate, filiform growth consisting mostly of small, discrete, greyish-white colomies, having a darker centre and a lighter feathery 
periphery; growth extends to bottom of tube. Slightly raised surface growth about $3 \mathrm{~mm}$. in diameter. Slight liquefaction after 6 weeks.

Broth.-7 days at $37^{\circ} \mathrm{C}$. Poor growth. Deposit of little greyish-white puff-balls, looking like colonies of moulds, and having a dense centre and a lighter periphery ; often cohering in groups of two or three. No turbidity ; no surface growth ; no odour. Later a white efflorescent surface growth may appear.

Loeffer's Serum. -7 days at $37^{\circ} \mathrm{C}$. Poor growth of isolated colonies. 21 days, moderate growth of heaped-up nodular colonies. No liquefaction.

Glucose Agar Slope. -7 days at $37^{\circ} \mathrm{C}$. Discrete colonies, raised, heaped-up, with wormcast surface, moist and glistening.

Glycerol Agar Slope.-12 days at $37^{\circ} \mathrm{C}$. Luxuriant, raised, confluent, greyish-white wormcast growth, very tough, adherent to medium, and difficult to emulsify.

Glycerol Egg. - 7 days at $37^{\circ} \mathrm{C}$. Mostly confluent growth of rounded, dome-like colonies with dull nodular surface. Very tough, adherent to medium, and difficult to emulsify. No liquefaction.

Potato -7 days at $37^{\circ} \mathrm{C}$. Discrete, heaped-up, nodular, yellowish-brown colonies. 18 days, heaped-up dry, chalky-white and greyish-brown, worm-cast colonies. Later may take on a rose-red colour.

MacConkey.-No growth on solid or liquid media.

Resistance.-Destroyed by moist heat at $60^{\circ} \mathrm{C}$. in 5 minutes.

Metabolism.-Aerobic; very slight growth on glycerol agar anaerobically. Optimum temperature $37^{\circ} \mathrm{C}$; grows at $20^{\circ} \mathrm{C}$. Forms sometimes a rose-red pigment on potato. Growth improved by glycerol and glucose.

Biochemical.-Ferments no sugars. L.M. turned slightly alkaline; may be peptonized. Indole - ; M.R. - ; V.P. - ; Nitrate reduction + ; $\mathrm{H}_{2} \mathrm{~S}-; \mathrm{NH}_{3}$ sl. + ; Catalase v. sl. + ; M.B. reduction -

Pathogenicity.-Subcutaneous inoculation into rabbits, guinea-pigs, mice, and cats causes a local nodule, which increases in size for a month and then retrogresses. Responsible for pale or ochroid variety of Madura disease in man.

\section{Actinomyces graminis Bostroem}

Isolation.-Isolated by Bostroem in 1891 from human actinomycosis. Subsequently isolated by numerous workers from different lesions in man and other animals.

Habitat.-Saprophyte living on grains and grasses. Often gains access to mouth and respiratory passages of man and other animals.

Morphology. - Agar 24 hours at $37^{\circ} \mathrm{C}$. Long filaments, $0 \cdot 6 \mu$ wide, showing true and false branching; long and short rods, and coccoid bodies. Sides parallel, ends rounded or tapering. Filaments are straight, wavy, or spirillar, and may or may not be segmented. Arranged in small loose groups, or in a mycelium. In broth ray forms are frequent, with compact centre and radiating filaments. Staining of rods and filaments is often irregular. Non-motile. Gram-positive. Nonacid-fast.

Agar Plate.-24 hours at $37^{\circ} \mathrm{C}$. Round, greyish-white, low convex, dull, opaque colonies with finely granular surface and erose edge. 7 days, rounded, up to $3 \mathrm{~mm}$. in diameter, umbonate, granular colonies, with raised, opaque, primrose-yellow, radially striated centre, and effuse, greyish, ground-glass periphery. Surface granular, edge feathery or rhizoid. Generally tough and adherent to agar, and difficult to emulsify. Colonies may be folded on surface and coral-like, or they may be undifferentiated with a nodular surface and lobate edge. Colour may be chalky-white, yellow or brown.

Agar Slope.-24 hours at $37^{\circ} \mathrm{C}$. Abundant, slightly raised, greyish-white, faintly translucent or opaque growth with dull, finely granular or mealy surface and entire or erose edge. 7 days, surface is whitish and moderately granular; growth may be heaped-up in places. 
Gelatin Stab. -7 days at $20^{\circ} \mathrm{C}$. Moderate filiform growth of confluent, greyish-white, feathery colonies; extending to bottom of tube. Slightly raised surface growth, $3 \mathrm{~mm}$. in diameter. After 3 weeks the growth near the surface is orange-pink. Liquefaction unusual.

Broth.-24 hours at $37^{\circ} \mathrm{C}$. Moderate growth ; ropy or membranous sediment, not disintegrating on shaking; ring growth and finely granular almost invisible surface pellicle; turbidity absent, or slight and finely granular. 7 days, thick surface pellicle, extending up sides of tube; dry, dull, and scaly with pinkish-yellow or orange nodules in places : heavy floccular deposit, pinkish in colour. No odour.

Glucose Agar Shake. -8 days at $37^{\circ} \mathrm{C}$. Good growth confined to surface, except for a few tiny colonies in upper $5 \mathrm{~mm}$. of medium. Surface growth is thick, raised, confluent, dull, greyish-white with several secondary colonies developing on it. Sometimes surface growth is heaped-up, yellowish-brown, and of worm-cast type with no colonies below surface. 21 days, growth is brick-red or yellowish-orange in colour.

Loefler's Serum. -24 hours at $37^{\circ} \mathrm{C}$. Good, raised, moist, glistening confluent growth with nodular surface and lobate edge. 24 days, no liquefaction.

Dorset Egg.-10 days at $37^{\circ} \mathrm{C}$. Good, confluent, raised, yellowish growth with nodular surface and edge formed of single colonies. 24 days, no liquefaction.

MacConkey's Agar. -6 days at $37^{\circ} \mathrm{C}$. Growth of small, $0 \cdot 1 \mathrm{~mm}$. in diameter, pinkish, opaque colonies; growth very poor compared to that on agar. In liquid medium there is good growth with a heavy granular deposit.

Potato. 24 hours at $37^{\circ} \mathrm{C}$. Poor, slightly raised, chalky-white growtl with powilery surface. Later, growth may turn yellowish-orange or ochre-brown.

Resistance.-Cultures remain viable for months.

Metubolism,-Aerobic. No growth under strict anaerobic conditions. Optimum temperature $37^{\circ} \mathrm{C}$; grows at $20^{\circ} \mathrm{C}$. No hremolysin for horse red cells. Yellowish, orange, or pink pigment formed, particularly in old cultures stood at room temperature. Growth is improved by glucose, sometimes by serum.

Biochemical.-No fermentation of sugars. Litmus milk turned slightly alkaline in 6 days; may be slowly peptonized. Indole - ; II.R. - ; V.P. - : Nitrate rerluction $+; \mathrm{NH}_{3}+; \mathrm{H}_{2} \mathrm{~S}$ very slight + ; Catalase + ; M. B. reduction -.

Prullogenicity.-Appears to be non-pathogenic to laboratory animals.

\section{Actinomyces muris}

Synonyms. - Streptothrix muris ratti Schottmüller; Streptobacillus moniliformis Levaditi.

Isolation. - Isolated by Schottmüller (1914) from human patients bitten by rats.

Habitet. - Natural parasite inhabiting the nasopharynx of rats (Strangeways 1933 ).

Morphology.-Loeffler's serum at $37^{\circ} \mathrm{C}$. Slender branching filaments, $0 \cdot 4-$ $0 \cdot 6 \mu$ wide, growing in interwoven masses. After 18-24 hours fragmentation of the filaments sets in, and many of the filaments are replaced by chains of bacillary or coccoid bodies. Very marked pleomorphism. Occasional filaments show spherical, oval, fusiP.B.

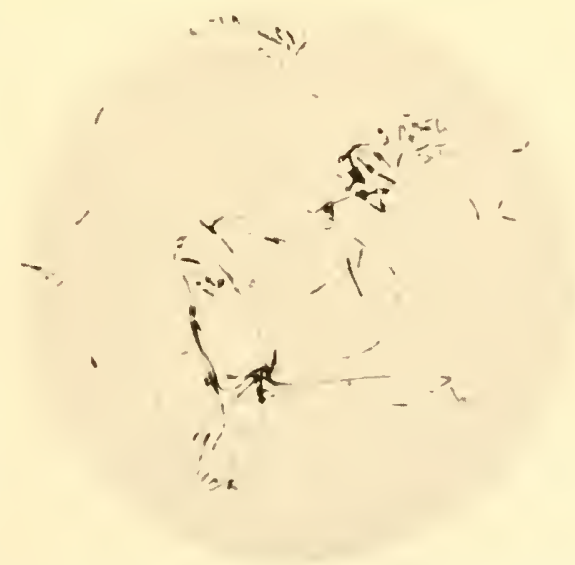

Fig. 64.-Actinomyces muris. From a Loeftier slope, 2 days, $37^{\circ} \mathrm{C}$. aerobically ( $\left.\times 1000\right)$. 
form or club-shaped swellings occurring terminally, sub-terminally, or in some other situation-hence the term "moniliformis." "These swellings may be 2-5 times the diameter of the filament, and may project from one side only. Accorting to Klieneberger (1942) the moniliform appearance and ecrtain other appearances are due to the pleuropneumonia or LI organism, which is a constant companion to Actinomyces muris. In the animal body the morphology is more regular and bacillary. Great irregularity in depth of staining. Non-motile. Usually described as Gram-negative, but may be Gram-positive in voung cultures. Non-acid-fast. (See Fig. 64.)

Nutrient Agar at $37^{\circ} \mathrm{C}$.- - No growth.

Glucose Agar at $37^{\circ} \mathrm{C}$.- -No growth.

Serum Agar Plate -2 days at $37^{\circ} \mathrm{C}$. Circular, greyish-yellow, low convex, almost waterclear, amorphous colonies, $0 \cdot 2-0 \cdot 3 \mathrm{~mm}$. in diameter, with smooth glistening surface and entire edge ; butyrous in consistency and easily emulsifiable. No differentiation. Little or no increase in size on further incubation.

Gelatin Stab. -7 days at $20^{\circ} \mathrm{C}$. No growth.

Nutrient Broth at $37^{\circ} \mathrm{C}$. No growth.

Serum Broth.-2 days at $37^{\circ} \mathrm{C}$. No turbidity. Abundant, greyish-white, coarsely granular sediment, looking like fluffy bread crumbs, miniature cotton balls, or tiny snow flakes, not disintegrating completely on shaking. No surface growth. No odour. Glucose Agar Shake.-7 days at $37^{\circ} \mathrm{C}$. No growth.

Loeff.er's Serum.-2 days at $37^{\circ} \mathrm{C}$. Discrete, circular, low convex colonies, similar to those on serum agar, but rather larger $-0.5-0.7 \mathrm{~mm}$. in diameter. 7 days; some colonies may show a differentiation into a slightly raised umbonate centre with a flatter periphery having an irregular or crenated edge; surface appears finely granular and rather dull. Growth may be confluent from the start, and appear slightly raised, colourless, with a glistening beaten-copper surface and a more or less entire edge. No liquefaction, even after 3 weeks.

Dorset Egg.-2 days at $37^{\circ} \mathrm{C}$. Similar to colonies on Loeffler's serum, but perhaps slightly smaller $-0 \cdot 3-0 \cdot 6 \mathrm{~mm}$. in diameter. No liquefaction, even after 3 weeks.

Horse Blood Agar. -2 days $37^{\circ} \mathrm{C}$. Colonies resemble those on serum agar. No hæmolysis. Potato. -7 days at $37^{\circ} \mathrm{C}$. No growth.

MacConkey's Agar. -7 days at $37^{\circ} \mathrm{C}$. No growth.

Grouth in Developing Egg.- Inoculated on to the chorio-allantoic membrane of the developing chick embryo, Actino. muris invades the embryo and becomes localized almost exclusively in the synovial lining of the joints, where it appears to grow mainly as an intracellular parasite; the embryo dies within 4 days (Buddingh 1944).

Resistance.-Destroyed in serum broth by heating to $55^{\circ} \mathrm{C}$. for 30 minutes. Dies out in culture very readily. Serum broth cultures may remain viable at $37^{\circ} \mathrm{C}$. for a week.

Metabolism.--Grows aerobically, but grows equally well or better under anaerobic conditions. Growth said to be improved by 10 per cent. $\mathrm{CO}_{2}$. Optimum temperature $37^{\circ} \mathrm{C}$.; little or no growth at $22^{\circ} \mathrm{C}$. No hæmolysin for horse red cells. No pigment formation. No growth on ordinary media, but growth occurs in presence of serum, ascitic fluid, or blood; not inproved by glucose or glycerol.

Biochemical.-Not thoroughly studied. In serum sugar media acid is produced within 3 days in glucose and salicin, sometimes in maltose and lactose. Litmus milk unchanged. Indole - ; M.R. - ; V.P. - ; Nitrate reduction - ; $\mathrm{H}_{2} \mathrm{~S}-$; catalase - ; M.B. reduction -

Antigenic Structure.-Different strains appear to be antigenically homogeneous.

Pathogenicity.-Responsible in man for one type of rat-bite fever-sometimes described as infectious erythema or Haverhill fever. May give rise to an epizootic disease in mice characterized by œdematous swelling of the feet and legs, arthritis, conjunctivitis, and lymphadenitis. Intraperitoneal inoculation of mice with $0.5 \mathrm{ml}$. of a serum broth culture is usually fatal in 1-2 days; no characteristic post-mortem 
appearances visible. Subcutaneous inoculation into one of the hind feet often leads to a more or less perfect reproduction of the natural disease. Comparatively avirulent for rats, guinea-pigs, and rabbits, though intravenous inoculation of culture into rabbits may sometimes lead to arthritis.

Variation.-Pleuropneumonia-like bodies are constantly associated with this organism (see p. 946); it is still doubtful whether they are variant forms or symbionts.

Note.-Dick and Tunnicliff (1918) isolated a similar organism-Actinomyces putorii--from a boy bitten by a weasel.

\section{A. Other Anaerobic Types.}

Tumnicliff (1926) describes a weakly Gram-positive motile anaerobic organism, which she isolated from a tonsillar granule. Smear preparations of the granule showed thick bacilli with rounded ends, filaments, tightly waved spirilla, and cocci. Sections stained with Giemsa showed bundles of filaments with the bacillary forms radiating from them. In anaerobic culture on ascitic fluid tissue medium and sheep-blood agar, rosettes and test-tube-brush-like forms appeared, similar to those in the original material. It is very doubtful whether this organism should be included in the Actinomyces group (see also Tunnicliff and Jackson 1930).

\section{B. Other Aerobic Types.}

\section{(1) Non-ACid-Fast 'TYPes}

Actinomyces capræ.--Described by Silberschmidt (1899) in 1897. It was isolated from the lung of a goat supposed to be suffering from tuberculosis. Consists of very thin, wavy filaments, showing a varying degree of branching; filaments segment into rod and coccoid forms. On agar, dry colonies, flattened in the centre with an irregular warty folded surface. In broth, surface growth of dry discoid colonies and a rough deposit. No change in litmus milk. Abundant growth on potato of rose-red colonies, later becoming chalky-white. No liquefaction of gelatin. Aerobic. Subcutaneous injection into rabbits produces an abscess. Intravenous injection sometimes causes tubercles in various organs. Guinea-pigs are rather more susceptible than rabbits (see also Galli-Valerio 1912).

Actinomyces somaliensis.-Described by Brumpt in 1906 (see Brumpt 1927). Was first isolated by Bouffard in French Somaliland from patients affected with mycetoma. Consists of long branching filaments with truncate or sometimes tapering ends. Grampositive. Grows on agar, but better on blood agar. On this mediun colonies are at first small, circular, convex and translucent, but after a few days they become irregularly heaped up, nodular, worm-cast, or crateriform; they are opaque, vary in colour from white, through yellowish-orange, to brown or black, often show radial segmentation which gives them a stellate appearance, are extremely tough in consistency and adherent to the medium, and have a peculiar odour. In broth no turbidity or surface growth, but a deposit of little greyish-white puff balls. On potato a white folded layer of growth, which in 5 to 6 days becomes yellow. Peptonizes milk. Ferments no sugars. Gives rise in man to mycetoma of the hand or foot. Lesions contain hard smooth yellowish-red granules, $1 \mathrm{~mm}$. in diameter, not dissociated by caustic potash.

\section{(2) Acid-Fast Types}

Actinomyces farcinicus.-Isolated by Nocard in 1888 from cattle suffering from farcy. Branching filaments growing in a mycelium. Gram-positive; feebly acid-fast (see p. 375). On agar it forms small, irregular, raised, opaque, yellowish-white colonies with a dull mammilated powdery surface. Dry, scaly, pale yellow plaques on potato. Irregular whitish masses in broth, some of which remain at the surface and others fall to the bottom. No liquefaction of gelatin. No change in litmus milk. No growth anaerobically. In cultures, the organism forms filamentous felted masses and diphtheroid-like bacilli. Cultures remain viable for 4 months at $37^{\circ} \mathrm{C}$.; killed by heat at $70^{\circ} \mathrm{C}$. in 10 minutes. Intra- 
peritoneal injection is fatal to guinea-pigs in 9 to 20 days; post mortem, miliary nodules over peritoneum, containing a little pus; in the pus are masses of bacilli. Intravenous injection of guinea-pigs causes the formation of generalized miliary nodules, particularly abundant in the lungs, liver, and spleen. Miliary nodules follow intravenous injection of cows and sheep. Rabbits, dogs, cats, horses, and asses are resistant to intravenous or intraperitoneal injection. Subcutaneous injection causes a slowly progressive abscess, which ulcerates and heals.

Actinomyces asteroides.-Isolated by Eppinger in 1891 from a brain abscess in a glassgrinder. Consists of threads showing true and false branching; threads may be long, or short and segmented; tiny rod forms also seen. In the body it forms long, granular, interlacing filaments with no ray or club formation. Gram-positive. Acid-fast, though not so strongly as the tubercle bacillus. Aerobic; no growth anaerobically. Destroyed by heat at $70^{\circ} \mathrm{C}$. in 5 minutes. On agar-at first whitish, later ochre-coloured, umbonate colonies, having a raised, dry, wrinkled, opaque centre and a moist glistening more translucent periphery with a mycelioid edge; whole colony star-shaped-hence the name asteroides-may be a central depressed crater; later, colour deepens to orange, and the wrinkling of the surface becomes more marked. Agar slope-raised ochre growth with a mealy surface and entire edge. Gelatin-very slow growth without liquefaction. Potato - red raised growth with a granular, and later wrinkled, surface ; a chalk-white bloom may develop due to a velvet-like upgrowth of fine filaments into the air. These uplifted filaments have terminal chains of coccoid bodies or spores, which, when transferred to broth, sprout and give rise to long filaments or star-like clusters (MacCallum 1902). Broth-white surface pellicle, which falls to the bottom, and is renewed several times; no turbidity. After subcutaneous, intraperitoneal, or intravenous injection, rabbits and guinea-pigs die in 5 days to 4 weeks. Post mortem, the viscera, especially lungs, liver, and spleen, are studded with small white nodules. Abscesses may develop in the muscles, kidneys, and other organs; these abscesses contain branching test-tube brush forms with laterally radiating clubs. An organism called Actinomyces variabilis with similar pathogenicity but different cultural reactions to Eppinger's strain was isolated by Cohn (1913) from the bladder of a man with pyuria.

Actinomyces gypsoides.--Isolated by Henrici and Gardner (1921) from the sputum of a woman. Acid-fast branching filaments were found in the sputum, sometimes in mycelial form. Agar slope-- thin greyish veil, soon becoming thick, opaque, and chalkywhite; surface dry and wrinkled; growth finely adherent to the medium and very brittle. Potato-growth similar to that on agar. Gelatin stab-surface growth only; liquefaction stratiform and complete in a week. Broth-small white flakes coalescing to form a thick, wrinkled, snow-white surface pellicle extending up sides of tube. Litmus milk-yellowish surface pellicle; milk is turned alkaline and curdled; litmus reduced; later digestion. Media containing peptone are darkened (tyrosinase). Growth improved by dextrose, maltose, and glycerol. No carbohydrates fermented. Intravenous injection into rabbits is fatal in 2 days; post mortem, minute abscesses in viscera, especially kidneys, which are studded with yellowish-white nodules. Intraperitoneal injection into guinea-pigs is fatal in 4 to 6 days; post mortem, small tubercle-like nodules over peritoneum ; omentum shrunken and studded with nodules.

An acid-fast strain described by Birt and Leishman (1902) gave a snow-white growth on solid media, peptonized milk, but did not liquefy gelatin. Another acid-fast strain described by Berestnew (see Feistmantel 1902) gave a grey to whitish growth, liquefied gelatin, but was non-pathogenic to laboratory animals. More recently, Goldsworthy (1937) has given a good description of an acid-fast strain isolated from a patient with pulmonary actinomycosis.

\section{Other Facultative aerobic Types.}

Described by Naeslund (1925), who isolated organisms of two different types from the human mouth. 
Type I consists morphologically of branching, and often very sinuous, relatively short threads, usually arranged in a radiating fashion; rods and granules are also present. In old cultures there is marked pleomorphism. No aerial spores. Chiefly Gram-positive, non-acid-fast. Culturally, growth in dextrose broth occurs in the form of round, oval, or ovoid colonies, white to yellowish-grey in colour, which appear at the bottom in about a week. On saliva dextrose agar a slightly shiny colourless film appears, becoming granular after a few days; some of the granules may develop into small greyish-white nodular adherent colonies, having a narrow translucent, finely striated margin. Little or no growth on gelatin, potato or milk. Optimum temperature for growth $37^{\circ} \mathrm{C}$.; no definite growth at $20^{\circ} \mathrm{C}$.

Type II consists of very long threads of fairly even thickness, showing typical but infrequent branching. No definite aerial spores formed. Gram-positive, but the greater part of the mycelium consists of Gram-negative elements with occasional Gram-positive segments. Non-acid-fast. Culturally, in saliva dextrose broth round, greyish, more or less translucent colonies develop at the bottom in about a couple of weeks. On saliva glucose agar growth at first occurs in a thin film, but in 1 to 2 weeks isolated pinhead colonies appear, hard or soft in consistence, and surrounded by a narrow translucent border. No definite growth on gelatin, potato, or in milk.

\section{ACTINOBACILLUS}

Lignières and Spitz (1902) isolated a non-motile, non-branching, Gram-negative bacillus from the lesions of cattle suffering from a disease which in many respects resembled actinomycosis. They called the organism the actinobacillus, and the disease to which it gave rise actinobacillosis. Two other organisms have since been described, having some points of similarity with this bacillus, and it is therefore convenient to consider them as forming a group to which the generic name Actinobacillus may be applied.

Definition. Actinobacillus Brumpt. (Emended from the American Committee's Report.)

Gram-negative, non-acid-fast rods, sometimes occurring in long chains or in unjointed filaments. In lesions in the animal borly no mycelium is formed, but at the periphery finger-shaped cells or clubs may be visible.

Type species is Actinobacillus lignieresi, Brumpt.

The classification we suggest is as follows:

Actinobacillus.

A. Aerobic and facultatively anaerobic. Actinobacillus lignieresi.

Actinobacillus actinomycetemcomitans.

B. Preferring raised $\mathrm{C}_{2}$ pressure. Actinobacillus actinoides.

A description follows of the separate organisms.

\section{Actinobacillus lignieresi}

For isolation see above. Appears to be a strict parasite. Synonym.-Probably the same as Bact. purifaciens (see Tumicliff 1941).

Morphology. - In young cultures it is a small rod-shaped organism; in older cultures it is cocco-bacillary, and various involution forms appear. In serum broth long streptobacillary forms are common. In glucose agar shake cultures long, tangled, unbrancherl filaments may be formerl, accompanied by smaller bacilli and coccoid bodies (Griffith 1916). Dimensions of the bacilli are given by Lignières and Spitz $(1902)$ as $1 \cdot 15-1 \cdot 25 \mu$ long by $0 \cdot 4 \mu$ broad. Non-motile; non-sporing; non-acidfast. Stains readily, especially with carbol fuchsin, and is Gram-negative; frequently shows bipolar staining. 
In lesions in the animal body small granules are found, which consist of tufts of radially disposed elubs similar to those in actinomyeosis. An important point of difference is that the centre of the granule is occupied not by a Gram-positive filamentous mycelium, such as is formed by Actinomyces bovis, but by minute Gram-negative bacilli, which may quite readily be overlooked. Though both the bacilli and the clubs formed by Actinobacillus lignieresi are Gram-negative, it is possible to differentiate between them by a modified Ziehl-Neelsen stain, as was pointed out by Bosworth (1923). If a section of affected tissue is stained with carbol fuchsin, decolorized for 20 to 30 scconds with $\mathrm{I}$ per cent. $\mathrm{H}_{2} \mathrm{SO}_{4}$, and counterstained with methylene blue, the clubs appear red and the bacilli blue. For pus, one of the best stains is glycerine piero-carmine, which stains the clubs yellow and the pus cells pink.

Cultivation.-Cultures are best obtained by grinding up infective pus in a mortar, and seeding on to agar. Growth oecurs readily under aerobic conditions, and less readily under anacrobie conditions. The optimum temperature for growth is $37^{\circ} \mathrm{C}$. : very slight growth oecurs at $20^{\circ} \mathrm{C}$.

On agar in 24 hours at $37^{\circ} \mathrm{C}$. small, circular, bluish-grey, translucent colonies with a smooth surface and an entire edge, up to $1.5 \mathrm{~mm}$. in diameter, are formed; further incubation results in a considerable increase in size-up to $4 \mathrm{~mm}$.- due to peripheral extension of the colony. On an agar slope the growth of freshly isolated strains is poor, consisting of small, discrete, translucent bluish colonies, or of a thin, dry, confluent layer of growth adherent to the medium. After cultivation for some time in the laboratory, the organism grows more readily, giving a confluent, filiform, viscous growth with a thickened edge.

In stab agar there is a whitish opaque spot at the surface; no growth occurs down the stab. In gelatin stab growth is very poor

EIG. 65.-Actinobacillus lignieresi. From a liver agar slope, 2 days, $37^{\circ} \mathrm{C}$. aerobically $(\times 1000)$.

and is not visible for some days. A small opaque spot appears at the surface; no growth occurs down the stab, and there is no liquefaction.

Coagulated serum is not a very favourable medium; only a thin whitish growth is formed.

On acid potato there is no growth. On alkaline potato a slight, glistening, greyish-yellow growth appears.

In peptone broth there is a slight mniform turbidity. In old cultures, a surface film may develop, and an abundant deposit. Growth in broth is improved by serum.

Resistance.-The organism is killed by heating to $62^{\circ} \mathrm{C}$. in 10 minutes. It rapidly succumbs to drying. Cultures do not live long, and should be transplanted every few days. Infected pus preserved in sealed tubes may remain virulent for a month or two.

Biochemical Reactions.-The fermentative ability of this organism is a little doubtful. Glucose, maltose, mannitol, and sucrose are generally rendered acid, though not markedly so, in a day, while lactose may be fermented later. Acid is produced in litmus milk, but no clot. Indole is formed, apparently in small quantity.

Antigenic Structure.-Little kuown. Tunnicliff (1941) states that bovine and ovine strains appear to be similar, hut that strains of either type may fail to agglntinate with a type serum. 
Pathogenicity.-No exotoxin is formed. The organism is responsible for Actinobacillosis in cattle. The virulence of different strains seems to vary considerably, and while cattle inoculation experiments are successful with some strains, they are completely negative with others (Magnusson 1928). Mlost workers, including ourselves, have been unable to produce any specific lesions in laboratory animals. The following statements, therefore, which are taken from Lignières and Spitz (1902), must be accepted with considerable reserve. Subcutaneous inoculation of pure cultures into cattle produces an abscess identical with those occurring spontaneously ; in the pus granules are found consisting of bacilli surrounded by clubs. Intraperitoneal inoculation of a whole agar culture is fatal to a guinea-pig in 12 to 24 hours. Post mortem there is an abundant turbid peritoncal exudate, rich in polymorphonuclear cells; the organisms can be cultivated from the exuclate, but rarely from the blood. Intraperitoneal injection of $\frac{1}{4}-\frac{1}{2}$ an agar culture into male guinea-pigs produces a typical Straus reaction. In 2 days the testicles are markedly inflamed, and the two layers of the tunica vaginalis are allherent; the scrotum is red, swollen, and tenrler. The animal loses weight, and dies in 5 to 7 days. Post mortem small purulent graunles, the size of a hemp seed, formed of a very thin membrane containing white or yellowish, thick, homogeneous pus, and seattered over the peritoneal serosa-particularly on the inferior surface of the diaphragm, the liver, spleen, and omentum. Around the testis there is a thick purulent exudate, gumming the two layers of the tunica vaginalis together. In the pus of these lesions tufts of elubs are found, thongh not in large numbers; they are rather smaller than those seen in cattle. Subeutancous inoeulation of gninea-pigs causes a local abscess, which may resolve; ulceration rarely occurs; clubs are not usually demonstrable in the pus.

Rabbits, cats, and dogs are considerably more resistant than guinea-pigs, but succumb to intravenous inoculation. Small lesions may develop in mice or rats after subcutaneous inoculation. Pigcons and fowls are resistant.

Actinobacillus actinomycetem-comitans.-Described by Klinger in 1912 under the name Bact. actinomycetem comitans. Found in lesions caused by Actinomyces bovis, as densely packed Gram-negative cocco-bacilli (Colebrook 1920). In culture the rod forms are $1 \cdot 0-1 \cdot 5 \mu$ long; the coccoid forms are $0 \cdot 6-0 \cdot 8 \mu$ in diameter. Intermediate forms are frequent. The organism is non-motile. In broth or liquid gelatin at $37^{\circ} \mathrm{C}$. it forms isolated, translucent granules, $0.5-1.0 \mathrm{~mm}$. in diameter, along the sides of the tube, most numerous near the surface; several hundreds of these colonies may develop. After some days they fuse into a greyish-white mass, forming a ring round the tube and a pellicle over the surface. The granules can be picked off the wall of the test-tube with a loop, but are very difficult to break up. Later they may become opaque and greyish-white. On agar it gives rise to small tough colonies, not unlike those of streptococei, adherent to the medium. The organism flourishes under both aerobic and anaerobic conditions. There is no growth at room temperature. Cultures live for 4 weeks. It is toxic on injection into rabbits, but does not set up a true infection.

Actinobacillus actinoides.-This organism was isolated by Smith in 1918 from the lungs of calves suffering from epizootic pneumonia, and called by him $B$. actinoides. In the animal body it appears as a minute Gram-negative bacillus arranged in groups. In the condensation water of coagulated serum it forms minute whitish flocculi, which consist of a central mass of radiating non-branching filaments ending peripherally in clubs. In tissue-agar cultures the organism grows as aggregations of rounded, ring-like bodies, $2 \mu$ in diameter, having a minute refringent speck on the periphery or near the centre. There are thus three distinet forms in which this bacillus occurs. Ilost strains are capsulated (Smith 1921b), but the capsule does not stain with the usual dyes. Growth occurs only under a raised pressure of $\mathrm{CO}_{2}-$ as in sealed tubes. On coagulated serum whitish flocculi appear in the condensation water in 3 days at $37^{\circ} \mathrm{C}$, and after several 


\begin{tabular}{|c|c|c|c|c|c|}
\hline 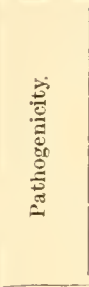 & 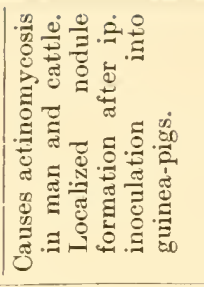 & 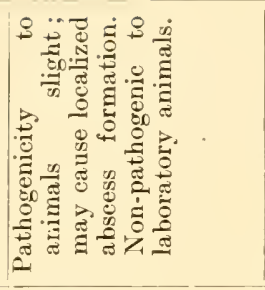 & 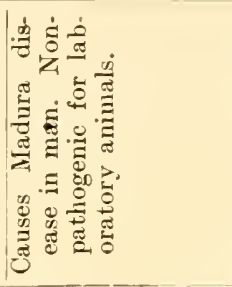 & 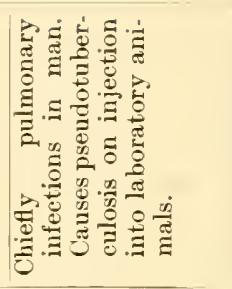 & 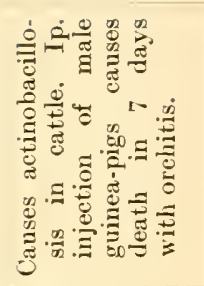 \\
\hline & 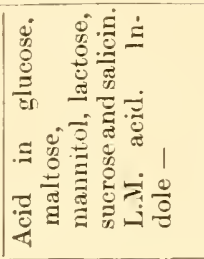 & 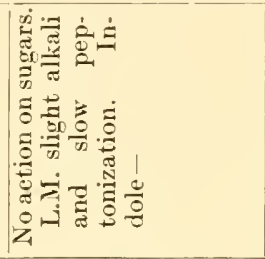 & 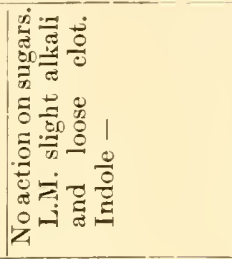 & 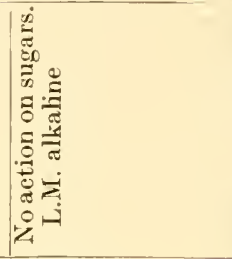 & 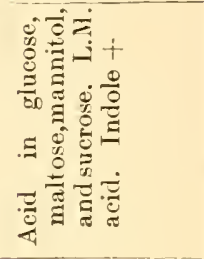 \\
\hline 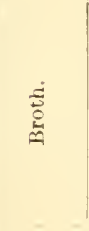 & 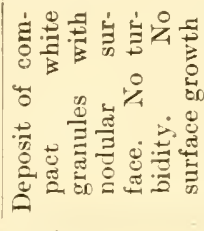 & 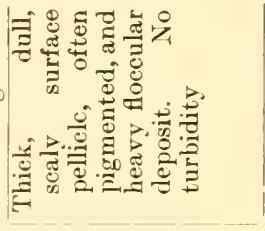 & 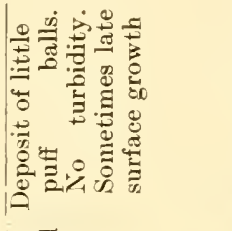 & 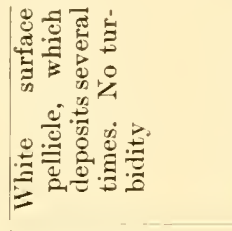 & 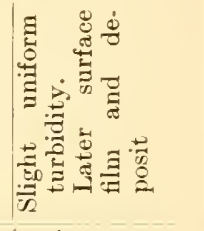 \\
\hline 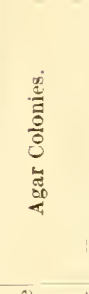 & 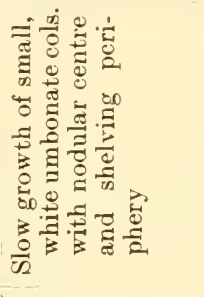 & 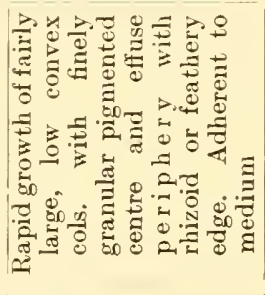 & 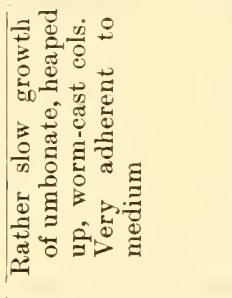 & 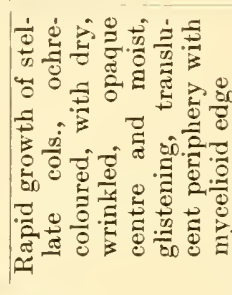 & 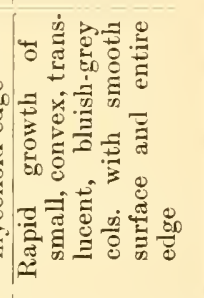 \\
\hline 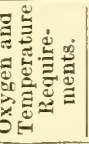 & 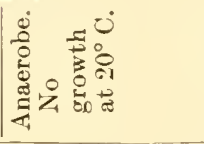 & 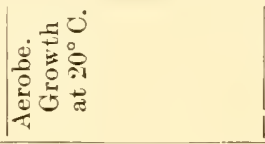 & 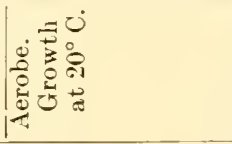 & 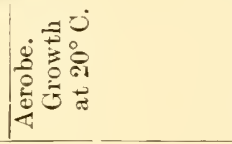 & 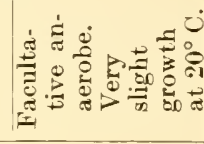 \\
\hline 常 & 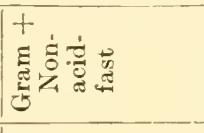 & | & 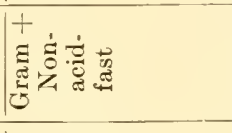 & 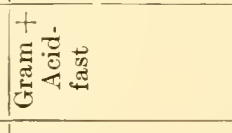 & 䨔 \\
\hline 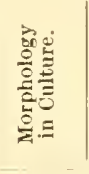 & 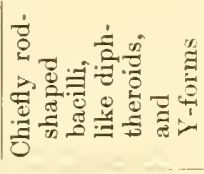 & 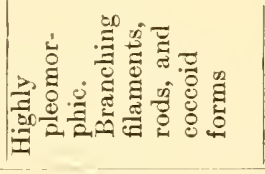 & 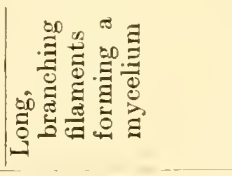 & 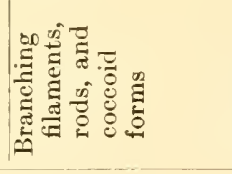 & 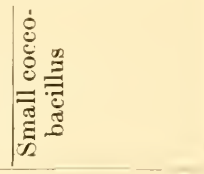 \\
\hline 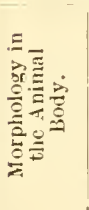 & 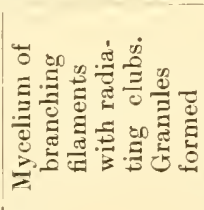 & 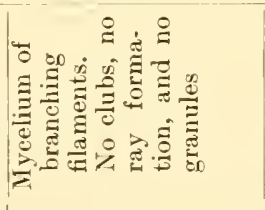 & 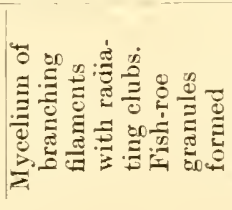 & 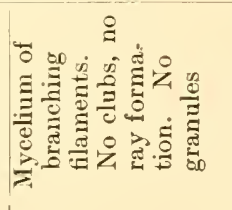 & 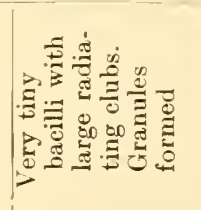 \\
\hline 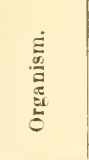 & 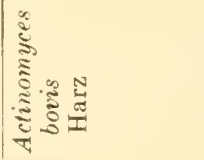 & 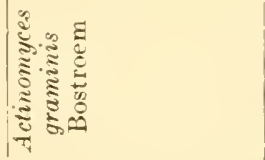 & 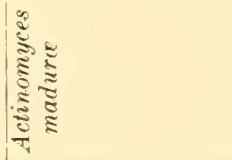 & 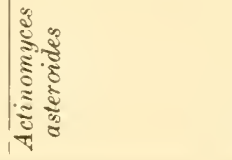 & 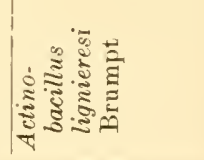 \\
\hline
\end{tabular}


weeks rery tiny, elevated, pointed-like colonies may appear on the slant. Growth may be obtained on agar to which a piece of guinea-pig's spleen has been added. No growth on ordinary media or on ascitic fluid. Non-pathogenic for laboratory animals on experimental injection. Subcutaneous injection into calves causes a large neerotic swelling with caseous contents; ulceration occurs in 4 weeks. Intratracheal injection into calves eauses small neerotic foci in the lungs, identical with those observed in the natural disease (Smith $192 \mathrm{~J} a$, see also Jones 1922). The fact that it has been isolated from the lungs of white rats suffering from pneumonia (Jones 1922) suggests that it may be a natural parasite of these animals. For further references to this organism see Smith $(1921 a, b)$. A similar organism, differing only in minor particulars has been isolated from the middle ear of white rats, in which it was eausing suppuration (Nelson 1930, 1931). The resemblance of Actinobacillus actinoides to Streptobacillus moniliformis (see p. 381) has been noted by Dienes and Edsall (1937); but in view of its reported lack of pathogenieity for laboratory animals and its baeillary morphology in the lungs of ealves, it is probably wiser to treat it separately for the moment.

\section{REEERENCES}

АокI, M. (1936 $\ell$ ) Z. ImmunForsh., 8\%, 196; (I936b) Ibit., 87, 24H).

Birt, C. and Leishman, W. B. (1902) J. Hyg., Camb., 2, 120.

BLAKE, F. G. (1916) J. exp. Med., 23, 39.

Bollinger, O. (1877) Zbl. med. Wiss., 15, 481.

Bostroem, E. (1891) Beitr. path. Anat., 9, 1.

Bosworth, T. J. (1923) J. comp. Path., 36, I.

Brumpt, E. (1927) "Précis de Parasitologir." Masson et Cie, Paris, p. 1201.

Bruns, H. (1899) Zbl. Bakt., 26, 11.

Buddingh, G. J. (1944) J. exp. lled., 80, 59.

Сону, T. (1913) Zbl. Bakt., 70, 290.

Colebrook, L. (1920) Brit. J. exp. Path., 1, 197; (1921) Lancet, i. 893.

Dick, G. F. and Turvicliff, R. (1918) J. infect. Dis., 23, 183.

Dienes, L. and Edsall, G. (1937) Proc. Soc. exp. Biol., N.Y., 36, 740.

Eppinger, H. (1891) Beitr. path. Anat., 9, 287.

Erikson, D. (1935) Spec. Rep. Ser. med. Res. Coun., Lond., No. 203; (1940) Ibit., No. 240.

Feistrantel, C. (1902) Zbl. Bakt., 31, 433.

Foulerton, A. G. R. (1910) Lancet, i. 551, 626, 769.

Galli-Valerio, B. (1912) Zbl. Bakt., 63, 555.

Goldsworthy, N. E. (I937) J. Path. Bact., 45, 17.

Goyal, R. K. (1936) "Contribution à l'éturle des Streptothricées." Jouve \& Cie, Paris.

Griffitil, F. (1916) J. Hyg., Camb., 15, 195.

Hassegawa, S., Nakanoto, T., Miyasaki, Y., ARluit, T', and Akrousi, ll. (1938) Jup. J. med. Sci., V, 3, 27.

Hellman, F. R. (1941) J. infect. Dis., 69, 32.

Henrici, A. T. and Gardner, E. L. (1921) J. infect. Dis., 28, 232.

Jones, F. S. (1922) J. exp. Hed., 35, 361.

Klieneberger, Eumy (1942) J. Hyg., Camb., 42, 48.5.

KliNger, R. (1912) Zbl. Baht., 62, 191.

Lentze, F. A. (1938) Zbl. Bakt., 141, 21.

Levaditi, C., Nicolat, S., and Poinclorx, P. (1925) C. R. Acad. Sci., 180, 1188.

Leevaditi, C., Selbie, R.-F., and Schoen, R. (1932) Ann. Inst. Pasteur., 48, 308.

Lieske, R. (1921) "Morphologie und Biologie der Strahlenpilze" (Actinomyceten) Gebrüder Borntraeger, Leipzig.

Lignières J. and Spitz, G. (1902) Bull. Soc. cent. Héd. Vét., 20, 487, 516; (1903) Arch. Parasit., Paris, \%, 428.

HacCallun, W. G. (190:) Zb7. Baht. 31, 5ะ9.

Iackie, T. J., liooyen, C. E. van, and Gilroy, E. (1933) Brit. J. exp. Path., 14, 132.

Magnusson, H. (1928) Acta. path. Microbiol. scund., 5, 170.

NAESLUND, C. (1925) Acta. path. Hicrobiol. scand., 2, 110.

Negroni, P. and Bonfroliol, H. (1937-38) Folin biol., No. 82, 1. 351.

Nelson, J. B. (1930) J. infect. Dis., 46, 64; (I931) J. Bact., 21, 18:3.

Nocard, E. (1888) Ann. Inst. Pasteur, 2, 293. 
ØRSkov, J. (1923) "Investigations into the Morphology of the Ray Fungi." Levin and Munksgaard, Copenhagen.

Parker, F. and Hudson, N. P. (1926) Amer. J. Path., 2, 357.

Report. (1917) J. Bact., 2, 505: (1920) Ibid., 5, 191.

Rooven, C. E. VAN (1936) J. Path. Bact., 43, 455.

Rosebury, T. (1944) Bact. Rev., 8, 189.

Schottǘller, H. (1914) Derm. W schr., 58, Supp., p. 77.

Setti, C. (1929) G. Bait. Immun., 4, 585.

Silberschmidt. (1899) Ann. Inst. Pasteur, 8, 841.

Slack, J. (1942) J. Bact., 43, 193.

Sмгтн, T. (1918) J. exp. Med., 28, 333 ; (1921 a) Ibid., 33, 441 ; (1921b) Ibid., 34, 593.

Strangeways, W. I. (1933) J. Path. Bact., 3\%, 45.

Tileston, W. (1916) J. Amer. med. Ass., 66, 995.

Tunnicliff, E. A. (1941) J. infert. Dis., 69, 52:

Tunnicliff, R. (1926) J. infect. Dis., 38, 366.

Tunnicliff, R. and Jackson, L. (1930) J. infect. Dis., 46, 12.

Vincent, H. (1894) Ann. Inst. Pasteur, 8, 129.

Waksman, S. A. (1918) J. infect. Dis., 23, 547.

Warsman, S. A. and Henrici, A. 'T'. (1943) J. Bact., 46, 337.

WolfF, M. and Israel, J. (1891) Virchows Arch., 126, 11. 


\section{CHAPTER 15}

\section{ERYSIPELOTHRIX AND LISTERELLA}

\section{DEFINITION.}

Rod-shaped organisms with a tendency to the formation of long filaments, which may show branching. The filaments may also thicken and show characteristic granules. No spores. Motility slight or absent. Gram-positive. Slight fermentative activities. Hicroaerophilic. Usually parasitic.

The type species is Erysipelothrix rhusiopathice, the causative organism of swine orysipelas.

The first member of this group to be described was the bacillus of mouse septicæmia, Erysipelothrix muriseptica; it was found by Koch in 1880 in the blood of mice that had been injected subcutaneously with putrefying blood. In 1882 Loeffler (1886) observed a similar bacillus in the blood vessels of the skin of a pig that had died of swine erysipclas. [It is possible that the bacillus observed four months previously by Thuillier (Pasteur and Thuillier 1883) in pigs dying of rouget was the same organism as that described by Loeffler, but this is not absolutely clear.] Another organism closely allied to Ery. rhusiopathiae was found by Rosenbach in cases of human erysipeloid. Subsequent workers have recorded the presence of Erysipelothrix in ontbreaks of polyarthritis in sheep and joint-ill in lambs, and in occasional infections of cattle, horses, turkeys, peacocks, and man (see Beaudette and Hudson 1936, Paterson and Heatley 1938, Greener 1939). There is also reason to believe that it is a not uncommon parasite of fish, though conclusive evidence of this is still lacking (Klauder 1926, 1932). Schoop (1936), for example, who recorded its isolation from fish, used the mouse inoculation method, so that it is impossible to be certain whether the organisms came from the fish or from latently infected mice.

In 1926 Murray, Webb, and Swann at Cambridge described a disease of rabbits characterized by a large-mononuclear leucocytosis, and caused by a small Grampositive non-sporing bacillus which they termed Bact. monocytogenes. The same organism has since been isolated by a number of workers from various diseases in animals and man characterized most often by a generalized infection tending to localize in the liver, myocardium, or central nervous system (see p. 1287). For this organism Pirie (1927) suggested the generic name of Listerella. Without for the moment discussing the appositeness or the validity of this name, we may point out that Erysipelothrix rhusiopathice and Listerella monocytogenes resemble each other in so many respects that it is convenient to describe them together.

Habitat. - Enongh has already been said to indicate the wide range of animals infected by these organisms. Though hoth of them appear to he mainly parasites, Ery. rhusiopathice is sid to be present in the slime surrounding the body of various fish (Klauder et al. 1926, Klander 1932, Schoop 1936), and in sewage derived from abattoirs (Hettehe 1937). 
Morphology.-The work of Spryszak and Szymanowski (1929), Meyn (1931), Redlich (1932), and Barber (1939) has made it clear that both Erysipelothrix and Listerella occur in a smooth and a rough form, each characterized by closely associated morphological and colonial appearances. In the smooth form they appear as small, straight or slightly curved, Gram-positive rods with rounder ends, about $0.8-2.5 \mu$ long and $0.3-0.6 \mu$ broad, arranged singly, in small packets or groups, or in short chains. In the rough form long filaments, up to $60 \mu$ or more, predominate, some of which are seen breaking down to form chains of bacilli. Erysipelothrix is more slender than Listerella and is non-motile. The motility of Listerella, however, which is said by some workers to be due to a single polar flagellum and by others to peritrichate flagella, is very sluggish. According to Seastone (1935) it is demonstrated best in a 4-hour glucose broth culture. Flagella are said to be developed better at $25^{\circ} \mathrm{C}$. than at $37^{\circ} \mathrm{C}$. (see Paterson 1939, Griffin and Robbins 1944).

Cultural Characters. - In the smooth form the colonies after 24 hours' incubation at $37^{\circ} \mathrm{C}$. are very small, circular, convex, amorphous, and water-clear, with a smooth glistening surface and entire edge. On further incubation Erysipelothrix colonies show little or no increase in size; those of Listerella become larger and less transparent. In the rough form the colonies are rather larger and flatter; their matt surface, curled structure, and fimbriate edge render them not unlike miniature anthrax colonies. On the whole Listerella colonies tend to be larger and less transparent than those of Erysipelothrix. In gelatin stab culture the growth of Erysipelothrix on first isolation is often of the lamp-brush type ; Listerella forms a filiform growth with no lateral outgrowths. Erysipelothrix fails to grow on MacConkey's medium; Listerella, according to Paterson (1937) and our own observations, forms small colonies on this medium, but Barber (1939) was unable to confirm this. Listerella forms a soluble hæmolysin; Erysipelothrix does not (Barber 1939), though hæmolysis may occur around colonies in blood agar.

Resistance.-Most strains of Erysipelothrix are killed by exposure to moist heat for 15 minutes at $55^{\circ} \mathrm{C}$. ; Listerella survives this temperature for 30 minutes, but is killed within 60 minutes (Barber 1939). Erysipelothrix is resistant to salting, pickling and smoking, and may remain alive in putrefying carcases for months. Hettche (1937) found that it survived for 4 to 5 days in drinking water, and for 12 to 14 days in sewage and aquarium water. Listerella is said to be fairly resistant to penicillin (Foley et al. 1944).

Growth Requirements and Metabolism.-The growth of both organisms is favoured by glucose, and to a less extent by blood and serum. According to Colella (1936) growth of Erysipelothrix occurs best in $0 \cdot 1$ per cent. glucose broth and 0.5 per cent. glucose agar; larger quantities of sugar were found to be inhibitory. A liver digest medium favours both organisms (Murray, Webb, and Swann 1926, Vawter 1937). On first isolation. Listerella, like Erysipelothrix, may give a band growth just helow the surface of a shake agar culture (Gibson 1935); whether this is due to a preference for $\mathrm{CO}_{2}$ or for a lowered partial pressure of oxygen is not clear. Both organisms are facultative anaerobes. Hutner (1942) found that the growth requirements of Listerella were simpler than those of Erysipelothrix; Listerella could grow in peptone water without serum or thioglycollate, whereas Erysipelothrix could not. Growth of Listerella is said to occur in an acid-hydrolysed "vitamin-free" medium containing glucose and inorganic salts, provided riboflavin, 
biotin, and hæmin are added (Porter and Pelczar 1941). Growth of both organisms occurs between about $15^{\circ}$ and $44^{\circ} \mathrm{C}$, but is best at $30^{\circ}$ to $37^{\circ} \mathrm{C}$.

Biochemical Characters.- The fermentative activity of Erysipelothix is less than that of Listerella. Neither organism forms gas. Erysipelothrix produces acid in glucose and lactose; Listerella produces acid in glucose, maltose, salicin, mannose, rhamnose, and dextrin, and slowly or in only small amount in lactose, sucrose and glycerol (see Deem and Williams 1936, Barber 1939, Julianelle 1941 , Harvey and Faber 1941). According to Barber (1939) and Julianelle (1941) Listerella gives a positive and Erysipelothrix a negative Voges-Proskauer reaction, though Harvey and Faber (1941) record a negative reaction for the strains of Listerella that they studied.

Antigenic Structure.-There is general agreement that Erysipelothrix and Listerella are antigenically distinct. Watts (1940), who studied 43 strains of Erysipelothrix, found that 38 appeared to belong to one antigenic type and 5 to another. Each group possessed a heat-stable specific antigen, and in addition two heat-labile antigeus, which were present in different proportions in the two groups and were responsible for cross-agglutination. Seastone (1935), Webb and Barber (1937), Schultz, Terry, Brice, and Gebhardt (1938), Paterson (1939, 1940a), and Julianelle and Pons (1939) have brought eridence to show the existence of some antigenic diversity among Listerella strains. Paterson recognizes four types, the division being made primarily on the $H$ and secondarily on the $O$ antigens.

Pathogenicity.-Both organisms have a wide range of pathogenicity for animals under natural conditions, and both may occasionally infect man (pp. 1285-7). The diseases to which they give rise will be described in Chapter 58; here we shall concern ourselves with their effect on laboratory animals. Three striking characteristics are possessed, though in differing degree, by Erysipelothrix and Listerella. Both produce a considerable monocytosis in rabbits, pin-point focal necroses in the liver of mice, and conjunctivitis in rabbits and mice. After intravenous inoculation into rabbits each of the organisms gives rise to a considerable monocytosis, which reaches its maximum in 3 to 7 days (Webb and Barber 1937, Barber 1939). In mice inoculated subcutaneously or intraperitoneally focal necroses of the liver, up to piuhead in size, are nearly always found post mortem in Listerella infections, but are much less numerous and striking in infections with Erysipelothrix. Conjunctivitis, on the other hand, is commoner in Erysipelothrix than in Listerella infections of mice (Barber 1939). If, however, the organisms are instilled into the conjunctival sac of the rabbit, or rubbed gently on the everted lid, Listerella gives rise to a severe conjunctivitis and keratitis, whereas Erysipelothrix causes a milder conjunctivitis without keratitis (Anton 1934, Graham, Hester, and Levine 1940, Julianelle 1941). According to Barber (1939), Listerella kills guinea-pigs but not pigeons; Erysipelothrix kills pigeons but not guinea-pigs. In assessing these differences it must be remembered that the virulence of both organisms is subject to variation, and that unless freshly isolated strains in the smooth phase are used the results of inoculation may be equivocal. In general it may be said that both organisms give rise to a septicæmic infection, and that Erysipelothrix has a tendency to localize in the skin, endocardium, and joints, and Listerella in the liver, myocardium, and central nervous system. Both Erysipelothrix and Listerella appear to be more virulent in the smooth than in the rough form (Schoening et al. 1938, Barber 1939). 


\section{Inoculation of Erysipelothrix into Animals}

Swine.-Loeffler (1886), who first isolated the swine erysipelas bacillus, failed to reproduce the disease in swine with pure cultures, but Schiitz (1886) later succeeded in doing so. Broth cultures injected subcutaneously proved fatal to two pigs, one animal dying in 3, the other in 4 days; there were typical findings at the necropsy, and the bacilli were recovered in pure culture from the blood and spleen, and from the pleural and peritoneal exudates. Artificial cultures rapidly lose their virulence for swine. Collins and Goldie (1940) produced polyarthritis by repeated intravenous inoculation of cultures. In addition there was a focal inflammatory polyarteritis, focal necrosis of the liver and nyocardium, lymphadenopathy, a monocytosis, and endocarditis, but skin lesions were never found.

The bacillus is pathogenic for mice, pigeons, and rabbits, but not for guinea-pigs.

MICE.- $0 \cdot 001-0 \cdot 1 \mathrm{ml}$. of a 24-hours' broth culture injected subcutaneously or intraperitoneally is usually fatal in 2 to 3 days. During life the mice develop conjunctivitis and their lids become glued together with a muco-purulent secretion; arching of the back is very common, and constipation is usual. Post mortem, the vessels of the skin and subcutaneous tissue are congested, the spleen is enlarged, and the lungs are bright red and cedematous. Bacilli are usually abundant in the blood and viscera; they are found particularly within the phagocytic cells, in which they appear to multiply (Tenbroeck 1920).

Pigeons. - $0.001-0 \cdot 1 \mathrm{ml}$. of a 24 -hours' broth culture inoculated intramuscularly proves fatal in 3 or 4 days as a rule. Death is often preceded by paralysis of the legs, dyspnoea, and convulsions. Post mortem, there is a black hæmorrhagic mass in the muscle at the site of inoculation; the spleen is enlarged; there are often punctiform hæmorrhages in the mucosæ and viscera; and there is almost constantly a clear lemon-yellow exudate in the pericardium (Crimi 1914). The bacilli are fairly numerous in the blood and organs.

RABBITS. - $0.5 \mathrm{ml}$. of a 24 -hours' broth culture inoculated intravenously sometimes proves fatal in 2 to 3 days. A marked œedematous swelling or erysipelatous rash develops in the injected ear, and there is a rise in temperature and a loss in weight. Post mortem, besides the rosy skin lesion, there is congestion of the viscera, and often a clear lemonyellow pericardial exudate; there may be large hæmorrhages into the lungs. The bacilli are scarce. If the disease is not acutely fatal, a monocytosis occurs, reaching its maximum in 3 to 7 days. In animals dying about this time occasional tiny focal necroses may be found in the liver. and areas of mononuclear cell reaction may be seen in sections of the spleen. Inoculation of the conjunctiva gives rise to conjunctivitis, which often proves fatal. After subcutaneous inoculation death seldom occurs.

\section{Inoculation of Listerella into Animals}

FisH.-Hettche (1937) and Brunner (1938) have shown that both freshwater and sea fish can be readily infected by feeding or by intraperitoneal inoculation with the bacilli. The organisms are widely distributed in the tissues and may be recovered after several weeks. They are particularly abundant in the kidneys and are excreted in the urine. The infection appears to be of the covert type, the fish showing no evidence of illness.

Mice.-Subcutaneous or intraperitoneal inoculation of 100 million living organisms of a highly virulent culture causes death in about 1 to 4 days. During life conjunctivitis sometimes occurs. At post mortem multiple tiny focal necroses arc found scattered throughout the liver. The organisms can be readily recovered from the spleen and heart blood.

RABBiTs.- Intravenous inoculation proves fatal in 24 hours or not for several days according to the dose and virulence of the strain. Animals surviving for some time develop a monocytosis, which reaches its maximum in about 3 to 7 days. Post-mortem examination of animals dying after a few days reveals the presence of multiple focal necroses in the liver, and rarely in the spleen and myocardium. Necrosis of the supra- 
renals is common. Oceasionally abscesses in the myocardium and inflammation of the meninges may be met with (see Burn 1935). After intraperitoneal inoculation, much the same lesions are found, lut in addition there is a sero-fibrinous peritonitis, with aloscesses containing thick white pus in the rolled-up omentum. The organisms can rarely be recovered from the blood stream. Instillation of a pure culture into the conjunctiva, or swabbing of the everted lid, gives rise to a severe conjunctivitis within 24 hours followed by keratitis; the animal itself rarely dies.

GuINEA-PIGs.-These animals die after inoculation with large doses. The lesions at necropsy are similar to those in mice. The organisms may be recovered from the spleen and sometimes from the heart blood.

CHICk EnBryos.-Paterson (1940b) has shown that Listerella gives rise to focal lesions on the chorio-allantoic membrane of chick embryos.

Classification.-In the Erysipelothrix group three species were differentiated by Rosenbach (1909)-muriseptica, porei, and erysipeloides. Ricknann (1909), however, pointed out that the morphological and cultural distinctions on which Rosenbach relied for differentiation were insufficient to serve as a means of classification; and since he found that all three organisms agglutinated to the same titre with immune sera, and exhibited the same pathogenicity to anima!s, he concluded that they should be regarded as belonging to a single species. To this the name Erysipelothrix rhusiopathice is now commonly applied.

For the organism isolated by Murray, Webl, and Swann (1926) and called nou-committally by then Bacterium monocylogenes, Pirie (1927) suggested the generic name Listerella in honour of Lord Lister. Apart from the inappositeness of this name-Lord Lister having neither discovered nor worked with this organism -it was too premature, since at that time no thorough comparison had been made between Bact. monocytogenes and Erysipelothrix, which it resembles in many respects (Topley and Wilson 1936).

Barber (1939) and Julianelle (1941) have now carried out a comparative study of these two organisms, and their observations seem to show that the relationship between them is even closer than was at first suspected. Thus, both organisms are small, Gram-positive rods, showing an extraordinary similarity in the morphological and cultural appearances of their smooth and rough forms. Both have much the same growth requirements and much the same degree of resistance. Both have a wide range of pathogenicity for animals, and both occasionally give rise to disease in man. In rabbits, experimental inoculation of either organism results in the development of a generalized infection accompanied by the appearance of conjunctivitis and, if the disease is not acutely fatal, of a considerable monocytosis; at post mortem focal necroses are found in the liver. Against these similarities, it may be said that Listerella is fatter and is motile, attacks rather more sugars, is antigenically distinct, and is fatal experimentally to guinea-pigs but not to pigeons, in contrast to Erysipelothrix which is fatal to pigeons but not to guinea-pigs.

No one who is familiar with systematic bacteriology and who has observed several strains of each of these organisms side by side can deny that the differences between them are less than those existing between different species of many other organisms elassified in the same genus, as, for example, between members of the Corymebucterium, Pasteurella, Bacillus, or Clostridium groups. The differences between Erysipelothrix and Listerella are those characteristic of specific, not of generic, differences; and it seems to us to be doing violence to the principles 
of bacterial taxonony to classify them in separate genera. Our proposal, therefore, is to include them both in the Erysipelothrix genus, calling them Ery. rhusiopathice and Ery. monocytogenes respectively. It may be pointed out that in any case the name Listerella is invalid, since it was given to a mycetozoan by Jahn in 1906 (see Pirie 1940).

\section{Erysipelothrix rhusiopathiæ}

Synonyms.-B. rhusiopathice suis Kitt; Erysipelothrix porci Rosenbach; B. erysipelatis suis.

Isolation.-Observed independently by Thuillier (Pasteur and Thuillier 1883) and Loeftler $(1886)$ in 1882 .

Habitat.-Found on the mucosæ and tonsils of swine.

Morphology. - Smooth form. Small, slender, straight or slightly curved rods, $0 \cdot 8-2 \cdot 0 \mu$ long and $0 \cdot 3-0 \cdot 4 \mu$ broad. Arranged singly, in small packets, in small groups, or in short chains. Rough form. Long chains of bacilli and interlaced filaments of variable length. Staining is fairly regular, but sometimes deeply-stained granules may be seen. Non-motile. Non-sporing. Gram-positive.
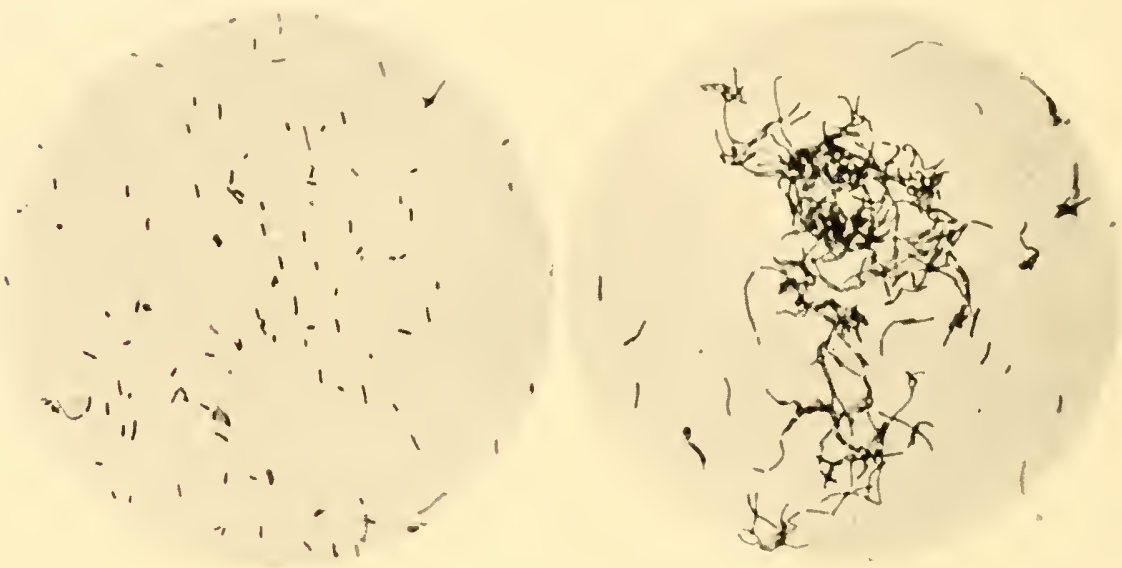

F1G. 66.-Erysipelothrix rhusioputhix.

L.tft: Smooth form. Right: Rough form. From a surface agar culture, 3 days, $37^{\circ} \mathrm{C}$. $(\times 1000)$.

Agar Plate.-24 hours, $37^{\circ} \mathrm{C}$. Smooth form. Round, convex, tiny, amorphous, waterclear colonies, $0 \cdot 1 \mathrm{~mm}$. in diameter, with smooth glistening surface and entire edge ; butyrous and easily emulsifiable. No increase in size on further incubation. Rough form. Rather larger and flatter, $0 \cdot 2-0 \cdot 4 \mathrm{~mm}$. in diameter, with a granular curled appearance and fimbriate edge; resemble miniature anthrax colonies.

Agar Slope.-24 hours, $37^{\circ} \mathrm{C}$. Very poor, partly confluent, slightly raised, colourless, transparent growth with an irregular surface due to imperfect fusion of individual colonies, and an edge which is very finely dentate or made up of single colonies.

1. Practically no change on further incubation; growth may become slightly viscous.

Gelatin Plates.-Deep colonies in 3 or 4 days resemble snow-flakes; they are very small, but when magnified 50 times they are seen to consist of a granular centre with branching threads radiating outwards.

Gelatin Stab.-Growth occurs slowly and is subject to considerable variation, apparently depending to some extent on the reaction of the medium. May be a simple 
filiform growth extending to the bottom of thetube. Usually lateral outgrowths occur; these may be ill-defined, looking like snow-flakes, nebulæ, or the conventional bursting bomb; or they may be definite branches producing a lampbrush appearance. The outgrowths may extend for a distance of only 2 or 3 $\mathrm{mm}$. from the stab, or they may reach the sides of the tube. The swooth form tends to remain restricted to the line of inoculation, while the rough form grows out laterally. No liquefaction. The lamp-brush form may not be obtained till after 2 or 3 subcultures in gelatin.

Broth. -24 hours, $37^{\circ} \mathrm{C}$. Smooth form. Slight uniform turbidity with very slight powdery deposit disintegrating on shaking. After a few days the broth may clear, and a viscous deposit become evident. Rough form. Little or no turbidity. Flocculi of varying size or tangled-hair-like masses of growth appear, and settle on the sides or bottom of the tube; they are difficult to disintegrate by shaking.

Loeffler's Serum. -7 days, $37^{\circ} \mathrm{C}$. Very poor, confluent, slightly raised, colourless growth, slightly better than on agar.

IIacConkey's Agar.- No growth.

Potato.-No visible growth.

Glucose Agar Shake.-Very tiny colonies throughout medinm; on first isolation there may be a band growth just below surface.

Resistance.-In broth cultures the bacilli are killed by moist heat at $55^{\circ} \mathrm{C}$. in 15 minutes. In meat they are highly resistant to salting, pickling and smoking, surviving for 1 to 3 months; they are likewise resistant to putrefaction, remaining alive and virulent for months in putrefying buried cadavers. Apparently succumb readily to drying, provided this is complete.

Metabolism.-Microaerophilic, but will grow under both aerobic and anaerobic conditions. Optimum temperature for growth is $30^{\circ} \mathrm{C}$.; grows between about $15^{\circ}$ and $44^{\circ} \mathrm{C}$. Growth favoured by glucose and slightly by blood. Hæmolysis occurs round deep colonies in 10 per cent. horse blood agar plates.

Biochemical. - Sugar reactions are variable. Usually forms

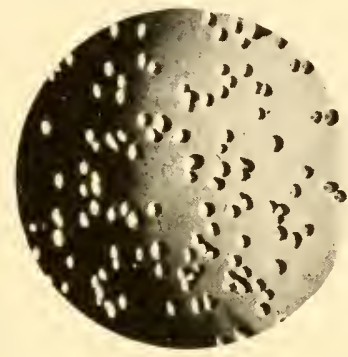

Fia. 67.-Erysipelothrix rhusiopathix.

Smooth form. Surface col onies on agar, if hours, $37^{\circ} \mathrm{C}(\times 8)$.

acid in dextrose and lactose, not in maltose, mannose, rhamnose, mamitol sucrose, dextrin, or salicin. L.M. no change or very slight acid. Indole negative, II.R. negative. V.P. negative. Nitrates slight reduction. Catalase \pm . M.B. reduction negative. $\mathrm{NH}_{3}$ negative. $\mathrm{H}_{2} \mathrm{~S} \pm$.

Antigenic Structure.-Evidence of two antigenic types, each possessing a heat-stable specific antigen, and two heat-labile antigens that are present in different proportions in the two groups and are responsible for cross-agglutination.

Puthogenicity. - Causes swine erysipelas in swine, and erysipeloid in man. Experimentally it is pathogenic for mice, pigeons, and rabbits, but not for guinea-pigs. A non-fatal dose inoculated into rabbits gives rise to a circulating monocytosis. Virulence for swine is said to fall on artificial cultivation. No exotoxin is producerl.

\section{Erysipelothrix monocytogenes}

Synonyms.-Bact. monocytogenes Murray, Webb and Swann; Listerella monocytogenes Pirie.

Isolation.-By Murray, Webb and Swann (1926) from rabbits snffering from a natural disease.

Habitat.-Widespread animal parasite. 
Horphology. - Simoth form. Small, straight or slightly eurved rods, 0.8-2.5 $\mu$ long and 0.5-0.6 " broad. Arranged singly, in pairs side by side or in V-form, in small packets, or in short ehains. Rough form. Long intcrlacing filaments, some of which slow segmentation into rods. Filaments are particularly abundant in cultures at room temperature. Sluggishly motile by polar, or according to some workers peritrichate, flagella. Stains fairly uniformly, but sometimes shows beading. Non-sporing. Gram-positive.

Agar Plate.-24 hours, $37^{\circ} \mathrm{C}$. Poor to moderate growth, having slightly acid smell reminiscent of the acne bacillus. Smooth form. Circular, low convex or convex, amorphous colonies, $0 \cdot 2-0.8 \mathrm{~mm}$. in diameter, with smooth mirror-like surface and entire edge; almost transparent by transmitted, greyish-yellow by reflected light; butyrous in consistency and easily emulsifiable. 48 hours. Slightly larger. 0.8-1.0 mm. in diameter, with tendeney to crinkling of edge. Older colonies are up to $1.5 \mathrm{~mm}$. in diameter, and have a brownish slightly granular centre and a translucent effuse periphery with a fincly erenated margin; when magnified, the colony has a poached-egg appearance. Rough form. 48 hours. Rather larger than the smooth form, $1 \cdot 5-2 \cdot 0 \mathrm{~mm}$. in diameter, flatter, with a matt surface. umbonate centre, and a finely fimbriate edge. After 5 days colony may reach $5 \mathrm{~mm}$. in diameter, and show a small, smooth, darker centre with a wide, more effuse, translucent, finely granular peripheral extension having a fimbriate edge.

Agar Slope.-24 hours, 37 C. 'Thin, raised, eonfluent, translncent growth with beatencopper surface and finely crenated edge. Growth becomes more abuodant on further incubation. Rongh form is similar, but shows a slightly spreading edge. Gelatin Stab. - Slow, filiform growth, not liquefying the gelatin; narrow surface growth. Broth.-24 hours, $37^{\circ} \mathrm{C}$. Smooth form. Slight to moderate turljidity, with slight powdery deposit disintegrating on shaking; no surface growth. Rough form. Little or no turbidity, with thread-like masses of deposit that are difficult to disintegrate.

Horse Blood Agar Plate. -24 hours, $37^{\circ} \mathrm{C}$. Circular, low eonvex colonies, $0 \cdot 7-1 \cdot 2 \mathrm{~mm}$. in diameter, with smooth mirror-like surface and entire or very finely crinkled edge; milky white by reflected light. Colonies are surrounded by a narrow zone of more or less complete $\beta$-hæmolysis.

Loeffler's Serum. -2 days, $37^{\circ} \mathrm{C}$. Thin, raised growth, like that on agar, with slightly beaten-copper surface and very finely crenated edge; no liquefaction, even after 3 weeks.

Mac'onkey's Agar. -24 hours, $37^{\circ} \mathrm{C}$. Circular, convex, colonrless eolonies, $0 \cdot 1-0 \cdot 4 \mathrm{~mm}$. in diameter, with smooth surface and entire edge. 5 days. No increase in size or change in colour, but surface tends to be slightly granular and edge slightly irregular.

Potato.-2 days, $37^{\circ} \mathrm{C}$. Slightly raised, greyish-white growth, with finely granular surface and very finely crenated edge.

Glucose Agar Shake.-2 days, $37^{\circ} \mathrm{C}$. Tiny colonies throughout medium. May be a band growth, on first isolation, just below surface.

Resistance.-Survives moist heat for 30 minutes at $55^{\circ} \mathrm{C}$., but is killed within 60 minutes. Cultures remain viable for several months.

Metabolism.- Aerobic and facultatively anaerobic. Optimum temperature for growth $30-37^{\circ} \mathrm{C}$.; growth occurs between $20^{\circ}$ and $44^{\circ} \mathrm{C}$. Growth favoured by glucose and liver extract, and to a less extent by blood and serum. $\beta$-hremolysis occurs around colonies on horse blood agar; weak soluble hæmolysin formed in broth cultures.

Biochemical.-Sugar reactions are variable. Usually produces acid in glucose, maltose, salicin, mannose, rhammose, and dextrin, and slowly or in only small amount in lactose, sucrose, and glycerol. L.M. slight acid. Indole negative. M.R. + . V.P. \pm . Nitrate reduction negative. M.B. reduction weakly positive. $\mathrm{H}_{2} \mathrm{~S}$ negative. $\mathrm{NH}_{3}$ in serum peptone water weakly positive. Catalase + . 
Antigenic Structure. - Probably 4 types, distinguished primarily on flagellar and secondarily on somatic antigens. Types I and 2 have the same II hut different $\mathrm{O}$ antigens. Types 3 and 4 have each specific $H$ and specific $O$ antigens.

Pathogenicity.-Causes disease in a wide variety of animals, including occasionally man. Experimentally it is pathogenic for mice, rabbits, guinea-pigs, but not pigeons. Non-lethal doses set up a monocytosis in rabhits. Virulence often falls on artificial cultivation. No exotoxin is producerl.

\section{REFERENCES}

Anton, W. (1934) Zbl. Bakt., 131, 89.

Barber, Mary (1939) .. Path. Bact., 48, 11.

Beaudette, F. R. anil Hudson, C. B. (1936) J. Amer. vet. med. Ass., 88, 475. Brtnner, Gertrud (1938) Zbl. Bakt., IIte Abt., 97, 457.

Burn, C. G. (1935) J. Bact., 30, 573.

Colella, C. (1936) Nuova Vet., 14,6.

Collins, D. H. and Goldie, W. (1940) J. Path. Bact., 50, 323.

Crimi, P. (1914) Ann. Staz. Mal. Best. Napoti, 2, 107.

Deen, A. W. and Willians, C. L. (1936) J. Bact., 32, 303.

Foley, E. J., Epstein, J. A., and LeE, S. W. (1944) J. Bact., 47, 110.

Gibson, H. J. (1935) J. Path. Bact., 41, 239.

Grahal, R., Hester, H. R., and Levine, N. I). (1940) J. infect. Dis., 66, 91.

Grenner, Averil W. (1939) Brit. J. Derm. Syph., 51, 372.

(iriffin, A. M. and liobrins, M. I. (1944) J. Bact.. 48, 114.

Harvey, P. C. and FAber, J. E. (1941) J. Bact., 42, 677.

Неттсне, Н. O. (1937) Arch. Hyg., 119, 178.

Hutner, \&. H. (1942) J. Bact., 43,6:9.

JuLIA Nelle, L. A. (1941) J. Bact., 42, 367, 385.

Julianelle, I. A. and Pons, C. A. (1939) Proc. Soc. exp. Biol., N.Y., 40, 364.

KlaUder, J. V. (1926) J. Amer. med. As.s., 86, 536; (1932) J. industr. Hyg., 14222.

Klauder, J. V., Righter, L. L., and HaRkins, M. J. (1926) Arch. Herm. S'yph., N.Y., 14,662 .

KocH, R. (1880) "Investigations into the Etiology of 'Traumatic Infective Diseases." New Sydenham Soc. London.

LOEFFlER. (1886) Arb. ReichsgesundhAmt., 1, 46.

Meyn, A. (1931) Zbl. Bakt., 122, 507.

Murray, E. G. D., WebB, R. A., and Swans, M. B. R. (1926) J. Path. Bact., $29,407$. Pasteur and Thulleter. (1883) C. R. Acad. Sci., 97, 1163.

Paterson, J. S. (1937) V'ct. Rec., 49, 1533; (1939) J. Path. Bact., 48, 25; (1940a) 1bid., 51,$427 ;(1940 b)$ Ibid., 51, 437 .

Paterson, J.S. and Heatley, T. G. (1938) Vet. J., 94, 33.

Pirie, J. H. H. (1927) Publ. S. Afr. Inst. med. Res., 3, 163; (1940) Nature, 145, 264. Porter, J. R. and l'elczar, M. J. (1941) J. Bact., 42, 141.

REDLICH, E. (1932) Z. InfektKr. Haustiere, 42, 300.

RickMañ. (1909) Z. Hyg. InfektKr., 64, 362.

Rosenbach, F. J. (1909) Z. Hyg. InfektKr., 58, 343.

Schoening, H. W., Gochenour, W. S., and Grey, C. G. (1938) J. Amer. vet. med. Ass., 92,61 .

Sсноор, G. (1936) Dtsch. tierürztl. W'schr., 44, 371.

Schultz, E. W., Terry, M. C., Brice, A. T., and Gebhardt, L. P. (1938) Proc. Soc. exp. Biol., N. Y., 38, 605 .

Sснӥтz. (1886) Arb. ReichsgesundhAmt., 1, 56.

Seastone, C. Y. (1935) J. exp. Med., 62, 203.

Spryszak, A. and Szymanowsit, Z. (1929) C. R. Soc. Biol., 100, 1151.

Tenbroeck, C. (1920) J. exp. Med., 32, 331.

Topley, W. W.C.and WiLsox, G.S. (1936) "The Principles of Bacteriology and Immunity," end ed., p. 709 .

Vaivter, L. R. (1937) J. Amer. vel. med. Ass., 90, 635.

Watts, P. S. (1940) J. Puth. Bact. 50, 355.

Weвв, R. A. and Barber, M. (1937) J. P'ath. Bact., 45, 523. 


\section{CHAPTER 16}

\section{MYCOBACTERIUM}

\section{Definition. Mycobacterium.}

Slender rods, which are stained with difficulty, but which, when once stained, are acid-fast. Cells are sometimes swollen, clavate, cuneate, or even branched. Non-motile. Gram-positive. No endospores. Growth on media slow. Aerobic. Several species are pathogenic to animals.

Type species is Mycobacterium tuberculosis.

The Acid-fast Bacteria.-The acid-fast bacteria are so called becanse of their ability, when once stained, to resist subsequent decolorization by mineral acids. The degree of acid-fastness varies with different members of the group, and in any single member is liable to alteration with changed environmental conditions; these differences are never so distinct or so constant as to serve as a reliable means of differentiating between the members of the group.

'The first member to be discovered was the leprosy bacillus in 1868 (see Hansen 1874). In 1882 came the discovery by Koch of the mummalian tubercle bacilli. The work of Smith (1898), Vagedes (1898), Ravenel (1901), Kossel, Weber and Heuss (1904, 1905), the English Royal Commission (Report 1911), and Park and Krumwiede (1910), during the years 1898-1910, showed that these mammalian hacilli could be divided into two types-the human and the bovine.

The discovery of the avian type of tubercle bacillus was due largely to the work of Rivolta (1889), Maffucci (1890, 1892), Cadiot, Gilbert and Roger (1890), Sibley (1890), and Straus and Gamaléia (1891), during the years 1889 to 1891 . 'The work of Sibley (1889), Bataillon, Dubard and Terre (1897), Ledoux-Lebard (1898, 1900), Friedmann (1903), and Küster (1905) from 1889 to 1905 served to differentiate a fourth type of tubercle bacillus-the cold-blooded type. In 1895 Johne and Frothingham described the organism which is now known as Johne's bacillus, and which is responsible for a chronic enteritis in cattle. The rat leprosy bacillus was discovered by Stefansky (1903) in 1901 at Odessa. During the years 1885 to 1906 a number of workers demonstrated the existence of the saprophytic acidfast bacilli-a gronp which, though able, under experimental conditions, to give rise in mammals to lesions closely simulating those of tuberculosis, does not appear capable of causing a definitely progressive disease. Amongst these bacilli the most important are (i) the butter bacillus Myco. butyricum-isolated by Rabinowitsch in 1897 from 23 out of 80 specimens of market butter examined in Germany, and since then by numerous other workers (Petri 1898, Korn 1899, 1900, Tobler 1901, Beck 1905, Pellegrino 1906); (ii) Moeller's Grass bacilli i and ii. Moeller isolated his first bacillus in 1898 from timothy-grass (Phleum pratense); hence it is generally referred to as Mycobacterium phlei i, or more familiarly as the 
timothy-grass bacillus; his second bacillus was isolated in 1899 from the dust of some plant material used as fodder; (iii) the Mist bacillus or Myco. stercoris; this was isolated by Moeller in 1901 from a dung-heap, and later from the frees of cows, donkeys, and other herbivora; it owes its name to the German term for manure (Mist); (iv) the Smegma bacillus, Myco. smegmatis; this was first described by Alvarez and Tavel in 1885, but was not obtained in pure culture till 1897, when Laser (1897) and Czaplewski (1897) cultivated it independently. This organism is present in varying numbers in smegma of both males and females; it has been found in the smegma of dogs (Pellegrino 1906).

Finally, in 1937 Wells isolated an acid-fast bacillus from voles (Microtus agrestis) suffering from natural tuberculosis. This organism differs in certain respects from the other mammalian types, and is probably best referred to as the murine type of tubercle bacillus or Myco. muris.

We shall describe the tubercle and saprophytic acid-fast bacilli together in the body of this chapter, but shall reserve the leprosy, rat leprosy, and Johne's bacillus for a separate description at the end.

Habitat.-The tubercle bacilli are essentially pathogenic; so far as we know they do not multiply naturally outside the animal body. The human, bovine, and murine bacilli give rise to mammalian tuberculosis (see Chapter 59). The avian type is found chiefly in birds, though it often infects pigs and occasionally cattle. It is sometimes present in hens' eggs (see Gloyne 1933). The cold-blooded type is responsible for disease in cold-blooded animals and fish. The saprophytic acid-fast bacilli are found in such diverse surroundings as butter, milk, smegma, grass, manure, and freces; they are also widely distributed in clust and water. The presence of metal seems to favour their growth, and they can almost invariably be found in scrapings from metal cold-water taps (Brem 1909, Beitzke 1910) and metal wind instruments (Jacobitz and Kayser 1910). They have been reported in cultures made from a gangrenous lung (Rabinowitsch 1900), from human fæces (Mironescu 1901), from the tonsils (Marzinowsky 1900, Beck 1905), from the nasal secretion (Karlinski 1901, Marchoux and Halphen 1912), from the intestinal contents of insects (Pellegrino 1906), from cow's milk (Albiston 1930), from a pleural exudate (Beaven and Bayne-Jones 1931), from pus (Bruynoghe and Adant 1933), from sputum (Cummins and Williams 1933), and from blood (Tiedemann 1931, Schwabacher $1933 a$ ). In view, however, of the frequency of acic-fast bacilli in dust, it seems probable that some of these organisms gained access to the cultures by air contamination and were not necessarily present in the material from which they were apparently derived. The leprosy bacillus is a specific parasite of man, and the rat leprosy bacillus of rats. Johne's bacillus infects cattle, and to a less extent sheep, in both of which it causes a chronic enteritis.

Morphology and Staining.--The acid-fast bacilli are rod-shaped organisms straight or slightly curved, with more or less parallel sides and rounded ends, they are arranged either singly, in small groups or bundles, or in groups of three or four with the individual bacilli lying at acute angles to each other, resembling diphtheria bacilli. Their size varies considerably according to the medium on which they are grown. In the animal body they are generally longer and thinner than in culture. Their length is $1-4 \mu$, but occasional forms as long as $8 \mu$ are seen; in breadth they vary from about $0 \cdot 3-0.6 \mu$. Long filamentous acid-fast bacilli have been described, but it is probable that these belong to the Actinomyces group (see Chapter 14). Numerous authors, however, have stated that 
the tubercle bacilli are capable of producing filaments-particularly in liquid media -but these forms are not encountered under ordinary conditions. Clubbed forms, resembling the typical clubs of the diphtheria bacillus, are not uncommon in culture; branched forms have been described by some authors, but are probably infrequent, except in the avian bacilli. Staining is either uniform or granular; in the latter type the granules may be restricted to the poles, or they may be evenly distributed throughout the length of the bacillus-the so-called beaded form. In bacilli that appear to be undergoing degeneration, the staining is often irregular both in depth and in situation. In young cultures it is common to find a certain proportion of non-acid-fast forms. The morphology of the developing organisms may be studied by Pryce's (1941) slide-cell technique of micro-culture.

On the average human bacilli tend to be long, thin, and curved, and to show granular staining, while bovine bacilli tend to be short, straight, and thick, and to show uniform staining. Their morphology, however, is so variable, and is so dependent upon environmental factors, that no weight can be attached to these

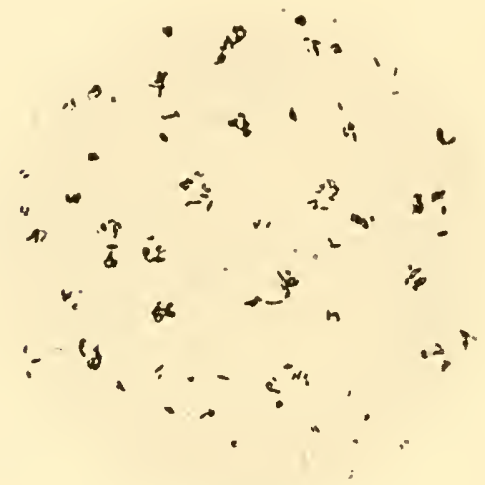

Fig. 68.-Myco. tuberculosis.

Glycerine egg-culture, 4 weeks, $37^{\circ} \mathrm{C}$., showing short, straight forms of bacilli $(\times 1000)$.

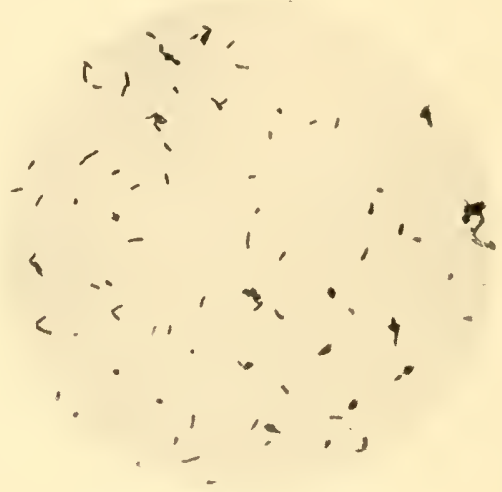

Fia. 69.-Myco, tuberculosis.

Glycerine agar culture, 4 weeks, at $37^{\circ} \mathrm{C}$., showing some short, straight forms, and some longer curved forms $(\times 1000)$.

criteria in the identification of individual strains. Nurine bacilli, according to Griffith (1942), are slender and often longer than human bacilli. Curved forms of the shepherd's crook, sickle, spiral, and S-type are abundant and characteristic. Some organisms show fine granulation and vacuolation along their whole length. The acid-fast bacilli are Gram-positive. Staining is not always easy, but with a 5 per cent. solution of gentian violet in alcohol and aniline oil, aided by gentle warming, it is usually possible to obtain satisfactory preparations. According to Kretschmer (1934), the Gram-positiveness is independent of treatment with iodine, and is closely bound up with the property of acid-fastness.

The organisms are resistant to simple solutions of the aniline dyes. To overcome this difficulty several methods of staining have been devised. Koch (1882) first stained the tubercle bacillus by immersion for 24 hours in an alkaline solution of methylene blue. Ehrlich (1882) improved on this by using aniline oil basic fuchsin or aniline oil methyl violet. By this means the bacilli were stained in 15 to 30 minutes and subsequently resisted decolorization with 33 per cent. $\mathrm{HNO}_{3}$ for a few 
seconds. He believed that the reason why the bacilli were resistant to ordinary stains was that they were surrounded by a capsule which was permeable only to alkalies. Ziehl (1882) showed that this conception was wrong; the bacilli could be stained quite satisfactorily by a dye of acid reaction. The stain he advocated was a 2 per cent. alcoholic methyl violet solution in carbolic acid water. Later Ziehl employed carbol-fuchsin. Neelsen increased the strength of phenol in the stain, and the Zieht-Neelsen method is the one that is now usually employed.

It consists in covering the film with earbol-fuchsin (basic fuchsin 1 part, absolute alcohol 10 parts, and 5 per cent. phenol in water 100 parts), and gently heating till the steam rises; the heating is continued for 5 to 15 mimutes, the water lost by evaporation being replaced by fresh stain. The film is then washed thoroughly in water, and treated with a 15-20 per cent. solution of a mineral aeid. If the film is from a pure culture, the effect of the acid will be merely to dissolve the excess stain; but if a film of tuberculous pus or a section of tuberculous tissue is being treated, the acid will turn the preparation yellow, indicating that the stain has been removed from the tissue cells, or from other organisms that may be present. The treatment with acid is eontinued for 5 to 10 minutes as a rule, till subsequent washing with water causes no more than a faint pink tinge to reappear. The film is then thoroughly washed in running water to remove all the acid. It is counterstained with a 1 per cent. aqueous solution of methylene blue for 5 minutes, after which it is washed and dried in the usual way. By this method the acid-fast hacilli are coloured red, white the tissue eells and all other organisms are coloured blue. Some workers prefer a yellow counterstain-usually 1 per eent. picrie acid. The success of this method depends partly upon the heat employed, which renders the waxy material in the tubercle bacillus more permeable to aqueons dyes, and partly on the phenol, whieh acts as a mordant.

Numerous other methods of staining have been described (Spengler 1907, Herman 1908, Mori 1911, Bozzelli 1914, Schulte-Tigges 1920, Kieffer 1921, Shoub 1923, Pottenger 1942).

The property of acid-fastness appears to be due to the presence in the bacilli of unsaponifiable wax (Anderson 1932). 'This substance was apparently first isolated by Aronson in 1898. It was referred to as one of the higher alcohols by Bulloch and Macleod (1904), and was termed "mykol" by Tamura (1913). The larger the amount of chloroform-soluble wax, the greater is the resistance of the bacilli to decolorization (Darzine 1932). The tubercle bacilli contain more of this substance than the saprophytic acid-fast bacilli (Table 25), and are therefore usually more strongly acid-fast. The degree of acid-fastness, however, is dependent on a number of factors, and no reliance should be placed on it in determining the particular type of organism under investigation. Not all workers are agreed on the simple chemical explanation of acid-fastness just given. Sordelli and Arena (1934), for example, state that it is a property of intact bacilli, and believe that it depends on the existence of a semi-permeable membrane around the organisms which allows fuchsin to diffuse in but does not allow acid fuchsin to diffuse out.

In formol-fixed tissue sections the bacilli of teu stain very poorly with ZiehlNeelsen. According to Fielding (1934), this is due to the development of an acid reaction following autolysis of the tissues, and can be overcome by fixing in a weakly alkaline solution of formol, or by staining with alkaline fuchsin.

Much's gramules.--Much (1907) brought evidence to show that under certain conditions the baeilli might be present in the tissues in the form of non-acid-fast granules. Starting from the observation that in the Perlsucht nodules of eattle, and in cold abseesses of man, 
it was often impossible to find acid-fast bacilli in films, even though the presence of tubercle bacilli could be shown by culture and by pathogenicity experiments, he devised a number of different staining methods to determine whether bacilli of any sort could be demonstrated microscopically. The method that he found most successful was to stain for 24 to 48 hours in aniline gentian violet or carbol methyl violet at a temperature of $37^{\circ} \mathrm{C}$., to treat with Lugol's iodine solution, and to decolorize by a mixture of absolute alcohol and clove oil, or by a dilute mineral acid, and a mixture of alcohol and acetone. Examining some miliary tubercles of a calf that had been injected with virulent bovine bacilli, he failed to find any bacilli in preparations stained with Ziehl-Neelsen ; but in preparations stained by his own method he found large numbers of fine rods in the tubercles, often accompanied by small rounded granules arranged singly, in pairs, or in short chains resembling beaded bacilli. The rods preponderated in the necrotic portions of the tubercles, the granules in the peripheral zones; both were coloured violet, and both were numerous within the cells. Small pieces of the lung were seeded on to the serum slopes, and incubated at $37^{\circ} \mathrm{C}$; smears were examined daily. In smears stained with Ziehl-Neelsen no bacilli were found for 6 days, when acid-fast rods appeared; but in smears stained by Much's method fine granules and rods were visible after 3 days. Small pieces of the lung injected subcutaneously into guinea-pigs gave rise to generalized fatal tuberculosis in 8 weeks; acid-fast bacilli were found in the tissues of the dead animals. Much obtained similar results with other tuberculous material. He concluded that-(i) There is a form of tubercle bacillus that is not stainable by Zichl. but is stainable by Much's method; it is granular. (ii) In tuberculous organs this granular form may be the only stainable form of bacillus present. (iii) The granular form may be accompanied by fine rods, which likewise do not stain with Ziehl. (iv) The granular forms are virulent. (v) There are transition forms between the Gram + granules, the fine Gram + rods, and the acid-fast rods and granules.

For a long time comparatively little attention was paid to Much's work, but of late years a number of observers have studied the growth of acid-fast bacilli in suitable culture preparations, and have demonstrated the presence of granular forms similar to those described by Much. The interpretation of these forms, however, has given rise to controversy. While Sweany (1928) and Kahn (1930) hold that they represent a stage in the life-cycle of the bacilli, Oerskov (1932) believes that they are products of degeneration. The bundles of extremely fine rods that Kahn described as forming part of the life-cycle are interpreted by Oerskov as crystals, formed partly from the medium and partly from bacillary products. According to Yegian and Porter (1944), many of the non-acid-fast forms are frank artefacts resulting from trauma to the organisms during the preparation of the film. These observers find that destruction of the integrity of the cell is accompanied by a loss of the acid-fast staining property. Young organisms are more readily destroyed than old, and the greater the effort spent in spreading the growth with a spatula on the slide, the more numerous are the non-acid-fast rods and granules seen microscopieally. The micro-motion pictures obtained by Wyckoff (1934) and Wyckoff and Smithburn (1933) show that the young bacilli increase in size before dividing, but that, as the culture ages, division continues without previous enlargement. The resulting organisms, therefore, become shorter and shorter, till true coccoid forms, staining intensely acid-fast, appear. Transplanted into a fresh medium, these short forms again give rise to typical bacilli. The sequence of events is so similar in general outline to the behaviour of nonacid-fast bacteria, that there seems no justification for postulating the existence of any special cycle of development.

Filtrable Forms of the Tubercle Bacillus.-Closely connected with the presence of Much granules is the existence of the so-called filtrable forms of the tubercle bacillus. Since Fontès' (1910) original observation, numerous workers have claimed to demonstrate under appropriate conditions the presence of filter-passing forms possessing a low degree of pathogenicity for guinea-pigs and constituting a special stage in the life-cycle of the organism. This subject was reviewed in the second edition of Topley and Wilson (pp. 
291-3), and the conclusion reached that no satisfactory evidence had been brought forward to prove the existence of such forms. The more recent findings of Soltys and Taylor (1944) support this conclusion; and there seems no justification for considering at length the significance of observations that were almost certainly the result of faulty technique.

Chemical Structure of Acid-fast Bacilli.-Largely owing to the work of Anderson and his colleagues (1927 et seq.) at Yale University, valuable information has been obtained in recent years on the chemical structure of the mycobacteria. Large quantities of bacilli of different types, grown on Long's (1926) synthetic medium, were extracted with a mixture of alcohol and ether, and the resulting extract was treated with chloroform and with acetone. In this way the lipoid material was separated into three fractions, consisting of glycerides, phosphatides, and wax. The alcohol-ether extract also contained a considerable amount of polysaccharide, and some basic compounds that could be precipitated by $\mathrm{HgCl}_{2}$ and by pliosphotungstic acid. The results of the fractionations are given in Table 25 .

\section{TABLE 25}

Percentage Fractions of Lipoid and other Material isolated from Alconol-Ether and Chloroforu Extracts of Acid-fast Baciliz (Chargaff, Pangborn, and Anderson $1931)$.

\begin{tabular}{|c|c|c|c|c|c|}
\hline & & \multicolumn{4}{|c|}{ Type of Organism. } \\
\hline & & $\begin{array}{l}\text { Human tubercle } \\
\text { bacillus H } 37 \text {. }\end{array}$ & $\begin{array}{l}\text { Avian tubercle } \\
\text { bacillus. }\end{array}$ & $\begin{array}{c}\text { Bovine tubercle } \\
\text { bacillus. }\end{array}$ & $\begin{array}{c}\text { Timothy grass } \\
\text { bacillus. }\end{array}$ \\
\hline Phosphatide & . & $6 \cdot 54$ & $2 \cdot 26$ & $1 \cdot 53$ & 0.59 \\
\hline Acetone-soluble fat & . & $6 \cdot 20$ & $2 \cdot 19$ & $3 \cdot 34$ & $2 \cdot 75$ \\
\hline Chloroform-soluble wax & . & $11 \cdot 03$ & $10 \cdot 79$ & $8 \cdot 52$ & $4 \cdot 98$ \\
\hline Total lipins . . . & . & $23 \cdot 78$ & $15 \cdot 26$ & $13 \cdot 40$ & $8 \cdot 37$ \\
\hline Polysaccharide. & . & 0.87 & $1 \cdot 02$ & $1 \cdot 09$ & $3 \cdot 90$ \\
\hline Dried bacterial residue & . & $75 \cdot 01$ & $83 \cdot 71$ & $85 \cdot 50$ & $87 \cdot 70$ \\
\hline
\end{tabular}

It will be observed that the total lipin content was highest in the human type of bacillus and lowest in the saprophytic acid-fast bacillus. The polysaccharide content, on the other hand, was arranged in the reverse order. Further analysis showed that the phospholipins contained saturated and unsaturated fatty acids and glycerophosphoric acid; moreover, on hydrolysis they yielded large amounts of water-soluble carbohydrates, of which mannose and inositol seemed to be the two most important. Besides palmitic, linoleic, and linolenic acids, there were two fatty acids of special interest. One, which was optically active and isomeric with cerotic acid, was termed phthioic acid ; the other, which was optically inactive and isomeric with stearic acid, was termed tuberculostearic acid. Of the waxy material, one portion was purified and found to be a white powder melting at $200^{\circ}-205^{\circ} \mathrm{C}$.; the remainder formed a yellowish salve-like mass which was called "soft wax." The purified wax yielded on hydrolysis about 56 per cent. of unsaponifiable wax; this corresponded to the higher alcohols of previous workers, and proved to be acid-fast. The purified wax also contained polysaccharides which on hydrolysis yielded a number of sugars includiug mannose, $d$-arabinose, and galactose (see also Gough 1932). The "soft wax" appeared to be a complex glyceride. From the acetone-soluble fat of the human tubercle bacillus a yellow pigment was isolated. This pigment, to which the name phthiocol has been given, is one of the hydroxynaphthaquinones, and is the oxidant of a reversible oxidation- 
reduction system whose $\mathrm{E}^{\prime}{ }_{0}$ is among the lowest reported for systems of biological origin (Ball 1934). The polysaccharides in the ether extract were apparently different from those in the phosphatide or wax.

A nitrogen-free and phosphorus-free polysaccharide was prepared by Hooper, Renfrew and Johnson (1934) by alkaline hydrolysis of the acetyl product of the crude carbohydrate from a protein-free ultrafiltrate. The molecular weight of the pure polysaccharide has been estimated by Seibert, Pedersen and Tiselius (1938) at 9,000 .

Besides the lipoid material and the polysaccharides, acid-fast bacilli contain proteins that are soluble in water. From cultures of tubercle bacilli on synthetic media Long and Seibert and their colleagues $(1926,1928)$ isolated various proteins, of which one appears to be the active principle of tuberculin. In this country Fough (1933) obtained evidence of the presence in cultures of human tubercle bacilli of two proteins having different chemical and immunological characters. The later work of Menzel and Heidelberger (1938), who fractionated the residue of frozen and dried bacilli after extraction in the cold with acetone and ether, revealed the great complexity of the proteins in the acid-fast bacilli. Differences were noted between the proteins of saprophytic acid-fast bacilli, avian bacilli, and mammalian bacilli. At least three antigenic components were found among the proteins separated from bacilli of the human type. The protein fraction, PPD-b3, which appears to be responsible for the tuberculin reaction, was found by Seibert, Pedersen and Tiselius (1938) to have a molecular weight of 16,000 and to contain 4.4 per cent. of polysaccharide and 3 per cent. of nucleic acid. Further purification by Seibert and Glenn (1941) resulted in the reduction of the nucleic acid to $1 \cdot 2$ per cent. and of the molecular weight to 10,500 .

The cellular reactions to the various fractions extracted from tubercle bacilli have been studied for many years by Sabin and her colleagues (for references, see Sabin 1938 and Sabin and Joyner 1938). They may be summarized briefly as follows.

Four types of reaction have been noted : $(a)$ exudation of neutrophiles from the blood vessels to the tissues; $(b)$ stimulation of the phagocytic mononuclear cells of the tissues; (c) multiplication of fibroblasts; and $(d)$ local increase in lymphocytes. The polysaccharide, when introduced into the tissues of suitable animals, called forth neutrophiles from the blood vessels, but had no further action. The lipins, in addition to evoking this reaction, stimulated the phagocytic mononuclear cells of the tissues. After injection of the phosphatide fraction tubercles developed consisting of epithelioid cells and of their multinucleated derivative, the Langhans giant cell; caseation sometimes followed. The waxes, higher alcohols, and hydroxy-aeits led to a multiplication of monocytes and their fusion into giant cells of the foreign body type. The reaction to the proteins was more complex. They gave rise not to one type of the phagocytic mononuclear cell, but to every type. They induced the formation of monocytes, of "stimulated" monocytes, of macrophages, of epithelioid cells singly and in tubercles, and of giant cells, both of the Langhans and the foreign body type. The degree of complexity of the reaction was least with the soluble proteins and greatest with the insoluble. A consistent increase in fibroblasts was found only after the injection of a wax-like fraction isolated from a saprophytic acid-fast organism. The new formation of lymphocytes was observed irregularly after injertion of the phosphatide and protein fractions. Reactions in general were greater in tubereulous than in normal animals.

The polysacelaride is non-toxic on intravenons injection into rabbits (Sabin et al. 1931), but like the protein it may play a part in the phenomenon of allergy. It reacts 
with a precipitating serum, though it is incapable of calling forth the production of antibodies (Laidlaw and Dudley 1925, Mueller 1926). The purest protein fractions appear to be practically non-antigenic; the fractions with larger molecular weight give rise on injection to antibodies (for reviews, see Wells and Long 1932, Anderson 1932, Sabin 1932, Calmette 1936, Seibert 1941, 1944).

\section{Cultural Reactions.}

The acid-fast bacilli vary in the ease with which they grow under artificial conditions. At one end of the scale are the saprophytic acid-fast bacilli and the cold-blooded tubercle bacilli, which grow well in 2 or 3 days on ordinary media; at the other end are the mammalian tubercle bacilli, which grow only on special media, and which may take 2 weeks or more to form a layer of growth visible to the naked eye; in between come the avian bacilli, which grow poorly or not at all on ordinary media, but which give a good growth in a few days on glycerinated media. The most satisfactory media for cultivation of the tubercle bacilli are inspissated serum, coagulated egg, and potato. The addition of 5 per cent. glycerine to these media is of great valne, enlancing the growth of all acid-fast organisms, with the exception of the murine and partial exception of the bovine tubercle bacillus. Its incorporation in nutrient agar or in broth renders these media suitable for the growth of human and hovine bacilli, and, except for the murine type, greatly improves the growth of other members of the group.

One of the striking characteristics of the acid-fast group is the friable tenacious consistency of the growth, and its adhesiveness to the medium. This is evident not only in the process of subculturing the organisms, when a stout platinum loop has to be used, but particularly in endeavouring to form a suspension of the organisms in saline or other fluid. Instead of giving rise to a uniform turbidity, they settle in granules to the bottom and leave the supernatant fluid clear; to produce a homogeneous suspension they must be ground up thoroughly in an agate mortar-a process that may take anything from half an hour to several days to complete. Similarly, it is difficult to make a uniform film of a culture for microscopic examination; even with a stout platinum loop it is impossible to break up the growth completely, and the film remains granular. As a rule the most difficult growths to emulsify are those of the human and bovine types; growths of the other types usually present less difficulty. Sometimes a creamy, almost butyrous, growth is formed by members of the avian and cold-blooded types, which can be emulsified rapidly; but even with these suspensions the disintegration of the bacillary clumps is rarely complete; against an illuminated dark-ground a fine granularity is visible to the naked eye.

Another feature that is common to all the tubercle bacilli, though not to the saprophytic acid-fast bacilli, is the rather pleasant, sweet, fruity odour of their cultures. This odour, so far as we know, is peculiar to the tubercle bacilli ; it is developed on all the usual media in which growth occurs, both solid and liquid; and is most readily detected in tubes that have been corked during incubation.

In studying the cultural reactions of the acid-fast bacilli it is important to realize that, on first isolation, the reactions of a given organism may seem to be anomalous. It is often not till two or three generations, and sometimes more, have been spent in artificial media that the characteristic reactions of the type develop. Thus a cold-blooded bacillus, when first isolated, may grow very poorly -almost like a human strain - but in 3 or 6 months in the laboratory it will become accustomed to its new surroundings, and instead of giving a poor discrete growth 
on glycerine egg in 4 weeks it will give a profuse confluent growth in 5 or 6 days.

Cultural Differentiation between the Human and Bovine Tubercle Bacilli.--For differentiating between the human and bovine types of tubercle bacilli it is desirable to seed a number of different media-serum, glycerine serum, egg, glycerine egg, glycerine agar, glycerine potato, and glycerine broth-with the organisms to be tested, preferably as soon after their isolation as possible. To obtain the maximum differentiation, the organisms used for seeding should never have been grown on a medium containing glycerine. It is advisable to inoculate two or three tubes of each medium, and to seal the tubes so as to prevent evaporation. For examining the growth in glycerine broth, flasks are most suitable, so that a large surface of

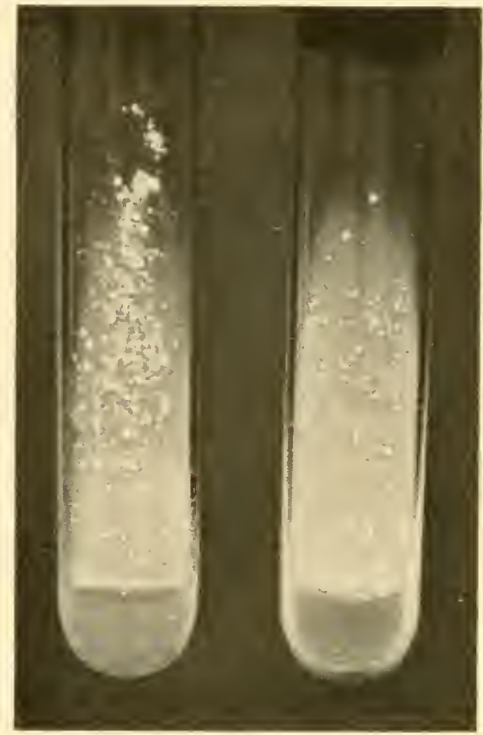

Fig. 70.-Myco. tuberculosis.

Human type. Left: Glycerine serum, 14 days, $37^{\circ} \mathrm{C}$. Right: Plain serum, 14 days, $37^{\circ} \mathrm{C}$., showing beneficial effect on growth of addition of glycerine.

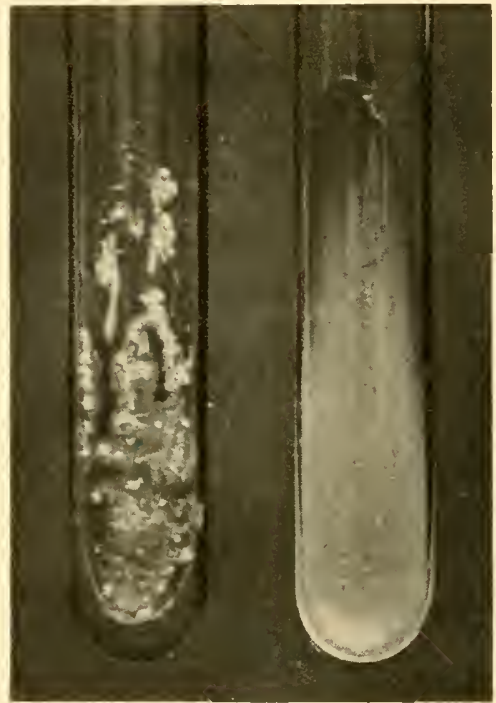

FIG. 71.-Myco.tuberculosis.

Left: Human type. Glycerine agar, 3 weeks, $37^{\circ}$ C. Right: Bovine type. Glycerine serum, 3 weeks, $37^{\circ} \mathrm{C}$.

medium is exposed to the air. Rapid methods of differentiating tubercle bacilli are to be regarded with caution. The method, for example, described by Jensen (1932), which claims to yield a result within 4 weeks, is open to several objections. Though possibly giving a high proportion of correct answers in experienced hands, it will not commend itself to those who realize how much bacteriology has suffered in the past from the use of faulty or inadequate technique.

On all media the growth of the human type is greater in amount than that of the bovine type; for this reason the human type is called eugonic, the bovine type dysgonic. On media containing no glycerine, such as coagulated serum or egg, the superior growth of the hmman bacillus is barely evident, but on media containing glycerine it is most striking. The effect of glycerine on growth is one of the most important of the differential characteristics; glycerine favours the growth 
of the human type, but has little or no effect on that of the bovine type (Figs. 71) and 71). Thus on glycerine agar the human bacillus gives a thick, confluent, wrinkled growth, while the bovine bacillus gives only a poor, effuse, groundglass growth, or may fail to grow at all. On glycerine potato again the human bacillus gives a raised, confluent, wrinkled or warty growth, while the bovine bacillus gives a poor, often discrete, effuse growth; and similarly with the other media.

Another point of distinction is that the human type often forms a yellowish or orange pigment, whereas the bovine type never does. The pigment is noticeable only on batches of serum that have a rich yellow colour. For this reason the serum should come from an old cow; pale serum from a young cow, or from another species of animal, is useless. On a goldenyellow serum the human type often produces a rich yellow or orangeyellow growth: the bovine type gives a non-pigmented growth. On a serum coloured only slightly yellow the growth of the human type is cream or light yellow in colour. The differentiation of the human and bovine types may take months to complete; often when the organisms are first isolated, they grow very poorly, and it is not till they have become accustomed to saprophytic conditions that they give the best growths of which they are capable.

Cultural Differentiation of the Murine from the Human and Bovine Bacilli.-The murine type, as exemplified by the vole bacillus, grows much more slowly than either of the other two mammalian types, taking four weeks or more

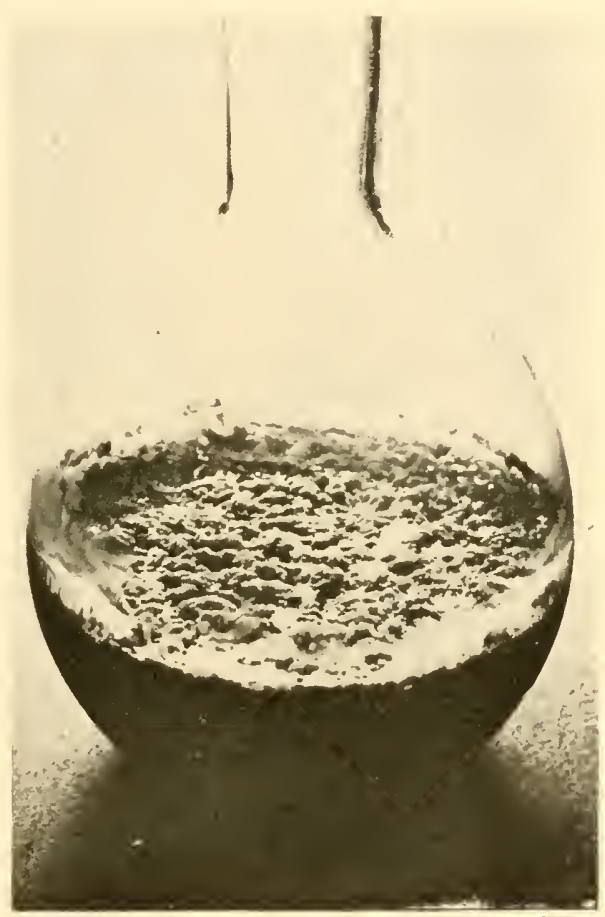

Fig. 72.-Myco. tuberculosis.

Human type. Glycerine broth, 6 weeks, $37^{\circ} \mathrm{C}$., showing thick, wrinklerl, surface growth, spreading up the sirles of the flask. to form colonies visible to the naked eye on egg medium. Little or no growth occurs in primary culture on media containing glycerine, and even in subculture growth is poor. Unlike the other two types, the murine bacillus grows in plain trypsinized broth, producing film-like colonies on the surface and a fine deposit. It grows likewise on potato without glycerine.

Cultural Differentiation between the Human and Avian Tubercle Bacilli.When first isolated from lesions in birds, the avian bacillus may closely resemble the human bacillus, but as a rule after a few generations in the laboratory it takes on a more rapid and luxuriant growth, so that differentiation on cultural grounds alone is rendered possible. There are certain avian strains, however, that remain 
permanently like the human type ; on solid media these strains are indistinguishable from human strains, but differentiation is generally possible by the growth in glycerine broth; in this medium the human bacillus forms a thick wrinkled surface pellicle (Fig. 72), whilst the avian bacillus grows at the bottom of the flask forming a granular deposit, or sometimes spreading out in a veil-like manner over the bottom, and part-way up the sides of the flask. Occasionally the avian bacilli give rise to a diffuse turbidity in broth. Why it is that some bacilli grow as a pellicle on the surface, others form a veil over the bottom, while still others grow diffusely, is not known. It may depend on the oxygen pressure most suitable for growth, or, as some workers think, on the surface tension of the medium. By lowering the surface tension to below 42 dynes, Larson (1926) states that it is possible to induce organisms, which usually form a surface pellicle, to grow diffusely or at the bottom of the flask.

Most avian strains grow more rapidly and more profusely than human strains, and tend on solid media to give a more creamy, homogeneous, and less granular growth than that of the human bacillus (Fig. 73). Though growth is favoured by glycerine, it is possible to get the avian bacillus to grow on simple media like nutrient agar or broth without the addition of any glycerine; this is more difficult with the buman bacillus. Another point of difference is that the human strains do not grow at a temperature above $40^{\circ} \mathrm{C}$, whereas the avian strains will grow up to $43^{\circ}$ or $45^{\circ} \mathrm{C}$. A point of not much importance is that avian cultures generally live longer than human ones; they may be found viable after 1 or even 2 years; human cultures are often dead in a couple of months, though occasionally they may survive for much louger. Another point of small importance is that a few avian strains when grown on glycerine egg medium give a faint pink-coloured growth; human bacilli never give a pink coloration.

Cultural Differentiation between the Avian and Cold-blooded Tubercle Bacilli.In culture the avian and the cold-blooded bacilli resemble each other closely. On first isolation they can easily be distiuguished by the difference in their optimum temperatures of growth, the avian bacillus growing best at about $40^{\circ} \mathrm{C}$., the coldblooded at $25^{\circ} \mathrm{C}$. After prolonged subculture in the laboratory this difference is partly obliterated; the cold-blooded bacillus comes to grow quite well at $37^{\circ} \mathrm{C}$, though the avian bacillus will rarely grow below $30^{\circ} \mathrm{C}$. Practical differentiation can therefore be made by growing the organisms at $25^{\circ} \mathrm{C}$.; if growth is as good at this temperature as at $37^{\circ} \mathrm{C}$., the strain is a cold-blooded one ; if growth occurs freely at $37^{\circ} \mathrm{C}$, but fails to occur at $25^{\circ} \mathrm{C}$., the strain is an avian one. Apart from the differences in optimum temperature there are no definite cultural characteristics distinguishing the two types, except the pink coloration of certain avian strains when grown on glycerine egg. Hinor differences such as the worm-cast growth of the cold-blooded type on glycerine potato, and the denser nature of the growth on the bottom of flasks of glycerine broth are not sufficiently constant to be reliable. As a rule the cold-blooded bacillus grows more rapidly than the avian bacillus; cold-blooded strains often give a definite layer of growth in 2 or 3 days : growth of avian strains is often not visible for 4 or 5 days.

Cultural Characteristics of the Saprophytic Acid-fast Bacilli.-These organisms grow rapidly at room temperature, giving rise in 2 or 3 days to a profuse confluent growth. Different strains vary in their cultural reactions, but on the whole their appearance on solid media is very characteristic. On glycerine agar there is a 
luxuriant, raised, dry growth, yellow, pink, or brick-red in colour, with a coarsely granular surface, resembling dry bread-crumbs (Figs. 71 and 75). This growth is unlike that of any of the tuberele bacilli. A few strains, however, are non-pigmented. In glycerine broth some strains give a turbidity, but most of them do not; there is generally a surface growth, which may be thick and wrinkled like the human tubercle bacillus, thick and coarsely granular, or more thin and delicate ; a deposit of granular material is usual. Growth occurs in gelatin, but no liquefaction is produced. The optimum temperature of growth varies, but is generally in the neighbourhood of $37^{\circ} \mathrm{C}$. All strains grow freely at room temperature ; several grow at $45^{\circ} \mathrm{C}$, while a few multiply even at $55^{\circ} \mathrm{C}$. (see Schwabacher 1933b).

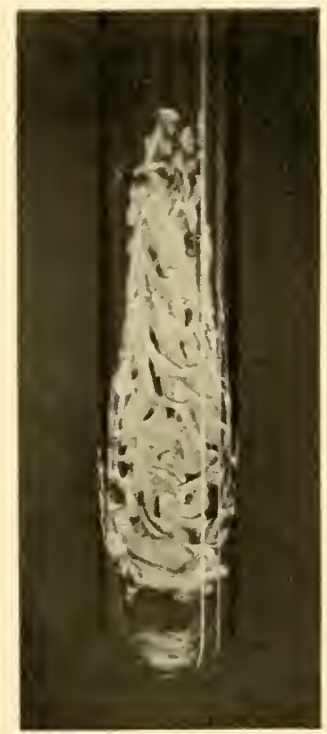

Fia. 73. - Myco. tuberculosis.

Avian type. Glycerine agar, 3 weeks, $37^{\circ} \mathrm{C}$., showing abundant growth of butter-cream type.

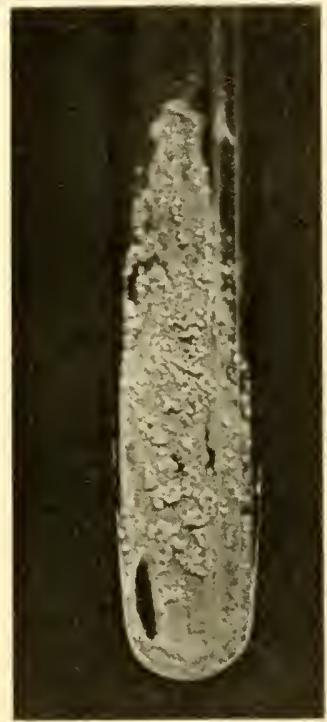

Fia. 74.-Myco. stercoris (Mist bacillus).

Clycerine agar, 3 weeks, $37^{\circ} \mathrm{C}$., showing abundant heaped-up growth.

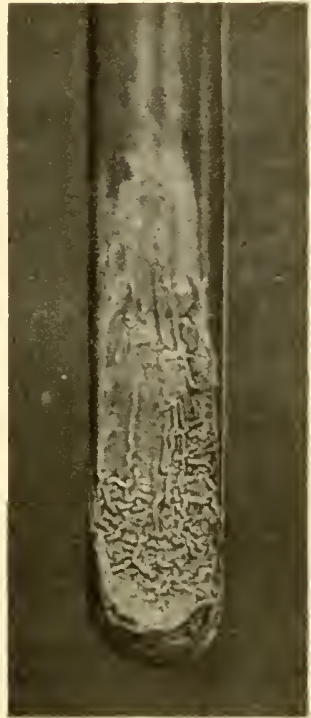

Fig. 75.-Myco. phlpi I

Glycerine agar, 3 weeks, $37^{\circ} \mathrm{C}$., showing abundant growth with wrinkled surface.

Variation.-The phenomenon of variation in acid-fast bacilli has occupied the attention of large numbers of workers in recent years. The field is a wide one, but so far the results of exploring it have not been fruitful. Many contributions to its study have been purely descriptive, and have been made without any apparent realization of its biological significance. The terms used by different workers to denote the variants they have observed have differed so widely that the nomenclature is in a state of the utmost confusion. Changes in virulence have been postulated on inadequate grounds, and attempts to measure small differences in virulence have often been confidently performed with numbers of animals quite insufficient to provide a definite answer.

One of the chief difficulties in work of this type lies in distinguishing between fixed and environmental variants. Our own experience suggests that a great many of the so-called variants are nothing more than adaptations to 
changed conditions. If, for example, a suspension of tubercle bacilli is inoculated simultaneously in equal quantities on to a number of different media, quite a variety of colonial types will develop (Fig. 76). If the different types are picked off and inoculated all on to the same medium, complete similarity of colonial appearance will often result, indicating that no fundamental biological change has been effected. What degree of fixity variant types may have, it is very difficult to ascertain, and we believe that before further progress is possible, a very careful study of the limits of transient environmental variation will have to be made. Without expressing any opinion on the degree of fixity of types, or of giving a critical review of the extensive bibliography that has grown up on the subject of variation, we shall confine ourselves to describing two main types possessing correlated morphological and cultural appearances.
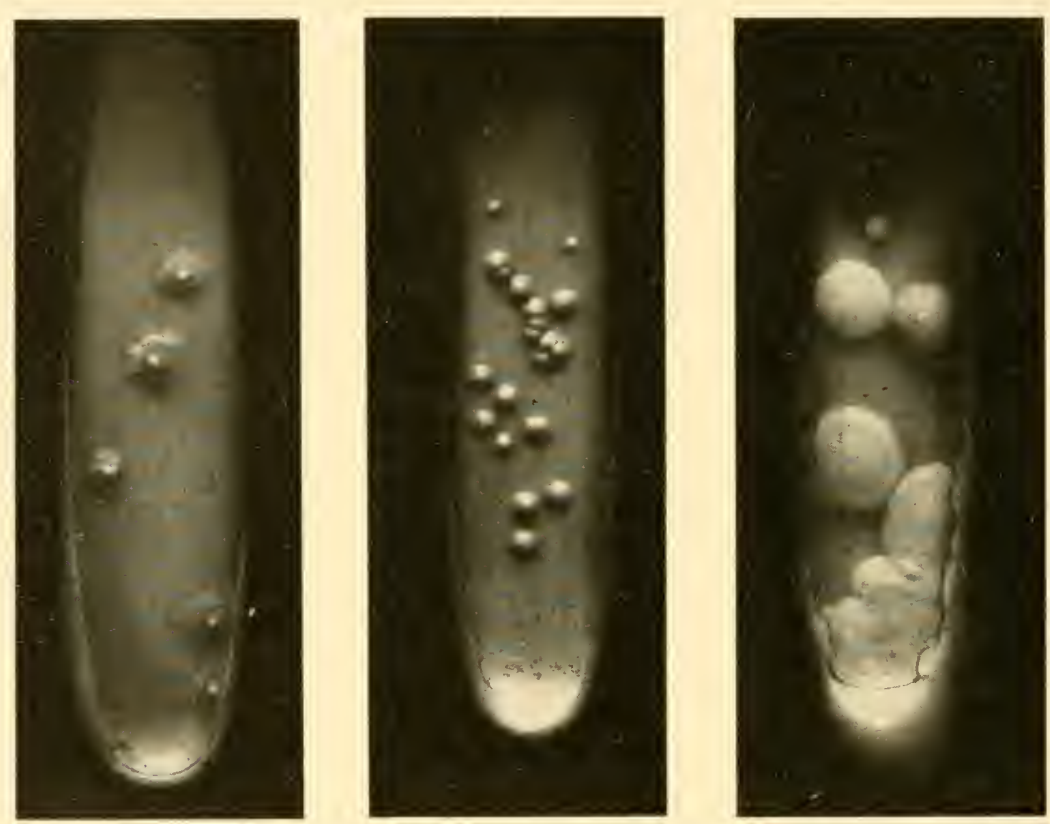

Fig. 76.-Myco. tuberculosis-bovine type.

Surface colonies on three different media, inoculated at the same time from the same suspension and incubated under identical conditions; 37 days, $37^{\circ} \mathrm{C}$. The figure illustrates the dependence of the colonial form on the nature of the medium.

Left: Modified Dorset egg medium. Middle: Egg yolk medium. Right: Egg yolk agar medium.

On Dorset or 5 per cent. glycerol egg medium many cultures of tubercle and saprophytic acid-fast bacilli produce colonies that are either smooth or rough. In stroke cultures the smooth forms give rise to a moist butyrous growth with a smooth glistening surface, while the rough forms produce a dry, rather friable growth, with a rough, dull, and often heaped-up surface, looking not unlike dry bread crumbs (Fig. 77). Rubbed up in water the smooth growth yields a fairly homogeneous suspension, the rough growth a granular suspension. Single colonies of the smooth form tend to be circular, convex, with a generally smooth surface and an entire edge; rough colonies are irregular in outline, are often 
heaped-up and convoluted, have a roughish granular surface, and tend to be surrounded by a spreading veil-like peripheral extension (Fig. 78). In glycerol broth the smooth forms may grow diffusely throughout the medium, they may form a thin, smooth surface pellicle, or they may cover the bottom of the flask with a reticulated veil-like growth; the rough forms usually grow as a thick wrinkled surface pellicle unaccompanied by turbidity. Morphologically, smooth strains consist of fairly long, curved, slender, sometimes beaded bacilli, lying more or less parallel to one another and occurring in bundles (Fig. 79); less often they are rather short, stout, or ovoid bacilli, staining evenly, and arranged singly or in groups (Fig. 80). Rough strains, on the other hand, consist of rather short, sometimes ovoid, bacilli or coccobacilli, arranged in Chinese letter forms and in dense masses (Fig. 81). This morphological difference in the arrangement of the smooth and rough types is similar to that described by Nutt (1927) and Wilson (1930) for Salm. typli-murium, and by Soule (1928) for $B$. anthracis and $B$. subtulis. It has been well pictured by Schwabacher (19336) and Wyckoff (1934) for saprophytic acid-fast and cold-blooded tubercle bacilli respectively. It depends essentially on the mode of division. The bacilli of the smooth type separate completely after division, and slip past each other so as to come to lie in parallel. The bacilli of the rough type exhibit an angular division, the organisms not separating completely but coming to lie at an obtuse angle to each other, resembling a greenstick fracture, or forming long tangled masses which become heaped-up and convoluted as growth continues. It is hardly necessary to add that many intermediate forms occur, partaking of some smooth and some rough characteristics.

Whether the smooth and rough types differ metabolically, antigenically, and in virulence, it is impossible to say definitely

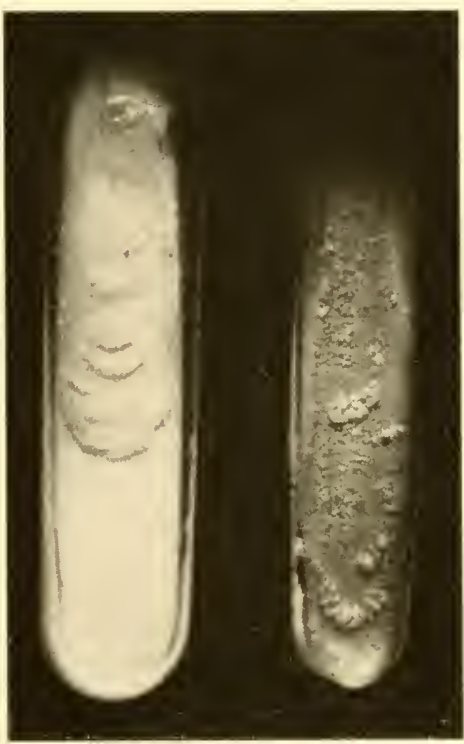

FIG. 77.--Saprophytic acid-fast bacilli.

Left : smooth strain, showing raised, confluent, butyrous growth, with smooth, glistening surface.

Right : rough strain, showing raised, confluent friable, heaped-up growth, with dry, dull, rough surface. Glycerol egg, 2 months, $37^{\circ} \mathrm{C}$. at present. Many workers assert that the smooth type of tubercle bacillus is more virulent than the rough, but further evidence is desirable on this point.

(References: Petroff 1927, Petroff, Branch, and Steenken 1927a, b, Petroff and Steenken 1930, 1935, Kraus and Gerlach 1929, Uhlenhuth and Seiffert 1930, Begbie 1930, 1931, Dreyer and Vollum 1931, Reed and Rice 1931a, b, Rice and Reed 1931, Rice 1931, Toda 1931, Seiffert 1932, Schwabacher 1933b, Seibert, Long, and Horley 1933, Wyckoff 1934, Meiszner and Prausnitz 1931, Steenken et al. 1931, Birkhaug 1935, Denys 1935, Shaffer 1935, Smithburn 1935.)

Resistance.-Acid-fast bacilli possess much the same degree of susceptibility to heat as other non-sporing bacteria, but a rather higher degree of resistance to chemical disinfectants. This behaviour is probably related to their content of

P.B. 
waxy substances, which are less permeable to cold than to warm aqueous solutions. There is evirlence that their resistance is more or less proportional to the amount

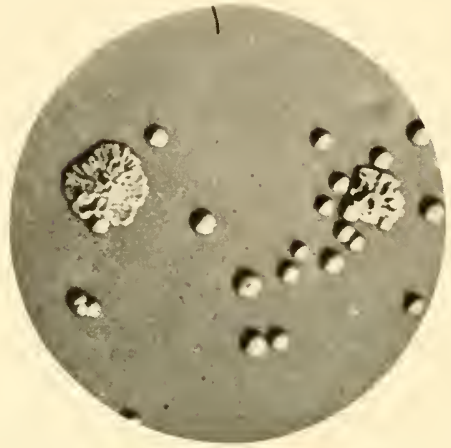

FIG. 78.-Myco. iuberculosis-avian type.

Surface colonies of smooth and rough forms. Petragnani medium, 7 weeks, $37^{\circ} \mathrm{C}$.

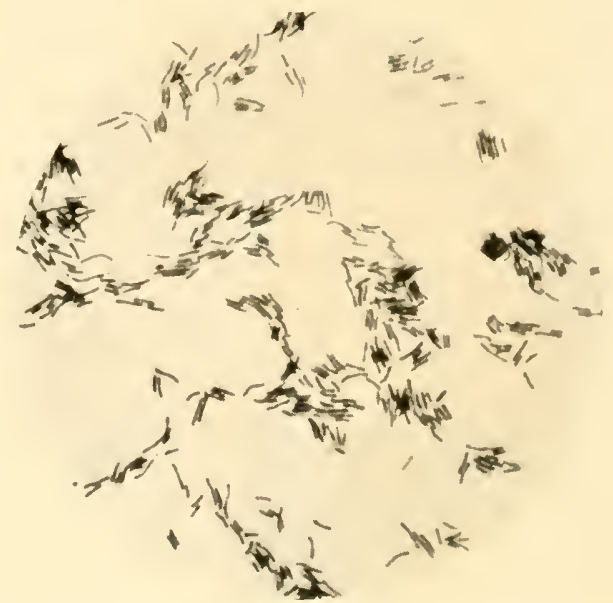

Fı. 79.-Glycerine egg, 1 month, $37^{\circ} \mathrm{C}$., show. ing long curved bacilli arranged in bundles. Smooth type of morphology.

of waxy material present (Shen 1934). Use is made of this differential susceptibility in isolating tubercle bacilli from contaminated material. Treatment of sputum, pus, or other products, with 15 per cent. antiformin or 15 per cent. sulphuric acid

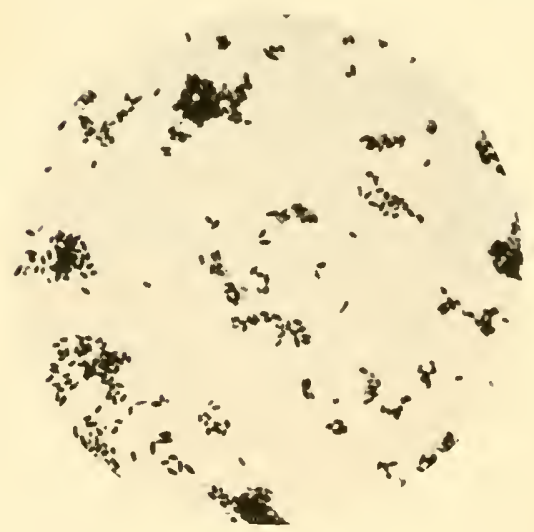

Fro. 80.-Glycerine egg, 1 month, $37^{\circ} \mathrm{C}$., showing fat ovoid forms arranged individually and in groups. Smooth type of morphology.

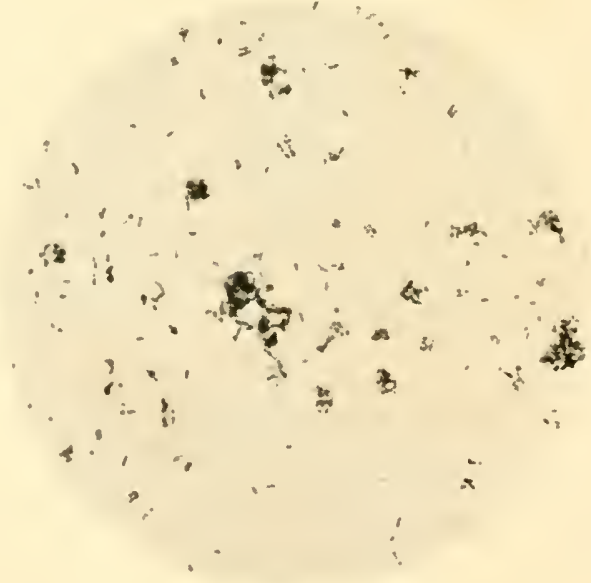

Fig. 81.-Glycerine egg, 1 month, $37^{\circ} \mathrm{C}$. showing mostly short irregularly stained rods, and cocco-bacilli, arranged singly, in Chincse lctter forms, and in dense elumps. Rough type of morphology.

for 5 to 20 minutes is often sufficient to destroy contaminating non-sporing bacteria without killing the tubercle bacilli, which can then be cultivated on suitable media. For the effect of relatively concentrated solutions of disinfectants reference may 
be made to Calmette (1936). With regard to weaker solutions Douglas and Hartley (1934) found that a saline suspension containing $1 \mathrm{mgm}$. of moist bovine bacilli per ml. was sterilized within 24 hours by exposure at room temperature to 0.5 per cent. phenol or 0.02 per cent. merthiolate. Phenol in 0.25 per cent. concentration failed to sterilize the suspension in 14 days, while 0.1 per cent. formol apparently just failed to do so. In our owu experience, using the cultural instead of the animal inoculation method, 0.5 per cent. phenol cannot be relied on to destroy all tubercle bacilli in 24 hours. In milk tubercle bacilli are killed in 20 minutes at $60^{\circ} \mathrm{C}$., provided it is contained in a closed vessel (for references, see Wilson 1942). In an open vessel a pellicle forms on the surface which protects the organisms to some extent, so that a few bacilli may escape destruction for an hour (Smith 1899, Oerskov 1925, Meanwell 1927). In polluted water kept in the dark at room temperature tubercle bacilli may remain alive for at least 3 months (Rhines 1935), while in soil or cow-dung exposed on pasture land during the summer and autumn in this country they may remain alive and virulent for 2 to 6 months (Williams and Hoy 1930, Maddock 1933). The organisms are comparatively resistant to drying, and provided they are protected from sunlight they may survive for months under suitable conditions. In books contaminated with the sputum of tuberculous patients, they may remain viable from 2 weeks to $3 \frac{1}{2}$ months (see Smith, C. R., 1942b). Numerous experiments have been performed to test the action of light on tubercle bacilli (Rochaix and Colin 1911, Mayer 1921, Caldwell 1925, Eidinow 1927, Mayer and Dworski 1932, Smith, C. R., 1912a). Most workers have found that they are rapidly destroyed, if spread in a thin layer, by bright sunlight, or by ultra-violet rays from a mercury vapour lamp. The most effective appear to be the short ultra-violet rays. Thus bacilli suspended in saline, when exposed in quartz flasks to rays of 2300-7620 A.U., were killed in 10 minutes; those exposed through window glass to rays of $3300-5720 \AA$.U. were not completely killed even in I hour. Probably the rays shorter than 3300 I.U. are the most lethal (Eidinow 1927). Blood, serum, and other proteins protect the bacilli against ultra-violet light.

Growth of tubercle bacilli in glycerine broth is inhibited by the addition of very small quantities of certain aniline dyes; thus a concentration of 0.0004 per cent. thioflavine or 0.0002 per cent. methylene blue entirely prevents growth; smaller quantities have little or no effect. Thymol is active in a concentration of 0.004 per cent.; most metals in colloidal suspension, however, are inactive even in a concentration of 10 per cent (Karwacki and Biernacki 1925).

Metabolism.--The optimum hydrogen-ion concentration for growth in 4 per cent. glycerine broth is said by Ishimori (1924) to lie between the following points :

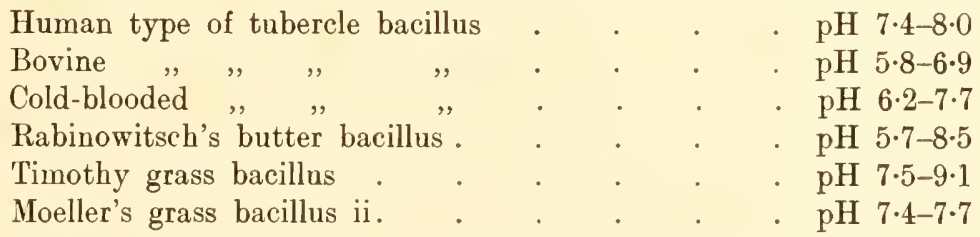

Dernby and Näslund (1922) found that growth of human and bovine tubercle bacilli in 3 per cent. glycerine veal broth occurred between $\mathrm{pH} 4.5$ and $8 \cdot 0$, the optimum being $\mathrm{pH} 6 \cdot 0-6.5$; their figures, it will be seen, do not agree with those 
of Ishimori, who found that the human preferred a more alkaline reaction than the bovine bacillus.

The optimum temperature for growth of the human, bovine, murine, and, generally the saprophytic acid-fast bacilli, is $37^{\circ} \mathrm{C}$.; for the avian $40^{\circ} \mathrm{C}$., and for the cold-blooded bacillus $25^{\circ} \mathrm{C}$. The human, bovine, and avian types do not grow below $30^{\circ} \mathrm{C}$, the cold-blooded and saprophytic acid-fast types grow freely at $20^{\circ} \mathrm{C}$. Many saprophytic acid-fast bacilli grow at $45^{\circ} \mathrm{C}$, and a few at $55^{\circ} \mathrm{C}$.

The tubercle bacillus is an aerobe; it will not grow under strictly anaerobic conditions. The optimum partial pressure of oxygen is saic to be $40-50$ per cent. for the human type (Novy and Soule 1925) and 60-70 per cent. for the avian and saprophytic acid-fast types (Uga 1935). Novy and Soule in their very careful study of the respiration of the tubercle bacillus found that $\mathrm{CO}_{2}$ had little effect on growth, and no inhibition occurred till a partial pressure of 60 per cent. was reached. Subsequent observations, however, by Rockwell and Highberger (1926) and others have shown that $\mathrm{CO}_{2}$ is beneficial for growth. For optimal development it is desirable to incubate cultures in a high partial pressure of oxygen and about 10 per cent. $\mathrm{CO}_{2}$. A few measurements have been made on the oxygen uptake of the bacilli (Dieckmann and Menzel 1932), and on the oxidation-reduction potential established in phosphate buffer solutions (Aksianzew 1933).

Moisture is an essential requirement of the tubercle bacillus in vitro. For good growth to occur, plenty of condensation water-supplied best by passing steam into the tube before inoculation-and an abundance of air are essential. Growth does occur in sealed tubes provided plenty of moisture is present, but it ceases after 3 or 4 weeks.

The effect of glycerine on the growth of the tubercle bacillus has been the subject of much controversy. Nocard and Roux in 1887, working apparently with an avian strain, were the first to notice the beneficial action of this substance. Since then it has been found that the addition of glycerine, generally in a concentration of 5 per cent., greatly increases the growth of all acid-fast bacilli with the exception of the murine, and to some extent the bovine tubercle bacillus. On this organism glycerine is not without effect; for it will enable it to grow-though very poorly -on agar, potato, or broth, on which no growth otherwise occurs. But the favourable action of glycerine on the bovine bacillus is not to be compared with that on other types; the addition of glycerine, for example, to serum or egg medium, makes no difference to the growth of the bovine bacillus, whereas it greatly increases the growth of all the other acid-fast bacilli.

The difference in the effect of glycerine on growth was made use of by Smith (1904-05) in the elaboration of a test for distinguishing between the human and bovine types. He found that if bovine tubercle bacilli were grown in glycerine broth, which had an acid reaction to phenol-phthalein, the acidity gradually decreased, till after full growth had occurred the reaction was about neutral ; in cultures of human bacilli, on the other hand, after an initial production of alkali, the reaction gradually became acid again. That is to say, the final reaction of a bovine culture was about neutral, of a human culture decidedly acid. This difference in the reaction curve of the two types of mammatian bacilli has been investigated by numerous workers, some of whom have confirmed, and some of whom have not confirmed, Smith's observations. Undoubtedly one of the reasons for this discrepancy is that most of the work was done before the days of accurate determination of acidity by measurement of the H-ion concentration was possible. Smith's view, put briefly, was that the human bacillus was able to utilize glycerine with the con- 
sequent production of acid, whereas the bovine bacillus was unable to use it, and so caused an increased hydrolysis of protein and an alkaline reaction. Whether Smith's view is correct or not, it is still impossible to say. The reaction curve of broth cultures is determined by so many factors, such as the production of $\mathrm{NH}_{3}, \mathrm{CO}_{2}$, amino-acids, and other substances, some of which are volatile, and some of which are not, that it is almost impos. sible to determine whether the production of acid is due to disintegration of the glycerine by the bacilli, or to hydrolysis of the protein material. Harden (1913), who made a special investigation of this subject, failed to obtain evidence of any definite distinction in biochemical behaviour between the human and the bovine types. Kendall, Day and Walker (1920), who have examined the question more recently, conclude that glycerine probably exerts a sparing action on the protein constituents of the medium in cultures of the human, but not in cultures of the bovine type; but, as noted elsewhere (p. 57), the existence of this protein-sparing effect is very doubtful. It is not possible to discuss this problem more fully (for a full discussion of the work up to 1917 see Cobbett 1917); but we may conchrde that as a practical means of differentiating the human from the bovine bacillus, determination of the glycerine-broth reaction curve is too complex for routine use.

Glucose seems to act in much the same way as glycerol (see Kiaffmaun 1932). The growth of tubercle bacilli is likewise improved by the addition of extracts of acid-fast bacilli to the medium; certain organisms, such as Johne's bacillus, will not grow at all unless such an extract is added (see p. 441). The tubercle bacillus has been grown in a number of synthetic media (see Wells and Long 1932); its growth is said to be increased by the addition of substances containing vitamin $B$, such as yeast, and of orange, tomato, or cabbage juice (Uyei 1927) (but see p. 66). Schmidt (1925) found 0.01 per cent. of iron chloride to farour growth, but Davies (1937) had the opposite experience. The effect of blood seems to be complex; tubercle bacilli grow well in liquid blood, particularly if lysed by saponin (Pryce 1941), but are generally inhibited by blood on the surface of a solid medium (Evans and Hanks 1939). Old potatoes that blacken on standing are more favourable for growth than new ones that do not (McCarter and Tatum 1937). Saprophytic acid-fast bacilli are said to grow in a simple solution of inorgamic salts containing neither carbon nor nitrogen (Bruner 1934, Gordon and Hagan 1937). There is evidence that tubercle bacilli can oxidize certain fats, such as those of olive oil and butter (Sédyeh and Seliber 1927).

Pigment production is very variable. On deeply coloured batches of ox serum the himan bacillus often forms a rich yellow or orange-yellow growth. A pink coloration on glycerol egg medium is not uncommon with avian strains, and according to Blacklock (1932) is sometimes given by rapidly growing bovine strains. Cold-blooded bacilli generally give colourless growths. On the other hand, the growth of nearly all the saprophytic acid-fast bacilli is accompanied by the production of a yellow, pink, orange, or brick-red pigment. On Sauton's medium a green coloration is not infrequently observed with cultures of mycobacteria ; its intensity varies from strain to strain (Lange 1932, de Grolier 1933, Kraus and Koref 1933). Pigment formation seems to depend on a number of different environmental factors, particularly the composition of the medium and the oxygen supply. Reed and Rice (1929) found that the addition of iron favoured its formation. On a glycerol agar medium containing 0.02 per cent. of ferric citrate, human, bovine, and avian types of tubercle bacilli, and saprophytic acid-fast bacilli, all formed pigment of varying depth, whereas on the same medium without iron many strains formed no pigment at all. Pigment production is not well developed at $37^{\circ} \mathrm{C}$.; it is best seen in 
cultures that have been incubated for some time at $37^{\circ} \mathrm{C}$., and then transferred to a dark cupboard at room temperature. In this respect the acid-fast bacilli resemble most other chromogenic bacteria.

The tubercle bacilli do not secrete a true exotoxin; but endotoxins are liberated on autolysis of the bacilli in broth cultures; these appear to be of complex constitution, and are more fully considered in the section on tuberculin (see Chapter 59). There is evidence that the endotoxins of all the acid-fast bacilli are closely alike; positive reactions may be obtained in animals infected with the mammalian type by injection of tuberculin made from the human, bovine, avian or cold-blooded type of bacillus (Ledoux-Lebard 1900, Wolbach and Ernst 1904). Intravenous injection of killed cultures of the mammalian, and especially of the avian tubercle bacilli, is often fatal to rabbits; Twort and Craig (1913) state that the smegma, mist and timothy-grass bacilli are also toxic for rabbits, whereas the cold-blooded tubercle and the butter bacilli are relatively non-toxic.

Biochemical Reactions.-Very little work has been done on the biochemical reactions of the acid-fast bacilli. Frouin and Guillaumie $(1923 a, b)$ brought evidence to show that human and avian bacilli could break down glucose, maltose, glycerol, and trehalose with the production of acid. Merrill (1930), who worked with one human, one avian, three cold-blooded, and several saprophytic acid-fast strains, and who made quantitative estimations of the sugar content of the medium before and after growth, found that glucose was utilized by all the strains, arabinose by all but one, sucrose by some, and lactose by none. The reaction, however, did not become acid, nor did any acid cleavage products accumulate. Instead, the medium became more and more alkaline during growth, owing to the production of $\mathrm{NH}_{3}$, though the rate at which it did so was less than in broth free from sugar. The conclusion was reached that the organisms oxidized the carbohydrates completely to $\mathrm{CO}_{2}$ and water.

Our own observations, based on an incomplete study of 7 cold-blooded and 36 saprophytic acid-fast strains, showed that there was no detectable acid production in any of the usual sugar media. Litmus milk was turned alkaline by the saprophytic acid-fast, but usually acid by the cold-blooded strains. In peptone water ammonia was produced in considerable quantity. Indole production was uniformly negative, though Rabinowitsch (1897) stated that it was sometimes positive. Catalase production was always positive. $\mathrm{H}_{2} \mathrm{~S}$ was formed in variable amount. The reduction of methylene blue in broth was weak or absent. The methyl red and. Voges-Proskauer reactions were consistently negative.

In milk tubercle bacilli are able to grow, but they produce in it no visible change.

Antigenic Structure.-By agglutination, absorption of agglutinins, and complement fixation, the acid-fast bacilli fall into four serological types-mammalian, avian, cold-blooded, and saprophytic acid-fast - the human and bovine types being indistinguishable (Tulloch et al. 1924, Cumming 1925, Wilson 1925, Griffith 1925, Furth 1926, Klopstock 1931, Kauffmann 1932). Direct agglutination is not sufficient to differentiate between the different types; absorption of agglutinins is essential. By this means it appears that there is an antigen common to the human, bovine, and avian types, but that the avian type also possesses an antigen which is not present in the mammalian types (Wilson 1925). Grüberg (1935) has reached similar conclusions on the basis of the precipitin test. Wulff (1925) concluded that by the absorption of agglutinins technique human and bovine strains could be separated, but his results still await confirmation. According to Wells (1944) 
the murine type is serologically indistinguishable from the human and bovine types. Observations suggest that group-specificity is determined by the polysaccharides, type-specificity by the proteins (Seibert 1941). There is evidence that the avian type may be divided serologically into a small number of sub-types (Wulff 1925, Furth 1926, Schaefer 1937, Harpoth 1938). It is possible that the cold-blooded and saprophytic acid-fast types are heterogeneous, but insufficient strains have as yet been examined to affirm this definitely.

It is noteworthy that serological methods are less valuable in classifying the tubercle bacilli than cultural methods; the bovine and human bacilli can be easily separated by their cultural reactions, yet serologically they form a homogeneous group. This affords an exception to the rule that serological examination is a far more delicate method of differentiation than cultural examination.

Pathogenicity and Experimental Infection of Animals.--Under natural conditions the human tubercle bacillus gives rise to disease mainly in man, monkeys, pigs, and occasionally in dogs and parrots; the bovine bacillus to disease in cattle, pigs, horses, man, and occasionally dogs, cats, and sheep; the murine bacillus to disease in voles and possibly other members of the family of Muridas the avian bacillus to disease in birds, and occasionally in pigs, sheep and cattle; and the cold-blooded bacillus to disease in cold-blooded animals and fish. Saprophytic acid-fast bacilli, though occasionally isolated from the tissues, rarely seem able to give rise to progressive disease (see also Chapter 59).

The virulence of tubercle bacilli is subject to variation. Though usually virulent on isolation, they not infrequently become more or less avirulent during subculture in the laboratory. Very little exact information, however, based on an arlequate number of animal tests, is available about the difference in virulence of freshly isolated strains of the same type, or about the factors that are responsible for changes in virulence occurring in vitio or in vivo.

The experiments of Villemin, recorded in 1868, furnished convincing proof of the transmissibility of human tuberculosis to animals. Material taken from different types of tuberculosis in man-catarrhal pneumonic forms, caseous pulmonary and chronic pulmonary tuberculosis, disseminated tuberculosis, scrofula, sputum, and the blood removed from a tuberculous patient after death-and introduced beneath the skin of rabbits, was successful in setting up the disease in 17 out of 21 animals; 3 of the 4 animals that did not contract tuberculosis died within a week of erysipelas. Villemin also transmitted bovine tuberculosis to rabbits, and made the important observation that material from bovine tuberculosis set up a more rapid and more generalized discase than material from human tuberculosis, when inoculated into these animals. Convineing though Villemin's experiments were, the final proof of the transmissibility of the clisease was not furnished till Koch in 1882 succeeded in producing tuberculosis in animals by injection of pure cultures of the tubercle bacillus. In 1898 Smith brought evidence to show that bovine tubercle bacilli were more virulent when injected into rabbits and calves than human bacilli. This difference was substantiated by other workers, in particular by Vagedes (1898), Ravenel (1901), the English Royal Commission on Tuberculosis (Report 1911), Kossel, Weber and Heuss (1904, 1905), and Park and Krumwiede (1910).

CATrLE.-The subcutaneous injection of $50 \mathrm{mgm}$. of bovine bacilli from young serum cultures into calves sets up an acute, rapidly generalizing disease, proving fatal in about 6 weeks to 3 months. At necropsy the main features are a local lesion, focal glandular swelling and abscess formation, generalized glandular lesions, and lesions in the viscera, 
most extensive in the lungs and spleen, and less extensive in the liver and kidneys. Smaller doses give less constant results. The intravenous injection of even $1 \mathrm{mgm}$. of bovine bacilli produces a severe and fatal tuberculosis. Cattle may likewise be infected with bovine bacilli by inhalation and feeding.

The subcutaneous injection of calves with human bacilli, in no matter what dosage, never gives rise to a progressive disease. At most there is a local abscess and swelling of the focal glands; when the animals are killed after 4 or 5 months, the lesions are minimal, and are often calcified. The intravenous injection of human bacilli may cause death by toxemia wilh acute infiltration and cedema of the lungs; but there is no true infection. The same toxæmia may be produced by the intravenous injection of avian bacilli, and even of the non-pathogenic saprophytic acid-fast bacilli.

The intramuscnlar inoculation of a calf with $80 \mathrm{mgm}$. of murine bacilli gave rise to a local alscess and to incouspicuous lesions in the regional lymphatic glands. Even $10 \mathrm{mgm}$. intravenously excited no more than a mild chronic retrogressive tuberculous reaction (Griffith and I lalling 1940).

The subcutaneous injection of calves with avian bacilli in a dose of $500 \mathrm{mgm}$. produces no more than a local lesion, with perhaps a caseous nodule in the nearest lymphatic gland. Intravenous inoculation, however, of even $5 \mathrm{mgm}$. may prove fatal in 2 to 3 wceks. Post mortem there are miliary tubercles in the lungs; the spleen, though showing no macroscopic tubercles, is enlarged and contains great numbers of tubercle bacilli visible microscopically.

Goats.-Bovine bacilli inoculated subcutaneously in a dose of I mgm. give rise to a fatal, generalized disease. Human bacilli, even in a dose of $100 \mathrm{mgm}$., cause no more than small retrogressive lesions. Avian bacilli in a dose of $100 \mathrm{mgm}$. rarely give rise to progressive disease; on the other hand the animals may remain chronically infected and excrete the bacilli in the milk for a long time (Griffith 1931).

SHEEP resemble goats in being highly susceptible to infection with bovine bacilli, moderately susceptible to infection with avian bacilli, and resistant to infection with human bacilli.

Pigs.-Injected subeutaneously, the bovine type in a dose of 10 to $50 \mathrm{mgm}$. produces rapidly fatal miliary tuberculosis. The human type in a dose of $50 \mathrm{mgm}$. produces a local lesion with slight dissemination. Avian bacilli injected subcutaneously do not give rise to progressive disease, but they may multiply and be disseminated through the internal organs, where they remain alive for some considerable time. Young pigs fed with bovine bacilli develop widespread disease and generally die; when fed with human bacilli they may develop extensive glandular and pulmonary disease. Though the human bacilli cause less severe and less extensive tuberculosis than bacilli of the bovine type, they are more pathogenic for pigs than they are for cattle.

PabBits.-Boine bacilli injected subcutaneously in a dose of $0 \cdot 1-1 \cdot 0 \mathrm{mgm}$. produce a generalized disease, fatal in 2 to 3 months. At necropsy a local castous lesion, caseous focal glands, innumerable little grey tubercles with caseating centres or larger irregular nodules in the lungs, numerons projecting hemispherical nodules in the cortex of the kidneys, and tubercles in the spleen and liver are found. Given intraperitoneally, even minute doses, such as $0 \cdot 000,000,001 \mathrm{mgm}$. of bovine bacilli, are said to prove fatal in 2 to 3 months (Cobbett 1917). Intravenous injection of 0.01-0.1 mgm. of bovine bacilli proves fatal in 3 to 6 weeks.

The subcutaneous injection of human bacilli in a dose of $1-100 \mathrm{mgm}$. never causes fatal tuberculosis. Human bacilli may, however, give rise to lesions in the lungs and kidneys. Usually the lungs contain a few small grey tubercles or caseous nodules, and the kidneys show a few miliary tubercles in the cortex. But tha acute fatal miliary tubereulosis seen after injection of bovine bacilli never results from the subcutaneous injection of human bacilli. Intravenous and intraperitoneal injections are less reliable for purposes of differentiation; $0.01 \mathrm{mgm}$. of human bacilli given intravenously rarely, if ever, proves fatal, but larger doses may give rise to a progressive fatal disease. Doses of $10-50 \mathrm{mgm}$. given 
intraperitoneally may likewise prove fatal on oceasion. Extensive lesions in the lungs and kidneys are often seen both after intravenous and intraperitoneal injectioin (see Cobbett 1932). The rabbit, in fact, possesses a considerable resistance to the human type of bacillus, but not so great as the ox or the goat. Rabbits are also fairly resistant to infection with murine bacilli. Intravenous inoculation of 0.1 to $1.0 \mathrm{mgm}$. may cause death from acute miliary tuberculosis. Smaller doses, $0.01 \mathrm{mgm}$. intravenously, or larger doses subentaneously, prodnce trivial lesions and the bacilli gradually die out; an abscess in the lung, however, may provide a nidus in which the bacilli may live for a long time. The avian bacillus is less virulent for rabbits than the bovine, but more virnlent than the human bacillus. Subcutaneous injection gives rise to a chronic disease; intraperitoneal injection of large doses to a rapidly fatal peritonitis; and intravenous injection to an acutely fatal infeetion. The macroseopic lesions caused by the avian bacillus are much less obvious than those cansed by the bovine bacillus; sometimes no tubereles are visible. After intravenous inoculation Yersin (1885) found the spleen greatly enlarged and the liver enlarged to a less extent; no macroseopie tubereles were seen, but mieroseopically there were numerous small tubereulous nodules in the liver and spleen containing large numbers of tuberele baeilli; the kidneys and lungs appeared practieally normal. This proliferation of the bacilli in the body without maeroscopic tuberele formation is known as the Yersin type of discase; it is seen both in rabbits and gninea-pigs injected with avian hacilli, and in rats injected with both manmalian and avian types. Chronic avian infeetions are generally characterized by infection of the joints (Griffith 1941c).

After injection of the bovine or human type of bacillus, no matter what route is chosen, the lesions in rabbits are most evident in the lungs and kidneys; the spleen and liver suffer less; the lymphatic glands, with the exception of the regional glands, hardly at all. Not infrequently the joints, mammary glands, and testes show lesions. For differentiating between bovine and human bacilli in the rabbit, the best doses to employ are $10 \mathrm{mgm}$. subeutaneously, $1 \mathrm{mgm}$. intraperitoneally, and $0 \cdot 01 \mathrm{mgm}$. intravenously (Cobbett 1917).

Feeding the rabbit with $1-10 \mathrm{mgm}$. of bovine bacilli sets up a disease that proves fatal in 2 to 3 months.

Guinea-pigs.-The guinea-pig is highly susceptible to experimental infection with bacilli of the human and hovine type. Indeed it has been stated that the subentaneous injection of a single tubercle bacillus may succed in producing disease-though only of a slow type showing little tendency to generalization (Wámoseher and Stoecklin 1927, Doerr and Gold 1932). Less than 10 living bacilli, even of virulent strains, cannot be relied upon to cause disease in every animal (Schwabacher and Wilson 1937). With between 10 and 100 bacilli intramuscular inoculation into the thigh usually sets up a slowly progressive disease, often characterized in the later stages by healing of the initial lesions. The bovine bacillus is rather more virulent than the human bacillus; this can he demonstrated. however, only by a series of comparative experiments in which carefully measured doses are introrluced. Table 26, from Griffith, quoted by Cobbett (1917), who gives an excellent review of the pathogenicity of tubercle bacilli to animals, illustrates this.

TABLE 26

Showing greater Virulence of Bovine than Human Bacilli to Guinea-pigs.

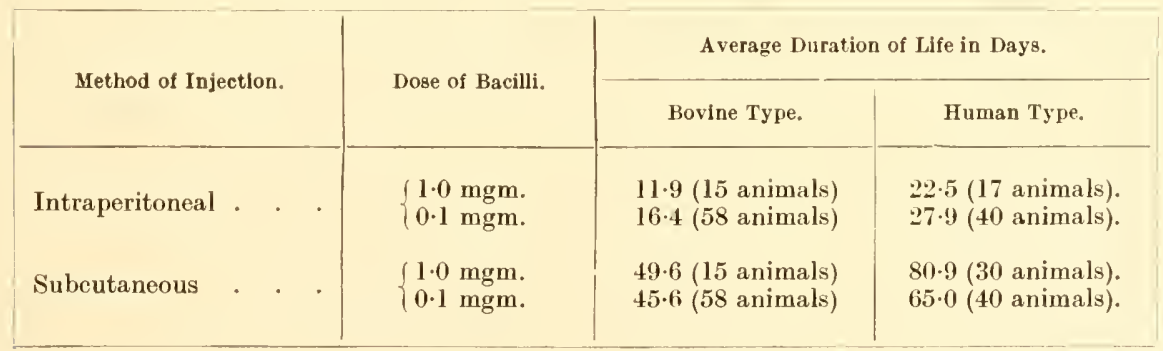


The subcutaneous injection-usually made in the left thigh - of either human or bovine bacilli, even in minute doses, is followed by death from generalized tuberculosis in about 6 to 15 weeks. At necropsy, there is a caseous local lesion; the superficial inguinal, and often the femoral, glands are much enlarged and easeous ; the sublumbar, portal, mediastinal, and bronchial glands are enlarged and generally easeous; the spleen is greatly enlarged, and is beset with irregular necrotic areas, varying considerably in size, of a yellowish-white waxy appearance; the liver is enlarged and contains smaller necrotic areas of a yellow or greenish colour; the lungs contain small numbers of rounded gelatinous tubercles. The most striking appearance is afforded by the necrotic areas in the spleen and liver ; these are peculiar to the guinea-pig. They are more marked after injection with bovine than with human bacilli. True tubercles are not often seen except in the very early stages of the disease, and in the lungs. It will be noted that the distribution of lesions is quite different in the guinea-pig from that in the rabbit. In the rabbit they are most marked in, and often confined to, the lungs and kidneys; in the guinea-pig the lymphatic glands, spleen, and liver bear the brunt of the disease ; the lungs are but slightly affected, and the kidneys practically never.

Intraperitoneal injection of human or bovine bacilli is followed by death in 2 or 3 weeks. Post mortem, there is a local caseous abscess in the abdominal wall; the superficial inguinal glands are enlarged and easeous; the omentum is rolled up, thickened, and easeous ; the portal and often the mediastinal glands are enlarged and caseous; and the spleen and liver may show small foci of necrosis. The effect of intravenous inoculation of minimal doses has been described by Fust (1938).

There is a disease that occurs naturally in guinea-pigs known as psendotuberculosis; it is caused by Pasteurella pseudotuberculosis (B. pseudotuberculosis rodentium). The lesions to which it gives rise include white rounded nodules in the spleen, liver, and mesenteric glands. These are unlike the necrotic lesions caused by the true tubercle bacillus, but may confuse those who are not well acquainted with the disease, especially since, when inoculated subcutaneously into normal guinea-pigs, the pseudotubercle bacillus gives rise to a local lesion and caseation of the focal lymphatic glands. The differential diagnosis can be made by microscopical and cultural examination. Microscopically, short, nonacid-fast, Gram-negative, bipolar-stained bacilli are seen, though often only in small numbers; culturally these organisms grow readily on ordinary media within 24 hours (see Chapter 32).

Murine bacilli are much less pathogenic for the guinea-pig than the other two mammakian types. Relatively large doses, $0 \cdot 1$ and $1 \cdot 0 \mathrm{mgm}$., intraperitoneally may cause death from acute or chronic atypical generalized tuberculosis; but smaller doses intraperitoneally, and subcutaneous doses up to $5 \mathrm{mgm}$., give rise to no more than retrogressive lesions remaining more or less localized to the site of inoculation.

Avian bacilli are very much less virulent for the guinea-pig than the human and bovine types. Subcutaneous injection is followed by a local abscess and swelling of the regional glands; death is unusual unless a large dose is given. Intraperitoneal injection of large doses is followed by death in a few weeks. Post mortem, the omentum is thickened, the spleen is large and red, there are minute grey points in the liver, and there may be fluid in the pleural cavities. Though neither after subcutaneous nor intraperitoneal injection are macroscopic tubercles visible, smears or cultures made from the spleen and liver will generally reveal the presence of tubercle bacilli-Yersin type of disease.

RATS AND Mice.-In the past these animals have been regarded as comparatively resistant to tuberculosis. More recent work, however, particularly with mice, seems to show that their resistance has been rather overestimated. Subcutaneous inoculation, except in large doses, seems to have little effect. Intraperitoneal inoculation of mice with $1 \mathrm{mgm}$. of human or bovine bacilli sets up a disease usually proving fatal in 3 to 4 weeks, while intravenous inoculation with $0.2 \mathrm{mgm}$. is often fatal in 2 to 3 weeks. Post mortem, the lungs are studded with translucent, gelatinous, miliary tubercles; similar tubercles are sometimes present on the pericardium; the spleen shows a variable degree of enlarge- 
ment but no macroscopic tubercle formation. Microscopically, the lungs contain enormous numbers of bacilli, while in smears of the spleen, liver, and kidneys the organisms are usually plentiful but not abundant. Smaller doses give rise to a less rapidly fatal disease, in which there is time for the pulmonary tubercles to become partly aggregated into irregular, hard, caseous masses. Small doses cannot be relied upon to cause progressive infection when injected intraperitoneally, but intravenous inoculation with as few as 10-100 living bacilli has, in our experience, often been followed by chronic disease. If the animals are killed within 3 months after such small doses, no tubercles may be visible in the lungs, but quantitative bacteriological examination leaves no doubt that the bacilli are actively proliferating (Schwabacher and Wilson 1937). Left to themselves, the animals may live for several months or even a year ; at post mortem some of them show miliary tuberculosis of the lungs. Mice may also be infected with very small numbers of bovine tubercle bacilli if these are inhaled in the form of an aerosol mist (Clover 1944). The virulence of the human and hovine types for mice seems to be very much the same (Lange 1922, Stamatin and Stamatin 1939). The avian type was found by Gunn and his colleagues (1934) never to cause fatal disease after intra venous inoculation of $0.25 \mathrm{mgm}$., hut our experience does not bear this out. We do, however, find that the avian bacillus is less virulent than the mammalian types. According to Lange (1922), tuberculous mice appear to be relatively non-allergic. Field mice (Arvicola arvalis) were found by Koch (1886) to be more susceptible than house mice; after subcutaneous inoculation they died in 4 to 6 weeks with extensive tuberculosis of the lungs, liver, and spleen.

VoLEs. - Wells (1938) found that the vole (Microtus agrestis) was useful for distinguishing between the human and the bovine types of tubercle bacilli. In his experience bovine bacilli in a dose of $0.001 \mathrm{mgm}$. intraperitoneally produced extensive and progressive disease. whereas human bacilli, even in a close of $1 \mathrm{mgm}$., produced no more than an insignificant lesion in the mesentery. Griffith $(1939 a, 1941 a, e)$ states that the vole is suseeptible to infection with all three manmalian types and with the avian type. The bovine type is the most virulent, giving rise when inoculated parenterally or introduced by feeding to generalized progressive tuberculosis, which runs a rapid course and is characterized by widespread caseation of the lymphatic glands and great multiplication of bacilli in the lesions. The human and the avian bacillus can multiply in the tissues of the vole, but have little tendency to produce macroscopic lesions. The murine type gives rise to generalized tuberculosis, which runs a more chronic course than that caused by the bovine type, and is distinguished by the occurrence in the areolar tissues of masses of necrotic or caseous material composed largely of acid-fast bacilli.

Other Animals. - The $d o g$ is relatively resistant to experimental tuberculosis, but young animals may be infected by intraperitoneal inoculation with the mammalian types. Horses and asses are also resistant; subcutaneous injection sets up no more than a local lesion. Cats are highly susceptible to bovine bacilli, slightly susceptible to avian, and resistant to human bacilli. Subcutaneous injection of $0.1 \mathrm{mgm}$. of bovine bacilli sets up invariably a rapidly fatal generalized tuberculosis. Honkeys and anthropoid apes are easily infected either by subcutaneous inoculation or by feeding with the human and bovine types, but are comparatively resistant to the avian type. The golden hamster (Cricetus auratus) is very susceptible to experimental infection with the hovine and slightly less so to the human type, both of which produce progressive generalized tuberculosis. The murine type causes generalized tuberculosis of slow development with the production of lesions that do not undergo necrosis or caseation. The avian type is the least virulent and rarely gives rise to macroscopic lesions (Griffith 1939b, 1941b). Ferrets in captivity brought up on raw milk sometimes contract tubereulosis. Experimentally they are susceptible to the bovine but only very slightly to the human type of bacillus (Dunkin, Laidlaw and Griffith 1929, Dalling and O'Brien 1935).

BIRDs. - With the exception of the parrot, the cockatoo, the canary, and possibly certain birds of prey, birds are resistant to infection with the mammalian bacilli. Sub- 
cutaneous, intraperitoneal, or intravenous injection of avian bacilli into fowls or pigeons gives rise to fatal tuberculosis. At neeropsy, the main lesions are found in the liver and spleen, which contain rather hard easeous tubercles well differentiated from the surrounding tissue. After feeding experiments, tubercles are often seen projeeting through the peritoneal covering of the intestine.

Cold-Blooded Avimals. - The cold-blooded type of tubercle baeillus is unable to set up progressive disease in mammals or probably birds; after subentaneous injection of large doses a local lesion may result, but the disease does not spread. Frogs, turtles, lizards, snakes, fish and other cold-blooded animals are susceptible to experimental inoeulation with the cold-blooded type, and are generally regarded as insusceptible to the mammalian and avian types. Griffith $(1941 \mathrm{c})$, however, states that the water or grass-snake (Trepidonotus natrix) is highly susceptible to experimental infection with the avian type. He (1941d) has also found that mammalian and avian bacilli, if inoculated into the dorsal sae of a toad, multiply slowly in the liver and may be reeovered 1, 2, or 3 years later. Subcutancous or intraperitoneal inoculation with cold-blooded baeilli gives rise to lesions that are often widely distributed throughout the viscera. Their nature depends on the site of inoculation and the time of survival. Soft, nodular lesions in the liver or lungs, filled with ereamy or easeous material, are not uncommon. Sometimes the liver is studderl with little greyish-white granules, almost confluent (see Ledoux-Lebard 1900, Friedmann 1903, Kïster 1905). Whether cold-blooded bacilli are pathogenie for birds is not clear. Aronson (1926) states that he isolated a cold-blooded bacillus from eertain salt-water fish - a sergcant-major (Abudefduf mauritii), three croakers (Micropogon undulatus), and two sea-bass (Centropristes striatus) — which proved pathogenic for pigeons.

Developing Chick embryo.- A few observations have been made on the growth of tubercle bacilli on the chorio-allantoic membrane of the devcloping ehick embryo. Aceording to Fite and Olson (1944), the lesions produced under these conditions by the human type of bacillus differ from those caused by the bovine and avian types. Though the method may be of differential value, it does not appear to be suited for estimating the virulenee of strains to their natural animal host.

Table 27 modified from Cobbett (1932), summarizes the reaction of different animals to the four main types of tubercle bacilli.

The saprophytic acid-fast bacilli are unable to set up a progressive infection in mammals or birds. Nevertheless, when injected intraperitoneally in fairly large doses, especially together with some fatty protective substance such as butter, they may give rise to extensive lesions closely simulating those of true tuberculosis (see Rabinowitsch 1897, Grassberger 1899, Hagan and Levine 1932). This appears to result from the dissemination of the bacilli in the tissues by the lymph stream and leucocytes, and the subsequent focal reaction of the tissues around them. True nodule formation and caseation may occur, and acid-fast bacilli are found microscopically in the lesions. After subcutaneous or intramuscular inoculation the lesions are usually confined to the local site and the regional lymphatic glands. The disease may be distinguished, however, from tuberculosis by culture and by further inoculation. Cultures made from the lesions on to glycerine agar will reveal a growth of saprophytic acid-fast bacilli in 2 or 3 days; while further inoculation into a guinea-pig, using a saline suspension of a small portion of one of the lesions, will prove innocuous. Histologically, the lesions caused by the saprophytic acid-fast bacilli show more exudation than proliferation; there is less tendency to caseation and more to suppuration ; polymorphonuclear are commoner than epithelioid cells; and typical giant cells with peripheral nuclei are rare (Rabinowitsch 1897). Saprophytic acid-fast bacilli injected intravenously 
'I'ABLE 27

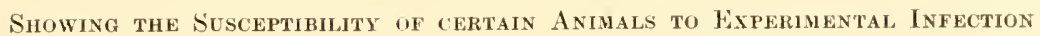
With the four main 'Types of Tubercle Bacilli.

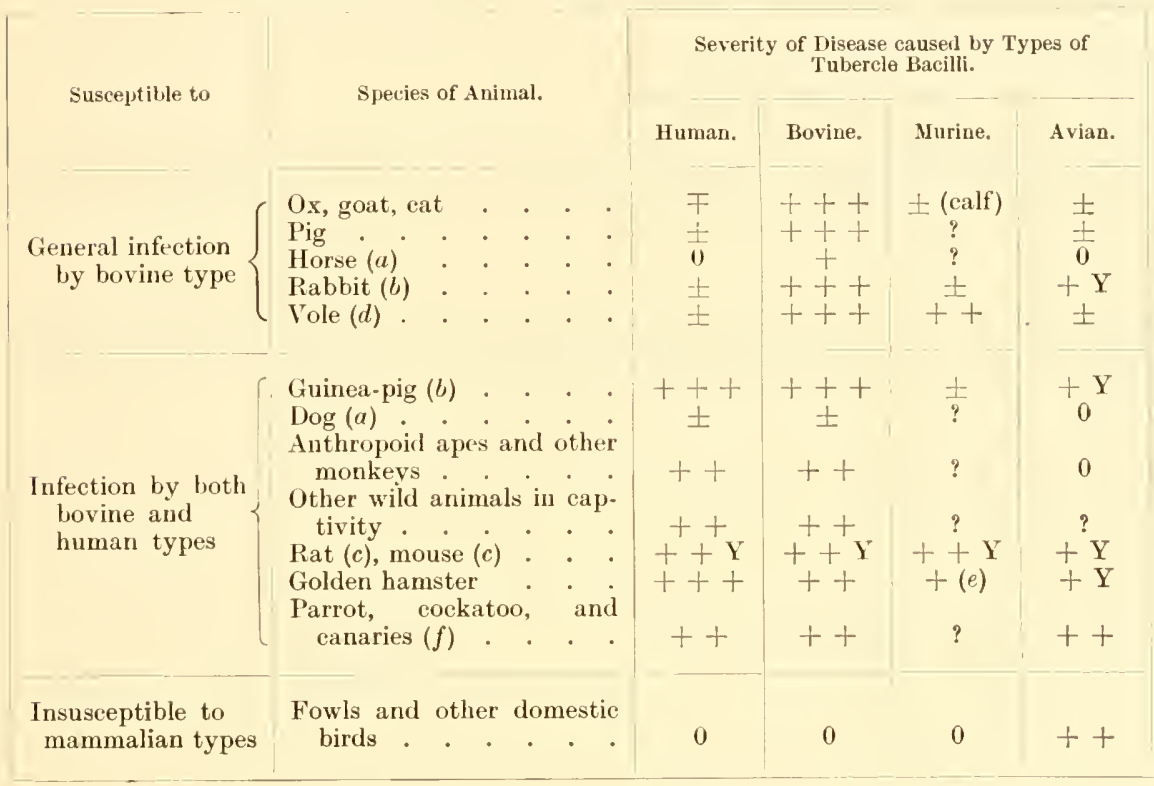

\section{干 A local retrogressive lesion.}

L Localized tuberculosis, with sometimes slight dissemination.

,,++++++ Different degrees of progressive tuberculosis.

$+\mathrm{Y}$ Tuberculosis of the Yersin type.

(a) Spontaneous tuberculosis relatively uneommon; difficult to infect experimentally.

(b) Extremely easy to infect experimentally, but seldom contracts tuberculosis naturally.

(c) Spontaneous tuberculosis is rare; after intravenous inoculation mice die with miliary tuberculosis of the lungs and an enlarged spleen containing tubercle bacilli in great numbers.

(d) Spontaneous infection with the murine type appears to be common. 'The natural disease, and the experimental disease caused by the murine type, are characterized by tuberculosis of the lungs and lymphatic system, and the occurrence of necrotic or easeous material in the subcutaneous areolar tissue reminiscent of rat leprosy. Experimental infection with the bovine type gives rise to acute generalized tuberculosis.

(e) Produces slowly progressing generalized tuberculosis without necrosis or caseation of the lesions.

( $f$ ) Canaries are said to be less susceptible to infection with the human and bovine types than parrots and cockatoos (Tulloch 1936).

into laboratory animals, or even into larger animals such as calves, may give rise to severe illness, sometimes followed by death (Kossel et al. 1904, Twort and Craig 1913). The bacilli do not multiply in the tissues, and therefore no true infection is set up ; the symptoms appear to be due to toxæmia following the liberation of endotoxins from the bacilli in contact with the tissues. Similar results can be obtained with dead bacilli. This toxæmia following the intravenous injection of living or dead acid-fast bacilli, whether of the saprophytic acid-fast or the true tubercle type, into susceptible animals, is a phenomenon that may lead to confusion if differentiation of the various types is attempted by the intravenous route. For this reason the subcutaneous method of injection is generally to be preferred. 
Type Differentiation by Pathogenicity.-At the risk of repetition we give for the sake of clearness the methods by which the three main types of tubercle bacilli may be differentiated.

The human and the bovine types of tubercle bacilli are able, when injected subcutaneously in minute doses, to give rise to a progressive and fatal disease in guinea-pigs. But whereas the bovine type is able likewise to produce a progressive, generalized, and fatal disease in rabbits, cats, voles, goats, and calves, the human bacillus cannot do so. This fundamental distinction can be elicited in practice only by strict regard to certain factors, such as dosage and route of inoculation. The experience of the Royal Commission showed that the best differentiating dose for calves was $50 \mathrm{mgm}$. subcutaneously, and for rabbits $10 \mathrm{mgm}$. subcutaneously. The bacilli are taken from a 1 to 3 -weeks old culture on inspissated serum and weighed moist. Under these conditions the bovine bacillus sets up a generalized fatal disease, while the human bacillus causes at most a localized and retrogressive disease, confined to the site of inoculation, and sometimes to one or more of the internal organs; often no lesions are visible at all. Animals vary so much in their susceptibility to experimental inoculation that it is advisable to inject two or three simultaneously, and to repeat the test on a fresh series of animals if the results are not precise. The time taken to produce death is probably not of such importance as the extent of the lesions at necropsy (Park and Krumwiede 1910) ; but generally speaking calves inoculated with a bovine strain may be expected to die in about 6 to 8 weeks, and rabbits in 6 to 12 weeks. A more rapid method of differentiation consists in the intravenous inoculation of rabbits with $0.001 \mathrm{mgm}$. or even less. In such a dose the human bacillus has little effect: the bovine kills the animal in 4 to 6 weeks, and at post mortem miliary tuberculosis is found affecting particularly the lungs and kidneys. According to Wells (1944), an equally satisfactory and perhaps even more rapid method consists in the subcutaneous inoculation of $1 \mathrm{mgm}$. into the Orkney vole (Microtus orcadensis). The human bacillus is practically nom-pathogenic to this animal, giving rise to no more than a local lesion with perhaps minimal lesions in the focal lymphatic nodes and occasionally a few scattered tubercles in the lungs, whereas the bovine bacillus causes generalized glandular and visceral tuberculosis, proving fatal as a rule in 3 to 5 weeks.

The separation of the avian from the mammalian bacilli is generally quite straightforward. The avian bacillus is pathogenic for fowls and pigeons, but not for the guinea-pig; the mammalian bacilli, on the contrary, are pathogenic for the guinea-pig, but not for fowls and pigeons. Cobbett (1917) recommends for differentiating purposes the injection of $10 \mathrm{mgm}$. of culture subcutaneously into guineapigs, and $10 \mathrm{mgm}$. intraperitoneally into fowls or pigeons. The mammalian bacilli prove fatal to guinea-pigs in about 4 to 8 weeks, and at necropsy there is generalized tuberculosis with extensive caseation of the glands and large necrotic areas in the spleen and liver. The avian bacilli often give rise to no lesions at all, or only to a slight local collection of caseous material ; but sometimes, if the animals are killed after a few weeks, the focal glands will be found to be hyperæmic, and the spleen enlarged and congested; smears of the spleen, liver, and lungs from these cases may reveal a few acid-fast bacilli (Straus and Gamaléia 1891). After injection with avian bacilli, fowls and pigeons die in a variable time, often considerably emaciated; if they are still alive at the end of 3 months they should be killed. Disease is most evident in the spleen, liver, and kidneys, which show numerous tubercles or hard caseous areas; bacilli are abundant in smears of these organs. 
The mammalian bacilli generally produce no recognizable lesions in fowls or pigeons, but occasionally a local abscess forms, which contains a few bacilli. Avian bacilli are pathogenic to rabbits if inoculated intravenously, giving rise to the Yersin type of disease (see p. 425). Griffith used to inject $0.01 \mathrm{mgm}$. intravenously into rabbits and $10 \mathrm{mgm}$. intramuscularly into hens. Sometimes, however, avian strains are encountered, particularly from pigs, that are pathogenic to fowls but not to rabbits.

Lesions caused by the Injection of Dead Tubercle Bacilli.-The injection into animals of dead tubercle bacilli, preferably killed by heat, gives rise to lesions of varying degrees of severity. Subcutaneous injection provokes merely a local abscess, but after intravenous injection the dead bacilli are deposited in the viscera, where they give rise to small follicular lesions closely resembling true tubercles, and sometimes undergoing caseation. This disease is known as Necro-tuberculosis (Miller 1905). Apparently the dead bacilli are attacked and broken down by the cells of the body and their intracellular toxins liberated; these toxins then act on the tissues, and give rise to the characteristic lesions of tuberculosis. For the production of necro-tuberculosis, virulent bacilli are not essential ; we have produced lesions in rabbits by the intravenous injection of dead avirulent bacilli, such as Calmette's B.C.G. strain.

Classification.-We have already indicated that the acid-fast bacilli may be divided into six types-human, bovine, murine, avian, and cold-blooded tubercle bacilli, and the saprophytic acid-fast bacilli. The distinction between the types rests chiefly on cultural appearances and pathogenicity, aided when necessary by serological reactions. These types are remarkably constant; so far as we are aware, no clear evidence has ever been produced to prove that one type may change into another. Numerous experiments have been made by different methods to bring this about, but no one has yet succeeded in doing so.

Nevertheless, the division of the mammalian tubercle bacilli is not always clearly defined. From time to time aberrant strains are encountered, which depart from the standard type either in cultural behaviour, in pathogenicity, or in both. The most fruitful source of these aberrant types is lupus; thus from 140 cases of lupus examined by A. S. Griffith (1924), no fewer than 99 yielded strains of aberrant type. Apparently, when growing in the skin, tubercle bacilli are liable to undergo a modification in virulence, so that both human and bovine types become less pathogenic for experimental animals. Aberrant types are encountered in other lesions, though much less frequently; thus Griffith (1916) found 4 aberrant strains amongst a total of 212 strains isolated from sputum, and 5 amongst 141 strains isolated from bone and joint disease (1916-17) ; Eastwood and F. Griffith (1916) likewise isolated 10 aberrant out of a total of 261 strains from cases of bone and joint disease. Aberrant strains have occasionally been found in animals, especially horses (A. S. Griffith 1924, Stableforth 1929).

The mammalian bacilli may be subdivided as follows (Table 28, p. 432).

Of these various types the attenuated eugonic human and the attenuated dysgonic bovine are the ones usually found in lupus; the attenuated dysgonic human and attenuated eugonic bovine are rare, and have been met with in lupus only. The dysgonic human type was the type isolated from sputum and from bone and joint disease, in the cases already referred to. On serum and glycerine serum it grows luxuriantly with the production of yellow pigment. But on glycerine agar and glycerine potato growth is very slow; an effuse grey glazed layer of growth 
TABLE 28. (Modified from A. S. Griffith 1924.)

Showing the Differential Characteristics of Mammalian Tubercle Bacilli.

\begin{tabular}{|c|c|c|c|}
\hline Human. & Classification. & Cultural Characteristics. & Virulence. \\
\hline 1 & Typical human . & $\begin{array}{l}\text { Eugonic on all glycerine } \\
\text { media. Pigmented growth } \\
\text { on bovine serum }\end{array}$ & $\begin{array}{l}\text { High for guinea-pig; low } \\
\text { for rabbit (standard } \\
\text { human virulence). }\end{array}$ \\
\hline 2 & Dysgonic human & $\begin{array}{l}\text { Dysgonic on glycerine agar, } \\
\text { potato, and broth. Pig- } \\
\text { mented growth on bovine } \\
\text { serum }\end{array}$ & Like (1). \\
\hline 3 & $\begin{array}{l}\text { Attenuated dys- } \\
\text { gonic human }\end{array}$ & Like (2) & $\begin{array}{l}\text { Very low for rabbit, and } \\
\text { lower for guinea-pigs and } \\
\text { monkeys than standard } \\
\text { luman strains. }\end{array}$ \\
\hline & $\begin{array}{l}\text { Attenuated eugonie } \\
\text { human }\end{array}$ & Like (1) & Like (3). \\
\hline Bovine. & & & \\
\hline 1 & Typical bovine . & $\begin{array}{l}\text { Dysgonic on all glycerine } \\
\text { media; no pigment for- } \\
\text { mation on bovine serum }\end{array}$ & $\begin{array}{l}\text { High for guinea-pig and } \\
\text { rabbit (standard bovine } \\
\text { virulence). }\end{array}$ \\
\hline 2 & Eugonic bovine . & $\begin{array}{l}\text { Growth better than typical } \\
\text { bovine strains, but not } \\
\text { equal to human }\end{array}$ & Like (1). \\
\hline 3 & $\begin{array}{l}\text { Attenuated eugonic } \\
\text { bovine }\end{array}$ & Like (2) & $\begin{array}{l}\text { Less than standard bovine } \\
\text { virulence for all animals, } \\
\text { but higher for calf and } \\
\text { rabbit than standard } \\
\text { human. }\end{array}$ \\
\hline 4 & $\begin{array}{l}\text { Attenuated dys. } \\
\text { gonic bovine }\end{array}$ & Like (1) & Like (3). \\
\hline Murine. & Typical murine. & $\begin{array}{l}\text { Grows very slowly, and dys- } \\
\text { gonic on glycerine media. }\end{array}$ & $\begin{array}{l}\text { High for voles, but very low } \\
\text { for guinea-pigs and rabbits. }\end{array}$ \\
\hline
\end{tabular}

occurs, on which a few large isolated warty and pigmented colonies may eventually develop. Similarly on glycerine broth there is only a thin layer of growth with an occasional raised island of irregular appearance. In virulence this variety resembles the standard human type, being high for the guinea-pig and low for the rabbit. These dysgonic human strains are most likely to be confused with the attenuated bovine strains; differentiation can be effected by making a sufficiently wide and prolonged series of tests. The dysgonic bovine strains do not give a yellow pigmented growth on bovine serum ; and their virulence is lowered not differentially but uniformly; that is to say, their virulence is lowered not only for the more resistant calf and rabbit, but also for the highly susceptible guinea-pig and monkey.

Whenever an aberrant strain is encountered, the possibility of the cultures being impure must always be considered. Thus the Royal Commission (Report 1911) examined 2 cultures that were eugonic on glycerine media and were highly virulent to calves and rabbits. By plating out these cultures they were able to separate off a dysgonic virulent strain from a eugonic strain of slight virulence for the calf and rabbit; in other words, the original cultures contained a mixture of bovine and human types.

Considerable attention has been paid to these atypical strains, because they have been regarded by some workers as evidence of instability of type. Griffith, who has had the greatest experience of these atypical strains, is, however, 
firmly of the opinion that they represent no more than modifications of the fixed types; they do not represent transitional forms between the types. By passage through suitable animals it is often possible to restore the normal properties of their type to these atypieal strains; but it has never yet proved possible to modify them in such a way that they change into a different type. Thus an attenuated dysgonie bovine strain, if passed through a series of rabbits, will often regain the standard virulence of the bovine type; it will not take on a eugonic growth and come to resemble the human type (Griffith 1924). The extensive observations of Jensen and Frimodt-Möller (1936) and of Frimodt-Möller (1939) are in general accord with Griffith's findings.

We may conclude therefore that the types of tubercle bacilli are fixed; that occasionally modifications due to residence in a particular environment may result ; but that no alteration of environment or any other factor has yet been successful under experimental conditions of changing an organism of one type into that of another.

Classification of the saprophytic acid-fast strains is very unsatisfactory. Numerons types have been described, and have been differentiated on morphological and cultural appearances, the degree of acid-fastness, the formation of pigment, and the optimum temperature of growth (see Haag 1927, Pinner 1932, Thomson 1932, Schwabacher 1933b, Gordon and Hagan 1938).

A large amount of detailed information, much of which has been overlooked by subsequent workers, on the various properties of the tubercle bacilli, is contained in the Reports $(1907,1909,1911,1913)$ of the Royal Commission on Tuberculosis. Those anxious to learn more about these organisms are advised to study these reports carefully, as well as the numerous subsequent publications of Dr. A. Stanley Griffith, many of which have appeared in the Journal of Pathology and Bacteriology and the Journal of Hygiene during the past thirty years.

\section{Míycobacterium tuberculosis, Human type}

Isolation.-By Koch in 1882 from human tuberculous lesions.

Habitat.-Strict parasite, causing tuberculosis in man, pigs, monkcys, dogs, and parrots. Morphology.-Rod-shaped organisms, 1-4 $\mu$ long and $0 \cdot 2-0 \cdot 8 \mu$ broad, straight or slightly curved, with parallel or irregular sides, and rounded ends; arranged singly or in small clumps; non-motile, non-sporing, and non-capsulated. Fail to stain with simple dyes except after prolonged exposure. Stain best with hot carbol-fuchsin ; resist decolorization with 25 per cent. $\mathrm{H}_{2} \mathrm{SO}_{4}$ and with absolute alcohol for 10 minutes. Gram-positive; staining may be even or granular; beaded forms are common. In the animal body the bacilli are larger than in culture. Non-acid-fast and clubbed forms are not uncommon in culture.

Coagulated $O x$ serum. -4 weeks, $37^{\circ} \mathrm{C}$. Thin, effuse, confluent, greyish-yellow growth, with a finely granular surface, looking like ground glass; colour may be golden yellow; consistency friable; emulsifies with great difficulty.

5 per cent. Glycerine $O x$ serum. -4 weeks, $37^{\circ} \mathrm{C}$. More luxuriant, thicker, raised, confluent, yellow or golden-yellow growth, with a coarsely granular surface ; irregularly heaped-up in places; often a granular film over the water of condensation. Consistency friable, emulsifies with great difficulty.

Dorset Egg. - 4 weeks, $37^{\circ} \mathrm{C}$. Rather poor, discrete or confluent, slightly raised, greyishyellow growth, with a finely granular surface.

5 per cent. Glycerine Egg. -4 weeks, $37^{\circ} \mathrm{C}$. More luxuriant, raised, confluent, greyish-yellow growth, with a coarsely granular surface; growth irregularly nodular and heapedup in places. 
5 per cent. Glycerine Agar. -4 weeks, $37^{\circ} \mathrm{C}$. Thick, raised, confluent, cream-coloured growth with a nodular or wrinkled surface.

5 per cent. Glycerine Potato. -4 weeks, $37^{\circ} \mathrm{C}$. Thick, raised, confluent growth, creamy or yellow in colour, with a wrinkled, nodular, or warty surface.

5 per cent. Glycerine Broth. -4 weeks, $37^{\circ} \mathrm{C}$. Greyish-white surface pellicle, often irregularly thickened in places. On further incubation the pellicle increases in thickness, develops a deeply wrinkled surface, and spreads for about $\frac{1}{2}$ inch up the sides of the flask. No turbidity, but often a slight granular deposit.

Plain Agar or Broth.-No growth, as a rule.

Resistance.-Cultures live for 4 to 8 weeks as a rule, but may remain viable for a year. Bacilli are killed by moist heat at $60^{\circ} \mathrm{C}$. in 15 to 20 minutes. In excised tissues, kept at $37^{\circ} \mathrm{C}$, they die in about a week. In dried sputum most of the bacilli die in a few days. Are fairly susceptible to sunlight and ultra-violet light. Moderately resistant to chemical disinfectants; in sputum may survive exposure to 5 per cent. phenol or antiformin for 24 hours.

Metabolism.-Growth occurs between $\mathrm{pH} 4.5$ and 8.0 ; optimum $\mathrm{pH}$ is 7.0-7.6. Optimum temperature $37^{\circ} \mathrm{C}$. ; very little growth, if any, below $30^{\circ} \mathrm{C}$. Growth occurs best in an atmosphere of 40-50 per cent. $\mathrm{O}_{2}$; no growth under strictly anaerobic conditions. Growth is improved by addition of glycerine and of dead acid-fast bacilli to the medium; and is said to be improved by substances rich in Vitamin B, and by very small quantities of iron salts. Golden-yellow pigment produced on glycerinated ox serum.

Biochemical.-Very little known about biochemical properties. Evidence that the bacilli can produce acid from glucose, maltose, lactose, sucrose, glycerol and trehalose.

Antigenic Structure.-By agglutination, absorption of agglutinins, and complement fixation the human bacilli are shown to form a homogeneous group indistinguishable from bovine tubercle bacilli, but easily distinguishable from avian, coldblooded, and saprophytic acid-fast bacilli.

Pathogenicity.-Produces tuberculosis in man, pigs, monkeys, dogs, and parrots. Experimentally, it is highly pathogenic for the guinea-pig, but not for the rabbit, cat, goat, or ox. A minute quantity injected subcutaneously into a guineapig's thigh causes death in 6 to 12 weeks. P.M. local caseous abscess ; enlargement and caseation of the inguinal, sublumbar, portal, axillary, and bronchial glands; enlargement of the spleen with production of irregular yellowish areas of necrosis ; enlargement of the liver with production of smaller, irregular, greenishyellow areas of necrosis; few rounded tubercles in the lungs. Tubercle bacilli are numerous in the local lesion and the inguinal glands; more scanty elsewhere.

The characters of the different types of tubercle bacilli, including the saprophytic acid-fast bacilli, are summarized in the table on pp. 435-7 (Table 29).

\section{The Leprosy Bacillus}

The causative organism of leprosy, which was one of the first micro-organisms shown to be pathogenic to man, was discovered by Hansen in 1868, though his published account did not appear till 1874. He observed it in the tissues of lepers, where it occurs in large numbers in the granulation tissue cells. The bacilli vary in size and shape; they may be straight or slightly curved, 1-8 $\mu$ in length, with parallel sides and rounded ends, arranged chiefly in clumps or bundles, and staining evenly; or they may resemble diphtheroids and show granular staining, confined to the poles or distributed throughout their length. They are not easily stained without a mordant; they are Gram-positive and strongly acid-fast. They are non-motile and non-sporing.

Though the leprosy bacillus was one of the first of the pathogenic organisms to 
TABLE 29

The Growth of the Various 'Types of Tubercle Bacilli on Different Media.

\begin{tabular}{|c|c|c|c|c|c|c|}
\hline & Human. & Bovine. & Murine. & Avian. & Cold-blooded. & $\begin{array}{l}\text { Saprophytle } \\
\text { Acid-fast. }\end{array}$ \\
\hline $\begin{array}{l}\text { Nutrient } \\
\text { agar. } \\
4 \text { weeks } \\
\text { at } 37^{\circ} \mathrm{C} \text {. }\end{array}$ & No growth & No growth & No growtl & $\begin{array}{l}\text { Poor, partly } \\
\text { confluent, } \\
\text { effuse, trans- } \\
\text { lucent, } \\
\text { ground-glass } \\
\text { growth, with } \\
\text { finely granu- } \\
\text { lar surface }\end{array}$ & $\begin{array}{l}7 d .22^{\circ} C . \\
\text { Moderate, } \\
\text { semi-conflu- } \\
\text { ent, raised, } \\
\text { irregularly } \\
\text { heaped-up } \\
\text { growth with } \\
\text { smooth or } \\
\text { granular sur- } \\
\text { face }\end{array}$ & $\begin{array}{l}7 \text { d. } 22^{\circ} \mathrm{C} \text {. or } \\
37^{\circ} \mathrm{C} \text {. Abun- } \\
\text { dant, conflu- } \\
\text { ent, raised, } \\
\text { irregularly } \\
\text { heaped-up, } \\
\text { pigmented } \\
\text { growth with } \\
\text { smooth, } \\
\text { granular, or } \\
\text { worm-cast } \\
\text { surface. }\end{array}$ \\
\hline $\begin{array}{l}\text { Broth. } \\
\qquad 4 \text { weeks } \\
\quad \text { at } 37^{\circ} \mathrm{C} .\end{array}$ & No growtli & No growth & $\begin{array}{l}\text { Usually no } \\
\text { growtls }\end{array}$ & $\begin{array}{l}\text { No turbidity, } \\
\text { or surface } \\
\text { growth ; but } \\
\text { a moderate } \\
\text { viscous mem- } \\
\text { brano-granu- } \\
\text { lar deposit, } \\
\text { partly dis- } \\
\text { integrating } \\
\text { on shaking }\end{array}$ & $\begin{array}{l}7 d .22^{\circ} \mathrm{C} . \\
\text { Similar to } \\
\text { avian type }\end{array}$ & $\begin{array}{l}7 \text { d. } 22^{\circ} C \text {. or } \\
37^{\circ} C \text {. No } \\
\text { turbidity ; } \\
\text { dull, dry, } \\
\text { scaly or } \\
\text { granular, } \\
\text { often pig- } \\
\text { mented, sur- } \\
\text { face pellicle, } \\
\text { and a viscous } \\
\text { membrano- } \\
\text { granular } \\
\text { deposit, } \\
\text { partly disin. } \\
\text { tegrating on } \\
\text { shaking. }\end{array}$ \\
\hline $\begin{array}{l}\text { Coagulated. } \\
\text { Ox serum } \\
4 \text { weels } \\
\text { at } 37^{\circ} \mathrm{C} \text {. }\end{array}$ & $\begin{array}{l}\text { Thin, effuse, } \\
\text { confluent, } \\
\text { greyish- } \\
\text { yellow } \\
\text { growth with } \\
\text { finely granu- } \\
\text { lar surface } \\
\text { like ground } \\
\text { glass }\end{array}$ & $\begin{array}{l}\text { Similar to the } \\
\text { human type, } \\
\text { but often } \\
\text { poorer }\end{array}$ & $?$ & $\begin{array}{l}\text { Similar to the } \\
\text { human type }\end{array}$ & $\begin{array}{l}7 \text { d. } 22^{\circ} C \text {. } \\
\text { Moderate, } \\
\text { confluent, } \\
\text { slightly } \\
\text { raised growth } \\
\text { with finely } \\
\text { nodular sur- } \\
\text { face }\end{array}$ & $\begin{array}{l}7 \text { d. } 22^{\circ} C \text {. or } \\
37^{\circ} C \text {. Abun- } \\
\text { dant, raised, } \\
\text { confluent, } \\
\text { often pig- } \\
\text { mented } \\
\text { growth, } \\
\text { irregularly } \\
\text { heaped up iu } \\
\text { places, and } \\
\text { with smooth } \\
\text { or nodular } \\
\text { surface. }\end{array}$ \\
\hline $\begin{array}{l}\text { Glycerine } \\
\text { Ox serum. } \\
4 \text { weeks } \\
\text { at } 37^{\circ} \mathrm{C} .\end{array}$ & $\begin{array}{l}\text { More luxuri- } \\
\text { ant, thicker, } \\
\text { raised, con- } \\
\text { fluent, } \\
\text { yellow or } \\
\text { golden- } \\
\text { yellow } \\
\text { growth, } \\
\text { with }\end{array}$ & $\begin{array}{l}\text { Similar to } \\
\text { growth on } \\
\text { plain ox } \\
\text { serum }\end{array}$ & $\begin{array}{l}\text { Similar to } \\
\text { the bovine } \\
\text { type, but } \\
\text { colourless }\end{array}$ & $\begin{array}{l}\text { Luxuriant, } \\
\text { raised, con- } \\
\text { fluent, yellow } \\
\text { or golden- } \\
\text { yellow growtb } \\
\text { with a } \\
\text { smooth } \\
\text { creamy sur- } \\
\text { face }\end{array}$ & $\begin{array}{l}\text { Similar to } \\
\text { growth on } \\
\text { plain serum, } \\
\text { but often } \\
\text { more } \\
\text { abundant }\end{array}$ & $\begin{array}{l}\text { Similar to } \\
\text { growth on } \\
\text { plain serum, } \\
\text { but often } \\
\text { more } \\
\text { abundant. }\end{array}$ \\
\hline
\end{tabular}


TABLE 29 (continued).

\begin{tabular}{|c|c|c|c|c|c|c|}
\hline & Human. & Bovine. & Murine. & A vian. & Coid-blooded. & $\begin{array}{l}\text { Saprophytic } \\
\text { Acld-fast. }\end{array}$ \\
\hline $\begin{array}{l}\text { Dorset } \\
\quad \text { egg. } \\
\quad 4 \text { weeks } \\
\text { at } 37^{\circ} \mathrm{C} \text {. }\end{array}$ & $\begin{array}{l}\text { Rather poor, } \\
\text { discrete or } \\
\text { confluent, } \\
\text { slightly } \\
\text { raised, } \\
\text { greyish- } \\
\text { yellow } \\
\text { growth, with } \\
\text { a finely } \\
\text { granular } \\
\text { surface }\end{array}$ & $\begin{array}{l}\text { Similar to } \\
\text { human } \\
\text { type, but } \\
\text { often poorer }\end{array}$ & $\begin{array}{l}\text { Very slow. } \\
\text { Just visible } \\
\text { in } 4 \text { weeks } \\
\text { as pearly } \\
\text { white hemi- } \\
\text { spherical } \\
\text { colonies, } \\
\text { sometimes } \\
\text { with narrow } \\
\text { frilled mar- } \\
\text { gin, or ocea- } \\
\text { sionally } \\
\text { with wide- } \\
\text { spread } \\
\text { outgrowth } \\
\text { having } \\
\text { fern-like } \\
\text { markings on } \\
\text { surface. } \\
\text { Colonies } \\
\text { may be } \\
\text { conical and } \\
\text { granular, } \\
\text { blackherry- } \\
\text { like, or } \\
\text { umbilicated. }\end{array}$ & $\begin{array}{l}\text { Similar to } \\
\text { human type, } \\
\text { but surface } \\
\text { is more } \\
\text { smooth and } \\
\text { less granular }\end{array}$ & $\begin{array}{l}7 d .22^{\circ} C, \\
\text { Moderate, } \\
\text { slightly } \\
\text { raised, } \\
\text { confluent } \\
\text { growth with } \\
\text { moist, } \\
\text { glistening, } \\
\text { finely granu- } \\
\text { lar surface }\end{array}$ & $\begin{array}{l}7 d .22^{\circ} C \text {.or } \\
37^{\circ} C \text {. Abun- } \\
\text { dant, con- } \\
\text { fluent, often } \\
\text { pigmented, } \\
\text { raised } \\
\text { growth witl } \\
\text { dull, dry, } \\
\text { finely } \\
\text { granular sur- } \\
\text { face; growth } \\
\text { may be } \\
\text { irregularly } \\
\text { heaped up. }\end{array}$ \\
\hline $\begin{array}{l}5 \% \\
\text { Glycerine } \\
\text { egg. } \\
4 \text { weeks } \\
\text { at } 37^{\circ} \mathrm{C} \text {. }\end{array}$ & $\begin{array}{l}\text { More luxuri- } \\
\text { ant, raised, } \\
\text { confluent, } \\
\text { greyish- } \\
\text { yellow } \\
\text { growth, with } \\
\text { coarsely } \\
\text { granular } \\
\text { surface; } \\
\text { growth } \\
\text { irregularly } \\
\text { nodular and } \\
\text { heaped up } \\
\text { in places }\end{array}$ & $\begin{array}{l}\text { Similar to } \\
\text { growth on } \\
\text { Dorset egg }\end{array}$ & $\begin{array}{l}\text { Sometimes no } \\
\text { growth. If } \\
\text { colonies do } \\
\text { develop they } \\
\text { are slightly } \\
\text { larger than } \\
\text { on egg, } \\
\text { ivory-white, } \\
\text { conical, with } \\
\text { smooth or } \\
\text { rough } \\
\text { surface, } \\
\text { and frilled } \\
\text { margin }\end{array}$ & $\begin{array}{l}\text { Sometimes } \\
\text { similar to } \\
\text { human type, } \\
\text { but usually } \\
\text { more luxuri- } \\
\text { ant, of a } \\
\text { creamy- } \\
\text { yellow or } \\
\text { slightly } \\
\text { pinkish } \\
\text { colour, with } \\
\text { a smoother } \\
\text { surface ; } \\
\text { growth often } \\
\text { resembles } \\
\text { butter- } \\
\text { cream }\end{array}$ & $\begin{array}{l}7 \text { d. } 22^{\circ} C \text {. } \\
\text { Good, raised, } \\
\text { confluent } \\
\text { growth, with } \\
\text { relatively } \\
\text { smooth sur- } \\
\text { face; often } \\
\text { resembles } \\
\text { butter- } \\
\text { cream }\end{array}$ & $\begin{array}{l}7 \text { d. } 22^{\circ} C . \text { or } \\
37^{\circ} C . \\
\text { Luxuriant } \\
\text { growth, } \\
\text { raised, dry ; } \\
\text { yellow, pink, } \\
\text { or brick-red } \\
\text { in colour, } \\
\text { with a } \\
\text { coarsely } \\
\text { granular sur- } \\
\text { face resem- } \\
\text { bling dry } \\
\text { bread- } \\
\text { crumbs. }\end{array}$ \\
\hline $\begin{array}{l}5 \% \\
\text { Glycerine } \\
\text { agar. } \\
\mathbf{4} \text { weeks } \\
\text { at } 37^{\circ} \mathrm{C} \text {. }\end{array}$ & $\begin{array}{l}\text { Good, some- } \\
\text { times lux- } \\
\text { uriant, gen- } \\
\text { erally con- } \\
\text { fluent, } \\
\text { raised, } \\
\text { ereamy- } \\
\text { white } \\
\text { growth with } \\
\text { a finely } \\
\text { granular, } \\
\text { scaly, or } \\
\text { wrinkled } \\
\text { surface }\end{array}$ & $\begin{array}{l}\text { Poor growth } \\
\text { of small, } \\
\text { discrete, } \\
\text { granular } \\
\text { colonies ; } \\
\text { sometimes } \\
\text { a thin, } \\
\text { effuse, con- } \\
\text { fluent film }\end{array}$ & $\begin{array}{l}\text { Extremely } \\
\text { thin trans- } \\
\text { lucent grey } \\
\text { layer, con- } \\
\text { sisting of } \\
\text { minute, } \\
\text { discrete, } \\
\text { flat, } \\
\text { glistening } \\
\text { colonies }\end{array}$ & $\begin{array}{l}\text { Sometimes } \\
\text { similar to } \\
\text { human type } \\
\text { but usually } \\
\text { more luxuri- } \\
\text { ant, creamy- } \\
\text { white, with a } \\
\text { paint-like } \\
\text { surface }\end{array}$ & $\begin{array}{l}\text { Luxuriant } \\
\text { growth in } \\
7 \text { days at } \\
22^{\circ} \mathrm{C} \text {.; } \\
\text { ereamy- } \\
\text { white and } \\
\text { paint-like }\end{array}$ & $\begin{array}{l}\text { Similar to } \\
\text { growth on } \\
\text { glycerine egg. }\end{array}$ \\
\hline
\end{tabular}


TABLE 29 (continued).

\begin{tabular}{|c|c|c|}
\hline & Human. & Bovine. \\
\hline $\begin{array}{l}5 \% \\
\text { Glyeerine } \\
\text { potato. } \\
4 \text { weeks } \\
\text { at } 37^{\circ} \mathrm{C} \text {. }\end{array}$ & $\begin{array}{l}\text { Luxuriant, } \\
\text { raised, con- } \\
\text { fluent, } \\
\text { cream- } \\
\text { coloured } \\
\text { growth, with } \\
\text { a nodular, } \\
\text { warty, or } \\
\text { worm-cast } \\
\text { surface }\end{array}$ & $\begin{array}{l}\text { Poor, dis- } \\
\text { crete or } \\
\text { confluent, } \\
\text { thin, effuse, } \\
\text { greyish } \\
\text { growth }\end{array}$ \\
\hline $\begin{array}{l}5 \% \\
\text { Glycerine } \\
\text { broth. } \\
\text { 8 weeks } \\
\text { at } 37^{\circ} C \text {. }\end{array}$ & $\begin{array}{l}\text { Thick, white } \\
\text { or eream- } \\
\text { colourer, } \\
\text { dull, } \\
\text { wrinkled } \\
\text { pellicle, } \\
\text { extending } \\
\text { up the sides } \\
\text { of the flask; } \\
\text { no turbidity; } \\
\text { slight grant- } \\
\text { lar or scaly } \\
\text { deposit }\end{array}$ & $\begin{array}{l}\text { 'lhin, greyish- } \\
\text { white film } \\
\text { over part of } \\
\text { surface, } \\
\text { slightly } \\
\text { nodular in } \\
\text { places. No } \\
\text { turbidity ; } \\
\text { slight finely } \\
\text { granular } \\
\text { deposit }\end{array}$ \\
\hline
\end{tabular}

\section{Antigenic By aggluti- structure nation, absorption of agglu- tinins, and complement. fixation, form a homogene- ous group}

Pathogeni- Natural discity ease in man, pigs, monkeys, dogs and parrots. Experiment. ally, pathogenic for guinea-pig and for mouse
Indistit guishable from the human group

Natural disease in bovines, pigs, cats, monkeys and man. Ex perimentally, pathogenic for guineapig, rabbit, cat, mouse, goat, vole, and calf

Saprophytic ?, but goor Sometimes $\quad 7 d .22^{\circ} \mathrm{C} . \quad 7 \mathrm{~d} .22^{\circ} \mathrm{C}$. or growth on similar to Profuse, $37^{\circ} \mathrm{C}$. Abunplain potato human type, raised, con- dant, raised, but usually fluent, confluent, profuse, crcam- often pig. confluent, coloured mented paint-like growth, with growth, with growth, with a coarsely coarsely slightly granular or nodular worm-cast surface surface

granular surface, looking like dry breadcrumbs.

Sometimes $\quad 4$ weeks $22^{\circ} \mathrm{C} .14$ days $22^{\circ} \mathrm{C}$. only a granu - No turbidity, or $37^{\circ} \mathrm{C}$. lardeposit at but thick Thick, the bottom; veil over the coarsely sometimes a bottom of the thick reil flask and over the bottom of the flask and part-way up the sides, made up of branching, interlacing colunns, like tripa. No turbidity ; no surface growth

Indistinguishable from the human groilp
Form a separ- Form a separate group ate group part-way up

the sides, made up of coarse inter-

lacing

columns ; no surface growth
Natural dis- Natural disease in voles. Experimentally pathogenic for voles, rats, mice and to a less degree for golden hamsters ease in birds and pigs. Experimentally, pathogenic for birds ; moderately pathogenic for rabbits, rats, and mice
Natural dis- Non-pathoease in cold- genic.

blooded animals and fish. Ex. perimentally, pathogenio for turtles, frogs, snails, lizards, and fish granular sur-

face pellicle, looking as if composed of dry breadcrumbs. May be yellowishwhite or pink : spreads up sides of tlask. No turbidity. Deposit of coarse granules.

Form a separate groupprobably divided into sub-groups. 
be described, very little more is known about it now than at the time of its original discovery. Numerous attempts have been made to cultivate it, and many different organisms have actually been isolated from the tissues of lepers, but it is by no means certain that the real causative organism of the disease has yet been grown in pure culture.

In 1901 Kedrowski cultivated, apparently from 4 cases of leprosy, a non-acid-fast diphtheroid bacillus showing true branching. In very young cultures, 10 to 14 hours old, most of the bacilli withstood decolorization with 5 per cent. $\mathrm{H}_{2} \mathrm{SO}_{4}$, but in older cultures the organisms were non-acid-fast except for the metachromatic granules. He stated that when injected into rabbits, the organisms became acid-fast after a residence of several weeks in the tissues. He thought that the bacillus belonged to the Actinomyces group, and that the acid-fast rods seen in human leprosy represented only one stage in the developmental cycle of a single pleomorphic species. In 1905 Émile-Weil obtained a growth of Gram-positive, acid-fast bacilli on a medium consisting of a mixture of glycerine glucose peptone agar and egg-yolk. Single colonies appeared about the 5th day, and increased in size till the 15th or 20 th day. Cultures were likewise successful in hens' eggs, the leprous juice being inoculated directly into the yolk of the whole egg. Subcultures were never obtained. In 1909 Clegg cultivated from 8 out of 10 lepers a weakly arid-fast chromogenic bacillus. The primary cultures were obtained in symbiosis with amcebæ and cholera vibrios; by heating these cultures to $60^{\circ} \mathrm{C}$. for 30 minutes, he obtained a pure culture of the acid-fast bacillus. The organisms, both morphologically and culturally, resembled the ordinary saprophytic acid-fast bacilli; they resisted decolorization with alcohol for 3 minutes, but were largely decolorized by 5 per cent. $\mathrm{HCl}$ in 2 minutes. Cultures on agar were of a bright orange colour. Local lesions resulted from injection of the bacilli into guinea-pigs, similar apparently to those following injection of such organisms as Myco. phlei and the smegma bacillus.

In 1910 Duval cultivated from 4 cases of leprosy a non-chromogenic acid-fast bacillus; cultures were made on an agar or banana medium enriched with a 1 per cent. solution of cysteine or tryptophan. Glistening white colonies 1-2 mm. in diameter appeared after 1 to 2 months at $32-33^{\circ} \mathrm{C}$, , but not at $37^{\circ} \mathrm{C}$. The organisms were nearly as acid-fast as the tubercle bacillus, but were decolorized by 30 per cent. $\mathrm{HNO}_{3}$ followed by 95 per cent. alcohol; they failed to grow on ordinary media. When injected, even in small numbers, subcutaneously or intraperitoneally into Japanese dancing mice, they gave rise to glistening white nodules, resembling miliary tubercles; these were disseminated throughout the body, but were especially numerous in the spleen and lymph nodes. These nodules resembled the human lesions very closely; they contained acid-fast bacilli in enormous numbers, chiefly intracellular in position. Injection into guinea-pigs, rabbits, rats, and mice was without effect. In the same year, Twort (1910) isolated an acid-fast bacillus from the nasal discharge of a leper by growth on an egg medium containing a glycerine extract of dead tubercle bacilli. Growth was not visible for 6 weeks. In 1911 Rost cultivated an acid-fast, chromogenic, highly pleomorphic bacillus from 3 cases of nodular leprosy at Rangoon. Primary isolation was obtained on a milk broth medium containing volatile alkaloids from rotten fish. Growth occurred in 3 days, and was yellow, pink, or orange-red in colour. A monkey injected repeatedly by different routes developed small nodules under the skin containing acid-fast bacilli. Using a medium similar to Rost's, but in which the rotten fish distillate was replaced by distilled water, Williams in 1911 cultivated two organisms from 5 cases of leprosy in Persia and Bombay; one was a non-acid-fast streptothrix, the other an acid-fast bacillus like Rost's. In old cultures of the latter organism in liquid media, a non-acid-fast diphtheroid appeared.

In 1912 Bayon (1911-12) isolated a non-acid-fast diphtheroid bacillus, sometimes showing branching, from a case of nodular leprosy in London; it was morphologically identical with Kedrowski's bacillus. Intraperitoneal injection into a rat caused a lump at the site of inoculation 3 months later the lump was punctured, and the fluid that 
was withdrawn showed leucocytes filled with acid-fast bacilli arranged in ray fungus form. Cultivation from this lump yielded a white, slowly growing, wrinkled culture of an organism that was moderately acid-fast, resisting 20 per cent. $\mathrm{HNO}_{3}$ for 3 minutes. From the same leper he also obtained a pleomorphic diphtheroid bacillus that resisted 2 per cent. $\mathrm{H}_{2} \mathrm{SO}_{4}$ for 3 seconds. Injection of this organism into a mouse produced lesions in the spleen, liver, and lungs; these lesions contained clumps of acid-fast bacilli. Bayon concludes that the bacillus of human leprosy has a non-acid-fast, a weakly acid-fast, and a fully acid-fast stage. In 1912 Duval and Wellman reported on 29 cases of leprosy; from 14 they isolated an acid-fast chromogenic bacillus like Clegg's; from 8 an acid-fast nonchromogenic bacillus similar to Duval's; and from 1 a non-acid-fast diphtheroid bacillus like Kedrowski's. Injection of the $\mathbf{2}$ acid-fast strains into a number of animals, including monkeys, caused small lesions, which, however, could not be differentiated from those produced by the saprophytic acid-fast bacilli. In 1912 Currie, Clegg and Hollmann stated that they had isolated by Clegg's method an acid-fast bacillus 16 times from 15 cases of leprosy. All strains were chromogenic, and were culturally similar to the saprophytic acid-fast bacilli ; they could be distinguished from these, however, by agglutination with a specific antiserum prepared by injection of the horse, though they were rarely agglutinated by the sera of lepers. A similar organism was isolated by MeCoy in 1914 from 11 out of 83 specimens of leprous tissue; cultures were obtained with about equal facility whether amœbæ were present or not. The organism was incapable of producing leprosy-like lesions in laboratory animals.

More recently a number of apparently successful attempts to cultivate the leprosy bacillus have been recorded. Shiga (1929) stated that by treating lepra nodules with 5 per cent. $\mathrm{H}_{2} \mathrm{SO}_{4}$ and inoculating them on to glycerol potato, he was able to obtain minute colony formation in abont 2 months. Subcultures were carried on for four generations. Schlossmann (1930, 1933) claimed to have obtained growth in sealed tubes of Martin's broth in 4 months, and Sonnenschein (1930) in sealed tubes of glycerol egg in 2 months. The most hopeful results, however, have been recorded by Soule and McKinley (1932), and Soule (1934). These workers inoculated saline suspensions of excised lepra nodules on to several different media, and following Wherry's (1930) recommendation, incubated the cultures in varying partial pressures of oxygen and carbon dioxide. In a number of instances they succeeded in obtaining colony formation after 6 weeks at $37^{\circ} \mathrm{C}$. Several media yielded growth, including glycerol potato, Dorset egg, Petroff egg, and hormone glycerol agar. The most favourable gaseous conditions were afforded by a mixture of 40 per cent. oxygen and 10 per cent. $\mathrm{CO}_{2}$. Sixty serial subcultures were made over a period of 6 years (McKinley and de Leon 1937). The colonies themselves were about $1 \mathrm{~mm}$. in diameter, heaped up, non-chromogenic, with a mucoid appearance and a loose filamentous border. Microscopically they consisted of acid-fast bacilli. Similar suceess is said to have attended the cultivation of the bacilli in a minced chicken embryo medium inculrated under suitable partial pressures of $\mathrm{O}_{2}$ and $\mathrm{CO}_{2}$ (McKinley and Verder 1933). Salle and Moser (1937) report snccess by both these methods, but Lowe and Dharmendra (1937) and Dharmendra and Lowe (1938) obtained eompletely negative results in an extensive trial. (For further review of bacteriology of leprosy, see McKinley 1934, Soule and MeKinley 1938.)

This résumé, which is by no means complete, illustrates the variety of organisms that have been cultivated from leprosy. It will be seen that they fall into three classes: (i) diphtheroid bacilli, which are either non-acid-fast or weakly acid-fast, (ii) chromogenic acid-fast bacilli, and (iii) non-chromogenic acid-fast bacilli. What relation, if any, these organisms bear to leprosy, it is at present impossible to say. We are faced with three possibilities: either (i) they are contaminating organisms that have nothing to do with the causation of leprosy; or (ii) they are different stages in the life-history of the true leprosy bacillus; or (iii) they are organisms whose presence is in some way associated with that of the true leprosy bacillus, which has 
not yet been cultivated. Agglutination tests carried out with the serum of lepers do not help in determining the rtiological significance of these organisms, for it has been found that tubercle bacilli and saprophytic acid-fast bacilli are agglutinated as well as the bacilli cultivated from leprosy (Duval and Wellman 1912). And since it is impossible to reproduce typical leprosy in laboratory animals by inoculation even with ground-up leprous material, it is clear that animal inoculation tests are likewise useless in deciding this question.

There is reason to believe that the non-chromogenic acid-fast bacilli cultivated by various workers were in fact the real leprosy bacillus. Examination, however, of the records reveals the fact that, though colonial development was obtainable in the first two or three subcultures, attempts to carry on the bacilli indefinitely in subculture almost invariably failed. Even in Soule and McKinley's work, which incidentally Schlossmann (1933), Duval and Holt (1934a), Holt (1934a, b), and Dharmendra and Lowe (1938) have failed to confirm, more and more difficulty was experienced in obtaining growth with each suceessive subculture. Duval (1910) many years ago brought evidence to show that leprosy bacilli were able to grow in artificial media only so long as some of the original human lepromatous tissue persisted in the culture, and that the bacilli derived their nutriment almost entirely from the autolytic prodncts of this human protein material (see also Duval 1934). These findings are supported by the more recent work of Schlossmann (1933). If they are correct, they seem to afford an explanation of many of the observed facts. Whether it will be possible, as Duval and Holt (1934b) anticipate, to replace the natural autolytic tissue products by proteinsplit substances derived from other sources, is still doubtful. We may conclude tentatively that the leprosy bacillus has been grown in culture, but that its indefinite subcultivation in artificial media has never been unequivocally demonstrated.

In these circumstances it is regrettable that a number of stock cultures in various collections are labelled Mycobacterium lepres, and that so much work has been expended in studying the various properties of these so-called leprosy bacilli. Many of the strains are undoubtedly ordinary saprophytic acid-fast bacilli, and their description under a false name can do nothing but cause confusion in the literature. (For attempts at experimental reproduction of leprosy, see Chapter 60.)

\section{The Rat Leprosy Bacillus}

This organism was first described by Stefansky (1903) in 1901 at Odessa, where it was giving rise to a leprosy-like disease in rats. The organisms, which have never yet been definitely cultivated, are 3-5 $\mu$ long, are often slightly curved, and have rounded ends. They are Gram-positive and strongly acid-fast, withstanding 5 per cent. $\mathrm{H}_{2} \mathrm{SO}_{4}$ and 95 per cent. alcohol for at least 5 minutes. Staining is often granular. According to Marchoux (1933) the rat leprosy bacillus is killed by exposure to moist heat at $60^{\circ} \mathrm{C}$. for 15 minutes. It cannot withstand drying, but it remains viable for 2 years or more in infected organs preserved in 40 per cent. glycerol at $0-6^{\circ} \mathrm{C}$. From the fact that human leprosy cannot be conveyed to rats, it is probable that the leprosy and the rat leprosy bacilli are different. There is reason to believe that some cases of human leprosy may be caused by the rat leprosy bacillus (see Chapter 60). (For experimental reproduction of the disease in rats, see Chapter 60.) 


\section{Johne's Bacillus}

T'his organism was found by Johne and Frothingham (1895) in a chronic disease of cattle characterized by massive infiltration of the intestinal tract. It was believed at first to be an avian type of tubercle bacillus, but subsequent work has rendered it clear that it is a different species of organism.

Morphologically it is a short, thick rod, 1-2 $\mu$ long, generally straight, with rounded ends and parallel or slightly bulging sides; it is non-motile, Grampositive, and strongly acid-fast. Staining is generally uniform, but may occasionally be granular. It was first isolated in pure culture by Twort in 1910, on a glycerine egg medium containing dead tuberele bacilli (Twort and Ingram 1912, 1913). Subsequently it was found that the saprophytic acid-fast bacilli could replace the tubercle bacilli, and that a glycerine extract of the organisms, or even liquid tuberculin, could be used (I·Fadyean ct al. 1912). Twort and Ingram advise the following medium: Cultures of Myco. phlei are killed by steaming; the growth is scraped off and dried; the bacilli are then ground in a mortar, and added in 0.5-1.0 per cent. concentration to Dorset's egg medium containing 4 per cent. of glycerine. On this medium primary cultures of Johne's bacillus consist of tiny, dull-white colonies, rarely visible to the naked eye in less than 4 weeks; they are more or less circular in shape, and may be discrete or confluent. As they grow older, they increase in size, become more elevated, and turn a dull yellowish-white colour; the edges remain thin, and from them numerous irregular striations rise towards the central peak. In subcultures the growth is more copious, more confluent, and may be slightly wrinkled. If the saline in Dorset's egg medium is replaced by peptone beef broth, the growth is still better; and if sheep's brain broth or horse liver extract is used, the growth cannot be distinguished from a Dorset's egg culture of human tubercle bacilli. On glycerine agar containing phlei extract the growth is slower and less vigorous. In glycerine broth with phlei extract added growth occurs in the form of a thin surface film, which may later show irregular areas of thickening. After several subcultures growth is more profuse, and may even occur in plain glycerine broth without the addition of phlei extract. Growth occurs between $28^{\circ} \mathrm{C}$. and $43^{\circ} \mathrm{C}$, the optimum temperature being $39^{\circ} \mathrm{C}$. In culture the bacilli are very short, often only about $1 \mu$ in length; there is no branching, but occasional club forms are seen. Primary cultures are best made by treating the washed intestinal mucosa with 20 per cent. antiformin for about 30 minutes, and seeding on to a glycerine egg medium containing dead phlei or tubercle bacilli (see Minett 1942). In what way the added organisms serve to promote the growth of Johne's bacillus is not known; they probably provide some nutrient substance, or enzyme, necessary for its metabolism; but that this substance is not specific to acid-fast bacilli is shown by the fact that alcoholic extracts of such diverse substances as currant. grapes, figs, oats, linseed, and the fungus Cantharellus aurantiacus, are all able to replace the acid-fast bacilli (Twort and Ingram 1914).

Whether the bacilli giving rise to Johne's disease in sheep are identical with those in cattle is not clear. Apparently they are more difficult to grow, and in primary culture may take 6 or 7 months to develop (Dunkin and Balfour-Jones 1935). On the other hand McEwen (1939) claims to have infected calves by feeding them with material from diseased sheep, so that the difference between the bovine and ovine bacilli may be less than Dunkin (1936) suggests. 
Reproduction of the Disease in Animals.-The injection intravenously, intraperitoneally, or subcutaneously of pure cultures of Johne's bacillus into bovine animals frequently gives rise to the typical disease, from the lesions of which pure cultures of the bacillus can be recovered (Twort and Ingram 1913). Feeding may also be successful. Sometimes goats and sheep can be infected by inoculation of pure cultures; lambs are said to be more susceptible than adult sheep (McEwen 1939). Reproduction of the disease in laboratory animals has not so far been successful. Johne and Frothingham (1895) and Twort and Ingram (1913) found that guinea-pigs, rats, and mice were refractory. Later Twort and Craig (1913) found that the intraperitoneal inoculation of 100-120 mgm. of bacilli into rabbits gave rise in the abdominal cavity to a few nodules which were slightly caseous; the animals remained perfectly well, and showed no signs of toxic disturbances. The same lesions were produced by Myco. phlei. Boquet (1925) found that the intraperitoneal injection of 5-10 $\mathrm{mgm}$. of culture into white rats gave rise to pin-head, greyish nodules on the surface of the peritoneum and omentum; these nodules contained pus very rich in bacilli. The mesenteric glands were enlarged; the tracheo-bronchial glands were enlarged, hard, and sclerotic, and contained enormous numbers of bacilli. Even more marked lesions were obtained when the injection was repeated in 15 to 20 days with a dose of 10-30 mgm. White mice developed similar but less chronic lesions. It is doubtful whether these changes can be considered specific for Johne's bacillus; similar lesions can often be produced by the saprophytic acid-fast bacilli. It is certain that no one has yet reproduced in laboratory animals the typical enteritis of the natural disease.

\section{REFERENCES}

Aksianzew, M. I. (1933) Z. Tuberk., 68, 249.

Albiston, H. E. (1930) Aust. vet. J., 6, 123.

Alvarez and Tavel. (1885) Areh. Phys. norm. Path., 6, 303.

Anderson, R. J. (1932) Physiol. Rev., 12, 166.

Anderson, R. J. and Colleagues. (1927 et seq.) 34 papers, mainly in $J$. biol. Chem., 1927-33, and 3 papers in $Z$. physiol. Chem. (1930), 191, 157, 166, 172.

Aronson, H. (1898) Berl. Klin. W schr., 35, 484.

Aronson, J. D. (1926) J. infect. Dis., 39, 315.

BaLL, E. G. (1934) J. biol. Chem., 106, 515.

Batalllon, E., Dubard, and Terre, L. (1897) C. R. Soc. Biol., 49, 446.

Bayon, H. (1911-12) Trans. R. Soc. trop. Med. Hyg., 5, 158.

Beaven, P. W. and Bayne-Jones, S. (1931) J. infect. Dis., 49, 399.

Веск, M. (1905) TuberkArb., 3, 145.

BegBie, R. S. (1930) Edint. med. J., 3\%. 187; (1931) Ibid., 38, 173.

Beitzke, H. (1910) Berl. klin. Wschr., 4\%, 1451.

BirkhadG, K. E. (1935) Ann. Inst. Pasteur, 54, 19.

Blacklock, J. W. S. (1932) Spec. Rep. Ser. med. Res. Coun., Lond., No. 172.

Boquet, A. (1925) C. R. Soc. Biol., 93, 219.

Bozzeltr, R. (1914) Ann. Staz. Mal. Best. Napoli, 2, 77.

Brem, W. V. (1909) J. Amer. med. Ass., 53, 909.

BRUNer, D. W. (1934) J. infect. Dis., 55, 26.

Broynoghe, R. and Adant, 11. (1933) C. R. Soc. Biol., 111, 1051.

Bulloch, W. and Macleod, J. J. R. (1904) J. Hyg., Camb., 4, 1.

Cadiot, P. J., Gilbert, A., and Roger, H. (1890) C. R. Soc. Biol., 42, 92.

CALDWELL, II. E. (1925) J. infect. Dis., 37, 465.

CaLmetTe, A. (1936) "L'infection bacillaire et la tuberculose ehez l'homme et chez les animaux." 4th ed. Masson et Cie, Paris.

Calmette, A., Valtis, J., Nègre, L., and Boquet, A. (1925) C. R. Acad. Sci., 181, 491.

Chargaff, E., Pangborn, M. C., and Anderson, R. J. (1931) J. biol. Chem., 90, 45.

Clege, M. T. (1909) Philipp. J. Sci., B, 4, 403. 
Coвbett, L. (1917) "The Causes of Tuberculosis" Cambridge; (1932) J. P'uth. Bact., 35,681 .

Comming, W. M. (1925) Tubercle, Lond., \%, 105.

Cummins, S. L. and Williams, E. M. (1933) Tubercle, Lond., 15, 49.

Currie, D. H., Clege, M. T., and Hollmand, H. T. (1912) Publ. Hlth Bull., Wash., No. 47.

Czaplewski, E. (1897) Münch. med. Wschr., 44, 1192.

Dalling, T. and O'Brien, R. A. (1935) Brit. med. J., i, 897.

Darzine, E. (1932) Ann. Inst. Pasteur, 49, 743.

Davies, R. (1937) J. Path. Bact., 45, 773.

Denys, P. (1935) "Contribution à l'étude de la variabilité du virus tubereuleux." Imprimerie Saint-Alphonse, Louvain.

Dernby, K. G. and Nüslund, C. (1922) Biochem. Z., 132, 393.

Dharmendra and Lowe, J. (I938) Indian J. med. Res.. 25, 835.

Dieckmans, H. and Ienzel, G. (1932) Z. Hyg. Infektkr., 113, 709.

DOERR, R. and GoLD, E. (1932) Z. ImmunForsch., 74, 7.

Douglas, S. R. and Hartley, P. (1934) Tubercle, Lond., 16, 97.

Dreyer, G. and Vollum, R. L. (1931) Lancet, i. 9.

Dunkin, G. W. (1936) I'roc. roy. Soc. Med., 30, 83.

Dunkin, G. W. and Balfour-Jones, S. E. B. (1935) J. comp. Path., 48, 236.

Dunkin, G. W., Laidlaw, P. P., and Griffith, A. S. (1929) J. comp. Puth., 42, 46.

Duval, C. W. (1910) J. exp. Med., 12, 619; (1934) Proc. Soc. exp. Biol., N.Y., 32, 498.

Duval, C. W. and Holt, R. A. (1934a) Proc. Soc. exp. Biol., N.Y., 31, 453; (1934b) Ibid., 31,828 .

Duval, C. WV. and Wellman, C. (19I2) J. infect. Dis., 11, 116.

Eastivood, A. and Griffith, F. (1916) J. Hyg., Camb., 15, 257.

EнrLich. (1882) Dtsch. med. ITschr., 8, 269.

Eidinow, A. (1927) Brit. med. J., ii. 160.

Émile-Weil, P. (1905) Ann. Inst. Pasteur, 19, 793.

Evans, B. and Hanks, J. H. (1939) Proc. Soc. exp. Biol., N.Y., 40, 112.

Fielding, J. W. (1934) Aust. J. exp. Biol., 12, 1.

Fite, G. L. and Olson, B. 1. (1944) Publ. Illth Rep., W'ash., 59, 1423.

Fontès, A. (1910) Mem. Inst. Osw. Cruz, 2, 141.

Friedmann, F. F. (1903) Zbl. Bakt., 34, 647, 793.

Erimont-Mö́lér, J. (1939) "Dissociation of 'Tubercle Bacilli. Investigations on the mammalian types including B.C.G." H. K. Lewis and Co., Ltd., London.

Frouin, A. and Guilladmie. (1923a) C. R. Soc. Biol., 88, 1002; (1923b) Ibid., 88, 1095.

Furth, J. (1926) J. Immunol., 12, 273.

Fust, B. (1938) Z. Hyg. InfehtKr., 120, 547.

GLover, R. E. (1944) Brit. J. exp. Path., 25, 141.

Gloyne, S. R. (1933) Bull. Hyg., Lond., 8, 39.

Gordon, R. E. and Hagan, W. A. (1937) Amer. Rev. Tuberc., 36, 549.; (1938) J. Bact., $36,39$.

Govor, G. A. C. (1932) Biochem. J., 26, 248; (1933) Ibid.. J., 2\%, 1049.

Grassberner, R. (1899) Münch. med. Wschr., 46, 341, 382.

Griffith, A. S. (1916) Lancet, i. 721 ; (1916-17) J. Path. Bact., 21, 54 ; (1924) Tubercle, Lome. 5, 569 ; (1925) Tubercl, Lond., 6, 4I7 ; (1931) J. comp. Path., 44, 144; (1939a) J. IIyg. Camb., 39, 244; (1939b) Ibid., 39, 154; (1941 a) Ibid., 41, 250; (1941b) Ibid., 41, 260; (1941c) Ibit., 41, 284; (1941d) Ibid., 41, 289; (194le) Tubercle, Lond., 22, 33; (1942) $J$. Hyg., Camb., 42, 527.

Griffith, A. S. and Dallina, T'. (1940) J. Hyg., Camb., 40, 673.

Grolier, A. De. (1933) C. R. Soc. Biol.. 113, 1506.

GrüNBERG, B. (1935) Z. Tuberk., 73, 197.

Gunn, F. D., Nungester, W. J., and Hougen, E. T. (1934) Proc. Soc. exp. Biol., N.Y., $31,527$.

HAAG, F. E. (1927) Zbl. Bakt., IIte Abt., 71, 1.

Hagan, W. A. and Levine, P. (1932) J. Amer. vet. med. Ass., 81, 723.

Hansen, G. H. A. (1874) Norsk. Mag. Laegevidensk.

Harden, A. (1913) Rep. roy. Comm. Tuberc., Lond., 6, part ii. Appendix.

Harpoth, H. (1938) Z. Tuherk.. 79, 140.

Herman, M. (1908) Ann. Inst. Pasteur, 22, 92.

Holt, R. A. (1934a) Proc. Soc. exp. Biol., N.Y., 31, 567 ; (1934b) Ibid., 31, 643.

Hooper, F. E., Renfrew, A. G., and Johnson, T. B. (1934) Amer. Rev. Tuberc., 29, 66.

IsHiмoRI, K. (1924) Z. Hyg. Infekt $K^{\prime}$., 102, 329.

JACOBITZ and KAYSER, H. (1910) Mïnch. med. Wschr., 5\%, 1175.

JENSEN, K. A. (1932) Zbl. Bakt., 125, 222.

Jansen, K. A. and Frimodt-Möller, J. (1936) Acta tuberc. Scand., 10, 83, 217.

Johne, H. A. and Frothingham, L. (1895) Dtsch. Z. Tiermed., 21, 438. 
KaHN, M. C. (1930) T'ubercle, Lond., 11, 202.

Ki.rLinski, J. (1901) Zbl. Bakt., 29, 521.

Karwacki, L. and Biernacki, S. (1925) Ann. Inst. Pasteur, 39, 476.

KadFFMAN, F. (1932) Z. IIyg. Infekthr., 114, 121.

liedrowski, W. J. (1901) Z. Hyg. InfektKr., 3\%, 52.

Kendall, A., Day, A., and Walker, A. (1920) J. infect. Dis., 26, 45.

Kineffer, J. (1921) Amer. Rev. Tuberc., 5, 662.

KLopstock, F. (193I) Klin. Jischr., 10,967.

Косн, R. (1882) Berl. Klin. II schr., 19, 221 ; (1886) "Microparasites in Disease." New Sydenhain Soc., London.

Korn, O. (1899) Zbl. Bakt., 25, 532; (1900) Ibid., 27, 481.

Kossel, H., Weber, A., and Heuss. (1901) TuberkArb., 1, 1 ; (1905) Ibid., 3, 1.

Krads, R. and Geriach, F. (1929) Z. Immun Forsch., 62, 339.

Kraus. R. and Koref, O. (1933) Z. Tuberk., 6\%, 42.

Kretschner, O. S. (1934) J. Lab. clin. Med., 19, 350.

KÜÜTER, E. (1905) Münch. med. Wschr., 52, 57.

l.aidlaw, P. P. and Dudley, H. W. (1925) Brit. J. exp. Puth., 6, 197.

LANGE, B. (1922) Z. IIyg. InfektKr., 98, 229.

l.ANGE, L. (1932) See Kolle, IT. (1932) Dtsch. med. I'schr., 58, 304.

l.arson, W'. P'. (1926) Lancet, ii. 1231.

l.ASER, H. (1897) Wünch. med. IV schr., 44, 1191.

Ledodx-Lebard. (1898) C. R. Sor. Biol., 50, 610; (1900) Ann. Inst. Pusteur, 14, 535.

Long, E. R. (1926) Amer. Rev. Tuberc., 13, 393.

Long, E. R. and Seibert, F. B. $(1926,1928)$ See series of I0 papers in Amcr. Rev Tuberc.; $13,1 \%$.

Lowe, J. and Dharmendra. (1937) Indian J. med. Res., 25, 329.

McCarter, J. R. and Tatum, E. L. (1937) Ann. Rep. Director, 1935-36, Agric. exp. Sta. Univ. Wisconsin, Bull., No. 438.

McCoy, G. W. (1914) Publ. Hlth Bull., Wash., No. 61, p. 27.

McEwen, A. D. (1939) J. comp. Path., 52, 69.

M'Fadrean, J., Sheather, A. L., and Edwards, J. T. (1912) J. comp. Palh., 25, 217., MCKInLey E. B. (1931) Medicine, Baltimore, 13, 377.

McKinley, E. B. and Leon, IT. DE (1937) Int. J. Leprosy, 5, 259.

McKinley, E. B. and Verder, E. (1933) Proc. Soc. exp. Biol., N.Y., 30, 659.

11 ADDOCK, E. C. G. (1933) J. Hyg., Camb., 33, 103.

II Affucci, A. (1890) Zbl. allg. Path. path. Anat., 1, 409 ; (1892) Z. IIyg. InfektKr., 11, 415. Marchoux, E. (1933) Rev. franç. Derm. Vénéréol., 9, 323.

Marchoux, E. and Halphen, E. (1912) C. R. Soc. Biol., 73, 249.

Marzinowskx, E. J. (1900) Zbl. Bukt., 28, 39.

MAYER, E. (1921) Amer. Rev. Tuberc., 5, 75.

Mayer, E. and Dwonski, M. (1932) Amer. Rev. Tuberc., 26, 105.

IIEANwell, L. J. (1927) J. Hyg., Camb., 26, 392.

Meiszner, I. and P'radsnitz, C. (1934) Zbl. Bakt., 132, 23.

Menzel, A. E. O. and Heidelberger, II. (1939) J. biol. Chem., 124, 89, 301.

Merrill, M. H. (1930) J. Bact., 20, 235.

Miller, J. (1905) J. Path. Bact., 10, 1.

MinetT, F. C. (1942) J. Path. Bact., 54, 209.

Mironesco, T. (1901) Z. Hyg. InfektKr., 37, 497.

Moeller, A. (1899) Dtsch. med. IYschr., 24, 376 ; (1899) Zbl. Bakt., 25, 369 ; (1901) Ibid., $30,513$.

Mori, N. (1911) Ann. Stuz. Mal. Best. Napoli, 1, 327.

Mvch, H. (1907) Beitr. lilin. Tuberk., 8, 85.

Mueller, J. H. (1926) J. exp. Med., 43, 9.

Nocard and houx. (1887) Ann. Inst. Pasteur, 1, 19.

Novy, F. G. and Soule, M. H. (1925) J. infect. Dis., 36, 168.

Nutr, II. M. (1927) J. IIyg., Cumb., 26, 44.

Oerskov, J. (1932) Zbl. Bakt., 123, 271.

Oerskov, J.-L. (1925) C. R. Soc. Biol., 92, 400.

Park, IV. H. and Krumiede, C. (1910) J. med. Res., 23, 205.

P'ellegrino, P. L. (1906) Ann. Igiene. (sper.), 16, 163.

Petri, R. J. (1898) Arb. ReichsgesundhAmt., 14, 1.

Petroff, S. A. (1927) Proc. Soc, exp. Biol., N.Y., 24, 632, 956.

Petroff, S. A., Branch, A., and Stennkev, W. (1927a) Proc. Soc. exp. Biol., N.Y., 25, 11; (1927b) Amer. Rev. Tuberc., 19, 9.

Petroff, S. A. and Stennkex, W. (1930) J.exp. Med., 51, 831 ; (1935) J. infect. Dis., $56,277$.

Pinner, M. (1932) Proc. Soc. exp. Biol., N.Y., 30, 214.

Pottenger, J. E. (1942) Amer. Rev. Tuberc., 45, 549, 558. 
Pryce, D. M. (1941) J. Path. Bact., 53, 327.

Rabinowitsch, L. (1897) Z. IIyg. InfektKr., 26, 90; (1900) Dtsch. med. IIschr., 26, 257.

Ravenel. (1901) Trans. Brit. Congr. Tuberc., 3, 553.

Reed, G. B. and Rice, C. E. (1929) J. Bact., 17, 407 ; (1931a) Canarl. J. Res., 4, 389; (1931b) Ibid., 5, 111 .

Report. (1907) Royal Commission on Tuberculosis, 2nd interim Rep., H.M. Stat. Off., London; (1909) Ibid., 3rd interim Rep.; (1911) Ibid., Final Rep.; (1913) Ibid., Final Rep., Appendix.

Rнines, C. (1935) Amer. Rev. Tuberc., 31, 493.

Rice, C. E. (1931) Canad. J. Res., 5, 375.

Rice, C. F. and Reed, G. B. (I931) Canad. J. Res., 5, 122.

Rivolta. (I889) G. anat. Fisiol., 1, 122.

Pochaix, A. and Colin, G. (1911) C. R. Acad. Sci., 153, 1253.

Rockwell, G. E. and Highberger, J. H. (1926) J. infect. Dis., 38, 92.

Rost, E. R. (1911) Sci. Mem. med. Sanil. Dep., India, No. 42, p. 7.

Sabin, F. R. (1932) Physiol. Rev., 12, 14l.; (1938) J. exp. Med., 68, 837.

Sabin, F. R., Doan, C. A., and Forkner, C. E. (1930) J.exp. Med., 52, Suppl. No. 3.

Sabin, F. R., Miller, F. R., Doan, C. A., and Wiseman, B. K. (193I) J. exp. Mell., 53,51 .

SABIN, F. R. and JoYNeR, A. L. (1938) J. exp. Med., 68, S53.

SALle, A. J. and Moser, J. R. (1937) Int. J. Leprosy, 5, 163, 253.

Schatefer, W. (1935) C. R. Soc. Biol., 120, 1185.

Schaefer, IV. (1937) Ann. Inst. Pasteur, 58, 385.

Schlossmane, K. (1930) Zbl. Bakt., 115, 474; (I933) Ibid., 128, 369.

Sснмірт, H. (1925) Zbl. Bakt., 94, 94.

Schulte-Tigges, H. (1920) Disch. med. Wschr., 46, I225.

Schwa Bacier, H. (1933a) Spec. Rep. Ser. med. Res. Coun., Lond., No. 182, 1. 104; (1933b) Ibid., p. 124 .

Schwabacher, H. and Wilson, G. S. (1937) Tubercle, Loml., 18, 44:.

Sédyeh, A. and Seliber, G. (1927) C. R. Soc. Biol., 97, 57.

Seibert, F. B. (1941) Bact. Rev., 5, 69; (1944) Chem. Rev., 34, 107.

Seibert, F. B. and Glens, J. T. (1941) Amer. Rev. Tuberc., 44, 9.

Seibert, F. B., Lono, E. R., and Morley, N. (1933) J. infect. I)is., 53, 175.

Seibert, F. B., Pedersen, K. O., and Tiselius, A. (1938) Amer. Rev. Tuberc., 38, 399.

SEIFFERT, W. (1932) Z. ImmunForsch., 74, 116.

Shaffer, M. F. (1935) J. Path. Bact., 40, 107.

SheN, T. H. (1934) J. Shanghai Sci. Inst., Sect. iv. 1, 157.

SHIG. K. (1929) Zbl. Bakt., 114, 511.

Sнош B, H. L. (1923) J. Bact., 8, 121.

Sibley, W. K. (18S9) Zbl. Bakt., 5, S31; (1890) Lancet, i. S04.

Surth, C. R. (1942a) Amer. Rev. Tuberc., 45, 334; (1942b) Ibid., 46, 549.

Sмiтн, T. (I89S) J. exp. Med., 3, 451; (1S99) Ibid., 4, 217 ; (1944-5) J. med. Res., 8, 253

Suithburn, K. O. (1935) J. exp. Med., 62, 645.

Soltys, M. A. and TAYior, A. W. (1944) J. P'ath. Bact., 56, 173.

Sonnenschein. C. (1930) Zbl. Bakt., 11\%, 284.

Sordelli, A. and Arena, A. (I934) C. R. Soc. Biol., 11\%, 63.

Soule, M. H. (1928) J. infect. Dis., 42, 93; (1934) Proc. Soc. exp. Biol., N.Y., 31, 1197.

Sodle, M. H. and McKinley, E. B. (1932) Amer. J.trop. Med., 12, 1, 44 I ; (1938) Amer. Ass. Advancement Sci., Symposium Ser., 1, 87. Science Press, N.Y.

SPENGLeR, C. (1907) Dtsch. med. Wschr., 33, 337.

Stableforth, A. W. (1929) J. comp. Path., 42, 91.

Sizmatin, N. and Stanatin, L. (1939) Ann. Inst. Pasteur, 63, 269.

Steen-ren, W., Oatway, W. H., and Petroff, S. A. (1934) J.exp. Med., 60, 515.

Stefansix, W. K. (1903) Zbl. Bakt., 33, 481 .

Straus, I. and Gamaléia, N. (1891) Arch. Med. exp., 3, 457.

Sweany, H. C. (1928) Amer. Rev. Tuberc, 17, 53.

TauURa, S. (1913) Z. physiol. Chem., 87, 85.

Thonson, H. II. (1932) Amer. Rev. Tuberc., 26, 162.

Tiedemann, H. J. (1931) Zul. Bakt., 122, 483.

ToBler, M. (1901) Z. Hyg. InfektKr., 36, 120.

TODA, T. (193I) Z. Hyg. InfektKr., 112, 463.

Tulloch, W. J. (1936) Edinb. med. J., 43, 144.

Tulloch, W. J., Munro, W. T., Ross, G. R., and Cumming, IV. M. (1924) Tubercle, Lond., $6,18,57,105$.

Twori, C. C. and Craie, T. (19I3) Zbl. Bakt., 68, 455.

Twort, F. W. (1910) Proc. roy. Soc., B, 83, 156. 
Twort, F. W. and Ineram, G. L. Y. (1912) Proc. roy. Soc., B, 84, 517 ; (1913) "A Monograph on Johne's Disease." London, (1914) Zbl. Bakt., 73, 277.

ÜgA, T. (1935) Jap. J. exp. Hed., 13, I67.

UhLENHUTH, P. and SEIFFERT, W. (1930) \%. Immuntorsch., 69, 187.

UYEI, N. (1927) J. infect. Wis., 40, 433.

VAGEDES, (1898) Z. Hyg. InfektKr., 28, 276.

Villemin, J. A. (1868) "Etudes sur la Tuberculose." Paris.

WÁmoscher, L. and Stoecklin, H. (1927) Zbl. Bakt., 104, Beiheft, p. 86.

Wells, A. Q. (1937) Lancet, i, 1221 ; (1938) Brit. J.exp. Path., 19, 324; (1944) Pers. comm.

Wells, H. G. and Long, E. R. (1932) "The Chemistry of Tuberculosis." Bailliere, Tindall \& Cox, London.

WHERrY, W. B. (1930) J. infect. Dis., 46, 263.

Willians, R. S. and Hoy, IV. A. (1930) J. Hyg., Camb., 30, 413.

Williams, T. S. B. (1911) Sci. Hem. med. Sanit. Dep., India, No. 42, p. 15.

Wilsox, G. S. (1925) J. Path. Bact., 28, 69; (1930) J. Hyg., Camb., 30, 40 ; (1942) "The Pasteurization of Milk." Ed. Arnold \& Co., London.

Wolbach, S. B. and Ernst, H. C. (1904) J. med. Res., 12, 295.

WULFF, F. (1925) Acta path. microbiol. scand., 2, 149.

Wyckoff, R. W. G. (1934) Amer, Rev. Tuberc., 29, 389.

Wrokoff, R. IV. G. and Smithburn, K. C. (1933) J. infect. Dis., 53, 201.

Yegian, D. and Porter, K. R. (1944) J. Bact., 48, 83.

Yersin, M. A. (1888) Ann. Inst. Pasteur, 2, "45.

ZieHL, F. (1882) Dtsch. med. Wschr., 8, 451. 


\section{CHAPTER 17}

\section{CORYNEBACTERIUM}

Definition. Corynebucterium.

Gram-positive rod-like forms, arranged usually in a palisade. Not acid-fast. Olten with club-shaped swellings at the poles, generally with imegularly staining segments or granules. Non-motile, non-sporing. Growing aerobically or under microaerophilic conditions, but often capable of anaerobic cultivation. Never forming gas in carbohydrate media, in which they may or may not produce acidity. They may or may not liquefy gelatin or serum. Some species produce a powerful exotoxin.

Type species, Corynebacterium diphtheria.

The generic name Corynebacterium was allotted by Lehmann and Neumann in 1896 to the group of bacteria containing the diphtheria bacillus and other species resembling it in morphology. By its derivation the name emphasizes the tendency to the formation of club-like forms that is characteristic of the type species and of several other species within the generic group. This name was accepted by the Committee appointed by the Society of American Bacteriologists (Winslow et al. 1920), and was adopted as the valid generic name in the monograph on diphtheria issued under the ægis of the Medical Research Council in 1923 (Andrewes et al.). It is gaining increasing currency in the literature and is very unlikely to be superseded. The summary of the generic characters, as recorded by the American Committee, was emended by the Bacteriological Committee of the Medical Research Council by the omission of aerobiosis as a generic character, the addition of a reference to the fermentative activities of the group, and the omission of any reference to toxin production. There appear to us to be advantages in calling attention to a striking biological character possessed by the type species and perhaps shared by some related species. With the restoration of this reference to toxigenicity we should adopt the summary of generic characters as given by the Committee of the Medical Research Council.

Although $C$. diphtheriae is universally accepted as the type species, it was not in fact the first to be described. Reymond and his colleagues in 1881, and again in 1883, described the isolation from the conjunctival sac of a bacillus which is now recognized, under the name of $C$.xerosis, as belonging to this genus. This organism was described more fully by Kuschbert and Neisser in the latter year, which also witnessed Klebs' description of the diphtheria bacillus in the diphtheritic false membrane. It was not until 1884, the year following, that Loeffler published his classical paper on diphtheria, and provided a description of the causative organism which afforded a standard of reference for all subsequent studies on this bacterial group. Any claims that might have accrued to $C$. xerosis on account of priority would in any case have been vitiated by the fact that it is quite impossible at the present time 
to be sure that Reymond, and Kuschbert and Neisser, were dealing with a single bacterial species. It is, moreover, equally impossible to identify with certainty any of the strains now labelled $C$. xcrosis with those described in the early 'eighties. The stage of technical development that bacteriology had then reached did not permit each new organism that was isolated to be fully studied and described. The importance of $C$. diphtherice as a human pathogen focussed attention on the differentiation of the species from others with which it might be confused. Our picture of the type species thus became more and more complete as time went on; but to the related diphtheroids little attention was paid beyond that necessary to determine their probable relation to the diphtheria bacillus itself. As Andrewes and his colleagues point out it is extremely difficult to determine which of the strains, to which specific names have from time to time been assigned, represent well-differentiated species. Some of those who have reviewed the group as a whole have been liberal in the distribution of titles (Graham-Smith 1908, Mellon 1917, Eberson 1918, Chalmers and Macdonald 1920). Here, as elsewhere,

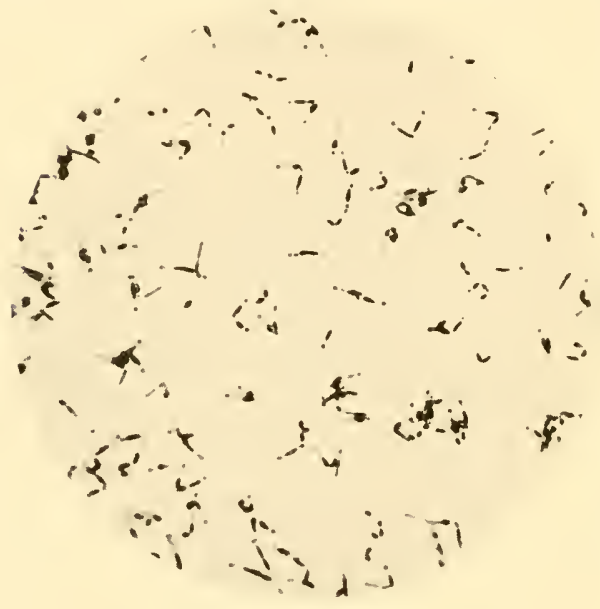

F'IG. 82.-C. diphtherio.

From 24-hours' culture on Loeffler's serum $(\times 1000)$. we propose to adopt a conservative view, and to list as species only those organisms which have been adequately described, and appear from this description to be reasonably well-differentiated. In discussing this question of classification and nomenclature in greater detail we may consider $C$. diphtheria as a species, since it has in fact been so regarded in most of the observations to which we shall refer. Recent work has, however, shown that it is divisible into three well-differentiated types, and that this differentiation is of considerable importance from the medical point of view. The discussion of this particular problem may, however, conveniently be dealt with in a separate section.

\section{Habitat.}

Though attention has in the past been concentrated almost entirely on the parasitic members of the Corynebacterium group, which live mainly on the skin and mucous surfaces of their animal host, there is reason to believe that some members are adapted to the saprophytic life. Diphtheroid bacilli are common in heated milk products (Mattick 1944), and appear to occur also in the soil. Many of the parasitic species are pathogenic, others form an important constituent of the normal bacterial flora of various hosts.

\section{Morphology.}

The club-form, from which the name is derived, is only one of many shapes which may be assumed by the individual cells of the type species, $C$. diphtheria. This organism is indeed characteristically pleomorphic. One of the most typical forms (see Fig. 82), in films prepared from a 24-hours' culture on Loeffler's serum, is that of a 
long, rather slender bacillus, of ten slightly curved, with rounded, somewhat swollen ends and sometimes with localized swellings elsewhere, and staining unevenly with such dyes as methylene blue: but in the same, or in other cultures, there will also be found much shorter forms, cells which stain solidly and evenly, cells in which the irregular staining takes the form of a series of transverse bars, and cells in which the combination of uneven staining and localized swellings gives to a single bacilius the appearance of a short chain of streptococci. This diversity of structure has led to attempts to classify the various forms of $C$. diphtherioe into definite types, indicated by names, numbers or letters, and to a description of individual strains in accordance with the predominant morphological form. It is quite true that different strains of this organism differ very markedly in morphology and that a notable frequency of one particular type of cell may characterize a particular strain throughout repeated subcultures. It seems, however, very doubtful whether it is wise to assign labels to different strains of this organism on the basis of morphological characters alone. Moreover, as Goldsworthy and Wilson (1942) have shown, the morphology of diphtheria bacilli varies greatly on different batches of Loeffler's serum, depending on its mode of preparation. A strain which, on one batch, develops into typical long, curved, granular forms may, on another batch, appear as short rods devoid of granules, closely resembling an ordinary diphtheroid bacillus.

The interest shown by the older workers in the morphology of the diphtheria bacillus as a criterion of differentiation from other members of the group was due mainly to the extensive use of stroke cultures on Loeffler's serum. Since the introduction of blood tellurite medium reliance has been placed to an increasing extent on the appearance of individual colonies, and morphology has been degraded to a secondary position. Furthermore, each of the three types of diphtheria bacillus now recognized, gravis, intermedius, and mitis, has been found to possess its own more or less characteristic morphology, with the result that microscopical examination is used more to distinguish between these three types than between the diphtheria bacillus and diphtheroid bacilli.

Another feature that characterizes $C$. diphtherice as a species, and serves to distinguish it from some, but by no means all, of the related "diphtheroids," is the presence of the metachromatic granules described by Babes (1886) and by Ernst $(1888,1889)$. These granules are coloured a reddish purple when a film preparation is stained with a suitable sample of methylene blue. They may be demonstrated more clearly by the differential stain devised by Neisser, or by one of its many modifications. A single cell may contain one or more of these granules, seldom more than half a dozen, usually two or three. When only one or two are present they show a definite tendency to be situated at one or both poles.

The arrangement of the bacilli in film preparations is at least as characteristic as the form of the individual cells. Adjacent cells tend to lie at any angle to one another, forming a $\mathrm{V}$ or an $\mathrm{L}$ according to the degree of angular displacement; and groups of such pairs form characteristic clusters, resembling Chinese letters, or cuneiform writing. It would appear, from the observations of Hill (1898-1902), that this particular arrangement results from incomplete separation at the moment of division, the daughter cells remaining attached at one point, and bending on this attachment as on a hinge as growth proceeds.

Finally, it may be noted that $C$. diphtheriae provided the first instance in which true branching was demonstrated in a bacillary species. The observations of Hill showed that this appearance was not an artefact, but could be observed to take 
place during the growth of the living cell. This character, among others, decided the American Committee to separate this genus from the Eubacteriales, and include it with some others in a family of the order Actinomycetales. It has, however, been noted in Chapter 2 that rudimentary branching has occasionally been observed in such typically bacillary forms as those of the genus Bacterium.

The morphology of other species of corynebacteria departs, to a greater or less extent, from that of $C$. diphtherice. Sometimes, as with $C$. ovis and $C$. murium, the resemblance may be so close that an experienced observer would be unable to distinguish either of these species from the true diphtheria bacillus on morphological appearances alone. Sometimes, as with C. hofmanni, the differences are so clear-cut that little difficulty arises.

It is characteristic of many species of diphtheroids that their morphology, as displayed in films from young cultures, is far less variable than that of $C$. diphtherice. A film of C.xerosis, for example, may show a marked resemblance to some of the average long forms of $C$. diphtherice, but while pleomorphism will usually be minimal in the former it will be marked in the latter. Similarly the socalled $C$. coryzce segmentosum may be very similar to the barred form which is sometimes assumed by $C$. diphtherice; but again pleomorphism is slight or absent.

With regard to the staining reactions of this group the bare statement that the corynebacteria are Gram-positive bacilli needs some qualification. The type species retains the stain to a sufficient degree to differentiate it quite clearly from the frankly Gram-negative bacteria; but it is decolorized by alcohol more easily than are many Gram-positive species. The metachromatic granules, on the other hand, retain the stain tenaciously; so that moderate overdecolorization, followed by the use of a red or brown counterstain, may give a picture very similar to that afforded by the use of Neisser's stain. Of the other species within this genus, C. hofmanni is very resistant to decolorization; so that the application of Gram's stain, followed by a prolonged exposure to alcohol (15 mins. or so), affords a useful differential criterion between these two species. Of the rest, some species behave as C. hofmanni, others as C. diphtherice. Absence of flagella and lack of motility are characteristic of the genus, as also is the absence of capsulation.

\section{Cultural Characters.}

With the exception of $C$. acnes, the members of this group grow on ordinary nutrient agar, though to a variable degree. Many of the diphtheroids, including C. hofmanni, develop freely, but the growth of all members is improved by the presence of natural animal protein. For several decades Loeffler's serum constituted the medium of choice. In stroke cultures on this medium there is a fairly abundant growth within 24 hours, having a moist, slightly creamy and sometimes faintly pigmented appearance. The degree of development, however, varies, and all transitions are seen between a slightly raised colourless film and a profuse succulent pigmented growth.

Since the introduction of blood tellurite medium attention has been concentrated mainly on the appearance of individual colonies, particularly in relation to the diphtheria bacillus. In $1931 \mathrm{McLeod}$ and his colleagues at Leeds (Anderson, Happold, McLeod and Thomson 1931) recorded the differentiation of two types of this organism. One, which was prevalent in severe cases, they called gravis ; the other, which was isolated from milder cases, they called mitis. In a subsequent report (Anderson, Cooper, Happold and McLeod 1933) they extended these observa- 
tions, and concluded that certain strains which they had found previously to correspond to neither the gravis nor the mitis type constituted a third type-now generally known as intermedius. Robinson and Marshall (1934) at Manchester, and numerous subsequent workers, have confirmed the accuracy of these observations. The three colonial types with their associated morphological, biochemical and pathogenic characters are now generally recognized as stable varieties of the diphtheria bacillus (see Siemens 1938).

A general description of the colonial appearance of the gravis, intermedius and mitis types is rendered peculiarly difficult because of the absence of a standard medium. McLeod and his colleagues originally used a heated rabbit-blood tellurite agar, on which the differentiation of the three variants was excellent. Later, however, it was found that some mitis and occasional gravis strains failed to develop on this medium. Numerous modifications have therefore been introduced. Rabbit and guinea-pig blood in 5-10 per cent. concentration give the best type differentiation, but are difficult to obtain in quantity. Sheep, horse and ox blood are less satisfactory, but they are improved either by heating the medium in which they are contained or by lysis of the red cells (Neill 1937, Hoyle 1941). Against the first method is the objection, noted above, that some strains, particularly of the mitis type, are inhibited by heated blood (Glass 1937, 1939a); against lysis there is the objection that the ability of the different variants to cause hrmolysis on blood agar plates is obscured. On the whole, therefore, we favour a medium made with unheated and unlysed blood containing sufficient potassium tellurite to prevent or restrain the growth of some of the organisms that are likely to be present in the nose or throat. A 10 per cent. sheep blood 0.5 per cent. glucose agar medium containing a final concentration of $0.03-0.04$ per cent. potassium tellurite is satisfactory in practice, though the type differentiation is not so good as on McLeod's medium.

The colonial appearances of the three types will be summarized more conveniently, along with their morphological, biochemical and other characters, in a later section of this chapter (p. 462). Suffice it to say here that diphtheroid bacilli also develop on a tellurite medium, forming colonies which are usually low convex, undifferentiated, about 1-2 mm. in diameter, varying in colour from pearl grey to jet black, with an entire edge, and a smooth, finely granular, or liquorice type of surface. Host of them can be easily recognized, but some strains form colonies so closely resembliug the gravis, intermedius or mitis types of the diphtheria bacillus that they can be distinguished from them only after careful study of their other characters.

Growth occurs readily in broth, but is seldom abundant. The degree of turlidity, pellicle formation, and the amount and nature of the deposit vary with different members of the group; with some species, as for instance the three types of diphtheria bacillus, they are of value in identification.

Growth in gelatin at room temperature is generally poor to moderate. A few members of the group, like C. ovis, C. pyogenes, and certain diphtheroids, liquefy the medium, but most members, including the diphtheria bacillus, do not. Wright (Report 1942) has drawn attention to gravis-like strains of a toxigenic diphtheroid bacillus that liquefy gelatiu in slope but not in stab culture.

Resistance.-C. diphtherice is readily killed by heat, suspensions of the bacilli failing to survive 10 minutes' heating at $58^{\circ} \mathrm{C}$. It is also easily destroyed by most of the usual antiseptics. It would appear to be relatively resistant to drying, 
though the evidence on this point is somewhat conflicting. Concerning the resistance of the various species of diphtheroid bacilli we have far less precise information; such data as are available suggest that they behave, in this respect, in much the same way as the type species. An exception must, however, be made for C. equi, which is reported by Karlson, Moses and Feldman (1940) to be unusually resistant to certain chemical disinfectants like oxalic acid that are used in the cultivation of tubercle bacilli from contaminated material. Most vegetative organisms are destroyed within 15 minutes by exposure to 1.5 per cent. oxalic acid; C. equi is said to resist even a 5 per cent. solution for an hour.

\section{Metabolism and Growth Requirements.}

The oxygen requirements of different species within this genus vary widely. Though all species are apparently able to grow in the absence of gaseous oxygen, some species, including $C$. diphtherice itself, develop far more freely under aerobic conditions, and display this preference by growing as a film or veil over the surface of a liquid medium. Other species, such as $C$. acnes and $C$.typhi, are microaerophilic.

The temperature range over which most members grow in artificial media extends from about $15^{\circ}$ to $40^{\circ} \mathrm{C}$, with an optimum at about $37^{\circ} \mathrm{C}$.

It has already been pointed out that the growth of all species is improved by the addition of a natural animal protein to the medium. Both blood and serum favour growth, but some members, particularly the mitis type of $C$. diphtheria, are inhibited by heated blood, as well as by blood treated by acid or alkali. According to Glass $(1939 a, b)$ this effect is apparent only in aerobic culture, and is abolished by the addition of sodium hydrosulphite or by anaerobic cultivation.

So far as growth on synthetic media is concerned, our information is as yet relatively scanty, in spite of a considerable mass of experimental clata (see Uschinsky 1893, 1897, Hadley 1907, Hadley and Gorham 1907, Koser and Rettger 1919, Davis and Ferry 1919, von Groer 1923, Hosoya and Kuroya 1923, Robertson 1924, Braun and Hofmeier 1927, Braun and Mündel 1927, 1929, Braun, Hofmeier and Mündel 1929, Maver 1930, Lindemann 1932, Ehrismann 1932-33, 1933, Hottinger and Hottinger 1933, Nitsch 1933, Schmidt 1933-34, Wadsworth and Wheeler 1934, Knight 1936). It may be summarized by noting (1) that $C$. diphtherice appears to be incapable of growth with ammonia as the sole source of nitrogen, carbon being supplied in an organic form ; (2) that growth, and toxin production, often occur when amino-acids are added to such a synthetic medium, and that, among these amino-acids, cystine, aspartic acid and perhaps tryptophan appear to be indispensable; and (3) that it is only certain "non-exacting" strains that are capable of growing in such a medium, and even with these strains toxin production is usually much less abundant than with a more adequate food-supply. Knight (1936) emphasizes the difficulty of assessing the real significance of many of the recorded findings in view of the great difficulty of obtaining most amino-acids in a state of chemical purity.

Attempts have been made (Bunker 1919, Hosoya et al. 1933, Mueller et al. 1933, Mueller $1935 a, b, c)$ to identify the additional substances required for active growth and toxin production by fractionating peptone, meat extract and other types of complex protein extracts or hydrolysates in which $C$. diphtherice is known to grow abundantly. Considerable progress has been made along these lines (see Chapter 3).

\section{Biochemical Reactions.}

The carbohydrates most frequently employed as test substrates for the differentiation of species within this genus are dextrose, maltose and saccharose. The type species, $C$. diphtheria, produces acid but no gas in dextrose and maltose, but does not attack saccharose. Other species, such as $C$. xerosis produce acid in all three 
sugars; others again, such as $C$. hofmanni, attack none of them. No species produces gas. Within the species $C$. diphtherice the gravis type ferments starch and glycogen; the mitis and intermedius types do not. In practice great care is necessary in the preparation of the medium if reliable results are to be obtained. A medium containing serum is almost indispensable, but it has certain drawbacks. The serum, for example, may contain sufficient fermentable carbohydrate to give a false reaction; this can be overcome by suitable buffering. Unheated horse serum may hydrolyse starch due to the presence of a natural diastase (Hendry 1938) ; this may be destroyed by heating. Not all samples of soluble starch are satio factory; each sample should be tested before use.

A suitable medium may be prepared by dissolving 0.5 per cent. peptone and $0 \cdot 1$ per cent. $\mathrm{Na}_{2} \mathrm{HPO}_{4}$ in $1,400 \mathrm{ml}$. of distilled water, steaming for 15 minutes, filtering, adjusting to $\mathrm{pH} 7 \cdot 4$, adding $250 \mathrm{ml}$. of horse serum, steaming for 20 minutes, adding $11 \mathrm{ml}$. of Andrade's indicator, adjusting to $\mathrm{pH} 7 \cdot 6-7 \cdot 8$, tubing in $3 \mathrm{ml}$. quantities, autoclaving at $10 \mathrm{lb}$. for 10 minutes, and adding to each tube separately sufficient of a sterile solution of the sugar in distilled water to give a final concentration of $0 \cdot 4$ per cent. starch or 1 per cent. of other sugars (Robinson 1940).

A more detailed and extended table of the fermentation reactions of ten named species within this genus, and of eleven unnamed diphtheroids examined by Barratt (see Andrewes et al. 1923) is appended to this chapter, but experience has revealed the existence of several more fermentative types. The fermentation of certain other substrates by different varieties of the type species is considered below.

We may note here that certain species and types within this group, for instance C. ovis, C. pyogenes and certain unnamed diphtheroids liquefy gelatin, while C. diphtheria and most diphtheroid organisms do not.

Many strains of $C$. diphtheria, but not all, produce areas of hæmolysis on bloodagar plates, and lyse red cells when these are added to broth cultures (Schwoner 1904, Costa et al. 1918, Goldie 1933). The red cells of the guinea-pig are the most sensitive, then those of the rabbit, horse, man, pig, mouse and sheep in this order. There is a conflict of evidence in regard to the production of a soluble hæmolysin. The earlier observers stated that culture-filtrates did not cause hæmolysis; but Goldie (1933) reports that cell-free filtrates are hæmolytic, and that their activity runs roughly parallei to their toxin content, though the hæmolysin is not neutralized by antitoxin. There is a similar conflict in regard to the heat-resistance of the hæmolytic agent, whether it be intracellular or extracellular. It was originally recorded as being inactivated by heating to $58^{\circ} \mathrm{C}$. for half an hour. Goldie states that it is not inactivated by boiling. Iysis around colonies on blood agar is of some value in distinguishing between the three types of the diphtheria bacillus. Most mitis strains are hæmolytic, intermedius strains are not, and gravis strains vary in their activity.

Two species of diphtheroids that are pathogenic for animals, $C$. ovis and $C$. pyogenes, also exert a hæmolytic action on the red blood corpuscles of various species, including the rabbit and horse. The other species and types within this genus that have been examined from this point of view appear to be non-hæmolytic.

Some diphtheroid strains form phosphatase; the true diphtheria bacillus never does. The production of this enzyme may be tested for on a medium containing phenolphthalein phosphate (see Bray 1944). 


\section{Antigenic Structure.}

In considering the antigenic structure of $C$. diphtheria and of the other species and types within this genus, we may note that those workers who have attacked this problem have, in most instances, approached it with a view to the identification of the type species, and its differentiation from related species, types and variants. It is, therefore, only in regard to the type species that onr knowledge is in any way detailed or systematized.

Almost all the studies on the antigenic structure of this group of organisms have been carried out by the method of agglutination; and the application of this technique soon showed that $C$. diphtherice is an antigenically heterogeneous species. Langer (1916) demonstrated the existence of two serological types, Durand (1918, 1920) six, Havens (1920) two, Smith (1923) seven, Eagleton and Baxter (1923) ten, and Scott (1923) eight; in all cases there remained strains that did not fall into any of the groups.

These conflicting results have been to some extent clarified by the more recent work of Ewing (1933) and of Robinson and Peeney (1936), who have shown that the antigenic structure of diphtheria bacilli differs according to whether they belong to the gravis, intermedius or mitis varieties. Ewing studied 106 gravis strains derived from Great Britain, Berlin and Khartoum, and was able to divide them by direct agglutination into four types, which she called A, B, C, and D. Robinson and Peeney studied 739 gravis strains derived from many different parts of the world, aud distinguished five antigenic types, which they called I, II, III, IV, and $\mathrm{V}$; the first four of these corresponded to Ewing's types A, B, C, and D. The antigens appear to be qualitatively distinct, so that absorption of agglutinins is not required to separate the types. On the other hand there does seem to be some group relationship between the gravis, intermedius, and mitis varieties, indicating the existence of a species-specific antigen. Types I and II strains have the characteristic cultural properties of gravis strains; Types III, IV, and V often differ from the classical description and may resemble the mitis variety. Types I and III seem to be restricted mainly to Great Britain; Type II has a world-wide distribution, but is uncommon in Great Britain; Type IV was the only type found by Robinson and Peeney in Egypt; and Type V was found only in the United States of America. No antigenic difference was detected between virulent and avirulent strains of gravis, though too few observations have yet been made to say whether loss of virulence is accompanied by change in antigenic structure.

Less attention has been paid so far to the intermedius and mitis types. Ewing (1933), however, mentions the occurrence of at least two antigenic types in a small series of intermedius, and of at least five antigenic types in a small series of mitis strains that she studied. By means of complement fixation, using alcoholic extracts of the organisms, Hoyle (1942) obtained evidence of the existence of two different lipoid antigens in diphtheria bacilli : $(a)$ a specific antigen characteristic of mitis but present also in small amount in gravis and intermedius; $(b)$ a nonspecific or group antigen present in large amount in gravis and intermedius and in small amount in mitis. C. hofmami contained the group antigen in large amount, as well as a specific antigen of its own.

Apart from Hoyle's observations, little antigenic relationship has been observed between C. diphtherice and other species of corynebacteria. Scott (1923) included in the series that he studied a number of strains of $C$. xerosis and $C$. hofmanni, but could detect no 
antigenic relationship between these species, or between either of them and any serological type of $C$. diphtherixe. Similarly, Bailey (1925) studied two strains of toxigenic $C$. diphtheria, two of $C$. hofmanni and three of $C$. xerosis, together with seventeen nontoxigenic strains of $C$. diphtherio. "He was unable to detect any significant antigenic relationship between these two species of diphtheroids and the diphtheria bacillus.

The fact that agglutination tests fail to reveal any antigenic relationship between $C$. diphtherix and the various diphtheroid organisms, does not, of course, mean that there is no sharing of antigenic components between them. It means only that, if such sharing exists, the shared antigens, either because of their position in the bacterium or for some other reason, are not concerned in the agglutination of the intact bacterial cells. It is not, therefore, surprising to find that certain antigenic components are distributed widely among the corynebacteria, and even among related genera. Thus Krah and Witebsky (1930) record that alcoholic extracts of the diphtheria bacillus, of certain diphtheroids, and of the tubercle bacillus, all fix complement in the presence of an antiserum prepared against any one of these organisms. Again, a strain of $C$. diphtherice that has undergone antigenic variation, and lost its type-specific surface antigen, may agglutinate with antisera prepared against diphtheria bacilli of other serological types, or against various diphtheroids. Neill and his colleagues (1931) have described such a strain.

\section{Toxin Production and Pathogenicity.}

The type species, $C$. diphtherice, is an important human pathogen, giving rise to a characteristic and often fatal disease, the lesions of which are, in the main, produced by the action of a powerful exotoxin. This diffuses throughout the body from the primary focus of infection, which is most frequently situated in the tonsillar region. The pathogenesis of diphtheria in man, and its diagnosis, prevention and treatment so far as these depend on bacteriological methods, are dealt with in Chapter 61. The characters of the specific toxin, its effects on certain laboratory animals, and its immunological relationships with toxins produced by other species and types within this genns, are, however, so important as differential criteria, that it is necessary to discuss them here.

The production of diphtheria toxin in artificial culture has been the subject of a large series of empirical observations. From these it is clear that optimal toxim production demands conditions which are not necessary for optimal growth.

Davis and Ferry (1919) tested the value of media prepared from beef infusion, peptone, and meat extract in various combinations with each other. The presence of beef infusion was found to be essential. This was later disproved by Wadsworth and Wheeler (1934), who devised a synthetic medium containing 2 per cent. of peptone, on which a toxin of high potency can be prepared (Eatrn 1936, Pappenheimer and Robinson 1937). Hartley and Hartley (1922) tested various specimens of peptone, and found that, while each brand of peptone gave a characteristic curve of toxin production, it was impossible to predict the value of any one brand by a preliminary chemical analysis. Hartley (I922) pointed out the superiority of a tryptic digest of horse muscle as a medium for the production of toxin, and his findings have been confirmed and extended by Watson and Langstaff (1927), who also confirm his observation that the value of such a medium is markedly influenced by the method of sterilization. In addition to growth-promoting substances. there are apparently toxin-inducing substances present, which are very lahile to heat when the $\mathrm{pH}$ is at nentrality, or on its alkaline side. Antoclaving at $\mathrm{pH} 8.0$ or over may completely destroy the value of a medium for toxin production. This may be avoided by filtration through a seitz press. followed by a short steaning. The addition to the medium of maltose, or certain other encrgy sources, considerably increases the toxin yicld, particularly if sodium acetate or lactate is also added (Pope 1932, Ramon and Berthelot 1932, Pope and Smith 1932, Pope and Healey 1933a, Pope and Linggood 1939). The 
iron content of the medium has to be carefully adjusted. (Pappenheimer and Johnson 1936). The initial reaction of the medium is of great importance, a fact which has been emphasized by many workers (Bunker 1919, Hartley 1922, Andrewes et al. 1923, Watson and Langstaff 1927). The most favourable starting reaction is at or just below $\mathrm{pH} 8 \cdot 0$, and it is important that, during the 7 to 10 days which elapse between sowing the culture and harvesting the toxin, the reaction should not swing far towards neutrality. The range over which growth of $C$. diphtherice takes place extends from about $\mathrm{pH} 5 \cdot 7$ to $\mathrm{pH} 8 \cdot 7$, but the zone over which toxin production occurs appears to be limited to $\mathrm{pH} \mathbf{7 \cdot 5}-\mathbf{8} \cdot \mathbf{2}$. The growth of the organism during its initial stages is associated with a slight production of acid, probably derived in part from nitrogenous constituents of the medium. Later there is a reversion in the alkaline direction, due to the splitting-up of these organic acids with the formation of carbonates. The balance between these metabolic activities is in part determined by the oxygen pressure to which the culture is submitted. Partly for this reason, partly perhaps for others, the slrape of the flask in which the medium is contained, the thickness of the layer of medium itself, and the type of plug used for closing the mouth of the flask, all exert an influence on the grade of toxin produced. It is also important to eliminate any movement which will prematurely break up the veil of growth which forms at the surface of the medium. In practice it is found that the best results are obtained by growing the organism in a shallow layer of medium, in a cylinclical bottle which is kept lying on its side, and is plugged loosely with gauze or cotton-wool (Bunker 1919, Hartley and Hartley 1922, Andrewes et al. 1923, Watson and Langstaff 1927. Pope and Healey 1933b). The cultures are incubated at $37^{\circ} \mathrm{C}$. for 7 to 10 days, at the end of which time phenol, or preferably toluol, is added in sufficient strength to ensure sterilization, the flasks are allowed to stand for 24 hours, and the contents are filtered. The crude toxin so obtained has now to be freed from the various non-toxic substances, such as toxoids and substances derived from the bacterial cells and the medium with which the pure toxin is contaminated. Progress has been made in the attempt to isolate diphtheria toxin in a pure form, but complete success has not yet been achieved. This is in part due to the fact that the toxin is very labile, and is readily inactivated or destroyed by heat or strong chemical reagents. It is probable that the specific material of a toxic filtrate-toxin and toxoid-constitutes a very small fraction of the total constituents of the crude filtrate (about 1 per cent. according to Glenny, 1925a). A considerable concentration can be achieved by precipitation with weak acids, or ammonium sulphate, or acetone, or by dialysis, or by fractional filtration through graded collodion membranes, or by adsorption on to aluminium hydroxide followed by elution, or by a combination of these methods (see Glenny and Walpole 1915, Watson and Langstaff 1926, Locke and Main 1928, Leulier et al. 1931, Bunny et al. 1931, Schmidt 1931, Schmidt et al. 1931, Tasman and Pondman 1931, Brandwijk and Tasman 1932, 1933, Zajdel 1932, Tasman and van Waasbergen 1932, Wadsworth et al. 1932. Leonard and Holm 1933, Wadsworth and Quigley 1934, Eaton and Bayne-Jones 1934, Goldie 1934). Eaton (1936), however, has shown that the toxin is denatured by acid, and therefore recommends a method of purification which does not necessitate the use of this reagent.

The evidence so far obtained suggests that diphtheria toxin is a heat-coagulable protein. The purest preparations contain about 16 per cent. nitrogen, 0.75 per cent. sulphur, 9 per cent. tyrosine, and 1.4 per cent. tryptophan. The isoelectric point is $\mathrm{pH} 4 \cdot 1$. The toxin is extremely sensitive to denaturation by solutions more acid than $\mathrm{pH} 6$ and by moderate heat. The amount of nitrogen per flocculating (Lf) unit is $0.00045 \mathrm{mgm}$, and the M.L.D. for guinea-pigs is about $0.0001 \mathrm{mgm}$. (Eaton 1936, Pappenheimer 1937, Pappenheimer and Robinson 1937). It is actively antigenic and, when injected into animals, stimulates the production of a powerful antitoxin. It can be converted into toxoid by suitable treatment with formalin; in this state it will cause a precipitate when mixed with specific anti- 
serum and will give rise to antibody production in animals, but is no longer toxic. It appears to be produced within the bodies of the bacilli, since toxin is liberated in bacterial suspensions submitted to treatment with sonic vibration (Morton and Gonzalez 1942).

The classical paper in which Loeffler (1884) first described the isolation and characters of the diphtheria bacillus, and the report by Roux and Yersin (1888) of the separation of the filtrable toxin, contain descriptions of the lesions produced by the living organism, or by its separated toxin, in a variety of laboratory animals. These original observations have since been extended by a host of experimental studies. It will suffice to note here that, among laboratory animals, the guinea-pig and the rabbit are the most susceptible, while rats and mice are extremely resistant. Dogs, cats, pigeons, and other birds appear to occupy an intermediate position. (See Loeffler 1884, 1890, Roux and Yersin 1888, Wernicke 1893, Goodman 1907, Coca et al. 1921, Glenny and Allen 1922, Andrewes et al. 1923). It may be noted that the bacillus appears to have little power of tissue invasion; whether the inoculum consists of a living culture, or of a toxic filtrate, death occurs as the result of a toxæmia in the strict sense. This general statement may require minor modification in regard to certain varieties of the diphtheria bacillus (see below). For our immediate purpose it will suffice to note the sequence of events that follow the injection of a living culture, or of a toxic filtrate, into the guinea-pig.

If a guinea-pig is inoculated subcutaneously into the flank with a dose of a virulent culture or of a toxic filtrate, of a size which will produce death within a few days, a soft œdematous swelling usually appears at the site of inoculation within 12 to 18 hours, and gradually extends. About the time the swelling appears, or shortly thereafter, the animal becomes obviously ill, developing a staring coat and sitting crouched in its cage. Death usually occurs between 18 and 96 hours, according to the size of the dose of culture or filtrate inoculated. With very large doses the time to death may be even shorter, but is never less than 10 to 14 hours (see Glenny 1925b). Animals that survive beyond the 4 th day may develop cachexia and paralysis, and die at some later period; but the pathogenesis of this condition appears to be essentially different from that of the acutely fatal toxæmia, and it is with the latter that we are here concerned. When a guinea-pig that has died within 4 days after a subcutaneous inoculation is examined post mortem, the typical findings are as follows:

At the site of inoculation is found an extensive area of gelatinous hæmorrhagic œdema, extending to the skin superficially, and deeply to the muscles or to the parietal peritoneal membrane. If the animal has survived for several days, the tissues in the more central parts of the cedematous area may be obviously necrotic. The regional lymph glands are usually swollen and congested. The peritoneum may contain a varying amount of fluid, which may be clear, cloudy or blood-stained. The abdominal viscera as a whole are congested; but the most striking lesion is the marked swelling and congestion of the adrenal glands. On macroscopical section there are seen to be scattered hæmorrhages, situated in the medulla, in the cortex, or in both. Sometimes all naked-eye distinction between cortex and medulla is lost. On opening the thorax a serous exudate will often be found in the pleural cavities, usually clear, sometimes cloudy or blood-stained. A pericardial effusion may or may not be present. Films prepared from such effusions reveal a marked preponderance of mononuclear cells.

It is of some interest to note the relative frequency of the more important lesions associated with acute diphtheritic toxæmia in the guinea-pig. Wright (1894) records the findings in 160 necropsies: a local lesion was present in 90 per cent. of the animals; congestion of the adrenals in 81.2 per cent. ; and a pleural effusion in 42.5 per cent. Barratt (1923) records the post-mortem findings in 50 guinea-pigs which died within 72 hours after 
the injection of $2 \mathrm{ml}$. of a virulent culture; œdema, of varying degree, was present at the site of inoculation in 94 per cent. of the animals; the adrenals were abnormal in all and are noted as pink in 4 per cent., red in 22 per cent., and deep red in 74 per cent.; a pleural exudate was present in 44 per cent.

For details of the histological changes associated with these lesions, reference may be made to the monograph published by the Medical Research Council. We may, however, note a few points which have a direct bearing on diphtheria as it occurs in man. Mollard and Regaud (1895) recorded the occurrence of degenerative changes in the myocardium in experimental diphtheria, and Flexner (1897) noted that fatty degeneration of the cardiac muscle was almost constantly present in animals which died within a short time after inoculation. There has been some discussion as to whether these changes are primary, or are a sequel to an initial reaction in the interstitial tissue, or to a primary lesion of afferent nerve fibres. The careful and detailed studies of Dudgeon (1906) gave a clear answer to this question, and afforded strong experimental support to the suggestion of Bolton (1905), that the direct action of diphtheria toxin on the cardiac muscle is the most important cause of acute cardiac failure in human diphtheria. Examining a large series of guineapigs, killed or dying in various stages of acute diphtheritic toxæmia, Dudgeon demonstrated the occurrence of fatty degeneration of the diaphragmatic muscle within 4 hours after inoculation, and of the cardiac muscle within 16 hours. Similar results have since been recorded by Jaffé (1920).

We may note also, since this method is now frequently employed in the identification of a toxigenic strain of $C$. diphtherice, that the intradermal injection of toxin, or of living bacilli, leads to a localized erythematous lesion, followed by necrosis (Römer 1909). This effect, as also the lethal action of the subcutaneous injection of larger doses, can, of course, be specifically neutralized by an antitoxic serum.

Although the production of this filtrable toxin, with its characteristic action in the guinea-pig and its property of being specifically neutralized by the homologous antitoxin, is one of the most important characters by which $C$. diphtherice is identified, there exist strains of bacilli that, while conforming in all other respects with the diphtheria bacillus, fail to form this filtrable toxin. These strains are commonly classed as non-toxigenic, or avirulent, diphtheria bacilli. Whether they should in all cases be assigned to this species is perhaps doubtful; but there can be no reasonable doubt that many of them, at least, are actually non-toxigenic variants of $C$. diphtheria. We have noted above that certain avirulent strains can be shown to be antigenically related to typical toxigenic strains, and the actual emergence of an avirulent variant from a virulent organism, under laboratory conditions, has been recorded by several observers.

Thus Crowell (1926), starting from a single-cell culture of a fully virulent strain, derived from this parent culture a series of daughter strains, one of which was entirely avirulent. All attempts to raise the virulence of this variant were without result. Cowan (1927) records the derivation of avirulent variants from 2 strains of virulent $C$. diphtherice, one of them the classical "Park 8 " which has yielded toxin to most laboratories in the world. These variants were "rough," in the sense that they formed small, raised, dense and granular colonies, and gave increased deposit in broth, with an absence of pellicle formation.

The diphtheria bacillus is not the only species of Corynebacterium that is pathogenic under natural conditions. C. ovis, C. murium, C. pyogenes, C. equi and $C$. renale certainly fall into this category. So probably does $C$. acnes.

A great variety of diphtheroir organisms have, at one time or another, been isolated from various tissues in man and animals. Sometimes the tissue from which the diphtheroid bacillus was isolated has been the site of some obvious lesion; 
sometimes it has been apparently healthy. It is exceedingly difficult, from the published reports, to determine whether these organisms have, or have not, played any pathogenic rôle; and in most instances they have not been studied in sufficient detail to allow of any systematic identification or classification. We shall therefore confine ourselves here to a brief description of the lesions produced by the named species referred to above.

C. ovis, often referred to as the Preisz-Nocard bacillus, (see Nocard 1889, Preisz 1894) causes caseous lymphadenitis in sheep and ulcerative lymphangitis in horses. It differs sharply from $C$. diphtherice in that it is a pyogenic organism, and invades the tissues. It resembles $C$. diphtherice in producing a filtrable toxin.

Nicolle, Loiseau and Forgeot (1912) have carefully recorded the lesions met with in guinea-pigs, which have died as the result of inoculating either living cultures of $C$. ovis or bacteria-free filtrates subcutaneously. In the former case, and where the dose of living culture has been so adjusted that the animal dies about the 25th day, subcutaneous abscesses develop in various situations during life. At necropsy, in addition to these superficial lesions, small granulomatous masses are found in the liver, spleen and lungs, and beneath the parietal peritoneum. In the male guinca-pig similar lesions are found in the tunica of the testis and epididymis Some of these lesions may have developed into large caseous or caseo-purulent masses.

When a guinea-pig is injected subeutaneously with a fatal dose of a toxic broth filtrate, death occurs within a few days, often in less than 24 hours, from an acute toxæmia. The necropsy findings in such cases are entirely different from those described above. There is a local, subcutaneous, inflammatory, gelatinous œedema at the site of inoculation, often hæmorrhagic in character. The abdominal viscera are congested, and often show small hæmorrhages, particularly in the stomach, large intestine, and kidneys. The latter may be almost black in colour. There is, however, no congestion of the adrenals, and no exudation into the pleura. Hall and Stone (1916) give a very similar picture.

Again, Petrie and McClean (1934) state that the effect produced by the injection of $C$. oris toxin into the skin of a guinea-pig differs from that produced by the injection of diphtheria toxin. The former gives rise to a definitely papular lesion, and if the dose of toxin injected is large the lesions become pustular. Aceording to Carne (I940), who defines the optimal conditions for toxin production, guinea-pigs, rabbits, sheep, goats, pigs, horses, oxen, dogs and cats are all sensitive to its action.

It has been shown by Bull and Dickinson (1935) that the pyogenic substance is largely contained in the bacterial cells, as is suggested by the observations of Nicolle and his colleagues, and that it is relatively thermostable. Suspensions of $C$. ovis, killed by heating at $60^{\circ} \mathrm{C}$. for 1 hour, no longer produce toxic death in susceptible animals, but they give rise to sterile abscesses when injected in adequate dosage.

It may be noted that the exotoxin of $C$. ovis is different from that of $C$. diphtheria. Nicolle and his colleagues found that an antitoxin prepared against the former gave specific protection against $C$. ovis toxin, whereas none was afforded by diphtheria antitoxin. Dassonville (1907), Hall and Stone (1916). Minett (1922a, b), and Barratt (1933) recorded some degree of protection by diphtheria antitoxin against the toxin of $C$. ovis; but the detailed study of Petrie and McClean (I934) leaves little doubt that these effects were due to the fact that the sera of normal horses may contain varying amounts of $C$. ovis antitoxin $(C$. ovis is a natural pathogen of the horse), and that the two toxins are immunologically quite distinct from one another.

Petrie and McClean have, however, found evidence of the existence of varieties of diphtheroid bacilli that are, in respect of certain characters, intermediate between $C$. diphtherice and $C$. ovis. These diphtheroids, all isolated by various observers from the 
human throat, were originally studied by Barratt (1933), who found them to have the power of liquefying gelatin, a property possessed by $C$. ovis but not by $C$. diphtherice. Petrie and McClean found that one of these strains produced both diphtheria toxin and a toxin that was immunologically related to that of $C$. ovis. The remaining strains produced only a toxin related to that of $C$. ovis. Two strains, similar to those of Barratt, have been described more recently by Wright at Liverpool (Report 1942). Both strains resembled the gravis type of diphtheria bacillus in their colonial appearance, fermentation of starch, and virulence to the guinea-pig, but differed from it in liquefying gelatin in slope culture at $22^{\circ} \mathrm{C}$., though not in stab culture at $22^{\circ} \mathrm{C}$. or $37^{\circ} \mathrm{C}$, in being inagglutinable with gravis type antisera, and in being apparently unaffected by diphtheria antitoxin; even 10,000 units of antitoxin failed to protect guinea-pigs against the usual test dose of culture, and the organisms were easily recoverable from the heart blood. One strain killed a rat injected intraperitoneally. Whether the toxin formed by these strains was neutralized by $C$. ovis antitoxin is not recorded.

C. pyogenes was first described by Lucet (1893), who isolated it from suppurative lesions in cattle. Similar organisms were isolated from similar lesions in cattle and swine by Grips (1898), Künnemann (1903) and Glage (1903). Since then they have been recorded by many workers (see Holth 1908, Poels 1912, Ward 1917). The lesions to which they most frequently give rise under natural conditions appear to be suppurative pneumonia, suppurative arthritis, and other suppurative lesions, including mastitis, the animals affected being cattle, pigs, sheep, and goats (see Merchant 1935, Magnusson 1938).

Among laboratory animals, the rabbit appears to be most susceptible, guineapigs less so, and mice relatively resistant (see Holth 1908, Ward 1917, Brown and Orcutt 1920).

The injection of living cultures of $C$. pyogenes into the rabbit is followed by the development of localized abscesses if the injections are given subcutaneously. If they are given intravenously, or if generalization occurs after a subcutaneous inoculation, abscesses develop in the bones and joints, less frequently in other organs.

C. ovis and $C$. pyogenes produce suppurative lesions in animals; both liquefy gelatin, and both produce hæmolysis. There is, however, a general consensus of opinion among those who have worked with them that they are different species. They tend to affect different animal hosts, and among laboratory animals, the guinea-pig is very susceptible to infection with $C$. ovis, but relatively resistant to C. pyogenes. There are also cultural differences. C. pyogenes liquefies gelatin rapidly and constantly, $C$. ovis slowly and irregularly. On a medium containing blood serum $C$. pyogenes gives small dewdrop colonies, which slowly enlarge and become granular in the centre. C. ovis gives colonies that are circular, umbonate and opaque, with a tendency to develop a yellowish pigment. Both organisms form a filtrable toxin. The toxin of $C$. pyogenes reaches its maximum concentration in culture after 48 hours at $37^{\circ} \mathrm{C}$. (Lovell 1937, 1944), that of $C$. ovis in about 5 days (Carne 1940). Lovell finds that the toxin of $C$. pyogenes is apparently identical with the hrmolysin; but the toxin of $C$. ovis, according to Came (1939), is unrelated to the hæmolysin. It would appear that the toxins of the two organisms are distinct; the results of cross-protection tests with antitoxin are, however, still awaited.

An organism that has sometimes been confused with C. pyogenes is C. equi, which was isolated by Magnusson (1923) in Sweden from foals affected with pyæmia. 
The differential characters of the two organisms are enumerated on p. 469 under C. equi.

Enderlen (1890-91) described a diphtheroid bacillus that he had isolated from the pus from a cow suffering from pyelitis. This organism has been named $C$. renale. A similar organism was isolated by Ernst $(1905,1906)$; and Jones and Little (1925) record an outbreak of infective cystitis and pyelitis in three dairy herds associated with the constant presence of a diphtheroid bacillus. In this last instance, at least, there would seem to be no reasonable doubt that the bacillus was roiologically related to the disease; though the same organism may often be isolated from the genital tract of healthy calves (Jones and Little 1930). There are as far as we know no records of the lesions produced by this organism in laboratory animals, though Jones and Little have reproduced the disease in cattle. Ernst, indeed, states that the bacillus isolated by him produced no lesions in any animal, and he therefore regarded it as devoid of pathogenic significance. No attempt has apparently been made to ascertain whether it produces a filtrable toxin. Its claim to rank as a separate species must rest in part on its predilection for the urinary tract in cattle, but mainly on its behaviour in the laboratory. It is recorded as not producing a hæmolysin and not liquefying gelatin, in both of which characters it differs sharply from C. pyogenes (Merchant 1935).

C. murium, which was first described by Kutscher (1894), gives rise to a natural disease in mice, and its pathogenic activity is apparently confined to this small laboratory animal. It has been injected without effect into the guineapig, rabbit, cat, dog, pigeon, hen, rat, goat, calf, sheep, cow and horse (Kutscher 1894, Bongert 1901), but Gundel, György, and Pagel (1932) have recorded spontaneous infections in rats on a vitamin-deficient diet. We may give here a brief description of the natural disease as well as of the results of experimental inoculation.

The natural disease has been described by Kutscher and by Bongert, and has been observed on many occasions by the present authors during necropsies on mice, though it is certainly relatively infrequent. The most characteristic lesion in the naturally-occurring disease is the presence of large, firm, caseous areas in the lung. In sections or films from these lesions the bacilli are usually abundant. Caseous nodules may be found in the liver, though they are less frequent. When present they project from the surface, in contradistinction to the necrotic areas seen in mouse typhoid. The lymphatic glands of the axilla, neck, mediastinum and mesentery may be enlarged and caseous ; but the pulmonary lesions are frequently the only obvious sign of disease. Occasionally the bacillus may be isolated from a single caseous gland, found at necropsy without any other detectable lesion.

The disease may readily be reproduced by inoculating mice with pure cultures of $C$. murium, or by administration per os. The findings at necropsy depend largely on the route of administration. After feeding, lesions develop in the mesenteric glands and in the liver. After intraperitoneal inoculation, which usually leads to death within a week, the peritoneum is found to be studded with minute tubercles, and there is a spreading granulomatosis, of very varying extent, involving the regional lymphatic glands, the liver, and less frequently the spleen. In our experience pulmonary lesions are much less frequent in the experimental than in the natural disease, though they occasionally occur. Bongert (1901) has called attention to the trivial lesions which may sometimes be found post mortem after experimental infection. In animals dying after subcutaneous inoculation the only detectable lesion may be a small caseous abscess at the site of inoculation. Seeking an explanation for this fact, he inoculated mice with filtrates of broth cultures, or with cultures killed by heat, and found that death resulted in every case, after about 10-14 days. No obvious lesions of any kind were found at necropsy. Mice fed with filtrates died in about the same time, and with the same absence of lesions. We can in part confirm these findings 
as regards the inoculation of filtrates, or of killed cultures, though our own results were far less uniform than those recorded by Bongert. It would appear that this organism produces an exotoxin which is fatal for mice. According to Bongert this toxin is relatively heatstable, since it withstands heating for 2 hours at $55^{\circ} \mathrm{C}$., or for a few minutes at $74^{\circ} \mathrm{C}$. ; but the particulars given are not sufficiently precise to allow of any definite conclusion with regard to the time or temperature required for inactivation.

The toxin of this organism has not, so far as we are aware, been compared with that of $C$. diphtherice or of $C$. ovis, but it seems exceedingly unlikely that there is any relationship, since the mouse is conspicuously resistant to diphtheria toxin, and the guinea-pig and rabbit, which are very susceptible to $C$. ovis, are resistant to $C$. murium.

It may be noted that Fischl, Koech and Kussat (1931) described an organism under the name of Corynebacterium arthritidis muris, which they isolated from the swollen ankle joint of a white mouse. On inoculation into the joints of normal mice and rats it gave rise to a similar arthritis. Little is known about this organism, but it appears to differ in some respects from $C$. murium.

There remains $C$. acnes, a diphtheroid organism described in the lesions of cutaneous acne by Unna (1896). It was first isolated by Sabouraud (1897), and has since then been studied by Gilchrist $(1900,1903)$, by Fleming (1909), and by Südmersen and Thompson (1909-10). Its claim to pathogenicity must rest in the main on its constant association with the disease in man. Südmersen and Thompson state that the two strains examined by them were pathogenic for the mouse but not for the guinea-pig; but their description of the lesions in the latter animal is extremely scanty. C. acnes is clearly marked as a distinct species from those described above by its peculiar growth requirements (see p. 471) and particularly by the fact that it is microaerophilic.

C. typhi, another microaerophilic diphtheroid, was isolated by Plotz (1914) from the blood in typhus fever. It is now generally admitted to be an example of a parasitic diphtheroid with no established pathological rôle (Olitsky 1921).

\section{The Gravis, Mitis and Intermedius Types of C. diphtheriæ.}

Attention has already been drawn on p. 450 to the recognition by HcLeod and his colleagues at Leeds (Anderson, Happold, McLeod and Thomson 1931) of three stable varieties of the diphtheria bacillus-the gravis, intermedius and mitis types. The characters of these three types, as recorded by Robinson $(1934,1940)$, and Cooper, Happold, HcLeod and Woodcock (1936), are summarized in Table 30.

This summary can do no more than serve as a guide to the recognition of the three types. On the whole the characters of the intermedius type are the most constant. In the identification of gravis strains starch fermentation is of particular value since, though it may be delayed beyond $2 t$ hours, it is seldom absent.

Sometimes it is difficult to distinguish not only between the three types, but between diphtheria bacilli and diphtheroids. Each of the types has one or more species of diphtheroid bacilli that closely resemble it. Space does not permit a detailed description of the differential characters of these organisms. In general, it will be found that the diphtheroid bacilli tend morphologically to be more regular in shape, size, depth of staining, distribution of bars or granules, and arrangement; diphtheroids lie often in palisades, whereas true diphtheria bacilli show the Chinese letter type of distribution, or, if they are in bundles, they have seldom the same regularity of arrangement as that of diphtheroids. The colonial differences are often very slight and, as they vary from one medium to another, they can be learned only by close observation and long experience. 
TABLE 30

Characters of the Types of Diphtheria Bacillos.

Morologeng on

Morphology on agar

Colonial appearance on tellurite blood agar.

Gravis.
Usually short rods,
resembling irregular
Hofmann. Staining
fairly uniform, few or
no granules, and often
a narrow unstained bar
dividing the rod un-
equally. Some degree
of pleomorphism with
irregularly barred,
snow-shoe and tear-
drop forms. May be
coceoid on first isola-
tion. Occasional strains
resemble intermedius
or mitis type.

An 18-hr. colony is 1-2 mm. q., circular, low convex, pearly grey or with greyish-black centre and paler semitranslucent periphery, with a smooth matt or rarely liquorice type of surface, and a commencing crenation of the edge. The colony is coherent, tending. when touched, to move as a whole on the surface of the medium; has a consistency of cold margarine, and breaks up radially into small masses that are not easily emulsitied. slight hrmolysis around colonies of some strains. In 2-3 days colony reaches $3-5 \mathrm{~mm}$. in diameter, is flattened with a slightly raised centre, is slatygrey or greyish-black in colour, often darker at the centre than at the periphery, has a frosted surface and a crenated edge, and shows radial striation, especially towards the margin. When striation and differentiation are well developed, the term "daisy head" colony is applicable.

\author{
Intermedius.
}

Usually long, irregularly barred rods, often with terminal club. bing. Granulation generally poor. Pleomorphism always present. Distinguished from diphtheroids, which may closely resemble them, by irregularity of size, shape, barring and arrangement. Some strains indistinguishable from mitis.

Colonies are uniform, small, discrete, delicate, almost misty in appearance, and under. go little increase in size between 24 and 48 hours. At 18 hours the colony is less than $1 \mathrm{~mm}$. q., is slightly raised, with or with. out umbonation, or is of sugar-loaf appearance; centre is greyishblack and generally darker than periphery; surface smooth or very finely granular, and edge entire or slightly spiky. Consistency is intermediate botween the brittle gracis and the butyrous mitis type. Hæmolysis never seen. At 45 hours colony is not much larger, has a dull granular centre and a smoother more glistening periphery, and is dark in colour except for a lighter ring near the edge-frog's-egg appearance. Edge may be entire or finely crenated. On further incubation, colony may enlarge and come to resemble a daisy-head colony of the gravis type.
Usually long, curved, pleomorphic rods with prominent metachromatic granules. Except for some shadow areas, protoplasm stains evenly. Some strains show barring, with or withont granules. Occasional strains are coccoid and others yeast-like.

Very variable in size, usually ranging between intermedius and gravis. At 18 hours colonies may be less than $1 \mathrm{~mm}$. q. up to $1.5 \mathrm{~mm}$. q. They are circular, convex, usually of a mushroomgrey colour, darker than that of gravis, though varying considerably. with a smooth, glistening surface and entire edge. Consistency is of soft butter, and emulsifiability is easy. Small ring of hamolysis is nsual. At 45 hours colony is $2-4 \mathrm{~mm}$. (.), undifferentiated, and dark greyish - black with sometimes a nar. row paler margin. On further incubation colony may become flatter witl a central elevation - poached. egg appearance--and the surface may become granular and contonred, or develop concentric rings or papular excrescences. 
TABLE 30-continued

\begin{tabular}{|c|c|c|c|}
\hline 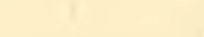 & Gravis. & Intermedius. & Mitis. \\
\hline $\begin{array}{l}\text { Growth in } \\
\text { broth. }\end{array}$ & $\begin{array}{l}\text { Appearance variable. } \\
\text { Usually surface pel- } \\
\text { licle and coarse granu- } \\
\text { lar deposit with little } \\
\text { or no turbidity. }\end{array}$ & $\begin{array}{l}\text { Appearance very con- } \\
\text { stant. In } 24 \text { hours } \\
\text { there is slight turbidity } \\
\text { witl little or no de- } \\
\text { posit. In } 48 \text { hours } \\
\text { brotl has cleared, and } \\
\text { there is a very finely } \\
\text { granular sediment, } \\
\text { which can be easily } \\
\text { dispersed on shaking. }\end{array}$ & $\begin{array}{l}\text { Appearance variable. } \\
\text { Usually diffuse even } \\
\text { turbidity, denser than } \\
\text { that of intermedius, } \\
\text { and moderate non- } \\
\text { granular deposit. Soft } \\
\text { pellicle may form on } \\
\text { further incubation. }\end{array}$ \\
\hline $\begin{array}{l}\text { Fermentation } \\
\text { of starch and } \\
\text { glycogen. }\end{array}$ & + & -1 & - \\
\hline $\begin{array}{l}\text { Antigenic } \\
\text { structure. }\end{array}$ & $\begin{array}{l}\text { At least } 5 \text { types recog- } \\
\text { nizable by direct ag- } \\
\text { glutination. Types I } \\
\text { and II form the clas- } \\
\text { sical daisy-head col- } \\
\text { onies; Types III, IV } \\
\text { and V approach nearer } \\
\text { to the mitis type. }\end{array}$ & $\begin{array}{l}\text { Little known, but prob- } \\
\text { ably at least } 2 \text { types } \\
\text { with specific antigens. }\end{array}$ & $\begin{array}{l}\text { Little known, but at } \\
\text { least } 5 \text { distinct types. }\end{array}$ \\
\hline $\begin{array}{l}\text { Animal } \\
\text { pathogenieity. }\end{array}$ & $\begin{array}{l}\text { Almost invariably viru- } \\
\text { lent to guinea-pigs. }\end{array}$ & $\begin{array}{l}\text { Almost invariably viru- } \\
\text { lent to guinea-pigs. }\end{array}$ & $\begin{array}{l}\text { Usually virulent to } \\
\text { guinea-pigs, but strains } \\
\text { from carriers are often } \\
\text { avirulent. }\end{array}$ \\
\hline
\end{tabular}

Note. $\quad y=$ diameter.

Fermentation reactions are often of help, since many diphtheroids either ferment sucrose or have no action at all on sugars. Some strains cannot be distinguished except by virulence tests, and even this method leaves the true nature of an avirulent mitis-like organism doubtful. Whether in identifying the individual types of

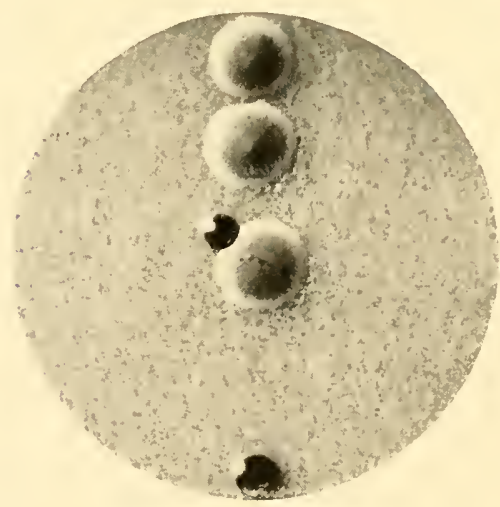

Fig. 83.-C. diphtherice.

Three colonies of gravis type and two of mitis type, on blood-tellurite-agar $(\times 8)$.

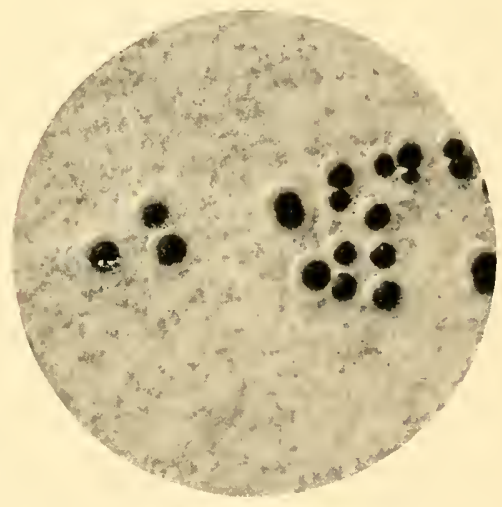

Fis. 34.-C. diphtherice.

Colonies of intermedius type on bloodtellurite-agar $(\times 8)$.

diphtheria bacilli, or in distinguishing between diphtheria bacilli and diphtheroid bacilli that closely resemble them, too much attention should never be paid to any one character. Often it is only by observing all the characters of a particular 
strain, and assigning to each character a weight which experience alone can provide, that a sound conclusion on its identity can be reached. Valuable help will be obtained by a careful study of McLeod's (1943) review.

\section{The Classification of Corynebacteria.}

It will be obvious from the foregoing discussion that the time has not yet arrived to attempt any general systematic classification of the large number of different types of corynebacteria that have been described. Certain organisms have, however, been studied in sufficient detail to make it clear that they deserve specific rank. Among these the type species, $C$. diphtheria, is of course pre-eminent. Among the non-pathogenic species parasitic to man $C$. hofmanni is a well-recognized species, and $C$. xerosis would probably fall into the same category. We follow Andrewes and his colleagues (1923) in excluding the so-called $C$. coryza segmentosum from the list of identifiable species. Including the various species and types, of human or animal origin, that have been dealt with above, this leaves us with the following named species within the genus: $C$. diphtheria (types, gravis, mitis and intermedius), $C$. hofmanni, C.xerosis, C. ovis, C. pyogenes, C. equi, C. renale, C. murium, C. acnes and $C$. typhi. For the rest, we are in entire agreement with Andrewes and his colleagues in believing that specific names should be withbeld from the numerous diphtheroids that have been described until they have been examined in greater detail and their identity more fully established.

We append a summarized description of the named species, and a tabular description of the fermentation reactions of the eleven types of diphtheroid bacilli differentiated by the Committee of the Medical Research Council (see Andrewes et al. 1923). Reference may also be made to a paper by Brooks and Hucker (1944), who divided 79 strains of animal diphtheroids into 3 groups on the basis of growth and biochemical reactions.

\section{C. diphtheriæ}

Observed by Klebs (1883); isolated and described by Loeffler (1884).

The morphology and staining reactions of this species have been described above, and the absence of motility and capsulation, common to the genus, has been noted.

'TYPE OF GRowth.-On Loeffler's serum, the colonies, after 24 hours' incubation at $37^{\circ} \mathrm{C}$, are about $1 \mathrm{~mm}$. in diameter, circular, convex, with a slightiy raised centre, a smooth or finely granular surface and an entire edge; granular in structure when viewed by transmitted light, butyrous in consistency. pale or deeper cream in colour, moderately opaque, and easily emulsifiable in water or saline. After 48 to 72 hours' incubation the colony shows a varying degree of enlargement, the centre becomes mure raised, more opaque, and deepens in colour, while the periphery remains flat, extends outwards and appears more transparent than the centre, giving the so-called "poached-egg" appearance.

On agar, growth is much less abundant, and the individual colonies are, for the most part, smaller, often having a diameter of $0.25 \mathrm{~mm}$. or less, after 24 hours' incubation at $37^{\circ} \mathrm{C}$. These small colonies, which are greyish-white in colour, convex with a raised central portion, and usually with an entire margin, are frequently mingled with a few larger, whiter colonies.

On gelatin, the growth is very similar to that on agar, but develops much more slowly owing to the lower temperature of incubation. In stab culture growth develops along the whole length of the needle-track without lateral outgrowths, and with a slight surface growth consisting of a raised central portion and a flatter periphery, sometimes showing an irregular margin. The medium is never liquefied.

On potato, growth is usually very scanty and often invisible to the naked eye. 
On tellurite blood agar plates, the colonial differences that characterize the gravis, mitis and intermedius types (see Table 30 ) can most readily be observed. These colonial differences are, however, also observable on certain other media, such as trypsin serumagar (Dudley et al. 1934).

Broth. See Table 30.

$C$. diphtherice is aerobic and facultatively anaerobic.

The optimum temperature for growth is in the near neighbourhood of $37^{\circ} \mathrm{C}$.; with a range from about $15^{\circ}$ to $40^{\circ} \mathrm{C}$. over which growth occurs.

Heat resistance is slight, a temperature of $58^{\circ} \mathrm{C}$. for 10 minutes sufficing to sterilize a suspension or broth culture.

Biochemical Reactions. - C. diphtherice ferments glucose, galactose, and maltose, with the production of acid but no gas. It has no action

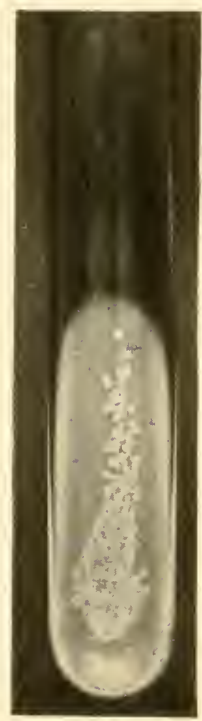

FIG. 85. $-C$. diph. therice.

24-hours' culture on Loeffler's serum. on saccharose, lactose or mannitol. Litmus milk is unchanged. Indole is not formed; but, according to the results obtained by Frieber (1921), C. diphtherio gives a colour reaction with sulphuric acid and potassium nitrite as a result of the formation of indoleacetic acid from tryptophan. This substance does not, however, give the colour reaction with paradimethylamidobenzaldehyde which is characteristic of indole itself. Nitrates are reduced. Gelatin is not liquefied.

The gravis type, in addition to the carbohydrate substrates referred to above, ferments dextrin, starch and glycogen. The mitis and intermedius types give irregular results with dextrin and do not ferment starch or glycogen.

The mitis type is usually hæmolytic, the gravis type is usually non-hæmolytic, and the intermedius type is consistently nonhæmolytic.

Antranic Structure. $-C$. diphtherice is divisible into a number of different antigenic types. The gravis, mitis and intermedius types differ antigenically from each other, and each is divisible into a number of antigenic sub-types.

Toxin Production and Pathogenicity.-C. diphtherice is pathogenic for man and for certain laboratory animals. It produces a powerful exotoxin with a characteristic action on the animal tissues (see above). Non-virulent strains of the mitis type are not uncommon.

\section{C. hofmanni}

Von Hofmann, in 1888, isolated from the throats of normal persons a diphtheroid bacillus which was probably identical with the species which now bears his name. The incompleteness of the earlier descriptions does not allow us to identify with any certainty the various strains which were, about this time, described under the general head of "pseudodiphtheria bacilli." The description which follows refers to a particular type of diphtheroid bacillus to which the name of $C$. hofmamni has been allotted by common consent. There are other forms of non-fermenting diphtheroid bacilli which possess quite a different morphology. These must, for the moment, be left unnamed, finding a temporary home in the appropriate groups of the fermentative types differentiated by the Committee of the Medical Research Council.

Morphology.- Short rods, 1.5 to $2 \mu$ in length, with parallel sides, rounded or slightly pointed ends, with a straight axis, and a single unstained central septum. Metachromatic granules are usually absent; if present they are few in number, small and inconspicuous. There is little or no tendency to pleomorphism. The bacilli are arranged in parallel rows, or in irregular groups, with the usual angular displacement of adjacent cells (see 
Fig. 56). The bacilli are more tenacious of the Gram stain than $C$. diphtherice, or than many other diphtheroids.

Growtr.-On Loefler's serum, after 24 hours at $37^{\circ}$ C., C. holmanni produces colonies which are larger than those of $C$. diphtherice, and whiter in colour. They vary in diameter from 1 to $1.5 \mathrm{~mm}$., and are circular, convex, smooth, and opaque, with an entire edge. They are homogeneous in structure, butyrous in consistency, and emulsify readily. After 48 hours they increase in size to a diameter of $2 \mathrm{~mm}$. or so, and the edge may become slightly erose. On agar this species, unlike $C$. diphtherice, grows readily and abundantly, forming colonies very similar to those produced on serum. In contrast to $C$. diphtherice, a confluent growth often occurs in primary culture, or in early subculture. In broth a moderate turbidity is produced, the growth gradually settling to the bottom as a powdery deposit. No pellicle is formed. In agar or in gelatin stab $C$. hofmanni produces little growth along the needle track, but a profuse surface growth. C. hofmanni is aerobic and facultatively anaerobic ; the optimal temperature for growth is in the neighbourhood of $37^{\circ} \mathrm{C}$. The resistance of this species to heat, or to antiseptics, has not been systemati. cally examined, but there is no evidence that it differs in these respects from the type species.

Biochemical Reactions.-C. hofmanni ferments none of the carbohydrates against which it has been tested, and these include all those referred to in the case of $C$. diphtherice. It produces no change in litmus milk, does not liquefy gelatin, and does not produce indole. Nitrates are reduced. It does not produce hæmolysis.

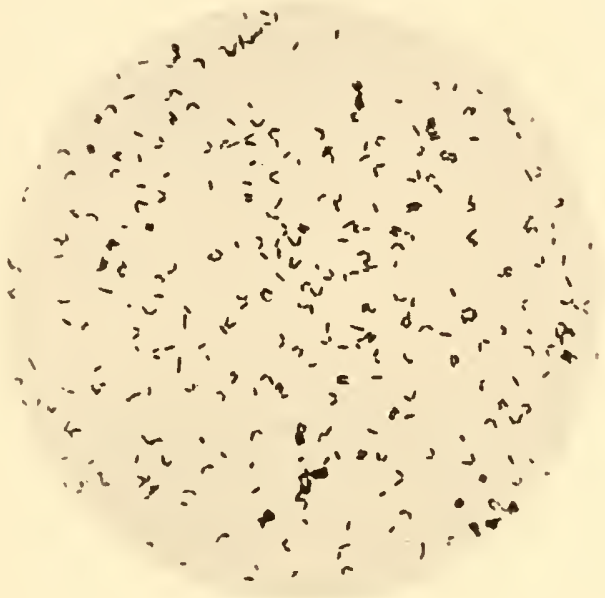

Fic. 86.-C.hofmanni.

From 24-hours' culture on Loeffler's serum $(\times 1000)$.

Antigenic Structure.-All that is known on this point is that the antigenic makeup of such strains of $C$. hofmanni as have been examined differs entirely from that of $C$. diphtherice on the one hand, and from $C$. xerosis on the other.

Toxin Prodoction and Pathogenicity.-C. hofmanni produces no toxin and is not pathogenic.

\section{C. xerosis}

An organism which possessed certain of the characters which we ascribe to $C$. xerosis was isolated from the conjunctiva by Reymond in 1881, and described somewhat more fully in 1883. The first detailed description of this organism was provided by Kuschbert and Neisser in 1883, if we may assume that these three records in fact refer to the same bacterial species. The original view, that $C$. xerosis was retiologically related to a particular conjunctival lesion, has been generally abandoned. Griffith (1901) states that this organism is the commonest bacterial inhabitant of the normal conjunctival sac. Andrewes and his colleagues record their doubt as to whether $C$. xerosis is even now sufficiently well characterized to deserve specific rank; and their scepticism appears fully justified. The description which follows must be regarded as summarizing the characters of those strains to which most observers would allot this particular name.

Morphology.-The form assumed by $C$. xerosis, in films from a culture grown on 
Loeffler's serum for 24 hours at $37^{\circ} \mathrm{C}$, is not unlike the bacillary form of $C$. diphtherice ; the differences consist in a preponderance of barred or segmented forms over the granular form, the infrequency of club forms, the relative infrequency and inconspicuousness of metachromatic granules, though these are often present in small numbers, and the relatively slight pleomorphism. The cells of this species retain the Gram stain more tenaciously than those of $C$. diphtheria.

Growth.-On Locfler's medium, or on agar, C. xerosis forms colonies which are smaller than those of $C$. diphtherice or of $C$. hofmanni; the margins may become irregular after 48 to 72 hours, and the colonies tend to adhere firmly to the medium during the later stages of growth. Broth remains clear, or shows a slight turbidity, while a granular deposit forms at the bottom of the tube. No pellicle is formed. The general conditions of growth, as regards temperature, oxygen pressure, etc., do not differ from those of the type species.

Biochemical Reactions.-C. xerosis produces acid in glucose, maltose and saccharose, but not in dextrin or mannitol. It does not acidify litmus milk, does not produce indole, and does not liquefy gelatin. Nitrates are reduced. It apparently forms no hæmolysin.

Antigenic Structure.-The antigenic constituents of those strains of $C$. xerosis which have been examined differ from those of $C$. diphtherice on the one hand, and from C. hofmanni on the other.

Pathogenicity.-There is no adequate evidence that $C$. xerosis is pathogenic.

\section{C. ovis}

\section{(Synonym. C. pseudotuberculosis ovis.)}

The Preisz-Nocard bacillus (see. Nocard 1889, Preisz 1894) was originally isolated from psendotuberculous lesions in sheep. Similar organisms have been isolated from ulcerative lymphangitis of horses by many workers. It resembles the type species very closely in its general morphology and behaviour.

Morphology.-Films from young cultures on Loeffer's medium show slender, clubbed bacillary forms, granular or segmented, often with numerous metachromatic granules, and exhibit a considerable degree of pleomorphism. The appearances are, in fact, indistinguishable from those presented by certain strains or cultures of $C$. diphtherice.

Growtн.-The organism grows well on Loeffler's medium, giving colonies which are cireular, umbonate, and opaque, with a tendency towards the develonment of a distinct yellowish pigment. As they enlarge they often develop a series of concentric rings round the raised centre. The growth is described as peculiarly friable, the colony breaking apart when touched by the needle (Hall and Stone 1916). The growth on agar is recorded as poor. Growth in broth is scanty, but there is definite pellicle formation. On blood agar, especially under anaerobic conditions, colonies are surrounded by a zone of hæmolysis. Moderate granular growth in gelatin with slight saccate liquefaction. Aerobic and facultative anaerobe, growing best at about $37^{\circ} \mathrm{C}$.

Biochemical Reactions.-Forms acid from dextrose, maltose, and glycerol. Accord. ing to most workers (Hall and Stone 1916, Minett 1922a, b, Andrewes et al. 1923) mannitol, lactose and sucrose are not fermented, but Carne (1939) in Austrahia reports that about half of his 133 strains from sheep fermented these sugars. Dextrin may be weakly acidified. Carne (1939) gives the following additional reactions for his strains: M.R. weakly positive, V.P. negative, $\mathrm{H}_{2} \mathrm{~S}$ formed by about half the strains. Gelatin is liquefied slowly and irregularly; nitrates reduced by some but not by all strains; indole not formed. C. ovis produces a hæmolysin, active against sheep, horse and rabbit corpuscles; according to Carne (1939) the hæmolysin is thermolabile, non-antigenic, unrelated to the exotoxin, and is linked to the cells so that it cannot be obtained free in a filtrate.

Antigenic Structure.-At present uninvestigated. 
Pathogenicity.-C. ovis is a natural pathogen of horses, sheep and perliaps cattle, and is pathogenic for rabbits and guinea-pigs, but not for pigeons or fowls. It produces a soluble toxin which differs from that of $C$. diphtherice (see above).

\section{C. pyogenes}

First described by Lucet in 1893 .

MorphologY.-The organism is a small, Gram-positive, pleomorphic, diphtheroid bacillus, frequently assuming an almost coccal form, staining irregularly with methylene blue, but apparently without metachromatic granules.

GrowTH.-Scanty on plain media, but improved by addition of blood or serum. Grows aerobically and anaerobically. Optimum temperature $37^{\circ} \mathrm{C}$.; little or no growth at room temperature. Optimum $\mathrm{pH}$ 7.5. On Loeffler's serum C. pyogenes forms minute colonies in 24 hours at $37^{\circ} \mathrm{C}$, which slowly enlarge, if incubation is continued, until they may reach a diameter of $2-3 \mathrm{~mm}$. ; the centre becomes granular and the medium is slowly liquefied, the liquefaction beginning as a small pit beneath each colony. On blood agar colonies, rarely exceeding $1 \mathrm{~mm}$. in diameter, are visible in 48 hours, surrounded by a zone of $\beta$-hæmolysis (Brown and Orentt 1920, Lovell 1937). In serum broth there is a granular growth without pellicle formation. On gelatin growth is slight, but the medium is slowly liquefied. No growth on MacConkey agar or on potato.

Brochemical Reactions.-Acid in glucose, maltose, and later in lactose; occasional strains ferment mannitol and sucrose ; dextrin and glycerol are also said to be fermented (Magnusson 1938). Litmus milk acidified and elotted within 3 days ; elot is later digested. Gelatin, coagulated serum, and coagulated egg albumin are gradually liquefied. Indole negative. Nitrates reduced to nitrites. Filtrable hremolysin produced, which is most active on horse and rabbit corpuscles and which is destroyed at $56^{\circ} \mathrm{C}$. in 30 minutes; appears to be identical with the toxin; reaches its maximum concentration in culture in 48 hours at $37^{\circ} \mathrm{C}$. (Lovell 1937,1941 ).

Resistance.- Rapidly killed at $57^{\circ} \mathrm{C}$., and very sensitive to disinfectants (Brown and Orcutt 1920).

Axtigenic Structure. - Brown and Orcutt studied 12 strains without finding any sharp difference in antigenic behaviour between them. Lovell (1937) studied 33 strains, and except for 5 strains which had been subcultured for some years and which contained a major and a minor antigen, found that, irrespective of animal origin, they appeared to be antigemically homogeneous.

Pathogenicity.--Under natural conditions prodnces suppurative lesions in cattle, pigs, sheep, and goats, but not in horses; is also pathogenie for the rabbit, producing suppurative lesions including arthritis. Experimentally, mice inoculated intraperitoneally with 100-1,000 million organisms die in a week with abscess formation in the omentum and liver. Rabbits inoculated subcutaneonsly develop localized abscesses; inoculated intravenously they develop abscesses, particularly in the bones and joints. C. pyogenes forms a weak toxin which, inocnlated intravenously into rabbits in a dose of 1 to $5 \mathrm{ml}$., produces convulsions and death within 30 minntes (Lovell 1937).

\section{C. equi}

This organism was isolated by Magnusson (1923) from foals affected with pyæmia. It has been confused with $C$. pyogenes, but differs from it in several respects, notably in its abundant growth on ordinary media, its pigment formation, its failure to liquefy coagulated serum, to lyse blook, or to ferment carbohydrates, and its pathogenicity for horses. The following description is taken from Magnusson (1938) and Karlson, Moses and Feldman (1940).

Morphology.-Fairly large, pleomorphic, Gram-positive bacillus, showing metachromatic granules; in pus and surface colonies, may appear coccoid. Reporterl by some workers to be partly acid-fast. 
Growth.-Grows frecly on ordinary media, forming large succulent colonies of irregular shape and pale pink colour. On potato the growth is moist, thick, and pale pink, later becoming deep reddish yellow. Slate to black colonies on tellurite blood agar. Grows well between $18^{\circ}$ and $37^{\circ} \mathrm{C}$.

Resistaxce. - Said by Karlson, Moses and Feldman (1940) to be unusually resistant to oxalie acid, which is sometimes used for the destruction of non-acid-fast bacilli.

Biochemical Reactions.-No fermentation of sugars. Poor growth in litmus milk with no obvious change. No hæmolysis. No liquefaction of coagulated serum or gelatin. Indole negative. Nitrates reduced to nitrites. Slight $\mathrm{H}_{2} \mathrm{~S}$ production. Catalase formed.

Antigenic Structure. - Strain specificity said to be marked, so that the agglutination reaction is of little help in identification. By complement fixation, however, a speciesspecific antigen ean be demonstrated (Bruner, Dimock, and Edwards 1939).

Pathogenicity.-Gives rise under natural conditions to pyæmia in foals, characterized by a suppurative bronchopneumonia with intense purulent infiltration of the adjoining lymph nodes, and sometimes to intestinal uleeration and abscess formation in the mesenteric lymph nodes. Probably non-pathogenic for swine, though frequently present in the submaxillary lymph nodes. Experimentally, the natural disease can be reproduced by intratracheal inoculation of foals. Subcutaneous inoculation produces local abscess formation with involvement of the focal lymph notes in horses, pigs, and goats. Gives rise to peritonitis, not always fatal, when inoculated intraperitoneally into guinea-pigs.

\section{C. renale}

This organism was first described by Enderlen (1890-91), but it is very doubtful whether all the strains of diphtheroid bacilli that have since been isolated from pyelitis in cattle were identical with the bacillus isolated by him; and it is by no means certain that the organism described by more recent workers is entitled to specific rank. It seems quite clear, however, that this organism differs in several ways from C. pyogenes, with which it has often been confused. The incomplete description that follows is taken mainly from the papers of Jones and Little (1925, 1930) and Merchant (1935) and is given with considerable reserve.

Monphology.-C. renale is a typical Gram-positive barred diphtheroid, showing numerous metachromatic granules, and considerable pleomorphism.

Growth.-On serum agar gives moist, raised colonies showing a pigmentation that varies from cream to yellow. Later the growth becomes drier.

Biochemical Reactions.-Most strains that have been examined have fermented dextrose alone, with the production of acid. According to Merchant (1935), lævulose and mannose may also be fermented. Gelatin is not liquefied. No hæmolysin is produced.

Antigenic Structure.-The few data available (see Merchant 1935) are insufficient to allow any adequate description.

Pathogenicity.-The organism has been isolated by several observers from cattle suffering from pyelitis: and Jones and Little $(1925,1930)$ have reproduced the disease experimentally in these animals by the injection of pure cultures. No data are available with regard to its pathogenicity for laboratory animals.

\section{C. murium}

(Synonym. C. pseudotuberculosis murium.)

Isolated from a mouse by Kutscher in 1894 and by Bongert in 1901. It has since been isolated by several observers (Andrewes et al. 1923).

Morphology.- In films from cultures on Loeffler's medium the appearances are very similar to those presented by $C$. ovis, or by some strains of $C$. diphtherice (sce Fig. 87). 
Growtr.-The type of growth on serum, agar, and gelatin appears to be very like that of $C$. diphtherice. Pellicle formation in broth has not been recorded, but suitable conditions have perhaps never been secured.

Biochemical Reactions.-Acid is produced from dextrose, maltose, and saccharose ; galactose, dextrin, lactose, and mannitol are unchanged. Litmus milk is unchanged, or shows a slight and transitory acidity. Indole is not formed. Gelatin is not liquefied. Nitrates are reduced.

Antigenic Structure.-At present uninvestigated.

Toxin Production and Pathogenicity.-C. murium is a natural pathogen of mice, and appears to be pathogenic for no other species, with the possible exception of rats. There is evidence that some of its effects are due to the action of a soluble toxin (see above).

\section{C. acnes}

A diphtheroid organism was described in the ordinary lesions of cutaneous acne by Unna in 1896, and was isolated in culture by Sabouraud (1897).

Morphology. - This has been generally reported as very variable, according to the medium employed, degree of acidity, oxygen pressure, etc. Craddock (1942) distinguishes two types on blood agar : in large colonies the organisms are short, thick, irregular in shape, and often clubbed; in small colonies the bacilli are longer, thinner and curved. Often weakly Gram-positive.

Growth.-C. acnes will grow aerobically if the medium contains serum or blood, and is acidified by the addition of lactic, or of hydrochloric acid, so as to fall within the $\mathrm{pH}$ range 6.2-6.8. Growth occurs better under anaerobic conditions, and is favoured by glucose, glycerol, blood, boiled blood, Fildes' extract of blood, and serum. On plain agar growth is poor

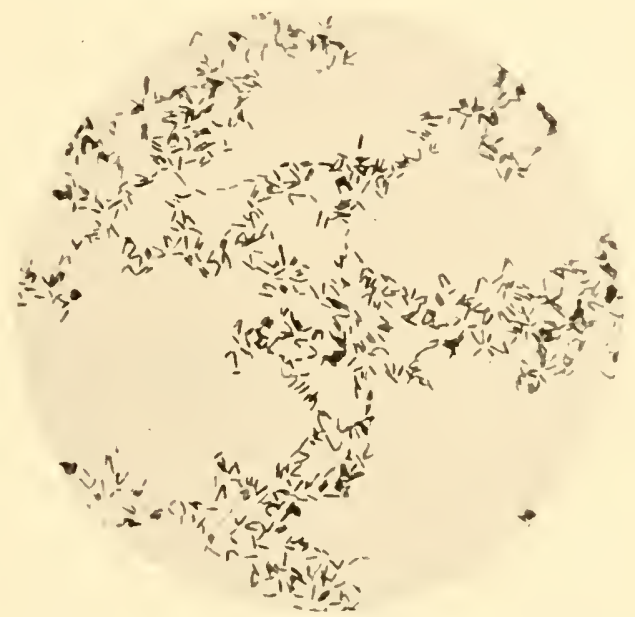

Fig. 87.-C. murium.

From 24-hours' culture on Loeffler's serum $(\times 1000)$. or absent. On a glucose agar plate anaerobically after 4 days at $37^{\circ} \mathrm{C}$. the colonies are circular, $0 \cdot 2-0 \cdot 4 \mathrm{~mm}$. in diameter, convex, amorphous, greyish-white, with a smooth glistening surface and an entire edge; they are butyrous in consistency, and emulsify easily. The growth has a sour smell. After 6 weeks, colonies may be coloured pink. On blood agar anaerobically Craddock (1942) describes two types: Type I forms a large heaped-up colony, yellowish-buff in colour, with a wide zone of hæmolysis ; Type II forms a small flat colony. In a glucose agar shake medium no growth occurs for about $10 \mathrm{~mm}$. below the surface ; there is then often a band growth for $10-20 \mathrm{~mm}$. below the surface, with discrete colonies to the bottom of the tube; the medium becomes milky and opaque. Loeffler's serum, no liquefaction. In glucose broth there is a slight turbidity after 3 days anaerobically, and a slight, finely granular sediment; after a week or so there is a heavy loose floccular deposit, which occupies the lower centimetre of the tube and disintegrates on shaking to give a moderate turbidity. Slight to moderate turbidity in cooked meat medium. No growth in gelatin stab culture at $22^{\circ} \mathrm{C}$.

Biochemical Reactions.-According to Südmersen and Thompson (1909-10) C. acnes produces acid from glucose, galactose, maltose, glycerol, and mannitol, but does not, 
ferment lactose. Of 2 strains examined by Südmersen and Thompson, one actively fermented saccharose, the other gave a late and slight acidity. In our experience acid is generally formed in glucose, maltose, and sucrose, sometimes in lactose, but not in mannitol. Slight acid in litmus milk. Indole negative. Nitrates reduced. Gelatin not liquefied.

Antigenic Structure.-According to Craddock (1942) two types can be distinguished by agglutination with specific rabbit sera.

Pathogenicity and Toxin Production.-It is generally believed that $C$. acnes is rtiologically related to the lesions from which it has been isolated. Experimentally it shows some degree of pathogenicity for the mouse.

\section{C. typhi}

Isolated by Plotz (1914) from the blood of patients suffering from typhus fever. Originally regarded by Plotz and his colleagues as atiologically related to the disease (Plotz, Olitsky and Baehr 1915), but now generally admitted to be an example of a parasitic microaerophilic diphtheroid, without any established pathological significance (Olitsky 1921).

Morphology is that of a small pleomorphic diphtheroid, with few elongated cells but numerous coccal forms. The rod-forms which occur may be straight or curved, with rounded or pointed ends. Metachromatic granules are present.

GrowTH.-C. typhi appears to demand more strictly anaerobic conditions for growth than $C$. acnes; but there appears to be no record of the result of acidification of the medium. It is recorded as giving a creamy-white growth on Loeffler's serum, glucose serum agar, or potato; the growth takes on a light brown colour in its later stages on the latter medium.

Biochemical Reactions.-Tested on ascitic agar containing 2 per cent. of the test carbohydrate, and incubated in Buchner tubes, $C$. typhi is stated to produce acid from glucose, maltose, and galactose, but not from mannitol, dextrin, lactose or saccharose.

Antigenic Structure unknown.

Pathogenicity.-Probably slight or absent.

We append, in tabular form (Table 31), the chief differential characteristics of the ten named species described above, and the fermentation reactions of the eleven groups of diphtheroid bacilli differentiated by the Committee of the Medical Research Council (Table 32). In both tables the + sign signifies the formation of acid. Since the fermentation reactions of this group are habitually tested in Hiss's serum-water medium, the formation of acid will usually be followed by the formation of a clot, after a longer or shorter period, though some strains which produce definite acidity in the presence of a particular carbohydrate fail to clot the medium.

With regard to the eleven groups of unnamed diphtheroids, the Committee note that there is no correlation between the source from which any strain was derived and its biochemical reactions. Thus, of the 15 strains which fall into Group I, ten came from the nose, one from the ear, two from the eye, one from an infected wound, and one from a specimen of pus. As already noted, there was no consistent relation between pigment production and fermentative activity. Thus one strain of Groups IV, VII and XI produced pigment, as did the single representative of Group IX. The four strains of Group VI, on the other hand, and the three strains of Group VIII all formed pigment; so that these two groups would appear to be characterized by a marked tendency to pigment production. It may be noted that none of the strains examined produced the pink or red pigments which have 


\section{TABLE 31}

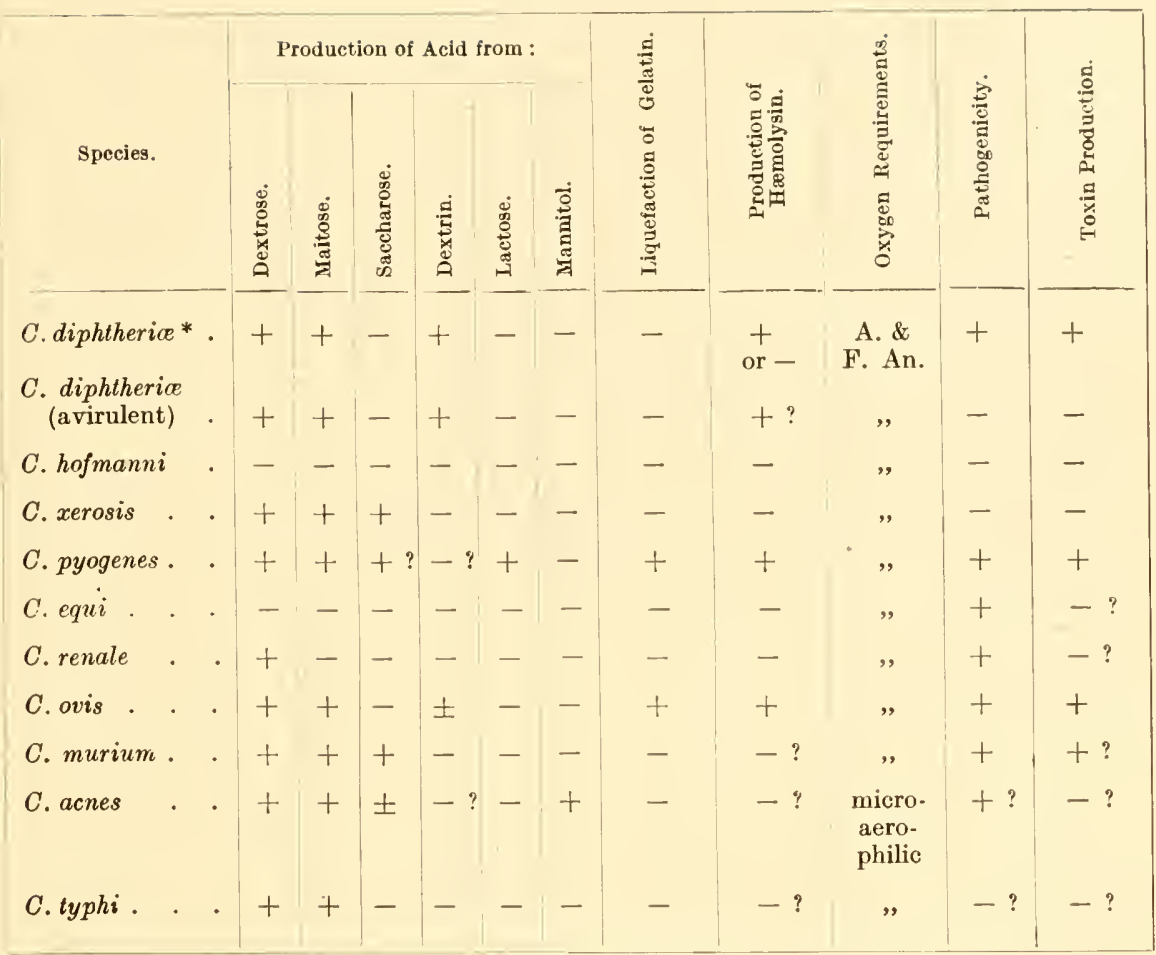

* See Table 30 for differentiation between gravis, mitis and intermedius.

been so frequently noted by some observers, and especially by those who have examined numerous strains from lymphatic glands and other tissues (Hoag 1907, Harris and Wade 1915). The Committee note that the strains selected have been tested on many occasions, and at long intervals, with scarcely varying results.

TABLE 32

Fermentation Reactions of 79 Unselected Strains of Diphtheroids, examined for Committee of Medical Research Council (see Andrewes et al. 1923).

\begin{tabular}{r|c|c|c|c|c|c|c|c}
\hline Group. & Dextrose. & Maltose. & $\begin{array}{c}\text { Galac- } \\
\text { tose. }\end{array}$ & $\begin{array}{c}\text { Sac- } \\
\text { charose. }\end{array}$ & Lactose. & Dextrin. & Mannitol. & $\begin{array}{c}\text { No. of Strains } \\
\text { in Group. }\end{array}$ \\
\hline I & + & - & + & + & - & - & - & 15 \\
II & + & - & - & + & - & - & - & 11 \\
III & + & + & - & - & - & - & - & 11 \\
IV & + & \pm & - & - & - & - & - & 6 \\
V & + & + & + & - & - & - & - & 4 \\
VI & + & + & + & + & + & - & - & 4 \\
VII & + & + & - & + & - & - & - & 5 \\
VIII & + & + & + & + & - & - & - & 3 \\
IX & + & + & + & + & + & + & - & 1 \\
X & + & - & + & + & - & - & + & 1 \\
XI & - & - & - & - & - & - & - & 18
\end{tabular}




\section{REFERENCES}

Anderson, J. S., Cooper, K. E., Happold, F. C., and McLeod, J. W. (1933) J. Path. Bact., 36, 169.

Anderson, J. S., Happord, F. C., McLeod, J. W., and Thomson, J. G. (1931) J. Path. Bact., 34, 667.

Andrewes, F. W. et al. (1923) Med. Res. Coun., "Monograph on Diphtheria." London. Babes, V. (1886) Bull. Soc. Anat., Paris, 61, 72.

BAILEY, G. H. (1925) J. Immunol., 10, 791.

Barr, M., Glenny, A. T., Pope, C. G., and Lingoood, F. V. (194I) Lancet, ii, 301.

Barratt, M. M. (1923) see Andrewes et al., p. 174; (1933) J. Path. Bact., 36, 369.

Bolton, C. (1905) Lancet, i. 278.

BonoERT. (1901) Z. Hyg. InfektKr., 37, 449.

BrandwiJk, A. C. and Tasman, A. (1932) Z. ImmunForsch., 77, 390 ; (1933) Ibid., 78, 540. BraUn, H. and HoFmeier, K. (1927) Klin. Wschr., 6, 690.

Braun, H., Hofmeier, K., and Mündel, F. (1929) Zbl. Bakt., 113, 530.

BraUn, H. and Mündel, F. (1927) Zbl. Bakt., 103, 182 ; (1929) Ibid., 112, 347.

Bray, J. (1944) J. Path. Bact., 56, 497.

Brooks, R. F. and Hucker, G. J. (1944) J. Bact., 48, 295.

Brown, J. H. and Orcutt, M. L. (1920) J.exp. Med., 32, 219.

Bruner, D. W.. Dimock, W. W., and Euwards, P. R. (1939) J. infect. Dis., 65, 92.

Bull, L. B. and Dićkinson, C. G. (1935) Aust. vet. J., 11, 126.

Bunker, J. W. (1919) J. Bact., 4, 217.

Bunny, W. E., Cianiarulo, J., and Kiamil, M. (1931) J. Immunol., 20, 417.

Carne, H. R. (1939) J. Path. Bact., 49, 313 ; (1940) Ibid., 51, 199.

Chalmers, A. J. and Macdonald, N. (1920) J. trop. Med. Hyg., 23, 85.

Coca, A. E., Russell, E. F., and Badohian, W. H. (1921) J. Immunol., 6, 887.

Cooper, K. E., Happold, F. C., McLeod, J. IV., and Woodcock, H. E. de C. (1936) Proc. R. Soc. Med., 29, 1029.

Costa, S., Troisier, J., and Dauvergne, J. (1918) C. R. Soc. Biol., 81, 89.

Cowan, M. L. (1927) Brit. J. exp. Path., 8, 6.

Crapdock, S. (1942) Lancet, i, 558.

Crowel., M. J. (1926) J. Bact., 11, 65.

Dassonville. (1907) Bull. Soc. cent. Méd. vét., 61, 576.

Davis, L. and FerRY, N. S. (1919) J. Bact., 4, 217.

DUdeEon, L. S. (1906) Brain, 29, 227.

Dudley, S. F., May, P. M., and O'Flynn, J. A. (1931) Spec. Rep. Ser. med. Res. Coun., Lond., No. 195.

Durand, P. (1918) C. R. Soc. Biol., 81, 1011 ; (1920) Ibid., 83, 613.

Eagleton, A. J. and Baxter, E. M. (1923) J. Hyg., Camb., 22, 107.

Eaton, M. D. (1936) J. Bact., 31, 367.

Eaton, M. D. and Bayne-Jones, S. (1934) j. Bact., 29, 56.

Eberson, F. (1918) J. infect. Dis., 23, 1.

Ehrismann, O. (1932-33) Zbl. Bakt., 127, 111 ; (1933) Z. Hyg. InfektKr., 115, 273.

Enderlen, E. (1890-91). Dtsch. Z. Thierheilk., 17, 325.

Ernst, W. (1888) Z. Hyg. InfektKr., 4, 25 ; (1889) Ibid., 5, 428; (1905) Zbl. Bakt., 39, 549,660 ; (1906) Ibid., 40, 79.

Ewing, J. O. (1933) J. Path. Bact., 37, 345.

Fischu, V., Коесн, M., and Kussat, E. (1931) Z. Hyg. InfektKr., 112, 421.

Fleming, A. (1909) Lancet, i. 1035.

Flexner, S. (1897) Rep. Johns Hopk. Hosp., 6, 259.

Frieber, W. (1921) Zbl. Bakt., 87, 254.

Gilchrist, T. C. (1900) Bull. Johns Hopk. Hosp., 9, 409 ; (1903) J. cutan. Dis., $21,107$. GraQE, F. (1903) Z. Fleisch-u. Milchhyg., 13, 166.

Grass, V. (1937) J. Path. Bact., 44, 235; (1939a) Ibid.. 48, 507; (1939b) Ibid., 49, 549. Glenny, A. T. (1925a) J. Hyg., Camb., 24, 301 ; (1925b) J. Path. Bact., 28, 251.

Glenny, A. T. and Allen, K. (1922) J. Path. Bact., 24, 61.

Glenny, A. T. and Walpole, G. S. (1915) Biochem. J., 9, 298.

Goldie, H. (1933) C. R. Soc. Biol., 112, 1210 ; (1934) Ibid., 116, 17.

Goldsworthy, N. E. and Wilson, H. (1942) J. Path. Bact., 54, 183.

Goodman, H. M. (1907) J. infect. Dis., 4, 509.

Graham-Simti, G. S. (1908) "Diphtheria." Nuttall and Graham-Smith, Cambridge.

Griffith, A. S. (1901). Rep. Thomps. Yates Lab. Univ. L'pool., 4, 99.

Grips. (1898) Z. Fleisch-u. Milchhyg., 8, 166.

Groer, F. von. (1923) Biochem. Z., 138, 13.

Gundel, M., György, P., and Pagel, W. (1932) Z. IIyg. InfehtKr., 113, 6:9.

Hadley, P. B. (1907) J. infect. Dis., 3, Suppl. No. 3., 95. 
HADley, P. B. and Gorham, F. P. (1907) Zbl. Bakt., Ref., 40, 392,

Hall, I. C. and Stone, R. V. (1916) J. infect. Dis., 18, 195.

Harris, IV. H. and WADE, H. W. (1915) J. exp. Med., 21, 493.

Hartley, P. (1922) J. Path. Bact., 25, 479.

Hartley, P. and Hartley, O. (1922) J. Path. Bact., 25, 458.

Havens, L. C. (1920) J. infect. Dis., 26, 388.

Hendry, Catherine B. (1938) J. Path. Bact., 46, 383.

HILL, H. W. (1898) J. Boston. Soc. med. Sci., 3, 86; (1899) Ibid., 4, 78; (1902a) J. med. Res., 7, 115 ; (1902b) Ibid., \%, 202.

HoAG, L. (1907) Boston med. surg. J., 15\%, 10.

Hofmann, G. von. (1888) Wien. med. Wschr., 38, 65, 108.

HoLth, H. (1908) Z. InfektKr. Haustiere, 3, 155.

Hosoya, S. and Kuroya, M. (1923) Sci. Rep. Inst. infect. Dis., Tokyo Univ., 2, 233.

Hosoya, S., Ozawa, E., and Tanaka, T. (1933) Jap. J. exp. Med., 11, 463.

Hottinger, A. and Hottinger, C. .(1933) Z. Kinderheilk., 54, 440.

Hoyle, L. (1941) Lancet, i, 175 ; (1942) J. IIyg., Camb., 42, 416.

JAFF́, R. (1920) Arb. Inst. exp. Ther. Georg Speyer Hause, Frank. am. M., 11, 5.

Jones, F. S. and Little, R. B. (1925) J. exp. Med., 42, 593 ; (1930) Ibid., 51, 909.

Karlson, A. G., Moses, H. E., and Feldman, W. H. (1940) J. infect. Dis., 67, 243.

KLEBS, E. (1883) Verh. Cong. inn. Med., Wiesbaden, 139.

Knight, B. C. J. G. (1936) Spec. Rep. Ser. med. Res. Coun., Lond., No. 210.

Koser, S. A. and Retteer, L. F. (1919) J. infect. Dis., 24, 301.

KraH, E. and Witebsky, E. (1930) Z. ImmunForsch., 66, 59.

KünNemann, O. (1903) Arch. wiss. prakt. Tierheilk., 29, 128.

Kuschbert and Neisser. (1883) Jber. schles. Ges. vaterl. Kult., 60, 50.

KUTSCher. (1894) Z. Hyg. InfehtKr., 18, 327.

LANGER, H. (1916) Zbl. Bakt., 78, 117.

Lehmann, K. B. and Neumann, R. O. (1896) "Atlas u. Grundriss. d. Bakt. u. Lehrb. d. spez. bakt. Diagnostik." 6th Ed., Munich.

Leonard, G. F. and Holm, A. (1933) J. infect. Dis., 53, 376.

Leulier, A., Sédallian, P., and Clavel. (1931) C. R. Soc. Biol., 107, 1136.

LindemanN, H. (1932) Z. Hyg. InfektKr., 113, 288.

Locke, A. and MAIN, E. R. (1928) J. infect. Dis, 43, 41.

LoefFler, F. (1884) Hitt. ReichsgesundhAmt., 2, 421 ; (1890) Zbl. Bakt., 7, 528.

Lovelt, R. (1937) J. Path. Bact., 45, 339 ; (1941) Ibid., 52, 295 ; (1944) Ibid., 56, 525.

Lucet, A. (1893) Ann. Inst. Pasteur, \%, 325.

McLeod, J. W. (1943) Bact. Rev., \%, 1.

Madousson, H. (1923) Arch. wiss. prakt. Tierheilk., 50, 22; (1938) Vet. Rec., 50, 1459.

Matтick, A. 'T. R. (1944) Pers. comm.

Maver, M. E. (1930) J. infect. Dis., 4\%, 384.

Mellon, R. R. (1917) J. Bact., 2, 269.

Merchant, I. H. (1935) J. Bact., 30, 95.

MinetT, F. C. (1922a) J. comp. Path., 35, 71; (1922b) Ibid., 35, 291.

Mollard, J. and Regaud, C. (1895) C. R. Soc. Biol., 2, 828.

Morton, H. E. and Gonzalez, L. M. (1942) J. Immunol, 45, 63.

Mueller, J. H. (1935a) Science, 81, $50 ;(1935 b)$ J. Bact., 29, 383 ; (1935c) Ibid., 29, 515.

Mueller, J. H., Klise, K. S., Porter, E. F., and Graybiel, A. (1933) J. Bact., 25, 509.

NeILl, G. A. W. (1937) J. Hyg., Camb., 37, 552.

Neill, J. M., Richardson, I. V., Fleming, W. L., Sugg, J. Y., and Gaspari, E. L. (1931) Amer. J. Hyg., 13, 499.

Nicolle, M., Loiseau, G., and Forgeot, P. (1912) Ann. Inst. Pasteur, 26, 83.

Nitsch, J. (1933) Z. Kinderheilk., 54, 470.

Nocard, E. (1889) C. R. Soc. Biol., 1, 608.

OLitsky, P. K. (1921) J. exp. Med., 34, 525.

Pappenheimer, A. M. (1937) J. biol. Chem., 120, 543.

Pappenheiner, A. M. and Johnson, S. J. (1936) Brit. J. exp. Path., 17, 335.

Pappenheimer, A. M. and Robinson, E. S. (1937) J. Immunol., 32, 291.

Petrie, C. F. and McClean, D. (1934) J. Path. Bact., 39, 635.

Plotz, H. (1914) J. Amer. med. Ass., 62, 1556.

Plotz, H., Olitsky, P. K., and Baenr, G. (1915) J. infect. Dis., 17, 1.

Poels. (1912) Tijdschr. Veeartsenijk., 39, 905.

Pope, C. G. (1932) Brit. J. exp. Path., 13, 207.

Pope, C. G. and Healey, M. (1933a) Brit. J. exp. Path., 14, 77 ; (1933b) Ibid., $14,87$.

Pope, C. G. and Linggood, F. V. (1939) Brit. J. exp. Path., 20, 297.

Pope, C. G. and Suтt, M. L. (1932) J. Path. Bact., 35, 573.

Preisz, H. (1894) Ann. Inst. Pasteur, 8, 231.

Ramon, G. and Berthelót, A. (1932) C. R. Soc. Biol., 110, 530.

Report (1942) Mon. Bull., Emerg. publ. Hlth. Lab. Serv., Feb., p. 12. 
Reymond, Colomiatri, and Perronotto. (1881) Cong. period. int.ophthalm. C. R. 1880, Milano, Annexes, 48; (1883) G. Accad. Med., Torino, 31, 519.

Robertson, R. C. (1924) J. infect. Dis., 35, 311.

Robinson, D. T. (1934) J. Path. Bact., 38, 551 ; (1940) Pers. comm.

Robinson, D. T. and Marshald, F. N. (1934) J. Path. Bact., 38, 73 ; (1935) Lancet, ii. 441.

Robinson, D. T. and Peeney, A. L. P. (1936) J. Path. Bact., 43, 403.

RöMer, P. H. (1909) Z. ImmunForsch., 3, 208.

Roux, E. and Yersiv, A. (1888) Ann. Inst. Pasteur, 2, 629.

Sabouradd, R. (1897) Ann. Inst. Pasteur, 11, 134.

SchMidT, H. (1933-34) Zbl. Bakt., 130, 391.

SсHм1Dт, S. (1931) Z. ImmunForsch., 'r1, 101.

Schmidt, S., Hansen, A., and Khaer, K. A. (1931) Ann. Inst. Pasteur, 46, 202.

SchWoNer, J. (1904) Zbl. Bakt., 35, 608.

Scotт, W. M. (1923) Rep. publ. Hlth med. Subj., Lond., No. 22.

Sifmens, B. W. L. (1938) "Bijdrage tot de Kennis der Typen van het Corynebacterium Diphtheria." Van Gorcum and Co., Assen.

Sмiтн, J. (1923) J. Hyg., Camb., 22, 1.

Süduersex, H. J. and Thompson, E. T. (1909-10) J. Path. Bact, 14, 224.

Tasman, A. and Ponduan, A. B. F. A. (1931) Z. ImmunForsch., 72, 245.

Tasman, A. and WaAsbergen, J. P. van. (1932) Z. ImmunForsch., 75, 164.

UnNa, P. G. (1896) "The Histopathology of Diseases of the Skin." Eng. Transl. by N. Walker, Edin.

Uschinsky, N. (1893) Zbl. Balt., 14, 316 ; (1897) Ibid., 21, 146.

WADSWorth, A. and Quigley, J. J. (1934) Amer. J. Hyg., 20, 225.

Wadsworth, A., Quigley, J. J., and Sickles, G. R. (1932) J. exp. Mled., 55, 815.

Wadsworth, A. and Wheeler, M. W. (1934) J. infect. Dis., 55, 123.

Wadsworth, A. and Wheeler, M. W. (1934) J. infect. Dis., 55, 123.

WARD, A. R. (1917) J. Bact., 2, 619.

Watson, A. F. and Langstaff, E. (1926) Biochem. J., 20, 763 ; (1927) J. Path. Buct., $30,383$.

WERNICKE. (1893) Arch. Hyg., 18, 192.

Winslow, C.-E. A., Broadhurst, J., Buchanan, R. E., Krumwiede, C., Rogers, L. A. and SuIт, G. H. (1920) J. Bact., 5, 191.

Wright, J. H. (1894) Boston med. surg. J., 131, 329.

ZAJDEL, R. (1932) C.R. Soc. Biol., 111, 1029 


\section{CHAPTER 18}

\section{FUSIFORMIS}

Definition. Fusiformis.

Obligate parasites. Anaerobic or microaerophilic. Cells frequently elongated and fusiform, staining somewhat unevenly. Filaments sometimes formed; non. branching; sometimes highly pleomorphic. Non-motile. No spores. Reaction to Gram variable, but mainly Gram-negative. Growth in laboratory media feeble.

A number of organisms, anaerobic or microaerophilic in their oxygen requirements, have been isolated by different workers from necrotic lesions in man and animals. The first of these was the so-called $B$. necrophorus, which was observed by Loeffler (1884) in calf diphtheria. In 1896 Vincent described a fusiform bacillus, frequently associated with a spirochæte, Trep. vincenti (see Chapter 79 ), in necrotic and ulcerative lesions of the throat and other tissues in human beings. Veillon and Zuber (1898), studying the bacterial flora of appendicitis and other suppurative lesions, described a number of non-sporing anaerobic bacilli, to which they gave the names of $B$. ramosus, B. serpens, B. fragilis, $B$. furcosus, and $B$. fusiformis; this last organism was apparently identical with the fusiform bacillus described by Vincent. Later work has revealed the frequent presence of organisms of this group in the mouth and on the teeth of man and certain animals (Tunnicliff 1906, Ellermann 1907, Varney 1927, Pratt 1927, Slanetz and Rettger 1933, Bachman and Gregor 1936, Pesch and Schmitz 1936, Spaulding and Rettger 1937, Hine and Berry 1937, Kelly 1944), and in the healthy alimentary canal of man (Eggerth and Gagnon 1933, Weiss and Rettger 1937, Misra 1938, Lewis and Rettger 1940, Dragstedt, Dack and Kirsner 1941). With the more general use of improved anaerobic methods they have been found in association with various human infections, especially in France and the United States (see Chapter 79).

Relatively little attention has been paid to the systematic study of these organisms, and their classification presents considerable difficulties. Castellani and Chalmers (1920) proposed a genus Bacteroides to contain obligatory anaerobic bacilli that did not form spores, and the genus has since been adopted in Bergey's manual, where it covers a variety of morphologically different organisms. The American Committee of Bacteriologists (see Report 1920) have described a genus Fusiformis, for the inclusion of organisms with certain characteristics resembling in some respects those possessed by the Corynebacterium and the Pfeifferella groups. As the definition of this genus (see above) seems to cover the main characteristics of the organisms we have mentioned, it seems permissible, at least for the moment, to include these organisms within it.

Group Characteristics.-The organisms of this group are typically rod-shaped; but their size is subject to considerable variation. They may be very short, or they 
may grow out into long filaments which are generally curved. Their width is also variable; not infrequently it is greater near the middle of the bacillus than at the end, giving the organism a fusiform appearance; the fusiform swellings may be as much as 4-5 $\mu$ in diameter. Occasionally the greatest diameter of the bacillus is at one end, so that the organism appears clubbed. Pleomorphism is a marked characteristic of some species. The organisms are arranged singly, in pairs endto-end, or in chains ; pseudo-filaments, showing evidence of subdivision are common. Some species show false branching, with the result that $\mathrm{V}$ - or $\mathrm{Y}$-forms are seen, but true branching does not occur. Some species are said to be motile, but the observations of recent workers render this doubtful. Spores are never formed. The organisms stain with the usual aniline dyes, but staining is often irregular. The reaction to Gram varies with different species. None of the organisms is acid-fast.

Culturally, growth occurs under anaerobic or microaerophilic conditions and is said to be favoured by the presence of 2 per cent. $\mathrm{CO}_{2}$. Little is known of their respiratory mechanism, but it seems probable that in fluid media they do not produce such low oxidation-reduction potentials as do many of the spore-bearing anaerobes (Dack and Burrows 1935). Some species grow readily in ordinary media, while others require the addition of natural animal protein. According to Slanetz and Rettger (1933), growth is stimulated by aqueous extracts of various vegetables, and a potato extract gelatin medium is recommended for the preservation of stock cultures. The optimum temperature for growth is about $37^{\circ} \mathrm{C}$.; some species will develop at $23^{\circ}$, others not below $30^{\circ} \mathrm{C}$. In solid media, colonies frequently do not become visible for 3 or 4 days, and usually remain small. For the isolation of the intestinal non-sporing anaerobic bacteria, Lewis, Bedell and Rettger (1940) recommend a glucose-cysteine agar medium at a $p \mathrm{H}$ between $6 \cdot 3$ and $7 \cdot 0$, enriched with yeast-extract and tomato juice, and state that growth is greatly improved by 10 per cent. $\mathrm{CO}_{2}$ in the anaerobic atmosphere. Little is known of the precise growth requirements of these organisms, though both pantothenic acid and pyruvic acid appear to be essential nutrients for a number of strains (West, Lewis and Militzer 1942).

The organisms are not particularly resistant; they are destroyed by exposure to moist heat at $55^{\circ} \mathrm{C}$. within an hour. The colonies of some species are highly oxygen-sensitive, dying withm an hour of exposure to air (Hine and Berry 1937). The fermentation reactions have been incompletely studied, but some species produce acid and gas in certain carbohydrate media.

The organisms of this group appear to be obligatory parasites, and may be cultivated from certain inflammatory processes, particularly those accompanied by necrosis and ulceration, as well as from the normal mouth, teeth, and fæees. There seems to be little doubt that some species are primarily responsible for the lesions from which they are isolated; but the aetiological rôle of others is probably more of a secondary nature. Many of the species are pathogenic to laboratory animals, producing necrotic lesions and death. Whether a true exotoxin is formed is not yet known.

Antigenically, little exact information is yet available about these organisms, but there is evidence that differences in antigenic structure do exist between different types.

The recorded investigations of these organisms are of two kinds: firstly, of organisms found in association with disease processes, and secondly, of organisms 
isolated from the normal flora of the mouth and intestines. Both Varney (1927) and Slanetz and Rettger (1933) divided fusiform bacilli of the normal mouth into four types, I to IV, on the basis of morphological and serological characteristies, but the divisions did not coincide. Agglutination tests revealed a high degree of strain-specificity, and a lesser degree of group-specificity. Weiss and Mercado (1938) extracted immunologically type-specific protein-like substances from organisms of Slanetz and Rettger's Types I, II and III, and also found some evidence of a group-specific carbohydrate in the organisms. Spaulding and Rettger (1937) later divided 84 strains of "fusobacteria" from many sources into two groups, Group I containing their previously described Types I, II and some of Type III, Group II containing the remainder of Type III and Type IV. In a limited cultural study of 193 human mouth strains, Pesch and Schmitz (1936) distinguished 6 types, but concluded that these were varieties of a single Bacterium fusiforme. Hine and Berry (1937) studied 104 mouth strains, dividing them into three species, $F$. nucleatus, $F$. polymorphus and $F$. dentium. The first two corresponded only very approximately to Spaulding and Rettger's Group I, consisting of short or long fusiform rods of limited saccharolytic activity, and $F$. dentium to Group II, consisting of long, relatively thick fusiform rods with marked saccharolytic activity.

Besides these fusiform bacteria of the mouth, there is a large group of Gramnegative non-sporing intestinal bacteria in the contents of the alimentary canal. It is clear from the work of Eggerth and Gagnon (1933), Weiss and Rettger (1937), Misra (1938) and Lewis and Rettger (1940) that these anaerobes are the predominant organisms in the human lower intestine, sometimes outmumbering Bact. coli by one hundredfold or more. They are biochemically and antigenically heterogeneous, though some association between morphological, biochemical and antigenic characters has been noted.

However, the relation of the normal flora of the alimentary canal to the organism responsible for infective lesions, and particularly to those species which were described in the early years of this century, is not yet clear. The intestinal species in man bear a morphological resemblance to some of the pathogenic species like $F$. funduliformis and certain fusiform bacilli but differ in biochemical reactions (hewis and Rettger 1940). Until the heterogeneity of both the pathogenic and the " normal" strains has been reduced by further study, speculation on cross-relationships within the group is unprofitable. (See Prévot 1938 for a taxonomic review of this difficult group).

We append descriptions of some of the named species that have been found in association with infective processes. In the first description we have assumed the substantial identity of $F$. necrophorus and Bacillus funduliformis, and retained the name $F$. necrophorus for the two (Dack et al. 1938, Kirchheiner 1940; see also Lemierre, Grumbach and Reilly 1936).

F. necrophorus.-Synonyms: Schmorl's bacillus, B. diphtherice vitulorum, Streptothrix cuniculi, Actinomyces necrophorus, Bang's necrosis bacillus, B. funduliformis, Bacteroides funduliformis. First observed by Loeffler in 1884 in calf diphtheria. He succeeded in producing necrotic lesions in mice by subcutaneous inoculation of the diphtheritic membrane, and in obtaining a primary culture of the organism from mice on calf serum ; but he failed to subculture it. Schmorl in 1891 encomntered apparently the same bacillus in a spontaneous epidemic amongst his laboratory rabbits, characterized by spreading necrosis of the lower lip. He inoculated mice with material from the rabbits, and obtained 
cultures of the organism from the mice. He classed it with the Streptothrix group under the Leptothrix or Cladothrix genera, and called it Streptothrix cuniculi. The organism has been described by a few other workers. (For a detailed description of it, see Orcutt 1930, Beveridge 1934.) Hallé (1898) named a bacillus associated with genital infections in man $B$. funduliformis, and later Teissier and his colleagues (Teissier et al. 1929, 1931) found the same organism in four cases of septicæmia in man. It probably corresponds to the species $\mathrm{C}$ described by Veillon and Zuber (1898) and its rôle in putrid infections of man has been confirmed by a number of workers (see Chapter 79). The human strains do not differ in any consistent way from the animal strains of $F$. necrophorus, except that they are usually less pathogenic for laboratory animals (Dack, Dragstedt and Heinz 1937) and tend to be pleomorphic.

Morphology.-In the diphtheritic membrane of calves, the bacilli appear as long threads-5 to 6 times as long as they are broad-arranged either in thick heaps or in long wavy rows. In the pleural or pericardial exudate of rabl,its dying of latial necrosis they usually appear as Gram-negative, highly pleomorphic bacilli, varying in shape from cocci to bacilli and long threads. In the thread forms there are often oval unstained portions arranged at regular intervals, looking like spores; but as they do not give the differential spore stain and are not particularly resistant to heat, they cannot be regarded as spores. The filaments may reach $80-100 \mu$ in length ; they are $0.75-1.5 \mu$ thick; one end is often narrow and pointed, the other thicker or almost fusiform. They may be surrounded by a capsular material. Branching does not occur. In culture the organisms appear as straight or curved rods, or as filaments. The ends are rounded, and the sides are generally parallel, though fusiform enlargements, including " ball " forms up to $5 \mu$ in diameter, are not uncommon. Except in young cultures, staining is irregular, and beaded forms are common.

Cultivation.-Schmorl cultivated the organism in deep inspissated sheep serum. It grows only under anaerobic conditions. Deep colonies in serum agar plates are round, pinhead in size, matt-white, with an entire edge; under a low power they have a thicker centre and an irregularly radiate periphery; under a high power the centre consists of a mesh-work of threads, and the periphery of streaming threads, which pass often for some distance into the surrounding medium. According to Shaw (1933), deep colonies in a semi-solid glucose serum agar medium have a grey, cotton-like, fluffy appearance, while in stiffer agar they are brown, circular or biconvex, with an entire edge. Surface colonies, after 2 days on a special serum agar medium (V.F.), made up with a peptic digest of ox muscle and liver, are circular, convex, about $1 \mathrm{~mm}$. in diameter, almost water-clear, and glistening; after 7 days they are differentiated into a convex centre and a narrow flat periphery with a slightly dentate edge; they are butyrous in consistency and easily emulsified (Beveridge 1934). In V.F. broth there is a dense turbidity, with a small dirty-white deposit, which increases as the medium clears on further incubation. Stab growth in serum begins in 24 to 40 hours near the bottom of the tube, and spreads upwards to within $\frac{1}{2}-1 \mathrm{~cm}$. of the surface; the growth is filiform with radiate branches; the serum is partly clouded, but not liquefied. There is no growth on potato. In glucose agar, Schmorl obtained growth only in the presence of a coccus. He quotes this as an example of metabiosisor the ability of one organism to grow in a particular medium only when another organism has prepared the medium for it, and rendered it suitable. V.F. gelatin (see above) is not liquefied. Turbidity and vigorous gas production occur in cooked heart medium and brain medium. A zone of hæmolysis, about $0.5 \mathrm{~mm}$. wide, is said to surround deep colonies in blood agar plates. Broth containing 10 per cent. of serum and a reducing agent like cysteine (0.1 per cent.) may support growth (Dack et al. 1938). Cultures, especially in liquid media, have a foul odour. Growth occurs between $30^{\circ}$ and $40^{\circ} \mathrm{C}$., with an optimum at $37^{\circ} \mathrm{C}$.

Brochemical and Metabolic.-Gas, and a variable amount of acid, are produced in glucose, maltose and lævulose. Fermentation of glycerol, galactose and sucrose is 
variable. Mannitol and lactose are not fermented. A soft clot is formed in 4-14 days in litmus milk. Indole + . $\mathrm{H}_{2} \mathrm{~S}+$. Nitrate reduction -. M.R. - . V.P. - . Methylene blue reduction + . Catalase weakly positive.

Antigentc Strocture.-Antigenic relationships exist within the group, and in some surveys the strains examined have fallen into a relatively few groups. As a whole, however, the species is antigenically heterogeneous (see Orcutt 1930, Beveridge 1934, Dack, Dragstedt and Heinz 1937, Henthorne, Thompson and Beaver 1936, Walker and Dack 1939).

Pathogenicity. - F. necrophorus appears to be responsible for several necrotic and gangrenous lesions in animals, such as calf diphtheria, labial necrosis of rabbits, and foot-rot of sheep, and occasionally for necrotic lesions in man. Organisms identical with or closely simulating $F$. necrophorus have been described by Harris and Brown (1927) in puerperal fever, by Dack, Dragstedt and Heinz (1936) in chronic ulcerative colitis, by Thompson and Beaver (1931) and Cohen (1932) in buman lung abscesses, by Beaver, Henthorne and Macy (1934) in hepatic abscesses, and by Lemierre (1936) as a cause of pyæmia and septicæmia (see also Necrobacillosis, Chapter 79).

Experimentally, the organism is pathogenic for rabbits and mice. It is non-patlogenic for guinea-pigs, dogs, cats, pigeons, and hens, though McCullough (1938) succeeded in infecting guinea-pigs deficient in vitamin $\mathrm{C}$ with human strains of $F$. necrophorus. Inoculated subcutaneously into the lower lip of rabbits, it gives rise to the typical necrotic disease (see p. 1788), and causes death in 4-12 days. Injected intraperitoneally into rabbits, it proves fatal in about the same time; p.m. the peritoneum is covered with large white masses, sticking the two layers of serosa together; similar masses of caseous material occur between the intestinal loops. Cultures can be obtained from the peritoneum. Intravenous inoculation into rabbits is fatal in about 8 days; post mortem, there is a fibrino-purulent pleurisy, a caseous bronchitis, and disseminated lobular pneumonia; the bacilli can be cultured from the affected organs. Histologically, the organisms give rise to intense inflammation, followed by a

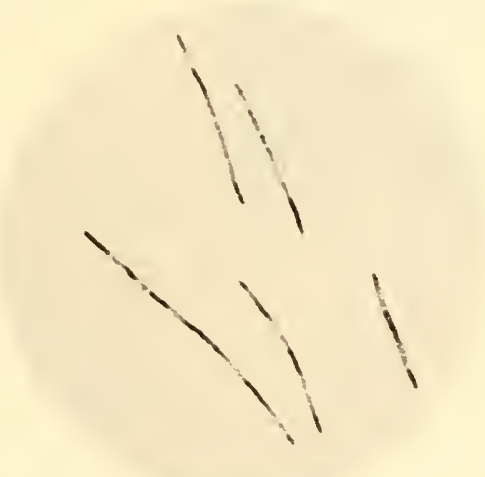

Fia. 88.-Fusiformis fusiformis.

From a surface culture on serum agar, anaerobically 2 days, $37^{\circ} \mathrm{C} .(\times 1000)$. rapid necrosis not only of the fixed tissue cells but also of the cells of the exudate; 110 liquefaction of the necrotic material occurs.

Mice inoculated subcutaneously at the root of the tail die after about 12 days in an emaciated condition. Two days after the inoculation, the local site is covered with a dry brownish crust; later a yellowish-grey discoloration of the borders of the inoculation wound becomes visible, and gradually spreads till at the time of death the whole of the lower third of the back is involved. A purulent conjunctivitis develops, and the lids are stuck together. Post mortem, the subcutaneous tissue around the wound and over the lower part of the back is converted into a tough, dryish, yellowish-white necrotic mass; the caseation involves the back muscles as well. The whole area is surrounded by codema. Numerous organisms are found in the caseous material. The internal organs appear normal.

In both rabbits and mice the organism is said to be found only in the affected parts, never in the blood or in those internal organs that are free from lesions, but on this question there is some doubt. The ability of the organism to produce lesions seems to be largely due to its production of a necrotizing endotoxin (Beveridge 1934). There 
is little evidence of the presence of a soluble substance of the exotoxin type (Scrivner and Lee 1934).

F. fusiformis.-This organism was first described by Vincent in 1896, who found it frequently associated with Trep. vincenti (see Chapter 38) in necrotic and ulcerative

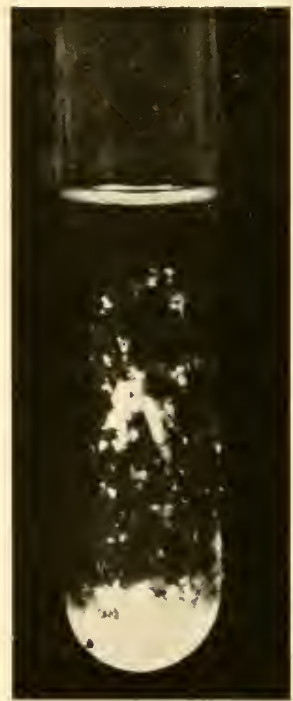

FIG. 89.-Fusiformis fusiformis.

Blood broth culture anaerobically, 3 days, $37^{\circ} \mathrm{C}$. showing floccular growth. lesions of the throat and other tissues. A similar, and possibly identical, organism was observed by Veillon and Zuber (1898) in appendicitis and other necrotic lesions of man, and named by them B. fusiformis. Smith (1933) has recorded its presence in tropical ulcer. In necrotic tissue the organisms appear as long rods, 5-12 $\mu$ in length, thickened in the middle, and with tapering or pointed ends. The axis is straight or curved. They are arranged singly or in pairs end-to-end, and are non-motile. They stain readily with the usual aniline dyes, and are Grampositive, but are decolorized if the treatment with alcohol is prolonged. In cultures the bacilli are pleomorphic (Leukowicz 1906); they vary greatly in size, and do not always show the typical fusiform shape. Long filamentous forms are common. The various morphological types of fusiform bacilli (see above) cultivated from the normal mouth of man and animals vary from thin, slightly pointed bacilli $0.4 \times 3-5 \mu$, to long and thick slightly pointed bacilli, $0.7 \times 6-25 \mu$. Tunnicliff $(1923,1933)$ considered the spirillar forms found in association with fusiform bacilli in lesions of the throat to be a phase of the fusiform type; and found that spiral forms were relatively common in "rough" variants of fusiform bacilli. Hine (1937) considers that there is no good evidence of a genetic relationship between fusiform bacilli and spirochætes.

The serological grouping of fusiform bacilli made by Rettger and his colleagues has been noted above. Bachmann and Gregor (1936) found two groups in mouth strains, corresponding respectively to filamentous, odour-producing bacilli associated with stomatitis, and to short diphtheroid bacilli, producing no odour in culture, found in healthy mouths.

The organism can be cultivated under anaerobic conditions at $37^{\circ} \mathrm{C}$., but not at room temperature, in serum agar or serum broth. The deep colonies appear in 2 days, and are 1-1 $\frac{1}{2} \mathrm{~mm}$. in diameter. The smallest colonies have a felt-like, branched appearance; the larger ones are round; and the largest of all are prismatic with angled projections, and are of a yellowish colour. On the surface of serum agar, the colonies are small and resemble those of streptococci, but on further incubation they may show a considerable peripheral extension, and come to resemble colonies of Cl. sporogenes (see p. 876). In serum broth, large white flocculi form in 24 hours, and later sink to the bottom (Ellermann 1904) (see Fig. 89). On plain agar there may be no growth at all, or a thin, very delicate whitish layer limited to the line of inoculation may develop

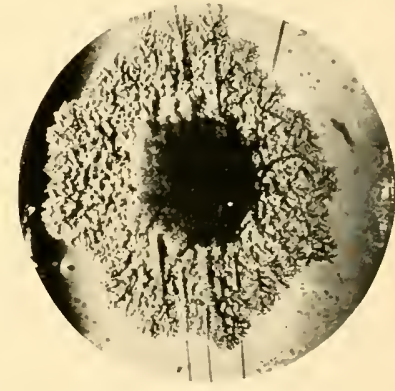

F'IG. 90.-Fusiformis fusiformis.

Surface colony on serum agar, anaerobically, 7 days, $37^{\circ} \mathrm{C}$. $(\times 8)$. Resembles Slanetz and Rettger's Type IV. after 48 hours (Weaver and Tunnicliff 1905). Loeffler's serum and Dorset's egg medium are both suitable. Neither is liquefied. Growth does not occur in media containing sugar, unless blood or ascitic fluid is added. Peters (1911) found that, in the presence of rabbit blood, acid was produced in glucose, maltose, mannitol, and lactose, but not in sucrose; there was no indole formation. Group I organisms of Spaulding and Rettger's 
fusiform organisms fermented only glucose and sucrose, were indole + , and $\mathrm{H}_{2} \mathrm{~S}+$ in the absence of cysteine; Group If organisms fermented glucose, sucrose, maltose, trehalose, lactose and raffinose, were indole negative and $\mathrm{H}_{2} \mathrm{~S}+$ only in the presence of cysteine. The place of $F$. fusiformis in these groups is not clear but it is more nearly related to Group II and to the Type IV of Slanetz and Rettger. Hine and Berry (1937) could find no such clear-cut association between fermentation reactions and cultural characters. The organisms do not form spores. They are killed in 15 minutes by moist heat at $55^{\circ} \mathrm{C}$., but resist 1 per cent. antiformin for 5 minutes. In cultures they may remain alive for 6 to 8 weeks. Injections subcutaneously or intramuscularly into rabbits and guinea-pigs may have little or no effect; at most an abscess is produced (Ellermann 1905).

F. ramosus.-Said by Veillon and Zuber (1898) to be one of the commonest organisms in appendicitis; has also been reported in human gas gangrene and bacteræmia (Lemierre, Reilly and Bloch-Michel 1937). Gram-positive, non-motile, non-sporing rod, slightly larger than Erysipelothrix rhusiopathice; arranged in pairs or short chains; in pus it is rather short but in culture it may form pseudo-filaments, which are really made up of numerous short rods. V-forms and $\mathrm{Y}$-forms are common. No growth occurs at room temperature; and at $37^{\circ} \mathrm{C}$. colonies do not become visible for 3 or 4 days. Surface colonies on agar are very fine, effuse, greyish-white, and translucent; later, they become granular and slightly cloudy. Deep colonies in agar are very small greyish points, which under low magnification are seen to be ovoid and granular with hatched borders; as the colony grows, it becomes greyish-yellow, and the edges become more defined. In broth it produces a uniform turbidity in 3 or 4 days with a greyish-white muddy deposit. Cultures have a characteristic œetid odour, but very little gas is formed. Strict anaerobe. Acid is formed in glucose, galactose and mannitol, and sometimes in lactose, saccharose and maltose. Lactose-fermenting strains clot litmus milk. Indole - . Gelatin not liquefied (Weinberg et al. 1937). Cultures live for over a month. Inoculated intravenously into rabbits, it causes death in some days by intoxication and cachexia. Subcutaneous inocculation into rabbits causes abscess formation with death in 8 to 10 days.

F. serpens.-Isolated by Veillon and Zuber (1898) from the pus of a mastoiditis in a child who died of pulmonary gangrene. Large, slightly motile, Gram-negative rod with rounded ends, arranged in pairs end-to-end, or in pseudo-filaments. Strict anaerobe. Grows best at $37^{\circ} \mathrm{C}$, , but develops at $20^{\circ} \mathrm{C}$. Surface colonies on agar after 48 hours are only just visible; later they form small cloudy translucent masses of greyish colour. Deep colonies in agar after 24 hours at $37^{\circ} \mathrm{C}$. are small, clear, rounded, greyish, granular masses with hatched edges, and sometimes a bouquet of filaments at one point on the periphery ; later they increase in opacity and the edges become better defined. In gelatin stab there is a filiform growth; liquefaction occurs down the whole length of the stab; the liquefied gelatin remains clear, and flocculi of growth fall slowly to the bottom forming a white deposit. Deep colonies in gelatin are round, greyish. and liquefy the gelatin slowly. In broth there is a rapid turbidity, followed by a clearing of the medium with the formation of a white deposit. Acid and gas are formed from glucose, lævulose, maltose, galactose and lactose. Indole -. No hæmolysin. Cultures have a foetid odour, but only a small amount of gas is evolved. Cultures remain viable for about 20 to 25 days. Pathogenic for mice, guinea-pigs, and especially rabbits, but not so pathogenic as $F$. ramosus.

F. fragilis.-Said by Veillon and Zuber (1898) to be the commonest organism in appen. dicitis. Non-motile, Gram-negative, rod with rounded ends, slightly smaller than the diphtheria bacillus; axis straight or slightly curved; arranged singly or in pairs endto-end. Strict anaerobe. Difficult to isolate. Surface colonies on agar are extremely fine, greyish, and translucent, and tend to undergo autolysis in a few days. Deep colonies in agar do not become visible for 3 or 4 days; they are very small, round, irregularly round, or ovoid, brownish-yellow, opaque, with an entire edge. Deep colonies in gelatin appear in 8 to 10 days; they are punctiform, yellow, granular, with an entire edge ; no 
liquefaction. Uniform turbidity in broth with a whitish deposit. Cultures have a fotid odour, but evolve little gas. Cultures in agar remain viable for less than a week. Subcutaneous inoculation into guinea-pigs produces abscess formation. The organism is more pathogenic for the rabbit, producing on subcutaneous inoculation an extensive phlegmon with sloughing of the skin, and death in 6 to 7 days. Intravenous inoculation of the rabbit is followed by death, but no organisms can be found in the tissues at necropsy; probably death is due to toxæmia, for the same result is brought about by killed cultures.

F. furcosus.-Not so common in appendicitis as $F$. fragilis and $F$. ramosus. In pus it is a very small rod, with one of its ends forming two little branches like a $\mathrm{Y}$. In culture it forms rods; many of these increase in length, and divide at one of their extremities into two branches, each terminated by a swelling; others form branches, which themselves divide. The bacilli and their branches are never very long; the swellings are rounded or pyriform, and are numerous. The whole organism is slightly larger than the tubercle bacillus. Non-motile and Gram-negative. Strict anaerobe. No growth at room temperature; at $37^{\circ} \mathrm{C}$. growth is not visible for 3 or 4 days. Surface colonies on agar are very fine grey points, scarcely raised at all from the medium; under low magnification they appear yellowish, very finely granular, and have transparent borders. Deep colonies in agar are so small and transparent that they are difficult to see; when magnified, they are round and yellowish, with delicate regular borders. A fine precipitate is formed in broth. Cultures have a slightly fœtid, sour odour, but evolve very little gas. Cultures remain viable for 15 to 20 days. Subcutaneous inoculation into a guinea-pig produces an abscess, which generally heals; sometimes death occurs after several weeks from cachexia.

F. melaninogenicus:-Isolated by Oliver and Wherry (1921) from the mouth, infected wounds, urine and fæces of the human subject. On hæmoglobin-containing media it forms a melanin-like pigment. According to Burdon (1928) it is a small Gram-negative, non-sporing diplococcobacillus growing feebly on blood media unless mixed with other organisms, when its growth is more profuse. The pigment develops slowly; after 1-2 days the colonies may be stained a pale brown; in $4-5$ days they are a deep black in colour. Cultures have a foul odour. By the time pigmentation is fully developed, the culture is often dead, which may account for the infrequency with which this organism has been isolated and its peculiar chromogenic character recognized. Weiss (1943) reported local inflammatory œdema, followed by necrosis, after intradermal injection of young cultures in rabbits, but no effect after intraperitoneal injection in mice.

Its pathogenicity is probably low. In man, it has been isolated from purulent lesions, usually along with other pathogemic bacteria, and sometimes from the blood.

\section{REFERENCES}

Bachmann, W. and Gregor, H. (1936) Z. ImmunForsch., 8\%, 238.

Beaver, D. C., Henthorne, J. C., and Macy, J. W. (1934) Arch. Path., 17, 493.

Beveridge, W. I. B. (1934) J. Path. Bact., 38, 467.

Burdon, K. L. (1928) J. infect. Dis., 42, 161.

Castellani, A and Chalmers, A. J. (1920) "Manual of Tropical Medicine." 3rd Ed., London.

Conen, J. (1932) Arch. Surg., 24, 171.

DACK, G. M. and Burrows, W. (1935) Proc. Soc. exp. Biol., N.Y., 32, 1441.

Dack, G. M., Dragstedt, L. R., and Heinz, T. E. (1936) J. Amer. med. Ass., 106, 7; (1937) J. infect. Dis., 40, 335.

Dack, G. M., Dragstedt, L. R., Johnson, R., and McCullough, N. B. (1938) J. infect. Dis., 62, 169.

Dragstedt, L. R., Dack, G. M., and Kirsner, J. B. (1941) Ann. Surg., 114, 653.

Egoerth, A. H. and Gagnon, B. H. (1933) J. Bact., 25, 389.

EllermanN, V. (1904) Zbl. Bakt., 37, 729 ; (1905) Ibid., 38, 383 ; (1907) Z. Hyg. InfektKr., $56,453$. 
Hallé, J. (1898) "Recherches sur la bacteriologie du canal génital de la femme." Thèse de Paris.

Harris, J. W. and Brown, J. H. (1927) Bull. Johns Hopk. Hosp., 40, 203.

Henthorne, J. C., Thompson, L., and Beaver, D. C. (1936) J. Bact., 31, 255.

HпN Е, M. K. (1937) J. infect. Dis., 61, 198.

Hine, M. K. and Berry, G. P. (1937) J. Bact., 34, 517.

KeLly, F. C. (1944) J. infect. Dis., 74, 93.

Kirchheiver, E. (1940) Ann. Inst. Pasteur., 64, 238.

Lemierre, A. (1936) Lancet, i. 701.

Lemierre, A., Grumbach, A., and Reilly, J. (1936) Bull. Acad.. Méd., Paris, 115, 945. Lemierre, A., Reilly, J., and Bloch-Michel, H. (1937) Bull. Acad. Méd., Paris, 117, 322.

LEU KowICZ, X. (1906) Zbl. Bakt., 41, 153.

Lewis, K. H., Bedell, M. and Rettger, L. F. (1940) J. Bact., 40, 309.

Lewis, K. H. and RetTGER, L. F. (1940) J. Bact., 40, 287.

LOEFFLER, F. (1884) Mitt. ReichsgesundhAmt., 2, 421.

McCullouah, N. B. (1938) J. infect. Dis., 63, 34.

Misra, S. S. (1938) J. Path. Bact., 46, 204.

Oliver, W. W. and Wherry, W. B. (1921) J. infoct. Dis., 28, 341.

Orcutt, M. L. (1930) J. Bact., 20, 343.

Pesch, K. L. and Schmitz, L. (1936) Zbl. Bakt., 136, 476.

Peters, W. H. (1911) J. infect. Dis., 8, 455.

Pratt, J. S. (1927) J. infect. Dis., 41, 461.

Prévot, A. R. (1938) Ann. Inst. Pasteur, 60, 285.

Report. (1920) J. Bact., 5, 191.

SchmorL, G. (1891) Dtsch. Z. Thiermed., 17, 375.

Scrivner, L. H. and LeE, A. M. (1934) J. Amer. vet. med. Ass., 85, 360.

Shaw, F. W. (1933) Zbl. Bakt., 129, 132.

Slanetz, I. IV. and Rettger, L. F. (1933) J. Bact., 26, 599.

Sirth, E. C. (1933) J. Hyg., Camb., 33, 95.

Spaulding, E. H. and Rettger, L. F. (1937) J. Bact., 34, 535, 549.

Teissier, P.. Reilly, J., Rivalier, E., and Layani, F. (1929) Paris méd., 71, 297.

Teissier, P., Reilly, J., Rivalier, E., and Stéfanesco. V. (1931) Ann. Méd., 30, 97.

Thompson, L. and Beaver, D. C. (1931) Proc. Mayo Clin., 6, 372.

Tunnicliff, R. (1906) J. infect. Dis., 3, 148; (1923) Ibid., 33, 147; (1933) Ibid., 53, 280.

VARNEY, P. L. (1927) J. Bact., 13, 275.

Veillon, A. and ZuBER, A. (1898) Arch. Méd. exp., 10, 517.

Vinoent, H. (1896) Ann. Inst. Pasteur, 10, 488.

WaLker, P. H. and DaCk, G. M. (1939) J. infect. Dis., 65, 285.

Weaver, G. H. and Tunnicliff, R. (1905) J. infect. Dis., 2, 446.

Wernberg, M., Nativelle, R., and Prévot, A. R. (1937) "Les Microbes Anaérobies." Masson et Cie., Paris.

Weiss, C. (1943) Surg., 13, 683.

Weiss, C. and IIercado, D. G. (1938) J. exper. Med., 67, 49.

Weiss, J. E. and Rettger, L. F. (1937) J. Bact., 33, 423.

West, R. A., Lewis, K. H. and Mllitzer, W. E. (1942) J. Bact., 43, 155. 


\section{CHAPTER 19}

\section{PFEIFFERELLA, AND CERTAIN ALLIED ORGANISMS}

\section{Definition (emended from the American Committee's Report).}

Small, slender, usually non-motile, Gram-negative rods, often staining irregularly and sonetimes forming threads or showing a tendency towards branching. Growth on all media is rather slow; gelatin may be slowly liquefied; fermentation of carbohydrates is very weak; characteristic brown honey-like growth on potato.

Type species is Pfeifferella mallei.

History.-The glanders bacillus was isolated by Loeffler and Schütz in 1882 (see Loeffler 1886) from a horse dying of acute glanders. An organism in many respects resembling $P f$. mallei was isolated by Whitmore and Krishnaswami (1912) from a glanders-like disease of human beings in Rangoon. It was designated $B$. pseudomallei by Whitmore (1913). Subsequently Stanton and Fletcher (1921, 1925) gave the name of Melioidosis to this disease and Bacillus whitmori to the causative organism.

The genus Pfeifferella was tentatively created by the American Committee as one of the genera intermediate in position between Actinomyces on the one hand and Mycobacterium on the other. The type species, and the only listed member of the group, is the glanders bacillus or Pf. mallei. The classification of this organism presents many difficulties, and it is by no means certain that the genus Pfeifferella will gain permanent recognition.

As regards the fermentative powers of $P f$. mallei, the American Committee's definition states that carbohydrates are not fermented. According to our observations, and those of Stanton and Fletcher (1932), acid is formed in glucose in 2 to 3 weeks; a small amount of acid, sufficient however to give a pink colour with Andrade's indicator, is produced in salicin. Moreover, there is a slow formation of acid in litmus milk, becoming apparent in about 5 days, and followed in 2 to 3 weeks by definite clotting.

In certain respects Whitmore's bacillus resembles $P f$. mallei, but it differs from it in many others. For the moment we assign it to the Pfeifferella group, entering a caveat that, should that genus attain a permanent place in systematic bacteriology, it is by no means certain that Whitmore's bacillus will be placed within it.

\section{Group Characteristics.}

Morphologically the two organisms are fairly similar; Pf. whitmori, however, is rather smaller, frequently shows bipolar staining, and is motile. In films of the smooth form, the organisms are arranged in long parallel bundles embedded in an interstitial substance, presenting a very characteristic appearance; with Loeffler's methylene blue, the interstitial substance stains blue, the bacilli bluish- 
red; films of the rough forms show no interstitial substance, and resemble $P f$. mallei more closely.

Culturally, the growth of $P f$. whitmori is more rapid and more profuse than that of Pf. mallei. On glycerol agar it may give a smooth mucoid or a corrugated and wrinkled growth. Stanton and Fletcher (1927) have also described an ultra-rugose variant, with an extremely corrugated surface, and a consistency so tenacious that if the colony is touched with a needle, it adheres to it and peels bodily off the medium. Growth in gelatin at $20^{\circ} \mathrm{C}$. is abundant, and liquefaction of the stratiform type is apparent in 4 or 5 days; $P f$. mallei, on the other hand, grows very poorly in gelatin at $20^{\circ} \mathrm{C}$. and never liquefies the medium, though according to Stanton and Fletcher (1925), if it is incubated

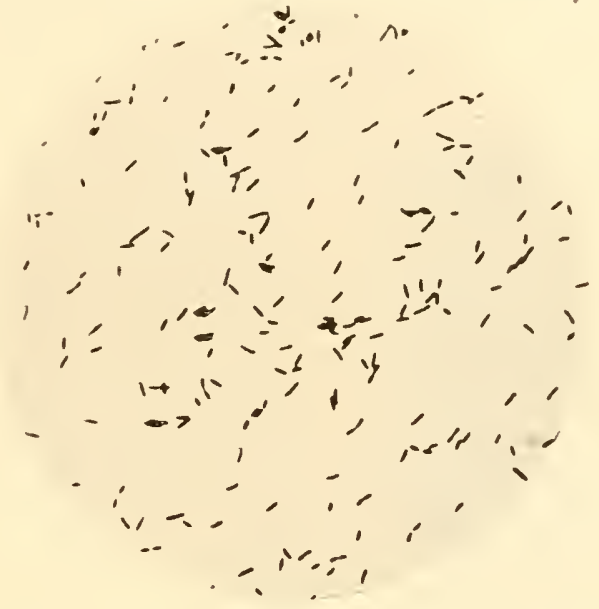

Fig. 91.-Pfeifferella mallei.

From an agar culture, 48 hours, $37^{\circ} \mathrm{C} .(\times 1000)$. at $37^{\circ} \mathrm{C}$., permanent liquefaction occurs in 4 to 6 weeks. In broth the growth of the smooth form of Pf. whitmori resembles that of $\mathrm{Pf}$. mallei; the rough form, however, gives rise to a wrinkled surface pellicle. On potato the smooth form gives a café-au-lait growth resembling that of $P f$. mallei, whereas the rough form gives a much

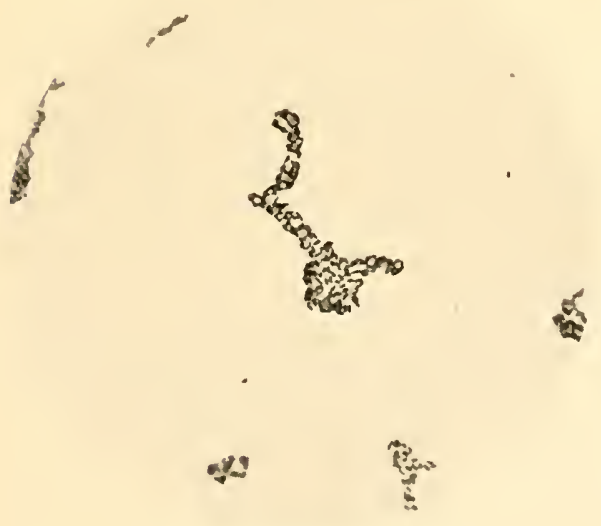

FIG. 92.-Pfeifferella whitmori.

From an agar culture, 24 hours, $37^{\circ} \mathrm{C} .(\times 1000)$. lighter growth of creamy or creamy-yellow colour. On HacConkey's agar, Pf. whitmori grows freely, forming red colonies, while Pf. mallei entirely fails to grow.

Biochemically, Pf. whimori is an active fermenter on first isolation, producing acid in glucose, mannitol, lactose, sucrose and dulcitol, and decolorizing Andrade's indicator; but after long subcultivation in the laboratory it loses its power of fermenting any sugars except glucose. In litmus milk acid is produced in 3 days; later the casein is precipitated, and may be digested; the litmus is partly decolorized. Pf. mallei, on the other hand, though it may produce acid and clot, never peptonizes the medium. Freshly isolated strains of Pf. whitmori liquefy blood serum and coagulated egg, but this property is often lost during cultivation in the laboratory. 
Pf. mallei has no digestive action on serum. Both organisms may produce a small amount of $\mathrm{H}_{2} \mathrm{~S}$, when tested by Huddleson's method on liver agar. (For the preparation of mallein see Chapter 62.)

Antigenically, the two organisms appear to resemble each other closely. Stanton and Fletcher (1925), who examined 5 strains of Pf. mallei and 14 strains of Pf. whitmori by agglutination, absorption, and complement fixation, found that the strains of $P f$. whitmori formed a homogeneous group, but that the 5 strains of $P f$.

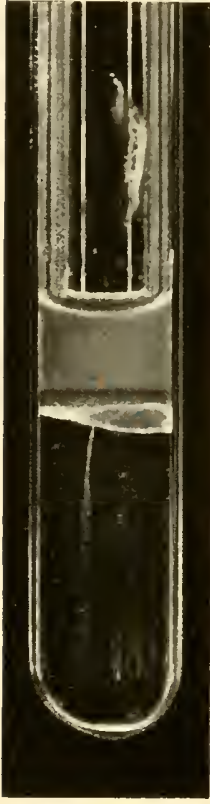

FIG. 93.Pfeifferella whitmori.

Gelatin stab culture, 10 days, $20^{\circ} \mathrm{C}$., showing stratiform liquefaction. malle $i$ fell into two groups. One of these groups was very closely related to, if not identical with, the $P f$. whitmori strains. It is important to note that the 2 strains of $P f$. mallei which resembled $P f$. whitmori were isolated from ponies in Java and India respectively; since they differed in certain respects from the other strains of $P f$. mallei, which were isolated in England and Egypt, it is just possible that they were variant strains of Pf. whitmori. According to de Moor and his colleagues (1932), strains of mallei and whitmori show cross-agglutination to titre, and complete cross-absorption, with specific sera. Cross-allergic reactions are also said to be obtainable in infected animals with mallein and the corresponding product prepared from cultures of $P f$. whitmori. Verge and Pairemaure (1928) have reported a positive complement-fixation reaction with the serum of a glandered horse in the presence of a whitmori antigen. Further work, however, is obviously desirable on the antigenic structure and relationship of these two organisms.

Pathogenically, Pf. mallei gives rise to disease in equines, while Pf. whitmori is a natural parasite of rodents. Both organisms can cause disease in man; both are infective for laboratory animals; and both are able to call forth the Straus reaction on intraperitoneal inoculation of male guinea-pigs.

Experimental Reproduction of Glanders in Animals.-Experimentally glanders may be reproduced in horses, asses, and mules by feeding with cultures of $P f$. mallei, and by subcutaneous inoculation. Sheep and goats a re easily infected, but cows and pigs are said to be resistant. Of laboratory animals the guineapig and the field mouse (Arvicola arvalis) are the most susceptible to experimental inoculation; rabbits and dogs are less so; rats, birds, and perhaps white mice, are comparatively resistant.

GuINEA-PIGS.-Loeffler (1886) made 85 experiments on guinea-pigs, and every animal developed the disease. Not all observers, however, are agreed on the uniform susceptibility of the guinea-pig; young animals seem to be more resistant than older ones.

After subcutaneous inoculation of a small amount of a pure culture, an abscess develops, which ulcerates in $\mathbf{5}$ days; the regional glands break down and discharge pus. At this stage the disease may become stationary or retrogress, but usually it advances. In the second week hard nodular foci are palpable in the testicles and epididymis; inflammation of the tunica vaginalis occurs, and the testicle becomes fixed to the overlying skin ; finally ulceration takes place with the discharge of purulent material. In female guinea-pigs the mammæ and labia may be inflamed. Swelling and inflammation of one or more joints is very common, sometimes leading to abscess formation and ulceration. Nodules often appear in the muscles, face, back, or beneath the periosteum of the bones. In about a third of the animals nodules appear on the nasal mucosa, and crusts collect around the 
external nares. Death follows in 1 to 8 weeks, generally in the 3 rd or 4 th week. At post mortem there is a local ulcer or scar ; the regional glands are swollen, and contain greyish. white purulent masses. Abscesses are found in the skin, subcutaneous tissues and around the joints. The lungs contain nodules, greyish-yellow and easily emulsifiable, particularly under the pleura. In the spleen there are numerous submiliary nodules of a greyish-yellow colour, projecting slightly above the surface. There are fewer nodules in the liver; these are more greyish-white in colour. The kidneys are free, but nodules may be seen in the suprarenals. Small, greyish-yellow nodules are found in the testicles, or larger foci, relatively firm with a caseous centre. The nasal mucosa is red and swollen, and may be covered with friable caseous masses. In recent lesions the bacilli can always be found, but in the older ones they are scanty.

After intraperitoneal injection the testicles swell in 2 to 3 days; by the 10 th day they are greatly enlarged; death occurs in a fortnight as a rule. The testicular lesion-Straus' reaction (Straus $1889 a$ ) - commences in the tunica vaginalis. The two layers of the serosa are covered with confluent yellowish-white granules of pinhead size. On the 3rd or 4th day the layers are united by a thick, purulent exudate rich in bacilli. The scrotal skin becomes inflamed and adherent to the underlying tissues; later ulceration occurs (see Panisset 1910). After intraperitoncal injection of organisms of lowered virulence the Straus' reaction may oceur without death ensuing.

Guinea-pigs have been infected by insufflation with powdered cultures (Babes 1891).

Field Mice.-After subcutaneous inoculation these animals die in 3 to 4 days. Post mortem, there is a greyish-green infiltration at the site of injection. The lymph vessels leading to the enlarged glands are studded with little greyish-white nodules. The spleen is greatly enlarged, and contains numerous yellowish-white nodules projecting slightly above the surface. There are several tiny nodules in the liver.

Woop Mice.-The wood mouse-Mus sylvaticus-is less susceptible than the field mouse-Arvicola arralis. After subcutaneous inoculation it develops a chronic disease not proving fatal for 3 to 6 weeks. Post mortem, the spleen is enlarged, and contains numerous pin-head, greyish spots or nodules; sometimes there is a fibrino-purulent exudate in the pleura, and enlargement of the lymphatic glands (Kitt 1887).

RABBITS.-Subcutaneous inoculation causes a local ulcerating lesion and swelling of the neighbouring lymphatic glands. If the animal is killed after a month, as well as the glandular swelling there may be a few greyish nodules in the lungs, and ulcers on the nasal mucosa. After intravenous inoculation, numerous nodules develop in the spleen and liver, but death may be delayed for some weeks.

White Mice axd White Rats.-These animals react to subcutaneous injection with a rapidly retrogressive local abscess. Occasionally an animal dies after about 7 weeks, and at post mortem shows caseous nodules in the spleen. Leo (1889) states that white mice can be rendered susceptible to glanders by being fed on a diet containing phloridzin. According to Sabolotny (1926), white mice are more susceptible than has generally been believed. In his experiments, after subcutaneous inoculations with a pure culture, the mice often died of an acute infection in 30 to 72 hours ; no macroscopic lesions were present at post mortem, but the bacillus could be recovered from the organs. After subcutaneous injection with glanders pus, they all died in 5 to 6 weeks of a chronic infection. Post mortem, the spleen was much enlarged and was riddled with nodules of varying sizes; there were also a few nodules in the liver and lungs.

DoGS AND CATS.-After subcutaneous inoculation in dogs a local abscess occurs, followed by ulceration; the disease apparently remains localized (Galtier 1881). Acute fatal glanders may follow intravenous injection of large doses of bacilli; numerous small subcutaneous nodules develop, and at post mortem lesions are found in the liver and spleen (Straus 1889b).

Cats are more susceptible ; after subcutaneous inoculation a local lesion occurs followed by death in 15 to 30 days; at post mortem nodules are found in the internal organs. 
Classification.-There are many points of resemblance between $\mathrm{Pf}$. mallei and the organisms of the Brucella group. Both this organism and Br.melitensis are small, non-motile, Gram-negative bacilli, developing slowly in culture media ; both give rise to a café-au-lait or chocolate-coloured growth on potato; both have very weak fermentative powers in the sugars; both give rise under natural conditions to acute or chronic disease in man and animals; both are pathogenic to guineapigs, setting up necrotic changes in the viscera; both may give the Straus reaction in male guinea-pigs; and both are characterized by their extraordinary infectivity for man in artificial cultures in the laboratory. There are, however, certain differences between them, the importance of which from the point of view of classification it is difficult to assess. Thus Br.melitensis is a definite cocco-bacillus; Pf. mallei, though often occurring in very short forms, is generally described as a slender rod, capable at times, especially in the animal body, of giving rise to long, and even branching, thread-like forms (Mayer 1900, Galli-Valerio 1900). In culture, however, it is not unusual for Pf. mallei to form short and almost ovoid bacilli, not unlike $\mathrm{Br}$. abortus. Antigenically, there is, in our experience, no apparent relationship between Pf. mallei and organisms of the Brucella group (Wilson 1934).

It may be pointed out that $P f$. whitmori bears certain points of resemblance to Friedländer's bacillus. Both these organisms are Gram-negative bacilli, and are surrounded by a capsule or an interstitial substance, which is not infrequently lost after artificial culture for some time. Both exhibit more or less similar appearances in culture, and both give rise to an extremely viscous deposit in broth. Both organisms give a café-au-lait growth on potato. Both are active fermenters of sugars on first isolation, and both may tend to lose this property after prolonged subculture in the laboratory. On the other hand, Pf. whitmori differs from Friedländer's bacillus in being motile, in liquefying gelatin, in failing to produce gas from carbohydrates, and in its capability of giving rise to the Straus reaction in the guinea-pig.

Thompson (1933) draws attention to certain morphological and cultural similarities between Pf. mallei and Pf. whitmori on the one hand and Actinobacillus lignieresi on the other, and states that the organisms are antigenically related. His protocols, however, furnish no evidence of specific cross-agglutination. The slight degree of agglutination that was observed may quite well have been due to normal agglutinins. Pf. mallei is frequently agglutinated by normal sera to a titre of $1 / 640$ or over, and the actinobacillus is often affected by normal sera, though usually to a lower titre. Thompson's suggestion that all three organisms should be classified together in the Mycobacterium group cannot be accepted until further evidence in support of it is forthcoming.

Some workers (Legroux and Djemil 1931, Legroux and Genevray 1933) regard $P f$. whitmori as being more nearly related to $P s$. pyocyanea than to $P f$. mallei, but the absence of pigment production by $P f$. whitmori, and its demonstrated antigenic affinity to $P f$. mallei, render this doubtful.

\section{Pfeifferella mallei}

Isolation.-By Loeffler and Schütz in 1882 (Loeffler 1886) from a horse dying of acute glanders.

Habitat.-Strict parasite ; found chiefly in equines and man.

Morphology.-Slender rod-shaped organism, about $1 \cdot 5-3 \mu \times 0 \cdot 3-0 \cdot 6 \mu$ broad. Straight or slightly curved, ends rounded, sides irregularly parallel or wavy; arranged singly, in pairs end-to-end, in parallel bundles, and in Chinese letter forms. In 
cultures there may be great variation in length and to a less extent in thickness; long filaments showing true or false branching, club, pear, flask, and other irregular forms have been described. Staining is usually uneven, deeply stained alternating with poorly stained or unstained areas; bipolar staining is common. Non-motile; non-capsulated; non-sporing; Gram-negative; non-acid-fast.

Agar Plate. -2 days at $37^{\circ} \mathrm{C}$. Round, convex, amorphous, translucent, greyish-yellow colonies, $0 \cdot 5-1 \cdot 0 \mathrm{~mm}$. in diameter, with smooth glistening surface and entire edge ; butyrous in consistency and easily emulsifiable. 9 days, colonies are 1-2 mm. in diameter, more opaque, and may have a very finely granular surface; sometimes the centre is coloured shightly brown.

Agar Slope.-2 days at $37^{\circ} \mathrm{C}$. Moderate, confluent, raised, greyish-yellow, translucent growth with beaten-copper surface and edge undulate or formed of single colonies. Medium unchanged. Not so profuse as growth of Bact. coli.

Gelatin Stab. -7 days at $20^{\circ} \mathrm{C}$. Poor to moderate, filiform growth, extending nearly to the bottom of the tube, and consisting of small discrete colonies; raised surface growth about $3 \mathrm{~mm}$. in diameter. No liquefaction. After 6 weeks the growth is often brownish in colour. If incubated at $37^{\circ} \mathrm{C}$, gelatin is said to be permanently liquefied in 4 to 6 weeks.

Broth. -2 days at $37^{\circ} \mathrm{C}$. Moderate growth with moderate uniform turbidity and a slight powdery sediment disintegrating completely. No surface growth. 14 days, ring growth is present, and there is a moderate, viscous deposit, very difficult to disintegrate.

Horse Blood Agar Plate.-3 days at $37^{\circ} \mathrm{C}$. Good growth of round, low convex, greyishgreen, opaque colonies, $1 \mathrm{~mm}$. in diameter. No hæmolysis, but plate is browned.

Loeffler's Serum.-3 days at $37^{\circ} \mathrm{C}$. Scanty growth, barely visible, of flat, discrete colonies, $0.3 \mathrm{~mm}$. in diameter. No liquefaction.

Potato. -2 days at $37^{\circ} \mathrm{C}$. Moderate, raised, greyish-yellow growth. After 4 days the colour deepens to café-au-lait, and in 10 days to chocolate.

MacConkey's Agar. -9 days at $37^{\circ} \mathrm{C}$. No growth.

Resistance.-Killed by moist heat in 10 minutes at $55^{\circ} \mathrm{C}$. Cultures dried on threads may live for 3 or 4 weeks; but infected pus or discharge, when clried, usually becomes sterile in a few days. Killed by 2 per cent. phenol in 1 hour, by $1 / 1000 \mathrm{HgCl}_{2}$ in 15 minutes, and by calcium hypochlorite with 2 parts of free chlorine per million in 30 minutes. In eulture the organism rarely survives longer than a month or 6 weeks.

Metabolism.-Aerobe; either no growth at all, or only very slight growth after 14 days, under strict anaerobic conditions. Optimum temperature $37^{\circ}$; little or no growth below $20^{\circ} \mathrm{C}$. On culture media it grows slowly; growth is often not apparent for 2 days. Tendency to formation of brownish pigment in cultures, especially on potato. No hæmolysin is produced.

Biochemical.-Acid, no gas in glucose, and sometimes very slight acid in salicin ; no other sugars fermented. L.M. slight acid; after 2 to 3 weeks a clot forms, and the litmus is partly decolorized at the bottom. Indole - ; M.P. - ; V.P. - ; nitrates reduced; $\mathrm{H}_{2} \mathrm{~S}$ slight $+; \mathrm{NH}_{3}+$; M. B. reduction - ; catalase slight + .

Antigenic Structure.-By agglutination, absorption, and complement fixation there appear to be at least two types of $P f$. mallei, of which one is related to $P f$. whitmori. Sakamoto (1930) has isolated from culture filtrates of $P f$. mallei a relatively non-specific nucleo-protein substance, and a soluble specific polysaccharide which gives a precipitation reaction with immune sera.

Pathogenicity.-Causes glanders or farcy in horses, mules, asses, and man. Experimentally the disease can be reproduced in equines, goats, cats, and guinea-pigs; sheep, rabbits, dogs, rats and mice are less susceptible; pigs and cattle are resistant. Intraperitoneal injection of the male guinea-pig is followed in 2 days by swelling of the testicles; after 3 or 4 days the two layers of the tunica vaginalis are gummed 
together by a thick, purulent, yellowish exudate. The scrotal skin becomes adherent, and ulcerates. Death in 14 days. P.M., besides the testicular lesions, there may be submiliary nodules in the spleen projecting above the surface, greyishwhite nodules in the lungs and liver, and subcutaneous or periarticular abscesses. Bacilli are present in the fresh lesions.

\section{Pfeifferella whitmori}

Isolation.-Isolated by Whitnore and Krishnaswami (1912) from human patients with melioidosis.

Synonyms.-B. pseudomallei, B. whitmori.

Habitat.-Parasite of rodents and man.

Morphology. - Small, slender rods, 1-2 $\mu$ long and $0 \cdot 1-0.5 \mu$ broad; sides parallel, ends rounded, axis straight; arranged singly, in pairs end-to-end, or sometimes in long parallel bundles, the bacilli being embedded in an interstitial substance Varia tions occur in depth of staining; bipolar staining common, especially in films from infected tissues. Motile; Gram-negative; acid-fast granules have been described in freshly isolated strains (Finlayson 1944). The short, oval, bipolar-stained rods are characteristic of the rongh form ; the longer, narrower rods, arranged in palisades with irregular staining and shadow forms, are characteristic of the smooth variant.

Agar Plate. 24 hours at $37^{\circ} \mathrm{C}$. Round, amorphous, low convex, translucent, greyishyellow colonies, 1-2 mm. in diameter ; smooth, ghistening surface and entire edge ; consistency mucoid; emulsifiability easy. 14 days, colonies are opaque, often coloured yellow, brown, or pinkish, and may have a wrinkled eentre.

Agar Slope.-24 hours at $37^{\circ} \mathrm{C}$. Abundant, confluent, raised, greyish-yellow, mucoid, spreading growth, with glistening, beaten-copper surface, and edge undulate or made up of single colonies. Growth more profuse than that of Bact. coli. Cultures have a mouldy, earthy smell.

Gelatin Stab.-10 days at $20^{\circ} \mathrm{C}$. Abundant, filiform growth, mostly of discrete colonies extending to bottom of tube. Stratiform liquefaction; between the liquefied and unliquefied portions of the gelatin there is a thick nodular pellicle of growth.

Broth.-24 hours at $37^{\circ} \mathrm{C}$. Good growth with moderate turbidity, and slight powdery deposit disintegrating completely; may be slight pellicle formation. 10 days, luxuriant growth with dense turbidity and a heavy viscous deposit disintegrating with difficulty. The rough form gives rise to a slight turbidity and a wrinkled surface pellicle. According to Nicholls (1930), the rough form produces about 0.2 per cent. oxalate (ealculated as calcium oxalate) in 4 days; the smooth form produces not more than about 0.01 per cent., but renders the medium alkaline- $-\mathrm{pH} 8 \cdot 4$.

Glycerol Agar. -3 days at $37^{\circ} \mathrm{C}$. May be (1) profuse mucoid growth with smooth glistening surface, or (2) profuse growth with dull, wrinkled, corrugated, or honeycombed surface.

Horse Blood Agar Plates. -3 days at $37^{\circ} \mathrm{C}$. Abundant growth of round, low convex, greyish-green colonies, 1-2 mm. in diameter.- No hæmolysis, except, perhaps, with freshly isolated strains.

Loeffler's Serum. - 3 days at $37^{\circ} \mathrm{C}$. Good, confluent, shightly raised, creamy growth with smooth surface and undulate edge. Liquefaction by freshly isolated strains after a variable number of days. Sometimes the growth itself appears to become liquefied, and runs down the slope.

Potato.-24 hours at $37^{\circ} \mathrm{C}$. S. type: good growth of a creamy or lemon-yellow colour not very easy to see; later the colour deepens to café-au-lait or chocolate. $R$. type: forms dry, dull, dirty-white growth.

MacConkey's Agar. -3 days at $37^{\circ} \mathrm{C}$. Abundant growth of red, opaque, low convex or umbonate colonies, $2-3 \mathrm{~mm}$. in diameter. 
Resistance.-Destroyed by moist heat at $56^{\circ} \mathrm{C}$. within 10 minutes. Killed within about 10 minutes by 1 per cent. phenol and 0.5 per cent. formol. Organisms may survive for a month or more in water, freces, and dried soil, and for a weck in putrefying carcasses.

Metabolism.-Aerobe ; very slight growth on agar and in broth after 14 days under strict anaerobic conditions. Optimum temperature for growth $37^{\circ} \mathrm{C}$. In culture it grows more rapidly and more abundantly than Pf. mallei. No hæmolysis of sheep or horse blood, except perhaps with freshly isolated strains. Tendency to formation of brownish pigment in cultures, especially on potato. Oxalates formed in broth by rough fornı.

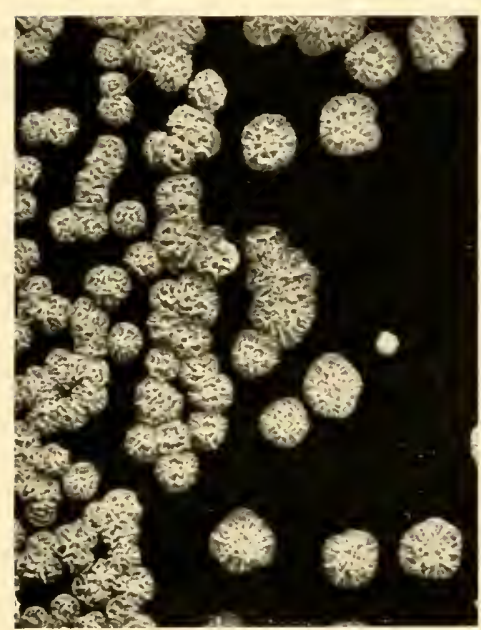

Fic, 94.-Pf. whitmori.

Surface colonies on glycerol agar plate. Rough form.

(After Stanton and Fleteher.)

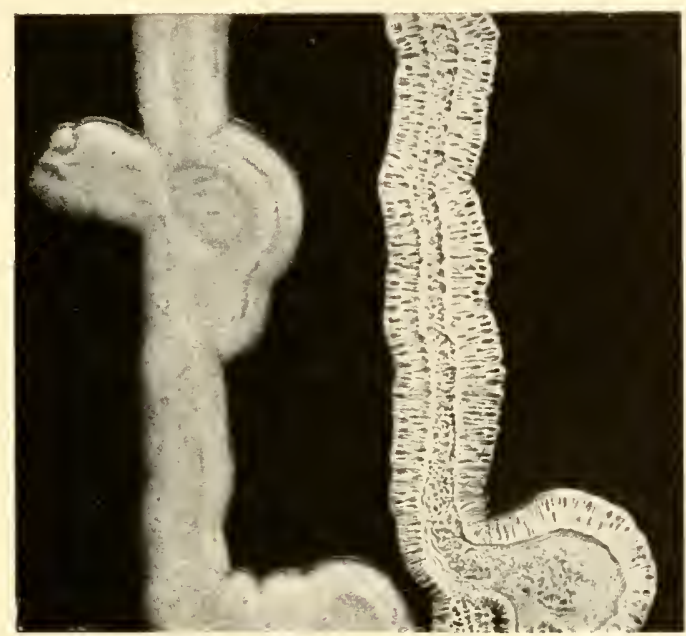

FIG. 95.-Pf. whitmori.

Growth on glyeerol agar. Left : smooth form. Right : rough form.

(After Stanton and Fletcher.)

Biochemical.-On first isolation it forms acid, no gas, in glucose, maltose, mannitol, lactose, dnlcitol, dextrin, and sucrose ; after long cultivation in the laboratory it may attack glucose only; Andrade indicator decolorized in 4-10 days. L.11. may be slightly acid in 3 days; casein is coagulated and may be digested; partial decolorization of litmus. Indole -; N.R. - ; V.P. - ; nitrates reduced ; $\mathrm{NH}_{3}+; \mathrm{H}_{2} \mathrm{~S} \pm$ when tested by Huddleson's method on liver agar; M.B. reduction + ; catalase + . Antigenic Structure.-By agglutination, absorption, and complement fixation cultures of Pf. whitmori form a homogeneous group, which is said to be closely allied to one group of $P f$. mallei.

Pathogenicity.-Causes melioidosis in man, rats, cats, dogs, guinea-pigs, rabbits, and occasionally horses. Experimentally, rodents can be infected by feeding, injection into the tissues, or by rubbing on the scarified skin. Produces abscess at site of inoculation, generalized adenitis, and nodular abscesses of the spleen and lungs. After ip. injection of a small quantity of culture into a male guinea-pig, swelling of the testicles occurs in 2 days with caseous exudate between the two layers of the tunica vaginalis. If a large dose is used, death may occur in 24 hours from septicæmia, before there is time for the Straus reaction to develop or for nodular lesions to appear in the viscera. Small doses prove fatal in about a week. Guineapigs are so susceptible that even minute numbers of bacilli brought into contact 
with the scarified skin may give rise to a fatal infection. Rabbits are as susceptible as guinea-pigs, but rats are more resistant. The subcutaneous inoculation of $0 \cdot 1 \mathrm{ml}$. of a 24-hour broth culture into a rabbit usually causes death in 3 weeks. Post mortem, there is local necrosis, and necrotic or purulent lesions are found in the lungs, spleen, liver, joints, bones, or testicles; purulent arthritis is common in the second week. Cats, monkeys, and goats can all be infected experimentally. The disease in the monkey is usually chronic, lasting 2 to 4 months, and is accompanied by severe emaciation. Horses are resistant (see Stanton and Fletcher 1932, de Moor et al. 1932, Nicholls 1934). According to Nicholls (1934), the rough type is the normal virulent form, while the smooth type is relatively avirulent if obtained completely free from rough organisms.

\section{Perez's Bacillus, or Cocco-bacillus fœtidus ozænæ.}

In 1899 Perez described, under the name of Cocco-bacillus foetidus ozcence a small organism that he had isolated from the nose of patients suffering from ozæna. His work was confirmed by numerous observers (Hofer 1913a, $b$, Ward 1916, 1917, Busson 1923, Olinescu and Atineu 1925a, $b$ ), but there is insufficient evidence to show that this organism is causally related to ozæna. Perez (1901, 1913) found his bacillus also in the saliva and nasal mucus of apparently healthy dogs.

To avoid confusion, it may be stated here that Perez's bacillus is different from the ozæna bacillus described by Loewenberg (1894) and by Abel (1896), which undoubtedly belongs to the Friedländer group (see Chapter 28).

The classification of Perez's bacillus is very doubtful; it is impossible to say to what group this organism will ultimately be assigned. Our reasons for including it in this chapter are to draw attention to certain points of resemblance that it shows to Pf. whitmori and $H$. bronchisepticus.

Morphologically, Perez's bacillus is a small, Gram-negative cocco-bacillus, which was originally described as being non-motile, but which is said by Ferry and Noble (1918) to be sluggishly motile, and by Ward (1917) occasionally to acquire motility in culture. In fluid media, and in old cultures on solid media, long, irregular, deeply staining, filamentous forms may appear. In broth, and on agar if the tubes are sealed, cultures have a heavy, sweet, unpleasant, or even nauseating odour, which is said by Ward to be due to volatile sulphur compounds. According to our own observations, agar cultures tend to wither after a few days, suggesting the occurrence of autolysis. In gelatin stab cultures of Perez's bacillus, fine lateral filaments often appear in 2 or 3 weeks, radiating from the central stab. On potato a creamy-white growth is formed. Biochemically, the organism produces acid and gas in glucose only; in litmus milk there may be a slight acidity; indole is produced. Antigenically, all strains are said by Ward (1917) to be homogeneous; but according to Olinescu and Atineu (1925a), this is doubtful. An antiserum to $H$. bronchisepticus is without action on Perez's bacillus, and vice versa (Ferry and Noble 1918). Both Perez's bacillus and H. bronchisepticus are parasites of the respiratory tract, and both seem to be secondary rather than primary agents in the causation of disease. It is probable that in the past they have been confused with each other. The following table (Table 33), modified from Ferry and Noble, enumerates the main differences between them.

An organism similar to Perez's bacillus has been isolated by Shiga (1922) from the nose of ozæna patients. The chief differences between the two are that Shiga's organism often ferments maltose and sucrose, as well as glucose; it sometimes peptonizes milk; it liquefies gelatin; and it is not agglutinated by a serum prepared against Perez's bacillus. Blane and Pangalos (1925) have isolated from the nose of ozæna patients a short, actively 
TABLE 33

Differentiation of H. Bronchisepticus from Perez's Bacillus.

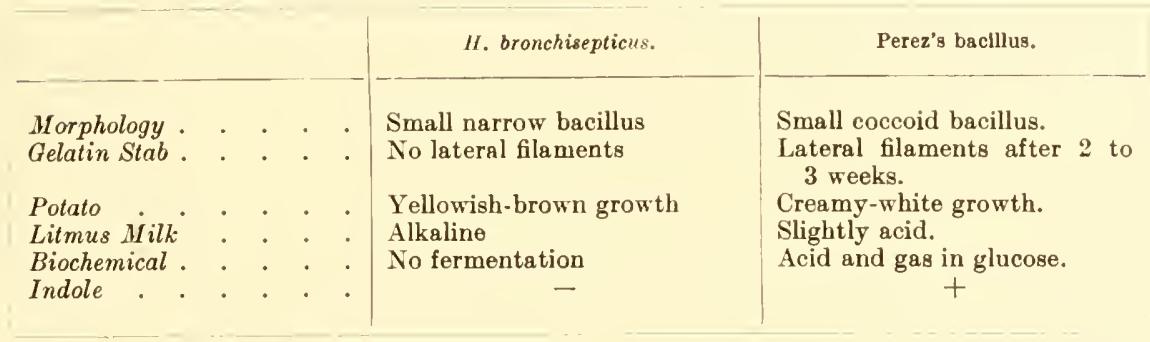

motile, Gram-negative bacillus, which they call $B$. ozogenes. It coagulates milk, ferments glucose, maltose, mannitol, lactose, and sucrose with the production of acid and gas, gives a variable indole reaction, and forms $\mathrm{H}_{2} \mathrm{~S}$.

\section{Cocco-bacillus fœtidus-ozænæ Perez}

Synonym.-Perez's bacillus.

Isolation.-By Perez in 1899 from nose of ozæna patients.

Habitat.-Nose of ozæna patients; saliva and nasal mucus of normal dogs. Probably strict parasite.

Morphology. - Small cocco-bacillus, 1-2 $\mu$ long by $0.5 \mu$ broad; some strains are coccoid, some are bacillary. Parallel or bulging sides, rounded ends; axis straight; arranged singly, in bundles of two or three members, and occasionally in pairs end-to-end. Long sinuous filaments and long straight or curved bacilli may be seen, especially in old cultures. Staining is fairly even. Non-motile or sluggishly motile. Non-capsulated. Gram-negative.

Agar Plate.-24 hours at $37^{\circ} \mathrm{C}$. Round, amorphous, low convex, greyish-white, translucent colonies, up to $1 \mathrm{~mm}$. in diameter; smooth glistening surface and entire edge; consistency butyrous or viscous; emulsifiability easy. 7 days, colonies are larger, 1-2 mm. in diameter, but owing to autolysis a re flattened, almost colourless, transparent, and adherent to the medium; surface is dull and very finely granular.

Agar Slope.-24 hours at $37^{\circ} \mathrm{C}$. Moderate, confluent, slightly raised, greyish-yellow, slightly translucent growth, with glistening beaten-copper surface and entire edge; condensation water consists of greyish-white mucoid material. 7 days, whole growth is flattened, and withered; surface dry and covered with secondary colonies.

Gelatin Stab.-7 days at $22^{\circ}$ C. Moderate, mostly confluent, filiform growth, greyish-white in colour, extending to bottom of tube. Surface growth $2-4 \mathrm{~mm}$. in diameter, slightly raised or flattened. No liquefaction, even after 3 weeks. After 2 to 3 weeks delicate feathery outgrowths from the stab appear in the upper half of the medium.

Broth. -24 hours at $37^{\circ} \mathrm{C}$. Moderate growth with moderate, uniform turbidity and a slight powdery deposit, disintegrating completely; heavy, unpleasant, sweetish odour. No surface growth.

Horse Blood Agar Plate.-24 hours at $37^{\circ} \mathrm{C}$. Abundant greyish-white growth. No hæmolysis, but plate is browned.

Potato. -7 days at $37^{\circ} \mathrm{C}$. Poor, confluent, slightly raised, creamy white growth with smooth surface; potato browned.

IIacConkey's Agar. - 24 hours at $37^{\circ} \mathrm{C}$. Good growth of small, round, low convex, yellowish colonies, not unlike those of Salm. typhi. 
Resistance.-Not known.

Metabolism.-Aerobe, facultative anaerobe. Optimum temperature for growth $37^{\circ} \mathrm{C}$, but growth occurs slowly at $20^{\circ} \mathrm{C}$. No hæmolysis of sheep's or horse's red corpuscles. Old cultures have a tendency to become brownish in colour. Footid odour, especially noticeable in broth or serum broth cultures, and in corked agar cultures.

Biochemical.-Acid and gas in glucose only. L.M. slight acid. Indole + ; M.R. - ; V.P. - ; nitrates reduced; $\mathrm{H}_{2} \mathrm{~S}+$; $\mathrm{NH}_{3}++$; M.B. reduced ; catalase + +. Antigenic. Structure.-Appears to be antigenically homogeneous, as tested by agglutination and complement fixation, but not many strains have been tested.

Pathogenicity.-Pathogenic for guinea-pigs, rabbits, and mice. After intravenous injection of rabbits a mucopurulent, sometimes hæmorrhagic, nasal discharge develops, containing large numbers of the bacilli. Chronic rhinitis may develop, with atrophy of the turbinate bones. Death occurs in from 24 hours to several weeks, according to the dose injected. Intraperitoneal injection of $0.25 \mathrm{ml}$. of a saline suspension off agar into guinea-pigs is fatal in 12 hours; post mortem, there is peritonitis with abundant hæmorrhagic exudate. A similar dose injected subcutaneously into mice is fatal in 48 hours; post mortem, there is gelatinous codema at local site, and enlargement of the spleen. Neither in guinea-pigs nor in mice can the bacillus be recovered from the blood.

\section{REFERENCES}

ABEL, R. (1896) Z. IIyg. InfektKr., 21, 89.

Babes, V. (1891) Arch. Méd. exp., 3, 619.

Blanc, G. and Pangalos, G. (1925) C. R. Soc. Bio?., 93, 1267, 1268.

Busson, B. (1923) Münch. med. Wschr., 70, 426.

Ferry, N. S. and Noble, A. (1918) J. Bact., 3. 499.

Finlayson, M. H. (1944) S. Afr. med. J., 18, 113.

Galli-Valerio, B. (1900) Zbl. Bakt., 28, 353.

Galtier, V. (1881) C. R. Acad. Sci., 92, 303.

Hofer, G. (1913a) Berl. klin. Wschr., 50, 2413; (1913b) Wien. klin. Wschr., 26, 1011.

KiтT, T. (1887) Zbl. Bakt., 2, 241.

Leoroux, R. and DJemil, K. (1931) C. R. Acad. Sci., 193, 1117.

Legroux, R. and Genevray, J. (1933) Ann. Inst. Pasteur, 51, 249.

LEO, H. (1889) Z. Hyg. InfektKr., \%, 505.

LoefFler. (1886) Arb. ReichsgesundhAmt., 1, 141.

LoewenberG. (1894) Ann. Inst. Pasteur, 8, 292.

Mayer, G. (1900) Zbl. Balt., 28, 673.

Moor, C. E. de, Soekarnen, and Walle, N. v. D. (1932) Geneesk. Tijdsch. Ned.-Ind., 24, 1618.

Nicholls, I. (1930) Brit. J. exp. Path., 11, 393 ; (1934) Ceylon J. Sci., Sect. D. med. Sci., $3,183$.

Olinescu, R. and Atineu, A. (1925a) C. R. Soc. Biol., 93, 740; (1925b) Ibid., 93, 741. Panisset, L. (1910) Rev. gén. Méd. vét., 15, 561.

Perez, F. (1899) Ann. Inst. Pasteur, 13, 937; (1901) Ibid., 15, 409 ; (1913) Berl. klin. Wschr., 50, 2411.

SaвоцотNY, S. S. (1926) Zbl. Bakt., 98, 37.

SAкамото, K. (1930) J. Immunol., 18, 331.

Shiga, M. (1922) Zbl. Bakt., 88, 521.

Stanton, A. T. and Fletcher, W. (1921) Proc. 4th. Congr. Far Eastern Ass. trop. Med. Hyg., 2, 196 ; (1925) J. Hyg., Camb., 23, 347 ; (1927) Ibid., 26, 31 ; (1932) Studies Inst. med. Res., F.M.S., No. 21.

Strraus, I. (1889a) Arch. Méd. exp., 1, 460 ; (1889b) Ibid., 1, 489.

Thомpson, L. (1933) J. Bact., 26, 221.

Verge, J. and Patremaure, O. (1928) C. R. Soc. Biol., 99, 182.

WARD, H. C. (1916) J. infect. Dis., 19, 153; (1917) Ibid., 21, 338.

Whitmore, A. (1913) J. Hyg., Camb., 13, 1.

Whitmore, A. and Krishnaswami, C. S. (1912) Indian med. Gaz., 47, 262.

Wirson, G. S. (1934) J. Hyg., Camb., 34, 361. 
CHAPTER 20

\section{AZOTOBACTER, RHIZOBIUM, NITROSONONAS, NITROBACTER, HYDROGENOMONAS, METHANOMONAS, CARBOXYDOMONAS, AND ACETOBACTER}

IN this chapter we group together a number of organisms playing an important part in the nitrogen metabolism of the soil.

\section{THE AZOTIFYING OR NITROGEN-FIXING BACTERIA}

DEFINTION.-Azotobacter.

Relatively large rods, or even cocci, sometimes almost yeast-like in appearance, dependent primarily for growth energy upon the oxidation of carbohydrates. Motile or non-motile; motile forms possess a tuft of polar Hagella. Obligate aerobes, usually growing in a film upon the surface of the culture medium. Capable of fixing atmospheric nitrogen when grown in solutions containing carbohydrates and deficient in combined nitrogen.

Type species. Azotobacter chroococcum, Beijerinck.

Isolated by Beijerinck in 1901. He described two species, Az. chroococcum, so called from the brown pigment to which it gives rise, and $A z$. agilis. The former is widespread in garden earth and in fruitful soil of all kinds; the latter was found in eanal water in Holland. Since then four other specics have been described-Az.vinelandii, $A z$. beijerincki, Az. woodstowni, and $A z$. vitreus; all of them inhabit the soil.

All species of Azotobacter are highly pleomorphic; the cells may be short, thick and rod-like, ellipsoidal, pyriform, or spindle-shaped ; when occurring in pairs they often look like giant diplococci. Their size varies from $4-7 \mu$ in length and $1.5-4 \mu$ in breadth. Giant involution forms, looking like

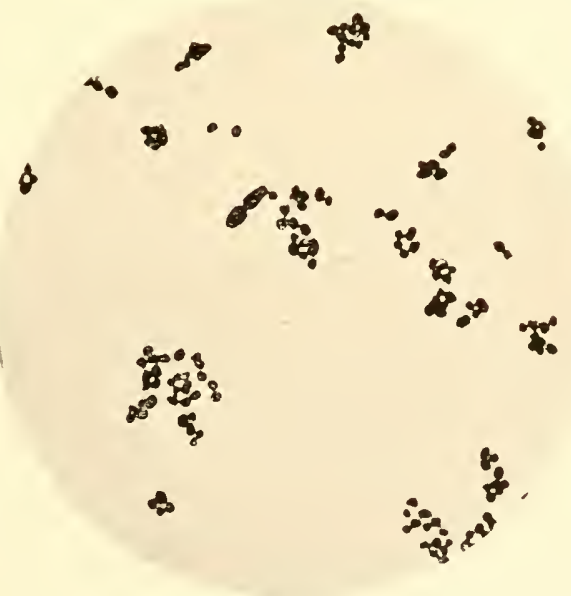

Fig. 16.-Azotobacter chroococcum.

From an agar culture, 3 days, $30^{\circ} \mathrm{C} .(\times 1000)$. amœeb or yeasts, are not uncommon. They are motile by one or more polar, or possibly peritrichate (Hofer 1944), flagella, and are Gram-negative.

The organism is strictly aerobic. It grows best in tap water containing 2 per 
cent. mannitol and 0.02 per cent. $\mathrm{K}_{2} \mathrm{HPO}_{4}$. If the medium is spread out in a thin layer in a wide-bottomed flask, Azotobacter chroococcum forms a surface pellicle in 2 or 3 days, which gradually becomes brown, and may later even turn black. Potassium, calcium, or sodium propionate in 0.5 per cent. solution may be substituted for the mannitol.

Of solid media, one of the best has the following composition :

\section{Mannitol \\ $\mathrm{K}_{2} \mathrm{HPO}$ \\ Washed Agar \\ Aq. Dist.}

$2 \mathrm{gm}$.
0.02
$2 "$,
$100 "$

Azotobacter grows best in solutions containing little or no combined nitrogen; according to Beijerinck (1901) ammonium salts are not easily assimilated, and peptone can be used only to a slight extent.

The peculiar property of this group of organisms is to fix atmospheric nitrogen and to convert it into ammonia, nitrites and nitrates (Beijerinck and van Delden 1902). To do this they must be supplied with a source of energy in the form of a suitable carbohydrate, such as mannitol. The energy gained from the oxidation of this substance is utilized in the fixation of nitrogen. Gainey (1918) found that in a synthetic mannitol medium inoculated with soil, about $8 \mathrm{mgm}$. of nitrogen were fixed for $50 \mathrm{ml}$. of medium. Not all strains of Azotobacter are capable of utilizing mannitol (Smith 1935). Azotobacter is more susceptible to acid than most soil organisms. Its growth limits are about $\mathrm{pH} 6 \cdot 5-8 \cdot 6$ in pure culture (Fred and Davenport 1918), but in soil it is probable that growth can occur down to about pH 6.0. Pigment formation is variable in Az. chroococcum; some variants form a brown pigment, others are achromogenic; intermediate forms occur (Omeliansky and Ssewerowa 1911). Pigment is formed only in the presence of a free supply of oxygen. We give a description of the type species Az. chroococcum, followed by notes on the lesser known species.

\section{Azotobacter chroococcum}

\section{Isolation.-By Beijerinck in 1901 .}

\section{Habitat.-Soil.}

Morphology.-Short, thick rods with rounded ends; large ovoid forms; forms like giant diplococci ; pear-shaped rods, and other forms. $4-5 \mu \times 1.5-2 \mu$. Slowly motile by polar flagella. Arranged singly, in pairs end-to-end, and in old cultures in packets. Cells often vacuolated; when grown on mannitol, cells may contain fat droplets. In surface membranes on liquid media cells are surrounded by a thick mucoid capsule. Large involution forms not uncommon. Non-sporing. Gramnegative. Non-acid-fast.

Agar Plate.-3 days at $30^{\circ} \mathrm{C}$. Round colonies, $1 \mathrm{~mm}$. in diameter, convex, with smooth, moist, glistening surface ; edge entire ; structure a morphous ; consistency butyrous, easily emulsifiable; often differentiated into an opaque brown centre and a translucent lighter periphery. After 6 days the colonies measure up to $3 \mathrm{~mm}$. in diameter; some remain homogeneous, undifferentiated and opaque; others show an opaque brownish centre, and a clear, slightly radiate periphery.

Agar Slope.-2 days at $30^{\circ} \mathrm{C}$. Moderate, confluent or partly confluent, slightly raised, translucent, greyish-yellow growth, with glistening, beaten-copper surface; edge formed of single colonies.

Gelatin Stab.-6 days at $20^{\circ} \mathrm{C}$. Poor to moderate, greyish-white, filiform growth, consisting chiefly of discrete colonies; extends $\frac{2}{3}$ way down tube; surface growth, 3-4 $\mathrm{mm}$. in diameter, with a lobate edge and irregular surface. No liquefaction. 
Broth. -2 days at $30^{\circ} \mathrm{C}$. Moderate growth, with moderate, finely granular turbidity, and slight, finely granular sediment, not disintegrating on shaking. After 6 days, surface ring growth.

Loeffler's Blood Serum.-6 days at $30^{\circ} \mathrm{C}$. Moderate, confluent, creamy growth, with slight yellowish colour; smooth, mirror-like surface; no liquefaction.

Potato.-10 days at $30^{\circ} \mathrm{C}$. Abundant, raised, opaque brown growth, with wrinkled honeycombed surface. During first few days, growth is yellowish, but later it becomes brown. Potato unchanged.

Resistance.--Killed by $55^{\circ} \mathrm{C}$. in 30 minutes.

Metabolism.-Aerobic. No growth anaerobically. Opt. temp. 28-30 ${ }^{\circ}$ C. Limits of $\mathrm{pH} 6 \cdot 5-8 \cdot 6$ in pure culture. Pigment; grown in mannitol and other suitable media, a surface scum is formed, which becomes brown, and later black. Pigment is insoluble in water, alcohol, $\mathrm{CHCl}_{3}$, ether, $\mathrm{CS}_{2}$, but slightly soluble in alkalies, which destroy it.

Nutritional. Grows best in 2-10 per cent. mannitol solutions, and in 0.5 per cent. solutions of $\mathrm{K}, \mathrm{Ca}$, or $\mathrm{Na}$ propionate. Growth on agar improved by 1 per cent. mannitol.

Biochemical.-Fixes atmospheric nitrogen, converting it into $\mathrm{NH}_{3}$, nitrite and nitrate. Capable of growing in media free from combined nitrogen but containing carbohydrates. Can utilize nitrates for its nitrogen supply; $\mathrm{NH}_{3}$ and peptone can be used only slightly.

Utilizes dextrose, maltose, mannitol, lactose, dextrin, starch, glycerol, alcohol, propionate, acetate, butyrate, citrate, lactate, malate, and succinate; gives off $\mathrm{CO}_{2}$. Indole - . M.R. - . V.P. - . Nitrates -. M.B. reduction -. Catalase +. $\mathrm{NH}_{3}$ very slight. L.M. partly decolorized, and rendered clearer and more fluid. Pathogenicity.--Nil.

Az. agilis is a large, oval organism containing granules and vacuoles, and provided with a bundle of polar flagella. The other four species of Azotobacter that have been described differ in minor respects from the type species $A z$. chroococcum.

Numerous non-sporing rod-shaped organisms, capable of fixing nitrogen, have been found in horse and cow dung by Fulmer and Fred (1917). The chief of these, which they call $B$. azophile, is a rod-shaped organism, $1.6 \times 0.8 \mu$, motile and Gram-positive. It gives a light-orange, wrinkled growth on agar, a heavy membranous surface growth in broth, and a brownish growth on potato. Gelatin is liquefied; milk is peptonized. Nitrates are reduced to nitrites and to gaseous nitrogen. Indole + . Strict aerobe. Grows well in mannitol solution, in which it fixes nitrogen in considerable quantities.

\section{RHIZOBIUM}

\section{Definition.-Rhizobium.}

Minute rods, motile when young. Specialized forms abundant and characteristic when grown under suitable conditions. Obligate aerobes, ${ }^{1}$ capable of fixing atmospheric nitrogen when grown in the presence of earbohydrates and in the absence of compounds of nitrogen. Produce nodules on the roots of leguminous plants.

Type species. Rhizobium leguminosarum, Frank.

The first member of this group was isolated by Beijerinck in 1888, who named it Bacillus radicicola. He found this organism in the root-nodules of leguminous plants, and in numerous specimens of soil and water of different origin; he noted its variable morphology; he described its cultural and biochemical reactions; and he showed how the bacilli in the "swarmer" stage penetrated

${ }^{1}$ This is not absolutely certain ; we have observed growth of one strain in broth, though not on agar, under strictly anaerobic conditions. 
the pore-spaces in the roots of certain plants. Though suspecting it of being capable of fixing atmospheric nitrogen, he was unable to demonstrate this conclusively. Numerous strains of Rhizobium have since been described, but as it is doubtful whether they are separate species, we shall confine ourselves to a description of Rhiz. leguminosarum.

In pure cultures on nutrient agar this organism occurs in the form of rods, $1.5-3 \mu \times 0.5 \mu$, but by varying the constituents of the medium it can be made to pass through a cycle of changes, described by Bewley and Hutchinson (1920) as follows : (1) Pre-swarmer stage, non-motile. When a pure culture is inoculated into a neutral soil solution, the organisms assume this form in 4 to 5 days. Diameter about $0.4 \mu$. (2) Pre-swarmer stage; larger, non-motile, cocci, $0.8 \mu$ in diameter. Appear in mannitol agar. (3) Swarmer stage; very actively motile. Cclls are ellipsoidal; $0.9 \mu \times 0.18 \mu$ (Beijerinck). They are so small as to be

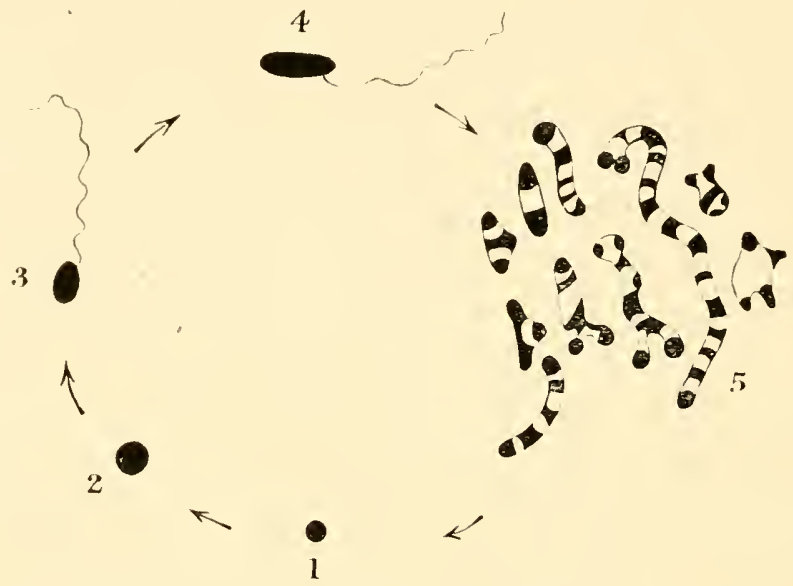

FIG. 97.-Rhizobium leguminosarum.

1. Pre-swarmer, first stage. 2. Pre-swarmer, sccond stage. 3. Swarmer. 4. Motile rod. 5. Highly vacuolated rods.

(After Bewley and Hutehinson.)

able to pass through a Chamberland filter. Appear in carbohydrate media. (4) Rod stage; motile. Appearance favoured by carbohydrates; as long as these are abundant, the organism remains in this stage. Dimensions $3.4 \mu \times 1 \mu$. (5) Stage of high vacuolation. In a neutral soil extract, or in a medium in which the carbohydrate has been exhausted, the organisms become highly vacuolated; the chromatin divides into a number of bands. Later these bands become rounded off and escape from the rod as the coccoid pre-swarmers (Fig. 97). The formation of the pre-swarmers may also be induced by the addition of calcium and magnesium carbonate to the medium, or by incubating the culture anaerobically. As Lewis (1938) points out, there is no reason to regard this succession of morphological changes as essentially different from that seen in other groups of bacteria, or as indicating the occurrence of a specialized form of reproduction; the vacuolated state appears to depend on the presence of fat globules.

Calcium phosphate causes a change from pre-swarmers to rods. Acid soils favour the production of highly vacuolated cells, and eventually kill the organisms. 
Slightly alkaline soils support vigorous growth without altering the morphology. Relatively high temperatures, $30-37^{\circ} \mathrm{C}$, prevent or postpone the change into the pre-swarming stage.

Inside the root nodules the organisms assume curious $\mathrm{Y}$-shaped, pyriform, and racket-like forms, known as bacteroids. Filaments may likewise be formed. In culture the bacteroids develop into rods (Fremlin 1898).

The properties of Rhiz. leguminosarum vary in accordance with the species of plant from which they are derived. Fred and Davenport (1918), who studied 21 strains from different Leguminosa, found a variation in their resistance to acids. Thus the strains from alfalfa and sweet clover had a limiting $\mathrm{pH}$ for growth of 4.9 ; for the garden pea and vetch of 4.7 ; for red clover and common beans of $4 \cdot 2$; for soya beans and velvet beans of $3 \cdot 3$; and for lupins of $3 \cdot 15$. All strains had much the same resistance to alkali. Incidentally their alkali tolerance was much greater than their acid tolerance.

Whether the strains from different plants should be regarded as varieties of one species is doubtful. Klimmer and Krüger (1914), who examined a number of strains from eighteen different species of Leguminosa by means of the agglutination, complement-fixation, and precipitation tests, were able to classify them into 9 different groups. Bushnell and Sarles (1939) likewise found a large number of serological types. In their experience little relationship was noted between the ability of strains from different plants to cross-agglutinate and to cross-infect (see also Fremlin 1898, Kleczkowski and Thornton 1944).

Rhiz. leguminosarum is an obligate aerobe, capable of fixing atmospheric nitrogen when grown in a medium free from combined nitrogen, but containing a fermentable carbohydrate. Such a medium is composed of :

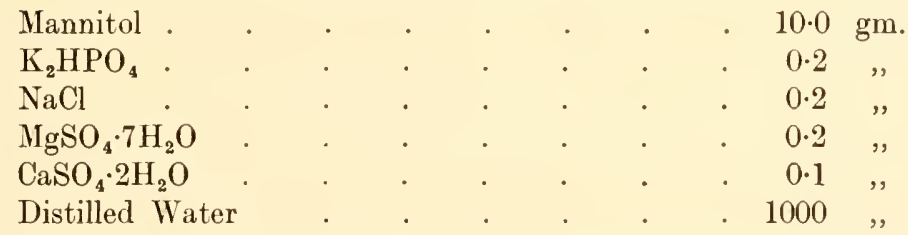

Inoculated into the root of a leguminous plant it gives rise to a nodule, from which it can be recovered in pure culture. Each variety is apparently specialized to attack its own or closely related species of plants; thus a strain isolated from a nodule on a pea (Pisum sativum) will produce nodules also on vicia, Lathyrus and Lens, but not on other legumes (Russell 1923).

We append a detailed description of Rhiz. leguminosarum.

\section{Rhizobium leguminosarum Frank}

Synonyms.-Bacillus radicicola; Ps. radicicola; nodule organism.

Isolation.-By Beijerinck in 1888 from root-nodules of leguminous plants.

Ecology.-Soil; found also in water.

Morphology.-Has a life-cycle with gross changes in morphology. Shows non-motile coccoid forms; very small, highly motile, ellipsoidal form (swarmer), $0.9 \mu \times 0 \cdot 2$ $\mu$; motile rods, $2-3 \mu \times 0.5 \mu$; large "vacuolated" rod and Y-forms. In root nodules shows filamentous forms, and $Y$-forms (bacteroids). Swarmer forms motile by single, long, polar flagellum. Vacuolated forms show bands of chromatin. Nonsporing; may be capsulated (see below). Gram-negative. Non-acid-fast. 
Agar Plate. -2 days at $25^{\circ} \mathrm{C}$. Round, $1 \mathrm{~mm}$. in diameter, low convex, greyish-yellow transparent colonies with smooth, glistening surface and lobate edge; structure amorphous; consistency butyrous; easily emulsifiable. Differentiated into a smooth, raised, darker centre, and a thin, effuse, radiate periphery. Some strains are said to form mucoid colonies, consisting of bacilli embedded in a mucinous material which appears to consist of a carbohydrate material, yielding glucose on inversion (Kramár 1921).

Agar Slope.-2 days at $25^{\circ} \mathrm{C}$. Moderate, partly confluent, slightly raised, greyish-yellow, translucent growth, with glistening, beaten-copper surface, and finely lobate edge.

Gelatin Stab. -5 days at $20^{\circ} \mathrm{C}$. Moderate, filiform growth extending to bottom of tube; slight surface growth $1 \mathrm{~mm}$. in diameter; no liquefaction except in very old cultures.

Broth. -2 days at $25^{\circ} \mathrm{C}$. Poor growth with slight turbidity, and slight powdery deposit disintegrating on shaking. 5 days ; moderate turbidity, slight surface ring growth, and moderate viscous deposit giving rise on shaking to dense turbidity.

Loeffer's Blood Serum. -6 days at $25^{\circ} \mathrm{C}$. Abundant, mostly confluent, raised, chromeyellow growth, slightly tinged with pink; smooth glistening surface. Medium not coloured; no digestion.

Potato.-6 days at $25^{\circ} \mathrm{C}$. Poor, effuse, confluent, whitish-pink growth, with smooth, moist, glistening surface; medium not coloured, or coloured slightly grey.

Resistance.-Killed at $60^{\circ} \mathrm{C}$. in 1 hour. Resists drying and freezing.

Metabolic.-Aerobic. No growth anaerobically on agar, but moderate growth in broth. Opt. temp. $25^{\circ} \mathrm{C}$. Pigment yellowish-pink, not marked, on certain media only.

Biochemical.-No acid or gas in glucose, maltose, mannitol, lactose, sucrose or salicin. Indole -. M.R. - . V.P. - . Nitrate reduction -. Catalase +. M.B. reduction $+. \mathrm{NH}_{3}+. \mathrm{H}_{2} \mathrm{~S}$ slight + . L.M. acid and clot in 21 days. Capable of fixing atmospheric nitrogen in the presence of carbohydrates and in the absence of combined nitrogen.

Antigenic Structure.-Can be divided by agglutination into numerous different groups. Pathogenicity.-Nil to man or animals. Produces nodules upon the roots of the Leguminosa.

\section{NITROSOMONAS}

\section{DEFINITION.-Nitrosomonas.}

Cells rod-shaped or spherical, motile or non-motile; motile forms possess polar flagella. Capable of securing growth energy by the oxidation of ammonia to nitrites. Growth on media containing organic substances is scanty or absent.

Type species. Nitrosomonas europcea, Winogradsky.

Winogradsky $(1890 a, b, c)$, finding that nitrification did not occur in a medium containing organic matter, seeded with soil, prepared the following solution:

Ammonium sulphate
Potassium phosphate
Pure water

\section{$1 \mathrm{gm}$.}

1,

To each $100 \mathrm{ml}$. was added $0.5-1.0 \mathrm{gm}$. of basic magnesium carbonate; when this medium was inoculated with soil, nitrification occurred satisfactorily, the ammonia being oxidized to nitrite. Five different types of organisms were found; one of them, which grew around the particles of carbonate at the bottom of the flask, failed to grow in gelatin on transplantation, but could be cultivated in a purely inorganic medium. This organism was obtained in pure culture, and was found to be responsible for nitrification. Morphologically it consists 
of ellipsoidal cells, intermediate between cocci and bacilli; its dimensions are $1 \cdot 1-1.8 \mu \times 0.9-1.0 \mu$. It is motile by a single long flagellum. It is arranged singly, or aggregated into a zoogloal mass by some slightly viscous substance; chains even of 3 or 4 members are rare.

Winogradsky found that this organism could grow in a medium devoid of all traces of organic matter. It must therefore obtain its carbon from the magnesium carbonate added to the medium; that is to say, it can assimilate the carbon of carbonic acid. It obtains its nitrogen from the ammonium sulphate, and oxidizes it to nitrite. From purely inorganic substances it can therefore synthesize organic matter-a process rarely accomplished independently of chlorophyll and sunlight.

Experiments showed that the amount of ammonia oxidized and the amount of carbon assimilated ran strictly parallel. For every $96 \mathrm{mgm}$. of nitrous acid formed it assimilated only $1 \mathrm{mgm}$. of carbon. This disproportion between the rapid oxidizing and the slow assimilating action of the organism explains why its growth is so slow.

Winogradsky and Omeliansky (1899) showed that Nitrosomonas europa a was very susceptible to the presence in the medium of organic nitrogenous substances such as peptone or asparagin. In fact the more nutritious a medium was for ordinary bacteria, the less suitable was it for the nitrite organism. It is possible, however, for it to grow in the presence of organic matter (Boullanger and Massol 1904, Fremlin 1914, Bonazzi 1919), but in artificial culture the results are not satisfactory. On the ordinary laboratory media, for example, growth is scanty or entirely absent. In the soil it is probable that its susceptibility to the presence of organic matter is less.

For the study of single colonies the best medium is a silicic acid gel poured into plates (Winogradsky 1891). After 3 or 4 weeks' incubation small, compact, sharply-defined colonies appear, of a brownish colour. Growth can be hastened by pouring a solution of ammonia over the plate.

Nitrosomonas europca is most active at a temperature of $25-30^{\circ} \mathrm{C}$, in a wellaerated medium contained in large flat-bottomed flasks, which are slowly agitated. The presence of scoria (cellular lava) in the medium is beneficial, apparently by increasing the surface exposed to the air (Boullanger and Massol 1903). Under such conditions in an inorganic solution it may oxidize as much as $169 \mathrm{mgm}$. of ammonia nitrogen to nitrite per litre of medium in 14 days (Bonazzi 1919). The accumulation of nitrite arrests the reaction. The type species is known as Nitrosomonas europaca, Winogradsky. Another similar organism, but of spherical shape, is called Nitrosococcus americanus; it is found in the New World.

Nitrosomonas is not by any means the only organism capable of forming nitrite from ammonia. Cutler and Mukerji (1931) isolated a number of organisms from soil that were able to perform this oxidation, though not so actively as Nitrosomonas. A full description of these organisms has not yet been published, but most of them were Gram-positive, non-sporing, non-motile rods, 1.4-1.9 $\iota$ long and 0.70-0.85 $\mu$ broad, which grew on ordinary agar, which oxidized various ammonium salts to nitrite, both in culture medium and in soil, which were unable to oxidize nitrite to nitrate, and which failed to grow in the absence of oxygen. Some strains liquefied gelatin and some did not. Nitrite formation was stimulated by the presence of $0 \cdot 1$ per cent. sucrose, but no growth occurred in pure sugar solutions. Unlike Nitrosomonas, which requires a distinctly alkaline medium, these organisms were able 
to form nitrite within a range of $\mathrm{pH} 4 \cdot 8-7 \cdot 3$. Similar organisms have been described by Cutler and Crump (1933), who found that no fewer than 104 out of 229 strains of bacteria isolated from bect sugar effluent were able to produce nitrite from ammonium salts. Fremlin $(1903,1914,1929-30)$ has worked for a long time on a very active nitroso-bacterium that grows in association with other organisms. S. Winogradsky and H. Winogradsky (1933) have described two further genera of nitrifying organisms-Nitrosocystis and Nitrosospira. Their article should be consulted not only for an account of these organisms, but also for much useful information on nitrifying bacteria in general.

\section{NITROBACTER}

DEFINITION.-Nitrobacter.

Cells rod-shaped, non-motile, not growing readily on organic media or in the presence of ammonia. Cells capable of securing growth energy by the oxidation of nitrites to nitrates.

Type species. Nitrobacter winogradskyi.

Nitrobacter winogradskyi was isolated by Winogradsky in 1891-the year after his discovery of Nitrosomonas europaca.

It is a small rod-shaped or pyriform organism, sometimes with one end drawn out or recurved. Size $0.5 \mu \times 0.25-0.3 \mu$. Non-motile. Arranged in more or less dense masses.

Winogradsky (1896) cultivated it in a medium of the following composition:

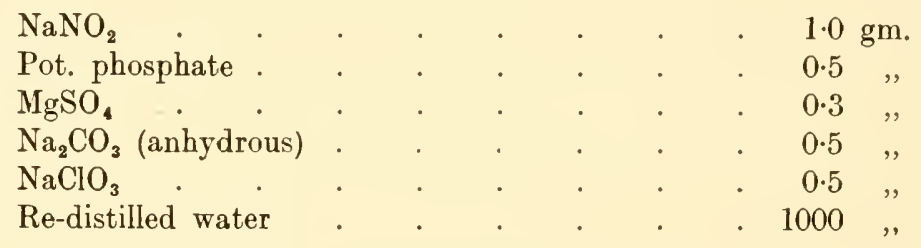

The medium is placed in a shallow layer in wide-bottomed flasks. Growth occurs in the form of a just pcrceptible gelatinous film at the bottom of the flask ; no turbidity appears. The nitrite is oxidized to nitrate. No organic nitrogen nor carbon is required. Indeed the presence of organic matter hinders its development, though not so markedly as that of the nitrite organism. On a washedagar medium made up with sodium nitrite, sodium carbonate, and potassium phosphate (Omeliansky 1899) single colonies are formed, light brown in colour and of irregular shape. Similar colonies, but smaller and more compact, appear on silicic acid gel plates. No growth occurs in broth.

In artificial culture the nitrate bacillus is very susceptible to the presence of ammonia; in soil it is less so. The accumulation of nitrate to the extent of $25 \mathrm{gm}$. per litre arrests the reaction (Boullanger and Massol 1903).

As well as the genera that we have described, there are numerous other organisms playing an important part in soil metabolism. We shall confine ourselves here to giving a definition of the genera Hydrogenomonas, Methanomonas, Carboxydomonas, and Acetobacter. 
DEFINITION.-Hydrogenomonas.

Monotrichate short rods, capable of growing in the absence of organic matter, and securing growth energy by the oxidation of hydrogen (forming water). Kaserer (1906), who first described the organism, states that his species will also grow well on a variety of organic substances.

Type species is Hydrogenomonas pantotropha. Niklewski (1908) described two additional species, Hyd. vitrea and Hyd. fava.

DeFinition.-Methanomonas.

Monotrichate short rods, capable of growing in the absence of organic matter, and securing growth energy by the oxidation of methane (forming carbon dioxide and water).

Type species is Methanomonas methanica (Söhngen 1906).

DeFinition.-Carboxydomonas.

Rod-shaped cells capable of securing growth energy by the oxidation of carbon monoxide (forming carbon dioxide).

The type species, Carboxydomonas oligocarbophila (Beijerinck and van Delden $1903)$, is described as non-motile.

DeFinition.-Acetobacter.

Cells rod-shaped, frequently in chains, non-motile. Cells grow usually on the surface of alcoholic solutions as obligate aerobes, securing growth energy by the oxidation of alcohol to acetic acid. Also capable of utilizing certain other carbonaceous compounds, as sugar and acetic acid. Elongated, filamentous, clubshaped, swollen, and even branched cells may occur as involution forms.

Type species is Acetobacter aceti (Thomson 1852).

\section{REFERENCES}

BeiJerinok, M. W. (1888) Bot. Ztg., 46, 724, 740, 756, 780, 796; (1901) Zbl. Bakt., II te Abt., 7, 561 .

BeiJerincK, MI. W. and Deldex, A. van. (1902) Zbl. Bakt., 9, 3 ; (1903) Ibid., 10, 33.

Bewley, W. F. and Hutchinson, H. B. (1920) J. agric. Sci., 10, 144.

Bovazzi, A. (1919) J. Bact., 4, 43.

Boullanger, E. and Massol, L. (1903) Ann. Inst. Pasteur, 17, 492 ; (1904) Ibid., 18, 181.

Bushnell, O. A. and Sarles, W. B. (1939) J. Bact., 38, 401.

Cutler, D. W. and Crump, L. M. (1933) Ann. appl. Biol., 20, 291.

Cutler, D. W. and Mukerji, B. K. (1931) Proc. roy. Soc., B, 108, 384.

Fred, E. B. and Davenport, A. (1918) J. agric. Res., 14, 317.

Fremlin, H. S. (1898) J. Path. Bact., 5, 389 ; (1903) J. Hyg., Camb., 3, 364; (1914) Ibid., 14, 149; (1929-30) Ibid., 29, 236.

FulMER, H. L. and Fred, E. B. (1917) J. Bact., 2, 423.

GaINey, P. L. (1918) J. agric. Res., 14, 265.

Hofer, A. W. (1944) J. Bact., 48, 697.

KASERer, H. (1906) Zbl. Bakt., IIte Abt., 16, 681.

KLeczkowski, A. and Thornton, H. G. (1944) J. Bacl., 48, 661.

KuIMMer, M. and KrÜGER, R. (1914) Zbl. Bakt., IIte Abt., 40, 256.

Kralír, E. (1921) Zbl. Bakt., 8\%, 401.

LewIS, I. II. (1938) J. Bact., 35, 573.

NikLewsei, B. (1908) Zbl. Bakt., IIte Abt., 20, 469.

Omeliansky, V. (1899) Zbl. Bakt., IIte Abt., 5, 537.

OMeliansky, W. L. and Ssewerowa, O. P. (1911) Zbl. Bakt., IIte Abt., 29, 643.

Russell, E. J. (1923) "The Micro-organisms of the Soil." London.

Sмiтн, N. R. (1935) J. Bact., 30,323.

SöHNGEN, N. L. (1906) Zbl. Bakt., IIte Abt., 15, 513.

Thomson, R. D. (1852) Liebigs Ann., 83, 89.

Winogradsky, S. (1890a) Ann. Inst. Pasteur, 4, 213 ; (1890b) Ibid., 4, 257 ; (1890c) Ibid., 4, 760; (1891) Ibid., 5, 92, 577; (1896) Zbl. Bakt., IIte Abt., 2, 329, 377, 429.

Winogradsky, S. and Oneliansky, W. (1899) Zbl. Bakt., IIte Abt., 5, 329, 377, 429.

Winogradsky, S. and Winogradsky, H. (1933) Ann. Inst. Pasteur, 50, 350. 


\section{CHAPTER 2I}

\section{PSEUDOMONAS}

Definition.-Pseudomonas.

Rod-shaped organisms, usually motile, by means of polar flagella. Generally Gram-negative. Non-sporing. Aerobic; some species are facultative anaerobes. Frequently produce a water-soluble pigment, which is yellow, green, blue, purple, or brown in colour, and which diffuses through the medium. Some species form a non-diffusible yellow pigment, and some species are photogenic. Fermentation of carbohydrates as a rule not active. Frequently gelatin-liquefiers, and active ammonifiers. Common in soil and water. Many yellow species are plant parasites.

Type species. Pseudomonas pyocyanea. (On grounds of priority the American Committee recommend that this organism should be called Ps. aeruginosa.)

Ps. pyocyanea was first isolated by Gessard in 1882 from "blue pus." Ps. fuorescens was described originally by Flügge (1896) under the name of Bacillus fuorescens liquefaciens. This organism appears to be closely related to Ps. pyocyanea; the possible differences between them will be discussed later.

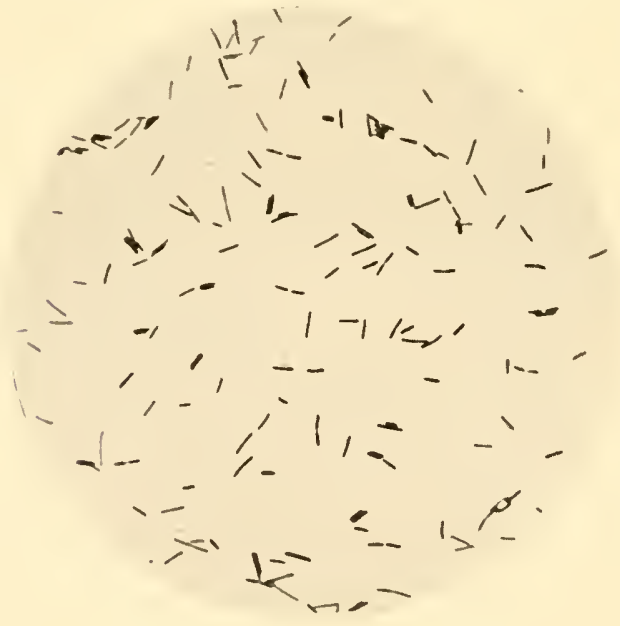

FIG. 98.-Pseudomonas fluorescens.

From an agar culture, 24 hours, $37^{\circ} \mathrm{C} .(\times 1000)$.

Morphology.-The organisms of this group are rod-shaped and rather slender. Their length is subject to considerable variation; even in a single strain some organisms may be very short, while others are long, or actually filamentous. The sides are parallel and the ends rounded. They are arranged singly, in small bundles, or in short chains. They are motile by one or more polar flagella; they are non-sporing; they stain readily with the ordinary aniline dyes, and are usually Gramnegative. They are non-acid-fast.

Cultural Appearances. - Growth occurs readily on the usnal media. Many species form a water-soluble pigment, which diffuses through

the medium. On potato $P$ s. pyocyanca and $P$ s. fuorescens give a pigmented growth, which frequently assumes a café-au-lait colour, not unlike that given by organisms of the Brucella and Pfeifferella groups, and V. cholerce. 
Resistance.-None of the members of the group forms spores, and none is particularly resistant to heat or chemical disinfectants. They all succumb on exposure in a water-bath to a temperature of $55^{\circ} \mathrm{C}$. in 1 hour. The greenish-yellow fluorescent bacilli-Ps. fuorescens and Ps. pyocyanea-are said to be more resistant than other vegetative organisms to ultra-violet light (Burge and Neill 1915). It is suggested that they are able to convert the short wave lengths into longer waves, and hence dispose of the energy of the absorbed waves, which would otherwise be spent in coagulating them. (see Chapter 5).

Metabolic and Biochemical Characters.-Many of the organisms are obligatory aerobes; others may give a very slight growth under anaerobic conditions. Growth of $P s$. pyocyanea in broth is accompanied by a fall in oxidation-reduction

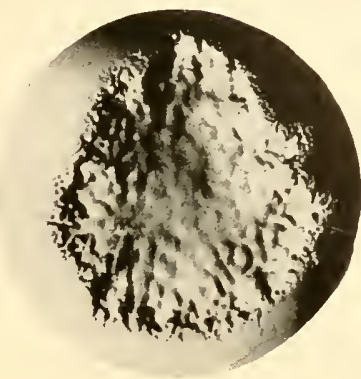

FIG. 99.-Pseudomonas pyocyanea. Surface colony on agar, 24 hours, $37^{\circ}$ C. $(\times 8)$. potential, which reaches a limiting value of between Eh $-0 \cdot 100$ and $-0 \cdot 200$ volt. The pigment pyocyanin constitutes a reversible oxidation-reduction system, and

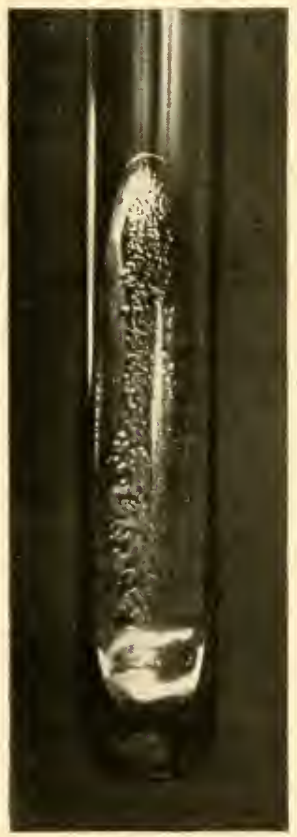

Fig. 100.-Pseudomonas fluorescens.

Agar slope culture, 48 hours, $22^{\circ} \mathrm{C}$. acts as a respiratory catalyst, but according to Reed and Boyd (1933) the changes of potential in culture are not dependent on the presence of this substance. The limiting temperatures for growth are about $0^{\circ} \mathrm{C}$. and $42^{\circ} \mathrm{C}$. Generally speaking, Ps. pyocyanea grows between $5^{\circ} \mathrm{C}$. and $42^{\circ} \mathrm{C}$., and has an optimum about $37^{\circ} \mathrm{C}$, whereas Ps. fluorescens grows between $0^{\circ} \mathrm{C}$. and $37^{\circ} \mathrm{C}$, and has an optimum about $25^{\circ} \mathrm{C}$. The fermentative power is usually weak; acid, but no gas, is formed by some species in certain sugars. According to Moltke (1927), 4 strains of Ps. pyocyanea which he examined failed to ferment any of the usual sugars. In our experience acid is generally formed from glucose, but from no other sugar. This is confirmed by Sandiford (1937), who examined 50 strains and found that glucose was the only sugar fermented. The formation of indole has been recorded by various observers, but, as Sandiford points out, a false reaction may result from the action of the acid in Böhme's reagent on the pigment produced by the organisms. If the oxalic acid method is used for testing, no indole formation can be demonstrated. Practically all strains liquefy gelatin and peptonize milk. The usual nitratereduction test at 5 days is negative with most strains, but this is often because the nitrite itself is reduced, resulting in the production of gaseous nitrogen or one of its compounds. If the test is carried out after one day, nitrites can be demonstrated (Ferramola and Monteverde 1939). All strains are said by Ferramola and Monteverde to utilize citrate as the sole source of carbon, but to be incapable of producing $\mathrm{H}_{2} \mathrm{~S}$. Ammonia is produced by all strains (Seleen and Stark 1943).

Pathogenicity.-With the exception of Ps. pyocyanea, members of the group 
are non-pathogenic to man. Ps. pyocyanea itself gives rise occasionally to suppurative processes and less often to generalized infection. Among the commonest manifestations are middle-ear suppuration in children, destructive lesions of the skin, sometimes described as ecthyma gangrenosum, in children and adults, and necrotic and ulcerative lesions of the alimentary mucosa. The respiratory tract, the eye, the joints, and the kidneys are sometimes affected. Wounds are often infected. There is also reason to believe that the organism plays a part in some cases of infantile diarrhœea. Infection may be primary or secondary, and is often acute and rapidly fatal. (For review of human infections see Waite 1908, Fraenkel 1917, and Lode 1929.)

Animals are rarely infected unless given large doses intravenously, when they may die of intoxication. Ps. pyocyanea, however, often produces fever and a local abscess after subcutaneous injection into rabbits; and, if highly virulent, it may prove fatal in 24 hours.

Group forming Bluish-green Pigment.-Ps. pyocyanea, first isolated in pure cuiture by Gessard (1882), is widely distributed in nature, being found in water, sewage, and sometimes on the normal skin, particularly of the axilla and perineum (Rủzička 1898). It is not infrequently found in wounds, where it gives rise to blue pus. It may invade the nasal fossæ, the middle ear, the meninges, the bronchi and other organs, and set up suppuration. In infants and young children it causes intestinal disturbances and diarrhøea; sometimes it enters the blood stream, and gives rise to a general infection (Williams and Cameron 1896). According to Pons (1927), Ps. pyocyanea is especially pathogenic in the tropics, where it is not infrequently responsible for typhoid-like infections and abscess of the liver.

Injected subcutaneously or intravenously into guinea-pigs or rabbits in a dose of $0.5-1.0 \mathrm{ml}$. of a 24 hours' broth culture, it may cause death in 24 to 48 hours; post mortem there is a hæmurrhagic œedema at the site of injection (after subcutaneous inoculation), small punctate hæmorrhages are seen in the stomach and intestine, and sometimes nephritis. The bacilli can be recovered from the blood, viscera, and urine. As much the same appearances result from the injection of dead cultures, it is probable that an endotoxin is responsible. Different strains vary in virulence; some do not kill for weeks, others not at all.

Little is known of the antigenic structure of this organism. Boivin and Mesrobeanu (1937), using their trichloracetic acid technique, isolated a glyco-lipoid complex or endotoxin containing a polysaccharide hapten. By inoculation of rabbits with the whole complex they obtained antisera which precipitated the homologous endotoxin and the polysaccharide hapten, and which agglutinated the homologous bacteria. The endotoxin, as a whole, killed mice, but the polysaccharide hapten was non-toxic on intraperitoneal inoculation, even in doses of $5 \mathrm{mgm}$. The authors conclude that the identity of the endotoxin with the somatic antigen of $P s$. pyocyanea is similar to that in members of the Salmonella group.

In culture, Ps. pyocyanea forms a bluish-green pigment. Gessard (1890, 1891, 1892) found that this pigment consists of two different substances. One, pyocyanin, is bluish-green, non-fluorescent, is formed in peptone media, and is soluble in both chloroform and water. The other, fluorescin, is greenish-yellow, fluorescent, is formed only in the presence of a phosphate, and is insoluble in chloroform, but soluble in water. By cultivation in different media he was able to obtain varieties 
that produced pyocyanin or fluorescin only, and some that were completely achromogenic. Fordos (1860) obtained pyocyanin in long blue crystals from a solution in chloroform. Wasserzug (1887) showed that its formation was prevented by several substances, such as 5 per cent. $\mathrm{KNO}_{3}, 8$ per cent. $\mathrm{KClO}_{3}, 0.5$ per cent. ammonium tartrate, 5 per cent. $\mathrm{NaCl}$, and by many disinfectants not strong enough to inhibit the growth of the organism. Jordan (1899) studied 7 strains of Ps. pyocyanea; 1 strain produced pyocyanin only, 5 both pyocyanin and fluorescin, and 1 fluorescin only. He found that the fluorescent pigment required for its formation both phosphate and sulphate, while neither of these substances was necessary for the production of pyocyanin. Both pigments are formed in suitable synthetic media. In old cultures a black pigment sometimes appears; this appears to be an oxidation product of pyocyanin. A yellowish-brown pigment, which may also be found in cultures, appears to be an oxidation product of the fluorescent substance. Boland (1899), who worked with a solution of pyocyanin in chloroform, found that it became yellow if exposed to sunlight. Apparently the chloroform was broken up, and chlorine set free, which oxidized the pyocyanin to pyoxanthose. He showed that pyocyanin was largely dissolved by $\mathrm{HCl}$, which turned it red, and pyoxanthose by 33 per cent. $\mathrm{H}_{2} \mathrm{SO}_{4}$, which turned it reddish-yellow. Turfitt (1936) found that the production of both pigments was favoured by 1 per cent. glycerol, of pyocyanin by 5 per cent. glycerol, and of fluorescin by asparagin.

More recently Wrede (1930) has determined the constitution of pyocyanin, and shown that it can be synthesized by the organism from lactic acid and salts. Chemically he regards it an entirely new type of dye, containing two pentavalent nitrogen atoms, but this requires confirmation (Michaelis 1935). It affords, moreover, the first instance in which phenazine derivatives have been found in nature. Its empirical formula is $\mathrm{C}_{26} \mathrm{H}_{20} \mathrm{~N}_{4} \mathrm{O}_{2}$. Wrede states that it dissolves poorly in cold, but readily in warm, water, as well as in chloroform, nitrobenzol, pyridine, and phenol. It is fairly resistant to acids and forms with them red-coloured salts. To alkali, on the other hand, in the presence of oxygen, it is much less resistant and is rapidly broken down.

Turfitt (1937) obtained a preparation of fuorescin by growth of Pseudomonas in synthetic liquid media and adsorption of the pigment on to "suma-carb," followed by electro-dialysis. The final product, which had an ash value of less than 0.4 per cent., was an amorphous greenish-brown powder, readily soluble in water, phenol, and acetic acid, but not in other organic solvents. A dilute aqueous alkaline solution showed a green fluorescence, becoming colourless and non-fluorescent on acidification. More concentrated alkaline solutions had a red colour and exhibited an intense green fluorescence. In alkaline solution the pigment showed a well-defined absorption band with its maximum at $410 \mu$; in acid solution the band was less evident and was nearer the shorter wave-lengths. The empirical formula was found to be $\mathrm{C}_{4} \mathrm{H}_{7} \mathrm{O}_{2} \mathrm{~N}$.

Summarizing, we may say that Ps. pyocyanea forms two pigments: (1) Pyocyanin ; a bluish-green pigment, soluble in both chloroform and water, from which it can be obtained in long blue crystals. For its production neither phosphate nor sulphate is required. (2) Fluorescin; a greenish-yellow fluorescent pigment, soluble in water but not in chloroform. For its production both phosphate and sulphate are required. In old cultures it may be oxidized to a yellowish-brown pigment. Ps. pyocyanea forms pyocyanin and fluorescin; Ps. fluorescens forms only fluorescin. Both pigments are themselves oxidation products of colourless 
substances. (For a careful study of the factors controlling the production of pyocyamin and other pigments, see Sullivan 1905-06.)

From Hadley's observations (1927) it appears fairly certain that the power of forming pyocyanin is subject to discontinuous variation. Under artificial conditions of cultivation, many strains of $P s$. pyocyanea tend to lose their ability to produce a bluish-green coloured growth on agar. If such strains are inoculated into broth, and plated out, it will be found that only a proportion of the colonies are coloured bluish-green; the remainder have merely the yellowish colour due to the presence of the fluorescent pigment. With many strains prolonged subcultivation is followed by the complete disappearance of the bluish-green variants, and their replacement by the yellowish variants. Once a strain has lost its power of producing pyocyanin, it is unable to recover it. According to Cataliotti (1935), cultivation in broth containing 2 per cent. zinc oxide or 1 per cent. zinc sulphate results after a few passages in suppression of pigment formation. The organisms, however, do not undergo dissociation, and when transferred back to normal media again give rise to pigment.

Emmerich and Löw (1899) and Emmerich, Löw and Korschun (1902) found that old cultures of Ps. pyocyanea were highly bactericidal to many organisms. They ascribed this action to an enzyme "pyocyanase." More recently Schoental (1941) has brought evidence to show that the antibacterial and lytic action is due not to enzymes but to pigments. Of these, the most active is a yellow pigment, $\alpha$-oxyphenazine, which can be isolated in crystalline form from old cultures. It has an antibacterial action similar to that of the flavines and is less toxic to tissue cells than pyocyanin. In a $1 / 20,000$ concentration in serum broth it inhibits growth of Gram-positive cocci for 24 hours, and in a 1/10,000 concentration organisms of the Neisseria, Corynebacterium, Proteus, Bacterium, Brucella and Clostridium groups. An oily substance, which she isolated from cultures of Ps. pyocyanea 2 to 3 months old, proved very active against $V$. cholerce, causing lysis in a $1 / 10,000$ dilution.

An interesting feature of $P$ s. pyocyanea is its ability both in culture and in the animal body to form hydrocyanic acid (Patty 1921).

We append a detailed description of this organism.

\section{Pseudomonas pyocyanea}

Synonyms.-Bacterium aeruginosum (Schröter) Migula. B. pyocyaneus, Gessard.

Habitat.-Intestinal canal, water, sewage, pus, sinuses, human skin; sometimes pathogenic to man.

Morphology.-Rods, $1 \cdot 5-3 \cdot 0 \mu \times 0.5 \mu$; axis straight, ends rounded, sides parallel, arranged singly, or in pairs and short chains; motile by 1-3 flagella at one pole. Nonsporing, non-capsulated. Gram-negative. Non-acid-fast.

Agar Plate.-2 days at $25^{\circ} \mathrm{C}$. Round colonies, 1-2 mm. in diameter, low convex, with smooth, moist, glistening surface; edge entire or undulate; structure amorphous; butyrous consistency, easily emulsifiable; fluorescent yellowish-green colour, translucent. After $\mathbf{5}$ days it is differentiated into a smooth, convex, translucent centre and a radially striated, effuse, transparent periphery, with an undulate, lobate, or villous edge. Medium coloured green.

Agar Slope.-2 days at $25^{\circ} \mathrm{C}$. Good growth, raised slightly, with beaten-copper surface, irregular edge, and sometimes clear phage-like areas; greenish-yellow, translucent. Medium green. 5 days: growth becomes effuse and scarcely visible.

Gelatin Stab.- Moderate filiform growth to bottom of stab; slow crateriform liquefaction. After 14 days the upper 1-2 cm. are digested in stratiform manner, and 
the fluid is turbid, yellowish-green, and sometimes granular; there may be saccate liquefaction around the filiform growth as well.

Broth. -2 days at $25^{\circ} \mathrm{C}$. Abundant growth, with dense turbidity ; yellowish-green colour' thick white ring growth and thin surface pellicle; slight powdery sediment, disintegrating on shaking. After $\mathbf{5}$ days there is an abundant, visco-floccular deposit, only partly disintegrating. Mawkish odour, like trimethylamine.

Blood Serum. -7 days at $25^{\circ} \mathrm{C}$. Good, confluent, slightly raised growth, of greenishyellow colour; medium is slightly green; medium is partly digested in $\mathbf{1 4}$ days.

Potato. -6 days at $25^{\circ} \mathrm{C}$. Abundant, slightly raised, confluent, greenish-brown growth with moist, glistening, contoured surface. Potato coloured green. Later, both the growth and the potato take on a brownish colour.

Resistance.-Destroyed by $55^{\circ} \mathrm{C}$. in 1 hour.

Metabolic.-Aerobic; no growth anaerobically. Opt. temp. $30-37^{\circ} \mathrm{C}$. ; limits $5-\mathbf{4 2} 2^{\circ} \mathrm{C}$. Forms a green pigment soluble in chloroform and in water, called pyocyanin; forms a greenish-yellow fluorescent pigment, soluble in water but not in chloroform. Nutritional: grows well on ordinary media; in synthetic media, both phosphate and sulphate are essential for production of fluorescent pigment.

Biochemical.-Acid, no gas, in glucose. Indole - ; a false reaction may be given by Böhme's reagent, the acid of which turns pyocyanin red. M.R. -, V.P. - , Nitrate reduction -. $\mathrm{H}_{2} \mathrm{~S}-. \mathrm{NH}_{3}$ production + . Catalase + . M.B. reduced. Starch diastase -. L.M. peptonization and decolorization complete in 5 days at $30^{\circ} \mathrm{C}$., may be slight preliminary clot; milk often turned green. Growth in citrate.

Pathogenicity.-Low pathogenicity to man, giving rise to diarrhœa and general infections in infants, and to suppuration. Cause of "blue pus." Gives rise to fever and local abscess after subcutaneous injection into rabbits.

Ps. fluorescens.-This organism is found in water, hail (Belli 1902), sewage, and has been isolated from lemonade (Thöni 1911). It is motile by one or more polar flagella. Many authors consider it a variety of $P s$. pyocyanea, which has become adapted to a purely saprophytic existence (Tanner 1918, Rủžčka 1898). The differential characters may be given as follows:

\section{Ps. pyocyanea.}

(1) Opt. temp. $37^{\circ} \mathrm{C}$. ; grows at $42^{\circ} \mathrm{C}$.

(2) Pyocyanin and fluorescent pigment formed.

(3) Liquefaction in gelatin stratiform and saccate.

(4) Pathogenic to rabbits and guinea-pigs.
Ps. fluorescens.

Opt. temp. $25^{\circ} \mathrm{C}$.; no growth at $42^{\circ} \mathrm{C}$.

Fluorescent pigment only formed.

Liquefaction in gelatin not always present : when present stratiform only.

Non-pathogenic to rabbits and guinea-pigs.

These differences are by no means constant. Further, since varieties of $P s$. pyocyanea may occur that fail to produce pyocyanin, and since achromogenic varieties of both organisms are not uncommon, differentiation of the two organisms is often impossible.

Jordan (1899) studied 58 strains of Ps. fuorescens from water. He found that 33 of them liquefied gelatin, and produced acid, clot, and peptonization in milk; 25 did not liquefy gelatin, and produced alkali in milk without coagulation. The variations in reaction that may occur can be judged from the fact that Tanner divided 42 strains, which he studied, into no fewer than 27 different groups.

Ps. fuorescens is generally non-pathogenic to animals, but it may give rise to a local abscess in rabbits and guinea-pigs. On the other hand, it is stated to be frequently pathogenic to plants, especially cultivated vegetables, such as carrots, cauliflowers and tomatoes, in which it causes areas of moist necrosis (Griffon 1909). 
Ps. cyanogena is a motile bacillus possessing polar flagella. It forms two pigments : one fluorescent, the other varying in colour from blue to brown or black. In milk with an acid reaction it gives rise to a bright blue colour. It is the cause of epidemics of "blue milk."

Pseudomonas denitrificans.-This organism was originally described by Christensen (1903-04) under the name of Bacillus denitrificans fuorescens. Two varieties were recognized, $A$ and $B$ : variety $A$ was isolated from garden earth, and was able to reduce nitrates to gaseous nitrogen; variety $B$ was isolated from horse dung, and was able to reduce nitrites, but not nitrates, to gaseous nitrogen.

Variety $A$ is a small bacillus, $0.5-1.25 \mu \times 0.5-0.7 \mu$. It is surrounded by a large capsule, measuring 2-5 $\mu$ in diameter. Appears to be slightly motile. Often shows bipolar staining. Is Gram-negative. Grows freely at $25^{\circ} \mathrm{C}$. Colonies on agar are 2-3 $\mathrm{mm}$. in diameter after 3 days, and are circular, with an entire edge; they have an opalescent sheen. In an agar stroke culture a whitish glistening growth is formed and the agar is coloured bright green. In gelatin stab there is a filiform growtb, and a whitish surface growth with a lobate edge; the gelatin is not liquefied. In a gelatin stroke culture there is a dirty-white layer of growth, which fluoresces brilliantly in transmitted light; the gelatin is coloured bright-green. In broth there is a dense turbidity, and a very thick wrinkled surface pellicle, which climbs up the walls of the tabe. In 0.2 per cent. nitrate broth a dense turbidity is produced, and a foam, due to the liberation of gaseous nitrogen, is seen, reaching its maximum in $\mathbf{4 0}$ hours; the nitrate is not completely destroyed, even in 3 weeks.

Variety $B$ is a larger bacillus, $1-3 \mu$ long by $0 \cdot 5-1 \cdot 25 \mu$ broad, and is surrounded by a capsule. Motility doubtful. Gram-negative. It is unable to reduce nitrates to nitrites, but is able to reduce nitrites to gaseous nitrogen. Colonies on agar are $\mathbf{2 . 5}-\mathbf{3 . 5} \mathbf{m m}$. in diameter after 2 to 3 days, and are flat, whitish, and so fluid that they may flow over the agar if the plate is stood on edge. In agar stroke culture there is a thinmish filiform grey growth, having an effuse iridescent peripheral extension; after 10 days the agar is slightly coffee-coloured. In gelatin stroke culture there is a greyish or slightly brownish layer of growth, which fluoresces strongly in transmitted light; the gelatin is coloured brown. In broth there is a dense turbidity, and a thin, iridescent surface pellicle.

Pseudomonas caviæ.-This name was suggested by Scherago (1937) for a capsulated organism that he isolated from the blood and viscera of young guinea-pigs dying from a rapid bacteræmic infection. Its general properties are as follows: Gram-negative rods, 1-5 $\mu$ long by $0 \cdot 6-0 \cdot 75 \mu$ broad, occurring singly and in pairs end-to-end. Actively motile by 1 to 3 polar flagella. Encapsulated when isolated from the animal body. Surface colonies on agar are 1-3 mm. in diameter, convex, smooth, iridescent and translucent, with a finely granular structure. In agar slope cultures the medium becomes greenishyellow in a week, and later turns brownish-yellow. Uniform turbidity in broth with a surface ring and pellicle and a heavy granular deposit, light yellow in colour. Infundibuliform liquefaction of gelatin, complete in 2 days. Scanty, glistening, light yellowish orange growth on potato. Aerobic, facultatively anaerobic. Grows well at $22^{\circ} \mathrm{C}$. and $37^{\circ} \mathrm{C}$. Acid in dextrose, maltose, mannitol, lactose, sucrose and salicin. Litmus milk acidified, coagulated, and partly peptonized. Indole and ammonia formed, but not $\mathrm{H}_{2} \mathrm{~S}$ or catalase. Nitrates reduced to nitrites. II.R. +, V.P. -, M.B. reduced. Reproduces the natural disease when inoculated parenterally into young guinea-pigs. Produces rapidly fatal septicæmia in mice inoculated intraperitoneally. Apparently non-pathogenic to rabbits. 


\section{REFERENCES}

BeLli, C. M. (1902) Zbl. Bakt., IIte Abt., 8, 445.

Boivin, A. and Mesrobeand, L. (1937) C. R. Soc. Biol., 125, 273.

Bola ND, G. W. (1899) Zbl. Bakt., 25, 897.

Burge, W. E. and Neill, A. J. (1915) Amer. J. Physiol., 38, 399.

Cataliotti, F. (1935) G. Batt. Immun., 15, 161.

Christensen, H. R. (1903-4) Zbl. Bakt., IIte Abt., 11, 190.

EmMerich, R. and Löw, O. (1899) Z. Hyg. InfektKr., 31, 1.

Emmerich, R., Löw, O., and Korschun, A. (1902) Zbl. Bakt., 31, 1.

Ferramola, R. and Monteverde, J. J. (1939) Bol. Obras san. Nacion, Buenos Aires, 3,272 .

FlÜGGE, C. G. F. IV. (1896) "Die Mikro-organismen," 3te Auflage, 2te Theil, p. 292. Leipzig.

Fordos, M. J. (1860) C. R. Acad. Sci., 51, 215, 326.

Fraenkel, E. (1917) Z. Hyg. InfektKr., 84, 369.

Gessard, C. (1882) C. R. Acad. Sci., 94, 563 ; (1890) Ann. Inst. Pasteur, 4, 88 ; (1891) Ibid., 5, 65 ; (1892) Ibid., 6, 801.

Griffon, E. (1909) C. R. Acad. Sci., 149, 50.

HadeEY, P. (1927) J. infect. Dis., 40, 74.

Jordan, E. O. (1899) J. exp. Med., 4, 627.

Lode, A. (1929) Kolle \& Wassermann Handb. path. Mikroorg., 6, 149. Gustav Fischer. Jena.

Michaelis, L. (1935) Chem. Rev., 16, 243.

Moltke, O. (1927) "B. proteus Vulgaris." Copenhagen.

Patty, F. A. (1921) J. infect. Dis., 29, 73.

Poss, R. (1927) Ann. Inst. Pasteur, 41, 1338.

ReEd, E. M. and Boyd, G. B. (1933) Canad. J. Res., 8, 173.

RŮžıčKa, S. (1898) Zbl. Bakt., 24, 11.

SANDIFORD, B. R. (1937) J. Path. Bact., 44, 567.

Scherago, M. (1937) J. infect. Dis., 60, 245.

Schoevtal, R. (1941) Brit. J. exp. Path., 22, 137.

Seleen, W. A. and Stark, C. N. (1943) J. Bact., 46, 491.

Sullivan, M. X. (1905-6) J. med. Res., 14, 109.

Tanner, F. W. (1918) J. Bact., 3, 63.

Тнӧхг, J. (1911) Zbl. Bakt., IIte Abt., 29, 616.

TurfitT, G. E. (1936) Biochem. J., 30, 1323; (1937) Ibid., 31, 21.2.

Waite, H. H. (1908) J. infect. Dis., 5, 542.

Wasserzug, E. (1887) Ann. Inst. Pasteur, 1, 581.

Williams, E. P. and Cameron, K. (1896) J. Path. Bact., 3, 344.

WREDE, F. (1930) Z. IIyg. Infektkrr., 111, 90. 


\section{CHAPTER 22}

\section{VIBRIO AND SPIRILLUM}

\section{VIBRIO}

\section{Definition.- V'ibrio.}

Short, curved, rigid rods, arranged singly or united into s-forms or spirals. Motile by a single polar flagellum, which is usually relatively short. (Some species may have two or three polar flagella.) Non-sporing. Usually Gram-negative. Aerobic and facultatively anaerobic. Many species liquefy gelatin and are active ammonifiers. Commonly found in water. Most species are saprophytic ; a few are pathogenic to man.

Type species. Vibrio cholerce.

The first member of this group to be described was $V$. cholerce, which was found by Koch (1886) in the dejecta of cholera patients. In 1888 Gamaléia (1888a) isolated a vibrio from the blood and intestinal contents of chickens dying from a cholera-like disease at Odessa; to this organism he gave the name of $V$. metchnikovi. During the next 10 years a large number of other vibrios, more or less resembling the cholera vibrio, were isolated from different sources, such as well, river, and sea water, the fæces of man and animals, cheese, and intestinal abscesses of pigs (Dunbar 1893, Smith 1894, Dieudonné 1894, Kutscher 1895, Gotschlich 1895, 1906, Ruffer 1907, Crendiropoulo 1912, Craster 1913). The differentiation of many of these organisms from $V$. cholerce proved impossible, until the introduction of the Pfeiffer test in 1894 (see Chapter 63). Even with the help of this test, it was not always possible to decide whether they were different species, or were merely variants of the main species. Since the chief interest of the vibriosat least to the medical bacteriologist-has been their relationship to $V$. cholera, it follows that a careful systematic study of the saprophytic species has not yet been made. For this reason it is premature to attempt a classification of the members of this group.

Morphology and Staining.-The vibrios are short, curved rods, looking like commas. In size they vary considerably, from about 1 to $5 \mu$ in length and about 0.3 to $0.6 \mu$ in breadth; the commas may appear long, thin, and delicate, or short, stunted and thick. They are arranged singly, in s-shaped or occasionally semicircular pairs, or in short chains. In fluid media spirals are often formed, and in old cultures a variety of forms may be seen; most of these are very small, looking like granules and staining poorly; but there are larger, swollen, shadow forms resembling bottles or clubs. When freshly isolated the resemblance to a comma is most striking; but after long subculture in the laboratory the vibrios frequently lose their curved shape, and are then not unlike coliform bacilli. The organisms are 
very actively motile by a single polar flagellum. They stain best with dilute carbol-fuchsin. They are Gram-negative.

Growth Requirements.-Growth occurs readily on the usual media. One of the most characteristic properties is the rapidity of growth in peptone water (1 per cent. peptone, 0.5 per cent. $\mathrm{NaCl}$ ). Multiplication occurs chiefly at the surface, where, after 6 to 9 hours, a delicate membrane is formed. There is very little turbidity as a rule; the deposit that forms appears to be derived from the surface pellicle.

The vibrios are markedly aerobic; they grow best in the presence of abundant oxygen. Under strictly anaerobic conditions some of the members fail to grow altogether; the majority give rise to a very slight growth on agar or in broth in about a week. The optimum temperature is $30-40^{\circ} \mathrm{C}$.; no growth occurs macroscopically under $16^{\circ} \mathrm{C}$.

For growth and survival a

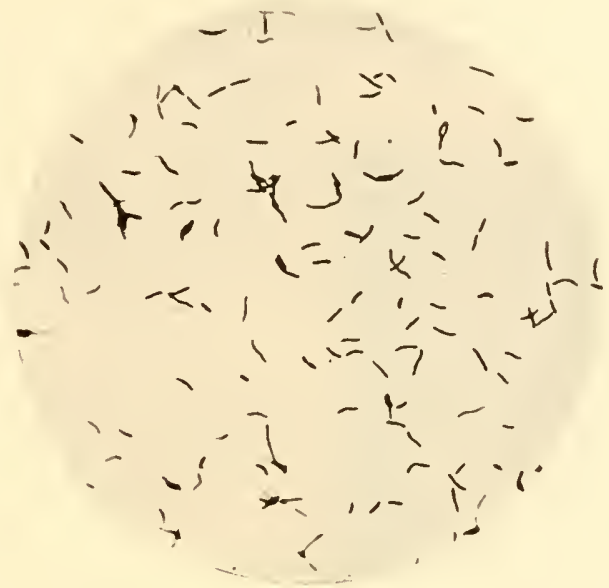

FIG. 101.-Vibrio cholere.

From an agar culture, 24 hours, $37^{\circ} \mathrm{C} .(\times 1000)$.

$\mathrm{H}$-ion concentration of $\mathrm{pH} 7 \cdot 6-8 \cdot 0$ is most suitable. The organisms have a high alkali, but a very low acid tolerance. Cultures containing a fermentable sugar are sterile in a day or two (Nobechi 1925).

A number of selective media have been devised for facilitating the isolation of $V$. cholerce from the frees.

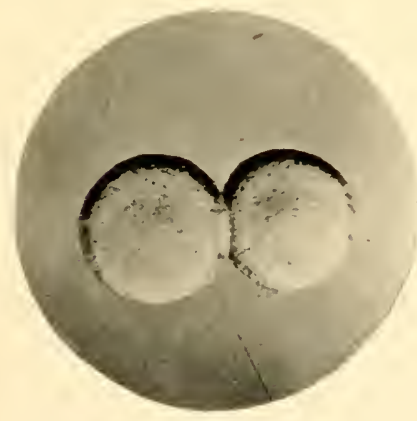

Fig. 102.-Vibrio cholere.

Surface colonies on agar, 24 hours, $37^{\circ}$ C. $(\times 8)$.

One of the best known of these-Dieudonne's medium (1909) - is prepared by adding normal $\mathrm{KOH}$ solution to an equal quantity of defibrinated ox blood, and heating to $100^{\circ} \mathrm{C}$. for half an hour. Thirty parts of this mixture are added to 70 parts of nutrient agar rendered neutral to litmus. According to Vedder and van Dam (1932), the medium should be allowed a day or two to "ripen." During this time $\mathrm{CO}_{2}$ is taken up from the air and $\mathrm{NH}_{3}$ is given off. The medium, when ready for use, should have a $p \mathrm{H}$ of 9.0-9.6. At a lower $\mathrm{pH}$ coliform and other organisms grow and the medium is no longer selective; at a higher $\mathrm{pH}$ the growth of the cholera vibrio itself is inhibited. Ottolenghi's medium (see Bocehia 1911) consists of ox bile to which 3 per cent. of a 10 per cent. solution of crystalline sodium carbonate has been added; steri-

lization is effected in the autoclave. Bandi's medium, suitable for cultivation of the cholera vibrio from water, is a peptone water solution containing dilute anticholera agglutinating serum; the vibrios multiply and fall to the bottom in clumps. Yen (1932-33) recommends a phenolphthalein starch medium for the isolation of the cholera vibrio. It depends on the unusual property possessed by this organism of rapidly fermenting starch in an alkaline solution (see Gordon 1906). 
Cultural Characters.-On agar the colonies are not distinctive; they may be either clear and amorphous, or finely gramular. Small, knob-like secondary colonies sometimes form in about a week on the surface of the parent colony. An effuse, transparent peripheral extension is not unusual. Crystals may form in the agar. Balteanu (1926) has described three colonial rariants in cultures of cholera and cholera-like vibrios. Variant (1) was rugose; (2) had a more opaque centre and a transparent periphery; and (3) was opaque. Variants 1 and 2 reverted to type when subcultured on agar; variant 3 reverted slowly in broth, but remained constant for a long time on agar. Variant 3 consisted of

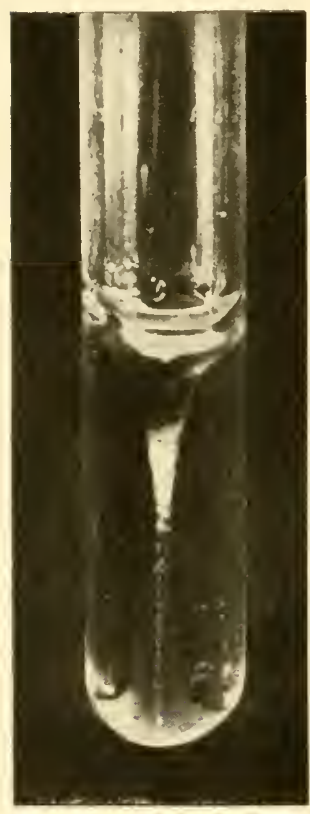

FIG. 103.-Vibrio cholerce.

Gelatin stab culture, 5 days, $22^{\circ} \mathrm{C}$., showing infundibuliform liquefaction. non-motile bacilli which had a mucoid envelope; the organisms contained a heat-stable antigen only and were apparently of the pure $O$ form (see antigenic structure). Though the rugose form has been regarded by some workers as an extreme rough form, the observations of White $(1938,1940 a)$ show that it is a peculiar variant characterized by the secretion of a diffuse intercellular gelatinous substance or of actual capsules. It is unstable, and is constantly tending to revert to the $\mathrm{S}$ or $\mathrm{R}$ form from which it is derived.

In gelatin stab many species produce liquefaction. On potato some of the members-including $V$. cholerce - give a raised growth of café-au-lait colour, resembling that of the Brucella group, Pfeiff. mallei, and Ps. pyocyanea. On MacConkey's medium growth is often poor; $V$. cholerce flourishes well on it, but the non-pathogenic members grow poorly. The colonies are colourless when young, but soon assume a pinkish-red appearance; the medium changes simultaneously to a darker red. The rate at which the colour alters depends on the organism observed; the colonies of $V$. cholerce may remain yellow for a week; those of the Nasik vibrio are bright red in 3 days.

On Loeffler's serum growth is plentiful, and is sometimes accompanied by slow liquefaction.

Resistance.- None of the vibrios forms spores. Their resistance to heat and disinfectants is low, and they are easily destroyed by drying (see Chapter 63). They are killed by heat at $55^{\circ} \mathrm{C}$. in 15 minutes or less (Kitasato 1889), and by 0.5 per cent. phenol in a few minutes. Dried on cover slips they perish in about 3 hours. Gastric juice containing more than 25 degrees of acidity (degrees equivalent to number of $\mathrm{ml}$. of $\mathrm{N} / 10 \mathrm{NaOH}$ necessary to neutralize $100 \mathrm{ml}$. of gastric juice) is said to kill cholera vibrios at once; in the absence of free acid the vibrios may survive for over 24 hours (Napier and Gupta 1942).

Biochemical Characters.-Acid, without gas, is generally formed within a day or two in glucose, maltose, mannitol and sucrose. Lactose is sometimes fermented after 10 or 14 days, and occasionally salicin. The Nasik vibrio ferments glucose only-at least in liquid media. Litmus milk may be unchanged; more often it is acidified; and sometimes it is clotted. Many species form indole, reduce nitrates to nitrites, and give the cholera-red reaction. This reaction is performed 
by adding pure sulphuric acid to a 24-hour peptone water culture of the organism; if positive, a red coloration appears almost immediately. It depends on the ability of the organism to produce indole and reduce nitrates, so that on the addition of sulphuric acid the nitroso-indole reaction occurs. Many organisms not belonging to the Vibrio group are able to form indole and reduce nitrates, but most of them break down the nitrite to $\mathrm{NH}_{3}$ (Maassen 1902). The reaction is given not only by $V$. cholerce, but by a number of other vibrios, so that it no longer has the diagnostic significance with which it was at first accredited. For its production it is essential to use a brand of peptone that contains a small amount of nitrate; not all peptones are suitable. Paladino-Blandini (1906) states that the indole and the nitrite must be formed in certain proportions; if either is in excess, the reaction fails: but if the culture is heated to $60-70^{\circ} \mathrm{C}$. for 15 minutes, the colour appears. All the members appear to form catalase and ammonia. Some reduce methylene blue, and some form $\mathrm{H}_{2} \mathrm{~S}$-though not for several days.

Nobechi (1925), who studied a large number of cholera and cholera-like vibrios, concluded that it was impossible to distinguish them by their biochemical reactions. Heiberg $(1935,1936 a)$, however, who studied 384 strains of vibrios, was able to classify them into six groups by the use of mannose, sucrose, and arabinose. He found that all true cholera vibrios fell into one group, which he calls Type I, but which, owing to the almost exclusive restriction of the word "type" to antigenic variants, we propose to refer to as Group $I$. His scheme of classification is as follows :

\begin{tabular}{|c|c|c|c|c|c|}
\hline & & & Mannose. & Sucrose. & Arabinose. \\
\hline Group I & . & .. & $A$ & A & - \\
\hline Group II & . & . . & - & $\mathrm{A}$ & - \\
\hline Group III & . & . . & A & A & A \\
\hline Group IV & . & . . & - & A & A \\
\hline Group V & . & . . & A & - & - \\
\hline Group VI & . & .. & - & - & - \\
\hline
\end{tabular}

Unfortunately Heiberg's series of strains was insufficiently representative. Numerous workers, such as Pollitzer (1935-36), Taylor, Read and Pandit (1936), Combiesco-Popesco and Cocioba (1936), Taylor and Ahuja (1938-39), and Pasricha, Chatterjee and Das (1938-39), who have repeated Heiberg's work, have found that, though all true agglutinable cholera strains fall into Group I, a considerable proportion of other vibrio strains from human sources and from water having no connection with cholera fall likewise into this group. It may be said, therefore, that a strain falling into Gromps II to VI may be excluded from the cholera species, but that a strain falling into Group I may or may not be a true cholera vibrio ; serological examination alone can determine its identity. It may be mentioned, however, that Taylor, Pandit and Read (1937) find that, whereas true cholera vibrios give a positive cholera red and a negative Toges-Proskaner reaction, the great majority of inagglutinable vibrios falling into Heiberg's Group I react positively to both tests - provided Barritt's (1936) modification is used for the 
V.P. test (see p 368). According to van Loghem (1938) the El Tor vibrio gives usually a positive V.P. reaction.

Hamolysin Formation.-Many members of this group form a hæmolysin acting on sheep, horse, or rabbit cells. This can be demonstrated either on plates or in a broth culture. In general, the cholera vibrio does not produce a soluble hæmolysin, while the EI Tor and many non-cholera vibrios are able to do so. For diagnostic purposes a standard technique is essential. Much confusion has in the past been due to differences in method of studying hæmolysis. Zimmermann (1932, 1933), working with 5 per cent. defibrinated sheep blood broth cultures, obtained conflicting results, but when he used a medium made up with peptone, asparagin, and ammonium lactate containing 4 per cent. sheep blood, and read his results after 48 hours' incubation at $37^{\circ} \mathrm{C}$., a clear differentiation between the non-hrmolytic cholera and the hæmolytic El Tor and non-cholera vibrios was apparent. Van Loghem (1932) states that sheep or goat's blood should be used; guinea-pig and rabbit red cells are too sensitive. He further points out that the cholera vibrio, though not forming a true soluble hæmolysin, does digest blood pigment; this property, which has been shown by Bernard, Guillerm and Gallut (1937) to be due to a ferment present in cultures but not in the bodies of the cholera vibrio, is responsible for the greenish discoloration around individual colonies on blood agar and for the complete clarification of the medium that occurs on further incubation. The El Tor vibrio digests blood likewise, but in addition it produces a soluble hæmolysin, the extraction of which has been recorded by Bernard, Guillerm and Gallut (1939). The observations of these workers suggest that the cholera vibrio forms a hæmolysin as well as a digesting ferment, but that it is mainly intracellular and, unlike that of the $\mathrm{El}$ Tor vibrio, does not diffuse out to any considerable extent into the medium. The identity of these two hæmolysins is suggested by the finding of Vassiliadis (1937) that the injection of non-hæmolytic cholera vibrios into animals gives rise to anti-hæmolysins for the El Tor vibrio as active as those prepared by injection of the El Tor vibrio itself.

Doorenbos (1936) finds that hæmolysis is very much more active in 8-hour than in 24-hour cultures. Many strains of true cholera vibrios that were nonhæmolytic after 24 hours produced hæmolysis of sheep or goat cells after 8 hours. With so many factors influencing hæmolysin production, it would clearly be dangerous to place too much weight on this characteristic as a means of differentiating between the vibrios.

The common routine method for testing hæmolytic activity is to grow the organism in broth for 3 days at $37^{\circ} \mathrm{C}$, to add $1 \mathrm{ml}$. of the culture to $1 \mathrm{ml}$. of a 5 per cent. suspension of washed goat or sheep red cells, to incubate for 2 hours at $37^{\circ} \mathrm{C}$., and to read the results after the tubes have been left in the cold overnight.

Toxin Production.-Nicati and Rietsch (1884) injected dogs intravenously with the filtrate of a broth culture of $V$. cholerce a week or more old. In their first series of experiments there were vomiting, defreation, and general depression, with recovery in an hour. In their second series there were dyspncea, vomiting, and paresis of the extremities, followed by recovery, or death in 12 hours. At necropsy in the fatal cases, ecchymoses were found in the duodenum and larger hæmorrhages in the stomach. Filtrates of young cultures were innocuous.

Pfeiffer (1892) likewise experimented with filtrates. He found that even $4 \mathrm{ml}$. of a 20-days' glycerine broth filtrate, injected intraperitoneally into guinea-pigs, had no 
more than a slight toxic effect. Dead vibrios, however, are very toxic. Pfeiffer (1892, 1895) found that the lethal dose of living vibrios on intraperitoneal injection into guinea-pigs was $1.5 \mathrm{mgm}$. of an 18-hours' agar slope culture. When the vibrios were killed by chloroform or thymol the lethal dose was $3-4.5 \mathrm{mgm}$.; when they were killed by drying, it was $6 \mathrm{mgm}$.; and when they were killed by heat at $55^{\circ} \mathrm{C}$. for an hour it was $10-20 \mathrm{mgm}$. While immune serum was able to protect a guinea-pig against several fatal doses of living vibrios, it possessed no more protective power than normal scrum against dead vibrios. From these experiments he concluded that in young cultures of $\mathrm{y}$. cholerce there was a specific toxic substance bound to the bacterial bodies; and that the immune substances in the antiserum were not antitoxic but bactericidal in their action.

Von Dungern (1895) working with one highly virulent strain of cholera and another of very low virulence found that the lethal dose of heat-killed organisms was the same in each instance. The toxicity of the cultures therefore bore no relation to their virulence.

Nanwaring, Boyd, and Okami (1923) perfused the mammalian heart with 2-7 days' culture filtrates of $V$. cholerce, added in 5-10 per cent. concentration to Locke's solution. Though non-toxic for the conducting and contractile tissues, the filtrates had a destructive effect on the capillary endothelium, as was evident from the cedema of the muscle and the hæmorrhages that occurred beneath the endocardium and pericardium.

We may conclude that the cholera vibrio does not secrete a true soluble exotoxin, but that it contains endotoxins which are liberated on the autolysis of the bacilli in culture or on the active disintegration of the bacilli by the cells of the animal body. The analogy that it presents with the meningococcus-another organism that readily undergoes autolysis - is very close, though the cholera vibrio is far more toxic.

Hahn and Hirsch (1929), working with El Tor and other hæmolytic vibrios, found that a soluble toxin was produced in peptone water cultures to which small quantities of glucose were added during growth, the reaction of the medium being kept alkaline by similar additions of $\mathrm{NaOH}$. The toxin, which passed through a Seitz filter, became demonstrable in 6-10 hours, and reached its maximum in 1-4 days. Bacterial counts indicated that the increase in toxicity of the culture coincided with the death of the organisms. The heat resistance of the toxin seemed to vary with different batches; sometimes it was destroyed in 2 minutes, at others not for 30 minutes, when exposed to a temperature of $100^{\circ} \mathrm{C}$. Injected intraperitoneally into guinea-pigs in a dose of $0.25 \mathrm{ml}$., the toxin had a marked effect on the temperature, which of ten fell to $30^{\circ} \mathrm{C}$. within $2-3$ hours. The animals became progressively weaker, paralysis developed in their hind legs, and they died in 6-10 hours. Post mortem, the findings consisted of a large exudate in the peritoneal cavity, fibrinopurulent deposits on the liver, and sometimes hyperæmia of the intestine. Injection of horses with increasing doses of toxin led to the appearance in the serum of antibodies capable of neutralizing, to some extent, the lethal action of the toxin for guinea-pigs. It is to be noted that true, non-hremolytic, cholera vibrios were almost completely devoid of toxin-producing power under the cultural conditions described (see also Andu and van Niekerk 1929). Takita (1939) found that the El Tor vibrio produced a true thermolabile exotoxin neutralizable by an antiserum according to the law of unltiple proportions. Hice inoculated intravenously with $0.01 \mathrm{ml}$. died within 24 hours. The exotoxin appeared to be different from the hæmolysin.

Antigenic Structure.-The antigenic structure of the vibrios has of late years received considerable attention. Kabéshima (1918), working with $V$. cholera, discovered the oceurrence of serological variants. Balteanu (1926) found a heatlabile $\mathrm{H}$ and a heat-stable $\mathrm{O}$ receptor in the cholera vibrio; immune serum prepared against organisms heated to $100^{\circ} \mathrm{C}$. for 2 hours contained only $\mathrm{O}$ agglutinins. This finding was confirmed and extended by Shousha (1931), Abloosh (1932), and Gohar (1932). These observations and a particularly careful study by Gardner and 
Venkatraman (1935) have done much to clarify the confusion resulting from the work carried out before the importance of flagellar and somatic antigens had been appreciated. The analysis is, however, by no means complete, and the scheme reproduced here must be regarded only as a working hypothesis, certain in the future to require considerable modification.

Attention has been concentrated mainly on the cholera and cholera-like vibrios, which we refer to for convenience as Group A. This group comprises organisms, most of which produce acid without gas in glucose, maltose, mannitol, and sucrose, but not in dulcitol, and which give the cholera-red reaction. All organisms of Group A possess a common $\mathrm{H}$ antigen. The major $\mathrm{O}$ antigens, on the other hand, of which six have already been differentiated, are much more specific and are used as a basis for the differentiation of Group A into sub-groups. The true cholera vibrios all appear to fall into sub-group I, which also contains most of the El Tor strains. Sub-groups II to VI contain organisms, referred to as paracholera and cholera-like, that have been isolated from cases of choleraic diarrhœa or from water. Thus, according to Gardner and Venkatraman, the true cholera vibrio is a nonhæmolytic organism containing the specific $O$ antigen of sub-group $I$; except by hæmolysin production it is indistinguishable from El Tor vibrios containing the same $\mathrm{O}$ antigen. A non-specific $\mathrm{O}$ antigen, shared to a variable extent by all the members of Group A, has also been described by these workers.

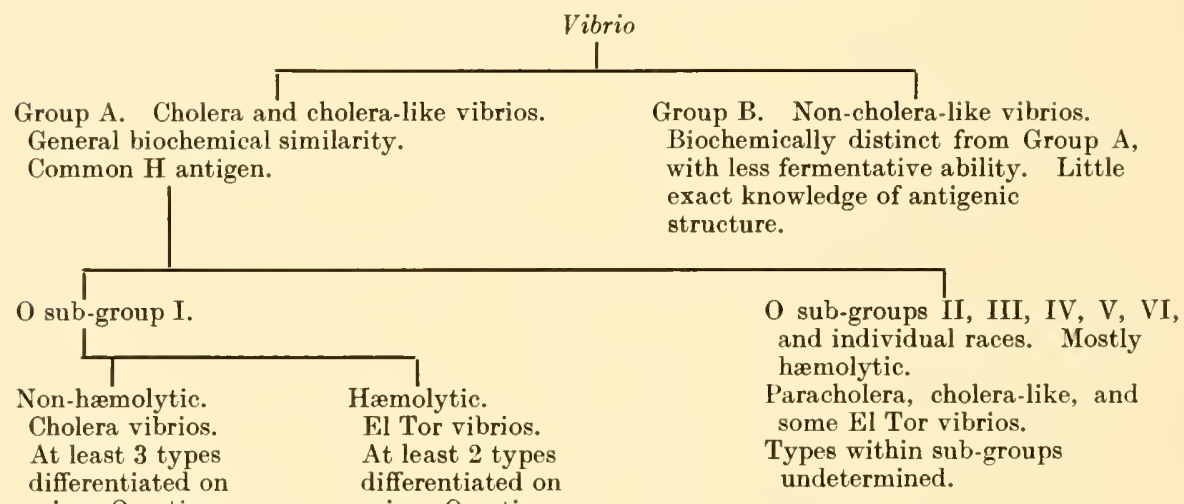

Fig. 104.

Chemical Analysis.-The chemical analysis of the Vibrio group has been intensively studied of late years by Linton and his colleagues in India (for early references see Linton, Shrivastava, and Mitra 1935), who followed up the work of Landsteiner and Levine (1927). They find that the vibrios can be classified into six groups on the basis of two protein and three polysaccharicle constituents (Table 34 ).

Proteins I and II show wide structural differences, but whether they are qualitatively distinct, or whether each is a mixture of several proteins, is not yet decided (Mitra 1938). The polysaccharides exist in the cell as acetyl compounds (Linton and Mitra 1936). On hydrolysis polysaccharide I yields galactose and aldobionic acid, polysaccharide II arabinose and aldobionic acid, whereas the polysaccharide complex III yields glucose only. It is doubtful how far the polysaccharides can be regarded as distinct compounds; Linton, Shrivastava and Seal (1938), for example, have found big differences in the physical, chemical and antigenic properties of preparations of a polysaccharide formed by a given vibrio grown on different media. Metabolic studics by Linton, Mitra and Mullick $(1936 a, b)$ 
TABLE 34

Chemical Classification of the Vibrio Group, according to linton and HIS COLLABORATORS.

\begin{tabular}{|c|c|c|c|}
\hline & Protein. & Polysaccharide. & Source of Origin of Vibrio. \\
\hline Group I . & I & I & Majority of strains from cholera cases. \\
\hline Group II . & I & II & $\begin{array}{l}\text { Some strains from cholera cases and some } \\
\text { water vibrios. }\end{array}$ \\
\hline Group III . & II & II & Mainly inagglutinable water strains. \\
\hline Group IV & II & I & $\begin{array}{l}\text { El Tor strains and some agglutinable } \\
\text { cholera strains found in India. }\end{array}$ \\
\hline Group V & 11 & III & Atypical cholera strains. \\
\hline Group VI & I & III & $\begin{array}{l}\text { Atypical cholera strains, including certain } \\
\text { old laboratory strains of true cholera } \\
\text { vibrios. }\end{array}$ \\
\hline
\end{tabular}

have shown that strains belonging to chemical Gromps I and VI have a high rate of respiration and of aerobic glycolysis; strains of Group II are moderately active, and those of Group III relatively imactive; strains of Groups IV and $V$ have a high respiration but a low aerobic glycolysis rate. In phosphate-buffered peptone water the final Eh of strains belonging to chemical Groups I, II, and VI is higher than of those belonging to Groups III, IV and V (Seal and Mitra 1939).

It is as yet too early to explain the antigenic behaviour of the Vibrio group in terms of chemical structure. As will be seen from Table 34, true cholera vibrios fall into chemical Groups I, II, IV and VI. In addition, it has been found by Linton, Mitra and Seal (1938-39) that the transition of a true cholera vibrio from the smooth to the rough or $\rho$ variant state may be accompanied by a change in its chemical grouping, such as from Group I to Group IV. Chemical and antigenic differences have also been observed in cholera strains isolated from different stages of a given epidemic (Linton, Shrivastava, Seal and Mookerji (1938-39).

In this country Bruce White has studied various fractions from members of the Vibrio group in particular relation to their antigenic structure. White (1935a) finds that, just as in the Salmonella group, the transformation of the smooth to the rough phase is accompanied by a loss of specific $\mathrm{O}$ antigens and the unmasking of a common rough antigen. In consequence, many organisms that are antigenically diverse in the sniooth state show a close similarity in the rough. White has established four rough antigenic groupings. Rough Group A contains strains derived from Gardner and Venkatraman's smooth O sub-group I; rough Group B from smooth O sub-group II; rough Group C from smooth $\mathrm{O}$ sub-groups III and IV; and rough Group D from unclassified smooth O sub-groups.

Variants in a further stage of degradation. known as $\rho$ variants, have been described by White $(1934 b, 1935 a)$. Organisms of this type have lost their dominant rough $\mathrm{O}$ antigen, and appear antigenically similar owing to the ummasking of a still deeper common $\rho$ antigen. Little is yet known of the chemical structure of this $\rho$ antigen, but it is very resistant to proteolytic digestion, and includes a polysaccharide hapten referred to by White $(1940 \mathrm{c})$ as $\mathrm{C} \delta$.

A study of the different types of cholera vibrios belonging to Gardner and Venkatraman's O sub-group I led Scholtens (1933) and Heiberg (1936b) to postulate the existence of two qualitatively distinct somatic antigens, A and B. Some strains appeared to contain A only, and some both A and B. White (1937b), on the other hand, following up his work of 1936, disagrees with this interpretation. On the basis of agglutination and 
absorption of agglutinins tests he believes that the Inaba (original), Hikojima (middle), and Ogawa (variant) strains of $V$. cholere, which have been extensively used for' serological work in the East, behave as if their heat-stable $O$ antigens had the general structure $A X, A B X$, and BX. By means of precipitin tests carried out with sera prepared by the inoculation of rabbits with polysaccharide extracts, he finds that there are at least four O receptor groups on the smooth polysaccharide of the cholera vibrio. Both the Inaba and the Ogawa strains possess these four groups, two of which are type specific and two of which are common to both types. In each type one of the receptors is alkali-labile and the other alkali-stable.

White $(1934 a, 1935 b)$ has described the occurrence of alcohol-soluble protein antigens, which he refers to as $\mathrm{Q}$ antigens. There is reason to believe that these substances play a part in the non-specific $\mathrm{O}$ agglutination of boiled vibrios, described by Gardner and Venkatraman.

White's studies were interrupted by the war, but it may be worth while summarizing his findings to date. From vibrio bodies he has isolated (I) a heat-labile protein antigen (White $1940 b)$; (2) a heat-stable protein antigen, possibly associated with a hapten $\mathrm{C} \gamma 2$ (White 1940d); (3) an alcohol-soluble Q protein fraction; $(4)$ the differential S, P and $\rho$ antigens with their respective polysaccharide haptens $\mathrm{C} \alpha, \mathrm{C} \beta$ and $\mathrm{C} \delta$; $(5)$ another hapten $\mathrm{C} \gamma \mathrm{l}$ is probably also of somatic origin ; and (6) another, the "rugose" hapten (White 1940a), has been derived from the intercellular secretion of rugose variant strains. Antibodies for all these components may occur in the sera of rabbits immunized with living cultures of $V$. cholerce.

From this brief summary it will be apparent that the fiudings of Bruce White and of Linton and his colleagues are hy no means easy to interpret, and that much further work will have to be done before the antigenic structure of even the cholera vibrio can be expressed in chemical terms. For a review of the chemistry and antigenic structure of the vibrios, see Linton (1940).

Pathogenicity.-The cholera vibrio causes Asiatic cholera in man. Metchnikoff's vibrio apparently is responsible for a choleraic disease in chickens (Gamaléia 1888a) - not for true chicken cholera, which is due to a member of the Pasteurella group. It is possible that $V$. phosphorescens may cause acute gastro-enteritis in man, but this has not been proved conclusively (Jermoljewa 1926).

A disease simulating cholera may be reproduced in guinea-pigs and new-born rabbits by certain experimental procedures (see Chapter 63). The cholera vibrio when given by mouth, or injected per rectum, is harmless to mice, rabbits, guinea-pigs, and monkeys. Intraperitoneal injection into guinea-pigs is fatal within 24 hours. If a small dose of vibrios- $-\frac{1}{4}$ loopful of an 18-hours' agar culture - is given, the animal dies of toxæmia, and at necropsy the peritoneal cavity is sterile. If a larger dose is given, $\frac{1}{2}$ loopful, cultures from the peritoneal cavity may be positive; and if a still larger dose is given, 1 or more loopfuls, the vibrios may be recovered also from the heart blood. Intraperitoneal injection of mice is fatal in 24 to 48 hours. Intravenous injection of young rabbits is fatal in 1 to 5 days. According to Botman (see van Loghem 1938) the rat is comparatively unaffected by the intraperitoneal inoculation of killed cholera vibrios, whereas it is susceptible to El Tor vibrios.

Metchnikoff's vibrio is more invasive than the cholera vibrio. Even after a small dose given intraperitoneally to guinea-pigs, the vibrios can be recovered from the heart's blood. It is fatal to guinea-pigs even when given subcutaneously ; under these conditions the cholera vibrio gives rise merely to a local abscess. Both guinea-pigs and chickens can be infected by feeding with $V$. metchnikovi. Moreover this organism is pathogenic to pigeons, on intramuscular injection, while the cholera 
vibrio is not, except occasionally in large doses (Wherry 1905). Pigeons injected intramuscularly with $\frac{1}{3}$ agar culture of $V$. metchnikovi die in about 8 hours with general septicæmia (Metchnikoff 1893). Intratracheal injection appears to be even more fatal, since not only guinea-pigs, pigeons, and fowls, but also rabbits may be infected by this route (Gamaléia 1888b). Deneke's Vibrio tyrogenus is pathogenic for the guinea-pig and the pigeon. Half an agar culture injected intraperitoneally into a guinea-pig was fatal in 6 hours, and a whole agar culture injected intramuscularly into a pigeon was fatal in 7 hours (Metchnikoff 1893). Finkler-Prior's Vibrio proteus resembles $V$. tyrogenus, but is slightly less virulent.

$\boldsymbol{V}$. phosphorescens is pathogenic for guinea-pigs, rabbits, and pigeons. About 500 million organisms injected intraperitoneally into guinea-pigs, intravenously into rabbits, or intramuscularly into pigeons proved fatal in 24 hours; vibrios were isolated post mortem from the heart's blood of the pigeons (Jermoljewa 1926). If a guinea-pig that has died after intraperitoneal injection is opened up and placed in the dark, the viscera are seen to exhibit a marked phosphorescence (Kutscher 1893).

Most other members of the group are non-pathogenic. The virulence of $V$. cholere is variable. Freshly isolated strains are more virulent than those kept in the laboratory. Moreover, even on isolation, the virulence of different strains to laboratory animals appears to vary. Haffkine (1892) stated that it was possible to raise the virulence by passing the organisms through the peritoneal cavity of gninea-pigs; between each injection the peritoneal exudate was exposed to the air for some time at room temperature. By growing the vibrio in broth in a constantly aerated atmosphere and subculturing every 2 or 3 days, the virulence was said to diminish. Gotschlich and Weigang (1895) also stated that the virulence might be raised by intraperitoneal passage through guinea-pigs.

Variation.-The occurrence of smooth, rough and rugose forms of the cholera vibrio has already been referred to in the sections on cultural and antigenic characters. Confusion has arisen from paying too much attention to colonial variation without a full study of antigenic and other properties. As White (1938) points out, the essential feature of rough variants is their absence of the specific smooth polysaccharide.

There is a widespread belief that cholera vibrios under unfavourable conditions, such as in water, may lose their specific characters and be transformed into some other type of vibrio. The alleged transmutation of vibrios in the laboratory by Linton (1935), Linton, Shrivastava, and Mitra (1934-35), Linton, Seal and Mitra (1938), and Taylor and Ahuja (1935-36) has tended to strengthen this belief. White $(1937 a)$, who has studied some of the strains before and after their alleged change, can find no evidence to support the conception of vibrionic transmutability. Until further studies have been made it is probably wiser to adopt a strictly conservative attitude toward the limits of variation within the different species of this group.

We append a summarized description of $\mathbf{T}$. cholerce, and brief notes on the characters of other species which have been described and named. (For general classification of members of this group, see Heiberg 1935.)

Synonym.-Comma bacillus.

\section{Vibrio choleræ}

Isolation.-Koch in 1884 (1886).

Inubitat.-Intestinal contents of cholera patients and carriers. 
Morphology.-Slightly curved bacillus, often resembling a comma. Varies considerably in size, $1.5-4 \mu \times 0.2-0.4 \mu$. One end often blunter than the other; ends rounded; axis generally curved; sides converging or parallel. Arranged singly, or in s-shaped pairs; sometimes short chains are found, and sometimes spirals. In the intestinal contents, arranged like fish in a stream. In old cultures the bacilli are very small, resembling granules, and stain poorly. Involution forms numerous. Actively motile by a single polar flagellum. Gram-negative. Nonsporing. Non-acid-fast.

Agar Plate.-24 hours at $37^{\circ} \mathrm{C}$. Round, 1-2 mm. in diameter, low convex, translucent, greyish-yellow oolonies with smooth, or finely granular, glistening surface and entire edge, and of amorphous or finely granular structure ; consistency butyrous ; easily emulsifiable. 7 days; slightly larger ; edge entire or undulate; surface sometimes studded with small, knob-like secondary colonies; colony is sometimes surrounded by a narrow, effuse, transparent peripheral extension. Crystals often formed in the medium.

Agar Stroke.-24 hours at $37^{\circ} \mathrm{C}$. Good, raised, translucent, greyish-yellow layer of growth, with smooth, glistening surface, and edge formed of single colonies. 7 days; surface sometimes studded with small, knob-like secondary colonies. Crystals often formed in the medium.

Gelatin Plate.-2 days at $23^{\circ} \mathrm{C}$. Round, $0.5 \mathrm{~mm}$. in diameter, amorphous, raised or low convex, greyish-white, opaque colonies, with smooth or slightly granular surface and entire or crenated edge. Zone of liquefaction around colony ; small flocculi of growth in liquefied gelatin.

Gelatin Stab.-3 days at $22^{\circ} \mathrm{C}$. Good filiform growth, confluent at top, discrete below, extending to bottom of tube. Infundibuliform or napiform liquefaction; thick, yellowish-brown pellicle on surface of liquid gelatin, and coarsely granular turbidity.

Broth. - 24 hours at $37^{\circ} \mathrm{C}$. Abundant growth with moderate turbidity, a slight powdery deposit, and a thick surface pellicle, breaking up on sbaking into coarse membranous and granular pieces.

Loeffler's Serum.-10 days at $37^{\circ} \mathrm{C}$. Good growth with partial liquefaction.

Horse Blood Agar Plates.-24 hours at $37^{\circ} \mathrm{C}$. Abundant growth ; colonies are surrounded for $2 \mathrm{~mm}$. by a zone of $\alpha$ - or $\beta$-hæmolysis.

Polato. - 7 days at $37^{\circ} \mathrm{C}$. Good, confluent, café-au-lait growth with smooth glistening surface.

MacConkey Plate.-24 hours at $37^{\circ} \mathrm{C}$. Good growth of clear, colourless colonies smaller than those on agar. After 7 to 9 days the colonies take on a reddish colour.

Resistance.-Not specially resistant. Easily killed by drying. Destroyed by heat at $55^{\circ} \mathrm{C}$. in 15 minutes. Dried on linen or threads they survive 1 to 3 days. Killed by 0.5 per cent. phenol in a few minutes. Survive in clean tap water up to 30 days, but perish in 24 hours in cesspool water.

Metabolism.-Strongly aerobic; very slight growth noticeable on agar and in broth after a week under strictly anaerobic conditions. Optimum temperature $37^{\circ} \mathrm{C}$. ; limits $16-42^{\circ} \mathrm{C}$. Optimum $\mathrm{pH} 7 \cdot 0-8 \cdot 0$. Limits for growth $\mathrm{pH} 6 \cdot 4-9 \cdot 6$. Growth favoured slightly by blood. Grows well and rapidly in peptone water. No soluble hæmolysin formed for sheep or goat cells. Proteolytic and diastatic ferments secreted.

Biochemical.-Acid, no gas, in glucose, maltose, mamitol, and sucrose in 1 to 3 days ; after 14 days there may be slight acid in lactose. L.M. acid, or acid and clot in 14 days. Indole + . Cholera-red reaction + . M.R.-. V.P. -. Nitrates reduced. $\mathrm{NH}_{3}+$. $\mathrm{H}_{2} \mathrm{~S}+$ in 14 days. Catalase + . M.B. reduction + .

Antigenic Structure.-All strains have a common $\mathrm{O}$ antigen, but 3 sub-types are distinguishable. Immume sera prepared by injection of rabbits, goats, or horses with living vibrios contain specific bacteriolysins, demonstrable by Pfeiffer's test.

Pathogenicity. - Causes Asiatic cholera in human beings. A similar disease may be repro- 
duced experimentally in new-born rabbits by feeding, and in young guinea-pigs by Koch's procedure. Pathogenic on $i p$. or $i v$. inoculation into guinea-pigs, rabbits, and mice, but not as a rule into pigeons. One loopful of an 18-hours' agar culture of a virulent strain injected ip. into a young guinea-pig is fatal within 24 hours. P.M. congestion of peritoneal and pleural cavities with some sero-sanguineous fluid. Small gut congested; may be fibrin over the abdominal viscera. Vibrios may or may not be cultivated from the peritoneal cavity. If a large dose is given the vibrios can be recovered from the peritoneal fluid and the heart blood. IV. injection of five loopfuls of an 18-hour agar culture of a virulent strain into rabbits is fatal in 48 hours or less. P.M. small gut congested and contains thin fluid. Vibrios generally recoverable from the blood. No true exotoxin formed, but disintegrated bodies of bacilli are very toxic to animals. Virulence rapidly falls on artificial cultivation.

Finkler-Prior's Vibrio proteus.-Isolated by Finkler and Prior (1884) from the old putrid excreta of a patient suffering from gastro-enteritis. Morphologically and culturally it resembles the cholera vibrio, but it can be differentiated by serological reactions, and by its failure to give the cholera-red reaction. Has frequently been found in water.

Deneke's Vibrio tyrogenus.-Found by Deneke (1885) in cheese. Resembles the cholera vibrio, but liquefies gelatin more rapidly, grows poorly or not at all on potato, and does not give the cholera-red reaction.

Vibrio metchnikovi.-Isolated by Gamaléia (1888a) from the blood and intestinal contents of chickens dying from a cholera-like disease at Odessa. Resembles the cholera vibrio very closely; gelatin is liquefied more rapidly; growth on MacConkey is poorer. Cholera-red and other biochemical reactions are identical with those of $V$. cholera. It is much more invasive when injected into animals, killing guinea-pigs injected subcutaneously, and pigeons injected intramuscularly (see Pathogenicity). Can be separated from $V$. cholerce by agglutination and Pfeiffer's reactions. Has been isolated from water.

Vibrio phosphorescens.-Isolated by Dunbar (1893) from the Elbe in 1893. Shown by Kutscher (1893) to exhibit phosphorescence in the dark. This occurs on ordinary media at $22^{\circ} \mathrm{C}$, reaching its maximum in 24 to 48 hours in gelatin, broth, or peptone water, and disappearing rapidly. It is a function of the living bacilli. Phosphorescence is not visible in cultures incubated anaerobically. V. phosphorescens grows in, and liquefies gelatin, more rapidly than $V$. cholerce. Grows very poorly or not at all on potato. Hæmolytic and diastatic; produces indole; gives acid in glucose, mannitol, lactose, and later maltose (Jermoljewa 1926). It has been isolated from human fæecs (Jermoljewa 1926).

El Tor Vibrio.--Isolated by Gotschlich (1906) in 1905 from six pilgrims who had died of dysentery or gangrene of the colon at the Tor quarantine station on the Sinai Peninsula. Forms soluble hæmolysin for sheep and goat cells. Gives atypical Pfeiffer reaction (Neufeld and Haendel 1907). Usually gives positive Voges-Proskauer reaction (van Loghem 1938). Killed vibrios injected intraperitoneally are said to be more toxic for the rat than killed cholera vibrios (see van Loghem 1938). According to Takita (1939) the El Tor vibrio produces a true thermolabile exotoxin, distinct from the lrmolysin, and proving fatal to mice on intraperitoneal inoculation. Relation to $V$. cholerce still doubtful (see Fig. 104).

Vibrio berolinensis.-Isolated by Neisser (1893) from water to which cholera vibrios had intentionally been added. Resembles the cholera vibrio closely; gelatin colonies are smaller and animal pathogenicity is low. But probably it is merely a variant of the true cholera vibrio. Similarly the "Vibrio ivanoff" (Ivanoff 1893), which was cultured from the fæees of a typhoid patient to which cholera vibrios had been added, and which differed in umimportant particulars from the cholera vibrio, is also a variant of the true oholera vibrio (Dieudonné 1894). 
Vibrio danublcus.-Isolated by Heider (1893) from the Danube canal. Resembles the cholera vibrio closely, but ean be differentiated by serological reactions.

Vibrio helcogenes.-Isolated by Fischer (1893) from the diarrhœal frees of a woman. Some of the mice inoculated subcutaneously developed ulcers of the skin ; hence the name.

The Massauah vibrio was isolated by Pasquale (1891) from the fæces of a patient who was probably not suffering from cholera. Resembles the cholera vibrio closely, but has four peritrichate flagella ; it is therefore not a true Vibrio. The Ghinda vibrio was isolated by Pasquale from water; it was regarded as a true cholera vibrio, but has since been shown to be distinguished from it by its immunity reactions.

The Nasik vibrio differs in several respects from $V$. cholera. It is less like a comma and is short and rather fat: arranged singly, and with great regularity; filamentous forms are common. The colonies on agar are more opaque than those of oholera. Infundibuliform liquefaction occurs in gelatin, later stratiform ; the liquefied gelatin is uniformly turbid and contains no flocculi. No growth under anaerobic conditions. Acid in glucose only; L.M. purple and clotted; indole - ; nitrate reduction-; $\mathrm{NH}_{3}+; \mathrm{H}_{2} \mathrm{~S}-$; Catalase ++ ; M.B. reduction - ; cholera-red reaction $-; \beta$-hæmolysis in horse blood agar plates in 4 days. Café-au-lait growth on potato. Broth cultures are very toxic to rabbits on intravenous injection (Kolle and Schürmann 1912).

Vibrio fetus.- This organism, on account of its special characteristics, must be considered separately. It was first isolated and described by M'Fadyean and Stockman in 1913 (see Report 1913), who found it in the uterine exudate of aborting sheep. Smith (1918) cultivated the same organism in America from the footuses of aborting cows (see Chapter 75); be named it $V$. fetus (Smith and Taylor 1919). In young cultures it is generally shaped like a comma, but later it assumes a spirillar appearance. It is questionable whether this organism should be classified as a Vibrio or as a Spirillum ; its characters partake of both groups. But since Smith has placed it with the vibrios, since it has a single polar flagellum, and since it is Gram-negative, it is perhaps best to regard it as belonging to the Vibrio group.

Morphologically, the smallest forms appear as minute, slender, s-shaped threads; the longest forms may stretch nearly across the field of the microscope. In length it is 1.5 to $5 \mu$ or more, and in breadth about 0.2 to $0.3 \mu$. A single organism shows one or two spirals ; the length of each spiral is about $2 \mu$, and the amplitude about $0.5 \mu$. In the long forms the spirals are drawn out, so that their length is far greater than their breadth. The short forms are sharply curved; the spirals often show an obtuse-angled curve. In young cultures the vibrios are actively motile by a single polar flagellum; in cultures a week old very few are motile. The organism is best stained with alkaline methylene blue, the staining being prolonged over-night. It is Gram-negative. In old cultures many of the organisms show granular degeneration.

For growth in artificial media, a reduced oxygen pressure is required. When first isolated, it will not grow on agar without the addition of blood or some other animal fluid. The growth is very delicate, and occurs at the edges of the slope between the agar and the glass; subsequently it spreads round the convexity of the agar. After some months of cultivation in the laboratory, a thin surface growth may be obtained. Growth in fluid media, even in blood broth, does not occur till the strain has become thoroughly accustomed to saprophytic conditions. There is no growth in gelatin, milk or potato. Sugars are not fermented, and there is no production of indole. In cultures, it lives for 2 to 20 weeks at room temperature, but dies rapidly in the ice-chest. Dried on threads, it lives for less than 3 hours. It is killed by $56^{\circ} \mathrm{C}$. in 5 minutes. The optimum temperature for growth is $37^{\circ} \mathrm{C}$. Antigenically it appears by agglutination to be homogeneous (Smith and Taylor 1919). It is non-pathogenic to laboratory animals. Under natural conditions it gives rise apparently to abortion in cattle and sheep. Experimental inoculation of pure 
cultures into pregnant cows may be followed by disease of the foetal membranes (Smith 1919).

A closely related organism, named Vibrio jejuni, has been described by Jones and Little (1931), and Jones, Orcutt, and Little (1931). It appears to be responsible for a disease of calves and older cattle, which may occur in epidemic form during the autumn and winter months, and is known as winter dysentery or black scours. The organisms are most abundant in the jejunum.

\section{SPIRILLUM}

DEFINITION.—Spirillum.

Rigid rods of spiral form, varying considerably in the number, length, and breadth of the spirals. Usually motile by means of a tuft of polar flagella ( 5 to 20), which are mostly semicircular in shape. The flagella occur at one or both poles; their number varies greatly, and is difficult to determine, since in stained preparations several are often united into a common strand. Generally Grampositive. Some species form a reddish-yellow, or greenish-yellow pigment. Found in water or putrid infusions.

Type species. Spirillum undula.

Not many orgauisms in this group have been described. One of the best known is Spirillum rubrum, which was isolated by Esmarch (1887) from a mouse that had been decomposing for 3 months under water.

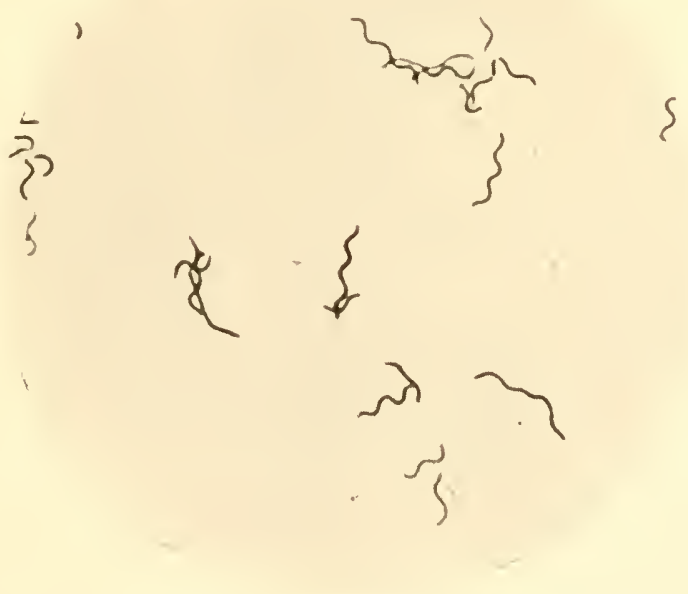

Fic. 105. -Spirillum rubrum.

From a broth culture, 2 days, $30^{\circ} \mathrm{C} .(\times 1000)$.

Morphologically the spirilla show considerable variation. Their length may vary from 1 to $50 \mu$, and the number of spirals from 1 to about 50 . The length of the individual spiral varies according to the species of organism ; in some spirilla the spirals are close set, each one being not more than about $1 \mu$ in length; in others they are looser, and may be $10 \mu$ or so in length. The width of the organisms 
varies from about 0.3 to $1.0 \mu$, and the amplitude of the spirals from about 0.8 to $2.0 \mu$. Even amongst different organisms of the same strain, there is often considerable variation in the number and size of the spirals; together with organisms showing regularly disposed spirals, there may be seen others of two or three times the length, with only one or two irregular undulations. In shape the whole organism may be straight, or it may be bent in one or more places, generally acutely. Filamentous forms are not uncommon. On agar or gelatin the spiral shape may be almost lost, and the organisms closely resemble vibrios. As a rule the curvature is very marked, and there is a tendency for the organisms to be arranged in pairs end-to-end with the concavities facing in the same direction, so as to present a scalloped appearance; s-shaped forms too are common. In young cultures the spirilla are motile-generally by tufts of flagella at the poles. Unlike the spirochaetes, the spirilla stain readily with the ordinary aniline dyes, and are usually Gram-positive. Growth is fairly free, though not abundant, on the ordinary media. Most of the water spirilla form a pigment of red, yellow, or greenishyellow colour. The pigment, at least of $S p$. rubrum, is formed most readily at a low oxygen pressure; it is well marked in the depths of gelatin stab cultures, and hardly noticeable on surface growths. The optimum temperature for growth is $25-30^{\circ} \mathrm{C}$. as a rule. Aerobic conditions are preferred; growth under strict anaerobiosis is very slight. None of the species forms spores, and none is particularly resistant to heat or disinfectants. The biochemical characteristics have not been fully studied. None of the members except Spirillum minus (see p. 1838) is pathogenic for man or animals. We append a description, based largely on personal observations, of Spirillum rubrum.

\section{Spirillum rubrum}

Isolation.-Esmarch 1887, from a mouse decomposing under water.

Habitat.--Water.

Morphology.-On solid media the organisms are sharply curved rods, $2-3 \mu \times 0.4 \mu$, arranged singly and in s-shaped or semicircular pairs. In fluid media long spirals are formed, $3-10 \mu$ or more in length. The axis of the spiral is straight, or bent sharply at a right-angle; the number of spirals varies from about 1 to 8 . Ends are sharp, drawn out, or sometimes blunt. Long thread-like forms also seen. Motile by bundles of flagella at both poles. Gram-positive.

Agar Plate. 2 days at $28^{\circ} \mathrm{C}$. Round, $0.4 \mathrm{~mm}$. in diameter, convex, amorphous, almost colourless and transparent colonies with smooth glistening surface and entire edge; consistency butyrous; easily emulsifiable. 7 days; rather larger and of a pinkish colour.

Agar Stroke.-2 days at $28^{\circ} \mathrm{C}$. Poor, slightly raised, and almost transparent, partly confluent growth, with irregular surface, and edge formed of single colonies. 7 days; slight pinkish coloration.

Gelatin Plate. 4 days at $23^{\circ} \mathrm{C}$. Small, $0 \cdot 3 \mathrm{~mm}$. in diameter, water-clear, convex colonies, with smooth surface and entire edge. No liquefaction. Deep colonies are pink.

Gelatin Stab.-5 days at $23^{\circ} \mathrm{C}$. Poor to moderate, filiform growth of very tiny diserete red colonies, extending nearly to bottom of tube. No surface growth: no liquefaction.

Broth.-2 days at $28^{\circ} \mathrm{C}$. Moderate growth with slight turbidity, and a pale pink floccnlogranular deposit, not disintegrating completely on shaking; no surface growth.

MacConkey. -7 days at $28^{\circ} \mathrm{C}$. No growth. 
Potato.-7 days at $28^{\circ} \mathrm{C}$. Poor growth of discrete or partly eonfluent red colonies.

Horse Blood Agar Plate.-2 days at $28^{\circ} \mathrm{C}$. Very small, low convex colonies; no hæmolysis.

Resistance.-Not specially resistant. Dried on silk threads, the organisms do not survive longer than 6 to 8 days.

Metabclism. Aerobio; grows very poorly uncier anaerobic conditions. Optimum temperature $25-30^{\circ} \mathrm{C}$. often little growth at $37^{\circ} \mathrm{C}$. Grows very poorly in peptone water. No hæmolysin formed. Red pigment formed, best under a low oxygen pressure.

Biochemical-No sugars fermented. L.M. unchanged. Indole--. Cholera-red reaction -. M.R. - . V.P. - . Nitrates not reduced. $\mathrm{NH}_{3}+. \mathrm{H}_{2} \mathrm{~S}-$. Catalase + . M.B. reduction-.

Pathogenicity.-Non-pathogenic to man or animals.

Other members of this group that have been described are Spirillum undula, Spirillum serpens, Spirillum volutans, and Spirillum minus (see p. 1838).

\section{REFERENCES}

Aвdoosh, Y. B. (1932) Brit. J. exp. Path., 13, 42.

ANDU, A. B. and NiEKERK, J. VAN. (1929) Zbl. Bulit., 112, 519

Balteanu, I. (1926) J. Path. Bact., 29, 251.

Bernard, P. N., Guilleri, J., and Gallut, J. (1937) C. R. Soo. Biol., 126, 180, 303, 394, 478,568 ; (1939) Ibid., 130, 23, 147, 228.

ВоссніА, I. (1911) Zbl. Bakt., 60, 434.

Conbiesco-Popesco, C. and Cocroba, 1. (1936) C. R. Soc. Biol., 124, 151.

Craster, C. V. (1913) J. infect. Dis., 12, 472.

Crendiropodlo. (1912) Conseil san. maritime quarant. d'Égypte.

DENEKE, T. (1885) Dtsch. med. Wschr., 11, 33.

Dieddonné. (1894) Zbl. Bakt., 16, 363; (1909) Ibid., 50, 107.

Doorenbos, W. (1936) C. R. Soc. Biol., 121, 128, 130.

DUNBaR. (1893) Dtsch. med. Wschr., 19, 799.

DUNGERN, voN. (1895) Z. Hyg. Infektkr., 20, 147.

EsMaRCH, E. (1887) Zbl. Bakt., 1, 225.

Finkler and Prior. (1884) Disch. med. Wschr., 10, 579, 632.

Fischer. (1893) Dtsch. med. Wschr., 19, 541, 575, 598, 627.

Gamaléja, M. N. (1888a) Ann. Inst. Pasteur, 2, 482; (1888b) Ibid., 2, 552.

Gardner, A. D. and Venkatraman, K. V. (1935) J. Hyg., Camb., 35, 262.

Gohar, M. A. (1932) Bril. J. exp. Path., 13, 371.

Gordon, M. H. (1906) Brit. med. J., ii. 197.

Gotsohlich. E. (1895) Z. Hyg. InfektKr., 20, 489; (1906) Ibid., 53, 281.

Gotsohlioh, E. and Weigang, J. (1895) Z. Hyg., InfektKr., 20, 376.

HAHN, M. and Hirsch, J. (1929) Z. Hyg. InfekiKr., 110, 355.

HafFkine, W. M. W. (1892) C. R. Soc. Biol., 44, 635, 671.

HeIBERG, B. (1935) "On the classification of Vibrio choleræ and the Cholera-like vibrios." Arnold Busck, Copenhagen; (1936a) J. Hyg., Camb., 36, 114; (1936b) Ibid., 36, 118.

HEIDER, A. (1893) Zbl. Bakt., 14, 341.

Iva NOFF, M. (1893) Z. Hyg. InfektKr., 15, 434.

JeRMOLJEIVA, S. (1926) Zbl. Bakt., 100, 170.

Jones, F. S. and LitTle, R. B. (1931) J. exp. Med., 53, 835, 815.

Jones, F. S., Orcutt, M., and Little, R. B. (1931) J. exp. Med., 53, 853.

Ka ве́SHIMA, T. (1918) C. R. Soc. Biol., 81, 618.

Kitasato, S. (1889) Z. Hyg. InfektKr., 5, 134.

Коон, R. (1886) "The Etiology of Cholera," New Sydenham. Soc., 115, 327.

Kolle, W. and Schürmann, IV. (1912) See Kolle and Wassermann Handb. path. Mikroorg. (1912-13) 4, 1.

KUTSCHER. (1893) Dtsch. med. Wschr., 19, 1301; (1895) Z. Hyg. InfektKr., 19, 461.

LANDSteiner, K. and Levine, P. (1927) J. exp. Hed., 46, 213.

Lrnton, R. W. (1935) Bull. Off. int. Hyg. publ., 27, $1105 ;$ (1940) Bact. Rev., 4, 261.

Linton, R. W. and Mitra, B. N. (1936) Indian J. med. Res., 24, 323.

Ijnton, R. W., Mitra, B. N., and Mullick, D. N. (1936a) Indian J. med. Res., 23, 589 (1936b) Ibid., 24, 317. 
Linton, R. W., Mitra, B. N., and Seal, S. C. (1938-39) Indian J. med. Res., 26, 329.

Jinton, R. W., Seal, S. C., and Mitra, B. N. (1938) Indian J. med. Res., 25, 575.

Linton, R. W., Shrivastava, D. L., and Mitra, B. N. (1934-35) Indian J. med. Res., 22, 633.

Linton, R. W., Simivastava, D. L., and Seal, S. C. (1938) Indian J. med. Res., 25, 569.

Linton, R. W., Shrivastaya, D. L., Sfal, S. C., and MlookerJi, S. P. (1938-39) Indian J. med. Res., 26, 41.

Loghem, J. J. van. (1932) Ned. Tijdschr. Geneesk., 76, 1939; (1938) Bull. Off. int. Hyg. publ., 30, 1520 .

MaASSEN, A. (1902) Arb. ReichsgesundhAmt., 18, 21.

Manwaring, W. H., Boyd, W. H., and OKami, S. (1923) J. infect. Dis., 32, 307.

МетонпікоғF, E. (1893) Ann. Inst. Pasteur, 7, 562.

Mitra, B. N. (1938) J. trop. Med. (Hyg.), 41, 37.

NAPIER, L. E. and GUPTA, S. K. (1942) Indian med. Gaz., 77, 717.

Neisser, M. (1893) Arch. Hyg., 19, 194.

NeUfELd and HAENDEl. (1907) Arb. ReichsgesundhAmt., 26, 536.

Nicati, W. and Rietsch, M. (1884) C. R. Acad. Sci., 99, 928.

Noвесні, K. (1925) J. Bact., 10, 197.

Pasquale, A. (1891) G. med. Eserc., 39, 1009.

Paladino-Blandini, A. (1906) Ann. Igiene (sper.), 15, 301.

Pasricha, C. L., Chatterjee, D. N., and Das, P. C. (1938-39) Indian J. med. Res., $26,33$.

Pfeiffer, R. (1892) Z. Hyg. InfektKr., 11, 393; (1894) Ibid., 18, 1 ; (1895) Ibid., 20, 198.

Pollitzer, R. (1935-36) Rep. nat. Quar. Serv., Shanghai, Ser. vi. p. 70.

Report. (1913) Rep. Dep. Comm. Epizootic Abortion, Part III. London.

RuFfer, M. A. (1907) Conseil san. mar. et Quarant. d'Égypte.

Soholtens, R. T. (1933) C. R. Soc. Biol., 114, 420, 422.

Seal, S. C. and Mitra, B. N. (1939) Indian J. med. Res., 26, 625.

Sнобsна, A. T. (1931) Bull. Off. int. Hyg. publ., 23, 1022.

Sмгтн, T. (1894) Zbl. Bakt., 16, 324; (1918) J. exp. Med., 28, 701 ; (1919) Ibid., $30,313$.

Sмith, T. and TAYLOR, M. S. (1919) J. exp. Med., 30, 299.

'Јакіта, J. (1939) Kitasato Arch. 16, 218.

TAylor, J. and AHUJA, M. L. (1935-36) Indian J. med. Res., 23, 95, 531 ; (1938-39) Ibid., $26,1$.

TAYlor, J., Pandit, S. R., and REad, W. D. B. (1937) Indian J. med. Res., 24, 931.

TAylor, J., Read, W. D. B., and Pandit, S. R. (1936) Indian J. med. Res., $24,349$.

VAssiliadis, P. C. (1937) "Etude sur la bactériologie des vibrions et l'épidémiolgie du choléra." 'Thèse, Louvain.

VeDder, A. and DAM, W. VAN. (1932) Zbl. Bakt., 126, 145.

Wherry, W. B. (1905) J. infect. Dis., 2, 309.

Whiте, P. B. (1934a) J. Path. Bact., 39, 529 ; (1934b) Ibid., 39, 530 ; (1935a) J. Hyg., Camb., 35, 347 ; (1935b) Ibid., 35, 498; (1937a) J. Path. Bact., 44, 490 ; (1937b) Ibid., 44, 706; (1938) Ibid., 46, 1; (1940a) Ibid., 50, 160; (1940b) Ibid., 50, 165 ; (1940c) Ibid., 51, 447 ; (1940d) Ibid., 51, 449.

YeN, A. C. H. (1932-3) Proc. Soc. exp. Biol., N.Y., 30, 884.

ZimmermanN, E. (1932) Zbl. Bakt., 127, 146; (1933) Z. ImmunForsch., 79, 219. 


\section{CHAPTER 23}

\section{NEISSERIA}

Definition.-Neisseria.

Gram-negative cocci, usually arranged in pairs. Strict parasites, often growing poorly on ordinary media, but growing well on serum media. Frequently pathogenic.

Type species is $N$. gonorrhoea.

The first member of this group to be described was the gonococcus; it was observed by Neisser in 1879 in the pus cells of patients with gonorrhoea, and was successfully cultivated by Bumm $(1885 a, b)$ and by Leistikow and Loeffler (Leistikow 1882) in 1882. Weichselbaum isolated the meningococcus from the cerebrospinal fluid of patients with cerebrospinal meningitis in the year 1887. In 1895 Jaeger described a similar organism, which he regarded as identical with the meningococcus, but which was almost certainly not this organism; it is now known as the Diplococcus crassus. R. Pfeiffer (see Flügge 1896) described the Micrococcus catarrhalis in 1896; he found it in the bronchioles and alveoli of children with broncho-pneumonia; it was carefully studied in 1902 by Ghon and H. Pfeiffer. In 1906 von Lingelsheim described a number of Gram-negative cocci in the nasopharynx of healthy and diseased persons; these included the Micrococcus pharyngis siccus, the Micrococcus pharyngis cinereus, the Diplococcus mucosus, and the Micrococcus pharyngis flavus $i, i i$, and $i i$. More recently Branham (1930) has added another member to the group, $Y$. flavescens. This organism was isolated from the spinal fluid of patients with epiclemic cerebrospinal meningitis.

Habitat.-With the exception of the gonococcus, which is the causative organism of gonorrhoea, those species of Gram-negative cocci which have been adequately described are found almost exclusively in the nasopharynx of healthy and diseased persons, or, in the case of the meningococcus, in the meninges and cerebrospinal fluid of patients with cerebrospinal fever.

Morphology.--The nembers of the group are all Gram-negative cocci, but they differ considerably in their morphology and arrangement, and in the ease with which they are decolorized by alcohol. Not only do they differ from one another, but the same organism may vary considerably according to environmental conditions; thus, in the body, the meningococcus and the gonococcus present an almost typical arrangement in the form of diplococci with flattened or slightly concave adjacent sides, but in culture they appear as oval or spherical cocci without the typical diplococcal arrangement. Most of the members of the group are arranged in pairs, tetrads, or small groups; but some members, such as $N$. pharyngis, 
appear frequently in the form of dense clumps with occasional isolated organisms. One difference in arrangement that serves to distinguish Gram-negative from Gram-positive diplococci is the way in which the main axis of the oval is directed; with the Gram-negative cocci this axis is always at right angles to the axis joining the two cocci; with the Gram-positive cocci it is often coincident with it. In other words, pairs of Gram-negative cocci are usually compressed laterally, Gram-positive cocci often longitudinally. As a rule the Gram-negative cocci are decolorized withont difficulty, but some members tend to retain the gentian violet, and so take on an indeterminate colour, which is most confusing. The most notable example of this is Jaeger's coccus or the Diplococcus crassus; in a single film Gram-positive and Gram-negative, and numerous other cocci with an indeterminate colour, are found lying side by side. One reason for the indeterminate staining of some of the Gram-negative cocci is the tendency they have to

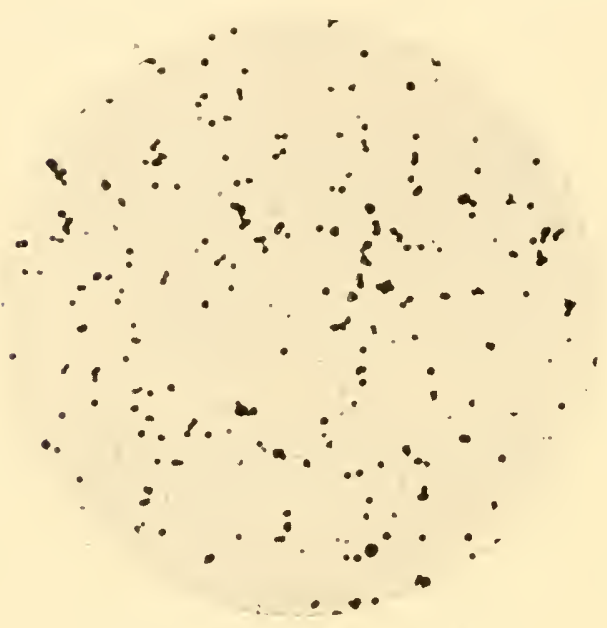

FIG. 106.-Neisseria meningitidis.

From a serum agar slope culture, 4 days, $37^{\circ} \mathrm{C}$. $(\times 1000)$.

formly. J. E. Gordon (1921) described a variety of $N$. catarrhalis in which the degenerative forms began to appear after the 4 th hour, but this is unusually early. Some workers have described the presence of Babes-Ernst bodies or metachromatic granules in members of this group. Elser and Huntoon (1909) state that the meningococcus, when stained with Loeffler's methylene blue, often shows a brightly stained central spot, whilst the remainder of the cell is scarcely coloured; with Neisser's stain the granules stain bluish-black, the cell body brown. Marx and Woithe (1900) found these granules in gonococci, but only in organisms taken from the florid stage of gonorrhoaa; they state that the whole cell may appear filled with granules. Capsules are demonstrable in some freshly isolated strains of meningococci (Clapp et al. 1935), and in the organism known as Diplococcus mucosus.

Growth Requirements.- Culturally many of the Gram-negative cocci are characterized by a reluctance to grow on ordinary media, especially fluid media. Most of the nasopharyngeal cocci will grow-though often poorly-on nutrient agar, 
but the meningococcus will not do so ; the addition, however, of a small quantity of blood or serum is sufficient to enable growth to occur. The most fastidious is the gonococcus; to cultivate this organism a great variety of media have been devised, the majority depending on the addition of some natural protein such as blood, serum, ascitic fluid, or hydrocele fluid to a basis of nutrient agar. The meningococcus and the gonococcus are not always easy to maintain in culture ; even though transplants are made every 2 or 3 days, the organisms not infrequently die out, and the strains are lost.

All the members of the group are aerobic; little or no growth occurs under strictly anaerobic conditions. Growth of the gonococcus is favoured by the addition of cystine or other source of - SH bodies (McLeod et al. 1927, Boor 1942). Many workers have stated that the meningococcus and the gonococcus grow best under a lowered oxygen pressure, and that their growth is improved by 10 per cent.

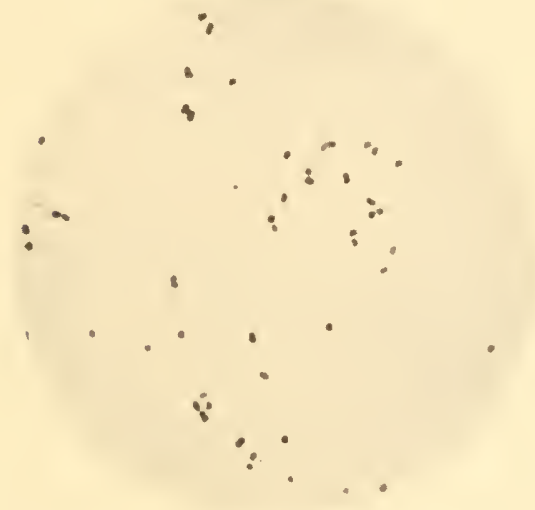

Fia. 107.-Neisseria gonorrheere.

From 'a blood agar slope culture, 24 hours, $37^{\circ} \mathrm{C} .(\times 1000)$.

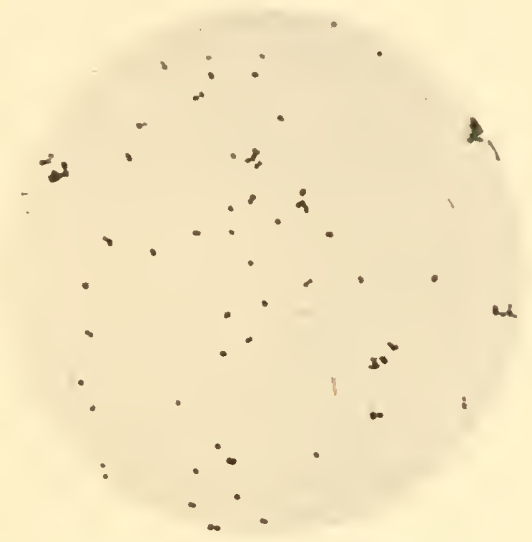

Fig. 108.-Neisseria pharyngis.

From a blood agar slope culture, 24 hours, $37^{\circ}$ C. $(\times 1000)$.

$\mathrm{CO}_{2}$ (Wherry and Oliver 1916, Chapin 1918, Kohman 1919, Ruediger 1919, Rockwell and McKhann 1921) ; but numerous other workers have failed to substantiate this (Cook and Stafford 1921, Erickson and Albert 1922, Torrey and Buckell 1922a).

When making experiments on the effect of altering the gaseous eonstitution of the atmosphere, it is very difficult to control all the factors concemed; the technique used may, for instance, ehange the moisture content of the atmosphere and the rate of evaporation from the medium. The presence of 10 per cent. $\mathrm{CO}_{2}$ alters the $\mathrm{H}$-ion concentration, and will interfere with the change in the reaction of the medium that normally occurs during growth; thus $\mathrm{CO}_{2}$ may be beneficial if the medium las been made too alkaline, and it may by its buffering action prevent the accumulation of acid. The failure to standardize these secondary faetors is probably sufficient to explain the diverse results obtained by different workers.

The work of Mcleod and his colleagues (1934), however, does suggest that growth is improved by the addition of 10 per cent. $\mathrm{CO}_{2}$, particularly that of freshly isolated strains. Glucose and glycerine have little or no beneficial effect; peptone in a 1-3 per cent. concentration seems to be favourable. The optimum $\mathrm{H}$-ion concentration for growth is about $\mathrm{pH} 7 \cdot 4-7 \cdot 6$; the limits within which growth 
will occur are comparatively narrow, but they depend largely on the constitution of the medium. The optimum temperature for all the members is $37^{\circ} \mathrm{C}$.; some of them, including the meningococcus and the gonococcus, will not grow at all below $30^{\circ} \mathrm{C}$. ; many of the nasopharyngeal cocci will grow at $22^{\circ} \mathrm{C}$, but not always on first isolation. The meningococcus forms a weak hæmolysin, reaching its maximum in trypagar cultures in about 4 days.

Some of the members-the pharyngis flava group-produce a greenish-yellow pigment on solid media, and occasionally a Gram-negative coccus is met with that forms a bright yellow pigment. Some of the Gram-negative cocci, particularly the gonococcus and the meningococcus, contain an active autolysin, which is destroyed by heating to $65^{\circ} \mathrm{C}$. for half an hour.

Cultural Characteristics.-The colonial appearances of all the Gram-negative cocci appear to be subject to considerable variation. Two different types of colony of both the meningococcus and the gonococcus have been described (Wassermann 1898, Lipschütz 1904, Atkin 1923, 1925, Cohn 1923); and S. P. Wilson (1928) and G. S. Wilson and Smith (1928) have observed and studied rough and smooth types of numerous nasopharyngeal cocci. In fluid media-broth and serum broth - growth is generally poor, and takes the form of a slight turbidity and a finely granular deposit, which disintegrates hardly at all on shaking; occasionally growth occurs on the surface.

Resistance.-The resistance of the Gram-negative cocci to inimical agencies is very low. In culture most of them die out in a few days; though if the organisms are seeded into ascitic agar stab tubes-preferably made up with 0.75 per cent. agar-prevented from drying, and kept in the incubator at $37^{\circ} \mathrm{C}$., they may live for weeks or even months. Though it is not known with certainty why the Gramnegative cocci die out in culture so quickly, it appears probable that they are killed by the amount of alkali produced; the production of $\mathrm{NH}_{3}$ and of alkaline carbonates of organic acids may apparently lower the $\mathrm{H}$-ion concentration of the medium to $\mathrm{pH} 8 \cdot 6-9 \cdot 0$, and thus bring about the death of the organisms (Phelon et al. 1927). The meningococcus and the gonococcus are killed by heating to $55^{\circ} \mathrm{C}$. in 5 minutes or less; they are very susceptible to desiccation, death occurring usually within an hour or two. Weak disinfectants, such as 1 per cent. phenol or $0 \cdot 1$ per cent. $\mathrm{HgCl}_{2}$, prove fatal in 1 or 2 minutes. The meningococcus and the gonococcus are both sensitive to the sulphonamides and to penicillin.

Biochemical Reactions.- Biochemically the members of the group are not very active; the production of acid in glucose, maltose, and sucrose is used as a means of classification. Other sugars, such as galactose, lævulose, and dextrin, are used by some workers, but those who have had most experience agree that they are unsatisfactory. Since many species of Neisseria will not grow on the ordinary peptone water sugar medium, it is necessary in testing their sugar reactions to add a small amount of serum, or to grow them on ascitic fluid agar containing litmus and 1 per cent. of the sugar. If serum is used, human or rabbit serum should be chosen, since horse, sheep and ox serum contain maltase, which may lead to a false reaction in the presence of maltose (Rosher 1936, Hendry 1938). Litmus milk is unaltered, except by the Diplococcus crassus, which turns it acid. Indole is not produced. The methyl-red test is weakly positive or frankly negative, according to whether or not the organism tested produces acid from glucose; as the increase in $\mathrm{H}$-ion concentration is rarely greater than to $\mathrm{pH} 6 \cdot 0$, the red colour 
developed with methyl red is usually faint. The Voges-Proskauer reaction is negative. Nitrates are not reduced. Catalase is produced, and all the members that have been tested give the oxidase reaction described by Gordon and M'Leod (1928).

Antigenic Structure.-Most attention has been concentrated on the meningococcus and the gonococcus. The meningococcus has been divided into four antigenic types-Types I, II, III and IV (see pp. 538-9); but the results obtained depend largely on the source from which the strains are obtained. In epidemic times most of the cocci isolated can be readily typed, but strains isolated from sporadic cases in non-epidemic times are frequently inagglutinable with any of the type sera. The gonococcus is even more irregular; clear types are difficult to establish. The majority of the strains appear to be related antigenically, and to fall more or less into one or other of two groups (Atkin 1925) (see pp. 545-7). Little work has been done on the other Gram-negative cocci ; one of the chief reasons for this is that most of them are auto-agglutinable, and homogeneous suspensions cannot be obtained. The complement-fixation test, however, seems to show that there is a group relationship between $N$. catarrhalis, N. pharyngis, the gonococcus and the meningococcus (see Oliver 1929, Price 1933).

Studies on the chemical fractionation of these organisms are still in their infancy. Boor and Miller (1934) and Miller and Boor (1934), amplifying the work of Zozaya (1931) and Zozaya and Wood (1932) (see p. 539), have extracted "nucleoproteins" and polysaccharides from various members of the group. By the precipitation reaction it was found that the nucleoproteins from the meningococcus, the gonococcus, and $N$. catarrhalis not only resembled each other closely, but also had an affinity with nucleoproteins extracted from pneumococci. Polysaccharides prepared from the meningococcus and the gonococcus reacted in high dilution with Type III antipneumococcal serum, as well as with antimeningococcal and antigonococcal serum. The polysaccharide extracted from $N$. catarrhalis reacted with antigonococcal but not with antimeningococcal serum.

Pathogenicity.-The meningococcus gives rise to rhinopharyngitis, to epidemic cerebrospinal meningitis, and to post-basic meningitis in children; by intraspinal injection of monkeys it is possible to produce a meningitis with pure cultures of the organism. The gonococcus gives rise in human beings to gonorrhoa, with all its complications, but it is impossible to reproduce this disease in animals.

Towards laboratory animals all the Gram-negative cocci behave in much the same way. Injected intraperitoneally in large doses into mice or guineapigs, they cause death in 1 to 3 days. Post inortem, there is a small amount of peritoneal exudate, and sometimes a little fibrin deposit on the organs; the spleen is slightly enlarged, and there is hyperæmia and degeneration of the viscera. The organisms can be cultivated from the peritoneal exudate, but rarely from the heart's blood. There is little multiplication of organisms inside the body; no true infection is set up, and death occurs from toxæmia. A similar result follows the injection of heat-killed organisms, though generally a rather larger dose is needed than of living cocci. It seems probable that the toxicity is due to some constituent of the nucleoprotein, since "nucleoprotein" extracted from meningococci and gonococci is almost as toxic to mice as are the dead organisms themselves (Boor and Miller 1934). 


\section{Classification}

The Gram-negative cocci, as a group, have been studied so little that it is impossible to lay down any satisfactory basis for classification. Apart from the meningococcus and the gonococcus, the definition of the different species is far from clear. This is due largely to the fact that the colonial appearances are subject to such great variation that the descriptions given of apparently the same species by different workers are often quite contradictory. The cultural descriptions, for example, of $N$. catarrhalis are most varied (Ghon and Pfeiffer 1902, Dunn and Gordon, M. H., 1905, von Lingelsheim 1906, Arkwright 1907, Gurd 1908, Elser and Huntoon 1909, Martin 1911, Netter and Debré 1911, Dopter 1921, Gordon, J. E., 1921), and the only sound basis for identification of this organism appears to be its failure to ferment any sugars. Again, in Germany and America, several chromogenic species have been described, forming a greenishyellow pigment, producing acid in glucose, maltose, and sometimes sucrose, and generally giving a smooth type of colony. In this country a large number of Gram-negative cocci have been isolated from the nasopharynx, giving the same sugar reactions, but quite devoid of pigment. Further it has been found that the

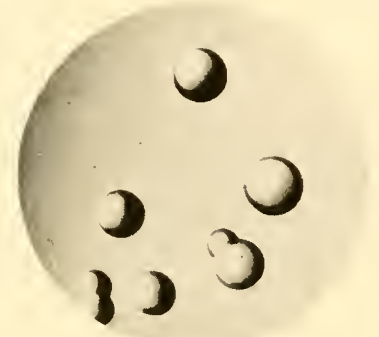

FIG. 109.-Neıseria meningitidis.

Surface colonies on serum agar, 24 hours, $37^{\circ} \mathrm{C} .(\times 8)$.

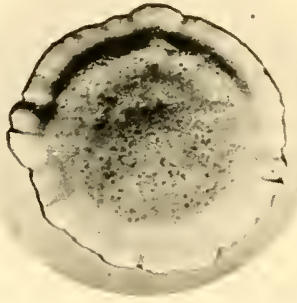

FIG. 110.- Yeisseria meningitidis.

Surface colony on serum agar, 7 days, $37^{\circ} \mathrm{C} .(\times \mathrm{S})$.

colonies formed are sometimes smooth and sometimes rough, and that an organism which gives a smooth colony on isolation may subsequently give a rough type of colony. The differentiation of those organisms giving rough colonies from N. pharyngis sicca is in our experience frankly impossible (Wilson, S. P., 1928, Wilson, G. S. and Smith 1928).

At the moment, therefore, it must be confessed that our ignorance is too great to allow of any satisfactory classification. For provisional purposes the classification on sugar reactions may be used, but this is subject to severe limitations. Briefly, it can be said that $N$. catarhalis ferments no sugars, the gonococcus ferments glucose, and the meningococcus gilucose and maltose; the other nasopharyngeal cocci give varied reactions, some being like the meningococcus, and others also fermenting sucrose. The Diplococcus crassus can be differentiated by its fermentation of lactose. When first isolated from the body the fermentative reactions of the Gram-negative cocci may be irregular. Nabarro (1917), for example, found that quite a number of meningococci from the cerebrospinal fluid of children with meningitis failed on first isolation to ferment 
either glucose or maltose. We ourselves have isolated organisms from the nasopharynx which fermented maltose, but not glucose. Other workers have observed similar irregularities in the behaviour of this group.

We append a detailed description of $N$. meningitidis and $N$.gonorrhose, together with some further notes on their differentiation; descriptions of those Gramnegative cocci which have received specific names; and a table giving particulars of the main differential criteria that have been relied upon by different workers in subdividing this group. We would add our personal opinion that there is, at present, little justification for the recognition of separate species among the Gram-negative cocci of the normal nasopharynx, with the possible exception of $N$. catarrhatis. We should, ourselves, combine the remaining types into a single species, with some appropriate name such as $N$. pharyngis, which we might define as follows:

Neisseria pharyngis.-Non-motile, Gram-negative diplococcus, arranged sometimes in tetrads and often in dense clumps. Grows on agar, giving rise to either rough or smooth colonies, which are generally coherent, tenacious, membranous, and friable, are difficult to emulsify, and are auto-agglutinable when suspended in saline. Grows in serum broth with the production of little or no turbidity, as a rule, and a coarsely granular sediment not disintegrating completely on shaking; a surface ring growth is not infrequently formed, particularly by the rough variants. A yellow, 'golden-yellow, or greenish-yellow pigment may be produced, but is variable in its appearance. The sugar reactions are subject to variation; glucose, maltose, or sucrose may be fermented with the production of acid. Aerobic; will not grow under strictly anaerobic conditions. Growth is best at $37^{\circ} \mathrm{C}$, , but will generally occur at $23^{\circ} \mathrm{C}$. Non-pathogenic on subcutaneous injection into mice; large doses intraperitoneally may cause death from toxæmia. The species is subject to great variation in colonial appearance, and, apart from the smooth and rough types, a smooth variant may occur that is of butyrous consistency and easy to emulsify, and also a mucoid variant containing eapsulated diplococei (Wilson, G. S. and Smith 1928).

\section{The Meningococcus}

Cultural Characters The meningococcus generally gives rise to a smooth typically lenticular colony. Atkin (1923), however, and more recently Rake (1933), have demonstrated the existence of colonial variants. The appearance of the colony depends on the nature of the medium, the age of the strain, and the antigenic type of the organisms. Freshly isolated strains of Group I generally form smooth colonies, which may be mucoid if the organisms are capsulated; on incubation for some days their edge may become crenated or dentate and secondary papillæ may appear on the surface. Organisms of Group II tend to form rather smaller colonies, and may assume a deep-yellowish tint on suitable media. Rough colonies, which are generally smaller than those of the smooth form, often appear in strains subjected to laboratory cultivation.

On primary isolation the meningococcus must be provided with such accessory growth factors as are present in blood, serum, milk, and other animal fluids, and in certain vegetable extracts (Lloyd 1916-17). After a few generations on such an enriched medium, it may sometimes be brought to grow on what are described as ordinary culture media, but its vitality under these conditions is uncertain (Murray 1929). Growth is usually favoured by the presence of 5 to 10 per cent. $\mathrm{CO}_{2}$. For preservation the meningococcus should be frozen and dried. If this is impossible, it should be maintained in ascitic fluid agar stabs or on Dorset egg slopes; the tubes should be corked to prevent evaporation and kept in the incu- 
bator. To conserve the virulence of the organisms they should be subcultured every two days on blood agar slopes, or preferably frozen and dried.

The meningococcus undergoes rapid autolysis; this is responsible for the swelling and loss of staining properties in cultures more than a few hours old. This property is destroyed by heating to $65^{\circ} \mathrm{C}$. for 30 minutes. If the organisms are suspended in saline, covered with toluol to prevent contamination, and incubated at $37^{\circ} \mathrm{C}$, autolysis is said to be nearly complete in 4 hours (Flexner 1907a). For this reason all suspensions intended for agglutination should be inactivated by heat.

Antigenic Structure.--Soon after the agglutination test was introduced for the identification of meningococci, it was noticed that different strains possessed varying degrees of agglutinability. Kutseher (1906), who employed the absorption test, observed that there was a marked difference between strains isolated from different sources, but he was unable to classify them by this method. In 1909 Elser and Huntoon found that 40 per cent. of meningococei were inagglutinable by a monovalent serum, and that these inagglutinable strains, which they term pseudo-meningococei, exhibited a reduced absorption capacity ; they further divided the psendo-meningococci by absorption into two sub-groups. In the same year Dopter (1909) noticed the presence in nasopharyngeal mucus of cocei resembling the meningococcus in morphology, cultural and fermentation reactions, but differing from it in their complete absence of agglutination with a meningococeal serum; these organisms he termed parameningococci. Arkwright, also in 1909, studied 25 strains of meningococei from cases oceurring in epidemic areas, and 20 strains from sporadic cases; he noticed not only that by agglutination and absorption the organisms could be roughly divided into groups, but that, serologically, the sporadic strains tended to deviate more from the type to which most strains conformed than did the epidemie strains. In 1914 Dopter and Pauron divided the parameningococei into 3 types, $\alpha, \beta$, and $\gamma$. Soon after the commencement of the War, Ellis (1915) examined 46 strains from 6 epidemic foci, and found that they fell by agglutination into 2 types, I and II, of which Type II was probably identical with Dopter's parameningococcus. Simultaneously Arkwright (1915) was able to classify 30 out of 35 strains from epidemic cases into 2 main groups, Types I and II, of which Type II, like Ellis's Type II, corresponded to Dopter's parameningococcus; of the remaining 5 strains, 3 were difficult to classify by agglutination, and 2 were intermediate between the two types. Gordon and Murray (1915) by using the absorption test found that 32 strains from the cerebrospinal fluid of epidemic cases fell sharply into 4 groups, which they called Groups I, II, III, and IV ; none of these groups, however. showed any relation to Dopter's parameningococcus. In 1917 Nicolle, Debains and Jouan (1918), using the agglutination test alone, were able to classify the meningococei into 4 types, called $\mathrm{A}, \mathrm{B}, \mathrm{C}$ and D. Gordon and Murray's Type I and III strains, as they are now generally referred to, corresponded to Dopter's Meningococcus and to Nicolle, Debain and Jonan's Type A, and their Type II and IV strains to Nicolle. Debain and Jouan's Type B. F. Griffith (1917), working at the Local Government Board laboratories, was able to divide his meningococei into two main groups by simple agglutination, Groups I and II ; his Group I corresponded roughly with Gordon and Murray's Types I and III, and his Group II with their Types II and IV. Scott (1917) similarly found that his strains fell into two groups.

Since 1918 observations, particularly in the United States, have served to show that no sharp line of demarcation can be drawn between different types of meningococci. Branham, Taft and Carlin (1931) and Branham (1932), it is true, were able to assign every one of 221 strains of meningococci isolated during a time of epidemic prevalence to one or other of Gordon and Murray's four types, but this was possible only after prolonged study involving examination of their agglntinability, their power to absorb agglutinins, and their agglntinogenic capacity. The lack of strict type specificity, and the readiness with which many strains 
undergo antigenic degradation, render classification by such means arbitrary and unconvincing. A change in the strains used for the preparation of typing sera can easily result in an apparent change in the type of organism under study.

Further observations by Branham and Carlin (1937) and others have led to the broad conclusion that two main groups can be distinguished by agglutination - Group I, which is mainly responsible for epidemic cases and tends to be antigenically homogeneous, and Group II, which is mainly responsible for sporadic cases and tends to be antigenically heterogeneous. This concept receives support from other methods of study such as chemical fractionation, and precipitation and capsular-swelling reactions.

The complement-fixation reaction has been used for classifying meningococci. Nicolle, Debains and Jouan (1918) found this reaction less specific than that of agglutination, whereas Bell (see Report 1920) and Butterfield and Neill (1920) regarded it as more specific. Evans (1920) studied the opsonin reactions of antimeningococcal serum. By this means she found that 63 strains fell sharply into 4 groups; there were 4 atypical strains. A fifth group could also be demonstrated, which was closely related to the other four; its members were able to effect a partial absorption of the sera prepared against strains of the other groups.

By chemical fractionation Rake and Scherp $(1933 a, b)$ have separated three fractions from meningococci. There is a carbohydrate or " $\mathrm{C}$ " substance, common to all meningococci and to some other micro-organisms, which is probably the same as that described by Zozaya (1931) and Zozaya and Wood (1932). There is a protein or " $\mathrm{P}$ " substance, which is also found in gonococci and Type III pneumococci. The third fraction is a sodium salt of a polysaccharide acid (Scherp and Rake 1935) and is responsible for the specificity of Types I and III strains. More recently Menzel and Rake (1942) have brought evidence to show that the specificity of Type II strains is determined by a protein substance. There appears to be no difference between the type-specific polysaccharide found in Types I and III.

The presence of the polysaccharide is generally demonstrated by the precipitation reaction, using as antigen a specially prepared extract of the organisms. Petrie (1932), however, has described a simple alternative method. It consists in growing the organisms on agar plates containing the homologous immune serum. Characteristic haloes develop around the colonies. These consist of a precipitate formed by the interaction of the specific polysaccharide, which has diffused out into the medium, with the homologous antibody.

It seems probable that the specific polysaccharide is also responsible for the capsular-swelling (Quellung) reaction demonstrated by Clapp, Phillips and Stahl (1935) in smooth Group I strains. A similar reaction with Group II strains was at first thought not to occur, but further observations by Cohen (1940) have shown that certain strains may exhibit capsular swelling and give a typical halo reaction in the presence of serum made with a capsulated Group II strain. Branham and Carlin (1942) have likewise described a separate sub-group of Group II strains, referred to as Group II alpha, which are capsulated, give a Quelling reaction, and are strongly antigenic, stimulating the production of antibodies with a specific protective action on mice.

Pathogenicity.--Mice.--In his original communication Weichselbaum (1887) observes that subcutaneous injection into mice is without effect. Injected intrapleurally with $0.5 \mathrm{ml}$. of a thick suspension from a 24-hours' agar culture, the mouse becomes ill, develops paralysis 
of the hind limbs, and dies in 1 to 2 days. Post mortem, there is a viscid, often hæmorrhagic, fluid in both pleural cavities ; the lungs are hyperæmic in places and may be covered with a false membrane; the spleen is generally enlarged and congested. The organisms are present in enormous numbers in the pus cells of the pleural exudate, and often, but not always, in the blood and the spleen, in both of which situations they remain mostly extracellular.

Intraperitoneal injection with the same dose kills the mouse in 18 to 24 hours. Post mortem, there is a small amount of sticky fluid in the peritoneal cavity, containing pus cells; the spleen is generally swollen and congested; cocci are found in varying numbers in the exudate, and in small numbers in the spleen and heart's blood.

Guinea-PIGs.-Weight for weight these animals are said to be somewhat more susceptible than mice (Rist and Paris 1904), but here again subcutaneous injection, even of massive doses, fails to give rise to a general infection; at most a small abscess is produced.

Intrapleural injection of $1 \mathrm{ml}$. of a thick suspension of a young agar culture causes death in 1 to 3 days. Post mortem, there is a thick exudate, poor in fibrin, in both pleural cavities; the lungs are thickened and dark red; the spleen is not enlarged. Cocei are found in the exudate, but are generally absent from the spleen and heart's blood.

Intraperitoneal injection causes death in 1 to 3 days. Post mortem, there is an exudate, clear or turbid, in the peritoneal cavity; on the rolled-up omentum and on the anterior surface of the liver there is a deposit of fibrin and pus; there are hæmorrhages into the mesentery, and into the visceral and parietal peritoneum; the adrenals are vividly congested and may be hæmorrhagic; the pancreas and surrounding tissues are œdematous. In the pleural cavities there is often an exudate of clear fluid; the lymphatic glands are swollen and congested. Cocci are found in moderate numbers in the peritoneal exudation, but are absent from the blood and viscera.

Sub-dural injection causes death in 20 to 24 hours; post mortem, there is cedema and congestion of the meninges, with pus at the site of injection; there is a large amount of clear fluid in the peritoneum, free from cocei (Albrecht and Ghon 1901). There is no nultiphication of organisms in the spinal fluid itself.

RABBiTs.-Subcutaneous, intrapleural, and intraperitoneal injections are generally without effect.

Intravenous injection with $1-4 \mathrm{ml}$. of a thick suspension of a young agar culture kills the animals in 1 to 4 days. Post mortem, apart sometimes from a few areas of congestion in the lungs, there is nothing abnormal to be found. No cocci are present in the blood stream.

Sub-dural injection into the skull occasionally causes death. Post mortem, there is congestion of the meninges; the cocei may be recovered in culture (IVeichselbaum 1887).

Intracisternal injection by the sub-occipital route is stated to give rise to cerebrospinal meningitis (Branham and Lillie 1932, Zdrodowski and Voronine 1932). To achieve success virulent cultures and young rabbits $(1,300-1,500 \mathrm{gm}$.$) are desirable. The disease may$ be acute and prove fatal in 24 hours, or sub-acute and cause death between the 2nd and 7 th days. Clinically, rigidity of the neck, retraction of the head, spasticity, and sensitiveness to touch, or progressive paralysis may be noted. At necropsy the brain and cord are markedly hyperæmic and are covered with a thin layer of purulent exudate. The cerebrospinal fluid may be almost clear, turbid, or frankly purulent. Meningococci can be recovered from the spinal fluid and usually also from the blood.

Doas.-Weichselbaum (1887) stated that he had succeeded, by sub-dural injection, in producing a pachy- and lepto-meningitis with acute encephalitis ; death occurred from a few hours to the 12th day after inoculation.

Monkeys. - Von Lingelsheim (1905) was apparently the first to reproduce the disease in monkeys. After intraspinal injection one monkey became ill in 6 hours; there was retraction of the head and opisthotonos, and death took place in 30 hours. At necropsy 
the pia mater was turbid along the vessels, with here and there small collections of pus. Meningococci were found in the pus and in the blood.

Flexner $(1907 b)$ made a number of experiments on monkeys, mostly Macacus rhesus. After intraspinal injection of $\frac{1}{2}$ to 1 agar slope he found that the monkeys became generally weak and apathetic; the head drooped so as to touch the floor of the cage; occasionally, however, it was retracted; death occurred in 18 hours to 4 days as a rule, and was not infrequently preceded by general convulsions. Post mortem, the chief lesions were leptomeningitis, particularly at the base of the brain, encephalitis and abscesses, hæmorrhages into the pia, inflammation of the dorsal root ganglia, and acute endarteritis of the vessels. The inflammation of the meninges extended into the membranes covering the olfactory lobes and along the dura mater into the ethmoid plate and nasal mucosa, which was often inflamed and beset with hæmorrhages. Diplococci were found in the meningeal exudate and in the nasal mucosa, but were not cultivated from the latter situation. They were also present in the sero-purulent fluid in the ventricles.

By giving small repeated doses Flexner succeeded in setting up a chronic meningitis lasting for several weeks. Post mortem, there was abundant exudate, rich in meningococci ; the foramen of Magendie was closed, and hydrocephalus and pyocephalus with ependymitis and dilatation of the ventricles were found. Neither in acute nor chronic cases did the internal organs, apart from the central nervous system, show any marked changes. Occasionally the organism is found in the blood stream.

Flexner found that not all the monkeys developed the disease. Those that did so generally died within 2 days, or else recovered after a severe illness. From his experiments it is clear that the disease is more acute in monkeys than in man.

M.Donald (1908) confirmed Flexner's results in monkeys. Though unable to infect rhesus monkeys, he obtained successes with Callithrix by sub-dural inoculation of cerebrospinal fluid from human cases of disease.

The experimental lesions which we have discussed are not produced with the regularity that one might expect, partly because the meningococci themselves vary considerably in virulence, and partly because the susceptibility of different animals-even of the same species-varies within a wide range.

The variations in virulence of the meningococcus depend on the source of origin of the strain, the length of time it has been isolated, the age of the subculture, the nature of the medium (Murray and Ayrton 1924), and doubtless on other factors. When freshly isolated, some strains are of sufficient virulence to kill mice inoculated intraperitoneally in a dose of about 100,000 organisms, but others are far less virulent. It has, however, been shown by Miller (1933, 1934-35) that if the culture is suspended in a solution of gastric mucin, as few as 2 to 10 organisms of a highly virulent strain suffice to kill a mouse. By frequent mouse passage, using organisms suspended in mucin, a given strain may be kept at its maximum degree of virulence. Alternatively it should be frozen and dried.

Toxin Production.-Flexner (1907a) pointed out that one of the earliest results of intraperitoneal inoculation into guinea-pigs was a marked fall of temperature. This is not the normal course in an acute infection, and he was led to conclude that the animals died from the effects of a poison liberated from the bodies of the organisms. Albrecht and Ghon (1901) found that filtered cultures were without effect on mice, but that 24-hour cultures heated to $65^{\circ} \mathrm{C}$. for 1 hour, when injected intraperitoneally into mice, produced death with the same picture as that found after injection of living cocci. Subsequent observers have found that the dose of dead and of living organisms necessary to kill mice is practically identical. Thus Neill and Taft (1920) found that 4,000 million living organisms injected intraperitoneally killed 6 out of 10 mice, whereas the same dose of dead cocci killed 5 out of 10 . M. H. Gordon 
(see Report 1920) showed that, when freshly isolated, the living cocci were fatal in smaller doses than the dead cocci, but after subculture for some time in the laboratory, the lethal doses tended to approximate. Petrie (1937), who has made a careful study of the endotoxin, finds that its effect on animals can be reproduced by injections of other Gram-negative cocci, and that it belongs to a group of nonspecific, non-antigenic, thermostable, bacterial poisons.

From these results, and from the fact that in animals dying from injection of living cocci the blood and viscera are frequently sterile, it would appear that the main cause of death is a toxæmia. Flexner (1907a) found that meningococci undergo very rapid autolysis in culture; as a result of this the endotoxins are liberated from the bodies of the organisms, and it is these which are responsible for the pathogenic effects in animals. This view is substantiated by the frequent occurrence of hæmorrhages on the serous membranes, of sterile transudates in the cavities of the body, and of the adrenal hæmorrhages which are found both in animals and in human beings dying from the disease (Maclagan and Cooke 1917, Petrie, 1937).

The endotoxin can be extracted from the bodies of the meningococci. One of the simplest means (M. H. Gordon, see Report 1920) is to grind $0.05 \mathrm{gm}$. of dried cocci in an agate mortar with $1.25 \mathrm{ml}$. of distilled water, to which after a few minutes $1.25 \mathrm{ml}$. of $\mathrm{N} / 20 \mathrm{NaOH}$ are added; the grinding is continued for about a minute. The cocci pass into solution on the addition of the alkali. The M.L.D. of the fluid thus obtained is generally $0 \cdot 1$ to $0 \cdot 15$ ml.- that is, an amount corresponding to about $2 \mathrm{mgm}$. of the dried cocci (see also Petrie 1937).

Though most workers have regarded the toxin of the meningococcus as essentially an endotoxin there seems to be little doubt that under certain conditions it can readily diffuse out into the medium. According to Ferry, Norton, and Steele (1931), hormone broth cultures of $\mathrm{pH}$ 6.6 incubated for 4-6 days contain a filtrable toxin, specific for each of the serological types of meningococci, as well as a group-specific toxin common to all four types. Ferry and Schornack (1934) and Maegraith (1935) have shown that these toxins, when inoculated by the intracisternal route into guinea-pigs, give rise to convulsions and death within 24 hours. It would serve no useful purpose to discuss how much of the toxic activity of these filtrates is due to substances secreted by the living organisms and how much to substances liberated from the dead organisms. There is evidence that the polysaccharide found in Types I and III is soluble in suitable media (Petrie 1932, Kirkbride and Cohen 1934), and it may be that the nucleoprotein to which Boor and Miller (1934) ascribe the toxicity of the meningococci is likewise soluble to a greater or less degree. The main conclusion is that for laboratory animals dead cocci are almost as fatal as living cocci, and that the tissue reactions are determined by toxic substances liberated from the organisms either before or after their death. (For a review of the meningococcus see Branham 1940).

\section{Neisseria meningitidis}

Synonyms.-Meningococcus; Diplococcus intracellularis meningitidis of Weichselbaum. Isolation.-From cerebrospinal fluid of patients with meningitis by Weichselbaum in 1887.

Habitat.-Strict parasite; found in nasopharynx of man. 
Morphology.-Oval or spherical cocci, $0.8 \times 0.6 \mu$, often arranged in pairs, with adjacent sides flattened; long axis of oval lies at right angles to axis joining the two cocci. In cultures great variation in size and in depth of staining occurs, due to autolysis; in the body the cocci are more regular aqd are generally intracellular. Non-motile; non-capsulated; Gram-negative.

Serum Agar Plate. 24 hours, $37^{\circ} \mathrm{C}$. Round, convex, bluish-grey, translucent, amorphous colonies, $1 \mathrm{~mm}$. in diameter, with smooth, moist, glistening surface and entire edge; consistency butyrous; easily emulsifiable. Colony is typically lenticular. Later, colonies increase in size, become more yellow and opaque, and may show a granular centre, and a radiate periphery.

Serum Agar Slope.-24 hours, $37^{\circ} \mathrm{C}$. Noderate, partly confluent, raised, greyish-yellow growth with smooth or irregular surface due to imperfect fusion of colonies; edge is undulate or made up of single colonies.

Gelatin Stab.-No growth.

Serum Broth. -24 hours, $37^{\circ} \mathrm{C}$. Poor to moderate turbidity with slight granular or viscous deposit. No surface growth.

Resistance.-Highly susceptible to inimical agencies. When dried, and kept at room temperature, cocci die in under 3 hours. Killed by moist heat at $55^{\circ} \mathrm{C}$. in less than 5 minutes. Killed by 1 per cent. phenol in 1 minute, and by $0 \cdot 1$ per cent. $\mathrm{HgCl}_{2}$ almost instantaneously. Sealed cultures kept at $37^{\circ} \mathrm{C}$. often live for 4 or 5 weeks, and occasionally for 2 or 3 months, but when kept at room temperature they generally die in a few days.

Metabolism.-Optimum $\mathrm{H}$-ion concentration is $\mathrm{pH} 7 \cdot 4-7 \cdot 6$. Optimum temperature for growth is $37^{\circ} \mathrm{C}$. ; little or no growth below $30^{\circ} \mathrm{C}$. Fails to grow on plain nutrient agar, but grows on trypagar, glucose agar, and agar to which blood, serum, or ascitic fluid has been added. Some strains show a slight formation of yellow pigment. Aerobe ; no growth under strictly anaerobic conditions ; growth favoured by 5 to 10 per cent. $\mathrm{CO}_{2}$. Produces a weak hæmolysin.

Biochemical-Produces acid, no gas, in glucose and maltose. No change in litmus milk. Catalase + ; methylene blue reduction + . M.R. - or weak + ; V.P. - ; indole $-; \mathrm{H}_{2} \mathrm{~S}-$.

Antigenic Structure.-Divided by agglutination and absorption of agglutinins into two main groups, Groups I and II. Some workers divide Group I into Types I and III and Group II into Types II and IV. Group II tends to be more leterogeneous than Group I.

Pathogenicity.-Responsible for sporadic and epidemic cerebrospinal meningitis in man. Experimentally, it is pathogenic to mice, guinea-pigs, and rabbits, if injected intraperitoneally in fairly large doses; causes death by toxæmia in 1 to 4 days; there is little or no multiplication of the organisms in the body.

\section{The Gonococcus}

Cultural Characters.-In culture the isolated cocci are round. According to Neisser (1882), as the spherical coccus grows, it becomes oval ; division occurs, and two cocci are formed, which cling closely together. These then separate a little, and each one grows and divides again, but in a plane at right angles to that of the first division, so that tetrads result. Each member of the tetrad divides in the same plane as that of the first division. The result is that four pairs of cocci are formed.

The gonococcus is the most difficult member of the group to cultivate. It was first grown by Leistikow and Loeffler (Leistikow 1882) on blood serum gelatin at $37^{\circ} \mathrm{C}$., and by Bumm (1885a) first on coagulated bovine or sheep serum at $30^{\circ}-34^{\circ} \mathrm{C}$, and later (Bumm 1885b) with more success on coagulated human serum. Since then 
a host of other media have been introduced; reference may be made to a few (Wertheim 1891, Kiefer 1895, Wassermann 1897, Thảmann 1900, Lipschütz 1904, Martin 1911, Vedder 1915, Hall 1916, Cole and Lloyd 1917, Thomson 1917, Clark 1920, Swartz and Davis 1920, Buschke and Langer 1921, Cook and Stafford 1921, Jenkins 1921, 1922, Costa and Boyer 1922, Erickson and Albert 1922, Kandiba 1922, Lorentz 1922, Torrey and Buckell 1922a, Torrey et al. 1922, Macnaughton 1923, Lebœuf 1924, Gordon, J., 1926).

According to Sordelli, Miravent and Negroni (1926), an excellent medium results from adding to nutrient agar 17 per cent. of liver extract. This extract is prepared by macerating ox liver in 5 per cent. $\mathrm{NaCl}$ solution for $2-4$ hours at $45^{\circ} \mathrm{C}$., raising the temperature gently to $60^{\circ} \mathrm{C}$., keeping it at $60^{\circ} \mathrm{C}$. for 10 minutes, and filtering first through paper, then through a Berkefeld candle. The liver extract, if kept in the ice-chest, remains potent for months.

As the result of long experience in routine cultivation, McLeod and his colleagues (1934) recommend the use of 10 per cent. heated blood agar of $\mathrm{pH} 7 \cdot 4$, prepared from broth in which the extraction of the meat has been carried out by Wright's (1933) method. The minimum amount of agar consistent with stability

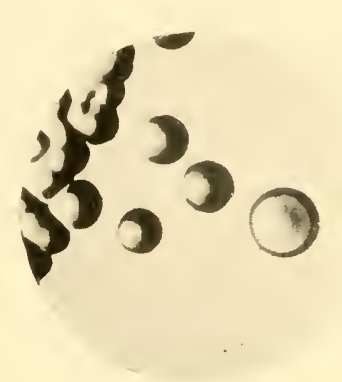

FIG. 111.-Neisseria gonorrhoece.

Surface colonies on serum agar, 24 hours, $37^{\circ}$ C. $(\times 8)$.

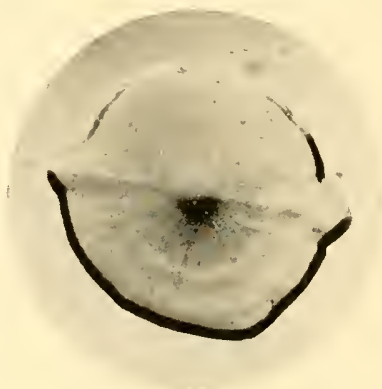

FIG. 112.-Neisseria gonorrhou.

Surface colony on serum agar, 7 days, $37^{\circ} \mathrm{C} .(\times 8)$.

is used. The cultures are incubated in air containing 8 per cent. $\mathrm{CO}_{2}$. For ordinary purposes a satisfactory medium is provided by nutrient agar containing 10 per cent. ascitic or hydrocele fluid, and having a $\mathrm{pH}$ of $7 \cdot 6$; the slopes or plates should be moist, and the air in the incubator should be saturated with water vapour. Stock cultures are best kept in a similar medium containing 0.75 per cent. agar, and put up in the form of stabs; these tubes should be corked and kept in the incubator. Media with a high amino-acid concentration are not usually satisfactory (Torrey and Buckell 1922a, Gordon, J. and M'Leod 1926), but the addition of - SH groups is beneficial (Boor 1942). Glucose does not improve growth.

Numerous workers (de Christmas 1897, Wassermann 1898, Lipschütz 1904, Gurd 1908, Martin 1911, Cohn 1923) have noticed that the cultural characters of the gonococcus are subject to variation. More recently Atkin (1925) has studied this phenomenon and found that, as with the meningococcus, there is a definite correlation between the serological type of the organism and its colonial appearance. By growing gonococci on thick trypagar plates of $\mathrm{pH} 7 \cdot 8$ he observed two different types of colony: Type I gave a large, irregularly round, flattened, translucent colony with an undulate edge, and a surface that in 5 days or so 
became covered with papillæ; Type II gave a smaller, round, low convex or raised, yellowish-white, opaque colony, with a slightly uneven surface, and an entire or faintly lobate edge; no papillse were formed. Serologically, colonies of Type I could be differentiated from those of Type II. Atkin found that the organisms in the papillæ of Type I colonies lived about twice as long as did those in the flat part; he therefore concludes that the papillæ arise as a reaction to an unfavourable environment, and represent the first step in the change over to Type II. In some colonies the papillæ actually fuse together, so that they constitute the entire colony; the papillated appearance is thus lost. During the course of subculture in the laboratory, there will be a constant selection of the longer-living organisms in the papillæ, so that eventually the strain will consist entirely of these organisms; when this process is complete, the strain will belong culturally, and probably serologically, to Type II. Type I colonies are usually observed when pus from acute gonorrhoa is plated out; Type II colonies are usually found in old laboratory cultures, or occasionally in chronic gonorrhoeal lesions.

Atkin would regard Type I as being highly parasitic, and Type II as a more saprophytic form of the gonococcus. Though he obtained evidence that, in the laboratory, Type I strains gradually acquired the properties of Type II strains, he was never able to follow the complete transition; probably this is a matter of months or years. It must not be supposed that gonococci can in practice be divided sharply into two colonial types; between the two main types there are probably numerous sub-types, each representing one stage in the process of transition. This accounts for the numerous arbitrary subdivisions that have been made on serological grounds by different workers (see below). But, broadly speaking, there appear to be two main centres around which the different strains may be grouped ; the type strains can be differentiated both by colonial form and serological behaviour. In these respects the gonococcus closely resembles the meningococcus.

Antigenic Structure.-AgGLutination.-The serological study of the gonococci may be said to have commenced with Bruckner and Cristéanu's work in 1906 (1906a). Using immune horse serum prepared by the injection of pure cultures of different strains, they found that the gonococci were agglutinated to a titre of about 1-750. A close relationship was established between the gonococcus and the meningococcus, both of which were agglutinated to nearly equal titre by a gonococcal serum (Bruckner and Cristéanu 1906b). Vannod in the same year (1906) found that immune gonococcal rabbit serum contained agglutinins for the gonococcus and to a less extent for the meningococcus. Torrey (1907) was the first to use the agglutination test for the serological differentiation of the gonococci. Working with 10 different strains and 8 immune sera, he found that there was a difference in the agglutinability of the gonococei ; and in conjunction with the absorption of agglutinins test he was able to divide the 10 strains into 3 groups. Such a differentiation was obviously of interest, but many subsequent workers failed to confirm this (Wollstein 1907, Vannod 1907, Thomsen and Vollmond 1921, Cook and Stafford 1921, Warren 1921). More recent work has, however, tended to show the essential correctness of 'Torrey's findings. Thus Pearce (1915) drew a distinction on the basis of direct agglutination between strains of gonococci isolated from infants-vulvovaginitis and ophthalmia-and those isolated from adults-acute urethritis. Hermanies (1921 $a$ ) studied 85 strains, and using the absorption of agglutinins test he was able to olassify them into 6 types, of which Types I and II contained the greatest numbers. Later $(1921 b)$ he subdivided his Type II strains into 4 races, $a, b, c$, and $d$. He found a considerable amount of lability in the antigenic structure of these races; some were simple, while others were more complex. Jötten (1921), by direct agglutination, classified 20 out of 27 strains into 4 groups, A, B, C, and D ; 7 remained ungrouped. The strains falling into Groups A and B were mostly from 
severe cases of disease with complications; those falling into Groups C and D were mostly from simple cases with no complications. He therefore established a correlation between the agglutinability and the virulence of his strains. Torrey, who together with Buckell (1922b) again studied the problem of agglutination, was able to a large extent to confirm his original conclusions. They used 77 strains of widely separated origin. By simple agglutination they were unable to obtain any definite grouping, but on the basis of absorption they found that their strains could be divided into 3 groups, which they called regular, intermediate, and irregular. The regular strains were most generalized as regards antigenic properties ; the intermediate strains were closely related to the regular types; the irregular strains exhibited marked individual variations. The regular strains were the most complex antigenically, and appeared on the whole to be more virulent for man ; the irregular strains were less complex, and appeared to be less virulent. These findings are analogous to those of Griffith with the meningococcus. Torrey and Buckell, however, point out that the antigenic structure of the gonococei is variable, and so prone to individualistic expression that grouping of the organisms into sharply defined types is not warranted.

Tulloch $(1923 a, b)$ studied 100 strains of gonococci. By simple agglutination he obtained no direct evidence of grouping, but by absorption he was able to classify them as follows:

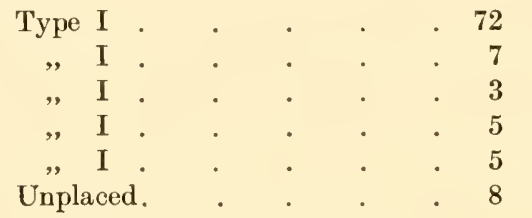

It will be seen that he classifies 92 per cent. in one group, of which 72 strains fall into one sub-group. Tulloch states that Gordon found 25 out of 30 strains by absorption of agglutinins to belong to a well-defined sub-group.

Atkin (1925) was able to classify gonococci into two serological types. Most strains isolated from cases of acute urethritis could be classified in Type I, whereas strains isolated from chronic infection, such as cervicitis or arthritis, generally belonged to Type II. Many strains agglutinated to a greater or less extent with serum of each type. Atkin suggests that in the body, during the process of a chronic infection, or in the laboratory during long periods of subcultivation, Type I may gradually change into Type II. The evidence for this, however, is admittedly inconclusive. Atkin's work does seem to reconcile to some extent the varying results of different workers, and to agree in particular with the findings of Torrey and Buckell.

Summarizing, we may say that the serological classification of the gonococcus is beset with difficulties; that there are probably two main types, of different degrees of antigenic complexity; that between these two main types there are a number of intermediate types, containing one or more antigens common to both main types; and that recently isolated strains from acute forms of the disease appear to belong chiefly to Type I, while old laboratory strains, or strains isolated from chronic disease, tend to belong to Type II.

Precipitins.-Torrey (1907) prepared a precipitinogen by filtering a 5-weeks' broth culture through a layer of sterile talc on filter paper. With this he obtained a precipitin reaction with immune gonococcal rabbit serum. Bruckner and Cristéanu macerated a culture in $0 \cdot 15$ per cent. $\mathrm{NaOH}$, which dissolved the gonococci in a few minutes; the solution was filtered through porcelain. They found a close relation between the gonococcus and the meningococcus; thus anti-gonococcal horse serum contained precipitins for both organisms; similarly with antimeningococcal goat serum. 
Complement Fixation.--Bruck (1906) was the first to study the presence of immune bodies in artificially prepared gonococcal serum. He showed that the injection of watery extracts of gonococe into rabbits ealled forth the production of specific antibodies, which were detectable by the complement-fixation test. Vannod (1906) found the complement-fixation test to be more suitable than agglutination for distinguishing between the gonococcus and the meningococcus, since no group reaction was apparent. Watabiki $(1910)$ was likewise able to distinguish between these two organisms by the complement-fixation test. Teague and Torrey (1907) showed that the serum of an animal immunized to one strain of gonococcus might not cause fixation of complement when tested against another strain; they concluded that the gonococei formed a heterogeneous family. Martin (1911) found both specific and group antibodies for the gonococcus and the meningococcus in their corresponding sera. The work of Oliver (1929) and Price (1933) indicates the existence of a group relationship between the gonococcus and certain other members of the Neisseria group. Pearce (1915) divided her strains into 2 groups.

Jötten (1921) found that the results of the complement-fixation test ran parallel with the agglutination test; by both he was able to place 20 out of 27 strains in 4 separate groups, A, B, C and D. Cook and Stafford (1921), on the other hand, were unable by the complement-fixation test to obtain any evidence of grouping amongst the gonococci. Thomsen and Vollmond (1921), though unable to classify their strains by direct agglutination, succeeded by employing the complementfixation test, with previous absorption of the sera, in dividing 26 strains into 4 different groups, $a, b, c$ and $d$. Of these, the group $b$ strains were most toxic for rabbits.

Bactericldins, Opsonins.-Torrey (1908), who studied the bactericidins present in normal and immune rabbit serum, found that gonococei varied in their susceptibility to these bodies. His experiments indicated too that there was a parallelism in the specificity of the results obtained by the complement-fixation and the bactericidin tests, both of which demonstrated the heterogeneity of the gonococcus group. The bactericidal action of the sera did not run parallel with the agglutination results. Martin (1910) found that the normal serum of the guinea-pig, rabbit, cat and man, was bactericidal to both gonococci and meningococci, though the action of guinea-pig and of human serum was more marked with meningococei than with gonococci. In immune sera the bactericidins were found to be relatively specific ; an immune serum prepared against the meningococcus had but little effect on the gonococcus, and vice versa. Jötten (1921), who divided the gonococci by agglutination and complement fixation into 4 groups, A, B, C and D, found that the strains of $\mathrm{A}$ and $\mathrm{B}$ groups were more resistant to the opsonins, tropins, and bactericidins present in normal serum than were the strains of $\mathrm{C}$ and $\mathrm{D}$ groups. This was correlated with the greater toxicity of the A and B groups to human beings.

The serological study of the gonococci therefore shows that the group is not absolutely homogeneous; that there are certain sub-groups in it; that these sub-groups are not very clearly defined; and that there is a close relation between the gonococcus and the meningococcus.

Later work, on the chemical fractionation of the gonococcus, by Boor and Miller (1934, 1944) and Miller and Boor (1934) suggests that the gonococci contain polysaccharide and nucleoprotein substances shared in part by other members of the Neisseria group (see also Stokinger et al. 1914). Casper $(1937 a, b)$, who recognizes two main antigenic types, has been able to separate a specific protein-free polysaccharide-like substance from each type, and to obtain type-specific allergic skin reactions with them in human subjects suffering from gonorrhœa. 
Pathogenicity.-The gonococcus is not only a strict parasite, but it is a specifically human parasite. Of the numerous attempts which have been made to reproduce gonorrhcea in animals other than man, not one has been successful (Leistikow 1882, Neisser 1882, Bumm 1885a, Nicolaysen 1897). Neither inoculation of pus nor of pure cultures on to the mucosa of the urethra or conjunctiva gives rise to disease, even in the anthropoid apes. Experiments on the lower animals, however, have shown that the injection of gonococci directly into the peritoneum or the blood stream frequently proves fatal, and that severe local inflammation may be set up by injecting the organisms into the anterior chamber of the eye or into a joint cavity.

Mice.-Subcutaneous injection of pure cultures is without effect. Intraperitoneal injection of a saline suspension of a young serum agar culture is often fatal in 24 hours. Post mortem, there is slight congestion of the peritoneum, and sometimes a small amount of viscous exudate, containing pus cells. Gonococci are found in varying numbers, both intra- and extra-cellular in position. They may frequently be cultivated from the peritoneum, and occasionally from the heart's blood. With smaller doses, many of the mice do not succumb for 2 to 3 days; at necropsy in these mice it is rare to find gonococci microscopically, and cultures are uniformly sterile. Some mice survive without showing signs of illness. Almost exactly the same results follow the injection of cultures which have been killed by heating to $70^{\circ} \mathrm{C}$. for 1 hour (Wassermann 1898). There is no increase of virulence by passage.

GUINEA-PIGS.-Intraperitoneal injection of $5 \mathrm{ml}$. of a 6-days' serum broth culture (Nicolaysen 1897), or of a 24-hours' growth on a Blake bottle of serum glucose agar (Wollstein 1907), kills the animals in 24 hours as a rule. Post mortem, there is congestion of the serosa, with small hæmorrhages; a little clear or turbid fluid in the peritoneal cavity ; œdema of the pancreas and surrounding tissues; congestion or hæmorrhage into the adrenals; a layer of fibrin and pus over the liver, spleen and omentum; sometimes clear fluid in the pleural cavities. Films from the peritoneum and omentum show varying numbers of polymorphonuclears, and diplococci situated intra- and extra-cellularly. Cultures from the peritoneum are generally positive. It will be seen that the post-mortem findings are similar to those following injection of guinea-pigs with meningococci; as a rule, however, larger doses of gonococci are required to produce the same effect. After some generations in vitro the gonococci lose their virulence, and become innocuous to guinea-pigs.

RaBBits.-Subcutaneous injection of $10 \mathrm{ml}$. of a 4 to 5 -days' serum broth culture (Maslovski 1900) gives rise to slight inflammatory swelling after 24 hours ; later suppuration occurs, so that in 10 days a small abscess is formed, containing thick, sterile pus. The temperature rises somewhat, and the animal loses weight. The same result follows the injection of heat-killed cultures. Smaller doses are without effect.

Intraperitoneal injection of large doses of gonococci, washed off young serum agar cultures, kills the animal in 24 hours. Post mortem, there is some peritoneal reaction, and the organisms may be cultivated from the peritoneum, and occasionally from the heart's blood. Bruckner and Cristéanu (1906c) claimed to have raised the virulence to such an extent that a rabbit injected intraperitoneally with $1 / 20$ of a serum agar slant died in 2-10 hours. These results have not been confirmed.

Intravenous injection produces fatal results with smaller doses. The gonococci may be recovered from the blood after 24 , sometimes after 48 hours. The results of different workers are, however, at variance. Vannod (1907), for example, injected living cocci in a dose of $5 \mathrm{ml}$. of an ascitic peptone broth culture intravenously into rabbits, and obtained practically no reaction.

Maslovski (1900) injected a few drops of a 3-day's serum broth culture into the anterior chamber of the eye of rabbits. The following day there was diffuse turbidity of the cornea, 
accompanied by hypopyon; in the pus cells gonococci could not be detected microscopically, but could be cultured for the first two days.

Nicolaysen (1897) injected an aqueous suspension of gonococci into the knee joint of a rabbit. Arthritis followed with abundant purulent exudate, which persisted for a week; no organisms could be demonstrated in it, however. The same result occurred when a culture killed by heat at $70^{\circ} \mathrm{C}$. for 1 hour was injected.

From these results it is seen that the gonococcus does not live for long in the animal body; cultures are rarely positive after 2 days, and then only when the injections are made directly into serous cavities or the blood stream. In fact it is doul,tful whether true proliferation occurs at all. De Christmas (1897), for instance, found that if the cocci were introduced, enclosed in collodion sacs, into the peritoneal cavity of laboratory animals, they failed to grow. The reaction following the injection of gonococci is due therefore, not to a true infection, but to a toxic action of the organisms. That this is true is abundantly clear from the numerous experiments on gonotoxin.

Toxin Production.-De Christmas (1897, 1900) recorded experiments from which he concluded that the gonococcus forms a true exotoxin. His results have not, however, been confirmed by other workers. Wassermann (1898), for instance, found that heat-killed cultures were as fatal to mice, injected intraperitoneally, as living cultures; on the other hand, filtered cultures proved innocuous, unless a filtrate of a 2 to 3 weeks' culture was used; even then, the filtrate was never as toxic as the whole culture. He grew the gonococci in 33 per cent. ascitic broth in a thin layer of fluid in flat-bottomed flasks. After a week, the cocei were collected, dried, and ground in an agate mortar; weighed quantities of the powder were suspended in water, and sterilized by steaming or in the autoclave. Injected intraperitoneally into mice this powder was fatal in a dose of $0.01 \mathrm{gm}$., death occurring in 24 hours or later. Injected into rabbits or guinea-pigs subcutaneously, it caused widespread doughy infiltration, often passing on to necrosis. Injected into the anterior chamber of the eye of rabbits, it caused corneal turbidity, hypopyon, and sometimes complete destruction of the eye. He was unable to extract the toxin by heat, by distilled water, or by $\mathrm{N} / 10 \mathrm{NaOH}$; the cocci still remained toxic. The toxin was not destroyed in the bacterial bodies by drying or by heat at $120^{\circ} \mathrm{C}$.; it was not destroyed by absolute alcohol, or by prolonged boiling; nor was he able to immunize rabbits or mice against it. These results point strongly to the conclusion that the toxin is an endotoxin - a body contained in the cell substance, and adhering strongly to it.

Schäffer (1897) found that filtered ascitic broth cultures, 2 to 6 days old, were without effect when injected in large doses-3-10 $\mathrm{ml}$.- -into guinea-pigs and rabbits. Maslorski (1900) likewise found that the filtrate of a 9-days' serum broth culture, injected subcutaneously into rabbits, gave rise to nothing but a slight rise of temperature and loss of weight. He concluded too that the toxic substance was an endotoxin. Scholtz (1900) came to the same conclusion. On the other hand, Vannod (1907) working with filtrates of 17-20-days' cultures in de Christmas's medium - concentrated veal broth containing 75 per cent. of ascitic fluid - found that they were fatal to rabbits injected intraperitoneally. Post mortem, there was a purulent effusion into the peritoneal cavity ; the serosa was congested and covered with purulent deposits; the suprarenals were enlarged and hyperæmic. Vannod, however, used large and repeated doses-a total of 18 to $24 \mathrm{ml}$. in 10 days to 3 weeks.

We may conclude that the gonococcus contains a toxin which can be extracted by grinding the dried organisms and suspending the resultant powder in water. In cultures in fluid media the toxin may be liberated from the cocci by autolysis; after 2 or 3 weeks' incubation, the organisms may have autolysed to such an extent that a certain amount of toxin may be present in filtrates of the cultures. The available evidence leaves little doubt that the toxin belongs to the class of so-called endotoxins. 


\section{Neisseria gonorrhœæ}

Synonym.-Gonococcus.

Isolation.-First described by Neisser in 1879; first cultivated by Bumm $(1885 a, b)$, and Leistikow and Loeffler (Leistikow 1882).

Habitat.-Strict parasite of man. Found in genito-urinary system of patients suffering from gonorrhøa.

Morphology.-Oval or spherical coccus ; $0.8 \mu \times 0.6 \mu$, frequently arranged in pairs, with adjacent sides flattened or slightly concave, resembling a pair of kidney beans; long axis of oval lies at right angles to axis joining the two cocci. In cultures great variation in size and in depth of staining occurs, due to autolysis; in the body the cocci are more regular, and are generally intracellular. Non-motile, non-capsulated, Gram-negative.

Serum Agar Plate. -24 hours, $37^{\circ} \mathrm{C}$. Round, convex, or slightly umbonate, greyishwhite, translucent, amorphous colonies, $0.5-1 \mathrm{~mm}$. in diameter, with smooth, glistening surface and entire edge; consistency butyrous or slightly viscid ; fairly easily emulsifiable. Later, colonies increase in size, and may develop a roughened surface and a crenated edge.

Serum Agar Slope. -24 hours, $37^{\circ} \mathrm{C}$. Rather poor, partly confluent, raised, greyishyellow growth with smooth surface; edge entire or formed of single colonies. Consistency often viscid.

Gelatin Stab.-No growth.

Serum Broth. -24 hours, $37^{\circ} \mathrm{C}$. Very poor growth with little or no turbidity, and a slight granular deposit, partly disintegrating on shaking.

Resistance.-Highly susceptible to inimical agencies. When dried, the cocci succumb in an hour or two. Killed by moist heat at $55^{\circ} \mathrm{C}$. in less than 5 minutes, and at $42^{\circ} \mathrm{C}$. in 5-15 hours (Carpenter et al. 1933). In serum cultures they are killed by $1 / 4000$ $\mathrm{AgNO}_{3}$ in $7 \frac{1}{2}$ minutes, and in pus in 2 minutes. Sealed cultures kept at $37^{\circ} \mathrm{C}$. may live for 4 or 5 weeks; when kept at room temperature, they die in a day or two.

Metabolism.-Optimum H-ion concentration for growth is $\mathrm{pH} 7 \cdot 5$. Optimum temperature for growth is $37^{\circ} \mathrm{C}$.; no growth under $30^{\circ} \mathrm{C}$. or over $38.5^{\circ} \mathrm{C}$. Fails to grow on plain agar as a rule; requires the presence of serum, blood, ascitic fluid, or hydrocele fluid; glucose is not beneficial. Aerobic, but growth is said to be improved by a lowered oxygen pressure, by presence of $-\mathrm{SH}$ groups, and by 10 per cent. of $\mathrm{CO}_{2}$ in the atmosphere. Little or no growth under strictly anaerobic conditions.

Biochemical.-Produces acid, no gas, in glucose. No change in litmus milk. Catalase + . M.B. reduction - M.R. - ; V.P. - ; indole - ; $\mathrm{H}_{2} \mathrm{~S}-$.

Antigenic Structure.-No clear definition into separate serological types; most strains appear by agglutination and absorption of agglutinins to belong to one or other of two main groups; numerous other less important groups.

Pathogenicity.-Responsible for gonorrhœa and ophthalmia neonatorum in man. Experimentally, it proves fatal to mice, guinea-pigs, and rabbits, if injected in large doses intraperitoneally; there is little or no multiplication of the organisms in the body.

Differentiation of the Gonococcus from the Meningococcus.-Morphologically the two organisms are very similar. In the body, both occur chiefly in pairs situated intracellularly. It is sometimes stated that the adjacent sides of the meningococci are flattened, whereas those of the gonococcl are concave, thus leaving an oval space between the two organisms. Possibly the meningococcus is slightly larger than the gonococcus in the body, though smaller in a 24-hours' culture in the laboratory (Wollstein 1907). Culturally the gonococcus is more dysgonic; it grows more slowly, forms smaller colonies, and grows on a narrower range of media than the meningococcus; the colonies are slightly viscous and do 
not emulsify so readily; colonies of the meningococcus, on the other hand, are butyrous and emulsify with the greatest of ease. Biochemically, the meningococcus produces acid in glucose and maltose, the gonococcus in glucose only; as pointed out on page 534 a medium containing human or rabbit serum should be used for testing the fermentation of maltose. The agglutination test is only of limited value, since there exists a group relationship between the two organisms (Bruckner and Cristéanu 1906b, Wollstein 1907, Elser and Huntoon 1909, Gordon, II. H., 1925). The meningococcus is more toxic to animals; tested by intraperitoneal injection on mice or guinea-pigs a smaller dose of meningococci than of gonococci is required, whether alive or dead, to cause death.

$\mathbf{N}$. catarrhalis. - This organism has been variously described by different workers. Ghon and Pfeiffer (1902), who first studied it fully, stated that in sputum it occurred in pairs, tetrads, or occasionally small groups; the organisms were shaped like coffee-beans, and were both intra- and extra-cellular. In culture they appeared larger, were generally in tetrads, and stained evenly. On agar after 24 hours, the colonies resembled in size those of Streptococcus pyogenes; they were convex, whitish-grey, with a glistening surface and an eaten edge. After 3 or 4 days they were 3 to $4 \mathrm{~mm}$. in diameter, and were differentiated into a prominent, more elevated, opaque, slightly brownish centre, and a thinner, grey, transparent, wave-like periphery with a crenated edge; the consistency was friable, and in saline they auto-agglutinated. A more or less similar description was given by von Lingelsheim (1906); he stated that the colony was smaller than that of a meningococcus colony, and that even on ascitic agar the diameter never exceeded 1-2 mm. Elser and Huntoon (1909) described two types of colony, one resembling Ghon and Pfeiffer's description, the other like a small meningococcus colony. J. E. Gordon (1921) described four types of colony : (1) like Ghon and Pfeiffer's; (2) similar to the first, but coloured pale yellow ; (3) small, flat, grey, translucent, amorphous colonies with a smooth glistening surface and entire edge; (4) almost pin-point, transparent glistening colonies with a smoothly rounded edge; morphologically, these consisted of giant cocci showing metachromatic staining. It seems clear that the colonial appearance of $N$.catarrhalis is subject to variation; in all probability both smooth and rough types are formed, similar to those of the meningococcus and the gonococcus. In gelatin stab culture there is a poor growth confined to the upper part of the tube; there is no liquefaction. There is no turbidity in broth, but a granular deposit is formed ; if the tube is kept still, a surface membrane may appear. Growth occurs within a range of $18^{\circ}$ to $42^{\circ} \mathrm{C}$, the optimum being at $37^{\circ} \mathrm{C}$. There is no development under anaerobic conditions. Growth is favoured by blood, serum, and ascitic fluid, but not by glycerine. The organism appears to be more resistant than the meningococeus or gonococcus; cultures are said to live for 4 or 5 months at $21^{\circ} \mathrm{C}$., if prevented from drying; the organisms may live in dried sputum for 27 days; they are killed by heating to $65^{\circ} \mathrm{C}$. for 30 minutes. No sugars are fermented. The virulence of this organism to laboratory animals is low. The rabbit is resistant, but guinea-pigs injected intraperitoneally with large doseshalf to one agar slope-die of toxæmia in about 24 hours. At post mortem there is a mild degree of peritonitis, slight enlargement of the spleen, and hyperæmia and degeneration of the viscera ; the organisms may be recovered from the peritoneal exudate, but rarely from the heart's blood. Heat-killed cultures are almost as fatal as living ones. 
N. pharyngis flava, i, ii and iii.-A number of Gram-negative cocei have been described whose characteristic feature is the formation of greenish-yellow colonies on agar or ascitic agar. von Lingelsheim (1906) and some other workers have called them Micrococcus or Diplococcus pharyngis flavus, and have differentiated them on the basis of colonial appearance and sugar reactions into three groups, i, ii and iii (Martin 1911, Report 1916, Dopter 1921). Elser and Huntoon (1909) have called them chromogenie cocei, and have divided them similarly into three groups $\mathrm{i}$, ii and iii; J. E. Gordon (1921) has likewise called them chromogenic cocei, and has divided them into six groups, i to vi. Elser and Huntoon's chromogenic iii and Gordon's chromogenic iii agree with von Lingelsheim's flava iii in fermenting glucose and maltose only; Elser and Huntoon's chromogenic ii and Gordon's iv agree with von Lingelsheim's i and ii in fermenting glucose, maltose and lævulose; Elser and Huntoon's chromogenic i agrees

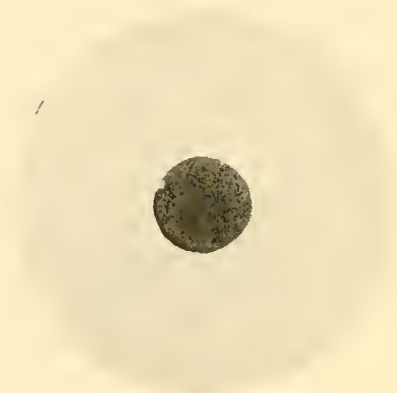

Fıg. 113.-Neisseria pharyngis.

Surface colony on agar, 24 hours, $37^{\circ} \mathrm{C}$. $(\times 8)$. Smooth type. with Gordon's chromogenic $\mathrm{v}$ in fermenting glucose, maltose, lævulose, and sucrose; but has no counterpart in von Lingelsheim's classification. Over and above these are Gordon's chromogenic i, which ferments glucose only, his chromogenic ii, which ferments glucose and lævulose, and his chromogenic vi, which ferments glucose, maltose, lævulose, sucrose, and lactose. Undoubtedly one of the reasons for the discrepancies in the sugar reactions is due to the fact that von Lingelsheim, who described the flava group, read all his reactions after 24 hours; this probably explains why he never observed the fermentation of sucrose. In colonial appearance von Lingelsheim's fava i and iii resemble the meningococcus, and flava ii $N$. catarrhalis ; Elser and Huntoon's chromogenic ii agrees with von Lingelsheim's flara i and iii. Gordon describes his organisms as giving colonies either like the meningococeus or like $N$. catarrhalis; his group vi gives pale yellow colonies, larger and more opaque than the others. In the face of these divergencies in cultural and biochemical reactions, it is clear that no fixed types can be described. Our own work has shown that the cultural appearances of the Gram-negative cocci from the nasopharynx are subject to great variation, and most workers agree now that lævulose is an unreliable sugar. Division therefore of these organisms either on

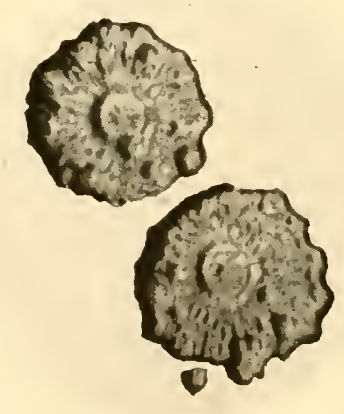

Fia. 114.-Teisseria pharyngis.

Surface colonies on agar, 5 days, $37^{\circ} \mathrm{C}$., showing differentiation. Secondary rough type $(\times 8)$.

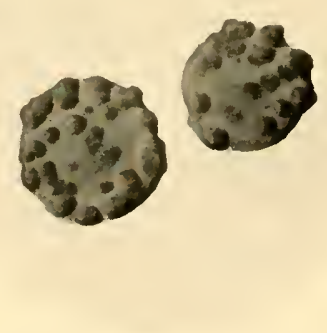

F16. 115.-Neisseria pharyngis.

Surface colonies on agar, 5 days, $37^{\circ} \mathrm{C}$., showing formation of secondary papillæ $(\times 8)$. 
colonial appearance or on the basis of lævulose fermentation is most unsatisfactory. We must await further work before a classification can be attempted.

N. pharyngis sicca.-According to von Lingelsheim (1906) this organism consists of fine Gram-negative diplococci. On agar it gives rise to an irregularly round, raised, opaque, slightly yellowish colony, up to $3 \mathrm{~mm}$. in diameter, with a dull, dry, deeply furrowed surface, and a crenated edge; the colony is very firm, often adherent to the medium, difficult to disintegrate, and impossible to emulsify; it is so coherent that it can be picked up bodily. von Lingelsheim says that it produces acid in glucose, maltose, and levulose, but other workers have found that it also ferments sucrose (Elser and Huntoon 1909, Gordon, J. E., 1921). It seems doubtful whether this organism should be regarded as a distinct species. Our own work suggests that it is merely a rough variant of one of the other nasopharyngeal cocei which ferments glucose, maltose, and sucrose. We have observed the formation by cocci giving these sugar reactions of smooth colonies when first isolated from the nose, and the appearance in later cultures of typically rough colonies indistinguishable from those described as being characteristic of $N$. pharyngis sicca.

N. pharyngis cinerea.-von Lingelsheim (1906) described this organism as consisting of plump cocei, arranged in pairs or more usually loose heaps. On agar it forms small, round, grey or greyish-white colonies, 1-1.5 mm. diameter, with an entire edge; under a low magnification their colour is brownish, and they appear coarsely granular. Some authors state that the colonies are dry, brittle, and opaque (Netter and Debré 1911). It ferments no sugars. This organism closely resembles $N$. catarrhalis and is probably merely a variety of it; it corresponds closely in description to Gordon's $\mathrm{N}$. catarrhalis sub-group III (Gordon, J. E., 1921).

Diplococcus mucosus. - This organism, which was isolated by von Lingelsheim (1906, 1908) from the nasopharynx and cerebrospinal fluid, is distinguished by its capsulation, growth on plain agar, growth at room temperature, and formation of mucinous colonies. Microscopically it consists of small diplo- and tetracocei surrounded by true capsules. Colonies are $1 \cdot 5-4 \mathrm{~mm}$. in diameter, convex, yellowish-grey, opaque, mucinous, and easily emulsified. Gelatin is not usually liquefied. McFarlan's (1941) and Bray and Cruickshank's (1943) strains grew on MacConkey's agar, fermented glucose only, and did not liquefy gelatin. Cowan's (1938) two strains grew on MacConkey's agar, produced late acid from lactose, and acid and clot in litmus milk. Most strains seem to be pathogenic for mice. The systematic position of this organism is still in doubt. It may be noted that an organism resembling Diploccus mucosus is not infrequently present in infected wounds and burns.

Diplococcus crassus.-This organism is not easy to define. It was first described by Jaeger (1895) as a meningococcus, but as it was said to form long chains in culture, to be Gram-positive in the cerebrospinal fluid and in pure cultures, to be able to grow on gelatin at room temperature, and to be highly resistant to drying, it is certain that it was not the meningococcus (Jaeger 1903a). On agar it forms rather small, greyishwhite, granular colonies, 1-1.5 mm. in diameter, with an entire edge. Growth occurs at $20^{\circ} \mathrm{C}$. Microscopically it consists of plump diplo- and tetracocci, some of which are Gram-positive and some Gram-negative; it appears in reality to be a Gram-positive organism that is very easily decolorized. Its sugar reactions differentiate it from all the other members of the group, since in addition to fermenting glucose, maltose, lærulose, and sucrose, it ferments lactose. It is said to be sometimes agglutinated by anti- 


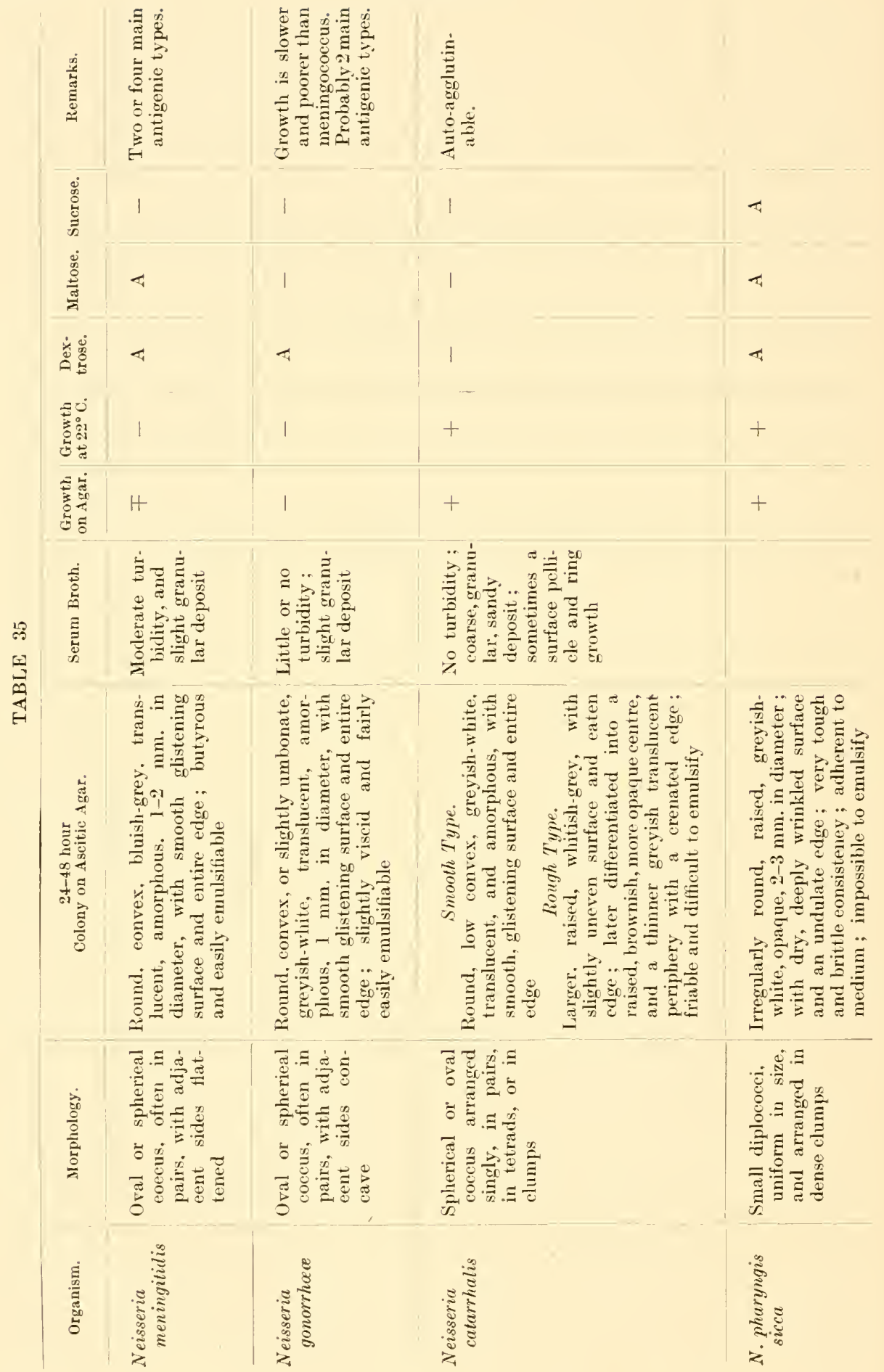




\begin{tabular}{|c|c|c|c|c|c|}
\hline & & & 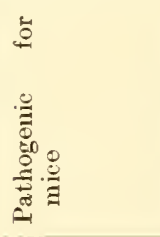 & 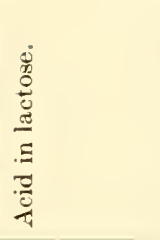 & 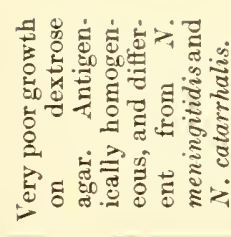 \\
\hline 1 & य & 1 & 1 & 4 & 1 \\
\hline$\pi$ & $\overrightarrow{4}$ & $\pi$ & 1 & 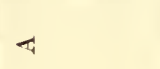 & 1 \\
\hline 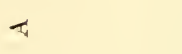 & द & 4 & $\checkmark$ & $\Psi_{4}$ & 1 \\
\hline+ & 1 & $H$ & + & + & $\sim$ \\
\hline+ & 1 & $H$ & + & + & 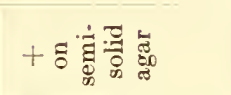 \\
\hline 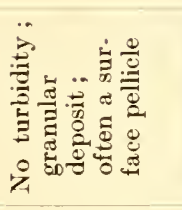 & 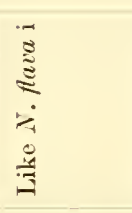 & 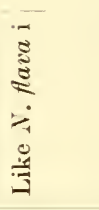 & 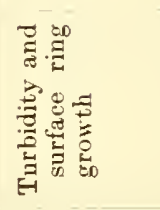 & & \\
\hline 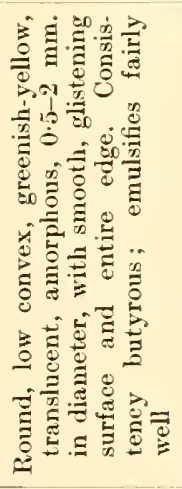 & 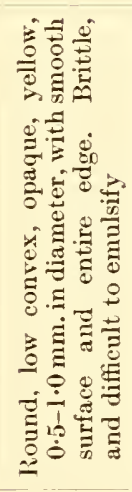 & 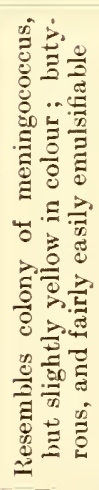 & 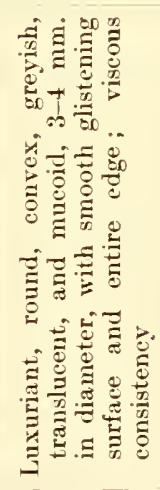 & 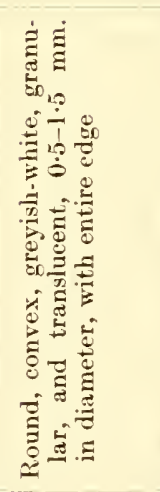 & 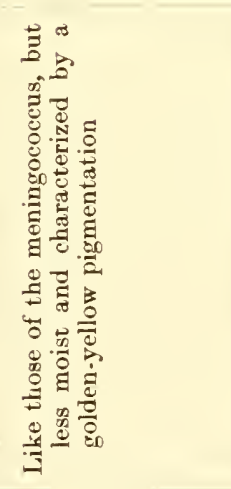 \\
\hline 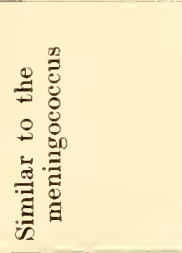 & 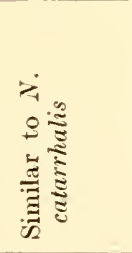 & 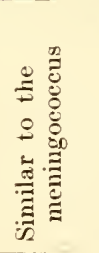 & 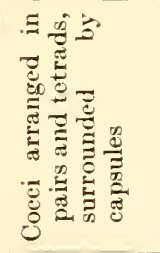 & 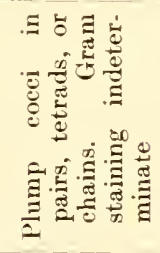 & 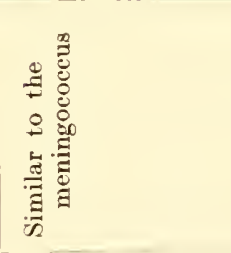 \\
\hline$\frac{\tilde{z}}{\tilde{z}}$ & 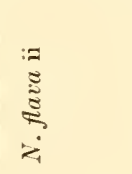 & 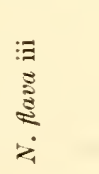 & 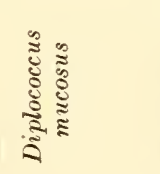 & 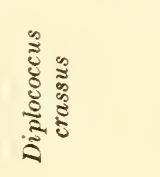 & 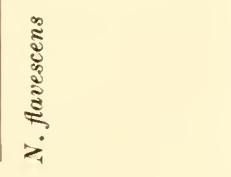 \\
\hline
\end{tabular}


meningococcal serum (Jaeger 1903b, $c$, Netter and Debré 1911, Dopter 1921). According to von Lingelsheim (1906) an antimeningococcal serum agglutinates Dip. crassus almost or quite to titre, whereas an anti-crassus serum agglutinates the meningococcus to only $\frac{1}{3}-\frac{1}{16}$ titre. According to Jaeger (1899) dricd cultures remain alive for 3 or 4 months.

N. flavescens.-This organism was isolated by Branhan (1930) from the spinal fluid of a number of patients suffering from epidemic cerebrospinal meningitis. It differs from the meningococcus chiefly in its production of pigment, its lack of fermentative action, and its antigenic constitution. In the spinal fluid it appears in the form of Gram-negative oval-shaped cocci arranged in flattened pairs; individual cells vary in size and depth of staining; giant forms are common. The organisms grow well on blood agar and semi-solid agar, but poorly on dextrose agar. They produce a golden-yellow pigment. They are without fermentative action on any of the usual carbohydrates. They are not agglutinated by type antimeningococcal sera, but constitute among themselves a serologically homogeneous group. Their ability to give rise under favourable conditions to meningitis appears to be unquestioned.

\section{REFERENCES}

Alereoht, H. and Ghon, A. (1901) Wien. klin. Wschr., 14, 984.

Arkwright, J. A. (1907) J. Hyg., Camb., 7, 145 ; (1909) J.Hyg., Camb., 9, 104; (1915) Brit. med. J., ii, 885.

Atkin, E. E. (1923) Brit. J. exp. Path., 4, 325 ; (1925) Ibid., 6, 235.

Boor, A. K. (1942) Proc. Soc. exp. Biol., N.Y., 50, 22.

Boor, A. K. and Hilter, C. P. (1934) J. exp. lled., 59, 63 ; (1944) J. infect. Dis., 75, 47.

Branhan S. E. (1930) Publ. Hlth. Rep., Wash., 45, 845; (1932) J. Immunol., 23, 49; (1940) Bact. Rev., 4, 59.

Branhait, S. E. and Carlin, S. A. (1937) J. Bact., 34, 275 ; (1942) Proc. Soc. exp. Biol., N.Y., 49, 141 .

BranhaM, S. E. and Lillie, R. D. (1932) Publ. Hlth Rep., Wash., 47, 2137.

Branham, S. E., Taft, C. E., and Carlin, S. A. (1931) Publ. Hlth Rep., Wash., 46, 897.

Bray, P. T. and Cruickshank, J. C. (1943) Brit. med. J., i. 601.

BRणCK, C. (1906) Dtsch. med. W schr., 32, 1368.

Brdckner, J. and Cristéand, C. (1906a) C. R. Soc. Biol., 60, 846 ; (1906b) Ibid., 60, 907; (1906c) Ibid., 60, 942.

Вомм, E. (1885a) Dtsch. med. Wschr., 11, 508; (1885b) Ibid., 11, 910.

BUSChKe, A. and Lavger, E. (1921) Dtsch. med. Wschr., 4\%, 65.

Butterfield, C. T. and Neill, M. H. (1920) Bull. U.S. hyg. Lab., No. 124, p. 11.

CARPENTER, C. M., Boak, R. A., MUCCI, L. A., and WarRen, S. L. (1933) J.Lab.clin. Med., 18,981 .

CASPER, W. A. (1937a) J. Bact., 34, 353; (1937b) J. Immunol., 32, 421.

Chapin, C. W. (1918) J. infect. Dis., 23, 342.

Christmas, J. De. (1897) Ann. Inst. Pasteur, 11, 609; (1900) Ibid., 14, 331.

Clapp, F. L., Philutes, S. W., and Stahl, H. J. (1935) Proc. Soc. exp. Biol., N.Y., 33, 302.

Clark, G. W. (1920) J. Bact., 5, 99.

Cohen, S. M. (1940) J. infect. Dis., 6\%, 74.

Coнs, A. (1923) Klin. Wschr., 2, 873.

Cole, S. W. and Lloyd, D. J. (1917) J. Path. Bact., 21, 267.

Cook, M. W. and StafFord, D. D. (1921) J. infect. Dis., 29, 561.

Costa, S. and Boyer, L. (1922) C. R. Soc. Biol., 8\%, 856.

Cowas, S. T. (1938) Lancet, ii. 1052.

Dopter, C. (1909) C. R. Soc. Biol., 6\%, 74; (1921) “L'Infection Méningococcique." Paris.

Dopter and PAURoN. (1914) C. R. Soc. Biol., 7\%, 231. 292.

Dunn, R. A. and Gordon, M. H. (1905) Brit. med. J., ii, 421.

ElLIS, A. W. M. (1915) Brit. med. J., ii, 880.

Elser, W. J. and Huntoon, F. M. (1909) J. med. Res., 20, 371.

Erickson, M. J. and Albert, H. (1922) J. infect. Dis., 30, 268.

Evans, A. C. (1920) Bull. U.S. hyg. Lab., No. 124, p. 45.

Ferry, N. S., Norton, J. F., and Steele, A. H. (1931) J. Immunol., 21, 293.

FERry, N. S. and Sohornack, P. J. (1934) J. Immunol., 26, 143.

FlexNer, S. (1907a) J. exp. Med., 9, 105; (1907b) Ibid., 9, 142. 
FlüGGE, C. (1896) "Die Mikroorganismen." Leipzig.

Gron, A. and Pfeiffer, H. (1902) Z. klin. Med., 44, 262.

Gordon, J. (1926) J. Path. Buct., 20, 319.

Gordon, J. and M·LeOD, J. W. (1926) J. Path. Bact., 29, 13 ; (1928) Ibid., 31, 185.

Gordon, J. E. (1921) J. infect. Dis., 29, 462.

Gordon, M. H. (1925) Spec. Rep. Ser. med. Res. Coun., Lond., No. 98, p. 105.

Gordon, M. H. and Murray, E. G. (1915) J. R. Army med. Cps., 25, 411.

Griffith, F. (1917) Rep. loc. Govt Bd publ. Hlth, New Ser., No. 111, p. 52.

GuRD, F. B. (1908) J. med. Res., 18, 291.

HaLl, I. C. (1916) J. Bact., 1, 343.

Hendry, C. B. (1938) J. Path. Bact., 46, 383.

Hermanies, J. (1921a) J. infect. Dis., 28, I32 ; (1921b) Ibid., 29, 11.

J JEGER, H. (1895) Z. Hyg. InfektKr., 19, 351 ; (1899) Dtsch. med. Wschr., 25, 472 ; (1903a) Zbl. Bakt., 33, 23 ; (1903b) Ibid., 33, 681 ; (1903c) Z. Hyg. InfektKr., 44, 225.

Jenkins, C. E. (1921) J. Path. Bact., 24, 160 ; (1922) Ibid., 25, 105.

JöTTEN, K. W. (1921) Z. Hyg. Infektkr., 92, 9.

KANDIBA, L. (1922) Z. Hyg. InfektKr., 96, 347.

KIEFER. (1895) Berl. klin. Wschr., 32, 332.

Kirkbride, M. B. and Сohen, S. M. (1934) Amer. J. Hyg., 20, 444.

Konman, E. F. (1919) J. Bact., 4, 571.

KUTSCHER, K. (1906) Dtsch. med. Wschr., 22, 1071.

LEBEUf, F. (1924) C. R. Soc. Biol., 90, 768.

LEISTIKow. (1882) Berl. klin. Wschr., 19, 500.

Lingelshein, W. vox. (1905) Dtsch. med. W'schr., 31, 1217 ; (1906) Klin. Jb., 15, 373 ; (1908) Z. Hyg. InfektKr., 59, 457.

LIPSCH ÜTZ, B. (1904) Zbl. Bakt., 36, 743.

LLoyd, D. J. (1916-17) J. Path. Bact., 21, 113.

Lorentz, F. H. (1922) Münch. med. I'schr., 69, 1695.

MlcFarlan, A. MI. (1941) J. Path. Bact., 53, 446.

Maclagan, P. W. and Cooke, W. E. (1917) J. R. Army med. Cps, 29, 228.

Mackaughton, F. G. (1923) J. Path. Bact., 26, 297.

Mcleod, J. W., Coates, J. C., Happold, F. C., Priestley, D. P., and Wheatley, B. (1934) J. Path. Bact., 39, 221.

Mcleod, J. W., Wheatley, B. and Phelon, H. V. (1927) Brit. J. exp. Path., 8, 25.

II'Donald, S. (1908) J. Path. Bact., 12, 442.

Maegraith, B. (1935) Brit. J. exp. Path., 16, 109.

Martin, W. B. II. (1910) J. Path. Bact., 14, 136; (1911) Ibid., 15, 76.

Marx, H. and Woithe, F. (1900) Zbl. Bakt., 28, 1, 33, 65, 97.

MasLovski. (1900) Zbl. Bakt., 27, 541.

Menzel, A. E. O. and Rake, G. (1942) J. exp. Med., 75, 437.

Mlitler, C. P. (1933) Science, 78, 340 ; (1934-35) Proc. Soc. exp. Biol., N.Y., 32, 1136, 1138,1140 .

Miller, C. P. and Boor, A. K. (1934) J. exp. Med., 59, 75.

IIurray, E. G. D. (1929) Spec. Rep. Ser. med. Res. Coun., Lond., No. 124, p. 15.

IIURray, E. G. D. and Ayrton, R. (1924) J. Hyg., Camb., 23, 23.

Nabarro, D. (1917) Rep. loc. Govt Bd. publ Hlth, New Ser., No. 114, p. 207.

Neill, M. H. and TAFT, C. E. (1920) Bull. U.S. hyg. Lab., No. 124, p. 93.

Neisser, A. (1879) Zbl. med. Wiss., 17, 497; (1882) Dtsch. med. W'schr., 8, 279.

Netter, A. and Debré, R. (1911) "La Méningite Cérébro-spinale." Paris.

NiCOLAYSEN, L. (1897) Zbl. Bakt., 22, 305.

Nicolle, N., Debains, E., and Jouan, C. (1918) Ann. Inst. Pasteur, 32, 150.

Oliver, J. O. (1929) J. Hyg., Camb., 29, 259.

Pearce, L. (1915) J. exp. Med., 21, 289.

Petrie, G. F. (1932) Brit. J. exp. Path., 13, 380 ; (1937) J. Hyg., Camb., 3\%, 42.

Phelos, H. V., Duthie, G. M., and I'Leod, J. W. (1927) J. Path. Bact., 30, 133.

Price, I. N. O. (1933) "The Complement Fixation Test for Gonorrhœa." London County Council.

Rake, G. (1933) J. exp. Med., 5\%, 549.

liake, G. and Scherp, H. W. (1933a) J. exp. Med., 58, 341 ; (1933b) Ibid., 58, 361.

Report. (1916) Spec. Rep. Ser. med. Res. Coun., Lond., No. 2; (1920) Ibid., No. 50.

RIST, E. and PARIS, A. (1904) Bull. Inst. Pasteur, 2, 338.

Rockwell, G. E. and IIcKhann, C. F. (1921) J. infect. Dis., 28, 249.

Rosher, A. B. (1936) Pers. comm.

RUEDIGER, E. H. (1919) J. infect. Dis., 24, 376.

SCHÄFFER, J (1897) Fortschr. Med., 15, 813.

Scherp, H. W. and RAKE, G. (1935) J. exp. Hed., 61, 753.

Sоноцтz, W. (1900) Zbl. Bakt., 2\%, 162.

ScotT, W. MI. (1917) Rep. loc. Govt. Bd. publ. Hlth, New Ser., No. 114, p. 111. 
Sordelli, A., Miravent, J. M., and Negroni, P. (1926) Rev. Inst. bact., B. Aires, 4, 636. Stokinger, H. F., Ackermas, H., and Carpenter, C. M. (1944) J. Bact., 47, 141.

Swartz, E. O. and Davis, D. M. (1920) J. Amer. med. Ass., 75, 1124.

Teague, O. and Torrey, J. C. (1907) J. med. Res., 17, 223.

Thalmañ. (1900) Zbl. Bakt., 27, 828.

Thomsen, O. and Vollmond, E. (1921) C. R. Soc. Biol., 84, 326.

Thouson, D. (1917) Brit. med. J., i. 869.

Torrey, J. C. (1907) J. med. Res., 16, 329 ; (1908) Ibid., 19, 471.

Torrey, J. C. and Buckell, G. T. (1922a) J. infect. Dis., 31, 125 ; $(1922 b) \mathrm{J}$. Imlmunol., $7,305$.

Torrey, J. C., Wilson, M. A., and Buckell, G. T. (1922) J. infect. Dis., 31, 148.

Tulloch, W. J. (1923a) J. State Med., 31, 501; (1923b) J. R. Army med. Cps, 40, $12,98$.

Vannod, T. (1906) Dtsch. med. Wschr., 32, 1984; (1907) Zbl. Bakt., 44, 10, 110.

VEDDER, E. B. (1915) J. infect. Dis., 16, 385.

Warren, S. H. (1921) J. Path. Bact., 24, 424.

Wassermanx, A. (1897) Berl. klin. Wschr., 34, 685; (1898) Z. Hyg. InfehtKr., 27, 298.

Watabiki, T. (1910) J. infect. Dis., 7, 159.

Weichselbaum, A. (1887) Fortschr. Hed., 5, 573, 620.

WERTHEIM, E. (1891) Dtsch. med. Wschr., 17, 1351.

Wherry, W. B. and Oliver, W. W. (1916) J. infect. Dis., 19, 288.

Wilson, G. S. and Smrth, M. M. (1928) J. Path. Bact., 31, 597.

Wilson, S. P. (1928) J. Path. Bact., 31, 477.

Wollstein, M. (1907) J. exp. Med., 9, 588.

Wright, H. D. (1933) J. Path. Bact., 37, 257.

Zprodowski, P. and Voronne, E. (1932) Ann. Inst. Pasteur, 48, 617.

Zozaya, J. (1931) J. exp. Med., 54, 725.

ZOzAYA, J. and Wood, J. E. (1932) J. infect. Dis., 50, 177. 


\section{CHAPTER 24}

\section{STREPTOCOCCUS}

Definition.-Streptococcus.

Spherical or ovoid cells, arranged in short or long chains, or in pairs. Usually non-motile. Non-sporing. Most species Gram-positive. Some species form capsules. Growth tends to be relatively slight on artificial media, and some species grow poorly in the absence of added native protein. Several species produce characteristic changes in media containing blood. Various carbohydrates are fermented, with the production of acid. Mast species fail to liquefy gelatin. Most species are aerobic and facultatively anaerobic ; some are anaerobic. Many species are normally parasitic on man or animals; some species are highly pathogenic, and some produce soluble toxins.

Type species. Streptococcus pyogenes.

The term Streptococcus was first used by Rosenbach in 1884, when describing a coccus, growing in chains, that had been isolated from suppurative lesions in man. To this organism he gave the name Streptococcus pyogenes. A chainforming coccus had, however, been described by Fehleisen in the previous year as the causative orgauism of erysipelas (Fehleisen 1883); and Pasteur, Chamberland and Roux, in 1881, had described a septicæmic infection in rabbits, resulting from the inoculation of these animals with human saliva, which probably affords the earliest recorded reference to the pneumococcus, although no clearly identifiable description of this species was published prior to the independent studies of Fraenkel and of Weichselbaum in 1886. In 1887 Nocard and Mollereau reported the experimental production of mastitis in the cow and goat, by the inoculation into the udder of a streptococcus isolated from the milk of a cow suffering from that disease. In 1888 Schütz described a streptococcus that he had isolated from the lesions of strangles in the horse. In more recent years, chain-forming cocci have been isolated from a variety of pathological conditions in man and animals, from the mouth or from the fæces of healthy subjects, from milk and various milk products, and from other sources.

The tendency to grow in chains of varying length gives to the members of this group a very characteristic morphology, and they possess in common other characters that appear to justify their inclusion in a single bacterial genus. The Committee of the Society of American Bacteriologists (Winslow et al. 1920) separated the pneumococcus from the main streptococcal group, by forming a genus Diplococcus, with Diplococcus pneumonice as the type species. It appears to the authors, for reasons which will become apparent, that this separation is undesirable, and the summary of generic characters as set out by the American Committee has been modified in the required sense. As so modified, and with other minor emendations, including the substitution of Str. pyogenes for Str. hamolyticus as the name of the type species, the description of these generic characters is as given above. 
The many attempts that have been made to evolve a satisfactory classification of the streptococci provide an admirable example of the difficulties with which the systematic bacteriologist is faced. As will be seen, there is no single criterion on which reliance can be placed, even in making a primary division into sub-groups that are themselves to be further divided by the application of other tests. It happens that, in this particular genus, one of the most useful differential criteria is provided by the changes induced by the growing organisms in media containing blood; but we shall note that this test, valuable as it is, cannot be too rigidly applied. Here, as elsewhere, we have to apply a variety of criteria selected, on the usual basis of statistical empiricism, as differentiating between groups each of which possesses several highly correlated characters. Here, as elsewhere, we find

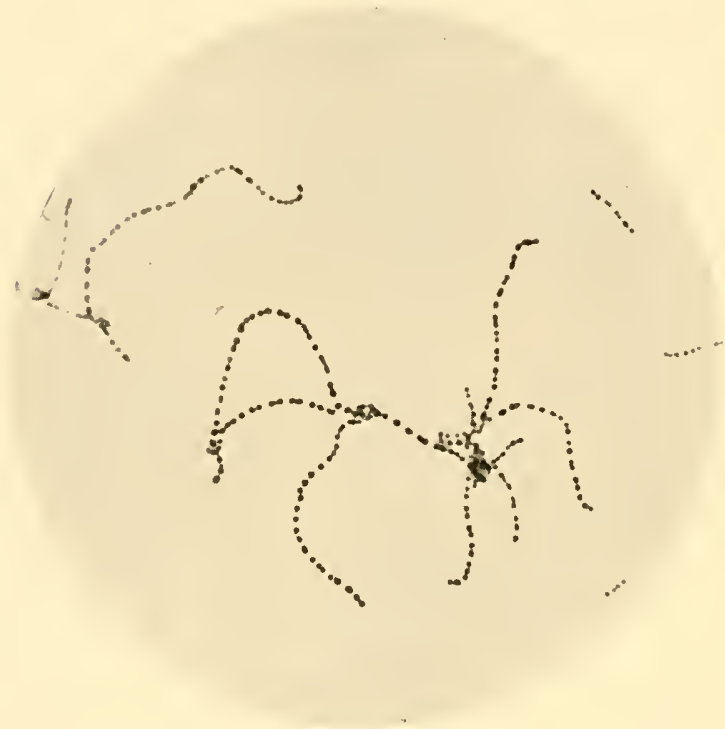

Fig. 117.-Str. pyogenes.

From 24-hours' culture on agar, showing long chains $(\times 1000)$. that the method of antigenic analysis is playing an increasingly important part in the differentiation and identification of those ultimate types, or varieties, for which we need distinguishing names or labels.

The most convenient method of discussing this problem will be to take various characters in turn, and see how far they enable us to differentiate between one species, or type, and another.

Morphology.-Taking the genus as a whole, the characters that might be regarded as supplying possible differential criteria are (1) the length of the chains formed, (2) the shape of the individual cells forming them, (3) the presence or absence of capsules, and (4) in the light of certain recent observations, the very occasional presence of flagella.

In the earlier days of bacteriology much attention was paid to chain formation as a differential criterion, and such names as Streptococcus brevis, Streptococcus longus, Streptococcus longissimus, and Streptococcus conglomeratus were coined to denote strains with the corresponding tendencies to grow in short chains, long chains, or chains which were very long or tangled. It has, however, come to be generally recognized (Thalmann 1912, Brown 1919) that these characters are often variable within a single strain ; so that, while the modal chain length of any species may be sufficiently characteristic to deserve inclusion in a description of the specific characters, it is quite useless for purposes of classification, and misleading when employed for purposes of nomenclature. Some species or groups, such as Str. pneumonice and the enterococcus, usually occur in pairs or very short chains and never form chains of any considerable length. Others, such as Str. pyogenes, 
usually occur in chains of eight or more cocci and often form long chains. Others again, such as the strains of the viridans type, are markedly variable in this respect, occurring both as very short chains, or pairs, and as chains of enormons length.

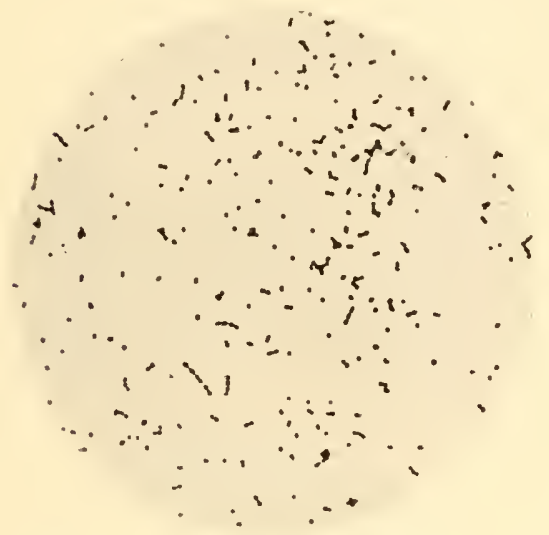

FiG. 118.-Viridans streptococci.

From 24-hours' culture in broth, showing short chains $(\times 1000)$.

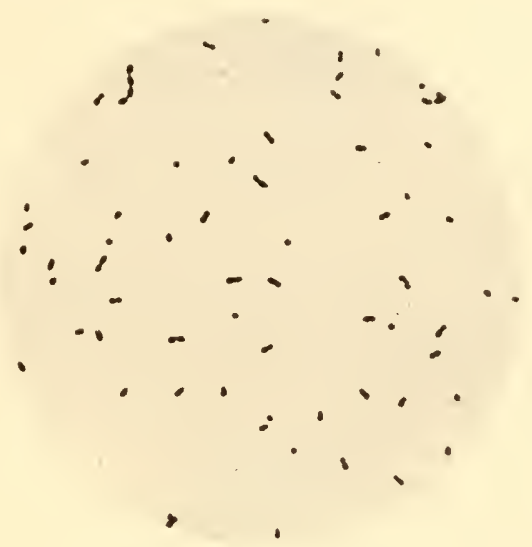

FIG. 119. -Str. pneumonice.

From 24-hours' culture on agar, showing (liplococci and short chains $(\times 1000)$.

The shape of the individual cocci forming the chain also varies, and there is a tendency for the cells of short-chained streptococei to be ovoid, with the long axis in the axis of the chain. When, as in the pnemmococcus, a majority of the cocei occur in pairs, each cell of a pair may be definitely lanceolate, the blunt ends being adjacent. The individual cells of longchained streptococci tend to approach more closely to the spherical form; or they may sometimes be compressed in such a way that the longer axis of the cell lies at right angles to the axis of the chain as a whole. Cell shape, like modal chain length, may vary from one species of streptococcus to another, but it forms no better criterion for systematic purposes. Occasional strains may take on a diphtheroid type of morphology, which may lead to errors in identification (see Lamanna 1944).

Capsule formation, when it occurs, is of greater value for classification. It is almost constantly shown by Str. pneumonice when growing within the tissues (see Fig. 120) and is absent in most other

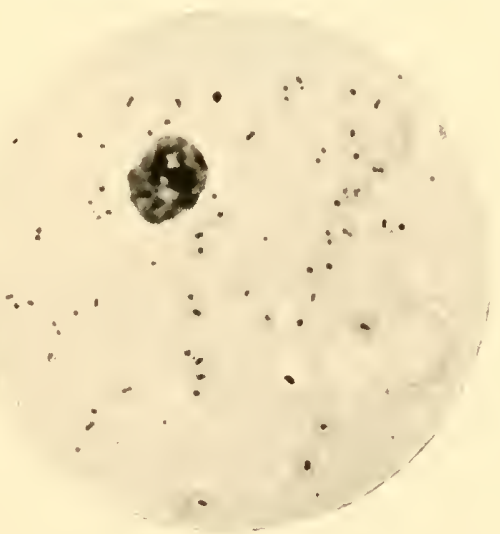

Fig. 120.-Str. pneumonice.

In peritoneal exudate of mouse, showing capsulation $(\times 1000)$. species, though not in all. Seastone (1934, 1943), however, has reported the presence of capsulation in Str. pyogenes during the first $2-2 \frac{1}{2}$ hours of growth in serum broth, though the capsules have usually disappeared by the 3rd or 4 th hour. Morison (1940) likewise finds that all recently isolated strains of this organism are 
capsulated to some degree. Capsulation is, however, very irregular, occurring in some types and not in others, and seems to be affected by the amount of hyaluronic acid in the substrate and the amount of hyaluronidase secreted. Generally speaking, capsulation and the secretion of hyaluronidase appear to be mutually exclusive (McClean 1941, 1942), and there is no constant association of capsulation with virulence.

From time to time, accounts have appeared in the literature of motile streptococci, but many of these have been based on somewhat inadequate observations, and the genus Streptococcus has, until recently, been regarded by most bacteriologists as composed exclusively of non-flagellated species. It is certain that flagellated forms are very rare; but two observers (Koblmiiller 1935, Pownall 1935) have recently given careful and detailed descriptions of motile strains of streptococci. In each case the organism described was apparently a motile variant of the species, or group, that is generally known by the name of "enterococcus."

Streptococci stain readily with the ordinary dyes; none of them is acid-fast; and the great majority are frankly Gram-positive. Some strains tend to lose the Gram stain if decolorization is prolonged; a few species, or varieties, have been described as frankly Gram-negative.

Cultural Requirements.--Some species of streptococci, such as Str. pneumonice and to a less extent Str. pyogenes, grow poorly on the simpler media of the laboratory when first isolated; though they can usually be trained to grow on these media after a limited number of subcultures. The growth of these species is markedly improved by the addition to the medium of such materials as blood or serum. A few species, or types, such as the enterococci, grow well in the presence of bile or bile salts, while most do not.

The optimal temperature for growth is, with most parasitic species, in the near neighbourhood of $37^{\circ} \mathrm{C}$. Some species found in milk have an optimum about $30^{\circ} \mathrm{C}$. The range over which growth occurs is, for the more sensitive species, somewhat restricted; $42^{\circ} \mathrm{C}$. or thereabouts marks the upper limit; growth is usually slow at temperatures below $30^{\circ} \mathrm{C}$. and often ceases below $20^{\circ} \mathrm{C}$. Some species, on the other hand, including certain streptococci found in milk, grow actively at temperatures of $45^{\circ} \mathrm{C}$. or over (see Sherman and Stark 1931). Such thermophilic types are of considerable economic importance in relation to pasteurization (see Chapter 93).

Most species are aerobic and facultatively anaerobic. Some are strict anaerobes, or microaerophilic. It will be more convenient to discuss the other characters of these anaerobic streptococci, which have not yet been studied in any detail, in a separate section of this chapter (p. 596). Pneumococci grow best in an atmosphere containing 10 per cent. carbon dioxide; some strains are said not to grow at all in its absence (see Fleming 1941, Kempuer and Schlayer 1942). Glutamine is said to be required for the growth of Group A but not of Group B hæmolytic streptococci (Fildes and Gladstone 1939). For a fuller account of the growth requirements see p. 66 .

These cultural requirements, while supplying useful ancillary evidence in identification, do not, except in the case of the anaerobic species and the markedly thermophilic streptococci, afford an adequate basis for any primary classification.

Growth Characters.- On solid media the streptococci tend to form small, discrete, slightly raised colonies, $1 \mathrm{~mm}$. or less in diameter. The modal colonial form varies in different species; and it may in some cases be sufficiently characteristic to assist 
in identification, particularly in recently isolated strains. Many strains of pneumococci, for instance, give characteristic "draughtsman" colonies, with an entire, sharply raised edge and a central depressed area. A Type III pneumococcus frequently gives a characteristic watery, or mucoid, colony. One type of hæmolytic streptococcus gives easily recognizable minute, clear colonies, and so on.

Many species grow poorly in gelatin; and in a gelatin stab such growth as occurs is mainly confined to the track of the needle. The only streptococci, apart from some of the anaerobic species, that are known to liquefy gelatin are certain strains of enterococci. When liquefaction occurs it is usually infundibuliform in type.

In broth or other liquid media many species of streptococci give a granular growth, the medium remaining clear and the granular masses collecting as a powdery deposit, or adhering to the sides of the tube. Although this type of growth shows a characteristically high frequency among certain species of streptococci, especially when first isolated, it is by no means constant; and the degree of granularity may vary over a wide range. In some cases a granular deposit may be associated with a

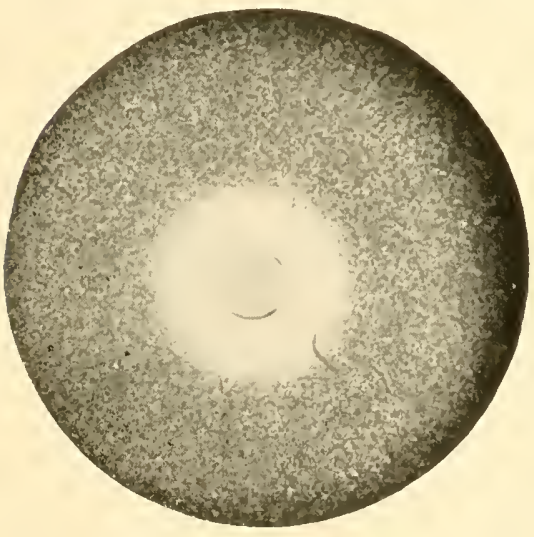

Fig. 121.-Str. pyogenes.

Surface colony on blood agar plate, showing zone of hæmolysis round colony $(\times 8)$.

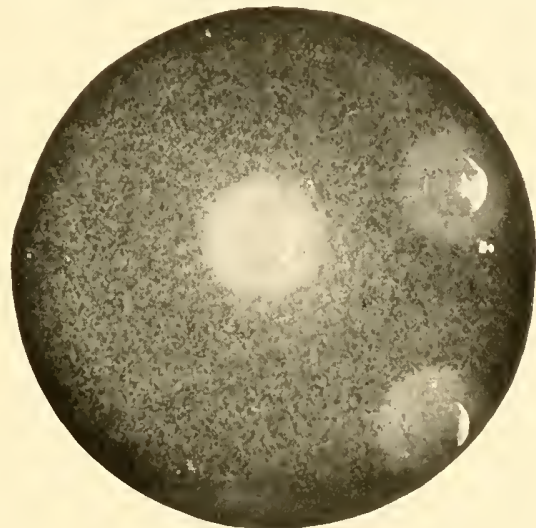

FIG. 122.-Str. pneumonice.

Surface colonies on blood agar plate, showing zones of discoloration and partial hiemolysis round colonies $(\times 8)$.

varying degree of turbidity of the medium; in others the growth, as a whole, may be distinctly though finely granular, the granules remaining dispersed throughout the medium. Any one strain may undergo marked changes in this respect on subculture ; if a strain which, on first isolation, gives a markedly granular growth, is subjected to repeated subculturing at short intervals, it is often possible to produce a diffuse growth within a limited number of generations. Granularity is usually more evident in cultures grown at $37^{\circ} \mathrm{C}$. than at $22^{\circ} \mathrm{C}$. In all cases the type of growth in a fluid medium is closely associated with the character of chain formation. A strain which is forming long chains will give a typically granular growth; if the growth becomes diffuse it will be found that the average chain length has diminished. Those species in which the diplococcal form predominates, as for instance Str. pneumonice or enterococci, usually give diffuse, non-granular growths in fluid media. A description of the variations in morphological appearance, colonial form, and type of growth in broth met with among the streptococci is given on page 594 . 
Metabolic Activities.-Most of the eariy attempts to classify the streptococci were based on their fermentation reactions in an empirically selected series of substrates. The behaviour of the different members of this genus in bloodcontaining media has, however, come to occupy so important a place as a criterion for primary classification, that it will be convenient to deal with it first.

\section{Classification Based on Changes Produced in Blood.}

Blood Plates.-Marmorek (1895) first noted the ability of certain strains of streptococci to lyse red blood corpuscles in vivo and in vitro, but it was Schottmïller (1903) who proposed that the ability to produce hæmolysis in vitro should be adopted as a differential criterion for purposes of classification. He noted that certain strains of streptococci produced clear zones of lysis when grown on blood agar plates, while others gave colonies which were surrounded by zones of greenish discoloration. For the former type Schottmiiller proposed the name Str. hamolyticus, for the latter Str. viridans. These observations formed the basis of a system of classification and nomenclature which has been developed by many

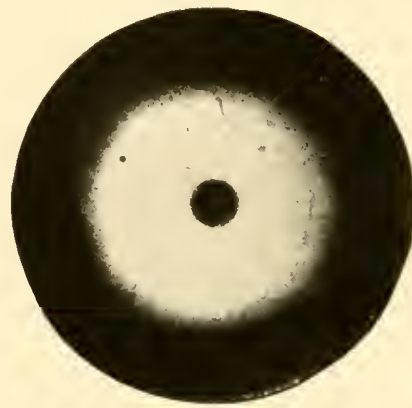

FIG. 123.-Str. pyogenes.

Deep colony in blood agar plate, showing wide zone of complete hiemolysis, and the sharply differentiated margin of the colony $-\beta$-hremolysis $(\times 8)$.

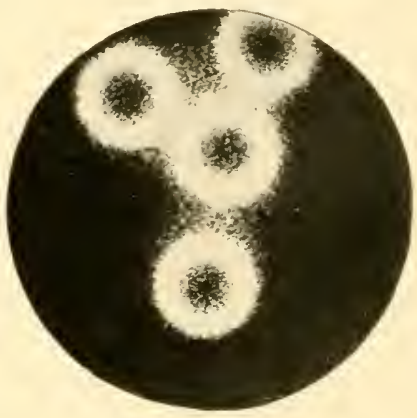

FIG. 124.-Viridans streptococci.

Deep colonies in blood agar plate, showing zone of discoloured cells round colony, obscuring margin, and zone of incomplete lysis beyond- $\alpha$-hæmolysis $(\times 8)$.

subsequent investigators. It has, indeed, been shown that the green-producing streptococci are not devoid of hæmolytic activity, though the zones which they produce on blood agar plates are different in kind, as well as in extent, from those formed round the colonies of the frankly hæmolytic strains. Mandelbaum (1907) emphasized the importance of microscopical examination of the colonies formed on blood agar plates, and noted that, while the colonies of the long pathogenic streptococci were surrounded by clear colourless zones, those of the viridans type, and also those of the pneumococcus, were surrounded by a zone of discoloured, non-hæmolysed corpuscles lying immediately next the colony, and an outer narrow hæmolysed zone containing only corpuscular shadows.

These phenomena were studied in much greater detail by Smith and Brown (1915), and by Brown (1919). The monograph by Brown, in which the appearances met with and the factors which determine or modify them are fully discussed, contains an admirable review of the literature dealing with the classification of streptococci up to 1919. In this monograph great emphasis is laid on the importance of employing a uniform and standardized technique, and, in particular, on the 
superiority of poured plates, and the observation of deep colonies, over plates which have been inoculated by surface spreading only.

The medium recommended by Brown consists of veal peptone agar containing 5 per cent. of horse blood. The agar is stored in tubes in $12 \mathrm{ml}$. amounts; when required for use a tube is melted and cooled to $45^{\circ} \mathrm{C}$; $0.66 \mathrm{ml}$. of horse blood is added and evenly mixed with the agar, the medium is inoculated with a loop or two of a 24 -hours' broth culture that has been so diluted as to give about 100 colonies; and is then poured into a Petri dish $9 \mathrm{~cm}$. in diameter, thus giving a layer about $2 \mathrm{~mm}$. thick. The plates, after preliminary drying in the inverted position with the plate tilted on the lid, are incubated at $37^{\circ} \mathrm{C}$. for 24 hours. They are then examined and the appearances noted. They are re-examined after another 24 hour's' incubation at $37^{\circ} \mathrm{C}$., and finally after a further 24 hours in the ice-chest. For the recognition of hæmolytic and non-hæmolytic strains in primary mixed cultures the use of blood agar plates with a surface inoculation usually suffices, and has certain advantages; but for the critical determination of the type of hæmolysis given by any strain, once it has been isolated in pure culture, the technique recommended by Brown should be strictly adhered to, at least in regard to the medium used and the examination of deep colonies. The use of horse blood is particularly important. It is well known that the red corpuscles of different animal species vary widely in their resistance to different hemolytic agents. In spite of this fact, many workers lave used the blood of the rabbit or ox or some other animal, in testing the hæmolytic activity of various species, or strains, of streptococei ; and it is probable that some at least of the discrepancies met with in the literature are due to this variable factor. It may, at times, be desirable to use the blood or red cells of some particular species in a given series of observations on streptococcal hæmolysin ; but, if the results are to be used for purposes of identification or classification, they should always be controlled by parallel tests made with horse blood.

Brown records four different types of reaction in blood agar plates, which he designates as follows:

$\alpha$. A somewhat greenish discoloration and partial hæmolysis of the blood corpuscles immediately surrounding the colony, forming a rather indefinitely bounded zone 1-2 mm. in diameter, outside of which is a second, narrow, clearer, not discoloured zone. Under the microscope many corpuscles are seen in the inner zone, and these are obviously discoloured, the discoloration varying in degree with different strains of streptococci. Very few corpuscles remain in the outer, clearer zone; and these are never discoloured. These typical appearances may fail to appear after 24 hours', or even after 48 hours' incubation, at the end of which time the narrow outer zone of hæmolysis may not have developed. In such cases this zone makes its appearance during the subsequent 24 hours in the ice-chest. If a plate, which has developed the typical appearances, is reincubated for 24 to 48 hours, and then placed in the ice-chest for a further 24 hours, a double series of rings will frequently develop, so that the colony is surrounded by a hazy discoloured ring, a clear hæmolysed ring, a second hazy ring, and a second clear ring. By repeating the whole process it is sometimes possible to develop three or more series of such rings.

$\beta$. The colonies are surrounded by sharply defined, clear, colourless zones of hæmolysis, 2-4 mm. in diameter. Under the microscope no corpuscles can be seen within this zone. The zones of $\beta$-hæmolysis develop more rapidly than those of the $\alpha$ type. They are often well developed after 18 hours' incubation. They extend slightly between the 24 th and 48th hour, but show no qualitative changes. They undergo no alteration or extension during the subsequent 24 hours in the ice-chest.

$\alpha^{\prime}(\alpha$ prime). The colonies are surrounded by a zone of hæmolysis, which is slightly hazy, and less sharply limited than in the case of true $\beta$-hæmolysis. The colony itself is not sharply defined, and examination with the microscope shows that the hæmolysed zone contains, throughout, a moderate number of unaltered corpuscles, which are most numerous 
in the immediate neighbourhnod of the colony. There is no discoloration. Unlike the zones of $\beta$-hæmolysis, a considerable extension of the zones may occur during the 24 hours in the ice-box. It is noted that some strains which produce $\alpha^{\prime}$-hæmolysis on horse blood agar may produce typical $\alpha$-hæmolysis on rabbit blood agar.

$\gamma$. The colonies develop in the blood agar without any change in the surrounding medium.

The $\beta$-hæmolytic strains of Smith and Brown correspond to Schottmüller's Str. hamolyticus. The $\alpha$-hæmolytic strains may be regarded as equivalent to his Str. viridans; though it may be noted that strains are encountered that produce the characteristic green coloration without the formation of a detectable zone of hæmolysis. The significance of the $\alpha^{\prime}$ type of hæmolysis is not clear. It seems to be of infrequent occurrence. There seems no good reason for attaching the label $\gamma$ to those streptococci that cause no change in blood media. Strains of this type have sometimes been referred to as "indifferent streptococci."

Soluble $\beta$-hæmolysins.-The terms $\alpha$ - and $\beta$-hæmolysis have attained general currency in bacteriological literature and serve a useful purpose. No effort has been made, however, to reconcile this usage with that of the terms " hæmolytic" and "non-hæmolytic" as applied to streptococci. By a "hæmolytic" streptococcus is meant almost always a strain that causes $\beta$-hemolysis on blood agar. By a "non-hæmolytic streptococcus" is meant a strain that either produces $\alpha$-hæmolysis, or gives rise to no change at all. Some workers would confine the term " hæmolytic streptococcus," or at least the specific name Str. homolyticus if that be used, to strains that, in addition to causing $\beta$-hæmolysis in blood agar, can be shown to produce a soluble hæmolysin, and this is an aspect of the problem that must be discussed in more detail.

Marmorek (1895) showed that cultures of certain strains of streptococci in a fluid medium had the power of lysing added blood corpuscles, and Besredka (1901) obtained hæmolytic filtrates from cultures of streptococei in heated rabbit's serum. Braun (1912) reported that all strains of streptococci which produced hæmolysis on rabbit blood agar plates gave rise to a filtrable hæmolysin when grown in rabbit serum broth. The factors which determine this hæmolysin production have been studied by McLeod (1912), Meader and Robinson (1920) and de Kruif and Ireland (1920). All these observers noted that hæmolysin production was absent or minimal in cultures grown in plain broth without the addition of serum. More recently Todd (1932) and Todd and Hewitt (1932) have shown that potent hæmolytic filtrates may be obtained by growing hæmolytic streptococci in a medium containing yeast extract, or in a special broth medium, sterilized by filtration instead of by autoclaving, and containing dextrose, sodium bicarbonate and sodium phosphate.

De Kruif and Ireland have carried out careful quantitative studies of the rate of hæmolysin production, and of its inactivation in the culture medium. They found that the hæmolytic titre of the supernatant fluid from their cultures after centrifugalization reached its maximum after 8 hours' incubation at $37^{\circ} \mathrm{C}$., and then rapidly declined. In many cases no hæmolysin could be detected after 14 hours; though, when the whole culture was tested instead of the supernatant fluid, some lytic action might persist up to the 24 th hour. A bæmolytic filtrate is completely inactivated by heating at $55^{\circ} \mathrm{C}$. for 30 minutes, and may lose most of its activity when incubated at $37^{\circ} \mathrm{C}$. for 2 hours or more (see McLeod 1912).

It has been shown by Neill and Mallory (1926) that streptolysin, when exposed to air at relatively low temperatures, undergoes an oxidation that is readily reversible by suitable chemical reagents. The hæmolysin is active in the reduced form, inactive in the oxidized. At higher temperatures $\left(55^{\circ} \mathrm{C}\right.$.) an irreversible inactivation occurs.

Todd $(1934,1938 a, 1939)$ has demonstrated the production of two kinds of hæmolysin. One, the $\mathrm{O}$ lysin, is oxygen-labile at ordinary temperatures, but can be re-activated by 
reduetion with 0.1 per cent. sodium hydrosulphite; provided that it is proteeted from the air, it remains stable in the ice-chest for years. The other, the S lysin, whieh can be extracted readily from streptocoeei by shaking with serum, is inaetivated, like the $\mathrm{O}$ lysin, by ineubation at $37^{\circ} \mathrm{C}$. for $2-4$ hours, but unlike the $\mathrm{O}$ lysin eannot be re-aetivated by reduction; it is very sensitive to both heat and acid and can be preserved only by storage at very low temperatures $\left(-73^{\circ} \mathrm{C}\right.$.). The 0 lysin produces hæmolysis rapidly and is antigenic in the free state: the S lysin produces hromolysis more slowly and is antigenic only when present in the organisms. Each is neutralized by a separate antibody. The $\mathrm{O}$ lysin is formed by strains belonging to Group $\mathrm{A}, \mathrm{C}$ (human), and G. The $S$ lysin appears to be formed by strains of all groups, but the type of lysin produced is group-speeifie, an antiserum to the $\mathrm{S}$ lysin of Group A strains failing to neutralize S lysin formed by other groups. Herbert and Todd (1944) have met with strains of streptococei produeing only $S$ and others only $O$ hæmolysin. On blood agar the $S$ strains formed $\beta$-hæmolytie colonies on the surface and in the depth, both ærobically and anærobically. The $O$ strains on rabbit blood agar-but not on horse blood, which eontains $\mathrm{O}$ antilysinformed $\beta$-lxæmolytic colonies only in the depth and only under ærobic eonditions. The $\mathrm{S}$ lysin was purified and found to be a lipo-protein hapten, incapable of stimulating the formation of antibodies.

The extreme lability of both $\mathrm{O}$ and $\mathrm{S}$ lysins in broth cultures at $37^{\circ} \mathrm{C}$. renders the ordinary titration method of assessing their potency unreliable. Special precautions have to be taken to prevent destruction of either lysin before we can assert that any given strain which produces $\beta$-hæmolysis on blood agar plates is incapable of forming a filtrable hæmolysin in a fluid medium.

Apart from the difficulties caused by the lability of the lysins, it may be noted that some strains of Str. pyogenes produce $\alpha$-hæmolytic colonies on blood agar, or sometimes completely non-hæmolytic colonies.

The first type has been studied by various workers. Fry (1933) showed that certain strains which formed $\alpha$-hæmolytic colonies on aerobic blood agar plates produced typical $\beta$-hæmolytie colonies when incubated anaerobically. Fuller and Maxted (1939) showed that two or three factors were concerned in this result. Under aerobic conditions $\beta$-hæmolytie colonies were formed if all reducing sugar was removed from the blood agar or if catalase was added to the medium. They bring evidence to suggest that in the $\alpha$-hæmolytic colonies peroxide is formed before the hrmolysin with the result that a green zone is produced. If the formation of the peroxide is prevented by anaerobic ineubation or is neutralized by catalase, then the hæmolysin produces typical $\beta$-hæmolytic colonies. The mode of action of the redueing sugar in the medium is not quite clear, but it appears to inhibit the formation of the hæmolysin. It may be noted that Fry's strains formed soluble hrmolysin in serum broth under acrobic conditions. For practical purposes it is advisable to incubate primary plate cultures anaerobieally, or both aerobically and anaerobieally.

The second type has been reported by Coburn and Pauli (1941) and Colebrook and his colleagues (1942). In a ward outbreak of streptoeoccal respiratory infection, Coburn and Pauli found that the causative organism, Type 12, occurred in two forms. One form gave rise to typical hæmolytie colonies at $37^{\circ} \mathrm{C}$; the other gave rise to hæmolytie colonies only at $22^{\circ} \mathrm{C}$. The second rariant appeared to be possessed of a higher degree of infectivity than the normal form. Colebrook's observations were made in a surgical ward in which typical $\beta$-hæmolytic colonies belonging to Group A Type 12 had been giving rise to septic complications. A series of cases was studied in which completely non-hæmolytic streptococei, both aerobically and anaerobically, were isolated from the wounds. These organisms proved also to belong to Group A Type 12. They formed no $\mathrm{S}$ hæmolysin, but most of them, when specially tested, were found to produce $\mathrm{O}$ hæmolysin. The frequency with which such strains occur is at present unknown, but they are believed to be uncommon. 
$\alpha$-hæmolysin.-When we turn to those streptococci that produce $\alpha$-hæmolysis on blood agar plates, we have two mechanisms to cousider: the reaction that causes the lysis of the red cells, and the reaction that causes the green coloration.

Cole (1914) described the presence in pneumococci of a labile intracellular hæmolysin which was liberated from the cells on autolysis. It was commonly stated by subsequent workers that the pneumococeus produced no filtrable hæmolysin in fluid media; and the agent causing $\alpha$-hæmolysis was supposed to be of some quite different nature. It has, however, been quite clearly demonstrated within recent years (see Neill 1926, Sickles and Coffey 1928, Cowan 1934, Todd 1934) that the pneumococeus, when grown under suitable conditions, produces a soluble hæmolysin of the oxygen-sensitive, heat-sensitive type, which undergoes reversible oxidation at low temperatures. Whether other streptococci that produce $\alpha$-hæmolysis wonld also elaborate a filtrable hæmolysin under suitable conditions is at present unknown.

Until recently the most widely accepted view in regard to the green coloration associated with $\alpha$-hæmolysis was that it was due to the formation of methæmoglobin or of some closely allied substance (Schnabel 1921, MeLeod and Gordon 1922, Rother 1925). It was shown by McLeod and Gordon that the pneumococcus produces hydrogen peroxide and that hydrogen peroxide will discolour heated blood agar, in which the blood catalase has been inactivated. The mechanisms involved in the production of hydrogen peroxicle by the pneumococcus, or by pneumococcal extracts, have been studied in considerable detail by Avery, Morgan and Neill (Avery and Morgan 1924, Morgan and Avery 1924, Avery and Neill 1924a, $b, c$, Neill and Avery 1924a, $b$, 1925, Morgan and Neill 1924, Neill 1925). The systems involved appear to include the catalysed oxidation-reduction and peroxidase mechanisms discussed in Chapter 3 , and the actual course of the reaction seems to be determined in the main by the oxygen pressure to which the reacting system is exposed. It is, however, clear that the formation of methrmoglobin is not itself the cause of the green pigmentation, unless the apparent greenness is due to an optical illusion resulting from a colour contrast; and, in view of the amount of catalase present in unheated blood, it is difficult to believe that hydrogen peroxide is the active agent in cultures on unheated blood agar plates.

This problem has been brought nearer solution by the studies of Hart and Anderson (1933) (see also Anderson and Hart 1934a). Working with the pneumococcus, they found that when small quantities of laked blood were added to broth cultures in the presence of an alkaline buffer solution, an olive-green precipitate was formed. This could be separated, washed, and dissolved in dilute alkali to give a green solution. Crystalline hæmoglobin, or methæmoglobin, gave the same green pigment when incubated under suitable conditions with pneumococcal cultures. The spectroscopic and chemical analysis of this pigment suggest that it is an iron-containing derivative of hæmoglobin. It is rapidly bleached by hydrogen peroxide, but it is not affected by reducing agents. An identical, or very similar, green pigment can be obtained by incubating laked blood, hæmoglobin, or methæmoglobin with various chemical reducing systems, such as ascorbic acid, cysteine-glucose, etc. From the results obtained with autolysed bacterial cells, washed bacteria, bacterial extracts, etc., it would seem that the green pigment results from the activity of a bacterial oxidationreduction system, one component of which is intracellular. This system is not peculiar to the pneumococcus; it is shared, not only by those streptococei that produce the green pigment on blood agar plates, but by Str. pyogenes which gives $\beta$-hæmolysis, and by the enterococci which usually produce no change on unheated blood media. It is also possessed by unrelated bacteria, such as Staph. aureus and Bact. coli. The production of green pigmentation by some species and not by others would seem to be due, not so much to the presence or absence of the necessary enzyme system as to secondary factors, themselves determined by the metabolic activities of the bacteria concerned, which sometimes permit this system to function, and sometimes suppress it. We have already noted that some strains of hæmolytic streptococci may produce $\beta$-hæmolysis under anaerobic 
conditions, while giving typical $\alpha$-hæmolysis with green coloration when cultivated aerobically.

Summarizing the observations recorded above we may say that a study of the appearance of the colonies on blood agar plates, together with a test for the production of a filtrable hæmolysin, enables us to divide streptococci into three main categories.

(1) Hamolytic streptococci.-These produce $\beta$-hæmolysis on blood agar plates. They may be differentiated into two sub-groups: $(a)$ those that produce a filtrable hæmolysin, and $(b)$ those that do not. Among strains of Str. pyogenes two variants are known. One produces $\alpha$-hæmolytic colonies acrobically but $\beta$-hæmolytic colonies anaerobically; it forms a soluble hæmolysin in broth aerobically. The other forms completely non-hæmolytic colonies, but produces a soluble hæmolysin of the $O$ type.

(2) Streptococci giving $\alpha$-hamolysis.

(3) Streptococci that have no action on blood media under the usual conditions of testing.

Classification Based on Fermentation Reactions.-The capacity of different species or types of streptococci to ferment different substrates played a very large part in the earlier attempts to separate this group of bacteria into its natural elements. The classification of Andrewes and Horder (1906), based largely on the earlier studies of Gordon (1902-03, 1903-04, 1905), and the more extensive and detailed classification proposed by Holman (1916) (see also Floyd and Wolbach 1914, Lyall 1914, Broadhurst 1915), depended almost wholly on a selected series of fermentation tests. It is hardly necessary to-day to set out these classifications in their original form; since few would now accept them as affording an adequate basis for the differentiation of named species or types. This does not of course mean that these fermentation tests are of no value in classifying the streptococci. The reverse is the case. In more recent years, however, the tendency has been to employ fermentation reactions as ancillary, rather than as primary differential criteria. When a particular substrate has been found to be of value in differentiating between certain related species or types, it has been used for this purpose, but has not necessarily been employed in the differentiation of other species within the genus. It will therefore be more convenient to consider the exact systematic significance of these fermentation tests after we have discussed certain other differential criteria, and, in particular, the results obtained by antigenic analysis : but it will be useful to summarize here the observations that have been made on the correlation between enzymic activities and natural habitat.

The early observations that streptococci isolated from pathogenic lesions in man usually ferment lactose and salicin, seldom, if ever, mannitol, raffinose or inulin, and give acid without clot in milk has been amply confirmed. The value of inulin fermentation as a differential test for the identification of the pneumococcus has also been firmly established. It is clear that this species almost always ferments this fructosan, while most other streptococci fail to do so. There has been a tendency to exclude from the species Str. pneumonice any strain that fails to ferment inulin ; but it is doubtful, as Berger and Silberstein (1926) point out, whether this test can be applied with such complete rigidity. As regards other substrates, the pneumococcus ferments lactose and usually raffinose, but not salicin or mannitol. Milk is acidified and frequently clotted.

There is some measure of agreement that the streptococcus commonly found in the human mouth, which is of the $\alpha$-hæmolytic type, ferments lactose, raffinose and salicin, but not mannitol, and usually forms a clot in milk. In addition, it has been pointed 
ont by Niven, Sniley and Sherman (1942) that, in general, the $\alpha$-hæmolytie streptococei fail to hydrolyse arginine with the production of ammonia, whereas the $\beta$-hrmolytic streptococci and the enterococci are able to do so.

There is also agreement that the fermentation of mannitol is characteristic of the streptococci that normally inhabit the human intestine (see Winslow and Palmer 1910, Fuller and Armstrong 1913, Hopkins and Lang 1914, Dible 1921, Bagger 1926, Meyer 1926, Meyer and Schönfeld 1926, Downie and Cruickshank 1928). These streptococei usually ferment salicin and lactose, seldom inulin or raffinose, and usually clot and decolorize litmus milk. A few of them are peculiar in liquefying gelatin. In relation to this group of streptococci an additional fermentation test was introduced by Rochaix (1924)-the fermentation of æsculin in a bile-containing medium. This reaction has been studied by Meyer and Schönfeld (1926) and by Weatherall and Dible (1929). It has been found by many workers to be of considerable differential value; but, as Weatherall and Dible point out, the inclusion of bile salts in the medium removes it from the ordinary category of fermentation tests, since bile salts inhibit the growth of many species of streptococci (see below) and in their absence æsculin is attacked, though often slowly, by many non-fæcal species or types.

Comparing the streptococci of man with those from other animals, several points of interest have emerged.

Winslow and Palmer (1910), while confirming the frequency of mannitol-fermenting streptococci in human fæces, noted their rarity in fæces from the cow or horse. They found also that raffinose-fermenting strains, while relatively uncommon in human fæces, were very common in the fæces of cattle. Fuller and Armstrong (1913) examined 349 strains of streptococci isolated from fæces- 123 from man, 129 from the horse and 97 from cattle. They found that 65 per cent. of the fæcal streptococci from man fermented mannitol, as compared with 2-3 per cent. of the bovine or equine fæcal strains. Raffinose. fermenting streptococci, on the other hand, were not found among the human fæcal strains, while 12 per cent. of the equine fæcal strains, and 73 per cent. of the bovine fæcal strains fermented this sugar. The equine fæcal strains were characterized by a frequent failure to ferment lactose.

The fact that streptococci isolated from horses, whether from suppurative lesions or from the mouths or intestines of normal animals, frequently fail to ferment lactose has been noted by many observers (see Jones 1919).

The importance of milk as a human food, and of the cow as a stock animal, has led to a careful and detailed study of the streptococei that occur in normal milk, or in the milk from diseased animals, or that have been isolated from the udder in acute or chronic mastitis.

A type of streptococcus that is almost constantly present in milk, and has been given the name Str. lactis, resembles in many ways the streptococens commonly found in human frees; and it will therefore be more convenient to deal with its fermentation reactions when considering its probable relation to that organism in a later section.

In regard to those streptococci that are associated with mastitis in the cow, acute or chronic, certain additional criteria, based on fermentation reactions, have been introduced within recent years in an attempt to differentiate the characteristically bovine strains from those of human origin. Among these are (1) the final $\mathrm{pH}$ produced in glucose broth (Avery, R. C., and Cullen 1919, Ayers and Mudge 1922, Frost et al. 1927, Minett et al. 1929, Avery, R. C. 1929a, $b$, Minett and Stableforth 1931, 1934, Lancefield 1933, Hare and Colebrook 1934); and (2) the capacity to hydrolyse sodium hippurate (Ayers and Rupp 1922 and other references above). It has been found that mastitis strains of human origin produce a final $\mathrm{pH}$ of $5 \cdot 0-5 \cdot 6 \mathrm{in}$ glucose broth, and fail to hydrolyse sodium hippurate. Bovine strains, on the other hand, produce a final $\mathrm{pH}$ of $4 \cdot 2-4.8$ in gheose broth, and hydrolyse sodium hippurate. It has also been found that the fermentation of trehalose and sorbitol afford a valuable criterion in the examination of a particular group of hæmolytic streptococei that are found both in men and animals-mainly horses. The human strains in the group usually ferment trehalose but not sorbitol ; the animal strains ferment sorbitol but not trehalose (Ogura 1929, Edwards 1932, 1933, Minett 1935a, b). 
Certain Other Biological Tests Employed in Classification.-Before discussing the important problem of the antigenic structure of the streptococci, we may deal briefly with certain other biological tests that have been found useful in identification and classification within this group.

It was shown by Neufeld (1900) that pneumococci are soluble in bile, while other species of streptococei are not.

The mechanism of the bile-solubility test has been studied in some detail by many subsequent observers. The pneumococeus is an organism that readily undergoes autolysis in culture; and Avery and Cullen (1923) have shown that extracts of washed pneumococei contain an enzyme that lyses the bacterial cells. This enzyme is inactivated by heating at $60^{\circ} \mathrm{C}$. for half an hour; but in its active state it is able to act on heat-killed pneumococci, though not on hæmolytic streptococei or other organisms. There seems little doubt (see Mair 1929) that the effect of bile is simply to accelerate this natural autolytic process. In regard to the active substance in the bile, many samples of ordinary commercial sodium taurocholate induce lysis, but the action is very variable, and Mair (1917) has shown that the most actively lytic bile constituent is sodium deoxycholate, a pure solution of which forms the most satisfactory test reagent (see also White 1929, Dowvie, Stent and White 1931). Other factors, besides the presence of bile-acids, are concerned in this test. It is usually stated (see Avery, R. C., and Cullen 1923, Mair 1929) that the optimum pH for autolysis lies between $\mathrm{pH} 6$ and $\mathrm{pH}$, and that the range for bile solubility is about the same, with the limitation that, since the bile-acids are thrown out of solution at $\mathrm{pH} 6 \cdot 5$. the test must be carried out in a solution more alkaline than this. It is, however, customary to employ a reaction of $\mathrm{pH} 7 \cdot 6$, or thereabouts; and it is also customary if a negative bile-solubility test is obtained, to add a drop of dilute alkali before regarding the test as negative. It seems doubtful whether this solubility in alkaline solutions is entirely dependent on the presence of bilc-acids. Dr. Edith Straker (unpublished), working in the authors' laboratory, has found that simple addition of alkali to a suspension of pneumococci lyses the great majority of recently isolated strains, though old laboratory strains are often more resistant.

The salt content, apart from acidity or alkalinity: also exerts a pronounced effect. Nicolle and Adil-Bey (1907) reported that the addition of magnesium sulphate favoured the lysis of pneumococci by bile salts, while Falk and Yang (1926) state that chlorides of monovalent cations inhibit lysis in low concentrations $(0 \cdot 004-1$ per cent.), but may accelerate it in high concentrations $(2 \cdot 0-\mathbf{4} \cdot 0)$. Anderson and Hart $(1934 b)$ studied this phenomenon in more detail. They found that there was a reciprocal relation between the concentration of sodium deoxycholate required to induce lysis and the concentration of sodium chloride in the suspending fluid. There was no evidence of any inhibition by the salt. Lysis occurred in the absence of sodium chloride, but as the concentration increased up to about 5 per cent., less and less deoxycholate was required to produce lysis.

Bile salts are not the only reagents that cause, or accelerate, the lysis of pneumococci; Downie, Stent and White (1931) noted that saponin had a similar effect. Klein and Stone (1931) noted the same fact and Klein $(1933,1935)$ has extended the study of this reaction. He has found that, in order to induce the lysis of pneumococei by saponin it is essential that there should be added to the medium in which the organism is grown either a sterol (cholesterol) or an animal fluid such as blood or serum. Under these conditions he has found all pneumococci to be saponin-soluble. Eight cultures of Str. hremolyticus or Str. viridans were found to be saponin-insoluble.

The question arises as to whether the "bile-solubility" test is to be regarded as a completely reliable criterion, bile-insoluble strains being excluded from the species Str. pneumonice. Neufeld originally regarded bile solubility as being characteristic only of recently isolated pneumococcal strains; but when the test is made with sodium deoxycholate and with due precautions as to the reaction of the inedium, etc., the consensus of opinion is that all, or almost all, strains of normal, smooth pneumococci undergo lysis. 
The behaviour of rough variants appears to be inconstant. Nany, probably most, rough strains are bile-soluble (Griffith 1923, Reimann 1927, Downie et al. 1931); but some are less readily lysed than normal, smooth strains, and a few are apparently quite insoluble. It seems clear that this test, properly performed, is one of the most reliable at our disposal. A smooth strain that proves insoluble in sodium deoxycholate should not be given the title Str. pneumonice without a clear statement that, because of this important divergence from the specific characters, the diagnosis must be regarded as uncertain.

The use of bile has provided another differential test within this group. Weissenbach (1918) noted that enterococci grew well in a medium containing 10 per cent. of bile, while Str. myogenes and other streptococci tested by him failed to grow in this medium. The observation that enterococei grow freely in high concentrations of bile has been confirmed by many subsequent observers; but it would appear that this character is not peculiar to this species, or group. The ability of other species and types to grow in bile-containing media (10 per cent. and 40 per cent.) has been studied by several workers (see Belenky and Popowa 1929, Hinett and Stableforth 1931, Lancefield 1933, Hare and Colebrook 1934, Hare 1935, Hare and Maxted 1935, Colebrook, Maxted and Johns 1935). The possible significance of this character in the classification of streptococei other than the enterococci will be more conveniently discussed in relation to the results obtained by antigenic analysis.

Another test that has been applied in the classification of this group is the ability to reduce, and thus decolorize, methylene blue added to milk, usually in a concentration of $1: 5,000$ (Avery, R. C. $1929 a, b$ ). This test would appear to depend in part on the ability of the organism to multiply in the presence of methylene blue, in part on the Eh that the growing organism induces. Many of the strains that reduce methylene blue have been isolated from animal sources or from milk. There is general agreement that strains of hæmolytic streptococei isolated from severe human infections fail to do so. Enterococci, however, reduce methylene blue actively (Kleckner 1935), even in a concentration of $1: 1,000$. Here again, the significance of this test must be considered in relation to the antigenic structure of the various species or types.

A fourth test that is of considerable value from the point of view of classification is that of heat resistance. It has long been known that heat-resistant streptococei occur in milk (see Ayers and Johnson 1910, 1913, Ayers, Johnson and Davis 1918); and Logan (1914) recorded the presence of heat-resistant streptococei in the human faeces. Houston and McCloy (1916) noted that heat resistance was characteristic of enterococci, and this observation has been amply confirmed by Dible (1921) and by many subsequent observers. It is usual to employ exposure to a temperature of $60^{\circ} \mathrm{C}$. for 20 to 30 minutes as an arbitrary test of heat resistance. The problem that arises in this connection is the identity or nonidentity of the milk and fæcal strains of heat-resistant streptococci; and this will be considered in a later section. Other tests, which will be considered under the classification of the enterococci, comprise ability to grow at $45^{\circ} \mathrm{C}$., at $\mathrm{pH} 9 \cdot 6$, and in the presence of 6.5 per cent. $\mathrm{NaCl}$.

Antigenic Structure.-It will be convenient to begin our discussion of antigenic structure with the pneumococcus, in part, because this is a well-defined species in which the complications referred to above do not arise, in part, because it was in fact one of the first species in which a division into immunologically different races, or types, was clearly demonstrated, and, in part, because the chemical study of antigenic structure was initiated with this organism, and we still know far more about the chemical differences that determine the antigenic specificity of the various types of pneumococci than we do about similar differences in any other bacterial species.

The Antigenic Structure of the Pneumococci.-Neufeld and Händel (1909) first demonstrated the existence of antigenically different types of pneumococci. They studied the protective effect of different antipneumococcal sera in mice, and 
found that a given serum would protect against the homologous strain of pneumococcus, but not against heterologous strains. Dochez, Avery and their colleagues (Dochez and Gillespie 1913, Dochez and Avery 1915, Avery 1915, Avery et al. 1917) later studied the antigenic relationships of a large collection of pneumococci, using the methods of direct agglutination and agglutinin absorption. They confined their attention, for the most part, to strains isolated from cases of lobar pneumonia in man, and among these they were able to recognize three well-differentiated types, Types I, II and III, leaving a large heterogeneous group unclassified. Lister (1916) carried out a similar study on strains isolated from cases of pneumonia among the mine-workers in South Africa. He was able to differentiate several antigenic types, which he labelled with letters instead of numbers. These observations have since been confirmed and extended by workers in many parts of the world, the three classical American types being generally accepted as the standard of reference; and it was not long before we obtained a reasonably adequate picture of the distribution of these three types in cases of lobar pneumonia, in other pneumococcal infections, in healthy contacts and in the population at large. For many years, however, no serious attempt was made to analyse the unclassified heterologous group, which formed a considerable proportion (25-50 per cent.) of the strains isolated from cases of lobar pneumonia, and the majority of those isolated from the upper respiratory tract of normal persons. It was the custom to refer to such strains as belonging to Group IV, an unfortunate nomenclature that became frankly misleading when the label was changed to "Type IV", which not infrequently happened.

Within recent years, Cooper and her colleagues (Cooper, Edwards and Rosenstein 1929, Cooper et al. 1932, Cooper and Walter 1935) have made a detailed study of this previously unclassified group. Among strains isolated from lobar and bronchopneumonia, from various other pneumococcal infections, and from normal persons, they have identified 29 new antigenic types, making 32 in all, including the classical Types I, II and III. Most of these types are, it should be noted, sharply differentiated from each other, so that they may be identified by direct agglutination. Since Cooper's study, further types have been described by other workers (see Kauffmann et al. 1940, Walter et al. 1941, Mørch 1942). Unfortunately two systems of labelling have been used, the one making use of numbers irrespective of antigenic components shared with other types, the other using letters in addition to numbers to bring out antigenic components possessed by different types in common. Eddy (1944) has described the cross-reactions that may be met with, and has suggested the use of a series of Arabic numbers ranging from 1 to 74 to cover the types known at present. We may be quite sure that there are still new types of pneumococci to be identified, and new labels to be given; but it seems likely that our present 74 types include most of those that are parasitic in man. Gundel and Schwarz (1932), for instance, report that, of 364 strains of pneumococci, isolated from sick or healthy children or adults, and containing no examples of the classical Types I, II and III, only 3 per cent. were unassignable to one or other of Cooper's new Types IV-XXXII.

It has long been recognized that the antigenic behaviour of intact pneumococci, in the normal smooth state, is probably determined by the nature of the capsules surrounding the bacterial cells. Neufeld (1902), for instance, noted that the capsules of pneumococci, when acted upon by a specific antiserum, become greatly swollen; and, within recent years, it has been shown that this phenomenon can be utilized in the identification of pneumococcal types (Neufeld and Etinger-Tulczynska 1931, Etinger-Tulczynska 1932, Armstrong 1932, Logan and Smeall 1932, Sabin 1933, Beckler and MacLeod 1934, Cooper and Walter 1935). 
A great advance was made in the study of the antigenic structure of the pneumococci. and, indeed, of bacteria in general, when Avery, Heidelberger and their colleagues attacked the problem from the chemical side (Heidelberger and Avery 1923, 1924, Avery and Heidelberger 1923, 1925, Avery et al. 1925, Heidelberger et al. 1925, Avery and Morgan 1925, Heidelberger 1927, Heidelberger and Goebel 1927). By suitable methods of extraction, followed by fractional precipitation, it was found possible to separate the capsular components that determine type-specificity in a state of chemical purity. These components were found to be complex polysaccharides; and some of the main chemical and physical characters of the capsular polysaccharides of the three classical types have been determined (see Table 36). Solutions of these polysaccharides, it will be noted, give specific precipitation, in high dilution, when mixed with the corresponding antisera.

TABLE 36

Characters of the Type-specteic Antigens of Pneumococci, after Avery and HEIDELBERGER.

\begin{tabular}{|c|c|c|c|c|}
\hline Type. & $\begin{array}{c}\text { Optical } \\
\text { Rotation. }\end{array}$ & $\begin{array}{l}\text { Per cent. } \\
\text { Nitrogen. }\end{array}$ & $\begin{array}{l}\text { Substances obtained } \\
\text { on Hydrolysis. }\end{array}$ & $\begin{array}{l}\text { Dilution giving } \\
\text { Specific Precipitation. }\end{array}$ \\
\hline I & $+300^{\circ}$ & 5 & $\begin{array}{l}\text { Galacturonic acid, and } \\
\text { amino-sugar derivative }\end{array}$ & $1: 6,000,000$ \\
\hline II & $+74^{\circ}$ & 0 & Glucose & $1: 5,000,000$ \\
\hline III & $-33^{\circ}$ & 0 & $\begin{array}{l}\text { Glucose and } \\
\text { Glucuronic acid }\end{array}$ & $1: 6,000,000$ \\
\hline
\end{tabular}

The chemical constitution of the capsular antigens of the new types of pneumococei differentiated by Cooper and her colleagues has yet to be determined, though a start has been made with this work (see Heidelberger and Kendall 193I, Brown and Robinson 1943). We may, however, safely assume that each pneumococcal type is characterized by a specific capsular polysaccharide that determines its antigenic behaviour. We may identify these types either by agglutination, or by the capsule-swelling reaction, or by precipitin tests carried out with an autolysate, or extract, of the pneumococcal cells.

Some, at least, of these pneumococeal polysaccharides may exist in immunologically different forms, or may be altered during the process of chemical extraction and purification. Thus, the studies of Enders (1930), and of Wadsworth and Brown (1931), showed the presence in Type I pneumococci of a specific antigenic component that differed in its immunological reactions from the specific capsular polysaccharide as ordinarily prepared. Avery and Goebel (1933) were able to show that this component is an acetylated form of the Type I capsular polysaccharide, and that it is apparently in this form that the polysaccharide exists in the normal bacterial cell. The acetyl groups are removed by the methods of extraction and purification that had been commonly employed, leaving a deacetylated polysaccharide that is still specific for the Type I pneumococcus, but has lost certain antigenic activities possessed by the normal acetylated form. It is important to note that the type-specificity of the capsular antigen is not destroyed by this particular chemical change ; and it seems quite likely that similar minor alterations in chemical structure may be induced during the extraction and purification of many other antigenic components.

This polysaccharide capsular component is not, of course, the only antigenic constituent of the pneumococcal cell. Tillett, Goebel and Avery (1930) have isolated another component that gives all the usual reactions of a polysaccharide, is not inactivated by peptic or tryptic digestion, and yields about 30 per cent. of reducing sugar on hydrolysis. It contains about $6 \cdot 1$ per cent. of nitrogen, and differs from the capsular polysaccharides in containing phosphoric acid. It is not type-specific, but appears to characterize the pneumococcus as a species. 
There is also (Avery and Heidelberger 1923) a nucleo-protein antigenic component, precipitable from extracts by acetic acid. It is probably situated deeply within the intact bacterial cell. It is shared by all pneumococei and by many other bacteria, including all those species of streptococei that have been examined.

The picture of the antigenic structure of the species Str. pneumonice that emerges from these studies may be tentatively outlined as follows: There is a central protoplasmic portion of the cell which, in its antigenic relationships, is neither species- nor type-specific. Situated probably at the cell surface, there is another component, mainly carbohydrate in nature, but containing nitrogen and phosphorus, that is specific for Str. pneumonice as a species. External to this, in the normal smooth forms, there is a capsule, composed wholly or in part of a polysaccharide that is specific for each pneumococcal type. There are, we must suppose, over seventy of these capsular polysaccharides within the pneunococcal species; probably there are many more. The antigenic behaviour, and to some extent the virulence, of the intact pneumococcal cells are, it should be noted, determined by these capsular antigenic components, so that they are of particular importance to the medical bacteriologist.

The Antigenic Structure of the Hæmolytic Streptococci.- ithen we come to study the antigenic structure of the hæmolytic streptococci we are on much more difficult and debatable ground ; in part because, as we have already indicated, it is by no means easy to define exactly what we mean by a hæmolytic streptococcus ; in part because, if we accept the usnal definition-the occurrence of $\beta$-hæmolysis on a blood agar plate-we shall include in our hæmolytic group, not one species, but several; in part because the technical difficulties of antigenic analysis are far greater than in the case of the pneumococcus. In spite of all these difficulties a great advance in our knowledge has been made during recent years; and, though we cannot as yet present any clear and detailed picture, we can provide a sketch-plan which, with the necessary modifications, will certainly provide the basis for any future, and more complete, classification.

To obtain a clear picture of the present position, it will be better to disregard the sequence in which our knowledge has been reached.

Group Relationships.-The observations of Hitcheock (1924) and of Lancefield $(1928,1933,1941)$ have revealed the presence of a number of different serologically active polysaccharides in hæmolytic streptococci from different sources. Instead of being responsible, as in pneumococci, for type-specificity, each polysaccharide is common to a group of organisms derived from a particular source. Thus, the majority of strains isolated from pathological lesions in man share the same polysaccharide and are classified as Group A. Strains from mastitis in the cow possess another polysaccharide and are classified as Group B. Strains from infections in lower animals possess still another polysaccharide and are classified as Group C and so on. Altogether 12 groups have now been recognized, each with its peculiar polysaccharide antigen (Table 37 ).

The association between the type of polysaccharide and the source of the strains is close, but not absolute. Human beings, for example, may be infected occasionally with organisms belonging to Groups B, C, D, and G, though Group A strains far ontnumber the rest. Moreover, when infection with these other types occurs, it is often confined to one part of the body. Thus, Group B strains are seldom found except in infections of the female genital tract; Group D strains are restricted mainly to cystitis and wound infections; and Group G strains to genito-urinary and occasional throat infections. 
TABLE 37

Group and Type Specific Antigens of Hzmolytic Streptococci (Modified from LANCEFJELD 1941).

\begin{tabular}{|c|c|c|c|c|}
\hline \multicolumn{2}{|c|}{ Group Specificity. } & \multicolumn{3}{|c|}{ Type Speciffity. } \\
\hline \multirow{2}{*}{ Serological group. } & \multirow{2}{*}{$\begin{array}{l}\text { Group-specifie } \\
\text { Carbohydrate } \\
\text { substances. }\end{array}$} & \multirow{2}{*}{$\begin{array}{l}\text { Specifie } \\
\text { types } \\
\text { recognized. }\end{array}$} & \multicolumn{2}{|c|}{ (Type-specific substances). } \\
\hline & & & Designated. & Chemical nature. \\
\hline A & \multirow{4}{*}{$\begin{array}{l}\text { Polysaccharides } \\
\text { immunologically } \\
\text { distinct for } \\
\text { each group }\end{array}$} & $\begin{array}{c}\text { At least } \\
30\end{array}$ & "MI" & $\begin{array}{l}\text { Proteins } \\
\text { Undetermined }\end{array}$ \\
\hline B & & Several & "S" & Polysaccharides \\
\hline C & & Several & - & Proteins \\
\hline $\mathrm{D}, \mathrm{E}, \mathrm{F}, \mathrm{G}, \mathrm{H}, \mathrm{K}, \mathrm{L}, \mathrm{M}, \mathrm{N}$ & & Several & "S" & Polysaccharides \\
\hline
\end{tabular}

The association between the type of polysaceharide and the hæmolytic power of the organisms is variable. So far as we know, most Group A strains give rise to typical $\beta$-hæmolysis on blood agar plates; but as already pointed out a few strains have been reported that are hæmolytic only under anaerobic conditions, or are frankly non-hæmolytic. In human infections we are generally on safe ground in disregarding non-hæmolytic streptococcal colonies; except under very unusual conditions, they are unlikely to be responsible for the ordinary manifestations of hæmolytic streptococcal infections. In Group B, however, the position is different. Stableforth (1932) found that about half the bovine strains he studied were non-hæmolytic, even though he could detect no immunological difference between the hæmolytic and the non-hæmolytic members of the group. Among the other groups, non-hæmolytic strains have been found with varying frequency. Special strains in Group C produce $\alpha$-hæmolytic colonies ; and one group, Group N, comprising strains of Str. lactis, appears to give rise to uniformly non-hæmolytic colonies. The evidence suggests that the possession of the group polysaccharide is a more constant and fundamental character than the ability to lyse blood cells. It is therefore becoming common to pay greater attention to the type of polysaccharide present in a given strain than to its hæmolytic power; though in practice, especially in medical laboratories, the observation of hæmolysis constitutes the first important differential criterion of presumptive pathogenicity.

The polysaccharides themselves, which are sometimes referred to as " $\mathrm{C}$ " substances, appear to form an integral part of the bacterial cell. To obtain them in solution, the organisms have to be disrupted. For this purpose, either Lancefield's (1933) acid-extraction method or Fuller's (1938) formamide method may be used. Their presence can be demonstrated by a simple precipitation reaction, using specially prepared antiserum. They seem to play no part in the agglutination of streptococci. There is little information yet on their chemical structure, but the polysaccharide of Group A strains is known to yield reducing sugars on hydrolysis, to be non-toxic for animals, and to be antigenic only when in combination with the cellular protein.

\section{Type Relationships.}

Group A.-The findings recorded by Dochez, Avery and Lancefield (1919) Bliss (1920, 1922), Gordon (1921), Eagles (1924) and Stevens and Dochez $(1926 a, b)$ 
made it possible to differentiate and identify many different antigenic types among hæmolytic streptococci isolated from human infections; and the more detailed and extensive studies of Griffith $(1926,1927,1928,1934,1935)$, Smith $(1926,1927)$, James (1926), McLachlan and Mackie (1928), Gumn and Griffith (1928), and Dora Colebrook (1935) have served to establish many of these types on a satisfactory serological basis. Griffith, who has been the main contributor to this particular problem, has differentiated by means of the slide agglutination reaction 27 types of pathogenic hæmolytic streptococci isolated from various lesions in man. Of these, 23 were later found to be members of Group A; Types 7, 20 and 21 fell into Group C, and Type 16 into Group G. Before he died, Griffith added three more types to Group A, bringing the total numbered types up to thirty. Further types have since been added.

Lancefield $(1928,1933)$ attacked the problem from another angle. She extracted type-specific antigens from the cocci by hot acid, and identified them by the precipitin test. On the whole, her results agreed with those of Griffith, but some discrepancies occurred. Further observations (Lancefield 1940, 1943, Swift et al. 1943, Lancefield and Stewart 1944, Watson and Lancefield 1944, Stewart et al. 1944, Zittle 1942, Elliott 1943, Krumwiede 1943) have shown that two separate antigens are concerned in the type-specificity of Group A strains. One, referred to as $\mathrm{M}$, is a protein : the other, referred to as $\mathrm{T}$, is of undetermined nature. The II antigen is present only in mucoid or matt colonies (see p. 595) and is most abundant in freshly isolated pathogenic strains; the $\mathrm{T}$ antigen is found not only in mucoid and matt, but also in the degraded glossy colonies containing avirulent organisms. Antibody to II seems to be responsible for the M precipitin reaction, for type-specific protection, and as a rule for part of the type-specific agglutination of matt variants. Antibody to $\mathrm{T}$ appears to be solely responsible for type-specific agglutination of glossy variants, and mainly responsible for type-specific agglutination of matt variants, but to be unconcerned in protection. The M antigen can be destroyed by peptic or tryptic digestion; heat-killed enzyme-treated organisms stimulate the production of $\mathrm{T}$ antibodies alone, so that pure $\mathrm{T}$ antibody can be obtained and the distribution of $\mathrm{T}$ antigen determined. In most types $\mathrm{M}$ and $T$ antigens occur together, but in a few types one or other is missing or is shared with some other type-hence the accasional discrepancies between the results of agglutination and precipitation tests.

For example, Types 10 and 12 contain serologically identical M antigens, but different $\mathrm{T}$ antigens. Closely related $\mathrm{T}$ antigens occur in Types $15,17,19,23$, and 30 , and another set of related $\mathrm{T}$ antigens occurs in Types 4, 24, 26, 28, 29, and 46. In some types, strains are encountered that contain no $\mathrm{T}$ antigen at all. Moreover, the amount of $\mathrm{M}$ and $\mathrm{T}$ antigen that is formed, or at least that can be detected by agglutination methods, depends on the temperature of incubation. There is evidence to suggest that the M substance is formed best at $37^{\circ} \mathrm{C}$., but is liable to inactivation at this temperature-possibly through enzyme action (Elliott 1944).

An additional complication to the precipitin reaction is that extracts may contain variable amounts of non-specific nucleo-proteins, which are common to hæmalytic streptococci, pneumococci, and streptococci of the viridans group. It may also be noted that there is at least one other non-type-specific protein (" $\mathrm{Y}$ ") about which little is as yet known. It will therefore be clear that the type determination of Group A streptococci is far from straightforward; nevertheless their general antigenic constitution is now fairly well understood.

P.B. 
Group B.-The existence of specific types in Group B was first established by Stableforth (1932), who found three serological types among strains from bovine mastitis ; these were sharply distinguished by their precipitation and agglutination reactions. The type-specific antigen was found by Lancefield $(1931,1938)$ to be, not a protein as in Group A, but a polysaccharide. By precipitation tests she was able to define four specific types. British and Australian investigators (Stableforth 1937, Stewart 1937, Simmons and Keogh 1940) have carried this study further by separating off, mainly by the agglutination and absorption of agglutinins technique, a number of sub-types. What the antigen or antigens concerned in the agglutination test may be is still a matter for conjecture, but so far as the four main types are concerned, there seems to be agreement between the results of the precipitation and agglutination tests (Slanetz and Naghski 1940, Simmons and Keogh 1940). The notation is still confused, but there is much to be said for Stableforth's suggestion of numbering the main types, as in Group A, and giving small letters to the sub-types, e.g. Type 1a, 1b, 1c, 1d, 2a, 3a, 3b, etc. Again, there is disagreement on the identity or non-identity of Group B strains of human and bovine origin. Further work will be required before discrepancies of this sort can be cleared up.

Group C.- It has alrealy been mentioned that three of the types of hæmolytic streptococci identified by Griffith, namely Types 7, 20, and 21, belonged to Group C. Five more types have now been differentiated by the agglutination test among human strains (Simmons and Keogh 1940), and five among equine strains (Bazeley and Battle 1940). There is reason to believe that the type-specific antigen is of protein nature.

Group D.-This group contains a number of organisms of the enterococcus type, the specific or varietal nature of which will be discussed later (p. 582). By agglutination several types have been distinguished by Japanese and other workers (for references see Grumbach and Schnetz 1938, Ehrismann-1943). Lancefield (1941) states that she has been able to define three distinct types. The typespecific antigens appear to be of polysaccharide nature.

Other Groups.-One type has been found in Group E (Lancefield 1941). In Group F Bliss (1937) established four types by precipitation and agglutination reactions, and in Group G one type, which was identical with F Type 1. Simmons and Keogh (1940) were able to distinguish a number of serological types among both the large-colony and the so-called minute forms of streptococci in Group $\mathrm{G}$. One type has been found in Group K (Lancefield 1941).

Antigenic Structure of the $\alpha$-Hæmolytic Streptococci.-We know little, as yet, about the antigenic structure of the many species and varieties of $\alpha$-hæmolytic streptococci that have been described. It seems quite clear that the viridans group of streptococci contains no such polysaccharide group antigens as have been found in the hæmolytic streptococei. On the other hand, specific types can be recognized within certain species. Sherman, Niven and Smiley (1943), for example, using the precipitation technique, have found that strains of Str. salivarius fall into two main types and an unknown number of other types. Solowey (1942) likewise, who studied 108 strains of viridans type from subacute bacterial endocarditis and 99 strains from human throats and extracted teeth, was able to distinguish at least eight different types. The systematic relationship between the different organisms has still to be worked out. 


\section{Properties, Classification and Nomenclature of the Hæmolytic Streptococci}

Before describing the pathogenicity of the various streptococci, it will be convenieut to summarize the main properties of the different groups, and to discuss the nomenclature of the organisms within each group. It must be emphasized once again that not all strains falling into Lancefield's groups form $\beta$-hæmolytic colonies on blood agar. The heading of this section is not strictly accurate; but until some more suitable term is proposed we shall include $\alpha$-hæmolytic and nonhæmolytic organisms possessing the same group polysaccharide as hæmolytic organisms under the general designation of hæmolytic streptococci.

\section{Group A.}

Streptococei belonging to this group share in common the Group A carbohydrate component described by Lancefield. They are divisible into a number of different serological types, either by agghtination reactions with absorbed sera, or by precipitin reactions carried out with type-specific antisera and suitably prepared bacterial extracts. The type-specific antigens concerned are protein in nature, and soluble in dilute acids. Of the 27 types deseribed by Griffith (1935), it would appear that four (Types 7, 20, 21 and 16) do not possess the group-specific carbohydrate, and should therefore be excluded from this group. Types 7, 20 and 21 belong, antigenically, to Group C; Type 16 to Group G (Hare 1935).

Group A streptococei, in addition to producing $\beta$-hæmolysis in blood agar plates, appear, without exception, to form a soluble hæmolysin.

In regard to their other biological and biochemical characters, Group A streptococei produce a final $\mathrm{pH}$ of $5 \cdot 0-5 \cdot 6$ in dextrose broth; they do not hydrolyse sodium hippurate ; they do not reduce methylene blue in milk; they ferment trehalose but not sorbitol; they may or may not grow in 10 per cent. bile agar, and seldom grow in 40 per cent. bile agar. These are the main differential group characters. In addition, Group A strains almost always ferment salicin and lactose, seldom mannitol, and very seldom raffinose or inulin. With the exception of Type 3 strains (Fuller and Naxted 1939, Hadley et al. 1941), peroxide is generally produced. A biochemical classification, based on the fermentation of starch, mannitol, and cellobiose, which is in concordance with serological typing, has been proposed by Keogh and Simmons (1940).

The strains that have been adequately identified as belonging to this group have, in the main, been derived from infections in man - tonsillitis, scarlet fever, cellulitis, erysipclas, puerperal fever, other types of septicæmia, acute broncho-pneumonia, otitis media and so on. They have also been isolated from the throat, nasopharynx, or nose, in normal persons. They have occasionally been isolated from cases of mastitis in cattle ; but in such instances there have usually been grounds for suspecting a human source of infection.

It is clear that streptococci falling into Group A possess all the characters that have been attributed to the classical Str. pyogenes; and the group itself is so well differentiated that it clearly requires a distinctive label. Should it be given specific rank? It is certain that we cannot include in a single species all the antigenic groups of hæmolytic streptococci $(\mathrm{A}-\mathrm{N})$. The only question is, into how many different species they should be divided. The balance of the evidence at present available appears to us to be in favour of recognizing the Group A strains as constituting a species in the generally accepted bacteriological sense. Its relation to the strains falling into other antigenic groups will be discussed in succeeding sections. There remains the question of the correct specific name. The name Str. homolyticus has obtained wide currency. It was used by the Committee of the Society of American Bacteriologists to denote the type species (Winslow et al. 1920), and we adopted it in the first edition of this book. 
But the knowledge that has accumulated during recent years seems to us to render the name inconvenient and misleading. If we adhered to it, we should no longer mean by Str. hcomolyticus a "hæmolytic streptococcus," or even a "streptococcus giving $\beta$-hæmolysis," but only a particular group of streptococci falling into that category. It therefore seems to us wiser to revert to the name Str.pyogenes, defining that name as equivalent to " a streptococcus of Group A," with the proviso that this definition may well have to be modified in the future.

Before leaving this group and turning to others we may note briefly the existence of certain strains that have been isolated from milk and from the throats of infected persons during milk-borne epidemics of tonsillitis (Davis and Rosenow 1912, Davis 1912, 1929, Stokes and Hachtel 1912, Capps and Davis 1914). These streptococci differ from the classical Str. pyogenes only in giving mucoid or semi-mucoid colonies on blood agar plates and in showing well-defined capsules. Because of this difference Davis allotted to them a separate specific name, Str. epidemicus. It seems very doubtful, however, whether this procedure is justified. Although Str. pyogenes is not a capsulated species in the same sense as Str. pneumonice, it not infrequently forms recognizable capsules when growing in the tissues, or during the first few hours of its growth in serum broth. Seelemann and Hadenfeldt (1932) compared a large number of strains bearing the label Str. epidemicus with typical strains of Str. pyogenes, and were unable to distinguish between them. On the basis of the available evidence we think that the name Str. epidemicus should be provisionally discarded, and that the strains bearing that label should be included in the species Str. pyogenes. It may be noted that there is some tendency for other organisms to produce capsules when growing in milk.

Similarly, there seems to us no adequate reason for allotting different specific names to strains that, while possessing all the essential characters of Str. pyogenes, differ from one another in regard to certain fermentation reactions. We should not, therefore, recognize a Str. infrequens, fermenting mannitol as well as salicin, or a Str. anginosus fermenting neither of these sugars. It is, indeed, by no means certain that all the strains to which these names have been attached are true hæmolytic streptococci of the pyogenes type.

\section{Group B.}

The streptococci of this group share in common a group-specific antigen that is carbohydrate in nature and differs from the group-specific antigen of Group A, or of any of the groups subsequently described. Group B streptococci are further divisible by precipitation or agglutination tests into four main antigenic types, and by absorption of agglutinin tests into a number of sub-types. The type-specific antigens within this group are apparently not acid-soluble proteins, but complex carbohydrates of a different chemical structure from the carbohydrate that determines group-specificity.

Streptococci falling within this group may or may not produce $\beta$-hæmolysis in blood agar plates. It would appear (see Stableforth 1932) that something over half of these strains are $\beta$-hæmolytic; but the exact proportion is still a subject of controversy. Brown $(1937,1939)$ has shown that the hæmolytic strains are characterized by the formation of a double zone of hæmolysis, which is best seen around deep colonies in rabbit blood agar incubated for 48 hour's at $37^{\circ} \mathrm{C}$. and refrigerated overmight. Although many hæmolytic Group B strains yield a soluble hæmolysin, it would seem that this character is much less constant than with Group A strains; and the titres of the filtrates obtained are usually much lower.

In regard to their other biological and biochemical characters, Group B strains differ from Group A strains in that they produce a lower final $\mathrm{pH}$ in glucose broth (4.2-4.8), hydrolyse sodium hippurate, and usually grow both on 10 per cent. and 40 per cent. bile agar. They resemble Group A strains in failing to reduce methylene blue in milk, and in fermenting trehalose but not sorbitol. Sucrose and glycerol are usually fermented (Simmons and Keogh 1940, Gunsalus and Sherman 1943), but mannitol, raffinose, sorbitol, 
and inulin are not; the reactions in lactose and salicin are variable (Brown 1939). It may be noted that a considerable proportion of Group B strains form pigmented cells, usually yellow or red in colour (Orla-Jensen 1919, Plummer 1941).

The great majority of Group B strains that have been adequately identified have been isolated from cases of mastitis in cattle, in many cases under conditions which have made it almost certain that they were the primary cause of the disease. They have occasionally been isolated from the normal human throat and vagina. They are very rarely pathogenic for man.

Group B, like Group A, clearly demands a label ; and we feel that it may provisionally be accorded specific rank. We follow Klimmer and Haupt, and Minett and his colleagues, in giving it the name Str. agalactice (Kitt 1893) (see Klimmer and Haupt 1930, Minett 1935b), in preference to the name Str. mastitidis contagiosa, assigned by Nocard and Mollereau (1887) to streptococci isolated from a similar source. There can, we think, be no doubt that, in defining this species, antigenic structure should take precedence over hæmolysin production as a systematic criterion, and that the specific name should be applied to the non-hæmolytic as well as to the hæmolytic strains. We should, therefore, describe hæmolysin production as being an almost constant character within the species Str. pyogenes, but a variable character within the species Str. agalactice; whether the nonhæmolytic strains of Str. agalactice should be classed as a distinct variety, the future must decide.

\section{Group C.}

The strains that fall within this group share a common group-specific polysaccharide antigen. The existence of a number of antigenic types can be demonstrated by agglutination. Of Griffith's original series, Types 7, 20 and 21 were later found to belong to this group. Five further types among human strains have been differentiated by Simmons and Keogh (1940), and five among equine strains by Bazclcy and Battle (1940). The type-specific antigen appears to be of protein nature. On blood agar the colonies tend to be surrounded by a rather larger zone of hæmolysis than colonies of Group A, the outer edge of the hæmolytic zone being hazy. Colonies of the typical Str. equi type are large, honey-coloured, and of very viscous consistency; if closely adjacent, they readily flow together, forming a streak of sticky material. Most, but not all, strains form a filtrable hæmolysin.

In regard to their other biological and biochemical characters Group C strains display certain significant differences among themselves. They produce in glucose broth a final $\mathrm{pH}$ intermediate between that produced by Group A strains on the one hand and by Group $B$ strains on the other; the range covered by the group as a whole appears to vary between $\mathrm{pH} 4.5$ and $\mathrm{pH} 5 \cdot 4$. No Group C strains hydrolyse sodium hippurate. Many of them grow on 10 per cent. bile agar, but few on 40 per cent. bile agar. On the basis of their action on trehalose and sorbitol Group C streptococci can be differentiated into three sub-groups (Edwards 1934). One of these sub-groups ferments neither trehalose nor sorbitol, another ferments sorbitol but not trehalose, a third ferments trehalose but not sorbitol.

There is a correlation between fermentation reactions and habitat that gives to these sub-groups an importance that they would not otherwise possess. Most of the strains belonging to the trehalose-negative, sorbitol-negative sub-group have been isolated from horses, particularly from cases of strangles, though they have also been obtained from infections in other animals. They are further differentiated from the gromps of streptococei that have been described above by their failure to ferment lactose. They share with these other gromps the ability to ferment salicin, and the inability to reduce methylene blue in milk. This sub-group clearly corresponds in its biochemical characters to the 
Str. equi of many authors. According to Bazeley and Battle (1940) all strains of this sub-group fall into one serological type-Type 1. The second sub-group ferments sorbitol but not trehalose, and fails to reduce methylene blue in milk. Serologically, it comprises two types. Type 2 is the more numerous, and is distinguished by fermenting lactose. It has been isolated from respiratory catarrh of horses, and from a variety of lesions in horses, eattle, guinea-pigs, rabbits and so on. Type 3 does not ferment lactose, and was found by Bazeley and Battle only in equine respiratory eatarrh.

There is as yet no evidence that streptococci belonging to this, or to the preceding, sub-group are pathogenic for man.

The third sub-group is characterized by the fermentation of trehalose but not of sorbitol. The strains belonging to this sub-group have been derived both from animal and human sources, and it seems clear that some strains at least are pathogenic for man. Three of Griffith's 27 types of human-pathogenic, hæmolytic streptococci belong to this C subgroup, not to Group A. All strains of this sub-group are recorded as fermenting salicin, but the action on lactose appears to vary. The results recorded by Edwards (1934) and by Hare (1935) suggest that many of the animal strains fail to ferment lactose, while almost all the human strains act on this sugar. Among equine strains Bazeley and Battle (1940) found that the great majority did not ferment lactose; these fell into their serological Type 4. A few, which did ferment lactose, fell into Type 5. Simmons and Keogh (1940), who studied 169 strains of human origin falling into the trehalose-positive sorbitolnegative sub-group, were able to divide them into seven further sub-groups on the basis of lactose, æsculin, amygdalin and raffinose fermentation; these bore some relation to the eight serological types-Types 7,20 and 21 and five new types-which they were able to distinguish by agglutination.

The labelling of Group C hæmolytic streptococci presents a problem of some difficulty. There is no specific name that can be applied to the group as a whole. One sub-group of strains, however, does seem to merit special attention, namely the group of trehalose, sorbitol and lactose-negative strains, responsible for strangles in horses, which produce characteristic colonies, and fall into Bazeley and Battle's serological Type 1. If Bazeley and Battle's findings are confirmed, then it may be justifiable to apply the specific designation Str. equi to this sub-group. The term Str. dysgalactio is in common use among veterinary bacteriologists to denote a non-hæmolytic streptococcus responsible for some cases of acute or subacute mastitis in cattle (see Minett 1936, Stableforth 1942). According to Stableforth (1945) this organism contains the Lancefield Group C carbohydrate, and should therefore be included with the other strains that we have just described. Its exact relationship, however, to these strains is still under study and the specific name that it has been awarded should be regarded as provisional only.

\section{Group D.}

Great confusion has existed in the past between $(a)$ the hæmolytic streptococci falling into Group D, (b) the enterococci-usually non-hæmolytic-isolated from human fæces, and $(c)$ the lactic streptococci-also non-hæmolytic-so frequently present in milk.

Group $D$ homolytic streptococci.-Lancefield $(1933,1941)$ has recognized a group of hæmolytic streptococci that are characterized by the possession of a specific polysaccharide antigen. These organisms are generally known as Group D hæmolytic streptococci. They have been isolated mainly from cheese and from human fæecs. Morphologically, they tend to assume the diplococcal rather than the streptococcal formation. On horseblood agar they give rise to $\beta$-hæmolytic colonies. Some, at least, of the strains are capable of producing a filtrable hæmolysin under special conditions (Todd 1934, Plummer 1941). 
They give a low final $\mathrm{pH}$ in glucose broth $(4 \cdot 0-4 \cdot 8)$. They grow freely on 10 per cent. and 40 per cent. bile agar. They reduce methylene blue in milk. They are heat-resistant, withstanding a temperature of $60^{\circ} \mathrm{C}$. for 30 minutes. They fail to hydrolyse sodium hippurate. They ferment lactose, salicin, nearly always mannitol, and usually but not always trehalose and sorbitol.

Enterococci.-Many bacteriologists, especially of the French school, have long recognized the occurrence in the human intestine of a characteristic streptococeus, usually occurring in pairs of ovoid cocei, sometimes in short chains. The characteristic morphology of this organism was described by Thiercelin in 1899, who called it the Enterococcus; and under that name it has made frequent appearances in later literature.

The interrelation of the streptococci of the human intestine has been stuctied by Dible (1921), whose paper on this subject affords an admirable example of the methods which should be employed in differentiating bacterial groups. He tested 134 strains of streptococci from human fæces as regards their behaviour in a large number of biological tests, including heat resistance (see Houston and McCloy 1916) and chain formation as well as various biochemical reactions; and measured the association between different pairs of reactions by calculating a statistical coefficient of association. Using one such coefficient, which gires the value of +1 where the association between two characters is absolute, 0 where there is no association, and -1 where the characters are mutually exclusive, he obtained the following values for the association between a particular series of characters among his 134 strains:

Heat resistance and mannitol fermentation

, ", " raffinose fermentation

$-0.85$

" " , " , chain formation .

$-0.96$

Mannitol fermentation and chain formation

Heat resistance, as here designated, was tested by the ability of the various strains to survive heating at $60^{\circ} \mathrm{C}$. for 30 minutes. Those organisms were classed as chain-formers which showed any wide departure from the diplococcal form. Dible thus succeeded in demonstrating the existence in human fæces of a characteristic group of organisms which possessed a predominantly diplococcal morphology, were unusually resistant to heat, almost always fermented mannitol, and very seldom fermented raffinose. It may be added that the streptococei belonging to this group constantly fermented salicin, very seldom fermented inulin, and gave good growth on gelatin at $22^{\circ} \mathrm{C}$. About 10 per cent. of them liquefied gelatin. None of them caused hrmolysis in blood agar, or produced a green pigment. In a later communication (Weatherall and Dible 1929), it was noted that some strains of enterococci, having all the characters referred to above, produced areas of hæmolysis when grown on blood agar plates; but no filtrable hæmolysin could be obtained by the usual methods of cultivation. It seems clear that these hæmolytic strains correspond to the "Group D" hæmolytic streptococci referred to above.

It has already been noted that streptococei from the human fæces grow freely in the presence of bile (Weissenbach 1918) and reduce methylene blue in milk. They have also been shown to produce a low final $\mathrm{pH}$ in glucose broth (Sherman and Stark 1931), to grow at both $10^{\circ}$ and $45^{\circ} \mathrm{C}$, and to develop in skimmed milk containing $1: 1,000$ methylene blue. Most strains belonging to this group produce no change on a blood agar medium, though a few have been described as forming $\alpha$-hæmolytic or $\beta$-hæmolytic colonies. According to Ehrismann (1943) the effect on blood is variable.

Lactic streptococci.-Günther and Thierfelder (1895) described the occurrence in milk of an organism which was responsible for spontaneous souring and clotting. They described the organism as a short bacillus; but Heinemann (1906), when investigating the bacterial flora of milk some ten years later, pointed out that a particular streptococeus, which was ahmost constantly present in fresh milk, was probably identical with the organism of Giunther and Thierfelder. Baehr (1910) confirmed the frequent presence of this streptococcus and noted that it produced a large amount of acid, and rapid clotting. Ruediger 
(1912) noted the absence of hæmolysis on blood agar plates. Sherman and Albus (1918), in a careful comparative study of strains of this organism, and of other strains referred to as Str. pyogenes, noted the following points of difference. The lactic-acid streptococcus grew predommantly as diplococci or short chains, it clotted milk within 24 hours, it produced high acidity in milk ( 0.75 per cent. or more measured as lactic acid), it grew well at $10^{\circ} \mathrm{C}$., but very poorly at $43^{\circ} \mathrm{C}$., and it rapidly reduced methylene blue, litmus, indigo carmine and neutral red. In each of these characters it differed sharply from Str. pyogenes. Ayers, Johnson and Mudge (1924) report that the lactic-acid streptococcus produces high acidity, rapidly clots and decolorizes litmus milk, and reduces methylene blue, or Janus green. This particular streptococcus has for long enjoyed specific rank, under the title Str. lactis; but it will be noted that many of its most striking characteristics are shared by enterococci. Ayers and Johnson (1924) carried out a careful comparative study of these two types, testing them as regards their reaction on blood agar, their morphology, their ability to withstand heating at $60^{\circ} \mathrm{C}$., their reaction in litmus milk and Janus green medium, their fermentation reactions, and the final $\mathrm{pH}$ attained. They were unable to detect any difference in behaviour, except that enterococci appeared to form acid somewhat less vigorously than Str. lactis. Kleckner (1935) also concludes that it is not possible to differentiate with certainty between lactic-acid streptococci and enterococci, though there are minor points of difference. Many strains of lactic-acid streptococci, for instance, fail to ferment mannitol.

The origin of these streptococei in milk is an unsolved problem. It seems clear that they are not normal inhabitants of the cow's udder, but find their way into the milk from some outside source (Sherman and Albus 1918, Ayers, Johnson and Mudge 1924). Stark and Sherman (1935) have recorded their common occurrence on certain plants.

The relation between these three groups of streptococci has become clarified as the result of recent work. In the first place it has been shown by Graham and Bartley (1939), Sherman, Smiley and Niven (1940), Seelemann and Nottbohm (1940), Shattock and Mattick (1943) and Ehrismann (1943) that the enterococci possess the specific carbohydrate antigen of Group D hæmolytic streptococci, but that the lactic streptococci do not. It would appear that the enterococci differ from Group D hæmolytic streptococci only in their failure as a rule to produce characteristic $\beta$-hæmolysis in blood agar. In the second place, careful comparison between strains of enterococci and of lactic streptococci has revealed differences in their resistance to heat and in certain growth characters that appear to be of some classificatory value. Sherman and his co-workers have been most active in this field. In 1934, Sherman and Stark found that enterococci withstood exposure in sterile skimmed milk to a temperature of $65^{\circ} \mathrm{C}$. for 30 minutes, grew vigorously at $45^{\circ} \mathrm{C}$, and developed in a lactose agar medium having a $\mathrm{pH}$ of 9.6 or containing 6.5 per cent. sodium chloride, whereas lactic streptococci did none of these things.

Tests of this sort, in which the result depends on a number of different factors besides the main one under examination, are seldom satisfactory, and it is not surprising therefore that the findings of subsequent workers have varied considerably. Shattock and Mattick (1943) find that resistance tests are best carried out in broth at $60^{\circ} \mathrm{C}$., that special precantions have to be taken to maintain a $\mathrm{pH}$ of $9 \cdot 6$ in lactose agar, and that growth of enterococci in the presence of 6.5 per cent. $\mathrm{NaCl}$ is often so poor as to render this test of little value. Ehrismann (1943) finds the heat-resistance test unreliable, some true enterococci proving susceptible, and some fæcal streptococei other than those belonging to Group D proving resistant. Hobbs (1939), working in our laboratory, noted a number of discrepancies in the behaviour of foccalis and lactis strains in the Sherman set of tests and Ehrismann (1943) in Germany made similar observations on frecal streptococci. According to Shattock (1945) the only two reliable tests are growth at $45^{\circ} \mathrm{C}$. and at $\mathrm{pH} \mathrm{9.6}$. 
Both tests should be conducted in glucose lemco broth. In the $45^{\circ} \mathrm{C}$. test the temperature of the water-bath must be controlled to $\pm 0 \cdot 1^{\circ} \mathrm{C}$; in the $\mathrm{pH} 9 \cdot 6$ test, the reaction of the medium must be adjusted immediately before use, the tubes should be incubated in an anærobie jar containing soda lime, and the $\mathrm{pH}$ of uninoculated control tubes should be checked by the glass electrode immediately before and after incubation. Provided that the tests are carried out under as standard conditions as possible, and that complete uniformity in every respeet is not insisted on, it is often possible to allocate individual strains to one or other group (Table 38).

TABLE 38

Differentiation of Str. facalis and Str. lactis

\begin{tabular}{|c|c|c|c|c|c|c|c|c|c|c|}
\hline & & & & & & & Growth & & & \\
\hline & $\begin{array}{l}\text { Haemolysis } \\
\text { on horse- } \\
\text { blool agar. }\end{array}$ & $\begin{array}{l}\text { Man- } \\
\text { nitol. }\end{array}$ & $\begin{array}{l}\text { Sor- } \\
\text { bitol. }\end{array}$ & $\begin{array}{l}\text { Suc- } \\
\text { rose. }\end{array}$ & $\begin{array}{l}\text { ance } \\
60^{\circ} \mathrm{C} \text {. } \\
30 \mathrm{~min} \text {. }\end{array}$ & $\begin{array}{l}\text { at } \\
45^{\circ} \mathrm{C} \text {. }\end{array}$ & pH $9 \cdot 6$. & $\begin{array}{l}\text { in } \\
6.5 \% \\
\text { Naci. }\end{array}$ & $\begin{array}{l}\text { Sero- } \\
\text { logical } \\
\text { Group. }\end{array}$ & Source. \\
\hline $\begin{array}{l}\text { Str. } \\
\text { focalis }\end{array}$ & Variable & + & + & + & + & + & + & + & D & $\begin{array}{l}\text { Hainly } \\
\text { fæces }\end{array}$ \\
\hline Str. lactis & $\begin{array}{c}\text { Usually } \\
\text { none }\end{array}$ & \pm & - & - & - & - & - & - & $\mathrm{N}$ & $\begin{array}{l}\text { Milk and } \\
\text { cheese }\end{array}$ \\
\hline
\end{tabular}

Since the lactic streptococci belong to a different serological group (Group N), they need not be considered here. Group D hæmolytic streptococci and enterococci require a little further discussion. Numerous species have been recognized and named among these organisms.

Str. zymogenes, for example, of MacCallum and Hastings (1899), which is hæmolytic, ferments mannitol, liquefies gelatin and digests casein, appears to correspond to the former group, and Str. facalis of Andrewes and Horder (1906) to the latter group. Str. liquefaciens of Orla-Jensen (1919) resembles Str. fxcalis, but liquefies gelatin and digests casein. Str. durans of Sherman and Wing (1937) is $\beta$-hrmolytic, grows at $50^{\circ} \mathrm{C}$., but does not usually ferment either mannitol or sorbitol. Str. glycerinaceus differs from Str. facalis in fermenting glycerol. Str. bovis and Str. inulaceus (see Orla-Jensen 1919, Ayers and Hudge 1923) resemble each other in fermenting raffinose but not mannitol ; their relation however, to the enterococci is still in doubt.

In considering the nomenclature of this group, we must determine whether any single species can be defined rigidly enough to enable it to be recognized and distinguished from other species by tests that yield consistent results. Unless we can do this, the use of a specific name tends to be misleading rather than helpful. We think that it is now possible to define broadly Str. facalis in this way. Ignoring the absence of hæmolysis, we see that the enterococci differ in no important respect from Group D hæmolytic streptococci. We should therefore define Str. faccalis as a coccus arranged in pairs or very short chains, growing in the presence of bile salt, usually resisting heat at $60^{\circ} \mathrm{C}$. for 30 minutes, almost always fermenting lactose, mannitol, salicin, and usually sucrose, trehalose and sorbitol, but not raffinose or inulin, reducing the dye and forming a solid clot in litmus milk, and possessing the specific Group D polysaccharide; subsidiary characters being the failure of most strains to hydrolyse sodium hippurate, the production of a low $\mathrm{pH}(4 \cdot 0-4 \cdot 8)$ in glucose broth, and the ability to grow at temperatures between $10^{\circ}$ and $45^{\circ}$ C., in skimmed milk containing $1: 1,000$ methylene blue, in lactose 
broth containing $1: 15,000$ potassium tellurite, and on lactose agar at a $\mathrm{pH}$ of $9 \cdot 6$ or containing 6.5 per cent. NaCl. It may be noted that Str. facalis possesses the unusual property among streptococci of being insensitive to penicillin (Fleming 1932). It seems to us very doubtful whether any other members of this group can be assigned specific rank, and we should agree with Shattock and Mattick (1943) in regarding for the present the zymogenes, liquefaciens and durans organisms as varieties of Str. focalis. Str. glycerinaceus has no claim even to a varietal name. The position of Str. bovis must await further observations.

Within the enterococcus group an attempt has been made to distinguish antigenic types, but so far little progress has been made. Lancefield (1941) states that she has been able to define three types, differing apparently in the nature of their polysaccharide antigen; and Grumbach and Schnetz (1938) claim to have distinguished seven types by agglutination. Whether these types occur within the species Str. focalis as we have defined it, or correspond to any of its varieties, it is at present impossible to say.

\section{Group E.}

The few strains belonging to this antigenic group were isolated from eows' milk by Lancefield (1933). In view of the small number of strains examined, it is too early to give any generalized description of the other group charaeters. Lancefield (1941) has recognized one antigenic type.

\section{Group F.}

This group has been differentiated by Lancefield and Hare (1935) and by Hare (1935). It possesses a characteristic group-specifie polysaccharide antigen, by means of which it may be identified. Bliss (1937) has established four antigenic types by the use of precipitation and agglutination tests.

Group $\mathrm{F}$ strains grow slowly on blood agar plates, forming minute pin-point transparent colonies surrounded by a narrow zone $(1 \cdot 5-1.8 \mathrm{~mm}$. in diameter) of $\beta$-hæmolysis. They are identical with the strains described by Long and Bliss (193t) as "minute hæmolytic streptococci". They do not, when tested by the usual methods, form a filtrable hæmolysin acting on horse blood, but according to Plummer (194I) they do form a filtrable hæmolysin for sheep blood.

Group F strains produce a final acidity in glucose broth of $\mathrm{pH} 4 \cdot 8-5 \cdot 2$. They do not hydrolyse sodium hippurate. They do not reduce methylene blue in milk. They do not grow either on 10 per cent. or on 40 per cent. bile agar. Some, but not all, strains ferment trehalose, none ferments sorbitol. All that have been tested ferment lactose and salicin.

The strains that have been identified as belonging to this group have been derived mainly from the human throat. There is some evidence that they may be responsible for occasional cases of tonsillitis, and perhaps for other infections of the respiratory tract.

The provisional label for this group is clearly "Group F hæmolytic streptococci," with a proviso that we are not insisting on the demonstration of the formation of a filtrable hæmolysin before admitting a streptococcus to the hæmolytic class.

\section{Group G.}

The strains of this group share a common group-specifie polysaccharide antigen (Lancefield and Hare 1935, Phummer 1935, Lancefield 1941). Hare (1935) notes that some sera produced hy the injection of Group C strains tend to give cross-precipitation with extracts from Group G strains - a circumstance that appears to be due to their possession of a common protein antigen (Lancefield 1941). Organisms from matt colonies of Groups C and $\mathrm{G}$ strains resemble each other in fermenting trehalose but not sorbitol, and in producing fibrinolysin and streptolysin O. A number of antigenic types have been dis- 
tinguished by Simmons and Keogh (1940) among both the large colony and the so-called minute forms of streptococci in this group; and Bliss (1937) has recognized one type, which was identical with her Group F Type 1.

As regards their other biological and hiochemical properties, there appears to be a conflict of evidence in regard to the final $\mathrm{pH}$ attained in glucose broth. Plummer (1935) gives this as $\mathrm{pH}+4 \cdot 4 \cdot 6$, whieh would place these strains in the "high-acid" group; but Lancefield and Hare (1935) and Hare (1935) give figures of $p \mathrm{H}+4 \cdot 6-5 \cdot 2$. Group G strains do not hydrolyse sodium hipporate. The majority are able to multiply on 10 per eent. bile agar, but few on 40 per cent. bile agar. They do not reduce methylene blue in milk. They ferment trehalose, but not sorbitol. All strains tested have fermented laetose, but the fermentation of saliein appears to be less constant than with Gronps $\mathrm{A}, \mathrm{B}$ and $\mathrm{C}$.

Most of the strains that have been identified as belonging to this group liave been isolated from man, a few from the monkey or the dog. Several of the human strains have been derived from normal persous, but it seems clear that some, at least, are pathogenie for man, though there is a suggestion that they seldom cause very severe infections. One of Griffith's 27 types-Type 16 - of human-pathogenic hremolytie streptocoeei falls into this group.

The labelling of this group should clearly be provisional. It shows obvious relationships to Group C, and, apart from its antigenic structure, to Group A. There seems no reason, at the moment, to allot to it any specific name. "Group G hæmolytic streptococci" will serve our immediate purpose.

\section{Groups $\mathrm{H}$ and $\mathrm{K}$.}

These two additional antigenic groups have been differentiated by Hare (1935). They appear to differ from each other, and from the other groups of læmolytic streptococei that have been described, in regard to their group-specific antigens. Those strains that have been examined have shown other cultural or biochemical characters that may have differential signifieance; but so few strains belonging to these two groups have yet been studied that it would be premature to describe their characters in any detail. They have all been isolated from the nose or throat of normal persons, and there is as yet no evidence that they are pathogenic. One antigenic type has been recognized in Group $\mathrm{K}$ (Lancefield 1941).

\section{Groups $L$ and $M$.}

These groups have been differentiated by Fry (1941), who has been kind enough to supply us with the following information. Most of the original strains studied were isolated by Dr. Tom Hare from animal sources.

The majority of Group L strains came from dogs and pigs, but two (Hooper and Krone) were isolated from the human throat by White, Rudd and Ward (1939) in Australia. The colonies are small, but have a wide zone of $\beta$-hæmolysis. A soluble hæmolysin is formed. There is no growth on bile agar, and no hydrolysis of sodium hippurate. Trehalose is fermented, and sometimes lactose, but not mannitol, salicin, or sorbitol.

Strains belonging to Group M came almost exelusively from the tonsil of the dog ; no human strains have yet been identified. Growth is extremely poor, and the organisms rapidly die out. On blood agar the colonies are very small, but are surrounded by a wide zone of $\beta$-hæmolysis. A soluble hæmolysin is formed, but it is weak and acts slowly. Lactose is fermented, but not trehalose, mannitol, salicin, or sorbitol. The formamide method of extraction cannot be used for this group, as heating much above $100^{\circ} \mathrm{C}$. destroys the precipitinogen.

\section{Group N.}

We have already pointed out, when discussing Group D streptoeocei (p. 582), that the lactic streptococci can be distinguished from the enterococei by a series of metabolic and heat-resistance tests, and by their failure to form the group-specific polysaccharide 
antigen common to the hæmolytic and non-hæinolytic members of Group D. The observations of Sherman, Smiley and Niven (1940), of Seelemann and Nottbohm 1940) and of Shattock and Mattick (1943) have shown that the lactic streptococci form a group-specific antigen of their own; and it therefore seems appropriate, in spite of the absence of hæmolysis caused by these organisms, to include them in the Lancefield series and assign them to Group N. There seems no reason why the chief representative of this group, the properties of which have abready been described, should not be awarded specific rank and referred to as Str. lactis. Whether the closely allied organism, called by Orla-Jensen (1919) Str. cremoris, is to be treated similarly is less clear. According to Yawger and Sherman (1937) it differs from Str. lactis in the slightly larger size of its cells, its greater tendency to form chains, its lower optimal temperature, its greater susceptibility to methylene blue, its failure to form ammonia in a 4 per cent. peptone medium, and certain other minor respects. These differences seem to be far more suggestive of environmental variation than of fixed hereditary characters, and it would be wise for the present to regard Str. cremoris as no more than a variant of Str. lactis.

The Classification of the $\alpha$-hæmolytic Streptococci.--It has been noted in preceding sections that streptococci giving $\alpha$-hæmolysis with a characteristic green coloration on blood agar plates, failing to produce a soluble hæmolysin, usually fermenting raffinose but not mannitol, and possessing certain other characters in common, can constantly be isolated from the human mouth and throat and from the frees of cattle. The problem that confronts us is whether these streptococci form a group or a species, and, if a group, whether the species of which that group is formed are sufficiently well differentiated to be allotted specific names.

Ayers and Mudge (1923) express the view that the $\alpha$-hæmolytic streptococci of the bovine intestine differ in certain minor characters from the $\alpha$-hæmolytic streptococci of the human mouth and throat; and reference to several of the papers quoted above will reveal a tendency to accord the bovine strains specific rank, under the name Str. bovis. It is, however, by no means clear that this procedure is justified, or on what differential characters the proposed nomenclature is to be based. Indeed there is some evidence to suggest that Str. bovis may be related to the Group D hæmolytic streptococei (see Shattock and Mattick 1943).

There is another streptococcus falling into this group that seems to merit separate consideration. Frendenreich (1897) isolated a streptococcus from Kefir, a form of fermented milk. This streptococeus has the usual characters of the viridans type (Sherman 1921, Ayers et al. 1921, Ayers and Rupp 1922). When tested in the ordinary way, with a Durham fermentation tube, this organism, like other streptococei, produces acid, but no gas, from various substrates. If, however, it is tested in the Eldridge fermentation tube in which it is grown in a shallow layer of fluid medium, freely exposed to the air, and the $\mathrm{CO}_{2}$ evolved is taken up by a standard solution of barium hydroxide, exposed in a connected tube of the same kind, as large an amount of $\mathrm{CO}_{2}$ is evolved from lactose as is given off when that sugar is fermented by Bact. coli. It would seem that the almost anaerobic conditions existing in the closed Durham fermentation tube inhibit the production of $\mathrm{CO}_{2}$ by the Kefir streptococcus. It is difficult to assess the real significance of this observation, since we have no knowledge of the way in which most species of non-gasproducing bacteria would behave if tested in the Eldridge tube, instead of in the closed Durham, or Smith, tubes in which they have in fact been tested; but no $\mathrm{CO}_{2}$ was formed in the Eldridge tube by such other strains of streptococei as were tested by Ayers and his colleagues, including strains isolated from the bovine faeces, and the lactic acid streptococcus.

There can, we think, be little doubt that the $\alpha$-hæmolytic streptococci of the viridans type will ultimately be separated into a number of distinct species or types ; 
but we doubt very greatly whether it is yet possible to define with any exactitude either an inclusive species Str. viriduns, or any of the fermentative types that have in the past been given specific names. We may, however, mention some of the properties autributed to the main types.

Str. salivarius.-This organism occurs in the human mouth and intestine. It is said to form short chains, to grow at $45^{\circ} \mathrm{C}$. but not at $10^{\circ} \mathrm{C}$, to produce very little greening on blood agar, to produce a low final acidity $(\mathrm{pH} 4 \cdot 0-4 \cdot 4)$ in glucose broth, to clot milk, to form a soluble levan from sucrose and raffinose, to give rise to large mucoid colonies on agar containing 5 per cent. of either of these sugars, to ferment lactose, raffinose and salicin, and to hydrolyse æseulin but not sodium hippurate (Niven et al. 1941, Sherman et al. 1943). Most workers record it as being without action on inulin, but Sherman, Niven and Smiley (1943) include this sugar among those that are fermented.

Str. mitis.-This organism also occurs in the human mouth. According to Sherman, Niven and Smiley (1943) it comprises a much less homogeneous group of strains than Str. salivarius. It does not usually grow at $45^{\circ} \mathrm{C}$., it forms good $\alpha$-hæmolytic colonies on blood agar, it does not produce so low an acidity in glucose broth as Str. salivarius, it often fails to clot milk, it synthesizes no polysaccharide from sucrose or raffinose, it does not form mucoid colonies on 5 per cent. sucrose agar, it usually ferments lactose and salicin but not as a rule raffinose or inulin, and it fails to hydrolyse sodium hippurate or, with some exceptions, æsculin.

Str. equinus.-This organism is found in the intestine of the horse. It is said to grow at $45^{\circ} \mathrm{C}$., to produce good greening on blood agar, to produce no polysaceharide from sucrose or raffinose, to hydrolyse æsculin but not hippurate, to grow on blood agar containing 30 per cent. bile, and to ferment saliein but not lactose. It is usually reported as fermenting raffinose, bnt Sherman, Niven and Smiley (1943) disagree with this statement.

Str. bovis.-This organism has already been mentioned under Group D hæmolytic streptococei and on p. 588.

Str. acidominimus.-This organism was described by Ayers and Mudge (1922), who isolated it from cows' milk and freces. It is also found in the vagima of the cow (Smith and Sherman 1939). It forms $\alpha$-hæmolytic colonies on blood agar, it fails to grow at $10^{\circ} \mathrm{C}$. or $45^{\circ} \mathrm{C}$, it has very weak fermentative properties producing a final $\mathrm{pH}$ in glucose broth of about $6 \cdot 2$, it has some hydrolysing effeet on hippurate but not nsually on æesculin, it ferments lactose and sucrose, and sometimes mannitol, but not as a rule salicin or raffinose, it has little or no action on litmus milk, and in milk containing $1: 10,000$ methylene blue it fails to grow.

Str. thermophilus.-This organism produces eompletely non-hæmolytic colonies on blood agar, but may conveniently be mentioned here. It was described by Orla-Jensen (1919) as one of the organisms that grows actively in milk at a temperature of $50^{\circ} \mathrm{C}$. It does not grow at $10^{\circ} \mathrm{C}$, it is not destroyed by heating to $63^{\circ} \mathrm{C}$. for 30 minutes, it forms long chains in milk, its colonies are of the pin-point type, it ferments laetose and sucrose, but not salicin, and it elots milk but has only slight reducing action on tlie litwus. Whether disaccharides are fermented by this organism without preliminary hydrolysis to monosaccharides has been discussed by Wright (1936) and Sherman and Stark (1938).

Omitting Str. thermophilus, which is non-hæmolytic, we feel that it is wiser at present to use the non-committal group term "viridans streptococci" for these organisms rather than the specific name Str. viridans. The strongest claim to specific rank appears to be possessed by Str. salivarus; and if the findings of Sherman and his colleagues are confirmed, namely that all strains of this species form a soluble polysaccharide from sucrose, it may be well to admit this claim. The other named organisms, however, are the object of so many discrepant reports that any attempt to accord them specific rank would in our opinion be premature. 


\section{Pathogenicity and Toxin Production.}

The various species of streptococci described above include some of the most important pathogens of man, and are responsible for many infections of economic importance among animals.

Str. pyogenes gives rise to numerous pyogenic and septicæmic infections in man (see Chapter 67), as well as being the cause of scarlet fever (see Chapter 66).

It is pathogenic for a number of laboratory animals, including the rabbit, the mouse and the guinea-pig; but the virulence of different strains for these animals is by no means uniform, and the guinea-pig is often relatively resistant. To obtain a highly virulent strain for the mouse, or for the rabbit, it is often necessary to test a large number of strains of human origin, or to adapt a strain to the new host by repeated passage. When a highly virulent strain has been obtained, its intravenous injection leads to a fatal septicæmia, frequently associated in the rabbit with suppurative lesions in the joints, and sometimes with an ulcerative endocarditis. Intraperitoneal injection is followed by a suppurative peritonitis leading to a septicæmic infection; while subcutaneous injection is followed by a localized abscess, with or withont a subsequent generalization. With some strains, so far at least as the rabbit is concerned, intradermal injection is followed by a spreading erysipelatous infection of the skin, or by a more severe dermal infection leading to necrosis; and an infection of the latter type not infrequently terminates as a septicæmia.

Str. pyogenes is of particular interest to the pathologist in that it combines the capacity for tissue invasion with the production of filtrable exotoxins.

One of these toxins, streptococcal hæmolysin, has already been considered. That this substance is toxic in the animal body, as well as being lytic for red blood corpuscles in vitro, there can be no doubt. Its minimal lethal dose is large (5-10 $\mathrm{ml}$. for the rabbit), but when administered intravenously in this amount it kills the animal within 24-36 hours with an associated hæmoglobinuria, and with evidence of intravascular hæmolysis at necropsy (McLeod and McNee 1913, Channon and McLeod 1929). Weld (1934, 1935) more recently described preparations of streptolysin, obtained by extracting the cocci with serum, that are fatal for mice in a dose of $0.1 \mathrm{ml}$. Streptococcal hæmolysin is, as we have seen, thermolabile, being inactivated at $58^{\circ} \mathrm{C}$. in 30 mins. As already noted, Todd (1934) has demonstrated the presence in lytic filtrates of two hæmolysins, one oxygen-sensitive, but reactivable by reduction, and another which is oxygen-stable but is very sensitive to heat and to acid. Both are toxic, the $\mathrm{S}$ type causing death by intravascular hæmolysis, the $O$ type probably causing death by some other means. Specific antibodies, having protective properties and showing no cross-neutralization, can be prepared against each type of hæmolysin (Todd 1938b).

In addition to their action on red blood corpuscles, filtrates of broth cultures of Str. pyogenes have a destructive action on polymorphonuclear leucocytes. This was first demonstrated by van der Velde (1894) in his studies on experimental infections with streptococci; and he named the active principle leucocidin. It was subscquently shown by Neisser and Wechsberg $(1900,1901)$ that this action could be demonstrated in vitro, since the streptococcal filtrates, in killing the leucocytes, deprive them of their power of reducing methylene blue. It was early noted that streptococcal leucocidin is relatively thermolabile, and most authors (see Nakayama 1920, Channon and McLeod 1929) have concluded that its thermolability is of the same order as that of streptococcal hæmolysin. Channon and McLeod conclude, from this and other observations, that streptococcal hæmolysin and leucocidin are identical; but Nakayama regards them as different, a view that is put forward more strongly by Evans (1931) and by Gay and Oram (1933), who dispute the previons findings in regard to the thermolability of streptococcal leucocidin, and state that it withstands heating at $70^{\circ} \mathrm{C}$. for 30 minutes. Todd (1942) has brought evidence to suggest that the leucocidin is identical with the oxygen-labile streptolysin $O$, and that it plays no part in the determination of virulence to mice. This problem must be regarded as awaiting final solution. 
The studies of Dick and Dick $(1924 a, b, 1925 a, b)$ on the ætiology of scarlet fever, and the observations of subsequent workers, have shown that Str. pyogenes produces another filtrable toxin, which is quite distinct from the hæmolysin and leucocidin. The properties of this toxin are described in some detail in Chapter 66. For our immediate purpose a brief summary will suffice.

The injection of this toxin in small amounts into the skin of persons susceptible to its action gives rise to a characteristic localized erythema. Its injection in larger amounts in particularly susceptible persons may result in a generalized erythematous rash, associated with fever and malaise ("miniature searlet fever"). Because of this action in man this component of streptococcal filtrates is known as the erythrogenic toxin, or scarlatinal toxin. It is toxic for the rabbit when injected intravenously, but its minimal lethal dose is very large $(5-10 \mathrm{ml}$. of an unconcentrated filtrate). It can be concentrated and partially purified by various methods (Hartley 1928, Pulvertaft 1928), but the M.L.D. remains relatively large $(0 \cdot 1-1 \mathrm{ml}$.), and the purification is certainly very incomplete. A skin reaction has been induced in certain laboratory animals, but none of them is as sensitive as man, and many are quite resistant.

The erythrogenic toxin differs sharply from streptococcal hæmolysin in being relatively heat resistant. It requires a temperature of $96^{\circ} \mathrm{C}$. for 45 minutes for complete inactivation. It is antigenic, producing an antitoxin that gives specific neutralization, and is immunologically distinct from antistreptolysin (Todd, Laurent and Hill 1933). The concentrated toxin can be titrated by the flocculation method, using specific antitoxin (Rane and Wyman 1937, Hottle and Pappenheimer 1941).

This does not exhaust the toxic armoury of Str. pyogenes. It has been shown (Tillett and Garner 1933) that pathogenic strains of hæmolytic streptococci produce a substance that dissolves human fibrin (see also Tillett, Edwards and Garner 1934, Tillett 1935). This fibrinolysin scems to be almost constantly present in strains of Group A hæmolytic strepto. cocei (Lancefield and Hare 1935, Hare 1935, Hare and Naxted 1935, Colebrook, Maxted and Johns 1935), and is formed by the matt-colony-producing strains of Groups C and G. The action appears to be relatively specific for the fibrin of certain animal species. Thus the fibrinolysin produced by many strains of Str. pyogenes dissolves human, but not rabbit fibrin. The fibrinolysin also acts on hmman fibrinogen, so altering it that it is no longer able to form fibrin. Garner and Tillett (1934) have been able to obtain partially purified streptococcal fibrinolysin from filtrates by aleohol precipitation, followed by adsorption and elution. The product obtained is remarkably heat resistant; its activity may be maintained after heating at $100^{\circ} \mathrm{C}$. for 50 minutes. It exerts no hydrolytic action on casein, gelatin or peptone. Its lytic action on human fibrin appears to be associated with a slight increase in the amino-nitrogen content of the reacting mixture.

Finally, Duran-Reynals (1933) has described the presence in lysates and filtrates of invasive streptococei of a spreading factor, which markedly increases the permeability of the rabbit's skin to suspensions of Indian ink, or to bacterial cells. A similar substance has been extracted by Chain and Duthie (1939) from the testicle, and has been shown to hydrolyse the muco polysaccharide, hyaluronic acid, found by Meyer and Palmer (1936) in vitreous humour and the umbilical cord, and by Kendall, Heidelberger and Dawson (1937) in the capsular substance of Group A streptococci. The enzyme hyaluronidase appears to be identical with the spreading factor. What part this enzyme plays in favouring the invasion of the tissues by streptococei, or what part the hyaluronic acid in the capsular substance plays in protecting the organisms from the cells or antibodies in the tissues of the host is still subject to discussion. According to McClean (1941) the development of capsules and hyaluronidase formation appear to be mutually exclusive. Seastone (1943) observed that hæmolytie streptococci from severe infections had a higher arerage muco-polysaccharide content than those from mild infections, suggesting that capsular material was favourable to the organism, but a relation between eapsule formation and virulence has still got to be proved. Hirst (1941), for example, found that decapsulation of the organisms with leech extract protected guinea-pigs and mice from fatal infection 
with Group C streptococci, but not with Group A streptococci. There is some evidence that the virulence of Group A strains is more closely related to the type-specific M protein than to the possession of a capsule (see Lancefield 1941). The importance of hyaluronidase is likewise doubtful. Neither Crowley (1944) who studied 376 strains of hæmolytic streptococci from human sources, nor Humphrey (1944) who studied 81 strains of pneumococci from consecutive cases of pneumonia, could find any relation between the amount of hyaluronidase produced and the apparent virulence of the strain.

In summary, Str. pyogenes produces the following toxins or aggressive substances (see Chapter 48) which, in one way or another, determine or are associated with its pathogenic activity : $(a)$ hæmolysin, $(b)$ leucocidin, $(c)$ erythrogenic toxin, $(d)$ fibrinolysin, $(e)$ hyaluronic acid, $(f)$ hyaluronidase, $(g)$ type-specific M protein.

Str. agalactiæ.-Our knowledge of the pathogenicity and toxigenicity of this species is, as yet, very incomplete. It produces mastitis in cattle, but, so far as our present knowledge goes, is not pathogenic for man. Its pathogenicity for the mouse, or the rabbit, is low (Minett and Stableforth 1931, Minett 1935b). Some, but not all, strains, produce a filtrable hæmolysin, which Todd (1934) has found to be of the oxygen-stable, non-antigenic type. Whether Str. agalactice produces a leucocidin is not known. It has been shown by Smith (1929) that strains of hæmolytic streptococci isolated from cattle may produce an erythrogenic toxin that is neutralized by scarlatinal antitoxin: but there is no evidence that these strains were Str. agalactice. No erythrogenic toxin was produced by the mastitis strains examined by Hinett and Stableforth (1931). Str. agalactice does not produce a fibrinolysin active against human fibrin (Lancefield and Hare 1935, Hare 1935), or against cattle fibrin. It produces hyaluronidase, but seldom forms capsules; when it does, the capsules do not consist of hyaluronic acid (MoClean 1941).

Str. pneumoniæ.-The pneumococcus is an important pathogen of man, giving rise to pneumonia, particularly the lobar form, sinusitis, otitis media, less frequently meningitis, suppurative arthritis, or peritonitis, and occasionally other infections.

It is highly pathogenic for the mouse and rabbit, rather less so for the guinea-pig. The cat, dog and chicken are relatively resistant. It is a characteristically invasive organism, causing a fatal bacteræmic infection when injected intravenously, an acute peritonitis followed by bacteræmia when injected intraperitoneally, localized suppuration followed by generalization when injected subcutaneously, and a spreading inflammatory lesion followed by generalization when injected intradermally in the rabbit. Different serological types of pneumococci may show differences in their virulence for different laboratory animals; for instance, many strains of Type III pneumococcus are relatively avirulent for the rabbit (Tillett 1927). Again, different strains belonging to the same type may show wide variations in virulence as judged by their minimal lethal dose. With a strain of maximal virulence the injection of 1-10 pneumococci into the peritoneum of a mouse will cause an infection that leads to death within 18-48 hours.

In contrast to Str. pyogenes, the pneumococcus is not an actively toxigenic organism, in the sense of producing filtrable toxins. Some observers have, indeed, regarded it as being devoid of this capacity, and as affording a typical example of a bacterium the pathogenicity of which is determined entirely by its powers of invasion, associated, of course, with the effects of those presumptive " endotoxins" (see Chapter 44) that must always be concemed in invasive bacterial infections. There is little doubt that this contrast is a true one, in the sense that filtrable toxins play a much more prominent part in Str. pyogenes infections than in those due to pneumococci; but the difference is not so absolute as it has been supposed to be.

We have seen that Str. pneumonice produces a filtrable hæmolysin when grown under optimal conditions. This is of the oxygen-sensitive type, and is antigenic. There is evidence (Todd 1934) that it bears some antigenic relationship to the oxygen-sensitive hæmolysin of Str. pyogenes, but is certainly not identical with it. Oram (1934) has described the presence in pneumococcal filtrates of a leucocidin, active for rabbit leucocytes. Like the streptococcal leucocidin described by Gay and Oram (1933), this substance is 
relatively thermostable. It is not inactivated in one hour at $70^{\circ} \mathrm{C}$., but is completely inactivated at $85^{\circ} \mathrm{C}$. for a similar period.

Several observers (Jukianelle and Reimann 1926, Reimann and Julianelle 1926, Mair 1928, Pittman and Falk 1930, Goodner 1931) have noted purpuric lesions following the injection of pneumococcal extracts and autolysates. The active agent would appear to be an "endotoxin," i.e. some constituent of the bacterial cell, rather than a soluble toxin. It may be noted that Avery and Goebel (1933) report that mice may develop purpura after injections of the acetylated form of the Type I pneumococcal polysaccharide, while this effect is not produced by the deacetylated form.

Pneumococci produce hyaluronidase in varying amount, but there appears to be no relation between their activity in this respect and their virulence (Humphrey 1944). Whether they form a fibrinolysin against human fibrin is not certainly known. The formation of the characteristic fibrin network in the alveoli in lobar pneumonia in man would seem to make it unlikely that the pneumococei concerned act vigorously on human fibrin; but it would appear that they are able to attack rabbit fibrin or fibrinogen. Goodner $(1931,1933)$ has recorded that the comema fluid, withdrawn from the spreading œdematous lesions caused by the injection of virulent pneumococei into the rabbit's skin, not only fails to clot, but retards the coagulation of normal rabbit's blood, and that a similar anticoagulant property is possessed by pneumococeal extracts and autolysates. The active substance is relatively thermostable, since it withstands heating to $70^{\circ} \mathrm{C}$. for 15 minutes. Such autolysates, when injected into the skin together with a slightly virulent strain of pneumococcus, greatly extend the area of the lesion produced.

None of the other groups, species, or types of streptococci that we have described above have been studied, in regard to their pathogenicity for laboratory animals, in the same detail as Str. pyogenes and Str. pneumonice. A few brief notes will give most of the information that we possess.

Streptococci of Group D.-'The organisms of this group are far less pathogenie than Str. pyogenes or Str. pneumonice. They have, however, occasionally been isolated from the blood stream in man, usually in cases of subacute endocarditis (see Chapter 68), rarely in other conditions. They are normal inhabitants of the intestinal traet, and are not infrequently present in suppurative alidominal lesions, though their pathogenic rôle is often doubtful. They are also not meommonly present in infections of the urinary tract. Their virulence for laboratory animals is usually low. Dible (1921) tested 83 fæcal strains by injection into mice, and 6 of these showed some degree of pathogenicity. It is very probable that such pathogenicity as this group possesses is confined to particular species, or strains; and that many strains, such for example as most of those isolated from milk or cheese, are altogether non-pathogenic. It may, perhaps, be noted that the hæmolytic strains belonging to this group do not produce a fibrinolysin active against human fibrin.

Streptococci of the Viridans Group.-The position of this group, in regard to pathogenicity, is very similar to that of Group D. Streptococci of the viridans type are of low virulence, both for man and for animals. In man they are frequently isolated from localized septic lesions in connection with the teeth and gums, and they are the most frequent cause of subacute bacterial endocarditis. Here again, it is probable that different species or types within the group vary considerably in their pathogenicity for different animal species. Many of them are probably quite avirulent. In none of them is the virulence high.

Very brief notes must suffice for the remaining labelled groups of hæmolytic streptococci, since our knowledge of their pathogenic potentialities is as yet in its earliest infancy. The data available are contained in the recent papers by Lancefield, Hare, and others, to which frequent reference has already been made. 
Hæmolytic Streptococci of Group C are certainly pathogenic. They have been frequently isolated from suppurative and acute inflammatory lesions in horses, cattle, guinea-pigs and other animals. They have also been isolated from human infections; but their pathogenicity for man appears to be lower than that of Str. pyogenes. The human pathogenic strains that have been examined produce a fibrinolysin active against human fibrin; the strains derived from animals do not.

Hæmolytic Streptococci of Group F.-The strains that have been identified as belonging to this group have been derived from minor infections of the respiratory tract in man, and from the normal human throat. Their possible pathogenie rôle must be regarded as sub judice; but their virulence would seem, in any case, to be of a low order. They do not produce a fibrinolysin active on human fibrin.

Hæmolytic Streptococci of Group G.-The streptococci of this group are certainly pathogenic. They have been isolated from tonsillitis, endocarditis and urinary infections in man (Macdonald 1939, Rantz 1942), from pneumonia in the monkey, and from otitis in the dog. They have also been isolated from the normal human throat. Such evidence as is available suggests that Group G, like Group C strains, have a definitely lower virulence for man than has Str. pyogenes. Group G strains produce a fibrinolysin acting on human fibrin.

Hæmolytic Streptococci of Groups E, $\mathbf{H}$ and $\mathbf{K}$.-There is as yet no evidence that the streptococci belonging to these groups are pathogenic.

Hæmolytic Streptococci of Groups L, M, and N.-Organisnis belonging to Groups $\mathrm{L}$ and $\mathrm{M}$ appear to be pathogenic for certain animals, especially the dog. Group N strains are apparently non-pathogenic.

\section{Variation in the Characters of Streptococci.}

It is not always possible from the records to identify the strain in which particular variations have been observed with one or other of the groups, species or types that have been defined in this chapter. It is, for instance, sometimes impossible to tell whether the term "hæmolytic streptococci" or Str. homolyticus, is equivalent to Str. pyogenes, in the sense in which we have used that term. In almost all the instances given below, however, the identity of the strain or strains concerned is not in doubt, and the reservation that it is necessary to make is little more than formal.

Variations in Str. pyogenes.-There are many reports in the literature of the appearance of non-hæmolytic, or $\alpha$-hæmolytic, variants in cultures derived from an originally hæmolytic strain. These reports have at times been regarded as invalidating hæmolysin production as a differential test; but in view of our more detailed knowledge of the factors that determine the action of streptococci on red blood corpuscles, it is clearly unnecessary to assume that the appearance, in a culture of a $\beta$-hæmolytic streptococcus, of a variant that gives typieal $\alpha$-hæmolysis, or no hæmolysis at all, on the surface of an aerobic blood agar plate affords an instance of the mutation of Str. pyogenes into a streptococcus of the viridans type, or into a completely non-hæmolytic form.

An illuminating example is given by Todd (1928b). By repeated mouse passage he was able to obtain, from a typical $\beta$-hæmolytic strain of streptococcus, a variant that produced no hæmolysis at all on the surface of aerobic blood agar plates. When grown anaerobically, this variant maintained full hæmolytic activity. Moreover, the aerobically non-hæmolytic variant not only inactivated its own hæmolysin when exposed to a free supply of oxygen, but, under the same conditions, inactivated the hæmolysin produced by the original hæmolytic strain, if the latter was grown in symbiosis with it. The appearance of non-hæmolytic variants in an originally hæmolytic strain has, it may be noted, been recorded by many other workers (see p. 567). 
Str. pyogenes varies considerably in colonial appearance. Adopting the terminology of Dawson, Hobby and Olmstead (1938), we may recognize the following forms : (a) mucoicl colonies, probably corresponding to the pseuto-glossy eolonies of Todd and Laneefield (1928). The organisms tend to be uniform in size, arranged in pairs or short chains, and to show capsulation when young. In broth, growth is diffuse or finely granular ; $(b)$ matt colonies, probably representing an intermediate stage between the mucoid and the smooth colony forms. The organisms are slightly pleomorphic, non-capsulated, and are arranged in short chains or small chums. The growth in broth is coarsely granular but not floceulent; $(c)$ smooth colonies, eorresponding to the glossy colonies of Todd (1928a). The organisms tend to be of uniform size and to be arranged in short chains. Growth in broth is diffuse or finely granular; (d) rough colonies, first described by Eagles (1928), flat and very irregular in outline. The organisms are large, pleomorphie, and arranged in long chains. Growth in broth is floeculent. The type-specific acid-soluble protein M is usually present in the mucoid, matt, and smooth forms, but not in the rough form. Its loss is associated with an absence of virulence to mice (Todd 1928a, Todd and Laneefield 1928, Lancefield and Todd 1928), but its presence does not necessarily signify that the strain is virulent. The ehange from mucoid or matt to rough or smooth corresponds to the $\mathrm{S} \rightarrow \mathrm{R}$ variation that occurs in many other baeterial species. According to Torłd (1930), who studied the influenee of oxygen pressure on this change, repeated subculture on solid media under aerobic conditions favours the appearance of smooth avirulent variants, but eultivation under anaerobic conditions prevents it. The effect of aerobiosis is apparently dependent on the formation of bacterial peroxide, the smooth variants being more resistant to this agent than the original matt virulent forms. In broth cultures the effeet of inereasing oxygen pressure by aeration is somewhat different; matt avirulent variants tend to appear muder anaerobic conditions, whereas the virulence of the original matt strain is maintained when the culture is freely aerated, even though smooth variants make their appearance. The change from matt to smooth is not associated with a loss of the power to produce hrmolysin.

Variations in other Groups of Hæmolytic Streptococci.-Variant eolonies of more or less similar type to those found in Str. pyogenes have been described in other groups of streptococci. The reader will find an illustrated deseription of them in the article by Dawson, Hobby and Olmsteat (1938). In addition, "minute" or dwarf colonies have been observed in Groups C and G by Long and BLiss (1934), I ancefield (1941) and Horton and Sommer (1944). Though, in general, the fermentation reactions of streptoeoeei are relatively stable, differences have been noted in the same speeies associated with variations in colony forms. For instanee, in Group C, the smooth and dwarf colony forms are said to ferment lactose and trehalose, but not sorbitol or mannitol: on the other hand, the mucoid colony forms ferment sorbitol and mannitol, but not laetose or trehalose (Morton and Sommer 1944).

Variations in Str. pneumoniæ.-The more important types of variation that are encountered in the pneumococcus have already been noted in Chapter 9. The $\mathrm{S} \rightarrow \mathrm{R}$ variation is here assoeiated with the loss of the eharaeteristic capsule, and with it the polysaccharide antigen that confers type-speeificity. Here, as elsewhere, the rough variants of pneumococci usually retain the characteristic bile solubility. They also retain the power of producing a hæmolysin and a leueodiein (Oram 1934).

We have also referred in Chapter 9 to the important observations of Griffith (1928) on the conversion of a smooth strain of pneumocoeeus, belonging to a partieular antigenic type, through the corresponding rough variant to a smooth strain belonging to a different antigenic type; and we have noted that these observations have been confirmed by several subsequent workers. Up to the present time this remains the only instance in which a transmutation of one normal bacterial type into another has been demonstrated under experimental eonditions; and it affords no grounds for the assumption that such types are unstable under natural conditions. 
In regard to the other groups or species, referred to in the present chapter, we know too little as yet of the variations to which they are subject to attempt any systematic description of them. The description of variants that fit iuto no general scheme tends to confusion rather than to the clarification of knowledge. We must not, of course, ignore the fact that such variations occur; but to assess their true significance we must wait until we can allot them their proper place in the picture of bacterial structure, which is being slowly but surely pieced together by modern methods of study.

\section{The Anaerobic Streptococci.}

All the species, or groups, of streptococci described above are aerobic and facultatively anaerobic. Cocci growing in short or long chains have, however, been isolated, which are either strictly anaerobic, or grow only under microaerophilic conditions.

These anaerobic streptococci are of considerable importance to the medical bacteriologist, since some, at least, are certainly pathogenic for man; and the studies of recent years have shown that organisms of this type are a frequent source of severe puerperal infection (see Chapter 66). Apart from puerperal sepsis, and puerperal septicæmia, most of the strains of anaerobic streptococci that have been isolated have been derived from suppurative or gangrenous lesions, which have often been noted as producing a foul or foetid odour (see Veillon 1893, Krönig 1895, Sternberg 1900, Rist 1901, Lowkewicz 1901, Silberschmidt 1902, Marwedel and Wehrsig 1915, Kissling 1924, 1929, Prévot 1924, 1925, 1933). The importance of these organisms in relation to puerperal septicæmia was first insisted on by Schottmüller $(1910,1928)$, though their presence in the genital tract during the puerperium had been noted by several earlier workers. Schottmüller's observations have since been extended and confirmed by many subsequent observers (Bingold 1921, 1932, Lehmann 1926, Harris and Brown 1929, Colebrook 1930, Colebrook and Hare 1933). It would seem (Natvig 1905, Wegelius 1909, Rosowsky 1912, Soule and Brown 1932, White, E. 1933) that these anaerobic streptococci form part of the normal flora of the female genital tract; and it seems possible (White, E. 1933) that this is their principal normal habitat. Such attempts as have been made to isolate them from the normal human throat or intestine have been unsuccessful.

It is certain that the anaerobic streptococei comprise many different groups, species or types; but the data available are as yet far too scanty to permit of any systematic classification or nomenclature. Reference to the papers by Prévot, and by Colebrook and Hare, will afford descriptions of several of the strains that have been isolated, and of certain differential criteria on which a future classification may in part be based.

It may be noted that many of these cocei are very small $(0 \cdot 3-0 \cdot 4 \mu)$; but the size tends to vary considerably in subculture (Colebrook and Hare 1933). Many but not all strains form abundant gas in fluid cultures, differing sharply in this way from the aerobic and facultatively anaerobic species that we have described above. Many, but not all strains produce an extremely foul odour.

Colebrook and Hare (1933) have studied the growth of 60 strains of anaerobic streptococei on blood agar, and have thus been able to distinguish four different types on the basis of rate of growtl, colony form, and changes produced in the medium. Only two of the 60 strains produced hæmolysis; three others gave characteristic coal-black colonies. The remaining 55 strains produced no change in the medium. About half the strains tested failed to ferment any test substrate; with the remainder it was not found possible to correlate the fermentation reactions with the colonial characters. A preliminary attempt at 
antigenic analysis, using antisera to eight of the strains, showed considerable diversity of antigenic structure, but many group reactions. The two main types differentiated on colonial appearances were shown to be antigenically distinct. Finally, those strains that were tested for heat resistance were found to be likilled by heating to $58-60^{\circ} \mathrm{C}$. for 30 minutes. Stone (1940), who studied 26 strains from parturient women, divided them into three groups according to their growth in $10 \mathrm{or} 40$ per cent. bile, and was able to demonstrate the presence of at least two acid-extractable antigens having some relation to those met with in the non-anærobic types of streptococei.

It may be noted that the pathogenicity of anaerobic streptococei for laboratory animals appears to vary widely. Wegelius (1909) produced small abscesses in the peritoneal cavity of mice. Marwede] and Wehrsig (1915), with one strain examined, produced an acutely fatal infection in a guinea-pig. Prévot (1925) records pathogenic lesions of a suppurative gangrenous or redematous type, sometimes fatal, with most of his strains. Harris and Brown (1929) found that three of 57 strains derived from cases of pnerperal fever killed mice within 24 hours. Colebrook and Hare (1933) tested seven puerperal strains by subcutaneous injections into mice. Two of them gave rise to small caseous foci at the site of inoculation, but none of the mice died.

Anaerobic pneumococei have also been described (Smith 1936).

We append a summarized description of the more important species, or groups, to which names, or labels, ean at the moment be attached.

\section{Species, Groups and T'ypes}

\section{Str. pyogenes}

Morpnologr.-Cocei, usually spheroidal, abont $0 \cdot 5-0.75 \mu$ in cliameter, arranged in chains of varying length, but usually including ten or more cocci. Capsules usually absent or poorly developed in tissues; frequently present in young serum broth cultures of freshly isolated strains. Non-motile. No spores. Gram-positive; not acil-fast.

Growtil Requirements.-When first isolated may grow poorly on ordinary nutrient agar. Growth is markedly improved by the addition of blood or serum. Optimal temperature $37^{\circ} \mathrm{C}$. Grows at temperature slightly over $40^{\circ} \mathrm{C}$. Poor growth below $20^{\circ} \mathrm{C}$, and usually fails to grow at $10^{\circ} \mathrm{C}$. Aerobic and facultatively anacrobic.

Type OF Growth.-On solid media after 24 hours' inculation, the colonies are small, about $0.5-0.75 \mathrm{~mm}$. in diameter, opaque, slightly raised, circular, with an entire margin, a slightly granular surface, and a granular structure, when viewed by transmitted light, showing some differentiation into a more opaque central portion, and a more transincent periphery. After further incubation (48 to 72 hours) the colony may extend in cliameter, and become differentiated into a raised central portion, smooth or contoured, and a flatter peripheral zone. The colonial forms presented by different strains are subjeet to considerable variation; and a plate from a single strain may show colonies of very varying appearance; particularly with regard to the smoothness, contouring, or granularity of the surface, and the degree of differentiation between the central and peripheral zones.

Streaked eultures on solid media give a relatively scanty growth, with a tendency for a majority of the colonies to remain discrete. The growth emulsifies easily, but usually gives a granular suspension.

In blood agar plates. - The colonies are surrounded by a zone of $\beta$-hæmolysis (see above), best seen in the deep colonies. A filtrable hamolysin is formed in fluid cultures, which is oxygen-labile. An oxygen-stable hamolysin is also produced.

In broth, or serum broth.--When first isolated, the growth may be finely gramular, or may form a powdery deposit at the bottom of the tube, or cling to its sicles. Turbidity of the medium may be moderate, or slight. After subculture, and particularly when repeatedly subcultured at short intervals, the turbidity may increase markedly, and the granular deposit decrease, or disappear ; but the turbidity almost always remains of a finely gramular 
type, and the growth seldom or never becomes evenly diffuse. There is no pellicle formation.

On gelatin the growth is slight, and the colonies are minute and punctiform.

In gelatin stab there is a slight growth along the track, with minimal growth on the surface. The gelatin is not liquefied.

On potato growth is very slight, and often not detectable by the naked eye.

Heat Resistance, and Viability.-Str. pyogenes is killed by heating to $55^{\circ} \mathrm{C}$. for 30 minutes. It tends to die out in subculture unless preserved under particularly favourable conditions, but it remains viable for long periods in the dry state.

Biochemical Reactions. - Str. pyogenes does not hydrolyse sodium hippurate; does not reduce methylene blue in milk; produces a final $\mathrm{pH}$ of $5 \cdot 0-5 \cdot 6$ in ghucose broth; ferments trehalose, lactose, saccharose, salicin and occasionally mannitol, with the formation of acid but no gas; does not ferment sorbitol, inulin or raffinose. Produces acid in litmus milk, but no coherent clot. Does not liquefy gelatin. Does not reduce nitrates. Does not form indole. Is not soluble in bile. Its growth is inhibited by bile.

Antigenic Structure.-Str. pyogenes possesses the group-specific polysaccharide antigen of Lancefield's Group A hæmolytic streptococei. It is differentiated into a large number of antigenic types by type-specific protein antigens; over 30 of these types have so far been identified by agghtination and absorption and by precipitation tests.

Pathogenicity ani Toxin Production.--Str. pyogenes produces a variety of infections in man, and more rarely in domestic animals. Some strains are highly pathogenic for the mouse or the rabbit, less so for the guinea-pig. It produces a soluble hæmolysin, a leucocidin, an erythrogenic toxin and a fibrinolysin acting on human fibrin.

\section{Str. agalactiæ}

Morphology, Growti Requirements and Type of Growth.-In these characters Str. agalactice does not differ significantly from Str. pyogenes; in many strains, however, the cells are yellow or red in colour.

ACTION ox BLOOD.-Between one-third and one-half of the strains of Str. agalactice that have been examined produce $\beta$-hæmolysis in blood agar plates. These strains produce a filtrable hæmolysin of the oxygen-stable type.

Heat Resistance.-Str. agalactice is killed by heating to $60^{\circ} \mathrm{C}$ for 30 minutes.

Biocilemical Reactions.-Str. agalactice hydrolyses sodium hippurate; it does not reduce methylene blue in milk; it produces a final $\mathrm{pH}$ of $4.2-4.8$ in ghucose broth; it produces acid in trehalose, sucrose, glycerol, and usually in lactose and salicin, but not in sorbitol, mannitol, raffinose or inulin ; it forms acid and clot in milk; it does not liquefy gelatin. It is not soluble in bile. Its growth is not inhibited by 10 per cent. bile, and usually not by 40 per cent.

Antigenic Structure.-Str. agalactioe possesses the group-specific polysaccharide antigen of Lancefield's Group B streptococci. It is separable into different antigenic types, four of which have so far been identified, by type-specific antigens which appear to be carbohydrate, not protein in uature. Sub-types of the four main types have been recognized.

Pathogenicity and Toxis Production.-Str. agalactice is an important cause of mastitis in cattle. Its pathogenicity for laboratory animals is low. Some, if not all, strains produce a filtrable hæmolysin. It does not produce a fibrinolysin acting on human fibrin. Whether it produces a leucocidin is unknown. There is no evidence that it produces an erythrogenic toxin.

\section{Str. pneumoniæ}

Morphology.-Ovoid or lanceolate cocei, arranged in pairs or short chains; when in pairs, the adjacent ends of the coci are usually huntly rounded, the opposite ends 
more acutely pointed. Some strains (particularly Type III) tend to form longer chains. As seen in films from the tissues, the pneumococcus shows a well-marked capsule; and this capsule is frequently retained in cultures on suitable media. Non-motile. No spores. Gram-positive. Not acid-fast.

Growth Requirements.-Grows poorly on ordinary media, especially when first isolated ; the addition of blood or serum to the medium greatly improves growth. Optimal temperature $37^{\circ} \mathrm{C}$, range of growth more restricted than with other species of Streptococcus. Usually no growth on gelatin at $20^{\circ} \mathrm{C}$. Aerobic and facultatively anaerobic. Growth of some strains improved by incubation in 10 per cent. $\mathrm{CO}_{2}$.

Type of Growth.-On solid media. Small, raised, circular colonies, 0.5-1 mm. in diameter, with a smooth surface, an entire edge, and very little differentiation. On a favourable medium, such as blood agar, the colonies are often characteristic ; the surface is flat and smooth, and the edges are sharply and steeply raised from the surface of the medium. In some cases the edge may be raised above the surface of the colony, forming a raised circumferential ring. Several adjacent colonies may become confluent, forming a raised area of growth with a flat, even surface and a sharply delimited edge. With longer periods of growth (4S-72 hours) the central portion of the colony often undergoes autolysis. Some strains (particularly Type III) give characteristic mucoid colonies. Old laboratory strains of pneumococci, particularly when grown on a relatively unfavourable medium such as ordinary nutrient agar, often give smaller colonies which lack the characteristic appearance of a recently isolated strain grown on a favourable medium. The consistency of the colonies is butyrous, and the growth emulsifies easily. In blood agar plates, the colonies are surrounded by a zone of $\alpha$-hæmolysis showing the characteristic green coloration. In a suitable fluid medium, a filtrable hæmolysin is formed, which is of the oxygen-labile type.

In broth, or serum broth, Str. pneumonice gives a diffuse turbid growth, with a slight deposit, increasing on prolonged incubation. No pellicle is formed.

On gelatin very slight growth, usually none at or below $20^{\circ} \mathrm{C}$.

Gelatin stab-very slight growth along track, with minimal surface growth. No liquefaction.

Potato-growth slight, or absent.

Heat Resistance and Viabilitx.--Str. pneumonice is sensitive to heat, being killed at a temperature of $55^{\circ} \mathrm{C}$. in 20 minutes or less. It is a relatively delicate organism, and dies out rapidly in artificial cultures unless maintained under particularly favourable conditions, as, for instance, in semi-solid agar to which blood has been added.

Biochemical Activities.-The pneumococcus produces acid, but no gas, from lactose, saccharose, and inulin, and usually from raffinose. Salicin is rarely férmented; when fermented, acid is not usually produced for some days. Mannitol is not fermented. Milk is acidified, and frequently clotted. Nitrates are not reduced. Indole is not formed. Gelatin is not liquefied. The pneumococcus is soluble in bile.

Antrigenic Structure.- The pneumococcus possesses a species-specific carbohydrate antigen, the presence of which is not detected by agglutination reactions carried out with normal smooth forms. The species is divided into a number of antigenic types by typespecific polysaccharide antigens contained in the capsules. Over seventy of these types have so far been identified.

Pathogenicity and Toxin Production.-The pneumococcus causes pneumonia and certain other infections in man. It is highly pathogenic for mice and slightly less so for rabbits. Guinea-pigs are rather more resistant, and cats, dogs, fowls and pigeons muoh more resistant.

The pneumococcus produces a soluble hæmolysin, and a leucocidin. It also produces a substance acting on rabbit fibrin or fibrinogen, and preventing the formation of a clot. 


\section{Streptococci of the Viridans Group}

Morphology.-Cocci in short or long chains, spheroidal or ovoid; when ovoid, long axis in axis of chain. Non-capsulated. No spores. Non-motile. Gram-positive. Not acid-fast.

Growth Requirements. - Most strains grow more readily on ordinary media than does Str. pyogenes or Str. pneumonia, but growth is usually improved by addition of blood or serum. Some strains, on the other hand, grow very poorly. Optimal temperature for most strains $37^{\circ} \mathrm{C}$. - the range of temperature for growth extends further than that of Str. pyogenes or Str. pneumonice in the downward direction. Aerobic and facultatively anaerobic.

TyPe of Growtr.-On solid media the colonies do not differ in any distinctive way from those of Str. pyogenes (see above). With streak cultures, the growth may be slightly more profuse, and more confluent.

In blood agar plates.-The colonies are surrounded by a zone of $\alpha$-hæmolysis, showing the characteristic green coloration.

In broth.-The type of growth varies with chain length. Many strains or varieties grow in short chains and produce a uniform, but slightly granular, turbidity in broth, with little or no deposit; but some strains grow in long chains and give growths which are indistinguishable from those of Str. pyogenes.

On gelatin, or in gelatin stab, the growth does not differ from that of Str. pyogenes, except that it may be slightly more profuse.

On potato, growth is slight, and often not detectable by the naked eye.

Heat Resistance.-Most strains are killed by heating at $55-58^{\circ} \mathrm{C}$. for 30 minutes. The general vitality is greater than that of Str. pyogenes.

Brochemical Reactions.-Milk is acidified, and often clotted. Most strains produce acid from lactose and saccharose, often from raffinose and/or salicin, rarely from inulin or mannitol. Nitrates are not reduced. Indole is not formed. Gelatin is not liquefied. Not soluble in bile.

Pathogenicity and Toxin Formation.-Viridans streptococci form no soluble toxin, nor hæmolysin. Usually non-pathogenic for laboratory animals other than the rabbit, in which some strains give rise to arthritis and valvular lesions. They are a common cause of subacute ulcerative endocarditis in man.

Differentiation withis the Grodp.-The viridans group of streptococci certainly contains more than a single species. It is possible that the common streptococcus of the human mouth, which has the peculiar property of producing a soluble levan from sucrose and raffinose and of forming large mucoid colonies when grown on agar containing 5 per cent. of these sugars, deserves specific rank with the title Str. salivarius; that the common streptococcus of bovine freces should be recognized as Str. bovis; and that a streptococcus isolated from Kefir, which produces $\mathrm{CO}_{2}$ from lactose when grown in Eldridge tubes should be known as Str. kefir. Our knowledge is, however, not yet sufficient to allow us to define species or to assign specific names with any degree of certainty.

\section{Str. fæcalis}

Morphology.-Oroid cocci, growing in pairs or short chains. Some strains resemble the pneumococcus in morphology, but possess no eapsule. More rarely, the appearance may be almost bacillary. Most strains non-motile, but a few motile strains have recently heen described. No spores are formed. Gram-positive and not acid-fast.

Growth Requirements.-Grows well on the ordinary laboratory media. Optimal temperature about $37^{\circ} \mathrm{C}$, but grows well up to $45^{\circ} \mathrm{C}$. and down to $10^{\circ} \mathrm{C}$. Aerobic and facultatively anaerobic.

TyPe of Growtr. On solid media, the colonies are somewhat larger than those of the species referred to above. After 24 hours the colonies are usually $0.75 \mathrm{~mm}$. in average 
diameter, and, on longer incubation, increase to a diameter of 1-2 nim. The colonies are smooth, cireular, low convex in elevation, with an entire edge; they have a homogeneous, or shightly granular structure, and show little differentiation. In streaked cultures the colonies tend to be confluent, and growth may appear as a uniform film. The growth is easily emulsified.

In blood agar-some strains, corresponding to Lancefield's Group D hæmolytic streptococci, give $\beta$-hæmolysis. They form no filtrable hæmolysin when tested by the ordinary methods, but a hæmolysin of the oxygen-stable type may be demonstrated by special cultural methods. Most strains are non-hæmolytic.

On MacConkey agar small pink colonies are formed.

In broth there is an abundant, diffuse growth, with a very shight deposit. No pelliele formation occurs.

On gelatin, there is a good growth, with colonies very similar to those produced on agar. Growth occurs at $10^{\circ} \mathrm{C}$. Most strains fail to liquefy the gelatin, but a few do so.

In gelatin stab, there is good growth along the track, with little surface growth. Some strains produce liquefaction, which is usually infundibuliform.

Grows in medium containing 10 or 40 per cent. bile, in lactose agar of $\mathrm{pH} 9 \cdot 6$ or containing 6.5 per cent. $\mathrm{NaCl}$, in lactose broth containing $1: 15,000$ potassium tellurite, and in milk containing $1: 1,000$ methylene blue.

Heat Resistance and Viability.-Heat resistant. Withstands a temperature of $60^{\circ} \mathrm{C}$. for 30 minutes. Survives in culture for a long time. Insoluble in bile. Insensitive to penicillin.

Biochemical Actrvities.-Usually fails to hydrolyse sodium hippurate. Produces a final $\mathrm{pH}$ of $4 \cdot 0-4.8$ in glucose broth. Produces acid in lactose, mannitol, salicin, and usually sucrose, trehalose and sorbitol, but not in raffinose or inulin. Rcduces the dye and produces acid and clot in litmus milk. Most strains reduce nitrates. Strains of the liquefaciens and zymogenes varieties liquefy gelatin and digest casein. Some strains produce $\mathrm{H}_{2} \mathrm{~S}$.

Antignenic Structure.-Possesses the Group D specific polysaccharide. There appear to be several antigenic types.

Pathogenicitr and Toxin Production.-Most strains a ppear to be non-pathogenic, or to possess a pathogenicity of a low order. They occasionally cause urinary infections in man, or infections in relation to the intestinal tract. They have occasionally been isolated from the blood stream, and are the cause of some cases of subacute endocarlitis. Most strains are non-pathogenic for laboratory animals. A few show some degree of pathogenicity. There is no evidence that any of the streptococci forms a filtrable toxin. Such strains as have been examined do not form a fibrinolysin acting on human fibrin.

Differentiation within the Group.-There appear to be a number of varieties of this organism, such as those called zymogenes, liquefaciens, and durans (see p. 585). Whether these have any special habitat apart from the human intestine is not known.

\section{Str. lactis}

This organism is found in milk and milk products, and on certain plants. Its differentiation from Str. feccalis has been discussed in the body of the chapter (sce p. 582). Suffice it to say that the main distinguishing characters are as follows: it does not grow at $45^{\circ} \mathrm{C}$. ; it does not grow in lactose agar of $\mathrm{pH} 9 \cdot 6$ or eontaining 6.5 per cent. $\mathrm{NaCl}$; it is killed by a temperature of $60^{\circ} \mathrm{C}$. within 30 minutes; it has no action on blood; mannitol, sucrose, and sorbitol are less readily fermented; and the organism possesses the Group N, not the Group D, specific polysaccharide. A cremoris variety has been described.

The characters of the other labelled groups or types of streptococci, as far as we yet know them, have been summarized in the body of this chapter. 


\section{REFEPENCES}

Anderson, A. B. and Hart, P. D'A. (1934a) J. Path. Bact., 39, 465; (1934b) Lancet, ii. 359. ANDREWES, F. and HoRDER, T. (1906) Lancet, ii. 708, 775, 852.

Armstrong, R. R. (1932) Brit. med. J., i. 187.

AvERy, O. T. (1915) J. exp. Med., 22, 804.

Avery, O. T., Chickerino, H. T., Cole, R., and Dochez, A. R. (1917) Monogr. Rockefeller Inst. med. Res., No. 7.

Avery, (1. T. and Cullen, G. E. (1919) J.exp. Med., 29, 215; (1923) Ibid., 38, 199.

Avery, O. T. and Goebel, W. F. (1933) J. exp. Med., 58, 731.

Avery, O. T. and Heidelberger, M. (1923) J.exp. Med., 38, 81 ; (1925) Ibid., 42, 367.

Avery, O. T., Heidelberger, M., and Goebel, W. F. (1925) J. exp. Med., $42,709$.

Avery, O. T. and Morgan, H. J. (1921) J. exp. Med., 39, 275, 289 ; (1925) Ibid., 42, 347.

Avery, O. T. and NeILl, J. M. (1924a) J.exp. Med., 39, 347; (1924b) Ibid., 39, 357; (1924c) Ibid., 39, 543.

Avery, R. C. (1929a) J. exp. Med., 50, 463; (1929b) Ibid., 50, 787.

Ayers, S. H. and Johnson, W. T. (1910) Bull. U.S. Dep. Agric., B.A.I., 126; (1913) Ibid., 161; (1924) J. infect. Dis., 34, 49.

Ayers, S. H., Johnson, W. T., and Davis, B. J. (1918) J. infect. Dis., 23, 290.

Arers, S. H., Johnson, IV. T., and Mudge, C. S. (1924) J. infect. Dis., 34, 29.

Ayers, S. H. and Mudoe, C. S. (1922) J. infect. Dis., 31, 40 ; (1923) Ibid., 33, 155.

AYERS, S. H. and RUPP, P. (1922) J. infect. Dis., 30, 388.

Ayers, S. H., Rupr, P., and Mudoe, C. S. (1921) J. infect. Dis., 29, 235.

BAEHR, J. (1910) Arch. Hyg., Berl., 72, 91.

Bagger, S. V. (1926) J. Path. Bact., 29, 225.

Bazeley, P'. L. and Battle, J. (1940) Aust. vet. J., 16, I40.

Beckler, E. and MacLeod, P. (1934) J. clin. Invest., 13, 90I.

Belenky, D. E. and Popowa, N. N. (1929) Zbl. Bakt., 113, 22.

Berger, E. and Silberstein, IV. (1926) Klin. Wschr., 5, 2307.

Besredka, A. (1901) Ann. Inst. Pasteur, 15, 880.

Bingold, K. (1921) Virchows Arch., 232, 22; (1932) Dtsch. med. Wschr., 5\%, 443.

BLISS, E. A. (1937) J. Bact., 33, 625.

BuIss, IV. P. (1920) Johns Hopk. Hosp. Bull., 31, 173 ; (1922) J. exp. Med., 36, 575.

Bradn, H. (1912) Zbl. Bakt., 62, 383.

Broadhurst, J. (1915) J. infect. Dis., 1\%, 277.

Brown, J. H. (1919) Monogr. Rockefeller Inst. med. Res., No. 9 ; (1937) J. Bact., 34, 35 ; (1939) Ibid., 3\%, 133.

Brown, R. and Robinson, L. K. (1943) J. Immunol., 47, 7.

CAPPS, J. A. and Davis, D. J. (1914) Arch. intern. Med., 14, 650.

Chain, E. and Duthie, E. S. (1939) Nature, Lond., 144, 977 ; (1940) Brit. J. exp. Path., 21,324 .

Channon, H. A. and McLeod, J. W. (1929) J. Path. Bact., 32, 283.

Coburn, A. F. and PAULI, R. H. (1941) J. exp. Med., 73, 551.

Cole, R. (1914) J. exp. Med., 20, 346.

Colebrook, Dora C. (1935) Spec. Rep. Ser. med. Res. Coun., Lond., No. 205.

Colebrook, L. (1930) Brit. med. J., ii. 134.

Colmbrook, L. and Hare, R. (1933) J. Obstet. Gynaec., 40, 609.

Colebrook, L., Elliott, S. D., Maxted, W. R., Morler, C. W., and Mortell, M. Lancet, ii. 30 .

Colebrook, L., Maxted, W. R., and Johns, A. M. (1935) J. Path. Bact., 41, 521.

Cooper, G., Edwards, M., and Rosenstein, C. (1929) J. exp. Med., 49, 461.

Cooper, G., Rosenstein, C., Walter, A., and Peizer, L. (1932) J. exp. Med., 55, 531.

Cooper, G. and Walter, A. W. (1935) Amer. J. publ. Hlth, 25, 469.

Cowan, S. T. (1934) J. Path. Bact., 38, 61.

Crowley, N. (1944) J. Path. Bact., 56, 27.

Davis, D. J. (1912) J. Amer. med. Ass., 58, 1852 ; (1929) Ibid., 93, 978.

Davis, D. J. and Rosenow, E. C. (1912) J. Amer. med. Ass., 58, 773.

Dawson, M. H., Новву, G. L., and Ormstead, M. (1938) J. infect. Dis., 62, 138.

Dible, J. H. (1921) J. Path. Bact., 24, 3.

Dick, G. F. and Dick, G. H. $(1924 a)$ J. Amer. med. Ass., 82, 265 ; (1924b) Ibid., 83, 84; (1925a) Ibid., 84, 802; (1925b) Ibid., 84, 1477.

Dochez, A. R. and Avery, O. T. (1915) J. exp. Med., 21, 114.

Dochez, A. R., Avery, U. T., and I Ancefield, R. C. (1919) J.exp. Med., 30, 179.

Dochez, A. R. and Gillespie, L. P. (1913) J. Amer. med. Ass., 61, 727.

Downie, A. W. and Cruichshank, J. (1928) Brit. J. exp. Path., 9, 171.

Downie, A. W., S'tent, L., and White, S. M. (1931) Brit. J. exp. Path., 12, 1,

Duran-Reynals, F. (1933) J.exp. Med., 58, 161. 
Eagles, G. H. (1924) Brit. J. exp. Path., 5, 199 ; (1928) Ibid.. 9, 330.

Eddy, B. E. (1944) Publ. Hllh. Rep., Wash., 59, 449, 451, I041.

Edwards, P. R. (1932) J. Bact., 23, 259; (1933) Ibid., 25, 527; (1934) Ibid., 27, 527. Ehrismanx, O. (1943) Arch. Hyg., Berl. 129, 116.

Elliott, S. D. (1943) Brit. J. exp. Path., 24, 159 ; (1944) Pers. comm.

ENDERS, J. F. (1930) J. exp. Hed., 52, 235.

ETINGER-Tulczy NSKA, R. (1932) Z. Hyg. Infehthr., 114, 769.

Evans, A. C. (1931) Publ. Hlth Rep., Wash., 46, 2539.

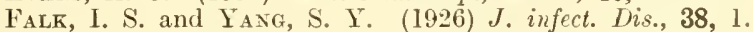

Fenleisen. (1883) "Aetiologie des Erysipels." Berlin.

Fildes, P. and Gladstone, G. P. (1939) Brit. J. exp. Puth., 20, 334.

Flemine, A. (1932) J. Path. Bact., 35, 83I ; (1941) Lancet, i. 110.

Floyd, C. and WOLBACH, S. B. (1914) J. med. Res., 29, 493.

Fraenkel, A. (1886) Z. klin. Med., 10, 401 ; (1886) Ibid., 11, 437.

Freudenreich, E. von. (1897) Zul. Bakt., IIte Abt., 3, 47.

Frost, W. D., Gumi, M., and Thomas, R. C. (1927) J. infect. Dis., 40, 698.

Fry, R. M. (1933) J. Path. Bact., 3\%, 337 ; (1941) I'ers. comm.

Fuller, A. T. (193s) Brit. J. cxp. Path., 19, 130.

Fuller, A. T. and Maxted, W. R. (1939) J. Path. Buct., 49, 83.

Fuller, C. A. and Armstrona, V. A. (1913) J. infect. Dis., 13, 442.

Garner, R. L. and TILletT, W. S. (1934) J. exp. Med., 60, 239.

GAY, F. P. and Orail, F. (1933) J. Immunol., 25, 501.

GOODNER, K. (193I) J. exp. med., 54, 847; (1933) Ibid.., 58, 153.

Gordon, M. H. (1902-3) Rep. loc. Govt Bd publ. Hlth, 32, 421 ; (1903-4) Ibid., 33, 388, 422 ; (1905) Lancet, ii. 1400 ; (1921) Brit. med. J., i. 632.

Grahair, N. C. and Bartley, E. O. (1939) J. Hyg., Camb., 39, 538.

Griffith, F. (1923) Rep. publ. Hlth med. Subj., Lond., No. 18; (1926) J. Hyg., Camb., 25, 385; (1927) Ibid., 26, 363; (1928) Ibid., 2\%, 113 ; (1934) Ibid., 34, 542; (1935) Ibid., 35, 23.

Grumbach, A. and Scinetz, A. (1938) Schweiz. Z. allg. Path. Bakt., 1, 59.

GUNDEL, M. and Schwarz, F. K. T. (1932) Z. Hyg. InfektKr., 113, 498.

Gunn, W. and Griffith, F. (1928) J. Hyg., Camb., 28, 250.

Gunsalus, I. C. and Sherman, J. II. (1943) J. Bart., 45, 15.5.

Günther, C. and Thierfelder, H. (I895) Arch. Hyg., Berl., 25, 164.

Hadley, F. P., Hadley, P., and Leatien, W. W. (1941) J. infect. Dis., 68, 264.

Hare, R. (1935) J. Path. Bact., 41, 499.

Hare, R. and Colebrook, L. (1934) J. Path. Bact., 39, 429.

Hare, R. and Maxted, W. R. (1935) J. Path. Bact., 41, 513.

Harris, J. W. and Brown, J. H. (I929) Johns Hopk. IIosp. Bull., 44, 1.

Hart, P. D'A. and Anderson, A. B. (1933) J. Path. Bact., 3\%, 91.

Hartley, P. (1928) Brit. J. exp. Path., 9, 259.

Heidelberger, M. (1927) Physiol. Rev., \%, 107.

Heidelberger, M. and Avery, O. T. (1923) J.exp. Med., 38, 73; (1924) 1bid., 40, 301.

Heidelberger, M. and Goebel, W. F. (1927) J. biol. Chem., 74, 613.

Heidelbereer, M., Goebel, W. F., and Averi, O. T. (1925) J. exp. Med., 42, 727.

Heidelberger, II. and Kendall, F. E. (1931) J. exp. Med., 53, 625.

Heinemann, P. G. (1906) J. infect. Dis., 3, 173.

Herbert, D. and Todd, E. W. (1944) Brit. J. exp. Path., 25, 242.

Hinst, G. K. (1941) J. exp. Med., 73, 493.

Нгтснсоск, С. Н. (1924) J. exp. Med., 40, 445.

Hoвbs, B. C. (1939) J. Dairy lies., 10, 35.

Holman, W. L. (1916) J. med. Res., 34, 377.

Hopkins, J. G. and Lang, A. (1914) J. infect. Dis., 15, 63.

Hottle, G. A. and Pappenheimer, A. M. (1941) J. exp. Med. 74, 545.

Houston, T. and McCloy, J. M. (1916) Lancet, ii. 632.

Humpireey, J. H. (1944) J. Path. Bact., 56, 273.

JAMES, G. R. (1926) J. Hyg., Camb., 25, 415.

Jones, F. S. (1919) J. exp. Med., 30, 159.

Julianelle, L. A. and Reinann, H. A. (1926) J. exp. Med., 43, 87.

Kauffuans, F., Morcir, E., and Sсhиith, K. (1940) J. Immunol., 39, 397.

KeMPNER, W. and SchlaYer, C. (1942) J. Bact., 43, 397.

Kendall, F. E., Heidelberger, M. and Dawson, M. H. (1937) J. biol. Chem., 118, 61.

Keogh, E. Y. and Sinnoxs, R. T. (19t(1) J. Path. Bart., 50, 137.

KissLing, K. (1924) Münch. med. I'schr., 71, It57; (1929) Ibid., 76, 1163.

Kiтt. (1893) see Minett (1935ל).

KLECKNeR, A. L. (1935) J. Lab. clin. Hed., 21, 111.

KLEIN, S. J. (1933) J. Bact., 26, 215; (1935) Ibid., 30, 43. 
Klein, S. J. and Stone, F. M. (1931) J. Bact., 22, 387.

Klimmer, M. and HAUPT, H. (1930) Ergebn. Hyg., 11, 354.

Kовlмüller, L. O. (1935) Zbl. Bakt., 133, 310.

KröNiQ. (1895) Zbl. Gynäk., 19, 409.

Kruif, P. H. DE and Ireland, P. M. (1920) J. infect. Dis., 26, 285.

Kromwiede, E. (1943) J. Bact., 46, 117.

Lamanna, C. (1944) J. Bact., 47, 327.

LANCEFIELD, R. C. (1928) J. exp. Med., 47, 91, 469, 481, 843, 857 ; (1933) Ibid., 57, 571 ; (1934) Ibid., 59, 441 ; (1938) Ibid., 67, 25; (1940) Ibid., 71, 521, 539 ; (1941) Harvey Lectures, Ser. 36, 251; (1943) J. exp. Med., 78, 465.

LaNCefield, R. C. and HaRe, R. (1935) J. exp. Med., 61, 335.

Lancefield, R. C. and Stewart, W. A. (1944) J. exp. Med., 79, 79.

LaNoefield, R. C. and Todd, E. W. (1928) J. exp. Med., 48, 769.

LehmanN, W. (1926) Münch. med. Wschr., 73, 233.

Lister, F. S. (1916) Publ. S. Afr. Inst. med. Res., No. 8.

Logan, W. R. (1914) J. Path. Bact., 18, 527.

Logan, W. R. and Smeall, J. T. (1932) Brit. med. J., i. 189.

Love, P. H. and Bliss, E. A. (1931) J. exp. Med., 60, 619.

Lowkewicz, X. (1901) Arch. med. Exp., 13, 633.

Lyall, H. W. (1914) J. med. Res., 30, 487.

MacCallum, W. G. and Hastings, T. W. (1S99) J. exp. Med., 4, 521.

IICClean, D. (1941) J. Path. Bact., 53, 13; (1942) Ibid., 54, 284.

Macdonald, I. (1939) Med. J. Aust., ii, 471.

Mclachla , D. G. S. and MaOkie, T. J. (1928) J. Hyg., Camb., 27, 225.

McLeod, J. W. (1912) J. Path. Bact., 16, 321.

McLeod, J. W. and Gordon, J. (1922) Biochem. J., 16, 499.

McLeod, J. W. and McNeE, J. W. (1913) J. Path. Bact., 17, 524.

MlaIr, W. (1917) J. Path. Bact., 21, 305; (1928) Ibid., 31, 215; (1929) " A System of Bacteriology," Med. Res. Coun., London, 2, 168.

Mandelbaum, M. (1907) Z. Hyg. InfektKr., 58, 26.

Marmorek, A. (1895) Ann. Inst. Pasteur, 9, 593.

MaRWedel and Wehrsia. (1915) Münch. med. Wschr., 62, 1023.

Meader, P. D. and Robinson, G. H. (1920) J. exp. Med., 32, 639.

MEYer, K. (1926) Zbl. Bakt., 99, 416.

Meyer, K.. and Palmer, J. W. (1936) J. biol. Chem., 114, 689.

MeYer, K. and SChÖNFELd, H. (1926) Zbl. Bakt., 99, 402.

MinetT, F C. (1935a) J. Path. Bact., 40, 357; (1935b) Proc. Twelfth Int. Vel. Cong., p. 511 ; (1936) J. Hyg., Camb., 35, 504.

Minett, F. C. and Stableforth, A. W. (1931) J. comp. Path., 44, 114 ; (1934) J. Dairy Res., 5, 223.

Minett, F. C., Stableforth, A. W., and Edwards, S. J. (1929) J. comp. Path., 42, 213 ; (1936) J. Hyg., Camb., 35, 504.

Morch, E. (1942) J. Immunol. 43, 177.

Morgan, H. J. and Avery, O. T. (1924) J. exp. Med., 39, 335.

Morgan, H. J. and Neill, J. M. (1924) J. exp. Med., 40, 269.

Morison, J. E. (1940) J. Path. Bact., 51, 401.

Morton, H. E. and Sommer, H. E. (1944) J. Bact., 47, 123.

Nakayama, Y. (1920) J. infect. Dis., 27, 86.

Natvig, H. (1905) Arch. Gynäk., 76, 701.

NeILL, J. M. (1925) J. exp. Med., 41, 299, 535 ; (1926) Ibid., 44, 199.

NemL, J. M. and Avery, O. T. (1924a) J.exp. Med., 39, 757; (1924b) Ibid., 40, 405, 423 ; (1925) Ibid., 41, 285.

NeILl, J. M. and Mallory, T. B. (1926) J. exp Med., 44, 241.

Neisser, M. and Wechsbera, F. (1900) Münch. med. W schr., 47, 1261 ; (1901) Z. Hyg. InfektKr., 36, 299.

NEUFELd, F. (1900) Z. Hyg. InfektKr., 34, 454; (1902) Ibid., 40, 54.

Nedfeld, F. and Etinger-TulczYNSKA, R. (1931) Z. Hyg. InfektKr., 112, 492.

NeUFeld, F. and HäNDeL, L. (1909) Arb. ReichsgesundhAmi., 34, 293.

Nicolle, M. and Adr-Bey. (1907) Ann. Inst. Pasteur, 21, 20.

Niven, C. F., Silley, K. L., and Sherian, J. M. (I941) J. Bact., 41, 479 ; (1942) Ibid., 43, 651 .

Nocard and Mollereau. (1887) Ann. Inst. Pasteur, 1, 109.

OGURA, K. (1929) J. Jap. Soc. vet. Sci, 8, 174.

OraM, F. (1934) J. Immunol., 26, 233.

ORLA-JENSEN, S. (1919) "The lactic acid bacteria." Copenhagen.

Pasteur, L., Chamberland, C., and Roux, E. (I881) C. R. Acad. Sci., 92, 159.

Pittman, M. and FALK, I. S. (1930) J. Bact., 19, 327. 
Plumaer, H. (1935) J. Bact., 30, 5; (1941) J. Immunol." 42, 91.

Pownall, N. (1935) Brit. J. exp. Path., 16, 155.

Prevot, A. (1924) "Les Streptocoques Anaerobes," 1'aris; (1925) Ann. Inst. Pasteur, 39, 417 ; (1933) Ann. Sci. Nat. (Sérics Botaniques), 15, 163.

Pulvertaft, R. J. V. (1928) Brit. J. exp. Path., 9, 276.

RANE, L. and IVYMAN, L. (1937) J. Immunol., 32, 321.

RaNTZ, L. A. (1942) J. infect. Dis., r1, 61.

Reimann, H. A. (1927) J. exp. Med., 45, 807.

Reimanj, H. A. and Julianelle, I. A. (1926) J. exp. Med., 43, 97.

Rist, E. (1901) Zbl. Bakt., 30, 287.

Rochaix, A. (1924) C. R. Soc. Biol., 90, 771.

RosenbacH, F. J. (1881) "Mikroorganismen bei den Wundinfektionskrankheiten." Wiesbaden.

Rosowsky, A. (1912) Zbl. Gynäh., 36, 4.

Rother, W. (1925) Dtsch. med. Wschr., 51, 1031.

Ruediger, G. F. (1912) Science, 35, 223.

Sabin, A. B. (1933) J. Amer. med. Ass., 100, 1584.

Schnabel, A. (1921) Z. Hyg. InfehtKr., 93, 175.

SсноттмÜLleR, H. (1903) Münch. med. Wschr., 50, 849, 909 ; (1910) Mitt. Grenzgeb. med. Chir., 21, 450 ; (1928) Münch. med. Wschr., 75, 1580, 1634.

Sснӥтz, IV. (1888) Arch. wiss. prakt. Tierheilk., 14, 456.

Seastone, C. V. (1934) J. Bact., 28, 481 ; (1943) J. exp. Med., ry, 21.

Seelemann, M. and Hadenfeldt, A. (1932) Zbl. Bakt., 126, 231.

Sembliane, M. and Notтвона, H. (1940) Zbl. Bakt., 146, 142.

Sнаттоск, P. M. F. (1945) Pers. comm.

Shatтock, P. M. F. and Mattick, A. T. R. (1943) J. Hyg., Camb., 43, 173.

SHERMAN, J. M. (1921) J. Bact., 6, 127.

Sherman, J. M. and Albus, W. R. (1918) J. Bact., 3, 153.

Sheritan, J. M., Niven, C. F., and Shimley, K. L. (1943) J. Bact., 45, 249.

Sherman, J. M., Surley, K. L., and Niven, C. F. (1940) J. Dairy Ści., 23, 529.

Sherman, J. M. and Stark, P. (1931) J. Bact., 22, 275; (1934) J. Nairy Sci., 17, 525; (1938) J. Bact., 36, 77

Sherman, J. M. and Wing, H. (1937) J. Dairy Sci., 20, 165.

Sickles, G. M. and Coffey, J. M. (1928) J. infect. Dis., 43, 490.

Silberschimt, W. (1902) Z. Hyg. InfektKr., 41, 427.

Simmons, R. T. and Keogir, E. V. (1940) Aust. J. exp. Biol. med. Sci., 18, 151.

Slanetz, L. W. and Naghski, J. (1940) J. infect. Dis., 66, 80.

Sмiтн, F. (1936) Brit. J. exp. Path., 1\%, 329.

Smith, F. R. and Sherman, J. M. (1939) J. infect. Dis., 65, 301.

Sмiтн, J. (1926) J. Hyg., Camb., 25, 165; (1927) Ibid., 26, 420 ; (1929) J. Path. Bact., $32,401$.

Surth, T. and Brown, J. H. (1915) J. med. Res., 31, 455.

SOLOWEy, M. (1942) J. exp. Med., \%6, 109.

Soule, S. D. and Brown, T. K. (1932) Amer. J. Obstet. Gynaec., 23, 532.

Stableforth, A. W. (1932) J. comp. Path., 45, 185; (1937) J. Path. Bact., 45, 263; (1942) Proc. R. Soc. Med., 35, 625; (1945) Pers. comm.

Stark, P. and Sherman, J. M. (1935) J. Bact., 30, 639.

Sternberg. (1900) Wien. klin. Wschr., 13, 551.

Stevens, F. A. and Dochez, A. R. (1926a) J.exp. Med., 43, 379 ; (1926b) Ibid., 44, 439. Stewart, D. F. (1937) J. Path. Bact., 45, 279.

Stewart, W. A., Lancefield, R. C., Wilson, A. T., and Swift, H. F. (1944) J.exp. Med., ษ9, 99.

Stokes, W. R. and Hachtel, F. W. (1912) Publ. Hlth Rep., Wash., Nov. 22.

Stone, M. L. (1940) J. Bact., 39, 559.

Swift, H. F., Wilsox, A. T., and Lancefield, R. C. (1943) J. exp. Med., 78, 127.

Thalitann. (1912) Zbl. Bakt., 66, 240.

Thiercelin. (1899) C. R. Soc. Biol., 5, 269.

Tillett, W. S. (1927) J. exp. Med., 45, 1093 ; (1935) J. Bact., 29, 111.

Tillett, W. S., Enwards, L. B., and Garner, R. L. (1934) J. clin. Invest., 13, 47.

Tillett, IV. S. and Garner, R. L. (1933) J. exp. Med., 58, 485.

Tillett, W. S., Goebel, W. F., and Avery, O. T. (1930) J. exp. Med., 52, 95.

Todd, E. W. (1928a) Brit. J. exp. Path., 9, 1; (1928b) J.exp. Med., 48, 493; (1930) Brit. J. exp. Path., 11, 368, 469, 480; (1932) J. exp. Med., 55, 267 ; (1934) J. Path. Bact., 39, 299 ; (1938a) Ibid, 47, 423; (19386) Brit. J. exp Path., 19, 367 ; (1939) J. Hyg., Camb., 39, I ; (1942) Brit. J. exp. Path., 23, 136.

Todd, E. W. and Hewite, L. F. (1932) J. Path. Bact., 35, 973.

TODD, E. W. and LANCEFIELd, R. C. (1928) J. exp. Med., 48, 751. 
Todd, E. W., Laurent, L. J. M., and Hrll, N. G. (1933) J. Path. Bact., 36, 201.

VeILlon, M. A. (1893) C. R. Soc. Biol., 40, 807.

VELDE, H. YAN DER. (1894) La Cellule, 10, 401.

Wadsworth, A. and lirown, R. (1931) J. Immunol., 21, 245.

Walter, A. W., Guevin, Y. H., Beattie, H. W., Cotler, H. Y., and Bucca, H. B. (1941) J. Immunol., 41, 279.

Watson, R. F. and Lancefield, R. C. (1944) J. exp. Med., 79, 89.

Weatherall, C. and Dible, J. H. (1929) J. Path. Bact., 32, 413.

Wegelius, W. (1909) Arch. Gynaek., 88, 249.

Weichselbaum, A. (1886) Med. Jb., i. 483.

Weissenbach, R. J. (1918) C. R. Soc. Biol., 81, 559, 819.

Weld, J. T. (1934) J.exp. Med., 59, \$3; (1935) Ibid., 61, 473.

White, C., Rudi, G. V., and Ward, H. K. (1939) Med. J. Aust. i. 96.

Whiте, E. (1933) J. Obstet. Gynaec., 40, 630.

Whiте, S. II. (1929) Biochem. J., 23, 1165.

Winslow, C.-E. A., Broadhurst, J., Buchanan, R. E., Krumwiede, C., Rogers, L. A., and Smith, G. H. (1920) J. Bact., 5, 191.

Winslow, C.-E. A. and Palmer, G. T. (1910) J. infect. Dis., \%, 1.

Wright, H. D. (1936) J. Path. Bact., 43, 487.

YaWger, E. S. and Shermax, J. M. (1937) J. Dairy Sci., 20, 205.

ZitTle, C. A. (1942) J. Immunol. 43, 31 . 
CHAPTER 25

\section{STAPHYLOCOCCUS, MICROCOCCUS, SARCINA, RHODOCOCCUS, AND LEUCONOSTOC}

\section{STAPH Y LOCOCCUS}

\section{DEFinition.-Staphylococcus.}

Spherical or ovoid, non-motile, Gram-positive cells, arranged in grape-like clusters on solid media, and in pairs, small groups, or short chains in liquid media. On agar the growth is of a golden, white, or yellow colour. Great variation in biochemical activities, hæmolytic power, and pathogenicity. Actual or potential parasites.

Type species is Staphylococcus aureus Rosenbach.

History.-The presence of micrococci in pus was noted by Koch in 1878 ; they were cultivated in a liquid medium by Pasteur in 1880; they were shown by Ogston in 1881 to be constantly present in acute and chronic abscesses; they were cultivated by him in eggs, and were found to be pathogenic to mice and guinea-pigs; but it was left to Rosenbach in 1884 to make a thorough study of the staphylococci, to obtain pure cultures on solid media, and to divide them into two species-Staphylococcus pyogenes aureus and Staphylococcus pyogenes albus. In the following year Passet (1885) added another species-Staphylococcus pyogenes citreus. In 1887 Biondi isolated two types from saliva, both pathogenic for laboratory animals; one of these was apparently identical with an organism described as $\boldsymbol{M}$. tetragenus by Koch and Gaffky (Gaffky 1883), who had found it in the sputum of patients suffering from pulmonary tuberculosis; the other was distinguished from Staphylococcus aureus by its diminutive size-0.3-0.5 $\mu$ in diameter-and the slowness with which it liquefied gelatin; to this he gave the name of Staphylococcus salivarius pyogenes. Welch (1891) noticed a white staphylococcus in stitch abscesses following the suturing of operation wounds; this he called the Staphylococcus epidermidis albus. Andrewes and Gordon (1905-6), who investigated a large number of cocci from different sources, found a special type commonly present in saliva which they named the Staphylococcus salivarius; this differed in many respects from the Staphylococcus salivarius pyogenes of Biondi ; they also found a coccus of peculiar characteristics present in scurf, but did not identify it by a special name. Winslow and Rogers (1906), in an attempt to arrive at a classification on a statistical basis, conducted a painstaking investigation into the Coccacex, and proposed a division into six genera, from which the original genus Staphylococcus was omitted. Later, however, Winslow, Rothberg and Parsons (1920) modified this classification, and reinstated the Staphylococcus in its old place, dividing the genus into six species. 
Habitat.-There are but few situations from which staphylococci may not be isolated. In the animal body they are normally found in the nose, on the skin, in saliva, in the intestinal contents, and in froces; they are frequently isolated from suppurative processes (see Pathogenicity); and they are present in varying numbers in air, water, milk, sewage, and on all articles liable to come in contact with these substances. Their main habitat appears to be the nose. Several workers (see Hallman 1937, McFarlan 1938, Gillespie et al. 1939) have shown that they are present in the anterior nares of a high proportion of normal persons and that 30 to 60 per cent. of persons are nasal carriers of potentially pathogenic staphylococci. Relatively harmless members of the albus species are commonly present on the skin, but the more dangerous aureus species is found on the hands of only a small proportion of healthy persons who are usually shown, on investigation, to be heavy nasal carriers (Gillespie et al. 1939, Miles et al. 1944). Staph. aureus has been reported in the milk of a high proportion of nursing mothers (Report 1942, Duncan and Walker 1942), but whether this is a normal condition or whether it occurs only in infected maternity wards is not clear. Ubiquitous as staphylococci are their natural habitat is the animal body, and it is the animal body that furnishes the main supply to the outside world.

Morphology.-The staphylococci consist of round or somewhat oval cells, having an average diameter of $0.8-1 \cdot 0 \mu$. The size is variable, not only from one species to another, but in members of the same species; it depends partly on the age of the culture and the nature of the medium on which it is grown. Some species are generally smaller than others; thus the average diameter of Staphylococcus aureus is $0.7-0.9 \mu$, whereas the salivary staphylococci are said to be larger, 1.0-1.2 $\mu$. All the members of the group are non-motile, nonflagellated, and non-sporing. They are usually described as non-capsulated.

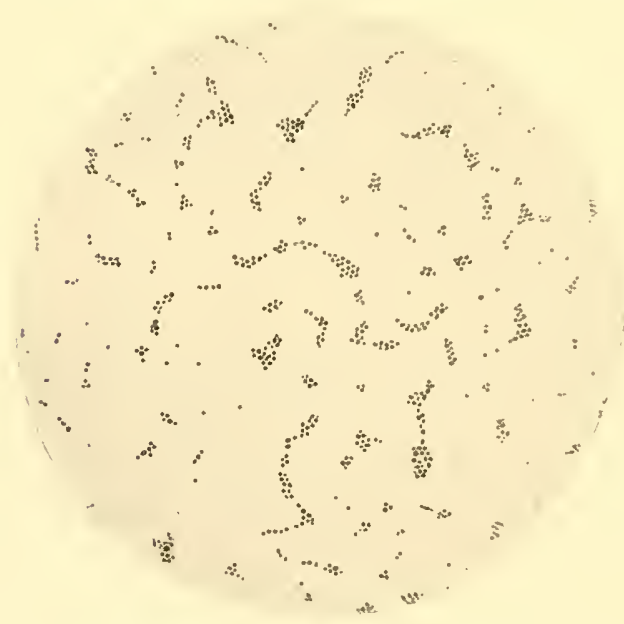

FIG. 125.-Staphylococcus aureus.

From an agar culture, 24 hours, $37^{\circ} \mathrm{C} .(\times 1000)$.

Lyons (1937), however, states that capsules are demonstrable in 3-hour broth cultures, but disappear as the culture gets older.

The true staphylococci are arranged in grape-like clusters, that is to say they form groups, the members of which are disposed in three planes of space without regard to any definite configuration. This distribution is best appreciated when a hanging drop preparation is examined-especially if a stereoscopic microscope is employed. The characteristic grouping into clusters is more evident on solid than in liquid media. Indeed in broth it is common to find the cocci occurring, not only in groups, but in pairs and in short chains; they are then liable to be mistaken for streptococci. This confusion may be accentuated owing to the fact that streptococci on solid media tend to lose their capacity for forming chains, and may develop in small clusters. 
The differentiation between these two types morphologically, therefore, is not always easy; on a single medium alone it may be impossible, but if the appearance of the coccus is studied in both liquid and solid media, not much difficulty will be experienced. The differential points are (1) that the chains formed by staphylococci rarely contain more than four members and (2) that the clusters formed by streptococci generally consist of aggregations of chains; the chain is the fundamental unit of the streptococcus.

Staining Reactions.-The staphylococci stain well with most of the aniline dyes, and are uniformly Gram-positive. It is not uncommon, however, to see them described as being sometimes negative. Winslow, Rothberg and Parsons (1920), in a study of 180 strains, encountered 5 Gram-negative strains, and the same authors make the generalization that, whereas the orange and white cocci are Gram-positive, the yellow and red ones, including the Sarcince, are Gram-negative. This discrepancy can be explained by the facts, firstly that different types of staphylococci do vary in their resistance to decolorization, and secondly that many strains which are Gram-positive in an 18 or 24 hours' culture become Gramnegative as they grow older. To obtain uniformity, therefore, it is essential to use a young culture-never more than a day old - and not to prolong unduly the process of decolorization. If these precautions are taken, it will be found that almost without exception the staphylococci, at any rate on first isolation, as also the sarcinæ, react positively to Gram's stain.

Cultural Reactions.- The staphylococci are among the easiest of micro-organisms to cultivate in vitro. Though some develop more slowly than others, particularly Staphylococcus citreus, they all give abundant growths.

In nutrient broth after 24 hours at $37^{\circ} \mathrm{C}$. there is a moderate to dense turbidity, with a moderate deposit of a powdery nature, which, on shaking, swirls up and disappears completely, increasing the turbidity. This is the usual picture. But some types, the salivary staphylococci, for example, form a thick, weedy, glutinous deposit, leaving the supernatant fluid clear. After 2 days' incubation, a surface ring growth is generally present. In no case is there any distinctive pigment formation in fluid media.

On nutrient agar, there is produced within 1 to 2 days a moderately thick, raised, confluent growth, with a moist, glistening, smooth, or somewhat honey-combed surface-due to the imperfect fusion of individual colonies; in most cases it is of butyrous consistency and easy to emulsify, but in the case of the salivary staphylococci, it is glutinous, adherent to the medium, and more difficult to emulsify. Pigment production is most obvious on agar at $22^{\circ} \mathrm{C}$.

On gelatin plates at $22^{\circ} \mathrm{C}$. development is slower, there being often no visible growth for 2 to 3 days;

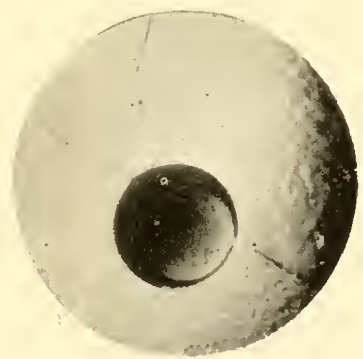

Fit. 126.-Staphylococcus aureus.

Surface colony on agar, 24 hours, $37^{\circ} \mathrm{C} .(\times 8)$. the colonies which are then formed are small and relatively unpigmented; later a zone of liquefaction may appear around them.

In gelatin stab cultures there is a filiform growth reaching to the bottom of the tube, and a surface growth of variable degree. Liquefaction may or may not occur; when it does, the usual type given by the white and golden cocci is 
infundibuliform or saccate, by the yellow cocci crateriform. The time of its appearance is likewise subject to a considerable amount of variation; it may be noticeable after 2 days, or it may be a fortnight ог even longer before it becomes apparent. Speaking broadly, it may be said that the pyogenic staphylococci liquefy gelatin early, the saprophytic ones late.

On potato there is a moderate confluent growth. Loeffler's serum is a good nutritive medium, on which pigment is well developed. On MacConkey's neutral red lactose bile salt agar, the colonies are very small, pale pink after 24 hours, and deep pink after 48 hours.

Working with pure cultures, Chapman and Berens (1935) and Chapman (1936) find that, on proteose peptone lactose agar containing a concentration of 1-300,000 crystal violet, white, violet, or orange colonies with a violet fringe may be formed. As a rule, the strains giving rise to either of the latter two types of colony are hæmolytic, produce coagulase, and are toxic to rabbits on intravenous inoculation, while strains forming white colonies are negative in all these respects. More recently, Chapman and his coleagues (1937) have described a proteose peptone lactose agar, $\mathrm{pH} 8 \cdot 6$, containing 0.017 per cent. bromthymol blue, that is said to inhibit the growth of non-pathogenic staphylococci ; and Chapman (1944) has described an alkaline bromthymol blue agar containing potassium tellurite for isolation of staphylococci from the fæces.

Variant colonial types-including rough and $\mathrm{G}$ forms-have been described by Hoffstadt and Youmans (1932), Bigger, Boland, and O'Meara (1927), and Swingle (1935).

Metabolism.-The staphylococci are facultative anaerobes, growing best in the presence of oxygen. On agar plates incubated anaerobically, the growth tends to spread out on the surface of the medium, so that the colonies instead of being

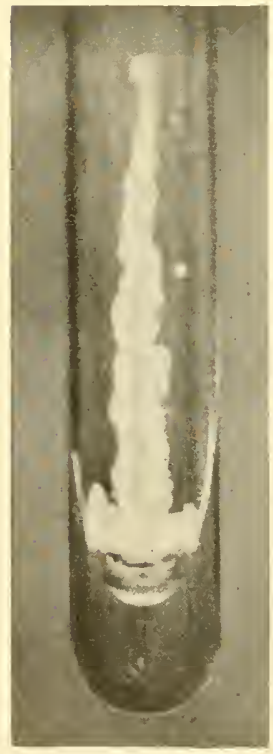

Fic. 127. - Staphy. lococcus aureus. Agar stroke culture, 24 hours, $37^{\circ} \mathrm{C}$. convex, are flat and effuse. Lubinski (1894) was the first to point out that no pigment is produced under anaerobic conditions, though, if a culture which has been incubated anaerobically is exposed to the air, it soon develops its characteristic colour. He found, however, that continuous anaerobic cultivation generally caused a strain to lose its power of producing pigment-a power which was not regained by subsequent cultivation in the presence of oxygen.

The limits of temperature between which growth is possible are wide, varying from about $12^{\circ}$ to $45^{\circ} \mathrm{C}$., but as a rule development is most rapid at $37^{\circ} \mathrm{C}$. A slightly alkaline medium of $\mathrm{pH} 7 \cdot 4-7 \cdot 6$ is preferable for the initiation of growth, but some growth will occur even at $\mathrm{pH} 4 \cdot 0-5 \cdot 0$. Growth is slightly increased by the addition of blood and glucose to the medium; serum has no beneficial effect. According to Hucker (1924a), the staphylococci are unable to use ammonium salts as their sole source of nitrogen. Hughes (1932), and later Knight (1935), both of whom have studied the nutritive requirements of Staph. aureus, describe the existence of an essential growth factor, which subsequent work (Knight 1937) has shown to contain vitamin $B_{1}$ and nicotinic acid. The organisms can now be grown aerobically on a medium containing only known chemical constituents, 
namely a mixture of glucose, salts and fourteen amino-acids, supplemented by the two vitamin constituents just mentioned (Fildes et al. 1936, Knight 1937). According to Kligler, Grossowicz and Bergner (1943), nicotinic acid is the essential factor in the glucose metabolism of Staph. aureus; thiamin $\left(B_{1}\right)$ acts as a catalyser in the oxidation of pyruvic acid. Riboflavin can apparently be synthesized by the organisms themselves (O'Kane 1941). As with other parasitic bacteria, organic sulphur is necessary for growth; this is usually obtained in the form of cystine, but methionine and sodium dithiodiacetate can be substituted (Fildes and Richardsoll 1937, Gladstone 1937). No growth occurs in the complete absence of $\mathrm{CO}_{2}$ (Gladstone et al. 1935). (See also Chapter 3).

Hewitt (1930) has shown that Staph. aureus, when grown in ordinary infusion broth, brings about a rapid fall in electrode potential to between Eh $-0 \cdot 1$ and $-0 \cdot 2$ volt. Owing to lack of peroxide formation the potential remains at a low level for a long time, showing no tendency to rise as it does with streptococci. In glucose broth the potential does not fall as low as in ordinary broth.

Pigment Production.-As already mentioned, the staphylococci are active pigment producers. Staphylococcus aureus forms a golden, Staphylococcus citreus a lemon-yellow pigment, while cultures of Staph. albus are of a porcelain-white colour. The development of the pigment and its actual tint depend, however, on several factors. As with many other organisms, the optimum temperature for pigment production does not coincide with the optimum temperature for growth; more pigment is produced at $22^{\circ} \mathrm{C}$. than at $37^{\circ} \mathrm{C}$.; if cultures that have been incubated at $37^{\circ} \mathrm{C}$. are subsequently left at room temperature, the colour is seen to deepen. Gelatin and broth are unsuitable. Oxygen is requisite for its development; under anaerobic conditions the growth is colourless. Carbon dioxide is said to favour its production, provided oxygen is also present (Lubinski 1894). An agar medium containing 33 per cent. milk is recommended for its study (see Christie and Keogh 1910).

A very important point to remember is that a given strain of staphylococcus under conditions of artificial cultivation may lose its power of producing pigment. The cause of this loss is unknown. It is most noticeable in the case of Staphylococcus aureus, which, when freshly isolated from the animal body, gives a rich golden pigment, but often loses this character on prolonged cultivation. This variation adds greatly to the difficulty of classification, and it must be emphasized that in the study of a particular strain, the property of pigment formation should be noted as soon after isolation as possible (Dudgeon 1908).

Gelatin Liquefaction.-There is a considerable amount of discrepancy in the reports of various authors as to the power of staphylococci to liquefy gelatin. Of 41 white strains examined by Gordon (1903-4), 24 liquefied gelatin. Kutscher and Konrich (1904) reported upon 57 strains of staphylococci, and found that all liquefied gelatin. Similarly with Klopstock and Bockenheimer (1904) who examined 30 strains, and with Fraenkel and Baumann (1905) who examined 36 strains. Dudgeon (1908) found that 44 out of 46 aureus strains and 35 out of 56 albus strains liquefied gelatin, while Winslow, Rothberg and Parsons (1920), in examining 180 strains, found that 67 per cent. of the aureus and 47 per cent. of the albus strains liquefied gelatin.

These discrepancies are to be explained partly by possible differences in the cocci examined and partly by the length of time during which growth was observed. 
There is general agreement that staphylococci isolated from pathological sources liquefy gelatin more frequently than those isolated from water, air, skin, etc.

There is likewise general agreement that the orange cocci are more rapid liquefiers than the white, and the white cocci more rapid than the yellow.

Summing up, it may be said that Staphylococcus aureus liquefies gelatin almost always, Staphylococcus albus frequently, and Staphylococcus citreus sometimes.

Resistance to Heat and Disinfectants.-The staphylococci are among the more resistant of the non-sporing organisms. In broth or agar tubes sealed with paraffin and kept in the ice-chest, cultures may remain alive for months. Dried on threads they retain their vitality for 3 to 6 months, and from dried pus they have been cultivated after 2 to 3 months. Many of them are heat-resistant, in that they will withstand a temperature of $60^{\circ} \mathrm{C}$. for half an hour. In pure culture they resist a concentration of 1 per cent. phenol for 15 minutes, but are killed by a concentration of 2 per cent. Mercuric chloride is a poor disinfectant for staphylococci; to kill them in 10 minutes a 1 per cent. solution is required. Many of the aniline dyes exert a strongly bactericidal action on the staphylococci-as indeed they do on most Gram-positive organisms. This selective action is made use of in certain technical procedures, such as the isolation of $B r$. abortus from milk, where it is endeavoured by the incorporation of a dye-gentian violet or crystal violet-to inhibit the growth of Gram-positive organisms. Use is also made of the great susceptibility of staphylococci to the violet dyes in the isolation of streptococci, whose susceptibility to these dyes is very much less, Garrod (1942) (see p. 135). Other dyes, of which malachite green appears to be the strongest and acid fuchsin the weakest, are also employed, usually in a concentration of about 1/10,000 (Oesterlin 1925). Most strains of staphylococci are sensitive to penicillin.

Biochemical Reactions. - The ability of the staphylococci to ferment sugars varies greatly according to the strain employed. For this reason it is not possible to classify them on this basis with anything like the same precision as, for example, the coliform group of bacilli. As a rule the golden cocci have the greatest fermentative power, the white are less active. There is a wealth of literature on the fermentative capacities of the staphylococci, with a corresponding difference of opinion amongst the various authors as to the importance of the different sugars. Thus, Andrewes and Gordon (1905-6) lay stress on the reactions in maltose, lactose, glycerol and mannitol. Winslow, Rothberg and Parsons (1920), on the other hand, come to the conclusion that the only sugar of differential value is lactose. Working with Staphylococcus aureus and albus, these authors found that 68 per cent. of the strains formed acid from glucose, 63 per cent. from maltose, 61 per cent. from sucrose, and 49 per cent. from lactose; salicin, inulin and raffinose were rarely fermented, mannitol and dulcitol never. With these findings most authors disagree, particularly with regard to mannitol, which is generally held to be attacked by Staphylococcus aureus, and frequently by Staphylococcus albus (Dudgeon and Simpson 1928). It is quite clear, however, that it is impossible to dogmatize on the reactions of any one strain. Dudgeon (1908), who examined 121 aureus and albus strains on a large number of sugars, found that very few agreed in giving identical results.

Similarly with litmus milk the reactions are variable. Studying 180 aureus and albus strains, Winslow, Rothberg and Parsons (1920) found that 75 produced acid, clot and peptonization, 60 acid, generally clot, and no peptonization, 22 alkali and peptonization, 16 alkali but no peptonization, while 7 produced no change. 
These findings are in agreement with those of other authors, except with regard to peptonization, which is less commonly reported.

The proteolytic activity of staphylococci is not very strong. Some strains are fibrinolytic (Saski and Fejgin 1937, Neter 1937), and some, particularly those of canine origin, can digest coagulated horse serum (Minett 1936). Lipase production has been reported by Orcutt and Howe (1922) and Christie and Graydon (1910). Most aureus strains produce hyaluronidase.

The methyl-red test is generally positive with the aureus and albus strains, negative with the citreus strains. The Troges-Proskauer reaction is given by most strains of Staphylococcus aureus. According to Winslow, Rothberg, and Parsons (1920), most strains of staphylococci reduce nitrates to nitrites. Hucker (1924a), on the contrary, found that, though 49 out of 50 aureus strains reduced nitrates, only 23 out of 152 albus strains were able to do so. Hydrogen sulphide is stated by Andrewes and Gordon (1905-6) to be formed in small quantity by the pyogenic staphylococei, in greater quantity by Staphylococcus albus. We have been unable to confirm this. Ammonia is produced by 89 per cent. of the golden and white strains (Winslow et al. 1920). Indole is apparently never produced (Hucker 1924a).

Antigenic Structure.-Studying agglutination of staphylococci by immune rabbits' sera, the early workers (Kolle and Otto 1902, Otto 1903, Pröscher 1903, Kutscher and Konrich 1904, Veiel 1904, Klopstock and Bockenheimer 1904, and Koch 1908) reported that they could be sharply divided into two types - the pathogenic and the saprophytic types. The great majority of strains isolated from purulent lesions in the human body were agglutinated by a serum prepared against one such strain, whereas the strains isolated from saprophytic sources were not agglutinated. The difference in titre to which agglutination occurred rather suggested that there might be one or more sub-groups within the main types. This suggestion was confirmed by later workers, who employed the more delicate test of absorption of agglutinins. Thus Julianelle (1922) found that the staphylococci could be divided into three types with two sub-groups, whereas Hine (1922), working with 81 strains, was able to classify them into two main types, each of which had at least three sub-groups. There was evidence to suggest that the pathogenic strains formed a more homogeneous serological group than the saprophytic.

More recently, Yonemura (1936) examined 324 strains from pathological sources and was able by the use of absorbed sera to divide them into nine main types; all but 22 of the strains, however, fell into three of these types. Blair and Hallman (1936) recognized three types by the absorption of agglutinins method. Cowan (1938, 1939a) likewise showed that it was possible by shde agglutination, using absorbed sera, to distinguish three main types among the pathogenic staphylococci; there is reason to believe, however, that each of these contains sub-types distinguishable by means of absorbed sera (Christie and Keogh 1940). Working in India, Goyle and Minchin (1940) were able to classify only 33 per cent. of pathogenic strains by Cowan's method. Considerably more work will have to be done before the serological typing of staphrlococci becomes of much practical use in epidemiological investigations.

Julianelle and Wieghard (1934, 1935), and Wieghard and Julianelle (1935), as the result of chemical fractionation of staphylococci, have isolated two polysaccharides each containing about 4 per cent. nitrogen, and distinguished from each other by optical rotation and by the type of sugar produced on hydrolysis. The first polysaccharide was extracted from pathogenic (A), the second from non-pathogenic 
(B), strains. The sera of rabbits that had been inoculated intravenously with whole cocci reacted specifically to a high titre with the polysaccharides when tested by the precipitation reaction. The polysaccharides were non-antigenic and non-toxic for rabbits and mice, but Type A gave rise on intradermal inoculation of patients with staphylococcal infections to an immediate skin reaction of the "wheal and erythema" type. Besides the carbohydrates, a complex protein substance was isolated from both pathogenic and non-pathogenic strains of staphylococci. This protein, though non-toxic to rabbits and mice, was antigenic, giving rise to precipitins that reacted, however, only with the protein and not with the polysaccharide substances. On intradermal inoculation into susceptible patients the protein evoked a skin reaction of the delayed inflammatory type. Serological observations showed that the protein was responsible for the speciesspecificity of staphylococci, while the type-specificity was determined by the soluble specific carbohydrate substances. For the differentiation of pathogenic from nonpathogenic strains it was essential to employ the precipitation reaction, using as an antigen either the extracted polysaccharide, the supermatant fluid of centrifuged young broth cultures, or an acid extract of the sedimented organisms. The agglutination reaction was affected by the group protein, and was unfitted for type differentiation. This work has been confirmed by Hegemanu (1937) and Peragallo (1937) ; but Thompson and Korazo (1937) have brought evidence of the existence of at least one further polysaccharide, which they term Type C; and Verwey (1940) claims to have isolated a type-specific protein from staphylococci. Durfee (1942) found that all strains agglutinating with Cowan's sera formed Julianelle's polysaccharide Type A.

In general, it may be said that the precipitin reaction is more suited for the differentiation of pathogenic from non-pathogenic strains, and the agglutination reaction for the sub-division of the pathogenic strains into types.

Staphylococci are known to be frequent carriers of bacteriophage. Fisk (1942) took advantage of this to develop a cross-culture method of bacteriophage typing. By this means he was able to classify 44 strains of Staph. aureus into 37 different groups. Our own observations have shown that, if potent lytic filtrates are prepared, staphylococci may be typed by a method similar to that used for the Vi phage-typing of typhoid bacilli. Already 21 different types have been established, and the method has proved of considerable value in epidemiological inquiries (Wilson and Atkinson 1945).

Toxin Production.- When grown under suitable conditions, certain strains of staphylococci give rise to a filtrable toxin having a series of effects which, though described a long time ago by such workers as van der Velde (1894), Denys and Havet (1898), von Lingelsheim (1899), Kraus and Clairmont (1900), and Neisser and Wechsberg (1901), have received intensive study during recent years. This activity followed largely on the reinvestigation of the problem by Parker in 1924. A toxic filtrate is hæmolytic, especially towards rabbit cells; it has a destructive action on leucocytes; when injected intradermally into the skin of the rabbit or the guinea-pig it gives rise to necrosis; and when injected intravenously into the rabbit or mouse it causes acute and fatal toxæmia.

Toxin formation is a property of pathogenic strains and is therefore limited mainly to the aureus type. Considerable variation exists between different strains, and if toxin is required on a large scale for immunological or other purposes it is important to select a strain with a high toxigenic capacity. Various methods are 
used in the production of the toxin. In a fluid medium it develops rather slowly. Thus Neisser and Wechsberg (1901), by testing broth cultures filtered at intervals, found that the toxin, as judged by the hæmolytic titre, was first demonstrable on the 4 th day of incubation at $37^{\circ} \mathrm{C}$., and that it rose to a maximum between the 10th and 14th days, after which it gradually diminished. Burnet's (1930) technique of growing the organisms on 0.5 per cent. nutrient agar for 24 hours in air containing 10-20 per cent. $\mathrm{CO}_{2}$, and extracting the toxin from the agar with saline, is very satisfactory, and is widely used, either in its original or a slightly modified form (Parish and Clark 1932, Dolman 1932). The medium used should have a reaction of between $\mathrm{pH} 6.0$ and 7.0 (Walbum 1922). Though the toxin can traverse a Seitz filter, some of its activity is lost in passage, and it is therefore advisable to separate the organisms from the toxin by centrifugation. A good toxin shonld hæmolyse a 1 per cent. suspension of rabbit cells in a dose of $0.0005-0.002 \mathrm{ml}$. : $0.001-0.005 \mathrm{ml}$. should cause necrosis on intradermal inoculation, and 0.25-0.8 $\mathrm{ml}$. per kilo injected intravenously should kill a rabbit within a few minutes (Burnet 1929, Gross 1931c, Parish and Clark 1932). Satisfactory toxins can be prepared by growth in a chemically defined medium (Gladstone 1938, Smith and Price 1938a).

Though Burnet (1929), Gross (1931c) and Gengon (1932) formed the opinion that the various activities of a toxic filtrate were due to one and the same toxin, subsequent workers have inclined to the opposite view. Without prejudicing the issue, we shall, for the sake of simplicity, describe each of the manifestations separately.

$\alpha$-hcemolysin.-This is active against rabbit, but not against human, red corpuseles, and causes lysis rapidly at $37^{\circ} \mathrm{C}$. It has some action on sheep red corpuscles, but this is destroyed by heating at $57^{\circ} \mathrm{C}$. for 30 minutes (Flaum 1938). It can be specifically neutralized in multiple proportions by an antiserum. Its resistance to heat is peculiar, since it is inactivated more readily by lower than by higher temperatures (Arrhenius 1907, Landsteiner and von Rauchenbichler 1909, Seiffert 1935-36, Kodama and Nisiyama 1938, Rigdon 1938a, Beumer 1939, Fulton 1943). According to Arrhenius (1907), lysin heated to $70^{\circ} \mathrm{C}$. loses a great part of its hæmolytic activity, but regains it if it is heated for a further 5 minutes to $100^{\circ} \mathrm{C}$. This fundamental observation was confirmed by Landsteiner and von Rauchenbichler (1909), using a temperature of $65^{\circ} \mathrm{C}$. for 30 minutes to inactivate the lysin. As the result of expcrimental observations they were led to believe that at the lower temperature the lysin enters into combination with a protein constituent, derived from the broken down organisms, the medium, or added serum, to form an inactive compound that can be destroyed by heating to a higher temperature, thus liberating the lysin. The failure to realize this unusual reaction to heat has led to much confusion by workers who have assumed that, because the lysin is apparently destroyed by heating for 2 hours at $57^{\circ} \mathrm{C}$, 1 hour at $60^{\circ} \mathrm{C}$, or 30 minutes at $65^{\circ} \mathrm{C}$, it is necessarily destroyed by boiling. According to Burnet (1931), Kodama and Nisiyama (1938), and Fulton (1943), a strong-toxin is not destroyed completely even in 30 minutes at $100^{\circ} \mathrm{C}$. Its activity is said to be inhibited by ascorbic acid in a concentration of $30 \mathrm{mgm}$. per ml. (Mercier 1938), and by azochloramid (Heise and Starin 1940). There is a close parallelism between the $\alpha$-hæmolysin content of a filtrate and (1) the dermonecrotic and lethal factors (see Panton and Valentine 1932, Levine 1939), (2) the ability of a given strain to reduce methylene blue (McBroom 1937), and (3) the leucocidin content, as estimated by the Neisser-Wechsberg method (Wright 1936), though this is still subject to dispute (see Flaum 1938). Morgan and Graydon (1936) have brought evidence to show that in most toxic filtrates the $\alpha$-lysin contains two antigenically distinct components, referred to as $\alpha_{1}$ and $\alpha_{2}$, which have different combining powers for antitoxin; both are dermonecrotic. Treatment of the toxin with $0.2-0.5$ per cent. formol at $37^{\circ} \mathrm{C}$. leads 
in the course of a few days to a disappearance of its hæmolytic and toxic properties, but not to that of its flocculating capacity with antitoxin (see Burnet 1931). Toxoid so pre. pared is antigenic and is sometimes used as a vaccine. Strains producing $\alpha$-lysin are predominantly of human origin.

$\beta$-lysin.-This acts on sheep, ox and human, but not on rabbit, red corpuscles, and causes lysis only after the tubes have stood at room temperature or in the ice-chest overnight-the so-called "hot-cold" lysis (Bigger 1933, Glenny and Stevens 1935). It is more resistant to formalin than the $\alpha$-lysin, and is usually stated to be more resistant also to heat, the degree of destruction at $57^{\circ} \mathrm{C}$. in half-an-hour being much less. According, however, to Kodama and Kojima (1939), it resembles $\alpha$-lysin in being inactivated more readily at low than at high temperatures. It is antigenically distinct from the $\alpha$-lysin, and can be specifically neutralized by a suitable antiserum. It is much less toxic than the $\alpha$-lysin to rabbits, guinea-pigs and mice (Bryce and Rountree 1936). Intradermal inoculation into guinea-pigs gives rise to no more than a transient erythema (Smith and Price 1938a). Strains producing $\beta$-lysin are predominantly of bovine origin (see Minett 1936, Slanetz 1942).

$\gamma$-lysin.- Smith and Price (1938b) have described the production by a strain of staphylococcus of a $\gamma$-toxin, which causes rapid lysis of the red corpuscles of a variety of animals including the rabbit and sheep, and delayed lysis of rat and guinea-pig corpuscles. It appears to be antigenically distinct from the $\alpha$ - and $\beta$-toxins, though it may have some relationship to the $\alpha_{2}$-toxin.

Leucocidin.-The study of the leucocidin content of toxic filtrates has been carried out by two different methods. In the Neisser-Wechsberg (N.W.) method the leucocidin is titrated by its ability to inhibit reduction of methylene blue by healthy rabbit leucocytes. The Panton-Valentine (P.V.) method, described by Panton and Valentine in 1932 and modified slightly from that of van der Velde (1894), depends on direct microscopic observation of the destructive action of the toxin on human leucocytes. According to the observations of Valentine (1936) and Wright (1936), it would appear that the N.W. leucocidin is identical with the $\alpha$-hæmolysin, but that the P.V. leucocidin is different from it. Flaum (1938), on the other hand, observed that a practically pure $\beta$-lysin might have a strong leucocidal effect, as tested by the N.W. method, and concluded that the N.W. leucocidin is not always identical with the $\alpha$-lysin. He suggests, however, that the P.V. leucocidin may be identical with the $\beta$-lysin. In his experience it proved to be more heat stable than the leucocidin associated with the $\alpha$-lysin. Proom (1937) considers that the $\alpha$-lysin is able to interfere with the respiratory activity of the leucocytes, as measured by the N.W. technique, but that it has not the same destructive action on the cell and nucleus as is manifested by the P.V. leucocidin. Weld and Mitchell (1942) state that the $\alpha$-lysin and the N.W. leucocidin agglutinate rabbit leucocytes, and that the agglutination reaction can be used instead of methylene blue reduction for measuring the N.W. leucocidin.

Enterotoxin.-Some strains of staphylococci, mostly of the aureus species, produce under favourable conditions an enterotoxin that is capable of giving rise to acute food poisoning in man (see Chapter 72). Our information on the properties of this substance is confusing and incomplete. There is no satisfactory method of titrating it, and there is some doubt about its heat stability. It is prepared best by growth in a semi-solid agar medium, such as that described by Dolman and Wilson $(1938,1940)$. The cultures should be incubated for about 40 hours in an atmosphere containing $10-30$ per cent. $\mathrm{CO}_{2}$, and should then be passed through cheese cloth and fine filter paper, and centrifuged at high speed. The clear supernatant fluid, which contains the enterotoxin, should be sterilized by gradocol filtration. Tests for its presence and its potency were originally made by feeding filtrates to human volunteers or to monkeys; but Dolman, Wilson and Cockcroft (1936) pointed out that, provided the $\alpha$ - and $\beta$-toxins were destroyed by heat or formalin, or were neutralized by antiserum, the enterotoxin could be demonstrated by its ability to give rise to vomiting and diarrhœa in kittens injected intraperitoneally. 
Inoculation of 1-3 ml. of a potent filtrate into a kitten weighing $350-700 \mathrm{gm}$. is followerl, as a rule within half-an-hour, by lassitude and weakness, unsteadiness of gait, and paroxysmal vomiting of the projectile type, associated with diarrhœe. The kitten may be ill for several hours, but usually recovers completely.

How far the kitten test can be regarded as a specific reaction to the enterotoxin is doubtful. Several workers (Rigdon 1938b, Jones and Lochhead 1939, Hammon 1941) have reported non-specific reactions after the intraperitoneal injection of control materials. Moreover, Fulton (1943), working in our laboratory, found little relationship between the ability of a filtrate to give rise to vomiting in kittens on intraperitoneal injection and to voniting in man when taken by the mouth. Other methods of demonstrating the enterotoxin have been suggested, such as the intravenous inoculation of rabbits (Kupchik 1937), of kittens (Davison, Dack and Cary 1938, Davison and Dack 1939), or of monkeys (Segalove and Dack 1941), or the feeding of kittens (Dolman, Wilson and Cockeroft 1936), but none has so far proved as reliable as the feeding of human rolunteers.

The nature of the enterotoxin is still in doubt. Dolman (1943) regards it as distinct from the $\alpha$-and the $\beta$-toxins. He finds that a pure $\beta$-toxin when injected intraperitoneally into the kitten gives rise to early and repeated vomiting, coming on sometimes within 5 minutes and followed by death some hours later. Vomiting caused by the enterotoxin, on the other hand, begins later and is not fatal. Though Slanetz (1942) suggests that the $\beta$-toxin and the enterotoxin are identical, this seens improbable, because a pure $\beta$-toxin is innocuous to man by the mouth (Fulton 1943, Dolman 1943). For the separation of the enterotoxin from the $\alpha$-toxin, reliance has been placed on the difference in their heat stability. The $\alpha$-toxin is said to be destroyed by heating at $65^{\circ} \mathrm{C}$. in 30 minutes, whereas the enterotoxin is said to withstand boiling for 15-30 minutes or longer. As Fulton (1943), however, points out, this is not a reliable method of differentiation, because the $\alpha$-toxin, though inactivated by heating at $65^{\circ} \mathrm{C}$., is morlerately resistant to boiling (see p. 615). It is at present very difficult to say whether the enterotoxin is distinct from the other toxins, or whether it is related to the $\alpha$-toxin; but the observation by Dolman (1944) of a strain that produced enterotoxin in the complete absence of $\alpha$-or $\beta$-toxin suggests that the enterotoxin is distinct from the hrmolytic toxins. The mode of action of the enterotoxin is not yet understood. The observations of Bayliss (1940) on the cat and of Richmond, Reed, Shaughnessy and Michael (1942) on the rabbit suggest that its action is peripheral rather than central, and that it affects either the sensory nerve endings or the smooth muscle of the small intestine.

Staphylocoagulase.-The ability of certain staphylococci to coagulate citrated or oxalated plasma was first described by Loeb (1903-04) and confirmed by Much (1908). Later it was studied by a number of other workers (von Darányi 1926, Gross 1931a, b, Stephan 1934, Vanbreuseghem 1934, Chapman et. al. 1934, Walston 1935).

For its demonstration about $0.1 \mathrm{ml}$. of an overnight broth culture of Staph. aureus, or of a broth suspension of an agar slope culture made up to the same density as a broth culture, is mixed with $0.5-1.0 \mathrm{ml}$. of a freshly prepared $1 / 10$ dilution of human or rabbit plasma in saline. 'The mixture is incubated at $37^{\circ} \mathrm{C}$. for $3-6$ hours ; if no clot has appeared by this time, it should be left overnight at room temperature and again examined. The plasma of other animals, such as the horse, ox, sheep, or guinea-pig may be used instead. If kept undiluted in the ice-chest under sterile conditions, citrated plasma remains suitable for several months (Fisk 1940). Since plasma may undergo spontaneous coagulation, a control tube containing plasma alone diluted with saline should always be put up, as well as tubes inoculated with a known coagulase-positive and a known coagulase-negative strain. Culture media contaiming fermentable carbohydrates should be avoided, since Neter (1937) has shown that under these conditions many strains form an anti-coagulaso 
which may inhibit the elotting of the plasma. Other sources of error have been pointed out by Gillespie (1943). According to Duncan and Walker (1942), the result of the test is influenced by the proportion of eulture to plasma in the mixture. The optimal proportion can be determined in the usual way by titrating a series of increasing dilutions of culture against a fixed volume of plasma, or of increasing dilutions of plasma against a fixed volume of eulture. Tests for the presence of coagulase may also be made by the slide method (Cadness-Graves et. al. 1943).

Though there is a high correlation between coagulase and $\alpha$-hæmolysin production, there seems little doubt that these two substances are distinct. The hæmolysin is destroyed in half an hour by exposure to a temperature of $56^{\circ} \mathrm{C}$., whereas the coagulase is not (Vanbreuseghem 1934, Cruickshank 1937, Smith and Hale 1944). The hæmolysin is absorbed by red corpuscles, the coagulase is not. An antibody to the hæmolysin can readily be obtained by the inoculation of rabbits, but not to the coagulase (Gross 1931a). Coagulase is formed almost exclusively by strains of Staph. aureus or by albus variants of aureus strains. Cruickshank (1937) maintains that coagulase production constitutes the most convenient and reliable single test for estimating the pathogenicity of a given strain. This contention is supported by a number of subsequent workers (Chapman et al. 1938, Marcuccio 1938, Gillespie, Devenish and Cowan 1939, Fairbrother 1940, Christie and Keogh 1940).

The method of coagulase action is not yet clearly understood. According to Smith and Hale (1944) coagulase itself is a thermostable substance, filtrable through a gradocol membrane having an A.P.D. of $0.31 \mathrm{~m} \mu$, but completely held back by one of $11 \mathrm{~m} /$. It appears to be the precursor of a thermolabile thrombinlike substance, the production of which depends on the participation of an activator present in the plasma of some animals, but deficient or lacking in others. The staphylocoagulase reaction resembles normal thrombin formation from prothrombin under the influence of thrombokinase except that calcium is not required.

Pathogenicity.--The staphylococci can be fairly sharply divided into pathogenic and non-pathogenic types. Thus the great majority of strains isolated from suppurative lesions in the animal body are found to be pathogenic for rabbits, and to a less extent for mice and guinea-pigs. On the other hand, the great majority of strains isolated from normal skin, air, water, dust, etc., are harmless to these animals. Sometimes the virulence of the pathogenic strains diminishes on prolonged cultivation, but this is not always so; even after years of subculture in the laboratory the virulence may remain intact. Moreover, by passage through rabbits, it is generally possible to raise the virulence of a strain which has become temporarily avirulent; with the saprophytic strains this is impossible. According to Lubinski (1894) the virulence of Staph. aureus for rabbits can be increased by growth under anaerobic conditions; growth in pure oxygen was said to have the reverse effect.

Man.-The staphylococei which are responsible for disease in the human body generally belong to the aureus or albus varieties; only occasionally can Staphylococcus citreus be incriminated. Staphylococcus aureus is more pathogenic to man than Staphylococcus albus; it gives rise to the severer lesions, such as osteomyelitis, pyæmia-sometimes associated with an infective endocarditis-mastitis, boils and abscesses in various parts of the body, and on occasion to a peculiarly fatal form of broncho-pneumonia (Finland, Peterson and Strauss 1942), whereas Staphylococcus 
albus is responsible for the milder inflammatory lesions, such as acne pustules, stitch abscesses, and other minor suppurative conditions of the skin. Staphylococci are frequently found in conjunction with other organisms, particularly in the chronic stages of gonorrhœa, in bronchitis, in post-influenzal pneumonia, and in catarrhal conditions of the nose and respiratory passages. Their exact significance in these cases is difficult to assess, but it is probable that they assist these other organisms in giving rise to suppuration.

Several observers have made personal experiments on themselves to test the pathogenicity of the staphylococci in pure culture. Thus Garré (see Neisser 1912) found that by rubbing staphylococci into the skin of his arm he was able to produce boils, which took a considerable time to heal. (For further information on Pathogenicity to man, see Chapter 67.)

Experimental Inoculation.-The numerous experiments which have been conducted on the pathogenicity of the staphylococci suffice to show that the only laboratory animals that can be artificially infected with ease are the rabbit, the mouse, and the guinea-pig. Of these undoubtedly the most susceptible is the rabbit.

Not all strains are equally pathogenic. As a rule the cocci which are isolated directly from suppurative processes in the body prove virulent, whereas those isolated from skin, air, water, etc., are avirulent. The most pathogenic are the golden strains; many of the white strains are pathogenic, though to a less degree; the yellow cocci are generally non-pathogenic.

RaBBits.-The subcutaneous injection of $1 \mathrm{ml}$. of a 24-hours' broth culture of Staph aureus gives rise to a local abscess, from which the organisms can be recovered. If the culture is mixed with an equal amount of 2 per cent. melted agar and inoculated intracutaneously into the rabbit's back, a spreading necrotic lesion occurs with little pus formation (Jackson, Nicholson and Holman 1940).

Intravenous injection of 0.1 to $0.5 \mathrm{ml}$. of a strain of Staph. aureus recently isolated from a suppurative focus generally proves fatal in 24 to 48 hours. Post mortem, there are hæmorrhages and bloody exudations on the serous membranes, and parenchymatous degeneration of the glandular organs; the cocci can be recovered from the blood stream.

Intravenous injection of a smaller dose-about 0.01 to 0-05 ml.-- gives rise to a pyæmic condition, accompanied by loss of weight and general weakness, and proving fatal in 1 to 6 weeks. Post mortem, multiple small or large circumscribed abscesses are found particularly in the kidneys, and less frequently in the myocardium, lungs, spleen, bone marrow, and costal cartilages. Sometimes regetations develop on the mitral and tricuspid valves and the chordæ tendiner, without any artificial wounding of these structures. Acute osteomyelitis of the long bones not infrequently develops. 'The cocci can be recovered from the suppurative lesions. In animals that recover, reparative processes occur.

Staphylococcus albus is usually much less pathogenic. Strains recently isolated from suppurative foci may cause death on intravenous injection of 1-2 ml. of a 24-hours' broth culture. Strains isolated from saprophytic sources are non-pathogenic unless given in large doses, when death occurs apparently from toxæmia.

Staphylococci which have been killed by heat at $60^{\circ} \mathrm{C}$. for 2 hours, if given in large doses-2-4 agar slopes - at repeated intervals of 10 days, may give rise to progressive cachexia with death in 2 or 3 weeks. Post mortem much the same changes are found as those following acute death from a living culture, namely a hæmorrhagic exudate in the peritoneal cavity, serous hæmorrhages, and parenchymatous degeneration of the glandular organs (Koch 1908). 
Mice and Guinea-pigs are much less susceptible than rabbits, and though death may follow the intraperitoneal injection of virulent cultures, there is frequently no more than a local abscess formation from which the animal recovers.

Protection Experiments.--Pröscher (1903), who attempted to prepare an immune serum suitable for prophylactic and therapeutic use, obtained some hopeful results by the injection of living, virulent staphylococci into goats and horses. $1.5 \mathrm{ml}$. of immune goat serum given subcutaneously protected rabbits against $0.5 \mathrm{ml}$. of a virulent broth culture injected intravenously 24 hours later. But if given at the same time as, or subsequently to, the injection of the cocci, it had but little or no effect. In spite of numerous studies by such workers as Forssman (1935-41), Blair and Hallmam (1935), Kitching and Farrell (1936), Lyons (1937), Downie (1937), Smith (1937), Cowan (1939b), and Valentine and Butler (1939), the mechanism of immunity in natural and experimental infections of man and animals with staphylococci still remains obscure. Serum prepared by the injection of rabbits or horses with toxoid, and later with toxin, has a high antitoxic titre, and may save the life of test animals inoculated prophylactically; but it seems to have little or no direct bactericidal effect, so that local abscess formation is not necessarily prevented. An international standard for staphylococcal antitoxin has now been laid down ; one unit is contained in $0.2376 \mathrm{mgm}$. of the standard preparation (Smith and Ipsen 1938). The potency of the serum is titrated by the hæmolytic method using rabbit red corpuscles, by the intracutaneous injection of guinea-pigs, and by the intravenous injection of mice.

\section{Classification}

Little useful purpose would be served by describing the numerous attempts that have been made to provide a satisfactory classification for the staphylococci. Those who are interested should consult the studies of Rosenbach (1884), Winslow and Rogers (1906), Dudgeon (1908), Kligler (1913), Winslow, Rothberg and Parsons (1920), Hucker (1924a), and Dudgeon and Simpson (1928). Nearly all workers are agreed that there is a gradation from the actively fermentative, gelatin-liquefying, pathogenic group, of which the type is Staph. aureus, down to the weakly fermentative, gelatin-non-liquefying, saprophytic group, of which Gordon's scurf staphylococcus is a typical representative. No sharp line of cleavage occurs, and no single property can be regarded as satisfactory as a basis for classification. Though there is something to be said for dividing the staphylococei into a pathogenic group, Staph. pyogenes (Cowan 1938), and a non-pathogenic group, Staph. saprophyticus (Fairbrother 1940), on the basis of coagulase production, we doubt whether there is much to be gained, partly because the production of golden pigment and coagulase are fairly highly correlated, partly because coagulase production has not yet been established as a certain indicator of potential pathogenicity and partly because pathogenicity is not a good criterion on which to establish specific differences. It is true that we recognize the name Streptococcus pyogenes, but here the differentiation is based on the firmer ground of antigenic structure. Until the serological study of the staphylococei has progressed further we think it best to keep to the time-honoured names of Staph. aureus, Staph. albus, and Staph. citreus, and taking account of more recent knowledge to define them tentatively as follows :

Staphylococcus aureus is a pathogenic species, producing suppurative lesions of 
varying severity in man and animals; usually it forms a golden-yellow pigment, but albus variants may be thrown off either in the body or in vitro. It produces toxic filtrates with hæmolytic, leucocytolytic, necrotizing, and lethal properties for the rabbit, and sometimes with irritating properties for the gastro-intestinal tract of man ; it almost always liquefies gelatin, ferments lactose and mannitol, and coagulates plasma; it contains a specific polysaccharide not possessed by nonpathogenic staphylococci ; its antigenic structure differs from that of the following species.

Staphylococcus albus is feebly pathogenic, or non-pathogenic; it is normally present on the skin, in the hair, and apparently in air, water and dust. It gives porcelain-white or indifferently coloured colonies, but never produces a yellow or golden pigment. It forms no toxin, or does so less frequently than Staph. aureus; though white variants of Staph. aureus are often highly toxigenic. It frequently liquefies gelatin, but less constantly than Staph. aureus. It often ferments lactose, but not mannitol ; it forms no coagulase; and it appears to contain, as a rule, a polysaccharide different from that in virulent Staph. aureus strains.

The third species, Staphylococcus citreus, is a non-pathogenic saprophyte. It produces a distinctive lemon-yellow pigment; it is doubtful whether it ever forms a toxin; it liquefies gelatin less frequently and less rapidly than the preceding species. It has little or no fermentative ability; and it forms no coagulase.

We append, for purposes of reference, a detailed description of Staph.aureus, together with some of the characters ascribed to those types of staphylococci which have, at various times, received specific names.

\section{Staphylococcus aureus Rosenbach}

Isolation.-First described fully by Rosenbach (1884).

Habitat.-Actual or potential parasite found in suppurative lesions of man, in the nose, on the normal skin, and in cow's milk.

Morphology. - Spherical cells, $0 \cdot 8-1 \cdot 0 \mu$ in diameter; in cultures on solid media the cocci are arranged in grape-like elusters; in broth they occur as small groups, pairs, and short chains of not more than four members. Stain well with the usual aniline dyes. Non-motile, Gram-positive, non-acid-fast.

Agar Plate. -24 hours, $37^{\circ} \mathrm{C}$. Circular colonies, 1-2 mm. in diameter, low convex, amorphous, opaque, and of a golden colour, having a smooth glistening surface and an entire edge; butyrous in consistency and easily emulsifiable. No differentiation visible.

Agar Stroke.-24 hours, $37^{\circ} \mathrm{C}$. Abundant, confluent, raised, golden-yellow, opaque growth, with a glistening, smooth or slightly contoured surface, and an entire or slightly undulate edge.

Gelatin Stab.-5 days, $22^{\circ} \mathrm{C}$. Abundant filiform growth reaching to bottom of stab; surface growth about $5 \mathrm{~mm}$. in diameter; liquefaction of infundibuliform or saccate type.

Broth.-24 hours, $37^{\circ} \mathrm{C}$. Moderate uniform turbidity with a moderate, powdery deposit, disintegrating readily on shaking; slight ring growth at surface.

Rabbit Blood Agar Plate. -48 hours, $37^{\circ} \mathrm{C}$. Good.growth. Blood is partly or completely hæmolysed around colonies.

MacConkey's Agar.-24 hours, $37^{\circ} \mathrm{C}$. Tiny, eonvex, pinkish colonies about $0.5 \mathrm{~mm}$. in diameter. Later they increase somewhat in size, and take on a deep red colour. 
Potato. -24 hours, $37^{\circ} \mathrm{C}$. Poor, yellowish, effuse growth. 5 days; moderate, confluent, slightly raised, golden-yellow growth.

Loeffler's Serum.-24 hours, $37^{\circ} \mathrm{C}$. Good, raised, confluent, golden-yellow growth.

Resistance.-May withstand moist heat at $60^{\circ} \mathrm{C}$. for 30 minutes ; generally killed in one hour. Destroyed by 2 per cent. phenol in 15 minutes.

Metabolism.-Aerobic, facultatively anaerobic. Growth occurs best at $37^{\circ} \mathrm{C}$., limits $12^{\circ}$ to $45^{\circ} \mathrm{C}$. Optimum $\mathrm{pH}$ for growth is $\mathbf{7 \cdot 4 - 7 \cdot 6}$. Pignent formed most readily at $22^{\circ} \mathrm{C}$; is not formed in cultures grown anaerobically. Filtrable hæmolysin produced; $\alpha$-lysin acts rapidly on rabbit and sheep red corpuscles ; $\beta$-lysin acts slowly on sheep corpuseles-hot-cold lysis. Coagulase produced which clots citrated human or rabbit plasma.

Biochemical Reactions.-Acid, no gas, in glucose, maltose, mannitol, lactose, and sucrose. L.M. acid, clot, and sometimes peptonization. Indole-. M.R. + . V.P. . Nitrates reduced to nitrites. II.B. reduction $+. \mathrm{H}_{2} \mathrm{~S}-. \mathrm{NH}_{3}+$.

Antigenic Structure.-Contains a polysaccharide A, demonstrable by precipitation. Divisible by agglutination with absorbed sera into three main types and a number of sub-types.

Pathogenicity.-Forms exotoxin with hæmolytic, leucocytolytic, skin-necrosing, and lethal properties for the rabbit. Some strains also form an enterotoxin acting on man. Staph. aureus is frequently responsible for suppurative lesions in the human body, such as boils and abscesses, acute osteomyelitis, infective endocarditis, pyæmia, etc. Experimentally, it is pathogenic for rabbits, less so for mice and guineapigs. $0 \cdot 1-0.5 \mathrm{ml}$. of a 24 -hours' broth culture injected intravenously into a rabbit is generally fatal in 24 to 48 hours; post mortem, hæmorrhages on the serous membranes; the cocci can be recovered from the blood stream. A smaller dose may not prove fatal for 1 to 6 weeks; post mortem, multiple abscesses are frequently seen, especially in the kidneys, less frequently in the myocardium, lungs, spleen, bone niarrow, and costal eartilages.

Staphylococcus albus.--Resembles Staph, aureus in many respects. For chief differences, see p. 621.

Staphylococcus citreus.-Resembles Staph. aureus in many respects. For chief differences, see p. 621 .

TABLE 39

\begin{tabular}{|c|c|c|c|c|c|c|c|c|}
\hline & Pigment. & $\begin{array}{l}\text { Glu- } \\
\text { cose. }\end{array}$ & $\begin{array}{l}\text { Mal- } \\
\text { tose. }\end{array}$ & $\begin{array}{l}\text { Man- } \\
\text { nitol. }\end{array}$ & $\begin{array}{l}\text { Lac- } \\
\text { tose. }\end{array}$ & $\begin{array}{l}\text { Suc- } \\
\text { rose. }\end{array}$ & $\begin{array}{l}\text { Gel. } \\
\text { liq. }\end{array}$ & $\begin{array}{l}\text { Path. } \\
\text { to Nan. }\end{array}$ \\
\hline $\begin{array}{l}\text { Staph. aureus Rosenbach } \\
\text { Staph. aurantiacus Schroeter } \\
\text { Staph. epidermidis Gordon } \\
\text { Staph. candidus Cohn } \\
\text { 1Staph. salivarius Gordon } \\
\text { Staph. candicans Flügge . } \\
\text { Scurf staphylococcus Gordon } \\
\text { Staph. citreus. . . . . }\end{array}$ & $\begin{array}{l}\text { Golden } \\
\text { Golden } \\
\text { White } \\
\text { White } \\
\text { White } \\
\text { White } \\
\text { White } \\
\text { Lemon } \\
\text { yellow }\end{array}$ & $\begin{array}{l}\text { A } \\
\text { A } \\
\text { A } \\
\text { A } \\
\text { A } \\
\text { A } \\
\text { A } \\
-\end{array}$ & $\begin{array}{l}\text { A } \\
\text { A } \\
\text { A } \\
\text { A } \\
\text { A } \\
\text { A } \\
- \\
-\end{array}$ & $\begin{array}{l}\mathrm{A} \\
\overline{-} \\
\bar{?} \\
\bar{?} \\
? \\
\mathrm{~A}\end{array}$ & $\begin{array}{l}\mathrm{A} \\
\bar{A} \\
\mathrm{~A} \\
- \\
- \\
- \\
-\end{array}$ & $\begin{array}{l}\text { A } \\
\text { A } \\
\text { A } \\
\text { A } \\
\text { A } \\
\text { A } \\
- \\
-\end{array}$ & $\begin{array}{l}+ \\
\pm \\
\pm \\
- \\
\bar{F}\end{array}$ & $\begin{array}{l}\text { High } \\
\text { High } \\
\text { Feeble } \\
\text { Feeble } \\
- \\
\text { Feeble } \\
- \\
-\end{array}$ \\
\hline
\end{tabular}

1 Viscid growth in broth. 


\section{MICROCOCCUS, SARCINA, RHODOCOCCUS AND LEUCONOSTOC}

In their monograph on the Systematic Relationships of the Coccacer, C.-E. A. Winslow and Anne R. Winslow (1908) emphasize that the white and golden cocci belong to a series of cocci, including the streptococci, which are essentially parasitic in nature and active in fermentative power, whereas the yellow and red forms, including the sarcinæ, are generally saprophytic in nature and possess a restricted fermentative power.

\section{DEFINITION.-Micrococcus.}

\section{Micrococcus}

Spherical or ovoid cells, non-motile, arranged in pairs, tetrads, or groups, but not in grape-like clusters or chains. Generally Gram-positive. Grow freely on ordinary media. Sometimes produce a yellowish pigment. Gelatin liquefaction is not constant, and is usually slow. Fermentative activities weak. Usually non-pathogenic to man or animals.

Type species is Micrococcus luteus Cohn.

The term Micrococcus was first used in a generic sense by Cohn (1875), who applied it to small spherical or oval non-motile organisms which occurred in chains or groups. This, it will be observed, was a comprehensive description; it was not long in fact before certain members, such as the streptococci and the staphylococci, were removed from it, and awarded generic names of their own. The term Micrococcus, as now used, is defined above.

As with most saprophytic organisms, the micrococci bave not been studied so fully as the pathogenic cocci-the streptococci and the staphylococci. It must therefore be made clear that, in the following account, we record the characters of certain micrococci which have been from time to time described by different workers, without committing ourselves to any judgment as to their claims to be accorded specific rank. In general, we have been guided in our selection by Hucker $(1924 b, 1928)$, who uses pigment production, gelatin liquefaction, nitrate reduction, and ability to use ammonium salts as the sole source of nitrogen, as his main differential criteria. To his reports we would refer the reader who requires more detailed information.

(1) Micrococcus luteus (Schroeter) Cohn.-Isolated by Schroeter (1875) from a potato on which a yellow growth was found; is a non-motile, Gram-positive coccus, $1 \cdot 0-1 \cdot 2 \mu$ in diameter, occurring in pairs, tetrads or small groups. It grows well on ordinary media, giving a smooth, lemon-yellow layer. Gelatin is not liquefied, though on this point various workers disagree. Nitrates are not reduced. The optimum temperature of growth is $25^{\circ} \mathrm{C}$. Its fermentative powers are weak, acid being formed in glucose, not in lactose. It is commonly found in air, water, milk and milk products. The pigment is insoluble in water.

(2) Micrococcus varians (Dyar) Migula (1900) is similar to $1 /$. luteus, but is differentiated from it by its ability to reduce nitrates.

(3) Micrococcus conglomeratus Flügge (Migula 1900). Forms large clumps of organisms, often in pairs. Generally Gram-positive. Liquefies gelatin and reduces nitrates. Forms an abundant light yellow growth on agar. Can utilize ammonium salts as the sole source of nitrogen.

(4) Micrococcus flavus.--Isolated by Prove (1884-87) from human urine. About $0.8 \mu$ in diameter, oceurring singly, in pairs or short chains. On gelatin gives colonies $5 \mathrm{~mm}$. in diameter by the 3rd day. Liquefies gelatin about the 12th day. Does not reduce nitrates. Forms a considerable amount of slimy matter. Is said to ferment certain 
carbohydrates. Grows between $6^{\circ} \mathrm{C}$. and $36^{\circ} \mathrm{C}$; optimum temperature $22^{\circ} \mathrm{C}$. Is distinguished by the fact that it forms a yellow pigment only when it is exposerl to light -sunlight or diffused daylight; when kept in the dark the growth is colourless.

(5) Micrococcus coronatus Flïgge.-Described by Flügge (1896). A coccus $1 \mu$ in diameter, occurring in groups or short chains, Gram-positive. On gelatin plates in two days it forms whitish points; around these there appears a fresh growth, which later becomes separated from the colony by a clear ring of liquefaction. The central part assumes a dark brown, the peripheral a yellowish-brown colour. It is the halo around the colony which gives the organism its name. Found in air.

(6) Micrococcus caseolyticus Evans (1916). This organism is similar in many respects to $M$. coronatus. It occurs in large clumps, is generally Gram-positive, and is found in milk, milk products, and in the udder of the cow. It produces a luxuriant white growth on agar slants. It liquefies gelatin, and reduces nitrates. It produces an acid curd in milk and peptonizes it rapidly. It generally utilizes ammonium salts as the sole source of nitrogen, and usually produces acid in glucose and lactose.

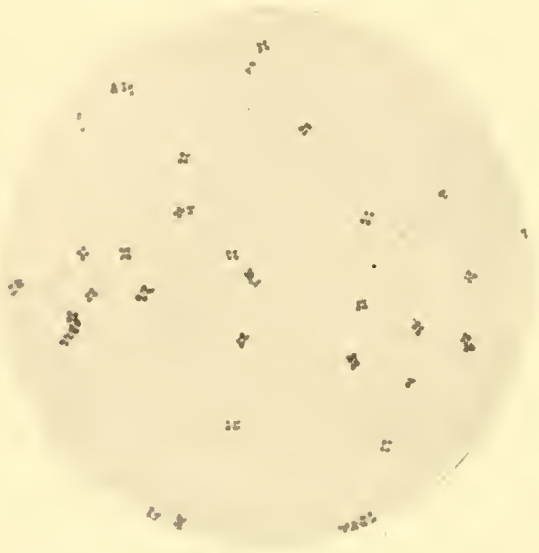

F1G. 128.-Micrococcus tetragenus. From an agar culture, 24 hours, $37^{\circ} \mathrm{C}$. $(\times 1000)$.

(7) Micrococcus radiatus Flügge. - Described by Flïgge (1896). Small coccus, less than $1 \mu$ in diameter, occurring in small groups, or sometimes short chains. On gelatin plates the colonies after 2 days are about $1 \mathrm{~mm}$. in diameter, whitish in colour, with a slightly irregular edge. During the next two days outgrowths occur radiating from the centre in an orderly manner, so that the colony assumes an appearance not unlike that of a starfish; meanwhile liquefaction sets in, and the centre sinks gently into the medium. The ends of the outgrowths are joined together by a ring of growth, from which after another two days fresh radiations may arise, and from these yet a third set. When fully grown, the colony has a diameter of $1 \cdot 0-1 \cdot 5 \mathrm{~cm}$. In a gelatin stab, the colonies down the stab send out horizontal shoots, giving the growth a feathery appearance; on the surface there is a slow liquefaction of infundibuliform type.

(8) Micrococcus ureæ Cohn (1875) is a spherical organism, occurring singly, in pairs, or in short chains. Diameter $0 \cdot 8-1 \cdot 0 \mu$. Gram-positive, though often weakly so. On agar it forms whitish, low convex, opaque colonies. Gelatin is not liquefied as a rule. Nitrates not reduced. Can utilize urea and ammonium salts as sole source of nitrogen. Found in stale urine.

(9) Micrococcus freudenreichii is closely related to $M$. urece. It is a facultative parasite. Cells occur singly or in clumps. Generally Gram-positive. Liquefies gelatin, but does not reduce nitrates. Does not usually produce sufficient acid to curdle milk. Can utilize ammonium salts, but not urea, as the sole source of nitrogen. Is one of the commonest non-pigment-producing micrococci in milk and dairy utensils.

(10) Micrococcus tetragenus Gaffky.-First described by Gaffky in 1883. It was isolated by Koch from lung cavities in patients with pulmonary tuberculosis. Grampositive, spherical organism, dividing in two planes at right angles to each other, so that tetrad forms are produced. In the human and animal body a capsule is formed. Grows freely on ordinary media. Optimum temperature for growth is $37^{\circ} \mathrm{C}$. The growth on agar is of a glutinous consistency, often adherent to the medium, and difficult to emulsify. 
Colonies are whitish in colour. Zone of lysis around colonies on blood agar. In broth a thick, weedy, glutinous deposit is formed, the supernatant fluid being comparatively clear. Gelatin is not liquefied. Nitrates are not reduced. Acid is said to be produced in glucose, maltose, lactose, and sucrose. The organism is occasionally pathogenic to man, and may even give rise to septicæmia. One of its most notable characteristics, differentiating it from other micrococci, is its high pathogenicity for the mouse. Subcutaneous or intraperitoneal inoculation leads to the production of a septicæmia, which proves fatal, according to circumstances, in 1 to 8 days. At post mortem there may be small abscesses in the spleen. The cocci are found in large numbers in the blood and tissues. Guinea-pigs are susceptible, though less so than mice. Non-capsulated avirulent and other variants have been described by Wreschner (1921) and Reimann (1936).

(11) Mierococcus buccalis is a small, non-motile, Gram-positive coccus, abont $0.5 \mu$ in diameter, isolated by Ozaki (1915) from the mouth. It is peculiar in being an obligate anaerobe. In stab glucose agar it gives a filiform growth with gas formation. There is turbidity in dextrose broth and a greyish sediment. It ferments glucose, maltose, lactose, and sucrose, but not mannitol, with the production of acid and gas. There is no liquefaction of gelatin; $\mathrm{H}_{2} \mathrm{~S}$ positive; indole negative. Its optimum temperature is $37^{\circ} \mathrm{C}$; there is no growth under $20^{\circ} \mathrm{C}$. It is non-pathogenic to laboratory animals. This eoccus differs in several important points - notably its active fermentative powers and its anaerobic nature-from most other micrococci, but as no special group is available for it as yet, we choose to place it here.

Other anaerobic micrococci have been described at various times, notably the Micrococcus gingiralis Ozaki (1912), the Micrococcus minimus Gioelli (1907), and the gas-producing organism Micrococcus aerogenes Schottmüller (1912). (For the last three references, see Ozaki 1915.)

\section{Definition.-Sarcina.}

\section{Sarcina}

Same definition as Micrococcus, except that cell division occurs, under favourable conditions, in three planes, so that cubical packets are formed.

The type species is Sarcina ventriculi Goodsir.

The first description of the sarcinæe was by Goodsir (1842), who found an organism arranged in cubical packets in the stomach of a patient suffering from gastric disease; to this he gave the name Sarcina ventriculi. Schroeter (1875) was the next to describe a sarcina-the Sarcina aurantiaca-and since then several others have been isolated, some from suppurative processes, but the majority from non-pathological sources, iucluding soil (see Smit 1933).

The following is a description of certain of the sarcinæ that have been described as separate species:

Sarcina ventriculi, Goodsir.-This, the first sarcina described, was not cultured till long after Goodsir (1842) had noticed it microscopically in the stomach contents.

Morphology.-Spherical coccus, $0.8-1.0 \mu$ in diameter, arranged in cubical packets and groups. In liquid media it occurs in pairs, small groups, and packets. Nonmotile. Gram-positive, but decolorizes easily. Non-acid-fast.

Agar Plate. -48 hours, $37^{\circ} \mathrm{C}$. Circular colonies, $1 \mathrm{~mm}$. in diameter, convex, amorphous, opaque, pale-yellow, with a smooth surface and entire edge; rather viscous in consistency, and easily emulsifiable.

Agar Slope. 48 hours, $37^{\circ} \mathrm{C}$. Moderate, confluent, raised, opaque, creamy-yellow growth with a smooth or contoured surface and an undulate edge.

Gelatin Stab.-Moderate filiform growth; no liquefaction.

Broth. -48 hours, $37^{\circ} \mathrm{C}$. Moderate uniform turbidity with a viscous deposit, disintegrating on shaking. No surface growth. 
Metabolic and Biochemical Activities.-Aerobic, facultatively anaerobic. Optimum temperature for growth $22-30^{\circ} \mathrm{C}$. Pigment formed most readily at $22^{\circ} \mathrm{C}$. No hæmolysin formed. Ferments no sugars. L.M. unchanged. M.R. - V.P. - M.B. reduction -.

Pathogenicity.-Non-pathogenic.

Sarcina Iutea Schroeter.-This organism, which was described by Schroeter (1875) as the Bacteridium aurantiacum, is a Gram-positive sarcina, giving a chrome-yellow growth on culture media. On agar the colonies are raised, yellow, coarsely granular, with an entire margin and a moist glistening surface. On potato they are dry, dull and granular. In broth there is an abundant yellow sediment with no turbidity. The optimum temperature of growth is $25^{\circ} \mathrm{C}$. It is found in air, soil and water.

Sarcina aurantiaca Flügge.-Described by Flügge (1896). Gram-positive spheres developing in packets. On gelatin plates forms slowly-growing orange-yellow colonies gradually liquefying gelatin. On agar slope, gives a thick reddish-yellow growth composed of single colonies; similarly on potato. In broth gives a turbidity with abundant sediment. Grows best at room temperature. Found in air and water.

Sarcina ureæ Beijerinck (1901) is a spherical organism, 0.7-1.2 $\mu$ in diameter, occurring singly, in pairs, and in packets. Said to be motile by peritrichate flagella and to form spores. Gram-positive. Resists heating to $100^{\circ} \mathrm{C}$. for 5 minutes. Gelatin not liquefied. Found in stale urine (see Gibson 1935).

Sarcina conjunctivæ Verderame.--Isolated by Verderame (1911) from the conjunctival sac of a girl suffering from acute conjunctivitis. Named by him Sarcina con, junctive citrea. Is stated to be Gram-negative. On agar after 24 hours the colonies are pinhead in size, round, bright-yellow, opaque, with an entire edge, and of butyrous consistency; after 48 hours they are $4 \mathrm{~mm}$. in diameter and lemon-yellow in colour. On an agar slope there is a thick creamy layer of growth after 2 days. Similar growth, but nore abundant on ascitic agar. In gelatin stab there is a surface growth of $2-3 \mathrm{~mm}$. in diameter after 24 hours, and a filiform growth of fine, light, greyish-yellow, opaque colonies: no liquefaction. On potato an abundant, creamy, lemon-yellow growth of rather dry appearance. In broth after 24 hours there is a very light turbidity, with a suspension in the liquid of fine flocculi which are easily broken up on shaking, and a cloudy viscous deposit of light yellow colour. Optimum temperature $37^{\circ} \mathrm{C}$, , but grows well at $14-18^{\circ} \mathrm{C}$. Facultative anaerobe. Forms acid in glucose, lævulose, maltose, lactose, sucrose and inulin, not in mannitol or galactose. $\mathrm{H}_{2} \mathrm{~S}$ positive. Indole negative. Nitrates not reduced. Non-pathogenic to mice or guinea-pigs.

Numerous other sareinæ have been described, such as Sarcina citrea Menge in 1892, and an unnamed Gram-negative Sarcina isolated by Nagano (1902) from the pus of an ovarian abscess. Ørskov (1930) draws attention to a sarcina found in the mouth and throat which, though non-motile itself, gives rise to motile cocci, each provided with a flagellum. The cocci themselves are not reproducible. For this remarkable organism the name Sarcina mirabilis is suggested.

\section{DEFINITION.-Rhodococcus.}

\section{Rhodococcus}

Spherical or ovoid cells occurring in groups or regular packets. Usually Grampositive, but are easily decolorized. Growth on agar abundant with formation of red pigment. Weak fermentative powers. Gelatin rarely liquefied. Nitrates generally reduced. Saprophytes.

Type species is Rhodococcus rhodochrous Zopf. The term Rhodococcus was first introduced by Zopf in 1891 (see Buchanan 1925).

Rhodococcus rhodochrous Zopf.-Found in water. Spherical organism, about $0 \cdot 8-\mathbf{I} \cdot 0 \mu$ in diameter, occurring singly, in pairs and in small groups. Gram-positive. Gives a confluent, raised growth of a carmine hue on agar. Thick, rose-red pellicle in broth, 
with a red flocculent sediment (Bergey 1923). L.M. slightly alkaline. Carmine-red streak on potato. Does not liquefy gelatin, but generally reduces nitrates. Aerobic. Optimum temperature $25^{\circ} \mathrm{C}$. Non-pathogenic.

Rhodococcus cinnabareus Flügge.-Described by Flügge as a large, spherical, Grampositive coceus, occurring in twos, threes, and fours; grows very slowly. On gelatin after 4 days the colonies are $0.5-1.0 \mathrm{~mm}$. in diameter; at first they are brick-red, later cinnabar-red in colour. In gelatin stab small white colonies are formed down the stab in 4 to 5 days; on the surface there is a large red knob of growth; no liquefaction. On potato the growth is even slower. Optimum temperature $25^{\circ} \mathrm{C}$. Does not reduce nitrates, and cannot utilize ammonium salts as the sole sonrce of nitrogen. Found in air and water.

Rhodococcus roseofulvus Fliigge.-Described by Babes as the cause of red sweat (see Flügge 1896). The cocei are oval in shape, $I \mu$ long by $0.6-0.8 \mu$ broad, and are bound by gelatinous material into a reddish zoogloeal mass. In the body they surround the hairs, particularly of the axilla, and impart a red coloration to the sweat. Gram-positive. Grows on egg white at $37^{\circ} \mathrm{C}$., forming a red pigment. Does not liquefy gelatin. Generally curdles milk and causes slight peptonization. Produces acid in glycerol and mannitol.

Rhodococcus agilis Ali-Cohen.--Isolated by Ali-Cohen (1889) from drinking water and named Micrococcus agilis. Peculiar in being motile, possessing one or two flagella. Occurs mostly in pairs, sometimes in short chains or in tetrads. Gram-positive; $1 \mu$ in diameter. Grows in all media at room temperature, forming a rose-coloured pigment. Liquefies gelatin slowly. Optimum temperature $25^{\circ} \mathrm{C}$. Found in water.

\section{Leuconostoc}

Definition.-Leuconostoc.

Spherical or ovoid cells, arranged in pairs and chains; the cocci are surrounded by a gelatinous envelope, which unites them into zoogloeal masses. Usually Grampositive, but decolorize easily. Saprophytes, usually growing in cane sugar solu. tions.

Type species is Leuconostoc mesenterioides van Tieghem.

The first organism of this group was described by Cienkowski in 1878 as the Ascococcus mesenterioides. This name was amended by van Tieghem in 1878 to Leuconostoc mesenterioides (see Flügge 1896). According to Hucker and Pederson (1930), to whom reference should be made for more detailed information, organisms of the genus Leuconostoc are found in slimy sugar solutions, in fermenting vegetables, and in milk and milk products. Morphologically, they are intermediate between the streptococci and the lactobacilli. They all produce about 45 per cent. lævolactic acid from glucose, 20 per cent. $\mathrm{CO}_{2}$, and 25 per cent. volatile products, including acetic acid and ethyl alcohol. They form mannitol from fructose and sucrose, and a levulan or dextran from sucrose.

Leuconostoc mesenterioides (Cienkowski) van Tieghem.-A spherical coccus, 0.9-I $2 \mu$ in diameter, occurring in pairs and in chains. Usually Gram-positive. The chains are surrounded by a thick, tough, gelatinous coating, which is stated by von Scheibler to consist of dextrin; the aggregation of several chains within their envelopes gives rise to large, oompact, gelatinous, zoogloeal masses. Develops on the surface of parsnip-root and beetroot solutions in the form of thick cakes of cartilaginous consistency (Flügge 1896). It likewise thrives on grape-sugar and cane-sugar solutions, provided nitrate and phosphate are added. Cultivated in peptone water, or in gelatin to which lactose or maltose has been added, it is morphologically similar to a streptococcus, no gelatinous envelope being formed; but if glucose or cane sugar is incorporated in the gelatin, then the characteristic zoogloeal masses appear. On either of these media there appears in 10 to 14 days a thick whitish mass of confluent colonies, having a glassy surface, looking 
rather like a layer of crystals. During the first week the growth is dry and of cartilaginous consistency, but later it becomes softer and moister, and assumes an almost pulp-like consistency. Ferments glucose, maltose, lactose, sucrose, mannitol and either arabinose or xylose, with the formation of acid and gas. Facultative anaerobe. Optimum temperature $25^{\circ} \mathrm{C}$. Is found in fermenting vegetable material and in sugar solutions.

Hucker and Pederson (1930) recognize two other species. Leuconostoc dextranicus ferments sucrose, but not pentoses, produces a moderate amount of slime from sucrose, and may be associated with either vegetable or dairy products.

Leuconostoc citrovorus does not ferment sucrose or pentoses, produces no slime from sucrose, and is generally associated with milk or milk products.

\section{REFERENCES}

Ali-Сонем, Сн. H. (1889) Zbl. Bakt., 6, 33.

Andrewes, F. W. and Gordon, M. H. (1905-6) Rep. loc. Govt Bd publ. Hlth Suppl., p. 543.

Arrhenius, S. (1907) "Immunochemie." Akad. Verlagsgesell., Leipzig.

BaYliss, M. (1940) J. exp. Med., "92, 669.

BeiJerinck, M. W. (1901) Zbl. Bakt., IIte Abt., \%, 33.

Bergey, D. H. (1923) "Manual of Determinative Bacteriology." Baltimore.

Beumer, J. (1939) C. R. Soc. Biol., 130, 283:

Bigaer, J. W. (1933) J. Path. Bact., 36, 87.

Bigger, J. W., Boland, C. R., and O'Meara, R. A. Q. (1927) J. Path. Bact., 30, 261.

Biond, D. (1887) Z. Hyg. InfektKr., 2, 194.

Blatr, J. E. and Hallinan, F. A. (1935) Proc. Soc. exp. Biol., N.Y., 33, 382 ; (1936) J. Bact., 31, 81 .

Bryce, L. M. and Rountree, P. M. (1936) J. Path. Bact., 43, 173.

Buchanan, R. E. (1925) "General Systematic Bacteriology." Baltimore.

Burnet, F. M. (1929) J. Path. Bact., 32, 717; (1930) Ibid., 33, 1; (1931) J. Path. Bact., $34,759$.

Cadness-Graves, B., Willians, R., Harper, G. J., and Miles, A. A. (1943) Lancet, i, 736.

Chapman, G. H. (1936) J. Bact., 32, 199 ; (1944) J. Bact., 4\%, 211.

Chapman, G. H. and Berens, C. (1935) J. Bact., 29, 437.

Chapman, G. H., Berens, C., Nilson, E. L., and Curcio, L. G. (1938) J. Bact., 35, 311.

Chapman, G. H., Berens, C., Peters, A., and Curcio, L. (1934) J. Bact., 28, 343.

Chapman, G. H., Lieb, C. IV., Berens, C., and Curcio, L. (1937) J. Bact., 33, 533.

Christie, R. and Graydon, J. J. (1940) Aust. J. exp. Biol., 19, Pt. 1, 9.

Christie, R. and KeOGH, E. V. (1940) J. Path. Bact., 51, 189.

Coнn, F. (1875) Cohns Beitr. Biol. Pflanz., 1, Hft. 2, 127.

Cowan, S. T. (1938) J. Path. Bact., 46, 31 ; (1939a) Ibid., 48, 169; (1939b) Ibid., 48, 545.

Cruickshank, R. (1937) J. Path. Bact., 45, 295.

DARÁNY, J. v. (1926) Zbl. Bakt., 99, 74.

Davison, E. and DACK, C. M. (1939) J. infect. Dis., 64, 302.

Davison, E., DaCK, G. M., and CARY, W. E. (1938) J. infect. Dis., 62, 219.

Denys, J. and Havet, J. (1898) La Cellule, 10, 7.

Devenish, E. A. and Miles, A. A. (1939) Lancet, i, 1088.

Dolman, C. E. (1932) Canad. publ. Hlth.J., 23, 125; (1943) Ibid., 34, 45 ; (1944) Ibid., 35,337 .

Dolman, C. E. and Wilson, R. J. (1938) J. Immunol., 35, 13 ; (1940) Canad. publ. Hlth. J., $31,68$.

Dolman, C. E., Wilson, R. J., and Cockcroft, W. H. (1936) Canad.publ. Hlth. J., $2 \%, 489$.

Downie, A. W. (1937) J. Path. Bact., 44, 573.

Dodgeon, L. S. (1908) J. Path. Bact., 12, 242.

Dudgeon, L. S. and Simpson, J. W. H. (1928) J. Hyg., Camb., 2\%, 160.

Duncan, J. T. and Walker, J. (1942) J. Hyg., Camb., 42, 474.

DuRfEe, O. (1942) J. Bact., 44, 589.

Evans, A. C. (1916) J. infect. Dis., 18, 437.

Fatrbrotiler, R. W. (1940) J. Path. Bact., 50, 83.

Fildes, P. and Richardson, G. M. (1937) Brit. J. exp. Path., 18, 292.

Fildes, P., Richardson, G. M., Knight, B. C. J. G., and Gladstone, G. P. (1936) Brit. J. exp. Path., 17, 481. 
Finland, M., Peterson, O. L., and Stradss, E. (1942) Arch. intern. Med., 70, 183. Fisk, A. (1940) Brit. J. exp. Path., 21, 311.

Fisk, R. T. (1942) J. infect. Dis., 71, 153, 161.

Fladr, A. (1938) Acta path. microbiol. Scand., Suppl. 35.

FLÜ̊øE, C. (1896) “Die Mikroorganismen," Part II, p. 96, 3rd edit. Leipzig.

Forssman, J. (1935-1941) Acta path. microbiol. Scand. Numerous references.

Fraenkel, C. and Baumann. (1905) Mï̈nch. med. Wschr., 52, 937.

Fulton, F. (1943) Brit. J. exp. Path., 24, 65.

GaFfry. (1883) Arch. klin. Chir., 28, 495.

Garrod, L. P. (1942) Brit. med. J., i, 290.

Gengou, O. (1932) Ann. Inst. Pasteur, 48, 135.

Gibson, T. (1935) Arch. Mikrobiol., 6, 73.

Gillespie, E. H. (1943) Mon. Bull. Emerg. publ. Hlth Lab. Serv., 2, 19.

Grlespre, E. H., Devenish, E. A., and Cowan, S. T. (1939) Lancet, ii. 870.

Gladstone, G. P. (1937) Brit. J. exp. Path., 18, 322; (1938) Ibid., 19, 208.

Gladstone, G. P., Fildes, P., and Richardson, G. N. (1935) Brit. J. exp. Path., 16, 335. Glenny, A. T. and Stevens, M. F. (1935) J. Path. Bact., 40, 201.

Goodsir, J. (1842) Edin. med. surg. J., 5\%, 430.

Gordox, M. H. (1903-4) 33rd ann. Rep. loc. Govt Bd publ. Hlth Suppl., p. 388.

Goyle, A. N. and Mixchin, R. L. H. (1940) Indian J. med. Res., 27, 611.

Gross, H. (1931a) Zbl. Bakt., 122, 354; (1931b) Ibid., 123, 212 ; (1931c) Z. ImmunForsch., $73, \mathrm{I} 4$.

Hallian, F. A. (1937) Proc. Soc. exp. Biol., N.Y., 36, 789.

Hamnon, W. I. (1941) Amer. J. publ. Hlth., 31, 1191.

Hegemann, G. (1937) Zbl. Bakt., 140, 108.

Heise, M. D. and STARIN, W. A. (1940) J. infect. Dis., 67, 70.

HewitT, L. F. (1930) Biochem. J., 24, 676.

HINE, T. G. II. (1922) Lancet, ii. 1380.

Hoffstadt, R. E. and Youmans, G. P. (1932) J. infect. Dis., 51, 216.

Hodker, G. J. (1924a) N.Y. St. agric. Exp. Sta., Tec. Bull., No. 100; (1924b) Ibid., No. 102 ; (1928) N.Y. St. agric. exp. Sta., T'ec. Bull., No. 135.

Hucker, G. J. and Pederson, C. S. (1930) N.Y. St. agric. Exp. Sta., Tec. Bull., No. 167. HUghes, T. P. (1932) J. Bact., 23, 437.

Jackson, S. H., Nicholson, T. F., and Holvan, W. L. (1940) J. Path. Bact., 50, 1.

Jones, A. H. and Lochhead, A. G. (1939) Food Res., 4, 203.

Julta Nelle, L。 A. (1922) J. infect. Dis., 31, 256.

Julianelle, L. A. and-Wieghard, C. W. (1931) Proc. Soc. exp. Biol. N.Y., 31, 947; (1935) J. exp. Hed., 62, 11, 31.

Kitchixa, J. S. and FARrell, L. N. (1936) Amer. J. Hyg., 24, 268.

Kligler, I. J. (1913) J. infect. Dis., 12, 432.

Klialer, I. J., Grossowicz, N., and Berganer, S. (1943) J. Bact., 46, 399.

Klopstock and BockenheIMer. (1904) Arch. klin. Chir., "'2, 325.

Knight, B. C. J. G. (1935) Brit. J. exp. Path., 16, 315; (1937) Biochem. J., 31, 731.

КоОН, J. (1908) Z. Hyg. InfektKr., 58, 287.

Kodalia, T. and Kojima, T. (1939) Kitasato Arch., 16, 36.

Kodama, T. and Nisirama, S. (1938) Kitasato Arch., 15, 247.

Kolle, W. and Oтto, R. (1902) Z. Hyg. InfektKr,, 41, 369.

Kraus, P. and Clatrmont, P. (1900) Wien. klin. Wschr., 13, 49.

KUРснIк, G. T. (1937) J. infect. Dis., 61, 320.

KUTSCHER and Konrich, F. (1904) Z. Hyg. InfektKr., 48, 249.

Landsteiner, K. and Radchenbichler, R. Vox (1909) Z. ImmunForsch., 1, 439.

Levine, B. S. (1939) J. Path. Bact., 48, 291.

Linoelsheim, W. von (1899) Beitr. exp. Ther., Heft. 1, 49.

LoeB, L. (1903-4) J. med. Res., 10, 407.

LUBINSKI, IV. (1894) Zbl. Bakt., 16, 769.

Lyons, C (1937) Brit. J. exp. Path., 18, 411.

McBroon, J. (1937) J. infect. Dis., 60, 364.

MicFarlan, A. II. (I938) Brit. med. J., ii, 939.

Marcuccio, G. (1938) Ri'v. sanit. sicil., 26, 621, 625, 629.

Mercier, P. (1938) C. R. Soc. Biol., 127, 297.

Migdla, W. (1900) "System der Bakterien," IIte Band, p. 1. Gustav Fischer, Jena.

Miles, A. A., Williams, R. E. O. and Clayton-Cooper, B. (1944) J. Path. Bact., 56, 513.

MinetT, F. C. (1936) J. Path. Bact., 42, 247.

Ilorgan, F. G. and Graydon, J. J. (1936) J. Path. Bact., 43, 385.

MUCH, H. (1908) Biochem. Z., 14, 143.

NaGANo, J. (1902) Zbl. Bakt., 32, 327. 
NeIsser, M. (1912) See Kolle and Wassermann, "Hdb. der path. Mikroorg.," IIte Abt. (1912-13), 4, 356.

Neisser, M. and Wechsbero, F. (1901) Z. Hyg. InfektKr., 36, 299.

Neter, E. (1937) J. Bact., 34, 243.

Ooston, A. (1881) Brit. med. J., i, 369.

Oesterlin, E. (1925) Zbl. Bakt., 94, 313.

O'KANE, D. J. (1941) J. Bact., 41, 441.

Orcutt, M. L. and Howe, P. E. (1922) J. cxp. Med., 35, 409.

Orskov, J. (1930) Acta path. Microbiol. scand. Suppl., 3, 519.

Oтто, R. (1903) Zbl. Bakt., 34, 44.

Ozaki, Y. (1915) Zbl. Bakt., \%6, 118.

Panton, P. N. and Valentine, F. C. O. (1932) Lancet, i. 506.

Parish, H. J. and Clark, W. H. M. (1932) J. Path. Bact., 35, 251.

PARKER, J. T. (1924) J. exp. Med., 40, 761.

Passet. (1885) Fort. Med., 3, 33.

Peragallo, I. (1937) G. Batl. Immun., 18, 577.

Proon, H. (1937) J. Path. Bact., 44, 425.

Pröscher. (1903) Zbl. Bakt., 34, 437.

Prove, O. (1884-7) Cohns Beitr. Biol. Pflanz., 4, 409.

Remann, H.A. (1936) J. Bact., 31, 385, 407.

Report (1942) Ion. Bull., Emerg. publ. IIth Lab. Serv., 1, Feb., p. 9.

Richuond, J. J., Reed, C. I., Shaughnessy, H. J., and Michael, V. (1942) J. Bact., 44, 201.

Rigdon, R. H. (1938a) J. Lab. clin. Mcd., 24, 142 ; (1938b) Proc. Soc. exp. Biol., N.Y., 38, 82.

Rosenbach, F. J. (1884). "Mikroorganismen bei den Wundinfektionskrankheiten des Menschen." Wiesbaden

SaSki, S. and FeJarn, B. (1937) C. R. Soc. Biol., 126, 139.

Schroeter, J. (1875) Cohns Beitr. Biol. Pflanz., 1, Heft. 2, 109.

Segalove, 11. and Dack, G. II. (1941) Food Res., 6, 127.

SEIFFERT, IV. (1935-36) Zbl. Bakt., 135, 100.

Slanetz, L. IV. (1942) J. Bact., 43, 105.

Sнiт, J. (1933) J. Path. Bact., 36, 455.

Suith, 11. L. (1937) J. Path. Bact., 45, 305.

Sirth, M. L. and IPsen, J. (1938) Quart. Bull. Illh. Org. L.o.X., 7, 845.

Suith, M. L. and Price, S. A. (1935a) J. Path. Bact., 4\%, 361 ; (1938b) Ibid., 4\%, 379.

Suiti, W. and Hale, J. H. (1944) Brit. J. exp. Path., 25, 101.

Stephan, F. (1934) Z. Hyg. InfektKr., 116, 550.

Swingle, E. L. (1935) J. Bact., 29, 467.

Thompson, R. and Khorazo, D. (1937) J. Bact., 34, 69.

Valentine, F. C. O. (1936) Lancet, i. 526.

Valentine, F. C. O. and Butler, E. C. B. (1939) Lancet, i, 973.

Vanbreusegheir, R. (1934) C. R. Soc. Biol., 116, 650.

VEIEL, F. (1904) Münch. med. Wschr., 51, 13.

VELDE, H. VAN DER. (1894) La Cellule, 10, ii. 401.

Verderane, P. (1911) Zbl. Bakt., 59, 377.

VerWey, W. F. (1940) J. exp. Med., Y1, 635.

WALBUM, L. E. (1922) Biochem. Z., 129, 367.

Walston, H. D. (1935) J. Hyg., Camb., 35, 549.

WELCH, W. H. (189I) Int. J. med. Sci., 102, 439.

Weld, J. T. and Mitchell, L. C. (1942) Proc. Soc. exp. Biol., N.Y., 49, 370.

Wieghard, C. W. and JUlianelle, L. A. (1935) J. exp. Med., 62, 23.

Wilson, G. S. and Atkinson, J. D. (1945) Lancet., i. 647.

Winslow, C.-E. A. and Rogers, A. F. (1906) J. infect. Dis., 3, 485.

Winslow, C.-E. A., Rothberg, W., and Parsons, E. I. (1920) J. Bact., 5, 145.

Wrnslow, C.-E. A. and Winslow, A. R. (1908) "The Systematic Relationship of the Coccaceæ." New York.

Wreschner, H. (1921) Z. Hyg. InfektKr., 93, 74,

Wrioht, J. (1936) Lancet, i, 1002.

Yoneudra, N. (1936) Z. ImmunForsch., 89, 392. 
CHAPTER 26

\section{CHROMOBACTERIUM AND ACHROMOBACTERIUM}

\section{CHROMOBACTERIUM}

\section{DeFinition.-Chromobacterium.}

Small, non-sporing, aerobic rods, usually motile and usually Gram-negative, producing a yellow, red, or violet pigment, which is generally insoluble in water. Saprophytic; commonly found in water or soil.

Type species. Chromobacterium violaceum.

Numerous organisms of this group have been isolated at various times from water, soil, sewage, and occasionally from contaminated food-stuffs. Chr. violaceum was described by Bergonzoni in 1881 (see Report 1920). Chr. prodigiosum was first observed by Bizio in 1823 (see Breed and Breed 1924), who found it in "bleeding polenta". Chr. aquatile was isolated from water by Frankland and Frankland in 1889 .

The classification of these organisms presents considerable difficulties. The American Committee of Bacteriologists has created a genus, which they call Erythrobacillus, for the inclusion of those bacilli which produce a red or pink pigment. The genus Chromobacterium they reserve for those bacilli which produce a violet pigment. As these organisms have received relatively little attention from bacteriologists, and as in consequence their properties have been incompletely studied, it would seem advisable for the moment to group all the aerobic non-sporing pigmentforming rods into the single genus Chromobacterium. With regard to the naming of the individual species within the genus, there is considerable confusion. One and the same organism has frequently been described under two names, and specific names have been given to organisms which appear to be merely varieties of existing species. We shall therefore describe in some detail those organisms which undoubtedly deserve specific rank, and shall refer briefly to others whose claim to this distinction is more doubtful.

Morphology. - The organisms of this group are small rods, varying in length from about 1.0 to $3 \cdot 0 \mu$, and in breadth from about 0.5 to $0.7 \mu$. Chr. prodigiosum may be regarded as an exception, since it is usually described as a small cocco-bacillus. It is necessary to point out, however, that the size of this organism is subject to considerable variation, and that even on the same type of medium a single strain may at one time give rise to cocco-bacilli, and at another to rod-forms indistinguishable from those formed by other members of Chromobacterium (Fig. 129). Motility is a frequent characteristic, and is dependent on the possession of peritrichate or sometimes polar flagella. Most members of this group are Gram-negative.

Cultural Reactions. - Growth occurs readily on the ordinary media; a particularly heary growth is generally observed in broth. 

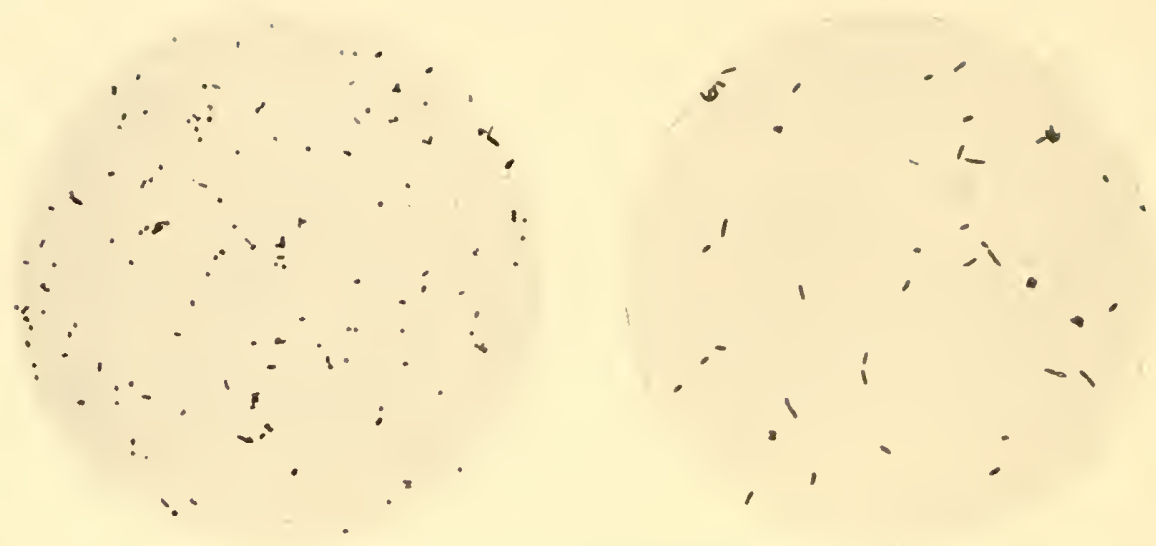

FIG. 129.-Chromobacterium prodigiosum.

From an agar culture, 24 hours, $37^{\circ} \mathrm{C} .(\times 1000)$.

Left.-Strain showing cocco-bacillary forms.

Right.-Same strain from a different culture showing definite rods.

The colonies on agar are usually homogeneous for the first day or two, and then become differentiated into a convex, pigmented, and

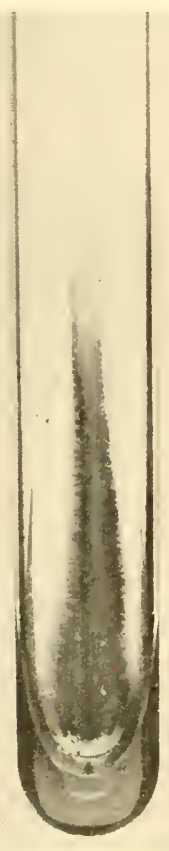

FIG. 130.-Chromobac. terium prodigiosum.

Agar stroke culture, 24 hours, $37^{\circ} \mathrm{C}$. relatively opaque centre, and an effuse, colourless, almost transparent periphery with an irregularly crenated edge. This differentiation results from the secondary outgrowth around the original colony of a film, which may be so thin as to escape observation unless a careful search is made for it.

In agar stroke cultures there is a raised, confluent, pigmented growth, with a smooth or beaten-copper surface, and an undulate or lobate edge.

In gelatin stab culture there is a moderate, filiform growth extending to the bottom of the tube, usually succeeded by liquefaction, which may occur rapidly or slowly. In general, the liquid is turbid and granular, and in the upper centimetre or so is pigmented; below this, out of contact with the air, the growth is colourless. A surface pellicle or ring growth, strongly pigmented, and a slightly pigmented deposit at the bottom of the liquefied gelatin, are not uncommon.

In broth there is a dense turbidity, generally with a pigmented ring growth or surface pellicle, and a slightly pigmented deposit, which frequently becomes viscous and is difficult to disintegrate on shaking. The broth itself often remains colourless. Multiplication of the bacilli seems to occur chiefly near the surface; a membrane forms in contact with the air, sinks to the bottom, and is replaced by a fresh membrane. This accounts for the heavy deposit that is generally present after a week.

Blood serum is frequently liquefied.

Resistance.-The organisms of this group are non-sporing, and show no par- 
ticular resistance to heat or disinfectants. They appear to be.killed by a temperature of $55^{\circ} \mathrm{C}$. in 1 hour.

Metabolism and Biochemical Characters.-All the members are markedly aerobic, growing best in the presence of an ample supply of oxygen. Growth under anaerobic conditions is poor, and is not accompanied by pigment formation.

Proteins are broken down readily as a rule; gelatin, casein, and often blood serum are digested. Carbohydrates are attacked freely by some, sparingly by others, generally with the production of acid only.

Growth can be obtained on synthetic media devoid of protein. The optimum temperature of growth is between $25^{\circ}$ and $37^{\circ} \mathrm{C}$., but some strains will grow as low as $0^{\circ} \mathrm{C}$.

All form catalase and reduce methylene blue. Indole is rarely produced; the II.R. and V.P. reactions are generally negative. Nitrates are often reduced to nitrites; and ammonia is generally formed from peptone. Formation of $\mathrm{H}_{2} \mathrm{~S}$ is slight or absent.

Pigment is produced only in the presence of oxygen, and at a suitable temperature. The optimum temperature for pigment formation does not necessarily correspond with that for growth. Thus many organisms grow best at $30-37^{\circ} \mathrm{C}$., but form little or no pigment, whereas at a lower temperature growth is poorer, but pigment formation is abundant. Pigment is developed best on the surface of solid media; in broth and in the depths of stab cultures there is little or none formed. Potato is a medium that may be specially recommended for the study of pigment production. As a rule pigment is formed most abundantly on primary isolation; after subculture in the laboratory for some time, the power to form it may diminish seriously, or be altogether lost. Not all bacilli in a given culture produce the same amount of pigment; some colonies are deeply coloured, others are faintly or partly coloured, while still others are completely colourless.

Sullivan (1905-6) states that the formation of pigment is dependent on the reaction of the medium, the temperature, the free access of oxygen, and the presence of certain salts. In synthetic media, asparagin, succinic, lactic and citric acids, when combined with an ammonium base, allow of growth and pigment formation. Malic, tartaric, and oxalic acids, combined with ammonia, allow of growth but not of pigment production. Acetic, uric, and formic acids, combined with ammonia, are unfavourable for both growth and pigment production. (This refers to solutions of 0.4 per cent. or under.) The salts favouring the production of pigment serve either to provide nutrient material, or to fix the acid produced, or to afford material for essential syntheses.

Pathogenicity.-The organisms of this group are essentially saprophytic. A few doubtful cases of suppuration in man have been described (see Schütz and Laun 1933), but generally speaking these organisms do not give rise to natural disease in man or in animals. On experimental inoculation into laboratory animals they prove harmless except in very large doses.

Variation.- It is convenient to mention here that the characters of a single strain are liable to considerable variation. Its cultural appearances are by no means uniform; colonies may vary in their size, shape, opacity, surface, and consistency.

In their ability to produce pigment there is likewise great variation. From 7 different strains of Chr. prodigiosum and Chr. kielense, Eisenberg (1914) isolated 
no fewer than 22 variants, differing in colour from dark red to red, orange-red, pink, pale-pink, pinkish-white, and colourless; most of these remained stable on subculture. From Chr. violaceum 5 variants were obtained. Eisenberg found that variants appeared most readily in ageing cultures, and more rapidly in fluid than in solid media.

Group Producing a Violet Pigment.-Chr. violaceum, the chief member of the group, is a common inhabitant of water. The violet pigment is soluble in alcohol, but insoluble in water, chloroform, or benzol. For its formation in synthetic media both magnesium sulphate and a phosphate are required. On subculture the organism frequently loses its pigment-forming power. We append a detailed description of this organism, together with brief accounts of some of the other violet-pigment-producing members.

Synonym.-B. violaceus.

\section{Chromobacterium violaceum}

Isolation.-Described by Bergonzoni in 1881 (see Text).

Habitat.-Water.

Iorphology.-Rods; $1.5-3 \cdot 0 \mu \times 0 \cdot 6 \mu$; axis straight, sides parallel, ends rounded; arranged singly. Motile by peritrichate flagella. Non-sporing; non-capsulated. Gram-negative, non-acid-fast. May show bipolar staining.

Agar Plate -2 days at $37^{\circ} \mathrm{C}$. Round colonies, $\frac{1}{2}-1 \mathrm{~mm}$. in diameter, low convex, violet

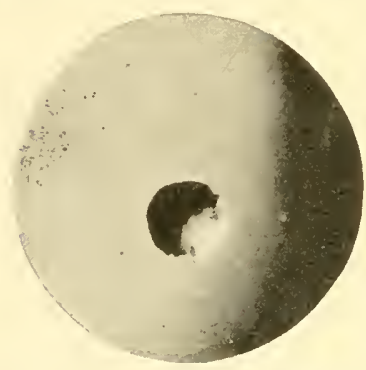

FiG. 131.-Chromobacterium violaceum.

Surface colony on agar, 48 hours, $22^{\circ} \mathrm{C} .(\times 8)$. coloured, translucent, with a smooth glistening surface and an entire edge; butyrous consistency and easily emulsifiable; structure appears floceular. After 5 days colonies are larger, 1-2 $\mathrm{mm}$. in diameter, have a finely lobate edge, and are differentiated into a dark convex centre, and a paler, flattened, radiate periphery.

Agar Slope.-2 days, $25^{\circ} \mathrm{C}$. Moderate growth, raised, violet, opaque, with glistening, smooth or beatencopper surface and an undulate edge. Medium unchanged.

Gelatin Stab. -5 days, $20^{\circ} \mathrm{C}$. Moderate, filiform growth to bottom of stab; violet surface growth; slow infundibuliform or saccate liquefaction; liquefied portion is granular and violet.

Broth.-2 days, $25^{\circ} \mathrm{C}$. Abundant growth, with dense colourless turbidity; a violet ring growth, and a moderate viscous deposit disintegrating on shaking. Mawkish odour.

Blood Serum.-9 days, $25^{\circ} \mathrm{C}$. Moderate, confluent growth, colourless, with an uneven surface. Digestion is very slow, or may be absent.

Potato. -6 days at $25^{\circ} \mathrm{C}$. Abundant, raised, confluent, creamy growth of violet colour, with smooth or contoured glistening surface.

Resistance.-Killed by $55^{\circ} \mathrm{C}$. in 1 hour.

Metabolism.-Aerobic ; slight colourless growth anaerobically. Opt. temp. $25^{\circ} \mathrm{C}$. Pigment formation best at $25-30^{\circ} \mathrm{C}$. Pigment: soluble in alcohol, insoluble in chloroform or water.

Nutritional.-Grows well on ordinary media. In synthetic media, pigment is formed only in the presence of $\mathrm{MgSO}_{4}$ and a phospluate.

Biochemical.-Sometimes acid in glucose and maltose. Indole - or slight + . M.R. - . V.P. - . Nitrate reduction +. $\mathrm{NH}_{3}+$. Catalase +. M.B. reduction.$+ \mathrm{H}_{2} \mathrm{~S}$ slight + . L.M. 6 days $25^{\circ} \mathrm{C}$. alkaline, violet ring growth; later may be coagulated, and slowly peptonized. 
Pathogenicity.-Non-pathogenic to man and animals.

Chr. janthinum, found in water and soil; is said to be motile by one or two polar flagella. Colonies on agar are at first milky white, but later become violet. Liquefaction of gelatin is slow. On broth it forms a violet surface pellicle. Optimum temperature $30^{\circ} \mathrm{C}$. It is doubtful whether this organism is a distinct species.

Chr. amethystium is a non-motile bacillus found in water. On agar the growth is first non-pigmented, later it assumes a dark violet colour with a wrinkled surface of metallic lustre. Gelatin is liquefied; a violet surface pellicle forms on the liquid. In broth a surface pellicle appears; the fluid itself is coloured brown. Optimum temperature $30^{\circ} \mathrm{C}$.

Chr. cœruleum is said to be motile by polar flagella. Colonies are spreading and of a bluish-grey colour. Slow liquefaction of gelatin. Greyish surface pellicle on broth. Milk is rendered sky-blue at the surface, and is digested with an alkaline reaction. The pigment is soluble in water and alcohol, but not in ether or chloroform. Optimum temperature $30^{\circ} \mathrm{C}$.

Group Forming a Pink or Red Pigment.-The most important member of this group is Chr. prodigiosum. It was first demonstrated by Bartolomeo Bizio in 1823 as a cause of "bleeding polenta" (Breed and Breed 1924). Infections of meat, fish, bread and other articles of food with this organism from time to time give rise to alarm. Klein (1894) describes an instance where the food in a large mercantile establishment in London became contaminated with it. In spite of the fact that the residents consumed pink meat for some days, they suffered from no apparent ill-effects. The pigment is soluble in absolute alcohol, ether, chloroform, benzol, and carbon disulphide, but is insoluble in water. An alcoholic solution when acted on by mineral acids is turned first carmine-red, then reddishviolet. Alkalies turn it brownish-yellow, and chlorine water, after turning it reddish-brown, then golden-yellow, finally decolorizes it. In the spectrum, blue and violet are completely absorbed, and an absorption band is seen in the green. Wrede (1930) gives its empirical formula as $\mathrm{C}_{20} \mathrm{H}_{25} \mathrm{~N}_{3} \mathrm{O}$, and says that only one of the nitrogen atoms will combine with acids. The pigment is destroyed in a few days by sunlight. It is very resistant to reduction, but is fairly easily oxidized. In synthetic media it is formed only in the presence of magnesium sulphate and a phosphate, preferably potassium phosphate (Kuntze 1900, and Sullivan 1905-06). Its formation is said to be inhibited by the presence of calcium salts (Bordet 1930). It is produced most readily at $22^{\circ} \mathrm{C}$., and according to Amako (1930) there is a close parallelism in cultures between the catalase content and the amount of pigment formed.

Hefferan (1904), whose detailed study is one of the most valuable on this group of bacteria, proposed the following classification.

Pigment insoluble in water :

Group I. Chr. prodigiosum, Chr. indicum, Chr. kielense, etc. Pigment red at first, later becomes darker-carmine or violet-red.

Group II. Chr. rubricum, Chr. ruber, etc. Develop more slowly. Pigment is orange-red or yellow-red, never becoming darker.

Group III. Chr. mycoides roseum, etc. Pigment is salmon-pink, coralpink, rose, or flesh-coloured.

Pigment soluble in water :

Group IV. Chr. lactis erythrogenes, Chr. rubefaciens, etc. Rose-red pigment. 
We append a detailed description of $C h r$. prodigiosum and $C h r$. indicum. For a description of other members of this group, see Hefferan (1904).

\section{Chromobacterium prodigiosum}

Synonyms.-Serratia marcescens Bizio. Micrococcus prodigiosus Cohn.

Isolation.-First described by Bizio in 1823 (see Text).

Hubitat.-Water and air; found on bread, meat, milk, potatoes and other food stuffs. Morphology. - Tiny rods, often oval or cocco-bacillary; $0.7-1.0 \mu \times 0.7 \mu$; sides convex, ends rounded, arranged singly and in groups; irregularity in shape frequent, there being generally some definitely bacillary forms present. Motile by $2-4$ peritrichate flagella. Non-sporing, non-capsulated. Gram-negative, non-acid-fast; bipolar staining not uncommon.

Agar Plate. -2 days, $25^{\circ} \mathrm{C}$. Round colonies, 1-2 mm. in diameter, low convex, smooth, glistening, paint-like surface, entire edge, amorphous structure; butyrous consistency, easily emulsifiable. Individual colonies vary from colourless to bright red in colour; all are translucent. 5 days. Colonies are larger, $2-4 \mathrm{~mm}$. in diameter,

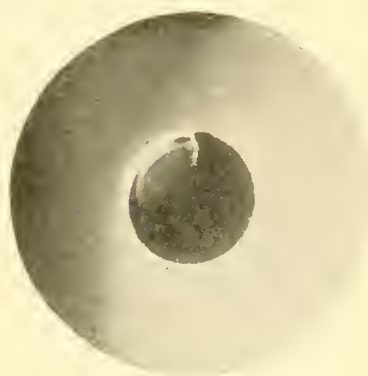

FIG. 132.-Chromobacterium prodigiosum.

Surface colony on agar, 24 hours, $37^{\circ} \mathrm{C} .(\times 8)$. surface radially striated, edge finely lobate; differentiated into a low convex, red, opaque centre, and an effuse, transparent, colourless periphery.

Agar Slope.-Abundant, raised, opaque, scarlet growth, with smooth, paint-like surface and lobate edge. Medium unchanged.

Gelatin Stab.-Abundant, filiform growth to bottom of tube. Liquefaction infundibuliform reaching to bottom of tube in 5 days at $20^{\circ} \mathrm{C}$.; liquid is pink at upper part and turbid; red ring growth at surface; pink floccular deposit; rest of growth uncoloured.

Broth. -2 days, $25^{\circ}$ C. Luxuriant, pink growth, with dense turbidity, a red ring growth, and a moderate, reddish, powdery or finely granular deposit, mostly disintegrating on shaking. After 5 days there is a red surface pellicle and a heavy flocculo-granular deposit. Mawkish odour, like trimethylamine.

Blood Serum. -7 days, $25^{\circ} \mathrm{C}$. Profuse growth; medium mostly liquefied; fluid is red and turbid.

Potato. -6 days at $25^{\circ} \mathrm{C}$. Abundant, thick, raised, confluent growth of dark maroon colour; surface papillate and slightly glistening. Potato not coloured.

MacConkey Plate.-24 hours at $37^{\circ} \mathrm{C}$. Circular, low convex, opaque, amorphous, pink colonies, $1 \mathrm{~mm}$. in diameter, with smooth glistening surface and entire edge.

Resistance.-Destroyed by $55^{\circ} \mathrm{C}$. for 1 hour.

Metabolic.-Aerobe; slight colourless growth anaerobically. Opt. temp. $25-30^{\circ} \mathrm{C}$. Grows at $37^{\circ} \mathrm{C}$, but no pigment produced. Pigment: blood-red, soluble in chloroform, not in water.

Nutritional.-Grows well on ordinary media. In synthetic media pigment is formed only in the presence of $\mathrm{MgSO}_{4}$ and a phosphate.

Biochemical.-Acid or acid and gas in glucose, maltose, mannitol, sucrose and salicin, occasionally in lactose. Indole -. M.R. - . V.P. +. Nitrates reduced. $\mathrm{NH}_{3}$ production + . Catalase + . M.B. reduction ++ . Starch diastase $-. \mathrm{H}_{2} \mathrm{~S}+$. L.M. clotted in 2 days, peptonized in 5 days ; fluid pinkish.

Pathogenicity.-Non-pathogenic to man; and to animals except in enormous doses. 


\section{Chromobacterium indicum}

Isolation.-By Koch from the stomach of an ape.

Morphology.-Slender, often eurved, bacillus, $2-4 \mu$ long by $0.6 \mu$ broad ; motile by peritrichate flagella. Gram-negative. Non-acid-fast.

Cultural Characters.-Grows readily on ordinary media.

Agar plate. -2 days at $25^{\circ} \mathrm{C}$. Colomies are circular, $1 \mathrm{~mm}$. in diameter, amorphous, low convex, translucent, and pink, with a smooth surface and entire edge ; butyrous in consistency and easily emulsifiable. After 5 days, there is usually a thin, transparent, colourless peripheral fringe with a finely lobate edge.

Gelatin. - 5 days at $20^{\circ} \mathrm{C}$. Abundant growth ; complete liquefaction of gelatin ; upper $\frac{1}{2} \mathrm{~cm}$. of the liquefied gelatin is densely turbid and bright red in colour; pinkish deposit at bottom of tube.

Broth.- -2 days, $25^{\circ} \mathrm{C}$. Luxuriant growth, with dense turbidity, a pink surface pellicle and ring growth, and an abundant, moderately granular, pink deposit, which disintegrates partly on shaking.

Potato. - 6 days, $25^{\circ} \mathrm{C}$. Noderate, slightly raised, confluent growth of café-aulait colour, having a smooth or finely granular, contoured, moist, glistening surface.

Loeffler's serum. -5 days, $25^{\circ} \mathrm{C}$. Abundant, confluent, raised, pinkish growth, with a glistening contoured surface; slight digestion, increasing with further incubation.

Biochemical Reactions.-Same as Chr. prodigiosum.

Pathogenicity.-Nil.

Chromobacterium kielense.-This organism, which was isolated from water, resembles Chr. prodigiosum very closely; it is not clear, in fact, that it is a separate species. It is said uniformly to produce both acid and gas in carbohydrate media; but as many strains of $\mathrm{Chr}$. prodigiosum also produce gas, this distinction is an imperfect one.

Group Forming a Yellow or Orange Pigment.-Chr. aquatile, an organism isolated by the Franklands (1889) from deep wells in the Kent chalk, is a typical example of this group. The yellow pigment is insoluble in water but dissolves in alcohol, ether and chloroform. In non-albuminous media it is developed slowly ; but it appears rapidly in a peptone solution containing magnesium sulphate and dipotassium hydrogen phosphate.

We append a detailed description of Chr. aquatile and Chr. typhi-flavum, together with brief notes on a few of the other members of this group. The identity of $C h r$. aquatile is not very certain, since the original description was incomplete. The present description is founded on a study of the strain of Chr. aquatile obtained from the National Collection of Type Cultures, London. Chr. typhi-flavum is the name we suggest for the organism commonly known as Bact. typhi flavum. Though suspected by certain German workers of being a pigmented variant of the true typhoid bacillus, the evidence, which has been critically reviewed by Cruickshank (1935), is quite insufficient to establish any such relationship.

\section{Chromobacterium aquatile}

Synonym.-Bacillus aquatilis Frankland.

Habitat.-Water.

Morphology.-Slender rod-shaped organism, $2.5 \mu \times 0.6 \mu$; axis straight, sides parallel, ends rounded; arranged in bundles; length irregular. Motile by peritrichate flagella. Non-sporing; non-capsulated. Gram-negative; non-acid-fast. 
Agar Plate.-2 days at $25^{\circ} \mathrm{C}$. Round colonies, 1-2 mm. in diameter, low convex, opaque, yellowish-grey colour, with a dull, dry, granular or rugose surface, and an entire edge; consistency slightly membranous but emulsification fairly easy. After 5 days, colony shows differentiation into finely granular, yellowish-brown, convex centre, and a clear almost transparent, effuse, and sometimes radiate periphery which has an erose or villous edge.

Agar Slope.-Abundant, confluent, opaque, yellowish, raised growth, with smooth glistening surface and a lobate or villous edge.

Broth. -2 days at $25^{\circ} \mathrm{C}$. Slight turbidity, with slight powdery deposit. After 5 days the growth is more abundant; there is a very slightly granular turbidity, and sometimes a surface pellicle and ring growth; moderate membranous deposit, disintegrating incompletely on shaking.

Gelatin Stab. -5 days at $20^{\circ} \mathrm{C}$. Good growth, extending to bottom of tube; gelatin shows commencing infundibuliform liquefaction; the liquefied gelatin shows a floccular turbidity, and is covered with a granular pellicle.

Potato. -6 days at $25^{\circ} \mathrm{C}$. Abundant, slightly raised, confluent, greyish-brown growth, with a dry, dull, worm-cast surface.

Loeffler's Serum.-5 days at $25^{\circ} \mathrm{C}$. Good, confluent, raised, yellowish-white growth, with wrinkled surface. 14 days, partial digestion.

Metabolism.-Strict aerobe; no growth under anaerobic conditions. Yellow pigment formed on agar, soluble in alcohol, ether, and chloroform, but insoluble in water. Optimum temperature for growth $25-30^{\circ} \mathrm{C}$.

Biochemical Characters.-Acid in glucose, maltose, mannitol, and sucrose. L.M. clot, peptonization, and partial decolorization in 3 days. Indole -. M.R. - V.P.t.

Nitrates reduced to nitrites. $\mathrm{NH}_{3}$ sl. $+. \mathrm{H}_{2} \mathrm{~S}+$. M.I. reduction + . Catalaset.

Pathogenicity. - Nil.

\section{Chromobacterium typhi-flavum}

Symonym.-Bucterium typhi flavum (Dresel and Stickl 1928).

Habitat.-Air, grass, plants. Has been found in normal human fæces and urine.

Morphology.-Slender, rod-shaped organism, $1-3 \mu \times 0.5-0 \cdot 7 \mu$ : axis straight, sides parallel, ends rounded; sometimes filaments up to $15-20 \mu$ in length; arranged

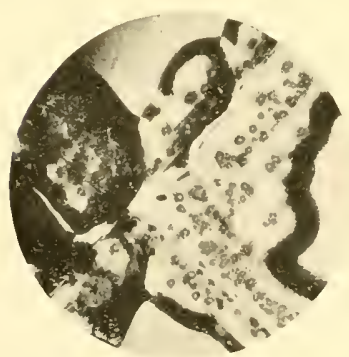

Fig. 133.-Chromobacterium typhi-flavum.

Surface growth on agar plate, showing the peculiar appearance caused by aggregations of the bacteria. One biconvex body is clearly visible. 24 hours, $37^{\circ} \mathrm{C}$. singly, in pairs end-to-end or in groups; in fluid media sausage-like aggregations may occur. Briskly motile when grown at $22^{\circ} \mathrm{C}$, but poorly or not at all at $37^{\circ} \mathrm{C}$. Flagella peritrichate. Non-sporing. Non-capsulated. Gram-negative; occasional bipolar staining. Non-acid-fast.

Agar Plate. -24 hours at $37^{\circ} \mathrm{C}$. Ready growth of colonies 1-2 mm. in diameter, round, low convex, amorphous, smooth, glistening, opaque, with entire edge ; consistency butyrous, emulsifiability easy. Ochre or rusty yellow pigment, not diffusing into the medium.

5 days.-Colonies are larger, 2-4 mm., differentiated frequently into a central plateau, often with a granular surface, and a smooth bevelled periphery; edge sometimes crenated.

In the centre of the colonies aggregated masses with radiating extensions of a granular nature, or biconvex bodies with a clear-cut margin, may often be seen with the lens by transmitted light. The granular structures are aggregations of organisms, known to the German workers as Bakterien-verbänden or symplasmata (Fig. 133). The biconvex bodies represent downgrowths of the colony into the medium. 
Agar Slope.-24 hours at $37^{\circ} \mathrm{C}$. Abundant, confluent, smooth, glistening, yellow, opaque growth, with entire or slightly undulate edge.

5 per cent. Glycerine Agar Slope.-Growth similar to that on agar slope but very mucoid in character.

Agar Shake.-Yellow surface growth with a few small colonies throughout the medium. Agar Pour-Plate.-Deep colonies are biconvex, sometimes with lateral knobs or projections. Broth.-24 hours at $37^{\circ} \mathrm{C}$. Uniform turbidity with a powdery white or yellowish deposit easily dispersed on shaking. After 5 days, increased turbidity with a fine surface seum or ring.

Horse-blood Agar Plate.-No hæmolysis. Colonies as on agar plate.

MacConkey's Agar.-24 hours at $37^{\circ} \mathrm{C}$. Growth hardly perceptible. 5 days.-Irregularly round colourless colonies with a rough surface and crenated edge.

Loeffler's Serum Slope.-24 hours at $37^{\circ} \mathrm{C}$. Good, confluent, glistening, yellow growth with smooth or slightly contoured surface and undulate edge. No liquefaction.

Potato.-3 days at $37^{\circ} \mathrm{C}$. Abundant, confluent, yellow, glistening, mucoid growth.

Gelatin Stab.-Filiform growth along line of inoculation. After 6-10 days liquefaction begins and progresses till the medium is entirely liquefied in the next 10-15 days. Liquefaction is infundibuliform; a yellow surface pellicle forms, and later sinks to the bottom.

Resistance.-Destroyed by $56^{\circ} \mathrm{C}$. in 10 minutes.

Metabolism.-Aerobic. Poor unpigmented growth anaerobically. Grows freely at $20-37^{\circ} \mathrm{C}$, with optimum nearer to $37^{\circ} \mathrm{C}$. Pigment ochre or rusty-yellow, insoluble in water and chloroform, partly soluble in alcohol and ether.

Biochemical Reactions.-Acid in glucose, mannitol, sucrose, salicin, rhamnose, arabinose, xylose ; maltose usually later. Lactose, inositol and dulcitol not fermented. Litmus milk neutral or transient acidity, becoming alkaline after 3-7 days ; occasional soft clot. Indole - M.R. +. V.P. - Nitrates reduced. Very slight $\mathrm{H}_{2} \mathrm{~S}$ production at $22^{\circ} \mathrm{C}$. Catalase + .

Antigenic Structure.-The organisms are antigenically heterogeneous. Both as regards flagellar and somatic antigens, sera prepared against any one strain will agglutinate the homologous strain, and usually several other strains. There is a tendency for the organisms to fall roughly into antigenically similar groups, the $\mathrm{H}$ antigens being more cosmopolitan than the 0 .

Pathogenicity.-It has been suggested that the organism is a variant of Salm. typhi, of potential pathogenicity to man, but this has not been substantiated. Pathogenic to mice only on injection of enormous doses.

There are several orgamisms in water and in soil belonging to this group, amongst which may be mentioned:

Chr. ochraceum.-Notile by polar flagella. Infundibuliform liquefaction in gelatin with a pale yellow, later ochre-coloured, deposit. On agar and potato a thin, ochre-yellow streak.

Chr. fuscum.-Non-motile; liquefies gelatin slowly or not at all. On agar and potato gives a thick, wrinkled, chrome-yellow growth.

Chr. aurantiacum.-Motile by peritrichate flagella. No liquefaction of gelatin. On agar and potato forms a light orange growth.

Chr. denitrificans.-Described by Burri and Stutzer (1895), who isolated it from horse fæces; called by them $B$. denitrificans I. Appears to be common in the soil. Rods with rounded ends, $1.5-2.5 \mu \times 0.75 \mu$. Actively motile. Gram-negative. Grows freely on ordinary media, more quickly at $37^{\circ} \mathrm{C}$. than at room temperature, and is aerobic. Colonies on agar are very thin and membranous, having a thicker centre; generally circular, but may have a lobate or irregularly erose edge. In broth there is a dense turbidity in 24 hours, with a reddish-white deposit disintegrating on shaking; surface 
ring growth. Produces large amount of gas in nitrate broth, reducing the nitrate to

free nitrogen. Non-pathogenic. This organism is not to be confused with Ps. denitrificans (q.v. Chapter 21).

\section{ACHROMOBACTERIUM}

\section{Definition.-Achromobacterium.}

Motile or non-motile, Gram-negative rods, usually small to medium in size, forming no pigment on agar, and varying in their fermentative ability. Optimum temperature for growth about $25^{\circ} \mathrm{C}$., but often good growth at $37^{\circ} \mathrm{C}$. Saprophytic ; commonly found in water, soil, and milk.

Organisms of this group are widespread in nature, but have so far received little systematic study. The public health bacteriologist meets them mainly in the analysis of water, milk, food, and soil, where they attract attention by their frequency on agar and gelatin plates incubated at $22^{\circ} \mathrm{C}$. or sometimes at $37^{\circ} \mathrm{C}$. They are mainly responsible for the formation of slime on stored meat (Haines 1933). They are differentiated from the Chromobacterium group mainly by their failure to form pigment. Discussion of their classification would at present serve no useful purpose, and all that we need do here is to give a brief account of their more common characteristics.

Morphologically they are Gram-negative, motile or non-motile rods, often of the size of coliform bacilli, but varying considerably in thickness; rather fat rods, and fat cocco-bacilli are quite common.

The optimum temperature for growth is about $20-25^{\circ} \mathrm{C}$. At $37^{\circ} \mathrm{C}$. growth is generally stated to be slight or absent, but in our experience abundant growth of these organisms is by no means infrequent in cultures made from certain foods. Most strains grow at $0^{\circ} \mathrm{C}$. (Coyne 1933) ; Haines (1933) found that the generation time in broth at this temperature was about 9 hours. The colonial appearances vary. After 24 hours at $22^{\circ} \mathrm{C}$. colonies on agar are about $0.5 \mathrm{~mm}$. in diameter, circular, smooth, convex, greyish white, and translucent with an entire edge ; after 5 days they are $3-5 \mathrm{~mm}$. in diameter, raised or low convex, greyish, opaque, with a smooth surface and entire edge, or sometimes with a beaten-copper surface and an irregular edge. Mucoid colonies are not uncommon, and seem to be formed most frequently by the short fat cocco-bacillary type. Colonies with central crateriform depressions, draughtsman-like colonies, colonies with a roughish surface, and colonies showing radial striation are sometimes met with. An aromatic odour may be noticeable.

In broth there is a uniform turbidity of varying degree with a powdery, granular, or viscous deposit. On potato a layer of growth is formed, which is sometimes mucoid or creamy, and which often takes on a café-au-lait appearance after a week or two.

Most, but not all, strains seem to grow in MacConkey's bile-salt medium. In liquid MacConkey they give rise to turbidity, but not usually to acid production ; on solid MacConkey they form small yellowish colonies, which after 5 days at $22^{\circ} \mathrm{C}$. may reach a diameter of $1-2 \mathrm{~mm}$. Their growth is not inhibited by concentrations of brilliant green and sodium tetrathionate, such as are used in the isolation of Salmonella organisms; and as they form non-lactose-fermenting colonies on MacConkey's medium, they may cause trouble in the search for pathogenic organ- 
isms in foods such as synthetic crean and made-up meat dishes suspected of being responsible for conveying food poisoning or enteric fever.

Litmus milk may be unchanged. More often it is turned alkaline, or peptonized. Some strains produce acid and curdle the milk. The litmus is often reduced to within a few millimetres of the surface.

Sugar reactions are variable. Most strains have little or no fermentative ability, but some produce acid in glucose, or in glucose, maltose, mannitol, sucrose, and salicin, less often in lactose. There is no record of gas production. Some strains give a positive M.R. or V.P. reaction, or both. Nitrates are not uncommonly reduced to nitrites, but indole is rarely formed. Haines (1933), who studied 132 strains isolated from meat, found that 67 strains fermented glucose and maltose, 59 fermented glucose only, whereas 6 failed to ferment even glucose. Gelatin may or may not be liquefied; in Haines's series 115 strains liquefied gelatin in 1 to 2 days, and 17 strains liquefied it slowly or not at all.

Little or nothing is known of their antigenic characteristics. It is probable that they are all non-pathogenic.

\section{REFERENCES}

Амако, T. H. (1930) Zbl. Bakt., 116, 494, 499.

Bordet, P. (1930) Ann. Inst. Pasteur, 45, 26.

Breed, R. S. and Breed, M. E. (1924) J. Bact., 9, 545.

Burri, R. and Stutzer, A. (1895) Zbl. Bakt., IIte Abt., 1, 257, 350.

Coyne, F. P. (1933) Proc. roy. Soc., B, 113, 196.

Cruicksha NK, J. C. (1935) J. Hyg., Camb., 35, 354.

Dresel, E. G. and Stickl, O. (1928) Dtsch. med. Wschr., 54, 517.

EisenberG, P. (1914) Zbl. Bakt., 73, 466.

Frankland, G. C. and Frankland, P. F. (1889) Z. Hyg. InfektKr., 6, 373.

Hatnes, R. B. (1933) J. Hyg., Camb., 33, 175.

Hefferas, M. (1904) Zbl. Bakt., IIte Abt., 6, 311, 397, 456, 520.

Klein, E. (1894) J. Path. Bact., 2, 217.

Kuntze, IV. (1900) Z. Hyg. Infekthr., 34, 169.

Report. (1920) Rep. Comm. Amer. Bacteriologists, J. Bact., 5, 191.

ScHÜTZ, F. and LAUN, H. (1933) Zbl. Bakt., 129, 124.

Sullivan, M. X. (1905-6) J. med. Res., 14, 109.

Wrede, F. (1930) Z. Hyg. InfektKr., 111, 531 . 


\title{
CHAPTER 27
}

\section{PROTEUS AND ZOPFIUS}

\author{
PROTEUS \\ Definition.-Proteus. \\ Highly pleomorphic rods, filaments and curved cells being common in young \\ cultures. Gram-negative. Actively motile. Characteristic spreading growth on \\ moist media. Often liquefy gelatin and often produce vigorous decomposition \\ of proteins. Ferment glucose and usually sucrose, but not mannitol or lactose, \\ with production of acid and gas. \\ Type species. $P$. vulgaris Hauser.
}

Habitat.-Organisms of the Proteus group have been known since the earliest days of bacteriology. They are widely distributed in nature, and constitute an important part of the flora of decomposing organic matter of animal origin. They are constantly present in rotten meat and in sewage, and very frequently in manure. Though often demonstrable in the fæces of man and animals, they are rarely found in large numbers except when the normal intestinal mechanism is deranged. They are not uncommon in garden soil and on certain vegetables, such as melons and celery (Cantu 1911), but it seems probable that their access to these materials results largely from contamination with sewage or manure.

Besides their wide saprophytic existence, Proteus bacilli are able under certain conditions to grow in the animal body and even to give rise to pathological disturbances. The rôle they play in summer diarrbœa is not yet entirely clear, but there is no doubt that in some outbreaks of this disease they multiply enormously in the intestinal canal, particularly of infants. This holds particularly true of Morgan's bacillus which, in the light of Rauss's (1936) recent work, must be regarded as belonging to the Proteus group. They are primarily responsible for some cases of cystitis, and they are to be met with as secondary invaders in infections of the bladder and in wounds. Many strains, referred to as Proteus $X$ strains, have been isolated from the urine, fæces, or blood of patients suffering from typhus fever, though their exact relationship to the ætiological agent of this disease is still obscure (see Chapter 83).

Morphology.-The organisms are rod-shaped, but are subject to great variation in size. In agar cultures after 24-48 hours, the majority are of the coliform type, 1-3 $\mu$ long by $0.4-0.6 \mu$ wide, though short fat cocco-bacillary forms are not uncommon (Figs. 136, 137). In young rapidly growing cultures, however, in which swarming (see p. 643) is apparent, many of the organisms are long, curved, and filamentous, reaching 10, 20, or even $30 \mu$ in length (Fig. 135). There is no very characteristic arrangement; the bacilli are distributed singly, in pairs, in short chains, in small bundles, or in larger bundles in which the members tend to be arranged concentrically, more or less simulating the isobars in a diagram of a 
cyclone. There is some variation in depth of staining. Except for non-flagellated $O$ variants, all members are actively motile by peritrichate flagella in young cultures. Neither spores nor capsules are formed. The reaction to Gram's stain is uniformly negative.

Cultural Characters.-Growth occurs freely on the usual media. One of the most characteristic properties of Proteus strains is their ability to "swarm" on solid media. Cantu (1911), who made a study of this feature, found that if an organism of the Proteus group was inoculated into the water of condensation of an agar slope, a rapid growth occurred, which spread over the whole surface, producing a uniform layer hardly distinguishable from the medium. This process has been more fully described by Moltke (1927, 1929). "Swarming" may be defined as a "progressive surface spreading by the microbes from the edge of the parent colony." It is best studied by touching the centre of an agar plate with a needle dipped in a Proleus culture. First of all

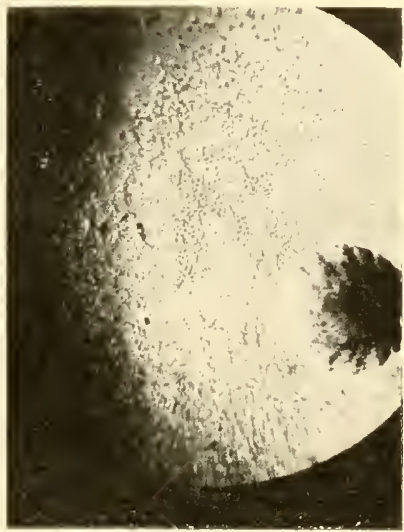

FId. 134.-Proteus vulgaris, showing swarming on agar, 6 hours, $37^{\circ} \mathrm{C} .(\times 8)$. a colony develops, and then after about 6 hours at $37^{\circ} \mathrm{C}$. a thin, effuse, ground-glass type of growth appears round the edge of the colony, and rapidly spreads over the whole plate (Fig. 131). If it is examined under the microscope, it is seen that, when swarming commences, long slender rods in continuous motion break away from the periphery of

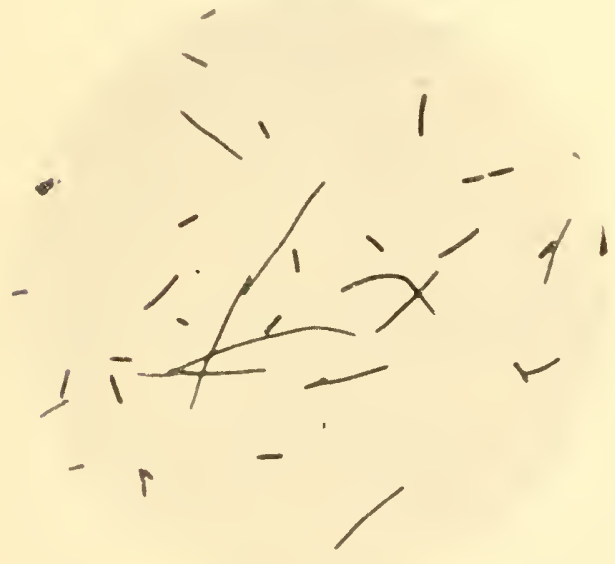

Fia. 135.-Proteus vulgaris.

From an agar culture, 6 hours, $37^{\circ} \mathrm{C}$., showing long filaments $(\times 1000)$. the colony, and, after travelling some distance from the parent colony, join neighlyouring lateral offshoots, to form arehes, which are rapidly filled with other rods from within. Whole rafts of rods tear loose from the peninsula so formed, and work across the agar, so that in a short time the colony is surrounded by an archipelago of islands and solitary organisms, all constantly in motion. The very long rod forms are the predominant feature in the picture; they form arches, islands, spirals, and question-mark forms (Fig. 135). Once the plate is completely covered with "swarmers", the long rods are replaced by quite short forms (Figs. 136 and 137). The property of swarming is observed only on the surface of solid media. In the depths of an agar shake or pour plate culture the colonies are compact. This circumstance may be made use of in the isolation of organisms mixed with Proteus bacilli (Fry 1932). Other methods for the inhibition 
of swarming consist in $(a)$ the addition to the medium of certain narcotic drugs such as chloral hydrate, morphine and sodium phenylethylbarbiturate (Krämer and Koch 1931, Lode and Howard 1932); chloral should be employed in a final concentration of $1 / 500-1 / 1000$; this method suffers from the disadvantage that many strains are relatively insensitive to the drugs used; $(b)$ the incorporation of 5-6 per cent. alcohol in the medium (Floyd and Dack 1939); this method is effective, but if used for the isolation of streptococci has the drawback of lysing the blood in the agar and inhibiting the growth of the streptococci; $(c)$ the addition to the medium of sodium azide in a final concentration of 1/5000-1/10,000 (Snyder and Lichstein 1940, Lichstein and Snyder 1941); in the higher concentration not only is spreading prevented, but the growth of Proteus is almost completely inhibited; the growth of streptococci is good, though the zone of hæmolysis around $\beta$-lytic colonies appears green and around $\alpha$-lytic colonies brown; $(d)$ the use of 6 per cent. agar (Hayward and Jiles 1943). Swarming is also suppressed

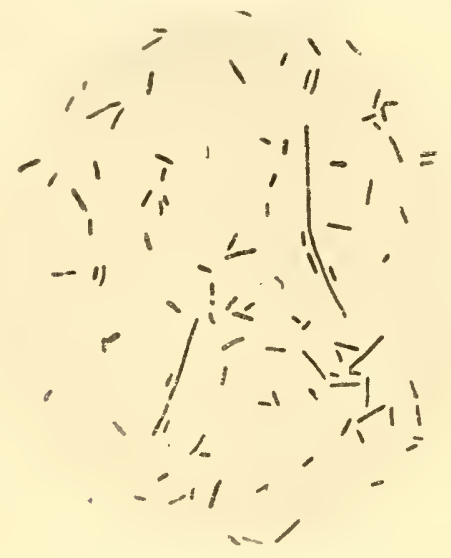

FIa. 136.- Proteus vulgaris.

From an agar culture, 24 hours, $37^{\circ} \mathrm{C}$, showing chiefly rod forms $(\times 1000)$.

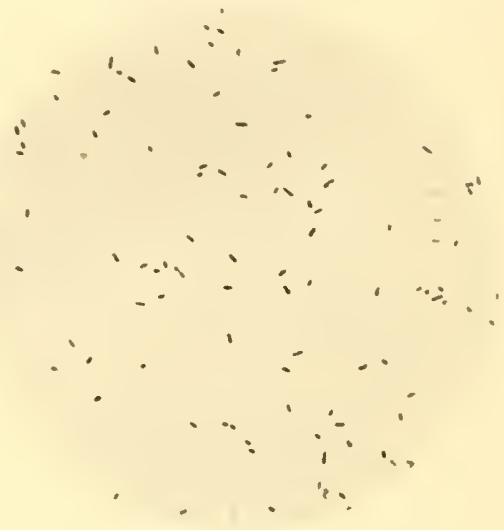

FIG. 137.-Proteus vulgaris.

From an agar culture, 48 hours, $37^{\circ} \mathrm{C}$., showing short rods only $(\times 1000)$.

on some of the media, such as Wilson and Blair's bismuth sulphite agar and Leifson's desoxycholate citrate agar, used for the isolation of the pathogenic intestinal Gram-negative bacilli. According to Lominski and Lendrum (1942) some "surface active agents," like the alkyl sulphates, have a strong anti-swarming power. ${ }^{1}$

Swarming is due essentially to the active motility of the bacilli. Non-motile O variants give rise to compact colonies which, according to Felix (1922), may be of three types: (1) smooth, translucent, homogeneous, with an entire edge; (2) granular and opaque with an irregular edge; (3) tiny colonies, barely visible to the naked eye after 24 hours.

Observation clearly shows that swarming on an agar plate is a discontinuous phenomenon. On a plate inoculated centrally and incubated at $37^{\circ}$ C., a thin layer of bacteria is present at the site of inoculation after about 4 hours. Swarming then begins, and in 6 hours the breadth of the growth is $1-1.5 \mathrm{~cm}$. Further progress outwards ceases, but the layer of growth becomes thicker. After 8 hours swarming again starts, and a fresh ring of growth appears. The alternation of swarming and

1 Certain quinone compounds have also been found to be effective in suppressing the growth of Proteus (see p. 1528). 
rest occurs regularly, a fresh ring of growth being formed about every 4 hours till the plate is covered. It is due to this periodic extension that the surface of the growth appears rippled or contoured (see Russ-Münzer (1935)).

Though the property of swarming is generally considered to be peculiar among aerobic bacteria to the Proteus group, it has been pointed out by Rauss (1936) that strains of Morgan's (1906) bacillus, which have not hitherto been included in this group, also exhibit the ability to swarm under appropriate conditions. On ordinary agar at $37^{\circ} \mathrm{C}$. this organism forms circumseribed colonies, but on solid media containing only 1 per cent. agar incubated at $20-28^{\circ} \mathrm{C}$., characteristic swarming occurs. Variant forms are described having a less marked power to spread, and these give rise to colonies distinguished by varying degrees of peripheral spread as well as by their structural appearance.

In broth Proteus gives rise to a uniform turbidity accompanied by a slight to moderate powdery deposit and a faint ammoniacal smell. Cantu (1911) states that a surface pellicle is never formed, while Wenner and Rettger (1919) and Yacob (1932) say that a thin fragile pellicle may develop in older cultures. Gelatin plate colonies are very characteristic (Cantu 1911).

Resistance.-Few observations appear to have been made on the resistance of Proteus bacilli. Our own limited experience has shown that they are readily destroyed by heat and disinfectants. Exposure to moist heat at $60^{\circ} \mathrm{C}$. for 1 hour is sufficient to sterilize a broth culture. A 1 per cent. phenol solution, inoculated with one million organisms per $\mathrm{ml}$, is found to be sterile within 30 minutes.

Metabolism.-The members of this group are aerobes and facultative anaerobes. Growth under strict anaerobic conditions is very poor, and certain enzymic activities may be suppressed. The optimum temperature for growth is about $34-37^{\circ} \mathrm{C}$., though rapid multiplication occurs above $20^{\circ} \mathrm{C}$. The limits of growth are between about $10^{\circ} \mathrm{C}$. and $43^{\circ} \mathrm{C}$.

Reports on the hæmolytic activity of Proteus are discrepant. Wemner and Rettger (1919) obtained uniformly negative, and Norton, Verder, and Ridgway (1928) uniformly positive results. In neither of these reports is the type of blood mentioned. Taylor (1928), using human blood, observed hæmolysis regularly within 24 hours in 1 per cent. blood broth, but not on 10 per cent. blood agar plates. Yacob (1932) used 5 per cent. rabbit blood agar plates, and found that all strains produced $\beta$-hæmolysis in 24-48 hours. As with many other organisms, it seems probable that the nature of the blood is an important factor in determining the result.

The main features that distinguish Proteus bacilli from other Gram-negative gelatin-liquefying rods are the production of $\mathrm{H}_{2} \mathrm{~S}$ and the active decomposition of urea (Moltke 1927). According to Wolf (1918-19), urea is broken down readily, as much as 45 per cent. of the total nitrogen of urine being transformed into ammonia. The production of indole and the digestion of serum proteins varies with different strains; as a rule these two properties are negatively correlated. The power to digest serum proteins is often lost during cultivation in the laboratory. Catalase is formed, but the oxidase reaction described by Gordon and McLeod (1928) is negative.

Since the Proteus bacilli are found most constantly in decomposing animal inatter, they are generally regarded as putrefactive organisms. Rettger and Newell (1912-13) dispute this. They define putrefaction as a "particular process of protein decomposition which is brought about through the agency of bacteria with the evolution of foul-smelling 
products which are characteristic of ordinary cadaveric decomposition." Amongst these decomposition products they consider mercaptan and the oxy-acids to be of particular significance ; indole, skatole, and $\mathrm{H}_{2} \mathrm{~S}$ are less characteristic. According to this definition the Proteus group would be classed amongst the, non-putrefactive bacteria. The connotation that Rettger and Newell attach to the term putrefaction is that it is essentially an anaerobic process; it is dependent therefore on the anaerobic growth of bacteria. Proteus bacilli, when grown under anaerobic conditions, do not digest proteins, and therefore cannot be regarded as capable of causing true putrefaction. They are, however, frequently associated with the anaerobes in putrefying organic material, and no doubt assist these greatly by using up oxygen and rendering the conditions suitable for their growth. It may be noted that this definition would not meet with universal acceptance.

Biochemical Reactions.-Acid and gas are formed from glucose, sucrose, glycerol, xylose, and almost always from salicin. Mannitol, lactose, dulcitol, starch, dextrin, sorbitol, and raffinose are never fermented. The action on maltose is variable and is of value in classification. Moltke (1927), who examined 194 strains, found that 37 fermented maltose, and 157 did not. The maltose-positive strains fermented sucrose and salicin within 24 hours, while the maltose-negative strains took 3-15 days to ferment sucrose and 10-21 days to ferment salicin. All but one of the maltose-positive strains produced indole, while the maltose-negative strains uniformly failed to do so. A negative correlation was observed between the fermentation of maltose and the ability to digest coagulated horse serum. In the definition of the group by Winslow and his colleagues (1920) it is stated that the gas produced from glucose and sucrose consists entirely of $\mathrm{CO}_{2}$. Mendel (1911), however, gives the gas ratio as $\mathrm{H}_{2}: \mathrm{CO}_{2}=6-8: 1$, while Yacob (1932) gives it as $3-4: 1$. These latter figures agree with our own findings. The fermentative astivity of Proteus is fairly constant, but occasional strains are anaerogenic on isolation (Edwards 1942), and others lose their power to attack certain sugars on prolonged cultivation. Proteus morgani is restricted in its fermentative power from the start. The action on litmus milk is subject to some variation. Usually a true rennet clot is produced, which retracts and squeezes out whey. Digestion of the clot sets in and is accompanied by progressive alkalinization of the medium. The litmus is reduced. The clot is often digested completely within a week, and the reaction is strongly alkaline. Slight initial acidity is sometimes observed, and not infrequently proteolysis may overshadow coagulation, so that there is not time for a definite clot to form. Most strains attack milk rapidly, imparting to it a yellow colour, a bitter soapy flavour and a foul odour, but some strains have little or no coagulative or proteolytic effect (Plähn 1937). The reaction to the methyl-red and Toges-Proskauer tests varies with different strains. Nitrates are reduced to nitrites. Methylene blue is decolorized slowly in broth cultures. Urea is decomposed with the production of ammonia--a reaction that may be used in the differentiation of Proteus from Salmonella (Ferguson and Hook 1943). Phenylalanine is broken down with the formation of phenylpyruvic acid (Henriksen and Closs 1938).

Antigenic Structure.-Our knowledge is both deficient and conflicting. Most workers (Cantu 1911, Wenner and Rettger 1919, Taylor 1928) who have prepared immune sera against different strains have found that a given serum may agglutinate either the homologous strain only or a number of heterologous strains as well. Any simple subdivision by agglutination or absorption of agglutinins has proved impossible. Moltke (1927), who paid special attention to the $\mathrm{H}$ and $\mathrm{O}$ antigens, 
found that by direct agglutination the swarming strains could be divided into 3 main groups, and that by absorption these main groups could be divided into a number of sub-groups. The non-swarming strains differed from the swarming strains in their absence of an $\mathrm{H}$ antigen, and differed among themselves in the type of their $O$ antigen. The relation of the vulgaris to the $X$ strains is still somewhat doubtful. The $O$ antigens of the $X$ strains differ from those of the vulgaris strains, but there appears to be a certain group relationship between the $\mathrm{H}$ antigens (Yacob 1932). Among the $O$ antigens of the $X$ strains there are at present three fairly well-defined groups, represented by the strains $O X 2, O X 19$, and $O X \mathrm{~K}$ (see Chapter 83). White (1933) has brought evidence to show that the $O$ antigen of $O X 19$ contains two receptors, one of which is alkali-labile and is mainly responsible for agglutination of this organism by an antiserum prepared against it, the other of which is alkali-stable, and is responsible for the reaction of the bacillus with the sera of patients suffering from typhus fever-the Weil-Felix reaction. Meisel and Mikulaszek (1933) and Castaneda $(1934,1935)$ have reported the extraction of soluble specific polysaccharide substances from Proteus $X$ strains. Castaneda's results agree closely with those obtained by White. They show that the alkalistable polysaccharide, referred to as $\mathrm{X}$, is common to both Proteus $X 19$ and Rickettsia prowazeki (see Chapter 39 ), while the alkali-labile polysaccharide, referred to as $\mathrm{P}$, is specific to Proteus $X 19$.

Our own observations on a limited number of strains suggest that there is a wide variety of $\mathrm{H}$ and $\mathrm{O}$ antigens in Proteus vulgaris, the $\mathrm{H}$ antigens being distributed to some extent independently of the $O$ antigens.

With regard to Proteus morgani, Rauss states that the $\mathrm{H}$ antigen tends to be group-specific, and the $\mathrm{O}$ antigen type-specific. In an examination of 48 strains, 7 types of $H$ antigen were differentiated, but as many as 17 types of 0 antigen. A relationship was found between the $\mathrm{H}$ receptor of one group of morgani and a strain of Proteus vulgaris.

Pathogenicity.--Proteus bacilli are frequently found in, and appear to be responsible for, a number of inflammatory and suppurative conditions in man. They are a very common cause of cystitis and may be isolated in pure culture from the urine of infected patients. They are not uncommonly found in abscesses, either alone or in combination with other organisms. Metchnikoff and his co-workers (see Chapter 71) found them almost constantly in the freces of infants with summer diarrhœa. They are frequently present, nsually as secondary invaders, in wounds and burns, where they probably favour the development of the pathogenic anaerobes. And they have been isolated from a variety of conditions, such as volvulus, peritonitis, croupous pneumonia, acute gastro-enteritis of the food-poisoning type (Wichels and Barner 1925, Plähn 1937, Cooper et al. 1941), empyema, gangrene of the lung, and septicæmia. Jensen (1913) considers them responsible for one form of epidemic calf dysentery ; and Wyss (1898) has encountered them in an epidemic disease of fish in Lake Zürich. They are also responsible for the black rot of eggs.

An organism described as $P$. hydrophilus, has been held responsible for the Red leg disease of frogs, but as this organism is monotrichate, does not swarm on agar, and does not decompose urea, it is very doubtful whether it should be included in the Protens group (see Kulp and Borden 1942).

The relation of Proteus X 19 to typhus fever is discussed in Chapter 83.

Their pathogenicity to laboratory animals is variable; virulent strains on introduction into the tissues are able to proliferate and invade the blood stream, 
Strains of lower virulence cause chronic inflammatory processes, either of the suppurative or of the infective granuloma type (Larson and Bell 1913, Wenner and Rettger 1919); the latter are best seen after intraperitoneal injection.

The inoculation intraperitoneally of $0.5-1.0 \mathrm{ml}$. of a 24 -hour broth culture of a virulent strain generally proves fatal to rats and mice in $18-48$ hours, and to guinea-pigs and rabbits in 1-7 days. Severe infections in rabbits are said to be characterized by extreme emaciation (Larson and Bell 1913). Though invasion of the tissues may occur on parenteral inoculation, Jensen (1913) states that in naturally infected calves, even when the organisms are seething in the gut, the blood and tissues remain sterile.

Classification.-Hauser (1885) divided the Proteus group into three species: (1) Proteus vulgaris; Gram-negative, liquefies gelatin, peptonizes fibrin, produces indole, and has a variable action on glncose and sucrose. (2) Proteus mirabilis; Gram-negative, more highly pleomorphic, and liquefies gelatin more slowly. (3) Proteus zenkeri; Gram-positive, does not liquefy gelatin, does not form indole, and fails to attack sugars. Proteus zenkeri was found to be very similar to an organism described two years previously by Kurth (1883) under the name of $B$. zopfi; ; as both of these organisms are Gram-positive, they had to be transferred to a separate genus, which is known as Zopfius.

Hauser's subdivision of the Proteus group on the basis of morphology, rate of liquefaction of gelatin, and indole production, has been found impracticable. The morphology is variable, depending especially on the medium and the age of the culture. The rate of liquefaction of gelatin is likewise variable; it is rapid with newly isolated strains, and is often much slower, or even absent, with strains that have been long under artificial cultivation. Indole production used to be tested by the nitroso-indole reaction; but as Berthelot (1914) has shown, this reaction is untrustworthy, and is given by indolacetic acid as well as by indole; when tested by Ehrlich's reagent, using the ether extraction method, it is found that the results are different, and that indole production is not nearly so constant a feature of Proteus as it was originally considered to be.

The most striking characteristics of the members of this group are their ability to swarm on solid media, their production of $\mathrm{H}_{2} \mathrm{~S}$, their decomposition of urea, their liquefaction of gelatin, and their failure to ferment lactose or any of the polyhydric alcohols. It should, however, be added that 0 variants occur which have lost their power of swarming, and that old strains may no longer liquefy gelatin.

The recent observations of Rauss (1936) suggest very strongly that Morgan's bacillus, which has hitherto occupied an invidious position in the Salmonella group, is closely related to Proteus. Its ability to swarm under suitable conditions, its frequent fermentation of xylose and its occasional fermentation of sucrose, its production of indole and $\mathrm{H}_{2} \mathrm{~S}$, its formation of alkali in litmus milk, the greater group specificity of its $\mathrm{H}$ and the greater type specificity of its $\mathrm{O}$ antigens, the group relationship of at least one of its $\mathrm{H}$ antigens to Proteus, its growth only under favourable conditions in the intestine of human beings, and its general pathogenicity for experimental animals - all bring it closely into line with organisms of the Proteus group. Its failure to liquefy gelatin or constantly to ferment sucrose must be considered in relation to the negative reactions obtained in these two respects with known Proteus strains, particularly those that have been long cultivated in the laboratory. Intermediate types that peptonize milk, and the organisms describerl by Magath (1928), which were isolated from cystitis and which liquefied gelatin but 
did not ferment sucrose, seem to show that too much stress should not be laid on any single biochemical characteristic. Rauss's conclusions are supported by the observations of Henriksen and Closs (1938), who found that both Proteus and Morgan's bacillus were able to break down phenylalanine with the formation of phenylpyruvic acid.

Until a more thorough study has been made, we consider that the most convenient method of classifying the Proteus group is on the basis of maltose fermentation and gelatin liquefaction, though we hesitate to ascribe specific names to the members of the sub-groups. The $X$ strains belong mostly to the maltosepositive sub-group, but the Kingsbury strain differs in this respect, as well as in its failure to liquefy gelatin.

(For review of the Proteus group, see Cantu 1911, Berthelot 1914, Wenner and Rettger 1919, Besson and Ehringer 1923, Moltke 1927, Taylor 1928, Yacob 1932, Mello 1938).

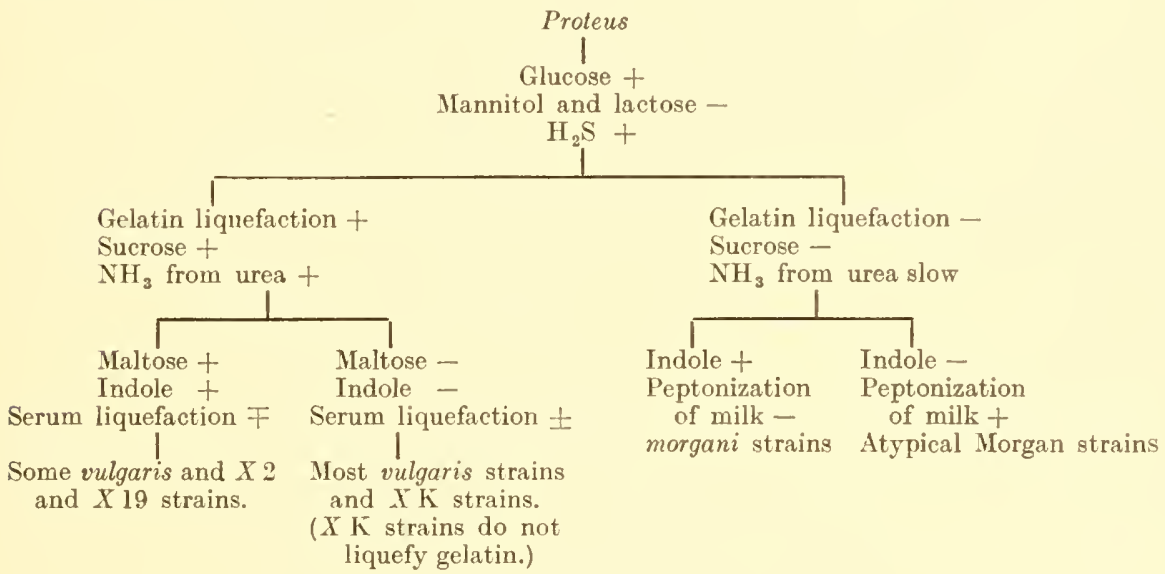

Fİ. 138.

This diagram must be regarded as merely tentative, and subject to both exceptions and alterations.

\section{Proteus vulgaris}

Synonym.-B. proteus vulgaris.

Isolation.--By Hauser in 1885 from putrefying material.

Habitat.-Putrefying animal and vegetable matter; often in fres, soil and infected wounds.

Morphology.-Straight or slightly curved rods, $1 \cdot 0-2 \cdot 5 \mu \times 0 \cdot 4-0 \cdot 6 \mu$, with parallel sides and rounded ends; arranged singly, in pairs end-to-end, and in short chains. In young swarming cultures, long curved filamentous forms are common. Considerable variation in length; ovoid forms in pairs may be seen, and in old cultures large bloated forms. Staining is fairly uniform, though variations in depth occur. Actively motile by numerous peritrichate flagella, though slightly motile forms with $\&$ flagella, two at each end, and non-motile forms devoid of flagella may occur. Gram-negative.

Agar Plates.- 24 hours, $37^{\circ} \mathrm{C}$. 'The whole plate is covered with a sliglitly raised layer of growth, which, but for a faint rippling or contouring of the surface and a marked 
odour, is easily overlooked. Sometimes indefinite primary colonies are seen, of variable diameter, having a smooth or slightly ringed draughtsman-like surface and an entire edge. The whole growth is translucent, of the same colour as the medium, butyrous, and easily emulsifiable. The complete layer of growth over the whole plate is due to swarming of the bacilli (see text). Non-flagellated $\mathrm{O}$ forms give rise to compact colonies.

Agar Slope.-If the organisms are inoculated into the condensation water, they swarm rapidly, and in about 8 hours at $37^{\circ} \mathrm{C}$. form a uniform, slightly raised, translucent growth with a glistening, faintly contoured surface over the whole slope There is a thick turbid growth in the water of condensation itself.

Gelatin Stab.-24 hours, $22^{\circ} \mathrm{C}$. Good filiform growth, consisting of discrete and confluent colonies, extending to the bottom of the tube; smooth raised surface growth 2 or $3 \mathrm{~mm}$. in diameter. Crateriform liquefaction is generally visible after 24 hours in newly isolated strains; liquefaction later becomes stratiform and is complete in 2 to 4 days. With old laboratory strains liquefaction is slower and may not be complete for 3 weeks; sometimes the power to liquefy gelatin is lost altogether. Occasionally very fine tangled branches grow out from the filiform stab. Sometimes the liquefaction is infundibuliform or saccate.

Broth. -24 hours, $37^{\circ} \mathrm{C}$. Moderate growth with a slight to moderate uniform turbidity, and a moderate powdery deposit, disintegrating completely on shaking. No surface growth. The growth increases only slightly on further incubation.

Glucose Agar Shake.-24 hours, $37^{\circ} \mathrm{C}$. Profuse growth of tiny colonies throughout medium, and layer of growth over whole surface. Numerous bubbles of gas throughout medium, sometimes blowing the agar up to the plug.

Horse Blood Agar Plates. -24 hours, $37^{\circ} \mathrm{C}$. Uniform growth over whole surface with indefinite single colonies. The blood is cleared, translucent, and of a slightly brownish colour. $\beta$-hæmolysis on 5 per cent. rabbit blood agar plates.

MacConkey Plates. -24 hours, $37^{\circ} \mathrm{C}$. Good growth of colourless, discrete or partly confluent colonies. The colonies may be smooth, but more often have a slightly roughish surface with an irregularly crenated, radially striated edge.

Loeffer's Serum.-Spreading growth over whole surface. On further incubation the serum is liquefied partly or completely, but this power of liquefaction is confined chiefly to maltose-negative strains. Newly isolated strains are more active liquefiers than old laboratory strains.

Dorset Egg. -24 hours, $37^{\circ} \mathrm{C}$. Spreading growth over whole surface. On further incubation digestion occurs, but does not usually proceed to completion.

Cooked Meat Medium. -5 days, $37^{\circ} \mathrm{C}$. Good growth with some bubbles of gas. No blackening or visible digestion occurs.

Potato. -5 days, $37^{\circ} \mathrm{C}$. Raised, confluent, glistening, greyish-brown growth. The potato itself takes on a café-au-lait colour.

Resistance.-Not specially resistant. Killed by moist heat at $55^{\circ} \mathrm{C}$. in 1 hour.

Metabolism.-Aerobe and facultative anaerobe. Growth under anaerobic conditions is poor; only a very thin, effuse, barely visible growth is formed on agar in 4 days at $37^{\circ} \mathrm{C}$. No digestion of protein media occurs under anaerobic conditions. Optimum temperature for growth $34^{\circ}-37^{\circ} \mathrm{C}$. Good growth occurs at $20^{\circ} \mathrm{C}$. A hæmolysin is formed, acting on rabbit blood. No pigment formed, except the café-au-lait pigment on potato. Growth is improved by the addition of glucose and of nitrates. No soluble toxin. formed.

Biochemical.-All strains produce acid and gas in ghcose, galactose, glycerol, and sucrose. Nearly all strains ferment saliein, and some ferment maltose. Lactose, mannitol, and mannose are never attacked. Old laboratory strains may lose their power of fermenting sucrose. Litmus milk. Alkaline; some strains coagulate the casein 
and then digest it ; others digest it without preliminary coagulation; the litmus is reduced. Indole is produced by the maltose-fermenting, but not by the maltose-negative strains. $\quad \mathrm{H}_{2} \mathrm{~S}++. \quad \mathrm{NH}_{3}++$. Catalase ++ . Methylene blue reduction + . Nitrates reduced to nitrites. M.R.. \pm . V.P. +, if Barritt's method is used. Urea is decomposed with the formation of $\mathrm{NH}_{3}$.

Antigenic Structure.-Incompletely worked out. By direet agglutination the swarming strains can be divided into 3 main groups, but several smaller groups are present; by absorption the main groups can be divided into sub-groups. No apparent relationship between the serological and the biochemical grouping. The $\mathrm{O}$ antigens tend to be type-specific. Among the $X$ strains the $O X 2, O X 19$, and $O X \mathrm{~K}$ strains are distinct.

Pathogenicity.-Produces no specific infection under natural conditions, but is frequently found in eystitis, infantile diarrhooa, and suppurative lesions generally. Is probably responsible for one form of calf dysentery. Virulence to laboratory animals is variable. Highly virulent cultures inoculated intraperitoneally into rabbits, rats, or guinea-pigs cause death in a few hours, presumably from toxæmia. Less virulent cultures cause emaciation with death in a week or more after intraperitoneal inoculation, and abscesses and inflammatory conditions lasting for months after subcutaneous inoculation. In fatal cases the organisms can generally be recovered from the blood and viscera.

Proteus morgani was isolated by Morgan (1906) from the stools of patients with summer diarrhœa. It is motile by $25-30$ peritrichate flagella. Motility is sometimes lost after long cultivation, but it may sometimes be restored by passage through broth at $20^{\circ} \mathrm{C}$. Though not swarming at $37^{\circ} \mathrm{C}$. on ordinary agar, it swarms readily at $20-28^{\circ} \mathrm{C}$. on 1 per cent. agar. Variant types, however, occur which are less actively motile, and which give rise to characteristic streaming colonies. The general cultural characters resemble those of the coliform group. Acid and a small amount of gas are produced in glucose peptone water within 24 hours. Xylose is often fermented with the production of acid only, while occasional strains are said to produce acid and a small amount of gas in sucrose after 10 days. Gelatin is not liquefied, but both indole and $\mathrm{H}_{2} \mathrm{~S}$ are formed abundantly. Litmus milk is turned alkaline. About 30 per cent. of strains give rise to a hæmolysin for sheep red cells. Antigenically, most workers (Lew is 1911-12, Kligler 1919, Thøjtta 1920, Jordan, Crawford, and IcBroom 1935), including ourselves, have noted the extraordinary heterogeneity of members of this species. Rauss (1936), who has made a careful study of this question, finds that the $\mathrm{H}$ antigen tends to be groupspecific and the $\mathrm{O}$ antigen type-specific. Seven $\mathrm{H}$ receptors and $17 \mathrm{O}$ receptors were differentiated in 48 strains. One of the $\mathrm{H}$ antigens in $P$. morgani is similar to one of the $\mathrm{H}$ antigens in $P$. vulgaris. The organism seems to be mainly parasitic and potentially pathogenic, assuming a considerable rôle in some outbreaks of infantile diarrhœa. It has been isolated from paratyphoid-like fevers (Havens and Mayfield 1930). Infections in birds, mammals, and reptiles are not uncommon (Lovell 1929), while in mice it may give rise to spontaneous epidemics of enteritis (Wilson 1927), especially in the late summer and autumn months. Experimentally, it produces a rapidly fatal infection in mice on intraperitoneal inoculation. It does not produce a soluble toxin.

Other types of bacilli were isolated by Morgan $(1906,1907)$, which are sometimes called after him, and which liffer from his No. I bacillus in their motility, their action on milk, or some other characteristic; but the term Morgan's bacillus is generally used to indicate the organism we have just described. 


\section{ZOPFIUS}

\section{DEFINITION.—Zopfius.}

Long rods, occurring in evenly curved chains. Gram-positive. Motile. Spiderweb growth on solid media. Facultative anaerobes. Carbohydrates and gelatin not attacked; hydrogen sulphide not formed.

Type species, Zopfius zopfii (Kurth) Wenner and Rettger; isolated originally from the intestinal tract of hens.

Organisms of this group are differentiated in several ways from those of the Proteus group. Apart from being Gram-positive, they ferment no carbohydrates, they form no $\mathrm{H}_{2} \mathrm{~S}$, they do not liquefy gelatin, and they do not exhibit the phenomenon of swarming.

Morphologically, rods are formed, about $3.5 \mu$ long by $0.8 \mu$ broad, having rounded ends and parallel sides, and occurring in long evenly curved chains; filamentous forms are common. The organisms are motile by peritrichate flagella (Fig. 12, p. 30).

On agar they form small indistinct colonies having, on magnification, a spider-web appearance; sometimes the colonies are thinnest at the centre and are surrounded by arborescent tufts. In gelatin stab arborescent lateral branches, interlacing freely, grow out from the stab. There is a slow, moderate growth in broth, with occasionally a thin fragile pellicle. Optimum temperature for growth is $25^{\circ} \mathrm{C}$.; growth occurs freely between $20^{\circ}$ and $30^{\circ} \mathrm{C}$., but is very poor at $37^{\circ} \mathrm{C}$. No sugars are fermented, and there is only a scanty growth in litmus milk with no visible change in the medium. On potato there is a moderate growth; the medium is darkened. Gelatin, serum, and egg are not digested. No indole or $\mathrm{H}_{2} \mathrm{~S}$ is formed, but there is some production of $\mathrm{NH}_{3}$.

\section{REFERENCES}

Berthelot, A. (1914) Ann. Inst. Pasteur, 28, 839, 913.

Besson, A. and Ehringer, G. (1923) Paris méd., i. 225.

Canto, C. (1911) Ann. Inst. Pasteur, 25, 852.

Castaneda, M. R. (1934) J. exp. Med., 60, $119 ;$ (1935) J. exp. Med., 62, 289.

Cooper, K. F., Davies, J., and Wiseman, J. (1941) J. Path. Bact., 52, 91.

EDWARDS, J. I. (1942) J. Hyg., Camb., 42, 238.

Felix, A. (1922) Z. ImmunForsch., 35, 57.

Ferguson, W. W. and Hook, A. F. (1943) J. Lab. clin. Hed., 28, 1715.

FLOY D, T. M. and DACK, G. M. (1939) J. infect. Dis., 64, 269.

Fry, R. M. (1932) Brit. J. exp. Path., 13, 456.

Gordon, J. and McLeod, J. W. (1928) J. Path. Bact., 31, 185.

HAUSER, G. (1885) "Ueber Fäulnisbakterien." Leipzig.

Havens, L. C. and MaYfield, C. R. (1930) J. prev. Med., 4, 179.

HaYward, N. I. and Niles, A. A. (1943) Lancet, ii, 116.

Hexriksen, S. D. and Closs, K. (1938) Acta path. microbiol. scand., 15, 101.

Jensen, C. O. (1913) "Handbuch der pathogenen Mikroorganismen." Kolle and Wassermann, 2te Autl., 6, 121.

Jordan, E. O., Crawford, R. R., and McBroom, J. (1935) J. Bact., 29, 130.

Kligler, I. J. (1919) J. exp. Med., 29, 531.

KRÄMER, E. and КОсH, F. E. (1931) Zbl. Bakt., 120, 452.

Kulp, W. L. and Borden, D. G. (1942) J. Bact., 44, 673.

KURтн, H. (1883) Bot. Ztg., 41, 369, 393, 409, 425.

Larson, W. P. and Bell, E. T. (1913) J. infect. Dis., 13, 510.

LewIs, G. J. (1911-12) 41 st Ann. Rep. loc. Govt Bd, M.O's Suppl. 265.

Lichstein, H. C. and SNyder, M. L. (1941) .J. Bact.. 42, 653.

Lode, A. and Howard, A. (1932) Zbl. Bakt., 124, 538.

Lominski, I. and Lendrun, A. U. (1942) J. Path. Buct., 54, 421.

Lovell, R. (1929) J. Path. Bact., 32, 79.

Magath, T. B. (1928) J. infect. Itis., 43, 181.

Mrisel, H. and Mikulaszek, E. (1933) C. R. Soc. Biol., 114, 364.

Mello, J. de T. (1938) Ann. Fac. Med. S. Paulo, 14, 247.

Mendel, J. (1911) Zbl. Bakt., IIte Abt., 29, 290. 
Moltke. O. (1927) "Contributions to the characterization and systematic classification of Bac. proteus vulgaris (Hauser)." Levin and Munksgaard, Copenhagen; (I929) Zbl. Balit., 111, 399.

MoraAn, H. DE R. (1906) Brit. med. J., i. 908 ; (1907) Ibid., ii. 16.

Norton, J. F., Verider, E., and Ridgway, C. (1928) J. infect. Dis., 43, 458.

Pläнs, O. (1937) Zbl. Baht., IIte Abt., 96, 19ij.

RAUSS, K. F. (1936) J. Path. Bact., 42, 183.

Rettoer, L. F. and Newelt, C. R. (1912-13) J. biol. Chem., 13, 341.

RUsS-MÜNZER, A. (1935) Zbl. Bakt., 133, 214.

SNyder, M. L. and Lichstein, H. C. (1940) J. infect. Dis., 6\%, 113.

TAYLOR, J. F. (1928) J. Path. Bact., 31, 897.

Тнјөтта, T. (1920) J. Bact., 5, 67.

Wenser, J. J. and Rettoer, L. F. (1919) J. Bact., 4, 331.

White, P. B. (1933) Brit. J. exp. Path., 14, 145.

Wichels, P. and BARNer, W. (1925) Med. Klin., 21, 1880.

Wrison, G. S. (1927) J. Hyg., Camb., 26, 170.

Winslow, C.-E. A., Broadhurst, J., Buchanan, R. E., Kromwiede, C., Rogers, L. A., and Sмгтн, G. H. (1920) J. Bact., 5, 191.

Wolf, C. G. L. (1918-19) J. Path. Bact., 22, 289.

Wrss, O. (1898) Z. Hyg. InfektKr., 2\%, 143.

YАсов, M. (1932) Indian J. med. Res., 19, 787. 


\section{OHAPTER 28}

\section{BACTERIUM}

\section{Definition.-Bacterium.}

Gram-negative, non-sporing rods: often motile, with peritrichate flagella. Some species capsulated. Easily cultivable on ordinary laboratory media. Aerobic and facultatively anaerobic. All species ferment dextrose with the formation of acid, or acid and gas. Many species are active fermenters of a wide range of carbohydrates and allied substrates. Typically intestinal parasites of man and animals, though some species may occur in other parts of the body, on plants, or in the soil. Many species are pathogenic.

Type species. Bacterium coli.

\section{Classification and Nomenclature.}

In the past the generic term Bacterium has been used to comprise a broad group of Gram-negative, non-sporing rods occurring in the intestinal canal of man and animals and on plants, and living either a saprophytic, commensal, or pathogenic existence. It was early realized that the members which were pathogenic to man and animals differed from most of the non-pathogenic forms in failing to ferment lactose. Of recent years the non-lactose-fermenting group has been subdivided, mainly on the basis of biochemical and antigenic characters, into a number of sub-groups, which have been given the generic names of Eberthella, Salmonella and Shigella. Of these, Eberthella, which comprises the typhoid bacillus, is so closely related to the Salmonella group, that we can see no useful purpose in maintaining its separate identity. We shall therefore deal in the following chapters with the Salmonella group, which comprises the typhoid, paratyphoid, and food-poisoning organisms, and the Shigella group containing the dysentery bacilli. A residue of strains fermenting lactose late, weakly, or not at all, and usually regarded as non-pathogenic, is classified in the relatively indeterminate group of paracolon bacilli and will be considered in the present chapter.

The classification and nomenclature of the lactose-fermenting organisms are subject to wide variation in opinion. On the one hand, there is a school, led by American workers (see Bergey et al. 1939), who would do away completely with the genus Bacterium, and substitute for it a number of genera-Escherichia, Aerobacter, and Klebsiella to include organisms of animal origin, and a single genus - Erwinia - to comprise the plant pathogens. On the other hand, there is a school, represented largely by workers in Great Britain but not without considerable support in the United States, which adopts for the present a conservative attitude and prefers to include all these organisms in a single genus-Bacterium. With this latter school we would identify ourselves.

In the definition of the genera mentioned above, reliance is placed for differential purposes largely on habitat, biochemical characters, and pathogenicity. 
Of these, neither habitat nor pathogenicity, though useful adjunets, can be regarded as satisfactory basal criteria for classification. The separation therefore of the group Erwinia from other coliform organisms merely on the hasis of plant pathogenicity is not sound taxonomical practice. If this property was closely associated with some more valid criterion, there would be something to be said for recognizing the Erwinia group; but though Elrod (1942) maintains that the secretion of an enzyme, protopectinase, which is responsible in plants for the production of soft rot, is characteristic of the Erwinia group, it is clear that this is merely another way of saying that these organisms are pathogenic to plants. Elrod's own observations on the biochemical characters of the plant pathogens support those of Dowson (1939) in showing that these organisms cannot be rlistinguished satisfactorily from coliform organisms of animal origin. Until some more stable differential eharacter is found, we prefer, therefore, to regard the coliform organisms of plant and animal origin as belonging to a single group.

The division of the organisms from animal sources into Escherichin, Aerobacter, and Klebsiella is, in our opinion, hardly more fortunate. The first two groups are morphologically and culturally indistinguishable; and their differences in biochemical behaviour, though worthy of specific recognition, are not of sufficient constancy or importance to serve as criteria for generic differentiation. Klebsiella, which comprises capsulated organisms found in the respiratory tract, is perhaps even less justified. Practically all coliform bacilli seem to be capable of forming capsules under favourable conditions; and in our experience there is no way of distinguishing a non-motile capsulated Granl-negative bacillus in the respiratory tract from a similar organism in the intestinal tract. Neither habitat nor capsule formation is sufficiently constant or distinctive to justify the recognition of the genus Klebsiella.

We would plead, therefore, for the maintenance of the Bucterium group as a convenient repository for a wide range of Gram-negative non-sporing bacilli that cannot be classed at present in either the Salmonella or Shigella groujs. As knowledge increases, it is probable that further genera will be split off. But international agreement is essential if the new genera are to receive universal recognition; without it they will merely add to the existing confusion.

The strict taxonomist is worried by the fact that the type species, Bacterium triloculare Ehrenberg, 1828, is no longer recognizable, and that the genus Bacterium is therefore invalid. On the other hand, the organism that has for years been regarded by bacteriologists as typical of this genus is the common coliform organism of the intestine, Bacterium coli. We see no reason why the genus should not be re-defined, with this organism as the type species, so as to include the numerous Gram-negative non-sporing bacilli that cannot be assigned to other recognized genera. Whether this genus is admitted as valid by the taxonomists, or is accepted temporarily for purposes of convenience (see Breed and Conn 1936), is a matter more of academic than practical importance. Our tentative definition of this genus follows closely that given by the American Committee (Winslow et al. 1917, 1920).

Morphology.-Neither the shape, size, structure or arrangement of the bacterial cells, nor the appearances presented by cultures on the ordinary solid or liquid media, afford any adequate criteria for the differentiation of speeies within this group.

The modal form of the individual cell is that of a bacillus, 2 to $3 \mu$ in length and $0.6 \mu$ in breadth, with parallel sides and rounded ends (see Fig. 139). By the usual methods of examination the cell appears to be almost devoid of internal 
structure. It stains evenly ; it forms no spores; and it shows no granules. It is Gram-negative, and non-acid-fast. This modal form is, however, widely departed from as regards the shape and size of the individual cells. Some strains are almost coccal in form, others st. ow long, sometimes filamentous bacilli. There is a tendency for the cocco-bacillary, or the elongated, form to predominate in any single strain, but some cultures show a wide diversity in this respect. Cell leugth is, indeed, a highly variable character in this group; and it is possible, as Barber (1907) has shown, to obtain long-celled strains of Bact. coli by simple selection of individual cells for successive subculture.

Rudimentary branching, with the formation of $\mathrm{Y}$ forms, followed by division at each of the three points of the $Y$, has been described by Hort (1920), and by Gardner (1925), as an occasional happening in some species of Bacterium.

Many species are motile; other species are non-motile. By the usual methods of staining, the flagella of motile species appear to be numerous and to have a

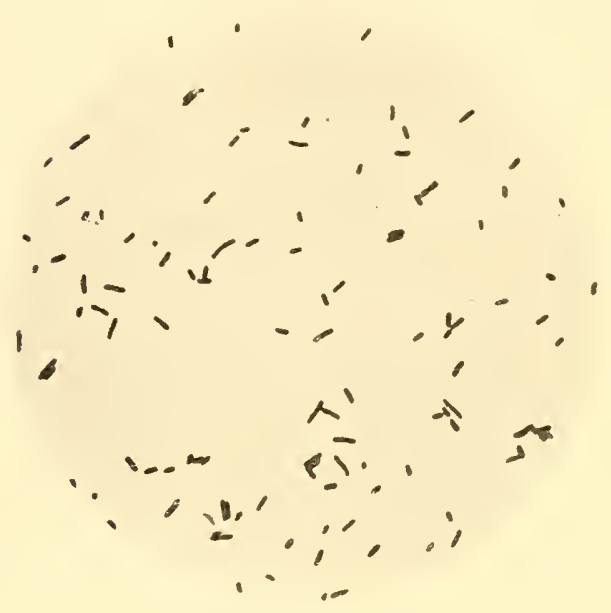

FIa, 139.-Bact. coli.

From 24-hours' culture on agar $(\times 1000)$. peritrichous arrangement. How far this appearance corresponds to reality is doubtful. Pijper (1938), for example, using solar dark-ground illumination, finds that the typhoid bacillus - an organism morphologically indistinguishable from Bact. coli - possesses only two flagella, attached one on each side of the body near the middle. They are broadly coiled spiral structures, which, when in action, become entwined to form a long tail by which the organism propels itself through the surrounding medium. There is evidence that each of these flagella is made up of a number of extremely fine threads; but, according to Pijper, there are only two flagella to each organism, with single opposite points of attachment. The peritrichous appearance disclosed by flagella stains is regarded as an artefact. How far Pijper's conclusions will be confirmed, it is impossible to say. Whether there are two flagella or several flagella is not likely to give rise to confusion, so long as it is recognized that they are attached to the sides of the rod and not, as in Pseudomonas, to the end (see Fig. 13, p. 31).

Before the Salmonella and the Shigella groups were separated from the Bacterium group, motility was of some importance in distinguishing, for instance, the typhoid and paratyphoid bacilli, which are nearly always motile, from the dysentery bacilli, which are consistently non-motile. In the present Bacterium group, however, motility is of far less differential value. It is true that some members, like Bact. coli, are usually motile, and that other members, like Bact. aerogenes, are usually non-motile; but the property of motility or non-motility is a characteristic of the individual strain, not of the species as a whole. Moreover, motile organisms may give rise to non-motile variants; though the reverse phenomenon, of normally non-motile species giving rise to motile variants, does not appear to occur. Motility 
is far too irregular and variable a character in the Bacterium group to serve as a criterion for specific separation.

Some species, such as Bact. friedländeri, are normally capsulated, and this character, when it occurs, has some differential value; but those bacilli which are normally capsulated, and form mucoid colonies when first isolated from the tissues, frequently lose the property of capsule formation during subculture on artificial media, while other normally non-capsulated species may acquire a capsule under particular conditions. It may be noted that many coliform strains isolated from milk are normally capsulated. Nost of these strains belong to the intermediate-aerogenes-cloacæ group, but some undoubted strains of Bact. coli form a capsule, and give rise to a mucoid growth on solid media. It may further be noted that the presence of a capsule is not incompatible with active motility.

Conditions of Growth.-The members of this group grow readily on the ordinary nutrient media of the laboratory, without the addition of any accessory substances.

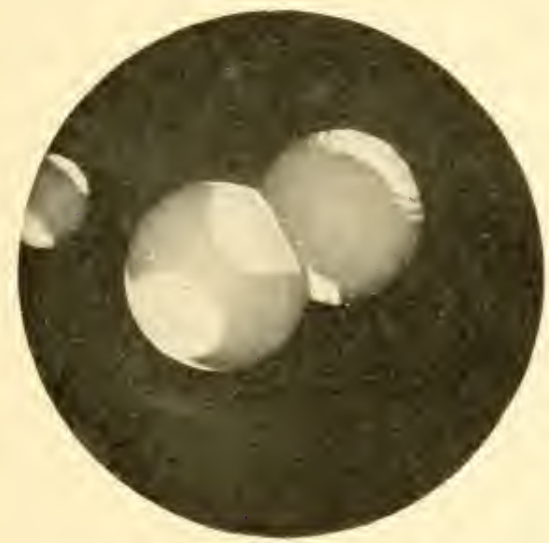

Fig. 140.-Bact. coli.

Colonies on agar plate after 24 hours $(\times 8)$.

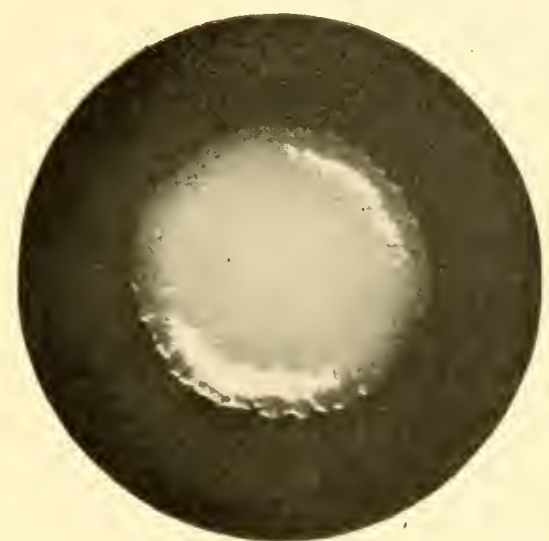

FIG. 141.-Bact. coli.

Larger and flatter type of colony on agar plate after 24 hours $(\times 8)$.

They are aerobic, and facultatively anaerobic, though the growth is usually far less copious under the latter conditions. The optimum temperature is, for most species, in the neighbourhood of $37^{\circ} \mathrm{C}$., and the range over which growth occurs is fairly wide, extending for most species from about $42^{\circ} \mathrm{C}$. as an upper limit to $18^{\circ} \mathrm{C}$. or lower.

There are, however, certain differences in behaviour that are of significance from the systematic point of view. Thus, Bact. aerogenes grows very poorly, or not at all, at a temperature of $44^{\circ} \mathrm{C}$, and differs in this respect from the closely related Bact. coli. Noreover, many strains of Bact. aerogenes have their optimum growth temperatures nearer $30^{\circ} \mathrm{C}$. than $37^{\circ} \mathrm{C}$.

It happens that the nutritional requirements of several species within this genus, and the enzymic mechanisms that they employ in their attack on various substrates, have been studied in considerable detail by the methods that have recently been introduced in the investigation of the biochemical activities of bacteria. Many of the results obtained in these studies have been described and discussed in Chapter 3. In the present chapter we may therefore confine our attention to such 
reactions as are of value in identifying the different species within the genus, or in distinguishing letween them.

Type of Growth.- The type of growth given by the varions species within this genus is very similar. When normal smooth strains are grown in broth a uniform turbidity develops, increasing rapidly during the first 12 to 18 hours of growth, and then more slowly up to 48 to 72 hours. Pellicle formation is rare and when present is very slight. A slight deposit forms as growth increases, and this is easily dispersed on shaking the tube.

On agar, the colonies are relatively large, with an average diameter of $2-3 \mathrm{~mm}$., but vary considerably in size. They may be circular, raised and low convex, with an entire edge and smooth surface; they may be flatter with a more irregular surface, and a more effuse and irregular edge, or they may assume the typical vine-leaf form which is commonly described as characteristic of Salm. typhi. Even with freshly isolated strains the range of variation is wide; and when old laboratory strains are under examination the most varied colonial forms may be seen. Apart from the possible appearance of rough variants, a single strain may show several different types of colony, if successive subcultures in broth are interspersed with platings and subculture of individual colonies.

As an exception to this general rule we may note that members of the Friedländer group, when freshly isolated, give rise to typically mucoid colonies. The differential value of this characteristic is diminished by the fact that certain other members, particularly those belonging to the intermediate-aerogenes-cloacæ group, may similarly give rise to this type of growth. It is very common in strains isolated from milk, and is often lost on subculture in the laboratory.

There are a few other growth characters which possess some differential valne. Thus, we may note the classical difference between the growth of Salm. typhi and Bact. coli on potato, the former being colourless and barely visible, the latter displaying a characteristic yellowish tint (Fremlin 1893). Again there is the socalled "nail-head" growth of Friedländer's bacillus when grown in stab culture in gelatin, due to the raised, circular, convex growth which sometimes develops on the surface above the inoculation-track; but this phenomenon is inconstant. Chromogenesis is not a common property of the group, but appears to be met with occasionally in individnal nembers, such as the organism described by Parr (1937) under the name of Bact. aurescens. It is a moot point, however, whether such organisms should not be classified in the genus Chromobacterium.

Resistance to Heat, and to various chemical Substances.--Most members of this group are killed by exposure to a temperature of $55^{\circ} \mathrm{C}$. for about 1 hour, or of $60^{\circ} \mathrm{C}$. for $15-20$ mimutes. So far as they have at present been studied, the various species within the genus do not differ from one another in any significant way. On the whole, the typical freal coli strains tend to have a slightly higher resistance to heat than the closely related members of the intermediate-aerogenes-cloacæ group. A small proportion of them are not completely destroyed by exposure to $60^{\circ} \mathrm{C}$. for 30 minutes in broth, or to pasteurization at $62 \cdot 8^{\circ} \mathrm{C}$. for the same time in milk (Henneberg and Wendt 1935, Wilson et al. 1935). Towards chlorine in water the aerogenes type tends to be slightly more resistant than the coli or intermediate types (Bardsley 1938b). In raw water stored under atmospheric conditions coliform bacilli may remain alive for weeks or months. On the whole Bact. aerogenes tends to survive rather longer than Bact. coli, but the results are influenced, among other things, by the temperature (see Platt 1935, Raghavachari and Iyer 19396). 
In freces stored at $0^{\circ} \mathrm{C}$. coliform organisms may be demonstrated for a year or more, the coli type being often gradually supplanted by the intermediate and aerogenes types (Parr 1938).

There are certain chemical substances which exert a definitely selective bactericidal or inhibitory action.

The typhoid bacillus is less resistant to the lethal action of mineral acids than is the colon bacillus. Winslow and Lochridge (1906) showed that the bactericidal effect was due to the action of the dissociated hydrogen ions; and found that the concentration required to bring about a 99 per cent. reduction in the viable organisms in a bacterial suspension was 2.94 per million in the case of Salm. typhi, and 7.49 per million in the case of Bact. coli.

Malachite green, in suitable concentration, kills Bact. coli or inhibits its growth without exerting the same effect on Salm. typhi (Loeffler 1903, I906, Lentz and Tietz 1903, 1905). There are other green dyes that have a similar selective action; and more recent studies (see Browning, Gilmour and Mlackie 1913, Krumwiede and Pratt 1914) have shown that brilliant green gives the best differential results. To this dye the bacilli of the para. typhoid group are most resistant, the typhoid bacillus is somewhat less resistant, while the dysentery bacilli, and still more the members of the Bact. coli group, are very susceptible.

Caffeine (Roth 1903, Hoffman and Ficker 1904) and lithium chloride (Gray 1931, Havens and Mayfield 1933) are other substances that inhibit the growth of Bact. coli in eoncentrations that have no effect on the typhoid bacillus; while cholesterol (see Manfredi 1917) appears to inhibit the growth of typhoid or paratyphoid bacilli in concentrations that permit the growth of Bact. coli.

Sodium desoxycholate in the presence of sodium eitrate inhibits the growth of coliform bacilli, while having little effect on organisms of the Salmonella and Shigella groups (Leifson 1935, Hynes 1942). Potassium tellurite, in suitable concentration, in the presence of iron alum, is said to inhibit coliform and salmonellae, but not the Flexner dysentery bacilli (Wilson and Blair 1941). Selenimm salts were found by Haendel (see Guth 1916, Leifson 1936) to inhibit coliform more than typhoid bacilli; and tetrathionate was found by Muller (1923) (see also Knox, Gell and Pollock 1943) to have much the same effect.

Differences of this kind have not, however, been employed for the purposes of identification or classification. They have, on the other hand, been extensively exploited in devising selective, or "enrichment," media for the isolation of the pathogenie species from freces or water. They are considered from this point of view in Chapters 69 and 92 .

Biochemical Activities.-From the first isolation of Bact. coli by Escherich, fermentation tests were found to provide the readiest method of distinguishing one species of Bacterium from another. It was soon found, for instance, that Bact. coli actively fermented lactose, while Salm. typhi did not (Chantemesse and Widal 1887, Smith 1890); and the production of acid and gas from glucose by Bact. coli, but of acid alone by Salm. typhi, was pointed ont by Chantemesse and Widal in 1891. The addition of a suitable indicator to the test media, to register acidity (Wurtz 1892), and the introduction of the simple fermentation tube as a test for gas production (Smith 1890, 1893, Durham 1898) greatly increased the facility with which a large series of comparative qualitative tests could be carried out. To dextrose and lactose other test substances have, from time to time, been added, such as the hexoses, fructose, lævulose and galactose; the disaccharides, maltose and saccharose; the trisaccharide, raffinose; polysaccharides, such as dextrin, starch and inulin ; the pentoses, arabinose and xylose ; the methyl-pentose, rhamnose; the hexahydric alcohols, dulcitol and mannitol; the glucoside, salicin; and the cyclohexanehexol, inositol. The reaction in litmus milk, the presence or 
absence of indole production in peptone water, and the production of hydrogen sulphide have serverl as additional differential criteria ; and other tests, such as the final $\mathrm{pH}$ attained in a dextrose-containing medium, the nature and amount of the gases evolved, or the production of some particular fermentation product, have been employed as aids to differentiation within particular sub-groups.

It soon became clear that the presence or absence of the power to ferment lactose, originally noted as differentiating Bact. coli from Salm. typhi, corresponded to a fundamental line of cleavage within this group. The lactose fermenters were, for the most part, found to be normal inhabitants of the intestinal tract of man or the higher animals or to exist on various plants or in the soil. They were active fermenters of many carbohydrates, including polysaccharides; they tended to clot milk, as well as acidify it; they frequently formed indole; and they tended to reduce various dyes (Dunbar 1892, Rothberger 1898). The non-lactose fermenters tended, as a class, to comprise the pathogenic species, producing intestinal infections in man and animals; and the range of their fermentative activity tended to be less extensive than that of the lactose fermenters, though most species attack a considerable number of substrates.

This early division of the genus into two broad sub-groups on the basis of the lactose fermentation has stood the test of time, although there are a few species or types for which some intermediate position must be found. As already noted, the genera Salmonella and Shigella have been created for the more important of the non-lactose fermenters. These organisms will be considered in separate chapters. In the remainder of the present chapter we shall devote ourselves to a consideration of the lactose-fermenting organisms, together with the indeterminate group of paracolon bacilli, which appear to be more nearly related to the coliform bacilli than to the Salmonella or Shigella organisms. Though no clear line of demarcation can be laid down between the coliform bacilli and the Friedländer group of bacilli, it will be convenient to describe them separately.

\section{The Coli-Aerogenes Group}

Biochemical Differentiation.-In his original description of Bact. coli, Escherich noted the occurrence of two types, one of which, Bact. coli, formed relatively long rods, was motile and clotted milk slowly, while the other, Bact. lactis aerogenes, formed shorter, plumper rods, was non-motile, and clotted milk more actively. Kruse (1894) emphasized the heterogeneity of the group covered by the term " B. coli " as usually employed, pointing out that it included a variety of related species, widely distributed as intestinal parasites and in water and soil. The use of a relatively small series of fermentation tests, including especially dextrose, lactose, sucrose, starch,inulin, action on litmus milk, and indole formation, resulted in the recognition of certain primary divisions within this group (Refik 1896, Grimbert and Legros 1900, Durham 1901, Jordan 1903). One of the groups so defined fermented polysaccharides, such as starch and inulin, and usually failed to form indole ; this corresponded with the Bact. lactis aerogenes type of Escherich and became established as a separate species, the lactis being usually omitted from the name. The second and third groups differed from Bact. aerogenes in failing to ferment starch and inulin, and in forming indole in peptone water. They differed from each other in regard to their action on saccharose. One, corresponding to the existing strains of Escherich's Bact. coli commune, failed to ferment this sugar; the other fermented it, and Durham (1901), who found it to occur more frequently than the saccharose-negative 
type, named it Bact. coli communius. The application of a more extended series of tests resulted in further subdivision of this group, and elaborate classifications were suggested by various observers on the basis of the results obtained (Bergey and Deehan 1908, MacConkey 1905, 1909, Jackson 1911). It may be noted that one important correlation between biochemical activity and natural habitat had already been detected. The Bact. aerogenes type was found to be a relatively infrequent inhabitant of the intestine, but was frequently isolated from certain grasses and from the soil, while Bact. coli commune and Bact. coli communius were noted to be typically intestinal parasites (Winslow and Walker 1907). This correlation was of practical as well as of theoretical importance. The presence or absence of "B. coli" in water supplies, and the relative number of this organism if present, soon came to be recognized as a very valuable iudication of the presence and degree of fæcal pollution (see Chapter 92), and it became very desirable, apart from any question of systematic classification, to differentiate between those types which were of intestinal origin, and those which might occur in unpolluted waters. The investigations of those who have been primarily concerned with the practical aspects of the bacteriological analysis of water supplies have added materially to our knowledge of this group.

Apart from the merely positive or negative results, as regards acid or gas production in the various sugars, certain observations made in the earlier days had indicated a difference in kind between the fermentation of one and the same carbohydrate by different strains of bacilli of the colon type. Thus Smith (1895), using the method of the fermentation tube, noted that gas was produced more rapidly and in greater amount by Bact. aerogenes than by Bact. coli; and a rough estimation of the ratio of $\mathrm{CO}_{2}$ to $\mathrm{H}_{2}$ in the gas evolved showed that this was higher with the former organism than with the latter. He noted also that the degree of final acidity was lower with Bact. aerogenes than with Bact. coli. In both respects Bact. cloace, a coliform organism isolated from sewage by Jordan (1890) and differentiated from all other types of coliform bacilli by its power of liquefying gelatin, corresponded with Bact. aerogenes. Russell and Bassett (1899) confirmed the differential value of a high or low $\mathrm{CO}_{2}: \mathrm{H}_{2}$ ratio, and noted that the high-ratio strains appeared to be normal soil forms, rather than intestinal parasites. This question was placed on an entirely new footing by the careful quantitative studies of Harden and his colleagues (Harden 1901, 1905, Harden and Walpole 1906), who showed that strains of coliform bacilli were divisible into two well-clefined classes. In one, typified by Bact. coli, the $\mathrm{CO}_{2}: \mathrm{H}_{2}$ ratio of the gas evolved gave a value closely approximating unity. In the other, typified by Bact. aerogenes, the $\mathrm{CO}_{2}: \mathrm{H}_{2}$ ratio gave a value of $2: 1$, or thereabouts. These observations have been amply confirmed by later observers.

Voges and Proskauer (1898) had described a colour reaction given by certain bacteria, but not by Bact. coli. It is obtained by adding a few drops of a strong solution of potassium hydrate to a culture grown in a dextrose medium. In a positive reaction a red, fluorescent coloration appears, which may develop relatively slowly. The nature of this reaction was elncidated by Harden and his colleagues (Harden 1906, Harden and Norris 1911), who showed that it depends on the production of acetylmethylcarbinol $\left(\mathrm{CH}_{3} \cdot \mathrm{CHOH} \cdot \mathrm{CO} \cdot \mathrm{CH}_{3}\right)$; this, in the presence of alkali and of atmospheric oxygen, is oxidized to diacetyl $\left(\mathrm{CH}_{3} \cdot \mathrm{CO} \cdot \mathrm{CO} \cdot \mathrm{CH}_{3}\right)$. which reacts with the peptone of the broth to give the red colour.

This reaction had been applied to the examination of the colon group by some 
of the observers referred to above, and it had been noted that Bact. aerogenes, as opposed to Bact. coli, gave a positive reaction (Durham 1901). MacConkey (1909) observed the great preponderance of Voges-Proskauer negative types among strains isolated from the frees; positive reactions were given by 11 of 178 strains isolated from human fæces, 8 of 67 strains from the fæces of the horse, and none of 87 strains from frees of the calf, goat, pig or goose.

The fundamental importance of the Voges-Proskauer reaction, and of the $\mathrm{CO}_{2}: \mathrm{H}_{2}$ ratio, as compared with the presence or absence of fermentation in particular carbohydrates other than lactose, was not however realized by the earlier investigators, so that the reaction was simply assigned a place among some selected series of tests, and V.P. positive and V.P. negative strains were often allocated to the same sub-group; though it was noted by Howe (1904) during the examination of strains of lactose-fermenting bacili derived from water, that there was almost perfect correlation between a positive V.P. reaction and the ability to produce large amounts of gas from dextrose.

Petruschky $(1889,1890)$ made the first attempt to measure, by titration, the degree of acidity produced by various members of the coli-typhoid group; while Smith (1895), as noted above, called attention to the low acid production of Bact. aerogenes as compared with Bact. coli. A great advance in the investigation of this aspect of bacterial metabolism was marked by the introduction of indicators, which rendered possible the ready determination of the hydrogen-ion concentration attained during the bacterial fermentation of any test substance. Clark aud Lubs (1915), on this basis, devised the methyl-red test for the differentiation of members of the coli-typhoid group. The addition of this indicator to five-day cultures in dextrose phosphate peptone water distinguishes between those strains which produce and maintain a high concentration of hydrogen-ions, and those which produce an initial lower concentration of hydrogen-ions and then cause reversion towards neutrality by the further decomposition of the organic acids to carbonates, and perhaps by the formation of ammonium compounds from proteins. The former type, such as Bact. coli, give a red coloration and are referred to as methyl-red positive; the latter, such as Bact. aerogenes, give a yellowish colour and are referred to as methyl-red negative. It soon became clear that there was a very high negative correlation between the methyl-red test and the VogesProskauer reaction (Levine $1916 a, b$ ), and a series of intensive studies soon placed on a firm foundation the conclusion, already propounded as a tentative hypothesis, that the lactose-fermenting coliform bacilli could be divided into two primary divisions on the basis of the $\mathrm{CO}_{2}: \mathrm{H}_{2}$ ratio, the Voges-Proskauer reaction, and the methyl-red test. The first of these, containing strains giving a $\mathrm{CO}_{2}: \mathrm{H}_{2}$ ratio of about $2: 1$, V.P. positive and M.R. negative, comprised the great majority of the strains isolated from plants, grain, and unpolluted soil or water. Such strains were relatively infrequent in material obtained from the intestines of man or animals. This group could be further subdivided, on the basis of gelatin liquefaction, into the non-liquefying form Bact. aerogenes, and the much less common liquefying form Bact. cloacre. The second group, containing strains giving a $\mathrm{CO}_{2}: \mathrm{H}_{2}$ ratio of approximately 1 : 1, V.P. negative and M.R. positive, contained the great majority of those strains isolated from the intestines of man or animals, as exemplified by Bact. coli commune or Bact. coli communius. This group was found to be further divisible, on the basis of the ordinary fermentation tests, along lines which will be considered later (Keyes 1909, Rogers et al. 1914, 1915, 1918, Johnson 1916, 
Hulton 1916, Levine 1916c, $d, 1917$, Burton and Rettger 1917, Chen and Rettger 1920, Winslow et al. 1919, Bardsley 1926, 1934, Pawan 1931).

Besides the division rendered possible by the tests we have just outlined into a coli group on the one hand and an aerogenes-cloace group on the other, further work revealed the occurrence of a third group of strains possessing properties intermediate between those of the two main groups. This group is, as yet, not completely defined, and is therefore most conveniently referred to as the "intermediate "group. Brown (1921) drew attention to the usefulness of a medium containing citrate for the differentiation of Bact. coli from Bact. aerogenes. Koser (1923, 1924, 1926a, $b$ ) devised a synthetic medium in which citrate was provided as the sole source of carbon. He found it possible to differentiate coliform bacilli into a M.R. +, V.P. - , citrate - coli type, a M.R. - , V.P. +, citrate + aerogenes type, and a M.R. +, V.P. - citrate + intermediate type. Examination of 104 soil strains from fields subjected to only chance pollution showed that $23 \cdot 1$ per cent. were of the coli, $67 \cdot 3$ per cent. of the aerogenes, and $7 \cdot 7$ per cent. of the intermediate type. Further work in numerous countries soon revealed the value of this test in differentiating the intermediate from the coli group (see Bardsley 1926, 1934, Pawan 1931).

As pointed out by Vaughn. Mitehell and Levine (1939), and Levine (1941), it is advisable to earry out the methyl-red test on a eulture incubated for 5 days at $30^{\circ} \mathrm{C}$., and the Voges-Proskaner test on a eulture incubated for not more than 2 days at $30^{\circ} \mathrm{C}$. At $37^{\circ} \mathrm{C}$, enltures of some strains of the aerogenes-cloaca group may fail to revert to alkaline ; and acetylmethylearbinol is either not formed or is destroyed (see Tittsler 1938). Some of the diserepaneies in the literature are doubtless aseribable to carrying out these tests under unfavourable conclitions. Speeial precantions have also to be taken with the citrate test. It may be noted that, aecording to a number of authors (Kiline 1935, Stuart, Griffin and Baker 1938, Parr 1939, Griffin and Stuart 1940), some strains of Bact. coli may acquire the power of utilizing eitrate.

In Table 40 we have summarized the results recorded by various observers (see Bardsley) with regard to the percentage of strains isolated from different sources, which give the particular reactions to which we have referred. We have included the indole reaction, because recent work suggests that it is particularly significant in relation to habitat. "It will be noted that figures are available for all tests only in the case of the strains derived from animal fæces; but it may safely be assumed that the strains with a high gas ratio would have given positive V.P. and negative M.R. reactions, and vice versa.

TABLE 40

Showing the Percentage of Lactose-fermenting, Coliforu Bacilli, from variods Sourees, wimch give the Reactions indicated (Various Authors).

\begin{tabular}{|c|c|c|c|c|c|c|}
\hline \multirow{3}{*}{ Source. } & \multicolumn{6}{|c|}{ Percentage. } \\
\hline & \multicolumn{2}{|c|}{$\mathrm{CO}_{2}: \mathrm{H}_{2}$} & \multirow{2}{*}{ M.R. 十。 } & \multirow{2}{*}{ V.P. +. } & \multirow{2}{*}{ Indole + . } & \multirow{2}{*}{ Citrate + . } \\
\hline & $2: 1$ & $1: 1$ & & & & \\
\hline $\begin{array}{l}\text { Frees (human and animal) } \\
\text { Sewage (and similar material) } \\
\text { Soil. } \\
\text { Grasses and grains } \cdot \cdot \cdot \cdot \cdot\end{array}$ & $\begin{array}{l}14 \cdot 37 \% \\
-\overline{-} \\
95 \cdot 18 \%\end{array}$ & $\begin{array}{c}85 \cdot 62 \% \\
\overline{4} \\
4.82 \%\end{array}$ & $\begin{array}{l}90 \cdot 40 \% \\
82 \cdot 74 \% \\
14 \cdot 70 \% \\
-\end{array}$ & $\begin{array}{l}7 \cdot 87 \% \\
17 \cdot 26 \% \\
80 \cdot 24 \% \\
-\end{array}$ & $\begin{array}{c}93 \cdot 03^{\circ} \% \\
33 \cdot 40_{\%}^{\circ} \\
-\end{array}$ & $\frac{6 \cdot 71 \%}{89 \cdot 6 \%}$ \\
\hline
\end{tabular}


Numerous other tests have been used for the differentiation of members of this group.

The fermentation of cellobiose, a glucoside derived from cellulose (see Jones 1924, Jones and Wise 1926), has been recommended for distinguishing Bact. aerogenes and intermediate strains, which ferment this substance, from Bact. coli, which fails to do so (see Koser 1926c, Tittsler and Sandholzer 1936). Too much rehance should not be placed upon the result of this test in the identification of individual strains. Batty-Smith (1942a), for example, who examined 600 strains of coliform bacilli, found that about 10 per cent. of Bact. coli strains fermented cellobiose, and that about one-third of the intermediate and aerogenes strains were without action upon it.

The production of $\mathrm{H}_{2} \mathrm{~S}$ in a proteose peptone ferric citrate agar medium was found by Vaughn and Levine (1934) to be characteristic of intermediate strains. Further observations by the same authors $(1936 a, b)$ showed that, if cysteine was incorporated in the medium, a high proportion of all coliform strains produced $\mathrm{H}_{2} \mathrm{~S}$, and that even without cysteine, the result was greatly influenced by the concentration of agar. If this test is to be of differential value, strict attention must be paid to the exact composition of the medium.

Leifson (1933) observed an almost perfect correlation between the V.P. reaction and the fermentation of sodium malonate.

The fermentation of polysaccharides, such as starch, by the aerogenes group was pointed out by Durham (1901) and confirmed by Levine (1918). It has been made use of by W. J. Wilson (1933) in the preparation of a medium for distinguishing Bact. aerogenes from Bact. coli. On the other hand, a medium containing sodium sulphite and rosolic acid in eertain proportions, and another containing hexamine, are said to favour Bact. coli at the expense of Bact. aerogenes (Wilson, W. J. 1933).

For the differentiation of Bact. aerogenes from Bact. cloacce gelatin liquefaction and the production of acid and gas from glycerol and starch are of chief value (Levine 1918). Bact. aerogenes is gelatin -, glycerol +, starch +, and Bact. cloacee gelatin +, glycerol -, starch -. Intermediate strains are said to be differentiated from strains of coli type, not only by their production of $\mathrm{H}_{2} \mathrm{~S}$, but also by their ability to form trimethyleneglycol in the anaerobic fermentation of glycerol (see Werkman and Gillen 1932).

Besides the effect on carbohydrates, nitrogen utilization has been explored in an attempt to discover reliable tests. Koser (1918), Chen and Rettger (1920), and numerous sub. sequent workers (see Bardsley 1926, 1934) found that aerogenes strains were able to utilize uric acid as their sole source of nitrogen, whereas strains of the coli and intermediate types were not. More recently Mitchell and Levine (1938) have examined other substances, such as yeast nucleic acid, allantoin, hydantoin, uracil, and urea. They find that aerogenes strains can make use of all these substances as their sole source of nitrogen, but that intermediate strains can use only urea, and coli strains only uracil.

Considerable help is afforded by a test introduced as long ago as 1904 by Eijkman $(1904,1914)$, who found that coli, but not aerogenes, strains were able to form gas in a glucose broth medium incubated at $46^{\circ} \mathrm{C}$. This test has had a chequered career, having been reported on favourably by some workers, and utterly condemned by others. Recent work (see Levine et al. 1934, Wilson et al. 1935) has rendered it clear that the success of the test depends on exact standardization of the incubating temperature, which must be adjusted to $43^{\circ}-45^{\circ} \mathrm{C}$. in the medium itself. The only satisfactory means of doing this is to incubate the tubes in a constant-temperature water-bath. The differential value of the test is greatly enhanced by the replacement of glucose by lactose. Our own experience suggests that MacConkey's lactose bile salt broth is the medium of choice. Using this medium in the way described, it was found that of 193 M.R. +, V.P. - citrate - indole + coli strains, 180 gave a positive $44^{\circ} \mathrm{C}$. MacConkey reaction, whereas of 303 other strains, 40 of which 
belonged to the indole-negative coli group, only 10 did so. The value of the method has now been amply confirmed (see Mackenzie and Hilton-Sergeant 1938, Dodgson 1938, Bardsley 1938a, Clegg and Sherwood 1939, Perry 1939, Raven, Peden and Wright 1940, Ferramola 1940, Clegg 1941, Sherwood and Clegg 1942, Batty-Smith 1942b, Stuart et al. 1942). Two discordant reports on the value of the test have appeared. Raghavachari and Iyer (1939a) found that about 50 per cent. of aerogenes-like strains in Madras waters, and Boizot (1941) about 10 per cent. in Singapore waters, were able to produce gas in MacConkey broth at $44^{\circ} \mathrm{C}$. Possibly this may be due to a higher optimal temperature for growth of aerogenes strains in the tropics than in temperate climates. With this reservation, however, we may say that the $44^{\circ} \mathrm{C}$. MacConkey test appears to be of greater value than any other single test in picking. out typical freal coli strains.

There can, we hink, be no doubt that the primary division of the lactose fermenters must be made on the basis of the gas ratio, methyl red, Voges-Proskauer, and citrate tests. By this means we obtain a primary classification into coli, intermediate, and aerogenes groups. The secondary division is more difficult. From an ecological standpoint, we believe that the indole, $44^{\circ} \mathrm{C}$. MacConkey, and gelatin liquefaction tests afford the most satisfactory grouping, but from a systematist's point of view there is much to be said in favour of classification on sugar reactions. Taking the first method of grouping, we may note that about 93 per cent. of strains from human and animal fæces produce indole, and that about 95 per cent. give a positive $44^{\circ} \mathrm{C}$. MacConkey reaction. On the other hand, indole-negative and $44^{\circ} \mathrm{C}$. MacConkey negative freal strains are uncommon. A positive indole test given by a citrate-negative strain, or a positive $44^{\circ} \mathrm{C}$. MacConkey test given by any strain, is therefore strongly suggestive of its fæcal origin. Though both intermediate, aerogenes and cloace strains are often present in mammalian fæces in small numbers (Cruickshank and Cruickshank 1931, Parr 1939), the balance of evidence strongly suggests that in this country, and in the United States (see Griffin and Stuart 1940), these organisms are primarily of non-fæcal origin. Their main habitat is still in doubt, but they are found most often in soil, grains, grasses, food-stuffs, and decaying vegetation. The classification reached by this method is of special value in the interpretation of water analysis tests and is depicted in Chapter 92.

With regard to the second method of grouping, the test substances that have been accorded special prominence are saccharose, dulcitol and salicin, the value of the last being emphasized by Kligler (1914a,b) and by Levine (1917). Winslow, Kligler and Rothberg (1919), in their excellent review of the classification of the whole coli-typhoid group, conclude that the types set out in Table 4I under their appropriate names are the only ones which are sufficiently well established to merit separate consideration.

It will be noted that, although the reactions in dulcitol and adonitol are included in the table, the species are adequately defined by the reactions in saccharose and salicin, together with the presence or absence of motility. It will also be noted that Bact. coscoroba differs from Bact. coli communius in being non-motile, Bact. immobile from Bact. coli commune in the same way, and Bact. grünthal from Bact. acidi-lactici only in being motile. Winslow and his colleagues express the view that the presence or absence of motility, taken alone, does not justify specific differentiation; and they suggest the recognition of four species, Bact. neapolitanum, Bact. coli communius, Bact. coli commune, and Bact. acidi-lactici, regarding the coscoroba, immobile, and grinthal types as varieties of the corresponding species. This appears to us a wise and conservative view. We should, ourselves, prefer to narrow the limits still further, regarding Bact. coli as a single species, and placing the neapolitanum, communius, commune and acidi-lactici types as varieties. 
TABLE 41

Fermentative Types of Bact. coli (Winslow et al).

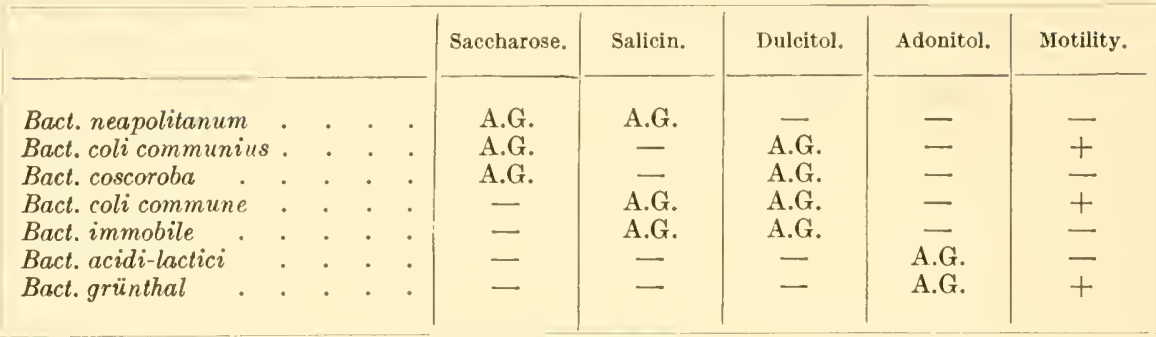

Serological Differentiation.--Several attempts have been made to study the antigenic structure of various strains of Bact. coli by the method of agglutination. The results display an extreme heterogeneity of antigenic factors (Mackie 1913, Mirra 1936, Bredenbröker 1938, Stuart et al. 1940). In some instances, as in the hæmolytic strains of Bact. coli isolated by Dndgeon from cases of acute urinary infection, there is evidence that antigenically homogeneous groups may be related to particular infective conditions (Dudgeon et al. 1921, 1922). The same suggestion is apparent in the work of Lovell (1937) on Bact. coli strains isolated from calves that had died from white scours. Using the precipitin test, he obtained evidence of the existence of two antigens: one, a soluble specific carbohydrate substance, associated with the capsule and probably similar to or identical with that previously described by D. E. Smith (1927); the other, of undetermined nature, contained in the body of the bacillus. Of 110 strains from 45 calves, he was able to place 79 in one of eight different types. It is interesting to note that some coliform strains contain $\mathrm{O}$ or $\mathrm{H}$ antigens found in members of the Salmonella group (see Chapter 30). The detailed antigenic analysis of the coliform bacilli remains for future study.

Stamp and Stone (1944) have described the presence of a common antigenreferred to as the $\alpha$-antigen-in many members of the coliform and paracolon group. It is more heat-stable than the $\mathrm{H}$-antigen, withstanding a temperature of $75^{\circ} \mathrm{C}$. for 1 hour, but less so than the $\mathrm{O}$-antigen, being inactivated at $100^{\circ} \mathrm{C}$. in 15 minutes. It is destroyed by exposure for 1 hour to alcohol at $65^{\circ} \mathrm{C}$. It is commonest in recently isolated strains, and tends to be lost on subculture. Agglutinins to the $\alpha$-antigen are not uncommon in rabbit sera, and may lead to confusion in the identification of suspected pathogenic organisms.

Pathogenicity.-The majority of the lactose-fermenting coliform bacilli appear to be non-pathogenic under ordinary conditions. The M.R. - , V.P. + group have their normal habitat on plants; the M.R. +, V.P.-, citrate + group appear to live in the soil ; while the M.R. +, V.P. - , citrate - forms are normal intestinal parasites. Under abnormal conditions these bacilli may canse acnte or chronic infective lesions in the urinary tract, or elsewhere (see Chapter 67). It seems probable also that they may sometimes play a part in the causation of enteritis, in man or in animals, though their role appears generally to be a secondary one. There is, however, some evidence that, in newly born animals, the advent to the intestine of Bact. coli, or of particular races of this organism, may result in a local enteritis or even general septicemia. Smith and Jittle (1922) and Smith and Orcutt (1925), for example, attributed the disease in newly born calves known 
as white scours, and the fatal septicæmia that may sometimes accompany it, to infection with Bact. coli (see also Lovell 1937). They found that the disease was prone to occur in calves fed on milk instead of colostrum. It is possible that the neonatal diarrhœa of human infants may result from the access to the intestine of specially virulent forms of Bact. coli, hut the available evidence is as yet insufficient to afford conviction (see Chapter 71). According to Gwatkin, LeGard and Hadwen (1938) bovine mastitis may occasionally be due to coliform bacilli.

The pathogenicity of most strains for laboratory animals is low. Very large doses administered intraperitoneally to the mouse, or intravenously to the rabbit, may prove fatal; but it seems likely that death results in these cases from a toxæmia rather than from a true infection. Occasionally strains of greater virulence are found.

Several observers have described the presence of soluble toxic substances in young broth cultures of Bart. coli (see, for instance, Steinherg and Ecker 1926, Smith and Little 1927, Smith, D.E., 1927, Rennebaum 1935). But most of these "toxins" have proverl to be heat-stahle, and there is no reason to believe that they differ from the toxic components that can be extracted, in larger quantity, from the bacterial cells. Since there is at the moment no clear evidence that the toxic substances that can he extracter from the colon bacillus differ in their action from those of other species within this genus, a discussion as to their probable nature may be deferred to a later section.

\section{The Friedländer Group}

There are certain species of lactose-fermenting coliform bacilli which cannot readily be placed in any of the groups which we have differentiated above. There are, for instance, the so-called capsulated bacilli, including Friedländer's pneumobacillus, Abel's bacillus of ozæna, the bacillus of rhinoscleroma, and others. There appears to be a preponderance of opinion that the organisms of this group are in some way related to Bact. aerogenes, principally because that organism is sometimes capsulated ; but the fermentation reactions, as described by those who have isolated and studied these capsulated coliform bacilli, appear to be extremely variable, and several observers have recorded the fermentation of saccharose, but not of lactose. The balance of evidence suggests that these strains should be included in the genus Bacterium, but it is convenient in the meantime to consider them in a separate sub-section of this group.

\section{Group Characteristics.}

Short, non-motile, non-sporing, capsulated Gram-negative rods, giving a profuse mucoid growth on solid media, and usually fermenting carbohydrates with the production of acid and gas. Usual habitat, respiratory tract of man and certain animals.

Under the general term $B$. mucosus capsulatus a large number of organisms have been described with the characteristics enumerated above. V. Frisch in 1882 isolated a capsulated bacillus from patients with rhinoscleroma. In 1883 Friedländer cultivated a similar organism-generally known as Friedländer's bacillus or Bact. friedländeri-from the lungs of patients who had died of pneumonia. loewenberg in 1894 and Abel in 1896 cultivated a similar organism from the nasal secretion of patients with ozæna. Besides these, several organisms have been described by other workers, such as B. pseudopneumonicus Passet, B. canalis 
capsulatus Mori, Proteus hominis capsulatus Bordoni-Uffreduzzi, B. capsulatus Pfeiffer (Pfeiffer 1889), B. mucosus capsulatus Paulsen, B. crassus sputigenus Kreibohm, B. buccalis muciferens Miller, the bacillus of sputum septicæmia Miller, the granuloma bacillus (Small ảnd Julianelle 1923), Bacterium mucogenum (Edwards, R. T. 1905), B. capsulatus (Wright and Mallory 1895), and Klebsiella paralytica, an organism isolated by Wallace, Cahn, and Thomas (1933) from a paralytic tickborne disease of moose. (For references see Fricke 1896, Bamforth 1928.) The ozæna bacillus described by Abel must not be confused with the non-capsulated coccobacillus deseribed by Perez (1899). (See Chapter 19.)

Habitat.-Friedländer's bacillus appears to lead a parasitic existence. The commonest situation in which it is found is the respiratory tract of man. It is an occasional inhabitant of the nasopharynx; it is found in diseased conditions of the nose, such as ozæna and rhinoscleroma; and it is sometimes present in the lungs in pneumonia, influenza, bronchitis, bronchiectasis, tuberculosis, and

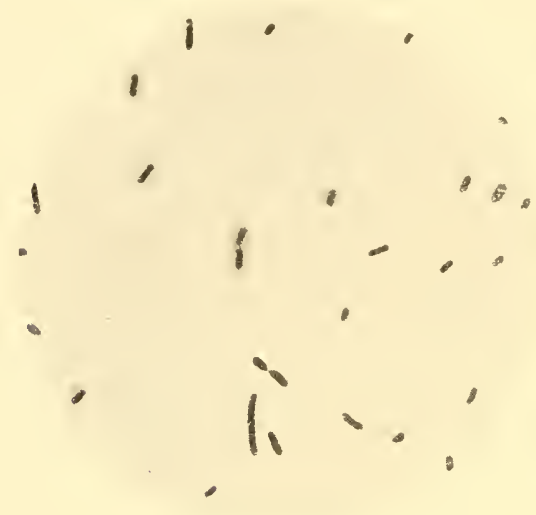

FiG. 142.-Friedländer's Bacillus.

From an agar culture, 24 hours, $37^{\circ} \mathrm{C}$. showing capsules $(\times 1000)$.

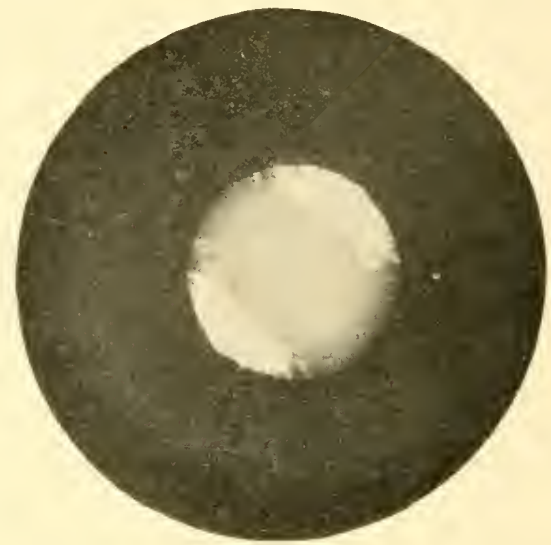

FıG. 143.-Friedländer's Bacillus.

Surface colony of mucoid type on agar, 24 hours, $37^{\circ} \mathrm{C} .(\times 8)$.

other diseases. It has been isolated from a large number of suppurative conditions in different parts of the body, such as pleurisy, appendicitis, cystitis, pyelonephritis, ulcerative endocarditis, endometritis, brain abseess, and general septicæmia (Perkins 1904), and was found by Dudgeon (1926) in 5.5 per cent. of fæces examined from normal and abnormal conditions.

Morphology.--In the body the organism generally takes the form of short, ovoid, diplobacilli, surrounded by large capsules, looking not unlike pneumococci. In culture it is pleomorphic. The usual form is a short, straight, thick rod, about 1-2 $\mu$ long and $0 \cdot 8 \mu$ wide, with parallel or bulging sides, and rounded or slightly pointed ends. The bacilli are usually in pairs end-to-end, or arranged singly. Besides this form there are several others of most varied appearance-thick curved sausage forms, clubbed forms, long sinuous filamentous forms, long straight rodsstaining regularly or irregularly. In most strains the organisms are surrounded by a capsule, apparent in cultures, which can be demonstrated by Gram's stain, or by any of the ordinary capsule stains. In some strains, however, the organisms, instead of being individually capsulated, are embedded in an interstitial mucoid 
substance, which stains less intensely than the bacilli. There are, moreover, noncapsulated variants, which morphologically and culturally resemble the coliform bacilli; these often become predominant in old cultures. The organisms are nonmotile and non-sporing. They stain easily, sometimes show bipolar staining, and are Gram-negative.

The capsule of these organisms is formed not only in the animal body but in culture. To demonstrate it in body fluids, it is sufficient to fix it by heat, and stain with carbol-fuchsin or methylene blue. To demonstrate it in culture, it is advisable to suspend the bacilli in a low dilution-not higher than $1 / 5$ - of serum, dry in air, and fix in a saturated solution of corrosive sublimate before staining (Toenniessen 1912).

Chemical examination of the capsule has revealed the presence of a nitrogenfree carbohydrate material, which according to Toenniessen (1921) and Kramár (1921) is a polysaccharide of galactose; but Heidelberger, Gocbel and Avery (1925) regard it as a polysaccharide containing glucose. The capsule appears to consist of gum. This substance may be separated from the bacilli in the following manner (Toenniessen 1921): The organisms are dried, suspended in water, to which 1 per cent. $\mathrm{KOH}$ is added, and heated until the gum covering passes into solution. On cooling, the undissolved bacilli form a sediment, and the opalescent supernatant fluid is pipetted off. This is acidified with acetic acid, and 3 volumes of 91 per cent. alcohol are added, when a heavy precipitate of the gummy substance occurs; this precipitate is purified by dissolving it in distilled water, and re-precipitating it with acid and alcohol. In the pure state it is a loose, snow-white powder, giving an opalescent solution in distilled water, acids, or alkalies; it is free from nitrogen, and does not reduce Fehling's solution. It has special antigenic properties, which will be referred to later.

Cultural Reactions.-One of the characteristic features of this group is the luxuriant, greyish-white, mucoid, almost diffluent, growth on agar. This results no doubt from the presence of the gummy envelope around the bacilli, containing a high proportion of water-92 per cent. (Toenniessen 1921). The condensation water on an agar slope is converted into a greyish-white mucoid mass. In broth the organisms grow freely, giving rise after a few days to a marked viscosity, so that the medium takes on the consistency of melted gelatin. Great stress used to be laid on the nail-headed growth in stab gelatin cultures; a circular, convex growth may occur on the surface, with a filiform growth in the stab, the whole resembling a round-headed nail. This appearance is not constant, and is given only by some strains; it depends too on the amount of gelatin in the culture; with small amounts, about 4 per cent., the surface growth is flat (Friedländer 1883). There is no liquefaction of the gelatin, but often a large napiform bubble of gas accumulates just beneath the surface, giving on first sight the appearance of liquefaction. On potato there is a moderate creamy-yellow mucoid growth, later turning to a buff or light café-au-lait colour.

The cultural appearances of the Friedländer group are subject to considerable variation. This is due to the production of variants which have different forms of growth from the parent strain. Toenniessen (1913) found that when the usual mucoid type was kept in the incubator and subcultured every few days, nonmucoid variants rapidly appeared. As the original colonies grew older, white, more or less translucent peripheral sectors developed, which on microscopical examination were found to consist largely of non-capsulated bacilli ; these could 
be subcultured, and obtained pure. The growth of these non-capsulated bacilli was no longer mucoid, but resembled the growth of Bact. coli. Regression to the mucoid type might occur-often suddenly. As well as this non-mucoid type, Toenniessen (1914) later observed three other variants. The mucoid, capsulated type may be regarded as the smooth form, and the non-mucoid, non-capsulated type as the rough form of Friedländer's bacillus (Julianelle 1926b). Dissociation may take other forms. Thus, according to our own observations, secondary colonies may appear in the substance or on the surface of the original colonies; or there may develop a jelly-like, translucent peripheral fringe showing slight radial striation; or sometimes the whole colony may dry up and wither away, leaving an effuse, transparent layer looking like ground glass aptly called by Collins (1924) "suicide" colonies. Beham (1912), Hadley (1927), and Goslings (1935) have recorded similar observations.

Resistance and Metabolism.-The organisms are killed by moist heat at $55^{\circ} \mathrm{C}$. in half an hour. They may survive drying for months (Loewenberg 1894). If kept at room temperature, cultures remain viable as a rule for weeks or months. They are aerobic; growth under strictly anaerobic conditions is very poor. There is no hæmolysis of horse's or sheep's red cells. The optimum temperature for growth is $37^{\circ} \mathrm{C}$., the limits are $12^{\circ}$ and $43^{\circ} \mathrm{C}$. Some strains form a slightly brownish pigment, most easily produced by growth on potato.

Biochemical Reactions.-The fermentation of sugars by members of this group is subject to considerable variation (Clairmont 1902, Perkins 1904, 1907, Edwards 1905, Page 1912, Fitzgerald 1914, Coulter 1917, Small and Julianelle 1923, Bamforth 1928, Edwards 1928, 1929, Julianelle 1930, 1935, Elbert and Guerkess 1930, Hay 1932, Wallace, Cahn and Thomas 1933, Morris and Julianelle 1934, Kliewe and Hsü 1935, Wilson et al. 1935, Goslings 1934, Osterman and Rettger 1941). Many strains produce acid and gas in glucose, maltose, mannitol, lactose, sucrose, and salicin. Several, however, do not attack lactose, and others either do not ferment sucrose, or ferment it late. Gas may be formed rapidly or not for several days; some strains do not form gas. Occasionally no sugars are fermented, but this lack of fermentative ability is uncommon except in strains that have been subcultured for a long time in the laboratory. In litmus milk also the reaction of different strains varies. Generally acid and clot are formed, but many strains do not produce sufficient acid to precipitate the caseinogen, while some strains produce no change at all. The litmus is occasionally decolorized.

There is fairly general agreement that biochemical reactions do not afford an adequate basis for classification, since most workers have been unable to discover any constant relationship between the biochemical activities of a given strain and its source of origin or antigenic structure. Goslings (1934) and Wielenga (1937), however, consider it possible to distinguish three species on the basis of biochemical activity. (a) The least active and the least variable comprises the rhinoscleroma strains, which have the following reactions: they form no gas from carbohydrates, they produce acid in glucose, maltose, mannitol, and adonitol in 24 hours, in sucrose within 10 days, and sometimes in glycerol, they do not ferment lactose, dulcitol, or amygdalin within 3 days, they have no action on litmus milk, they usually produce alkali in neutral peptone water in 10 days, they are M.R. positive and V.P. negative, they do not grow in eitrate or $d$-tartrate, they do not grow in fresh ox bile, and they form no $\mathrm{H}_{2} \mathrm{~S}$. (b) The second group, comprising the ozæna strains, is similar in most respects, but there is more variability between the different members. Most strains fail to produce gas from carbohydrates, but some do ; they produce acid and sometimes gas from glucose, maltose, mannitol, and adonitol in 24 hours, many strains from lactose or amygdalin within 3 days, but the majority fail to ferment sucrose within 10 days and none ferments dulcitol, many strains produce acid and some- 
times clot in litmus milk, they usually produce allali in neutral peptone water in 10 days, they are H.R. positive and V.P. negative, some strains grow in citrate, most strains grow in fresh ox bile, and many strains form $\mathrm{H}_{2} \mathrm{~S}$. (c) The third, according to Goslings (1934), comprises the Friedländer and linet. urrogenes strains. These are the most active biochemically, growing freely in fresh bile, producing gas from carbobydrate media, fermenting lactose and amygdalin within 3 days and suerose within 10 days, turning nentral peptone water either slightly acid or alkaline, reducing litmus, utilizing sodium citrate and $d$-tartrate, but not forming $\mathrm{H}_{2} \mathrm{~S}$. De Graaff (1936) and Wielenga (1937) would distinguish Friedländer's bacillus from Bact. aerogenes, though they are not in complete agreement on the criteria that should be used for this purpose.

Of reactions not studied by Goslings or Wielenga, the indole test is generally negative; nitrates are reduced to nitrites; ammonia is formed from peptone; methylene blue is generally reduced in broth; gelatin is not liquefied; and there is usually an abundant production of catalase. All these reactions, however, apply mainly to Friedländer and aerogenes strains; the behaviour of rhinoscleroma and ozæna strains has been less thoroughly studied. According to Hay (1932), one of the most characteristic features of the mucosus capsulatus group is their ability to ferment inositol, but this is likewise shared by Bact. aerogenes.

Antigenic Structure.-Till quite recently no satisfactory division of the Friedländer group had been made on the basis of antigenic structure. The maiu difficulty was due to the fact that, though injection of the capsulated bacilli iuto rabbits is able to give rise to an agglutinating serum, this serum has little action except on non-capsulated organisms; several attempts were therefore made to rid the bacilli of their capsules. Porges' method (1905) was one of the most successful. He suspended an agar culture in $10 \mathrm{ml}$. of saline, filtered through paper: mixed it with a quarter of its volume of $\mathrm{N} / 4 \mathrm{HCl}$ solution, and heated for 15 minutes; it was cooled rapidly, and neutralized with $\mathrm{N} / 4 \mathrm{NaOH}$ solution. The resultant suspension was homogeneous and non-viscous, and agglutinated with a specific immune serum. Though this method undoubtedly removes the capsules, it often renders the bacilli spontaneously agglutinable or agglutinable by normal serum. Streit (1906) found that if the bacilli were grown on potato or potato agar, they gradually lost their capsules, and became more agglutinable. Small and Julianelle (1923) obtained the same result by growing them on agar for 24 hours, storing the cultures in the ice-chest, and subculturing monthly; after 1 to 2 years many of the strains had lost their capsules. Agglutination tests made with non-capsulated bacilli obtained in these ways gave, however, very inconstant results; nor could a method of analysis in which the natural capsular antigens were disregarded be accepted as satisfactory. (Streit 1906, Beham 1912, Fitzgerald 1914, Coulter 1917, Small and Julianelle 1923).

Further work in America (Avery et al. 1925, Heidelberger et al. 1925, Julianelle $1926 a, b, c)$ has largely cleared up the confusion. It would appear that the immunological reactions of the Friedländer group are similar to those of the pneumococci, depending on the presence in the cell of two entirely different factorsa polysaccharide in the capsule responsible for the type-specificity, and a nucleoprotein in the soma responsible for the species-specificity. According to Julianelle (1926a) there are three serological types, distinguishable by agglutination, absorption, precipitin, and protection tests, and a heterogeneous group $(\mathrm{X})$ of strains that have not yet been antigenically differentiated. If a serum is prepared against Type A by injection of heat-killed encapsulated organisms, it will agglutinate encapsulated bacilli of its own type, but not those of any other type; 
similarly with Types B and C. The serum contains an antibody that reacts specifically with the polysaccharide fraction in the capsule; and as the polysaccharide varies in the different types, the serum against each type is specific. The polysaccharide of Types A and B has been isolated, and has been found to flocculate in the presence of a specific serum. If the bacilli are deprived of their capsules, they lose their specificity, and agglutinate equally, though only to a low titre, with sera prepared against any type.

The nucleo-protein can be separated from the dissolved bacilli by precipitation with acetic acid in the cold. It gives rise to a species antibody, which does not react with the capsulated bacilli or with the polysaccharide, but which agglutinates capsule-free cells of any type, and reacts with the nucleo-protein of any type.

A serum prepared by injection of smooth, capsulated bacilli contains antibodies to the polysaccharide and the nucleo-protein; a serum prepared by injection of rough, non-capsulated bacilli contains only one antibody-active against the nucleo-protein. For the classification of the Friedländer group into types it is essential to use smooth bacilli both for the preparation of sera and for agglutinating antigens. Unless this rule is strictly observed, the results will be unsatisfactory. Failure to realize this principle probably accounts for the discrepant results of the earlier workers.

The capsulated bacilli stimulate the production not only of type-specific agglutinins and precipitins, but also of type-specific protective bodies. Thus a serum prepared by injection of capsulated bacilli of Type A will protect mice against intraperitoneal injection of Type $\mathrm{A}$, but not against injection of bacilli of Types B or C. The nucleo-protein does not stimulate the production of protective bodies; a serum, therefore, prepared by injection of capsule-free bacilli of any type is unable to protect mice against infection with capsulated bacilli of any type. It is important to note that the polysaccharide in the pure state, though precipitable by immune anti-S serum of the homologous type, is unable to stimulate the production of immune bodies; it must be present in combination with the proteins of the cell. The nucleo-protein, on the other hand, is able by itself to stimulate the production of non-specific antibodies. The polysaccharide is present in quite young cultures of Friedländer's bacillus; so that filtrates of these cultures may be used as antigens in the precipitin test.

A few tabular results may make these relations clear.

TABLE 42

Anti-smooth Serum acting on Shooth Capsulated Bacilli and on Rough Non-capsu. lated Bacilli, or on Smooth Bacilli that haVe been artificially deprived of their CAPSULES.

Anti-S Serum,

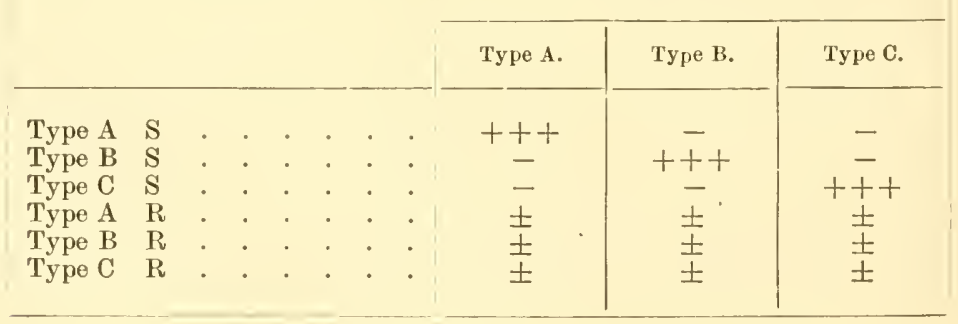


TABLE 43

Anti-rodall Serum, or an Anti-protein Serum, aoting on Shooth Capsulated and Rough Non-Capsulated Bafilli.

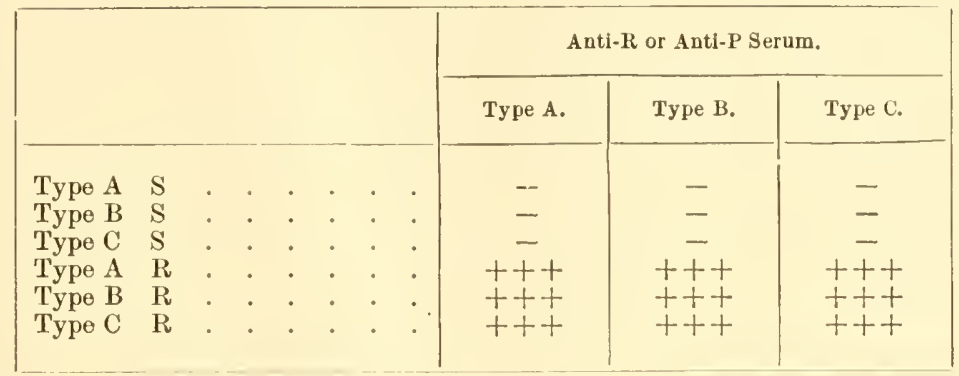

It is of interest to note that the B type of Friedländer's bacillus is similar immunologically to Type II pneumococcus. The specific polysaccharide in the B type, in conjunction with the protein fraction, stimulates the formation of an immune serum that will agglutinate pneumococcus Type II, and will protect mice against infection with it; similarly pneumococcus Type II serum will agglutinate Friedländer Type B and protect mice against infection with it. The polysaccharides in the two organisms appear to be closely alike, though not absolutely identical (Avery et al. 1925). A Friedländer Type B organism will not, however, absorb the agglutinins from pneumococcus Type II serum, nor a pneumococcus Type II organism from a Friedländer Type B serum. This probably indicates that though the polysaccharides are alike, the protein fractions of the organisms are different.

According to Tomášek (1925), Quast (1926), Prica (1930), and Neuber (1934) the rhinoscleroma bacillus can be distinguished by agglutination and complement fixation from Friedländer's and the ozæna bacillus. Morris and Julianelle (1934), however, who examined 10 strains of the rhinoscleroma bacillus, were unable to distinguish this organism antigenically from strains of Friedländer's Type C. 'This observation is supported to some extent by Goslings and Snijders (1936), who found that all rhinoseleroma strains had the same capsular and the same somatic antigens; the capsular antigen was practically indistinguishable from the Type C Friedländer antigen.

According to Julianelle (1935) the ozcena bacillus can be distinguished by agglutination and absorption of agglutinins from Friedländer and rhinoseleroma bacilli. Of 19 ozæna strains studied, 12 fell into one group, 2 into another, and the remaining 5 were antigenically heterogeneous. Goslings and Snijders (1936), likewise, concluded that there were two, and possibly three, different capsular antigens among ozæna strains; these they labelled D, E, and (F). All strains, however, appeared to share a common somatic antigen. Wielenga (1937) reports on three strains that possessed the somatic antigen of Bact. ozcence and the Type C capsular antigen of Bact. friedländeri.

Prášek and Prica (1933) state that they have been successful in extracting a soluble specific carbohydrate-containing substance, which is probably a galactan, from the capsules of the ozæna and the rhinoscleroma bacillus. The substances from the two organisms were quite distinct, and showed no cross-precipitation when tested against the heterologous immune sera. They were likewise distinct from the polysaceharide extracted from a Friedländer's bacillus.

The relation between Friedländer and aerogenes strains is still obscure. Julianelle (1937), who studied 3 strains of Bact. aerogenes, found that they shared a common somatic 
antigen, which was different from the Fricdländer somatic antigen, but that each had a distinct capsular antigen. One of the capsular antigens was specific; one was related to pneumococcus Type II, and one to both pneumococcus Type II and Friedländer Type B. Goslings and Snijders (1936), Wielenga (1937), and Osterman and Rettger (1941) likewise found considerable antigenic complexity in the aerogenes group. According to Goslings and Snijders, even the somatic antigen varies in different strains of Bact. aerogenes, being sometimes similar to that of the rhinoscleroma bacillus, sometimes to the ozæna bacillus, and sometimes to neither. The capsular antigen of one strain was similar to that of Friedländer Type B, but the rest were different from any of the $\mathrm{A}$ to $\mathrm{F}$ capsular antigens.

Studying capsulated strains of diverse origin, Edwards (1929) was able to divide them into three groups by agglutination: (1) strains, chiefly from human pneumonia and pleurisy, identical with Julianelle's Type A; (2) strains from metritis of mares, together with some strains of Bact. aerogenes isolated from soil, water, or milk, and an oceasional strain of human origin, identical with Type B; (3) some soil strains of Bact. aerogenes and an inguinal granuloma strain. Julianelle (1930) ol,tained evidence that most Type A strains were of human, and most Type B strains of animal origin.

It is clear that the relationship of these various capsulated organisms to each other will not be understood until a sufficient number of fully representative strains of each group have been examined by serological and other methods.

Pathogenicity.-Organisms of this group are frequently encountered in diseases of the respiratory tract in man. As a rule they appear to act chiefly as secondary invaders, but it is possible that they are sometimes responsible for the primary disease (see Bhatnagar and Singh 1935). They are not uncommonly isolated from suppurative processes of various kinds throughout the body; occasionally they invade the blood stream, and give rise to septicæmia. Jampolis and his colleagues (1932) record a hospital outbreak of infectious diarrhøa in infants, accompanied by severe constitutional disturbance and a high case mortality. Friedländer's bacillus was isolated from the nasal secretions, stomach contents, and stools of most of the patients, and appeared to be the primary cause of the condition. Webster $(1928,1930)$ has described a spontaneous respiratory epidemic in mice caused by Friedländer's bacillus ; Edwards (1928) has isolated this organism from metritis of mares; while Wallace, Cahn, and Thomas (1933) have found it in a paralytic disease of moose. It has been held responsible for the disease of human beings known as granuloma inguinale; but according to Anderson, DeMonbreun and Goodpasture (1945), this disease is due to a different organism, cultivable in embryonic egg yolk, for which the name Donovania granulomatis is suggested.

Experimentally the virulence of Friedländer bacilli is subject to considerable variation. The smooth type is pathogenic, the rough type non-pathogenic for laboratory animals (Toenniessen 1914, Julianelle 1926b, Webster 1928). Other variants have differing grades of pathogenicity. Many observers have drawn attention to the differences in pathogenicity between Friedländer's bacillus, the ozæna bacillus, the rhinoscleroma bacillus, and other members of this group, and have endeavoured to make use of these differences in classification. Unfortunately the protocols in the literature are not sufficiently definite to enable one to assert that there is any constant difference between these organisms. It is therefore possible that the actual virulence of any strain depends upon the proportion of smooth bacilli in the culture. When this consists entirely of the smooth type, it is usually virulent; in proportion as the rough type appears, the virulence falls; and when the smooth has been replaced completely by the rough type, the culture proves avirulent. On the whole it would appear that Friedländer Types A and B bacilli are highly pathogenic to mice when injected intraperitoneally, while Friedländer Type C, ozæna, and rhinoscleroma strains are usually non-virulent. 
The smooth type is capsulated; the rough type is not. It might therefore be thought that virulence depends on capsule formation. Toenniessen (1914) discusses this possibility, but concludes that the association between capsule formation and virulence is fortuitous. He isolated, for example, one variant which, thougl non-capsulated, was highly virulent. He states that old cultures of the smooth type, in which the capsules have largely become autolysed, have just the same virulence for mice as fresh young capsulated cultures. Moreover, Julianelle's Friedländer Type C strains, though capsulated, were non-virulent. It would appear, therefore, that capsule formation is of ten associated with, but is not essential to virulence.

After subcutaneous injection of a very small dose-about $0.0000001 \mathrm{ml}$. of a 24-hours' broth culture of a virulent strain-into mice, the animals die in 12 to 72 hours. Post mortem, there is a local exudate, the focal glands are swollen, and the spleen is cnlarged. Capsulated bacilli are found in the blood and viscera (Pfeiffer 1889, Fricke 1896, Tocnniessen 1914).

Guinea-pigs are refractory to subcutancous, but succumb to intraperitoneal injection, death occurring in 12 to 72 hours. The fatal dose is about $0.01 \mathrm{ml}$. of a 24 -hours' broth culture. Post mortem, there is a viscous exudate in the peritoneum ; the splcen may be enlarged, and the suprarenals hæmorrhagic. The bacilli are found in large numbers in the blood and viscera.

Rabbits appear to be more resistant, but they succumb after intravenous or intraperitoneal injection with a dose of about $0.1 \mathrm{ml}$. of a broth culture. Intraperitoneal inoculation is likewise fatal to pigeons.

Classification.--The demarcation of this group from other groups of capsulated bacilli, and the sublivision of the group itself, are both in a very unsatisfactory state. In the first place it is doubtful what relation capsulated organisms of the aerogenes and intermediate type have to Friedländer's bacillus. It is usual to regard Bact. aerogenes as a saprophyte of grains, the intermediate types of coliform bacilli as saprophytes of soil, and the Friedländer-ozæna-rhinosileroma group as parasites of man and animals. But the fact that most Friedländer strains of respiratory origin are indistinguishable from straius of intermediate I type (see (hapter 92), and that many strains found in cystitis appear to be identical with Bact. aerogenes, renders dangerous any attempt to separate these organisms on the basis of habitat alone. It is difficult to avoid the conclusion that all these organisms should be classified in a single group, but whether that group should be called Aerobacter, Encapsulatus, or Klebsiella is very doubtful. For the moment we prefer to keep them within the wide Bacterium genus.

Attempts to make subdivisions within the group must necessarily await the definition of the group itself. Though Goslings (1934) and Wielenga (1937) are convinced of the value of biochemical tests in distinguishing between the scleroma, ozæua, and pneumonice types, it is clear from a careful study of their papers that, apart perhaps from the scleroma group, there is so much variation between the different members within each group as to make the classification of any indivitual strain of unknown origin often impossible. At the moment a study of antigenic structure seems to hold out the only promise of throwing any light on this problem. A careful comparison of adequate numbers of freshly isolated strains from different sources is urgently called for. Until this is done, it will be impossible to decide whether ozana and rhinoseleroma strains are specifically distinct from strains of Friedländer, or whether they are merely types of the same species differing in the polysaccharide constituents of their capsule. Any such attempt should include a thorough study of intermediate and aerogenes strains. 
Mention should perhaps be made here of an organism that appears to be responsible for joint ill of foals, and that is referred to by a variety of names, such as $B$. nephritidis equi, B. equirulis, Bact. viscosum equi, and B. pyosepticus equi. Morphologically, this organism is a pleomorphic Gram-negative bacillus occurring singly, in streptococcus-like chains, and as filaments. Some authors describe it as capsulated, others as non-capsulated. It forms tenacious colonies on agar, gives rise to a very viscous sediment in broth, gives a nail-head growth in gelatin stab without liquefaction, ferments glucose, maltose, mannitol, lactose, and sucrose with the production of acid but not gas, is M.R. - , V.P. - , citrate - indole reduces nitrates to nitrites, produces acid, and sometimes clot, in litmus milk, is antigenically heterogeneous, and is non-pathogenic to laboratory animals. The normal form on isolation is said to be mucoid, but a non-mucoid variant is sometimes cultivated directly from foals, though more of ten it is seen only as the result of in vitro variation. (For references see Edwards 1931, 1932.) The exact relationship of this organism to the members of the Friedländer group is doubtful.

A detailed description of Friedländer's bacillus is given on p. 680 .

\section{The Paracolon Group}

There remain a number of organisms which, for one reason or another, cannot be included in any of the lactose-fermenting groups already described, or in either of the non-lactose-fermenting groups to be considered in the next two chapters. These organisms ferment lactose late, weakly, irregularly, or not at all. Some constantly give rise to non-lactose-fermenting variants. Some produce gas abundantly, and some in only small quantity; others are completely anaerogenic. A few species are pathogenic for man; others are under suspicion; and others again are almost certainly non-pathogenic. Some are found in frees, some in water, some in soil, and some in other situations.

This heterogeneous collection of organisms we propose to refer to as paracolon bacilli. They constitute a group that appears to be intermediate between the coliform bacilli on the one hand and the non-lactose-fermenting Salmonella and Shigella bacilli on the other. From the coliform bacilli they are distinguished mainly by their late fermentation of lactose, or by their failure to ferment it at all. From Salmonella and Shigella they differ mainly in fermenting either lactose, sucrose, or salicin and in their lack of the particular antigens that characterize these two groups.

Paracolon bacilli have frequently been isolated from the fæces of persons suffering from enteric-like infections, enteritis and cystitis. Occasionally an organism belonging to this group has been isolated from the blood stream. In very many instances, however, they have been cultivated from the fæces of normal persons (see Sandiford 1935); and it seems doubtful whether they have any real significance as primary infecting agents in epidemic infections of the enteric or dysenteric type, though there can be no doubt that some species at least possess pathogenic potentialities when they invade the tissues from the intestinal tract. The great majority of the organisms included in this group have, it may be noted, been isolated in the tropics; and there seems no doubt (Sandiford 1935) that they are a more common constituent of the normal intestinal flora under tropical than under temperate conditions.

The classification of these strains raises problems of considerable difficulty. Various schemes have been suggested (Chalmers and Macdonald 1916, Castellani and Chalmers 1920, Castellani 1938); but these are not in accordance with the general lines that we have discussed above, and in the authors' opinion lay too much stress on minor differences in fermentative ability. Stuart, Wheeler, Rustigian and Zimmerman (1943), who have subjected several members of this group to the series of tests used for differentiating 
the coliform bacilli-M.R., V.P., indole, citrate, and cellobiose-find that they can be divided roughly into three main groups according to whether they resemble the coli, intermediate, or aerogenes types. Within the separate sub-divisions many of the strains were found to be antigenically similar, but a number remained that could not be classified by serological means. For the sake of example the fermentation reactions of a few of the paracolon bacilli that have received names are set out in Table 44. For a fuller description of their characters the reader is referred to papers by Castellani (1902-14, 1907, 1912, 1938), Castellani and Chalmers (1920), Archibald (1911), Chalmers and Macdonald (1916); but it must be understood that the nomenclature of all these organisms may have to be revised when a satisfactory basis of classification is eventually laid down.

More recently Sevitt (1945), who studied 108 strams of paracolon bacilli, mostly isolated from cases of infantile diarrhœa, found that they could be classified into four groups on their reactions in sucrose and dulcitol. Serologically five main antigens could be defined, though many strains were heterogeneous. Some strains, besides containing one or more of the A-E series of antigens, were characterized by the additional inclusion of a Shigella alkalescens antigen, or of antigens found in the Flexner or Newcastle groups.

It is of interest to note that Dudgeon has recorded a considerable series of cases of acute urimary infection, associated with a pyrexial reaction simulating enteric fever, and caused by a late-lactose-fermenting coliform bacillus. Lactose is fermented slowly, saccharose is unchanged, mannitol and dulcitol are fermented with the production of acid and gas, and litmus milk is rendered acid and usually clotted (Dudgeon 1924. Dudgeon and Pulvertaft 1927). It may be noted that this organism is hromolytic for human red cells, and that Dudgeon and his colleagues (Dudgeon et al. 1921, 1922) have shown that strains of Bact. coli from the freces, or from the urine in cases of cystitis, may be divided into hæmolytic and non-hæmolytic types. The hæmolytic strains are particularly frequent in certain types of acute urinary infection. It may clearly be necessary to elaborate our classification, when these, or other tests, have been applied to the group as a whole. Paracolon bacilli have likewise been isolated by Webb (1937) in Nauritius from the urinary tract of patients suffering from enteric-like ferers, pyelitis, or cystitis, but they differed in some respects from the organism described by Dudgeon. Agglutinins were usually demonstrable in the serum of the patients in titres varying from $1 / 25$ to $1 / 250$.

The strains that have from time to time been described as Bact. coli anaerogenes, on account of their failure to produce gas from ccrtain carbohydrates, are probably related either to some members of this gromp, or to the late-lactose-fermenting types of the Shigella group.

The curious organism described by Massini (1907) as Bact. coli mutabile is itself a non. lactose-fermenting strain, but is characterized by the property of giving rise to lactose fermenting mutants, which show no tendency to revert to the parent form. This species has been discussed in some detail in Chapter 9. It may be noted that Dulaney and Michelson (1935) have recently described a severe epidemic of diarrhoa in infants, apparently eaused by this organism. It is clearly allied to the paracolon group of Bacteria ; though the exact relationship remains obscure.

It will be conrenient to describe at this point an organism originally isolated by Castellani (1912), and studied in nore detail by Khaled (1923). This species, Bact. asiaticum, is a non-lactose-fermenter, and on this criterion would be excluded from the coliform group. In saccharose, however, it forms both acid and gas; and in its ability to attack this sugar it differs sharply from the gas-forming, non-lactose-fermenting bacilli of the paratyphoid group that will be considered in Chapter 30. It differs from them also in its ability to form indole. Bact. asiaticum is a motile bacillus having the usual characters of the genus. It ferments dextrose, mannitol and saccharose with the formation of acid and gas, but produces no change in lactose or dulcitol. It acidifies, but does not clot milk. It usually forms indole. It does not liquefy gelatin. It appears to be a cause of enteric-like infections in man, particularly in the tropics. 
TABLE 44

The Fermentation Reactions of some Paracolon Bacilli.

\begin{tabular}{|c|c|c|c|c|c|c|c|c|c|c|c|c|c|}
\hline & 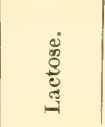 & 兽 & 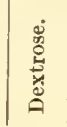 & 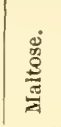 & 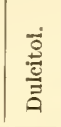 & 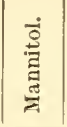 & 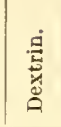 & 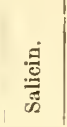 & 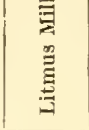 & $\frac{\stackrel{0}{0}}{\stackrel{\Xi}{\Xi}}$ & $\stackrel{4}{\vdots}$ & 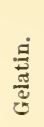 & 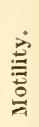 \\
\hline Bact. columbense. & $\begin{array}{c}\mathrm{O} \\
\text { or } \mathrm{S}\end{array}$ & $\mathrm{O}$ & A.G. & A.G. & A.G. & A.G. & A.G. & A.G. & $\begin{array}{l}\text { A }(\mathrm{S}) \\
\text { or } \\
\text { alk. } \\
\text { or D }\end{array}$ & + & - & - & + \\
\hline Bact. giumai. . & $\begin{array}{c}\text { A } \\
\text { (G.v.s.) }\end{array}$ & $\mathrm{O}$ & A.G. & A.G. & 0 & 0 & $\begin{array}{l}\text { A.G. } \\
\text { (S) }\end{array}$ & A.G. & $\underset{\text { alk. }}{\text { A }}$ & + & - & - & 一 \\
\hline Bact. khartoumense & $\begin{array}{l}\text { A.G. } \\
\text { (Slow) }\end{array}$ & $\mathrm{O}$ & A.G. & A.G. & A.G. & A.G. & 0 & A.G. & A & + & - & - & 一 \\
\hline Bact. wesenbergi & A & A.G. & A.G. & & A & A & & & A & + & - & - & + \\
\hline
\end{tabular}

Notes.-O or $\mathrm{S}=$ no fermentation, or very slight.

A.G. (S). = slight acid and gas.

A (G.V.s.) = acid, very little gas.

$\mathrm{A}(\mathrm{S})$, or alk., or $\mathrm{D}=$ slight acid, or alkalinity, or decolorized.

A alk. $(S)=$ acid, reverting to slight alkalinity.

\section{Bact. coli}

Isulation.--Isolated from fæces by Escherich (1885).

Habitat.-The intestinal tract of man and animals.

Morphology. - Bacilli with parallel sides and rounded ends, varying in length from almost coccal forms to long rods. The predominating form is a short rod about $2-3 \mu$ long and $0.6 \mu$ in breadth. The bacilli stain evenly, form no spores, and are not usually capsulated. They are Gram-negative and not acid-fast. Some strains are actively motile, with peritrichate flagella. Some are non-flagellated and non-motile.

Agar Plates. -24 hours at $37^{\circ} \mathrm{C}$. Bact. coli forms circular, low convex, smooth, colourless colonies, about $1-3 \mathrm{~mm}$. in diameter, with a finely granular structure, and an entire edge. The consistency is butyrous, and the growth emulsifies readily. The typical form is often departed from. The surface may be more contoured, and the edge less regular and more effuse.

Broth.-There is abundant growth with a uniform turbidity, increasing up to 24-72 hours, with a slight, powdery deposit that disperses readily on shaking, and sometimes with a minimal degree of surface growth.

Gelatin Stab.-There is good growth along the track, and moderate growth on the surface. The medium is not liquefied.

Blood Agar Plate.-The medium in the neighbourhood of the colonies is discoloured, and there may be hæmolysis. Different strains may vary widely in their action on blood-containing media.

MacConkey's Lactose Agar.-Bact. coli gives rise to circular, smooth, convex colonies, similar to those formed on ordinary agar, but coloured red as a result of the action of the acid formed from the lactose on the neutral red that diffuses into the colony from the medium.

Potato.-There is slight but obvious growth, with a cream or faintly yellow colour.

Resistance.-Bact. coli is usually killed by exposure to a temperature of $60^{\circ} \mathrm{C}$. for 15 minutes, or of $55^{\circ} \mathrm{C}$. for 1 hour, but some strains are more resistant. It is less resistant than members of the Salmonella genus to the action of certain green dyes, particularly brilliant green. 
Growth Requirements.-Bact. coli grows readily on all ordinary laboratory media. The optimum temperature for growth is in the neighbourhood of $37^{\circ} \mathrm{C}$., but growth oceurs over an extended range, from about 15 to $45^{\circ} \mathrm{C}$.

Biochemical.-Produces acid and gas in dextrose, maltose, mannitol, lactose, xylose, rhamnose, and arabinose, but not in dextrin, starch, inositol, or as a rule cellobiose. Sucrose, salicin, raffinose, glycerol, and dulcitol are attacked by some strains, but not by others. Acidifies and clots milk, reduces nitrates, and nearly always forms indole. M.R. + ; V.P. - ; Koser's citrate - ; gas production in MacConkey's broth at $44^{\circ} \mathrm{C}$. + . $\mathrm{H}_{2} \mathrm{~S}$ is not usually formed, but the result depends to some extent on the medium.

Antigenic Structure.-Bact. coli is an antigenically heterogeneous species, the antigenic structure of which has not yet been studied in any detail.

Pathogenicity.-Bact. coli is a normal inhabitant of the intestine of man and other animals. In certain circumstances it may play a pathogenic rôle, sometimes in the intestine itself, more commonly in organs or tissues anatomically related to it, such as the gall-bladder. It is a frequent cause of infection of the urinary tract in man.

Varieties.-On the basis of the fermentation reactions, Bact. coli may be divided into the following varieties :-

var. commune ferments salicin but not saccharose.

var. communius ferments sacharose but not salicin.

var. neapolitanum ferments both saccharose and salicin.

var. acidi-lactici ferments neither saccharose nor salicin.

Some authorities recognize further varieties which are differentiated by being nonmotile.

\section{Bact. aerogenes}

Bact. aerogenes differs from Bact. coli in the following points. Morphologically the rods are often shorter and plumper and they are occasionally capsulated. They may be motile or non-motile. The colonies on agar are more convex, smoother and often mucoid. The deposit in broth is often more viscous. Growth is rather more abundant at lower temperatures, less abundant at temperatures over $37^{\circ} \mathrm{C}$., and usually very slight, or absent, at temperatures of $42-44^{\circ} \mathrm{C}$. Inositol, cellobiose, dextrin and starch are frequently fermented. Most strains fail to form indole. There is a more abundant formation of gas, but a lower acidity. The $\mathrm{CO}_{2}: \mathrm{H}_{2}$ ratio is high, approximately $2: 1$. The VogesProskauer reaction is positive. The methyl-red reaction is negative. Growth occurs with citrate as the only source of carbon. Gas is not produced in MacConkey broth at $44^{\circ} \mathrm{C}$. The normal habitat of this species is not known with eertainty. It is common on grains and plants, and is often found, though usually only in small numbers, in the frees of man and animals ; the balanee of evidenee, however, is against its being primarily an intestinal parasite.

\section{Bact. cloacæ}

Bact. cloace resembles Bact. aerogenes, except that it is usually motile, is seldom capsulated, liquefies gelatin, but does not ferment glycerol or starch. Its distribution in nature appears to be the same.

\section{Intermediate Forms}

The existence of strains that are intermediate between Bact. coli and Bact. aerogenes has been noted in the text, and their characters have been described (see p. 663). They are distinguished (1) from Buct. coli mainly in being citrate-positive, in fermenting cellohiose, in being usually indole-negative, and in failing to form gas in MacConkey broth at $44^{\circ} \mathrm{C}$., and (2) from Bact. aerogenes in being II.R. +, V.P. - , and in failing to utilize uric acid as their sole souree of nitrogen. In this country they appear to be distributed chiefly in the soil. 


\section{Friedländer's bacillus}

Synonyms.--Pneumobacillus, Bact. friedländeri, Bact. pneumonia, B. pneumonia, B. mucosus capsulatus, Encapsulatus pneumonix, Klebsiella meumonice.

Isolation.-Isolated by Friedländer in 1883 from the lungs of patients dying of pneumonia.

Habitat.-Chiefly a parasite. Found in the nose, mouth, and intestine of normal persons ; in the lungs of patients with pneumonia, influenza, and tuberculosis, and other respiratory diseases; and in suppurative conditions of other parts of the body.

Morphology.-Short, thick, oval rod, about $1-2 \mu$ long and $0 \cdot 5-0 \cdot 8 \mu$ broad. Axis straight, sides parallel or bulging, ends rounded, arranged singly and in pairs end-to-end. Some strains are highly pleomorphic, curved rods, sausage forms, and long wary filaments being found in culture; in the body diplobacilli, very like pneumococci, are commonest. Considerable variation in staining, particularly of pleomorphic forms. Non-motile. A capsule is present even in cultures ; it contains a nitrogenfrce polysaccharide of glucose. Gram-negative.

Agar Plate.-24 hours at $37^{\circ} \mathrm{C}$. Round, amorphous, convex, greyish-white, faintly translucent, mucoid colonies, 1-2 mm. in diameter, with smooth, glistening surface and entire edge; consistency mucoid, emulsifiability easy. 7 days, larger, from 3-10 $\mathrm{mm}$. in diameter, raised, with flattened surface, sometimes studded with secondary colonies, and slightly undulate edge; sometimes differentiated into an opaque porcelain-white centre and a less opaque, jelly-coloured periphery showing more translucent radial sectors. A non-mucoid variant occurs.

Agar Slope.-24 hours at $37^{\circ} \mathrm{C}$. Abundant, raised, faintly translucent, greyish-yellow, mucoid, almost diffluent growth, with glistening, smooth or beaten-copper surface, and entire or undulate edge.

Gelatin Stab. 7 days at $20^{\circ} \mathrm{C}$. Moderate, filiform, greyish-white growth, confluent in the upper part, extending to bottom of tube. Convex, mucoid surface growth about $3 \mathrm{~mm}$. in diameter-nail-headed appearancc. No liquefaction, even after 4 weeks, but a large napiform gas bubble often appears near the surface. Sometimes numerous lateral outgrowths occur from the stab after 3 or 4 weeks.

Broth. -24 hours at $37^{\circ} \mathrm{C}$. Moderate growth with moderate uniform turbidity, and a slight, powdery deposit disintegrating easily. Ring growth at surface. 7 days, heavy turbidity with abundant, viscous deposit; marked ring growth. The culture is viscous in consistency.

Glucose Agar Shake.-24 hours at $37^{\circ} \mathrm{C}$. Multiple tiny colonies throughout medium, more numerous near the top. Medium is torn into rifts with gas.

Horse Blood Agar Plate.-24 hours at $37^{\circ} \mathrm{C}$. Convex, milky-white colonies, $1 \mathrm{~mm}$. in diameter, with smooth surface and entire edge. No hæmolysis, but plate is browned.

MacConkey's Agar. -24 hours at $37^{\circ} \mathrm{C}$. Reddish colonies, 1-3 mm. in diameter. 7 days, round, convex or umbonate, red colonies with smooth surface and entire or lobate edge.

MacConkey's Fluid Medium. -7 days at $37^{\circ} \mathrm{C}$. Moderate turbidity; magenta colour ; sometimes gas formation.

Potato.-24 hours at $37^{\circ} \mathrm{C}$. Yellowish, confluent, mucoid growth. 7 days, abundant, raised, mucoid, creamy, buff-yellow, or café-au-lait growth, with smooth, slightly pitted, or nodular surface.

Resistance.-Killed by moist heat at $55^{\circ} \mathrm{C}$. in half an hour. Cultures at room temperature live for months.

Metabolism.-Aerobe. Very slight growth in 10 days under strict anaerobic conditions. Grows luxuriantly in culture. No hæmolysis of sheep's or horse's red cells. Tendency to form brownish pigment on potato.

Biochemical.-Highly variable. Acid, and generally gas, in glucose, maltose, mannitol, lactose, sucrose, and salicin. Inositol is said to be fermented. L.M. acid, or acid and clot. Indole $\mp$; M.R. + ; V.P. - ; nitrates reduced; $\mathrm{NH}_{3}++$; 
$\mathrm{H}_{2} \mathrm{~S}$ - ; M.B. reduced ; catalase ++ ; Koser's citrate + ; gas in MacConkey broth at $44^{\circ} \mathrm{C}$. negative. (For biochemical characters of rhinoscleroma and ozæna strains, see p. 670).

Antigenic Structure.-By agglutination, absorption, and precipitin tests there are three types, A, B, and C of Friedländer's bacillus, and a heterogeneous group $\mathrm{X}$. 'The specificity of the types is determined by the carbohydrate fraction in the capsule. The B type is similar inmunologically to Type II pneumococcus. (For antigenic structure of rhimoscleroma and oziena strains, see p. 673).

Pathogenicity. - Is found in lesions of the respiratory tract and in suppurative conditions generally in the human body. Experimentally, it is highly pathogenic to mice on intraperitoneal or intravenous injcction. Rabbits are less susceptible, but often succumb to intravenous or intraperitoneal injection. Types A and B are highly virulent for mice; Type $\mathrm{C}$ is avirulent. After intraperitoneal injection of a very small quantity of broth culture mice die in 18 to 24 hours; P.M. viscous exudate in peritoneum; capsulated bacilli numerous in blood, lungs and spleen.

\section{The Paracolon Group}

The organisms of this group, which differ from Bact. coli in fermenting lactose late, weakly, irregularly, or not at all, and from the Salmonella and Shigella groups in fermenting either lactose, sucrose, or salicin, in usually producing indole, and in their antigenic struc. ture, have been described on pp. 676-8.

\section{REFERENCES}

AвEL, R. (1896) Z. Hyg. InfektKr., 21, 89.

Anderson, K., DeMlonbreun, W. A. and Goodpasture, E. W. (1945) J. exp. Med., 81, 25.

Archibald, R. G. (1911) 4th Rep. Wellcome trop. Res. Lab.

Avery, O T., Heidelberoer, M., and Goebel, W. F. (1925) J. exp. Mcd., 42, 709.

Bамғовтн, J. (1928) J. Hyg., Camb., 2\%, 343.

Barber, M. A. (1907) Kansas Univ. Sci. Bull., 4, 1.

BARDSley, D. A. (1926) J. Hyg., Camb., 25, 11; (1934) Ibid., 34, 38; (1938a) Ibid., 38, 309; (1938b) Ibid., 38, 721.

BatтY-Smith, C. G. (1942a) J. Path. Bact., 54, 45; (1942b) J. Hyg., Camb., 42, 55.

BeHAM, L. II. (1912) Zbl. Bakt., 66, 110.

Bergey, D. H., Breed, R. S., Murray, E. G. D., and Hitchens, A. P. (1939) "Manual of Determinative Bacteriology." 5th Edit. Baillière, Tindall and Cox, London.

Bergey, D. H. and Deenan, S. J. (1908) J. med. Res., 19, 175.

Bhatnagar, S. S. and Singh, K. (1935) Indian J. med. Res., 23, 337.

Borzot, G. E. (1911) J. IIyg., Camb., 41, 566.

BredenBRöker, J. (1938) Arch. Hyg., Berl., 119, 289.

Breed, R. S. and Conn, H. J. (1936) J. Bact., 31, 517.

Brown, H. C. (1921) Lancet, i. 22.

Browning, C. H., Gilmour, W., and Mackie, T. J. (1913) J. Hyg. Camb., 13, 335.

Burton, L. V. and RETTGER, L. F. (1917) J. infect. Dis., 21, 162.

Castellani, A. (1902-14) Ceylon med. Rep. Colombo; (1907) J. Hyg., Camb., 7, 1 ; (1912) J. trop. Med. Hyg., 15, 161 ; (1938) Ibid., 41, 325, 344, 362.

Castellani, A. and Chalmers, A. J. (1920) Ann. Inst. Pasteur, 34, 600.

Chalmers, A. J. and Macdonald, N. (1916) Lancet, ii. 139.

Chantemesse, A. and Widal, F. (1887) Arch. Physiol. norm. path., 9, 217; (1891) Bull. Méd., 5, 935 .

Chen, C. C. and Rettger, L. F. (1920) J. Bact., 5, 253.

Clatrmont, P. (1902) Z. Hyg. InfektKr., 39, 1.

Clark, IV. M. and Lubs, H. A. (1915) J. infect. Dis., 17, 160.

Clege, L. F. L. (1941) J. Path. Bact., 53, 51.

Clego, L. F. L. and Sherwood, H. P. (1939) J. Hyg., Camb., 39, 361.

Collins, G. (1924) See Hadley, P. (1927) J. infect. Dis., 40, 1.

Covlter, C. B. (1917) J. exp. Med., 26, 763.

Cruickshank, J. and Cruickshank, R. (1931) "A System of Bacteriology," Med. Res. Coun., Lond., 8, 353.

Dodgson, R.W. (1938) Proc. R. Soc. Med., 31, 925. 
Dowson, IV. J. (1939) Zbl. Bakt., iite Abt., 100, 177.

Dudgeon, L. S. (1924) J. IIyg., Camb., 22, 348; (1926) Ibid., 25, 119.

Dudgeon, I. S. and Pulvertaft, R. J. V. (1927) J. Hyg., Camb., 26, 285.

DUDGEoN, L. S., WORDLEY, E., and BAWTrEe, F. (192I) J. Hyg., Camb., 20, 137 ; (1922) Ibid., 21, 168.

Dulaney, A. D. and Micielson, I. D. (1935) Amer. J. publ. Illh., 25, 1241.

DUNBaR, IT. (1892) Z. Hyg. Infekthr., 12, 485.

DurhaM, H. E. (IS98) Brit. med. J., i, 1357; (I90I) J. exp Hed., 5, 353.

EDwardos, 1'. R. (1928) J. Buct., 15, 245 : (1929) Ibid., 1\%, 339; (1931) Kentucky agric. Exp. Str. Bull., No. 320; (1932) J. infect. Dis., 51, 268.

Edwards, R. T. (1905) J. infect. Dis., 2, 431.

Eijkman, C. (1904) Zbl. Bakt., 37, 436, 742: (1914) Ibid., IIte Abt., 39, 75.

Elbert, B. J. and Guerkess, W. M. (1930) Ann. Inst. Pasteur, 44, 548.

Elrod, R. P. (1942) J. Bact., 44, 433.

Escherich, 'T. (1885) Fortschr. Hed., 3, 515, 547.

Ferramola, R. (1940) Amer. J. publ. Hith., 30, 1083.

Fitzgerald, J. G. (19I1) J. infect. Dis., 15, 268.

Fremlin, H. (1S93) Arch. Hyg., Berl., 19, 295.

Fricke. C. (1S96) Z. Iygg. Infehthr., 23, 380.

Friedländer, C. (1S83) Fortschr. Hed., 1, 715.

Gardner, A. D. (1925) J. Path. Bact., 28, 189.

Gosuings, W. R. O. (1934) Zbbl. Bakt., 133, 33 ; (1935) Ibid., 134, 195.

Goslings, W. R. O. and Snijners, E. P. (1936) Zbl. Balt., 136, I.

GraAfF, IV. C. DE (1936) Ant. v. Leewwenhoek ned. Tijdschr. Hyg., 3, 18.

Gray, J. D. A. (1931) J. Path. Bact., 34, 335.

Griffin, A. M. and Stuart, C. A. (1940) J. Bact., 40, 83.

Grimbert, L. and Legros, G. (1900) C. R. Soc. Biol., 52, 491.

Guth, F. (1916) Zlu. Bakt., 9y, 487.

Gwatkin, R., LeGard, H. M., and Hadwen, S. (1938) Canad. J. comp. Med., 2, 155.

HADLEY, P. (1927) J. infect. Dis., 40, 1.

Harden, A. (1901) J. chem. Soc., 99, 610 ; (1905) J. Hyg., Camb., 5, 485; (1906) Proc. roy. Soc., $B$, yry, 424 .

Harden, A. and Norris, D. (1911) Proc. roy. Soc., B, 84, 492.

Harden, A. and Walpole, G. S. (1906) Proc. roy. Soc., B, 7y, 399.

Havens, L. C. and Mayfield, C. R. (1933) J. infect. Di.s., 52, 157.

Hay, H. R. (1932) J. IIyg., Camb., 32, 240.

Heidelberger, M., Goebel, W. F., and Avery, O. T. (1925) J. exp. Med., 42, 701.

Hennebera, $W^{7}$. and Wendt, H. (1935) Zbl. Bakt., iite Abt., 93, 39.

Hoffian, IV. and Ficker, M. (1904) Hyg. Rundsch., 14, 1.

Hort, E. C. (1920) J. Hyg., Camb., 18, 369.

Howe, F. (1904) Zbl. Bakt., 36, 484.

Hultox, F. (1916) J. infect. Dis., 19, 606.

Hynes, M. (1942) J. Path. Bact., 54, 193.

JaCkson, D. D. (1911) J. infect. Dis., 8, 241.

Jampolis, M., Howell, K. M., Calvin, J. K., and Leventhal, M. L. (1932) Amer. J. Dis. Child., 43, 70.

Johnson, B. R. (1916) J. Bact., 1, 96.

Jonts, H. N. (1924) Science, 60, 455.

Jones, H. N. and WISE, J. E. (1926) J. Bact., 11, 359.

Jordan, E. O. (1S90) Report on Water Supply and Sewerage, Mass. Bd. Hlth., Pt. 2, p. $\$ 36 ;$ (1903) Science, 3, 1 .

Jolianelle, L. A. (1926a) J.exp. Med., 44, 113; (1926b) Ibid., 44, 683; (1926c) Ibid., 44, 735; (1930) Ibill., 52, 539; (1935) J. Bact., 30, 535 ; (1937) J. Immunol., 32, 21.

KeYEs, F. G. (1909) J. med. Res., 21, 69.

KhaLED, Z. (1923) J. IIyg., Camb., 21, 362.

KLIEWE, H. and Hsü, I. (1935) Z. ImmunForsch., 86, 481.

Kligler, I. J. (1914a) J. infect. Dis., 14, 81 ; (1914b) Ibid., 15, 187.

KLINE, E. K. (1935) Amer. J. publ. Hlth., 25, 833.

Knox, R., Gell, P. G. H. and Pollock, M. R. (1943) J. IIyg., Camb., 43, 147.

Koser, S. A. (1918) J. infect. Dis., 23, 377 ; (1923) J. Bact., 8, 493; (1924) Ibid., 9, 59 ; (1926a) J. Amer. IVat. H'ks. Ass., 15, 641; (1926b) J. Bact., 11, 409; (1926c) J. infect., Dis., 38, 506 .

Kramá, E. (1921) Zbl. Bakt., 8\%, 401.

Krumwiede, C. and Pratt, J.S. (1914) J.exp. Mel., 19, 501.

Kruse, W. (1894) Z. Hyg. Infehtkr., 1\%, I.

Leifson, E. (1933) J. Bact., 26, 329 ; (1935) J. Path. Bact., 40, 581 ; (1936) Amer. J. Hyg., 24, 423 . 
Lentz, O. and Tietz. J. (1903) Hünch. med. Wschr., 50, 2139; (1905) Klin. Jb., 14, 495.

Levine, II. (1916q) J. Burt., 1, 87; (1916b) Ibid., 1, 153; (1916c) J. infect. Dis., 18, 358; (1916d) Ibid., 19, 773; (1917) Amer. I. publ. Ilth., \%, 784; (1918) J. Bact., 3, 253; (1941) Amer. J. publ. Hlth., 31, 351.

Levine, M., Epstein, S. S., and Taughs, R. H. (1934) Amer. J. publ. Hlth, 24, 505.

LOEFFler, F. (1903) Dtsch. med. Wrshr., 29, 36; (1906) Ibid., 32, 289.

Loewenberg. (1894) Ann. Inst. Pasteur, 8, 292.

Lovell, R. (1937) J. Path. Bact., 44, 125.

MacConkey, A. (1905) J. Hyg., Camb., 5, 333 ; (1909) Ibirl., 9, 86.

Mackenzie, E. F. W. and Hilton-Sergeant, F. C. (1938) J. R. A rmy med. Cps., y0, 14, 73.

Mackie, T. J. (1913) J. Path. Bact., 18, 137.

Manfredi, L. (1917) Rif. Med., 33, No. 35, 849.

Massini, R. (1907) Arch. Hyg., Berl., 61, 250.

Mirra, G. (1936) Ann. Med. mav. colon., 42, 205.

Mitchell, N. B. and Levine, M. (1938) J. Bact., 36, 587.

Morris, M. C. and Julianelle, L. A. (1934) J. infect. Dis., 55, 150.

Muller, L. (1923) C. R. Soc. Biols, 89, 434.

Neuber, E. (1934) Arch. Derm. Syph., Wien., 1\%0, 154.

Osterian, E. and Rettger, L. F. (1941) J. Bact., 42, 699, 7:1.

PAge, C. G. (1912) J. med. Res., 26, 489.

Parr, L. W. (1937) Proc. Soc. exp. Biol., N.Y., 35, 563 ; (1938) Amer.J. publ. Hlth, 28, 445 ; (1939) Bact. Rev., 3, 1.

Pawan, J. L. (1931) J.trop. Med. Hyg., 34, 229, 267, 288, 310, 317, 345, 360, 350, $391,413$.

Perez, F. (1899) Ann. Inst. Pasteur, 13, 937.

Perkins, R. G. (1904) J. infect. Dis., 1, 241; (1907) Ibid., 4, 51.

Perry, C. A. (1939) Food Res, 4, 381.

Petruschky, J. (1889) Zbl. Bakt., 6, 625, 657; (1890) Ibid., \%, 49.

PFEIfFer. (1889) Z. Hyg. Infekthr., 6, 145.

PiJper, A. (1938) J. Path. Bact., 4\%, 1.

Platt, A. E. (1935) J. Hyg.. Camb.. 35, 437.

Porges, O. (1905) Wien. klin. Wischr., 18, 691.

Prášek, E. and Prica, M. (1933) Zbl. Brakt., 128, 381.

Prica, M. (1930) Zbl. Bakt., 115, 334.

Quast, G. (1926) Zbl. Bakt., 97, 174.

Raghavachari, T. N. S. and Irer, P. I. S. (1939a) Indian J.med.Res., 26, 867 ; (1939b) Ibid., 26, 877 .

Raven, C., Pedex, D., and Wright, H. D. (1940) J. Path. Bact., 50, 257.

Refik, E. (1896) Ann. Inst. Pasteur, 10, 242.

RennebauM, E. H. (1935) J. Bact., 30, 625.

Rogers, L. A., Clark, W. M. and Davis, B. J. (1914) J. infect. Dis., 14, 411.

Rogers, L. A., Clark, IV. M., and Evans, A. C. (1914) J. infect. Dis., 15, 99 ; (1915) Ibid., 1\%, 137.

Rogers, L. A., Clark, W. M., and Lubs, H. A. (1918) J. Bact., 3, 231.

Rотн, E. (1903) Hyg. Rundsch., 13, 489.

Rothberoer, C. J. (1898) Zbl. Bakt., 24, 513.

Russeli, H. L. and Bassett, V. H. (1899) Proc. Amer. publ. Iflth Ass., 25, 570.

SANDIFORD, B. R. (1935) J. Path. Bact., 41, 77.

SevitT, S. (1945) J. Hyg., Camb., 44, 37.

Sherwood, H. P. and ClegG, L. F. L. (1942) J. Hyg., Camb., 42, 45.

Small, J. C. and Julianelle, L. A. (1923) J. infect. Jis., 32, 456.

Sнттн, D. E. (1927) J. exp. Med., 46, 155.

Sмiтн, T. (1890) Zbl. Baht., \%, 502 ; (1893) Misc. Invest. infcet. parasit. Dis. Dom. Animals, $8^{\circ}$ Washington, 53 ; (1895) Amer. J. med. Sci., 110, 283.

Smith, 'T. and Little, R. B. (1922) J.exp. Med., 36, 1s1. 453; (1923) Ibid., 3\%, 671; (1927) Ibid., 46, 123.

Sнитн, T. and Orcutт, MI. I. (1925) J. exp. Hed., 41, 89.

Stamp and Stone, D. II. (1944) J. Hyg., Camb., 43, 266.

Steinberg, B. and Ecker, E. E. (1926) J. exp. Med., 43, 443.

Streit, H. (1906) Zbl. Balit., 40, 709.

Stuart, C. A., Baker, M., Zmimeritax, A., Brown, C., and Stone, C. M. (1940) J. Bact., $40,101$.

Stcart, C. A., Griffin, A. M., and Bakfr, M. E. (1938) J. Bact., 36, 391.

Stuart, C. A., Wheeler, K. Ml., Rustigian, R., and Zimmerman, A. (1943) J. Bact., 45, 101.

Strart, C. A., Zimiermax, A., Baker, M., and Rustigian, P. (1942) J. Bact., 43, 557.

Tittsler, R. P. (1938) J. Bact., 35, 157.

Tittsler, R. P. and SANDholzer, L. A. (1936) J. Bact., 31, 301. 
Toenniessen, E. (1912) Zbl. Bakt., 65, 23 ; (1913) Ibid., 69, 391 ; (1914) Ibid., 73, 241 ; (1921) Ibid., 85, 225.

ToMÁš, V. (1925) Zbl. Bakt., Ref., 79, 564.

VAUGhN, R. and Levine, M. (1936a) J. Bact., 31, 24; (1936b) Ibid., 32, 65.

Vaughn, R., Mitcheli, N. B., and Levine, M. (1939) J. Amer. Water Works Ass., $31,993$.

Voges, O. and Proskauer, B. (1898) Z. Hyg. InfektKr., 28, 20.

Wallace, G. I., Cahn, A. R., and Thomas, L. J. (1933) J. infect. Dis., 53, 386.

WЕвв, J. L. (1937) J. Hyg., Camb., 37, 307.

Webster, L. T. (1928) J. exp. Med., 47, 685; (1930) Ibid., 52, 909.

WerkmaN, C. H. and Gillen, G. F. (1932) J. Bact., 23, 167.

WIELENGA, D. K. (1937) "Onderzoekingen over het Scleroma respiratorium en de Groep der Kapselbakteriën." N.V. Noord-Hollandsche Uitgeversmaatschappij, Amsterdam.

Wilson, G. S., Twiga, R. S., Wright, R. C., Hendry, C. B., Cowell, M. P., and Maier, I. (1935) Spec. Rep. Ser. med. Res. Coun., Lond., No. 206.

WILson, W. J. (1933) J. Hyg., Camb., 33, 404.

Wilson, W. J. and Blair, E. M. Me V. (1941) Brit. med. J., ii, 501.

Winslow, C.-E. A., Broadhurst, J., Buchanan, R. E., Krumwiede, C., Rogers, L. A. and Sмiтн, G. H. (1917) J. Bact., 2, 505 ; (1920) Ibid., 5, 191.

Winslow, C.-E. A., Kligler, I. J., and Rothberg, W. (1919) J. Bact., 4, 429.

Winslow, C.-E. A. and Lochridge, E. E. (1906) J. infect. Dis., 3, 547.

Winslow, C.-E. A. and Walker, L. T. (1907) Science, 26, 797.

Wright, J. H. and Mallory, F. B. (1895) Z. IIyg. InfektKr., 20, 220.

Wortz, R. (1892) Arch. Méd. exp., 4, 85. 


\section{CHAPTER 29}

\section{SHIGELLA}

\section{Definition.-Shigella.}

Gram-negative, non-motile rods, $2-3 \mu$ long by $0 \cdot 5-0 \cdot 7 \mu$ broad. Non-capsulated; non-sporing. Ferment a variable number of earbohydrates with the production of acid. Lactose is not attacked except by a few species, and then not for two days or more. Reduce nitrates to nitrites, form ammonia but not liydrogen sulphide, are Voges-Proskauer negative, and fail to grow in Koser's citrate. Facultative anaerobes. Some species are antigenically related. At least one species produces a toxin. Most speeies are pathogenie to man, giving rise to dysentery or sometimes acute gastro-enteritis. Found, as a rule, in the intestinal traet of human dysentery patients and eontaets.

Type speeies, Shigella shige.

Without entering here into the rexed question of classification (see p. 696), we propose to discuss in this chapter the following organisms or groups of organisms: (1) Sh. shigce, described by Shiga $(1898 a, b, 1901)$ in Japan, and Kruse $(1900,1901)$ in Germany; (2) Sh. schmitzi, isolated by Schmitz (1917) in Rumania, and called B. ambiguus by Andrewes (1918); (3) Other non-mamnitol fermenters described by Dudgeon and Urquhart (1919) in Macedonia, by Large and Sankaran (1934) and by Sachs (1943) in India, by Hazen (1938) in the United States, and by Ali (1938) in Egypt, and referred to as para-Shiga bacilli; (4) Sh. flexneri, described by Flexner $(1900 a, b)$ in the Philippines, and Strong and Musgrave (1900) in Manila; (5) The organism isolated by Clayton and Warren $(1929 a, b)$ known as the Newcastle bacillus, by Downie, Wade and Young (1933) known as the Manchester bacillus, and by Boyd $(1931,1932)$ in India known as 88 ; (6) A group of mannitol fermenters resembling $S h$. flexneri biochemically, but differing from it antigenically, described by Boyd $(1931,1932,1936,1938)$ in India under the names 170, P288, and D1; (7) Sh. alkalescens, described by Andrewes (1918); (8) Sh. sonnei, a late-lactose-fermenting organism defined by Sonne (1915) in Denmark, though almost certainly described by previous workers in the United States and Germany (see Koser et al. 1930, Bojlén 1934) and probably identical with Kruse's Type E bacillus; (9) Sh. dispar, a late-lactose-fermenting organism described by Andrewes (1918), and possibly related to the organisms described by Castellani $(1907,1912 b)$ as $B$. ceylonensis $B$ and $B$. madampensis. A few other organisms of less importance will receive occasional mention.

Morphological and cultural Characters.-Dysentery bacilli are non-motile, Gram-negative organisms indistinguishable morphologically from members of the Bacterium group. Their cultural characteristics are likewise insufficiently distinctive to require separate description from those of the Bacterium and Salmonella groups. It may be noted, however, that the colonies of Sonne's bacillus tend to 
be larger and more opaque than those of the shiga-Flexner types. On primary cultivation from the frees this organism forms colonies on agar that are circular, convex, $2 \mathrm{~mm}$. in diameter, colourless, fairly translucent, with a smooth surface and entire edge ; when viewed against a dark background they appear whitish and relatively opaque. Some colonies show, at one or more portions of the periphery, a small tangled-hair-like projection, giving to the colony a bursting bombshell appearance (Braun and Weil 1928). Sometimes on first isolation, but nearly always in early subculture, a second type of colony is seen, which is larger, raised, translucent, with a coarsely ground-glass surface and an irregularly undulate or crenated edge; when viewed against a dark background it appears clearer and less opaque than the first type. These two types of colony, which consist of organisms differing antigenically and in certain other respects, have been described by several workers (Mita 1921, Orskov and Larsen 1925, Leuchs and Plochmann 1927, Fyfe 1927, Large 1929, Camn and de Navasquez 1931, Thjotta and Waaler 1932, Johnston and Kaake 1932, Waaler 1935, Glynn and Starkey 1939). They are generally regarded as corresponding to smooth and rough forms; though, as Bridges (see Report 1939) points out, the antigenic change that accompanies the colonial transition resembles in some respects the specific-group rather than the smooth-rough type of variation.

Morphological and cultural variants of the dysentery bacilli have been described by several workers. Differences have often been noted in the antigenic structure, saline and acid agglutinability, and pathogenicity for laboratory animals of the rough and smooth variants. Indeed, as in members of the Salmonella group, antigenic structure is of far more importance in defining smooth and rough types than the colonial appearance. (For references to variation see Arkwright 1921, Carver 1921, Isabolinsky 1926, Koser and Styron 1930, Braun and Baake 1930, Kobayashi et al. 1931, Johnston and Kaake 1932, Wyckoff 1933, Waaler 1935.)

Resistance and Metabolism.-The members of this group are not specially resistant. They are killed by a temperature of $55^{\circ} \mathrm{C}$. in 1 hour, by 0.5 per cent. phenol in 6 hours, and by 1 per cent. phenol in about 15-30 minutes. When dried on linen and kept in the dark at room temperature, they survive for from 5 to 46 days (Vaillard and Dopter 1903, Roelcke 1938). In garden earth at room temperature in the dark they survive for 9 to 12 (lays (Roelcke 1938). In naturally infected frecs kept alkaline and prevented from drying they may remain alive for some days, but in stools that are allowed to become acid through growth of coliform or other bacilli, they perish often in a few hours. On the whole, Sonne's bacillus is more resistant to inimical agents generally than the Shiga or Flexner bacillus.

They are aerobes and facultative anaerobes. Their optimum temperature is about $37^{\circ} \mathrm{C}$. According to Braun and Weil (1928) Sonne's bacillus grows as readily at $45^{\circ} \mathrm{C}$. as at $37^{\circ} \mathrm{C}$, and more readily at $10^{\circ} \mathrm{C}$. than most coliform bacilli. Shiga's bacillus is characterized by its inability to form catalase.

With the exception of some strains of Sh. alkalescens, none of the members appears capable of producing a hrmolysin active against sheep corpuscles.

Biochemical Reactions.-Sh. shigx, Sh. schmitzi, and members of the paraShiga group produce acid from glucose; the remaining members, with the exception of some strains of the Newcastle bacillus, also ferment mannitol. Hence, the primary classification of the group is into mamitol and non-mannitol fermenters. $S h$. sonnei and $S h$. dispar produce acid from lactose ; the fermentation, however, 
is slow and is not usually apparent for 2 to 10 days. Lactose-fermenting papillie often develop on the original lactose-negative colonies olstained by primary plating. Sucrose is also fermented late, though, according to Forsyth (1933), Sh. dispur acidifies sucrose before lactose. Sucrose is attacked by a small minority of Flexner strains after subculture in the laboratory for a variable length of time, but never on first isolation. Dulcitol is fermented rapidly by $S h$. alkalescens, and slowly by the Newcastle bacillus and the dispar-like organism known as Bact. ceylonense $B$. The Newcastle bacillus is distinguished from other members by its ability to form gas in glucose and dulcitol. The quantity of gas is very small, often amounting to not more than a bubble in a Durham fermentation tube, and may be evident only on first isolation. The closely allied Manchester bacillus forms a trace of gas in mannitol as well as in glucose and dulcitol; but the antigenically similar organism met with in India and in the United States, known as Type 88 , forms $n$ o gas at all. Sh. alkalescens is distinguished from Sh. flexneri by the fermentation of dulcitol and xylose; but according to de Assis (1939a), the substance of greatest differential value is 5 per cent. glycerol, which is always attacked by the alkalescens and not by the flexneri type. Sh. alkalescens is said by Wood and Keeping (1944) to be unique among the dysentery bacilli in its ability to form trimethylamine from ehohine. Sh. dispar ferments xylose and sorbitol, thus differing from $S h$. sonnei; it must be pointed out, however, that xylose-fermenting strains of Sh. somei do occur (Bojlén 1934, Cruickshank and Swyer 1940), though the great majority of strains isolated in Great Britain and the United States lack the power to ferment this sugar. In litmus milk there is generally a slight acidity for a few days. The reaction may remain permanently acid and go on to clotting, as with Sh. sonnei and Sh. dispar, or it may revert to nentral, as with sh. shige, Sh. schmitzi, and Sh. flexneri. Nany strains of flexneri, after a preliminary acidity, turn milk alkaline. Sh. alkalescens produces an initial and lasting alkalinity.

Indole formation is of some differential importance, serving to distinguish Schmitz's from Shiga's bacillus, and Sh. dispar from Sh. somei. The Newcastle bacillus does not form indole, but Sh. alkulescens does. As regards the Flexner group, Gettings (1919) found that of 285 strains tested, 158 produced indole, and 127 did not. The methyl-red test is of limited ralue; if the cultures are incubated at $37^{\circ} \mathrm{C}$, it may help to distinguish the positive Sh. dispar from the negative Sh. sonnei; but, according to Bamforth (1934), Sh. sonnei may give a positive result if it is incubated at $30^{\circ} \mathrm{C}$.

All strains reduce nitrates to nitrites; none forms $\mathrm{H}_{2} \mathrm{~S}$; none grows in Koser's citrate; and none gives a positive Voges-Proskauer reaction. A list of biochemical reactions is given in Table 45. (For reference to the more recent studies on these reactions, see Lester 1926, Smith and Fraser 1928, Kerrin 1928, Nelson 1930, Bojlén 1930, 1934, Johnston and Brown 1930, Buchanan and Roux 1930, Koser et al. 1930, Cam and de Navasquez 1931, Welch and Mickle 1932, 1934, Downie et al. 1933, Forsyth 1933, Bamforth 1934, Whitehead and Seott 1934, Large and Sankaran 1934, Mandry 1935, McGinnes et al. 1936, Boyd 1931, 1932, 1936, 1938, Hazen 1938, Ali 1938, Hardy et al. 1940, Sachs 1943).

Antigenic Structure.-The serological behaviour of the dysentery bacilli is complicated. Of the non-mannitol fermenters the Shiga group is homogeneous; all strains of Sh. shiga are agglutinated by a specific serum prepared against any one strain. An anti-shigæ serum has some agglutinating action on- Schmitz's bacillus and on some strains of the Flexner group. A serum prepared against Schmitz's 


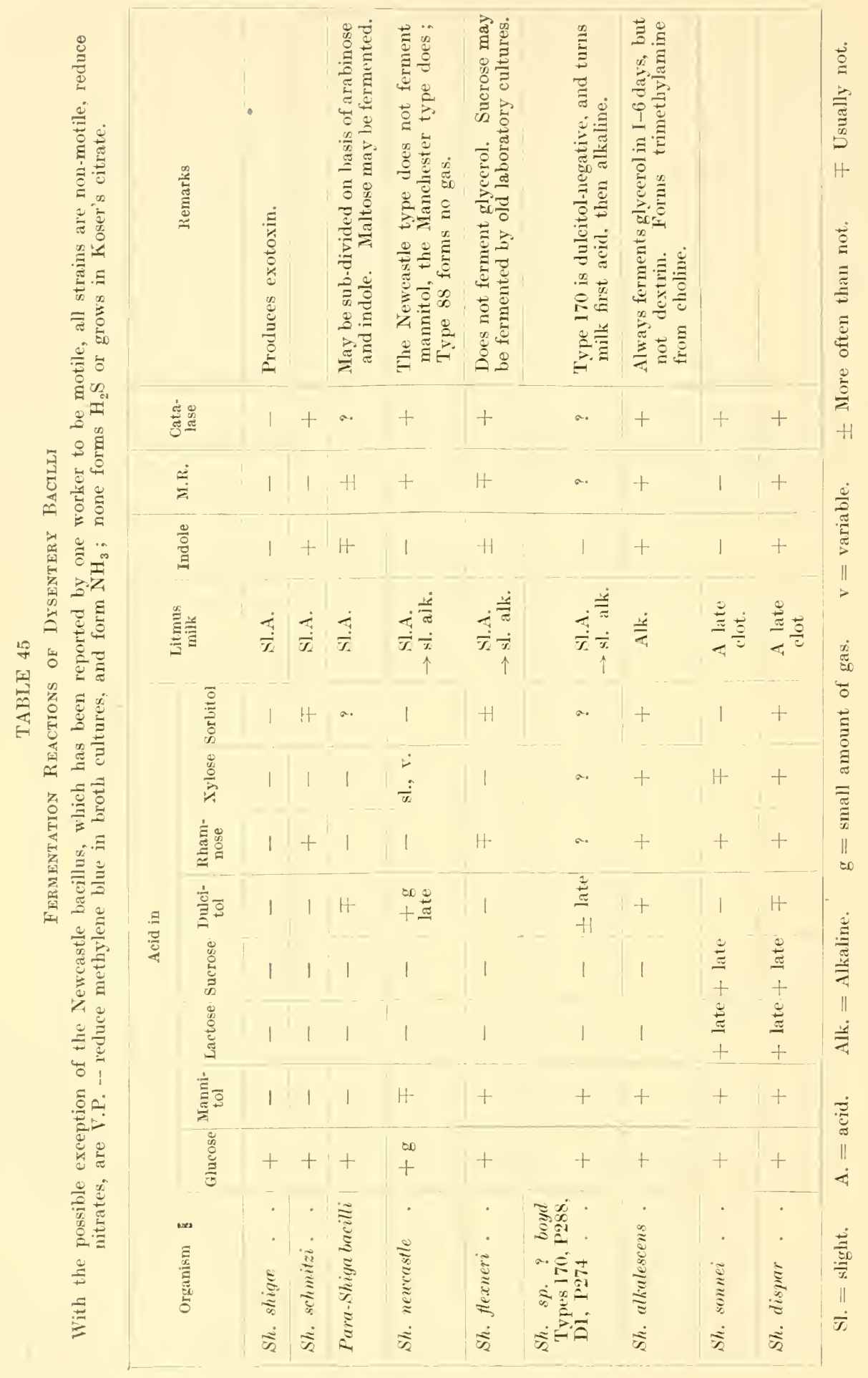


bacillus will agglutinate a Shiga bacillus to $\frac{1}{4}$ or $\frac{1}{2}$ titre; but antigenically Schmitz's bacillus and Shiga's bacillus are easily distinguishable; a Shiga bacillus cannot absorb the agglutinins from a serum prepared against Schmitz's bacillus, nor a Schmitz bacillus from an anti-shigæ serum. According to Schütze (1944) Shiga strains vary in their agglutinability; hypoagglutinable strains may be rendered more agglutinable by growth at $20-26^{\circ} \mathrm{C}$., by heating in saline to $60^{\circ} \mathrm{C}$. for 1 hour, or by adding 0.5 per cent. phenol.

Schmitz's bacillus used to be regarded as antigenically homogeneous, but the observations of Boyd (1935) suggest that the freshly isolated organisms contain two antigens which, for convenience, may be referred to as type and group. It is believed that after some time in the laboratory the type antigen is lost and the group alone remains. A serum, therefore, prepared against freshly isolated bacilli will agglutinate both recent and old strains; a serum prepared against old strains will have little or no action on recent strains. The picture seems to be very similar to that which will be described below for the 103 type of Flexner's bacillus, though it is still doubtful whether the variation is to be regarded as of the type and group or the smooth and rough order.

Little is yet known of the antigenic structure of the para-Shiga group of dysentery bacilli. Durgeon and Urquhart (1919), who studied 11 strains, found them to be antigenically alike. Similar findings were reported by Hazen (1938). Ali (1938), however, was able by agglutination and absorption of agglutinins to distinguish four serological groups among 8 strains; and Sachs (1943) was able to distinguish eight serological groups among 107 strains (see also Large and Sankaran 1934, Christenson and Gowen 1944). It would appear that the para-Shiga group of bacilli is far from being antigenically homogeneous. None of the members appears to be related to either Sh. shiga or Sh. schmitzi.

The mannitol-fermenting group has long been recognized as antigenically heterogeneous. The work of Gettings (1919), Murray (1918), and Andrewes and Inman (1919) afforded a picture of the Flexner bacillus as containing at least four antigenic components.

According to Andrewes and Inman, each of these components, which they refer to as $\mathrm{V}, \mathrm{W}, \mathrm{X}$ and $\mathrm{Z}$, is represented to some extent in every strain, but in any given strain there is usually a preponderance of one antigen over the rest. In certain races, $\mathrm{V}, \mathrm{W}$, and $\mathrm{Z}$, there is so great a preponderance of a single antigenic component, different in each instance, over the rest, as to make them behave like distinct serological types; each race requires its own antiserum for adequate agglutination. The $\mathrm{X}$ race is peculiar in that it will not agglutinate with any sera but its own; yet it is able to give rise to a serum that will agglutinate not only $\mathrm{X}$ races, but also $\mathrm{Z}$, and, to a certain extent, $\mathrm{V}$ races. The agglutinins corresponding to each of these four types cannot be more than partially absorbed by the others. Andrewes and Inman found at least two sub-races, VZ and WX; these were members of the $\mathrm{V}$ and $\mathrm{W}$ races respectively, but contained so large a proportion of a second antigenic constituent as to modify their serological behaviour. One race, which is called $Y$, and which corresponds to the original Y-strain of Hiss and Russell (1903), contains a more evenly balanced mixture of $\mathrm{V}, \mathrm{W}$, and $\mathrm{Z}$ components, with a small amount of $\mathrm{X}$. For this reason a serum prepared against a $\mathrm{Y}$ strain is more cosmopolitan than the rest, having a wide range of agglutination (Fig. 144).

This conception has been challenged by the work of Boyd (1931, 1932, 1936, 


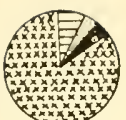

Flexner $V$.

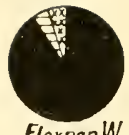

FlexnerW.

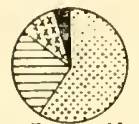

Flexner $X$.

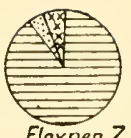

Flexner $Z$. Flexner Y.

Fig. 144.-Diagram representing the Variation in Antigenio STRUCTURE OF Shigella flexneri.

(After Andrewes.)

1938) in India. Studying a strain of dysentery bacillus known as 103, which was biochemically similar to Flexner's bacillus, but which, when newly isolated, was not agglutinated by antisera to the $\mathrm{V}, \mathrm{W}, \mathrm{Y}$, and $\mathrm{Z}$ races, and only very feebly by an $\mathrm{X}$ antiserum, Boyd observed that after some time in artificial culture it gave rise to two types of colony. One of them, referred to as 103A, was in all respects identical with the smooth circular colonies of the freshly isolated strain, and was virtually inagglutimable by $\mathrm{V}$ to $\mathrm{Z}$ antisera. The other, referred to as 103B, was slightly larger than normal and somewhat rongh in ontline, and was agglutinated readily by antisera of the $\mathrm{V}$ to $\mathrm{Z}$ group. This variant differed further from $103 \mathrm{~A}$ in that it bred true, whereas $103 \mathrm{~A}$ behaved like the parent strain in consistently giving rise to a variant of the 103B type. Further study showed that a serum prepared against $103 \mathrm{~A}$ agghtinated both $103 \mathrm{~A}$ and $103 \mathrm{~B}$, but that a serum prepared against $103 \mathrm{~B}$ had practically no action on $103 \mathrm{~A}$. Absorption of agglutinin experiments confirmed the suggestion that $103 \mathrm{~A}$ contained two antigens a type and a group-but that $103 \mathrm{~B}$ contained only the group antigen. Since 103B grew uniformly in broth and was perfectly stable in saline, Boyd concluded that the variation he had observed was more of the phasic than of the smooth-rough order. It differed from the type-group variation seen in the flagellar antigens of organisms of the Salmonella genus in that it was irreversible. The time at which the $\mathrm{A} \rightarrow \mathrm{B}$ variation occurs varies greatly from strain to strain. Sometimes the parent culture may remain stable for months or years; at others it throws off $B$ variants on first subculture or even in the body.

Similar observations were made with other strains of Flexner's bacillus, such as P119 and the 88 strain of the Neweastle-Manehester bacillus. Absorption of agglutinin experiments seemed to show that the $\mathrm{V}$, $\mathrm{W}$, and $\mathrm{Z}$ races each contained
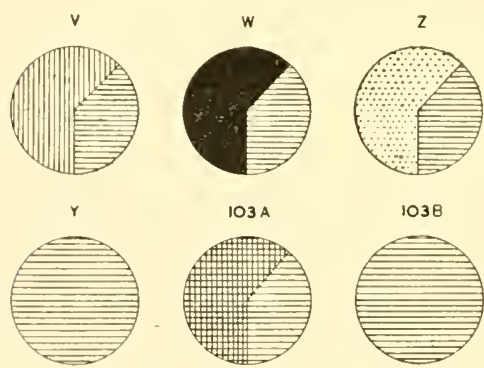

$103 \mathrm{~A}$

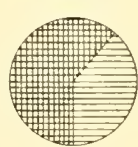

1038

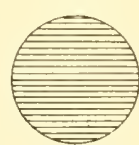

Fig. 145.-DIAGRAM REPRESENTING THE Variation in Axtigenic Structure of Shigella flexneri.

The different components of the eomposite group antigen has been omitted for the sake of simplicity.

(After Boyd.) a specific and a group antigen. The $\mathrm{Y}$ race contained group antigen only. The group antigen was not completely homogeneous; in fact Boyd obtained evidence of at least six different components. The $\mathrm{X}$ race was at first thought to be similar to the others, but later Boyd (1940) came to regard it not as a separate race but as an incomplete variant of $\mathrm{Z}$. It will be seen that in contradistinction to Andrewes and Inman who regarded $\mathrm{V}, \mathrm{W}, \mathrm{X}$, and $\mathrm{Z}$ as each containing the same four antigens, one of which alone was dominant in each race, Boyd believes that $\mathrm{V}, \mathrm{W}$, and $\mathrm{Z}$ share some components of a common group antigen but that their specific antigens are distinct (Fig. 145). On 
this view, Boyd (1940) would classify all strains containing the group antigen, including the Newcastle bacillus, as belonging to the Flexner variety, numbering the different races according to their specific antigen, and would include other strains devoid of the group antigen but biochemically similar to Flexner's bacillus in a new species for which the name of boyd has been suggested.

Thus:

\begin{tabular}{|c|c|c|}
\hline & $\begin{array}{c}\text { Old } \\
\text { designation }\end{array}$ & $\begin{array}{c}\text { New } \\
\text { designation }\end{array}$ \\
\hline Sh. flexneri & $\mathrm{V}$ & I \\
\hline , & IV & II \\
\hline , & Z & III \\
\hline , & 103 & IV \\
\hline , & P119 & $\mathrm{V}$ \\
\hline ,", & Newcastle-Manchester-88 & VI \\
\hline Sh. boyd & 170 & I \\
\hline,$\quad$, & P288 & II \\
\hline, & Dl & III \\
\hline
\end{tabular}

It seems to us very doubtful whether the Newcastle bacillus should be included in the Flexner group. Though it may share some of the component antigens of this group, it differs sufficiently in its biochemical characters to justify its being allocated provisionally to a species of its own.

Boyd's work, which is of fundamental importance, has been substantially confirmed by Wheeler $(1944 a, b)$ in the United States. Wheeler has extended the six component factors of the Flexner group antigen to nine; he agrees that Type $\mathrm{X}$ has no specific antigen and therefore camnot be regarded as a type; and he finds that the specific antigen of the $\mathrm{W}$ race may be combined with two different sets of group antigen components.

Weil, Black and Farsetta (1944), neglecting the presence of minor antigens, found no group antigen common to the Flexner types, and therefore proposed that the Flexner and Boyd groups should be regarded as belonging to a single series and classified according to the nature of the primary antigen. Fourteen types were distinguished in this way, labelled I to XIV. Neither Wheeler nor Weil and his colleagues seem to have recognized the form of variation described by Boyd in his strains 103 and P119. Weil worked with a relatively few strains, many of them isolated a considerable time previonsly, and it would therefore be unwise to lay too much stress on his findings. All that we can say at present is that Boyd's classification, though admittedly imperfect, appears to provide us with the best working model for the serological examination of the Flexner dysentery group. Takita (1937) observed in Flexner bacilli a variation similar to that in 103, but proceeding in the opposite way. It is probable that the discrepancy is apparent not real, depending on the reverse way in which the variants were labelled.

Before leaving Flexner's bacillus it may be noted that several workers have studied its complex antigenic constitution, particularly Kruse and his colleagues (1907) and Lentz and Prigge (1931) in Germany, and Aoki (1921, 1923) in Japan. Sartorius and Reploh (1931, 1932), Clauberg (1932), Kemper (1933), and Nagakura (1937) have endeavoured to relate the different types established by various workers to each other, but with only partial success. Further observations will be required before we reach a satisfactory understanding of the problem that this organism presents. 
It is now known that some strains of the Flexner $Y$ variety contain somatic antigens that are found in the Salmonella group. The presence of the vi.xiii combination has been demonstrated by Bornstein, Saphra and Danicls (1941), who point out that care must be taken in the identification of strains on the basis of agglutination.

The antigenic structure of Sh. alkalescens is still in doubt. According to Neter (1938) and Neter and Heide (1940), it contains two antigenic components, one specific and the other shared with the Flexner bacillus. De Assis (19396) found that two serological types conld be established, distinguished by their specific antigens, but that both shared the same group antigen with Sh. flexneri. Archer (1942) noticed that Sh. alkalescens might form two types of colony; against a dark ground one appeared clear, the other opaque. Clear colonies were readily agglutinated; opaque colonies were hypoagglutinable, but were rendered almost completely agglutinable by boiling. Boyd (1938) observed the occurrence of cross-agglutination between Sh. alkalescens and an organism known as P274, which was biochemically similar to Flexner's bacillus, but was not antigenically related to it. Rothstadt, Fenner and Baker (1942) in Australia have brought strong evidence to show that P274 is capable of giving rise to dysentery, and have suggested that it should be classified with the Boyd variety. These results are conflicting. On the one hand, there are observations pointing to the possession by Sh. alkalescens of the group Flexner antigen and on the other to its relationship to the P274 type. From a study of this organism Stuart, Rustigian, Zimmerman and Corrigan (1943) conclude that there is a species-specific antigen $A$, a major antigen $\mathrm{B}$ found also in paracolon and coliform bacilli, a minor antigen $\mathrm{C}$ found in $S h$. flexneri and in paracolon and coliform bacilli, and minor antigens D and $\mathrm{E}$ found again in paracolon and coliform bacilli. These workers observed a series of strains showing biochemical reactions ranging from those of the typical $S h$. alkalescens on the one hand to those of Bact. coli on the other, and their results render it doubtful how far $S h$. alkalescens can as yet be defined as a species. An antigenic relationship between $S h$. alkalescens and some paracolon bacilli was also noted by Sevitt (1945).

Sonne's bacillus presents less difficulty. As has been mentioned, this organism forms two types of colony which, as Large (1929) showed, are serologically distinct. A serum prepared against organisms in the smooth colony phase agglutinates both the smooth and the rough colony organisms, whereas a serum prepared against organisms in the rough colony phase agglutinates only the rough colony organisms. There is a close analogy with the 103 strain of Flexner's bacillus. For practical identification of the organism it is important that a serum should be used containing both types of agglutinins. Sh. dispar is antigenically heterogeneous, though Carpenter (1944) found that 38 ont of 44 strains he studied fell into one type.

It may be noted that the agglutination of dysentery bacilli occurs rather slowly, and that it is advisable to incubate all tests for $4-6$ hours at $50^{\circ} \mathrm{C}$., and to delay the final reading till the tubes have been left at room temperature overnight. This holds particularly for the newcastle, alkalescens, sonnei, and dispar types.

Apart from the serological differentiation of the dysentery bacilli, certain indirect tests may be used to distinguish between the types, such as Michaelis's (1917) acid agglutination test, and susceptibility to action of the bacteriophage. The acid agglutination test depends on the different $\mathrm{H}$-ion concentrations necessary for flocculation. Using the particular range employed by Michaelis, Andrewes and 
Inman (1919) found that Schmitz's bacillus, Sh. alkalescens, and Sh. dispar were agglutinated, whereas $S h$. shigce and Sh. flexneri were not. The test is only a rough one, and it is doubtful whether the information it furnishes justifies its use under ordinary conditions. On the other hand, the susceptibility of different types of dysentery bacilli to the action of certain phages appears to be very much more specific. According to Burnet and McKie (1930), whose article should be consulted for further details, bacteriophages that are active against Flexner bacilli can be divided into four main groups. One of these groups is capable of lysing bacilli only in the smooth phase, while the other three may or may not lyse smooth strains but generally lyse rough strains of all types. Characteristic differences in their sensitivity to a series of phages are presented by the $V, W, X, Y$, and Z types of $S h$. flexneri.

Chemical Fractionation.-Early observations on the chemical structure of the antigens of the dysentery bacilhus revealed the presence of specific polysaccharides in Sh. shigoe (Kurauchi 1929, Meyer 1930, 1931, Morgan 1931). Sh. flexneri, and Sh. sonnei (Kurauchi 1929). The more recent work of Boivin and Mesrobeanu (1937a-h, 1938), Mesrobeanu and Boivin (1937), and Haas (1937, 1938 ), carried out by use of the trichloracetic acid technique, shows that the smooth, but not the rough, somatic antigen of the Shiga and Flexner bacilli contains a polysaccharide hapten, which is linked to nitrogenous and lipoid compounds to form a complete antigen. Similar polysaccharides have been demonstrated in Sh. schmitzi (Haas 1938c), Sh. sonnei (Haas 1938b), and Sh. alkalescens (see Weil 1943). The still more recent studies of Morgan (1936, 1937) and Morgan and Partridge (1940, 1941), who used the less drastic method of extraction of acetone-treated bacterial cells with diethylene-glyeol, secm to show that the complete smooth somatic antigen of Shiga's bacillus consists of a specific polysaccharide hapten, a non-antigenic conjugated protein, and a non-antigenic phospholipin of the cephalin type. The polysaccharide is strongly dextrorotatory and yields 97 per cent. of reducing sugars on acid hydrolysis. When combined with the conjugated protein, it forms a powerful antigen. It is interesting to note that the conjugated protein extraeted from Shiga's bacillus appears to be identical with that found in the somatic antigen of Salmonella typhi. The polysaccharide not only forms a precipitate in the presence of a specific antiserum, but neutralizes specifically the hæmolytic action of shigce heterophile antibody (see Chapter 8) on sheep red corpuscles in the presence of complement (Meyer and Morgan 1935).

Toxin Formation by Dysentery Bacilli.-A large amount of work has been carried out on the formation of toxin by members of the dysentery group, particularly by $S h$. shiga.

In 1903 Conradi prepared an antolysate of dysentery bacilli-probably Shiga's bacillus - which he found to be toxic for rabbits and guinea-pigs. An 18-hours' culture was suspended in saline, and incubated for 24 to 48 hours at $37.5^{\circ} \mathrm{C}$.; after centrifugalization the yellowish supernatant fluid was removed, diluted with 5 times its volume of salime, and filtered through a Berkefeld candle; the filtrate was tested for sterility, and then concentrated to $1 / 10-1 / 50$ of its bulk at $35^{\circ} \mathrm{C}$. This product, when injected intravenously into rabbits or intraperitoneally into guinea-pigs in a dose of $0.1 \mathrm{ml}$, proved fatal in about 48 hours. In rabbits death was precerled by diarrhœa, collapse, and paralysis of the legs; in guinea-pigs by a rapid fall of temperature and collapse. At necropsy Conradi found in both animals congestion of the intestine, mucus and blood adhering to the mucosa, and frequently small bæmorrhages of the mucous and the serous coats. When a smaller dose was injected into rabbits, the animals lived for 4 to 6 days; and he found that post mortem the mucosa of the last third of the large intestine was swollen, blackish-red in colour, and ulcerated in several places.

Neisser and Shiga (1903) confirmed Conradi's results, and noted in addition that the 
toxic substances were precipitated by alcohol and ether, and largely destroyed by heat at $75^{\circ} \mathrm{C}$. Todd (1904) in this country was able to show that 4 to 6 weeks' cultures of Shiga's bacillus contained a soluble toxin, which was highly active on rabbits and horses, but to a much less extent on guinea-pigs, rats, and mice. Flexner's bacillus proved incapable of giving rise to a soluble toxin. Dopter (1905), studying the histological appearances of rabbits dying of paralysis subsequent to injection with 24-hours' broth cultures of $S h$. shigce, observed definite lesions in the spinal cord, consisting chiefly of chromatolysis of the anterior horn cells, sometimes with small interstitial hæmorrhages and focal necroses of the grey matter. The lesions occurred as frequently after the injection of toxin as of bacilli. Further work by Kraus and Dörr (1905) led to the conclusion that Shiga's bacillus gave rise to two toxins: (1) a soluble toxin, present in 8-10-days' broth cultures and in filtered saline suspensions of 24-hours' agar cultures; this was fatal to rabbits but not to guinea-pigs, and gave rise to the production of a specific neutralizing antitoxin; (2) an insoluble toxin present in the bacterial bodies, which was fatal both to rabbits and to guineapigs. No soluble toxins were found in cultures of Flexner's bacillus.

Flexner and Sweet (1906), using a modification of Conradi's method, obtained a toxin from 24-hours' agar cultures of Shiga's bacillus, which, injected intravenously into rabbits, gave rise to diarrhœe, paralysis, convulsions, and death. The paralysis began in the upper limbs and extended at times to the lower limbs. Sometimes the animals survived for 10 days after the extremities were paralysed; they lay on one side in a position of opisthotonos. Post mortem, small hæmorrhages were seen in the brain, and softening of the grey matter in the spinal cord. In the intestine there was congestion of the serosa; the walls of the gut, especially of the cæcum and appendix, were thickened and cedematous, the mucosa was yellowish-white and thrown into deep folds, which were sometimes covered by a pseudo-membrane or stippled with hæmorrhages. The mesenteric glands were swollen, cedematous, and congested. Heat at $81^{\circ} \mathrm{C}$. for 1 hour destroyed the toxin. These observations were largely confirmed by Bessau (1911), who concluded that there were two different toxins-one a paretic or neuro-toxin causing paralysis of the muscles, the other a marasmic or intestinal toxin causing a fall in temperature, diarrhœa, and chronic marasmus. The paretic toxin was neutralized by antitoxin, the marasmic toxin was not. Further, rabbits were affected by both toxins, whereas in guinea-pigs the paretic toxin was without effect.

Bessau's conclusions were supported by the observations of Olitsky and Kligler (1920), who concluded that Sh. shigce formed two toxins: one a neurotoxin acting on the central nervous system of the rabbit and identified by Olitsky and Kligler as an exotoxin; the other having a specific affinity for the intestine and regarded by Olitsky and Kligler as an endotoxin.

This conception was challenged by Okell and Blake (1930), who found that toxin was not liberated from the cell bodies in the absence of autolysis, and therefore concluded that there was only one toxin produced, namely an endotoxin. The more recent chemical work, however, of Boivin and Mesrobeanu $(1937 a-h, 1938)$ leaves little doubt that the dual conception is correct. By means of the trichloracetic acid method these workers were able to demonstrate the presence in cultures of Shiga's bacillus of (1) a thermostable glycolipoid somatic antigen, which they regarded as an endotoxin having an enterotoxic effect, and (2) a thermolabile protein substance, which they regarded as an exotoxin having a neurotropic effect. The exotoxin is specific to Shiga's bacillus, and may be formed by either antigenically smooth or rough strains of this organism (Haas 1937, Istrati 1938, Steabben 1943, Olitzki et al. 1943); the endotoxin is similar to the smooth somatic antigens found in other members of the dysentery group (Haas 1938a, $b, c$ ) and in the Salmonella group (see p. 724), and is absent from antigenically rough strains.

It is perhaps unfortunate that the terms endotoxin and exotoxin have been used to refer to the two types of toxic substances produced by Shiga's bacillus. The so-called exotoxin is closely bound up with the cell bodies and, as the observations of Okell and Blake (1930) showed, (see also Olitzki, Bendersky and Koch 1943), is not excreted 
by the living baeilli but appears free in the culture medium only after antolysis of the dead cells has begmn. Again the implied distinction between enterotoxin and neurotoxin is misleading, since it is apparent that the so-called exotoxin, besides causing paralysis of the limbs, leads also to serious changes in the intestinc, perhaps by causing vasoconstrietion of the intestinal blood vessels (Penner and Bernheim 1942). For the present it is better to refer to the so-called exotoxin by Steabben's term, neuro-enterotoxin.

Snmmarizing our present knowledge, we may say that Sh. shigce gives rise to a specific soluble neuro-enterotoxin, which is present in broth cultures about a week old, in filtered autolysates of 21 -hour agar cultures, and in the dried bacterial hodies. It is destroyed by a temperature of $75^{\circ}-80^{\circ} \mathrm{C}$. maintained for an hour. If prepared by a method favouring bacillary autolysis (Hansen 1936, Takita 1939), it proves fatal to mice inoculated intravenously in a dose of about 0.001 to $0.01 \mathrm{ml}$. It is fatal to the rabbit, causing paralysis of the limbs, diarrhoa and collapse ; but has less action on the guinea-pig, in which diarrhoea and collapse alone are produced. It is a protein, and may be precipitated by trichloracetic acid (Boivin and Mesrobeanu 1937a), and to a less extent by the addition of 40 per cent. solid ammonium sulphate (Blake and Okell 1929). It gives rise to, and is neutralized by, a specific antitoxin, which combines with it in constant proportions. In addition, smooth strains of Shiga's bacillus contain a toxic somatic antigen, which is a lipopolysaccharide conjugated with a protein, resembling similar smooth antigens in the Salmonella and in other members of the shigella group.

With the partial exception of Schmitz's bacillus, the high toxicity of Sh. shige is not rivalled by other dysentery bacilli. The ground-up bacterial bodies of Sh. flexneri, Sh. sonnei, and Sh. dispar prove fatal on intravenous inoculation of rabbits, but only in a dose that is about 20 times greater than the corresponding fatal dose of dried Shiga cells. Schmitz's bacillus is variable in its toxicity. According to Buchwald (1939), it may give rise to a thermolabile neurotoxin, precipitable by trichloracetic acid from old broth cultures, and causing paralysis of the extremities when inoculated into mice and rabbits; but it appears to be rather less potent than that formed by Shiga's bacillus. Sh. alkalescens is said to be non-toxic.

Pathogenicity.-Sh. shigx, Sh. schmitzi, Sh. flexneri, the Neweastle bacillus, Sh. somei, and a number of types described by Boyd (1938, 1940), undoubtedly give rise to dysentery in man. The rôle of Sh. alkalescens is still doubtful, though it may be responsible for infections of the urinary tract. Sh. dispar appears to be non-pathogenic. The evidence in favour of the pathogenicity of some members of the para-Shiga group is strong, but further observations are desirable. (For fuller information see Chapter 70.)

With the exception of captive monkeys, which may carry Sh. Alexneri in the gut (see Lovell 1929), and which may sometimes, as on a vitamin-deficient diet (Verder and Petran 1937, Janota and Dack 1939), develop dysenteric symptoms (Preston and Clark 1938, David and Schirl 1939), and with the possible exception of dogs which may be infected with either Shiga's or Flexner's bacillus (Dold 1916), animals do not appear to suffer from dysentery. It is not possible to reproduce the typical disease, as it occurs in man, by experimental inoculation or feeding of the ordinary laboratory animals. Nevertheless many dysentery organisms, especially $S h$. shiga, are toxic to rabbits, horses, and mice, and to a less extent to guinea-pigs. After subcutaneous inoculation into rabbits, dogs, and young pigs 
the living bacilli may become localized in the intestine and give rise to catarhal and necrotic lesions, which often prove fatal (Vaillard and Dopter 1903).

RABBIrs.-A small dose-0.01 mgm. of a 24-hours' agar culture of Sh. shigo-injected intravenously proves fatal in 1 to 4 days. Death is preceded by diarrhœa, paresis or total paralysis of the extremities, and collapse. Post mortem, there may be hæmorrhages into the subcutaneous tissue and peritoneum; the intestinc, especially the cæcum and colon, is congested and may show submucous hæmorrhages. The mucosa itself is congested, œdematous, and sometimes studded with petechiæ(Vaillard and Dopter 1903, Amako 1908). In the lumen of the gut there is often mucoid or bloody fluid. A similar picture is seen after injection of dead bacilli in larger quantity, or of toxin. If a smaller dose of bacilli is given, there may be time for nccrosis and actual ulceration of the intestine to occur. The living bacilli can be recovered from the mucosa and from the corresponding mesenteric glands. Subcutaneous injection has much the same effect as intravenous, but the animals survive longer. The lesions following injection of Flexner's and Sonne's bacillus are not unlike those produced by $s h$. shige, if a sufficient dose is given, but they are rarely so severe.

Mice.- 0.1 mgm. of a 24-hours' culture of Sh. shige injected intraperitoneally or subcutaneously kills the animal in 1 to 4 days. At necropsy there may be no evident ehange, or there may be catarrhal inflammation of the intestine with watery mucus in the gut. Sh. flexneri and Sh. sonnei often prove fatal on intraperitoneal inoculation of large doses.

Guiven-PIGs.-These animals are less susceptible, weight for weight, to Sh. shiga than are rabbits and mice. The lesions produced by subcutaneous or intraperitoneal injection of living bacilli vary. After a fatal dose there may be no marked macroscopic changes, or there may be intestinal lesions similar to those found in rabbits. Death may be produced by large intraperitoneal doses of Flexner's or Sonne's bacillus.

Other Animals.-By giving a cat $\frac{1}{2}$ drop of croton oil, and injecting a whole agar slope of Shiga's bacillus directly into the stomach, Shiga (1898) succeeded in setting up diarrhœa for a week; the animal passed grey, slimy stools, from which the bacilli could always be cultivated. It died 4 weeks later; at necropsy there was congestion of the rectal mucosa, and a covering of mucus over the whole of the large gut. The bacillus was recovered from the cæcum and large intestine. Most workers have failed completely to reproduce true dysenteric lesions in cats, dogs, rabbits, or monkeys either by injection per os or per rectum, though the feeding of monkeys with large doses of Flexner's bacillus may give rise to severe dysenteric symptoms (see Dack and Petran 1934).

Classification.- Space does not permit of a description of the numerous attempts that have been made to afford a satisfactory elassification of the dysentery bacilli. These may be summarized by saying that Lentz (1902) in Germany first perceived the difference between the mannitol and the non-mannitol fermenters; that Hiss (1904) in the United States, later supported by Bojlén (1934) in Denmark, suggested a classification on fermentation reactions; that Kruse and his colleagues (1907) in Germany realized the value of serologieal methods of elassification, and had the merit of pointing out the antigenic complexity of the mannitol-fermenting group; that Shiga (1908) and Amako (1908) in Japan combined fermentation and serological methods; that Gettings (1919), Murray (1918), and Andrewes and Inman (1919) in England arrived independently at results agreeing closely with each other, and showed that the non-mannitol-fermenting Shiga baeillus was antigenically distinct and homogeneous, whereas the mannitol-fermenting bacilli, of which Flexner's bacillus was the main example, were heterogeneous and divisible into four types according to the preponderance of one or other of the antigenic components $\mathrm{V}, \mathrm{W}, \mathrm{X}$, and $\mathrm{Z}$; and that Boyd (1931, 1932, 1936, 1938, 1940) reached a different conclusion on the antigenic structure of the mannitol- 
fermenting group, finding that some strains shared a group antigen and that others did not.

All workers are now agrecd that the main lines of cleavage are (1) between the mannitol and the non-mannitol fermenters, and (2) in the mannitol-fermenting group between the non-lactose and the late-lactose fermenters. Opinion varies mainly in respect of the differentiation of antigenic types from species, and the nomenclature to be employed.

In the non-mannitol-fermenting group, Sh. schmitzi is distinguished from Sh. shiga by fermenting rhamnose, producing indole, and being antigenically distinct. The Newcastle bacillus may or may not ferment mamnitol ; the nonmannitol-fermenting strains can be distinguished readily from Sh. shigce or Sh. schmitzi by their production, on first isolation, of a bubble or two of gas in tubes of glucose and dulcitol, and by their different serological behaviour. Besides these species, there is a group of organisms, at present ill-defined, which resemble Sh. shige or Sh. schmitzi biochemically, but differ from both in their antigenic structure. These organisms have been described by Dudgeon and Urquhart (1919) as para-Shiga bacilli. Since they vary in their fermentation of arabinose, their production of indole, and their serological reactions, it is impossible to say at present whether they should be classified as a single species, Sh. parashigce, and subdivided into types, or whether more than one species will be required. We prefer to leave the matter open and refer to them sinply as para-Shiga bacilli.

In the non-lactose-fermenting subdivision of the mannitol-fermenting group, Sh. alkalescens differs from most strains of Sh. flexneri in fermenting dulcitol, xylose, sorbitol, and, according to de Assis (1939a) most important of all, glycerol. It is, moreover, susceptible to acid agglutination. The flexneri types are peculiarly difficult to classify satisfactorily at present. The old division on antigenic structure into $\mathrm{V}, \mathrm{W}, \mathrm{X}$, and $\mathrm{Z}$ types has been challenged by Boyd $(1938,1940)$, who does not recognize the $\mathrm{X}$ type, and who proposes to replace the letters $\mathrm{V}, \mathrm{W}$, and $\mathrm{Z}$ by the Roman numerals i, ii, iii and to include under the term Bact. dysenterice, Flexner, three other types iv, v, and vi, corresponding to his strains 103, P119, and 88. All of these types, according to Boyd, share a common group antigen. Other strains, biochemically similar to Flexner's bacillus but not containing the Flexner group antigen, he would put into a separate species, labelled by his own name, and divide them on an antigenic basis into Bact. dysenterio, Boyd, Types i, ii, and iii, corresponding to his strains 170, P288, and D1.

This raises serious difficulties both of classification and of nomenclature. Leaving nomenclature until later, we think that there is a good precedent for classifying antigenic variants of the same species into types distinguished by numerals; for this reason we would welcome the replacement of the letters $V$, $\mathrm{W}$, and $\mathrm{Z}$ by numerals. We think also that there is justification for classifying biochemically similar, but antigenically distinct, organisms as separate species, and we see no objection to the use of the term boyd as a specific name. When, however, we consider the constitution of the flexneri species, we are faced with two difficulties, namely, should the $\mathrm{X}$ type be omitted, and should strain 88 , which is a mannitol-fermenting variant of the Newcastle bacillus, be included ? In his 1938 paper Boyd concluded provisionally that $\mathrm{X}$ contained a distinctive type antigen, and it was not till 1940 that he decided that this type was merely a variant of $Z$. Previous workers have had little difficulty in recognizing the $\mathrm{X}$ type, and the evidence on which it has been degraded might be considered as unconvincing. 
Wheeler $(1944 a, b)$, lowever, agrees with Boyd in this matter. The gas-producing, dulcitol-positive, indole-negative Newcastle bacillus, is sufficiently distinctive to justify the award of specific rank. Its possession of the group Flexner antigen is no more a reason for including it in the flexneri species than the common possession of a group antigen between Sh. alkalescens and Boyd's strain P274 is sufficient to justify ranking these two biochemically distinct organisms in the same species. On the whole, we would accept Boyd's general thesis, but would prefer to accord specific rank to the Newcastle bacillus, and to delay the final numbering of the flexneri types till the position of $\mathrm{X}$ is cleared up.

When we come to consider the definition of the boyd species, we are again in difficulties, because we know far too little as yet to decide what strains to place in it. Provisionally, we may accept Type 170 as boyd i, P288 as boyd ii, D1 as boyd iii, and P274 as boyd iv, but until the species can be properly defined, we are on very unsure ground.

In the lactose-fermenting subdivision of the mannitol-fermenting group, $S h$. dispar ferments xylose and sorbitol, produces indole, and is generally M.R. +, while Sh. sonnei is negative in all these respects. Sonne's bacillus appears to be antigenically homogeneous, and to differ from Sh. dispar, which is antigenically heterogeneous. In contrast to most other workers, Bojlén (1934), who has made a study of Sonne dysentery in Denmark, maintains that many strains ferment xylose. He divides the Sonne group into six sub-groups on the basis of maltose and xylose fermentation. Maltose fermentation is admitted to be irregular, except with freshly isolated strains, and two of the sub-groups are differentiated from two of the others merely by their slightly delayed fermentation of certain sugars. The justification advanced for such a procedure is that in any given closed epidemic the strains isolated belonged to one sub-group. It is very doubtful whether, in the absence of other correlated properties, attention should be paid by systematists to minor fermentative activities that may be characteristic of strain rather than of type differences. The fact, moreover, that xylose-positive strains of Sonne appear to be uncommon outside of Denmark suggests that Bojlén's classification should be accepted with reserve until it has received confirmation from other countries.

There is in the lactose-fermenting sub-group an undefined series of strains usually referred to by the term Bact. coli anaerogenes. Some of these strains are undoubtedly of the Sonne or dispar type (see Nabarro 1923, 1927 ; Koser et al. 1930). Others are distinguished from these organisms by the fact that they produce small quantities of gas, often late, in glucose, maltose, mannitol, sucrose, or salicin, maltose being one of the commonest. Organisms of this type are not infrequently found in milk (Wilson et al. 1935), and appear to be related more nearly to the coli-ærogenes than to the dysentery group. Some ancerogenes strains are motile, and some liquefy gelatin.

Nomenclature.-The old practice of regarding all dysentery bacilli as belonging to a single species, Bact. dysenteria, has clearly nothing to support it. If the numerous differences between the Shiga, Flexner, and Sonne bacillus are insufficient to justify the award to each of specific rank, then it is impossible to decide on what grounds species should ever be separated. Just as much reason exists for classifying the colon and the typhoid bacillus in a single species as for uniting the Shiga and the Somne bacillus. In the second edition of this book we adopted the expedient of classifying the dysentery bacilli in the genus Bacterium and awarding them the specific names of shiga, flexneri, and sonnei, etc. There is 
still, in our opinion, good reason for continuing this mode of classification, but there is now perhaps reason for proceeding still further in the taxonomic differentiation of these organisms. As we have already explained in the previous chapter, the term Salmonella has got for practical purposes to be accepted generically, in spite of the logical inconsistencies which its adoption leads to. If we accept this, then we are forced to separate off the dysentery bacilli from the Bacterium genus, and classify them under a separate generic name, such as Shigella. The specific names can then follow easily. American workers have adopted the specific name ambigua in place of schmitzi, and of paradysenterice in place of flexneri.

Both Schmitz's and Flexner's bacillus are so well known by the names of their discoverers that it is a pity not to perpetuate this association in the nomenclature of the dysentery group. The strict systematist may object that the terms ambigua and paradysenterice have priority, but this argument has little power to move us. The primary purpose of nomenclature is utility, and to insist on an inapt and uninformative specific name merely on grounds of botanical convention is to forget that the Sabbath was made for man and not man for the Sabbath. The classification and nomenclature that we would suggest as being the most valuable for teaching purposes is given diagrammatically in Fig. 146.

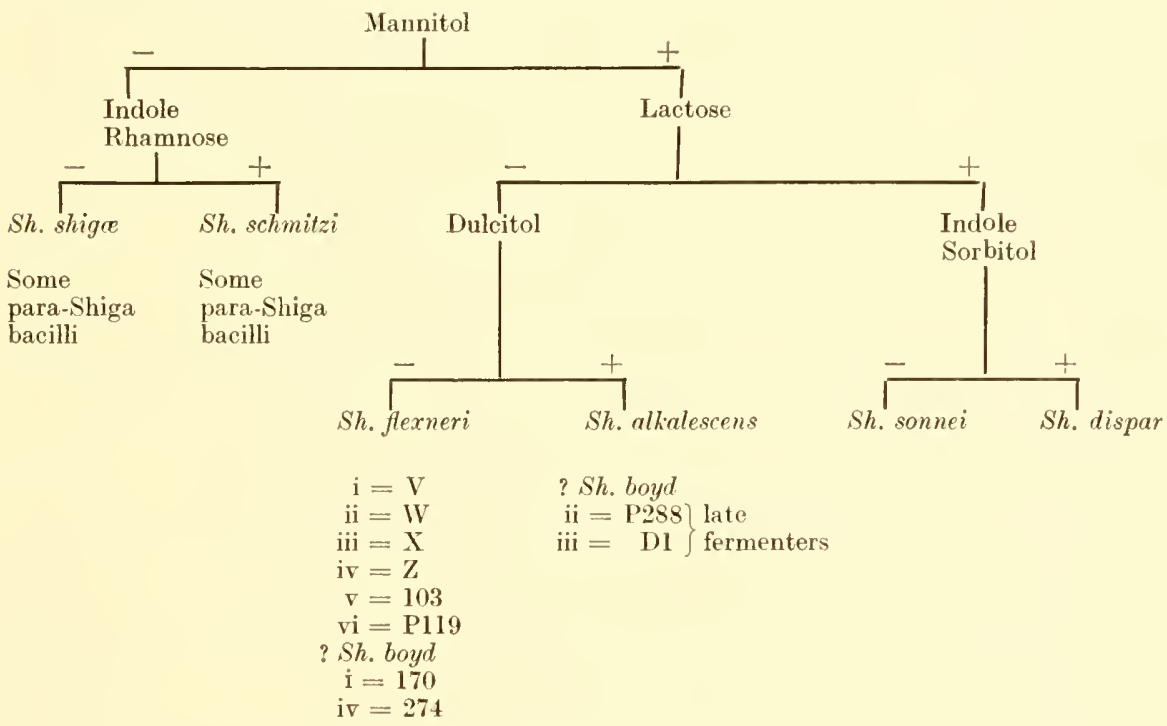

Fig. 146.-Tentative Classification of the Dysentery Bacilli.

Note.-Shigella newcastle may or may not ferment mannitol, and has been omitted from the figure.

For two recent reviews of the dysentery bacilli reference may be made to Neter (1942) and Weil (1943).

\section{REFERENCES}

ALI, M. (1938) J. Egypt. med. Ass., 21, 650.

Амако, T. (1908) Z. Hyg. InfektKr., 60, 93.

ANDrewes, F. W. (1918) Lancet, i. 560.

Andrewes, F. W. and Inman, A. C. (1919) Spec. Rep. Ser. med. Res. Coun., Lond., No. 42. AokI, K. (1921) Tokohu J. exp. Med., 2, 142; (1923) Ibid., 4, 12. 
Archer, G. T. L. (1942) J. R. Army med. Cps., 79, 109.

Arkwright, J. A. (1921) J. Path. Bact., 24, 36.

Assis, A. DE (1939a) O Hospital, 15, 447; (1939b) Ibid., 15, 655.

BamForth, J. (1934) J. Hyg., Camb., 34, 69.

BeCK, A. and BuCkLE, D. F. (1939) J. Hyg., Camb., 39, 311.

Bessad, G. (1911) Zbl. Bakt., 5\%, 27.

Blake, A. V. and Okell, C. C. (1929) J. Path. Bact., 32, 121.

Boivin, A. and Mesrobeanu, L. (1937a) C. R. Acad. Sci., 204, 302; (1937b) Ibid., 204, 1759 ; (1937c) C.R. Soc. Biol., 124, 442; (1937d) Ibid., 124, 1078 ; (1937e) Ibid., 125, 796 ; (1937f) Ibid., 126, 222; (1937g) Ibid., 126, 323; (1937h) Ibid., 126, 652; (1938) Ibid., 128, 446.

BoJlén, K. (1930) C. R. Soc. Biol., 103, 613 ; (1934) "Dysentery in Denmark." Bianco Lunos Bogtrykkeri A/s, Copenhagen.

Bornstein, S., SAPHra, I., and Daniels, J. B. (1911) J. Immunol. 42, 401.

Boxd, J. S. K. (1931) J. R. Army med. Cps., 5\%, 161; (1932) Ilid., 59, 241, 331 ; (1935) Ibid., 64, 289; (1936) Ibid., 66, 1 ; (1938) J. Iygg., Camb., 38, 477 ; (1940) Trans., R. Soc. trop. Med. Hyg., 33, 553.

BrauN, H. and BAAKE, F. (1930) Zbl. Bakt., 116, 462.

BraUN, H. and Wem, A. J. (1928) Zbl. Bakt., 109, 16.

Buchanan, G. and Roux, P. (1930) J. med. Ass. S. Africa, 4, 685.

BuCHWALD, H. (1939) Z. ImmunForsch., 96, 445.

BurNet, F. and MlKie, M. (1930) J. Path. Bact., 33, 637.

Cann, L. W. and Navasquez, S. DE. (1931) J. Hyg., Camb., 31, 361.

Carpenter, P. L. (1944) J. Bact., 4\%, 419.

Carver, J. R. (1921) Lancet, i. 687.

Castellani, A. (1907) J.Hyg., Camb., \%, 1; (1912a) J.trop. Med. Hyg., 15, 162 ; (1912b) Zbl. Bakt., 65, 262.

Christensen, W. B. and Gowen, G. H. (1944) J. Bact., 47, 171.

Cla berg, K. W. (1932) Zbl. Bakt., 124, 23.

Clayton, F. H. A. and Warren, S. H. (1929a) J. Hyg., Camb., 28, 355 ; (1929b) Ibid., $29,191$.

Conradi, H. (1903) Dtsch. med. Wschr., 29, 26.

Cruickshank, R. and Swyer, R. (1940) Lancet, ii. 803.

Dack, G. M. and Petran, E. (1934) J. infect. Dis., 55, I.

David, H. and SchIrL, A. (1939) Zbl. Bakt., 144, 43.

DoLd, H. (1916) Dtsch. med. Wschr., 42, 811.

Dopter, C. (1905) Ann. Inst. Pasteur, 19, 353.

Downie, A. W., WAde, E., and Young, J. A. (1933) J. Hyg., Camb., 33, 196.

Dudgeon, L. S. and Urquhart, A. L. (1919) Spec. Rep. Ser. med. Res. Coun., No. 40, p. 25.

Flexner, S. (1900a) Zbl. Bakt., 28, 625; (1900b) Bull. Johns Hopk. Hosp., 11, 231.

Flexner, S. and Sweet, J. E. (1906) J. exp. Med., 8, 514.

Forsyth, IV. L. (1933) J. trop. Med. Hyg., 36, 65.

Fyfe, G. M. (1927) J. Hyg., Camb., 26, 271.

Gettings, H. S. (1919) Spec. Rep. Ser. med. Res. Coun. Lond., No. 30.

GlynN, J. H. and Starkey, D. H. (1939) J. Bact., 3\%, 315.

HAas, R. (1937) Z. ImmunForsch, 91, 254; (1938a) Ibid., 92, 355 ; (1938b) Ibid., 94, 239 ; (1938c) Ibid., 94, 480.

HaNSEN, A. (1936) Biochem. Z., 28\%, 35.

Hardy, A. V., Watt, J., Kiolodny, M. H., and DeCapito, T. (1910) Amer. J. publ. Hlth, $30,53$.

HAZEN, E. L. (1938) J. infect. Dis., 63, 330.

Hiss, P. H. (1904) J. med. Res., 13, 1.

Hiss, P. H. and Russelt, F. F. (1903) Med. Nevs, 82, 289.

Isabolinsky, II. and Gitowitsch, W. (1926) Zbl. Bakt., 9\%, 148.

Istrati, G. (1938) C. R. Soc. Biol., 129, 1010.

JAnota, M. and DACK, G. M. (1939) J. infect. Dis., 65, 219.

Johnston, M. M. and Brown, A. (1930) Canad. publ. Hlth J., 21, 394.

Johnston, M. M. and KaAke, M. J. (1932) Canad. publ. IIlth J., 23, 159.

KeMPER, F. (1933) Zbl. Bakt., 130, 265.

Kerrin, J. C. (192S) J. IIyg., Camb., 28, 4.

Kobayashi, R., OmkUbo, H., Оhno, J., Ide, II., Nakamura, B., Machida, S., KobaYashi, E., Matsmioto, I., and Matsuraia, S. (1931) Kitasato Arch. 8, 99.

Koser, S. A., Reiter, D. O., Bortniker, E., and Swingle, E. L. (1930) J. prev. Med., $4,477$.

Koser, S. A. and Strron, N. C. (1930) J. infect. Dis., 47, 443.

Kraus, R. and DörR, R. (1905) Wien. klin Wschr., 18, 1077.

KRUSE, W. (1900) Dtsch. med. Wschr., 26, 637 ; (1901) Ibid., 27, 370, 386. 
Kruse, Rittershads, Kemp, and Metz. (1907) Z. Hyg. InfektKr., 57, 417.

Kurauchi, K. (1929) See Ando, K. (1929) J. Immunol., 17, 555.

LARGE, D. T. M. (1929) J. R. Army med. Cps., 52, 1.

Large, D. T. M. and Sankaran, O. K. (1934) J. R. Army med. Cps., 63, 231.

LENTZ. (1902) Z. Hyg. InfektKr., 41, 559.

Lentz, O. and Prigge, R. (1931) Kolle, Kraus and Uhlenhuth's "Handbuch der pathogenen Mikroorganismen." Gustav Fischer, Jena. 3te Aufl., 3, 1377.

LeSTER, V. (1926) Acta path. microbiol. scand., 3, 696.

Leuchs, J. and Plochmann, E. (1927) Zbl. Bakt., 104, 347.

Lovell, R. (1929) Proc. roy. Soc. Med., 22, 820.

McGinnes, G. F., McClean, A. L., Spindel, F., and Maxcy, K. F. (1936) Amer. J. Hyg., 24,552 .

MaNdry, O. C. (1935) Puerto Rico J. publ. Hith, 10, 308.

Mesrobeane, L. and Boivin, A. (1937) C. R. Soc. Biol., 124, 439.

MEYER, K. (1930) Z. ImmunForsch., 68, 98; (1931) Ibud., 69, 134, 499.

MeYer, K. and Morgan, W. T. J. (1935) Brit. J. exp. Paih., 16, 476.

Michaelis, L. (1917) Dtsch. med. W'schr., 43, 1506.

Mrta, K. (1921) J. infect. Dis., 29, 580.

Morgan, W. T. J. (1931) Brit. J. exp. Path., 12, 62 ; (1936) Biochem. J., 30, 9019 ; (1937) Ibid., 31, 2003.

Morgan, W. T. J. and Partridae, S. M. (1940) Biochem. J., 34, 169 ; (1941) Itid., 35, 1140.

Murray, E. G. D. (1918) J. R. Army med. Cps, 31, 257, 353.

Nabarro. D. (1923) J. P'uth. Bact., 26, 429; (1927) Illid., 30, 176.

NAGAKURA, K. (1937) Z. ImmunForsch., 90, 139.

NeIsser, M. and Shiga, K. (1903) Dtsch. med. Wschr., 29, 61.

Nelson, R. L. (1930) J. Bact., 20, 183.

Neter, E. (1938) J. Immunol., 35, 339 ; (1942) Bact. Rev., 6, 1.

Neter, E. and Heide, A. M. $(1940)$ Amer. J. Hyg., 31, B., 69.

Okell, C. C. and Blake, A. V. (1930) J. Path. Bact., 33, 57.

Ouitsky, P. K. and Kligler, I. J. (1920) J. exp. Med., 31, 19.

Olitzki, L., Bendersky, J., and Косн, P. K. (1943) J. Immunol., 46, 71.

Orskov, J. and Larsen, A. (1925) J. Bact., 10, 473.

Penner, A. and Bernhem, A. I. (1942) J. exp. Med., \%6, 271.

Preston, W. S. and Clark, P. F. (1938) J. infect. Dis., 63, 238.

Report. (1939) "Antigenic variation in the organisms of bacillary dysentery, etc." Standards Laboratory, Oxford.

ROELCKE, K. (1938) Z. Hyg. InfektKr., 120, 307.

Rothstadt, L. E., Fenner, F., and Baker, B. A. (1942) Pers. comm.

SACHS, A. (1943) J. R. Army med. Cps., 80, 92. [Wrongly deseribed as Saclss, H.]

Sartorius, F. and Reploir, H. (1931) Klin.W Schr., 10, 2216; (1932) Zbl. Bakt., 126, 10.

Sснмiтz, K. E. F. (1917) Z. IIyg. InfektKr., 84, 449.

Schüтze, H. (1944) J. I'ath. Bact., 56, 250.

SevitT, S. (1945) J. Hyg., Camb., 44, 37.

Shiga, K. (1898a) Ztl. Baht., 23, 599 ; (1898b) Ibid., 24, 817, 870, 913 ; (1901) Dtsch. med. Wschr., 2\%, 741, 765, 783; (1908) Z. Hyg. Infekthr., 60, 75.

Simth, J. and Fraser, A. H. (1928) J. l'ath. Bact., 31, 511.

SonNe, C. (1915) Zbl. Bakt., '75, 408.

Steabben, D. (1943) J. Hyg., Camb., 43, 83.

Strong, R. P. and llusarave, W. E. (1900) J. Amer. merl. Ass., 35, 498.

Stuart, C. A., Rustigian, R., Zimmerman, A., ani Corrigan, F. V. (1943) J. Immunol., $47,425$.

TAKITA, J. (1937) J. Hyg., Camb., 37, 271 ; (1939) Kitasato Arch. 16, 174.

'THuotTa, T. and WaALer, E. (1932) J. Bact., 24, 301.

Todd, C. (1904) J. Hyg., Camb., 4, 480.

VALliard, L. and Dopter, C. (1903) Ann. Inst. Pasteur, 1\%, 463.

Verder, E. and Petran, E. (1937) J. infect. Dis., 60, 193.

WaAler, E. (1935) "Studies on the Dissociation of the Dysentery Bacilli." I Kommisjon Hos Jacob Dybwad, Oslo.

WeIL, A. J. (1943) J. Immunol., 46, 13.

Weil, A. J., Black, J. and FARSETTA, K. (1944) J. Immunol., 49, 321, 341.

WeLCH, H. and MrckLe, F. L. (1932) Amer. J. publ. Hlth, 22, 263; (1934) Ibid., 24, 219.

WheEler, K. M. (1944a) J. Immunol., 48, 87 ; (1944b) Amer. J. publ. Hlth., 34, 621.

WhiteHEAD, H. and SCOTT, W. M. (1934) Lancet, ii. 248.

Wilson, G. S., Twige, R. S., Wrigit, R. C., Hendry, C. B., Cowell, M. P. and Maier, I. (1935) Spec. Rep. Ser. med. Res. Coun., Lond., No. 206.

Wood, A. J. and Keeping, F. E. (1944) J. Bact., 47, 309.

WyскоF, R. W. G. (1933) J. exp. Med., 5\%, 165. 


\section{CHAPTER 30}

\section{SALMONELLA}

Defintion.-Salmonella.

Gram-negative, non sporing rods, usually $1-3 \mu$ long and 0.5-0.7 $\mu$ broad. Primarily intestinal parasites, widely distributed in man, mammals, and birds. With few exceptions all species are motile, by peritrichate flagella. Easily cultivable on ordinary media. Aerobic and facultatively anaerobic. Apart from a few species that form acid only, acid and gas are produced from glucose, mannitol, dulcitol, and sorbitol. Lactose, sucrose, adonitol, and, except rarely, salicin are not fermented. Indole and acetylmethylcarbinol are not formed. Gelatin is seldom liquefied. $\mathrm{H}_{2} \mathrm{~S}$ production is usual. The species are closely related to each other by somatic and flagellar antigens; most species are diphasic. Pathogenic for man, animals, birds, or all three, giving rise to food poisoning, enteritis or typhoid-like infections.

Nomenclature.-In Chapter 28 we have already mentioned our decision to split off the Salmonella and Shigella sub-groups from the wide group of Gramnegative non-sporing rods previously classified under the genus Bacterium. The justification for this decision is one of expediency. That the close relation of their antigenic components and the type of disease to which they give rise serve to differentiate the salmonella and the dysentery bacilli from the ordinary coliform bacilli will not be questioned; but whether it is justifiable to give these subgroups generic rank and to distinguish each of the antigenic types by a specific name, when in the Streptococcus group the main sub-groups are not given generic rank and the antigenic types are treated as varieties and labelled by numbers, is very questionable. That the term "species" is being used with two different connotations is clear enough, and we make no attempt to defend such inconsistency. On the other hand, we may plead that bacterial taxonomy is still in a process of evolution; that there is as yet no general agreement on the definition of the terms "genus" and "species" ; and that, until a final ruling is laid down by some properly constituted international committee, we, as writers of a textbook of bacteriology, must be free to select such names from among those that have been proposed as will best serve to aid the student in the recognition of the various groups of bacteria that he has to study.

So far as the term Salmonella is concerned, a special sub-committee of the International Society of Microbiology (Report 1934) recommended the adoption of the terminology introduced by Kauffmann, which recognized the generic status of the Salmonella group and the specific rank of each of the antigenically distinguishable types. Since then the number of recognized types has more than doubled. There are now over 130 specifically named serological types, around which an extensive literature has grown up. It is therefore almost inconceivable that any international committee on nomenclature appointed in future would 
suggest such changes in definition as would necessitate the degradation of the generic term Salmonella to specific rank, and the numbering as varieties of all the present named species. Convenience and expediency nust be our justification for adopting the recommendations of the Salmonella sub-committee in face of the obvious illogicality of using a different system of nomenclature for members of the Streptococcus group.

If, however, we decide to employ Salmonella as a generic term, we must take care to define it as far as possible on the same general principles as bave guided us in defining other genera. The lines of demarcation in biology can rarely be sharp. It is dangerous to rely, therefore, on any single character in the identification of a bacterium. Here we find ourselves in conflict with Kauffmann (1941), who lays down the following definition: "Salmonella bacteria are Gram-negative bacteria which, on the ground of their antigenic structure, can be included in the Kauffmann-White scheme." If we rely solely on antigenic constitution, as Kauffmann does, we shall be logically compelled to include in the Salmonella genus every organism, no matter how different it may be in other characters, that contains a single antigen hitherto recognized in the scheme of classification drawn up by Kauffmann and White. Already numerous strains of coliform and paracolon bacilli have been found to contain one or more of the $\mathrm{H}$ or $\mathrm{O}$ antigens of Salmonella (Habs and Arjona 1935, Gard 1937, Gard and Eriksson 1939, Schiff, Bornstein, and Saphra 1941, Saphra and Silberberg 1942, Peluffo, Edwards, and Bruner 1942, Leon 1942, Wheeler et. al. 1943, Edwards, Cherry and Bruner 1943) ; and some of the Salmonella $\mathrm{O}$ antigens have been recognized in strains of Flexner dysentery bacili (Bornstein, Saphra, and Daniels 1941), and even in members, of the Pasteurella gronp (Schiitze 1928, Pirosky 1938). To transfer these organisms, which differ in fermentation and pathogenic characters, to the Salmonella genus, merely because their constituent antigens happen to share some of the necessarily limited groupings with those common among the salmonellæ, is not only to lose all sense of proportion, but to sanction a principle in bacterial nomenclature that cannot but lead to progressively increasing confusion.

We have felt it important to define the Salmonella genus in terms of fermentative activity, antigenic constitution, and pathogenicity, so as to exclude the dysentery, the coliform, and the paracolon bacilli. Our definition is necessarily tentative. Apart from their consistently negative reaction to Gram's stain, there is practically no criterion to which all strains conform. As with so many other groups of bacteria, the decision whether a given organism should or should not be classified as a Salmonella must be determined by a careful consideration of all its properties; unless the majority of these are in agreement with those laid down in the definition, it would be better for the present to treat it as a member of the wide Bacterium group. (For reviews of the Salmonella group see Tesdal 1938, Kauffmann 1941, Bornstein 1943.)

Habitat.-There is no reason to doubt that members of the Salmonella group are primarily intestinal parasites. It is true that they may be found in the blood, the lymphatic nodes, the ovary, the eggs of fowls, and other situations such as water and sewage (see Ferramola, Monteverde, and Leiguarda 1943); but their commonest location appears undoubtedly to be the intestine of man, mammals, and birds. The chief reservoirs of infection are fowls and pigs. From fowls over 40 different species have now been isolated in the United States and Panama alone, and from pigs nearly 30 (Edwards and Bruner 1943). Rodents, ruminants, 
and carnivores are not uncommonly infected. Except for one or two members, like the typhoid bacillus, that are non-pathogenic to animals, man does not seem to act as an important reservoir of infection; the invading organisus are quickly thrown off, and the chronic carrier state is unusual.

Morphology.--The shape, size, structure, and arrangement of the bacterial cells do not differ materially from those in the Bacterium group. The usual length is $2-3 \mu$ and the usual width $0 \cdot 6 \mu$; but considerable deviation from these modal values is found under different environmental conditions and in different cultural variants. With the exception of Salm. gallinarum and its variant pullorum, all species are motile; though individual non-motile strains of the typhoid bacillus, for example, may be encountered occasionally in the body, and non-motile variants may be thrown off under cultural conditions in the laboratory. Motility, however, is such a general characteristic that failure of a strain to exhibit it on primary isolation from the body must be regarded as almost sufficient in itself to exclude it from the Salmonella group. Whether the flagella are peritrichate, which has

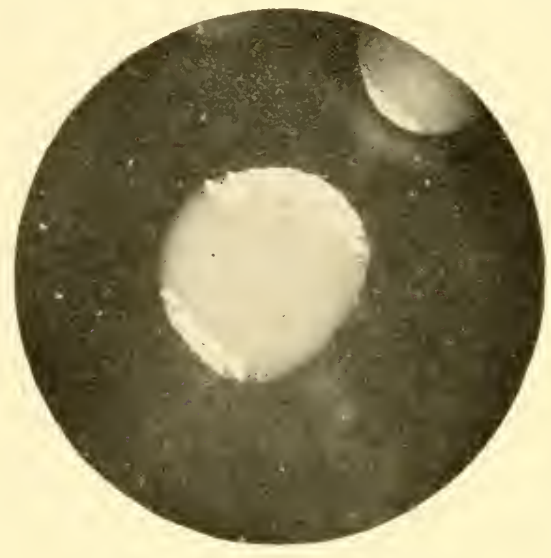

FIG. 147.-Salm. enteritidis.

been the conclusion drawn by most workers from a study of fixed and stained bacilli, or whether, as appears possible from Pijper's $(1938,1940)$ studies of living typhoid bacilli by sunlight darkground photomicrography, they are disposed one on each side of the bacillus, is disputable, and is for our purpose more of academic than of practical importance.

Capsules are not ordinarily formed, but many species, notably Salm. paratyphi $B$, may give rise to mucoid colonies in which the individual organisms are surrounded by a polysaccharide-containing capsular material (Fletcher 1918). The formation of a capsule by the typhoid bacillus has been described by Kühnemann (1911), Carpano (1913), Marrassini Colony on agar plate after 24 hours $(\times 8)$. (1913), Shimidsu (1913), and Gay and Claypole (1913).

Cultural Characters. - The members of this group grow readily on ordinary nutrient media and cannot be distinguished from coliform bacilli. A few species, however, such as paratyphi $A$, abortus-ovis, typhi-suis, sendai, and pullorum, grow less abundantly and form but a thin layer of growth on an agar slope. On brilliant green agar plates the difference is particularly noticeable, the growth being both slower and less abundant than that of other members of the group. Salm. typhi and Salm. rostock likewise grow poorly on brilliant green agar, though developing fairly well on ordinary nutrient agar. In broth, smooth strains give rise to a uniform turbidity, increasing rapidly during the first 12 to 18 hours of growth, and then more slowly up to 48 to 72 hours. Pellicle formation is rare, and when present is slight. A deposit forms as growth increases; this disperses readily on shaking, leading to an increase in the turbidity of the culture.

On agar, the colonies are relatively large, with an average diameter of $2-3 \mathrm{~mm}$., but they vary considerably in size. They may be circular and low convex with a smooth surface and entire edge; they may be flatter with a less regular surface 
and a more effuse serrated edge; or they may assume the vine-leaf form, which used to be regarded as characteristic of Salm. typhi. Dwarf colony forms are sometimes met with. They were first described by Jacobsen in 1910, and have since been reported on by several workers (Mellon and Jost 1926, W. J. Wilson 1938, Morris, Sellers, and Brown 1941, Morris, Barnes, and Sellers 1943). On ordinary agar the colonies after 24 hours' incubation are only about $0.2-0.3 \mathrm{~mm}$. in diameter. According, however, to the original observations of Jacobsen (1910), which have since been confirmed many times, colonies of more normal size are formed on media containing assimilable sulphur compounds.

As has already been noted in the previous section, certain species, notably Salm. paratyphi $B$, give rise under favourable conditions to a mucoid growth. Sometimes the colonies are mucoid after 24 hours' incubation; they are about twice the size of normal colonies and resemble large drops of mucilage (Fletcher 1918). More often the mucoid appearance is developed as a secondary phenomenon after prolonged incubation. Thus, if an agar plate is inoculated in three or four

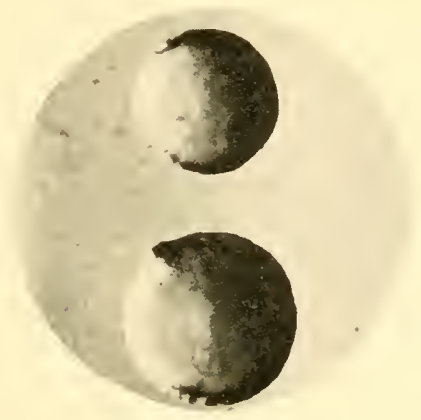

Fig. 148.-Salm. typhi.

Surface colonies on agar, 24 hours, at $37^{\circ} \mathrm{C} .(\times 8)$.

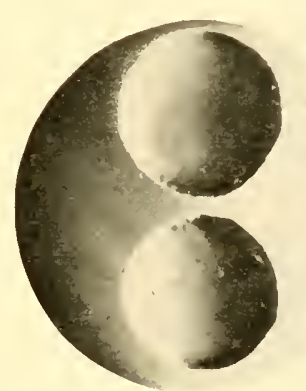

FIG. 149.-Salm. typhi-murium.

Surface colonies on agar, 24 hours, at $37^{\circ} \mathrm{C} .(\times 8)$.

places with the point of a needle, and after one day's incubation at $37^{\circ} \mathrm{C}$. is left at room temperature for a few days, large colonies are formed characterized by a depressed centre surrounded by a luxuriant mucoid wall. The "mucoid wall test", or Schleimwall-Versuch, described originally by Müller (1910), is of some differential value, being common with most freshly isolated $d$-tartrate-negative strains of Salm. paratyphi $B$ and generally negative with Salm. typhi-murium (Kauffmann 1941). The mucoid material contains a polysaccharide (BirchHirschfeld 1935), which appears to be antigenically homogeneous no matter by what species of Salmonella it is formed.

Resistance to Heat and to various Chemical Substances.-Most members of this group are killed by exposure to a temperature of $55^{\circ} \mathrm{C}$. for about 1 hour, or of $60^{\circ} \mathrm{C}$. for $15-20$ minutes. Many observations have been made on the resistance of salmonellæ to different chemical reagents, chiefly in an endeavour to prepare selective media on which the growth of coliform bacilli would be inhibited. Malachite green, in suitable concentration, kills Bact. coli or inhibits its growth without exerting the same effect on Salm. typhi (Loeffler 1903, 1906, Lentz and Tietz 1903, 1905). There are other green dyes that have a similar selective action; 
and the studies of Browning, Gilmour, and Mackie (1913) and of Krumwiede and Pratt (1914) have shown that brilliant green gives the best results. To this dye bacilli of the paratyphoid group are most resistant, the typhoid bacillus is somewhat less resistant, whereas the dysentery bacilli, and still more the coliform and paracolon Jacilli, are very susceptible. Caffeine (Roth 1903, Hoffman and Ficker 1904) and lithium chloride (Gray 1931, Havens and Mayfield 1933) are other substances that inhibit the growth of Bact. coli in concentrations that have no effect on the typhoid bacillus. Sodium tetrathionate is now being extensively used to favour the growth of salmonellæ at the expense of the coliform bacilli (Muller 1923, Schäfer 1934-35, Jones 1936)--an action that appears to be due not to any inhibitory action it possesses on coliform bacilli, but to the ability of most organisms of the Salmonella group) to reduce this substance and use it as

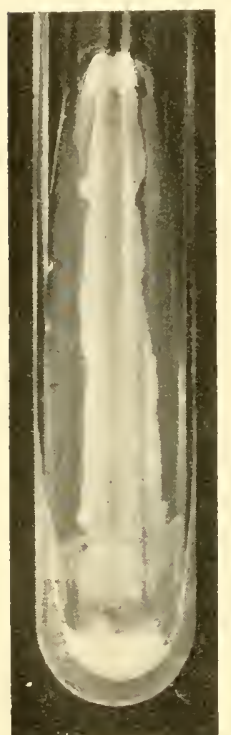

FiG. 150.-Salm. typhi

24-hours' culture on agar slope. a source of energy (Pollock, Knox, and Gell 1942). Sodium desoxycholate, in the presence of certain other substances, inhibits the growth of coliform but not of dysentery or salmonella bacilli, and is being used increasingly in the preparation of selective media for the isolation of intestinal pathogens (Leifson 1935, Hynes 1942). Selenium salts are also of value for the same purpose (Guth 1916, Leifson 1936).

Biochemical Activities.-As stated in the definition of the genus, the members of this group do not ferment lactose, sucrose, or adonitol. Salicin, also, is rarely fermented, and then only late. On the other hand, glucose, mamitol, dulcitol, and sorbitol, and almost invariably maltose and dextrin, are fermented. Apart from a few species, like Salm. typhi and Salm. gallinarum, gas is formed, though anaerogenic variants are not uncommon. Among the ordinary sugars, arabinose, xylose, trehalose, and inositol are useful for the differentiation of species. As examples, we may quote the failure of Salm. cholerce-suis to ferment arabinose or trebalose, which is of value in distinguishing it from Salm. paratyphi C, and the failure of Salm. zagreb to ferment inositol, which is of value in distinguishing it from Salm. saint-paul. The power to ferment rhammose often varies with different strains of the same species; this is sometimes made use of in the separation of epidemiological types, as, for instance, in Salm. typhi-murium (Edwards and Bruner 1940a).

Also of value in the differentiation of species are the organic acids, which were introduced by Brown, Duncan, and Henry (1924, 1926). Those commonly used are $d$-tartrate, $i$-tartrate, $l$-tartrate, sodimm citrate, and sodium mucate. Failure to ferment $i$-tartrate is fairly common; the other four acids are acted upon by most species.

In the past considerable attention has been devoted to certain special reactions. One of these, first described by Stern (1916), consists in growing the organisms under test iu a fuchsin sulphite glycerol meat-extract medium. Some organisms, known as "Sternpositive," produce in this medium a deep lilac colour within 3 days. If the medium is pink to red after a week, the strain is regarded as "Stern-negative." An intermediate group of strains, sometimes regarded as giving a weakly positive reaction, turn the medium deep red, purple or lilac in between 3 to 7 days. The reaction is apparently due to the formation of an aldehyde. It is certainly not due solely to acid formation. 
Fermentation Reactions of Salmonelia Group (monfen from Kiuffiann 1941)

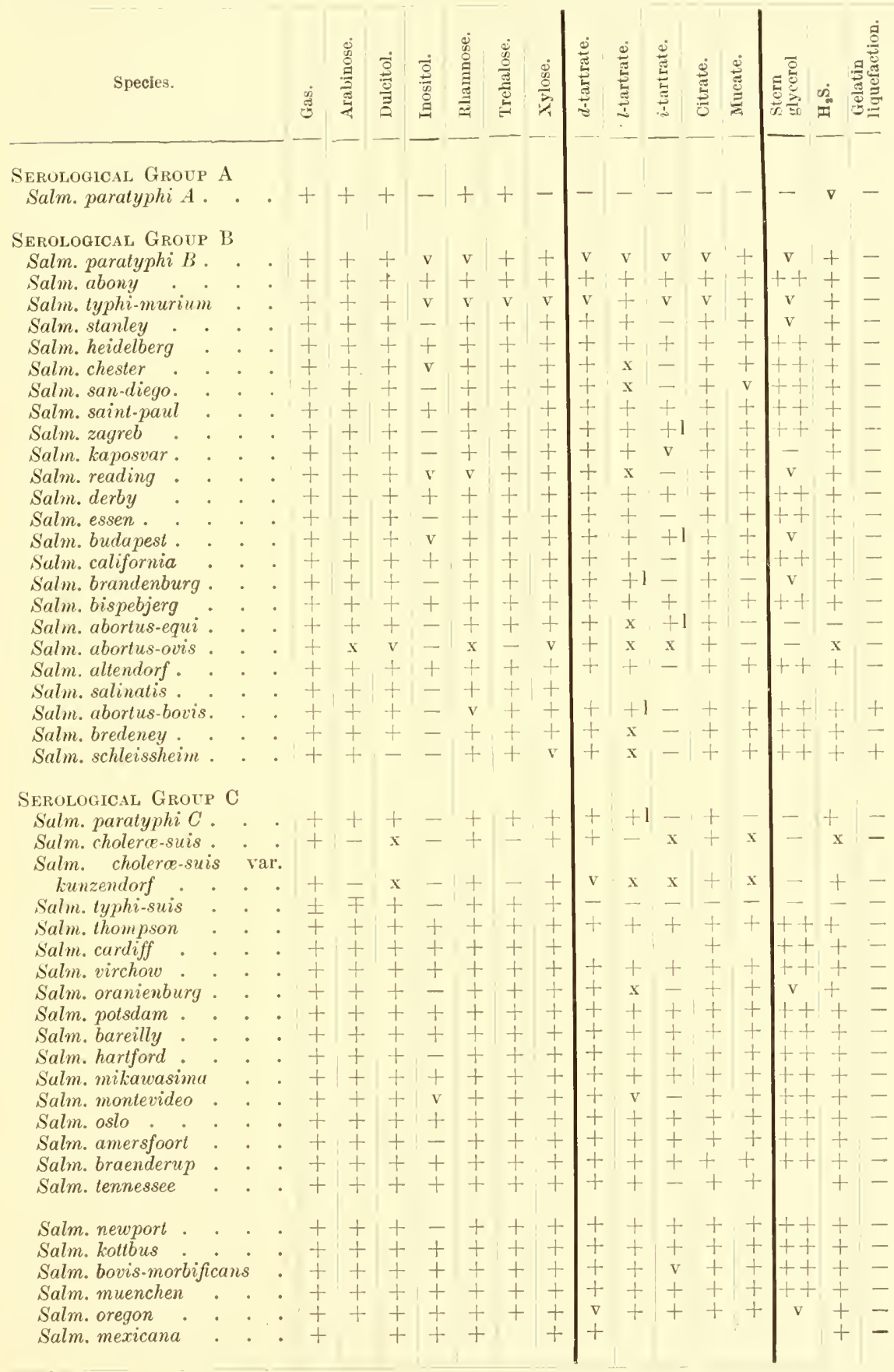


Fermentation Reactions of Salmonella Group (modified from Kauffand 1941)

Species.

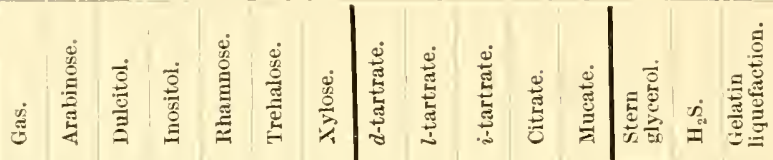

Serological. Grote Ccontinued.

Salm. manhattan

Salm. narashino

Salm. glostrup

Salm. litchfield.

Salm. duesseldorf

Salm. bonariensis

Salm. amherstiana

Serological Group D

Salm. typhi.

Salm. enteritidis var. danysz var, essen. var. chaco

Salm. dublin

Salm. rostock.

Salm. moscow

Salm. blegdam .

Salm. berta.

Salm. eastbourne

Salm. sendai

Salm. onarimon

Salm. dar-es-salaam

Salm. goettingen

Salm. panama.

Salm. claibornei

Salm. javiana

Salm. pullorum

Salm. gallinarmm

Serological Group E

Salm. london

Salm. give.

Salm. uganda

Salm. anatum

Sulm. muenster

Salm. nyborg

Salm. vejle.

Salm. amager

Salm. zanzibar

Salm. shangani

Salm. meleagridis

Salm. lexington

Salm. newington

Salm. selandia

Salm. new-brunswick

Salm. illinois

Salm.taksony .

Salm. senftenberg

var. newcastle

Salm. niloese

Salm. simsbury

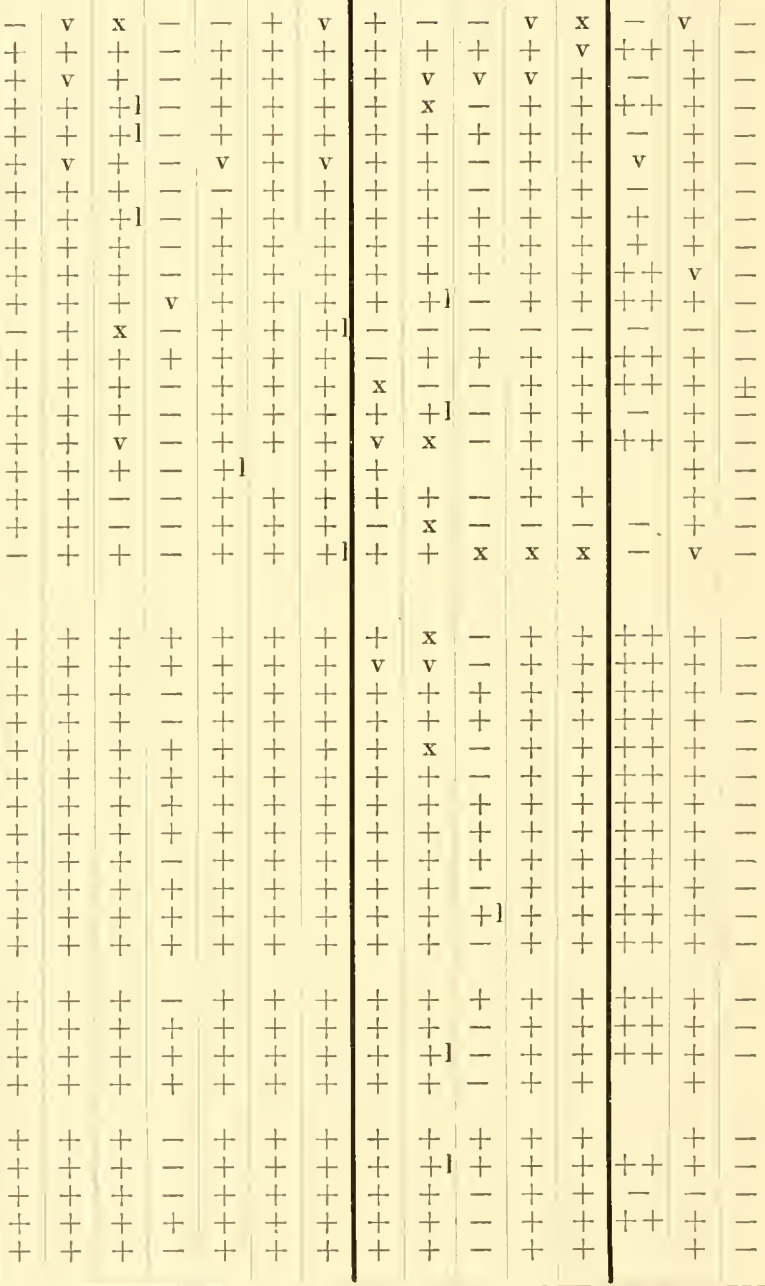




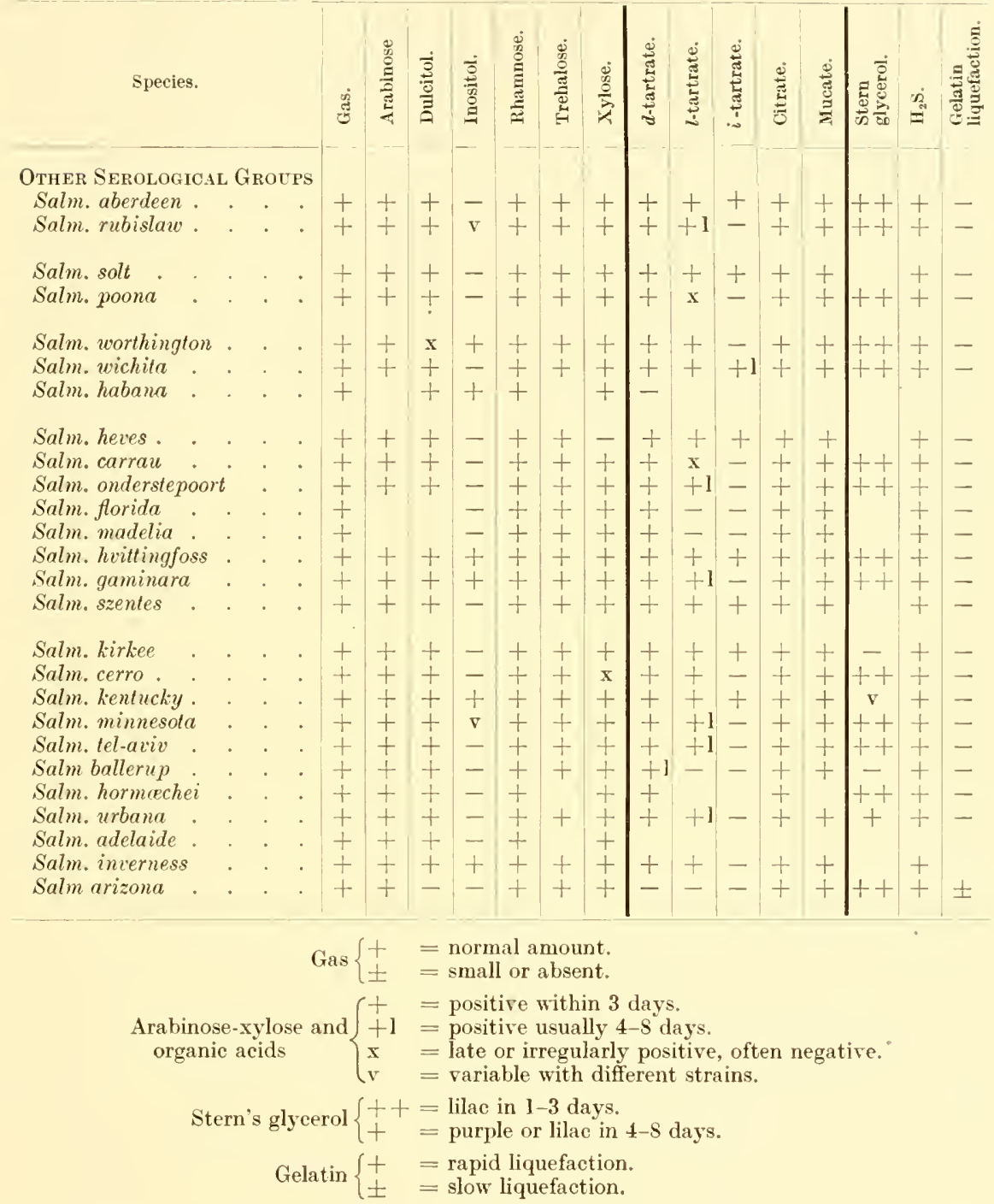

The second reaction was described by Bitter, Weigmann, and Habs (1926). It is essentially a test of the ability of the organism to grow in a synthetic medium containing ammonium salts as the main source of nitrogen. A 1 per cent. solution of sugar, of which the most generally useful is rhamnose, is added to test the fermentative power of the strain under these conditions. Strains that grow readily ferment the sugar and are referred to as "ammonium-strong." Strains that grow poorly and fail to ferment the sugar are referred to as "ammonium-weak." The result of the test may be negative, not because the organism cannot grow in an ammonium medium, but because, like some strains of Salm. paratyphi $B$, it fails to ferment rhamnose. In practice, the inoculated medium is incubated for 20 hours at $37^{\circ} \mathrm{C}$; methyl red is then added to determine 
whether or not the $\mathrm{pH}$ has been lowered to the point at which this indicator gives its characteristic red colour. Instead of the liquid medium, a citrate agar medium introduced by Simmons (1926) may be employed. This has been extensively used by Hohn and Herrmann $(1936 b, c)$. According to Kauffmann (1941), less reliance can be placed on the ammonium reaction than on the fermentation of organic acids. The ammonium reaction and the reaction in Stern's medium are, however, useful at times, as, for instance, in the differentiation of the fermentative types of Salm. enteritidis (Kauffmann 1935b).

In litmus milk the great majority of species produce transitory acidity followed by permanent alkalinity in 3 days, but with a few species, like Salm. paratyphi $A$, Salm. abortus-ovis, Salm.typhi-suis, Salm.typhi, Salm. sendai, and Salm. pullorum, the reaction in 3 days is neutral or slightly acid. On the whole little information of differential value is gleaned from a study of the reaction in litmus milk, and Kauffmann (1941) has now ceased to include this medium in his series of routine tests.

With the exception of variant colonies of Salm. eastbourne, and very occasional variants of Salm. enteritidis and Salm. panama (see Seligmann and Saphra 1943), indole production is uniformly negative. The majority of strains form $\mathrm{H}_{2} \mathrm{~S}$, but Salm. typhi-suis, Salm. sendai, Salm. gallinarum var. duisburg, and Salm. senftenberg var. newcastle fail to do so. $\mathrm{H}_{2} \mathrm{~S}$ formation is variable with Salm. typhi and Salm. paratyphi $A$, some strains being positive and others negative. Gelatin liquefaction is very uncommon, but Salm. abortus-bovis and Salm. schleissheim liquefy it rapidly and Salm. dar-es-salaam and Salm. arizona very slowly. Urea is not decomposed.

Antigenic Structure.-As has already been noted, the classification of the Salmonella group is now firmly based on the antigenic structure of the species of which it is composed. The arrangement of the various antigenic components in the cells and flagella of the salmonellæ has already been described in Chapter 8 in relation to the general problem of antigenic structure, and in Chapter 9 in relation to the study of bacterial variation. Before considering the different species that have been differentiated by this method of analysis, we may, however, recall the relevant facts and consider some of them in greater detail.

In the Salmonella gromp of bacilli we are dealing, in the main, with flagellated organisms. We therefore have to consider both the $\mathrm{H}$ (flagellar) and $\mathrm{O}$ (somatic) antigens. By appropriate methods we can test for these components separately. A formolized broth culture of a flagellated species readily agglutinates in the presence of the homologous $H$ agglutinins, but responds very poorly, if at all, to the homologous $O$ agglutinins. The reason for this behaviour is, perhaps, that the formalin fixes the flagella over the bacterial surface in such a way that the somatic antigens are no longer exposed to the action of the $O$ agglutinins. To test for the $\mathrm{O}$ antigens we can employ a bacterial suspension that has been treated with hot alcohol, thus removing or inactivating the $H$ antigens.

In respect of their flagellar antigens many of the species with which we are concerned are diphasic ; that is to say, their flagella may assume two alternative forms. Originally the two phases were referred to as specific and group; but later, when another type of phase variation was discovered, the so-called $\alpha-\beta$ variation, this nomenclature became no longer generally applicable. It is now usnal to call the two phases 1 and 2 , and to restrict the terms specific and group to organisms in which Phase 1 contains a, b, c, etc., antigens and Phase 2, 1, 2, 3, etc., antigens. Speaking generally, Phase 1 tends to contain more specific, and Phase 2 less specific, antigens; but it will be convenient to reserve a full description of 
the variations in the antigenic constitution of the two phases till a little later in this chapter (p. 716). It is sufficient to remark here that in Phase 1 there are one or more antigenic components, and in Phase 2 there are two or more.

The somatic antigens are monophasic, though occasionally a given strain may differ from the type species in its lack of one of the two, three, or four antigenic components which most nembers possess.

The identification and labelling of the Salmonella antigens was initiated by White $(1926,1929 a, b)$, and continued and extended by Kauffmann $(1929 a, b$, $1930 b, 1931,1934 a)$. In their earlier studies the two investigators used a different system of labelling, so that descriptions given in the English and German papers during the period can be correlated only by the aid of an appropriate key giving the equivalent numbers and letters in the two systems (see Lovell 1932 $a$ ). Recently, however, the terminology introduced by Kauffmann has been adopted for general use by a special sub-committee of the International Society for Microbiology (Report 1934); and each member of the Salmonella group is now allotted an antigenic formula based on this system of notation.

The somatic $(\mathrm{O})$ antigens are aecorded Roman numerals. The flagellar antigens of Phase 1 are accorded small letters; these antigens have already illustrated the limitations of an alphabetical notation by exceeding twenty-six in number. By convention, those discovered later than the antigen that received the label y have been accorded an additional distinguishing numeral, $z_{1}, z_{2}$, and so on. The flagellar antigens of Phase 2 are labelled in two different ways. At first they were accorded arabic numerals, but later it was found that the second phase of certain species uniformly contained the antigens $\mathrm{e}$ and $\mathrm{n}$, often associated with $\mathrm{x}$ or with one of the $\mathrm{z}$ series of antigens. It thus happens that Phase 2 may contain antigenic components of the $1,2,3 \ldots$ series, or of the $e, n, \ldots$ series. The position is confusing, because both the e and some of the $z$ antigenic components may be found in the first phase. More recently, strains have been described in which Phase 2 contains neither the $1,2,3 \ldots$ nor the e, n... series, but instead antigenic components that are characteristic of Phase 1.

A few examples may be given to illustrate the use of this notation. The formula for Salm. paratyphi A, whieh is a monophasic flagellated baeillus, existing only in Phase I is [I], II, XII . . . a - . The square braekets indieate that the I somatic antigen may be missing. The three dots after XII mean that traces of other somatic antigens are present, but are exeluded from the antigenie formula for the sake of simplicity. The a represents the flagellar antigen, and the dash following it shows that there is no second phase. The formula for Salm. paratyphi $B$ is $[\mathrm{I}], \mathrm{IV},[\mathrm{V}], \mathrm{XII} \ldots \mathrm{b} \leftrightarrow 1,2 \ldots$ The square brackets show that antigenic components $I$ and $V$ may be missing; $b$ is the flagellar antigen of Phase 1 , and 1,2 ... the flagellar antigens of Phase 2. Again, the dots following XII and 2 indicate an abbreviation of the antigenic formula. The doubletipped arrow shows that the two flagellar phases are reversible. The formula for Salm. amersfoort is $\mathrm{VI}_{1}, \mathrm{VI}_{2}, \mathrm{VII} \ldots \mathrm{d} \longleftrightarrow \mathrm{e}, \mathrm{n}, \mathrm{x} \ldots$. It will be seen that there are two components to the VI somatic antigen; and that, as indieated by the presence of the $\mathrm{e}, \mathrm{n}$ antigens in Phase 2, the organism shows the $\alpha-\beta$ type of phase variation (see p. 716). The formula for Salm. meleagridis is III.X.XXVI. e, h neither the $1,2,3 \ldots$ nor the $\mathrm{e}, \mathrm{n}$. . . series of antigens, but two antigenic components, 1 and $w$, that are normally found in Phase 1 of other organisms. Salm. gallinarum, which is non-motile, has the simple formula [I], IX, XII . . - . - the two dashes showing that there is neither a first nor a scoond flagellar phase. An organism, so far met with only in Phase 2, is Salm, abortus-equi, which has the formula IV, XII . . - . e, n, x; the dash shows that Phase 1 is absent. 
One of the most interesting features of the Salmonella group is the way in which the same antigenic components recur in different combinations. Thus, different $\mathrm{O}$ antigens are found in combination with the same $\mathrm{H}$ antigens. The same set of Phase 2 antigens are replaced by different Phase 1 antigens, when different diphasic species change from the group to the specific or from the $\beta$ to the $\alpha$ phase. The same set of antigenic components recurs in different combinations in Phase 2 of different species, and so on. The implications of these facts in relation to the evolution of the Salmonella group are discussed by White (1926).

The Kauffmann-White Diagnostic Scheme.-It is clear that the observed distribution of these antigenic components forms a basis for a natural scheme of classification, and this has been adopted in the Kauffmann-White scheme, proposed for international adopuion by the Salmonella Sub-committee (Report 1934). It seems reasonable to regard the antigenic structure of the bacterial cell as more fundamental than the antigenic structure of the appended flagella. The Salmonella group, as a whole, has therefore been divided into sub-groups, each of which shares a common somatic antigen. Where more than one somatic antigen is present, one of these antigens is regarded as determining the sub-group to which the species concerned shall be allocated. Thus, Group B consists of those organisms that possess the $\mathrm{O}$ antigen $\mathrm{IV}$, or the antigens IV or $\mathrm{V}$. Group $\mathrm{D}$ consists of those organisms that possess the $O$ antigen $I X$, and so on. The group letter, it should be noted, forms no constituent part of the name of any species or of its antigenic formula. The groups display the natural relations of the different salmonellæ, but the antigenic components that determine those relationships are labelled according to the Kauffmann convention. It should, perhaps, be recalled that there is a fundamental difference between this scheme of classification and that adopted in describing the antigenic structure of other bacterial groups. A "Group B haemolytic streptococcus", for instance, means a haemolytic streptococcus that possesses the Group B antigen, and that antigen has no other label.

It must be made clear that the Kauffmann-White scheme is essentially a scheme for the differentiation in practice of the various species. It is not, nor does it pretend to be, a record of the complete antigenic structure of each organism. The antigenic constitution of most organisms is far more complex than is suggested by the formulæe given in the table. Only the major antigens or those antigens that are of differential importance are recorded. It follows that, owing to the possession of minor somatic or flagellar antigens, cross-agglutination may occur between organisms which, judged by the table, possess no common factor. It follows, too, that as further members of the Salmonella group are discovered alterations will have to be made in some of the present formulæ if the diagnostic value of the table is to be preserved.

Another warning must be issued. Nany of the antigenic components identified by a single numeral or letter are themselves complex, cousisting of two or more fractions. For instance, the $\mathrm{V}$ and VI somatic antigens each comprise two portions labelled $V_{1}$ and $V_{2}$ and $V I_{1}$ and $V I_{2}$. The XII somatic antigen contains three portions, $\mathrm{XII}_{1}, \mathrm{XII}_{2}$, and $\mathrm{XII}_{3}$. The d flagellar antigen contains five partial antigens, $d, d_{1}, d_{2}, d_{3}$, and $d_{4}$. The fact, therefore, that two species are represented in the table as containing, for example, the $d$ antigen, does not mean that their d antigens are necessarily identical. One of the species may contain one pair of the $d$ fractions and the other species another pair, so that though both species will be agglutinated by an anti-d serum prepared against all the fractions, 
KatfFu.inn-White Sounda.

II-Antigen.

Group.

'Type.

o-Antigen.

1 Pliase.

2 Phase.

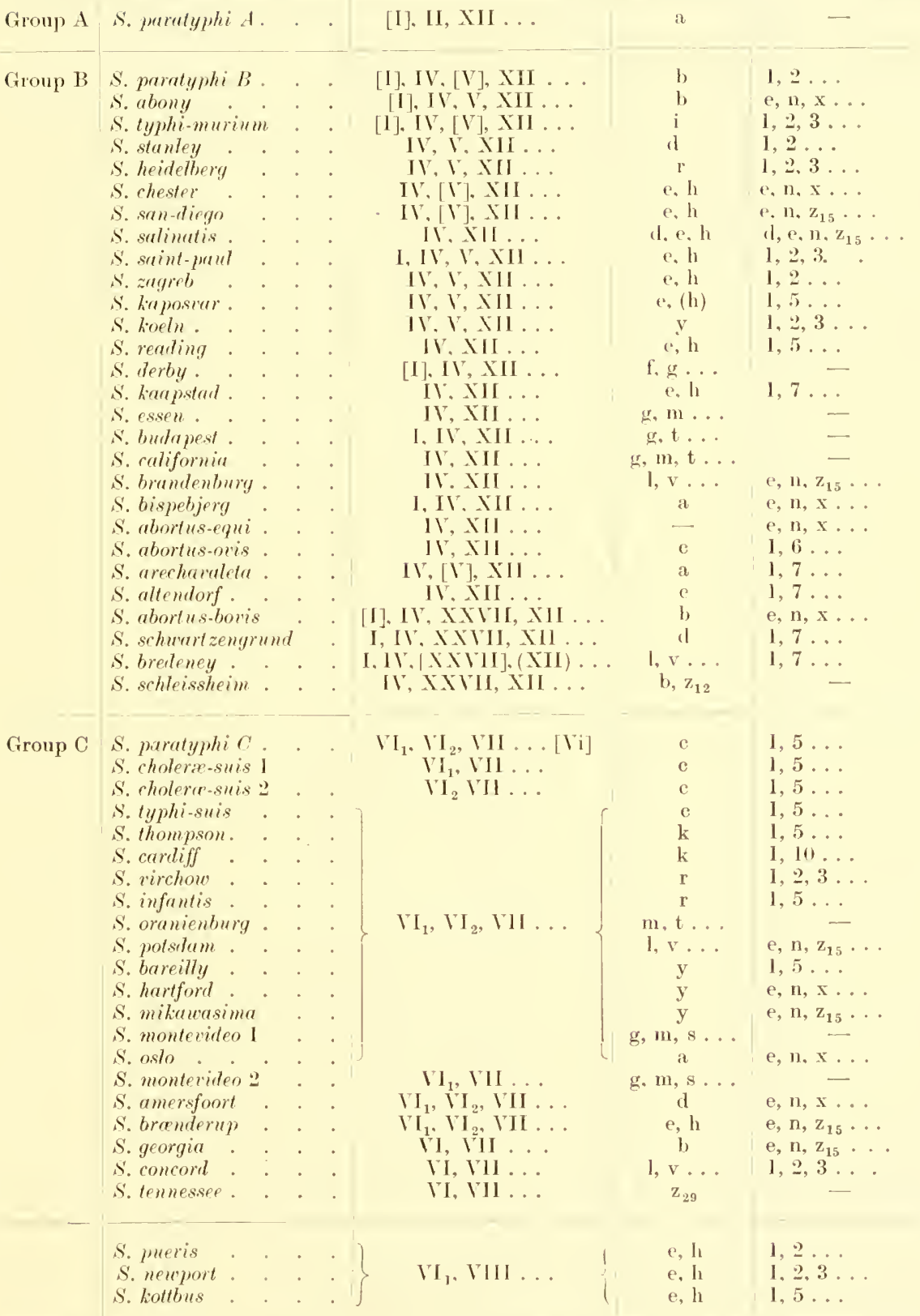


TABLE 47 (continued).

\begin{tabular}{|c|c|c|c|c|c|c|}
\hline \multirow{2}{*}{ Group. } & \multirow{2}{*}{\multicolumn{2}{|c|}{ Type. }} & & \multirow{2}{*}{ o-Antigen. } & \multicolumn{2}{|c|}{ H-Antigen. } \\
\hline & & & & & 1 Phase. & 2 Phase. \\
\hline $\begin{array}{c}\text { Group C } \\
\text { (contd.) }\end{array}$ & $\begin{array}{l}\text { S. bovis-morbifical } \\
\text { S. muenchen } \\
\text { S. oregon } \\
\text { S. mexicana } \\
\text { S. manhattan : } \\
\text { S. narashino } \\
\text { S. glostrup. } \\
\text { S. litchfield : } \\
\text { S. duesseldorf : } \\
\text { S. bonariensis : } \\
\text { Unnamed type } \\
\text { S. virginia. } \\
\text { S. amherstiana. }\end{array}$ & $\begin{array}{l}\text { ans } \\
: \\
: \\
: \\
: \\
: \\
:\end{array}$ & 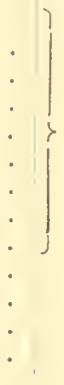 & $\begin{array}{l}V I_{1}, V I I I \ldots \\
\\
\text { VI, VIII ... } \\
\text { VI, VIII . . } \\
\text { VIII . . . } \\
\text { (VIII) }\end{array}$ & $\begin{array}{c}\mathrm{r} \\
\mathrm{d} \\
\mathrm{d} \\
\mathrm{d} \\
\mathrm{d} \\
\mathrm{a} \\
\mathrm{z}_{10} \\
\mathrm{l}, \mathrm{v} \ldots \\
\mathrm{z}_{4}, \mathrm{z}_{24} \\
\mathrm{i} \\
\mathrm{e}, \mathrm{h} \\
\mathrm{d} \\
1,(\mathrm{v}) \ldots\end{array}$ & $\begin{array}{l}1,5 \ldots \\
1,2 \ldots \\
1.2,3 \\
1,2,4 \ldots \\
1,5 \ldots \\
\text { e, n, x } \ldots \\
\text { e, n, } \mathrm{z}_{15} \ldots \\
1,2,3 \ldots \\
\mathrm{e}, \mathrm{n}, \mathrm{x} \ldots \\
1,2 \ldots \\
1,6 \ldots\end{array}$ \\
\hline Group D & 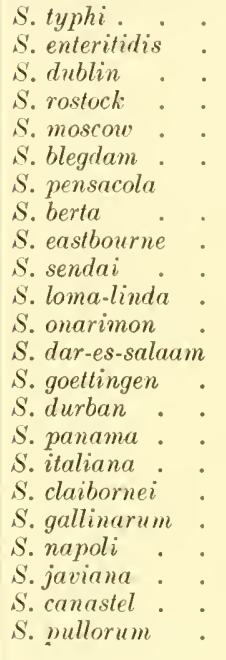 & $\begin{array}{l}: \\
: \\
: \\
: \\
: \\
: \\
: \\
: \\
:\end{array}$ & 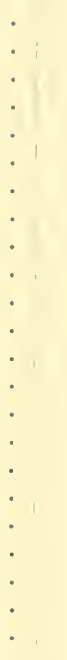 & 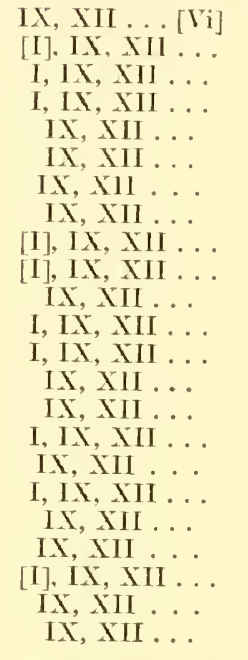 & 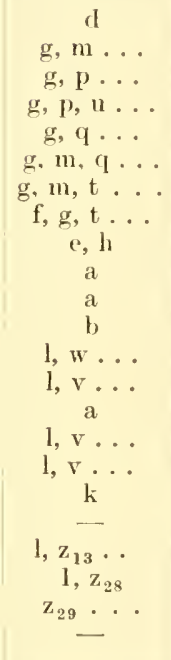 & $\begin{aligned} &= \\
&= \\
&= \\
&= \\
& 1,5 \ldots \\
& 1,5 \ldots \\
& \mathrm{e}, \mathrm{n}, \mathrm{x} \ldots \\
& \mathrm{l}, 2 \ldots \\
& \mathrm{e}, \mathrm{n} \ldots \\
& \mathrm{e}, \mathrm{n}, \mathrm{z}_{15} \ldots \\
& \mathrm{e}, \mathrm{n}, \mathrm{z} 15 \\
& 1,5 \ldots \\
& 1,11 \ldots \\
& 1,5 \ldots \\
& \mathrm{e}, \mathrm{n}, \mathrm{x} . \\
& 1,5 \ldots \\
& 1,5 \ldots \\
&\end{aligned}$ \\
\hline \multirow[t]{2}{*}{ Group E } & $\begin{array}{l}\text { S. london : } \\
\text { S. give : : } \\
\text { S. uganda : } \\
\text { S. anatum : } \\
\text { S. muenster: } \\
\text { S. nyborg : } \\
\text { S. vejle: : } \\
\text { S. amager: : } \\
\text { S. zanzibar: : } \\
\text { S. shangani : } \\
\text { S. meleagridis: } \\
\text { S. lexington : } \\
\text { S. velterreden: }\end{array}$ & $\begin{array}{l}: \\
: \\
: \\
: \\
:\end{array}$ & $\begin{array}{l}. \\
\dot{0} \\
\dot{0} \\
\dot{0}\end{array}$ & $\mathrm{III}, \mathrm{X}, \mathrm{XXVI}$ & $\begin{array}{c}\text { I, r } \cdots \\
\text { l, r } \ldots \\
\text { l, } z_{13} \\
\text { e, h } \\
\text { e, h } \\
\text { e, h } \\
\text { e, h } \\
y \\
\text { k } \\
\text { d } \\
e, h \\
z_{10} \\
\text { r }\end{array}$ & $\begin{array}{l}1,6 \ldots \\
1,7 \ldots \\
1,5 \ldots \\
1,6 \ldots \\
1,5 \ldots \\
1,7 \ldots \\
1,2,3 \ldots \\
1,2,3 \ldots \\
1,5 \ldots \\
1,5 \ldots \\
1, \ldots \ldots \\
1,5 \ldots \\
\mathrm{z}_{6}\end{array}$ \\
\hline & $\begin{array}{l}\text { S. newington } \\
\text { S. selandia } \\
\text { S. new-brunswick } \\
\text { S. cambridge } \\
\text { S. illinois. }\end{array}$ & : & . & III, XV & $\begin{array}{c}\mathrm{e}, \mathrm{h} \\
\mathrm{e}, \mathrm{h} \\
1, w \ldots \\
1, \ldots \ldots \\
\mathrm{z}_{10}, \ldots\end{array}$ & $\begin{array}{l}1,6 \ldots \\
1,7 \ldots \\
1,7 \ldots \\
\text { e, h } \ldots \\
1,5 \ldots\end{array}$ \\
\hline
\end{tabular}




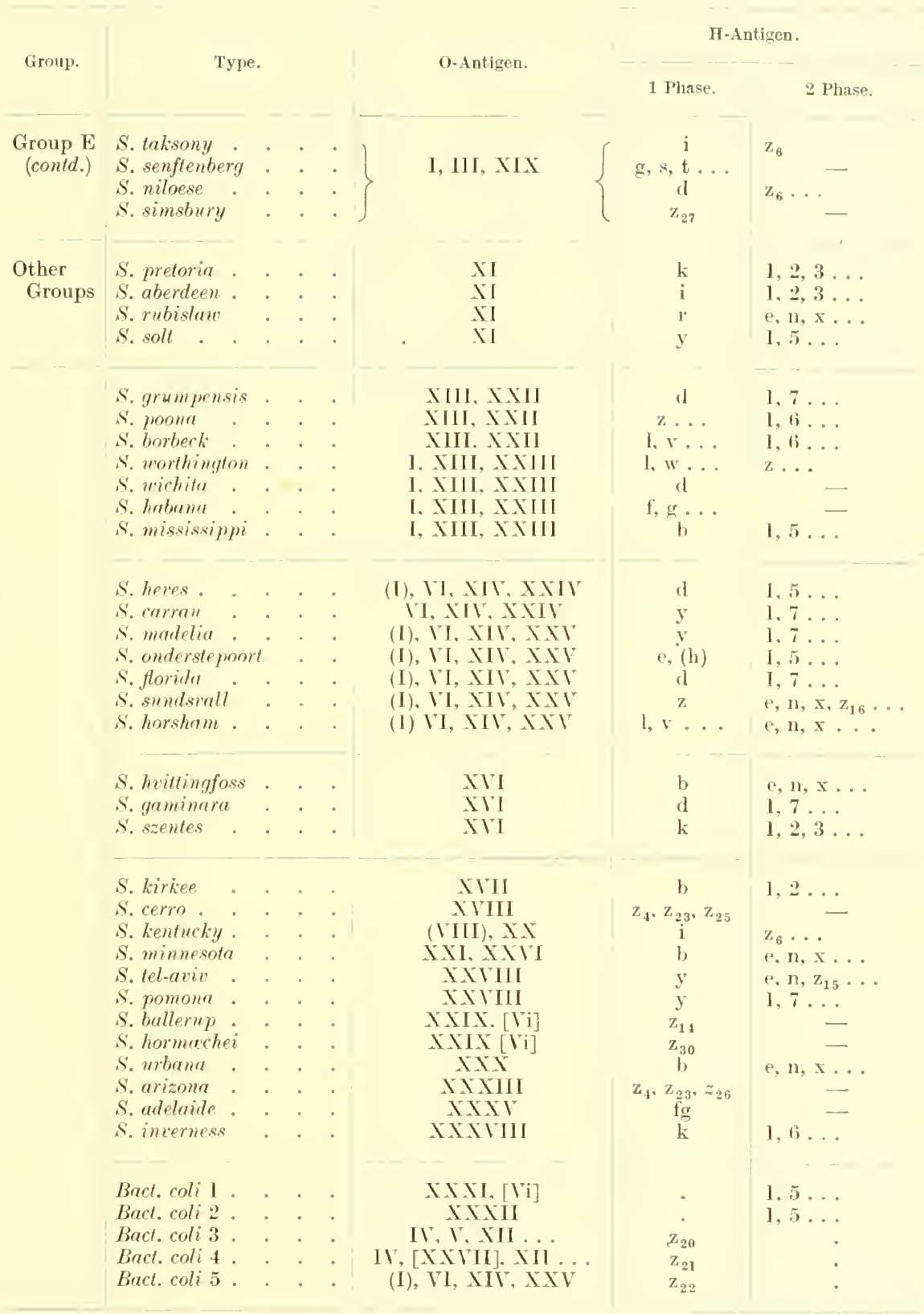

Numerous other coliform and paracolon bacilli containing $O$ or $H$ antigens found in the Salmonella group have been deseribed. Salmonella $U$ antigens have likewise been met with in members of the Flexner dysentery and the Pasteurella groups.

[ ] = These antigens may be missing.

()$=$ Only a part of the antigen is present.

. = Very much abbreviated formula.

. = Not fully investigated. 
absorption of such a serum with one species will not remove the agglutinins for the other species. The $\mathrm{g}$ and $\mathrm{t}$ antigens are likewise complex (Edwards, Bruner, and Hinshaw 1940). In the descriptions of the individual species we have recorded many of the minor and the partial antigens that do not appear in the KauffmannWhite scheme.

This is not the place to describe the practical identification of Salmonella species. For information on this subject readers are referred to publications by Kauffmann (1939b, 1941) and Edwards and Bruner (1942c).

\section{Antigenic Variation.}

The variations that occur within this group have been studied by many observers; and these studies have been particularly fruitful in adding to our knowledge of the mechanisms of bacterial variation as a general phenomenon. For this reason they have been discussed in some detail in Chapters 8 and 9. There is no need to repeat that discussion here, but for the sake of clarity and completeness, it will be well to give a brief résumé of the antigenic variations that may be met with in the Salmonella group.

Phase variation affecting the $\mathbf{H}$ antigens.-In specific-group phase variation, which was described by Andrewes (1922, 1925), the flagella may assume two alternative antigenic forms. The antigenic components of the speeific phase are either peculiar to the particular species concerned or are shared by only a few other species. On the other hand, the group phase contains antigenic components that are shared by many other species. Any given culture of a particular diphasic strain may consist entirely of bacilli in the specific phase, or entirely of bacilli in the group phase, or may contain representatives of both phases. A bacillus in one phase, though always capable of giving rise to descendants in the alternative phase, usually maintains a constant phase over a number of generations. If, therefore, we prepare plate eultures of a diphasic organism and make numerous subcultures, each from a single colony, we should as a rule obtain some suspensions in the specific, others in the group phase. If these are killed by the addition of formalin after 18-24 hours' growth, there will usually not have been time for any change in phase to oceur.

The $\alpha-\beta$ phase variation, which was described by Kauffmann and Mitsui (1930), consists in a variation that does not differ in principle from specific-group variation. Instead, however, of Phase 2 containing the antigenic components 1, 2, 3, etc., it contains the antigenic components $\mathrm{e}, \mathrm{n}$, etc. It will be noted that the antigens in Phase 1 are similar in both types of variation, being of the a, b, c . . . type, but that in Phase 2 they differ.

A special type of $\alpha-\beta$ phase variation has been described by Edwards and Bruner (1938), which may perhaps be regarded as a third type. It was found that, in Salm. worthington, Phase 2, instead of containing the 1, 2, 3 . or the e, n . . type of antigens, contained an antigenic component of the a, b, c . . series, which had previously been met with only in Phase 1. In other words, organisms of this type have what, for practical purposes, may be regarded as two alternative first phases.

It must be realized that, in organisms showing diphasic variation, strains may occur which remain persistently in one phase-nsually Phase $\mathbf{2}$-and in which the alteruative phase can be demonstrater only by growth in the presence of immune serum containing agglutinins to the Phase 2 antigens. Salm. thompson, for example, is almost invariably in the non-specific phase on first isolation and the $\mathrm{k}$ antigen of Phase $\mathrm{I}$ may not be demonstrable till after two or three subcultures in the presenee of an anti-group serum.

Artificial phuse variation is a phenomenon that appears at present to be more of academic than of practical importance, though its significance must remain donbtful till further work has been earried ont. It was observed by Kauffmann (1936c) that, if a certain strain of Salm. typhi was cultured in broth containing agglutinating serum to the normal 
$\mathrm{d}$ antigen, this antigen was lost and replaced by a new antigen $\mathrm{j}$, which had never been met with in typhoid bacilli under natural conditions. Similarly, the $b, z_{12}$ antigens of Salm. schleissheim may be replaced by a new antigen, $\mathrm{z}_{5}$. In both these organisms, which are monophasie, the antigen appearing in the induced phase belongs to the series, a. b, e ... commonly found in Phase 1. Bruner and Edwards (194la), however, were able to induce in the monophasic Salm. paratyphi $A$ a second phase containing the 1, 5 ... antigens, which are characteristic of the group series of antigens of Phase 2. An even more remarkable series of induced variations was recorded by Edwards and Bruner (1939) in Salm. abortus-equi. This organism exists normally only in Phase 2, in which it possesses the antigens $\mathrm{e}, \mathrm{n}, \mathrm{x}$. . B By growth in appropriate sera a Phase 1 , containing the antigen a, was induced, and a Phase 3 , containing the antigen $z_{5}$. All three phases were reversible. It is by no means always easy to decide whether a new phase that appears as the result of growth in immune serum is to be regarded as an artificial phase or as the alternative phase of an organism that is generally, though not invariably, monophasic. Legitimate difference of opinion may exist on this point. For example, Salm. cholerce-suis var kunzendorf was for many years regarded as a well-established monophasic type oceurring in the group phase. When it was found, however, by Bruner and Edwards (1939) that a specific phase could be induced containing the same antigen c as Salm. cholerce-suis, it was decided to omit the kunzendorf type from the Kauffmann-White scheme on the ground that it was merely a group phase variant of the parent strain.

Variation in the $\mathrm{Vi}$ antigen.- There is a type of variation that concerns the $\mathrm{Vi}$ antigen (see p. 1525). The $\mathrm{H}$ and $\mathrm{O}$ antigens remain unaffected. Kauffmamn (1935a) found that typhoid bacilli might exist in three forms: (1) the $V$ form, which contains the full quota of $\mathrm{Vi}$ antigen and is inagglutinable by $\mathrm{O}$ serum ; (2) the $\mathrm{VW}$ form, which contains some $\mathrm{Vi}$ antigen but not enough to inhibit $\mathrm{O}$ agglutinability ; and $(3)$ the $\mathrm{W}$ form, which contains no $\mathrm{Vi}$ antigen and is fully agglutinable by $\mathrm{O}$ serum. A similar variation in the $\mathrm{Vi}$ antigen may be observed in Salm. paratyphi $C$ and Salm. ballerup. The $\mathrm{V} \rightarrow \mathrm{W}$ variation appears to be irreversible (Craigie and Brandon 1936b).

Variations in the 0 antigens.- In some strains containing two or more $\mathrm{O}$ components, some colonies may be found in which one of the components is missing or developed to only a slight extent. The two antigenic components most likely to be affected are I and XII. Within the same strain there are $\mathrm{I}++, \mathrm{I}+$ and $\mathrm{I} \pm$ colonies, the tendeney being for the $\mathrm{I}++$ to pass over to the $\mathrm{I} \pm$ type. The XII antigen is complex, containing $\mathrm{XII}_{1}, \mathrm{XII}_{2}$, and $\mathrm{XII}_{3}$ fractions. According to Kauffmann (1941), it is the $\mathrm{XII}_{2}$ fraction that is most likely to be lost or weakened. A rather different type of variation is noticed in organisms, like Salm. paratyphi $B$ and Salm. typhi-murium, containing the $I V . V$ antigenic components. In some strains the $V$ antigen is missing, not merely from certain colonies but from the whole strain. Strains of Salm. paratyphi $B$ showing this so-called loss variation seem to be commoner in carriers than in acute cases; and strains of Salm. typhi-murium showing the same loss variation are, according to Edwards and Bruner (1940a), usually derived - at least in the United States-from pigeons.

The $\mathbf{O H} \rightarrow \mathbf{0}$ variation.-Flagellated strains may occasionally give rise to variants that contain only the $\mathrm{O}$ antigen. As a rule, this type of variation is irreversible. Some strains, like Salm. gallinarum and Salm. pullorum, are permanently non-flagellated. The $\mathrm{OH} \rightarrow \mathrm{O}$ variation eannot readily be induced in the laboratory. The production of $\mathrm{O}$ forms by growth on phenolized agar is not a true impressed variation, since there is a rapid reversion to the normal flagellated form when the organisms are inoculated on to ordinary media.

The smooth $\rightarrow$ rough variation.-This type of variation has already been described at sufficient length in Chapter 9. So far as the Salmonella group is concerned, it consists essentially in a loss of the normal smooth somatic antigen, with the appearance of a new, rough antigen, having far less specific properties than the smooth antigen and conferring on the bacilli a sensitivity to salt solution which renders them unstable in suspension. 
The flagellar antigens are unaffected. This type of variation seems to occur rather more freqnently in nature, is common in strains maintained through many generations on ordinary laboratory media, and can readily be indnced by several different proceduresthe prolonged incubation of a broth culture, growth in the presence of antibodies acting on the smooth somatic antigens, subjection to the action of an anti-smooth phage, and so on. To prevent its occurrence, strains should be dried from the frozen state in vacuo, or, if this is not practicable, kept on a dry Dorset egg medium in the ice-chest and subcultured as infrequently as possible. Moist solid media and sugar-containing media should be avoided.

It is of interest to note that the rough polysaccharide antigen appears to be limited to nembers of the Salmonella group. It is possessed by all those strains that have been examined, but not by other species of bacteria, such as coliform or dysentery bacilli (White 1929a).

Many of the observations recorded by workers in this field indicate quite clearly that the $\mathrm{S} \rightarrow \mathrm{R}$ variation is not an "all-or-none" process so far as the culture as a whole is concerned. It is gradual or step-like. A smooth strain may lose some of its normal somatic antigen and uncover some of its rough antigen, or develop it to replace the smooth, so that it will respond to both an anti-smooth and an anti-rough serum. As has already been noted (see Chapters 8 and 9 ), variation by loss may proceed further than the $\mathrm{R}$ form, giving rise to the $\rho$ forms of White (1932), in which the R polysaccharide is lost.

Variation of the mucoid or M antigen.-Mention has already been made (p. 705) of the formation by some members of the Salmonella group, notably Salm. paratyphi B, of mucoid colonies containing a nitrogen-free polysaccharide (Birch-Hirsehfeld 1935). This so-called mucoid or M antigen (Kauffmann 1935a, 1936a) is formed best at room temperature, is resistant to heating at $60^{\circ} \mathrm{C}$. for 1 hour and to formalin, but is destroyed partly or completely by heating at $100^{\circ} \mathrm{C}$. for 2 hours, and by treatment with 96 per cent. alcohol or normal $\mathrm{HCl}$ at $37^{\circ} \mathrm{C}$. for 20 hours. The presence of the antigen is best demonstrated by the use of living suspensions and an anti-M serum. The mixtures should be incubated for 2 hours at $37^{\circ} \mathrm{C}$. and overnight at room temperature. Sera usually have a low titre, seldom exceeding 1/160. Only one M antigen has yet been met with.

Variation of the $\mathrm{X}$ antigen.-The $\mathrm{X}$ antigen was first observed by Topley and Ayrton (1924) in suspensions of Salm. typhi-murium, Salm. newport, and Salm. enteritidis which had been grown in broth for some days at $37^{\circ} \mathrm{C}$, but has since been found to develop in almost all the salmonellæ studied. Like the M antigen, it is non-specific. It is thermostable, resisting heat at $100^{\circ} \mathrm{C}$. for 30 minutes, and is unaffected by 0.25 per cent. formalin. It occurs in both rough and smooth strains. Its appearance is most common in old broth cultures, though it may be present in broth cultures grown for the norma length of time, or even in suspensions from agar, especially if the surface is moist. Agglutination resembles the granular somatic type, but the flakes are as a rule slightly coarser and tend to lie along the sides of the agglutimation tube. They become apparent in 2 to 4 hours, but continue to increase for some time. High-titre serum is easy to prepare by the inoculation of rabbits with a culture rich in $\mathrm{X}$ agglutinogen. The importance of the $\mathbf{X}$ antigen lies in the confusion it may cause in routine serum agglutination tests should any of the suspensions contain this antigen or any of the sera contain anti- $X$ agglutinins. In practice, few workers seem to have met with the $\mathrm{X}$ antigen, but in one or two laboratories its presence has caused considerable trouble. It is always wise to make certain that standard agglutinable suspensions are free from it (see Cruickshank 1939).

Fermentative Varieties of Antigenic Strains,--Another point that perhaps needs some comment is the recognition of fermentative varieties that are antigenically identical. As a systematic procedure this is a reversal of the common finding, in which different antigenic types can be recognized in a species that is homogeneous as regards its enzymic activities. Many of the fermentative differences that have 
been accepted as defining separable varieties are very slight. Sometimes they are revealed only by the use of a special medium or a special reaction. The definition and labelling of these fermentative types has been carried out by a few observers, particularly by Kauffinann, who would extend the process even further than many other workers would be prepared to do (see Kauffmann and Burón 1935). The significance of these types must, we think, remain very doubtful until we have far more extensive data than are at present available. At the same time the existence of the differences in enzymic activity can clearly not be ignored, if only because the evidence that exists to-day, scanty as it is, suggests that they are correlated with natural habitat and natural pathogenicity.

To take, as an example, the fermentative varieties of Salm. enteritidis, Kauffmann (1935b) records the origin of small samples of these strains. Of 8 strains of Salm. enteritidis examined by him, 7 were isolated from man, one from a gninea-pig. Of 22 strains of Salm. enteritidis var. danysz, 13 strains came from human infeetions, 9 from rats or from the Ratin virus used for the extermination of these vermin. The 8 strains of Salm. enteritidis var. chaco all came from infected soldiers in the Chaco war. Of 7 strains of Salm. enteritidis var. essen, some came from human infections, some from infections in ducks. Again, strains of Salm. paratyphi $B$ that ferment $d$-tartrate were found by Kristensen and Kauffmam (1937) in patients suffering from enteritis; strains causing typical paratyphoid fever do not usually ferment $d$-tartrate. Strains of Salm. typhi-murium that infect European dueks and chickens do not as a rule ferment rhamnose in opposition to those found in the United States, which are rhamnose-positive (Edwards and Bruner 1940a).

If the fermentative abilities described are found to be constant for any particular strain, and if the varieties differentiated by them are found to show signifieant differences in their natural distribution, there will clearly be a good case for providing them with separate labels. At the moment it is wisest to regard these labels as provisional.

The Bacteriophage Method of typing Salmonella Strains.-Of far greater value, though of more restricted application, is the methor of subdividing antigenic types into sub-types by means of the baeteriophage method of typing introduced by Craigie and Brandon $(1936 a, b)$ and Craigie and Yen (1938). It was known that salmonellse were susceptible to the action of bacteriophages, and that these were closely related to the type of $\mathrm{O}$ antigen present; but owing to the wide communal sharing of the same $O$ antigen, bacteriophages of this sort were useless for the differentiation of sub-types. Soon after the discovery by Felix and Pitt $(1934 a, b)$ of the $\mathrm{Vi}$ antigen of the typhoid bacillus, several workers established the existence of bacteriophages acting specifically on the $\mathrm{V}$ form of the bacillus. The special contribution of Craigie and his co-workers was their observation of a peculiar adaptability possessed by one particular anti- $\mathrm{Vi}$ phage. When this phage was grown on typhoid strains of different origin, races of bacteriophage were obtained that were found to have developed a high degree of specificity for the particular strain on which they had been propagated. By means of a series of bacteriophages prepared in this way, Craigie and Yen were able to classify nearly all of 592 strains of typhoid bacilli into six bacteriophage types. The evidence obtained from a study of the origin of the strains revealed a high degree of correlation between the bacteriophage type and the epidemic source. These observations have since been abundantly confirmed, so that the bacteriophage method of typing is rapidly becoming a routine procedure in the investigation of outbreaks of typhoid fever. The degree of specificity seems to be almost complete, and the results are more constant and reliable than those of typing by the fermentative method. 
As already mentioned, however, the method is subject to limitations. In the first place, not all strains of typhoid bacilli possess a $\mathrm{Vi}$ antigen or possess it in sufficient quantity to enable the bacteriophage to act satisfactorily. For example, in Craigie and Yen's series of 706 strains, 42 belonged to the $W$ form and 72 to the VW form. The remaining 592 strains belonged to the $\mathrm{V}$ form, possessing a fully developed $\mathrm{Vi}$ antigen ; of these, 98.6 per cent. were successfully typed. The proportion of $W^{T}$ and $T W$ forms was higher in this series, which contained many strains that had been isolated months or years previously, than is commonly found in routine laboratories handling freshly isolated strains, of which usually ouly about 5 per cent. are found to be lacking in $\mathrm{Ti}$ antigen (Craigie and Brandou 1936b, Rauss 1939b). Another limitation is that only species of Salmonella containing the Ti antigen can be typed. This is a very serious limitation since, according to our present knowledge, the distribution of this antigen is restricted to Salm. typhi, Salm. paratyphi $C$, Salm. ballerup, and Salm. hormachei.

More recent observations, however, suggest that some other species possess either a $\mathrm{Vi}$ antigen or another type of antigen which is susceptible to differential attack by the bacteriophage. Felix and Callow (1943), for example, have been able to develop a series of bacteriophages acting on Salm. paratyphi B, and have by this means succeeded in dividing strains of this organism into four bacteriophage types, which correspond closely with the epidemiological types.

The technical methods used in the bacteriophage typing of typhoid and paratyphoid bacilli camnot be described here. Table 48, however, illustrates the susceptibility of typhoid bacilli to the different types of bacteriophage.

It will be noticed that Type A strains are sensitive to all bacteriophage type strains, but that the remaining types possess a fairly high degree of specificity. Further types and sub-types are constantly being described. Types $\mathrm{L}$ and M and sub-types $\mathrm{B}_{3}, \mathrm{~B}_{4}, \mathrm{D}_{3}, \mathrm{E}_{2}$, and $\mathrm{F}_{2}$ have since been added; and Felix (1943) in this country has reported three new sub-types $\mathrm{D}_{4}, \mathrm{D}_{5}$ and $\mathrm{L}_{2}$, and a new type labelled provisionally No. 91. As more strains of typhoid bacilli are studied from different envirommental conditions, it is probable that even further bacteriophage types will be discovered. Sufficient, however, are already known to render this method of study profoundly valuable in the field of epidemiological inquiry. More recently, Craigie (I942) has modified his original scheme, but the principles of his method remain unaltered.

Chemical Structure of the Salmonella antigens.- We may add a brief note on our knowledge, such as it is, of the chenical constitution of the antigens on which the present classification of the Salmonella group is based. Of the nature of the flagellar antigens we as yet know nothing, beyond the hints conveyed by heat lability and sensitiveness to extraction with alcohol.

Of the somatic antigens we know rather more. Furth and Landsteiner (1928, 1929) isolated several different chemical components from bacilli of the Salmonella group. Some of these were polysaccharides. The studies of White $(1929 a, b$, 1931) make it clear that the somatic antigens-those labelled I, II, III and so on in our antigenic formulæ-are polysaccharides, or have a polysaccharide component; and further evidence on similar lines has been recorded by subsequent observers (Casper 1928-29, Combiesco et al. 1930, Basilewsky and Remgild 1935). More recent studies by Freeman, Challinor, and Wilson (1940) and Morgan and Partridge (1942) on Salm. typhi suggest that the $O$ antigen of this orgauism is a polymolecular complex formed of a specific polysaccharide, a protein, and a 
phospholipin. The protein component appears to be chemically and immunologically very similar to the conjugated protein prepared by Morgan and Partridge (1940) from the specific somatic antigen of Sh. shiga. According to Freeman (1943), the somatic antigen of Salm. typhi-murium chemically resembles that of Salm. typhi.

TABLE 48

REACtions OF TYPE strans of Salm. typhi to CRITICAL TEST DILUTIONS OF TYPE bacteriuphage straiss (From Cralgie aNd Yex 1938).

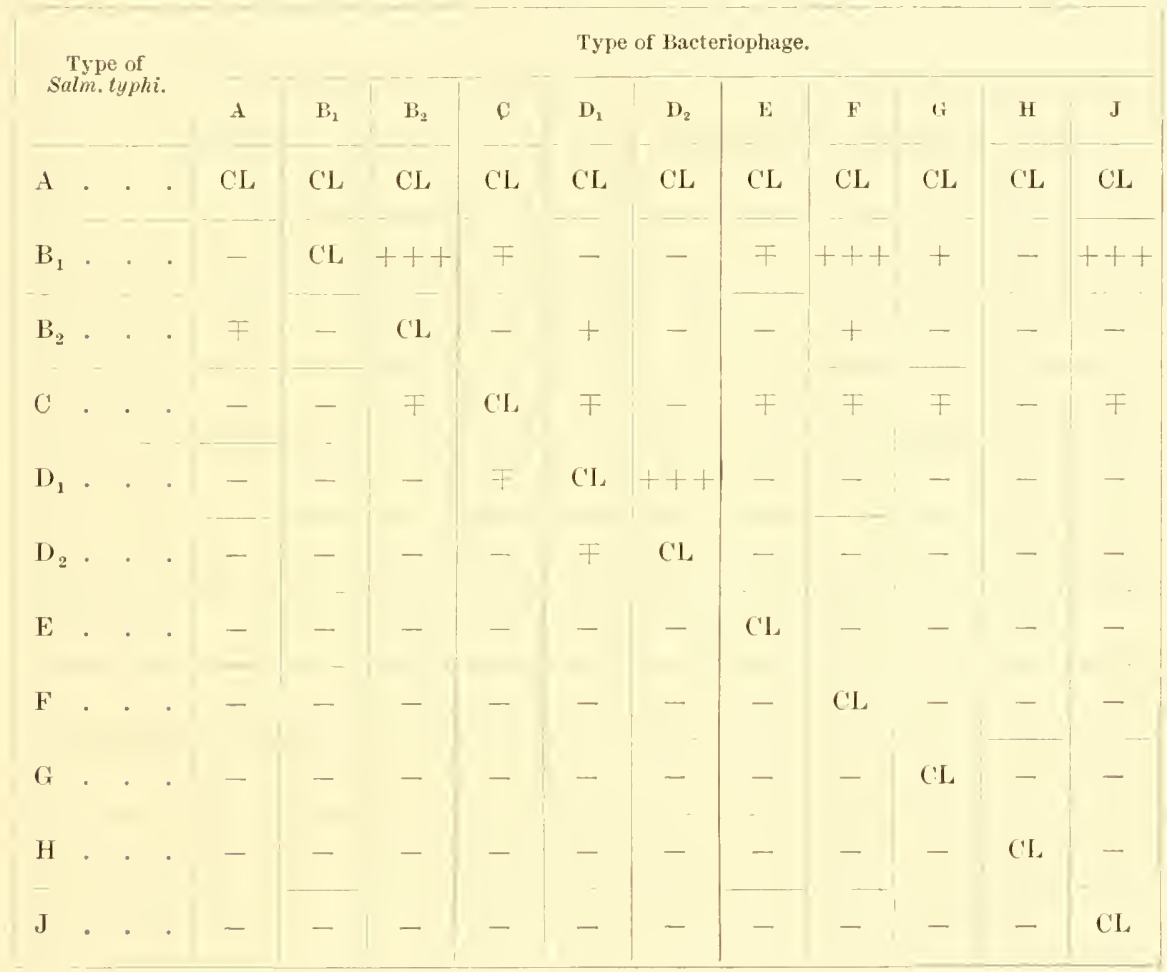

$\mathrm{CL}=$ Confluent Lysis. $\quad+++=$ Numerous diserete plaques of subnormal size. $F=$ Few plaques, subnormal in size except in Type C strains.

Pathogenicity and Toxin Production.-As has already been pointed out (p. 703), members of the Salmonella group are widely distributed in the animal kingdom. Broadly speaking, the type of disease with which these organisms are associated is either an enteritis or a septicæmia. In man, a few species, like Salm. typhi and Salm. paratyphi $B$, give rise to a disease characterized by a fairly long incubation period and the predominance of septicæmic over intestinal symptoms. The great majority, however, of the Salmonella species produce acute gastro-enteritis of the food-poisoning type in children and adults, or acute enteritis in infants characterized by a short incubation period and the predominance of intestinal, over septicæmic symptoms. Nevertheless, a disease apparently starting as an enteritis, may become a septicæmia, which runs a typhoid-like course or is associated with localized manifestations of disease in the meninges, bones, joints, or other 
situation. As in animals and birds, a proportion of persons who become infected may harbour the organism for a varying length of time without showing any signs of disease. Though certain types of Salmonella are far commoner in man than others, it is now clear that practically every species so far described is capable of infecting human beings. Even Salm. gallinarum, which has been regarded as restricted to fowls, has been isolated from a human case of disease. It is impossible here to describe in detail the natural pathogenicity of members of the Salmonella group for man and animals, but some information in this respect will be found in the descriptions of the individual species on pp. 726-44.

Experimental observations on the pathogenicity of Salmonella for laboratory animals have been confined to a relatively few species, such as Salm. typhi, Salm. typhi-murium and Salm. enteritidis. It will be sufficient in this chapter to give a brief description of the effects produced in experimental aninals by some of the better known species, and a short discussion of the nature of the toxic substances that these organisms produce.

\section{Salm. typhi.}

If massive doses of living typhoid bacilli are administered by the mouth to chimpanzees, it is possible to produce a disease that is very similar to typhoid fever in man (Metchnikoff and Besredka 1911).

Administration of typhoid bacilli by the mouth to ordinary laboratory animals (rabbit, guinea-pig, rat or mouse) does not give rise to an infection of this type, or usually to any harmful result at all.

The intraperitoneal or intravenous injection of living typhoid bacilli in adequate doses induces a fatal infection, and the bacilli can be recovered from the blood and tissues post mortem. The effect of such injections would seem to be in part toxæmic, in part dependent on the multiplication of the bacteria in the body. With some strains of typhoid bacilli it may be necessary to inject 1,000 million living bacilli or more into the peritoneum of a mouse to produce a fatal result. A low multiple of this dose $(5,000-10,000$ million bacilli) will usually cause a purely toxæmic death when the bacilli have been killed by heat before inoculation. A highly virulent strain will induce a fatal infection following the intraperitoneal injection of 50 million bacilli, or even a little less. But there is no evidence that the typhoid bacillus possesses an ability to multiply freely in the blood or tissues of small laboratory rodents when injected in small doses, such as would certainly prove fatal in the case of a frankly invasive organism.

There is nothing characteristic in the findings at necropsy in a mouse, or guinea-pig, that has died as the result of an intraperitoneal injection of living typhoid bacilli. There is, of course, the usual inflammatory reaction in the peritoneum, sometimes associated with subserous hæmorrhages; and the organism which has become generalized throughout the body may be recovered from the blood or from any of the tissues. Following the intravenous injection into the rabbit of doses of living typhoid bacilli too small to produce a rapidly fatal infection, the bacilli tend to localize themselves in certain situations, particularly in the gall-bladder. But experiments of this type will be more conveniently considered in Chapter 69, in relation to the pathogenesis of enteric infections in man.

\section{Salm. typhi-murium.}

The effects produced by the administration of living cultures of this organism to the small rodents of the laboratory are entirely different from those produced by the typhoid bacillus. We are dealing with a natural pathogen of these animals, which gives rise in them to a characteristic disease, usually known as mouse typhoid. This disease is produced when living cultures of Salm. typhi-murium are given by the mouth as well as when they are administered by subcutaneous or intraperitoneal injection, though the time to death is longer in the former case than in the latter. The organism has a very definite 
invasive power for the tissues of mice and of other laboratory rodents. A virulent strain will kill 50 per cent. or more of mice injected intraperitoneally with a dose of 100 bacilli, as compared with the 50-100 million that are required in the case of a virulent typhoid bacillus. Mice dying within 2 to 3 days after the injection of a moderate dose of a virulent strain will be found to have succumbed to an acute septicæmia with few obvious lesions; but mice dying after the more usual period of 5 to 10 days often show characteristic lesions, including a varying degree of splenic enlargement, often associated with the presence of small necrotic foci, larger and very characteristic necrotic lesions in the liver, and sometimes scattered pneumonic patches in the lungs, associated with a scanty pleural exudate. These lesions have been described in some detail by many observers (see, for instance, Seiffert, Jahncke and Arnold 1928). The spread of infection from the intestine, and the subsequent involvement of the various tissues, have been studied and described by Müller (1912) and by Orskov and his colleagues (Orskov, Jensen and Kobayashi 1928, Orskov and Moltke 1928, Orskov and Lassen 1930). These experiments will be considered in some detail in Chapter 47.

One other point should be emphasized. The disease produced in the mouse by Salm. typhi shows no tendency to spread by contact from mouse to mouse. The disease produced by Salm. typhi-murium is highly contagions, and there are few laboratories that have not experienced serious epidemics of this infection among their normal stock of mice or guinea-pigs.

\section{Salm. enteritidis.}

It need only be said that this species, particularly the danysz variety, behaves in much the same way as Salm. typhi-murium. Whether all varieties of Salm. enteritidis are equally pathogenic for small rodents, as judged by experimental infection in the laboratory, wo do not know.

\section{Salm. paratyphi B.}

The main interest of this species, from our present point of view, is that it occupies a position in some ways intermediate between that of Sulm.typhi and Sulm. typhi-murium. It is a natural pathogen of man but not of rodents, but antigenically it is very closely related to the mouse-typhoid bacillus. When injected into mice it kills them in far smaller doses than does Salm. typhi, thongh it is less virulent than Salm.typhi-murium. When administered by the mouth, as Orskov and his colleagues have shown, it has a limited ability to invade the tissues and multiply in them; but it rarely gives rise to fatal infections.

It is probable that many other species of Salmonelle will be found to pussess high or moderate virulence for laboratory animals, and in some instances we already know this to be the case. Sulm. cholera-suis, for instance, is highly virulent for the rabbit. But the mapping-out of the relative virulence, or pathogenicity, of all the 130 or more species or varieties of Salmonella that have so far been described, and of the new arrivals that may well be imminent, will clearly be a laborious undertaking, and one that is perhaps not very likely to be embarked on in the near future.

The Toxins of the Salmonella Group.-As we have noted above, the injection of killed typhoid bacilli, in adequate dosage, will produce toxæmic death in the uswal laboratory animals; and the "toxins" of the typhoid bacillus were studied by several of the earlier bacteriological workers (Pfeiffer 1894, Sanarelli 1894, Chantemesse 1897, Brieger 1902).

Macfadyen and Rowland (1901, 1903), by grinding typhoid bacilli in the frozen state and subsequently extracting them with $0 \cdot 1$ per cent. $\mathrm{KOH}$, prepared a solution which was highly toxic for rabbits but only slightly toxic for guinea-pigs. Meyer and Bergell (1907) obtained an extract of typhoid bacilli that was toxic for rabbits by suspending the bacilli from agar cultures in slightly alkaline distilled water, and filtering the extracts through Chamberland candles after they had stood for 48 hours at room temperature. 
Conradi (1906) found that a filtrate from an autolysed saline suspension of Salm. typhi, after evaporation at $35^{\circ} \mathrm{C}$. to one-tenth of its original volume, was toxic for guinea-pigs in a dose of $0.2 \mathrm{ml}$. Yamanouchi (1909) reported that filtrates of cultures of Salm. typhi in 5 per cent. peptone water were toxic for rabbits in a dose of $0.5-1 \mathrm{ml}$. per kilo bodyweight. Arima (1912) grew Salm. typhi on weakly alkaline agar, suspended the growth in saline and later separated the bacillary bodies by centrifugation. The supernatant fluid was pipetted off and examined separately. The bacillary bodies were washed three tines, ground up, and extracted with saline. Both solutions were toxic for rabbits, less so for guinea-pigs and mice. Douglas (1921) showed that tryptic digests of acetoneextracted typhoid bacilli were highly toxic. The statements with regard to the thermostability of these toxic extracts or filtrates are somewhat conflicting. But most workers report them as heat-resistant. Taken as a whole these observations indicate that typhoid bacilli elaborate toxic substances which are, in the main, retained within the bacterial cell, though they are liberated into the surrounding medium when autolysis occurs. There is, at the moment, no evidence that the typhoid bacillus produces an exotoxin in the ordinary sense of that term.

Most other salmonellæ that have been examined behave, in this respect, in the same way as Salm. typhi, though the toxicity of killed cultures or extracts of the different species may show significant differences in toxicity for laboratory animals, particularly the rabbit (White 1926, 1929b).

The two natural pathogens of rodents, Salm. typhi-murium and Salm. enteritidis, may be considered in rather more detail from this point of view, since it happens that we have recently obtained some knowledge of the constitution of one, at least, of their toxic products.

Ecker (1917), Ecker and Richardson (1925), Branham (1925), Ecker and Rimington (1928) and Ienten and King (1930) noted the toxicity for rabbits or mice of broth filtrates of Salm. typhi-murium and of certain other salmonellæ; and Casper (1928-29) prepared a toxic extract of the bacterial bodies by extraction with antiformin. Ecker and Rimington (1928), Menten and King (1930) and Casper (1928-29) were able, by various methods of adsorption or precipitation, to obtain a partially purified toxic fraction from these crude filtrates or extracts. The fractions so obtained were thermostable. The purification was very incomplete, and though qualitative tests indicated the presence of polysaccharide constituents, no serious attempt was made to determine the chemical constitution of the toxic components. Martin (1934) showed that the greater part of the toxic material present in a broth culture of Salm. typhi-murium was contained in the bacterial cells; and that these constituted the most favourable starting material for obtaining a purified toxin. His method of purification is considered below.

Independent studies on the immunological properties of chemical fractions isolated from Salm. typhi-murium and Salm. enteritidis by Boivin and his colleagues (1933, 1934, 1935) and by Raistrick and Topley (1934), Delafield (1934) and Martin (1934) have thrown further light on this problem. These studies were undertaken, in the main, with the object of obtaining a chemically pure immunizing substance from these organisms. The point that concerns us here is that fractions obtained by both groups of workers were found to be highly toxic.

Boivin and his colleagues obtained their fractions by extraction with trichloracetic acid, followed by alcohol precipitation. Raistrick and Topley extracted the bacilli with acetone, digested them with trypsin, and submitted the tryptic digest to fractional precipitation with alcohol.

The final product obtained by both groups of workers was free from protein, as judged by the ordinary chemical tests, but contained a polysaccharide component that gave rise to reducing sugars on hydrolysis. Nitrogen and phosphorus were also present, and the results of micro-combustion analysis, taken with various qualitative tests, suggested that the active fraction consisted of a complex polysaccharide combined with a phosphatide. The polysaccharide can be separated from the phosphatide residue by heating with weak acetic acid. When this is done, the polysaccharide is entirely, and the phosphatide 
almost, non-toxic. The unhydrolysed toxie material, as prepared and tested by Martin (1934), had an average lethal dose for the mouse of $0.5 \mathrm{mgm}$. The substance obtained by Boivin and his colleagues is reported as being slightly more toxic.

A point of considerable interest in regard to these findings is that there seems little doubt that the toxic substance is itself the somatic antigen of the bacterial cell. Rabbits immunized with the purified fractions produce characteristic somatic agglutinins. The polysaccharides isolated from salmonella organisms by previous workers, and identified with the somatic antigens, or with their hapten constituents, were in most cases found to be non-toxic. But this was probably because the method of preparation had resulted in the splitting of the polysaccharide from the phosphatide component.

The same series of experiments have given an indication of one of the ways in which these toxic substances produce their effects. It had already been shown by several workers (see Chapter 44) that natural infections in man, and experimental infections in animals, might be associated with an increase in the concentration of sugar in the blood; and Menten and King (1930) had found that rabbits injected with the fraction isolated by them from Salm. typhi-murium developed hyperglycæmia.

Delafield (1934), who had already made a careful study of the chemical changes occurring in the blood of the rabbit following the injection of killed suspensions of various bacteria (see Chapter 44), was able to show that the purified fractions prepared by Raistrick and Topley induced a marked hyperglycæmia, followed in many cases by a fall in blood sugar far below the normal level. These findings were confirmed by Boivin and Mesrobeanu (1934b).

These observations suggest that the toxic substances isolated from Salm.typhi-murium and Salm. enteritidis are representatives of a group of antigenic components, possessing very similar toxic properties but differing widely in the chemical structure that determines their antigenic specificity, and that bacterial components of this type are very widely distributed among different bacterial groups. Delafield (1931, 1932) found that a wide variety of Gram-negative bacteria, including Bact. coli, Bact. aerogenes, Sh. shigce, Salm. typhi, Salm. typhi-murium, II. influenze, H. bronchisepticus, Past. muriseptica, Proteus vulgaris and the meningococcus, induced hyperglycæmia in rabbits, while the Gram-positive species studied did not; and Boivin and Mesrobeanu (1935b) have isolated fractions of the same type as those obtained from Salm. typhi-murium from a variety of bacilli of the coli-typhoid group, though the toxicity of most of these fractions has not yet been determined.

There is, of course, no reason to suppose that these particular substances are the only toxic constituents of the bacteria in which they occur. It is possible that there are many others. They do, however, afford examples of "endotoxins" that are definable chemical substances, and not merely crude extracts containing all the multitudinous components of the bacterial cells from which they were derived.

We append a detailed description of the general characters of a typical member of the Salmonella group-Salm. paratyphi B-and a summarized description of each of the different species.

\section{Salm. paratyphi B}

Synonyms.-Bact. paratyphosum B, Bact. schottmülleri.

Isolation.-From cases of enteric infection in man by Achard and Bensaude (1896) and Schottmüller $(1900,1901)$.

Habitat.-Almost entirely a parasite of the luman intestinal tract.

Morphology.-Bacilli with parallel sides and rounded ends, usually $2-3 \mu$ long and 0-6 $\mu$ broad. Gram-negative; non-acid-fast. Non-sporing; usually non-eapsulated, but may form a mucoid envelope. Actively motile; flagella are stated to be peritrichate, but of this there is some doubt, 
Agar Plate.-24 hours at $37^{\circ} \mathrm{C}$. Commonest type of colony is circular, low convex, grevish-yellow, faintly translucent, $1-3 \mathrm{~mm}$. in diameter, with smooth surface and entire edge; butyrous in consistency and emulsifies easily. Surface may be irregular though smooth, and edge may be coarsely undulate or finely but irregularly indented. Colonies that are left at room temperature, after preliminary incubation at $37^{\circ} \mathrm{C}$., may develop a so-called mucoid wall.

Agar Slope. -24 hours at $37^{\circ} \mathrm{C}$. Abundant, raised, faintly translucent, greyish-yellow growth with glistening, smooth or beaten-copper surface and entire or undulate edge.

Gelatin Stab. -7 days at $20^{\circ} \mathrm{C}$. Good filiform growth, with moderate growth on the surface. No liquefaction.

Broth.-24 hours at $37^{\circ} \mathrm{C}$. Abundant growth with uniform turbidity, which continues to increase slightly for 2-3 days. Slight to moderate powdery deposit, disintegrating readily on shaking. No surface growth.

MacConkey's Agar. 24 hours at $37^{\circ} \mathrm{C}$. Yellow colonies, 1-3 mm. in diameter, often of vine-leaf type.

Desoxycholate Citrate Agar.-24 hours at $37^{\circ} \mathrm{C}$. Colourless colonies, 1-2 mm. in diameter, sometimes with a black centre.

Hilson and Blair's Agar.-48 hours at $37^{\circ} \mathrm{C}$. If closely packed, colonies are about $1 \mathrm{~mm}$. in diameter, and appear faintly greenish; if discrete with room to spread, they are larger. flatter. and darkish in eolour with a metallic sheen.

Resistance. - Killed by moist heat at $55 \mathrm{C}$. in half an hour. Cultures at room temperature, or preferably on egg medium in the ice-chest, live for months.

Vetabolism.-Aerobe and faeultative anaerobe. Grows hetween about $15^{\circ}$ and $42^{\circ} \mathrm{C}$., best at $37^{\circ} \mathrm{C}$. Nlay form a mucoid, capsular material at room temperature. Grows well in Bitter's medium containing ammonium salts as the main source of nitrogen. No hæmolysin formed.

Biochemical.-Acid and gas in glucose, maltose, mannitol, sorbitol, dulcitol, arabinose, trehalose and xylose, but not in lactose, sucrose, salicin or adonitol. Reactions variable in rhamnose and inositol, in $d-, l$ - and $i$ - tartrates, and in citrate ; mucate generally fermented in one day. Variable reaction in Stern's medium. Occasional variants fail to form gas. Fermentative sub-division based mainly on failure to ferment d-tartrate or rhamnose. $\mathrm{H}_{2} \mathrm{~S}+$. Indole negative. II.R. +. V.P. - . Nitrates reduced. $\mathrm{NH}_{3}+$. M.B. reduced. Catalase ++ . Koser's eitrate Gas formation in MacConkey broth at $44^{\circ} \mathrm{C}$. negative.

Antigenic Structure.-Diphasic flagellated bacillus. Antigenic formula [I], IV, [V], XII . . b variety-are said to be commoner in carriers than in acute cases.

Pathogenicity.-Gives rise as a rule to paratyphoid fever in human beings; but may be responsible for acute gastro-enteritis, followed or not after some days by the usual febrile disease. Strains from cases of gastro-enteritis often ferment $d$-tartrate, which strains from cases of paratyphoid fever seldom do. Has oceasionally been isolated from domestic animals, but is not known to cause disease in them under natural conditions. Inoculated intraperitoneally into mice in a dose of about 500 million bacilli, it produces death in 1 to 3 days. Post mortem there is some peritonitis and enlargement of the spleen. The bacilli can be recovered in culture from the spleen and heart blood.

\section{GROUP A.}

Salm. paratyphi A A.F. [I], II, XII ... a-.

Isolated from enteric fever in man (Gwyn 1898. Schottmüller 1900. 1901, Brion and Kayser 1902). Canses enterie fever, but not aente gastro-enteritis, in man. Has once been isolated from a pig (Broudin 1927), but is not a natural pathogen of animals. Growth is poorer than that of most other salmonellæ. Different strains vary in their $\mathrm{H}_{2} \mathrm{~S}$ pro- 
duction. Occasional strains fail to form gas. By growth in immune serum an artificial second phase containing the 1,5 antigens, and an artificial third phase containing antigen $z_{11}$, have been demonstrated by Bruner and Edwards (1941 a).

\section{GROUP B.}

Salm. paratyphi B A.F. [I], IV, [V], XII . . b

Isolated from cases of enteric fever in man (Achard and Bensaude 1896, Schottmïller $1900,1901)$. The I antigen is present only in some strains. The $\mathrm{V}$ antigen is generally present, but may be missing, particularly in strains from carriers (Kauffmann 1934c); such strains have been named Salm. parutyphi $B$ var. odense. Strains in which the nonspecific phase is missing are sometimes referred to as Salm. paratyphi $B$ var. java. Canses enteric fever in man, and sometimes an acute, but mild, enteritis. The strains causing enteric fever form a mucoid wall and do not ferment $d$-tartrate; the strains causing simple enteritis do not form a mucoid wall but do ferment $d$-tartrate. The java variety is distinguished by its inability to ferment $i$-tartrate; it appears to be unusnally common in Panama (Edwards and Bruner 1943). By means of suitable bacteriophages several sub-types have been distinguished by Felix and Callow (1943). Kíristensen and Bojlén (1929) have described a number of fermentative sub-types based on the use of rhamnose and inositol. Nay rarely fail to form gas. Salm. paratyphi $B$ is mainly a human pathogen. but its isolation has occasionally been recorded from the mesenteric nodes of normal pigs (Hormaeche and Salsamendi 1936, 1939), from animals slaughtered for human consumption (Bartel 1938), and from chickens (Eclwards and Bruner 1943).

An organism, describer by Gard (1938) as a new Salmonella type under the name of Salm. abortus-canis, with the antigenic formula $I V, V, X I I \ldots b \longleftrightarrow \mathrm{z}_{7}$, $\mathrm{z}_{8}$, is regarded by Kauffmann (1941) not as a true type, but as an artificial variant of Salm. paratyphi B.

Salm. abony A.F. [I], IV, V, XII . . b «e, n, x . .

Isolated from the freces of normal persons by Rauss in Budapest, and described by Kauffmann (1940a). Is distinguished from Salm. abortus-bovis by absence of the XXVII antigen, by its fermentation of inositol and of $i$-tartrate, and by its failure to liquefy gelatin. Salm. schleissheim, with which it may be confused, contains the XXV1I somatic and the $z_{12}$ flagellar antigen, fails to ferment dulcitol, inositol, or $i$-tartrate, and liquefies gelatin. Salm. abony can be distinguished from the $d$-tartrate positive strains of Salm. paratyphi $B$ only by the differences in its Phase 2 flagellar antigens. Its pathogenicity for man is doubtful.

Salm. typhi-murium A.F. [I], IV, [V], XII . . i $\longleftrightarrow 1,2,3 \ldots$

A natural pathogen of rodents, particularly mice, in which it causes a typhoid-like disease (Loeffler 1892). This organism is identical with Bact. aertrycke, which was originally isolated from a case of acute gastro-enteritis (food poisoning) in man (de Nobele 1898). It is under the name of Bact. aertrycke that it appears in almost all the recent and current literature; so that this synonym is an important one to remember. It is also identical with $B$. pestis cavice, described by Wherry (1908) as the cause of an epidemic disease in guinea-pigs, and with the bacillus that Nocard (1893) isolated from a parrot suffering from psittacosis, and named Bact. psittacosis under the mistaken impression that it was the cause of that disease. The same organism is frequently referred to in German literature as the "Breslau bacillus." Strains in which the $\mathrm{V}$ antigen is missing are sometimes referred to as the copenhagen (Kauffmann 1934c) or the storrs (Edwards 1935) variety; this type is common in American pigeons. Those in which the non-specific phase alone can be demonstrated are sometimes referred to as the binns variety (Schütze 1920); it should be noted, however, that by growth of the binns varicty in the presence of group serum, Bruncr and Edwards (1939) were able to demonstrate the existence of a specific phase in all strains. It shares its $\mathrm{H}$ antigens with Salm, aberdeen. Numerous fermentative sub-types have been described. In Europe strains from ducks and chickens usually fail to ferment rhamnose, inositol, $d$-tartrate, or citrate, but non-rhamnose-fermenting strains 
are uncommon in American ducks (Edwards and Bruner 1940a). Occasional strains fail to form gas.

Salm.typhi-murium is pathogenic for man as well as for animals and is the most frequent cause of outbreaks of salmonella food poisoning. It commonly gives rise in man to an acute gastro-enteritis, not infrequently fatal, but it occasionally causes a prolonged fever of the enteric type. Although it has most often been recorded as causing epidemic disease in mice and rats (see, for instance, Meyer and Matsumura 1927), it is naturally pathogenic for many other animal species. In addition to eausing infections in guinea-pigs and parrots, it has been isolated from epidemies in sheep (Bruns and Gasters 1920, White 1929t, Lovell 1932b), chicks (Doyle 1927, Edwards 1929, 1939), pigeons (Beaudette 1926, Lesbouyries and Verge 1932, Cernaianu and Popovici 1933), turkeys (Rettger et al. 1933), canaries (Beaudette 1926), and silver foxes (Benedict et al. 1941). In Germany, it is by no means uncommon in cattle, in which it may cause severe enteritis (Lütje 1937, Bartel 1938). It also causes infections in ducks, and has been isolated from ducks' eggs (Scott 1932, 1933, Dalling and Warrack 1932, Lovell 1932b), and from American dried egg. It has been demonstrated in the mesenteric glands of normal pigs (Hormaeche and Salsamendi 1936. 1939, Scott 1940, Varela and Zozaya 1942, Rubin et al. 1942), and has sometimes been isolated from pigs that have died of swine fever (see Lovell 1932b). It may be responsible for enteritis in cats (van Dorssen 1937).

Salm. stanley A.F. IV, V, XII ... d $\longleftrightarrow 1,2 \ldots$

Isolated from cases of food poisoning, and examined by Schïtze (1920), Savage and White (1925). White (1926), and Kanffmann (1931). (See also Boecker and Silberstein 1932, Kauffmann $1930 a, 1934 a$.) The partial antigens present in the specific phase are $\mathrm{d}$ and $\mathrm{d}_{3}$, of which the $\mathrm{d}$ is sharer with Salm. amersfoort, Salm. muenchen, Salm. typhi and Salm. gaminara. It is not known to be a natural pathogen of animals, but it has been isolated from imported American spray-dried egg in Great Britain.

Salm. heidelberg A.F. IV, V, X1I . . r $\longleftrightarrow 1,2,3 \ldots$

Isolated from a case of food poisoning by Habs (1933). (See also Kauffmann 1934a, Kauffmann and Silberstein 1934.) It has since been found in the mesenteric lymph nodes of normal pigs in Mexico (Varela and Zozaya 1942).

Salm. chester A.F. IV, [V], XII . . e, h $\longleftrightarrow$ e, n, x . .

Isolated by Grace of Chester from the frees of patients suffering from gastro-enteritis in a mental institution, and described by Kauffmann and Tesdal (1937-38). Has since been found in other European countries (Kauffmann 1941). Has been isolated from cases of infantile diarrhcea in Uruguay (Hormaeche, Peluffo and Aleppo 1940), from cases of gastro-enteritis in the United States (Bornstein, Saphra and Strauss 194I) and in Panama (Edwards and Bruner 1943), and from the mesenteric nodes of healthy pigs in Uruguay (Hormaeche and Salsamendi 1939). It is indistinguishable from Salm. san-diego except in the $\beta$ phase, in which chester is said to have the formula e, $\mathrm{n}, \mathrm{x}, \mathrm{z}_{15}, \mathrm{z}_{17}, \mathrm{z}_{19} \ldots$ and san-diego e, $\mathrm{n}, \mathrm{z}_{15}, \mathrm{z}_{17}, \mathrm{z}_{19} \ldots$

Salm. san-diego A.F. IV, [V], XII . . e, h

Isolated originally from an outbreak of food poisoning. Described by Kauffmann $(1940 d)$. Also found in a case of enteritis in the United States, and in New York sewage (Borustein and Saphra 1942), in a lealthy pig in Uruguay (see Kauffmann 1941), and in fowls in the United States (Edwards and Bruner 1943). For differentiation from Salm. chester, see above.

Salm. salinatis A.F. IV, X1I . . d, e, $\mathrm{h} \longleftrightarrow \mathrm{d}, \mathrm{e}, \mathrm{n}, \mathrm{z}_{15} \ldots$

Isolated from rat freces near Salinas, California. Described by Edwards and Bruner (1942 $\alpha$ ). Is antigenically complex, having $d$ and $\mathrm{e}$ in both phases. By cultivation in the presence of agglutinating serum to Salm. typhi the d antigen is lost from each phase, and the organism becomes biochemically and antigenically indistinguishable from Salm. san-diego. 
Salm. saint-paul A.F. I, IV, V, XII ...e, h $\longleftrightarrow 1,2,3 \ldots$

Isolated from the liver of a turkey poult in the United States. Described by Edwards and Bruner (1940b). Also isolated from freces and urine of a patient with an intermittent fever in New York City (Bornstein and Saphra 1942).

Salm. zagreb A.F. IV, V, XII . . e, h $\longleftrightarrow 1,2 \ldots$

Isolated at Zagreb. Described by Kauffmann (1941).

Salm. kaposvar A.F. IV, V, XII . . e, (h) $\longleftrightarrow 1,5 \ldots$

Isolated from fæces of 3 members of a family who were suffering from gastro-enteritis and described by Rauss (1941) in Hungary. Phase 1 is similar to that of Salm. onderstepoort in that only part of the $\mathrm{H}$ antigen is present.

Salm. koeln A.F. IV, V, XII . . y $\rightarrow 1,2,3 \ldots$

Not to be confused with the koeln variety of Salm. dublin.

Salm. reading A.F. IV, XII ... e, $\mathrm{H} \bullet \longleftrightarrow 1,5 \ldots$

Isolated from the Reading water supply (Sehütze 1920). Has since been isolated from freces of cases or carriers in relation to outbreaks of gastro-enteritis (Kauffmann $1930 a, 1931,1934 a)$, Boecker and Silberstein 1932), from the mesenteric nodes of healthy pigs (Scott 1940), and from an epidemic among laboratory guinea-pigs (see Lovell 1932b).

Salm. derby A.F. [I], IV, XII ...f, g . . -

Isolated from eases of food poisoning at Derby by Peekham (1923). (See also Sarage and White 1925, White 1926, Kauffmann 1931, 1934a). Kanffmann (1937a) pointed out that in some strains the $I$ antigen was absent. Has since been isolated from cases of infantile diarrhcea (Hormaeehe, Peluffo and Aleppo 1936, 1940), from the mesenteric nodes of healthy pigs (Hormaeche and Salsamendi 1936, 1939, Edwards, Bruner and Rubin 1940, Rubin et al. 1942, Varela and Zozaya 1942), from turkeys (Edwards 1939), and from imported American spray-dried egg in Great Britain. Occasional strains fail to form gas. The flagellar antigens comprise also $\mathrm{z}_{8}$.

Salm. kaapstad A.F. IV, XII . . e, h $\longleftrightarrow 1,7 \ldots$

Salm. essen A.F. IV, XII . . g, m . . - .

Isolated from the stools of an infant who had been suffering for 3 days from enteritis (Hohn and Herrmann 1936a). Not to be eonfused with the essen fermentative sub-type of Salm. enteritidis (see p. 736).

Salm. budapest A.F. I, IT, XII . . g, t . . -

Isolated in Hungary from the stools of 3 persons who had been suffering for a week from enteritis, and from 3 healthy carriers (Rauss 1939a). The presence of the I antigen was pointed out by Kauffmann (1940b). Aecording to Rauss (1939a), the flagellar antigen consists of $\mathrm{g}, \mathrm{t}, \mathrm{z}_{8}$ and $\mathrm{z}_{9}$; this may be confused with that of Salm. senftenberg, which eonsists of $g, s, t, z_{8}$ and $z_{9}$, though, of course, the $O$ antigens of the two organisms are quite different. The $\mathrm{t}$ antigen of budapest is said to be different from that of oranienburg (Edwards, Bruner and Hinshaw 1940). Non-pathogenic to miee by the mouth.

Salm. california A.F. IV, XII . . g, $\mathrm{m}, \mathrm{t} \ldots$.

Isolated from young turkeys suffering from paratyphoid fever. Described by Edwards, Bruner and Hinshaw (1940). Later isolated from fowls (Hallmann et al. 1942), and from American spray dried egg in Great Britain. Both the $g$ and the $t$ antigens are eomplex. Non-pathogenic to monkeys by the mouth.

Salm. brandenburg A.F. IV, XII . . l, v $\leftrightarrow$ e, $11, z_{15} \ldots$

Isolated from a case of acute gastro-enteritis eoming on after a meal of raw ham (Kauffmann and Mitsui 1930). Since isolated from the frecs of other cases of gastroenteritis, and from the blood of patients suffering from a pyrexial disease (Černozubov et al. 1936-37). Not known to be a natural pathogen of animals.

Salm. bispebjerg A.F. I, IV, XII ... a $\longleftrightarrow e, n, x \ldots$

Isolated from the stools of a 71-year-old man suffering from acute gastro-enteritis and bronchopneumonia (Kauffmann 1936b). 
Salm. abortus-equi A.F. IV, XII . . - e, n, x . .

Isolated by Kilborne (1893) from a case of abortion in a mare. Has since been isolated or studied by Smith (1893), de Jong (1913), Meyer and Boerner (1913), Good and Corbett (1913), van Heelsbergen (1914), Murray (1919), and Fitch and Billings (1920). For antigenic structure see White (1926) and Kauffmann (1931, 1934a). Phase 2 contains antigens $z_{16}$ and $z_{19}$ in addition to e, $n, x$. Edwards and Bruner (1939) have been able by growth of Salm. abortus-equi in the presence of suitable immune sera, to induce two artificial phase variations: one, referred to as Phase 1, containing the a antigen of Salm. paratyphi $A$; the other, referred to as Phase 3 , containing an antigen $\mathrm{z}_{5}$ which was similar to, though not identical with, a similar antigen in the artificially induced phase of Salm. schleissheim. It should be pointed out, however, that neither Phase 1 nor 3 has been met with under natural conditions. Oceasional strains fail to form gas. Salm. abortus-equi is a natural pathogen of the horse, eausing abortion in mares. It is not known to have caused infection in man.

Salm. abortus-ovis A.F. IV, XII . . c $\longleftrightarrow 1,6 \ldots$

Isolated by Schermer and Ehrlich (1921) from cases of abortion in sheep. For antigenic structure see Lovell (1931) and Liaffmann (1931, 1934a). Compared with most other organisms of the Salmonella group, this organism grows poorly. Many of the usual sugar reactions are negative, late, or irregular. Not known to infect man or any animal other than the sheep.

Salm. arechavaleta A.F. IV, [V], XII . . a $\longleftrightarrow 1,7 \ldots$

Described by Hormaeche and Peluffo (see Kauffmann 1941). Has been found in the Panama Canal zone in patients suffering from gastro-enteritis. Found once since in a case of gastro-enteritis (Edwards and Bruner 1943).

Salm. altendorf A.F. IV, XII . . c $\longleftrightarrow 1,7 \ldots$

Isolated by Hohn (1940) from the fæces of a woman suffering from severe febrile diarrhcea.

Salm. abortus-bovis A.F. [I], IV, XII, XXVII . . b

Isolated by Bernard (1935-36) from the lymph nodes and viscera of two cows that had aborted, and from the fæces of another cow and a bull. Studied by Kauffmann $(1937 b)$; (see also Kauffmani 1941). Only part of the XII antigen is present. Phase 2 also contains $\mathrm{z}_{16}$. For differentiation from Salm. abony see above. Like Salm. schleissheim, it liquefies gelatin, but is distinguished from it by its absence of $z_{12}$ in Phase 1 , by its possession of a second phase, and by its fermentation of dulcitol. Has been isolated in Hungary from the fæces of a patient with a mild typhoid-like fever. The strain from this source was in Phase 2, and some of the colonies lacked the I antigen (Rauss 1941).

Salm. schwartzengrund A.F. I, IV, XXVII, XII . . d $\longleftrightarrow 1,7 \ldots$

Salm. bredeney A.F. I, IV, [XXVII], (XII) . . 1, $\mathrm{X} \longleftrightarrow 1,7 \ldots$

Isolated in Germany from a case of diarrhea and vomiting, and from a patient suffering from osteomyelitis of the leg. Studied by Kauffmann (1937a). Has since been found in Uruguay by Hormaeche, Peluffo and Aleppo (1940) in eases of infantile diarrhea, and by Hormaeche and Salsamendi (1939) in the mesenteric nodes of normal pigs. Found also in the United States in turkeys by Edwards (1939), in the mesenteric lymph nodes of pigs by Rubin, Scherago and Weaver (1942) and in retail meat by Cherry, Scherago and Weaver (1943). Isolated in Great Britain from imported American spray-dried egg. Is non-pathogenie io mice by the mouth.

Salm. schleissheim A.F. IV, XXV1I, XII . . b, $\mathrm{z}_{12} \ldots$ -

Isolated from the viscera of a bovine animal in Germany. Studied by Kauffmamn and Tesdal (1937-38). Contains part of the XII antigen. The b antigen is not quite identical with the b antigen of Salm. paratyphi $B$. An artificial second phase, $z_{5}$, may be demonstrated by culturing in immune serum eontaining the antibody to $\mathrm{b}$ antigen. Liquefies gelatin. For differentiation from Salm. abortus-bovis sce above. Is apparently 
able to give rise to acute gastro-enteritis in man, since about 40 persons were taken ill after eating a meat dish containing Salm. schleissheim, and the organism was isolated from their fæees (see Kauffmann 1941).

\section{GROUP C}

Salm. paratyphi C A.F. $V I_{2}, V I_{2}$, VII $\ldots[V i], \mathrm{c} \longleftrightarrow 1,5 \ldots$

Isolated from cases of enteric fever in man, mainly in Eastern Europe; more recently in British Guiana. It has received many other names and, in particular, is often referred to as "Hirsehfeld's Bacillus," or the "Eastern European type of Bact. paratyphosum C" (see Weil 1917, Neukirch 1918, MacAdam 1919, Mackie and Bowen 1919, Hirsehfeld 1919, Schiitze 1920, Dudgeon and Urquhart 1920, Andrewes and Neave 1921, Weigmann 1925a, $b$, Iwaschenzoff 1926, White 1926, Kauffmann 1931, 1934a, Giglioli, 1930). Kauffmann (1935a) demonstrated the presence of a, Vi antigen in some strains. Its apparent identity with the Vi antigen of Salm. typhi was shown by Kauffmann (1936a) and Rouchdi (1938). The two antigens can, however, be distinguished by the bacteriophage technique (Scholtens 1937). The Vi antigen seems to play no part in determining the high pathogenicity of Salm. paratyphi $C$ for the mouse. 'This organism is an important pathogen of man, giving rise to enteric fever that is often associated with septic lesions. It is not known to be a natural pathogen of animals.

Salm. choleræ-suis A. $\mathbb{F}^{\prime}, V I_{1}, V I I \ldots c \longleftrightarrow 1,5 \ldots$ or

$$
\mathrm{Vl}_{2}, \mathrm{VIl} \ldots \mathrm{c} \leftrightarrow 1,5 \ldots
$$

The American hog-cholera bacillus, isolated by Salmon and Smith (1885, 1886), from the former of whom the name Salmonella is derived. Though hog cholera is now known to be a virus disease (see p. 1963), Salm. cholerce-suis is a common secondary invader in this disease. It is probably the commonest salmonella found in pigs. Either in the diphasic form or in the non-specific phase (kunzendorf variety), it has been isolated from the mesenteric lymph nodes of apparently normal pigs in Great Britain by Scott (1940), in South America by Hormaeche and Salsamendi (1939), in Mexico by Varela and Zozaya (1942), and in the United States by Rubin, Scherago and Weaver (1942) and Edwards and Bruner (1943). It is also commonly found in silver foxes (Benedict et al. 1941, Edwards and Bruner 1943), and has been isolated from cattle (Lütje 1939). Occasionally present in imported American dried egg. In the past it has been usual to refer to the diphasic $\mathrm{H}_{2} \mathrm{~S}$-negative variety as the American type, and to the $\mathrm{H}_{2} \mathrm{~S}$-positive varicty, which exists in the group phase only, as the European or kunzendorf type. More extensive observations, however, have rendered it doubtful whether this distinction should be maintained. It is elear that their geographical relationship is subject to numerous exceptions, so that the names European and American had better be dropped. Whether the two organisms should be awarded separate varietal names is less clear. Kauffmann (1941) is in favour of abolishing the term kunzendorf altogether on the ground that numerous workers have been able to demonstrate Phase 1 in kunzendorf strains by growing them in immune serum. On the other hand, the two organisms usually differ in their $\mathrm{H}_{2} \mathrm{~S}$ production, and - what is probably more important-their natural pathogenicity. As Edwards and Bruner (1943) have shown, Salm. cholerce-suis is far more invasive than the kunzendorf variety; it can often be isolated from the heart blood of infected animals, whereas the kunzendorf variety remains confined to the intestine or mesenteric lymph nodes. Both organisms are closely related to Salm. paratyphi $C$; they are distinguished from it by their failure to ferment arabinose and trehalose, and to some extent by their lack of the $\mathrm{Vi}$ antigen. Of their pathogenicity to human beings there is no doubt. Numerous outbreaks of food poisoning have been ascribed to them (Clauberg 1931, Boecker and Silberstein 1932, Kauffmann 1934a, 1941). A typhoid-like fever may result from infection, sometimes complicated by pneumonia, arthritis, purulent meningitis, or bacterial endocarditis (Boycott and McNee 1936, Harvey 1937, Goulder et al. 1942, Schwabacher, Taylor and White 1943). 
Salm. typhi-suis A.F. $\mathrm{VI}_{1}, \mathrm{VI}_{2}, \mathrm{VII} \ldots \mathrm{c} \longleftrightarrow 1,5 \ldots$

This organism was isolated from young pigs suffering from a typhoid-like disease by GLässer $(1909,1910)$. The monophasic type, which exists in the group phase only and is sometimes known as the voldagsen variety, was isolated from a similar disease in pigs by Dammann and Stedefeder (1910). These organisms represent the "Ferkeltyphus" bacillus of German literature (see also Neukirch 1918, Andrewes and Neave 1921, White 1926, Kauffmann 1931, 1934a, Bartel 1938). Salm. typhi-suis is almost antigenically identical with Salm. cholerce-suis. It differs, however, in growing poorly on ordinary media and in its fermentation reactions. It forms gas very slowly and sparsely ; it usually fails to ferment mannitol or does so late; it does not attack eitrate, mucate, or the tartrates; and it produces a slight permanent acidity in litmus milk. It also ferments trehalose, which Salm. choleree-suis does not. The specific phase can be demonstrated in the voldagsen variety by growth in presence of immune serum. So far as is known, Salm. typhi-suis does not naturally infect animals other than the pig, nor does it give rise to disease in man. It seems to be very uncommon in the United States (Bruner and Edwards 1940).

Salm. thompson A.F. $\mathrm{VI}_{1}, \mathrm{VI}_{2}$, VII ... $\mathrm{k} \longleftrightarrow 1,5 \ldots$

Isolated from cases of food poisoning in man by Scott (1926), and since by numerous other workers. The berlin variety, which exists in the group phase, was isolated from eases of food poisoning by Kauffmann (1929a, $b$; see also Kauffmann 1931, 1934a, Boecker and Kauffmann 1930, Kauffmann and Mitsui 1930, Clauberg 1931, Seligmann and Clauberg 1932, Boecker and Silberstein 1932). Salm. thompson is very common in Great Britain, where it is about the third most frequent Salmonella type met with in outbreaks of food poisoning. It was demonstrated in the mesenteric lymph nodes of normal pigs by Scott (1940), and was found once in a rat by Khalil (1938) at Liverpool. Knorr (1931) isolated it from Chinese egg yolk. In the United States it appears to be uncommon (Edwards and Bruner 1943). Has been isolated from imported American dried egg.

Salm. cardiff A.F. VI, VII . . $\mathrm{k} \longleftrightarrow 1,10 \ldots$

Isolated in South Wales from a case of acute gastro-enteritis, and recognized as a new type by Taylor at Oxford and Edwards in Kentucky (Taylor, Edward, and Edwards 1945).

Salm. virchow A.F. $\mathrm{VI}_{1}, \mathrm{VI}_{2}, \mathrm{VII} \ldots \mathrm{r} \longleftrightarrow 1,2,3 \ldots$

Isolated from a case of acute gastro-enteritis in Germany. Antigenic structure determined by Kauffmann $(1930 b, 1931,1934 a)$. May give rise to a prolonged febrile disease.

Salm. infantis A.F. $\mathrm{VI}_{1}, \mathrm{VI}_{2}, \mathrm{VII} \ldots \mathrm{r} \longleftrightarrow 1,5 \ldots$

Isolated from the blood of an infant in New Haven Hospital suffering from diarrhœa and mild fever. Described by Wheeler and Borman (1943). Bioehemically indistinguishable from Salm. virchou:

Salm. oranienburg A.F. $V I_{1}, V I_{2}, V I I \ldots \mathrm{m}, \mathrm{t} \ldots$. .

Isolated from frees of normal persons and of patients suffering from gastro-enteritis (see Kauffmann, 1930b, 1931, 1934a, Clauberg 1932, Seligmann and Clauberg 1932, Boecker and Silberstein 1932). Its first demonstration in animals was by Edwards (1936), who found it responsible for a disease of baby quail in the United States. It has been found in chickens in the United States (Edwards 1939); judging from the frequency with which it has been isolated from American dried egg imported into Great Britain, it must be a common parasite of fowls. In luman beings it gives rise to infantile diarrhoea (Hormaeche, Peluffo and Aleppo 1940), and to gastro-enteritis in adults. Since the introduction of American dried egg in 1941, numerous sporadic cases of infection with this organism and at least one outbreak have been reported in England.

Salm. potsdam A.F. $V I_{1}, V I_{2}, V I I \ldots 1, \mathrm{v} \longleftrightarrow \mathrm{e}, \mathrm{n}, \mathrm{z}_{15} \ldots$

Isolated from cases of food poisoning. Examined by Kauffmamn and Mitsui (1930; see also Kauffmamn 1931, 1934 . Clauberg 1931, Seligmanu and Clauberg 1932). Contains the $\mathrm{z}_{17}$ and $\mathrm{z}_{19}$ antigens in Phase 2 in addition to $\mathrm{z}_{15}$. Has bcen found in imported American spray-dried egg. 
Salm. bareilly A.F. VI,$V_{2}$, VII $\ldots y \longleftrightarrow 1,5 \ldots$

Isolated in India from mild enteric infections (Bridges and Scott 1931). Since found in infantile diarrhcea in the United States (Bornstein, Saphra and Strauss 194l), and in typhoid-like cases in Hungary (Rauss 1941) and in Canada (Wyllie 1943). Demonstrated in chickens and turkeys in the United States (Edwards 1939), and in the mesenteric lymph nodes of normal pigs (Edwards, Bruner and Rubin 1940, Rubin et al. 1942). Has been isolated from imported American dried egg, and from cases of food poisoning in Great Britain.

Salm. hartford A.F. VI $, V I_{2}, V I I \ldots y \leftrightarrow e, n, x \ldots$

Isolated from the frees of a 7l-year-old man who suffered from abdominal cramps, followed by diarhoea for a week (Edwards and Bruner 194la). ['hase 2 contains $\mathrm{z}_{16}$ and $\mathrm{z}_{19}$ in addition to $\mathrm{e}, \mathrm{n}, \mathrm{x}$.

Salm. mikawasima A.F. $V I_{1}, V_{2}, V I I \ldots y \longleftrightarrow e, n, z_{15} \ldots$

Isolated from rat's freces in Japan. Described by Hatta (1938), and studied by Hormaeche and by Kauffmann (see Kauffmann 1941). Is closely related to Salm. hartford, from which it differs in the possession of the $z_{15}$ instead of the $\mathrm{x}$ antigen in Phase 2, and in the fermentation of inositol. Phase 2 also contains $\mathrm{z}_{19}$. Non-pathogenic to mice by the mouth..

Salm. montevideo A.F. $V I_{1}, V I_{2}, V I I \ldots g, m, s \ldots$. or

$$
\mathrm{VI}_{1}, \mathrm{VII} \ldots \mathrm{g}, \mathrm{m}, \mathrm{s} \ldots \text {. }
$$

Isolated by Hormaeche and Pehuffo (1936) in Uruguay from the frees of a monkey suffering from chronic enterocolitis, from an infant with chronic enterocolitis, from a pulmonary abscess in a child with enteritis, and from the mesenteric lymph nodes of normal pigs. Since found in Uuruguay in eases of infantile diarthcea (Hormaeche, Peluffo and Aleppo 1940) and in the mesenteric lymph nodes of pigs (Hormaeche and Salsamendi 1936, 1939); in the United States in two gall-bladder carriers (Erlwards and Bruner 1943), in a patient with an attack of mild febrile diarrhœe (Schiff and Saphra 1940), in chickens (Jungherr and Claney 1939) and in turkeys (Edwards 1939), in eases and outbreaks of food poisoning and in imported American spray-dried egg in Great Britain; in Denmark and in Palestine (see Kauffmann 1941). The Danish strain lacked the $V I_{2}$ antigen and differed in some cultural respects.

Salm. oslo A.F. $\mathrm{VI}_{1}, \mathrm{VI}_{2}, \mathrm{VII} \ldots \mathrm{a} \longleftrightarrow \mathrm{e}, \mathrm{n}, \mathrm{x} \ldots$

Isolated by Tesdal (1937) from fæces of patients with gastro-enteritis in five different places in Norway. Phase 2 also contains $z_{16}$ and $z_{19}$.

Salm. amersfoort A.F. VI $I_{1}, V I_{2}, V I I \ldots d \longleftrightarrow e, n, x \ldots$

Isolated from the heart blood of a 7-day-old chick in the Transvaal; the chickens at the time were suffering from a severe infectious disease accompanied by a high case mortality. Described by Henning (1937). Also isolated, together with Salm. typhimurium, by Schiff and Strauss (1939b) in the United States from a patient suffering from a typhoid-like ferer. The $d$ antigen in Phase 1 consists of the partial antigens $d, d_{2}$ and $d_{3}$. Phase 2 also contains $\mathrm{z}_{16}$ and $\mathrm{z}_{19}$. Pathogenic for chickens and mice on intraperitoneal injection.

Salm. braenderup A.F. $\mathrm{VI}_{1}, \mathrm{VI}_{2}, \mathrm{VII} \ldots \mathrm{e}, \mathrm{h} \longleftrightarrow \mathrm{e}, \mathrm{n}, \mathrm{z}_{15} \ldots$

Isolated by Kauffmann and Henningsen (1938) in Denmark from the fieces of a 54 -yearold man suffering from enteritis and from his cat, which had died of diarrhœa. Sinee found in South Africa (see Kauffmann 1941). Phase 2 also contains $\mathrm{z}_{17}$ and $\mathrm{z}_{19}$.

Salm. georgia A.F. VI, VII . . b

Isolated from healthy earrier (Morris, Brim and Sellers 1945).

Salm. concord A.F. VI, VII . . I, $\mathrm{v} \longleftrightarrow 1,2,3 \ldots$

Isolated in Massachusetts; also in England by Taylor at Oxford from a case of acute gastro-enteritis in a child living on a farm, and from her cat. 
Salm. tennessee A.F. VI, VI $I_{2}$, VII . . . $\mathrm{z}_{29} \ldots$. .

Isolated from the fæces of a healthy food handler employed in a fraternity house, a number of whose ocenpants were suffering from food poisoning (Bruner and Edwards 1942a). lsolated from eases of food poisoning in Great Britain, and from imported American spray-dried egg. Apparently monophasic. A traee of $\mathrm{z}_{10}$ may be present.

Salm. pueris A.F. VI 1, VIII . . e, $\mathrm{b} \longleftrightarrow 1,2 \ldots$

Isolated from the fæees of a boy at New Haven Hospital suffering from gastro-enteritis. Described by Wheeler and Borman (1943). Biochemically indistinguishable from Salm. newport.

Salm. newport A.F. VI ${ }_{1}$, VIII . . e, $\mathrm{h} \longleftrightarrow 1,2,3 \ldots$

Isolated from cases of food poisoning in man (Sehuitze 1920). Corresponds to Paratyphus $\beta 2$ of Weil and Saxl (1917). (See also White 1926, Kauffmann 1929a, 1931, 1934a, Schütze 1930, Clauberg 1931, Seligmann and Clauberg 1932, Boecker and Silberstein 1932.) One of the commonest types met with in outbreaks of food poisoning in Great Britain. Isolated by Khalil (1938) from rats at Liverpool, from cases of infantile diarrhca in Uruguay (Hormaeche, Peluffo and Aleppo 1936, 1940), from chickens and turkeys in the United States (Edwards 1939), from retail meat in the United States (Cherry, Scherago and Weaver 1943), and from the mesenteric lymph nodes of normal pigs in England (Scott 1940) and in Urugnay (Hormaeche and Salsamendi 1936, 1939). The puerto-rico variety (Jordan 1934, Kauffmann 1934a) exists only in the non-specific phase, but by cultivation in the presence of an immune serum the specific phase may be obtained from it (Bruner and Edwards 1939). Has been isolated from imported American spray-dried egg.

Salm. kottbus A.F. VI $I_{1}$, VIII . . e, $\mathrm{h} \longleftrightarrow 1,5 \ldots$

Isolated from cases of acute gastro-enteritis (Kauffmann 1934a). Differs from Salm. newport, of which it used to be eonsidered a variety, in the non-specific phase.

Salm. bovis-morbificans A.F. VI 1 , VIII . . $\mathrm{r} \longleftrightarrow 1,5 \ldots$

Isolated from an infected cow by Basenau (1894). Has also been isolated from gastroenteritis in man (see White 1926, Sladden and Scott 1927, Kauffmann and Mitsui 1930, Kauffmann 1931, 1934 $a, 1941$, Clauberg 1931, Seligmann and Clauberg 1932, Boecker and Silberstein 1932). Not very common in cattle (Bartel 1938, Liitje 1939). Cultured from imported American spray-dried egg in Great Britain.

Salm. muenehen A.F. $\mathrm{VI}_{1}$, VIII . . d $\longleftrightarrow 1,2 \ldots$

Isolated by Mandelbaum (1932) from a fatal case of gastro-enteritis (see also Silberstein 1932, Kauffmann 1934a, 1941). Found by Hormaeche, Peluffo and Aleppo (1940) in infantile diarrhcea in Uruguay. Isolated from mesenteric lymph nodes of normal pigs in Uruguay by Hormaeele and Salsamendi (1939), and from chickens in the United States (Edwards 1939). Sometimes present in importerl American spray-dried egg. Appeared to be responsible for an epizootic among guinea-pigs in New York City (Bornstein, Saphra and Stranss 194l). The $d$ antigen contains the partial antigens $d, d_{3}$ and $d_{4}$.

Salm. oregon A.F. VI $I_{1}$ VIII ... d $\longleftrightarrow 1,2,3 \ldots$

Isolated from a turkey and from the mesenterie lymph nodes of normal pigs in the United States. Also found in imported American spray-dried egg. Deseribed by Rubin (1940) as a variant of Salm. muenchen, but regarded by Edwards and Bruner (1941b) as a new type. The $\mathrm{d}$ antigen in the specific phase is identical with that of manhattan, gamimara and shangani, but differs to some extent from that of muenchen, typhi and stanley.

Salm. mexicana A.F. VI, VIII . . d $\longleftrightarrow 1,2,4 \ldots$

Isolated in Mexico from the mesenteric glands of a subject at post mortem, and from the frees of a child with diarrhoa (Varela, Zozaya and Olarte 1943).

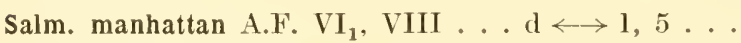

Isolated from a chicken and from a turkey in the United States. Found also in imported American spray-dried egg. Described by Edwards and Bruner (1911b). Isolated also 
from a human source in Massachusetts (Bornstein and Saphra 1942). For the nature of the d antigen, see Salm. oregon.

Salm. narashino A.F. $V I_{1}$, VIII . a a $\longleftrightarrow e, n, x \ldots$

Accorling to Kauffmann (1941) this organism was isolated in 1937 from the blood and freces of a patient with a typhoid-like disease in Japan, and its antigenic structure was determined by Nakaguro and Yamashita. Phase 2 contains also $z_{16}$ and $z_{19}$.

Salm. glostrup A.F. VI $I_{1}$, VIII . . $\mathrm{z}_{10} \longleftrightarrow \mathrm{e}, \mathrm{n}, \mathrm{z}_{15} \ldots$

Isolated by Kauffmann and Henningsen (1939) from the frees of patients in a family outbreak of gastro-enteritis, and from the fæces and blood of their dog, which was ill at the same time. Phase 2 contains also $z_{17}$ and $z_{19}$. Non-pathogenic to mice by the mouth.

Salm. litchfield A.F. VI, VIII ... $1, \mathrm{v} \longleftrightarrow 1,2,3 \ldots$

Isolated from the liver of a turkey poult in the United States. Described by Edwards and Bruner (1940b). A strain isolated many years previously from a case of food poisoning in man was later found to belong to this type. Also isolated from American spray-dried egg in Great Britain.

Salm. duesseldorf A.F. VI $\mathrm{V}_{1}$, VIII ... $\mathrm{z}_{4}, \mathrm{z}_{24}$.

Isolated in Germany from two patients in hospital-a boy of 3 years, and a man of 40 years, who died (Hohn 1940).

Salm. bonariensis A.F. VI $I_{1}$, VIII . . i i $\longleftrightarrow e, n, x \ldots$

Isolated from the mesenteric gland of a normal pig (Nonteverde 1942). Since met with in a normal human carrier (Edwards and Bruner 1943). Antigenic structure not yet completely worked out.

Salm. virginia A.F. VIII ...d-.

Studied by Seligmann and Saphra (see Edwards 1945).

Salm. amherstiana A.F. (VIII), $1,(\mathrm{v}) \ldots \leftrightarrow 1,6 \ldots$

Isolated from the liver of one of a group of poults affected with a fatal disease. This is the only known salmonella to contain the VIII antigen alone, and even this is incomplete. It is perhaps doubtful whether it should be included in Group C. Described by Edwards and Bruner (1942b).

\section{GROUP D.}

Salm. typhi A.F. IX, XII ... [Vi], d-.

The cause of typhoid fever in man. Its general characters have been referred to in previous sections. The great majority of freshly isolated strains contain the Vi antigen. The $d$ antigen contains the partial antigens $d$ and $d_{1}$. Dwarf colonies, containing the $O$ but not the $\mathrm{H}$ or $\mathrm{Vi}$ antigens, nay occur. Artificial phase variation may follow growth in the presence of a d antiserum, the $d$ antigen being replaced by $\mathrm{j}$ (Kauffmann 1936c); the new phase is referred to as Phase 3. Though many strains of Salm. typhi-suis, Salm. sendai and Salm. gallinarum fail to produce gas from glucose, Salm. typhi is unique among the Salmonella group in never forming gas. Sub-types based on the presence or absence of fermentation of xylose, arabinose, $d$-tartrate, and soctium citrate bave been described, but for epidemiological purposes the method of bacteriophage typing is of greater usefulness. Does not appear to infect animals under natural conditions. (For properties of Vi antigen see p. 1525 and a short review by Almon 1943).

Salm. enteritidis A.F. [I], IX, XII ...g, m . . - .

Referred to sometimes as the gärtner or jena variety. Isolated from the spleen of a fatal case of food poisoning, which affected 58 persons at Frankenhausen who had eaten the flesh of an emergency-slaughtered cow (Gaertner 1888). Since isolated from numerous sporadic cases and outbreaks of food poisoning in different parts of the world (see White 1926, 1930, Kauffmann 1930b, 1931, 1934a, Warren and Scott 1930, Boecker and Silberstein 1932, Boecker 1936). The full formula of the flagellar antigen is $g, 0, m, z_{1}, z_{2}$. 
Salm. enteritidis has four fermentative varieties (see Table 46): the original, gärtner, or jena variety, just described ; the danysz variety, sometimes known as the Ratin bacillus; the essen variety; and the chaco variety. The danysz variety was isolated by Danysz (1900) from an epidemic of mouse typhoid in field mice. The Ratin strain of this variety was cultured from the urine of a sick child by Neumann in 1902 (see Report 1934, Kauffmann 1934a). Both the Ratin and the Liverpool "viruses" are prepared with this variety (Leslie 1942). The essen variety was isolated from cases of gastro-enteritis in man, from ducks, and from ducks' eggs by Hohn and Herrmann (1935a, $b$ ) and described as Salm. moskau; it is not to be confused with Salm. essen, which belongs to the B group of Salmonella (see p. 729), nor with Salm. moscow (see below). The chaco variety was isolated by Savino and Menéndez (1934) from cases of continued ferer in the Chaco war.

Apart from the chaco variety, which has so far been met with only in man, the natural habitat and frequency distribution of the other three varieties of Salm. enteritidis is subject to doubt. So many workers who have isolated Salm. enteritidis from cattle, horses, pigs, chickens, ducklings, rats, and other sources have failed to differentiate between, or make no mention of, the different fermentative varieties that much of the available information is difficult to interpret. The jena variety appcars to be responsible for most outbreaks of food poisoning due to Salm. enteritidis that follow the consumption of meat. It does not, however, appear to be very common, and it constituted only 1.06 per cent. of 1,690 Salmonella strains isolated from domestic animals commonly used for food (Barthel 1938). It seems probable, also, that it may give rise at times to purulent meningitis (Guthrie and Montgomery 1939). The danysz variety appears to be predominantly a parasite of rodents; it has been isolated from silver foxes suffering from distemper or paratyphoid (Benedict et al. 1941). Its pathogenicity for man has been disputed, but a number of cases and outbreaks of gastro-enteritis in man have now been described in which the causative rôle of this organism and its origin from rat "virus " are well established (see Leslie 1942); some of the cases have proved fatal. The essen variety appears to be characteristically a parasite of ducks (Jansen 1937), but is eapable of giving rise to acute gastro-enteritis in human beings. (For further information on the varieties of Salm. enteritidis, see Kauffmann 1941.)

Salm. dublin A.F. I, IX, XII ...g, p...-

Sometimes known as the kiel variety of Salm. enteritidis. Isolated on several occasions from calf diarrhoea and described under the name "paracolon bacillus," but not adequately differentiated till examined by White (1930). (See also Warren and Scott 1930, Smith and Scott 1930, Ströman and Örn 1932, Kauffmann 1930b, 1931, 1934a, Bosworth and Lovell 1931, Boecker and Silberstein 1932, Hohn and Herrmann 1935a). In Europe its principal habitat appears to be calves, less frequently older cattle. It is very common; for instance, it constituted 394 ont of 456 Salmonella strains from cattlc studied by Lütje (1939); 314 of the 394 strains were isolated from calves or occasionally foetuses, and only 72 from adult cattle. It formed 78.37 per cent. of Bartel's (1938) series of 1,690 Salmonella strains isolated from domestic animals commonly used for food. In the United States it seems to be found principally in silver foxes, and only seldom in cattle (Edwards and Bruner 1943). It has been isolated from fowls suffering from enteritis (Lütje 1937), and from the mesenteric lymph nodes of normal pigs (Scott 1940). In man it may give rise to a continued fever of the enterie type (Smith and Scott 1930), and sometimes to meningitis and cholecystitis (Guthrie and Montgomery 1939). As a cause of acute gastro-enteritis in man it is less common than the jena variety of Salm. enteritidis, but it has been responsible for more than one milk-borne outbreak of the acute food-poisoning type (Conybeare and Thornton 1938, Tulloch 1939). In calves it is responsible for enteritis (Bosworth and Lovell 1931) - the so-called calf-diarrhøa-but in adult cattle it affects more often the udder, lungs or heart, giving rise to inflammation. Chronic carriers are not unknown in cattle. In the past many strains of this organism have probably been described as Salm. enteritidis. Two fermentative varieties have been described, accra and koeln. Occasional variants fail to form gas. 
Salm. rostock A.F. I, IX, XII ...g, p, u ...-

Isolated from cattle. First differentiated on basis of fermentation reactions (Bahr $1930 a, b)$. Antigenic structure studied by Kauffmann $(1930 b, 1931,1934 a, 1935 b$ ). (See also Ströman and Örn 1932, Hohn and Hermann 1935a). It appears to be relatively uncommon in cattle in Germany (Bartel 1938, Lütje 1939), and has not yet been isolated from human beings.

Salm. moscow A.F. IX, XII ...g, q ....-

Isolated mainly in Russia from febrile infections in man (Weigmann 1925a, $b$, Iwaschen. zoff 1926). It has since been isolated from cases of gastro-enteritis in man (Hohn and Herrmann 1935a, Kauffmann 1935b), and from a cow and a horse (Kauffmann 1935b). Its structure was first determined by Hicks (1930) (see also Kauffmann 1930b, 1931, 1934a, $1935 b$ ). The full formula for its flagellar antigen is $\mathrm{g}, \mathrm{o}, \mathrm{q}, \mathrm{z}_{3}$. It is doubtful whether it can give rise primarily to a typhoid-like disease in man ; most of the Russian strains appear to have been eases of relapsing fever in which Salm. moscow was a secondary invader. Not to be confused with Salm. enteritidis var. essen, which was first described as Salm. moscau (Hohn and Herrmann 1935a).

Salm. blegdam A.F. IX, XII ...g, m, q ... -

Isolated from the blood of a patient in Denmark suffering from pneumonia ; described by Kauffmann (1935b). Does not appear to have been met with again.

Salm. pensacola A.F. IX, XII ... g, m, t ...

Studied by Edwards (1945).

Salm. berta A.F. IX, XII ... f, g, t . . -

Isolated from the pooled mesenteric lymph nodes of normal pigs in Uruguay and reported to a local society in 1937; described by Hormaeche, Peluffo and Salsamendi in 1938. Studied also by Kauffmann (1937b). $\mathrm{H}$ antigen contains also $z_{7}$ and $z_{8} \cdot H_{2} \mathrm{~S}$ production irregular. Pathogenic to rats by mouth, and by subcutaneous and intraperitoneal injection when given in large doses.

Salm. eastbourne A.F. [I], IX, XII . . e, h $\longleftrightarrow 1,5 \ldots$

Isolated from a case of enteric fever (Leslie and Shera 1931). (See also Kauffmann 1934a.) Subsequently met with in the United States and in Norway. Bornstein and Saphra (1942) examined a strain that had been isolated from the spinal fluid of a child who died of meningitis. Also found in animals. Some strains are peculiar in producing indole. Inositol fermentation is irregular, and occasional variants fail to form gas.

Salm. sendai A.F. [I], IX, XII . . . a

Isolated from cases of enteric ferer in Japan (Aoki and Sakai 1925). Antigenic structure determined by White (1926) and Kauffmann (1931, 1934a). Since isolated from a patient with a typhoid-like fever in Georgia (Bornstein and Saphra 1942). Closely related to $S a l m$. paratyphi $A$, from which it differs in containing the ix antigen, in possessing a group phase (which can, however, be artificially induced in Salm. paratyphi $A$ by growth in the presence of immune serum), in fermenting xylose, and in forming little or no gas from dextrose. Resembles Salm. paratyphi $A$ in growing more poorly than most other Salmonella strains.

Salm. loma-linda A.F. IX, XII ... a $\longleftrightarrow e, n, x \ldots$

Salm. onarimon A.F. I, IX, XII . . b

Isolated from the stools of a healthy woman in Japan. Described by Kisida (1940). Closely resembles Salm. paratyphi $B$, and like that organism it forms a mucoid wall. Was later isolated by Anzai and Tsurumi (1940) from the blood of a patient with a typhoid. like disease which began with dysenteric symptoms.

Salm. dar-es-salaam A.F. I, IX, XII . . . I, w $\longleftrightarrow$ e, n . .

Isolated from the urine of a febrile patient in Dar es Salaam. Antigenic structure determined by White (1926). (See also Kauffmann and Mitsui 1930, Kauffmann 1931, $1934 a, 1940 \mathrm{c})$. Peculiar in that it liquefies gelatin. Variable in its ability to grow on an ammonium base medium. Full formula of Phase 2 is $e, n, z_{18}, z_{18} \ldots$ 
Salm. goettingen A.F. IX, XII . . I, v

Deseribed by Hohn (1940) in Germany ; origin not stated, except that it was isolated locally. Studied by Kauffmann (1940c).

Salm. durban A.F. IX, XII ... a $\longleftrightarrow e, n, z_{15} \ldots$

Salm. panama A.F. I, IX, XII . . I, v $\longleftrightarrow 1,5 \ldots$

Isolated by Jordan (1934) from a food-poisoning outbreak. Studied by Kauffmann $(1934 a, 1937 a)$. Found by Schiff (1938) in Now York City in 4 cases of infantile diarrhoa, 2 of which proved fatal; and by Schiff and Strauss (1939b) in three further infections, one of which was in an adult. Isolated from cases of infantile diarrhœa in Uruguay by Hormaeche, Peluffo and Aleppo (1940). Was responsible for $6 \cdot 1$ per cent. of 2,285 Salmonella infections in the United States and Panama in which the causative organism was studied by Elwards and Bruner (1943). It is found in animals, but its natural habitat is not known. Strains vary in their ability to ferment $d$-tartrate, and in the rate at which they attack dulcitol. Occasional strains are said to form indole (Seligmann and Saphra 1943).

Salm. italiana A.F. IX, XII . . 1, $\nabla \leftarrow 1,11 \ldots$

Studied by Bruner (see Edwards 1945).

Salm. claibornei A.F. I, IX, XII ... $\mathrm{k} \longleftrightarrow 1,5 \ldots$

Isolated from a patient suffering from gastro-enteritis at Camp Claiborne, Louisiana, and described by Wilcox and Lennox (1944).

Salm. gallinarum A.F. IX, XII .......

Isolated from fowls suffering from fowl typhoid (Klein 1889). (See also Moore 1895, Pfeiler and Rehse 1913, Pfeiler and Roepke 1917, White 1926, Kauffmann 1934a, $b$ ). Differs from other members of Salmonella group in being non-flagellated and possessing therefore only an $\mathrm{O}$ antigen. Forms little or no gas from dextrose; fermentation of other sugars is relatively slow and feeble. Salm. gallinarum appears to be seldom pathogenic to man, but a fermentative variant, known as duisburg, which fails to attack $d$-tartrate or to produce $\mathrm{H}_{2} \mathrm{~S}$, may be responsible for cases of acute gastro-enteritis (see Müller 1933, Kauffmann 1934b). A more important fermentative variant, often regarded as a separate type and called Salm. pullorum, is responsible for the widespread disease of chickens known as bacillary white diarrhœea. Whether this organism is antigenically identical with Salm. gallinarum is still under discussion. It was isolated by Rettger (1900), and has been studied by numerous workers (see Rettger and Harvey 1908, Rettger 1909, Smith and Tenbroeck 1915, Gage and Martin 1916, Krumwiede and Kohn 1917, Rettger and Koser 1917, Hadley et al. 1917, Mulsow 1919, Winslow et al. 1919, St. JohnBrooks and Rhodes 1923, White 1926, Kauffmann 1931, 1934a, b). Salm. pullorum differs from Salm. gallinarum in producing gas in dextrose, and in failing to ferment maltose, dulcitol, dextrin or $d$-tartrate ; it also grows more poorly. Hinshaw, Browne and Taylor (1943), however, who studied 300 strains of Salm. pullorum, found that 43 strains produced acidity in maltose in 24 hours, and that a further 158 produced some degree of acidity within 4 weeks. Of the total 300 strains, 40 failed to produce gas in dextrose. It is mainly a parasite of chickens, but has been isolated from sparrows (Dalling, Mason and Gordon 1928), silver foxes (Benedict et al. 1941), pigs, mink and man (Edwards and Bruner 1943), and also from imported American spray-dried egg.

Salm. napoli A.F. IX, XII ... l, $\mathrm{z}_{13} \leftrightarrow \mathrm{e}, \mathrm{n}, \mathrm{x} \ldots$

Salm. javiana A.F. [I], IX, XII . . I, $\mathrm{z}_{28} \longleftrightarrow 1,5 \ldots$

Isolated from fæces of a child suffering from gastro-enteritis in Batavia, and from human carriers in Panama. Deseribed by Edwards and Bruner (1942b). Closely related to Salm. panama, but differs in the specific phase. The $\mathrm{z}_{28}$ antigen contains part of the $\mathrm{z}_{13}$ factor of uganda. Not to be confused with the java variety of Salm. paratyphi B.

Salm. canastel A.F. IX, XII . . $\mathrm{z}_{29} \leftrightarrow 1,5 \ldots$

Studied by Bruner and Randall (see Edwards 1945). 


\section{GROUP E.}

Salm. london A.F. III, X, XXVI, I, $\mathrm{X} \longleftrightarrow 1,6 \ldots$

Isolated by White (1926) from a patient at Reading with gastro-enteritis and described as Type L. Later called london by Kauffmann. (See also Kauffmann 1930b, 1931, 1934a, 1939a, Kauffmann and Silberstein 1934.) Cultivated by Černozubov, Filipović and Staval (1936-37) in Jugoslavia from the frees of three patients who developed febrile diarrhœa, accompanied by the passage of blood and mucus, after eating sausage. Found by Hormaeche, Peluffo and Aleppo (1936) in infantile diarrhœa in Uruguay. Isolated from mesenteric glands of normal pigs in Uruguay (Hormaeche and Salsamendi 1939) and in Great Britain (Scott 1940). Also isolated from chickens in the United States (Edwards 1939), and from imported American spray-dried egg in Great Britain.

Salm. give A.F. III, $\mathrm{X}, \mathrm{XXVI}, 1, \mathrm{v} \longleftrightarrow 1,7 \ldots$

Isolated from a patient in Spain with long-standing diarrhcea. Described by Kauffmann (1937b). Since isolated from gastro-enteritis in a child in the United States (see Bornstein, Saphra and Strauss 1941), from mesenteric lymph nodes of healthy pigs in the United States (Rubin, Scherago and Weaver 1942), and from chickens in the United States (see Mallmann et al. 1942). Found also in imported American spray-dried egg.

Salm. uganda A.F. III, X, XXVI, 1, $z_{13} \longleftrightarrow 1,5 \ldots$

Isolated from the spleen of a fatal case of pyrexia of unknown origin in Uganda. Described by Kauffmann $(1940 c)$. The 1 antigen is not identical with that of london and dar-es-salaam, nor are the 1,5 antigens identical with the 1, 5 antigens of Salm. thompson. Phase 1 contains small amounts of antigens $\mathrm{v}$ and $\mathrm{w}$. Distinguished biochemically from london by its fermentation of $i$-tartrate, its more rapid fermentation of $l$-tartrate, and its failure to ferment inositol.

Salm. anatum A.F. III, X, XXVI, e, $h \longleftrightarrow 1,6 \ldots$

Isolated from an epidemic intestinal infection of ducklings, known as keel disease (Rettger and Scoville 1919, 1920). (See also Elwards and Rettger 1927, Lovell 1932b, Kauffmann 1934a, Kauffmann and Silberstein 1934). Nany early strains deseribed under this name were really Salm. typhi-murium. Has been isolated by Hormaeche, Peluffo and Aleppo $(1936,1940)$ from infantile diarrhoea in Uruguay; by Rauss (1941) in Hungary from the freces of healthy persons; by Edwards (1939) in the United States from chickens and turkeys ; by workers in Great Britain from eases of food poisoning aud from imported American spray-dried egg; from mesenteric lymph nodes of normal pigs in the United States by Rubin, Scherago and Weaver (1942) and in Mexico by Varela and Zozaya (1942); and from silver foxes in the United States (Benedict et al. 1941).

Salm. muenster A.F. III, X, XXVI, e, $\mathrm{h} \longleftrightarrow 1,5 \ldots$

Isolated from a food-poisoning outbreak due to raw horse-flesh. Described by Kauffmanu and Silberstein (1934). Also met with in eases of infantile diarrhoa in Uruguay (Hormaeche, Peluffo and Aleppo 1940). Differs biochemically from Sulm. anatum in fermenting inositol and in failing to ferment $i$-tartrate.

Salm. vejle A.F. III, X, XXVI, e, $\mathrm{h} \longleftrightarrow 1,2,3 \ldots$

According to Kauffmann (1941) this organism was isolated by Moller at Copenhagen from acute gastro-enteritis, and described by Harhoff.

Salm. a mager A.F. III, X, XXVI, $\mathrm{y} \longleftrightarrow 1,2,3 \ldots$

Isolated in Denmark from the fæces of a patient suffering from enteritis. Deseribed by Kauffmann (1939a). Non-pathogenic to mice by the mouth.

Salm. shangani A.F. III, X, XXV1, $\mathrm{d} \longleftrightarrow 1_{2} 5 \ldots$

Isolated from frees of a patient with febrile diarrhoea and a commencing miscarriage at Zanzibar. Described by Kauffmann (1939a). The d antigen is not identioal with that of typhi. Non-pathogenic to mice by the mouth. 
Salm. meleagrldls A.F. III, X, XXVI, e, h

(Name derived from meleagris-a kind of guinea-fowl; pronounced mĕlěăgridis). Isolated in Minnesota from outbrcaks of infection in turkey poults; later found in Massachusetts, Michigan and California. Described by Bruner and Edwards(1941b). Isolated also from a patient with a typhoid-like fever in Venezuela and from New York sewage (Bornstein and Saphra 1942); from the mesenteric lymph nodes of normal pigs in Mexico (Varela and Zozaya 1942); from German soldiers in Norway (see Kauffmann 1941); and from American dried egg in England. As in Salm. worthington, the H antigens in Phase 2 are common in Phase 1 of other Salmonella types.

Salm. nyborg A.F. III, X, XXVI, e, $\mathrm{h} \longleftrightarrow 1,7 \ldots$

Isolated in Denmark from a case of acute enteritis in a child of 6 years. Described by Kristensen and Bojlén (1936) and studied by Kauffmann (1937b). The $O$ antigen is not completely identical with that of Salm. anatum, as an anatum serum after absorption with nyborg still contains agglutinins for anatum and for other organisms containing the III, X, XXVI combination.

Salm. zanzibar A.F. III, X, XXVI, $\mathrm{k} \longleftrightarrow 1,5 \ldots$

Isolated from freces of a healthy carrier in Zanzibar. Described by Kauffmann (1939a). The $\mathrm{k}$ antigen is identical with that of thompson. The $\mathrm{O}$ antigen is slightly aberrant. Non-pathogenic to mice by the mouth.

Salm. lexington A.F. III, X, XXVI, $\mathrm{z}_{10} \longleftrightarrow 1,5 \ldots$

Isolated from mesenteric lymph nodes of normal pigs. Described by Edwards, Bruner and Rubin (1940). (See also Rubin et al. 1942.) According to Kauffmann (1941) an organism having the same antigenic formula was found independently by Erber in the Dutch East Indies and described as Salm. batavia. The O antigen of Salm. lexington is slightly aberrant and contains a special factor in addition to the III, X, XXVI factors.

Salm. weltevreden A.F. III, X, XXVI, $\mathrm{r} \longleftrightarrow \mathrm{z}_{6} \ldots$

Salm. newington A.F. III, XV, e, $\mathrm{h} \longleftrightarrow 1,6 \ldots$

Isolated by Rettger from ducklings in Connecticut, and described by Edwards (1937). Also isolated from 3 cases of gastro-enteritis in man in the United States and from sewage (Bornstein and Saphra 1942) ; from chickens and turkeys in the United States by Edwards (1939); from the mesenteric lymph nodes of normal pigs in Uruguay (Hormaeche and Salsamendi 1939) and in the United States (Rubin et al. 1942); from silver foxes in the United States (Benedict et al. 1941); and from imported spray-dried egg in Great Britain. Phase 2 contains 4 in addition to the 1 and 6 factors. Under the name of Salm. tim, Kauffmann (1937b) described a variant of newington, isolated from two patients suffering from gastro-enteritis in Denmark. It is differentiated from newington by the greater complexity of the $\mathrm{e}, \mathrm{h}$ antigen, and by its late or inconstant fermentation of maltose and dextrin.

Salm. selandia A.F. III, XV, e, h

Isolated from the freces of a young sailor, belonging to S.S. Selandia, who on a long voyage to Asia and Australia had suffered from repeated diarrhoea. On his return to Denmark be developed fever, lung symptoms, and diarrhœa following constipation. Described by Kauffmann (1937b). Also found in Havana (Seligmann, Saphra and Wassermann 1944).

Salm. new-brunswick A.F. III, XV, I, $\mathrm{v} \longleftrightarrow 1,7 \ldots$

Isolated from a baby chick. Described by Edwards (1937). Found also in mesenteric lymph nodes of normal pigs in the United States (Rubin et al. 1942). Two strains have been isolated in Denmark, one from a woman with acute gastro-enteritis, the other from a patient who had returned from the tropics and was suspected of having malaria (Kauffmann 1941). 
Salm. cambridge III, XV . . l, w $\leftrightarrow e, \mathrm{~h} \ldots$

Isolated from a soldier suffering from Sonne dysentery. Studied by Taylor (unpublished).

Salm. illinois A.F. III, XV, $\mathrm{z}_{10} \longleftrightarrow 1,5 \ldots$

Isolated from pigs in Illinois, Hungarian partridges in Michigan, and turkeys in Minnesota. Described by Edwards and Bruner (194lc). Some doubt about the constitution of the $\mathrm{O}$ antigen, which is given as (III), (XV), XXXIV by Edwards and Bruner, but as III, XV by Kauffmann (1941).

Salm. taksony A.F. I, III, XIX . . . i $\longleftrightarrow \mathrm{z}_{6}$

Described by Rauss (1943). O antigen is identical with that of Salm. senftenberg.

Salm. senftenberg A.F. I, III, XIX, g, s, t . . - .

Isolated by Kauffmann (1929c), though not under the name of Salm. senftenberg, from an 8-year-old boy suffering from acute gastro-enteritis. (See also Kauffmann 1930b, $1934 a, 1941$, Kauffmann and Mitsui 1930, Boecker and Silberstein 1932). Found also in the United States in young turkeys suffering from an epidemic disease (Edwards 1937), and in chickens (Edwards 1939); in a human carrier in the United States and in Chinese egg (Bornstein and Saphra 1942); in retail meat in the United States (Cherry, Scherago and Weaver 1943); and in the mesenteric lymph nodes of normal pigs in Mexico (Varela and Zozaya 1942). Isolated from imported American spray-dried egg in Great Britain. Included in the flagellar antigens are $\mathrm{z}_{8}$ and $\mathrm{z}_{9}$. Salm. senftenberg var. newcastle was isolated from the fæces of a healthy woman under conditions that precluded any opinion as to its pathogenic rôle (Warren and Scott 1930). It differs from senftenberg in failing to produce $\mathrm{H}_{2} \mathrm{~S}$ and to ferment glycerol and $i$-tartrate.

Salm. niloese A.F. I, III, XIX, $d \longleftrightarrow z_{6} \ldots$

Isolated from the freces of a patient suffering from acute gastro-enteritis in Denmark, and later found repeatedly in similar cases. Described by Kauffmann (1939a). The $\mathrm{d}$ antigen is not identical with that of Salm. typhi, but the $\mathrm{z}_{\mathrm{b}}$ antigen is identical with that of Salm. kentucky. Non-pathogenic to mice by the mouth.

Salm. simsbury A.F. I, III, XIX, $\mathrm{z}_{27} \ldots \ldots$.

Isolated from normal human frecs in the United States (Bruner and Edwards 1942a).

\section{OTHER GROUPS.}

Salm. aberdeen A.F. XI, i $\longleftrightarrow 1,2,3 \ldots$

Isolated from a case of acute gastro-enteritis in an infant (Smith 1934). Since found in chickens in the United States (Mallmann et al. 1942).

Salm. rubislaw A.F. XI, $\mathrm{r} \longleftrightarrow \mathrm{e}, \mathrm{n}, \mathrm{x} \ldots$

Isolated from the fæces of a boy in Scotland who was suffering from enteritis (Smith and Kauffmann 1940). The $\mathbf{r}$ antigen is identical with that of virchow, and the $\theta, \mathrm{n}, \mathbf{x}$ antigens with those of abortus-equi. Also found in American sewage (Bornstein and Saphra 1942).

Salm. pretoria A.F. XI, $k \leftarrow 1,2,3 \ldots$

Salm. solt A.F. XI, $y \longleftrightarrow 1,5 \ldots$

Isolated from fæces of a normal person. Described by Rauss (1943). $O$ antigen is identical with that of Salm. aberdeen.

Salm. grumpensis A.F. XIII, XXII, $d \longleftrightarrow 1,7 \ldots$

Salm. poona A.F. XIII, XXII, z ..

Isolated from a case of acute gastro-enteritis in an infant (Bridges and Scott 1935).

Salm. borbeck A.F. XIII, XXII, 1, $\mathrm{X} \longleftrightarrow 1,6 \ldots$

Isolated from the fæces of a child suffering from typhoid fever in Germany (Hohn and Herrmann 1940). 
Salm. worthlngton A.F. I, XIII, XXIII, l, w $\longleftrightarrow \mathrm{z} \ldots$

Isolated from a young turkey and a chick in Minnesota (Edwards and Bruner 1938). Found also in the United States in human freces (Bornstein and Saphra 1942, Borman et $a l .1943$ ) and in the mesenteric lymph nodes of normal pigs (Rubin et al. 1942). Isolated from imported American spray-dried egg in Great Britain. The $\mathrm{z}$ antigen is not quite identical with that of poona.

Salm. wichita A.F. I, XIII, XXIII, d . . -

Isolated from the freces of infants in Kansas who were suffering from neonatal diarrhoea (McKinlay 1937), and studied by Schiff and Strauss (1939a).

Salm. habana A.F. I, XIII, XXIII, f, g ...-

Isolated from cerebrospinal fluid, blood and fæces during the course of a hospital outbreak of purulent meningitis among new-born babies in Havana; all the infants admitted to hospital, 21 in number, died (Schiff and Saphra 1941). Since found in imported American spray-dried egg. Like all Salmonella strains containing the $\mathrm{g}$ antigen, it is monophasic. The flagellar antigen is not quite identical with that of derby; there appears to be an extra minor antigen in both organisms.

Salm. mississippi A.F. I, XIII, XXIII, b $\longleftrightarrow 1,5 \ldots$

Isolated from the fæecs of a normal human carrier (Edwards and Bruner 1943).

Salm. heves A.F. (I), VI, XIV, XXIV ...

Described by Rauss (1943).

Salm. carrau A.F. VI, XIV, XXIV, y $\longleftrightarrow 1,7 \ldots$

Isolated from the mesenteric lymph node of a normal pig in Uruguay (Hormaeche, Peluffo and Salsamendi 1938). Also found in infantile diarrhœa (Hormaeche, Peluffo and Aleppo 1940). It is probable that the XIV antigen of onderstepoort (see below) consists of two fractions, one of which is specific and the other common to carrau. Hormæche, Peluffo and de Pereyra (1944) find that Salmonella strains having factor 7 in Phase 2 must be divided into two sub-groups; sub-group i possesses a factor that is lacking in sub-group ii. Sub-group i contains arechavaleta, altendorf, florida, gaminara, kaapstad and pomona. Sub-group ii contains bredeney, carrau, grumpensis, selandia, give, new-brunswick, nyborg and madelia.

Salm. onderstepoort A.F. (I), VI, XIV, XXV, e, (h) $\longleftrightarrow 1,5 \ldots$

Isolated from 2 sheep in South Africa. Described by Henning (1936). (For the constitution of the XIV antigen, see Salm. carrau.) Is pathogenic to mice inoculated intraperitoneally.

Salm. florida A.F. (I), VI, XIV, XXV, d $\longleftrightarrow 1,7 \ldots$

Isolated from fæces of a patient suffering from febrile diarrhœea. Described by Cherry, Edwards and Bruner (1943). The d antigen is not quite identical with that of Salm. typhi, Salm. oregon or Salm. muenchen.

Salm. madelia A.F. (I), VI, XIV, XXV, y

Isolated fiom the liver of a poult. Described by Cherry, Edwards and Bruner (1943). The $y$ antigen is identical with that of Salm. bareilly.

Salm. sundsvall A.F. (I), VI, XIV, XXV . . z $\longleftrightarrow$, $\mathrm{n}, \mathrm{x}, \mathrm{z}_{16} \ldots$

Isolated in Scandinavia. Responsible in England for an outbreak of gastro-enteritis. Also found in imported American spray-dried egg.

Salm. horsham A.F. (I), VI, XIV, XXV . . l, v $\leftrightarrow$ e, n, x . .

Isolated in England from imported spray-dried egg powder. Studied by Taylor (unpublished).

Salm. hulttingfoss A.F. XVI, $b \longleftrightarrow e, n, x \ldots$

Isolated in Norway from the fæces of patients who were suffering from acute gastroenteritis following the consumption of soft cheese, and from the cheese itself; one patient had a typhoid-like disease that lasted for a week (Tesdal 1936, 1938). Also found in 
fowls in the United States (Mallmann et al. 1942). The $\mathrm{O}$ antigen was given originally as XIII. Non-pathogenic to mice by the mouth.

Salm. gaminara A.F. XVI, $d \longleftrightarrow I, 7 \ldots$

Isolated from a child in Urugnay suffering from dysenteriform enteritis (Hormaeche and Peluffo 1939). Hormaeche and Peluffo, who analysed the d antigen of this and other salmonellie, give the following distribution:-Salm. gaminara $\mathrm{d}, \mathrm{d}_{2}, \mathrm{~d}_{3} ;$ Salm. stanley $\mathrm{d}, \mathrm{d}_{3}$; Salm. amersfoort d, $\mathrm{d}_{2}, \mathrm{~d}_{3}$; Salm. muenchen $\mathrm{d}, \mathrm{d}_{3}, \mathrm{~d}_{4}$; Salm. typhi $\mathrm{d}, \mathrm{d}_{1}$. The non-specific phase contains an antigen that has not been met with in other species of Salmonella. Pathogenic for rats by the mouth and subcutaneously, if given in large doses, and for rabbits intravenously and subcutaneously. The non-specific phase is said to be more toxic than the specific phase.

Salm. szentes A.F. XVI, $k \leftrightarrow 1,2,3 \ldots$

Deseribed by Rauss (1943). O antigen appears to be identical with that of Salm. hvittingfoss.

Salm. kirkee A.F. XVII, b $\longleftrightarrow 1,2 \ldots$

Isolated from the stools of an infant in India suffering from diarrhne of the dysenteric type (Bridges and Dunbar 1936). The $\mathrm{O}$ antigen was given originally as XIV.

Salm. cerro A.F. XVIII, $z_{4}, z_{23}, z_{25}-$.

Isolated in 1936 from the pooled mesenteric lymph nodes of normal pigs in Uruguay. Later isolated from infants suffering from enteritis and sometimes rhino-pharyngitis (Hormaeche, Peluffo and Aleppo 1941). Found in imported American spray-dried egg. Shares the $\mathrm{z}_{4}$ antigen with duesseldorf and arizona.

Salm. kentucky A.F. (VIII), XX, i $\longleftrightarrow \mathrm{z}_{6} \ldots$

Isolated from a chicken in the United States suffering from enteritis (Edwards 1938), and from pheasants (Edwards 1939). Found also in a human case of gastro-enteritis in the United States (Bornstein and Saphra 1942); in a mild case of febrile diarrhœa in Palestine and in camels in Palestine (Olitzki 1942); and in imported American spray-dried egg in Great Britain. The Palestine strains differed from the American kentucky strains in minor fermentative respects.

Salm. minnesota A.F. XXI, XXVI, b $\leftrightarrow \mathrm{e}, \mathrm{n}, \mathrm{x} \ldots$

Isolated from a turkey poult (Edwards and Bruner 1935). (See also Kauffmann 1939a). Found by Hormaeche, Peluffo and Aleppo (1940) in infantile diarrhrea in Uruguay. Isolated from American spray-dried egr. The b antigen is not completely identical with that of Salm. paratyphi B.

Salm. tel-aviv A.F. XXVIII, $\mathrm{y} \longleftrightarrow \mathrm{e}, \mathrm{n}, \mathrm{z}_{15} \ldots$

Isolated from a sick cow, and from an epidemic disease of chickens accompanied by a 50 per cent. case mortality in Palestine. Studied by Kauffmann (1940a).

Salm. pomona A.F. XXVIII, $y \leftrightarrow \rightarrow 1,7 \ldots$

Salm. ballerup A.F. XXIX, [Vi], $\mathrm{z}_{14}-$.

Isolated from the fæces of a woman in Denmark who had a history of gastro-enteritis lasting for sereral weeks (Kauffmann and Moller 1940). Somatic antigen contains also a small amount of the XIX fraction related to senftenberg. Salm. ballerup forms two types of colony. The relatively stable $V$ form, which is smooth and opaque, contains a Vi antigen: the unstable $\mathrm{W}$ form, which is smooth and translucent, does not. The $\mathrm{Vi}$ antigen is the same as that in Salm. typhi and Salm. paratyphi C, and will immunize mice against the $V$ form of Salm. typhi (Kauffmann and Moller 1940). Similarly, rabbit serum eontaining $\mathrm{Vi}$ antibodies to Salm. ballemp affords passive protection to mice against inoculation with Salm. typhi (Longfellow and Luippold 1943). Both the V and the W forms of Sulm. ballerup are relatively non-pathogenic to mice by the mouth and intraperitoneally. An extra flagellar antigen $z_{31}$ is said to be present (Monteverde and Leiguarda 1944). 
Salm. hormæchei A.F. XXIX, [Vi], z $z_{30}-$.

Isolated from sewage at Buenos Aires and described by Monteverde and Leiguarda (1944). Resembles Salm. ballerup in the variability of its Vi antigen, which may be present or absent. Contains in addition to $z_{30}$ the flagellar antigen $z_{31}$, but this is subject to quantitative fluctuation. Non-pathogenic for guinea-pigs on intraperitoneal inoculation, but large doses inoculated intraperitoneally kill mice in 24 hours, and the organism can be recovered from the heart blood.

Salm. urbana A.F. XXX, b $\longleftrightarrow e, n, x \ldots$

Isolated in the United States from the colon of a pig dying of hæmorrhagic enteritis, and from the gut of a dead chicken (Edwards and Bruner 194la). Found also in patients suffering from enteritis in Massachusetts (Bornstein and Saphra 1942). Contains some XVI antigen, which it shares with hvittingfoss. The $\mathrm{b}$ antigen is incomplete, so that absorption with Salm. urbana of a serum prepared against Salm. paratyphi $B$ leaves a considerable part of the $b$ antibody behind. Phase 1 probably contains an antigen, as yet unidentified, in addition to $\mathrm{b}$. Phase 2 contains the $\mathrm{z}_{16}$ antigen; the $\mathrm{e}, \mathrm{n}, \mathrm{x}, \mathrm{z}_{16}$ complex is shared with abortus-bovis and minnesota.

Salm. arizona A.F. XXXIII, $\mathrm{z}_{4}, \mathrm{z}_{23}, \mathrm{z}_{26} \ldots$ - .

Isolated in the United States from certain reptiles by Caldwell and Ryerson (1939). Studied by Kauffmann (see Kauffmann 1941). Resembles Salm. dar-es-salaam in slowly liquefying gelatin. Is peculiar in sometimes fermenting lactose, though not for 2 weeks or so. Cultures appear to be partly rough, and the antigenic structure is therefore still in doubt. Appears to be pathogenic for certain reptiles, and is highly pathogenic for guinea-pigs and rabbits. Has since been isolated from a woman suffering from high fever, diarrhoea and vomiting (Seligmann, Saphra and Wassermann 1944).

Salm. adelaide A.F. XXXV, f, g -

Isolated from fæces during life, and from liver and spleen post mortem, of a man suffering from enteritis in Australia. Described by Atkinson (1943). Isolated on at least four occasions in England from cases of gastro-enteritis-one of them in a German prisoner-of-war. Original strain said not to ferment sorbitol, but English strains all ferment this sugar.

Salm. inverness A.F. XXXVIII, $\mathrm{k} \longleftrightarrow 1,6 \ldots$

Isolated from a normal food handler in Florida and described by Edwards and Hughes (1944). Possesses a somatic antigen not previously described. The $\mathrm{k}$ antigen is the same as that in Salm. thompson.

\section{Other organisms containing antigens of the Salmonella group.}

Since Habs and Arjona (1935) described a paracolon bacillus containing part of one of the Salmonella O antigens, and Gard (1937) and Gard and Eriksson (1959) described coliform bacilli containing Salmonella $\mathrm{H}$ antigens, several workers have reported the occurrence of $\mathrm{O}$, less often of $\mathrm{H}$, antigens in paracolon, coliform and Flexner dysentery bacilli (Bornstein, Saphra and Daniels 1941, Schiff, Bornstein and Saphra 1941, Kauffmann 1941, Saphra and Silberberg 1942, Peluffo, Edwards and Bruner 1942). These workers alone have met with about 50 strains. Some of these strains contain only part of one $\mathrm{O}$ antigen; others contain a complete complement of $\mathrm{O}$ antigens and are indistinguishable from such Salmonella types as onderstepoort, worthington, or carrau. At one time only $\mathrm{H}$ antigens common to the non-specific phase of diphasic Salmonella types were found; but Peluffo, Edwards and Bruner (1942) have now met with paracolon bacilli containing antigens, such as $z_{4}$, that are present in monophasic types. Four strains at least have been described that contain a Vi antigen apparently identical with that present in Salm. typhi, Salm. paratyphi $C$, and Salm. ballerup.

The fermentative behaviour of these coliform and paracolon bacilli varies considerably. Some of them form acid and gas in lactose within 24 hours, others not till after some days; some form acid only, and some fail to attack lactose altogether. Most of them fail to ferment dulcitol and tartrates; many attack sucrose or salicin or both; and a 
few of them form indole or liquefy gelatin. None of them has yet been shown to be pathogenic to man, but there is strong reason to believe that some are pathogenic to animals (Edwards, Cherry and Bruner 1943).

Of the Flexner dysentery bacilli, two strains were shown by agglutination, absorption, and production of antibody tests to contain $O$ antigens VI and XIII, and 14 other strains were agglutinated by sera containing VI and XIII antibodies (Bornstein, Saphra and Daniels 1941).

Though Kauffmann (1941) has included five types of coliform and paracolon bacilli in his diagnostic table under the name of Salmonella coli 1, 2, 3, 4, 5, there seems little justification for regarding these organisms as nembers of the Salmonella group. Our present knowledge suggests that Salmonella antigens are by no means uncommon in the Bacterium group. In our own laboratory we have met several such strains; and it may well be that, when they are looked for, they will be found to be widely distributed. As we have already pointed out, it is doing violence to the principles of taxonomy to include every organism in the Salmonella group merely beeause it contains some antigenic fraction that has hitherto been regarded as peculiar to this group. The main interest of these organisms is that they serve as pitfalls for the unwary; and that unless a proper study of the cultural and biochemical behaviour of every strain suspected of being a Salmonella is made, in addition to a serological examination, they are liable to give rise to false positive results from time to time. Admittedly, there are intermediate strains such as those deseribed by Edwards, Cherry and Bruner (1943), some of which appear to be pathogenic, which are at present very difficult to classify; but it will be wise for the moment to maintain a conservative attitude, and to include in the Salmonella group only those organisms possessing the general, as well as the antigenic characters, of accepted members of this group.

\section{REFERENCES}

Achard, C. and Bensaude, R. (1896) Bull. Mém. Soc. méd. Hôp., 13, 820.

Alson, L. (1943) Bact. Rev., 7, 43.

ANDrew Es, F. W. (1922) J. Path. Bact., 25, 505; (1925) Ibid., 28, 345.

Andrewes, F. W. and Neave, S. (1921) Brit. J. exp. Path., 2, 157.

AxzaI, H. and Tsurumi, H. (1940) Kitasato Arch., 17, 106.

AOKI, K. and SAKAI, K. (1925) Zbl. Balt., 95, 152.

ARIMA, R. (1912) Zbl. Bakt., 63, 424.

Atrinson, N. (1943) Aust. J. exp. Biol. med. Sci., 21, 171.

BAHR, L. (1930a) Dtsch. tierärztl. Wschr., 38, 145; (1930b) Ibid., 38, 165.

Bartel, H. (1938) Tierärztl Rdsch., 44, 601.

Basenau, F. (1894) Arch. Hyg., Berl., 20, 242.

Basilewsey, B. G. and RemaLd, W. I. (1935) Z. ImmunForsch., 85, 10.

Beaudette, F. R. (1926) J. Amer. vet. med. Ass., 68, 642.

BeNeDict, R. G., MCCOY, E., and WisnickY, W. (1941) J. infect. Dis., 69, 167.

BERNARD, H. (1935-36) Z. Hyg, InfektKr., 11\%, 352.

BIROH-HirschfELd, L. (1935) Z. Hyg. InfektKr., 11\%, 626.

Bitter, L., Weigmany, F., and Habs, H. (1926) Münch. med. Wrschr., 73, 940.

BOECKER, E. (1936) Zbl. Bakt., 135, 501.

BoECKER, E. and KadfFuan, F. (1930 Zbl. Bakt., 116, 458.

Boecker, E. and Silberstein, W. (1932) Zbl. Bakt., 125, 257.

Boivin, A. and Mesrobeant, L. (1933) C. R. Soc. Biol., 112, 76; (1934a) Ibid., 115, 304, 309 ; (1934b) Ibid., 11\%, 273; (1934c) C. R. Acad. Sci., 198, 2211; (1935a) C. R. Soc. Biol., 118, 612; (1935b) C. R. Acad. Sci., 201, 168.

Boivin, A., MIesrobeand, I., and Miesrobeand, L. (1933a) C. R. Soc. Biol., 113, 490; (1933b) Ibid., 114, 307; (1934a) Ibid., 115, 306 ; (1934b) Ibid., 11\%, 271 ; (1935) Arch. roum. Path. exp. Microbiol., 8, 45.

Borman, E. K., Wheeler, K. II., West, D. E., and Mickle, F. L. (1943) Amer. J. publ. Hlth, 33, 127.

BoRnsteIn, S. (1943) J. Immunol., 46, 439.

Bornstein, S. and SAPHra, I. (1942) J. infect. Dis., 71, 55.

Bornstein, S., Saphra, I., and Daniels, J. B. (1941) J. Immunol., 42, 401.

Bornstein, S., Saphra, I., and Stradss, L. (1941) J. infect. Dis., 69, 59.

Bosworte, T. J. and I.oveld, R. (1931) Univ. Camb. Inst. Animal Path., 2nd Ann. Rep., p. 21 . 
Boycott, J. and McNeE, J. W. (1936) Lancet, ii. 741.

BranHaM, S. E. (1925) J. Infect. Dis., 37, 291.

Bridges, R. F. and Dunbar, L. (1936) J. R. Army med. Cps, 67, 289.

Bridges, R. F. and Scott, W. M. (1931) J. R. Army med. Cps., 56, 241 ; (1935) Ibid, 65,221 .

BriegER, I. (1902) Dtsch. med. Wschr., 28, 477.

Brion, A. and KAYser, H. (1902) Münch. med. Wschr., 49, 611 .

Broudin, L. (1927) C. R. Soc. Biol., 9\%, 1589.

Brown, H. C., Duncan, J. T., and Henry, T. A. (1924) J. Hyg., Camb., 23, 1 ; Lancet, i. 117.

Browning, C. H., Gilmour, W., and Mackie, T. J. (1913) J. Hyg., Camb., 13, 335.

Bruner, D. W. and Edwards, P. R. (1939) J. Bact., 3\%, 365 ; (1940) Agric. Exp. Sta. Univ. Kentucky, Bull. No. 404; (194la) J. Bact., 42, 467 ; (1941b) Amer. J. Hyg., 34, B., 82; (1942a) Proc. Soc. exp. Biol., N.Y., 50, 174; (1942b) J. Immunol., 44, 319.

Bruns, H. and Gasters. (1920) Z. Hyg. InfeltKr., 90, 263.

Caldwell, M. E. and Ryerson, D. L. (1939) J. infect. Dis., 65, 242.

Carpano, M. (1913) Zbl. Bakt., 70, 42.

Casper, W. (1928-29) Z. Hyg. InfeltKr., 109, 170.

Cernaianu, C. and Popovici, I. (1933) C. R. Soc. Biol., 112, 829.

Črnozubov, N., Filipović, D., and Stavel, J. (1936-37) Zbl. Bakt., 138, 460.

Chantemesse, A. (1897) C. R. Soc. Biol., 49, 96, 101.

Cherry, W. B., Edwards. P. R., and Broner, D. W. (1943) Proc. Soc. exp. Biol. N.Y., 52,125 .

Cherry, W. B., Scherago, M. and Weaver, R. H. (1943) Amer. J. Hyg., 37, 211.

Cladberg, K. W. (1931) Klin. Wschr., 10, 540 ; (1932) Zbl. Bakt., 124, 23.

Combiesco, D., Stamatesco, S., and Sord, E. (1930) Arch. rom. Path. exp. Microbiol. 3, 189.

Conradi, H. (1903) Dtsch. med. Wschr., 29, 26; (1906) Ibid., 32, 58.

Conybeare, E. T. and Thornton, L. H. D. (193S) Rep. publ. Hlth med. Subj., Min. Hlth, Lond., No. 82.

Chaigie, J. (1942) Canad publ. Hith J., 33, 41.

Craigie, J. and Brandox, K. F. (1936a) J.Path. Bact., 43, 233 ; (1936b) Ibid., 43, 249.

Craigie, J. and Yen, C. H. (1938) Canad. publ. Hlth. J., 29, 448, 484.

Cruickshank, J. C. (1939) J. Hyg., Camb., 39, 224.

Dalling, T., Mason, J. H., and Gordon, W. S. (1928) Vet. Rec., 8, 329.

Dalling, T. and Warrack, G. K. (1932) J. Path. Bact., 35, 655.

Dammann and Stedefeder. (1910) Arch. wiss. prakt. Tierheilk., 36, 432.

Danysz, J. (1900) Ann. Inst. Pasteur, 14, 193.

Delafield, 11. E. (1931) J. Path. Bact., 34, 177 ; (1932) Ibid., 35, 53 ; (1934) Brit. J. exp. Path., 15, 130.

Donssen, C. A. vaN. (1937) Dtsch. tierärztl. Wschr., 45, 196.

Dodglas, S. R. (1921) Brit. J. exp. Path., 2, 175.

DOYLE, T. 11. (1927) J. comp. Path., 40, 71.

Dudgeon, L. S. and Urquhart, A. L. (1920) Lancet, ii. 15.

ECKER, E. E. (1917) J. infect. Dis., 21, 541.

Ecker, E. E. and Richardson, M. L. (1925) J. infect. Dis., 37, 538.

EскеR, E. E. and Rimington, C. (1928) J. Hyg., Camb., 27, 44.

Edwards, P. R. (1929) J. infect. Dis., 45, 191 ; (1935) J. Bact., 30, 465 ; (1936) Ibid., 32, 259 ; (1937) J. IIyg., Camb., 37, 384; (1938) J. Hyg., Camb., 38, 306; (1939) Proc. 7th World's Poultry Congr., p. 271 ; (1945) Pers. comm.

Edwards, P. R. and Brdner, D. W. (1938) J.Hyg., Camb., 38, 716 ; (1939) J. Bact., 38, 63 ; (1940a) Agric. Exp. Sta. Univ. Kentucky, Bull. No. 400 ; (1940b) J. infect. Dis., 66, 218 ; (194la) J. infect. Dis., 69, 220 ; (1941b) Amer. J. Hyg., 34, See. B., 121 ; (1941c) Proc. Soc. exp. Biol. N.Y., 48, 240 ; (1942a) J. Bact., 44, 289; (1942b) J. Immunol., 44, 319 ; (1942c) Agric. exp. Sta. Univ. Kentucky, Circular No. 54. (1943) J. infect. Dis., 72,58 .

Edwards, P. R., Bruner, D. W., and Hinshaw, W. R. (1940) J. infect. Dis., 66, 127.

Edwards, P. R., Bruner, D. W., and Rubin, H. L. (1940) Proc. Soc. exp. Biol., N.Y., 44, 395.

Edwards, P. R., Cherry, W. B., and Bruner, D. W. (1943) J. infect. Dis., 73, 229.

Edwards, P. R. and Hughes, H. (1944) Proc. Soc. exp. Biol., N.Y., 56, 33.

Edwards, P. R. and Rettger, L. F. (1927) J. Bact., 13, 73.

Felix, A. (1943) Brit. med. J., i, 435.

Felix, A. and Callow, B. R. (1943) Brit. med. J., ii, 127.

Felix, A. and Pitr, R. M. (1934a) J. Path. Bact., 38, 409; (1934b) Lancet, ii. 186.

Ferramola, R., Monteverde, J. J. and Leiguarda, R. H. (1943) Bol. Obras sanit. Nación, Aug. No. 74, p. 103.

Fitch, C. P. and Billings, W. A. (1920) J. Bact., 5, 469. 
Fletcher, W. (1918) Lancel, ii. 102.

Freeman, G. G. (1943) Biochem., J., 37, 601.

Freeman, G. G., Challinor, S. W., and Wilson, J. (1940) Biochem. J., 34, 307.

Furth, J. and Landsteiner, K. (1928) J. exp. Med., 47, 171 ; (1929) Ibid., 49, 727.

GaERTNER. (1885) KorrespBl. ärztl. Ver. Thüringen, 1\%, 573.

GAGE, G. E. and Martin, J. F. (1916) J. med. Res., 34, 149.

GARD, S. (1937) Z. IIyg. InfektKr., 120, 59; (1938) Ibid., 121, 139.

GARD, S. and ERIKSson, E. J. (1939) Z. Hyg. InfcktKr., 122, 54.

Gay, F. P. and Claypole, E. J. (1913) Arch. intern. Med., 12, 621.

Giglioli, G. (1930) J. IIyg., Camb., 29, 273.

GLäSSER, K. (1909) Dtsch. tierärztl. W'schr., 16, 513 ; (1910) Zbl. Bakt. Ref., 45, 612.

Good, E. S. and CorbeTt, L. S. (1913) J. infect. Dis., 13, 53.

Goulder, N. E., Kingsland, H. F., and Janeway, C. A. (1942) New Engl. J. Med., 226, 127.

GraY, I. D. A. (1931) J. Puth. Bact., 34, 335.

GutI, F. (1916) Zbl. Bukt., $7 \%, 487$.

Guthrie, K. J. and Moxtgomery, G. L. (1939) J. P'ath. Bact., 49, 393.

GWyn, N. B. (1898) Johns. Hopk. Hosp. Bull., 9, 54.

HABS, H. (1933) Zbl. Bakt., 130, 367.

Habs, H. and ARJona, E. (1935) Zbl. Bakt., 133, 204.

Hadley, P. B., Caldwell, D. W., Elkins, 11. W., and Lambert, D. J. (1917) R. I. agric. Exp. Sta. Bull., No. 172.

HaRVEY, A. II. (1937) Arch. intern. Hed., 59, 118.

HatTa, S. (1938) Jap. J. exp. med., 16, 201.

Havess, L. C. and MaYfield, C. R. (1933) J. infect. Dis., 52, 157.

Heelsbergen, T. van. (1914) Zbl. Bakt., 72, 38.

Hexsing, M. W. (1936) J. Hyg., Camb., 36, 525; (1937), Ibid. 37, 561.

Hrcks, E. P. (1930) J. Hyg., Camb., 29, 446.

Hinshaw, W. R., Browne, A. S., and TAYlor, T. J. (1943) J. infect. Dis., 72, 197.

HiRSCHFELD, L. (1919) Lancet, i. 296.

HofFman, IV. and Ficker, M. (1904) IIyg. Rundsch., 14, 1.

Hoнs, J. (1940) Zbl. Bakt., 146, 215.

Holin, J. and Herrma NN, W. (1935a) Zbl. Bakt., 133, 183 ; (1935b) Ibid., 134, 277 ; (1936a) Zbl. Bakt., 135, 505 ; (1936b) Z. Hyg. InfektKr., 11\%, 722 ; (1936c) Ibid., 118, 656 ; (1940) Zbl. Bakt., 145, 209.

Hormaeche, E. and Peldffo, C. A. (1936) Arch. urug. Med., 9, 673; (1939) Ibid., 14, 217.

Hormaeche, E., Peldffo, C. A., and Alepro, P. L. (1936) Arch. urug. Med, 9, 113 ; (1940) Arch. Pediat. Urug., 11, s; (1941). Arch. urug. Med., 19, 125.

Hormaeche, E., Peleffo, C. A. and Pereyra, V. R. De. (1944) J. Bact., 4\%, 323.

Hormaeche, E., Peluffo, C. A., and Salsamendi, R. (1938) Arch. urug. Med., 12, 377.

Hormaeche, E. and Salsamendi, R. (1936) Arch. urug. Med., 9, 665; (1939) Ibid., 14, 375.

Hynes, M. (1942) J. Path. Bact., 54, 193.

Iwaschexzoff, G. (1926) Arch. Schiffs- u. Tropenhyg., 30, 1.

JACOBSEN, K. A. (1910) Zbl. Bakt., 56, 208.

JANSEN, J. (1937) Bull. Hyg., Lond., 12, 139.

JoNes, E. R. (1936) J. Path. Bact., 42, 455.

JoNG, D. A. DE. (1913) Zbl. Bakt., 6\%, 148.

Jordan, E. O. (1934) Amer. J. trop. Hed., 14, 27.

Jungherr, E. and Claxcy, C. F. (1939) J. infect. Dis., 64, 1.

Kadffmann, F. (1929a) Zbl. Bakt., Ref., 94, 282; (1929b) Z. Hyg. InfektKr., 110, 537 ; (1929c) Ibid., 109, 427, 491 ; (1930a) Ibid., 111, 210 ; (1930b) Ibid., 111, 221, 233, 247 ; (1931) Zbl. ges. Hyg., 25, 273; (1934a) Ergebn. IIyg. Bakt., 15, 219; (1934b) Zbl. Baki., 132, 337 ; (1934c) Z. Hyg. InfehtKr., 116, 368 ; (1935a) Ibid., 116, 617 ; (1935b) Ibid., 11\%, 431 ; (1936a) Ibid., 11\%, 778; (1936b) Ibid., 118, 540; (1936c) Ibid., 119, 103; (1937a) z. Hyg. InfektKr., 119, 356; (1937b) Ibid., 120, 177 ; (1939a) Acta path. microbiol. scand., 16, 347; (1939b) Ibid., 16, 278 ; (1940a) Ibid., 17, 1; (1940b) Ibid., 17, 135 ; (1940c) Ibid., 1\%, 189 ; (1940d) Ibid., 1\%, 429 ; (1941) "Die Bakteriologie der SalmonellaGruppe." Einar Munksgaard, Copenhagen.

KaUfryan, F. and Burón, F. A. (1935) Z. Hyg. InfektKr., 11\%, 650.

Kaufriann, F. and Henningsen, E. J. (1938) Z. Hyg. InfektKr., 120, 640; (1939) Acta path. microbiol. scand., 16, 99.

Kauffiann, F. and Mitsui, C. (1930) Z. Hyg. Infektkr., 111, 749.

Kauftuane, F. and Moller, E. (1940) J. Hyg., Camb., 40, 246.

Kauffmann, F. and Silberstein, W. (1934) Zbl. Bakt., 132, 431.

KaUfrmanx, F. and Tesdal, MI. (1937-38) Z. Hyg. InfektKr., 120, 168.

KhalIL, A. I. (1938) J. Hyg., Camb., 38, 75.

Kilborne, F. L. (I893) Misc. Invest. infect. parasit. Dis., $8^{0}$ Washington, 49. 
KISIDA, S. (1940) Kitasato Arch. 1\%, 1.

KueIN, E. (1889) Zbl. Bakt., 5, 689.

Knork, M. (1931) Arch. IIyg., Berl., 105, 237.

Kristensen, M. and Bojlén, K. (1929) Zbl. Bakt., 114, 86; (1936) Z. Hyg. InfektKr., $120,177$.

Kristensen, M. and Kadffuan,, F. (1937) Z. Hyg. InfektKr., 120, 149.

Krumwiede, C. and Kohn, L. A. (1917) J. med. Res., 36, 509.

Krunwiede, C. and Pratt, J. S. (1914) J. exp. Med., 19, 501.

KüHnemanN, G. (1911) Zbl. Bakt., 5\%, 497.

Leifson, E. (1935) J. Path. Bact., 40, 581 ; (1936) Amer. J. Hyg., 24, 423.

LEnTz, O. and Tietz, J. (1903) Mïnch. med. Wschr., 50, 2139 ; (1905) Klin. Jb., 14, 495.

LEON, A. P. (1942) Rev. Inst. Salub. Enferm. trop., 3, 273.

Lesbouyries and Verge. (1932) Bull. Acad. Vét. France, 5, 294.

Leslie, P. H. (1942) J. Hyg., Camb., 42, 552.

Leslie, P. H. and Shera, A. G. (1931) J. Path. Bact., 34, 533.

Loeffler, F. (1892) Zbl. Bakt., 11, 129 ; (1903) Dtscr. med. Wschr., 29, 36 ; (1906) Ibid., 32,289 .

Longfellow, D. and Luippold, G. F. (1943) Amer. J. Hyg., 37, 206.

Lovell, R. (1931) J. Path. Bact., 34, 13; (1932a) Bull. Hyg., 7, 405; (1932b) Vet. Reo., 12, 1052 .

LÜTJE. (1937) Dtsch. tierärztl. Wschr., 45, 242 ; (1939) Ibid., 47, 227, 246, 257.

MacAdan, W. (1919) Lancet, ii. 189.

Macfadyen, A. and Rowland, S. (1901) Zbl. Bakt., 30, 753 ; (1903) Proc. roy. Soc., 71, 77.

MACKIE, E. P. and Bowen, C. J. (1919) J. R. Army med. Cps, 33, 154.

McKinlay, B. (1937) Amer. J. Dis. Child., 54, 1252.

Malliani, W. L., Ryff, J. F., and Matthews, E. (1942) J. infect. Dis., 90, 253.

MandelbaUm. (1932) Mïnch. med. Wschr., 79, 1566.

Marrassini, A. (1913) Zbl. Bakt., \%1, 113.

Martin, A. R. (1934) Brit. J. exp. Path., 15, 137.

Mellon, R. R. and Jost, E. L. (1926) J. Immunol. 12, 331.

Menten, M. L. and Kino, C. G. (1930) J. infect. Dis., 46, 275.

Metchnikoff, E. and Besredka, A. (1911) Ann. Inst. Pasteur, 25, 193.

Meyer, K. and Bergell. (1907) Berl. klin. Wschr., 54, 568.

Meyer, K. F. and Boerner, F. (1913) J. med. Res., 29, 325.

Meyer, K. F. and Matsumura, K. (1927) J. infect. Dis., 41, 395.

Monteverde, J. J. (1942) Nature, 149, 472.

Monteverde, J. J. and Leiguarda, R. H. (1944) Bol. Obr. sanit. Nac., 8, 168.

Moore, V. A. (1895) U.S. Dept. Agri. Bur. Animal Ind., 8, 71.

Morgan, W. T. J. and Partridge, S. M. (1940) Biochem. J., 34, 169 ; (1942) Brit. J. exp. Path., 23, 151.

Morris, J. F., Barnes, C. G., and Sellers, T. F. (1943) Amer. J. publ. Hlth., 33, 246.

Morris, J. F., Brim, A. and Sellers, T. F. (1944) Amer. J. publ. Hlth., 34, 1277.

Morris, J. F., Sellers, T. F., and Brown, A. W. (1941) J. infect. Dis., 68, 117.

Muller, L. (1923) C. R. Soc. Biol., 89, 434.

MÜLler, M. (1912) Zbl. Bakt., 62, 335.

MÜLler, R. (1910) Dtsch. med. Wschr., 36, 2387; (1933) Münch. med. Wschr., 80, 1771.

Mulsow, F. W. (1919) J. infect. Dis., 25, 135.

Murray, C. (1919) J. infect. Dis., 25, 341.

NeURIRCH, P. (1918) Z. IIyg. InfektKr., 85, 103.

Nobele, De. (1898) Ann. Soc. Méd. Gand., Y2, 281.

Nocard. (1893) Cons. Hyg. Publ. Sal. Dept. Seine. Séance. Mar. 24.

Olitzki, L. (1942) J. Hyg., Camb., 42, 547.

Orskov, J., Jexsen, K., and KobaYASH, K. (1928) Z. ImmunForsch., 55, 34.

Orskov, J. and LASSEN, H. C. A. (1930) Z. ImmunForsch., 6\%, 137.

Orskov, J. and Moltke, O. (1928) Z. ImmunForsch., 59, 357.

Рecknai, C. F. (1923) J. Hyg., Camb., 22, 69.

Peluffo, C. A., Edwards, P. R., and Broner, D. W. (1942) J. infect. Dis., 70, 185.

PFEIfFER, R. (1894) Dtsch. med. Wschr., 20, 598.

Pfeiler, W. and Rehse, A. (1913) Z3l. Bakt., 68, 174.

Pfeiler, W. and Roepke, E. (1917) Zbl. Bakt., 79, 125.

Pijper, A. (1938) J. Path. Bact., 47, 1; (1940) J. biol. photogr. Ass., 8, 158.

Pirosky, I. (1938) C. R. Soc. Biol., 128, 347.

Pollock, M. R., Krox, R., and Gell, P. G. H. (1942) Nature, 150, 94.

Raistrick, H. and Topley, W. W. C. (1934) Brit. J. exp. Path., 15, 113.

RAuss, K. (1939a) Z. ImmunForsch.,95, 489; (1939b) Ibid., 9\%, 281; (1941) Zbl. Bakt., 147,253 ; (1943) Z. Immun Forsch., 103, 220.

Report (1934) Salmonella Subcommittee of Nomenclature Comm. int. Soc. Microbiol., J. Hyg., Camb., 34, 333. 
Rettaer, L. F. (1900) N. Y. med. J., 71, 803 ; (1909) J. med. Res., 21, 115.

Rettger, L. F. and Harvey, S. C. (1908) J. med. Res., 18, 277.

Rettaer, L. F. and Koser, S. A. (1917) J. med. Res., 35, 443.

Rettger, L. F., Plastridoe, W. N., and Cameron, R. (1933) J. infect. Dis., 53, 272. Rettger, L. F. and Scoville, M. M. (1919) Abst. Bact., 3, 8; (1920) J. infect. Dis., 26, 217.

Rotн, E. (1903) Hyg. Rundsch., 13, 489.

Rouchd, M. (1938) C. R. Soc. Biol., 128, 1022.

RuBIN, H. L. (1940) J. Bact., 40, 463.

Rubin, H. L., Scherago, M., and Weaver, R. H. (1942) Amer. J. IIyg., 36, 43.

Salion, E. and Sirth, 'T. (1585) Ann. Rep. Bureau Animal Industry; (1886) Amer. mon. micr. J., \%, 204.

Sanarelli, J. (1894) Ann. Inst. Pasteur, 8, 193, 353.

Saphra, I. and Silberberg, M. (1942) J. Immunol., 44, 129.

Savage, W. G. and White, P. B. (1925) Spec. Rep. Ser. med. Res. Coun., Lond., No. 91.

Savino, E. and Menéndez, P. E. (1934) Rev. Inst. Bact., 6, 347.

SснӓғER, IV. (1934-35) Zbl. Bakt., 133, 458.

SCherder and EHRLICH. (1921) Berl. tierärzll. Wschr., 37, 469.

Schiff, F. (1938) J. Amer. med. Ass., 111, 2458.

SCHIF, F., Bornstein, S., and SAPHRA, I. (1941) J. Immunol., 40, 365.

SchIFF, F. and SaPhra, I. (1940) J. infect. Dis., 66, 97 ; (1941) Ibid., 68, I25.

Schiff, F. and Strauss, L. $\quad(1939 a)$ J. infect. Dis., 65, 125 ; (1939b) Ibid., 65, 160.

Scholtens, R. T. (1937) J. Hyg., Camb., 3\%, 315.

SchotтMÜLleR, H. (1900) Dtsch. med. Wschr., 26, 511 ; (1901) Z. IIyg. InfektKr., 36, 368.

Sснӥтzе, H. (1920) Lancet, i. 93 ; (1928) Arch. Hyg., Berl., 100, 181 ; (1930) Brit. J. exp. Path., 11, 34.

Schwa Bacher, H., TAylor, J. and White, M. H. G. (1943) Brit. med. J., ii, 358.

scotr, W. M. (1926) J. Hyg., Camb., 25, 39s; (1932) J. Path. Bact., 35, 655; (1933) Bull. Office int. Hyg., 25, fase. 5 ; (1940) Proc. R. Soc. Med., 33, 366.

Seiffert, G., Jahncke, A., and Arnold, A. (1928) Zbl. Bakt., 109, 193.

Seligmann, E. and Clauberg, K. W. (1932) Zbl. Bakt., 125, 266.

Seligmane, E. and Saphra, I. (1943) Amer. J. Hyg., 38, 223.

Seligmann, E., Saphra, I, and Wasserhann, M. (1944) Amer. J. Hyg., 40, 2.27.

SHmios, K. (1913) Zbl. Bakt., \%1, 338.

Silberstein, W. (1931) Zbl. Bakt., 122, Beiheft 131 ; (1932) Z. Ilyg. InfektKr., 114, 124.

Simmons, J. S. (1926) J. infect. Dis., 39, 209.

Sladden, A. F. and Scott, W. M. (1927) J.Hyg., Camb., 26, 111.

Sнiтн, J. (1934) J. Hyg., Camb., 34, 351.

Sirth, J. and Kauffinan, F. (1940) J. IIyg., Camb., 40, 122.

Sмith, J. and Scotт, W. M. (1930) J. Hyg., Camb., 30, 32.

Smith, T. (1893) Misc. Invest. infect. parasit. Dis. Dom. Animals, $8^{0}$ Washington, 53.

Smith, 'T. and Ten Broeck, C. (1915) J. med. Res., 31, 503.

St. John-Brooks, R. and Rhodes, M. (1923) J. Path. Bact., 26, 433.

STERN, W. (1916) Zbl. Bakt., 78, 481.

Ströman, R. and ÖRN, C. (1932) Zbl. Bakt., 126, 340.

'TAYLOR, J., EDWARD, D. G.ff., and EDWARDs, P. R. (1945) Brit. med. J., i. 368.

Tesdal, M. (1936) Z. Hyg. InfektKr., 118, 533; (1937) Ibid., 119, 28; (1938) "Die Salmonellagruppe mit besonderer Berücksichtigung der Salmonellinfektionen in Norwegen." Olaf Norli, Oslo.

Topley, W. W. C. and Ayrton, J. (1924) J. Hyg., Camb., 23, 198.

Tulloch, W. J. (1939) J. Hyg., Camb., 39, 324.

VARELA, G. and ZozayA, J. (1942) Bull. Hyg., Lond., 1\%, 721.

Varela, G., Zozaya, J. and Olarte, J. (1943) Bull. Hyg., Lond., 18, 411.

Warren, S. H. and ScotT, W. M. (1930) J. Hyg., Camb., 29, 415.

Weigmann, F. (1925a) Zbl. Bakt., 95, 396; (1925b) Ibid., 9\%, Beiheft. 299.

WEח, E. (1917) Wien. klin. Wschr., 30, 1061.

WeIL, E. and SAxL, P. (1917) Wien. klin. Wschr., 30, 519.

WheEler, K. M. and Borman, E. K. (1943) J. Bact., 46, 481.

Wheeler, K. M., Stuart, C. A., Rustigian, R., and Borman, E. K. (1943) J. Immunol., 47, 59 .

Wherry, W. B. (1908) J. infect. Dis., 5, 519.

White, P. B. (1926) Spec. Rep. Ser. med. Res. Coun., Lond., No. 103 ; (1929a) J. Path. Bact., 32, 85; (19296) Med. Res.Coun., "System of Bacteriology," 4, 86 ; (1930) J. Hyg., Camb., 29, 443 ; (1931) J. Path. Bact., 34, 325 ; (1932) Ibid., 35, 77.

Wilcox, K. S. and Lennox, E. K. (1944) J. Immunol., 49, 71.

Wilson, W. J. (1938) J. Hyg., Camb., 38, 507.

Winslow, C.-E. A., Kligler, I. J., and Rothberg, W. (1919) J. Bact., 4, 429.

WyLlie, J. (1943) Canad. publ. Hlth J., 34, 82.

YaMaNodCHI, T. (1909) C.R. Soc. Biol., 66, 1050. 


\section{CHAPTER 31}

\section{LACTOBACILLUS}

\section{Definition.-Lactobacillus.}

Rods, often long and slender, sometimes pleomorphic. Gram-positive. Nonmotile and non-sporing. Growth on surface media poor. Some members grow best at $40^{\circ}-44^{\circ} \mathrm{C}$. Usually produce acid from carbohydrates, as a rule lactic; some members form gas in addition. Little or no proteolytic activity. Most members are microaerophilic and facultative anaerobes; some members are strict anaerobes. Readily destroyed by heat, but unusually resistant to acid. Not known to be pathogenic to man or animals. Widely distributed in fermenting vegetable and animal products.

Type species is Lactobacillus caucasicus, Beijerinck.

History.-The first organism of this group was isolated by Kern in 1881 from the fermented milk of the Caucasus, known as Kefir. The name he gave it was Dispora Kaukasica, but later it was called Bacillus Kaukasicus, and is now known as Lactobacillus caucasicus. As Kern did not give a complete description of the organism, and as it is impossible to be certain of its identity, it is somewhat unfortunate that it has been adopted as the type species. A similar bacillus was observed by Döderlein in 1892 in the acid vaginal secretion of pregnant fomen; this is usually known as Döderlein's bacillus, but is also known as $B$. vaginalis and $B$. crassus. Slender Gram-positive bacilli were observed microscopically in the stomach contents of patients with gastric carcinoma by Oppler in 1895, working in the clinic of Dr. Boas at Berlin ; this organism, which was not cultivated, is generally known as the Boas-Oppler bacillus. In 1900 Moro $(1900 a, b)$ cultivated a similar bacillus from the fæces of breast-fed infants ; this organism he called $B$. acidophitus; his findings were confirmed in the same year by Finkelstein (1900). Tissier, also in 1900, isolated two new organisms of the same group from the fæces of infants, to which he gave the names $B$. bifidus and $B$. exilis. In 1905 Grigoroff, working in Massol's laboratory, isolated from Kissélo-mléko, better known as Yoghurt, the fermented milk of Bulgaria, three organisms to which he gave the names A, B, and C; the first of these is now known as Lactobacillus bulgaricus or Massol's bacillus. Similar bacilli have been found by other workers in a number of fermented milks, chiefly the Armenian Mazun, the Sardinian Gioddu, and the Egyptian Leben raib (Cohendy 1906, White and Avery 1910); they have also been isolated from ordinary market milk and from human milk (Moro 1900a, Heinemann and Hefferau 1909, Sherman and Stark 1927). Bacilli of this group have been cultivated by Mereshkowsky and his pupils (Mereshkowsky 1905, 1906, Petrow 1907) from the fæces of a large series of invertebrates, fishes, and mammals; by Heinemann and Hefferan (1909) from human saliva and gastric juice, from soil, and from a number of different foods, such as 
bran, silage, cornmeal, and olive-juice; by McIntosh, James and Lazarus-Barlow (1922, 1924) from carious teeth; and by Kendall (1910) from sewage. Eggerth and Gagnon (1933) and Eggerth (1935) drew attention to the presence in normal adult faces of Gram-positive and Gram-negative anaerobic bacilli, which they described under the generic name of Bacteroides. There seems little doubt that the Gram-positive members are closely related to the lactobacilli and they will therefore be described in this chapter.

Morphology.-The members of this group are in general fairly large, non-motile, non-sporing, Gram-positive bacilli. They are arranged singly, in pairs end-to-end, in chains, and sometimes in palisades (Fig. 151). Some members are markedly pleomorphic, especially in old cultures, forming clubbed, knobbed, curled, spiral, candle-flame, vacuolated, whorled, and filamentous forms, and frequently showing irregular, granular, or beaded staining. In some species the bacilli tend to be arranged at angles to each other, giving rise to $\mathrm{Y}$-forms, which may simulate true branching. Another characteristic of some members is the formation of lateral offshoots or buds, either directly adherent to the parent cell, or connected with it by a short stem; these buds may themselves be bifid.

Cultivation. - These bacteria do not as a rule grow well on the usual laboratory media ; their growth is much improved by the addition of whey or glucose. Surface colonies show a good deal of variation, but on the whole conform to one or other of the two types described by Mereshkowsky $(1905,1906):(1)$ round or navicular, pinhead in size, opaque, whitish, and surrounded by an areola of turbid agar;

(2) round or irregularly round, less than pinhead in size, greyish, translucent colonies with a finely erose edge, and with no areola around them; microscopically these colonies are of typically rhizoid structure. Deep colonies in glucose agar likewise tend to be either compact, with an entire edge or sometimes a single lateral knob (Rettger and Horton's (1914) Y type), or curled, rhizoid, and feathery, looking like a tuft of hair or moss (Rettger and Horton's $\mathrm{X}$ type). Intermediate types of colony are not uncommon. The compact and feathery types of colony are referred to by some writers as "smooth" and "rough"

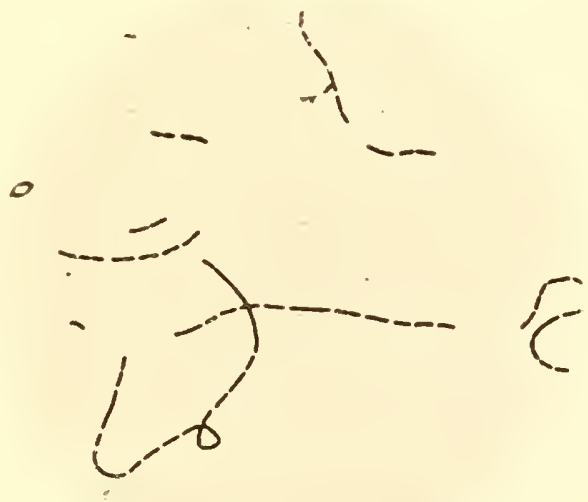
respectively, but since the par-

ticular type of colony formed seems to depend largely on environmental conditions, and since there seems to be little relationship between the colonial type and any other important characteristic of the organisms, it is probably wiser to refrain from the use of terms that have now come to possess a wider connotation. A very characteristic appearance is the turbidity or milkiness of the agar produced in shake plates or tubes; it is a variable characteristic, however, 
and has little or no differentiating value amongst the members of the group. Growth in gelatin is either poor or absent; liquefaction never occurs.

Resistance and Methods of Isolation from Natural Sources.-The organisms are not particularly resistant to heat, and are generally destroyed by an exposure to $60^{\circ} \mathrm{C}$. for half an hour. One of their most striking features, which gives to them the names acidophilic, acid-resisting, or aciduric, is their ability to survive in concentrations of acid that usually prove fatal to other non-sporing bacteria. It is this characteristic that is generally made use of in their isolation.

One of the most successful methods is to incubate the material for 1 to 3 days in 0.5 per cent. acetic acid broth, and subsequently to plate on 2 per cent. glucose agar. McIntosh and his co-workers (1922) recommend incubating the material in broth of $\mathrm{pH} 3.5$ for 24 hours, then subculturing into a series of broth tubes varying in $\mathrm{pH}$ from 3.0 to 4.5 , and plating after a further 24 hours. Kendall (1910) made three consecutive subcultures in acid broth, and plated the last on dextrose agar containing $0 \cdot 2$ per cent. sodium oleate, which is said to improve the growth. The acid may be added to the tubes directly, or the organisms may be seeded into a medium containing a fermentable carbohydrate; the acid produced in this medium is usually sufficient to kill off most other micro-organisms. Cruickshank (1925) recommends, for the isolation of L. bifidus, inoculating the freces into a deep tube containing $20 \mathrm{ml}$. of 1 per cent. glucose or lactose broth together with a small piece of fresh sterile rabbit kidney; the kidney is added to promote anaerobiosis, but does not appear to be essential; the medium is covered with a vaseline seal. The culture is incubated for about a week at $37^{\circ} \mathrm{C}$. and then plated on to 1 per cent. glucose agar or Loeffler's serum, which is incubated aerobically and anaerobically. After 48 hours on the anaerobic plates, greyish pinhead colonies appear. The only other organism that is likely to develop under these conditions is the enterococcus, which forms larger whitish colonies. Another method that may be émployed for the isolation of the acid-resistant bacteria is the use of Veillon tubes (Veillon and Zuber 1898, Rettger and Cheplin 1921), containing 2 per cent. glucose agar or whey agar ; the different organisms adapt themselves to the varying oxygen pressure in the medium, and form characteristic colonies, which may be picked off with ease. The tomato broth medium described by Kulp (1927) has given very favourable results; if the primary cultures are plated on tomato agar, single colony isolations are frequently successful.

In our experience (Crowley et al. 1941) direct plating of consecutive dilutions on the glucose liver blood agar medium recommended by Eggerth and Gagnon (1933) has proved satisfactory for the isolation of lactobacilli from fæces. Lewis, Bedell and Rettger (1940) have described a glucose cysteine agar medium which, likewise, seems well suited for this purpose. The presence of added $\mathrm{CO}_{2}$ is beneficial for growth (Kulp 1926, Cruickshank 1934, Lewis et al. 1940).

Metabolism.-Most of the members are microaerophilic or facultative anaerobes. For their isolation fairly strict anaerobic conditions are often, though not always, necessary. After a few subcultures many of them can be brought to grow aerobically, but others remain persistently obligate anaerobes. Peroxide is produced without, or with only very small quantities of, catalase. This probably accounts for the poor viability of the organisms in media not maintaining a low oxidationreduction potential. Gillespie and Rettger (1938) noted a difference between different species in the intensity of their reducing activity. Oral lactobacilli, for example, were found in a particular medium to reduce the Eh to about $-240 \mathrm{mv}$, whereas $L$. acidophilus of intestinal origin, $L$. bifidus, Döderlein's bacillus and L. bulgaricus reduced it to only about - $140 \mathrm{mv}$. According to Curran, Rogers, and Whittier (1933), the optimum growth temperature is $37^{\circ}-40^{\circ} \mathrm{C}$.; the range 
of growth with most strains is $25^{\circ}-46^{\circ} \mathrm{C}$., but certain strains, particularly those of the casei type, may grow even at $10^{\circ} \mathrm{C}$. Though some of the earlier workers (Rodella 1901) stated that an alkaline was preferable to an acid medium, later workers (Morishita 1929, Weiss and Rettger 1934, Longsworth and MacInnes 1935) have found that both growth and acid production occur best in the neighbourhood of $\mathrm{pH} 6 \cdot 0$, the optimal range being about $\mathrm{pH} 5 \cdot 4-6 \cdot 8$. Pantothenic acid, riboflavin and pyridoxin appear to be important accessory growth factors (Snell, Strong and Peterson 1939). No pigment is produced, but deep colonies in glucose agar often develop a brownish centre, and the agar itself is frequently clouded. Hæmolysin production is variable and has not been studied fully. No toxins are formed. The organisms have very little effect on proteins, and growth on protein media without carbohydrates is very poor. Peterson, Pruess and Fred (1928) have found that they do possess some proteolytic action, as judged by the quantitative estimation of non-protein and amino-acid nitrogen, but according to Kendall and Haner $(1924 a, b)$ this is very slight; no indole, scatole, or histamine is formed.

On the other hand they are very active in fermenting carbohydrates. The acid produced from lactose is partly fixed, consisting of lævo- or dextro-rotatory or inactive.lactic acid, and partly volatile, consisting of formic, acetic, and butyric acids in the ratio of $6: 3: 1$ (Curran, Rogers and Whittier 1933). The proportion of volatile to fixed acids varies with different strains from about 4-20 per cent. According to Barker and Haas (1944), however, the intestinal members of the group do not produce lactic acid from lactate, but volatile fatty acids. This type of butyric acid fermentation differs from others in that no appreciable amount of molecular hydrogen and very little $\mathrm{CO}_{2}$ are formed. Malic acid is said to be produced by $L$. odontolyticus in greater quantity than lactic acid (McIntosh et al. 1924). Gas production is not detectable by the ordinary Durham fermentation tube, except with $L$. acidophil-aerogenes (Torrey and Rahe 1915), which produces 4-6 volumes of $\mathrm{H}_{2}$ to 1 of $\mathrm{CO}_{2}$. Curran, Rogers, and Whittier (1933), however, have shown that most strains produce small quantities of gas from fermentable carbohydrates. The formation of lactic acid from glucose does not require the presence of oxygen or lead to the production of $\mathrm{CO}_{2}$. Consequently $\mathrm{CO}_{2}$ is not a major product of fermentation with most strains. An exception to this rule is furnished by $L$. pentoaceticus, which is able to oxidize lactic to acetic acid (Hunt 1933). In this process one molecule of $\mathrm{O}_{2}$ is used and one molecule of $\mathrm{CO}_{2}$ produced. Hence $\mathrm{CO}_{2}$ constitutes a more important product of fermentation with this organism than with the other members of the group. The usual products of fermentation, such as alcohol, acetone, acetylmethylcarbinol, and butylene glycol, are not formed (Bertrand and Duchacek 1909). For all practical purposes the organisms may be considered as of the obligatory saccharolytic type.

Biochemical.-There is considerable variation in the sugars fermented. Glucose and lactose are fermented by practically all strains, maltose and sucrose by a high proportion, mannitol, salicin, and raffinose by a small proportion, while dextrin, inulin, dulcitol, and starch are rarely fermented. Strains of $L$. bifidus are said, however, to ferment inulin (Weiss and Rettger 1934), and strains isolated from soil and grain are said not to ferment lactose (Hunt and Rettger 1930). Both these statements await confirmation. L. pentoaceticus, the organism described by Fred, Peterson, and Davenport (1919) from silage, sauerkraut, and manure, is peculiar in its ability to ferment xylose. According to Weinstein and Rettger (1932), it is 
further distinguished from most of the other members of the group by its failure to ferment lactose or to curdle milk. Cruickshank (1934) says that Döderlein's bacillus ferments glycogen with the production of lactic acid in a few days, while strains of acidophilus, odontolyticus, and bifidus take 7-10 days to ferment it ; bulgaricus does not ferment it at all. It is very doubtful whether the reactions of individual species and strains are constant; according to some authors they are not (McIntosh et al. 1924, Day and Gibbs 1928). Most, but not all, the members produce acid in milk, often in sufficient quantity to precipitate the casein in the form of a loose clot, which does not contract and express whey; the litmus is frequently decolorized, especially in the lower part of the tube. The rate at which clotting is produced is of some slight differential significance. White and Avery (1910) divided the acid-resistant organisms obtained from milk into two types; their Type A produced a large quantity of lactic acid in milk-2.7 to 3.7 per cent.; their Type B produced a smaller quantity-1.2 to 1.6 per cent. The most active acid producer is $L$. bulgaricus; the least active $L$. bifidus; $L$. acidophilus occupies an intermediate position.

Antigenic Structure.-The serological reactions of these organisms have been incompletely studied, and so far no satisfactory classification of the group has been possible by agglutination or absorption. Generally speaking, the members of a single species show a considerable amount of heterogeneity (Kendall and Haner 1924b, Lash and Kaplan 1926, Thomas 1928, Howitt 1930). McIntosh and his co-workers (McIntosh et al. 1924), however, observed a marked group reaction between members of the acidophilus and acidophilus-odontolyticus types; and Cruickshank (1925) and Weiss and Rettger (1934) found a close relationship between L. bifidus and L. acidophilus. Thomas (1928) found that Döderlein's bacillus had some relation to $L$. acidophilus, but none to $L$. bulgaricus. Working with oral strains, many of which had been isolated several months previously, Harrison, Zidek and Hemmens (1939) extracted a carbohydrate-containing substance from lactobacilli, and by means of precipitating antisera prepared by the inoculation of rabbits with heat-killed bacilli were able to divide the strains into four serological types and a heterogeneous group.

Pathogenicity.-None of the members appears to be pathogenic to man or animals. It is true that, in fermentative diarrhœa, acid-resisting bacteria may be present in large numbers in the stools, but whether they are responsible for initiating the diarrhœa, or whether they merely take advantage of the abnormal conditions prevailing in the intestine to multiply abundantly, is not clear. Their numbers increase in the intestine when lactose or dextrin are given in considerable quantities in the diet (Rettger and Cheplin 1921, Cannon and McNease 1923); and because, under these conditions, they tend to replace the proteolytic flora, their administration along with these sugars has been advocated for therapeutic purposes.

There is reason to believe that the oral lactobacilli play some part in the development of dental caries. The opinion that this disease is due to the action of acids formed in the mouth from retained food was put forward a long time ago by Robertson (1835), Tomes (1873), and others. It was not, however, till the appearance of Niller's (1889) monograph, in which it was maintained that lactic acid resulting from the bacterial fermentation of starch and sugar was primarily responsible for decalcification and solution of the enamel, that serious suspicion was thrown on the acid-forming flora of the mouth. Though the streptococci have received attention from a number of workers, (see Snyder 1939), it 
seems more probable that the lactobacilli, on account of their greater acid production and their ability to grow at a low $\mathrm{pH}$, are implicated. Kligler (1915) and Snyder (1939) noted the increased numbers of $L$. acidophilus in the mouths of people with caries. MeIntosh, James and Lazarus-Barlow $(1922,1924)$ isolated two varieties of lactobacilli from carious teeth, which they named $L$. odontolyticus $I$ and $I I$. In glucose broth cultures these organisms produced a final $\mathrm{pH}$ of 2.2 to 3.4 ; and it was found that teeth left in these cultures gradually became decalcified, the change being evident in 7 weeks. There is evidence to suggest that refined sugar in the diet is a more potent predisposing factor to caries than other forms of carbohydrate, but the exact mechanism by which it acts is still unknown. The effect of the acid is limited, of course, by the structural integrity and proper calcification of the enamel, which in their turu depend on nutritional factors (see McCollum 1941).

The repeated inoculation intravenously of very large doses of lactobacilli into rabbits is said to be followed by the development of joint lesions. A mucopurulent exudate is found in the joints, and cultures can be obtained for a week after the last inoculation (Howitt and van Meter 1930). It is doubtful, however, whether the organisms actually multiply within the tissues under these conditions.

\section{Classification}

Classification is at present unsatisfactory, partly because of some difference of opinion as to which organisms should be included in the Lactobacillus group, and partly by the absence of suitable criteria to serve as a basis of sub-division. There seems to us goor reason for including the Gram-positive members of the non-sporing anaerobic bacilli isolated by Eggerth and Gagnon (1933) from the human intestine in the Lactobacillus rather than in the Bacteroides group, and in this we are supported by King and Rettger (1942). When we look for differential characters for use in classification we are in serious difficulties. Morphological and colonial appearances vary considerably, and metabolic properties and fermentation reactions tend to show continuous, as opposed to discontinuous, variation; so that it is difficult to do more than select modal points around which strains can be grouped. Whether it is justifiable to assign specific rank to these type organisms is doubtful, but provided not too many types are recognized, and provided it is realized that many of them are tentative, convenience alone affords a strong plea for their characterization by name.

The most serious attempt to classify the lactobacilli is undoubtedly that made by Curran, Rogers, and Whittier (1933). As the result of a very careful study, particularly of their metabolic characteristics, these workers were able to divide 103 strains of varied origin into three groups. Group A strains produced inactive lactic acid, i.e., equal quantities of dextro- and lævo-acid, from whey, failed to grow above $43^{\circ}-46^{\circ} \mathrm{C}$. or as low as $20^{\circ} \mathrm{C}$., fermented raffinose but not manuitol, and on agar plates formed either the fuzzy $(\mathrm{X})$ type of colony, or a mixture of fuzzy and compact $(\mathrm{Y})$ types. As a rule they gave rise to more $\mathrm{CO}_{2}$ and a larger proportion of volatile to fixed acids, and grew in higher concentrations of phenol and indole (see Kulp 1929), than the members of the second group. Group B strains produced an excess of dextro-lactic acid, grew as high as $43^{\circ}-50^{\circ} \mathrm{C}$. and as low as $10^{\circ}-15^{\circ} \mathrm{C}$, fermented mannitol but not raffinose, and formed either the compact $(Y)$ type of colony, or a mixture of compact and fuzzy (X) types. They were less active than 
Group $\mathrm{A}$ strains in the production of volatile acids and $\mathrm{CO}_{2}$, and were inhibited by relatively dilute solutions of phenol and indole. Group $\mathrm{C}$ strains differed from Group $\mathrm{A}$ in growing at $20^{\circ} \mathrm{C}$. and in a number of minor particulars. Of the 103 strains, Group A comprised 58, Group B 30, and Group C 15. With regard to their source of origin, about three-quarters of the intestinal strains belonged to Group A, while over half of the dental strains belonged to Group B. Group A probably represented the typical L. acidophilus; Group B probably included $L$. bulgaricus and L. casei ; while Group C comprised a heterogeneous collection of strains whose identity was doubtful, and whose classification into one group was largely a matter of temporary convenience. Lewis and Rettger (1940) suggest a classification of the anaerobic members into three groups, A, B and C-which do not, of course, correspond to the similarly named groups of Curran, Rogers and Whittier - based on morphology, minimum growth temperature, resistance to heat, and the production of gas from fermentable carbohydrates.

The identity of $L$. bulgaricus has often been under discussion. Sherman and Hodge (1940) state that it differs from $L$. acidophilus in the following respects. Freshly isolated strains of $L$. bulgaricus grow at $50^{\circ} \mathrm{C}$, but are unable to grow in serial culture in a simple medium containing 1 per cent. each of lactose, peptone and yeast extract, or in broth of $\mathrm{pH} 7 \cdot 8$, or in broth containing 2.5 per cent. sodium chloride. L. acidophilus, on the other hand, will not grow above a temperature of $48^{\circ} \mathrm{C}$., but will grow under the other conditions described. It need hardly be mentioned that minor physiological differences of this type, however useful in practice, can afford no satisfactory basis for permanent classification.

A classification of the gas-producing strains has been suggested by Pedersen (1938) based primarily on arabinose fermentation, and secondarily on the fermentation of lactose, sucrose and raffinose. L. brevis (Synonym: L. pentoaceticus; B. acidophil-aerogenes) ferments arabinose, and varies in its effect on the other three sugars; its growth range is $10^{\circ}$ to $45^{\circ} \mathrm{C}$, and its optimum $30^{\circ}$ to $35^{\circ} \mathrm{C}$. $L$. fermenti does not ferment arabinose, but usually ferments the three other sugars ; its growth range is $15^{\circ}$ to $50^{\circ} \mathrm{C}$, and its optimum $35^{\circ}$ to $40^{\circ} \mathrm{C}$. L. buchneri (Synonym : B. wehmeri) usually ferments arabinose, lactose, sucrose and raffinose ; its growth range is $10^{\circ}$ to $48^{\circ} \mathrm{C}$., and its optimum $44^{\circ}$ to $48^{\circ} \mathrm{C}$. L. pastorianus (Synonym: L. berolinensis) usually ferments arabinose, lactose, sucrose and raffinose; its optimum temperature for growth is $27^{\circ}$ to $32^{\circ} \mathrm{C}$. No satisfactory description, however, appears to have been given of this organism.

Barker and Haas (1944) find that the Gram-positive, anaerobic, non-sporing intestinal bacteria break down lactate with the production of volatile fatty acids, and they therefore suggest that they should be classified in a separate genus, Butyribacterium. Further work will clearly be required to find out whether there is, in fact, a sharp cleavage in their type of metabolism among the organisms hitherto classified as lactobacilli. In the meantime, we may point out the coufusion that is likely to be caused by using the termination "bacterium" for anaerobic bacilli; it would be much better to reserve this term for won-sporing aerobic rods. Much the same criticism applies to the termination "bacillus," and it is unfortunate that the generic name Lactobacillus should have been chosen for organisms which are mainly anaerobic or microaerophilic. It would be better if the name could be replaced by one more suitable, such, for instance, as Lactiformans.

As has frequently happened with other groups, several members have been accredited with specific names without an adequate description being given of them. Moreover 
it seems probable that some species that have been called by different names are in reality identical. It seems likely that Döderlein's bacillus, for example, is the same as $L$. acido. philus (Heinemann and Ecker 1916, Thomas 1928), and for that reason we have not given a separate description of it. Similarly, according to Schlirf (1926), Goadby's B. necrodentalis is identical with $L$. acidophilus. It is doubtful whether the Boas-Oppler bacillus is a separate species; it may quite well be identical with, or a variety of, $L$. acidophilus (Heinemann and Ecker 1916). L. bulgaricus is quite possibly the same as the type species L. caucasicus, of which no adequate description has ever been given (IVhite and Avery 1910). Cruickshank (1925) suggests that $L$. exilis is the aerobic phase of $L$. bifidus; but from Tissier's (1900) original description this seems doubtful. Weiss and Rettger (1934) were unable to detect any greater difference between strains of $L$. bifidus and $L$. acidophilus than existed between the individual strains themselves, and they would therefore regard $L$. bifidus as a variant of the species of which $L$. acidophilus is the central type. The organisms described by McIntosh and his colleagues as L.odontolyticus $I$ and II have received considerable study. Morishita (1929), Rosebury, Linton, and Buchbinder (1929), Howitt (1930), and Hadley, Bunting, and Delves (1930) have failed to find any clear distinction between oral and intestinal strains of $L$. acidophilus. Curran, Rogers and Whittier (1933), on the other hand, conclude that the lactobacilli in carious teeth do not all belong to one species and are not usually of the acidophilus type. Gillespie and Rettger (1938) also have noted differences between the oral and the intestinal strains of lactobacilli. For this reason we shall describe the odontolyticus strains separately from $L$. acidophilus.

A number of lactic-acid-forming bacilli were isolated from cheese by von Freudenreich and Thöni (1903), and named Bacillus casei by Orla-Jensen (1904). These organisms have not been fully described, and we do not propose to deal with them further here. They are frequently found in milk (Sherman and Stark 1927). Another organism, described by Pederson (1936) as Lactobacillus plantarum, is widely distributed in fermenting plant and animal products. The differentiation of this organism from Lactobacillus casei is not very clear.

With regard to the nomenclature of this group, the term "acid-resisting" is frequently employed, and, though correct, it is open to the objection that it may cause confusion with the acid-fast bacilli. The term "acidophilic" is justifiable, but is unfortunately a hybrid. Probably Kendall's (1910) term "aciduric" (able to endure acid), which has the advantages of not being a hybrid, of not being hyphenated, and of being technically correct, is the best one to employ.

Workers studying this group may consult the annotated bibliography on $L$. acidophilus drawn up by Frost and Hankinson (1931). Those interested in the chemical constitution of this organism are referred to a series of papers by Crowder and Anderson (1932, 1934a, b).

While emphasizing again the impossibility of classifying these organisms satisfactorily at present, we give a differential table of some of the main species, pointing out, however, that it is to be used only as a very rough indication of the characteristics of these organisms, Table 49, p. 758.

\section{Lactobacillus acidophilus}

Synonyms.-Probably identical with Döderlein's bacillus, which is sometimes called $B$. vaginalis or $B$. crassus.

Isolation.-Isolated by Moro (1900b) in 1900 from the freces of breast-fed infants.

Habitat.-Found in milk; the fæces of bottle-fed infants, and often of adults; the frees of nearly all mammalia, and of many fish and invertebrates; in saliva and carious teeth. 


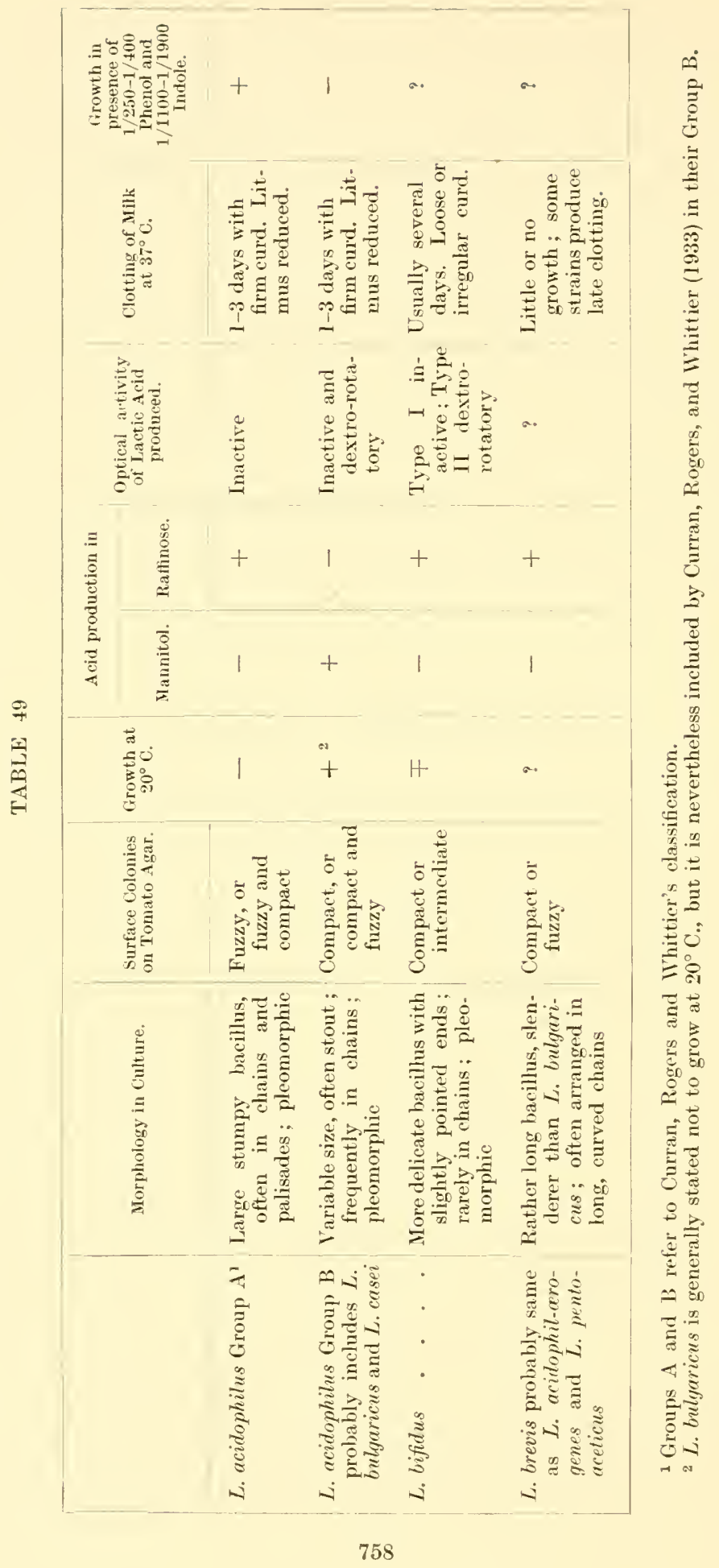


Morphology.-In fæces it is a large stumpy bacillus of variable length and fairly constant breadth, generally straight, with parallel sides and rounded ends. On agar plate cultures, after 48 hours at $37^{\circ} \mathrm{C}$., it forms fairly thick rods, $1-3 \mu$ long by $1 \cdot 0 \mu$ broad, straight or slightly curved, with parallel sides and rounded or slightly truncated ends; arranged singly, in pairs end-to-end, in short chains, and in palisades. On glucose agar longer chains and filamentous forms are common. In broth and litmus milk cultures the bacilli are thinner, $0 \cdot 6-0 \cdot 8 \mu$ broad. Considerable variation in morphology on artificial

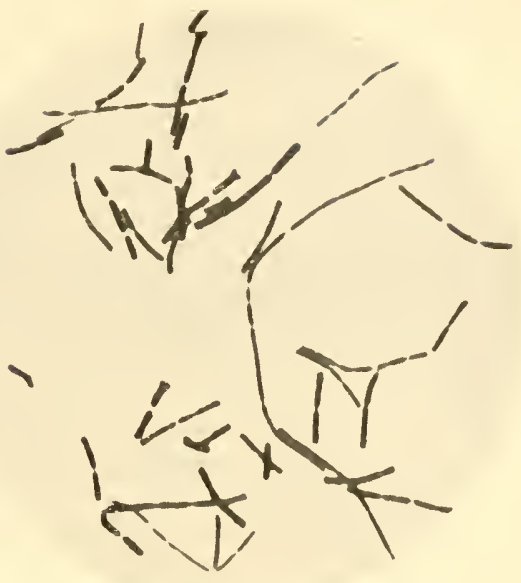

F16. 152.-Lactobacillus acidophilus.

From an agar culture, 48 hours, $37^{\circ} \mathrm{C} .(\times 1000)$. media; forms with markedly curved extremities, curled forms, forms with bulbous extremities, clubbed forms, filamentous forms, and large swollen oval forms in pairs are not uncommon. Under anaerobic conditions long curved filamentous forms, often with pointed, swollen, or spatulate ends are seen. Non-motile. Gram-positive. Staining is uniform in young cultures, but irregularly stained, bipolar-stained, and beaded forms are met with in old cultures. Non-acid-fast.

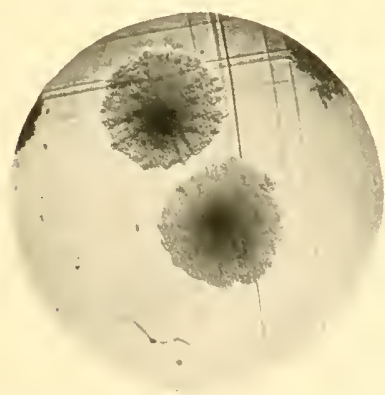

FIG. 153.-Lactobacillus acidophilus.

Surface colony on agar, 4 days, $37^{\circ} \mathrm{C}$., showing differentiation $(\times 8)$.

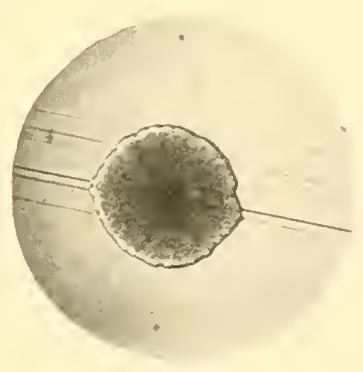

FIa. 154.-Lactobacillus acidophilus.

Surface colony on agar, 4 days, $37^{\circ} \mathrm{C} .(\times 8)$.

Agar Plates.-Great variability in colonial appearance. After 48 hours at $37^{\circ} \mathrm{C}$. one of the commonest is a small, irregularly round, raised, colourless, transparent colony, about $0.5 \mathrm{~mm}$. in diameter, of coarsely frosted-glass structure, with a dull uneven relief-map-like surface and a fimbriate or curled edge; later, differentiation occurs into a thicker darker centre, and a thinner periphery consisting of delicately curled and branched hair-like streamers-not unlike a colony of $\mathrm{Cl}$. tetani. Other forms are feathery colonies, rosette-like colonies, smooth colonies with entire or slightly fimbriate edges, and opaque striated colonies. 
Agar Slope.-24 hours, $37^{\circ} \mathrm{C}$. Poor filiform growth of small transparent granular colonies, 0.25 to $0.5 \mathrm{~mm}$. in diameter. Sometimes after a few days a thick greyish-yellow secondary growth occurs, having a contoured surface, and an edge from which thin translucent branching tufts, looking like sea-anemones, project.

2 per cent. Glucose Agar Shake. -48 hours, $37^{\circ} \mathrm{C}$. Good growth throughout tube of small, spherical, lenticular, or lobulated colonies, $0.25 \mathrm{~mm}$. in diameter, having an entire edge ( $\mathrm{Y}$ type), or of small irregular spherical colonies with a fuzzy, filamentous border ( $\mathrm{X}$ type). The agar is clouded and has an almost milky look. After a week the colonies are $0.5 \mathrm{~mm}$. in diameter, are porcelain-white in colour, and look like colonies of moulds.

Gelatin Stab.-7 days, $22^{\circ} \mathrm{C}$. Very poor greyish-white filiform growth ; no surface growth ; no liquefaction.

Broth. -48 hours, $37^{\circ} \mathrm{C}$. Poor to moderate growth with a very slight, only just perceptible turbidity, and a moderate flocculn-granular deposit disintegrating to some extent on shaking; sometimes the deposit sticks to the walls of the tube.

2 per cent. Glucose Broth.-Growth is better than in plain broth. Sometimes after a week an enormous loose flocculent deposit forms, filling the lowest $1 \mathrm{~cm}$. of the tube; it disintegrates partly on shaking, producing a dense turbidity.

Potato.-No growth.

Horse Blood Agar Plates. - 48 hours, $37^{\circ} \mathrm{C}$. Small discrete colonies, similar to those on agar. No hæmolysis.

Glucose Blood Liver Agar Plates.-48 hours, $37^{\circ} \mathrm{C}$. Flat, dingy colonies with a serrated edge. MacConkey Plate.-Results discrepant. Cruickshank (1931) says that all strains grow on this medium, but this has not been our own experience.

Resistance.-Not particularly resistant. Killed by moist heat at $56^{\circ} \mathrm{C}$. in 30 minutes. Very resistant to acids, living for 1 to 3 days in broth containing $0.5-1.0$ per cent. acetic or lactic acid. Glucose broth cultures at $37^{\circ} \mathrm{C}$. remain viable for about a fortnight.

Metabolism.-Often microaerophilic on first isolation. Grows better under aerobic than anaerobic conditions. Grows slightly or not at all at $20^{\circ} \mathrm{C}$; ; optimum temperature for growth $37^{\circ} \mathrm{C}$. Forms no pigment and no toxin. Does not lyse horse blood. Growth is improved by glucose and by whey, but not by blood serum. Grows best at $\mathrm{pH} 6 \cdot 0$, but will grow even at $\mathrm{pH} 5 \cdot 0$.

Biochemical.-Sugar reactions variable. Produces acid in glucose and in lactose; often in maltose and sucrose; sometimes in mannitol, salicin, and raffinose; less frequently in other sugars. L.M. acid and clot in 24 to 48 hours; the clot is really an acid precipitate, and does not contract; on shaking it breaks up into flocculent masses; the litmus is at first reduced at the bottom of the tube only, but later the decolorization spreads upwards. Indole negative. M.R. positive. V.P. negative. Nitrates reduced slightly or not at all. Catalase very weak positive. $\mathrm{H}_{2} \mathrm{~S}$ negative. $\mathrm{NH}_{3}$ negative.

Antigenic Structure.-Not studied fully. By agglutination numerous groups can be made out, having little affinity with each other. Some group relationship to $L$. bifidus.

Pathogenicity.-Non-pathogenic to man or to laboratory animals.

\section{Lactobacillus odontolyticus I}

Isolation.-Isolated by McIntosh, James, and Lazarus-Barlow in 1922 from carious teeth and from saliva, and called B. acidophilus odontolyticus $I$.

Morphology.-Thin bacillus, 2-3 $\mu$ long by $0.75 \mu$ broad, occurring singly, in pairs, or chains, and in palisades. Non-motile. Gram-positive.

Agar Plates. -48 hours, $37^{\circ} \mathrm{C}$. Small, round, greyish, opaque colonies, $0 \cdot 6-1 \cdot 0 \mathrm{~mm}$. in diameter, with a finely granular appearance and an entire edge. On serum agar the colonies are larger up to $2 \mathrm{~mm}$. in diameter. 
Gelatin Stab.-Grows to the bottom. No liquefaction.

Gelatin Agar Shake.-Deep colonies are roughly biconvex or tam-o'-shanter-shaped.

Broth.-Uniform turbidity; sometimes the growth settles to the bottom.

Resistance.-Not particularly resistant. Killed by $56^{\circ} \mathrm{C}$. in 25 minutes, and by 2 per cent. phenol in $7 \frac{1}{2}$ minutes. Highly resistant to acids; will withstand incubation for 24 hours at $37^{\circ} \mathrm{C}$. in broth of $\mathrm{pH} 3 \cdot 5$. No growth above $\mathrm{pH} 9 \cdot 1-9 \cdot 6$.

Metabolism.-Aerobe and facultative anaerobe.

Biochemical.--Sugar reactions variable. Usually produces acid in glucose, maltose, salicin . and lactose, but not in sucrose, dextrin, dulcitol, or raffinose. L.M. acid and clot in 2 to 3 days ; lower $\frac{2}{3}$ of tube decolorized. Indole negative. Final $\mathrm{pH}$ in glucose broth cultures is about $\mathrm{pH} 2 \cdot 75$. Chief acid formed is malic acid; lactic acid is formed in only a very small amount. Methyl-red positive. Voges-Proskauer negative. Nitrates not reduced. Catalase very slight positive. $\mathrm{NH}_{3}$ negative. Antigenic Structure.-Appears to be fairly homogeneous, and to be closely related to $L$. odontolyticus 11 , and to $L$. acidophilus.

Pathogenicity.-Suspected of being responsible for production of dental caries. Nonpathogenic to laboratory animals.

\section{Lactobacillus odontolyticus II}

solation.--Isolated by McIntosh, James, and Lazarus-Barlow in 1922 from carious teeth and from saliva, and called B. acidophilus odontolyticus $I I$.

Morphology.-Rather short bacillus, 1-2 $\mu$ long by $0.5 \mu$ broad, usually arranged in short chains. Often very pleomorphic, coccal forms being mixed with bacillary forms in the same chain; may closely resemble a streptococcus. Non-motile. Grampositive.

Cultural Reactions.-Similar to odontolyticus $I$.

Resistance and Metabolism.- Similar to odontolyticus $I$.

Biochemical.-Variable sugar reactions. 7 out of 18 strains produced acid in glucose, lactose, and sucrose. L.M. Some strains produce acid and clot, others have no action on it. Indole negative.

Antigenic Structure.-Closely allied to odontolyticus $I$ and to L. acidophilus.

Pathogenicity.-Like odontolyticus $I$.

\section{Lactobacillus brevis}

Synonyms.-Probably L. pentoaceticus; $B$. acidophil-aerogenes.

Isolation.-By Torrey and Rahe in 1915 from the fæces of human beings, sheep and hens. Ilabitat.-Common in fermenting plant and animal products.

Morphology. - Variable morphology. Size given by some authors as $1.5-11.5 \mu$ long by $0.8 \mu$ broad, and by others as $1-4 \mu$ long by $0.6-0.8 \mu$ broad; often arranged in long, curved strings. Non-motile. Gram-positive. Stain uniformly with Loeffler's methylene blue.

Cultural Reactions.-On glucose oleate agar it forms either (1) small, round or navicular, opaque, whitish colonies surrounded by an areola of turbid agar: or (2) tiny, round, translucent, greyish colonies with a finely erose edge; on microscopic examination these appear typically rhizoid. In glucose broth a growth forms adherent to the bottom and sides of the tube; on shaking this gives rise to a dense turbidity.

Resistance.-Highly resistant to acids; will remain alive in a glucose broth culture at $37^{\circ} \mathrm{C}$. for 1 week.

Metabolism.-Microaerophilic. Range of growth $10^{\circ}-45^{\circ} \mathrm{C}$; optimum temperature $30^{\circ}-35{ }^{\circ} \mathrm{C}$. 
Biochemical.-Produces acid and gas in glucose, maltose, arabinose and xylose ; fermentation variable of lactose, sucrose, mannose, raffinose and mannitol.

The gas ratio is $4 \mathrm{H}_{2} / 1 \mathrm{CO}_{2}$ or $6 \mathrm{H}_{2} / \mathrm{ICO}_{2}$. L.M. grows poorly or not at all; some strains produce partial clotting in 2 to 3 weeks.

Antigenic Structure.-Appears to be more homogeneous than the non-gas-forming group.

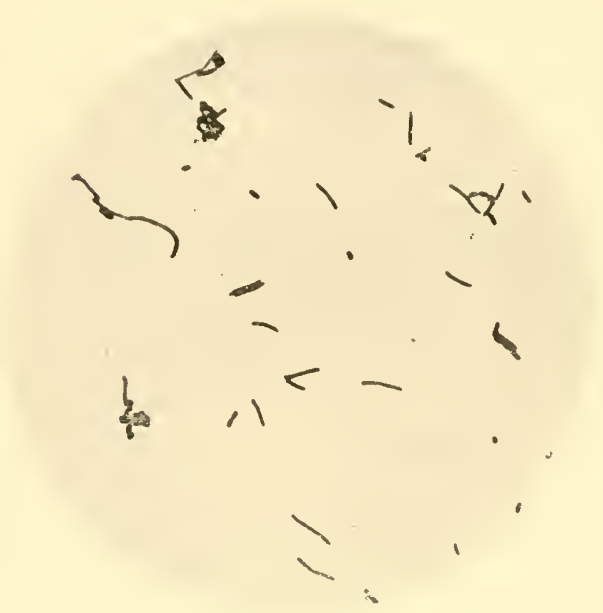

FIG. 155.-Lactobacillus bifidus.

From a glucose agar culture, 7 days, $37^{\circ} \mathrm{C} .(\times 1000)$.

\section{Lactobacillus bifidus I}

Isolation.-Isolated by Tissier in 1900 from the freces of breast-fed infants.

Habitat.-Common in the freces of breast-fed, and much less common in those of bottle-fed infants. Sometimes present in the frees of adults and of animals. In breast-fed infants during the first few weeks of life it may form 99 per cent. of the frecal flora.

Morphology. - In freces it is a delicate baeillus, about $4 \mu$ long and $0 \cdot 7 \mu$ broad, with tapering pointed ends; arranged in pairs end-toend, with the distal ends pointed and the proximal ends swollen; they generally lie parallel to one

another, rarely intertwined. Two or three bacilli often radiate from a single point, forming a $\mathrm{Y}$-shaped structure, simulating branching ; clubbed forms and forms ending in knobs are not uncommon. Often arranged in palisades or Chinese letters. General appearance is not unlike a diphtheroid bacillus. In young cultures bacilli with slightly pointed ends of varying length, arranged singly or in pairs end-to-end, are usual. In older cultures longer clubbed forms, geniculate forms, bifid forms showing false branching, forms ending in knobs, forms with lateral buds, bladder forms, candle-flame forms, and filamentous forms may appear. Both in fæces and in eulture there is a striking pleomorphism. The absence of chain formation is noteworthy. Non-motile. In young cultures staining is fairly uniform, but in older cultures and in fæces irregular, granular, and beaded staining are common. Gram-positive in young cultures; later Gram-negative forms appear. Non-acid-fast.

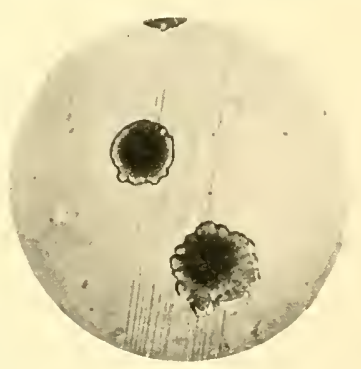

F1a. 156.-Lactobacillus bifidus.

Surface colonies on glucose agar, 6 days, $37^{\circ} \mathrm{C}$. $(\times 8)$.

Agar Plate.-No growth.

Glucose Agar Plate.-48 hours, $37^{\circ} \mathrm{C}$. Small round low convex colonies, $0.5 \mathrm{~mm}$. in diameter, showing under the microscope a delicately granular structure, a brownish opaque centre, a thinner translucent periphery, and a finely crenated edge (Fig. 156).

Glucose Agar Shake Tubes.-3 days, $37^{\circ} \mathrm{C}$. Small, greyish-brown, lenticular or ovoid colonies, 1-2 $\mathrm{mm}$. in diameter, extending up to within about $3 \mathrm{~cm}$. of the surface, where they may form a ring. At first the edge of the colony is entire, but after 4 or 5 days a lateral projection or bud often develops from one of the faces. 
Gelatin Shake.-If incubated at $37^{\circ} \mathrm{C}$. for 24 hours, and then left at room temperature for some days, fine discrete colonies appear; no liquefaction.

Broth. -24 hours, $37^{\circ} \mathrm{C}$. Granular turbidity.

Glucose Broth.-24 hours, $37^{\circ} \mathrm{C}$. More abundant growth; in 3 or 4 days the organisms fall to the bottom of the tube, producing an abundant loose flocculo-granular deposit, easily disintegrated on shaking.

Blood Agar. - White colonies surrounded by a greenish halo of $\alpha$-hæmolysis.

Glucose Blood Liver Agar Plates. - 48 hours, $37^{\circ} \mathrm{C}$. Raised, globular, opaque colonies, 1-3 mm. in diameter, buff to reddish-brown in colour.

Resistance.-Cultures live for about a month at room temperature. Broth cultures are killed by heat at $55^{\circ} \mathrm{C}$. in half an hour, and at $70^{\circ} \mathrm{C}$. in 5 minutes. Is resistant to acids; will withstand $0 \cdot 5-1 \cdot 0$ per cent. acetic or lactic acid in broth for 2 to 3 days.

Metabolism. - Strict anaerobe on first isolation; later it may be grown in air. Very slight or no growth at $20^{\circ} \mathrm{C}$; ; optimum temperature $37^{\circ} \mathrm{C}$. No pigment or toxin formed. $\alpha$-hæmolysis on blood agar. Growth improved by glucose, serum, and blood.

Biochemical.-Sugar reactions rariable. Produces acid in glucose, mannose, maltose, inulin, and generally in lactose, sucrose, salicin, and raffinose; sometimes in mannitol and dextrin; occasionally in dulcitol; not in arabinose, xylose or melezitose. Produces mainly lactic acid, of the inactive variety, and some acetic acid. L.M. grows well and produces an acid clot. Indole negative. Methyl-red positive. Voges-Proskaner negative. Nitrates rery slight reduction, or none at all. Catalase very slight positive. $\mathrm{NH}_{3}$ slight production or none at all.

Antigenic Structure.-Not fully worked out. By agglutination they appear to fall into more than one group. Some relationship to L. acidophilus.

Pathogenicity.-Non-pathogenic to man and laboratory animals.

\section{Lactobacillus bifidus II}

Srovoxyas: Bucteroides bifidus (Eggerth, Group II, 1935); Bacterium bifidum (OrlaJensen ; see Orla-Jensen et. al. 1936).

Differs from L. bifidus I mainly in the following characters. It is found in the intestine of the adult living on a mixed diet, in which it may form quite a high proportion of the total flora. It branches more readily, and continues to Jranch on subculture. It is an obligate anaerobe, and rarely becomes accustomed to aerobic conditions. Deep colonies in tomato agar have an entire, not a filamentous, edge (Weiss and Rettger 1938). Mannose is not usually fermented, but most strains ferment arabinose, xylose, and melezitose. It produces lactic acid, of the dextro-rariety, and about 50 per cent. of acetic acid. It appears to be antigenically more strain-specific.

\section{Lactobacillus bulgaricus}

Synonyms.-Massol's bacillus. Probably identioal with L. caucaszcus.

Isolation. - Isolated by Grigoroff in 1905 from kissélo-mléko, the fermented milk of Bulgaria ; described originally as "Bacillus A."

Habitat.-Found in milk, particularly the fermented milks of Bulgaria, Turkey, Egypt, and Sardinia.

Morphology.-Large rods, $2-20 \mu$ long and about I $\mu$ broad, with parallel sides and slightly rounded ends; arranged singly or in short chains. Non-motile. Gram-positive. Two morphological types are described by White and Avery (1910) in whey. Type A consists of chains of short bacilli with oral or reniform nodules extruding from the cell substance; the bacilli stain uniformly; Type B forms long bacilli arranged singly, having spherical bodies attached to the cell wall, not stemmed nodules as in A; the bacilli show intense granular staining with Loeffler's methylene blue or Neisser's stain. 
Agar Plate.-No growth.

Whey Agar Plate.-Usual type of colony is irregularly round, greyish-white, $0.5-1.5 \mathrm{~mm}$. in diameter, of loose curled structure with a streaming filamentous edge ; microscopically these colonies are typically rhizoid. Sometimes, especially in old laboratory cultures, a rounder, more regular colony is formed, with a smooth or slightly fissured surface and an entire edge.

Whey Agar Shake Tubes.-Deep colonies are lenticular or umbilicated, whitish incolour, and $1 \mathrm{~mm}$. in diameter.

Whey Agar Stab.-Filiform growth, beaded, later with horizontal ramifications; no surface growth; medium is clouded.

Gelatin.-Not liquefied.

2 per cent. Glucose Broth.-Heavy uniform turbidity.

Potato.-No growth.

Resistance.-Killed by moist heat at $60^{\circ} \mathrm{C}$. in 1 hour. Is very resistant to acids; gluoose broth cultures at $37^{\circ} \mathrm{C}$. remain viable for about 6 days.

Metabolism.-Facultative anaerobe; is said by some authors to prefer anaerobic conditions. No growth at $15^{\circ} \mathrm{C}$. ; grows very slightly at $25^{\circ} \mathrm{C}$.; growth is poor under $35^{\circ} \mathrm{C}$.; optimum temperature for growth is $44-45^{\circ} \mathrm{C}$; usually grows at $50^{\circ} \mathrm{C}$. No pigment, toxin, or hæmolysin formed. Is resistant to acids, but grows best in a neutral or slightly alkaline medium. Difficult to cultivate; growth in most media is feeble, and when freshly isolated it will grow only on media containing whey or malt, or in milk.

Biochemical.-Sugar reactions described differently by different authors. Is generally considered to produce acid in glucose, lactose, and sometimes lævulose, but not in maltose, sucrose, mannitol, or raffinose. Lactic acid is the chief acid formed from fermentable carbohydrates. L.MI. acid and coagulation in 18 hours at $37^{\circ} \mathrm{C}$; the clot does not contract. Indole negative. White and Avery's Type A in milk produces $2 \cdot 7-3 \cdot 7$ per cent. of lactic acid of the inactive variety; Type B produces only $1.2-1 \cdot 6$ per cent. of lactic acid, of the lævo-rotatory variety.

Antigenic Structure.-Nothing known.

Pathogenicity.-Non-pathogenic to man and animals.

Grigoroff's Bacillus C.-Isolated from kissélo-mléko, the fermented milk of Bulgaria, by Grigoroff in 1905. Is a streptobacillus forming short rods arranged in chains of four to twenty members. Gram-positive. Culturally it resembles $L$. bulgaricus, but is more heat resistant, requiring an exposure of 1 hour at $70^{\circ} \mathrm{C}$. to kill it. Produces acid in glucose, lævulose, lactose, sucrose, and glycerol, but not in maltose, mannitol, or dulcitol. Produces acid and clot in milk, the acid being inactive lactic acid.

Lactobacillus exilis.--Isolated by Tissier in 1900 from the fæces of children on milk or mixed diets. It is a thin, straight, slender bacillus, arranged singly, in pairs, or in chains of four or five elements; non-pleomorphic. Non-motile. Gram-positive. On agar plates it forms very tiny bluish colonies with entire edges in 48 hours, no bigger than the point of a needle. On glucose agar they may reach $0.5 \mathrm{~mm}$. in diameter. In broth there is a slight filamentous deposit without turbidity. No growth in gelatin. In deep glucose agar small, oval, regular colonies with entire edges are formed throughout the tube. Cultures die out in 10 to 15 days. Grows at $20^{\circ} \mathrm{C}$., but better at $37^{\circ} \mathrm{C}$. Grows both aerobically and anaerobically. Milk is coagulated in 8 to 10 days; there is no retrac. tion of the clot. Non-pathogenic for mice. Differs from $L$. acidophilus in being slender and constant in morphology, in forming small regular colonies in agar shake cultures, and in being less resistant to acid. Cruickshank (1925) regards $L$. exilis as probably the aerobic phase of $L$. bifidus.

The Boas-Oppler Bacillus.-Observed microscopically in the stomach contents of patients with gastric carcinoma by Oppler in 1895, working in Boas' chinic in Berlin. He did not succeed in cultivating it. In stomach contents it occurs as a rather slender bacillus, 
arranged in long threads and zigzags; the bacilli may be so numerous as to fill every space between the other elements in the field. Has since been isolated by Heinemann and Ecker (1916) from the stomach contents of patients with gastritis, gastric ulcer, carcinoma, and pernicious anæmia. It probably occurs in moderate numbers in normal gastric juice. In culture the organism is a large, rather slender bacillus, showing granular staining. Gram-positive. Forms compact colonies as a rule, but colonies with woolly edges have been described. Does not ferment maltose. Produces acid in milk. Is of the low acid-producing type-White and Avery's Type B.

\section{REFERENCES}

BARKER, H. A. and HAAS, V. (1914) J. Bact., 47, 301.

Bertrand, G. and Dechacek, F. (1909) Ann. Inst. Pasteur, 23, 402.

Cannon, P. R. and McNease, B. W. (1923) J. infect. Dis., 32, 175.

Cohendy, II. (1906) C. R. Soc. Biol., 58, 558.

Crowder, J. A. and ANderson, R. J. (1932) J. biol. Chem., 97, 393 ; (1934a) Ibid., 104, $399 ;(1934 b)$ Ibid., 104, 487.

Crowley, N., Downie, A. W., Fulton, F., and Wilson, G. S. (1941) Lancet, ii. 590.

Cruickshank, R. (1925) J. Hyg., Camb., 24, 241 ; (1931) J. Hyg., Camb., 31, 375; (1934) J. Path. Bact., 39, 213.

Curray, H. R., Rogers, L. A., and Whittier, E. O. (1933) J. Bact., 25, 595.

DAY, A. A. and GibBs, W. M. (1928) J. infect. Dis., 43, 97.

DöDERLEIN. (1892) "Das Scheidensekret und seine Bedeutung fūr das Puerperalfieber." Leipzig.

EgGerth, A. H. (1935) J. Bact., 30, 277.

EGGerTh, A. H. and Gagnon, B. H. (1933) J. Bact., 25, 389.

Finkelstein, H. (1900) Dtsch. med. Wschr., 26, 263.

Fred, E. B., Peterson, W. H., and Davenport, A. (1919) J. biol. Chem., 39, 347.

Freudenreich, E. von and Thöni, J. (1903) Zul. Bakt., IIte Abt., 10, 305, 340.

Frost, W. D. and Hankinson, H. (1931) "Lactobacillus acidophilus." Davis-Greene Corp., Milton, Wis.

Gillespie, R. IV. H. and RetTaer, L. F. (1938) J. Bact., 36, 621.

GrigorofF, S. (1905) Rev. med. Suisse rom., 25, 714.

Hadley, F. P., Bunting, R. W., and Delves, E. A. (1930) J. Amer. dent. Ass., 17, 2041.

Harrison, R. W., Zidek, Z. C., and Hemmens, E. S. (1939) J. infect. Dis., 65, 255.

HeINemans, P. G. and Echer, E. E. (1916) J. Bact., 1, 435.

Heinemanx, P. G. and Hefferan, M. (1909) J. infect. Dis., 6, 304.

Howite, B. (1930) J. infect. Dis., 46, 351.

Howit, B. and Meter, M. vas. (1930) J. infect. Dis., 46, 368.

Hunt, G. A. (1933) J. Bact., 26, 341.

Hent, G. A. and Rettaer, L. F. (1930) J. Bact., 20,61.

Kendall, A. I. (1910) J. med. Res., 22, 153.

KENDALL, A. I. and HANer, R. C. (1924a) J. infect. Dis., 35, 77; (1924b) Ibid., 35, 89.

KerN. (1881) Bull. Soc. Nat. Moscou. No. 3.

King, J. W. and Rettger, L. F. (1942) J. Bact., 44, 301.

KLIGLER, I. J. (1915) J. allied dent. Soc., 10, 141, 2S2, 445.

KULP, W. L. (1926) Science, 64, 304; (1927) Ibid., 66, 512 ; (1929) J. Bact., 17, 355.

Lash, A. F. and Kaplax, B. (1926) J. infect. Dis., 38, 333.

Lewis, K. H., Bedeli, M., and RetTGer, L. F. (1940) J. Bact., 40, 309.

Lewis, K. H. and RetTGer, L. F. (1940) J. Bact., 40, 287.

Longsworth, L. G. and MACINNES, D. A. (1935) J. Bact., 29, 595.

IICColluM, E. V. (1941) Nature, 147, 104.

MoIntosh, J., James, W. W., and Lazarus-Barlow, P. (1922) Brit. J.exp. Path., 3, 138 ; (1924) Ibid., 5, 175.

MereshrowskY, S. S. (1905) Zbl. Bakt., 39, 380, 584, 696 ; (1906) Ibid., 40, 118.

MIILER, W. D. (1889) "Die Mikroorganismen der Mundhöhle." G. Thieme, Leipzig.

Morishita, T. (1929) J. Bact., 18, 181.

Moro, E. (1900a) Jb. Kinderheilk., 52, 38; (1900b) Wien. klin. Wschr., 13, 114.

Oppler, B. (1895) Disch. med. Wschr., 21, 73.

ORLA-JENSEN. (1904) Zbl. Bakt., IIte Abt., 13, 161, 291, 428, 514, 604, 687, 753.

Orla-Jensen, S., Orla-Jensen, A. D., and Winther, O. (1936) Zbl. Bakt., IIte Abt., 93, 321.

Pederson, C. S. (1936) J. Bact., 31, 217 ; (1938) Ibid., 35, 95.

Peterson, W. H., Proess, L. M., and Fred, E. B. (1928) J. Bact., 15, 165. 
PETROW, N. P. (1907) Zbl. Bakt., 43, 349.

Rettger, L. F. and Cheplin, H. A. (1921) "A Treatise on the Transformation of the Intestinal Flora with Special Reference to the Implantation of Acidophilus." New Haven.

Retrger, L. F. and Horton, G. D. (1914) Zbl. Bakt., 73, 362.

ROBERTson, W. (1835) "A practical treatise on the human teeth, etc." Quoted from Miller (1889).

RodelLA, A. (1901) Zbl. Bakt., 29, 717.

Rosebury, T., Linton, R. W., and Buchimder, L. (1929) J. Bact., 18, 395.

SoHLIRF, K. (1926) Zbl. Bakt., 97, 104.

Sherman, J. M. and Hodge, H. M. (1940) J. Bact., 40, 11.

Sherman, J. M. and Stark, C. N. (1927) J. Bact., 13, 60.

Sneld, E. E., Strong, F. M., and Peterson, W. H. (1939) J. Bact., 38, 293.

SNYDER, M. L. (1939) J. dent. Res., 18, 497.

Thomas, S. (1928) J. infect. Dis., 43, 218.

Trssier, H. (1900) "Recherches sur la flore intestinale des nourrissons." Paris.

Toмes, J. (1873) Dent. Surg., p. 734. Quoted from Miller. (1889).

Torrey, J. C. and Rahe, A. H. (1915) J. infect. Dis., 17, 437.

Veillon, A. and Zuber, A. (1898) Arch. Méd. exp., 10, 517.

Weinstein, L. and Rettaer, L. F. (1932) J. Bact., 24, 1.

Weiss, J. E. and Rettger, L. F. (1934) J. Bact., 28, 501 ; (1938) J. infect. Dis., 62, 115.

White, B. and Avery, O. T. (1910) Zbl. Bakt., IIte Abt., 25, 161. 
CHAPTER 32

PASTEURELLA

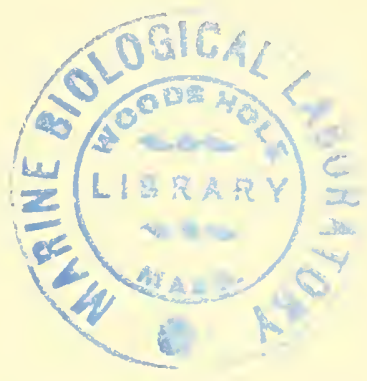

\section{Definition.-Pasteurella.}

Small, Gram-negative, ovoid bacilli, showing bipolar staining. Aerobio and facultatively anaerobic. Powers of carbohydrate fermentation relatively slight ; no gas produced. Gelatin not liquefied. Parasites in man and animals, producing characteristic infections.

The type species is Pasteurella aviseptica.

Isolation.--The first member of this group was isolated by Kitt in 1878 from an epidemic disease affecting wild hogs and deer. Similar organisms have been isolated from several species of animals and birds suffering from a disease known as hæmorrhagic septicæmia. It has become customary to give a specific name to each organism, corresponding to the animal from which it was derived; thus we have Past. aviseptica from fowls, Past. lepiseptica from rabbits, Past. suiseptica from pigs, Past. vituliseptica from calves, Past. oviseptica from sheep, Past. boviseptica from cattle, and Past. muriseptica from mice (not to be confused with Erysipelothrix muriseptica). Such a nomenclature is purely arbitrary, and is clearly unjustifiable from the sytenratic point of view. The differentiation of species within this group, as in all others, must depend on the detailed study of an adequate sample of strains. Such data as are available do not suggest that the strains from the various animal species, which are liable to natural pasteurellosis, are themselves specifically distinct. In the description which follows we have taken Past. aviseptica as a type of the hæmorrhagic septicæmia group; but, as we point out in a later section, it is doubtful whether this name will survive as a designation for a distinct species.

Malassez and Vignal (1883) were apparently the first to describe pseudotuberculosis in the guinea-pig. Several workers recorded the finding of a bacillus in this disease (see Chapter 73), chief amongst whom was Pfeiffer (1890), who named it $B$. pseudotuberculosis. It is not to be confused with Corynebacterium pseudotuberculosis ovis, described by Preisz and Nocard as the cause of pseudotuberculosis in sheep (see Chapter 17), or with Corynebacterium pseudotuberculosis murium, described by Kutscher (1894) and Bongert (1901) as the cause of pseudotuberculosis in mice (see Chapter 17).

The plague bacillus, Past. pestis, was isolated almost simultaneously by Kitasato (1894) and by Yersin (1894), from buman patients suffering from plague.

These three organisms resemble each other in so many characters, and appear to be so closely related, that they may well be considered as falling within a single genus.

Morphology and Staining.-All the members of the group are small, ovoid bacilli, with convex sides and rounded ends; there is no characteristic arrange- 
ment; they are generally disposed singly, in pairs, in short chains, or in small groups. The most striking feature is their pleomorphism, which is most noticeable with Past. pestis, least with the hæmorrhagic septicæmia bacilli. Though well marked on ordinary media, it is best brought out by cultivation on nutrient agar containing 3 per cent. sodium chloride. The growth on this medium is poor; microscopically, as well as the usual ovoid or short bacillary forms, there will be found shadow forms, filamentous snake-like forms, club forms, large yeast-like globules, and other irregular forms. Past. pestis and Past. pseudotuberculosis are larger and more ovoid than Past. aviseptica, but their shape varies considerably with the medium on which they are grown. Generally speaking, the bacilli of all three species tend to be ovoid and to show bipolar staining when taken from smooth colonies, and to be more bacillary, filamentous, or pleomorphic, without bipolar staining, when taken from rough colonies, but many exceptions occur.

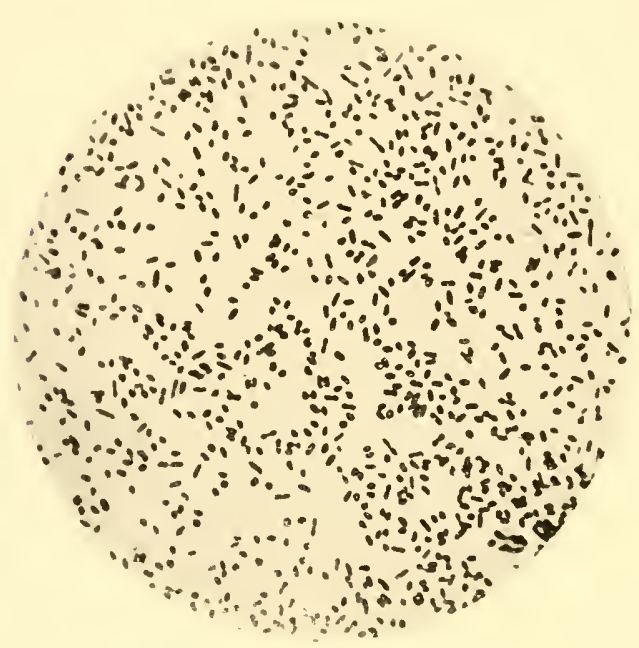

Fig. 157.-Pasteurella pestis.

From an agar culture, 3 days, $37^{\circ} \mathrm{C} .(\times 1000)$.

Past. pestis and Past. aviseptica are non-motile. Past. pseudotuberculosis, on the other hand, though usually non-motile in cultures incubated at $37^{\circ} \mathrm{C}$., is often motile in broth cultures grown for 18 hours at $20-22^{\circ} \mathrm{C}$. The possession of motility by this organism is of considerable value in differentiating it from Past. pestis, with which it may easily be confused (Arkwright 1927). The value of this test, however, is limited. Weitzenberg (1935), for example, made observations on 25 strains of pseudotuberculosis, and found that, though all were motile at room temperature, some were very poorly so, and had to be examined repeatedly before their motility could be definitely established. A negative result, therefore, on a single examination cannot be regarded as conclusive. Both Levinthal (1930) and Weitzenberg (1935) have demonstrated the presence of flagella on Past. pseudotuberculosis. The usual number appears to be 1-2, arranged at one or both poles.

In the animal body Past. pestis may form a true capsule with a definite edge (Kitasato 1894). More often it is surrounded by a gelatinous envelope of ill-defined capsular material, which is soluble in weak alkalies (Rowland 1914a). The same envelope may be formed in artificial media, especially in 10 per cent. inactivated horse serum broth incubated at $36^{\circ} \mathrm{C}$. According to Schütze (1932a), it develops best at $37^{\circ} \mathrm{C}$., poorly at $26^{\circ} \mathrm{C}$, and not at all at $20^{\circ} \mathrm{C}$. By serological methods it can be shown to contain a special antigen distinct from that present in the body of the organism. Past. aviseptica may show an indefinite capsule in the animal body. From this capsule Hoffenreich (1928) has extracted a polysaccharide, which, though unable to give rise to precipitins on injection into rabbits, yet reacts to a high titre with a specific precipitating serum. Priestley $(1936 a, c)$ has shown that an envelope substance, apparently similar to that formed by 
the plague bacillus, can be demonstrated in cultures of virulent, but not of avirulent, strains of Pasteurella septica. It reaches its maximum development after 24 hours at $37^{\circ} \mathrm{C}$, and then gradually disappears. Temperatures above and below $37^{\circ} \mathrm{C}$. tend to inhibit its formation. Antigenically it is distinct from the somatic substances.

Bipolar staining is very common and gives to the ovoid bacilli a characteristic appearance. The rod forms often stain irregularly, appearing as granular or barred forms. The reaction to Gram's stain is uniformly negative.

Cultural Characteristics.-Moderate growth occurs on the ordinary media. Past. pseudotuberculosis and Past. aviseptica grow fairly rapidly, giving a confluent growth on agar after 24 hours; Past. pestis, on the other hand, develops more slowly, and gives a less abundant grow th, often barely noticeable after this time. The agar colonies of Past. pestis and Past. pseudotuberculosis resemble each other in many respects, and are characterized by the effuse, clear or slightly granular, peripheral extension that occurs after 2 to 4 days' growth (Figs. 158, 159); the

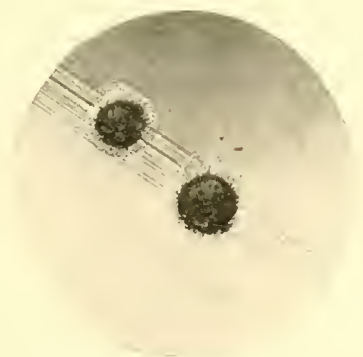

FIG. 158.-Pasteurella pestis.

Surface colonies on agar. 3 days, $37^{\circ} \mathrm{C}$., showing differentiation, and effuse edge $(x s)$.

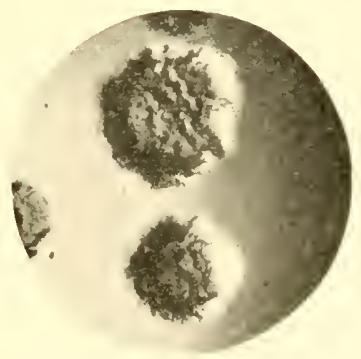

FIG. 1.59.-Pasteurella pseudo. tubercutosis.

Surface colonies on agar, 24 hours, $37^{\circ} \mathrm{C}$., showing irregular granular surface and effuse edge $(\times 8)$.

colonies of Past. pseudotuberculosis, however, develop more rapilly, and are larger and more gramular. With further incubation the central raiserl part of the colony may assume a ringed or draughtsman-like appearance. On moist agar the colonies, especially of Past. pestis, are of viscous consistency, and tend to adhere to the medium (Eastwood and Griffith 1914).

Colonial variants have been described for each member. From Past. pestis Gotschlich (1912) obtained round, slimy, undifferentiated colonies, which were poorly agglutinable and proved avirulent for rats; they subsequently reverted to the normal virulent type. Variant types, including smooth compact, smallfringed, large irregular, and "sunflower" types, have been described by a number of other workers (see Bessonowa, Sémikoz, and Kotelnikow 1927, Pirie 1929, Burgess 1930, Bessonowa and Lenskaja 1931, Bhatnagar 1940a), but the exact form produced seems to be so influenced by environmental factors that it is probably better not to refer to these types by the terms rough and smooth. Kakehi (1915-16) described two variants of Past. pseudotuberculosis ; A was almost transparent and had a bluish, glimmering appearance ; B was greyish-white and opaque. A rendered broth turbid, B did not. Zlatogoroff and Moghilewskaja $(1928 a, b)$ 
have similarly encountered two variants. On first isolation from the animal body, the organisms formed smooth colonies, consisting of short bacilli, which often showed well-marked hipolar staining; on plates seeded from old broth cultures, rough colonies developed, with a dull wrinkled surface and a lobate or crenated edge ; morphologically these colonies consisted of larger, often longer, bacilli, which did not exhibit bipolar staining. These two variants likewise differed in their biochemical and serological characters (see below). In cultures of Past. lepiseptica De Kruif $(1921,1922 a, b, 1923)$ found two different types; Type D grew diffusely in broth, formed rather opaque, fluorescent colonies on serum agar, and was highly virulent for rabbits; Type $\mathrm{G}$ gave a granular deposit in broth, formed translucent bluish colonies with little fluorescence, and was completely avirulent. The D type gave rise to $G$ variants, but the $G$ type did not revert to $D$. A mucoid variant of intermediate virulence has been described for Past. lepiseptica by Webster and Burn (1926), while colonies similar in many respects to the $D$ and $G$ types have heen recorded for Past. aviseptica by Anderson, Coombes, and Mallick (1929), Morch and Krogh-Lund (1930), and Hughes (1930).

In broth Past. pestis causes little or no turbidity, but gives rise to a deposit of fine flocculi ; with Past. pseudotuberculosis there is no turbidity but a deposit of coarse flocculi ; with Past. aviseptica there is a uniform turbidity with a powdery deposit (Zlatogoroff 1904). These differences are not entirely constant. A noteworthy feature is that whereas growth of all three organisms on an agar slope reaches its maximum in 2 to 4 days, in broth growth continues for 7 to 10 days, or, at room temperature, for several weeks. An old broth culture is almost clear; there is a heavy deposit which is difficult or impossible to disintegrate, and there may be a surface pellicle and ring; in cultures of Past. aviseptica the deposit is viscous; in cultures of the other two it is usually floccular or membranous.

If Past. pestis is seeded into broth covered with melted butter or oil, and the flask is allowed to remain undisturbed

FiG. 160 .

Pasteurella muriseptica.

Surface colony of smooth type on agar, 24 hours. $37^{\circ} \mathrm{C} .(\times 8)$. in the incubator, growth occurs in the form of stalactites depending from the under-surface of the droplets. This property is not peculiar to the plague bacillus, nor is it possessed by all strains of that species. Past. pseudotuberculosis may likewise give a stalactite growth in broth.

None of the members liquefies gelatin; in a stab culture there is a surface layer, and a filiform growth extending to the bottom of the tube. In the case of Past. pestis little feathery projections sometimes occur from the stab into the surrounding gelatin.

Potato is not a suitable medium. Past. pestis and Past. aviseptica give little or no growth (Kitasato 1894, Magnusson 1914, Tanaka 1926). Past. pseudotuberculosis either gives no growth at all or else forms a thin, yellowish layer, which may later turn brown (Preisz 1894).

In bile salt media-MacConkey's liquid or solid medium-Past. pestis and Past. pseudotuberculosis give a slight but definite growth, which disappears in the course of 2 or 3 days, owing presumably to autolysis of the bacilli ; Past. aviseptica fails entirely to grow.

Resistance.-None of the members is highly resistant to inimical agencies. Broth cultures are killed by heat at $55^{\circ} \mathrm{C}$, and by 0.5 per cent. phenol, within 15 
minutes. Agar plate cultures exposed to sunlight are sterilized in 3 or 4 hours (Ogata 1897). Dried on threads and kept at room temperature in a desiccator the bacilli survive for not more than a few days. In bubo juice, dried on a cover slip, Past. pestis dies in under 4 days (Kitasato 1894); but in dry flca fæces kept at room temperature $\left(66^{\circ}\right.$ F.) it may survive for 5 weeks (Eskey and Haas 1940). The blood of animals dying from hæmorrhagic septicæmia remains virulent in the dried state for about 3 weeks; blood which is allowed to putrefy in a glass tube may remain virulent for 100 days (Ostertag 1908). Bacilli in cultures or infected organs, kept in the ice-chest, may survive for months. According to Francis (1932), Past. pestis, in an infected guinea-pig's spleen kept in pure nentral glycerol at $-15^{\circ} \mathrm{C}$., may retain its virulence for years.

Metabolism.-The bacilli have a wide range of growth. Past. pestis and Past. pseudotuberculosis can grow to some extent at very low temperatures-according to Trumansky and his colleagues (1935) even at $0^{\circ} \mathrm{C}$. Their upper limit of growth is about $43^{\circ} \mathrm{C}$. Different observers disagrec about their optimum temperature for growth, but $30^{\circ} \mathrm{C}$. is generally considered to be the most favourable. Growth, however, at both $24^{\circ} \mathrm{C}$. and $37^{\circ} \mathrm{C}$. is often nearly as good as at $30^{\circ} \mathrm{C}$. According to Sokhey and Habbu (1943), Past. pestis grows in nutrient broth between - $2^{\circ}$ and $+45^{\circ} \mathrm{C}$. ; at 27 to $28^{\circ} \mathrm{C}$. - its optimum temperature-growth is five times as rapid as at $37^{\circ} \mathrm{C}$. Pasteurella septica has a rather narrower range of growth, and develops best at $37^{\circ} \mathrm{C}$.

All the members are aerobes and facultative anaerobes. Working with Past. lepiseptica, Webster $(1924 a, 1925)$ and Webster and Baudisch (1925) found that the $\mathrm{D}$ or smooth variant would not grow in plain broth unless large numbers of organisms were introduced-about 100,000 per $\mathrm{ml}$. ; whereas the G or rough variant grew if only a few organisms were introduced. But if a trace of rabbit blood, or an iron compound with strongly catalytic properties, was added to the medium, or the partial pressure of oxygen was lowered mechanically, growth of the D rariant occurred with the smallest inoculum. Schütze and Hassanein (1929) made similar olservations on Past. pestis. They found that the difficulty of obtaining growth from small inocula on agar plates.could be overcome by the addition of a small amount of blood or of 0.025 per cent. sodium sulphite to the medium, or by incubation under anaerobic conditions. Their conclusion that the organisms were sensitive to oxygen was confirmed by Wright (1934), who found that plague bacilli were destroyed fairly rapidly on the surface of agar plates if exposed at $37^{\circ} \mathrm{C}$. to a partial pressure of oxygen exceeding 1 per cent. Aerobic surface cultivation was, however, successful if $0 \cdot 1$ per cent. of blood, 10 per cent. of serum, or $0 \cdot 05$ per cent. of sodium sulphite was added to the agar. Under these conditions growth was more profuse aerobically than anaerobically. For the growth of Past. pestis Rao (1939) found that three amino-acids, proline, phenylalanine, and cystine, were required; glucose and lactate were the two best sources of carbon and energy with which to supplement media. Growth was stimulated by glycine, hrmatin, cozymase, thiamin and nicotinic acid; hæmatin appeared to be essential for the aerobic growth of plague bacilli when these were present in only small numbers.

On horse blood agar plates there is no hæmolysis around the colonies, but the whole plate is slightly cleared and browned. When a suspension of bacilli is incubated with sheep's red cells there is again no hæmolysis, but the oxyhæmoglobin is reduced to hæmoglobin. In this respect all members behave alike.

No true exotoxm is formed (Hadley 1918). Old broth cultures are, however, 
very toxic to animals, suggesting that endotoxins are liberated by the autolysis of the bacilli.

Biochemical Reactions.-(MacConkey 1908, Vourloud 1908, Magnusson 1914, Kakehi 1915-16, Besemer 1917, Brooks and Rliorles 1923, Pons 1925, Colas-Belcour 1926, Csontos 1926, Tanaka 1926, Morch and Krogh-Lund 1931.)

Past. pestis and Past. pseudotuberculosis produce acid, without gas, in glucose, maltose, mannitol, and salicin within 14 days; salicin is not usually fermented for about 10 days. Some strains of Past. pestis fail to attack maltose, and some strains of Past. pseudotuberculosis ferment sucrose. Past. pseudotuberculosis is stated (Colas-Belcour 1926, Zlatogoroff and Moghilewskaja 1928a) to form acid from glycerol, but so also do some strains of Past. pestis (Bessonowa 1928). Bessonowa (1930), and Russo (1939), say that Past. pseudotuberculosis ferments rhamnose, while Past. pestis does not; Kauffmann (1932), however, says that Past. pestis may ferment rhamnose, though its reaction in this sugar is inconstant. Past. aviseptica produces acid in glucose, mannitol and sucrose; some types also produce acid in maltose. Tanaka (1926) found that Past. bubaliseptica (from buffaloes) formed acid in lactose and clotted milk; the same observation was made by Magnusson (1914) for a reindeer strain, and by Besemer (1917) for a calf strain of Pasteurella. We have encountered a strain of Past. lepiseptica that fermented lactose, and to a less extent salicin. Newsom and Cross (1932) found differences in the fermentation of arabinose, dulcitol, and glycerol; and Rosenbusch and Merchant (1939) were able on the hasis of fermentation of xylose, arabinose, or both, to divide 114 strains of Past. septica of diverse origin into three sub-groups, each of which showed a considerable degree of antigenic homogeneity. In practice great care is required in determining the fermentation reactions, since growth in sugar peptone water media is very poor.

In plain broth Past. pestis produces alkali ; the maximum production is not reached for 6 to 8 weeks (Bannerman 1908) in a litre flask of medium. In peptone water containing 0.5 per cent. glucose the hydrogen-ion concentration reached in 7 days is $\mathrm{pH} 4.6-4.9$ for Past. pestis, $\mathrm{pH} 4.6-4.8$ for Past. pseudotuberculosis, and $\mathrm{pH}$ 5.6-6.1 for Past, aviseptica (Otten 1926). That is to say the first two give a positive, the last a negative methyl-red reaction. But in peptone water containing 0.05 per cent. glucose the final hydrogen-ion concentration is $\mathrm{pH}$ 5.1-5.5 for Past. pestis, 7.0-7.3 for Past. pseudotuberculosis, and $\mathrm{pH} 5 \cdot 8-6 \cdot 1$ for Past. aviseptica. After exhausting the sugar Past. pseudotuberculosis would therefore appear to produce alkali more rapidly than the other two. According to Zlatogoroff and Moghilewskaja (1928a), cultures of the rough variant form of Past. pseudotuberculosis return to neutral more rapidly than those of the smooth variant form. It is not yet certain whether the different end-reactions in glucose broth can be used for differentiating between Past. pestis and Past. pseudotuberculosis; according to d'Aunoy (1923) the results obtained depend to some extent on the initial H-ion concentration of the medium.

Past. aviseptica forms indole and gives a negative M.R. reaction ; Past. pestis and Past. pseudotuberculosis form no indole, and give a positive M.R. reaction. Past. pestis and Past. aviseptica have no action on litmus milk; Past. pseudotuberculosis turns it slightly alkaline. All members reduce nitrates, form ammonia and a small amount of $\mathrm{H}_{2} \mathrm{~S}$, and give a positive catalase test. According to our observations Past. pestis does not reduce methylene blue, whereas Past. aviseptica and Past. pseudotuberculosis are able to do so. In confirmation of this Iwanowsky and Sassy- 
kina (1930) have found that saline suspensions of Past. pseudotuberculosis reduce Schardinger's reagent (formolized M.B. solution) much more rapidly than those of Past. pestis. Past. aviseptica is stated to form phenol in peptone water (BunzlFedern 1891).

Antigenic Structure.-Antigenically all the members of the group are closely related. An agglutinating serum prepared against any one of them is said to act not only on its homologous strain but to a less extent on the heterologous strains. It is stated that the relationship between Past. pestis and Past. pseudotuberculosis is very close, and that it is often impossible to distinguish between them by direct agglutination. Past. aviseptica is not so closely allied, but nevertheless it may give a definite group reaction with sera prepared against either of the other members. This, however, has not been our experience; using low titre sera we have had no difficulty in distinguishing between the three species by direct agglutination.

According to Schütze (1932a), there are only two antigens in the plague bacillus, one corresponding to the envelope and the other to the somatic substance. The envelope antigen is developed best in cultures grown at $37^{\circ} \mathrm{C}$. and is heat-labile ; the somatic antigen is formed as well at $20^{\circ} \mathrm{C}$. as at $37^{\circ} \mathrm{C}$. and is beat-stable. Both antigens may be present in avirulent as well as in virulent forms, though variants lacking the power to form the envelope substance have been described (Schütze 1939). Wats, Wagle, and Puduval (1939) find that organisms provided with an envelope agglutinate more rapidly than those containing only the somatic antigen; they differ also from these in forming larger flakes of varying size, and a voluminous deposit, which is easily homogenized by shaking. The antigenic structure of the flagellated Past. pseudotuberculosis is more complex. Arkwright (1927) has shown that agar cultures incubated for $2 t$ hours at 18 to $22^{\circ} \mathrm{C}$. contain a heat-labile $\mathrm{H}$, and a heat-stable $\mathrm{O}$ antigen. The $\mathrm{H}$ antigen is apparently associated with the flagella, and is destroyed by boiling for half an hour, though not by exposure to $56^{\circ} \mathrm{C}$. for a similar length of time; it agglutinates in the form of loose flocculi ; the $\mathrm{O}$ antigen is apparently associated with the bacterial bodies, is not destroyed by boiling for 1 hour, and agglutinates in the form of granules. Further work by Schuitze (1928, 1932b), Zlatogoroff and Moghilewskaja (1928a), Kauffmann (1932), and Bhatnagar (1940b) seems to show that the flagellar antigen, which is formed only in cultures grown at $26^{\circ} \mathrm{C}$. or lower, is common to all strains within the species, but that the somatic antigen is more complex. One somatic antigen is shared with the plague bacillus; another is closely related to the $O$ antigen present in Salm. paratyphi $B$ and related organisms of the Salmonella group. In addition, there appear to be one or more type-specific antigens that characterize individual strains or groups of strains. According to Bhatnagar (1940b) a serum prepared against the plague bacillus will agglutinate Past. pseudotuberculosis by virtue of the antibody to their common somatic antigen, but a serum prepared against Past. pseudotuberculosis will not agglutinate plague bacilli grown at $37^{\circ} \mathrm{C}$. because the somatic antigen is protected by the envelope substance.

The antigenic structure of the members of the Pasteurella septica group of organisms is still very uncertain. With regard to Past. muriseptica, we have found two distinct types, distinguishable either by direct agglntination or by absorption of agglutinins. The two types were further distinguished by the fact that one type fermented maltose and the other did not. Using the complementfixation reaction, Lal (1927) found that there was a cross-reaction between different 
members of the hæmorrhagic septicæmia group, but that differences in the degree of fixation were usually sufficient to enable strains coming from one animal source to be separated from those from another. Cornelius (1929), by means of the agglutinin-absorption test, classified 17 out of 26 strains of Pasteurella of diverse origin into 4 groups; the remaining 9 strains defied classification. Very similar results have been recorded by Yusef (1935) using the precipitation test. Priestley's $(1936 a, c)$ observations suggest that Past. septica resembles Past. pestis in forming a heat-labile envelope antigen and a heat-stable somatic antigen. It differs from the plague bacillus in that the envelope antigen appears to be present only in virulent strains, though this requires confirmation; moreover, the somatic antigen is not the same in all members of the species. Hoffenreich (1928) reported the isolation of a polysaccharide hapten that reacted to high titre with a specific precipitating serum. By means of the trichloracetic acid technique, Pirosky $(1938 a, c, d)$ has now extracted from smooth and rough variants four different glycolipoid antigens; judged by cross-precipitation tests one of these antigens was found to be related to the $\mathrm{Vi}$ antigen of the typhoid bacillus, and another to the $O$ antigen shared by Salm. typhi and Salm. enteritidis. Unfortunately, the relation between Pirosky's glycolipoid antigens and the envelope and capsular antigens is still far from clear.

Immune sera can be prepared for all the members by injection of rabbits or horses with living or dead organisms (Haffkine 1905). If living organisms are used, a weakly virulent culture should be chosen for the first few injections. The sera have prophylactic, and to a less extent curative, properties for laboratory animals (Yersin et al. 1895, Chamberland and Jouan 1906). Schütze's (1932c, 1934) observations suggest that the protect:ve power of a plague vaccine is largely dependent on the presence of the envelope antigen. Working with rats he found that a plague culture grown at $37^{\circ}$ C.- a temperature at which the envelope antigen is well developed-was considerably more potent for purposes of immunization than one grown at $26^{\circ} \mathrm{C}$ - - a temperature which is less favourable for the formation of the capsular material. Moreover, heating of a culture to $100^{\circ} \mathrm{C}$. for 15 minutes destroyed the envelope substance and rendered the vaccine useless, while exposure to a temperature of $56^{\circ} \mathrm{C}$. for 30 minutes had no such deleterious effect. Sokhey and Maurice (1935), however, working with mice, found that cultures grown at $25^{\circ} \mathrm{C}$. were as effective as those grown at $37^{\circ} \mathrm{C}$. According to Schütze (1939), the explanation of this discrepancy lies in the relative importance of the two antigens for the type of animal under study. In the protection of rats and guineapigs a large part is played by the envelope antigen, but in mice this antigen appears to be of less importance.

It has been stated that it is possible, by the use of a pseudotuberculosis vaccine, to immunize rats and guinea-pigs against infection with virulent plague bacilli (MacConkey 1908, McCoy 1911, Report 1915). Zlatogoroff (1904), on the other hand, was unable to immunize animals against plague with a pseudotuberculosis antigen, or against pseudotuberculosis with a pestis antigen. He also found that a specific anti-plague serum would protect guinea-pigs against plague, but not against pseudotuberculosis. Boquet (1937) was able, by the use of avirulent bacilli, to protect guinea-pigs against infection with virulent Pust. pseudotuberculosis.

As regards the hæmorrhagic septicæmic group, most workers agree that a strain from one animal can be used to vaccinate against infection with strains from other animals (Chamberland and Jouan 1906, Magnusson 1914). A fowl-cholera 
strain, Past. aviseptica, for example, will protect mice against infection with a strain from swine plague or pleuropneumonia of calves. Likewise an immune serum prepared against one strain is said to protect against all other strains. Schirop (1908), however, obtained evidence of varietal differences in the species; according to him protection cas be realized with certainty only by the use of inonovalent sera (see also Priestley 1936b.)

Pathogenicity.-Past. pestis and Past. pseudotuberculosis cause disease in rodents ; Past. pestis is also pathogenic for man. The hæmorrhagic septicæmia group is pathogenic for a large number of animals and birds, but only very exceptionally for man (see Chapter 73).

The virulence of all three organisms is subject to considerable variation, and appears to be determined, at least in part, by the particular variant that has gained the ascendancy. The $\mathrm{D}$ variant of Past. lepiseptica is highly virulent, the $\mathrm{G}$ variant comparatively avirulent. There is evidence that the smooth type of Past. pseudotuberculosis is more virulent for guinea-pigs than the rough type (Zlatogoroff and Moghilewskaja 1928a), though, according to Boquet (1937), this relationship is fortuitous. There are several reports on variations in virulence of the plague bacillus occurring under natural and experimental conditions. (See Yersin, Calmette, and Borrel 1895, Report 1906, McCoy 1911, Rowland 1914b, Eberson 1917, Pirie 1929, Burgess 1930.) Many of the statements are conflicting, rendering it impossible at the moment to draw any definite conclusions on the relation of virulence to colonial appearance or antigenic structure. Further study of this problem by careful quantitative methods is required. For maintaining the virulence of the plague bacillus, Sokhey (1939b) recommends growing the organisins on 5 per cent. ral,bit blood agar for 4 days at $26^{\circ}-32^{\circ} \mathrm{C}$., sealing the tubes in the flame, and storing them at $4^{\circ} \mathrm{C}$.

\section{Experimental Reproduction of Plague in Animals.}

Bubonic plague can be reproduced in rodents and monkeys by experimental inoculation. Dogs, cats, pigs, cattle, sheep, goats, and horses are difficult to infect; birds, with the exception of sparrows, are completely resistant. The disease is said to occur naturally in camels; but these animals are refractory to experimental inoculation (Zabolotny 1923). Even the rodents show great variation in susceptibility to infection. The less resistant members succumb rapidly, whereas the more resistant ones either fail to develop the disease, or else develop a subacute or chronic type. Spencer (1921) found in America that about 30 per cent. of the rats from a plague-free district were resistant to subcutaneous inoculation of Past. pestis. According to Sokhey (1939a), the white mouse is the most susceptible laboratory animal, but Otten (1938) on the contrary maintains that the guinea-pig, in spite of its greater weight, is more susceptible than the mouse.

Pneumonic plague has been produced in rats and also in marmots (Spermophilus citellus) by causing them to inhale cultures of Past. pestis (Eberson and Wu Lien Teh 1917, Wı Lien Teh and Eberson 1917).

Rats. Subculaneous injection of a very small number of virulent plague bacilli leads to death in 2 to 8 days. Post mortem, there is necrosis and cedema at the site of inoculation; the reginal lymph glands are swollen and surrounded by a hamorrhagic infiltration of the subcutaneous tissue. Glands in other parts of the body are often eongested and swollen; the spleen may be enlarged and dark red; the liver and lungs are hyperæmic, and sometimes a pleural exudate is seen. Baeilli are found in large numbers in the local 
lesion and the nearest glands; they are usually irregular in size and shape, exhibit bipolar staining, and may show involution forms. They are often present in the spleen and in the blood. If the animal lives for a week, small, irregular, necrotic foci may be observed in the spleen and liver.

The German Plague Commission (Report 1899) had apparently no difficulty in infecting rats by the mouth, but other workers have not been so successful. They fed the animals with a drop of plague culture or with the cadaver of a rat that had died of plague. Death followed uniformly in 2 to 3 days. Post mortem, three types of lesion were found. (a) Most commonly enlargement and congestion of the submaxillary and suprahyoid glands, with the general picture of septicæmia. (b) Less often primary infection of the stomach and intestine, numerous punctiform hæmorrhages round the pylorus, swelling and hæmorrhagic infiltration of the lymph follicles and mesenteric glands, which were often quite large, and contained innumerable plague bacilli. (c) Quite frequently an aspiration pneumonia; the lungs showed inflammatory foci of varying size, containing large numbers of plague bacilli; the spleen was enlarged, and the liver hyperæmic.

The English Plague Commission had less success with feeding. Of the wild rats of Bombay only 38 per cent. were found to be susceptible when fed on the carcasses of dead plague rats. In the Punjab the proportion was nearly 70 per cent. The lesions found in rats infected by feeding were of a similar type to those in rats infected naturally. Two striking differences, however, were present. (a) In naturally infected plague rats the bubo is in the neck; a mesenteric bubo was not encountered in 5,000 examinations: in the case of fed rats the bubo is generally in the mesentery. (b) In naturally infected rats the stomach and intestines show no marked pathological changes: in rats infected by feeding wellmarked lesions are found in the intestines - hæmorrhages in the stomach wall 3 per cent., congestion of intestines 27 per cent., enlargement of Peyer's patches 31 per cent.

The nasal mucosa and conjunctiva are favourable spots for inoculation in rats (Report 1899). A trace of infective material smeared over the conjunctiva proves fatal in 3 to 4 days. Post mortem, there is swelling of the cervical lymph glands, enlargement of the spleen, and frequently numerous hæmorrhages in the stomach and jejunum. The appearances are in fact similar to those of an animal dying after oral infection. Contamination of the nasal mucosa is frequently followed by an inhalation pneumonia.

The English Plague Commission (Report 1912, p. 287) were able to reproduce the lesions of chronic or-as they prefer to call it - of resolving plague in rats by inoculating large numbers of animals with small doses, and retaining the survivors for 3 weeks after inoculation. The chief lesions found were chronic buboes, necrotic areas in the spleen, and chronic abscesses in the spleen or more rarely the liver.

MICE.-The mouse reacts to inoculation in much the same way as the rat. After subcutaneous inoculation the septicemia is very marked; the blood and internal organs swarm with bacilli. Infection can be accomplished by feeding, if a sufficiently large dose of a virulent culture is used.

Guinea-Pigs.-Guinea-pigs are highly susceptible to plague, dying in 2 to 5 days after subcutaneous injection of a pure culture. Post mortem, there is a necrotic focus at the site of injection surrounded by intense congestion and cedema; the regional lymph glands are swollen and embedded in a bloody oedema; their interior is soft and necrotic. There is enlargement and congestion of the spleen, which is often studded with miliary, soft, grey nodules up to $1 \mathrm{~mm}$. in diameter, sometimes projecting above its surface, and containing large numbers of bacilli. The suprarenals may be congested (Yersin 1894). The liver may be peppered with tiny neerotic foci, and occasional small necrotic nodules are visible in the lungs. Sometimes there is a pleural effusion. Guinea-pigs can be infected by the cutaneous route. If the plague material is rubbed on the shaven skin of the abdomen, an inflammatory reaction appears in the neighbourhood, marked by a slight reddening and the formation of umbilicated pustules in which plague bacilli are present (Dieudonné and Otto 1912). After a few days the regional glands swell and death occurs in 4 to 5 days. The post-mortem signs are similar to those after subcutaneous inoculation. 
Intraperitoneal injection is fatal in 24 to 36 hours. Post mortem, there is a rich fibrinous exudate, containing enormous quantities of plague bacilli. Infection by the mouth, nasal mucosa, and vaginal mucosa is not constant.

The animals are very sensitive to conjunctival infection. By inhalation or intratracheal inoculation it is possible to set up an acute primary pneumonia. Symptoms appear in 48 hours, and death occurs in about 72 hours. At necropsy there is a confluent bronchopneumonia with œdema or commencing necrosis of the lung tissue (Bessonowa, Kotelnikow, and Sémikoz 1927, Bablet and Girard 1933). However infection occurs, the organisms sooner or later gain access to the blood stream. At post mortem they can be isolated from the blood, spleen, liver, lung, and bone marrow. After bacteræmia has developed, the organisms may often be found in the bile, urine, and less frequently in other excretions (Sémikoz, Bessonowa, and Kotelnikow 1927).

RABBits are less susceptible to plague than rats and guinea-pigs, but they can generally be infected by subcutaneous inoculation (Dieudonné and Otto 1912).

Monkeys vary in susceptibility. The German Commission (Report 1899) infecter IIacacus radiatus by subcutaneous and intraperitoneal inoculation, and by feeding. This species was not nearly so susceptible, however, as Presbytes entellus (Semnopithecus entellus) which succumbed after minute quantities of plague culture subcutaneously.

\section{Experimental Reproduction of Pasteurellosis in Animals.}

Most animals are susceptible to experimental infection with Pasteurella. On primary isolation, a strain from one specios of animal may prove of low infectivity for other species (Karlinski 1890), but this is by no means always true. Magnusson, for example, found that his reindeer organism was pathogenic to mice, guinea-pigs, rabbits, sheep, dogs, and pigeons. The most suitable animals for inoculation are the mouse, rabbit and pigeon.

Mice.- Subcutaneous inoculation of a small quantity of a 24-hours' broth culture proves fatal in 24 to 48 hours. Post mortem, there may be local œedema and congestion, with practically no other signs. Microscopically the bacilli are found in large numbers in the blood and viscera. If very few organisms are injected, or a culture of relatively low virulence is used, the mouse does not die for 2 to 8 days, or even longer ; at necropsy there is a fibrino-purulent pericarditis, a layer of fibrin over the pleura, partial consolidation of the lungs, and not infrequently a purulent exudate in the peritoneum. Bacilli are plentiful in the blood and organs. Intraperitoneal inoculation is more rapidly fatal.

$\mathrm{R}_{\mathrm{ABBITS}}$ can be infected by subcutaneous, intraperitoneal, intravenous, intratracheal, or intranasal inoculation. Death occurs in 2 to 5 days as a rule after intraperitoneal injection, with lesions similar to those in mice. In addition there may be a hæmorrhagic tracheitis (Magnusson 1914), and hyperæmia of the kidneys and intestine (Poels 1886). Intranasal insufflation of the bacilli is often followed by snuffles or pleuro-pneumonia (Beck 1893, Webster 1924b, 1926), and sometimes by purulent otitis media (Smith and Webster 1925).

Pigeons are very susceptible to intravenous or intraperitoneal, less so to intramuscular, injection. Death occurs in 24 to 48 hours. Bacilli are abundant in the blood.

\section{Experimental Reproduction of Pseudotuberculosis in Animals.}

The term pseudotuberculosis is used to refer to a number of diseases that are caused by different organisms (see Chapter 73). We shall restrict ourselves here to the lesions due to Past. pseudotuberculosis. The experimental disease can be produced in guinea-pigs, rabbits, rats, mice, and according to Pallaske (1933), to whom reference should be made for a detailed account of the lesions produced, in cats, pigeons, canaries, and turkeys. For laboratory purposes the guinea-pig is the most suitable animal to study. 
Subcutaneous or intramuscular inoculation of the guinea-pig is followed by a disease which, depending on the dose and the virulence of the strain, may be acute, subacute, or chronic. The acute disease resembles plague and is fatal in a few days. The differential diagnosis can be made only by cultivation and a thorough study of the organism responsible. Macroscopically, the focal lymphatic glands tend to be more affected in plague than in pseudotuberculosis. The subacute disease proves fatal in about 2 weeks, and the chronic in 3 weeks or longer. Post mortem there is a caseous local swelling, the regional lymphatic glands are enlarged, and there are nodules varying in number, size, and degree of caseation, in the spleen, liver, and lungs. If the animal lives for 3 weeks or so, the nodules are usually very conspicuous. They are more or less spherical, greyish-white in colour, and 0.2 to $3 \cdot 0$ $\mathrm{mm}$. in diameter; they project above the surface of the organ, and in the liver they show no particular localization for the free border, as do the necrotic areas in rodent typhoid infection. Microscopically the bacilli are generally present in considerable numbers at the site of inoculation and in the regional glands, and they can be readily cultivated from all the lesions. The disease can also be reproduced by feeding, which is the natural methor of infection. Death occurs in 1 to 3 weeks. Nodules of varying size are found in the intestinal wall, the mesenteric glands are enlarged and often caseous, and nodules may be present in the spleen, liver, and lungs.

Classification and Identification.-The members of this group resemble each other very closely; between Past. pestis and Past. pseudotuberculosis the similarity is so great that the identification of a given strain is not always easy. Agglutination and precipitation may be of assistance, especially if supplemented by the absorption test. The production of alkali in litmus milk by Past. pseudotuberculosis, and its comparative harmlessness for white rats (Report 1912, p. 350), are two differential tests that are sometimes recommended. Past. pseudotuberculosis is often motile in broth cultures incubalted for 18 hours at $20-22^{\circ} \mathrm{C}$. whereas Past. pestis is uniformly non-motile. The greater rapidity and luxuriance of growth of Past. pseudotuberculosis is usualy very striking, particularly if studied on nutrient agar plates incubated aerobically for 24 hours. Under these conditions Past. pestis shows very slight confluent growtl restricted to the first line of inoculation. Single colonies are rarely seen mnless a strong reducing substance is present in the inoculum. On the other hand colonies of Past. pseudotuberculosis reaching 0.5 or $1 \mathrm{~mm}$. in diameter are usually evident over the whole plate. The hæmorrhagic septicæmia bacilli can be differentiated from Past. pestis and Past. pseudotuberculosis by their fermentation of sucrose, their production of indole, and their negative methyl-red reaction; it must be noted, however, that sucrose is fermented by certain strains of Past. pseudotuberculosis. According to Brigham and Rettger (1935), Past. pestis and Past. pseudotuberculosis grow on potato at $20^{\circ} \mathrm{C}$., while Past. septica does not. Between the hremorrhagic septicrmia strains of different animal origin, there appears to be no constant characteristic of diagnostic value. Most workers therefore agree that these bacilli form a single group, though it appears possible, using a careful techrique, to make out antigenic differences between them. At present each member is given a specific name; probably it would be better to call them all by one specific name, such as Pasteurella septica, and to indicate the animal origin where necessary.

A group of strains in many respects resembling Pust. septica, but differing from it in others, have been described by Jones (1921), Tweed and Edington (1930), Newsom and Cross (1932), and Rosenbusch and Merehant (1939). These strains are characterized by the production of $\beta$-hæmolysis on horse or rabbit blood agar, failure to produce indole, and non-pathogenicity to mice and rabbits; most of 
them appear to ferment maltose, lactose, dextrin and inositol in addition to glucose mannitol and sucrose. It is very doubtful whether these strains form a true soluble hæmolysin; the clearing on a blood agar plate is insufficient evidence of this. If it should be proved that a true hæmolysin is formed, it may be necessary to divide the hæmorrhagic septicæmia group of organisms into a non-hæmolytic and a hæmolytic sub-group, and award each sub-group a specific name.

We may note here that Dungal (1931) in Iceland, studying acute contagious pneumonia in sheep, cultivated an organism which resembled the hæmorrhagic septicæmia group, but differed from it in the following respects. It was Grampositive in young broth cultures, and tended to be definitely rod-shaped, $1-3 \mu$ in length ; it failed to grow at $22^{\circ} \mathrm{C}$., in gelatin or broth ; it grew on MacConkey agar ; it fermented maltose ; it did not produce indole ; it died out in 2 to 5 days in culture at room temperature, and it was pathogenic to mice, but not to rabbits or guinea-pigs. The organism was said to be non-hrmolytic, differing in this respect from the hrmolytic group of organisms described by Newsom and Cross, some of which were likewise isolated from sheep suffering from pneumonia. In Australia, Beveridge (1937) isolated a bacillus, very similar to that described by Dungal, from sheep suffering from lesions of the digestive organs and lungs.

TABLE 50

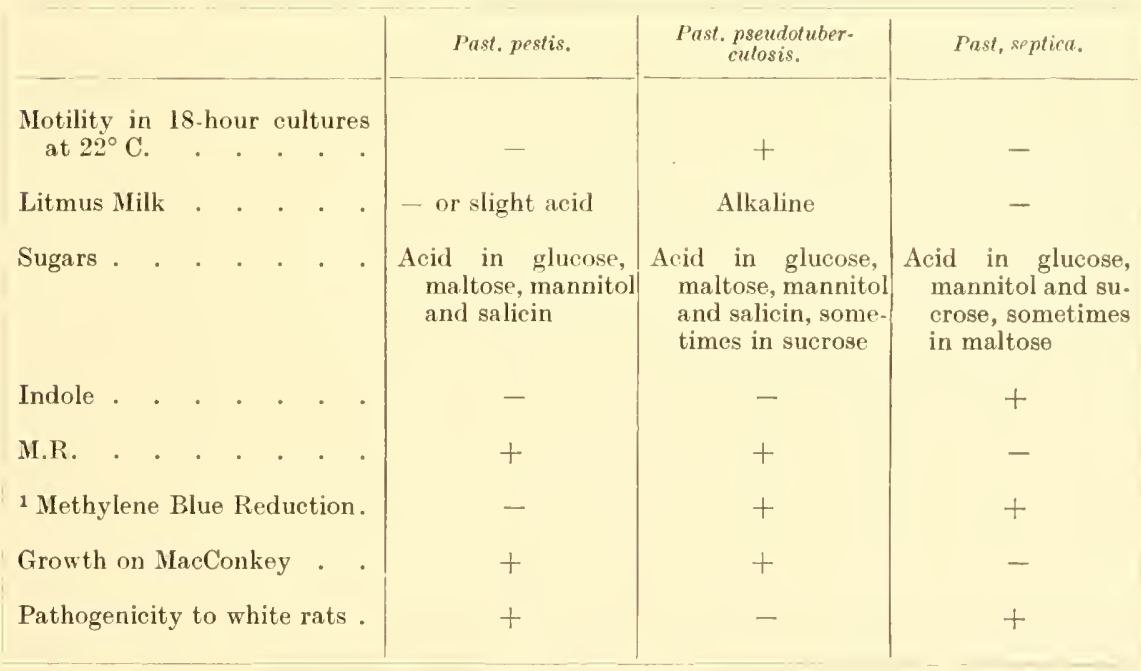

1 Personal observations on a relatively few strains.

Fedorova and Lalazarow (1935) described a Pasteurella-like organism, which they isolated from a spontaneous epidemic among mice in the outskirts of Astrakhan. The organisms were 1-2 $\mu$ long by $0.5 \mu$ wide, Gram-negative, non-motile, and capsulated. Acicl was formed in lactose, mannitol, and dextrin, and acid plus gas in glucose. Sucrose was unchanged. No indole was formed. The organism was highly pathogenic for mice, but had no effect on rabbits, pigeons, or rats injected by various routes. It is doubtful whether this organism should be placed in the Pasteurella group. 


\section{Pasteurella pestis}

Isolation.--Independently by Kitasato (1894) and by Yersin (1894).

Habitat.-Parasite of rodents and man.

Morphology. -Small, straight, ovoid bacillus, $1.5 \mu \times 0.7 \mu$, with rounded ends and convex sides; arranged singly, in short chains, or in small groups. Shows high degree of pleomorphism, especially in buboes and on 3 per cent. salt agar; there is every degree of variation in depth of staining, and clubs, shadow forms, snake-like filaments, coccoid forms, yeast-like forms, and numerous others may be seen. Nonmotile. Non-sporing. In the animal body a true capsule may be formed; in culture media there is often a slimy envelope around each bacillus. Shows bipolar staining, is Gram-negative, and non-acid-fast.

Agar Plate. -24 hours at $37^{\circ} \mathrm{C}$. Very small, 0.1-0.2 mm. in diameter, round, glistening, transparent, colourless, finely granular, umbonate colonies, with smooth or finely granular surface and an entire or delicately notched edge; differentiated into a raised centre and a flat periphery.

5 days at $37^{\circ} \mathrm{C}$. Larger, up to $4 \mathrm{~mm}$. in diameter, with a raised, sometimes ringed, nearly opaque, greyish-yellow centre and a flat or shelving, finely granular, translucent, greyish-white periphery ; consistency is butyrous or viscous, emulsifiability easy; sometimes a secondary ring of growth is seen. Variant colonies occur.

Deep Agar Shake.-5 days at $37^{\circ} \mathrm{C}$. Maximum growth at the surface; numerous round, transparent, colourless, punctiform colonies, visible with a hand lens, scattered throughout the medium.

Agar Stroke.-24 hours at $37^{\circ} \mathrm{C}$. Poor, slightly raised, translucent, greyish-yellow, glistening growth, with a wavy or frosted-glass surface, and an irregularly lobate edge. Growth increases very little with subsequent incubation.

Gelatin Stab.-7 days at $22^{\circ} \mathrm{C}$. Good, filiform growth, confluent at top, discrete below, extending to bottom of tube, and sometimes sending out little feathery projections into medium. Surface growth is raised, $5 \mathrm{~mm}$. in diameter, with a slightly lobate edge. No liquefaction.

Broth. -24 hours at $37^{\circ} \mathrm{C}$. Moderate growth ; little or no turbidity; a floccular or powdery deposit, not disintegrating completely on shaking. Later the flaky deposit increases and may crawl up the sides of the tube; a delicate surface pellicle often forms. If butter or oil is floated on the medium, stalactites grow down from the under-surface of the droplets.

Loeffler's Serum. - 24 hours at $37^{\circ} \mathrm{C}$. Fairly good, confluent growth, better than that on agar.

Horse Blood Agar Plate. -2 days at $37^{\circ} \mathrm{C}$. Colonies are similar to those on agar but show less tendency to differentiation and peripheral spread. No hæmolysis; whole plate is shightly eleared and browned.

Potato.-7 days at $22^{\circ} \mathrm{C}$. Usually a thin layer of growth.

MacConkey Plate.-24 hours at $37^{\circ} \mathrm{C}$. Very shight, effuse, confluent growth, just visible to the naked eye. Colonies disappear after 2 or 3 days, owing presumably to autolysis.

Resistance.-Fairly susceptible to inimical agencies. Killed by drying in a day or two, by heat at $55^{\circ} \mathrm{C}$. in 5 minutes, by 5 per cent. phenol immediately, and by 0.5 per cent. phenol in 15 minutes. Agar plate cultures exposed to sun are sterilized in 1 to 5 hours. Cultures in the ice-chest may survive for months.

Metabolism.-Aerobic, facultative anaerobe. Requires low $\mathrm{O}-\mathrm{R}$ potential for initiation of growth. Opt. temp. $27-28^{\circ} \mathrm{C}$. ; limits $-2^{\circ}$ to $+45^{\circ} \mathrm{C}$. Opt. $\mathrm{pH} 7 \cdot 2$; limits $\mathrm{pH} 5 \cdot 0-9 \cdot 6$. Forms alkali in broth. Growth favoured slightly by serum, uninfluenced by glucose; partly inhibited by glycerol. No hæmolysis. 
Biochemical.-Acid, no gas, in glucose, maltose, mannitol, salicin, arabinose, xylose, and sometimes rhamnose and glycerol within 14 days. L.M. unaltered or turned slightly acid; Indole -; M.R. +; V.P. - ; Nitrates +; $\mathrm{NH}_{3}+$; Methylene blue reduction -; $\mathrm{H}_{2} \mathrm{~S}$ very slight + ; Catalase ++ .

Antigenic Structure.-Appears to possess a heat-labile envelope antigen and a heat-stable somatic antigen. The somatic antigen is similar to one of the somatic antigens in Past. pseudotuberculosis. Immune sera with protective and curative properties for animals can be prepared by injection of horses with living or dead bacilli.

Pathogenicity.-No true exotoxin formed. Virulence subject to considerable variation. Causes plague in man and rodents. Experimental inoculation reproduces disease in mice, rats, guinea-pigs, rabbits, marmots, ground squirrels, and other rodents; also in monkeys. Dogs, cats, pigs, cattle, sheep, goats, and horses are difficult to infect. Birds, with exception of sparrows, are completely resistant.

Subcutaneous inoculation of a 24 -hours' broth culture into a mouse or guineapig is fatal in 2 to 5 days. P.M. necrotic local lesion surrounded by congestion and codema. Regional glands enlarged and embedded in bloody odema ; they are soft and necrotic on section. Spleen firm, slightly enlarged and congested; may contain miliary, soft, grey nodules; liver peppered with tiny necrotic foci. Microscopically, bacilli found in abundance in local lesion and bubo; smaller numbers in spleen and heart's blood.

\section{Pasteurella septica}

Isolation.-First member of the Pasteurella group isolated by Kitt in 1878. Pastariseptica isolated by Pasteur in 1880.

Habitat.-Parasites of domestic and wild animals and birds.

Morphology.-Very small, $0 \cdot 7-2 \mu \times 0.3-0 \cdot 6 \mu$, ovoid bacilli, with straight axis, slightly convex sides, and rounded ends; arranged singly, in pairs, or in small bundles. In smears from the animal body the organisms are regular, oroid, and evenly distributed; on agar cultures they are more rod-shaped and often show pleomorphism. Non-motile, non-sporing. May form a capsule in aninal body, and an envelope in artificial media. Shows bipolar staining. Gram-negative and nonacid-fast.

Agar Plate. -24 hours at $37^{\circ} \mathrm{C}$. Round, $0 \cdot 5-1 \cdot 0 \mathrm{~mm}$. in diameter, low convex, amorphous, greyish-yellow, translucent colonies, with smooth, glistening surface and entire edge; consistency butyrous; emulsifiability easy.

5 days at $37^{\circ} \mathrm{C}$. Up to $6 \mathrm{~mm}$. in diameter, differentiated into a brownish, finely granular, sometimes ringed or striated, nearly opaque centre and a clearer, smooth, homogeneous, greyish-yellow translucent periphery.

Deep Agar Shake. -5 days at $37^{\circ} \mathrm{C}$. Thick surface growth; numerous, punctiform, undifferentiated colonies scattered throughout medium.

Agar Stroke. -24 hours at $37^{\circ} \mathrm{C}$. Noderate, confluent, raised, greyish-yellow, translucent growth, with glistening, wavy or beaten-copper surface and finely lobate edge.

Gelatin Stab. -7 days at $22^{\circ} \mathrm{C}$. Good, filiform growth, confluent at top, discrete below, extending to bottom; raised surface growth, $5 \mathrm{~mm}$. in diameter, with crenated edge, no liquefaction.

Broth. -24 hours at $37^{\circ} \mathrm{C}$. Moderate growth with slight turbidity, and a slight powdery or viscous deposit. Later the turbidity increases, and a heavy, viscous deposit forms, disintegrating partly on shaking but leaving irregularly-sized wisp-like masses of growth in suspension. An incomplete surface pellicle forms with an inconspicuous ring growth.

Loeffler's Serum. -24 hours at $37^{\circ} \mathrm{C}$. Good confluent growth, similar to that on agar. 
Horse Blood Agar Plate. -2 days at $37^{\circ} \mathrm{C}$. Good growth similar to that on agar; no hæmolysis, but blood plate is slightly cleared and browned.

Potato--7 days at $22^{\circ} \mathrm{C}$. No visible growth.

MacConkey Plate. -5 days at $37^{\circ} \mathrm{C}$. No visible growth.

Resistance.-Very susceptible to inimieal agencies; killed by heat at $60^{\circ} \mathrm{C}$. in a few minutes; by 0.5 per cent. phenol in 15 minutes.

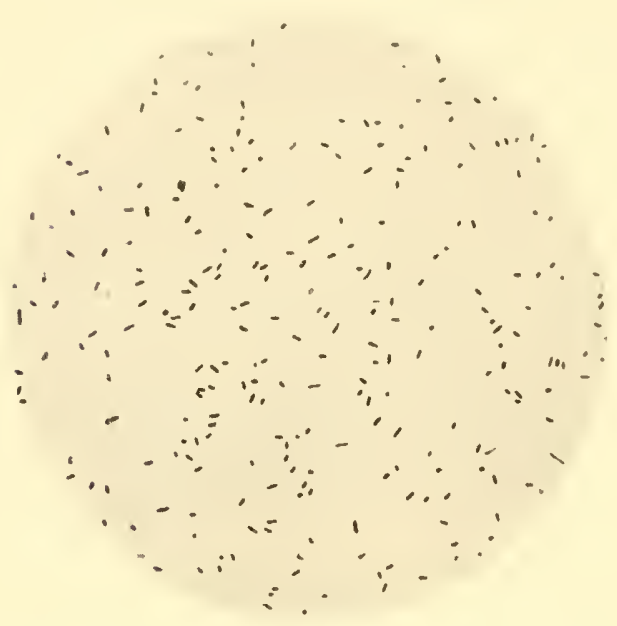

Fia. 161.--Pasteurella septica.

From an agar culture, 24 hours, $37^{\circ} \mathrm{C} .(\times 1000)$.

Netabolism.-Aerobe, facultative anaerobe. May require low $\mathrm{O}-\mathrm{R}$ potential on first isolation. Opt. temp. $37^{\circ} \mathrm{C}$, limits $12^{\circ}$ $43^{\circ} \mathrm{C}$. Growth improved slightly by serum, uninfluenced by glucose, slightly inhibited by glycerol. No hæmolysis, but see p. 778.

Biochemical.-Acid, no gas, in glucose, mannitol, sucrose, and sorbitol within 14 days; one type forms acid in maltose. Some strains produce acid in arabinose, xylose, and glycerol. L.M. ınchanged ; Indole + ; M.R. - ; V.P. - ; Nitrates reduced; $\mathrm{NH}_{3}$ very slightly +; M.B. reduction $++; \mathrm{H}_{2} \mathrm{~S}+$; Catalase ++ .

Antigenic Structure.-At least two types distinguishable by agglutination. Probably one envelope antigen, but more than one somatic antigen. Specific sera agglutinate Past. pestis and Past. pseudotuberculosis to a certain extent. Immune sera with protective and curative properties for animals can be prepared by injection of horses with living or dead bacilli.

Pathogenicity.--No true exotoxin produced. Virulence subject to alteration. Causes fowl cholera in birds. Other members of this group produce hæmorrhagic septicæmia in pigs, cattle, sheep, rabbits, mice, rats, reindeer, buffaloes, and other animals. Experimental inoculation reproduces the disease in these animals. Subcutaneous inoculation of a 24 -hours' broth culture into a mouse proves fatal in 18 to 72 hours. P.M. local cedema and congestion; often no other signs ; microscopically bacilli present in enormous numbers in blood and viscera. If a small dose is given and the animal does not die for 4 to 7 days, there is often a fibrino. purulent pericarditis, a layer of fibrin over the pleura, and partial consolidation of the lungs. Bacilli are numerons in blood and organs.

\section{Pasteurella pseudotuberculosis}

Synonym.-B. pseudotuberculosis rodentium.

Isolation.-First observed by Malassez and Vignal in 1853, named B. pseudotuberculosis rodentium by Pfeiffer (1890) in 1889 .

Hulitut.-Parasite of rodents, particularly guinea-pigs.

Morphology. - Small, pleomorphic cocco-bacillus varying greatly in length and shape. Some strains consist of regular ovoid or coccoid organisms, $0.8-2.0 \mu \times 0.8 \mu$, with convex sides, rounded ends, and straight axis ; arranged singly. Other strains consist of rod-shaped organisms, $1 \cdot 5-5 \cdot 0 \mu \times 0 \cdot 6 \mu$, with parallel sides, rounded ends, and straight or curved axis ; arranged singly, in groups, or in short chains. Long curved filaments are not uncommon (Fig. 162). Motile in broth cultures at $22^{\circ}$ C. Non-sporing. Non-capsulated. Ovoid forms show bipolar staining; rod 
forms show great irregularity of staining; the barred and granular type of staining is very common. Gram-negative, and non-acid-fast.

Agar Plate-24 hours at $37^{\circ} \mathrm{C}$. Round, $0.5-1.0 \mathrm{~mm}$. in diameter, umbonate, granular, translucent, greyish-yellow colonies, with dull, finely granular or beatencopper surface and entire edge ; butyrous consistency ; easily emulsifiable; differentiated into a raised, more opaque centre and a flat, clearer periphery with radial striation. A rough variant with an irregular surface and a crenated edge also occurs.

Deep Agar Shake.-5 days at $37^{\circ} \mathrm{C}$. Heavy surface growth. No colonies beneath surface.

Agar Stroke. -24 hours at $37^{\circ} \mathrm{C}$. Moderate, confluent, raised, greyish - yellow, translucent growth, with glistening, wavy or beaten-copper surface and an irregularly lobate edge.

Gelutin Stab. -7 days at $22^{\circ} \mathrm{C}$. Good filiform growth, confluent at top, discrete below, extending to bottom of tube. Raised surface growth, $5 \mathrm{~mm}$. in diameter, with finely lobate edge. No liquefaction.

Broth.- 2t hours at $37^{\circ} \mathrm{C}$. Moderate growth withont turbidity and

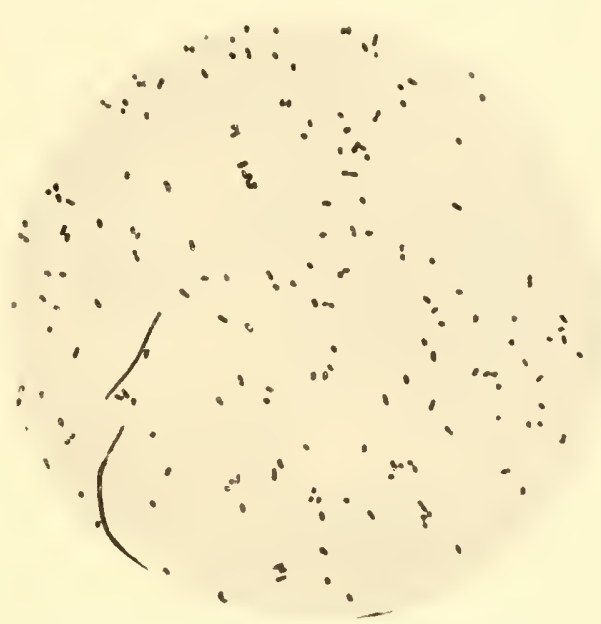

FIG. 162.-P'Asteurella pseudotuberculosis. From an agar culture, 24 hours, $37^{\circ} \mathrm{C} .(\times 1000)$. a viscous deposit disintegrating on shaking. Later the broth elcars and a lieavy, flocculo-membranous deposit forms, partly disintegrating on shaking. Incomplete surface and ring growth.

Loeffler's Serum.-24 hours at $37^{\circ} \mathrm{C}$. Confluent growth, not so good as on agar.

Horse Blood Agar Plute.-2 days at $37^{\circ} \mathrm{C}$. Good growth, but colonies are more compact and less differentiated than on agar. No hæmolysis; whole plate is slightly cleared and browned.

Potato. -7 days at $22^{\circ} \mathrm{C}$. A thin yellowish membrane, which subsequently terns brown.

Mac Conkey Plate-2t hours at $37^{\circ} \mathrm{C}$. Very slight effuse confluent growth. Colonies disappear after a few days, owing presumably to autolysis.

Resistance. - Fairly susceptible to inimical agencies. Killed by moist heat at $60^{\circ} \mathrm{C}$., in 10 minutes.

Metabolism. - Aerobe, facultative anaerobe. Opt. temp. $30^{\circ} \mathrm{C}$, linits $5-43^{\circ} \mathrm{C}$. Growth uninfluenced by serum and glucose, slightly inhibited by glycerol. No hæmolysis.

Biochemical.-Acid, no gas, in glucose, maltose, mannitol, salicin, arabinose, xylose, rhamnose, and glycerol within 14 days, sometimes in sucrose. L.M. usually slight alkali formation; Indole - ; M.R. + V.P. - N Nitrates +; $\mathrm{NH}_{3}+$; I.B. reduction $++; \mathrm{H}_{2} \mathrm{~S} \perp$ : Catalase ++ .

Antigenic Structure.-Apparently contains (1) a heat-labile flagellar antigen, which is formed only in cultures grown below about $25^{\circ} \mathrm{C}$.; (2) a heat-stable somatio antigen similar to that of the plague bacillus; (3) a heat-stable somatic antigen sinilar to that in Salm. paratyphi B; (4) possibly other relatively strain-specific somatic antigens. A plague serum does not protect animals against infection with Past. pseudotuberculosis. 
Pathogenicity.-No tme exotoxin formed. Virulencesubject to alteration. Causes pseudotuberculosis in rodents, especially guinea-pigs. Experimental inoculation reproduces the disease in rodents. Subcutaneous injection of a 24-hour broth eulture into a guinea-pig proves fatal in 1 to 3 weeks. P.MI. caseous local swelling, enlargement of regional glands, and nodules in spleen, liver, and lungs. Microscopically the bacilli are numerous in the local lesion and glands.

\section{REFERENCES}

Anderson, L. A. P., Coombes, M. G., and Mallick, S. M. K. (1929) Indian J. med. Res., 17,611 .

Arkwright, J. A. (1927) Lancet, i. 13.

D'Aunor, R. (1923) J. infect. Dis., 33, 391.

Bablet, J. and Girard, G. (1933) C.R. Soc. Biol., 114, 471.

Bannerman, W. B. (1908) Sci. Mem. med. sanit. Dep. India, New Ser., No. 33.

BECK, M. (1893) Z. IIyg. InfektKr., 15, 363.

BESEMER, A. 11. (1917) J. Bact., 2, 177.

Bessonowa, A. (1928) Rev. Microbiol. Saratov, \%, 336 ; (1930) Zbl. Balt., 119, 32.

Bessonowa, A., Kotelnikow, G., and Sémikoz, F. (1927) C. R. Ist. Congr, antipest. U.R.S.S., p. 485 .

Bessonowa, A. and Lenskaja, G. (1931) Zbl. Bakt., 119, 430.

Bessonowa, A., Sénikoz, T., and Kotelnikow, G. (1927) Rev. Microbiol. Sarator, 6, 472.

Beveridge, W. I. B. (1937) Aust. vet. J., 13, 15.

Bhatnagar, S. S. (1940a) Indian J. med. Res., 28, 1 ; (1940b) Ibid., 28, 17.

BONGERT. (1901) Z. Hyg. Infekthr., 3\%, 449.

Boquet, 1. (1937) Ann. Inst. Pasteur, 59, 341.

Brighal, G. D. and Rettger, L. F. (1935) J. infect. Dis., 56, 225.

Brooks, R. St. J. and Rinodes, H. (19:3) J. Path. Bact., 26, 433.

Bunzl-Federn, H. (1891) Z Zl. Bakt., 9, 787.

Burgess, A. S. (1930) J."Hyg., Camb., 30, 165.

Chamberland and Jouan. (1906) Ann. Inst. Pasteur, 20, 81.

Colas-Belcour, J. (1926) C. R. Soc. Biol., 94, 238.

Cornelius, J. T. (1929) J. Path. Bact., 32, 355.

Csontos, J. (1926) Zbl. Bakt., 97, 178.

Dieudonné, A. and OtTo, R. (1912) See Kolle and Wassermann's "Hdb. path. Mikroorg," IIte Abt., 1912-13, 4, 155.

Dengal, N. (1931) J. comp. Path., 44, 126.

Eastwoon, A. and Griffith, F. (1914) J. Hyg., Camb., 14, 285.

Eberson, F. (1917) J. infect. Dis., 20, 180.

Eberson, F. and Wu Lien Ter. (1917) J. infect. Dis., 20, 170.

Eskey, C. R. and HaAs, V. H. (1940) Publ. Hllth. Bull., JVash., No. 254.

Fedorova, T. and Ladazarow, G. (1935) Rev. Hicrobiol. Saralov, 14, 55.

Francis, E. (1932) Publ. Hlth Rep., Wash., 4\%, 1287.

Grrard, G. (1936) Ann. Méd., Pharm. colon., 34, 235.

Gotschlich, E. (1912) See Kolle and Wassermann, "Hdb. path. Mikroorg.," IIte Abt., $1912-13,1,167$.

HADLEY, P. (1918) J. Bact., 3, 277.

Haffise, IV. M. (1905) Sci. Mem. med. sanit. Dep. India, New Ser., No. 20.

HOFFENREICH, F. (1928) Zbl. Bakt., 108, 87.

Hughes, T. P. (1930) J. exp. Med., 51, 225.

Iwanowsky, N. and Sassykina, T. (1930) Zul. Bakl., 11\%, 535.

Jones, F. S. (1921) J. exp. Med., 34,561.

Kakehi, S. (1915-16) J. Path. Bact., 20, 269.

KARLinski, J. (1890) Zll. Bakt., $7,335$.

Kauffuand, F. (1932) Z. Hyg. InfektKr., 114, 97.

Kitasato, S. (1894) Lancet, ii. 428.

KrUIF, P. H. DE (1921) J.exp. Iled., 33, 773 ; (1922a) Ibid., 35, 561 ; (1922b) Ibid., 36, 309 ; (1923) Itid., $3 \%, 6+7$.

Kutscher. (1894) Z. IIyg. InfektKr., 18, 327.

LAL, R. B. (1927) Amer. J. Hyg., \%, 561.

Levinthal, W. (1930) Z. Hyg. InfektKr., 111, 140.

MacConkey, A. T. (1908) J. Hyg., Camb., 8, 335.

McCoy, G. W. (1911) Publ. Hlth Bull., Wash., No. 43.

I Aanusson, H. (1914) Z. InfektKr. Haustiere, 15, 61.

Malassez, L. C. and Vignal, W. (1883) Arch. Physiol. norm. path., 3rd series, 2, 369. 
Mørch, J. R. and Krogh-Lund, G. (1930) C. R. Soc. Biol., 105, 319; (1931) Z. Hyg. InfektKr., 112, 471 .

Newsom, I. E. and Cross, F. (1932) J. Amer. vet. med. Ass., 80, 711 .

OGata, H. (1897) Zbl. Bakt., 21, 769.

Ostertac, R. (1908) Z. Infelitr. Haustiere, 4, 1.

Otten, L. (1926) Zbl. Balt., 98, 484; (1935a) P. V. Séances Comité permanent Off. int. Hyg. publ., Session d'Octobre, 1934, p. 93 ; (1935b) Bull. Off. int. Hyg. publ., 29, 1542 ; (1938) Meded. Dienst. Folksgezondh. Jed.-Ind., 2\%, I11.

Pallaske, G. (1933) Z. InfektKr. Haustiere, 44, 43.

Pfeiffer, A. (1890) Zbl. Bakt., \%, 219.

Pirie, J. H. H. (1929) Publ. S. Afr. Inst. med. Res., 4, 203.

Prrosky, I. (193sa) C. R. Soc. Biol., 127, 98 ; (1938b) Ibid., 127, 966 ; (1938c) Ibid., 128, $346 ;(1938 d)$ Ibid., 128, 347.

Poels, J. (1886) Fortschr. Med., 4, 388.

Poss, R. (1925) Ann. Inst. Pasteur, 39, 884.

Preisz, H. (1894) Ann. Inst. Pasteur, 8, 231.

Priestley, F. W. (1936a) Brit. J.exp. Path., 17, 374; (19366) J. comp. Path., 49, 340 ; (1936c) Ibid., 49, 348.

RAO, M. S. (1939) Indian J. med. Res., 27, 75, 617, 833.

Reports. (1899) Germ. Plague Comm., Arb. ReichsgesundhAmt, 16, 1 ; (1906) Eng., Plague Comm., J. Hyg., Camb., 6, 421; (1912) Engl. Plague Comm., J. IIyg., Camb., 12, Suppl., p. 287 ; (1915) Ibid., 14, Suppl., p. 754.

Rosenbusch, C. T. and llerchant, 1. A. (1939) J. Bact., 37, 69.

Rowland, S. (1914a) J. Hyg., Camb., Engl. Plague Comm., 13, Suppl., 418 ; (1914b) Ibid., 440.

Russo, E. (I939) O Hospital, 16, 57.

Schirop, H. (1908) Zbl. Baht., 47, 307.

Sснüтze, H. (1928) Arch. Hyg., 100, 181 ; (1932a) Brit. J.exp. Puth., 13, 284; (1932b) Ibid., 13, 289; (1932c) Ibid., 13, 293 ; (1934) Ibid., 15, 200 ; (1939) Ibid., 20, 235.

Schütze, H. and Hassanein, M. A. (1929) Brit. J. exp. Path., 10, 204.

Sémikoz, F., Bessonowa, A., and liotelnikow, G. (1927) C. R. Ist. Congr. antipest. U.R.S.S., p. 488 .

SMth, D. T. and Webster, L. T. (1925) J. exp. Med., 41, 275.

Sокнет, S. S. (1939a) Indian J. med. Res., 27, 341 ; (1939b) Ibid., 27, 363.

Soкнеу, S. S. and HAввU, M. K. (I943) J. Bact., 46, 25.

Sokнey, S. S. and Maurice, H. (1935) Bull. Off. int. Hyg. publ., 27, 1534.

Spencer, R. R. (1921) Publ. Hlth Rep., Wash., To. 36, p. 2836.

Tanaka, A. (1926) J. infect. Dis., 38, 421 .

Tumansky, W., Müller, U., Bokalo, A., Wedistschew, S., and Sabinin, A. (1935) Rev. Microbiol. Saratov, 14, 128.

Tweed, W. and Edingtox, J. W. (1930) J. comp. Path., 43, 234.

Vourloud. (1908) Zbl. Bakt., 45, 97, 193.

Wats, R. C., Wagle, P. M., and Puduval, T. K. (1939) Indian J. med. Res., 27, 373.

Webster, L. T. (1924a) Proc. Soc. exp. Biol., 22, 139; (1924b) J.exp. Med., 40, 109, 117; (1925) Ibid., 41, 571 ; (1926) Ibid., 43, 555.

Webster, I. T. and BAUDisch, O. (1925) J. exp. Hcd., 40, 473.

Webster, L. T. and Burn, C. G. (1926) J. exp. Med., 44, 343, 359.

WeitzenberG, R. (1935) Zbl. Bakt., 133, 343.

Wright, H. D. (1934) J. Path. Bact., 39, 381.

Wo Lien Teh, and Eberson, F. (1917) J. Hyg., Camb., 16, 1.

Yersin. (1594) Ann. Inst. Pasteur, 8, 662.

Yersin, Calmette, and Borrel. (1895) Ann. Inst. Pasteur, 9, 589.

YUsef, H. S. (1935) J. Path. Bact., 41, 203.

Zabolotiy, D. (1923) Ann. Inst. Pasteur, 3\%, 618.

Zlatogorofe, S. J. (1904) Zbl. Bakt., 3\%, 345, 513, 654.

Zlatogoroff, S. I. and MoghilewskaJA, B. I. (1928x) Ann. Inst. Pasteur, 42, 1615 ; (1928b) C. R. Soc. Biol., 99, 506. 


\section{CHAPTER 33}

\section{HÆMOPHILUS}

\section{Definition.-Hamophilus.}

Minute rods, sometimes almost coccal, sometimes thread-like; may be highly pleomorphic. Usually non-motile. Non-sporing, Gram-negative, non-acid-fast. On first isolation dependent for grow th on some factor, or factors, contained in blood or in plant tissues. Some species retain this dependence after prolonged cultivation on laboratory media. Some species are obligatory aerobes, or grow very poorly under anaerobic conditions. All known species appear to be obligatory parasites, inhabiting particularly the upper respiratory tract; and most of the described species or types are pathogenic.

Type species. H. influenzo.

Since the isolation and description by Pfeiffer $(1892,1893)$ of the bacillus which, though not the primary causal organism of influenza, is closely associated with that disease, several other small, Gram-negative bacilli have been described, which share with it certain characteristic growth requirements. In their final report on classification and nomenclature, the American Committee (see Winslow et al. 1920) grouped these species together under the generic name of Hamophilus. The generic definition suggested in the Committee's report opens the door more widely than insistence on a close similarity in behaviour to the type species would allow ; and such species as the Bordet-Gengou bacillus of whooping cough, the MoraxAxenfeld bacillus of angular conjunctivitis, and Ducrey's bacillus of soft sore, have been included, by certain writers, within this generic group. Such an extension of the term "hæmophilic bacilli," whether in the form of a generic name or as a convenient appellation for a charactcristic bacterial group, has been opposed by Kristensen (1922) and by Fildes (1923). It seems clear that their objection is valid if the character from which the name is derived is to retain a decisive differential significance. To include all the species referred to, it would be necessary to define the genus on some other basis, with a sub-group characterized by the particular growth requirements that $H$. influenze displays. There is no reason why we should not do this.

The two most important growth factors in blood which determine the hæmophilic nature of Pfeiffer's bacillus have been identified, the one as a co-enzyme, the other as an iron-containing pigment which presumably supplies the tetrapyrrole compounds necessary for the synthesis of cytochrome and related substances (see Chapter 3). But if we confine the term hæmophilic bacilli to strains that need one or both of these substances for growth, we may be excluding organisms which can synthesize the growth factors, but which are nevertheless closely related. Horeover, the existence of organisms which synthesize small, sub-optimal amounts of these growth factors provides a link between the definitely hæmophilic bacilli 
and those, like the bacillus of whooping cough, for which the growth factors are not essential as nutrients.

Ducrey's bacillus in any event is hæmophilic on the narrower definition, but it is doubtful whether the Morax-Axenfeld bacillus would be included in this genus by any such redefinition, and we have therefore described it in Chapter 37, together with others that cannot at present be assigned to any named bacterial genus. The Bordet-Gengou bacillus cannot, we think, be dealt with in the same way. It resembles $H$. influenze so closely in morphology, in habitat, and in many other ways, that it would certainly be placed in close association with it by any systematic definition that did not rely exclusively on a narrow nutritional criterion.

In 1911, Ferry in the United States described a short bacillus that he had isolated from the respiratory tract of dogs in the early stage of distemper; to this organism he gave the name of Bucillus bronchicanis. A similar organism had been described as early as 1896 by Galli-Valerio, by Tartakowsky (1897-98) in 1898, by Strada and Traina in 1900 as B. preumonice caviarum, by Martini in 1900 as B. pulmonum glutinosus, and by Selter in 1906 as B. cavisepticus mobilis ; Ferry, however, was the first to study it fully. Later Ferry (1912, 1912-13) found the same bacillus in a guinea-pig epizootic, and in monkeys and rabbits; he therefore changed its name to $B$. bronchisepticus. This organism has in recent years been included in the Brucella group, whose type species, Br. melitensis, it resembles in both individual and colonial morphology, and in its inability to ferment carbohydrates. However, the conspicuous degree of antigenic similarity letween $B$. bronchisepticus and $H$. pertussis that has become evident in the past seven years demands a reconsideration of its classification. Antigenic relationships alone do not establish a taxonomic relationship, but in this case other resemblances are sufficiently good to warrant provisional inclusion of $B$. bronchisepticus in the group containing $H$. pertussis. Thus, $B$. bronchisepticus produces in guineapigs lesions sinilar to those produced by $H$. pertussis in rabbits and puppies (Smitl 1913, Mallory and Hornor 1912, Nallory, Hornor and Henderson 1912). Both $H$. pertussis and $B$. bronchisepticus are natural pathogens in the upper respiratory tract, and, according to Rhea (1915) B. bronchisepticus lesions in rabbit lungs are similar to the lung lesions of human whooping cough. And, finally, the general cultural resemblances between $H$. pertussis and $B$. bronchisepticus are as close as those between B. bronchisepticus and Br. melitensis (Chapter 34). We have therefore provisionally included both the Bordet-Gengou bacillus and $B$. bronchisepticus in the genus, and have emended the generic definition accordingly (see Lwoff 1939). It should, however, be pointed out that H. bronchisepticus grows very much more readily than any other member of the Hamophilus groupso much so that it has been classified by some workers with the paracolon bacilli. Such a difference, taken together with the free growth on plain nutrient agar and the possession of motility, renders the classification of this organism in the Hcmophilus group necessarily tentative.

Morphology.- H. influenza, as originally described by Pfeiffer (1893), and as most commonly seen in strains recently isolated from cases of influenza, is a short rod, so short as to be almost coccal. It is very small, $1-1.5 \mu$ by $0 \cdot 3-0.4 \mu$, with rounded, sometimes rather pointed ends. In some cultures these cocco-bacillary forms are the only forms seen. More usually, among these predominating short forms, are found a proportion of longer bacilli, and a few long thread forms. In other cultures, the cocco-bacilli may be relatively scanty, or altogether absent; 
and longer and somewhat stouter rods may predominate. Other strains, again, may present an entirely different picture, the bacilli being thin, long, wavy or curved and sonetimes lying together in tangled masses. In films prepared from strains showing any of these diverse morphological types, but especially those which show some proportion of thread forms, it is not umusual to encounter large, spherical swollen bodies, often attaining a diameter of $2-3 \mu$, or even more. These are sometimes attached to the end of a thread, sometimes laterally, and sometimes apparently at the end of a short lateral stalk (Wade and Manalang 1920, Kristensen 1922). Another form which is occasionally met with consists of a long thread with an enormous fusiform swelling, situated centrally or towards one end.

This morphological diversity raises a problem of considerable difficulty from the point of view of classification. Any one of the types we have referred to may predominate in a single strain; and two strains may yield a microscopical picture so different that it is difficult to believe that we are dealing with a single bacterial

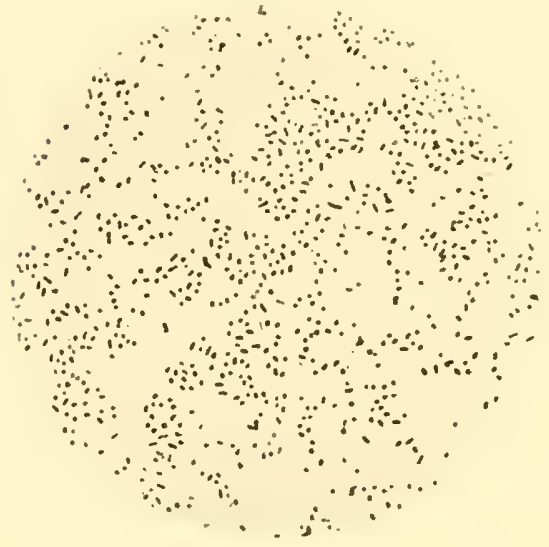

Fig. 163.- H. influenzo.

From 24-hours' culture on Fildes' agar, showing typical cocco-bacillary forms $(\times 1000)$.

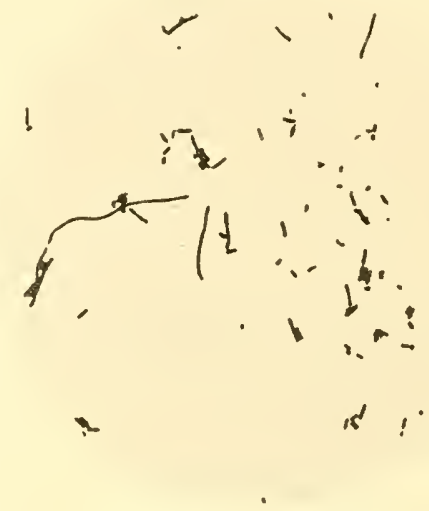

FIG. 164.-H. influenza.

From 24-hours' culture on Fildes' agar, showing short and long bacillary forms $(\times 1000)$.

species. The only test of the real significance of such morphological differences is their constancy ; and this test is not so easy to apply as might at first sight appear. It is quite certain that many strains maintain their morphological individuality over long periods of artificial cultivation, involving numerous successive subcultures; and Dible (1924) regards these morphological characters as sufficiently well differentiated, and sufficiently stable, to justify the recognition of several different varieties, or types. Many of those who have studied this group, on the other hand, have rejected morphological criteria as a basis of classification within the group, on the following grounds. If a large series of strains is carefully examined the variation in form is not found to be discontinuous. In a small sample of strains it is easy to obtain an appearance of discontinuity; but, unless one classes all strains which depart from the typical cocco-bacillary form in a single heterogeneous group, it is not possible to define a limited number of morphological categories, to which all strains can be definitely assigned. Between the minute, short bacilli, and the long tangled threads, there exists a long range of intermediate forms. Moreover, while some strains are morphologically homogeneous, others display, in the 
same culture, a heterogeneous mixture of cocco-bacilli, rods, and long threads. Finally, though many strains retain their morphological eharacters unchanged for weeks, months, or longer periods, others show quite definite changes in form after a few cultures. Wollstein (1915) has noted the frequeney with which the coccobacillary type acquires the power of forming threads in artificial culture; and there has been a very general tendency to discard the old conception of a para-influenza bacillus, differentiated on purely morphological grounds (Pfeiffer 1893), in favour of the tentative view that such differences, unless associated with other well-marked characters, must be disregarded by the systematist (Kristensen 1922). Snith (1931) records a careful study of a series of strains isolated from the human nasopharynx. In almost every instance morphological variation occurrerl after a varying period of cultivation in artificial media, the most usual change being from the short cocco-bacillary form to longer bacilli, or to curved or jointed filaments. This change was in general associated with a change in colony form (see below).

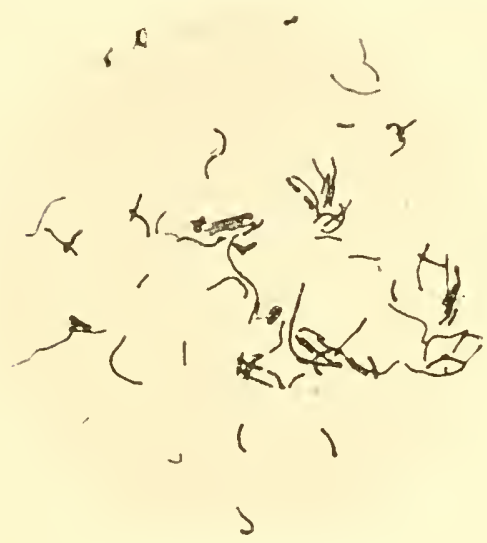

FIa. 165.-H. influenze.

From 24-hours' culture on Fildes' agar, atypical form, showing long, curved bacillary forms $(\times 1000)$.

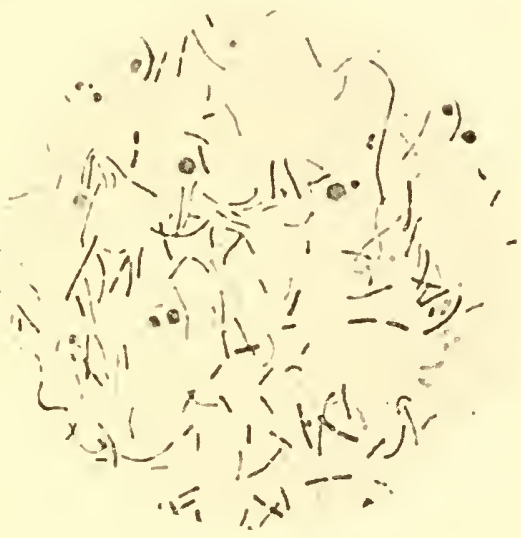

Fia. 166.-H. influenzoe.

From 24-hours' culture on Fildes' agar, showing thread forms, and large spherical borlies $(\times 1000)$.

There is general agreement that it is possible to make a rough classification of strains into two groups, "typical" and "atypical" on the basis of morphology alone; and there are indications that, apart from certain strains isolated from cases of meningitis (see Cohen 1909, Ritchie 1910, Henry 1912, Wollstein 1915), the typical morphology predominates among strains isolated from pathological conditions. The studies of Pittman (1931), which will be discussed more fully in relation to the problem of antigenic structure, have emphasized the possible importance of this distinction. But the evidence at present available suggests that typical and atypical morphology are associated with a change presenting many analogies to the smooth $\rightarrow$ rough type of variation, rather than permanent char acters serving to differentiate stahle varieties or types.

The organisms of this genus form no spores and are, with the exception of $H$. bronchisepticus, non-flagellated. They are usually deseribed as non-capsulated, but since Pittman's (1931) description of a "smooth" eapsulated form of virulent $H$. influenzo it is now clear that capsulation is not an uncommon feature within the group. 
H. influenzoe stains with some difficulty, and many of the ordinary bacteriological dyes are unsuitable for this purpose. Dilute carbol-fuchsin applied for 5 to 15 minutes usually gives satisfactory results. All species within this genus are frankly Gram-negative and non-acid-fast.

It may be added that it is impossible to differentiate any given strain of $H$. pertussis from $H$. influenzo on grounds of morphology alone. If, however, large samples of strains are compared, certain modal differences may be observed. $H$. pertussis displays a more constant morphology than $H$. influenzce, and there is a marked tendency towards the predoninance of the short oval form of cell. Longer bacillary or thread forms may occur, but they are relatively uncommon.

Cultural Characters. Growth Requirements.- Since certain nutritional requirements provide the criteria by which this genus has been defined, and also the basis on which many of the species included within it are differentiated from one another, it will be convenient to discuss this aspect of their behaviour before dealing with their type of growth, enzymic activities, antigenic structure or pathogenicity. The most characteristic feature of the hamophilic bacilli is their failure to grow in the absence of certain factors which are present in blood. The ability of the influenza bacillus to grow on blood agar, and its inability to grow on agar, with or without the addition of serum, or other native protein, has been noted by all workers from Pfeiffer onwards. Grassuerger (1897) described another phenomenon - that of satellitism-which is highly characteristic of $H$. influenzo. In cultures on blood agar plates, streaked with sputum or with bronchial secretion, he noted the appearance of relatively large colonies of the influenza bacillus, with a slightly granular central portion. These large colonies ( $1 \mathrm{~mm}$. or more in diameter) always developed in the immediate vicinity of a colony of staphylococcus. Studying this phenomenon in greater detail, Grassberger streaked agar plates with a suspension of $H$. influenzo mixed with a small quantity of blood, and then inoculated the central portion of the plate with a trace of a pure culture of Staph. aureus. After 24 hours' incubation such plates showed a well-defined zone of colonies of $H$. influenzo, surrounding each colony of staphylococcus. A similar result was obtained with Staph. albus, Staph. citreus, and certain other chromogenic micrococci. These observations have been repeatedly confirmed (see Davis 1921, Kristensen 1922, and Fig. 167).

The inability of $H$. influenza to grow on serum agar indicates that some constituent of the red cells is essential for growth; and it was at first assumed that this constituent was hæmoglobin itself. More detailed study, however, showed that the addition to an agar medium, prepared with water or with peptone solution, of pure crystallized hæmoglobin does not suffice to ensure growth (Ghon and von Preyss 1904, Thalimer 1914, Davis 1917, Olsen 1920, Fildes 1921). It would appear (Fildes 1921) that the growth-promoting substance derived from the blood pigment is methæmoglobin, or hæmatin, rather than hæmoglobin itself. Hæmatin is more active in this respect than the other derivatives which have been tested; while hæmatoporphyrin is inactive (Fildes 1921). There is, however, another factor which comes into play, besides the presence of some suitable iron-containing pigment. Davis (1917) showed that $H$. influenzoe required for its growth the presence of two distinct substances: one contained in, or derived from, hæmoglobin; the other present in the tissues of various plants and animals, and synthesized by most bacterial species other than $H$. influenza. This second factor he likened to a vitamin. This substance, as compared with the factor provided by blood pigment, 
is relatively thermolabile; it is inactivated by heating to $120^{\circ} \mathrm{C}$. for 30 minutes. Both substances are present in blood. The label " $\mathrm{X}$ factor" . is generally applied to the substance present in blood pigments, the label " $V$ factor " to the relatively thermolabile substance provided by animal or vegetable tissues or by most bacterial cells (see also Thjotta 1921, Thjotta and Avery 1921, Davis 1921, Fildes 1922, 1923, 1924, Kristensen 1922, Valentine and Rivers 1927).

Pittman (1935) brought evidence to show that $\mathrm{V}$ factor was closely concerner in oxidation-reduction processes of the growing cell. With regard to $\mathrm{X}$ factor, early observations (Olsen 1920, Fildes 1921) suggested that its growth-promoting activity was correlated with its ability to act as a peroxidase ; but not all peroxides promote the growth of $H$. influenza, and certain iron compounds without peroxidase activity will act as $\mathrm{X}$ factor (Baudisch 1932). All such iron compounds that have been tested in this respect have shown catalase activity (see Davis 1921, Welster and Baudisch 1925, Bourn 1927, Baudisch 1932, Knight 1936). H. infuenzc grows poorly under anaerobic conditions, but Kopp (1927-28), Eirund (1929) and Anderson (1931) have recorded the anaerobic growth of certain strains. In these conditions, the organism grows in the absence of $\mathrm{X}$ factor. These facts together suggest that $\mathrm{X}$ factor is closely associated with the aerobic respiration of the bacillus.

It is now apparent from the work of the Lwoffs (Lwoff and Lwoff $1937 a, b, c$ ) that $\mathrm{V}$ factor is one of two co-dehydrogenases, di- and tri-phospopyridine nucleotide (see Chapter 3), and that the $\mathrm{X}$ factor is hæmin, which, they conclude, is normally utilized by $H$. influenza for synthesis of cytochrome, cytochrome-oxidase, catalase and peroxidase.

These observations clearly provide an explanation of the satellitism described by Grassberger. The staphylococci, and many other organisms of greater synthetic ability than the hæmophilic bacilli, synthesize the co-dehydrogenase, which diffuses into the medium and stimulates the growth of bacilli that require it. They explain, too, the anaerobic growth of $H$. infuenze without the help of $\mathrm{X}$ factor, since in the absence of molecular oxygen there is no need for that part of the cytochrome system which protects the organism against the inhibitory effect of oxygen (see Chapter 3).

It should be noted that Hoagland and his colleagues (1942) have found in blood an unidentified factor which stimulates the growth of $H$. influenzoe in the presence of optimum amounts of $\mathrm{X}$ and $\mathrm{V}$ factors.

Among the species included within this genus, besides $I$. influenzo itself, are (1) the bacillus associated with conjunctivitis, described by Koch (1887) and Weeks (1887), and commonly known as the "Koch-Weeks bacillus"; (2) the bacillus isolated by Friedberger (1903) from the prepuce of dogs, named by him $B$. homoglobinophilus canis and now known as $H$. canis ; (3) the organism isolated by Shope (1931) (see also Lewis and Shope 1931) from swine influenza and named by him $I I$. influenzce-suis; (4) the causative organism of whooping cough, described by Bordet and Gengou (1906) and named by them B. pertussis, now known as H. pertussis; (5) the causative organism of soft sore described by Ducrey as the probable cause of soft chancre (see ('hapter 79) and named $H$. ducreyi by Lwoff and his colleagues (Lwoff and Pirosky 1937, Lwoft 1939); (6) the bacilli described by Rivers (1922a) as $H$. para-influenze, associated with acute pharyngitis and bacterial endocarditis in man; and (7) the causative organism of broncho-pneumonia in rodents and dogs, described by Ferry (1912-13) as B. bronchisepticus and now 
named $H$. bronchisepticus. The nutritional requirements of most of these species have been studied in some detail by various observers, with results that may be summarized as follows :

With regard to the Koch-Weeks bacillus, the statements in the literature are somewhat contradictory (see Kristensen 1922); thus, some authors have stated that this organism grows on ascitic agar and hydrocele-fluid agar. Later studies by Fildes (1923) and by Knorr (1924), however, show quite clearly that the strains, isolated by them from cases of muco-purulent conjunctivitis, behave in exactly the same way as $I I$. influenze, and require both the $\mathrm{X}$ and the $\mathrm{V}$ factor.

H. canis is unable to synthesize the $\mathrm{X}$ factor, and is therefore dependent on hæmatin, or some similarly-acting substance. It can, however, synthesize the $\mathrm{V}$ factor, and hence it does not show the

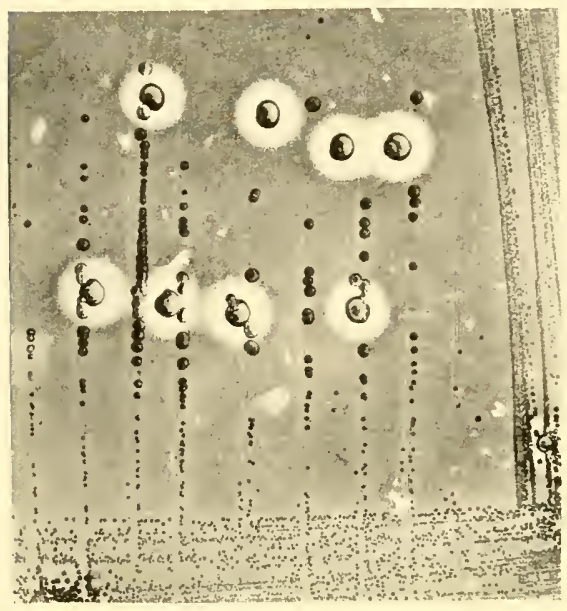

FIG. 167.

Showing satellite growth of $H$. infuenzce in the neighbourhoor of hæmolytic colonies of Staph. aureus on blood agar. phenomenon of satellitism, but can itself induce the formation of satellite colonies of $H$. influenzce (see Friedberger 1903, Krage 1910, Odaira 1911, Rivers 1922b, Kristensen 1922, Fildes 1923, Valentine and Rivers 1927). H. ducreyi resembles H. canis in its growth requirements (Lwoff and Pirosky 1937). Khairat (1940) has described a hæmophilic bacillus from a human endocardial lesion having the same $\mathrm{X}$ and $\mathrm{V}$ factor requirements as $H$. canis.

H. influenzo-suis requires both the $\mathrm{X}$ and $\mathrm{V}$ factors for its growth, resembling in this as in almost all other respects the human influenza bacillus (Lewis and Shope 1931).

H. para-influenze requires $\mathrm{V}$ but not $\mathrm{X}$ factor for growth. It shows the phenomenon of satellitism. H. pertussis and $H$. bronchisepticus differ from the other species within this genus in being capable of growth in the absence of both the $\mathrm{X}$ and $\mathrm{V}$ factors. For primary isolation of $H$. pertussis Bordet and Gengou (1906) employed an agar medium containing blood, glycerine and potato extract, and this medium, with various minor modifications, is still used for this purpose. Even on first isolation $H$. pertussis and $H$. influenzo show differences in their nutritional requirements. The former grows well on media containing large amounts of hlood and vegetable extract, poorly on media containing the $\mathrm{X}$ and $\mathrm{V}$ factors in the absence of other blood or tissue constituents; the latter grows better on Fildes' or Levinthal's medium than on media of the Bordet-Gengou type (see Gundel and Schlüter 1933). On subculture in the laboratory these differences become more marked. $H$. pertussis can readily be trained to grow on serum agar, and, with slightly more difficulty, on ordinary agar. There is, however, general agreement that on such media the organism rapidly loses its natural virulence (see below). These relations are clearly shown in Table 51. 
TABLE 51

Showng Growth of certain Bacterial Species in Peptone Water, with and WITHOUT $\mathrm{X}$ AND $\mathrm{V}$ FACTORS.

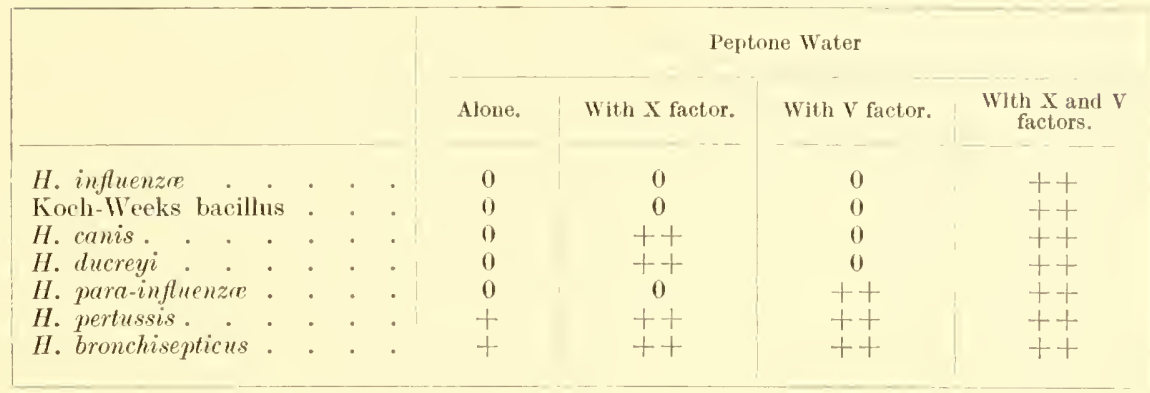

It may be convenient at this point to indicate the various media that are, at the present time, employed in the study of the hrmophilie bacilli.

Ordinary blood agar is by no means a satisfactory medium from this point of view ; far better results are obtained with media in which the red cells have been broken up, and their modified contents distributed throughout the medium. The well-known " ehocolate" agar, prepared by adding blood to melted agar, raising the mixture to the boilingpoint for 3 minutes, and then preparing slopes or plates from the chocolate-coloured mass, is a considerable improvement on the ordinary blood agar plate, but it shares the disadvantage that the medium is opaque.

The medium devised by Levinthal (1918) has the great advantage of being colourless and transparent. It is prepared by adding 5 per cent. of defibrinated rabbit or human blood to melted agar in a flask, and raising it to the boiling-point over a flame, with several shakings. The precipitate of eoagnlated blood and serum protein is allowed to settle, and the clear supernatant fluid is carefully decanted, or may be filtered through sterile glass-wool. The medium may be, for safety, sterilized by a further short heating, but must not be subjected to prolonged sterilization in the steamer.

Fildes (1920) has introduced a peptic digest of blood, which is preserved with chloroform and may be added to broth, or melted agar, as required. This medium, which is transparent and has the colour of ordinary broth or agar, gives eopions growths of $H$. influenza, and inhibits the growth of many other organisms. It is admirably suited for the primary isolation of the influenza baeillus. The ability to support the optimal growth of hæmophilic baeilli is not a property of blood from all species of animals. For example, on horse blood media, $H$. pertussis loses its smooth eharaeters more readily than on media made with human or sheep blood ('Toomey and Takaes 1938). The influenza bacillus may be even more susceptible to species variations in blood. Thus, Krumwiede and Kuttner (1938) deseribe thermolabile substances inhibiting the growth of $H$. influenzce and $H$. para-influenze in sheep, goat, bovine and human blood, but not in the blood of the rat, rabbit or gninea-pig.

For primary eulture from such a souree as the nasopharynx advantage may be taken of the selective action of penicillim, which inhibits the growth of Gram-positive coeci and of diphtheroid bacilli but has almost no action on $I$. influenze or $I$. pertussis (see Chapter 77).

With regard to other conditions of growth, the optimum temperature for $H$. influenza is in the neighbourhood of $37^{\circ} \mathrm{C}$. The minimal temperature for growth lies between $20^{\circ}$ and $25^{\circ} \mathrm{C}$. 'The same conditions hold for other hæmophilie bacilli. There is general agreement that $H$. infuenzo grows far better under aerobic than 
under anaerobic conditions. Statements with regard to its ability to develop under strictly anaerobic conditions are somewhat contradictory (see Kristensen 1922). Fildes (1921) states that $H$. influenzo gives good initial anaerobic growth on a suitable medium, but quickly dies out.

Cultural Reactions. Type of Growth.- The type of colony given by H. influenzo. on solid media varies widely with the kind of medium employed. On blood agar it forms tiny transparent, pin-point colonies, sometimes flat, and tending to become confluent, sometimes more convex, and with less tendency to confluence. On a more favourable medium, such as Levinthal's agar, and especially Fildes' agar, the colonies are far larger. After 24 hours' incubation they attain a diameter of $0.5-0.8 \mathrm{~mm}$. They are cirenlar in outline, raised and dome-shaped, with a slightly splayed-out, entire edge. The surface is usually smooth; the colony is translucent; there is little differentiation; and the growth emulsifies easily (Fig. 168).

On further incubation, and in many cases during the first 24 hours of growth, the colony becomes differentiated into a central portion with a granular or contoured surface, an intermediate flattened portion, and a sharply bevelled periphery

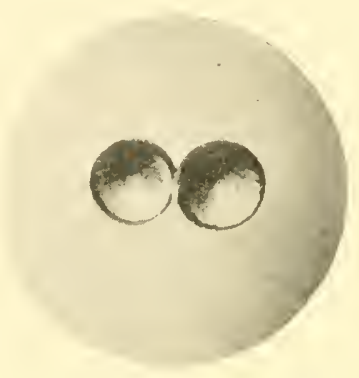

FIG. 168.- H. influenzie.

Colonies on Fildes' agar after 24 hours $(\times 8)$.

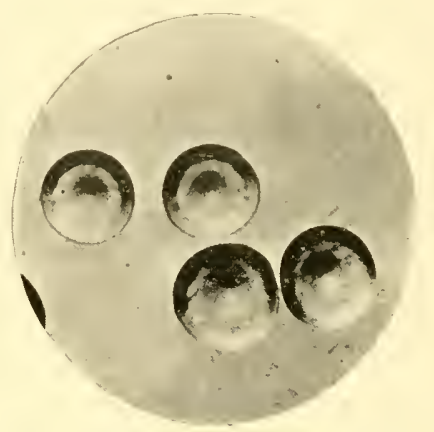

FIG. 169.-H. influenze.

Colonies on Fildes' agar after 48 hour's $(\times 8)$.

with a narrow splayed-out edge. Between the 24th and 48th hours there is usually a considerable enlargement of the colonies, which may attain a diameter of $1-1.5 \mathrm{~mm}$. This increase in size results in the formation of a flatter colony, retaining a raised central boss, sometimes smooth, sometimes granular or contoured (Fig. 169). Some colonies may be, from the start, flatter and more granular ; others may remain raised, conical and smooth, with little central differentiation. Kristensen (1922). lays considerable stress on such colonial differences, especially those which develop on blood agar, and apparently regards them as more important than cellular differences in morphology, in distinguishing "typical " from " atypical " strains. Smith (1931), in the study referred to above, noted a definite but not absolute correlation between morphology and colony form. The morphologically typical strains tended to give, on Fildes agar, smooth colonies with little differentiation. The morphologically atypical straius, or variants, tended to produce a more gramular colony, with earlier and more considerable differentiation.

The "smooth" strains described by Pittman (1931) have distinctive colonial characters. Levint hal's agar gives rather better differentiation than Fildes'. Smooth strains give relatively large colonies, sometimes attaining $3 \mathrm{~mm}$. in diameter. They 
are slightly opaque, and viewed by obliquely transmitted light they are iridescent. This iridescence is, perhaps, their most characteristic property. The surface is smooth and slightly mucoid in appearance. The edge is entire.

These "smooth" strains are described by Pittman as readily giving rise to " rough" variants producing colonies of varying granularity and differentiation, the organisins composing them being in the form either of short bacilli, or of longer rods or threads. The rough variants were never capsulated.

The relation of Pittman's " smooth" strains to the strains isolated by other workers from infective conditions, or from the normal respiratory tract, raises a problem in terminology which is at the moment difficult to solve. Her observations have been confirmed, in whole or in part, by several subsequent workers, and there can be little doubt that the strains she has differentiated correspoud to a form of II. influenze that is frequently associated with acute infections in man. If, however, we accept Pittman's colonial differentiation as a criterion separating "smooth" from "rough" strains, we must include among our " roughs " many, probably the majority, of those strains that give, on Fildes' or Levinthal's agar, colonies of the type that earlier observers had generally regarded as "smooth," and that consist of morphologically typical cocco-bacillary organisms. These difficulties may be resolved if we accept three forms of $H$. infuenzo, the mucoid capsulated II form with a characteristic specific soluble substance, an $\mathrm{S}$ form without a capsule, and $\mathrm{R}$ forms (Chandler, Fothergill and Dingle 1937, 1939) (see p. 799). The balance of evidence is, we think, in favour of accepting Pittman's nomenclature, but this question will be discussed more fully in relation to antigenic structure.

$H$. canis forms colonies that are at first indistinguishable from those of $I$. influenza, but as they grow older they become larger and distinctly more opaque.

$H$. pertussis, when grown on Bordet-Gengou medium, also forms colonies that, during the early stages of growth, may resemble those of $H$. influenzo. But when incubation is prolonged beyond 24 hours the colonies become larger, more opaque, and greyish in colour, a form that is never assumed by $H$. influenzo. They are also smoother, more shining and more distinctly dome-shaped. The combination of slight opacity, greyness of hue, and shining surface, gives them an appearance that has not inaptly been compared to that of a bisected pearl. They have also been compared to drops of mercury; but this overstates their metallic appearance. A confluent row of colonies has been compared to an "aluminium streak," and this simile again is not inapt. As already noted, $H$. bronchisepticus grows much more readily than other members of the group, forming quite well developed colonies on plain nutrient agar within 24 hours.

In liquid media, such as Fildes' broth, the majority of strains of II. influenze give rise to a uniform turbidity, with or without a slight powdery deposit. Some, on the other hand, give a flocculent deposit, with a varying degree of turbiclity of the supernatant fluid. There is, as would be expected, a correlation between morphology and type of growth in a fluid medium. Cocco-bacillary strains give a uniform turbidity. Many of those showing long bacilli, or twisted and convoluted threads, give flocculent growths.

$H$. canis gives a diffuse growth with a slight deposit. So does $I$. perlussis.

Resistance. $-H$, influenze is killerl by exposure to a temperature of $50-55^{\circ} \mathrm{C}$. for 30 minutes. $I$. canis and $H$. pertussis behave similarly.

Hæmolysin Production.-Before considering the fermentation of carbohydrates, or other substrates, it will be convenient to discuss the hæmolytic activity of the 
influenza bacillus, since this character serves to divide the species into two distinct types.

Pritchett and Stillman (1919) noted the occurrence, among a large sample of cultures of hæmophilic bacilli isolated from cases of influenza and from normal persons, of a small proportion of strains which produced a well-defined zone of hæmolysis on blood agar. These strains were morphologically of the bacillary or thread type. They were studied in greater detail by Stillman and Bourn (1920); and their occurrence has been noted by many subsequent workers. Kristensen (1922) studied several strains of these hæmolytic bacilli, and noted that some of them seemed less dependent on the presence of hæmoglobin than $H$. influenzox. On the other hand, the majority of his strains showed well-marked satellitism; thus demonstrating their dependence on the $V$ factor. Fildes (1924) found that 13 of 14 hæmolytic strains grew in the presence of the $V$ factor alone. In morphology these strains are, for the most part, definitely atypical, showing numerous threads, and coarser bacillary forms than are commonly encountered in $H$. influenzoe itself. The colonies produced by these strains tend to assume the characters which Kristensen regards as atypical, being more opaque and more friable than the typical form. Another striking characteristic of these hæmolytic strains is their tendency to die out in subculture, a character which has been noted by subsequent observers (Dible 1924). Kristensen notes that the power to cause hremolysis is maintained unaltered by those strains which survive artificial cultivation for considerable periods; and that there is no tendency for other strains to acquire this property. Dible (1924) studied 67 strains of hemophilic baeilli isolated from the nasopharynx of normal persons, and found 14 of them to be hemolytic. Five of these strains resembled the cocco-bacillary form of $H$. infuenzo, with the single exception that the bacilli were a little larger, and rather more definitely bacillary : the other 9 were atypical, in forming larger colonies, giving a flocculent growth in broth, or departing widely from the cocco-bacillary form. Valentine and Rivers (1927) report that the majority of these hæmolytic strains require the $\mathrm{V}$ factor only for their growth, while a minority require both the $\mathrm{X}$ and $\mathrm{V}$ factors; a proportion of non-hæmolytic strains of hæmophilic bacilli require the $\mathrm{V}$ factor only. Valentine and Rivers proposed the name $H$. parainfluenzce for the hæmophilic organisins requiring only $V$ factor, irrespective of hremolysin production. It is clear from their descriptions that organisms resembling Pfeiffer's bacillus in cultural characters and growth requirements may or may not form hæmolysin. We propose to adopt their nomenclature, and distinguish $I$. para-influenzo from $H$. influenzoe by $\mathrm{X}$ and $\mathrm{V}$ factor requirements and sub-divide each if necessary into hæmolytic and non-hremolytic varieties (see Miles and Gray 1938).

Biochemical Activities.-The study of the fermentation reactions of $H$. influenza, and of other hæmophilic bacilli, has been retarded by the difficulty experienced in preparing a medium which allows of copious growth, and has, at the same time, the transparency and absence of colour which are essential, if changes in hydrogen-ion concentration are to be detected by the usual methods. Some of the media which have been devised within recent years are, however, well adapted for this purpose.

Levinthal (1918) added various carbohydrates to the agar medium which he devised, tinted it with litmus, and tested the fermentative ability of several strains of $H$. infuenzoe. He noted acid production from glucose, but not from lævulose, lactose, mannitol or maltose. Messerschmidt, Hundeshagen and Scheer (1919), using a similar technique, noted slight acid production in glucose, but not in mannitol, lactose, or saccharose.

Stillman and Bourn (1920) employed a liquid medium prepared by adding an extract of boiled rabbit blood to peptone water, and carried out a careful series of tests on 119 strains of $I I$. influenzee and 29 hæmolytic strains. Hore than 90 per cent. of the 119 nonhæmolytic strains produced acid from dextrose and galactose, and reduced nitrates; 73 per cent. produced acid from lævulose, and about 25 per cent. from maltose, saccharose 
and dextrin; no strain fermented mannitol or lactose; 53 per cent. produced indole. Of the 29 hæmolytic strains, all fermented dextrose and reduced nitrates, the majority fermented maltose and saccharose, 3 fermented galactose, 10 levulose, and 15 dextrin; none fermented mannitol or lactose; 3 produced indole; and 4 formed gas. It would appear that the hæmolytic, as compared with the non-hæmolytic strains, ferment maltose and saccharose more frequently, galactose and levulose less frequently, and seldom produce indole; but the number of hæmolytic strains examined was small. Stillman and Bourn specifically note that they obtained irregular results when they carried out repeated tests on the same strains. Fildes (1924) notes that the hæmolytic strains studied by him fermented glucose, saccharose, and maltose ; but not lactose, dulcitol, or mannitol. The nonhæmolytic strains of $H$. influenze which he examined did not ferment any of these sugars.

Kristensen (1922) carried out a considerable number of fermentation tests, but obtained almost entirely negative results. It seems probable that these were due to an unsatisfactory technique.

Dible (1924), using a technique which did not differ essentially from that employed by Stillman and Bourn, obtained results which he regarded as sufficiently sharp and constant to afford a basis for a tentative grouping of his strains; though he notes that, of 25 strains which were retested after 8 months, 9 showed changes in their fermentation reactions. In 8 cases this change involved a loss of the power to ferment one or more carbohydrates; in the remaining instance a strain, previously inactive, was found to ferment glucose. It may be noted that, of 14 hremolytic strains, 9 fermented glucose and lævulose, none galactose, 8 saccharose, and 6 maltose, while none formed indole. Of 6 non-hæmolytic strains, which Dible excludes from the species $H$. influenzee on account of their bacillary or thread-like morphology, 4 fermented glucose, 4 levulose, 5 galactose, 4 saccharose and none maltose, while none produced indole. Of 45 strains which showed the typical minute bacilli and cocco-bacilli, 38 fermented glucose, lævulose and galactose, none fermented saccharose or maltose, while 16 produced indole. Dible's results thus tend to confirm those of Stillman and Bourn with regard to the frequency of saccharose fermentation, and infrequency of indole formation, among the hæmolytic as compared with the non-hæmolytic strains.

In regard to the relation between morphology and fermentation reactions among the non-hæmolytic strains, Smith (1931) records observations on 143 strains isolated from the nasopharynx of normal persons. There was no clear-cut fermentative separation between morphologically typical and atypical strains, but, in conformity with the results recorded by other workers, it was found that the typical strains showed a more restricted enzymic activity than the atypical. Thus, $22 \cdot 6$ per cent. of the atypicals fermented saccharose, as compared with $4 \cdot 3$ per cent. of the typicals. The correlation between typical morphology and ability to form indole was further confirmed; 63.9 per cent. of the cocco-bacillary strains were indole-producers, as against 18.0 per cent. of the morphologically atypical strains.

Later work with para-influenzal strains makes it clear that the hæmolytic influenzal strains referred to ahove and the strains isolated in an epidemic of pharyngitis by Lamont (1926) had the biochemical reactions of H. para-influenzoe. Miles and Gray (1938) found that all of 12 hæmolytic strains of this organism were alike in fermenting dextrose, lævulose, maltose and sucrose, and in producing no indole. The reaction of 9 non-hemolytic strains of $H$. para-influenzo were variable, though all fermented dextrose and sucrose and two were indole-positive.

On the basis of hæmolysin production, indole production and other fermentative reactions, taken in conjunction with morphology and dependence on the $\mathrm{X}$ and $\mathrm{V}$ factors, we may, then, recognize several different types of $H$. influenza.

(1) Typical $H$. influenza-requiring both $\mathrm{X}$ and V factors, showing a predominantly cocco-bacillary morphology, not producing hæmolysis, usually showing a restricted range of enzymic activities, particularly in failing to ferment saccharose, and usually producing indole. 
(2) Atypical $H$. influenze - requiring both $\mathrm{X}$ and $\mathrm{V}$ factors and not producing hæmolysis, but differing from typical strains in showing a predominantly bacillary or filamentous morphology, fermenting saccharose and some other substrates more frequently, and less frequently producing indole.

(3) A small group of hæmolytic influenza bacilli that require both the $\mathrm{X}$ and $\mathrm{V}$ factor for growth.

We have not, it will be noted, made any reference in the classification given alove to Pittman's "smooth" strains. These will be further considered in relation to antigenic structure.

The $H$. para-influenze strains resemble the atypical $H$. influenzex strains in morphology in fermenting saccharose and other substrates more frequently, and in less frequently producing indole. The fermentation reactions of $H$. ducreyi have received little attention. Khairat's (1940) organism, resembling it in requiring $\mathrm{X}$ but not $\mathrm{V}$ factor, fermented dextrose, sucrose, maltose and lactose in 2-3 days, and the polysaccharides dextrin, glycogen and starch in 9-10 days.

The fermentation reactions of $H$. canis were studied by Rivers (1922b), who records the formation of acid in dextrose, lævulose, galactose, mannitol, saccharose, and xylose, but not in maltose, lactose, dextrin, arabinose or glycerol. Indole was produced by all strains examined and nitrates were reduced. Fildes (1924) states that $H$. canis ferments glucose, saccharose and mannitol; but not lactose, dulcitol or maltose.

The strains of $H$. influenzoe-suis tested by Lewis and Shope (1931) are recorded as producing no change in dextrose, lactose, saccharose, mannitol, dulcitol, glycerol, inulin or arabinose. They produced no indole. They reduced nitrates. It has been noted that some observers have recorded similar negative results with strains of $H$. influenze, and it is doubtful whether this apparent absence of enzymic activity should be taken as differ. entiating the swine influenza bacillus from the human type in the absence of further evidence. Kirchenbauer (1934), who has studied several strains of this organism, confirms the reduction of nitrates and the absence of indole production.

$H$. pertussis, which is sharply differentiated from $H$. infuenzee in other ways, has been recorded by Stillman and Bourn (1920) as failing to ferment dextrose, lævulose, galactose, maltose, saccharose, dextrin, mannitol, lactose or inulin, as producing no indole and as failing to reduce nitrates. It produces a hazy zone of hæmolysis. H. bronchisepticus resembles $H$. pertussis in fermenting none of the commonly used carbohydrates.

It may be added that $H$. infuenzo-suis and $H$. canis are non-hrmolytic.

Many of the fermentative reactions within this group appear to be so irregular that they have little value in classification, or in the identification of particular strains. We may, however, tabulate for purposes of reference the reactions that have actually been observed, using + and - signs as rough indicators of the frequency with which the various substrates are attacked (Table 52).

TABLE 52

Showing the Fermentation Reactions of various Species, or Groups, of Henophilic Bacilli, and of the Pertussis Bacillus of Bordet and Gengou.

\begin{tabular}{|c|c|c|c|c|c|c|c|c|c|c|}
\hline & $\begin{array}{l}\text { Dex- } \\
\text { trose. }\end{array}$ & $\begin{array}{l}\text { Lævu- } \\
\text { lose. }\end{array}$ & $\begin{array}{c}\text { Galac- } \\
\text { tose. }\end{array}$ & $\begin{array}{l}\text { Mal- } \\
\text { tose. }\end{array}$ & $\begin{array}{l}\text { Lac- } \\
\text { tose. }\end{array}$ & $\begin{array}{c}\text { Sac- } \\
\text { charose }\end{array}$ & $\begin{array}{l}\text { Man- } \\
\text { nitol. }\end{array}$ & $\begin{array}{l}\text { Dex- } \\
\text { trin. }\end{array}$ & $\begin{array}{l}\text { Indole } \\
\text { formed. }\end{array}$ & $\begin{array}{c}\mathrm{Ni}- \\
\text { trates } \\
\text { reduced }\end{array}$ \\
\hline H. influenzos . & + & + & + & -+ & - & -+ & - & -+ & +- & + \\
\hline H. para-influenzæ. & + & +- & -+ & + & - & + & - & +- & -+ & + \\
\hline H.canis. . & + & + & + & - & - & + & + & - & + & + \\
\hline 11. pertussis . . & - & - & - & - & - & - & - & - & - & - \\
\hline
\end{tabular}




\section{The Antigenic Relationships of the Hæmophilic Bacilli.}

Taking first $H$. influenzo, as a speeies, the peculiarity that has emerged from most of the recorled attempts at serological analysis is its extreme antigenic heterogeneity.

By direct agglutination with 20 antisera, followed by absorption tests where necessary, Park, Williams and Cooper (1918) could find only four identical pairs among 160 strains. Valentine and Cooper (1919) record a similar experience. Among 10 strains isolated at autopsy, tested against the 10 homologous antisera, no two were identical. Among 73 miscellaneous strains tested against 18 antisera no two were identical. Among 54 strains isolated from a group of marines, and tested against 18 antisera, 2 strains from different individuals were identical. It was noted in this group that strains isolated from the same individual on different days were usually, but not always, identical. Among 28 strains isolated from the inmates of an orphan asylum, there was one pair of identical strains. Of 6 strains isolated from the members of a single family, all of whom had contracted infuenza at about the same time, no two strains were identical.

This extreme heterogeneity, as judged by agglutination tests, has been amply confirmed by numerous workers (Rivers and Kohn 1921, Yabe 1921, Anderson and Sichultz 1921, Cooper et al. 1921, Povitsky and Denny 1921, Kristensen 1922, Knorr 1924, Iizuka 1938, and others). Iizuka, for example, records more than 50 different agglutinating types among 249 strains isolated from sick and healthy persons.

The actual sigmificance of these earlier observations has been rendered very doubtful by the observations of Pittman (1931).

Among 97 strains of influenza bacilli isolated from various sources, she noted 15 that produced colonies of a characteristic "smooth" type (see above). All these 15 strains were isolated from sources, or under conditions, which indicated that they were playing a pathogenic rôle. In addition to forming a characteristic colony, the bacilli of these "smooth" strains were found to be capsulated. When tested by agglutination at $37^{\circ} \mathrm{C}$. these 15 strains were found to fall into two antigenic types $\mathrm{A}$ and $\mathrm{B}$, one containing 12 strains, the other 3 . This specificity was not apparent if the reactions were carried out at a higher temperature, a possible reason being the loss of the bacterial capsules. It was also found possible to separate from these 15 strains a soluble specific substance, apparently carbohydrate in nature, and presumably associated with the capsule. Precipitin tests carried out with this material gave the same antigenic grouping as the agglutination tests carried out at $37^{\circ} \mathrm{C}$. These smootlu strains, in artificial culture, readily gave rise to non-capsulated rough variants, usually with a bacillary or filamentous morphology. The rough variants no longer produced the soluble specific substance, nor did they conform to the antigenic grouping of the smooth parent strains. These findings were confirmed, in their essential points at least, by several subsequent workers (see Dochez et al. 1932, Wright and Ward 1932, Platt 1937). Later work has shown that characteristic "smooth" strains are commonly found among bacilli isolated from infections of the meninges. Among respiratory strains, either from infected or healthy persons, the "smooth" types are less common. Mulder (1937) for example, found 7 in 90 sputum strains, and Platt (1937) 16 in 86 nasopharyngeal strains. Respiratory strains tend to be serologically heterogeneous, whereas "smooth" meningeal strains tend to be homogeneous (Fothergill and Chandler 1936, Wilkes-Weiss 1937, Platt 1937). The homogeneity is not complete. Pittman has divided these capsulated smooth strains into six serological types, a, b, c, d, e and $\mathrm{f}$. Type $\mathrm{b}$ occurs with the greatest frequency, but all are rare in the normal upper respiratory tract (see, e.g., Silverthorne et al. 1943).

As in the pneumococci, type specificity depends on a polysaccharide component in the capsule of the organism (Platt 1937, Alexander and Heidelberger 1940). If we follow Chandler, Fothergill and Dingle (1939) and designate capsulated forms as $\mathrm{M}$ or mucoid forms, then both the $\mathrm{S}$ and $\mathrm{R}$ forms, which bave no type-specific capsular substances, display extreme antigenic heterogeneity. According to Platt (1939) individual non- 
capsulated strains appear to possess a relatively specific protein component $\mathrm{P}$, and a protein component M, which is common to most strains of $H$. influenza. It may be noted that several earlier workers found that complement-fixation tests gave evidence of the presence of an antigenic relationship that was not revealed by agglutination (Wollstein 1919, Bieling and Weichbrodt 1920, Kristensen 1922).

It is elear that the ehange that Pittman deseribes as occurring in her smooth strains falls within the definition that we arlopted for the $S \rightarrow R$, or the $\mathrm{M} \rightarrow \mathrm{S} \rightarrow \mathrm{R}$, variation in Chapter 8 , the loss of the surface antigen which determines type-specificity in the normal, virulent form of a bacillus. Pittman's capsulated forms are apparently the most virulent pathogenic type of $I I$. infuenzo; they are isolated commonly from the meninges in one of the severest of human infections caused by the organism ; and Gordon, Woodeock and Zimmemann (1944) report that meningeal infections with strains not among Pittman's types are less severe. They are also the most mouse-virulent of the observed varieties (Fothergill and Chandler 1936, Chandler et al. 1937, 1939, Raettig 1940). The implication that most of the strains isolated from the nose and throat of normal persons are in an intermediate or a "rough" phase, must, therefore, we think, be accepted. We must also, it would appear, accept the view that the antigens that dominate the rough forms in this bacterial speeies are more heterogeneous than those present in the normal smooth phase, a finding that differs from that recorded for most other groups.

It will be noted that, under this definition, all the strains referred to in preeeding sections as "atypical " and many, probably the great majority, of those referred to as "typieal," would be elassed as rough variants. The antigenic strueture of the para-influeuza baeilli has not yet been submitted to any special study; but it seems very unlikely that any of them would fall into Pittman's " smooth " category. Whether they are in any way antigenically different from the non-hæmolytic rough forms we do not know. Miles and Gray (1938) found an antigenic relationship between a proportion of the strains of non-hæmolytie H. para-influenzo they studied.

The Koch-Weeks bacillus, in its serological relationships as in all its other characters, appears to be indistinguishable from $H$. influenze. Knorr (1924) has found that different strains of this organism show marked antigenic heterogeneity, while some strains are identical with certain strains of the influenza bacillus.

A small sample of strains of $H$. influenzce-suis exammed by Lewis and Shope (1931) showed the same type of antigenic heterogeneity that is encountered among the ordinary strains of human influenza bacilli. Comparison with a few strains of $H$. influenzce of human origin did not reveal any example of antigenic identity, though there was some overlapping in cross-agglutination tests. Similar findings are recorded by Kirchenbaner (1934). These observations were made before the publication of Pittman's findings, so that there was no differentiation between smooth and rough strains.

W'e have as yet no information in regard to the antigenic relationships of $H$. canis.

$H$. pertussis differs from $H$. influenze in that all recently isolated smooth strains appear to belong to a single antigenic type. Moreover, it would seem that all straius, when first isolated from the body on an optimal medium, are in the smooth state. The behaviour of these strains on artifieial culture raises points of considerable interest.

Bordet and Sleeswyk (1910) noted that recently isolated strains of $H$. pertussis, grown on the Bordet-Gengou medium, all agglutinated with a serum prepared against any one 
of them. Strains that had been trained to grow on agar, however, failed to agglutinate with the sera prepared against the recently isolated strains, and sera prepared against the agar strains failed to agglutinate the strains grown on the Bordet-Gengon medium. These observations were confirmed and extended by Bordet (1912). The change in antigenic structure was ascribed to the medium, but it was noted that a similar change might occur on a blood-containing medium after repeated subculture. Host observers have confirmed Bordet's findings that all recently isolated strains belong to a single serological type (see Kristensen 1922, 1927). A few have recorded the existence of two different types among strains maintained permanently on a blood-containing medium (Krumwiede et al. 1923); but there is little doubt that such findings have been due to the slow occurrence of an antigenic variation that takes place more rapidly when smooth strains are grown on an unsuitable medium. Leslie and Gardner (1931) made a careful study of 32 strains of $H$. pertussis, none of which had been regarded as rough variants. They found that these strains fell into four different antigenic groups, to which they refer as Phases I, II, III and IV. Of 20 recently isolated strains 18 fell into Phase I, and 2 were intermediate between Phase I and Phase II. Of 7 laboratory strains that had been maintained on an egg medium, 3 were in Phase III and 4 in Phase IV. Of 5 other laboratory strains, one was intermediate between Phases II and III, 3 were in Phase III and 1 in Phase IV. Studies by later workers (Shibley and Hoelscher 1934, Toomey et al. 1935) have been in general agreement with Leslie and Gardner's findings, though they regarded the varions phases as arbitrary stages in the course of an $S \rightarrow R$ variation, depending on the amount of Phase I antigen on the bacillary surface (see also Toomey, Takacs and Ranta 1936, Toomey and Takacs 1937). Flosdorf, Dozois and Kimball (1941) on the other hand, find, like Leslie and Gardner, that the phases differ qualitatively, and suggest the following antigenic structure for three of the four phases they studied:

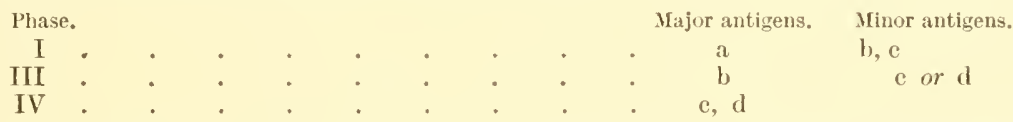

They also described a new Phase $\mathrm{X}$, related to III and IV. Taking these studies as a whole it seems safe to conclude that $H$. pertussis, in the form in which it exists in the tissues or in recent cultures on Bordet-Gengon medium, belongs to a single, homogeneous antigenic type; but that an $\mathrm{S} \rightarrow \mathrm{R}$ variation occurs somewhat readily, even in cultures kept on a blood-containing medium, and very readily on less favourable media. This variation is apparently step-like, so that intermediate stages exist between the normal smooth form, which corresponds to Leslie and Gardner's Phase I, and the fully developed rough form which corresponds to their Phase IV. It was noted by Leslie and Gardner that there is no very obvious and striking colonial difference between the rough and the smooth forms, nor any constant and measurable difference in salt sensitiveness, though strains in their Phase III and Phase IV are, on the average, rougher in colonial appearance and less stable in saline suspensions than strains in Phase I or II. The S form is capsulated (Lawson 1933). The capsular substance, which determines the agglutination of the $\mathrm{S}$ form by smooth antisera, is readily removed by washing (Miller 1937). Flosdorf, Kimball and Chambers (1939) and Flosdorf and Kimball $(1940 a, b)$ have studied this soluble agglutinogen extensively. It removes agglutinating antibodies from smooth antisera. It may be liberated by sonic vibrations. It is non-toxic and induces agglutinins in the rabbit and stimulates pertussis immunity. By tryptic digestion of $H$. pertussis, Cruickshank and Freeman (1937) obtained a carbohydrate-containing water-soluble fraction capable of inducing active immunity to experimental infection in mice. It is probable that this fraction is the same as the "agglutinogen " of later workers, mixed perhaps with some toxin. Smolens and Mudd (1943) obtained a large yield of agglutinogen by acid extraction of the bacilli.

Eldering and Kendrick (1937, 1938) and Bradford and Slavin (1937) isolated an organism from cases of whooping cough which differed from $H$. pertussis in producing definito 
hæmolysis on Bordet-Gengou medium, in growing more profusely and developing a brownish pigment on nutrient agar, and in producing a large amount of catalase. The strains isolated were antigenically homogeneous, and cross-agglutinated with Phase $\mathrm{I} H$. pertussis and $H$. bronchisepticus. Like $H$. pertussis, the organism was serologically characterized by a readily extractable, non-toxic and stable agglutinogen, having a minor antigenic component in common with H. pertussis (Flosdorf, Bondi and Dozois 1941, Bondi and Flosdorf 1943). This organism is now gencrally known as $H$. parapertussis.

The antigenic relationship of the agglutinogens of these three organisms is paralleled to some extent by an antigenic relationship of their toxins (see below). There are cultural resemblances between the three, and all are associated with infections of the lung in the higher mammals. These facts justify the provisional inclusion of $\mathrm{Br}$. bronchiseptica in the group containing $H$. pertussis, and we have accordingly implemented the suggestion of Eldering and Kendrick and of Evans and Maitland (1939), and renamed Br. bronchiseptica, H. bronchisepticus (see also Watanabe 1938).

It is clear that, as far as whooping cough is concerned, any bacillary material used for whooping cough inoculation must be derived from organisms in Phase I, so treated that the very soluble agglutinogen is not removed during the preparation of the vaccine; it may also be necessary to include in the vaccine the major antigens of $H$. parapertussis.

Finally, we may note that such comparative tests as have been performed show little if any antigenic relationship between $H$. pertussis and H. influenze (Odaira 1911, Shiga et al. 1913, Winholt 1915, Olmstead and Povitsky 1916, Kristensen 1922, Schlüter 1936).

\section{Pathogenicity and Toxin Production.}

The probable rôle of $H$. influenze in human influenza, which is now known to be a virus disease, is considered in Chapter 74. It is a common cause of sinusitis, alone or in association with the pneumococcus, an occasional cause of meningitis, almost always in children, and a rare cause of ulcerative endocarditis. The KochWeeks bacillus has been isolated from epidemics of conjunctivitis in many parts of the world, children being mainly infected. As, however, there is no known method by which this organism can be distinguished from $H$. influenze, it seems unnecessary to regard it as a different species.

$H$. para-influenze is occasionally associated with acute pharyngitis; it is a rare cause of ulcerative endocarditis, though probably less rare than $H$. influenzae itself (Miles and Gray 1938), and it is occasionally found in infected wounds and sinuses.

There appears to be little doubt that $H$. ducreyi is responsible for soft chancre, and for the buboes which are sometimes associated with the primary lesion.

$H$. bronchisepticus is essentially parasitic, giving rise to lesions in the respiratory tract of dogs, monkeys, guinea-pigs and other laboratory animals: it is occasionally found in the nasopharynx of man. It appears to be a secondary invader in dogs suffering from distemper, being frequently responsible for the pulmonary complications of the disease (M'Gowan 1911, Laidlaw and Dunkin 1926). Spooner (1938) found it playing a similar rôle in a spontaneous distemper-like disease of ferrets.

$H$. pertussis is the cause of whooping cough, and as such is one of the more important human pathogens. $H$. parapertussis appears to be responsible for a minority of cases of whooping cough. Unlike $H$. influenze, $H$. pertussis seems seldom to play a harmless parasitic rôle.

Both $H$. pertussis and $H$. bronchisepticus elaborate at least one toxin. H. pertussis toxin was first described by Evans and Maitland (1937), who extracted it 
from ground-up bacilli. It was lethal on intravenous injection into guinea-pigs. and produced areas of necrosis on intradermal injection in the rabbit. Evans and Maitland's preparations also contained the agglutinogen; this proved to be distinct from the toxin, since antisera to the extract were protective against experimental infection, agglutinated bacillary suspensions, but had no antitoxic activity as judged by their ability to modify skin neerosis induced by the toxin. The toxin was easily destroyed by formalin, was unstable at $37^{\circ} \mathrm{C}$, and was rapidly destroyed at $55^{\circ} \mathrm{C}$. A similar toxin with very similar properties was olstained from $I I$. bronchisepticus (Evans and Maitland 1939) and from H. parapertussis (Brueckner and Evans 1939). The toxin, presumably owing to its marked instability, did not at first appear to be antigenic. Evans $(1910,1942)$ later found that formolized toxin was antigenic, and that antitoxin prepared against it neutralized the toxins of $H$. pertussis, $H$. parapertussis and $H$. bronchisepticus. It is of practical interest that he was unable to induce antitoxin formation in the rabbit by the injection of whole bacilli, but found that toxic extracts of the bacilli were antitoxinogenic (see also Katsampes, Brooks and Bradford 1942). These observations have been confirmed and extended (Flostorf, Bondi and Dozois 1941, Ehrich, Boudi, Mudd and Flosdorf 1942, Eldering 1911, 1942). Flosdorf and his colleagues distinguish in $H$. pertussis a feebly antigenic thermostable toxin in addition to the thermolabile toxin of Evans and Maitland. Toxin is produced by all phases of $H$. pertussis, though most abuudantly by Phase I (see also Wood 1940, Roberts and Ospeck 1942). Eldering obtained toxic fractions from $H$. pertussis, H. purapertussis and $I$. bronchisepticus, and demonstrated varying degrees of cross-protection against infection by living bacteria, in animals actively immunized by the fractions. It is probable that her results reflect the antigenic similarity of both toxins and agglutinogens present in the extracts.

The mode of action of the toxin is at present obscure. Ehrich and his colleagnes (1942) describe generalized degenerative ehanges in the viscera of intoxicated rabbits, particularly in the lymphoid tissue. Given intratracheally, the heat-labile toxin produees in the lungs of rabbits a severe cedematons reaction, followed by a eharacteristic aceumu. lation of macrophages in the alveoli, of lymphoeytes round the blood ressels, and severe necrosis in scattered areas - a histological picture not unlike that found in the lung in whooping congh; antitoxin protected rabbits against this effect (Sprunt and Martin 1943). Though the precise rôle of toxin and antitoxin in infection with $H$. pertussis is not yet fully understood, the protective action of antitoxin in experimental infection appears to be limited to neutralizing the toxin eontained in the infecting dose, and thus reducing the likelihood of the organisms establishing a foothold in the tissues. Thus, Anderson and North (1943) protected mice against an intraperitoneal injection of $H$. pertussis with antitoxin, but not with antibacterial sera. Systemically administered, antitoxin had no effect in amimals infected by the nasal route, but antibacterial serum was effective. In their hands toxin had no aggressive action (see Chapter 48) in nasal infections. It should be noted, however, that North and his eolleagues (1939) found that in intranasally infected mice the protective effect of human sera from pertussis eonvalescents could not be aecounted for simply in terms of agglutinin or toxin-neutralizing power, though the effect as a whole appeared to be antibacterial. The labile toxin, however, may have immunizing properties, for in a later study (194l) of active immunization they found that whole bacilli treated with the minimum of heat $\left(58^{\circ} \mathrm{C}\right.$. for $8 \mathrm{~min}$.) or with phenolic preservatives, induced a higher degree of aetive immunity than bacilli preserved with formalin or heated to $60^{\circ} \mathrm{C}$. for one hour. Evans (1944) confirmed the inability of intravenous antitoxin to protect mice against intranasal infections, but found that antitoxin mixed with the bacteria before instillation into the nose lowered their infectivity, 
suggesting that in these circumstances antitoxin had an anti-aggressive effect. Ospeck and Poberts (1944) were able to protect mice and rabbits against live bacilli or toxin by previously administered antitoxin. (For active immunization of laboratory animals by $H$. pertussis and various fractions of the organism, see also Cruickshank and Freeman 1937, Niller and Silverberg 1939, Nishulow et al. 1939, Silverthorne 1940, Silverthorne and Cameron 1942, Holm and Bunney 1942, Lapin 1942, Strean et al. 1941).

H. influenzo-suis plays an important part in swine influenza (see Chapter 74). $H$. canis was isolated by Friedberger (1903) from 19 of 20 dogs suffering from a suppurative inflammation of the prepuce, but he was unable to reproduce the disease with it, and concluded that it was a harmless parasite of the preputial sac. It has also been isolated from normal dogs by Krage (1910), Kristensen (1922), Rivers (1922b), and Kirchenbauer (1934).

\section{Experimental Infections.}

H. influenzox.-Attempts to produce an infection resembling influenza in man by experiments on human volunteers or on the higher apes are considered in Chapter 74 .

As regards the usual laboratory animals, the injection of large doses of living culture (the growth from $\frac{1}{2}$ to 1 blood-agar slope suspended in saline) into the peritoneum of rabbits, guinea-pigs, or mice, often results in death within 24-48 hours. At necropsy petechial hrmorrhages may be found, scattered over the peritoneum, and sometimes over the pleura. The suprarenals may be congested or hæmorrhagic. The organisms can be recovered from the peritoneal cavity, but not often from the heart's blood. The cause of death seems to be a toxæmia, rather than an invasive infection (see Pfeiffer 1893, Delius and Kolle 1897, McIntosh 1922). Similar results may be obtained with filtrates of cultures in liquid media, and these may produce death on intravenous injection into rabbits or guinea-pigs, though relatively large doses $(0.5-5 \mathrm{ml}$.) are usually required (see Parker 1919, Ferry and Houghton 1919, Wollstein 1919, McIntosh 1922). There is no evidence that these filtrates contain an exotoxin in the usually accepted sense. In view of Pittman's observations and of her reports that her smooth strains are more virulent than the usual rough strains, it is of interest to note that many observers have recorded wide variations in virulence when a number of strains are tested by the intraperitoneal injection of living cultures. McIntosh (1922), for instance, found that only a small minority of recently isolated strains proved to be of high virulence when tested in this way. It would seem also that strains of $H$. influenzce isolated from cases of meningitis are usually far more virulent for laboratory animals than strains isolated from the respiratory tract, and that some of these meningeal strains have definite invasive powers (Cohen 1909, Henry 1912, Wollstein 1915). The incorporation of the intraperitoneal infecting dose in mucin-a techmique which has been successfully applied to enhancing the virulence of meningococci (see Chapter 23)-increases the virulence of $H$. influenzox; small doses produce a fatal septicæmia in mice against which anti-influenzal horse serum is protective (Fothergill, Dingle and Chandler 1937) (see also Silverthorne 1940). Certain strains of $H$. influenzoe of human origin give rise to a fatal infection after intracerebral injection of about 2,000 organisms into mice; other strains, and strains of $H$. para-influenzoe are non-virulent by this route (de Torregrosa and Francis 1941).

H. pertussis. - The effect of the intraperitoneal injection of this organism into rabbits or guinea-pigs is very similar to that of $H$. influenzo. Here again large doses are required to produce death, and the infection seems to be toxæmic rather than invasive (Bordet and Gengou 1907, 1909, Wollstein 1909). Leshie and Gardner (1931) carried out a careful series of experiments in which they determined the relative toxicity of suspensions of strains of $H$. pertussis, antigenically in Phase I, II, III or IV, by intraperitoneal injections in guinea. pigs. They found that the minimal lethal dose of strains in Phase III or IV (rough strains) was twenty to thirty times greater than the minimal lethal dose of strains in Phase I or II (smooth, or relatively smooth strains). 
The intranasal instillation of $H$. pertussis into anæsthetized mice produces a patchy or diffuse interstitial pneumonia, leucocytic infiltration round vessels and bronchioles, proliferation of the bronchiolar epithelium, and mucous secretion in the bronchioles containing masses of bacteria (Bumet and Timmins 1937, Bradford 1938). The histological picture in many respects resembles that of the lung in human pertussis, and clearly offers a near approach to the natural disease for immunological study. For intraperitoneal infection, the normally low virulence of $H$. pertussis by the intraperitoneal route may be enhanced by starch (Powell and Jamieson 1937) or mucin (Silverthorne 1938). Witebsky and Salm (1937), using rabbits injected intradermally, produced inflammatory lesions followed in $2-3$ days by necrosis.

After intratracheal inoculation, Culotta, Harvey and Gordon (1935) produced in three monkeys a disease with a 10-day incubation period, a catarrhal stage, and a febrile coughing stage not unlike human pertussis. By similar means Sprunt, Martin and MeDearman (1938) produced an interstitial pneumonia in the monkey, characterized by a mononuclear cell reaction, and accompanied by a lymphocytosis; and North and his colleagues (1940) induced in Macacus monkeys an infection which by the seventh day resulted in a sticky tracheal and bronchiolar exudate full of $H$. pertussis, and pulmonary congestion with conspicuous fibrinous and cellular infiltration, both interstitially and in the alveoli. None of the monkeys developed a cough.

H. influenzo-suis.-In association with a filtrable virus (see Chapter 74) this organism is an important natural pathogen of swine, and the disease can be experimentally produced in these animals. In relation to the small animals of the laboratory this organism appears to behave much in the same way as $H$. influenzo. Large intravenous injections may be fatal for rabbits, and large intraperitoneal injections for guinea-pigs or mice; but the results are very irregular, and there appear to be great differences in the virulence, or toxicity, of different strains (see Lewis and Shope 1931, Kirchenbauer 1934).

$H$. canis.-The data with regard to the pathogenicity of this species for laboratory animals are extremely scanty. Rivers (1922b) notes that the intraperitoneal injection of I ml. of a 24 -hours' culture in blood broth failed to kill a monse ; $2 \mathrm{ml}$. intraperitoneally did not kill a small guinea-pig; I ml. intravenously did not kill a small rabbit.

$H$. ducreyi.-Tomasczerski (1903) was successful in reproducing the disease in human subjects with pure cultures. In man, progressive purulent lesions follow the intradermal injection of cultures; and it is apparently a common practice to separate $H$. ducreyi from contaminating saprophytes in genital material by injecting it intradermally into the patient (see Cunha 1939). Ulcerative lesions have followed the inoculation of monkers and rabbits with cultures several generations removed from primary isolation. (Relenstierna 1921, Nicolle 1923).

H. para-influenza.-This organism appears to be non-pathogenic for laboratory animals.

\section{Variation.}

The available data with regard to variation in the genus Homophilus have already been referred to in the discussion of antigenic structure and of pathogenicity.

Both $H$. influenzce and $H$. pertussis give rise in artificial culture to variants that are essentially of the rough type. It would, indeed, seem that these species are peculiarly liable to undergo this change. The evidence suggests that rough strains of $H$. influenza occur very commonly in the normal nasopharynx, so that the smooth $\rightarrow$ rough variation must be supposed in this case to be of frequent occurrence when the organism is living in its normal habitat. In the case of $H$. pertussis we have, at present, no evidence that rough variations occur among recently isolated parasitic strains; but there is much evidence to suggest that the production of rough variants is readily induced by growing the organism on a relatively unfavourable medium. 
We have, as yet, no evidence with regard to variation in the other species of this group.

\section{H. influenzæ}

Isnlation.--Isolated by Pfeiffer (1892) from cases of influenza in man.

Habitat.-Strict parasite, living particularly in the upper respiratory tract of man.

Uorphology.-In its typical form $H$. influenzec is a tiny cocco-bacillus ( $1-1.5$ by $0.3-0.4 \mu$ ). According to Pittman (1931), the bacillus in its virulent smooth form is capsulated. Most strains, even when first isolated from the tissues are non-capsulated, but it is possible that these should be regarded as rough variants. Among any large sample of strains, or in any one strain during prolonged subculture in the laboratory, wide departures from the typical morphology will usually be found. Longer bacillary forms and definitely filamentous forms often oceur, and the latter may show angular bendings or sinuous curves. In the filamentons forms globular or ovoid swellings are not uncommon. The organism is non-flagellated, and forms no spores. It stains feebly with many of the ordinary bacteriological dyes, more readily with dilute earbol-fuchsin. It is Gram-negative and not acid-fast.

Growth requirements. - H. influenzoe requires both the $\mathrm{X}$ factor and $\mathrm{V}$ factor for its growth. It grows far more readily under aerobic than under anaerobic conditions, and it would appear that some strains are incapable of prolonged anaerobic subcultivation. The optinal temperature for growth is in the neighbourhood of $37^{\circ} \mathrm{C}$.

Growth on Solid Media.-On Fildes' or Levinthal's medium the usual type of colony produced by $H$. influenze is transparent, or slightly opaque, circular and domeshaped, or slightly conical, with a slightly splayed-out entire edge. At the end of 24 hours' growth at $37^{\circ} \mathrm{C}$. these colonies usually attain a diameter of $0.5-0.8 \mathrm{~mm}$. On further incubation, and in some cases during the first 24 hours, the colony becomes differentiated into a central portion with a granular or contoured surface, an intermediate flattened portion, and a sharply bevelled periphery with a narrow splayedout edge. During the second 24 hours of growth the colony usually enlarges to a diameter of $1-1.5 \mathrm{~mm}$. There is a tendency, which is not absolute, for differentiation to occur carlier, and to be more pronounced, in strains that have an atypical morphology. The growth is butyrous and emulsifies easily.

Some strains, described by Pittman (1931) as smooth, and by Chandler, Fothergill and Dingle (1939) as mucoid, give colonies that differ from those described above in having a smooth, undifferentiated, slightly mucoid surface. They have an entire edge. They tend to attain a larger size (1-3 $\mathrm{mm}$. in diameter). They are slightly opaque; and, when viewed by obliquely transmitted light, they are iridescent Strains that give this type of colony show antigenic characters, and differences in pathogenicity, which are in accord with the view that they represent the "smooth" phase of the organism, while the more frequently encountered strains, having the colonial appearances previonsly described, are in the nonmucoid or the rough state, and are not iridescent.

Growth in Liquid . Hedia.-In a suitable liquid medium most strains of $H$. influenze give rise to a uniform turbidity, with or withont a slight powdery deposit. Some give a more flocculent deposit. The latter usually show an atypical morphology, and the colonial appearances associated with the more advanced stage of rough variation.

Resistance. $-H$. influenzo is killed by an exposure to a temperature of $50-55^{\circ} \mathrm{C}$. for 30 minutes.

Biochemical Activities.-H. influenzce usually ferments dextrose, though not vigorously, producing acid withont gas. Lactose and mannitol are never fermented. The action on maltose, saccharose and dextrin varies. Smooth and morphologically typical strains tend not to attack these substrates. The rougher, morphologically atypical strains ferment them rather more frequently. The production of indole shows a high correlation with otlier characters that differentiate between relatively 
smooth and relatively rough strains; a high proportion of the former, including both Pittman's smooth strains and the more "typical" strains isolated from the normal nasopharynx, produce indole, but only a small proportion of the latter. All strains reduce nitrates.

$H$. influenzce, as that species is here defined, does not as a rule produce hæmolysis, which has been recorded only in a very few strains.

Antigenic Structure.--The majority of smooth strains, as defined by Pittman (193I), fall into 6 well-differentiated antigenic types which appear to be characterized by specific polysaccharide surface antigens, sometimes occurring in a capsular form. The more common rough, or partially rough, strains are antigenically heterogeneous.

Pathogenicity.-Pathogenic for man, particularly in association with virus infections, or with other bacterial diseases. Produces toxic death when injected in large doses into laboratory animals, and infective death when injected in small doses together with mucin.

\section{The Koch-Weeks Bacillus}

There is no known way in which this organism can be distinguished from $H$. influenzce. The fact that strains so labelled have been isolated from the conjunctiva does not seem to warrant the allotment of a separate specific name.

\section{H. influenzæ-suis}

Isolated from cases of swine influenza in which it is associated with a filtrable virus. The characters of this species as recorded by Lewis and Shope (1931) differ from the human strains of $H$. influenze only in that no carbohydrates are fermented, and no indole is produced. The number of strains as yet examined does not, however, justify any definite generalized statement on this point. (See also Kirchenbaner 1934.)

\section{H. para-influenzæ}

Strains of this organism differ from $H$. influenze in requiring the $V$ factor but not the $\mathrm{X}$ factor for their growth, and in fermenting maltose, saccharose and often dextrin. In this last respect they resemble the rougher strains of $H$. influenze, except that their action on maltose is far more consistent. Their individual and colonial morphology often resembles that of "atypical" $H$. influenze strains. Some are hæmolytie, others are not, and a few strains produce indole. In man hæmolytic strains tend to be associated with acute pharyngitis and both the hæmolytic and non-hæmolytic strains with ulcerative endocarditis (Russell and Filckes 1928, Fox 1935, Stnart-Harris et al. 1935, Miles and Gray 1938). The organism survives only $2+4$ days on solid eulture media.

\section{H. canis}

Isolated by Friedberger (1903) from the prepuce of dogs. It is apparently parasitic, but not pathogenic. It differs from $H$. influenze in the following ways: It requires the $\mathrm{X}$ factor, but not the $\mathrm{V}$ factor, for its growth. On solid media it forms colonies that are at first indistinguishable from those of $H$. influenzce, but later become larger and more opaque. As regards its fermentation reactions it ferments dextrose, saccharose and manmitol, produces indole and reduces nitrates. In its fermentation of mannitol it differs from both typical and atypical strains of H. influenzee.

\section{H. ducreyi}

Morphologically in the purulent diseharge from the ulcerated surface of the lesion the organisms appear as small ovoid rods, arranged in pairs, in groups, or in chains lying parallel to one another. Several forms may, however, be assumed. Thus, it may appear as a short rod with parallel sides and rounded ends, staining evenly; or it may be ovoid or navicular in shape with marked bipolar staining; 
or it may occur in pairs end-to-end, having a dumb-bell appearance. In size the bacillus is about $1 \cdot 1-1.5 \mu$ long by $0.6 \mu$ broad (Stein 1928). It may be intra or extracellular in position. It does not form spores; it is Gram-negative, and non-acid-fast. In cultures on solid media the organisms appear as isolated individuals, in groups, and in short chains ; in fluid media very long chains are frequently formed, and in certain media it produces a pellicle with dependent "stalactites" of growth (Cunha 1939, 1943).

The organisms may be cultivated by inoculating scrapings from the floor of the ulcer on to a medium consisting of 3 per cent. agar contaiming 20-33 per cent. defibrinated rabbit's blood: the medium should be prepared on the day of inoculation, and should be distributed into wide tubes having a large surface exposed to the air (Nicolle 1923, Reenstierna 1923, Nicolle and Durand 1924). Several tubes should be inoculated, and incubated at $35^{\circ} \mathrm{C}$. Colonies appear in 24 hours, and may be picked off for purification. On blood agar after 24 hours the colonies are circular, $0.5-1.0 \mathrm{~mm}$. in diameter, low convex, greyish-white and ghistening, with a smooth surface and entire edge ; after 2 to 3 days, they may reach a diameter of $2 \mathrm{~mm}$., and the surface may show a crateriform depression. According to Hunt (1935), growth occurs best in sealed tubes, suggesting that it is favoured by an increased partial pressure of $\mathrm{CO}_{2}$.

The necessity for blood in the medium, and a low partial oxygen pressure is stressed by Sanderson and Greenblatt (1937). Watanabe (1939) confirmed the necessity for blood; rabbit blood was best, followed by that of the goat, sheep, ox or man. He observed no growth stimulation by $\mathrm{CO}_{2}$.

According to Lwoff and Pirosky (1937) $H$. ducreyi requires $\mathrm{X}$ but not $\mathrm{V}$ factor for growth. Only small quantities of hæmin are required. The growth of some strains in the absence of blood or serum in the medium (Hababou-Sala 1925, de Assis 1926) may be attributed to the presence of small but sufficient quantities of hæmin in nutrient broth.

Another medium that is recommended for primary isolation consists of 1 part of 5 per cent. glycerine agar and 4 parts of Besredka's egg medium. On this medium the colonies are said to be round, transparent, and of a rose mother-ofpearl colour (Hababou-Sala 1925). After preliminary incubation at $35^{\circ} \mathrm{C}$., cultures are said to remain viable at room temperature for about a month.

In Martin's broth, to which 20 per cent. of defibrinated rabbit's blood has been added, the organism develops rapidly, forming granules, which are suspended in the liquid or become attached to the walls of the tube. After a few days, an incomplete film may form on the surface. Cultures in this medium remain viable in the incubator for at least 10 days.

For preserving the organism, it should be inoculated into a medium consisting of 0.25 per cent. of nutrient agar, I per cent. starch, and 20 per cent. of defibrinated rabbit's blood. Cultures on this medium remain alive for a month at incubator temperature, and for a similar period at room temperature, provided they are previously incubated for 5 days.

H. ducreyi is not specially resistant : it is killed by moist heat at $55^{\circ} \mathrm{C}$. within an hour, and by 0.5 per cent. phenol in a comparatively short time.

The fermentation reactions of $H$. ducreyi do not appear to have received much attention. Serologically, suspensions from blood-agar cultures are agglutinated by a specific antiserum; this reaction may be used for identification.

$H$. ducreyi is naturally pathogenic for man. Monkeys have been successfully infected. The organism has a low pathogenicity for chick embryos (Anderson and Snow 1940).

A hæmophilic organism resembling $H$. canis and $H$. ducreyi in requiring only $\mathrm{X}$ factor, but requiring an excess of $\mathrm{CO}_{2}$ in the atmosphere for its growth, has been isolated from an ulcerative endocardial lesion in man (Khairat 1940). 


\section{H. pertussis}

Isolation.--Isolated by Bordet and Gengou (1906) from cases of whooping cough, and now recognized as the causal organism of that disease.

Morphology.-H. pertussis bears a general resemblance to $H$. influenzae in its morphology. The cell-form is more constant, being usually of the short bacillary type. Longer bacillary or thread forms may occur, but they are relatively uncommon.

Growth Requirements. $-H$. pertussis is not dependent on either the $\mathrm{V}$ factor or the $\mathrm{X}$ factor for growth. On first isolation it requires a complex medium, the most suitablo being that devised by Bordet and Gengou, containing blood, potato extract and glycerol. It can, however, be trained to grow on agar. The optimal temperature for growth is in the near neighbourhood of $37^{\circ} \mathrm{C}$.

Growth on Solid Media.-On the Bordet-Gengou medium H. pertussis gives smooth, domeshaped, glistening colonies, with an entire edge. They are more opaque than those of $H$. influenzo, and are greyish as well as glistening. They have been likened not inappropriately to a bisected pearl, less appropriately to a small drop of mercury. When fully developed they tend to be rather larger than the colonies of $H$. influenzoe; but they develop more slowly and the characteristic appearances described above are often not obvious in less than 48-72 hours' incubation.

Growth in Liquid Media.-In serum $H$. pertussis gives a uniform turbidity with a slight deposit, which is sometimes slightly flocculent.

Resistance. $-H$. pertussis is killed by exposure to a temperature of $55^{\circ} \mathrm{C}$. for 30 minutes. Biochemical Activities.-H. pertussis does not ferment any sugar. It does not form indole, or reduce nitrates. It produces a hazy zone of hæmolysis.

Antigenic Structure.-H. pertussis in the normal smooth phase constitutes a single antigenic type. In artificial culture, particularly on a relatively unfavourable medium, it gives rise to rough, or partially rough, variants, with a different antigenic structure. The antigen characterizing the $\mathrm{S}$ form, and the endotoxin of $H$. pertussis are serologically related to the corresponding substances in $H$. parapertussis and $H$. bronchisepticus.

Pathogenicity.-H. pertussis is the cause of whooping cough in man. Injected in large doses into laboratory animals it gives rise to a fatal toxæmic infection very similar to that produced by $H$. influenzce. Introduced intranasally or intratracheally, it produces a fatal broncho-pneumonic infection.

\section{H. parapertussis}

An organism isolated by Eldering and Kendrick (1937) from cases of whooping cough in the United States, which differs from $H$. pertussis $(a)$ in producing a brown pigment on certain blood media, $(b)$ in producing catalase, and $(c)$ in having an S somatic antigen and an endotoxin which are apparently distinct from, though related to, those in $H$. pertussis.

\section{H. bronchisepticus}

Synonyms.-B. bronchisepticus, Br. bronchiseptica.

Isolation.-By Ferry (1911) in the United States, and by M'Gowan (1911) in Edinburgh from dogs affected with distemper.

Habitat.-Strict parasite, occurring in several different species of animals, and sometimes in man.

Morphology.-Similar to $H$. pertussis, but is motile by peritrichate flagella.

Cultural Characteristics.-Grows fairly well on nutrient agar media, producing small, round, convex amorphous colonies, with smooth glistening surface, of butyrous consistency. Grows best under aerobic conditions; no growth under strictly anaerobic conditions. In agar shake cultures, growth is almost entirely on the surface. Some strains are hæmolytic. 


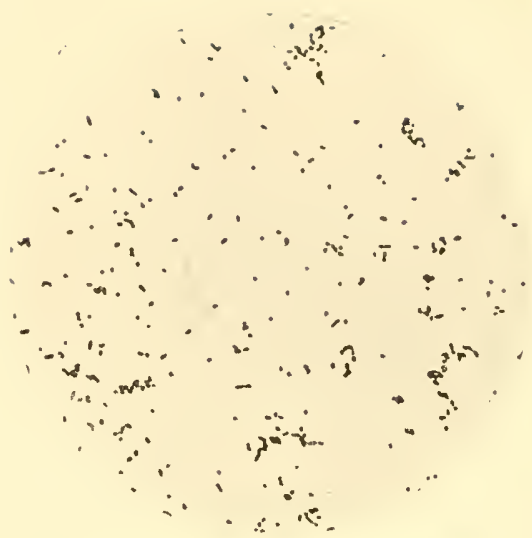

Fus. 170.-Hemophilus bronshisepticus.

From an agar culture, 24 hours, $37^{\circ} \mathrm{C}$. $(\times 1000)$.

Resistance.--Similar to $H$. pertussis.

Biochemical Activities.-Hæmolysin produced, active on red corpuscles of rabbit, dog and guinea-pig. Grows freely on $\mathrm{Mac}-$ Conkey. No earbohydrates fermented. Produces marked alkalinity in litmus milk. Nitrates often reduced. $\mathrm{H}_{2} \mathrm{~S}-$, $\mathrm{NH}_{3}$ very slight production or none at all. Catalase +++ . Grows in Koser's citrate.

Antigenic Structure.-The surface antigen of the $\mathrm{S}$ form, and its endotoxin, are serologieally homogeneous, and related to the corresponding substances in $H$. pertussis and 11. parapertussis.

l'athogenicity.-Frequent cause of bronehomeumonia in rodents, and of bronchopmeumonia complicating distemper in dogs. Experimentally, intraperitoneal

inoenlation of guinea-pigs with $0.5 \mathrm{ml}$. of a 24 -hours' broth culture causes death in 24 to 48 hours. Post mortem, there are small hæmorrhages on the peritonem. and a viseid translucent exudate forming pseudo-membranes on the liver, spleen. and the less mobile parts of the intestine. The bacilli are easily recovered from the peritoneal cavity, but with difficulty from the hlood, liver and lungs. Subcutaneous inoculation produces only a loeal lesion. Feeding and inhalation are without effect. The organism is non-pathogenic to mice. It rapidly loses its virulence in culture.

\section{REFERENCES}

Alexander, H. E. and Heinelberger, M. (1940) J.exp. Med., \%1, 1.

Anderson, G. and North, E. A. (1943) Aust. J. exp. Biol. med. Sci., 21, 1.

Anderson, K. and Snow, J. S. (1940) Amer. J. Path., 16, 269.

ANDERSOs, L. R. (1931) Amer. J. Hyg., 13, 164.

Andersox, R. A. and Scheltz, O. T. (1921) J. exp. Med., 33, 653.

Assis, A. DE. (1926) C. R. Soc. Biol., 95, 1008.

BAUDISCH, O. (1932) Biochem. Z., 245, 265.

Bieling, R. and Weichbrodt, R. (1920) Dtsch. med. H'schr., 46, 1183.

Bondi, A. and Flosdorf, E. W. (1943) J. Immunol., 47, 315.

Bordet, J. (1912) Zbl. Bakt., 66, 276.

Bordet, J. and Gevgou, O. (1906) Ann. Inst. Paste ur, 20, 731; (1907) Ibid., 21, 720; (1909) Ibid., 23, 415.

Bordet, J. and Sleeswyk. (1910) Ann. Inst. Pasteur, 24, 476.

Bourv, J. M. (1927) J. infect. Dis., 41, 294.

Bradford, W. L. (1938) Amer. J. Path., 14, 377.

Bradford, W. L. and Slavin, B. (1937) Amer. J. publ. Hith, 2\%, 1277.

Brueckner, I. E. and Evans, D. G. (1939) J. Path. Bact., 49, 563.

Burnet, F. M. and Tmuns, C. (1937) Brit. J. exp. Path., 18, 83.

Chandeer, C. A., Fothergill, L. D., and Divale, J. H. (1937) J. exp. Hed., 66, 789; (1939) J. Bact., 3\%, 415.

Cohen, C. (1909) Ann. Inst. Pasteur, 23, 273.

Cooper, G. M., Mishulow, L., and BLANC, N. E. (1921) J. Immunol., 6, 25.

('ruickshank, J. C. and Freeman, G. (†. (1937) Lancet, ii. 567.

Culotta, ( : S., Harvey, D. F, and Gordon, E. F. (1935) J. Pediat. 6, 743.

Cunha, R. (1939) Zbl. Bukt., 144, 508; (1943) O Ilospital, 23, 393.

Davis, J. D. (1917) J. infect. Dis., 21, 392 ; (1921) Ibid., 29, 178, 187.

Delius, W. and Kolle, W. (1897) Z. IIyg. InfektKr., 24, 327.

Dible, J. H. (1924) J. Path. Bact., 2\%, 151. 
Dochez, A. R., Mills, K. C., and Kneeland, Y. JR. (1932) Proc. Soc.exp. Biol., N.Y., $30,314$.

Ehrich, IV. E., Bondi, A., Mudo, s., and Flostorf, E. W. (1942) Amer. J. med. Sci., 204, 530 .

Eirund, A. (1929) Zbl. Bakt., 111, 195.

Eldering, G. (1941) Amer. J. Hyg., 34, B., 1; (1942) Ihid., 36, 294.

Eldering, G. and Kendrick, P. (1937) J. Bact., 33, 71 ; (1938) Ibid., 35, 561.

Evans, D. G. (1940) J. Path. Bact., 51, 49 ; (1942) Lancet, i. 529; (1944) J. Path. Bact., $56,49$.

Evans, D. G. and Maitland, H. B. (1937) J. Path. Bact., 45, 715 ; (1939) Ibid., $48,67$.

Ferry, N. S. (1911) J. infect. Dis., 8, 399; (1912) Tet. J., 68, 376; (1912-13) Amer. vet. Rev., 41, 77.

Ferry, N. S. and Houghtor, E. M. (1919) J. Immunol., 4, 233.

Fildes, P. (1920) Brit. J. exp. Path., 1, 129 ; (1921) Ibid., 2, 16; (1922) Ibid., 3, 210; (1923) Ibid., 4, 265 ; (1924) Ibid., 5, 69.

Fleming, A. (1929) Brit. J. exp. Puth., 10, 226.

Fleming, A. and Maclean, I. H. (I930) Brit. J. exp. Path., 11, 127.

Flosnorf, E. II., Bonni, A., and lozors, 'T'. F. (1941) J. Immunol., 42, 133.

Flosdorf, F. W., Bonde, A., Felton, H., and IlcGunness, A. (! (1942) J. Pediat., $21,625$.

Flosdorf, E. W., Dozois, T. F., and Kingall, A. ( . (1941) J. Buct., 41, 157.

Flosdorf, E. W. and Kmbale, A. C. $\quad(1940 a)$.J. Immunol., 39, "287; (1940b) Ibil., 39, 475.

Flosdorf, E. W., Kimball, A. C., and Chambers, L. A. (1939) Proc. Soc. cxp. Biol., N.Y., $41,122$.

Fothergill, L. D. and Chandler, C. A. (1936) J. Immunol., 31, 401.

Fothergill, L. D., Dingle, J. H., and Chandeer, C. A. (1937) J. exp. Med., 65, 721. Fox, W. W. (1935) J. Amer. med. Ass., 105, 876.

Friedberger, E. (1903) Zbl. Bakt., 33, 401.

Galli-Valerio, B. (1896) Zbl. Bakt., 19, 694.

Ghon, A. and Preyss, W. vor. (1904) Zbl. Bakt., 35, 531.

Gordon, J., Woodcock, H. E. de C., and Zinnemann, K. (1944) Brit. mcd. J., i. 779.

Grassberger, R. (1897) Z. Hyg. Infelthr., 25, 453.

Gundel, M. and Schlüter, IV. (1933) Zbl. Bakt., 129, 461.

HaBABOU.SALA, J. (1925) C. R. Soc. Biol., 92, 498.

Henry, H. (1912) J. Path. Bact., 1\%, 174.

Hodgland, C. I., Ward, S. M., Gilder, H., and Shank, R. E. (1942) J.exp. Med., \%6, 241.

Holi, A. and BUNNEY, W. E. (1942) J. Immunol., 44, 33.

Hunt, G. A. (1935) Proc. Soc. exp. Biol., N.Y., 33, 293.

IIZUKA, A. (1938) Z. ImmunForsch., 94, 312, 318.

Katsampes, C. P., Brooks, A. H. and Bradford, IV. L. (1942) Proc. Soc. exp. Biol., N.Y.,

49,615 .

Khairat, O. (1940) J. Path. Bact., 50, 497.

KirchenbaUER, H. (1934) Z. InfektKr. Haustiere, 45, 273.

Knight, B. C. J. G. (1936) Spec. Rep. Ser. med. Res. Coun., Lond., No. 210.

KNoRR, M. (1924) Zbl. Bakt., 92, 371, 385.

KосH, R. (1887) Arb. ReichsgesundhAmt., 3, 62.

КорР, H. (1927-8) Zbl. Bakt., 105, 54.

Krage, P. (1910) Z. InfektKr. Haustiere, \%, 380.

Kristensen, M. (1922) "Hæmoglobinophilie Bacteria." Copenhagen; (1927) C. R. Soc. Biol., 96, 355.

Krumwede, C., Mishulow, L., and Oldenbusch, C. (1923) J. infect. Dis., 32, 22.

Krumiede, E. and Kuttner, A. G. (1938) J.exp. Med., 67, 429.

Laidlaw, P. P. and Dunkin, G. IV. (1926) J. comp. Path., 39, 222.

Lamont, J. A. (1926) Canad. med. Ass. J., 16, 1447.

LAPIN, J. H. (1942) J. Pcdiat, 20, 161.

Lawson, G. M. (1933) Amer. J. Dis. Child., 47, 1454.

Leslie, P. H. and Garder, A. D. (1931) J.Hyg., Camb., 31, 423.

LevinthaL, W. (1918) Z. Hyg. InfektKr., 86, 1.

Lewis, P. A. and Shope, R. E. (1931) J. exp. Med., 54, 361.

Lwoff, A. (1939) Ann. Inst. Pasteur, 62, 168.

Lwoff, A.and Lwoff, M. (1937a) Proc. roy. Soc., B, 122, 352, 360 ; (1937b) Ann. Inst. Pasteur, 59, 129 ; (1937c) C. R. Acad. Sci., 204, 1510.

Lwoff, A. and Pirosky, 1. (1937) C. R. Soc. Biol., 124, 1169.

I'Gowan, J. P. (1911) J. Path. Bact., 15, 372.

IIcIntosh, J. (1922) Spec. Rep. Ser. med. Res. Coun., Lond., No. 63.

Mallory, F. B. and Hornor, A. A. (1912) J. mcd. Res., 27, 115.

Mallory, F. B., Horvor, A. A., and Henderson, F. F. (1912) Ibid., 27, 391. 
Martini, E. (1900) Arch. Hyg., Berl., 38, 114.

Messerschmidt, T., Hundeshagen, K., and Scheer, K. (1919) Z. Hyg. InfektKr., 88, 552.

Miles, A. A. and Gray, J. (1938) J. Path. Bact., 47, 257.

Muller, J. J. (1937) Proc. Soc. exp. Biol. N.Y., 3\%, 45.

Miller, J. J. and Silverbera, R. J. (1939) J. infect. Dis., 65, 16.

Mishulow, L., Ḱlein, I. F., Liss, M. II., and Leifer, L. (1939) J. Immunol., 37, 17.

MULDER, J. (1937) Acto med. scand., 91, 320.

Nrcolle, C. (1923) C. R. Soc. Biol., 88, 871.

Nicolle and Durand. (1924) Arch. Inst. Pasteur, Tunis, 13, 243.

Norti, E. A., Anderson, G., and Graydon, J. J. (1941) Merl. J. Aust., 21, 589.

North, E. A., Keogh, E. V., Anderson, G., and Williams, S. (1939) Aust. J. exp. Biol. med. Sci., 1\%, 275.

North, E. A., Keogh, E. V., Christie, R., and Anderson, G. (1940) Aust. J. exp. Biol. med. Sci., 18, 125.

ODATRA. (1911) Zbl. Balt., 61, 289.

Olistead, M. and Povitzky, O. R. (1916) J. med. Res., 33, 379.

Olsen, O. (1920) Zbl. Bakt., 85, 12.

Ospeck, A. G. and Roberts, M. E. (1941) J. infect. Di.s., 74, 22.

Park, W. H., Williams, A. W., and Cooper, G. (1918) Proc. Soc. exp. Biol., N.Y., 16, 120.

PARKer, J. T. (1919) J. Amer. med. Ass., r2, 476.

Pfelffer, R. (1892) Dtsch. med. Wschr., 18, 28; (1893) Z. Hyg. Infelikr., 13, 357.

Ptttulan, M. (1931) J. exp. Med., 53, 471; (1935) J. Bact., 30, 149.

Platt, A. E. (1937) J. Hyg., Camb., 3\%, 98; (1939) Aust. J. exp. Biol. med. Sci., $17,19$.

Povitzky, O. R. and Denny, H. T. (1921) J. Immunol., 6, 65.

Powell, H. M. and Jameson, W. A. (1937) J. Immunol., 32, 153.

Pritchett, I. W. and Strllaian, E. G. (1919) J. exp. Med., 29, 259.

RaETtig, H. (1940) Zbl. Bakt., 145, 386.

Reenstierna, J. (1921) Acta derm-venereol., Stockh., 2, 1; (1923) Arch. Inst. Pasteur, Tunis, 12, 273.

RнеA, I. J. (1915) J. med. Res., 32, 471.

Rrtchie, J. (1910) J. Path. Bact., 14, 615.

Rrvers, T. MI. (1922a) Johns Hopk. Hosp. Bull., 33, 429; (1922b) J. Bact., 7, 579.

Rivers, T. M. and KoHN, L. A. (1921) J. exp. Med., 34, 477.

Roberts, M. E. and Ospeck, A. G. (1942) J. infect. Dis., r1, 264.

Russell, D. S. and Fildes, P. (1928) J. Path. Bact., 31,651.

Sanderson, E. S. and Greenblatt, R. B. (1937) Sth. med. J., 30, 147.

SCHLÜTER, IV. (1936) Zbl. Bakt., 136, 362.

SELTer, H. (1906) Z. Hyg. InfehtKr., 54, 347.

Shiblex, G. S. and Hoelscher, H. (1934) J. exp. Med., 60, 403.

Shiga, K., ImaI, N., and Eguchi, C. (1913) Zbl. Bakt., 69, 104.

Sноре, R. E. (1931) J. exp. Hed., 54, 349.

Silverthorne, N. (1938) Canad. J. publ. Illth, 29, 233 ; (1940) Ibid., 31, 560.

Siluverthorne, N. and Cameron, C. (1942) J. Pediat., 20, 1.

Sillverthorne, N., Cameron, C., and Paterson, M. (1943) Canad. J. publ. Hlth, 34, 175.

Sinverthorne, N. and Paterson, M. (1943) Canad.J. publ. Hlth., 34, 178.

Sнттн, М. M. (1931) J. IIyg., Camb., 31, 321.

Sмiтн, T. (1913) J. med. Res., 29, 291.

Smolens, J. and MUdD, S. (1943) J. Immunol., 47, 155.

Stillian, E. G. and Bourn, J. M. (1920) J. exp. Med., 32, 665.

Spooner, E. T. C. (1938) J. Hyg., Camb., 38, 79.

Sprunt, D. H. and Martin, D. S. (1913) Amer. J. Path., 19, 255.

Stein, R. O. (1928) See Kolle and Wasserman, "Hdb. path. Mikroorg.," IIte Aufl., 1928-9, $6,185$.

Strada, F. and Traina, R. (1900) Zbl. Bakt., 28, 635.

Strean, L. P., Lapointe, D. and Dechene, E. (1941) Canad. med. J. Ass., 45, 326.

Studurt-Harris, C. H., Wells, A. Q., Rosher, A. B., Mackie, F. P., and Wilson, G. S. (1935) J. Path. Bact., 41, 407.

Tartakowsky, M. G. (1897-8) Arch. Sci. biol., St. Pétersb., 6, 263.

Thalmer, W. (1914) Z6l. Bakt., ry, 189.

Tнлютта, T. (1921) J. exp. Med., 33, 763.

Thjøтta, T. and Avery, O. T. (1921) J. exp. Med., 34, 97, 455.

ToMASCZEWskI, E. (1903) Z. Hyg. InfektKr., 42, 327.

Toomey, J. A., Ranta, K., Robey, L., and McClelland, J. E. (1935) J. infect. Dis., $5 \%, 49$.

Tooney, J. H. and Takacs, W. S. (1937) J. infect. Dis., 60, 41 ; (1938) Ibid., 62, 297.

ToomeY, J. A., TAkacs, IV. S., and Ranta, K. (1936) J. infect. Dis., 59, 326. 
Valentine, E. and Cooper, G. M. (1919) J. Immunol., 4, 359.

Valentine, F. C. O. and Rivers, T. M. (1927) J. exp. Med., 45, 993.

Wade, H. W. and Manalang, C. (1920) $J$. exp. Med., 31, 95.

WATANABE, S. (1939) Kitasato Arch. 16, 1.

Watanabe, I. (1938) Jap. J.exp. Hed., 16, 529.

Webster, L. T. and BAUdisch, O. (1925) J. exp. Med., 42, 473.

WeEks, J. E. (18S7) Arch. Augenheilk., 17, 318.

Wilkes-IVeiss, D. (1936) Proc. Soc. exp. Biol. N.Y., 35, 289 ; (1937) J.infect. Dis., 60, 213. WINHOLT, W. (1915) J. infect. Dis., 16, 389.

Winslow, C.-E. A., Broadhurst, J., Buchanax, R. E., Kromwiede, C., liogers, I. A. and Sмттн, G. H. (1920) J. Bact., 5, 191.

Witebsky, E. and Salm, H. (1937) J.exp. Med., 65, 43.

Wollsteix, M. (1909) J. exp. Med., 11, 41; (1915) Ibid., 22, 445; (1919) Ibid., 30, 555. WOOD, M. L. (1940) J. Immunol., 39, 25.

Wright, J. and W' WRD H. K. (1932) J.exp. Med., 55235.

YABE, S. (1921) Brit. J. exp. Path., 2, 197. 


\section{CHAPTER 34}

\section{BRUCELLA}

Definition.--Brucella.

Small, non-motile, non-sporing, Gram-negative cocco-bacilli. Grow rather poorly on ordinary media, or may require special media. Aerobic; no growth under strict anaerobic conditions. Growth often improved by $\mathrm{CO}_{2}$. Little or no fermentative action on carbohydrates. Usually tend to produce alkali in litmus milk, and a brown pigmentation on potato. Strict parasites, occurring in man and animals, and producing characteristic infections.

Type species. Brucella melitensis.

History.-The first member of the group, $\mathrm{Br}$. melitensis, was isolated in 1887 by Bruce from the spleen of patients who had died of Malta fever. At that time, and for a long time afterwards, the bacillary nature of the organism was not recognized; in all the older textbooks it is therefore deseribed as a micrococeus. The organism finds its natural habitat in the goat and the sheep. It may, however, infect other animals. In man it gives rise to undulant fever. It is fairly wislely distributed throughout the world.

The discovery of the second member, Br. abortus, was made by Bang of Copenhagen in 1897. Working in eonjunction with Stribolt, he isolated the organism from cows suffering from infectious abortion, and by a series of experiments demonstrated its specific rôle in this disease. The organism is parasitie in cattle. To a less extent it infects certain other animals. In man it gives rise to undulant fever. It is perhaps even more widespread than $\mathrm{Br}$. melitensis, having been found in praetieally every country of the world.

The third nember of the group, Brucella tulurensis, was isolated by MeCoy and Chapin in 1912 from a plague-like disease among rodents in California, and was called by them Bacterium tularense. It infects ground-squirrels, jack-rabbits, and other rodents, and occasionally gives rise to a disease in man called tularæmia.

The fourth member, Br. suis, was isolated by Tramm(1914) from the foetus of a sow. It is a natural parasite of pigs, in which it gives rise to a disease frequently characterized by inflammatory lesions in the reproductive organs. It may oeeasionally infect other animals. In man it shares with Br.melitensis and Br. abortus the ability to produee undulant fever. It appears to be very mueh less widespread than these two organisms, its chief home being in the large hog-raising distriets of the middle western states of North America. In Denmark, Br. suis strains have been isolated by Thomsen $(1931,1934)$, whieh differ in certain respects from those found in the United States; they will be referred to as the Danish porcine type. The American type has been found cecasionally in Europe (see Thomsen 1934), and has been reported from Brazil (Neiva, 1934), the Argentine, and Australia (King 1934). 
Nomenclature.-We have no space to discuss the early confusion of terminology that existed over members of this group. The whole position was altered when Evans in 1918 drew attention to the essential similarity of the organisms which at that time were described as Micrococcus melitensis and Bacillus abortus. Nor do we propose to discuss the validity of the generic name Brucella suggested for them by Meyer and Shaw (1920) and by Feusier and Meyer (1920) in honour of Sir David Bruce. This is so appropriate and has met with such universal approval that no other term seems likely to enter into serious competition with it. There are, however, certain points that require discussion. The three organisms isolated from goats, cattle, and pigs respectively are so closely allied that their differentiation can be accomplished only with difficulty. The question is, therefore, whether they should be regarded as varieties of one species, or should be ranked as separate species. Both proposals have their advocates. On the whole we favour the latter course, mainly for the sake of convenience. We shall therefore refer to these three

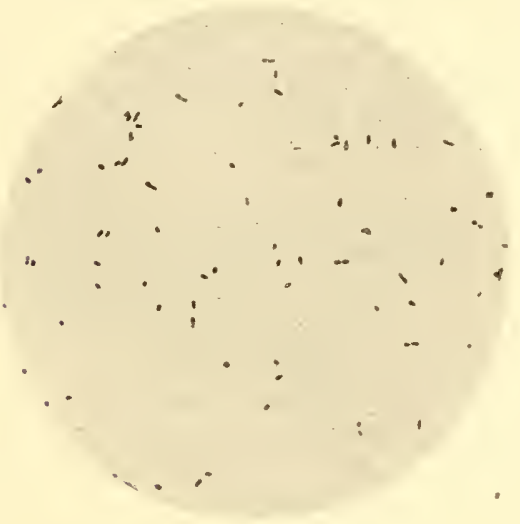

FIG. 171.-Brucella abortus.

From an agar culture, 24 hours, $37^{\circ} \mathrm{C}$., showing very short bacillary forms $(\times 1000)$.

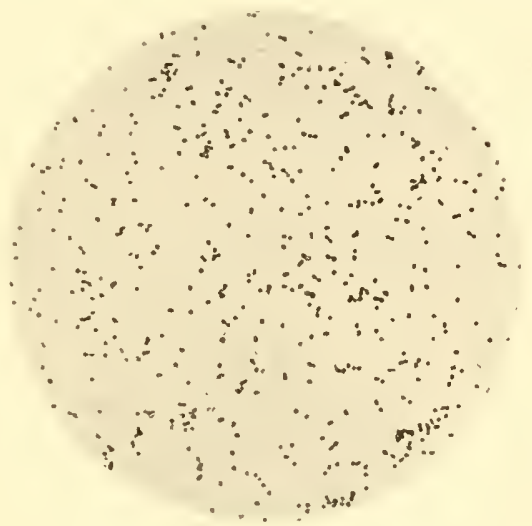

FIG. 172.-Brucella melitensis.

From an agar culture, 2 days, $37^{\circ} \mathrm{C}$., showing mainly coccal forms $(\times 1000)$.

organisms as $B r$. melitensis, $B r$. abortus and $B r$. suis. It must be pointed out, however, that the differences between the American and Danish porcine types are almost as great as those between the porcine and bovine types, and the decision to treat them as varieties of $B$ r. suis is purely arbitrary.

The names paramelitensis, para-abortus, and parasuis are frequently used to refer to inagglutinable strains of Brucella, corresponding most closely to the melitensis, abortus, and suis types. So long as the so-called para-strains were regarded as distinct species, no objection could be raised to this terminology; but now that they are known to be merely rough variants of the original smooth forms, this practice is no longer justifiable and merely serves to confuse the nomenclature. We shall refer to these, therefore, as rough metitensis, abortus, or suis strains, as the case may be.

The inclusion of Br.tularensis in this group is largely tentative. Reimann (1932), along with several other workers, would assign it to the Pasteurella group on account of its bipolar staining, its solubility in 1/800 sodium ricinoleate, and its transmission by insect vectors. On the other hand its general morphology, the beneficial effect 
of $\mathrm{CO}_{2}$ on its growth, its cytotrophism (see Buddingh and Womack 1941), its failure to develop anaerobically, its production of $\mathrm{H}_{2} \mathrm{~S}$, its very weak fermentative ability, its antigenic affinity to $\mathrm{Br}$. melitensis and $\mathrm{Br}$. abortus, and its high pathogenicity for mau in the laboratory qualify it perhaps even better for inclusion in the Brucella group. Since, however, it has not been studied with the same thoroughness as the other members, we shall exclude it from the following description of the group characteristics, and describe it separately at the end of the chapter.

Morphology and Staining.-The bacilli are short and slender; the axis is straight; the ends are rounded; the sides may be parallel or convex outwards. In length they vary from about 0.6 to $1.5 \mu$, and in breadth from 0.5 to $0.7 \mu$. The short forms may appear as oval cocci, or, if they are about to divide, as diplococri. As a rule they are arranged singly, in pairs end-to-end, or in small groups; sonetimes short ehains of 4-6 members may be seen, especially in liquid media. Owing to the frequent coceoid appearance, their bacillary nature may be in doubt, but it may be noted that in size they are smaller than any of the Gramnegative cocci. Moreover, when arranged in pairs, their long diameter is in the same axis as that in which they are lying, in distinction to the Gram-negative diplococci, whose long axis is generally at right angles to that in which they are lying.

Br. melitensis is generally considered to be more coccal in form than Br. abortus, and for this there is some justification. The difference in size and shape, however, is so slight as to render it impossible to distinguish with certainty between individual strains. Duncan (1928) has pointed out that these organisms, when grown on agar or glucose agar, show no marked morphological differences; but if they are cultivated on a relatively rich medium, such as Fildes' peptic digest blood agar, the $B r$. abortus strains frequently develop long bacillary forms, reaching 2.0 or $3.0 \mu$ in length, whereas $B r$. melitensis strains usually retain their coccal shape and rarely exceed $1.0 \mu$ in length.

The organisms stain fairly well with the ordinary dyes. Bipolar staining is not uncommon, and occasionally irregularity in the depth of colour is seen. In old cultures irregular forms may be noted. They are Gram-negative, non-acid-fast, non-motile and non-sporing. The presence of capsules in freshly isolated smooth strains has been described (Huddleson 1940, Miekle 1940).

Cultural Reactions.-Apart from their different $\mathrm{CO}_{2}$ requirements, the members of this group resemble each other closely in their cultural characteristics. None of them is difficult to grow; none grows profusely. On agar the colonies are small, translucent and undifferentiated. In broth there is a moderate turbidity with a slight powdery or viscous sediment, which disintegrates completely on shaking; after about 2 weeks in the incubator or at room temperature the deposit becomes extremely viscous, and can be disintegrated only with difficulty. According to Thomsen (1933), if the organisms are grown in flasks of broth instead of in tubes the suis and $\mathrm{CO}_{2}$-sensitive abortus types give rise in 1 to 3 weeks to a mealy or scaly surface pellicle and a heavy deposit that is difficult to disintegrate by shaking. Aerobic abortus strains form no pellicle, but produce a uniform turbidity and a slight deposit that is easily disintegrated. Strains of $\mathrm{Br}$. melitensis give rise to a fairly dense turbidity, a moderately heavy deposit, and a granular, usually incomplete, surface growth. Growth in gelatin is poor, and is unaccompanied by liquefactiou. Perhaps the most striking peculiarity is the yellowish colour that develops on potato 
in 2 to 3 days, deepening to a café-au-lait or chocolate tint in the course of a fortnight. Individual strains vary in the depth of colour they produce, some giving a darker brown than others. The pigment is not confined to the layer of growth; it spreads throughout the potato. It will be recalled that a brown growth on potato is also given by $P f$. mallei, Pf. whitmori, V. cholerce, and Ps. pyocyanea, and certain other organisms. A similar but less intense brownish colour is sometimes noticeable in old agar slope cultures, particularly of Br. melitensis (Kristensen 1931) ; it is not sufficiently constant, however, to be of differential value.

Huddleson, Hasley, and Torrey (1927) and Huddleson and Winter (1927) have observed the development of erystals of ammonium magnesium phosphate in cultures of Brucella on liver agar incubated aerobically, but not in 5-10 per cent. $\mathrm{CO}_{2}$. They regard their formation as being due to the production of ammonia by the growing organisms, and to its combination with the magnesium phosphate in the medium. In their experience crystals are formed much more rapidly by melitensis and paramelitensis than by abortus strains. Our own experience (Wilson 1933) bears out their observations to some extent, but does not suggest that the differences between different types are suffieient to be of value in the identification of individual strains. Incidentally American suis strains appear to be most active in the production of ammonia.

Attention has been drawn by de Santis (1933) to the different appearances presented on Petragnani's egg medium. Strains of $\mathrm{Br}$. melitensis are said to grow on this medium, and usually to change the colour from light yellowish-green to dark green. Br. abortus strains, on the other hand, generally fail to develop. The behaviour of Br. suis strains is doubtful, but in our limited experience growth is not infrequent. 'The reliability of this test for differential purposes is still under discussion (see Menzani 1934, Tosatti 1934, Messieri 1935, Vittone 1935, Pagnini 1935, Foresti 1935). The general opimion seems to be that it is less satisfactory than the $\mathrm{H}_{2} \mathrm{~S}$ and dye tests (see later). According to Schwarzmaier (1936), Br. melitensis grows on Petragnani's medium both with and without malachite green, $\mathrm{Br}$. suis grows only without malachite green, and $\mathrm{Br}$. abortus fails to grow under either condition. The mode of preparation of the medium appears to be of importance. If it is heated to a temperature above $80^{\circ} \mathrm{C}$. for too long the normal inhibitory action of the egg albumin on the bovine and porcine strains is destroyed, thus allowing some development of these organisms to occur (de Santis 1935). According to Martini (1935), the inhibitory effect of the egg albumin on abortus strains can be removed by the addition of sufficient $\mathrm{HCl}$ to lower the reaction of the finished medium from $\mathrm{pH} 8.4$ to $\mathrm{pH} 6 \cdot 8$. If this is true, it suggests that the effeet may be related to the greater necessity of abortus strains for $\mathrm{CO}_{2}$.

Growth is rather slow, and unless a fairly heavy inoculum is made, colonies are not usually visible for 2 days or even longer. In broth the maximum turbidity is not reached for a week or more. On the whole the American porcine strains probably g've the best, and the Danish porcine strains the poorest growth, the melitensis and abortus strains occupying an intermediate position. The behaviour, however, of different strains of the same type is subject to so much variation that no reliance can be placed on this character for differential purposes.

$B r$. abortus, Br. melitensis and Br. suis, when inoculated on to the chorioallantoic membrane of the developing chick embryo, are able to multiply and to bring about death of the embryo in a few days with lesions in the spleen and liver. All three organisms grow intracellularly-Br. melitensis in the ectodermal epithelium, Br. abortus and Br. suis in cells of mesodermal origin and in the vascular endothelium. Rough strains are non-invasive (Goodpasture and Anderson 1937, Buddingh and Womack 1941, de Ropp 1944). 
The colonial appearance of Brucella depends on the smoothness or roughness of the strain. The difference betwcen smooth and rough colonies is not great, and is best brought out by examination under a binocular plate mieroseope using obliquely transmitted light. Colonies of antigenically smooth strains of $\mathrm{Br}$. abortus on potato agar are small, bluish and transhucent with regular margins and a smooth ghistening surface. The individual cells are uniformly short rods arranged singly. Colonies of antigenically rough strains are of much the same size as the smooth colonies, but are less convex, more opaque, and have a dull granular appearance. The individual cells are usually somewhat larger than those of the smooth type, and occasional long slender rods may be observed. Intermediate colonial and morphological forms have also been described (Mingle and Mantei 1941). The colonial differenees are intensified on glycerol glucose agar. On this medium $\mathrm{S}$ and $\mathrm{R}$ colonies, if examined by obhque transmitted light against a dark background, produce the appearance of an irregular mosaic of light and dark, curved and angular areas (Henry 1933).

Growth Requirements. - Growth is generally improved by the addition of natural animal protein to the medium. The most satisfactory media, particularly for the growth of $\mathrm{Br}$. abortus and $\mathrm{Br}$. melitensis, are liver extract agar-first described by Holth (1911), subsequently by Stafseth (1920), and frequently referred to as Huddleson's medium -2 per cent. glycerol agar (Zeller and Stockmayer 1933), Fleming's (1919) chocolate agar (Henry et al. 1932), 5 per cent. serum agar and Bacto-tryptose agar. Zobell and Meyer (1932) have described a synthetic medium in which the metabolism of Brucella strains may be studied. Br. melitensis and Br. suis are said not to grow in an amino-acid glucose inorganic salt medium unless nicotinamide, thiamin and pantothenic acid are added ; Br. abortus requires biotin as well (Kerby 1939, Koser et al. 1941, Koser and Wright 1942).

The range of temperature consistent with growth is $20^{\circ}-40^{\circ} \mathrm{C}$. ; the optimum being about $37^{\circ} \mathrm{C}$. At $20^{\circ} \mathrm{C}$. growth is very slow. The effect of $\mathrm{H}$-ion concentration is rather difficult to dissociate from that of $\mathrm{CO}_{2}$. Many strains of $\mathrm{Br}$, abortus require for their optimum development a concentration of 5-10 per cent. $\mathrm{CO}_{2}$ in the atmosphere. This has the effect of turning an alkaline medium acid. For the growth of these organisms an initial $\mathrm{H}$-ion concentration of $\mathrm{pH} 6 \cdot 6$ is desirable. The other members of the group usually grow as well on an alkaline as on a slightly acid medium ; but since, as will be pointed out directly, even melitensis strains are often benefited by a small amount of extra $\mathrm{CO}_{2}$, it is advisable for practical purposes to adjust media to $\mathrm{pH} 6 \cdot 6-6 \cdot 8$.

$\mathbf{C O}_{2}$ Requirements.-One of the most interesting features of the Brucella group is their peculiar respiratory behaviour. Ever since its original isolation by Bang (1897), Br. abortus has presented certain difficulties in cultivation. No growth occurs on a solid medium under aerobic conditions. If, however, the tube is suitably sealed (Preisz 1903) (see Fig. 173), or if it is attached by rubber tubing to another tube inoculated with an organism such as B. subtilis (Nowak 1908), growth occurs after a delay of a few days. These observations were generally interpreted as showing that $\mathrm{Br}$. abortus was microaerophilic, and could not grow till the partial pressure of oxygen over the culture had been lowered to a suitable extent. A sinilar interpretation was also placed on the fact that, when inoculated into a serum agar gelatin shake medium, it grew in the form of a band situated about $\frac{1}{2} \mathrm{~cm}$. below the surface (Fig. 174).

Credit is due to Huddleson (1921) for showing that this organism requires for its derelopment a partial pressure of $\mathrm{C}_{2}$ higher than that normally present in the 
atmosphere (0.03-0.04 per cent.). He found that if slopes of $\mathrm{Br}$. abortus were incubated in a glass jar containing 10 per cent. $\mathrm{CO}_{2}$, good growth occurred in 24 hours, while under aerobic conditions there was no growth at all. Analysis of the gas over a culture of $B$. subtilis revealed the presence of $\mathrm{CO}_{2}$, and it was therefore concluded that the success of Nowak's method depended on the evolution of this gas rather than on a decrease in the partial pressure of oxygen. Further work by Smith (1924) and McAlpine and Slanetz (1928b) confirmed the importance of $\mathrm{CO}_{2}$. Smith showed that development was much better in an atmosphere of 10 per cent. $\mathrm{CO}_{2}$ than in sealed tuhes, and that in agar shake cultures, either sealed or incubated in an atmosphere of 10 per cent. $\mathrm{CO}_{2}$, growth occurred, not in a band below the surface as Bang (1897) and his co-worker Stribolt had found, but on the surface itself.

In spite of these observations, it was not easy to understand why growth shonld occur in sealed tubes, or why, in the absence of added $\mathrm{CO}_{2}$, growth in shake tubes should occur in a band below the surface.

A fuller study of the gaseous requirements of $\mathrm{Br}$. abortus (Wilson 1931 $a$ ) showed (1) that the organism wonld not grow anaerobically even in the presence of added $\mathrm{CO}_{2}$, nor aerobically in its absence; (2) that growth would oceur in partial pressures of oxygen varying from $0 \cdot 5-99.0$ per cent., provided a minimum of 0.5 per cent. $\mathrm{CO}_{2}$ was added, and in partial pressures of $\mathrm{CO}_{2}$ varying from $0 \cdot 5-98.0$ per cent., provided a minimum of 0.5 per cent. oxygen was added; (3) that the optimum partial pressure of oxygen for development was about 21 per cent., i.e., that normally present in the atmosphere, and of $\mathrm{CO}_{2}$ about 10 per cent. No evidence was obtained to suggest that a partial pressure of oxygen lower than that normally present in the a tmosphere was beneficial to growth. It seemed clear, therefore, that neither growth in sealed tubes nor the band phenomenon

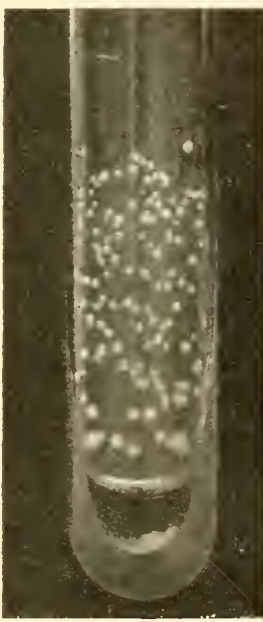

Fia. 173.-Brucella abortus.

(ilycerine agar slope culture, 3 days, $37^{\circ}$ C., in corked tube, showing character of growth on direct isolation from the tissues.

in shake tubes could be due to a preference of the organism for microaerophilic conditions. Further observations (Wilson 1930) showed that $\mathrm{CO}_{2}$ was given off by burning cotton-wool plugs, and to a less extent by heated paraffin wax, rubber stoppers, and sealing wax. Analysis of the gas inside sterile sealed tubes revealed the presence of $\mathrm{CO}_{2}$ in amounts varying from about 1-3 per cent.- - a proportion ample to initiate growth in inoculated tubes. The larger the number of organisms inoculated, the less need was there for additional $\mathrm{CO}_{2}$, since the organisms themselves produced a certain amount of this gas. But with inocula of any size, growth was always most rapid and luxuriant when the partial pressures of oxygen and $\mathrm{CO}_{2}$ most nearly approached the optima.

Similarly, evidence was brought (Wilson $1931 b$ ) to suggest that the band phenomenon in shake tubes was due to the necessity of an adequate concentration of $\mathrm{CO}_{2}$ (Fig. 174). It was found that this gas was given off to a certain extent by the organisms themselves, and to a still greater extent by certain media, particularly those containing serum. Growth could not occur at the surface, because the $\mathrm{CO}_{2}$ was given off into the atmosphere; nor could it occur in the depths of the medium, because the conditions were anaerobic. It therefore commenced in a zone as near the surface as was consistent with the maintenance of an adequate partial pressure of $\mathrm{CO}_{2}$. If the tube was sealed, or was incubated in an atmosphere of 10 per cent. $\mathrm{CO}_{2}$ then growth occurred at the surface, where the optimum partial pressure of oxygen existed. This explanation, when slightly amplified, was found 


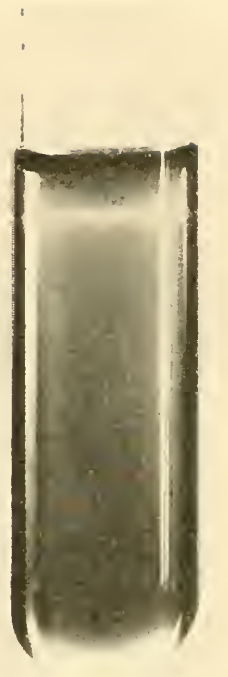

Fig. 174.-Br. abortus.

Growth in form of band situated 6-8 mm. below the surface. Bang's medium, $\mathrm{pH} 7 \cdot 0$, incubated aerobically. gelatin agar serum

to fit the numerous observations on variation in the distance of the band from the surface, and on the so-called double zone phenomenon, in which two bands of growth, separated from each other by apparently unaltered medium, are visible.

The demand for an increased partial pressure of $\mathrm{CO}_{2}$ is particularly characteristic of abortus strains. Not all strains, however, of this organism require it (see Smith 1924, 1926b). The Southern Rhodesian strains (Bevan 1930), for example, grow quite well under ordinary rerobic conditions. Moreover, even strains that need extra $\mathrm{CO}_{2}$ on isolation frequently become adapted, after a variable time in the laboratory, to do without it. The growth of many strains of $\mathrm{Br}$. melitensis is greatly benefited by incubation in an atmosphere of 5-10 per cent. $\mathrm{CO}_{2}$, though some growth always occurs in ordinary air. The porcine strains, both American and Danish, appear to be least dependent upon $\mathrm{CO}_{2}$. The addition of this gas to the air never improves their growth and sometimes actually inhibits it. How the $\mathrm{CO}_{2}$ acts is not definitely known. Alteration in the $\mathrm{H}$-ion concentration of the medium does not seem to be the explanation. It has been suggested that the gas passes through the cell wall and brings about a change in the intracellular $\mathrm{H}$-ion concentration or oxidation-reduction potential, which is necessary for the initiation of growth. As Gladstone, Fildes, and Richardson (1935) have shown, $\mathrm{CO}_{2}$ seems to be required in a greater or less degree by practically all bacteria, and presumably plays an important part in their metabolism (see Chapter 3).

Cultivation in the Presence of Dyes.-To Huddleson and Abell (1928) and Huddleson $(1929,1931)$ we owe a valuable method of distinguishing between the melitensis, abortus, and suis types, depending on their ability to grow in the presence of certain aniline dyes. Without entering into the detailed technique of the method, we may say that the general procedure is to prepare plates of liver agar, $\mathrm{pH} 6 \cdot 6$, containing $1 / 30,000$ and $1 / 60,000$ thionin, $1 / 25,000$ and $1 / 50,000$ basic fuchsin, $1 / 50,000$ and $1 / 100,000$ methyl violet, and $1 / 100,000$ and $1 / 200,000$ pyronin. The dyes used must be obtained from the National Aniline Chemical Company of New York, or standardized against these dyes. The organisms are inoculated rather heavily on to the plates, which are then incubated for 3 days aerobically, or in 10 per cent. $\mathrm{CO}_{2}$, according to the probable nature of the strains under examination. Strains of $\mathrm{Br}$. melitensis usually grow to some extent in the presence of all four dyes ; $B r$. abortus strains are inhibited by thionin, but grow freely in the presence of the other three; $B$ r. suis strains grow well in the presence of thionin, but are in. hibited by basic fuchsin, methyl violet, and pyronin. Though this is the general behaviour of the three types, there is considerable variation between different strains of the same type, especially those coming from different localities (Meyer and Zobell 1932, Wilson 1933). Some strains of melitensis, for example, may grow very poorly on the thionin, methyl violet, or pyronin plates. Southern Rhodesian strains 
of $\mathrm{Br}$. abortus often have a rather greater resistance to thionin than abortus strains from other sources. The Danish suis strains are more susceptible to all dyes than the American suis strains, though their differential susceptibility is the same; for this reason they must be tested on plates containing only half the dye concentrations just given. If reliance is placed exclusively on this method of differentiation, confusion will not infrequently result between strains of different types. If on the other hand, it is used, as we believe it should be used, in conjunction with other methods, it will be found of considerable value. No other method, it may be noted, is so useful in distinguishing between bovine and porcine strains. The method has been subjected to some criticism (Saitta 1929, Meyer and Eddie 1930, Marshall and Jared 1930, Cerruti 1932, Maggiora-Vergano 1932), but most workers have reported on it very favourably (Kristensen and Holm 1929, Kristensen 1931, Taylor, Lisbonne and Roman 1932, Grumbach and Grillichess 1932, Meyer and Zobell 1932, Wilson 1933, Olin and Lindström 1934, Pagnini 1934, di Mino 1935).

Resistance.-The members of this group exhibit the usual susceptibility of vegetative bacteria to heat and disinfectants. In aqneous suspensions of moderate density they are destroyed by heating for about 10 minutes at $60^{\circ} \mathrm{C}$, and by exposure for about 15 minutes to 1.0 per cent. phenol. In milk they are readily destroyed by holder pasteurization. In agar cultures kept sealed at $0^{\circ} \mathrm{C}$. they generally live for at least 1 month, and often for considerably longer. Considerable attention has been paid to their resistance under natural conditions, and much information on this subject will be found in the Report of the Mediterranean Fever Commission (1905-07). So many factors determine the exact outcome of any given observation under natural conditions that it is dangerous to draw general conclusions from the data so collected. In favourable circumstances, however, Br. melitensis may remain alive for 6 days in urine, 6 weeks in dust, and 10 weeks in water or soil. $\mathrm{Br}$. abortus may survive for 7 months in infected uterine exudate kept at about freezing-point (Bang 1897). In raw milk at room temperature it seems to die out fairly rapidly with the production of acid. Acid production also seems to be the cause of its rapid death in butter and cheese; the organisms can rarely be found in these articles for more than a few days (Smith 1934, Pullinger 1935). It may live for a month in ice-cream (Thompson 1933). Br. suis may live on sacking for 4 weeks and in sterile fæces for 100 days in the dark (Cameron 1932, 1933).

Metabolism and Biochemical Properties.-The effect of temperature and H-ion conditions on growth has already been considered. All the members require the presence of oxygen; most strains of $\mathrm{Br}$. abortus require in addition a partial pressure of $\mathrm{CO}_{2}$ considerably higher than that found in atmospheric air. Under ordinary aerobic conditions of incubation broth cultures become markedly alkaline, owing to the production of ammonia. Litmus milk is turned weakly alkaline. Occasional hæmolytic strains of Br. melitensis have been described (Forni 1927), but usually neither the melitensis, abortus, nor suis strains have any lytic action on blood. The effect of bile-salt on growth has not been studied fully; on MacConkey's medium strains of Br. abortus, $\mathrm{Br}$. melitensis, and $\mathrm{Br}$. suis generally give rise to small non-lactose-fermenting colonies after 3 or 4 days.

In ordinary sugar media no fermentation is observable. Unlike most pathogenic organisms, the members of this group are unable to produce obvious acid even from glucose. However, quantitative observations have shown that some melitensis and American suis strains, if grown in 1 per cent. glucose peptone water, may utilize 5-20 per cent. of the glucose within a week, while abortus strains are unable to use 
more than 2 per cent. The acid produced is more than neutralized by the alkali formed as the result of protein breakdown, so that it is not detected by the usual indicators. McAlpine and Slanetz (1928a) have recommended the glucose utilization test as a means of differentiating between the abortus, melitensis, and suis types. but most workers have found it unreliable, and it has now been generally discarded. There is evidence that arabinose and xylose are fermented by members of the Brucella group (Mallardo 1930, Coleman et al. 1930, McNutt and Purwin 1931, Silberstein 1932), but the reaction is of no differential significance.

The methyl red and Voges-Proskaner tests are negative. No indole is formed. According to Zobell and Meyer (1932), all types reduce nitrates to nitrites. Nitrites a re also rapidly reduced, so that the Griess-Ilosvay test on nitrate broth cultures may be negative. American suis strains are more active than the other types in reducing nitrites. Anmonia is produced to a variable extent from peptone, urea, and asparagin. Catalase is formed, being strongest with $\mathrm{Br}$. suis and weakest with Br. abortus. According to Huddleson and Stahl (1943), the degree of catalase activity is closely associated with virulence. The reducing action of these organisms is comparatively weak (Habs 1930), and in broth cultures methylene blue is often not decolorized. Tuttle and Huddleson (1934) found that liver extract broth cultures showed a negative drift to a limiting potential after 8 days of +0.15 to +0.09 volt. $B r$. suis appeared to be slightly more active than the abortus or melitensis types, but the difference was insufficient to be of value in species identification (see also Bau and Wang 1935-36). It may be noted that some strains of $\mathrm{Br}$. abortus reduce basic fuchsin. Huddleson (1931) thought that this was a property of non-pathogenic strains, but our observations do not bear this out.

$\mathbf{H}_{2} \mathbf{S}$ Production.- One of the most important differential criteria, to which attention was first drawn by Huddleson and Abell (1927), and Huddleson (1929), is the production of $\mathrm{H}_{2} \mathrm{~S}$. This test should be carried out on liver agar using lead acetate papers (see p. 369). Freshly isolated strains of $\mathrm{Br}$. abortus and $\mathrm{Br}$. suis (American variety) give off $\mathrm{H}_{2} \mathrm{~S}$ for at least the first 4 days, while strains of melitensis produce either none at all, or only during the first 24 hours of incubation. The Danish variety of $\mathrm{Br}$. suis forms no $\mathrm{H}_{2} \mathrm{~S}$. In the laboratory, abortus and American suis strains sometimes lose their ability to produce $\mathrm{H}_{2} \mathrm{~S}$; the test should therefore be made as soon after isolation as possible. The interpretation of this test can be summed up by saying that, while failure of a given strain to produce $\mathrm{H}_{2} \mathrm{~S}$ beyond the first day does not exclude its being of abortus or American suis type, the continued production of $\mathrm{H}_{2} \mathrm{~S}$ after the first day affords a strong presumption that it is not of melitensis or Danish suis type. This test has now been widely used, and to those who have realized its limitations it has given satisfaction (Favilli 1930, Kristensen 1931, Taylor, Lisbonne, and Roman 1932, Zobell and Meyer 1932, Zeller and Stockmayer 1933, Wilson 1933, Olin and Lindström 1934, Pagnini 1934, di Mino 1935).

Antigenic Structure.-It wonld be idle to recapitulate here the confusion that reigned for so long over the antigenic relationship of members of this group. Previous to 1918, when Evans demonstrated an antigenic affinity between Br. melitensis and $\mathrm{Br}$. abortus, most workers had concerned themselves with comparison of melitensis and so-called paramelitensis strains, while for many years subsequently progress was hindered by a failure to realize the difference in antigenic structure between strains in the smooth and rough phases. 
The early work of Sergent, Gillot, and Lemaire (1908), and Nègre and Raynaud $(1912 a, b)$, demonstrated the existence of strains morphologically and eulturally resembling $\mathrm{Br}$. melitensis but failing to agglutinate to more than a fraction of the titre with an anti-melitensis serum. These irregular strains were given the name of paramelitensis. Later on, so-called para-abortus strains were encountered, and these were believed to represent merely a special antigenic type of $\mathrm{Br}$. abortus. Further study, however, by such workers as Favilli $(1926 a, b)$, Ross (1927a), Valenti (1927), Vidal and Abella (1928), de Antoni (1929), Zdrodowski et al. (1930), Pampana (1931) and Pandit and Wilson (1932), showed that paramelitensis and para-abortus strains were agglutinable by non-specific agents, particularly acid, salt, peptone, and certain aniline dyes, whereas freshly isolated strains of $\mathrm{Br}$. melitemsis and $\mathrm{Br}$. abortus were unaffected by these agents under similar conditions. Moreover, it was found that continued cultivation in broth, or better still in broth containing immune serum, led to a transition of melitensis and abortus strains into paramelitensis and paraabortus respectively. The transition, it may be noted, occurs much more readily with melitensis than with abortus strains, and evidence of its commencement is often noticeable within a very short time of isolation, even when the organisms are kept on solid media: 'There seems to be little doubt that this change, which may be accompanied by alterations in colonial appearance and by a decrease in virulence for laboratory animals, is essentially a manifestation of the $S \rightarrow R$ variation. The antigenic change concerned is not yet clearly understood, but there is evidence that it involves a loss of the specifie smooth antigen. Strains of different degrees of roughness are encountered, varying from those that agglutinate to titre with a smooth serum but are slightly susceptible to non-specific agglutination to those that are unaffected by a smooth serum and are incapable of remaining homogeneously distributed even in cold saline. Moreover the degree of roughness of a given strain appears to vary from one culture to another. Onoe roughness has appeared, it persists, or recurs after an intervening period of apparent smoothness. Roughness may be tested for by boiling in saline for 2 hours (thermoagglutination test), by incubation at $37^{\circ} \mathrm{C}$. with $1 / 500$ or $1 / 1,000$ acriflavine (Alessandrini and Sabatucci 1931, Pampana 1931), by agglutination with an antiserum prepared against a completely rough strain of the corresponding type, or by the ease of phagocytosis by normal leucocytes (Munger and Huddleson 1938). Little work has so far been done on the antigenic structure of the rough types. Our own incomplete observations suggest that, though there may be a common antigen to paramelitensis, para-abortus, and parasuis types, there are certain differences between them. One important practical point is that partly rough strains are liable to be agglutinated non-specifically by the sera of normal persons, and particularly of those suffering from certain febrile diseases (see Mohr 1935), and are therefore liable to lead to an erroneous diagnosis of undulant fever in routine serological work.

Turning now to the differentiation of $\mathrm{Br}$. melitensis, Br. abortus, and Br. suis on the basis of antigenie structure, we are faced with a mass of conflicting reports most of which may be summarized by saying, either that no difference was found between the three organisms, or that they fell serologically into a number of different types (see Feusier and Meyer 1920, Burnet 1925, Evans 1925a, b, Ross 1927b, Cerruti 1927, Kristensen and Holm 1929, Bieling 1930, Kristensen 1931, Francis 1931, Plastridge and McAlpine 1932). The reason for this contusion is probably due to the failure of most of these workers to realize the disturbance caused by 
antigenic variation of the $\mathrm{S} \rightarrow \mathrm{R}$ type. If, as Wilson and Miles (1932) showed, care is taken to exclude all but absolutely smooth strains, then it is possible by means of quantitative agglutinin-absorption tests to differentiate between $\mathrm{Br}$. melitensis on the one hand and $\mathrm{Br}$. abortus and Br. suis on the other. The antigenic picture so obtained is represented in Fig. 175. It will be seen that all three types contain the same two antigens, but with a different quantitative distribution, the II antigen being in excess in the melitensis, the $\mathrm{A}$ antigen in the abortus and suis types. By carefully adjusting the absorbing dose to the titre of the serum, it is generally possible to absorb out all the minor agglutinins without removing more than a fraction of the major agglutinins. The resulting serum is therefore monospecific,

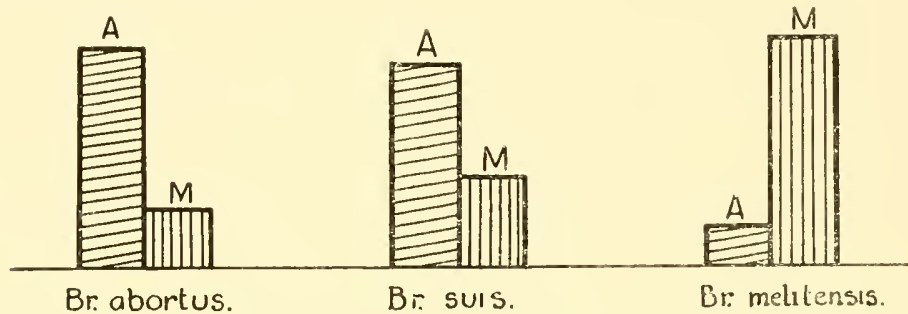

Fia. 175. - Sohematio Representation of Antighnic Structure of Brucella Strains.

and will agglutinate only those strains in which the corresponding antigen is predominant. Usually an absorbing dose standardized by opacity to match $3,000 \times 10^{6}$ coli per.ml. is satisfactory for absorbing a serum diluted to $1 / 32-1 / 64$ of its titre, but preliminary adjustment may be necessary before a monospecific serum can be obtained. With such a serum direct agglutination tests can be put up against strains of unknown type, and their antigenic identity established.

Why it is that a serum, from which the whole of the minor and part of the major agglutimins have been absorbed, will agglutinate only those organisms in which the corresponding antigen predominates, is rather puzzling. Why is it, for example, that a melitensis serum, from which the minor A agglutinin has been absorbed by an abortus or suis strain, will agglutinate only melitensis strains? On general grounds, abortus and suis strains, each of which contains a certain amount of M antigen, might be expected to agglutinate with such a serum to at least a quarter or half titre, since there must be ample M agglutinins in the serum to satisfy the limited number of MI receptors on the organisms. The fact that no agglutination does occur suggests that the quantitative and spatial distribution of the satisfied M receptors is such that any considerable degree of adhesion between adjacent organisms is improbable. This explanation is borne out by the observations of Miles (1939), who finds that the antigenic A:M ratio in $\mathrm{Br}$. abortus is about $20: 1$, and in $B r$. melitensis about $1: 20$. If, therefore, the minor antigenic surface constitutes only about 5 per cent. of the total, it may be supposed on the two-stage hypothesis (see Chapter 7) that its sensitization is insufficient to decrease the salt stability of the suspension beyond the critical point, or on the lattice hypothesis that the linkages between the minor antigenic groups on the heterologous bacteria are insufficient to hold the individual bacteria in clumps.

The conclusions reached on the antigenic structure of these organisms have received confirmation from the work of Miles (1933), who has shown that the melitensis may be separated from the abortus-suis types by the optimal proportion agglutination method, and from that of Habs (1933), Habs and Sievert (1935), 
Sievert (1936), Olin and Lindström (1934), Olin (1935), and Veazie and Meyer (1936), using the method of quantitative absorption.

As a means of typing unknown strains, direct agglutination with monospecific serum is one of the simplest and most rapid methods. Like other tests, however, for differentiation of this group, it cannot be relied upon entirely, because, as Wilson (1933) has shown, certain strains, particularly from the South-East of France, may possess the antigenic structure of abortus, while having the biochemical and pathogenic characteristics of melitensis.

Several workers claim to have established an antigenic affinity between members of the Brucella group and those of the Pasteurella, Proteus, or Pfeifferella groups. Most of these conclusions have been based on the observation that a given serum agglutinated strains of both Brucella and some other group. By itself this is, of course, quite insufficient evidence on which to base any such conclusion, since it takes no account of ron-specific agglutination or of the presence of specific agglutinins to two different organisms co-existing in the same serum. Absorption and other experiments have entirely failed to confirm the conclusions of these workers (for references see Priestley 1933, Wilson 1934).

Chemical Fractionation.-The reported results of chemical fractionation of Brucella strains are to some extent conflicting, especially with regard to the fractions exhibiting antigenic activity. The chemical characterization of many of the protein, nucleo-protein and polysaccharide fractions isolated varies greatly, and it is hard to judge the degree of purification, both chemical and serological, that has been achieved in each fraction. The more recent work has shown that a precipitiu titre, by a constant-antibody titration, of the order of $1: 5$ million represents the linit of purification so far achieved in the main antigenic fractions of this group.

The fractions isolated by the earlier workers had precipitin titres of $1: 2000$ to $1: 50,000$, and were clearly impure (Favilli and Biancalani 1932, 1934, Topping 1934, Gwatkin 1935, Reiter 1936, Schapira 1936, Higginbotham and Heathman 1936). These substances appeared to be polysaccharide in nature, and when fractions from different species were compared serologically they proved to be mainly group-specific, and displayed only minor degrees of species specificity. Huddleson and his colleagues (Huston, Huddleson and Hershey 1934, Hershey, Huddleson and Pennell 1935) isolated mactive proteins, polysaccharides and lipoids from the thrce Brucella species, whose proportions varied with the species studied. They also found a substance "S," free from protein and polysaccharide, precipitating with antisera at $1: 2,000,000$, which was readily extractable from $\mathrm{Br}$. melitensis, but was apparently bound to protein in $\mathrm{Br}$. abortus and $\mathrm{Br}$. suis. The " $\mathrm{S}$ " substance appeared to be related to the specific soluble substances isolated by Favilli and Biancalani $(1932,1934)$. The technique of treatment of the bacterial cell with trichloracetic acid developed by Boivin for the extraction of " complete " antigens from salmonellæ (see Chapter 8) was applied to Br. melitensis by Lisbonne and Monnier (1936) and to all three species of Brucella by Pop and his colleagues (Pop et al. 1938, Damboviceanu et al. 1938, Vanghelovici et al. 1938). (See also Davoli 1938, Stahl and Hamann 1941). The solutions obtained by dialysing the trichloracetic acid extracts were antigenic, toxic and precipitated in titres up to $1: 100,000$. The reported nitrogen content varied from 0.4 to 8 per cent.; lipins $10-17$ per cent., and reducing sugars on hydrolysis, 4-20 per cent. Pennell and Huddleson (1937), by digestion of acetone-dried organisms, extracted toxic and antigenic "endo-antigens" with a precipitin titre of 1:5 million which in many respects resembled the "complete" antigen obtained by the Boivin technique. They found certain chemical differences between the endo-antigens 
from the three species, and considerable cross-precipitin reactions between the three endo-antigens and their respective antisera. $\mathrm{Br}$. abortus and $\mathrm{Br}$. suis endo-antigens reacted similarly, though not identically, and Br. melitensis endo-antigen was more sharply distinguished from the other two (Pennell and Huddleson 1938). Huddleson (1943) has more recently reported that neither the "complete" antigen nor the "endo-antigen," which are both antigenic, will induce a significant degree of active immunity to experimental infection in the guinea-pig, but that a watery extract of crushed living $\mathrm{Br}$. abortus or Br. suis is highly effective against infection with Br. abortus.

Miles and Pirie $(1939 a, b)$ attempted to separate from $\mathrm{Br}$. melitensis the $\mathrm{A}$ and $\mathrm{M}$ antigens postulated by Wilson and Mliles (1932). By progressive degradation of antigenic material obtained by the gentlest possible treatment of the bacterium, they prepared a series of substances. The most degraded product was a formyl amino-polyhydroxy compound containing earbohydrates with a probable molecular weight of about 3.300. This substanee inhibited agglutination of Br. melitensis by homologous antibody, but did not itself react with antisera, i.e. it was an inhibiting hapten. Next in complexity was a substance analogous with the "endo-antigens" described above. It was antigenic, toxic, and precipitated with antisera in a dilution of $1: 5$ million. Its molecular weight was about 1,000,000. On hydrolysis it yielded a phospholipin, phosphate, and the formyl amino compound. This antigen was in turn derived from a more polymerized material combined with a protein-like material; and finally, this last substance, combined with one-third to one-quarter its weight of a mixture of lipins and phospholipins, constituted the relatively unstable native antigen. The native antigen, which comprised about 10 per cent. of the dry weight of the cell, was in a state of greater aggregation than the simple antigen, and weight for weight was more antigenic and less toxic. The native antigen consequently appears to be a large complex of a protein-like substance, with two sets of phospholipins differing in their readiness of separation from the complex, the second of which, together with a polymerized formyl amino-polyhydroxy compound, determines the specificity and antigenicity of the main antigen of $\mathrm{Br}$. melitensis. In the light of these findings it seems probable that the antigenic fractions of brucellae prepared by trichloracetic acid extraction or tryptic digestion of the bacilli consisted of mixtures in varying proportions of native antigen and its various degradation products. Niles and Pirie failed to separate the A and M antigens, but, as in previous work, they obtained evidence that the antigens of the S-forms of $\mathrm{Br}$. abortus and $\mathrm{Br}$. melitensis showed a qualitative similarity, but a quantitative difference in distribution.

The apparent efficacy of live as compared with dead vacines, led Priestley (1938) to search-without success - for a labile antigen in Br. abortus, similar, perhaps, to the Vi antigen of Salm.typhi. Topping (1934) isolated substances of a nucleo-protein nature from $\mathrm{Br}$. abortus and $\mathrm{Br}$. melitensis, whieh were feebly reactive with heterologous and homologous sera. The reactivity may have been due to contamination with the main soluble antigen. Stahl, Pennell and Huddleson (1939) extracted non-toxic "proteinnucleates" from all three species; those from is strains were serologically reactive, but only group-specific, and constituted about 14 per cent. of the dry weight of the cell; those from $\mathrm{R}$ strains constituter about 18 per cent. of the weight of the cell, and were serologically inactive. The serological reactivity of the $\mathrm{S}$ protein-nucleates was associated with the protein, and not with the nucleic acid part of the fractions. Stahl (1941) found 5-6 per cent. of lipins in the dry cells. They were non-toxic and serologically inactive.

Pathogenicity.-Br. melitensis, $B r$. abortus, and $B r$. suis are all infective for man and animals. Thongh undulant fever is the most characteristic result of infection in man, numerous other clinical manifestations oceur. Often the infection remains latent, causing no recognizable symptoms of disease. Since we cannot carry out large-scale experiments on man under similar conditions, it is impossible to make any definite statement on the comparative virulence of these three organisms, but the limited observations on human volunteers of Morales-Otero (1929, 
$1930,1933)$, a careful study of the available epidemiological data, and the frequency with which laboratory infections occur, suggest that $\mathrm{Br}$. melitensis is the most pathogenic and $\mathrm{Br}$. abortus the least pathogenic of the three types. The American suis type appears to occupy an intermediate position. The Danish suis type, on the other hand, is probably even less pathogenic than Br. abortus, since there is no record of its ever having been responsible for disease in man.

Under natural conditions Br. melitensis is pathogenic for goats and sheep, giving rise to an infection which may be acute and accompanied by abortion, but is more frequently chronic and detectable only by bacteriological examination of the milk, blood, or urine, or by allergic skin tests. In areas where infected goats or sheep are numerous, cows may also become infected (Taylor, Virlal, and Roman 1934). No symptoms of disease are manifest, the animals do not abort when pregnant, but the organisms are often excreted in the milk.

Both varieties of $\mathrm{Br}$. suis are pathogenic for pigs, in which they give rise to a disease sometimes accompanied by abortion. Like $B r$. melitensis, the American type of $\mathrm{Br}$. suis may infect cows and be excreted in the milk (Huddleson 1934, Beattie and Rice 1934). Horses, dogs, and fowls are occasionally infeeted.

$\mathrm{Br}$. abortus probably has the widest range of pathogenicity. Besides being responsible for the almost universal and economically important disease of contagious abortion in cattle, it occasionally infects other animals, such as horses and dogs, and less often sheep and goats. In the United States it is said to give rise to fairly extensive infection of fowls and other birds (Emmel and Huddleson 1929, 1930, Emmel 1930), though the evidence for this is not wholly satisfactory. Guineapigs have also been found infected under natural conditions (Manzullo 1935).

All three species are infective to a variable extent for laboratory animals. On the whole the guinea-pig appears to be the most susceptible, but rabbits, rats, and mice can often be infected. In the guinea-pig the disease produced by parenteral inoculation with moderate doses is usually chronic and retrogressive. The brunt of the infection is borne by the reticulo-endothelial system. The resulting lesions are relatively inconspicuous, and consist mainly of a non-hyperæmic enlargenent of the lymphatic glands, some degree of enlargement of the spleen, and the presence of a variable number of circular necrotic foci in the spleen and liver. In male guineapigs abscess formation is not uncommon in the testicle or epididymis, and intraperitoneal inoculation is sometimes followed by a Straus reaction. Occasionally the bones, joints, or other organs may be affected. The lesions are extremely variable in size and number, and may be completely absent on naked-eye inspection. In infections with $\mathrm{Br}$. suis (American variety) the necrotic lesions tend to be few in number, large in size, and purulent in consistency. The lesions in melitensis and abortus infections, on the other hand, are smaller, more numerous, and generally non-purulent, except in the testicle. Numerous statements have been made about the relative virulence of the three main types for guinea-pigs, but workers who have had the widest experience are the most cautious in drawing conclusions. At the moment it is probably safe to conclude that there is no satisfactory method of distinguishing between them on the basis of pathogenicity to laboratory animals. Kristensen (1931) and Bang (1931) regard the Danish suis type as probably the least virulent, while Thomsen (1934) regards it as slightly more virulent than Br. abortus. Its differentiation from the other members is not practicable by guinea-pig inoculation. The rough, so-called paramelitensis, para-abortus, and parasuis varieties are comparatively avirulent to guinea-pigs. Kritschewski and Halperin (1934) state 
that a suspension of $B r$. abortus has a powerful stimulating effect on the uterine muscle of the virgin guinea-pig. According to Jadassohn, Riedmüller, and Schaaf (1934), the Schultz-Dale technique may be used to differentiate between the different types of Brucella, the reaction in sensitized guinea-pigs being type specific.

Little work has been done on monkeys, but the observations of Huddleson and Hallman (1929), Weigmann (1931), and Zeller, Beller, and Stockmayer (1934) suggest that melitensis and American suis strains are more virulent than abortus strains. (For reproduction of disease in the larger animals see Chapter 75.)

No exotoxin is formed, but the intraperitoneal inoculation of mice with very large numbers of virulent $B r$. abortus brings about death within a few days. The toxicity of the organisms is said to be destroyed by heating to $56^{\circ} \mathrm{C}$. for 20 minutes, and to be related to the virulence of the strain (Priestley and McEwen 1938).

\section{Pathogenicity of $\mathrm{Br}$. melitensis for Small Animals.}

GUINEA-PIGs.- Though death within a few days may follow intracerebral inoculation (Durham 1898, Eyre 1905), or intraperitoneal inoculation with large doses, the disease set up by intramuscular or cutaneous inoculation is chronic, retrogressive, and rarely proves fatal. Small numbers of organisms cannot be relied on to cause infection. Most of the animals continue to gain weight. If they are killed 6 weeks after inoculation, the following lesions may be found. Occasionally there is a local abscess eontaining creamy pus. The regional and the more distal lymphatio glands often show a certain amount of hyperplasia of the pale bloodless variety. The spleen may show a variable degree of enlargement, and may contain a number of circular, greyish-yellow necrotic foci, $0 \cdot 1-0.5$ $\mathrm{mm}$. in diameter, rarely projecting above the surface. Similar foci, usually few in number, may be present in the liver. Sometimes abscesses are found in connection with the joints or bones. The organisms can be cultivated most readily from the glands, spleen, and bonemarrow. The blood usually contains agglutinins in fairly high titre. The lesions are very variable, and may not be detectable by naked-eye examination. The diagnosis must always be made by testing the blood serum for agglutinins and by cultivation of the causative organism from the tissues. A titre of $\mathbf{1 / 2 5}$ or over is strongly suggestive of infection. The intradermal test with a nucleo-protein extract may be used during life for diagnostic purposes, but reliance must never be placed on it alone. After 6 weeks the diagnosis becomes less easy, because the infection tends to retrogress. Guinea-pigs may also be infected by feeding, by conjunctival or nasal instillation, and by inoculation of the scarified skin. Sometimes they contract the disease naturally from their fellows. (For a description of the histopathology of the disease, see Fabyan 1912.)

RABBits appear to be rather less susceptible, but otherwise the infection runs much the same course as in guinea-pigs. The lymphatic system is less affected, and the organisms can rarely be demonstrated in the blood stream. Agglutinin formation is common, but the intradermal reaction is negative.

RATs AND MICE.- There is little information about the effect of inoculation of rats or mice with $\mathrm{Br}$. melitensis, but there is reason to believe that these animals are slightly susceptible to infection (see Singer-Brooks 1937).

MoNkeys may be infected either by feeding or by subcutaneous inoculation. Frequently an intermittent fever is set up, simulating in many respects undulant fever. If the animals are killed after a few weeks, there may be some enlargement of the lymph glands and spleen ; occasionally necrotic lesions are found in the lungs or liver. Agglutinins are demonstrable in the serum, and the organisms can often be recovered from the tissues (see Bruce 1893, Hughes 1893, Horrocks and Kennedy 1906, Huddleson and Hallman 1929, Weigmann 1931, Zeller, Beller, and Stockmayer 1934).

(References to pathogenicity of $B r$. melitensis for small animals: Durham 1898, Eyre 1905, 1908-09, Nicolle and Conseil 1909, Burnet 1922, Zdrodowski et al. 1930, Rainsford 1933.) For reproduction of disease in larger animals see Chapter 75. 


\section{Pathogenicity of Br. abortus for Small Animals.}

In GUINEA-PIGS a disease is set up closely resembling that caused by $\mathrm{Br}$. melitensis. After intramuscular inoculation a local suppurating lesion is rare, but abscess formation in the testis or epididymis is not uncommon. A mild infection can be produced in RABBITs, RATS and MICE. The morbid anatomy and histopathology of the disease have been described by Fabyan (1912). In MICE inoculated subcutaneously with 10-1000 million organisms a retrogressive disease is set up. The organisms can be demonstrated in the regional lym. phatic glands and the spleen for a month or so, and agglutinins are present in the blood serum. Hice may also be infected by feeding with large doses. RATs may be infected by feeding as well as by intraperitoneal inoculation, and the organisms nay be excreted for a time in the urine and fæces, but unless very large doses are used the infection retrogresses (Ber 1936, Sandholm 1938, Bosworth 1938). According to Emmel and Huddleson $(1929,1930)$, fowls can be infected by feeding or parenteral inoculation. The birds stop laying and develop severe diarrhœa. There is a gradually increasing pallor of the head, comb, and wattles, emaciation, and often paralysis and death. The course of the disease ranges from about 2 to 14 weeks. Post mortem, the main lesions consist of a necrotic enteritis and degenerative changes in the liver and kidneys. The majority of other workers, however, who have studied this question, have found that, on the whole, fowls are resistant to infection except with large doses administered parenterally (MoNutt and Purwin 1930, 1932, van Roekel et al. 1932, Beller and Stockmayer 1933). With smaller doses the organisms can rarely be recovered from the tissues. In the absence of direct cultural experiments, a rise in the agglutinin titre cannot be interpreted as necessarily indicative of infection. The conclusions of Emmel and Huddleson require confirmation before being accepted.

Monkeys may be infected with $\mathrm{Br}$. abortus, but they are less susceptible to it than to infection with $\mathrm{Br}$. melitensis. (References to pathogenicity of Br. abortus for small animals : Schroeder and Cotton 1911, Smith and Fabyan 1912, Emmel and Huddleson 1929, 1930, Morales-Otero 1930, McNutt and Purwin 1930, 1932, Bang 1931, Pagnini 1932, Henry, Traum, and Haring 1932, Henricsson 1932, Helms, Holm, and Orskov 1932, Rainsford 1933, Ber 1933, Olin and Lindström 1934, Huddleson 1934, Thomsen 1934, Feldman and Olson 1935, Singer-Brooks 1937, Scorgie 1938). For reproduction of disease in larger animals, see Chapter 75 .

\section{Pathogenicity of $\mathrm{Br}$. suis for Small Animals.}

Experimentally, this organism gives rise in GUINEA-PIGS to a disease closely resembling that caused by $\mathrm{Br}$. melitensis. Local abscess formation is rare, but in infections by the American type large suppurating lesions, few in number, are not uncommon in the spleen, liver, lymph glands, testicles, and joints. The Danish type appears to be less virulent than the American type. Br. suis appears to resemble $\mathrm{Br}$. abortus in its infectivity for MICE, RABBITS, and FowLs, but there is little exact information available. For MICE $\mathrm{Br}$. suis is said to be more virulent than Br, abortus (Singer-Brooks 1937). For MonkEYs it appears to be perhaps even more virulent than Br. melitensis. (References to pathogenicity of Br. suis for small animals: Smith 1926a, Hardy et al. 1930, Cotton 1932, Thomsen 1934, Huddleson 1934, Feldman and Olson 1935.) For reproduction of disease in larger animals, see Chapter 75 .

Variation.-It has already been mentioned that under artificial conditions of cultivation Brucella strains, particularly of the melitensis type, tend to undergo a change which is characterized by a gradual loss of specific, and gradual increase of non-specific, agglutinability, together with a decrease in virulence to animals. This change appears to be an example of the smooth $\rightarrow$ rough variation. Whether the change is accompanied by any corresponding alteration in the morphological and colonial appearances of the organisms is still a little doubtful, though there is reason to believe that some change does occur. The descriptions of various workers, 
however, many of whom have used different media, are so difficult to summarize, that we shall content ourselves with giving references to some of the more important papers on this subject (Henry 1928, 1933, Plastridge and McAlpine 1930, Marshall and Jared 1930; 1931, Morales-Otero 1931, Grumbach and Grillichess 1932). The growth of smooth and rough forms in the presence of dyes appears to be very much the same, but there is some evidence that the $\mathrm{S} \rightarrow \mathrm{R}$ variation may be accompanied by a decrease in biochemical activity, particularly in the production of $\mathrm{H}_{2} \mathrm{~S}$. Though it is true that some rough abortus and American suis strains still produce $\mathrm{H}_{2} \mathrm{~S}$, it is equally true that many do not. Since practically all freshly isolated smooth strains produce $\mathrm{H}_{2} \mathrm{~S}$, it seems not improbable that the loss of this property on continued subcultivation is a manifestation of the $\mathrm{S} \rightarrow \mathrm{R}$ variation.

Classification and Identification.-As has already been pointed out, the inclusion of Br. tularensis in the Brucella group is largely tentative. This organism may be distinguished from the other three members on the basis of morphological, cultural, biochemical, antigenic, and pathogenic properties.

The main difficulty lies in distinguishing between $\mathrm{Br}$. melitensis, Br. abortus, and $B r$. suis. This difficulty is accentuated by the fact that within each species there are a number of sub-types differing from one another in minor respects and approaching closely to the sub-types of adjacent species (see Meyer and Zobell 1932, Wilson 1933). These sub-types are often associated with some special topographical distribution. Melitensis strains, for example, from Malta, may differ from those from Palestine or from the South of France. Southern Rhodesian abortus strains differ from European or American strains. The American suis strains differ from the Danish strains, and so on. For purposes of identification, therefore, the fullest possible examination is required, and no strain should be definitely allocated to a particular species without a careful study by all available bacteriological methods, including $\mathrm{CO}_{2}$-sensitivity, growth in the presence of dyes, $\mathrm{H}_{2} \mathrm{~S}$ formation, antigenic analysis, and if possible virulence. Once the infecting type has been firmly established, help is often afforded by a knowledge of the animal source and country of origin. For instance, in this country Br. suis has never been found, and $\mathrm{Br}$. melitensis has been observed only once among cattle on a small number of farms in the Midlands (see Duke 1940, Wilson 1940), so that any indigenous strain of Brucella isolated from mau, horses, dogs, or cattle, is probably of the abortus type. In any country, strains isolated from sheep or goats are usually of the melitensis type, from pigs of the suis type, from cattle, horses, and dogs of the abortus type, while strains isolated from man usually belong to that type which is most prevalent in the neighbouring animal population. Exceptions, however, are not uncommon, so that too much reliance should not be placed on this particular aid to identification.

Though there are numerous sub-types of Brucella with particular geographical locations, indicating that the members of the group are relatively labile and responsive to environmental changes, no one has yet succeeded in converting one type into another. Even prolonged residence of the melitensis and suis types in cows, and of the abortus type in sheep, scems to have no effect on the type of organism introduced. For practical purposes, therefore, the main types can be regarded as constant. Table 53 summarizes the chief differential features of members of this group. (Useful reviews of the Brucella group will be found in papers by Kristensen 1931, Taylor, Lisbonne, and Roman 1932, Zeller 1933, Habs 1933, Wilson 1933, Huddleson 1934, Thomsen 1934, Olin and Lindström 1934.) 
TABLE 53

Classification of Brucella Group

\begin{tabular}{|c|c|c|c|c|c|c|c|c|}
\hline \multirow{2}{*}{ Type. } & \multirow{2}{*}{ Usual Habitat. } & \multirow{2}{*}{$\begin{array}{l}\text { Growth } \\
\text { in } \\
\text { absence } \\
\text { of extra } \\
\mathrm{CO}_{2} \text {. }\end{array}$} & \multicolumn{4}{|c|}{ Growth in presence of } & \multirow{2}{*}{$\begin{array}{c}\mathrm{H}_{2} \mathrm{~S} \\
\text { Forma- } \\
\text { tion. }\end{array}$} & \multirow{2}{*}{$\begin{array}{l}\text { Antigeni- } \\
\text { cally. }\end{array}$} \\
\hline & & & Thionin. & $\begin{array}{c}\text { Basic } \\
\text { Fuchsin. }\end{array}$ & $\begin{array}{l}\text { Methyl } \\
\text { Violet. }\end{array}$ & Pyтоniu. & & \\
\hline melitensis & Goats, sheep & + & + & + & + & + & - & melitensis \\
\hline abortus . . & Cows, horses & - & - & + & + & + & + & abortus \\
\hline American suis & Pigs & + & + & - & - & - & + & abortus \\
\hline Danish suis. & Pigs & + & + & - & - & - & - & abortus \\
\hline
\end{tabular}

\section{Brucella melitensis}

Synonyms.-M. melitensis, Alkaligenes melitensis.

Isolation.-Isolated by Bruce (1887) from the spleen of patients dying of Malta fever.

Habitat.-Strict parasite living in goats, sheep and man.

Morphology. - Small bacilli, 0.6-1.2 $\mu$ long $\times 0.5-0.7 \mu$ broad, coccoid forms abundant. Axis straight, ends rounded, sides bulging or parallel. Arranged singly, in pairs end-to-end, in small groups, or-especially in liquid media-in short chains of four to six members. Non-motile. Bipolar staining not uncommon. Gramnegative.

Agar Plate. -48 hours at $37^{\circ} \mathrm{C}$. Small, round, convex, amorphous colonies about 0.5 $\mathrm{mm}$. in diameter. Smooth, glistening surface, entire edge, translucent, greyish-white by reflected light, almost colourless by transmitted light; consistency butyrous ; emulsification easy. 6-day colonies slightly larger, and greyisli-yellow. No differentiation.

Agar Stroke. -48 hours at $37^{\circ} \mathrm{C}$. Poor to moderate, partly confluent, slightly raised, translucent growth, with pitted surface, and edge formed of single colonies. After a week the agar is turned brownish and crystals may appear.

Gelatin Stab.-10 days at $22^{\circ} \mathrm{C}$. Poor to moderate, filiform, greyish-white growth, consisting of very small colonies closely packed; extends to bottom of tube. No surface growth and no liquefaction.

Broth.-24 hours at $37^{\circ} \mathrm{C}$. Poor growth with slight turbidity; no surface growth and no deposit. After 10 days there is an abundant growth with moderate turbidity, and a moderate powdery deposit disintegrating completely on shaking. Later the deposit becomes rery viscous, and almost impossible to disintegrate.

Loeffler's Serum. -48 hours at $37^{\circ} \mathrm{C}$. Moderate, slightly raised, chiefly confluent growth of yellowish colour. No liquefaction.

Potato.-6 days at $37^{\circ} \mathrm{C}$. Thin, mostly confluent growth of greyish-brown colour. After 14 days the growth has a café-au-lait or chocolate colour.

Shake Agar. 4 days at $37^{\circ} \mathrm{C}$. Growth of tiny, discrete colonies, situated at the surface, or some distance below the surface; exact position depends on the $\mathrm{CO}_{2}$-sensitivity of the strain.

Liver Agar Plates containing Dyes.-3 days at $37^{\circ} \mathrm{C}$. Usually some growth in presence of thionin, basic fuchsin, methyl violet, and pyronin, but reaction varies considerably according to source of origin of strain.

MacConkey Agar Plate.- 7 days at $37^{\circ} \mathrm{C}$. Small, circular, convex, amorphous, yellowish colonies, $0 \cdot 1-1 \cdot 0 \mathrm{~mm}$. in diameter, with smooth surface and entire edge. May appear slightly mucoid. 
Resistance.-Not specially resistant. Killed by moist heat at $60^{\circ} \mathrm{C}$. in 10 minutes, and by 1.0 per cent. phenol in about 15 minutes. In the dried, powdered eondition they may survive for 3 months. Sealed agar slope cultures at room temperature may remain alive for 1-6 months.

Metabolism.-Aerobic; no growth under strictly anaerobic conditions. Growth is often improved by 10 per cent. $\mathrm{CO}_{2}$. Opt. temp. $37^{\circ} \mathrm{C}$. ; limits $20-40^{\circ} \mathrm{C}$. Opt. H-ion concentration $\mathrm{pH} 6 \cdot 6-7 \cdot 4$. Growth slightly improved by glucose, glycerine, liver extract, blood and serum. Brown pigment formed on potato and sometimes in old agar cultures. Broth turned alkaline-to $\mathrm{pH} 8.0$ or even higher. Growth in all media is relatively slow. Some growth on MacConkey's medium. Does not hæmolyse blood.

Biochemical.-No carbohydrates fermented. L.M. turned slightly alkaline. Indole - ; II.R. - ; V.P. - ; Nitrates and nitrites reduced. $\mathrm{NH}_{3}$ sometimes $+; \mathrm{H}_{2} \mathrm{~S}-$; I.B. reduced; catalase + .

Antigenic Structure.-Only one serological type known. Appears to contain the same antigens as $\mathrm{Br}$. abortus and $\mathrm{Br}$. suis, but in different quantitative proportions. Provided absolutely smooth strains are used, it may be differentiated from $\mathrm{Br}$. abortus and $\mathrm{Br}$. suis by quantitative absorption of agglutinins. The rough variant, incorrectly called $\mathrm{Br}$. paramelitensis, is agglutinable by non-specific agents, but not by a serum prepared against the smooth form.

Pathogenicity.-Causes undulant fever in man, and a septicæmic infection of goats and sheep, sometimes accompanied by abortion. May infect cows and be excreted in the milk. Experimentally, it is pathogenic to a variable degree for man, goats, sheep, monkeys, and the small laboratory animals. The rough variant is avirulent.

\section{Brucella abortus}

Isolation.-By Bang (1897) from cows with infectious abortion.

Habitat.-Strict parasite, occurring in cattle, horses, dogs, and man.

Morphology.-Similar to that of type species, but usually more bacillary; rods reach $1.5 \mu$ in length, or on special media even $3.0 \mu$. May be capsulated.

Cultural Characters.-Similar to those of type species, except that growth of the bovine type, whether isolated from eattle or from man, usually occurs only in the presence of added $\mathrm{CO}_{2}$, preferably 5-10 per cent. In shake agar cultures growth occurs 1-2 $\mathrm{mm}$. or more below the surface, and extends downwards for $\frac{1}{2}-1 \mathrm{~cm}$. Old laboratory cultures grow freely under aerobic conditions; no growth under strictly anaerobic conditions.

Resistance.-Similar to type species. In uterinc exudate kept in the ice-chest it survives for 9 months. May live in sterile water for 3 or 4 months. Readily killed in milk by holder pasteurization. Nay survive in ice-cream for a month.

Metalolism.-Similar to type species, but as most strains on isolation require $\mathrm{CO}_{2}$, the optimum $\mathrm{H}$-ion concentration of media is about $\mathrm{pH} 6 \cdot 6$. Brown coloration in old agar cultures less common than with $\mathrm{Br}$. melitensis.

Biochemical. - Similar to type species, but nearly all strains on isolation produce $\mathrm{H}_{2} \mathrm{~S}$ in liver agar for at least 4 days.

Antigenic Structure.-Appears to possess the same antigens as Br.melitensis, but distributed in different quantitative proportions. Provided absolutely smooth strains are used, it may be differentiated from $\mathrm{Br}$. melitensis by quantitative absorption of agglutinins. The rough variant, incorrectly referred to as $\mathrm{Br}$. para-abortus, is agglutinable by non-specific agents.

Pathogenicity.-Causes epizootic abortion in cattle, fistulous withers in horses, and a mild septicæmic infection in dogs. Is said to infect rats. Gives rise to undulant fever in man. Experimentally, it is pathogenic to a variable degree for man, cattle, horses, dogs, fowls, monkeys, and the small laboratory animals. The rough variant is avirulent. 


\section{Brucella suis}

Isolation.-Amcrican type by 'Traum (19I4) from the fotus of a sow, and Danish type by Thomsen (1931).

Habitat.-Strict parasite, occurring in pigs and man.

Morphology.- Similar to $\mathrm{Br}$. abortus.

Cultural Characters.--Similar to those of type species, except that growth is never improved by addition of $\mathrm{CO}_{2}$. In shake agar cultures growth occurs on the surface. No growth under strictly anaerobic conditions. The American type grows rather more freely than the Danish type. On liver agar plates both types grow in the presence of thionin, but are inhibited by basic fuchsin, methyl violet, and pyronin. The Danish type has the same differential susceptibility as the American type, but is rather more susceptible to all dyes; consequently half the usual concentrations of dye should be employed when testing it.

Resistance.-Similar to that of type species and Br. abortus.

Metabolism.- Similar to type species, but grow th is not improved by $\mathrm{CO}_{2}$. Brown coloration in old agar cultures less common than with $\mathrm{Br}$. melitensis.

Biochemical.-Similar to type species, but American type produces $\mathrm{H}_{2} \mathrm{~S}$ in liver agar for at least 4 days; Danish type produces no $\mathrm{H}_{2} \mathrm{~S}$.

Antigenic Structure.-Appears to possess the same antigens as Br. melitensis, but distributed in quantitatice proportions nearer those of Br. abortus than Br. melitensis. Provided absolutely smooth strains are used, it may be differentiated from $\mathrm{Br}$. melitensis, but not from $\mathrm{Br}$. abortus, by quantitative absorption of agglutinins. The rough variant, incorrectly referred to as $\mathrm{Br}$. parasuis, is agglutinable by nonspecific agents.

Pathogenicity.-Gives rise to a disease of pigs, which may be accompanied by abortion, and to undulant fever in man. May infect cows and be exereted in the milk. Experimentally, it is pathogenic to a variable degree for man, pigs, cows, monkeys, and the small laboratory animals. Possibly pathogenic to some degree for horses and dogs. The rough variant is avirulent.

\section{Brucella tularensis}

This organism is a tiny, non-motile, Gram-negative bacillus, which was isolated by McCoy and Chapin in 1912 from rodents suffering from tularemia (see Chapter 75). In the animal body, it occurs as a coccoid or rod-shaped organism surrounded by a clear area, which probably represents a capsule. The diameter of the organism is $0.3-0.7 \mu$ long by $0.2 \mu$ wide; the diameter with the capsule is $0 \cdot 4-1 \cdot 0 \mu$ by $0 \cdot 3-0 \cdot 5 \mu$. The organisms stain best with carbol-fuchsin or aniline gentian violet; with methylene blue they stain very poorly and show no capsule. In culture, coccoid forms alone are seen (Wherry and Lamb 1914); a capsule is visible if the organisms are mixed with serum. No growth occurs in the usual media. It was first cultivated on Dorset's egg, but later it was found that coagulated egg yolk was more satisfactory (McCoy 1912). On this medium the maximum growth is reached in 2 days; it is pale, translucent, slightly mucoid, and pearly in appearance, not easily distinguishable from the medium; it is readily emulsifiable. Growth occurs also on glucose blood agar, glucose serum agar, and blool agar slopes, provided that a piece of rabbit's spleen is rubbed over the surface and then left in the condensation water (Francis and Lake 1922); and on agar to which 0.02 per cent. of cystine is added (Francis 1922, 1923). On these media the organism should be subcultured every other day, but on egg yolk it may remain viable for 3 months (Wherry and Lamb 1914). Shaw and Humicntt (1930) recommend a medium composed of brain veal infusion agar, $\mathrm{pH} 7 \cdot 6$, containing 5 per cent. rabbit serum, 1 per cent. dextrose, and 0.05 per cent. cystine, while Kudo 
(1934) prepares a mixture of 60 per cent. egg yolk and 40 per cent. rabbit serum sterilized at $70^{\circ}-75^{\circ} \mathrm{C}$. on 3 suceessive days. Liquid media can also be used (Tamura and Gibby 1943); Steinhans, Parker and McKce (1944) recommend for this purpose a medium containing 1 per cent. dextrose, $0 \cdot 15$ per cent. cystine, and 0.5 per eent. hæmoglobin. Br. tularensis grows well in the developing ehick embryo, multiplying particularly in the ectodermal epithelial cells. When inoeulated on to the chorio-allantoic membrane, it leads to the death of the embryo in 3-4 days. In both these respects it resembles Br. melitensis (Buddingh and Womack 1941, Ransmeier 1943). Under suitable conditions the organism is said to produce acid in glucose and glycerol, and usually in maltose, mannose and lævulose (Downs and Bond 1935, Francis 1942); but the acid produced seems to be very slight, and may possibly be of the order of that formed by melitensis and American suis strains.

Moist heat at $55^{\circ}-60^{\circ} \mathrm{C}$. is fatal in 10 minutes. Antigenically $\mathrm{Br}$. tularensis is allied to the other members of the Brucella group. Francis and Evans (1926) found that a serum prepared against $\mathrm{Br}$. tularensis agglutinated $\mathrm{Br}$. melitensis and $\mathrm{Br}$. abortus to about $\frac{1}{4}$ or $\frac{1}{6}$ of the titre. Neither organism was able, however, to absorb the homologous agglutinins from a tularensis serum. Br.tularensis was agglutinated to a low titre by anti-melitensis and anti-abortus sera, but was unable to absorb the homologous agglutinins from these sera. The 3 strains of $B r$.tularensis examined appeared to be antigenically homogeneous.

Under natural conditions it gives rise to tularæmia in rodents-especially groundsquirrels and jack-rabbits-and occasionally in man. Sheep are sometimes affected (Parker and Dade 1929). Experimentally the disease can be reproduced in groundsquirrels, gophers, guinea-pigs, rabbits, mice, and monkeys ; rats are more resistant (Dieter and Rhodes 1926); cats, dogs, and pigeons appear to be immune. Feeding, nasal instillation, cutaneous, subcntaneous, intraperitoneal, and conjunctival infection are all successful. After subcutaneous infection of the guinea-pig, death oceurs in 5 to 8 days. Post mortem there is a whitish membrane-like area at the site of inoculation; the regional lymphatic glands may be enlarged and caseous; the spleen is enlarged, very dark in colour, and contains discrete, yellowish-white, caseous granules up to $1 \mathrm{~mm}$. in diameter, projecting slightly above the surface; there are numerous granules in the liver; foeal neerotic areas are sometimes present in the bone marrow (Lillie and Francis 1933); the lungs are rarely involved. The bacilli are present in large numbers in the blood and organs; as little as $0.000,000,1$ $\mathrm{ml}$. of the heart's blood may prove infective for fresh animals. The virulence of the organism may decline in culture, so that instead of causing an acute or subacute disease in guinea-pigs, it gives rise to a chronic disease from which the animal often recovers (McCoy 1912, Foshay 1932). Strains of lowered virulence have also been isolated directly from ticks (Davis et al. 1934). The organism is extremely dangerous to handle in the laboratory, and large numbers of workers have contraeted the infection.

\section{REFERENCES}

Alessandrint, A. and Sabatudot, M. (1931) Ann. Igiene (Sper.), 41, 29, 852. Antoni, V. DE. (1929) Boll. Ist. sieroter. Milano, 8, 651.

BANo, B. (1897) Z. Thiermed., 1, 241.

Bang, O. (1931) 2 me Congr. int. Path. comp., i. 95.

BAU, K. H. and WANG, K. (1935-36) Z. Hyg. InfektKr., 11\%, 399.

Beattie, C. P. and Rioe, R. M. (1934) J. Amer. med. Ass., 102, 1670.

Beller, K. and Stockmayer, W. (1933) Dtsch. tierärztl. Wschr., 41, 551.

Ber, A. (1933) Z. InfektKr. Haust., 44, 129; (1936) C. R. Soc. Biol., 122, 845.

BEVAN, L. E. W. (1930) Brit. med. J., ii. 267.

Bieling, R. (1930) Z. Hyg. InfektKr., 111, 728.

Bosworth, T. J. (1938) J. comp. Path., 50, 345.

Bruce, D. (1887) Practitioner, 39, 161; (1893) Ann. Inst. Pasteur, \%, 289. 
Boddingh, G. J. and WomAck, F. C. (1941) J. exp. Med., 74, 213.

Burnet, E. (1922) Arch. Inst. Pasteur Afrique nord, 2, 165; (1925) Arch. Inst. Pasteur Tunis, 14, 247.

Cameron, H.S. (1932) Comell I'eterinarian, 22, 212; (1933) Rep. N.Y. St. vet. Coll. 1931-32, No. 18.

Cerruti, C. F. (1927) Boll. Ist. sieroter. Milano, 6, 425 ; (1932) Ibid., 11, 400.

Coleman, M. B., Owen, H. H., and DaceY, H. G. (1930) J. lab. clin. Med., 15, 641.

Cotton, W. E. (1932) J. agric. Res., 45, 705.

Damboviceanu, A., Barber, C., Pop, A., and Marinov, I. (1938) C. R. Soc. Biol., 12\%, 736

Davis, G. E., Philip, C. B., and Parker, R. R. (1934) Amer. J. Hyg., 19, 449.

Davoli, R. (1938) Boll. Sez. ital. Soc. int. Hicrobial., 10, 124.

Dieter, L. V. and Rhodes, B. (1926) J. infect. Dis., 38, 541.

Downs, C. M. and Bond, G. C. (1935) J. Bact., 30, 485.

Duke, F. IV. (1940) Lancet, ii. 517.

Duncan, J. T. (1928) Trans. roy. Soc. trop. Med. Hyg., 22, 269.

Durhal, H. E. (1898) J. Path. Bact., 5, 377.

Emane, M. W. (1930) J. Amer. vet. med. Ass., 76, 452, 564.

Enmel, M. IV. and Huddesson, I. F. (1929) J. Amer. vet. med. Ass., 75, 578 ; (1930) Ibid., 76, 449.

Evans, A C. (1918) J. infect. Dis., 22, 580 ; (1925a) Amer. J. trop. Med., 5, 419; (1925b) Bull. U.S. hyg. Lab., No. 143.

Eyre, J. IV. H. (1905) Rep. Comm. Medit. Fev., Lond., Part II, p. 67 ; (1908 9) Proc. roy. Soc. Edin., 29, 537.

FавтаN, M. (1912) J. med. Res., 26, 441.

Favill, G. (1926a) Sperimentale, 80, 41 ; (1926b) Ibid., 80, 396; (1930) Ibid., 84, 287.

Favili, G. and Blancalani, G. (1932) Sperimentale, 86, 357 ; (1934) Itid., 88, 337.

Feldman, W. H. and Olson, C. (1935) J. infect. Dis., 5\%, 212.

Feuster M. L. and Meyer, K. F. (1920) J. infect. Dis., 2\%, 185.

Fleming, A. (1919) Lancet, i. 138.

Foresti, C. (1935) Nuova Vet., 13, 11.

Forni, G. (1927). G. Bait. Immun., 2, 823.

Foshay, L. (1932) J. infect. Dis., 51, 280.

Francis, E. (1922) Publ. Hlth Rep., Wash., No. 17, 37, 987 ; (1923) Ibid., No. 25, 38, 1391 ; (1931) Ibid., 46, 2416; (1942) J. Bact., 43, 343.

Francis, E. and Evans, A. C. (1926) Publ. Hlth Rep., Wash., No. 26, 41, 1273.

Francis, E. and Lake, G. C. (1922) Publ. Hlth Rep., Wash., No. 3, 37, 83.

Gladstone, G. P., Fildes, P., and Richardson, G. M. (1935) Brit. J. exp. Path., 16, 335.

Goodpasture, E. W. and Anderson, K. (1937) Amer. J. Path., 13, 149.

Grombach, A. and Grilliohess, R. K. (1932) Zbl. Balt., 126, 321.

Gwatkin, R. (1935) Canad. J. Res., 12, 115, 133.

Haвs, H. (1930) Zbl. Bakt., 116, 89; (1933) Zbl. ges. Hyg., 28, 481.

HaBs, H. and Sievert, L. (1935) Dtsch. med. Hschr., 61, 1398.

Hardy, A. V., Jordax, C. F., Borts, I. H., and Hardy, G. C. (1930) Nat. Inst. Hlth. Bull., No. 158.

Helus, T., Holi, P., and Orskov, J. (1932) Z. ImmunForsch., 75, 55.

Henricsson, E. (1932) "Epizootischer Abortus und Undulantfieber." Isaac Marcus Boktryckeri-Aktiebolag, Stockholm.

Henry, B. S. (1928) Proc. Soc. exp. Biol., N.Y., 26, I01 ; (1929) Ibid., 2\%, s ; (1933) J. infect. Dis., 52, 374, 403.

Henry, B. S., Traum, J., and Harino, C. M. (1932) Hilgardia, 6, 355.

Hershey, A. D., Huddleson, I. F., and Pennell, R. B. (1935) J. infect. Dis., $5 \%, 183$.

Higginbothai, M. and Heathian, L. S. (1936) J. infect. Dis., 59, 30.

Hоцтн, H. (1911) Z. InfektKr. Haustiere, 10, 207.

Horrocks, W. H. and Kennedy, J. C. (1906) Rep. Comm. Medit. Fev., Lond., Part IV, p. 37.

Huddleson, I. F. (1921) Cornell veterinarian, 11, 210; (1929) Mich. State College, agric. Exp. Sta., Tec. Bull., No. 100 ; (1931) Amer.J. publ. Hlth, 21, 491 ; (1934) "Brucella Infections in Animals and Man." Commonwealth Fund, New York; (1940) J. Amer. vet. med. Ass., 96, 708; (1943) Tech. Bull. Mich. agric. Exp. Sta., No. 182, 45.

Huddleson, I. F. and Abell, E. (1927) J. Bact., 13, 13; (1928) J. infect. Dis., 43, 81.

Hoddleson, I. F. and Hallian, E. T. (1929) J. infect. Dis., 45, 293.

Hodnleson, I. F., Hasley, D. E., and Torrey, J. P. (1927) J. infect. Dis., 40, 352.

Huddeson, I. F. and Stahl, IV. H. (1943) Tech. Bull. Mich. agric. Exp. Sta., No. 182, p. 57.

Huddleson, I. F. and Winter, O. B. (1927) J. infect. Dis., 40, 476.

Huoнes, M. L. (1893) Ann. Inst. Pasteur, 7, 628. 
Hoston, R. C., Huddleson, I. F., and Hershey, A. D. (1934) Tech. Bull., Mich. agric. Exp. Sta., No. 137.

Jadassohn, W., Riedmüller, L., and SchaAf, F. (1934) Klin. Wschr., 13, 879.

Kerby, G. I'. (1939) J. Bact., 37, 495.

ǨIne, K. O. C. (1934) Aust. vet. J., 10, 93.

Koser, S. A., Breslove, B. B., and Dorfman, A. (1941) J. infect. Dis., 69, 114.

Koser, S. A. and Wrignt, M. H. (1942) J. infect. Dis., 71, s6.

KRISTENSEN, M. (193I) Zbl. Bakt., 120, 179.

Kristensen, M. and Holm, P. (1929) Z Zbl. Baht., 112, 281.

Kritschewski, I. L. and Halperin, E. P. (1934) Z. ImmunForsch., 82, 421.

KंUdo, M. (1934) Jap. J. exp. Med., 12, 371.

Lillie, R. D. and Francis, E. (1933) Publ. Hlth Rep., Wash, 48, 1127.

Lisbonne, H. and Monnier, P. (1936) C. R. Soc. Biol., 123, 1114.

McAlpine, J. G. and Slanetz, C. A. (1928a) J. infect. Dis., 42, 66, 73; (1928b) Ibid., 43, 232.

MoCoy, G. W. (1912) Publ. Hlth Bull., Wash., No. 53, p. 17.

McCoy, G. W. and Chapin, C. W. (1912) J. infect. Dis., 10, 61.

McNutt, S. H. and Purwin P. (1930) J. Amer. vet. med. Ass., 30, 350; (1931) J. infect. Dis., 48, 292 ; (1932) J. Amer. vet. med. Ass., 81, 641.

Maggiora-Vergano, A. (1932) Boll. Ist. sieroter. Milano, 11, 400.

Mallardo, C. A. (1930) J. trop. Med. Hyg., 33, 125.

Manzullo, A. (1935) Fol. biol., No. 47, 211.

Marshall, M. S. and Jared, D. (1930) Proc. Soc. exp. Biol., N.Y., 27, 525 ; (1931) J. infect. Dis., 49, 318.

Martini, G. (1935) Boll. Ist. sieroter Milano, 14, 431.

Menzani, C. (1934) Nuova Vet., 12, 83.

Messieri, A. (1935) Nuova Vet., 13, I, 13.

Meyer, K. F. and EdDie, B. (1930) J. lab. clin. Med., 15, 447.

MeYER, K. F. and Shaw, E. B. (1920) J. infect. Dis., 2\%, 173.

MeYer, K. F. and Zobell, C. E. (1932) J. infect. Dis., 51, 72.

MiскLе, W. A. (1940) J. infect. Dis., 66, 271.

Miles, A. A. (1933) Brit. J. exp. Path., 14, 43 ; (1939) Ilid., 20, 63.

Mliles, A. A. and Pirie, N. W. (1939a) Brit. J. exp. Path., 20, 83, 109, 278 ; (1939b) Biochem. $J ., 33,1709,1716$.

Mingle, C. K. and Manthei, C. A. (1941) Amer. J. vet. Res., 2, 181.

Mrno, G. DI. (1935) Boll. Ist. sieroter. Milanese, 14, 123.

Монг, IV. (1935) Z. ImmunForsch., 86, 235.

Morales-Otero, P. (1929) Porto Rico J. publ. Hlth. trop. Med., 5, 144; (1930) Ilid., 6, 3. (1931) Porto Rico J. publ. Hlth trop. Med., 7, 233 ; (1933) J. infect. Dis., 52, 54.

M UNGER, M. and Huddleson, I. F. (1938) J. Bact., 35, 255.

Nègre, L. and Raynaud, M. (1912a) C. R. Soc. Biol., Y2, 791; (1912b) Ibid., "22, 1052.

Nerva, C. (1934) Brasil-Medico, 48, 421.

Nicolle, C. and Conseil, E. (1909) C. R. Soc. Biol., 6\%, 267.

Nowak, J. (1908) Ann. Inst. Pasteur, 22, 541.

OLrN, G. (1935) "Studien über das Undulantfieber in Schweden." Isaac Marcus Boktryekeri-Aktiebolag, Stockholm.

Ouin, G. and LindströM, B. (1934) Zbl. Bakt., 131, 257.

Pagnini, U. (1932) G. Batt. Immun., 9, 1004; (1934) Boll. Ist. sieroter. Milano, 13, 145 ; (1935) G. Batt. Immun., 15, 847.

Pampana, E. J. (1931) Ann. Igiene (Sper.), 41, 537.

Pandit, S. R. and Wilson, G. S. (1932) J. Hyg., Camb., 32, 45.

PAREER, R. R. and DADE, J. S. (1929) Publ. Hlth Rep., Wash., 44, 126.

Pennell, R. B. and Huddleson, I. F. (1937) Tech. Bull. Mich. agric. exp. Sta. No. 156, 1 ; (1938) J. exp. Med., 68, 73, 83.

Plastridge, W. N. and McAlpine, J. G. (1930) J. infect. Dis., 46, 315 ; (1932) J. infect. Dis., 50, 555 .

Pop, A. Damboviceane, A., Barber, C. and Marinov, I. (1938) C. R. Soc. Biol., 12\%, 733.

Preisz, H. (1903) Zbl. Bakt., 33, 190.

Priestley, F. W. (1933) J. comp. Path., 46, 38; (1938) Vet. Rec., 50, 137.

Priestley, F. W. and McEwen, A. D. (1938) J. comp. Path., 51, 282.

Pullinger, E. J. (1935) Lancet, i. 1342.

RajNSFORD, S. G. (1933) Irish J. med. Sci., April, p. 150.

Ransmeter, J. C. (1943) J. infect. Dis., "r2, 86.

Reimann, H. A. (1932) Amer. J. Hyg., 16, 206.

REITER, D. O. (1936) J. infect. Dis., 58, 45.

Report. (1905-7) Comm. Mediterranean Fever, Parts I, III, IV. Harrison and Sons, London. 
Roekel, H. van, Bullis, K. L., Flint, O. S., and Clarke, M. K. (1932) J. Amer. vet. med. Ass., 80, 641 .

RopP, R. S. DE. (1944) J. comp. Path., 54, 53.

Ross, G. R. (1927a) J. IIyg., Camb., 26, 279 ; (1927b) Ibid., 26, 403.

SAITTA, S. (1929) G. Batt. Immun., 4, 307.

Sandholn, A. (1938) Z. InfektKr. Haust., 53, 201.

Santis, M. DE. (1933) Boll. Ist. sieroter. Milano, 12, 846 ; (1935) Ibid., 14, 113.

Schapira, G. B. (1936) Sperimentale, 90, 450.

Schroeder, E. C. and Cotton, W. E. (1911) Bur. Animal Ind., 28th Ann. Rep., p. 139.

Schwarzmaier, E. (1936) Z. InfektKr. Haust., 49, 309.

Scorgie, N. J. (1938) J. Path. Bact., 46, 165.

Sergent, E., Gillot, V., and Lematre, G. (1908) Ann. Inst. Pasteur, 22, 209.

Shaw, F. W. and Hunnioutr, T. (1930) J. Lab. clin. Med., 16, 46.

Sievert, L. (1936) Z. ImmunForsch., 89, 249.

Silberstein, W. (1932) Z. Hyg. InfektKr., 114, 177.

Singer-Brooks, C. H. (1937) J. infect. Dis., 60, 265.

Sмiтн, J. (1934) J. Hyg., Camb., 34, 242.

Sмгтн, Т. (1924) J. exp. Med., 40, 219; (1926a) Ibid. 43, 207; (1926b) Ibid., 43, 317.

Sмitth, T. and FabYaN, M. (1912) Zbl. Bakt., 61, 549.

Stafseth, H. J. (1920) Mich. agric. exp. Sta., Tec. Bull., No. 49.

Stahl, W. H. (1941) Tech. Bull. Mich. agric. Exp. Sta., No. 177, 29.

Stahu, W. H. and Hamann, E. E. (1941) Ibid., No. 177, 17.

Stahl, W. H., Pennell, R. B. and Huddeeson, I. F. (1939) Ibid., No. 168, 1.

Steinhaus, E. A., Parker, R. R., and McKee, M. T. (1944) Publ. Hlth. Rep., Wash., $59,78$.

Tamura, J. T. and Gibby, I. W. (1943) J. Bact., 45, 361.

Thylor, R. M., Iisbonne, M., and Roman, G. (1932) Ann. Inst. Pasteur, 49, 284.

Taylor, R. M., Vidal, L. F., and Roman, G. (1934) C. R. Soc. Biol., 116, 132.

Thomsen, A. (1931) Rev. gén. Méd. vét., 40, 457; (1933) Zbl. Bakt., 130, 257; (1934) "Brucella Infection in Swine." Acta path. microbiol. Scand., Suppl. No. 21.

Thompson, R. (1933) Canad. med. Ass. J., 29, 9.

Toppina, L. E. (1934) J. Path. Bact., 39, 665̃.

Tosatti, E. (1934) Pathologica, 26, 247.

Traum, J. E. (1914) Rep. Chief Bur. Anim. Industry, p. 30.

Tuttle, C. D. and Huddenon, I. F. (1934) J. infcet. Dis., 54, 259.

VALENTI, E. (1927) Biochim. Terap. sper, 14, 77.

Vangielovici, M., Damboviceanu, A., Barber, C., and Pop, A. (I938) C. R. Soc. Biol., $12 \%, 738$.

VEAZIE, L. and MEYeR, K. F. (1936) J. infect. Dis., 58, 280.

Vidal, J. and Abet.la, R. (1928) C. R. Soc. Biol., 99, 1271.

Vittone, R. (1935) Boll. Sez. ital., Soc. int. Microbiol., \%, 277.

Weigmann, F. (1931) Zbl. Bakt., 121, 318.

Wherry, W. B. and LaMb, B. H. (1914) J. infect. Dis., 15, 331.

Wilson, G. S. (1930) Brit. J. exp. Path., 11, 157; (1931a) Ibi.., 12, 88; (1931b) Ibid., 12,152 ; (1933) J. Hyg., Camb., 33, 516; (1934) Ibid., 34, 361 ; (1940) J'et. Rec.. 52, 737.

Wilson, G. S. and Milles, A. A. (1932) Brit. J. exp. Path., 13, 1.

Znrodowski, P., Brenn, H., and Voskressenski, B. (1930) Ann. Inst. Pasteur, 45, 768. Zeller, H. (1933) Münch. lierärztl. Wschr., 84, 337, 349, 361, 373, 389.

Zeller, H., Beller, K., and Stockmayer, W. (1934) Münch. tierärztl. Wschr., 85, 143. ZeLier, H. and StockMaYer, W. (1933) Z. InfektKr. Haustiere, 44, 67.

Zobele, C. E. and Meyer, K. F. (1932) J. infect. Dis., 51, 91, 99, 109, 344, 361. 


\section{CHAPTER 35}

\section{BACILLUS}

\section{AEROBIC SPORE-BEARING BACILLI}

\section{Definition.-Barillus.}

Aerobic, spore-bearing rods, usually Gram-positive. Often oceur in long threads, and form rhizoid colonies. Form of rod, as a rule, not greatly changed at sporulation. Liquefy gelatin. Mostly saprophytes.

Type species. Bacillus subtilis.

\section{INTRODUCTORY}

The aerobic spore-bearing Bacillus forms one division of the family Bacillacea while the anaerobic spore-bearing Clostridum forms the other. As many of the organisms in the former division are widely distributed, being found in air, soil, water, milk, dust, fish-meal, wool, fæces, and other situations, it is not unnatural that they were among the first micro-organisms to be studied; but as there are large numbers of different species, almost all of which are devoid of pathogenic action, it follows from the way in which bacteriological investigation has been directed mainly along medical, veterinary, or agricultural lines, that our knowledge of these organisms is far from complete.

Some authors have separated the group into two divisions, the one containing $B$. anthracis and the closely allied pseudoanthrax bacillus, the other containing $B$. subtitis and other saprophytic forms. As, however, $B$. subtilis may itself be confused with $B$. anthracis, this division is of no real value. We shall treat all the members as belonging to one single group-the group Bacillus-reserving the term "pseudoanthrax bacillus" as an inexact but convenient designation for any organism of the aerobic spore-bearing group that is liable to be confused with the true $B$. anthracis.

There is a large group of thermophilic bacilli found in milk, manure, and other situations which grow best at temperatures round about $60^{\circ} \mathrm{C}$. We do not propose to describe these organisms, but references to them will be found in a paper by Wilson and his colleagues (1935). Not all of them belong to the Bacillus group; many species appear to be streptococci or non-sporing rods.

\section{Group Characteristics}

Morphology and Staining.-Nembers of this group are rod-shaped organisms varying in size from abont $3 \mu \times 0.4 \mu$ to $9 \mu \times 2 \mu$. The sides are parallel, the axis straight or slightly curved, the ends either truncated, as in $B$. anthracis, or more usually convex. Their arrangement varies considerably; though single and diplobacillary forms predominate, they may be arranged in chains, often of con- 
siderable length, or in groups. Long, unjointed filaments are characteristic of some species, notably of the anthrax bacillus. Irregular forms, consisting mainly of poorly-stained thin bacilli, or of club- or bottle-shaped bacilli, are not uncommon. With a few exceptions, of which $B$. anthracis is the most important, all the members are motile by about 4-12 peritrichous flagella. Spores are present in all; and are formed only in the presence of oxygen; they vary in shape from spherical to ellipsoidal, and may appear at the equator, subterminally, or at the very end of the bacillus. In some members their diameter does not exceed that of the bacillus, but in others the rod is swollen to resemble a clostridium. Capsules are met with in only one member, $B$. anthracis, and then only when it is growing in the animal body, or in media rich in animal protein. The organisms are usually Gram-positive, but considerable variation may be shown; some are strongly positive, others weakly positive, and a few frankly negative. When stained with varions dyes, it is generally possible to distinguish areas of uneven staining; in large bacilli a number of small particles are seen, quite distinct from spores. Some of these particles appear to consist of fat (see Burdon et al. 1942), others of volutin or glycogen. By a few authors the volutin granules have been regarded as nuclear material scattered diffusely through the cell; the evidence against this contention has already been given in Chapter 2. None of the vegetative bacilli is acid-fast, though, in the sporing condition, they resist decolorization for a short time with weak acids and alcohol.

Cultural Reactions.--Growth is free on all the ordinary media. Single colonies on agar are generally large, varying from 2 to several millimetres in diameter. Some have a finely granular, mealy appearance, others are membranous and thrown into wrinkles. In broth there is a tendency towards the formation of a surface scum, with or without turbidity, or of a heavy flocculent or membranous deposit. Gelatin is

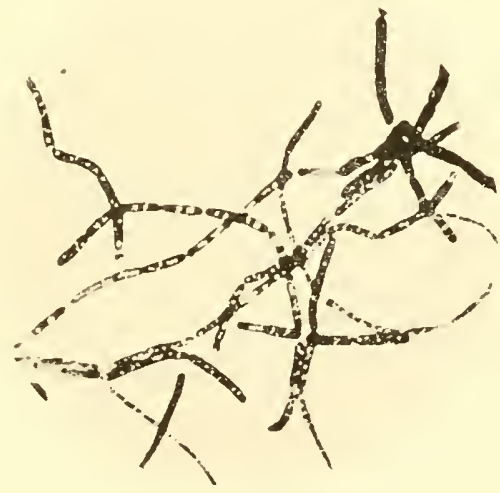

FIG. 176.-B. megatherium.

From an agar slope culture $(\times 1000)$. usually liquefied rapidly. In a stroke agar culture the growth is raised and confluent, and generally of membranous consistency, rendering emulsification difficult. Growth is not improved by the addition of blood, serum, or glucose. Variant colonial types have been described for several members of the group. Some nembers form motile colonies (see $B$. rotans p. 855).

Resistance.-In the vegetative condition the bacilli are killed by moist heat at a temperature of $55^{\circ} \mathrm{C}$. in 1 hour. The spores vary greatly in resistance; some, like those of $B$. anthracis, are destroyed by boiling for about 10 minutes; others, like those of $B$. subtilis, may withstand boiling for hours. All are killed by stean under pressure at $120^{\circ} \mathrm{C}$. in 40 minutes. Similarly with disinfectants the resistance varies; $\mathrm{HgCl}_{2}$ even in a $1 / 1000$ solution may fail to kill anthrax spores in less than 70 hours (Poppe 1922). Potassium permanganate, on the other hand, in a 4 per cent. solution kills them in 15 minutes, and a 3 per cent. solution of hydrogen peroxide in 1 hour. Generally speaking, the spores are 
extremely resistant to chemical disinfectants, with the exception of those substances which act by oxidation.

Metabolic and Biochemical Reactions.--Some members form a pigment, generally brownish-yellow in colour, oceasionally pink. On the whole, pigment formation is not a striking characteristic, and tends to appear late. The optimum temperature for growth varies from $25^{\circ} \mathrm{C}$. to $37^{\circ} \mathrm{C}$.; few grow below $12^{\circ} \mathrm{C}$., and excluding the thermophilic bacilli which grow at $60^{\circ} \mathrm{C}$, none grows above $55^{\circ} \mathrm{C}$. According to Lamanna $(1940 a)$ the small-celled species, like $B$. subtitis, $B$. vulgatus, and $B$. mesentericus, have an average minimum and maximum temperature about $5^{\circ} \mathrm{C}$. higher than the large-celled species like $B$. cereus. As regards oxygen pressure, they are aerobic or facultatively anaerobic. A high partial pressure of $\mathrm{CO}_{2}$ tends to inhibit growth of $B$. subtitis (Levine 1936), and to favour capsulation but to restrict sporulation of $B$. anthracis (Sterne 1937).

On carbohydrate media the majority of members form acid only, but a few, like $B$. asterosporus, $B$. polymyxa, and $B$. acetoathylicum produce gas. Most of the members ferment glucose, maltose and sucrose ; some are able to attack mannitol and salicin. Lactose is rarely fermented. A diastatic ferment capable of inverting starch is secreted by some. A proteolytic ferment for gelatin is produced by nearly all, and by a few for blood serum. A true rennet-like clot is often formed in litmus milk, and is subsequently digested; the litmus is reduced. Some strains peptonize milk without actually clotting it. The reaction becomes alkaline. Both the catalase test and the oxidase reaction deseribed by Gordon and McLeod (1928) are usually positive. Methylene blue is reduced in broth. Some members are able to produce $\mathrm{H}_{2} \mathrm{~S}$, and some to reduce nitrates to nitrites. Indole is not produced. A powerful filtrable hæmolysin is formed by one member of the group B. megatherium - but many species are said to be hæmolytic (Poppe 1922). Highly active bactericidal substances, called tyrocidin and gramicidin (see Chapter 6) appear to be formed by several different members of the group (Dubos 1939, Dubos and Cattaneo 1939, Dubos and Hotchkiss 1941).

Antigenic Structure.-Most of the serological work has been carried out with a view to separating $B$. anthracis from the other members of the group. A precipitating serum prepared against the anthrax bacillus will react not only with its homologous antigen, but also with the pseudoanthrax bacilli, though in a lower titre; conversely the anthrax bacillus will react in a low titre with a serum prepared against some of the pseudoanthrax bacilli. A similar group reaction is noticeable in the complement-fixation test (Poppe 1922). From this we gather that the aerobic spore-bearing bacilli form a group, the members of which are closely related antigenically; the differentiation of $B$. anthracis, at least, can be carried out on a quantitative basis.

An attempt has been made by Sievers and Zetterberg (1940), with some slight success, to classify other members of the aerobic spore-beariug group by precipitation and complement-fixation tests. The most important antigenic study, however, of recent years is that of Howie and Cruickshank (1940) who, working with $B$. cereus, $B$. mesentericus, and certain other members of the group, were able to show that the vegetative bacilli and the spores contained different antigens, thus confirming the previous general conclusions of Defalle (1902) and Mellon and Anderson (1919). Pure anti-spore sera conld be obtained by injecting rabbits with a suspension of spores practically free from bacilli, or with a suspension of organisms in which the bacillary antigen had been destroyed by autoclaving for 
20 minutes at $15 \mathrm{lb}$, or by absorbing out the anti-bacillary agglutinins fron a mixed serum. No attempt was made in this work to study the relationship of different members of the group to each other, but in a paper published shortly after Howie and Cruickshank's, Lamanna (1910b) brought evidence to show that $B$. subtitis, $B$. vulgatus, and $B$. mesentericus along with $B$. agri, belonging to the small-celled species, could be separated serologically from each other by means of their spore antigens (see also Lamanna 1942). Separation of members of the large-celled species was less successful, but a broad distinction could be drawn between $B$. cereus and $B$. megatherium.

Pathogenicity.-Speaking generally, we may say that the anthrax bacillus is highly pathogenic for most animals; and that most other members are nonpathogenic for all animals. This statement, however, must be qualified. Under natural conditions the anthrax bacillus gives rise to diseasc in man, cattle, sheep, and certain other of the domesticated animals; under experimental conditions it is pathogenic for the laboratory animals. Under natural conditions other species of Bacillus rarely give rise to disease, but an exception must be made for $B$. subtilis, which may cause severe eye lesions, notably iridocyclitis and panophthalmitis (Axenfeld 1908), and which may occasionally invade the blood stream of patients whose powers of resistance are lowered by the attack of some fatal disease (Sweany and Pinner 1925). Occasionally too, infections such as meningitis and pneumonia may be due to pseudoanthrax bacilli (Senge 1913, Wilamowski 1912). There is evidence that some members, when allowed to grow excessively in food, may produce toxic substances capable of giving rise on ingestion hy man to gastro-enteritis (see Chapter 72). Under experimental conditions, the pseudoanthrax bacilli are non-pathogenic for all laboratory animals except mice, and for these animals only when injected intraperitoneally in a large dose-1-3 loopfuls of an agar eulture. B. megatherium is, however, definitely toxic, and is able to kill guinea-pigs injected intraperitoneally in less than 24 hours. This is due to the formation of a hromolysin. The fact that some pseudoanthrax bacilli may on occasion prove pathogenic to man and animals, and that after long subculture $B$. anthracis may lose its virulence for laboratory animals, suggests that there may be a gradual transition from the non-pathogenic to the pathogenic state (but see p. 845). A pseudoanthrax bacillus, described as $B$. tropicus, has been isolated from mice inoculated with the blood of patients suffering from "coastal fever" in Queensland, but its relation to the disease is doubtful (see Heaslip 1941).

Classification.-This is very difficult, and any classification adopted is bound to be arbitrary. Some authors divide the group on the basis of motility, others on the character and situation of the spore, others on cultural characteristics, and others on several properties taken together. To each of these methods there are objections, and agreement is still far from being reached. In two of the most recent studies, for example, the criteria used for classification differ widely, with necessarily contradictory results. De Soriano (1935), who made a systematic study of 206 strains, proposes a classification based primarily on whether or not the spore causes deformation of the bacillus, and secondarily on morphological, cultural and biochemical characteristics. Lamanna (19f0a), on the other hand, who studied 105 strains, suggests a classification based primarily on the size of the hacterial cell, and secondarily on the mode of germination of the spore. Thus he distinguishes first of all between small-celled aml large-celled species. The small- 
celled species are subdivided into those that germinate (1) by shedding their spore coat equatorially or at the pole or (2) by comma-shaped expansion. The largecelled species all germinate by absorption of the spore coat. Unfortunately this brings many of Lamanna's large-celled species, like B. mycoides, B. cereus, B. megatherium, and many of his small-celled species, like $B$. subtilis, $B$. vulgatus, and $B$. mesentericus into the same primary subdivision, suggested by de Soriano, of species whose spores do not cause bulging of the vegetative portion of the cell. Some hope is held out by the serological studies of Howie and Cruickshank (1940) and Lamanna (1940b), who have shown that the spore antigen is distinct from the bacillary antigen, that a classification of the non-pathogenic members of the group may ultimately be based on antigenic characteristics.

To the medical and veterinary student the chief interest is in the differentiation of $B$. anthracis from the non-pathogenic species; this will be discussed under the description of $B$. anthracis which is given below.

\section{Bacillus anthracis.}

Named B. anthracis by Cohn (1875b) and Bacteridium by Davaine (1864). This bacillus is non-motile, forms capsules in the animal body and sometimes on artificial media, and grows on agar in characteristic long, segmented, parallel or interwoven chains. The spores are ellipsoidal or oval in shape, are found equa-

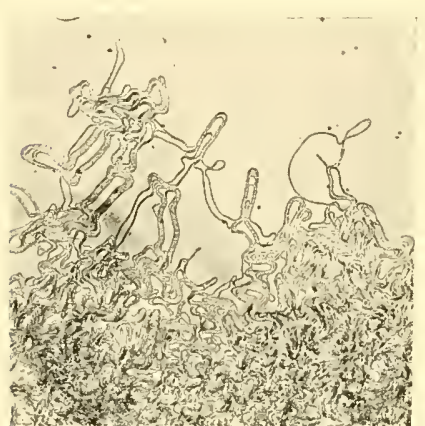

FIG. 177.-B. anthracis,

Edge of eolony on agar to show curled hair-lock appearance $(\times 18)$. torially, and germinate by polar rupture. It is interesting to note that the anthrax bacillus was the first micro-organism in which the presence of resistant spores was demonstrated. Spores are never found in the animal body during life, and in culture appear more slowly than those of the other members of the group. They seem to be formed under conditions unfavourable to continued growth of the vegetative bacilli. Their appearance can be hastened by the addition of distilled water, 2 per cent. sodium chloride, and other salts (Bongert 1903). According to Bordet and Renaux (1930), sporulation is inhibited by the presence of calcium chloride and favoured by its absence. Cultures grown on oxalated agar often come to consist mainly of spores, while those grown on agar to which $\mathrm{CaCl}_{2}$ has been added may lose their spore-forming power completely.

The curled hair-lock appearance of single colonies on agar or gelatin is characteristic, but may be closely simulated by $B$. subtitis. Microscopically this is seen to be due to the growth of the bacilli in long interwoven chains. Growth, particularly in broth, at a temperature of $42 \cdot 5^{\circ} \mathrm{C}$. for some days, causes the appearance of several different vuriants; some have tough, well-defined capsules, give rise on agar to the typical curled colonies, and are highly virulent; some have soft poorly-defined capsules, form thin, shining colonies on agar, and are slightly virulent ; others are non-capsulated, give rise to smooth, round, convex, glistening, mucoid colonies on agar, and are entirely avirulent (Preisz 1911, Nungester 1929a, b, Bordet and Renaux 1930). Capsulation is favoured by growth in air containing 
10-25 per cent. of $\mathrm{CO}_{2}$ (Ivánovies 1937). Some variants are asporogenous; it was these which attracted Pasteur's attention, and which he considered to be avirulent. Preisz (1911) has, however, shown that, though there is a definite correlation between capsule formation and virulence, there is none between spore formation and

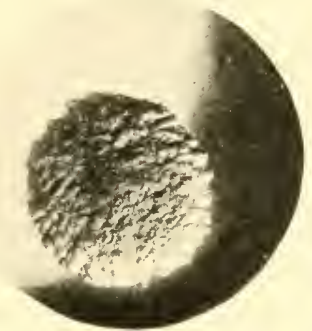

Fis. 178.-B. anthracis.

Smooth type of colony. Agar, 24 hours, $37^{\circ} \mathrm{C} .(\times 8)$

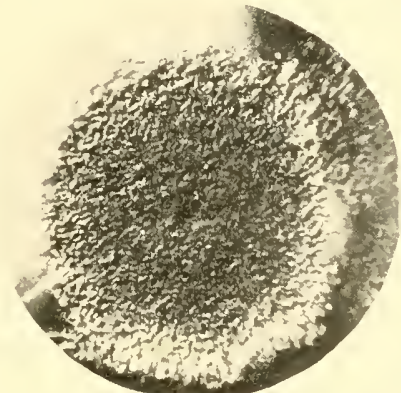

FIa. 179.-B, anthracis.

Rough type of colony. Agar, 24 hours, $37^{\circ} \mathrm{C} .(\times 8)$.

virulence. Virulent strains may be either sporogenous or asporogenous ; similarly with avirulent strains. Asporogenous varieties may appear spontaneously in cultures incubated at the usual temperature (Behring 1889), or in cultures containing weak antisepties, such as 1/2,000 potassium dichromate, or 1 1,000 phenol (Roux 1890). Such varieties, when arising from a virulent strain, are themselves fully virulent, though prolonged contact with weak antiseptics may eventually lower their virulence. The normal highly virulent bacillus forms a large, rough colony of frosted glass appearance with a curled edge ; morpholngically it consists of bacilli arranged in chains (Figs. 179, 181). The avirulent bacillus forms a smaller, smoother type of colony, with a slightly crenated edge ; morphologically it consists of bacilli arranged singly, in pairs end-to-end, or in small bundles (Figs. 178, 180).

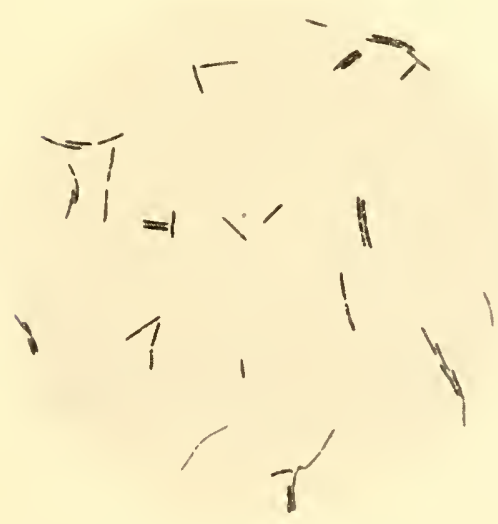

Fia. 180.-B. anthracis.

Fromı smooth colony on agar, 24 hours, $37^{\circ} \mathrm{C} .(\times 1000)$.
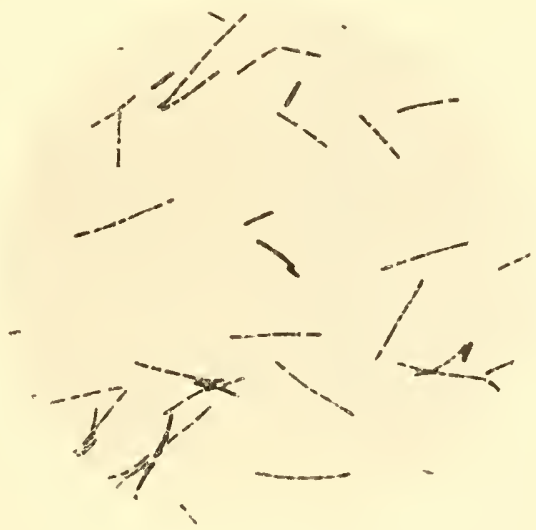

FIG. 181.-B. anthracis.

From rough colony on agar, 24 hours. $37^{\circ} \mathrm{C} .(\times 1000)$. 
In the dry state the spores may remain alive for 12 years or more (Pasteur 1881a). According to Murray (1931), a saline suspension containing $1,000,000$ spores per ml. is sterilized by moist heat at $90^{\circ} \mathrm{C}$. in 15 to 45 minutes, at $95^{\circ} \mathrm{C}$. in 10 to 25 minutes, and at $100^{\circ} \mathrm{C}$. in 5 to 10 minutes.

The antigenic structure of the anthrax bacillus has received considerable attention. As early as 1921 Kramár obtained evidence that the capsule contained a glycoprotein probably belonging to the class of pseudomucins. A soluble specific carbohydrate, incapable of giving rise to antibodies on injection into animals, but reacting to a high titre with anti-anthrax serum, was extracted from anthrax bacilli by Combiesco, Soru, and Stamatesco (1929). A similar substance was prepared by Schockaert (1929). It is, however, mainly to the work of Tomesik (1930), Tomesik and Szongott (1932, 1933), Tomcsik and Bodon (1934, 1935), Bodon and Tomcsik (1934), Sordelli and Deulofeu $(1930,1933)$ and Sordelli, Deulofeu, and Ferrari (1932) that our knowledge of the antigenic structure of this organism is due. There appear to be two main antigens; one a protein-like substance present in the capsule, the other a polysaccharide substance present in the body of the organism. Specific precipitins for both the protein-like and polysaccharide constituents are found in serum prepared by the injection of animals with capsulated bacilli, and each can be differentially absorbed in the usual way. In animals infected with anthrax both antigens can be demonstrated in the tissues by the use of precipitating sera. Later observations by Ivánovics and Erdös (1937), and Ivánovics and Bruckner (1937a, b, 1938), have shown that the capsular substance contains a group-specific hapten common to $B$. anthracis and certain other members of the Bacillus group. It appears to consist of a polypeptide of high molecular weight containing $d(-)$ glutamic acid. It reacts in high titre with a precipitating serum prepared by the inoculation of rabbits with heat-killed capsulated anthrax bacilli, but cannot by itself stimulate the formation of antibodies (Tomcsik and Ivánovics 1938). The somatic polysaccharide is said by Ivánovics (1940) to consist of glucosamine and galactose with acetic acid attached to the molecule.

The anthrax bacillus is naturally pathogenic mainly to the herbivora and to man, but occasionally it attacks other animals. Experimentally it proves fatal to the mouse, guinea-pig, and rabbit, less often to the rat. The larger the dose, the shorter is the time to death. Subcutaneous injection of 1 loopful of an 18 hours' agar culture kills mice, guinea-pigs, and rabbits in about 12 to 30 hours; a smaller dose, 1/100 of a loupful, kills them in 30 to 40 hours ; a still smaller dose, 1/2,000,000 of a loopful, kills mice in 96 hours, guinea-pigs in 56 hours, and rabbits in 104 hours (Sobernheim 1897). Other members of this group never kill in such small doses.

Post mortem, there is a gelatinous hæmorrhagic local œedema; the viscera are congested, the blood is dark red and coagulates less firmly than usual; the spleen is enlarged, dark red, and very friable. Microscopically, the bacilli are found in large numbers in the local lesion, in the blood, and in the thoracic and abdominal viscera; they are confined almost entirely to the interior of the capillaries, where their numbers may be so great as to cause obstruction to the blood flow; the tissues themselves are rarely penetrated. Though the disease terminates in a septicæmia, it is not till 4 or 5 hours before death, as a rule, that the bacilli actually gain access to the blood stream.

Infection may also be successfully achieved by cutanẹous, intracutaneous, intramuscular, intraperitoneal or intravenous injection, or by feeting. The most certain route is the intramuscular, the least certain the oral (Sobernheim and 
Murata 1921). In general it requires a large dose to produce a fatal infection by the mouth (Giovanardi 1931). Post mortem in eases of oral infection, in addition to the enlargement of the spleen and the occurrence of septicæmia, the intestinal mucosa is seen to be covered with small furuncular swellings, through which the bacilli have gained access to the blood. Of the three animals the mouse is the most susceptible and the rabbit the least, the guinea-pig occupying an intermediate position. This difference in susceptibility is scarcely noticeable, except with a strain of weakened virulence (see Chapter 66). Rats are more difficult to infect than other rodents, but are said to succumb easily if fatigued by continuous exercise on a revolving drum (Charrin and Roger 1890). They may develop a chronic disease after subcutaneons injection, which does not prove fatal for 4 or 5 weeks. Dogs may be infected by subcutaneous injection, though not uniformly. Birds, with the exceptiun of sparrows and young doves, and cold-blooded animals are resistant, likewise Algerian sheep (Chauveau 1880a, b). (See Davaine 1863a, b, 1864, Koch 1877, Frank and Lubarsch 1892, Sobernheim 1897, Oppermann 1906, Balteano 1922, Poppe 1922, Basset 1925, Katzu 1925, Muller 1925, Sanarelli 1925.) The experimental reproduction of the disease in larger animals is considered on p. 1736.

Pasteur $(1881 b, c)$ found that by growing the anthrax bacillus at $42.5^{\circ} \mathrm{C}$. for about a month, he was able to lower its virulence to such an extent that it proved harmless to all animals except new-born guinea-pigs. By successive passage through these animals, the bacillus gradually regained its virulence till it was able to kill 2, 3, and 4-day, and later fully grown guinea-pigs; eventually its virulence was entirely restored. From the work of Preisz (1911) it would appear that this resumption of virulence is due not to a gradually increasing virulence of the individual organisms, but to an alteration in the proportions of virulent and avirulent bacilli in the culture. He found that the effect of incubating a virulent culture at $42.5^{\circ} \mathrm{C}$. was to cause the appearance of variants that were no longer virulent to animals, so that in one and the same culture both virulent and avirulent bacilli were found side by side. The longer the incubation, the higher was the proportion of avirulent bacilli. After a month or more the culture consisted almost entirely of avirulent variants, and on injection into mice proved to be harmless; the virulent bacilli that were still present were too few to cause death. But if such a culture is injected into a new-born guinea-pig, these few virulent bacilli may be just sufficient to overcome the very low resistance of the animal ; in consequence they proliferate, and during the course of successive passages increase in proportion relatively to the avirulent variants, till eventually the culture consists almost entirely of the virulent type. The modern conception of the essential heterogeneity of single strains, i.e. the presence in one and the same strain of organisms showing sharp discontinuous variations in virulence, not only has more evidence in its favour than the older conception of the simultaneous and equal raising or lowering of all the bacilli in the strain, but explains more easily the variations in virulence that are noted consequent on altered environmental conditions.

Several observers (Hankin 1889, Martin 1890, Marmier 1895, Standfusz and Schnauder 1925) have shown that

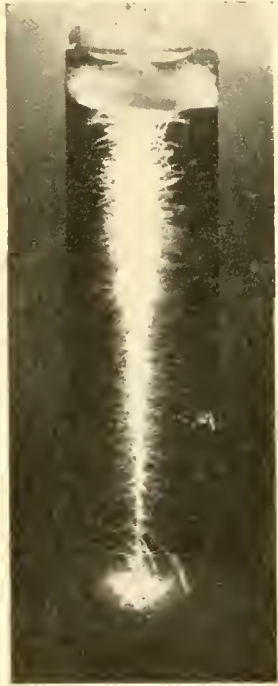

Fig. 182.-B. anthra. cis.

In gelatin stab culture, 3 days, $22^{\circ} \mathrm{C}$., showing inverted fir-tree growth, with commencing lique. faction. 
when $B$. anthracis is grown in a broth culture toxic albumoses are formed, which prove fatal on injection into animals; Martin also found a toxic alkaloid. There is no evidence that a true exotoxin is formed; the toxic substances appear to be formed largely by the disintegration of the proteins in the medium. Aoki and Yamamoto (1939) bring evidence to suggest that B. anthracis forms an endotoxin which is capable of activating spores in vivo. The formation of a hæmolysin has been asserted by some authors, and denied by others; it is possible that different strains may vary in this respect. There is certainly no hæmolysin produced for cattle or horse blood, but there is evidence to suggest that one may be formed for sheep, goat and rabbit blood (Poppe 1922).

The main criteria of value in the differentiation of $B$. anthracis from those bacilli which may be confused with it may be given in tabular form:

\section{$B$. anthracis.}

1. Non-motile.

2. Capsulated.

3. Grows in long chains.

4. No turbidity in broth.

5. Inverted fir-tree growth in gelatin.

6. Polysaccharide precipitin reaction strongly positive.

7. Pathogenic to laboratory animals.

8. Liquefaction of gelatin slow.
Anthrax-like or so-called pseudoanthrax bacilli. Generally motile.

Non-capsulated.

Grow in short chains.

Frequent turbidity in broth.

Fir-tree growth absent or atypical.

Polysaccharide precipitin reaction weakly positive.

Non-pathogenic to laboratory animals. Liquefaction of gelatin rapid.

An inverted fir-tree growth in gelatin is given by some strains of pseudoanthrax bacilli, but the branches are thick and interlaced, quite different from the regular, delicate, lateral outgrowths of $B$. anthracis. It is sometimes stated that the anthrax bacillus does not show hæmolysis on blood agar plates, whereas the pseudoanthrax bacilli form colonies surrounded by a zone of hæmolysis. This depends on the type of blood used, and is at best an uncertain criterion for differentiation.

When freshly isolated from the animal body, the anthrax bacillus rarely canses difficulty in identification, but after prolonged subculture in the laboratory it may lose several of its important characteristics, such as capsule formation, the inverted fir-tree growth in stab gelatin, and its pathogenicity for laboratory animals, and may then be very difficult to classify. Nevertheless bacilli have been described which have given rise to an anthrax-like disease in man and other animals, yet which have not conformed to the usual criteria of $B$. anthracis (Schulz 1901, Wilamowski 1912, Senge 1913). Such bacilli have usually been classed as pseudoanthrax bacilli, but it is probable that some at least have been variants of the real B. anthracis, similar to those described by Preisz (1911).

The characters of certain species of Bacillus are summarized below.

\section{Bacillus anthracis}

Synonyms.-Bactéridie du charbon, Milzbrandbacillus.

Hubitut.-Parasitic in man, eattle, sheep and other animals.

Morphology.--Rods, $3-8 \mu \times 1-1 \cdot 2 \mu$. Straight or slightly curved, ends truncate; on agar plates arranged characteristically in very long, segmented, parallel, or interwoven chains. Unjointed filaments not infrequent in cultures. In blood of animals mostly in pairs or chains of 3 or 4 . Spores equatorial, ellipsoidal, not bulging; polar germination; not formed in animal body. Non-motile. Capsule found in 
animal body, and on serum media; lost on agar; surrounds entire chain of bacilli. Gram-positive. Non-acid-fast.

Agar Plate.-Irregularly round, 2-3 $\mathrm{mm}$. in diameter, raised, dull, opaque, greyishwhite, plumose colonies, with a tessellated or reticular structure, an uneven surface and a curled edge. Membranous consistency, emulsifiability difficult; colony consists of parallel interlacing chains of bacilli, and is characteristic. After about a week irregular round scales appear on the surface of the colony. Several colonial variants have been described.

Agar Slope.-Thick, raised, spreading, greyish-yellow growth, with an uneven surface and an undulate edge showing little curled projections ; moist and slightly glistening. Looks as if there were innumerable tiny air bubbles beneath the surface. After about a week irregular round scales appear on the surface of the growth.

Gelutin Stab.-Poor filiform growth followed by outgrowth of delicate lateral extensions, longest at the upper part of the culture, giving an inverted fir-tree or lamp-brush effect. Liquefaction crateriform; occurs very slowly.

Broth.-No turbidity, or very fine floccular turbidity; nioderate floccular deposit, consisting of interwoven threads, and disintegrating partly on shaking. No surface growth.

Blood Serum.-Abundant, creamy-yellow, confluent, eurled growth with uneven surface. No liquefaction.

Potato.-Raised, dry, greyish-white, slightly spreading growth, with undulate or serrated edge.

Resistance.-Spores killed by boiling in 10 minutes. In dry state remain alive for years. Metabolic.-Aerobic; facultative anaerobe. Opt. temp. $37^{\circ} \mathrm{C}$. Limits $12^{\circ}$ to $44^{\circ} \mathrm{C}$. Pigment none. Hæmolysis: some strains are stated to hæmolyse sheep's red cells. Nutritional; grows well on ordinary media; growth not improved by blood, serum or glucose.

Bivehemical.-Acid, no gas, in glucose, maltose, sucrose, and later in salicin ; final $\mathrm{pH}$

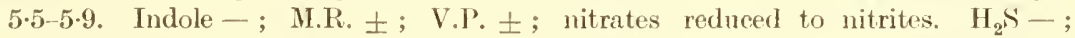
$\mathrm{NH}_{3}++$; methylene blue reduced; catalase + . Litmus milk coagulated and decolorized; later peptonized.

Autigenic Structure.-There is a capsular polypeptide-containing antigen and a somatic polysaccharide-containing antigen; the former has some group relationship to capsular antigens found in certain other members of the Bacillus gromp. Buth antigens react specifically with precipitating antisera.

Pathogenicity.-Naturally pathogenic to man, cattle, sheep (not Algerian), goats, pigs, and camels; rarely to carnivores. Experimentally mice, guinea-pigs, and rabbits, injected $s c$. or $i m$., die in 12 to 40 hours; p.m., hæmorrhagic local exudate, enlarged spleen, and bacilli in blood. Rats less susceptible. Birds, except sparrows and young pigeons, cold-blooded animals, and fish are resistant.

\section{"Bacillus anthracoides," or "Bacillus pseudoanthracis."}

Bacilli more or less closely resembling the anthrax bacillus have been isolated by numerous workers from such substances as soil, water, meat-, fish- and bonemeal, wool, dust, oil-cake, and less frequently from animals and man. 'These organisms have frequently been termed $B$. pseudoanthracis or $B$. anthracoides. Reference to the available papers renders it evident that more than one species has been described. We do not propose to consider these organisms in more detail, since their differential diagnosis from the anthrax bacillus has already been dealt with. It is sufficient to point out that most of them are motile, non-capsulated, form spores abundantly within 24 hours on agar, produce an even turbidity or a surface pellicle in broth, give rise to colonies on agar which are less curled and have fewer and less regular outgrowths at the edges, are more 
resistant to heat, form alkali in litmus milk, and are generally non-pathogenic, though sometimes they may be fatal to mice and even guinea-pigs on intraperitoneal inoculation in fairly large doses. The classification of these organisms is at present impossible; they seem to range from avirulent variants of $B$. anthracis, on the one hand, to virulent variants of $B$. subtilis on the other. For some of the strains which have been described, see Hueppe and Wood (1889), Hartleb and Stutzer (1897), Schulz (1901), Bainbridge (1903), Wilamowski (1912), and Grierson (1928) ; and for two useful reviews see Pokschischewsky (1914) and Poppe (1922).

\section{B. subtilis.}

Great confusion has prevailed, and in fact still prevails, over this organism. It was described by Ehrenberg in 1838, who found it in hay infusion, as Vibrio subtitis, and by Cohn $(1875 a)$ as Bacillus subtilis. Neither of the descriptions was sufficiently full to enable the organism to be distinguished from others that simulate it closely, and in consequence organisms that are almost certainly different from Cohn's original bacillus have been identified with this organism. In an extensive investigation of the Bacillus group, Lawrence and Ford (Ford 1916) in America gave this name to an organism that differs in several important particulars from that given by Cohn. The bacillus described by the German workers is fairly large, 3-4 4 long by $1 \mu$ thick, may form threads, is actively motile, forms anthrax-like colonies on agar, gives rise to a thick, wrinkled surface nembrane in broth, liquefies blood serum, and gives a thick, yellowish-white, creamy growth on potato, later appearing as if strewn with dry, white granules. The bacillus described by the American bacteriologists is one of the smallest of the aerobic spore-bearing bacilli, is $2 \mu$ long by $0.4 \mu$ broad, does not usually form threads, is sluggishly motile, forms dry, hard, glassy colonies on agar, adherent to the medium, gives rise to a thin branching scum in broth, later becoming more dense, fails to liquefy blood serum, and on potato gives a luxuriant, warty, pink growth.

According to Conn (1930), there are two different types of bacilli commonly caller B. subtilis, one forming small spores which germinate equatorially (Marburg type), the other forming larger spores showing polar germination (Michigan type). Conn advances reasons to prove that the Marburg type is the original and genuine type, while Soule (1932) maintains that the classical B. subtilis is represented by the Michigan type. At the second international Microbiological Congress the Marburg type was officially accepted as the type strain (St. John-Brooks and Breed 1937). Unfortunately, however, evidence on serological and other grounds has since been adduced by Lamanna (19406) to show that the Marburg type strain is really a strain not of $B$. subtilis but of $B$. vulgatus (see also Lamanna 1942, Knaysi and Gunsalus 1944).

In the following summary of the properties of $B$. subtilis we have described the small-celled type of organism showing equatorial germination of the spore, which was in the mind of the bacteriological nomenclature committee of the Congress. For some of its properties we have drawn on the description given by de Soriano (1935), Lamanna $(1940 a, b)$ and on our own personal observations.

Variant colonies with different bacillary morphology have been described by a number of workers. According to Soule (1928), there is a rough and a smooth type closely simulating the corresponding type of $B$. anthracis (see p. 843). Graham (1930), who, like Soule, probably worked mainly with strains of the Michigan type, described four variants, two of which were motile and two usually non-motile. 
Variants I and III formed smooth, circular, shiny colonies with regular margins; II formed "medusa-head" colonies; while IV formed slightly irregular colonies with an uneven surface and a rather granular texture. All four variants had the same heat-stable somatic antigen. Variants I and II had in addition a common heat-labile flagellar antigen.

Bacillus subtilis Cohn emendavit Prazmowski Synonyms.-Hay baeillus.

Habitat.-Hay, dust, milk, soil, water.

Morphology. - Slender rods, $1.5-3 \mu \times 0.5-0.8 \mu$, straight or curved, rounded ents, oeeurring singly or in short chains. Actively motile by $8-12$ peritriehate flagella. Non-capsulated. Spores are oval, $1.5 \mu$ $\times 0.6 \mu$, formed sub-terminally, do not eause bulging of the cell, and germinate equatorially without sphtting along the transverse axis; appear

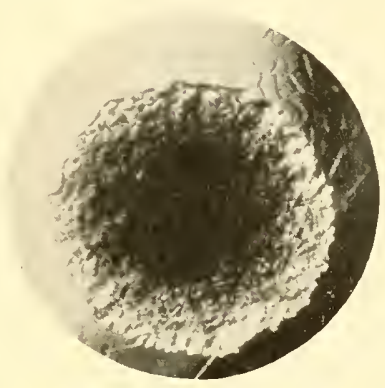

Fig. 183.-B. subtilis.

Smooth type of eolony. Agar 24 hours, $37^{\circ} \mathrm{C} .(\times 8)$. on agar in 18 hours. Gram-positive. Non-acid-fast.

Agar Plate.--Irregularly circular colonies, 4-6 $\mathrm{mm}$. in diameter, slightly raised, greyishyellow, and having a darker erumbly centre surrounded by a lighter periphery with a curled edge. Surface is finely granular; membranous or friable consistency; adherent to medium; emulsification rather diffieult. Resemble anthrax colonies.

Agar Slope.-Abundant, confluent, greyish-white, raised, opaque, sometimes wrinkled growth, with an undulate and finely serrated edge and a mealy surface. Membranous consisteney, adherent to medium, emulsifying fairly easily.

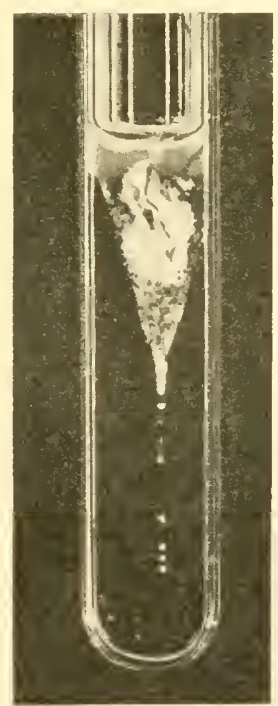

Fia. 184.-B. sub. tilis.

In gelatin stab culture, 4 days, $20^{\circ} \mathrm{C}$., showing infundibuliform liquefaction.
Gelatin Stab. - Filiform growth with rapid infundibuliform or saceate liquefaction; thick white membrane on surface adhering to the sides of the tube.

Broth.-Moderate turbidity, slight deposit, with formation of a thick wrinkled surface membrane adhering to the walls of the tube; pellicle often sinks to the bottom.

Blood Serum.-Thick folded membrane; liquefaction.

Potato.-Thick, yellowish-white, raised, dull, creamy growth, later sprinkled with dry white granules, giving a mealy appearance.

Biochemical.-Acid, no gas, in glucose, maltose and sucrose. Indole -. M.R. - ; V.P. F; nitrates reduced to nitrites. $\mathrm{H}_{2} \mathrm{~S}-; \mathrm{NH}_{3}+$; methylene blue reduced; catalase + . Litmus milk partially clotted, peptonized, and decolorized from above downwards. Starch is hydrolysed.

Metabolic-Aerobe, facultative anaerobe Opt. temp. $37^{\circ} \mathrm{C}$. Limits $15-55^{\circ} \mathrm{C}$.

Pigment.-Cream to ehestnut-biown.

Nutritional.-Grows freely on ordinary media; growth not improved by blood, serum, or glucose.

Hamolysis.- $\beta$-type on horse blood agar plates given by some strains.

Resistance.-Spores withstand boiling for hours.

Antigenic Structure-Little exact knowledge available. Motile strains appear to have a heat-labile flagellar and a heatstable somatic antigen. Spore antigen is different from bacillary antigen. 
Pathogenicity.-May give rise to conjunctivitis, iridochoroiditis, and panophthalmitis in man. Occasionally invades the blood stream in cachectic diseases. $1 \mathrm{ml}$. 24-hours' broth culture sometimes proves fatal to mice injected intraperitoneally; subcutaneous inoculation into rats occasionally gives rise to a local infiltration, and to submiliary abscesses in the lungs. Most strains are non-pathogenic.

\section{B. mesentericus and B. vulgatus}

Flügge (1886) first described an organism, generally known as the potato bacillus, under the name $B$. mesentericus vulgatus. There is a tendency now to regard B. mesentericus and B. vulgatus as two distinct species, though Flynn and Rettger (1934) think that they are variants of a single species. Even the most recent descriptions, such as those of de Soriano (1935) and Lamanna (1940c), are not in entire harmony, and it is difficult in the absence of international agreement to do more than reproduce the characters that have been assigned to these organisms.

A variety, by some regarded as a distinct species, of $B$. mesentericus is $B$. mesentericus fuscus (Flügge) or the brown potato bacillus. It resembles $B$. mesentericus, but differs from it in being slightly smaller, in having less tendency to thread formation, and in forming on agar and potato a thinner, greyish-brown layer of growth. A red potato bacillus was described by Globig (1888) as $B$. mesentericus ruber. It is sometimes known as B. globigii Migula. On potato it forms a reddish wrinkled growth of a tough viscous consistency. There is also a black potato bacillus, first described as $B$. mesentericus niger, and sometimes known as B. aterrimus Lehman and Neumann; on solid media, especially on potato, it gives a characteristic thick, wrinkled, black growth. A bacillus, $B$. mesentericus panis viscosi, sometimes known as B. panis, is responsible for ropy bread.

\section{Bacillus vulgatus (Flügge) Trevisan}

Synonyms.-B. mesentericus vulgatus; potato bacillus.

Habitut.-Found in dust, soil, water, milk.

Morphology.--Rather slender, about 2-4 $\mu \times 0.75 \mu$; slightly rounded ends, occurring singly and in short chains. Motile by peritrichate Hagella. Spores ovoid, $1.5 \mu$ $\times 0.6 \mu$, formed sub-terminally, do not, cause bulging of the cell, and germinate equatorially with splitting along the transverse axis. Noncapsulated. Gram-positive. Nonacil-fast.

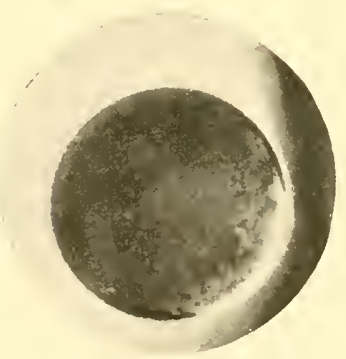

F19. 185.-B. vulgatus.

Smooth type of colony. Agar, 24 hours, $37^{\circ} \mathrm{C} .(\times 8)$.

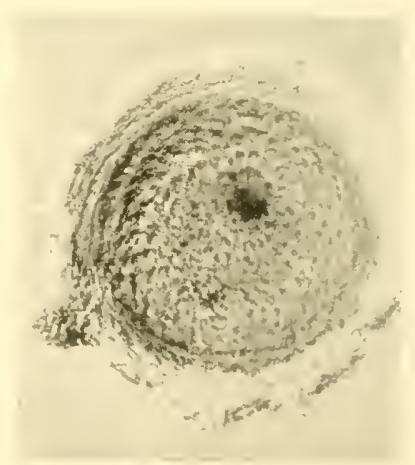

Fio. 186.-B. vulgatus.

Same colony as in Fig. 185, after 48 hours incubation, showing marked irregularity of surface and edge, and raised peri. pheral ring $(\times 8)$. 
Agar Plate.-Round or oval, $4 \mathrm{~mm}$. in diameter, raised greyishyellow, opaque colonies with entire edge. Surface is smooth in eentre and tends to be wrinkled towards periphery. Consistency membranous or friable; not adherent to medium; emulsification difficult.

Agar Slope.-Profuse, confluent, raised, greyish-yellow, dull, opaque, wrinkled growth, with finely granular surface, and smooth or undulate edge; membranous and difficult to emulsify. Membrane on water of condensation.

Gelatin Stab.-Filiform growth; rapid hiquefaction, infundibulosaccate, with heavy surface membrane and deposit.

Broth.-Forms thick surface scum, which falls to the bottom, but re-forms; turbidity moderate at first, later clears, with heavy tough membranous deposit.

Blood Serum.-Moderate, confluent, greyish-yellow growth, with uneven or nodular surface; slight liquefaction.

Potato.-Thick, creamy yellow, coarsely wrinkled, viscous growth, surface mealy and yellowish.

Metabolism.-Aerobe, facultative anaerobe. Opt. temp. $37^{\circ}$ C. Fia.187.-B.vulgatus. Limits $12-55^{\circ} \mathrm{C}$.

Biochemical.-Acid in glucose and sucrose, but not lactose or mannitol. Indole - ; M.R. - ; V.P. F ; nitrates reduced to nitrites. $\mathrm{H}_{2} \mathrm{~S}-$; $\mathrm{NH}_{3}+$; methylene blue not reduced;

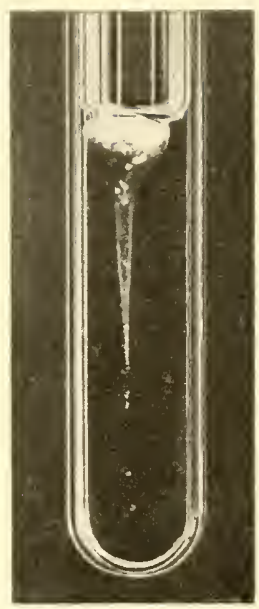

In stab gelatin, 4 days, $20^{\circ} \mathrm{C}$., showing $n$ apiform liquefaction. catalase + . Litmus milk decolorized, and peptonized from above downwards. Starch is hydrolysed.

Pigment. - Slight yellowish or brownish-red.

Humolysis. - $\beta$-hæmolysis on horse blood agar plate by some strains.

Nutritional.-Grows freely on ordinary media. Growth not improved by blood, serum, or glucose.

Antigenic Structure. - Spore antigen differs from that of B. subtilis. According to Sievers (1942), the vegetative bacilli may be sub-divided into at least four groups by means of preeipitation tests.

Pathogenicity.-1 ml. 24-hours' broth culture may kill mice injected intraperitoneally. Otherwise non-pathogenic.

\section{Bacillus mesentericus (Flïgge) Migula}

Synonyms.-B. mesentericus vulgatus; $B$. mesentericus fuscus; potato bacillus.

Habitat.-Found in dust, soil, water, milk.

Morphology. Slender rods, $1-3 \mu \times 0.5-0.7 \mu$; rounded ends, occurring singly or in pairs. Motile. Spores ovoid, $\mathbf{1}-\mathbf{1} \cdot \mathbf{5} \mu \times 0.6 \mu$. formed sub-terminally, do not canse bulging of the cell, and germinate by comma-shaped expansion (Lamanna 1940b). Non-capsulated. Gram-positive. Non-acid-fast.

Agar Plate.-Raised, whitish, irregular colonies of moderate size, with smooth glistening surface and entire or undulate edge.

Agar slope.-Abundant, glistening, smooth, whitish growth with undulate edge.

Gelatin Stab.-Filiform growth with slow stratiform liquefaction.

Broth.-Marked turbidity without formation of pellicle and with cottony sediment, followed gradually by sedimentation and clearing.

Potato.-Abundant, glistening, whitish growth.

Metabolism.-Aerobe. Opt. temp. $37^{\circ} \mathrm{C}$. Limits $12-55^{\circ} \mathrm{C}$.

Biochemical.-Acid in glucose, maltose, mannitol and sucrose. Indole - ; M.R. -- ; V.P. F; nitrates not reduced to nitrites; $\mathrm{H}_{2} \mathrm{~S}-$; catalase +; Litmus milk decolorized, coagulated, and slowly peptonized. Starch not hydrolysed. 
Pigment.--None.

Antigenic Structure. - Spere antigen is different from that of B. sublitis or R. rulgutus.

\section{B. megatherium de Bary.}

First described by de Bary in 1884, who found it on cooked cabbage leaves. It is one of the largest members of the Bacillus group, and occurs in dust, soil, air, milk, and water. Morphological and colonial variants have been described by Knaysi (1933). According to Rettger and Gillespic (1935), the well-known morphological pleomorphism of this organism is governed largely by envirommental conditions, particularly oxygen starvation. It forms a powerful hæmolysin (Todd 1901, 1902, Warden et al. 1921), most active towards the red corpuscles of man, monkey, and the guinea-pig; it appears in broth cultures at $37^{\circ} \mathrm{C}$. on the 2 nd or $3 r d$ day, increases to a maximum on the 6th or 7 th day, and then diminishes slowly. Oxygen is essential for its production. The hæmolysin, which can be filtered through a Pasteur-Chamberland candle, deteriorates rapidly on keeping, and like many other true toxins is destroyed by heating at $56^{\circ} \mathrm{C}$. for half an hour. Subcutaneous injection into guinea-pigs gives rise to a large local swelling with subsequent necrosis. On intravenous injection into guinea-pigs it gives rise to hæmoglobinuria, but is not fatal except in large doses-about $10 \mathrm{ml}$. Antihæmolysin can be prepared by injection of the hæmolysin into goats. Warden, Connell and Holly (1921) found that when $2 \mathrm{ml}$. of centrifuged broth culture were injected intraperitoneally into guinea-pigs, the animals died in less than 12 hours. Post mortem the abdomen was distended, the peritoneum congested, there was hæmolysed blood in the peritoneal cavity, and bloody fluid in the lumen of the gut, in the pleural cavities and over the thighs. They bring evidence to suggest that the toxin and the hæmolysin are one and the same body.

Bacillus megatherium de Bary

Synonyms.-Probably represents some strains known as B. anthracoides or B. pseudoanthracis.

Habital.-Found in dust, soil, water, milk.

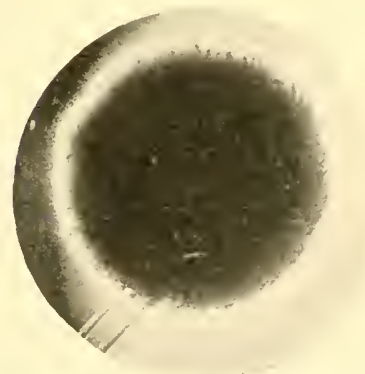

Fio. 188.-B. megatherium.

Surface colony on agar, 36 hours, $37^{\circ}(\mathrm{C} .(\times 8)$.

Morphology.-Large, rod-shaped, 3-9 $\mu \times 1.2 \mu$. Long unsegmented forms are common, and shadow forms appear early. Ends slightly rounded, axis curved; occurs singly, in pairs and in chains. Cells contain fat globules. Motile by 4-8 peritrichate flagella. Spores equatorial, oval, or ellipsoidal, not bulging ; germination by absorption of spore coat. Non-capsulated. Gram-positive. Non-acid-fast.

Agar Plate-Round, 3-5 mm. in diameter, raised, dull, greyish-white, opaque colonies with entire edge and finely granular surface, sometimes radially striated; may show differentiation into raised opaque centre and thin translucent periphery; membranous consisteney; emulsifiability fairly easy. After about a week irregular round scales appear on the surface of the colony, similar to those on anthrax colonies.

Agar Slope.-Profuse, moist, raised, glistening, greyish-yellow, creamy growth with smooth surface and entire edge; butyrous consistency; sometimes may show parallel raised ridges like contour lines; emulsifies easily. After about a week irregular round scales appear on the surface of the growth, similar to those of anthrax. 
Gelatin Stab.-Abundant filiform growth with infundibuliform or saccate liquefaction; no surface membrane.

Broth.-Moderate, finely floccular, turbidity, with slight ring growth and a powdery deposit, later becoming heavy and viscous.

Blood Serum.-Abundant, moist, creamy, yellowish growth, with granular structure and finely contoured surface. No liquefaction.

Potato.-Thick, greyish-yellow, mealy growth.

Resistance.-Spores are said to withstand 18 lbs. steam pressure for 1 hour; killed by 20 lbs. for I hour.

Metabolism.-Aerobe, facultative anaerobe. Opt. temp. $35^{\circ} \mathrm{C}$. Limits $10-45^{\circ} \mathrm{C}$.

Pigment.-None.

Hamolysis.-Powerful hæmolysin produced, acting especially on the red cells of man, monkeys, and guinea-pigs.

Toxin.-The hæmolysin is fatal to laboratory animals.

Nutritional.-Grows well on ordinary media; not improved by blood, serum, or glucose.

Biochemical.-Acid, no gas, in glucose, maltose, and sucrose. Indole - ; M.R. - ; V.P. F. Nitrates reduced to nitrites, slight; $\mathrm{NH}_{3}+; \mathrm{H}_{2} \mathrm{~S}-$; methylene blue reduced; catalase + . Litmus milk sometimes clot, followed by peptonization and decolorization. Starch is hydrolysed.

Pathogenicity.-Non-pathogenic under natural conditions. The hrmolysin is fatal in 1-2 ml. doses to mice and guinea-pigs injected intraperitoneally. P.M. hæmorrhagic exudate in peritoneum and pleura.

\section{B. mycoides Flügge.}

First described by Flügge (1886); common in milk, water, soil, and dust. Is easily distinguishable from other members by its typical rhizoid growth on agar. It is a highly proteolytic organism, which is said to convert half the protein nitrogen of the medium into ammonia; when growing in the soil it therefore plays an important part in the process of denitrification. Some strains are said to secrete a highly active proteolytic ferment capable of lysing cultures of certain bacteria (Schubert 1928). Variant morphological and colonial types have been described by Lewis $(1932,1933)$ and den Dooren de Jong (1933).

\section{Bacillus mycoides}

Synonyms.-B. ramosus Eisenberg. Root bacillus.

Habitat.-Found in milk, water, soil and dust.

Morphology.-Rod-shaped, $3-5 \mu \times 1.0 \mu$; truncated or slightly rounded ends, occurring singly, in pairs, small groups, and chains; long unjointed threads not uncommon. Motile by peritrichate flagella. Spores are large, equatorial and ellipsoidal, measuring $1.8 \mu \times 0.8 \mu$, not bulging; germinate by absorption of spore coat. Noncapsulated. Cells store fat as reserve material. Gram-positive. Non-acid-fast.

Agar Plate.-Large, spreading, raised, greyish-white, dull, opaque and rhizoid colonies, with finely granular surface; denser nuclei, dark in colour, are visible from which the peripheral shoots arise ; membranous consistency; emulsification fairly easy.

Agar Slope.-Abundant, confluent, spreading, rhizoid, opaque growth, greyish-white and slightly glistening; surfaee honeyeombed, due to the presence of arborescent ridges forming a raised network. Gruwth penetrates the medium, and is hence firmly adherent to it.

Gelatin Stub.-Arborescent, filamentous growth ; saccate liqnefaetion; clearing of gelatin witl formation of a deposit and a surface membrane.

Broth.-No turbidity; firm, sometimes wrinkled, surface membrane, depositing later.

Blood Serum.-Luxuriant, rhizoid growth; no digestion, 
Potato.-Abundant, mealy, greyish-brown, growth of viscous consistency; surface granular.

Resistance.-Spores are said to withstand $15 \mathrm{lbs}$. steam pressure for 1 hour. Killed by 20 lbs. in half an hour.

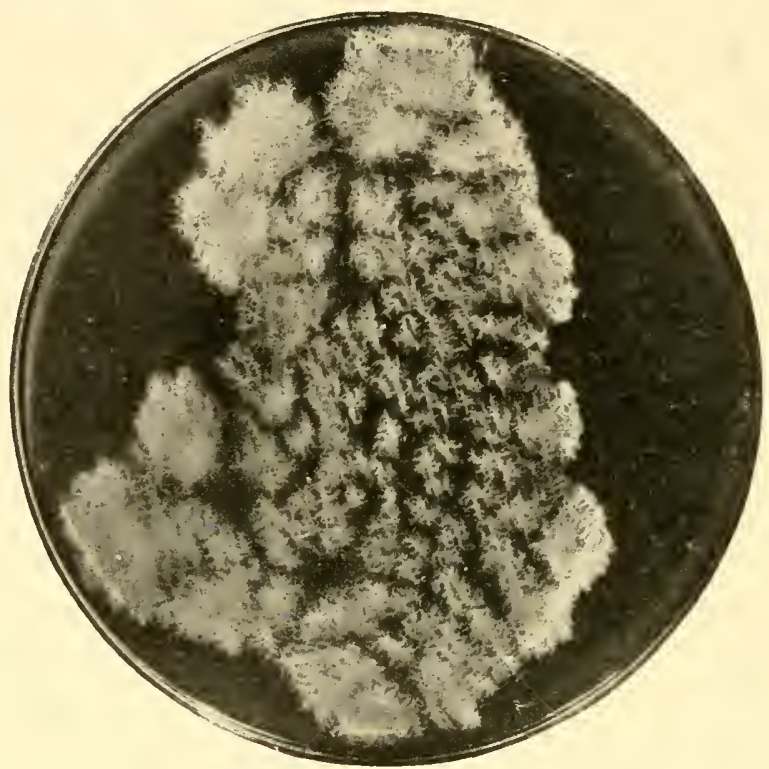

Fig. 189.-B. mycoides.

Surface growth of rhizoid type on agar, 3 days, $30^{\circ} \mathrm{C}$. (悉ths natural size.)

Metabolism.-Aerobe; facultative anaerobe. Opt. temp. $30^{\circ} \mathrm{C}$.; limits $10-40^{\circ} \mathrm{C}$. Pigment none. Some strains secrete a lysin capable of dissolving certain bacteria. Hamolysis.-None on horse blood agar plates.

Nutritional.-Grows fairly well on ordinary media; growth not improved by blood, serum, or glucose, but augmented by nitrates.

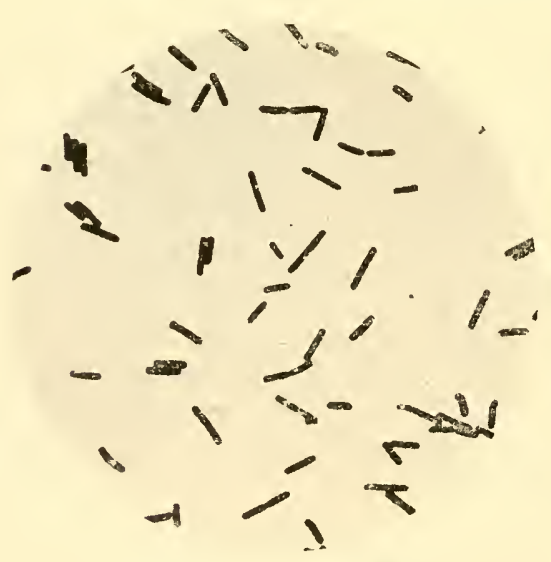

FIa. 190.-B. mycoides.

From an agar slope culture, 2 days, $30^{\circ} \mathrm{C}$. $(\times 1000)$.

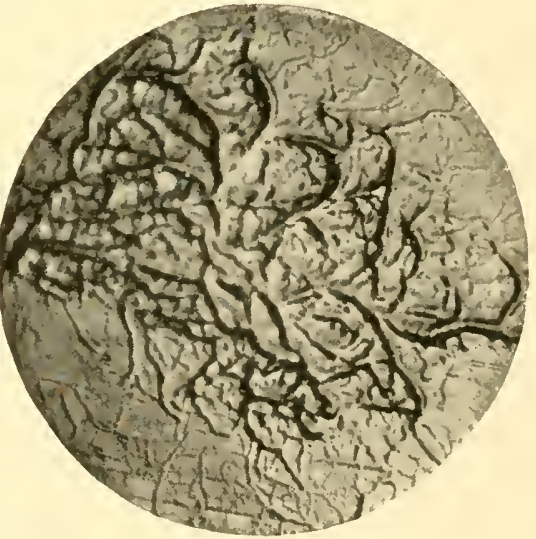

FIa. 191.-B. mycoides.

Central part of a surface colony on agar, 3 days, $30^{\circ} \mathrm{C}$., showing rhizoid structure $(\times 8)$. 
Biochemical.--Acid, no gas, in glucose, maltose and sucrose. Indole - ; M.R. -- ; V.P. Ғ ; nitrates $+; \mathrm{NH}_{3}+; \mathrm{H}_{2} \mathrm{~S}$ slight + ; metlylene blue reduced; catalase + . Litmus milk, slow peptonization and decolorization. Starch is hydrolysed. Pathogenicity.-Non-pathogenic to man and animals.

\section{B. cereus Frankland}

This organism is one of the large-celled species. Its exact identity is doultful. lamanna (1940c), who studied 31 strains, found three distinct physiological gromps, and concluded that $B$. cereus represents a group of organisms rather than a single speeies. It is generally described as an organism $3-7 \mu \times 1-1.2 \mu$, forming ovoir, non-bulging, snb-terminal spores. Large, smooth, irregular, very finely granular colonies on agar with a rhizoid periphery. Abundant, smooth, glistening, whitish growth on agar slope. Very marked turbidity in broth with formation of a surface pellicle and a cottony reposit. Filiform growth in gelatin with fine ramified offshoots; infundibuliform liquefaction. Range of grow th $10^{\circ}-40^{\circ} \mathrm{C}$. Forms acid in glncose and maltose; Indole - ; $\mathrm{H}_{2} \mathrm{~S}-$; V.P. - ; nitrates usually reduced to nitrites; litmus milk decolorized, congulated, and slowly peptonized; stareh hydrolysis variahle. The spore antigen seems to differ from that of $B$. megutherium.

\section{B. rotans.}

The interest of this organism, which was described by Roberts (1935), lies in the fact that its colonies are motile. 'Two sorts of co-ordinated motility are displayerl. rotation and migration. Rotation may occur either clockwise, or anti-clockwise, and is common in the early stages of colony formation. Later the whole colony migrates, pursuing an involverl and sometimes spiral course, leaving behind it a few cells to mark its snail-like track. Unlike Proteus, this organism moves freely even on a dry surface. Other organisms showing colonial migration have been described by Simith and Clark (1938), RussMünzer (1938), Shimn (1938), and Turner and Eales (1941) under such names as B. circulans and $B$. alvei. The organism studied by Turner and Eales formed colonies that migrated at $37^{\circ} \mathrm{C}$. at the rate of $2.5 \mathrm{~mm}$. per minute.

As well as the more common representatives, there are large numbers of other organisms that have been described, such as $B$. polymyxa Nigula, $B$. albolactus Migula, B. fusiformis Gottheil, B. cohorens Gottheil, B. terminalis Migula, B. petasites Gottheil, B. tumescens Zopf, and B. graveolens Gottheil. For their description the reader is referred to the publications of Loeffler 1887, Globig 1888, Hueppe and Wood 1889, Flügge 1896, Lehmann and Neumann 1896, Hartleb and Stutzer 1897, Chester 1901, Gottheil 1901, Schulz 1901, Bainbridge 1903, Neide 1904, Neufeld 1913, Poppe 1913, 1922, Senge 1913, Pokschischewsky 1914, Ford 1916, Laubach 1916, Bergey 1939, and to the monograph of de Soriano, 1935.

\section{REFERENCES}

AxENFEL, T. (1908) "The Bacteriology of the Eye." London.

AокI, K. and Yаманото, K. (1939) Z. ImmunForsch., 95, 374.

Bainbridge, F. A. (1903) J. Path. Bact., 8, 117.

Balteano, L. (1922) Ann. Inst. Pasteur, 36, 805.

BARY, H. A. DE. (1884) "Vergleichende Morphologie und Biologie der Pilze, Mycetozoen, und Bakterien." Leipzig.

BASSET, J. (1925) C. R. Soc. Biol., 93, 1513, 1515, 1517.

BEHRINA. (I889) Z. IYy. InfektKr., 7, 171.

Berger, 1). H. (1939) "Manual of Determinative Bacteriology." 5th erl. William Wilkins, Baltimore.

Bodon, G. and Tomcsik, J. (1934) Proc. Soc. exp. Biol., N. Y., 32, 122.

BoNgert, J. (1903) Zbl. Bal.t., 34, 497, 623, 772.

Bordet, J. and Renaux, E. (1930) Ann. Inst. Pasteur, 45, 1. 
Burdon, K. L.. SтоKes, .J. (., and Kimbrough, С. E. (1942) J. Bact, 43, 717.

Charrix, A. and Roger, G. H. (1890) Arch. Physiol. norm. path., 22, 273.

Chauveau, A. (1880a) C. R. Acad. Sci., 91, 33 ; (1880b) Ibid., 91, 618.

Chester. (1901) "A Manual of Determinative Baeteriology." New York.

Cohs, F. (1875a) Cohn's Beitr. Biol. Pflanz., 1, Heft 2, y. 175 ; (1875b) Ibid., 2, Heft 3, p. 141 .

Combiesco, D., Soru, E., and Stamatesco, S. (1929) C. R. Soc. Biol., 102, 124.

Conn, H. J. (1930) J. infect. Dis., 46, 341.

Davaine, C. (1863a) C. R. Acad. Sci., 5\%, 220 ; (1863b) Ibid., 5\%, 351 ; (1864) Ibid., 59, 393.

Defalle, Wr. (1902) Ann. Inst. Pasteur, 16, 756.

Dooren De Jong, L. E. Dex. (1933) Arch. Mikrobiol., 4, 36.

Duвos, R. J. (1939) J. exp. Med., \%0, 1, 11.

Dubos, R. J. and Cattaneo, C. (1939) J. exp. Mcd., \%0, 249.

Duвos, R. J. and Hotchiss, R. D. (1941) J. exp. Med., r3, 629.

EHRENBERG. (1838) "Infusionsthierchen als vollkommene Organismen." Leipzig.

FlügGE, C. G. F. W. (1886) "Die Mikroorganismen." Leipzig. (1896) Ibid., 3te Aull., Vol. 2. Leipzig.

Flyne, C. S. and Rettger, L. F. (1931) J. Bact., 28, 1.

lord, W. W. (1916) J. Bact., 1, 273.

Frank, (i. and Lubarsch, O. (1892) Z. IIyg. Infekt Frr., 11, 259,

Giovanard, A. (1931) Krankheitsforschung, 9, 13.

Globig. (1888) Z. Hyg. InfektKr., 3, 322.

Gordon, J. and Mcleod, J. W. (1928) J. Path. Bact., 31, 185.

Gotтheil, O. (1901) Zbl. Bakt., IIte Abt., \%, 430, 449, 529, 627, 680, 717.

Graham, N. C. (1930) J. Path. Bact., 33, 665.

Grierson, A. M. M. (1928) J. IIyg., Camb., 27, 306.

Hankin, E. H. (1889) Brit. med. J., ii, 810.

Hartleb, R. and Stutzer, A. (1897) Zbl. Bakt., IIte Abt., 3, 81, 129, 179.

Heaslip, W. G. (1911) Med. J. Aust., ii, 536.

Howif, J. W. and Cruicksnank, J. (1940) J. Path. Bact.. 50, 235.

Hueppe, F. and Woon, G. C. (1889) Berl. klin. Wschr., 26, 347.

Ivánovics, G. (1937) Zbl. Bukt., 138, 449 ; (1941) Z. ImmunForsch., 97, 402.

Iránovics, G. and Brockner, T. (1937a) Z. ImmunForsch., 90, 304; (1937b) Ibid., 91 175 ; (193s) Ibid., 93, 119.

Ivánovics, G. and ERDös, L. (1937) Z. ImmunFursch., 90, 5.

Katzu, S. (1925) Zbl. Bakt., 94, 165.

KNAYsi, G. (1933) J. Bact., 26, 623.

Knaysi, G. and Gunsalus, 1. C. (1944) J. Bact., 47, 381.

Kосн, R. (1877) Cohn's Beitr. Biol. Pflanz., 2, 277.

Kramár, E. (1921) Zbl. Bakt., 8\%, 401.

Lamanxa, C. (1940a) J. Bact.. 39, 593 ; (1940b) Ilid., 40, 347 ; (1940c) J. infcct. Dis., $67,193,205 ;(1942) \mathrm{J}$. Bact., 44, 611.

LAUBACH, C. H. (1916) J. Bact., 1, 493.

Lehmann, K. and Neumann, R. (1896) "Atlas und Grundriss der Bakteriologie, ete." J. F. Lehmann, Munieh.

Levine, P. P. (1936) J. Bact., 31, 151.

Lewis, I. M. (1932) J. Bact., 24,381 ; (1933) Ibid., 25, 359.

LOEFFLER, F. (1887) Berl. kilin. Wschr., 24, 607, 620.

Marmier, L. (1895) Ann. Inst. Pasteur, 9, 533.

Martin, S. (1890) 20th Rep. loc. Govt Bd publ. Hlth, Suppl., p. 255.

IIellon, R. R., and Anderson, L. M. (1919) J. Immunol., 4, 203.

Muller, L. (1925) C. R. Soc. Biol., 93, 1243.

Murray, T. J. (1931) J. infect. Dis., 48, 457.

NeIDE, E. (1904) Zbl. Bakt., IIte Abt., 12, 1, 161, 337, 539.

Neufeld. (1913) Zbl. Bakt., Ref. Beiheft., 5\%, 279.

Nungester, IV. J. (1929a) J. infect. Dis., 44, 73; (1929b) Ibid., 45, 214.

Oppermann. (1906) J. comp. Path., 19, 264.

Pasteur, L. (1881a) C. R. Acad. Sci., 92, 209; (1881b) Ibid., 92, 429; (1881c) Ibid., 92,666 .

Pokschischewsky, N. (1914) Arb. ReichsgesundhAmt., 4\%, 541.

Poppe, K. (1913) Zbl. Bakt., Ref. 57, 277; (1922) Ergebn. Hyg. Bakt., 5, 597.

Preisz, H. (1911) Zbl. Bakt. 58, 510.

Rettger, I. F. and Gillespie, H. I. (1935) J. Bact., 30, 213.

Roberts, I. I. (1935) .J. Bert., 29, 229.

Roux, E. (1890) Ann. Inst. Pasteur, 4, 25.

Russ-Münzer, A. (193s) Zbl. Balit., 142, 175.

今't. Joll-Brooks, R. and BREE1, R. ド. (1937) J. Buct., 33, 44 ). 
Sanarelli, G. (1925) Ann. Inst. Pasteur, 39, 209.

Soноcкаert, J. (1929) Arch. int. Méd. exp., 5, 155.

Schuber't, J. (1928) Zbl. Bakt., 108, 151.

ScHulz, R. (1901) Zbl. Balit., 30, 582.

Senge, J. (1913) Zbl. Balit., '70, 353.

Shins, L. E. (1938) J. Bact., 36, 419.

Sievers, O. (194:) J. Bact., 43, 305.

Sievers, O. and Zetterberg, B. (1940) J. Bact., 40, 45.

Sмith, N. R. and Clark, F. E. (1938) J. Bret., 35, 59.

SOBERNHEIM, G. (1897) Z. Hyg. InfehtKr., 25, 301.

Sobernheim, G. and Murata, H. (1924) Z. IIyg. InfektKr., 103, 691.

Sordelli, A. and Deulofeu, V. (1930) C. R. Soc. Biol., 105, 721; (1933) Folia biol., No. $26-27$, p. 121.

Sordelli, A., Deulofed, V., and Ferrari, J. (1932) Folia biol., No. 11, p. 45 ; No. 20 , p. 93 and 94 .

Soriano, A. 11. de. (1935) Rev. Inst. bact., B. Aires, 6, 507.

Soule, M. H. (1928) J. infect. Dis., 42, 93 ; (1932) Ibid., 51, 191.

Standfusz, R. and Schnadder, F. (1925) Zbl. Bakt., 95, 61.

STERNe, M. (1937) Onderste poort J. vet. Sci., 8, 271; (1939) Ibid., 13, 307.

Sweany, H. C. and Pinner, M. (1925) J. infect. Dis., 37, 340.

Topn, C. (1901) Lancet, ii. 1663; (1902) Trans. path. Soc., Lond., 53, 196.

Toмсsiк, J. (1930) Z. IIyg. Infekthr., 111, 119.

Tomcsik, J. and Bopos, G. (1934) Z. ImmunForsch., 83, 426 ; (1935) Ibid., 84, 308.

Toucsik. J. and Ivínovics, G. (1938) Z. Immun Forsch., 93, 196.

Tomcsik, J. and Szongot', H. (1932) Z. ImmmnForsch., 76, 214; (1933) Ibid., 78, 86.

Turner, A. W. and Eales, (. E. (1941) Aust. J.exp. Biol., 19, 161.

Warden, C. C., Connell, J. T., and Holly, L. E. (1921) J. Bact., 6, 103.

Wilamowski, B. I. (1912) Zbl. Bakt., 66, 39.

Wilson, G. S., Twigg, R. S., Wright, R. C., Hendry, C. B., Cowell, M. P., and Maier, I. (1935) Spec. Rep. Ser. med. Res. Coun., Lond., No. 206, I. 376. 


\section{CHAPTER 36}

\section{CLOSTRIDIUM}

\section{THE SPORE-BEARING ANAEROBES}

\section{DEFINITION,-Clostridium.}

Anaerobic or microaerophilic rods, producing endosprores, which are usually wider than the vegetative organisms in which they arise-so-callerl clostridium forms. Generally Gram-positive. Often decompose protein media and often ferment carbohydrates. Many species form exotoxins, and many are pathogenic.

Type species is Clostridium butyricum Prazmowski.

Before the war of 1914-18, the study of the spore-bearing anaerobes had been undertaken fitfully and by imperfect methods; much attention had been paid to their pathogenicity, but little to their general biological characters. One and the same organism had received many different names, and many organisms with the same name undoubtedly belonged to different species. The only two organisms about which no doubt existed were the two that formed a highly potent toxin, recognizable by the specific effects they produced on injection into animals - namely $\mathrm{Cl}$. tetani and $\mathrm{Cl}$. botulinum. It was not till the exigencies of war rendered an intensive study of the anaerobes necessary, and till the introduction of McIntosh and Fildes' jar made it feasible to obtain pure cultures with relative ease, that the obscurity surrounding this group was dispersed.

Most of the older workers had failed to realize the difficulty inherent in obtaining pure cultures of the anaerobic bacilli. The new technique, especially by enabling plate cultures to be made, revealed at once the impurity of many of the classical strains, and provided a means for the preparation of single-colony cultures. For the first time a distinctive account was provided of the main species, which made possible their identification, and which disposed of many spurious eharacters that had been attributed to them. Incidentally fresh species were discovered. (For references on the production - of anaerobiosis see Liborius 1886, Frankland 1889, Smith 1890, Tarozzi 1905, Smith et al. 1905-06, Laidlaw 1915, MeIntosh and Fildes 1916, Report 1917, Holker 1918-19, Rockwell 1921, Varney 1926, Wilson 1928, Hall 1929a, Dickens 1934, McClung et al. 1935).

Habitat.-The anaerobes are widely distributed in nature, but their main habitat is undoubtedly the soil. Some of them appear to be common inhabitants of the intestinal canal of man and animals. Cl. welchii, for example, is uniformly present in human frecs; $\mathrm{Cl}$. tetani has been found in about 10-40 per cent. of fæcal specimens of domestic animals; $\mathrm{Cl}$. sporogenes is frequently, and $\mathrm{Cl}$. histolyticum occasionally present. It has been held by some that the intestinal canal is the main habitat of certain of the anaerobes, particularly $\mathrm{Cl}$. tetani, and that their presence in the soil can be explained by fæcal contamination. The fact that this 
organism is found in virgin soil taken far from human or animal habitations renders this view improbable. It would seem more likely that the primary habitat of the majority of the anaerobes is the soil; that they are ingested frequently with vegetables and fruit; and that some of them are able to adapt themselves temporarily or permanently to a life in the intestinal canal.

Their presence in soil and fæces accounts for their frequent appearance in dust, milk and sewage. In spite of the fact that they lead a saprophytic existence, several of these species are causally related to well-recognized diseases in man and a nimals.

Morphology.-The anaerobes are endowed with a pleomorphism that renders their identification on a morphological basis very difficult, and often frankly impossible. Not only may an organism assume different shapes under changing environmental conditions, but under one and the same set of conditions it may present very different forms.

Like the aerobic spore-bearing bacilli they are large, rod-shaped organisms. In length they vary from about $3 \mu$ to 7 or $8 \mu$, but long filamentous forms are quite common. Their breadth varies from about 0.4 to $1.2 \mu$. The vegetative bacilli are straight or curved, their sides are parallel, and their ends rounded or somewhat truncated. Most are arranged singly, but some occur in pairs or in chains, others in bundles the nembers of which are arranged parallel to each other. Irregular forms include navicular or boat-shaped organisms ; citron forms shaped like a lemon with a small knob at each end; large, swollen, non-sporing rods or "orgonts"; snake-like filaments; deeply stained bulb-like types; and a great variety of so-called involution forms varying both in shape and in depth of staining. Autolysis frequently sets in with the commencement of sporulation so that shadow forms are numerous, particularly in certain species.

Sporulation is common to all members, but there is considerable variation in the readiness with which it occurs. $C l$. sporogenes, for example, spores readily on all media; $\mathrm{Cl}$. welchii only in media free from a fermentable carbohydrate, and then inconstantly. All the pathogenic members are able to form spores in the animal body, though $\mathrm{Cl}$. welchii does so rarely.

It has been customary to classify the anaerobes according to the shape of the spore and the position in the rod at which it appears. Thus we have (1) those with an equatorial or subterminal spore; (2) those with an oval terminal spore; and (3) those with a spherical terminal spore. This division is useful for certain purposes, but it must not be used too rigidly. It is common, for instance, to find organisms that usually form subterminal spores giving rise to spores that are strictly terminal. The distinction between a spherical and an oval terminal spore may also be a matter of the utmost nicety.

The spores of most members are wider than the vegetative bacilli; they therefore confer on the organism a distinctive appearance according to the position in which they arise. If they are formed at the equator the clostridium is spindleshaped; if subterminally club-shaped; with an oval terminal spore the organism may look like a tennis racket; with a spherical terminal spore like a drum-stick.

With the exception of $C l$. welchii, all the members are motile, by peritrichate flagella. Motility, however, is often difficult to demonstrate, especially in artificial cultures and in strains that have been subcultured for some time. Young cultures in broth or cooked meat medium, not more than 6 to 24 hours old, are the most suitable for examination. If these are negative, the organisms should be examined 
in the tissue fluids of injected animals. The usual coverslip method is satisfactory in most instances, but if this fails, examination should be eondueted in a closed capillary tube that has been inoeulated with a young broth culture and kept at $37^{\circ} \mathrm{C}$. for about half an hour. As is eustomary with large organisms, motility is rarely well marked, and is usually of the slow and stately variety, in eontrast to the rapid, darting movements of smaller organisms such as Salm. typhi.

$\mathrm{Cl}$. butyricum and $\mathrm{Cl}$. welchii are the only members possessing a capsule; the eapsule of $\mathrm{Cl}$. welchii is noticeable in the animal body, and sometimes in eultures eontaining serum.

Staining Reactions.-All members stain readily with the usual dyes. Great irregularity is noticeable in the depth of staining, especially in cultures more than a day or two old. Sometimes metachromatic granules are noticeable, or points of more intense coloration. Provided young eultures are examined, the bacilli are all Gram-positive. Some species rapidly lose this property, and some can be decolorized if the alcohol is applied for too long. In the early stages of spore formation, the position of the spore is often marked by an area of intense staining; as it matures, however, the spore presents a colourless eentre surrounded by a peripherally stained ring.

Cultural Reactions.-On solid media growth is relatively slow, and takes the form of a thin, effuse, often spreading film, which may be difficult to distinguish from the underlying medium.

The tendency to film formation is promoted by moisture. On first isolation $\mathrm{Cl}$. septicum, and partieularly $\mathrm{Cl}$. tetani, tend to spread rapidly over a moist surface. If $C l$. tetani is inoeulated into the condensation water of an agar slope, it will in the course of a day spread over the whole medium; the film is so thin that, were it not for the dentate edge presented at the upper end of the slope, where the medium is drier, it might easily eseape detection. Advantage may be taken of this faet in the isolation of this organism (Fildes 1925a). The spreading of clostridia ean be inhibited by the incorporation of certain chemicals in the solid media. The majority of these, however, are to some extent bacteriostatic ; inhibition of spreading without bacteriostasis, as Hayward (Miles and Hayward 1943) has shown, may be achieved by inereasing the concentration of agar up to about 6 per cent. Certain elostridia also produce on agar motile daughter-eolonies, which rotate and wander over the surface of the medium (Turner and Eales 1941). Concentrated agar is less effective as an inhibitor of this type of spreading.

Agak Plates.-Single colonies are rounded, generally effuse, and present erenated, fimbriate, or rhizoid edges. $C l$. welchii, which is one of the less strict anaerobes, forms low convex colonies with an entire edge; $\mathrm{Cl}$. sporogenes and $\mathrm{Cl}$. histolyticum may form umbonate eolonies with a raised centre and a flat periphery. The colonial appearances are often eharacteristic, but some species give rise to variants which not only are unlike the typical colony, but which strongly suggest the oceurrence of contamination. Several different types of colony may be formed, for example, by $\mathrm{Cl}$. sporogenes.

Glucose Agar Shake Cultures.-These are commonly employed for studying the form of deep colonies, and by many workers for the preparation of pure cultures. Exeept near the surface, growth occurs throughout the medium ; this is frequently disrupted and blown upwards by the development of gas. Single eolonies are rounded or lenticular in shape, and lenticular forms may later develop irregular tufts of growth, sprouting from the edge or the poles of the leuses; sometimes 
they are differentiated into an opaque centre and a translucent periphery; their edge may be entire, but is more often woolly, erose, or presents that curious reticular filamentous appearance of a cigarette thrown into water. There is a general correspondence between the form of surface and deep colonies. Thus round, entire-edged and raised surface colonies usually correspond to opaque and lenticular colonies in deep agar; irregular or coarsely rhizoidal to opaque and lumpy; delicately rhizoidal to fluffy; and spreading colonies to deep colonies like a snowflake. Again, surface colonies with central papillæ usually correspond to deep colonies with a marked central opacity.

Blood Agar Plates.-On these, not only is the colonial form characteristic, but the degree and type of hæmolysis afford a useful differentiating feature between the members of the group. Hæmolysis is well marked after 3 -days' incubation at $37^{\circ} \mathrm{C}$.; if the plates are then stored in a dark cupboard at room temperature it often continues to increase. With a thick seeding the whole plate may be completely decolorized.

Many organisms give hæmolysis of the $\alpha$-prime type after 3 days' incubation (see Chapter 24); after a further 3 days this passes into the fully developed $\beta$-variety. In some cases, it is possible to specify the hæmolytic factors concerned. For instance, the relatively wide zone of hæmolysis produced by toxigenic strains of $\mathrm{Cl}$. welchii on rontine horse blood agar is usually due to the 0 -toxin (p. 866) ; if the action of $\theta$-toxin is suppressed by $\theta$-antitoxin, a narrower ill-defined zone of partial hrmolysis is revealed, due to $\alpha$-toxin. The action of the $\alpha$-lysin is greatly enhanced by calcium ions. Some strains of $C l$. welchii produce only the $\alpha$-type of hæmolysis (Evans 1945).

Cooked Meat Medium.-Most of the members grow well in this medinm. All render the fluid turbid to some extent, and most produce gas. The proteolytic members turn the meat black and may obviously digest it; the saccharolytic members do not digest the meat, and frequently turn it pink. Varying reactions are recorded in this medium, depending on the strain used, the batch of medium, and the length of incubation. Both in this medium and in other media the proteolytic members form characteristic foul and pervasive odours, while in cultures of the saccharolytic members there is no odour or, if there is, it is not foul.

Coagulated Serum and Coagulated EgG.--These media are used for testing the proteolytic powers. None of the saccharolytic organisms is able to liquefy them.

Gelatin.-At $23^{\circ} \mathrm{C}$. most members grow poorly. In stab culture $\mathrm{Cl}$. tetani gives a characteristic fir-tree growth, followed later by liquefaction. At $37^{\circ} \mathrm{C}$. growth is improved, and is generally accompanied by permanent liquefaction.

Resistance.-In the sporing stage all the members present a marked but variable resistance to heat, drying, and disinfectants. Thus the spores of $\mathrm{Cl}$. botulinum withstand boiling for 3 or 4 hours, and even at $105^{\circ} \mathrm{C}$. are not killed completely in less than 100 minutes. $C l$. odematiens is a little less resistant than $\mathrm{Cl}$. botulinum (Hoyt, Chaney and Cavell 1938). On the other hand, spores of $\mathrm{Cl}$. welchii are said to be destroyed by boiling in less than 5 minutes (Headlee 1931). Cl. sporogenes can survive exposure for 8 days to a 5 per cent. phenol solution. In dried earth or dust $C l$. tetani may live for years. Stock cultures of most members in cooked meat medium remain viable for montlis; some, such as $\mathrm{Cl}$. fallax and $\mathrm{Cl}$. cochlearium, are more delicate and require transferring frequently. 
Metabolism.-Up till within recent years it was generally believed that members of this group were unable to grow except when oxygen was rigidly excluded from the medium. Though free oxygen does inhibit their growth, and may actually destroy organisms in the non-sporing state, it is quite possible to obtain growth of anaerobic bacteria in the presence of air provided a sufficiently low oxidationreduction potential is established in the medium. This can be done by including substances in the medium which will take up molecular oxygen and bring about a fall in the Eh below that necessary for the initiation of growth. Many such substances are available, some of which act mainly by absorbing oxygen, others of which are chiefly responsible for the establishment of a low Eh after the molecular oxygen has been nearly used up or removed by mechanical means. Sulphites, reduced iron compounds, unsaturated fatty acids, activated glucose, cysteine, glutathione, ascorbic acid, thioglycollic acid, and metallic iron are examples of some of the substances commonly added to media to bring about the requisite anaerobic conditions. Cooked meat is an example of a medium that affords excellent conditions for anaerobic growth even when incubated aerobically. Its virtue lies in its containing (1) unsaturated fatty acids, which take up oxygen, the reaction being catalysed by the hæmatin of the muscle, and (2) glutathione, which brings about a negative $\mathrm{O}-\mathrm{R}$ potential corresponding to an Eh of about -0.2 volt (Lepper and Martin 1929, 1930). Fildes (1929) has shown that for the germination of tetanus spores an Eh in the medium approximating to +0.01 volt at pH 7.0 is required; this corresponds to the zone of complete reduction of thionin. It is probable that similar conditions determine the growth of most other anaerobes. $C l$. histolyticum, $\mathrm{Cl}$. tertium and $\mathrm{Cl}$. camis, however, are exceptions. These organisms are microaerophilic rather than anaerobic, and can grow to a limited extent aerobically, though they are said to be incapable of forming spores under these conditions (Hall and Duffett 1935). Once growth has started, most anaerobic organisms appear to bring about a rapid fall in the $\mathrm{O}-\mathrm{R}$ potential of the medium, probably owing to the production of a more active reducing system than that present in the medium itself. The Eh frequently falls to below $-0 \cdot 4$ volt. According to Gillespie and Rettger (1938) the final Eh reached by the various clostridia in a given medium may be useful in species characterization. As has just been pointed out, in the presence of powerful reducing systems growth may continue even though considerable quantities of oxygen are gaining access to the medium.

We have discussed the exact nutritive requirements of certain clostridia and the problems of anaerobiosis at some length in Chapter 3. The earlier work of Fildes and his colleagues (see Fildes 1935, Fildes and Knight 1933, Knight and Fildes 1933, Fildes and Richardson 1935, Pappenheimer 1935, Knight 1936) and of Stickland $(1934,1935)$ on essential nutrients of clostridia and the modes of their utilization has been developed to the point where it is clear that the majority of pathogenic clostridia are heterotrophs, requiring a battery of amino-acids, carbohydrates, and vitamins for growth in artificial media. Moreover, the energyproducing mechanisms, especially of those clostridia that depend mainly on aminoacid breakdown for their energy, have been studied in some detail (see, for example, Gale 1940, Woods and Trim 1942, Clifton 1942, Guggenheim 1944). A small concentration of $\mathrm{CO}_{2}$ seems to be as essential for the growth of the anaerobic as it is for so many of the aerobic bacteria (Gladstone, Fildes, and Richardson 1935). In addition, the growth of some clostridia is greatly improved by a con- 
centration of $\mathrm{CO}_{2}$ of the order of 2-10 per cent. (Rockwell 1924, Dack et al. 1927, Aitken et al. 1936).

On ordinary media growth of the anaerobes is poor compared with that of the aerobic spore-bearers. Some strains grow better than others- $\mathrm{Cl}$. welchii, $\mathrm{Cl}$. bifermentans, $\mathrm{Cl}$. botulinum; some give poorer growths-Cl. chauvoei, $\mathrm{Cl}$. cochlearium.

Glucose favours the saccharolytic species; blood or serum improves the growth of all. The optimum $\mathrm{H}$-ion concentration for growth is about $\mathrm{pH} 7 \cdot 0$ to $7 \cdot 4$ (Reddish and Rettger 1924).

On media containing bile salts, such as MacConkey's medium, growth of $\mathrm{Cl}$. sporogenes, $\mathrm{Cl}$. botulinum, $\mathrm{Cl}$. histolyticum, $\mathrm{Cl}$. welchii, $\mathrm{Cl}$. tetani, and $\mathrm{Cl}$. septicum is accompanied by a greenish fluorescence. In our experience, $\mathrm{Cl}$. chauvœi and $\mathrm{Cl}$. oedematiens have failed to grow on this medium.

Most of the members with which we are dealing here grow best at about $37^{\circ} \mathrm{C}$., though many of them are capable of growing at temperatures of $20^{\circ} \mathrm{C}$. and even lower. There is a group of thermophilic clostridia which have an optimum temperature about $50^{\circ}-60^{\circ} \mathrm{C}$, and which sometimes do not grow at all below $30^{\circ} \mathrm{C}$.

Hжmolysin Production.-Apart from their action on blood agar plates, many of the anaerobes, such as $\mathrm{Cl}$. tetani, $\mathrm{Cl}$. welchii, $\mathrm{Cl}$. septicum, $\mathrm{Cl}$. odematiens, and $C l$. chauvoi, produce filtrable hæmolysins capable of dissolving sheep's red blood corpuscles. Kerrin (1930) states that atoxic strains of $\mathrm{Cl}$. tetani produce as powerful a hæmolysin as do toxic strains, and that normal rabbit, horse, and human serum have a very strong antihæmolytic effect. For the detection of hæmolysins, care must be taken to buffer the hæmolytic systems at the $\mathrm{pH}$ of optimum activity (Walbum 1938). Fibrinolysins are formed by some species (Carlen 1939, Reed, Orr and Brown 1943) and leucocidins by others.

Biochemical Reactions. - The action on sugars is of some value in differentiating the anaerobes, and constitutes one basis of classification. Great care must be exercised in carrying out the tests, since even with known stock strains the results are often irregular and must be repeated two or three times before they can be relied on. Some clostridia decolorize indicators irreversibly, so that the formation of acid in a fermentation-tube should always be tested by the addition of fresh indicator to a sample of the culture.

Reed (1942) points out that both indole formation from tryptophan, and the reduction of nitrates to nitrites, depend on the relative rates of breakdown of the original substrates, and of substances formed from them. Thus, most clostridia reduce both nitrates and nitrites, and only if the reduction of nitrates is the quicker of the two processes will a positive test for nitrites be obtained.

One of the striking features of the anaerobic bacteria is the large amount of gas that they are able to produce even in media free from fermentable carbohydrates. Thus Wolf and Harris (1917) found that $C l$. welchii in casein water produced $90 \mathrm{ml}$. of gas per litre of medium, and in peptone water $186 \mathrm{ml}$. Cl. sporogenes formed $1,044 \mathrm{ml}$. of gas per litre of casein water in 157 hours, and in peptone water $360 \mathrm{ml}$. in 24 hours. The gas consists of $\mathrm{CO}_{2}$ and $\mathrm{H}_{2}$ in different proportions according to the species of anaerobe. The addition of a fermentable carbohydrate to the medium increases the gas production. Acids are formed as the result of the fermentation; with $\mathrm{Cl}$. welchii rather more than 50 per cent. are volatile-mostly butyric acid. Ammonia appears to be formed in large quantities by the proteolytic, and in much smaller quantities by the saccharolytic anaerobes.

An attempt has been made (Anderson 1924) to classify the anaerobes on the 
basis of their gaseous metabolism. Growth in plain peptone water results in the production of $\mathrm{CO}_{2}$ and $\mathrm{H}_{2}$ in different proportions; the $\mathrm{CO}_{2} / \mathrm{H}_{2}$ ratio is said to be high with the proteolytic and low with the saccharolytic members. Thus for Cl. histolyticum it is over 91, for $\mathrm{Cl}$. sporogenes $36 \cdot 9$, for $\mathrm{Cl}$. botulinum $18 \cdot 3$, for Cl. tetani 1.17, for $\mathrm{Cl}$. septicum 0.98 , and for $\mathrm{Cl}$. welchii $0 \cdot 4$.

Litmus milk is a useful medium for differentiation (see Wolf and Harris 1917, Weinberg and Séguin 1918, Wolf 1918-19, 1919-20, Report 1919, Anderson 1924, Wagner et al. 1924). Spray (1936) introduced iron-litmus milk and iron-gelatim as differential media, in which reactions with the iron, notably blackening of the medium, provide several useful distinctive characters among clostridia (see Table 57).

It has been stated that none of the anaerobes is able to form catalase (Adamson 1919-20). This statement probably needs modification; we have obtained evidence of its production by $\mathrm{Cl}$. sporogenes and $\mathrm{Cl}$. histolyticum, though only in small amounts.

Antigenic Structure.-Antisera have been prepared against a number of species, and agglutination and complement-fixation reactions have been carried out. Difficulty has often been experienced in the preparation of stable suspensions ; there is a great tendency for auto-agglutination to occur. The work of Felix and Robertson (1928) showed that the motile species of anaerobes contained thermolabile $\mathrm{H}$ and thermostable $\mathrm{O}$ antigens, similar to those described for so many of the aerobic bacteria. It was thought that type specificity, as determined by agglutination, was dependent on the $\mathrm{H}$ antigen, and group specificity on the $\mathrm{O}$ antigen.

The more recent work of Henderson and others, however, seems to show that the position is rather more complex. With $\mathrm{Cl}$. septicum, for example, Henderson (1934) finds that the most convenient subdivision is made on the basis of the $\mathrm{O}$ antigen. Though there is considerable overlapping in different strains, there appear to be four specific O receptors. These four primary groups can be further subdivided according to the type of the $\mathrm{H}$ antigen. Henderson (1932) states that there is an $\mathrm{O}$ antigen common to the ovine and the bovine strains of $\mathrm{Cl}$. chauvoei, but that the $\mathrm{H}$ antigen is complex, differing to some extent aecording to the animal source and the country of origin of the strain (see also $\mathrm{HcE}$ wen 1926, Roberts 1931). The relation between $\mathrm{Cl}$. septicum and $\mathrm{Cl}$. chancoi is not very clear, but the work of Weinberg. Davesne, Mihailesco, and Sanchez (1929) and Kreuzer (1939) suggests that the two organisms are closely related antigenically. (1. tetani is divisible into at least 10 types, all of which possess a common $\mathrm{O}$ antigen, and a second $\mathrm{O}$ antigen is present in Types I, III, VI, VII, VIII and X. Type specificity depends on a flagellar antigen. Cl. tetanomorphum has a minor somatic antigen in common with Cl. tetani (Wilsdon 1931, Gumnison 1937, NacLemnan 1939). Attempts to form serological groups of $\mathrm{Cl}$. welchii have met with varying success. Test antisera usually reaet fully with the homologous strain, and only with a few heterologous strains (Henriksen 1937, Duffett 1938, Orr and Reed 1940). There is some evidence that the four toxigenic types (see below) differ in their bacterial antigens (Freuzer 1939), but each type is antigenically heterogeneous. Thus Henderson (1940) distinguished two kinds of somatic antigen, a heat-stable $\mathrm{O}$, and a heat-labile (L), antigen. He found strain-specific $\mathrm{O}$ antigens in Type A, but no $\mathrm{L}$ antigen ; 13 strains of Type $\mathrm{B}$ fell into two $\mathrm{O}$-antigenic groups, and into 7 L-antigenic groups; all his Type Cstrains had a common $\mathrm{O}$ antigen but no Lantigen; and there were various $\mathrm{O}$ and $\mathrm{L}$ antigenic gromps in 'Type D. Rodwell (1941) found a similar variety of $\mathrm{O}$ antigens in the four types. In $\mathrm{Cl}$. welchii the specificity of agglutma. tion among $\mathrm{S}$ forms appears to depend on the nature of the capsular substance, which contains polysaccharides. On the whole, the serological reactions of antigenic extracts of eapsular substance are as heterogeneous as the agghtination reactions of the baeilli 
(Meisel 1938, Orr and Reed 1940, Sree and MeCoy 1944). The S $\rightarrow$ R variation in Cl. welchii is accompanied by a loss of speeific $O$ antigen (Henderson 1940).

Cl. botulinum is divisible into seven groups aecording to the flagellar antigens, and the proteolytic strains appear to possess a common 0 antigen (Sehoenholz and Meyer 1925, McClung 1937).

Among the proteolytic species we may note that $\mathrm{Cl}$. sporogenes can be divided into at least two groups. Serological tests have been useful in resolving some problems of identity of various species. Thus it appears from the work of Clark and Hall (1937) and Stewart (1938) that $\mathrm{Cl}$. sordellii may be considered as a variety of Cl. bifermentans. (For other examples, see the review of MeCoy and McClung 1938). One of the more interesting results of serological study is the confirmation of a cultural and biochemical relationship between $\mathrm{R}$ variants of $\mathrm{Cl}$. histolyticum and $\mathrm{Cl}$. sporogenes (Smith 1937, Hoogerheide 1937); its significance is not clear.

Toxin Formation.- It is remarkable that, with the exception of the diphtheria bacillus, the organisms forming powerful exotoxins belong almost entirely to the group of anaerobic spore-bearing bacilli. Two of them- $\mathrm{Cl}$. botulinum and $\mathrm{Cl}$. tetani-give rise to toxins more poisonous than any other substances with which we are acquainted. It has been calculated that the most powerful toxin of $\mathrm{Cl}$. tetani would kill a man in a dose of $0.25 \mathrm{mgm}$., and of $\mathrm{Cl}$. botulinum in a dose of $0.0084 \mathrm{mgm}$.

The formation of a powerful exotoxin does not appear to be associated with the proteolytic activity of the organism. Soluble diffusible toxins have been described in only two proteolytic species, $\mathrm{Cl}$. bifermentans and $\mathrm{Cl}$. histolyticum. Whether the toxins are formed intra- or extra-cellularly is still unkmown. Stark, Sherman, and Stark (1928) have found that, if bacteria-free filtrates of $\mathrm{Cl}$. botulinum are added to sterilized skim milk in suitable proportions and incubated for 4 days at $37^{\circ} \mathrm{C}$., a considerable increase in toxicity occurs, suggesting that enzymes present in the filtrate have formed fresh toxin from some constituent of the milk. A number of clostridial toxins resemble the diphtheria toxin in that they can be detoxified by formaldehyde with the formation of an antigenic toxoid that can be used for active immunization (see Chapters 77,78 ). The preparation of these toxins requires attention to a number of factors with which we have no space to deal. But their properties are important, and must be considered briefly. It should be emphasized that many of the properties described are those of toxic culture filtrates, not of isolated substances. Filtrates from cultures of $\mathrm{Cl}$. welchii, for example, have been resolved into a number of components, and it is probable that other "toxins" hitherto referred to for convenience as single substances will prove to be mixtures.

Tetanus Toxin.-This varies in potency; a good filtrate will kill a mouse in a dose of $0.00001 \mathrm{ml}$. It is destroyed by heat at $65^{\circ} \mathrm{C}$. for 5 minutes, but if dried it will resist $120^{\circ} \mathrm{C}$. for 1 hour. Exposure to $55^{\circ} \mathrm{C}$. for 1 hour is said to destroy the greater part of its toxicity, while having little effect on its antitoxin-combining power (Tschertkow 1929). It is destroyed by direct sunlight in about 15 hours at $40^{\circ}$ C. ; exposure to diffuse daylight results in a gradual weakening of the toxin. If precipitated with ammonium sulphate, dried over sulphuric acid, ground to powder, and preserved in the dark at $5^{\circ} \mathrm{C}$. in vacuum tubes under phosphorus pentoxide, the toxin will remain unchanged for 2 years or more. 0.55 per cent. $\mathrm{HCl}, 0.3$ per cent. $\mathrm{NaOH}$, and 70 per cent. alcohol each destroy the toxin in 1 hour. The toxicity can be modified by iodine trichloride, by formol and (Velluz 1936) by carbon disulphide; these reagents are used in serum institutes for weakening 
the toxin prior to injection of animals. Tetanus toxin is not absorbed from the intact alimentary canal ; there is evidence that it is destroyed by the digestive juices. It combines with and is neutralized by specific antitoxin. There is evidence, based on a lack of parallelism in the toxicity of culture filtrates of $\mathrm{Cl}$. tetani for different species of laboratory animals, that there is more than one component in tetanus toxin. These hypothetical components, however, appear to have a similar antigenic specificity (Ipsen 1940-41, Smith, M. L., 1942-43). Petrie (1942-43) found corresponding variations in the activity of antitoxins, and concluded that crude tetanus toxin contained varying proportions of a "primary" toxin molecule, and an antigenic variant of it. From somewhat similar experiences Friedemann and Hollander (1943) postulated qualitative differences between tetanus toxins from various sources.

The toxin of $\mathrm{Cl}$. botulinum resembles tetanus toxin in many respects, but is more resistant to heat and to acids. Thus it requires for its destruction a temperature of $80^{\circ} \mathrm{C}$. for half an hour. Normal hydrochloric acid fails to destroy it even in 24 hours, but normal soda destroys it rapidly. It is non-dialysable. The potency of the toxin varies; it has been possible to obtain filtrates with a M.L.D. for a guinea-pig of $0.000001 \mathrm{ml}$, but this is exceptionally strong. It is often said to be the only exotoxin that can be absorbed from the alimentary canal, but the recent work on enterotoxæmic diseases of sheep (see Chapter 78) suggests that the $\varepsilon$-toxin of $C l$. welchii shares this property.

The general properties of the toxins of $\mathrm{Cl}$. welchii, $\mathrm{Cl}$. septicum, and $\mathrm{Cl}$. odematiens may be considered together. They are all moderately thermolabile, being destroyed by heating to $70^{\circ} \mathrm{C}$. for 30 to 60 minutes. They are likewise destroyed by weak concentrations of acids. When toxic filtrates are injected into guineapigs or mice they give rise to a gelatinous oedema and a varying amount of necrosis. Weinberg and Combiesco (1930) state that welchii toxin lyses the red blood corpuscles, producing hæmoglobinuria, causes focal areas of necrosis in the kidney and liver, and leads to an increase in blood pressure, which may in its turn be responsible for hæmorrhages in various parts of the body.

Before discussing the toxins of $\mathrm{Cl}$. welchii in particular, we must refer briefly to the four main varieties of the species, each associated with a different disease (see Chapter 78) ; these are the classical $\mathrm{Cl}$. welchii of human gas gangrene, " $\mathrm{Cl}$. agni," causing lamb dysentery, "Cl. paludis," causing the sheep-disease " Struck," and " $\mathrm{Cl}$. ovitoxicum," causing an infectious enterotoxæmia in sheep. The claim of the last three to specific status has not yet been established. Each variety, however, was shown by Wilsdon $(1931,1933)$ to produce toxic filtrates that could be distinguished by their content of a number of toxic components.

Glenny and his colleagues (1933) identified five scparate toxic components in culturefiltrates of Wilsdon's types, $\alpha, \beta, \gamma, \delta$, and $\varepsilon$. The existence of these components was confirmed by a number of workers (Bosworth and Glover 1935, Borthwick 1935, Mason 1935, Weinberg anả Guillaumie 1936, Dalling and Ross 1938, Duffett 1938, Stewart 1940, Taylor and Stewart 1941). Of these five components, Cl. welchii Type A was at first thought to contain only $\alpha$. Prigge $(1936,1937)$ and Ipsen and his colleagues (Ipsen 1939, Ipsen et al. $1939 a, b)$ found two components, $\alpha$ and $\zeta$, and in one Type A strain, a third component, which they designated $\eta$ (see also Nagler 1940). It is now clear that Prigge's $\zeta$ toxin is equivalent to Glenny's $\alpha$, and British workers (see Dalling and Stephenson 1942) have recently adopted a convention whereby Prigge's $\alpha$ and $\zeta$ are designated $\theta$ and $\alpha$ respectively. The $\eta$ toxin of Ipsen retains its original designation. There are, therefore, seven toxic components to consider, $\alpha, \beta, \gamma, \delta, \varepsilon, \theta$ and $\eta$. Space forbids more than a 
brief outline of the properties and relationships of the $C l$. welchii toxins. For a full discussion the reader is referred to the exhaustive review of Oakley (1943) from which, with certain modifications, we reproduce Table 54, showing the toxins present in the different filtrates, and Table 55, showing the properties of the toxins.

TABLE 54

Giving the Distribution of Toxic Components in Culture Filtrates of $C l$. welchii (Oakley 1943)

\begin{tabular}{|c|c|c|c|c|c|c|c|c|}
\hline \multirow{2}{*}{ Variety of $\mathrm{Cl}$. welchii. } & \multirow{2}{*}{$\begin{array}{l}\text { Wilsdon's } \\
\text { Type. }\end{array}$} & \multicolumn{6}{|c|}{ Toxin. } & \multirow[b]{2}{*}{$\theta$} \\
\hline & & $\alpha$ & $\beta$ & $\gamma$ & $\delta$ & $\varepsilon$ & $\eta$ & \\
\hline 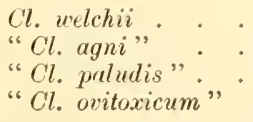 & $\begin{array}{l}A \\
B \\
C \\
D\end{array}$ & $\begin{array}{l}++ \\
+ \\
+ \\
+\end{array}$ & $\begin{array}{l}-\bar{t} \\
++t \\
++t\end{array}$ & $\begin{array}{l}- \\
+ \\
+\end{array}$ & $\begin{array}{l}- \\
+ \\
+\end{array}$ & $\frac{\overline{t+}}{\overline{+}+}$ & $\begin{array}{c}(+) \\
? \\
? \\
?\end{array}$ & $\begin{array}{l}+ \\
?+ \\
?+ \\
?+\end{array}$ \\
\hline
\end{tabular}

It will be seen that the $\alpha$ toxin predominates in Type $A, \beta$ and $\varepsilon$ in Type $\mathrm{B}, \beta$ and $\delta$ in Type $C$ and $\varepsilon$ in Type D filtrates. The distinctions, both qualitative and quantitative, between the types are not absolute. Mason (1935), for instance, records the loss of ability to produce $\varepsilon$-toxin in Type B strains, Borthwick (1937) a similar loss in Type D strains, and Taylor (1940) a loss of $\beta$-toxigenicity in a Type B strain. The properties of the toxins are summarized in lable 55.

\section{TABLE 55}

Giving the Properties of the Toxins of $\mathrm{Cl}$. welchii (Oakley 1943)

\begin{tabular}{|c|c|c|c|c|c|c|c|}
\hline & Toxin. & & Hæmolytic. & Lethal. & Necrotizing. & Lecithinase. & Effect of heat. \\
\hline$\alpha$ &.$\quad$. & . & + & + & + & + & Thermostable. \\
\hline$\theta$ & .. & . & + & + & + & - & 'Thermolabile. \\
\hline$\eta$ & . . & . & - & + & - & - & \\
\hline$\beta$ &.$\quad$. & . & - & + & + & - & Thermolabile. \\
\hline$\gamma$ &.$\quad$. & . & - & + & - & - & \\
\hline$\delta$ & .. & . & + & + & - & - & \\
\hline$\epsilon$ & . & . & - & + & + & - & $\begin{array}{l}\text { Culture filtrate thermostable. } \\
\text { Intestinal fluid thermolabile. }\end{array}$ \\
\hline
\end{tabular}

The $\alpha$-toxin is a thermostable substance, lethal for mice, guinea-pigs, rabbits, pigeons and sheep, and when given intradermally, produces a necrotic lesion. It is hæmolytic for the red cells of most laboratory animals excepting the horse and the goat, and is a powerful lecithmase. The lecithinase activity of $\alpha$-toxin is of great interest, since it is the first known instance of an exotoxin dependent upon a demonstrable enzyme for its activity. Its discovery dates from the demonstration by Nagler (1939) that toxic filtrates of all four types of $\mathrm{Cl}$. welchii produced an opalescence in human sera, and that the reaction was specifically inhibited by antisera to Type A filtrates (see also Seiffert 1939). 'The reactivity ran parallel to the toxin content of filtrates. Macfarlane, Oakley and Anderson (1941) demonstrated a similar action of $\alpha$-toxin on extract of egg yolk, and suggested that both phenomena were due to an enzymic splitting of lipo-protein complexes in serum and egg-yolk respectively; and that hæmolysis by $\alpha$-toxin might also be due to its action on bipo-proteins in the red cell envelope. The identity of $\alpha$-toxin and lecithinase was further established by Macfarlane and Knight (1941), who demonstrated a quantitative splitting of lecithin by $\alpha$-toxin, into phosphocholine and a glyceride, and the necessity for $\mathrm{Ca}$ or $\mathrm{Mg}$ ions in the reaction. The lecithinase activity of a filtrate may be used as 
a measure of its $\alpha$-toxin content, and for comparisons of the neutralizing power of $\alpha$-antitoxins (for details see Nagler 1939, Oakley and Warrack 1941, van Heyningen 194la, Crook 1942). Lecithinise produetion in fluid and on agar cultures of Cl. welchii may be detected by incorporating human serum or egg-yolk extract in media containing a sufficiency of free calcium. Other bacteria, mainly spore-bearing bacilli, produce lecithinase-like substances (Nagler 1939, Hayward 1941, Crook 1942) but with one exception, Cl. bifermentans (Hayward 1943), none is fully neutralized by $\alpha$-antitoxin.

The $\theta$-toxin las been found only in Type A filtrates. It is a strong hæmolysin, and is also lethal and necrotizing. Todd (194I) has shown that it is thermolabile and oxygenlabile. The activity of oxidized $\theta$-toxin is restored by reducing agents containing sulphydryl groups. This property of reversible oxidation is shared by the O-streptolysin of Str. pyogenes (Chapter 24). There is also an antigenic relationship between the two substances, each being to a large extent neutralized by antisera prepared against the other (Todd 1941). $\theta$-toxin may be removed from mixtures with $\alpha$-toxin by adsorption on to susceptible red cell stromata (van Heyningen 1941 $b$; see also Gale and van Heyningen 1942).

The $\beta$-toxin is not hæmolytic; it is lethal to mice, producing on intravenous injection a spasmodic twitching rapidly followed by death; given intradermally in guinea-pigs and rabbits it produces necrotic lesions. The $\gamma$-toxin is neither necrotizing nor hæmolytic. Its existence in filtrates can be proved only by its lethal activity in mice, after the other toxins have been neutralized by appropriate antitoxins. The $\delta$-toxin is hæmolytic and lethal and. like $\gamma$-toxin, is detected after neutralization of other components by antitoxins. The $\varepsilon$-toxin, which predominates in Type D filtrates, is not hæmolytic, but is both lethal and necrotizing. Large doses in mice produce spasmodic twitching similar to those following injections of $\beta$-toxin. Smaller doses, after a latent period of several days, produce paralysis. The $\varepsilon$-toxin is produced by Type $D$ strains as a thermostable relatively non-toxic substance, which is activated by trypsin and other proteolytic enzymes with the formation of the thermolabile toxin (Gill 1933, Bosworth and Glover 1935). In disease produced by Type D strains (see Chapter 78) the activating enzyme is apparently supplied by the infected animal, though according to Turner and Rodwell (1943) in favourable conditions extracellular proteinases of the bacillus itself will activate the toxin. It should be noted that culture filtrates of Type A strains may also contain large amounts of a hyaluronidase (McClean 1936; see Chapters 44, 78).

The toxin of $\mathrm{Cl}$. adematiens is the most potent of the gas-gangrene toxins; the average M.L.D. for a mouse is about $0.0002 \mathrm{ml}$. ; of welchii toxin the M.L.D. is about $0.25 \mathrm{ml}$; ; and of $\mathrm{Cl}$. septicum toxin about $0.005 \mathrm{ml}$.

Toxic filtrates of $\mathrm{Cl}$. septicum produce a marked liquefactive necrosis of muscle ; lethal doses, when given intravenously, produce intense capillary engorgement and interstitial hæmorrhages in the heart, with hyaline degeneration of the muscle fibres, and a toxic nephrosis in the kidney of experimental animals (Pasternack and Bengtson 1936). Filtrates may also contain a hæmolysin and a hyaluronidase. The hæmolysin has usually been regarded as distinct from the lethal toxin; but Bernheimer (1944) has produced filtrates in which the lethal and the hæmolytic activities are substantially parallel.

Toxic filtrates of $\mathrm{Cl}$. odematiens contain a potent toxin that produces intense gelatinous odema in muscle. There are few gross changes in the organs following a lethal intravenous dose of toxin, but degenerative changes, particularly in the spleen and kidney, have been observed (Pasternack and Bengtson 1940). Traces of lecithinase, a hyaluronidase and a hæmolysin are sometimes present.

Cl. histolyticum usually produces a weakly toxic filtrate that contains an active proteolytic enzyme. Toxigenic strains of $\mathrm{Cl}$. bifermentans have been described under the name of $\mathrm{Cl}$. sordellii; the filtrates are moderately toxic. All the toxins 
of these five organisms associated with gas gangrene give rise to specific antitoxins on injection into suitable animals.

$\mathrm{Cl}$. chauvoi under suitable conditions forms a weak toxin that is very heatlabile, being destroyed in 5 minutes by exposure to a temperature of $52^{\circ} \mathrm{C}$. Injected intravenously into mice in a dose of $0.025-0.5 \mathrm{ml}$., it causes respiratory embarrassment and death within a few minutes. It is also toxic to guinea-pigs, though not rabbits, on intravenous inoculation. Subcutaneous inoculation into mice and guinea-pigs is not fatal, but produces a local blood-stained oedema (Kerrin 1934).

It is interestıng to note that all the different groups of $C l$. tetani give rise to identical toxins; the antitoxin prepared against any one type will neutralize the toxins of all types. With $\mathrm{Cl}$. botulinum it is otherwise. Type A toxin is different from Type B toxin. By agglutination Type A strains can be divided into 4, and Type B into 3 groups (Starin and Dack 1923), but the divisions do not appear to be clear-cut. Three further types have been described, C, D, and E, which appear to differ in the type of toxin produced.

Pathogenicity.-The pathogenicity of the anaerobes appears to depend almost entirely on their toxin production. Cl. tetani, for example, multiplies locally, and does not invade the body. $C l$. botulinum is not even a parasite ; it is apparently unable to grow in the tissues, and its pathogenic effects are determined by the formation of toxin in food-substances prior to their ingestion. Cl. oedematiens remains almost confined to the site of inoculation. $\mathrm{Cl}$. welchii and $\mathrm{Cl}$. septicum become generalized in the final stages of an infection, but they multiply only locally before the death of the animal. Tetanus, botulism, and to a large extent gas gangrene are intoxications.

\section{Pathogenicity of $\mathrm{Cl}$. botulinum for Laboratory Animals.}

Monkeys.-Van Ermengem (1897) fed a Macacus rhesus with $5 \mathrm{ml}$. of a preparation of macerated ham, which was known to be toxic. Symptoms developed in 12 hours, and consisted of restlessness, crying, coughing, and sneezing; later there was a secretion of viscid mucus in the nose and mouth, leading to transient suffocation; the pupils were dilated, reacting weakly to light. The animal became motionless, its head rrooped, its eyes were fixed and half covered by the lids. Death occurred after 24 hours from the time of feeding. At necropsy the stomach, the bases of the lungs, and the meninges were congested, and petechial hrmorrhages were noticed on the arachnoid and throughout the brain and medulla.

CATs.-The typical toxæmia may be reproduced in cats by feeding, but more certainly by subcutaneous injection of cultures or of toxin. After a latent period of about 24 hours the animal becomes quiet, loses its interest in external objects, and may refuse food. In 2 or 3 days the characteristic paralyses appear, giving a peculiar facies to the animal. Its general aspect is stupid, the lids remain open, the eyes fixed in a glassy stare, the pupils dilated and sluggish in their reaction to light. The animal sits in a dark corner, moves little, and when disturbed takes a few uncoordinated steps across the cage and drops down as if exhausted. Its head droops and its tongue protrudes. Thick, viscous secretion fills the throat and nose, and causes severe paroxysmal attacks of suffocation relieved by a hoarse croup-like cough. The mew takes on a dull tone, and is succeeded by complete aphonia. For the first 2 or 3 days milk is accepted, but later owing to the dysphagia or complete aphagia it is left untouched; when delivered by a pipette into the mouth it is not swallowed, but trickles down the trachea and causes choking. No urine or freces are voided. The animal remains susceptible to sensory impressions till the end, but is unable to express its emotions in any way. Death occurs after a week or more, according to the dose, and is apparently due as much to starvation as to the lethal effect of the 
toxin. Occasionally life may be prolonged for 3 or 4 weeks, and recovery may eventually take place. At necropsy no local lesion is visible at the site of injection; the mucosa of the small and large intestine is hyperæmic. The kidneys are congested, and the liver may show areas of degeneration. Clear urine distends the bladder. In the lungs, which are very congested, there may be infarets or areas of hepatization. Sometimes œedema or hæmorrhages of the central nervous system may be observed, especially round the fourth ventricle. Cultures of the organs are usually sterile.

Dogs are very much less susceptible than cats, but they may succumb to the disease after subcutaneous injection of toxin, or occasionally after feeding with large doses. Mice and guinea-pigs are highly susceptible, and succumb in $1-4$ days.

\section{Pathogenicity of $\mathbf{C l}$. tetani for Laboratory Animals.}

Tetanus can be reproduced by the inoculation of pure cultures, or of the toxin into mice, rats, guinea-pigs, rabbits, goats, horses and monkeys. Cats and dogs are more resistant; birds and cold-blooded animals are highly resistant. The most susceptible animal, calculated on the amount of toxin per gram of body weight necessary to prove fatal on injection, is the horse. This is about 12 times as susceptible as the mouse; the guinea-pig is 6 times, and the monkey 4 times, as susceptible as the mouse (von Lingelsheim 1912, Sherrington 1917). On the other hand, the rabbit is twice, the dog 50 times, the cat 600 , and the hen 30,000 times as resistant as the mouse (Kitasato 1891, von Lingelsheim 1912).

Mrce.-After the subcutaneous injection of a small quantity of toxin or of pure culture into the mouse near the root of the tail, symptoms develop in about 12 to 24 hours. The spasms start near the site of injection, and spread to the rest of the body, till the animal dies in a state of general tonic contraction. The first symptom noticed is a stiffening of the tail, which becomes erect and is turned towards the side of inoculation; the hinder extremity of that side becomes stiff, followed later by rigidity of the opposite leg. The contractions pass to the imuscles of the trunk, and the mouse develops kyphosis or pleurothotonos. Next, the fore-legs become involved, and finally trismus and opisthotonos set in. The contractions occur spasmodically and are succeeded by intervals of rest, during which the animal lies exhausted; in this phase they can be readily excited by the slightest touch or a breath of air. Death follows in about 24 hours. Post mortem there is little to be seen. There may be slight congestion and cedema round the site of inoculation, and the spleen may be somewhat enlarged. An exudate of fluid. sometimes blood-stained, may be seen in the pleura or peritoneum. After injection of a pure culture, the bacilli can generally be cultivated from the local site, but are difficult to find under the microscope. The heart's blood and viscera are sterile.

Gutnea-pigs.-The experimental disease in guinea-pigs follows much the same course in about the same time as in mice.

RaBbits.-After subcutaneous or intramuscular injection the incubation period in rabbits is at least 24 , and generally 36 hours; death does not occur for 3 or 4 days. The general tetanic spasms are more marked than in mice or guinea-pigs (Rosenbach 1856).

\section{Pathogenicity of $\mathrm{Cl}$. welchii for Laboratory Animals.}

Intramuscular injection of about $0.2 \mathrm{ml}$. of an 18-hours' glucose broth culture into the thigh of a guinea-pig usually results in gas gangrene with death in 12-18 hours. Post mortem, there is a large, brawny, crepitant swelling at the site of inoculation, covered with a dark-red, teuse layer of skin. The muscle is pale and is undergoing liquefactive necrosis. In the subcutaneous tissue around the local lesion and spreading up to the abdomen, reaching sometimes to the sternum and over to the opposite thigh, is a collection of slightly blood-stained fluid and gas smelling of hydrogen sulphide. The suprarenal glands are often congested, so that the normally sharp differentiation of cortex from medulla 
becomes obscured. Microscopically, the organisms are present in large numbers in the local effusion and in much smaller numbers in the blood stream. Sporing forms are absent. An eren more typical picture of gas gangrene can be obtained by the injection of $\mathrm{Cl}$. welchii intramuscularly into pigeons (Bull and Pritchett 1917a). Mice are less susceptible than guinea-pigs.

\section{Pathogenicity of $\mathrm{Cl}$. septicum for Laboratory Animals.}

Intramuscular injection of about $0 \cdot 1 \mathrm{ml}$. of a 36 -hours' glucose broth culture into a guinea-pig causes death in 12 to 24 hours. Post mortem, there is a blood-stained gaseous œdema at the site of inoculation, spreading up over the abdominal wall, with collections of gas in the groins and axillæ. The thigh and abdominal muscles are soft and deep red in colour. In the pericardial and peritoneal eavities there may be some fluid; the suprarenals are congested, but not so markedly as in animals infected with $\mathrm{Cl}$. welchii. Microscopically the exudate shows large numbers of motile rods and usually the characteristic naricular or citron forms. Most characteristic are the long curved filaments found on the peritoneal surface of the liver. A similar picture can be reproduced by the inoculation of mice.

\section{Pathogenicity of Cl. œdematiens for Laboratory Animals.}

Intramuscular injection of about $1 \mathrm{ml}$. of a 24-hours' glucose broth culture into a guinea-pig or mouse produces death in 1 to 2 days. Post mortem, the muscles at the site of inoculation are very congested, purplish-red in colour, and infiltrated with small bubbles of gas. There is a spreading, gelatinous œelema, sometimes slightly blood-tinged, extending over the thigh. The abdominal muscles are unaltered. Microscopically, bacilli are found in small numbers in the odema fluid, and on the peritoneal surface of the liver: cultures from the heart's blood may or may not be positive.

It will be seen that the action of these last three organisms varies in certain particulars. $\mathrm{Cl}$. welchii gives rise to a large amount of gas, $\mathrm{Cl}$. cedematiens to very little. The œdema fluid of $C l$. oedematiens infections is practically clear, of $C l$. welchii infections slightly blood-tinged, and of $\mathrm{Cl}$. septicum infections strongly -blood-tinged. With $\mathrm{Cl}$. welchii the muscles are pale pink, with $\mathrm{Cl}$. adematiens purplish-red, and with $\mathrm{Cl}$. septicum intensely and deeply red. Human cases of gas gangrene differ too in certain respects; as a rule either odema or, more rarely, gas production is dominant; occasionally both are apparent. The particular form in any individual case is determined by the nature of the organisms present.

\section{Classification}

Although it is clear that the time is not yet ripe for any rigid classification of the anaerobic bacilli, we can recognize certain well-differentiated types, which should clearly be accorded specific rank. Table 56 presents a rough classification of the organisms chiefly associated with gas gangrene and similar infections in man, which were considered by the Anaerobic Committee of the Medical Research Council (Report 1919) to be separate species. We may note, however, that the characters differentiating $\mathrm{Cl}$. parasporogenes from $\mathrm{Cl}$. sporogenes are hardly of sufficient importance to entitle it to classification as a separate species; it may prove on further investigation to be merely a variant of the latter organism. Moreover the identity of $\mathrm{Cl}$. butyricum with the organism originally described by Pasteur seems to be very doubtful; it is unfortunate that this organism has been selected as the type species. In many respects it resembles $\mathrm{Cl}$. fallax and $\mathrm{Cl}$. multifermentans. A few organisms have since been studied in sufficient detail to provide an adequate description of their biological characters and to differentiate them 
clearly and unmistakably from other forms which have been described and ramed. Some of these we have included in Table 57 and others are described at the end of the chapter. Nevertheless, a large number of named forms remain, whose place in the Clostridium group is still a matter of conjecture, as reference to the monograph of Weinberg, Nativelle and Prévot (1937) will show. Prévot (1938) rejects the genus Clostridium as incapable of covering all the anaerobic sporebearing bacilli, and proposes to create four families and nine genera, of which Clostridium is one; this classification in our opinion places too much weight on morphological distinctions. The recognition of variation of the $\mathrm{S} \rightarrow \mathrm{R}$ type and detailed serological studies are helping to resolve some taxonomic difficulties, but it is elear that a large number of clostridia so far studied are antigenically heterogeneous, and that the variety of antigens, $H$, thermostable $O$, labile $O$, etc., is probably as great as that displayed by the salmonellæ.

TABLE 56.

Giving a Classification of the Clostridia.

\begin{tabular}{|c|c|c|c|c|c|}
\hline \multirow{2}{*}{ Spores. } & \multicolumn{2}{|c|}{$\begin{array}{l}\text { Both Proteolytic and } \\
\text { Saccharolytic Properties. }\end{array}$} & \multirow{2}{*}{$\begin{array}{c}\text { Slight Pro- } \\
\text { teolytic but } \\
\text { no Saccharo- } \\
\text { lytic Proper- } \\
\text { ties. }\end{array}$} & \multirow{2}{*}{$\begin{array}{l}\text { Saccharolytic } \\
\text { but no Proteo- } \\
\text { lytic Properties. }\end{array}$} & \multirow{2}{*}{$\begin{array}{l}\text { Neither Pro- } \\
\text { teolytic nor } \\
\text { Saccharolytic } \\
\text { Properties. }\end{array}$} \\
\hline & $\begin{array}{l}\text { Proteolytic } \\
\text { Predominating. }\end{array}$ & $\begin{array}{l}\text { Saccharolytic } \\
\text { Predominating. }\end{array}$ & & & \\
\hline $\begin{array}{l}\text { Equatorial or } \\
\text { Subterminal }\end{array}$ & $\begin{array}{l}\text { Cl. sporogenes } \\
C l \text {. parasporo- } \\
\text { genes } \\
\text { Cl. histolyticum } \\
\text { Cl. aerofotidum } \\
\text { Cl. bifermen- } \\
\text { tans } \\
C l . \text { botulinum }\end{array}$ & $\begin{array}{l}\text { Cl. welchii } \\
C l \text {. septicum } \\
C l \text { chauvœi } \\
\text { Cl. oedematiens }\end{array}$ & - & $\begin{array}{l}C l . \text { fallax } \\
C l . \text { butyricum } \\
C l . \text { multifer. } \\
\text { mentans }\end{array}$ & - \\
\hline $\begin{array}{l}\text { Oval and Ter- } \\
\text { minal }\end{array}$ & - & - & - & Cl. tertium & $\begin{array}{l}\text { Cl. cochlear- } \\
\text { ium }\end{array}$ \\
\hline $\begin{array}{l}\text { Spherical and } \\
\text { Terminal }\end{array}$ & - & - & Cl. tetani & $\begin{array}{l}\text { Cl. tetanomor- } \\
\text { phum } \\
\text { Cl. sphenoides }\end{array}$ & - \\
\hline
\end{tabular}

(For classification see Weinberg and Séguin 1918, Report 1919, Heller 1921, and Hall 1922.)

The grouping of the species within the genus Clostridium presents even greater difficulties. Whether the primary division should be made on morphological grounds-mainly on the shape and position of the spore-or on physiological grounds - mainly on the relative activity of proteolytic and saccharolytic fermentation-must at the moment remain a matter of choice. We give in Table 57 (pp. 874, $875)$ the more important characters of the recognized species. We also append a summarized description of each of a number of recognized species. It should be noted that we have omitted to discuss clostridia like $\mathrm{Cl}$. acetobutylicum (see Chapters 3 and 6), which are of interest to the biochemist, and have made only a brief reference to the clostridia associated with the spoilage of camned foods. Of particular interest is Cl. thermosaccharolyticum (McClung 1935), which is an apparently non-pathogenic thermophilic organism having an optimum temperature 
of growth between $50^{\circ}$ and $60^{\circ} \mathrm{C}$., associated with the type of spoilage of nonacid canned foods known in the United States as "hard swell" (see also Paine 1931, McCoy 1937). In addition to the common proteolytic clostridia of the Cl. sporogenes type associated with food spoilage, we may note an organism resembling $\mathrm{Cl}$. welchii (McClung and Wheaton 1936), and an organism resembling $\mathrm{Cl}$. odematiens. which Haines and Scott (1940) found associated with "bone taint" of cattle carcases. (For practical keys to the separation of the clostridia see Spray 1936, Reed and Orr 1941).

\section{Clostridium butyricum}

Synonyms._Clos. pasteurianum, B. amylobacter, Granulobacter saccharobutyricum. Pasteur's Vibrion butyrique.

Isolation.--Apparently first described by Prazmowski (see Report 1919). Possibly identical with Pasteur's Vibrion butyrique, described fully by Winogradsky in 1902

Habitat.-Soil.

Morphology.-Rods, $3-4 \mu \times 0.7 \mu$; parallel sides, flattened ends, axis straight or slightly eurved; arranged singly or in pairs end-to-end; considerable variation in length. Motile by peritrichate flagella. Spores oval, subterminal, measuring $1 \cdot 6 \mu$ $\times 1 \cdot 3 \mu$; $\operatorname{rod}$ becomes spindleshaped. Germination polar. Capsule formed on agar. Cells store glycogen; stain yellow with iodine. Gram-positive.

Agar Plate. -2 days, $30^{\circ} \mathrm{C}$. Circular colonies, $0.5-1 \cdot 0 \mathrm{~mm}$. in diameter, low convex, amorphous, faintly translucent or opaque, greyish-white, with smooth glistening surface and entire edge ; butyrous eonsisteney and easily emulsifiable. After 6 days the colonies are slightly larger.

Agar Stroke. -4 days at $30^{\circ} \mathrm{C}$. Very poor growth of discrete, irregular colonies, slightly raised, and water-clear. On 0.5 per cent. mannitol agar there is a moder-

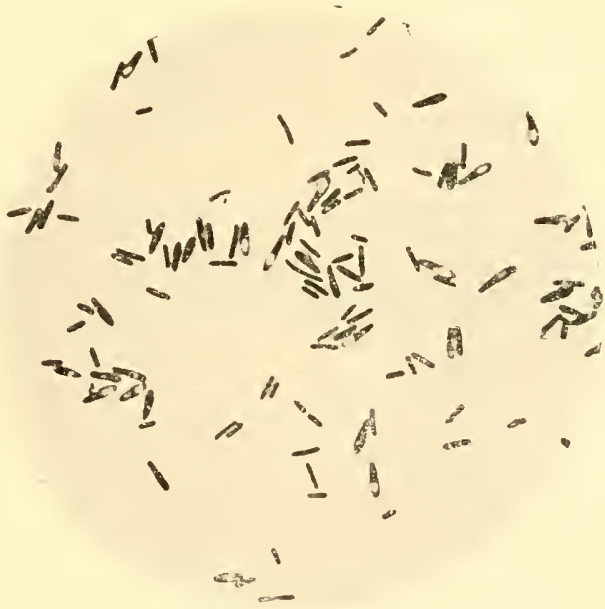

Fig. 192.-Clostridium butyricum.

From a surface agar culture, anaerobically, 5 days, $37^{\circ} \mathrm{C}$. $(\times 1000)$.

ate, raised, greyish-white, opaque growth, consisting chiefly of diserete colonies with a moist, glistening, smooth surface.

Gelatin Stab.-No growth.

Glucose Agar Shake.-2 days at $30^{\circ} \mathrm{C}$. Good growth ; the medium is disrupted by large bubbles of gas, and blown up the tube. Colonies are yellowish-grey, opaque, biconvex with elear-cut edges, and about $1 \mathrm{~mm}$. in diameter.

Broth.-2 days at $30^{\circ} \mathrm{C}$. Poor to moderate growth, with slight turbidity, and slight, very finely granular deposit; after 6 days, moderate turbidity, and moderate powdery deposit, disintegrating completely on shaking.

Loeffer's Blood Serum.-2 days at $30^{\circ} \mathrm{C}$. Moderate, raised, eonfluent, colourless growth with irregularly contoured surface. No digestion.

Cooked Meat Medium. -5 days, $37^{\circ}$ C. Marked turbidity; no digestion. 


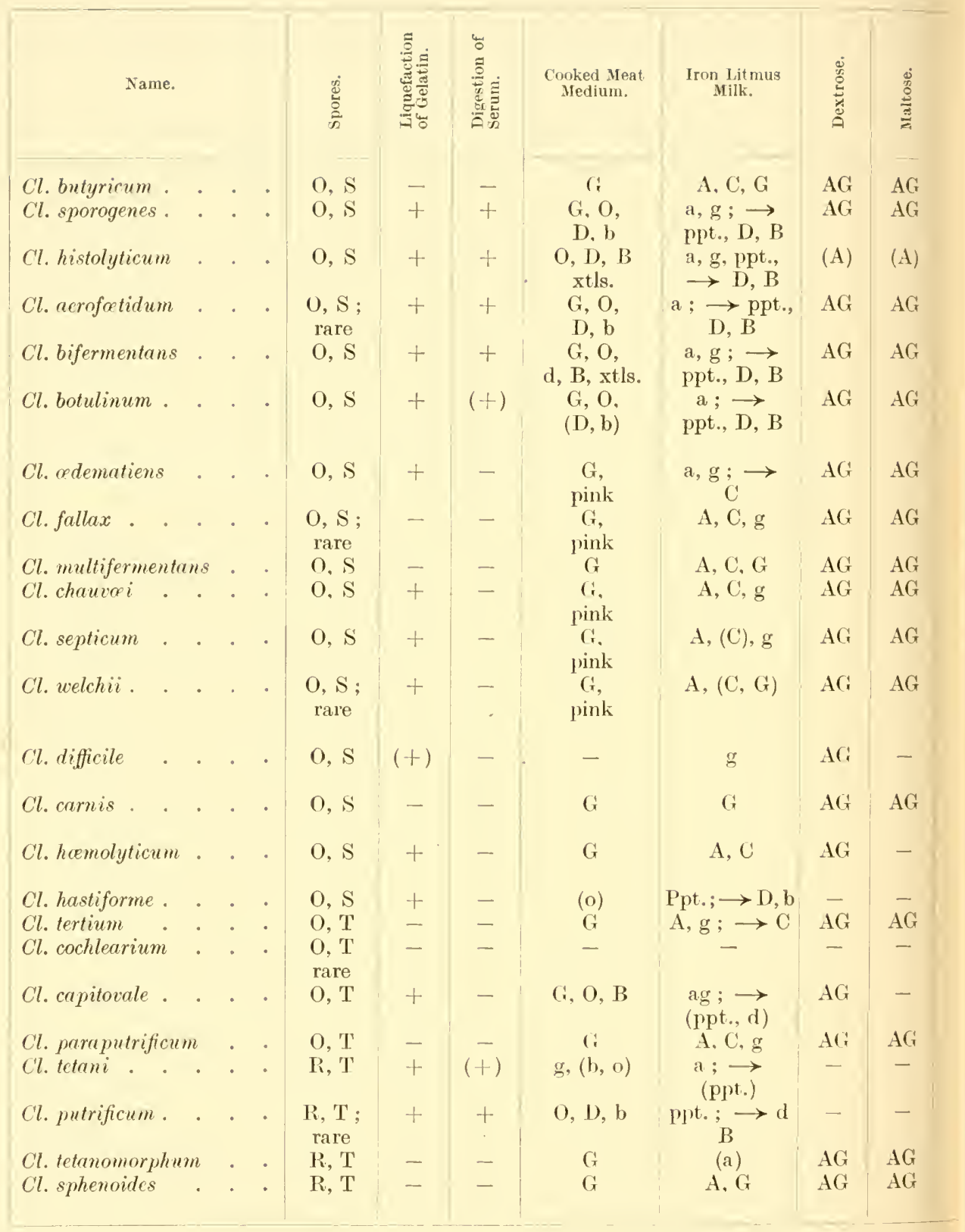

A or $\mathrm{a}=$ acid ; $\mathrm{B}$ or $\mathrm{b}=$ blackening ; $\mathrm{C}$ or $\mathrm{c}=\operatorname{clot} ; \mathrm{D}$ or $\mathrm{d}=$ digestion ; $\mathrm{G}$ or $\mathrm{g}=$ gas ; ppt. = precipitate $; \mathrm{R}=$ round (spherical) $; \mathrm{S}=$ subterminal (or equatorial) $; \mathrm{T}=$ terminal; Brackets indicate that the activity is not a characteristic of all members of the species. Rare $=$ rarely observed microscopically in cultures. 


\begin{tabular}{|c|c|c|c|c|c|c|c|}
\hline 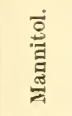 & 总 & 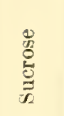 & 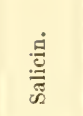 & 离 & Exotoxin. & $\begin{array}{l}\text { Patho- } \\
\text { genicity to } \\
\text { Guinea-pigs. }\end{array}$ & Remarks. \\
\hline $\begin{array}{l}? \\
-\end{array}$ & $\mathrm{AG}$ & $A C_{-}$ & $\begin{array}{l}\mathrm{ACr} \\
(\mathrm{ACr})\end{array}$ & $\begin{array}{l}(\mathrm{AG}) \\
(\mathrm{AG})\end{array}$ & $\begin{array}{l}- \\
-\end{array}$ & - & $\begin{array}{l}\text { Possesses a capsule. } \\
\text { Tanillin violet test }+ \text {. }\end{array}$ \\
\hline- & - & - & - & - & + & $(+)$ & Feeble aerobic growth. \\
\hline- & $A G$ & - & $(\mathrm{AG})$ & - & - & - & \\
\hline - & - & - & (AG) & $\mathrm{AG}$ & $(+)$ & $(+)$ & Only toxigenic strains are patho- \\
\hline- & $(\mathrm{AG})$ & - & $(\mathrm{AG})$ & $(\mathrm{AG})$ & $+t+$ & + & $\begin{array}{l}5 \text { types, } \mathrm{A}, \mathrm{B}, \mathrm{C}, \mathrm{D} \text { and } \mathrm{E} \text {, each } \\
\text { with a different toxin. Some } \\
\text { strains not proteolytic. }\end{array}$ \\
\hline - & - & 一 & - & $(\mathrm{AG})$ & +++ & + & \\
\hline (AG) & $A G$ & $\mathrm{AG}$ & $\mathrm{AG}$ & - & + & $(+)$ & $\begin{array}{l}\text { Pathogenic only when freshly } \\
\text { isolated. }\end{array}$ \\
\hline - & $A G$ & $A G$ & $A G$ & $A G$ & - & - & \\
\hline- & $\mathrm{AG}$ & $A G$ & - & - & $+t$ & + & \\
\hline- & $\mathrm{AG}$ & - & $A G$ & - & ++ & + & \\
\hline- & $\mathrm{AC}$ & $\mathrm{AG}$ & $(\mathrm{AG})$ & $(\mathrm{AG})$ & ++ & $(+)$ & $\begin{array}{l}\text { Possesses a capsule. Non-motile. } \\
\text { Four types, each with a differ- } \\
\text { ent combination of toxins. }\end{array}$ \\
\hline $\mathrm{AG}$ & - & - & $\mathrm{AG}$ & - & + & + & \\
\hline- & $A G$ & Ar: & $\mathrm{AG}$ & - & + & + & Feeble aerobic growth. \\
\hline- & - & - & - & $\mathrm{AG}$ & $+t$ & + & \\
\hline- & - & - & - & - & - & - & \\
\hline$\stackrel{A G}{-}$ & $\stackrel{A G}{-}$ & $\stackrel{A G}{-}$ & AG & - & - & - & Grows aerobically. \\
\hline 一 & - & - & - & - & - & - & Indole $(+)$ \\
\hline - & $\mathrm{AG}$ & $\mathrm{AG}$ & $\mathrm{AC}$ & - & - & - & \\
\hline- & - & - & - & - & $+t+t$ & $(+)$ & Indole + . \\
\hline- & 一 & - & - & - & - & - & \\
\hline- & - & - & - & - & - & - & \\
\hline $\mathrm{AG}$ & $\mathrm{AG}$ & - & $A G$ & - & - & - & Indole $(+)$. \\
\hline
\end{tabular}

O (under "Spores") $=$ oval; 0 or o (under "Cooked Meat Medium ") $=$ foul odour ; $\mathrm{xtls}=$ late deposit of white crystals. Small-letter symbols indicate a slighter degree of change. Under exotoxin,,,,++++++++++ indicate the relative potency of toxic filtrates. 
Potato.-14 days at $30^{\circ} \mathrm{C}$. No definite growth visible on the potato itself, but there is a considerable evolution of gas from the liquid in which the potato is soaked; the liquid is turbid.

II etabolic.-Obligate anaerobe. Opt. temp. $30-40^{\circ} \mathrm{C}$. Pigment none. Nutritional : grows best in sugar solutions. Growth on agar improved by 1 per cent. mannitol. Non-proteolytic.

Biochemical.-Forms acid and gas in dextrose, lævulose, maltose, galactose, lactose, sucrose, inulin, dextrin and starch. Fermentation of mannitol variously reported. Indole - ; II.R. + ; V.P. - ; nitrates reduced ; II.B. reduction - ; eatalase - ; $\mathrm{NH}_{3}$ slight + ; L.M. acid and clot. Can fix atmospheric nitrogen in presence of a fermentable sugar; the sugar is broken down with the formation of butyric and acetic acids, $\mathrm{CO}_{2}$, and $\mathrm{H}_{2}$. Can utilize $\mathrm{NH}_{3}$, peptone, and asparagin as its source of nitrogen.

Pathogenicity.-Nil.

\section{Clostridium sporogenes}

1solation.-Deseribed by Metelnikoff in 1908 .

Habitat.-Fond in soil, and in frees of man and animals.

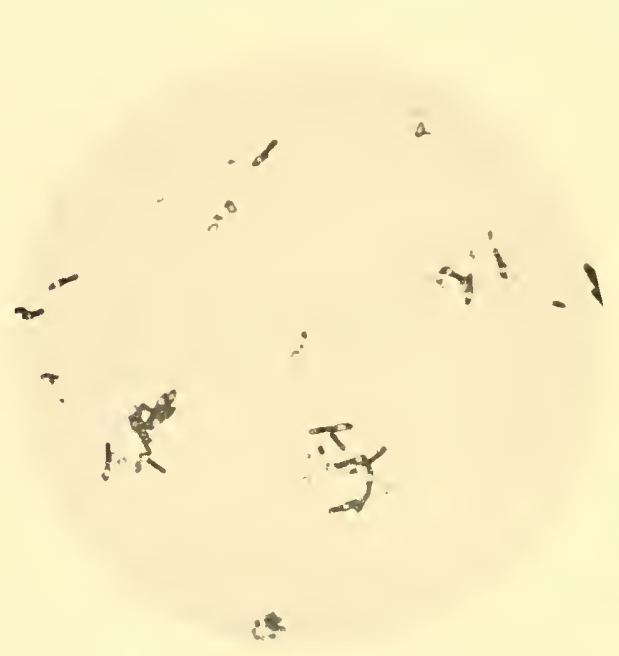

Fig. 193.-Clostridium sporogenes.

From a surface agar culture anaerobically, 2 lays, $37^{\circ} \mathrm{C} .(\times 1000)$.
Morphology. - Rod-shaped, 3-6 $\mu \times 0.5$ $\mu$, parallel sides, rounded ends, axis straight or slightly curved, arranged singly, in pairs, and small groups; long filaments occasionally formed. Spores

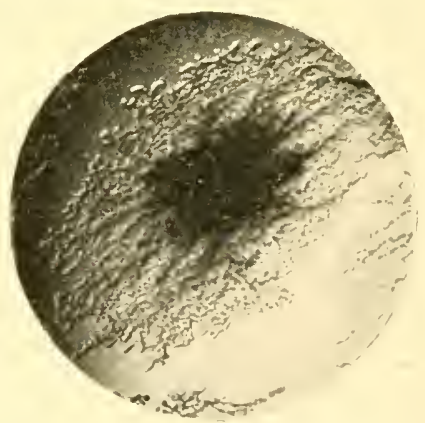

FIG. 194.-Clostridium sporogenes.

Surface colony on agar anaerobically, 4 days, $37^{\circ} \mathrm{C} .(\times 8)$.

freely; spores are oval, subterminal, and wider than bacillus; free spores numerous. Motile. No capsule. Strongly Gram-positive, except in old cultures.

Agar Plate. 4 days at $37^{\circ} \mathrm{C}$. Irregularly round colonies, growing from a central focus like B. mycoides; 2-6 $\mathrm{mm}$. in diameter, effuse or slightly umbonate, and rhizoid; surface covered by arborescent ridges, edge rhizoid; rather dull, greyish-yellow by reflected, bluish-grey by transmitted light; butyrous and easily emulsifiable; differentiated into brownish opaque centre and bluish translucent periphery.

Deep Glucose Agar.-4 days at $37^{\circ} \mathrm{C}$. Abundant gas formation; medium driven to plug and disrupted. Colonies throughout medium; rounded, $0.5-2 \mathrm{~mm}$. in diameter, with opaque brown centre and a woolly translucent periphery.

Horse Blood Agar. -3 days at $37^{\circ} \mathrm{C}$. Colonies $3 \mathrm{~mm}$. in diameter, consisting of tangled rhizoids growing from a raised, glistening centre, $0.5 \mathrm{~mm}$. in diameter; on moist 
media, smooth lobate outgrowths may arise from a rhizoidal centre. Occasionally round dew-drop colonies are formed. Hamolysis for 2-4 mm. around colony. Agar Slope.-4 days at $37^{\circ} \mathrm{C}$. Morlerate, confluent, effuse, glistening, greyish-yellow, translucent growth, with beaten-copper surface and cigarette-in-water edge.

Gelatin. -2 days at $37^{\circ} \mathrm{C}$. Liquefied.

Broth. -4 days at $37^{\circ} \mathrm{C}$. Good growth with moderate turbidity, and moderate powdery or irregularly granular sediment, not disintegrating completely; strong putrid odour.

Loeffer's Serum.-15 days at $37^{\circ} \mathrm{C}$. Serum digested and rendered turbid; medium dark blue.

Coagulated Egg.-15 days at $37^{\circ} \mathrm{C}$. Poor, effuse growth; slight digestion. In alkaline egg broth the white coagulum is digested.

Cooked Meat Medium.-15 days at $37^{\circ} \mathrm{C}$. Heavy growth with dense turbidity; gas production; meat digested and blackened; putrid odour.

Resistance.-Withstands moist heat at $100^{\circ} \mathrm{C}$. for 10 to 150 minutes, at $105^{\circ} \mathrm{C}$. for 4 to 45 minutes, and at $110^{\circ} \mathrm{C}$. for 1 to 12 minutes.

Metabolic.-Anaerobic, but not strictly so. Opt.temp. $37^{\circ} \mathrm{C}$. Hæmolysis on horse blood agar plates. Hrmolyses human but not sheep's red cells. Nutritional: grows well on ordinary media, and in media containing very little nutrient material, such as tap water containing fragments of coagulated egg white. Certain amino. acids, such as tryptophan, leucine, phenylalanine, tyrosine, and arginine, as well as the sporogenes vitamin, are essential. Growth not improved by glucose. Green fluorescent colonies on MacConkey plate.

Biochenical.-Acid and gas in glucose and maltose. No action on mannitol, lactose, or sucrose. Some strains ferment salicin. Indole - ; vanillin violet + (Spray 1936) ; I.R. - ; V.P. - ; nitrites not produced in nitrate broth; $\mathrm{NH}_{3} \pm ; \mathrm{H}_{2} \mathrm{~S}+++$; 11.B. reduction - ; catalase weak + . Litmus milk : casein precipitated and almost completely digested in 15 days ; reduction, and marked alkaline reaction; acid in young cultures.

Antigenic Structure.-Can be divided by agglutination into at least two groups.

Pathogenicity.-Not naturally pathogenic. Experimentally is non-pathogenic to laboratory animals, but enhances the pathogenicity of other anaerobes, such as $\mathrm{Cl}$. welchii, in mixed cultures. No exotoxin formed, but a broth filtrate is toxic to guineapigs in a dose of $1 \mathrm{ml}$; this is due apparently to a volatile substance, possibly an ammonium base. Forms a fibrinolysin.

(See von Hibler 1908, Wolf and Harris 1917, Weinberg and Séguin 1918, Report 1919, Hall 1922, de Smidt 1924, Weinberg and Ginsbourg 1927, Knight and Fildes 1933, Fildes and lichasdson 1£35, Stickland 1934, 1935, Pappenheimer 1935, Spray 1936.)

\section{Clostridium histolyticum}

Isolation.-Described by Weinberg and Séguin in 1916 (1916, 1918).

Habitat.-Soil; possibly intestinal canal of man and animals.

Morphology.-Rod-shaped, 3-5 $\mu \times 0.5-0 \cdot 8 \mu$; parallel sides, rounded ends, axis generally straight; occur singly and as diplobacilli. In cultures more than a day old irregular forms appear-long curved filaments, and irregularly stained forms. Spores are readily formed in all media; they are oval, subterminal, and wider than the bacillus; become free in old cultures. Motile by about 20 peritrichate flagella. Gram-positive in young cultures. No capsule.

Agar Plate. -4 days at $37^{\circ} \mathrm{C}$. Variable. Colonies may be delicate and flat with erenated edges; or may be cuttle-fish-like, umbonate, amorphous, and glistening, with very finely granular surface and a fimbriate edge; greyish-white by reflected light, bluish-grey by transmitted light; differentiated into opaque yellowish centre and greyish translucent periphery; butyrous and easily emulsifiable. 
Deep Glucose Agar Shake-4 days at $37^{\circ} \mathrm{C}$. No gas. Abundant growth through out medium. Colonies are $1 \mathrm{~mm}$. in diameter, irregularly round, opaque, brown with blunt, coral-like projections with very fine woolly ends.

Horse Blood Agar Plate. 4 days at $37^{\circ} \mathrm{C}$. Irregularly round eolonies, slightly raised, 2-3 mm. in diameter, with irregularly lobate edge, and smooth or pitted surface. No hæmolysis.

Agar Slope.-4 days at $37^{\circ} \mathrm{C}$. Moderate, partly confluent, effuse, glistening, translucent, greyish-yellow growth with beaten-copper surface and delicate fimbriate edge.

Gelatin.-Liquefied in 3 days at $37^{\circ} \mathrm{C}$.

Broth. - 4 days at $37^{\circ} \mathrm{C}$. Moderate growth, with moderate turbidity, and a granulopowdery deposit partly disintegrating; slight footid odour.

Loeffler's Serum. - 15 days at $37^{\circ} \mathrm{C}$. Almost eompletely digested; the fluid is almost clear. Coagulated Egg.-15 days at $37^{\circ} \mathrm{C}$. Partly digested; butt turned bluish-green.

Cooked Meat Medium.-15 days at $37^{\circ} \mathrm{C}$. Abundant growth; meat digested and slightly blackened; long column of slightly turbid fluid; gas produced; a deposit of white tyrosine crystals occurs, increasing with age. Slightly footid odour.

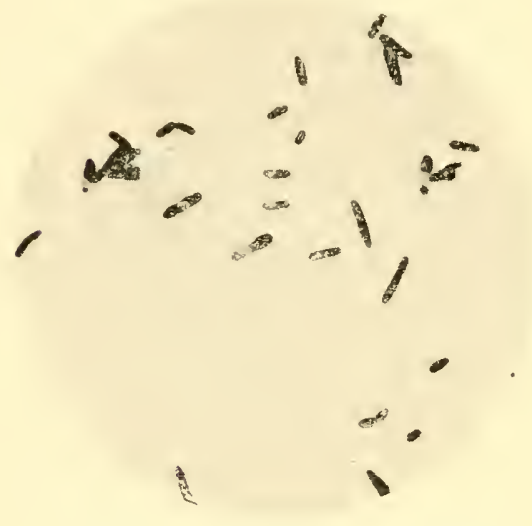

FIG. 195.-Clostridium histolyticum.

From a surface culture on agar anaerobically, 6 days, $37^{\circ} \mathrm{C} .(\times 1000)$.

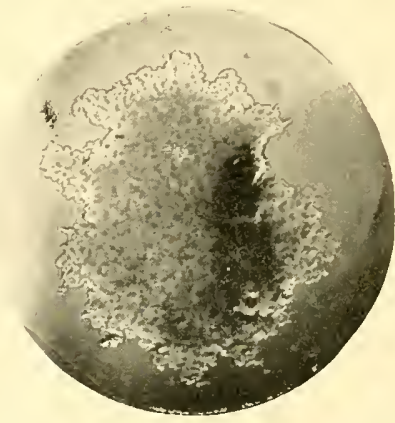

F10. 196.-Clostridium histolyti. cum.

Surface colony on agar anaerobi. eally, 2 days, $37^{\circ} \mathrm{C} .(\times 8)$.

Resistance.-Killed in 6 minutes at $105^{\circ} \mathrm{C}$. (moist heat).

Metabotism.-Microaerophilic. Opt. temp. $37^{\circ} \mathrm{C}$. No hiemolysis on horse blood agar plates. Hrmolyses human but not sheep's red cells. Nutritional : grows well in ordinary media; growth not improved by glucose. Green fluorescent colonies on MacConkey plate. Forms a fibrinolysin.

Biochemical.--Some strains produce acid, but no gas, in glucose, and occasionally in maltose. No fermentation of mannitol, lactose, sucrose or saliein; sometimes no acid produced at all. Indole - ; M.R. - ; V.P. - ; nitrates not reduced; $\mathrm{NH}_{3}$ - ; $\mathrm{H}_{2} \mathrm{~S}+++$; II.B. reduction - ; catalase + weak. Litmus milk : casein precipitated and digested; reduction; after 8 to 10 days it is transformed into a elear, amber-coloured fluid.

Antigenic Structure.-Agglutinating sera seem to act chrefly on homologous strains. Antitoxin can be prepared by injection of toxin into horses.

Pathogenicity.-Exotoxin is said to be formed in very young cultures. Natural pathogenicity doubtful; appears often in gangrenous processes in man. Experimentally, strains vary in pathogenicity; susceptible animals are guinea-pig, rabbit, and mouse. Bacillus is actively proteolytio and digests living tissue. $1 \mathrm{ml}$. of a young 
broth culture injected intramuscularly into a guinea-pig causes digestion of skin and muscles, and a hæmorrhagic liquefaction of the softer parts of the limb. This digestion may spread over the abdomen, and death occur during the next 12 to 24 hours; or recovery may follow with more or less complete necrosis of the limb.

The fluid contains no gas and is not putrid.

(See Weinberg and Séguin 1918, Report 1919, Hall 1922, Torrey 1925, Weinberg et al. 1926.)

\section{Clostridium botulinum}

Isolation.-By van Ermengem from ham in 1896 (1896, 1897). Several other organismhave since been isolated from botulism-like diseases in animals. These are some, times referred to as $\mathrm{Cl}$. parabotulinum, but it is probably better to give them letters using $\mathrm{A}$ and $\mathrm{B}$ to refer to the two main toxigenic types. Type $\mathrm{C}_{\alpha}$ was isolated by Bengtson $(1922 a, b, 1923)$ and by Graham and Boughton $(1923 a, b)$ in the United States from chickens and ducks; Type $\mathrm{C}_{\beta}$ by Seddon (1922) in Australia from cattle; Type D by Theiler and his colleagues (1926) in South Africa from cattle, and Type E by Theiler (1928) in South Africa from horses. The relation between Types C, D and E is not yet entirely clear, but Types $\mathrm{C}$ and $\mathrm{E}$ are said to be identical. Following description refers chiefly to Types $A$ and $B$.

Habitut.- Widely distributed in soil, both virgin and cultivated. Not infrequently present in intestinal tract of domestic animals.

Murphology.-4-day agar slope at $37^{\circ} \mathrm{C}$. Rather large, stout rods, $4-6 \mu \times 0.9 \mu$; axis straight, parallel sides, and slightly rounded ends; arranged singly, or sometimes in pairs or chains. Variations in depth of staining. Spores are oval, wider than the bacillus, thick-walled, and situated at or near the end. Free spores are numerous. Some strains spore readily, others hardly at all. Spores formed best in sugar-free media. Sluggishly motile by 4-8 peritrichate flagella. No capsule. Gram-positive in young cultures.

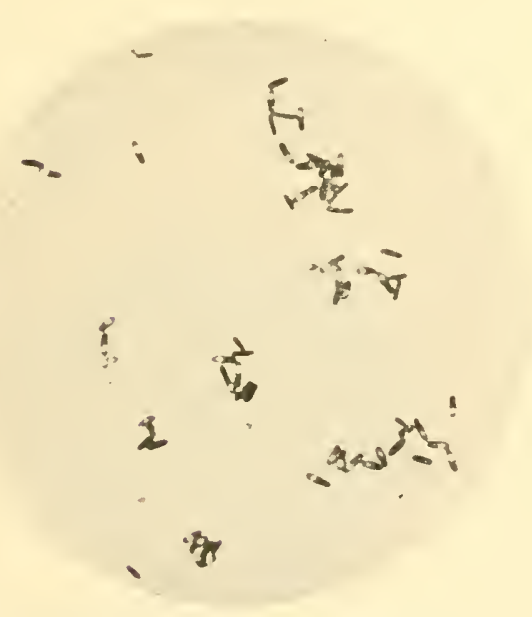

FIo. 197.-Clostridium botulinum.

From a surface agar culture anaerobically, 2 days, $37^{\circ} \mathrm{C} .(\times 1000)$.
Agar Plate. -4 days at $37^{\circ} \mathrm{C}$. Irregularly round, 5-10 $\mathrm{mm}$. in diameter, glistening, translucent, effuse, filamentous colonies, with an alternately smooth and granular surface (due to crossing of fila-

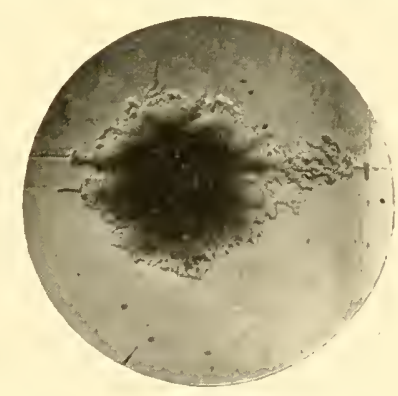

FIG. 198.-Clostridium botulinum.

Surface colony on agar, anaerobically, 2 days, $37^{\circ} \mathrm{C} .(\times 8)$.

ments), and an indefinite, fimbriate, reticular edge. Greyish-yellow by reflected, bluish-grey by transmitted light. Butyrous consistency, easily emulsifiable. Differentiated into thicker, browner centre, and thinner, more translucent peri- 
phery. Single colonies often difficnlt to obtain owing to tendency to spread. Variant types have been deseribed on blood agar (Schoenholz 1928).

Deep Glucose Agar Shake. I days at $37^{\circ} \mathrm{r}$. Abundant gas formation ; medium disrupted and driven up to plug. Colonies throughout medium, varying in size. Large colonies are 1-2 $\mathrm{mm}$. in diameter, having an opaque, brown, spherical or biconvex centre and a large, clear, more translucent, cigarette-ill-water edge. Another type consists of a thin, translucent disc with an eccentric opaque nucleus, the edge of the disc being indented at the point nearest the nucleus; the disc may contain gas bubbles. Types C, D and E form woolly colonies without a central nucleus.

Agar Slope. -4 days at $37^{\circ} \mathrm{C}$. Moderate, confluent, effuse, greyish-yellow, translucent growth with a very finely granular surface, and an irregular villous edge.

Gelatin. -7 days at $37^{\circ} \mathrm{C}$. Completely liquefied

Broth. -4 days at $37^{\circ} \mathrm{C}$. Abundant growth with dense turbidity and a moderate, powdery and granular deposit, mostly disintegrating. Rancid odour. Types C, D and E cause little turbidity, but form a flaky deposit, sticking to the sides of the tube. Loefler's Serum.-15 days at $37^{\circ} \mathrm{C}$. Moderate growth of small, discrete colonies; digestion by $\mathrm{A}$ and $\mathrm{B}$, but not by $\mathrm{C}$ and $\mathrm{D}$ types.

Horse Blood Agar Plates. -3 days at $37^{\circ} \mathrm{C}$. Irregularly round, $2-3 \mathrm{~mm}$. in diameter, umbonate colonies, with smooth centre and curled or fimbriate periphery; zone of hæmolysis, sometimes co-extensive with the colony, sometimes larger; around the colony blood is clear, transparent, and brown.

Coagulated Egg.-21 days at $37^{\circ} \mathrm{C}$. Very poor growth. Butt turned bluish-green, and egg partly digested by $\mathrm{A}$ and $\mathrm{B}$, but not by $\mathrm{C}$ and $\mathrm{D}$ types.

Cooked Meat Medium.-15 days at $37^{\circ} \mathrm{C}$. Abundant growth; gas produced; long column of slightly turbid fluid with digested meat beneath; blackening. Putrid odour. No digestion by $\mathrm{C}, \mathrm{D}$ or $\mathrm{E}$ types.

Resistance.-Spores are destroyed by dry heat at $180^{\circ} \mathrm{C}$. in 5 to 15 minutes. Moist heat at $100^{\circ} \mathrm{C}$. destroys them in 5 hours, at $105^{\circ} \mathrm{C}$. in 100 minutes, and at $120^{\circ} \mathrm{C}$. in 5 minutes (but see p. 1616). Gelatin cultures remain viable for a year or more.

Metabrlic.-Strict anaerobe. Opt. temp. $35^{\circ} \mathrm{C}$. Grows well at $20^{\circ} \mathrm{C}$. $\alpha$-prime hæmolysis on horse blood agar plates. Hæmolysis of human, but not of sheep's red cells. Types A and B are generally proteolytic, digesting gelatin, serum, egg, and meat; Types C and D digest gelatin only. Nutritional: grows fairly well on ordinary media; growth not improved by glucose; tryptophan and the sporogenes factor are both required. Green fluorescent colonies on MacConkey plates. Powerful exotoxin produced, specific to each type.

Biochemical.-Type A gives acid and gas in glueose, maltose and salicin; Types B and $\mathrm{C}$ do not ferment salicin. Types $\mathrm{A}$ and $\mathrm{B}$ ferment glycerol; Type $\mathrm{C}$ does not. Indole - ; M.R. - ; V.P. - ; nitrate reduction - ; $\mathrm{NH}_{3}+; \mathrm{H}_{2} \mathrm{~S}++$; methylene blue reduction - ; catalase - . Litmus milk: fine precipitate of casein, with almost complete digestion in a fortnight; litmus reduced, reaction alkaline.

Antigenic Structure.-Two main types, A and B, distinguished by their toxin production.

Antitoxin to $\mathrm{A}$ does not neutralize toxin of $\mathrm{B}$, nor vice versa. By agglutination and complement fixation the two types can also be distinguished, and are found to contain 3 or 4 sub-types each. Three other types, C, D and E, have been described forming separate specific toxins.

Puthogenicity.-Types A, $\mathrm{B}$ and E cause botulism in man. Type $\mathrm{C}_{\alpha}$ causes limberneck in ehickens and ducks; $\mathrm{C}_{\beta}$ causes one type of forage poisoning in horses in Australia and U.S.A.; Calso causes botulism in equines in South Africa ; D causes lamziekte in cattle in South Africa. (For fuller description see Chaptcr 72.) The organism itself is a saprophyte and does not multiply in the body; it acts entirely by its toxin. Injected subcutaneously, a broth culture of Type A or B is fatal to guinea-pigs, mice, rabbits, cats, monkeys, and often chickens in 1 to 4 days; symptoms are 
musenlar paralysis, dilatation of the pupils, shallow breathing, intense salivation, prostration and death. The toxin is the most powerful known, and may kill a mouse in a dose of $0.00001 \mathrm{ml}$.

(See Kempner 1897, v. Hibler 1908, Leuchs 1910, Dickson 1918, Weinberg and Séguin 1918, Burke 1919a, b, Graham and Brueckner 1919, Report 1919, Shippen 1919, Edmonson et al. 1920, Orr 1920, 1922, Bengtson 1921, 1922a, b, 1923, 1924a, Nevin 1921, Weiss 1921, Coleman 1922, 1923, Coleman and Meyer 1922, Dubovsky and Meyer $1922 a, b$, Esty and Meyer 1922, Hall 1922, Meyer and Dubovsky 1922a, $b$, $c$, Schoenholz and Meyer 1922, 1924, Seddon 1922, Tanner and Dack 1922, Graham and Boughton $1923 a, b$, Hall and Davis 1923, Starin and Daek 1923, 1924, 1925, Dozier 1924, Easton and Meyer 1924, Pfenninger 1924, Starin 1924, Wagner 1924, Wheeler and Humphreys 1924. Dickson et al. 1925, Tanner and Twohey 1926, Theiler et al. 1926, Weinberg and Ginsbourg 1927, Theiler 1928, Schoenholz 1928, Stark, Sherman, and Stark 1928, Robinson 1929, Graham and Thorp 1929, Lommel and Gunnison 1929, Kerrin 1930, Gunnison and Meyer 1930, Sommer and Sommer 1932, Gunnison and Coleman 1932, Fildes 1935, Gunnison, Cummings and Meyer 1936-37, Hazen 1937, 1942).

\section{Clostridium œdematiens}

Isolation.-Described by Weinberg and Séguin in 1915.

Synonyms.-Probably identical with Novy's B. oedematis maligni II, or Cl. novyi J (Novy 1894), Zeissler and Raszfeld's (1929) B. gigas, and Kraneveld's (1930) baeillus of osteomyelitis bacillosa bubalorum. Scott, Turner and Vawter (1933) propose the terms 'Type A, B and C for the classieal $C l$. oedematiens, $B$. gigas and Kranefeld's bacillus respectively.

Habitat.-Soil.

Morphology.-Prod-shaped, 3-10 $\mu \times 0.8-1 \cdot 0 \mu$, not unlike $C l$. welchii, but longer; 'Types B and $C$ strains may be as large as 4-20 $\mu \times 1-2 \mu$; sides parallel; ends rounded; axis straight or curved; arranged singly, in pairs or chains; jointed filaments not uncommon. Spores formed freely in all media; they are large, oval and subterminal, generally free. Notile by 20 or more peritrichate flagella; but motility is observed only under strictly anaerobic conditions. No capsule. Gram-positive in young cultures.

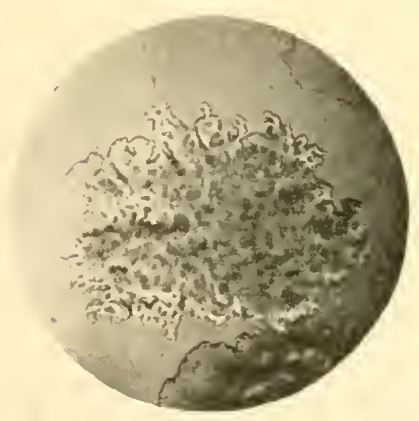

Fig. 199.-Clostridium øedema. tiens.

Surface colony on agar anaerobically, 2 days, $37^{\circ} \mathrm{C} .(\times 8)$.

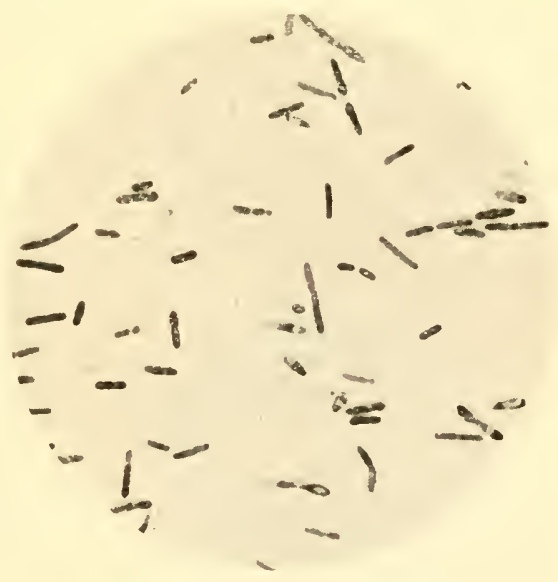

FIG. 200.-Cl. odematiens.

From a surface agar culture anaerobically 2 days, $37^{\circ} \mathrm{C} .(\times 1000)$.

Agar Plate.- 4 days at $37^{\circ} \mathrm{C}$. Irregnlarly round colonies, $2-3 \mathrm{~mm}$. in diameter, effuse, filamentous or curled, glistening, translucent with finely sponge-like surface and 
irregularly lobate edge with very fine dentations; greyish-yellow by reflected, greyish-blue by transmitted light; butyrous and easily emulsifiable.

Deep Glucose Agar Shake. -4 days at $37^{\circ} \mathrm{C}$. Good growth, gas produced, and agar disrupted. Colonies throughout medium, varying in appearanee; usually resemble snowflakes; some have an opaque, brownish centre with a finely filamentous or fluffy periphery ; some have the appearance of a conventional bursting grenade.

Horse Blood Agar Plates. -3 days at $37^{\circ} \mathrm{C}$. Large spreading colonies, as on nutrient agar; and round, slightly umbonate colonies, with entire or undulate edge and a finely granular surface; about $3 \mathrm{~mm}$. in diameter. Zone of $\beta$-hæmolysis coincident with colony. Centre of colony is more opaque, periphery more translucent. Agar Slope.-4 days at $37^{\circ} \mathrm{C}$. Thin spreading film of growth, or poor to moderate, partly confluent, slightly raised, glistening, greyish-yellow growth with finely granular surface, and an edge made up of single colonies.

Gelatin.-3 days at $37^{\circ} \mathrm{C}$. Liquefaction.

Broth.-4 days at $37^{\circ} \mathrm{C}$. Poor growth with no turbidity and a granulo-powdery deposit, partly disintegrating; slight rancid odour. In glucose broth there is an early turbidity, which clears after a day or two with the deposit of the organisms in a flocculent mass.

Loeffler's Serum.--15 days at $37^{\circ} \mathrm{C}$. Moderate, confluent, slightly raised growth; no digestion.

Coagulated Egg.-15 days at $37^{\circ} \mathrm{C}$. No digestion.

Cooked Meat Medium.-15 days at $37^{\circ} \mathrm{C}$. Moderategrowth ; fluid turbid; gas produced, meat turned slightly pink or bleached; no digestion; rancid odour.

Resistance.-Destroyed by moist heat at $105^{\circ} \mathrm{C}$. in 6 minutes.

Metabolic.-Strict anaerobe. Opt. temp. $37^{\circ} \mathrm{C} . \quad \beta$-hæmolysis on horse blood agar plates. Hæmolyses human and sheep's red cells. Nutritional : grows fairly well in ordinary media; growth improved by glucose. No growth in bile-salt media. Toxin produced. Forms a fibrinolysin.

Biochemical.-Acid and gas in glucose, and maltose, not in mannitol, lactose, sucrose, or salicin. Glycerol fermented by Type A but not by Type B or C strains. Indole - ; I.R. - ; V.P. - ; nitrites not produced in nitrate broth; $\mathrm{NH}_{3}-; \mathrm{H}_{2} \mathrm{~S}+$; II.B. reduction - ; catalase - . Litmus milk: acid production in 1 to 5 days with gas formation; after 10 to 30 days a clot appears in the form of fine flocculi. No digestion.

Antigenic Structure.-Agglutinins act on homologous strains only; auto-agglutination frequent. Antitoxin can be produced by injection of horses.

Pathogenicity.-Produces a potent exotoxin. One agent in causation of gas gangrene in man. Responsible for one type of braxy in Europe, for black disease in Australia, and for a non-fatal osteomyelitis of the humerus and femur of Dutch East Indian buffaloes. Experimentally it is pathogenic for guinea-pigs, mice and rabbits. 0.25-1 $\mathrm{ml}$. of a 24-hour broth culture injected intramuscularly into a guinea-pig causes death in 24 to 48 hours. P.II. muscles are red and softened; little gas production, but a spreading gelatinous cedema. Bacilli found at site of inoculation, and occasionally on surface of liver. Blood cultures may or may not be positive. (See p. 871.)

(See Weinberg and Séguin 1918, Report 1919, Wolf 1919-20, Hall 1922, Turner 1930, Mieszner, Meyn and Schoop 1931, Kraneveld 1930, Zeissler and Kraneveld 1929, Kraneveld and Djaenoedin 1933, Scott, Turner and Vawter 1933, Djaenoedin and Kraneveld 1936, Turner and Eales 1941.)

\section{Clostridium chauvœi}

Isolation.-First distinctive description by Arloing, Cornevin and Thomas in 1879 (Arloing et al. 1887).

Habitat.-Lives in soil. 
Morphology.-4-day agar plate at $37^{\circ} \mathrm{C}$. Rod-shaped, 3-8 $\mu \times 0 \cdot 6 \mu$, with parallel sides and rounded ends. Short filaments are not uncommon. On serum or in meat medium navicular and swollen forms are seen. Axis straight or slightly curved; arranged singly, in small gromps, or in short chains. Variation in depth of staining; some organisms show chromatic granules near the poles. Spores are elongated oval, subterminal, and wider than the bacillus. Clostridial forms are lemon- or pear-shaped. Motile. Gram-positive, but weakly so after 4 days. No capsule. On surface of liver of infected animals it is found singly or in pairs, not in chains or filaments like $\mathrm{Cl}$. septicum.

Agar Plate. -4 days at $37^{\circ} \mathrm{C}$. Irregularly round, $4-8 \mathrm{~mm}$. in diameter, granulo-filamentous, effuse, transparent colonies, difficult to see; surface is glistening and very finely granular ; edge is fern-like and irregularly dentate ; greyish by reflected, and bluish-grey by transmitted light; consistency butyrous, easily emulsifiable; no differentiation.

Deep Glucose Agar Shake. - 4 days at $37^{\circ} \mathrm{C}$. Abundant growth of diserete colonies throughout medium, exeept for $3 \mathrm{~mm}$. below surface. Colonies are irregularly round, 0.5-1 $\mathrm{mm}$. in diameter, with an opaque brownish centre and a lighter, translucent, plumose periphery, with an irregularly erose edge. Moderate gas formation; medium split slightly in 4 or 5 places.

IIorse Blood Agar Plate. -3 days at $37^{\circ} \mathrm{C}$. Colonies irregularly round, $3-6 \mathrm{~mm}$. in diameter, effuse, transparent, with granulo-filamentous structure, and entirc or rhizoid edge. No definite hæmolytic zone, but plate is cleared slightly.

Agar Slope. 4 days at $37^{\circ} \mathrm{C}$. Poor, confluent, effuse, transparent growth, difficult to see. Surface smooth or very finely granular, edge fern-like and irregularly dentate. Gelatin.-Liquefaction complete in 14 days at $37^{\circ} \mathrm{C}$.

Broth.-4 days at $37^{\circ} \mathrm{C}$. Poor growth, no turbidity, slight powdery deposit disintegrating on shaking; weakly rancid odour. In young cultures a turbidity is noticeable, but this clears as the bacilli sediment.

Loeffler's Serum.-15 days at $37^{\circ} \mathrm{C}$. Effuse, confluent growth; no digestion.

Coagulated Egg. - 15 days at $37^{\circ} \mathrm{C}$. Poor, effuse, confluent growth; no digestion.

Cooked Meat Medium.-15 days at $37^{\circ} \mathrm{C}$. Very slight or no turbidity; some gas production; meat turned pink. Raneid odour.

Resistance.-Dried on silk threads spores are destroyed by steam in 38 to 48 minutes.

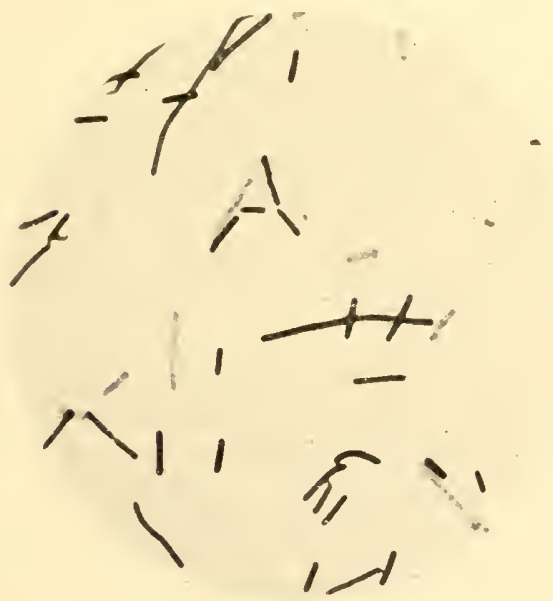

Fia. 201.-Clostridium chauvoi.

From a surface agar culture, anaerobically, 2 days, $37^{\circ} \mathrm{C} .(\times 1000)$.
Metabolic.-Strict anaerobe. On the whole grows poorly. Opt. temp. $37^{\circ} \mathrm{C}$. Very slight hæmolysis on horse blood agar plates. Hæmolyses human and sheep's red cells. Forms a fibrinolysin.

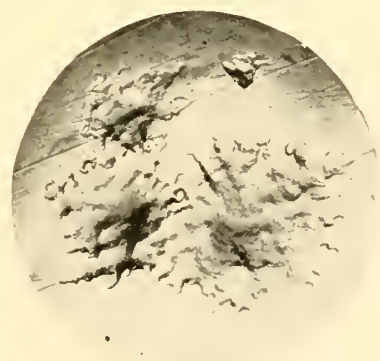

FIG. 202.-Clostridium chauvoi.

Surface colonies on agar anaerobi. cally, 6 days, $37^{\circ} \mathrm{C} .(\times 8)$. 
Nutritional.-Grows poorly in ordinary media; growth improved by glucose, and by heart extract; very poor growth in easein digest broth. No growth in bilesalt media. Weak toxin produced.

Biochemical.-Acid and gas in glucose, maltose, lactose, and suerose, not in mannitol or salicin. Indole - ; II.R. - ; V.P. - ; nitrate reduction +; $\mathrm{NH}_{3}-; \mathrm{H}_{2} \mathrm{~S}+$; M.B. reduction - ; catalase - Litmus milk: variable; sometimes no change; sometimes slight acid production, and partial precipitation of casein.

Antigenic Structure.-Appears by agglutination to be serologically homogeneous. Agglutinating sera prepared against $\mathrm{Cl}$. chauvoei agglutinate this organism, but do not agglutinate $\mathrm{Cl}$. septicum except to very low titre. By complement fixation $\mathrm{Cl}$. chauvoi and $\mathrm{Cl}$. septicum appear to be closely related. Antitoxin is specific, protecting against $\mathrm{Cl}$. chanv:ei but not against $\mathrm{Cl}$. septicum.

Pathogenicity.-Exotoxin produced. Causes blackleg in cattle, and less often in sheep. Non-pathogenic to man. Experimentally it is fatal to guinea-pigs and less often to mice; rabbits and pigeons are fairly resistant. $0.25 \mathrm{ml}$. of a 24 -hour culture in Hibler's medium injected intramuscularly kills a guinea-pig in 24 to 48 hours; p.m. slightly blood-stained serous exudate at site of injection; abdominal muscles are deep red and contain numerous small gas bubbles. Cl. chan wei can be recovered from local lesion, peritoneal cavity, and heart blood.

(See Kitt 1887, Nocard and Roux 1887, Roux 1858, Kitasato 1889a, 1890, Leclainche and Vallée 1900, Eisenberg 1907, Markoff 1911, Landau 1917, Weinberg and Séguin 1918, Haslam and Lumb 1919, Report 1919, Heller 1920, Goss et al. 1921, Gaiger 1922, 1924, Hall 1922, Weinberg and Ginsbourg 1927, Weinberg and Mihailesco 1929, Roberts 1931, Hendersoln 1932, Kerrin 1934.)

\section{Clostridium septicum}

Isolation.-Described by Pasteur and Joubert in $\mathbf{1 8 7 7 .}$

Synonyms. - B. oedematis maligni, Koeh (I881). Vibrion septique, Pasteur.

Habitut.-Found ehiefly in soil.

Morphology.-Rod-shaped, of variable length and thickness; on agar cultures, $2-6 \mu \times$ 0.4-0.6 $\mu$; sides parallel, ends rounded, axis straight or curved, arranged singly, in pairs, and in short ehains. On peritoneal surface of dead guinea-pig it forms long jointed filaments. In tissue exudates and in fluid media containing fresh tissue there are navicular or eitron forms with pale swollen bodies and deeperstaining pointed extremities. In agar cultures there is marked pleomorphism; organisms vary in size, shape, and depth of staining, large numbers of shadow forms are seen. Spores readily formed, and are oval, subterminal, and slightly wider than bacilli; often found free. Motile by 4-16 peritrichate flagella. No capsule. Gram-positive in young cultures, but often frankly Gram-negative in 4 to 5 days.

Agar Plate. -4 days at $37^{\circ} \mathrm{C}$. Irregularly round, having a general cigarette-in-water appearance, $10 \mathrm{~mm}$. in diameter, effuse, filamentous, translucent colonies, with finely honeycombed surface due to crossing of numerous filaments, and fimbriate edge; greyish by reflected, bluish-grey by transmitted light; butyrous and easily emulsifiable. No definite differentiation but filaments are less dense at periphery. Recently isolated strains tend to form continuous spreading films.

Deep Glucose Agar Shake.-4 days at $37^{\circ} \mathrm{C}$. Abundant gas formation; nedium disrupted and driven up nearly to plug. Numerous colonies throughout medium, varying in appearanee; most usual type is delicate, arboreseent, and floeculent ; sometimes opaque with an irregularly dentate, well-defined edge, from which later woolly filamentous outshoots appear.

Horse Blood Agar Plates. -3 days at $37^{\circ}$ C. $\quad \alpha$-prime hæmolysis; after 6 days hæmolysis is of $\beta$-type. 


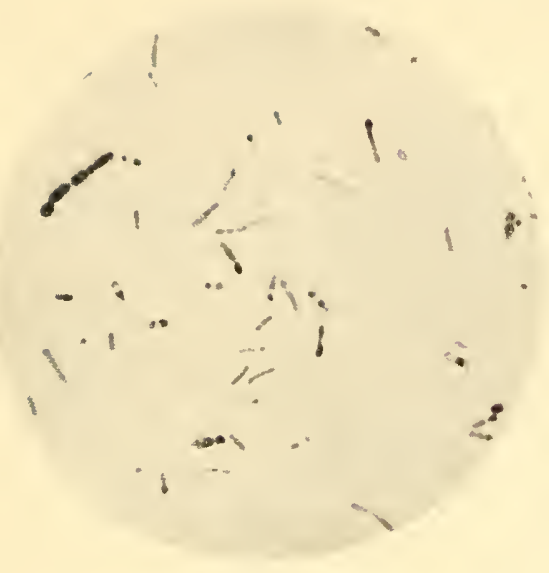

FIG. 203.-Cl. septicum.

From a surface agar culture anaerobically, 2 days, $37^{\circ} \mathrm{C} .(\times 1000)$.

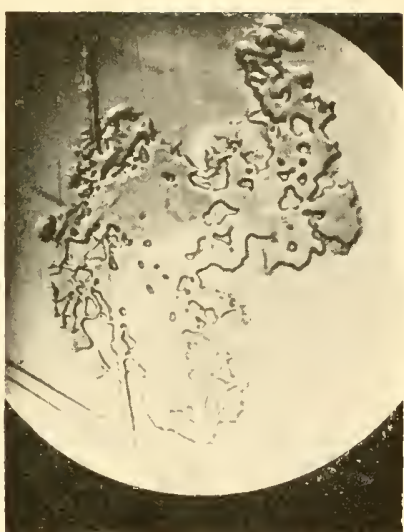

FIG. 204.-Clostridium septicum.

Surface colony on agar anaerobically, 2 days, $37^{\circ} \mathrm{C} .(\times 8)$.

Agar Slope.-4 days at $37^{\circ} \mathrm{C}$. Scanty to moderate, effuse, translucent, glistening, greyishyellow growth, forming little islands, each with a coarsely erose edge; surface smooth or very finely granular.

Gelatin. -7 days at $37^{\circ} \mathrm{C}$. Liquefied.

Broth.-4 days at $37^{\circ} \mathrm{C}$. Poor to moderate growth with slight turbidity and a moderate, powdery deposit disintegrating completely; slight rancid odour.

Loeffler's Serum.-15 days at $37^{\circ} \mathrm{C}$. Fairly good confluent growth; no liquefaction.

Coagulated Egg. -15 days at $37^{\circ} \mathrm{C}$. Fairly good, partly confluent growth, with a moderately granular surface; no digestion.

Cooked Meat Medium.-15 days at $37^{\circ} \mathrm{C}$. Moderate growth with slight turbidity; gas production; meat turned pink; no digestion; rancid odour.

Resistance.-Not recorded.

Metabolic.- Strict anaerobe. Opt. temp. $37^{\circ} \mathrm{C} . \alpha$-prime, and later $\beta$-hæmolysis on horse blood agar plates. Hæmolyses human and sheep's red cells. Nutritional: grows fairly well on ordinary media; growth improved by glucose. Green fluorescent colonies on MacConkey plate. Toxin produced. Forms a fibrinolysin.

Biochemical.-Acid and gas in glucose, maltose, lactose and salicin, not in mannitol or sucrose. Indole - ; M.R. - ; V.P. - ; nitrates reduced; $\mathrm{NH}_{3}$ slight $+; \mathrm{H}_{2} \mathrm{~S}+$; M.B. reduction - ; catalase -. Litmus milk : acid and elot and some gas; the clot does not form for 3 to 6 days.

Antigenic Structure.-By agglutination four groups can be distinguished on basis of $O$ antigen; further subdivision is possible on basis of $\mathrm{H}$ antigen. Some cross-agglutination and much cross-complement-fixation with $\mathrm{Cl}$. chauvoi strains. Antitoxin appears to be specific.

Pathogenicity.-Exotoxin produced. One agent in production of gas gangrene in man. Causes blackleg and braxy in sheep, and sometimes blackleg in cattle. Experimentally, it is pathogenic to guinea-pigs, mice, rabbits, and pigeons. Pathogenicity is retained for years in subculture. $0 \cdot 01-0.5 \mathrm{ml}$. of a 24-hour glucose broth culture injected intramuscularly into gainea-pigs causes death in 12 to 24 hours. P.M. blood-stained oodema and gas production; muscles intense deep red in colour and softened; sometimes fluid in peritoneum and pericardium. Motile rods and navicular forms at site of injection, and long, jointed, snake-like filaments on peritoneal surface of liver. (See p. 871.) 
(See ron Hibler 1908, Meyer 1915, Weinberg and Séguin 1918, Wolf 1918-19, Report 1919, Heller 1920, Gaiger 1922, 1924, Hall 1922, Weinberg and Ginsbourg 1927, Henderson 1934.)

\section{Clostridium welchii}

Synonyms.-B. aerogenes capsulatus, B. phlegmonis emphysematosa, B. perfringens, $B$. saccharobutyricus immobilis, $B$. enteritidis sporogenes, Granulobacillus butyricus. $B$. cadaveris butyricus, $B$. vagince emphysematosa, Achalme's bacillus.

Isolation.-First complete description by Welch and Nuttall in 1892, who isolated it from a cadaver. Various organisms closely resembling $\mathrm{Cl}$. welchii, but differing from it in type of toxin production, have been isolated from diseased sheep, and called the lamb dysentery bacillus (Dalling 1926), Cl. paludis (McEwen 1930), and Cl. ovitoxicum (Bennetts 1932). Exact relation of these organisms to $\mathrm{Cl}$. welchii and to one another is doubtful, but Wilsdon's (1931) classification into A, B, C, and D types corresponding respectively to the welchii, lamb dysentery, paludis, and ovitoxicum types may be accepted provisionally.

Habitat.-Found in soil, water, milk, dust, sewage, and intestinal canal of man and animals.
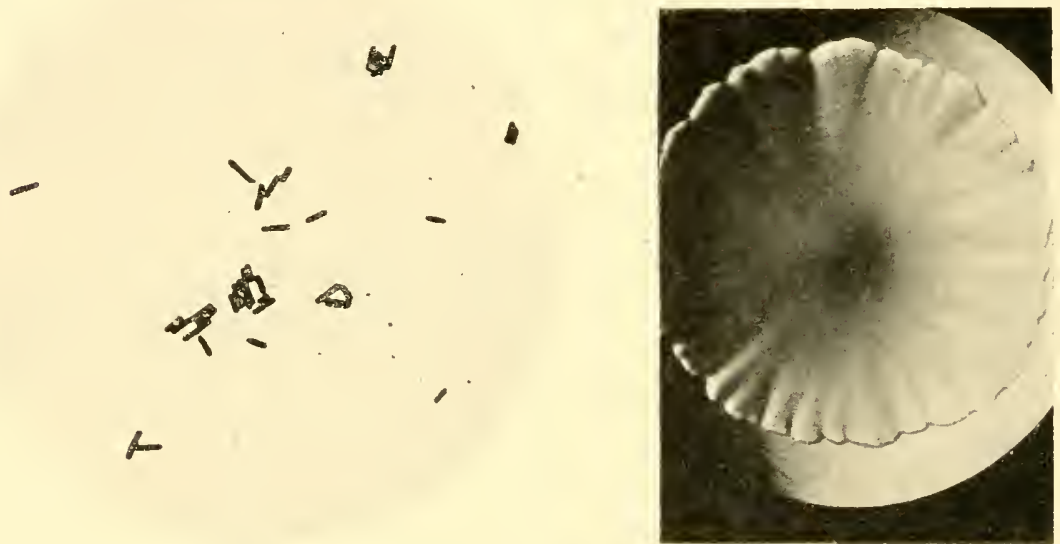

Fı. 205.-Clostridium welchii.

From a surface agar culture, anaerobically, 2 days, $37^{\circ} \mathrm{C} .(\times 1000)$.

F10. 206.-Clostridium welchii.

Surface colony on agar anaerobically, 5 days, $37^{\circ} \mathrm{C} .(\times 8)$.

Morphology.-Rather stout rods, varying considerably in length; 4-8 $\mu \times 0.8-1 \cdot 0 \mu$; sometimes shorter and more slender; filaments not uncommon. Parallel sides, ends truncated or slightly rounded; axis straight. Arranged singly, often side by side forming small bundles. Variation in depth of staining; involution forms - clubs, filaments, tadpoles, granular forms-frequent in old cultures. Spores large, oval, and subterminal. Sporulation occurs more readily with some strains than with others, and is favoured by an alkaline reaction; does not occur below $\mathrm{pH} 6 \cdot 6$, and hence is unusual in media containing a fermentable carbohydrate. Spores are seldom seen in cultures, but are commonly formed under natural conditions. Non-motile. Capsules formed in animal body.

Agar Plate. 4 days at $37^{\circ} \mathrm{C}$. 2 main types of colony formed. One round, $2-4 \mathrm{~mm}$. in diameter, low convex, amorphous, greyish-yellow, opaque, with smooth surface and entire edge; butyrous and easily emulsifiable. Other is umbonate, and is differentiated into an opaque brownish centre and a lighter, more translucent, 
radially striated periphery with a crenated edge (Fig. 206). Other variant forms have been described, differing in morphology, colonial appearance, and sometimes toxicity (Sordelli, Prado, and Ferrari 1932, McGaughey 1933, Livesay 1933, Stevens 1935).

Deep Glucose Agar Shake.-4 days at $37^{\circ} \mathrm{C}$. Abundant gas, medium ruptured and driven nearly to plug. Numerous colonies throughout medium; they are biconvex, $1 \mathrm{~mm}$. long, opaque, with an entire edge.

Horsc Blood Agar. -3 days at $37^{\circ} \mathrm{C}$. Round colonies, 2-5 nm. in diameter, umbonate, greyish-white, with opaque raised centre, and a translucent flattened periphery; surface smooth ; entire edge. Zone of $\beta$-hæmolysis for $1-3 \mathrm{~mm}$. around colony in most strains (see p. 861).

Agar Slope. -4 days at $37^{\circ} \mathrm{C}$. Good growth consisting of discrete colonies.

Gelatin. 2 days at $37^{\circ} \mathrm{C}$. Complete liquefaction.

Broth. -4 days at $37^{\circ} \mathrm{C}$. Good growth with moderate turbidity, and moderate powdery deposit, disintegrating completely. Slight sour odour.

Loeffler's Serum.-15 days at $37^{\circ} \mathrm{C}$. Good confluent, slightly raised growth with crenated edge; no digestion.

Coagulated Egg. -15 days at $37^{\circ} \mathrm{C}$. Fairly good, confluent, slightly raised growth; no digestion.

Cooked Meat Medium.-15 days at $37^{\circ} \mathrm{C}$. Good growth; fluid slightly turbid; gas evolved; meat turned pink; no digestion; acid reaction; sour odour.

liesistance.-Cultures in fermentable carbohydrate media die in a few days owing to the effect of the acid produced. In sugar-free protein media, in which spores have formed, the organisms may live for months. A suspension containing a million spores per ml. is sterilized in 30 minutes at $90^{\circ} \mathrm{C}$. and in 5 minutes or less at $100^{\circ} \mathrm{C}$. (Headlee 1931).

Metabolic.-Fairly strict anaerobe. Opt. temp. $37^{\circ} \mathrm{C}$. Hæmolysins and leucocidins are formed by some, but not by all strains. Usually gives $\beta$-hæmolysis on horse blood agar plates; hæmolyses human and sheep's red cells. Nutritional: grows fairly well on ordinary media; growth greatly improved by I per cent. glucose. Green fluorescence on MacConkey plate. Toxin produced. Forms a fibrinolysin.

Biochemical.-Acid and gas in glucuse, maltose, lactose, sucrose, and occasionally salicin ; not in mannitol ; some strains ferment imulin, some glyeerol. Indole - ; M.R. + ; V.P. - ; nitrates slight reduction; $\mathrm{NH}_{3}$ slight + ; $\mathrm{H}_{2} \mathrm{~S}++$; M.B. reduction - ; eatalase - Litmus milk : acid, gas, clot--stormy fermentation-occurring in 12 to 48 hours in a proportion of strains. Type A, C, and D strains form acrolein from glycerol.

Antigenic Structure.-By agglutination no elear-eut grouping is apparent; considerable overlapping of antigens. No obvious relation between agglutination results and typing of strains by toxin-antitoxin or biochemical methods. Antitoxic serum can be readily prepared in horses.

Pathogenicity. - Apparently 7 different exotoxic substances produced, of which up to 5 may be produced by one strain. Chief agent in causation of gas gangrene in man. May play a part in causation of enteritis, appendicitis, and puerperal fever. Causes gas gangrene in animals, especially sheep. Experimentally, great

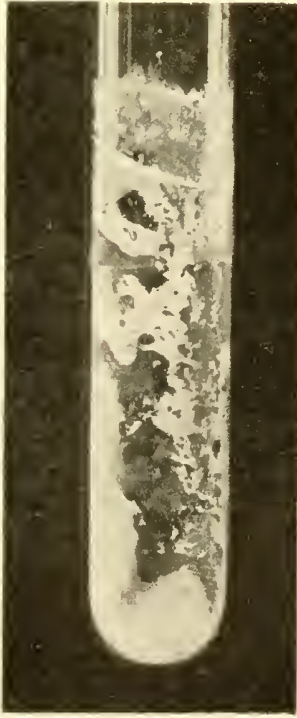

F10. 207.-Clostridium welchii.

Culture in litmus milk anaerobically, $\quad 24$ hours, $37^{\circ} \mathrm{C}$. , showing stormy fermentation. 
variation in pathogenicity of different strains. Washed bacilli or spores are nonpathogenic. $0 \cdot 1-1 \cdot 0 \mathrm{ml}$. broth culture injected intramuscularly into guinea-pig causes local tumefaction, spreading œdema, and death in 24 to 48 hours. P.M. suprarenal glands congested. Also pathogenic to mice, pigeons, and less so to rabbits (see p. 870). B type is responsible for lamb dysentery, C type for "struck"-an enteritis of shee $\mathrm{P}$-and D type for an enterotoxæmic disease and for pulpy kidney disease of sheep (see Chapter 78).

(See Kamen 1904, Simonds 1915a, b, c, Robertson 1916, Bull 1917, Bull and Pritchett 1917a, b, De Kruif and Bollman 1917, De Kruif et al. 1917, Weinberg and Séguin 1918, Report 1919, Bengtson 1920, ('aulfeild 1920, Hall 1922, Humphreys 1924, Dalling 1926, Weinberg and Ginsbourg 1927, Howard 1928, Weinberg 1929, Weinberg and Combiesco 1930, McEwen 1930, Torrey, Kahn and Salinger 1930, Headlee 1931, Mason, Ross and Dalling 1931, Wilsdon 1931, 1933, Bennetts 1932, Glenny et al. 1933, Walbum and Reymann 1933, McGaughey 1933, Livesay 1933, Weinberg and Guillaumie 1936.)

\section{Clostridium tetani}

Isolation.-Deseribed by Nicolaier in 1884; isolated by Kitasato in 1889 (1889b).

Ilabitat.-Found in soil-especially cultivated soil-and in the intestine of man and animals.

Morphology.-4-day agar slope at $37^{\circ} \mathrm{C}$. Rods, $2-5 \mu \times 0.5 \mu$; eonsiderable variation in length; long, curved, filamentous forms are not uncommon. Axis straight, sides parallel, ends rounded; arranged singly and occasionally in chains. Variation in depth of staining. Spores spherical, terminal, and wider than the bacillus, giving characteristic drum-stick appearance. Sluggishly motile; peritrichate flagella. No capsule. Strongly Gram-positive in young eultures. In early stages, spores stain solidly; later, only the thin wall stains; spores rarely become free. Bizarre involution forms appear in old cultures.

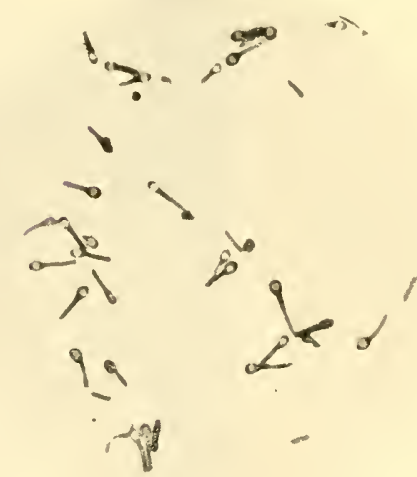

FIG. 208.-Clostridium tetani.

From a surface agar eulture anaerobically, 7 days, $37^{\circ} \mathrm{C}$., showing ring form of staining $(\times 1000)$.

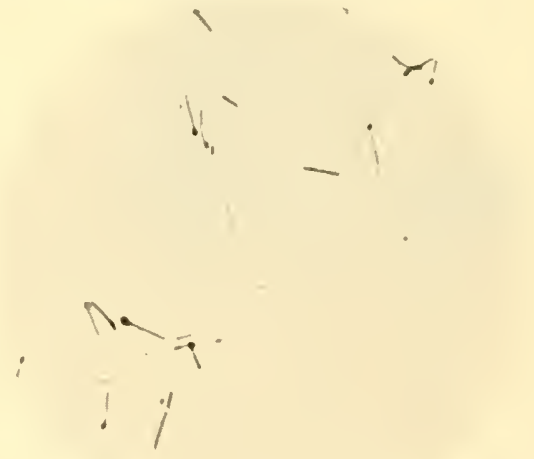

F1G. 209.-Clostridium tetani.

Froma broth eulture anaerobically, 7 days, $37^{\circ} \mathrm{C}$., showing the spores stained solidly $(\times 1000)$.

Agar Plate.-4 lays at $37^{\circ} \mathrm{C}$. Irregularly round, 2-5 mm. in diameter, effuse, glistening, translucent, greyish-yellow colonies with irregularly granular surface, and illdefined edge, showing filamentous, curled projections; structure very finely granular or filamentous; butyrous consistency, emulsifying easily. Some strains form colonies differentiated into thicker, translucent, yellowish-brown centre, and thimner, transparent, almost colourless periphery. Whole colony has 
a fuzzy appearance. Isolated colonies of the normal motile type of $\mathrm{Cl}$. tetani are extremely difficult to obtain, owing to the tendency of the organisms to spread in a proteus-like or a delicately rhizoidal film over the surface of the agar; but it is stated that non-motile variants give rise to separate discrete colonies, even on moist media.

Deep Glucose Agar Shake.-4 days at $37^{\circ} \mathrm{C}$. Slight gas formation; medium disrupted in 2 or 3 places. Colonies scattered thronghont medium, except at surface; they are romided, 1-2 mm. in diameter, with sometimes a brownish opaque centre and a lighter more transhucent periphery; fuzzy, filamentous, branching edge.

Agar Slope. 4 days at $37^{\circ} \mathrm{C}$. Scanty to moderate, greyish-yellow, almost transparent, effuse growth, stretching to sides of tube, with very finely granular, glistening surface. If inoculated into condensation water, spreads rapidly over the whole surface of the agar, and presents a filamentous edge at the top.

Gelatin Stab.-10 days at $23^{\circ} \mathrm{C}$. Poor growth of fir-tree appearance. At $37^{\circ} \mathrm{C}$. liquefies gelatin in a week.

Broth.-4 days at $37^{\circ} \mathrm{C}$. Poor to moderate growth with slight turbidity, and a finely granular deposit, not disintegrating on shaking. Odour of manure.

Loeffer's Serum. -4 days at $37^{\circ} \mathrm{C}$. Poor, effuse, mostly discrete growth; serum may be softened or disintegrated after several days, but no true liquefaction occurs.

Horse Blood Agar Plates. -3 days at $37^{\circ} \mathrm{C}$. Rounded,

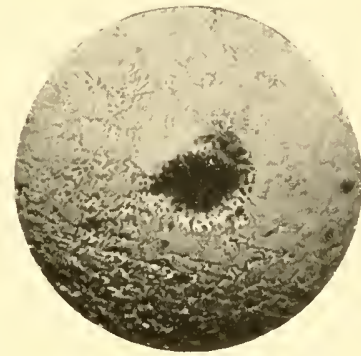

F19. 210.-Clostridium tetani.

Surface colony on agar anaerobically, 2 days, $37^{\circ} \mathrm{C}$., show ing parent colony surrounded by an effuse outgrowth $(\times 8)$. $2-5 \mathrm{~mm}$. in diameter, effuse, greyish, translucent colonies, with finely granular surface and lobate or fimbriate edge. Sometimes zone of $\alpha$-prime hæmolysis, diam., 1-5 mm., later passing into the $\beta$-type.

Coagulated Egg.-4 days at $37^{\circ} \mathrm{C}$. Poor, effuse, partly confluent growth; no digestion. Cooked Meat Medium. -4 days at $37^{\circ} \mathrm{C}$. Good growth; fluid shows moderate turbidity; some gas production; no blackening, and no digestion of meat. Slight blackening is said to occur after some weeks.

Resistance.-Spores resist boiling for 15 to 90 minutes. Killed at $105^{\circ} \mathrm{C}$. in 3 to 25 minutes; killed by 5 per cent. phenol in 15 hours.

Metabolic.-Strict anaerobe. Opt. temp. $37^{\circ} \mathrm{C}$. Grows poorly at $20^{\circ} \mathrm{C}$. $\alpha$-prime or $\beta$-hæmolysis on blood agar plates. Hæmolysin acts on R.B.C. of rabbit, goat, sheep, horse, and many other animals. Nutritional: grows fairly well on ordinary media; growth improved by blood and serum; not by glucose. TuIloch's exhausted medium is particularly suitable. Produces green fluorescence on MacConkey's agar. Possesses slight proteolytic properties.

Biochemical.-Ferments no sugars. Indole + ; M.R. - ; V.P. - ; nitrites not produced in nitrate broth ; $\mathrm{NH}_{3}$ slight $+; \mathrm{H}_{2} \mathrm{~S}-$; M.B. reduction - ; catalase -. Litmus milk: reduction of litmus, usually slight precipitation of casein, sometimes no change. Said to form phenol.

Antigenic Structure.-Differentiated by agglutination into at least 10 types, of which Types I and III appear to be the commonest in this country. Toxin formed by all types is identical and is neutralized by antitoxin prepared against any one type. Anti. bacterial sera contain agglutinins and opsonins specific to each type.

Pathogenicity.-Potent exotoxin formed. Naturally pathogenic to man and horses in particular. Experimentally, mice, guinea-pigs, and rabbits are susceptible, dying of tetanus in 1 to 4 days after subcutaneous irjection of a broth culture. Washed spores or bacilli are innocuous. The toxin is very potent, and will kill a mouse in a dose of $0.0001 \mathrm{ml}$. Birds are resistant. (See Chapter 77.) 
(See Rosenbach 1886, Behring and Kitasato 1890, Kitasato and Weyl 1890, Kitasato 1891, Toledo and Veillon 1891, Behring 1892, Vaillard and Rouget 1892, Usohinsky 1893, Pizzini 1898, Madsen 1899, Ritehie 1901, Rosenau and Anderson 1908, von Hibler 1908, Metchnikoff 1908, Smith 1908, Noble 1915, Corbitt 1918, Weinberg and Séguin 1918, Report 1919, Tulloch 1919, Hall 1922, Heller 1922a, b, Anderson 1924, Bengtson 1924b, Deseombey 1924, Fildes 1925 $a$, $b$, Weinberg and Ginsbourg 1927, Tschertkow 1929, Fildes 1929, Knight and Fildes 1930, Kerrin 1930, 1934, Belin 1931, Mutermilch et al. 1933.)

\section{Notes on Less Important Strains (see Table 57).}

Cl. aerofœtidum.-Deseribed by Weinberg and Séguin (1918). Small slender bacillus, 3-5 $\mu$ long, slightly motile, and weakly Gram-positive. Spores are subterminal, and are not readily formed. Surface colonies are round and transparent; deep colonies are small and irregular. Non-pathogenic to guinea-pigs.

Cl. bifermentans.-Isolated in 1902 by Tissier and Martelly from putrefying meat, and named $B$. bifermentans sporogenes. So called from its being the first anaerobe shown to decompose both sugars and proteins. Probably identical with $\mathrm{Cl}$. centrosporogenes, described by Hall (1922), McCoy and McClung (1936) and Clark and Hall (1937), and, according to Stewart (1938), with Cl. sordellii (synonym B. oedematis sporogenes) isolated by Sordelli $(1922,1923)$ in Buenos Aires from a human case of gas gangrene (see also Weinberg, Davesne and Lefranc 1931, Hall and Scott 1927). Gram-positive rod, $3 \cdot 6-6 \cdot 0 \mu \times 1 \cdot 2-1.5 \mu$, motile by peritrichate flagella, readily forming central or subterminal oval spores. Uniform turbidity in broth with filament production, and viscous deposit, which is easily disintegrated on shaking; nauseous odour. Surface colonies on horse blood agar are round, crenated, or irregular, and occasionally hæmolytic ; deep colonies are round or irregular and opaque with a woolly periphery. Gelatin liquefied rapidly. Coagulated serum disintegrated or liquefied. Cooked meat medium digested, blackened, with a late deposit of small rounded masses of white crystals. Ferments glucose and maltose, but not lactose, saccharose, or mannitol. Forms a fibrinolysin. Pathogenic strains (equivalent to $\mathrm{Cl}$. sordellii) produce a fairly powerful toxin which is destroyed by $30^{\circ} \mathrm{C}$. in 60 minutes. Both toxigenie and non-toxigenic strains produce a lecithinase, specifically neutralized by the $\alpha$-antitoxin of $\mathrm{Cl}$. welchii (Hayward 1943). $0.01 \mathrm{ml}$. of a 48 -hour glucose broth culture of a pathogenic strain injected intramuscularly kills a gninea-pig in 2 days with lesions similar to those eauscd by $\mathrm{Cl}$. oedematiens. Appears to be morphologically, culturally and antigenically more or less identical with $\mathrm{Cl}$. adematoides, an organism isolated from a wound infection by Heleney, Humphreys and Carp (1926-27, 1927). (See Humphreys and Meleney 1927-28, Hall, Rymer and Jungherr 1929, Hall and Scott 1931, Levenson 1936.)

CI. capitovale.--Isolated by Snyder and Hall (1935) from various situations including the pleural fluid of a sheep that had died of gas gangrene, the heart blood and peritoneal fluid at post mortem of cases of septic infection in human beings, and the fæces of normal infants. It is a slender, motile, Gram-positive rod with rounded ends, measuring $2.0-2.5 \mu \times 0.5-() \cdot 8 \mu$, and forming terminal oval spores. It gives rise on blood agar to minute, cireular or irregularly circular, transparent, non-hæmolytie surface eolonies. It produces acid and gas in glucose, but not in maltose, mannitol, lactose, sucrose, or salicin. It liquefies gelatin, is mildly proteolytic, clots milk irregularly and sometimes digests the clot, is non-pathogenic to guinea-pigs and rabbits inoculated subcutaneously, and appears to be antigenically homogeneous. For its differentiation from $\mathrm{Cl}$. paraputrificum, $\mathrm{Cl}$. caloritolerans, and $\mathrm{Cl}$. cochlearium, see Snyder and Hall 1935). Serologically distinct from Cl. paraputrificum (Snydei 1936).

Cl. earnis.-Described by Klein (1903). A similar bacillus, pathogenie for rabbits, "von Hibler vi," was isolated from soil by von Hibler (1908). Hall and Duffett (1935) consider the two to be identical. Gram-positive rod, sluggishly motile by peritriehate flagella, 1.5 to $4.5 \mu \times 0.5-0.7 \mu$. Readily forms subterminal elongated spores, slightly wider than the bacillus, which later appear to be terminal. Microaerophilic. Surface 
colonies round and transparent, later flat and lobate; deep colonies lenticular. Uniform turbidity in broth, and later, granular, then mucoid deposit. Ferments glucose, maltose, lactose, saccharose and salicin, but not mamitol. Litmus milk unchanged. Coagulated serum and gelatin not liquefied. Indole - Produces a soluble exotoxin. Pathogenic to mice, white rats and rabbits, with production of an oedematous and congested local lesion.

Cl. cochlearium.-Described by McIntosh (1917) as Type III C, and named B. cochlearis by Douglas, Fleming and Colebrook (1920) on account of its likeness to a spoon. Its claim to the rank of a species confirmed by MacLennan (1939). Slender rod, Gram-positive only in young cultures, $3-5 \mu \times 0 \cdot 5-0 \cdot 6 \mu$, actively motile by peritrichate flagella. Spores formed late and in small numbers; are oval, terminal and twice the width of the bacillus. Surface colonies are translucent, round, with delicately crenated cdge; deep colonies are lenticular, and may develop polar tufts of growth. Non-hæmolytic, non-saccharolytic, non-proteolytic. Coagulated serum and gelatin not liquefied. $\mathrm{NH}_{3}-$; indole - ; V.P. - , M.R. - ; M.B. reductase - ; catalase - ; $\mathrm{H}_{2} \mathrm{~S}+$. Antigenically homogeneous. Non-pathogenic to guinea-pigs.

Cl. difficile.-Described by Hall and O'Toole (1935) and Snyder (1937). Isolated from the fæces of normal infants. Strictly anaerobic, large Gran-positive rod with subterminal elongated spores of about the same width as the bacillus. Colonies irregular, flat, rough and non-hæmolytic. Ferments glucose, lævulose, xylose, salicin, mannose and mannitol. Does not ferment galactose, lactose, saccharose, raffinose, inulin or glycerol. $\mathrm{H}_{2} \mathrm{~S} \pm$; Indole - ; coagulated serum not liquefied; late liquefaction of gelatin by a few strains. Some strains produce a filtrable, thermolabile, antitoxinogenic toxin which induces local œdema and convulsions in guinea-pigs. The toxin is lethal on injection into the cat, dog, rat, guinea-pig, rabbit and pigeon, but has no effect by mouth in the rat. guinea-pig and dog. The bacteria are antigenically heterogeneous, the toxin apparently antigenically homogeneous.

Cl. fallax was found by Weinberg and Séguin (1918) in infected wounds and gas gangrene, It is a motile, Gram-positive bacillus, $1.2-5 \mu \times 0.6 \mu$ in diameter, with rounded ends, straight axis, arranged singly. Spores, which are rarely formed, are subterminal or central, oval and wider than the bacillus. Surface colonies round and transparent, later raised, irregular and more opaque; deep colonies lenticular, irregular or bean-shaped. Uniform turbidity in broth, later granular, then mucoid, deposit. Ferments glucose, maltose, lactose, saccharose and salicin; mannitol fermentation variously reported. Litmus milk, coagulation in 7 days. Coagulated serum and gelatin not hiquefied. Indole -; (see Duffett 1935). Produces a soluble toxin. When freshly isolated, is pathogenic for mice and guinea-pigs.

Cl. hastiforme isolated by Cunningham (1930-31, 1931) and by MacLennan (1939), who accorded it the rank of a species. A Gram-positive rod, 2-6 $\mu \times 0 \cdot 3-0 \cdot 6 \mu$, sluggishly motile by peritrichate flagella. Readily produces oval subterminal spores which, together with a minute terminal tip of bacillary protoplasm, constitute the spear-head shape from which the organism is named. Surface colonies are non-hremolytic, minute, transparent and round, later becoming irregular; deep colonies round and coarsely filamentous. Gelatin liquefied 7-10 days; coagulated serum not liquefied. Non-saccharolytic. $\mathrm{H}_{2} \mathrm{~S}-$; Indole - ; $\mathrm{NH}_{3}-$. Antigenically homogeneous. Hayward (personal communication) has met with strains resembling $\mathrm{Cl}$. hastiforme which are hæmolytic on horse blood agar.

Cl. haemolyticum.-Described by Vawter and Records $(1926,1931)$ as the cause of red-water disease or bacillary hæmoglobinuria of cattle in the United States, and by Sordelli, Ferrari, and Prado (1930) in South America. Named B. hoemolyticus by Hall $(1929 b)$. It is a fairly large bacillus, $3 \cdot 0-5 \cdot 6 \mu \times 1 \cdot 0-1 \cdot 3 \mu$, occurring individually, in pairs, and occasionally in short chains. Spores are oval, terminal or subterminal. Sluggishly motile by 6-16 peritrichate flagella. Gram-positive in young cultures. Deep colonies in agar are at first lenticular, later fluffy. Gelatin is liquefied, but otherwise no 
proteolytie action. Acid and gas produeed in glucose, acid and clot in litmus milk. Complete hæmolysis on blood agar plates in 24 hours. A toxin is produced. By agglutination most strains appear to be antigenically homogeneous. Intramuscular inoculation of guinea-pigs with a toxic culture gives rise to an extensive bloorly œedema, sometimes accompanied by hæmoglobinuria. Rabbits also susceptible. A disease simulating in many respects the natural disease can be reproduced in cattle by injection of toxic cultures.

Cl. multifermentans (tenalbum.) -Described by Stoddard $(1919 a)$; isolated from a case of gas gangrene. Resembles $\mathrm{Cl}$. septicum morphologically, especially in its formation of citrons, but is non-pathogenic. Gram-positive, motile bacillus with subterminal spores. Surface colonies are large, round with slightly irregular edges; after several days they become white and opaque, and rise up from the surface. Deep colonies are white and opaque, irregular or biconvex, with projecting outgrowths. Acid and gas in glucose, maltose, lactose, sucrose, and salicin; acid and clot in L.M. Non-proteolytic. Nonpathogenic to guinea-pigs.

Cl. putrificum.-Described by Bienstock (1884, 1899, 1901), who isolated it from freces. Appears to have been a slender Gram-positive rod with spherical or oval terminal spores, which digested proteins but had no action on carbohydrates. Its identity has been in doubt (for critical discussion see Hall and Snyder 1934. Hartsell and Rettger 1934, Morgan and Wright 1934). Many workers regard it as identical with $\mathrm{Cl}$. cochlearium, but this is disputed by Hartsell and Rettger and by MacLennan (1939). According to MacLennan, Cl. putrificum is a slender bacillus, Gram-positive only in young cultures, $0 \cdot 3-0 \cdot 5 \mu$ in diameter, forming long tangled threads in old cultures, with slowly developing, large round terminal spores. Sluggishly motile by peritrichous flagella. Colonies are irregularly round, transparent, with filamentous or delicately fimbriate edge ; non-hrmolytie. Deep colonies are minute, spherical and hairy. Non-saceharolytic; $\mathrm{H}_{2} \mathrm{~S}+$; M.R. - ; M.B. reduetion - ; catalase - ; liquefies coagulated serum and gelatin in 7-20 days and blackens cooked meat medium slightly. Three serological groups, which are distinct from $\mathrm{Cl}$. cochlearium.

Cl. paraputrificum.--According to Hall and his colleagues (Hall and Snyder 1934, Snyder 1936, Hall and Ridgeway 1937) this organism, which was described by Bienstock (1906), is probably identical with Escherich's "Köpfchenbakterien," von Hibler's ix bacillus, Rodella's iii bacillus, and Kleinschmidt's (1934) B. innutritus. It is found in the frees, particularly of infants, both normal and ill-nourished, and is a slender, motile, Gram-variable bacillus with terminal oval spores. It is non-proteolytic; it ferments glucose, maltose, lactose, sucrose, and salicin, but not mannitol or xylose, with the production of acid and gas. It is non-pathogenic for guinea-pigs and rabbits.

CI. parasporogenes.-Described by McIntosh (1917). Resembles Cl. sporogenes, but deep colonies in agar shake cultures are biconvex or irregular in shape. Also forms specific agglutinins, which do not aet on Cl. sporogenes. Non-pathogenic to guinea-pigs.

Cl. sphenoides.-Isolated by Douglas, Fleming and Colebrook (1920) from wounds. So called from the wedge-shape of the sporing bacillus. Small, motile, weakly Grampositive; regetative bacilli are fusiform in shape and arranged in pairs end-to-end. Spores are large and round, appear subterminally, but soon become strictly terminal. Surface colonies are round with entire edges. Pathogenicity not examined.

CI. tertium.-Deseribed by Henry (1917). Resembles, but is probably different from, $\mathrm{Cl}$. paruputrificum (see above). Thin, slightly curved bacillus, 3-5 $\mu$ long, sluggishly motile, Gram-positive, often showing granular staining. Spores freely, giving rise to large, oval, elongated terminal spores. Surface colonies are rounded, delicate, iridescent, and almost transparent. with entire or slightly crenated edge. Deep colonies are small, biconvex or irregular in shape. Ferments mannitol and xylose. Non-pathogenic to guinea-pigs. (See von Hibler 1908, Hall and Matsuma 1924). Aceording to Hall and Duffett (1935), both $\mathrm{Cl}$. tertium and $\mathrm{Cl}$. histolyticum are microaerophilic rather than strictly anaerobic, but spores are formed only under anaerobic conditions. 
Cl. tetanomorphum.-Deseribed by MeIntosh (1917) as Type IX. Morphologically resembles $C l$. tetani, but differs in cultural and hiochemical characteristics ; fails to form a speeific toxin. Surface colonies are small, flat, irregularly round, and almost transparent; deep eolonies are small and imegular in shape, but are not woolly or branched. Non-pathogenic to guinea-pigs.

\section{REFERENCES}

Adauson, R. S. (1919-20) J. Path. Bact., 23, 241.

Aitken, R. S., Barling, B., and Miles, A. A. (1936) Lancet, ii. 780.

Anderson, B. G. (1924) J. infect. Dis., 35, 213, 244.

Arloing, S., Corvevis, C. E., and Thomas, O. (1887) "Le charbon symptomatique du bœuf," 2nd edition. Paris.

Behring. (1892) Z. Hyg. InfektKr., 12, 45.

Behring and Kitasato. (1890) Dtsch. med. Wschr., 16, 1113.

Belin, M. (1931) C. R. Soc. Biol., 105, 840.

Bengtson, I. A. (1920) Bull. U.S. hyg. Lab., No. 122; (1921) Publ. Hlth Rep., Wash., No. 29, p. 1665; (1922a) Publ. Hlth Rep., Wash., 37, 164; (1922b) Ibid., 37, 2252; (1923) Ibid., 38, 340; (1924a) Bull. U.S. hyg. Lab., No. 136; (1924b) Ibid., No. 139.

Bennet'ts, H. W. (1932) Aust. Counc, sci. industr. Res., Bull. No. 57.

Bernheimer, A. W. (1944) J. exp. Med., 80, 309, 321, 333.

Bıеnstock, B. (1884) Z. klin. Med., 8, 1 ; (1899) Arch. Hyg., 36, 335 ; (1901) Ibid., 39, 390 ; (1906) Ann. Inst. Pasteur, 20, 407.

Borthwick, G. R. (1935) Zbl. Bakt., 134, 2s9; (1937) Brit. J. exp. Path., 18, 475.

Bosworth, T. J. and Glover, R. E. (1935) Proc. R. Soc. Med., 28, 1004.

Boll, C. G. (1917) J. exp. Med., 26, 603.

Bull, C. G. and Pritchett, I. W. (1917a) J.exp. Med., 26, 119 ; (1917b) Ibid., 26, 867. Burke, G. S. (1919a) J. Bact., 4, 555 ; (1919b) J. Amer. med. Ass., \%2, 88.

Carlex, S. A. (1939) Proc. Soc. exp. Biol., . . I., 40, 39.

Caulfend, A. H. W. (1920) J. infect. Dis., 2\%, 151.

C'Lark, F. E. and Hall, I. C. (1937) J. Buce, 33, 23.

Cliftox, C. E. (1942) J. Bact., 44, 179.

Colemax, G. E. (1922) J. infect. Dis., 31, 556 ; (1923) Ibid., 33, 384.

Colemax, G. E. and Meyer, K. F. (1922) J. infect. His., 31, 622.

Corbitt, H. B. (1918) Bull. U.S. hyg. Lab., No. 112.

Crook, E. 11. (1942) Brit. J. exp. P'ath., 23, 37.

Cunninghai, A. (1930-3I) Zbl. Bakt., IIte Abt., 82, 25, 481; (1931) Ibid., 83, 1, 22, 219.

Dack, G. M., Starin, W. A. and Werner, M. (1927) J. infect. Dis., 40, 525.

Dalling, T. (1926) J. comp. Path., 39, 148.

Dallixg, T. and Ross, H. E. (193s) J. comp. Path., 51, 235.

Dalling, T. and Stephexson, M. (1942) Nature, Lond., 149, 56.

Descombey, P. (1924) C. R. Soc. Biol., 91, 239.

Dickens, P. F. (1934) U.S. nav. med. Bull., 32, 267.

Dickson, E. C. (1918) Monogr. Rockefeller Inst. med. Res., No. 8.

Dicksos, E. C., Burke, G. S., Beck, I)., and Johnston, J. (1925) J. infect. Dis., 36, 172. Djaexoedin, R. and Kiraneveld, F. C. (1936) Ned-Ind. Bl. Diergenesk, 48, 290.

Dodglas, S. R., Flening, A., and Colebrook, L. (1920) Spec. Rep. Ser. med. Res. Coun., Lond., No. 57.

Dozier, C. C. (1924) J. infect. Dis., 35, 105.

Duborsky, B. J. and IIEYer, K. F. (1922a) J. infect. Dis., 31, 501 ; (1922b) Ibid., 31, 595.

Duffett, N. D. (1935) J. Bact., 29, 573; (1935) Univ. Colo. Stud., 26, 46.

Easton, L. J. and MeYer, K. F. (1924) J. infect. Dis., 35, 207.

Edsoxdson, R. B., Giltiner, L. T., and Thos, C. (1920) Arch. intern. Med., 26, 357.

Eisenberg, P. (1907) C. R. Soc. Biol., 62, 613.

Ermengem, E. van. (1896) Zbl. Baht., 19, 442; (i897) Z. Hyg. InfehtKr., 26, 1.

Esty, J. R. and Meyer, K. F. (1922) J. infect. Dis., 31, 650.

Evans, D. G. (1945) J. Path. Bact., 57, 75.

Felix, A. and Robertson, M. (1928) Brit. J. exp. Path., 9, 6.

Fildes, P. (1925a) Brit. J.exp. Path., 6, 62; (1925b) Ibid., 6, 91 ; (1929) Brit. J. exp. Path., 10, 151 ; (1935) Brit. J. exp. Path., 16, 309.

Fildes, P. and Knight, B. C. J. G. (1933) Brit. J. exp. Path., 14, 343.

Fildes, P. and Richardson, G. M. (1935) Brit. J. exp. Path., 16, 326.

Frankland, P. F. (1889) Z. Hyg. InfektKr., 6, 13.

Friedemans, U. and Hollander, A. (1943) J. Immurol., 4\%, 23, 29.

GaIGer, S. H. (1922) J. comp. Path., 35, 191, 235; (1924) Ibid., 37, 163. 
Gale, E. F. (1940) Bact. Rev., 4, 135.

Gale, E. I. and Heyningen, WV. E. van, (1942) Biochem. J., 36, 624.

GIIL, D. A. (1933) Tot. J., 89, 399.

Gillespie, R. W. H. and Rettger, I. F. (1938) J. Buct., 36, 605.

Gladstone, G. P., Fildes, P., and Richardson, G. M. (1935) Brit. J. exp. Puth., 16, 3 35.

Glenny, A. T., Barr, M., Llewellyn-Jones, M., Dalling, T., and Ross, H. E. (1933)

J. Path. Bact., 3\%, 53 .

Goss, L. W., Barbarin, R. E., and Haines, A. W. (1921) J. infect. Dis., 29, 615.

Graham, R. and Boughtox, T. B. (1923a) Abstr. Bact., 1, 29 ; (1923b) Ibid., 7, 30.

Grahai, R. and Broeckner, A. I. (1919) J. Bact., 4, 1.

GrahaM, R. and Thorp, F. (1929), J. Immunol., 16, 391.

GugGENHEL, K. (1944) J. Bact., 4\%, 313.

Gunnison, J. B. (1937) J. Immunol., 32, 63.

Gunnison, J. B. and Colemax, G. E. (1932) J. infect. Dis., 51, 542.

Gunnison, J. B., Cummings, J. R., and Meyer, K. F. (1936-37) Proc. Soc. exp. Biol., N.Y., $35,278$.

Gunsison, J. B. and Meyer, K. F. (1930) J. infect. Dis., 46, 335.

Haines, R. B., and ScotT, W. J. (1940) J. Hyg., Camb., 40, 154.

HaL, I. C. (1922) J. infect. Dis., 30, $445 ;(1929 a) \mathrm{J}$. Bact., 17, 255; (1929b) J. infect. Dis.

45, 156.

HaLL, I. C. and Davis, N. C. (1923) J. exp. Med., 3\%, 585.

Hall, I. C. and DufFetT, N. D. (1935) J. Bact., 29, 269.

Hall, I. C. and Matsuma, K. (1924) J. infect. Dis., 35, 502.

Hall, I. C. and Ridaway, D. (1937) J. Bact., 34, 631.

Hali, I. C., Rymer, M. R., and Jungherr, E. (1929) J. infect. Dis., 45, 42.

Hall, I. C. and Scott, A. L. (1927) J. infect. Dis., 41, 329 ; (1931) J. Bact., 22, 375.

HaLl, I. C. and Swyder, M. L. (1934) J. Bact., 28, 181.

Hall, I. C. and O'Toole, E. (1935) Amer. J. Dis. Child., 49, 390.

Hartsell, S. E. and Rettger, L. F. (1934) J. Bact., 27, 497.

Hastam, T. P. and Lumb, J. W. (1919) J. infect. Dis., 24, 362.

HaYward, N. J. (1941) Brit. med. J., 1, 811 ; (1943) J. Path. Bact., 55, 285.

Hazen, E. L. (1937) J. infect. Dis., 60, 260; (1942) Proc. Soc. exp. Biol., N.Y., 50, 112. HEADLEE, M. R. (1931) J. infect. Dis., 48, 468.

Heller, H. H. (1920) J. infect. Dis., 27, 385 ; (1921) J. Bact., 6, 521 ; (1922a) J. infect. Dis., 30, 18 ; (1922b) Ibid., 30, 33.

Henderson, D. W. (1932) Brit. J. exp. Path., 13, 412 ; (1934) Ibid 15, 166 ; (1940)

J. Hyg., Camb., 40, 501 .

Henriksen, S. D. (1937) Acta. path. microbiol. Scand., 14, 570.

Henry, H. (1917) J. Path. Bact., 21, 344.

Herningen, W. E. van. (194la) Biochem. J., 35, 1246 ; (1941b) Ibid., 35, 1257.

Hibler, E. voN. (1908) "Untersuchungen über die pathogenen Anaëroben." Jena.

Holker, J. (1918-19) J. Path. Bact., 22, 28.

Hoogerheide, J. C. (1937) J. Bact., 34, 387.

Howard, A. (1928) Ann. Inst. Pasteur, 42, 1403.

Hoyt, A., Chaney, A. L., and Cavell, K. (1938) J. Bact., 36, 639.

Honphreys, F. and Meleney, F. L. (1927-8) Proc. Soc. exp. Biol., N.Y., 25, 611.

Humphreys, F. B. (1924) J. infect. Dis., 35, 282.

Ipsen, J. (1939) Bull. Hlth. Org., L.o.N., 8, 825; (1940-41) Ibid., 9, 447, 452.

Ipsen, J. and Davoli, R. (1939a) Bull. Hlth Org. L.o.N., 8, 833.

Ipsen, J., Sirth, M. L., and Sordell, A. (1939b) Ibil., 8, 797.

KAMEN, L. (1904) Zbl. Bakt., 35, 686.

KEMPNER, W. (1897) Z. Hyg. InfektKr., 26, 481.

Kerrin, J. C. (1930) Brit. J. exp. Path., 11, 153 ; (1934) J. Path. Bact., 38, 219.

Kitasato, S. (1889a) Z. Hyg. InfektKr., 6, 105; (1889b) Ibid., 7, 225; (1890) Ibid., 8, 55 ; (1891) Ibid., 10, 267.

Kitasato, S. and Weyl, Тн. (1890) Z. Hyg. InfektKr., 8, 41, 404.

Кітт, T. (1887) Zbl. Bakt., 1, 684, 716, 741 .

Klein, E. (1903) Zbl. Bakt., 35, 459.

Kleinschint, H. (1934) Mschr. Kinderheilk., 62, 14.

К піянт, B. C. J. G. (1936) Spec. Rep. Ser. med. Res. Coun., Lond., No. 210.

KNight, B. C. J. G. and Flldes, P. (1930) Biochem. J., 24, 1496; (1933) Brit. J. exp. Path., 14, 112.

Косн, R. (1881) Mitt. ReichsgesundhAmt., 1, 49.

Kraneveld, F. C. (1930) Ned-Ind. Bl. Diergencesk., 42, 564.

Kraneveld, F. C. and DJaenoedin, R. (1933) Ned.Ind. Bl. Diergeneesk, 45, 80.

Kredzer, E. (1939) Z. ImmunForsch., 95, 345.

KroIF, P. H. DE, Adams, T. W., and Ireland, P. M. (1917) J. infect. Dis., 21, 580.

KRUIF, P. H. DE and Bollman, J. L. (1917) J. infect. Dis., 21, 588. 
LAIDLAW, P. P. (1915) Brit. med. J., i. 497.

LANDAU, H. (1917) Zbl. Bakt., 79, 417.

Leclainche, E. and Vallée, H. (1900) Ann. Inst. Pasteur, 14, 202.

Lepper, E. and Martin, C.J. (1929) Brit.J.exp.Path., 10, 327; (1930) Ibid., 11, 137, 140.

LeUCHS, J. (1910) Z. Hyg. InfektKr., 65, 55.

Levenson, S. (1936) C. R. Soc. Biol., 121, 221.

LiBoRIUS, P. (1886) Z. IIyg. InfektKr., 1, 115.

LiNGelshem, voN. (1912) See Kolle and Wassermann's " Hdb. path. Mikroorg." IIte Abt. $(1912-13), 4,737$.

LIVESAY, H. R. (1933) J. infect. Dis., 53, 125.

Lomel, J. and GunNison, J. B. (1929) J. Immunol., 16, 403.

IoClean, D. (1936) J. Path. Bact., 42, 477.

McClung, L. S. (1935) J. Bact., 29, 189 ; (1937) J. infect. Dis., 60, 122.

McCluxg, L. S., McCor, E., and Fred, E. B. (1935) Zbl. Bakt., IIte Abt., 91, 225.

McClung, L. S. and Wheaton, E. (1936) Food Research, 1, 307.

MoCoy, E. (1937) J. Bact., 34, 321.

McCoy, E. and McClung, L. S. (1936) J. Bact., 31, 557 ; (1938) Bact. Rev., 2, 47.

IcEwen, A. D. (1926) J. comp. Path., 39, 253 ; (1930) Ibid., 43, 1.

Macfarlane, M. G. and KNight, B. C. J. G. (1941) Biochem. J., 35, 884.

Macfarlane, R. G., Oakley, C. L., and Anderson, C. G. (1941) J. Path., Bact., 52, 99.

McGadohey, C. A. (1933) J. Path. Bact., 36, 263.

IcIntosh, J. (1917) Spec. Rep. Ser. med. Res. Coun., Lond., No. 12.

McIntosh, J. and Fildes, P. (1916) Lancet, i, 768.

MacLennan, J. D. (1939) Brit. J. exp. Path., 20, 371.

MAdSEN, T. (1899) Z. Hyg. InfektKr., 32, 214.

Markoff, W. N. (1911) Zbl. Bakt., 60, 188.

Mason, J. H. (1935) Onderstepoort J. vet. Sci., 5, 363.

Mason, J. H., Ross, H. E., and Dalline, T. (1931) J. comp. Path., 44, 258.

Meisel, H. (1938) Z. ImmunForsch., 92, 79.

Meleney, F. L., Humphreys, F. B., and Carp, L. (1926-7) Proc. Soc. exp. Biol., N.Y., 24, 675 ; (1927) Surg. Gynec. Obstet., 45, 775.

Metchnikoff, E. (1908) Ann. Inst. Pasteur, 22, 929.

MeYER, K. F. (1915) J. infect. Dis,, 1\%, 458.

Mleyer, K. F. and Dubovsky, B. J. (1922a) J. infect. Dis., 31, 541 ; (1922b) Ibid., 31, $559 ;(1922 c)$ Ibid., 31, 600.

Mieszner, H., Mern, A., and Schoop, G. (1931) Zbl. Bakt., 120, 258.

Miles, A. A. and Hayward, N. J. (1943) Lancet, ii. I16.

Morgan, E. L. and Wright, H. D. (1934) J. Path. Bact., 39, 457.

Mutermilch, S., Belin, M., and Salayox, E. (1933) C. R. Soc. Biol., 114, 1005.

Nagler, F. P. O. (1936) Z. ImmunForsch., 89, 477 ; (1937) Ibid., 91, 457 ; (1939) Brit. J. exp. Path., 20, 473; (1940) Z. ImmunForsch., 97, 273.

Nevin, M. (1921) J. infect. Dis., 28, 226.

Nicolaier. (1881) Dtsch. med. IT schr., 10, 812.

Noble, W. (1915) J. infect. Dis., 16, 132.

Nocard and Roux. (1887) Ann. Inst. Pasteur, 1, 257.

Novy, F. G. (1894) Z. Hyg. Infekthr., 17, 209.

OAKLeY, C. L. (1943) Bull. Hyg. Lond., 18, 781.

Oakley, C. L. and Warrack, G. H. (1941) J. Path. Bact., 53, 335.

OrR, J. H. and ReEd, G. B. (1940) J. Buct., 40, 441.

Orr, P. F. (1920) Abstr. Bact., 4, 10 ; (1922) J. infect. Dis., 30, 118.

PaINe, F. S. (1931) Zbl. Bakt., IIte Abt., 85, 122.

Pappenheimer, A. I. (1935) Biochem. J., 29, 2057.

Pasternack, J. G. and Bengtson, I. A. (1936) Nat. Inst. Hith Bull., No. 168; (1940) Publ. Hlth. Rep., Wash., 55, 775.

Pasteur and Joubert. (1877) Bull. Acad. Méd., 6, 781.

Petrie, G. F. (1942-43) Bull. Hlth Org., L.o.N., 10, 113.

Pfenninoer, W. (1924) J. infect. Dis., 35, 347.

Pizzini, L. (1898) Zbl. Bakt., 24, 890.

Prévot, A. R. (1938) Ann. Inst. Pasteur, 61, 72.

Prigge, R. (1936) Z. ImmunForsch., 89, 477; (1937) Ibid., 91, 457.

Redish, G. F. and Rettger, L. F. (1924) J. Bact., 9, 13.

Reed, G. B. and OrR, J. H. (1941) War Med., 1, 193.

Reed, G. B., OrR, J. H., and Brows, H. J. (1943) J. Bact., 46, 475.

REed, R. W. (1942) J. Bact., 44, 425.

Reports. (1917) Spec. Rep. Ser. med. Res. Coun., Lond., No. 12 ; (1919) Ibid., No. 39.

Riтсніе, J. (1901) J. Hyg., Camb., 1, 125.

Roberts, R. S. (1931) J. comp. Path., 44, 240. 
Robertson, M. (1916) J. Path. Bact., 20, 327.

Robinson, E. M. (1929) 15th Ann. Rep. Dir. vet. Serv. Union S. Afr., 1, 111.

Rock Well, G. E. (1924) J. infert. Dis., 35, 581.

Rodweld, A. W. (1941) Aust. I'et. J., 1\%, 58.

Rosenau, M. J. and Anderson, J. F. (1908) Bull. U.S. hyg. Lab., No. 43.

Rosenbacir. (1886) Arch. klin. Chir., 34, 306.

Roux, E. (1888) Ann. Inst. Pasteur, 2, 49.

Sacquépée, E. (1915) Pr. méd., 23, 183.

Schoenholz, P. (1928) J. infect. Dis., 42, 40.

Schoenholz, P. and Meyer, K. F. (1922) J. infect. Dis., 31, 610 ; (1924) Ibid., 35, 361 ; (1925) J. Immunol., 10, 1.

Scotr, J. P., Turner, A. W., and Vawter, L. R. (1933) Proc. 12th int. vet. Congr. N.Y., p. 168 .

Seddon, H. R. (1922) J. comp. Path., 35, 147.

SeIfFERT, G. (1939) Z. ImmunForsch, 96, 515.

Sherrington, C. S. (1917) Lancet, ii. 964.

SHIPPEN, L. P. (1919) Arch. intern. Med., 23, 346.

Simonds, J. P. (1915a) J. infect. Dis., 16, 31 ; (1915b) Ibid., 16, 35 ; (1915c) Monogr. Rockefeller Inst., No. 5.

DE SMidt, F. P. G. (1924) J. Hyg., Camb., 22, 314.

Sмiтt, L. (1937) J. Bact., 34, 409.

Sмith, M. L. (1941-42) Bull. Hlth Org., L.o.N., 10, 104.

Sмiтн, T. (1890) Zbl. Bakt., 7, 502; (1908) Trans. Chicago path. Soc., 7, 1.

Smith, T., Brown, T. H. R., and Walker, E. L. (1905-6) J. med. Res., 14, 193.

SNyder, MI. L. (1936) J. Bact., 32, 401 ; (1937) J. infcct. Dis., 60, 223.

SNyder, M. L. and HaLl, I. C. (1935) Zbl. Bakt., 135, 290.

Sommer, H. and Sommer, E. W. (1932) J. infect. Dis., 51, 243.

Sordelli, A. (1922) C. R. Soc. Biol., 87, 838; (1923) Ibid., 89, 53.

Sordelli, A., Ferrari, J., and Prado, M. (1930) Rev. Inst. bact., B. Aires, 5, 797.

Sordelit, A., Prado, M., and Ferrari, J. (1932) Folia biol., B. Aires, Nos. 14 and 15, pp. 58,63 .

Spray, R. S. (1936) J. Bact., 32, 135.

Starin, W. A. (1924) J. infect. Dis., 34, 148.

Starin, W. A. and Dack, G. M. (1923) J. infect. Dis., 33, 169 ; (1924) Ibid., 34, 137 ; (1925) Ibid., 36, 383 .

Stark, C. N., Sherman, J. M., and Stark, P. (1928) J. infect. Dis., 43, 565.

Stevens, F. A. (1935) J. infect. Dis., 5\%, 275.

Stewart, S. E. (1938) J. Bact., 35, 13; (1940) Publ. Illth Rep., Wash., 55, 753.

Stickland, L. H. (1934) Biochem. J., 28, 1746 ; (1935) Ibid., 29, 288, 889.

Stoddard, J. L. (1919a) Lancet, i. 12; (1919b) J. exp. Med., 29, 187.

Svec, M. H. and IICCoy, E. (1944) J. Bact., 48, 31.

TANNER, F. W. and DACK, G. M. (1922) J. infect. Dis., 31, 92.

Tanner, F. W. and T'wohey, H. B. (1926) Zbl. Bakt., 98, 136.

TARozZI, G. (1905) Zbl. Bakt., 37, 619.

TAYLOR, A. W. (1940) J. comp. Path., 53, 50.

TaYlor, A. W. and Stewart, J. (1941) J. Path. Bact., 53, 87.

Theiler, A. (1928) 13th and 1'th Rep., Director vet. Educat. Res., S. Africa, p. 47.

Theiler, A., Viluoen, P. R., Green, H. H., Do Toit, P. J., Mieier, H., and Robinson, E. M. (1926) 11 th and 12 th Rep., Director vet. Educat. Res., S. Africa, Part ii. p. 821.

Tissier, H. and Martelly. (1902) Ann. Inst. Pasteur, 16, 865.

Todd, E. W. (1941) Brit. J. exp. Path., 22, 172.

Toledo, S. and VeILlon. (1891) Zbl. Bakt., 9, 18.

Torrey, J. C. (1925) J. infect. Dis., 36, 517.

Torrey, J. C., KaHN, M. C., and Salinger, M. H. (1930) J. Bact., 20, 85.

TschentKow, L. (1929) Z. ImmunForsch., 63, 262.

Tulloch, W. J. (1919) J. Hyg., Camb., 18, 103.

Turner, A. W. (1930) Aust. Coun. sci. industr. Res., Bull., No. 46.

Turner, A. W. and Eales, C. E. (1941) Aust. J. exp. Biol. med. Sci., 19, 167.

'Turner, A. W. and Rodwell, A. W. (1943) Aust. J. exp. Biol. med. Sci., 21, 17, 27.

Uschinskx, N. (1893) Zbl. Bakt., 14, 316.

VAILlard, L. and RoveEt, J. (1892) Ann. Inst. Pasteur, 6, 385.

VARNEY, P. L. (1926) J. Lab. clin. Med., 11, 1.

VAWTER, L. R. and Records, E. (1926) J. Amer. vet. med. Ass., 68, 494; (1931) J. infect. Dis., 48, 581.

Velluz, L. (1936) C. R. Acad. Sci., 203, 471, 498.

WAGNer, E. (1924) J. infect. Dis., 35, 353.

Wagner, E., Dozier, C. C., and MeYer, K. F. (1924) J. infect. Dis., 34, 63.

WalbUM, L. E. (1938) J. P'ath. Bact., 46, 85. 
Walbum, L. E. and Reymann, C. G. (1933) J. Path. Bact., 36, 469.

WeinberG, M. (1929) Bull. Inst. Pasteur, 27, 529, 577.

Weinbern, M. and Conbiesco, N. (1930) Ann. Inst. Pasteur, 45, 547.

Weinberg, M., Davesne, J., and Lefranc, M. (1931) C. R. Soc. Biol., 10\%, 506.

Weinberg, M., Davesne, J., Mihailesco, M., and Sanchez, C. (1929) C. R. Soc. Biol., 101, 907.

Weinberg, M. and Ginsbourg, B. (1927) "Données récentes sur les microbes anaérobies et leur rôle en pathologie." Masson et Cie., Paris.

Weinberg, M. and Guillaumie, M. (1936) C. R. Soc. Biol., 121, 127.;.

Weinberg, M. and Minailesco, M. (1929) Ann. Inst. Pasteur, 43, 140 s.

Weinberg, M., Nativelle, R., and Prévot, A. R. (1937) "Les Microlu's Anaérobies." Masson et Cie, Paris.

Weinberg, Renard, C., and Davesne, J. (1926) C. R. Soc. Biol., 94, 813.

Weinberg and Séguin, P. (1915) C. R. Soc. Biol., 78, 274; (1916) C. R. Acad. Sci., 163, 449 ; (1918) "La gangrène gazeuse." Paris.

Weiss, H. (1921) J. infect. Dis., 28, 70.

Welch, W. H. and Nutrall, G. H. F. (1892) Johns Hopk. Hosp. Bull., 3, 81.

Wheeler, M. W'. and Hunphreys, E. M. (1924) Johns Hopk. Hosp. Bull., 35, 305.

Wilsdon, A. J. (1931) 2nd Rep. Director, Inst. Anim. Path., Camb., p. 53; (1933) Ibid., 3rd Rep., p. 46.

Wilson, G. S. (1928) J. Path. Bact., 31, 113.

Winogradsky, S. (1902) Zbl. Bakt., IIte Abt., 9, 43, 107.

Woods, D. D. and Trim, A. R. (1942) Biochem. J., 36, 501.

Wolf, C. G. L. (1918-19) J. Path. Bact., 22, 115 ; (1919-20) Ibid., 23, 254.

Wolf, C. G. L. and Harris, J. E. (1917) J. Path. Bact., 21, 386.

Zeissler, J. and Kraneveld, F. C. (1929) Arch. wiss. prakt. Tierheilk., 60, 441.

Zeissler, J. and Raszeld, L. (1929) Arch. wiss. praht. Tierheilh., 59, 419. 


\section{CHAPTER 37}

\section{MISCELLANEOUS BAC'TERIA}

WE include in this chapter a number of organisms which, for one reason or another, cannot justifiably be allotted to any of the named groups. ${ }^{1}$

\section{The Morax-Axenfeld Bacillus.}

This organism, which is responsible for subacute or angular conjunctivitis in human beings, was described independently by Morax (1896) and Axenfeld (1897). The name B. lacunatus was suggested for it by Eyre (1900).

Morphology. - In films of the conjunctival secretion it occurs in the form of rods, 2-3 $\mu$ long and I $\mu$ broad, with parallel or slightly convex sides and rounded ends. The bacilli oceur in pairs, placed end-to-end, and sometimes in short chains. They are found free in the secretion, or within the polymorphonuclear and desquamated epithelial cells. They are non-motile, non-sporing, Gram-negative, and except for the absence of a capsule, they closely resemble Friedländer's pneumobacillus. In old cultures pleomorphic forms are numerous, ranging in size and shape from short stunted diplococcal forms to long. jointed or filamentous, sometimes fusiform threads (Eyre 1900).

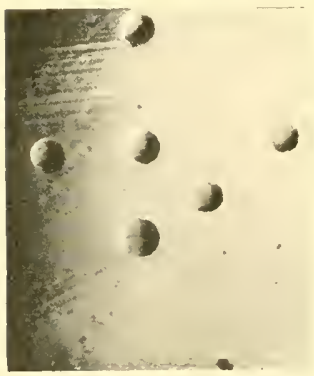

Fig. 211.-Morax-AxENFELD BACILLUS.

Surface colonies on Fildes' agar plate, 24 hours, $37^{\circ} \mathrm{C} .(\times 8)$.

Cultivation.-Growth occurs only in the presence of some natural animal protein, such as serum, blood, or ascitic fluid; there is no development on ordinary nutrient agar or potato nor in broth, milk, or gelatin. Development is best on Fildes' agar and on serum or egg medium; it is poor on blood agar and very poor on chocolate agar. The organism is said to grow only between $30^{\circ}$ and $40^{\circ} \mathrm{C}$., and to be strictly aerobic. Our own limited experience suggests that some growth may occur below $30^{\circ} \mathrm{C}$, and that under anaerobic conditions on favourable media slight but definite growth may be evident. On serum agar plates after 24 hours at $37^{\circ} \mathrm{C}$. the colonies are round, up to $1 \mathrm{~mm}$. in diameter, raised, greyish, and translucent; during the next few days they increase in size, reaching 2-5 mm. in diameter, and become differentiated into a slightly raised opaque whitish centre, and a thin, translucent periphery with a lobate edge; the medium becomes pitted owing to liquefaction of the serum. On Loeffler's serum no actual colonies are visible, but the whole surface is covered with pits of liquefaction-hence the term lacunatus. On Fildes' agar after 24 hours at $37^{\circ} \mathrm{C}$. the colonies are water-clear, amorphous, low convex, $0.4 \mathrm{~mm}$. or so in diameter, with a smooth glistening surface and entire edge ; after 4 days they are larger, and are differentiated into a smooth, raised, central papilla and a wide, effuse, granular, dull, transparent, peripheral extension with an irregularly undulate margin. The central papilla may be so small and the peripheral portion so transparent that the colonies are difficult to see. By transmitted light they have a frosted-glass appearance. On horse blood

1 The terms Bact. pneumosintes and Bact. granulosis are used for convenience, but it must be pointed out that neither of these organisms belongs to the Bacterium group as defined on p. 654 . 
agar the eolonies of some strains are surrounded by a fairly wide zone of incomplete hæmolysis, though no soluble hæmolysin is formed in fluid media (Oag 1942). On Dorset egg low convex colonies are formed, which lead in a few clays to slight pitting of the surface. There is no growth on MacConkey agar. In serum broth after 24 hours at $37^{\circ} \mathrm{C}$., there is a uniform turbidity; later a greyish-white deposit appears, and increases for 6 to 10 days, after which the medium elears, the whole of the growth sinking to the bottom of the tube.

Resistance, Biochemical Reactions, and Pathogenicity.-Cultures in serum broth live for weeks at $35^{\circ} \mathrm{C}$, , but die in 48 hours if left at room temperature. The bacillus is killed by moist heat at $58^{\circ} \mathrm{C}$. in 15 minutes, and is apparently very susceptible to zinc salts, which have an almost specific action in the treatment of angular conjunctivitis. In our experience no sugars are fermented. Gelatin may be hquefied, and litmus milk becomes very slowly alkaline, though according to Oag (1942) acid is produced. Nitrates are reduced. Catalase is negative. No indole is formed in serum broth cultures, even after 7 days. According to Oag (1942) antigenie differences can be demonstrated by agglutination between hæmolytic and non-hæmolytic strains. The organism is nonpathogenio to laboratory animals, whether inoculated into the conjunctival sac or direetly into the tissues; but a drop of culture instilled into the conjunctival sac of a healthy human volunteer gives rise in about 5 days to a typical attack of angular conjunetivitis.

A similar organism, B. duplex, was described by Petit in 1900 , who isolated it from cases of conjunctivitis associated with corneal ulceration. It differs from the Morax. Axenfeld bacillus mainly in its ability to grow on media without the addition of natural animal protein (though no growth may occur in ordinary broth), in its development at room temperature, in its liquefaction of gelatin, and in its more active digestion of Loeffler's serum, which it liquefies in 3 or 4 days. It is also said to be antigenically distinet (Audureau 1940). On ascitic agar it gives rise to convex, not umbonate, eolonies, which are greyish in colour, viscous, and not so translueent as those of the Morax-Axenfeld bacillus.

Another organism, which is closely related to, if not identical with, the Morax-Axenfeld baeillus, was isolated by Jones and Little (1923) from eattle suffering from acute conunctivitis, sometimes complicated by corneal ulceration. It is saicl to liquefy gelatin, and to produce alkali in litmus milk.

Classification.- The classifieation of these organisms is still in doubt (see Chapter 33). Lwoff (1939) suggested that they should be grouped in a new genus, ealled Moraxella. Audureau (1940) aecepts this suggestion and reeognizes three main species, lacunata, duplex and lwoffi. Moraxella lacunata grows only in the presenee of serum; Moraxella duplex grows in the absence of serum but not in synthetic media; and Moraxella lwoffi, which was isolated by Audureau from patients affected with conjunctivitis, will grow in a synthetie medium free from protein and amino-aeids, provided a little carbohydrate is added to provide energy.

\section{Bacterium pneumosintes.}

Isolated by Olitsky and Gates (1921 $a, b, c, 1922 a, b)$ from the nasopharyngeal washings of patients in the early stage of influenza, and since observed by certain other workers (Hall 1926). It is a very small organism and is capable of passing through Berkefeld $\mathrm{N}$ and $\mathrm{V}$ candles.

Morphology.- In Smith-Noguchi medium on first isolation, it is described as consisting of minute bodies, arranged singly, in pairs, or short chains; its length is given as $0 \cdot 15-0 \cdot 3 \mu$, and its breadth as two or three times less. After subculture for some time in the laboratory, the organisms appear in dextrose peptone broth as plump rods with rather pointed ends, arranged in pairs end-to-end, and in fairly long chains. It may be $0.5-1.0 \mu$ long; it stains more deeply in the middle than at the ends. Our own observations on one of Olitsky and Gates's original strains, grown anaerobically for 12 
days on blood agar, show that it is a very small straight rod-shaped organism, $0.5-1 \cdot 0 \mu$ long by $0.2-0.3 \mu$ broad, with parallel sides and rounded ends; it is arranged singly, in pairs end-to-end, and in small dense groujs. No irregular forms are seen ; it stains regularly with the usual aniline dyes, is Gram-negative, and non-motile (Fig. 212).

Culturalix, the organism can be isolated only in Smith-Noguchi medium (human ascitic fluid containing a piece of sterile rabbit kidney, and covered with a vaseline seal); but after three or four subcultures in this medium it can be brought to grow anaerobically on blood agar, chocolate agar, Fildes' agar, Bordet's medium, and certain other media.

Smith-Noguchi Medium at $37^{\circ} \mathrm{C}$ - -A faint liaze becomes visible around the kidney about the 5th day, and reaches a maximum about the 8 th day, when it is $3 \mathrm{~cm}$. deep. These appearances are not characteristic; they often appear in uninoculated tubes.

Horse Blood Agar. -7 days, $37^{\circ}$ C. Round,

F19. 212.-- Bacterium pneumosintes.

From a surface growth on blood agar anaerobically, 12 days, $37^{\circ} \mathrm{C}$. $(\times 1000)$. convex, milky-white opaque colonies, $0.5 \mathrm{~mm}$. in diameter, of amorphous structure, and with a smooth glistening surface and an entire edge; butyrous in consistency and easily emulsified; no hæmolysis.

Horse Blood Agar or Chocolate Agar Slope.-12 days, $37^{\circ} \mathrm{C}$. Slightly raised, partly confluent, glistening growth with an irregular surface, due to imperfect fusion, and an edge made up of very tiny discrete colonies.

Bordet's Medium.-12 days, $37^{\circ} \mathrm{C}$. Slightly raised, confluent, glistening growth with a finely pitted surface.

Glycerol Egg. -7 days, $37^{\circ} \mathrm{C}$. Good, slightly raised, glistening, viscous growth with a smooth surface and an entire edge.

Loeffler's Serum. -7 days, $37^{\circ} \mathrm{C}$. Similar to growth on glycerol egg, but not so abundant.

Trypsinized Heart Agar Slope, and Glycerol Agar Slope.-7 days, $37^{\circ} \mathrm{C}$. Rather poor, slightly raised, confluent, nearly colourless, translucent growth with an irregular surface due to imperfect fusion; edge made up of single colonies.

Trypsinized Heart Broth. -7 days, $37^{\circ} \mathrm{C}$. Poor to moderate, uniform turbidity, with a moderate, highly viscous deposit, which, on shaking, coheres in a ropy mass and is difficult to disintegrate. No surface growth; slightly aromatic odour.

Fildes' Broth. -7 days, $37^{\circ} \mathrm{C}$. No turbidity, but slight powdery deposit disintegrating on shaking. After 14 days the deposit is viscons, coherent, and difficult to disintegrate.

Nitrate Broth. -7 days, $37^{\circ} \mathrm{C}$. Slight turbidity; moderate, viscons deposit, disintegrating completely; no surface growth.

Serum Broth.-Growth similar to that in heart broth.

Coli-Broth. -5 days, $37^{\circ} \mathrm{C}$. (Prepared by growing a strain of Bact. coli in I per cent. dextrose broth till the first sign of turbidity appears; the culture is then steamed for half an hour.) Moderate to dense turbidity; moderate viscous deposit, very difficult to disintegrate.

Resistance.-Cultures in Smith-Noguchi medium, after 5 to 7 days' incubation at $37^{\circ} \mathrm{C}$, remain viable at room temperature for $2 \frac{1}{2}$ years. Cultures in coli-broth become sterile in about a week. The organisms withstand freezing, and drying in vacuo, and appear to remain alive for a long time when dried. Infected lungs of rabbits, kept in 50 per cent. glycerol at $4^{\circ} \mathrm{C}$., remain virulent for 9 months. The organism is destroyed by moist heat at $56^{\circ} \mathrm{C}$. in 30 minutes, and by chloroform vapour in 1 to $1 \frac{1}{2}$ hours. 
Metabolism. - Striet anaerobe. Grows best at $37^{\circ} \mathrm{C}$. ; no growth at room temperature. When used to artificial conditions, it grows best on Fildes' agar and blood agar; it also grows well in coli-broth. In our experienceit does not grow on the $\mathrm{V}$ or $\mathrm{X}$ factors, either alone or in combination (see Chapter 33). Does not hæmolyse horse blood. Optimum $\mathrm{pH}$ for growth $7 \cdot 6$; limits $7 \cdot 0-8 \cdot 0$.

Brochemical.-Acid is produced in dextrose. Indole negative. Nitrates not reduced. Catalase negative. Hethylene blue reduction negative.

Serological.-Agglutinins are formed by injection of cultures into rabbits, but a serological study of different strains of the organism has not been made.

Pathogenicity.- - Was originally suspected of being responsible for human influenza, but is now regarded as having no causal relationship to this disease. Is mildly pathogenic for rabbits, but loses its virulence after artificial culture for some time in the laboratory. Injection of mass cultures intratracheally into rabbits produces a rise of temperature in 24 hours; usually a conjunctivitis, and a mononuclear leueopænia. The symptoms disappear in 2 to 3 days, and the rabbit recovers. If killed during the reaction, the rabbits are found to have voluminous lungs, affected with codema and emphysema. Numerous hæmorrhages, discrete or diffuse, are seen on the surface of the lungs. 'The pleura is not involved. On section the lungs drip a frothy, blood-stained fluid, and hæinorrhages are seen scattered through the parenchyma. The trachea and bronehi contain a muco-purulent exudate, covering an exfoliated and hæmorrhagic epithelium. The organisms may be recovered from the lungs in pure culture, using Smith-Noguchi medium. Huch the same reaction is produced by intratracheal moculation of guinea-pigs. Nonpathogenic to monkeys, injected intratracheally or subconjunctivally.

Several other tiny anaerobic bacilli have been isolated from the nasopharynx of healthy and diseased persons, differing from Bact. pneumosintes in cultural reactions, pathogenicity, and eertain other respects (Olitsky and Gates 1922b, Gates 1926). Olitsky and McCartney (1923) found them in the nasal washings of persons with colds and in normal persons. Mills, Shibley and Dochez (1928) found them in 75 per cent. of normal persons. This high pereentage was observed only when buffered broth was used for the nasal irrigation: Ringer's fluid gave much lower figures. The bacilli were of variable morphology, and appeared by agglutination tests to belong to several different types. In this country Garrod (1928) has demonstrated similar filter-passing, anaerobic, Gramnegative organisms in the upper respiratory. tract of both healthy and diseased subjects. He states that morphologically they appear either as very tiny cocci, about $\frac{1}{5}$ the diameter of a staphylococcus, or as very short, sometimes curved bacilli. These organisms can be cultivated anaerobically on rabbit blood agar.

\section{Bacterium granulosis.}

This organism was isolated by Noguchi (1927) from American Indians suffering from trachoma. It is a very small Gram-negative bacillus, with a tendency to pointed ends and a diploid arrangement. In young blood-agar cultures it measures $0.8-1 \cdot 2 \mu$ in length and $0 \cdot 2-0 \cdot 3 \mu$ in breadth. In older cultures large irregular involution forms are common. In the condensation water of blood-agar slopes the organism is actively motile by a single polar flagellum. It has a superficial resemblance to $C$. xerosis, but it is smaller, Gramnegative and motile. It was first eultivated on semi-solid leptospiral medium, in which it gives rise to a diffuse nebulous greyish-white growth in the uppermost centimetre, extending for some distance down the stab. No growth oceurs on agar, but on horse blood agar incubated at $30^{\circ} \mathrm{C}$. minute, shiny, almost transparent or slightly greyish, eircular, convex colonies are evident in 48 hours, which later increase in size and acquire a greyish opalescence and a viscons consistency. Growth occurs best at $30^{\circ} \mathrm{C}$, and harlly at all at $37^{\circ} \mathrm{C}$; the lower limit appears to be about $15^{\circ} \mathrm{C}$. The optimum II-ion concentration for growth is $\mathrm{pH} 7 \cdot 8$, and the range $6 \cdot 8-8 \cdot 8$. No growth oceurs umler strict anaerobie conditions. When freshly isolated the organism is devoid of fermentative activity, but after cultivation in the laboratory for some months it is said to produce acid from a number of sugars. 
Indole is not formed; nitrates are reduced to nitrites; and gelatin is not liquefied. It is destroyed by heat at $57^{\circ} \mathrm{C}$. in 10 minutes (Olitsky 1930). The organism is non-pathogenic for laboratory animals, but when inoculated subconjunctivally into rhesus monkeys it gives rise to a granular conjunctivitis simulating trachoma (see Chapter 85). (For a fuller description of this organism see Noguchi 1928, Tilden and Tyler 1930.)

A Gram-negative bacillus simulating Bact. granulosis in many respects has been described under the name of Bact. simice by Olitsky, Syverton, and Tyler (1933), who isolated it from monkeys suffering from spontaneous conjunctival folliculosis. Like Bact. granulosis, which it resembles in size, it is motile by a single polar flagellum, produces a nebulous opacity in leptospiral medium, fails to grow anaerobically, has an optimum growth temperature of $30^{\circ} \mathrm{C}$, and is non-pathogenic for laboratory animals. It differs, however, from this organism in having a capsule, in growing on plain nutrient agar, in fermenting different carbohydrates, in failing to reduce nitrates, and in showing no agglutination with granulosis antisera. What relation Bact. granulosis and Bact. simice bear to Ducrey's bacillus, and to the less exacting members of the Hamophilus group, has still to be determined.

\section{Bact. alkaligenes and Vibrio alkaligenes.}

Petruschky (1896) isolated from human frees an organism that produced an alkaline reaetion in certain media, and was named by him Buct. foccalis alkaligenes. It was described as a Gram-negative bacillus, and has long been included by most authorities in the genus Bacterium under the name of Bact. alkaligenes. Though it appears to be an almost constant inhabitant of the intestinal tract of man, this organism may give rise to infections of the enterie type (Petrusehky 1896, Hirst 1917, Khaled 1923).

The characters usually ascribed to Bact. alkaligenes are as follows: The cells are very variable in size, but are usually longer and thimner than those of Bact. coli $(5-7 \mu \times 0 \cdot 4 \mu)$, and the ends are less definitely rounded. The bacilli are motile, with peritrichate flagella. The colonies on agar are flatter than those of Bact. coli, and more contoured, with a raised central portion and a spreading undulate edge. Neither acid nor gas is produced in any of the usual test substrates. Litmus milk is rendered strongly alkaline. A characteristic brown colour is produced on potato.

Many of these characters are very unlike those of the genus Bacterium, and doubts have been frequently expressed as to its real systematic affinities. A recent study by Nyberg (1935) raises even stronger doubts as to whether the strains that have been described under this name can be regarded as forming a bacterial species. Nyberg examined with great care 134 strains labelled Bact. alkaligenes, and was able to distinguish among them two quite distinct forms, and a number of less well-differentiated types. The form for which he would reserve the name Bact. alkaligenes is a short, thick bacillus, usually nonmotile or very feebly motile, but possessing in most cases poorly formed peritrichate flagella. It fails to ferment dextrose, lævulose, lactose, maltose, saccharose, rhamnose, xylose, arabinose, mannitol, sorbitol, dulcitol, inositol or salicin. It does not form indole, and it produces no change in milk. Of the 134 strains examined, 71 were of this type. Nyberg notes that this description does not agree with that given of Bact. alkaligenes in many books and papers, and that it is impossible to be certain that his strains correspond to the organism originally isolated by Petruschky. He eonsiders, however, that they probably belong to the same species, and that the name must certainly be given to them and not to the type to which his other strains belong.

This second type is a long, thin, slightly curved rod, actively motile by lophotrichate flagella. It is therefore not a bacillus, but a vibrio. Unlike the former type, it renders a dextrose medium slightly but definitely alkaline. Nyberg regards this organism as identical with the Vibrio alkaligenes of Lehmann and Neumann (1896); and there would seem no doubt that this is its proper name. It also seems very probable, as Nyberg 
emphasizes, that many of the strains that have been isolated by various workers, and have been given the description summarized earlier, were Vibrio alkaligenes, not Bacterïum alkaligenes.

It is worth noting that, as pointed out by Conn (1942), failure to produce acid in a glucose medium may be due either (1) to non-fermentation, or (2) to utilization of the glucose so completely that there are no by-products capable of giving an acid reaction except $\mathrm{CO}_{2}$, which, in a buffered medium, will not be detected.

\section{Bartonella, Eperythrozoon, and Grahamella}

Various bodies have been described by different workers in close association with the red blood corpuseles of man and animals suffering usually, though not always, from certain types of anæmia. The evidence that Bartonella and Eperythrozoon are living reproducible micro-organisms capable of giving rise under favourable conditions to disease is now very strong, but considerably less is known about the Grahamella, though there is increasing reason to believe that these bodies also are definite bacteria. The interest that Bartonella muris particularly has stimulated of recent years is due to the remarkable part played by the spleen in the normal defence mechanism of the host (see Chapter 79).

Some anthors regard the organism causing human Oroya fever as generically distinet from the organisms responsible for infective anæmia of animals. They would classify the first as Bartonella, the remainder as Hamobartonellu. The distinctions between them are summarized as follows. Bartonella, besides invading red blood corpuscles, is able to develop in fixed tissue cells and to canse a skin eruption; it is moreover insusceptible to arsenic preparations. Hcemobartonella, on the other hand, is said not to grow outside the blood, it rarely produces disease without remoral of the spleen, and the disease it causes is influenced by arsenotherapy (see Weinman 1944). Whether these differences are sufficient to justify the establishment of a separate genus for the animal bartonellæ is very doubtful, and for the present we shall group them all under the one genus Bartonella. Though we shall refer to only three species of Bartonella, it may be mentioned that numerous other species have been described, infecting guinea-pigs, voles, squirrels, hamsters, opossums, cattle, buffaloes, and other animals. For a review of Bartonella and Eperythrozoon the reader is referred to the monograph by Weinman (1944).

\section{Bartonella bacilliformis.}

This organism is a small bacillus, which invades the red blood cells, and is responsible for Peruvian Oroya fever and for verruga peruana (see Chapter 79). It was called Bartonella bacilliformis by Strong and his colleagues (1915) in honour of Barton, who was one of the first to observe the bacillus in the red cells. Noguchi and Battistini in 1926 cultivated the organism, and reproduced a disease in monkeys bearing a close resemblance to the natural disease in human beings. The organism was recovered in pure culture from the blood of the injected monkeys.

Morphologically, in culture, it is a small pleomorphic bacillus, varying in length from $0 \cdot 3-2.5 \mu$ and in breadth from less than 0.2 to as much as $0.5 \mu$. Dumb-bell forms predominate, and coccoid forms are common. It is arranged singly and in dense masses. It is motile, Gram-negative, and stains reddish-violet with Giemsa. Cultivation can be effected on a variety of media, such as the semi-solid serum lremoglobin agar medimm used for leptospiræ, or a blood glucose cystine agar (Jiménez 1940), or a 2 per cent. proteose agar to which 25 per cent. of fresh defibrinated blood or serum from the rabbit or sheep is added, together with 0.2 per cent. of an ascorbic acid glutathione solution (Geiman 1941). Though a high proportion of natural animal protein favours growth, it does not 
appear to be essential. Jiménez (1940), for example, states that good growth was obtained on 1 per cent. glycerol infusion agar provided the $X$, though not the $V$, factor (see Chapter 33 ) was added. On solid media growth may occur in 4-5 days, either as mimute, circular, clear, mmeoid colonies, or as an opaque, finely granular, mucoid film that has a tendeney to outgrow the original boundaries of the inoculum (Jiménez 1940). The organism is acrobic, grows well at $25-37^{\circ} \mathrm{C}$, though hest at about $25-28^{\circ} \mathrm{C}$. prefers a pH of $7 \cdot 8$, and survives in cultures for 1 to 4 months. Pinkerton and Weinman (1937) found that in tissue cultures the organisms, mulike Ricketsia. grew extracellularly as well as intracellularly. (krowth oceurs also in the allantoic fluid of the developing chick embryo incubated at $25^{\circ}-25^{\circ} \mathrm{C}$. but this medium is unsuitable for serial cultivation (Jiménez

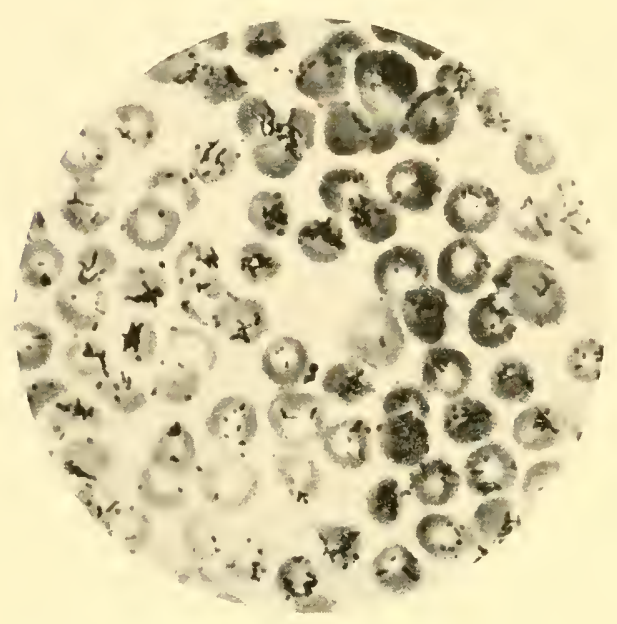

Fra. 213.-Bartonella muris.

Organisms in red blood cells of rat $(\times 1000)$. [From specimen kindly supplied by the late Professor J. G. Thomson.]

medium. The blood is not hemolysed. Cultures on solid media lave a sweet odour resembling canned pineapple. The optimum temperature for growth is $25^{\circ} \mathrm{C}$. In infected blood the organisms are destroyed by exposure to a temperature of $57^{\circ} \mathrm{C}$. for 30 minutes (Ford and Eliot 1929), but remain virulent in the frozen state for at least 11 weeks (Kessler 1942). Pure cultures inoculated into splenectomized rats give rise to anæmia (see Chapter 79). The organism is fairly common in rats, eausing an infection which normally remains latent, but which can be activated by splenectomy, by poisons such as toluylenediamine, and by certain infections (for general description see Landa and Marcus 1928, Marmorston-Gottesman and Perla 1932, Kikuth 1931, 1934).

\section{Bartonella canis.}

A similar organism las been deseribed under this name by Kikuth (1928), which is responsible for infectious anemia of dogs. In the red cells the organisms are very pleomorphic, large and small, coccoid and rod-shaped forms being seen. Kikuth was unsuccessful in cultivating them on artificial media. (See Kikuth 1929, Pérard 1929, Lwoff and Provost 1929. Regendanz and Reichenow 1932, and Clapter 79.)

Besides the species alrearly mentioned, Bartonella has been found infecting various rodents in different parts of the world, monkeys and some other mammals in America, and cattle in Algeria and Palestine (see Pittaluga 1938). Whether these organisms deserve specific rank or not must await further study. 


\section{Eperythrozoon coccoides.}

This is a parasite of mice which was discovered independently by Dinger $(1928,1929)$, and Schilling (1928). A considerable proportion of normal mice appear to be infected, but the organisms are rarely found unless the spleen is removed. Two to four days after splenectomy the organisms appear in the blood in the form of rings or dises stuck on to the external surface of the red blood corpuscles, and staining a bluish-purple colour with Giemsa. Unlike Bartonella muris, they have a preference for polychromatic red cells. They may persist in the blood for weeks or months, their numbers varying from time to time. Beyond producing a slight degree of anæmia, they seem to be without any very definite effect on the animal. Hice infected with Eperythrozoon coccoides are susceptible to Bartonella muris, showing that the two organisms are distinct (see also MeCluskie and Niven 1934, Sehwetz 1934, Marmorston 1935). In contrast to Bartonella, Eperythrozoon is round in shape with numerous annular and disc-like elements; rods are rare and are seldom in chain formation. The organisms lave not yet been eultivated. Infeetion appears to be spread by lice. Numerous other animals, besides mice, are liable to infection with Eperythrozoon (see Weinman 1944).

\section{Grahamella.}

This parasite was first described by Graham-Smith (1905) at Cambridge, who observed it in the red blood cells of 10 per eent. of moles that were being examined for Piroplasma. Since then it has been found in the blood of several other animals. The organisms appear as longer or shorter rods of irregular contour lying within the red corpuseles. Though resembling Bartonella, they are much coarser, and more like ordinary bacteria. With Giemsa they take on a blue rather than a rectdish tint. Only oeeasional red cells are affected. Aeeording to Jettmar (1932) they ean be cultivated on serum blood agar. They appear to be non-pathogenic and to have no effect on the health of the host. In the rat Vassiliadis (1935) las been able to transmit infection from one animal to another in series. In most animals splencetumy has little or no influence on infection, though the rat is said to constitute an exception (Vassiliadis 1935). Eetoparasites, such as lice, are probably responsible for the natural transmission of infection. Unlike Bartonelln muris, Grahamella seems to be resistant to arsenic (see also Kikuth 1934).

\section{REFERENCES}

Auduread, A. (1940) Ann. Inst. Pasteur, 64, 1:6).

Axenfeid, T. (1897) Zbl. Baht., 21, 340 .

Covs, H. J. (1942) J. Bact., 44, 353.

Dinger, J. E. (1928) Ned. Tijdschr. Geneesk., No. 48, 72, 5903; (1929) Zbl. Balt., 113, 503

Exre, J. W. (1900) J. Path. Bact., 6, 1.

FORD, W. W. and Eliot, C. P. (1928) $\%$ exp. Med., 48, 475.

Garrod, L. P. (1928) Brit. J. exp. Path., 9, 155.

Gates, F. L. (19:6) J. exp. Med., 44, 787.

Geivin. Q. II. (1941) Proc. Soc. exp. Biol., N.Y., 4\%, 329,

Grahan-Shith, G. S. (1905) J. Hyg., Camb., 5, 453.

HaLl, M. W. (1926) J. exp. Med., 44, 539.

Hirst, L. F. (1917) J. R. Army med. Cps, 29, 476.

JetTMAR, H. M. (1932) Z. P'irasitenk., 4, 254.

Jiménez, J. F. (1940) P'roc. Soc. exp. Biol., N.Y., 45, 40z.

Jiménez, J. F. and Budolxa11, G. J. (1940) Proc. Soc. exp. Biol., T.Y., 45, 546.

Jones, F. S. ant Littile, R. B. (1923) $J$. ғxp. Med.. 38, 139.

Kessler, IT. R. (194:) Proc. Soc. exp. Biol., Y.Y., 49, 238.

Khaled, Z. (1923) J. Hyg., Camb., 21, 362.

Kiкuтн, W. (1928) Klin. Wrshr., 7, 1729; (1929) Zhl. Bakt.. 113, I: (1931) Z. ImmunForsch., 73, 1: (1934) Proc. roy. Soc. Hed., $2 \%, 1241$.

LAUDA, E. and MAReUs, F. (19:8) Zbl. Bukt., 10\%, 104.

Lehiann, K. and NeUmanN, R. (1596) "Atlas und Grundriss der Pakteriologie, ete." J. F. Lehmann, Wunich. 
Lwoff, A. (1939) Ann. Inst. Pasteur, 62, 168.

Lwoff, A. and Provost, A. (1929) C. R. Soc. Biol., 101, 8.

MoCluskie, J. A. W. and Niven, J. S. F. (1934) J. Path. Bact., 39, 185.

Marmorstow, J. (1935) J. infect. Dis., 56, 142.

Marmorston-Gottesman, J. and Perla, D. (1932) J. exp. Med., 56, 763.

MIAYER, M. (1921) Arch. Schiffs-u. Tropentuyg., 25, 150.

Mills, K. C., Shibley, G. S., and Dochez, A. R. (1928) J. exp. Med., 47, 193.

Morax, V. (1896) Ann. Inst. Pasteur, 10, 337.

Noquchi, H. (1926) J. exp. Med., 44, 533, 697, 715, 729; (1927) J. Amer. med. Ass., 89, 739; (1928) Monograph on Trachoma; J. exp. Mfed., 48, No. 2, Suppl. No. 2.

Noguchi, H. and Battistini, T. S. (1926) J. exp. Med., 43, 851.

Nybera, C. (1935) Zbl. Bakt., 133, 443.

OAG, R. K. (1942) J. Path. Bact., 54, 128.

OLitsky, P. K. (1930) Trans. 35th ann. Meeting Amer. Acad. Ophthal. Oto-Laryngol., p. 225.

Olitsky, P. K. and Gates, F. L. (1921a) J.exp. Med., 33, 125 ; (1921b) Ibid., 33, 361 ; (1921c) Ibid., 33, 713; (1922a) J. exp. Med., 35, 813; (1922b) Ibid., 36, 501.

OltTsky, P. K. and MoCartney, J. E. (1923) J.exp. Med., 38, 427.

Olitsky, P. K., Syverton, J. T., and Tyler, J. R. (1933) J. exp. Med., 5\%, 871.

Pérard, C. H. (1929) C. R. Soc. Biol., 100, 1111.

Petit, P. (1900) "Recherches cliniques et bactériologiques sur les infections aiguës de la cornée." Thèse de la Faculté de Médecine de Paris. G. Steinheil, Paris.

Petruschis, J. (1896) Zbl. Bakt., 19, 187.

Prnkerton, H. and Weinman, D. (1937) Proc. Soc. exp. Biol., N.Y., 37, 587.

Pittaluga, G. (1938) Bull. Inst. Pasteur, 36, 961.

Regendanz, P. and Reichenow, E. (1932) Arch. Schiffs-u. Tropenhyg., 36, 305.

Schilling, V. (1928) Kilin. Wschr., \%, 1853.

SCHWETZ, J. (1934) Zbl. Bakt., 132, 211.

Strong, R. P., Tyzzer, E. E., Brues, C. T., Sellards, A. W., and Gastiaburu, J. C. (1915) Rep. 1st Expedition S. America, 1913. Harvard Univ. Press, Cambridge, Mass.

Tilden, E. B. and Tyler, J. R. (1930) J. exp. Med., 52, 617.

Vassiliadis, P. (1935) Ann. Soc. belge Méd. trop., 15, 279.

Weinman, D. (1944) Trans. Amer. philosoph. Soc., 33, Pt. III, 243. 


\section{CHAPTER 38}

\section{THE SPIROCHÆTES}

TнЕ name Spirochæte was first given by Ehrenberg in 1833 to a large flexible motile organism occurring in water; it is now used as a general term for all elongated, motile, flexible organisms that are twisted spirally around their long axis. Though the spirochætes vary greatly in size, they all possess certain features in common : thus they possess no flagella; they exhibit no antero-posterior polarity (i.e. they can move either forwards or backwards); they contain no colouring matter and no cyanophycin granules; they show no definite localized nucleus : they divide transversely, division being either simple or multiple; and they exhiljit no sexual phenomena of reproduction. These properties bring them closely into line with the Bacteria, to which they are more nearly related than to the Protozoa. Indeed, as Dobell (1912) points out, while there are many features in which they differ from the Protozoa, there is only one feature that differentiates them from the bacteria, namely motility without flagella. Even this difference is now subject to doubt, since studies by the electron microscope on some of the treponemata have revealed filamentous bodies that may be interpreted as flagella (Mudd, Polevitzky and Anderson 1943, Wile and Kearney 1943).

Without entering into the disputed question of their classification, it is convenient for descriptive purposes to divide the spirochætes into four groups-Spirochata,
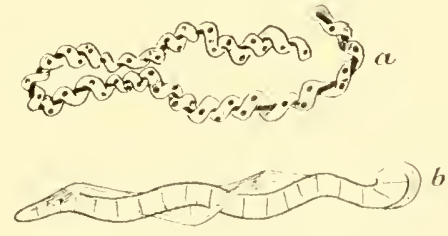
Cristispira, Treponema, and Leptospira.

Spirochaeta. - The members of this group possess an axial fibre, around which the body is twisted in a spiral manner, in just the same way as a spiral staircase is built round the newel. The organism possesses a series of regular primary spirals; during motion a series of secondary waves may be superimposed on these, but whatever form the organism as a whole may assume, the primary spirals remain intact. Metachromatic granules of volutin are distributed uniformly throughout the length of the organism. The type species, Spirochata plicatilis Ehrenberg, is usually 200-500 $\mu$ in length and 0.5-0.7 $\mu$ in thickness (Wenyon 1926). The number of primary spirals is 100 to 250 , the distance between successive turns being about $2 \mu$. So far no members of this group have been found to be capable of causing disease. 
Cristispira.-The peculiar characteristic of members of this group is the possession of a band-like membrane or crista, which runs in a spiral manner along the organism. This membrane is extremely thin; its width is much the same as that of the organism itself, except at the ends, where it narrows down to fuse with the surface pellicle. The body of the organism is divided into chambers by septa of thickened cytoplasm, and on either side of each septum, or sometimes distributed irregularly through the more fluid cytoplasm in the chambers, are a number of metachromatic granules. The type species, Cristispira balbianii, is found in the crystalline style of the oyster ; it is about $45-100 \mu$ long and $1-1.5 \mu$ broad (Wenyon 1926). Other species have been found in a similar situation in other Mollusca.

Treponema.-Members of this group possess neither an axial fibre nor a crista. According to some observers the body is divided up into chambers like that of a Cristispira, but this is by no means certain. The presence of metachromatic granules is doubtful. The organism shows a number of primary spirals, which may be closely or loosely wound ; during movement secondary turns may develop but at rest these disappear so that the organism is straight. The ends may be rounded or pointed, and in some members it is possible to distinguish a thin drawnout filament at the ends; this is probably not a true flagellum, but the remains of the thin connecting bridge of cytoplasm that is seen during transverse division. Electron micrographs (Wile et al. 1942, Wile and Kearney 1943, Mudd, Polevitzky and Anderson 1943) show the presence of a delicate cell wall, or periplast, enclosing the inner protoplasm. Dense granules, $40-90 \mathrm{~m} \mu$ in diameter, are often seen in the protoplasm; and large, irregular, spheroidal bodies, $150-500 \mathrm{~m} \mu$ in diameter, may occur attached to the cell near the end, or connected with it by a short stalk. What have been interpreted as true flagella may be seen, often in groups of four, along the sides or near the ends, of Trep. pallidum and Trep. macrodentium. Treponemata are widely distributed; numerous species have been described in water, in the gut of certain insects such as white ants and cockroaches, and in the large gut of the toad; in human beings they are found in the month, sometimes in the alimentary tract and the bronchi, around the urethral orifice, in certain ulcerating conditions of the skin, in condylomata, in the blood of patients with relapsing fever, and in the manifold lesions of syphilis. They vary considerably in size; thus Treponema termitidis Leidy is $20-60 \mu$ long and $0.5 \mu$ broad; on the other hand, Treponema parvum may be only $3 \mu$ in length and $0.2 \mu$ in thickness (Dobell 1912).

Leptospira.-The members of this group possess neither axial filament nor crista; their cytoplasm is not obviously chambered. They show, however, a large number of closely wound, primary spirals; this differentiates them from the treponemata, the spirals of which are fewer and less closely wound. Moreover, the leptospiræ frequently have their ends turned round at a sharp angle to the rest of the body. When the organism is at rest the ends appear characteristically hooked; but when it is in motion, rotating round its long axis, the ends take on the appearance of button-holes, the narrow pointed end of the button-hole being attached to the body of the organism, the wider and rounded end being free. During motion secondary curves often appear and disappear in rapid succession, giving the organism a resemblance to a $\mathrm{C}, \mathrm{O}, \mathrm{S}$, or other curved letter. The primary spirals are absolutely regular, and remain intact throughout all the various contortions executed by the organism as a whole. The leptospiræ are widely distributed in water, and can be easily demonstrated in it by simple cultural methods 
(Hindle 1925). They are found in the tissues of patients with Weil's disease and other leptospiral infections. In length they vary from about 6-85 $\mu$, but are generally about $9-12 \mu$; their thickness is only about $0 \cdot 1 \mu$. The length of the middle portion is variable, but the hooked ends are always of about the same size, suggesting that growth may occur only in the middle portion (Zuelzer 1925). No internal structure can be distinguished in electron micrographs, nor can flagellumlike bodies, similar to those observed in some of the treponemata, be seen (Morton and Anderson 1943).

The brief account that has been given of the different groups is necessarily dogmatic, and it may well be that further work will necessitate a revision of our present classification.

\section{General Characteristics of the Spirochetes}

Morphology.-With the exception of the structures seen in electron micrographs of some of the treponemata (see p. 908), spirochætes are generally regarded as being withont flagella; they are nevertheless motile. Three kinds of movement are generally described: (1) Movements of flexion, in which the whole organism undergoes a change in shape. As a rule the natural shape of a spirochrte at rest is straight; but during movement all sorts of twists and turns may develop, one form following another in rapid succession, but each one tending to return to the normal straight form. During these movements of flexion the primary spirals remain unaltered. (2) Movements of rotation around the long axis; these are difficult to see unless the ends of the organism are bent at an angle to the main axis, when the rotatory movement is especially apparent. When the rotation is very rapid, and when as in Leptospira the ends are hooked, the spirochæte may take on the appearance of a spiral thread with a button-hole at each end. (3) Movements of translation, by which the organism changes its position, moving from one place to another. This change in place is probably dependent upon the rotatory movement, which acts like a propeller driving the organism forwards or backwards according to the direction of the rotation.

Different spirochætes vary greatly in their activity; some, for example, exhibit very active movements of flexion, lashing furiously in various directions, but making very little progress from their original position; others dart rapidly hither and thither, rendering their ocular pursuit almost impossible.

Multiplication occurs by transverse fission. It is not clear, however, whether the organism always divides in the middle giving rise to two shorter spirochætes of equal length, or whether division occurs at times asymmetrically. According to Séguin's (1930) observations on Trep. calligyrum and Trep. gallinarum, and Manouélian's (1940) observations on Trep. pallidum, asymmetrical division, at least in cultures, is not uncommon, the shorter cell seen after separation having only two, one, or even less than one complete spiral. Again, whether the large knob-like bodies seen attached to some of the treponemata play any part in reproduction is still doubtful.

Generally speaking, the spirochætes are more difficult to stain than the bacteria. Methylene blue, which is usually a satisfactory bacterial stain, leaves many of the spirochætes unstained. It has been suggested that the spirochætes are devoid of nucleo-protein, and to the absence of this substance has been ascribed the failure of the treponemata and all the known leptospiræ to stain with this dye. On the other hand, the fact that an organism does stain with methylene blue is no proof 
that it contains nucleic acid (see Zuelzer 1925). The spirochætes that stain with methylene blue are generally coloured blue by Giemsa, whereas those that do not are generally coloured red. But the exact tint that results from Giemsa's stain depends to some extent on the medium in which the organism is grown; thus in the blood, Trep. recurrentis stains blue with occasional reddish granules, whereas in culture it stains red. In the larger spirochætes it is possible by such stains as Giemsa's and iron hæmatoxylin to bring out the finer details of structure, but in the smaller ones this is practically impossible. The reaction to Gram's stain is negative, though this stain is rarely used in practice.

For the demonstration of spirochætes in tissue-sections Levaditi's method is one of the most successful. It depends on the ability of the organisms, when treated with silver nitrate followed by reduction with a formol-pyrogallic acid mixture, to become impregnated with metallic silver, and therefore to appear black. Fo the demonstration of spirochætes in films the Fontana method of silver impregnation is most useful.

Many species, particularly the leptospiræ, are able to pass through the usual bacterial filter candles. This property they owe to their extreme tenuity. Exact measurements by Hindle and Elford (1933), made with graded collodion membranes, show that the width of Trep. pallidum is about $0 \cdot 2 \mu$ and of Lepto. biflexa $0 \cdot 1 \mu$. Use is often made of their filtrability to separate them from contaminating bacteria.

Cultivation.- The cultivation of spirochætes in vitro is not so simple as that of most bacteria. Nearly all the methods that have proved snccessful involve the use of a medium containing native animal protein, such as blood, serum, or ascitic fluid. Whether this acts chemically as a nutrient material, or physically as a protective colloid preventing the organisms from being poisoned by the products of their own metabolism, is not clear. A further requirement for many spirochrtes is a low oxygen pressure; this is obtained by culturing them in narrow tubes containing a high column of medium ; or by adding a piece of sterile kidney, which produces a zone of anaerobiosis in its neighbourhood (Theobald Smith's method). It is usual to cover the medium with a layer of paraffin oil ; this was at first believed to prevent the ingress of oxygen, but it appears now that this explanation is incorrect. The beneficial action of the oil probably depends partly on the prevention of evaporation of water from the medium, and partly on the prevention of the loss of $\mathrm{CO}_{2}$ from the medium, which would otherwise become progressively more alkaline (Gates and Olitsky 1921, Kligler and Robertson 1922). Spirochætes have been cultivated mainly in liquid or in semisolid media. Colony production ou the surface of solid media under aerobic or anaerobic conditions has been reported by a few workers (Twort 1921, Gates 1923, Aksjanzew-Malkin 1933, Séguin and Vinzent 1938), but in general little success has been obtained with this method.

Multiplication occurs rather slowly, and may not be evident for a week or more. When a clear medium is used, growth may be evident from the appearance of a faint turbidity; but generally microscopical examination is necessary, particularly for motility and signs of transverse division. Most spirochætes seem to prefer a slightly alkaline medium, about $\mathrm{pH} 7 \cdot 2-7 \cdot 6$. Subculture has to be performed every few days as a rule, the exact time depending on the particular organism. In young cultures the organisms are actively motile and under dark-ground illumination appear uniformly refractile; but in older cultures when degeneration sets in, they lose their motility, tend to agglutinate into clumps, and become granular ; 
the granules, some of which result from a change in the cytoplasm within the organism, and some of which are probably particles of the culture medium adhering to their exterior, are more highly refractile than the rest of the spirochrte, and show up as bright, glistening points. Some observers consider that these granules are not the result of degeneration, but are analogous to bacterial spores, affording a means of continuing life under unfavourable environmental conditions. The evidence in favour of this view is not convincing.

The isolation of spirochretes in pure culture from material in which they are accompanied by bacteria often presents great difficulty. Various methods may be tried, such as rapid subculture, making use of the ability of some species to grow out from the line of inoculation in stab cultures, picking deep colonies, choosing media made selective by the addition of a dye or some other substance, filtration, animal inoculation, and so on (see Séguin and Vinzent 1938, Schüffner 1940, Wichelhausen and Wichelhausen 1942).

With some organisms, such as Leptospira icterohcemorrhagia, in vitro culture is remarkably successful; but with organisms such as Trep. pallidum and Trep. recurrentis it is not so satisfactory. For this reason these organisms are generally preserved by in vivo culture; that is to say, they are injected into a susceptible animal, which henceforth becomes a chronic carrier, and which can be drawn upon at will for a fresh supply of infective material. A modification of the animal inoculation method, which has proved successful in the cultivation of relapsing fever spirochætes and Lepto. icterohcmorrhagice, is growth in the developing chick embryo (see Morrow et al. 1938, Chabaud 1939, Oag 1939, Soule 1912).

Resistance and Metabolism.-The resistance of spirochæes to inimical agencies is no greater and generally less than that of the vegetative bacteria. Dry heat, moist heat, and desiccation prove quickly fatal, as do comparatively low concentrations of the chemical disinfectants. It is generally stated that spirochres are lysed by a 10 per cent. solution of sodium taurocholate (see v. Prowazek 1907), and all, with the exception of the leptospiræ, by a 10 per cent. solution of saponin (Noguchi 1917). Some of the highly parasitic members, such as Trep. pallidum, are unable to survive outside the animal body for more than an hour or two. Indeed this particular organism is extremely susceptible to heat, being destroyed in an hour at $41.5^{\circ} \mathrm{C}$. Advantage is now being taken of this property to sterilize the organisms in the tissues by exposure to fever-heat temperatures (see Chapter 81 ). Spirochætes in infected tissues may be preserved alive for several months by keeping them in the frozen state, preferably at about $-78^{\circ} \mathrm{C}$. (Jahnel 1937, Turner 1938).

Practically nothing is known about the metabolism of spirochætes. Scheff (1935), who made observations on two strains of Trep. pallidum, one of Trep. recurrentis, and one of Trep. anserinum, stated that, when these organisms were grown in a medium containing glucose, they broke down the sugar to lactic acid and $\mathrm{CO}_{2}$ without using up any of the oxygen.

Antigenic Structure.-Antigenically, spirochætes behave very much as bacteria. They give rise on injection to agglutinins, spirochætocidins, spirochætolysins, and protective antibodies. Agglutination tests are often conducted by the microscopic method, and observations made on the loss of motility and the clumping of the organisms, which may occur in radiate fashion. Similarly the destruction and lysis of the organisms that frequently follow contact with a highly immune serum are generally watched under the microscope. The macroscopic method, however, 
is now used cxtensively for study of the leptospirs, and antigenic types are determined by cross-agglutination, and sonetimes cross-absorption, tests. Protective bodies in serum are tested for in the usual way by animal inoculation.

A valuable method of study is afforded by the test described by Rieckeuberg (1917), known sometimes as the thrombocytobarin reaction or the adhesion phenomenon. It depends on the fact that if a suspensiou of blood platelets or bacteria is added to a mixture of a spirochæte and its specific antiserum, and the preparation is observed under dark-ground illumination after preliminary incubation at $30^{\circ} \mathrm{C}$. for 20 minutes, the platelets or bacteria are seen to have become adherent to the spirochrtes in the form of small clumps. In the presence of a non-specific serum no such clumping or adhesion occurs. By this reaction it is possible to distinguish rapidly between two such closely-allied forms as Lepto. icterohcemorrhagice and Lepto. hebdomadis (Brown and Davis 1927). Living motile spirochætes are required and fresh complement must be present. The specific antibody is destroyed by heating to $72^{\circ} \mathrm{C}$. for 30 minutes (Inoue 1930). Pfeiffer's test and cross-immunity protection tests in animals are likewise of value in distinguishing between closely allied species, and even between variants of the same species.

Pathogenicity.-The virulence of spirochætes appears to be subject to considerable variation. Many members are strictly parasitic and give rise to infections in man or animals, while others are saprophytic and appear to be devoid of any pathogenic effect. There is evidence, however, that the virulence of the parasitic nembers may undergo change as the result of residence in the body of the host. In relapsing fever, for example, the strains that appear in the blood at the second or third relapses may differ antigenically from the strain responsible for the original attack, and by virtue of this change are able to multiply in the tissues of a host that has become immunized to the original parent strain. Again, strains of certain spirochrtes, such as Trep. pallidum, may be brought by passage to grow readily in an animal which at first resists their invasion. Trep. pertenue, the organism that is responsible for yaws, is regarded by many observers as merely a variety of Trep. pallidum which, by residence in the negro, has developed dermotropic affinities (Parham 1922). Residence outside the body of certain parasitic strains may apparently be accompanied by a fall in virulence, which renders them indistinguishable from naturally saprophytic strains. Thus Lepto. icterohamorrhagice, if kept in water, may become indistinguishable from Lepto. biflexa (Zuelzer 1925). Whether the virulence of naturally saprophytic species ever becomes increased so as to render them pathogenic for man is doubtful. Baermann and Zuelzer $(1927,1928)$ have brought a considerable amount of evidence to show that Lepto. biflexa may be transformed by repeated animal passage into Lepto. icterohamorrhagice. Their findings, however, are not in harmony with the experience of most other workers nor with the epidemiological picture of Weil's disease, and it seems probable that such instances are due to the recovery in virulence of a real icterohamomhagice strain.

Though the spirochætes may be classified into the free-living, the commensal, and the pathogenic types, it must be realized that there is no sharp line of demarcation between the three groups. An organism that is pathogenic in one animal may be purely commensal in another, and an organism that is highly pathogenic at one time to a particular host may at another give rise to no more than a latent infection.

We append a description of some of the members that are of most interest to the student of medical and veterinary bacteriology. 


\section{Treponema recurrentis}

Isolation.-Observed by Obermeicr (1873) in the blood of paticnts with European relapsing fever.

Morphology.-Actively motile spiral organisms, varying considerably in length but usually $10-20 \mu$ long. Series of 5-10 fairly regular but loose primary waves; each spiral is $2-3 \mu$ long and about $1 \mu$ in amplitude (Fig. 215). The width is usually given as $0 \cdot 2-0 \cdot 3 \mu$ (Wenyon 1926, Hindle 1931), but this is probably an underestimate. Personal observations on the organisms in blood have suggested that $0.4 \mu$ more nearly represents their true diameter. ${ }^{1}$ This is supported by the observations of Tilden (1937), who found that the limiting pore size for filtration through Elford's gradocol membranes was $0.57 \mu$. After transverse fission the two new organisms may remain conmected by a remnant of the periplast. Stains purplish-red with Giemsa. Organisms are said to be shorter ancl thimer in young culture, thicker and longer in old (Plotz 1917).

Cultivation.-First successful cultivation reported by Noguchi (1912e), who seederl a few drops of eitrated blood from the heart of an infected mouse or rat into a tube containing $15 \mathrm{ml}$. of unheated and unfiltered ascitic or hydrocele fluit and a small piece of sterile ral,bit's kidney. The blood was taken from the animal 48 to 72 hours after inoculation. Multiplication of the spirochates in the cultures was visible in 2 to 3 days, and reached its maximum abont the 7 th to the 9 th day. No change was noticeable in the medium, but actively motile spirochetes could be found in every field, arranged either singly, in chains, or in masses. After about the 9th day a sudden decrease in their numbers occurred, and spherical bodies and irregular protoplasmic masses appeared, indicating that the organisms were undergoing degeneration. Subcultures were most successfully made on the 4th to the 9th days. Other workers (Plotz 1917, Kligler and Robertson 1922, Sinton 1924, Lapidari and Sparrow 1928, Yuan-Po 1933, Scheff 1935), using Noguchi's technique or more often a modification of it, have claimed to cultivate relapsing fever spirochates in vitro, but the results appear to have been very irregular (see Morocker 1929); no one appears yet to have succeeded in establishing a culture that can be continued indefinitely in the laboratory (see Sonle 1942). On the other hand, growth can readily be obtained by inoculation of the chorio-allantoic membrane of the developing chick embryo (Chaband 1939, Oag 1939, Soule 1942). The spirochaetes produce no change in the membrane itself, but invade the blood of the embryo, where they may be demonstrated by the usual methods. Some strains prove fatal to the embryo; others do not.

Resistance and Metabolism.-Resistance is apparently similar to that of the more susceptible vegetative bacteria. Said to remain viable in clotted blood for 6 clays at room temperature and for at least 100 days at $0^{\circ} \mathrm{C}$. (Wynns and Beck 1935). Little is known about metabolism. According to Scheff (1935), glucose is broken down with production of lactic acid and $\mathrm{CO}_{2}$, but no oxygen is used up. A moderate partial pressure of oxygen, however, is required for growth ; there is no multiplication under strict anaerobic conditions.

${ }^{1}$ Mr. J. E. Barnard, F.R.S., has kindly measured for us a strain of Trep. duttoni; he finds it to be $0 \cdot 35 \mu$ in diameter. 
Antigenic Structure.-Little exact information. Some evidence that "relapse" strains differ from the parent strain.

Pathogenicity.-Causes European relapsing fever in human beings. Infection can be transmitted to monkeys, rats, and mice, but not to rabbits or guinea-pigs. In monkeys the disease runs much the same course as in man. Two or three days after subcutaneous inoculation with the patient's blood a pyrexial attack occurs, lasting for 3 or 4 days ; two, three, or four relapses may occur at intervals of 2 to 8 days, each relapse lasting from 1 to 4 days (Norris et al. 1906). After intraperitoneal inoculation of mice the organisms appear in the blood within 24 hours, and persist for 3 to 4 days; they then disappear for several days, after which a relapse may occur ; three or four relapses may follow each other, separated by an interval of about 7 days (Novy and Knapp 1906). As many as 10 to 50 organisms may be present per field during the first infection, but in the relapses only 1 or 2 organisms are seen as a rule. Intraperitoneal inoculation of white rats is followed by the appearance of spirochætes in the blood in about 40 hours; they disappear about 2 days later. The spirochætes are found not only in the blood, but in all the organs of the body. Infection is never fatal. Novy and Knapp (1906) state that rats never relapse.

Numerous strains of relapsing fever spirochætes have been isolated in different parts of the world. As these exhibit certain antigenic differences from Trep. recurrentis, they have been regarded as different species and named accordingly. Thus we have Trep. dultoni of Central Africa, Trep. novyi of America, Trep. kochi of East Africa, Trep. carteri of India, and several others (see p. 1804).

\section{Treponema anserinum}

Described by Sakharoff (1891), who observed it in the blood of infected geese. Morphologically it closely resembles Trep. recurrentis. In blood its mean length is about $14 \mu$, and the mean number of coils about five (Knowles et al. 1932). The organism was cultivated by Noguchi (1912g), using his ascitic-fluid rabbit kidney medium. Growth reaches its maximum about the 5th day, after which degeneration sets in; death is usually complete in 3 weeks. Growth occurs best at $30^{\circ} \mathrm{C}$. Subcultures should be made every 4 days. In eulture the organism is said to be $8-16 \mu$ long, $0 \cdot 3 \mu$ wide, and to show rounded spirals, each of which is about $1.8 \mu$ long and $1 \mu$ in amplitude. According to Landauer (1931) and to Knowles and his colleagues (1932), one of the best media for its cultivation is that devised by Galloway (1925); this consists of coagulated egg white to which dilute inactivated serum is added. In early cultures blood is advantageous. No growth occurs anaerobically. Trep. anserinum is pathogenic for birds, but not for rodents. Intramuscular inoculation of fowls with $0.5 \mathrm{ml}$. of infected blood gives rise to acute spirochætosis in 24 hours. A high mortality results. Spirochætes are numerous in the blood (Knowles et al. 1932). Cross-spirochreticidal and cross-protection tests in chickens suggest that fowl spirochrtes may be subdivided into different antigenic groups (Kligler et al. 1938).

\section{Treponema vincenti}

Described by Vincent $(1896,1899)$, who observed it in the throat of patients suffering from Vincent's angina. This organism is very delicate, about 5-10 $\mu$ long, and has 3 to 8 irregular spirals. In cultures filamentous forms are common. It stains poorly but uniformly, is Gram-negative, and is actively motile. It can be cultivated under anaerobic conditions in serum agar or in serum broth. Growth occurs most readily at $37^{\circ} \mathrm{C}$.; there is no growth at room tempcrature. In serum agar, colonies appear in 3 days, and are very tiny and tenacious (Ellermann 1904). Injected subcutaneously into guinea-pigs, the organisms are generally without effect (Tunnicliff 1906). It is not clear whether Trep. vincenti is responsible for the necrotic lesions in luman beings in which it is found, or whether it is a mere secondary invader. Since the organism may sometimes be demonstrated in the depths of the infected tissues, it is possible that it may possess actual 
invasive properties (Ellermann 1907). It is very frequently found in association with a characteristic fusiform bacillus, likewise described by Vincent (1896, see Chapter 18). It has becn suggested (Tunnicliff 1906) that Trep. vincenti and the fusiform bacilus represent two phases of the same organism; but the balance of evidence is definitely against this view.

\section{Treponema pallidum}

Isolation.-Described by Schandinn and Hoffmann (1905), who observed it in chancres and inguinal glands of syphilitic patients (see Schuberg and Schlossberger 1930).

Morphology. - Thin, delicate spirochæete with tapering ends. Its length varies from 4-14 $\mu$, and its breadth is about $0.2 \mu$. It contains a number of regular primary spirals, which appear rather sharp and angular, and each of which is a little over $1 \mu$ in length. During motion secondary curves may appear and disappear in rapid succession, but the primary spirals remain undisturbed. The organism is actively motile; the movements were originally described by Schaudinn and Hoffmann (1905) as being of 3 types: (1) rotation round

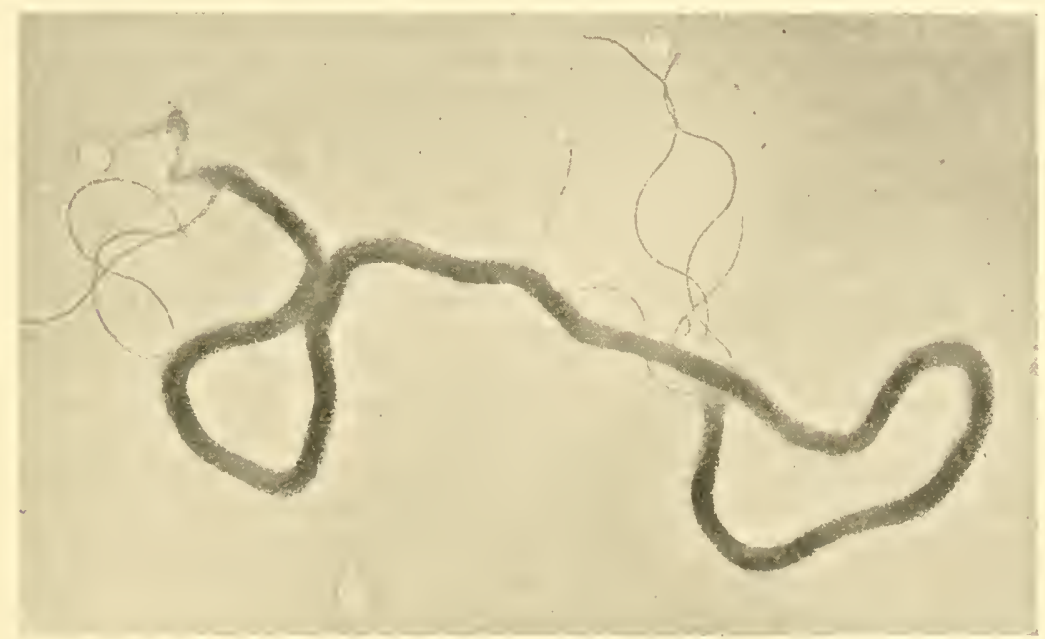

Fag. 216. - Electron micrograph of Treponema pallidum, showing what ajpear to be flagella $(\times 21,000)$. [Kindly supplied by Dr. Stuart Hudd.]

the longitudinal axis; (2) backward and forward movements; (3) flexion movements of the whole body, resulting in the production of secondary waves. The rotation or spinning movement is responsible for the backward or forward movements; the primary spirals act like the blades of a jropeller and drive the organism forward. No flagella can be demonstrated by ordinary methods, but according to Mndd, Polevitzky and Anderson (1943) and Wile and Kearney (1943) flagella, often four in number, may be seen in electron micrographs distributed along the sides or near the ends of the organism. In cultures the morphology is not so regular as in the animal body; Nognchi (1912c) has described three types of pallidum-the thicker, the normal, and the thinner type. Whether these types are constant, or merely represent fluctuations round a mean, is not known ; Noguchi favours the former view. The organism stains rose-red with Giemsa. The organisms are held back by gradocol nembranes having a pore size of $0 \cdot 4 \mu$; their narrowest diameter is therefore about $0 \cdot 2 \mu$ (Hindle and Elford 1933, Tilden 1937).

Cultivation.-Schereschewsky (1909) was the first to cultivate Trep. pallidum in vitro, but he did not succeed in obtaining pure cultures. Noguchi in 1911 was the first to do this. He used a medium of serum water to which a piece of sterile rabbit tissue had been added; 
the medium was seeded with a fragment of syphilitic rabbit's testicle, and the whole was covered with a layer of liquid paraffin. Incubation was earried out anaerobically at $37^{\circ} \mathrm{C}$. The primary mixed culture was later purified by growth in sernm agar stabs containing fresh rabbit tissue; in this medium a hazy zone around and above the tissue became pereeptible in alont 3 days due to proliferation of the spirochetes; by subenlture from this zone he eventually sueceeded in obtaining pure cultures of the organism. The following year Noguchi (1912b) succeeded in cultivating Trep. pallidum directly from human lesions. The medium he used consisted of a mixture of 2 parts of nutrient agar and 1 part of ascitic or hydrocele fluid, put up in tubes $20 \times 2 \mathrm{~cm}$. in size, each containing at the bottom a piece of sterile rabbit kidney or testicle. Material from a chancre, condyloma, or skin papule was inoculated into the tube, and was covered with liquid paraffin. The organisms produced a slight haze round the kidney and could be pieked off for purification. In fluid medinm growth occurred very slowly and continued for several weeks. The pure cultures were inoculated into monkeys, and proved to be pathogenic. According to Gates (1923), surface colonies may be obtained on 6 per cent. rabbit blood agar plates incubated anaerobically. Colonies are said to be well developed in about a week at $37^{\circ} \mathrm{C}$., and to be surrounded by a zone of complete hæmolysis. Other media have since been used with apparent snceess (Gates 1923, Weiss and Wilkes-Weiss 1924, Hoder 1930, Aksjanzew-Malkin 1933). It must be pointed out, however, that several reputable workers have entirely failed to cultivate Trep. pallidum. Among them is Jahnel (1934), who maintains that the so-called cultures of this organism are in fact cultures of a saprophytic spirochæte (see also Göhring 1940). According to Kast and Kolmer (1940), no one has yet succeeded in devising an in vitro method of cultivation in which the virnlence of the organisms for the rabbit is preserved. The difficulty in practice of obtaining in vitro cultures is so great that for preserving the organism it is usual to employ in vivo methorls. Brown and Pearce (1921b) found that if rablits are infected with syphilis, the organisms are carried to the lymphatic glands and remain there indefinitely. When the strain is required for use, a popliteal gland is excised, ground up in a mortar with saline, and injected intratesticularly into fresh rabbits. Kolle and Schlossberger (1928) have moreover shown that Trep pallidum remains alive in the tissues of miee for an indefinite period, and can be recovered at any time from the glands, spleen, or brain. Using this method they made three passages through mice in 19 months, and fonnd that the organisms remained fully virulent for rabbits.

Resistance.-Very susceptible to heat. According to Boak, Carpenter, and Warren (1932), saline suspensions of infected rabbit testicle are sterilized by exposure to $39^{\circ} \mathrm{C}$. for 5 hours, $40^{\circ} \mathrm{C}$. for 3 hours, $41^{\circ} \mathrm{C}$. for 2 hours, and $41.5^{\circ} \mathrm{C}$. for 1 hour. Dies ont rapidly in stored blood, unless frozen, so that the chances of transmitting syphilis with stored blood, plasma, or serum are very small (see Selbie 1943).

Antigenic Structure.-Little known. Noguchi and Akatsu (1917), using agglutination and complement fixation, obtained evidence of an affinity of Trep. pallidum to Trep. calligyrum. They also observed a certain amount of heterogeneity between different strains of pallidum. There is said to be a strain-specificity in cultures of pallidum (see Georgi el al. 1929), but in view of the serions criticisms that have been made on the sup. posed cultivation of this organism, it would be dangerous to lay too much stress on this statement.

\section{Pathogenicity of Treponema pallidum for Animals.}

RABBits.- Haensell in 1881 was the first to prodnce keratitis in rabbits by inoculation of syphilitic material into the anterior cham ber of the eye. His observations were neglected for over 20 years, when they were confirmed by Bertarelli in 1906; the syphilitic nature of the lesions was proved by the demonstration in them of Treponema pallidum. The receptivity of the rabbit's eye has been confirmed by numerous workers. Bertarelli (1907, 1908) moreover showed that it was possible to carry over syphilis from one animal to another. According to Uhlenhuth and Nulzer (1913) inoculation of a small piece of 
syphilitic rabbit's cornea into the anterior chamber of the eye of a fresh rabbit is followed by complete healing of the loeal wound in 5 to 10 days. After 3 to 6 weeks, as a rule, pericorneal congestion commenees, followed by pannus and keratitis. The keratitis increases to an acme, after which retrogression and healing occur; this process may take weeks or months to complete, and may be interrupted by a relapse. Only a certain proportion of rabbits develop keratitis. The lesion is very much easier to produce by inoculation of rabbit than of human syphilitic material. Successive passages of the virus through the eye of rabbits resulted in an inerease of virulence, manifested by a reduction in the incubation period from 6 to 8 weeks to 4 to 5 weeks.

Syphilis may also be conveyed to rabbits by inoculation into the testicle; this method of transference was first successfully used by Parodi (1907). As with ocular injection, the implantation of human syphilitic material gives much less constant results than of that from the rabbit. Uhlenhuth and Mulzer (1913), for example, inoculated 27 rabbits intratesticularly with human syphilitic material - the juice from primary chancres-and obtained only 5 positive reactions. But after 15 passages through the rabbit the virulence had so increased that inoculation was almost uniformly successful, and the severity and extent of the disease were correspondingly greater. Brown and Pearce (1920a), using the method of intratesticular inoculation of gromd-up syphilitic rabbit's testicle suspended in saline, were likewise uniformly successful in producing the disease. After an incubation period of about 3 to 4 weeks the testicle commences to swell, and soon reaches the size of a pigeon's egg ; the inflammation also affects the epididymis and cord. Sometimes a small superficial erosion may develop at the site of inoculation, covered with a dry yellowish-brown adbesive erust; or an actual chancre may appear. According to Brown and Pearce (1920a) the testicular reaction pursues a cyclic or relapsing course, periods of active progression alternating with periods of quiescence or retrogression; these phases apparently correspond with the variations in the number of spirochrtes in the lesion. The length of time that the testicle is inflamed varies; the lesion may disappear in 6 weeks, or it may last for over a year.

The method of intracutaneous or subcutaneous injection of rabbit syphilitic material into the scrotum, introdnced by Tomasczewski (1910), gives rise after an incubation period of about a fortnight to a typical primary ehancre with a central necrotic area and indurated edges. Sometimes a diffuse lesion of the scrotum follows. These scrotal lesions are invariably accompanied by marked inguinal lymphadenitis. The scrotal infection may spread, and numerous secondary lesions develop, lasting from 1 to 18 months.

Following on scrotal or testicular infection, generalized lesions may develop affecting practically any structure of the body (Brown and Pearce 1920b, 1921 a, Brown et al. 1921). Thus there may be : papular or erythematous eruptions on the skin, sometimes appearing in successive crops; granulomatous lesions of the skin passing on to ulceration; alopecia, onychia, and paronychia; necrotic and ulcerative lesions of the mucosæ and mueocutaneous borders; localized lesions of the periosteum, bone, cartilage, tendons, and tendon sheaths, including such typical manifestations as destruction of the nasal septum and separation of the epiphyses; conjunctivitis, keratitis, and iritis. Generalized lesions of syphilis may also be produced by the intravenous or intracardial injection of rabbits a few days old (Uhlenhuth and Mulzer 1913). For cliscussion of immunity in rabbits see p. 1165, Chapter 51 .

Monkeys.-The experiments of Metehnikoff and Roux (1903, 1904a, $b, 1905)$ amplified the earlier observations of Klebs in 1875-77 (see Klebs 1932), and showed that syphilis might be transmitted to the anthropoid apes, and with less certainty to monkeys. Of the apes the chimpanzee appeared to be the most susceptible. Altogether they inoculated 22 chimpanzees (Troglodytes niger and T. calvuss) witl syphilitie material, either of human origin or derived from experimental animals, and succeed od in producing disease in all of them. Inoculation was performed by searification of the genitals, the thigh, or the eyebrow. After an incubation period of 15 to 49 days, generally 4 weeks, a primary chancre developed at the site of inoculation, and was followed in a few days by swelling of the focal lymph glands. Many of the animals developed lesions of secondary syphilis 
3 to 9 weeks after the appearance of the chancre; these comprised a papular eruption on the skin, palmar psoriasis, mucous plaques of the lips, tongue, and palate, and enlargement of the spleen. Occasionally very severe syphilis developed, accompanied by alopecia, skin eruptions, emaciation, paresis of the hind-limbs, or even death. No lesions of tertiary syphilis were ever found; but it is to be noted that most of the animals died of bronchopneumonia before they had been many weeks under observation, and in these tertiary lesions had no time to develop. In macaques secondary lesions were never observed. According to Uhlenluth and Vulzer (1913) apes are more difficult to infect than rabbits. These workers were successful in conveying human syphilis to rabbits, from rabbits to monkeys, and from a monkey back to rabbits.

Other Animals.-For studying syphilitic lesions, chimpanzees and rabbits are the most useful experimental animals. Infection can, however, be conveyed to certain other animals, such as pigs, guinea-pigs, rats and mice. According to Tani, Kakishita, and Saito (1930), intratesticular inoculation of guinea-pigs gives rise to no obvious lesions, but intracutaneous inoculation, particularly into the perineal fold, is followed in about 11 days by the developiment of a swelling which persists for about 7 wecks (see also Mulzer and Hahn 1930). In rats and mice a symptomless infection is usually produced, similar to that often seen in guinea-pigs. The spirochæetes remain latent in the tissues for months, as can be shown by inoculation of rabbits (Kolle and Schlossberger 1926. 1928). Occasionally, however, a local chancre may be produced by inoculation of the scarified skin of the ano-scrotal region (Bessemans and de Potter 1930, 1931).

\section{Treponema cuniculi}

First observed by Bayon (1913). Responsible for a distase linown as " rabbit syphilis." Morphologically very similar to Trep. pallidum, but tends to be slightly longer and thicker. According to Noguchi (1922), dimensions are : length $7-30 \mu$, arerage $13 \mu$; width $0.25 \mu$; length of spirals $1-1.2 \mu$; amplitude of spirals $0.6-1.0 \mu$. Like Trep. pallidum it stains rose-red with Giemsa. Inoculation of infective material on to the scarified skin of the genital region is followed, after an incubation period of 2 to 8 weeks, by characteristic lesions (see Chapter 81).

\section{Notes on certain other Treponemata found in the Human or Animal Body.}

Treponema pertenue.-Described by Castellani (1905). Responsible for yaws. Morphologically indistinguishable from Trep. pallitum. Exact relation to this organism not yet fully understood.

Treponema refringens.--This organism was first described by Schaudinn and Hoffmann (1905) in their original report on the discovery of Trep.pallidum. It was observed in cases of syphilis complicated with such lesions as balanitis, ulcers, and papillomata, and in non-syphilitic lesions such as gonorrhœal papillomata. Noguchi (1912d) cultivated it from a condyloma. According to him it grows luxuriantly in the deeper part of an ascitic agar tube, forming hazy colonies, denser than those of Trep. pallidum, which gradually extend from the deeper parts of the tube to the more superficial. It is an anaerobe; no growth occurs within $2 \mathrm{~cm}$. of the surface. Growth becomes visible in 4 days at $37^{\circ} \mathrm{C}$. and proceeds for some weeks. The addition of fresh tissue is not essential. In culture the organism is $6-24 \mu$ long by $0 \cdot 5-0 \cdot 75 \mu$ broad ; the middle part of the organism is wavy, but the two extremities are more regularly and deeply curved. The ends are pointed. It is non-pathogenic for rabbits and monkeys.

Treponema calligyrum.--This organism was observed by Noguchi in 1913 in two cases of condyloma, one syphilitic, the other not. Pure cultures were obtained by the ascitic agar stab method. In this medium growth is sinilar to that of Trep.refringens; the hazy colonies are more dense and diffuse than those of Trep. pallidum. In culture the organisms are 6-14 $\mu$ long by $0 \cdot 35-0 \cdot 4 \mu$ wide. The primary spirals are regular and deep; the length of each spiral is $1-6 \mu$, and the amplitude 1 to $1.5 \mu$. The apex of the curve is not 
sharp and pointed as in pallidum, but more or less rounded. It is non-pathogenic for rabbits and monkeys.

Treponema phagedenis.-This organism was cultivated by Noguchi (1912f) from a phagedenic ulcer on the labium of a woman. Growth occurred in ascitic agar medium under anaerobic conditions in the absence of kidney tissue. In the original lesion the spirochætes were $4-30 \mu$ in length and $0.75 \mu$ in thickness; in culture their length was less variable, being $10-15 \mu$. The number of spirals varies from one to eight, and there is great variation in the length of each spiral. Some organisms appear nearly straight. The ends are pointed, but not drawn out. Other spirochretes have been described in ulcerative lesions round the genital regions, such as Trep. balanitidis, Trep. pseudo-pallidum, and Trep. gangrenosa nosocomialis (see Noguchi 1912f).

Spirochætes in the Human Mouth.-Spirochrtes of different types have been described in the mouth; they can generally be seen in scrapings from between the teeth. Sometimes organisms morphologically indistinguishable from Trep. pallidum are found. Noguchi (1912a) succeeded in cultivating what he regards as two separate species. Trep. microdentium is a short spirochæte about $3-4 \mu$ long by $0.25 \mu$ wide, having shallow rectangular curves of constant size. The ends are drawn out and pointed. In culture it is said by Séguin and Vinzent (1938) to be an actively motile organism, 4-7 $\mu$ long, having $6-12$ welldefined regular spirals. In serum agar tissue medium it forms a haze near the bottom of the tube, gradually becoming denser and spreading upwards till it is within $2-3 \mathrm{~cm}$. of the surface. Growth is anaerobic. Trep. macrodentium is a larger organism, varying from $3-8 \mu$ long by $0 \cdot 7-1 \cdot 0 \mu$ broad in young cultures, and having $2-8$ irregular shallow curves; the ends taper off abruptly. In older cultures the organisms are longer and thinner. In serum agar tissne medium growth occurs under anaerobic conditions in the form of a faint almost transparent laze. For methods of isolating and culturing the mouth spirochetes, reference may be made to papers by Séguin and Vinzent (1938), Kast and Kolmer (1940), and Wichelhausen and Wichelhausen (1942).

Vinzent and Daufresne (1934), working mainly with pure cultures, have provisionally classified the mouth spirochætes into groups, which they label A to G. Group B corresponds to Trep. microdentium and Group $\mathrm{F}$ to Trep. macrodentium.

Treponema cobayae.-Found by Knowles and Basu (1935) in the blood of guinea-pigs. Blood parasite belonging to the relapsing fever group. Thin, delicate spirochæte, 13.5-23 $\mu$ in length, with finely tapering ends; average length of spirals $3.6 \mu$. Can be cultivated in Galloway's medium. Inocnlation of guinea-pigs with infected blood is followed, after an incubation period of 2 to 6 days, by a febrile disease accompanied by the presence of spirochætes in the blood. Fully virulent strains kill 30-60 per cent. of inoculated animals. Relapses may occur in animals that recover from the first attack. White rats and rabbits are also susceptible to infection.

Blood spirochrtes have been described in other animals, such as the rabbit and the mouse (see Knowles and Basu 1935).

\section{Leptospira icterohæmorrhagiæ}

Isolation.-First adequate description given in 1915 by Inada and his colleagues in Japan (see Inada et al. 1916), who observed it in the blood and tissues of patients with Weil's disease.

Synonyms.-Spirocheta icterohomorrhagice; Spirochata icterogenes.

Morphology.-Very delicate organism whose morphology can be studied satisfactorily only by dark-ground illumination. The spirals are too fine to be properly resolved in stained preparations. In length it is about $6-12 \mu$, and $0 \cdot 1-0 \cdot 15 \mu$ in thickness ; forms as short as $4 \mu$ and as long as $25 \mu$ may sometimes be observed. It contains a number of perfectly regular closely-wound spirals, each of which is about $0.5 \mu$ long or even less, and has an amplitude of $0.5 \mu$. Near the extremities the spirals become even closer. Secondary waves commonly appear during motion, but the spirochæte has a marked 
tendency to straighten itself out again. Apart from the primary spirals, which are set more closely than in any other group of spirochætes, the most characteristic feature of Lepto. icterohcemorrhagice is its sharp, tapering, hooked ends, which are set at an angle to the main axis, giving the whole organism a resemblance to the letter $\mathrm{C}$ or $\mathrm{S}$. During the rotation that occurs when the organism is moving, these hooked ends are whirled round so rapidly that the organism appears to be furnished with a button-hole or an eye-splice at each extremity (Fig. 217). In fluid media the spirochrotes may become entangled with each other and give rise to the characteristic picture of a "nest." This appears as a highly refractile ball composed of hundreds of interlaced organisms, some of which project radially from the circumference (Taylor and Goyle 1931). In dry-fixed films of blood or urine all sorts of forms may be seen, bearing a resemblance to the letters $\mathrm{C}, \mathrm{S}, l$, or $b$. Degeneration forms with thick, blunt, straight ends are not uncommon. According to Kaneko and Okuda (1917), the morphology of the spirochætes in man is less regular than in the guinea-pig ; they are often shrunken and atrophic, of varying thickness, with greater rigidity and less regular curves; they may show circunscribed thickenings at two or three points, or they may resemble chaius of granules. 'These irregular forms may perhaps result from the action of immunc bodies on the organisms.

In suitable preparations the spirochates may be stained by Giemsa or by one of the silver impregnation methods. In stain ${ }^{\circ}$ films the primary spirals are not visible. Several observers have found that leptospire my pass through Berkefeld eandles (Inada et al. 1916. Baner 1927, Buchanan 1927, Dimitroff 1927). Lut the results are variable. According to Hinlle and Elford (1933), the organisms are held back by collodion membranes

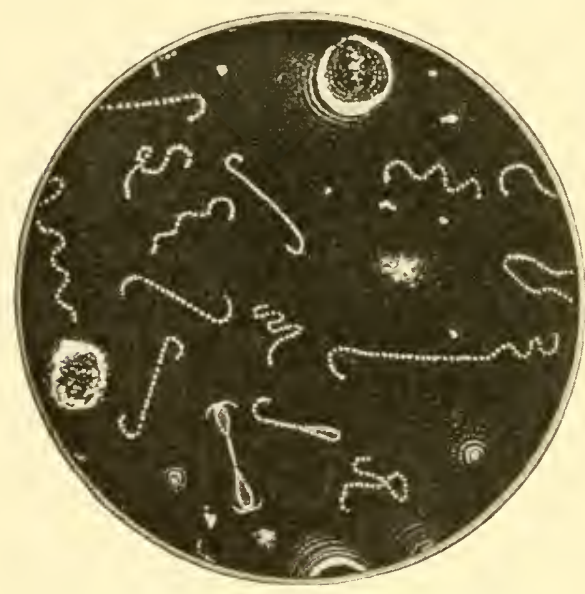

FIG. 217.-Lepto. icterohomorrhagice.

Diagrammatic drawing showing primary and secondary spirals, and hooked and buttonhole ends. (After Wenyon.) having a porosity of $0.25 \mu$; this suggests that their diameter is $0 \cdot 1 \mu$.

Cultivation.-Leptospira icterohomorrhagice was first cultivated by the Japanese workers (Inada et al. 1916) in Noguchi's ascitic fluid kidney medium. Subsequently Noguchi (1917) devised other media that were simpler to make and more satisfactory in practice. The first consists of rabbit serum 1 part, Ringer's solution 3 parts, and citrated rabbit plasma 0.5 parts; the medium should be put up in tubes about $\frac{3}{8}$-inch in diameter, and may or may not be covered with liquid paraffin. The second medium is similar to the first, but 0.5 1.0 parts of slightly alkaline 2 per cent. agar are added, at a temperature of $60-65^{\circ} \mathrm{C}$., the whole medium being well mixed. From this semi-solid medium the citrated rabbit plasma may be omitted if necessary. The NoguchiWenyon medium is likewise satisfactory; it is made by mixing 9 parts of saline with 1 part of 2 per cent. nutrient agar, and adding 20 drops of fresh rabbit's blood to each $10 \mathrm{ml}$. of the autoclaved medium cooled to $50^{\circ} \mathrm{C}$.; the tubes are not shaken. Very good results are obtained with Fletcher's (1928) medium. This is prepared by heating a 12 per cent. solution of rabbit serum in distilled water to $50^{\circ} \mathrm{C}$, adding $6 \mathrm{ml}$. of 2.5 per cent. mutrient agar to every $100 \mathrm{ml}$. of serum-water mixture, arljusting the reaction to $1 \mathrm{ll} 7 \cdot 4$, tubing in $5 \mathrm{ml}$. quantities, and sterilizing at 56 ( $\because$ for 1 hom on 2 sncessive days. Acording to Gardner (1943 $\ell$ ), the simplest am most satisfactory medium consists of a 12 per cent. solution of heat-inactivated Seitz-filtered rabhit sermm in glass-distilled water. For inoculation, $0.1 \mathrm{ml}$. of infected 
guinea-pig blood or liver, or rat-kidney suspension should be used. In subeulturing, 0.5-1.0 ml. should be earried over to $5 \mathrm{ml}$. of fresh medium. The tubes should be ineubated at $25-310^{\circ} \mathrm{C}$.; growth oceurs at $37^{\circ} \mathrm{C}$., but degeneration rapidly sets in. Subcultures should be made every 4 to 6 weeks, and kept at $25^{\circ} \mathrm{C}$. The optimum $\mathrm{pH}$ for growth is 7.6. The organism is acrobic; in Nognchi's serum media, growth oceurs at the top, giving rise to a slight haze, which stops abruptly a few centimetres from the surface. Lepto. icterohomorrhagice can also be cultivated on the chorio-allantoic membrane of the developing chick embryo (Morrow et al. 1938, Chabaud 1939). The organisms become demonstrable in the blood of the allantoic artery in 4 or 5 days; the embryo generally dies within 7 days. Pure cultures of Lepto. icterohomorrhagice may be obtained from contaminated material by inoeulating $0 \cdot 5-1.0 \mathrm{ml}$. intraperitoneally into a guinea-pig, withdrawing blood by heart puncture ten minutes later, and culturing it in a suitable medium (Schiiffner 1940); the leptospiræ invade the blood more rapidly than the accompanying bacteria.

Resistance.-Leptospira icterohomorrhagice is killed by moist heat at $50-55^{\circ} \mathrm{C}$. in half an hour; it can withstand freezing. It is very sensitive to acid, being destroyed by human gastric juice in 30 minutes; it will not grow in an even slightly acid medium. 'l'he organisms are rendered motionless in 10 to 15 minutes by $\mathbf{l} / 2000 \mathrm{HgCl}_{2}$ and are gradually dissolved. They are rapidly destroyed by bile. In defibrinated blood kept at room temperature in the light, the organisins remained virulent for 7 days, and in decomposing liver for 27 hours (Uhlenhuth and Fromme 1916). In infected guinea-pig liver kept in the ice-chest they remained virulent for 26 days (Buchanan 1927).

Antigenic Structure.-Though it was thought at one time that there was but a single species of Leptospira, namely Lepto. icterohamorrhagice, further experience has revealed the occurrence of several types differing in their antigenic structure, pathogenicity to guinea-pigs, and the nature and severity of the disease to which they give rise in man. Nany of these types have been given speeific names. The original icterohamorrhagive species can be distinguished antigenically from other leptospiral strains that are pathogenic for human beings. Its relation to water spirochetes is still under discussion. Baermann and Zuelzer (1928) found that water strains were not agglutinated by the sera of convalescents from Weil's disease, or by the sera of animals inoculated experimentally with Lepto. icterohomorrhagice, and that sera prepared against avirulent water strains did not agglutinate Weil strains. Brown and Davis (1927), using the adhesion test (see p.912), found that Lepto. icterohcemorrhagice from rats or man behaved alike, while Lepto. biflexa was antigenically distinct. Vaecination of guinea-pigs with cultures of Lepto. biflexa failed to immunize them against Lepto. icterohcemorrhagice (Uhlenhuth and Znelzer 1921). According to Baermann and Zuelzer (1928), however, some water strains, the virulence of which has been raised by animal passage, behave antigenically like Lepto. icterohamorhagice. Some of the confusion is probably due to the lack of homogeneity among water strains. The majority of these are saprophytes and belong to the species Lepto. biflexa. Some of them, however, appear to be real icterohcemorrhagice strains, either in their normal virulent condition or in a degenerate avirulent condition. Passage through animals may succeed in restoring these to full virulence, rendering them indistinguishable antigenically from typical icterohomorrhagice strains of parasitic origin. Since animals not infrequently act as leptospiral carriers, it is possible that apparent changes in antigenic structure and virulence brought about by passage are due to the isolation of an organism from the animal different from that which was inoculated. Caution must therefore be exereised in drawing conclusions from the type of evidence advanced by Baermann and Zuelzer (1928) and Znelzer (1930).

\section{Pathogenicity of Lepto, icterohæmorrhagia for Animals.}

Lepto. icterohcemorrhagice is highly pathogenie for young guinea-pigs, whether given intraperitoneally, subeutaneousty, cutaneously, or by the month. The golden hamster is likewise very susceptible (Morton 1942, Larson 1944). Rabbits, rats, and mice are only 
slightly susceptible, and usually remain perfectly well after inoculation (Martin and Pettit 1919). Cats, dogs, pigs, sheep, hens, pigeons, and monkeys are said to be refractory (Uhlenhuth and Fromme 1916, Martin and Pettit 1919), but later evidence suggests that Lepto. icterohemorrhagice is responsible for some cases of infectious jaundice in the dog (Dhont et al. 1934).

Intraperitoneal injection of guinea-pigs with 1-2 $\mathrm{ml}$. of infected human blood or groundup rat's kidney is followed by an illness lasting for 5 to 12 days, and terminating in death. The chief symptoms of the disease are fever and jaundice. The fever commences the day after inoculation, reaches its acme in a few days, falls to normal, and finally to subnormal just before death. Jaundice first becomes visible when the temperature begins to fallusually on the 4th or 5th day ; it increases till death, and is often accompanied by choluria. Anæmia and conjunctival congestion are frequent, and external hæmorrhages from the rectum, nose, and genitals may occur. Blood counts reveal a lymphocytosis during the first few days of the disease, and an anæmia (Buchanan 1927). Spirochætes appear in the

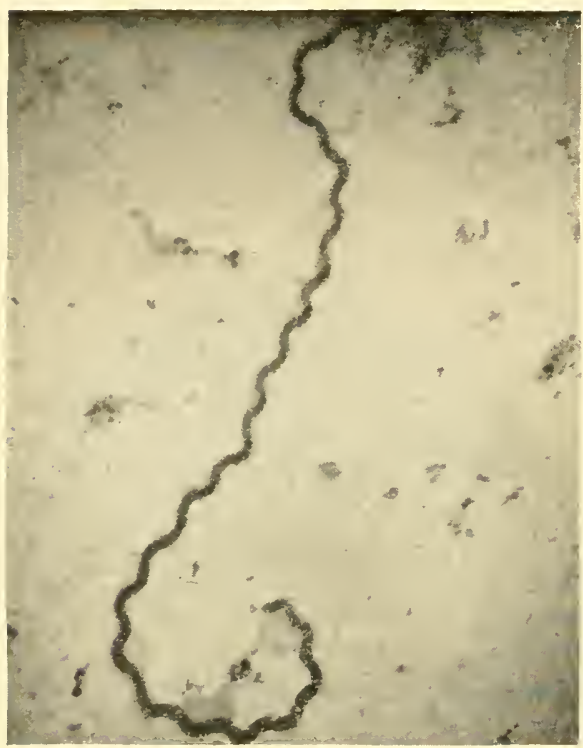

FIG. 218.-Leptospira icterohomorrhagice.

Electron micrograph $(\times 12,000)$.

Kindly supplied by Dr. H. E. Morton and Dr. T. F. Anderson. blood about the 4 th day, but are not easy to find microscopically. Post mortem, the animal shows generalized jaundice; there are hæmorrhages into various parts of the body, particularly the lungs, intestinal walls, retroperitoneal tissues, and fatty tissues of the inguinal region. The hæmorrhages in the lungs form irregular spots of varying size, sharply demareated from the surrounding tissue - giving the lungs a resemblance to the mottled wings of a butterfly (Inada et al. 1916). The spleen is enlarged and congested ; the kidneys show an acute parenchymatous nephritis and capsular hæmorrhages; the suprarenals are often enlarged and hæmorrhagic. Histologically the chief lesions are cloudy swelling of the liver, sometimes accompanied by focal necroses, acute parenchymatous nephritis, endothelial cell proliferation in the spleen and lymph glands, and hæmorrhages in practically every structure of the body (Buchanan 1927). Spirochætes are most numerous in the liver, and are best demonstrated by dark-ground illumination. They occur in the spaces between the cells, and when numerous are arranged about the cells like a garland. Their appearance is different from that seen under dark-ground illumination; they are short and thick; the primary spirals and tapering extremities are not evident, and numerous irregular undulations are seen. They are found in smaller numbers in the kidneys and adrenals.

By passage from guinea-pig to gumea-pig the virulence of the organism can apparently be increased. Stokes (Stokes et al. 1917), for example, found that the average time to death of animals inoculated intraperitoneally with human blood was 10 days, but that when passage strains were used it was only 5 days. Noguchi (1917) likewise noticed a reduction in survival time after passage of a strain through guinea-pigs. 


\section{Notes on other species of Leptospira}

Leptospira, Rachmat type.--Found in eases of human infection in the Dutch East Indies. It is antigenically distinct from Lepto. icterohcemorrhagice; some strains are closely allied to Lepto. antumnalis, Akiyami A type (see Walch-Sorgdrager 1939). It is virulent for guinea-pigs, in which it usually causes jaundice. Its natural animal host remains unknown.

Leptospira bataviæ. -This organism, which is sometimes referred to as the Swart van Tienen type, canses sporadic infections of human beings in Batavia, Borneo and the Celebes. It is antigenically distinguishable from other types. It is moderately virulent for the guinea-pig. Its natural host is Rattus norvegicus.

Leptospira, Salinem type.-This type is also found in the Dutch East Indies, where it causes sporadic cases of leptospiral infection in Sumatra. It is of moderate virulence for guinea-pigs. It appears to be carried by the rat, Rattus brevicaudatus.

Leptospira hebdomadis.-Described by Ido, Ito and Wani (1918, 1919). Gires rise to the 7-day fever of Japan. Differs antigenically from Lepto. icterohcemorrhagice, and is less virulent for the guinea-pig. It is avirulent for rats and mice in the laboratory. It is a natural parasite of the field-mouse, Hicrotis montebelloi. Is apparently identical with the akiyami $B$ type found in the autumn fever of Japan.

Leptospira akiyami and Leptospira autumnalis.-The identity of these organisms is still under discussion. The first was isolated from cases of Autumn fever or Akiyami in Japan, the second from a disease known as Hasamiyami. Two types of organism from Akiyami have been described. The A type appears to belong to the Rachmat group; the B type is apparently identical with Lepto. hebdomadis (Stéfanopoulo and Hosoya 1928, Yang and Theiler 1930, Inoue 1930). The organism isolated from Hasamiyami is very similar to the akiyami $A$ type. According to Walch-Sorgdrager (1939), akiyami $A$, autumnalis and Rachmat share a common antigen, but are not identical. Both akiyami $A$ and antumnalis are fairly virulent for guinea-pigs, of ten producing jaundice. The natural host of akiyami $A$ is a field-mouse, Hicrotus montebelloi, of Lepto. autumnalis a field-mouse, Apodemus speciosus.

Leptospira australis A and B.-These two types were isolated from cases of coastal fever in Queensland (Lumley 1937). The A type is antigenically distinct from other strains; the B type appears to be related to the Salinem type of the Dutch East Indies (Walch-Sorgdrager 1939). Both the Australian types are moderately pathogenic for guinea-pigs, and both are carried by rats- $R$. culmorum.

Leptospira pomona.-This organism was isolated from a patient affected with the 7-day fever of Queensland (Ciayton and Derrick 1937). It is antigenically distinct from the other strains. Its virulence both for human beings and guinea-pigs is low. It is said by Johnson (1943) to cause endemic infection of pigs and cattle in the coastal district of southern Queensland.

Leptospira mitis.-This organism was also isolated from a febrile patient in Queensland (Johnson 1942). It is antigenically distinct from other types, appears to be moderately virulent for guinea-pigs and, like Lepto. pomona, causes an endemic infection of pigs and cattle. It is not to be confused with the Leptospira mitis, which was described by Mino (1939) as being responsible for infection in rice-field workers in Italy, and which was shown to be identical witls Lepto. batavice.

Leptospira grippotyphosa.-This organism is the cause of a widespread disease in eastern Europe known as swamp or mud fever. It was eultivated by Korthof (1932) in a peptone rabbit serum salt mixture. It differs antigenically from other leptospiræ, and is not very pathogenic to guinea-pigs, but it may infect mice on passage (WalchSorgdrager 1939). Its natural animal carrier is still unknown, though voles are suspected. 
Leptospira, Andaman A and B types.-Organisms belonging to these two types were isolated by Taylor and Goyle (1931) from patients suffering from a febrile disease in the Andaman islands. Illness was confined mainly to adnlts working in mud or in marshy districts in the autumn. The A type is antigenically distinet, and is of only moderate virulence for the guinea-pig. The B type appears to be identical with Lepto, grippotyphosa (Walch-Sorgdrager 1939).

Leptospira sejroe.--Isolated by Petersen and Christensen (1939) from the blood of a fisherman in the small Danish island of Sejrö. It is antigenically related to Lepto hebdomadis, but absorption experiments show it to be distinct. Its virulence for guinea-pigs is low. Its natural host appears to be a mouse, Mus spicilegus.

Leptospira oryzeti.--This organism was isolated from rice-field workers in Italy (Babudicri 1938,1939$)$. It appears to be antigenically related to Lepto. batavice, but as it has not yet been properly studied its claim to specific rank is still in doubt.

Leptospira canicola. - This is a natural parasite of dogs, in which it gives rise to a disease more often associated with uremia than with jaundice (see Chapter 82 ). It was first distinguished from Lepto. icterohomorrhagies by Klarenbeck and Sehïfner (1933). It differs from Lepto, icterohemorrhagice in its antigenic structure and its lower virulenee for quinea-pigs on first isolation. After passage through guinea-pigs, its virulence may increase. No jaundice is produced, but in animals that survive leptospire may be excreted in the urine for months. Oceasionally it infects human beings, but it is not a normal parasite of rats (see Walch-Sorgdrager and Schüffner 1938). Gartner (1943b) thinks that there may be a type of leptospira infecting dogs and man in Great Britain differing from Lepto. canicola, but the evidence on this point is not yet conclusive.

Leptospira javanica. - Found in field-rats and eats in the Dut ch East Indies (see WalchSorgdrager 1939). Has not so far been isolated from human beings.

Leptospira biflexa.--Usually referred to by German workers as Spirochøta pseudoicterogenes. Widespread saprophyte found mainly in water. Described originally by Wolbach

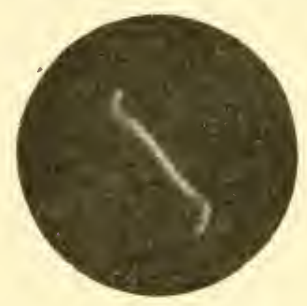

FIG. 219.-Lepto. biflexa.

Dark-ground illumination $(\times 1500$ ca. $)$.

lifficult Hindle (19.2) four that if ou ml of water were adled to a Petri clish eontaining a portion of human freces about the size of a pea, and incubated at $25^{\circ}-30^{\circ} \mathrm{C}$. in the dark, Lepto. biflexa was generally observable microscopically in 10 days, and was abundant in 20 days. The leptospire were able to pass through an L5 candle. These observations formed the basis of several methods of isolation (Bauer 1927, Mochtar 1928, von Vagedes 1935). The general principle is to filter the water through a suitable candle and eultivate the filtrate. Is antigenisally distinct from Lepte. icterohcemorrhagice. A specifie prefipitating substane of earbohydrate nature has been extracted from it by Hindle and White (1931). Is non-pathogenie for animals. Is believed by certain workers to be an avirulent form of Lepto. ictrohtomorhagior. which ean be rendered virulent by suitable animal passage. Balance of evidence is against this view. 


\section{Spirillum minus}

Sometimes relerred to as spirochelu morsus muris. Deseribed by Futaki and his colleagues $(1916,1917)$ as the cause of rat-bite fever in man. According to Robertson (1924), it is a spirillum and not a spirochete, and its correct name is spirillum minus. Appears to be a natural parasite of rats, which act as healthy carriers of the organism. Morphologically the spirillum is short, rather thick, and has tapering ends, provided with one or more flagella. It is $2-5 \mu$ long, motile, and has regular rigid spirals, each of which is about $1 \mu$ in length. The movements are rapid-like those of a vibrio. It is readily stained by ordinary aniline dyes, such as Loeffler's methylene blue, and by Giemsa. Cultures may be obtained in Shimamine's medium, but sucessive transfers have not been successful. The organism gives rise to one type of rat-bite fever in man. Intraperitoneal inoculation of infective human material into mice is followed by no clinical evidence of disease, but spirilla appear in the blood after 5 to 14 days. They are scaree at first, bnt later they increase, though they never become numerous; it is uncommon to find two organisms in the same field (Theiler 1926). They persist indefinitely, though only in small numbers. Rats behave like mice, but the number of spirilla in the blood is fewer. Intraperitoneal inoculation of guinea-pigs produces a febrile disease. After an incubation period of 6 to 15 days spirilla appear in small numbers

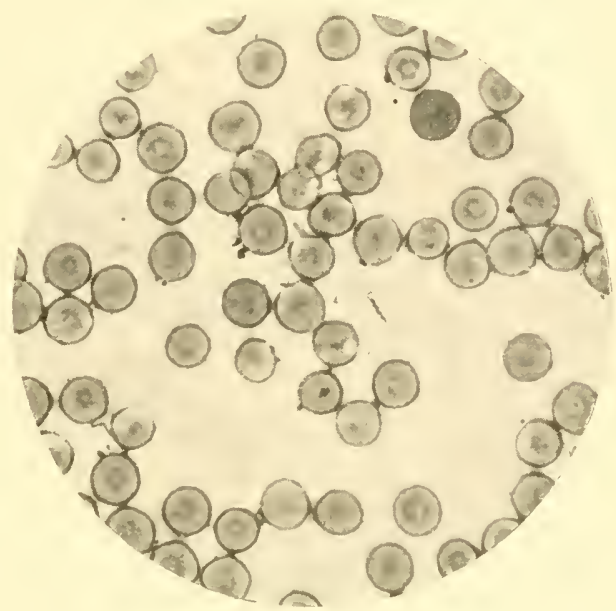

FIG. 220.-Spirillum minus.

In film of blood of experimentally infecterl mouse. Giemsa. $(\times 1000)$. [From specimen kindly sup. plied by the late P'rof. J. G. T'homson.] in the blood, and pyrexia sets in accompanied by enlargement of the lymplı glands. There may be a marked inflammation of the subeutaneous tissue in the ano-genital region, involving the scrotal sacs, perianal tissue, and prepuce in males and the labia and perianal tissue in females. Later, after 3 or 4 weeks, alopecia, ulceration of the skin, and chronic eonjunctivitis and keratitis may occur. The disease is generally chronie, lasting from about 2 to 4 months, but sometimes death occurs in the first 5 weeks (Ishiwara et al. 1917). Spirilla can be demonstrated in the blood, lymph glands, spleen, kidney, adrenal, and subcutaneous tissue. In Robertson's (1924) experience spirilla were never demonstrable in the blood, even by mouse inoculation, nor did any of the guinea-pigs die. Rabbits may be infected, but are less suitable for diagnostic purposes than mice or guinea-jigs. Monkeys are also susceptible (Inada el al. 1916).

\section{REFERENCES}

Aksjanzew-Malinin, S. (1933) Zbl. Baht., 129, 405.

Babodieri, B. (1938) Policlinico, Sez. prat., 45, 1774; (1939) Riv. Parassitol., 3, 93.

Baermans, G. and Zuelzer, M. (1927) Klin. Wschr., 6, 979 ; (1928) Zbl. Bakt., 105, 345.

BaUer, J. H. (1927) Amer. J. trop. Med., 7, 177.

BAYON, H. (1913) Brit. med. J., ii. 1159.

Bertarelli, E. (1906) Zbl. Bakt., 41, 320 ; (1907) Ibid., 43, 238, 448 ; (1908) Ibid., 46, 51. Besselins, A. and Potter, F. De. (1930) C.R. Soc. Biol., 104, 818; (1931) Ibid., 10\%, 279.

Boak, R. A., Carpenter, C. M., and Warren, S. L. (1932) J. exp. Med., 56, 725.

Brown, H. C. and Davis, L. J. (1927) Brit. J. exp. Path., 8, 397. 
Brown, W. H. and Pearce, L. (1920a) J.exp. Med., 31, 475, 709, 729, 749 ; (1920b) Ibid., 32, 445, 473, 497; (192la) Ibid., 34, 167; (1921b) Ibid., 34, 185.

Brown, W. H., Pearce, L., and Witherbee, IV. D. (1921) J. exp. Med., 33, 495, 515, 525.

Buchanan, G. (1927) Spec. Rep. Ser. med. Res. Coun., Lond., No. 113.

Castellani, A. (1905) Brit. med. J., ii, 1280.

Chabaud, A. (1939) Bull. Soc. Path. exot., 32, 483.

Clayton, G. E. B. and Derrick, E. H. (1937) Med. J. Aust., i. 647.

Dhont, C. M., Klarenbeek, A., Schüffiner, W. A. P., and Voet, J. (1934) Ned. Tijdschr. Geneesk., 78, 5197.

DimitrofF, V. T. (1927) J. infect. Dis., 40, 508.

Dobell, C. (1912) Arch. Protistenk., 26, 117.

Ellermann, V. (1904) Zbl. Bakt., 37, 729; (1907) Z. Hyg. InfektKr., 56, 453.

Fletcher, IV. (1928) Trans. roy. Soc. trop. Med., 21, 265.

Futaki, K., Takaki, I., Taniguchi, T., and Osumi, S. (1916) J.exp. Med., 23, 249 ; (1917) Ibid., 25, 33.

Galloway, I. A. (1925) C. R. Soc. Biol., 93, 1074.

Gardenr, A. D. (1943a) Mon. Bull. Emerg. publ. Hlth Lab. Serv., 2, 40 ; (1943b) Lancet, ii., 157.

GATES, F. L. (1923) J. exp. Med., 3\%, 311.

Gates, F. L. and Olitsky, P. K. (1921) J. exp. Med., 33, 51.

Georoi, F., Pradsnitz, C., and Fischer, O. (1929) Klin. Wschr., 8, 2007.

Göhring, G. (1940) Z. ImmunForsch., 98, 90.

Haensell, P. (1881) v. Graefes Arch. Ophthal., 2\%, 93.

Hindle, E. (1925) Brit. med. J., ii. 57 (1931) "A System of Bacteriology," Med. Res. Counc., 8, 148.

Hindle, E. and Elford, W. J. (1933) J. Path. Bact., 3\%, 9.

Hindle, E. and White, P. B. (1934) Proc. roy. Soc., B, 114, 523.

Hoder, F. (1930) Z. ImmunForsch., 68, 256.

Ido, Y., Iто, H., and Wani, H. (1918) J.exp. Med., 28, 435 ; (1919) Ibid., 29, 199.

Inada, R., Ido, Y., Hoki, R., Kaneko, R., and Iтo, H. (1916) J.exp. Med., 23, 377.

INOUE, S. (1930) Zbl. Bakt., 117, s0.

Ishiwara, K., Ohtawara, T., and Tamura, K. (1917) J.exp. Med., 25, 45.

JAHNEL, F. (1934) Klin. Wschr., 13, 550; (1937) Ibid., 16, 1304.

Johnson, D. W. (1942) Hed. J. Aust., i. 431 ; (1943) Brit. med. J., ii. 659.

KANEKU, R., and OKUDA, K. (1917) J. exp. Med., 26, 325.

KAst, C. C. and Kolmer, J. A. (1940) Amer. J. Syph., 24, 671.

Kllarenbeek, A. and Schüffner, W. A. P. (1933) Ned. Tijilschr. Geneesk., Yy, 4271.

Ḱlebs, A. C. (1932) Science, \%5, 191.

Kligler, I. J., Hermoni, D., and Perek, M. (1938) J. comp. Path. Therap., 51, 206.

Kligler, I. J. and Robertson, O. H. (1922) J. exp. Med., 35, 303.

K nowles, R. and BasU, B. C. (1935) Indian J. med. Res., 22, 449.

K vowles, R., Gupta, B. M. D., and Basu, B. C. (19.32) In lian J. mod. Res., Memoir No. 22,

Kolle, IV. and Schlossberger, H. (1926) Dtsch. med. Wschr., 52, 1245 ; (1928) Ibid., $54,129$.

Korthof, G. (1932) Zbl. Bakt., 125, 429.

Landauer, E. (1931) Ann. Inst. Pasteur, 47, 667.

Lapidari, 11. and Sparrow, H. (1928) Arch. Inst. Pasteur Tunis, 1\%, 191.

Larson, C. L. (1944) Publ. Illth. Rep., Wash., 59, 522.

Lumley, G. F. (1937) Med. J. Aust., i. 654.

Manoú́ltan, Y. (1940) Ann. Inst. Pasteur, 64, 439.

Martin, L. and Pettit, A. (1919) "Spirochétose ictérohémorragique." Paris.

Metchnikoff, E. and Rodx, E. M. (1903) Ann. Inst. Pasteur, 17, 809; (1904a) Ibid., 18, 1 ; (1904b) Ibid., 18, 657 ; (1905) Ibid., 19, 673.

Mino, P. (1939) Policlinico, Sez. med., 46, 410.

Mochitar, A. (1928) Zbl. Bakt., 10\%, 374.

Moroder, J. (1929) Arch. Schiffs.-Tropenhyg., 33, 603.

Morrow, G., Syverton, J. T., Stiles, W. W., and Berry, G. P. (1938) Science, 88, 384.

Morton, H. E. (1942) Proc. Soc. exp. Biol., N.Y., 49, 566.

Morton, H. E. and Anderson, T. F. (1943) J. Bact., 45, 143.

Mudd, S., Polevitzkx, K., and Anderson, T. F. (1943) J. Bact., 46, 15.

Mulzer, P. and HahN, C.-F. (1930) Arch. Hyg., 103, 95.

Noquchi, H. (1911) J. exp. Med., 14, 99 ; (1912a) Ibid., 15, 81 ; (1912b) Ibid., 15, 90; (1912c) Ibid., 15, 201 ; (1912d) Ibid., 15, 466; (1912e) Ibid., 16, 199; (1912f) Ibid., 16, 261 ; (1912g) Ibid., 16,620; (1913) Ibid., 17, 89; (1917) Ibid., 25, 755; (1922) Ibid., 35, 391.

Noguchi, H. and Akatsu, S. (1917) J. exp. Med., 25, 765.

Norris, C., Pappenheimer, A. M., and Flournox, T. (1906) J. infect. Dis., 3, 266. 
Novy, F, G. and Knapp, R. E. (1906) J. infect. Dis., 3, 291.

OAg, R. K. (1939) J. Puth. Bact., 49, 339.

Oberveier, O. (1873) Berl. klin. W schr., 10, 152, 378, 391, 455.

P'ARHAil, J. C. (1922) Amer. J. trop. Llicd., 2, 341.

Parodi, U. (1907) Zbl. Bakt., 44, 428.

Petersen, B. and Christensen, H. I. (1939) C. R. Soc. Biol., 130, 1507.

Plotz, H. (1917) J. exp. Med., 26, 37.

Prowazek, S. von. (1907) Arb. Gesundh Amt., Berl., 26, 23.

Rieckenberg, H. (1917) Z. ImmunForsch., 26, 53.

Robertson, A. (1924) Ann. trop. Mled. Parasit., 18, 157.

Sakharoff, N. (1891) Ann. Inst. Pasteur, 5, 564.

Schaudins, F. and Hoffuann, E. (1905) Arb. ReichsgesundhAmt, 22, 527.

ScheFF, G. (1935) Zbl. Bakt., 134, 35.

Schereschewsky, J. (1909) Dtsch. med. W'schr., 35, 835, 1260, 1652.

Schuberg, A. and Schlossberger, H. (1930) Klin. Wschr., 9, 499.

SCHÜFFNER, W. (1940) Z Zl. Bakt., 145, 341.

SÉgulv, P. (1930) C. R. Soc. Biol., 104, 247, 836.

Séguin, P. and Tinzent, R. (1938) Ann. Inst. Pasteur, 61, 255.

Selbie, F, R. (1943) Brit. J. exp. Path., 24, 150.

Sinton, J. A. (1924) Indian J. med. Res., 11, 825.

Socle, II. H. (1942) Publ. Amer. Ass. Advanc. Sci., No. 1s, p. 53.

Stéfanopollo, G. J. and Hosoya, S. (1928) C. R. Soc. Biol., 98, 1317.

Stokes, A., Ryle, J. A., and Tytrer, W. H. (1917) Lancet, i. 142.

Tani, Kakishita, M., and Saito, K. (1930) Zbl. Bukt., 11\%, 73.

TAYlor, J. and Goyle, A. N. (1931) Indian med. Res. Hemoirs, No. 20.

TheILER, II. (1926) Amer. J. trop. Lled., 6, 131.

'T'Illdex, E. B. (1937) J. Bact.. 33, 307.

Tonasczewski. (1910) Dtsch. med. Wschr., 36, 1025.

TunviclifF, R. (1906) J. infect. Dis., 3, 148.

Terner, 'T. 13. (1938) J. exp. Med.. 6\%, 61.

Twort, F. W. (1921) Lancel, ii. 798.

Uhlexheth and Fromie. (1916) Berl. klin. Wrschr., 53, 269.

Uhlenhuth, P. and IIUlzer, P. (1913) drb. ReichsgesundhAmt., 44, 307.

UhleNHUTII and Zuelzer. (192I) Zbl. Bakt., 85, Beiheft, $1+1$.

Taredes, K. von. (1935) Zbl. Bakt., 133, 401.

Viveext, H. (1896) Ann. Inst. Pasteur, 10, 488; (1899) Ibid., 8, 609.

Vinzent, R. and Daufresne, I. (1934) C. R. Soc. Biol., 116, 490.

Walch-Sorgdrager, B. (1939) Bull. Hlth Org., L.o.N., 8, 143.

WALCH-Sorgdrager, B. and SCHÜFFNER, W. (1938) Zbl. Baht., 141, 97.

Weiss, C. and Wilkes-Weiss, D. (1924) J. infect. Dis., 34, 212.

Wexyon, C. M. (1926) "Protozoology," ii. London.

Wichelifatsen, $\mathrm{O} . \mathrm{W}$. and Wichelhausen, R. H. (1942) J. dent. Res., 21, 543.

Wile, U. J. and Kearney, E. B. (1943) J. Amer. med. Ass., 122, 167.

Wile, U. J., Picard, R. G., and Kearny, E. B. (1942) J. Amer. med. Ass., 119, sso.

Wolbach, S. B. and Brvaer, C. A. L. (1914) J. med. Res., 30, 23.

Wrons, H. L. and BeCK, M. D. (1935) Amer. J. publ. Hlth, 25, 270.

YANo, K. and Thenler, II. (1930) Amer. J. trop. Hed., 10, 407.

YUax-Po, L. (1933) Kitasato Arch., 10, 78.

Zuelzer, M. (1925) "Die Spirochäten." "Handbuch der pathogenen Protozoen." Band 3, 1627. Leipzig; (1928) Zbl. Bakt., 105, 384; (1930) Arch. Hyg., 103, 282. 


\section{CHAPTER 39}

\section{RICKET'TSIA}

\section{DeFinition,--Rickettsia.}

Small, Gram-negative, bacterium-like organisms, usually less than half a micron in diameter. More or less pleomorphic. Stain rather poorly witlı aniline dyes, but well with Giemsa. Natural inhabitants of intestinal canal of arthropods; usually occupy an intracellular position. Some species are parasitic in higher animals and are pathogenic for man. The type species is Rickettsia prowazeki.

Rickettsia is the name given to certain small bacteria-like bodies which are found in the alimentary canal of insects and other arthropods, and which are frequently associated with disease in man and animals. Definition of the group presents several difficulties. Some workers, like Zinsser (1937), would automatically exclude any organism such as $R$. melophagi that has been cultivated on artificial media. Further, they would insist that, when growing in the animal body or in tissue cultures, only such organisms as multiply intracellularly should be regarded as true rickettsiæ. Most species appear to be unable to pass through the ordinary bacterial filters, though there are exceptions, as with $R$. burneti. Since, however, it is often difficult to obtain a homogeneous suspension of the organisms free from cellular material, too much weight should not be placed on this characteristic. It seems clear that the rickettsiæ occupy a position in between the smallest bacteria, like Bartonella, and the filtrable viruses. The fact that they can be resolved microscopically by visible light, and that they are held back by membranes which allow most of the filtrable viruses to pass throngh, brings them into line with the bacteria; but their failure to grow on ordinary culture media, and their predilection for intracellular multiplication, show that their metabolic requirements are more akin to those of the filtrable viruses.

We shall not attempt to define the gemus too closely, nor shall we exclude from the present chapter organisms, such as $R$. quintana, merely because they appear to grow extracellularly. Mention will be made of $R$. conjunctiva, $R$. canis, $R$. bovis, and $R$. ovina, even though it is doubtful whether these organisms are natural inhabitants of arthropods, or even whether they are living organisms at all.

The first-named species was described by da Rocha-Lima in 1916, who found these bodies in lice taken from patients with typhus fever; he proposed the name of Rickettsia prowazekii in honour of Ricketts and of Prowazek, both of whom died of typhus fever while investigating the disease. The second species was described by Töpfer (1916), also in 1916, in lice taken from patients suffering from Wolhynian fever-better known as Trench fever; this species has been given the alternative names of Rickettsia quintana-on account of the 5-day febrile parox- 
ysms characteristic of this disease-and $R$. wolhynica; it appears to be closely related to, if not identical with, $R$. pediculi, which was found by da Rocha-Iima in the apparently normal human body louse. A third species, which was first describer by Ricketts as far back as 1909 in Rocky Mountain spotted fever, has been called Dermacentroxenus rickettsi by Wolbach; as this organism appears to belong to the Rickettsia group, we shall refer to it as Rickettsia rickettsi. Sellards in 1923 claimed to have cultivated a fourth species, $R$. nipponica, from animals experimentally infected with tsutsugamushi fever in Japan. This speeies has been given the alternative names of $R$. tsutsugamushi, $R$. akamushi, and $R$. orientalis; but, in spite of Philip's (1943) argument that the name $R$. nipponica is ruled out becanse of the reported cultivation of this organism on artificial media, there still seems insufficient reason for disearding it. If every organism on which a fallacious observation has been made must be re-named, bacterial taxonomy will become even more perplexing than it is already.

Cowdry (1925) described a fifth species, $R$. ruminuntium, as the cause of heart-water of sheep, goats and cattle in South Africa. A murine type of typhus virus, sometimes referred to as $R$. mooseri, was discovered as the result of epidemiological and bacteriological studies undertaken by American and Mexican workers (for references see p. 1845). More recently, the observations of Derrick (1937) and Burnet and Freeman (1937) in Australia and of Davis anc Cox (1938) in the United States have revealed a rickettsia, $R$. burneti ( $R$. diaporica), as the cause of a febrile disease known as $Q$ fever.

Besides these six or seven species, which have been found in association with disease of

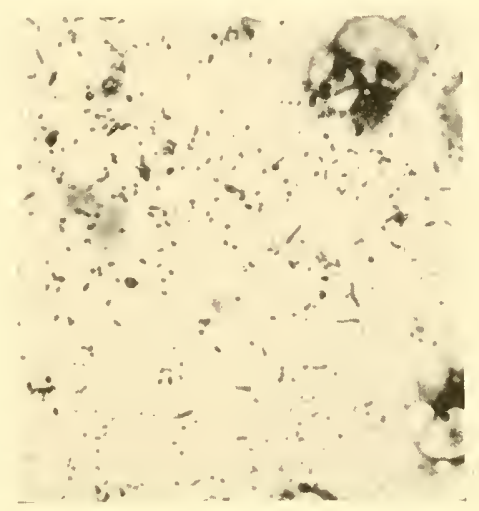

FIt. 221.-Rickettsia melophagi.

Smear preparation from gut of sheppked. Giensa. ( $\times 1000)$. mammals, over forty other, apparently non-pathogenic, species of Rickettsia have been lescribed in various insects and other arthropods. One or two of these will receive brief mention at the end of this chapter, together with a few pathogenic organisms whose relationship to the Rickettsia group is still in doubt.

As the rickettsiæ have been found both in blood-sucking and in non-bloodsucking insects it seems probable that they are primarily inhabitants of the alimentary canal of insects, and that infection of insects occurs by contamination with infected excreta (Hindle 1921). Some species are found not only in the lumen of the alimentary canal, but also in the epithelial cells lining the canal. A further invasion of the body may occur, leading to infection of the salivary glands and other tissues. Most species appear to be transmitted hereditarily to successive generations through infection of the eggs. There is a marked host specificity. Some species appear to have reached a perfect equilibrium with their insect host, and sometimes with the animal on which the insect is a parasite, such as in the system comprised by the murine typhus virus, the rat flea, and the rat. Others, however, which by some workers (see Zinsser 1937, Burnet 1942) are supposed to have developed later in the evolutionary period, are pathogenic for their insect hosts, killing a high proportion of them-as, for instance, the classical typhus rickettsia and the louse. A few species appear to have become adapted to an alternate 
existence in insects and in animals; infection of insects occurs in these cases by blood-sucking. There is, however, no evidence to suggest that a separate cycle of development occurs in either host, as is so frequently observed with the protozoa.

Morphologically, in the gut of the louse rickettsixe appear like very small cocci, diplococci, or short bacilli. Their size is generally given as 0.3-0.5 $\mu$ long by $0.3 \mu$ broal, but the bacillary forms may reach a length of 1.5 or even $2.0 \mu$. According to Elford and van den Ende (1944) the size of $R$. prowazeki, judged by ultra-violet photomicrographs, is $0.6-1.8 \mu$ long by $0.45 \mu$ broad. Gradocol membrane experiments likewise suggest that the width is not less than $0.4-0.5 \mu-$ a valne greater than that which has previously been quoted. Their arrangement is variable; single forms may occur; diploid forms are very common; and the small coccoid forms are often grouped in dense masses. Under

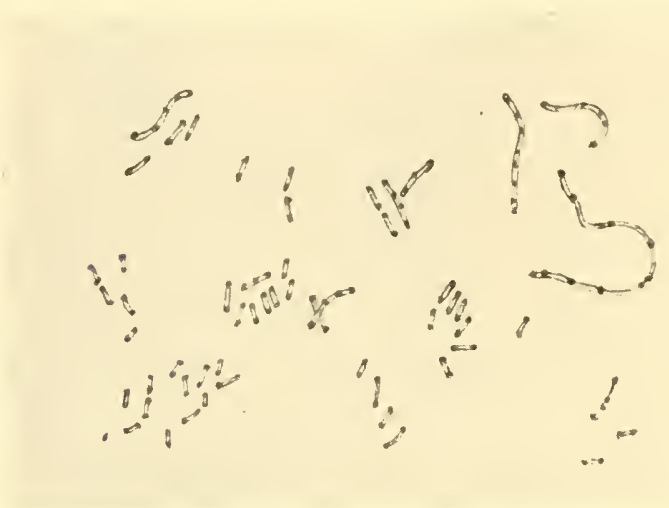

FIG. 222.-Rickettsia prowazeki.

Smear preparation from louse's gut, showing bacillary forms, and occasional thread-like chains. Magnification 2,000 diameters (approx.). (After Wolbach, Todd and Palfrey.) certain conditions, they may form intracellular homogeneous or granular inclusion bodies not unlike those seen in psittacosis (Pinkerton and Hass 1932, Begg et al. 1944). By the electron microscope they appear, like bacteria, to possess a limiting membrane, enclosing a substance of moderate opacity ; dense granules may be seen in some forms (Plotz et al. 1943, Weiss 1943). Most species are non-motile. On the whole they stain poorly with the ordinary aniline dyes, but Castaneda (1930), Lépine (1932), Laigret and Auburtin (1938), and Macchiavello (1941) have shown that good results may be obtained with methylene blue, thionin, and fuchsin, provided the stain is made up with a suitable buffer or mordant. Macchiavello's stain, in particular, gives excellent results with smears, though it is not so successful for tissue sections. For general purposes, Giemsa is probably the best stain; with this the rickettsix appear as purple cocco-bacilli, or frequently as bipolarstaining rods; sometimes they seem to be surrounded by a paler-staining substance. They are uniformly Gram-negative. When very few in number, they cannot be recognized with certainty, since their resemblance to minute particles of detritus is too close; but when they are present in large numbers, their recognition is comparatively easy. It is by reason of the small numbers in which they occur in the blood of infected men and animals, that their demonstration in this medium has only rarely been accomplished. Arkwright, Bacot and Duncan (1919-20) lay down the following criteria for the recognition of Rickettsia in the louse's gut: (1) its minute size, $0.3-0.5 \mu \times 0.3 \mu$; (2) its irregularity in shape, round, oval, diplococcal, and bipolar-stained bacillary forms being seen; (3) its occurrence in very large numbers, or even masses, especially on flakes of solid material in the excreta; (4) its well-stained appearance and purple colour with Giemsa. 
Cultivation and Metabolism.-None of the pathogenic species has yet been cultivated apart from living cells. Of the commensal species, $R$. melophagi, found in the sheep-ked, is said to have been grown on blood agar. Noguchi (1926) claimed to have cultivated some of the commensal rickettsiæ, found in ticks, on leptospiral medium containing 0.2 per cent. of a carbohydrate; when 1 per cent. of agar was added, and the medium was slanted, surface colomies were obtained. Working with the rickettsix from Rocky Mountain fever and from typhus, Wolbach and Schlesinger (1923-24) succeeded in obtaining growth in tissue cultures. The organisms survived and multiplied only in the endothelial cells. Primary cultures remained alive and virulent for 1 to 2 weeks as a rule, and later-generation cultures for 2 to 4 weeks. Nigg and Landsteiner (1930) showed that cultivation of the typhus virus could be accomplished in a medium, similar to that described by Maitland and Maitland (1928) for vaccinia virus, which contains living but not actively proliferating cells. A study by Zinsser and Schoenbach (1937) of the growth of rickettsiæ in such a medium showed that multiplication of these organisms did not begin till after the tissue cells had ceased growing actively. This was different from the behaviour of viruses, which multiply most during the stage of tissue cell growth. Burnet (1938) has made similar observations, and would regard this difference in metabolism between the rickettsiæ and filtrable viruses as of classificatory value. Another method used successfully in the cultivation of the filtrable viruses, namely growth on the chorio-allantoic membrane of the developing chick embryo, was found by da Cunha (1934) to be applicable to rickettsiæ. An even more successful method is that described by Cox (1941) of growing rickettsix in the yolk sac of the developing chick embryo. Pure, or practically pure, strains of $R$. prowazeki may be obtained by Weigl's method of intrarectal injection of body lice with infective material. The intestine of the louse is practically free from ordinary bacteria, so that it serves as an almost sterile medium for the cultivation of rickettsiæ. In practice, in vivo cultivation in the tissues of a susceptible animal, such as the testicle of the guinea-pig or rabbit, is frequently employed for preserving strains of typhus virus; according to Kodama and Takahashi (1931), viruses kept in this way undergo no change in antigenic structure or pathogenicity. Certain rickettsial strains, such as $R$. nipponica, can be cultivated in the anterior chamber of the rabbit's eye (Nagayo et al. 1930). After an incubation period of 4 to 8 days iritis develops, similar to that occurring naturally in tsutsugamushi fever. Histological examination reveals the presence of peculiar corpuscles in the endothelial cells of Descemet's membrane, consisting apparently of colonies of rickettsiæ. The animal method of cultivation has been applied particularly to $R$. prowazeki and $R$. mooser in an effort to provide heavy suspensions of rickettsiæ for serological and vaccination purposes. The mouse's lung has proved particularly useful for this purpose, the animals being inoculated intranasally (Okamoto 1937, Castaneda 1939, Durand and Sparrow 1940, Giroud and Panthier 1942).

Little is yet known about the growth requirements of Rickettsia, but interesting observations have been made. Pinkerton and Hass (1932) found that in plasma tissue cultures $R$. prowazeki grew best at $32^{\circ} \mathrm{C}$. This behaviour may be related to the preference that some species of rickettsiæ show, after inoculation into the peritoneal cavity of the guinea-pig, for growth in the scrotal sac, where the temperature is lower, rather than for the general peritoneal cavity. In the Maitland medium growth occurs equally well at $32^{\circ} \mathrm{C}$. and at $37^{\circ} \mathrm{C}$. This apparent dis- 
crepancy may be due to the circumstance that in plasma tissue cultures the cells are multiplying actively, whereas in the Maitland medium mitotic division is rarely seen. From these observations l'inkerton (1934) drew the conclusion that typhus rickettsic grow best in cells which are metabolizing slowly. That this explanation is correct is rendered probable by the work of Zinsser and Schoenbach (1937) and Burnet (1938). Thus Zinsser and Schoenbach found that in tissue culture rickettsix underwent no appreciable multiplication till the tissue cells had ceased to grow actively. Viruses, on the other hand, multiplied most abundantly during the stage of tissue cell growth. Burnet (1938) made similar observations on the rickettsia of $Q$ fever. It would appear that, in this respect, there is an important difference between the metabolism of Rickettsia and of filtrable viruses. The reason why rickettsiæ grow better at $32^{\circ} \mathrm{C}$. than at $37^{\circ} \mathrm{C}$. in plasma tissue cultures, and in the scrotal sac than in the peritoneal cavity, is probably not because they prefer the lower temperature in itself, but because tissue cell metabolism is less rapid at this temperature than at $37^{\circ} \mathrm{C}$. Further observations by Burnet and Freeman (1941) on egg membrane cultures suggest that rickettsix grow only in regions where there is an abundant supply of oxygen. The authors recall the fact that the rickettsiæ are essentially parasites of the vascular endothelium, which is rich in oxygen, and explain their better growth in the later than in the early stages of tissue cultures on the ground that, not till active tissue growth is over, does the oxidation-reduction potential of the cells rise sufficiently high to enable rickettsiæe to multiply. Other factors that favour growth of rickettsiæ in the animal body, such as riboflavin deficiency, benzol poisoning, and $\mathrm{X}$-ray irradiation, possibly act in the same way, namely by lowering intracellular metabolism.

Though most of the pathogenic species of Rickettsia seem to require intracellular conditions for growth, $R$. nipponica and $R$. burneti are both said to be capable of growth outside the cells though not, of course, in a cell-free medium. A further difference in the metabolism of different species is shown by the fact that the typhus group of rickettsiæ multiply exclusively in the cytoplasm, whereas the spotted fever group $(R$. rickettsi) grow best within the nucleus.

The Resistance of Rickettsia has not been fully studied. The rickettsiæ of typhus, Rocky Nountain fever, and heartwater are said to be easily inactivated by heat, drying, and chemical disinfectants, but $R$. quintana is said to be more resistant (Cowdry 1926). The Trench fever Committee (Bruce 1921), however, found that the infectivity of louse excreta was destroyed by exposure to moist heat for 20 minutes at $60^{\circ} \mathrm{C}$., and to dry heat for the same time at $100^{\circ} \mathrm{C}$. Arkwright and Bacot (1923) found that $R$. prowazeki remained virulent for 11 days in louse excreta which had been kept dry at room temperature. The viability of rickettsio in infected tissues and in tissue cultures, as judged by their infectivity, seems to be considerably affected by the temperature at which they are kept. Spencer and Parker (1924), working with $R$. rickettsi, found that certain tissues remained infective in pure glycerol for as long as 10 months if preserved at $-10^{\circ} \mathrm{C}$. Nigg (1935), working with $R$. mooseri, found that tissue cultures in a serum-Tyrode mixture remained alive and virulent for several months at $37^{\circ} \mathrm{C}$. and at $-20^{\circ} \mathrm{C}$, but generally died out in a week or two at the intermediate temperatures of $20^{\circ} \mathrm{C}$. and $-4^{\circ} \mathrm{C}$. Sterile skim milk is said to be a good suspending agent for rickettsiæ that are to be preserved in the dried state (Topping 1910); and used as a diluent it maintains the virulence of $R$. prowazeki at $26^{\circ}-28^{\circ} \mathrm{C}$. for 24 hours (Anderson 
1944). In serum broth at $\mathrm{pH} 6 \cdot 0-8 \cdot 5$ rickettsial suspensions may be stored for months at $-77^{\circ} \mathrm{C}$. with little alteration in potency (Elford and van den Ende 1944).

Antigenic Structure.-The difficulty of obtaining suspensiors of rickettsiæ free from admixture with cells and other bacteria has rendered the study of the antigenic structure of these organisms peculiarly difficult. Ledingham (1920) and others have shown that inoculation of infective material or of rickettsial suspensions into rabbits is followed by the appearance of specific agglutinins. Advantage has been taken of this circumstance to study the relationship of the pathogenic rickettsix to Proteus $X$ strains. Without entering here into the practical performance of the Weil-Felix test (see (hapter 83), it may be mentioned that the serum of patients suffering from typhus and typhus-like diseases frequently agglutinates Proteus OX 19 or one of its variant strains, OX 2 or OX K. Castaneda and Zia (1933), studying R. prowazeki and Proteus $I 19$ by the agglutination and absorption of agglutinins technique, found that these organisms behaved as if each possessed a specific and a group somatic antigen. White (1933), using in addition the precipitation test, obtained evidence of the existence in X 19 of two distinct heat-stable somatic receptors: (1) an alkali-labile receptor (Castaneda's $\mathbf{P}$ factor), which is mainly responsible for the agglutination of this organism by its own antiserum; (2) an alkali-stable receptor (Castaneda's $\mathrm{X}$ factor), which is responsible for the reaction of this organism with the sera of typhus patients. W'hite's conclusion received confirmation from the further work of Castaneda $(1934,1935)$, who was successful in extracting specific soluble substances of polysaccharicle nature from X 19 and R. prowazeli. These substances have already been referred to as $\mathrm{P}$ and $\mathrm{X}$. It appears, therefore, as if Proters X 19 and $R$. prouazeki possess a common alkalistalle antigenic factor $(X)$, of polysaccharide nature, which is responsible for the Weil-Felix reaction. In addition, Proteus X 19 contains a specific alkali-labile receptor, also apparently of polysaccharide nature, which plays no part in this reaction (see Chapter 27). Whether $R$. prowazeki contains a specific receptor of its own similar to the P factor of Proteus X 19 is not yet clear, but there seems little doubt from Castanedit and Zia's work that it possesses a heat-labile antigen, behaving in much the same way towards the heat-stable antigen as the Vi antigen of the typhoid bacillus does to the $\mathrm{O}$ antigen. Topping (1944) has reported the existence of a "soluble" substance present in the supernatant fluid after ether extraction and centrifugation of yolk sac cultures of $R$. prowazeki possessing apparently the same antigenic properties as those of the intact organisms.

The relationship of the different types of typhus virus to each other, and to the viruses of Rocky Mountain spotted fever, tsutsugamushi fever, fièvre boutonneuse, tick-bite fever and $\mathbf{Q}$ fever has leen studied partly by serological methods and partly by cross-protection tests in living aninals. The interpretation of the results is so closely bound up with the Weil-Felix reaction and with the clinical and epidemiological characteristics of these diseases that it is proposed to defer further discussion of this subject to Chapter 83. Suffice it to say that there appear to be at least three major receptors in Proteus $\mathrm{I}$ strains, represented by the $O X 19$, $O X 2$, and $O X \mathrm{~K}$ types, which correspond to similar receptors in rickettsial strains isolated from different typhus and typhus-like diseases. Ciuca and his colleagues (1938) have shown that all three receptors are of glycolipoid nature and can be extracter from the bolies of the bacilli by the trichloracetic acid method.

Pathogenicity.-As already mentioned, there are five or six known pathogenic species for man and one for cattle. Leaving aside this last species, $R$. ruminantium, 
about which comparatively little is known, we may refer briefly to the reproduction of the various rickettsial diseases in animals.

Pathogenicity of $\mathbf{R}$. prowazeki for Animals.-A febrile disease simulating typhus can be reproduced in apes, monkeys, and guinea-pigs by the classical louse-borne type of virus; rabbits and rats are relatively resistant to inoculation. According to Nicolle, Conor and Conseil (1911), chimpanzees are more sensitive than macaques; subcutaneous inoculation of $1 \mathrm{ml}$. of human blood is generally sufficient to infect chimpanzees, but for macaques $4-5 \mathrm{ml}$. intraperitoneally are required. The blood of human patients is most virulent towards the end of the fever, but it is said to be virulent from 2 days before the onset to 2 days after the decline of the fever (Arkwright et al. 1919-20).

After inoculation of typhus blood into monkeys there is an incubation period of about a week, followed by a rise of temperature, which continues to ascend gradually for some days, just as in man; the temperature is maintained for 7 to 10 days, and then falls rapidly. A period of hypothermia may succeed, followed by a return to normal temperature. Accompanying the fever there are general constitutional symptoms, such as anorexia, ruffled coat, and conjunctival congestion; on the $3 \mathrm{rd}$ or 4 th day a rash sometimes breaks out on the face. Death may occur. During the early part of the fever there is a leucopenia, followed by a return to normal; the leucocytes continue to rise, passing above normal during convalescence, and not returning to normal till about a month after inoculation. The disease can be passed indefinitely through monkeys. A single attack, provided it is severe, produces a solid immunity; but after a mild attack the immunity is less marked. Instead of typhus blood, monkeys can be infected with a suspension of guinea-pig brain tissue, or with ground-up lice or louse excreta. Arkwright, Bacot and Duncan (1919) brought evidence to show that the monkey-louse, Pedicinus longiceps, became infected by feeding on typhus monkeys, or after rectal injeotion of typhus blood, and was able to transmit the disease to normal monkeys. The infected lice were found to contain rickettsiæ; Pedicini from non-inoculated monkeys never contained rickettsiæ.

Guinea-pigs can be infected by virus from man, the louse, or infected guinea-pigs or monkeys. The incubation period is generally 6 to 14 days, but it may extend to 26 days (da Rocha-Lima 1920a); it is longer after subcutaneous than after intraperitoneal injection. The disease is characterized mainly by fever. The rectal temperature rises from $102^{\circ}$ to $103^{\circ} \mathrm{F}$. at the end of the incubation period, remains at between $103^{\circ}$ and $106^{\circ} \mathrm{F}$. for 3 to 14 days, and then falls to normal. According to Grünfeld, Serebrjannaja, and Neumann (1933), there is a mononuclear leucocytosis reaching its maximum as the fever declines; the mononuclear cells rise from 2 per cent. to between 6 and 14 per cent. The animals recover, and are subsequently immume to a fresh inoculation. If killed, there is little to be seen macroscopically beyond shight enlargement and darkening of the spleen, and sometimes slight congestion of the testicles, which may be covered with a gelatinous exudate. Microscopically, both in man and in guinea-pigs the main lesions are found in the blood capillaries, especially those in the skin, skeletal muscles and central nervous system. They consist of thromboses with perivascular accunulations of cells, often accompanied by small hæmorrhages. In the central nervous system characteristic nodules are found, simulating tubercles. The primary lesion is in the endothelial cells lining the walls of the capillaries. Rickettsiæ have been demonstrated in the lesions of the skin, kidneys, testicles, brain, and other organs in man (Wolbach et al. 1922). The height and duration of the fever in guinea-pigs is variable, and great care should be taken before concluding that it is definitely caused by the typhus virus. Ecker and IVeed (1932) and Badger $(1933 a, b)$ point out that symptoms very suggestive of infection with $R$. prowazeki or $R$. rickettsi may be produced in guinea-pigs by certain organisms of the Proteus and Salmonella groups. Cultural, serological and cross-immunity tests may all be required to establish the real causative agent in any given febrile condition. According to Arkwright and Bacot (1923), 
the most certain way of establishing that an attack of fever in the guinea-pig is really due to the typhus virus is to inject lice intra-rectally with a suspension of the guinea-pig's platelets, and observe the development of rickettsiæ in the excreta. Infected guinea-pigs, it may be noted, do not give a positive Weil-Felix reaction, though natural agglutinins to Proteus OX 19 are sometimes present in a titre of $1 / 25$ or less.

The typhus virus can be passed from man to monkeys, from monkeys to guineapigs, and from guinea-pigs to monkeys.

Pathogenicity of R. mooseri for Animals.-The murine typhus virus gives rise in guinea-pigs to a disease differing in certain respects from that caused by the classical louse-Jorne virus (Pinkerton 1929, 1931, Zinsser and Castaneda 1930). After intraperitoneal inoculation with the murine type the temperature rises rather earlier, about the 4th to 6th day, though the actual height reached may be less than with the louseborne type. The scrotal and testicular reaction caused by the murine type, first describerl by Neill (1917) when investigating Mexican typhus, is much more intense, and microseopical examination reveals the presence of large numbers of rickettsiæ-sometimes known as Mooser (1928) bodies-in the tunica vaginalis. On the other hand, nodular lesions in the brain are more frequent in louse-borne than in murine type infections. $R$. mooseri is further distinguished from $R$. prowazeki by its ability to give rise after intraperitoneal inoculation to a febrile disease in rats, accompanied by proliferation of rickettsiæ in the scrotal sac ; $R$. prowazehi causes a completely inapparent infection in these animals. Moreover, $R$. mooseri causes a heavy infection of the lnng after intranasal inoculation into rats or mice (see Castaneda 1939), whereas $R$. prowazeki usually grows much less abundantly. Incidentally, it may be noted that, when growing in the lungs of rats or rabbits, the murine virus may give rise to intracellular inclusion borlies of the morula type, consisting of colonies of rickettsiæ (Begg et al. 1944). Cross-immunity tests indicate that the two types of virus are very closely related (Mooser and Dummer 1930, Nicolle and Laigret 1932, Zinsser and Castaneda 1934). More recent work-mainly unpublished - suggests, however, that they are not antigenically identical.

Pathogenicity of $\mathbf{R}$. rickettsi for Animals.-The disease produced in guinea-pigs by inoculation of Rocky Mountain spotted fever virus is similar to that caused by the typhus virus, but is much more severe. After intraperitoneal inoculation with the Western type, the incubation period is usually only 2 to 4 days. The temperature rises rapidly to about $106^{\circ} \mathrm{F}$, and death usually occurs within a week. From the 3rd or 4 th day of the fever swellings and hæmorrhages of the scrotum and ears occur, which may go on to necrosis. Post-mortem examination shows a considerable enlargement of the spleen, and frequently a marked scrotal reaction with rickettsial bodies in the tunica vaginalis. The Eastern type is said to be less virulent, but both viruses produce a characteristic rash in the monkey (Badger 1933c).

Rabbits can be infected with the Rocky Mountain virus ; they develop a febrile disease ; rabbits inoculated with the typhus virus do not react at all. It is interesting to note, as indicating the closeness of the relationship between the two viruses, that rabbits experimentally infected with the Rocky Mountain virus may develop agglutinins to Proteus $X 19$ and give a positive IVeil-Felix reaction; rabbits inoculated with the typhus virus likewise develop agglutinins-usually to a rather higher titre (Munter 1928). But inoculation of a rabbit with typhus is said not to protect it against subsequent inoculation with Rocky Iountain virus; indicating that though both viruses closely resemble each other antigenically, they are distinguishable by their virulence and by their immunizing properties. Experiments on guinea-pigs, however, indicate that inoculation with either virus provides a certain amount of protection against subsequent inoculation with the other (Breinl 1928). White mice and rats are said to develop a symptomless infection after intraperitoneal inoculation with Rocky Mountain spotted fever virus (Fukuda 1929), but after intranasal inoculation they develop pulmonary lesions in which considerable numbers of rickettsiæ are present (Durand and Giroud 1940, Durand and Sparrow 1940). 
Pathogenicity of R. nipponica for Animals.- The viruses of tsutsugamushi fever, mite fever, and scrub typhus appear to be elosely related. They are not as a rule very infective for guinea-pigs, though occasional strains may prove highly fatal, giving rise to aseites and splenic enlargement after intraperitoneal inoculation. In white rats they produce an inaprarent infection. One of their most striking properties is their ability to give rise to an acute reaction. characterized by eirenm-comeal injection, iritis, turbidity of the aqueous hmour. panmus, and the presence of rickettsial bodies in Desecmet's membrane, on inoculation into the anterior chamber of the eye of rabbits (Nagayn et al. 1931, Lewthwaite and Savoor $1934,193(i)$. The sermm of the animals often agghtinates Protens O.X K 10 days or so after inoculation. The viruses of this group are further distingnished by the uleration and hubo formation which they cause on intracutaneous injection of monkeys. The virus of fievre houtomneuse appears to be the only other known Rickettsin that can produce a marked local lesion in monkeys. After intraperitoneal inoculation with infeetive material from the rabhit's eye, mice may dic in a fortnight or so; post mortem, there is a sticky fluid in the peritonenm enntaining large numbers of rickettsix.

Pathogenicity of $\mathbf{R}$. burneti for Animals. - This organism may eause a fobrile reaction in monkeys inoculatel intracutaneously or subentaneously. No loeal lesion oceurs in the skin similar to that seen after intracutaneous inoeulation with $R$. nipponicn (Burnet and Freeman 1937). Inoculated intraperitoneally into guinea-pigs, it gives rise, after an incubation period of 2 to Is days depending on the dose-to a febrike non-fatal disease of 4 to 6 days' duration. If the animal is killed during the height of the infection, the spleen is found to be enlarged. The serotal sae is seldom affected. Strains of Ameriean origin are more virulent than those of Australian origin, frequently produeing a fibrinous exudate around the spleen in which large numbers of rickettsixe can be demonstrated mieroscopically. The liver of guinea-pigs inoculated with either strain ean be shown by inoenlation to be highly infective (Derrick 1937, Dyer 1939. Burnet and Freeman 1939).

Hice, rats and rabbits are all susceptible. but suffer from an inaplarent infection. In mice killed 7 to 10 days after a heavy intraperitoncal inoenlation, the liver is enlarged and pale. and the spleen is often greatly enlarged, tense, and of a uniform deep red eolour. Rickettsiæ are present in smears from both organs (Burnet and Freeman 1937, 1938). Hice inoenlated intranasally may develop pneumonitis. characterized by irregularly distributed nodules of consolidation. in which large numbers of rickettsiae ean be demonstrated (Findlay 1942).

Pathogenicity of R. quintana for Animals. - We have little exact knowledge of the behavionr of the virus of trench fever in laboratory animals. Da Rocha-Lima (1920b) states that a small proportion of guinea-pigs may develop a low undulating fever after inoculation with material containing this organism, but that infection cannot be transmitted by passage to fresh animals.

\section{Notes on a few miscellaneous organisms.}

R. rocha-limæ.-This organism was described hy Weigl in 1921. It is apparently a parasite of the boly louse. It is distinguished from $R$. pediculi by morphological peculiarities and its variability (see also p. 1852). Introduced into a stock of lice, it may spread with extraordinary rapidity (IVeigl 1939). There is reason to believe that it may give rise to a bacteræmia in man. It has been regarded by some anthors as the cause of trachoma, but the evidence in favour of this is unconvincing (see Chapter 85). What relation $R$. rocha-lime has to similar rickettsix found in lice, such as $R$. weigli and others, is still doubtful (see Herzig 1939).

R. conjunctivæ.--Coles $(1931,1935,1936)$ in Sonth Africa described a rickettsialike organism in the conjunctival epithelium of sheep, goats, and cattle. Sometimes its presence was accompanied by inflammation. A similar organism was 
met with by Donatien and Lestoquard (1937a) in their study of an epidemic of conjunctivitis among sheep in Algeria. The organism has not been fully examined; from its description it appears rather large; 110 arthropod vector is known; and it is donbtful whether it belongs to the Rickellsia group. According to (iiroud and Panthier (1939) there is no such organism as $R$. conjunctiva ; the bodies that have been mistaken for it are the result of bacteria undergoing phagocytosis, and can be found in the conjunctiva of normal cattle.

R. canis, R. ovina, and $\mathbf{R}$. bovis.-These organisms, which were described hy Donatien and Lestoquard $(1935,1936 a, b, 1937 b)$ in Algeria, are believed to be parasites of the mononuclear colls. $R$. canis is said to be responsible for a severe disease of dogs; the other two species are said to give rise to a relatively mild disease in their respective hosts. There is evidence that infection is transmitted by ticks. Whether they are true micro-organisms and, if so, whether they belong to the Rickettsia group, or should be classified in some other genus, must await further sturly. Again, their relation to the intracellular bodies found by Kurloff in the mononuclear colls of the guinca-pig's blood is doubtful (see Mochovski 1937).

Classification.--Most of our knowledge on the relationship of the pathogenic rickettsix to each other has been gleaned from an examination of the sera of patients and experimentally inoculated rabbits and from cross-immunity experiments in animals. The results of these will be more conveniently dealt with in Chapter 83. Suffice it to say here that, excluding $R$. quintana about which our information is very slight, the rickettsise pathogenic to man may be classified broadly into four groups: the typhus gromp, the spotted fever group, the tsutsugamushi group and the $\mathrm{Q}$ fever group. Within each of these groups there are types of Rickettsia, sometimes with a circumseribed geographical distribution, that differs from each other in their insect host, in their virulence for man and for experimental animals, and in their apparent antigenic structure. Whether these types should be regarded as species or as varieties of the main type it is at present impossible to decide. (For general reviews on the rickettsiæ see Arkwright 1924, Wolbach 1925, 1941, Cowdry 1926, Pinkerton 1942, and for useful technical information on their study see Clavero and Gallardo 1943).

\section{REFERENCES}

Anderson, C. R. (1941) J. Bact., 4\%, 519.

ARK WRIGHT, J. A. (1924) J.R. Army med. Cps, 42, 447.

Arkwright, J. A. and Bacot, A. W. (1923) Brit. J. exp. Path., 4, 70.

Arkwright, J. A., Bacot, A., and Duncan, F. M. (1919) Trans. Soc. trop. Med. Hyg., 12, $61 ;(1919-20)$ J. Hyg., Camb., 18, 76.

Badger, L. F. (1933a) Amer. J. trop. Med., 13, 179 ; (1933b) Pubt. Hith Rep., Wash., 48, 677; (1933c) Amcr. J. publ. Illth., 23, 19.

Bega, A. M., Fituton, F., and Ente, M. van den. (1944) J. Prath. Bract., 56, 109.

Breint, F. (1928) J. infect. Dis., 42, 48.

Bruce, D. (1921) J. Ilyg., Camb., 20, 258.

Burnet, F. H. (1938) Aust. J. exp. Biol., 16, 19: (1942) Herl. J. Aust., ii. 129.

Burnet, F. M. and Freeman, M. (1937) Med. J. Aust., ii. 2999; (1938) Ibid., ii. 1114 (1939) Ibid., ii. 587 ; (1941) J. Immunol., 40, 405.

Castaneda, MI. R. (1930) J. infect. Dis., 4\%, 416 ; (1934) J.exp. Med., 60, 119 ; (1935) Ibid., 62, 289; (1939) Amer. J. Path., 15, 467.

Castaneda, M. R. and Zia, S. (1933) J. exp. Med., 58, 55.

Ciuca, M., Mesrobeanu, l., Pidenski, A., and Mrnteanu, G. (193s) C. R. Soc. Biol., $12 \%, 1414$.

Clavero, G. and Gallarno, F. P. (1943) "Tecnicas de Laboratorio en el Tifus exantemático." l'rensa española, Madriol.

Coles, J. D. W. A. (1931) 17 th Rep., Dircetor vet. Strv., South Africa, p. 175; (1935) Onderstepoort J., 4, 389; (1936) J.s. Afr. vet. med. Ass., \%, 221. 
Cowdry, E. V. (1925) J. exp. Med., 42, 231, 253; (1926) Arch. Path. Lab. Med., 2, 59. Cox, H. R. (1941) Science, 94, 399.

CunHa, A.-M. DA. (1934) C. R. Soc. Biol., 11\%, 392.

Davis, G. E. and Cox, H. R. (1938) Publ. Hlth Rep., Wash., 53, 2259.

Derrick, E. H. (1937) Med. J. A ust., ii. 281.

Donatien, A. and Lestoquard, F. (I935) Bull. Soc. Path. exot., 28, 4 Is : (1936a) Ibid., 29, 105; (1936b) Ibid., 29, 1057; (1937a) Ibid., 30, 18; (1937b) Arch. Inst. Pasteur Algér., 15, 142.

Durand, P. and Grodod, P. (1940) Arch. Inst. Pasteur Tunis, 29, 234.

Durand, P. and Sparrow, H. (1940) Arch. Inst. Pasteur Tunis, 29, 1.

Dyer, R. E. (1939) Publ. Illth Rep., Wash., 54, 1229.

ECKER, E. E. and WeED, L. A. (1932) J. infect. Dis., 50, 484.

Findlay, (6. II. (1942) Trans. R. Soc. trop. Hed. Hyg., 35, 2 I3.

FukUda, Y. (1929) Zbl. Bakt., 111, 408.

Grroud, P. and Panthier, R. (1939) Bull. Soc. Path. exol., 32, 464; (1942) Anu. Inst. Pasteur, 68, 137.

Grünfeld, A. A., Serebrjannaja, A. I., and Neumanx, M. W. (1933) Zk\%. Bakt., 129, 56.

Herzig, A. (1939) Zbl. Bakt., 143, 299, 303.

Hinde, E. (192I) Parasitology, 13, 152.

Kodaia, M. and Takahashi, K. (1931) Zbl. Bakt., 119, 311.

Laigret, J. and Auburtis, P. (193s) Bull. Soc. Path. exot., 31, 790.

Ledinghan, J. C. G. (1920) Lancet, i. 1264.

LÉPINE, P. (1932) C. R. Soc. Biol., 109, 1162.

Lewthwaite, R. and Savoor, S. R. (1934) Trans. 9 th Congr., Far East. Assoc. trop. Med., Nanking, 1, 249; (1936) Brit. J. exp. Path., 1\%, 1.

Nacchinello, A. (1941) See Zinsser (194I), p. 896.

Mattland, H. B. and Maitland, M. C. (1928) Lancet, ii. 596.

Mochkovski, C. (1937) C. R. Soc. Biol.. 126, 379.

Mooser, H. (1928) J. infect. Dis., 43, 241, 261.

Mooser, H. and DumMer, C. (1930) J. exp. Med., 51, 189.

MUNTer, H. (1928) Z. Hyg. Infekthr., 109, 124.

Nagayo, M., MiYagawa, Y., Mitamura, T., Tamiya, T., Sato, K., Hazato, H., and Imamura, A. (1931) Jap. J. exp. Med., 9, 87.

Nagayo, M., Tamiya, T., Mitamura, T., and Sato, K. (1930) C. R. Soc. Biol., 104, 637.

Neml, H. H. (1917) Publ. Hlth Rep., Wash., 32, 1105.

Neitz, W. O. (1940) J. S. Afr. vet. med. Ass., 11, 15.

Nicolle, C., Conor, A., and Cosseil, E. (1911) Ann. Inst. Pasteur, 25, 97.

Nicolle, C. and Lateret, J. (1932) Arch. Inst. Pasteur, Tumis, 21, 251.

Nige, C. (1935) J. exp. Med., 61, 17.

Nigo, C. and Landsteiner, K. (1930) Proc. Soc. exp. Biol., N.Y., 28, 3.

Noguchi, H. (1926) J. exp. Med., 43, 515.

Окамото, Y. (1937) Kitasato Arch., 14, 23, 99, 113.

Philip, C. B. (1943) Amer. J. Hyg., 3\%, 301.

Pinkerton, H. (1929) J. infect. Dis., 44, 337; (1931) J. exp. Med., 54, 181 ; (1934) Arch. exp. Zellforsch., 15, 125; (1942) Bact. Rev., 6, 37.

Pinkerton, H. and Hass, G. M. (1932) J. exp. Med., 56, 131, 145, 151.

Plotz, H., Siadel, J. E., Anderson, T. F., and Chambers, L. A. (1943) J. exp. Med., $7 \%, 355$.

Ricketts, H. T. (1909) J. Amer. med. Ass., 52, 379.

Rocha-Lima, H. DA. (1916) Berl. klin. Hschr., 53, 567; (1920a) Prowazek's "Handbuch der pathogenen Protozoer," ii, 990 ; (1920b) Ibid., 1031.

Sellards, A. W. (1923) Amer. J. trop. Med., 3, 529.

Spencer, R. R. and Parker, R. R. (1924) Publ. Hlth Rep., Wash., 39, 55.

Töpfer, H. (1916) Münch. med. Wschr., 63, 1495.

Toppisc, N. H. (1940) Publ. Hlth. Rep., Wash., 55, 545; (1944) Ibid., 59, 1671.

WEISS, L. J. (1943) J. Immunol. 47, 353.

WEIGL, R. (I939) Zbl. Bakt., 143, 291.

White, P. B. (1933) Brit. J. exp. Path., 14, 145.

Wolbach, S. B. (1925) J. Amer. med. Ass., 84, 723 ; (1941) "Virus and Rickettsial Diseases." Harrard Univ. Press, Camb., Mlass., p. 789.

Wolbach, S. B. and Schlesinger, M. J. (1923-24) J. med. Res., 44, 231.

Wolbach, S. B., Tond, J. L., and Palfrey, F. W. (1922) "Report of Typhus Research Commission of League of Red Cross Societies to Poland." Harvard Univ. Press., Cambridge, Mass.

Zinsser, H. (1937) Amer. J. Hyg., 25, 430 ; (1941) "Virus and Rickettsial Diseases," p. 872. Harvard Univ. Press, Cambridge, Mass.

Zinsser, H. and CASTANeDA, M. R. (1930) J. exp. Med., 52, 649; (1934) Ibid., 58, 471. Zinsser, H. and Schoensach, E. B. (1937) J. exp. Med., 66, 207. 


\section{CHAPTER 40}

\section{THE PLEUROPNEUMONIA GROUP OF ORGANISHS}

\section{Tentative Definition.}

Microscopically visible, extremely pleomorphic organisms showing granules, rings, coccoid forms, filaments and other bizarre forms. Some forms can pass coarse bacterial filters. Size of smallest elements varies from about 125 to $250 \mathrm{~m} \mu$. Non-motile. Stain poorly with ordinary bacterial stains, but well with Giemsa. Gram-negative. Grow in nutrient media in absence of living tissue cells. Facultative anaerobes. Form characteristic minute colonies on suitable solid media. Parasitic species require a high concentration of animal protein in the medium. Readily destroyed by heat. Apparently no special resistance to glycerol. Some species are bile-soluble. Antigenic specificity is usual, but not complete. Ability to give rise to inclusion bodies in tissnes rery doubtful. Considerable degree of host specificity. Immunity following disease docs not appear to be specially lasting.

Though the organism responsible for pleuropneumonia of cattle (see Chapter 84) was recognized and cultivated by Nocard and Roux as long ago as 1898, it is only within the past few years that a number of closely related organisms, some pathogenic, some saprophytic, have been described, and that the importance of a large group of organisms possessing unusual and distinctive properties has been realized. The complex morphology of the pleuropneumonia organism was described by Bordet (1910) and by Borrel and his colleagues (1910). The fact that Berkefeld filtrates often proved infective afforded ground for the belief that it was a filtrable virus. The more recent observations, however, of Barnard (1926), Smiles (1926), Orskov (1927), Nowak (1929), Wroblewski (1931), Ledingham (1933), Klieneberger (1934), Tang et al. (1935, 1936), Turner (1935), and Merling-Eisenberg (1935) have rendered it probable that only the tiny granular or elementary forms, and the plastic filamentous forms, are capable of passing through coarse filters.

The second organism of this group was described by Bridré and Donatien $(1923,1925)$, who isolated it from sheep infected with contagious agalactia (see Chapter 84). In 1935 Klieneberger reported the occurrence in cultures of Streptobacillus monitiformis (Actinomyces muris) of a pleuropneumonia-like organism, now referred to as L1, living apparently in symbiosis with the bacillus. This discovery formed the start of a fruitful series of investigations. By means of the technique that Klieneberger described for their cultivation, she and numerous other workers during the following years succeeded in isolating, mainly from rats and mice, a number of different species of pleuropneumonia-like organisms. The existence of similar saprophytic organisms was demonstrated in sewage by Laidlaw and Elford (1936), and confirmed by Seiffert $(1937 a, b)$. 
The systentic position of these organisms has given rise to much discussion. The curious association of L1 with Streptobacillus moniliformis has been, in particular, the subject of wide speculation. Though Klieneberger herself regards it as a symbiont, other workers, notably Dienes (1939) and Heilman (1941), maintain that it is merely a variant form of the bacillus. It is too early as yet to form any sound judgment on these conflicting views. The fact, however, that none of the numerous other pleuropneumonia-like organisms has been found accompanying a given bacillus does tend, in our opinion, to support Klieneberger's contention. In this regard the close association of the genetically distinct fusiform bacilli and spirochetes found in Vincent's angina will be recalled to mind.

Apart from their peculiar mode of reproduction, the pleuropneumonia group of organisms seems to fall in lictween the bacteria on the one hand and the Rickettsin and filtrable virus group of organisms on the other. They are distinguished from Rickettsia mainly by their extreme pleomorphism and by their ability to grow on nutrient media in the absence of living cells. From bacteria they seem to differ less fundamentally, but the filtrability of their smallest elements and their limited metabolic powers assign them a place at the lowest end of the bacterial scale. What relation they bear to the group of cocco-bacilliform bodies believer by Nelson $(1936 a, b, 1937,1940)$ to be responsible for fowl coryza and for infectious catarrh of mice and rats it is impossible to say, though it may be noted that the organisms described by Nelson were less pleomorphic and grew only in the presence of living cells.

The nomenclature and classification of the pleuropneumonia group of organisms present grave difficulties. Ledingham (1933) would place them in the family Actinomyectacece, Turner (1935) in a new order of Borrelomycetales, and Sabin (1941) in a new class of Paramycetes. Klieneberger and Smiles (1942) agree with Sabin that their inclusion among the Schizomycetes is hardly justifiable. Until we know more about the genetic relationship of these organisms to the common bacteria, it seems wiser to refrain from premature commitments and to suffer the discomfort of using a clumsy, thongh comprehensive, ten like the pleuropneumonia group.

In the remainder of this chapter we shall give a fairly full description of the original type species, and brief accounts of the others. Those who wish for further information should consult the review by Sabin (1941), to which we ourselves are indebted.

\section{The Organism of Pleuropneumonia}

Cultivation.-This organism, which is sometimes referred to as Asterococcus mycoides (Borrel et al. 1910), can be cultivated on a number of different media, but most workers have used serum broth or serum agar. Growth is said to occur under both aerobic and anaerobic conditions; according to Turner (1935), microaerophilic conditions are most suitable. The optimum temperature for development is $37^{\circ} \mathrm{C}$. ; no growth occurs below $30^{\circ} \mathrm{C}$. (Tang et al. 1935). With freshly isolated strains, 2-3 day serum broth cultures often contain distinctive mucoid islands and threads visible to the naked eye, while dark-ground examination may reveal the prestence of minute colonies, the smallest of which is about $12 \mu$ in diancter ('Tang of al. 1936). With older strains only a general clouthess of the medium is seen. On solid media dew-drop colonies appear in 5 or 6 days. Under the microscope these are often umbonate, and consist of a yellowish-brown granular 
centre surrounded by a smooth transparent peripheral extension (Fig. 223). Welldeveloped colonies may reach a diameter of $2 \mathrm{~mm}$. Cultivation is also suecessful on the chorio-allantoie membrane of the developing chick embryo (Tang et al. 1936). Swift (1941), however, fomm that both the organism of pleuropnemmonia itself and seven other strains of the same group grew better on dead membranes, prepared by freezing the embryo for an hour with try ice, than on living membranes-a point of possibly some differential importance from Rickettsia and the filtrable viruses. On living membranes no constant macroscopic lesions were produced, and the embryo was not killed.

Morphology.-The morphology of the organism is influenced by a number of factors, particularly the age of the culture and the methor of examination. Growth of organisms of the pleuropneumonia group appears to be accompanied by the

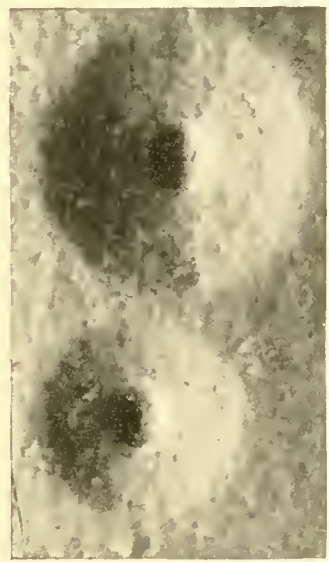

Fig. 23.- Organisil of Pleuropneumonia.

Surface colonies on serum $\operatorname{agar}(\times 140)$.

(After Tang et al.)

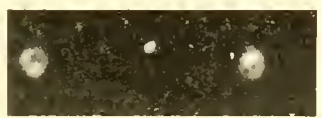

Fic. 224.-ORGanisM OF Pleuropneumonia.

Elementary bodies, granules, or conjdioids. Dark-ground illumination $(\times 3600)$. (After Turner.)

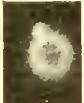

Fif. 225.--Organish of Pleuropneumonia.

Spheroid showing unipolar germination. Dark-ground illumination $(\times 3600)$. (After Turner.)

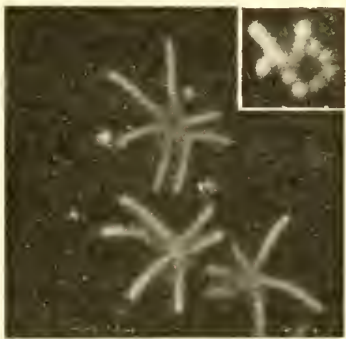

Fir. 226 (Inset).- Organism of Pleuropneumonia.

Spheroid showing multipolar germination. Dark-ground illumination $(\times 3600)$.

(After Turner.)

Fig. 227.-ORganisM of Pleuropnedmonia.

Multipolar germination, showing how the buds have moved away from the parent spheroid and are developing into filaments. Dark-ground illumination ( $x$ 3600). (After Turner.)

formation of large amounts of cholesterol and cholesterol esters from the serum in the medium (Partridge and Klieneberger 1941). These bodies are present as the myelin forms of lecithin, and assume the most bizarre shapes (Williams 1941). In addition, there is reason to believe that the protoplasm of the organism itself is in some stages of its development peculiarly plastic, and is readily distorted by external pressure or tension. Since many workers have used different inethods of examining cultures, and since most of these methods have entailed a risk of distorting the microbial elements, it is not surprising that the observations recorded have often been diverse, conflicting, and difficult to interpret. Hore recently Klieneberger and Siniles (1942) and Klieneberger (1942) have described methods for examining cultures in situ, either by reflected light on a dark ground using annular oblique incident illumination, or by fixation and staining. 'The use of these methods suggests that the developmental process of the pleuropneumonia groul of organisms is simpler than had formerly been believed, and that the 
majority of bizarre forms described by previous observers (see Ledingham 1933, Turner 1935, Tang et al. 1935) were not essential morphological stages of growth, but were either myelin forms or the result of distortion of the plastic elements of the organism. Agreement, however, has not yet been reached on the true morphology and mode of development of the pleuropneumonia organism, and we shall therefore give $(a)$ one account based on the older methods of examination of slide preparations by transmitted light on a dark ground or by the staining of impression films, and (b) another based on the more recent methods of examination of the organisms in situ, either by reflected light on a dark ground or by fixation and staining.

(a) By Older Methods of Examination.-At the risk of undue simplification, we

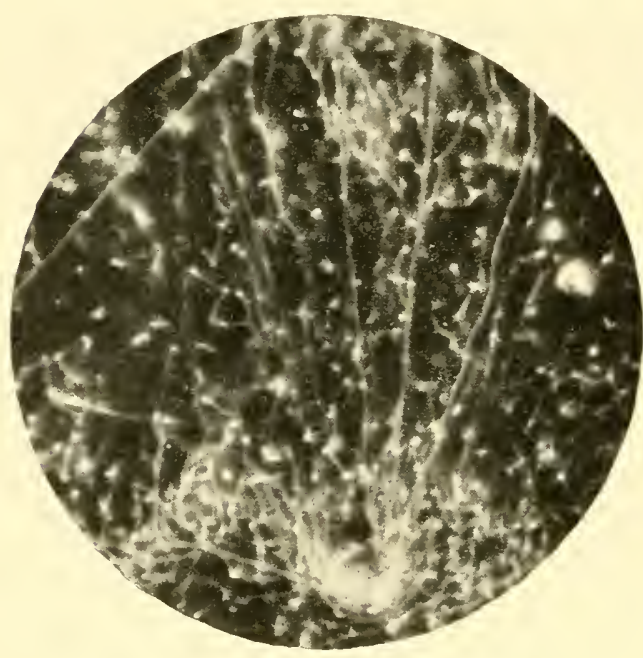

Fig. 228.-Organisu of Pleuropneumonia.

Stage of ramification, showing nodes of protoplasmic condensation which form the starting-point for the outgrowth of fresh filaments. Dark-ground illumination ( $\times$ 1114). (After Tang et al.)

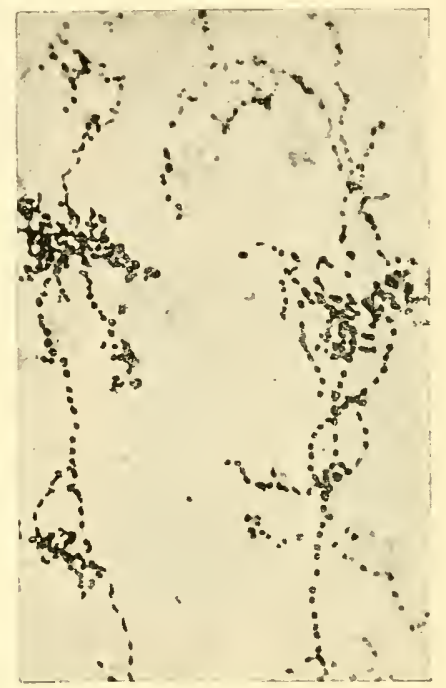

FIG. 229.-ORGANISM OF Pleuropneumonia.

Stage of chain formation, showing condensation of protoplasm at multiple points of filament. Giemsa. ( $\times 1640)$. (After Tang et al.)

shall follow Tang and his colleagues (1935) in describing five morphological stages in the growth of this organism. (a) Granular stage. Small granules, coccoid, diplococcoid, and cocco-bacillary bodies are seen, usually $0 \cdot 15-0 \cdot 4 \mu$ in diameter. Turner refers to them as "conidioids." They stain deeply with Giemsa, and may be regarded as a resting stage (Fig. 224). (b) Filamentous stage. On inoculation into a fresh medium the granular bodies grow into spheroids about $0 \cdot 4-0.8 \mu$ in diameter, and develop on their periphery one or more spherical buds (Figs. 225, 226). These gradually move away from the parent body, but remain attached to it by a filament (Fig. 227). In old strains this filament is usually very short, and resembles with its terminal bud a sporing tetanus bacillus. In freshly isolated strains, however, enormously long filaments develop, sometimes crossing several fields of the microscope. Endomycelial protoplasmic streaming is often notice- 
able. The filaments stain very poorly with Giemsa. (c) Stage of ramification. During the process of streaming, areas of protoplasmic condensation appear at various points in the filament, and form the starting-point for the outgrowth of fresh filaments. A tangled branching mycelium is the result (Fig. 228). In older strains this stage is lacking. (d) Stage of chain formation. In this stage the streaming protoplasm condenses rapidly at inultiple points, so that the filament takes on a streptococcal appearance (Fig. 229). (e) Stage of disintegration. The chains break up, and set free a multitude of granular and coccoid forms, thus conpleting the cycle of development. In old cultures, in which the filamentous stage is lacking, the buds in the second stage become detached from the parent body, and either grow out again into spheroids which themselves start budding, or remain in the granular phase.

It will be seen that reproduction occurs partly by budding, and partly by fragmentation. Turner (1935) has described no fewer than five different methods of reproduction, and the reader who is interested will do well to refer to his beautifully illustrated paper. Again, reference must be made to Ledingham (1933) and

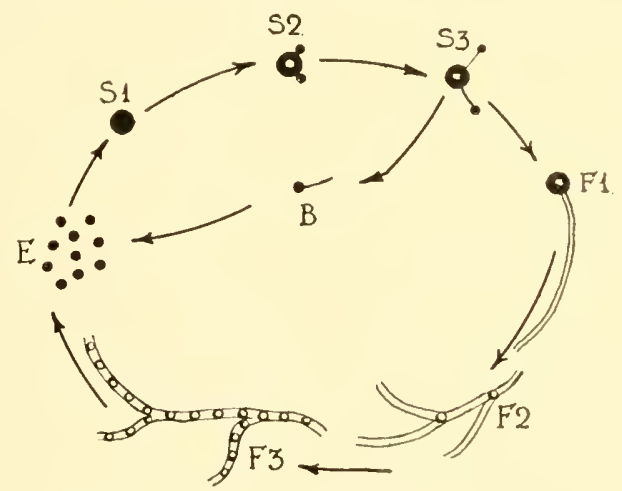

Fig. 230.-Organisir of Pleuropneumonia.

Diagrammatic representation of developmental cycle according to Ledingham (1933), Klieneberger (1934), Turner (1935), and Tang and his colleagues (1936). Outer circle represents freshly isolated strain forming filaments. Inner circle represents old laboratory strain not forming filaments.

E. Elementary bodies, granules, or conidioids.

$\mathrm{S}_{1}$. Spheroid.

$\mathrm{S}_{2}$. Bipolar germination of spheroid.

$\mathrm{S}_{3}$. Spheroid with buds still attached to it by very short filaments-drum-stick appearance.

$\mathrm{F}_{1}$. Long filament growing out from spheroid.

$\mathrm{F}_{2}$. Filament showing ramification.

$\mathbf{F}_{3}$. Filament showing protoplasmic condensation, preparatory to liberation of condensed particles as elementary bodies.

B. Buds detached from spheroid, and becoming elementary bodies.

Klieneberger (1934) for the mode of origin of the vibrionic forms, chromatic nodes, large oval swollen bodies, and other elements that are seen in preparations from colonies on solid media, and to Tang and his colleagues (1936) for a description of the apparently rare amcboid and giant ring forms. Fig. 230 represents diagrammatically the apparent sequence of development.

(b) By Newer Methods of Examination.-The picture obtained by the newer methods of examination, referred to on p. 941, is much simpler. Following Klieneberger and Smiles (1942), we may describe two methods of multiplication: 
(1) segmentation, and (2) the formation of elementary corpuscles within a body smrouncel by a liniting memlorane. (1) Starting as an elementary corpuscle or gramule (Fig. 23la), the organism grows into a small resolvable sphere, which may take on an irregular $(b)$ or even filamentons $(c)$ shape. This then divides into a variable number of segments $(d, e)$, which may or may not separate from each other. (2) Starting in the segments resulting from (1b), darkly stained bodies (f), probably consisting of nuclear material (see Klieneberger 1942), appear. These then divide, forming multiple elementary corpuscles within a body surrounded by a limiting membrane $(g)$. Later, each of these corpuscles is liberated, apparently surrounded by a small portion of cytoplasm (h). This stage, it may be remarked, recalls to mind the formation of merozoites in the developmental cycle of the malarial parasite. later still, the cytoplasmic sheath is lost. and the elementary corpuscles remaining ( $($ ) are indistinguishable from those from which the original cycle, describerl in (1), began.
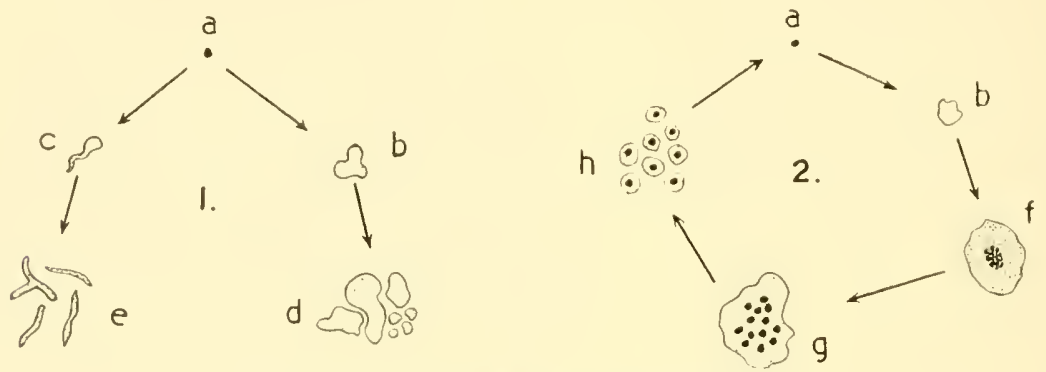

Fig. 231.-Organism of Peetropnetimonia.

Diagrammatie representation of developmental cyele aceording to Klieneberger and Smiles (1942) and Klieneberger (1942).

1. Proliferation by segmentation.

2. Proliferation by formation of elementary corpuseles.

(a) Elementary granule.

(b) Siphere.

(c) Simall filament.

(d) Segments resulting from division of $(b)$.

(c) Filaments resulting from division of $(c)$.

(f) Nucleated bodies.

(g) Mnltiple granules within a limiting memlurane resulting from division of $(f)$.

(h) Liberated gramules still surrounded by eytoplasm.

\section{Resistance, Metabolism, Biochemical Reactions, and Antigenic Structure.-} According to Tang and his colleagues (1935), serum broth cultures may remain viable for 45 days at $37^{\circ} \mathrm{C}$, and for 98 dlays at $0^{\circ}-5^{\circ} \mathrm{C}$. The organisms are bilesoluble, particularly in the filamentous stage, but are very resistant to ultra-violet irradiation and to the photodynamic action of methylene bhe (Tang et al. 1936). They ferment ghlucose, maltose, and dextrin, and to a less extent sucrose, with the production of acid, but not mannitol, lactose, or salicin. Hæmoglobin is reduced by freshly isolated strains. Warren (1942) states that growth is prevented by 10 per cent. $\mathrm{CO}_{2}$, that a hemolysin is produced, that methylene blue is decolorized in the presence of lactate, and that the time-potential curve differs from that of the $\mathrm{L}$ group of organisms. Little is known of the antigenic structure of this organism, but there is some evidence, based mainly on cross-protection tests, of the existence of more than one immunological type. 
Pathogenicity. - The organism is naturally pathogenic to cattle. Experimentally the subcutaneous inoculation of $0.5-1.0 \mathrm{ml}$. of infected lymph or a virulent culture produces in 8 to 25 days a tense, hot, painful, inflammatory swelling accompanied by high fever and often followed by death. Incision of the skin over the affected part is followed by the exudation of a clear straw-coloured fluid, often amounting to several litres. Post mortem, the connective tissue meshes of the lungs are distended with an immense quantity of clear yellow fluid, which is here and there coagulated into gelatinous trembling masses. Microseopical examination of the freshly collected fluid reveals the presence of forms similar to those seen in culture ; the mycelial phase appears to predominate (Turner 1935). It is interesting in this counection to note that only freshly isolated strains showing the filamentous phase of development are fully virulent. There may be a little serous exudate in the pleural cavity, and the thoracic and inguinal lymphatic glands may be affected. According to Daubney (1935), the typical disease can he reproduced by inocualtion into the jugular vein of lymph or culture mixed with a few millilitres of 10 per cent. agar. The amboli are held up in the lungs and form the starting-point of the discase. Goats and buffaloes appear to be susceptible to experimental iuoculation, but laboratory animals are resistant. (Nocard and Roux 1898, Tang et al. 1935).

\section{The Organism of Contagious Agalactia}

This organism was isolated by Bridré and Donatien $(1923,1925)$ from infected sheep (see Chapter 84). It has been studied hy Nowak and Wroblewski (1930), Wroblewshi (1931), Ledingham (1933), and Nowak and Lominski (1934). Its general characters are so similar to those of the pleuropneumonia organism that we do not propose to describe them separately. The disease can be produced experimentally by inoculation with pure cultures. The goat is more susceptible than the sheep. The subcntaneous inoculation of $0.5-1.0 \mathrm{ml}$. of a pure culture is followed in 4 to 7 days by the appearance of a small local swelling which disappears during the following week. After a further incubation period of 1 to $t$ weeks, localizing lesions appear in the joints, cornea, and, in lactating females, the udder. The amount of milk secreted diminishes, and a yellowish purulent fluid takes its place. Laboratory animals appear to be insusceptible.

\section{Other Organisms of the Pleuropneumonia Group}

It is unnecessary to describe in detail the individual features of the numerous other nembers of the pleuropnemmonia group that have been isolated. All of them agree in being pleomorphic, though differences are evident in the range and shape of the elements that are formed. All the parasitic species require for growth, particularly on solid media, a high proportion of animal protein. For this purpose a 30 per cent. serum broth or agar of $\mathrm{pH} 7 \cdot 6-8 \cdot 0$, to which 5 per cent. of boiled blood may be added, is generally suitable. The optimum reaction for growth appears to he about $\mathrm{pH} 7 \cdot 8-8.0$; below $\mathrm{pH} 7 \cdot 0$ growth generally ceases. The appearance and rate of development of colonies on solid media differ to some extent with different species. Most members grow better aerobically than anaerobically, but the organism isolated from dogs and the Lt strain from rats are exceptions. Incubation in 10 per cent. $\mathrm{C}^{\mathrm{O}} \mathrm{O}_{2}$ is said to enhance the growth of $\mathrm{L} 1$, but to inhibit that of other L organisms (Warren 1942). Fermentation of sugars is very weak, and the final $\mathrm{pH}$ reached is seldom lower than $7 \cdot 0$. Hæmolysis and 
reduction of hæmoglobin result from the growth of some strains. Antigenically there is a considerable degree of specificity, though there is evidence of some group relationship among many of the members that have been studied (Klieneberger 1940). The parasitic species often seem to lead a harmless commensal existence in the body of their host, though under certain conditions they may give rise to lesions of various types, of which arthritis is one of the commonest. At least one species, L5, forms an exotoxin acting on the central nervous system. Virulence is apt to decline rapidly in artificial culture. Pathogenicity is limited usually to a single host species. Some members are susceptible in the tissues to organic gold salts (Collier 1939a. Findlay et al. 1939).

The saprophytic species, isolated from sewage, earth, and other situations, differ from the parasitic species mainly in their simpler growth requirements-a high proportion of animal protein being unnecessary for their development-in their alility to multiply at $22^{\circ} \mathrm{C}$., in their antigenic structure, and in their absence of pathogenicity. We append brief notes on some of the commoner strains.

\section{Pleuropneumonia Strains in Dogs.}

Shoetensack (1934) isolated in pure culture an organism, sonetimes referred to as Asterococcus canis, from nasal secretion, lung, and liver of dogs suffering from distemper. Two antigenic types are recognized (Klieneberger 1938, 1940). The pathogenicity of these strains to dogs and their relationship to distemper are in doubt.

\section{Pleuropneumonia Strains in Rats.}

L1 strains. - Klieneberger (1935) described the isolation of a pleuropneumonia-like organism from cultures of Streptobacillus moniliformis, which is a normal parasite of the nasopharynx of rats (see p. 385). She obtained it in pure culture, and showed that it had the same colony type, characterized by a central granular part embedded in the agar medium and a flatter peripheral zone, as the organism of pleuropneumonia. It was filtrable through Berkefeld $V$ candles. By itself it appeared to be non-pathogenic. From the lung of one rat it was isolated independently of Streptobacillus moniliformis (Klieneberger 1938). Though Klieneberger maintained that it was a symbiont, Dienes (1939, 1942), Heilman (1941), Smith (1941), and Brown and Nunemaker (1942), regard it as a variant of the bacillary organism. Klieneberger's (1942) most recent observations bring further evidence in favour of the symbiotic view. She shows that the developmental cycle of the L1 organism appears to be almost identical with that of the organism of pleuropneumonia as described by Klieneberger and Smiles (1942); and that the results of cross-absorption tests made between Streptobacillus moniliformis and Ll are most easily explicable on the assumption that the two organisms are antigenically distinct.

L3 strains. - Klieneberger and Steabben (1937) isolated this organism from the bronchiectatic lesions that are so common in the lungs of old rats. It is antigenically distinct from Ll (Klieneberger 1940). Though abscess formation results from subcutaneous inoculation of pure cultures into half-grown mice, it has so far proved impossible to reproduce the natural disease in rats (Klieneberger and Steabben 1940).

L4 strains.-This organism was isolated first by Woglom and Warren (1938) from subcutaneous abscesses in rats following inoculation with a rat sarcoma strain. Its relation to the pleuropneumonia group was not recognized till, later in the same year, Klieneberger (1938) reported the isolation of an organism, which she called L4, from the swollen submaxillary gland of a rat. It is antigenically distinct from Ll or L3. When inoculated subcutaneously or intraperitoneally into rats, it gives rise to abscess formation. Inoculated intravenously with cells or agar it produces a serere arthritis, particularly in young rats. An organism, labelled L7, was isolated by Findlay, Mackenzie, MacCallum, and Klieneberger (1939) from spontaneous polyarthritis in the rat, but this organism was later shown by Klieneberger $(1939 a)$ to be identical with L4. Its relation to the organisms described by Collier (19396) and Beeuwkes and Collier (1942), which were like- 
wise isolated from polyarthritis in the rat, and to the organisms cultivated by Preston (1942) from the swollen joints and middle ear of rats, is still in doul,t. Gold salts appear to be of prophylactic value in protecting against arthritis caused by the inoculation of L4 into rats (Collier 1939a, Findlay et al. 1940).

\section{Pleuropneumonia Strains in Mice.}

Pleuropneumonia-like organisms in the mouse's brain were demonstrated independently by Findlay and his collaborators (1938) in this country while investigating lymphocytic choriomeningitis, and by Sabin (1938) in the United States during the course of experiments with Toxoplusme. Several other strains, biologically and immunologically distinct, have since been isolated. Except in very young mice, they seem to be normal parasites of the conjunctiva, nose, and brain (Sabin and Johnson 1940).

L5 and Type A strains.-L5 was isolated by Findlay and his colleagues (1938) from the brains of mice suffering from rolling disease. A similar, if not identical, organism was isolated by Sabin (1938), who showed that it produced a true exotoxin having strongly neurolytic properties. Intracerebral inoculation of the organism gives rise, usually after an incubation period of $2-3$ days, to a disease characterized by rolling morements on the long axis of the body. Some mice die, and at post mortem extensive necrosis and lysis are found of the posterior pole of the cerebellum. Apparently the same organisms have been isolated from the lungs by Sullivan and Dienes (1939).

L6 strcins.-These strains were isolated by Findlay and his colleagues (1939) from the brains of mice that had been inoculated intracerebrally with the blood of splenectomized mice containing Eperythrozoon coccoides. They differ colonially and antigenically from L5 strains.

M55 strain.-This organism was isolated from the swollen joint of a mouse by Jahn (see Klieneberger 1940). It is responsible for one type of mouse arthritis.

$B, C, D$, and $E$ strains.-These organisms were isolated by Sabin $(1938,1939 a, b)$ and by Sabin and Johnson (1940) from the conjunctiva, respiratory tract, and brain of mice. All four types are able to give rise to arthritis on intravenous inoculation. What relation they bear to the L6 and $\mathbf{H} 55$ strains is not yet known.

Edward's strains. - Edward (1940) isolated 7 strains from the lungs of normal mice. Some evidence was obtained that they might give rise to pneumonic lesions after nasal instillation, but they did not produce arthritis.

\section{Pleuropneumonia-like Strains in Guinea-pigs.}

The guinea-pig so far has not been a fruitful source of pleuropneumonia-like organisms. Klieneberger (1940) isolated a strain, in conjunction with Streptobacillus moniliformis, from abscesses in the neck, but circumstances prevented its proper study. Smith (1941) likewise reported the isolation of Streptobacillus moniliformis from alsscesses in guinea-pigs, but was unable to separate a pleuropneumonia-like strain from the bacillary organisms.

\section{Pleuropneumonia-like Strains in Man.}

Dienes and Edsall (1937) isolated a strain from a suppurating Bartholin's gland in a woman who was working in the laboratory with rats. Subsequently Dienes (1940) demonstrated pleuropneumonia-like strains in the cervical secretion of five patients suffering from pelvic infections. Whether these organisms are pathogenic or not is still undetermined. The frequent association of pleuropmeumonia-like organisms with arthritis in rats and mice has naturally raised hopes that they may be responsible for human rheumatism. In spite, however, of preliminary suggestive findings by Swift and Brown (1939), practically all attempts to demonstrate such a relationship have proved unsuccessful (see Sabin 1941).

\section{Saprophytic Pleuropneumonia-like Strains.}

Laidlaw and Elford (1936) described the isolation of three pleuropneumonia-like strains, A, B and C, from London sewage by the inoculation of suitable filtrates into Fildes' broth. The organisms grew best at $30^{\circ} \mathrm{C}$. in Hartley's horse digest broth, pH $8 \cdot 0$, to which Fildes' peptic digest of red cells had been added. They fermented no sugars 
and were nom-pathogenic to animals. Strains $\mathrm{A}$ and $\mathrm{C}$ were antigenically distinct ; strain B was more closely related to A than to C. Pirie (1937) found certain metabolic differences between strains $A$ and $C$. Similiar organisms lave been isolated by Seiffert $(1937 a, b)$ from soil, manure, and related substances. 'These saprophytic forms can grow in the absence of high concentrations of natural animal proteins.

\section{REFERENCES}

BARNARD, J. E. (1926) J.R. micr. Soc., p. 253.

BeEUWKes, H. and Collier, H. A. (1942) J. infect. Jis., 70, 1.

Bordet, J. (1910) Ann. Inst. Pasteur, 24, 161.

Borrel, Dujardin-Beaumetz, Jeantet, and Jovan. (1910) Ann. Inst. Pasteur, 24, 168.

Bridré, J. and Donatien, A. (1923) C.R. Acad. Sci., 17\%, 841 (1925) Ann. Inst. Pasteur, 39,925 .

Brown, T. II. and Nunemaker, J. C. (194:) Johns Ilopk. Hosp. Bull, \%0, 20I.

C'oldier, W. A. (1939k) Z. ImmunForsch., 95, 132; (19396) J. I'uth. Bact., 48, 579.

Datbney, R. (1935) J. comp. Path., 48, 83.

Dienes, L. (1939) J. infect. Di.s., 65, 24: (194(1) Proc. Soc. rop. Biol., I.Y., 44, 46s; (1942) J. Bact., 44, 37.

Dienes, L. and Eisald, G. (1937) Iror. Soc, exp. Biel., Y.Y., 36, 740.

EDWARI, D. G. ff. (1940) J. P'ath. Bact., 50, 409.

Elfurd, W. J. and Ende, M. van den. (1944) Brit. J. exp. Path., 25, 2213.

Findlay, G. M., Mackenzie, R. D., MacCallum, F. U., and Klieneberger, E. (1938) Luncet, ii. 1511; (1939) Lancet, ii. 7.

Findeay, G. M., Mackenzie, R. D., and Maccallum, F. O, (1940) Brit. J.exp. Path. 21,13 .

Heilman, F R. (1941) J. infect. Dis., 69, 32, 45.

Kliteneberger, E. (1934) J. Path. Bact., 39, 409 ; (1935) Ibid., 40, 93 ; (1936) Ibid., 42, 587 ; (1938) J. Hyg., Camb., 38, 458 ; (1939a) Ibid., 39, 260 ; (19396) J. Path. Bact., 49, 451 ; (1940) J. Hyg., Camb., 40, 204; (1942) Ibid., 42, 4\$5.

Kuneneberger, E. and Sulles, J. (1942) J. IIyg., Camb., 42, 110.

Klieneberger, E. and Steabres, D. B. (1937) J. Hyg., Camb., 3\%, 143; (1940) Ibid., $40,223$.

Laidlaw, P. P. and Filford, W. J. (1936) Proc. roy. Soc, $B, 120,292$.

Ledingham, J. C. G. (1933) J. Path. Bact., 37, 393.

Merlixg-Eisfenberg, K. B. (1935) Brit. J. exp. Path., 16, 411.

Nelson, J. B. (1936a) J.exp. Hed., 63, 515 ; (1936b) Ibid., 64, 749; (1937) Ibid., 65, 851 ; (1940) Ibid., 72, 655.

Nocard and Rodx. (1898) Ann. Inst. Pasteur, 12, 240.

Nowak, J. (1929) Ann. Inst. Pasteur, 43, 1330.

Nowak, J. and Lominski, I. (1934) Ann. Inst. Pasteur, 53, 438.

Nowak, J. and Wroblewski, W. (1930) Trans. Congr. int. Microbiol., 1, 619.

Orskov, J. (1927) Anu. Inst. Pasteur, 41, 473.

Partridge, S. M. and Ḱlieneberger, E. (1941) J. Puth. Buet., 52, 219.

Pirie, A. (1937) Brit. J. exp. Path., 18, 96.

Preston, W. S. (1942) J. infect. Dis., 70, 180.

SABIN, A. B. (1938) Science, 88, 189, 575; (1939a) Ibid., 89, 228; (19396) Ibid., 90, 1s; (1941) Burt. Rer., 5, 1, 331.

Sabin, A. B. and Johnson, B. (1940) Proc. Soc. exp. Biol., N.Y., 44, 569.

Seiffert, G. (1937a) Zul. Bakt., 139, 337 ; (1937b) lbid., 140, Beilheft, p. 168.

Shoetensack, II. (1934) Kitasato Arch., 11, 277.

Smiles, J. (1926) J. R. micr. Soc., p. 257.

Sмгтн, W. (1941) J. Path. Bact., 53, 29.

Suluivan, E. R. and Dienes, L. (1939) I'roc. Soc. exp. Biol., X.Y., 41, 620.

SwIFT, H. F. (1941) J. exp. Ved., 74, 557.

SwIFT, H. F. and Brown, T. II. (1939) Science, 89, 271.

TANG, F, F., WEJ, H., and Edgar, J. (1936) J. Path. Buct., 42, 45.

Tang, F. F., Wei, H., McWhirter, D. L., and Edgar, J. (1935) J. Path. Bact., 40, 391.

Turner, A. W. (1935) J. Path. Bact., 41, 1.

Warrex, J. (1942) J. Bact., 43, 211.

Williaus, S. (1941) Aust. J. exp. Biol., 19, 25\%.

WogloM, W. H. and Warren, J. (1938) Science, 8\%, 370.

Wroblewski, W. (1931) Ann. Inst. Pasteur, 4\%, 94. 


\section{CHAPTER 41}

\section{THE ANIMAL VIRUSES: GENERAL PROPERTIES}

\section{Tentative Definition of Pathogenic Forms}

Minute bodies which are usually invisible by ordinary microscopic methods of examination, which have a diameter of less than $0 \cdot 2 \mu$, which ean often be filtered through candles and membranes impermeable to ordinary bacteria, which have not yet been cultivated in cell-free media but which multiply freely in the presence of susceptible cells in vitro or in vivo, which generally have a high resistance to glycerol, which frequently invade one particular species of host and tend to affect one particular tissue, which give rise to characteristic inclusion bodies in the tissues, and which cause a latent or overt infection often followed by a lasting immunity.

There is a large group of diseases affecting man, animals, insects, and plants, which have most or all of the characteristics of infectious diseases, yet in which no visible microscopical organism has been satisfactorily demonstrated. That these diseases are infectious is shown by the fact that it is usually possible to reproduce them in normal hosts by inoculation, not only of the ground-up diseased tissue, but also of cell-free extracts or filtrates of the tissue. Since these cell-free extracts are most commonly obtained by filtration, the practice has grown up of referring to the specific infecting agents contained in them as "filtrable viruses." This term is sanctioned by usage; but it is necessary to point out that as not all these viruses have yet been shown to be filtrable, and as the exact standardization of filtration has not yet proved possible, the term "filtrable" is necessarily a loose one. With increasing knowledge of the properties of viruses, the present tendency is to omit all qualifying adjectives, and to speak of them simply as viruses. Alternative names, which have from time to time found favour with some workers, but which have now been generally discarded, are "ultramicroscopic viruses," "protista," "chlamydozoa," and "strongyloplasms."

\section{Methods of Study}

Filtration.-The ability of a particulate body to pass through a filter is not a simple function of the relation of the size of the borly to the size of the pores; that is to say, a filter is not a mere mechanical sieve. Several factors other than the size of the filter pore determine whether a given body will pass through it or not. Thus, according to Rivers (1928), "the electrical charge on the virus, the electrical charge on the filter, the adsorption of the virus by aggregates of protein or by cell letritus, the amount of protein or other substances in the virus emulsion, the temperature at which the filtration is conducted, the amount of negative or positive pressure employed, the duration of filtiation, and other factors 
... serve to influence the results of all filtration experiments." The thickness of the filter also makes a considerable difference.

The ordinary porons bacterial filter consists of a positively charged alkaline earth cation, and a negatively charged silicate anion. If a simple basic dye, such as methylene blue, which consists of an organic coloured cation united to an inorganic anion, is passed through a filter, a large amount of the dye will be adsorhed. The explanation of this is that the organic colonred cation cuters into combination with the silicate anion in the filter, forming an insoluble dye-silicate, which is retained; the soluble salt, such as $\mathrm{NaCl}$ or $\mathrm{KCl}$, formed by the union of the alkaline earth cation in the filter with the inorganic anion of the dye, passes through. A similar phenomenon is observed in protein solutions. In solutions more aeid than the isoelectric point of the protein, the dissociated protein is chiefly in the form of multivalent eations eapable of entering into combination with the silicate anions in the filter, and forming an insoluble compound, which is retained. On the other hand, in solutions more alkaline than the isoelectric point, the dissociated protein is chiefly in the form of multivalent anions, capable of entering into combination with the alkaline earth cations in the filter with the formation of soluble salts, which pass through. This is probably why enzymes, toxins, and viruses appear to pass more readily through filters in weakly alkaline than in aeid solutions (see Mndd 1922-23, 1928).

The nature of the suspending fluid plays an important part in determining the result. Several workers have noted that viruses pass much more readily through filters when the suspension is made up with broth or serum than with saline or phosphate buffer (Grinnell 1929, Ward 1929, Sawyer and Frobisher 1929, Tallerman 1929, Marie and Urbain 1930, Galloway and Elford 1931). The mode of action of the broth is not known with certainty, but according to Elford (1933) it appears to be closely related to the ability of this medium to stabilize the dispersion of a lyophilic colloid. Soap has the opposite effect. Another factor, which is of special importance in comparing the filtrability of two different strains of virus, is the initial concentration of virus. The greater the number of virus particles present in the suspension, the more likely is virus to be found in the filtrate (Galloway and Elford 1931).

Cataphoresis experiments on such viruses as vaccinia, fowl-pox, foot-and-mouth, rabies, yellow fever, myxoma, and Rous sarcoma have agreed in showing that most viruses carry a negative charge in neutral or nearly neutral suspensions (Douglas and Smith 1928, Findlay 1930, Hindle and Findlay 1930, Poppe and Busch 1930, Natarajan and Hyde 1930, Sichert-Modrow 1930, Sankaran et al. 1934). It is true that Olitsky and Boëz (1927) stated that the foot-and-mouth virus carried a positive charge up to $\mathrm{pH} 8 \cdot 0$, but the results of these workers have not been confirmed. Most viruses have been studied over a range of about $\mathrm{pH} 5.0$ to $9 \cdot 0$; they have been found to be negatively charged up to about $\mathrm{pH} 7 \cdot 6$, though the exact location of the isoelectric point has varied from $\mathrm{pH} 7 \cdot 0$ with the yellow fever virus to $\mathrm{pH} 9 \cdot 3$ with the virus of myxoma. Not too much attention, however, should be paid to these measurements, since most of them have been carried out in the presence of tissue protein. Beard, Finkelstein and Wyckoff (1938), who worked with a relatively pure suspension of vaccinial elementary bodies, found their isoelectric point to be between $\mathrm{pH} 4 \cdot 6$ and $4 \cdot 3$. The nature of the charge carried will affect, to some extent, the passage of the virus through a filter. Incidentally, use may be made of the electric charge carried by the virus to free it from other material in a tissue suspension, or at any rate to obtain it in a more concentrated form. (Douglas and Smith 1928, Sankaran et al. 1934). 
It is important to realize that the mere passage of an organism through a filter candle does not justify its inclusion in the group of filtrable viruses. Even under conditions of careful experimentation, small organisms, particularly slender flexible and motile organisms such as spirochætes, frequently appear in small numbers in the filtrate ; and conversely, the mere failure of an organism to pass through a filter candle does not justify its exclusion from the group of filtrable viruses. Some viruses, for example, such as those of varicella and herpes zoster, have not yet been shown to be filtrable, yet there is little doubt from what is known of their other properties that they should be included in this group. The term "filtrable virus" is one connoting a number of properties, the most important of which have already been defined at the head of this chapter.

Ultrafiltration.--In recent years ultrafiltration has been introduced. In this process, thin collodion membranes are prepared with a given size of pore, the size being determined largely by the concentration of collodion used. In the development of these filters Elford $(1931,1933)$ has played a prominent part. Starting from the earlier work of Bechhold, he has been able by the use of appropriate solvent mixtures, and by the careful standardization of his technique, to prepare a scries of membranes of very regular and accurately graded porosities by means of which determinations of the size of many of the commoner viruses have been successfully made, and subsequently confirmed by other workers (see also Elford, Grabar, and Ferry 1935, Duclaux and Amat 1938). These filters-Gradocol membranesapproach nearer to the mechanical sieve than do ordinary filter candles; they appear to be less influenced by the various secondary factors which we have mentioned, and to be capable, when properly used, of sorting out particles very largely according to their size; though the influence of the pH of the suspending fluid, and of the electrical charge carried, must still be taken into account.

In calculating the size of the particle from the average poro diameter through which it just fails to pass, the effect of adsorption has to be considered. This effect is most influential in membranes with very small pores (Table 58). Thus a particle held back by a membrane with an average pore diameter of $30 \mathrm{~m} \mu$ probably has a diameter of $10-15 \mathrm{~m} \mu$, while one held back by a membrane of 1,000 $\mathrm{m} \mu$ probably has a diameter of $0 \cdot 75-1 \cdot 0 \mu$ (Elford 1933). This relationship, however, is disputed by Markham, Smith and Lea (1942) (see also Cox and Hyde 1932, Asheshov 1933 on ultrafiltration).

\section{TABLE 58}

Relation of Size of Retained Particle to average Pore Diameter of Gradocol MemBRANES (Elford 1933)

\begin{tabular}{|c|c|}
\hline $\begin{array}{c}\text { Membrane average pore } \\
\text { diameter. }\end{array}$ & Size of Retained Particle. \\
\hline $\begin{array}{c}\mathrm{m} \mu \\
10-100 \\
100-500 \\
500-1,000\end{array}$ & $\begin{array}{l}(0.33-0.5) d \\
(0.5-0.75) d \\
(0.75-1.0) d\end{array}$ \\
\hline
\end{tabular}

$d=$ average pore diameter of limiting membrane for optimum filtration conditions.

Microscopical Examination.- There are difficulties in the microscopical examination of viruses. It has already been pointed out in Chapter 2 that under ordinary 
conditions of examination it is impossible to resolve particles less than $0 \cdot 2 \mu$ in diameter. Resolution, it will be remembered, is limited by the numerical aperture of the objective and the wave-length of the light used. Since there are serious difficulties in increasing the numerical aperture of the objective, it follows that the only way to resolve very small bodies is to use a wave-length shorter than any present in the visible speetrum. Resolution, however, is not always required, and considerable attention has been devoted of recent years to methods for rendering small bodies visible. Though certain filtrable viruses may be demonstrated in seetions or smear preparations, their study is greatly facilitated by olotaining them in a suspension relatively free from tissue cells and other gross matter. Usually this is done by differential centrifugation, sometimes aceompanied by filtration. The suspension can then be examined by one of the following methods.

I. Firing and staining with a suitable dye.-Numerous workers, among whom Ledingham (1931) has been one of the foremost, have used this method. The dyes chosen are most frequently Giemsa's stain, or one of its modifications. By this means minnte particles - the so-called elementary bodies - may be rendered visible in appropriate preparations. Since, however, it is impossible to demonstrate very small particles by transmitted light, even when they are deeply stained, it follows that this method is limited to the larger virnses. Its most conspienons success has, in fact, been aehieved hitherto with the virus of vaecinia, the diameter of which is about $0.15 \mu$. It has proved of particular value in the microscopical observation of agglutination, where, of eourse, visibility, and not resolution, of the aggregating particles is alone required.

II. Dark-ground examination using visible light.-Provided the particles under examination can scatter enongh light, and there is a sufficient difference of refractive index between them and the medium in which they are suspended, this method enables very small particles to be rendered visible, even though they are incapable of resolution. It provides a useful means of direet microscopic olservation of virus particles.

III. Photography in ultra-violet light.- Barnard (1925) has been the ehief exponent of this method. After preliminary examination by method II, photographs are taken at partieular wave-lengths in the ultra-violet spectrum (see Chapter 2). Either transmitted or dark-ground illumination may be used. The former suffers from the disadvantage that the ability of viruses to absorb light is very low, and the image so obtained is smaller than it otherwise would be. With dark-ground illumination there is strong contrast, and sharply defined images are obtainable, though their size tends to be slightly too large. With a wave-length of $257 \mathrm{~m} \mu$, particles as small as $75 \mathrm{~m} \mu$ ean be aetually resolved. Their approximate size ean then be determined from the mean of the images given by transmitted and dark-ground illumination. Theoretically, this method is open to almost unlimited extension, but in praetice great teehnieal diffieulties are encountered.

Fluorescence microscopy. - This makes use of the ability of certain bodies to transmute the short invisible waves of ultra-violet light to longer visible waves (see Clauberg 1939).

V. Anmular oblique incident illumination.-In this method a relatively opaque body, like the ehorio-allantoie membrane, ean be examined by oblique illumination from above (see Himmelweit 1938).

VI. Electron microscope.-More recently this has provided a tool that bids fair to outstrip any method hitherto known. Though technical defects prevent 
the realization of the full potentialities of this instrunent, it is said to be possible now, under optimal conditions, to resolve particles as small as $1 \mathrm{m \mu}$ and to use magnifications up to 200,000 times (see Marton 1941, Stanley and Anderson 1941, Sharp et al. 1942, 1913, 1944, Luria et al. 1943, Taylor et al. 1943).

Centrifugalization.-There are considerable meelianical difficulties in constructing a machine that is sufliciently powerful to throw down very fine suspended particles; this difficulty is increased if the suspending fluid, as is usually the case, has a speeific gravity greater than water. The eentrifugal force of a machine varies with the square of the rate of rotation, and directly with the distance of the centrifuged material from the centre of the plate. But neither of these factors can be increased indefinitely, beeause with increasing rate of rotation, and witl inereasing diameter of the plate, a vibration develops that very largely counteracts the centrifugal force. Numerous other mechanical factors, such as the airresistance and the heat generated in the machine, come into play when high speeds are developed, and limit the rate and time during which the machine may be run. Nevertheless, serious attempts have been made in recent years to overcome these

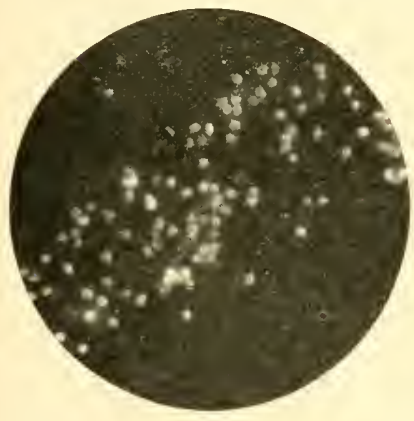

$a$

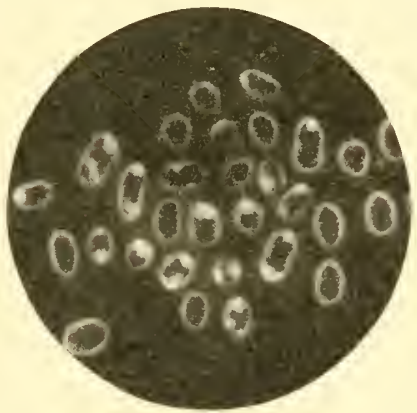

$b$

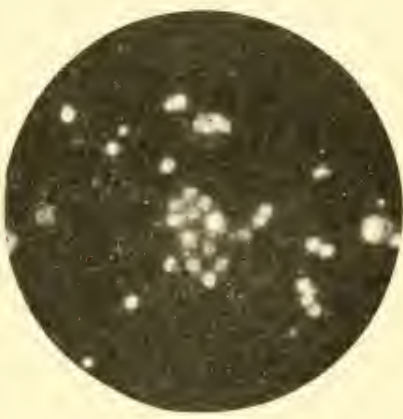

FIG. 232.-ON LEFT $(a)$, ELEMENTARY BODHES OF VACCINIA FROM RABBIT TESTIS, AND ON RIGHT (c) ELEMENTARY BODIES OF CANARY POX; IN THE HIDLE (b) Chromo. prodigiosum FOR COMPARISON. PHOTOGRAPHED in ULTRA-VIOLET Lifht ( 3200$)$. (After Barnard.)

difficulties, and very real progress has been registered. The introduction of higher speer electric motors, of the Lundgren angle centrifuge, and of the spinning-top centrifuge of Henriot and Huguenard $(1925,1927)$ (see also McIntosh 1935, McIntosh and Selbie 1937) have each contributed to this end. Indeed with the spinningtop centrifuge, in which friction is diminished to a minimum, speeds of 80,000 r.p.m. have been reported. The greatest advance, however, has been marte by Svedberg and his colleagues (1934) (see also Svellberg 1937), who have devised a centrifuge capable of revolving at 160,000 r.p.m. In this machine the rotor carries a cell which contains a column, $8 \mathrm{~mm}$. in height, of the fluid to be centrifuged, situated $36 \mathrm{~mm}$. from the centre. The cell has windows of erystalline quartz to allow of serial photographs being taken to register the progress of sedimentation. The rotor is driven by two twin turbines fed with oil at a pressure of $15 \mathrm{kgm}$. per sq. $\mathrm{cm}$. Rotation takes place in an atmosphere of hydrogen at $25 \mathrm{~mm}$. pressure, so as to limit air friction and contection currents. In the original resign (Svedberg and Nichols 1927) 240 litres of oil were required per minute to drive the turbines, and 7 litres of oil per minute to lubricate and cool the bearings. The cost of this machine has so far prohibited most laboratories from testing it, but in 
Svedberg's hands it has been used with conspicuous success in estimating the molecular weight of proteins.

Bauer and Pickels $(1936,1937)$ have devised a high-speed centrifuge combining some of the principles of the Henriot and Huguenard and some of the Svedberg model. Elford (1936) has shown that, by introducing a capillary tube into the flnid to be centrifuged and collecting the virus particles on blotting paper at the bottom, much of the difficulty eaused by "mixing" ean be overcome. Schlesinger (1936) has adapted the Sharples centrifuge for the concentration of virus by using a thin layer of suspension in a hollow cylinder rotating vertically, so that the total distance the virus particles have to travel is a fraction of a millimetre. MeIntosh and Selbie (1940) have modified the Sharples centrifuge so as to permit the collection of the virus particles on a sheet of cellophane; this method has the advantage that a continuous stream of the virus to be purified can be fed to the machine. The preparation of pure virus suspensions is sometimes facilitated by preliminary tryptic digestion of the matrix in whieh they are embedeled.

Not only is it possible, now, with some of the high-speed centrifuges, to throw down completely the larger viruses, but their approximate size can be calculated from measuring their rate of sedimentation. Bechhold and Schlesinger (1931) have worked out a formula from which the size of evenly dispersed spherical particles submitted to a constant centrifugal force may be determined. Further, by measuring the rate of concentration, it can be ascertained whether the particles are of uniform size. It can be shown. for instance, that the logarithm of the concentration of particles in the supernatant fluid is proportional to the length of time of centrifugation. If they are of unequal size, the larger particles will be thrown down rapidly, and the curve formed by plotting the logarithms of the concentrations against time will not be a straight line.

The larger viruses, of $0 \cdot 1-0 \cdot 2 \mu$ in diameter, can be thrown down completely under suitable conditions in about half an hour by a centrifuge revolving at 10,000 r.p.m. (see Amies 1933), while particles of about $60 \mathrm{~m} \mu$, such as the staphylococcal bacteriophage, require a speed of 40,000 r.p.m. maintained for 1 to $1 \frac{1}{2}$ hours (McIntosh 1935). Where centrifuges of only 3,000 r.p.m. are available, and it is desired to concentrate the suspension, the virus may sometimes be adsorbed on to kaolin, animal charcoal or blood corpuscles, and the deposit subsequently suspended in a protein-free medium (see Levaditi and Nicolau 1923, Gins and Krause 1923, Tang 1932, Francis and Salk 1942). Tiruses vary, however, in their reaction to different adsorbing agents (Lewis and Andervont 1927), and this method is therefore not always successful. The purity of centrifuged virus suspensions is dependent on the number of particles of foreign matter present resembling in sedimentation rate that of the virus which is being concentrated. Methods for determining the degree of purity have been suggested by Smadel, Rivers and Pickels (1939) and Luria 1940).

Morphology.- Information on the shape of filtrable virus particles has been furnished by Barnard, who has been successful in photographing some of the larger viruses in ultra-violet light. One of the most carefully studied is that of ectromelia, a virus with a diameter of about $120 \mathrm{~m} \mu$ (Barnard and Elford 1931). This organism is coccoid, and frequently occurs in pairs. Isolated organisms are spherical and highly refractile, the refractivity apparently decreasing with shortening of the wave-length used for illumination. Reproduction is by binary fission, and elongation is evident before division. The final separation of the two organisms takes place quickly, but a very fine connecting filament may be 
left between them. Ultra-violet photographs show a more highly refractive outline corresponding to the periphery of the cell, and often an increased density at the poles. Whether these appearances are due to the presence of a cell-wall and to polar condensation of the cytoplasm respectively, or are merely the effects of interfacial phenomena, it is impossible to say. The foot-and-mouth virus, which is among the smallest of the viruses, is, according to Barnard (1937), rodshaped, the length being sometimes as much as three times the breadth.

TABLE 59

\section{Approximate Sizes of Filtrable Viruses}

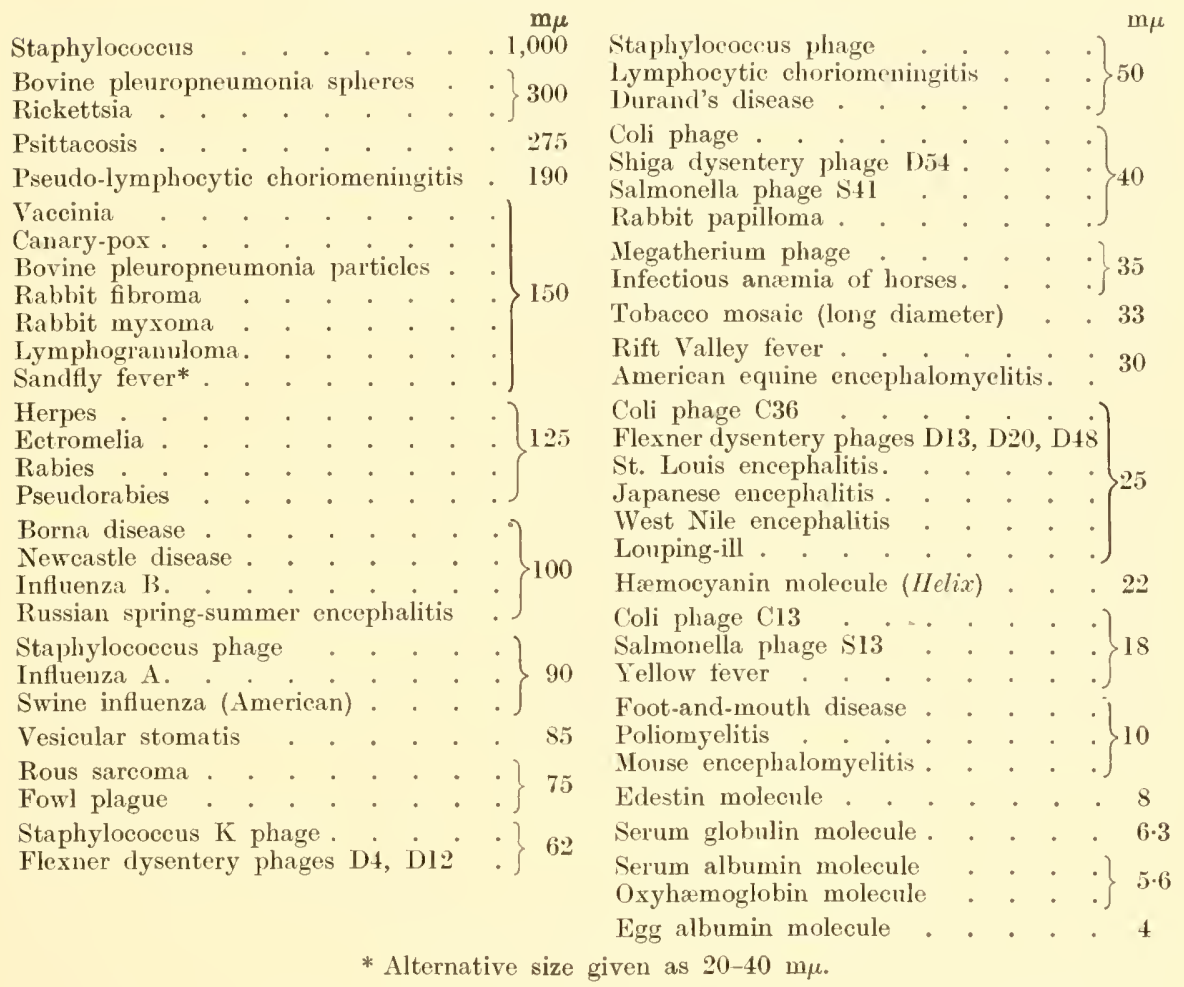

Further information on the shape, size, and structure of the viruses is being afforded by the electron microscope. By its use the elementary bodies of vaccinia appear to be brick-shaped, to possess some sort of limiting membrane, and to contain fine circumscribed areas of greater density arranged like the spots on dice (Green et al. 1942) (see Fig. 233).

The size of many viruses has now been calculated, mainly from data based on the use of Elford's gradocol membrane technique, and to a less extent from the microscopical photographs of Barnard, the electron micrographs of American workers, high-speed centrifugation, and the rate of diffusion of virus particles in a suitable medium. These different methods do not always agree in their results. By the filtration method, for example, the figure reached tends to correspond to the size of the smaller particles, by the centrifugation technique to the 
size of the larger particles. The size of the smaller viruses may be overestimated loy ultra-violet microseopy, because of failure of perfect resolution (see Barnard 1937). Electron micrographs vary somewhat according to the mode of preparation of the film. Conclusions drawn from the rate of sedimentation in a gravitational field are affected ly the density of the virus particles, and density appears to be variable and is difficult to determine with accuracy (see Smadel et al. 1938). Nevertheless, the degree of concordance is sufficient to justify us in assigning with some confidence mean particle diameters to the more important viruses. In Table 59 the size of the commoner viruses and some of the bacteriophages is

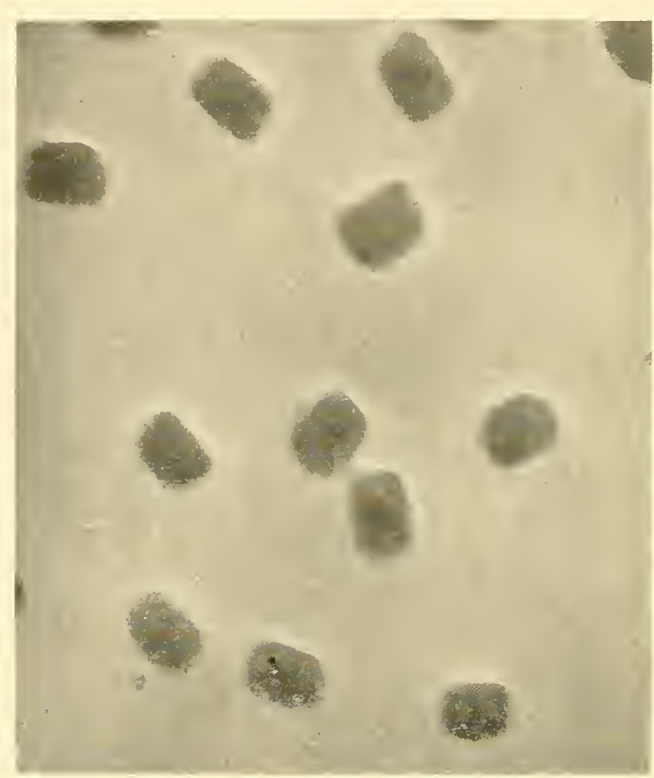

Fig. 233.-Electron Micrograpi of Vaccinia Tirus Elementary Bodies $(\times 26,1000)$.

[From Green el al., 19.2.] compared with Staphylococcus on the one hand and the larger protein molecules on the other.

The method of reproduction of the filtrable virmses is still in doubt. The evidence so far obtained seems to favour binary fission. EisenlyergMerling (1943) has described a conplex life cycle for the vaccinia virus, but his observations and the conclusions he draws from them must await confirmation.

Wicrochemical amalysis of vacrinial elementary bollies has revealed the presence of ash, carlohydrate, fat, and nitrogen, a part of which is undoubtedly in the form of protein (Hughes et al. 1935). Further observations by Hoagland, Smadel and Rivers (1910) have led to the ilentification of neutral fat, phospholipin, reducing sugar after hydrolysis, and thymonucleic acid. MeFarlane and his colleagues (1939) believe that there is a shell

lipin arranged around the particle in two or three loose layers, but no real membranc. These results suggest that the composition of the larger virus particles is essentially similar to that of ordinary bacteria. The nature of the smaller viruses is in greater doubt, Stanley's.work (see p. 966) indicating that they may be no more, in fact, than macromolernles of protein. (For a general review of the properties of vaccinial elenentary borlies, see smadel and Hoagland 1912.)

Habitat.- With the exceptions noted below, all the filtrable viruses at present known are associated with living cells, whether in the animal or the vegetable kingdom. This does not mean that they are never found apart from disease processes, for their presence has been demonstrated in healthy carriers; but it does mean that they are essentially parasitic. The existence, however, of saprophytic viruses is suggested hy the work of Barnard (1930). Hitherto the only satisfactory criterion of the presence of a virus has consisted in the production of characteristic lesions in a susceptible animal by a snitably prepared filtrate-a technique, it may be remarked, that automatically excludes the discovery of a saprophytic virus 
or of a completely avirulent variant of a parasitic virus. Barnarl, however, by ultra-violet photography has been able to detect the presence of minute, cultivable bodies, about $150 \mathrm{~m} \mu$ in diameter, in sterile tubes of serum broth.

Many of the viruses in the animal body appear to show a particular affinity for special tissues, such as nervous tissue or skin ; this resembles the affinity manifested by many of the known bacteria for special tissues. Even, however, when the lesions are confined to one tissue, the virus can frequently be demonstrated in other parts of the body. There is evidence, too, that the tissue localization is more apparent than real, depending on the mode of infection. Thus Ledingham (1924) found that in rabbits the virus of vaccinia, which usually affects the skin, was able to give rise to nodules on the peritoneum after direct inoculation into the spleen or the abdominal cavity. As well as their selective tissue localization, many viruses exhibit a species-specificity, giving rise to lesions only in one particular species of animal. Thus Cole and Kuttner's salivary gland virus is active only in guinea-pigs, Virus iii only in rabbits, and so on. On the other hand, there are viruses, such as those of rabies and foot-and-mouth disease, which are pathogenic not only to different species but also to widely separate groups of animals. Possibly too much weight has been laid in the past on the species-specificity of the viruses. It is now clear that most of the viruses are capable of infecting several different species of animal under experimental conditions.

Apart from the presence of a virus in a healthy carrier free from all clinical symptoms of disease, it has been shown that a virus may remain latent in the tissues after causing an initial infection. Thus, according to Gastinel and Reilly (1928) the herpes virus can sometimes be demonstrated in the brain of guinea-pigs that have recovered from a keratitis caused by inoculation of the cornea. Its presence gives rise to no symptoms, but can be shown by inoculation of the brain on to the cornea of a normal guinea-pig. It is possible that an attack of intercurrent disease, or some artificial procedure such as vaccination, may activate such a latent virus, and cause it to give rise to clinical disease. In some virus infections, such as yellow fever, there is reason to believe that the virus, after causing an attack of the disease, remains latent in the tissues for years, if not for the patient's whole life. By this means a lasting immunity is maintained (see Rivers 1943).

Whether the filtrable viruses occupy an intra- or an extracellular position in the body is not certainly known, but the indirect evidence so far accumulated suggests that their growth and multiplication occurs actually within the cells. The rinderpest virus, for example, appears to be contained within the leucocytes; by centrifugalization of the blood, the virus is found to be concentrated mainly in the lencocytic layer. Similarly with the virus of fowl-plague, Todd (1928) found that in centrifuged blood the concentration of the virus was 100 times greater in the leucocytic layer than in the clear plasma or the washed red cells. Moreover there is evidence that for their multiplication the filtrable viruses often prefer young newly-formed cells; many of the viruses acting on the skin, for example, give rise to lesions first along the lines of scarification, where repair is taking place. Further evidence in favour of this view is that certain viruses, e.g. Virus iii and vaccinia, have been found to grow in a transplantable rabbit tumour, in which the cells are in process of active multiplication, and to survive longer in the tumour than in the healthy tissues of the rabbit (Rivers and Pearce 1925). The observations of Perdrau and Todd (1936) on the lower susceptibility 
of viruses to ultra-violet light in the presence of cells, particularly dividing cells than in their absence also suggest that viruses oceupy an intracellular position and multiply most readily in growing cells. How far, in fact, the viruses are cytotropic and how far they are cytotrophic is a matter for dispute - if in fact any clear distinction can be drawn between these two properties. Goodpasture (1930) believes that actual growth occurs only in the living cells of the body, while Ledingham (1932) is not prepared to go to this length. Probably they take advantage of ferment action in the body cells, and receive their nutritive material in a partly digested state. If this is so, then viruses, including the bacteriophage, must be among the most dependent parasites of which we have knowledge in the unicellular world.

Inclusion Bodies.-Histological examination of the lesions occurring in filtrable virus diseases often reveals the presence within the cytoplasm or the nucleus, or sometimes both, of peculiar bodies whose nature is at present unknown, and which are usually referred to as "inclusion bodies." The appearance of these bodies varies in different diseases, and often in the same disease in different animals (Figs. 234, 235). The bodies may be rounded, oval, pyriform, or irregular in shape;
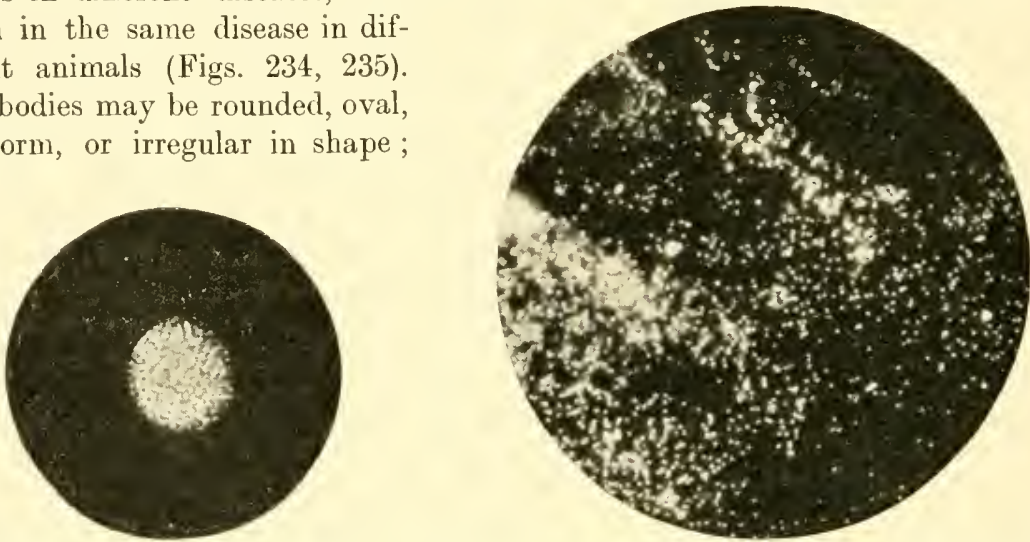

Ectromelia virus on left (Fig. 234), inclusion body from foot of mouse; on right (Fig. 235), inclusion body after maceration, showing the liberated elementary bodies. Photographed in visible light $(\times 1250)$. (After Barnard.)

their substance may be hyaline or granular; in structure they may be homogeneous, or they may contain one or more, often several, elementary corpuscles; in their staining reactions they may be basophilic or acidophilic, and within the same inclusion body the granules or elementary corpuscles may stain differently from the ground substance; lipoid substances staining with osmic acid are sometimes found. In many diseases affecting the skin, such as fowl-pox, human variola, and the common wart, the formation of inclusion bodies is restricted to the epidermis, but in others, such as zoster, varicella, and venereal herpes, they are found both in the epidermis and in the corium. Moreover, according to Lipschïtz (1925), only certain layers of the epidermis may be affected; thus in the common wart, inclusion bodies are found in the prickle-and horn-cell layers but not in the basal cell layer. Inclusion bodies can be produced experimentally only by the inoculation of living viruses; they are not formed after inoculation of dead viruses, even though the latter have immunizing properties, e.g. vaccinia and herpes. After inoculation of the virus, the inclusion bodies appear at different times in different infections. Thus in common warts, the nuclear inclusion bodies are demonstrable 
only in the earliest stages; in herpetic keratitis of rabbits the inclusion bodies appear within the first 24 hours; in venereal herpes they are best seen on the third day, and so on. There appears to be some relationship between the presence of inclusion bodies and the infectivity of the tissue ; in herpetic keratitis of rabbits, for example, it is said that with the disappearance of the nuclear inclusion bodies the disease ean be no longer propagated. Experimentally, the formation of inclusion bodies can be stimulated by the inoculation not only of infected tissue extracts, but often of filtered cell-free material. They can, moreover, often be demonstrated in tissue cultures (Andrewes 1929, Rivers et al. 1929).

The earlier workers regarded these inclusion bodies as protozoa, and pictured them as varying stages of an elaborate life-cycle. Subsequently they were believed to represent cellular degeneration products due to nucleolar extrusion, vacuolation of the cytoplasm, and other processes consequent on the attack of the virus. Von Prowazek regarded them as of a dual nature, consisting of micro-organisms embedded in material deposited around them as the result of a reaction of the cell protoplasm; for these bodies the term "Chlamydozoa"-literally cloak animals-was proposed. It is now, however, becoming increasingly clear that intracellular inclusion bodies are essentially colonies of the infecting virus. Since Woodruff and Goodpasture $(1929,1930)$ showed that the Bollinger inclusion body of fowl-pox consisted of 10,000-20,000 minute Borrel bodies, and that a single Bollinger body, washed free from surrounding virus, was capable of giving rise to a typical fowl-pox lesion on skin inoculation, it has been difficult to regard inclusion bodies as other than intracellular aggregations of elementary virus particles. By tryptic digestion, by maceration, by surface tension, or other means it has now been shown that the inclusion bodies of ectromelia (Barnard and Elford 1931), vaccinia (Ledingham 1931, Paschen 1932), and psittacosis (Bedson and Bland 1932, 1934) contain masses of elementary bodies which are apparently responsible for giving rise to characteristie intracellular changes. Moreover, the formation of inclusion bodies from elementary bodies has now been watched experimentally in the chorioallantoic membrane and the rabbit's cornea after inoculation with vaccinia virus (Herzberg 1936, Tang and Wei 1937, Himmelweit 1938, Bland and Robinow 1939). Whether the intranuclear acidophilie bodies, which are so common in infections caused by nentrotropie viruses, are of the same nature as the intracellular but extranuclear bodies has not yet been made clear either by morphological study or by the micro-incineration technique (see Cowdry 1933). There is some evidence that they result from flocculation of the nuclear colloids (Findlay 1939). Whatever the structure of inclusion bodies may be, however, there is no doubt whatever of their significance; their presence in the tissue is a sure sign of infection, and is made use of in the rontine diagnosis of certain of the filtrable virus diseases, such as rabies. (For a pictorial review of inclusion bodies see Findlay and Ludford 1926, and for a general account of their properties see Goodpasture 1929, 1929-30, Ledingham 1935. See also Figs. 298-302.)

Cultivation.-With the possible exception of saprophytic viruses cultivable in serum broth, the filtrable viruses have proved refractory to cultivation in the absence of living cells. In 1915 Noguchi succeeded in obtaining pure cultures in vivo of vaccinia virus, by growing it in the testicles of rabbits and bulls. The virus, obtained from skin scrapings, was first freed from bacteria by suitable means, and was then inoculated intratesticularly into rabbits. Transfers were made every four days. Several passages were necessary before the virus became adapted to growth 
in the testicle, but subsequently transfers were made without difficulty, and the virus reached its maximum multiplication in the testicle after 4 or 5 days; it remained stationary in amount till the 8th day, and then decreased till after 5 weeks its presence could no longer be detected. By testicular cultivation, the virus did not lose its affinity for the skin; both the testicular and the skin strains gave similar reactions in the skin, cornea, and testicles of rabbits, and in the skin of human beings. In 1925 Parker and Nye succeeded in growing the vaccinia and herpes viruses in tissue cultures prepared with normal rabbit testis and plasma. This was confirmed by Carrel and Rivers in 1927 working with the vaccinia virus. Infected rabbit testicle was ground up in a mortar, added to chick-embryo pulp, left for 24 hours in the ice-chest, and then inoculated into a Carrel flask containing a coagulum of hen plasma. The cultures were washed every 2 or 3 days with Tyrode's solution, and were nourished with a dilute fowl-embryo extract. After periods varying from 1 to 4 weeks the contents of the flasks were withdrawn, ground up in a mortar, and titrated on rabbits by the intradermal method. It was found that the cultures, which were seeded with 25 to 250 intradermal units of virus per ml., contained after incubation for a week between 10,000 and 100,000 units per ml., showing that actual multiplication of the virus had occurred. Haagen (1928) reported a modified method of in vitro cultivation of vaccinia virus in the presence of rabbit testicle, rabbit plasma, and rabbit spleen extract. Using this method, he carried the virus through 37 passages in a period of about 8 months. During the first 5 days of each subculture the virus multiplied about 1,000 times, and during the whole period its virulence remained approximately constant. Findlay (1928) reported similar success in the cultivation of the fowl-pox virus by Carrel's method. Craciun and Oppenheimer (1926) cultivated vaccinia virus in association with embryonic guinea-pig's cornea, and carried through nine successive transfers during a period of 71 days. Finally the in vitro cultivation of vaceinia virus in the apparent absence of proliferating cells was reported by the Maitlands (1928). Infected rabbit testicle was ground up, diluted with Tyrode's solution, added to minced hen's kidney, placed in the cold room for 4 hours, and then cultivated in a Carrel flask containing hen's serum. The flasks were incubated at $37^{\circ} \mathrm{C}$., and subcultures made about once a week. Four passages were made in all. A 1/625 dilution of the last subculture, which represented a dilution of $1 / 625 \times 10^{8}$ of the primary inoculum, produced vaccinia on inoculation into rabbits; the original inoculum was active only in a 1/2500 dilution. Living tissue was, of course, not excluded, but no evidence of its multiplication was obtained. Various modifications of the Maitland technique, such as cultivation in agar slant cultures (Kurotchkin 1939), wide tubes (Findlay and MacCallum 1940), and roller tubes (Feller et al. 1940) have been successfully introduced.

The function of the tissue cells in the cultivation of viruses has not been established, though much is known about the various factors that influence growth (see Hallauer 1938). Zinsser and Schoenbach (1937) observed that development of the equine encephalitis virus reached its maximum in 3 to 4 days, whereas that of Rickettsia was considerably later-6th to 8th day. They therefore drew the tentative conchusion that the metabolism of viruses and of rickettsiæ differed, the former multiplying best during the period of active growth of the tissue cells, the latter after the cells had begun to die off. Maitland and Laing (1941) have brought evidence to suggest that with vaccinia virus the cells exert two separate functions, one to initiate growth, the other to maintain growth once the virus 
has started to multiply. That the growth factors supplied by the cells are relatively specific for different viruses is suggested by Andrewes' (1942) observation that growth of one strain of a given virus may render the tissue culture medium unsuitable for growth of another strain of the same virus added 24 hours later, though not for a strain of a different virus. This is analogous to experience in the growth of bacteria on lifeless media, and suggests that some substance required for the growth of a particular species is soon exhausted. As Rivers (1932) points out, many viruses in tissue culture exhibit both a species and a cellular specificity. Fowl plague virus multiplies only in the presence of chick embryo skin and brain, not in cultures of fibroblasts; moreover, avian tissue appears to be essential.

None of the filtrable viruses has yet been cultivated in the absence of living cells. It is true that Eagles and McClean (1931) and Eagles (1935) state that they have grown vaccinia virus in a cell-free medium, but their results have not so far been confirmed (Maitland et al. 1932, Rivers and Ward 1933). It may indeed be questioned whether such highly parasitic organisms as the viruses appear to be are provided with sufficient enzyme systems to enable them to grow in the absence of the cellular activity of their host. The successful cultivation of the pathogenic viruses on lifeless media may well have to await the reproduction in vitro of the complete ferment mechanism of the living cell.

Most of the common viruses have now been grown in tissue culture. Another method, which has come into increasing prominence of late years, makes use of the developing hen's egg. It was introduced by Ogston in 1881 for the cultivation of bacteria, and was re-discovered by Woodruff and Goodpasture (1931) fifty years later. Inoculation of the chorio-allantoic membrane is the most generally useful way of applying this technique. Some viruses, like vaccinia and psittacosis, produce characteristic lesions or pocks on the membrane; others, like fowl plague and vesicular stomatitis, kill the embryo before local lesions have had time to develop; others, like Rift Valley fever and influenza, produce both local membrane lesions and characteristic effects on the embryo; and others, like poliomyelitis, rabies, and foot-and-mouth disease viruses, fail to grow on the egg membrane at all. Burnet has been particularly fertile in recognizing the potentialities of this method, and readers who are interested in its technical details and general application would do well to consult his monograph (1936). (See also Stevenson and Butler 1939, Burnet and Faris 1942, Dunham and MacNeal 1942.) Inoculation into the yolk sac or the amniotic sac, or even directly into the embryo, may be used for certain purposes.

Resistance.-The filtrable viruses vary considerably in their resistance to nocuous agencies. Generally speaking, they resemble the vegetative bacteria more closely than the spore-bearing organisms; that is to say they are generally destroyed by exposure to moist heat at $55-60^{\circ} \mathrm{C}$. within half an hour, and succumb to fairly low concentrations of chemical disinfectants. On the whole, they appear to be more resistant than the vegetative bacteria to chemical agencies, but it must be remembered that experiments can never be performed in the complete absence of cellular, or at any rate, of protein material ; their apparently greater resistance may, therefore, be due to the protective action of substances in the medium.

The effect of desiccation varies, partly with the method employed, and partly with the particular virus in question. The Foot-and-Mouth Disease Research Committee (Report 1927) found that filtered vesicle fluid from the guinea-pig, if dried rapidly on slides at $37^{\circ} \mathrm{C}$., was often inactivated immediately; on the other

P.B. 
hand, if dried slowly at room temperature, and kept at room temperature over $\mathrm{H}_{2} \mathrm{SO}_{4}$, it survived for 3 to 6 months. Noguchi (1918) found that, when dried, vaccinia virus remained alive for over a year, but its virulence was considerably decreased. Haagen (1939), on the other hand, observed no loss of virulence in a year when mouse brain infected with vaccinia was dried over calcimm chloride and kept in sealed glass vessels in the ice-chest. One hour's exposure, to the August sun, of the foot-and-mouth virus, dried on a glass slide, inactivated it. Most viruses appear to be very resistant to cold. Vaccinia virus withstands a temperature of $-180^{\circ} \mathrm{C}$. for months, and even repeated freezing and thawing fails to destroy it. Frozen and dried, they may live for months (Sawyer et al. 1929, Wooley 1939). Vaccinia virus in dry powdered form withstands dry heat at $100^{\circ} \mathrm{C}$. for 5 to 10 minutes. Moist heat at $55-60^{\circ} \mathrm{C}$. for half to one hour is fatal to most viruses, but blood from swine fever is said to withstand a temperature of $58^{\circ} \mathrm{C}$. for at least 2 hours; it is inactivated, however, within an hour by a temperature of $78^{\circ} \mathrm{C}$. Some viruses, like the poliomyelitis virus, are rapidly killed by ultra-violet light. Many are also susceptible to photodynamic action (see Chapter 5), and succumb in a few minutes when exposed to a concentration of about 1/100,000 methylene blue in the presence of daylight (Perdrau and Todd 1933, Herzherg 1933, Shortt and Brooks 1934). Alpha rays, X-rays, and gamma rays are all lethal (Lea and Salaman 1942).

Most viruses exhibit a fairly high resistance to glycerol, and one of the best methods of preserving infectious tissue is to suspend it in 50 per cent. glycerolated saline, cover it with liquid paraffin, and store it in the ice-chest (Perdrau 1927). Pure glycerol destroys the viruses fairly rapidly as a rule; thus Noguchi (1918) found that vaccinia virus was destroyed by pure glycerol at $4^{\circ} \mathrm{C}$. within 24 hours, though in 40 per cent. glycerol it survived for about 6 months. The preservative action of glycerol probably depends on the inhibition it exerts on autolysis of the infected tissue (Rivers 1928).

Survival in distilled water, saline, or Ringer's solution, varies considerably. In the ice-chest many viruses will survive for a long time, but most of them perish rapidly if kept at room temperature or $37^{\circ} \mathrm{C}$. The foot-and-mouth virus in saline rarely survives at $37^{\circ} \mathrm{C}$. for more than 24 hours; and the lymphocytic choriomeningitis virus is non-infective within 3 hours at $20^{\circ} \mathrm{C}$. (Lépine et al. 1937). Susceptibility to oxidation may be chiefly responsible for this behaviour. At low temperatures the presence of tissue cells seems to be beneficial to the survival of viruses, but at higher temperatures the reverse is probably true. Amies (1934), for example, found that vaccinia virus remained virulent much longer at $37^{\circ} \mathrm{C}$. when stored in the form of a suspension of elementary bodies than in tissue culture. In general, the presence of serum or 0.5 per cent. agar is beneficial for survival, as is also storage under anaerobic conditions (Zinsser and Tang 1929, Zinsser and Seastone 1930, McClean and Eagles 1931). The optimum H-ion concentration for survival of the foot-and-mouth virus and the vaccinia virus is $\mathrm{pH} 7 \cdot 6$; between $\mathrm{pH} 4.0$ and 3.0 vaccinia virus is rendered non-infective within about an hour (Beard et al. 1938).

Disinfectants.-Vaccinia virus in testicular suspension is said to survive in 0.5 and 1.0 per cent. phenol solutions for over a year at $4^{\circ} \mathrm{C}$., but to be destroyed by 2 per cent. phenol within 24 hours, and by a $1 / 30,000$ solution of iodine within 1 hour at $37^{\circ} \mathrm{C}$. (Noguchi 1918). Likewise, ectromelia virus in a suspension of mouse's liver will remain virulent in 0.5 per cent. phenolized saline at $4^{\circ} \mathrm{C}$. for 
several months. Purified influenza virus, on the other hand, is destroyed by 0.5 per cent. phenol at $4^{\circ} \mathrm{C}$. within a week (Knight and Stanley 1941). Gordon (1925) found that vaccinia virus was destroyed by 50 per cent. ethyl alcohol, 50 per cent. methyl alcohol, and 50 per cent. acetone within an hour at room temperature; 20 per cent. ethyl alcohol, 10 per cont. methyl alcohol, 10 per cent. acetone, and 20 per cent. ether failed to destroy it in 24 hours; even 50 per cent. ether did not destroy it completely in this time. Potassium permanganate was found to be extremely viricidal, destroying it even in a $1 / 10,000$ solution within an hour at room temperature. Chloroform is said to be very much more destructive than ether, alcohol, or acetone (Reynals 1928). The foot-and-mouth virus is resistant to concentrations of phenol, lysol, toluol, hydrogen peroxide, chlorine, iodine, acetone, and chloropicrin that rapidly destroy vegetative bacteria; but it is killed by $0 \cdot 1$ per cent. formol at $26-27^{\circ} \mathrm{C}$. in 24 hours, and by 2 per cent. antiformin or 0.4 per cent. $\mathrm{HgCl}_{2}$ within 21 hours. The effect of bile salts varies according to the species of virus (Smith 1939). Influenza A and louping-ill viruses are inactivated almost instantaneously by exposure at room temperature to a final concen. tration of 1/1,000 sodium deoxycholate, whereas vaccinia and ectromelia viruses are unaffected after 2 hours. Inactivation of susceptible viruses is thought to be due to lysis. Certain soaps and unsaturated fatty acids, and some synthetic detergents, have been found to have a strong destructive action on influenza virus (Stock and Francis 1940, Knight and Stanley 1914).

With the very doubtful exception of the lymphogranuloma virus (MacCallum and Findlay 1938, Rodaniche 1942), the mouse pueumonia virus, and the viruses of trachoma and inclusion blemnorrhoea (Rake et al. 1942), the viruses are insusceptible to sulphonamides. Penicillin likewise appears to be without action.

Metabolism.-Practically nothing is known about the metabolisus of the filtrable viruses, one of the great hindrances being the impossibility of cultivating them in the absence of tissue cells. In purified suspensions of vaccinia virus Parker and Smythe (1937) could demonstrate no oxygen consumption. On the other hand Mlacfarlane and Salaman (1938) and Mlacfarlane and Dolby (1940), though finding no evidence of dehydrogenase activity, were able to demonstrate the presence of phosphatase and catalase; both ribonucleic acid and adenylic acid were rapidly hydrolysed. It is not yet clear, however, whether these enzymes form an integral part of the elementary bodies or are derived from the host tissues (see Smadel and Hoagland 1912). The ability to oxidize cysteine has been traced to the presence of copper in vaccinial elementary bodies (Hoagland et al. 1941). Flavin and biotin have also been found (see Rivers 1943). What part these various enzymes play in the metabolism of the larger viruses under natural conditions, it is too early to say.

Antigenic Structure.-Although the study of the antigenic structure of viruses is as yet in its infancy, enough has been learned to show that, in this respect, viruses differ in no essential way from bacteria. The presence of precipitins in the blood serum of animals inoculated with vaccinia virus has been reported by several workers (see Sobernheim 1925). Gordon (1925) found agglutinins and complementfixing bodies in the serum of rabbits inoculated with vaccinia, active up to a dilution of $1 / 100-1 / 200$; both antibodies were specific in the sense that they reacter solely with vaccinia and variola virus suspensions, and gave no reaction with varicella virus, sterile pus, or brain suspensions from encephalitis lethargica. In general, 
precipitins have been demonstrated more frequently than agglutinins or complement-fixing bodies.

Recent work, particularly with vaccinia, has revealed an antigenic complexity in the viruses similar to that present in many bacteria. The elementary bodies of vaccinia appear to contain two agglutinogens, one of which, $\mathrm{L}$, is destroyed by exposure to $56^{\circ} \mathrm{C}$. for one hour, the other of which, S, withstands a temperature of at least $95^{\circ} \mathrm{C}$. for this time. By suitable methods precipitinogens can be extracted from infected matcrial and from tissuc cultures which appear to currespond to the agglutinogens in the elementary bodies (Craigie 1932, 1935, Smith 1932, Craigie and Wishart 1934a, b, 1936, Ch'en 1934, Salaman 1934, Parker and Rivers 1937, Smadel et al. 1940a). It was thought at first that the two antigens were quite separate, and that the heat-stable precipitinogen was a polysaccharide hapten; but more recent work has shown that both the $\mathrm{L}$ and the $\mathrm{S}$ substances are contained in a single protein molecule (Shedlovsky and Smadel 1942, Shedlovsky et al. 1943, Smadel et al. 1943). In addition, two further antigens have been demonstrated in vaccinial elementary bodies. One is the so-called NP or nucleoprotein antigen described by Smadel, Rivers and Hoagland (1942), and demonstrable by precipitation. The other is the $\mathrm{X}$ antigen, which can be demonstrated by agglutination, using a serum from which the LS and NP antibodies have been removed by absorption (Craigie and Wishart 1936, 1938, Smadel et al. 1942). Soluble antigens, capable of reacting in complement-fixation or precipitin tests, have been demonstrated in other viruses, such as the virnses of lymphocytic choriomeningitis and lymphogranuloma.

A further analogy with bacteria is afforded by the demonstration of multiple antigenic types in a single species of virus. For example, at least three distinct types of foot-and-mouth virus, differing in their infectivity, have been found (Vallée and Carré 1922, Waldmann and Trautwein 1926). Several immunologically distinct types of poliomyelitis have now been differentiated; some of these are so sharply defined that the serum of monkeys convalescent from infection with one type will not protect against another (see Sabin 1941).

The existence of a group antigenic relationship such as that demonstrated between the viruses of influenza and swine influenza (see Chapter 74) is again reminiscent of the antigenic morphology of bacteria.

The inoculation of many viruses into suitable animals is followed by the appearance of neutralizing antibodies in the serum. The mode of action of these antibodies forms a constant subject of discussion. Whether they destroy the virus, whether they merely inactivate it, whether they sensitize it, or whether they fail even to combine with it, is still not known with certainty. Most of the evidence seems to be in favour of the occurrence of a slow union between virus and antibody leading to sensitization or actual destruction (see Chapter 55).

The type of antibody called forth by different viruses varies. The only antibody to polionyelitis virus of which we have knowledge is a neutralizing antibody. On the other hand, vaccinia virus stimulates the production of agglutinins, precipitins, and complement-fixing bodies as well as neutralizing antibodies. In some instances, as in the vaccinia and lymphocytic choriomeningitis viruses, the neutralizing antibodies appear to be distinct from the other antibodies (Salaman 1937. Smadel et al. 1940b); and we are still ignorant of the nature of the antigen against which the neutralizing antibody is active. In general, neutralizing antibodies are of most help in revealing antigenic affinities and differences between 
closely related viruses, but there are exceptions. The relationship, for example, between the Eastern and Western types of equine encephalomyelitis viruses is said to be brought out by the complement-fixation, but not by the neutralization test (Havens et. al. 1943).

Pathogenicity.--The pathogenicity of different viruses for different hosts varies greatly. Some, like the vaccinia and the rabies virus, have a wide range of pathogenicity; others, like the foot-and-mouth and the encephalitis viruses, have a narrow range; and still others, like the measles and mumps viruses, seem to be pathogenic for one species alone. Some attack only mammals, some only birts, and some both mammals and birds. One curious feature of most of the viruses is their ability to grow in the embryonic or associated colls of the developing hen's egg, in spite of the fact that the chickell, once it is hatched, is resistant to all the pure mammalian viruses. In becoming adapted to different hosts viruses often undergo minor variations; there is evidence, for example, that the numerous animal poxes, with the possible exception of fowl-pox, are due to varieties of one and the same virus (Zwick 1924). On the other hand, the properties of a virus may be considerably altered. Thus, inoculation of the calf with variola virus, and subsequent transference by passage through calves, modifies the virus in such a way that when reinoculated into human beings it gives rise not to smallpox but to vaccinia. Passage of the street virus of rabies through the brain of rabbits gives rise to the production of a fixed virus, which, though it kills rabbits on intracerebral inoculation more rapidly than the street virus, is yet much less virulent than the street virus on subcutaneous inoculation (Levaditi et al. 1924). This example illustrates another characteristic that is frequently observed in the study of viruses, namely, their adaptation not only to one particular host, but to one special tissue or route of inoculation. Findlay (1936), whose review on variation in the animal viruses should be consulted, is of opinion that variants are of two types: (a) variants associated with pathological lesions mlike those produced by the parent strains, but without any great antigenic difference; $(b)$ variants associated with pathological lesions like those produced by the parent strains, but with considerable antigenic difference, more often quantitative than qualitative in character. Some changes, such as the conversion of rabbit fibroma into myxoma, may justly be regarded as mutations; the majority are to be regarded as changes of the environmental type.

Infection in most of the filtrable virus diseases appears to occur by direct contagion, the infective-material gaining access to the body either by the nasopharynx or sometimes by the skin, as in rabies. In certain diseases the virus is inoculated into the blood stream by an insect vector; yellow fever, for example, is carried by the mosquito Aëdes aegypti, dengue fever by Aëdes aegypti and Aëdes albopictus, and Pappataci fever by the sandfly, Phlebotomus papatassii. Laboratory infections are not infrequent, especially with yellow fever, psittacosis, Rift Valley fever, and louping-ill. Little is yet known about the mechanism of infection, but studies of the vaccinia virus suggest strongly that the elementary bodies constitute the infecting agents, and that in virulent strains even one elementary body may suffice to cause infection (Parker 1938, Parker et al. 1941, Smadel et. al. 1939).

In general, the cellular lesions produced by viruses are of one or other of two kinds. Either the cells are stimulated so that the tissues become hyperplastic, as in the fibromata, papillomata, warts and poxes; or they may lue damaged so severely that they die, as in the hepatic necrosis of yellow fever and Rift 
Valley fever. A preliminary hyperplasia may be succeeded, as in the poxes, by necrosis.

The Nature of Viruses.-Up till a few years ago the evidence was becoming increasingly stronger that the animal viruses were essentially minute microorganisms. Nicroscopical, filtration and centrifugal observations left little doubt that they consisted of relatively large particles which, in each virus, were of fairly uniform size. The agreement between the results of these three methods of examination was, in fact, so good as to make it necessary to conclude that, if the visible coccoid bodies were not the infective units of the virus, then the virus must consist of particles of the same size which, for some reason or other, were invisible either by direct observation or by ultra-violet photography (Dale 1935). In their morphological appearance, in their formation of discrete colonies under suitable conditions - as on the chorio-allantoic membrane of the developing chick embryo-in their complex antigenic structure, in their ability to stimulate the production of different types of antibody, in their pathogenicity and their selective tissue localization, in their capacity for variation, in their neutralizability by specific antiserum, and in their power to give rise to immunity to fresh infection, some of the better-studied viruses were, apart from their small size and their failure to grow on lifeless media, indistinguishable from ordinary bacteria.

In 1935, however, just when this view was gaining general acceptance, Stanley in the United States made the startling announcement that he had succeeded in crystallizing the tobacco mosaic virus. From subsequent work carried out in the United States and in Great Britain (see Stanley 1938a, b, $c, d, 1941$, Bawden 1943), it became clear that this virus, which produces a disease in the Turkish tobacco plant, apparently consists of large nucleoprotein molecules, about ten times as long as they are wide, having a molecular weight of about 17 million, and capable of fitting together lengthwise to form needle-shaped crystals $20-30 \mu$ long. The high molecular weight of this substance-greater than even the largest hæmocyanin molecules-its ability to produce disease in a quantity as small as one thousand-millionth of a gram, combined with the almost perfect parallelism between the amount of protein estimated chemically and the virus activity estimated biologically, set it apart from any other known protein. It was further shown that variants of this virus had a slightly different chemical structure from the parent virus, and that chemical alterations brought about in the molecule by laboratory methods led to changes in its pathogenic properties.

The shock produced by Stanley's discovery was somewhat mitigated when it was pointed out (Takahashi and Rawlins 1935, Bawden et al. 1936, Bernal and Fankuchen 1937) that the crystals were not true crystals, but two-dimensional liquid crystals formed as the result of linear aggregation of smaller thread-like bodies showing anisotropy of flow. Under these conditions it did not appear necessary to abandon the conception of viruses as living micro-organisms merely because some of them could arrange themselves in orderly rows (Andrewes 1938). When it was shown, however, by Bawden and Pirie (1938) that the bushy stunt virus of tomatoes formed typical three-dimensional dodecahedral crystals, apparently composed of spherical isotropic particles, this way of escape from the dilemma proposed by Stanley looked less promising.

How are we to reconcile the complex bacteria-like structure and behaviour of the larger animal viruses with the apparently pure nucleoprotein molecules of the plant viruses? The explanation favoured by many workers (Green 1935, 
1938, Laidlaw 1938, Gortner 1938) is that the viruses represent decadent forms of organisms that have become progressively degraded through long persistent parasitism. At the upper end of the scale are representatives, such as the psittacosis and the vaceinia viruses, that contain protein, fat, and carbohydrate, and that differ from bacteria chiefly in their loss of power to synthesize some factor or factors essential for their growth and multiplication: at the lower end of the scale are representatives, such as the tobacco mosaic virus, that consist of pure nucleoprotein, and that are entirely dependent on living cells for all their ferment and autosynthetic activities-inert chemical complexes which become living only when bathed in functioning protoplasm. This view has something to be said for it; it serves to reconcile the apparent break between the living and the non-living. It is, however, not without difficulties of its own. Since the tobacco mosaic virus can grow in a number of plants, belonging even to different families, in which the composition of the cell protoplasm is known to be different, it follows that its huge nucleoprotein molecule must be built up from relatively simple chemical substances. This presupposes a degree of synthetic organizing ability hitherto associated with living cells alone. Acceptance of this view opens up once again the whole question of spontaneous generation. If a protein molecule can, in contact with living matter, reproduce itself and undergo variations each of which is attended by specific biological changes and each of which is genetically transmissible, it is difficult to avoid the conclusion that new bodies, presenting the characters of living matter, must be constantly appearing in Nature. How also are we to regard structure in relation to size? The foot-and-mouth virus, for example, which is about $12 \mathrm{~m} \mu$ in diameter, is so similar in almost every respect to the virus of vesicular stomatitis, which has a particle size of $85 \mathrm{~m} \mu$, that it is difficult to avoid the conclusion that they are structurally alike. Yet the footand-mouth virus is smaller-at least in one diameter-than the tobacco mosaic virus, the length of which is estimated to be about $30 \mathrm{~m} \mu$. Must we assume that the foot-and-mouth virus, which incidentally has room for only about ten protein molecules, is organized like one of the larger animal viruses, in spite of its being smaller than a plant virus which is known to consist of a single macromolecule?

One thing is clear. Before we can reach any conclusion on the nature of filtrable viruses, we shall have to re-define our terms. At the larger protein level the words "living" and "non-living" have lost their conventional meaning (see Pirie 1937). It is difficult, even in Science, to avoid the common solecism of attempting to force new facts into a conception that has no reality as such, but has been formed merely as an abstraction from other previously known facts; and it is time for us to realize that our concept of "life " is too crude to be used in relation to the infinitely small. Whatever the viruses are-micro-organisms reproducing themselves by binary fission, huge nucleoprotein molecules multiplying by autocatalysis, or something else still-there is no doubt that they present us at the moment with one of the most fascinating and fundamental problems of the biochemical world.

We have said nothing in this chapter about the filtrable tumours. Here again we are on very difficult ground. There is evidence suggesting that virus particles are essential to their reproduction, and that tumours both of avian and mammalian origin are caused, to some extent at least, by infecting particles having many of the characteristics of the known filtrable viruses (see Gye 1925, Andrewes 1934, Ledingham and Gye 1935, and Chapter 89). 


\section{REFERENCES}

Amies, C. R. (1933) Lancet, i. 1015; (1934) Brit. J. exp. Path., 15, 180.

Andrewes, C. H. (1929) Brit. J. exp. Path., 10, 188; (1934) Lancet, ii. 63, 117 ; (1938) Proc. roy. Soc. Med., 31, 203; (1942) Brit. J. exp. Path., 23, 214.

Asheshov, I. (1933) J. Bact., 25, 323, 339.

BarNarn, J. E. (1925) Lancet, ii. 117 ; (1935) Brit. J. exp. Path., 16, 129 ; (1937) Proc. roy. Soc., B., 124, 107.

Barnard, J. E. and Elford, IV. J. (1931) Proc. roy. Soc., B, 109, 360.

BAuer, J. H. and Pickels, E. G. (1936) J. exp. Med., 64, 503 ; (1937) Ibid., 65, 565.

BAwDen, F. C. (19.3) "Plant Viruses and Virus Diseases," 2nd edit. Chronica Botanica Co., Waltham, Mass.

Bawden, F. C. and Pirie, N. W. (1938) Brit. J. exp. Path., 19, 251.

Bawden, F. C., Pirie, N. IT., Bernal, J. D., and Fankuchex, I. (1936) Nature, Lond., 138,1051 .

Beari, J. W., Finkelstein, H., and Wrakoff, R. W. G. (1938) J. Immunol., 35, 415. Bechitold, H. and Schlesinger, M. (1931) Biochem. Z., 236, 387.

Beoson, S. P. and Bland, J. O. IV. (1932) Brit. J.exp. Path., 13, 46I ; (1934) Ibid., 15, 243.

Bernal, J. D. and Fankuchen, I. (1937) Nature, Lond., 139, 923.

Bland, J. O. W. and Robisow, C. F. (1939) J. Path. Bact., 48, 351.

Burnet, F. M. (1936) Spec. Rep. Ser. med. Res. Coun., No. 220.

BORnET, F. M. and FARIS, D. D. (1942) J. Buct., 44, 241.

Carrel, A. and Rivers, T. M. (1927) C. R. Soc. Biol., 96, 848.

Ch'en, W. K. (1934) Proc. Soc. exp. Biol., N.Y., 32, 491.

ClaUBerg, K. IT. (1939) Klin. Wschr., 18, 632.

Cownry, E. V. (1933) Amer. J. Path., 9, 149.

Cox, H. R. and Hyde, R. P. (1932) Amer. J. Hyg., 16, 667.

Craciun, E. C. and Oppexheimer, E. H. (1926) J.exp. Med., 43, 815.

Craigie, J. (1932) Brit. J. exp. Path., 13, 259; (1935) J. Immunol., 29, 70.

Craigie, J. and Wishart, F. O. $(1934 a)$ Brit. J. exp. Path., 15, 390; (1934b) Trans. roy. Soc. Can., Section v. 91 ; (1936) J. exp. Hed., 64, 803, 819; (1938) J. Bact., 35, 25.

Dale, H. H. (1935) Huxley Nemorial Lecture. Macmillan \& Co., Ltd., London.

Douglas, S. R. and Simti,, W. (1928) Brit. J. exp. Palh., 9. 213.

Duclaux, J. and Amat, M. (1938) C. R. Acad. Sci., 206, 1475.

Dunilam, W. B. and MacNeaL, W. J. (1942) J. Bact., 44, 413.

Eagles, G. H. (1935) Brit. J. exp. Path., 16, 188.

Eagles, G. H. and Mlclean, D. (1931) Brit. J. exp. Path., 12, 97.

Eisenberg-Merling, K. B. (1943) Brit. J. exp. Path., 24, 240.

Elford, W. J. (1931) J. Path. Bact., 34, 505; (1933) Ploc. roy. Soc., B, 112, 384; (1936) Brit. J. exp. Path., 1\%, 399.

Elford, T.. J., Grabar, P., and Ferry, J. D. (1935) Brit. J. exp. Path., 16, 583.

Feller, A. E., Enders, J. F., and Werler, T. H. (1940) J. exp. Med., 72, 367.

Fixdlay, G. M. (1928) Brit. J. exp. Path., 9, 28; (1930) Brit. J. exp. Path., 11, 109 ; (1936) J. R. microscop. Soc., 56, 213 ; (1939) Brit. med. J., i. 257.

Findlay, G. M. and Lodford, R. J. (1926) Brit. J. exp. Path., \%, 223.

Findlay, G. II. and MacCallum, F. O. (1940) Lancet, ii. 163.

Francis, T. and SALK, J. E. (1942) science, 96, 499.

Galloway, I. A. and Elford, W. J. (1931) Brit. J. exp. Path., 12, 407.

Gastinel, P. and Reilly, J. (1928) Bull. Méd., 42, 839.

Gixs, H. A. and Krause, C. (1923) Ergebn. allg. Path. path. Anat., 20, ii. 805.

Goodpasture, E. IV. (1929) Arch. Path., \%, 114 ; (1929-30) Harvey Lectures ; (1930) Zbl. Gies. Neurol. Psychiat., 129, 599.

Gordon, M. H. (1925) Spec. Rep. Ser. med. Res. Coun., Lond., No. 98.

GorTNER, R. A. (1938) Science, 8\%, 529.

Green, R. G. (1935) Science, 82, 443; (1938) Biodynamica, No. 39, 1.

Green, R. H., Anderson, 'T. F., and Sundel, J. E. (1942) J. exp. Med., 75, 651.

Grinnell, F. B. (1929) J. Bact., 18, 175.

Gye, IV. E. (1925) Lancet, ii. 109.

HaAGer, E. (1928) Zbl. Balt., 109, 31 ; (1939) Ibid., 143, 283.

Hallauer, C. (1938) Doerr and Hallauer's "Handbuch der Virusforschung," i. 369.

Havens, W. P., Watson, D. W., Green, R. H., Javin, G. I., and Siladel, J. E. (1943) J. exp. Med., ry, 139.

Henriot, E. and Huguenari), E. (1925) C. R. Acad. Sci., 180, 1389; (1927) J. Phys. Radium, 8, 433.

HerzberG, K. (1933) Z. ImmunForsch., 80, 507; (1936) Zbl. Bakt., 136, 257.

Himielweit, F. (1938) Brit. J. exp. Path., 19, 108. 
Hindle, E. and FindeaY, G. M. (1930) Brit. J. exp. Path., 11, 13 t.

Hoagland, C. L., Smadel, J. E., and Rivers, T. M. (1940) J.exp. Med., 71, 737.

Hoaglaxd, C. L., WARD, S. M., Smadel, J. E., and Rivers, T. M. (1941) J. exp. Med., $74,69$.

Hughes, T. P., Parker, R. F., and Rivers, T. M. (1935) J. exp. Med., 62, 349.

KNIGHT, C. A. and Stanley, IT. M. (1944) J. exp. Med., \%9, 291.

Kírotchein, T. J. (1939) Proc. Soc. exp. Biol., N.Y., 41, 407.

Laidlaw, P. P. (1938) "Virus Diseases and Tiruses." Camb. Univ. Press.

Lea, D. E. and Salamax. M. H. (1942) Brit. J. exp. Path., 23, 27.

Ledixghair, J. C. G. (1924) Brit. J. exp. Path., 5, 332 ; (1931) Lancet, ii. 525 ; (1932) Brit, med. J., ii. 953; (1935) Johns Hopkins Hosp. Bull., 56, 247, 337 ; 16id., 5\%, 32.

Ledixghay, J. C. G. and Gye, IT. E. (1935) Lancet, i. 376.

Lépine, P., Sautter. T., and Kreis, B. (1937) C. R. Soc. Biol., 124, 42.2.

Levaditi, C. and Nicolau, S. (1923) C. R. Soc. Biol, 88, 66.

Ievadit, C., Nicolau, S., and Schoen, R. (1924) C. R. Soc. Biol., 91, 423.

Lewis, M. R. and Andervont, H. B. (1927) Amer. J. Hyg., 7, 505.

LipschüTz, B. (1925) Zbl. Balit., 96, 222.

Luria, S. (1940) Ann. Inst. Pasteur, 64, 115.

Luria, S. E., Delbrüris, M., and Anderson, T. F, (1943) J. Bect., 46, 57.

MacCallum, F. O. and Findlat, G. M. (1938) Lancet, ii. 136.

McClean, D. and Eagles, G. H. (1931) Brit. J. exp. Path., 12, 103.

IcFarlane, A. S., Macharlane, M. G., Aires, C. P., and Eagles, G. H. (1939) Brit. J. exp. Path.. 20, 485.

Macfarlane, M. G. and Dolbr, D. E. (1940) Brit. J. exp. Path., 21, 219.

Macfarlane, M. G. and Salainax, M. H. (1938) Brit. J. exp. Path., 19, 184.

McIntosh, J. (1935) J. Path. Bact., 41, 215.

McIntosh, J. and Selbie, F. R. (1937) Brit. J.exp. Path., 18, 162; (1940) Ibid., 21, 153.

Maitlaxd, H. B. and Laine, A. W. (1941) J. Path. Bact., 53, 419.

Maitland, H. B., Laing, A. W., and Lyth, R. (1932) Brit. J. exp. Path., 13, 90.

Mattland, H. B. and Mattland, M. C. (1928) Lancet, ii. 596.

Marie, A C. and Urbain, A. (1930) C. R. Soc. Biol., 103, 866.

Markham, R., Surth, K. M. and LeA, I). (1942) Parasitology, 34, 315.

Marton. L. (1941) J. Bact., 41, 397.

MudD, S. (1922-3) Amer. J. Physiol., 63, 429 ; (1928) "Filterable Virnses." 'T. M. Rivers, p. 55. Baillière, Tindall \& Cox, London.

Natarajan, C. V. and Hyok, li. R. (1930) Amer. J. Hyg., 11, 652.

Noguchi, H. (1915) J. exp. Med., 21, 539; (19I8) Ibid., 27, 425.

Oaston, A. (1ss1) Brit. med. J., i. 369.

OLIтsкY, P. K. and Bö̈z, L. (1927) . . exp. Med., 45, 685.

Parker, F. and Nye, R. N. (1925) Amer. J. Path., 1, 325, 337.

PARker, R. F. (1938) J. exp. Med., 6\%, 725.

Parker, R. F., Bronson, L. H., and Green, R. H. (1941) J. exp. Med., 74, 263.

Parker, R. F. and Pivers, T. M. (1937) J.exp. Med., 65, 213.

Parker, R. F. and Sirthe, C. V. (1937) J.exp. Med., 65, 109.

Paschen, E. (1932) Zbl. Bakt., 124, 89.

Perdrau, J. R. (1927) Brit. J. exp. Path., 8, 167.

Perdrat, J. R. and Todd, C. (1933) Proc. roy. Soc., B., 112, 288 ; (1936) Ibil., 121, 253.

Pirie, N. IV. (1937) "Perspeetives in Biochemistry," Camb. Univ. Press.

Poppe, K. and Busch, G. (1930) Z. ImmunForsch., 68, 510.

Rake, G., Shaffer, M. F., and Tingeson, P. (1942) Proc. Soc. exp. Biol., N.Y., 49, 545.

Report. (1927) 2nd Progr. Rep. Foot and Mouth Dis. Res. Comm. Ministry Agric. Fish., Lond.

ReYNals, F. D. (1928) J.exp. Med., 47, 389.

Rrvers, T. M. (1928) "Filterable Viruses," p. 3. Baillière, Tindall \& Cox, London; (1932) Physiol. Rev. 12, 423 ; (1943) "Virus Diseases," p. 3. Cornell Univ. Press, Tthaea, N.Y.

Rivers, T. M., HaAgen, E., and Muckenfuss, R. S. (1929) J.exp. Med., 50, 665.

Rivers, T. M. and Pearce, L. (1925) J. exp. Med., 42, 523.

Rivers, T. M. and WARD, S. M. (1933) J.exp. Hed., 5\%, 51, 741.

RoDANiche, E. C. (1942) J. infect. Dis., 70, 58.

Sabin, A. B. (1941) J. Amer. med. Ass., 11\%, 267.

Salaman, M. H. (1934) Brit. J. exp. Path., 15, 381 ; (1937) Ibid., 18, 245.

Sankaran, G., Iyengar, K. R. K., and Beer, IV. A. (1934) Indian J. med. Res., 21, 909.

Sawyer, W. A. and Frobisher, M. (1929) J. exp. Med., 50, 713. 
Sawyer, W. A., Lloyd, W. D. M., and Kitchen, S. F. (1929) J. exp. Med., 50, 1.

Schlesinger, MI. (1936) Nature, 138, 549.

Sharp, D. G., Taylor, A. R., Beard, D., and Beard, J. W. (1912) Proc. Soc. exp. Biol., N.Y., 50, 20.5; (1943) Arch. Path., 36, 167.

Sharp, D. G., Taylor, A. R., Mclean, I. W., Beard, D., Beard, J. W., Feller, A. E., and Dingle, J. H. (1944) J. Immunol., 48, 129.

Shedlotsky, T. Rothen, A., and Shadel, J. E. (1943) J. exp. Med., 7\%, 155.

Shedlovsky, T. and Smadel, J. E. (1912) J. exp. Med., 75, 165.

Sirortт, H. E. and Brooks, A. G. (1934) Indian J. med. Res., 21, 581.

Sichert-Hodrow, I. (1930) Zbl. Bakt., 119, 12.

SMadel, J. E. and Hoagland, C. I. (1942) Bact. Rev., 6, 79.

Smadel, J. E., Hoagland, C. L., and Shedlovsky, T. (1943) J. exp. Med., ry, 165.

Smadel, J. E., Lavin, G. I., and Dobos, R. J. (1940 $)$ J. exp. Med., r1, 373.

SMADEL, J. E., WALL, M. J., and BAIRD, R. D. (1940b) Ibid., r1, 43.

Shadel, J. E., Pickels, E. G., and Shedlovsky, T. (1938) J. exp. Med., 68, 607.

Smadel, J. E., Rivers, T. M., and Hoagland, C. L. (1942) Arch. Path., 34, 275.

Smadel, J. F., Rbvers, T. M., and Pickels, E. G. (1939) J. exp. Med., 70, 379.

Sмiтн, W. (1932) Brit. J. exp. Path., 13, 434; (1939) J. Path. Bact., 48, 557.

Sobernheim, G. (1925) Ergebn. Hyg., $\%$, 133.

Stanley, W. M. (1938a) J. phys. Chem., 42, 55 ; (1938b) Bull. New York Acad. Med., 14, 398; (1938c) J. appl. Phys., 9, 148; (1938d) Amer. Nat., 72, 110 ; (1941) "Virus Diseases," p. 35. Cornell Univ. Press, Ithaca, N.Y.

Stanley, W. M. and Anderson, T. F. (1941) J. bial. Chem., 139, 325.

Stevenson, IV. D. H. and Butler, C. G. (1939) Rep. publ. Illth med. Subj., Min. Hlth, Lond., No. 87.

Stock, C. C. and Francis, T. (1940) J. exp. Med., 71, 661.

SvedberG, T. (1937) Nature, Lond., 139, Suppl., 1051.

Svedberg, T., Boestad, G., and Eriksson-Quensel, I. B. (1934) Nature, 134, 98.

Svedberg, T. and Nichols, J. B. (1927) J. Amer. chem. Soc., 49, 2920.

Takahashi, W. N. and Rawlins, T. E. (1935) Science, 81, 299.

Tallerman, K. H. (1929) Brit. J. exp. Path., 10, 360.

TANG, F. F. (1932) J. Bact., 24, 133.

TANG, F. F. and WeI, H. (1937) J. Path. Bact., 45, 317.

Taylor, A. R., Sharp, D. G., Beard, D., Beard, J. W., Dingle, J. H., and Feller, A. E. (1913) J. Immunol., 4\%, 261.

ToDd, C. (1928) Brit. J. exp. Path., 9, 19.

Vallée, H. and Carré, H. (1922) C. R. Acad. Sci., 1\%4, 1498.

IWalduand, O. and Tradtwein, K. (1926) Berl. tierärztl. Wschr., 42, 569.

Ward, H. K. (1929) J. exp. Med., 50, 31.

Woodruff, A. M. and Goodpasture, E. W. (1931) Amer. J. Path., \%, 209.

Woodruff, C. E. and Goompasture, E. W. (1929) Amer. J. Path., 5, 1; (1930) Ibid., 6, 713.

Wooley, J. G. (1939) Publ. Hlth Rep., Wash., 54, 1077.

Zinsser, H. and Schoenbach, E. B. (1937) J. exp. Med., 66, 207.

Zinsser, H. and Seastone, C. V. (1930) J. Immunol., 18, 1.

Zinsser, H. and TANG, F. (1929) J. Immunol., 1\%, 343.

Zivick. (1924) Dtsch. tierärztl. W'schr., 32, 643. 


\section{N D E X}

All organisms are listed under the generic names given in the Classificatory Chart on p. 319. Since many rod-shaped organisms have in the past leen known under the generie name of Bacillus, irrespective of whether or not they belonged to this genus, we have for the convenience of the reader included most of them under the heading Bacillus (nonitalieized). This heading should therefore be consulted by those who are doubtful to what genus a given organism belongs.

de Aar disease, 1648

Abel-Loewenberg bacillus, 494, 667, 1789

Abortin. See Brucellin

Abortion, contagious, in cattle, 1708

diagnosis of, 1711

epizootic, of mares, 1968

human, due to $\mathrm{Br}$. abortus, 1698

infection of ealves in, 1711,1716

infection of milk in, 1709,1713

infectious, in cattle and sheep due to Vibrio fetus, 1720

mode of infection in, 1710

prophylaxis of, 1715

by eradication, 1715

by vaccination, 1716

Abortus fever. See Undulant fever

Accessory food factors in bacterial growth. See Vitamins

Acetobacter aceti, 505

Acetyl-choline, relation to anaphylactic shock, 1150

Achahme's bacillus. See Clostridium welchii

Achromobacterium, 640, 641

Acid agglutination, 213, 692

Acid-fast bacilli. See Mycobacterim

Acid-fastness, 38 cause of, 407

Acidophilic bacteria, definition of, 757. See also Lactobacillus

Acids, effect of on bacteria, 118, 2017. See also under $p \mathrm{H}$

Aciduric bacteria, definition of, 757. See also Lactobacillus

Acne bacillus, 471, 1403, 1492, 1505, 1506

Acriflavine, disinfection by, 135

for distinguishing smooth and rongh organisms, 823

Actinobacillosis, 389, 1270, 1279-1281

differentiation of, from actinomycosis, 1280

pneumonia in calves due to Actinobacillus actinoides, 1281

Actinobacillus, 389-393

actinoides, 391, 1270

causing pneumonia in calves, 1281

in otitis media of rats, 1499

actinomycetem-comitans, 391, 1:274

lignieresi, 389, 1270

discase due to, 1279

tabulated reactions of, 392
Actinomyces, 373-389

acid-fast types of, $375,381,387$

aerial hyphæ of, 377

antigenic structure of, 379

biochemical characteristics of, 378

classification of, 380

cultural reactions of, 376

culture clubs of, 375

Drusen of, 375

growth requirements of, 378

habitat of, 374

human and bovine types of, 379

in mouth and salivary calculi, 1272

morphology of, 374

non-acid-fast types of, 375, 381, 387

pathogenicity of, 379

pigment formation of, 379

resistance of, $37 \mathrm{~s}$

spores of, 375

tabular differentiation of, 392

tissue clubs of, 375

variation in, 379

asteroides, 388,1275

bovis, 373 et seq., 382, 1270

capre, 387,1276

farcinicus, 387, 1276

graminis, 373 et seq., 384

gypsoides, 388,1275

madurce, 376 et seq., 383, 1275

muris, 381, 385, 939, 1277, 1910

necrophorus. See Fusiformis

putorii, 387

somaliensis, 387

variabilis, $38 s$

Actinomycetes, antibacterial substances formed by, 175

Actinomycetin, 176

Actinomycin, 176

Actinomycosis, 1269-1278

differentiation of from actinobacillosis, 1280

Drusen in, 1270, 1272

due to Actinomyces bovis, 1270-1274 in cattle, 1271

due to aerobic Actinomyces, 1270, 12751276

cattle farcy, 1276

in goats and horses, 1276

in man, 1275

Nadura disease, 1275 
Actinomycosis, due to facultative acrobic Actinomyces, 1276-1278

infective arthritis of mice, 1278 rat-bite fever, 1276

in swine, 1271

mode of infection in, 1271

Activated sludge method in sewage purification, 2033

Active immunity. See Immnnity, active

Adaptive enzymes in bacteria, 293

Adbesion phenomenon, 912, 1833

Adjuvant substances for enhancement of antigenic response, $1115,1116,1123$

Adsorption in antigen-antibody reactions, 199 ,

$216-219,226,240,273-275$

Aerobacter. See Bacterium xrogenes

Aerobes, obligatory, 70, 360 enzyme systems of, 70

Aerobic and anaerobic incubation, use of in obtaining pure cultnres, 354,360

Aerobic spore-bearing bacilli. See Bacillus

Aerobiosis, 70 et seq. in identification of bacteria, 360

Aesosols, 2007-2009

African horse sickness, 1965

Agalactia, contagious, of goats, 945, 1867

Agar, 10

Agglntinating serum, use of in obtaining pure cultures, 355

Agglutination, 211. See also Antigen-antibody reactions and under individual discases

Agglutinins, See also Antigen-antibody reactions

absorption of, by bacteria, 212, 273

cold, in virns pneumonias, 1877

formation of in response to infection, 1120

to primary stimulns, 1106

to repeated injections, 1113

natural, 1085 et seq.

for various organisms. Sce under individual diseases

protective action of, 1058

Agglutinogens, 195. See also Antigens, bacterial

Aggressins, bacteria], 1068-1074

antigens, relation of to, 1072

anti-opsonic effect of, 1069-1071

pnenmococcal polysaccharides as, 1678

polysaccharide haptens as, 1072

Air, bacteriology of, 2002-2010

contamination of, 2004

droplet infection of, 1312, 2003

infection of, measures for controlling, 2005-2010

liquid, effect of on bacteria, 112

Aliyami, 1834

Alastrim, 1884, 1889

Alcohol, effect of on immunity, 1209

Alcoholized T.A.B. vaccine, 1553

Alcohols, effect of on bacteria, 132

Alexine, 193, 196

Alimentary tract, bactericidal mechanisms in, 1020

normal flora of, 1986

importance of, 1989

origin of, 1989

suppurative lesions of, 1497
Alkalics, effect of, on bacteria, 120

on bactericidal action of gastric juice, 1021-1023

Allantiasis. See Botulism

Allergy, 1160-1168

bacterial. 1162

relation to immunity, $1163,1201,1330$

infection-immunity in, 1165

Koch's phenomcnon as example of, 1161, 1326

tuberculin reaction, as example of, 1161 . 1326

virus discases, in relation to, 1241

Alpha rays, effect of on bacteria, 110

$\alpha$-hydroxyphenazine, 173

Aluminium salts, cffect on antigenic stimulus, 1115

Alum-precipitated toxoid in diphtheria ins. munization, 1393

Amboceptors, 198, 225, 228

Amino-acids, as essential nutrients for bacteria, 65

rôle of in bacterial mntrition, 56,62

Amino-acridine compounds in chemotherapy, 172

Ammonia, methods of examination for, 369

Anæmia, infections, of $\operatorname{dogs}, 904,1798$

of horses, 1837,1966

of rats, 904, 1797

Anaerobes, enzyme systems of, 70

facultative, 70,360

obligatory, 70,360

Anacrobic bacilli, species of. See Fusiformis and Clostridium

Anaerobic infections of animals, 1778-1783

black disease, 1780

Cl. gigas in, 1781

relation of liver flukes to, 1781

blackleg, 1778

braxy, 1779

German type, 1780

Northern type, 1779

due to $C l$. uelchii, 1781-1783

enterotoxæmia, infectious, of sheep, 1782

lamb dysentery, 1781

pulpy kidney disease of lambs, 1782

"struck" in sheep, 1782

gas gangrene, 1778

malignant œedema, 1778

Anaerobic spore-bearing bacilli. See Clos. tridium

Anaerobic streptococci, 596, 1484, 1772, 1996

Anaerobiosis, 70 et seq.

in identification of bacteria, 354,360

possible relation of hydrogen peroxide to, 72

rôle of oxidation-rednction potential in, 71

Anamnestic reaction, 1122

diagnosis of cnteric fever in inoculated persons in relation to, 1539

Anaphylactic shock, 1136-1151

acetyl-choline in, 1150

anaphylactoid shock, relation of to, 1144

anaphylatoxin in, 1143

desensitization in, 1142

differcnt antisera, effectiveness of in, 1140 
Anaphylactic shock, hapteus, rôle of in, 1141 listamine shock, relation of to, 1141, 1148 himoral hypothesis of, I 143 in the dog, 1138

in the guinea-pig, 1137,1147

in the rabbit, 1138

in various animals, 1139

induction period in, 1136

inhibition by antihistamine sera, 1150

by histaninase, 1150

by normal sera, 1148

mechanism of, 1142-1151

cellular hypothesis of, 1145

Schultz-Dale technique in study of, 1146

passive sensitization in, 1140

peptone shock, relation of to, 1144

reversed passive anaphylaxis in, 1140

sensitization to, factors effecting, 1136

Anaphylactoid shock, 1144

Anaphylatoxin, 1143

Anaphylaxis, 1136-1151, 1156. See also Allergy, Anaphylactic shock and Hypersensitiveness

Arthus' phenomenon in, 1151

genetic factors in, 1159

local reactions in, 1151,1156

relation of to immunity, 1146,1164

Anatoxin, 1392

Angina, Vincent's, 914, 1810

Angular conjunctivitis, 898

Aniline dyes, effect of on bacteria. See Dyes

Animal discases. See under name of disease and spccies of animal

Animal poxes. Sce Poxes, animal

Animals, anaerobic infections of. See Anaerobic infections of animals

Anions, effect of on bacteria, 124

Antagonizers of chemotherapentic agents, 1.59, $161,165,166$

Anthrax, 1730-1744

Ascoli test in, 1738

chemotherapy in, 1743

cutaneous, susceptibility to, 1183, 1184

diagnosis of, 1737

experimental production of in animals, 1736

in animals, 1731,1735

infection of milk in, 1735,1740

in man, 1733,1735

local immunity to, 1181

mode of infection and spread, 173:

by carth-worms, 1732

by flies, 1733

by food-stuffs, 1732

natural immunity to, 1739

prevention of, 1740

scrum treatment of, 1743

symptomatic. See Blackleg

vaccination against, 1740

wool-sorters' disease in, 1733, 173t

Antibacterial immunity. See Immunity, antibacterial

Antibacterial inclex, 162

Antibacterial substances, 155, 17:2

from bacteria, 173,174

from moulds, 17.5
Antibacterial substances, susceptibility to in relation to Gram's stain, 38, I70

Antibiotic agents, 155, 175

Antibodies. See also under Antigen-antibody reactions

availability in tissues of host, 10.58

blocking, in allergic reactions, 1159

distribution of in tissues, 1058

electrophoretic fractionation, $242, \mathbf{2 4 6}$, $248,1030,1092,1385$

fate of, after injection, 1124

formation of, $1101 \mathrm{et}$ seq.

adjuvants, effect of on, 1115

agglutiuins $\mathrm{O}$, formation of after administration of antigen by mouth, 1117

anamnestic reaction in, 1122

as evidence of specific infection, 1002 a vitaminosis, effect of on, 1192,1198 , 1204

blockade, effect of on, 1103

by synthesis in contact with antigens, 1127,1128

different antigens, in response to, 1108

dispersal of inoculum. effect of on, 1115

dosage, effect of on, 1107

in skin, 1102

in lymphocytes, I 105

in tissue cultures, 1105

in various organs, 1102,1104

in vitro, 1128

local, 1101

mechanism of, 1127

metallic salts, effect of on, 1115, 1122

multiple antigens, in response to, 1109

negative phase in, 1116

non-specific stimulus in relation to, 1120

oral administration of antigen in response to, 1117

physiological fate of, 1127

pneumococcal polysaccharides, in response to, 1684

primary stimulus in response to, 1106 protective action of in vivo, conditions of, 1057

repeated injections, effect of on, 1113 reticulo-endothelial system, rôle of in, I 102

secondary stimulus in response to, 1110

spleen, rôle of in, 1103

sulphonamides, effect of on, 1213

synthesis in vitro of, 1128

transference of via colostrum, 1077 , 1088 via placenta, 1077, $108 \mathrm{~s}$

various factors, effect of on, 1104

various tissues, rôle of in, 1105

virus injections in, 1241

volume of inoculum, effect of on, 1114

general properties of, 194, 242

"heavy," 24.j

leterophile. See Forssman antibody 
Antibodies, modification of, by chemical means, 247

by enzymes, 247

by physical means, 247

molecular weight of, 243,245

monospecific, homogeneity of, 249

natural, 1075-1097

age in relation to, 1091

antibacterial antibodies, 1085, $1092-$ 1096

agglutinins, 1085 el seq.

Brucella infections, 1699

in latent Salmonella infections, 1533

bactericidins, 1086

complement-fixing, 1086

immunological significance of, 1095

origin of, 1092-1095

antitoxins, 1075

in blood of normal horses, 1084

immunological significance of, 1084

origin of, 1076-1084

environment in relation to, 1080 et seq.

rate of development under different conditions, 1083

viricidal, 1226

possible inclusion of antigens in, 1127

protein nature of, 242 et seq.

purification of, 243

reagins, relation of to, 1157

serum-globulins, relation of to, 244

site of formation of, 1101-1106

unity or diversity of, 248-252, 285

valency of, 250

various, names of, 195

Antibody-forming apparatus, 1101-1131. See

Antibodies, formation of

Antibody globulin, 242

varieties of, 245

Anti-endotoxins, 1008

failure of law of multiple proportions in action of, 1009

Antiformin, use of in obtaining pure cultures, $354,418,1321$

Antigen-antibody reactions, 192-265

adsorption in, 199, 216-219, 226, 240, 273275

agglutination, 211

cohesive force in relation to, 214

electric charge in relation to, 213

electrolytes in relation to, 213

in diagnosis. See under individual diseases

optimal antigen-antibody ratio in relation to, 212

potential difference in relation to, 214 tests of lattice hypothesis in, 221

alexine, 193,196

antigen and antibody, combination of, in varying proportions, 205, 216, 219, 227 240

antigenic specificity, surface structure in relation to, 263

Arrhenius and Madsen's theory of, 199, 239
Antigen-antibody reactions, bactericidins, 228 bacteriolysis, 192, $22 \mathrm{~S}$

Neisser-Wechsberg phenomenon in, 228

Pfeiffer's reaction, 192, 1050, 1425

bacteriotropins, 194, 235

complement, relation of to, 236

electric charge in relation to action of, 235

relation of to opsonins, 235

Bordet's theory of, 199

capsular swelling reaction, 241

combination in varying proportions, 205 complement, 193, 196

nature of, 229

unity or diversity of, 229

complement fixation, 232

in diagnosis. See under individual diseases

optimal conditions for, 233

relation of to precipitin reaction, 233

differences in antigenic structure in rela-

tion to, 285

efficacy of in vivo, 1061

Ehrlich's theory of, 196

electrolytes, effect of on, 200, 207, 211,

213,226

Freundlich isotherm in relation to, 218

hæmolysis, 193, 224

supposed rôle of amboceptors in, 225

temperature, effect of, on, $2: 27$

haptens, inhibition of precipitation by, 257

hydrophile and hydrophobe states in rela. tion to, 209

lattice hypothesis of, 208,220

law of multiple proportions in, 217

mass law in, 219

microscopic appearance of specific aggregates, 223

miscellaneous manifestations of, 240

nature of union in, 263

opsonins, 194, 235

complement, relation of to, 236

electric charge in relation to action of, 235

relation of to bacteriotropins, 235

optimal antigen : antibody ratio in relation to, 201 et seq., 212

relation of constant-antigen ratio to constant-antibody ratio, 203-207

relation of to chemical equivalence, 204

precipitin reaction, 200

effect of various factors on, 200

clectrolytes, effect of, on, 200

in standardization of antipneumococcal serum, 168I

optimal antigen-antibody ratio in relation to, 201

relation of to complement fixation, 233

tests of lattice hypothesis in, 221

qualitative aspects of, $242-265$

quantitative aspects of, 201-241

reversibility of, 216

effect of salts on, 214

"side-chain" theory of, 196 
Antigen-antibody reactions, specificity of, 252

terminology employed in relation to, 194 et seq.

toxin-antitoxin reaction, 238-240

antitoxin, unit of, 238

Danysz phenomenon in, 216,240

Ehrlich's phenomenon in, 239

epitoxoids in relation to, 239 toxin. $\mathrm{L}_{0}$ and $\mathrm{L}_{+}$doses of, 239 Minimal Lethal Dose of, 238 unit of antitoxin, 238

"two-stage" hypothesis of, 208 unitarian hypothesis of, 248

unity or diversity of antibodies, 248,252

Antigenic structure of bacteria, 273-285. See also under individual species

bacterial variation in relation to, 276

bacteriophage activity in relation to, 338

chemical methods in determination of, 279

classification in relation to, 314

determination of by sclective absorption of agglutinins, 273

- clifferent antigen-antibody reactions in relation to, 285

fallacies in determination of, $274,275,281$

flagellar antigens, $276,283,284$

group and specific phases of, 279

Forssman antigen in, 282, 1090

$\mathrm{H}$ antigens, gromp and specific phases of, 278

heterophile antigen in, 282

localization of antigens in bacterial cell, 283

0 antigens, 277

optimal proportions, in determination of, 276

roughness in relation to, 277,285

sharing of antigens by different bacteria. 282

smoothness in relation to, 277,285

somatic antigens, 276,283

Antigenicity, of denatured protein, 261

of gelatin, 260

of proteins, 259

rôle of carbohydrate in, 261

Antigens. See also Antigenic structure of bacteria and Antigen-antibody reactions atopens, relation of to, $1154 \mathrm{et} \mathrm{seq.}$

bacterial. See under Antigenic structure of bacteria

aggressins, relation of to, 1071

bacteriophage. in relation to, 338

endotoxins, relation of to, $100 \mathrm{~s}$

relation of to active immunization, 1055

basis of antigenicity of, 259

basis of specificity of, 252

chemical nature of in relation to stimu-

lation of antibody production. 1119

different, effect of simultaneous injection of, 1109

filtrable viruses, of, 1240

Forssman, 1089

in bacteria, 282,1090

general properties of, 194
Antigens, haptens, 258

aggressive action of in pmeunonia, 1071,1679

anaphylaxis, rôle of in, 1141

antibody formation, stimulation of, by, 1119,1684

bacterial polysaccharides as, 258

complex, 258

in filtrable viruses, 1237

in Forssman antigens, 1090

in human group A substance, 1090

inhibition of precipitation lyy, 257

relation of to complete antigens, $25 \mathrm{~s}$ simple, 258

masked, 262

nature and properties of, 25:

organ specific, 252

persistence in body, effect on immunity of, 1129

possible inclusion of in antibodies, 1127

proteins in relation to full antigenicity of, 260,1119

species, specific, 252

synthetic, $253-256$

diazo reaction in preparation of, 253

indncing type-specific immunity against pneumococei, 1054

valency of, 262

various, names of, 195

Antipneumococcal immunity, experimental, in rabbit, $1037,1048-1052$

Antisepsis, Lister's studies on, 11

Antitoxic immunity, 1029-1033. See Immunity, antitoxic

Antitoxin. See also under individual diseases avidity of, 1030

natural, 1075 et seq. See Antibodies, natura!

route of administration of, effect of, 1124, 1763

unit of, 238

Antiviral antibodies, 1237

Antiviral immunity. See Immunity, antiviral

Appendicitis, 1497

hacteriology of, 1497

isolation of Fusiformis from, 477 et seq.

Arhenius and Madsen's theory of antigenantibody reactions, 199,239

Arsenic compounds in chemotherapy, 155, 156 , 171

Arsenic-fast strains of spirochætes in relapsing fever, 1808

Arthritis in rheumatism, 1509 et seq.

in joint ill, $676,1284,1496$

infective of mice, 386,1278

purulent, 1496

Arthus' phenomenon, 1151

Ascococcus mesenterioides, 627

Ascoli's thermoprecipitin reaction, 1738

Aspergilli, 175

Aspergillic acid, 177

Asterococcus canis, 946

mycoides, 940

Asthma, hypersensitiveness in relation to, 1153

Atopens, 1154 et seq. simple compounds as, 1155

Aujesky's disease, 1941 
Australian X encephalitis, 1918

Auto-disinfecting mechanism of alimentary tract, 1020, 1988

of conjunctivæ, 1025

of genital tract, 1025

of nose and naso-pharynx, 1023

of skin, 1019

Autotrophic bacteria, 61

Autoxidizable substances in bacterial meta. bolism, 48

Autumn diarrhoea in cattle, 17:0

fever of Japan, 1828, 1836

fever of Quecnsland, 1828, 1837

Avian spirochrtosis, Is08

Avidity of antitoxin, 1030

Azotolacter, 497-199

agilis, 499

beijerincki, 497

chroococcum, 497, 498

vinclandii. 497

vitreus, 497

woodstowni, 497

B.C.G., 1306, 1331, 1337, 1347

$B$ encephalitis, 1917

$B$ virus of myelitis, 1921

Babes-Ernst granules, 23

in $C$. diphtherix, 449

in Neisscria, 532

Bacillemia. See Bacleræmia

Bacillary hæmoglobinuria of cattle, 891

Bacillary white diarrhoea of chicks, 1555

Bacillus, Bang's abortion. See Brucella abortus

Bang's necrosis. See Fusiformis necrophorns

of Abel-Loewenberg, 494, 667, 1789

of Achalme, 886

of Boas-Oppler, 750, 757, 764

of Bordet-Gengou. See IIæmophilus per. tussis

of Calmette-Guérin (B.C.G.), 1306, I331, 1337,1347

of Danysz. See Salmonella enteritidis of Denekc. See Vibrio

of Döderlein, 750 et seq., 757, 1996

of Ducrev, 791 et seq., 807, 1790

of Finkler-Prior. See Vibrio

of Flexner. See Shigella

of Friedländer. See Bacterinm

of Gaertner. See Salmonella enteritidis

of Glässer. See Salmonella typhi-suis

of Grigoroff, 764

of von Hibler (IX). See Clostridium tertium

of Hirschfeld. See Salmonella paratyphi $C$ of Hiss, 689

of Hofmann. See Coryncbacterium

of Johne. See Mycobacterium

of Koch-Wiceks, 791 et seq., 807, 1496

of Massauah. See Vibrio

of Mlassol. See Lactobacillus bulgaricus

of Morax-Axenfeld, 898

of Morgan. See Proteus morgani

of Nasik. See Vibrio

of Nocard, 1873

of Perez, 494
Bacillus of Petit, 899

of Preisz-Nocard. See Corynebacterium ovis

of Rodella (III). See Clostridium tertium of Schmitz. See Shigella

of Schmorl. See Fusiformis necrophorus of Shiga. See Shigella

of Sonne. See Shigella.

of Sordelli. See Clostridium bifermentans of Voldagsen. See Salmonella typhi-suis var. voldagsen

of Welch. See Clostridium

of Whitmore. See Pfeifferella

of leprosy. See Mycolacterium lepræ

of mouse septicæmia. See Erysipelothrix muriseptica

of ozæna. See Ozæna

of rat leprosy. See Mycobacterium of rat leprosy

of soft chanere, 791 et seq., 807,1790

of sputum septicæmia, 668

of timotliy grass. See Mycohactorium phlei

abcrdcen. See Salinonella

abony. See Salmonella

abortus. See Brucella

abortus-bovis. See Salmonella

abortus-equi. See Salmonella

abortus-ovis. See Salmonella

accra. See Salmonella dublin

acetobutylicum. See Clostridium

acidi-lactici. See Bacterium

acidophil-rerogenes. See Lactobacillus

acidophilus. See Lactobacillus

acnes. See Corynebacterium

actinuides. See Actinobacillus

actinomycetem-comitans. See

Actinobacillus

adelaide. See Salmonella.

ærofœtidus. See Clostridium

arogenes. See Bacterium

xrogenes capsulatus. See Clostridium welchii

ærtrycke. Seo Salmonella typhi-murium æruginosus. See Pseudomonas pyocyanea agilis. See Azotobacter

alkalescens. See Shigella

alkaligenes. See Bacterium

altendorf. See Salmonella

amager. Sec Salmonella

ambiguns. Sce Shigella schmitzi

amersfoort. See Salmonella

amethystius. See Chromobacterium

amherstiana. See Salmonella

amylobacter. See Clostridium

anatum. See Salmonella

aquatilis. See Chromobacterium

arechavaleta. See Salmonella

arizona. See Salmonella

arthritidis muris. See Corynebaclerium

asiaticus. Sce Bacterium

aurantiacus. Sce Chromobacterium

avisepticus. See Pasteurella

azophile, 499

ballerup. See Salmonella

bareilly. See Sulnonella

batavia. See Salmonella 
Bacillus beijerincki. See Azotobacter beılin. See Salmonella thompson berolinensis. See Vibrio and Lactobacillus berta. See Salmonella

bifermentans. See Clostridium

bifermentans sporogenes. See Clostridium bifermentans

bifidus. See Lactobacillus

binns. See Salmonella typhi-murium

bispebjerg. See Salmonella

blegdam. See Salmonella

bonariensis. See Salmonella

borbeck. See Salmonella

botulinus. See Clostrilium

bovimorbiticans. See Salmonella

bovisepticus. See Pasteurella

brænderup. See Salmonella

brandenburg. See Salmonella

bredeney. See Salmonella

brevis. See Lactobacillus

bronchicanis. See Hæmophilus bronchisepticus

bronchisepticus. See Hæmophilus

bubalisepticus. See Pasteurella

buccalis muciferens, 668

buchneri. See Lactobacillus

budapest. See Salmonella

bulgaricus. See Lactobacillus

butyricus. See Clostridium and Mycobacterium

cadaveris butyricus. See Clostridium welchii

california. See Salmonella

caloritolerans. See Clostridium

cambridge. See Salmonella

canalis capsulatus, 667

canastel. See Salmonella

capitovalis. See Clostridium

capsulatus, 668

cardiff. See Salmonella

carnis. See Clostridium

carrau. See Salmonella

casei. See Lactobacillus

caucasicus. See Lactobacillus

caviæ. See Pseudomonas

cavisepticus mobilis, 787

centrosporogenes. See Clostridium

cerro. See Salmonella

ceylonensis, 685

chaco. See Salmonella

chauvœi. See Clostridium

chester. See Salmonella

choleræ. See Vibrio

choleræ-suis. See Salmonella

chroococcus. See Azotobacter

claibornei. See Salmonella

cloacæ. See Bacterium

cochlearius. See Clostridium

coeruleus. See Chromobacterium

coli. See Bacterium

coli-anaerogenes. See Bacterium

coli-communior. See Bactcrium

coli-communis. See Bacterium

coli-mutabilis. See Bacterium

columbensis. See Bacterium

concord. See Salmonella

copenhagen. See Salmonella typhi-murium
Bacillus coryzæ segmentosus, 450 coscoroba. See Bacterium crassus. See Döderlein's bacillus crassus sputigenus, 668 cuniculi, 479

cyanogenus. See Pseudomonas danubicus. See Vibrio danysz. See Salmonella dar-es-salaam. See Salmonella denitrificans. See Chromobacterium denitrificans fluorescens. See Pseudo. monas

derby. See Salmonella difficilis. See Clostridium

diphtheriæ. See Corynebacterium

diphtheriæ vitulorum. See Fusiformis necrophorus

dispar. See Shigella

dublin. See Salmonella

ducreyi. See Hæmophilus

duesseldorf. See Salmonella

duisburg. See Salmonella

duplex, 899

durban. See Salmonella

dysenteria. See Shigella

eastbourne. See Salmonella

El Tor. See Vibrio

enteritidis. See Salmonella

enteritidis sporogenes. See Clostridium welchii

cqui. See Corynebacterium

equirulis. See Bacterium

erysipelatis suis. See Erysipelothrix rhu. siopathize

erysipeloides. See Erysipelothrix

essen. See Salmonella

europous. See Nitrosomonas

exilis. See Lactobacillus

frealis alkaligenes. See Bacterium

fallax. See Clostridium

fermenti, 756. See Lactobacillus

fetus. See Vibrio

flavescens. See Neisseria

flavus. See Nitrobacter

flexneri. See Shigella

florida. See Salmonella.

fluorescens. See Pseudomonas

fluorescens liquefaciens. See Pseudo. monxs

foctidus ozænæ. See Perez's bacillus

fragilis. See Fusiformis

friedländeri. See Bacterium, Friedländer group of

funduliformis. See Fusiformis necrophorus

furcosus. See Fusiformis

fuscus. See Chromobacterium

fusiformis. See Fusiformis and Bacillus

gallinarum. See Salmonella

gaminara. See Salmonella

gärtner. See Salmonella enteritidis

georgia. See Salmonella

gigas. See Clostridium oedematiens

giumai. See Bacterium

give. See Salmonella

glostrup. See Salmonella

gocttingen. See Salmonella 
Bacillus granulosis. See Bacterium grumpensis. See Salmonella grïthal. See Bacterium habana. See Salmonella hremoglobinophilus canis. See Hæmo. philus canis

hrmolyticus. See Clostridium

hartford. See Salmonella

hastiforme. See Clostridium

heidelberg. See Salmonella helcogenes. See Vibrio histolyticus. See Clostridium hofmannii. See Corynebacterium hormæchei. See Salmonella horsham. See Salmonella hvittingfoss. See Salmonella icteroides. See Salmonella typhi-murium illinois. See Salmonella immobilis. See Bacterium indicus. See Chromobacterium infantis. Sce Salmonella influenzæ. See Hamophilus inmutritus. See Clostridium inverness. See Salmonella italiana. See Salmonella janthinus. See Chromobacterium java. See Salmonella paratyphi $B$ javiana. See Salmonella jena. See Salmonella enteritidis kaspstad. See Salmonella. kaposvar. See Salmonella kaukasicus. See Lactobacillus caucasicus kentucky. See Salmonella khartoumensis. See Bacterium kiel. See Salmonella kielensis. See Chromobacterium kirkee. See Salmonella koeln. See Salmonella kottbus. See Salmonella kunzendorf. See Salmonella choleræ-suis lactis ærogenes; See Bactcrium aerogenes lactis erythrogenes. See Chromobacterium lacunatus, 898

leguminosarum. See Rhizobium lepisepticus. See Pasteurella lepræ. See Mycobacterium lexington. See Salmonella iignieresi. See Actinobacillus litchfield. See Salmonella london. See Salmonella loma-linda. See Salmonella luteus. See Micrococcus madampensis, 685

madelia. See Salmonella mallei. See Pfeifferella manchester. Sce Shigella neucastle manhattan. See Salmonella melaninogenicus. See Fusiformis meleagridis. See Salmonella melitensis. See Brucclla mexicana. See Salmonella mikawasima. See Salmonella. minnesota. See Salmonella. mirabilis. See Proteus mississippi. See Salmonella moniliformis. Sce Actinomyces muris monocytogenes. See Erysipelothrix
Bacillus montevideo. See Salmonella morbificans bovis. See Salmonella bovismorbificans

morgani. See Proteus

moscow. See Salmonella

moskau. See Salmonella enteritidis

mucosus capsulatus. See Bacterium,

Friedländer group of

muenchen. See Salmonella

muenster. See Salmonella

multifermentans. See Clostridium

multifermentans tenalbus. See Clostridium

muris. See Actinomyces and Mycobacterium

murisepticus. See Erysipelothrix and Pasteurella

murium. See Corynebacterium murium mycoides roseus. See Chromobacterium napoli. See Salmonella

narashino. See Salmonella neapolitanus. See Bacterium necrodentalis. See Lactobacillus acido. philus

necrophorus. See Fusiformis

nephritidis equi. See Bacterium

new-brunswick. See Salmonella

newcastle. See Shigella, and Salmonella senftenberg

newington. See Salmonella

newport. See Salmonella

niloese. See Salmonella

novyi I. See Clostridium odematiens

nyborg. See Salmonella

ochraceus. Sce Chromobacterium

odense. See Salmonella paratyphi B

odontolyticus I and II. See Lactobacillus wedematiens. See Clostridium

œdematis maligni. See Clostridium septicum

œedematis maligni II. See Clostridium odematiens

cedematis sporogenes. See Clostridium bifermentans

cedematoides. See Clostridium

onarimon. See Salmonella

onderstepoort. See Salmonella

oranicnburg. See Salmonella

oregon. See Salmonella

oslo. See Salmonella

ovisepticus. See Pasteurella

ozogenes, 495

panama. Sce Salmonella

para-abortus. See Brucella

paracholeræ. See Vibrio

para-influenzæ. See Hærmophilu.

paramelitensis. See Brucella

parapertussis. See Hrmophilus

paraputrificus. See Clostridium

para-Shiga. See Shigella

parasporogenes. See Clostridium

parasuis. See Brucella

paratyphosum. See Salmonella

pasteurianus. See Lactobacillus and Clostridium

pensacola. See Salmonetia

pentoaceticus. See Lactobacillus 
Bacillus perfringens. See Clostridium welchii pertussis. See Hæmophilus

pestis. See Pasteurella

pestis caviæ. See Salmonella typhimurium

phlegmonis emphysematosæ. See Clostridium welchii

phlei I and II. See Mycobacterium

phosphorescens. See Vibrio

plantarum. See Lactobacillus

pneumonix. See Bacterium, Friedländer group of

pneumoniæ caviarum. See Hæmophilus bronchisepticus

pneumosintes. See Bacterium

pomona. See Salmonella

poona. See Salmonella

porci. See Erysipelothrix rhusiopathit,

potsdam. See Salmonella

pretoria. See Salmonella

prodigiosus. See Chromobacterium

protens vulgaris. See Proteus

pseudomallei. See Pfeifferella whitmori

pseudopneumonicus, 667

pseudotuberculosis. See Pasteurella,

Corynebacterium murium, and Corynebacterium ovis

psittacosis. See Salmonella typhi-murium pueris. See Salmonella

puerto-rico. See Salmonella newport

pullorum. See Salmonella

pulmonum glutinosus. See II imophitus bronchisepticus

purifaciens. See Bacterium

putrificus. See Clostridium

pyocyaneus. See Pseudomonas

pyogenes. See Corynebacterium

pyosepticus equi. See Bnclerium

radicicola. See Rhizobium

ramosus. See Fusiformis

reading. See Salmonella

renalis bovis. See Corynebacterium renal rhinoscleromatis, 667 et seq., 1789

rhusiopathia. Sce Erysipelothrix

rostoek. See Salmonella

rubefaciens. See Chromobacterium

ruber. See Chromobacterium

rubislaw. See Salmonella

rubricus. See Chromobacterium

saccharobutyricus imuobilis. See Clos. tridium welchii

saint-paul. See Salmonella

salinatis. See Salmonella

san-diego. Sce Salmonella

sanguinaritum. See Salmonella gallinarum

schleissheim. See Salmonella

schwartzengrund. See Salmonella

selandia, See Salmonella

sendai. See Salmonella

senftenberg. See Salmonella

septique. See Clostridium septicum

serpens. See Fusiformis

sliangani. See Salmonella

shigr. See Shigella

simiæ. See Bacterium

simsbury See Salmonella

smegmatis. See Mycobacterium
Bacillus solt. See Salmonella sounei. See Shigellu

sordellii. See Clostridinm bifermentans

sphenoides. See Clostridium

sporogenes. See Clostridium

stanley. See Salmonella

stercoris. See Mycobacterium

storrs. See Salmonella typhi-murium

suipestifer. See Salmonella choleræ-su is

suis. See Brucella

suisepticus. See Pasteurella

sundsvall. See Salmonella

szentes. See Salmonella

taksony. See Salmonella

tcl-aviv. See Salmonellu.

temnessee. See Salmonella

tertius. See Clostridium

tetani. See Clostridium

tetanomorphus. See Clostridium

thermosaccharolyticum. See Clostridium.

thompson. See Salmonella

tim. See Salmonella newington

tuberculosis. See Mycobacterium

tularensis. See Brucella

trphi. See Salmonetla

typhi-exanthematici. See Corynebacterium typhi

typhi-flavus. See Chromobacterium

typhi-murimm. See Salmonella

trphi-suis. See Salmonella

typhosus. See Salmonella

uganda. See Salmonella

urbana. See Salmonella

vagine enphysematosæ. See Clostridium welchii

vaginalis. See Döderlein's bacillus

vejle. See Salmonella

vinelandii. See Azotobacter

violaceus. See Chromolacterium

virchow, See Salmonella

virginia. See Salmonella

viseosus equi. Sce Bacterium

vitreus. See Azotobacter

vitulisepticus. See Pasteurclla.

voldagsen. See Salmonellutyphi-suis

vulgaris. See Proteus

wehmeri, 756

welehii. See Clostridium

weltevreden. See Salmonella

wesenbergi. See Bacterium

whitmori. Ste Pfeifferella

wichita. See Sulmonella

winogradskyi. See Nitrobacter

woodstowni. See Azotobacter

worthington. See Salmonella

xerosis. See Corynebacterium

zagreb. See Salmonella

zanzibar. See Salmonella

zenkeri. See Proteus

zopfii. See Zopfius

Bacillus, 838-855

characters of, $838-842$

classification of, 841

thermophilic speeies of, 838

albolectus, 855

anthracis, 838 et seq., 842-847

antigenic structure of, 281.844 
Bacillus anthracis, $\beta$-lysins, effect of on, 1173 pathogenicity of, $844,1730 \mathrm{et}$ seq. smooth and rough forms of, 301 , 843

tabulated differentiation of from pseudoanthrax bacilli, 846

variants of, 842,845

anthracoides, $838,846,847$

aterrimus, 850

ccreus, 855

cohorens, 855

fusiformis, 855

globigii, 850

graveolens, 855

megatherium, 839 et seq., 852

mesentericus, 851

mesentericus niger, 850

mesentericus panis viscosi, 850

mesentericus ruber, $\$ 50$

mycoides. 853

panis, 850

petasites, 855

polymyxa, 855

pseudoanthracis, 838 et seq., 846, 847

rotans, 85.5

subtilis, 838 et seq., 849

terminalis, 855

tumescens, 855

vulgatus, 850

Bacteræmia, 1490

after operative procedure, 1515

oral sepsis associated with, 1515

suppression of in immune animal, 1047

in normal animal, 1037

Bacteria, aggressive action of, 1068-1074 antigenic structure of, 273-285. See also

Antigenic structure of bacteria

arrangement of, 35

autotrophic, 61

cell wall of, 27

chemical constitution of, 42-45

classification of, $310-323$

epidemic strains of, 1260,1471

examination of, methods used in systema. tic, 364-372

gaseous requirements of, 69

beterotrophic, 61

identification of, 357

importance of for life, 1989

infectivity of in relation to virulence, 1261-1263

intestinal, as food-stuffs, 1991

life-cycle of, 34

metabolism of, 45-74

modes of division of, $33-36$

in rough variants, 300

morphology of, 16-39

nomenclature of, $310-323$

nutritional requirements of, 61

reproduction of, $33,80-99$

respiration of in relation to growth, 85

size of, $16,84,358$

staining of, 37

temperature requirements of, 360

variation of, 288-307. See Variation of

bacteria

virulence of. See Virulence of bacteria
Bactericidal action of normal and immune serum, 192

Bactericidal substances, use of in obtaining pure cultures, 354

Bactericidins, 228. See also Antigen-antibody reactions natural, 1086

Bactéridie, 1730

Bacteridium. See Bacillus anthracis aurantiacum, 626

Bacteriolysis, 192, 228. See also Antigenantibody reactions

Bacteriophage, 325-347 absorption of, specific, by bacteria, 334 antigenie property of, 341

antiphage sera, 342

autolytic theory of, 328

bacterial precursor of, 337

carrier strains among bacteria, 345

different types of, $3+0$

classification of, 343

differentiation of by testing against resistant bacteria, 340

by various methods, 343

discontinuous multiplication of, 333

ecology of, 345

enzyme theory of, $32 \mathrm{~s}$

genetic theory of, 328

growth of bacteria in relation to, 327

luabitat of, 327,345

identification of bacteria by, 338

in relation to Flexner bacilli, 693

killing of bacteria by, without lysis, 334

lysogenic strains of bacteria, 346

lytic action of, 327

calcium in relation to, 333

citrate in relation to, 333

inhibition of by bacterial antigens and haptens, 339

mechanism of, 333

methods of demonstrating, 326

microscopical observation of, 336

quantitative study of, 333

metabolism of, 332

multiplicity of, 328

nature of, 327

plaques formed by, 326

different types of, 343

size of in relation to size of bacteriophage, 344

physical state of, 329

production and bacterial growth, 337

resistance to various agents, 332

resistant strains of bacteria in relatiom to. 340

rôle of in infection and immunity, 1215

size of, 330

specificity of, 338

relation of to antigenic structure of bacteria, 338

spores, bacterial, in relation to, $3+7$

symbiotic with bacteria, 346

variation of, 345

variation of bacteria, as cause of, 305 , 340

virus theory of, 327

Bacteriostasis, 159 
Bacteriotropins, 194, 235. See also Antigenantibody reactions

Bacterium, 654-681

biochemical activities of, 659-666

elassification of, 654

('ultural appearances of, 657

dyes, etc., action of on, 659, 705, 15271528

morphology of, 655

nomenclature of, 654

resistance of, 658,2027

Salmonella antigens in, 715,744

acidi-laetici, $665-666,679$

verogenes, 655 et seq.. 679

differentiation of from Bact. coli and intermediate group, 660 665. 2023 2024

alkaligenes, 902

asiaticum, 677

asophile, 499

cloncr, $66 \mathrm{I}$ et seq.. 679

eoli, 655 et seq., 678

antigenic structure of, 666

differentiation of from Bact. aerogenes and intermediate group, 660-66.5, 2023-2024

cnzyme systems of, 59 et seq.

fermentative types of, 666,2024

habitat of, $660,66 \mathrm{I}, 663$

hæmolytic and non-hæmolytic types of, 1499

in food poisoning, 1606

in pyogenic infections, 1497-1503

in wound infection, 1501

lysogenic strain of, 346

pathogenicity of, 666

summarized description of, 678

toxins of, 667,1606

coli-aerogenes group of, 660-667

in water, 2023-2024

coli-anacrogenes, 677

coli-commune, 660-661,665, 666, 679

coli-communius, $661,665,666,679$

coli-mutabile, 297, 677

columbense, 678

coscoroba, 665-666

dysentery group of. See Shigella

equirule, 676

frecalis alkaligenes, 902

Friedländer group of, 667-676, 680

broncho-pneumonia in relation to, 1667

capsule of, chemieal emstitution of, 669

classification of, 675

pathogenicity of, 674

suicile colonies of, 670

giumai, 678

granulosis, 901, 187s

grünthal, 665-666

immobile, 665-666

intermediate group of coliform bacilli, $663-667,679,2023-2024$

khartoumense, 678

lactis aerogenes. See Bact, aerogenes monoeytogenes. See Erysipelothrix morgani. See Proteus
Bacterium. mucosus capsulatus, 667. Spe

Bacterium, Friedländer group of neapolitanum, $665,666,679$

nephritidis equi, 676

огæ⿱ $x, 670,673,1789$

paracolon group of, 676,681

pneumosintes, 899

purifaciens, 1499

pyosepticum equi, 676

ritinoscleromatis, $670,673,1789$

simix, 902

triloculare, 65.5

tularense. See Brucella

typhi-flavum. See Chromolactorium.

viscosum equi, 676

wesenbergi. 678

Bacteroides, 477, 751, 75.5 funduliformis, 479

Bail's hypothesis of limiting population density, 94

Balantidium coli, dysentery caused by, 1561

Bang's abortion bacillus. See Brucella abortus

Bang's necrosis bacillus. See Fusiformis necrophorus

Barbone of buffalocs, 1647

Burtonella, 903

diseases due to, 1796-1798

bacilliformis, 903

in Oroya fever, 1797

canis, 904

in infectious anxmia of dogs, 1798

muris, 903,904

in infectious anæmia of rats, 1797

Bats, in relation to rabies, 1935

Benzene, effect of on immunity, 1209

Beta lysins, 1173

rays, effect of on bacteria, 110

$\beta$ alanine as bacterial vitamin, 67,164

Bile, differential effect of on growth of streptococci, 572

Bile-solubility of pneumococcus, 571

Biochemical reactions of bacteria, methods of examination for, 367-369 variation in, 292

Bios hypothesis of lag, 84

Biotin as bacterial vitamin, 69

Bitter's medium for fermentation test, 709

Black disease, 882,1780

Black rot of eggs, 647

Blackleg, 884,1778

Blackquarter, 1778

Bleeding, effect of on antibody formation, 1122

Blennorrhoe, inclusion, 1880

Blockade, effect of on antibody formation, 1103

Blood, anaphylactic reaction in rabbits, 1149 , 1151

bacteria normally present in, 1998

bactericidal power of, non-specific induction of inerease in, 1175

Blood capillaries; permeability to foreign matter, 1042

cerebral. permeability to foreign matter, 1060, 1754, 1764

Blood culture. See under individual diseases groups, hnman, 1087

in relation to Schick reaction, 1079

Rh factor in, 1088 
Blood stream, removal of bacteria from, 1037

1047. See also Immunity, antibacterial

Blue-tongue of sheep, 1967

Boas-Oppler bacillus, 750, 757, 764

Bollinger's granules, 1492

inclusion bodies, 959, 1896

Bone taint, 873

Bones and joints, suppurative lesions in, 1496

Boosting dose. See Secondary stimulus

Bordet-Gengou bacillus. See IIæmophitus per. tussis

Bordet's theory of antigen-antibody reactions. 199

Borna disease, 1921

Bornholm disease, 1960

Borrel bodies, 959, 1896

Borrelomycetales, 940

Botriomycosis, 1270, 1271, 1492

Botulism, 1612-1623

diagnosis of, 1617

distribution of botulinum in nature, 1615

epidemiology of, 1613

factors influencing development of $\mathrm{Cl}$. botu-

linum in canned foods, 1616

in animals, 1619-1623

mode of infection of food in, 1615

prophylaxis and treatment of, 1618-1619

type of food causing. 1614

Boyd's dysentery bacilli, 685 et seq.

Bradsot. See Braxy

Branching forms of bacilli, 33

Braxy, 1779

German type of, 1780

meadow type of, 1780

Northern type of, 1779

stall type of, 1780

Brazilian spotted fever, 1847

Breast-feeding in relation to infantile diarrhoa, 1582,1586

Brilliant green, in cultures from enteric fever, 1527,1528

Brill's disease, 1811, 1842

Broucho-pneumonia, 1666 secondary. 1666-1668 virus, 1876

Brucella, 814-834

antigenic structure of, 822

band phenomenon in agar-shake cultures of, 819

biochemical properties of, 821

carbon dioxide requirements of, 818

chemical fractionation of, 825

classification and identification of, 830 tabulated, 831

crystal formation in cultures of, 817

cultivation of in presence of dyes, 820 , 1714

cultural reactions of, 816

differentiation of, on Petragnani's egg medium, 817

growth of in sealed tubes, 819

growth requirements of, 818

$\mathrm{H}_{2} \mathrm{~S}$ production by, 822

infections, 1692-1722. See also Undulant fever, Contagious abortion in cattle, and Tularaemia
Brucella, infections of cattle, 1708

of cats, 1719

of dogs, 1719

of fowls, 1719

of goats, 1695, 1697, 1706

of horses, 1718

of $\operatorname{man}, 1692$

of rats, 1720

of sheep, 1697, 1706

of swine, 1702,1717

in Hodgkin's disease, 1981

morphology of, 816

nomenclature of, 815

pathogenicity of, 826

relation of to Pasteurella. 825

to Pfeifferella, 490, 825

to Proteus, $8: 5$

resistance of, 821

staining of, 816

variation of, 829

abortus, 814 et seq., 832

institutional outbreaks due to, 1701

isolation of from milk, 1713

pathogenicity of for small animals, 829

undulant fever due to, 1697

bronchiseptica. See IIæmophitus bronchisepticus

melitensis, 814 et seq., 831

infection of cattle. $1697,1710,1711$

pathogenicity of for small auimals, 828

undulant fever due to, 1693

para-abortus, 815, 823

paramelitensis, $815,822,823$

parasuis, 815,823

suis, 814 et seq., 833

infection of cattle, 1710, 1711

pathogenicity of for small animals, 829

undulant fever due to, 1702

tularensis, 814, 815, 833

disease in man due to, 1720

relation of to Pasteurella, 815

Brucellergin, 1705

Brucellin test in diagnosis of contagious abortion in cattle, 1713

of undulant fever, 1705

Bubo, climatic, 1869

Buffaloes, osteomyelitis of, 1781

Bulbar paralysis in cattle, 1621

due to pseudorabies virus, 1941

Bullis fever, 1846

Bumble-foot of chickens. 1349

Butter bacillus. See Mycobacterium butyricum

in relation to undulant fever due to $\mathrm{Br}$. abortus, 1701

Butyribacterium, 756

Calcium as kataphylactic agent, 1206, 1750, 1773

effect of on spore formation, 291

inhibition of pigment formation by, 635

relation of to bacteriophage activity, 333

rôle of in tetanus infection, 1206, 1750

Calculi salivary, Actinomyces in, 1272 
Calf diphtheria, 479,1787

Calmette-Guérin's bacillus (B.C.G.), 1306 , $1331,1337,1347$

Calves. See Cattle

Canary-pox, 953, 1896

Canine typhus of Lukes, 1837

Capillaries, cerebral, permeability of, 1060 , 1754,1764

per'meability of, 1042

Capsules, bacterial, 29

aggressive action of bacteria, in relation to, 1072

hyaluronic acid in, 1072

in relation to virulence, $300,575,842$, 1072

Carbohydrate constituents of bacteria, 44 metabolism of bacteria, $\tilde{l} 1$

Carbohydrates, action of bacteria on, 52-55 protein-sparing effect of in bacterial metabolism, 57

synthesis of, by bacteria, 55

Carbon dioxide, as essential nutrient for bacteria, 61,69

use of in blood culture, 1491, 1703

Carbon dioxide : hydrogen ratio, in differentiation of coli-ærogenes group, 661

in growth of Brucella, 818

rôle of in bacterial giowth, 69

Carboxydomonas oligocarbophila, 505

Carrier epidemic, 1251. See also Carriers under individual diseases

Carrier types of pnemmococci, 1673

Carriers, bacterial. See under individual diseases herd infection in relation to, 1246 in experimental epidemics, 1253

Catalase, methods of examination for, 369 protective action of in relation to bydrogen peroxide, 72

Cataphoresis of bacteria, 213 of filtrable viruses, 950

of serum proteins, 242, 246, 248, 1030, 1092,1385

Catarrh, malignant, of cattle, 1966

Cathode rays, effect of on bacteria, 108

Cations, effect of on bacteria, 123

Cats, Brucella infection in, 1719

distemper in, 1877

enteritis in, 1963

tuberculosis in, 427, 429, 1343

Cattle, actinomycosis in, 1271, 1276 anaerobic infections of. See Anaerobic infections of animals

autumn diarrhœa in, 1720

bacillary hæmoglobimuria of, 891

blackleg in, $884,885,1778$

botriomycosis in. 1492

botulism in, 1621

Brueella infections of, 1708-1716

with $\mathrm{Br}$. abortus. See Abortion, contagious, in cattle

with $\mathrm{Br}$. melitensis, 1697, 1710, 171]

with $\mathrm{Br}$. suis, 1710, 1711

bulbar paralysis of, 1621

cow-pox of, 1888

diphtheria in, 1390

diphtheria of calves due to $F$. necro. phorus, 1787
Cattle, diphtheroid infections of $458-462$, 1403

dry bible of, 1621

ephemeral fever of, 1966

epizootic pneumonia of calves, 391, 1281

farcy of, 387,1276

ictero-hæunoglobinuria of, 1621

impaction paralysis of, 1621

influenzal pnemmonia of calves, 1662. See also Scours

Johne's disease of, 441, 1363

lamziekte of, 880,1621

malignant catarrh of, 1966

mastitis in, 1493. See Mastitis in cattle

Midland disease of, 1621

plague, 1965

pleuropneumonia of, $939,1647,1867$

pneumonia of calves due to Actino-

bacillus actinoides, 1281

pyelitis in, 461

pyelonephritis in, 1500

red-water disease of, 891

Salmonella infections of, 1600

scours in, 527, 667, 1662. Sec also [n-

fluenzal pncumonia of calves

tuberculosis in, $1317, \mathrm{3} 342-1348$

Cellobiose, in differentiation of coli-rerogenes group, $66 \pm$

Cellular hypothesis of anaphylactic shock, 1145 immunity, rôle of in virus infections, 1:34 reactions in bacterial infection, 1007

Cellulitis, 1493 anaerobic, 1771

Centrifugation, factors affecting, $95:$

Centrifuges, types of, 953

Cerebrospinal meningitis. See Meningitis

Chancre, luard, 1812 et seq. soft, 1790

Cheese in relation to typhoid fever, 1542 to undulant fever, 1701

Chemical changes produced by bacteria, 51 et seq.

Chemical constitution of bacteria, 42

Chemistry of bacterial antigens. See under individual organisms

Chemotaxis in pyogenic infections, 1007

Clemotherapeutic activity, acidic or basic groups, 170

and relation to chemical structure, 156 , 169

in the sulphonamide series, 167

as interference with essential metabolites, 162

surface activity and chain length, 170, 171

Chemotherapeutic agents, acquired resistance of micro-organisms to, $156,160,172,18 \mathrm{I}$

antagonizer-inhibitor systems, 159, 161

nicotinamide and sulphathiazole, 165

nicotinic acid and pyridine sulphonamide, 163

pantothenic acid and pantoyltaurine, 164

$p$-aminobenzoic acid and the sulphonamides, 161

thiamin and pyrithiamin, 164

antagonizers of, $159,161,165$

as inhibitors of bacterial growth, 159 
Chemotherapeutic agents, cross-resistance of micro-organisms to, 161,172 gramicidin, 174

penicillin, 179

sulphonamide compounds, 157

sulphone compounds, 171

Chesney's hypothesis of lag, 84

Chicken-pox, 1901

Chickens. See Fowls

Chick-Martin method of standardizing disin. fectants, 147

Chicks, bacillary white diarrhœa in, 1555

Chlamydozoa, 949

Chlorine, effect of on bacteria, 128, 149, 2030

Cholecystitis. 1497. See also Gall-bladder, infection of

Cholera, 1418-1428. See also Vibrio cholera diagnosis of, 1424

Pfeiffer's reaction in, 1425

epidemiology of, 1421

examination of suspected convalescents from, 1426

experimental reproduction of, 522,1420

healthy carriers in, 1424

laboratory infections in, 1420

mode of spread in, 1422

of fowls, 1646

prophylaxis and treatment of, 1426

by bacteriophage, 1427

by chemotherapy, 1428

by serum therapy, 1428

by vaccination, 1426

Cholera-red reaction, 516

Choriomeningitis, lymphocytic, 1932 psendo-lymphocytic, 1932, 1934 swineherds' disease, 1932, 1934

Chromobactcrium, 631-640

group forming pink or red pigment, 635 forming violet pigment, 634

forming yellow or orange pigment, 637

pigment formation by, 633 et $s e q$.

amethystium, 635

aquatile, 631, 637

aurantiacum, 639

cœruleum, 635

denitrificans, 639

fuscum, 639

indicum, 635, 637

janthinum, 635

kielense, 635, 637

lactis erythrogenes, 635

mycoides roseum, 635

ochraceum, 639

prodigiosum, 631 et seq., 636

rubefaciens, 635

ruber, 635

rubricum, 635

typhi-flavum, 637, 638

violaceum, 631 et seq., 634

Chromogenesis. See Pigment formation

Circulation of blood, removal of hacteria from, 1037, 1047

Citrate, relation of to bacteriophage activity, 333

in differentiation of coli-ærogenes group. 663
Citrinin, 177

Cladothricosis, 1270

Cladothrix asteroides. See Actinomyces

Classification of bacteria, 310-323

antigenic structure in relation to, 314

criteria of, 311

nomenclature in, 313

Clavacin, 177

Clavatin, 177

Clavelée, 1895

Claviformin, 177

Climatic bubo. See Lymphogranuloma inguinale

\section{Clostridium, 858-893}

antigenic structure of, 864

biochemical reactions of, 863

classification of, 871

tabulated, 872, 874-875

cultural reactions of, 860

hæmolysin production by, 863

in appendicitis, 1497,1777

infections duc to, 1746-1783

metabolism of, 862

morphology of, 859

pathogenicity of, 869

resistance of, 861

thermophilic species of, 872

toxin formation by, 865-869

aerofotidum, 872, 890

acetobutylicum, 162, 872

bifermentans, 890, 1771-1772

botulinum, 858 et seq., 879

in relation to botulism in animals, 1619

in $\operatorname{man}, 1613$

pathogenicity of for laboratory animals, 869

toxin of, 866

butyricum, 858 et seq., 873

capitovale, 874,890

caloritolerans, 890

carnis, 862, 874, 890

chauvœi, 863 et seq., 852, 1778 toxin of, 869

cochlcarium, 861, 863, 872, 874, 891

difficile, 874, 891, 1987

fallax, 861, 872, 874, 891

gigas, 881, 1781

hæmolyticum, 891

hastiforme, 874, 891

histolyticum, 858 et seq.. 877, 1771

multifermentans tenalbum, 872.892

novyi 1 . See C'l. odematiens

oedematiens, 861 ct seq., S81, 1771-1777, 1780

pathogenicity of for laboratory animals, 87

toxin of, 868

cedematoides, 890

parabotulinum, 879

in relation to botulism in animals, 1620

paraputrificum, 892

parasporogenes, 871, 872, 892

pasteurianum. Sce Cl. butyricum

putrificum, 892 
Clostrilium septicum, 860 et seq.,884, 1771, 1778 1780

pathogenicity of for laboratory animals, 871

toxin of, 866

septique. See Cl. septicum

sordellii. See Cl. bifermentans

sphenoides, 872, 874, 892

sporogenes, 858 et seq., 876. 1771

tertium, 862, 892

tetani, 858 et seq., 888, 1746-1767

distribution of in nature. 17.48

pathogenicity of for laboratory animals, 870

toxin of, 865

tetanomorphum, 872, 874, 893

thermosaccharolyticum, 872

welchii, 858 et seq., 886

hyaluronidase of, 868,1013

in suppurative lesions of the urinary tract, 1500

in wound infection, 1501

infections due to, $1750,1771,1781$

infections in sheep, due to, 1781 . See also Sheep

lecithinase of, 867,1774

pathogenicity of for laboratory animals, 870

toxins of, 866

Coagulase, in bacterial infections, 1012

Cocco-bacillus fœetidus-ozænæ, 494, 495

Co-enzymes, rôle of in bacterial metabolism, 49

Cold, effect of on bacteria, 112

the common, 1653

vaccination against, 1654

Coli-ærogenes group, 660-667. See also Bacterium

dilferential count of, 2023

intermediate group of, 663,2024

Coliform bacteria, differential count of, 2023

Collodion filters, 951

Collodial reactions, in relation to antigenantibody reactions, $199, \mathbf{2 0 7}$

Colonies, bacterial, 36 description of, 359,364

Colony form, variation in, 299

Colorado tick fever, 1846

Colostrum, transference of antibodies in, 1077 , 1092,1782

Comma bacillus. See Vibrio choleræ

Common cold, the. See Cold, the common

Complement, 193, 196

analysis of guinea-pig, 230

analysis of human, 231

components of in relation to opsonins, 237

end-piece of, 229

fourth component of, 230

mid-piece of, 229

nature of, 229

opsonins in relation to, 235

relation to vitamin intake, 231

rôle of components in fixation, 233

rôle of in hæmolysis, 225

splitting of, 229

symbols for the components, 230

third component of, 230

unity or diversity of, 229
Complement fixation, 193, 232. See also Antigen-antibody reactions and under intividual discases

Complement-fixing antibodies. See under Antigen-antibody reactions natural, 1086

Complementophilic group, 198

Concentration exponent $n$ of disinfectants, 140

Conglutination test in diagnosis of glanders, 1414

Conjunctiva, normal flora of, 1998

Conjunctival sac, lemoval of bacteria from, 1025

Conjunctivitis, 1496 ancular, 898

follicular in monkeys, 1878

swimming bath, 1880

Constant-antibody optimal ratio, 202

Constant-antigen optimal ratio, 202

Contagious abortion in cattle. See Abortion, contagious in cattle

Contagious pustular dermatitis of sheep, 1897

Correlated variations in bacteria, 290

Corynebacterium, 447-473

antigenic structure of, 454

classification of, 465

diseases caused by, 1403. See also Diphtheria

fermentation reactions of, 452 table of, 473

morphology of, 448

pathogenicity of, 455

phosphatase production by, 453

strains of, intermediate between $C$. diphtheriæe and $C$.ovis, 459

toxin production by, 455

acnes, 462, 471, 1403, 1492

arthritidis muris, 462

coryzæ segmentosum, 450

diphtheriæ, 447 et scq.

distribution of in tissues, 1369

gravis type of, 449, 450, 462, 1370

in relation to diphtheria in man, 1370

growth requirements of, 452

hæmolysis produced by, 453

in milk, 1391, 2039

in wound inlection, 1502

inhibition of, by heated blood, 451, 452

intermedius type of, 449, 451, 462, 1370

in relation to diphtheria in man, 1370

lesions produced by in guinca-pigs, 457

lysogenic strains of, 346

mitis type of, $449,450.462,1370$

ill relation to diphtheria in man, 1370

morphology of, 448

pathogenicity of, 455

resistance of, $45 \mathrm{l}$

summarized description of, 465

tabular differcntiation of types of, 463-464 
Corynebacterium diphtherix, tellurite medium for growth of, 450-451

toxin of, 455

action of on adrenals, 458 on heart muscle, 458

gravis, mitis and intermedius types in relation to, 1375

M.L.D. of, 238,1384

virulence of in relation to toxigenicity, 1372

virulence tests on, 1375

virulent and avirulent strains of, 458,1375

equi, 452, 460, 469

hofmanni, 450, 453, 466, 473

murium, 450, 461, 470, 473, 1404

ovis, 450, 451, 453, 459, 460, 468, 478, 1403,1649

diseases due to, 1403

relation of to $C$. pyogenes, 460

toxin of, 459

pseudotuberculosis murium. See $C$. murium

pseudotuberculosis ovis. See $C$. ovis

pyogenes, 45l, 453, 460, 469, 473

diseases due to, $1403,1494,1496,1500$

in arthritis of animals, 1496

in mastitis, 1494

in pyelonephritis of cattle, 1500

relation of to $C$. ovis, 460

renale, $458,465,470,473$

typhi, 462, 472

xerosis, 447 et seq., 467, 473

Cough plate in diagnosis of whooping cough, 1663

Counting of bacteria, 80-82

technique of total count, 80

centrifugal method, 81

Eberle's method, 80

Helber chamber method, s1

opacity method, 81

Wright's method, 80

technique of viable count, 81

dilution method, 81

plating method, 82

roll-tube method, 82

surface plate method, 82

Cow. See Cattle

Cow-pox, 1888

Cresols, effect of on bacteria, 133

Cristispira, balbianii, 908

Cross-infection of wounds, 1502

Crystals, mixed, formation of as analogy to antigen-antibody reactions, 261

Cultural reactions, in identification of bacteria, 359

of bacteria, terms used in description of, $364-366,369$

Culture media. See under name of medium

Cultures, pure, methods of obtaining, 351-357

by aerobic and anaerobie incubation, 354

by agglutinating serum, 355

by dilution, 351

by filtration, 355

by heating, 354

by indicator media, 356
Cultures, pure, methods of obtaining, by Koch's plating method, 352 by motility, 353

hy optimum temperature, 354

by pathogenicity, 356

by selective bactericidal substances, 354

by selective media, 355

by shake-tube methor, 353

by single-cell methods, 357

by Veillon tube, 353

Cystitis, 1499

Cytochrome-oxidase, rôle of in bacterial oxida. tions, 48, 60

Cytochrome systems of bacteria and anaero. biosis, 71

Cytophilic group, 198, 225

Dale technique in study of anaphylactic shock, 1146

Danysz phenomenon, 216,240

Danysz's bacillus. See Salmonella enteritidis

Dark-ground illumination, 18 in study of filtrable viruses, 952

Decline phase of bacterial growth, 83, 97

Deer-fly fever. See Tularæmia

Dehydrogenases, bacterial, 47, 52, 59 inactivation of, 59

Deneke's vibrio, 523, 525

Dengue fever, 1958

Dental caries, 754

Dermacentroxenus rickettsi. See Rickettsia rickettsi

Dermatitis, contagions of sheep, 1897

Descriptive terms for bacteria, 36t-366, 369372

Desiccation, effect of on bacteria, 111 on filtrable viruses, 961

for preserving stock cultures, 112

Desoxycholate, for lysing pneumococci, 571 Leifson's medium, 659, 1528

Deuteroalbumose, effect of on antibody formation, 1123

" Diamonds," 1283

Diaphragm, action of in intraperitoneal infections, 1040

Diaplyte vaccine, 1337

Diarrhœa borne by milk, 2049 summer, 1580, 1585, 2049. See also Enteritis of infancy white bacillary of chicks, 1555

Diastase in serum, 453

Diazo reaction in preparation of synthetic antigens, 253

Dick test, 1465,1467 age groups in relation to, 1078, 1080 native populations, in, 1081 puerperal fever in relation to, 1481

Diet as determining intestinal flora, 1990 effect of on immunity, 1190-1202

Dieudonné's medium, 515, 1424

Dilution method of counting, 81 coliform bacilli in water, 2022 use of in obtaining pure cultures, 351

Diminishing returns, law of in antibody production, 1113 
Diphtheria, 1368-1403

antitoxin, avidity of, 1030,1385

concentrated, 1379

in blood of normal horses, 1081 of normal persons, $1075-1085$

in vivo/in vitro ratio of, 1385

refined, 1379

standardization of, 1383

bacillus. See Corynebacterium

borne by milk, 1390, 2039, 2049

carrier : case ratio in, 1249

carriers in, 1248

a virulent strains in, 1083

effect of active immunization on, I25I

rate of clearing of. 1387

treatment of, 1386

diagnosis of, 1373

different grades of immunity to, 1250

epidemiology of, 1246-1251, 1386

experimental production of, in Schick-

positive reactor's, 1085

gravis, intermedius and mitis types of

$C$. diphtherix in relation to 1370,1382

immunization against.

active, 1249, 1391-1400

active and passive, 1401

effect of on carrier rate, 1399

passive, 1401

results of, 1398

in domestic animals, 1390, 1403

in pre-school children, 1399

in schools, 1248-125l

institutional epidemics of, control of, I 101

Moloney reaction in relation to, 1393, 1396

natural immunization against, 1246-1251

pathogenesis of, 1369

prophylactics against, 1391

standardization of, 1402

prophylaxis in, 1391-1403

Schick test in relation to, 1248, 1376, 1393-139s

spread of, by animals, 1390

by dust, 1389

by fomites, 1389

by milk, 1390,2049

susceptibility to, measurement of, 1376

toxin in relation to type of bacillus, 1372

Lf, Lo, L+, and Lr dose of, 1384

M.I.D. of, 238,1384

M.P.D. of, 1384

Schick dose of, 1377, 1384

treatment of with antitoxin, 1378

virulence tests in, 1375

Diphtheroid bacilli. See Coryncliacterium

in animal diseases, 1403

in liuman disease, 1403

Diplococcus crassus, 531, 532, 553

intracellularis meningitidis. See Neisseria meningitidis

mucosus, 553

pharyngis flavus. See Neisserin

pneumoniæ. See Streptococcus pneumoniæ

Disinfectants concentration exponent $n$ of, 142

emulsified, 133

gaseous, 149

liquid, 149
Disinfectants, practical application of, $\mathbf{1 4 9}$ solid, 150

standardization of, 145

temperature coefficient of, 143

Disinfection, 101-150

by acids, 114,118

by alcohols and ethers, 132

by alkalies, 114, 120

by cathode rays, 108

by chloroxylemols, 134

by cold, 112

by desiccation, 111

by distilled water, 117

by dry heat, 112

by dyes, 134

by electricity, 107

by electromagnetic waves, 103

by essential oils, 136

by flaming, 113

by gases, 149

by heavy metals, 121

by moist heat, 113

by mould products, 172

by phenols and cresols, 133

by photodynamic sensitization, 106

by radium, 109

by Röntgen rays, 108

by salts, 120 et seq.

by soaps and synthetic detergents, 130

by solid disinfectants, 150

by sonic and supersonic waves, 110

by steam, 114

by sulphonamides, 157

by sunlight, 102, 104

by ultra-violet light, 102

dynamics of, 137

effect of concentration of disinfectant on, 140

of hydrogen-ion concentration on, 114, 118

of lipoid content of bacteria on, 149

of organic matter on, 134, 135

of proteins on, 149

of temperature on, 1.43

of variations in bacterial resistance on, 140

reaction velocity of, 137

Dispora Kaukasica. See Lactobacillus caucasicus

Dissociation, bacterial, 299. See also Variation of bacteria

Distemper of cats, 1877. See also Feline enteritis of dogs, foxes and ferrets, 1961 encephalitis in, 1961

Distilled water, effect of on bacteria, 117

Division of bacteria, different methods of, 33

Döderlein's bacillus, 750 et seq., 757, 1996

Dogs, anaphylactic shock in, 1138

Brucella infection of, 1719

distemper of, 1961

experimental pneumonia of, 1669

in relation to fièvre boutonneuse, 1848

infectious anæmia of, 904,1798

leptospiral jaundice of, 1837

streptococcal lymphadenitis in, $\mathbf{1 4 9 6}$

Stuttgart plague in, 1837

tuberculosis in, 427, 1342. 1343

typhus of Lukes in, 1837 
Donovania granulomatis, 1790

Dormaney of baeteria, 98

Dose-response curve in measurement of toxicity, 995-998

Droplet infection, 1312, 2003, 2006

Droplet nuclei

fate in respiratory tract, 1024

Drug idiosynerasies, 1153

Drug-fast micro-organisms, 156, 160, 180

Drug-fast strains of pyogenie bacteria, 1504

Drusen in aetinomyeosis, $375,1270,1272$

Dry bible of eattle, 1621

Ducks, disease of due to $\mathrm{Cl}$. botulinum, 1622 eggs of in relation to food poisoning, 15981599

keel disease of, 1599

Salmonella infection of, 1598

spirochætosis of, 1809

Ducrey's baeilhus, 791 et seq., 807, 1790

Durand's disease, 1961

Durchseuchung hypothesis of origin of natural antibodies, 1081-1083

Dust infection, 1312, 2003, 2005

Dyes, baeteriostatic effect of on bacteria. 134 , $356,659,1527-1528$

suseeptibility to, of Gram-positive and

Gram-negative baeteria, 134,356 use of in differentiation of Brucelia; 8:0

Dysentery, amœbic, 1561

bacillary, 1561-1576

antiserum. use of in, 1571

definition of unit of, 1575

asylum, 1565

bacteriology of, 1566-1570

bacteriophage in treatment of, 1576

borne by milk, 1565, 2049

carriers of, 1570

chemotherapy of, 1576

diagnosis of, 1571

epidemiology of, 1562

flies in relation to, 1563

laboratory infection in man, 1571

prophylaxis of, 1573

raceination against, 1574

winter, of calves, 527

balantidial, 1561

Dysentery group of bacteria, 685-699. See Shigella

Earthworms in spread of anthrax. 1732

Eetromelia, 1278, 1909

active immunization against, 1258

experimental epidemies of, 1256

Egg, dried, Sulmonella in, 728-743, 1602

Eggs, blaek rot of, 617

for cultivation of viruses, 961

Eh, 50

Salmonella infection of, 1598, 1599

relation of to anaerobic growth of bacteria, 71

Ehrlich's phenomenon in toxin-antitoxin reaction, 239

method of diphtheria antitoxin standardization, 1383

theory of autigen-antibody reactions, 196

Eijkman's test, in differentiation of coliærogenes group, 664, 2023
Ekiri, 1568

El Tor vibrio, 518 et seg., 525

Electric eharge in relation to agglutination, 214,264

to phagocytosis, 235

Electrieity, effect of on bacteria, 107

Electrolytes, effeet of on antigen-antibody reactions, 200

Electromagnetic waves, effect of on bacteria, 103

Electrophoresis. See Cataphoresis

Elementary bodies, 958, 1887

Encephalitis in animals, 1921-1925

Borna disease, 1921

horses, foxes, mice, chickens, and sheep, $1922-1924$

in distemper, 1961

louping-ill, 1925

meningo-encephalitis of rabbjts and guinea-pigs, 1924

Encephalitis in man, 1915-1921

Australian X type. 1918

Bwamba fever, 1920

equine type, 1918

Japanese, summer, or B type, 1917

lethargica, 1915

Russian spring-summer type, 1918

St. Louis type, 1916

secondary type, 1894, 1921

Semliki Forest type, 1920

West Nile type, 1918

Encephalitis post-vaecinal, 1894, 1921

Encephalitozoon cuniculi, 1925

Endo medium, 1528

Endocarditis, bacterial, 1514

in swine erysipelas, 1283

lenta, subaeute, 1515

Endotoxins, 1008

antigens bacterial, relation of to, 1010

changes produced by in blood sugar, 1011

See also Toxins, bacterial

Find-piece of complement, 229

Entamoeba histolytica, dysentery eaused by, 1561

Interic fever, 1519-1555

aetive immunization against, 1550, 1554

antibody response in, 1520

bacteræmia in, 1520

bacteria eausing, 1526

carriers in, 1543

detection of by $\mathrm{Vi}$ test, 1547

gall-bladder infections in relation to, 1522

chemotherapy in, 1555

diagnosis of, 1526-1540

by agglutination reaction, 1529

by blood culture, 1527

by eultivation from faeces, 1527

natural agglutinins in relation to, 1532

previous inoculation in relation to, 1537

effect of general sanitation on, 1548

epiclemiology of, 1540

flies in relation to, 1543

mesenterie glands, infeetion of in, 1522

wilk in relation to, 1542,2049 
Entcric fever, pathogenesis of, 1520-1525 . period of disease in relation to contact infection, 1546

relapses in, $\mathbf{1 5 2 5}$

route of infection in, 1523

shell fish in relation to, 1541,2031

tonsillar infection in, 1524

treatment of with specific antisera, 1554

$\mathrm{Vi}$ antigen in relation to, 1525, 1547

water-borne epidemics of, 1541, 2031

Enteric infections in animals, 1555

Enteritis, feline, 1963 of animals. Sce Enteric infections

Enteritis of infancy, 1580-1590. See also Enteric fever and Food poisoning associated with organisms of doubtful pathogenicity, 1585

with parenteral infection, 1589

due to known pathogenic organisms, 1584 due to toxic substances of bacterial origin, 1588

epidemiology of, 1580

influence of breast-feeding on, 1582

neonatal diarrbœa in, 1583

of protozoal origin, 1588

prophylaxis and treatment of, 1589

summer diarrhœa, 1585

Enterococcus. See Streptococcus, focalis group of

Enterotoxæmia of shecp, 1782

Enterotoxic substances produced by Sal. monella. 1607

Entiqué, 1647

Enzootic hepatitis, 1959

Enzymes, bacterial, acting on Type III pueumococcal polysaccharide, 173,1682

adaptation of, 293

" adaptive," 293

"constitutive," 293

mutation of, 295

nature and integration of, 59

rôle of in bacterial metabolism, $46 \mathrm{ct}$ seq.

site of action of, 59

variation of, 295

Eperythrozoon, 903, 905 coccoidcs, 905

Epidemic strains of bacteria, 1261

Epidemiology, experimental, 1252-1264

carriers and latent infections, 1253

epidemic strains of bacteria, 1261

genetic factors in, 1254

immunizing strains of bacteria, 1262

natural immunization, 1251

selection in, 1254

spatial distribution in, 1263

variation of bacteria in relation to, 1260

Epitoxoids, 239

Epizootic hepato-enteritis of guinea-pigs, 1960 lymphadenitis in guinca-pigs, 1495 pneumonia of calves, 391,1281

Ergophore group, 198

Errors of random sampling, $981 \mathrm{et} \mathrm{seg}$.

Erwinia, definition of, 654,655

Erysipelas, 1479

chemotherapy in, 1485

experimental in rabbit, 1180-1182

local immunity to. $1180-1182$
Erysipelas of swine. See Swine

Erysipeloid, 1285

Erysipelothrix, 395403

rough types of, 396

smooth types of, 396

erysipeloides, 399

monocytogenes, 395 et seq., 401

disease due to, 1286, 1791

muriseptica, 395 et seq., 767, 1286

porci, 399

rhusiopathix, 171, 395 et seq., 400

disease due to, $395,1283-1285$

Erythema arthriticun epidemicum, 1277

Erythema, infective, 386, 1277 nodosum, 1332

Erythrobacillus, 631

Erythrogenic toxin of Streptococcus pyogenes, $591,1465-1469$

Esbach reagent for inhibiting Proteus, 1528

Escherichia. See Bacterium coli

Essential oils, effect of on bacteria, 136

Esthiomène, 1870

Ether extraction, effect of on antigen and antibody, 207

Ethers, effect of on bacteria, 132

Euglobulin. See Globulins

Exanthematic fever of Marseille, 1848

Exhaustion hypothesis in relation to bacterial growth, 94-97

Exotoxins, 1007-1011. See also Toxins, bacterial

Experimental epidcmiology. See Epidemio$\log y$, experimental

Freces, normal flora of, $1987 \mathrm{et} \mathrm{seq}$.

Famine fever. Sce Relapsing fever

Farcy of cattle, 387,1276

Fat metabolism of bacteria, 58

Fatigue. effect of on resistance, 1202

Fats, effect of on antigenic stimulus, 1115 lipins and waxes in bacteria, 45, 409 synthesis of, by bacteria, 59

Febris quintana or wolhynica, 1851

Feline enteritis, 1963

Ferkeltyphus, bacillus of. See Sulmonella typhi-suis

Fermentation, study of by Pasteur, 2 reactions in identification of bacteria, 361

Ferret, distemper in, 1962 influenza in, 1656, 1659, 1660

Fibrinolysin in bacterial infection, 1012 produced by Str. pyogenes, 591

Fibromatosis, infectious, of rabbits, 1979

Field mice or voles in relation to leptospiral infections, 1834-1836

to tsutsugamushi fever, 1849

Fièvre boutonneuse, 1848

Fildes' medium for growth of $H$. influenzæ, 793

Filters, bacterial, 949 collodion, 951 gradocol, 951, 955

Filtrable forms of bacteria, 35, $40 \mathrm{~s}$ viruses. See Viruses, animal

Filtration, factor's affecting, 949 use in obtaining pure cultures, 355,910

Finkler-Prior's vibrio, 5:5

Fixation of bacteria in tissues, 1044, 1045 
Flagella, bacterial, 30

Flagellar antigens. See Antigenic structure of bacteria

Elaming in disinfection, 113

Flavacin, 177

Flavine, disinfection by, 135, 1776

Elavoprotein, rôle of in bacterial oxidation, 50

Eleas, in rtiology of plague, 1628 et seq. in relation to murine typhus fever, 1845

Flexner's bacillus. See Shigella

Flies in relation to anthrax, 1733

to dysentery, 1563

to enteric fever, 1543

to limberneck of chickens, 1622

to summer diarrhoa, 1586

Fluorescin, 508-510

Foals, pyæmia in, 1404

Food poisoning, 1592-1612. See also Botulism chemical, 1592

diagnosis of, 1608

due to spray-dried cgg, 1598, 1602, 1604

to "virus" preparations, 1603

epidemiology of, 1593

general bacteriology of, 1595

"infection" type of, 1596

caused by eggs, 1598, 1599

caused by milk, 1602,2049

mode of infection of food in, 1602

human carriers, 1603

infected animals, 1598-1600, 1602,1603

rats and mice, 1600,1603

Salmonella types in, 1597

symptomatology of, 1592

type of food causing, 1601

investigation of outbreaks of, 1608

prophylaxis of, 1610

"toxin" type of, 1593, 1604

due to staphylococci, 1605

to various organisms, $\mathbf{1 6 0 6}$

Foot-and-mouth disease, 1903-1907. See also

Chapter 55

experimental reproduction of, 1904

immunity in. 1906

mechanical factors in relation to lesions of, 1242

relation of to vesicular exanthema of swine, 1908

Ring-Impfung in, 1907

virus of, 1905

Foot-rot of sheep, 481, 1787

Forage poisoning in horses, 880,1620

Formaldehyde, effect of on bacteria, 143 hypersensitiveness to, 1155

Formol toxoid in diphtheria immunization, 1392

in tetanus immunization, 1765

Forssman antibody, anaphylactic shock in relation to, 1141

antigen and antibody, 1089

antigen in bacteria, 282

Fowls, bacillary white diarrhcea in chicks, 155.5

blue comb of, 1970

botulism of, 1622

Brucella infection of, 1714

bumble-foot of, 1349

cholera of, 1646
Fowls, choleraic disease of, 522

coryza of, 1799

infectious bronchitis of, 1970

infectious laryngo-tracheitis of, 1969

leucrmia of, 1976

limberneck in, 880,1622

Newcastle disease of, 1658, 1969

paralysis of, 1976

plague or pest of, 1968. See also Chapter 55

pox and roup of, 1896, 1970. See also Chapter 55

Rous sarcoma of, 1977

Salmonella infection of, 1599

tuberculosis in, 428, 1343, 1348

Foxes, encephalitis of, 1923

Freezing, effect of on bacteria, 112

Frei test, 1871

Freundlich isotherm, $21 \mathrm{~S}$

Freundlich series, 129

Friedländer's bacillus. See Bacterium, Fried. länder group of

Frogs, red leg disease of, 647

Fumigacill, 177

Fumigatin, 177

Fusiformis, 477-484

group characteristics of, 477

growth requirements of, $47 \mathrm{~S}$

in infections of man, 1497, 1498, 1786

in mouth, 479

fragitis, 477,483

furcosus, 477,484

fusiformis, 477,482

melaninogenicus, 361, 484

necrophorus, 477,479

in calf diphtheria, 481,1787

in foot-rot of sheep, 481,1787

in human infections, 4S1, $17 \mathrm{~S} 6$

in labial necrosis of rabbits, 481, 1788

rumosus, 177,483

serpens, 477,483

Gaertner's bacillus. See Salmonella enteritidis Gall-bladder, infection of, 1497, 152.2

Gall-stones, effect of in promoting infection, 1497

Gamma globulin in measles prophylaxis, 1952

Gaol fever. See Typhus fever, classical

Garnet method of standardizing disinfectants, 146

Gas gangrene, 882, 885, 857, 1770-1783

active immunization against, 1777

chemotherapy of, 1776

hyaluronidase in, 1773, 1774

in animals, 1778

in man, 1770 et seq.

antisera to, 1775

preparation and standardization of, 1776

bacteriology of, 1770

diagnosis of, 1774

mode of development of, 1772

prophylaxis and treatment of, 1775

lecithinase in, 1774

reproduction of in animals, 1772

Gas ratio, in differentiation of coli-ærogenes group, 661 et seq. 
Gaseous disinfectants, 149

requirements of bacteria, 69

Gastro-enteritis. See Enteritis of infancy and Food poisoning

Gastric juice, bactericidal action of, 1021, 1987

Geese, septicæmia of due to Trep. anserinum, 1808

Genera, bacterial, summarized descriptions of, $318-3 \geq 3$

Generation index, 89

Generation time, 89 of different organisms, 92 ?

Genetic factors in anaphylaxis and hypersensitiveness, 1159

in immunity, 973

to Pasteurella and Salmonellu, 974, 975,1254

to tubercle bacillus, 975 , 1298

Genital tract, factors determining normal flora of, $1025,1210,1996$

Genus, bacterial, 316

Gerbilles, plague in, 1637

German measles, 1952

Germicides, practical application of, 149. See also Disinfectants and Disinfection

Giardia lamblia, enteritis due to, 1588

Gigantic acid, 177

"Ginger " paralysis, 1592

Gioddu, 750

Glanders, 1408-1415

and farcy, 1108

control of in horses, 1415

diagnosis of, 1411

epidemiology of in animals, 1408 in man, 1411

experimental reproduction of in animals, 488,491

immunity in, 1414

latent, 1409

mallein test in, 1412

prophylaxis of, 1414

Glandular fever, I791

Glässer's bacillus. See Salmonella typhi-suis

Gliotoxin, 177

Globulins, antiviral antibodies in relation to, 1240

serum, nature of, 242

molecular weight of, 243

Glossary of terms employed in describing bacteria, $364-372,369$

Glycolipoid antigens, 280, 1008

Goats, actinomycosis of due to aerobic Actinomyees, 1276

Brucella infection of, $1695,1697,1706$

contagious agalactia of, 945,1867

tuberculosis in, $424,429,1343$

Gonococcin, 1459

Gonococcus. See Neisseria gonorrhoex

Gonorhoea, 1454-1460. See also Neisseria gonorrhoeæ

chemotherapy in prevention and treatment of, 1459

diagnosis of, 1456

in adults, 1454

in children, 1455

prerention and treatment of, 1459 pyrotherapy in, 1160
Gonorrhœa, reproduction of in man, 1456

Gonotoxin, 549, 1456

Gradocol membranes, 951, 955

Grahamella, 903, 905

Gramicidin, 174

Gram's stain, 38

in identification of hacteria, 359

Granules, Babes-Ernst, 23, 449, 532

Bollinger's, 1492

glycogen and starch, 23

metachromatic, 23,532

Much, 407

Paschen, 959, 1887

volutin, 23

Gramulobacillus butyricus. See Clostridium welchii

Granulobacter saccharobutyricum. See Clostridium butyricum

Granuloma inguinale, 1790

Granuloma venereum, 1790

Gravis type of $C$. diphtheria. See Corynebacterium diphtheriæ

Grigroroff's Bacillus C., 764

Group and specific phases of flagellar $(\mathrm{H})$ antigens, 278

Growth and death of bacteria, 80-99

Growth curve of bacteria, 82

decline phase, $83,97-98$

lag phase, 82, 83-88

logarithmic phase, $82,88-94$

stationary phase, $83,94-97$

Growth of bacteria

effect of oxygen on, 96

of $\mathrm{pH}$ on, 72

of temperature on, 72

without multiplication, 84, 2045

Guamieri corpuscles, 1887

Guinea-pig, normal, antitoxin in blood of, 1084

Guinea-pigs, anaphylactic shock in, 1137 epizootic hepato-enteritis of, 1960

epizootic lymphadenitis of, 1495

infectious paralysis of, 1932

meningo-encephalitis of, 1925

pneumococeal pncumonia of, 1499

psendotuberculosis of, 1649

salivary gland virus of, 1943

tuberculosis in, $425,429,1325$

$H$ agglutinins in diagnosis of enteric fever, $1529-1540$

in relation to antityphoid inoculation, 1537

$H$ antigens, 277,283

$\mathrm{H} \rightarrow \mathrm{O}$ variation, $29 \mathrm{I}$

Hæmin as bacterial vitamin, 68

Hæmobartonella, 903

Hremolysins, 224. Sce also Antigen-antibody reactions. For hamolysins produced by various bacteria see under bacterium in question

Hæmolysins, natural, 1089

Hæmolysis, 193, 224. See also Antigen-antibody reactions

hot-cold type, 616

Hæmolytic streptococci. See Streptococcus, hæmolytic group of 
Ilæmophilus, 786-810

antigenic relations of, 799

broncho-pneumonia in relation to, 1666

cultural characters of, 790

experimental infections with, 804-805

fermentative reactions of, 796

in lioch-Woeks conjunctivitis, 1496

in endocarditis, 1515

in wound infection, 1502

morphology of, 787

variation of, $80 \overline{5}$

bronchisepticus, 494, 495, 787 et seq., 809

causing pneumonia in animals, 1686

in distemper, 1962

relation to Perez's bacillus, 495

toxins of, 802

canis, 791 et seq., 807

ducreyi, 791, 807

influenzx, 786-\$07

antigenic structure of, 799

broncho-pneumonia in relation to, 1666

fermentation reactions of, 796

growth requirements of, 790

hæmolytic strains of, 795 et seq., 807

in meningitis, 1450

in normal nasopharynx, 1993

in sinusitis, 1499

nucoid type of, 799

pathogenicity of, 802

phases of, 801

rôle of in epidemic influenza, 1656

in swine influenza, 1661

smooth type of, $79+$ et seq., 789

summarized description of, 806

type of growth of, 794

$\mathrm{X}$ and $\mathrm{V}$ factors in relation to growtl of, 791

influenzæ-suis, 791 et seq., 807

para-influenzæ, 791 et seq., 807

parapertussis, 801, 809

pertussis, 787, 791 et seq., 809

antigenic structure of, 800

in relation to immunization against whooping cough, 1664

in whooping cough, 1662

pathogenicity of, 802

summarized description of, 809

toxins of, 802

Hæmorrhagic septicæmia. See Pasteurellosis

Hail, bacteria in, 2014

Haptens, 258. See under Antigens

Haptopliore group, 197

Hasamiyami, I 834

Haverhill fever, $356,1277,1278$

Haverhillia multiformis, 1277

Hay bacillus. See Bacillus subtilis

Hay fever, 1153

Heart, congenital malformations of, in relation to bacterial endocarditis, 1515

Heart-water fever, 929,1862

Heat. See also Temperature and Disinfection effect of on subsequent multiplication of bacteria, 116

use of in obtaining pure cultures, 354

Hedgehogs, foot-and-mouth virus in, 1903

Helber chamber, 81
Helvolic acid, 177

Hepatitis, enzootic, 1959

Hepatitis, infectious necrotic, 1780

Hepatitis, infective, 1793

relation of to serum and arsenic jaundice, I794-1796

Hepato-enteritis of guinea-pigs, 1960

Herd infection and herd immunity, 1245-1266

Heredity of immunity, 973 et seq. in tuberculosis, 1298

d'Herelle's phenomenon. See Bacteriophage

Herpes febrilis, 1897. See also Chapter 55 encephalitis in, 1898

keratitis in, I897, 1899

relation of to $\mathrm{B}$ and pseudo-rabies viruses, 1921

to encephalitis lethargica, 1916

Herpes genitalis, 1900

Herpes zoster, 1900 relation of varicella to, 1901

Heterologous, meaning of in relation to antigen-antibody reactions, 274

Heterophile antigen and antibody, 1089 antigen in bacteria, 282

Heterotrophic bacteria, 61

von Hibler's bacillus IX. See Clostridium tertium

Hiccup, epidemic, 1915

Hirschfeld's bacillus, 731. See Salmonella paratyphi $C$.

Hirst test, 1658, 1969

Hiss- $Y$ bacillus, 689

Histaminase, relation of to anaphylactic shock, 1150

Histamine, action of on skin, 1149

antisera to, inhibition of, anaphylactic shock by, 1150

as kataphylactic agent, 1208

production of by tissues, 1149

shock, IIt4 anaphylactic and anaphylactoid shock, relation of to, 1149

Histiocytes in relation to antibody formation, $1103-1106$

to local immunity, 1185

phagocytosis of bacteria by, 1035 et seq.

Historical outline of bacteriology, 1-15

Hodgkin's disease, 198I

Hofmann's bacillus. See Corynebacterium

Hofmeister series, 129

in relation to inhibition of agglutination, 214

inactivation of complement, 230

Hog cholera, 1963

Homologous, meaning of in relation to anti. gen-antibody reactions, 274

Hormones, relation of to immunity, 1210

Horses, actinomycosis due to aerobic Actinomyces, 1276

African sickness of. 1965

botriomycosis in, 1492

botulism in, 880,1620

Brucella infection of, 1718

contagious pustular stomatitis in, 189.

epizootic abortion of, 1968

foragc poisoning in, 880,1620

grass disease or sickness of, 1621 
Horses, infectious anæmia of, 1837, 1966 influenza in, 1662

joint ill of foals, 676

normal, diphtheria antitoxin in blood of, 1084

staphylococcal antitoxin in blood of, 1084

periodic ophthalmia of, 1943

strangles of, 1496

swamp fever of, 1966

tetanus of, 1767

tuberculosis in, 427, 429, 1342

ulcerative lymphangitis of, 1403

vesicular stomatitis of, 1907

Hot-cold lysis, 616

Hoyle's medium, 451, 1374

Humidity, effect of on resistance, 1203

Hyaluronidase formation, 59I, 593

in bacterial infections, 1013

produced by various bacteria, 1013

protective action of, 1072

Hyaluronic acid in bacterial capsules, 1072 in tissues, 1013

Hydrocyanic acid, production of by $P s$. pyocyanea, 510

Hydrogen peroxide, production of by bacteria, 72

toxic action of on bacteria, 72

Hydrogen sulphide, methods of examination for, 369

Hydrogen-ion concentration, 72. See also pH effect of on disinfection, 114, 118

Hydrogenomonas flava, 505

pantotropha, 505

vitrea, 505

Hydrogen-transport in bacterial metabolism, 47

Hydrophile state in relation to antigenantibody reactions 209

Hydrophobe state in relation to antigenantibody reactions, 209

Hyperemesis hiemis, 1798

Hypersensitiveness, 1152-1160. See also Allergy and Anaphylaxis

active and passive sensitization in man, 1153

bacterial, 1162

desensitization in, 1162-1164

relation of, to immunity, 1163

reaction of, inhibition by blocking antibody, 1159

desensitization in, 1160

genetic factors in, 1159

Prausnitz-Küstner reaction in, 1156

I.N.I. agent, 1875

Ice, bacteria in, 2014

Ice-cream, 1542, 1565, 1604, 2049

Ichthyosismus. See Botulism

Ictero-hæmoglobinuria of cattle, 1621

Identification of bacteria, 357-363

Idiosyncrasies, the, 1153

Immunity. See also Antibodies, Antigens, Antigen-antibody reactions and under individual diseases acquired, 976
Immunity, active, 973

in experimental epidemics, 1252-1260

induction of by particular antigens, 1055

alcohol, effect of on, 1209

allergy in relation to, 1160, 1330-1336

antibacterial, 1034-1065

antibodies, relation of to, $105 \mathrm{I}$ different, relation of to, 1054

antigenic specificity of, 1054

antipneumococcal in rabbit, 1037, 1047

synthetic antigens in induction of, 1054

bactericidal and bacteriolytic reactions in relation to, 1046

efficacy of, 1057

histiocytes in relation to, 1035

leucocytes in relation to, 1036

macrophages in relation to, 1034

microphages in relation to, 1039

natural acquirement of, $\mathrm{I} 062$

passive transference of, 1048

reactions of immunized as compared with normal animals in, 1047

reticulo-endothelial system in rela. tion to, 1034 et seq.

antitoxic, 1029-1033

antitoxin in, 1030

avidity of, 1030

time of administration, importance of, 1031

endotoxins, relation of to, 1032

in relation to invasive infections, 1031

antiviral, active, 1229

cellular and humoral factors in, 1234 mechanisms of, 1232

passive, 1231

bacteriophage in relation to, 1215

benzene, effect of on, 1209

calcium as kataphylactic agent in relation to, 1206

cellular, 1053

in relation to local, 1187

congenital, 973, 1077

placenta, relation of to, 1077

diet, effect of on, 1190-1202

enhancement of by non-specific substances, 1214

fatigue, effect of on, 1202

genetic, 973, 1078, 1079, 1298

genetic factors in, 1254

in experimental epidemics, 1254

grades of, 976

herd, 1245-1266

hormones effect of on, 1210

bumidity, effect of on, 1202

inanition, effect of on, 1201

infection, existing as result of, 1165

infection-, 1165-1166. See also Infectionimmunity

innate, 973

local, 1180-1188

non-specific factors in, 1185

measurement of, 981-994

statistical methods, use of in, 9831001 
Immunity, mechanisms that hinder access of bacteria to tissues, 1019-1027

effect of A-avitaminosis on, 1191

non-specific factors in, 1173-1178

partial, 977

passive, 973

in relation to antibacterial immunity, 1048

to antitoxic immunity, 1029

penicillin, relation of to, 184

persistence of antigen in body in relation to, 1129

relation of to anaphylaxis, 1146

response to chilling as test of, 1203

seasonal variations in, 1205

Shwartzman phenomenon, relation of to, 1177

starvation, effect of on, 1193, 1201

sulphonamide drugs, relation of to, 1211

temperature, external, effect of on, 1202

tissue, in relation to local, 1187

ultra-violet rays, effect of on, 1204

various types of, 971-978

virus diseases, in relation to, 1225-1242

vitamins, effect of on, 1190-1199

Immunization, active, 1257. See also under individual diseases

in experimental epidemics, 1257

relation of different antigens to, 1055

resulting from infection, 1062

combined active and passive, 1117, 1401 concentrated, 1115

natural, in experimental epidemics, 1254

side-to-side inoculation in, 1231

Impaction paralysis of cattle, 1621

Impetigo contagiosa, 1493

Impressed variations in bacteria, 290, 291

Inanition, effect of on resistance, 1201

Inclusion blennorrhœea, 1880

Inclusion bodies, 958, 1228. See also Bollinger inclusion bodies, Borrel bodies, Guarnieri corpuscles and Paschen granules

Indicator media, use of in obtaining pure cultures, 356

Indole, methods of examination for, 367

Infantile paralysis. See Poliomyelitis

Infants, enteritis of. See Enteritis of Infancy

Infection, bacterial, 1002 et seq.

by the mouth, experimental, 1045

cellular reactions in, 1007

mechanisms of, 1002-1016

mechanisms that hinder access of

bacteria to tissues, 1019-1027

of wounds, 1500-1503

sequence of immunological events in, 1062

herd, 1245-1267

latent, herd infection in relation to, 1246

Infection-immunity, 1165, 1813

Infectivity, bacterial, relation of to virulence, 1260

Influenza, 1655-1662

active immunization against, 1659

antiviral sera in, 1660

bacillus. See Hæmophilus influenzæ

diagnosis of, 1659

Hirst test in, 1658
Influenza in ferret, $1656,1659,1660$

in swine and other animals, 1661-1662

relation of human to swine virus in, 1656 1657

rôle of $H$. influenzæ in, 1656

virus of, 1650-1659

Influenzal meningitis, 1450

Insect vectors of infection. See under F'leas, Flies, Lice, Mosquitoes, Ticks and individual diseases

Interference phenomenon, 1236

Intermedius ty pe of $C$. diphtherix. See Coryne. baclerium diphtherix

of coli-ærogenes, 663,2024

Intestinal bacteria

as food-stuffs, 1991

destruction of vitamins by, 1992

effect of sulphonamides on, 1993

synthesis of vitamins by, 1991

Intestinal obstruction, clostridial infections in, 1777

Intestinal toxæmia, 1990

Intestines, bactericidal mechanisms in, 1021, 1988

importance of micro-organisms in, 1989

local immunity of, 1183

normal flora of, 1986-1992

Involution forms of bacteria, 33

Irradiation, effect of on resistance, 1203

Isoagglutinins, 1089

Isolation, effect of on incidence of infective disease, 1265

Isolysins, 1089

Jaeger's coccus, 531, 532, 553

"Jake" paralysis, 1592

Japanese, or B, encephalitis, 1917

Japanese River fever, 1849

Jaundice, arsenic, 1795

catairhal, 1794

epidemic, 1793

infective, 1793

leptospiral in animals, 1837

in man, 1828-1837

serum, 1794

Joest-Degen corpuscles, 1922

Johne's bacillus. See Mycobacterium johnei

Johne's disease, 441, 1363

bacteriology of, 441

johnin reaction in, 1365

tuberculin reaction in, 1365

Johnin, 1365

Joint ill in foals, bacillus associated with, 676 in lambs, 1284

streptococcal, of lambs, 1496

Joints, pyogenic infections of, 1496. See also Arthritis

Jones and Handley's medium for inhibiting Proteus, 1528

K.H. reaction in diaguosis of glanders, 1414

Kahn test, 1819 interpretation of, 1820 et seq.

Kataphylaxis, 1206, 1750, 1773

Kauffmann-White schema, 712-716

Kedani, 1849

Keel disease of ducks, 1599 
Kefir, 750

Kenya fever, 1848

Keratitis herpetica, 1899

Keratoconjunctivitis, epidemic, 1902

Kidney, pulpy disease of lambs, 1782 pyogenic infections of, 1499

Kikuyu, diet and resistance of, 1200

Kissélo-mléko, 750, 763, 764

Klebsiella, 654, 655

Koch, 9 et seq.

Koch's phenomenon as example of allergy, 1161,1326

Koch's postulates, 1002

Koch-Weeks bacillus, 791 et seq., 807 in conjunctivitis, 1496

Kupffer cells, phagocytic action of, 1035 et seq.

LD50 dose of lethal substances, 995

Lf and Lr dose of toxin, 1381

Lo and $\mathrm{L}+$ dose of toxin, 239,1384

Labial necrosis of rabbits, 481, 1788

Laboratory infections in cholera, 1420

in dysentery, 1571

in glanders, 1411

in louping-ill, 1925

in lymphocytic choriomeningitis, 1933

in psittacosis, 1872

in scarlet-fever, 1464

in tularæmia, 1721

in typhus ferer, $928, \mathbf{1 8 1 6}$

in undulant ferer, 1701, 1702

Lactiformans, 756

Lactobacillus, 750-765

antigenic structure of, 754

biochemical reactions of, 753

classification of, 755

metabolism of, 752

methods of isolation of from natural sources, 752

pathogenicity of, 751

resistance of, 752

acidophil-ærogenes, 753, 758, 761. See also $L$. brevis

acidophilus, 750 et seq., 757, 1987, 1991, 1996

berolinensis, 756

bifidus, 750 et seq., 762, 763, 1987

brevis, $756,758,761$

buchneri, 756

bulgaricus, 750 et seq., 763, 1990

casei, 756,757

caucasicus, 750, 757, 763

exilis, $750,757,761$

fermenti, 756

odontolyticus $I$ and $I I, 753$ et seq., 760, 761

pastorianus, 756

pentoaceticus, 753. See also L. brevis

plantarum, 757

wehmeri, 756

Lag phase of growth, 83

Lakes, bacteria in, 2015

Lambs. See Sheep

Lamziekte, 880, 1621

Laryngo-tracheitis of fowls, 1969

Latent infection. See under individual diseases
Latent infection, in relation to herd infection, 1246

Lattice hypothesis of antigen-antibody reactions, 208, 220

Leben raib, 750

Lecithinase in bacterial infection, 867, 1774

Leeuwenhoek, van, 2

Leifson's desoxycholate citrate medium, 1528 sodium selenite merlium, 1528

Lemming fever, 1721

Lemon's formula, 90

Lepromin, 1361

Leprosy, 1358-1363

bacillus. See Mycobacterium lepræ

bacteriology of, 434-440

due to rat leprosy bacillus, 1359

in rats. See Rats

Wassermann reaction in, 1360

Leptospira, 908 et seq., 919-924

in water, 1829-1831

infections due to, 1828-1838

tabulated diseases due to, 1835

akiyami, 923, 1834, 1836

Andaman A and B types, 924, 1834, 1836

australis $A$ and $B, 923,1836$

autumnalis, 923, 1834, 1836

bataviz, 923,1834

biflexa, $921,924,1831$

canicola, 924, 1837

grippo-typhosa, 923, 1836

hebdomadis, 923,1836

icterohæmorrhagiæ, 911, 912, 919

in canine jaundice, 1837

in infection of rats, 1830

in Weil's disease, 1828

javanica, 924

mitis, 923

oryzeti, 924

pomona, 923,1837

Rachmat type, 923, 1834

Salinem type, 923, 1834

sejroe, 924,1836

Swart van Tienen type, 923, 1834

Leucæmia of fowls, 1976

Leucocidin, pneumococcal, 592

staphylococcal, 616

streptococcal, 590

Leucocytes, in relation to immunity, 1036

killing of bacteria by without phagocy tosis, 1175

in antiviral immunity, 1233

rôle of in immunity, 1174

variation in phagocytic power of, 1174

Leuconostoc citrovorus, 628

dextranicus, 628

mesenterioides, 627

Leukins, 1173

Leukotaxine, 1044

Levinthal's medium for growth of $H$. influenzæ, 793

Lice in relation to epidemic typhus fever, 18411844

to infectious anæmia of rats, 1798

to relapsing fever, 1805

to trench fever, 1851

Life-cycle of bacteria, 34,87

Light, effect of on bacteria, 102 
Limberneck in chickens, 880, 1622

Lindner's initial bodies in trachoma, 1878

Lipopolysaccharide antigens. See Glycolipoid antigens

Liquid air, effect of on bacteria, 112

Lister, 11

Listerella, 395-403, 401. See Erysipelothrix monocytogenes

Lister's method of counting bacteria, 81

Litmus milk, changes produced by bacteria in, 367

Liver flukes in causation of black disease, 1781

Local anaphylactic reactions, 1151

Local formation of antibodies, 1101

Local immunity, 1180-1188. See also Immunity, local

Localization of bacteria, 1005, 1045

Loewenberg's bacillus, 494, 667, 1789

Logarithmic phase of growth, 82, 88-94

Lombriz of sheep, 1646

Lone Star fever, 1846

Louping-ill, 1925

Lübeck catastrophe, 1306, 1341

Lukes, canine typhus of, 1837

Lungs, relative sterility of normal, 1024

Lygranum, 1871

Lymph capillaries, permeability to foreign matter of, 1042

Lymph glands. See Lymph nodes

Lymph nodes, fate of bacteria in, 1043

fate of viruses in, 1045

filtering action of, 1043

formation of antibodies in, 1104

Lymphadenitis, epizootic, of guinea-pigs, 1495 streptococcal, of dogs, 1496

Lymphadenoma, 1981

Lymphatic blockade, 1044

system, availability of antibody in, 1061

Lymphocytic choriomeningitis, 1932

Lymphogranuloma inguinale, 1869

Lymphopathia venereum, 1869

Lysis. See Bacteriolysis, Hæmolysis and Bacteriophage

Lysozyme, 1020, 1022, 1175

in tears, 1025

in tissues, 1998

MacConkey's medium in cultures from enteric fever, 1527

in water analysis, 2022

McLeod's medium, 451, 1374

Macrophages, 1035. See also Histiocytes

Mad itch, 1941

Madura disease, 1275

Malignant catarrh of cattle, 1966 œdema, 1778 pustule, 1733

Mallein test, 1412

Malta fever, 1693-1697. See also Undulant fever, due to $\mathrm{Br}$. melitensis

Maltase in serum, 367,534

Manchester bacillus. See Shigella newcastle

Marfanil, 158, 167

Marseille, exanthematic fever of, 1848

Masai, diet and resistance of, 1200
Mass law, in antigen-antibody reactions, 219

in enzyme adaptation, 293

Massauah vibrio, 526

Massol's bacillus. See Lactobacillus bulgaricus

Mastitis, suppurative in nursing mothers, 1492

Mastitis in cattle, 1493

C. pyogenes associated with, 1494

staphylococci associated with, 1493, 1494 streptococci associated with, 581, 1493, 1494

Mazun, 750

M-concentration of living organisms in culture, 94

Measles, 1949-1952. See also Chapter 55 latent infection in, 1226 prophylaxis of, 1950-1952 reproduction of in animals, 1949

Meconium, bacteria in, 1989

Media indicator, use of in obtaining pure cultures, 356

selective, use of in obtaining pure culture, 355

solid, introduction of by Koch, 10

Mediterranean fever. See Undulant fever

Melioidosis, 1415

Melitin reaction in diagnosis of undulant fever, 1705

Membrane, cytoplasmic, of bacterial cell, 27

Meningitis, 1431-1451

acute, lymphocytic type of, 1932 aseptic, 1932

cerebrospinal, 1431-1449. See also Neisseria meningitidis

carrier epidemic in, 1434

carrier rate in, 1434,1442

effect of overcrowding on, 14341436

chemoprophylaxis in, 1443

chemotherapy in, 1448

diagnosis of, 1438

by examination of petechial skin lesions, 1441

epidemiology of, 1432-1438

mode of spread of, 1433

prophylaxis of, 1441

rhinopharyngitis in, 1434

routes of infection in, 1436

direct extension from nose, 1436

hæmatogenous, 1437

serum treatment of, 1444-1448

vaccination in, 1444

influenzal, 1450

in Weil's disease, 1829, 1837

pneumococcal, 1449

streptococcal, 1449

tuberculous, 1290,1449

uncommon forms of, 1451

Meningococcus. See Neisseria meningitidis

Meningo-encephalitis of rabbits, 1916, 1924

Meningo-pneumonitis virus, 1876

Metabolic properties of bacteria, 360,367

Metabolism of bacteria, 42-74 mechanisms of, 46 et seq.

Metachromatic granules, $23,449,532$

Metallic salts, effect of in antibody formation, 1115,1122

Metals, effect of on bacteria, 120-130 
Methanomonas methanica, 505

Methods of examination of bacteria, 364-369

Methyl red test, 368,662

Methylene blue, use of in study of oxidationreduction reactions, 50

Methylene blue reduction, methods of examination for, 369

test in examination of milk, 2045

Mexican typhus, 1841, 1845

Mice, anaphylaxis in, 1139

choriomeningitis in, 1933

ectromelia of, 1278, 1909

in experimental epidemics, 12531264

epidemic in, due to Pasteurella-like organism, 779

field. See Field mice

in relation to food poisoning, $\mathbf{1 6 0 0}$

infectious catarrh of, 1799

infective arthritis of, 386,1278

influenza of, 1799

pasteurellosis of, 777,1254

plague in, 1637

pseudotuberculosis of, 461,1649

tuberculosis in, 426, 429

typhoid in. See Mouse typhoid

Michaelis's acid agglutination test, 692

Micrococcus, 623-625

ærogenes, 625

agitis, 627

ascoformans, 1492

buccalis, 625

caseolyticus, 624

catarrhalis. See Neisseria

conglomeratus, 623

coronatus, 624

flavus, 623

freudenreichii, 624

gingivalis, 625

luteus, 623

melitensis. See Brucella

minimus, 625

pharyngis cinereus. See Neisseria

pharyngis flavus, i, ii and ii. See Neisseria

pharyngis siccus. See Neisseria

prodigiosus. See Chromobacterium

radiatus, 624

tetragenus, 607, 624

urex, 624

varians, 623

Microphages, 1039. See also Polymorphonuclear leucocytes

Micromonospora, 382

Microscopy, electron, 18

in study of filtrable viruses, 952

limitations of, 18, 951-953

Midland cattle disease, 1621

Mid-piece of complement, 229

Milk, bacterial flora of, 2036-2039

pathogenic bacteria in, 2038

Actinomyces muris, 1278

anthrax bacilli, 1735

Brucella abortus, 1700, 1713, 2038

Brucella melitensis, 1695, 1697, 1707, 1711

Brucella suis, 1702, 1711
Milk, bacterial flora of, pathogenio bacteria in, Corynebacterium diphtheriæ, 1390, 2039 foot-and-mouth virus, 1905

Staphylococcus aureus, 1494, 1605, 2039

streptococci, 1471, 1494, 2039

tubercle bacilli, 1317, 1344, 2038

bacteriological grading of, 2041-2049

cleanliness, safety, keeping quality and pasteurizability of, 2039

designations of, 2048

diseases borne by, 2049. See also individual diseases

diar'rhœe and dysentery, 1565, 1588, $1590,2049,2050$

diphtheria, I390, 2049, 2050

enteric fever, 1542, 2049, 2050

food poisoning, 1602, 1605, 2039

Haverhill fever, 1278

poliomyelitis, 1927

rat-bite fever, 1278

scarlet fever and other streptococcal infections, 1471, 2039, 2049, 2050

tonsillitis, 1478, 2049, 2050

tuberculosis, 1289, 1317, 1336, 2049

undulant fever, 1695, 1697, 1700, 2049

dried, as dietary factor in relation to immunity, 1200

foot-and-mouth virus in, 1905

grades of

accredited, tuberculin tested, pasteurized, heat-treated, 2047-2049

sterilized, 2053

litmus, changes produced by bacteria in, 367

pasteurization of, $1318,1319,1336,1588$,

2048, 2051

production of clean milk, 2037

of safe milk, 2050-2053

Milk-borne disease. See Milk, diseases borne by

"Miniature" scarlet fever, 1465

Mineral salts, possible effect of on resistance, 1201

waters, bacteria in, 2015

Minimal Hæmolytic Dose (M.H.D.) of complement, 227

of hæmolysin, 227

Minimal Lethal Dose (M.L.D.) of toxin, 238 of diphtheria toxin, 1384

Miscellaneous bacteria, group of, 898-905

Mist bacillus. See Mycobacterium stercoris

Mite fever, 936, 1849

Mitis type of $C$. diphtherix. See Corynebac. terium diphtheriæ

Mitogenetic rays, 98

Moeller's grass bacilli i and ii, 404

Moloney reaction, 1393,1396

Molluscum contagiosum, 1978

Mongoose, in relation to rabies, 1936

Mongoose virus, 1876

to virus pneumonia, 1876

Monkeys, anaphylaxis in, 1139

experimental pneumococcal pneumonia of, 1668

follicular conjunctivitis of, 1878

tuberculosis in, $427,429,1343$

P.B. 
Monomolecular reaction, similarity of disinfection to, 137-140

Mononucleosis, infectious of man, 1791 of rabbits, 1286

Morax-Axenfeld bacillus, 898

Moraxella, 899

Morgan's bacillus. See Proteus morgani

Morphology of bacteria, 16-39

in identification, 358

variations in, 290

Morvine, 1414

Moscow typhus, 1845

Mosquitoes in spread of dengue fever, 1958 of yellow fever, 1953

Motility, use of in obtaining pure cultures, 353

Mould products, miscellaneous, 179

Mouse pneumonia virus, 1876

Mouse septicæmia, bacillus of, 395,1286

Mouse typhoid, experimental epidemics of, 1253 et seq.

Mouth, removal of bacteria from, 1020

Much granules, 407

Mucin, effect of on bacterial infection, 1209

Mucus, rôle of in removal of bacteria, 1022

Mud fever. See Swamp fever

Muller's tetrathionate medium, 1528

Mumps, 1952

Mutation, bacterial, 295. See also Variation of bacteria

Myalgia, epidemic, 1960

Mycetoma, 387. See also Madura disease

Mycobacterium, 404-442

acid-fast bacteria, 404

antigenic structure of, 422

avian types of, 404

biochemical reactions of, 422

chemical structure of, 409

classification of, 431

cold-blooded types of, 404

cultural reactions of, 411

habitat of, 405

mammalian types of, 404, 431

metabolism of, 419

morphology of, 405

of voles. See Mycobaclerium muris

optimum hydrogen-ion concentration for growth of, 419

optimum temperature for growth of, 420

oxygen requirements of, 420

pathogenicity of, 423

resistance of, 417

rough and smooth forms of, 416

saprophytic types of, 404, 414

staining of, 405

tabulated reactions of, $435-437$

toxin production by, 422

variation of, 415

butyricum, 404

johnei, 404, 441

lepræ, 404,434

muris, 405-406, 411, 413, 420, 423-429, $432,435-437$

of rat leprosy, 404,440

phlei $\mathrm{i}$ and $\mathrm{ii,} 404$

smegmatis, 405

stercoris, 405
Mycobaclerium tuberculosis, 404 et seq., 433

aberrant types of, 431

attenuated types of, 431

avian and cold-blooded types of, cultural differentiation of, 414

bovine type of, 404

cellular reactions to products of, 410 , chemical structure of, 409

differential characteristics of, tabulated, 432, 435-437

dysgonic type of, 412,431

effect of glycerine on growth of, 420

eugonic type of, 412,431

filtrable forms of, 408

human and avian types, differentiation of, by pathogenicity, 430 culturally, 413

serologically, 422

human and bovine types of, differentiation of, by pathogenicity, 430

culturally, 412, 420

human type of, 404

in suppurative lesions of the urinary tract, 1500

lesions caused by dead bacilli, 431

minimal infecting dose of for guineapigs, 425

Much granules of, 407

murine type of, differentiation of, 413 , 435-437

pathogenicity of, 423-428

pigment formation by, $413,414,421$

summarized description of, 433

supposed life-cycle of, 408

tuberculin formation by, 410, 1327

Yersin type of disease caused by, 426

Myelitis, due to $\mathrm{B}$ virus, 1921

due to herpes virus, 1921

due to rabies virus, 1921,1935

Mykol, 407

Myositis, clostridial, 1771. See Gas gangrene

Myxomatosis, infectious, of rabbits, 1978

Nairobi disease of sheep and goats, 1967

Nanukayami, 1834

Nasal mucosa, effect of temperature and humidity on, 1203

Nasik vibrio, 516,526

Nasopharynx, normal flora of, 1992 et seq.

Nastin in treatment of leprosy, 1361

Native populations, Schick and Dick reactions among, 1080-1082

tuberculosis in, 1296

Natural antibodies. See Antibodies, natural

Neapolitan fever. See Undulant fever due to $\mathrm{Br}$. melitensis

Necrobacillosis, 1786

in animals, 1787

in $\operatorname{man}, 1786,1788$

Necrosin, $10 \pm 4$

Necrosis bacillus, 479. See also Fusiformis necrophorus

Necrotuberculosis, 431

Negative phase in antibody production, 1116

Negri corpuscles, 1937

Neill's medium, 451, 1374 


\section{Neisseria, 531-556}

antigenic structure of, $535,538,545$

biochemical reactions of, 531 maltase in serum, 534

chemical fractionation of, $535,539,547$

classification of, 536

growth requirements of, 532

pathogenicity of, $535,539,548$

pigment formation by, 534, 536, 556

resistance of, 534

tabulated reactions of, 554,555

variation in, 534, 536, 537, 541

catarrhalis, 531 et seq., $\mathbf{5 5 1}$

flavescens, 531, 556

gonorrhoæ, 531 et seq., 543-550

differentiation of from $N$. meningitidis, 550

disease caused by, 1454-1460

in suppurative lesions of the urinary tract, 1500

media for cultivation of, 543

toxin production by, 549

meningitidis, 531 et seq., 537-543

autolysin of, 538

differentiation of from $N$. gonorrhoex, 550

disease caused by, 1431-1449

toxin production by, 541

pharyngis, $535,537,553$

pharyngis cinerea, 531,553

pharyngis flava $i$, $i i$ and $i i i, 531$ et seq., 552

pharyngis sicca, 531 et seq., 553

Neisser-IVechsberg phenomenon, 228

Neonatal diarrhœa, 1583

Newcastle bacillus, 690 . See Shigella

Newcastle disease of fowls, 1658, 1969

Niacin. See Nicotinic acid

Nicotinic acid as bacterial vitamin, 67, 163

Nicotinamide, 165

Nitrate reduction, methods of examination for, 368

Nitrates, effect of on anaerobic growth, 54

Nitrobacter, 504 winogradslyi, 504

Nitrogen-fixing bacteria, 497

Nitrogenous constituents of bacteria, 43 metabolism of bacteria, 55

Nitrosococcus americanus, 503

Nitrosomonas, 502-504 europoea, 503

Nocardia, 374,382

Nocard's bacillus, 1873

Noma, 1810

Nomenclature of bacteria, 310-323

Normal curve, 985

Normal flora of body, 1984-1999

alimentary tract, 1986-1992. See also Intestines

factors affecting, 1988

blood and internal organs,. 1998

conjunctiva, 1998

factors affecting, 1984-1985

mouth, 1986

respiratory tract, 1992-1996

persistence of species in, 1995

skin, 1997

technique of examination, 1984
Normal flora of body, tonsils, 1992 urethra, 1997 vagina and vulva, 1996

Nose, arrest of bacteria in, 1023 normal flora of, 1992 et seq.

Notatin, 178

Nuclear apparatus of bacteria, 19 as genetical postulate, 289

Nucleic acid, effect of on antibody formation, 1123

Nuclein, increase of opsonic power following injection of, 1175

Null hypothesis, in statistical reasoning, 1000

Nutritional requirements of bacteria, 61 and essential nutrients, 63 and evolutionary series, 62 and growth stimulants, 63 as basis of classification, 312

Oagglutinins in diagnosis of enteric fever, $1529-1540$

in relation to antityphoid inoculation, 1537

O antigens, 277,283

Ohara's disease. See Tularæmia

Oils, animal, effect of on bacteria, 137 essential, effect of on bacteria, 136

Omentum, rôle of in peritoneal infections, 1040 Oosporosis, 1270

Opacity of a bacterial suspension, factors determining, 81,85

Ophthalmia neonatorum, 1455, 1460

Ophthalmia, periodic, of horses, 1943

Opsonins, 194, 235. See also Antigen-antibody reactions

increase of, induced by injection of nuclein, 1175

non-specific, 235,237

Optimal antigen-antibody ratio in agglutination, 212

in precipitin reaction, 201 et seq. with constant antibody, 202, 204 with constant antigen, 202, 204

relation of to chemical equivalence, 204 of constant-antigen ratio to constantantibody ratio, 202,209

Optimal proportions in agglutination, 213 in complement fixation, $\mathbf{2 3 3}$ in precipitation, 201

Oral sepsis in relation to endocarditis, 1515

Organic acids as disinfectants, 119 as substrates for fermentation tests, 706

Ornithosis, 1874

Oroya fever, 903, 1796

Osmotic effect of salts on bacteria, 127

Osteomyelitis, 1496, 1506

Osteophagia, 1621

Otitis media in rats, $\mathbf{1 4 9 9}$

Owls, virus disease of, 1971

Oxalates in metabolism of Pf. whitmori, 493

Oxalates, production of by $P f$. whitmori, 492493

Oxidase, bacterial, 48

Oxidation-reduction potential, measurement of, 50 reactions in bacterial metabolism, 50, 71 
Oxygen, effect of on growth of bacteria, 96 on survival of bacteria, 2017

variation in requirement of, by bacteria, 360

Oxygen lack, effect of on antibody formation, 1122

Oysters, 2031

Ozæna, 494, 1788

Abel-Loewenberg bacillus in, 667 et seq.

bacillus of Perez in, 494

Ozone, effect of on bacteria, 149

Pahvant Valley fever. See Tularæmia

Pantothenic acid as bacterial vitamin, 67, 164

Pantoyltaurine, 164

Papillomatosis of rabbits, infectious, 1980 oral, 1980

Paracholera vibrios, 520

Para-aminobenzene sulphonamide. See Sulphanilamide

Para-aminobenzoic acid, as bacterial vitamin, 162

as antagonizer of sulphonamide compounds, 161

Paracolon bacilli, 676, 715

Salmonella antigens in, 715, 744

$\mathrm{Vi}$ antigen in, 715,744

Para-influenza bacilli, 791 et seq.

Paralysis, bulbar due to pseudorabies virus, 1941

infectious, of guinea-pigs, 1932

Parameningococcus, 538

Para-Shiga bacilli. See Shigella

Paratyphoid fever. See Enteric fever

Parotitis, epidemic, 1952

Parrots, psittacosis in, 1872 tuberculosis in, $427,429,1343$

Paschen granules, 959, 1887

Passive anaphylaxis, 1140 immunity. See Immunity, passive

Pasteur, 2 et seq.

Pasteurella, 767-784

antigenic structure of, 773

biochemical reactions of, 772

classification and identification of, 778

colonial variants of, 769

cultural characteristics of, 769

differential characteristics of, tabulated, 779

hæmolytic group of, 778

in experimental epidemics, 1252-1259

metabolism of, 771

organisms resembling, in acute contagious pneumonia of sheep, 779

in epidemic in mice, 779

pathogenicity of, 775

resistance of, 770

Salmonella antigens in, 703, 773

variations in virulence of, 775

$\mathrm{Vi}$ antigens in, 774

aviseptica, 767 et seq.

boviseptica, 767

bubaliseptica, 772

lepiseptica, 767, 770,771

muriseptica, 767

oviseptica, 767
Pasteurella pestis, 767 et seq., 780

disease caused by, 775, 1627-1646

pseudotuberculosis, 767 et seq., 782

disease caused by, 777, 1649

motility of, 768

septica, 767 et seq., 778, 781

diseases caused by, 777, 1616-1649

suiseptica, 767

vituliseptica, 767

Pasteurellosis, 1646-1649

experimental epidemics of in mice, 1254 experimental reproduction of in animals, 777

of rats, 1639

Pasteurization of milk, 1318, 1319, 1336, 1588, 2048, 2051

Pathogenicity in identification of bacteria, 362 of various bacteria. See under individual bacterium

methods, use of in obtaining pure cultures, 356

Patulin, 177

Paul-Bunnell test, 1792

Paul's test in diagnosis of smallpox, 1888

Pemphigus neonatorum, 1492

Penatin, 178

Penfold's hypothesis of lag, 84

Penicidin, 178

Penicillia, 175

Penicillic acid, 178

Penicillin, 175, 179-185

acquired resistance of bacteria to, 180

action of in amimal body, 184

assay of, 181

discovery of, 175

laboratory use of, 182

mode of action of, 182

range of activity of, 175,180

use of in obtaining pure cultures, 356, 1664

Penicillinase, 180

Peptone shock, 1144 possible relation of histamine to, 1151

Perez's bacillus, 494

Periostitis, 1496

Peritoneal cavity, experimental infections in, 1039

passage of bacteria from to blood stream, 1041

Peritonitis, 1498 clostridial infections in, 1777

Peroxidases in bacterial metabolism, 48

Pertussis. See Whooping Cough and Hæmophilus

Petit's bacillus, 899

Pfeifferella, 486-496

antigenic structure of, 488

classification of, 490

group characters of, 486

pathogenicity of, 488

relation of to Actinobacillus, 490

mallei, 486 et seq., 490 disease caused by, 1408-1415

whitmori, 486 et seq., 492

disease caused by, 1415

oxalate production by, 492-493

Pfeiffer's reaction, 192, 1425, 1806

$\mathrm{pH}$, effect of on bacterial growth, 72 
Phagocytes, 1039

possible protective action of on bacteria, 1060

Phagocytosis, 1039. See also Opsonins, Bacteriotropins and Immunity, antibacterial

aggressins, inhibition of by, 1069

effect of protein deficiency on, 1201

electric charge in relation to, 235

genetically determined, 975

rôle of opsonins in, 193

variations in phagocytic power of leucocytes, $117 t$

Phelps' method of standardizing disinfectants, 148

Phenols, effect of on bacteria, 133

Phlebitis, 1491

Phlebotomus fever, 1959

Phlebotomus noguchi in relation to verruga peruana, 1797

Phosphatase, production of by diphtheroid bacilli, 453

test for heated milk, 2051

Photodynamic sensitization, effect of on bacteria, 106

on bacteriophage, 107

Photomierography, use of ultra-violet light in, 18,952

Photosynthesis in bacteria, 61

Pigeon-pox, 1896

Pigment formation by bacteria, effect of salts on, $633,635,637$

in identification of bacteria, 361

inhibition of by calcium, 635

variation in, 299

Pigments, bacterial, rôle of in metabolism, 73 in photosynthesis, 61

Pigs. See Swine

Placenta, relation of to congenital immunity, 1077, 1092

Plague, 775, 1627-1646

chemotherapy in, 1646 .

diagnosis of in man, 1641

in rats, 1631,1638

thermoprecipitin test in, 1639, 1641

differential diagnosis of from tularæmia, 1722

differentiation of from pseudotubcreulosis, 1640

epidemiology of, 1627

experimental reproduction of in animals, 775

fleas in ætiology of, 1628 et seq.

in animals, other than rats, 1637

in rats, 1628 et seq.

experimental transmission of, 775 , 1631

increasing immunity of rats to, 1635 , 1641

mode of spread of in Bombay, 1628, 1634

in Egypt and other parts, 1636I638

pneumonic, 1638

prophylaxis of, 1642

serum treatment in, 1645

Plakins, 1173
Plate method of counting bacteria, 82

in milk, 2043

in water, 2021

use of in obtaining pure cultures, 352

Platelets, rôle of in clearing mechanism of blood stream, 1036

Pleurodynia, epidemic, 1960

Pleuropneumonia group of organisms, 939-948

cultivation of, 940

diseases caused by, 945-948 1867

morphology of, 941-944

organism of contagious agalactia, 945 , 1867

other characters of, $944-945$

strains of, in dogs, 946

in guinea-pigs, 947

in $\operatorname{man}, 947$

in mice, 947

in rats, 946

lettered and numbered types of, 946,947

saprophytic pleuropneumonialike, $9+7$

of cattle, 1867

due to Pasteurella, I647

of swine, 1964

Pneumobacillus. See Bacterium, Friedländer group of

Pneumococcal meningitis, 1449

polysaccharides. See Streptococcus pneumoniæ

septicæmia, experimental study of in rabbits, 1037,1048

Preumococcus, 561. See Streptococcus pneumonix

Pneumonia, 1668-1686

active immunization against, 1683

aggressive action of haptens in, 1071, 1679

antipneumococcal serum, and sulphonamide drugs, combined treatment of with, 1682

from horses, 1675-1677

from rabbits, 1678

standardization of, 1679

treatment of with, in animals, 1669 in $\operatorname{man}, 1675-1679$

atypical, 1876

bacteræmia in, 1670

chemotherapy of, 1681

contagious of sheep, 779,1648

enzyme treatment of, 1682

experimental in animals, 1669

frequency of different pneumococcal types in, 1671 et seq.

in animals, due to $I I$. bronchisepticus 1686 due to Pasteurella, 1616-1648

in guinea-pigs, 1499

in rats, 1499

infectious of goats, 1646

mortality of in relation to infecting type, I671-1673

polysaccharides, excretion of in, 1678

relation of " carrier" types to, 1673 et seq. as a contagious disease, 1674

skin reactions to pneumococcal polysac. charides, 1685 
Pneumonia, Type III, treatment of with bacterial enzyme, 1682

typing of infecting pneumococcus in, 1678

vaccination against, 1683

vitamin A in relation to, 1195

viruses in, 1875

Pneumonic plague, 1638

Poisoning, food. See Food poisoning ptomaine, 1595

Polar staining, 37

Poliomyelitis, 1926-1932. See also Chapter 55 latent infection in, 1226 natural antibodies to, 1226

Polysaccharide antigens of various bacteria, 279. For particular bacteria see under organisms in question

Polysaccharides bacterial, as aggressins, 1071, 1679

as haptens, 258

excretion of, in disease, 1678

in stimulation of antibody production, 1119,1684

Porges' method for capsule removal, 671

Portal pyæmia, 1491

Potential difference in relation to agglutination, 214

to phagocytosis, 235

Poxes, animal, 1895-1897

Prausnitz-Küstner reaction in hypersensitive. ness, 1156

Precipitin reaction, 200. See also Antigenantibody reactions

Precipitins. See under Antigen-antibody reactions

formation of, 1109

protective action of, 1057

Preisz-Nocard bacillus. See Corynebacterium ovis

Pre-school child, immunization of against diphtheria, 1399

Primary stimulus, effect of on antibody formation, 1106

Proactinomycin, 178

Probability in relation to assessment of significance, 983 et seq.

Probability tables in counting bacteria, 82, 2022

Promin. See Sulphone

Prontosil. See Sulphonamido-crysoïclin

Propamidine, 172

Protein constituents of bacteria, 43 metabolism of bacteria, 55

Proteins, antigenic differences between, $\mathbf{2 5 3}$ effect of chemical treatment on antigenic structure of, 253 relation of to full antigenicity, 260

Protein-sparing effect of carbohydrates in bacterial metabolism, 57

Proteus, 642-651

classification of, 648

diseases associated with, 1497, 1498, 1500, 1501,1586

hæmolytic activity of, 645

in relation to putrefaction, 645

to summer diarrhœa, 1586

prevention of swarming of, 644,1528
Proteus, swarming of, 643-645

hydrophilus, 647

mirabilis, 648

morgani, 642 et seq., 651

vulgaris, 642 et seq., 649

in wound infection, 1501

$X, 612,647$ et seq., $933,1853-1858$

zenkeri, 648

Protista, 949

von Prowazek-Halberstaedter inclusion bodies in trachoma, 1878

Pro-zone in agglutination, 212

in bacteriolysis, 228

in precipitation. See Optimal antigen: antibody ratio, 201 et seq.

Pscudoanthrax bacillus. See Bacillus pseudoanthracis

Pseudo-lymphocytic choriomeningitis, 1932, 1934

\section{Pseudomonas, 506-512}

group of forming bluish-green pigment, 508 in peritonitis, 1498

in summer diarrhœa, 1586

in suppurative lesions of urinary tract, 1500

in wounds, 1501, 1503

æruginosa. See Ps. pyocyanea

caviæ, 512

cyanogena, $5 \mathrm{I} 2$

denitrificans, 512

fluorescens, 506 et seq., 511

pyocyanea, 506 et seq., 510

antigenic structure of, 508

differentiation of from $P s$. fluorescens, 511

pathogenicity of for animals, 508,511 pigment formation by, 508, $5 \mathrm{I} 0$

variation of, 508,510

radicicola. See Rlizobium leguminosarum

Pseudorabies, 1941

relation of to $B$, and herpes viruses, 1921

Psendotuberculosis, $426,777,1403,1404,1649$ clarlothrichicha, 1275

differentiation of from plagne, 1640

experimental reproduction of in animals 777

of $\operatorname{man}, 1650$

of mice, 461,1649

of pigs, 1650

of rodents, 1649

of sheep, 468, 1403, 1649

Pseudotuberculous enteritis. See Johne's disease

Psittacosis, 1872-1875

in fowls, pigeons and petrels, 1872, 1874

in psittacine birds, 1872 et seq.

-like diseases, 1875

Ptomaine poisoning, 1595

Puerperal fever, 1480 -I 485 anaerobic streptococci in relation to, 1484 chemotherapy in, 1485

Dick test in relation to, 1480

intrinsic and extrinsic infection in, 1481 prophylaxis in, 1483

serum treatment of, 1484

various bacteria in relation to, 1485

vitamin $\mathrm{A}$ in relation to, 1194 
Pulpy kidney disease of lambs, 1782

Pure cultures of bacteria, methods of obtaining. See Cultures, pure

Pyæmia, 1491 in foals, 1404

Pyæmia, portal, 1491

Pyelitis, 1499

Pyelonephritis in cattle, 1500 in $\operatorname{man}, 1499$

Pyobacillosis, chrouic, of sheep, 1499

Pyocyanin, 508-510 as respiratory pigment, 54

Pyogenic infections, 1490-1506

Pyrotherapy in treatment of gonorrhœa, 1460 of syphilis, 1824

Pyridine sulphonamide, 163

Pyrithiamine, 164

$\mathrm{Q}$ fever, 1850

Quarter-evil, 1778

Queensland, coastal fever of,

B. tropicus in, 841

Leptospira in, 1834,1837

7-day fever of, 923,1837

Rabbits, anaphylactic shock in, 1138

experimental pneumococcal pneumonia of, 1669

infectious fibromatosis of, 1979

infectious mononucleosis of, 1286

infectious myxomatosis of, 1978

infectious papillomatosis of, 1980

labial necrosis of, 479,1788

meningo-encephalitis of, 1924

oral papillomatosis of, 1980

pox of, 1896

snuffles in, 1647

syphilis in, experimental, $916,1165,1814$ 1816

natural, 1825

tuberculosis in, 424, 429, 1343

virus-III infection of, 1909. See also Chapter 55

Rabies, 1935-1941. See also Chapter 55

ascending myelitis in, 1935

due to vampire bats, 1935

fixed virus of, 1936

mode of infection of central nervous system in, 1936

Negri corpuscles in, 1937

Radium, effect of on antibody formation, 1104 on bacteria, 109

Rain, bacteria in, 2013

Ramon precipitin reaction, 202

in standardization of diphtheria antitoxin, 1384

Rat-bite fever due to Actinomyces muris, 386 , 1276

due to Spirillum minus, 925, 1276-1278, 1838

Ratin bacillus, 736,1603

Rats, Brucella infection of, 1720

experimental pneumococcal pneumonia of, 1669

in ætiology of plague, 1627 et seq. in relation to food poisoning, 1603 in relation to typhus fever, 1845
Rats, in relation to Weil's disease, 1830 increasing immunity of to plague, 1635 , 1641

infectious anæmia of, 904,1797

leprosy of, 404, 440, 1361

otitis media in, 393,1499

pasteurellosis of, 1639

plague in, 1631,1638

pneumonia of, $393,1281,1499,1699$

tuberculosis in, 426,429

typhoid of, 1600

"Ray-fungus," 373

Reaction velocity of disinfection, 137. Seealso Disinfection

Reagins, 1157 et seq.

Receptors, 196

different orders of, $19 \mathrm{~s}$

Red-water disease of cattle, 891

Rejuvenation, relation of to lag phase, 8t-88

Relapse strains of spirochrtes, 1806

Relapsing fever, 1803-1808

arsenic-fast strains of spirochætes in, 1808

immunity to, 1805

Pfeiffer's phenomenon in, 1806

prophylaxis and treatment of, 1808

relapse strains of spirochetes in, 1806

reproduction of in animals, 914, 1807

transmission of, 1804

Treponema recurrentis in, 913, 1803 named varieties of, 1804

Reproduction of bacteria, 33

Resistance, 981. See Immunity

of bacteria to various agents, 101-150 methods of examination for, 359 , 366

variations in, in relation to disinfection, 140

Resolution, optical, factors determining, 18

Respiration, bacterial, 46

in relation to growth, 85

Respiratory activity of bacteria in relation to growth, 85

infections, 1653-1686

Respiratory tract, normal distribution of bacteria in, 1023

normal flora of, 1992

Reticulo-endothelial system, antibodies, formation of, rôle of in, 1102

antiviral immunity, rôle of in, 1232, 1233

in relation to antibacterial immunity, 1034 et seq.

Reversed anaphylaxis, 1140

$\mathrm{Rh}$ factor in human blood, immunological significance of, 1088

Rheumatic fever, 1509-1516

hypersensitive reactions in, 1511,1513

relation of to Vitamin $\mathrm{C}, 1197$

streptococci in relation to, 1509-1511

Str. pyogenes in relation to, 1511-1514 virus, as cause of, 1514

Rhinopharyngitis in meningitis, 1434, 1436

Rhinoscleroma, 1789 bacillus of, 667 et seq.

Rhizobium, 499-502 leguminosarum, 499-502

Rho-variants $(\rho)$ of Salmonella bacilli, 303 of vibrios, 521 
Rhodococcus agilis, 627 cinnabareus, 627 rhodochrous, 626 roseofulvus, 627

Riboflavin as bacterial vitamin, 66

Rickettsia, 928-937

antigenic structure of, 933

classification of, 937

cultivation of, 931, 960

diseases caused by, 1841-1862

habitat of, 929

metabolism of, 931

morphology of, 930

pathogenicity of, 933-936

relation of to Proteus $X, 1857$

resistance of, 932

akamushi. See $R$. nipponica

bovis, 937,1862

burneti, 929, 932, 936, 1850

canis, 937, 1862

conjunctivæ, 936,1862

conori, 1848

diaporica. See R. burneti

melophagi, 929,931

mooseri, 929 et seq., 935,1845

nipponica, 929 et seq., 936, 1849

orientalis. See $R$. nipponica

ovina, 937, 1862

pediculi, 929,1852

prowazeki, 647, 928 et seq., 934, 1841 et seq.

louse-borne type of, 934, 1841

murine type of. See $R$. mooseri

quintana, 928, 936, 1851

rickettsi, 929 et seq., 935, 1846

rocha-limæ, 936, 1844, 1852

ruminantium, 929,1862

trachomatis, 1879

tsutsugamushi. See R. nipponica

wolhynica. See R. quintana

Pideal-Walker drop method of standardizing disinfectants, 146

Rieckenberg reaction. See Adhesion phenomenon

Rift Valley Fever, 1959

Rinderpest, 1965. See also Chapter $5 \tilde{}$

Ringer's solution, 126

Ring-Impfung, 1907

Rivers, bacteria in, 2015 self-purification of, 2020

Rocky Mlountain spotted fever, 935, 1846

Rodella's bacillus III. See Clostridium tertium

Rodent diseases, bacteriology of. See under Rats, Mice, Guinea-pigs, etc.

Rodent typhoid, 1600

Rodents, plague in gerbilles, ground squirrels, field and multimammate mice, spermophiles and tarbagans, 1637

Rolling disease, 947

Roll-tube method of counting bacteria, 82

Römer's method of performing tuberculin reaction, 1328

of titrating diphtheria antitoxin, 1383 of titrating diphtheria toxin, 1376,1377

Röntgen rays, effect of on bacteria, 108

Rough variants of bacteria, 299

mode of division of, 300

salt-sensitivity of, 299
Roughness in relation to antigenic structure, 277,283

Roup, 1895, 1896

Rous sarcoma of chickens, 1977

Rovida's tube, 353

Rubella, 1952

Ruys' medium, 1528

Sakushu fever, $183 t$

Saliva, 1986

bactericidal activity of, 1021 normal flora of, 1986

Salivary calculi due to Actinomyces, 1272 gland virus of guinea-pigs, 1943

Salmonella, 702-745

animal reservoirs of, 1598

antigenic structure of, $710-719,726-745$

antigenic types, fermentative varieties of, 718

antigenic variation of. See also Variation $\alpha-\beta$ phase, 279,716

artificial phase, 716

$\mathrm{H}$ antigens, 716

M antigen, 718

$\mathrm{O}$ antigens, 717

$\mathrm{OH} \rightarrow \mathrm{O}, 717$

smooth $\rightarrow$ rough, 277,717

specific-group phase, 278,716

$V$ i antigen, 717

$\mathrm{X}$ antigen, 718

bacteriophage method of typing of, 719 , 1548

biochemical activities of, 706,718

chemical fractionation of, $280,720,724$

composite antigens in, 712

d, 712, 743. See Salm. gaminara

$\mathrm{v}$, vi, and xii, 712,717

7, 742. See Salm. carrau

cultural characters of, 704

genetic resistance to, 975

habitat of, 703

in birds and animals, 1598-1601

in food poisoning, 1594-1604

in the tonsils, 1524, 1604

in typhoid fever. See Chapter 69

morphology of, 704

mucoid colonies of, 704, 705

nomenclature of, 702

pathogenicity of, 721, 1519 et seq., 1584, 1596-1601

production of enterotoxic substances by, 1607

resistance to heat and chemicals, 659, 705, 1527,1528

summarized description of different species of, $725-744$

toxins of, $721,723,1595,1607$

Vi antigens in, 713-715, 717, 1525. See also $\mathrm{Vi}$ antigen

aberdeen, 709, 715, 741, 1597

abony, 707, 713, 727

abortus-bovis, 707, 713, 730

abortus-canis, 727. See Salm. paratyphi B

abortus-equi, 707, 713, 730

abortus-ovis, 707, 713, 730

accra, 736. See Salm. dublin

adelaide, $709,715,744,1597$ 
Salmonella aertrycke. See Salm. typhi-murium altendorf, 707, 713, 730

anager, 708, 714, 739

amersfoort, 707, 713, 733

amherstiana, 708, 714, 735

anatum, 708, 714, 739, 1597-1599, 1602

arechavaleta, 713,730

arizona, $709,715,744$

ballerup, 709, 715, 743

bareilly, 707, 713, 733, 1526, 1597, 1602

batavia, 740. See Salm. lexington

berlin, 732. See Salm. thompson

berta, 708, 714, 737

binns, 727. See Salm. typhi-murium

bispebjerg, 707, 713, 729

blegdam, 708, 714, 737

bonariensis, 708, 714, 735

borbeck, 715, 741

bovis-morbificans, 707, 714, 734, 1597

braenderup, 707, 713, 733, 1597

brandenburg, 707, 713, 729

bredeney, 707, 713, 730

budapest, 707, 713, 729

california, 707, 713, 729

cambridge, 714, 741

canastel, 714, 738

cardiff, $707,713,732,1597$

carrau, 709, 715, 742

cerro, 709, 715, 743

chaco, 708, 736. See Salm. enteritidis

chester, 707, 713, 728, 1597

choleræ-suis, 707, 713, 731, 1526, 1597

claibornei, 708, 714, 738

coli, Types $\mathrm{i}-\mathrm{v}, 715,745$

concord, 713, 733, 1597

copenhagen, 727. See Salm.typhi-murium

danysz, 708, 736. See Salm. enteritidis

dar-es-salaam, 708, 714, 737

derby, 707, 713, 729, 1597

dublin, 708, 714, 736, 1597

duesseldorf, 708, 714, 735

duisburg, 738. See Salm. gallinarum

durban, 714, 738

eastbourne, 708, 714, 737, 1597

enteritidis, 708, 714, 723, 735, 1526, 1597 lysogenic strains of, 346

essen, 707, 713, 729, 1597. See also Salm. enteritidis

florida, 709, 715, 742

gallinarum, 708, 714, 738, 1597

gaminara, 709, 715, 743

gärtner, 735, 736. See Salm. enteritidis

georgia, 713,733

give, 708, 714, 739, 1597

glostrup, 708, 714, 735

goettingen, 708, 714, 738

grumpensis, 715, 741

habana, 709, 715, 742

hartford, 707, 713, 733

heidelberg, 707, 713, 728

heves, 709, 715, 742

hormæchei, 709, 715, 744

horsham, 715, 742

hvittingfoss, $709,715,742$

illinois, 708, 714, 741

infantis, 713, 732

inverness, 709, 715, 744
Salmonella italiana, 714, 738

java, 727. See Salm. paratyphi B.

javiana, 708, 714, 738, 1597

jena, 735, 736. See Salm. enteritidis

kaapstad, 713, 729

kaposvar, 707, 713, 729

kentucky, 709, 715, 743, 1597

kiel, 736. See Salm dublin

kirkee, 709, 715, 743

koeln, 713, 729. See also Salm. dublin

kottbus, 707, 713, 734

kunzendorf, 707, 731. See Salm. choleræsuis

lexington, 708, 714, 740

litchfield, 708, 714, 735

loma-linda, 714, 737

london, 708, 714, 739, 1597

madelia, 709, 715, 742

manhattan, 708, 714, 731

meleagridis, 708, 714, 740, 1597, 1602

mexicana, 707, 714, 734

mikawasima, 707, 713, 733

minnesota, 709, 715, 743

mississippi, 715, 742

montevideo, 707, 713, 733, 1597, 1602

moscow, 708, 714, 737, 1526

moskau, 736. See Salm. enteritidis

muenchen, 707, 714, 734, 1597

muenster, 708, 714, 739

napoli, 714, 738

narashino, 708, 714, 735

new-brunswich, 708, 714, 740, 1597

newcastle, 741. See Salm. senftenberg

newington, 708, 714, 740, 1597

newport, 707, 713, 734, 1597

niloese, 708, 715, 741

nyborg, 708, 714, 740

odense, 727. See Salm. paratyph $B$.

onarimon, 708, 714, 737

onderstepoort, $709,715,742$

oranienburg, $707,713,732,1597,1602$

oregon, $707,714,734$

oslo, 707, 713, 733, 1597

panama, 708, 714, 738, 1597

paratyphi $A, 707,713,726,1526$ et seq.

paratyphi $B, 707,713,723,725,727$,

1526 et seq.

paratyphi $C, 707,713,731$, I526 et seq.

pensacola, 714, 737

pestis cavix, 727. See Salm. typhi-murium

pomona, 715, 743

poona, 709, 715, 741

potsdam, 707, 713, 732, 1597

pretoria, 715,741

pueris, 713,734

puerto-rico, 734. See Salm. newport

pullorum, 708, 714, 738, 1555, 1597

reading, 707, $713,729,1597$

rostock, 708, 714, 737

rubislaw, 709, 715, 741

saint-paul, 707, 713, 729, 1597

salinatis, 707, 713, 728

san-diego, 707, 713, 728, 1597

schleissheim, 707, 713, 730

schwartzengrund, 713, 730

selandia, 708, 714, 740

sendai, 708, 714, 737 
Salmonella shangani, 708, 714, 739

senftenberg, 708, 715, 741, 1597

simsbury, 708, 715, 741

solt, 709, 715, 741

stanley, 707, 713, 728, 1597

storrs, 727. See Salm. typhi-murium

sundsvall, 715, 742, 1597

szentes, 709, 715,743

taksony, 708, 715, 741

tel-aviv, 709, 715, 743

tennessee, 707, 713, 734, 1597

thompson, 707, 713, 732, 1597

tim, 740. See Salm. newington

typhi, 708, 714, 722, 735, and Chapter 69

in the tonsils, 1524

typhi-murium, 707, 713, 722, 727, 1597, 1600

typhi-suis, 707, 713, 732

uganda, 708, 714, 739

unnamed type, 714

urbana, 709, 715, 744

vejle, $708,714,739$

virchow, 707, 713, 732, 1597

virginia, 714, 735

voldagsen, 732. See Salm. typhi-suis

weltevreden, 714, 740

wichita, 709, 715,742

worthington, 709, 715, 742, 1597

zagreb, 707, 713, 729

zanzibar, 708, 714, 740

Salt agglutination, 214

Salt sensitivity of rough variants, 299

Salts, antagonistic effect of, 125

effect of on bacteria, 120 et seq.

on other germicides, 127

on pigment formation, $633,635,637$

mode of action of, 127

susceptibility of Gram-positive and Gramnegative bacteria to, 125

Sampling errors, assessment of, 981 et seq.

Sandflies, in relation to verruga peruana, 1797

Sandfly fever, 1959

São Paulo typhus, 1847

Saponin solubility of pneumococcus, 571

Saprophytic acid-fast bacilli, 405 et seq.

chemical structure of, 409

classification of, 433

cultural characteristics of, 414

effect of glycerine on growth of, 420

pathogenicity of, 428

pigment formation by, 415,421

Saprophytic viruses, 956

Sarcina aurantiaca, 625, 626

conjunctivæ, 626

conjunctivæ citrea, 626

lutea, 626

mirabilis, 626

ureæ, 626

ventriculi, 625

Satellitism of $H$. influenzx, 790

Scarlet fever, 1462-1478

active immunization against, 1473

antitoxic sera, prophylactic treatment with, 1475

standardization of, 1476

therápeutic treatment with, 1475 , 1477
Scarlet fever, carriers of, 1469

chemotherapy of, 1477

convalescent serum in, 1477

diagnosis of, 1467

Dick test in, 1078, 1080, 1465, 1467

epidemiology of, 1469

experimental production of, 1464

laboratory infection with, 1464

milk in relation to, $1471,2039,2049$

"miniature," 1465

mode of spread of, 1471

prophylaxis in, 1472

Schultz-Charlton reaction in, 1465

tonsillitis, relation of to, 1478

treatment of by chemotherapy, 1477 with convalescent serum, 1477

types of Str. pyogenes associated with, 1463

Schick test, 1075 et seq., 1376

age groups in relation to, 1076

antibody content of blood in relation to, 1377

blood groups, relation of, to, 1079

conversion rate, 1396

environmental conditions in relation to, 1080

experimental production of diphtheria in positive reactors to, 1085

immunological significance of, 1075-1085

in control of active immunization, 13931396

in diphtheria convalescents, 1395

in indigenous populations, 1075-1085

natural immunization in relation to, 1248

Schick dose of toxin in, 1377, 1381

susceptibility of Schick-positive reactors to infection, 1377

Schizomycetes, classification of, 310

Schmitz's bacillus. See Shigella schmitzi

Schmorl's bacillus. See Fusiformis necro. phorus

Schools, diphtheria immunization in, 12481251,1399

Schultz technique in study of anaphylactic shock, 1146

Schultz-Charlton reaction, 1465

Scours, black, 527 white, 667,1662

Scrapie, 1942

Scrub typhus, 1849,1856

Scurf staphylococcus, 622, 1997

Sea water, bacteria in, 2015

Seasonal variations in immunity, 1205

Secondary stimulus, formation of antibodies in response to, 1110

Selenium salts, $659,706,1528$

Sensitizer, Bordet's conception of in hæmolysis, 193

Septic sore throat borne by milk 1471, 1478, 2049

Septicæmia, bacteriology of, 1490

hæmorrhagica. See Pasteurellosis

Serological reactions, in identification of bacteria, 361. See also under Antigen-antibody reactions

Serotherapy. See under individual diseases

Serotoxins, in anaphylactic shock, 1143 
Serratia marcescens, 636

Serum, diastase in, 453 maltase in, 367,534

Serum-fast strains of spirochætes in relapsing fever, 1806, 1808

Serum reactions. See Antigen-Antibody reactions

Serum sickness, 1152

Seven-day fever of Japan, 1834 of Queensland, 1837

Sewage, activated sludge process of purification, 2033

bacteriology of, 2032

pleuropneumonia-like organisms in, 939, 947

Shake-tube method, use of in obtaining pure cultures, 353

Sheep, anaerobic infections of. See Anaerobic infections of animals anthrax of, 1731

black disease of, 1780

blackleg in, 884, 885,1778

blue-tongue of, 1967

braxy of. See Braxy

Brucella infection of, 1697, 1706

caseous lymphadenitis of, 459 . See also

Pseudotuberculosis

chronic pyobacillosis of, 1499

contagious agalactia of, 945,1867

contagious pneumonia of, acute, 779, 1648

contagious pustular dermatitis of, 1897

enzootic hepatitis of, 1959

foot-rot of, 481,1787

infections of with $C l$. welchii, 1781

infectious enterotoxæmia, 178?

lamb dysentery, 1781

pulpy kidney disease, 1782

"struck," 1782

infectious necrotic hepatitis of, 1780

Johne's disease of, 1363

joint-ill in lambs, 1284, 1496

lombriz of, $16 \pm 6$

louping-ill of, 1925

Nairobi disease of, 1967

pox of, 1895

psendotuberculosis of, 1403, 1649

scrapie of, 1942

tuberculosis in, $424,429,1343$

Shell fish, 2031

Shell fish in relation to enteric fever, 1541

Shiga's bacillus. See Shigella

Shigella, 685-699

antigenic structure of, 687

bacteriophage action on, 693

biochemical reactions of, 686 tabulated, 688

chemical fractionation of, 693

classification of, 696

colonial types of, 686

differentiation of by Michaelis' acid agglutination test, 692

disease due to, 1561-1576, 1584-1585, 1598

hæmolysin formation by, 686

netabolism of, 686

morphology of, 685

nomenclature of, 685,698
Shigella, pathogenicity of, 695, 1561 et seq.

resistance of, 686

smooth-rough or type-group variation in, $686,690,692$

toxin-formation by, 693

alkalescens, 677, 685 et seq., 1569

dispar, 685 et seq., 1569

flexneri, 685 et seq., 1561 et seq., 1585, 1598

relation of to Salmonella group, 692, 744,745

newcastle, 685 et seq., 1569

paradysenterix, 699

para-Shiga group of, 685 et seq., 1568

schmitzi, 685 et seq., 1567

shigæ, 685 et seq., 1566

sonne $i, 685$ et seq., 1568, 1585

sp.? boyd Types 170, P288, D1, P274, 685, $688-690,697-699,1569$

Shop typhus, 1845,1856

Shwartzman phenomenon, 1176

relation to pathogenesis of infection. 1177

"Side-chain" theory, 196

Side-to-side inoculation, 1231

Silica as kataphylactic agent, 1207

Silicosis, in relation to tuberculosis, 1208

Single cell methods, use of in obtaining pure cultures, 357

Sinusitis, 1499

Site of formation of antibodies, 1101-1106

Size of bacteria, 16

increase in, 841

variation of with age, 84 in relation to opacity, 81

Skin, bactericidal action of, 1019

formation of antibodies in, 1102

local immunity of to streptococcal infection and staphylococcal infection, 1181, $1182,1184,1185$

normal flora of, 1997

of infants, sensitivity of in relation to Dick test, 1078

staphylococcal and streptococcal infections of, 1492-1493

Slime-fever. See Swamp fever.

Smallpox, 1884-1895

elementary bodies in, 958,1887

Guarnieri corpuscles in, 1887

immunity in, 1888. See also Chapter 55

laboratory diagnosis of, 1887

mode of infection in, 1884

Paschen's granules in, 1887

Paul's test in diagnosis of, 1888

relation of cow-pox and vaccinia to, 18881890

reproduction of in animals, 1855

serum against, 1895

types of, classical, 1884 variola minor or alastrim, 1884

vaccination against, 1890 complications of, 1894

foot-and-mouth virus in vaccine, 1894 protective effect of, 1891

Smegma bacillus. See Mycobacterium smeg. matis

Smooth $\rightarrow$ Rough $(\mathrm{S} \rightarrow \mathrm{R})$ variation, 299

Smoothness and roughness in relation to immunity, 1055 
Smoothness in relation to antigenic structure, 277,285

Sneeze, droplets in, 2003

Snotsiekte, 1966

Snow, bacteria in, 2014

Snuff, in treatment of nasal diphtheria carriers, 1386

Snuffles in rabbits, 1647

Soft chancre, 1790

Sodium desoxycholate, 571, 659, 706, 1528

Somatic antigens, 276, 283

Sonic waves, effect of on bacteria, 110

Sonne's bacillus. Sce Shigella sonnei

Sordelli's bacillus. See Clostridium bifermentans

Species, bacterial, 316

Specific and group phases of flagellar (H) antigens, 278

Specificity of antigen-antibody reactions, 242

Spectrum, diagrammatic representation of, 103

Spinulosin, 178

Spirillum, 527-529

minus, 529, 925, 1276-1278, 1838

rubrum, 527, 528

serpens, 529

undula, 527, 529

volutans, 529

Spirochæta, characters of, 907

morsus muris. See Spirillum minus plicatilis, 907

Spirochætes, 907-925. See also Spirochæta, Cristispira, Leptospira and Treponema general characteristics of, 909-912 in human mouth, 919

Spirochætosis a vian, 1808 in animals, 1809

Spirochætosis icterohæmorrhagica. See Weil's disease

Spleen, effect of removal on antibody formation, 1103 on Bartonella infection, 903,1797

Spontaneous generation, controversy on, 4

Spore formation, effect of calcium on, 291 variation in, 291

Spores, as antigens, 279,840

bacterial, 24

bacteriophage in relation to, 347

chromatinic bodies of, 25

germination of, 25

Sporogenes vitamin, 69

Spotted fever, due to Neisseria meningitidis. See Meningitis

due to Rickettsia rickettsi, 935,1846

Spreading-factors in bacterial infection, 1012

Staining of bacteria, 37

Staining reactions in identification of bactcria, 358

Standard deviation, 985

Standard error, 987

of a difference, 987, 994

Standardization of antitoxin, 238. See also under individual diseases and organisms of disinfectants. See Disinfectants

Standardization of various reagents. See under individual reagents

Staphylocoagulase, 617

\section{Staphylococcus, 607-622}

antigenic structure of, 613

antiserum to, 620,1504

bacteriophage typing of, 614

biochemical reactions of, 612

capsulation of, 608

chemical fractionation of, 613

classification of, 620

coagulase formation by, 617

cultural reactions of, 609

enterotoxin of, 616, 1605

habitat of, 608

hæmolysin production by, 615,616 $\alpha$-hæmolysin, 615

$\beta$-lysin, 616 $\gamma$-lysin, 616

in broncho-pneumonia, 1667

in food poisoning, 1604-1608

in suppurative lesions, 1490-1506

leucocidin production by, 616

metabolism of, 610

morphology of, 608

pathogenicity of, 618

pigment production by, 611

resistance of, to heat and disinfectants, 612

satellite growth of $H$. influenzæ in relation to, 790

toxin production by, 614, 1605

toxoid in vaccination, 1506

vaccines of in treatment, 1505

albus, 607 et seq., 620, 622

aurantiacus, 622

aureus, 607 el seq., 621

in wound infection, 1501-1503

candicans, 632

candidus, 622

citreus, 607 et seq., 620, 622

epidermidis, 622

epidernidis albus, 607

pyogenes albus, 607

pyogenes aureus, 607

pyogenes citreus, 607

salivarius, 607,622

salivarius pyogenes, 607

Starvation, effect of on resistance, 1201

Stationary phase of growth, 83, 94

Statistical methods, use of, 980-1001

Sterilization, early work on, 7

of milk, 2053

Stern reaction, 706

Stomach, bactericidal mechanisms of, 1020 . normal flora of, 1987

Stomatitis, aphthous, 1897 contagious pustular of horses, 1895

herpetic, 1897

vesicular of horses, 1907

Strangles, 581, 582, 1496 of horses, 1496

Straus's reaction, 489,490

Streptobacillus moniliformis. See Actinomyces muris

Streptococcus, 559-601

anaerobic species of, 596, 1772, 1996

in gas gangrene, 1772

in puerperal fever, 1484

in septicæmia, 1491

antigenic structure of, $572-588$ 
Streptococcus, bile, effect of on growth of, 572 classification of, $579-589$

by changes produced on blood media, 564

by fermentation reactions, 569

growth characters of, 562

growth requirements of, 562

hæmolysis produced by, 561-569

hæmolytic group of, antigenic structure of, $575-588$

chemical structure of, $279,280,574$ 577

Group A, 575, 576, 579. See also Str. pyogenes

Group B, 575, 576, 578, 580. See also Str. agalactix

Group C, 575, 576, 578, 581, 594

Group D, 575, 576, 578, 582, 593. See also Str. frecalis

Group E, 576, 578, 586, 59t

Group F, 576, 578, 586, 591

Group G, 575, 576, 578, 586, 594

Group H, 576, 587, 594

Group K, 576, 578, 587, 594

Group L, 576, 587, 594

Group MI, 576, 587, 594

Group N, 576, 587, 594. See also Str. lactis

in normal nasopharynx and tonsils, 1993, 1994

matt and glossy forms of, $301,577,595$

non-hæmolytic variants of, 567

smooth and rough forms of, 300,594 , 595

heat resistance of, 572,583

in appendicitis, 1497

in cholecystitis, 1497

in mastitis, 1494

in peritonitis, 1498

in sinusitis, 1499

in suppurative lesions of the urinary tract, 1500

methylene blue, reduction of by different species of, 572

morphology of, 560

motile, 562

pathogenicity of, 590

toxin production of, 590

variation in, 594

acidominimus, 589

agalactiæ, 581, 598, 1494

in mastitis, 1494

pathogenicity of, 592,1494

bovis, $585,586,588$

cremoris, 588

durans, 585

dysgalactix, 582

epidemicus, relation of to Str. pyogenes, 580 equi, 582

equinus, 589

frcalis group of, 572, 583, 593, 600. See also Group D and Str. frecalis

classification of, 583

endocarditis in relation to, 1514

heat resistance of, 572

in water, 2013, 2025, 2028, 2029

relation of to Str. lactis, $\mathbf{5 8 5}$
Streptococcus glycerinaceus, 585

hæmolyticus, 559. See Str. pyogenes

inulaceus, 585

lefir, 588

lactis, 576, 601

in milk, 2037

relation of to frecalis group, 583-585

liquefaciens, 585

mitis, 589

pneumonix, 559 et.seq., 592, 598

antigenic structure of, 572

antigenic types of, 1670 transmutation of, 305

antigens of, 279

bile solubility of, 571

capsular swelling reaction of, 241

capsule formation by, 561

chemical structure of, 574

diseases caused by, 1496, 1498, 1499, 1666-1686

frequency of types in health and dis. ease, 1670-1675

hæmolysin of, 568,592

hyaluronidase production by, 593

in normal nasopharynx, 1993-1994

morphology of, 560

pathogenicity of, 592

polysaccharide antigens of, 574

aggressive effects of, 1071

skin reaction to in pneumonia, 1685

stimulation of antibody formation by, 1119,1684

Type III, bacterial enzyme acting on, 173, 168:

rough form of, 300,595

saponin solubility of, 571

typing of in pneumonia, I678

variations in, 595

pyogenes, 559 et seq., 579, 590, 597

$\alpha$ - and non-hæmolytic colonies of, 567

antigenic structure of, $280,575-577$, 579

capsule formation by, 561

chemotherapy in infections with, $1477,1485,1504$

erythrogenic toxin of, $591,1464-1469$

in relation to toxæmic infection, 1031

fibrinolysin produced by, 591

hæmolysins of, 566,590

hyaluronic acid production by, 591

hyaluronidase production by, $59 \mathrm{I}$

in wound infection, 1501-1503

infections due to, 1462-1506, 15I1, 1666

leucocidin of, 590

local immunity to, 1180-1182

$\mathrm{M}$ and $\mathrm{T}$ antigens of, 577

pathogenicity of, 590

presence of in normal throat, 1469 ,

1993-1994

spreading factor of, 591

toxins produced by, 590

typing of, by agglutination, 577

by precipitation, 577

variation in, 594 
Streptococcus salivarius, 589

thermophilus, 589

viridans, $561-566$

viridans group of, $564-566,578,588,593$, 600

in blood after tooth extraction, 1515

in endocarditis, 1514

zymogenes, 585

Streptolysin, 566, 590

rheumatic fever, in relation to, 1512

Streptomyces, 382

Streptomycin, 178

Streptothricin, 175, 179

Streptothrix. See Actinomyces and Fusiformis

cuniculi. See Fusiformis necrophorus

maduræ. See Actinomyces

moniliformis. See Actinomyces muris

muris ratti. See Actinomyces muris

Strongyloplasms, 949

Stuttgart dog plague, 1837

Subacute bacterial endocarditis, 1515

Suicide colonies, 670

Sulphadiazine, 157

Sulphaguanidine, 157

Sulphanilamide, 157

Sulphapyridine, 157

Sulphathiazole, 157

Sulphonamide drugs, 157

action of on respiratory enzymes of bacteria, $\mathbf{1 6 6}$

on tissue defences, 1212

antagonizers of, $159,160,161,165$

chemotherapeutic activity and chemical structure of, 167

mode of action of in vitro, 159,165

in vivo, 161, 1211

Woods-Fildes lyypothesis of, 162

relation of to antibody formation, 1212 to immunity, 1211

resistance, acquired, of bacteria to, 160 , 161

Sulphonamido-crysoildin, 156, 158

Sulphone compounds in chemotherapy, 171

Sulphur bacteria, 61

Sulphur dioxide, effect of on bacteria, 149

Summer diarrhoea. See Enteritis of infancy

Sunlight, effect of on bacteria, 102-104 on media, 102

Supersonic waves, effect of on bacteria, 111

Suppurative lesions, See Pyogenic infections

Surface plate method of counting, 82

Surface structure in relation to antigenic specificity, 263

Susceptibility to disinfectants in relation to age, 85

Swamp fever, 923,1836 of horses, 1966

Swine, actinomycosis in, 1271 anaerobic infections of. See Anaerobic infections of animals

blackleg of, 1778

botriomycosis in, 1492

Brucella infection of. See Brucella infection of swine

erysipelas, 395, 1283-1285

fever, 1963

hog cholera in, 1963
Swine, influenza, 1661

plague, 1646

pleuropneumonia of, 1964

pseudotuberculosis of, 1650

rouget of, 395

tuberculosis in, 424, 429, 1343, 1348

vesicular exanthema of, 1908

Swineherds' disease, 1932, 1934

Symplasmata, 638

Synthetic antigens, 253-258. See also Antigens

Syphilis. 1812-1825

diagnosis of, 1816-1824

by animal inoculation, 1817

by microscopical examination, 1816

by serum reactions, 1817-1824 interpretation of, 1820

immunity to, 1165, 1813

in rabbits, experimental, 916, 1814-1816 natural, 1825

prophylaxis and treatment of, 1824

pyrotherapy in, 1824

T antigen of Salmonella bacilli, 303

Tabardillo, 1841, 1845

Tears, bactericidal action of, 1025

Teeth, bacteræmia following extraction of, 1515

Tellurite media for diphtheria bacilli, 450, 463, 1374

Temperature. See also Disinfection. on antibody formation, 1204

on antigen-antibody reactions, 200

on bacteria in water, 2016

on bacterial growth, 72

on bacterial growth rate, 91

on hæmolysis, 227

on keeping quality of milk, 2037

external, effect of on resistance to infection, 1202

in identification of bacteria, 360

optimum, use of in obtaining pure cultures, 354

Terms used in description of bacteria, glossary of, 369

of cultural reactions of bacteria, 36t-367

of morphological appearance of bacteria: 364

Tetanus, 1746-1767

absorption and mode of action of toxin in 1752-1755

antitoxin to, 1759

calcium salts in relation to, 1206

cephalic, 1747

diagnosis of, 1758

idiopathic, 1750

immunity to, 1755

acquired, 1757

natural, 1755

in animals, 1767

in $\operatorname{man}, 1746$

epidemiology of, 1746

distribution of tetanus bacilli in nature, 1748

incidence, 1746

incubation period, 1747, 1761

mortality, 1747 
Tetanus, in man, prophylaxis and treatment of, 1758-1767

by toxoid, 1765

treatment of by prophylactic injection of antitoxin, 1761

by therapeutic injection of antitoxin, 1762

mode of infection in, 1749

post-operative, 1751

neonatorum, 1746

puerperal, 1746

reproduction of in animals, 1749

splanchnic or visceral, 1747

toxoid, active immunization by, 1765 standardization of, 1767

Tetrathionate broth in cultures from enteric fever, 1528

Therapeutic index, 155

Thermal death point of bacteria, 115

Thermophilic bacilli, 838 clostridia, 872

Thermoprecipitin test in anthrax, 1738

Thiamin, 164 as bacterial vitamin, 64,66

Thorium $\mathrm{X}$, effect of on antibody formation, 1104

Thread method of standardizing disinfectants, 145

Thrombocytobarin reaction, 912,1833

Thrushes, plague of, 1968

Thunberg tube, use of in study of oxidationreduction reactions, 47

Tick fever, 1803. See also Relapsing fever and Typhus fever

due to Brucella tularensis, 1720

due to Richettsia, 1816

due to Treponema, 1803

due to unknown cause, 1846

Tick paralysis, 1846

Tick-borne diseases, 1846

Ticks in relation to avian spirochætosis, 1809 to louping-ill, 1925

to Nairobi disease, 1967

to relapsing fever, 1803-1805

to spotted fever, 1846-1848

Timothy-grass bacillus. See Mycobacterium phlei

Tissue cultures, formation of antibodies in 1105

of Rickettsia, 931

of filtrable viruses, 959-961

Tissue immunity in relation to local immunity, 1187

Toluol, effect of on antibody formation, 1104

Tonsillitis, 1478

chemoprophylaxis in, 1479

chemotherapy in, 1485

rheumatic fever in relation to, 1511

Tonsils, flora of, 1524, 1604, 1992-1996

Total count of bacteria, 80

Toulon typhus, 1845

Toxæmia, intestinal, 1990

Toxicity, measurement of, 995

Toxigenicity, bacterial, variation in, 306

'Toxin, Lf and Lr dose of, 1384

Lo and $\mathrm{L}+$ dose of, 239,1384

Minimal Lethal Dose of, 238, 1384
Toxin-antitoxin mixture in diphtheria immunization, 1392

Toxin-antitoxin reaction, 238-241. See also Antigen-antibody reactions

Toxins, bacterial, 1007-1011. Sce also under individual species

constitution of, 1009

mode of action of, 1010. See also under various bacteria

Toxoid, 238

as prophylactic agent in diphtheria, 1392 in tetanus, 1765

Toxoid-antitoxin floccules in diphtheria immunization, 1393

Toxoid-antitoxin mixtures in diphtheria immunization, 1393

Toxophore group, 198

Trachoma, 1878-1880

Bacterium granulosis in, 901, 1878

Rickettsia trachomatis in, 1879

virus origin of, 1879

'Transmutation, of bacteria, 305

of fibroma to myxoma, 1980

of papilloma to carcinoma, 1980

Trench fever, 936,1851

Treponema, 908

anserinum, 911, 914

in avian spirochætosis, 1808

buccalis, 1986

calligyrum, 918

carteri, 1804

cobayæ, 919

cuniculi, 918

in rabbit syphilis, 1825

duttoni, 1804

hispanicum, 1804

intermedium, 1986

kochi, 1804

macrodentium and microdentium, 908, 919

novyi, 1804

pallidum, 908 et seq., 915-918

filtrable form of, 1816

in syphilis, 1812

parvum, 908

persicum, 1804

pertenue, $912,918,1825$

phagedenis, 919

recurrentis, $910,912,913$

in relapsing fever, 1803

refringens, 918

in syphilitic lesions, 1817

sogdianum, 1804

termitidis, 908

usbekistanicum, 1804

venezuelense, 1804

vincenti, 914

in Vincent's angina, 1810

Trypaflavine, 300. See also Acriflavine

Trytophan as bacterial vitamin, 67, 163 rôle of in bacterial nutrition, 65

Tsutsugamushi fever, 1848

Tubercle bacillus. See Mycobacterium tuberculosis

Tuberculin, 410, 1327

reaction, $1161,1300-1302,1324,1328$

in cattle, 1345

in contact children, 1303, 1307 
Tuberculin, reaction, iu Johne's disease, 1365 in guinea-pigs, 1326,1328 methods of eliciting, 1324 relationship of to anaphylaxis, 1161 , 1330

Tuberculosis, 1289-1349

allergy in, 1161, 1324, 1326-1336 relation of to immunity, 1330

bacillæmia in, 1322

borne by milk, 1317, 2038

diagnosis of, 1319

due to bovine tubercle bacillus, 1289, 1311,1317

epidemiology of, 1291

factors affecting mortality from, 1293 race and civilization, 1296

fatality of infection in early life, 1306

frequency of in human beings, 1290, 12991308

heredity in, 975,1298

immunity in, 1326

in animals, $1342-1349$

in cats and dogs, 427, 429, 1342, 1343

in cattle, 1342-1348

in fowls and other birds, 427, 429, 1348

in goats and sheep, 424, 429, 1343

in guinea-pigs, $425,429,1325$

in horses, $427,429,1343$

in mice and rats, 426,429

in rabbits, $424,429,1343$

in swine, $424,429,1342,1348$

latent, 1303

mortality from, 1292

of bovine origin, 1317 et seq.

of human origin, methods of infection in, 1308

prevention of infection, 1336

prophylactic vaccination against, 1336

pulmonary, 1309 et seq. childhood and adult types, 1314 due to droplet infection, 1312 to dust infection, 1312 endogenous and exogenous infection in, 1316

focal lesions in, 1314 pathogenesis of, 1314

silica as kataphylactic agent in relation to 1207,1294

therapeutic immunization against, 1341

Tularæmia, 1720

laboratory infections in, 1721

lemming fever, 1721

Tumours, filtrable, 1976-1981

Twort-d'Herelle phenomenon. See Bacteriophage

"Two-stage" hypothesis of antigen-antibody reactions, 208

Tyndallization, 7, 114

Typhoid fever. See Enteric fever

Typhoid-paratyphoid group. See Salmonella

Typhus fevers, 1841-1862. See also Rickettsia Brazilian spotted fever, 1847

Brill's disease, 1841, 1842

classical type of, $934,1842,1845,1854$

classification of, 1852,1854

diagnosis of, 1853-1858

by complement-fixation test, 1856
Typhus fevers, diagnosis of, by infection test, 1857

by virus neutralization test, 1857

by Weil-Felix test, 1853-1858

exanthematic fever of Marseille, 1848

experimental in guinea-pig, avitaminosis in relation to, 1197

fièvre boutonneuse, 1848

flea-borne, 1845

gaol fever, 1812

Kenya fever, 1848

louse-borne, 1842

Mexican typhus, 1845

mite typhus, 936,1849

murine type of, $935,1845,1854$

of Lukes in dogs, 1837

pathology of, 1844

prophylaxis and treatment of, 1858

by serum in, 1861

by vaccination, 1859

Q fever, 1850

Rocky mountain spotted fever, 935, 1846

São Paulo typhus, 1847

serub typhus, $936,1849,1856$

shop typhus, 1815, 1856

South African tick fever, 1848

tabardillo, 1845

tick-borne, 1846-1848

trench fever, 1851

tropical typhus of Malaya, 1845, 1849, 1855

tsutsugamushi fever, 936, 1848

Weil-Felix reaction in, 1853-185s

Tyrocidin, 174

Tyrothricin, 174

Ulcerative lymphangitis of horses, 1403

Ulcus molle, 1790

Ultrafiltration, 951

Ultramicroscopic viruses. See Viruses animal

Ultra-violet light, disinfecting action of, 105 effect of on bodily resistance, 1203 on proteins, 105 on toxins, 105

use of in photomicrography, 18, 952

Undulant fever, 1692-1706

diagnosis of, 1702

due to $B r$, abortus, 1697

abortion in, 1698

butter and cheese infections in, 1701 contact infections in, 1701

institutional outbreaks of, $170 \mathrm{I}$

laboratory infections in, 1701, 1702

latent infections in, 1699

milk infections in, 1700

tonsillar infections in, 1703

due to $\mathrm{Br}$. melitensis, 1693

epidemiology of, in Malta, 1694

in other countries, 1697

eradication of by stopping of goats' milk, 1695

infection of fæces and urine in, 1694, 1703

due to $B r$. suis, 1702

milk in relation to, $1695,1699,1700,1706$

prophylaxis and treatment of, 1706 
Unimolecular reaction, similarity of disinfection to, 137-140

Unit of antitoxin, 238

Unitarian conception of antibodies, 248, 285

Uracil, 71

Urethra, normal flora of, 1997

Uric acid, fermentation of in differentiation of coli-aerogenes group, 664

$\mathrm{V}$ factor in relation to growth of $H$. influenzæ, 790-793

Vaccination, 1888-1895 complications of, 1894

Vaccine therapy. See under individual diseases

Vaccinia, 1884-1895. See also Smallpox and Chapter 55

elementary bodies in, 953,1887

generalized, in man, 1894 in rabbit, 1228

presence of virus in circulating blood, 1233,1889

Vagina, normal flora of, 1996

Variation of bacteria, 288-307. See under individuul species for variation in each $\alpha$ and $\beta$ phases of antigens, 279, 716 as a guide to antigenic structure, 276 bacteriophage as cause of, 327,340 biochemical reactions, in relation to, 292 colony form, in, 299

correlated variations, 290

$\mathrm{H} \rightarrow \mathrm{O}$ variation, $276,291,717$,

impressed variations, 290

in relation to enzyme systems, 295

in size, 358 to epidemic spread, 1260

during growth, 85

induction of, 304

morphology in, 290

mucoid variants, 300,718

pigment production, in, 299

$\rho$ variants, 303

smooth $\rightarrow$ rough $(S \rightarrow R)$ variation, 277 , $278,299,717$

production of by growth in immune sera, 304

specific and group phases of flagellar $(\mathrm{H})$

antigens, 278,716

spore-formation in, 291

terminology employed in, 288

transmutation, 305

virulence in, 303,306

Varicella, 1901

Variola. See Smallpox

Veillon tube, use of in obtaining pure cultures, 353,752

Verruga peruana, 903, 1796, 1797

Vesicular exanthema of swine, 1908

Vesicular stomatitis of horses, 1907

$\mathrm{Vi}$ antigen in Bacterium coli, 715

Pasteurella, 774

Salm. ballerup, 743

Salm. hormæchei, 744

Salm. paratyphi $B, 720$

Salm. paratyphi $C, 731$

Salm.typhi, 717, 719, 720, 735, 1525, 1547

Viable count of bacteria, 81

\section{Vibrio, 514-527}

antigenic structure of, 519

biochemical characteristics of, 516

chemical analysis of, 520

fermentative classification of, 517

hæmolysin formation by, 518

pathogenicity of, 522

resistance of, 516

variation of, $516,521,523$

alkaligenes, 902

berolinensis, 525

cholerx, 514 et seq., 523

disease caused by. See Cholera

selective media for isolation of, $\mathbf{5 1 5}$, 1424

toxin production by, 518

lanubicus, 526

Deneke. See Vibrio tyrogenus

El Tor, 518 et seq., 525, 1419

fetus, 526,1720

Finkler-Prior. See Vibrio proteus

helcogenes, 526

ivanoff, 525

jejuni, 527

IIassauah, 526

metchnikovi, 514 et seq., 525

Nasik, 516, 526

paracholeræ, 520

phosphorescens, 522, 523, 525

proteus, 523, 525

Vibrio subtilis. See Bacillus

tyrogenus, 523, 525

Vibrion butyrique. See Clostridium butyricum septique. See Clostridium septicum

Vincent's angina, 914, 1810

Viricidal antibodies, natural, 1226

Virulence of bacteria, 1005

measurement of, 995

relation of to infectivity, 1260

roughness and smoothness in relation to, 301

variation in, 303,306

Virulins, 1070

Virus diseases, 1869-1981. See under in. dividual diseases

allergy in relation to, 1241

characterized by catarrhal or generalized infection, 1949-1971

by lesions of the nervous system, 1915-1943

by lesions of the skin, 1884-1910

by tumour formation, 1976-1981

histopathology of, 1228

immunity to, 1225-1242

lymphogranuloma-psittacosis group of 1869-1880

mechanical factors in relation to lesions of, 1242

mechanism of infection in, 1227

spreading factor in relation to, 1242

Virus infections, mechanisms of, 1227

Virus pneumonias, 1875 cold agglutinins in, 1877

"Virus" preparations, 1603

Viruses, animal, 949-967

affinity of for special tissues, 957, 1228

antigenic structure of, 963,1240 
Viruses, cultivation of, 959

diseases due to, 1869-1981. See also under individual diseases

distribution of in tissues, 1227

habitat of, 956

inclusion bodies formed by, 958, 1228

intracellular position of, 958, 1228

killed, as immunizing agents, 1229

latent infections with, 957, 1226

masked, 1889

metabolism of, 963

morphology of, $95 \pm$

nature of, 966

pathogenicity of, 965

pueumonia due to, 1875-1877

presence of in circulating blood, 1228, 1889 in leucocytes, 957,1233

resistance of, 961

saprophytic forms of, 956

size of, tabulated, 955

study of by cataphoresis, 950

by centrifugation, 953

by filtration, 949

by microscopy, 951

by ultrafiltration, 951

Virus-III infection of rabbits, 1909. See also Chapter 55

Viscera, bacteria normally present in, 1998

Vitamin $B_{1}$. See Thiamin

Vitamin requirements of bacteria, 66 sporogenes, 69

Vitamins, influence of on immunity, 1190-1199 synthesis and destruction of by intestinal bacteria, 1991

vitamin A, 1191

vitamin B, 1195

vitamin C, 1196

vitamin D, 1198

Voges-Proskauer reaction, 368, 661

Voldagsen's bacillus. Sce Salmonella typhisuis var, voldagsen

Voles. See Field mice

bacillus of. See Iycobacterium muris susceptibility of to tubercle bacilli, 427 , 430

Volutin granules, 23, 832

in $C$. diphtheriæ, 449

Vulva, normal flora of, 1996

Vulvo-vaginitis in children, 1455

Warts, common and venereal, 1978

Wassermann test, 1817 interpretation of, 1820 et seq.

Water, bacterial flora of, 2012

factors determining, 2013-2021

bacteriological analysis of, 2021-2030

classification of coliform organisms, 2024

coliform count, differential, 2023 presumptive, 2022

examination for freal streptococci,

$C l$, welchii and pathogenic organ-

isms, 2025

interpretation of, 2025

plate count in, 2021
Water, bacteriological analysis of, standards of purity in, 2029

diseases carried by, 2031

distilled, effect of on bacter a, 117

Leptospira in, 1830, 1831

self-purification of, 2020

supplies in relation to enteric fever, 1541

swimming bath, 2030

Weil-Felix reaction, 1853-1858

Weil's disease, 1828-1834

diagnosis of, 1832

differential diagnosis of from epidemic jaundice, 1833

Leptospira biflexa in, 1831

Leptospira canicola in, 1837

leptospiræ in water in relation to, 1831

leptospiral jaundice in animals, 1837

meningitis in, 1829,1837

mode of transmission of, 1830 infection of rats in relation to, 1830

occupational incidence of, 1829

prophylaxis and treatment of, 1833

relation of to other leptospiral infections 1831-1837

water-borne, 1830

Welch's bacillus. See Clostridium

Whitmore's bacillus. See Pfeifferella

Whooping cough, 1662-1666 active immunization against, 1661 diagnosis of, 1663

serum treatment of, 1666

Wilson and Blair's medium, 1528

Winter dysentery of calves, 527 vomiting disease, 1798

Wolhynian fever, 1851

Woods-Fildes hypothesis, 162

Worms, in spread of anthrax, 1732

Wound contamination, 1501

Wound infection, 1500-1503 sources of pyogenic bacteria in, 1502-1503

Wright's method of counting bacteria, 80

X factor in relation to growth of $H$. influenzæ, 790-794

nature of, 791

$\mathrm{X}$ and $\mathrm{V}$ factors in relation to infection, 1208

Xerophthalmia, 1191

X-rays, effect of on antibody formation, 1104 on bacteria, 108

Yaws, 1825

Yellow fever, 1953-1958. See also Chapter 55 jaundice due to vaccination against, 1957 jungle type of, 1954 viscerotomy service in, 1954

Yersin type of tuberculosis, 426

Yoghurt, 750

Ziehl-Neelsen stain, in identification of bacteria, 359,407

Zone phenomenon in agglutination, 212 in specific precipitation, 201

Zopfius, 652 zopfii, 648, 652 





19.

3.

if 190

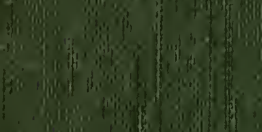

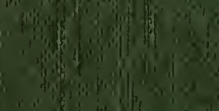

is

and

40 ment

$\sin 8)^{2}$

if

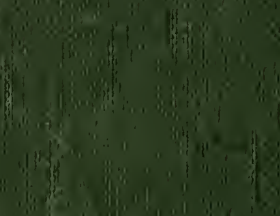

(1)

sind:

(1)

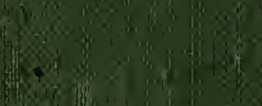

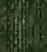

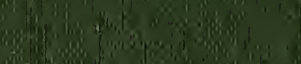
(1) 6. 50 -
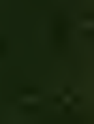

(1)

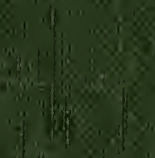

